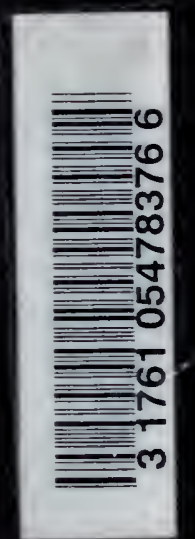



Digitized by the Internet Archive in 2008 with funding from Microsoft Corporation 





\section{MANUAL OF ANATOMY}

\section{SYSTEMATIC AND PRACTICAL, INCLUDING}

EMBRYOLOGY

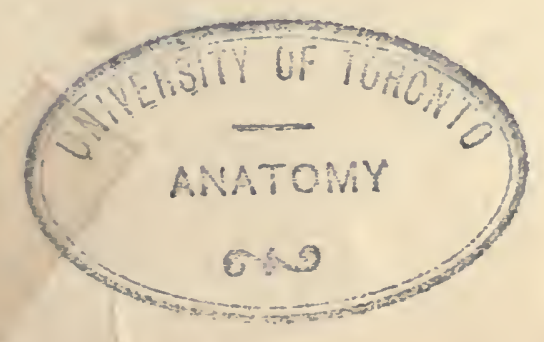





\section{MANUAL OF ANATOMY}

\section{SYSTEMATIC AND PRACTICAL, INCLUDING EMBRYOLOGY}

BY

A. M. BUCHANAN, M.A., M.D., C.M., F.R.F.P.S. Glas. PROFESSOR OF ANATOMY IN THE ANDERSON COLLEGE OF MEDICINE, GLASGOW ; EXAMINER IN ANATOMY FOR THE TRIPLE QUALIFICATIOX OF THE SCOTTISH LICENSING BODIES: EXANINER IN ANATONY FOR THE DENTAL DIPLOML, AND EXAMINER IN ANATOMY (HUMAN AND COMPARATIVE) FOR THE FELLOWSHIP, OP THE ROYAL FACLLTY OF PHYSICIANS AND SURGEONS OF GLASGOW; EX-EXAMINER IN ANATOMIY TO THE UNIVERSTTY OF GLASGOW; FORIERLY SENIOR DEATONSTRATOR OF ANATOMY IN THE UNIVERSITY OF GLASGOW

WITH 675 ILLUSTRATIONS, MOSTLY ORIGINAL AND IN COLOURS

TH:RD EDITION

TORONTO

THE MACMILLAN COMPANY OF CANADA, LTD. 1916 
170 NIISYJAIN oLb! 9 7n $x$ 부릭

$$
\begin{aligned}
& \text { QM } \\
& 23 \\
& B 83 \\
& 1916
\end{aligned}
$$




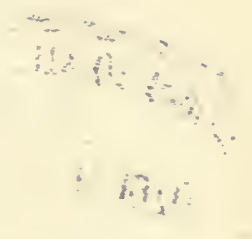

DEDICATED BY PERMISSION

IO THE

\section{RIGHT HONOURABLE LORD LISTER, O.M.,}

$$
\text { LL.D., D.C.L., F.R.S., Erc., }
$$

IN GRATEFUL ACKNOWLEDGMENT OF THE

FOWERFUL INFLUENCE WHICH HE EXERCISED OVER THE

INTELLECTUAL CULTURE OF HIS GLASGOW STUDENTS, OF WHOM THE AUTHOR WAS ONE. 



\section{PREFACE TO THIRD EDITION}

EVERY section of this manual has been carefully and completely revised. Some have been partially, and many, such as the Nervous Srstem and its Development, have been entirely rewritten. It has still been considered advisable to retain the old anatomical nomenclature until such time as a standard nomenclature has been fixed and generally accepted. The Appendix, however, containing a large number of the terms of the Basle nomenclature, has been retained for those who wish to refer to them. The subject of Embryology is still partially dealt with by appending to the description of each organ a concise account of its development. Over and above this an entirely new and complete chapter, dealing with General Embryology, has been added, with the view of elucidating many points in the various concise developments. This chapter has been specially and skilfully illustrated.

The author has to acknowledge with deep gratitude the valuable assistance which he has so willingly received from his demonstrator, Dr. Peter Thomson, M.A., B.Sc., in preparing this edition for the press. He has also to thank the publishers for the great labour which they have kindly undertaken in various directions, and for the great care which they have bestowed upon the publication of this edition.

Many new figures have been added, all of which have been executed by the well-known anatomical artist, Mr. James T. Murray, Edinburgh, who has done his work with rare excellence, and whom the author desires to thank most cordially. He also begs to thank all those authors who have kindly and generously granted permission to use their figures.

The Axderson College of Medicine,

Glasgow,

January, 19r6. 



\section{PREFACE TO SECOND IMPRESSION}

THE First Edition of this Manual has been so favourably received, and was published such a short time ago, that it has seemed unnecessary to make any very important alterations, or to effect a thorough and complete revisal in the meantime, in view of the early call for a Second Edition. In reprinting this Second Impression it has been considered advisable not to allow the Manual to remain out of print (as a revised edition throughout would necessitate), it being the only text-book of its kind which adheres to the old anatomical nomenclature.

The feeling among many teachers of anatomy seems to be setting against the adoption of the Basle nomenclature in its entirety. Moreover, among students there is a great deal of dissatisfaction at its use on account of its being so cumbrous, and occasionally not quite accurate. It has therefore been considered advisable to retain the old nomenclature, which is so simple and so correct for all practical purposes, until such time as a standard nomenclature has been fixed and generally accepted. The Appendix containing a large number of the terms of the Basle nomenclature has, however, been retained for the use of those who may desire to refer to it.

The errata and omissions, noted in the First Edition, have been put right, and certain critical remarks have received willing attention.

The subject of the ossification of bones, now so much modified, has been made more in conformity with the views of recent investigators, and in certain cases will be doubtless more acceptable to students than previous accounts.

\section{The Axderson College of Medicine, Glasgow, May, Igr4.}





\section{CONTENTS}

OSTEOLOGY
ARTHROLOGY





\section{A MANUAL OF ANATOMY}

\section{OSTEOLOGY}

THE skeleton is the hard 'dry' osseous and cartilaginous framework of the body. It consists in the adult of 200 bones, exclusive of the small bones of the ear and sesamoid bones, most of which are held together by ligaments. The functions of the bones are as follows: (I) they impart shape to the body; (2) they support the soft parts; (3) they protect important organs ; and (4) ths $y$ afford attachment to the muscles. In the performance of tis latter function the bones are to be regarded as passive organs or locomotion, the active organs being the muscles-that is to say, the bones act as levers to the muscles. There are two varieties of skeleton, namely, endoskeleton and exoskeleton. " In the former, as in man, the bones are covered by soft parts. In the latter, as in crustacea, the bones are so disposed as to lie upon the surface, where they form a case which contains the soft parts of the animal. The human skeleton is arranged in two divisions, axial and appendicular. The axial skeleton comprises the head and trunk, and the appendicular represents the limbs. The head is composed of 23 bones, including the hyoid bone. The trunk is made up of the vertebral column, containing 26 separate bones in adult life; the sternum; and the ribs, with their costal cartilages, 24 in number, thus making .5I bones in all in the trunk. The appendicular skeleton comprises the two upper or pectoral limbs, each containing 32 bones, exclusive of sesamoid bones, and the two lower or pelvic limbs, each containing 3I bones, also exclusive of sesamoid bones.

\section{Descriptive Terms.}

Ankylosis: bony union between two bones which are normally separate.

Apophysis ('grow from '): this is any process or swelling on a bone.

Capitellum : a small head.

Caudal: towards the tail. 
Cephalic : towards the head.

Clinoid: like the knob of a bedpost.

Condyle (' knuckle'): a small round eminence covered by cartilage -in reality a capitellum.

Cotyloid : cup-like.

Diaphysis ("grow between '): this term is applicable to long bones. It is the shaft of the bone, or the part which grows between the epiphyses.

Dorsal : pertaining to the back aspect. In man ventral and dorsal are synonymous with anterior and posterior in the erect posture.

Epiphysis ('grow upon'): this is a process of bone which has a secondary centre of ossification, and which is attached for a time to the principal part of the bone by cartilage, but subsequently becomes consolidated.

Facet (Fr. facette, ' a little face '): a small plane articular surface.

Fissure : a cleft or slit.

Foramen : an opening in the sense of a hole.

Fossa : a shallow depression.

Glenoid: like a shallow socket.

Groove : a furrow or gutter.

Head : a rounded eminence, covered by cartilage, at the extremity s a bone.

Hiatus : an opening in the sense of a gap.

Malleolus : a small hammer.

Meatus (pl. meatûs): a passage or canal.

Medullary: pertaining to the medulla or marrow.

Neck : a constricted portion supporting a head.

Neural : pertaining to the nervous system.

Sinus or antrum: a cavity in the interior of a bone.

Spine: this is a sharp-pointed process, and in certain bones is erroneously applied.

Styloid: pen-like.

Trochan'er ('running round' or 'rolled'): a prominence wlich has a rolled or wheel-like arrangement, and which runs round a portion of a bone.

Trochlea : a pulley.

Tubercle ('swelling '): a small rounded prominence on a bone.

Tuberosity: an exaggerated tubercle.

Ventral : pertaining to the belly aspect of the body.

Mesial plane: this is the imaginary longitudinal plane which divides the body into two very nearly symmetrical lialves, right and left. It extends from the mesial line on the ventral aspect to the mesial line on the dorsal aspect.

Sagittal: this means pertaining to the antero-postcrior mesial plane with which it is parallel, and it practically means dorsoventral or antero-posterior.

Coronal: this means intersecting the antero-posterior mesial plane at right angles in a vertical direction, and it is practically synonymous witl transverse. 
Internal or mesial: near to the mesial plane; and external or lateral: farther away from the mesial plane.

Preaxial and postaxial: these terms were originally used in connection with the embryo. Preaxial, proximal, or cephalic means towards the head (superior), and postaxial, distal, or caudal means towards the tail (inferior).

Morphology.-This is the science which investigates the form and structure of parts and organs in reference to the series of changes through which they pass during their development. In a morphological sense parts of man's body may be compared with one another, or parts of the body of one animal may be compared with apparently similar parts in the body of another animal, and in this way their actual identity may be established.

Homology.-Parts or organs which are represented in different animals are said to be homologous (homogenetic) when they are constructed on the same type and have the same embryonic origin, although they may differ in function. For example, the upper limb of man and the fore-limb of a quadruped are homologous (homogenetic). Parts in the body of man are said to be serially homologous (homodynamic) when they are repeated. For example, the humerus is serially homologous with the femur.

Analogy.--This is identity in function, but not necessarily in structure. The gills of a fish are analogous with the lungs of man, though differing in structure.

Segmental Type.-This consists in the repetition longitudinally of a series of segments similar to each other-e.g., vertebræ, and ribs.

Chemical Composition of Bone.-Osseous tissue belongs to the connective tissues, and it consists of an organic matrix or ground substance, impregnated with mineral matter. The mineral matter is composed chiefly of calcium salts, and so it petrifies the ground substance. The organic matrix is usually spoken of as the animal matter, and it forms about 33 per cent. of the entire bone. The mineral matter is spoken of as the earthy matter, and it forms about 67 per cent. of the bone.

The animal matter imparts flexibility and elasticity to the bone, and is composed of very delicate fibres, which are collected into bundles, held together by cement substance. The fibres consist of collagen, which is converted into gelatin by boiling. The animal matter can be separated from the earthy matter by steeping a bone for some time in dilute hydrochloric acid, the effect of the acid being to dissolve out the earthy matter. When bone is so treated it is said to be decalcified. There is left a tough, flexible, elastic substance, which can be bent and twisted in various directions, and even tied into a knot, but no amount of force applied to it, or pressure laid upon it, would cause it to break. A bone when so treated retains its original shape, but it loses weight to the extent of about two-thirds, and it also loses its property of hardness, so that it is incapable of bearing weight. 
The earthy matter imparts hardness and rigidity to the bone, and is composed principally of calcium salts, of which the most abundant is calcium phosphate, there being about 57 per cent. of this salt present. Besides this there are calcium carbonate in the proportion of about 7 per cent., and calcium fuoride in the proportion of about I per cent. In addition to the calcium salts there are about I per cent. of magnesium phosphate, and about $\frac{1}{2}$ per cent. of sodium chloride. The earthy matter may be separated from the animal matter by burning a bone. The first effect of the heat is to char the animal matter, which is

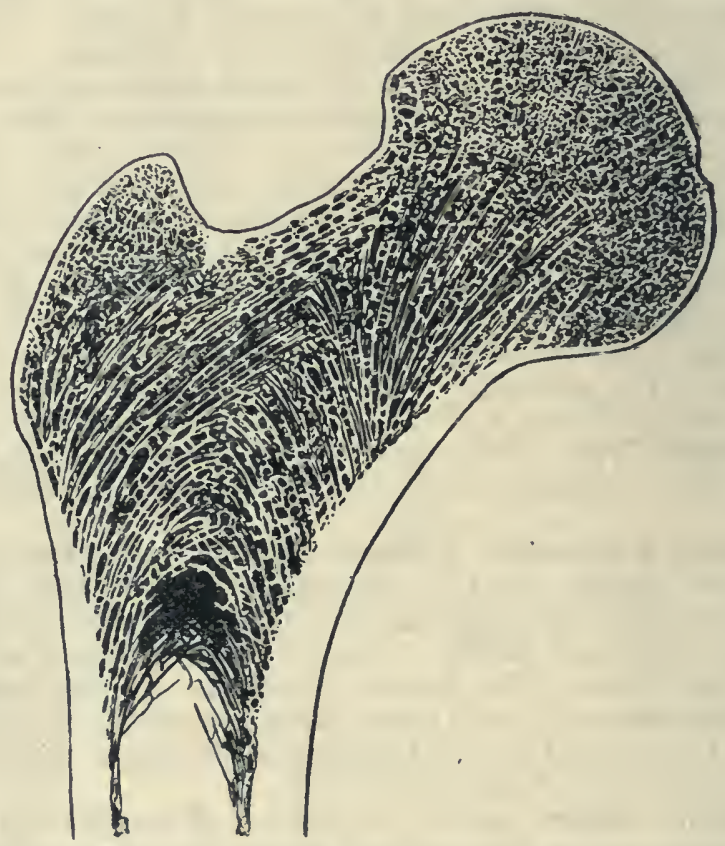

Fig. 1.-Longitudinal Section through the Upper End of the Femur, Showing Compact and Cancellated Osseous Tissues.

subsequently consumed. When so treated a bone is said to be calcined, and the process is spoken of as combustion or calcination. There is left a white, chalk-like, very brittle substance, which, if of small size and carcfully handled, retains its original shape. The slightest rough liandling, however, will cause it to break, or crumble into a coarse powder. Calcined bone undergoes no change in shape, but it loses weight to the extent of about one-third. It also loses its flexibility and elasticity. The only property it now possesses is hardness. As stated, it is also very brittle, and, by reason of this, it cannot be bent nor twisted in 
the slightest degree. The alimal and earthy matter, as they form bone, are intimately combined.

The proportion of about one-third of animal matter to about two-thirds of earthy matter applies to the healthy adult. In young children the relative amount of animal matter is much greater, so that in them the bones are very tough and elastic, but not very hard, and therefore not capable of bearing much weight. In old age there is a relatively large amount of earthy matter present. The bones of old persons, therefore, are very hard and brittle, but not very tough and elastic ; hence the frequency of fractures in old persons from slight causes.

Structure of Bone.-There are two varieties of osseous tissue, namely, compact, and spongy or cancellated. Compact osseous tissue is so named because its constituents are so closely packed together that the bone appears to the naked eye (macroscopic)

A

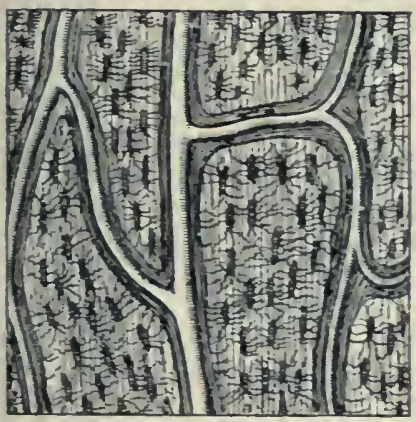

B

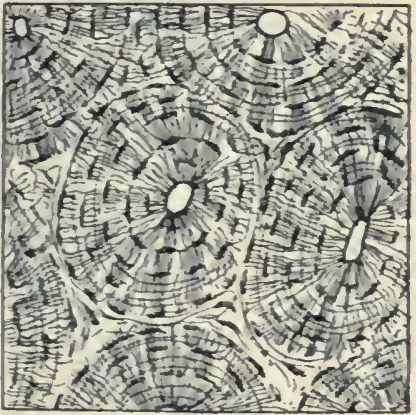

Fig. 2.-Structure of Compact Bone.

A, Longitudinal section, showing Haversian canals; B, Transverse section, showing Haversian systems.

to be. dense and close like ivory. Spongy or cancellated osseous tissue, on the other hand, presents an open porous appearance like a sponge. It is called cancellated because it resembles latticework. These two varieties of osseous tissue merge very gradually into one another.

When compact bone is viewed under the microscope in thin transverse section it presents a number of small round or oval openings, and when viewed in thin vertical section it presents short longitudinal tukes, called Haversian canals. These pervade every part of compact bone, and the innermost open into the marrow canal of long bones, whilst the outermost open by minute orifices on the external surface. They range in diameter from $\frac{1}{1000}$ to $\frac{1}{200}$ inch, the average being $\frac{1}{500}$ inch. The smallest lie nearest the external surface, and the largest are nearest the marrow canal. They are very short and longitudinal in direction, and they com- 
municate freely with one another by connecting canals, some of which are oblique and others transverse. These connecting canals are very small, having a diameter of only $\frac{1}{2000}$ inch. The Haversian canals thus form a freely intercommunicating system of tubes throughout compact bone. The largest canals contain each an arteriole, a radicle vein, one or two lymphatics, and a small amount of marrow tissue. The smallest canals contain only one bloodvessel, which is of the nature of a capillary. Thosc nearest the external surface also contain very delicate thread-like processes of the periosteum. Nerve fibrils have been demonstrated in bone by Kölliker, Rémy, and Variot.

The bone around the Haversian canals is arranged in the form of concentric plates, called Haversian or concentric bone lamella. In transverse section these appear as concentric rings, and in longi-

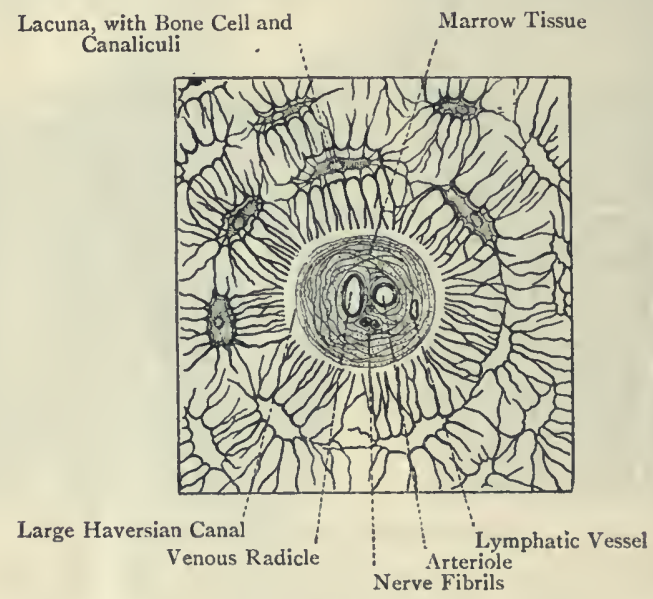

Fig. 3.-Diagram of a Haversian System, minus the Concentric Bone Lamelle.

tudinal section as parallel lines. In the interspaces between the systems of Haversian or concentric lamellæ there are intermediate, interstitial, or ground lamella, and near the external surface there are circumferential or peripheral lamella, which are parallel with the surface. Some of the canals which pierce the circumferential lamellix are devoid of concentric lamellæ, and are knowll as Volkmann's canals. In the interspaces between the Haversian or concentric lamellæ there are small fusiform cavities, known as the bone lacunce. These are about $\frac{1}{2000}$ inch in length, and, like the lamelle, they are arranged concentrically round the Haversian canals. Radiating from these lacunæ there are minute channels, called bone canaliculi, which pass through perforations in the lamellæ, and so serve to connect the various lacune with one another. Some of those radiating from the innermost ring of lacune communicate 
directly with the Haversian canal. The canaliculi thus constitute a system of intercommunicating channels which maintain a connection directly and indirectly between a given Haversian canal and the lacunæ arranged concentrically around it, and traverse the lamellæ in their course. Each lacuna contains a protoplasmic nucleated cell, called the bone cell, which almost completely fills it, and sends off processes into the canaliculi communicating with it. The canaliculi contain nutritive fluid derived from the arteriole. A given Haversian canal, with its concentric bone lamellæ, concentric bone lacunæ, and canaliculi, constitutes a Haversian system, and compact bone is simply an aggregation of such systems, with, in addition, the intermediate and circumferential lamellæ. Certain lamellæ are perforated perpendicularly by fibres which thus bind them together. These fibres are known as the perforating fibres of Sharpey. Some of them are composed of white fibrous tissue, and others of elastic tissue, and those nearest the periphery are connected with the periosteum from which they are derived. Others, however, more deeply placed, have no apparent direct connection with the periosteum. The perforating fibres are absent from the lamellæ of the Haversian systems.

Cancellated bone is composed of very slender trabecula, which are arranged in a reticular manner so as to enclose spaces, known as the medullary spaces. These spaces, in the recent state, are filled with marrow. The trabeculæ consist of superimposed lamellæ of compact bone. The strongest lamellæ are disposed in the direction in which the greatest pressure has to be borne, and these are known as pressure lamella. Other lamellæ which intersect these, are spoken of as tersion lamella. The use of cancellated bone is to impart sufficient strength without adding unduly to weight.

Classification of Bones.-Bones are arranged in four classes, as follows: Long; short; tabular; and irregular.

A long bone consists of a shaft and two articular extremities. The shaft is more or less cylindrical, and contains a marrow canal, which is surrounded principally by compact bone. The articular extremities are composed of cancellated tissue, except at the surface where there is a thin shell of compact bone. Long bones are found in the appendicular skeleton.

A short bone consists mainly of cancellated tissue, except at the surface where there is a thin covering of compact bone. Short bones are more or less oblong in shape, and are found in the carpus and tarsus.

A tabular bone is composed of two plates or tables of compact bone, which enclose between them cancellated tissue. The scapula, ilium, and tegmental bones of the skull belong to this class. In the case of the latter the cancellated tissue is called diploë.

An irregular bone is one which is so irregular in form and in the relative distribution of the compact and cancellated tissues as to be 
excluded from any of the preceding classes. The vertebræ belong to this class.

Ossifleation.-All bones are originally membranous. Some of them-for example, the tegmental bones of the cranium and most of the bones of the face-ossify in membrane, but the majority pass through a cartilaginous stage before becoming ossified. There are, therefore, two modes of ossification, namely intramembranous and intracartilaginous, and bones are consequently spoken of as membrane- and cartilage-bones.

Centres of Ossification.--These are primary and secondary. The primary centre, which as a rule appears early in intra-uterine life,

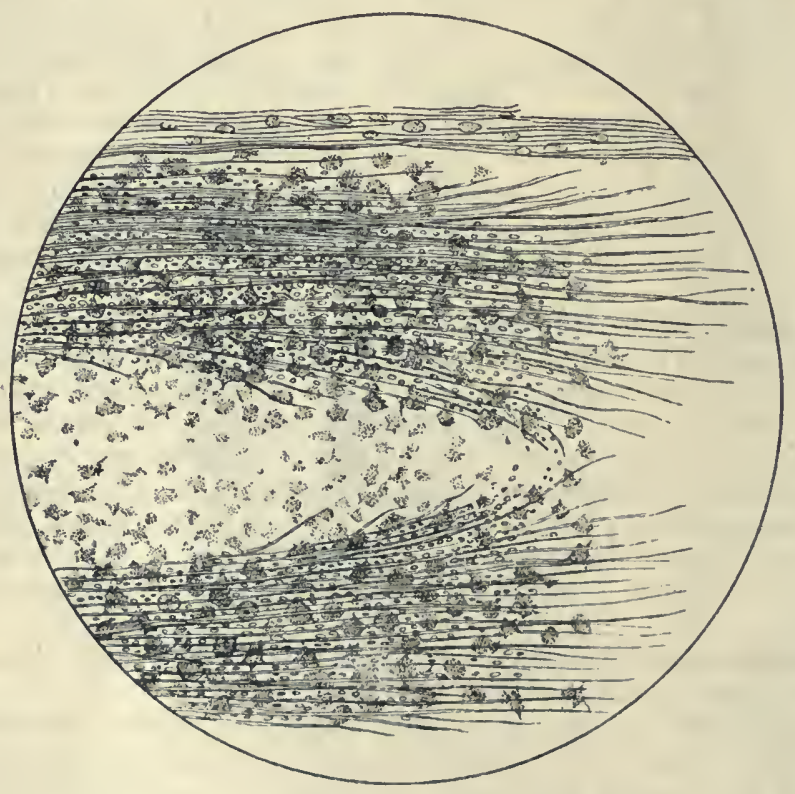

Fig. 4.-Ossification in Membrane.

is that from which the ossification of the principal part of the bone proceeds. This, in the case of a long bone, is the shaft or diaphysis. il he secondary centres, which for the most part do not appear until after birth, are those from which the ossification of outgrowtlis of a bone proceeds, these forming what are known as the epiphyses.

Ossifleation in Membrane.-In this mode of ossification the bone is preceded by fibrous tissue. The fibres of this tissue are known as osteogenetic fibres, and they are arranged in small bundles. The tissue is very vascular, and contains many nucleated cells, called osteoblasts. At the centre of ossification the osteogenetic fibres, which have a covering of osteoblasts, become calcified, and bony spicula are thus formed, which radiate towards the circumference of the bone. These radiating spicula are connected at frequent intervals, and 
so build up a bony reticulum. As the osteogenetic fibres grow and shoot out, they carry with them coatings of osteoblasts, and the process of calcareous incrustation goes on, so that the bony spicula increase in length, and gradually approach the periphery. During this process of spicular bony formation many of the osteoblasts are left behind, and become imprisoned in the lacunar spaces of the forming bone, where they represent the future bone cells. The ossification of a membrane bone is thus effected by means of osteogenetic fibres plus osteoblasts, the fibres acting as outrunners and becoming calcified.

Ossifieation in Cartilage. - The cartilage is covered by a membrane, called the perichoudrium, which corresponds to the periosteum, and the process of ossification takes place in three stages.

First Stage.- In this stage the ossification is partly endochondral, and partly ectockondral or on the surface beneath the perichondrium. In the endochond ral form the cartilage cells at the centre become enlarged, and the intervening matrix becomes calcified. Above and below the centre the cartilage cells are arranged in long columns, directed towards each extremity. The matrix between these columns becomes calcified by an extension of the calcareous matter at the centre, which now surrounds the cellcolumns. The spaces in the calcified matrix, which contain these columns, are known as the primary areole. At the same time, ectoehondral cr subperichondral ossification is proceeding in a manner similar to what takes place in membranous ossification, that is to say, by osteogenetic fibres, osteoblasts, and calcareous impregnation. In this way several layers of bone are laid down at the surface beneath the perichondrium, and these constitute the circumferential lamellx. During this process some of the osteoblasts are detained in lacunar spaces, and form the bone cells.

Second Stage. - This is known as the stage of irruption. The inner or osteogenetic layer of the perichondrium bursts in through openings in the circumferential lamella, in the form of osteogenetic fibres, osteoblasts, and osteoclasts or bone destroyers. These incursions reach the calcified matrix, and the osteoclasts now commence their destructive work. The cartilage cells of the primary areolæ, as well as the walls of these areolx, are absorbed, and larger spaces, called secondary areolx or medullary spaces, are formed in the original calcified matrix. These spaces contain osteogenetic fibres and osteoblasts, and the latter now build up lamellæ of bone.

Third Stage. - This is a repetition of the preceding two stages. The cartilage cells arrange themselves in rows; the intervening matrix becomes calcified, and invests them; the enclosed cartilage cells atrophy and give rise to primary areolx; the osteoclasts produce partial absorption of the calcified cartilage, giving rise to medullary spaces; and the osteogenetic fibres and osteoblasts build up lamellz of bone. Simultaneously with these processes, subperiosteal ossification is going on. The medullary canal is due to absorption by the osteoclasts of the osseous tissue in the centre of the shaft.

The ossification of the epiphyses of a bone is endochondral.

Periosteum.-The periosteum is a fibrous, vascular membrane, which closely invests bones, except where there is articular cartilage, 
at the margin of which it ceases. It consists of two layers--outer and inner. The fibres of the outer layer are arranged closely, and it is therefore dense. The inner layer consists chiefly of ramifying elastic fibres. Between the inner layer and the surface of the shaft of a long bone there is, especially during the development and growth of the bone, a layer of subperiosteal areolar tissue. Within its meshes cells, called osteoblasts, accumulate during the period of growth. These cells emerge from the outermost Haversian canals along the course of the entering arteries, and they represent the bone cells of the bone-lacunæ of compact bone which have migrated outwards. The osteoblasts take an important part in the formation of bone during its growth.

The periosteum is richly supplied with arteries which, after ramifying, enter the outermost Haversian canals, along with fine processes of the inner periosteal layer. The periosteum serves (I) as a bed in which the arteries subdivide before entering the bone, and (2) to give a firm hold to tendons and ligaments. It also takes part in ossification and regeneration of bone. When the periosteum is stripped from a bone the uncovered portion is liable to necrosis and exfoliation.

Marrow or Medulla.-The marrow fills the marrow canals of long bones and the medullary spaces of cancellated bone, and it also sends processes into the innermost Haversian canals. It is composed of a reticular fibrous matrix, which is pervaded by many bloodvessels and cells, the latter being called myelocytes (marrow cells). There are two kinds of marrow, yellow and red, which differ as to the character of the cells. In yellow marrow most of the cells have become transformed into fat cells, so that the marrow resembles adipose tissue. Red marrow contains very few fat cells. Many of its cells are colourless, protoplasmic, nucleated cells, which resemble the leucocytes of the blood, though of larger size, and like them are capable of amoboid movement. Other reddisls cells, called erythroblasts, are present, which are the sources from which large numbers of red blood corpuscles are formed. In addition to these two sets of cells, there are large multinucleated, protoplasmic cells, called the myeloplaxes of Robin, which play an important part in the absorption of bone.

Yellow marrow is found in the marrow canals of long bones, whilst red marrow occurs in (I) articular ends of long bones; (2) medullary spaces of cancellated bone; (3) bodies of the vertebræ; (4) sternum; (5) ribs; and (6) the diploë of the cranial bones. Marrow serves the following uses: (I) it (red marrow) is an important blood-forming organ (red corpuscles); (2) it contributes to the nourishment of bone; and (3) it serves as a liglit packing material for all hollow spaces within bones, with the exception of the air-sinuses in the bones of the head.

The wall of the marrow canal of all long bones and that of the medullary spaces of all cancellated bone are lined with a very delicate layer of areolar tissue, which is richly provided 
with bloodvessels. This is known as the endosteum or medullary membrane.

Osseous tissue is richly supplied with bloodvessels, which are derived from the periosteum and marrow.

\section{THE BONES OF THE TRUNK.}

\section{A. The Vertebral Column.}

The vertebral column is composed of thirty-three vertebræ in the young subject, and these in the adult are divided into two classes, namely, true or movable, and false, immovable, or fixed. The true vertebræ are those which, though connected by ligaments, are quite distinct from each other, so that a limited amount of movement is allowed between them. The false vertebræ are those

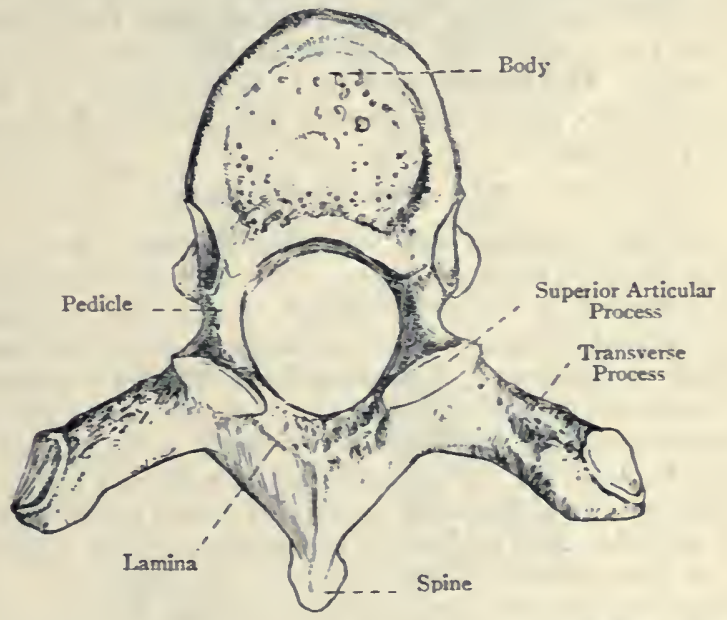

Fig. 6. -The Sixth Thoracic Vertebra (Superior View).

which, though distinct up to a certain period of life, subsequently become ankylosed. The true vertebræ are subdivided into three groups-cervical, thoracic, and lumbar. The false vertebræ are subdivided into two groups-sacral, and coccygeal.

Component Parts of a Complete Vertebra.-A complete vertebra is composed of a body or centrum; a neural arch, consisting of two pedicles and two laminæ (neurapophyses); a spinous process, or neural spine; two transverse processes; four articular processes (zygapophyses), two superior and two inferior; and a spinal or neural foramen. The body forms the anterior or ventral part of the bone, and is somewhat disc-shaped. The neural arch consists of two halves, the anterior portion of each being the pedicle, and the posterior portion the lamina. The pedicles 
present, above and below, the superior and inferior vertebral notches. The spinous process is formed by the fusion of the two laminæ in the median line posteriorly. The transverse processes project outwards, one at either side, from the neural arch at the junction of the pedicle and lamina. The articular processes, two superior and two inferior, project upwards and downwards from the junction of the pedicle and lamina at either side, and they are covered by cartilage. The spinal or neural foramen is enclosed by the body and neural arch. It is bounded in front by the posterior surface of the body, on either side by a pedicle and lamina, and behind by the fusion of the laminæ to form the spinous process.

Structure of a Vertebra.-The body is composed of cancellated tissue, covered by a thin layer of compact bone. The chief lamellæ are disfosed in almost vertical curves, the convexities of which are directed towards the periphery. Crossing these there are horizontal lamellæ, which are nearly parallel with the superior and inferior surfaces. The cancellated tissue is permeated by venous channels which converge to the two large foramina on the posterior surface of the body. The neural arch and its processes are chiefly composed of compact bone, the amount of cancellated tissue being for the most part small.

\section{The Cervical Vertebræ.}

The cervical vertebræ are seven in number, and they occupy the region of the cervix or neck. The distinctive character of all cervical vertebræ is the presence of an aperture at either side of the body, called the costo-transverse fo-amen. The first or atlas, the second or axis, and the seventh or vertebra prominens have such pronounced characters that they require a special description.

A Typical Cervical Vertebra.--The body is small and elongated from side to side. The superior surface presents at either side an antero-posterior.lip, the inner surface of which is sloped towards the superior surface, and embraces the corresponding bevelled lateral border of the inferior surface of the body above. The whole surface is thus concave from side to side, and the posterior lip is on a slightly higher level than the anterior. The inferior surface is bevelled laterally, and its anterior lip is on a lower level than the posterior. The inferior surface is convex from side to side, and concave from before backwards. The superior and inferior surfaces give attachment to the intervertebral discs. The anterior surface is convex from side to side, and concave from above downwards. It is covered by the anterior common ligament, and it presents a number of nutrient foramina. The posterior surface is flat, and presents several nutrient foramina, two of which, one at either side of the middle line, are large, for the escape of the venæ basis vertebræ. The posterior surface is related to the posterior common ligament. Each lateral surface forms the inner boundary of the costo-transverse foramen.

The pedicles spring at cither side from the posterior part of the 
lateral surface of the body, where each encroaches rather nearer the upper than the lower surface. They are smooth and almost cylindrical, and their direction is outwards and backwards. Above and below each pedicle there is a well-marked vertebral notch, the superior being narrower and slightly shallower than the inferior. The superior notch lodges a spinal nerve. When two vertebræ are in position the contiguous vertebral notches, at either side, form an intervertebral foramen.

The laminz spring each from a pedicle. They are compressed
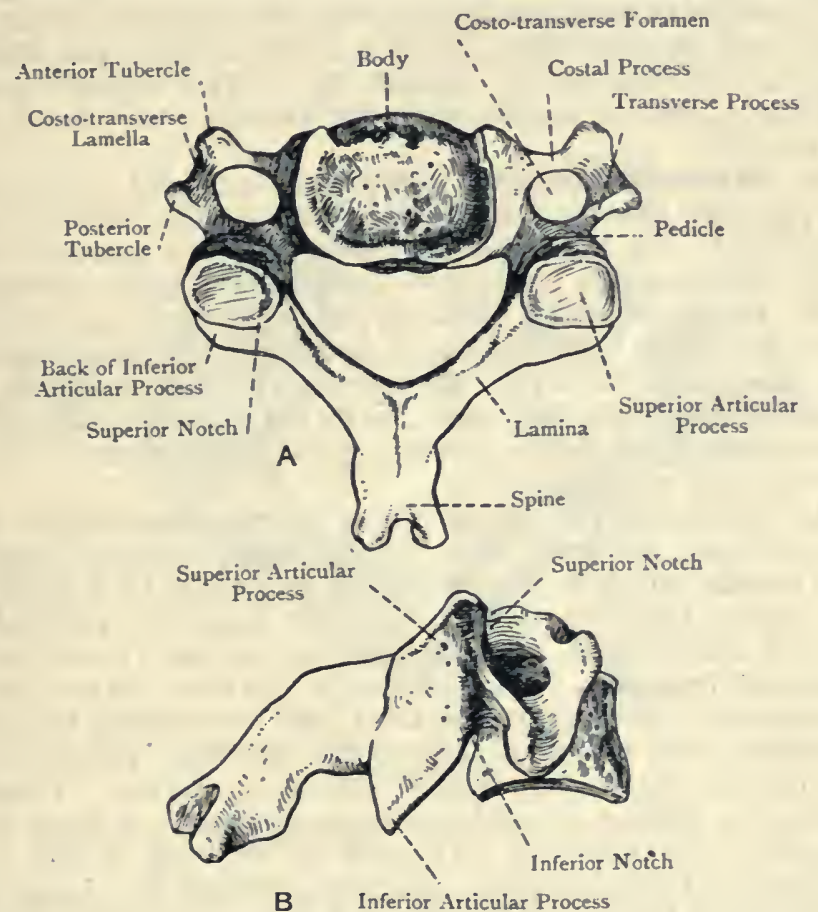

Fig. 7.-The Fifth Cervical Vertebra.

A, Superior view; B, Lateral view.

from before backwards, and their direction is backwards and inwards to the middle line, where they fuse, and so give rise to the spinous process. The upper border and adjacent portion of the posterior surface of each lamina give attachment to the ligamentum subflavum connecting it to the lamina above, whilst the lower part of the anterior surface near the lower border gives attachment to the ligamentum subflavum connecting it to the lamina below.

The spinous process is formed by the fusion of the two laminæ. 
It is triangular, and its direction is backwards and slightly down. wards. Superiorly it presents an antero-posterior ridge for an interspinous ligainent, and inferiorly a grooved surface, also for an interspinous ligament. It terminates behind in a bifid extremity, which, in the case of the third, fourth, and fifth vertebræ, presents a distinct triangular notch. The bifurcated extremity gives attachment to the deep fibres of the ligamentum nuchæ. The cervical spinous processes are very short, except those of the sixth and seventh, especially the latter, and in this way backward flexion or over-extension of the neck is not interfered with.

The articular processes spring from the junction of the pedicle and lamina at either side. Each is nearly circular, the plane being oblique, and the surface almost flat. The superior pair look backwards and upwards, and the inferior pair forwards and downwards.

The transverse processes (diapophyses) spring at either side from the junction between the pedicle and lamina, and are serially homologous with the transverse processes of a thoracic vertebra. Each terminates in a projection, known as the posterior tubercle.

The costal processes (pleurapophyses) project outwards from either side of the body anteriorly, and are serially homologous with the vertebral part of a rib. Each terminates in a projection, known as the anterior tubercle, which, with the posterior tubercle of the corresponding transverse process, gives attachment to the intertransversales muscles.

The transverse and costal processes are connected, at a short distance from the body, by a plate of bone, called the costo-transverse lamella, which is deeply grooved superiorly for a spinal nerve, this groove being continuous with the superior vertebral notch. There is thus formed, at either side, an aperture, called the costotransverse foramen. This foramen is circular, vertical in direction (except in the case of the axis), and it transmits the following structures: the vertebral artery; the vertebral plexus of veins; and the vertebral sympathetic plexus of nerves. Though the foramen is present in each transverse process, it does not give passage to the foregoing structures in the case of the seventh. The vertebral vein, however, may pass through it. In many cases an additional foramen of small sizc is present on one or both sides, lying behind the main foramen, and when this is so, it transmits a small vein. The costo-transverse foramina of either side, when in position, build up a canal, which is open in each intertransverse space.

The neural foramen is situate! behind the body, and is triangular, with the angles rounded off. It is of larger size than in the thoracic or lumbar vertebræ, its direction is vertical, and it lodges the spinal cord with its membranes.

The Atlas.- The atlas is the first cervical vertebra, and is so named because it supports the head. Its distinctive characters are the absence of a body and spinous process. It has the form of a ring, narrow in front and wide behind, and its component parts 
are as follows: an anterior arch: a posterior arch; two lateral masses; and a ring.

The anterior arch is a curved plate of bone which connects the antero-internal parts of the lateral masses. It is compressed from before backwards, convex in front, and concave behind. The ancricr surface presents at its centre a conical prominence, called the anderior tubercle. This gives attachment at either side to a portion of the longus colli muscle, and its central part receives the accesscry ligament. The posterior surface presents at its centre a circular concare facet, called the odontoid facet, for articulation with the anterior surface of the odontoid process of the axis. The upper border gives attachment to the anterior occipito-

Anterive Tubercle

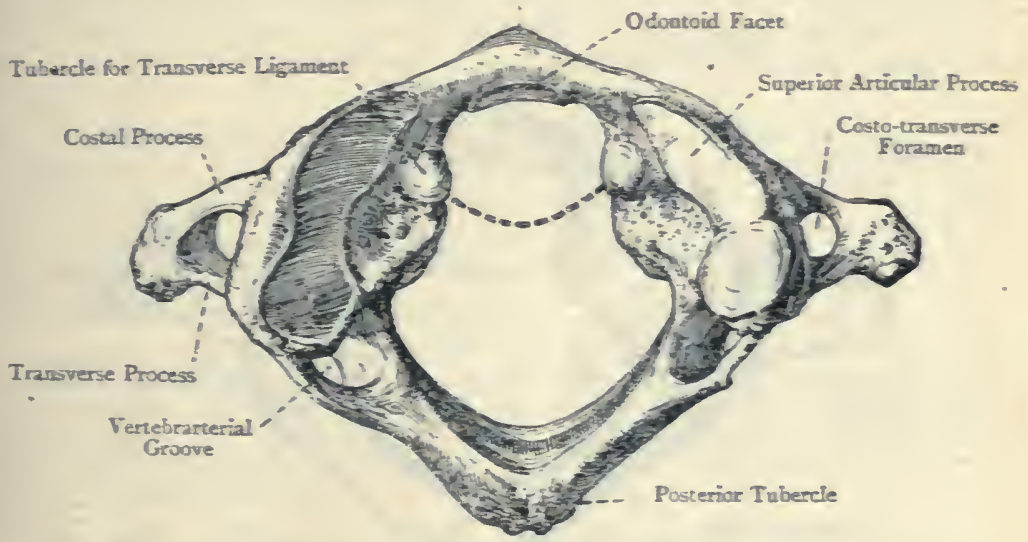

Fig. 8.-THE AtLAS (StPERTOR VIEW).

(The Vertebrarterial Groove on this bone was convinted into a Foramen or both sides.)

atlantal ligament, and the lower to the anterior atlanto-axial ligament.

The posterior arch is serially homologous with the laminx of other vertebra. It springs at either side from the back part of a lateral mass, from which it sweeps backwards and inwards. The part close to the lateral mass at either side is flattened from above downwards. It presents on its upper surface a shallow depression, called the vertebrarterial grooze, which lodges the vertebral artery and suboccipital nerve. This groove is sometimes converted into a foramen on one or both sides by a spiculum of bone extending from the back part of the superior articular process to the posterior arch behind the groove. The vertebrarterial groove is serially homologous with the superior vertebral notch of other vertebræ, but, unlike them, it lies behind the superior articular process. The inferior surface of the posterior arch, behind each lateral mass, 
presents a shallow vertebral notch, which lies behind the inferior articular process. The centre of the posterior arch presents the posterior tubercle, which is the only representative of a spinous process. At either side of this tubercle the rectus capitis posticus minor arises. The upper aspect of the posterior arch gives attachment to the posterior occipito-atlantal ligament, and the lower aspect to the posterior atlanto axial ligament.

The lateral masses support the superior and inferior articular processes, and laterally the transverse and costal processes spring from them. The anterior surface of each gives partial origin to the rectus capitis anticus minor. The in ternal surface of each presentsanteriorly a tubercle for the transverse ligament. The superior urticular processes are oval and deeply concave, to articulate with the condyles of the occipital bone. Their long axes are directed backwards

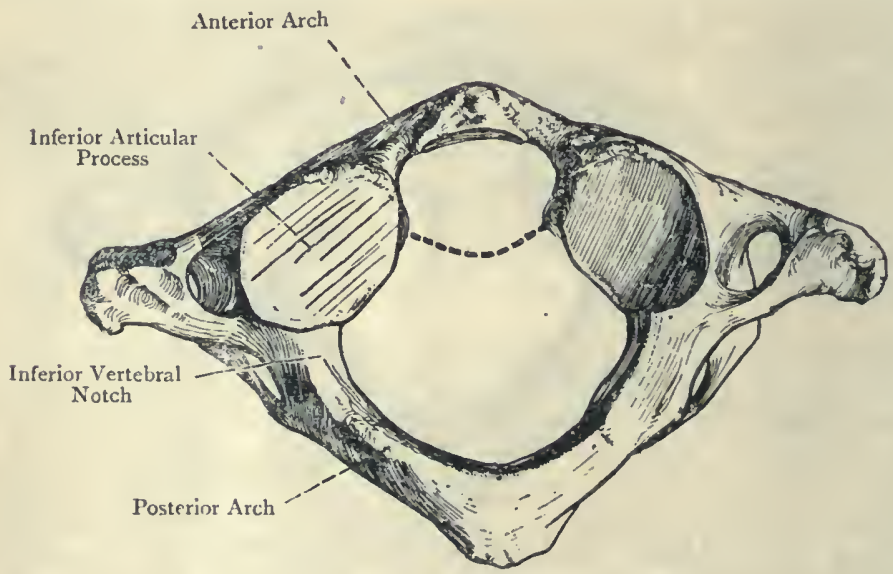

Fig. 9.-The Atlas (INferior View).

and outwards, so that they converge in front and diverge behind. Anteriorly they reach as far as the anterior arch, and posteriorly they overhang the vertebrarterial grooves on the posterior arch to a slight extent, but they do not extend farther back than about the centre of the ring. The plane of each is sloped down. wards and inwards, and the direction of the surface is upwards and inwards. The movement between them and the occipital condyles is one of flexion and extension, or nodding. Sometimes one or both of them may be divided by a groove into two circular facets. The inferior articular processes are circular and slightly concave. The plane of each is sloped upwards and inwards, and the direction of the surface is downwards and inwards. They articulate with the superior articular processes of the axis, and the movement allowed is rotation. The articular processes of the atlas, being placed in front of the points of exit of the spinal 
nerves, do not correspond in position with the articular processes of succeeding vertebræ (with the exception of the superior pair of the axis). They occupy a position corresponding with the pedicular portions of the bodies of vertebræ, and in this way the superincumbent weight is transmitted to the vertebral bodies.

The transverse and costal processes spring from the side of each lateral mass, and, external to the costo-transverse foramen, the costo-transverse lamella and the anterior and posterior tubercles are more or less fused into one long irregular mass, though the posterior tubercle usually remains conspicuous. The upper surface of this mass at its front part gives origin to the rectus capitis lateralis, and at its back part to the obliquus capitis superior, whilst the lower surface at its back part gives insertion to the obliquus capitis inferior. The costo-transverse foramen is of large size in order to guard against the vertebral artery being compressed during the rotatory movements of the bone upon the axis.

The ring of the atlas, in the recent state, is divided into two compartments by the transverse ligament. The anterior small division is called the odontoid compartment, and it lodges the odontoid process of the axis. The posterior large division represents the neural foramen of other vertebræ, and it lodges the spinal cord with its membranes.

Varieties.-(I) The posterior arch may be incomplete at the centre, the deficiency being bridged over by fibrous tissue. (2) The costal process may be incomplete, the deficiency in the costo-transverse foramen being filled by fibrous tissue. (3) There is sometimes an additional small foramen on either side, a little behind the costo-transverse foramen, for the passage of the suboccipital radicles of the vertebral plexus of veins.

The Axis.-The axis is the second cervical vertebra, and is so named because its odontoid process, which is the distinctive character of the bone, forms a pivot on which the atlas, supporting the head, rotates. From the presence of this process the axis is sometimes called the vertebra dentata.

The odontoid process (processus dentatus) springs from the superior surface of the body, and represents the body of the atlas. It is constricted and somewhat circular close to the body, this part being called the neck. Above this it expands into a head, which tapers off at either side by two sloping surfaces, forming by their convergence an antero-posterior ridge, known as the summit. The anterior surface presents a circular convex facet, called the atlantal facet, for articulation with the odontoid facet on the posterior surface of the anterior arch of the atlas. The posterior surface presents a shallow transverse groove for the play of the transverse ligament of the atlas. The lateral sloping surfaces on either side of the summit give attachment to the lateral odontoid ligaments, whilst the summit itself gives attachment to the middle odontoid ligament. 
The superior surface of the body is occupied by the odontoid process and portions of the superior articular processes. The inferior surface differs from that of other cervical vertebræ only in the greater downward projection of its anterior lip. The anterior surface presents a median vertical ridge which bifurcates inferiorly into diverging lips, enclosing a small triangular surface. On either side of the median ridge the surface is depressed, and gives attachment to a portion of the longus colli muscle. The other surfaces of the body present no:hing peculiar.

The pedicles are concealed above by the superior articular processes. Each, on its inferior aspect, presents a wide and deep inferior vertebral notch, which is placed in front of an inferior articular process. The superior vertebral notches, which are very shallow, are situated on the upper borders of the laminz, and, like

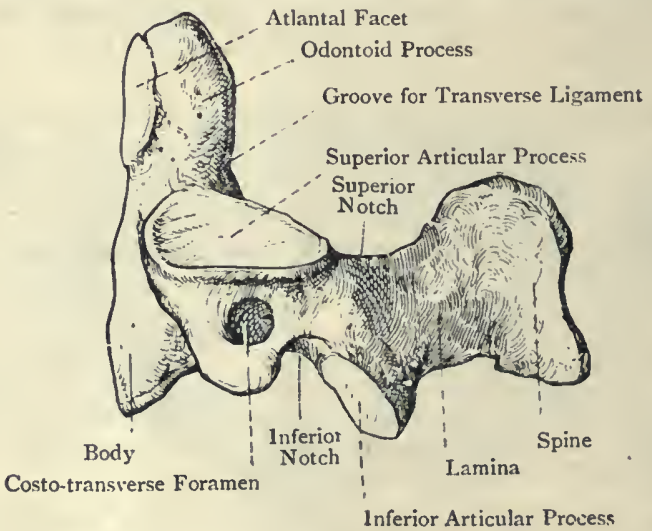

Fig. io.-The Axis (Lateral View).

those of the atlas, are placed behind the superior articular processes.

The laminæ are massive, and give attachment by their upper borders to the posterior atlanto-axial ligaments, whilst their anterior surfaces, near the lower borders, give attachment to ligamenta subflava, as in other vertebræ.

The spinous process is massive. Its direction is backwards, and it terminates in two strong tubercles, separated inferiorly by a triangular cleft. Each of these tubercles gives attachment to some of the deep fibres of the ligamentum nuchæ, and to the following muscles from above downwards: the rectus capitis posticus major; the obliquus capitis inferior; and the highest portion of the semispinalis colli.

The superior articular processes are situated on the upper surface of the pedicle at either side, the upper surface of the costal process, and a portion of the superior surface of the body, upon which 
latter it encroaches very near to the odontoid process. The plane of each is sloped outwards and downwards. The surface is slightly convex from before backwards and circular, its direction being upwards and outwards. They articulate with the inferior articular pro. cesses of the atlas, and the movement allowed is rota. tion. The inferior articular processes differ from those of most vertebræ only in being situated principally upon the lower borders of the laminæ. The superior pair, like all four articular processes of the atlas, being placed in front of the points of exit of the spinal nerves, do not correspond in position with the superior articular processes of succeeding vertebræ, but

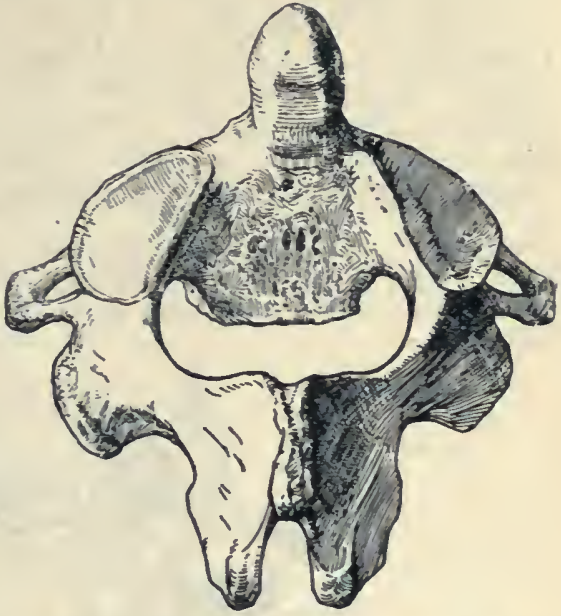

Fig. II.-The Axis (SUperior View). occupy a position corresponding with the pedicular portions of the bodies of vertebræ.

The transverse processes are very short, and are directed outwards and downwards. Each terminates in a single tubercle.

The costal processes also terminate in tubercles, and the costotransverse lamellæ are not grooved superiorly.

The costo-transverse foramen is directed upwards and outwards, the reason of this obliquity being as follows: when the atlas and axis are in position each costo-transverse foramen in the atias lies farther out than that in the axis. In order, therefore, to obviate any sudden and undue bend in the vertebral artery, the foramen in the axis is directed obliquely upwards and outwards so as to guide the vertebral artery gradually to the foramen in the atlas.

There is nothing peculiar about the neural foramen.

Varieties.-(I) The summit of the odontoid process may present a facet, indicating an articulation with the anterior margin of the foramen magnum of the occipital bone, which in such cases presents a prominence known as the middle occipitai condyle. (2) The odontoid process may, in very rare cases, remain separate from the body, thus forming the os dentatum. (3) An odontoid process in two halves has been recorded.

The seventh cervical vertebra.-The distinctive character of this vertebra is the great length of its spinous process, which is the only cervical $s_{;}$ine that can readily be felt beneath the integument of the neck. On account of this outstanding prominence the seventh cervical is known as the vertebra prominens. The spinous process 
is directed straight backwards, and terminates in a single large tubercular eminence. The other characters of this vertebra to be noted are as follows: the antero-posterior measurement of the body exceeds that of other cervical vertebræ; the transverse process is massive and comparatively long; the posterior tubercle is very distinct, but the anterior is rudimentary, or wanting; the costo-transverse foramen is of small size, and does not transmit the vertebral vessels and vertebral sympathetic plexus. The vertebral vein, however, may pass through it.

Varieties.-(I) The costal process may remain separate from the transverse process, thus giving rise to a cervical rib. (2) The costal process may be wanting on one or both sides, in which cases there is no costo-transverse foramen.

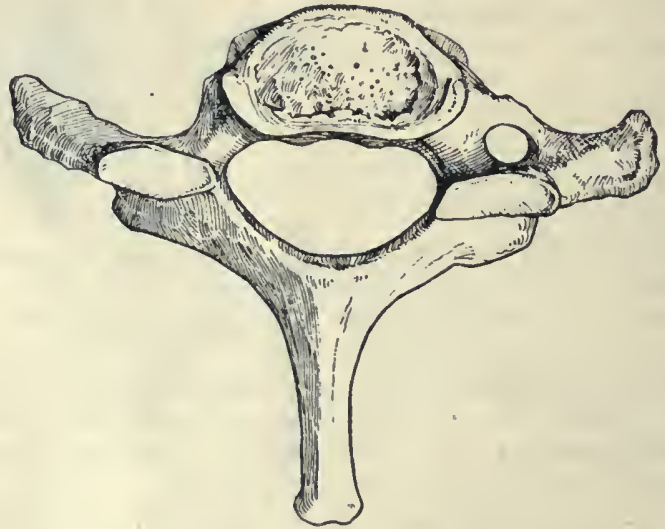

Fig. I2.-The Seventh Cervical Vertebra (Superior View). (The Costal Process of the Left Side was undeveloped in this vertebra.)

It is to be noted that the sixth cervical vertebra is peculiar in the following respects: the spinous process, like that of the vertebra prominens, terminates in a single large tubercular eminence; and the tubercle of each costal process, known as the anterior tubercle, is of large size, and is called the carotid tubercle of Chassaignac.

The cervical vertebræ receive their blood-supply from branches of the vertebral arteries.

\section{The Thoracic Vertebræ.}

The thoracic vertebræ are twelve in number, and their distinctive character is the presence of one or more facets on either side of the bodies for articulation with the heads of ribs. The first, 
tenth, eleventh, ard twelfth (sometimes also the ninth) are peculiar, and require separate descriptions.

A Typical Thoracic Vertebra.-The body is larger than that of a cervical vertebra, but smaller than that of a lumbar. When viewed from above or below it is cordate or heart-shaped, being broad aid hollowed out behind, ard narrow and rounded off in front. The posterior depth of the body exceeds the anterior, in adaptation to the backward curve of the vertebral column in the thoracic region. The superior and inferior surfaces present a raised rim round the circumference, due to the original epiphysial plate, and this renders the whole of each surface slightly concave from the periphery towards the centre. The anterior and

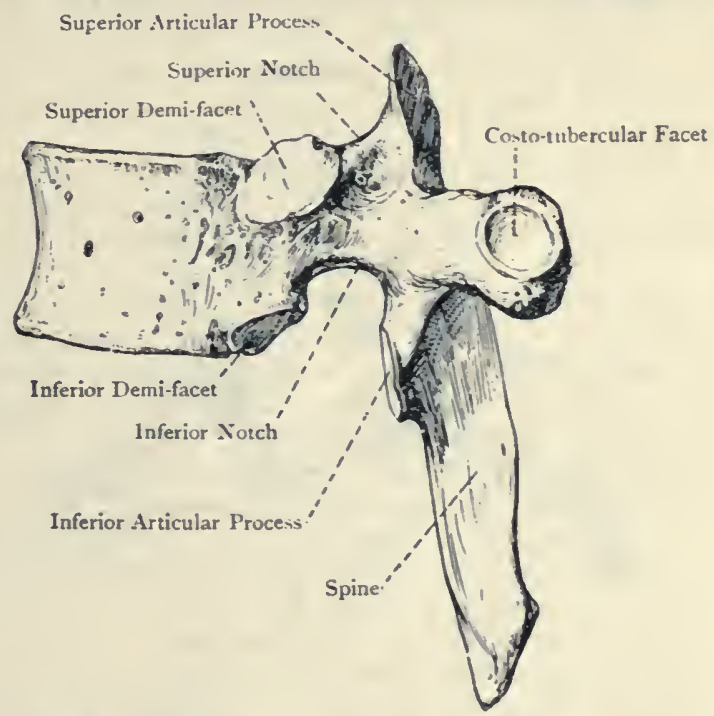

Fig. 13.-The Sixth Thoracic Vertebra (Lateral View).

lateral surfaces merge gradually into each other, and are concave from above downwards, the entire antero-lateral surface being convex from side to side and pierced by numerous nutrient foramina. Each lateral surface, close to the neural arch, presents two articular demi-facets, superior and inferior, of which the superior is the larger, and is situated upon the pedicular portion of the body, the inferior smaller one being just in front of the lower part of the inferior vertebral notch. These demi-facets are for articulation with the heads of the ribs, and are called the costocapitular facets. When two vertebrie are in position the superior demi-facet of the lower vertebra and the inferior demi-facet of the upper form an articular cavity for the head of a rib. The posterior surface of the body is concave from side to side, and presents 
nutrient foramina, as in the cervical vertebræ. The superior and inferio: surfaces are related to the intervertebral discs, and the anterior and posterior surfaces are related to the anterior and posterior common ligaments.

The pedicles spring from either lateral extremity of the posterior surface of the body, and their upper borders are very nearly on a level with its superior surface. Each pedicle is laterally compressed, and is directed backwards and slightly outwards. The superior vertebral notches are shallow, and each is usually bounded in front by a transverse neuro-central lip. The inferior vertebral notches are deep and wide.

The laminæ are short, deep, and compressed from before backwards, their planes being sloped downwards and backwards. The

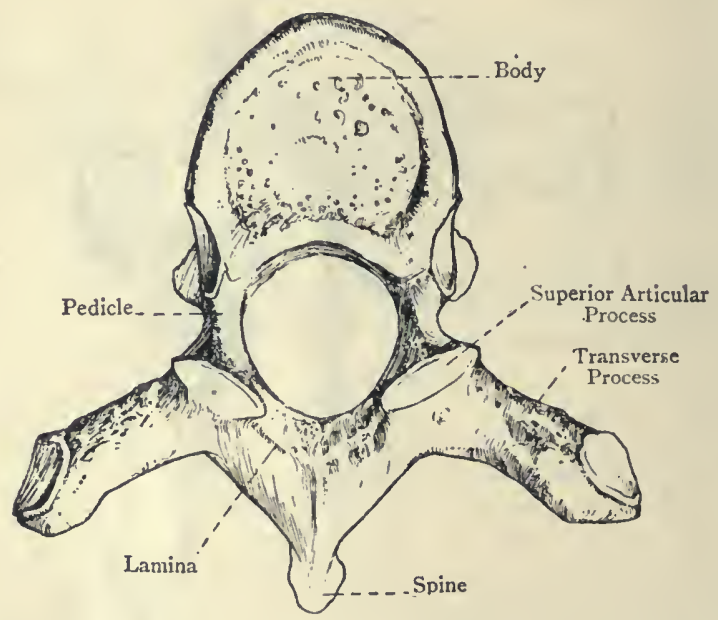

Fig. I4.-The Sixth Thoracic Vertebra (Superior View).

markings for the ligamenta subflava are the same as in cervical vertebræ.

The spinous process is triangular or bayonet-shaped. Its direction is downwards and slightly backwards, and it terminates in a sloping border ending below in a sharp point. The spinous processes of the central thoracic vertebræe are imbricated or overlapping.

The articular processes are nearly circular, their surfaces are flat, and their planes are almost vertical. The superior pair project upwards from the junction between the pedicles and laminæ, and they look backwards and slightly upwards and outwards. The inferior pair are placed on the anterior surfaces of the laminx, and they look forwards and slightly downwards and inwards. 
The transverse processes spring from the junction of the pedicles and lamina, and each is directed outwards and backwards. They are long and club-shaped, being somewhat constricted at their bases, but expanding into knob-like enlargements at their extremities. The anterior surface of the extremity of each presents a circular concave facet, called the costo-tubercular facet, for articulation with the tubercle of a rib. The posterior surface of the extremity gives attachment to the posterior costo-transverse ligament. The anterior surface of the transverse process faces the posterior surface of the neck of a rib, and gives attachment to the middle costo-transverse or interosseous ligament. This region corresponds with the costo-transverse foramen in a cervical vertebra. The lower border of the transverse process gives attachment to the superior costo-transverse ligament, which connects it with the crest, or upper border of the neck, of the rib below. The transverse process is serially homologous with a cervical transverse process.

The neural foramen is almost circular, and is of smaller size than in the cervical or lumbar vertebræ.

Peculiar Thoracic Vertebræ.--These are the first, tenth, eleventh, and twelfth (sometimes also the ninth).

The Fir'st Thoracic Vertebra.- This vertebra closely resembles the seventh cervical, as, indeed, do one or two below it. Its distinctive character is the presence on each side of the body of one entire facet close to the upper part, and situated on the pedicular portion, for the head of the first rib, and one demi-facet close to the lower part for a portion of the head of the second rib. With the exception of the inferior articular and transverse processes, this vertebra in other respects closely corresponds with the seventh cervical, with this difference, that the antero-posterior lips of the superior surface of the body of the seventh cervical are replaced by transverse lips lying in front of the superior vertebral notches. The inferior articular and transverse processes are similar to those of a typical thoracic vertebra, each transverse process having the usual costo-tubercular facet on the anterior surface of its extremity.

The Tenth Thoracic Vertebra.-This vertebra has usually one entire facet on either side, mainly on the pedicle, for the head of the tenth rib. This facet, however, may only be a three-quarter facet, if the ninth thoracic vertebra is normal. It has, usually, a costo-tubercular facet on the anterior aspect of the extremity of each transverse process for the tubercle of the tenth rib, but this facet may be wanting. The body and spinous process of this vertebra show indications of the lumbar type, its other characters being thoracic.

The Eleventh Thoracic Vertebra.-This vertebra has an entire facet on the outer surface of each pedicle for the head of the eleventh rib, but there is no facet on the transverse process, which has become short and stunted, the tendency to the club shape being, however, 
still perceptible. The lumbar type of the bone is more pronounced than in the case of the tenth.

The Twelfth Thoracic Vertebra.-This, like the eleventh, has an entire facet on the outer surface of each pedicle for the head of the

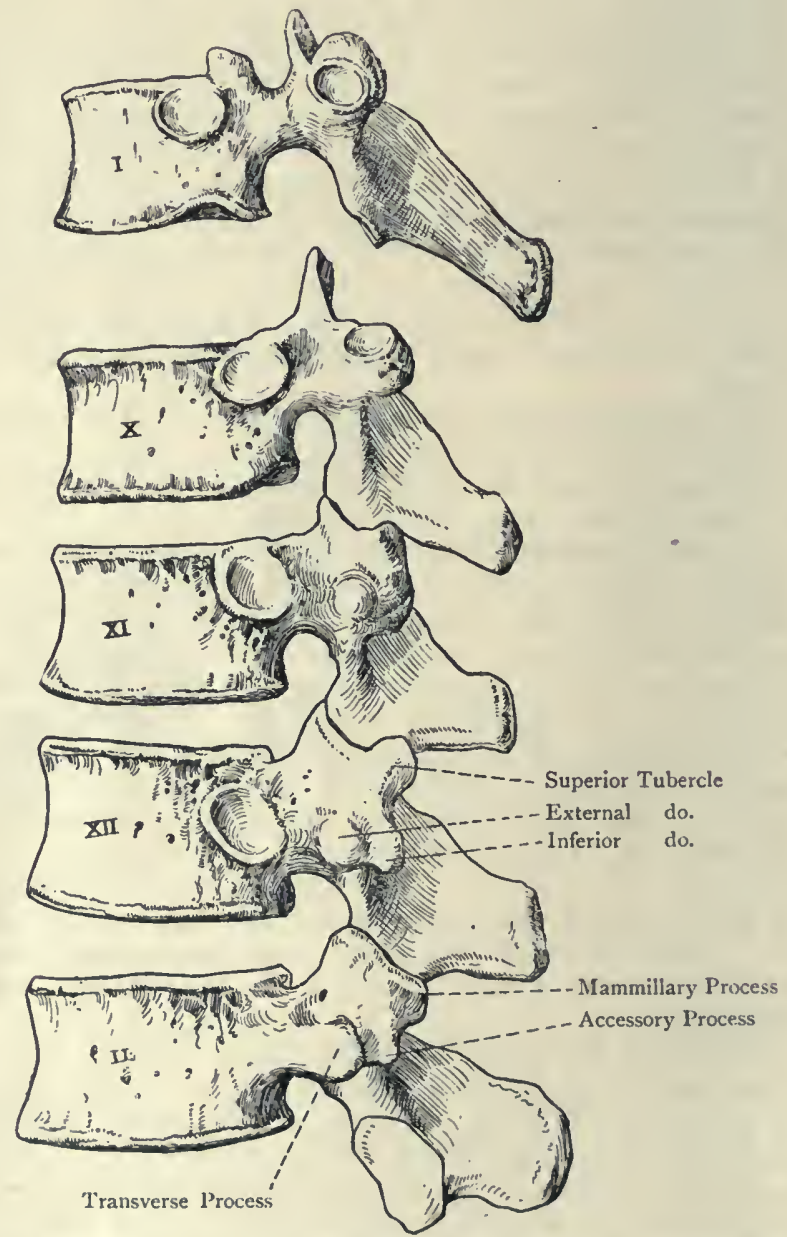

lig. I 5.-The Peculiar Thoracic Vertebre and the First Lumbar VERTEBRA.

twelfth rib, and no facet on the transverse process: The transverse processes are very stunted, and each presents three tuberclesexternal, superior, and inferior-a condition which also manifests itself, though not so conspicuously, in the transverse processes of the eleventh, and even the tenth. The external tubercle, along 
with the twelfth rib, is serially homologous with a lumbar transverse process, the superior with a lumbar mammillary process, and the inferior with a lumbar accessory process. The superior articular processes are thoracic in type, whilst the inferior are like those of a lumbar vertebra, being convex and directed outwards and forwards, or away from each other. Sometimes the superior articular processes are also lumbar in type, being concave and looking inwards and backwards, or towards each other. When this is so, the inferior articular processes of the eleventh thoracic vertebra are also lumbar in type. The twelfth thoracic vertebra very closely resembles a lumbar vertebra, from which, however, it differs in having a facet on the outer surface of each pedicle.

The thoracic vertebræ receive their blood-supply from the intercostal arteries.

\section{The Lumbar Vertebræ.}

The Iumbar vertebræ are five in number, and are so named because they occupy the region of the loins. They are the largest of the true vertebræ, and their negative characters are-the absence of a costo-transverse foramen in the transverse process; and the absence of any kind of costal facet on the side of the body. They increase in size from above downwards, the fifth being the largest, but, as this vertebra has certain distinctive characters, it will be separately described.

A Typical Lumbar Vertebra.--The body, when viewed from above or below, is reniform, being flattened from above downwards, convex transversely over its antero-lateral surface, and slightly concave transversely on its posterior surface. It is wider from side to side than from before backwards. The anterior depth is slightly greater than the posterior, in adaptation to the forward curve of the vertebral column in the lumbar region. There is no facet on either side of the body.

The pedicles are short, strong, and directed backwards. The superior vertebral notches are shallow, the inferior being deep and wide.

The laminæ are short, thick, and deep, and their planes are almost vertical.

The spinous process is axe-shaped, its-direction being straight backwards, and it terminates in a round elongated border.

The articular processes are strong. The superior pair project upwards from the junction of the pedicles and laminæ, and the inferior pair project downwards from the lower borders of the laminæ. The superior pair are concave, their planes being vertical, and their direction being inwards and backwards, so that they almost face each other. They stand wide apart, so as to embrace the inferior articular processes of the vertebra above. On the posterior border of each there is a nipple-shaped projection directed backwards and slightly upwards, called the manmillary process (me'a- 
pophysis), which corresponds with the superior tubercle of the lower thoracic transverse processes. The inferior articular processes are convex, their planes being vertical, and their direction being outwards and forwards, so that they look away from each other. They are nearer to each other than the superior pair, and are reccived between the superior pair of the vertebra below.

The transverse processes are comparatively slender, except in the case of the fifth; they are directed outwards and slightly backwards, and they increase in length from the first to the fourth. Each is spatula-shaped, being compressed from before

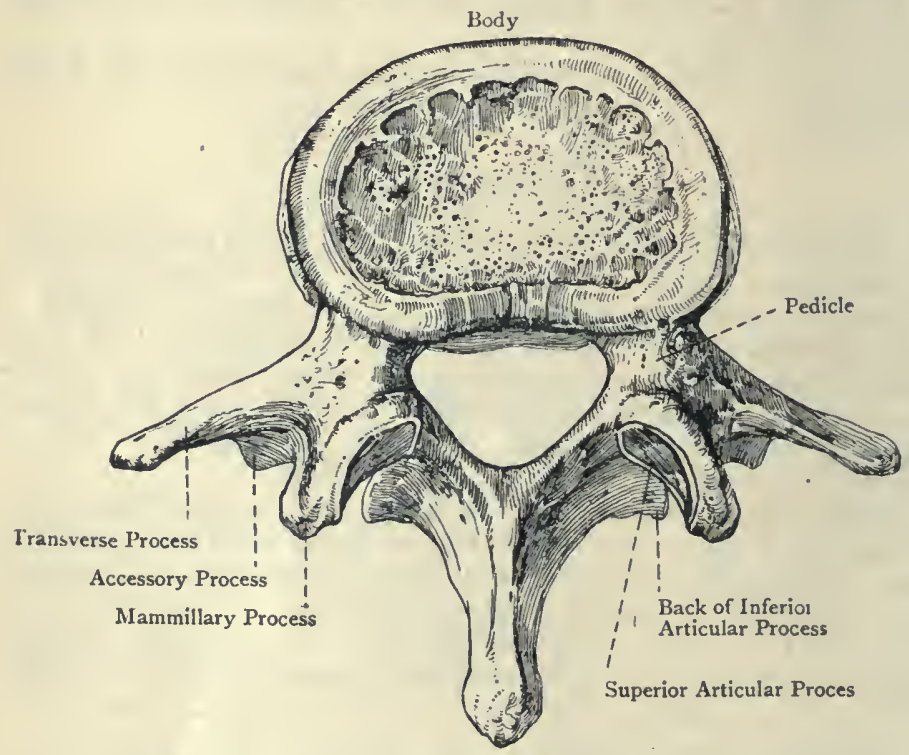

Fig. 16.-The Third Lumbar Vertebra (Superior View).

backwards, and terminates in a short round border. It represents the vertebral portion of a rib, and therefore constitutes the costal element of the vertebra. Situated on the posterior aspect of the base of the transverse process, just external to and below the lower border of the superior articular process, there is a small sharp projection directed downwards, called the accessory process (anapophysis), which is the rudiment of the true transverse process, and is serially homologous with the inferior tubercles of the lower thoracic vertebræ and the other thoracic transverse processes. In the case of the fourth and fifth lumbar vertebræ the transverse process becomes shifted on to the pedicle, and even slightly on to the body. Between the base of the transverse process and the accessory process posteriorly there are a few nutrient 
foramina which correspond with the costo-transverse foramen in cervical vertebræ.

The lumbar transverse processes (costal elements) of man ace serially homologous with the ribs, and also, in the case of the lower thoracic vertebræ, with the external tubercles of the transverse processes. In the lumbar region each transverse prosess (costal element) has fused with the accessory process (true transverse process), and so the costo-transverse foramen in the transverse process of a cervical vertebra is represented only by a few nutrient foramina.

The neural foramen is larger than in the thoracic vertebræ, but not so large as in the cervical, its shape being triangular with rounded angles.

The Fifth Lumbar Vertebra.-The distinctive characters of this

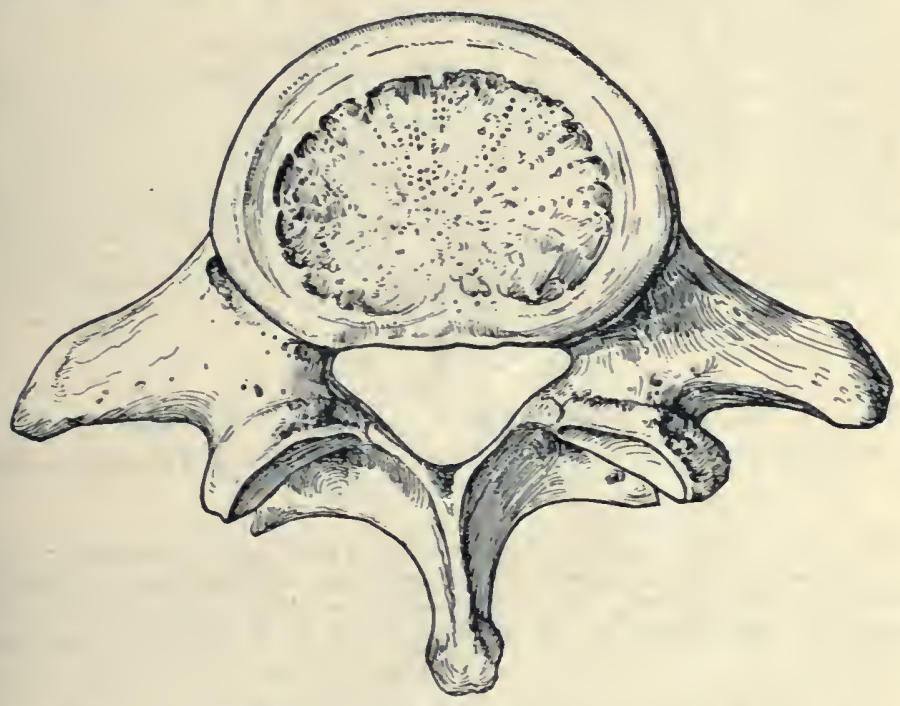

Fig. I7.-The Fifth Lumbar Vertebra (Superior View).

vertebra are as follows : (I) it is the most massive of all the lumbar. vertebræ; (2) the greater depth of the body in front is more conspicuous than in the others; (3) the transverse processes are thick and conical; and (4) the inferior articular processes are wide apart.

The lumbar vertebræ receive their blood-supply from the lumbar arteries.

\section{Ossification of the True Vertebræ.}

Each true vertebra ossifies in cartilage from three primary, and five secondary, centres. One primary centre is for the principal part of the body, and two are for the neural arch and its processes, including also a small portion of the body at either side adjacent to the pedicle. The centres for the neural arch appear 
about the seventh week of intra-uterine life at the junction of the pedicles and lamine, and from these ossification invades the neural arch, with its processes, and the adjacent portions of the body. The centre for the principal part of the body appears about the eighth week in the portion of cartilage dorsal to

A
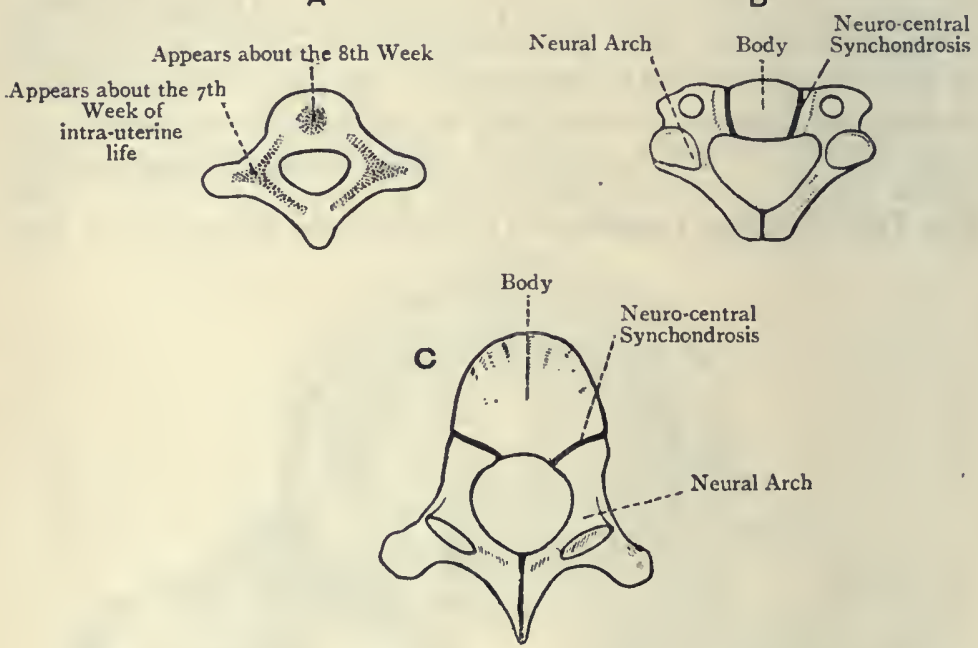

Fig. I8.-OSsification of the True Vertebre.

A, Cervical Vertebra at the Third Month; B, Cervical Vertebra at Birth ; C, Thoracic Vertebra at Birth.

the notochord. It is usually single at first, but it soon assumes a bilobed form, and so it comes to surround the notochord, which becomes constricted, and ultimately disappears. This nucleus may be double, and, if this character persists, the body ossifies in two separate parts, or, if one nucleus should be

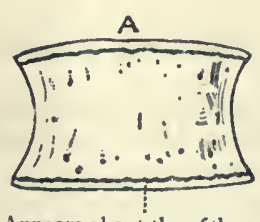

Appears about the $x 6$ th, and joins about the 25 th, Year

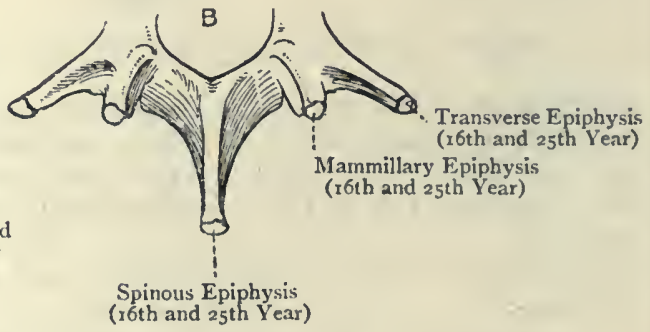

( $x 6$ th and 25 th Year)

IïIg. I9.-Lumbar Vertebra, SHOWing the EPIfHyses. A, The Body; B, The Neural Arch.

arrested, only one-half of the body ossifies (Turncr). At birth a vertebra is composed of three osseous parts, connected by cartilage, namely, the principal part of the body, and the two halves of the neural arch, each bearing a small portion of the body. The lamina unite behind in the first year, except in the 
axis, where the union is delayed until the forth year, and the neural arch joins the body in the third year. The cartilaginous union between the neural arch and the body at either side is called the neuro-central synchondrosis. In the thoracic vertebræ the superior demi-facets lie behind this, and so they are shown to be placed on the pedicular portion of the body. All vestiges of this synchondrosis have disappeared prior to the sixth year. The secondary centres, five in number, appear about the sixteenth year, and they are consolidated about the twenty-fifth year. One appears at the extremity of the spinous process, one at the extremity of each transverse process, and the other two take the form of epiphysial plates, one on the upper surface and the other on the urder surface of the body. In the case of the seventh cervical vertebra, and sometimes one or two above it, the costal process has a special centre which appears before birth, and it may be developed into a cervical rib. The transverse process (costal element) of the first lumbar has occasionally a special centre, and in these cases it may be developed into a lumbar rib. The lumbar mammillary processes are ossified from special secondary centres. The fifth lumbar has sometimes four centres for the neural arch, two at either side, one of which is for the pedicle, transverse process, and superior articular process, and the other for the lamina, inferior articular process, and one-half of the spinous process. These parts may fail to unite, in which cases the neural arch presents a synchondrosis on either side, situated between the superior and inferior artisular processe: (Turner). Sometimes the laminæe of the fifth lumbar fail to unite, and so a space is left, bridged over by fibrous tissue.

The Atlas. - The atlas has three centres of ossification, two for the latera] masses and posterior arch, appearing in the seventh week of intra-uterine life, and one (sometimes two) for the anterior arch, which does not appear until the

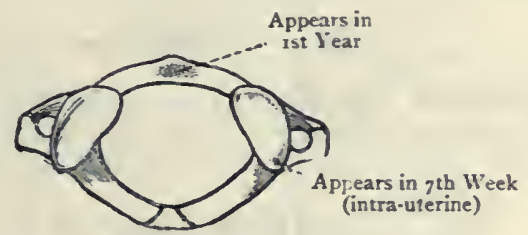

Fig. 20.-Ossification of the Atlas.

first year. The two halves of the neural arch usually join towards the end of the third year, there being sometimes a special osseous deposit at the place of junction. The two halves, however, may remain separate throughout life, the interval being bridged over by fibrous tissue. The anterior arch joins the lateral masses in the sixth year. The anterior arch represents the hypochordal brace of the first vertebral bow.

The Axis.-Excluding the odontoid process, the axis has three primary centres, like an ordinary vertebra, two for the neural arch appearing about the seventh week, and one (sometimes two) appearing in the lower part of the common cartilage of the body and odontoid process in the fourth month. In the upper part of this common cartilage two centres, laterally disposed, appear in the fifth month for the odontoid process, and these unite into one centre about the sixth month. At birth the axis is composed of four osseous parts connected by cartilage, namely, a body, an odontoid process surmounted by cartilage, and two halves of the neural arch. The odontoid process joins the body about the fourth year. The two halves of the neural arch join each other. and the arch joins the body, in the fourth year. The apical part of the odontoid process has a special centre appearing in the fourth year, and it joins the rest of the process in the twelfth year. The body of the axis has the usual epiphysial plate on the under surface of the body, but there is no such plate 
on the upper surface. The union between the odontoid process and the body is indicated by a small cartilaginous disc in the centre, which persists until advanced life. The odontoid process is to be regarded as the original body of the atlas for the following reasons: (I) in the embryo the notochord passes through its cartilage; (2) the notochord presents a swelling between

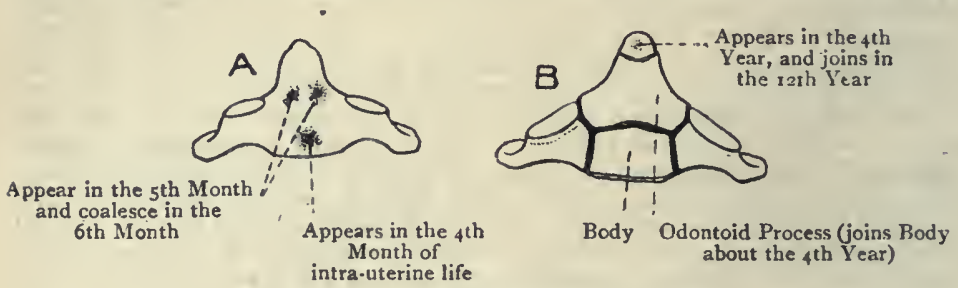

Fig. 21.-Ossification OF the Axis.

A, At the Fifth Month; B, at the Fourth Year.

the cartilage of the odontoid process and that of the body of the axis, as it does in the case of other vertebræ; (3) there is a cartilaginous disc concealed within the odontoid process, which is persistent until advanced life; (4) the odontoid process has two primary centres of ossification; (5) in chelonians it forms a separate ossicle; (6) a permanently separate odontoid process in man has been recorded.

\section{The False Vertobre.}

The false vertebræ are usually nine in number, the upper five of which form the sacrum, and the lower four the coccyx.

\section{The Sacrum.}

The sacrum lies below the fifth lumbar vertebra, and is wedged in between the ossa innominata, where it forms the greater part of the posterior wall of the pelvis, its direction being downwards and backwards. The sacral vertebræ diminish in size from above downwards, which renders the bone triangular, the base being upwards.

The ventral or pelvic surface, which is directed downwards and forwards, is concave from above downwards, and from side to side. It presents along the centre a solid mass, representing the ankylosed bodies and ossified intervertebral discs, which is marked by four transverse ridges situated at the places of junction. Superiorly it presents a projecting lip, called the promontory. On either side there is a row of anterior sacral foramina, four in number, which diminish in size from above downwards, and are directed outwards and forwards from the intervertebral foramina, by means of which they communicate with the sacral canal. They transmit the anterior primary divisions of the first four sacral nerves.

The lateral masses are situated external to the anterior sacral 
foramina at either side, and each is marked anteriorly by four transverse grooves, which prolong outwards the foramina and lodge the transmitted nerves. The pyriformis muscle arises from the front of each lateral mass by three slips, which are interposed between, and lie external to, the foramina. The lateral masses are formed by the fusion of the pedicles, transverse processes, and costal elements of the sacral vertebre.

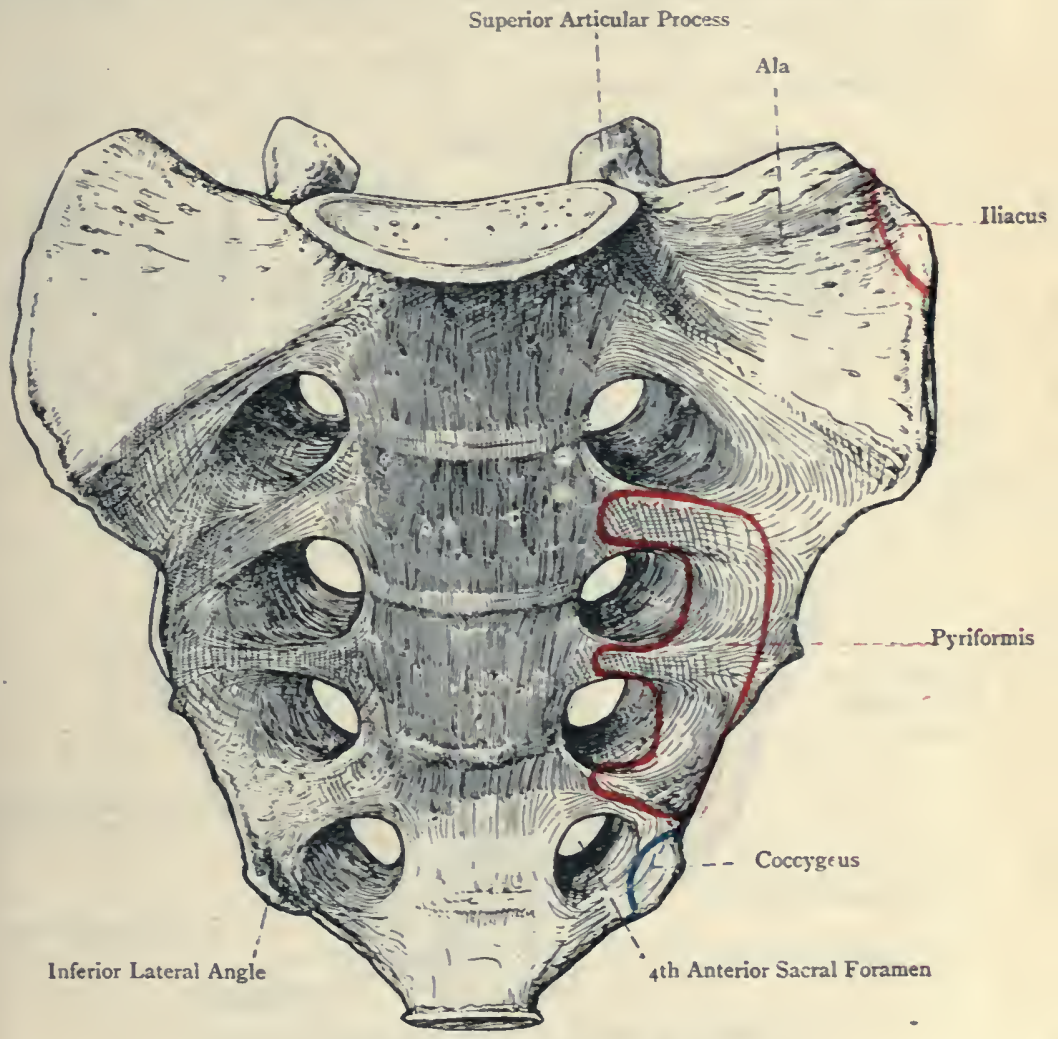

Fig. 22.-The SAcrum (ANTERIOR View.)

The dorsal surface, which is directed upwards and backwards, is irregularly convex and narrower than the ventral. In the middle line it presents four eminences, which may be distinct, or fused to form a ridge, representing the spinous processes of the upper four sacral vertebræ. The spinous process of the fifth vertebra is absent, the development of its laminæ having been arrested, and there is thus left a triangular opening, which is the outlet of the sacral canal, to be presently described. On either side of the median 
interval thus bridged over at either side represents a fifth intervertebral foramen, through which the fifth sacral nerve passes. The solid portion external to the posterior foramina at either side is the lateral mass, and it presents a row of four tubercles, each of which is situated external to a posterior foramen. These represent the transverse processes of the lower four sacral vertebræ. The boundaries of the triangular outlet of the sacral canal are the spine of the fourth sacral vertebra above, and the imperfect laminæ of the fifth sacral and the sacral cornua at either side. It

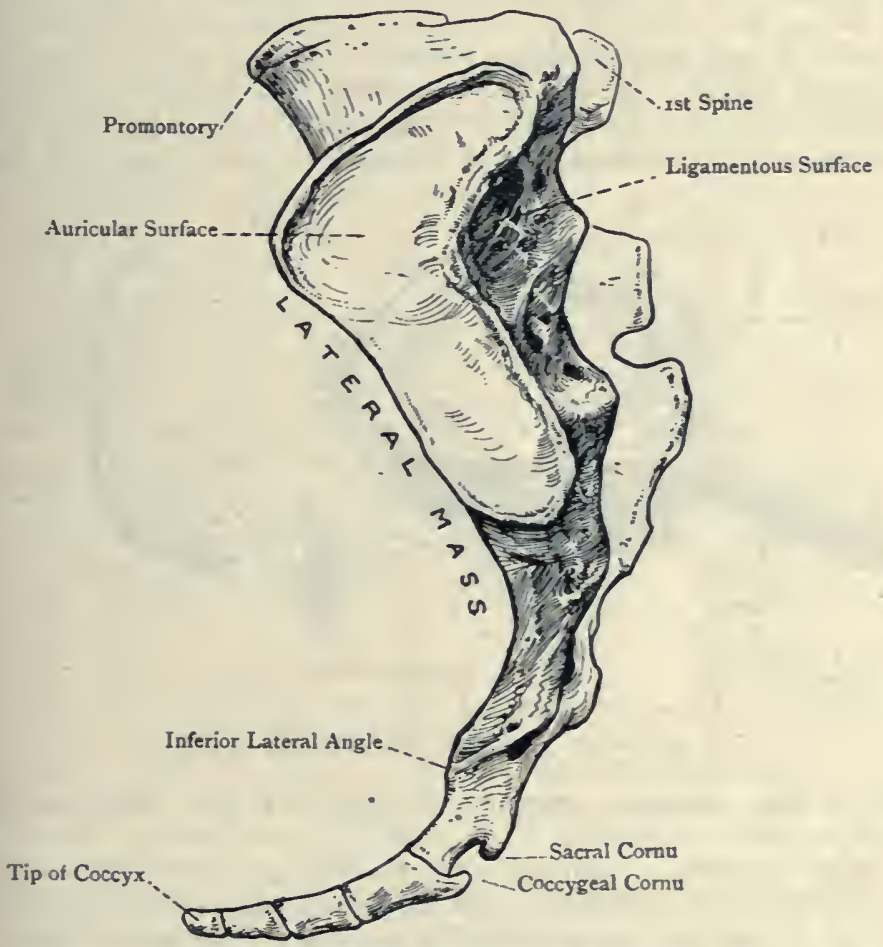

Fig. 24.-The Sacrum (Left Lateral View).

transmits the fifth pair of sacral nerves and the two coccygeal nerves.

The lateral surface is broad above and narrow below. The upper part is divided into two portions-articular and non-articular. The articular division, anterior in position, is covered by cartilage, and is shaped like an ear, on which account it is called the auricular surface. It articulates with the iliac portion of the os innominatum, and extends over at least the first two sacral vertebræ. The nonarticular division, posterior in position, is rough and irregular for 
the attachment of the posterior sacro-iliac ligament, and it is known as the ligamentous surface. The lower part of the lateral surface corresponds with at least the lower two sacral vertebræ, and may include more or less of the third. It gives attachment to fibres of the great and small sacro-sciatic ligaments and a portion of the coccygeus muscle, whilst the adjacent portion of the posterior aspect gives origin to fibres of the gluteus maximus. Inferiorly the lower part is thinned away to a mere margin, and presents a process, called the inferior lateral angle. Below this is the transverse process of the first coccygeal vertebra when that is in position, a notch being thus formed on the side of the fifth sacral vertebra. The inferior lateral angle inclines towards the coccygeal transverse process, with which it is usually connected by a ligament, though in some cases the two processes become ankylosed. There is thus constructed a fifth anterior sacral foramen at either side for the

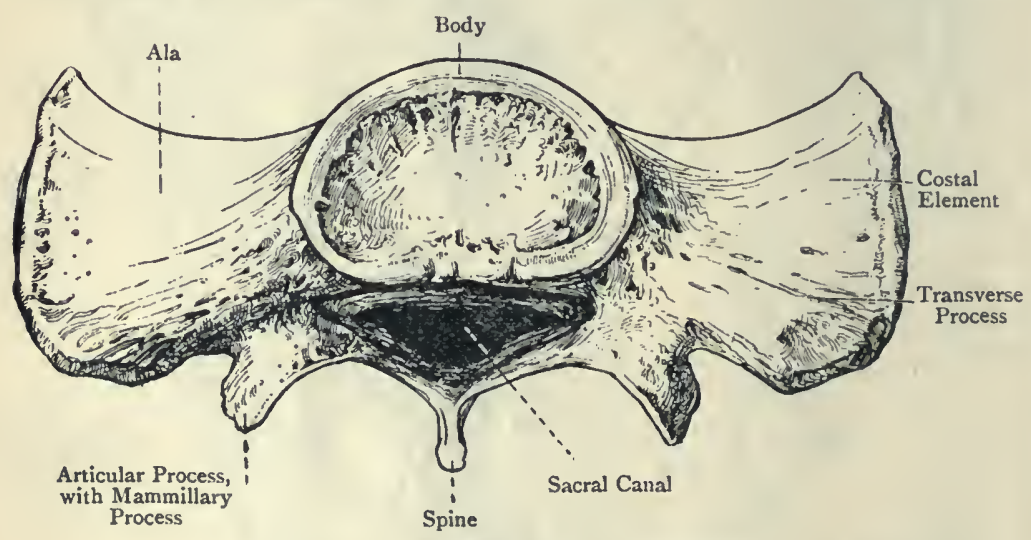

Fig. 25.-The Base of the Sacrum.

passage of the anterior primary division of the fifth sacral nerve.

The base presents a central and two lateral divisions. The central division corresponds in its characters with the superior surface of a lumbar vertebra. Each superior vertebral notch lodges a fifth lumbar spinal nerve, and the superior articular processes stand wide apart. The lateral divisions of the base are called the alce. Each ala is triangular with the apex directed backwards. The alar surface is depressed, concave from side to side, and convex from behind forwards. It gives attacliment to fibres of the iliacus, the lateral lumbo-sacral and anterior sacroiliac ligaments, and it supports the lumbo-sacral nervous cord and the internal iliac vessels. The ala is formed by the fusion of the pedicle, transverse process, and costal element of the first sacral vertebra.

The apex is transversely oval, and articulates with the first 
coccygeal vertebra, with the intervention of an intervertebral disc until advanced life, when ankylosis takes place.

The sacral canal is situated behind the bodies of the first four sacral vertebræ, as a rule. It is triangular in the upper part, but somewhat crescentic below. It is closed in front by the ankylosed bodies, and behind by the ankylosed laminæ. Along each side it presents four intervertebral foramina for the passage of nerves. These are bounded externally by the lateral mass, but each opens on the ventral and dorsal surfaces by the anterior and posterior sacral foramina, which represent the limbs of a capital V, the apex of which corresponds with an intervertebral foramen. The superior aperture or inlet is large, triangular, and wide transversely. It represents the spinal or neural foramen of the first sacral vertebra. The inferior aperture or outlet is comparatively small and somewhat triangular, and is usu-

Appear about the $18 \mathrm{th}$ Year, the $25^{\text {th }}$ Year. ally situated on the back of the body of the fifth sacral vertebra. It is bounded above by the tubercle which represents the spinous process of the fourth sacral vertebra, and on either side by (I) the imperfectly developed lamina of the fifth sacral vertebra, and (2) the sacral cornu. The outlet transmits the fifth pair of sacral nerves and the two

Appears about the 16 th Year, and joins about the $25^{\text {th }}$ Year

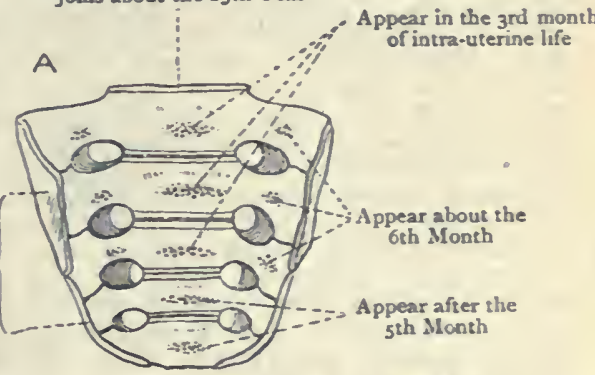

Neural Arch

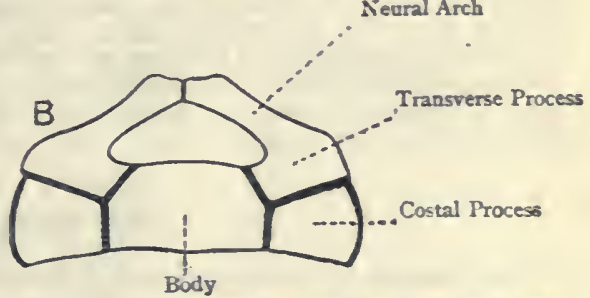

Fig. 26.-OSsification of The Sacruy. A, Anterior View; B, First Sacral Vertebra in early life (Superior View). coccygeal nerves. The contents of the canal are the sacral and coccygeal nerves, and the filum terminale of the spinal cord.

The sacrum derives its blood-supply from the lateral sacral and middle sacral arteries.

Articulations.-Superiorly with the fifth lumbar vertebra, inferiorly with the coccyx, and at either side with the os innominatum.

Varieties.-(I) The number of sacral segments may be six, or more rarely four. Increase in the number is usually due to the incorporation of the first coccygeal vertebra, or sometimes the fifth lumbar. The decrease may be due to the fifth sacral vertebra forming a part of the coccyx, or to the first sacral forming a sixth lumbar. (2) The bodies of the first and second sacral vertebræ may remain permanently separate, though ankylosis has taken place in all their other parts. (3) The first sacral vertebra may be normal on one side, 
but on the other side it may remain separate from the second, and present the characters of a fifth lumbar. (4) The number of sacral spines may be reduced from four to three, two, or one, or they may be entirely absent. As a consequence of this, the sacral canal, which usually opens on the back of the fifth sacral vertebra, may do so on the back of the fourth, third, second, or first, so that in some cases it may be entirely open posteriorly. (5) The sacrum is liable to much variety as regards the extent of its vertical curve.

Characters of the Female Sacrum.-In the female the sacrum is smoother, shorter, broader, less curved, and is set more backwards than in the male.

Ossification.-The sacrum ossifies in cartilage from thirty-five centres. Each segment has three primary centres, one for the body and two for the neural arch. The centre for the body appears in the third month of intrauterine life in the case of the first three, and after the fifth month in the last two. The centres for the neural arches appear about the sixth month. The neural arches join the bodies, in order from below upwards, from the second to the sixth year. The union of the laminæ takes place from the eighth to the twelfth year. It, however, fails in the lowest, and sometimes in those higher up. The anterior parts of the lateral masses of the first three vertebræ, which represent the costal elements, have separate centres, which appear about the sixth month. These join the neural arches before uniting with the bodies, the latter union taking place rather later than the union between the neural arches and the bodies. Each vertebra has two annular circumferential epiphysial plates, superior and inferior, which begin to ossify about the sixteenth year. On each side of the sacrum there are two epiphyses, an upper for the auricular surface, and a lower for the sharp edge below, which appear abont the eighteenth year. Consolidation begins about the eighteenth year, and proceeds from below upwards, union taking place earlier between the segments of the lateral masses than between the bodies. In the latter case the ossification invades the intervertebral discs, but in the former it is direct union. The union is complete about the twenty-fifth year, at which period also the lateral epiphyses join the bone.

\section{The Coccyx.}

The coccyx is composed as a rule of four rudimentary vertebræ, and it lies below the apex of the sacrum, which constitutes its only articulation. The direction of the bone is downwards and forwards, and its elements diminish in size from above downwards. It is triangular.

The first coceygeal vertebra is compressed from before backwards, broad above, narrow below, concave in front, and convex behind. The superior and inferior surfaces are transversely oval, and the lateral borders are sloped downwards and inwards. Two processes project upwards from the dorsal surface at either side, called the cornua, which articulate with the sacral cornua, usually by ligaments, but sometimes directly. Each lateral border presents, superiorly, a projection, called the transverse process, which inclines towards the inferior lateral angle of the sacrum, and is usually connected to it by a ligament, which is sometimes ossified.

The second coccygeal vertebra presents traces of transverse processes and cornua, whilst the third and fourth are reduced to mere nodules. 
The muscular attachments of the coccyx are as follows: the gluteus maximus to the back of the upper three segments close to the lateral border; the sphincter ani externus to the tip; the posterior fibres of the levator ani and a portion of the coccygeus to the lateral border. The great and small sacro-sciatic ligaments

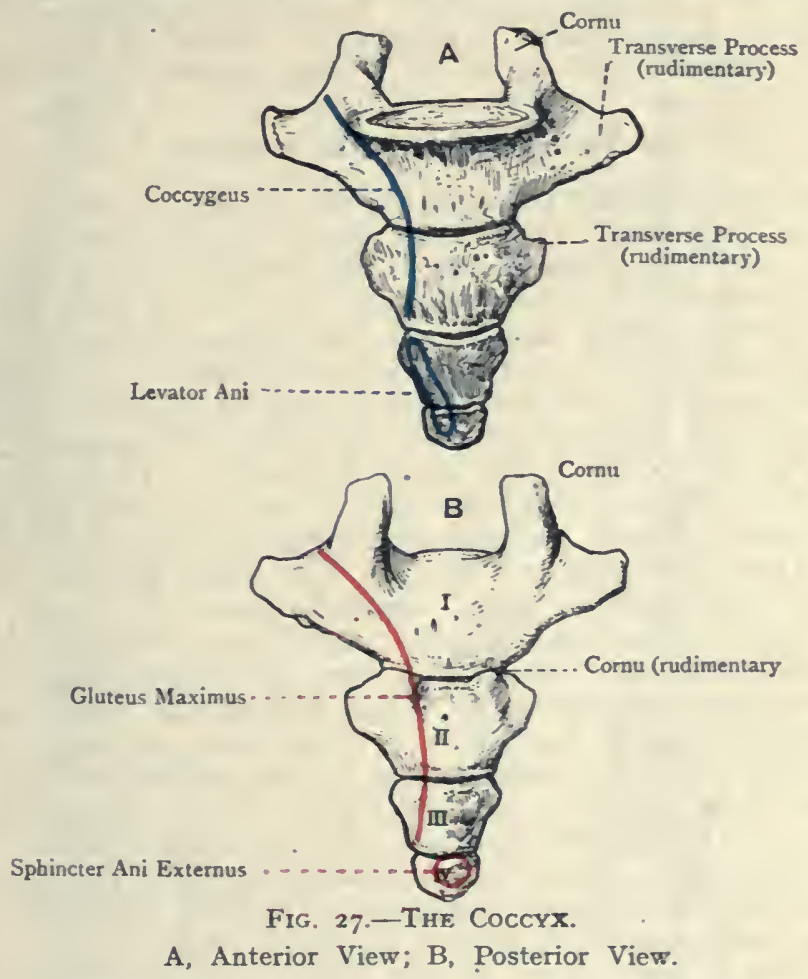

are partially attached to the lateral border of the first coccygeal vertebra.

The coccyx derives its blood-supply from the lower lateral sacral and middle sacral arteries.

Varieties.-The number of coccygeal segments may be increased to five, due either to the addition of an extra nodule, or to incorporation of the fifth sacral segment. The number may be reduced to three, due either to incorporation of the first coccygeal segment with the sacrum, or to suppression of one of the nodules.

Ossification.-The coccygeal vertebre are cartilaginous at birth. Each vertebra has one primary centre and two secondary centres, the first vertebra having an additional pair of secondary centres.

Primary Centres. - The primary centre for the first vertebra appears from the second to the fifth year; that for the second vertebra from the sixth to the tenth year, and those for the third and fourth vertebræ about pubsrty. 
Secondary Centres.-Each vertebra has two secondary centres for the epiphysial plates - one on the upper surface, and the other on the inder surface of the rudimentary vertebra (centrum or body). In addition, the first coccygeal vertebra has two special secondary centres, one for each cornu.

Union of the four coccygeal vertebræ takes place from below upwards, as in the sacrum. It commences shortly after the eighteenth yeav, and is not completed until the thivtieth year or later, the last two coccygeal vertebræ to join being the first and second. In advanced life it is not uncommon to find the coccyx ankylosed to the sacrum, thus forming one sacro-coceygeal bone.

\section{The Vertebral Column as a Whole.}

The vertebral column supports the other parts of the skeleton, directly or indirectly. Its average length is about 28 inches in the male, and rather less in the female. When viewed from the front, it presents four pyramids. The first extends from the axis to the seventh cervical vertebra, its base being downwards. The second extends from the first to the fourth thoracic vertebra, its base being upwards. The third extends from the fifth thoracic to the fifth lumbar, its base being downwards. The fourth extends from the base of the sacrum to the tip of the coccyx. These pyramids are due to the differences in breadth of the bodies in different parts.

The column presents certain curves, which are arranged in two groups, antero-posterior and lateral. The antero-posterior group comprises four curves, named cervical, thoracic, lumbar, and pelvic. The cervical and lumbar curves have their convexities directed forwards, and the thoracic and pelvic curves have their convexities directed backwards. The lumbar and pelvic curves meet rather abruptly and form a projection, called the sacrovertebral angle, which is estimated at II 7 degrees in the male and I30 degrees in the female. The curves impart springiness or elasticity to the column, and so guard it against shock. The thoracic and pelvic curves are associated with the thoracic and pelvic cavities, the capacity of which they serve to increase. They appear in early fœtal life, and are known as primary curves. The cervical and lumbar curves do not appear until after birth, and are known as secondary or compensatory curves. The primary curves are brought about by the greater depth posteriorly of the thoracic and sacral bodies, whilst the compensatory curves are largely due to the intervertebral discs, though in the lumbar region the greater depth of the bodies anteriorly, especially in the fifth lumbar, must also be taken into account.

The lateral group comprises two curves. One is situated in the upper thoracic region, with its convexity directed towards the right side in right-handed persons, and it is to be regarded as due to the greater use made of the right arm. To compensate for this curve there is another slight curve in the upper lumbar region, with the convexity to the left.

When viewed anteriorly, the column presents the bodies, which form the pyramids already described. When viewed laterally, it presents the sides of the bodies, pedicles, intervertebral foramina, and articular and transverse processes. The intervertebral foramina 


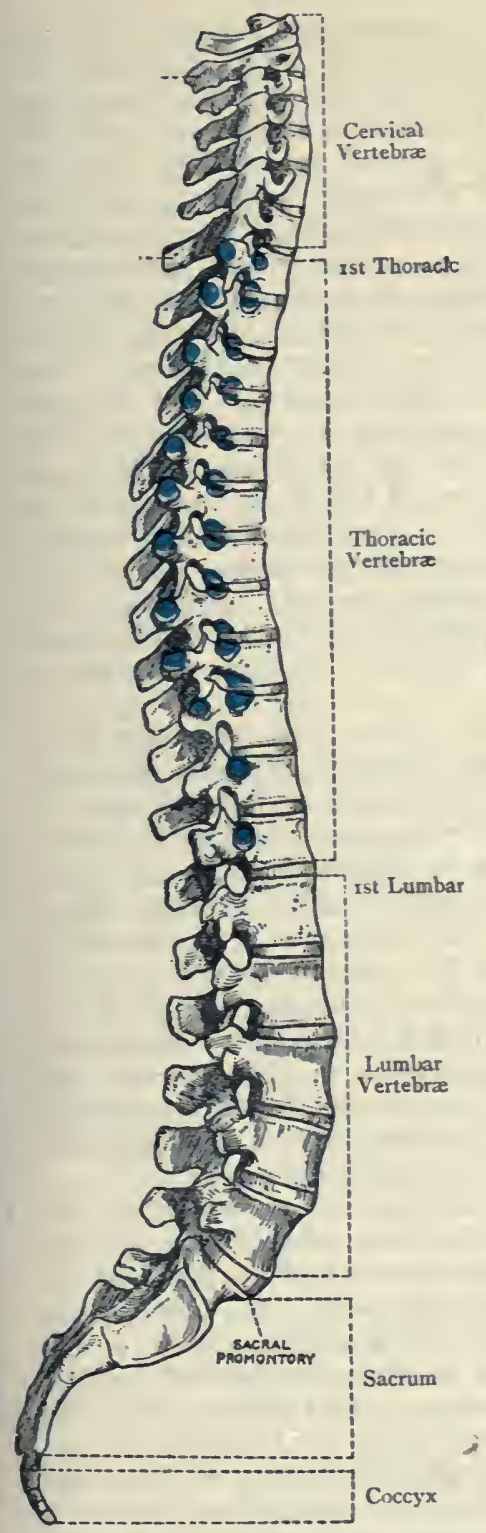

F!g. 28A.-The Vertebral Columin (LATERAl View).

(The Blue Markings represent the Facets on the Bodies and Transverse Processes.)

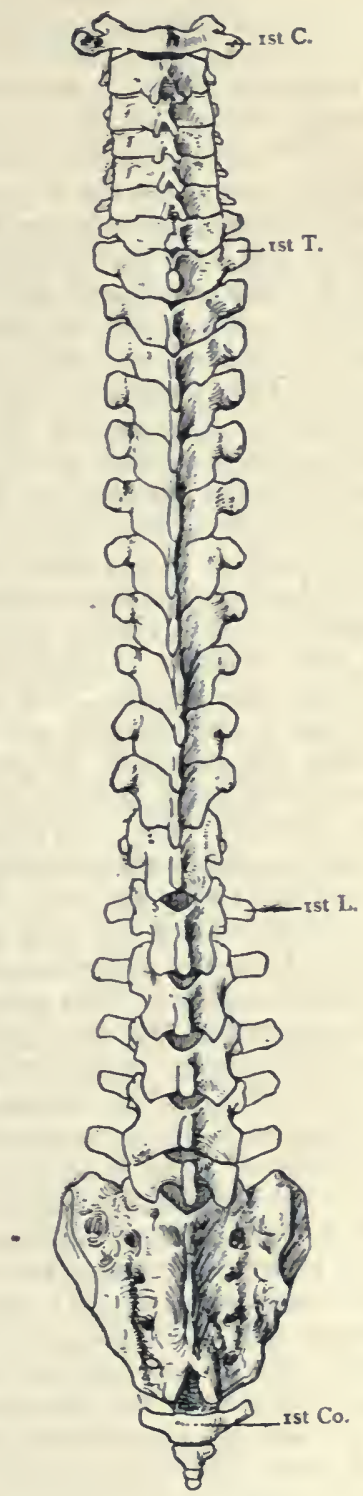

Fig. 28B.-The Vertebral Coluun (POSTERIOR VIEW). 
are formed by the apposition of the superior and inferior vertebral notches of contiguo's pedicles. They lead outwards from the spinal canal, and each transmits a spinal nerve. They increase in size from above downwards until the sacrum is reached, in which, though hidden at either side of the central mass, they diminish in size from above downwards. In this region each intervertebral foramen opens on the front and back of the sacrum by means of an anterior and posterior sacral foramen, the arrangement thus formed resem'ling a capital $\mathrm{V}$, the apex being at an intervertebral foramen. On the lateral aspect of the thoracic portion of the column are seen the costo-capitular facets, which are twelve in number. The first is situated on the upper part of the side of the first thoracic body. The second to the tenth inclusive are situated on the contiguous margins of the bodies of the vertebræ, each being formed by the small inferior demi-facet of the upper body and the large superior demi-facet of the lower. The eleventh and twelfth are situated on the sides of the corresponding pedicles. The tenth facet may belong entirely to the tenth thoracic vertebra. The thoracic transverse processes, except the eleventh and twelfth (and sometimes the tenth), are faceted in front at their extremities for the tubercles of the ribs.

When the column is viewed from behind the following parts are seen : the spinous processes; the laminæ; the articular processes; the backs of the transverse processes; and the dorsum of the sacrum and coccyx. The cervical spines, except the sixth and seventh, are short, so as not to interfere with backward flexion or over-extension of the neck. The middle thoracic spines are imbricated, and the lumbar spines stand out horizontally. On either side of the spines there is the vertebral groove, which is occupied by the deep muscles of the back, the deepest being the multifidus spinæ. This groove is bounded internally by the spines, and externally by the transverse processes in the cervical and thoracic regions, and by the mammillary tubercles in the lumbar region. The floor is formed by the laminæ, and its continuation over the back of the sacrum is known as the sacral groove.

The spinal canal is situated behind the bodies of the vertebrx, and is formed by the neural foramina of all the vertebræ except the fifth sacral and four coccygeal. It commences at the level of the atlas, and it terminates as a rule upon the back of the body of the fifth sacral vertebra. It adapts itself to the various curves of the column, and is large and triangular in the cervical and lumbar regions, small and circular in the thoracic, and triangular in the upper part, but crescentic in the lower part, of the sacral region. It contains the spinal cord and its membranes as low as about the level of the disc between the first and second lumbar bodies, and a copious plexus of vessels. Below the level just mentioned it contains the filum terminale of the spinal cord and the leash of nerves known as the cauda equina, with their coverings. The duramatral covering or theca ccases by taking attachment to the back 
of the second sacral body, and the filum terminale passes on to be attached to the back of the fifth sacral or first coccygeal vertebra.

\section{B. The Ribs.}

The ribs (costæ) are twelve in number at either side, and are arranged in two groups, true or sternal, and false or asternal. The true ribs are those which articulate directly with the sternum by their costal cartilages, and they represent as a rule the first seven at either side. The false ribs are those which have no direct articulation by their costal cartilages with the sternum, and they represent, as a rule, the last five at either side. The last two false ribs, eleventh and twelfth, are called the free or floating ribs, because their costal cartilages stand quite clear of each other and of the tenth. The ribs are elastic, and increase in length from the first to the seventh, whence they decrease to the twelfth. The first is the broadest, and the twelfth the narrowest. Their direction is at first downwards, outwards, and slightly backwards, then downwards and forwards, and finally inwards. The upper ribs are not so oblique as those lower down, the most oblique being the ninth. With the exception of the first rib, the surfaces of the others are vertically disposed posteriorly, but in front they are sloped downwards and forwards, and this circumstance renders most of them twisted.

A Typical Rib.-A typical rib presents for consideration a head, neck, tubercle, shaft, and sternal extremity.

The head (capitulum) forms the posterior or vertebral extremity, and is slightly expanded. It presents an irregularly flat surface and an anterior margin. The surface is marked by two oblique facets, upper and lower, which are separated by a horizontal ridge. The lower or primary facet is the larger of the two, and articulates with the large upper or primary demi-facet of the lower of the two thoracic bodies with which the head is connected. The upper facet articulates with the small lower demi-facet on the side of the upper thoracic body, and the intervening ridge gives attachment to the interarticular ligament. The anterior margin gives attachment to the anterior costo-central ligament.

The neck is about I inch long, and is compressed from before backwards. Its anterior surface is smooth and covered by the costal pleura. Its posterior surface, which is rough, faces the anterior surface of the lower thoracic transverse process, or that with which its tubercle articulates, and it gives attachment to the middle costo-transverse or interosseous ligament. Its superior border forms a sharp lip, called the crest, which gives attachment to the superior costo-transverse ligament. Its inferior border may show traces of the subcostal groove.

The tubercle is situated on the external surface of the rib at the outer extremity of the neck, and presents two divisions, articular and non-articular. The articular division, inferior and slightly 
internal in position, presents a somewhat oval facet for articulation with that on the front of the extremity of the lower thoracic transverse process. The non-articular division, superior and slightly

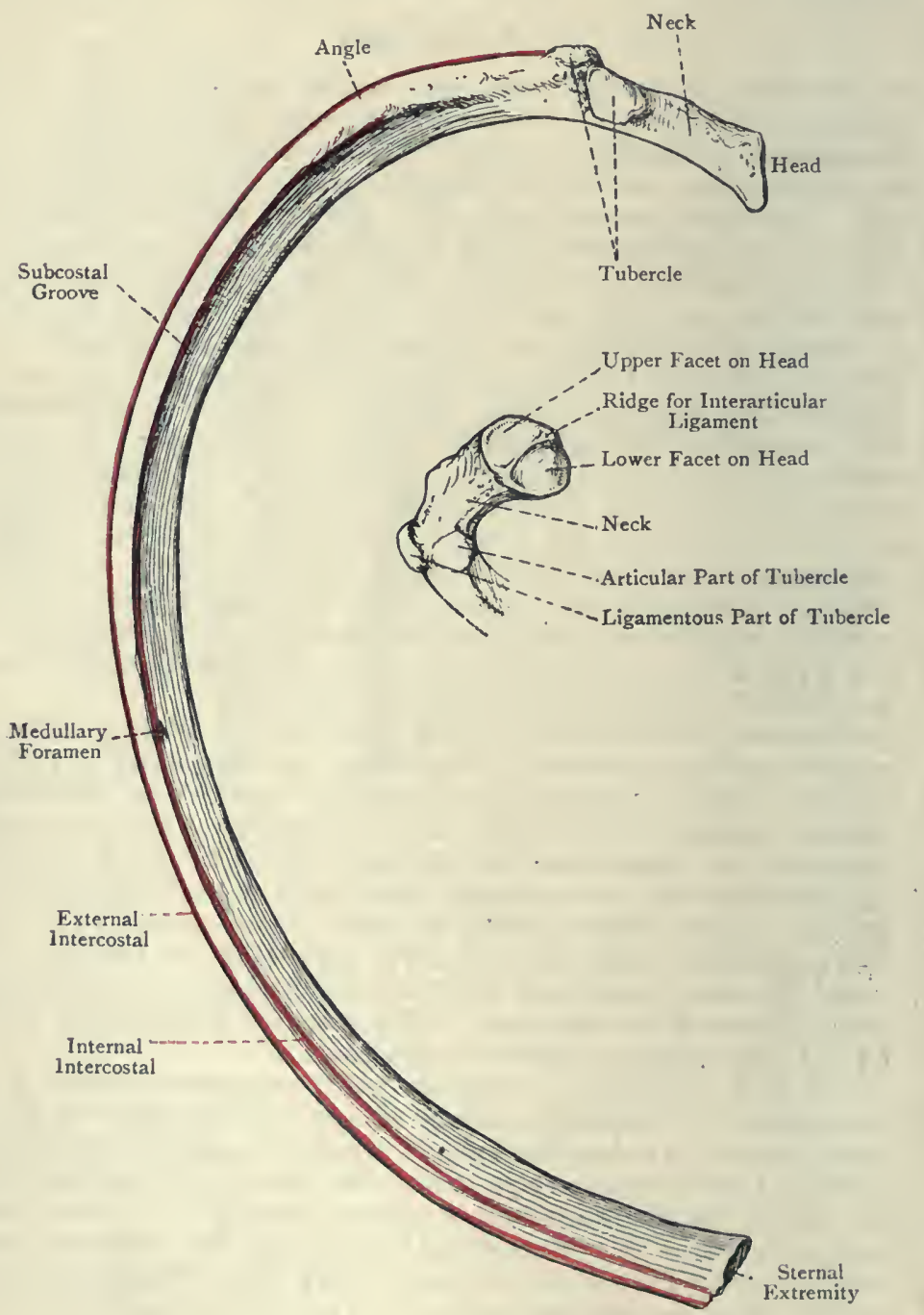

Fig. 29.-The Sixth Left Rib (Internal View).

external in position, gives attachment to the posterior costo-transverse ligament.

The shaft is curved and twisted. It presents two surfaces and 
two borders. The external surface is convex, and its plane is vertical behind, but oblique in front, being here sloped downwards and forwards. Opposite the greatest bend of the rib it presents an oblique ridge, directed downwards and outwards, called the angle, for a tendinous slip of the erector spinæ. The surface between the tubercle and the angle gives attachment to the longissimus dorsi. Near to the anterior extremity (about 2 inches from it) the external surface presents another oblique ridge directed downwards and outwards, known as the anterior angle, where the rib describes a slight curve. The internal surface, which is concave, is covered by the costal pleura. At its lower part it presents the subcostal groove, to be presently described. The superior border is thick and round behind, but thin and sharp in front. Its outer lip gives attachment to an external intercostal muscle, and its inner lip to an internal intercostal, a collateral intercostal artery lying between the two muscles. The inferior border is for the most part sharp and wiry. Immediately within and above it there is the subcostal groove, which commences behind at the tubercle and disappears over the anterior fifth. Posteriorly the groove belongs to the inferior border. Its upper lip is rounded and gives attachment to an internal intercostal muscle, whilst the lower lip gives attachment to an external intercostal. The nutrient foramen is situated in the subcostal groove a little anterior to the centre of the bone, and the canal to which it leads is directed towards the head. It gives passage to a branch of the intercostal artery which lies in the groove. The contents of the groove from above downwards are an intercostal vein, artery, and nerve.

The anterior or sternal extremity presents an oval pit, which is almost vertical in direction, for the costal cartilage.

The Peculiar Ribs.- These are the first, second, tenth, eleventh. and twelfth.

The First Rib.-This is the shortest, broadest, and flattest of all the ribs, and its curve is very distinct, but there is no twist. The head is small, and presents a nearly circular facet for articulation with the entire facet on the body of the first thoracic vertebra. The neck is narrow, and compressed from above downwards. The tubercle, which is large, is situated on the external border at the junction of the neck with the shaft. Being placed opposite the greatest bend of the bone, it takes the place of the angle, and presents the usual articular and non-articular portions, the former being for the first thoracic transverse process.

The shaft is broad and compressed from above downwards, its surfaces being superior and inferior, whilst the borders are internal and external. The superior surface close to the anterior extremity gives attachment to the tendon of the subclavius and the costo-coracoid ligament. Farther back there are two oblique grooves, separated to a limited extent by a tubercle or spine for the scalenus anticus. The anterior groove is shallow, and lodges the subclavian vein, whilst the posterior deeper groove 
is occupied by the third part of the subclavian artery and the trunks of the brachial plexus of nerves. Behind the posterior groove, and extending as far back as the tubercle, there is a rough impression for the insertion of the scalenus medius. The inferior surface is flat and covered by the costal pleura. Near the external border it gives attachment to the internal intercostal muscle of the first space. The internal border, which is thin and concave, gives attachment to Sibson's fascia. Fully I inch from

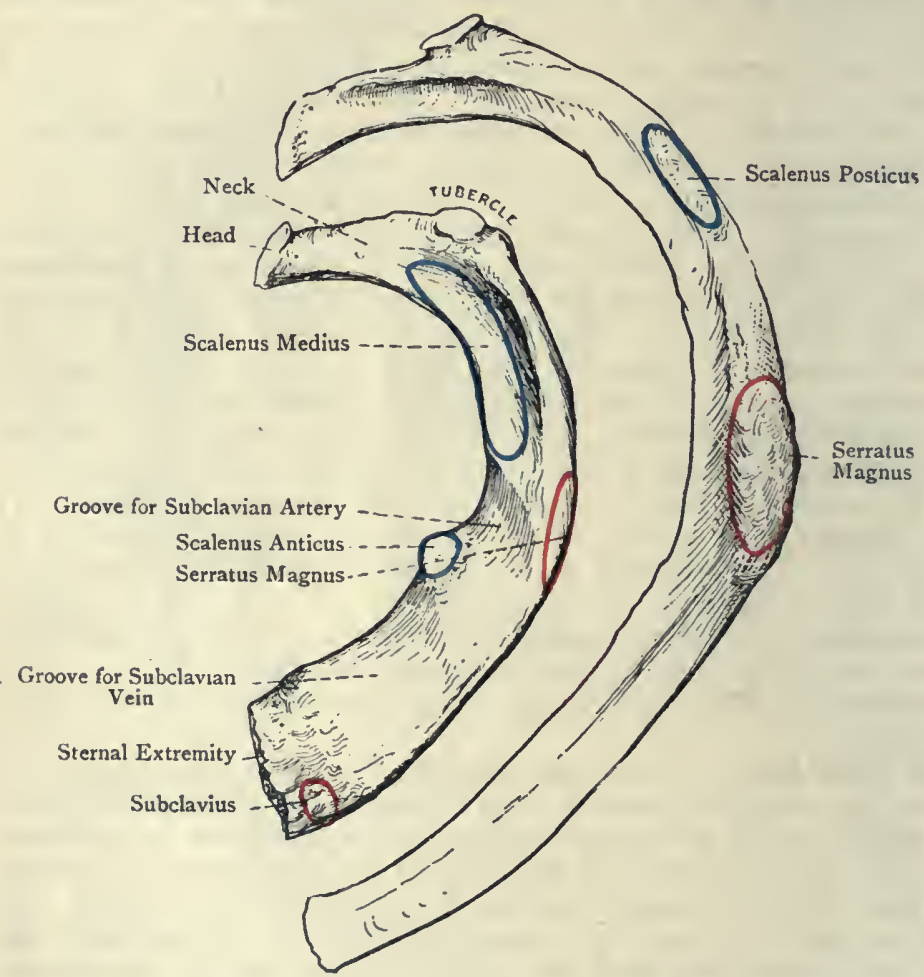

Fig. 30.-The First and Second Ribs of the Left Side

(SUPERIOR VIEW).

the anterior extremity this border presents a projection, called the scalene tubercle or spine (tubercle of Lisfranc), for the insertion of the scalenus anticus: It encroaches slightly on the adjacent part of the superior surface, and is inclined backwards. The external border is convex. It gives attachment to the external intercostal muscle of the first space, and a portion of the first serration of the serratus magnus at a point opposite the groove for the subclavian artery, where the external border is often prominent. The 
anterior extremity presents the usual oval pit for the first costal cartilage, its direction being horizontal from before backwards The first rib has no subcostal groove.

The Second Rib.-The surfaces of the shaft of this rib occupy a transitional plane between that of the first and those of the succeeding ribs. It is practically destitute of a twist. The neck is compressed fromabovedownwaris, and from before backwards. The distinctive character of the bone is the presence on its supero-external surface, near the centre, of a rough oval eminence for a portion of the first and the second slips of the serratus magnus. Behind this impression the surface gives insertion to the scalenus posticus.

The Tenth Rib.-This bone may or may not be peculiar. If the body of the ninth thoracic vertebra has a lower demi-facet, there is nothing peculiar about the head of the tenth rib. If, however, the lower demi-facet is wanting on the ninth thoracic body, the head of the tenth rib has only one facet for that on the body and pedicle of the tenth thoracic vertebra. The tubercle has usually an articular facet for the tenth thoracic transverse process, but this may be awanting. The angle and subcostal groove are well marked.

The Eleventh Rib.-The head of this rib has one facet for that on the pedicle of the eleventh thoracic vertebra. There is a slight tubercle, destitute of an articular facet, a faint angle, and an equally faint subcostal groove. The anterior extremity is pointed, and only tipped with a costal cartilage, which is free.

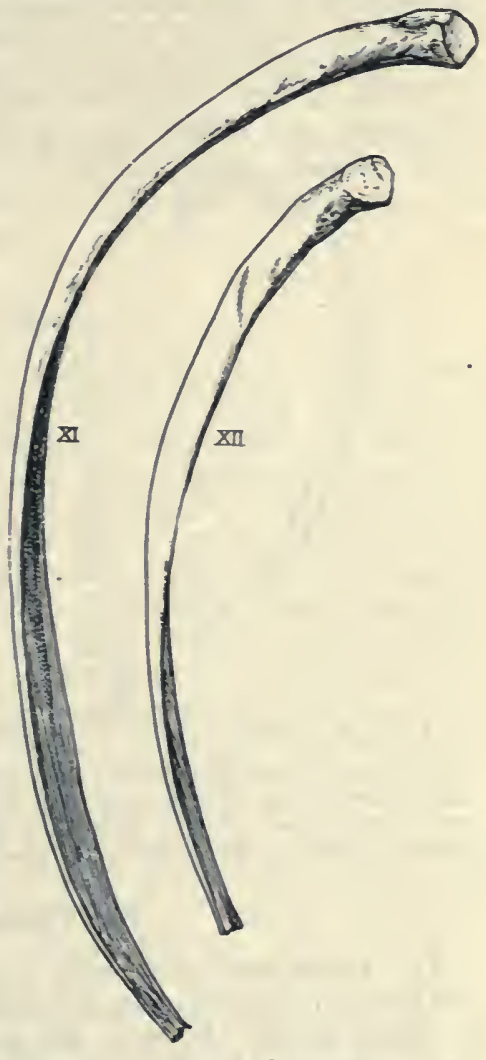

Fig. 31. - The Eleventh aNd TWELFTH RIBS OF THE LEFT SIDE (INFERIOR VIEW).

The Twelfth Rib. - This is a very short bone. Its head has one facet for that on the pedicle of the twelfth thoracic vertebra. The tubercle, angle, and subcostal groove are awanting. The shaft is very narrow, and terminates anteriorly in a pointed extremity, which is merely tipped with a free costal cartilage. The lower border of the shaft has a rough, sharp outline, and gives attachment to a portion of the quadratus lumborum muscle, whilst the upper border, especially towards the back part, is smooth and round. 
The ribs are supplied with blood by branches of the intercostal arteries.

Structure.-A rib is composed of loose cancellated tissue surrounded by compact bone.

Varieties.-(1) The number may be increased to thirteen on one or both sides, and the supernumerary rib may be cervical or lumbar. If cervical, it is developed in connection with the costal process of the seventh cervical vertebra. It may join the shaft of tha first thoracic rib, or it may reach the sternum. If lumbar, it is developed in connection with the costal element of the first lumbar vertebra, is usually very short, and does not articulate with the body of that

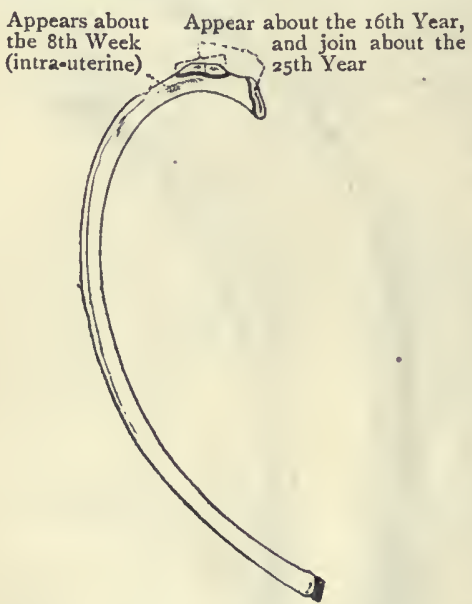

Fig. 32.-Ossification of a Rib. vertebra. (2) In rare cases the number may be decreased by one, at the expense of the twelfth rib. (3) The ribs are subject to variety in form as follows: (a) the vertebral end of the first thoracic rib may be joined by a cervical rib, or by the vertebral end of the second rib, in which cases the variety known as bicipital rib occurs; (b) the anterior extremity of a rib may be bifurcated; (c) adjacent ribs may be connected by small plates of bone.

Ossification.-An ordinary rib has one primary centre and three secondary centres. The primary centre for the shaft appears about the sixth week near the angle: Ossification proceeds so rapidly along the shaft that by the fourth month the shaft is completely ossified. The secondteenth year. One gives rise to the head, and of the other two one is for the rough part of the tubercle and the other for its articular part. The head and two parts of the tubercle join the shaft about the twenty-fifth year. The two secondary tubercular centres are absent in the eleventh and twelfth ribs.

\section{The Costal Cartilages.}

The costal cartilages, which are composed of hyaline cartilage, are twelve in number on either side. The outer extremity of each is received into the oval pit on the anterior extremity of a rib, and is there maintained in position by the continuity which takes place between the periosteum of the rib and the perichondrium of the cartilage. The inner extremities of the true ribs articulate with the side of the sternum by means of synovial joints, except in the case of the first, which is directly united to the presternum without the intervention of a synovial membrane. The eighth as a rule, ninth, and tenth do not reach the sternum, and they articulate with each other by synovial joints, each cartilage being widened at the place of articulation, where it sends downwards a process to the upper border of the cartilage below. In this way interchondral joints are formed between these cartilages, as well 
as between the eighth, seventh, sixth, and sometimes the fifth. The eleventh and twelfth cartilages are mere nodules tipping the corresponding ribs, and they have no articulation with each other, nor has the eleventh with the tenth. The cartilages increase in length from the first to the seventh, beyond which they gradually diminish to the twelfth. They diminish in breadth from above downwards. The direction of the first cartilage is inwards and downwards, and that of the second horizontally inwards, whilst the succeeding ones, except the eleventh and twelfth, incline more and more upwards as they pass inwards. Prior to middle life the first costal cartilage undergoes superficial ossification underneath the perichondrium, and so a thin shell of bone is formed around it. In advanced life this condition may be met with in the other

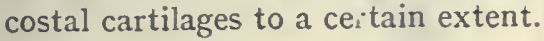

\section{The Sternum.}

The sternum or breast-bone is situated in the middle line of the anterior wall of the thorax, where it articulates on either side with the first seven costal cartilages, and superiorly with the clavicle. It occupies an oblique plane, which is directed downwards and forwards, forming an angle with the vertical of about 20 degrees. It is compressed from before backwards, of unequal width at different parts, and more or less curved from above downwards, the convexity being directed forwards, and being very pronounced in the condition known as 'pigeon-breast.' The bone is originally composed of six segments, called sternebræ. The first sternebra forms the manubrium (" handle ') or presternum. The succeeding four sternebræ form the body, gladiolus, or meso-sternum, and the sixth sternebra forms the ensiform or xiphoid process (xiphi. sternum), otherwise known as the metasternum.

The presternum is irregularly four-sided, and broader above than below. It presents two surfaces and four borders. The anterior surface is convex from side to side, and concave from above downwards. It gives origin at either side to a portion of the pectoralis major, and at its upper and outer part to the sternal head of the sterno-cleido mastoid. Between the latter point and the clavicular depression on the upper border it gives attachment to the anterior sterno-clavicular ligament. The posterior surface is concave. At its upper and outer part it gives origin to portions of the sterno-hyoid and sterno-thyroid, the former being the higher of the two, and close to the clavicular depression it gives attachment to the posterior sterno-clavicular ligament. The superion border over its middle portion presents the interclavicular or semilunar notch, which gives attachment to fibres of the interclavicular ligament. At either side of this there is a large, oval, concavoconvex articular surface for the clavicle, which is directed upwards, outwards, and slightly backwards, an interarticular fibro-cartilage intervening between the bones. Close to the inner end of each 
clavicular depression there may be found, on the anterior aspect, an ossicle, known as the episternal bone. The inferior border, which is short and straight, articulates with the mesosternum, a disc of fibro-cartilage intervening. In this situation there is a transverse

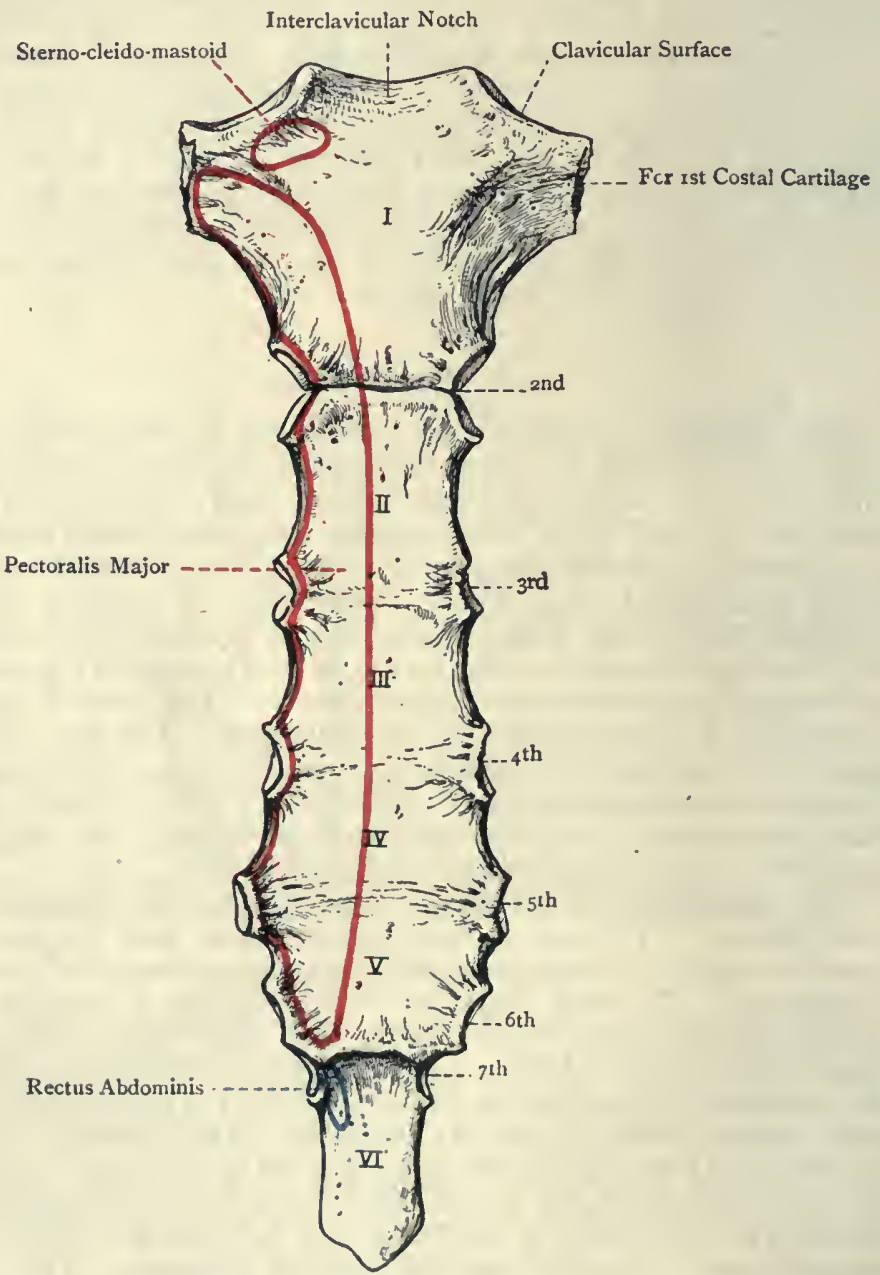

Fig. 33.-The Sternum (Anterior Surface).

elevation, called the sternal angle, which serves as a guide to the second rib at either side. Each lateral border slopes downwards and inwards. The upper part presents a triangular depression for the first costal cartilage, and close to the lower part a demi-facet for a portion of the second costal cartilage. 
The mesosternum presents two surfaces and four borders. The interior surface is marked by three transverse lines, which indicate the places of junction of the original four sternebræ. At either side it gives origin to a large portion of the pectoralis major. The posterior surface presents traces of the highest transverse line, but the lower two have usually become effaced. Adjacent to each lateral border it gives origin to a portion of the triangularis sterni, as high as the level of the third costal facet. Each lateral border presents a series of costal facets, disposed as follows: close to the upper extremity there is a demi-facet for a portion of the second costal cartilage; opposite each of the three transverse lines there is an entire facet for the third, fourth, and fifth costal cartilages; and on the side of the fourth segment of the body there are usually one entire facet and one demi-facet lying close together, the former being for the sixth costal cartilage, and the latter for a portion of the seventh. Altogether there are usually four entire facets and two demi-facets on each lateral border, the demi-facets being situated one at either extremity. Sometimes, however, the inferior demi-facet is replaced by an entire facet for the whole of the seventh costal cartilage. Each of the upper three entire facets is made up of the contiguous demi-facets of two adjacent sternebræ, as in the bodies of most of the thoracic vertebræ. The superior border of the meso. sternum articulates, as stated,

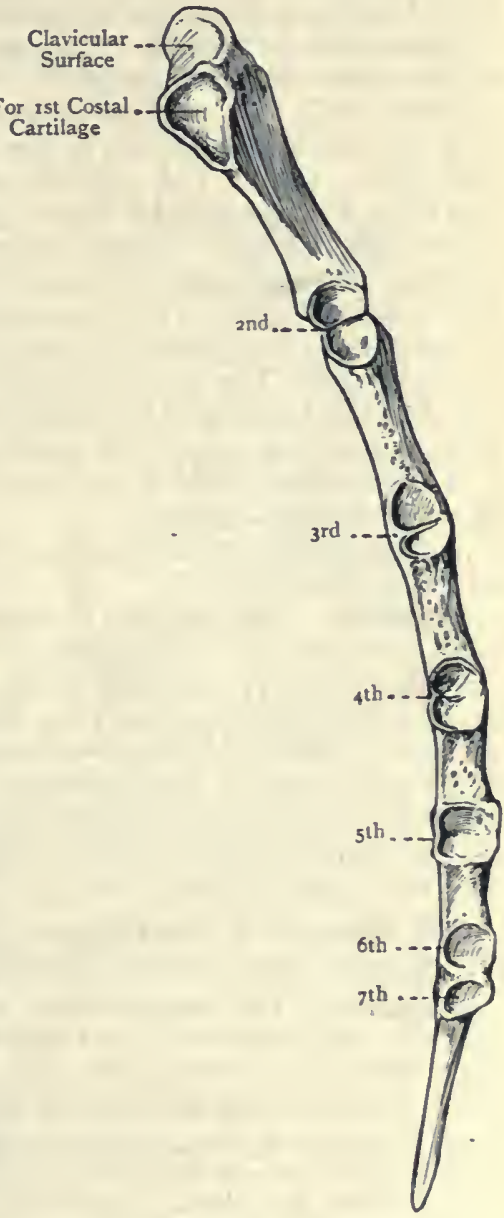

Fig. 34,-The Sternum (LATERAL View).

with the presternum. The inferior border, which is very narrow, articulates with the metasternum, an intersternebral disc intervening until about the firtieth year, when osseous union usually takes place.

The metasternum is subject to much variety as regards condition, 
direction, and form. It may be entirely osseous, or osseous above and cartilaginous below. Its typical direction is downwards between the seventh pair of costal cartilages, but it may have an inclination forwards, backwards, or even to one side. It is narrow from side to side, and compressed from before backwards. It may terminate in a thin transverse border, in a sharp point, or in a bifurcated extremity. The anterior surface lies at the bottom of the infrasternal depression. The posterior surface gives origin at either side to a portion of the triangularis sterni, and inferiorly it gives origin to a portion of the diaphragm, usually in the form of two fleshy slips. The superior border articulates with the mesosternum, and the inferior border gives attachment to the linea alba. Each lateral border usually presents superiorly a demi-facet for a portion of the seventh costal cartilage, but this may be transferred to the fourth segment of the mesosternum. In rare cases there may be an entire facet for the eighth costal cartilage, this being constant in early life. The lateral border gives insertion at either side to some of the fibres of the internal oblique aponeurosis, and occasionally, at its upper part, to a portion of the rectus abdominis.

The sternum derives its blood-supply from branches of the internal mammary artery.

Articulations. - With the clavicle and first seven costal cartilages, at either side.

Structure.-The sternum is composed of cancellated tissue covered by a thin layer of compact bone.

Varieties.-(I) The sternum is sometimes characterized by its shortness, breadth, and great depression in its lower part. This condition is liable to be met with in cobblers. (2) A sternal foramen may be present in the mesosternum, usually in the third or fourth segment. (3) A sternal foramen may be present in the inetasternum. (4) In very rare cases the sternum may be intersected from end to end by a sternal fissure, in which cases the heart and pericardium are left uncovered (ectopia cordis). (5) The costal cartilages may articulate with the stcrnum asymmetrically.

The Sternum of the Female.-The bone is usually shorter than in the male, the shortness affecting the mesosternum.

Ossification.-The sternum ossifies in cartilage from a variable number of centres. There is usually one centre for the presternum, which appears in the sixth month of intra-uterine life. Sometimes there are two, placed one above the other, and there may be as many as six, placed thus . . The first segment of the mesosternum usually ossifies from one centre, appearing in the seventh month, though there may be two, disposed laterally. The second, third, and fourth segments of the mesosternum usually ossify from two centres each, which are disposed laterally and remain separate for some time, but subsequently unite as a rule. There may, however, be only one mesial centre for each of these segments. In the second segment they appear in the eighth month, in the third just before birth, and in the fourth during the first year. The metasternum ossifies from one centre, which appears in its upper part from the third to the sixth year, though it may be delayed to a later period. The lower three segments of the mesosternum unite in order from below upwards, the union commencing about puberty and being completed shortly afterwards. The first segment of the mesosternum joins the remainder about twenty-five. The metasternum unites with the mesosternum 
about forty, but the presternum usually remains permanently separate unless in advanced life, when it may become ankylosed to the mesosternum.

The sternal cartilage from which the bone is developed consists originally of two elongated strips, each of which bears the cartilages of nine ribs. The strips are separated for some time by a median fissure, but fusion subsequently takes place, and so a single sternal cartilage is formed. The eighth costal cartilage usually loses its connection with the sternum, though it may articulate, permanently with the inetasternum. The ninth costal cartilage at either side is regarded as dividing into two parts, one of which

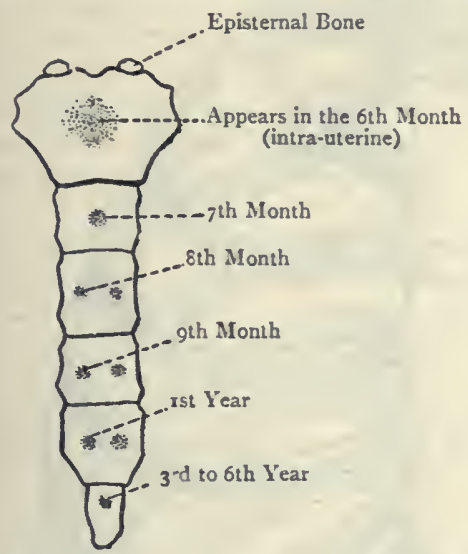

Fig. 35A-OSSification of THE StERNUM.

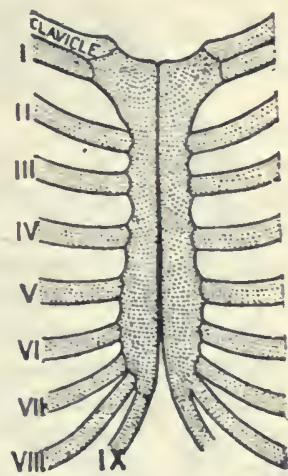

FIG. 35B.-DEVELOPMENT OF THE STERNUM

(Modified from Ruge).

remains connected with the sternal cartilage and forms with its fellow the metasternum, whilst the other acquires a connection with the eighth costal cartilage. If the parts of the ninth costal cartilages, which remain connected with the sternal cartilage, do not unite with each other over their whole extent, a bifurcated metasternum is the result. They usually, however, unite wholly, or sometimes in such a manner as to leave a foramen at the centre. A sternal fissure is due to the permanent separation of the two original cartilaginous strips, which, as a rule, unite to form the sternal cartilage. A sternal foramen in the second, third, or fourth segment of the mesosternum is due to ossification from two collateral centres failing to meet at the median line.

Sometimes two ossicles, called the episternal bones, are met with at either side of the interclavicular notch of the sternum. These are developed in connection with the suprasternal ligaments, which extend between the inner end of each clavicle and the upper end of the sternum.

\section{The Thorax as a Whole.}

The thorax constitutes an osseous and cartilaginous cage which lodges the heart and lungs, along with important bloodvessels and nerves, as well as the trachea and œesophagus. It is bounded anteriorly by the sternum, with the costal cartilages and anterior extremities of the first eight or nine ribs; posteriorly by the bodies of the thoracic vertebræ and the vertebral extremities of the ribs 
from the heads to the angles; and laterally by the ribs beyond their angles. It is conical, the truncated apex being directed upwards, and it is somewhat flattened from before backwards.

The superior aperture is bounded in front by the upper border of the presternum and the first costal cartilages, on either side by

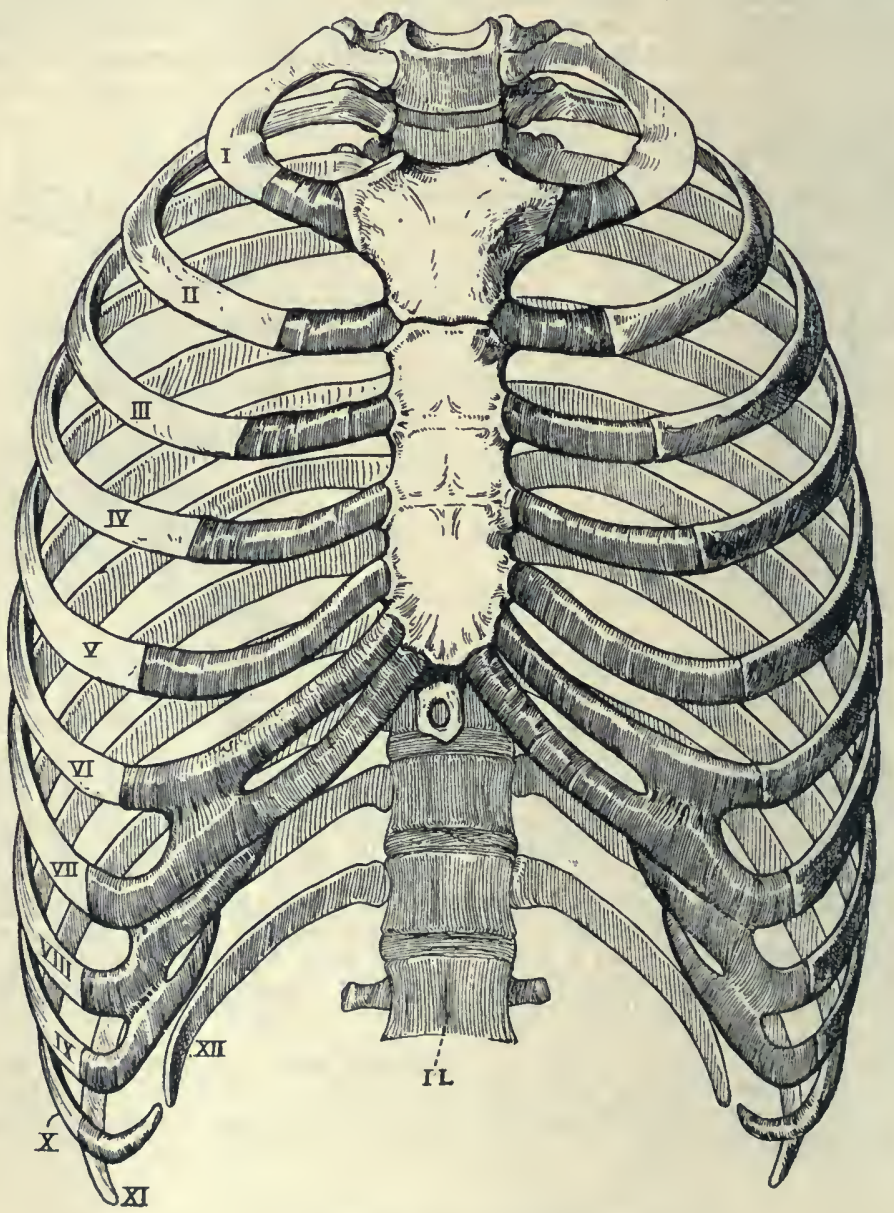

Fig. 36.-The Thorax (ANTERIOR View).

the first rib, and behind by the body of the first thoracic vertebra. Its transwerse measurement exceeds the antero-posterior, and it is reniform, due to the forward projection of the first thoracic body. Its plane is oblique, being sloped downwards and forwards, so that the upper border of the presternum is on a level with the disc between the second and third thoracic bodies. The superior 
aperture transmits the following structures: the apical parts of the lungs and pleura:, the trachea and oesophagus, the pneumogastric, sympathetic, and phrenic nerves, the terminal part of the innominate artery, the left common carotid and left subclavian arteries, and the right and left innominate veins. In early life it also transmits the thymus gland.

The inferior aperture is of large size, and is bounded posteriorly by the twelfth thoracic body, laterally by the twelfth rib at either side, and anteriorly by a line, at either side, connecting the costal cartilages from the twelfth to the seventh inclusive. These two lines constitute the subcostal angle, within which the metasternum is situated. The inferior aperture is occupied by the diaphragm, which presents certain openings for the passage of important structures.

The cavity, on either side of the thoracic bodies, presents an elongated groove, called the pulmonary groove, which lodges the thick posterior border of a lung. The cavity has the following diameters, namely, vertical, transverse, and antero-posterior. The vertical diameter extends from the superior aperture to the inferior. The transverse diameter extends from the centre of a given intercostal space to the centre of the corresponding space of the opposite side. The antero-posterior diameter extends from the anterior to the posterior wall, and is necessarily of less extent in the median line than at either side, on account of the projection formed by the thoracic bodies, its increase at each side being due to the presence of the pulmonary groove. The cavity is increased in all these diameters during inspiration, and diminished during expiration.

The intercostal spaces are eleven in number at either side. They increase in length from the first to the fifth, and are occupied for the greater part of their extent by the external and internal intercostal muscles.

The thorax of the female is rather shorter than that of the male, and is not so much flattened from before backwards.

In early life the thorax is flattened from side to side, and its height is relatively less than in the adult.

\section{The Notochord and the Development of the Vertebral Column, Ribs, and Sternum.}

\section{Notochord.}

The notochord forms the primitive basis of the axial skeleton, and around it the bodies of the vertebra are developed. It is a solid cylindrical rod of cells, derived from the cephalic end of the primitive streak, and it occnpies the median line, corresponding to the centres of the bodies of the future vertebræ. It lies along the ventral aspect of the neural tube, which constitutes the primitive tubular nervous system, and along the dorsal aspect of the archenteron, or primitive intestinal cavity. The cephalic end of the notochord is situated on the ventral aspect of the mid-brain, and corresponds to the posterior part of the pituitary region of the base of the future cranium. From this region it extends to the caudal end of the future axial skeleton, 
where it is continuous with the wall of the neurenteric canal. On either side of it there are the mesodermic somites.

The notochord is of temporary duration, and a considerable part of it is

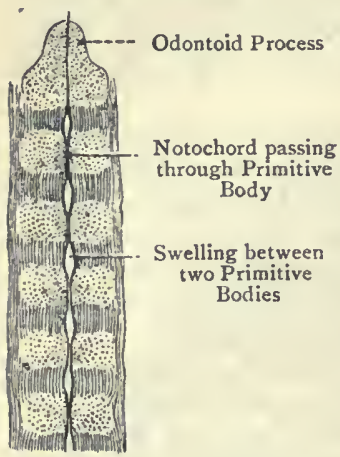

Fig. 37.-The NotoCHORD (CERVICAL REGION).

hollow. The cells of its walls, however, are pro-

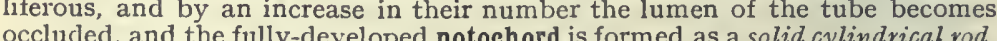

\section{Development of the Vertebral Column.}

The notochord forms the axis round which the vertebral column is developed, and the formation of the notochord may be regarded as constituting the first or notochordal stage. The notochord and the neural tube, which lies along its dorsal aspect, become surrounded by mesenchyme (mesoderm), and this undergoes chondrification and subsequently ossification. As the process of ossification proceeds, a great part of the notochord becomes constricted at regular intervals, where the bodies of the vertebræ are undergoing ossification, and these portions of it ultimately disappear. The parts of it, however, round which the intervertebral discs are formed persist and constitute the central pulp of each disc, as stated.

In addition to the notochordal stage of development, there are three other stages-namely, membranous, cartilaginous, and osseous.

Membranous Stage.-The notochord and neural tube become surrounded, as stated, by a mesenchyme, which forms a membranous sheath, spoken of as the skeletogenous sheath. This sheath constitutes the primitive membranous vertebral column. It is derived from the sclerotomes of the mesodermic somites which lie along either side of the notochord. Each sclerotome is the innermost segment of a somite, and the successive sclerotomes lie serially on either side of the notochord and close to it. They are formed of mesenchyme, and their cells multiply very rapidly. As they do so, they extend ventralwards, dorsalwards, cephalicwards, caudalwards, and mesially, between the notochord and the neural tube. In this manner the notochord becomes surrounded by a sheath. On either side this sheath furnishes two prolongations, which pass dorsalwards, one on either side of the neural tube, and meet over its dorsal aspect. There are thus formed continuous sheaths for the notochord and neural tube, and so the chordal or skeletogenous sheath is formed. The cells of the sclerotomes also extend in an outward direction, and these lateral extensions lie between, and separate, contiguous muscle-segments, each of these being a segment of a mesodermic somite, in which it lies external to the sclerotome. These lateral extensions are called the intersegmental septa, or ligamenta intermuscularia. 
The skeletogenous sheath soon acquires great density at regular intervals namely, opposite the intersegmental areas, which lie between the muscle. segments. Each of these dense parts is curved, somewhat like a horseshoe, and is known as the vertebral bow, or scleromere. The central part of the bow lies on the ventral aspect of, or below, the notochord, and is called the hypochordal bar. The lateral extremities of the bow divide, each into two limbs, dorsal and ventral. The dorsal limbs, called the neural prcesses, right and left, extend dorsalwards, one on either side of the neural tube, on

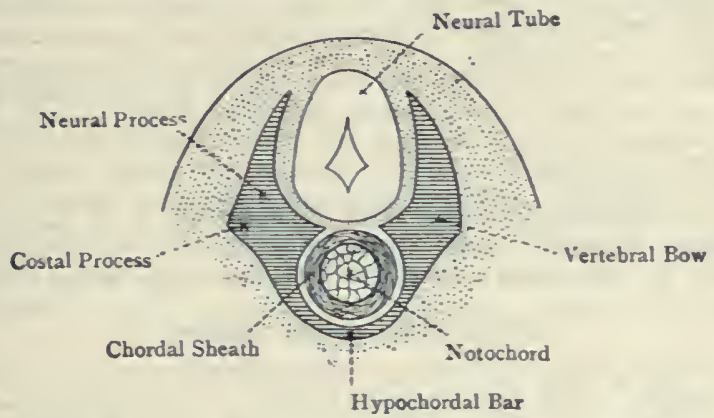

Fig. 38.-The Vertebral Bow (Schematic).

the dorsal aspect of which they meet. They form the primitive neural arches of the vertebra. The ventral limbs, called the costal processes, extend ventralwards on either side. In the thoracic region they form the primitive ribs; in the cerrical region, the primitive costal processes of the cervical vertebræ; in the lumbar region, the primitive transverse processes (costal elements) of the lumbar vertebra; and in the sacral region they form the primitive costal elements of the upper three sacral vertebræ.

The vertebral bows are arranged in regular series, opposite the intersegmental areas, each having on either side a neural process passing dorsalwards, and a costal process extending ventralwards. The neural processes, right and left, of a vertebral bow, as just stated, meet on the dorsal aspect of the neural tube, and they form the primitive neural arch of a vertebra The costal processes, as just stated, give rise to the following parts:

Thoracic Region :

The Ribs.

\section{Cervical Region :}

The Costal Processes.
Lumbar Region:

Transverse Processes Costal Elements).

Sacral Region :

Costal Elements of Upper Three Sacral Vertebræ.

The hypochordal brace or bar (central part of the vertebral bow) dis. appears, except in the atlas, of which it forms the anterior arch.

Cartilaginous Stage.- The skeletogenous sheath undergoes chondrification at regular intervals opposite the intersegmental areas and the portions of the muscle-segments which limit these areas, or, it may be, entirely opposite the muscle-segments. In each part two nodules of cartilage appear, one on each side of the notochord. These rapidly surround the chord, and so form the cartilaginous body of a vertebra. The neural processes of the lateral extremities of a vertebral bow, which lie on either side of the neural tube and coalesce along its dorsal aspect, also undergo chondrification and form the cartilaginous neural arch of a vertebra, as well as the processes connected with that arch. In this manner a cartilaginous sheath is constructed for the neural tube, which tube to a large extent beçomes converted into the spinal cord. 
The skeletogenous (niesenchymatous) tissue between the cartilaginous bodies of successive vertebræ forms the intervertebral discs, and the skeletogenous tissue between the successive cartilaginous neural arches forms the ligamenta subflava. bræ.

Osseous Stage. - For the stage of ossification see Ossification of the VerteDevelopment of the Ribs.

The ribs are developed from the mesenchyme of the costal processes of the lateral extremities of the vertebral bows in the thoracic region, which processes invade the intersegmental septa or ligamenta intermuscularia between contiguous muscle-segments. They are at first curved membranous rods, which subsequently undergo chondrification and form the cartilaginous ribs, these in turn undergoing ossification, and giving rise to the osseous ribs. They are intersegmental in position, and are confined to the thoracic region of the trunk. In the other regions their development is arrested, but rudiments of them persist. Thus, as stated, in the cervical region they are represented by the costal processes of cervical vertebra; in the lumbar region, by the transverse processes (costal elements) of lumbar vertebræ; and in the sacral region, by the costal elements of the upper three sacral vertebræ.

\section{Development of the Sternum.}

The ventral extremities of the upper nine cartilaginous ribs, on either side, become expanded, and these portions unite. In this manner an elongated strip of cartilage is formed on either side, each of which bears nine cartilaginous ribs. These strips are known as the hemisternal cartilages, and by their union a slngle sternal cartilage is formed. This cartilaginous sternum undergoes ossification in the manner described in connection with the sternum as a bone.

The eighth and ninth cartilaginous ribs, on either side, lose their connection with the hemisternal cartilages, and the portions of these hemisternal cartilages, which are contributed by the expanded ventral or anterior ends of the eighth and ninth cartilaginous ribs, give rise, by their union, to the cartilaginous metasternum (ensiform process).

In exceptional cases ossification may take place in each hemisternal cartilage independently, and under these circumstances the hemisterna may remain permanently separate, thus giving rise to the extremely rare condition of sternal fissure.

The two halves of the cartilaginous metasternum usually unite along their entire extent. Their distal ends, however, may remain permanently separate, and so give rise to a bifurcated metasternum. Again, the two halves may unite in such a manner as to leave a permanent metasternal foramen. The presence of a sternal foramen is explained under Varietios of the Sternum.

\section{II.-THE BONES OF THE HEAD.}

The head or skull is supported on the upper end of the vertebral column, and is divisible into the cranium and face. The cranium or brain-case is composed of eight bones, namely, the occipital, two parietals, frontal, two temporals, sphenoid, and ethmoid. The face, which protects organs of special sense, such as the eyes, the olfactory mucous membrane, and the tongue, is composed of the following fourteen bones, the majority of which are arranged in pairs: the two superior maxillæ, two malars, two nasals, two lachrymals, two inferior turbinates, two palate bones, the vomer, and the inferior maxilla. All the bones of the skull, except the inferior maxilla, are immovably united by sutures. 


\section{The Occipital Bone.}

The occipital bone is so named because it is situated against the posterior and inferior parts of the cranium. It is quadrilateral and curved, its long axis extending from above downwards and forwards. At its lower and anterior part there is a large opening, called the foramen magnum, by which the cranial cavity communicates with the spinal canal. The bone is divisible into four parts, which meet around this opening. The part behind is called the tabular portion, that in front ine basilar process, and the part at either side the condylar portion.

The tabular portion presents two surfaces, three angles, and four borders. The posterior or external surface is convex and projected at its centre into the external occipital protuberance, from which a median ridge, called the external occipital crest, passes downwards and forwards to the foramen magnum. The protuberance and crest give attachment to the ligamentum nuchæ. Arching outwards on either side from the protuberance to the lateral angle there is the superior curved line, the convexity of which is directed upwards. The two lines with the protuberance divide this surface into an upper or interparietal and a lower or supra-occipital part. A little above each superior curved line there is the highest curved line, which has a bold curve with the convexity upwards, and gradually subsides in the superior curved line externally. Between these two lines there is a semilunar area, over which the bone is smooth and dense. The highest curved line gives attachment to the epicranial aponeurosis internally, and to fibres of the occipitalis externally. The superior curved line gives origin over about its inner third to the trapezius, and externally to fibres of the occipitalis, whilst over its outer half, or more, it gives insertion to the sterno-cleido-mastoid, immediately below which the splenius capitis is inserted over about the outer third. The portion of this surface above the highest curved lines is smooth, convex, and covered by the epicranial aponeurosis. The portion below the superior curved lines, which is rough and irregular, is divided into two equal lateral parts by the crest, and each of these is subdivided into an upper and lower portion by the inferior curved line, which extends from the centre of the crest to the extremity of the jugular process. The space between the superior and inferior curved lines gives insertion internally to the complexus, and externally, from above downwards, to the splenius capitis and obliquus capitis superior. The inferior curved line gives insertion over its outer part to the rectus capitis posticus major. The inner third of this line and the surface between that extent of it and the foramen magnum give insertion to the rectus capitis posticus minor.

The anterior or internal surface is irregularly concave and divided into four fossæ by two ridges-a longitudinal, extending from the superior angle to the foramen magnum, and a transverse, extending from one lateral angle to the other. At the point where these two 
ridges intersect there is the internal occipital protuberance. The upper half of the longitudinal ridge gives attachment to a portion of the falx cerebri, and is marked by a groove for the superior longitudinal venous sinus, this groove being confined to one side of it, usually the right. The lower half is sharp and wiry, and is called the internal occipital crest. It gives attachment to the falx cerebelli,

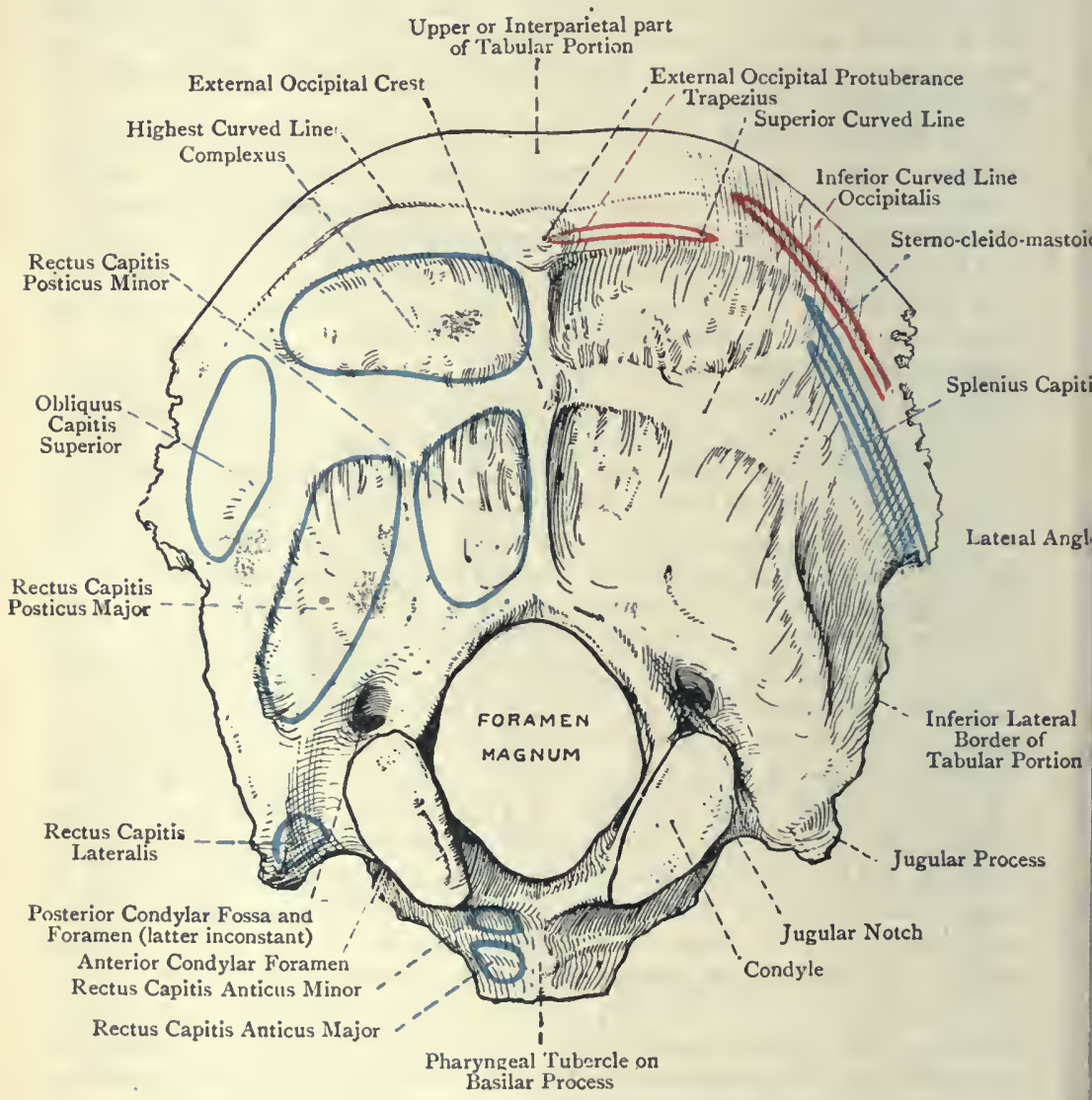

Fig. 39. - The Occipitar. Bone (External View).

and is occasionally grooved for the occipital venous sinus. Near the foramen magnum it divides into two parts, which diverge as they pass to that opening, and enclose between them the vermiform fossa, which receives a part of the vermiform process of the cerebellum. The transverse ridge gives attachment to the tentorium cerebelli, and is deeply grooved along each half for the lateral venous sinus. On one side of the internal occipital protuberance, usually 
the right, there is a wide depression, at which point the longitudinal groove is continued into the corresponding lateral groove. This depression lodges the torcular Herophili, which is a dilatation formed where the superior longitudinal sinus bends sharply to become continuous with the right lateral sinus. The four fossæ are arranged in a superior pair, called superior occipital or cerebral, and an inferior pair, called inferior occipital or cerebellar. Each cerebral fossa presents a number of digitate impressions for the convolutions of the occipital lobe of the cerebrum, which is lodged in it. The cerebellar fossæ, which are separated by the internal occipital crest, are smooth, but may show transverse striations. They are much thinner than the cerebral, and lodge the hemispheres of the cerebellum.

The angles are superior and two lateral. The superior angle forms the highest part of the bone, and fits in between the posterosuperior angles of the parietals. The lateral angles are situated at either end of the transverse ridge on the internal surface.

The borders are two superior and two inferior. Each superior border extends from the superior angle to the lateral angle, and is serrated for the posterior border of the corresponding parietal. Each inferior border extends from the lateral angle to the jugular process, and is faintly serrated for the mastoid portion of the temporal.

The basilar process (basi-occipital) is a compressed quadrilateral mass, which projects forwards and upwards in front of the foramen magnum. Its superior surface presents a broad median depression, called the basilar groove, which is sloped downwards and backwards to the foramen magnum, and lodges the medulla oblongata. At either side of this groove there is a narrow groove for the inferior petrosal venous sinus The inferior surface presents at its centre the pharyngeal tubercle for the fibrous raphé of the pharynx. On either side of this tubercle there is a rough, oblique impression for the insertion of the rectus capitis anticus major, and between the outer part of this impression and the foramen magnum the surface gives insertion to the rectus capitis anticus minor. The anterior border is thick, rough, and truncated, and up to the twentieth year it articulates with the body of the sphenoid by synchondrosis, but thereafter ankylosis takes place. The posterior border, which is thin, smooth, and concave, bounds anteriorly the foramen magnum, and sometimes presents a third occipital condyle of small size for articulation with the tip of the odontoid process of the axis. This border gives attachment to the middle odontoid or suspensory ligament. Each lateral border is thick and rough for the petrous portion of the temporal.

The condylar portions (exoccipitals) are placed on either side of the foramen magnum, where they extend as far back as its posterior margin, and very nearly as far forwards as its anterior margin. Each bears on its under surface a condyle. The condyles are oval, convex, and covered by cartilage, and they articulate with the superior articular processes of the atlas. Their long axes are 
directed forwards and inwards, and the direction of each surface is downwards and slightly outwards. They do not extend farther back on the lateral margins of the foramen magnum than the level of the centre, and the front part of each belongs to the basilar portion. The circumference of a condyle gives attachment to the capsular ligament of the corresponding occipito-atlantal joint, and on the inner aspect of each there is a tubercle for the lateral odontoid or check ligament. External to the front of each condyle is the

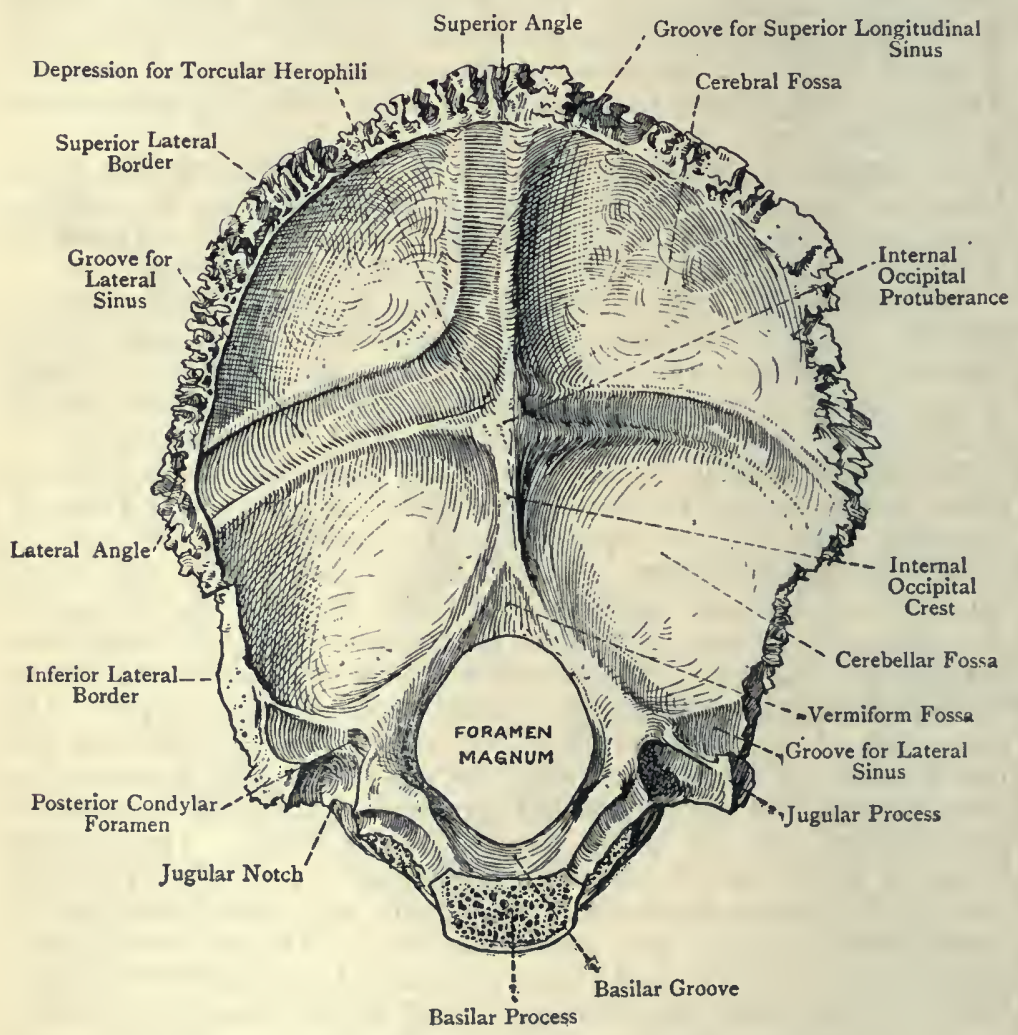

Fig. 40.-The Occhpital Bone (Internal View).

anterior condylar foramen, which opens forwards and outwards from the cranial cavity. It transmits the hypoglossal nerve and a meningeal branch of the ascending pharyngeal artery. Behind each condyle is the posterior condylar fossa, which may be pierced by a posterior condylar foramen, on one or both sides, for an emissary vein passing between the intracranial lateral sinus and the extracranial suboccipital venous plexus. The part external to the condyle is called the jugular process, which lies above the 
transverse process of the atlas, and is homologous with it. Posteriorly it is continuous with the tabular portion, and anteriorly it presents the jugular noteh, which, with the jugular fossa of the petrous portion of the temporal, forms the jugular foramen. Superiorly it presents a short, but deep and wide, groove for a portion of the lateral venous sinus just before it leaves by the jugular foramen. This groove may be pierced by a posterior condylar foramen. Inferiorly it gives attachment to the rectus capitis lateralis, and may send downwards a projection towards the transverse process of the atlas, which represents the paramastoid process of comparative anatomy. Externally the jugular process articulates with the jugular facet on the petrous portion of the temporal by synchondrosis up to the twenty-fifth year, after which ankylosis takes place.

The foramen magnum is situated at the lower and anterior part of the bone, and is oval, its long axis extending from before backwards. The inferior margin, in front of the condyles, gives attachment to the anterior occipito-atlantal ligament, and, behind them, to the posterior occipito-atlantal ligament. The foramen transmits the medulla oblongata and its membranes, the spinal accessory nerves, the vertebral arteries, the anterior and posterior spinal arteries, and parts of the cerebellar amygdalæ.

The chief blood-supply of the bone is derived from the occipital and posterior auricular arteries.

Articulations.-Superiorly with the parietals, laterally with the temporals (mastoid and petrous portions), anteriorly with the sphenoid, and inferiorly with the atlas, and in rare cases with the odontoid process of the axis.

Structure.-The occipital, being a tabular bone, is composed of two tables of compact bone, with cancellated tissue, called diploë, between them.

Varieties.-(1) There may be a minute foramen piercing the external occipital protuberance for an emissary vein, which passes between the intracranial torcular Herophili and one of the tributaries of the extracranial occipital yein. (2) The upper division of the tabular portion may be separate, representing the interparietal bone of comparative anatomy, and it may be in one piece, or in two or more. (3) The semilunar area between the highest and superior curved lines may be prominent, constituting the torus occipitalis transversus. (4) The anterior condylar foramen may be double on its cranial aspect. (5) There may be a third occipital condyle on the anterior margin of the foramen magnum. (6) There may be a paramastoid process on the under aspect of the jugular process. (7) The condyle may be divided into two parts, anterior and posterior. (8) There may be an intrajugular process on the front of the jugular notch, which may extend as far as the petrous portion of the temporal.

Ossification.-The bone is developed in four parts. The tabular portion usually ossifies from four centres, which appear around the internal occipital protuberance about the eighth week of intra-uterine life. Two are deposited in cartilage, one for each cerebellar fossa, which soon fuse and give rise to the lower or supra-occipital division. The other two are deposited in membrane, one in each cerebral fossa, which also soon fuse and give rise to the upper or interparietal division. Indeed, as a general rule, all four ultimately blend. There may, however, be two other centres for the interparietal portion, placed 
on either side of the middle line not far from the future superior angle, which occasionally remain as separate ossicles, or they may fuse and give rise to the pre-interparietals. The interparietal portion may remain separate from the supra-occipital, with which it may be connected by a suture, or they may be separated by a partial fissure. Fissures, which persist for some time after birth, intersect the tabular part at the superior and lateral angles, and a membranous interval extends from the protuberance to the foramen magnum in early life, which remains for some weeks, after which it is replaced by bone.

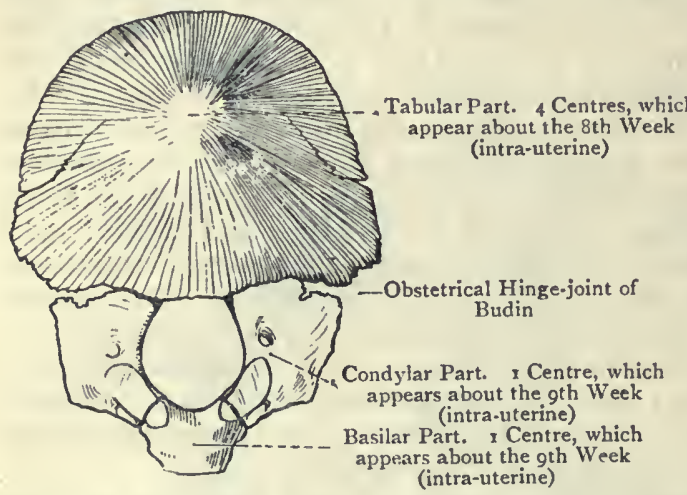

Fig. 4I.-Ossification of the Occipital Bone.
It is in this latter situation where an encephalocele may occur. The basilar and condylar parts have each one centre appearing in cartilage about the ninth week, the anterior part of each condylar portion deriving its ossification from the basilar centre. At birth the bone is in four parts, connected by cartilage. Union between the tabular and condylar portions is completed by the fourth year, and the condylar and basilar portions unite about the sixth year. After the twentieth year

the basilar portion joins the sphenoid, and at the twenty-fifth year the jugular process becomes ankylosed to the petrous portion of the temporal.

Obstetrical Hinge-Joint of Budin.-At birth the tabular or squamous portion of the occipital bone is connected with the two condylar portions by a band of cartilage. This region is known as the obstetrical hinge-joint of Budin. The connection is such as to allow of limited swinging or see-saw movements in front of, and behind, the cartilaginous band, by which movements the diameters and form of the child's head are liable to be modified during labour.

\section{The Parietal Bones.}

The parietal bones are so named because they form a large part of the cranial wall. They lie between the frontal and occipital, and superiorly they articulate with each other by the sagittal or interparietal suture. Each bone is quadrilateral and curved, and presents two surfaces, four borders, and four angles. The external surface is convex, and near its centre is more elevated than elsewhere, this part, from which ossification originally proceeds, being called the parietal eminence. A little below this the surface is crossed from before backwards by two curved lines, called the superior and inferior temporal ridges, the narrow space between which is smoother and more glistening than the rest of the surface. The part above the superior ridge is covered by the epicranial aponeurosis, and the ridge itself gives attachment to the temporal fascia. The inferior temporal ridge limits the origin of the 
temporal muscle, and the portion between it and the inferior border, which is vertically striated and called the planum temporale, forms a part of the temporal fossa, and gives origin to fibres of the temporal muscle. Near the superior border, about an inch in front of the postero-superior angle, is the parietal foramen, for an emissary vein which passes between the intracranial superior longitudinal sinus and one of the tributaries of the extracranial occipital vein.

The internal surface is concave, its deepest part, opposite the parietal eminence, being known as the parietal fossa. This surface presents a number of digitate impressions for the convolutions of

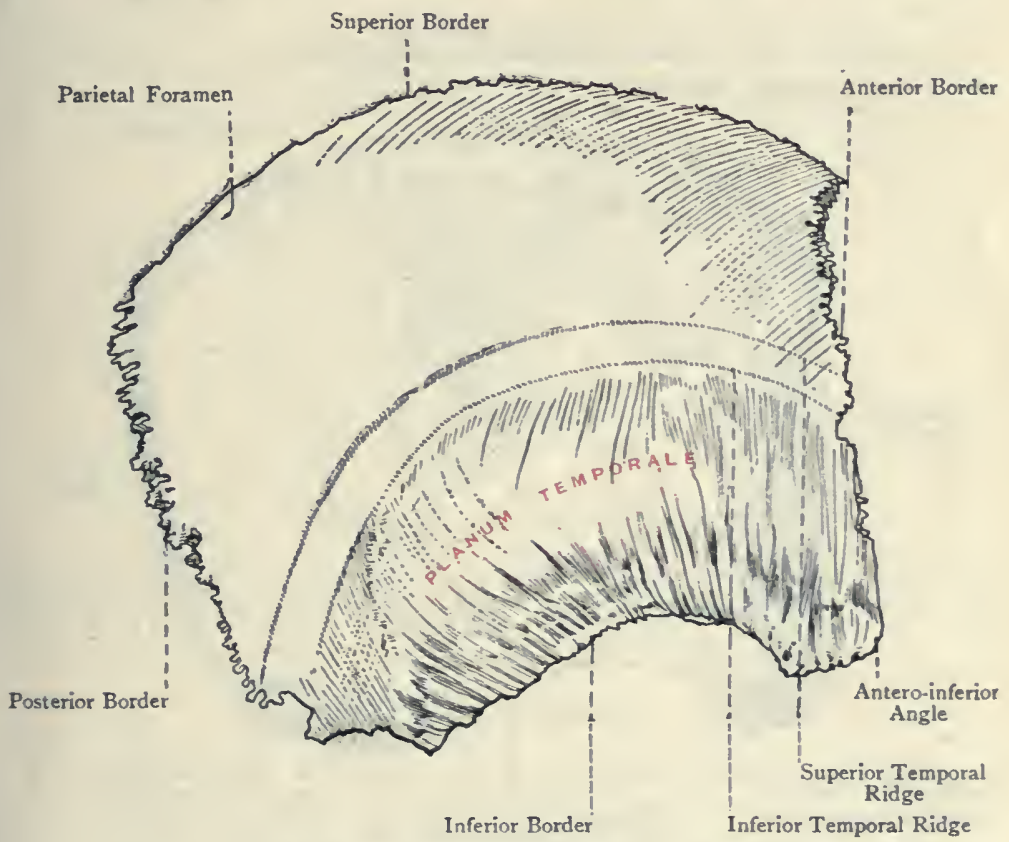

Fig. 42.-The Right Parietal Bone (External View).

the parietal and part of the frontal lobes of the cerebrum, and a system of branching meningeal grooves for the divisions of the middle meningeal artery. These commence as two grooves, each of which soon becomes arborescent. The anterior, the larger of the two, commences on the inner surface of the antero-inferior angle, where it may be bridged over into a short canal, and the posterior starts from the centre of the inferior border. Superiorly, close to the superior border, there is a half groove which, with that of the opposite bone, lodges the superior longitudinal venous sinus. Along the course of this groove, but external to it, are several depressions, best marked in old persons, which lodge the 
Pacchionian bodies. Close to the postero-inferior angle there is a short groove for part of the lateral venous sinus.

Borders.-The posterior, anterior, and superior borders are serrated. The posterior border articulates with the occipital; the superior, with its fellow; and the anterior with the frontal. The anterior border is bevelled below at the expense of the inner plate, where it overlaps the frontal, and it is slightly bevelled above at the expense of the outer plate, where it is overlapped by the frontal. The inferior border, which is the shortest, is for the most part concave and markedly bevelled at the expense of the outer plate,

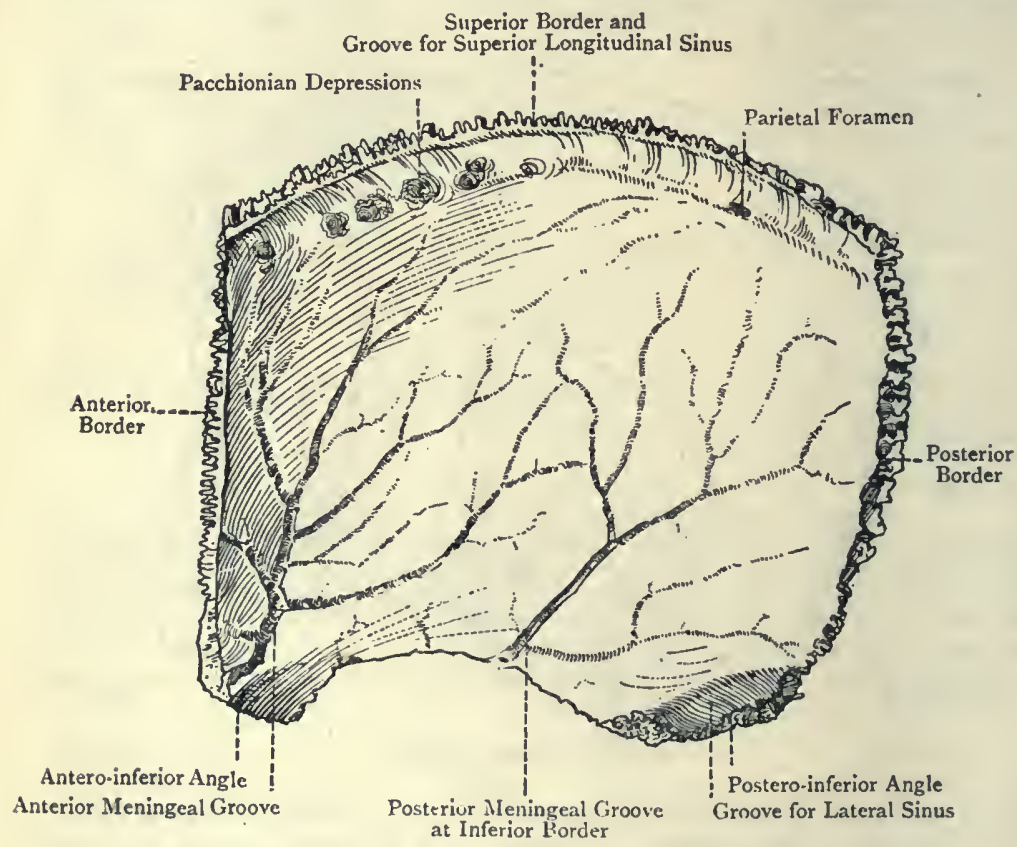

Fig. 43.-The Right Parietal Bone (Internal View).

where it is overlapped by the squamous portion of the temporal. Posteriorly, however, it is serrated for the superior border of the mastoid portion of the temporal.

Angles.-The antero-superior angle, with its fellow, lies in the situation of the original anterior fontanelle. The postero-superior angle, with its fellow, occupies the region of the original posterior fontanelle. The postero-inferior angle is truncated, and articulates with the mastoid portion of the temporal, being also recognised by the short groove for the lateral venous sinus on its inner aspect. The antero-inferior angle is prolonged and pointed, and articulates with the great wing of the sphenoid, being also 
recognised by the large anterior meningeal groove on its inner surface.

The bone receives its chief blood-supply from the middle meningeal artery internally, and the occipital externally.

Articulations.-Posteriorly with the occipital, superiorly with its fellow, anteriorly with the frontal, antero-inferiorly with the sphenoid, and inferiorly with the temporal.

Structure.-It is a characteristic tabular bone.

Varieties.-(I) The bone may persist in two parts, upper and lower, connected by an antero-posterior suture. (2) The parietal foramen may be absent on one or both sides.

Ossification.-The parietal ossifies in membrane from two centres, which appear about the seventh week in the region of the future parietal eminence, one above and the other below it, and soon coalesce. The ossification radiates

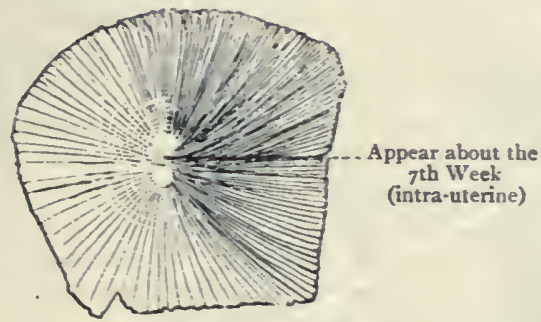

Fig. 44.-Ossification of the Parietal Bone.

from this point in such a manner as to leave a notch on the upper border a little in front of the postero-superior angle, which forms one-half of the sagittal fontanelle of the earlier half of foetal life.

\section{The Frontal Bone.}

The frontal bone forms the forehead and greater part of the roof of each orbit, and it lies in front of the parietals. It is divisible into a frontal portion and two orbital plates, the latter being situated inferiorly, where they are separated by the ethmoidal notch.

The frontal portion presents two surfaces, external and internal. The external surface, which is smooth and convex, presents a little below its centre, on either side, an elevation, called the frontal eminence. Below this, and separated from it by a shallow groove; there is the curved superciliary ridge which supports the eyebrow. This ridge is prominent internally, but it subsides externally. It supports the upper half of the orbicularis palpebrarum, and internally it gives origin to the corrugator supercilii, whilst the surface above each ridge supports the frontalis and part of the epicranial aponeurosis. Between the two superciliary ridges is an elevation, called the nasal eminence or glabella. Below each ridge is the curved supra-orbital arch, which is most prominent over its 
outer two-thirds. At the junction of the inner third and outer two-thirds is the supra-orbital notch, sometimes a foramen, for the passage of the supra-orbital nerve and artery. Occasionally there is a frontal notch, inside the normal notch, for a branch of the supra-orbital nerve. The extremities of the supra-orbital arch form the external and internal angular processes. The external process is stout and serrated for articulation with the malar. The internal process is faintly marked, and lies by the side of the nasal

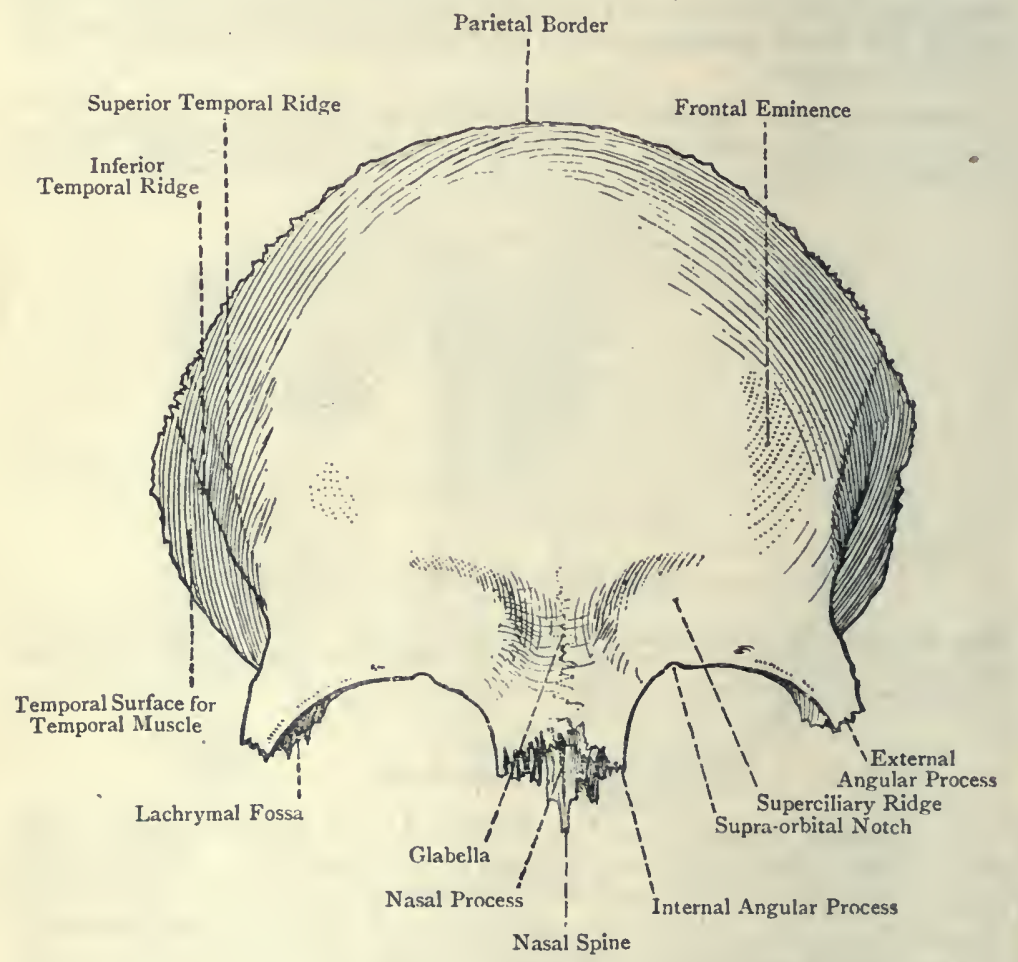

Fig. 45.-The Frontal Bone (Anterior View).

notch, where it articulates with the lachrymal, and gives origin to some fibres of the orbicularis palpebrarum.

On the lateral aspect of the external surface there are two curved lines, called the superior and inferior temporal ridges, the superior being faint and the inferior bold. They spring from the external angular process, and arch upwards and backwards to become continuous with the corresponding ridges on the parietal. The superior gives attachment to the temporal fascia, and the inferior limits the temporal muscle, which arises from it and the surface below, this latter forming a part of the temporal fossa. Below the glabella 
on the under aspect there is a rough, semilunar, serrated surface for articulation with the nasal bones and nasal processes of the superior maxillæ, and behind this is the nasal notch, bounded at either side by the internal angular process. Within the notch is the nasal process, which supports the nasal bones, and projecting downwards from it is the sharp nasal spine, which articulates in front with the upper part of the crest of the nasal bones, and behind $w$. th the vertical plate of the ethmoid. This spine enters into the nasal septum. On either side of the spine is the ala, which is grooved to take part in the roof of the corresponding nasal fossa.

The internal or cerebral surface of the frontal portion is concave, and in the middle line presents a groove, called the frontal sulcus, which lodges a part of the superior longitudinal venous sinus. On either side of the upper part of this groove there are a few depressions for the Pacchionian bodies. In eriorly the groove is replaced by the frontal crest, which terminates at the foramen cæcum. This foramen is sometimes partly formed by the crista galli of the ethmoid, and it may be closed below, or it may transmit an emissary vein, which passes between the intracranial superior longitudinal sinus and the veins of the roof of the nose. The internal surface presents numerous digitate impressions for the convolutions of the frontal lobes of the cerebrum, and laterally there are a few meningeal grooves, transversely disposed, for branches of the middle meningeal arteries.

The supero-lateral or parietal border of the frontal portion is serrated for the parietal bones. Superiorly it is slightly bevelled near the middle line at the expense of the inner plate, where it overlaps the parietal, and at either lower extremity it is distinctly bevelled at the expense of the outer plate, where it is overlapped by the parietal. Internal to its lower termination at either side there is a rough triangular surface. which is serrated for the great wing of the sphenoid.

The orbital plates, thin and brittle, project backwards in a curved manner from the supra-orbital arches, and are widely separated by the ethmoia.l noteh, which is occupied by the cribriform plate of the ethmoid. Each is triangular, with the truncated apex directed backwards and inwards, and presents two surfaces and three borders. The superior or cerebral surface is irregularly convex, and marked by digitate impressions for the convolutions of the orbital surface of the frontal lobe, which rests upon it. The inferior or orbital surface, smooth and concave, forms the principal part of the roof of the orbit. Within the external angular process is the lachrymal fossa, which lodges the lachrymal gland, and near to the internal angular piucess is the small trochlear fossa, which gives attachment to the trochlea of the superior oblique muscle of the eycba!l.

The borders are anterior, external, and internal. The anterior border represents the supra-orbital arch, and is free. The external border is sharp and irregular, and its dilection is backwards and 
inwards. It forms a right angle with its fellow of the opposite side, and abuts against the great wing of the sphenoid. The internal border is directed from before backwards, is parallel with its fellow of the opposite side, and forms the lateral boundary of the ethmoidal notch. It is bevelled at the expense of the lower plate, and the bevelled surface presents several excavations, which close in the ethmoidal cells on the upper border of the lateral mass of the ethmoid. This surface is crossed by two transverse grooves, anterior and posterior, which, with similar grooves on the contiguous part of the ethmoid, form the anterior and posterior ethmoidal (internal orbttal) canals. These open on the inner wall

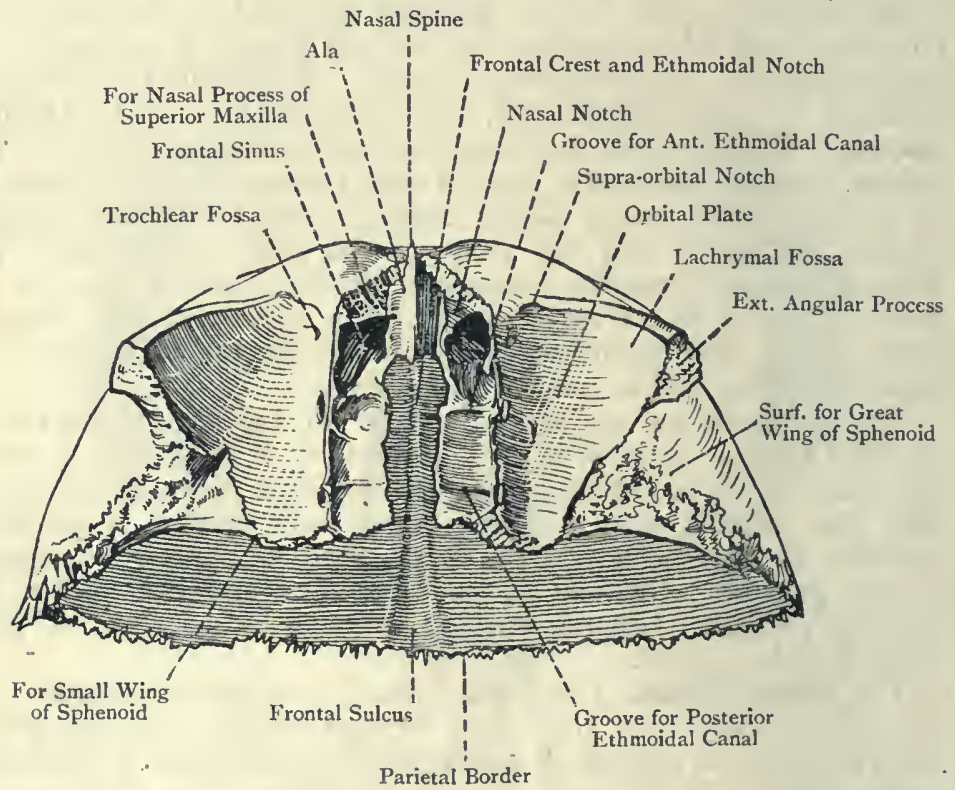

Fig. 46.-The Frontal Bone (Inferior View).

of the orbit, and the anterior gives passage to the nasal nerve and anterior ethmoidal vessels, whilst the posterior transmits the posterior ethmoidal vessels and spheno-ethmoidal nerve. The truncated apex of the orbital plate articulates with the small wing of the sphenoid.

In front of the anterior ethmoidal groove on either side is the opening of the frontal air sinus. Each leads into a cavity within the bone, which extends outwards from near the middle line for a variable distance behind the superciliary ridge. The sinuses are separated by a median septum, and may be unilocular or multilocular. In the latter case the subdivisions may extend back- 
wards for a variable distance within the roof of the orbit. Each sinus is lined by mucous membrane continuous with that of the corresponding nasal fossa, with which it communicates by a passage called the infundibulum.

The bone derives its blood-supply from the middle meningeal, meningeal branches of the internal carotid, and anterior and posterior ethmoidal arteries internally, and the supra-orbital and frontal arteries externally.

Articulations. - These are twelve in number, as follows: posleriorly with the two parietals above, and the sphenoid (great and small wings) below; by the external angular processes with the two malars; between the orbits with the two nasals, two superior maxillæ, and two lachrymals; and in the middle line with the lateral masses and vertical plate of the ethmoid.

Structure.-It is a tabular bone. The orbital plates, being destitute of diploë, are thin and translucent, except in those cases where extensions of the frontal air sinuses invade them.

Varieties.-( $\mathrm{s}$ ) There may be a persistent frontal suture, called the metopic suture, this condition being known as metopism. (2) Wormian bones are sometimes met with at the centre of the supero-lateral border in the region of the anterior fontanelle, and, if these remain permanent, they give rise by their union to a breg matic bone.

Ossification.-The frontal ossifies in membrane from two centres, one for each half, which appear about the seventh week of intra-uterine life in the situation of the future frontal eminences. At birth the bone consists of two halves united by membrane, and in the course of the first year they become united by a vertical frontal or metopic suture. This suture gradually becomes obliterated from below upwards, and usually disappears about the sixth

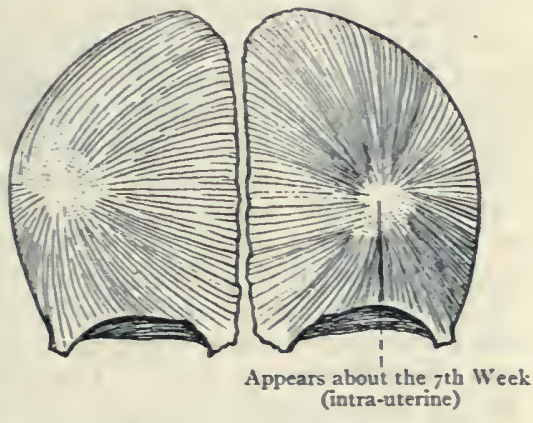

Fig. 4i.-OSSIFICATION OF THE Frontal Bone. year, though slight traces may persist above and below, especially in the latter situation. Three pairs of secondary centres are described, two mesially; placed for the nasal spine, one at either side in the region of the future trochlear fossa, and one for eacli external angular process. The frontal air sinuses begin to appear about the seventh year, but they do not attain any size till after puberty. They are rather larger in the male than in the female.

\section{The Temporal Bones.}

The temporal bones (ossa temporis) are so named because the hair over the temple is the first to become gray, thus indicating advance in life. Each bone is situated on the lateral aspect of the head below the parietal. For convenience of description each is divided into three parts, namely: the squamous portion, which bears the 


\section{zygoma; the mastoid portion; and the petrous portion, which} bears inferiorly the styloid process.

The squamous portion (squamo-zygomatic) lies almost vertically, and presents two surfaces, outer and inner, and a superior border. The outer surface is convex towards its centre, and forms a large part of the temporal fossa. It gives origin to fibres of the temporal muscle, and is marked by a groove for the middle temporal artery, which extends upwards and slightly forwards from a point just above the external auditory meatus to the superior border. The inner surface, which is concave, is related to the temporo-sphenoidal lobe of the cerebrum, and presents a few digitate impressions and meningeal grooves. The superior border is much arched, and describes about two-thirds of a circle. Except over the lower part of its anterior portion, it is markedly bevelled at the expense of the inner plate for the parietal, which it overlaps. Anteriorly over its lower part it is thick and serrated for the external border of the great wing of the sphenoid. The place of junction of the squamous and petrous portions is indicated at the lower part of the inner surface of the former by the narrow petro-squamous groove or suture.

The zygoma (jugal process) springs from the lower part of the outer surface of the squamous portion. Its base is compressed from above downwards, and directed outwards. It then undergoes a twist, and is directed forwards in a curved manner, being laterally compressed. This part of it presents two borders, two surfaces, and an extremity. The superior border, sharp and convex, extends farther forwards than the inferior, and gives attachment to the temporal fascia in two divisions. The inferior border gives origin to fibres of the masseter. The outer surface is convex and subcutaneous, whilst the inner, which is concave and looks towards the upper part of the zygomatic fossa, gives origin to fibres of the deep part of the masseter. The extremity is bevelled at the expense of the lower border, and serrated for the malar. The base of the zygoma presents two roots, anterior and posterior. The anterior root, which is continuous with the inferior border of the process, is directed inwards in front of the glenoid fossa. It is at first narrow, but subsequently thick and convex, where it is covered by cartilage. This portion is called the eminentia articularis, and in front of it there is a small triangular area which looks into the zygomatic fossa. The posterior root, which is continuous with the superior border of the process, passes backwards above the external auditory meatus, then between the squamous and mastoid portions, where it is known as the supramastoid crest, and finally it turns upwards, where it forms part of the posterior boundary of the temporal fossa. In front of the external auditory meatus it sends downwards a short offshoot, which lies between the external auditory meatus and the anterior part of the glenoid fossa. This is called the postglenoid process, and is sometimes referred to as the middle root of the zygoma. 
On the outer surface of the zygoma, above the place where the anterior root becomes continuous with its lower border, there is a projection, called the preglenoid tubercle, which gives attachment to the external lateral ligament of the temporo-maxillary articulation.

Behind the anterior root is the glenoid fossa, which extends on to the tympanic plate. It is elongated from before backwards and inwards, and is divided into two parts by the fissure of Glaser. The anterior part, which belongs to the squamo-zygomatic portion

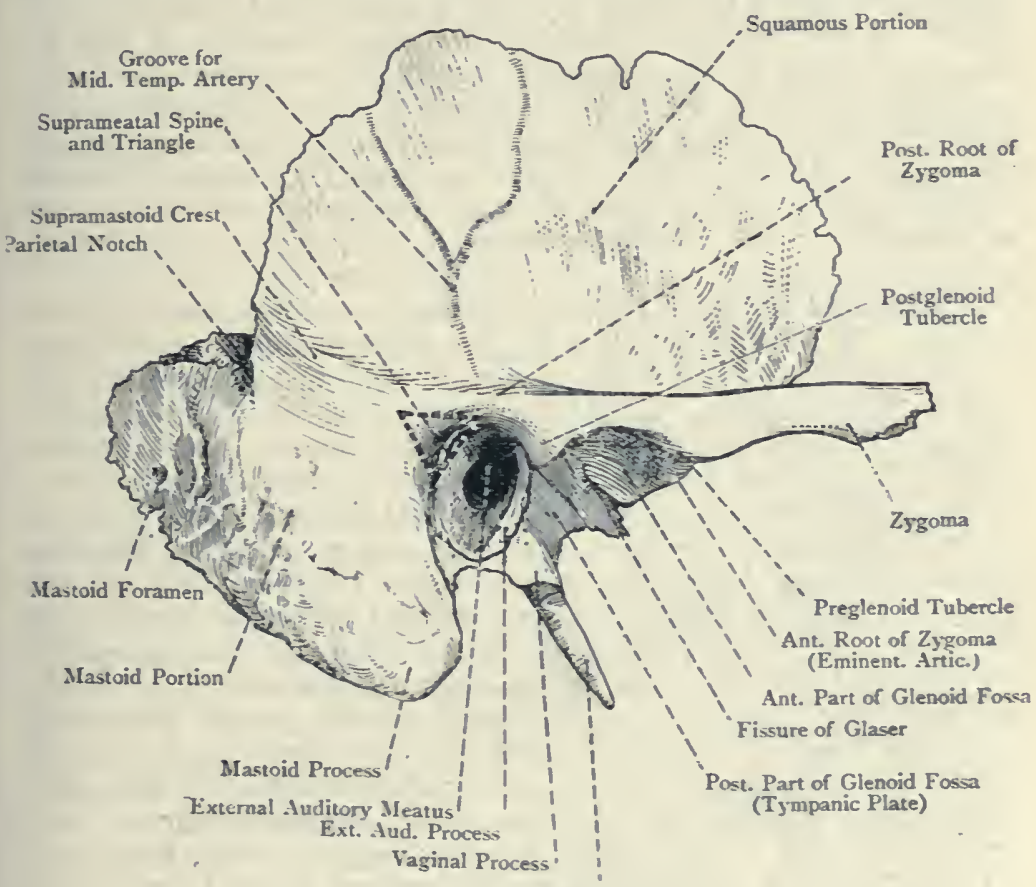

Styloid Process

Fig. 48.-The Right Temporal Bone (External View).

of the bone, is covered by cartilage, and is triangular, with the apex at the preglenoid tubercle and the base at the Glaserian fissure. It is deeply concave, and is bounded anteriorly by the eminentia articularis, externally by the commencement of the posterior root of the zygoma, and posteriorly from without inwards by the postglenoid process and Glaserian fissure. It articulates with the condyle of the inferior maxilla when the mouth is closed, an interarticular fibro-cartilage intervening; but, when the mouth is open, the condyle with the fibro-cartilage moves forwards on to the eminentia articularis. The posterior part of the glenoid fossa is situated behind the 
Glaserian fissure, and is formed by the tympanic plate, which separates it from the external auditory meatus. It is shallow, non-articular, and quadrilateral, and it lodges the deep part of the parotid gland.

The fissure of Glaser, which is the remains of the petro-tympanic fissure, is closed in its outer part, and is divided into two internally by means of a thin plate which descends from the tegmen tympani, and forms the chief part of the outer wall of the canals for the osseous part of the Eustachian tube and tensor tympani muscle. Between this plate and the tympanic plate the processus gracilis of the malleus is located internally, and there is a small opening leading to the tympanic cavity for the passage of the tympanic branch of the internal maxillary artery and the anterior ligament of the malleus (so-called laxator tympani muscle) or band of Meckel. At the inner end of the Glaserian fissure is another minute opening leading from the tympanic cavity, called the canal of Huguier, or iter chordæ anterius, which transmits the chorda tympani nerve.

The mastoid portion is so named from the mastoid process which it bears. It is limited above by the supramastoid crest and its own superior border, in front by the external auditory meatus and auricular or tympano-mastoid fissure, and behind by its posterior border. It presents two surfaces and two borders. The external surface, rough and convex, is prolonged downwards behind the external auditory meatus into the mastoid process, which presents on its inner surface two grooves. The outer, called the digastric groove, is deep, and gives origin to the posterior belly of the digastric; and the inner, called the oceipital groove, is narrow, and lodges the occipital artery. The upper part of the outer surface of the mastoid process gives origin over its posterior half to the retrahens auriculam and part of the occipitalis, in this order from before backwards; and lower down, it gives insertion to the sternocleido-mastoid, splenius capitis, and trachelo-mastoid, in this order from above downwards. At the upper and back part of the mastoid portion, a little below the supramastoid crest, there may be the remains of the squamo-mastoid suture directed downwards and forwards, indicating the line of junction of the squamo-zygomatic and basal part of the petrous portions. Directly in front of the root of the mastoid process there is an important depressed area, called the suprameatal triangle (Macewen), which is bounded as follows: above by part of the posterior root of the zygoma, below by the postero-superior part of the external auditory meatus, and behind by a vertical line connecting the upper and lower boundaries, which line is continuous with the posterior part of the external auditory meatus. In the lower part of the suprameatal triangle is the suprameatal spine, a sharp, antero-posterior scale of bone, which gives attachment to a portion of the cartilage of the external ear. The outer surface of the mastoid portion presents several small nutrient foramina, and often there is a large opening, called 
the mastoid foramen, usually placed near the posterior border, for a large emissary vein, which passes between the lateral sinus internally and the outermost tributary of the occipital vein, or the posterior auricular vein externally. In addition to these, there is the minute arterial fissure on the outer surface of the mastoid process below its centre for the mastoid branch of the occipital artery.

The inner surface presents the deep sinuous sigmoid groove, which lodges a part of the lateral venous sinus, and from which the mastoid foramen opens. The genu or bend of this groove and its descending limb lie behind the mastoid antrum.

The superior border, thick and serrated, articulates with the back part of the inferior border of the parietal. Near its anterior part it presents the parietal notch, which receives a portion of the parietal bone. The posterior border, also thick and serrated, articulates with the inferior border of the tabular portion of the occipital.

The interior of the mastoid portion contains a number of cavities lined with mucous membrane, called the mastoid air cells. These open into an irregular chamber, known as the mastoid antrum, which is situated at the upper part of the posterior wall of the tympanum, and is lined by mucous membrane continuous with that of the tympanum and mastoid cells. The upper part of the antrum communicates with the upper part or attic of the tympanum by an opening which faces that of the Eustachian tube, but the lower part is shut off from the tympanic cavity, and its floor is on a lower level than the floor of that cavity, which explains the difficulty in the drainage of fluid. The roof of the antrum, called tegmen antri, is continuous with the tegmen tympani, and both enter into the formation of the middle fossa of the base of the skull. The outer wall is formed by the squamo-mastoid junction in the region of the suprameatal triangle; the floor and inner wall are constructed by the petro-mastoid portion; and the posterior wall represents that part of the mastoid portion which bears the genu or bend and descending limb of the sigmoid groove. The mastoid cells extend from the antrum into the mastoid portion in a backward and downward direction, and are subject to variety as regards number and size. They are arranged in two groups-horizontal or superior, and vertical or inferior. The former are purely pneumatic or air cells, but the latter are of two kinds, the upper ones being pneumatic, and the lower ones, which extend to the tip of the mastoid process, being diploëtic and of large size. Superiorly the cells extend forwards over the roof of the external auditory meatus, upwards as high as the supramastoid crest, and inwards for a certain distance into the petrous portion. They may also extend into the jugular process of the occipital in old persons. At birth the mastoid cells are not dereloped, but the antrum is present.

The petrous portion is so named from its rocky consistence, and its direction is inwards and forwards into the base of the skull. It 
has the shape of a four-sided pyramid, and presents four surfaces (one of which is concealed by the tympanic plate), four borders, an apex, and a base. The surfaces are superior, posterior, inferior, and aliterior.

The superior surface, which has an inclination forwards, looks into the middle fossa of the base of the skull, and towards its outer part presents a few digitate impressions for convolutions of the temporosphenoidal lobe of the cerebrum. Near the apex is the Gasserian depression for the Gasserian ganglion, and below this is the outlet of

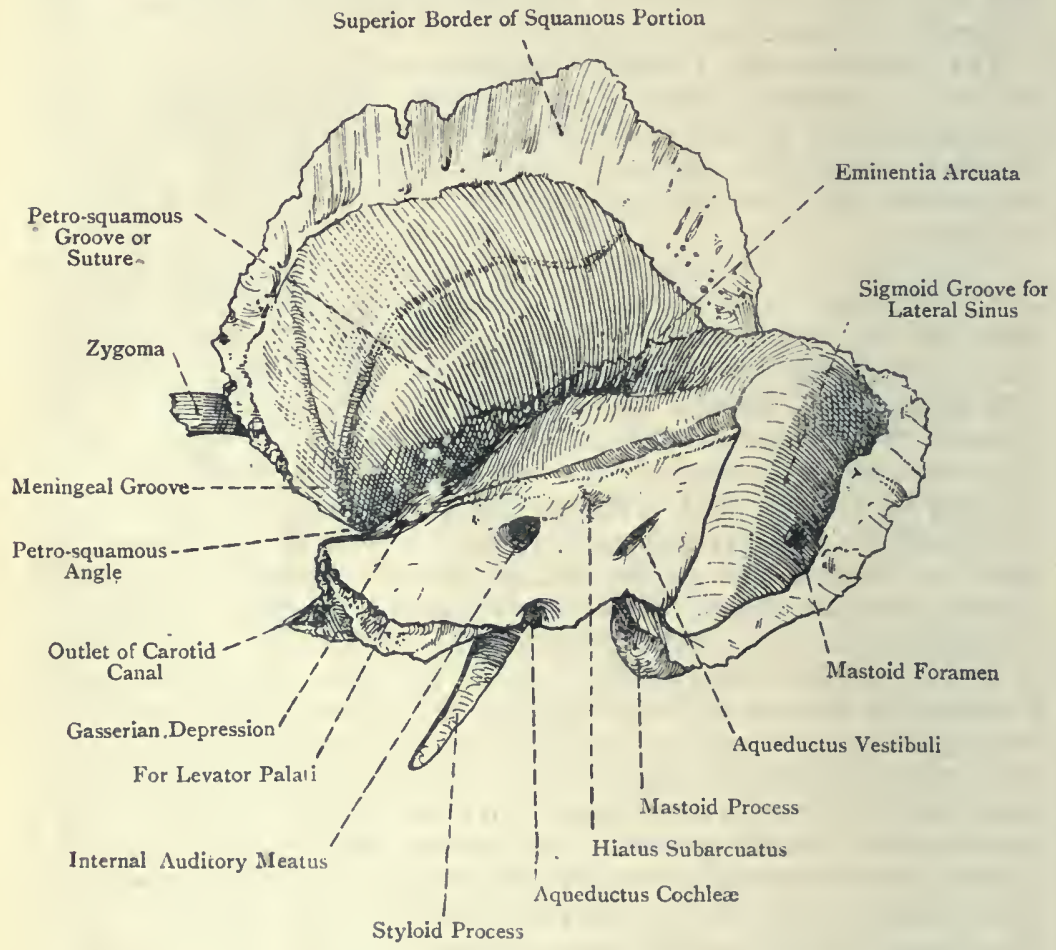

Fig. 49.-The Right Temporal Bone (Internal View).

the carotid canal for the internal carotid artery. Proceeding backwards and outwards, there is a small groove leading to a foramen very nearly in line with the petro-squamous angle, called the hiatus Fallopii, within the thin margin of which there may be two openings. The inner and larger of these openings leads to the commencement of the aqueduct of Fallopius, and thence to the internal auditory meatus, and it transmits the great superficial petrosal nerve, along with the petrosal branch of the middle meningeal artery. The outer opening, of small size, also leads to the aqueduct of Fallopius, 
and transmits the external superficial petrosal nerve. This latter, however, with the nerve, is inconstant. Another small groove marks the upper wall of the canal for the tensor tympani muscle at the petro-squamous angle, and leads backwards and outwards, parallel with the preceding groove, to a minute foramen, situated a little external to the hiatus Fallopii, called the accessory hiatus, which transmits the small superficial petrosal nerve. Behind and external to the hiatus Fallopii is an elevation, called the eminentia arcuata, which coincides with the position of the superior semicircular canal of the internal ear. Between this eminence and the hiatus Fallopii internally and the petro-squamous fissure externally there is a plate of bone, called the tegmen tympani, which forms the roof of the tympanic cavity and of the canal for the tensor tympani muscle.

The posterior surface, which looks backwards and inwards, forms part of the posterior cranial fossa. It presents about its centre a large opening, which leads into a short canal, called the internal auditory meatus, for the passage of the facial and auditory nerves, the pars intermedia of Wrisberg, and the auditory artery. At the deep end of this meatus there is a perforated plate of bone, known as the lamina cribrosa, which is divided into an upper and a lower fossa by a transverse ridge, called the falciform crest. The upper fossa presents at its anterior part a special foramen which leads into the aqueduct of Fallopius, and by this foramen the facial nerve leaves the meatus. The remainder of the upper fossa is known as the superior vestibular area, and it is pierced by the nerves and arteries destined for the utricle and the ampullæ of the superior and external semicircular canals. The lower fossa contains the cochlear area, which is pierced by the cochlear nerves and arteries, the inferior vestibular area for the nerves and arteries to the saccule, and the foramen singulare for the nerves and arteries to the ampulla of the posterior semicircular canal. The aqueduct of Fallopius, for the facial nerve, extends from the deep end of the internal auditory meatus to the stylo-mastoid foramen, between which points it takes a very circuitous course. It passes at first horizontally outwards between the cochlea and vestibule to the inner wall of the tympanum, then it bends sharply backwards, lying above the fenestra ovalis, and finally, making another abrupt bend, it descends in the angle between the inner and posterior walls of the tympanum to the stylo-mastoid foramen. The hiatus Fallopii leads from the commencement of the aqueduct to the superior surface of the petrous portion, and, as stated, transmits the great superficial petrosal nerve. The aqueduct, as it descends behind the tympanum, communicates with the canal of the pyramid by an opening through which the nerve to the stapedius reaches that muscle, and below the pyramid it presents another opening, called the iter chordæ posterius, by which the chorda tympani nerve passes into the tympanum.

About $\frac{1}{4}$ inch external to the opening of the internal auditory 
meatus there is a narrow fissure, overhung by a thin scale of bone, called the aqueductus vestibuli, which contains a small artery and vein, and the ductus endolymphaticus. Close to the superior border, about midway between the opening of the internal auditory meatus and the aqueductus vestibuli, there is a depression containing a small opening, known as the fossa subarcuata, which represents the floccular fossa of early life.

The inferior surface appears on the exterior of the base of the skull. Near the apex there is a large rough surface which gives origin to fibres of the levator palati, and behind this a circular opening, called the carotid foramen, which is the inlet to the carotid

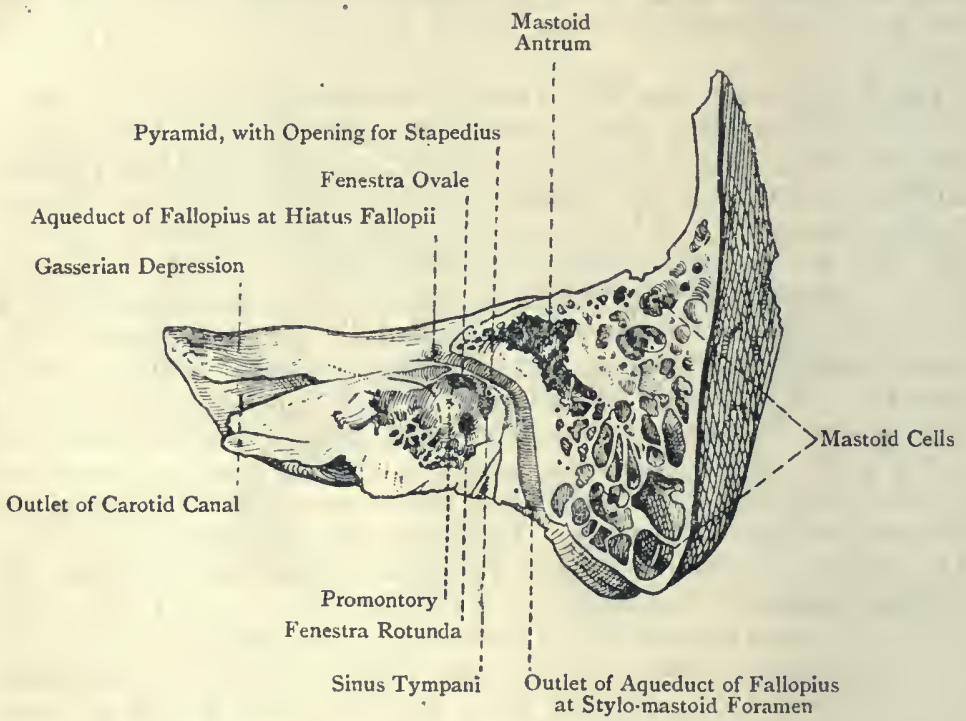

Fig. 50.-Section through the Petrous and Mastoid Portions of the Temporal Bone, showing the Tympanum and Mastoid Cells.

canal. This canal, which transmits the internal carotid artery and the carotid sympathetic plexus, passes at first vertically uproards, and then, bending at a right angle, it passes horizontally forwards and inwards, to open at the apex, below the Gasserian depression, into the foramen lacerum medium. On the posterior wall of the vertical portion of the canal is a minute foramen for the tympanic branch of the carotid sympathetic plexus and tympanic branch of the internal carotid artery. Behind the carotid foramen is the jugular fossa, which, with the jugular notch of the occipital, forms the jugular foramen. On the outer wall of this fossa, near the root of the styloid process, there is the opening of the auricular canaliculus for the auricular branch (Arnold's nerve) of the pneumogastric. On the carotid ridge, between the carotid foramen and 
the jugular fossa, is the opening of the tympanic canaliculus for the tympanic branch (Jacobson's nerve) of the glosso-pharyngeal and the tympanic branch of the ascending pharyngeal artery. Behind the jugular fossa, internal to the stylo-mastoid foramen, is the rough jugular facet for articulation with the extremity of the jugular process of the occipital, by synchondrosis up to the twenty-fifth year, after which ankylosis takes place. External to the jugular facet is the styloid process, immediately behind

Squamous Portion

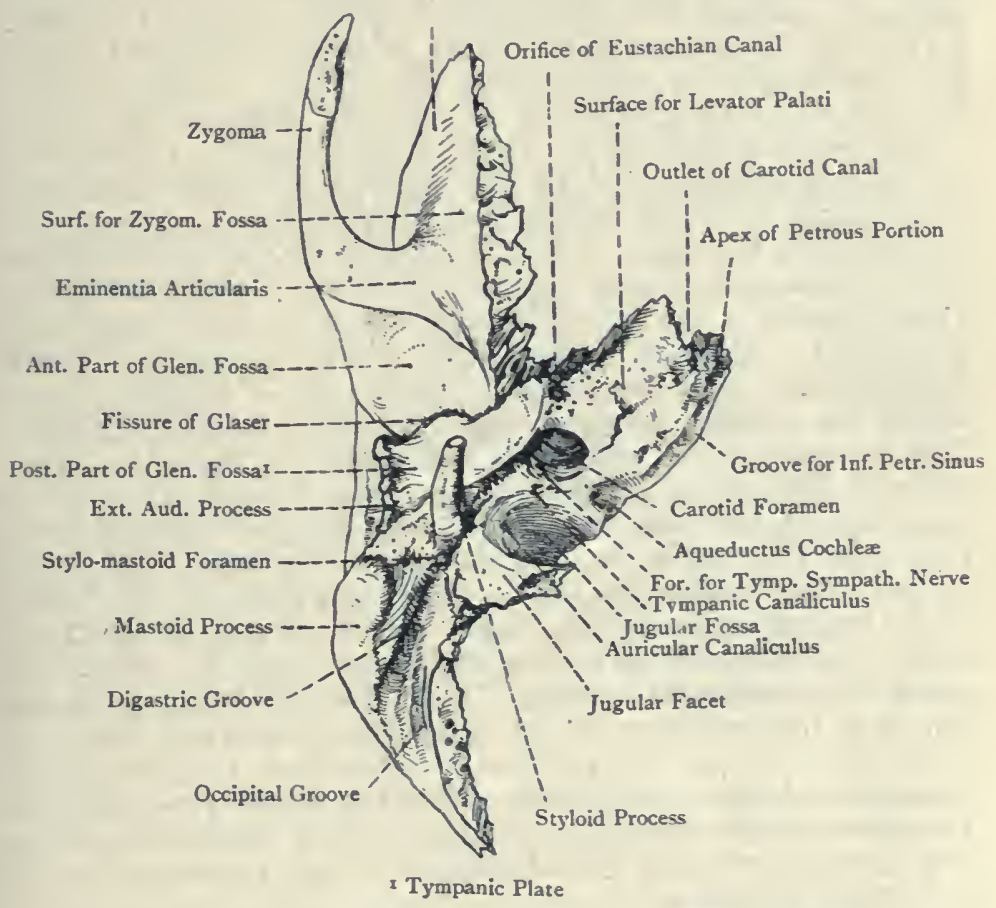

Fig. 51.-The Right Temporal Bone (Inferior View):

the root of which is the stylo-mastoid foramen. This foramen is the outlet of the aqueduct of Fallopius, and by it the facial nerve makes its exit, whilst the stylo-mastoid branch of the posterior auricular artery passes in. A little behind the stylomastoid foramen, between the mastoid process and tympanic plate, is the auricular or tympano-mastoid fissure for the exit of Arnold's nerve.

The anterior or tumpanic surface, which is concealed by the tympanic plate, looks into the tympanic cavity, of which it 
forms the posterior and inner walls, and it presents the mastoid antrum.

The borders are anterior, superior, posterior, and inferior. The anterior border separates the superior from the anterior or tympanic surface. It is very short, and forms an acute angle with the squamous portion within which the posterior pointed extremity of the great wing of the sphenoid is received. This angle presents an opening. called the Eustachian orifice, and the canal to which it leads is divided into two compartments by a thin transverse shelf of bone, scooped out on its under surface, called the processus cochleariformis. The upper small compartment lodges the tensor tympani muscle, and the lower large one forms the osseous part of the Eustachian tube. Both of these canals lead upwards and backwards to the anterior part of the tympanic cavity. The superior border, which is the longest, separates the superior from the posterior surface. It gives attachment to the tentorium cerebelli, and is grooved for the superior petrosal venous sinus. The inner part of this border frequently presents a process, which projects over the upper end of the groove for the inferior petrosal venous sinus, and gives attachment to the petro-sphenoidal ligament. This ligament connects it with the lateral border of the dorsum sellæ of the sphenoid (which may present a superior petrosal process), and, if it ossifies, it bridges over a foramen through which the inferior petrosal sinus and sixth cranial nerve pass. The posterior border separates the posterior from the inferior surface. Its outer part, opposite the jugular fossa, enters into the formation of the jugular foramen, and its inner part presents a groove for the inferior petrosal sinus, and articulates with the side of the basilar portion of the occipital. In line with the opening of the internal auditory meatus it presents a triangular depression, which encroaches on the inferior surface and leads to a small canal, called the aqueductus cochleæ. This aqueduct transmits a small vein from the cochlea to the inferior petrosal sinus, and also a communication between the perilymph of the scala tympani and the subarachnoid space, sometimes called the ductus perilymphaticus. The inferior border separates the inferior from the anterior surface, and coincides with the line of contact of the tympanic plate with the petrous portion.

The apex of the petrous portion presents on its anterior aspect the Gasserian depression superiorly, and the outlet of the carotid canal inferiorly.

The base is the part of the petrous portion which appears on the external surface, and it presents a large opening leading into the external auditory meatus. This opening is oval, its long axis lying vertically, and it is bounded above by the posterior root of the zygoma, whilst the remainder of its circumference is formed mainly by the external auditory process of the tympanic plate. The external auditory meatus is formed chiefly by the tympanic and squamous portions, but also slightly by the mastoid portion. Its direction 
is inwards, slightly forwards, and finally downwards, its length being rather more than $\frac{1}{2}$ inch ( 14 millimetres). It leads to the tympanum; and its deep end, which is nearly circular, is closed by the membrana tympani. This membrane is placed obliquely, and forms an acute angle with the lower wall and an obtuse angle with the upper, so that the floor of the meatus is longer than the roof, the anterior wall being also longer than the posterior. Its floor presents a slight elevation at the centre, where the passage is narrower than elsewhere, this portion being called the isthmus.

The tympanic plate is situated behind the fissure of Glaser, and is quadrilateral. It presents two surfaces and four borders. The external surface forms the posterior part of the glenoid fossa, and lodges the deep portion of the parotid gland. The internal surface forms the anterior, inferior, and part of the posterior, walls of the external auditory meatus, and the anterior and inferior walls of the tympanum, and at its inner or deep end it presents a groove, deficient above, for the membrana tympani, called the sulcus tympanicus. The outer border forms the external auditory process, and is curved and rough for the cartilage of the pinna. The inner border is situated immediately outside the bony part of the Eustachian tube, and is short and irregular. The upper border bounds the fissure of Glaser posteriorly, and the lower border forms at its back part the vaginal process, which ensheathes the base of the styloid process externally. The tympanic plate sometimes presents a small opening at its centre, called the foramen of Huschke.

The styloid process, which is cylindrical and tapering, starts from a point immediately in front of the stylo-mastoid foramen, and is directed downwards and inwards. The muscular and ligamentous relations of the process are as follows: The stylopharyngeus muscle arises from the inner aspect of the base; the stylo-hyoid muscle from the posterior and outer aspect of the process near its base; the stylo-glossus muscle from the front of the process near its tip; the stylo-mandibular ligament is attached to it just below the stylo-glossus; and the stylo-hyoid ligament is attached exactly to the tip.

The blood-supply of the bone is chiefly derived from the following sources: The squamous portion receives externally branches from the anterior and posterior deep temporal arteries of the internal maxillary, and internally branches of the middle meningeal. Other arterial twigs enter the bone at definite points, as follows: internal auditory from the basilar, through the internal auditory meatus; petrosal from the middle meningeal, through the hiatus Fallopii; stylo-mastoid from the posterior auricular, through the-stylo-mastoid foramen; tympanic from the internal maxillary, through the Glaserian fissure; tympanic from the ascending pharyngeal, through the tympanic canaliculus; tympanic from the internal carotid, through the foramen on the posterior wall of the vertical portion of the carotid canal; the mastoid from the occipital, through the arterial fissure on the outer surface of the 
mastoid process; and twigs from the mastoid-division of the posterior auricular, through the foramina on the outer surface of the mastoid portion.

Articulations.--These are usually five in number, as follows: posteriorly and internally with the occipital, superiorly with the parietal, anteriorly with the sphenoid and malar, and externally with the condyle of the inferior maxilla, the latter being a movable articulation. Sometimes the temporal articulates with the frontal, giving rise to a fronto-squamosal suture.

Structure.--The squamous portion is thin, and is practically composed of two plates of compact bone. The mastoid portion is thick, and, as stated, contains the mastoid antrum and mastoid cells. The petrous portion is remarkable for its hardness, and it contains all the divisions of the organ of hearing, except the cartilaginous pinna on the outer side of the head. Thus it contains (I) the osseous external auditory meatus; (2) the tympanum or middle ear, with its three ossicles, malleus, incus, and stapes, etc.; and (3) the osseous labyrinth or internal ear, which contains the membranous labyrinth, consisting of the utricle, saccule, semicircular canals, and membranous cochlea. It also contains, for a certain distance, extensions of the mastoid cells.

Varieties.-(I) Foramen of Huschke in the centre of the tympanic plate, due to imperfect ossification. (2) Absence of petro-squamous suture. (3) Absence of the foramen within the hiatus Fallopii for the external superficial petrosal nerve.

Ossification.-The temporal bone is developed in three parts, namely; squamosal, tympanic, and petrosal. The squamosal and tympanic elements are formed in membrane, and the petrosal in cartilage. The squamosal gives rise to the squamo-zygomatic portion, and the upper and front part of the mastoid portion; the tympanle forms the tympanic annulus; and from the petrosal are developed the petrous portion and the greater part of the mastoid portion. It is to be noted that the mastoid portion is not an independent part developmentally, but belongs chiefly to the petrous and partly to the squamosal portions. The centre for the squamosal appears towards the end of the second month of intra-uterine life in the region of the root of the zygoma, and from this ossification extends upwards into the squamosal, forwards into the zygoma, and inwards into the glenoid fossa in front of the Glaserian fissure. From the posterior part of the squamosal a downward growth of bone takes place below the supramastoid crest, called the postauditory process, which forms the outer wall of the mastoid antrum, and gives rise to the upper and front part of the mastoid portion. The centre for the tympanic element appears towards the end of the third month of intra-uterine life in the lower part of the external membranous wall of the tympanum, and from this is developed the tympanic annulus. This ring forms about five-sixths of a circle, the deficiency being above, where it is closed by the squamosal, and within the circumference of the ring there is a groove for the membrana tympani. Previous to birth the extremities of the ring become ankylosed to the squamosal, and the tympanic plate is formed by an outward growth from it, so that it is ultimately located at the deep end of the external auditory meatus. The petrosal element or periotlc cartilaginous capsule is developed from four centres, which appear towards the end of the fifth month, and from which ossification proceeds rapidly, union between the four centres being effected by the end of the sixth month of intra-uterine life. These centres are cal!ed opisthotic, pro-otic, pterotic, and epiotic, in the order of their appear- 
ance. The opisthotic centre appears on the promontory on the inner wall of the tympanum, from which point ossification extends downwards around the fenestra rotunda, and forms (I) the floor of the vestibule, (2) the lower part of the fenestra ovalis, (3) the floor or the internal auditory meatus, (4) the greater part of the bony investment of the cochlea, (5) the carotid canal, and (6) the floor of the tympanum. The pro-otic centre appears near the inner limb of the superior semicircular canal in the region of the eminentia arcuata, and from it are formed (I) the bony investment of the superior semicircular

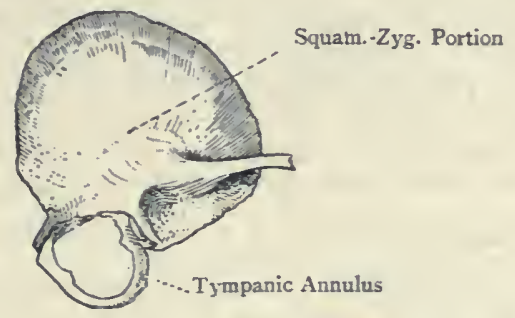

Internal Auditory Meatus
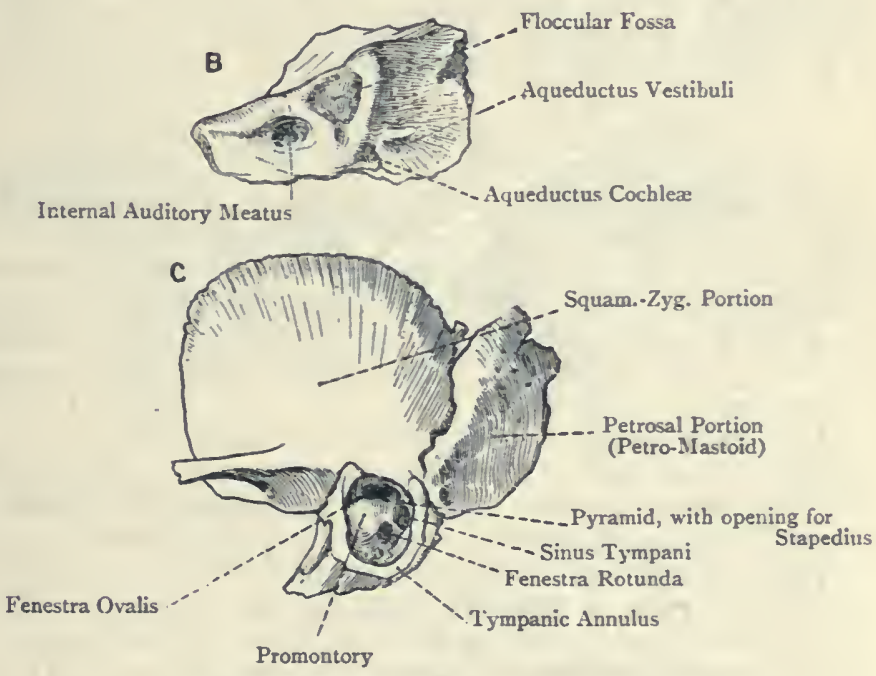

Fig. 52.-The Temporal Bone in Early Life.

A, Squamo-Zygomatic Portion and Tympanic Annulus; B, Petrosal Portion; C, The Bone at Birth.

canal, (2) the roof of the vestibule, (3) the roof of the cochlea, (4) the roof of the internal auditory meatus, (5) the upper part of the fenestra ovalis, and (6) the upper and inner part of the mastoid portion. The pterotic centre (BlandSutton) appears over the outer limb of the external semicircular canal, and from it are formed ( $\mathrm{I}$ ) the covering of the external semicircular canal, and (2) the tegmen tympani. The epiotic centre, sometimes double, appears in the region of the back part of the posterior semicircular canal, and from it the lower part of the mastoid is formed, as well as the investment of the posterior semicircular canal. At the period of birth (the tympanic having previously joined the squamosal) the temporal bone is composed of two parts-(I) a united 
squamo-zygomatic and tympanic, and (2) a petrosal, a plate of cartilage intervening, and these unite in the course of the first year. At birth the bone is of loose consistence, the mastoid portion is flat, the external auditory meatus is undeveloped, the tympanic annulus and membrana tympani are on a level with the exterior of the bone, the glenoid and jugular fossæ are shallow, the floccular fossa is conspicuous, and the hiatus Fallopii is an open groove. The tympanic plate now becomes formed in fibrous tissue, by the extension of osseous matter outwards from two tubercles on the anterior and posterior parts of the outer aspect of the tympanic annulus superiorly. As these tubercles grow, they meet and enclose an opening in the floor of the external auditory meatus, which usually becomes closed before the period of puberty, but it may persist throughout life as the foramen of Huschke. The mastoid antrum is present at birth, and is of large proportionate size, its outer wall being very thin. The mastoid process becomes developed in the course of the second year, and the antrum becomes relatively smaller, its outer wall at the same time becoming thicker. The mastoid cells do not appear until the approach of the period of puberty.

Styloid Process.-This process is developed separately from the upper end of the cartilage of the second visceral arch. It has two centres of ossification, one for the tympano-hyal or basal part appearing before birth, which soon joins the rest of the bone, and the other for the stylo-hyal appearing in the second year. The latter portion does not attain marked development until after puberty, and its union with the tympano-hyal usually takes place in adult life, but it sometimes persists in an independent condition.

\section{The Sphenold Bone.}

The sphenoid bone is so named from the wedge-like position which it occupies in the base of the skull, where it lies with its long axis placed transversely. It enters into the formation of the anterior, middle, and posterior, fossæ of the base, the temporal and nasal fossæ, and the orbits. It consists of a central portion or body, two great wings, two small wings, and two pterygoid processes.

The body presents six surfaces-superior, inferior, anterior, posterior, and two lateral, one at either side. Within the body are two large cavities, called the sphenoidal air sinuses, each of which opens on the anterior surface by a small circular aperture.

The superior surface presents at its centre a depression, called the sella turcica or pituitary fossa, for the pituitary body or hypophysis cerebri, and in the fœtus it is pierced by the superior opening of the cranio-pharyngeal canal. In front of the sella turcica is the olivary eminence, which indicates the place of junction of the presphenoid and postsphenoid portions, and anterior to this is a transverse furrow, called the optic groove, both of which support the optic commissure or chiasma. The groove leads at either side to the optic foramen, by which the optic nerve leaves the cranial cavity, and anteriorly it is limited by a transverse ridge, called the limbus sphenoidalis. In front of the limbus (border) is a smooth elevated platform, called the jugum sphenoidale, which is continuous laterally with the superior surface of the small wing, and jresents at either side the olfactory groove for the oltactory bulb. The anterior border of the jugum is projected in the middle line into the ethmoidal spine, which articulates with the posterior 
margin of the cribriform plate of the ethmoid. The sella turcica is bounded posteriorly by a prominent quadrilateral plate of bone, called the dorsum sellæ or dorsum ephippii (back of the saddle), which is directed forwards and upwards. The antero-inferior surface of this plate overhangs the sella turcica, and the posterosuperior surface, called the clivus (slope), is inclined downwards and backwards to become continuous with the basilar groove of the occipital. The clivus lodges the upper part of the pons Varolii and the basilar artery. The antero-superior border of the dorsum sellæ presents at either side the posterior clinoid process for a portion

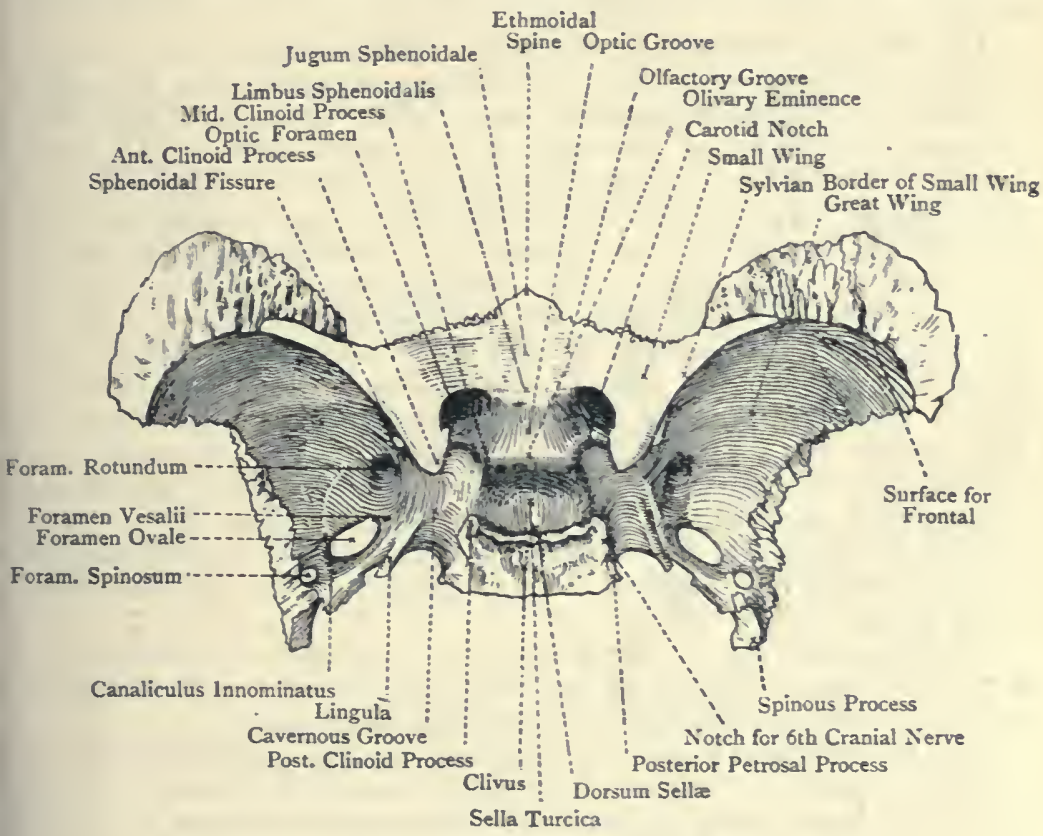

Fig. 53.-The Sphenoid Bone (Superior View).

of the tentorium cerebelli and the interclinoid ligament, which latter connects it with the anterior clinoid process, and is sometimes ossified. On each lateral border of the dorsum selle, a little below the posterior clinoid process, is a notch, which transmits the sixth cranial nerve. At the lower end of each lateral border there is a projection, called the posterior petrosal process, which articulates with the apex of the petrous portion of the temporal, and bounds internally the foramen lacerum medium.

The inferior surface of the body is limited at either side by the internal pterygoid plate of the pterygoid process. In the middle line it presents a vertical, antero-posterior ridge, called 
the rostrum, which is continuous with the sphenoidal crest on the anterior surface, and is received into the cleft between the alæ of the vomer. At either side of the rostrum there is a thin scale of bone projecting inwards. for a short distance from the root of the internal pterygoid plate, called the vaginal process, which articulates with the ala on the upper border of the vomer, and with it covers the greater part of the inferior surface of the body at either side of the middle line. On the inferior surface of this process there is a groove, which is converted by the sphenoidal process of the palate bone into a canal, called the pterygo-palatine canal, for the passage of the pharyngeal nerve and pterygo-palatine artery.

The anterior surface presents in the middle line a vertical ridge, continuous above with the ethmoidal spine and below with the rostrum, called the sphenoidal crest, which articulates with the perpendicular plate of the ethmoid and forms part of the nasal septum. On either side of this crest the surface is divided into two parts, outer and inner. The outer part is rough, and articulates with the back part of the lateral mass of the ethmoid and the orbital process of the palate bone. The inner part presents the opening of the sphenoidal air sinus of its own side, with the margins of which the sphenoidal turbinate or spongy bone articulates. When this bone is in position the opening of the sinus is small and circular, and is placed superiorly, but when the bone has been removed the opening is of large size and irregular outline. It communicates anteriorly with the spheno-ethmoidal recess of the nasal fossa above and behind the superior meatus. The part of the anterior surface which presents the opening of the sphenoidal air sinus enters into the formation of the roof of the corresponding nasal fossa.

The posterior surface is rough and truncated. It articulates with the basilar process of the occipital by synchondrosis up to the twentieth year, after which ankylosis takes place.

The lateral surface gives attachment to the great wing and a portion of the small wing. Anteriorly, beneath the small wing, it forms the inner boundary of the sphenoidal fissure and the back part of the inner wall of the orbit. Above the attachment of the great wing it presents a winding groove, called the cavernous or carotid groove, which contains the cavernous venous sinus and the internal carotid artery. The direction of this groove is from behind forwards, and its deepest part is placed posteriorly, where it is bounded internally by the posterior petrosal process, and externally by the lingula sphenoidalis or anterior petrosal process. This latter process is a sliarp scale of bone which projects backwards in the angle between the great wing and body.

The small or orbital wings (orbito-sphenoids) extend almost horizontally outwards on a level with the anterior part of the upper surface of the body. Each arises by two roots-an upper, which is expanded and compressed from above downwards, 
and is on a level with the anterior part of the upper surface of the body; and a lower, slender and compressed from before backwards, which arises from the anterior part of the side of the body. The wing is triangular and flattened from above downwards. The superior surface, smooth and somewhat concave, forms the back part of the anterior cranial fossa. The inferior surface overhangs the sphenoidal fissure, and forms the back part of the roof of the orbit. Externally the wing ends in a slender, pointed extremity, which lies very near the great wing, but does not as a rule touch

\section{Opening of Sphenoidal Sinus Ethmoidal Spine}

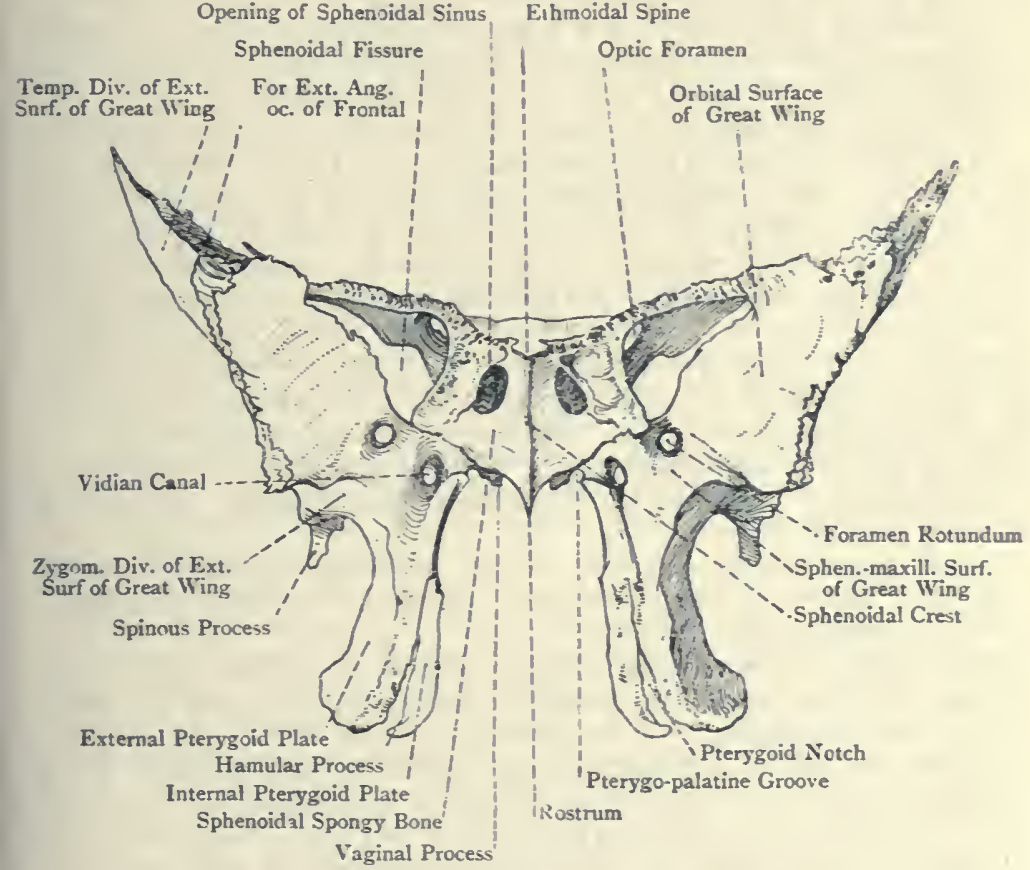

Fig. 54--The Sphenoid Bone (ANterior View).

it, though it may do so. The anterior border is thin and serrated for the orbital plate of the frontal. The posterior border, smooth, thick, and round, corresponds with the Sylvian fissure of the cerebrum, from which circumstance it is known as the Sylvian borde: It forms at either side the line of demarcation between the anterior and middle cranial fossæ, and terminates internally in the anterior clinoid process for a portion of the tentorium cerebelli and the interclinoid ligament.

Between the anterior clinoid process and the side of the olivary eminence is the semicircular carotid notch, which is the anterior 
termination of the carotid groove, and lodges the internal carotid artery.

On either side of the body, close to the inner side of the anterior extremity of the carotid groove and posterior to the carotid notch, opposite the anterior clinoid process, there is usually a small tubercle, called the middle clinoid process. - It is connected with the anterior clinoid process by the carotico-clinoid ligament, which bridges over the carotid notch. When this ligament undergoes ossification a carotico-clinoid foramen is formed, through which the internal carotid artery ascends after leaving the carotid groove.

In front of the carotid notch, between the upper and lower roots of the small wing, there is a circular aperture, called the optic foramen, which leads forwards and outwards into the orbit, and transmits the optic nerve and the ophthalmic artery.

The great or temporal wings (alisphenoids) extend outwards, upwards, and forwards from the sides of the body. The posterior part of each projects backwards, and ends in a pointed extremity, which is received within the petro-squamous angle of the temporal bone. From this extremity a sharp projection extends downwards for a short distance, called the spinous process or alar spine, which presents a groove on its inner aspect for the chorda tympani nerve. Anterior to this groove and encroaching on the posterior border of the great wing, is another groove for the cartilaginous part of the Eustachian tube. The spinous process gives attachment to (I) the spheno-mandibular ligament, (2) some fibres of the tensor palati, and (3) the anterior ligament of the malleus, or band of Meckel.

Each great wing presents three surfaces-superior, anterointernal, and external; and four borders-posterior, external, anterior, and internal.

The superior or cerebral surface, which at its front part rises almost vertically upwards, is concave, and enters into the formation of the lateral division of the middle cranial fossa. It supports the temporo-sphenoidal lobe of the cerebrum, and presents a few digitate impressions, whilst externally it is grooved for a branch of the middle meningeal artery. This surface presents several important foramina. At the anterior part of its attachment to the side of the body, just below the inner end of the sphenoidal fissure, is the foramen rotundum, which is directed from behind forwards and transmits the superior maxillary division of the fifth cranial nerve. A little behind and external to this foramen is the foramen ovale, of large size and opening vertically down. wards, for the passage of the inferior maxillary division and the motor root of the fifth cranial nerve, the small meningeal artery, an emissary vein from the cavernous sinus, and sometimes the small superficial petrosal nerve. Internal and anterior to the foramen ovale, between it and the lingula sphenoidalis, there is sometimes a small opening, called the foramen Vesalii, which leads to the scaphoid fossa on the outer side of the root of 
the internal pterygoid plate, or to the pterygoid fossa external to the scaphoid fossa. It transmits a small emissary vein from the cavernous sinus. Behind and external to the foramen ovale is the small circular foramen spinosum, close to the spinous process, which opens vertically downwards. It transmits the middle meningeal artery and a recurrent branch of the inferior maxillary nerve, and is sometimes incomplete posteriorly. Internal to this foramen, between it and the foramen ovale, there is sometimes a small opening, called the canaliculus innominatus, for the small superficial petrosal nerve.

The antero-internal surface is divisible into a large orbital portion and a small spheno-maxillary portion. The orbital division is quadrilateral, smooth, and slightly concave, and it forms the greater part of the outer wall of the orbit. The spheno-maxillary division is situated at the lower and inner part above the root of the pterygoid process. It is pierced by the foramen rotundum, and lies in the posterior wall of the spheno-maxillary fossa.

The external or iemporo-zygomatic surface is elongated from above downwards, and is continuous with the outer surface of the external pterygoid plate of the pterygoid process. Towards its lower part it is crossed by the infratemporal crest, which divides it into a large upper and a small lower portion. The upper or temporal division, which is directed outwards, forms part of the temporal fossa, and gives origin to fibres of the temporal muscle. The lower or zygomatic division looks downwards into the zygomatic fossa, and gives origin to the upper head of the external pterygoid muscle. At its lower and back part it presents the openings of the foramen ovale and foramen spinosum.

The posterior border extends from the spinous process to the body, passing in its course behind the foramen ovale. Over its inner two-thirds it bounds the foramen lacerum medium anteriorly, and over its outer third, where it becomes serrated, it articulates with the petrous portion of the temporal, the two forming a groove for the cartilaginous part of the. Eustachian tube. The external border separates the superior or cerebral from the external or temporozygomatic surface. It is serrated behind, where it is bevelled at the expense of the upper or inner plate, but in front it is squamous and bevelled at the expense of the outer plate. The entire border articulates with the squamous portion of the temporal. The anterior border or malar crest separates the orbital and temporal surfaces. Its direction is downwards and inwards, and it is sharp and irregular for the malar. The internal border is situated between the orbital and cerebral surfaces. Its direction is backwards and inwards, ar d it forms the lower boundary of the sphenoidal fissure. About its centre it presents a small spine, which gives origin to fibres of the lower head of the external rectus muscle of the eyeball. The great wing antero-superiorly becomes thick and expanded, and it here presents a rough, triangular, serrated surface for the frontal. At the outer end of this surface there is another small 
triangular, : errated impression, for the antero-inferior angle of the parietal.

The sphenoidal fissure, also called the foramen lacerum anterius or orbitale, is situated between the great and small wings. It is triangular, and its direction is inwards and downwards. It is bounded above by the small wing, below by the internal border of the great wing, and internally by the anterior part of the side of the body, whilst externally it is closed by the frontal, or, it may be, the meeting between the two wings. It leads from the middle cranial fossa to the orbit, and transmits the following structures: the third cranial nerve, the fourth, the three branches of the ophthalmic division of the fifth (namely, frontal, lachrymal, and nasal), and the sixth cranial nerves, the sympathetic root of the ciliary ganglion,

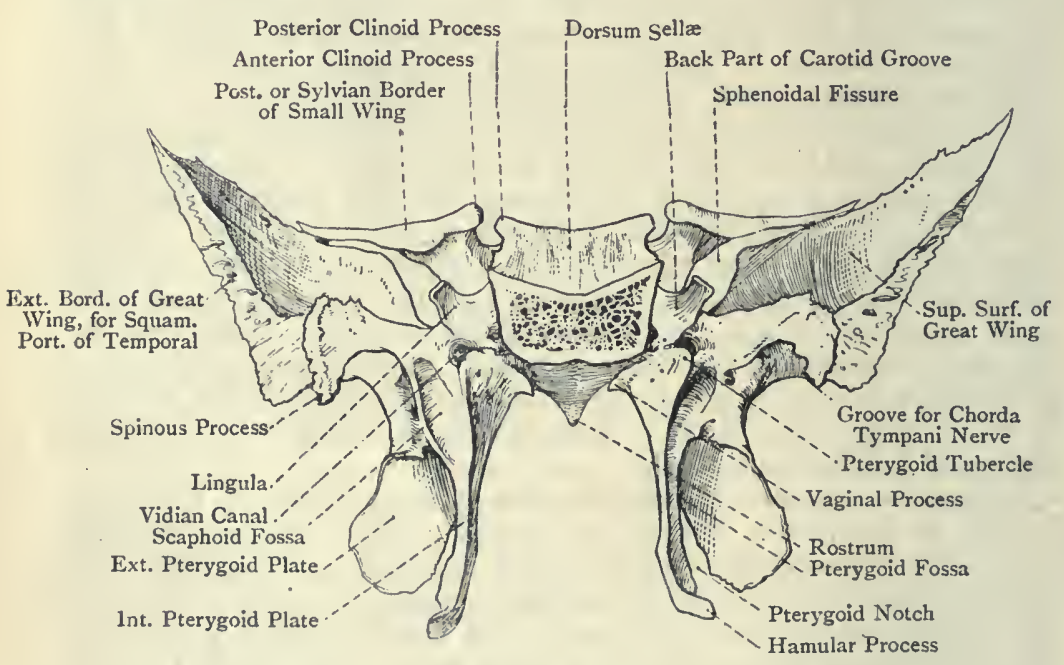

Fig. 55:-The Sphenoid Bone (Posterior View).

the superior and inferior ophthalmic veins, the orbital branch of the middle meningeal artery, and a portion of the dura mater to form the orbital periosteum.

The pterygoid processes project downwards from the junction of the body and great wings. Each is composed of two plates, external and internal, united in front to form a thick round border, except inferiorly, where they are separated by the pterygoid notch, which receives the pyramidal process or tuberosity of the palate bone. At the upper end of the anterior border a triangular surface opens out, which lies in the posterior wall of the spheno-maxillary fossa, and presents the anterior orifice of the Vidian or pterygoid canal. Posteriorly the two plates diverge, and enclose between them the pterygoid fossa, which contains the internal pterygoid and tensor or circumflexus palati muscles. 
The external pterygoid plate is broader and shorter than the internal, and is directed backwards and slightly outwards. Its outer surface looks into the zygomatic fossa, and gives origin to the lower head of the external pterygoid muscle. Its inner surface looks into the pterygoid fossa, and gives origin to the internal pterygoid muscle. The posterior border usually presents towards its upper part a sharp spine, from which the pterygo-spinous ligament extends backwards and outwards to the spinous process. This ligament sometimes becomes ossified, and a foramen is then formed, called the pterygo-spinous foramen, for the passage of muscular branches of the inferior maxillary nerve. "Sometimes there is another spine towards the lower end of this border for another pterygo-spinous ligament.

The internal pterygoid plate, narrower and longer than the external, is prolonged inferiorly into the hamular process, which is inclined outward, its outer and inferior aspects being smooth and grooved for the play of the tendon of the tensor or circum. flexus palati. Superiorly this plate is inflected as the vaginal process, which articulates with the ala of the vomer, and presents on its under surface a groove forming part of the pterygo-palatine canal already referred to. The outer surface of the internal pterygoid plate looks into the pterygoid fossa, and is related to the tensor or circumflexus palati. The inner surface forms the back part of the outer wall of the nasal fossa. The posterior border at its upper end presents the pterygoid tubercle, which has the posterior end of the Vidian canal above and external to it. Between this tubercle and the Vidian canal on the one hand, and the pterygoid fossa on the other, is the scaphoid fossa, which gives origin to the tensor or circumflexus palati. On the posterior border of the internal pterygoid plate, below the lower pointed end of the scaphoid fossa, is the Eustachian spine or processus tubarius, which supports the cartilage of the Eustachian tube. The lower third of the posterior border and the hamular process give origin to fibres of the superior constrictor muscle of the pharynx, and the hamular process also gives attachment to the pterygo-mandibular ligament. The anterior border articulates with the posterior border of the perpendicular plate of the palate bone.

The Vidian or pterygoid canal pierces the bone from before backwards at the junction of the internal pterygoid plate and body on either side. Its anterior orifice appears on the posterior wall of the spheno-maxillary fossa, below and internal to the anterior orifice of the foramen rotundum, and posteriorly it opens on the anterior wall of the foramen lacerum medium, above and external to the pterygoid tubercle. It gives passage to the Vidian nerve and artery.

Summary of Openings in the Sphenoid Bone.-(I) Sphenoidal fissure, between small and great wings ; (2) optic foramen, between the two roots of the small wing; and, in the great wing, (3) foramen rotundum ; (4) foramen ovale ; (5) foramen Vesalii (inconstant); (6) foramen spinosum; (7) canaliculus innominatus (inconstant); 
and (8) Vidian or pterygoid canal, the last-named being between the internal pterygoid plate and the body. All these openings are common to each side.

The sphenoidal air sinuses are situated within the body, and are two in number, right and left. They are separated from each other by a septum, which is usually slightly bent to the left side. The sinuses are-at least, after adult life-usually multilocular, and they may extend backwards so as to invade the basilar process of the occipital, especially in old age. Each sinus may even extend slightly into the attached portion of the great wing. They are lined with mucous membrane, which is continuous with that of the nasal fossæ, and each opens anteriorly by a small circular aperture into the spheno-ethmoidal recess above and behind the corresponding superior meatus.

The sphenoidal turbinate or spongy bones (sphenoidal turbinals or bones of Bertin) are situated on the anterior and inferior surfaces of the body of the sphenoid, of which they form a large part. In the adult they are blended with the sphenoid and adjacent parts of the ethmoid and palate bones, but in early life they are quite distinct. Each has the form of a three-sided, hollow pyramid, the apex of which is directed backwards and downwards to the front part of the vaginal process, whilst the base is in contact with the back part of the lateral mass of the ethmoid. The inferior surface looks into the posterior part of the roof of the nasal fossa, and it converts the spheno-palatine notch on the upper border of the perpendicular plate of the palate bone into a foramen. The external surface appears on the inner wall of the spheno-maxillary fossa, and a portion of it is sometimes seen on the inner wall of the orbit, behind the os planum of the ethmoid. The superior surface is in contact with the anterior and inferior surfaces of the front part of the body of the sphenoid. It is at the upper part of this surface, on either side of the middle line, where the openings of the sphenoidal air sinuses ultimately appear as small circular apertures. When the sphenoidal spongy bones are broken away these openings are of large size and irregular outline.

The blood-supply of the bone is derived from branches of the deep temporal arteries externally, the middle and small meningeal internally, and the Vidian, pterygo-palatine, and spheno-palatine branches of the internal maxillary, as these traverse their respective passages.

Articulations - The sphenoid articulates with fourteen bones, as follows: occipital, two temporals, two parietals, frontal, ethnloid, two sphenoidal turbinates, two malars, two palates, and vomer. It sometimes also articulates with the superior maxillæ.

Structure. - The body of the bone is excavated into two air sinuses,

Varieties.-(I) Middle clinoid process. (2) Carotico-clinoid foramen. (3) Ossification of interclinoid ligament between anterior and posterior clinoid processes. (4) The lateral margin of the dorsum sellæ may present a superior 
petrosal process for the attachment of the petro-sphenoidal ligament, which connects it with a projection sometimes present on the inner part of the superior border of the petrous portion of the temporal. This ligament, which is sometimes ossified, bridges over a foramen through which the inferior petrosal venous sinus and sixth cranial nerve pass. (5) Pterygo-spinous foramen. (6) Foramen ovale and foramen spinosum are sometimes incomplete. (7) Foramen Vesalii. (8) Canaliculus innominatus. (9) The cranio-pharyngeal canal may remain persistent, opening into the pituitary fossa.

Ossification.-The sphenoid is developed in cartilage, with the exception of the internal pterygoid plates, which are developed in fibrous tissue. ${ }^{*}$ The bone is originally divided into two parts-presphenoid, representing the part of the body in front of the olivary eminence, and the small wings; and postsphenoid, including the part of the body behind the olivary eminence, the great wings, and the pterygoid processes. The postsphenoid division is developed from lour pairs of centres. One pair appear in the eighth week of intra-uterine life, one at either side in the great wing between the foramen

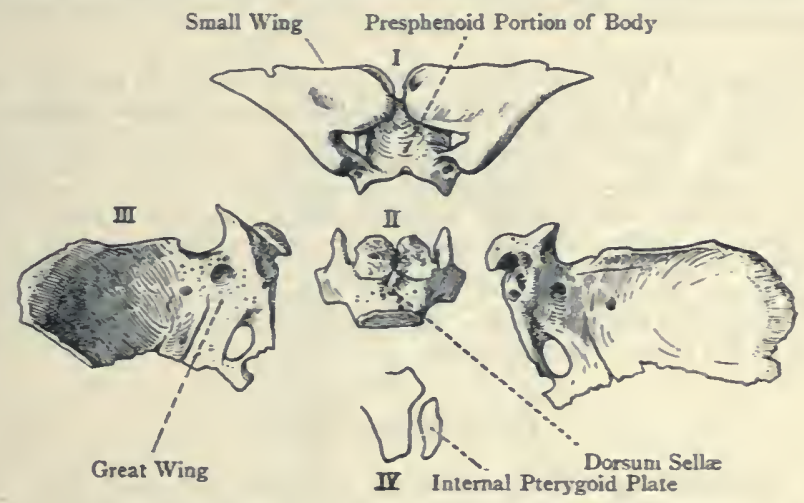

Fig. 56.-The Sphenotd Bone in Early Life.

I. Presphenoid Division; II, Postsphenoid Portion of Body ; III, Great Ving and Pterygoid Process; IV, Elements of Pterygoid Process.

rotundum and foramen ovale, and from this ossification extends outwards in to the great wing and downwards into the external pterygoid plate. Another pair appear about the same time in the sella turcica on either side of the cranio-pharyngeal canal, from which ossification extends around the canal. gradually constricting it, and finally leading to its closure. At this time another pair (sphenotics of Bland-Sutton) appear, one at either side, for the lingula. In the fourth month (ninth or tenth week, Fawcett) another pair of centres appear in fibrous tissue, one at either side, for the internal pterygoid plate, which nnites with the external pterygoid plate before the sixth month. The presphenoid division is developed from two pairs of centres. Two appear in the ninth week, one at either side, external to the optic foramen, for the small wing. Another pair appear in the eleventh week internal to the optic foramina for the presphenoid portion of the body. The latter pair soon unite with each other, and also with those for the small wings. The presphenoid division, bearing the small wings, joins the postsphenoid division shortly before birth in the region of the olivary eminence. At birth the place of junction is indicated by a wide depression on the under

* According to recent observations made by Fawcett the external pterygoid plates are also developed in fibrous tissue. 
aspect of that eminence, which may even extend through it and give rise to a small foramen on its upper surface.

At birth the bone is composed of three parts-a central, representing the presphenoid and postsphenoid portions of the body, the former bearing the small wings; and two lateral, each of which represents a great wing bearing a pterygoid process. In the first year the lingula joins the great wing, and the wing and body unite. About the same time the small wings come together and blend over the anterior part of the upper surface of the presphenoid portion of the body, where they give rise to a smooth, elevated, flat platform, called the jugum sphenoidale.

In fotal life a canal, called the cranio-pharyngeal canal, leads downwards from the sella turcica into the body, and contains a process of the dura mater. This canal is the remains of a cleft originally present in the base of the skull, through which a diverticulum of the buccal epiblast, known as the pouch of Rathke, originally passed upwards to form the anterior lobe of the pituitary body.

The sphenoidal air sinuses do not appear until after the seventh year.

In some animals the presphenoid and postsphenoid portions remain permanently separate, and the internal pterygoid plates form the pterygoid bones.

The sphenoidal turbinate bones commence to ossify in the fifth month of intra-uterine life. At birth each partially envelops a small extension of the nasal mucous membrane, and by the third year it has surrounded it in the form of a bony capsule, except anteriorly, where an opening, called the sphenoidal foramen, is left. Subsequently a portion of this capsule becomes absorbed, and its place is taken by the presphenoid, which latter, after the seventh year, is gradually invaded by the original extension of the nasal mucous membrane. The sphenoidal spongy bones become ankylosed to the ethmoid about the fourth year, and are sometimes regarded as belonging to that bone. By the twelfth year they have become united to the sphenoid, and also to the palate bones.

\section{The Ethmoid Bone.}

The ethmoid bone is situated at the anterior part of the base of the skull, where it lies in the middle line in front of the sphenoid. A portion of it occupies the ethmoidal notch between the orbital plates of the frontal, whence the greater part of the bone projects downwards to take part in the formation of the orbits and nasal fossæ. The only portions of the bone visible in the interior of the base are the cribriform plate and crista galli. It is irregularly cubical, its long axis being directed from before backwards, and it is remarkable for its lightness, which is due to the great number of enclosed air cells, these being surrounded by very thin plates of bone. It is composed of four parts, namely, a cribriform plate, a perpendicular plate, and two lateral masses.

The cribriform plate connects the upper borders of the lateral masses, and enters into the formation of the middle division of the anterior cranial fossa, where it occupies the ethmoidal notch of the frontal bone. In the middle line anteriorly it presents an upward extension of the perpendicular plate, called the crista galli. This is a stout, triangular, laterally-compressed process, which presents a smooth, sloping posterior border, for the falx cerebri. The anterior border, short and vertical, is somewhat narrow above, but soon expands into two alar processes, for the frontal bone, 
and it here sometimes completes the foramen cæcum. The posterior border is prolonged backwards as a median ridge, and on either side of this ridge and the crista galli is the olfactory groove, which lodges the olfactory tract and bulb. Each half of the cribriform plate, which lies in the roof of the corresponding nasal fossa, is pierced by foramina for the filaments of the olfactory bulb. The foramina in each half are arranged in three sets, as follows-a middle set, which are simple perforations, and an internal and external set, which lead into small canals. These canals descend on the perpendicular plate and inner surface of the lateral mass respectively, branching and opening out as they descend. All the foramina lead to the upper part of the corresponding nasal fossa. At the anterior and inner part of each half of the cribriform plate, close to the side of the crista galli, near its anterior border,

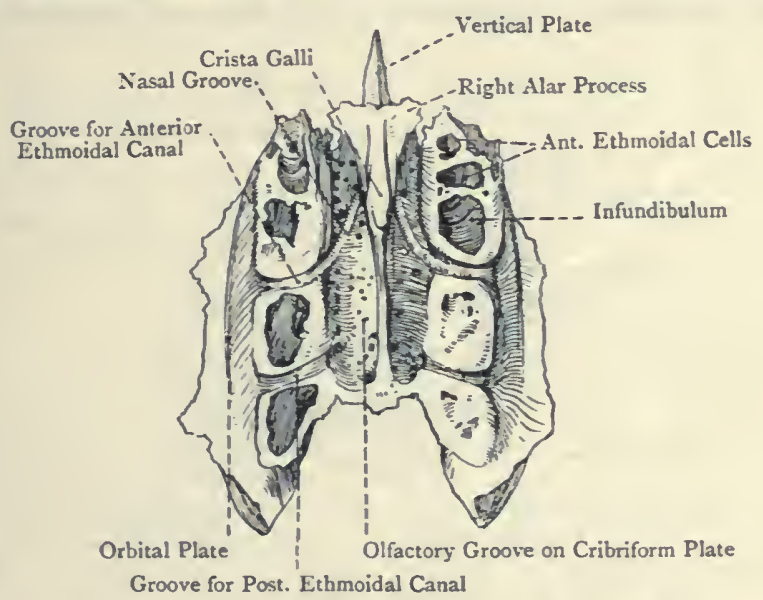

Fig. 57.-The Ethmoid Bone (Superior View).

there is an antero-posterior fissure, called the nasal slit, which transmits the nasal branch of the ophthalmic nerve and nasal branch of the anterior ethmoidal artery to the nasal fossa. . Leading backwards and outwards from this slit to the anterior, ethmoidal groove on the upper border of the lateral mass is the rasal groove, also for the nasal nerve. The posterior border of the cribriform plate articulatt.....un the ethmoidal spine of the sinenoid.

The perpendicular plate (mesecimuviä; sutends downwards from the cribriform plate in the middle line. It lies between the lateral masses, where it forms about the upper third of the nasal septum, and it is usually inclined more to one side than the other. It is very thin and irregularly quadrilateral. The superior border projects above the cribriform plate and forms the crista galli. The inferior border articulates, in front, with the septal cartilage of the nose, and behind, with the alæ of the vomer in the intervening cleft, 
aspect of that eminence, which may even extend through it and give rise to a small foramen on its upper surface.

At birth the bone is composed of three parts-a central, representing the presphenoid and postsphenoid portions of the body, the former bearing the small wings; and two lateral, each of which represents a great wing bearing a pterygoid process. In the first year the lingula joins the great wing, and the wing and body unite. About the same time the small wings come together and blend over the anterior part of the upper surface of the presphenoid portion of the body, where they give rise to a smooth, elevated, flat platform, called the jugum sphenoidale.

In fœtal life a canal, called the cranio-pharyngeal canal, leads downwards from the sella turcica into the body, and contains a process of the dura mater. This canal is the remains of a cleft originally present in the base of the skull, through which a diverticulum of the buccal epiblast, known as the pouch of Rathke, originally passed upwards to form the anterior lobe of the pituitary body.

The sphenoidal air sinuses do not appear until after the seventh year.

In some animals the presphenoid and postsphenoid portions remain permanently separate, and the internal pterygoid plates form the pterygoid bones.

The sphenoidal turbinate bones commence to ossify in the fifth month of intra-uterine life. At birth each partially envelops a small extension of the nasal mucous membrane, and by the third year it has surrounded it in the form of a bony capsule, except anteriorly, where an opening, called the sphenoidal foramen, is left. Subsequently a portion of this capsule becomes absorbed, and its place is taken by the presphenoid, which latter, after the seventh year, is gradually invaded by the original extension of the nasal mucous membrane. The sphenoidal spongy bones become ankylosed to the ethmoid about the fourth year, and are sometimes regarded as belonging to that bone. By the twelfth year they have become united to the sphenoid, and also to the palate bones.

\section{The Ethmoid Bone.}

The ethmoid bone is situated at the anterior part of the base of the skull, where it lies in the middle line in front of the sphenoid. A portion of it occupies the ethmoidal notch between the orbital plates of the frontal, whence the greater part of the bone projects downwards to take part in the formation of the orbits and nasal fossæ. The only portions of the bone visible in It 2 interior of the base are the cribriform plate and crista galli. of theirregularly cubical, its long axis being directed from before inner mals, and it is remarkable for its lightness, which is due to behind thrumber of enclosed air cells, these being surrounded by the postero-intes of bone. It is composed of four parts, namrui, it may be, willate, a perpendicular plate, and two $1,+. .$. At the lower part of $\mathrm{rm}$ plate connests, tho. $y_{w}$ the os planum, there is a deep channel, etros.: them before backwards, which forms the middle meatus of the nose, and is limited below by the inferior rolled border of the inferior turbinate process. This groove turns upwards in front, under cover of the anterior part of the inferior turbinate process, and is continued into the infundibulum which communicates with the frontal sinus of the same side. The anterior ethmoidal cells open into the ascending part of the middle meatus, whilst the middle ethmoidal cells and the antrum of Highmore 
and it here sometimes completes the foramen cæcum. The posterior border is prolonged backwards as a median ridge, and on either side of this ridge and the crista galli is the olfactory groove, which lodges the olfactory tract and bulb. Each half of the cribriform plate, which lies in the roof of the corresponding nasal fossa, is pierced by foramina for the filaments of the olfactory bulb. The foramina in each half are arranged in three sets, as follows-a middle set, which are simple perforations, and an internal and external set, which lead into small canals. These canals descend on the perpendicular plate and inner surface of the lateral mass respectively, branching and opening out as they descend. All the foramina lead to the upper part of the corresponding nasal fossa. At the anterior and inner part of each half of the cribriform plate, close to the side of the crista galli, near its anterior border,

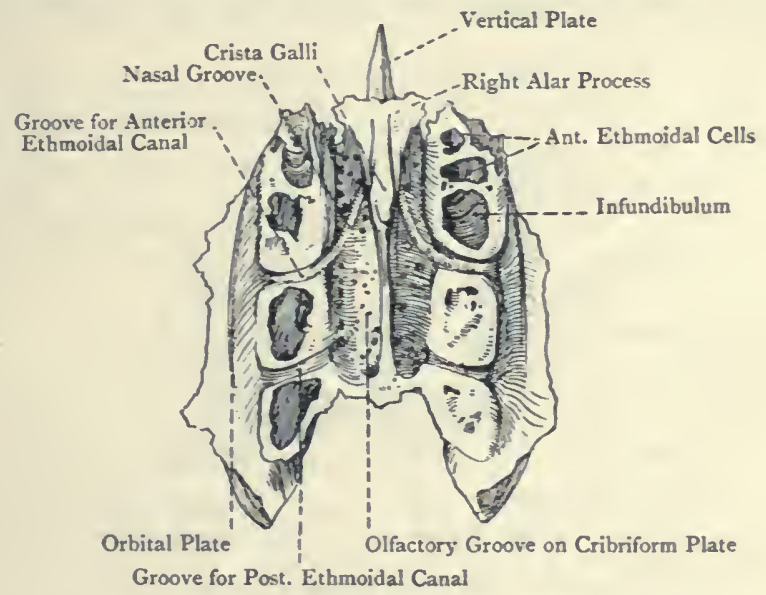

Fig. 57.-The Ethmoid BoNe (Superior View).

there is an antero-posterior fissure, called the nasal slit, whicl transmits the nasal branch of the oph thalmic nerve and nasal bratus. of the anterior ethmoidal artery to the nasal fossa. .ra, and backwards and outwards from this slit to the anterior, rroove on the upper border of the lateral mass is the r margin of the oiv: the nasal nerve. The posterior border of $t$ depressions upon it, and conveis thic ethmoidal spine of the sithese depressions this border presents two transveise vifends do bout half an inch apart, which, with corresponding grooves on the orbital plate of the frontal, form the anterior and posterior ethmoidal or internal orbital canals. These open upon the inner wall of the orbit, and the anterior transmits the anterior ethmoidal vessels and the nasal nerve, whilst the posierior gives passage to the posterior ethmoidal vessels and the spheno-ethmoidal nerve. The inferior border, which is free on the outer wall of the nasal 
fossa, is formed by the lower border of the inferior turbinate process. Anteriorly it articulates with the superior turbinate crest of the superior maxilla, and posteriorly with the ethmoidal or superior turbinate crest of the palate bone. The anterior border, like the superior, presents depressions, which form air cells when the lachrymal and nasal process of the superior maxilla are in position. This border projects slightly in advance of the front of the os planum, and from the lower part of this projecting portion there springs the uncinate process. This is a long, thin, curved plate which extends downwards, backwards, and slightly outwards into the anterior part of the middle meatus. In its course it crosses the opening of the antrum of Highmore in the superior maxilla, and thus forms part of the inner wall of that air sinus. The lower border of the process presents two spur-like projections, between which the border is markedly concave. The posterior terminal spur articulates with the ethmoidal process of the inferior turbinate bone. The posterior border of the lateral mass presents a few depressions, closed by the sphenoidal spongy bone and orbital process of the palate bone, which latter process becomes ankylosed with it about the fourth year.

The ethmoidal cells are contained within each lateral mass, and are lined with mucous membrane, which is continuous with that of the nose. They are arranged in three sets-anterior, m:ddle, and posterior. The anterior ethmoidal cells, along with the frontal sinus of the same side, open by a common passage, already described as the infundibulum, into the ascending front part of the middle meatus; the middle ethmoidal cells open into the horizontal part of the middle meatus; and the posterior ethmoidal cells open into the superior meatus.

The bone receives its blood-supply from the anterior and posterior ethmoidal branches of the ophthalmic, and the sphenopalatine branch of the internal maxillary.

Articulations.-The ethmoid articulates with fifteen bones, as follows: (I) frontal (nasal spine and orbital plates); (2) sphenoid (ethmoidal spine and sphenoidal crest); (3) two sphenoidal spongy bones; (4) two nasal bones (nasal crest); (5) vomer (cleft between alæ) ; (6) two palate bones (ethmoidal or superior turbinate crests and orbital processes) ; (7) two lachrymals (upper part of internal surface) ; (8) two superior maxillæ (nasal processes, orbital plates, and opening of each antrum); and (9) two inferior turbinates (ethmoidal processes).

Structure.-The lateral masses are excavated into many thinwalled air cells, and the crista galli contains a small amount of cancellated tissue.

Ossification. - The ethmoid is developed in cartilage from three centres. Two of these appear in the fifth month of intra uterine life, one in each os planum, from which ossification extends in to the superior and inferior turbinate processes. At birth the lateral masses are ossified, but the perpendicular plate and crista galli are cartilaginous. In the first year a centre appears at the base of the crista galli, and from this ossification extends upwards into 
that process, downwards into the perpendicular plate, and outwards into the cribriform plate, into which latter osseous matter also extends inwards from each lateral mass. The three original parts unite about the fitth year. The osseous ethmoidal cells usually make their appearance about the third year.

\section{The Superior Maxillary Bones.}

The superior maxillary bone forms, with its fellow, a large part of the face, and, besides supporting the upper teeth of its own side, it enters into the formation of the orbit, nasal fossa, and hard palate. It is composed of a central portion or body, and four processes-nasal, malar, alveolar, and palatal.

The body is excavated into a large cavity, called the antrum of Highmore or maxillary air sinus, and it presents four surfacesantero-external, postero-external, superior, and internal.

The antero-external or facial surface is limited above by the infra-orbital border, below by the alveolar border, internally by the mesial border, presenting the nasal notch, and externally by the malar process and a ridge of bone extending downwards from it to the first molar alveolus. It presents inferiorly five ridges, coinciding with the roots of the incisor, canine, and bicuspid teeth, of which that of the canine is conspicuous, and is called the canine ridge. Internal to this ridge is the incisor or myrtiform fossa (like a myrtle-berry), which gives origin internally to the depressor alæ nasi, and externally to a deep slip of the orbicularis oris, whilst above, and external to, the latter the compressor naris arises. External to the canine ridge is the canine fossa, which, at its upper part, gives origin to the levator anguli oris, the bone being here thin and translucent in front of the antrum. Above the canine fossa, near the infra-orbital border, is the infra-orbital foramen, which is the outlet of the infra-orbital canal, and transmits the infra-orbital nerve and vessels. Immediately above this foramen the levatc: labii superioris arises. The mesial border of the facial surface presents the deep nasal notch, at the lower and inner part of which is a sharp projection, forming, with its fellow, the anterior nasal spine, below which the border is vertical.

The postero-external or zygomatic surface is situated behind the malar process and the ridge connecting that process with the first molar alveolus. Superiorly it is limited by the posterior border of the orbital surface, inferiorly by the molar portion of the alveolar border, and posteriorly by the posterior border of the bone. It looks into the zygomatic and spheno-maxillary fossæ, and its outline is convex. Towards the centre it presents the openings of two or three posterior dental canals, which lead to the molar. alveoli, and transmit branches of the posterior superior dental nerve and artery. At the lower and back part this surface gives rise to the tuberosity, which lies above and behind the last molar tooth. This tuberosity articulates with the pyramidal process of the palate bone, and gives origin to some fibres of the internal pterygoid muscle. 
The superior or orbital surface is triangular, smooth, and slightly concave, and it forms the greater part of the floor of the orbit. This portion of the bone is known as the orbital plate. It presents the infra-orbital groove, which, commencing at the posterior border in a notch, ultimately becomes converted into the infra-orbital canal. This canal transmits the infra-orbital nerve and vessels. From its posterior part the middle dental canal, for the middle superior dental nerve and artery, passes downwards and forwards

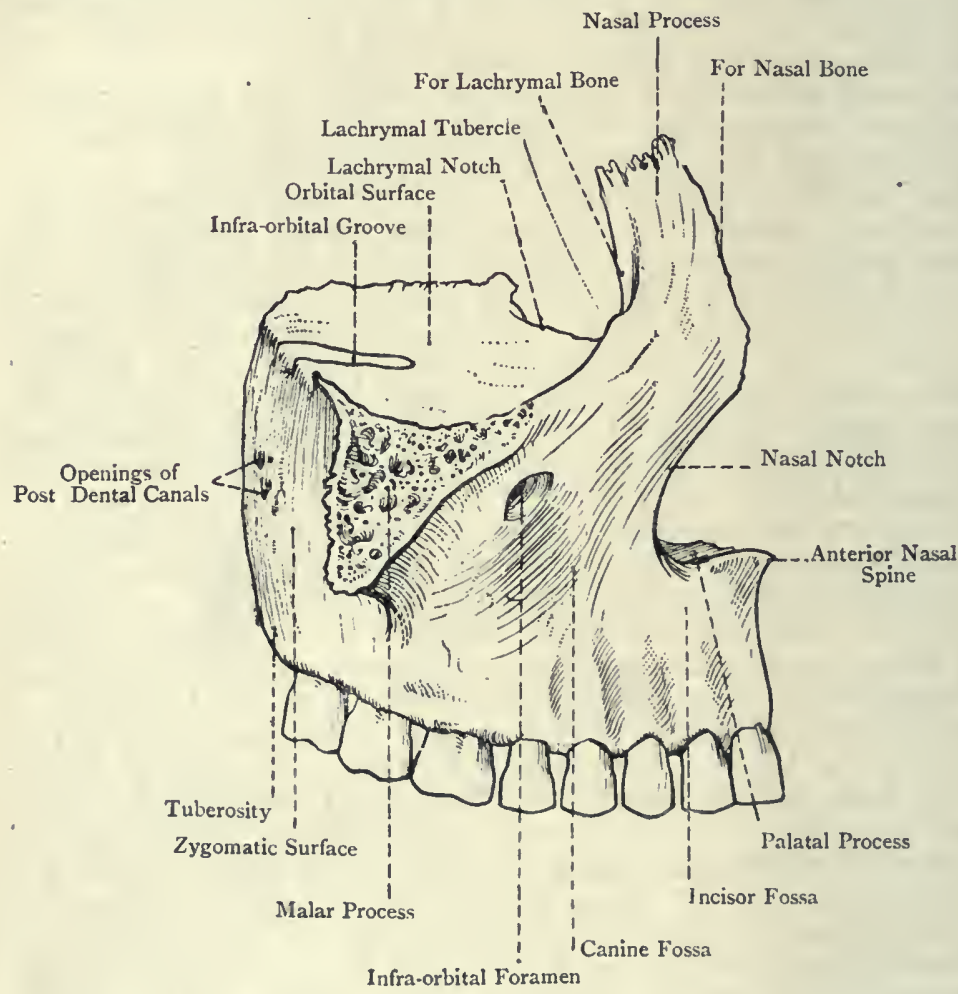

Fig. 60.-The Right Superior Maxillary Bone (External View).

to the bicuspid alveoli, lying at first in the postero-external wall of the antrum, and subsequently in the antero-external wall. This canal is often for the most part a groove. The anterior dental canal, for the anterior superior dental nerve and artery, descends in a branching manner from the anterior part of the infra-orbital canal to the incisor and canine alveoli, lying in the antero-external wall of the antrum. At the anterior and inner part of the orbital plate, external to the lachrymal groove, there is a slight depression which gives origin to the inferior oblique muscle of the eyeball. 
The borders of the orbital surface are anterior, posterior, and internal. The anterior border coincides with the infra-orbital border. The posterior border, which has an inclination outwards, forms the anterior boundary of the spheno-maxillary fissure, and presents a notch representing the commencement of the infraorbital groove. The internal border, antero-posterior in direction, presents, behind the nasal process, the lachrymal notch for the lachrymal bone, and behind this it articulates, from before backwards, with the lower border of the os planum of the ethmoid and the orbital process of the palate bone. This border presents a few depressions which close in ethmoidal cells.

The internal or nasal surface forms part of the outer wall of the nasal fossa. It is limited in front by the mesial border of the bone, behind by the posterior border, above by the internal border of the orbital surface, and below for the most part by the palatal process. It presents the opening of the antrum of Highmore, in front of which is the deep lachrymal groove, directed downwards, outwards, and backwards, and, after a course of about $\frac{1}{2}$ inch, opening into the front part of the inferior meatus of the nose. This groove is converted posteriorly and internally into the lachrymal canal by the lachrymal and inferior turbinate bones, and it transmits the nasal duct. In front of the lachrymal groove is a slightly oblique ridge, called the inferior turbinate crest, for articulation with the inferior turbinate bone, and below this is a smooth concave surface which forms the anterior part of the inferior meatus: Above the crest is the commencement of another smooth surface, which extends upwards on to the inner aspect of the nasal process, and forms the outer wall of the atrium of the middle meatus. Behind the opening of the antrum the internal surface articulates with the perpendicular plate of the palate bone, and it presents, from the centre downwards, a groove, directed downwards and forwards, which, with the palate bone, forms the posterior palatine canal for the great or anterior palatine nerve, and the superior or descending palatine artery. Above the opening of the antrum are a few depressions on the internal border of the orbital surface, forming ethmoidal cells.

The nasal or frontal process ascends vertically from the mesial part of the facial surface above the nasal notch. It is somewhat triangular, and presents two surfaces and three borders. The external surface is continuous with the facial surface of the body, and gives attachment to the orbicularis palpebrarum, tendo oculi, and levator labii superioris alæque nasi. The internal surface forms part of the outer wall of the nasal fossa, and, at its back part superiorly, it presents one or two depressions, completing cells on the anterior border of the lateral mass of the ethmoid. The surface is crossed obliquely backwards and upwards by a ridge, called the agger nasi (mound) or superior turbinate crest, which represents the naso-turbinal of most mammals (Schwalbe). This crest bounds superiorly the atrium of the middle meatus, and 
articulates posteriorly with the anterior extremity of the inferior turbinate process of the ethmoid. Above the agger nasi there is a groove, called the sulcus olfactorius. The superior border is short, thick, and serrated for the frontal. The anterior border is sharp and articulates with the nasal. The posterior border is thick, and in. its lower part presents a continuation of the lachrymal groove which here lodges the lachrymal sac. The lips of this part of the groove are sharp, the inner articulating with the lachrymal, and the outer, which is crescentic, being continuous

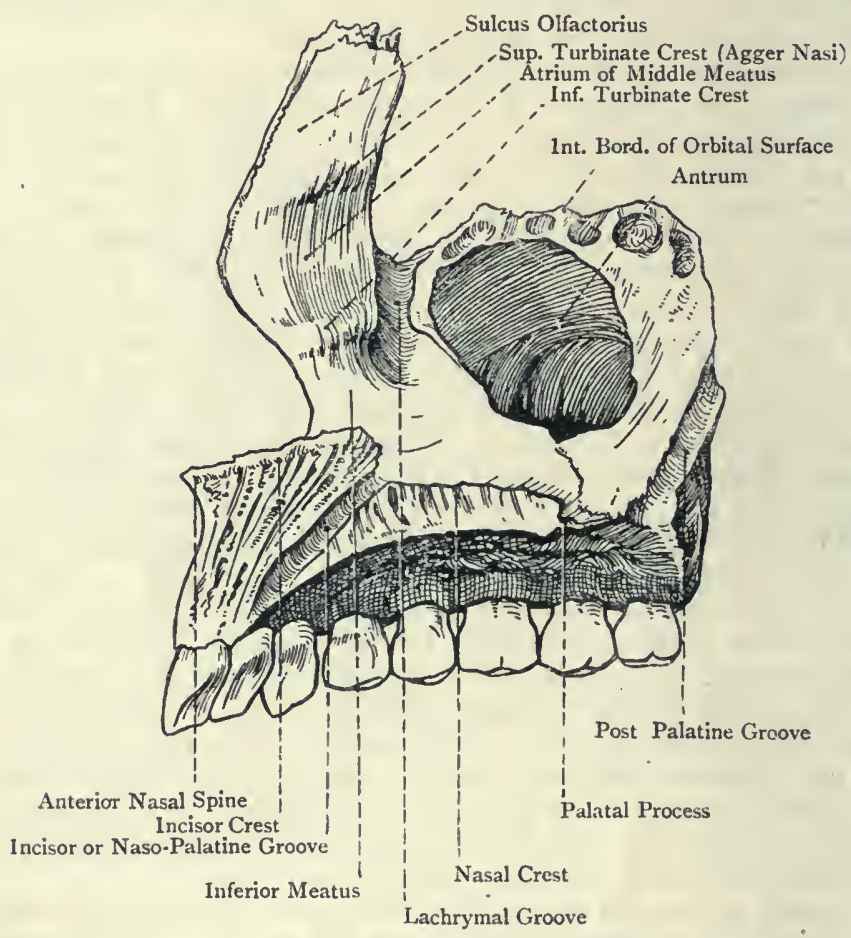

Fig. 6i.-The Right Superior Maxillary Bone (Internal View).

with the infra-orbital margin, at which point there is a projection, called the lachrymal tubercle.

The malar process is stout and triangular. Its anterior surface is continuous with the facial surface of the body, and its posterior with the zygomatic surface, whilst the superior surface is rough and slightly serrated for the malar.

The alveolar process forms the dependent part of the bone, and is thick and curved, being convex externally and concave internally. The outer plate is known as the labial plate; and the inner as the lingual. The two plates are widely separated, and 
the intervening space is partitioned off into alveoli or sockets by septa which pass between the two plates. The number of alveoli in the adult bone is as a rule eight, and they gradually narrow towards their upper or deep ends, where they are perforated by foramina for the nerves and arteries of the teeth. They lodge the roots of the teeth, which, in order from the middle line outwards and backwards, are as follows: central incisor, lateral incisor, canine, first bicuspid, second bicuspid, and first, second, and third molars. The alveoli correspond in shape with the roots of the teeth, the canine being the deepest. The outer surface of the alveolar border, over the extent of the three molar sockets, gives origin to fibres of the buccinator.

The palatal process is situated on the internal surface of the body, from which it projects horizontally inwards, and, with its fellow, it forms three-fourths of the hard palate. It is quadrilateral, and presents two surfaces and four borders. The superior surface forms three-fourths of the floor of the nasal fossa, and is smooth, concave, and covered in the recent state by the nasal mucous membrane. The inferior surface forms a part of the hard palate, and is rough, arched, and covered in the recent state by the buccal mucous membrane. It presents several depressions for the palatal mucous glands, and is perforated by several nutrient foramina. Laterally it is marked by a groove, directed from behind forwards, for the nerve and artery which reach the hard palate through the posterior palatine canal. The posterior border stops short of the back part of the alveolar border, and is short and serrated for the horizontal plate of the palate bone. The anterior border, superiorly, forms the lower part of the nasal notch. The external border is attached to the body. The internal or mesial border is faintly serrated, and articulates with its fellow. At the place of meeting it is elevated into a ridge, forming, with that of its fellow, the nasal crest, which is grooved to receive the lower border of the vomer. This mesial ridge becomes prominent in front, where it forms the incisor erest, which is projected to constitute, with its fellow, the anterior nasal spine. It supports the septal nasal cartilage, and the anterior extremity of the vomer lies behind it.

Close to the outer side of the incisor crest the palatal process is pierced by an opening leading into a canal, which is bounded internally by a thin plate of bone, and descends to the front part of the hard palate, being ultimately converted into a groove, due to its inner thin wall becoming deficient. This passage is variously known as the incisor or naso-palatine canal, or canal of Stensen (Steno), and the two canals, right and left, in the articulated condition form inferiorly a large orifice, called the anterior palatine fossa. This fossa, which is somewhat diamondshaped, is situated in the middle line of the hard palate, behind the central incisor teeth. On looking into it from below four foramina are seen, two of which are placed in the middle line, 
where they lie in the intermaxillary suture. These are known as the foramina of Scarpa, and they transmit the naso-palatine nerves, the left nerve passing through the anterior, which usually communicates with the left nasal fossa, and the right through the posterior, which usually communicates with the right nasal fossa. The other two foramina are situated one at either side, and are known as the foramina of Stensen, and the canal into which each leads opens superiorly on the floor of the corresponding nasal fossa, close to the outer side of the incisor crest. Each of Stensen's canals transmits a branch of the superior or descending palatine artery from the anterior palatine fossa to the nasal fossa. The inner wall of Stensen's canal, on each side, represents the mesial palatal process of the premaxilla or intermaxillary bone, and also a portion developed from the prepalatine centre. The canals of Stensen correspond to the incisor foramina of many animalse.g., the ruminantia, in which they are of large size, and each opens independently on the front part of the hard palate as a large aperture, there being no anterior palatine fossa. In such animals each incisor foramen leads up to the orifice of Jacobson's organ, which is a supplementary organ of smell. In man the canals of Stensen are the remains of a communication which existed in early fotal life between the nasal and buccal cavities.

Passing transversely outwards from the anterior palatine fossa at its back part to the interval between the lateral incisor and canine teeth a suture is always present in early life, and may persist in the adult, which indicates the line of junction of the maxillary portion proper and the premaxilla or intermaxillary bone, the latter representing the part which bears the central and lateral incisor teeth. This intermaxillary portion forms an independent bone in many animals.

The antrum of Highmore or maxillary air sinus is situated within the body of the bone, and is of large size, its capacity in health being equal to about 2 drachms. It has the shape of a four-sided pyramid, and is lined with mucous membrane continuous with that of the nasal fossa. The apex corresponds to the malar process, and the base represents the internal or nasal aspect. The superior wall or roof is formed by the orbital plate. The inferior wall or floor is formed by that portion of the alveolar border which contains the molar and second bicuspid alveoli, and, in some cases, the first bicuspid alveolus also. It is often very irregular, due tc projections of the upper ends of the alveoli, and in some cases the root of the first molar, and, it may be, that of the second, projects into the antral cavity. The antero-external wall is formed by the facial surface, and is thin and translucent over the region of the canine fossa. It contains the anterior, and the lower part of the middle, dental canals. The postero-external wall is formed by the zygomatic surface, and it contains the upper part of the middle dental canal. The opening of the antrum, which is large and irregular, is situated on the base or nasal aspect. In the articu- 
lated skull its size is considerably diminished by the perpendicular plate of the palate bone behind, the maxillary process of the inferior turbinate below, and above this by the uncinate process of the ethmoid. The opening is further curtailed by the adjacent mucous "membrane. Under these circumstances it is reduced to a small aperture, situated near the upper part, which opens into the middle meatus of the nasal fossa. The antrum is usually unilocular, but it may be partially divided into compartments.

The bone derives its blood - supply from many sources, such as posterior dental, infra-orbital, middle and anterior dental, facial, frontal, superior or descending palatine, and naso-palatine arteries.

Articulations.-The superior maxilla articulates with nine bones, as follows: malar, nasal, frontal, lachrymal, ethmoid, inferior turbinate, palate, vomer, and its fellow of the opposite side. In addition to these it may articulate with the pterygoid process and great wing of the sphenoid.
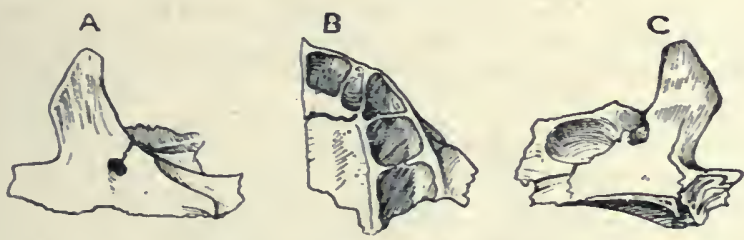

Fig. 62.-The Fotal Superior Maxillary Bone.

A, External View, showing the Infra-orbital Groove and Foramen, with Fissure; B, Inferior View, showing the Incisor Fissure and Alvecli; C, Internal View, showing the Antrum and Incisor Fissure.

Structure.-The bone contains the maxillary air sinus. For the most part cancellated tissue is absent, except in the alveolar process around the alveoli.

Ossiffeation.-The superior maxillary bone is ossified in membrane. Accorling to Mall and Fawcett it has two centres-maxillary proper and premaxillary, which appear about the sixth week, and join about the third month. The centre referred to as maxillary proper appears in the region of the future canine alveolus, and from it ossification proceeds backwards into the malar process, upwards into the posferior half of the nasal process, inwards into the posterior three-quarters of the palatal process, and downwards into the alveolar border, excluding the incisor portion.

The premaxillary centre gives rise to the premaxilla, which lies on the mesial side of the maxilla proper and bears the upper incisor teeth. It also gives rise to (I) the anterior fourth of the palatal plate, and (2), according to Fawcett, the anterior half of the nasal process. It is to be noted that, whilst the anterior half of the nasal process is ossified from the premaxillary centre, the posterior half of that process (bearing the lachyrmal groove) is ossified from the maxillary proper centre.

Besides the premaxillary centre there is an infravomerine centre, according to Rambaud and Renault. This centre lies beneath the anterior part of the vomer, and it gives rise to the infravomerine part of the bone, which forms the mesial wall of Stensen's canal. The line of union between the premaxilla 
and maxilla proper is indicated by the premaxillary suture on the palatal surface of young bones, which may, though somewhat rarely, persist in adult life. This suture extends outwards and forwards from a point directly behind the lower end of Stensen's canal to the alveolar border between the lateral incisor and canine alveoli.

The premaxilla of each side forms an independent bone in many animals. It may be developed in two parts from separate centres of ossification-an inner for the portion bearing the central incisor alveolus, and an outer for the portion containing the lateral incisor alveolus, and these two portions may remain separate. The inner portion is known as the endognathion, and the outer portion as the mesognathion, whilst the remainder and greater part of the bone is referred to as the maxilla proper.

The varieties of alveolar cleft palate are explained by a reference to these conditions of the bone. In mesial cleft palate the two premaxillæ (right and left) are separated by a mesial cleft. Lateral cleft palate may occur in two forms - the maxilla proper or excgnathion and the entire premaxilla may fail to unite, and the cleft is situated between them, and invades the alveolar border between the lateral incisor and canine alveoli; or the premaxilla may exist in two parts, inner or endognathion, and outer or mesognathion, and the cleft may be between these two, in which case it invades the alveolar border between the central and lateral incisor alveoli. These conditions may occur on one or both sides.

In the earlier stages of intra uterine life there is no trace of the antrum, and the alveolar border lies close to the infra-orbital border. In the course of the fourth month, however, the antrum makes its appearance as a shallow depression on the inner aspect of the bone, and, as this increases, it gradually separates the orbital, alveolar, and palatal portions. In the process of development the alveolar border first presents an elongated furrow, called the dental groove, on either side of which a plate grows downwards, forming the labial and lingual plates. The groove is thus converted into a trench with these ramparts on either side. Subsequently these plates are connected by a number of septa, which intersect the trench and break it up into alveoli. At this stage these are only five in number for each bone, and the canine alveolus is the first to be partitioned off. In early life the bone contains the temporary teeth, which are five in number on either side, but in the adult, as stated, it contains eight alveoli for the eight permanent teeth.

\section{The Malar Bones.}

The malar or cheek-bone is situated between the external angular process of the frontal and zygoma of the temporal on the one hand, and the malar process of the superior maxilla on the other, where it separates the orbit from the temporal fossa. It is quadrilateral, and presents two surfaces, four processes, and four borders. The external surface is convex, and near its centre there is the malar tuberosity. Above this is the malar foramen for the passage of the malar branch of the temporo-malar or orbital nerve, and a branch of the lachrymal artery. The portion of this surface close to the zygomatic process gives origin to the zygomaticus major, and the lower and anterior part to the zygomaticus minor. The internal surface, which is concave, looks into the temporal fossa above and zygomatic fossa below, and it is overhung superiorly by a curved plate of bone, called the orbital process. Anteriorly it presents a rough, slightly serrated, triangular area for the malar process of the superior maxilla. The orbital process projects backwards and inwards, in a curved manner, from the 
upper part of the internal surface on a level with the orbital border. It is triangular, and its superior or orbital surface presents a sweeping concavity, which enables it to form the front part of the outer wall of the orbit, and a portion of the floor. This surface is pierced by one or, it may be, two openings. If there is one, it ultimately leads to two canals-malar, which opens on the external surface, and temporal, which opens on the temporal division of the internal surface, as a rule near the frontal process. These canals transmit the malar and temporal branches of the temporo-malar or orbital nerve. If there are two, each leads to its own canal. The inferior surface of the orbital process, which is convex, forms the anterior part of the temporal fossa. The rough margin of the process articulates by its superior part with the anterior border or malar crest of the

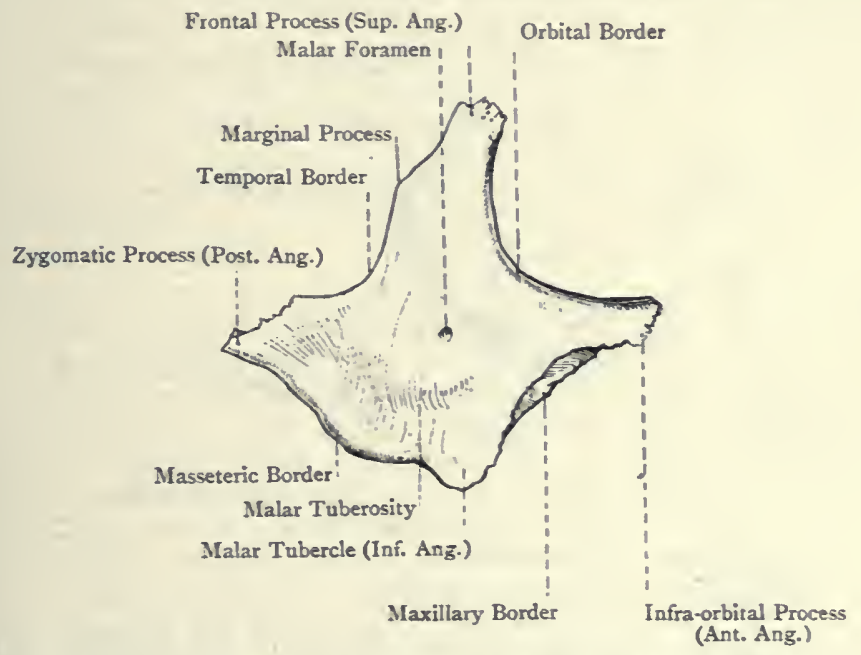

Fig. 63.-The Right Malar Bone (External View).

great wing of the sphenoid, and below with a part of the orbital plate of the superior maxilla. The part of this border between the sphenoidal and maxillary portions sometimes closes the anterior and outer extremity of the spheno-maxillary fissure, and thus intervenes between the great wing of the sphenoid and the superior maxilla.

The processes are four in number-superior, posterior, inferior, and anterior. The superior or frontal process is stout and prominent. Its direction is vertically upwards, and it terminates in a thick serrated extremity for the external angular process of the frontal. The posterior or zygomatic process is short and usually blunt. Its direction is backwards, and it terminates in a serrated extremity for the zygoma of the temporal. The inferior or maxillary process is blunt and truncated. Its direction is downwards, and 
it articulates with part of the malar process of the superior maxilla. The anterior or infra-orbital process is slender and pointed. Its direction is forwards, and it articulates with the superior maxilla near the infra-orbital foramen.

The borders are four in number-temporal, masseteric, maxillary, and orbital. The temporal border extends from the frontal process to the zygomatic, and is directed backwards. It is sinuous, and continuous with the upper border of the zygoma, and it gives attachment to the temporal fascia. Near the frontal process it usually presents a slight prominence, called the marginal process, to which a stout slip of the temporal fascia is attached. The masseteric border extends from the zygomatic process to the maxillary process, and looks downwards. It is thick, rough, and continuous with the lower border of the zygoma, and it gives origin

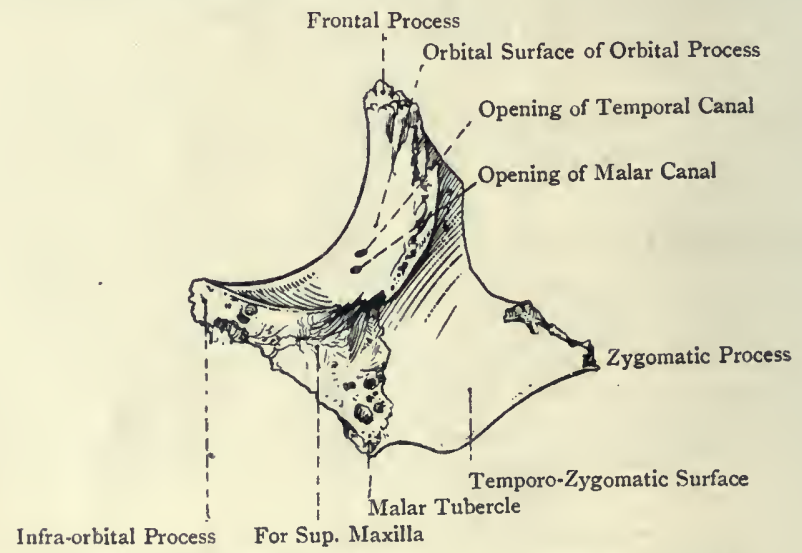

Fig. 64.-The Right Malar Bone (Internal View).

to the anterior fibres of the superficial part of the masseter. The maxillary border extends from the maxillary process to the infraorbital process, and it looks forwards and slightly downwards. It is rough and slightly concave, and, together with the rough, slightly serrated, triangular area on the internal surface adjacent to it, articulates with the malar process of the superior maxilla. The orbital border extends from the infra-orbital process to the frontal, and is smooth, round, and concave. Its direction is outwards and upwards, and it forms a large part of the circumference of the orbit.

The bone derives its blood-supply from the lachrymal, anterior deep temporal, and transverse facial arteries.

Articulations.-The malar articulates with four bones, as follows: superiorly with the frontal and sphenoid, posteriorly with the temporal, and anteriorly with the superior maxilla.

Structure.-The bone is mainly composed of compact tissue, the amount of cancellated tissue being small. 
Varieties. - The bone may persist in two parts connected by a suture, which may be horizontal or vertical. It sometimes persists in three parts.

Ossification.-The malar is developed in membrane from three centres, which appear in the eighth week of intra-uterine life, and they unite at the end of the fourth month. These centres are called premalar, postmalar, and hypomalar. If all three centres should fail to unite, then a tripartite malar is the result. If the premalar and postmalar unite, and the hypomalar remains separate, a bipartite malar persists with a horizontal suture. If the postmalar and hypomalar unite, and the premalar remains separate, the suture is vertical. A bipartite malar occurs with great frequentcy amongst the Japanese, and from this circumstance the bone is known as the os Japonieum.

\section{The Nasal Bones.}

The nasal bone, which articulates with its fellow by its mesial border, forms with it the bridge of the nose. It lies in front of the nasal process of the superior maxilla, where it enters into the formation of the face and nasal fossa. The bone is elongated from above downwards, and presents two surfaces and four borders. The anterior or facial surface is smooth, concavo-convex from above downwards, and convex from side to side. Near its centre it usually presents a minute foramen for the passage of a small vein from the nose to the commencement of the facial vein. This surface supports the common aponeurosis of the pyramidalis nasi and compressor naris muscles. The posterior or nasal surface is rough superiorly, where it articulates with the nasal process of the frontal. Elsewhere it is smooth and concave from side to side, and in the recent state is covered by the nasal mucous membrane. It is traversed longitudinally near the centre by the nasal groove for the nasal nerve.

The superior border is short, thick, and serrated for the nasal notch of the frontal: The inferior border is thin and expanded for the upper lateral nasal cartilage. It usually presents the nasal notcl, which is situated near its inner end. The mesial border articulates with its fellow. It is usually rather shorter and thicker than the external, and projecting backwards from it is a ledge of bone, which, with its fellow,

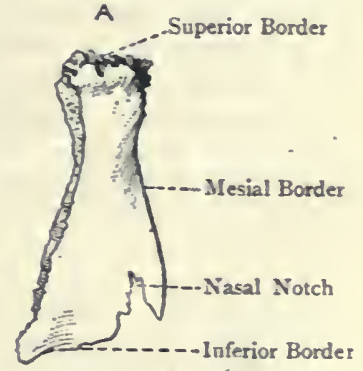

B

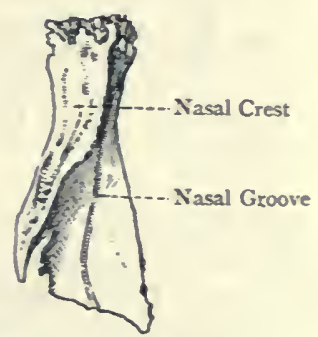

Fig. 65.-ThE RIGHT Nasal Bone.

A, Anterior View; B, Posterior View. forms the nasal crest for articulation with the nasal spine of the frontal and the anterior border of the perpendicular plate of the ethmoid. The external border, long and thin, is finely serrated for the nasal process of the superior maxilla. 
The bone receives its blood-supply from the angular branch of the facial, and the nasal and anterior ethmoidal branches of the ophthalmic, arteries.

Articulations.-The nasal articulates with four bones, as follows : superiorly with the frontal, externally with the superior maxilla, internally with its fellow, and posteriorly with the ethmoid and again with the frontal.

Structure.-The bone is composed of compact tissue, and is therefore dense.

Ossification.-The nasal is developed in membrane from one centre, which appears about the eighth week of intra-uterine life.

\section{The Lachrymal. Bones.}

The lachrymal or tear-bone is situated at the anterior part of the inner wall of the orbit, where it lies behind the nasal process of the superior maxilla, and in front of the os planum of the ethmoid. It is very thin and scale-like. From its resemblance in this sense to a finger-nail, it is known as the os unguis. It is quadrilateral and presents two surfaces and four borders, the inferior border being recognised by its presenting a hamular and a descending process. The external or orbital surface is traversed by the lachrymal crest, which is nearer the anterior than the posterior border, and

Superior Border

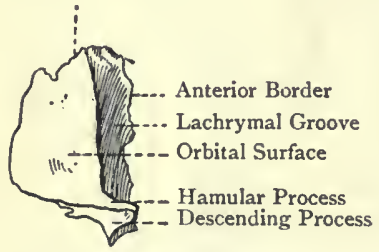

Fig. 66.-The Right LachRYMAL BONE (EXTERNAL VIEW).

part of the inner wall of the orbit. The lachrymal crest gives origin to the tensor tarsi muscle, and inferiorly terminates in a hook-like projection, called the hamular process. This process is curved in a forward direction, and is received into the lachrymal notch at the front part of the internal border of the orbital plate of the superior maxilla, where it bounds externally the superior orifice of the lachrymal canal. It articulates with the lachrymal tubercle of the superior maxilla. The internal surface presents a vertical furrow corresponding with the position of the lachrymal crest on the external surface. Superiorly it articulates with the front part of the lateral mass of the ethmoid, where it helps to close ethmoidal cells, and forms part of the infundibulum. Inferiorly it forms part of the outer wall of the nasal fossa, and looks into the middle meatus. 
The superior border is short, and articulates with the internal angular process of the frontal. The inferior border, behind the lachrymal crest, articulates with the internal border of the orbital surface of the superior maxilla, whilst in front of the crest it forms, as stated, the descending process, and articulates with the lachrymal process of the inferior turbinate. The anterior border articulates with the inner lip of the lachrymal groove on the posterior border of the nasal process of the superior maxilla. The posterior lorder articulates with the anterior border of the os planum of the ethmoid.

The bone derives its blood-supply from the nasal and anterior ethmoidal branches of the ophthalmic artery.

Articulations. - The lachrymal articulates with four bones, as follows: superiorly with the frontal, anteriorly with the superior maxilla, inferiorly with the inferior turbinate, and again with the superior maxilla, and posteriorly with the ethmoid.

Structure.-The bone consists of a thin translucent plate.

Ossification.-The lachrymal is developed in membrane usually from one centre, which appears during the third month of intra-uterine life.

\section{The Inferior Turbinate Bones.}

The inferior turbinate or spongy bone (maxillo-turbinal) sometimes called the inferior concha, is situated on the outer wall of the

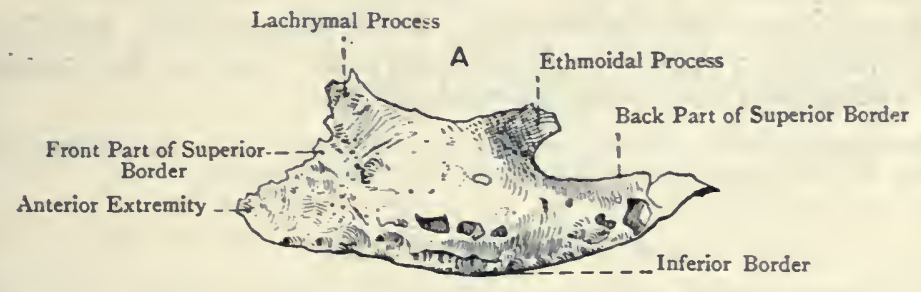

B

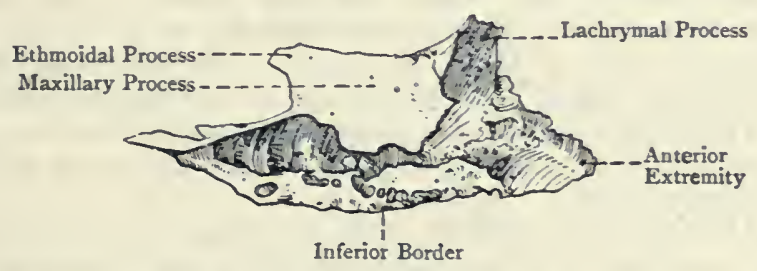

Fig. 67.-The Right Inferior Turbinate Bone.

A, Internal View ; B, External View.

nasal fossa, where it overhangs the inferior meatus, and is in series with the inferior turbinate process (middle spongy bone) of the ethmoid. It is elongated from before backwards, and presents 
two surfaces, two borders, and two extremities. The externat surface is concave, and is overhung above, over about its middle third, by the maxillary process. It looks towards the outer wall of the nasal fossa. The internal surface is irregularly convex, pitted, and marked by a few antero-posterior grooves. It bulges into the rasal fossa, and limits inferiorly the middle meatus.

The superior border, which is attached, slopes downwards and forwards in front, where it articulates with the inferior turbinate crest of the superior maxilla. Behind this it presents a slight concavity, limited in front by the lachrymal process, which articulates with the descending process of the lachrymal, and forms part of the lachrymal canal. Behind the concavity is the ethmoidal process for the uncinate process of the ethmoid. The portion of the superior border between these two processes is folded downwards and outwards into a thin plate, called the maxillary process, which forms part of the inner wall of the antrum of Highmore below the opening of that cavity. Behind the ethmoidal process the superior border slopes downwards and backwards, and articulates with the inferior turbinate crest of the palate bone. The inferior border is convex, thick, pitted, and free. The anterior extremity is short and stunted, whilst the posterior is long, slender, and pointed.

The bone receives its blood-supply from the spheno-palatine branch of the internal maxillary, and anterior ethmoidal branch of the ophthalmic, arteries.

Articulations.- The inferior turbinate articulates with the following four bones: superior maxilla, lachrymal, ethmoid, and palate.

Structure.-The bone is light and porous.

Ossification.-The inferior turbinate is developed in cartilage from one centre, which appears in the fifth month of intra-uterine life.

\section{The Palate Bones.}

The palate bone enters into the formation of the hard palate, the outer wall of the nasal fossa, and the floor of the orbit. It consists of a horizontal and perpendicular plate, which meet at a right angle, and of three processes, namely, pyramidal process or tuberosity, situated at the meeting of the two plates posteriorly, and orbital and sphenoidal processes, situated at the upper extremity of the perpendicular plate, where they are separated by the spheno-palatine notch.

The horizontal or palatal plate is quadrate, and presents two surfaces and four borders. The superior or nasal surface is smooth and concave from side to side. It forms the posterior fourth of the floor of the nasal fossa, and is covered in the recent state by the nasal mucous membrane. The inferior or palatal surface forms the posterior fourth of one-half of the hard palate, and near its posterior border it presents a short transverse ridge, which gives insertion to a portion of the tendon of the tensor palati. The anterior border 
is serrated for the posterior border of the palatal process of the superior maxilla. The posterior border is concave and sharp. It gives attachment to one-half of the soft palate, and at its inner extremity it forms a backward projection, which, with its fellow, constitutes the palatal or posterior nasal spine, for the attachment of the azygos uvulæ muscle. The external border is attached, and meets the perpendicular plate at a right angle. On its outer aspect posteriorly it is excavated by the lower part of the posterior palatine canal. The internal border is thick and serrated, and articulates with its fellow, forming an upward elevation, called the nasal crest. This crest is continuous with that of the palatal processes of the superior maxillæ, and, like it, is grooved superiorly for a portion of the inferior border of the vomer.

The perpendicular plate rises upwards from the outer border of the horizontal plate. It is long and thin, and presents two

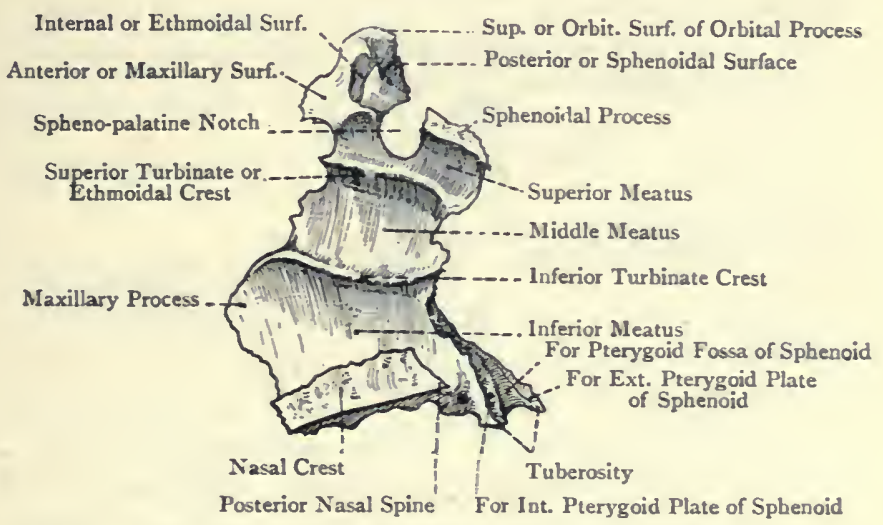

Fig. 68.-The Right Palate Bone (Internal View).

surfaces and four borders. The internal surface forms part of the outer wall of the nasal fossa, and is crossed from before backwards by two ridges. The lower ridge is called the inferior turbinate crest, and articulates with the posterior sloping part of the superior border of the inferior turbinate bone. The upper ridge, which crosses the roots of the orbital and sphenoidal processes, is called the ethmoidal or superior turbinate crest, and it articulates with the inferior turbinate process of the ethmoid. Below the inferior turbinate crest is a smooth groove, which forms part of the inferior meatus of the nose; beiween the inferior and superior turbinate crests is another groove, which forms part of the middle meatus; and above the superior turbinate crest there is a third groove, which forms part of the superior meatus. The external or maxillary surface, towards its upper and posterior part, forms the inner boundary of the pterygo-maxillary fissure, and leading downwards from this part is a groove, which, with ine superior maxilla, forms 
the posterior palatine or palato-maxillary canal for the great or anterior descending palatine nerve and the superior or descending palatine artery. In front of this groove the external surface articulates with the inner surface of the superior maxilla behind the opening of the an'rum. Behind the groove the external surface articulates inferiorly with the posterior border of the superior maxilla, and superiorly with the internal pterygoid plate of the sphenoid.

The anterior border of the perpendicular plate presents, just below the inferior turbinate crest, a leaf-like projection, called the maxillary process, which closes the lower and back part of the opening of the antrum of Highmore. Superiorly it articulates with the ethmoid, and inferiorly with the superior maxilla. The posterior border articulates superiorly with the anterior border of the internal pterygoid plate of the sphenoid, and inferiorly it

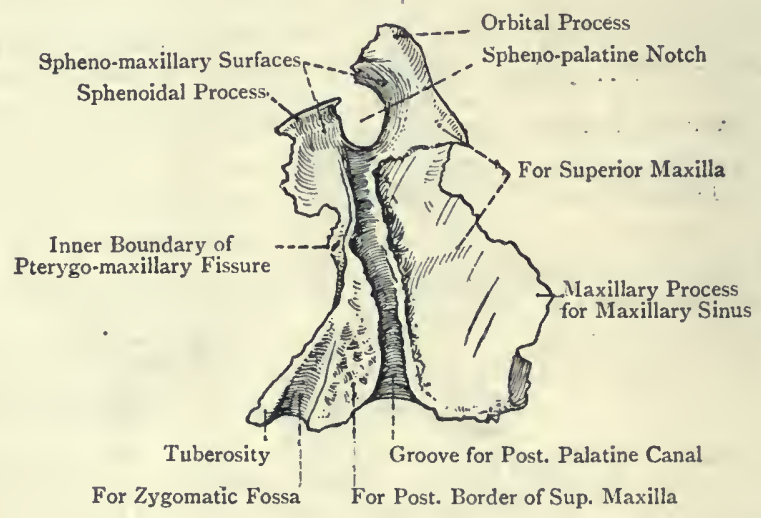

Fig. 69.-The Right Palate Bone (External View).

is prolonged into the tuberosity. The inferior border is attached, and maets the horizontal plate. The superior border presents the orbital and sphenoidal processes and the spheno-palatine notch, to be presently described.

The pyramidal process or tuberosity projects backwards, downwards, and outwards from the meeting of the horizontal and perpendicular plates, and is received into the pterygoid notch of the sphenoid. Posteriorly it presents three grooves. The central groove forms part of the pterygoid fossa, and gives origin to fibres of the internal pterygoid. The grooves on either side are rough, and articulate with the anterior borders of the corresponding pterygoid plates. The tuberosity on its inferior aspect, close to the horizontal plate, presents two small openings, which are the orifices of the posterior and external accessory palatine canals, the latter being the smaller of the two, and inconstant. These canals transmit the posterior and external descending palatine 
nerves and arteries. Internally the tuberosity gives origin to a few fibres of the superior constrictor muscle of the pharynx. Externally there is a small free surface, which looks into the zygomatic fossa, between the pterygoid process of the sphenoid and the tuberosity of the superior maxilla.

The orbital process surmounts the anterior border of the perpendicular plate.' It is of large size, and presents six surfaces, three of which are articular. The superior or orbital surface forms a small part of the floor of the orbit posteriorly. The external or spheno-maxillary surface looks into the spheno-maxillary fossa. The anterior or maxillary surface is continuous with the external surface of the perpendicular plate, and articulates with the back part of the internal border of the orbital surface of the superior maxilla. The internal or ethmoidal surface, which is excavated, articulates with the lower border of the os planum of the

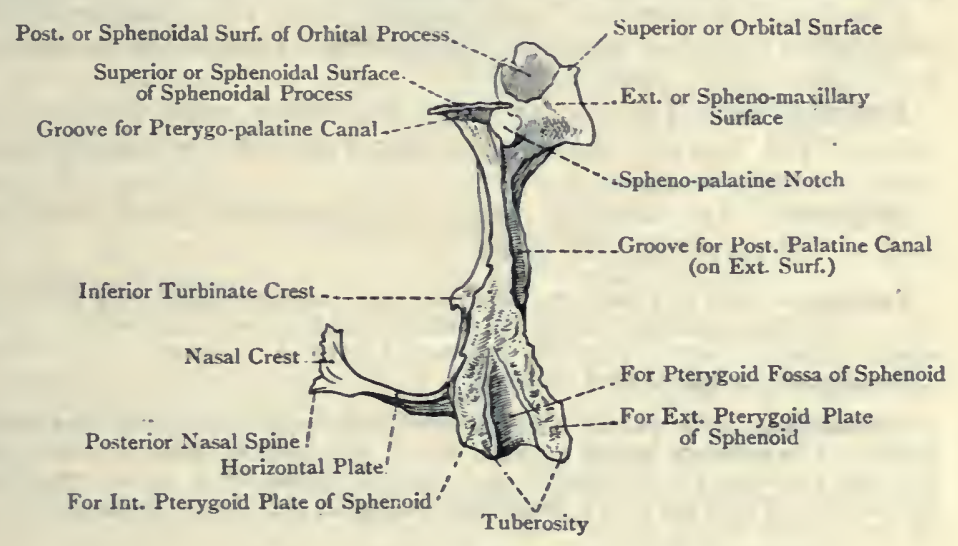

Fig. 70.-The Right Palate Bone (Posterior View).

ethmoid at its back part, close to the postero-inferior angle. The posterinr or sphenoidal surface small and excavated, articulates with the front of the body of the sphenoid, and, as a rule, communicates with the sphenoidal air sinus. The inferior or nasal surface is continuous with the internal surface of the perpendicular plate, and forms part of the outer wall of the nasal tossa at its upper and back part, whele it overhangs the groove for the superior meatus.

The sphenoidal process surmounts the posterior border of the perpendicular plate, and is slightly incurved. It presents three surfaces, and three borders. The superior or sphenoidal surface, which is grooved, articulates with the inferior surface of the body, and the vaginal process, of the sphenoid. The groove on this surface, with that on the under surface of the vaginal process, forms the pterygo-palatine canal for the pharyngeal nerve and pterygopalatine artery. The internal or nasal surface is scooped out, and 
has an inclination downwards as well as inwards. It forms part of the outer wall and roof of the nasal fossa. The external or spheno-maxillary surface forms part of the inner wall of the sphenomaxillary fossa. The anterior border bounds the spheno-palatine notch posteriorly, and may be projected over it to join the orbital process. The posterior border articulates with the internal pterygoid plate of the sphenoid. The internal border articulates with the ala of the vomer.

The spheno-palatine notch is situated between the orbital and sphenoidal processes, and is converted into a foramen usually by the inferior surface of the body of the sphenoid, representing the part formed by a sphenoidal spongy bone. It leads from the spheno-maxillary fossa into the superior meatus of the nose, and transmits the internal branches of the spheno-palatine or Meckel's ganglion, and spheno-palatine artery.

The bone derives its blood-supply from the spheno-palatine, descending palatine, and pterygo-palatine branches of the internal maxillary artery.

Articulations.- The palate bone articulates with six bones, as follows: the superior maxilla, inferior turbinate, ethmoid, vomer, sphenoid, and its fellow.

Structure.-The bone is very thin, especially over the upper part of the perpendicular plate.

Varieties.-(1) The groove for the posterior palatine canal may be bridged over. (2) The external accessory palatine canal may be absent. (3) The spheno-palatine notch may be converted into a foramen by a forward extension of the sphenoidal process.

Ossiffcation.-The palate bone is ossified in membrane from one primary centre. The primary centre appears about the seventh week, at the angle of junction between the horizontal and vertical plates, or in the vertical plate (Fawcett). There may be a secondary centre for the orbital process.

\section{The Vomer.}

The vomer is situated in the median plane, and forms part of the septum of the nose. It presents two surfaces, four borders, and an anterior extremity. 'The surfaces are disposed laterally, and each looks into the corresponding nasal fossa. Traversing each there is a groove, directed forwards and downwards, for the naso-palatine nerve.

The superior border is characterized by two thick, everted alæ, separated by a groove, which receives the rostrum of the sphenoid, Each ala by its upper aspect fits against the inferior surface of the body of the sphenoid, and the lateral margin of each meets the vaginal process of that bone, and also articulates with the internal border of the sphenoidal process of the palate bone. The inferior border is irregular, and is received into the groove which marks the nasal crests of the palatal plates of the superior maxillæ and palate bones. The anterior border is sloped downwards and 
forwards, and it may present two alæ, but these are very thin, and lie near each other, being separated by a narrow cleft. These characters are always more pronounced in earlier life. The cleft in its lower part receives the septal nasal cartilage, and superiorly the perpendicular plate of the ethmoid fits into it, being usually ankylosed with one or both alæ. In many cases, however, the anterior border is simply grooved. The posterior border is sharp, and aimost vertical, and lies between the posterior nares. The anterior extremity forms a short irregular lip, which touches the back parts of the incisor crests of the superior maxillæ.

The bone receives its blood-supply from the spheno-palatine branch of the internal maxillary artery.

Articulations. -The vomer articulates with six bones, as follows: the sphenoid, two palate bones, eth- Posterior Border moid, and superior maxillæ. In addition to these, it articulates with the septal nasal cartilage.

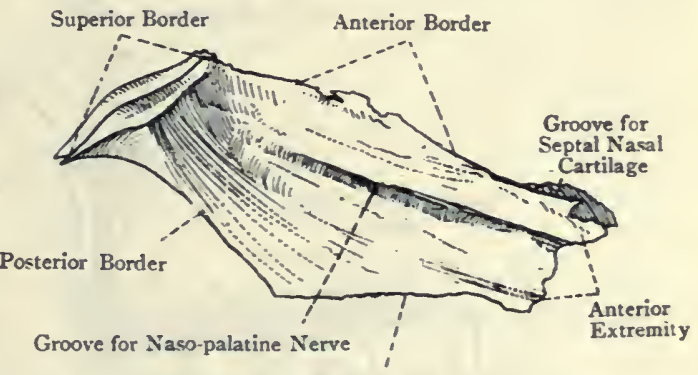

Inferior Border

Fig. 71.-The Vomer (Lateral View).

Structure.-The vomer is composed of two thin ptates of compact bone, which are blended into one, except superiorly, and, it may be, to a certain extent anteriorly.

Varieties. - The bone is often much deflected to one or other side, more frequently the left, and so it may curtail the cavity of the nasal fossa to which it is deflected.

Ossification.-The vomer is developed in membrane from two centres, which appear about the eighth week of intra-uterine life. The centres unite below in the third month, and form a groove in which the septal nasal cartilage lies. The laminæ forming the lips of the groove continue to grow upwards and forwards, and subsequently fuse, the enclosed cartilage becoming absorbed. Ultimately there are left the alæ on the superior border, and, it may be, on the anterior border, which are permanent indications of the original bilaminar condition of the bone.

\section{The Inferior Maxillary Bone.}

The inferior maxillary bone or mandible supports the lower teeth, and articulates at either side with the anterior part of the glenoid fossa of the temporal in a freely movable manner. It has the shape of a horse-shoe, and consists of a central horizontal portion, called the body, and two upright portions, called the rami.

The body is arched, being convex in front and concave behind, and it presents two surfaces and two borders. The external surface presents a slight median vertical ridge over its upper two-thirds, which marks the symphysis or place of union of the two halves 
of which the bone is originally composed. This ridge bifurcates at the lower third, and its two divisions, diverging, pass to the lower border, where each terminates in the mental tuberele. Between these diverging divisions there is a triangular elevated surface, called the mental protuberance or chin. On either side of the symphysis is the incisor fossa, which gives origin to the levator menti and a deep slip of the orbicularis oris. A little external to this fossa is the mental foramen, which opens outwards from the inferior dental canal, and transmits the mental nerve and vessels. This

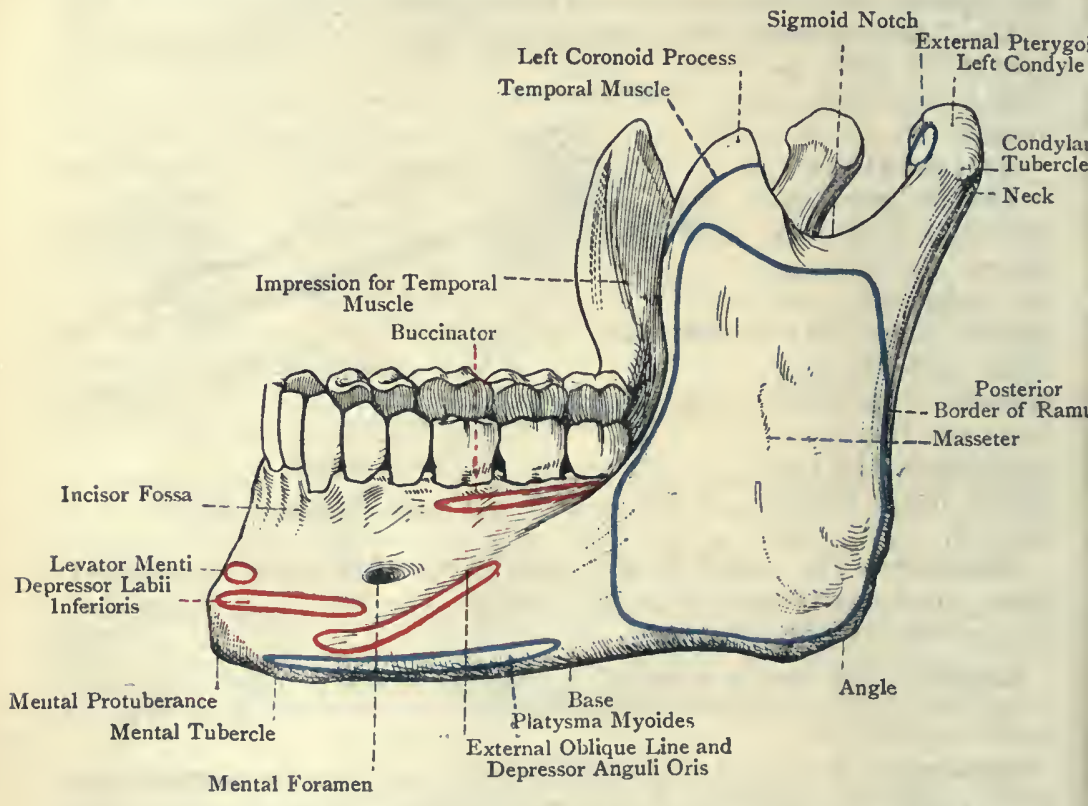

Fig. 72.-The Inferior Maxillary Bone (External View).

foramen is in line with the septum between the two bicuspid alveoli, and in the adult it is midway between the superior and inferior borders. Below it is the external oblique line, which extends from the mental tubercle to the lower extremity of the anterior border of the ramus. This line gives origin to the depressor anguli oris. The lower part of the external surface, from near the symphysis to about the level of the mental foramen, gives origin to the depressor labii inferioris.

The internal surface presents a slight median groove over about its upper two-thirds, which coincides with the symphysis. Lower down there are four small projections, called collectively the genial spines, which are arranged in pairs on either side of the middle line. The upper spine gives origin, at either side, to the genio-hyoglossus, and the lower to the genio-hyoid. Close to the lower border, at either side of the symphysis, is the oval digastric impression, 
which gives origin to the anterior belly of the digastric. Coinciding with the position of the external oblique line there is the internal oblique line or mylo-hyoid ridge. This commences near the symphysis below the lower genial spine, and, passing obliquely backwards and upwards, it terminates a little behind the last molar alveolus. It gives origin to the mylo-hyoid muscle over its whole length, whilst at its upper and back part it gives attachment to some fibres of the superior constrictor muscle of the pharynx and the pterygo-mandibular ligament. Below the posterior part of this

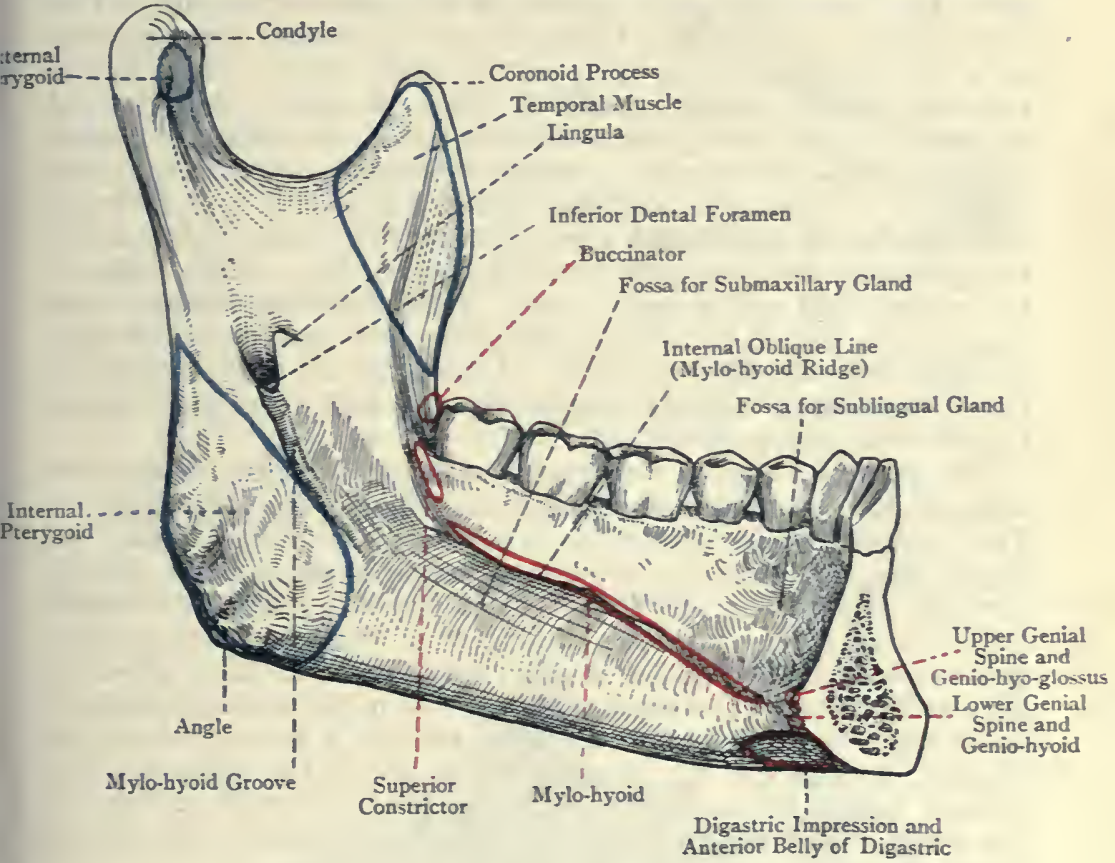

Fig. 73.-The Left Half of the INferior Maxillary Bone (INTERNAL VIEW).

ridge is the submaxillary fossa for the submaxillary gland, and above its anterior part is the sublingual fossa for the sublingual gland.

The superior or alveolar border is excavated into sixteen alveoli or sockets, eight in each half of the bone, which correspond with those in each superior maxilla. The outer surface of the alveolar border, over the extent of the three molar alveoli at either side, gives origin to some fibres of the buccinator. The inferior border or base terminates, at either side, on a level with the anterior border of the ramus. It projects more than the superior border, and gives insertion on its outer aspect to a portion of the platysma myoides. Near its termination it is marked by a short vertical groove for the facial artery. 
The ramus rises, at either side, from the extremity of the body. It is compressed from without inwards and presents two surfaces and four borders. The external surface gives insertion to the masseter, and, in the vicinity of the angle, it presents a few oblique ridges for the tendinous bands of that muscle. The internal surface presents, a little below its centre, the inferior dental foramen, which is on a level with the summit of the crown of the third molar tooth. This foramen leads to the dental canal, which traverses the bone to near the symphysis, and from which, near its anterior part, the mental foramen opens on the external surface. This canal lodges the inferior dental nerve and vessels, and communicates with the foramina which open on the extremities of the fangs of the teeth. The inferior dental foramen presents anteriorly and internally a thin, sharp plate of bone, called the lingula. Behind the lower end of the latter is a short crescentic margin on the inner aspect of the foramen, and proceeding downwards and forwards from this is the mylo-hyoid groove, which terminates a little below the posterior extremity of the mylo-hyoid ridge, and transmits the mylo-hyoid nerve and artery. The spheno-mandibular ligament is attached to the lingula and to the crescentic margin behind it. Between the inferior dental foramen and the angle there is a rough impression, often strongly ridged, which gives insertion to the internal pterygoid.

The anterior border is continuous with the external oblique line opposite the third molar alveolus, and is shorter than the posterior. The posterior border meets the inferior border, thus forming the angle, which, in muscular subjects, is strongly marked and slightly everted. Externally and internally it presents rough impressions for portions of the masseter and internal pterygoid respectively, and between these muscles it gives attachment to the stylo-mandibular ligament. The angle is obtuse, and in the adult amounts on an average to I20 degrees. In early infancy it is as much as I5O degrees, and in old age it amounts to about I 40 degrees. The inferior border is continuous with the inferior border or base of the body. The superior border presents the sigmoid notch, the coronoid process, and the condyle.

The sigmoid notch communicates with the zygomatic fossa, and transmits the masseteric nerve and artery to the deep surface of the masseter.

The coronoid process surmounts the anterior border of the ramus, and is triangular and compressed from without inwards. Its external surface gives insertion to fibres of the masseter, and its internal surface, as well as the superior and anterior borders, to part of the temporal muscle. The internal surface is marked by a ridge which extends downwards on the internal surface of the ramus, not far from the anterior border, to a point on the inner side of the last molar alveolus, where it becomes continuous with the mylo-hyoid ridge. The temporal muscle continues to take insertion into this ridge, as well as into the elongated triangular depression between it and the anterior border of the ramus. 
The condyle surmounts the posterior border of the ramus. It is oval and convex, and it articulates with the anterior part of the glenoid fossa of the temporal, an interarticular fibro-cartilage intervening. Its long axis is oblique, so that the axes of the two condyles, if sufficiently prolonged inwards and slightly backwards, would meet near the anterior margin of the foramen magnum. Externally the condyle presents a projection, called the condylar tubercle, for the external lateral ligament of the temporo-mandibular articulation. Below the condyle is the neck, which presents anteriorly a depression for the insertion of the greater part of the external pterygoid muscle.

The bone receives its chief bloodsupply from the inferior dental branch of the internal maxillary artery. Other sources are the facial, and the sublingual branch of the lingual.

Articulations. - With the glenoid fossæ of the temporal bones.

Structure.-The inferior maxilla is

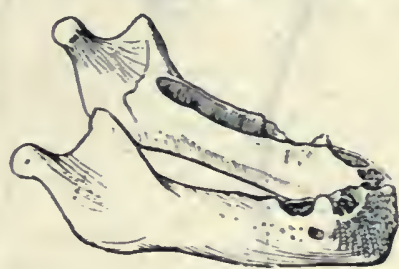

Fig. 74A.-THE INFERIOR Maxillary Bone at Birth. composed of two dense plates of compact bone, which are particularly strong in the region of the base, but become thinner superiorly at the alveolar border. Between these plates there is cancellated tissue with wide meshes.

Ossification.-The mandible is a mixed bone, being chiefly a membrane bone, but in part also a cartilage bone. It is ossified in connection with Meckel's cartilage and its fibrous investment. Each half of the bone has one centre (Low and Fawcett), which appears about the sixth week of intra-

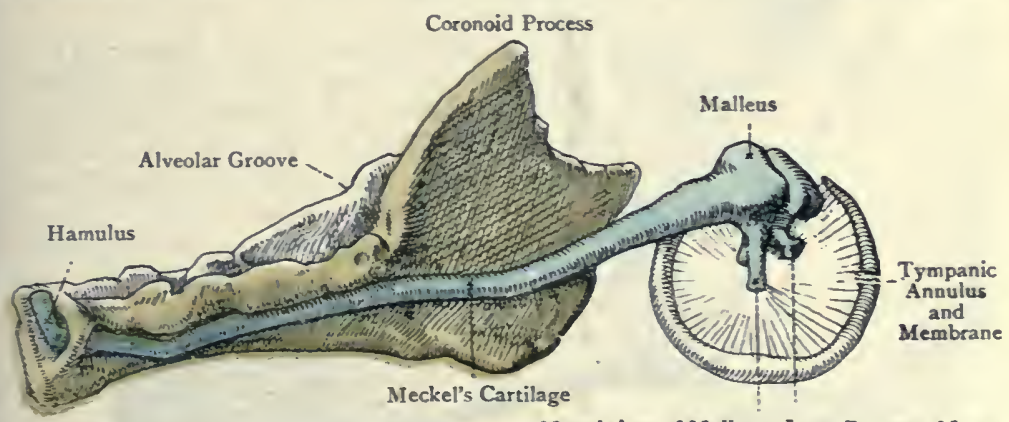

Manubrium of Malleus Long Process of Incus

Fig. 74B.-Meckel's Cartilage: (From Keibel and Mlall. After KollyaNa).

uterine life, being only preceded by the primary centres for the clavicle. It is deposited in the membrane which covers the outer surface of Meckel's cartilage in the region of the future mental foramen. From this centre onehalf of the bone is ossified, chiefly in membrane, but also in cartilage, namely, the mesial end of Meckel's cartilage, and certain other accessory cartilages. The original centre gives membranous origin to (I) the walls of the alveoli and 
dental canal, (2) the basilar border and angle, and (3) the ramus as high as the interior dental foramen. The mesial part of Meckel's cartilage is invaded by osseous extension from the primordial membrane-bone formed from the single centre, the mesial part of Meckel's cartilage becoming incorporated with the bone so formed, and constituting the incisor portion of the mandible.

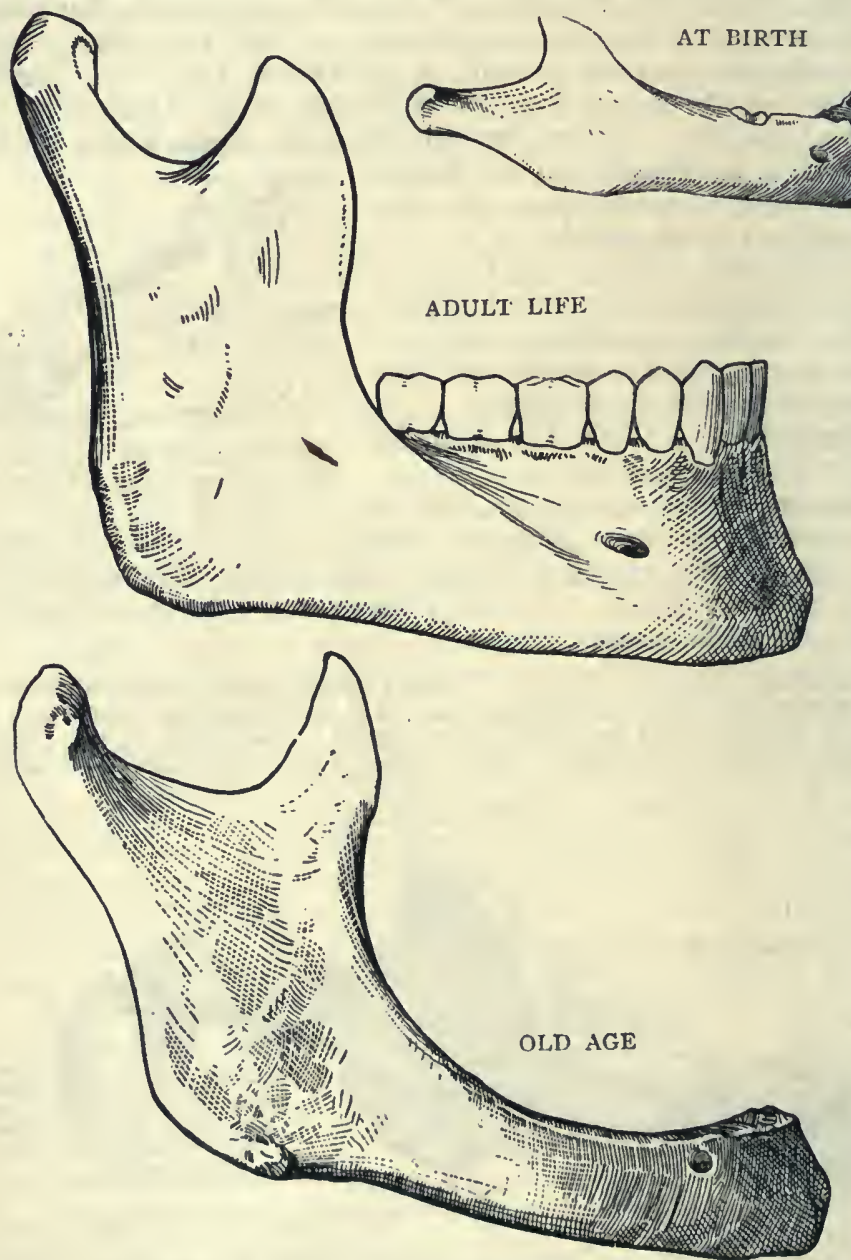

Fig. 75. - The Inferior Maxillary Bone at Different Periods of Life.

The accessory cartilages, which are distinct from Meckel's cartilage, are as follows: (I) Condylar, (2) coronoid, and (3) symphysial. All these accessory cartilages become surrounded and invaded by osseous extension from the primordial membrane-bone formed from the single centre, and they become incorporated with the parts of the mandible so formed.

The condylar cartilage gives rise to (I) the condyle, and (2) the posterior 
part of the ramus as low as the inferior dental foramen. The coronoid cartilage gives rise to (1) the coronoid process; and (2) the anterior part of the ramus as low as the inferior dental foramen. The symphysial cartilage gives rise to the limited symphysial part of the mandible.

At birth the mandible consists of two halves, connected at the symphysis by fibrous tissue. In the course of the first year osseous union takes place, which is completed towards the end of the first year or beginniog of the second year.

Meckel's cartilage 'extends on either side downwards and forwards from the periotic cartilaginous capsule to the median line, where it meets its fellow. It is surrounded by a fibrous investment. The proximal end of the cartilage gives rise to the malleus and incus, two of the three ossicles of the tympanum. The part of the cartilage between the periotic cartilaginous capsule and the inferior dental foramen disappears, and the membranous investment of this part persists as the spheno-mandibular ligament. The part of the cartilage between the inferior dental foramen and the mental foramen also disappears, and its membranous investment undergoes ossification from a single centre, and gives rise to ( 1 ) the greater part of one-half of the body of the mandible (incisor and symphysial parts excepted), and (2) the lower half of the ramus as high as the inferior dental foramen. The mesial part of Meckel's cartilage. when ossified, becomes the incisor part of the mandible.

At birth the inferior border is but little developed, and the body is consequently shallow. The rami are very short, so that each condyle is nearly on a level with the upper border of the symphysis, and the coronoid process is rather longer than the condyle. The mental foramen is nearer the inferior than the superior border, and the angle amounts to 150 degrees or more. Subsequently the bod $y$ increases in depth, the rami lengthen, the angledecreases, and the mental foramen gradually assumes a position midway between the superior and inferior borders. In old age, after the bone becomes edentulous. the alveolar border undergoes absorption, the body consequently becomes shallower, the mental foramen lies near the superior border, the rami droop backwards, and each angle becomes increased to about 140 degrees. For the development of the alveolar border, and its relation to the milk-teeth, see the superior maxilla.

\section{The Hyoid Bone.}

The hyoid bone is situated in the median line of the neck, between the chin and the thyroid cartilage of the larynx, with which latter it is connected by means of the thyro-hyoid membrane and thyro-hyoid ligaments. It is closely connected with the base of the tongue, and is hence known as the os lingua. In its development it is associated with the skull, and it is suspended from the lower ends of the styloid processes of the temporal bones by two fibrous bands, called the stylo-hyoid ligaments (epi-hyals). It consists of a central portion or body and two pairs of cornua, great and small.

The body is elongated transversely, compressed from before backwards, and quadrilateral. Its surfaces, which are anterior and posterior, occupy an oblique plane, being sloped downwards and for- 
wards. The anterior surface is convex, and is crossed transversely by a ridge, which divides it into an upper and a lower part. At the middle line this is intersected at right angles by a vertical ridge, which, however, is often incomplete, being sometimes confined to the upper half, and sometimes to the lower. At the place of intersection of the two ridges there is a slight projection, called the hyoid tubercle. Each half of the anterior surface is thus mapped out into an upper and a lower irregular muscular division. The upper division, provided the upper border is not very thick, gives attachment to the genio-hyoid and genio-hyo-glossus, and the lower division to the digastric, stylo-hyoid, and mylo-hyoid. The posterior surface is concave, and is covered by the thyro-hyoid membrane as it ascends to be attached to the superior border, a synovial bursa intervening. This surface is related to the epiglottis.

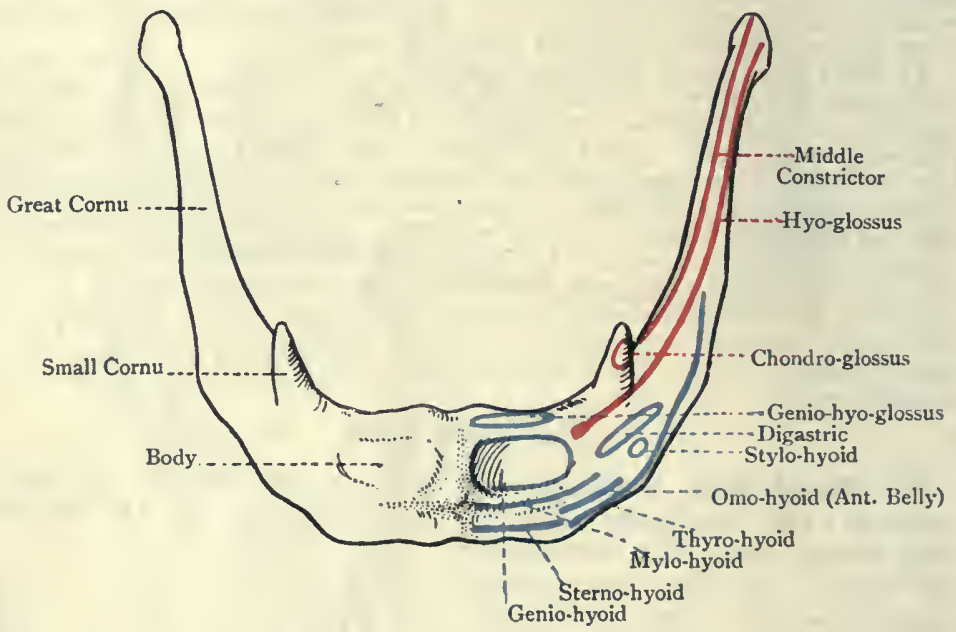

Fig. 77.-The Hyoid Bone, showing its Muscular Attachments.

The superior border is somewhat thick, and occasionally is really a surface, in which cases it gives attachment to the genio-hyoglossus, whilst its posterior lip gives attachment to the thyro-hyoid membrane. The inferior border is sharp, and gives insertion to the sterno-hyoid, anterior belly of the omo-hyoid, and thyro-hyoid muscles. Each lateral border is connected with a great cornu.

The great cornua project upwards and backwards from the lateral borders of the body. Each is compressed from above downwards, and gradually diminishes in size to its termination, where it ends in a small tubercle for the attachment of the thyro-hyoid ligament. It gives attachment to fibres of the thyro-hyoid, hyo-glossus, middle constrictor muscle of the pharynx, and the thyro-hyoid membrane. Each great cornu is connected with the body by synchondrosis up to middle life, after which ankylosis usually takes place. 
The small cornua are short conical nodules, each of which projects upwards and backwards from the junction between the body and great cornu. Its tip gives attachment to the stylohyoid ligament, which is sometimes ossified, a possible condition to be borne in mind during digital examination of the upper part of the pharynx. Elsewhere it gives attachment to the middle constrictor muscle of the pharynx, and sometimes to the chondroglossus. The small cornua may be wholly or partially cartilaginous, and they articulate with the body by a synovial joint, unles. in advanced life, when ankylosis usually takes place.

Ossification.-The hyoid bone is developed in connection with the second and third visceral arches. The cartilaginous bar of the second visceral arch is known as the hyoid bar, and is continuous with its fellow at the median line. The cartilaginous bar of the third visceral arch is known as the thyro-hyoid bar, and at the median line it blends with the junction of the hyoid bars. With the foregoing proviso, the hyoid bone is developed from five, or, it may be, six centres. One, or, it may be, two are deposited during the last month of intra-uterine life at the place of fusion of the two hyoid bars. If there are two centres they soon join, and give rise to the greater part of the body of the hyoid bone or basi-hyal. About the same time a centre appears at either side in the thyro-hyoid bar of the third visceral arch, and from these centres are developed the great cornua or thyro-hyals, and the adjacent portions of the body. In the course of the first year the two remaining centres appear, one at either side, in the lower or mesial part of each hyoid bar, and these centres give rise to the small cornua or cerato-hyals. The great cornua join the body in middle life, but the small cornua do not join until advanced life. The stylo-hyoid ligaments may become ossified more or less completely, and so represent the epi-hyal bones of many animals.

\section{The Skull as a Whole.}

\section{Sutures.}

The only bone of the skull which has movable articulations is the mandible. Each condyle of that bone articulates with the anterior part of the glenoid fossa of the corresponding temporal bone, with the intervention of an interarticular fibro-cartilage, the articulation so formed being called the temporo-mandibular joint.

The other bones of the skull, for the most part, are in close contact with each other, a small amount of fibrous tissue being interposed, which is continuous with the periosteum. These articulations are called sutures. Certain cranial bones, however, are separated at first by a plate of hyaline cartilage, the articulation being called synchondrosis. This, however, is a temporary joint, inasmuch as ankylosis takes place after a certain period of life. It applies to (I) the articulation between the basilar process of the occipital and the body of the sphenoid, and (2) the articulation between the jugular process of the occipital bone and the jugular impression on the inferior surface of the petrous portion of the temporal bone.

The occipito-parietal or lambdoid suture connects the superior lateral borders of the tabular part of the occipital bone with the 
posterior borders of the two parietal bones. The two limbs of the suture, right and left, become continuous with each other at the superior angle of the tabular part of the occipital bone, from which point each limb passes outwards and downwards. The margins of the bones are strongly serrated, and along the course of the suture supernumerary ossicles, called Wormian bones, are frequently present.

The occipito-mastoid suture, on either side, connects the inferior lateral border of the tabular part of the occipital bone with the posterior border of the mastoid part of the temporal bone. It is one of the bifurcations of the lower end of the corresponding limb of the occipito-parietal suture, the other bifurcation being the parieto-mastoid suture. Its direction is downwards and forwards.

The interparietal or sagittal suture connects the superior borders of the two parietal bones. It is serrated, and occupies the median

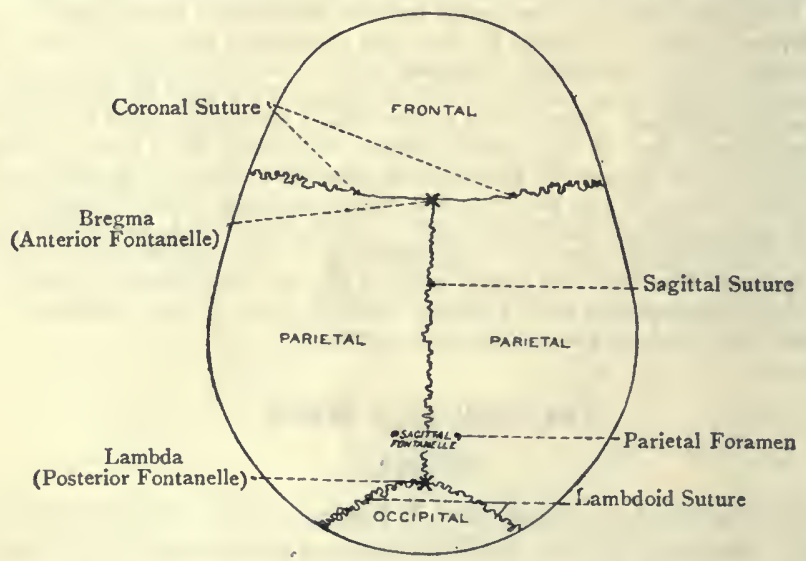

Fig. 78.-Sutures of Superior Surface of Skull.

line, its direction being from behind forwards. Posteriorly it meets the lambdoid suture, the place of meeting constituting the lambda, which indicates the situation of the posterior fontanelle of early life. From the lambda three sutures radiate-namely, the interparietal suture and the two limbs of the occipito-parietal suture. Anteriorly the interparietal suture meets the coronal- suture, the place of meeting constituting the bregma, which indicates the situation of the anterior fontanelle of early life. The part of the sagittal suture in the region of the two parietal foramina is usually less serrated than elsewhere, and is the first part to show signs of obliteration. It constitutes the obelion, which indicates the situation of the sagittal fontanelle of early intra-uterine life.

The fronto-parietal or coronal suture connects the supero-lateral or parietal border of the frontal portion of the frontal bone with the anterior borders of the two parietal bones. It crosses the 
superior surface of the skull in an arched manner, extending on either side as low as the superior border of the great wing of the sphenoid. As it crosses the median line it is joined by the anterior end of the interparietal suture. The place of junction is, as stated, called the bregma, which indicates the position of the anterior fontanelle of early life. From the bregma three sutures radiatenamely, the interparietal suture and the two limbs, right and left, of the fronto-parietal suture. Each limb of the fronto-parietal suture is serrated, but more so over the central part than the upper and lower parts, and more so over the upper part near the median line than over the lower part, in which two latter situations overlapping takes place. Superiorly the frontal bone slightly overlaps the parietal bune, and inferiorly the parietal bone distinctly overlaps the frontal bone, the opposed bevelled surfaces in the latter region being ridged. This latter portion of the coronal suture is spoken of as a limbous suture.

The metopic or frontal suture, which connects the two halves of the frontal bone during the first year of life, may persist throughout life. Under these circumstances, it extends in the median line from the bregma to the centre of the nasal notch of the frontal bone. Superiorly it is continuous with the interparietal suture, and inferiorly with the internasal suture. Even though the suture may not persist entirely, traces of it may be present inferiorly and superiorly, especially inferiorly. As a rule, however, the suture is entirely obliterated by the end of the sixth year of life.

The parieto-mastoid suture connects the posterior part of the inferior border of the parietal bone with the superior border of the mastoid part of the temporal bone. Its direction is from behind forwards, and, as stated, it is one of the bifurcations of the lower end of the corresponding limb of the occipito-parietal suture. It is serrated, and anteriorly is continuous with the posterior end of the squamo-parietal suture. It is on a level with the upper border of the zygoma. The point, situated at its posterior extremity, coincides with the asterion, which indicates the situation of the postero-lateral or mastoid fontanelle of early life. From this point three sutures radiate-namely, the corresponding limb of the occipito-parietal suture, the parieto-mastoid suture, and the occipitomastoid suture. Near the anterior part of the parieto-mastoid suture there is the parietal notch on the superior border of the pars mastoidea, which coincides with the entomion.

The parieto-squamosal suture connects the greater part of the inferior border of the parietal bone with the superior border of the squamous part of the temporal bone. It is arched, the convexity being directed upwards, and after having descended posteriorly, it becomes continuous with the parieto-mastoid suture. Anteriorly it is continuous with the spheno-parietal suture. The parieto-squamosal suture presents a marked contrast to the foregoing serrated sutures, inasmuch as it is a squamous suture, the squamous part of the temporal overlapping the lower border of the parietal bone. 
The spheno-parietal suture connects the posterior part of the superior border of the great wing of the sphenoid with the anteroinferior angle and anterior part of the inferior border of the parietal bone. Its direction is from behind forwards. Posteriorly it is continuous with the parieto-squamosal suture, and anteriorly with the spheno-frontal suture. The spheno-parietal suture, like the parieto-squamosal, is a squamous suture. The region of the sphenoparietal suture coincides with the pterion. The spheno-parietal suture may be very short, or entirely absent, in which latter case the lower and posterior part of the frontal bone articulates directly with the squamous part of the temporal bone, thus excluding the antero-inferior part of the parietal bone from any articulation with the great wing of the sphenoid: In such cases the pterion is situated at the posterior end of the spheno-fronal suture. In all cases the guide to it is the lower end of the posterior border of the

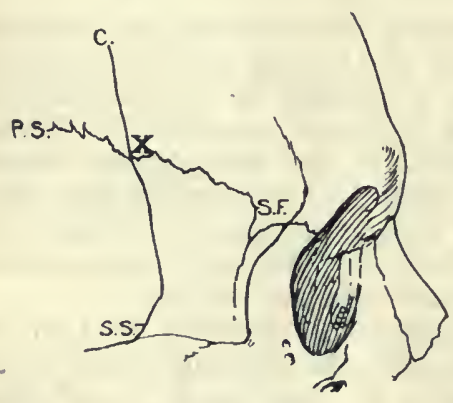

Abnormal.

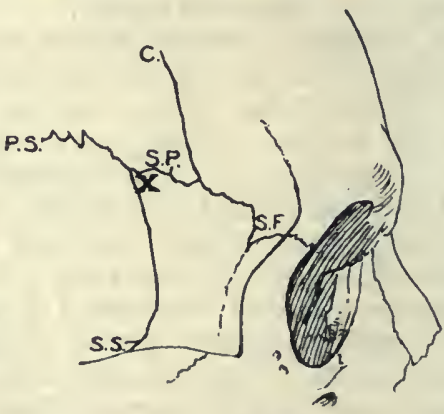

Normal.

Fig. 79.-Sutures of Right Lateral Surface of Skull.

S.P., Spheno-parietal; S.F., Spheno-frontal; P.S., Parieto-squamosal; $\mathrm{X}$, Pterion; C., Coronal; S.S., Spheno-squamosal.

external angular process of the frontal bone, the pterion being situated about I $\frac{1}{2}$ inches behind it, and about 2 inches above the zygomatic arch. The pterion indicates the situation of the anterolateral or sphenoidal fontanelle of early life. In this region a Wormian bone, called the epipteric bone, is sometimes met with.

The fronto-squamosal suture is not constant. When present, it connects the lower and posterior part of the frontal bone with the upper and anterior portion of the squamous part of the temporal bone. Its direction is vertical, and it is usually continuous with the terminal part of the coronal suture. It excludes the anteroinferior part of the parietal bone from any articulation with the great wing of the sphenoid.

The spheno-frontal suture, which is a squamous suture, connects the superior border of the great wing of the sphenoid bone with the lower part of the lateral aspect of the frontal bone. Posteriorly it is continuous with the spheno-parietal suture, and in cases where 
that suture is absent, with the parieto-squamosal suture. Anteriorly it is continuous with the fronto-malar suture.

There are two other spheno-frontal sutures. One connects the rough triangular surface on the antero-superior part of the great wing of the sphenoid bone with the rough triangular surface on the frontal bone behind the external angular process. This is a harmonic suture. The other spheno-frontal suture (orbito-spheno-frontal suture) will be described under the orbital sutures. It also appears, however, in the anterior fossa of the internal surface of the base of the skull.

The spheno-squamosal suture connects the external border of the great wing of the sphenoid bone with the antero-inferior border of the squamous part of the temporal bone, its direction being downwards and backwards as far as the inner end of the fissure of Glaser. Its upper part forms a squamous suture, but its lower part is serrated.

The zygomatico-malar suture connects the extremity of the zygomatic process of the temporal bone with the posterior or zygomatic process of the malar bone. It lies obliquely, being directed downwards and backwards, and it is finely serrated.

The fronto-malar suture will be described under the orbital sutures.

The fronto-maxillary suture, which is serrated, connects the lateral part of the nasal notch of the frontal bone with the superior border of the nasal process of the superior maxilla. It lies almost transversely.

The fronto-nasal suture connects the mesial part of the nasal notch of the frontal bone with the superior border of the nasal bone. It lies transversely, and is serrated. Across the median line it is continuous with its fellow of the opposite side, and laterally it is continuous with the fronto-maxillary suture. The point corresponding to the meeting of the two fronto-nasal sutures coincides with the nasal point or nasion.

The internasal suture connects the mesial borders of the nasal bones. Its direction is vertical, and it is a harmonic suture. The lower end of the suture coincides with the rhinion.

The naso-maxillary suture connects the external border of the nasal bone with the anterior border of the nasal process of the superior maxilla. Its direction is almost vertical, and it is finely serrated.

The malo-maxillary suture connects the rough triangular area at the anterior part of the internal surface of the malar bone with the rough superior surface of the malar process of the superior maxilla. The opposed surfaces are rough, and a false suture is formed of the harmonic variety. The orbito-malo-maxillary suture will be described under the orbital sutures.

The intermaxillary suture is only partially seen on the facial surface of the skull. This part extends from the centre of the anterior nasal spine to the point of meeting of the alveolar margins of the superior maxillæ. The palatal part of the suture connects the mesial borders of the palatal plates of the superior maxillæ. Its direction is antero-posterior, and it is a harmonic suture. 
The interpalatal suture connects the mesial borders of the horizontal plates of the palate bones. It is continuous with the inter maxillary suture, and is a harmonic suture.

The transverse palato-maxillary suture connects the anterior border of the horizontal plate of the palate bone with the posterior border of the palatal plate of the superior maxilla. Its direction is transverse, it is continuous with its fellow of the opposite side, and it is a finely serrated suture.

The vertical palato-maxillary suture connects the lower part of the anterior border of the perpendicular plate of the palate bone with the internal surface of the superior maxilla behind the opening of the antrum of Highmore. It is a harmonic suture. The orbitopalato-maxillary suture will be described under the orbital sutures.

The incisor or premaxillary suture is usually of temporary duration. It connects the incisor or premaxillary part of the superior maxilla with the superior maxilla proper. It extends from a point immediately behind the lower end of Stensen's canal to the alveolar border at a point between the lateral incisor and canine alveoli, its direction being outwards and forwards. It is always present in early life, and traces of it may be present in adult life on the inferior surface of the palatal plate of the superior maxilla.

The pterygo-maxillary suture connects the lower part of the anterior border of the pterygoid process of the sphenoid with the lower part of the posterior border of the superior maxilla. Its direction is vertical, and it is a harmonic suture. The pterygomaxillary fissure lies above it.

The petro-squamosal suture connects the petrous and squamous parts of the temporal bone, and is situated on the internal or cerebral surface of the bone. Its direction is antero-posterior, and it extends from the petro-squamosal angle anteriorly to the parietal notch (entomion) on the superior border of the pars mastoidea posteriorly, where it meets the squamo-mastoid suture. It is a harmonic suture, and ankylosis usually takes place in the course of the first year of life. The suture, however, may persist until puberty, or later. Even though ankylosis takes place at the normal period of time, it may occur in such an imperfect manner that the suture is drilled by several channels. Under the foregoing circumstances pyogenic infection may extend from the tympanic cavity to the cerebral meninges and brain. The petro-squamosal venous simus of fœtal life extends along the petro-squamosal suturc.

The squamo-mastoid suture connects the mastoid and squamous parts of the temporal bone, and is situated on the external surface of the bone. Its direction is almost vertical, and it extends from the parietal notch (entomion) on the superior border of the pars mastoidea anteriorly to a point behind the posterior limb of the tympanic annulus. The lower part of the suture intervenes between the contiguous part of the pars mastoidea and the post-meatal plate of the pars squamosa. which latter forms the supra-meatal triangle and outer wall of the antrum. Superiorly it meets the petro- 
squamosal suture. The squamo-mastoid suture is a harmonic suture, and ankylosis usually takes place in the course of the first year of life. The suture, however, may persist until puberty, or even throughout life. Even though ankylosis takes place at the normal period of life, it may occur in such an imperfect manner that the suture is drilled by several channels. Under these circumstances, pus may find an exit from the tympanic cavity to the exterior.

The petro-sphenoidal suture connects a portion of the anterior border of the petrous part of the temporal bone with the outer portion of the posterior border of the great wing of the sphenoid. It lies obliquely, its direction being inwards and forwards.

The petro-basilar suture connects the inner half of the posterior border of the petrous portion of the temporal bone with the lateral border of the basilar portion of the occipital bone. Its direction is forwards and slightly inwards, and it is a harmonic suture. Superiorly it is grooved for the inferior petrosal venous sinus.

Orbital Sutures.-The orbito-spheno-frontal suture connects the anterior border of the small wing of the sphenoid with the posterior border of the orbital plate of the frontal. It lies transversely at the back part of the roof of the orbit, and is finely serrated.

The orbito-malo-maxillary suture connects the lower part of the posterior border of the orbital plate of the malar with the posterointernal boundary of the rough superior surface of the malar process of the superior maxilla. It is situated on the outer part of the floor of the orbit, and extends forwards and inwards from the anterior end of the spheno-maxillary fissure to the infra-orbital margin at a point above, and slightly internal to, the infra-orbital foramen. It is a limbous suture, the orbital plate of the malar slightly overlapping the contiguous part of the superior maxilla.

The orbito-palato-maxillary suture connects the orbital process of the palate bone with the back part of the internal border of the orbital plate of the superior maxilla. It is situated on the posterior part of the floor of the orbit. Its direction is antero-posterior, and it is a harmonic suture.

The orbito-palato-ethmoidal suture connects the orbital process of the palate bone with the back part of the inferior border of the os planum, or orbital plate, of the ethmoid. It lies on the back part of the inner wall of the orbit, where that wall meets the floor. Its direction is antero-posterior, and it is a harmonic suture.

The spheno-malar suture connects the anterior border or malar crest of the great wing of the sphenoid with the upper part of the posterior border of the orbital plate of the malar. It is situated towards the front part of the outer wall of the orbit. Its direction is vertical, and it is a limbous suture, the orbital plate of the malar slightly overlapping the malar crest of the sphenoid.

The fronto-malar suture connects the external angular process of the frontal bone and the short serrated border directly behind that process with the superior or frontal process of the malar and the 
superior border of its orbital. plate. A portion of this suture lies on the facial surface of the skull, and the other part lies on the anterior and upper part of the outer wall of the orbit. Its direction is antero-posterior, and it is a serrated suture. It is in line with the spheno-frontal suture.

The lachrymo-maxillary suture connects the anterior border of the lachrymal bone with the inner lip of the lachrymal groove on the posterior border of the nasal process of the superior maxilla. It lies on the anterior part of the inner wall of the orbit. Its direction is vertical, and it is a harmonic suture. The place of meeting between the vertical lachrymo-maxillary and almost horizontal fronto-maxillary sutures coincides with the dacryon.

The lachrymo-ethmoidal suture connects the posterior border of the lachrymal bone with the anterior border of the os planum, or orbital plate, of the ethmoid. It lies on the inner wall of the orbit. Its direction is vertical, and it is a harmonic suture.

The orbito-spheno-ethmoidal suture connects the posterior border of the os planum, or orbital plate, of the ethmoid with the sphenoidal spongy bone, or with the anterior margin of the lateral surface of the body of the sphenoid bone. It lies vertically on the back part of the inner wall of the orbit, and it is a harmonic suture.

There are two other spheno-ethmoidal sutures, which, however, are not orbital. One of these connects the ethmoidal spine of the sphenoid bone and the contiguous portions of the anterior border of the superior surface of the body with the posterior border of the cribriform plate of the ethmoid. It lies in the anterior fossa of the internal surface of the base of the skull, and is irregularly continuous, on either side, with the orbito-spheno-frontal suture, which connects the small wing of the sphenoid with the orbital plate of the frontal. It is a limbous suture, and it may be called the basal sphenoethmoidal suture. The remaining spheno-ethmoidal suture connects the sphenoidal crest of the sphenoid with the posterior border of the perpendicular plate of the ethmoid bone. It is situated at the back part of the osseous nasal septum, its direction being vertical, and it is a harmonic suture. It may be called the septal spheno-ethmoidal suture.

The fronto-lachrymal suture connects the internal angular process of the frontal bone and the contiguous part of the outer lip of the bevelled and excavated inner border of its orbital plate, which lies close behind the internal angular process, with the superior border of the lachrymal bone. It is situated on the anterior part of the inner wall of the orbit, where that wall meets the roof; its direction is antero-posterior, and it is a harmonic suture.

The fronto-ethmoidal suture connects the inner bevelled and excavated border of the orbital plate of the frontal bone with the superior excavated border of the lateral mass of the ethmoid. It is situated on the inner wall of the orbit, where that wall meets the roof. Its direction is antero-posterior, and it is continuous with the fronto-lachrymal suture. It may be regarded as a muchmodified harmonic suture. The anterior and posterior ethmoidal, or internal orbital, canals lie transversely along its course.

The ethmo-maxillary suture connects the inferior border of the os planum, or orbital plate, of the ethmoid with the inner border 
of the orbital plate of the superior maxilla. It is situated on the inner wall of the orbit, where that wall meets the floor, and its direction is antero-posterior. It is parallel to the fronto-ethmoidal suture, and is a harmonic suture.

Schindylesis.-There are only a few examples of this variety of synarthrosis, or immovable joint, in the skull. These are as follows: (I) The articulation between the inferior border of the vomer and the cleft which lies along the nasal crests of the palatal plates of the superior maxillary and palate bones; (2) the articulation between the rostrum of the sphenoid and the cleft on the superior border of the vomer between the two thick, everted alæ; and (3) the. articulation between the inferior border of the perpendicular plate of the ethmoid with the narrow cleft on the anterior border of the vomer.

Gomphosis.-This variety of synarthrosis is illustrated in the impaction of the fangs of the teeth within the alveoli of the superior and inferior maxillæ.

\section{Synchondroses.}

The following are examples of this temporary form of synarthrosis:

Synchondrosis Spheno-Occipitalis.-This is situated between the posterior surface of the body of the sphenoid and the anterior surface of the basilar part of the occipital bone.

Synchondrosis Petro-Occipitalis.-This is situated between the jugular impression on the inferior surface of the petrous part of the temporal bone and the extremity of the jugular process of the occipital.

Synchondrosis Spheno-Petrosa.-This is situated between the posterior border of the great wing of the sphenoid and the anterior part of the petrous portion of the temporal bone.

There are also intra-occipital and intra-sphenoidal synchondroses until the ossification of these bones is completed.

\section{The Posterior Region of the Skull.}

The posterior region (norma occipitalis) is formed by the posterior parts of the parietal bones and the upper or interparietal division of the tabular part of the occipital. It is limited above by a line connecting the parietal eminences, and below by the superior curved lines of the occipital, whilst laterally it is limited by a line connecting the parietal eminence with the lateral angle of the tabular part of the occipital. A little above the centre it presents the lambda, which is the place where the sagittal meets the lambdoid suture in the situation of the posterior fontanelle of early life. Radiating from the lambda there are three sutures: One passes upwards and forms the posterior part of the sagittal or interparietal suture. The other two, diverging, pass outwards and downwards, and form together the lambdoid or occipito-parietal suture. About I inch above the lambda, at either side of the sagittal suture, is the 
parietal foramen, and the point where the horizontal line connecting the parietal foramina intersects the sagittal suture is known as the obelion, which coincides with the situation of the sagittal fontanelle of early fœtal life. This part of the sagittal suture is less serrated than elsewhere, and is the first to show signs of obliteration. At the lower part of the posterior region in the middle line is the external occipital protuberance, which is known as the inion. A little above this is the occipital point, which is the part in the median plane at the greatest distance from the glabella of the frontal. The tabular part of the occipital may present an occipital suture, if the interparietal division persists as a separate bone.

\section{The Superior Region.}

The superior region (norma verticalis) varies in shape. It may be oval with its long axis antero-posterior, and broader behind than in front. Such skulls are called dolicocephalic, and in them the zygomatic arches are usually visible at either side from above, a condition known as phenozygous, In other cases the superior region assumes a circular shape, due to the broadening of its anterior part. Such skulks are called brachycephalic, and in them the zygoma ic arches are usually concealed from above, a condition known as cryptozygous, Some skulls are intermediate between the dolicocephalic and brachycephalic, and ar: known as mesaticephalic. The bones which enter into the superior region are the upper part of the frontal and the anterior parts of the parietals. It is limited in front by a line connecting the frontal eminences, behind by a line connecting the parietal eminences, and on either side by the superior temporal ridges of the parietal and frontal bones. The highest point is situated in the course of the sagittal suture, and is called the vertex. The sutures in this region are usually two in number, coronal and sagittal, but there is sometimes a third, namely, the metopic or frontal. The coronal or fronto-parietal suture lies between the frontal and parietal bones. The anterior part of the sagittal or interparietal suture meets the coronal suture from behind, and the place of junction is known as the bregma, which coincides with the anterior fontanelle of early life. If there is a metopic or frontal suture present it connects the two halves of the frontal bone, and is a continuation of the sagittal suture as far as the fronto-nasal suture. The superior region, as viewed from above, reveals certain parts of the posterior region, namely, the posterior portions of the parietals, with the parietal foramina and obelion, the lambda, the interparietal portion of the occipital, and the lambdoid suture.

\section{The Anterior Region.}

The anterior region (norma frontalis et facialis) is limited above by a line connecting the frontal eminences, and below by the lower 
border of the inferior maxilla. It is formed by a portion of the frontal, the nasals, superior maxillæ, malars, and inferior maxilla. It is subdivided into two portions, frontal and facial.

The frontal division is limited laterally by the superior temporal ridge, below by the supra-orbital border at either side of the median line, and by the fronto-nasal and fronto-maxillary sutures close to the median line. It presents the frontal eminences, superciliary ridges, external and internal angular processes, supraorbital notches, or it may be foramina, all on either side of the median line, and the nasal eminence or glabella at the median line. between the two superciliary ridges. The most prominent point of the glabella is known as the antinion. The meeting of the two fronto-nasal sutures is known as the nasion or nasal point. The centre of a line drawn from one temporal ridge to the other across the narrowest part of the frontal region is known as the ophryon.

The upper part of the facial division presents the openings of the orbits. . These cavities are separated at the median line by the bridge of the nose, which is formed by the nasal bones and the nasal processes of the superior maxillæ, whilst externally each orbit is limited by the malar bone and the external angular process of the frontal. The point at the inner angle of the orbit where the horizontal fronto-maxillary suture meets the vertical lachrymomaxillary suture is known as the dacryon, and the lower part of the internasal suture is known as the rhinion. Below the nasal bones is the anterior nasal aperture. It is bounded on either side by the nasal notch on the mesial border of the suferior maxilla, and above by the inferior borders of the nasal bones, whilst inferiorly in the median line is the anterior nasal spine in two halves. The central point of the base of this spine is known as the subrasal point. The anterior nasal aperture is the common external opening of the two nasal fossæ, which are separated by a septum composed of bones and cartilage. An inspection of each nasal fossa will reveal two bulging prominences on its outer wall, the lower of which is formed by the inferior turbinate bone, and the upper by the inferior turbinate process of the ethmoid. Below the former is the inferior meatus, whilst between the two is the middle meatus. The outer wall, from its irregularity, thus presents a marked contrast to the floor, which is smooth and unbroken. The osseous septum is, as a rule, deflected to one side, most commonly the left, thus diminishing the capacity of the left fossa. Below the anterior nasal aperture are the alveolar borders of the superior maxillæ, which lodge the upper teeth. The point where the anterior margins of these two borders meet in the median line is known as the alveolar point. Below these borders is the entrance to the buccal cavity, and below this is the alveolar border of the inferior maxilla, which lodges the lower teeth. The middle point of the anterior lip of the lower border of the inferior maxilla is known as the mental point or gnathion.

The superior maxillæ having a wider range than the inferior 
maxilla, the upper teeth slightly overlap the lower. According to the degree of projection of the maxillary bones, skulls are spoken of as orthognathous, prognathous, or mesognathous.

The chief small foramina of the anterior region are as follows, from above downwards at either side : supra-orbital, at the junction of the outer two-thirds and inner third of the supra-orbital arch of the frontal (which in most cases is a notch); infra-orbital, in the superior maxilla near the infra-orbital margin; mental, in the inferior maxilla in line with the septum between the bicuspid alveoli; and malar, situated above the malar tuberosity. The supra-orbital, infra-orbital, and mental foramina are in the same perpendicular line, and transmit the following important sensory nerves, in order from above downwards: supra-orbital, infraorbital, and mental, which are branches of the ophthalmic, superior maxillary, and inferior maxillary divisions of the Gasserian ganglion on the sensory root of the fifth cranial nerve. The malar foramen transmits the malar branch of the temporo-malar or orbital nerve, from the superior maxillary division of the fifth.

The sutures in the anterior region are as follows: fronto-malar, fronto-maxillary, lachrymo-maxillary, fronto-nasal, internasal, nasomaxillary, malo-maxillary, and intermaxillary.

The Orbits. - The orbits have the shape of four-sided pyramids, their bases being directed forwards and outwards, and their apices backwards and inwards. The inner walls are nearly parallel, and occupy an antero-posterior plane, but the outer walls diverge, the plane of each being directed forwards and outwards, so that they almost fo:m a right angle with each other. Each orbit is lined with periosteum, which is continuous with the dura mater through the sphenoidal fissure, and it contains the eyeball, with the ocular muscles, nerves, and bloodvessels, the lachrymal gland, and a large amount of fat. It presents an apex, a base, and four sides or walls. The apex, which is directed backwards and inwards, is formed by the inner end of the sphenoidal fissure, and just above and internal to this is the optic foramen. The base is free, and is directed forwards and outwards. Its circumference presents the fronto-malar suture externally, the malo-maxillary inferiorly, and the fronto-maxillary internally. The walls are superior, inferior, external, and internal.

The superior wall or roof, which is thin and brittle, is formed mainly by the orbital plate of the frontal, and behind this by the small wing of the sphenoid. It is smooth and concave. Within the external angular process it p:esents the lachrymal fossa for the lachrymal gland, and near the internal angular process the trochlear fossa, which gives attachment to the cartilaginous pulley of the superior oblique muscle of the eyeball.

The inferior wall or floor is fo med by three bones, namely, the orbital surface (orbital plate) of the superior maxilla, external to which is a part of the orbital process of the malar, whilst posteriorly is the orbital process of the palate bone. The floor is thin, and sepa- 
Anterior Ethmoidal Canal,

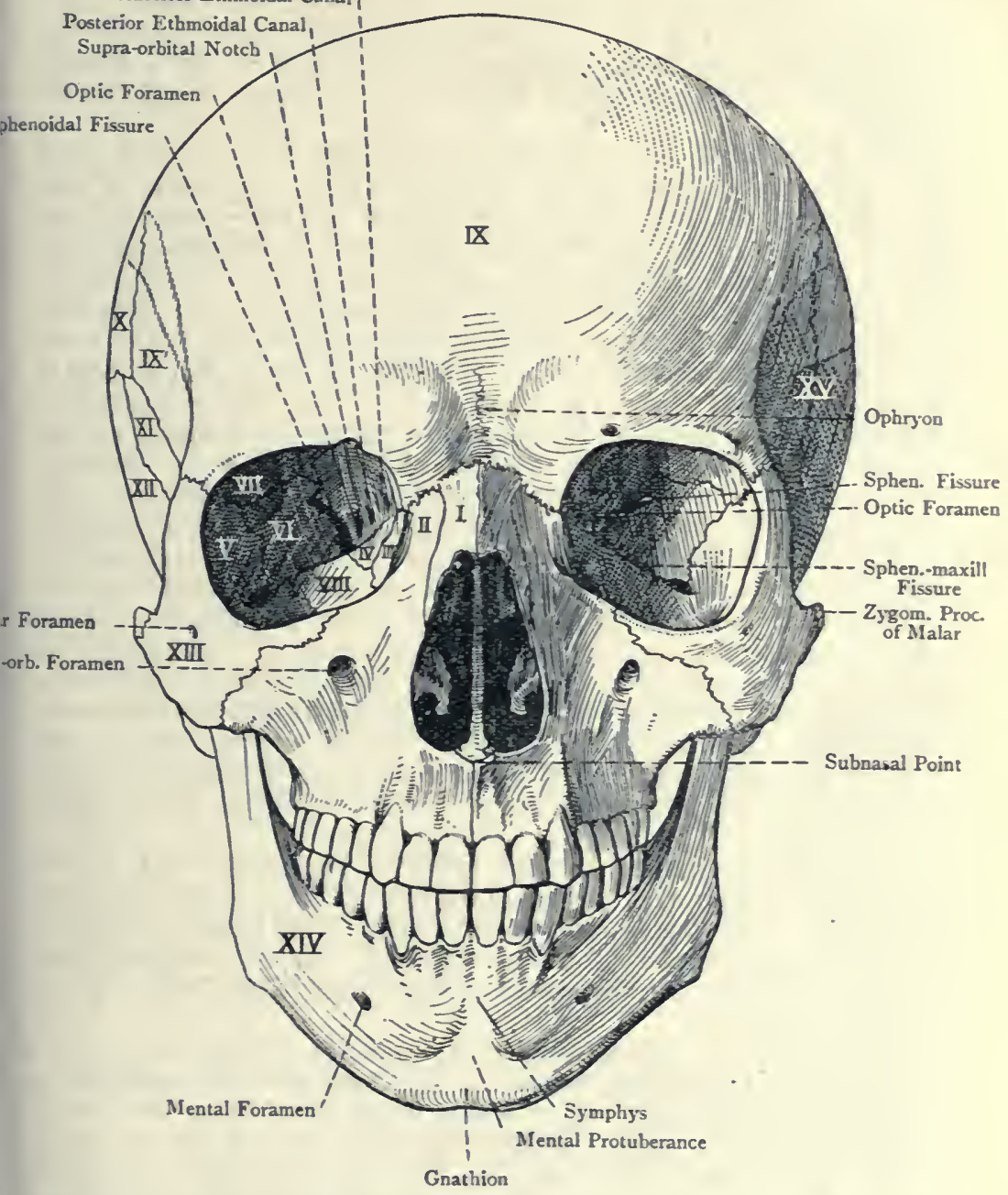

Fig. 80.-The Anterior Region of the Skull (Norma Frontalis et Facialis).

I, Nasal. II, Nas. Proc. of Sup. Maxilla. III, Lachrymal. IV, Ethmoid (Os Planum). V, Orbital Proc. of Malar. VI, Orb. Surf. of Great Wing of Sphenoid. VII, Orb. Plate of Frontal. VIII, Orb. Surf, of Sup. Maxilla. IX, Frontal. IX $\mathrm{X}^{1}$, Temp. Div. of Frontal. X, Parietal. XI, Great Wing of Sphenoid. XII, Squam. Port. of Temporal. XIII, Malar. XIV, Inf. Maxilla. XV, Temporal Fossa. 
ratej the orbit from the subjacent antrum of Highmore. It is traversed from behind forwards by the infra-orbital canal, which posteriorly is a groove. At its anterior and inner part is the upper orifice of the lachrymal canal, and external to this is a small depression which gives origin to the inferior oblique muscle of the eyeball.

The outer wall looks forwards and inwards, and is formed mainly by the orbital surface of the great wing of the sphenoid, and in front of this by a part of the orbital process of the malar. Between the outer wall and the floor is the spheno-maxillary fissure, the front part of which communicates with the zygomatic fossa, and the back part with the spheno-maxillary fossa. Between the outer wall and the roof, towards the posterior part, is the outer portion of the sphenoidal fissure. The part of the orbital process of the malar which forms the front part of this wall presents two foramina (sometimes one) leading to the malar and temporal canals.

The inner wall is almost vertical, and looks directly outwards. It is formed by four (sometimes five) bones, in the following order from before backwards: (I) the nasal process of the superior maxilla; (2) the lachrymal; (3) the os planum or orbital plate of the ethmoid ; and (4) the anterior part of the lateral surface of the body of the sphenoid. If there are five bones, the fifth is a portion of the sphenoidal spongy bone, which would lie behind the os planum of the ethmoid. Between the inner wall and roof, in the ethmo-frontal suture, are the openings of the anterior and posterior ethmoidal or internal orbital canals. At the anterior part of this wall is the lachrymal groove, which lodges the lachrymal sac, and behind this is the lachrymal crest, which gives origin to the tensor tarsi muscle.

The orbital sutures are as follows: superiorly, the orbitosphenoidal; inferiorly, the malo-maxillary and palato-maxillary; externally, the spheno-malar; and internally, from before backwards, the lachrymo-maxillary, ethmo-lachrymal, and ethmo-sphenoidal, all of which three are disposed vertically, and ethmo-frontal, which is antero-posterior.

The orbit has ten (sometimes nine) openings communicating with it. (I) The sphenoidal fissure or foramen lacerum anterius or orbitale, the wide inner end of which forms the apex of the cavity, whilst the narrow outer part lies between the roof and the outer wall. This fissure transmits $(a)$ the third nerve, the sympathetic filament to the lenticular ganglion, the fourth, the three branches (frontal, lachrymal, and nasal) of the ophthalmic division of the fifth, and the sixth, cranial nerves; $(b)$ the superior and inferior ophthalmic veins; (c) the orbital branch of the middle meningeal artery ; and (d) a portion of the dura mater. (2) The optic foramen, situated above and internal to the apex, for the optic nerve and the ophthalmic artery, along with a plexus of sympathetic nerve fibres. (3) The supra-orbital notch (or it may be foramen), on the supra-orbital border, for the supra-orbital nerve 
and vessels. (4) The opening of the infra-orbital canal, on the floor, transmitting the infra-orbital nerve and vessels. (5) The opening of the temporal canal, and (6) the opening of the malar canal, both on the outer wall, for the branches of the temporo-malar or orbital nerve from the superior maxillary division of the fifth cranial nerve. (The temporal and malar openings may be combined into one.) (7) The spheno-maxillary fissure, at the junction of the outer wall and floor, which transmits the superior maxillary nerve to become the infra-orbital, and the infraorbital vessels. (8) The lachrymal canal, at the anterior part of the inner wall, for the nasal duct. (9) The anterior ethmoidal canal, and (IO) the posterior ethmoidal canal, both situated on the inner wall, the former transmitting the nasal nerve and anterior ethmoidal vessels, and the latter the posterior ethmoidal vessels and the spheno-ethmoidal nerve.

Eight muscles take their origin within each orbit. The four recti arise from a fibrous ring surrounding the optic foramen. The levator palpebræ superioris arises above and in front of the optic foramen, and the superior oblique arises internal to the last named. The inferior oblique arises from the depression at the anterior and inner part of the floor, external to the orifice of the lachrymal canal, and the tensor tarsi arises from the lachrymal crest behind the lachrymal groove.

The Nasal Fossæ.- The nasal fossæ are two in number, right and left, and they lie on either side of the median plane. They extend horizontally from before backwards, opening on the face as the anterior nares by means of the anterior nasal aperture, and communicating posteriorly with the naso-pharynx by the posterior nares. The vertical and antero-posterior dimensions of each fossa greatly exceed the transverse. The two fossæ are separated from each other by a partition, called the septum nasi, which is partly osseous and (in the recent state) partly cartilaginous. Each fossa presents a roof, floor, and two walls, inner and outer.

The roof over its central part is horizontal, but in front and behind it is inclined dowrwards. Six bones enter into its formation. The central portion is formed by one-half of the cribriform plate of the ethmoid. The sloping anterior part is formed by the grooved ala of the frontal bone, by the side of the nasal spine, and the posterior surface of the nasal bone. The sloping posterior part is formed by portions of the anterior and inferior surfaces of the body of the sphenoid, the ala of the vomer, and a part of the spherioidal process of the palate bone. The central part of the roof is perforated by the foramina of one-half of the cribriform plate, including the nasal slit, and, at its back part, the aperture of the sphenoidal air sinus opens into the spheno-ethmoidal recess.

The floor is smooth and concave from side to side. Over its anterior three-fourths it is formed by the palatal process of the superior maxilla, and over its posterior fourth by the horizontal plate of the palate bone. Near its anterior extremity, close to 
the incisor crest of the superior maxilla, is the upper opening of Stensen's canal.

The inner wall is known as the septum nasi. The osseous septum is formed by ten bones, in the following order, as nearly as possible, from below upwards: the nasal crests of the palatal processes of the superior maxillæ and palate bones; the vomer; the perpendicular plate of the ethmoid; the rostrum of the

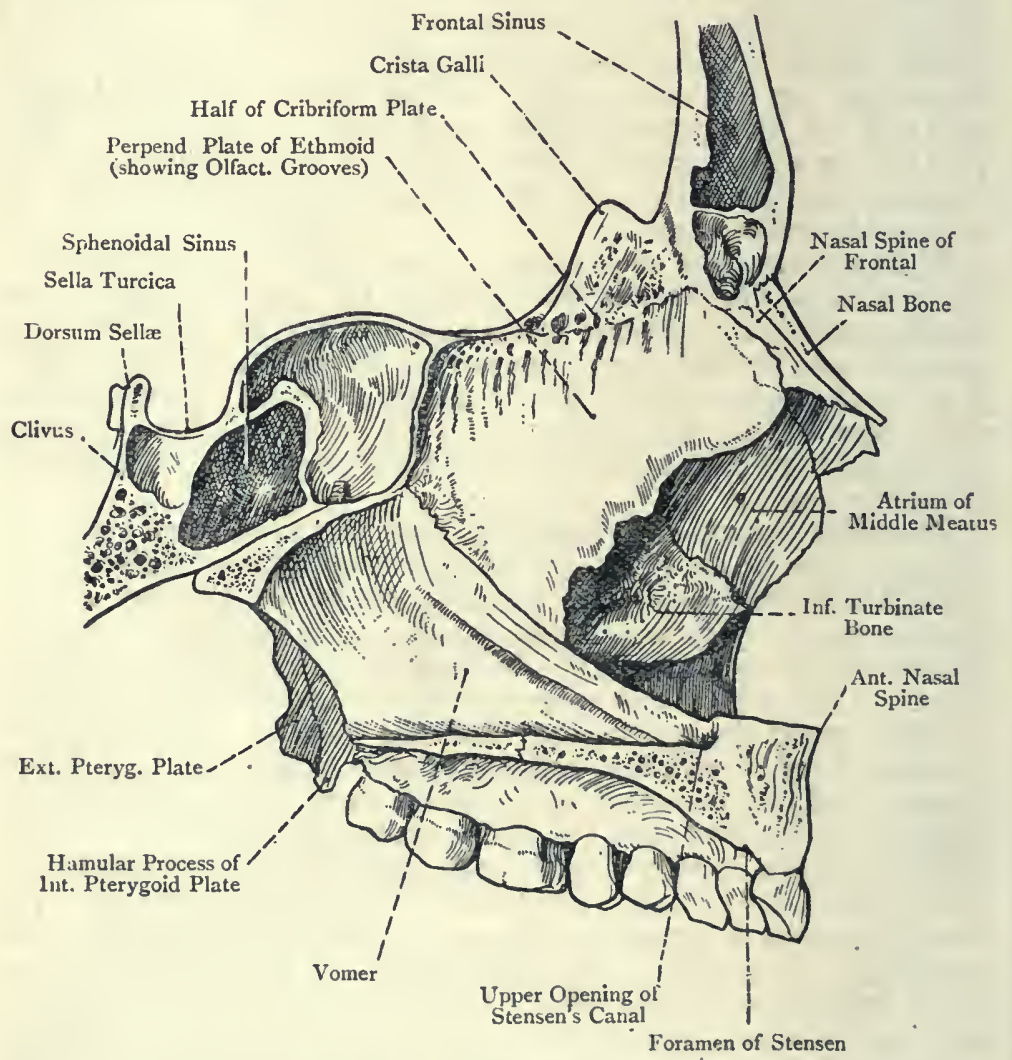

Fig. 8I, -Sagittal Section of the Anterior Part of the Skull to the Right of the Nasal Septum.

sphenoid; the nasal crest of the nasal bones; and the nasal spine of the frontal. The anterior border of the osseous septum presents a triangular deficiency, which is occupied in the recent state by the septal cartilage. The posterior border is formed by the posterior border of the vomer, which lies between the posterior nares. As previously stated, the septum is usually deflected to one side, most commonly the left. 
The outer wall is characterized by great irregularity, and is formed by seven bones, in the following order, as nearly as possible, from before backwards: (I) the nasal; (2) the nasal process of the superior maxilla; (3) the lachrymal; (4) the internal surface of the lateral mass of the ethmoid, presenting the superior and inferior turbinate processes (superior and middle spongy bones); (5) the inferior turbinate or spongy bone, which lies below the last named; (6) the perpendicular plate of the palate kone, together with parts of its orbital and sphenoidal processes; and (7) the internal pterygoid plate of the pterygoid process of the sphenoid.

The bulging projections on this wall are produced by the superior and inferior turbinate processes of the ethmoid and the inferior turbinate bone, in this order from above downward;, and the deep channels thereby formed are known as the meatis. These are three in number-superior, middle, and inferior. The superior meatus is situated towards the back part of the outer wal!, where it lies between the superior and inferior turbinate processe; of the ethmoid. It is comparatively short, and is directed obliquely forwards and upwards. The posterior ethmoidal cells open into it anteriorly, and the spheno-palatine foramen posteriorly. The middle meatus, which is longer than the superior, lies between the inferior turbinate process of the ethmoid and the inferior turbinate bone. At its anterior part it turns upwards, and is continued into the passage known as the infundibulum, which communicates with the frontal air sinus of its own side. The ascending part also communicates with the anterior ethmoidal cells. The middle portion-communicates with the middle ethmoidal cells, and presents the opening of the antrum of Highmore. The inferior meatus, which is the longest of the three, lies between the inferior turbinate bone and the floor of the nasal fossa. Near its anterior part is the lower orifice of the lachrymal canal, which lodges the nasal duct.

The Air Sinuses.-These are hollow cavities lined with mucous membrane, which are contained within the following bones: the frontal, sphenoid, ethmoid, superior maxillæ, and mastoid portions of the temporals. They communicate directly with the nasal fossæ, except the mastoid cells, which at either side are in communication with the tympanum, that in turn being connected by means of the Eustachian tube with the naso-pharynx, at a point external to the posterior naris. The maxillary air sinus or antrum of Highmore appears about the fourth month of intra-uterine life, but the other air sinuses do not appear until childhood, and they. do not show much development until the period of puberty (see the individual bones). In old age they all tend to become enlarged.

The frontal sinus (through means of the infundibulum) and the anterior ethmoidal cells open into the ascending front part of the middle meatus. The middle ethmoidal cells and the maxillary sinus open into the central portion of the middle meatus. The 
posterior ethmoidal cells open into the superior meatus, and the sphenoidal sinus opens into the spheno-ethmoidal recess.

The foramina which perforate the cribriform plate of the ethmoid transmit the filaments of the olfactory bulb, and are arranged in three sets, as follows: a middle set, which are simple perforations, and an external and internal set, which lead into small canals. These canals descend on the inner surface of the lateral mass and corresponding part of the perpendicular plate respectively, branching and opening out as they descend. The nasal slit transmits the nasal nerve and anterior ethmoidal artery. The spheno-palatine foramen leads from the spheno-maxillary fossa, and transmits the internal nerves of Meckel's ganglion and the spheno-palatine artery.

The anterior nares are the orifices by which the nasal fossæ open on the face through means of the anterior nasal aperture. Each naris is semipyriform, and is bounded above by the lower border of the nasal, externally by the nasal notch of the superior maxilla, and inferiorly by the premaxillary portion of that bone.

The posterior nares or choanæ are situated at the posterior extremities of the nasal fossæ, between the pterygoid processes of the sphenoid, and they communicate in the recent state with the naso-pharynx. They are oblong from above downwards, and their plane is oblique, being directed downwards and slightly forwards. Each naris is bounded externally by the internal pterygoid plate of the sphenoid, internally by the posterior border of the vomer, which separates the two nares, inferiorly by the posterior border of the horizontal plate of the palate bone, and superiorly by the vaginal process of the sphenoid, ala of the vomer, and sphenoidal process of the palate bone.

\section{The Lateral Region.}

The lateral region (norma lateralis) is formed by portions of the frontal, parietal, sphenoid, temporal, malar, superior maxilla, and inferior maxilla. It is somewhat triangular, with the base directed upwards. The base represents, for the most part, the superior temporal ridge, and corresponds with a curved line connecting the external angular process of the frontal with the lateral angle of the tabular part of the occipital. In front and behind it is limited by lines connecting the extremities of the base with the ramus of the inferior maxilla in the vicinity of the angle. This region presents the zygomatic arch, and, from behind forwards, are seen the mastoid process, opening of the external auditory meatus and suprameatal triangle, condyle of the inferior maxilla, lying in the anterior part of the glenoid fossa, eminentia articularis, and the sigmoid notch and coronoid process of the inferior maxilla, the latter lying within - the front part of the zygomatic arch. The central point of the orifice of the external auditory meatus is known as the auricular point, and the outer side of the angle of the inferior maxilla is 


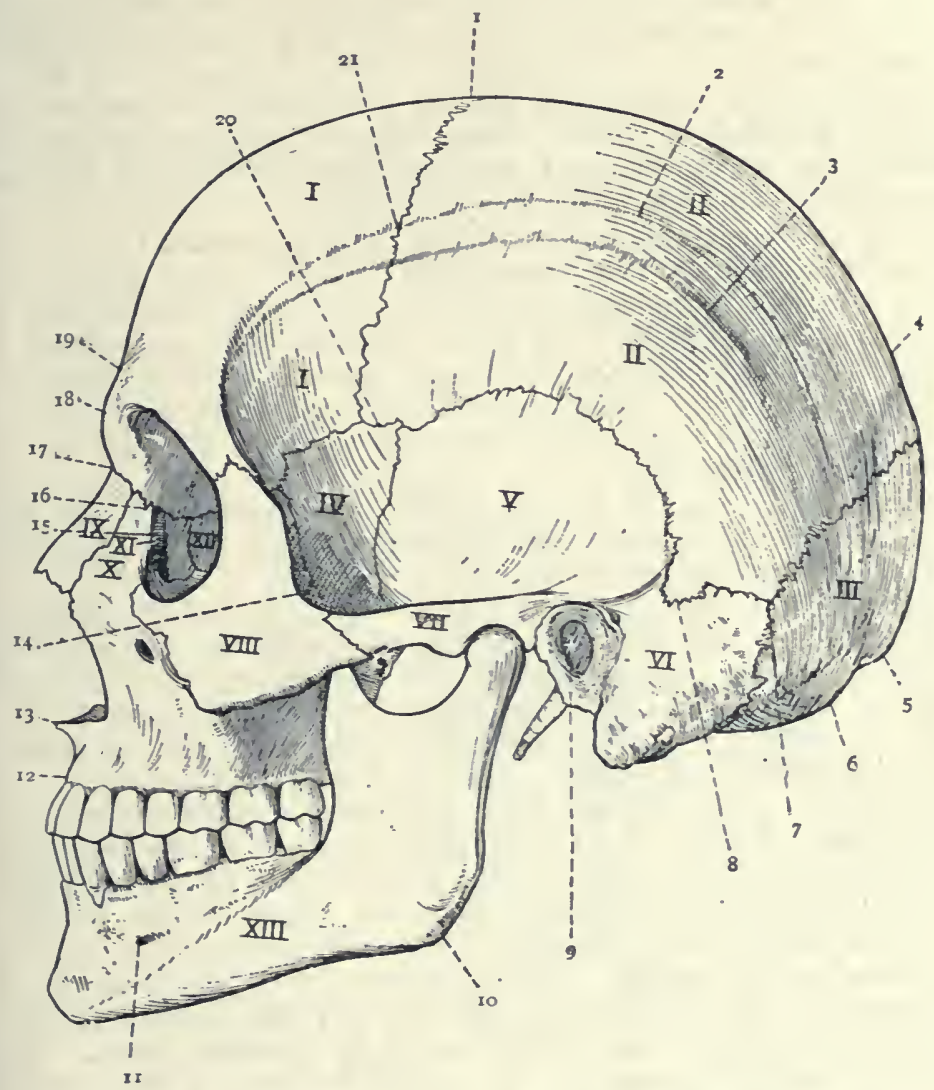

Fig. 82.--The Lateral Region of the Skull (Norma Lateralis).

I, I, Frontal ; II, II, Parietal ; III, Occipital ; IV, Great Wing of Sphenoid : V, Squamous Portion of Temporal ; VI, Nastoid Portion of Temporal ; VII, Zygoma ; VIII, Malar ; IX, Nasal ; X, Superior Maxilla (Nasal Process); XI, Lachrymal ; XII, Ethmoid (Os Planum); XIII, Inferior Maxilla.

I, Bregma ; 2, Superior Temporal Ridge; 3, Inferior Temporal Ridge; 4. Obelion; 5, Occipital Point ; 6, Inion ; 7, Asterion ; 8, Entomion; 9. Auricular Point ; 10, Gonion; I1, Mental Foramen; 12, Alveolar Point ; 13, Subnasal Point ; I4, Jugal Point ; 15, Lachrymal Groove ; 16, Dacryon; 17, Nasion; 18, Glabella; 19, Ophryon; 20, Pterion; 21, Stephanion. 
known as the gonion. The point, situated at the angle which the posterior border of the frontal process of the malar makes with the superior border of its zygomatic process, is known as the jugal point.

The sutures in this region are the fronto-malar, spheno-malar, fronto-sphenoidal, spheno-parietal, squamo-sphenoidal, coronal, squamous or squamo-parietal, temporo-malar (zygomatic), parietomastoid, and a portion of the occipito-mastoid.

The point where the superior temporal ridge crosses the coronal suture is known as the stephanion, and the point where the parieto-mastoid, occipito-mastoid, and lambdoid sutures meet is known as the asterion. The latter coincides with the position of the postero-lateral fontanelle in early life. The point near the anterior part of the parieto-mastoid suture, where a process of the parietal is received into the parietal notch of the mastoid, is known as the entomion.

The lateral region is divided by the zygomatic arch into the temporal and zygomatic fossæ, the former being above the arch, and the latter within and below it.

The temporal fossa is bounded above by the superior temporal ridges of the frontal and parietal, and below by the upper border of the zygomatic arch externally, and the infratemporal crest of the great wing of the sphenoid internally. It is formed by five bones, as follows: in front by the orbital process of the malar, above by the lower portions of the frontal and parietal, and below by the temporal division of the outer surface of the great wing of the sphenoid and the squamous portion of the temporal. The place where the parietal, frontal, great wing of the sphenoid, and squamous portion of the temporal are related to one another, and more particularly the region of the spheno-parietal suture, is known as the pterion, which coincides with the position of the antero-lateral fontanelle of early life. In this situation a Wormian bone is sometimes present, called the epipteric bone. The temporal fossa gives origin to the temporal muscle as high as the inferior temporal ridge, and the temporal fascia, which covers that muscle, is attached to the superior temporal ridge.

The zygomatic fossa is situated below the level of the infratemporal crest of the sphenoid. It is bounded externally by the ramus of the inferior maxilla and the inner surface of the zygomatic arch, and, between the two, it communicates with the exterior by means of the sigmoid notch. Internally it is bounded by the external pterygoid plate of the sphenoid. Superiorly it is limited by the zygomatic surface of the great wing of the sphenoid below the infratemporal crest, where it presents the foramen ovale and foramen spinosum, and by a small part of the squamous portion of the temporal. Anteriorly its wall is formed by the lower portion of the internal surface of the malar and the zygomatic surface of the superior maxilla, which latter presents the openings of the posterior dental canals. Its superior limit is 
closed, as a rule, by the malar, but sometimes by the great wing of the sphenoid, which may here articulate with the superior maxilla. Internally it is bounded by the zygomatic surface of the orbital process of the palate bone. The fissure leads into the orbit, and transmits the superior maxillary nerve to become the infraorbital, the infra-orbital vessels, the temporo-malar or orbital branch of the superior maxillary nerve, the orbital branches of Meckel's ganglion, and a communicating vein which passes between the inferior ophthalmic vein and the pterygoid plexus.

The pterygo-maxillary fissure lies vertically between the anterior border of the pterygoid process of the sphenoid and the posterior border of the superior maxilla, at their upper ends. Inferiorly the fissure is closed by the approximation of the bones forming its lips, a part of the tuberosity of the palate bone usually intervening between them, though direct articulation sometimes takes place between the pterygoid process and the superior maxilla. Internally the fissure is bounded by the perpendicular plate of the palate bone. It transmits the internal maxillary artery to the spheno-maxillary fossa. The pterygo-maxillary fissure meets the spheno-maxillary fissure at a right angle, and situated deeply within this angle is the spheno-maxillary fossa.

The boundaries of the spheno-maxillary fossa are as follows: anteriorly, the zygomatic surface of the superior maxilla at its inner and back part superiorly; posteriorly, the base of the pterygoid process of the sphenoid, and the lower and inner part of the anterior surface of its great wing; internally, the perpendicular plate of the palate bone, with its orbital and sphenoidal processes; and superiorly, the under surface of the body of the sphenoid. The contents of the fossa are the third part of the internal maxillary artery, the superior maxillary nerve, and the spheno-palatine or Meckel's ganglion, along with their branches. Two fissures communicate with this fossa, namely, the sphenomaxillary, leading into the orbit, and the pterygo-maxillary, opening into the zygomatic fossa. It also communicates with the.superior meatus of the nose by means of the spheno-palatine foramen on its inner wall.

The foramina which open into the spheno-maxillary fossa are as follows: three on the posterior wall, in the following order from above downwards, and from without inwards: the foramen rotundum for the superior maxillary nerve, the Vidian or pterygoid canal for the Vidian nerve and vessels, and the pterygo-palatine canal for the pharyngeal nerve and pterygo-palatine vessels. On the internal wall is the spheno-palatine foramen for the internal branches of Meckel's ganglion and the spheno-palatine artery. Inferiorly is the opening of the posterior palatine canal for the great or anterior descending palatine nerve and the descending palatine artery. In this situation there may also be the openings of the posterior and external accessory palatine canals for the posterior and external descending palatine nerves, but these openings usually branch off 
from the main canal. Anteriorly is the spheno-maxillary fissure. Externally the fossa communicates with the zygomatic fossa through the pterygo-maxillary fissure.

\section{The Inferior Region.}

The inferior region or external base (norma basilaris), from which the inferior maxilla is excluded, is limited in front by the central portions of the alveolar borders of the superior maxillæ, and behind by the superior curved lines of the occipital. At either side it is limited by the lateral portion of the alveolar border of the superior maxilla, and by a line connecting the tuberosity of that bone with the lateral angle of the tabular part of the occipital. It is very irregular, and presents three divisions-anterior, middle, and posterior.

The anterior division forms the hard palate, and resembles a horseshoe. It is bounded in front and laterally by the alveolar borders of the superior maxillæ, and behind by the posterior borders of the horizontal plates of the palate bones. The posterior border presents in the middle line the posterior nasal spine in two halves, from which the azygos uvulæ muscle arises. At either side of this it is sharp and concave for the attachment of the soft palate. The bones forming the hard palate are the palatal processes of the superior maxillæ over the anterior three-fourths, and the horizontal plates of the palate bones over the posterior fourth. The surface is vaulted, and is intersected by two sutures, middle palatal and transverse palatal. The middle palatal suture extends from the alveolar point to the posterior nasal spine, and indicates the meeting of the palatal plates of the superior maxillæ and palate bones of opposite sides. The transverse palatal suture crosses the middle one at right angles about $\frac{1}{2}$ inch in front of the posterior border, and externally it turns backwards to end at the posterior palatine foramen. It indicates the meeting of the palatal process of the superior maxilla and the horizontal plate of the palate bone of either side.

In young skulls two additional sutures are present, called maxillo-premaxillary, each of which extends from the posterior part of the anterior palatine fossa to the interval between the lateral incisor and canine teeth. Each of these sutures corresponds with the place of junction of the maxilla proper and the premaxilla.

The hard palate presents several openings. At the anterior extremity of the middle palatal suture is the diamond-shaped anterior palatine fossa. Within this are four openings, two being placed laterally, one at either side, called the foramina of Stensen (incisor foramina), and two in the median line in the intermaxillary suture, called the foramina of Scarpa, anterior and posterior respectively. Each of the former transmits a branch of the descending 


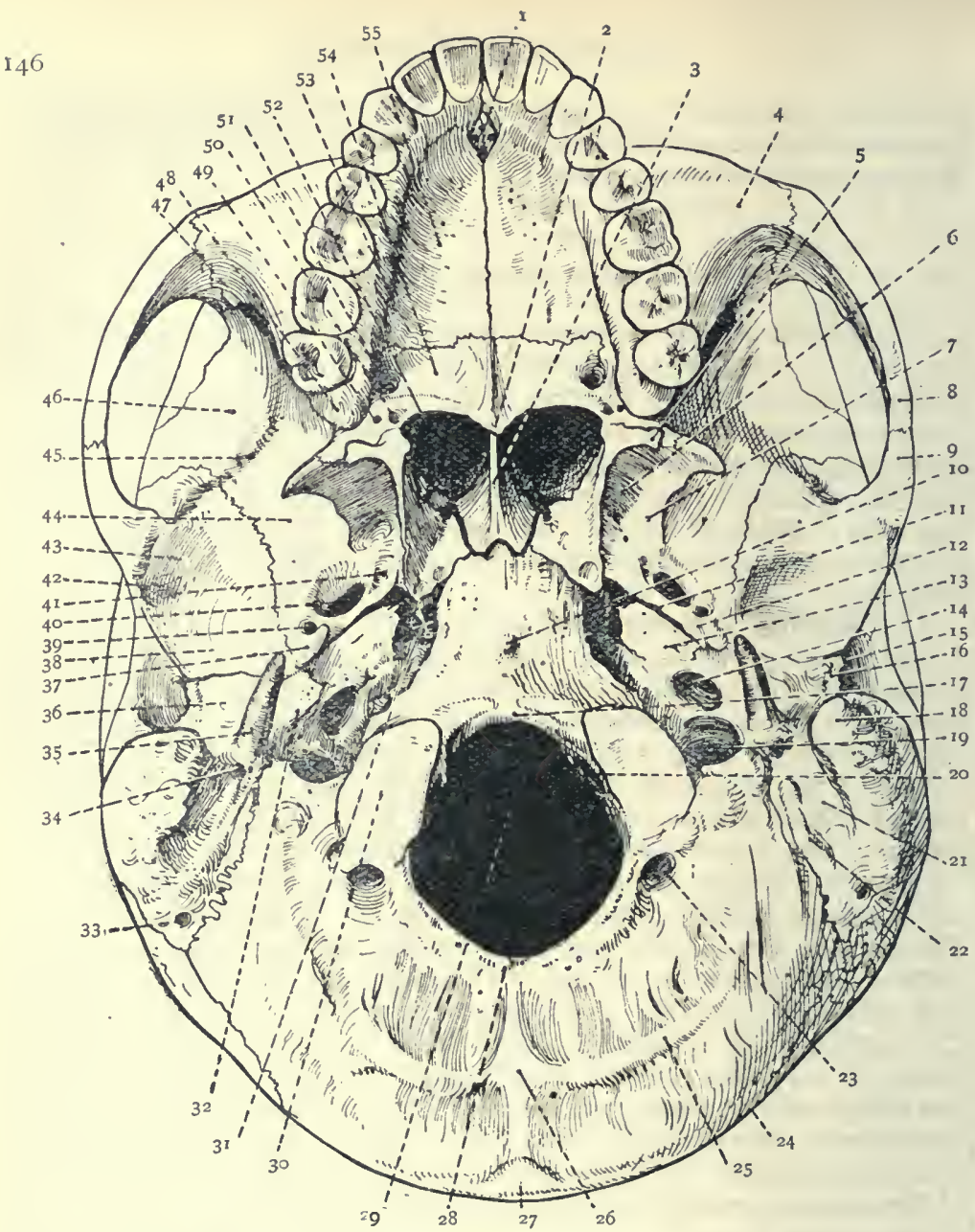

Fig. 84.-The External Base of the Skull.

1. Ant. Palatine Fossa

2. Post. Nasal Spine

3. Post. Border of Vonier

4. Facial Surf. of Sup. Maxilla

5. Hamular Process of Int. Pterygoid

Plate of Sphenoid

6. Pterygoid Fossa

7. Ext. Pterygoid Plate

8. Zygomatic Process of Malar

9. Zygoma of Temporal

I0. Pharyngeal Tubercle (pointer crosses Foram. Lacerum Med.)

II. Eustachian Groove

12. Groove for Chorda Tympani Nerve

13. Petrous Portion of Temporal (Origin of I,evator Palati)

14. Carotid Foramen

15. Iixt. Auditory Meatus

16. lixt. Auditory Process

17. Basion
18. Mastoid Process

19. Jugular Foramen

20. Ant. Condylar Foramen

21. Digastric Groove

22. Occipital Groove

23. Post. Condylar Foramen

24. Sup Curved Line of Occipital

25. Inf. Curved Line

26. Ext. Occipital Crest

27. Ext. Occipital Protuberance

28. Opisthion

29. Foramen Magnum

30. Right Occipital Condyle

3I. Foram. Lacerum Medium at +

32. Vaginal Proc. of Tymp. Plate 33. Mastoid Foramen

34. Stydo-Mastoid Foranten

35. Styloid l'rocess

36. ' 'ympanic Plate (Post. part of Glenoid Fossa)
37. Spinous Proc. of Sphenoid 38. Ant. part of Glenoid Fossa 39. Foramen Spinosum 40. Foramen Ovale 41. Foramen Vesalii

42. Preglenoid Tubercle

43. Eminentia Articurlaris

44. Zygonatic Fossa

45. Infratemporal Crest

46. Temporal Division of Gre Wing of Sphenoid

47. Spheno-Maxillary Fissure

48. Tuberosity of Sup. Maxilla

49. Ext. Access. Palat. Foramı

50. " Post. Access. Palat. Foram

51. Post. Palatine Foramen

52. Right Post. Naris (pointer ridge for Tensor Palati)

53. Croove for Descend. Palat.

54. Horiz, Plate of Palate Bon

55. Palat. Proc. of Sup. Maxil 
palatine artery from the palate to the nasal fossa, whilst each of the latter transmits the naso-palatine nerve from the nasal fossa to the hard palate, the anterior, which usually opens from the left nasal fossa, containing the left nerve, and the posterior, which usually opens from the right nasal fossa, containing the right nerve. Internal to the last molar alveolus at either side is the posterior palatine foramen, which is the outlet of the posterior palatine or palato-maxillary canal, and through which the great or anterior descending palatine nerve and the descending palatine ressels pass. Leading forwards from this foramen there is a groove for the transmitted structures. A little behind the posterior palatine foramen is the posterior accessory palatine foramen for the posterior de cending palatine nerve, and outside this is the external accessory palatine foramen for the external descending palatine nerve, but the last-named foramen is inconstant. Besides the foregoing openings, there are a number of nutrient foramina: Over its anterior three-fourths the hard palate presents several depressions for the palatal mucous glands, and extending inwards from the back part of the posterior palatine foramen at either side is a transverse ridge, which gives partial insertion to the tensor palati muscle.

The middle division extends from the posterior border of the hard palate to a transverse line on a level with the anterior margin of the foramen magnum. Laterally it is limited by a line extending from the tuberosity of the superior maxilla to the styloid process of the temporal. It is on a higher level than the anterior division, and its central or basilar part is known as the guttural fossa. The bones forming it at either side are the tuberosity of the palate bone, the pterygoid process, and a small part of the great wing, of the sphenoid, and the inferior surface of the petrous portion of the temporal. The central part is formed by the basilar process of the occipital, the body and vaginal processes of the sphenoid, the superior border and alæ of the vomer, and the sphenoidal processes of the palate bones.

Anteriorly it presents the posterior nares, already described, and . at either side of these openings is the pterygoid fossa, which is bounded internally by the internal, and externally by the external, pterygoid plate of the sphenoid, the fossa being completed inferiorly by the tuberosity of the palate bone.

In a line extending backwards and outwards from the external pterygoid plate to the styloid process the following parts are seen, in order from before backwards: foramen ovale; foramen spinosum; spinous process of the sphenoid; internal border of the tympanic plate of the temporal, forming posteriorly the vaginal process; and styloid process. Inside the foregoing line anteriorly is the Eustachian groove, which lies obliquely between the great wing of the sphenoid and the apical part of the petrous portion of the temporal. This groove lodges the cartilaginous part 
of the Eustachian tube, and, when followed outwards and backwards, it leads to the Eustachian canal in the angle between the squamous and petrous portions of the temporal.

On either side of the basilar process of the occipital is the foramen lacerum medium. It lies between the basilar process, the apex of the petrous portion of the temporal, and the great wing of the sphenoid near the root of the pterygoid process. In the recent state it is closed below by fibrous tissue, which is pierced by a meningeal branch of the ascending pharyngeal artery, and one or more emissary veins from the cavernous sinus.

In a line extending backwards and outwards from the foramen lacerum medium are the following markings on the inferior surface of the petrous portion of the temporal: the rough surface from which the levator palati arises; the carotid foramen, which transmits the internal carotid artery and the ascending branch of the superior cervical ganglion of the sympathetic; a minute foramen, on the posterior wall of the vertical portion of the carotid canal, for the tympanic branch of the carotid sympathetic plexus and tympanic branch of the internal carotid artery; the jugular fossa, which forms part of the jugular foramen; the tympanic canaliculus, on the ridge between the carotid foramen and jugular fossa, for the tympanic branch (Jacobson's nerve) of the glossopharyng@al and the tympanic branch of the ascending pharyngeal artery; and the auricular canaliculus, on the outer wall of the jugular fossa, for the auricular branch (Arnold's nerve) of the pneumogastric. Between the petrous portion of the temporal and the jugular process of the occipital is the foramen lacerum posterius or jugular foramen, which lodges the commencement of the internal jugular vein, and transmits the following structu es : the glosso-pharyngeal, pneumogastric, and spinal accessory nerves; the inferior petrosal sinus; and meningeal branches of the ascending pharyngeal and occipital arteries.

External to the front of the occipital condyle is the anterior condylar foramen for the hypoglossal nerve and a meningeal branch of the ascending pharyngeal artery. The under surface of the basilar process of the occipital presents the pharyngeal tubercle.

The posterior division is limited in front by a transverse line on a level with the anterior margin of the foramen magnum. and behind by the external occipital protuberance and the superior curved line at either side. It is formed by the supra occipital and condylar portions of the occipital, and the mastoic portions of the temporal bones.

In the middle line is the foramen magnum, which transmits the medulla oblongata and its membranes, the spinal accessory nerves the vertebral arterics, the anterior and posterior spinal arteries, anc parts of the cerebellar amygdalæ. The centre of the anterio margin of the foramen magnum is known as the basion, and th centre of the posterior margin as the opisthion. Proceeding outward 
from this foramen are the occipital condyle, jugular process, occipital groove for the occipital artery, digastric groove for the posterior belly of the digastric, and the mastoid process. Behind the occipital condyle is the posterior condylar fossa, in which there may be a posterior condylar foramen for the passage of an emissary vein from the lateral sinus. Behind the foramen magnum is the supra-occipital portion of the occipital, which presents the external occipital crest in the median line, and the inferior curved line extending outwards on either side from its centre.

\section{The Interior of the Cranium.}

A sagittal or antero-posterior section of the skull a little to one side of the median plane shows the septum nasi already described. Along, and at either side of, the vault of the cranium is the groove for the superior longitudinal venous sinus, which extends from before backwards, and on either side of its parietal portion are the Pacchionian depressions. The internal openings of the parietal foramina may be seen, as well as the branching system of meningeal grooves, and digitate impressions. The basi-cranial, basifacial, and basi-bregmatic axes are to be studied from this section. The basi-cranial axis represents a line drawn upwards and forwards from the basion to the spheno-ethmoidal suture. The basi-facial axis corresponds with a line drawn from the spheno-ethmoidal suture to the subnasal point. The angle formed by these two axes is known as the cranio-facial angle. The basi-bregmatic axis represents a line drawn vertically from the basion to the bregma.

The most instructive coronal or transverse section is one made in the plane of the basi-bregmatic axis. Such a section gives important views of the parts within the petrous portion of the temporal, such as the external auditory meatus, tympanum, and vestibule.

When a horizontal section has been made on a level with the occipital point and the most prominent part of the glabella, the vaulted roof of the cranium is removed. This is called the calvaria, or skull-cap, and it is formed by portions of the frontal, parietals, squamous portions of the temporals, and occipital. The outer plate is strong, except over the temporal region, but the inner is brittle and readily cracked, from which circumstance it is known as the vitreous (glassy) plate. Between the two plates there is cancellated tissue, here called diploë. The interior of the calvaria presents branching meningeal grooves, digitate impressions, and along the middle line the groove for the superior longitudinal venous sinus, with depressions at either side for the Pacchionian bodies. The openings of the parietal foramina may be noted. 


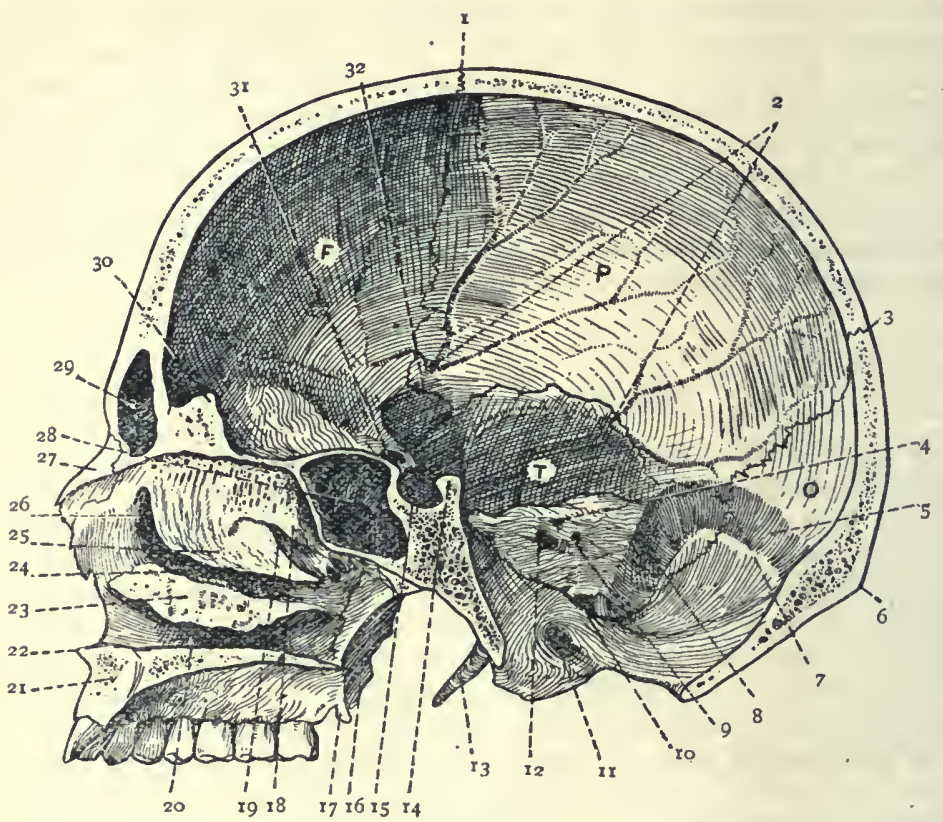

Fig. 85.-Sagitral Section of the Skull to the Right of the Median Plane.

F, Frontal; P, Parietal; O, Occipital; T, Temporal.

1. Coronal Suture

2. Anterior and Posterior Meningeal Grooves

3. Lambdoid Suture

4. Hiatus Subarcuatus

5. Sigmoid Groove

6. Inion (External Occipital Protuberance)

7. Asterion

8. Sigmoid Groove

9. Aqueductus Vestibuli

ro. Jugular Foramen ri. Anterior Condylar Foramen

r2. Internal Auditory Meatus

13. Styloid Process

14. Dorsum Sella

5. Sella Turcica

16. External Pterygoid Plate

77. Hamular Process

18. Superior Turbinate Process of Ethmoid.

19. Superior Meatus

20. Inferior Meatus

21. Right Superior Maxilla

\author{
22. Anterior Nasal Spine \\ 23. Inferior Turbinate Bone \\ 24. Middle Meatus \\ 25. Inferior Turbinate Process \\ of Ethmoid \\ 26. Infundibulum \\ 27. Nasal Bone \\ 28. Right Sphenoidal Sinus \\ 29. Right Frontal Sinus \\ 3o. Crista Galli \\ 3x. Optic Foramen \\ 32. Pterion
}

\section{The Internal Base of the Skull.}

The internal base forms the floor of the cranial cavity, and is of very irregular outline and thickness. The thickest and densest parts are the petrous portions of the temporals. The mastoid portion of the temporal and the basilar part of the occipital are also thick. The thinnest parts are the cribriform plate of the ethmoid and the orbital plates of the frontal, but the central portions of the cerebellar fossæ of the occipital are also thin, sometimes remarkably so. The interior of the base is divided into three fossæ-anterior, middle, and posterior.

Anterior Fossa.-The floor of this fossa is formed by the orbital 
plates of the frontal, the cribriform plate of the ethmoid, and the small wings, jugum sphenoidale, and ethmoidal spine of the sphenoid. It is limited posteriorly by the posterior or Sylvian border of the small wing of the sphenoid at either side, and by the limbus sphenoidalis in the centre. It is subdivided into a central and two lateral parts.

The central portion, which is depressed, is formed by the cribriform plate of the ethmoid and the ethmoidal spine and jugum of the sphenoid. In the middle line it presents the crista galli, which gives attachment to the falx cerebri. In front of this is the foramen cæcum, which, when pervious, transmits an emissary-vein passing between the intracranial superior longitudinal sinus and the veins of the roof of the nose. At each side of the crista galli are the nasal slit for the nasal nerve and anterior ethmoidal artery; the cribriform foramina for the filaments of the olfactory bulb; the cranial opening of the anterior ethmoidal canal for the anterior ethmoidal artery and nasal nerve; and the cranial opening of the posterior ethmoidal canal for the posterior ethmoidal artery and spheno-ethmoidal branch of the nasal nerve. The last two openings are situated at the outer side of the cribriform plate, external to the olfactory groove which marks it. Directly beneath each half of the cribriform plate is the corresponding nasal fossa.

Each lateral portion of the anterior fossa is irregularly convex, and forms the roof of the orbit. It is formed by the orbital plate of the frontal and the small wing of the sphenoid. It is very thin, and, except over the small wing of the sphenoid, it presents digitate impressions for the convolutions of the orbital surface of the frontal lobe of the cerebrum, which it supports. The sutures in the anterior fossa are the fronto-ethmoidal, fronto-sphenoidal, and sphenoethmoidal.

Middle Fossa.-This fossa is on a lower level than the anterior. It is bounded in front by the posterior or Sylvian border of the small wing of the sphenoid at either side, and by the limbus sphenoidalis in the centre. Behind, it is limited by the superior border of the petrous portion of the temporal at either side, and by the dorsum sellæ of the sphenoid in the centre. It presents a central and two lateral divisions. The central division is formed by the superior surface of the body of the sphenoid, posterior to the limbus sphenoidalis. Each lateral division, which is much depressed, is formed anteriorly by the superior surface of the great wing of the sphenoid, externally by part of the squamous portion of the temporal, and posteriorly by the superior surface of the petrous portion of that bone. It lodges the temporo-sphenoidal lobe of the cerebrum, and it presents the following sutures: the spheno-parietal; squamous or squamoparietal ; squamo-sphenoidal ; and petro-sphenoidal.

The central division presents the following parts: the optic groove and olivary eminence for the optic commissure; the optic foramen of each side for the optic nerve and ophthalinic artery; the anterior clinoid process of each side; the sella turcica or 
pituitary fossa for the pituitary body; the cavernous or carotid groove, at either side of the sella turcica, for the cavernous venous sinus and internal carotid artery, the latter being accompanied by the cavernous sympathetic plexus of nerves, and having the sixth cranial nerve on its outer side; the middle clinoid process of each side (sometimes connected with the anterior, which it faces); the dorsum sellæ; the posterior clinoid process of each side, at either lateral angle of the dorsum sellæ; and the notch for the sixth cranial nerve, on each side of the dorsum sellæ a little below the posterior clinoid process. The central division corresponds with the interpeduncular region at the base of the cerebrum:

Each lateral division is marked by merirgeal grooves and digitate impressiors, and presents the following openings: the sphenoidal fissure, or foramen lacerum anterius or orbitale, for the third cranial nerve, the fourth, the three branches of the ophthalmic division of the fifth (namely; frontal, lachrymal, and nasal), and the sixth cranial nerves, the sympathetic root of the ciliary ganglion, the superior and inferior ophthalmic veins, the orbital branch of the middle meningeal artery, and a portion of the dura mater to form the orbital periosteum; the foramen rotundum, leading to the spheno-maxillary. fossa, and transmitting the superior maxillary division of the fifth cranial nerve ; the foramen ovale, leading to the zygomatic fossa, and transmitting the inferior maxillary division and the motor root of the fifth cranial nerve, the small meningeal artery, an emissary vein from the cavernous sinus, and occasionally the small superficial petrosal nerve ; the foramen Vesalii (inconstant), leading to the scaphoid fossa at the root of the internal pterygoid plate, or to the pterygoid fossa external to the scaphoid fossa, and transmitting an emissary vein from the cavernous sinus; the foramen spinosum, leading to the zygomatic fossa, and transmitting the middle meningeal artery and a recurrent branch of the inferior maxillary nerve; and the foramen lacerum medium situated between the basilar process of the occipital, the apex of the petrous portion of the temporal, and the great wing of the sphenoid near the root of the pterygoid process. The posterior opening of the Vidian canal, which leads to the spheno-maxillary fossa and transmits the Vidian nerve and artery, is to be fourd on its anterior wall, while the carotid canal for the internal carotid artery, with a plexus of sympathetic nerve fibres, opens on its external wall.

The superior surface of the petrous portion of the temporal presents the following markings: the Gasserian depression, near the apex, for the Gasserian ganglion ; the hiatus Fallopii, to which a groove conducts for the great superficial petrosal nerve and the petrosal branch of the middle meningeal artery; (within this opening there may be a small one for the external superficial petrosal nerve); the accessory hiatus, to which a groove conducts, for the small superficial petrosal nerve; the eminentia arcuata, 


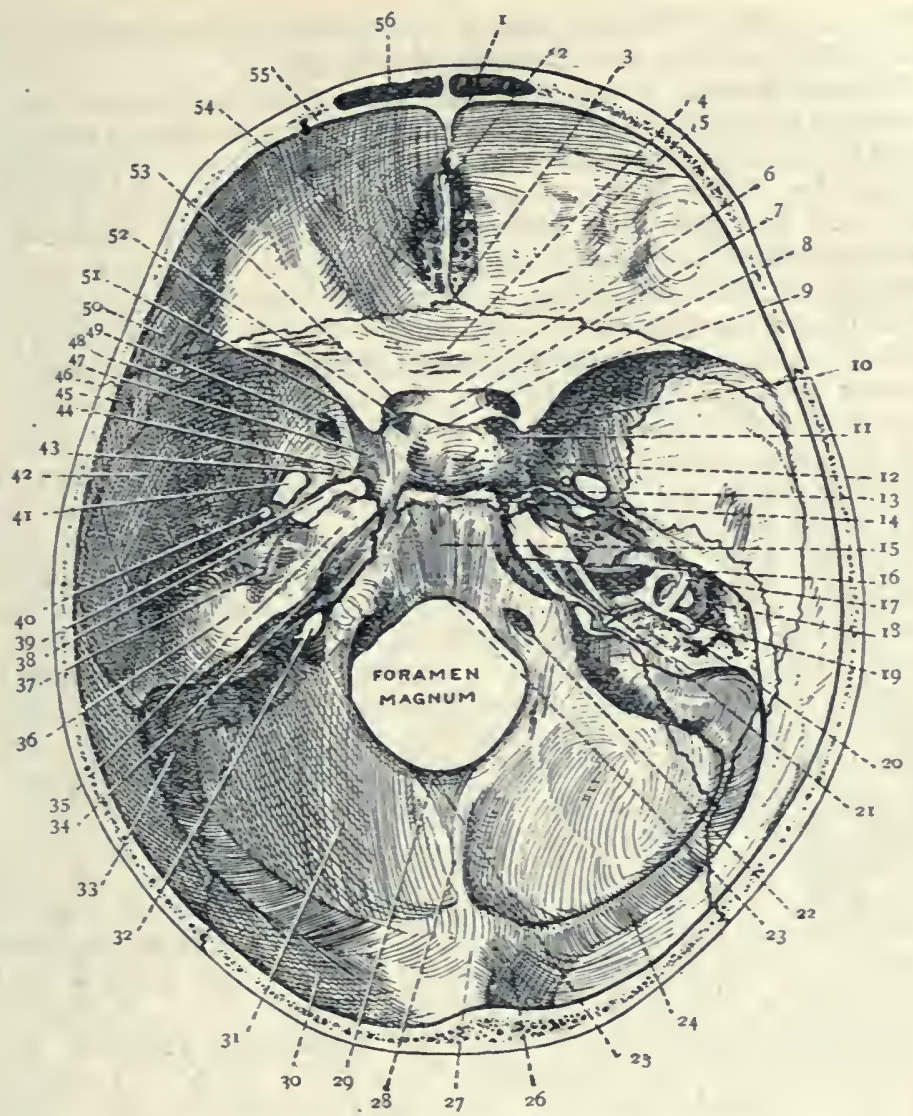

Fig. 86.-The Internal Base of the Skull.

(The Suprior Surface of the Petrous Portion of the Right Temporal Bone has been removed to expose the Internal Auditory Meatus and Semicircular Canals.)

x. Foramen Cacum

2. Crista Galli

3. Ethmoidal Spine of Sphenoid

4. Jugum Sphenoidale

5. Anterior Fossa

6. Limbus Sphenoidalis

7. Optic Groove

8. Olivary Eminence

9. Sylvian Border of Small Wing of Sphenoid

ra Anterior Clinoid Process

1x. Middle Clinoid Process

12. Sella Turcica

r3. Posterior Clinoid Process

r4. Dorsum Sella

15. Basilar Groove

16. Groove for Inf. Petrosal Sinus

17. Superior Semicircular Canal 18. External Semicircular Canal
89. Posterior Semicircular Canal 2a. Internal Auditory Meatus (laid open)

21. Opening of Internal Auditory Meatus

22. Anterior Condylar Foramen

23. Basion

24. Groove for Lateral Sinus

25. Vermiform Fossa

26. Torcular Herophili

27. Int. Occipital Protuberance

28. Internal Occipital Crest

29. Opisthion

30. Cerebral Fossa

31. Cerebellar Fosa

32. Jugular Foramen

33. Sigmoid Groove

34. Internal Auditory Meatus

35. Gasserian Depression

36. Eminertia Arcuata
37. Hiatus Fallopii

38. Accessory Hiatus

39. Foramen Lacerum Medium

4a Foramen Spinosum

4x. Foramen Ovale

42. Middle Fosso

43. Foramen Vesalii

44. Lingula of Sphenoid

45. Parietal Bone

46. Coronal Snture

47. Cavernous Groove

48. Pterion

49. Foramen Rotundum

50. Orbital Plate of Frontal

5 . Sphenoidal Fissure

52. Carotid Notch

53. Optic Foramen

54. Posterior Ethmoidal Canal

55. Anterior Ethmoidal Canal

56. Left Frontal Sinus 
which coincides with the position of the superior semicircular canal of the internal ear ; and the tegmen tympani.

Posterior Fossa.-This fossa is on a lower level than the middle. It is limited in front by the dorsum sellæ of the sphenoid in the centre, and the superior border of the petrous portion of the temporal at either side. Behind, it is limited by the internal occipital protuberance and the groove at either side for the lateral venous sinus, which groove also marks its lateral extent. It lodges the pons Varolii, medulla oblongata, and cerebellum. The bones which form it are as follows : the dorsum sellæ of the sphenoid; the basilar, condylar, and supra-occipital portions of the occipital ; the petrous and mastoid portions of the temporal; and the posteroinferior angle of the parietal. It presents the following sutures: the occipito-mastoid ; parieto-mastoid; and petro-basilar. The following parts are to be noted: the basilar groove, the upper part of which lodges the pons Varolii and basilar artery, whilst the lower part contains the medulla oblongata; the foramen magnum, which transmits the medulla oblongata and its membranes, the spinal accessory nerves, and the vertebral, anterior spinal, and posterior spinal, arteries; the anterior condylar foramen, on either side of the foramen magnum, for the hypoglossal nerve and a meningeal branch of the ascending pharyngeal artery; the internal occipital crest, which gives attachment to the falx cerebelli, and is occasionally grooved for the occipital process of the cerebelnear the foramen magnum this crest presents hemispheres of the meatus, on the posterior temporal, for the posterior surface of the petio of the internal auditory Wrisberg, and aucial and auditory nerves, pir trous portion of the $\frac{1}{4}$ inch external auditory artery; the aqueductus ve ciars intermedia of the ductus en the preceding, for a small artery ancsa, atibuli, about the flocturaticus; the fossa subarcuata, repr bein, and the floccular fossa of early life, situated close to the superior $u$ mesenting of the petrous portion, about midway between the opening he apeborder foramal auditory meatus and aqueductus vestibuli. opening ' $\mathrm{ng}$ of of the of amen or foramen lacerum posterius, between the jugla jusposterigular The occipital and petrous portion of the temporal. lie obliquiar foramen is divided into three compartments, wh fourc lie obliquely from behind forwards and compartments, whicarotid ch external compartment transmits the inwards. The poster on its become the internal jugular vein, the lateral venous sinus 1 ascending pharyngeal artery. the glosso-pharyngeal, pneumogastric and compartment transmits near and the antero-internal compartment and spinal accessory nerves; hich petrosal venous sinus. The antero-internassage to the inferior and be more or less completely isolated by mean compartment may this process passing between the occipital means of the intrajugular ficial the temporal. 
The posterior fossa is grooved by the following venous sinuses: the lateral sinus, which extends from the internal occipital protuberance to the jugular foramen, grooving in its sinuous course the tabular part of the occipital, the postero-inferior angle of the parietal, the mastoid portion of the temporal, and the jugular process of the occipital, (opening from which there is usually the mastoid foramen, and occasionally the posterior condylar foramen); the superior petrosal sinus, which grooves the superior border of the petrous bone; the inferior petrosal sinus, along the course of the petro-basilar suture; and the occipital sinus, which sometimes grooves the internal occipital crest.

\section{Wormian Bones.}

The Wormian bones (so named after Wormius) are supernumerary bones which are frequently met with in the course of the cranial sutures, and occasionally in the face, as in the region of the lachrymal bones, and at the outer extremity of each spheno-maxillary fissure. From their position in the course of sutures they are known as ossa suturarum. They are for the most part of small size and triangular outline, and are hence sometimes spoken of as ossa triquetra. They are usually due to the appearance of special ossific centres. Their most common situation is along the course of the lambdoid suture, where they may form a regular chain. The superior angle of the occipital sometimes persists as a Wormian bone, called pre-interparietal, which may be double. One is often found between the antero-inferior angle of the parietal and great wing of the sphenoid in the region of the pterion, and it is known as the epipteric bone. If the metopic or frontal suture is persistent one or more Wormian bones may be present along its course, and, if at the upper part, they may give rise by their persistence and union to a bregmatic bone. In the condition known as chronic hydrocephalus Wormian bones of large size are present in great numbers along the cranial sutures.

\section{The Skull at Birth.}

The skull at birth is remarkable for its large size, and for the great development of the cranium as compared with the face. The face is equal to one-eighth of the cranium, whereas in the adult it is equal to one-half. The occipital, parietal, and frontal regions are well developed, the parietal and frontal eminences are very conspicuous, and the mastoid processes are absent. The bones are not united by sutures, but are connected by fibrous tissue, continuous with the periosteum externally and dura mater internally. Membranous intervals exist between the angles of certain bones, these being called fontanelles from the pulsation, or wellingup sensation, which can be felt there. They are six in number, two being placed in the median line, anterior and posterior, and two at either side, antero-lateral and postero-lateral. The anterior fontanelle is situated between the antero-superior angles of the 
parietals and the superior angles of the two halves of the frontal. It is large and diamond-shaped, and it is not completely closed, as a rule, until towards the end of the second year. The posterior fontanelle is situated between the postero-superior angles of the parietals and the superior angle of the occipital. It is small and triangular, and is usually closed at, or shortly after, birth, but the surrounding bones are still movable. The antero-lateral and postero-lateral fontanelles correspond with these angles of the parietal. The antero-lateral is situated between the parietal,

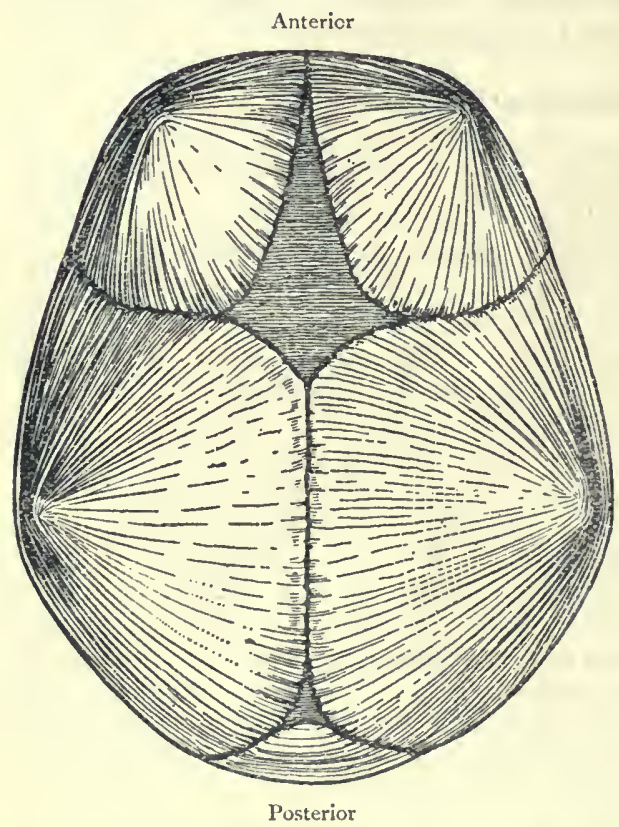

Fig. 87.-The Sielle at Birth, Showing the Anterior and Posterior Fontanelles, and the Parietal Eminences. sphenoid, frontal, and squamous portion of the temporal, whilst the postero-lateral is situated. between the parietal, occipital, and mastoid portion of the temporal. For the sagittal fontanelle of the earlier part of fœtal life, see p. 63 .

The skull increases rapidly in sire during the first six years, and a further marked increase commences on the approach of puberty, which is associated with the development of the air sinuses. The latter increase affects chiefly the frontal and facial regions. In old age the cranial bones become thinner, the air sinuses undergo enlargement, and the sutures show indications of obliteration.

Characters of the Female Skull.- The bones are smaller and smoother than in the male. The mastoid processes, superciliary ridges, and glabella are less prominent. The frontal and occipital regions are not so well developed relatively to the parietal. The cranial capacity is rather less. The face as a rule is narrower, and bears a smaller proportion to the cranium.

\section{Racial Peculiarities of the Skull.}

In comparing the skulls of different races attention has to be directed to the following points : the capacity of the cranium, its circumference, its relative length, breadth, and height, the degree of forward elongation of the jaws, and the shape of the anterior nasal and orbital apertures. The cranial capacity: may be ascertained by filling the skull with shot, or various kinds of seeds, and 
then measuring the contents in a graduated vessel. The capacity ranges from about 60 to iro cubic inches, and, according to their capacity, skulls are divided into three groups, namely, mesocephalic, with a capacity of about 85 cubic inches, as in Chinese ; megacephalic, with a capacity exceeding that, as in Europeans; and microcephalic, with a capacity under it, as in Australians.

In regard to craniometry the following craniometrical terms may here be summarized :

Alveolar point $=$ the point of meeting of the anterior margins of the alveolar borders of the superior maxillæ.

Antinion = the most prominent point of the glabella.

Asterion = the point where the parieto-mastoid, occipito-mastoid, and lamb doid sutures meet.

Auricular point $=$ the centre of the opening of the external auditory meatus. Basion = the centre of the anterior margin of the foramen magnum.

Bregma = the point of junction of the sagittal and coronal sutures.

Dacryon $=$ the point where the horizontal fronto-maxillary suture meets the vertical lachrymo-maxillary suture.

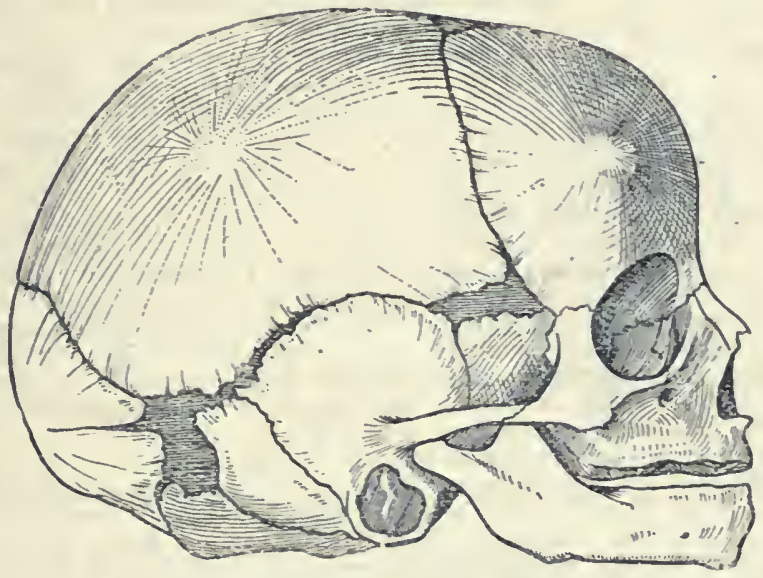

Fig. 83.-The Skull at Birth, showing the Antero-laterai. and Postero-lateral Fontanelles.

Entomion = the point near the anterior part of the parieto-mastoid suture where a process of the parietal is received into the parietal.notch of the mastoid.

Glabella $=a$ point midway between the superciliary ridges of the frontal.

Gnathion, or mental point = the middle point of the anterior lip of the lower border of the inferior maxilla.

Gonion = the outer side of the angle of the inferior maxilla.

Inion = the external occipital protuberance.

Jugal point $=$ a point situated at the angle which the posterior border of the frontal process of the malar forms with the superior border of its zygomatic process.

Lambda $=$ the meeting of the sagittal and lambdoid sutures.

Nasion, or nasal point = the meeting of the two fronto-nasal sutures.

Obelion = the point where the horizontal line connecting the parietal fora. mina intersects the sagittal suture.

Occipital point = the part of the occipital in the median plane at the greatest distance from the glabella.

Ophryon=the centre of a line drawn from one temporal ridge to the other across the narrowest part of the frontal region.

Opisthion = the centre of the posterior margin of the foramen magnura. 
Pterion $=$ the region of the spheno-parietal suture.

Rhinion = the lower part of the in ternasal suture.

Stephanion $=$ the point where the superior temporal ridge crosses the coronal suture.

Subnasal point $=$ the centre of the base of the anterior nasal spine.

The horizontal circumference of the cranium represents the measurement at the level of a plane passing through the most prominent part of the glabella in front, the pterion laterally, and the occipital point behind.

The greatest length represents the measurement from the most prominent part of the glabella to the occipital point. The greatest breadth represents the transverse measurement at the level of the most prominent parts of the temporal fossæ above the supramastoid crests. The proportion of greatest breadth to greatest length is the index of breadth, or cephalic index. In civilized races about 7 inches represents an average length, and about $5 \frac{1}{2}$ inches an average breadth. According to their cephalic index, skulls are arranged in three classes, namely, brachycephalic (broad and short), with a cephalic index over 80, as in Malays, etc. ; mesaticephalic (intermediate), with an index of 75 to 80, as in Europeans and Chinese ; and dolicocephalic (long and narrow), with a cephalic index below 75, as in Kaffirs and Fijians.

The height of the skull represents the measurement from the basion to the bregma, and its proportion to the length is the index of height, or vertical index. Its average in civilized races corresponds with the breadth.

The longitudinal are of the skull represents the measurement from the nasion to the opisthion carried over the roof, and the basi-nasal length represents the measurement from the basion to the nasion. These two measure ments, plus the distance between the basion and the opisthion, represent the vertical circumference of the cranium in the median plane. The degree of projection of the jaws is ascertained from the gnathic or alveolar index. This index represents the proportion of the basi-alveolar length to the basinasal. According to the gnathic index, skulls are arranged in three classes, namely, orthognathous (straight and upright jaw), with a gnathic index below 98, as in Europeans ; mesognathous (intermediate jaw), with an index of from 98 to 103 , as in Chinese and Japanese; and prognathous (forward jaw), with an index over 103 , as in Australians.

The form of the anterior nasal aperture is ascertained from the nasal index. This represents the proportion of the greatest transverse measurement of the aperture to the height, which latter is the measurement from the nasion to the subnasal point. According to their nasal index, skulls are arranged in three classes, namely, leptorhine (narrow nose), with a nasal index below 48, as in Europeans; mesorhine (intermediate nose), with an index of from 48 to 53 , as in Chinese and Japanese ; and platyrhine (broad nose), with an index above 53, as in Australians and Kaffirs.

The form of the orbital aperture is ascertained from the orbital index, which represents the proportion of the height to the width of the orbital aperture. There are three varieties of orbital index, namely, megaseme (great index), when it exceeds 89 , as in the Chinese; mesoseme (intermediate index), when it is between 89 and 84 , as in Europeans ; and microseme (small index), when it is below 84, as in Australians.

\section{Delormities of the Skull.}

The most common cause of cranial deformities is premature synostosis or osseous union of bones which are normally separate, the result being closure or obliteration of certain sutures. When the sagittal suture becomes prematurely obliterated transverse growth is arrested, and, to compensate for this, increased growth takes place at the coronal and lambdoid sutures. The anteroposterior diameter of the cranium is greatly increased, and the vault assumes a boat-like sliape. This variety is known as scaphocephalus. When the coronal suture becomes prematurely obliterated, increased growth takes place upwards; ancl the vertical diameter is greatly increased. This variety is known as acrocephalus (pointed liead). When one-half of the coronal or lambdoid suture becomes prematurely obliterated, oblique deformity takes place, this 
form being known as plagiocephalus (oblique or awry head). When the metopic or frontal suture becomes prematurely obliterated, growth is arrested in the frontal region, and the skull assumes a triangular shape. This variety is known as trigonocephalus. When premature obliteration of the sutures at the base of the skull takes place, the deformity known as eretin skull results. This is characterized by enlargement of the cranium (except in the occipital region), which becomes very heavy, and assumes an irregular, somewhat conical shape, with the apex at the sagittal suture. It is associated with mental dulness, idiocy, and stunted growth, and the general condition is known as cretinism.

\section{Development of the Skull.}

The skull is composed of two divisions, which are genetically distinct from each other. One division represents the cranium, which is developed from the mesenchyme (mesoderm), around the cephalic part of the notochord. The other division, which includes the face and the hyoid bone, represents the visceral-
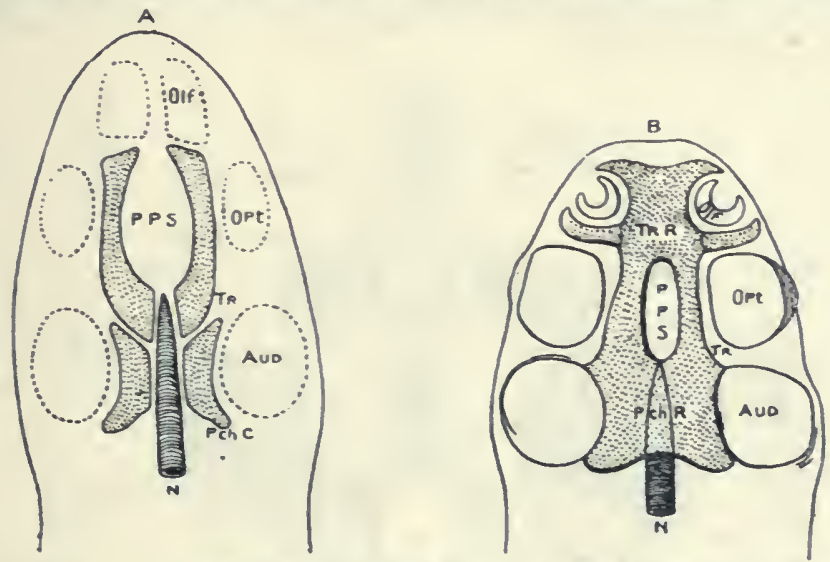

Fig. 89.-Diagrams of the Primitive Cartilaginous Cranium (WIEDERSHEIM).

A. First Stage. $-N$, Notochord; PchC, Parachordal Cartilage; Tr, Prechordal Cartilage; PPS, - Primitive Pituitary Space; Olf, Opt, Aud, positions of Organs of Smell, Sight, and Hearing.

B. Second Stage.-N, Notochord; PchR, Parachordal Region (Basilar Plate); TrR, Fusion of Prechordal Cartilages; PPS, Primitive Pituitary Space; TrR, Prechordal Region; Olf, Opt, Aud, as in A.

arch skeleton, and is developed, for the most part, from the first, second, and third visceral arches. The stages in the development of the skull are, as in the vertebral column, membranous, chondral or cartilaginous, and osseous.

\section{The Cranium.}

The cranium, as just stated, is developed from the mesenchyme which surrounds the cephalic part of the notochord.

This chord, after passing through the future odontoid process of the axis, extends forwards to the posterior part of the pituitary region of the base of the cranium, where the dorsum sellæ of the sphenoid bone is subsequently developed. In this situation it lies on the ventral aspect of the mid-brain, and within the sharp ventral bend described by the fore-brain. The cephalic part of the notochord carries along with it the skeletogenous sheath of mesenchyme. 
Membranous Stage.-The cranium is at first entirely membranous, and is formed by the mesenchyme which surrounds the cephalic part of the notochord. This mesenchyme expands forwards laterally, and then backwards, so as to form a membranous capsule for the cerebral vesicles. This capsule forms the primitive membranous cranium, which presents a base and a vault, or tegmental part.

Chondral or Cartilaginous Stage.-The base of the primitive membranous cranium undergoes chondrification, but the vault or tegmental portion is not involved in this process. Having, therefore, regard to the development of the bones which construct the osseous cranium, the membranous craniun is to be considered under two aspects-chondral and membranous.

Chondrocranium.-The chondrocranium involves the basal part of the primitive membranous cranium, which has become converted into cartilage. Chondrification gives rise to a single plate of cartilage, extending from the basi-occipital to the ethmoidal region: This basal cartilage may be regarded as consisting of two segments-chordal and prechordal. The chordal segment embraces the cephalic part of the notochord, and the prechordal segment is in front of the notochord.

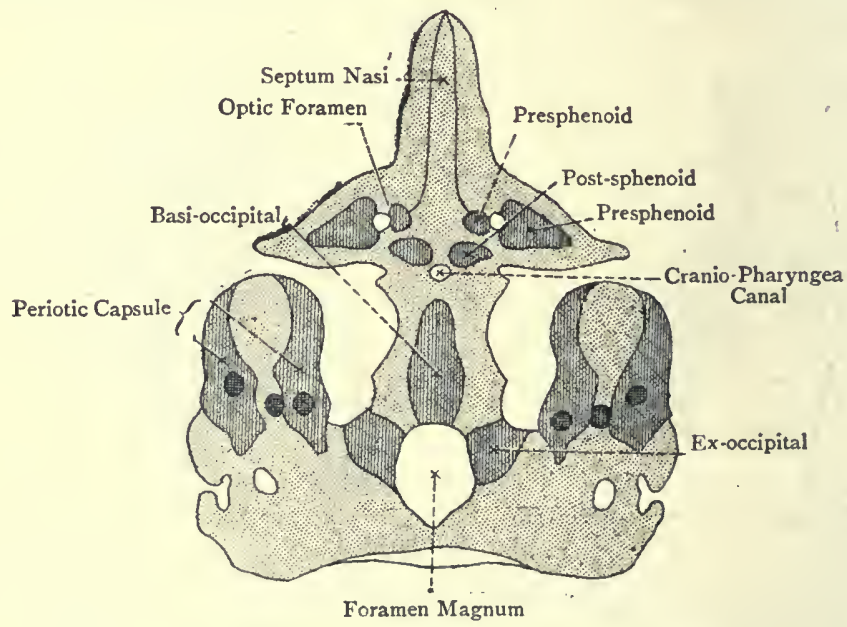

Fig. 90.-The Chondrocranium (After Huxley).

The chordal segment represents the parachordal cartilages of certain animals. The prechovdal segment represents the prechordal cartilages, or trabeculæ cranii of Rathke, present in certain animals. In man, however, it is a continuous cartilage, which is interrupted at the centre by a membranous interval, representing the primitive pituitary space. In this region the cranial cavity communicates with the primitive mouth, or stomodxum, from which latter the pouch of Rathke ascends to form the anterior lobe of the pituitary body.

The chordal segment, as stated, embraces the cephalic end of the notochord, but the chord soon disappears, and the cartilage of the chordal segment extends backwards, so as to embrace the foramen magnum and involve the supra-occipital region.

The prechordal segment is continuous posteriorly with the chordal segment. Anteriorly, in front of the primitive pituitary space, the basal plate of cartilage (prechordal segment) here spreads out laterally. Subsequently. the pituitary space becomes closed by the encroachment upon it, from either side, of the prechordal cartilage.

On each side of the chordal segment of the basal cartilage is the periotic cartilaginous capsuie. 
The basal cartilage is conveniently divided, for purposes of development, into three regional parts, which, in order from behind forwards, are as follows: (1) basi-occipital, (2) basisphenoid, and (3) ethmoidal. In addition to these three parts there are the two periotic cartilaginous capsules, right and left.

The basi-occipital cartilage gives rise to the following parts of the occipital bone-namely, (1) the basi-occipital, or basilar process; (2) the exoccipitals, or condylar portions; and (3) the supra-occipital, or lower division of the tabular portion.

The basisphenoid cartilage gives rise to the following parts of the sphenoid bone-namely: (I) the basisphenoid, or posterior part of the body, inclusive of the sella turcica; (2) the alisphenoids, or great wings; and (3) the external pterygoid plates.

The ethmoidal cartilage gives rise to osseous parts as follows: the posterior portion gives rise to (I) the presphenoid, or anterior part of the body of the sphenoid, and (2) the orbito-sphenoids, or small wings of the sphenoid.
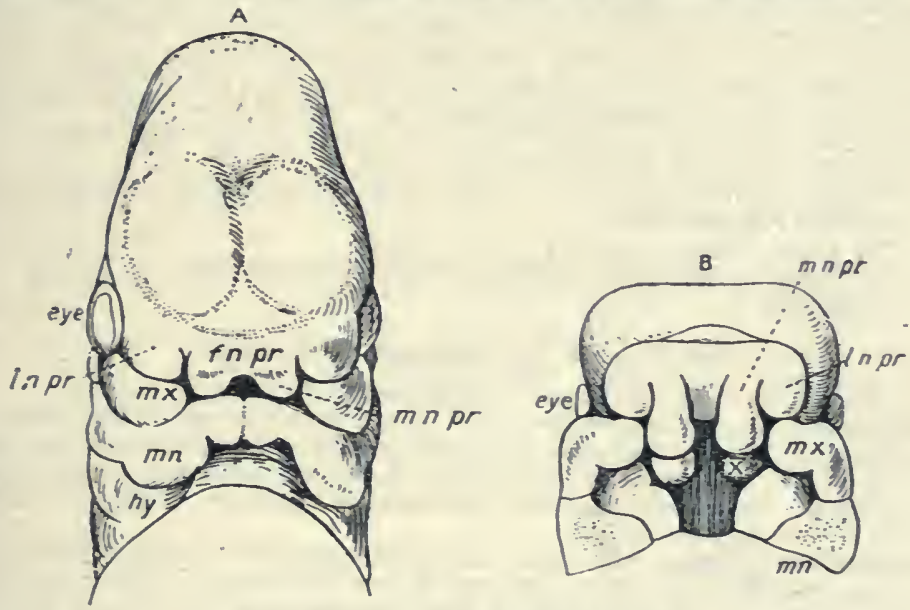

Fig. 91.-HeAd OF AN EMBRYo (His).

A, View from above.-B. View showing roof of mouth after removal of the Mandible. $f n p r$, Fronto-Nasal Process; $m n p r$, Mesial Nasal Process, terminating in Globular Process : lnpr, Lateral Nasal Process: $m x$, Maxillary Process; $m n$, Mandibular Arch; $h y$, Hyoid Arch. The $x$ in $B$ indicates the Nasal Lamina.

The anterior portion gives rise mesially to the mesethmoid, or crista galli, and perpendicular plate of the ethmoid, as well as to the septal cartilage of of the nose. It is also prolonged anteriorly at its centre into the frontonasal process, of which it forms the core.

Each lateral part of the anterior portion of the ethmoidal cartilage gives rise to the following parts-namely, (1) the ethmo-turbinal, or lateral mass of the ethmoid, inclusive of the superior and middle conchæ of the ethmoid; (2) the inferior concha; and (3) the sphenoidal turbinate bone of one side.

Each periotic eartilaginous capsule gives rise to the greater part of the petro-mastoid portion of the temporal bone.

The ethmoidal division of the basal cartilage undergoes considerable modification. It spreads out laterally, so, that it forms a broad plate. On either side in the region of each olfactory pit it assumes a deeply-curved crescentic outline, and forms the cartilaginous nasal capsule, this being the part which gives rise on either side to the ethmo-turbinal, inferior concha, and sphenoidal 
turbinate bone. Though known as the ethmoidal division or region, it gives rise not only to the ethmoid, etc., but also to the presphenoid and alisphenoids posteriorly, whilst anteriorly and mesially it dips down to form the septal nasal cartilage, and is prolonged as the core of the fronto-nasal process.

Those bones of the cranium which are ossified from centres appearing in the basal sheet of cartilage, or chondrocraniun,, are called endochondral or cartilage bones, and the following is a summary of them:

1. Occipital, except the interparietal part, or upper division of the tabular portion.

2. Sphenold, except the two internal pterygoid plates, but including the two sphenoidal turbinate bones.

3. Eth moid.

4. Petro-Mastoid portion (greater part) of the temporal bone.

Mombranous Cranium, or Tegmental.-As previously stated, the primitive membranous cranium undergoes chondrification along its basal part. The tegniental part is not involved in this process, but forms a connective-tissue membrane, which subsequently gives rise to certain cranial bones. From the fact that these bones are ossified fron centres which appear in this $\mathrm{mem}$. brane they are called membrane-bones, and, inasmuch as they constitute the covering of the cranial vault, they are known as tegmental bones. The following is a summary of these bones:

1. The Interparietal, or upper division of the tabular portion of the Occipital.

2. The two Parietal bones.

3. The Frontal bone (originally in two halves).

4. The Squamo-Zygomatic portion of each Temporal bone, as well as the pars tympanica.

\section{The Visceral Arch Skeloton of the Skull.}

The visceral arch skeleton comprises the face and the hyoid bone.

The Face.-The structures concerned in the development of the bones of the face are-(1) The fronto-nasal process, and the ethmoidal division of the basal sheet of cartilage which constitutes the chondro-cranium; and (2) the first visceral, or mandibular, arch, right and left, along with the maxillary process of each of these arches.

Fronto-Nasal Process.-This process occupies a median position, and consists of a central part or core, and an external covering. The core is cartilaginous, and is a downward and forward extension of the anterior part of the ethmoidal division of the basal sheet of cartilage which forms the chondrocranium. The external covering of the process is derived from the primitive cerebral capsule on the ventral aspect of the fore-brain, and is therefore membranous, being formed by mesenchyme.

The process grows downwards and forwards, and from either side of its base a bud grows in a downward and forward direction, this bud having the same structure as the parent process.

The fronto-nasal process then becomes somewhat complicated. The central portion of it is called the mesial nasal process, and this terminates below in two well-marked protuberances, one on either side of the median line, called the globular processes. The lateral bud on either side becomes the lateral nasal process. The fronto-nasal process, then, consists of (I) an undivided upper portion, (2) a mesial nasal process, terminating in two globular processes, and (3) two lateral nasal processes, right and left. As seen superficially on the embryonic face, the mesial nasal process and its two globular processes lie between the widely-separated ventral or mesial extremities of the two maxillar processes, and form the cephalic, or superior, boundary of the primitive mouth, or stomodxum. The globular processes at first stand distinctly apart from each other, and each globular process is separated from the corresponding maxillary process by a groove which leads to the nasal pit of that side.

The lateral nasal, process is separated from the globular process by the nasal pit, and external to the lateral nasal process is the optic or ocular. de- 
pression. The position of parts, then, is as follows: The globular process forms the inner boundary of the nasal pit, and the lateral nasal process forms its outer boundary, the optic or ocular depression lying on the outer side of the lateral nasal process. In other words, the lateral nasal process lies between the nasal pit internally and the optic or ocular depression externally. The two nasal pits communicate widely with the primitive mouth or stomodrum. Inferiorly the lateral nasal process is contiguous to the ventral part of the maxillary process, from which it is at first separated by a groove, called the oculo-nasal, naso-optic, or lachrymal sulcus, which extends from the ocular depression to the nasal pit. This sulcus subsequently disappears, but it indicates the position of the future lachrymal sac and nasal duct. The lateral nasal process joins the maxillary process, and the two fuse with the globular process.

Fronto-Nasal Process. Parts developed from it. (I) Globular Process.Each globular process, as stated, consists of a core of cartilage, covered by a connective-tissue investment. The cartilaginous core disappears, and from the connective-tissue investment of each process the following parts are developed: (a) The incisor or premaxillary portion of the superior maxilla; (b) the anterior part of the palatal plate of the superior maxilla-that is to say, the part in front of the incisor or premaxillary suture through means of the nasallamina; and $(c)$ the portion of the upper lip, which corresponds to the premaxilla. The portion of the mesial nasal process which lies between the two globular processes gives rise superiorly to the dorsum of the nose, and inferiorly to the lower and anterior part of the nasal septum, which part is called the columella nasi.

A prolongation of each globular process takes place in a backward direction in the form of a plate. These two plates are called the nasal lantina. At first they are some distance apart, but they gradually come together, and each forms the anterior part of the palatal plate of the corresponding superior maxilla-that is to say, the part in front of the incisor or premaxillary suture, and belonging to the premaxilla.

(2) Lateral Nasal Processes.-Each of these, as stated, consists of a core of cartilage, covered by a connective-tissue investment. The cartilaginous core gives rise to the upper and lower lateral nasal cartilages. The connectivetissuc investment gives rise to the ala nasi, and the nasal and lachrymal bones.

First Visceral, or Mandibular Arches, and their Maxillary Processes.Simultaneously with the foregoing developmental changes, the mesoderm of each mandibular arch is undergoing differentiation. From the dorsal or proximal part of each mandibular arch a budding of its mesoderm takes place. This outgrowth gives rise to the well-marked maxillary process, which is for the most part membranous.

Maxillary Processes.- The two maxillary processes grow in a ventral and mesial direction, below the ocular depressions, and, as seen superficially, on the primitive face, they lie transversely, one on either side of the primitive mouth. Their ventral or mesial extremities are widely separated from each other, and the mesial nasal process, with its two globular processes, lies between them, forming the cephalic or superior boundary of the primitive mouth. The lateral nasal process is contiguous superiorly to the ventral part of the maxillary process, the two being separated by the oculo-nasal, naso-optic, or lachrymal groove, all as previously stated. Inferior to the maxillary process is the corresponding mandibular arch.

The upper border of the maxillary process is joined by the lateral nasal process, the oculo-motor or naso-optic groove becoming obliterated, and being replaced deeply by the lachrymal sac and nasal duct. The ventral extremity of the maxillary process abuts against, and fuses with, the globular process. As the result of this fusion between the maxillary process and globular process, the external orifice (anterior nares) of the nasal pit of the corresponding side (now developing into the nasal fossa) is cut off from direct communication with the primitive mouth, but the nasal pit itself still remains in free communication with that cavity. 
The mesodermic maxillary process gives rise to-(i) The chiel part of the superior maxilla, and the chief part of its palatal plate, the premaxilla and anterior part of the palatal plate being developed from the mesodermic investment of the globular process and its nasal lamina: (2) the corresponding part of the upper lip; (3) the malar bone; (4) the palate bone ; and (5) the internal pterygoid plate of the sphenoid. As has been stated, the lateral nasal process gives rise to the ala nasi, and the nasal and lachrymal bones.

From the inner surface of each maxillary process there grows, in a mesial direction, a shelf-like plate of mesoderm. The two plates, right and left, are known as the palatal shelves, and they meet along the median line. Each gives rise to (1) the horizontal plate of the palate bone, and (2) the palatal plate of the superior maxilla, except its anterior or premaxillary part, which is developed from the nasal lamina of the globular process. In this manner the hard palate is formed, and the primitive mouth, or stomodreum, is now divided into two chambers, upper and lower. The upper chamber represents the two nasal fossæ, which open anteriorly through the anterior nares, and communicate posteriorly with the naso-pharynx through the posterior nares. 'The lower chamber constitutes the permanent mouth. The wide communication between the original nasal pits and the primitive mouth now becomes considerably curtailed, and is only represented in adult life by the canals of stensen in the region of the anterior palatine fossa.

Each maxillary process at its dorsal or proximal end-that is to say, where it springs from the mandibular arch-contains for a short distance an offshoot from the cartilaginous bar of the mandibular arch, this prolongation being known as the pterygo-paiatine bar. The bar soon disappears, but its mesodermic investment gives rise to (I) the internal pterygoid plate of the sphenoid, and (2) the perpendicular plate of the palate bone.

Mandibular Arches. - The two mandibular arches having parted with their maxillary processes, meet each other at the median line, and form the caudal or posterior boundary of the primitive mouth. The cartilaginous bar of each arch is called Meckel's cartilage. The mesial portion of this cartilage gives rise to the incisor part of the inferior maxilla or mandible. The chief part of the body of the mandible, however, and the lower part of its ramus, as high as the inferior dental foramen, are developed from the mesodermic investment of that portion of Meckel's cartilage which intervenes between the incisor part and the level at which the cartilage gives off the pterygo-palatine bar. This mesodermic investment also gives rise to one-half of the lower lip.

From the foregoing description it will be evident that the first visceral or mandibular arches build up the chief part of the facial portion of the visceral-arch skeleton of the skull, as shown by the following summary:

The maxillary process of each mandibular arch gives rise to (I) the chief part of the superior maxilla, including the chief part of its palatal plate; (2) the malar bone; and (3) the palate bone, including its horizontal plate. the mandibular arch itself gives rise to the greater part of the mandible.

The premaxillary portion of the superior maxilla, and the premaxillary part of its palatal plate, are developed from the globular process of the mesial nasal process and the nasal lamina of the globular process. The nasal bone and the lachrymal bone are developed from the lateral nasal process of the fronto-nasal process.

Each inferior turbinate bone, as already stated, like the lateral mass of the ethmoid (ethmo-turbinal), is developed in the lateral portion of the ethmoidal division of the basal sheet of cartilage, or chondrocranium, from which it becomes differentiated.

The ethmoid bone, though tabulated as a cranial bone, is chiefly facial in its disposition, inasmuch as it forms (1) a large part of the inner wall of the orbit, (2) a large part of the outer wall of the nasal fossa, and (3) a large part of the nasal septum. The only parts of the bone which belong to the base of the cranium are the cribriform plate and the crista galli. All parts of the bone are deycloped from the ethmoidal division of the chondrocranium, as already stated.

There now only remain for consideration the vomer and the styloid process of the temporal bone. 
Vomer.-This bone is developed in the mesoderm, which nvests the 'posterior and inferior parts of the septal cartilage of the nose, that cartilage being derived from the ethmoidal division of the basal sheet of cartilage or chondrocranium.

Styloid. Process of Temporal Bone.-Both parts of this process-namely, the tympano-hyal and stylo-hyal-are developed from the proximal or dorsal end of the hyoid bar (Reichert's cartilage) of the second visceral or hyoid arch.

Summary. - The first visceral or mandibular arches contribute the following bones to the facial portion of the visceral-arch skeleton: (I) The greater part of each superior maxilla; (2) the greater part of the inferior maxilla or mandible; (3) the two malar bones; and the two palate bones.

The fronto-nasal process and the ethmoidal division of the basal cartilage, or chondrocranium, contribute the following bones: (I) Premaxillary portion of each superior maxilla; (2) the two nasal bones; (3) the two lachrymal bones; and (4) the two inferior turbinate bones.

The vomer, as just stated, belongs to the mesodermic investment of the posterior and inferior part of the septal cartilage of the nose, which cartilage is derived from the ethmoidal division of the chondrocranium.

The greater part of the hard palate is derived from the palatal shelves of the two maxillary processes of the mandibular arches, but the premaxillary part is formed by the nasal laminx of the globular processes of the mesial nasal process of the fronto-nasal process.

\section{The Hyoid Bone.}

The hyoid bone forms a part of the visceral-arch skeleton of the skull, and the structures concerned in its development are the second and third visceral arches of each side.

Second Visceral or Hyoid Arch.-The cartilage of this arch is known as the hyoid bar, or cartilage of Reichert, and its distal or ventral segment gives rise to the small cornu or cerato-hyal.

Third Visceral or Thyro-hyoid Arch.-The distal or ventral end of the cartilage of this arch is connected with that of its fellow of the opposite side by a median piece of cartilage, which acts as a bond of union between them, and is from that circumstance called the copula. The copula also connects the right and left hyoid bars. The body of the hyoid bone, or the basi-hyal, is developed from the copula; and each great cornu, or thyro-hyal, is developed from the cartilaginous bar of the corresponding third visceral arch.

The bones of the visceral-arch skeleton of the skull, genetically considered, are (1) membranous, (2) complex or mixed-that is to say, partly membranous and partly cartilaginous-and (3) cartilaginous.

Membrane Bones :
Superior Maxillæ.
Malars.
Nasals.
Lachry mals.
Palate Bones.
Vomer.

Osseous Stage.--The osseous stage in the development of the skull is treated under each individual bone.

\section{THE TEETH.}

The teeth are divided into two sets, namely, the temporary, milk, or deciduous, which belong to early infancy, and the permanent, which replace the temporary. The temporary teeth are twenty in number - ten upper, five in each superior maxilla, and ten lower, five in each half of the inferior maxilla. The number of permanent teeth is thirty-two-sixteen upper, and sixteen lower. 

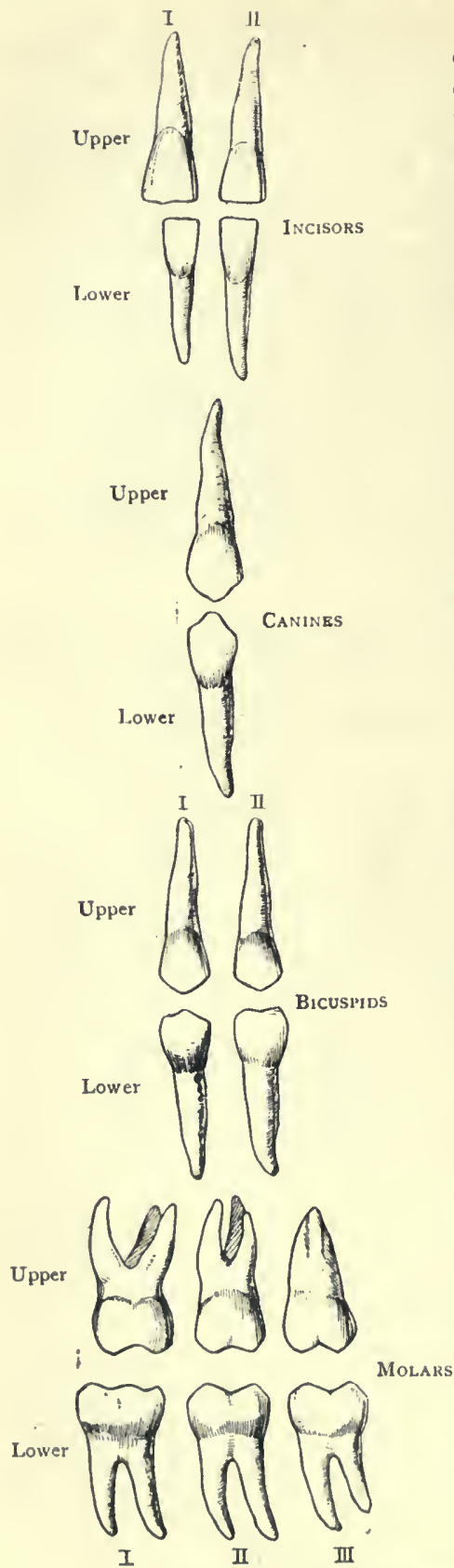

Fig. 92.-The Permanent Teetu
The Permanent Teeth.-Proceeding from the median line in a direction outwards and backwards, the permanent teeth are as follows: mesial incisor, lateral incisor, canine, first bicuspid, second bicuspid, and first, second, and third molars. The third molar is known as the dens sapientia or wisdom tooth. Each tooth is composed of the following parts: the crown, which is the part above the gum; the root, which is the part embedded in the alveolus; and the neck, which lies between the crown and root. The surface of a tooth which looks towards the lip is called labial, and that looking towards the tongue lingual, whilst of the opposed surfaces one is called mesial or proximal, and the other lateral or distal.

The Incisors.--There are eight incisor teeth-two in each upper jaw, and two in each half of the lower jaw. In each case the two incisors are known as mesial and lateral respectively, and they are called 'incisor' because their crowns are so formed as to be adapted for the division of food. The cutting edges of the crowns of the upper incisors are bevelled behind, and those of the lower incisors in front.

The upper mesial incisor is larger than the lateral, and it gradually tapers from the cutting edge of the crown to the root. The length of the crown exceeds its breadth. The labial surface is slightly convex, whilst the lingual surface is concave. The lingual surface presents near the gum a ridge, called the basal ridge, or cingulum. The mesial angle of the cutting edge of the crown is longer or more projecting than the lateral angle.

The root is long, tapering, conical, and slightly flattened on either side. 
The upper lateral incisor is smaller than the mesial, which it for the most part resembles. The lateral angle of the cutting edge of the crown is rounded off, and the cingulum is more prominent than in the upper central incisor.

The lower mesial incisor is smaller than the lateral, and narrower than the upper mesial incisor. The cingulum, if present, is not well marked. The root is much compressed laterally.

The lower lateral incisor is larger than the mesial, which it for the most part resembles. The presence of a cingulum is rare. The root is longer than that of the central incisor, and on each flattened lateral surface there may be an indication of a longitudinal groove.

The Canines.-There are four canine teeth-one in each upper jaw, and one in each half of the lower jaw.

The crown of a canine tooth is larger than that of an incisor. It is somewhat conical, and terminates in a cusp, from which circumstance the canine teeth are known as the cuspidate teeth. The labial surface of the crown is convex.

In the upper eanines the lingual surface of the crown usually presents a slight median ridge, which extends from the cusp to a ridge which represents the cingulum. The root is long, thick, and almost round.

The lower canines have no median ridge on the lingual surface, which may be slightly concave. The cingulum is absent. The root is shorter than in the upper canines.

The Bicuspids, or Premolars.-There are eight bicuspid teethtwo in each upper jaw, and two in each half of the lower jaw.

Upper Bicuspids. - The crown is quadrilateral, and its labial border is longer than the lingual. It has two cusps-labial and lingual-both of which are prominent. The labial cusp is the larger and broader of the two, and the cusps are separated by a deep transverse fissure. The labial and lingual surfaces of the crown are convex, and there is no cinguhm. The root is single, much compressed laterally, and usually marked on either side by a longitudinal groove.

The first upper bicuspid has frequently two slender fangs. This occurs so often that it may be regarded as the normal condition in that tooth.

The second upper bicuspid differs from the first in the following respects: (I) Its crown is larger, and approaches more nearly the molar type; and (2) its lingual cusp is larger and broader than that of the first upper bicuspid, so that its labial and lingual cusps are of nearly equal size.

Lower Bicuspids.-These teeth are smaller than the upper bicuspids. The labial cusp has an inclination inwards towards the mouth, and the lingual cusp is depressed. The cusps are connected by a low-lying ridge. The root is single, round, and tapering.

The second lower bicuspid differs from the first in the following respects: (I) Its crown is larger, and approaches more nearly the 
molar type; and (2) its lingual cusp is stout, and not so much depressed as that of the first lower bicuspid.

The second lower bicuspid has occasionally two fangs.

The Molars (Multicuspidate Teeth).-There are twelve molar, or grinding teeth-three in each upper jaw, and three in each half of the lower jaw.

First and Second Upper Molars.-The crown is somewhat square, the angles being rounded off. The grinding surface is furnished with four cusps, situated at each angle of the square, two of them being labial and two lingual. The anterior lingual cusp is the largest, and it is connected with the posterior labial cusp by a stout oblique ridge. The labial cusps are longer or more projecting than the lingual cusps, which applies to all the upper molars. The fissure between the two labial cusps and that between the two lingual cusps appear for a short distance on the labial and lingual surfaces of the crown respectively. The posterior lingual cusp is occasionally suppressed.

The root has three fangs- two labial and one lingual. The lingual fang is the largest and most divergent, its direction being obliquely inwards towards the tongue.

Third Upper Molar.-This tooth is subject to much variety. Its crown is usually of smaller size than that of the first and second. The two lingual cusps of the crown are frequently confluent. The three fangs are blended together to form a tapering cone.

Lower Molars.-The first lower molar is the largest. The crown is somewhat square, and its grinding surface is provided with five cusps. Four of these are placed at the angles of the square, and are separated from each other by a crucial fissure. The posterior limb of this fissure bifurcates, and the fifth cusp lies in the angle of bifurcation. This cusp may be in the median line, but it usually lies towards the labial aspect, from which aspect it can readily be seen. The lingual cusps are longer or more projecting than the labial cusps, which applies to all the lower molars. The fissure which separates the two labial cusps usually passes for a short distance on to the labial surface of the crown, but the fissure between the two lingual cusps only occasionally passes on to the lingual surface of the crown. The root has two fangs-anterior and posterior. Each fang is much compressed from before backwards, and in most cases each presents a slight backward curve.

The second lower molar bears a general resemblance to the first. The fifth cusp is often absent, and, when present, it is of small size. The two fangs of the root are frequently blended together.

The third lower molar is of larger size than the third upper molar. Its crown is large, and is usually provided with five cusps. Its root may have two scparate fangs, or these may be confluent. In the latter case a groove indicates the double condition of the root.

Dental Arches.--The alveolar borders of the superior and inferior maxillary bones, and the upper and lower teeth, are so disposed as to form two, almost similar, curves. When the mouth is closed, 
the summits of the crowns of the upper and lower teeth are brought together, the line of contact being known as the dental articulation. A line drawn through this articulation forms the best means of determining the breadth of the crown of each tooth.

The crowns of the upper eight anterior teeth-namely, the four incisors, two canines, and the two first bicuspids-are broader than the crowns of the corresponding lower eight anterior teeth, measured according to the foregoing line.

The crowns of the lower eight posterior teeth-namely, the six molars and the two second bicuspids-similarly measured, are broader than the crowns of the corresponding upper eight posterior teeth.

The difference in breadth between the crowns of the upper and lower eight anterior teeth is equal to the difference in breadth between the crowns of the lower and upper eight posterior teeth.

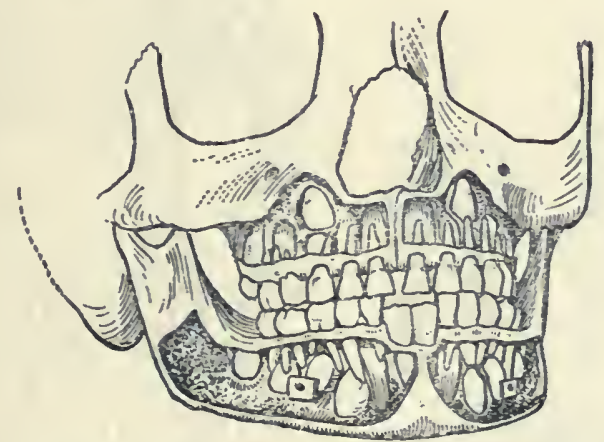

Fig. 93.-The Superior and Inferior Maxillary Bones at the Seventh Year, showing Most of the Temporary Teeth, and the Permanent TEETH ABOUT TO REPLACE THEM.

The First Permanent Molars and the Permanent Lower Central Incisors are in position.

The result is that, if the dental arches could be straightened out, the crowns of the upper and lower sets of teeth would have the same measurement as regards breadth.

The crowns of the upper six anterior teeth-namely, the four incisors and the two canines-overlap the crowns of the corresponding lower six anterior teeth.

The Temporary Teeth.-Proceeding from the median line, these are as follows on either side: two incisors, central and lateral, one canine, and two molars, first and second. Their necks are more constricted than in the permanent set. The incisors and canines resemble, for the most part, those of the permanent set, but they are smaller. The molars, which are replaced by the permanent bicuspids, exceed them in size, the second molars being particularly large. The first upper molar has three cusps-two labial and one 
lingual, the second upper and first lower molars have each four, and the second lower molar has five.

Eruption of the Teeth. - The eruption of particular teeth of the lower jaw precedes that of the corresponding teeth of the upper jaw, and the periods are as follows:

\section{Temporary Teeth.}

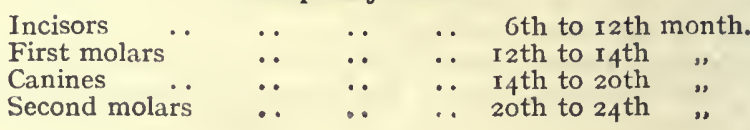

B

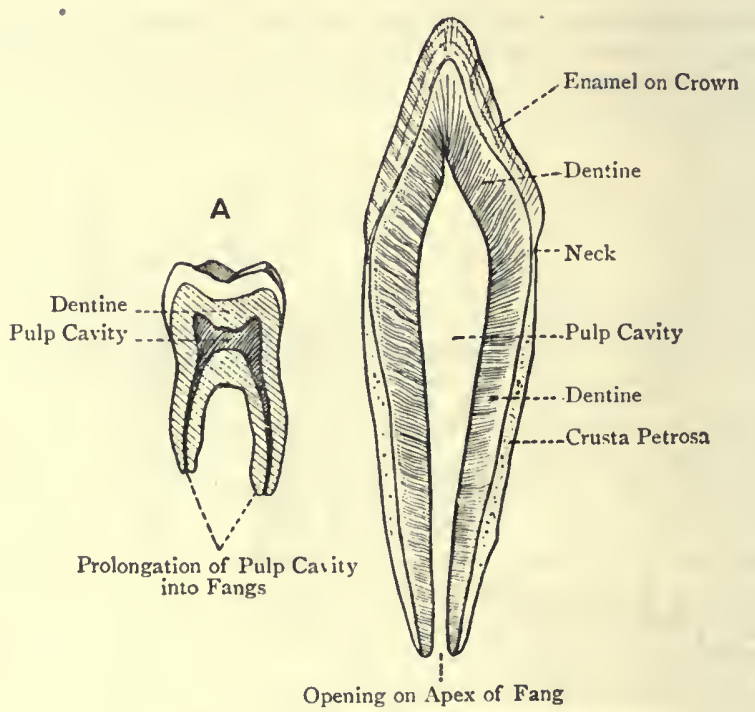

Fig. 94.-Sections of Teeth.

A, Lower Molar; B, Lower Incisor.

\section{Permanent Teeth.}

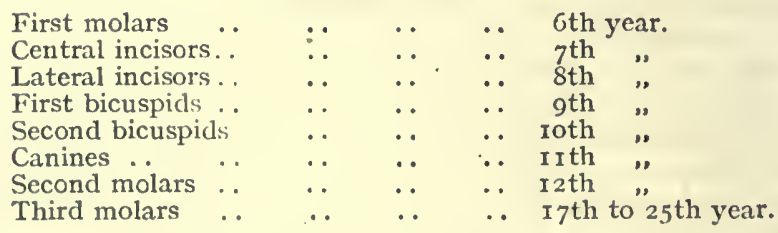

About the sixth year is the period at which most teeth are present in the jaws, there being the twenty temporary teeth, and all the permanent, except the four wisdom tecth, (namely, twenty-eight), making in all forty-cight. 
Structure of a Tooth.- The crown of a tooth contains a central cavity, called the pulp eavity, which is occupied by the dental pulp. The shape of the pulp cavity corresponds with that of the crown, and it extends into the root, and as many fangs as compose it, terminating in a small opening on the apex of the fang. The cavity also extends for a little into the cusps of the bicuspids and molars, and in the incisors it is continued into each angle of the crown. The wall of the cavity presents a number of openings, which lead into the dentinal tubules. The dental pulp is composed of a matrix of connective tissue, containing bloodvessels, nerves,

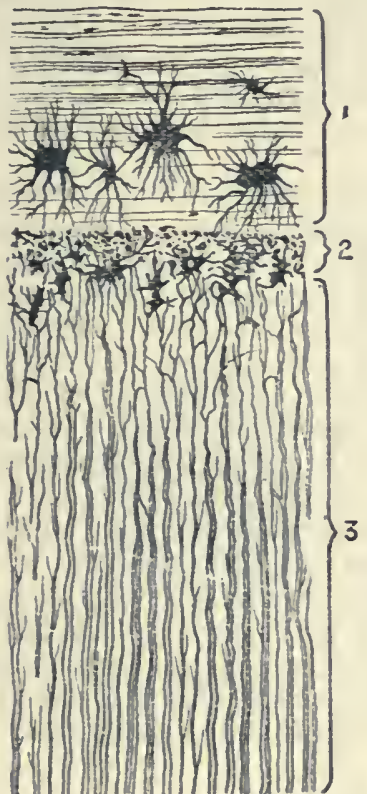

Fig. 95:-Section of the FANG of A ТоОтн.

I, Cement; 2, Granular Layer of Purkinje: 3. Dentinal Tubules.
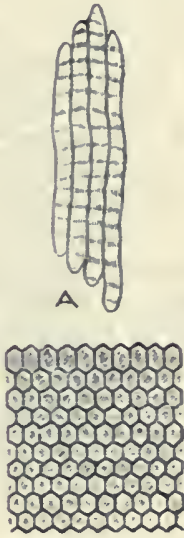

B

Fig. 96.-Enamel Prisms.

A, Four prisms (Longitudinal View); $B$, Surface of the Enamel.

cells, and fibres, which latter seem to be processes of the cells. It is destitute of lymphatics. The cells are scattered throughout the matrix, and at the surface of the pulp they form a continuous layer, being there known as the odontoblasts. This layer is sometimes spoken of as the membrana eboris. The pulp is very vascular and sensitive, its vessels and nerves reaching it through the minute openings at the apices of the fangs.

The substance of the tooth is formed of three tissues, namely, ivory or dentine, enamel, and cement or crusta petrosa. The 
dentine forms the principal part of the tooth, surrounding the pulp cavity and its prolongations; the enamel covers the exposed part or crown; and the cement covers the root.

Dentine.-This bears a resemblance to bone, but contains rather less animal, and more earthy, matter, the proportion in Ioo parts being about 28 of animal matter to 72 of earthy. The dentine has a striated appearance, due to the fact that it is traversed by a number of minute branched channels, called the dentinal tubules, which radiate in a curved manner outwards from the pulp cavity to the deep aspect of the enamel and cement. These tubules contain processes of the odontoblasts which form the membrana eboris, and are known as Tomes' fibres. The part of the dentine adjacent to the enamel and cement is known as the granular layer of Purkinje. It presents a number of irregular spaces, known as the interglobular spaces, which are surrounded by minute globules of calcareous matter.

Enamel.-This caps and protects the dentine of the crown. It is exceedingly hard, which is due to the fact that it contains no animal matter-at least, to any appreciable extent. It consists of solid hexagonal prisms, which are marked by transverse striations. These are received by their deep extremities into depressions on the dentine, and are placed vertically on the summit of the crown, but horizontally on its sides. At the period of eruption of a tooth, and for some little time thereafter, the enamel of the crown is covered by a thin membrane, called the enamel cuticle, or Nasmyth's membrane.

Cement, or Crusta Petrosa.-This covers the dentine which forms the root of the tooth. It is true bone, and contains lacunæ and canaliculi, but it is destitute of Haversian canals.

The root of a tooth is maintained in its alveolus by the peridental membrane or dental periosteum, which covers the cement, and lines the wall of the alveolus, being continuous with the gum at the neck of the tooth. The articulation is called gomphosis.

\section{Development of the Teeth.}

The teeth are calcified papillæ of the gingival mesoderm, their crowns being covered by calcified ectodermic cells. Three structures are involved in the development of a tooth-namely, (I) the enamel organ, which furnishes the enamel; (2) the dental papilla, which gives rise to the dentine and dental pulp; and (3) the dental sac, from the inner layer of which the cement is formed. The enamel organ is developed from the ectoderm, whilst the dental papilla and the dental sac are of mesodermic origin.

The development of the temporary teeth commences as ingrowths of the epithelium along the margins of the embryonic jaws into the mesodernic tissue of the jaws. Each of these ingrowths, which consists of epithelial cells, soon gives rise to two vertical layers-an outer or labial, and an inner or lingual. The outer layer is called the labio-dental lamina, and the inner layer is the true dental lamina. The labio-dental or labio-gingival Iamina is concerned with the lip and gum, and it presents superficially a furrow, called the labio-dental or labio-gingival groove, which divides it into two parallel ridges-labial for the lip, and gingival for the gum. T'he labiogingival gronve itself deepens, and separates the lip from the gum. 
The true dental lamina is concerned with the enamel. Along its attachment to the oral epithelium a furrow is formed, called the dental groote, and its deep margin is received into the mesoderm of the embryonic jaw.

The true dental laminæ, which are of ectodermic origin, are at first unbroken, but they soon become broken up into separate masses, called enamel organs, there being twenty of these for the temporary teeth, ten upper and ten lower. Each enamel organ appears as a thickening or protuberance of ectodermic cells on the deep margin of the true dental lamina, which protuberance soon becomes flask-shaped, the neck being directed superficially. This neck soon disappears, and the connection between the enamel organ and the ectodermic cells of the free surface of the gum is thereby severed. Moreover, the enamel organs become isolated from one another.

The dental papilla appears as an elevation of the mesoderm of the floor of the dental groove, subjacent to each enamel organ. The number of papillæe, therefore, corresponds to the number of enamel organs. As the dental papilla grow's, it becomes capped by the enamel organ, which also invests it laterally. The enamel organ thus becomes moulded over the dental papilla in the forn of a cap.

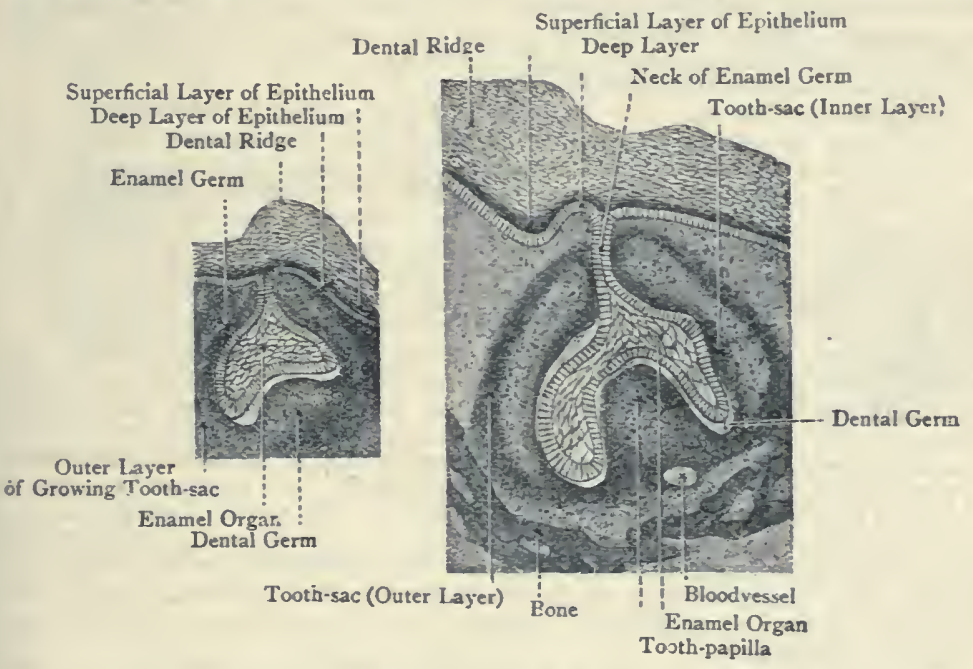

Fig. 97--Development of Tooth (Frey, AFter Thiersch),
Vertical Transverse Section of UPPER Jaw.

Enamel Organ.-As stated, the enamel organ is of ectodermic origin, being derived from the true dental lamina, which, in turn, is derived from the ectodermic cells covering the surface of the gum. The chief cells of the enamel organ are situated deeply, and lie close to the surface of the dental papilla. They are long columnar cells, which are called enamel cells, adamantoblasts, or ameloblasts, and they give rise to the enamel prisms.

Formation of Enamel Prisms.-Each enamel cell gives rise to one enamel prism. The deep part of each cell undergoes calcification. The superficial ends of the enamel cells do not become calcified, but form a delicate membrane, called the enamel cuticle, or Nasmyth's membrane, which covers the crown of the tooth for a short time after its eruption.

Dental Papilla.-As stated, the dental papilla is of mesodermic origin, and it gives rise to the dentine and dental pulp. Essentially, the papilla is a 
collection of mesodermic cells, wlich becone transformed into connective tissue, this being permeated by bloodvessels and nerves.

The cells at the surface of the papilla become enlarged, and are called odontoblasts. These cells are identical with the osteoblasts of developing bone, and they form a continuous layer, known as the membrana eboris. From these cells the dentine is laid down. The central portion of the dental papilla, consisting of connective tissue, bloodvessels, and nerves, constitutes the dental pulp.

Formation of Dentine.-The dentine is laid down in successive layers. Each layer consists at first of a fibrous dentinal matrix, called prodentine, the fibres of which are continuous with those of the dental pulp. Subsequently the matrix undergoes calcification by the deposits of calcareous matter. During the formation of these layers the odontoblasts become shifted inwards toriards the centre, and they leave behind them fine processes, which become invested by the calcified fibrous matrix of the dentine. In this manner the dentinal tubules are formed, with processes of the odontoblasts within them, which represent the fibres of Tomes. The dentine so formed constructs a case for each dental papilla. The central part of the papilla, consisting of connective tissue, bloodvessels, and nerves, forms, as stated, the dental pulp. The peripheral part of the dentine has several small uncalcified areas, which lie between globular masses of calcareous matter. These areas represent the interglobular spaces of the adult tooth.

Dental Sac.-This sac, like the dental papilla, is of mesodermic origin. As the dental papilla is undergoing differentiation into dentine and dental pulp, the mesodermic connective tissue around the papilla bccomes condensed into a follicle, called the dental sac, which encloses both the dental papilla and the corresponding enamel organ. The developing tooth (enamel organ and dental papilla) thus becomes isolated, the enamel organ having previously lost its connection with the ectoderm of the gum by rupture of its neck. The part of the dental sac which is related to the crown of the tooth is connected with the surface of the gum by a band of connective tissue, called the gubernaculum dentis. This part of the sac, along with the gubernaculum, disappears. The part of it which is related to the root of the tooth undergoes important developments. It consists of two layers-inner and outer. The inner layer gives rise to the cement, or crusta petrosa, whilst the outer layer, remaining fibrous, forms the alveolo-dental periosteum, or peridental membrane.

Permanent Teeth. - The process of development of the permanent teeth is similar to that of the temporary teeth. The enamel organs of those permanent teeth (teeth of succession) which are to replace the temporary teethnamely, the permanent incisors, canines, and bicuspids-are developed as thickenings or protuberances of the true dental laminæ, as in the case of the temporary teeth, and they grow inwards on the lingual aspects of the temporary enamel organs.

The enamel organs of the three permanent molar teeth spring from a backward prolongation of the true dental lamina.

Teeth Present at Birth.-At the period of birth the gums contain forty-four teeth. These consist of--(I) the twenty temporary teeth; (2) the twenty permanent teeth, which are to replace the temporary teeth; and (3) the four first permanent molar teeth. At birth the second and third permanent molar teeth, eight in all, are not yet developed.

Eruption of the Temporary Teeth.-As the permanent teeth grow, they exercise pressure upon the fangs of those temporary teeth which they are about to replace. This continued pressure leads to partial absorption of the fangs of the temporary teeth. These teeth, therefore, become loosened in their alveoli, and as the permanent teeth continue to advance, the loosened temporary teeth are pushed out by the advancing permanent teeth. Osteo. clasts also take part in the absorption of the fangs. 


\section{THE BONES OF THE UPPER LIMB.}

The upper limb is arranged in four divisions, namely, the pectoral or shoulder-girdle, brachium or arm proper, antibrachium or forearm, and manus or hand. The shoulder-girdle consists of the clavicle and scapula, the brachium comprises the humerus, the antibrachium is composed of the radius and ulna, and the hand is subdivided into a carpus, comprising eight bones, a metacarpus, consisting of five bones, and phalanges, which number fourteen.

\section{The Clavicle.}

The clavicle or collar-bone is situated at the lower part of the neck anteriorly, where it lies above the first rib, and it extends outwards and backwards from the upper border of the presternum to the acromion process of the scapula. The bone presents two curves, an inner or sternal, occupying two-thirds, with its con-

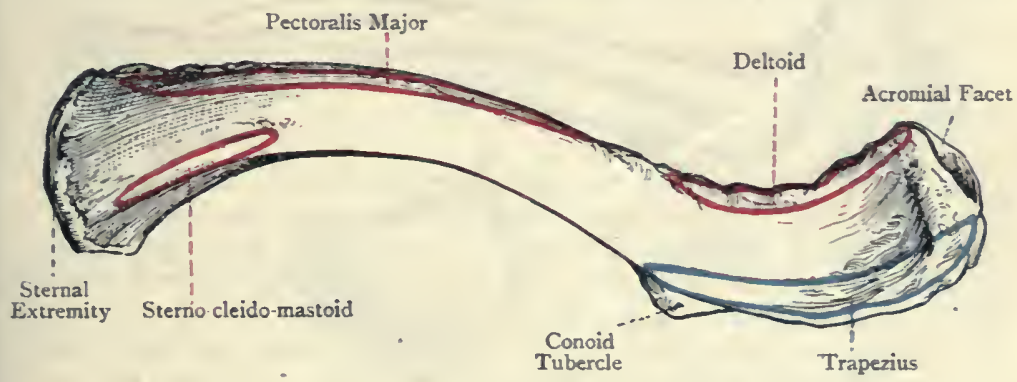

Fig. 98.-The Right Clavicle (Superior View).

vexity directed forwards, and an outer or acromial, extending over the outer third, with its convexity directed backwards. These curves impart elasticity to the bone. The clavicle is divided into a shaft and two articular extremities.

The shaft is somewhat quadrilateral over its inner two-thirds, and compressed from above downwards over its cuter third. The superior surface is for the most part narrow, but externally it becomes broad. At its inner end, near the postero-superior border, it presents a rough ridge, about $I \frac{1}{2}$ inches long, for the origin of the clavicular head of the sterno-cleido-mastoid. At its outer expanded part it is encroached upon by the tendinous fibres of the trapezius and deltoid. Elsewhere it is covered by the skin, fascia, and platysma myoides. The anterior surface is convex over its inner two-thirds, and concave over its outer third, where it is reduced to a mere rough border. Over the inner half it is rough for the origin of the clavicular portion of the pectoralis major, and over its outer marginal third it gives origin to the clavicular portion of the deltoid. At the inner end of the deltoid impression there is sometimes a pointed projection, known as the deltoid spine. The 
posterior surface is concave over its inner two-thirds, and convex over its outer third, where it is narrowed into a rough border. 'The inner two-thirds overhang the subclavian vessels and trunks of the brachial plexus, whilst the outer marginal third gives insertion to the upper fibres of the trapezius. At the inner end of the impression for these fibres, opposite the deltoid spine, there is a conical projection which extends on to the inferior surface for a little, called the conoid tubercle, for the conoid ligament. About the centre of the posterior surface there is the medullary foramen for the medullary artery, which is a branch of the suprascapular. The canal to which the foramen leads is directed outwards. The foramen may be situated on the inferior surface in, or close to, the subclavian groove, or there may be two foramina, one on the posterior and one on the inferior surface, about an inch apart.

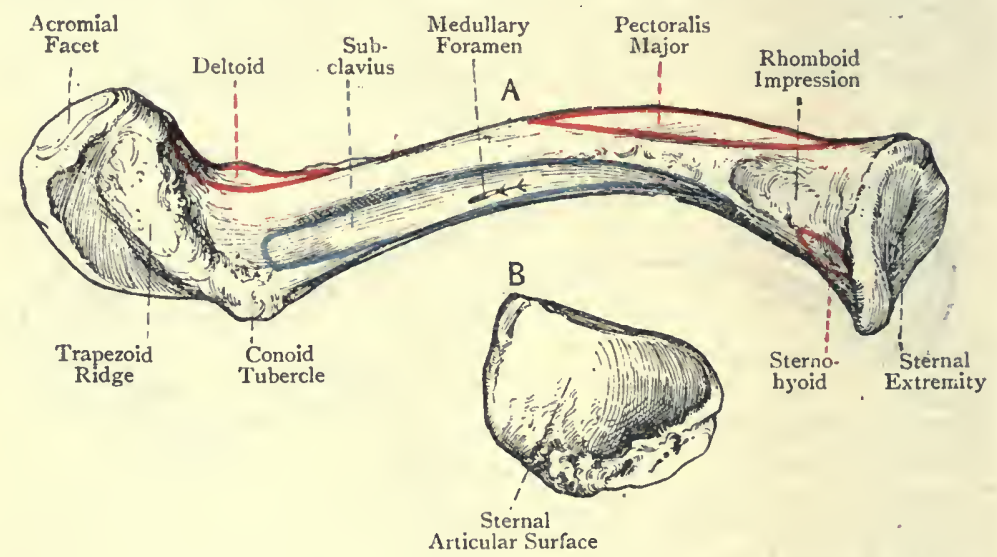

Fig. 99.-The Right Clavicle.

A. Inferior View ; B. Sternal Extremity.

Close to the sternal end the posterior surface gives partial origin to the sterno-hyoid. The inferior surface presents near its sternai end the rhomboid impression, about an inch long, for the costoclavicular or rhomboid ligament. External to this there is the subclavian groove, which extends from the rhomboid impression to near the conoid tubercle, and gives insertion to the subclavius. The groove is bounded by two lips, anterior and posterior, to which the costo-coracoid membrane is attached in two laminæ. At the outer extremity of the inferior surface there is a rough oblique line, called the trapezoid ridge, which extends forwards and outwards from the conoid tubercle, and gives attachment to the trapezoid ligament. The conoid tubercle is more fully seen here than on the posterior border, and is situated at the postero-internal extremity. of the trapezoid ridge.

The borders of the clavicle over its outer third are anterior and posterior. The enterior border bifurcates, over the inner two-thirds, 
into an antero-superior and antero-inferior border, which enclose between them the anterior surface. The posterior border bifurcates, over the inner two-thirds, into a postero-superior and posteroinferior border, the latter forming the posterior lip of the subclavian groove. The antero-superior and postero-superior borders limit the superior surface, the postero-superior and postero-inferior limit the posterior surface, the postero-inferior and antero-inferior limit the inferior surface, and, as stated, the antero-inferior and antero-superior limit the anterior surface.

The sternal extremity is enlarged and covered by cartilage. As viewed on end, it is somewhat triangular, and presents a prominent posterior angle which is directed downwards, inwards, and backwards. The surface is concave from before backwards, and convex from above downwards, and it articulates with the clavicular impression on the upper border of the presternum, an inarticular fibro-cartilage intervening. The circumference of the sternal end is rough for the sterno-clavicular and interclavicular ligaments, except inferiorly, where there is a narrow strip for the first costal cartilage.

The acromial extremity presents an oval facet for the acromion process of the scapula.

The clavicle receives its blood-supply from the suprascapular artery and the thoracic axis.

Articulations.--Internally with the presternum and first costal cartilage, and externally with the acromion process of the scapula.

Structure.-The exterior is composed of compact bone which is thickest towards the centre, and the interior of coarse cancellated tissue, the principal lamellæ being disposed longitudinally. The clavicle has no medullary canal, but, towards the centre of the shaft, the medullary spaces of the cancellated tissue are of large size.

Varieties.-(1) There may be a deltoid spine. (2) The superior surface may present a small slit-like aperture, variously situated, for one of the descending branches of the cervical plexus of nerves.

The Clavicle of the Female.-The bone is smoother, more slender, straighter, and more cylindrical over its inner two-thirds, than that of the male.

Ossification.-The clavicle, which is the earliest bone to ossify, has two primary centres and one secondary centre. It is preceded by a rod of con-

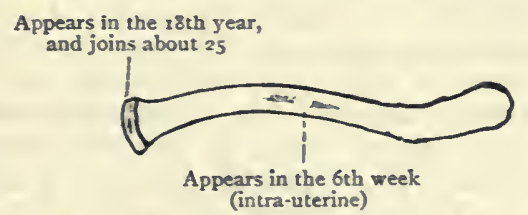

Fig. IOO-Ossification of the Clavicle.

nective tissue. Within the two halves of this rod collections of "precartilaginous tissue' are formed, and within these at their contiguons ends the primary centres appear about the 6 th week. Subsequently the precartilag. 
inous collections fuse, and thereafter the primary centres coalesce Ossifi. cation from these two centres proceeds at first in the precartilaginous tissue, but subsequently in the cartilage to which this tissue gives place. The primary centres may fail to join, with the result that the clavicle may persist in two halves.

The secondary centre appears in the cartilage of the sternal end about the 2oth year, and this epiphysis joins the shaft about the 25th year (Mall and Fawcett).

The law of ossification applicable to bones having a shaft and one epiphysis is as follows: the medullary foramen and the canal to which it leads are directed towards that extremity which has no epiphysis. This law is illustrated in the clavicle and the metacarpal, metatarsal, and phalangeal bones.

\section{The Scapula.}

The scapula or shoulder-blade is situated on the posterior aspect of the thorax, where it extends from the second to the seventh rib, being separated by muscles from the thoracic wall. It consists of a body and three processes, namely, a spine, an acromion and a coracoid process.

The body is a thin triangular plate, and it presents two surfaces, three borders, and three angles. The anterior surface, venter, or subscapular fossa is concave, and the bone forming it is for the most part thin, except near the external or axillary border, where there is a thick, round, elongated ridge. It gives origin to the subscapularis, except (I) along the anterior aspect of the base from the superior to the inferior angle, where the serratus magnus is inserted, and (2) over the front of the neck. The venter is crossed by three or four oblique ridges, which extend upwards and outwards from the base, and give attachment to tendinous septa intersecting the subscapularis muscle.

The posterior surface or dorsum is irregularly convex, and is divided into two unequal parts by the spine. The upper division, along witl the superior surface of the spine, forms the supraspinous fossa. It represents about one-fourth of the dorsum, and gives origin over its inner two-thirds to the supraspinatus. In the region of the neck it presents a nutrient foramen for a branch of the suprascapular artery. The lower division, along with the inferior surface of the spine, forms the infraspinous fossa, and it represents about threefourths of the dorsum. Towards the external or axillary border it presents an elongated concavity, external to which is an oblique line extending from its upper end downwards and inwards to the base near the inferior angle. The infraspinous fossa, as far out as this oblique line, gives origin to the infraspinatus, except at the upper and outer part, and it presents a nutrient foramen superiorly, close to the spine near the centre, for a branch of the dorsalis scapulæ artery. The oblique line marks off impressions for the teres muscles and dorsalis scapula artery, as follows: the teres minor arises from about the upper two-thirds, near the centre of which there is a groove for the dorsalis scapule artery, and the 
teres major arises from about the lower third. The impression for the latter muscle is oval; it extends on to the back of the inferior angle, and it is separated from the impresssion for the teres minor by a short rough line. This line gives attachment to an intermuscular septum which separates the teres muscles, and the oblique line to a septum which separates these muscles from the infraspinatus. The supra- and infraspinous fossæ communicate with each other by means of the great scapular notch, which lies outside

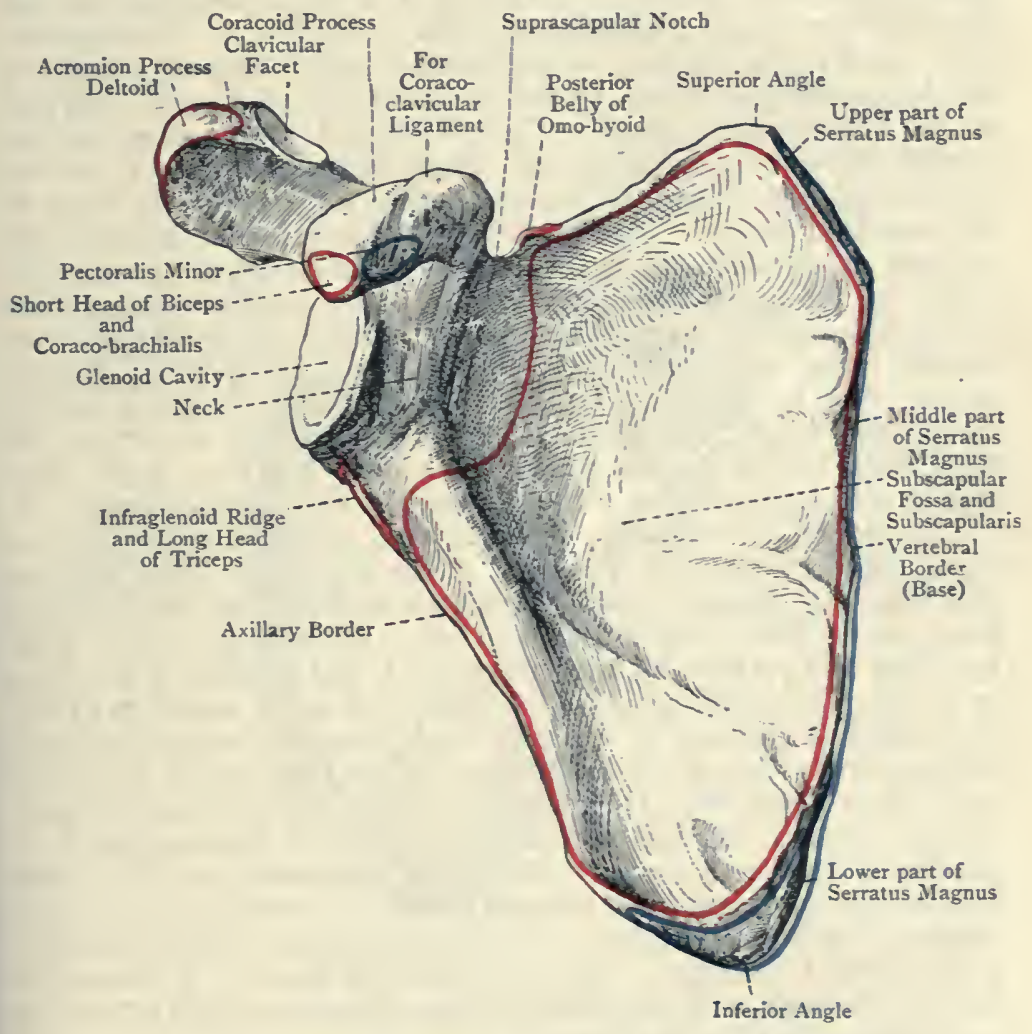

Fig. Iot.-The Right Scapula (Anterior View).

the short external border of the spine, and transmits the suprascapular artery and nerve.

The borders are superior, internal or vertebral, and external or axillary. The superior border, which is the shortest and thinnest, extends from the superior angle to the coracoid process. Close to that process it presents the suprascapular notch. This is converted into a foramen by the suprascapular or transverse ligament, which sometimes undergoes ossification. The supra- 
scapular nerve passes backwards beneath the ligament, and the suprascapular artery over it, whilst the posterior belly of the omo-hyoid arises from its inner part and from the adjacent portion of the superior border. The internal or vertebral border is known as the base. It is the longest, intermediate in thickness, and extends from the superior to the inferior angle. It is convex, and is divisible into three parts. One part represents the base of the small triangular surface by which the spine arises from the vertebral border, and it gives insertion to the rhomboideus minor; another extends from this to the superior angle, and gives insertion to the levator anguli scapulæ; and the third extends downwards to the inferior angle, and gives insertion to the rhomboideus major. On the ventral aspect close to this border there is a long narrow linear impression, which widens towards the superior and inferior angles, and gives insertion to the serratus magnus. The external or axillary border, which is the thickest and intermediate in length, extends from the inferior angle to the lower margin of the glenoid cavity. Below that cavity it presents a rough impression, an inch long, called the infraglenoid ridge, which gives origin to the long head of the triceps, and a little below this a groove for the dorsalis scapulæ artery, which also marks the dorsal aspect. The ventral aspect of the bone close to this border presents a groove over the upper twothirds, which gives origin to many fibres of the subscapularis.

The angles are superior, inferior, and external. The superior angle, which is thin, is situated at the meeting of the superior and vertebral borders, and it forms the highest part of the body, being on a level with the second rib. Its ventral aspect gives insertion to a part of the serratus magnus, and its edge to a portion of the levator anguli scapulæ. The inferior angle, somewhat thick and round, is situated at the meeting of the vertebral and axillary borders, and it forms the lowest part of the bone, being on a level with the seventh rib. Its ventral aspect gives insertion to a part of the serratus magnus, and its dorsal aspect gives origin to a portion of the teres major. Below the impression for the latter muscle there is sometimes a rough semilunar marking for a slip of origin of the latissimus dorsi. The external angle, which is massive, is situated at the upper end of the axillary border. It forms the head of the bone, and supports the glenoid cavity, which articulates with the head of the humerus.

The glenoid cavity, so named from its shallowness, is pyriform, with the narrow end upwards, and its direction is outwards and forwards. Its margin is slightly elevated and rough for the glenoid ligament, and immediately outside the margin the capsular ligament of the shoulder-joint is attached. Superiorly it presents a small rough elevation, called the supraglenoid tubercle, which gives origin to the long head of the biceps. The neck is the constricted portion which extends from the suprascapular notch to a point immediately above the infraglenoid ridge, and it is most evident posteriorly, 
where it forms, with the external border of the spine, the great scapular notch. In this latter situation there are numerous foramina for branches of the suprascapular artery.

The spine is situated on the dorsum of the bone, which it crosses in a direction outwards and slightly upwards. It commences at the vertebral border in a flat triangular surface, over which the tendon receiving the lower fibres of the trapezius glides, with the intervention of a bursa. It soon becomes very prominent, and at its outer

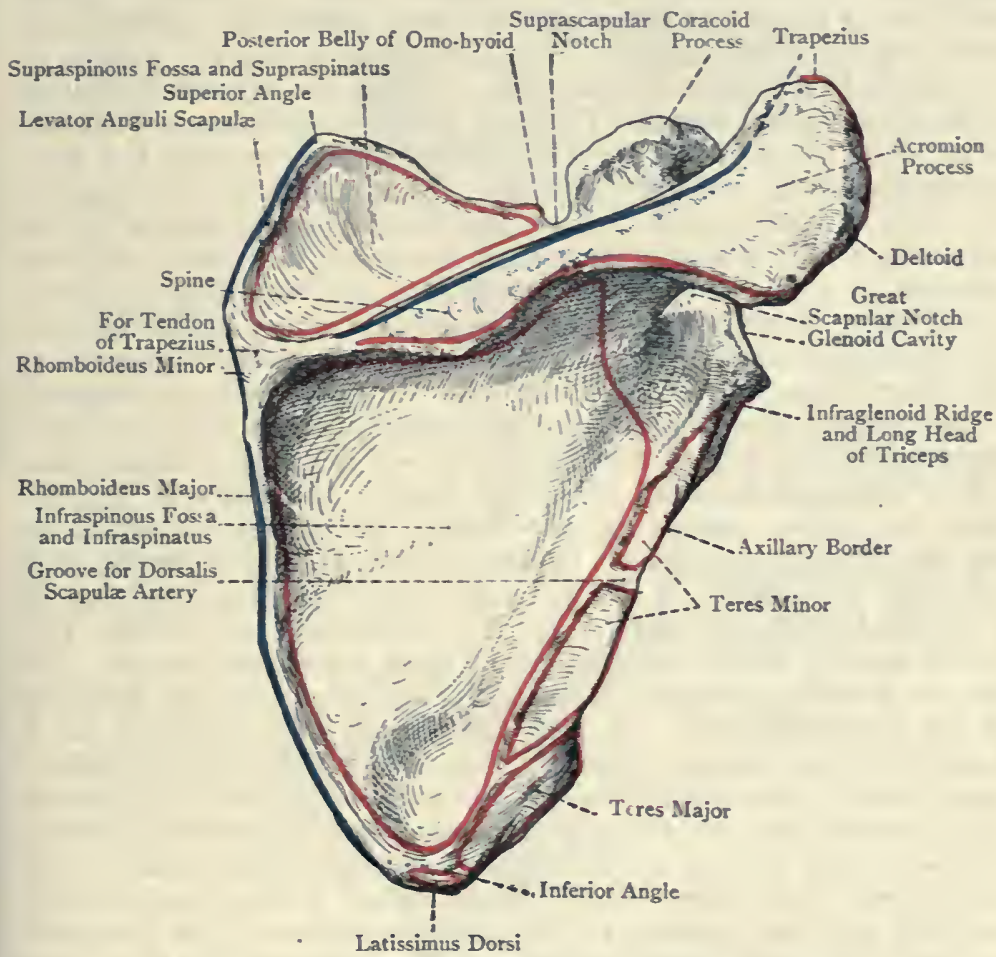

Fig. I02,-The Right Scapula (Posterior View).

extremity it undergoes a slight twist and forms the acromion process. It is triangular, and compressed from above downwards. The superior surface forms part of the supraspinous fossa, and gives origin to fibres of the supraspinatus, whilst the inferior surface forms part of the infraspinous fossa, and gives origin to fibres of the infraspinatus. The external border, which is short, bounds internally the great scapular notch. The anterior border is continuous with the body of the bone. The posterior border or crest presents an upper lip, which gives insertion to part of the trapezius, a lower lip, giving partial 
origin to the deltoid, and an intervening rough surface which is encroached upon by the tendinous fibres of these two muscles. The upper lip is often very projecting at its inner end, where the tendon receiving the lower fibres of the trapezius is inserted.

The acromion process is situated at the outer extremity of the spine, and its direction is outwards, upwards, and forwards, so as to overhang the glenoid cavity. It is somewhat triangular, and is compressed from above and behind downwards and forwards. The postero-superior surface, which is rough, gives origin at its lower and inner part to some fibres of the deltoid, and elsewhere is subcutaneous. The antero-inferior surface, which is smooth and concave, overhangs the glenoid cavity, and is related to the subacromial bursa. The outer border is continuous with the lower lip of the posterior border of the spine, where there is a projection called the acromial angle, and this outer border gives origin to a portion of the deltoid. The inner border is continuous with the upper lip of the posterior border of the spine, near which it gives insertion to a part of the trapezius, whilst near the tip of the acromion it presents an oval facet for the outer extremity of the clavicle. The upper and lower margins of this facet are rough for the acromioclavicular ligaments. The tip or apex of the process is situated at the meeting of the outer and inner borders. The acromion process is pierced by many arterial twigs derived from the acromial rete.

The coracoid process, which is strong and curved, springs from the upper aspect of the head, immediately external to the suprascapular notch. It is directed at first upwards and forwards for about $\frac{1}{2}$ inch, and then, bending sharply, it is directed forwards and outwards to terminate in a blunt tip. The ascending portion is compressed from before backwards. Its anterior surface is related to the subscapularis, and the posterior to the supraspinatus. Its outer border gives attachment to a portion of the coraco-humeral ligament, and the inner border, which bounds the suprascapular notch externally, gives attachment at its upper part to the suprascapular or transverse ligament. The horizontal portion of the process is compressed from above downwards. Its antero-internal border, which is long and convex, and the adjacent portion of the superior surface, give insertion anteriorly to the pectoralis minor, whilst posteriorly they give attachment to the costo-coracoid membrane and ligament. The postero-external border, which is short, receives the fibres of the coraco-acromial ligament, and gives attachment to a portion of the coraco-humeral ligament. At the back part of the antero-internal border there is the conoid impression for the conoid ligament. On the back part of the superior surface there is the trapezoid ridge for the trapezoid ligament, which ridge extends forwards and outwards from the conoid impression. The inferior surface of the horizontal portion is smooth and concave. The tip or apex, which is blunt, gives origin to the conjoined short head of the biceps and coraco-brachialis. 
The coracoid process of man represents the coracoid bone of monotremata and lower vertebrates.

The scapula derives its blood-supply from the dorsalis scapulæ, suprascapular, and posterior scapular, arteries.

Articulations.-By its acromion process with the outer extremity of the clavicle, and by the glenoid cavity with the head of the humerus.

Structure.-The scapula is a flat or tabular bone, and is composed

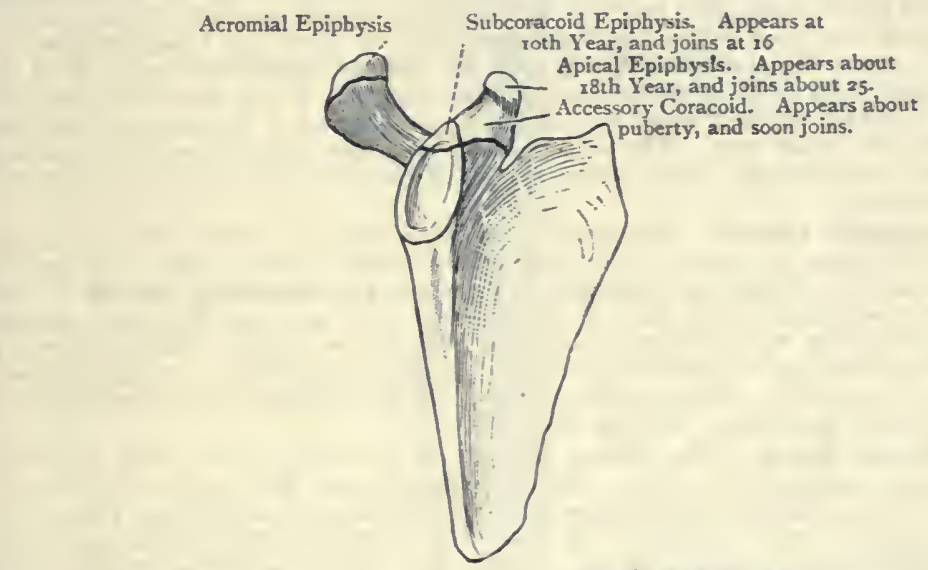

Coracoid Element. Appears in Ist Year, and joins at 15

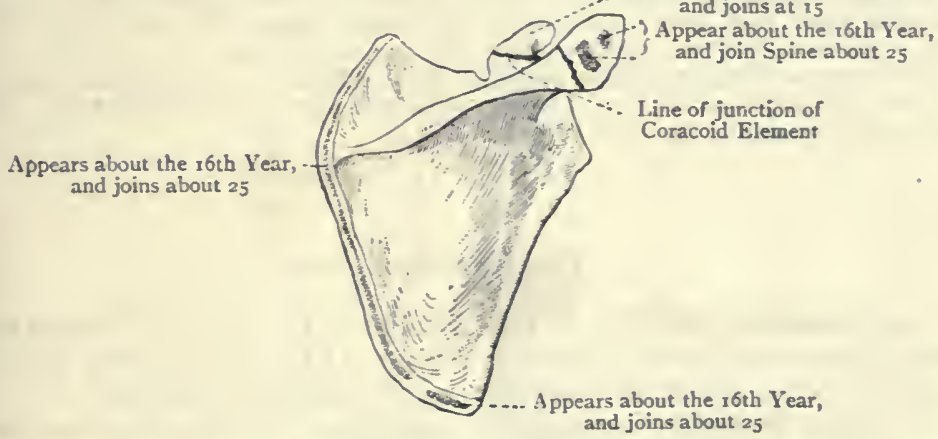

Fig. 103.-Ossification of the Scapula.

of two tables of compact bone. In the head, axillary border, inferior angle, and processes there is cancellated tissue between the two tables, but in the central portions of the supra- and infraspinous fossæ there is none, and the two tables coalesce, so that the bone is very thin and transparent.

Varieties.-(I) Suprascapular foramen. (2) Fenestrated scapula, presenting one or more perforations in the subscapular fossa. (3) Separation of 
the acromion process, which may be connected with the spine by a plate of cartilage, or by fibrous tissue. (4) Imperfect ankylosis of the coracoid process, which, however, is extremely rare.

Ossification.-The scapula has one primary centre and eleven secondary centres. The primary centre appears in the body near the neck about the 8 th week.

The coracoid process, cartilaginous at birth, has four secondary centrescoracoid, accessory coracoid, supracoracoid, and apical. The coracoid centre appears in the end of the Ist year, and gives rise to the chief part of the process, which joins about the I $5^{\text {th }}$ year. The accessury coracoid centre appears about puberty, and soon joins the main coracoid. It forms the triangular part of the process which enters slightly into the extreme upper and inner part of the glenoid cavity. The supracoracoid centre appears about the 18 th year, and forms a thin laminar epiphysis on the upper surface of the process. It gives about the 25 th year. The apical centre appears about the 18 th year,and forms an epiphysis which caps the tip of the process, and joins about the $25^{\text {th }}$ year.

Acromion Process.-The inner or basal portion is ossified from the spine, which in turn is ossified from the primary centre for the body. The greater portion constitutes an epiphysis, which has two secondary centres. These appear about the 16 th year and soon join. The acromial epiphysis usually joins the rest of the process about the 25 th year or earlier. Union, however, may not take place, and then the acromial epiphysis forms a separate acromial bone, connected with the rest of the process by cartilage or by fibrous tissue, and this condition may simulate a fracture.

Glenoid Cavity. - The fundus or bed of this cavity is ossified from the primary centre for the body, and its extreme upper and inner part is formed from the accessory coracoid centre. Besides these the cavity has two special secondary centres-suparior and inferior. The superior glenoid or subcoracoid centre appears about the loth year, and it joins the fundus or bed of the fossa about the 16 th year. The inferior glenoid centre appears about the I6th year, and forms the glenoid epiphysial plate. It joins about the zoth year, and gives rise to the slight concavity of the fossa.

The other secondary centres are allocated as follows: (1) Posterior border of spine; (2) inferior angle; (3) base. These centres appear about the 16th year, and join about the $25^{\text {th }}$ year.

\section{The Humerus.}

The humerus extends from the shoulder to the elbow, its direction being downwards and slightly inwards. It is a long bone, and is divisible into a shaft and two extremities, upper and lower.

The upper extremity includes the head, anatomical neck, great and small tuberosities, commencement of the bicipital groove, and surgical neck. The head, which is almost hemispherical, is smooth, convex, and covered by cartilage. Its direction is upwards, inwards, and backwards, and it articulates with the glenoid cavity of the scapula. The anatomical neck is the constriction immediately beyond the cartilage of the head. It is best marked above, especially between the head and great tuberosity, and it gives attachment to the capsular ligament of the shoulder-joint. It is 


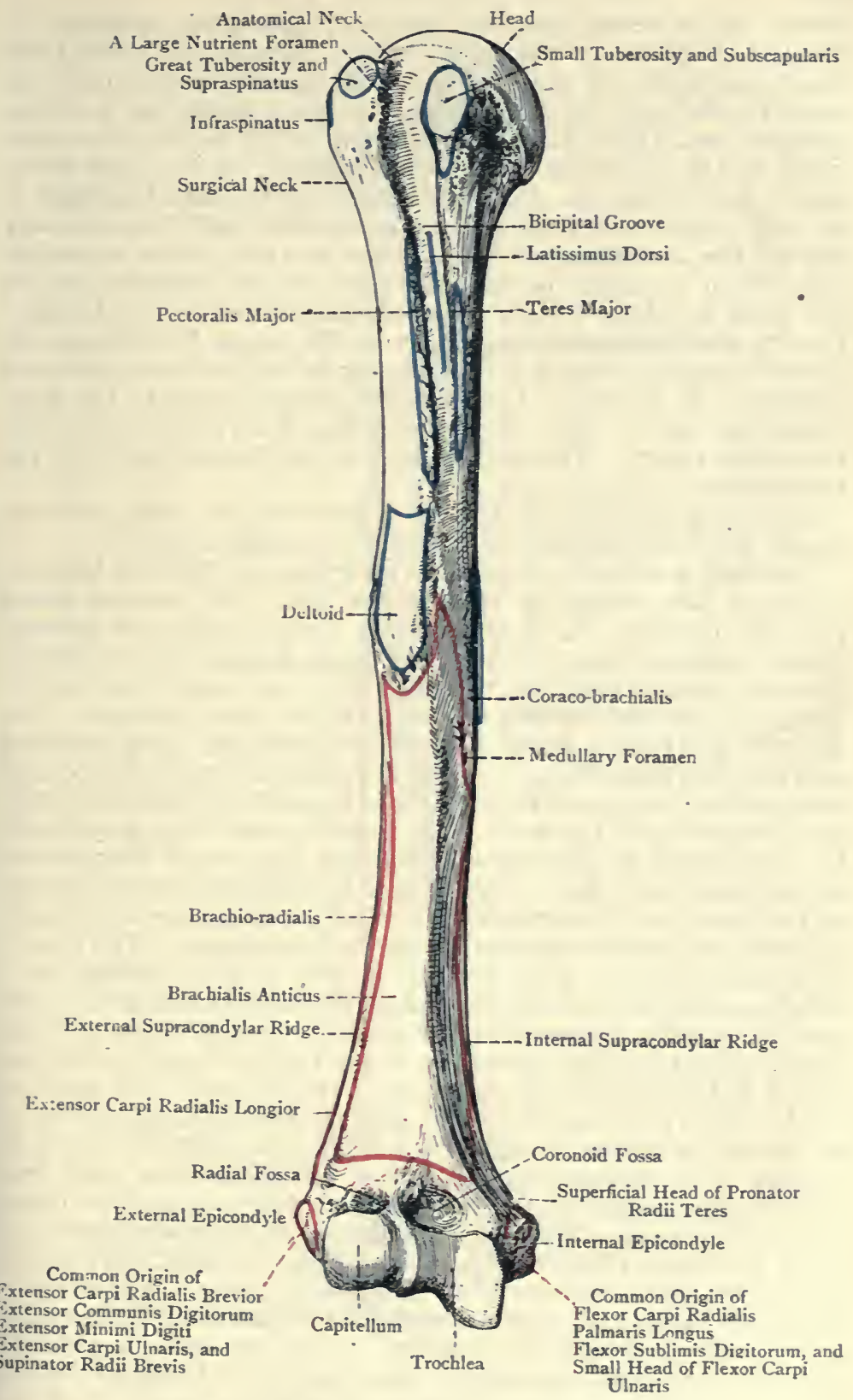

Fig. 104-The Right Humerus (Anterior View). 
pierced by numerous nutrient foramina: The great tuberosity is situated obliquely on the outer surface, immediately beyond the anatomical neck. It presents three flat muscular impressions-an upper for the insertion of the supraspinatus, a middle for the infraspinatus, and a lower for the teres minor, which latter muscle continues to take insertion into a rough marking on the shaft for at least $\frac{1}{2}$ inch below the lower impression. The small tuberosity is an oval prominence situated on the anterior aspect, immediately beyond the anatomical neck. It gives insertion to the subscapularis, which continues to take insertion into the adjacent part of the shaft for about $\frac{1}{2}$ inch. The commencement of the bicipital groove, which lodges the long tendon of the biceps, lies between the two tuberosities, where it is bridged over by the transverse humeral ligament. It presents a large nutrient foramen close to the great tuberosity for an offset of the ascending branch of the anterior circumflex artery. The surgical neck is the constriction below the tuberosities.

The upper extremity of the humerus receives its principal bloodsupply from the anterior and posterior circumflex arteries.

The shaft is almost cylindrical in its upper half, but it is laterally expanded and triangular in its lower half. The anterior aspect presents superiorly the bicipital groove, which commences between the tuberosities, where it is deep, and passes downwards and slightly inwards, terminating about the junction of the upper and middle thirds. It is bounded by two rough ridges, outer and inner. The outer bicipital ridge, which is the more prominent, gives insertion over about its lower three-fourths to the pectoralis major, this portion being called the pectoral ridge. It is in line with the anterior border of the lower half of the shaft. The inner bicipital ridge gives insertion over about its lower two-thirds to the teres major, this portion being known as the teres ridge. It is in line with the internal border of the lower half of the shaft. The floor of the groove over about its middle third gives insertion to the latissimus dorsi. The groove is occupied by the long tendon of the biceps, invested by a tubular prolongation of the synovial membrane of the shoulder-joint ; and the ascending branch of the anterior circumflex artery. The outer aspect of the shaft presents a rough V-shaped mark, called the deltoid impression, for the insertion of the deltoid. The point of the $\mathrm{V}$, which is embraced by two slips of the brachialis anticus, is at the centre of the shaft, whence it extends upwards for about 2 inches. Its anterior margin is in line with the pectoral ridge, and its posterior margin bounds superiorly the lower part of the spiral groove. On the inner aspect of the shaft, about the centre, there is a rough line about $\mathrm{x}$ inches long, placed in the course of the internal border, for the insertion of the coraco-brachialis. Immediately below this line is the principal medullary foramen for the nutrient or medullary branch of the brachial artery, the canal to which it leads being directed downwards. The posterior and external aspects of the upper part of the shaft present a winding groove, called the 


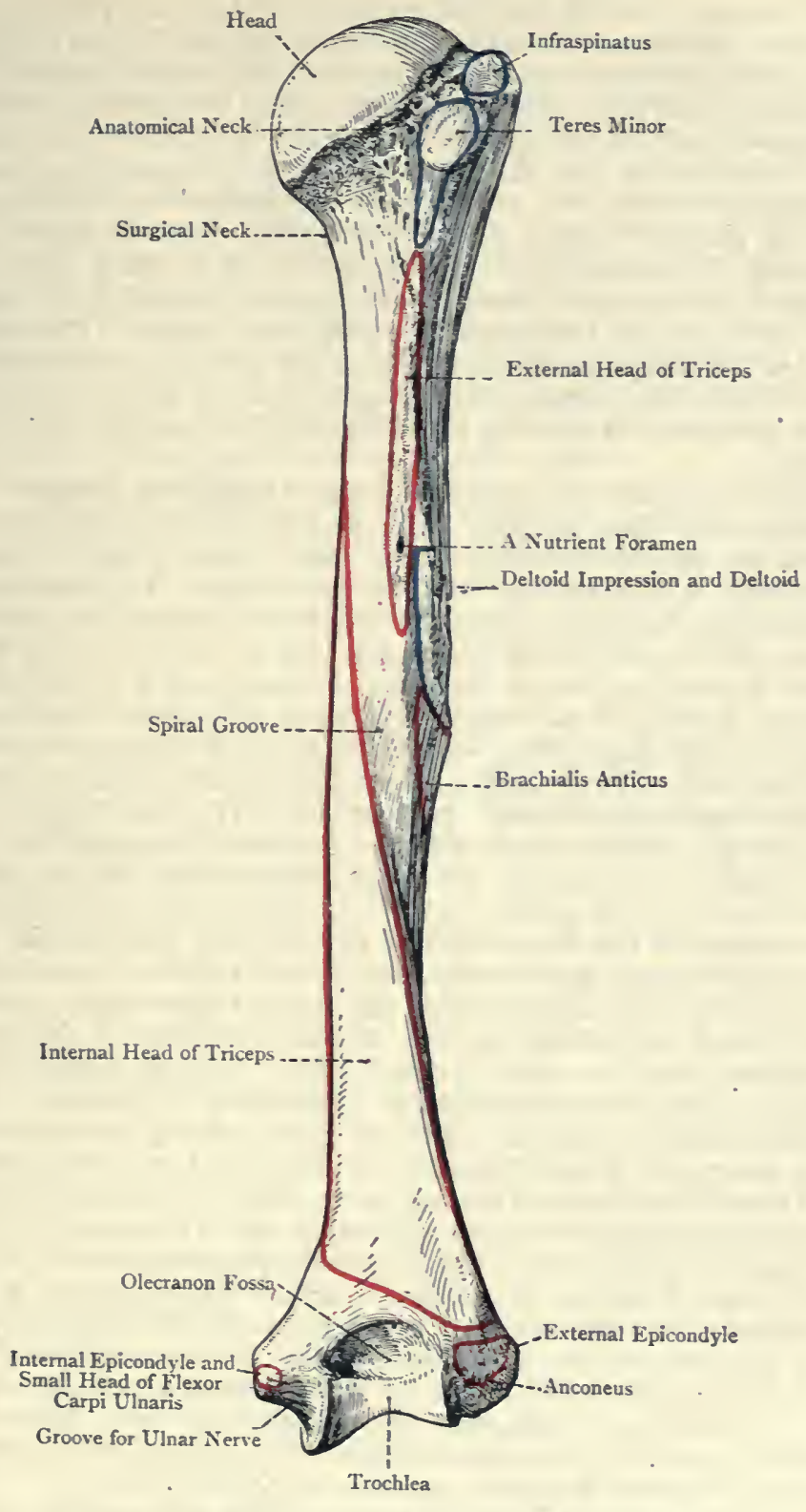

Fig. IO5.-The Right Humerus (Posterior View). 
spiral groove, for the musculo-spiral nerve and superior profunda vessels. It commences in the upper third posteriorly, and is directed downwards and forwards on to the external surface, where it terminates a little below and behind the apex of the deltoid impression. The lower part of this groove is occupied by a pointed slip of the brachialis anticus. In the upper part of the groove there is usually a nutrient foramen for a branch of the superior profunda artery. On the posterior aspect of the shaft, over about its upper third, external to the spiral groove, there is a rough marking which gives origin to the external head of the triceps, extending as high as the lower part of the insertion of the teres minor. The internal head of the triceps commences to arise in a pointed manner from the back of the shaft internal to the spiral groove, where it reaches a little above and behind the lower border of the tendon of the teres major.

The lower half of the shaft, being triangular, presents three surfaces and three borders. The posterior surface, which is flat, terminates at the olecranon fossa, and it gives origin to most of the fibres of the internal head of the triceps. The external and internal surfaces, as well as the anterior border which separates them, give origin to the brachialis anticus. The anterior border, which is round, separates the lateral surfaces, and is in line with the anterior margin of the deltoid impression, and, above this, with the outer bicipital ridge. The external border is called the external supracondylar ridge. It is sharp and prominent, and extends from the external epicondyle to the spiral groove. It gives attachment to the external intermuscular septum. Anteriorly its upper two-thirds give origin to the brachio-radialis (supinator radii longus), and the lower third to the extensor carpi radialis longior. Posteriorly it gives origin to the inner head of the triceps. The internal border forms the internal supracondylar ridge, and is not so prominent as the external. It commences at the internal epicondyle, and it can be followed up through the line for the insertion of the coracobrachialis into the inner bicipital ridge. It gives attachment to the internal intermuscular septum. Anteriorly it gives origin to the brachialis anticus, posteriorly to the inner head of the triceps, and in its lower part to some fibres of the superficial head of the pronator radii teres. The internal surface of the shaft, in front of the internal supracondylar ridge and about $2 \frac{1}{2}$ inches above the internal epicondyle, sometimes presents a sharp spur-like projection directed downwards, called the supracondylar process. When present it gives attachment to a fibrous band which passes to the internal epicondyle, and gives origin to a third head of the pronator radii teres. In such cases the band forms an arch through which the median nerve passes, and frequently the brachial artery. The supracondylar process represents a portion of bone which forms a supracondylar foramen in many carnivora-e.g., the felida.

The lower extremity presents at either side the internal and external epicondyles, and inferiorly a transversely elongated articular 
surface covered by cartilage, and divided by a vertical curved ridge into an external part, called the capitellum, and an internal, called the trochlea. Above the capitellum in front there is a rough transverse depression, called the radial fossa. Above the trochlea in front is the coronoid fossa, and above it posteriorly is the olecranon fossa.

The internal epicondyle (epitrochlea) is very prominent, and is directed inwards and slightly backwards. Its lower part and the adjacent portions of its anterior and posterior aspects give attachment to the internal lateral ligament of the elbow-joint. Its anterior aspect gives origin to the common tendon of the superficial head of the pronator radii teres, flexor carpi radialis, palmaris longus, part of the flexor sublimis digitorum, and one head of the flexor carpi ulnaris. Behind the internal epicondyle, close to the trochlea, is the ulnar groove, through which the ulnar nerve passes.

The external epicondyle is much less prominent than the internal. Anteriorly it gives origin to the common tendon of the extensor carpi radialis brevior, extensor communis digitorum, extensor minimi digiti, extensor carpi ulnaris, and supinator radii brevis. Posteriorly it presents an impression for the anconeus, and inferiorly a depression near the capitellum for the external lateral ligament of the elbow-joint.

The capitellum mainly takes the form of a rounded eminence. It is limited to the anterior and inferior aspects of the bone, and articulates with the cup-shaped depression on the head of the radius. Internal to the rounded portion there is a groove for the play of the inner convex part on the head of the radius. The radial fossa receives the anterior margin of the head of the radius in complete flexion of the elbow-joint.

The trcchlea is pulley-shaped, and turns completely round from the front to the back of the bone, becoming rather broader posteriorly. It is concave from side to side, and convex from before backwards. The internal border is more prominent and thicker than the external, and extends lower down. As viewed from before, the borders are inclined downwards and slightly inwards, but posteriorly they incline upwards and slightly outwards, and so the trochlea is here brought into the centre of the bone. The trochlea articulates with the great sigmoid cavity of the ulna. The coronoid fossa receives the coronoid process of the ulna in flexion of the elbow-joint, and the anterior ligament is attached just above it. The olecranon foss $a$, much larger than the coronoid, receives the olecranon process of the ulna in extension of the joint, and its margins give attachment to the posterior ligament. The portion of bone which separates the two fossæ is thin, and is sometimes perforated by a foramen, called the supratrochlear foramen.

In the vicinity of the lower extremity there are numerous nutrient foramina for branches of the superior and inferior profundæe and anastomotica magna of the brachial, radial and ulnar recurrent, and interosseous recurrent, arteries. 
Articulations.-Superiorly with the scapula, and inferiorly with the radius externally, and ulna internally.

Structure.-The shaft is composed of compact bone, which is thicker at the centre than at the extremities. It contains a medullary canal lined with a thin coating of cancellated tissue. The articular extremities are filled with cancellated tissue, except at the surface, where there is a thin layer of compact bone.

Varieties.-These are (I) a supracondylar process, and (2) a supratrochlear foramen.

Ossification.-The humerus ossifies in cartilage from one primary, and seven secondary, centres. The primary centre appears at the middle of the shaft in the eighth week of intra-uterine life. At birth the shaft is ossified, but the

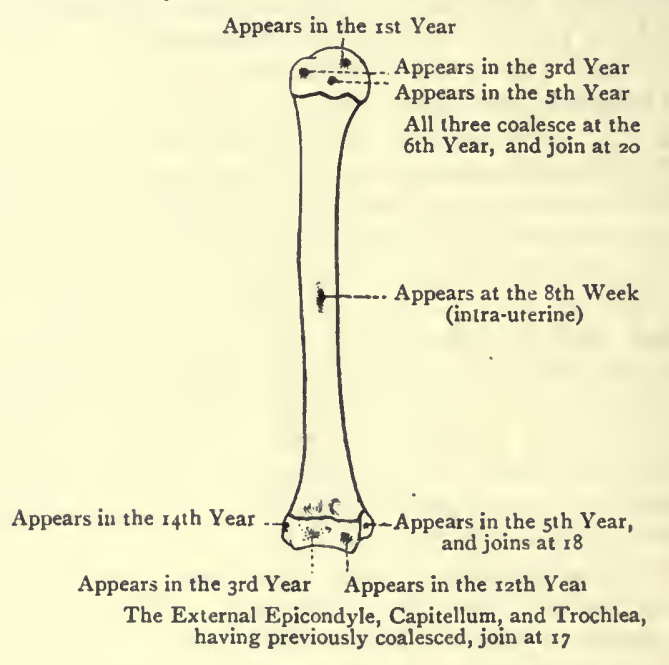

Frg. 106.-Ossification of the Humerus.

extremities are cartilaginous. The superior extremity is ossified from three secondary centres. The centre for the head appears in the first year (sometimes just before birth), that for the great tuberosity in the third year, and that for the small tuberosity in the fitth year. The upper epiphyses join to form one compound epiphysis in the sixth year, and this unites with the shaft in the twentieth year. The lower extremity is ossified from four secondary centres, as follows: one for the capitellum and outer half of the trochlea appearing in the third year; one for the internal epicondyle in the fith year; one for the inner half of the trochlea in the twelfth year; and one for the external epicondyle in the fourteenth year. The trochlea, capitellum, and external epicondyle join to form one compound epiphysis, and this unites with the shaft in the seventeenth year. The internal epicondyle unites with the shaft, as an independent epiphysis, in the eighteenth year.

The law of ossifleation applicable to long bones with an epiphysis or epiphyses at either end is as follows: the epiphysis or epiphyses, 
at the en $l$ towards which the medullary foramen and the canal to which it leads are directed, are the last to show signs of ossification, but they are the first to join the shaft. The only exception to this rule occurs in the fibula.

\section{The Radius.}

The radius is the external bone of the forearm (which is assumed to be in a position of supination). It is parallel with, and shorter than, the ulna, and extends from the elbow to the wrist. It is a long bone, and is divisible into a shaft and two extremities.

The upper extremity, which is small, presents a head and neck. The head is disc-shaped, and covered by cartilage, both on its upper surface and circumference. The upper suirface, at its centre, presents a depression which articulates with the rounded portion of the capitellum of the humerus in flexion of the elbow-joint. Around this depression the surface is convex, especially on the inner side, and this portion glides on the inner grooved part of the capitellum. The circumferential cartilage is deeper on the inner aspect than elsewhere, and this portion articulates with the small sigmoid cavity of the ulna, whilst the remainder plays within the orbicular ligament. The constricted portion below the head is called the neck. It is cylindrical, and its upper part is embraced by the orbicular ligament, whilst beyond this on the outer aspect it gives insertion to a few fibres of the supinator radii brevis. The upper extremity presents several nutrient foramina for branches of the radial recurrent and interosseous recurrent arteries.

The shaft increases in size from above downwards, and is curved, the convexity being directed outwards and slightly backwards. This curve imparts elasticity to the bone, and guards it against the shocks to which it is so much exposed from the fact that it supports the hand. The shaft is triangular, and presents superiorly, on its antero-internal aspect just below the neck, an oval eminence, called the bicipital tuberosity. This is divided vertically into two parts, a rough posterior portion which gives insertion to the tendon of the biceps, and a smooth anterior part which is separated from that tendon by a bursa. Below the bicipital tuberosity the shaft presents three borders and three surfaces. The anterior border extends from the-lower and anterior part of the bicipital tuberosity to the anterior border of the styloid process. In its upper third it crosses the shaft obliquely downwards and outwards, this portion of it being called the anterior oblique line. This line limits externally the insertion of the supinator radii brevis, and internally the origin of the flexor longus pollicis, whilst its prominent edge gives origin to the thin radial portion of the flexor sublimis digitorum. The internal or interosseous border commences at the lower and back part of the bicipital tuberosity, and near the lower extremity of the shaft it divides into two ridges, which pass to 


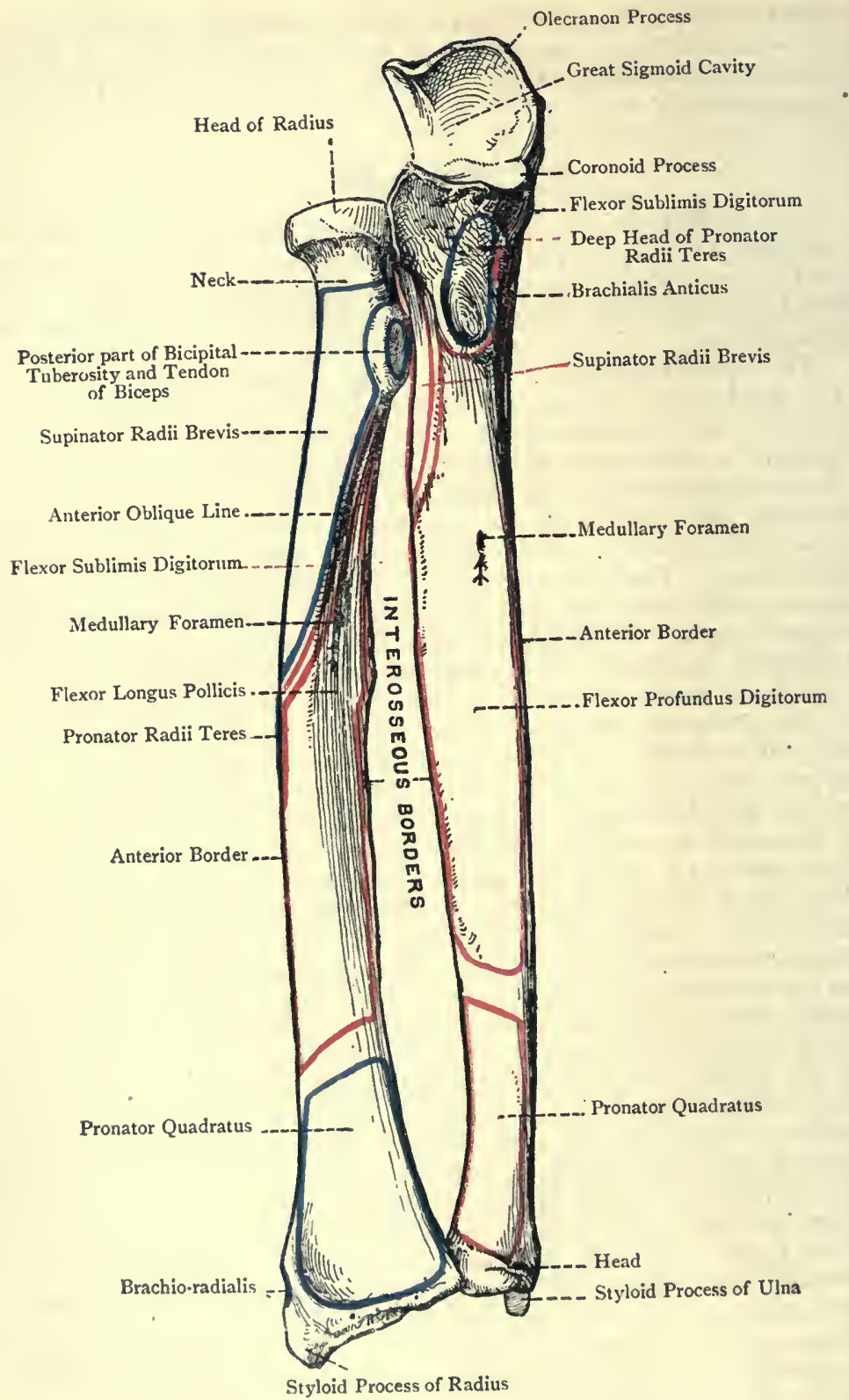

Fig. 107.-The Right Radius and Ulna (ANterior View). 
the anterior and posterior margins of the sigmoid cavity. At its commencement it is round and indistinct, and immediately below the bicipital tuberosity it gives attachment to the oblique ligament. Over the rest of its extent it is sharp and wiry for the attachment of the interosseous membrane, which is also connected with the posterior of the two lower divisions. The posterior border extends from the back of the bicipital tuberosity to the prominent radial tubercle about the centre of the posterior border of the lower extremity. In its upper third it crosses the shaft obliquely downwards and outwards, this portion of it, which is prominent, being called the posterior oblique line. This line limits the insertion of the supinator radii brevis above, and the origin of the extensor ossis metacarpi pollicis below.

The anterior surface is situated between the anterior and internal or interosseous borders. In the upper two-thirds it is concave, and gives origin to the flexor longus pollicis. In the lower third it is flat and expanded, and this portion gives insertion to the pronator quadratus, except close to the anterior border of the lower extremity, where it gives attachment to the anterior ligament of the wrist-joint. The anterior surface presents the medullary foramen about the junction of the upper and middle thirds. The direction of the canal to which it leads is upwards, and it gives passage to the medullary branch of the anterior inter. osseous artery. The portion of bone between the anterior oblique line, the lower part of the front of the neck, and the bicipital tuberosity gives insertion to a portion of the supinator radii brevis The external surface is situated between the anterior and posterios borders. It is convex from above downwards, and from side to side. In its upper third it gives insertion to the supinator radii brevis; at its centre there is a rough impression, fully an inch long, for the insertion of the pronator radii teres; and below this it supports the tendons of the extensores carpi radialis longiol et brevior, and is crossed obliquely by the tendons of the extensor ossis metacarpi pollicis and extensor brevis pollicis (primi internodii pollicis). The external surface also supports the brachio-radialis, which is inserted into its lower extremity close to the base of the styloid process. The posterior surface is situated between the posterior and internal or interosseous borders. Above the posterior oblique line it is covered by the supinator radii brevis, which takes insertion into its outer half. Below the posterior oblique line it is concave over about the middle third, where it gives origin from above downwards to the extensor ossis metacarpi pollicis and extensor brevis pollicis. The lower third is broad and convex, and it supports the tendons of the extensor longus pollicis, extensor communis digitorum, and extensor indicis.

The lower extremity is large and pentagonal, as viewed from below. Externally it presents the styloid process, internally the sigmoid cavity, and inferiorly the carpal articular surface, the latter two being covered by cartilage. The styloid process projects down. 


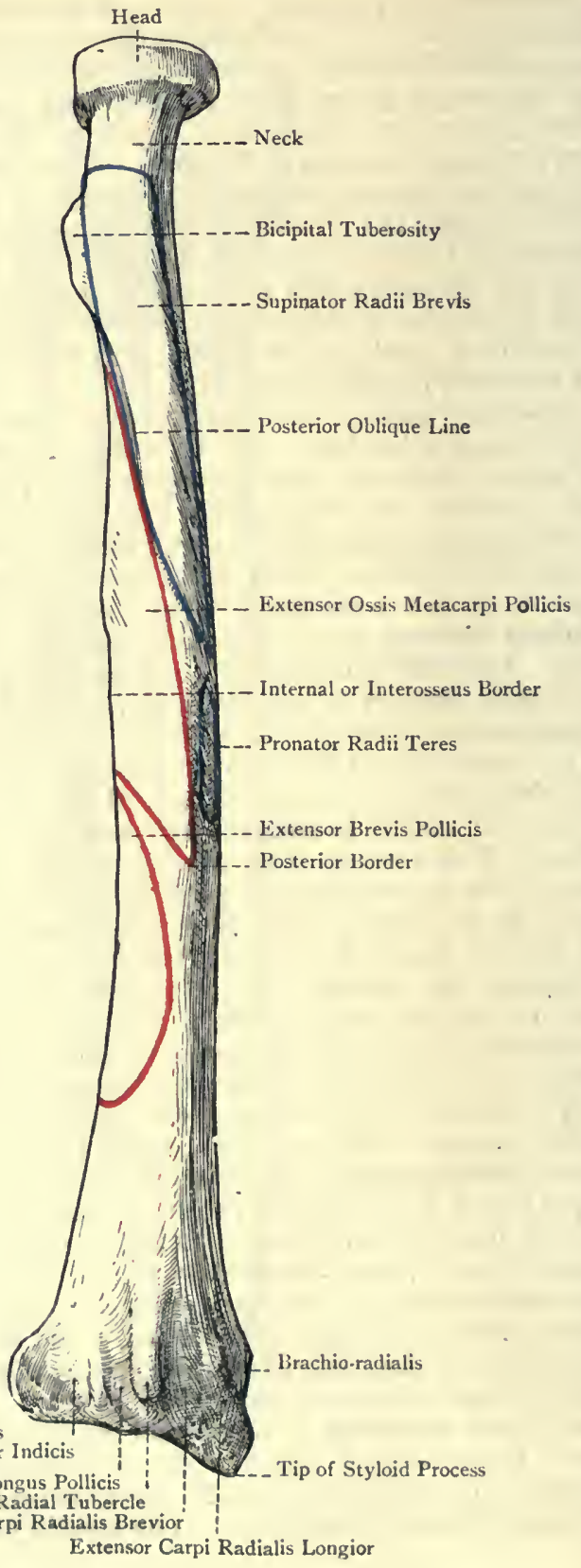

Extensor Cummunis Digitorum and Extensor Indicis

Extensor Longus Pollicis Radial Tubercle
Extensor Carpi Radialis Breviot

Extensor Carpi Radialis Longior

Fig. 108. - The Right Radius (Posterior Surface). 
wards as a stout conical process, terminating in a round tip which gives attachment to the external lateral ligament of the wrist-joint. The inner surface is covered by the cartilage of the carpal articular surface. The outer surface presents a groove directed downwards and slightly forwards, and subdivided into two compartments, the outer of which transmits the tendon of the extensor ossis metacarpi pollicis, and the inner that of the extensor brevis pollicis. This groove is separated from the pronator surface in front by a prominent ridge which gives attachment to the posterior annular ligament. The anterior surface supports a portion of the radial artery.

The sigmoid cavity is concave from before backwards, and articulates with the outer convex surface of the head of the ulna.

The carpal articular surface is of large size, and its plane is oblique, being sloped outwards and a little downwards. It is concave from before backwards, and from side to side, and is divided into two parts by an antero-posterior elevation. The outer division is triangular, its cartilage being prolonged on to the inner surface of the styloid process, and it articulates with the scaphoid bone. The inner division is quadrilateral, and articulates with the semilunar bone. It is separated from the sigmoid cavity by a sharp concave margin, which gives attachment to the base of the triangular interarticular fibro-cartilage. Immediately above the anterior border there is a rough surface for the attachment of the anterior ligament of the wristjoint. The posterior border is on a slightly lower level than the anterior, and is irregularly convex. It presents about its centre a prominent elevation, called the radial tubercle, and is divided into three grooves - outer, middle, and inner. The outer groove, which is broad, is bounded externally by a ridge which separates it from the groove on the outer surface of the styloid process, and internally by the radial tubercle. It is usually subdivided by a faint line into two compartments, the outer of which transmits the tendon of the extensor carpi radialis longior, and the inner that of the extensor carpi radialis brevior. The middle groove, narrow and deep, is directed from above downwards and outwards, and is bounded externally by the radial tubercle, which slightly overhangs it, and internally by an oblique ridge separating it from the inner groove. It transmits the tendon of the extensor longus pollicis (secundi internodii pollicis). The inner groove is single, and transmits the tendons of the extensor communis digitorum and extensor indicis. It is separated from the sigmoid cavity by a sharp ridge which, with the ulna in position, bounds a groove for the tendon of the extensor minimi digiti. The ridges separating the grooves give attachment to deep expansions of the posterior annular ligament, which, with the ligament, convert the grooves into fibro-osseous canals.

The lower extremity presents several nutrient foramina for branches of the anterior and posterior interosseous arteries, and anterior and posterior carpal arches. 
Articulations.-Superiorly with the capitellum of the humerus and small sigmoid cavity of the ulna, and inferiorly with the head of the ulna, scaphoid, and semilunar.

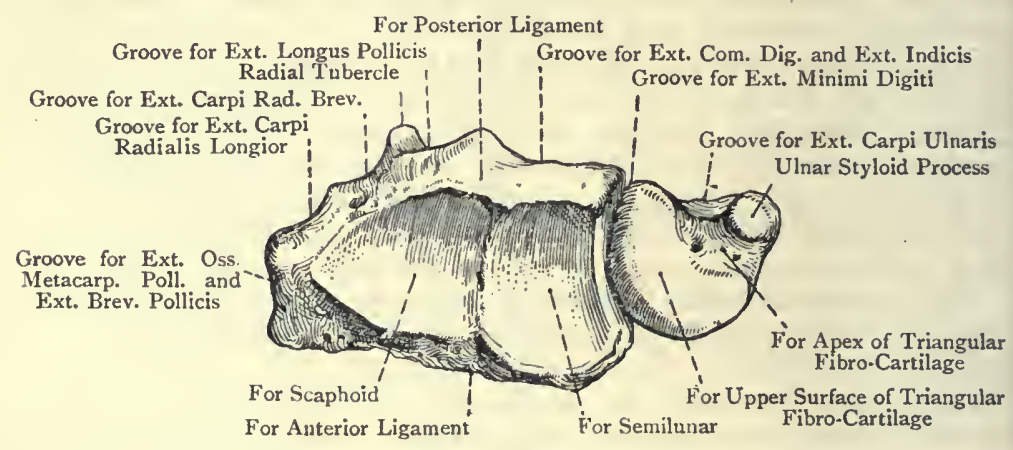

Fig. 109.-The Lower Ends of the Left Radius and Ulna AS SEEN FROM BELOW IN PRONATION.

(The Dorsal Aspect is upwards.)

Structure.-This is similar to that of long bones. At the lower extremity the cancellated tissue extends upwards for about $I_{2}^{\frac{1}{2}}$ inches above the styloid process, this level being the site of Colles' fracture.

Ossification.-The radius ossifies in cartilage from one primary, and two secondary, centres. The primary centre appears at the middle of the shaft in the eighth week of intra-uterine life. At birth the shaft is ossified, but the extremities are cartilaginous. The secondary centre for the lower extremity appears in the second year, and that for the head in the fifth year. The

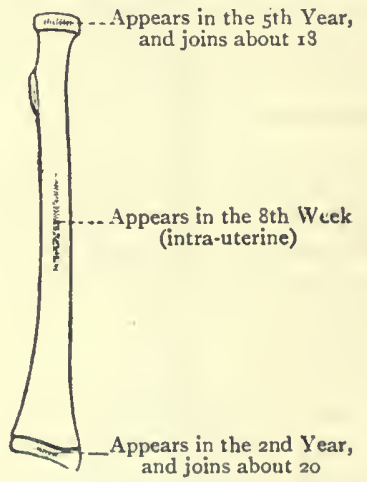

Fig. iro.-Ossification of the Radius.

upper epiphysis joins the shaft about the eighteenth year, and the lower epiphysis about the twentieth year. Sometimes the rough part of the bicipital tuberosity forms an epiphysis, in which cases there is a special secondary centre for it, appearing about the fourteenth year, and joining the rest of the shaft very soon thereafter. 


\section{The Ulna.}

The ulna is the internal bone of the forearm (which is assumed to be in a position of supination). It is parallel with, and longer than, the radius, and extends from the elbow to the wrist, being separated from the latter joint by the triangular interarticular fibro-cartilage. It is a long bone, and is divisible into a shaft and two extremities, the upper of which is of large size.

The upper extremity presents the olecranon and coronoid processes, and the great and small sigmoid cavities. The olecranon process forms the highest part of the bone, and is curved forwards at its upper part. It is largely subcutaneous. Superiorly it presents a broad, flat, quadrilateral surface, at the back part of which is a rough elevation for the insertion of the triceps. In front of this there is a smooth area where a bursa intervenes between that muscle and the bone. At its anterior part, near the anterior margin, there is a narrow transverse impression for part of the posterior ligament of the elbow-joint. The superior surface is limited anteriorly by a sharp convex border, projected at its centre into a process, called the beak, which overhangs the upper part of the great sigmoid cavity, and is received into the olecranon fossa of the humerus in extension of the joint. The anterior surface is directed downwards and forwards, and forms the upper part of the great sigmoid cavity. The posterior surface, smooth, flat, and triangular, is subcutaneous, and covered by a bursa. The inner surface presents a tubercle for the ulnar head of the flexor carpi ulnaris, and the inner border gives attachment to the posterior part of the internal lateral ligament of the elbow-joint. The outer surface gives insertion to a portion of the anconeus, and the outer border gives attachment to fibres of the posterior ligament of the joint.

The coronoid process is triangular, and projects forwards. The superior surface forms the lower and anterior part of the great sigmoid cavity. The inferior surface is rough and concave, and the roughness is prolonged upon the anterior surface of the shaft for about an inch, giving rise to a triangular impression, the inner half of which gives insertion to the brachialis anticus. The upper part of this surface, close to the anterior border of the process, affords attachment to the anterior ligament of the elbow-joint. The lower pointed portion presents externally a rough prominence, called the tuberosity, which gives insertion to fibres of the brachialis anticus, and attachment to the oblique ligament. The anterior margin is sharp, convex, and curved slightly upwards, and it is projected at its outer part into a process, called the beak, which is feceived into the coronoid fossa of the humerus in flexion of the dbow-joint. The inner border gives attachment to the anterior portion of the internal lateral ligament, and at its upper part it presents a tubercle for the ulnar head of the flexor sublimis digitorum. Leading downwards from this there is a short ridge for the origin of the deep head of the pronator radii teres, below which 
a slip of the flexor longus pollicis sometimes arises. Behind the flexor sublimis tubercle there is a depressed surface which gives origin to the highest fibres of the flexor profundus digitorum. The outer surface presents the small sigmoid cavity.

The great sigmoid cavity, which articulates with the trochlea of the humerus, when viewed from the side, forms half a circle. The upper half of the cavity is formed by the anterior surface of the olecranon process, and the lower half by the upper surface of the coronoid process. It is constricted at the centre by a notch at either side, which marks the meeting of the olecranon and coronoid processes, the inner notch being bridged over by a fibrous band with which the middle part of the internal lateral ligament blends. The cartilage of the cavity is sometimes broken up at this part by a narrow, rough, transverse interval. Extending from the beak of the olecranon to the beak of the coronoid there is a longitudinal elevation, which divides the cavity into two lateral halves, the inner being concave from side to side, and the outer convex.

The small sigmoid cavity, which is situated on the outer surface of the coronoid process, is concave from before backwards, and ar. ticulates with the inner aspect of the head of the radius. Its anterior and posterior margins give attachment to the cornua of the orbicular ligament.

The upper extremity presents several nutrient foramina for branches of the anterior and posterior ulnar recurrent and interosseous recurrent arteries.

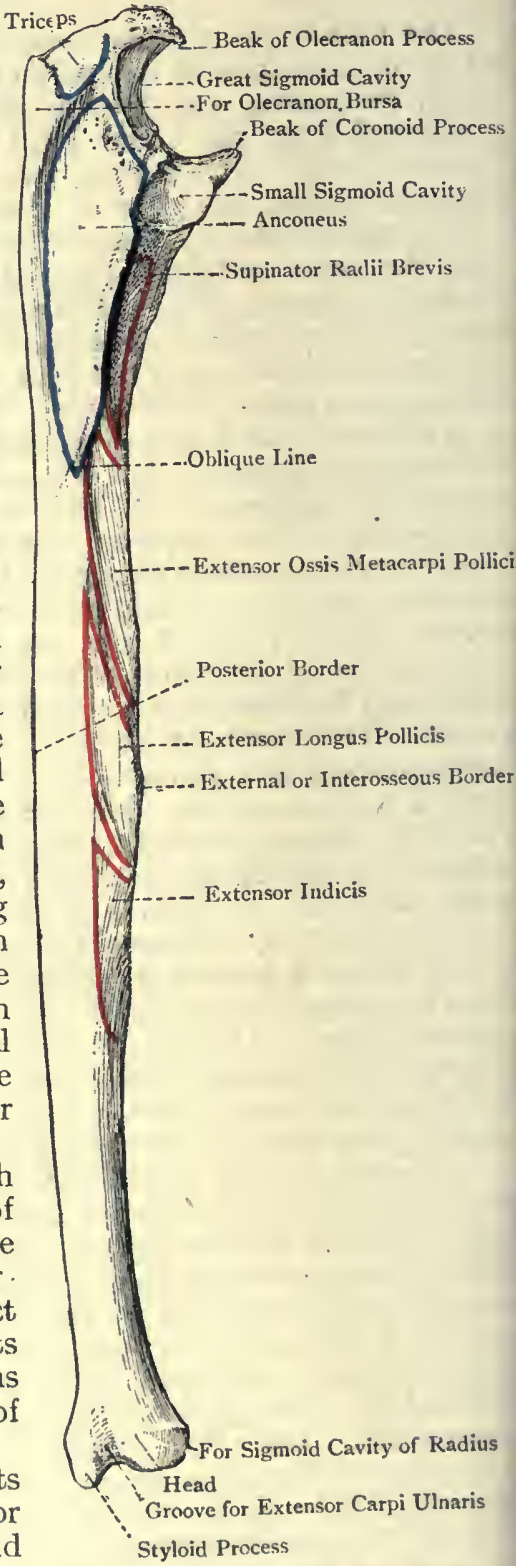

Fig. III.-ThE Right UlNa (Posteriar Surface). 
The shaft diminishes in size from above downwards, and is triangular in its upper three-fourths, where it is slightly curved with the convexity directed backwards. In the lower fourth it is slender and subcylindrical, being flattened in front. It presents three borders and three surfaces. The anterior border extends from the flexor sublimis tubercle on the inner margin of the coronoid process to the front of the styloid process. Over its upper three-fourths it is round, and gives origin to fibres of the flexor profundus digitorum. Over its lower fourth it is sharp, and gives origin to the pronator quadratus. The posterior border, which is subcutaneous, extends from the apex of the triangular subcutaneous surface on the back of the olecranon to the back of the styloid process. Over its upper two-thirds it gives attachment to a strong aponeurosis, which gives common origin to the flexor, and extensor, carpi ulnaris, and flexor profundus digitorum. The external or interosseous border extends from the apex of the bicipital hollow, about two inches below the small sigmoid cavity, to the outer aspect of the head. Over the middle three-fifths of the shaft it is sharp and prominent, but over the lower fifth it is very faint. It gives attachment to the interosseous membrane.

The anterior surface is situated between the anterior and interosseous borders. It is concave over its upper three-fourths, and gives origin to part of the flexor profundus digitorum. The lower fourth is flat, and gives origin to the pronator quadratus. This surface presents the medullary foramen a little above the centre, the direction of the canal to which it leads being upwards. It gives passage to the medullary branch of the anterior interosseous artery. The internal surface is situated between the anterior and posterior borders. Over its upper two-thirds it gives origin to part of the flexor profundus digitorum, the lower portion being subcutaneous. The posterior surface is situated between the posterior and interosseous borders, and is directed backwards and outwards. It presents superiorly the oblique line, which extends from the supinator ridge on the posterior margin of the bicipital hollow to the posterior border at the junction of the upper and middle thirds. The triangular portion above this line is called the anconeal surface, which extends over the outer surface of the olecranon. It gives insertion to the anconeus. The posterior surface, below the oblique line, is divided into two lateral parts by a vertical ridge. The inner portion supports the extensor carpi ulnaris, and the outer gives origin, from above downwards, to the extensor ossis metacarpi pollicis, extensor longus pollicis, and extensor indicis. On the outer aspect of the shaft superiorly there is a triangular depression, which commences immediately below the small sigmoid cavity, and extends downwards for about 2 inches. It is bounded in front and behind by prominent lips, the anterior of which passes above into the outer margin of the coronoid process, and the posterior into the posterior margin of the small sigmoid cavity. The upper part of the posterior lip, which is prominent, 
is called the supinator ridge, and it gives origin to a part of the supinator radii brevis. The two lips form by their meeting the commencement of the external or interosseous border. The anterior part of this surface superiorly receives the bicipital tuberosity of the radius, with the tendon of insertion of the biceps, in pronation, whilst the posterior part gives origin to fibres of the supinator radii brevis.

The lower extremity is small, and presents a head and styloid process. These are separated behind by a groove for the tendon of the extensor carpi ulnaris, and below by a rough pit which gives attachment to the apex of the triangular interarticular fibro-cartilage. The outer aspect of the head is convex, and covered by cartilage for articulation with the sigmoid cavity of the radius, a portion of the synovial membrane, called membrana sacciformis, intervening. The inferior surface, also covered by cartilage, is flat, and is related to the upper surface of the triangular interarticular fibrocartilage, with the intervention of another portion of the membrana sacciformis.

The styloid process, of small size and subcutaneous, projects downwards from the posterior and inner part of the head (mainly from the back part), and it terminates in a round tip which gives attachment to the internal lateral ligament of the wristjoint.

The lower extremity presents several nutrient foramina for branches of the anterior and posterior interosseous arteries.

Articulations. - Superiorly with the trochlea of the humerus, and the inner aspect of the head of the radius; inferiorly with the sigmoid cavity of the radius, and the triangular interarticular fibro-cartilage, the latter structure separating it from the cuneiform or pyramidal bone.

Structure.-This is similar to that of. long bones.

Ossiffeation.-The ulna ossifies in cartilage from one primary, and two secondary, centres. The primary centre appears at the middle of the shaft in the eighth week, and from it the shaft, coronoid process, and greater part of the olecranon process are ossified. At birth the shaft and coronoid process are ossified, but the greater part of the olecranon and the lower extremity are cartilaginous. The secondary centre for the lower extremity appears in the fourth year, and from it

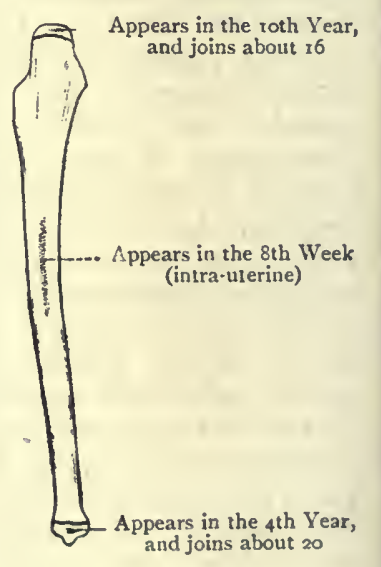

Fig. II2.-Ossification OF THE ULNA. the head and styloid process are ossified. The secondary centre for the upper extremity appears in the tenth year, and may involve only a thin scale on the summit of the olecranon process, or it may afford ossification to a third, or even a half, of that process. The upper epiphysis joins about the sixteenth year, and the lower about the twentieth year. 


\section{The Carpus.}

The carpus or wrist is composed of eight short bones, which are arranged in two rows, there being four bones in each row. The rows are called first or proximal, and second or distal. The bones of the first row, from without inwards, are called scaphoid, semilunar, cuneiform, and pisiform, whilst those of the second row, in a similar order, are named trapezium, trapezoid, os magnum, and unciform.

\section{The Scaphoid Bone.}

The scaphoid bone, which is characterized by its boat-like shape, lies with its long axis oblique, the broad end being directed upwards and inwards, and the narrow end or prow downwards, outwards and forwards. Superiorly it presents a convex articular.surface for the radius, which encroaches on the dorsal aspect. Inferiorly it also presents a convex articular surface directed downwards, out-

A

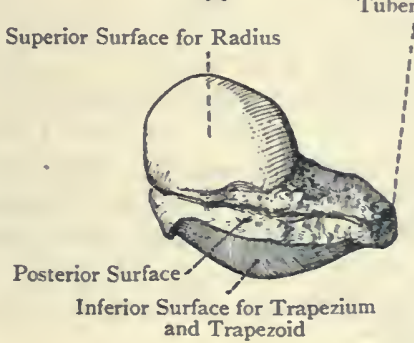

B

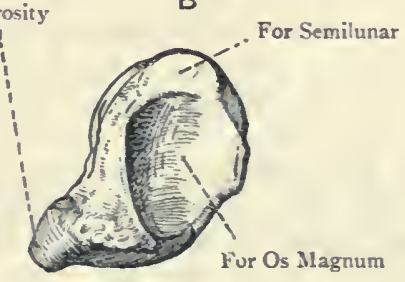

Fig. II3.-The Right Scaphold Bone.

A, Posterior View; B, Internal View.

wards, and backwards, which likewise encroaches on the dorsal asfect, and is divisible into two parts-an outer for the trapezium, and an inner for the trapezoid. The internal surface presents two articular facets-a superior, crescentic, narrow from above downwards, and looking inwards, for the semilunar, and an inferior, large, concave, and directed downwards as well as inwards, for the outer side of the head of the os magnum. The external aspect takes the form of a rough border, extending from the radial surface to the tuberosity, and giving attachment to the external lateral ligament of the wrist-joint. At its lower end there is a prominent tuberosity, directed forwards, which gives attachment to fibres of the anterior annular ligament and abductor pollicis. The palmar surface is rough and triangular. The dorsal surface, being encroached upon by the superior and inferior convex articular surfaces, is reduced to a rough oblique groove.

Articulations.- Superiorly with the radius, injeriorly with the trapezium and trapezoid, and internally with the semilunar and os magnum. 


\section{The Semilunar Bone.}

The semilunar or lunar bone is characterized by the crescentic concavity on its inferior surface. Superiorly it presents a quadr:lateral, convex, articular surface for the radius, which encroaches on the dorsal aspect. The inferior surface is deeply concave from before backwards. The greater part of it articulates with the upper surface of the head of the os magnum, and the narrow inner strip with the upper border of the unciform. The external surface,

A

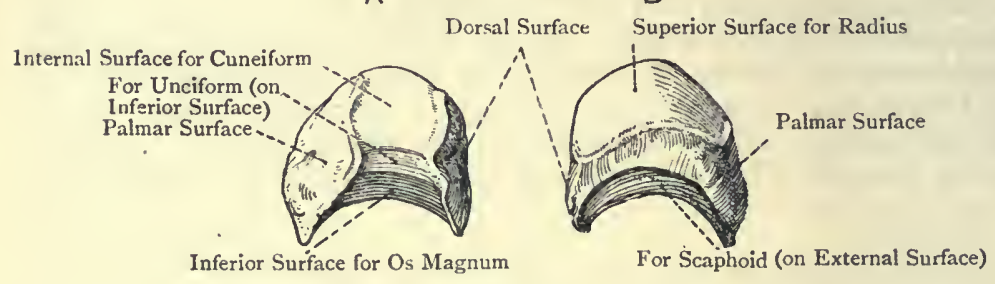

Fig. il4.-The Right Semilunar Bone.

A, Internal View; B, Supero-external View.

narrow from above downwards, presents a crescentic facet for the scaphoid. The internal surface, which is inclined downwards and outwards, is deep from above downwards, and presents a. semi-oval facet for the cuneiform. The palmar and dorsal surfaces are rough, the former being large, convex, and quadrilateral, and the latter small and flat.

Articulations.-Superiorly with the radius, inferiorly with the os magnum and unciform, externally with the scaphoid, and internally with the cuneiform.

\section{The Cuneiform Bone.}

The cuneiform or pyramidal bone is characterized by its resemblance to a wedge, or pyramid, and it lies obliquely with the base

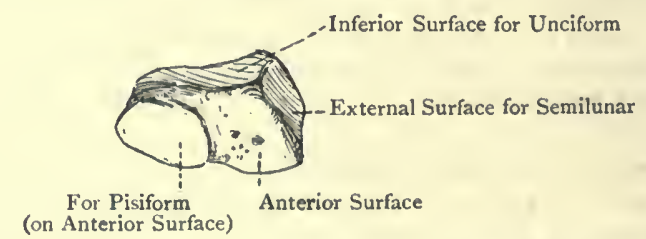

- Fig. 1i5.-The Right Cuneiform or Pyramidal Bone

(ANTERIor, External, and InfERior SUrfaces).

directed outwards and upwards. The external surface, which corresponds with the base, presents a semi-oval facet for the semi- 
lunar. The internal surface, which represents the rounded apex, is of limited extent, and rough for the internal lateral ligament of the wrist-joint. The palmar surface presents a circular, slightly convex facet, which occupies rather more than the inner and lower half, and articulates with the pisiform, the rest of the surface being rough. The supero-posterior surface is divisible into two parts, outer and inner. The outer portion, which is close to the base, presents a convex facet for the inferior surface of the triangular interarticular fibro-cartilage. The inner portion is marked by two rough oblique grooves, superior and posterior, separated by a ridge which is dorsally placed. The inferior surface presents a large triangular facet, concavo-convex from without inwards, for the unciform.

Articulations.--Superiorly with the triangular interarticular fibro-cartilage, inferiorly with the unciform, externally with the semilunar, and anteriorly with the pisiform.

\section{The Pisiform Bone.}

The pisiform bone is characterized by its resemblance to a pea, and is placed in front of the cuneiform, which constitutes its only articulation. It is irregularly round, except posteriorly, where it presents over its upper three-fourths a circular, slightly concave facet for the cuneiform, the lower fourth being non-articular. The

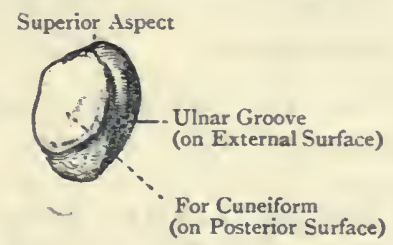

Fig. ir6.-The Right Pisiform Bone (Postero-external View).

long axis of the bone is directed downwards and slightly outwards. The palmar surface gives attachment superiorly to the flexor carpi ulnaris, inferiorly to the pisi-uncinate and pisi-metacarpal ligaments, and abductor minimi digiti, and externally to a portion of the anterior annular ligament. The internal surface is irregularly convex, and the external presents the ulnar groove, which lodges the ulnar nerve and artery.

\section{The Trapezium.}

The trapezium is the external bone of the second row, and is characterized by a groove and ridge on its palmar surface, and a saddle-shaped facet on its inferior surface. It is polyhedral, 
and its long axis is directed downwards and inwards. The superior surface presents a semi-oval, concave facet for the scaphoid. The inferior surface presents a saddle-shaped facet, concave from side to side, convex from before backwards, and directed outwards as well as downwards, for the base of the first metacarpal bone. The internal surface has two facets-an upper, which is large and concave, for the trapezoid, and a lower, which is small, for the base of the second metacarpal bone. The external surface is broad, pentagonal, and rough. The palmar surface, rough and elongated from above downwards and inwards, is broad above and narrow below. Superiorly it presents a deep groove, directed downwards and inwards, which transmits the tendon of the flexor carpi radialis, and external to this groove a prominent ridge, called the tuberosity, which gives attachment to the anterior annular ligament, abductor pollicis, and opponens pollicis. The

A

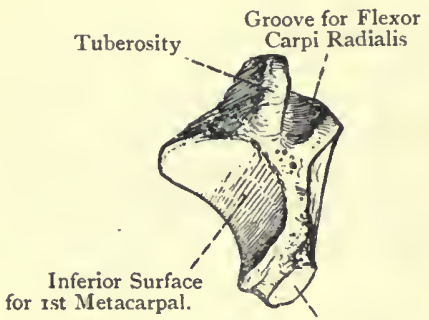

For and Metacarpal
B

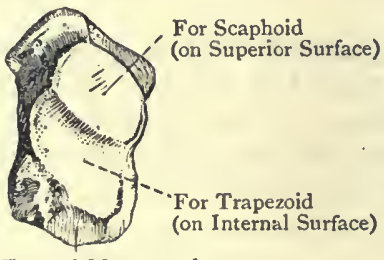

For 2nd Metacarpal (on Internal Surface)

Fig. II7.-The Right Trapezium.

A, Antero-inferịor View ; B, Supero-internal View.

dorsal surface is broader than the palmar, and its inferior and internal angle is much elongated towards the base of the second metacarpal bone, with which it articulates by the small facet on its inner aspect.

Articulations.- Superiorly with the scaphoid, inferiorly with the first metacarpal, and internally chiefly with the trapezoid, but also with the second metacarpal.

\section{The Trapezoid Bone.}

The trapezoid bone somewhat resen?bles the trapezium, but it is destitute of a groove and tuberositv. Its antero-posterior diameter is longer than the transverse. The palmar surface is small and pentagonal, and it gives origin to fibres of the adductor obliquus pollicis. The dorsal surface is large and has its internal and inferior angle elongated towards the styloid process of the third meta- 
carpal bone. Both of these surfaces are rough for ligaments. The superior surface presents a quadrilateral, concave facet, elongated from before backwards, for the scaphoid. The inferior surface is characterized by a large saddle-shaped facet, convex from side to side and concave from before backwards, for the base of the second metacarpal bone. The external surface has a convex facet for the trapezium, below which there is a rough triangular surface with the base directed anteriorly. The internal surface is concave from before backwards, and its anterior portion presents a facet for the os magnum.

A

For Trapezium

(on External Surface)

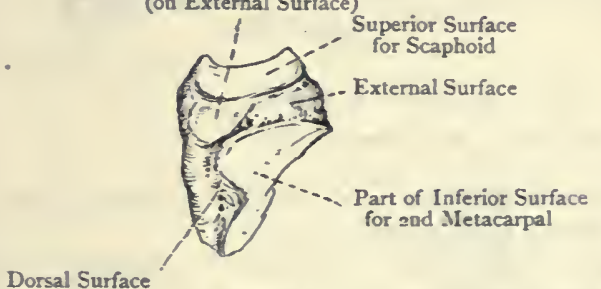

Fio. 118.-The Right Trapezoid BoNe.
B

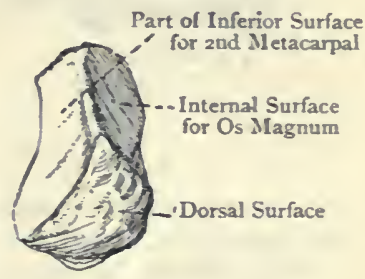

A, External View; B, Posterior View.

Articulations. - Superiorly with the scaphoid, inferiorly with the second metacarpal, externally with the trapezium, and internally with the os magnum.

\section{The Os Magnum.}

The os magnum is the largest bone of the carpus, its distinctive characters being that it is composed of a head, neck, and body. The superior and external aspects of the head are convex, and merge gradually into each other. The cartilage of the superior aspect is prolonged more behind than in front, and articulates with the semilunar. The external aspect of the head articulates with the scaphoid. The internal aspect of the head is flat, and presents the commencement of the facet for the unciform bone. The neck is mainly present in front and behind.

The body is quadrilateral, and narrower in front than behind. The palmar and dorsal surfaces are rough, the former giving origin to fibres of the adductor obliquus pollicis. The external surface, which is con inuous with the outer convex aspect of the head, presents anteriorly a facet for the trapezoid. The internal surface presents at its back part the lower portion of the facet for the unciform, which is here narrow. The inferior surface is narrow in front, but broad behind, and the internal of the two posterior angles is elongated downwards and inwards. This surface presents 
three facets. The middle one is the largest, and articulates with the third metacarpal bone. The external one is a narrow, concave strip for the second metacarpal bone. The internal one, small

A

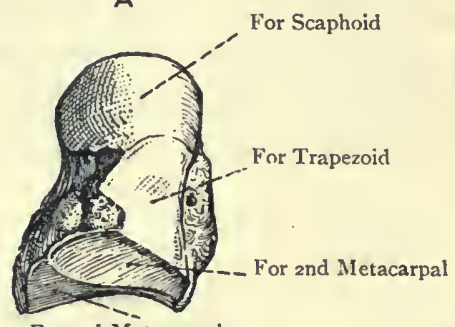

For 3rd Metacarpal
B

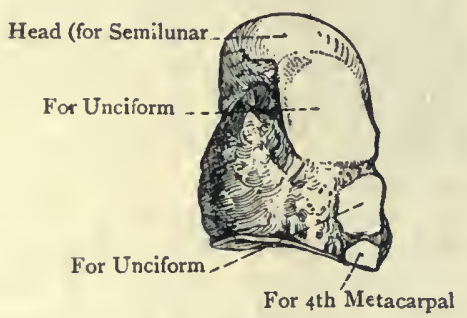

Fig. I19. - The Right Os Magnum.

A, External View; B, Internal View.

and circular, tips inferiorly the projecting postero-internal angle, and articulates with the fourth metacarpal bone.

Articulations.- Superiorly with the semilunar, superiorly and externally with the scaphoid, inferiorly with the second, third, and fourth metacarpal bones, externally with the trapczoid, and internally with the unciform.

\section{The Uneiform Bone.}

The unciform bone is characterized by a hook-like process on its palmar surface. It is triangular, or wedge-shaped, and lies with its base downwards. The superior extremity presents a narrow facet for the semilunar. The inferior surface is divided by an anteroposterior ridge into two quadrilateral facets, the outer of which articulates with the fourth, and the inner with the fifth, metacarpal bone. The palmar surface, which is rough, presents at its lower and inner part a prominent curved projection, called the unciform process. This process is laterally compressed, the external surface being concave and the internal convex, so that the direction of the curve is outwards. Its borders are superior, inferior, and anterior. The internal surface gives origin to the flexor brevis, and opponens, minimi digiti, and close to the root it presents the ulnar groove for the deep branches of the ulnar artery and nerve. The anterior border gives attachment to the anterior annular and pisiuncinate ligaments. The dorsal surface is extensive and rough. The external surface presents an elongated facet, broad above and narrow below, where it is confined to the back part, for articuliation with the os magnum. The supero-internal surface is concavo-convex from below upwards, and articulates with the cuneiform. The internal border, situated at the meeting of the supero-internal and inferior surfaces, is narrow and rough, its direction bcing from before backwards. 
Articulations.-Superiorly with the semilunar, supero-internally with the cuneiform, inferiorly with the fourth and fitth metacarpal bones, and externally with the os magnum.

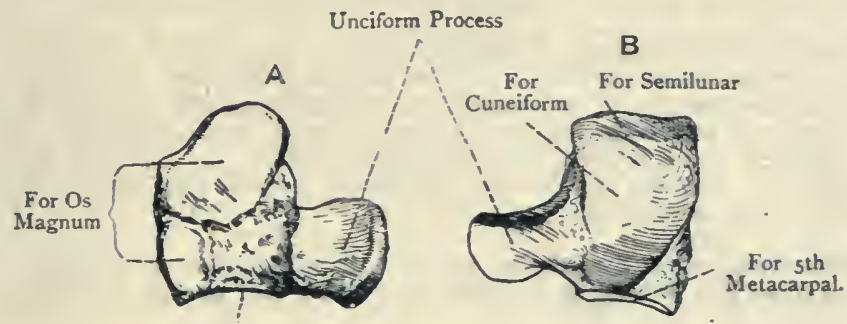

Inferior Aspect

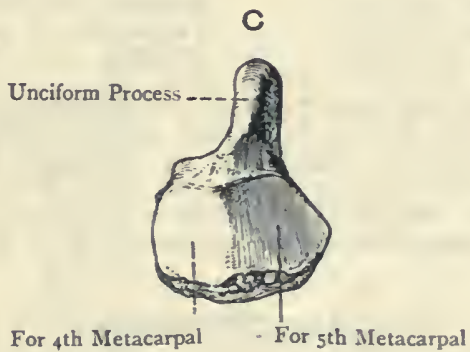

Fig. I20.-The Right Unciform Bone.

A, External View; B, Supero-internal View ; C, Inferior View.

The carpus as a whole is narrower above than below. The dorsal aspect is irregularly convex, and the dorsal surfaces of the bones of the first row (exclusive of the pisiform) are narrow, but in the second row they are broad, this being reversed on the palmar aspect. It is to be noted that the postero-internal angles of the trapezium, trapezoid, and os magnum are distinctly elongated. The palmar aspect is rendered concave by the tuberosities of the scaphoid and trapezium externally, and the pisifo-m bone and unciform process internally. These projections give attachment to the anterior annular ligament, which with the palmar concavity forms a fibroosseous canal for the passage of the flexor tendons and median nerve. The superior aspect, which is directed backwards as well as upwards, is convex, and articulates with the radius and triangular interarticular fibro-cartilage. The inferior or metacarpal aspect is somewhat undulating. The inferior surface of the first row is for the most part deeply concave, but externally it is convex. The superior surface of the second row is concavo-convex from without inwards, the concavity being formed by the trapezium and trapezoid, into which the scaphoid convexity above is received, whilst the con- 
vexity is formed by the os magnum and unciform, and is received into the concavity above.

Structure.-The carpal bones are each composed of cancellated tissue, covered by a thin shell of compact bone.

Varieties. - The number of carpal bones is sometimes increased to nine, which is brought about in one or other of the following ways : $(a)$ the scaphoid, semilunar, trapezium, or os magnum may be divided into two parts; $(b)$ the styloid process at the base of the third metacarpal, or the hook-like process of the unciform, may remain an independent ossicle; or (c) there may be a persistent os centrale, situated on the dorsal aspect between the scaphoid, trapezoid, and os magnum.

Ossification. - The carpal bones are all cartilaginous at birth. Each ossifies from one centre, in the following order, and at the following periods approximately :

Os magnum, ist year.

Uncilorm, 2nd year.

Cuneilorm, 3 rd year.

Semilunar, 5 th year.
Trapezium, 5 th year.

Scaphoid, 6 th year.

Trapezoid, 7 th year.

Pisilorm, I 2 th year.

The Os Centrale.- This appeats as an independent cartilage in the second month of intra-uterine life on the dorsal aspect of the carpus between the cartilaginous scaphoid, trapezoid, and os magnum. As a general rule it joins the cartilage of the scaphoid in the third month, but it may ossify independently, and remain persistent, as in the water tortoises and many apes. It is the representative of the navicular or scaphoid bone of the tarsus.

\section{The Metacarpus.}

The metacarpus supports the phalanges, and is composed of five long bones, which are named numerically from without inwards, that of the thumb being the first. Each bone is divisible into a shaft and two extremities, proximal and distal. The shaft is triangular, except in the first, in which it is compressed from before backwards. It is longitudinally concave on the palmar aspect, and presents three surfaces, two lateral and a dorsal. The lateral surfaces give attachment to interosseous muscles; and are separated from each other by an anterior border. The dorsal surface over its proximal third presents a median ridge, which in the case of the fifth metacarpal is placed towards the inner side. Over the distal two-thirds the ridge bifurcates, its divisions passing each to the dorsal tubercle on the side of the head, and enclosing between them a flat triangular surface.

The head or distal extremity, which articulates with a first phalanx, is convex, and covered by cartilage, except laterally. The cartilage is prolonged farther on the palmar than on the dorsal surface, and terminates anteriorly in a concave border, the extremities of which form small cornua. Laterally the head is compressed, and presents at either side a dorsal tubercle and palmar depression for the lateral metacarpo-phalangeal ligament.

The base or proximal extremity is irregularly quadrilateral, being broader on its dorsal than palmar surface, and it articulates 
superiorly with the carpus, and at either side with its fellows, except in the case of the first.

The First Metacarpal Bone.-This is shorter than any of the others, and its shaft is compressed from before backwards. The palmar aspect, which has an inclination inwards, has the anterior border placed nearer the inner than the outer side. The outer margin and adjacent part of the palmar aspect give insertion to the opponens pollicis, and the inner margin over its proximal half gives origin to the outer head of the first dorsal interosseous. The dorsal surface is slightly convex, and is destitute of the ridge which characterizes the others. The head is elongated transversely, and presents on its palmar surface two shallow depressions for the sesamoid bones. The base is transversely oval,

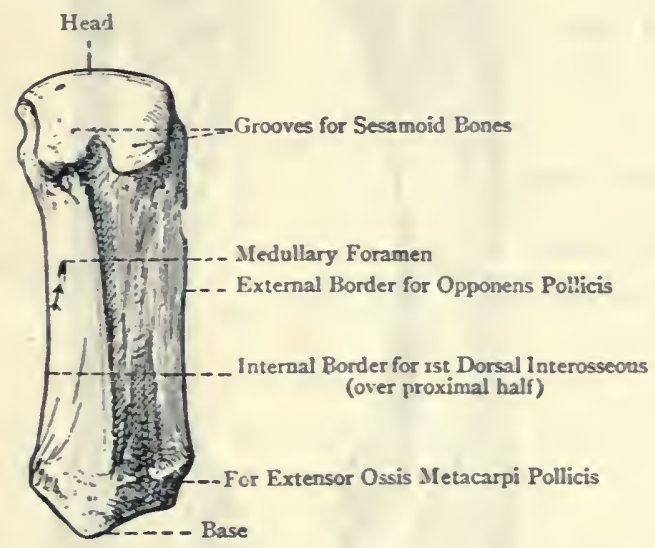

Fig. 121.-The First Right Metacarpal Bone (Palmar View).

and has a saddle-shaped articular surface for the trapezium, which is concave from before backwards, and convex from side to side. Externally it presents a tubercle for the insertion of the extensor ossis metacarpi pollicis, and internally it gives origin to the deep head of the flexor brevis pollicis.

Articulations.-Superiorly with the trapezium, and inferiorly with the first phalanx of the thumb, and the two sesamoid bones.

The Second Metacarpal Bone.-This is the longest. Iis base, which is the largest, is deeply excavated superiorly for the trapezoid, being concave from side to side. Internal to this, it rises into a prominent border, which presents a faceted strip for the os magnum, and externally at the back part there is a small facet for the trapezium. The inner side presents an antero-posterior facet, notched at the centre of its lower border, for the third metacarpal. The palmar aspect gives insertion to the principal part of the tendon of the flexor carpi radialis, and origin to a portion of the adductor obliquus 
pollicis. The dorsal aspect at its outer part gives insertion to the extensor carpi radialis longior, and at its inner part to a small slip of the extensor carpi radialis brevior, there being a notch between the two impressions. The shaft gives origin to the first and second dorsal, and first palmar, interossei.

Articulations.- Superiorly with the trapezium, trapezoid, and os magnum, internally with the third metacarpal, and inferiorly with the first phalanx of the index finger.

A

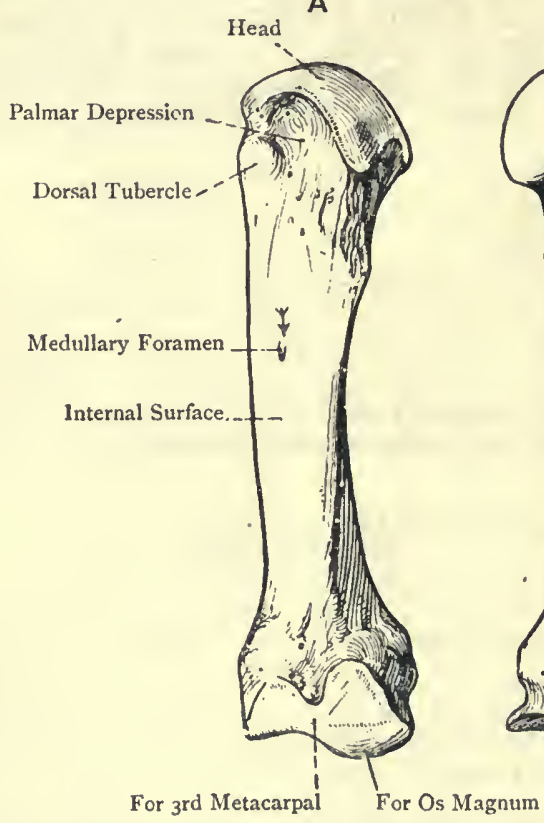

B

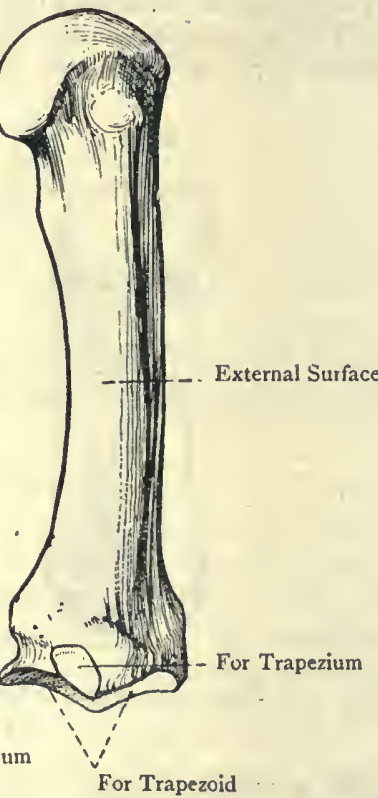

Fig. 122.-The Sećond Right Metacarpal Bone.

A, Internal View; B, External View.

The Third Metacarpal Bone.- This is next in length to the second. Its distinctive character is the styloid process at the base. The superior surface of the base articulates with the os magnum. The outer side presents an antero-posterior facet, notched at its lower border, for the second metacarpal. The inner side presents two circular facets, separated by a rough vertical groove, for the fourth metacarpal. The palmar aspect gives insertion to a slip of the flexor carpi radialis, and origin to a portion of the adductor obliquus pollicis. The dorsal aspect externally gives insertion to the principal part of the extensor carpi radialis brevior, and it is projected upwards at its outer angle into the styloid process. The anterior border of the shaft, over its distal two-thirds, gives origin to the 
adductor transversus pollicis, and the shaft also affords origin to the second and third dorsal interossei.

Articulations.-Superiorly with the os magnum, and the second and fourth metacarpal bones, and inferiorly with the first phalanx of the middle finger.

The Fourth Metacarpal Bone.-This is shorter than the third, and its base is small. The outer side of the base presents two circular facets, separated by a rough vertical groove, for the third metacarpal. The inner side has a semi-oval facet for the fifth metacarpal. The superior surface presents two facets. One is large for the unci-
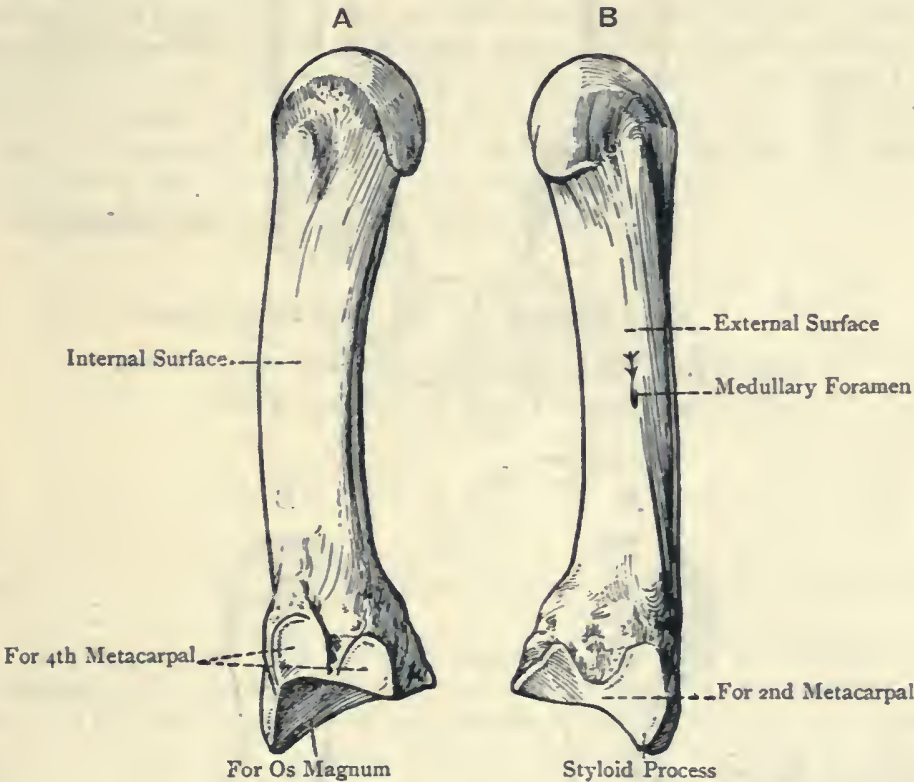

Fig. 123.-The Third Right Metacarpal Bone.

A, Internal View; B, External View.

form, whilst the other, situated at the outer and posterior part, is small for the os magnum. The shaft gives origin to the third and fourth dorsal, and second palmar, interossei.

Articulations.-Superiorly with the os magnum, unciform, and third and fifth metacarpals, and inferiorly with the first phalanx of the ring finger.

The Fifth Metacarpal Bone.-This is shorter than the fourth, but longer than the first. The superior surface of the base presents a quadrilateral facet for the unciform. The outer side has a semi-oval facet for the fourth metacarpal, and the inner side presents a rounded tubercle for the insertion of the extensor carpi ulnaris. The inner 


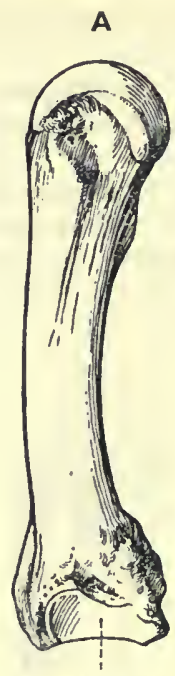

For 5 th Metacarpal
B

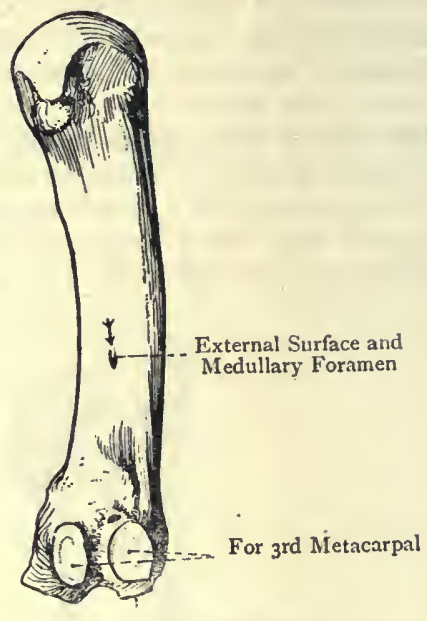

For Os Magnum

Fig. I24.-The Fourth Right Metacarpal Bone.

A, Internal View; B, External View.
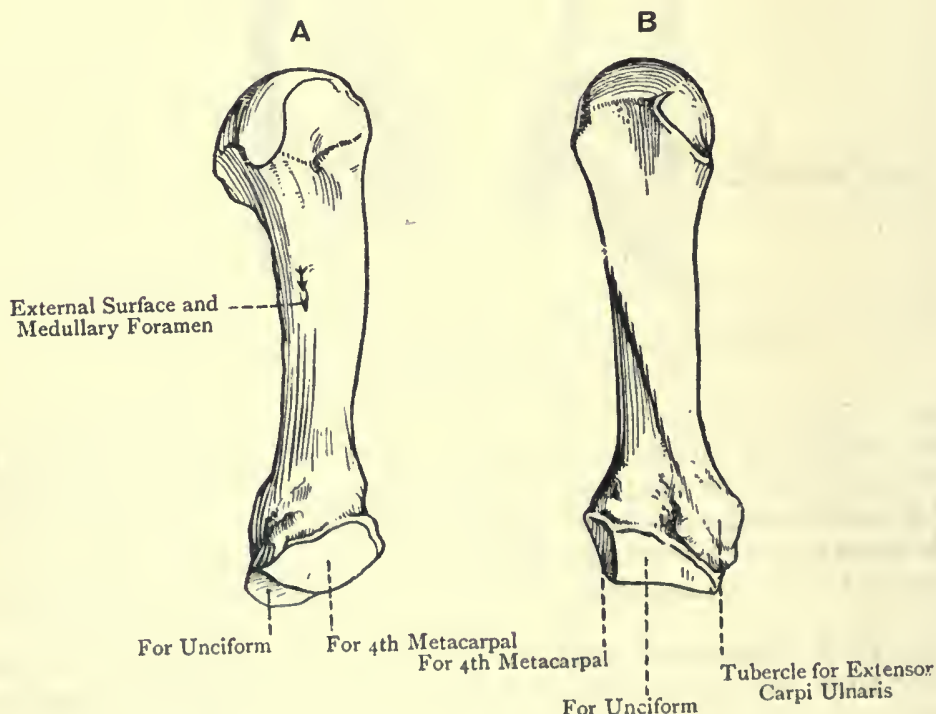

Fig. 125.-Tile Fifth Right Metacalpal Bone.

A, External View ; B, Dorsal View. 
margin of the shaft gives insertion to the opponens minimi digiti, and the shaft also affords origin to the fourth dorsal, and third palmar, interossei.

Articulations.-Superiorly with the unciform, externally with the fourth metacarpal, and inferiorly with the first phalanx of the little finger.

Each metacarpal bone presents a medullary foramen, that of the first, and usually that of the second, being situated on the inner or ulnar side of each shaft, whilst those of the third, fourth, and fifth are situated on the outer or radial side.* The foramen of the first and the canal to which it leads are directed downwards towards the head, but those of the other four are directed upwards towards the base. The medullary artery of the first metacarpal is furnished by the arteria princeps pollicis, those of the second and third are branches of the first palmar interosseous, that of the fourth is furnished by the second palmar interosseous, and that of the fifth by the third palmar interosseous, arteries.

Structure.-The structure is that of a long bone.

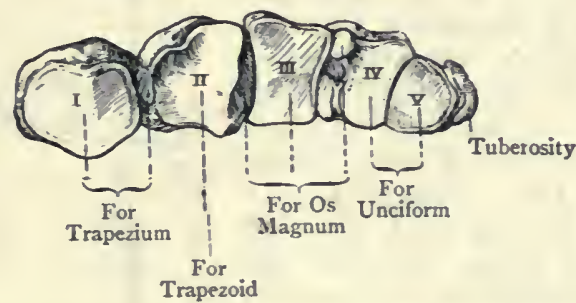

Fig. I26.-The Bases of the Right Metacarpal Bones (Superior View).

The metacarpus as a whole is concave from side to side, and also longitudinally, on its palmar aspect, whilst the dorsal aspect is convex. The first metacarpal stands off at an angle from its fellows, and occupies a more anterior plane, thus fitting it for the important movement of opposition on the part of the thumb. The other four metacarpals lie very nearly parallel with each other. They articulate with one another by their bases, but diverge slightly towards their heads, where they are connected on their palmar aspects by the transverse met: icarpal ligament. Between the five bones there are four intervals, called interosseous spaces, the first being that between tho first and second bones.

\section{The Phalanges.}

The phalanges are also known as ossa internodia, from their position between the joints of the fingers. The fingers, of which they form the framework, are called pollex or thumb, index, middle,

* Of I00 second metacarpal bones examined, 59 had the medullary foramen on the inner side, and $4 \mathrm{I}$ on the outer. 
ring, and little, respectively. The number of phalanges is fourteen, three for each of the four inner fingers, and two for the thumb. They are arranged in rows, both longitudinally and transversely, and they diminish in length from above downwards. They are distinguished as first or proximal, second or intermediate, and third, distal, or ungual, except in the case of the thumb, where the second is wanting.

The First Phalanx.-The shaft is compressed from before backwards, flat and concave longitudinally on the palmar aspect, and convex on the dorsal. The palmar surface presents at either side a rough border for the sbeath of the flexor tendons. The proximal end or base is enlarged, and presents superiorly a concave articular surface, transversely oval, for the head of a metacarpal bone, and at either side a slight tubercular enlargement. The distal end is small, and presents a trochlear surface, grooved at the centre and elevated at either side of this into a small condyle, for articulation with the second phalanx, except in the case of the thumb, where it articulates with the ungual phalanx. The cartilage of the distal end is prolonged more on the palmar aspect than on the dorsal. At either side it presents a depression for the lateral interphalangeal ligament.

The Second Phalanx.-This is shorter and smaller than the first, from which it is distinguished by having on its proximal end or base two shallow articular depressions, separated by a median anteroposterior ridge, for the distal end

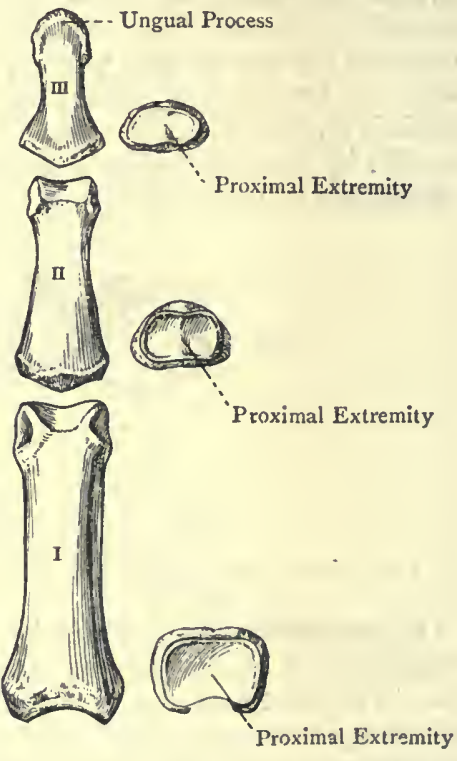

Fig. I27.-The Phalanges of the Middle Finger (Dorsal View). of the first phalanx. The distal end resembles that of a first phalanx, except that it is smaller. The shaft resembles that of a first. Its palmar surface presents at the centre of the lateral borders two rough impressions, one at either side, which give insertion to the divisions of a superficial flexor tendon. The dorsal surface of the base is marked by a rough transverse ridge for the insertion of the middle division of a common extensor tendon.

The Third Phalanx.-This is of small size. The proximal end or base resembles that of a second phalanx, and has in front a rough transverse ridge for the insertion of a deep flexor tendon, whilst the dorsal surface gives insertion to the two lateral divisions of a common extensor tendon. The distal end presents a rough, tapering, convex 
border, the roughness being continued for a little on the palmar aspect. This roughness, which is semilunar, is called the ungual process, and it supports the nail and the tissues forming the pulp of the finger.

The two phalanges of the thumb are of large size.

Special Muscular Attachments.-The base of the proximal phalanx of the thumb gives insertion externally to the abductor pollicisand superficial head of the flexor brevis pollicis, internally to the deep head of the flexor brevis pollicis, adductor obliquus pollicis, and adductor transversus pollicis, and posteriorly to the extensor brevis pollicis. The base of the distal phalanx gives insertion anteriorly to the flexor longus pollicis, and posteriorly to the extensor longus pollicis. The base of the proximal phalanx of the index finger gives partial insertion to the first dorsal interosseous externally, and the first palmar interosseous internally. The base of the proximal phalanx of the middle finger gives partial insertion to the second dorsal interosseous externally, and the third dorsal interosseous internally. The base of the proximal

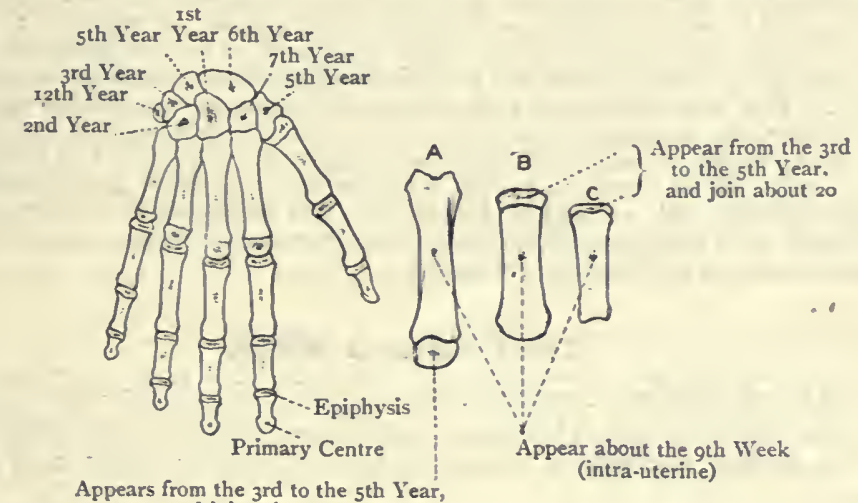

Fig. 128.-Ossification of the Bones of tHe Hand.

A, 2nd Metacarpal ; B, Ist Metacarpal ; C, Ist Phalanx.

phalanx of the ring finger gives partial insertion to the second palmar interosseous externally, and the fourth dorsal interosseous internally. The base of the proximal phalanx of the little finger gives partial insertion to the third palmar interosseous externally, and insertion to the abductor minimi digiti (partially) and flexor brevis minimi digiti internally.

The medullary foramen of each phalanx is situated on the palmar aspect of the shaft, not far from the distal end. It may be single, in which case it is mesially placed, or there may be two, one close to each lateral border. In all cases the direction of the foramen and the canal to which it leads is downwards towards the distal end. The medullary arteries are furnished by the corresponding palmar digital arteries.

Structure.-The structure is that of a long bone. In the terminal phalanges the medullary canal is replaced by cancellated tissue with wide meshes. 
The Sesamoid Bones. - These are usually two in number, and are placed on the palmar aspect of the head of the first metacarpal bone. They are originally nodules of cartilage, one of which is developed in the tendon of insertion of the superficial head of the flexor brevis pollicis, and the other in that of the adductor obliquus pollicis. Similar ossicles are sometimes met with on the palmar aspects of the heads of the second and fifth metacarpal bones:

Ossification of the Metacarpal Bones and Phalanges.-Each of these bones ossifies in cartilage from one primary, and one secondary, centre. The primary centre appears about the ninth week of intra-uterine life at the middle of the shaft, except in the case of the ungual phalanges, in which it appears at the distal end. In the inner four metacarpal bones the epiphysis is placed at the distal end or head, but in the first metacarpal bone (that of the thumb), and in all the phalanges, it is placed at the proximal end or base. The secondary centre in each case appears from the third to the fith year, and the epiphyses are united to their respective shafts about the twentieth year. The first metacarpal bone has sometimes a thin distal epiphysis, as well as a proximal, which begins to ossify about the eighth year, and joins soon thereafter; and the second metacarpal bone has sometimes a proximal epiphysis, as well as a distal. The first metacarpal bone, being a phalanx as regards its epiphysial ossification, is regarded by some as the proximal phalanx of the thumb, according to which view the bone wanting in the thumb is the metacarpal bone. The styloid process of the third metacarpal bone has sometimes a special centre, and, instead of joining the rest of the bone, it may remain persistent as a supernumerary bone of the carpus, or it may unite with the postero-internal angle of the trapezoid.

\section{The Hand as a Whole.}

The hand presents two surfaces-dorsal and palmar-two borders -outer and inner-and two extremities-proximal and distal.

The dorsal surface is convex, both longitudinally and transversely.

The palmar or volar surface is concave, both longitudinally and transversely.

The outer or radial border is in line with the thumb or pollex, and is constructed by the scaphoid or navicular, trapezium, and the phalanges of the thumb.

The inner or ulnar border is in line with the little finger, and is constructed by the cuneiform or pyramidal, unciform, and the phalanges of the little finger. This border presents the tubercle on the inner side of the proximal end or base of the fifth metacarpal bone for the tendon of the extensor carpi ulnaris.

The proximal extremity is formed by the scaphoid or navicular, semilunar, and cuneiform or pyramidal bones.

The distal extremity is formed by the third or ungual phalanges.

It is to be noted (I) that the thumb is the shortest of all the digits; (2) that it stands off at an angle from its fellows; and (3) that it occupies a more anterior plane than its fellows, in which respects it presents a striking contrast to the great toe.

The palmar aspect of the carpus is rendered concave by the 
tuberosities of the scaphoid and trapezium externally, and the pisiform and the hook of the unciform internally. These four projections give attachment to the anterior annular ligament. This ligament, along with the palmar concavity of the carpus, constructs a fibro-osseous passage, called the anterior palmar canal. This canal transmits (I) the tendons of the flexor sublimis, and flexor profundus digitorum, and the median nerve lying within the large inner compartment of the great palmar bursa ; and (2) the tendon of the flexor longus pollicis lying within the small outer compartment of the great palmar bursa.

The tuberosity of the scaphoid gives partial attachment to the anterior annular ligament, and partial origin to the abductor pollicis muscle.

The tuberosity of the trapezium, which has the form of a ridge, gives partial attachment to the anterior annular ligament, and partial origin to (I) the abductor pollicis, (2) the opponens pollicis, and (3) the superficial head of the flexor brevis pollicis.

The groove on the palmar surface of the trapezium transmits the tendon of the flexor carpi radialis, which lies in a special compartment in the outer part of the anterior annular ligament, and is invested by a special synovial membrane.

The pisiform bone gives attachment to (I) part of the anterior annular ligament, (2) the tendon of the flexor carpi ulnaris, (3) the pisi-uncinate and pisi-metacarpal ligaments, and (4) the abductor minimi digiti muscle.

The hook of the unciform gives attachment anteriorly to part of the anterior annular ligament and the pisi-uncinate ligament, and internally partial origin to the flexor brevis minimi digiti and opponens minimi digiti. The ulnar groove on the internal surface of the hook, close to its root, transmits the deep branches of the ulnar artery and nerve. 
Abd. Pollicis and Superf. Head of Flex. Brev. Poll. Extensor Brevis Pollicis -

Deep Head of Flex. Brev. Poll., Add. Obliq. Poll., and Add. Trans. Poll.

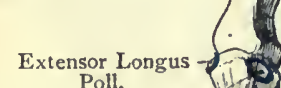

\section{Poll.}

Flexor Longus Poll. -

$$
\text { Pisi-uncinate Ligament }
$$

Abductor Pollicis Opponens Pollicis 、 Superficial Head of Flexor
Brevis Pollicis

Ext. Ossis Metacarpi Poll,-

Deep Head of Flex. Brevis Pollicis-

Opponens Pollicis-

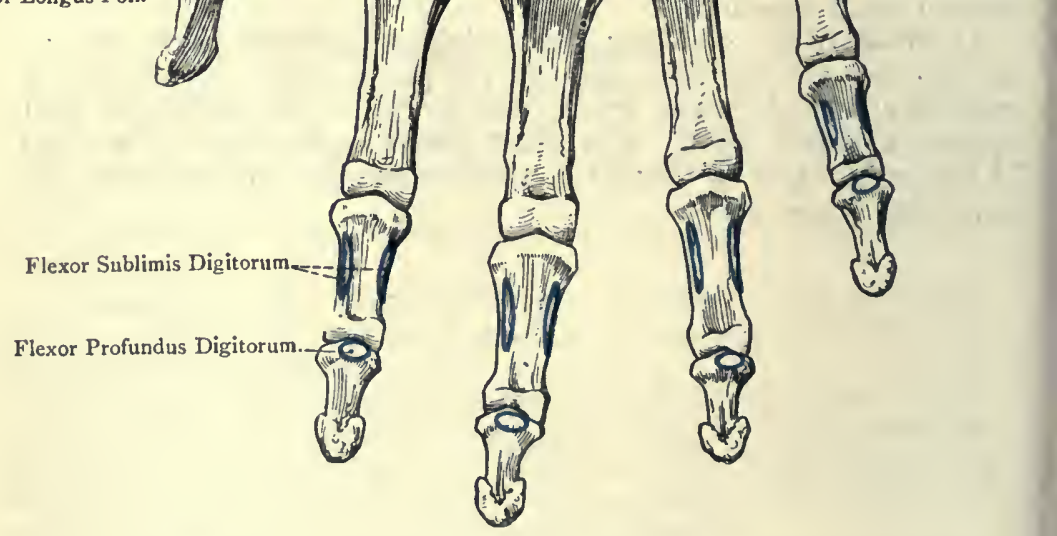

Fig. 129.-The Right Hand (Palmar Surface). 


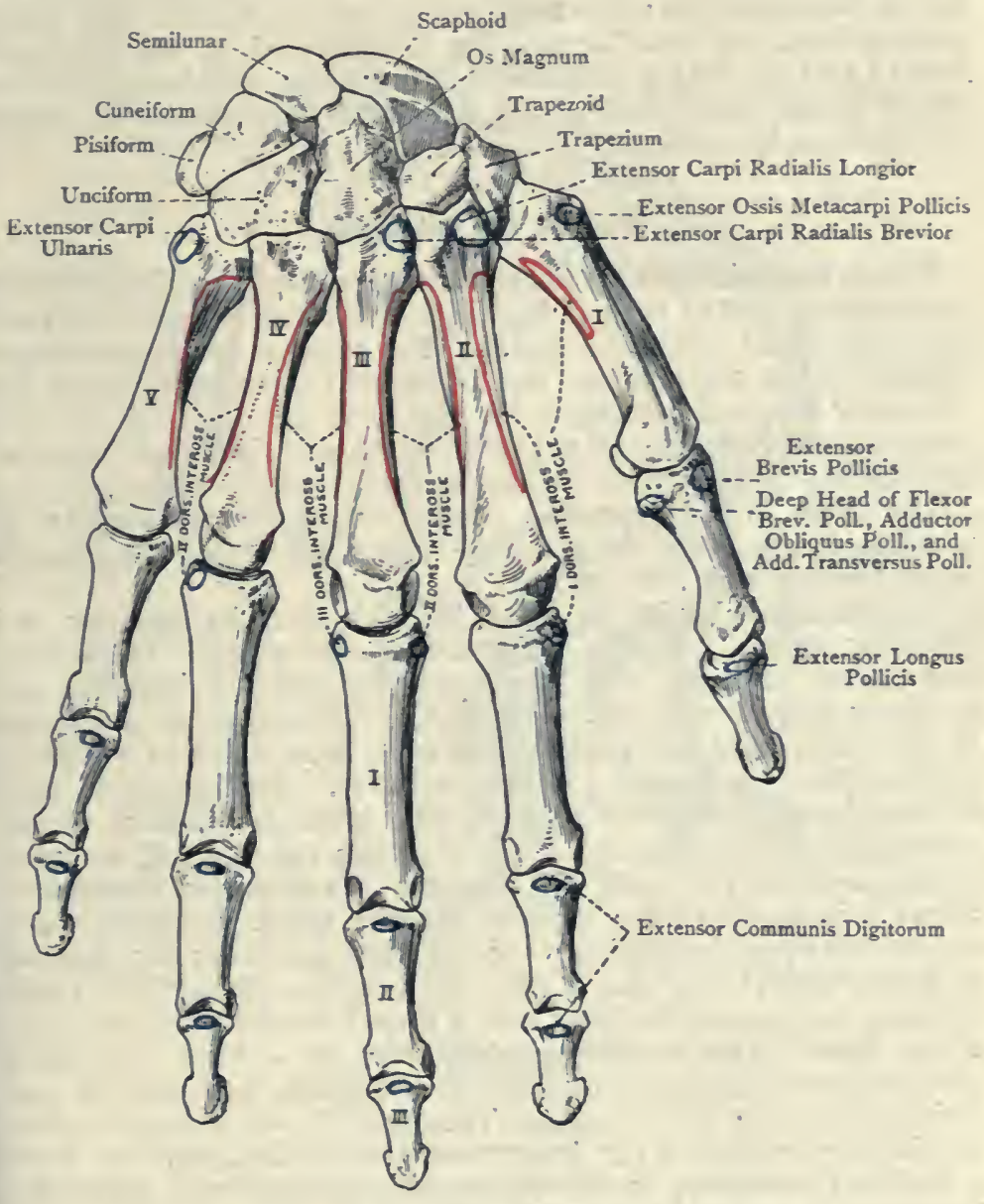

Fig. 130.-The Right Hand (Dorsal Surface). 


\section{THE BONES OF THE LOWER LIMB.}

The lower limb is arranged in four divisions, namely, hip, or pelvic girdle, thigh, leg, and foot. The pelvic girdle consists of the os innominatum; the thigh comprises the femur, with which is associated the patella; the leg is composed of the tibia and fibula ; and the foot is subdivided into a tarsus, consisting of seven bones, a metatarsus, comprising five bones, and phalanges, which are fourteen in number.

\section{The Os Innominatum.}

The os innominatum (os coxæ) forms the lateral, and one half of the anterior, wall of the pelvis. It is much twisted, quadrilateral, and constricted about the centre. The external surface is characterized by the acetabulum, and below and internal to this is the obturator foramen. In early life the bone is composed of three parts-ilium, ischium, and os pubis - which unite in the acetabulum, and in the adult it is described under these three divisions.

The ilium is the expanded portion above the acetabulum, of which it forms rather less than the upper two-fifths. It presents three borders and two surfaces.

The superior border or crest is thick over its anterior and posterior thirds, but thin over the middle third. It presents two curves-anterior with the concavity directed inwards, and posterior with the concavity outwards. Anteriorly it terminates in the anterior superior spine, which gives attachment to Poupart's ligament and a portion of the sartorius. Posteriorly it ends in the posterior superior spine, which gives attachment to the oblique sacro-iliac ligament. The crest has two lips and an intervening space. The outer lip presents a tubercular prominence about 3 inches from the anterior superior spine. Over its whole extent this lip gives attachment to the iliac fascia lata ; for $I \frac{1}{2}$ inches in front, to the tensor fasciæ femoris; over its anterior half, to the obliquus externus abdominis; and a little behind this, to the latissimus dorsi. The intervening space over its anterior two-thirds gives origin to the obliquus internus abdominis, and over its posterior fifth, to the erector spinæ. The inner lip over its anterior twothirds gives origin to the transversalis abdominis, and for about 2 inches posteriorly, to the ilio-lumbar ligament and quadratus lumborum. Immediately within the inner lip, over its anterior twothirds, the fascia transversalis and fascia iliaca take attachment.

The anterior border extends from the anterior superior spine to the ilio-pectineal eminence. Superiorly it presents the anterior interspinous notch, the upper part of which gives partial origin to the sartorius. Below this notch is the anterior inferior spine, which gives origin anteriorly to the straight head of the rectus femoris, and inferiorly, to the ilio-femoral ligament. Internal to this spine 
Transversalis Abdominis Crest

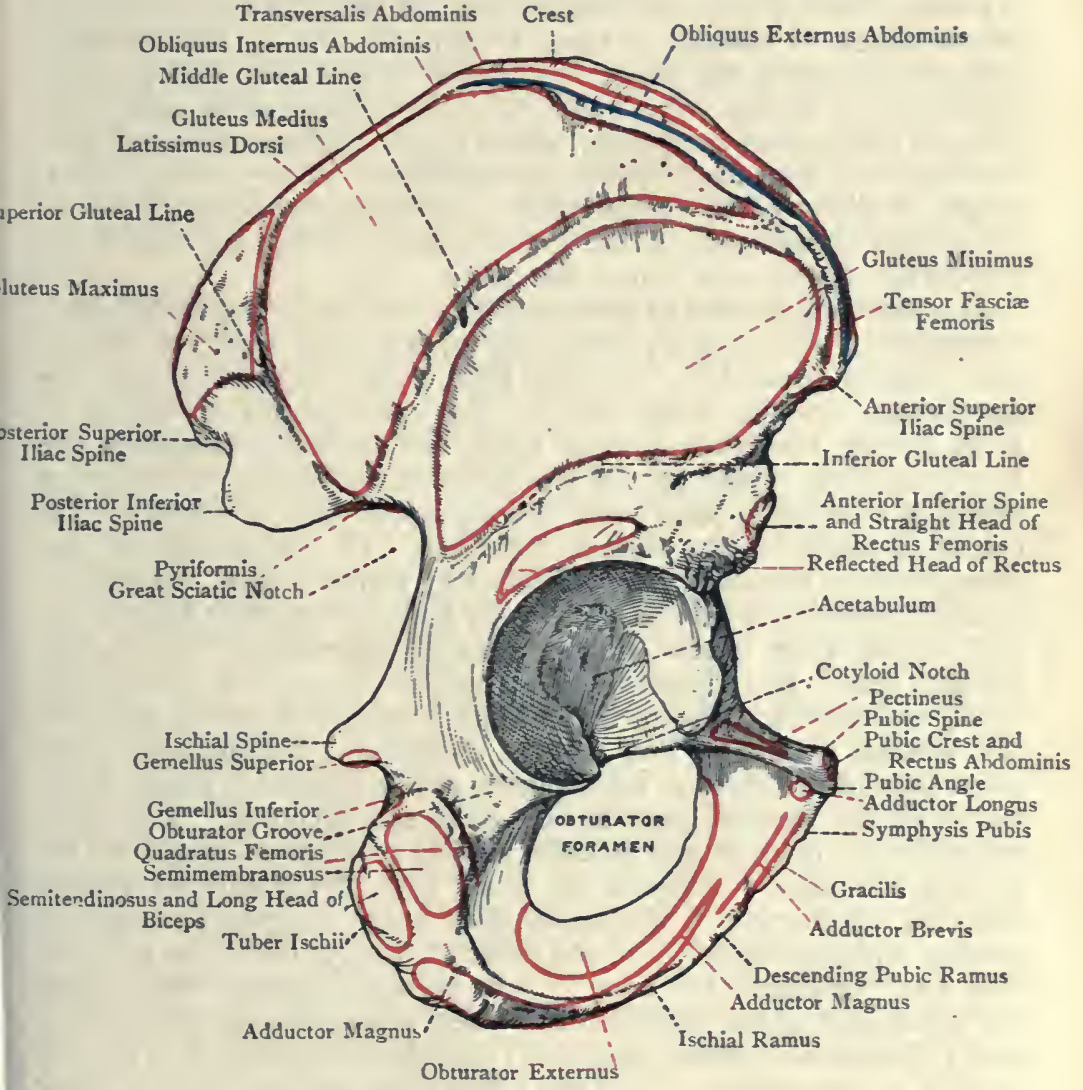

Fig. I31.-The Right Os Innominatum (External View). 
there is a groove for the passage of the ilio-psoas, and internal to the groove is the ilio-pectineal eminence, which marks the junction of the ilium and superior pubic ramus. This eminence gives attachment to the ilio-pectineal intermuscular septum, and, it may be, partial insertion to the psoas parvus.

The posterior border extends from the posterior superior spine to a point a little below the deepest part of the great sciatic notch, where there is usually a faint transverse line on the external surface, indicating the place of junction of the ilium and ischium. Superiorly it presents the posterior interspinous notch, and below this the posterior inferior spine, which gives attachment to the great sacro-sciatic ligament, whilst immediately below this it gives origin to fibres of the pyriformis. Inferiorly the posterior border forms the upper part of the great sciatic notch, over which the pyriformis passes as it leaves the pelvis.

The external surface or dorsum ilii, concavo-convex from behind forwards, is traversed by three ridges, called the superior, middle, and inferior gluteal, or curved, lines. The superior gluteal line commences at the outer lip of the crest about 2 inches in front of the posterior superior spine, and passes downwards and forwards to the upper part of the great sciatic notch. The surface which it cuts off is semilunar, and its upper part is rough for the origin of fibres of the gluteus maximus. The middle gluteal line commences at the outer lip of the crest $I \frac{1}{2}$ inches behind the anterior superior spine, and passes backwards and downwards to the upper part of the great sciatic notch, where it terminates close to the superior line. The surface included between the middle gluteal line, crest, and superior gluteal line, which is falciform, gives origin to the gluteus medius. The inferior gluteal line commences at the lower part of the anterior interspinous notch, whence it passes backwards to the deepest part of the great sciatic notch. The space between the inferior and middle gluteal lines gives origin to the gluteus minimus. Between the front part of the inferior gluteal line and the margin of the acetabulum there is a short transverse groove, which gives origin to the reflected head of the rectus femoris. The iliac portion of the bone is very thin and translucent toward the upper part of the middle third, where it is sometimes perforated, and it presents a strong rounded ridge, leading upwards from the margin of the acetabulum to the tubercular eminence on the outer lip of the crest. There is also a strong bar of bone extending from the upper margin of the acetabulum to the auricular surface on the internal aspect.

The internal surface is divisible into an anterior, and a posterior, portion. The anterior division, which represents two-thirds, is subdivided into a small lower, and large upper, part by the iliac portion of the ilio-pectineal line, the direction of which is forwards and downwards. The part below and behind the line enters into the lateral wall of the pelvis, and gives origin to a portion of the obturator internus. The part above the line is extensive and concave, and 
forms the iliac fossa, which lodges the iliacus muscle. The iliac portion of the ilio-pectineal line gives attachment to the fascia iliaca, and for about an inch posteriorly to the parietal pelvic fascia. It may also give partial insertion to the psoas parvus near the ilio-pectineal eminence. The posterior division is subdivided into auricular, ligamentous, and muscular portions. The auricular division, antero-inferior in position, is broad in front and narrow behind, where it extends over the inner aspect of the posterior inferior spine. It is covered by cartilage in the recent state, and articulates with the auricular surface of the sacrum. The ligamentous division, situated above and behind the auricular, presents an elevation, called the tuberosity, for the posterior sacro-iliac ligament, its surface being marked by several pits. The muscular division, placed superiorly, gives origin to fibres of the multifidus spinæ.

The ischium forms the lower and back part of the bone, and is divisible into a body, tuberosity, and ramus. The body contributes rather more than two-fifths to the acetabulum, and forms the greater portion of its non-articular part. It is somewhat triangular, the truncated apex being directed downwards, and its surfaces are internal, external, and posterior. The internal surface extends from near the centre of the ilio-pectineal line to the ischial spine, and is narrow above, but widens inferiorly before reaching the spine. Its place of junction with the ilium is indicated by a line passing from the ilio-pectineal eminence backwards and downwards to a point a little below the deepest part of the great sciatic notch. Its junction with the superior pubic ramus is marked by a line passing from the ilio-pectineal eminence downwards to the posterior margin of the obturator foramen about $\frac{3}{4}$ inch below its upper end. This surface gives origin to part of the obturator internus. The external surface enters into the acetabulum, and between that cavity and the tuberosity it presents an obturator groove for the tendon of the obturator externus. The upper part of this groove gives attachment to the ischio-capsular ligament. The posterior surface is limited externally by the brim of the acetabulum, behind by the posterior border, and below by the upper border, of the tuberosity. At the lower part it presents a portion of the obturator groove, and it supports the pyriformis, and the sciatic nerves and vessels.

The borders, like the surfaces, are internal, external, and posterior. The internal border forms a portion of the posterior margin of the obturator foramen, and is sharp for the obturator membrane. It separates the internal from the external surface. The external border forms the posterior part of the margin of the acetabulum, and gives attachment to a part of the cotyloid ligament. The posterior border is continuous with that of the ilium. Superiorly it forms the lower part of the great sciatic notch, below which it presents a projection, called the spine, which has an inward curve towards the pelvis, and gives attachment to the 
following structures: the small sacro-sciatic ligament at the tip, the levator ani, coccygeus, and white line of the pelvic fascia on the inner surface, and the gemellus superior along the lower border. The external surface (back) supports, from within outwards, the pudic nerve, internal pudic vessels, and nerve to the obturator internus.

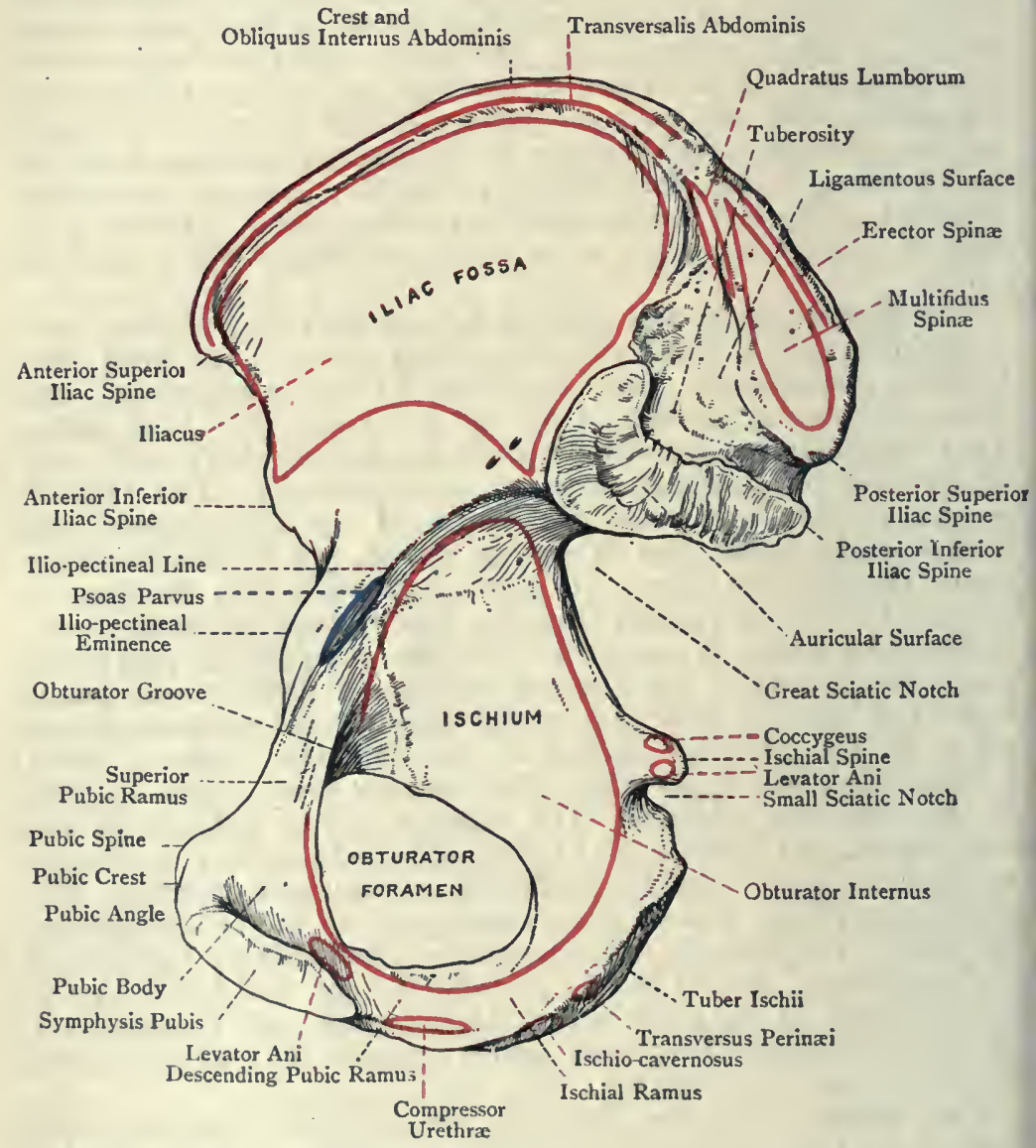

Fig. 132.-The Right Os Innominatum (Internal View).

Below the spine is the small sciatic notch, which is covered by cartilage in the recent state for the play of the tendon of the obturator internus.

The tuberosity (tuber ischii) forms the thick dependent part, and supports the body in the sitting posture. The upper border limits inferiorly the obturator groove and small sciatic notch, and 
in the latter situation it gives origin to the gemellus inferior. The inner border is prominent and sharp, and gives attachment to the great sacro-sciatic ligament. The outer border gives origin to the quadratus femoris. The anterior border is sharp and prominent, and forms the lower part of the posterior margin of the obturator foramen. The surfaces are postero-inferior, external, and internal. The postero-inferior surface lies between the outer and inner borders, and is divided into two parts. The posterior portion is somewhat quadrilateral, and is subdivided into two parts by a diagonal line directed downwards, forwards, and outwards. The upper and outer part gives origin to the semimembranosus, and the lower and inner, to the conjoined long head of the biceps and semitendinosus. The inferior portion, which is in line with the inner margin of the ramus, is rough and triangular, and gives origin to fibres of the adductor magnus. The external surface is situated between the outer and anterior borders, and supports the obturator externus. The internal surface is placed between the inner and anterior borders. It looks towards the ischio-rectal fossa, and gives origin to fibres of the obturator internus.

The ramus is the compressed portion which extends upwards and inwards, from the tuberosity, on the anterior aspect of the obturator foramen, where it joins the inferior pubic ramus, the place of meeting being indicated externally by a rough ridge. The outer border is sharp for a portion of the obturator membrane, and forms part of the anterior margin of the obturator foramen. The inner border is thick, and anteriorly it is rough and everted, more so in the male than in the female, for the attachment of the fascia of Colles, crus penis, and ischio-cavernosus muscle. In the female this part gives attachment to the crus clitoridis and erector clitoridis. Elsewhere the inner border gives attachment to the triangular ligament and compressor urethræ. The outer surface gives origin, from within outwards, to portions of the adductor magnus and obturator externus. The inner or pelvic surface gives attachment to part of the obturator internus and parietal pelvic fascia. At its lower part, near the inner border, there is a sharp ridge which gives attachment to the falciform process of the great sacro-sciatic ligament.

The os pubis lies in the anterior wall of the pelvis, and is composed of a body and two rami, superior and inferior. The body is compressed from before backwards, and occupies an oblique plane, which is directed downwards and backwards. It presents three surfaces-anterior, posterior, and internal. The anterior or femoral surface has an inclination downwards. At its upper and inner part, below and external to the pubic angle, it gives origin to the adductor longus, and, lower down, to the following muscles, in order from within outwards-gracilis, adductor brevis, a small portion of the adductor magnus, and obturator externus. The posterior or pelvic surface has an inclination upwards, and gives attachment from without inwards to the obturator internus, 
parietal pelvic fascia, levator ani, and pubo-prostatic ligament. The internal surface is oval, and occupies an oblique plane, which is directed downwards and backwards. It is covered by cartilage, and articulates with its fellow to form the symphysis pubis, a plate of fibro-cartilage intervening.

The borders are external and superior. The external border, which is sharp, looks into the obturator foramen, and gives attachment to part of the obturator membrane. The superior border or crest is thick, and about an inch long. At its outer extremity is the pubic spine, which may be blunt or sharp, for the attachment of Poupart's ligament, and internally is the pubic angle, which surmounts the internal surface. The crest gives attachment to the conjoined tendon, pyramidalis, and outer head of the rectus abdominis.

The inferior ramus passes downwards and outwards, and corresponds in all respects with the ischial ramus, which it joins. Its anterior surface gives origin, from within outwards, to the gracilis, adductor brevis, adductor magnus, and obturator externus. The structures attached to the posterior surface are portions of the obturator internus and parietal pelvic fascia.

The superior ramus extends outwards and upwards from the body to the ilio-pectineal eminence and anterior part of the acetabulum, of which latter it forms one-fifth. It lies above the obturator foramen, and is triangular. Superiorly, at the back part, is a prominent ridge, representing the pectineal portion of the ilio-pectineal line, which leads to the pubic spine, and gives attachment to the following structures : the pubic lamina of the fascia lata, pectineus, Gimbernat's ligament, and conjoined tendon. In front of this line is the superior or pectineal surface, which is sloped downwards and forwards, and is triangular. It supports the pectineus, and is limited antero-inferiorly by the obturator crest, which extends from the pubic spine to the anterior margin of the cotyloid notch. The inferior surface presents the obturator groove for the obturator vessels and nerve, the direction of which is downwards, forwards, and inwards. The posterior surface gives partial origin to the obturator internus.

The acetabulum or cotyloid cavity is situated on the outer surface of the bone, and is directed downwards, outwards, and forwards. It is deep and circular, and articulates with the head of the femur. The ischium forms rather more than two-fifths of it, the ilium rather less, and the os pubis the remaining fifth. It is surmounted by a prominent brim, upon which the cotyloid ligament is set, except at the anterior and inferior part, where there is the cotyloid notch, this being bridged over by the cotyloid and transverse ligaments: The capsular ligament is attached to the bone just outside the brim. The interior is divided into two parts - articular and nonarticular. The articular portion is covered by cartilage, which is arranged in the form of a horseshoe, and surrounds the circumference, except opposite the cotyloid notch. The non-articular part, 
which is formed mainly by the ischium, is depressed, and lodges the Haversian gland of the hip-joint.

The obturator or thyroid foramen lies below, and internal to, the acetabulum, its boundaries being formed by the ischium and os pubis. Its long diameter is directed downwards and outwards, and it is oval in the male, but triangular, with rounded angles, in the female. Its circumference is sharp for the obturator membrane, which closes the opening, except opposite the obturator groove superiorly, where it converts that groove into the obturator canal.

The great and small sciatic notches are situated on the posterior border of the bone, and are separated from each other by the spine of the ischium. The great notch is formed partly by the ilium, and partly by the ischium; and the small notch lies between the ischial spine and tuber ischii.

In the recent state these notches are converted into foramina by the great and small sacro-sciatic ligaments. For the structures which pass through these foramina, see the description of the gluteal region.

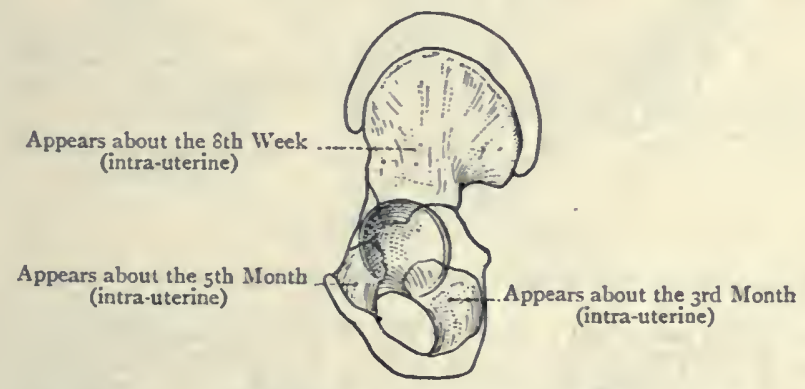

Fig. 133.-Ossification of the Os Innominatum.

The os innominatum is pierced by a great number of nutrient foramina for arteries, the chief of which are situated as follows: along the inner aspect of the crest for branches of the deep circumflex iliac; in the iliac fossa near the auricular surface, where there are one or two for branches of the ilio-lumbar; on the external surface of the ilium, and around the margin of the acetabulum for branches of the gluteal; between the acetabulum and tuber ischii for branches of the obturator; on the ilio-pectineal eminence for branches of the deep circumflex iliac; and over the body of the os pubis for branches of the obturator, and of the pubic branches of the common femoral.

Articulations.-Posteriorly with the sacrum, externally with the femur, and internally with its fellow.

Ossification.-The os innominatum is ossified in cartılage from 3 primary and 9 secondary centres. The primary centres are iliac, ischial, and pubic. The iliac centre appears in the 8 th week; the ischial centre in the 3 rd nonth:

The ischial and inferior pubie rami join about the 8 th year. 
Acetabulum.-The superior pubic ramus is shut out from this cavity for some time by a triangular portion of cartilage, called the pars acetabularis. From its apex there is prolonged backwards a strip of cartilage (ilio-ischial). The entire cartilage resembles the letter $Y$, laid on its side. The open part of the $\mathrm{Y}$ is directed forwards, and forms the pars acetabularis. The $\mathrm{Y}$ cartilage has 3 secondary centres. One, called the acetabular centre, appears in the I 2 th year in the pars acetabularis, and ossification is completed by the I 4 th year. It then forms a distinct bone, called the os acetabuli, which joins the superior pubic ramus about the I6th year.

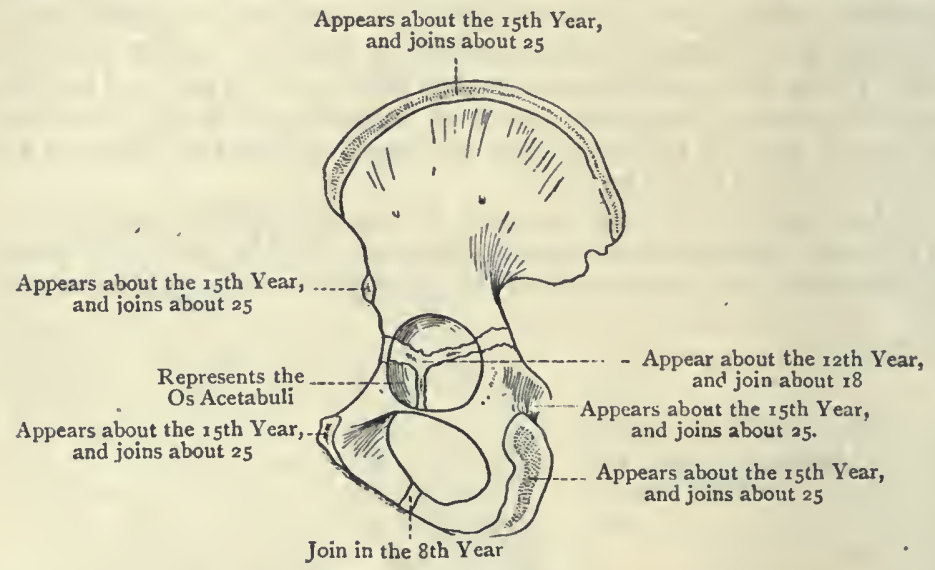

Fig. 134.-OSSification of the Epiphyses of the Os Innominatum.

The other two secondary centres for the $Y$ cartilage appear about the I4th year, one of them in the ilio-ischial strip or stem of the $Y$; and the other at the meeting of the two limbs and stem of the Y. The ossification of the bottom of the acetabulum is completed from the 16 th to the I8th year.

Other Secondary Centres.- These are as follows: (I) One for the crest, one for the anterior inferior iliae spine, one (a thin scale) for the surface of the tuber ischil, and one for the spine of the ischium, each of these centres appearing about the I 5 th year; (2) one for the puble spine, and one for the pubic angle, each of these two centres appearing about the 18th year. These epiphyses usually join about the 25 th year.

\section{The Pelvis.}

The pelvis is formed by the ossa innominata, sacrum, and coccyx, the hip-bones constructing the anterior and lateral walls, whilst the sacrum and coccyx lie in the posterior wall. It is divided into two parts, called false pelvis and true pelvis, the division being effected by a plane passing through the upper border of the symphysis pubis, ilio-pectineal line, and sacral promontory.

The false pelvis, which lies above this plane, is formed by the iliac fossæ, and constitutes a part of the abdomen proper.

The true pelvis is situated below the plane referred to, and presents a brim or inlet, a cavity, and an outlet. The brim is formed in front by the upper border of the symphysis pubis, behind by the 
sacral promontory, and between these two points by the following parts from before backwards - the angle, crest, and spine of the os pubis, the ilio-pectineal line, and the antero-inferior border of

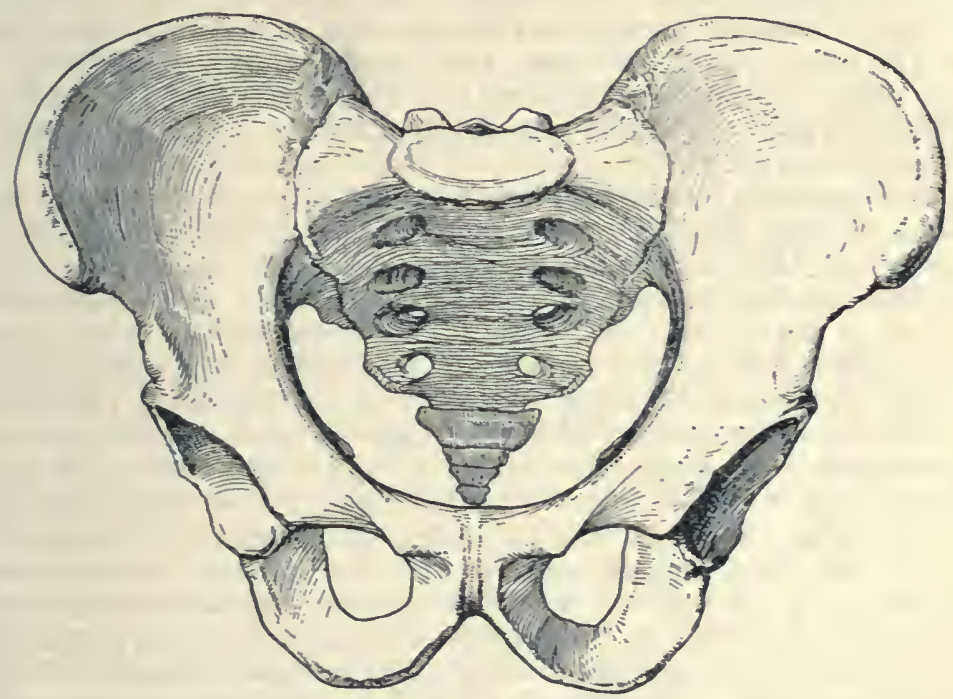

Fig. I35.-The Male Pelvis.

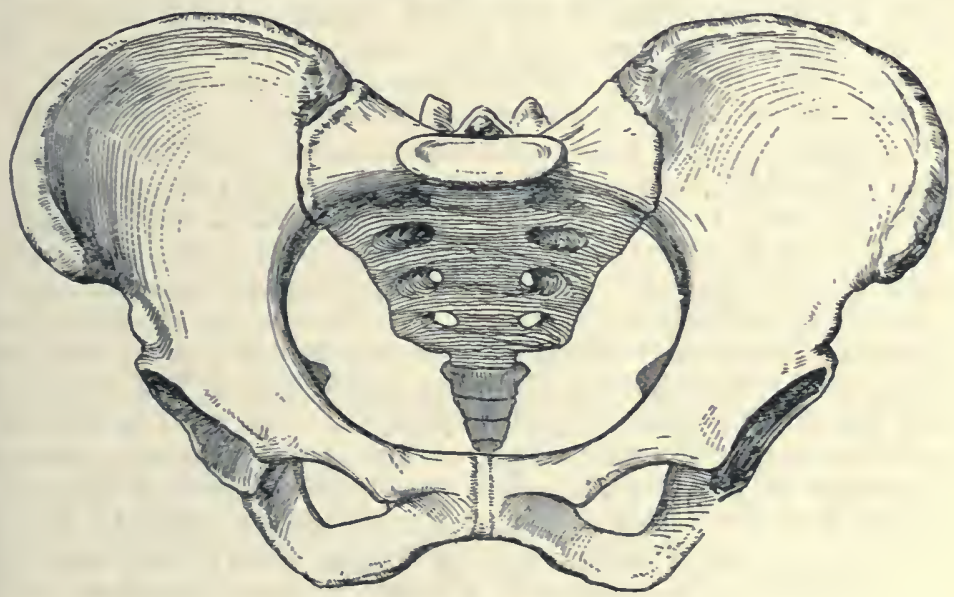

Fig. I36.-The Female Pelvis.

the ala of the sacrum. In the male it is cordate, the base of the heart, which is encroached upon by the sacral promontory, being directed backwards. In the female it is oval, the long diameter 
being transverse. The diameters of the brim are antero-posterior or conjugate, transverse, right oblique, and left oblique. The anteroposterior or conjugate diameter extends from the upper border of the symphysis pubis to the sacral promontory; the transverse, from one ilio-pectineal line to the opposite, across the widest part of the brim; and the oblique, from one sacro-iliac articulation to the ilio-pectineal eminence of the opposite side. The oblique diameters are called right and left from the sacro-iliac articulations whence they extend.

The cavity is bounded in front by the bodies and rami of the pubic bones, behind by the sacrum and coccyx, and laterally by an extensive osseous plane, formed chiefly by the pelvic surface of the ischium, but also by that of the ilium, and terminating below in the incurved ischial spine. It is shallow in front, where its depth is from $I_{2}^{1}$ to 2 inches, but deep behind, where it measures about $5 \frac{1}{2}$ inches, following the curve of the sacrum. The plane of the anterior wall is oblique, being directed downwards and backwards. The posterior wall is curved, and at its upper part looks mainly downwards. The lateral wall is divided into two parts by an indistinct line extending downwards and backwards from the ilio-pectineal eminence to the spine of the ischium. These parts are spoken of as the anterior and posterior inclined planes of the ischium, the anterior looking slightly forwards and the posterior slightly backwards. The conjugate diameter of the cavity extends from the centre of the symphysis pubis to the upper margin of the third sacral segment; the transverse, from a point corresponding to the lower margin of the acetabulum on one side to the corresponding point on the other; and the oblique, from the centre of the great sacro-sciatic foramen on one side to the centre of the obturator membrane on the other.

The outlet presents three prominences, namely, the tuber ischii at either side, and the tip of the coccyx in the median line posteriorly. Its boundaries, at either side from before backwards, are as follows : the lower border of the symphysis pubis, inferior ramus of os pubis, ramus of ischium, tuber ischii, great sacro-sciatic ligament in the recent state, and tip of the coccyx. In front of an imaginary line connecting the ischial tuberosities is the subpubic arch, which is bounded at either side by the ischio-pubic ramus, and above by their meeting to form the subpubic angle. The arch is occupied by the triangular ligament of the urethra, and its plane is directed downwards and backwards. The conjugate diameter of the outlet extends from the lower border of the symphysis pubis to the tip of the coccyx, the transverse from one tuber ischii to the other, and the oblique from the middle of the lower border of the great sacrosciatic ligament on one side to the place of union between the inferior pubic and ischial rami on the other.

The Inclination of the Pelvis.--In the erect posture the plane of the pelvic brim forms with the horizontal an angle of from 50 to 60 degrees, and the base of the sacrum is about $3 \frac{3}{4}$ inches above the 
upper border of the symphysis pubis. The brim is therefore directed upwards and forwards. An idea of this obliquity may be obtained by placing a pelvis against a wall in such a way that the anterior superior iliac spines and the upper border of the symphysis pubis will touch the wall so as to lie in the same vertical plane. A line connecting the tip of the coccyx with the lower border of the symphysis pubis forms with the horizontal an angle of about II degrees, and the tip of the coccyx is about $\frac{3}{4}$ inch above the subpubic angle. The direction of the outlet is downwards and backwards, principally downwards when the coccyx is extended. The plane of the symphysis pubis forms with the horizontal an angle of from 35 to 40 degrees. It is worthy of note that the sacrovertebral angle is estimated at II 7 degrees in the male, and as much as 130 in the female.

The Axes of the Pelvis.-The axes represent imaginary lines intersecting the planes of the brim, cavity, and outlet at right angles through their central points. The axis of the brim corresponds with a line drawn from the umbilicus to the sacro-coccygeal articulation, and its direction is downwards and distinctly backwards. The axis of the outlet represents a line drawn from the sacral promontory through the centre of the outlet, and its direction is downwards and very slightly backwards. The axis of the cavity intersects planes having different inclinations, and is necessarily curved, the concavity being directed forwards. It is described as 'the perpendicular of a line drawn from the middle of the symphysis pubis to the centre of the sacro-coccygeal curve.' The average measurements of the axes of the female pelvis are as follows :

\begin{tabular}{|c|c|c|c|c|c|c|}
\hline Brim & , & & & Conjugate. & Transperse. & Obliqua. \\
\hline Cavity & 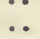 & $\because$ & $\because$ & $5^{42}$ & & 5 \\
\hline Outlet & . & .. & $\cdots$ & 5 & $4 \frac{3}{4}$ & $4 \frac{3}{3}$ \\
\hline
\end{tabular}

Sexual Differences.-The differences in the two sexes are as follows :

\section{Female.}

Bones smoother and more slender. Acetabula wide apart.

True pelvis wider and shallower.

Obturator foramen triangular.

Ischial tuberosities wider apart and everted.

Span of subpubic arch wide.

Inner border of ischio-pubic ramus comparatively smooth.

Brim transversely oval.

Ilia more vertical.

False pelvis narrower.

Sacral promontory less projecting.

Sacrum broader, shorter, and straighter.

Coccyx more movably articulated with sacrum.

Symphysis pubis shallower.

\section{Male.}

Bones rongher and more massive

Acetabula not so wide apart.

True pelvis narrower and deeper.

Obturator foramen oval.

Ischial tuberosities not so wide apart and inverted.

Span of subpubic arch narrow.

Inner border of ischio-pubic ramus strongly marked and everted.

Brim cordate.

Ilia less vertical.

False pelvis wider.

Sacral promontory more projecting.

Sacrum narrower, longer, and more curved.

Coccyx less movably articulated with sacrum.

Symphysis pubis deeper. 
The Pelvis of the Child.-The pelvis is of small size in the child. The iliac alæ are expanded, and the cavity is of small dimensions, so that a large part of the urinary bladder in both sexes lies in the hypogastric region of the abdomen. The sacro-vertebral angle is relatively greater, and the pelvis has consequently a greater inclination.

\section{The Femur.}

The femur extends from the hip to the knee, its direction being downwards, inwards, and slightly backwards. It is a long bone, and is divisible into a shaft and two extremities, upper and lower.

The upper extremity presents a head, neck, and two trochanters, great and small, together with anterior and posterior intertrochanteric lines. The head forms more than half a sphere, and its direction is upwards, inwards, and slightly forwards. Its surface is smooth and covered by cartilage, except at a point behind and below the centre, where it presents a rough depression. The ligamentum teres is attached to the upper part of this depression, which presents a small foramen for the passage of a nutrient artery. The head encroaches more upon the upper surface of the neck than on the under.

The neck forms with the shaft an angle of about I25 degrees on an average, the range being from Iro to $x 40$. It is greater in the male than in the female. The direction of the neck is upwards, inwards, and slightly forwards. It is expanded at either extremity, especially towards the shaft, and it presents four aspects-anterior, posterior, superior, and inferior, of which the posterior and inferior are more extensive than the other two. The anterior aspect is on the same plane with the anterior surface of the shaft, from which it is separated by the anterior intertrochanteric line. This line passes downwards and inwards, and it presents at either extremity the superior cervical, and inferior cervical, tubercle. The anterior intertrochanteric line gives attachment along its cervical aspect to the anterior part of the capsular ligament of the hip-joint, including the ilio-femoral band. Its inferior relations are the vastus externus over about the upper third, and the crureus over about the lower two-thirds. The anterior aspect of the neck is entirely intracapsular, and is more or less ridged, being closely covered by the retinacula of the capsular ligament. The posterior aspect is of greater extent than the anterior, and is smooth and concave. It is separated from the shaft by the posterior intertrochanteric line, which presents at its junction with the posterior border of the great trochanter the quadrate tubercle, whence the linea quadrati, for the quadratus femoris muscle, descends. Only the upper two-thirds of this aspect are intracapsular and covered by retinacula, the capsular ligament being very loosely attached along the junction of the middle and lower thirds. The lower 


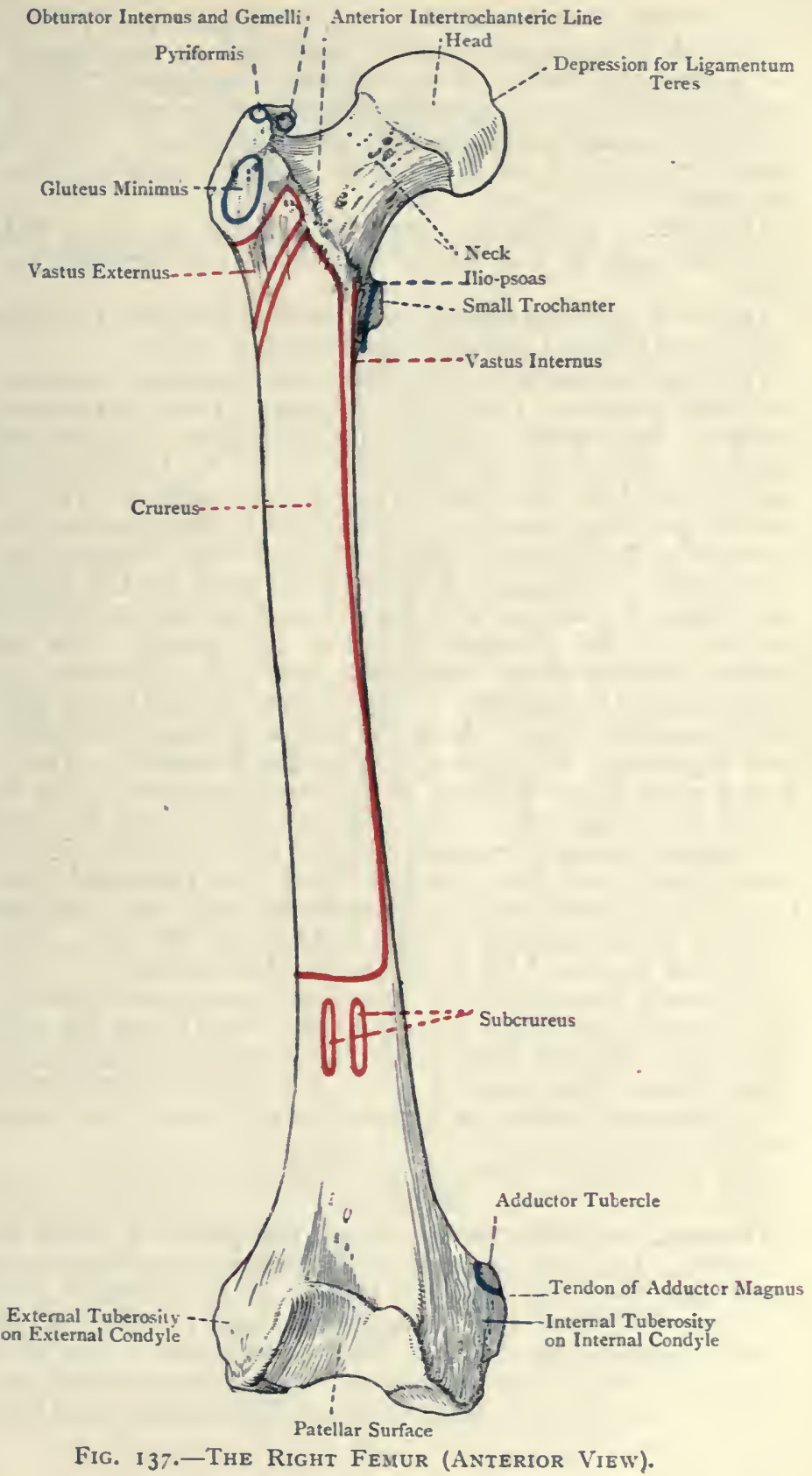


third is therefore extracapsular, and at its outer part it presents a horizontal groove which leads to the digital or trochanteric fossa, and lodges the tendon of the obturator externus. The superior aspect forms a short, almost horizontal border, which has a slight inclination downwards to the great trochanter. The inferior aspect forms a long, concave border, ending inferiorly at the small trochanter.

The neck is pierced by numerous nutrient foramina, which are more abundant and of larger size above and behind than elsewhere.

The head and neck receive their blood-supply from the obturator, sciatic, and external and internal circumflex, arteries.

The great trochanter is a quadrilateral eminence continuous with the outer surface of the shaft. It presents three surfaces and four borders. The external surface is marked by an oblique impression which extends from the postero-superior to the antero-inferior angle, and gives insertion to the gluteus medius. The anterior surface presents, towards its lower part, an impression for the insertion of the gluteus minimus. The internal surface at its lower part presents the digital or trochanteric fossa for the insertion of the obturator externus, whilst above and in front of this it affords insertion to the obturator internus and gemelli. The superior border, which is almost horizontal, meets the posterior border at a right angle, the pointed projection thus formed being called the postero-superior angle. Near the centre of this border there is an oval impression for the insertion of the pyriformis. The inferior border gives origin to fibres of the vastus externus. The anterior border skirts the front of the great trochanter, and ends above at the superior cervical tubercle. It also gives origin to fibres of the vastus externus. The posterior border is prominent and continuous with the posterior intertrochanteric line, the quadrate tubercle being situated at the junction of the two. The great trochanter is pierced by several nutrient foramina.

The small trochanter is a conical projection which springs from the posterior and inner aspects of the bone where the neck and shaft join. It gives insertion to the ilio-psoas, some of the fibres of the iliacus being inserted below and in front of it, where there is a depressed triangular surface lying between the prominence and the spiral line.

The trochanters receive their blood-supply from the circumflex arteries.

The shaft is longitudinally curved, the convexity being directed forwards. Its girth is least at the centre, and it expands at either end, more especially the lower. It is triangular in section in the middle third, the lateral angles being rounded off, and the posterior, formed by the linea aspera, prominent. Over its upper and lower thirds it is somewhat subcylindrical. The posterior aspect presents over its middle third a bold ridge, called the linea 


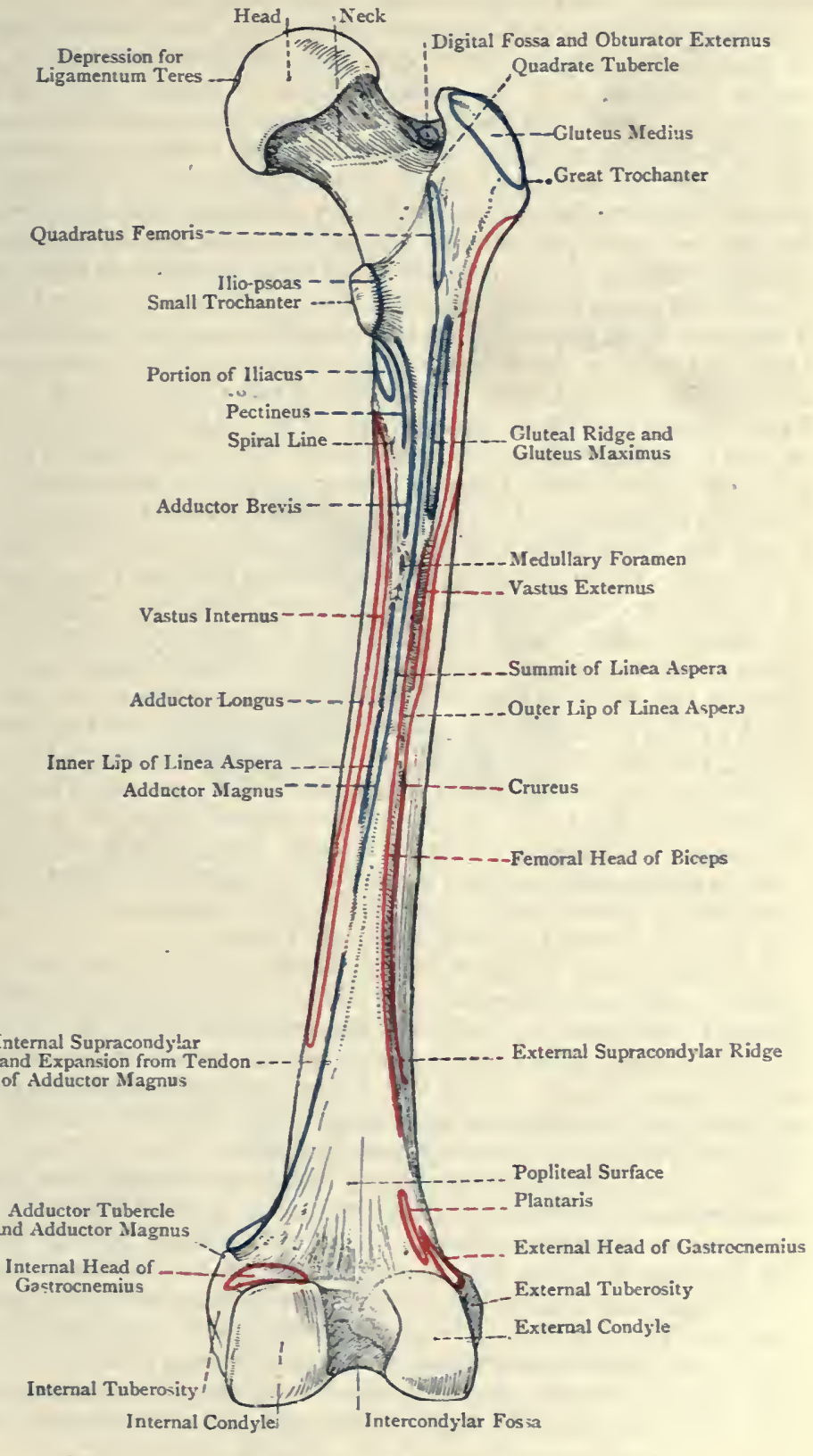

Fig. i38.-The Right Femur (Posterior View). 
aspera, which has two lips, outer and inner, and a narrow intervening space. The outer lip gives attachment, from behind forwards, to the short head of the biceps, external intermuscular septum, vastus externus over its upper half, and crureus over its lower half. The inner lip gives attachment, from behind forwards, to the adductor magnus, adductor longus, internal intermuscular septum, and vastus internus. A little above the centre of the shaft, close to the inner lip, is the medullary foramen for a branch of the second, or third, perforating artery, the direction of the foramen and the canal to which it leads being upwards towards the head.

Over the upper third of the shaft the lips of the linea aspera diverge. The outer lip is prolonged to the base of the great trochanter posteriorly, and over about its lower 3 inches it is conspicuously rough, this portion being known as the gluteal ridge, which gives insertion to the lower part of the gluteus maximus. Close to the outer side of this ridge the vastus externus takes origin, and close to its inner side the upper fibres of the adductor magnus take insertion. The inner lip bifurcates. One division is prolonged in a winding manner round the inner aspect of the shaft, passing in front of the small trochanter and terminating at the inferior cervical tubercle, where it passes into the anterior intertrochanteric line. This winding division is called the spiral line, and it gives origin to the upper fibres of the vastus internus. The other division is prolonged to the back of the small trochanter, and it gives insertion over its upper third to the pectineus, and over its whole extent to the adductor brevis, the latter being behind the former. The relation of muscles at the back of the upper end of the shaft, from the small trochanter outwards to the outer margin of the gluteal ridge, is as follows: ilio-psoas; pectineus; adductor brevis; lower fibres of quadratus femoris; adductor magnus ; gluteus maximus ; and vastus externus. The narrow intervening space of the linea aspera between its two lips is in line with the linea quadrati above.

Over the lower third of the shaft the lips of the linea aspera diverge widely, and are prolonged to the condyles as the external and internal supracondylar ridges. These enclose between them a flat triangular area, called the popliteal surface, which is also known as the trigonum femoris. Over this region the periosteum is very thin, and this part of the bone is predisposed to necrotic changes. The popliteal surface forms the upper part of the floor of the popliteal space. The external supracondylar ridge gives attachment over its whole extent to the external intermuscular septum, and over about its upper two-thirds to the short head of the biceps and the crureus. For a short distance below, it gives origin to the plantaris, and immediately external to this, to fibres of the outer head of the gastrocnemius. The internal supracondylar ridge is interrupted about an inch below its commencement by a slight groove, which is produced by the femoral vessels, and at its lower extremity, close to the internal condyle, there is a projection called the alductor tubercle, for the 
insertion of the tendon of the adductor magnus. Superiorly, for about an inch, this ridge gives insertion to a portion of the adductor magnus, and below the femoral groove to an expansion from its tendon.

The shaft presents three surfaces, anterior and two lateral, but these merge gradually into one another, except posteriorly in the situation of the linea aspera over the middle third. The anterior and external surfaces over about their upper three-fourths give origin to the crureus. The internal surface is non-muscular, and presents a characteristic elongated bare strip, which is merely covered by the vastus internus. The lower fourth of the anterior surface at its upper part gives origin to the subcrureus, and lower

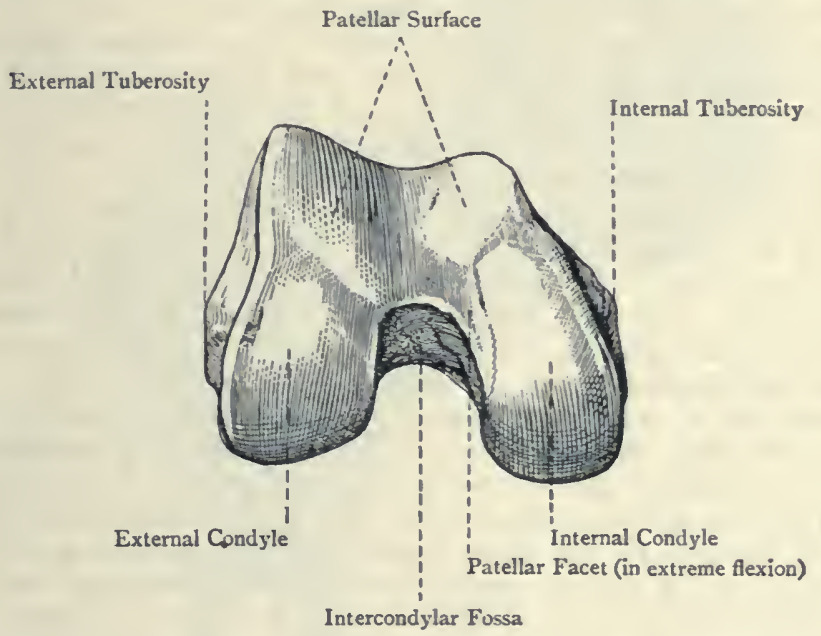

Fig. 139.-The Lower Extremity of the Right Femur.

down is covered by the suprapatellar bursa, and the pouch which the synovial membrane of the knee-joint sends upwards above the patellar surface of the bone.

The lower end of the shaft presents many nutrient foramina for the passage of branches of the anastomotica magna of the superficial femoral, and the articular branches of the popliteal, arteries.

The lower extremity presents an extensive articular surface, which is divided into three parts-anterior or patellar, and two postero-inferior or condylar. All three surfaces are continuous in front, but the condylar surfaces are widely separated behind by the intercondylar fossa. The patellar surface is trochlear, and presents a vertical groove with a convexity on either side. The groove is to the inner side of the centre, and the part external to it is broader, more prominent, and extends higher, than the internal part. The 
upper border is therefore sloped inwards and slightly downwards. The greater forward prominence of the outer part of the surface explains why the patella is inclined inwards in extension of the knee-joint.

The condyles are convex from before backwards and from side to side. Posteriorly they become prominent, and on this aspect the external condyle extends a little higher than the internal. As viewed from below the external condyle is broad and short, the internal being long and narrow. When the femur is held vertically the internal condyle projects lower down than the external, and this brings the two condyles upon the same horizontal plane when the bone occupies its natural sloping position. The outer border of the external condyle is very nearly in the same line with the outer border of the patellar surface, and the outer border of the internal condyle is in the same line with the inner border of the patellar surface. The inner border of the internal condyle has a convex outline, and at its anterior part it turns outwards to the patellar surface. For the most part the condyles are parallel, the exception being the front part of the internal condyle, which inclines outwards to meet the patellar surface.

The demarcation between the condylar surfaces and the patellar surface is clearly marked at either side. The external condyle is separated from the patellar surface by a slightly elevated line and groove, extending outwards and slightly forwards from the front and outer part of the intercondylar fossa to the outer border of the cartilaginous surface, where there is a depression which receives the anterior part of the external semilunar fibro-cartilage during extension of the knee-joint. The internal condyle is separated from the patellar surface by a line and groove, extending from near the front and inner part of the intercondylar fossa forwards and slightly inwards to the inner border of the cartilaginous surface, at a point about $I$ inch below the inner end of the upper border of the patellar surface. At this latter point there is a depression which receives the anterior part of the internal semilunar fibro-cartilage during extension of the knee-joint. The line and groove just referred to do not extend quite close to the intercondylar fossa. The groove subsides, but the line sweeps backwards in a curved manner along the outer part of the inner condylar surface, thus marking off a narrow semilunar zone from the general tibial surface. This zone lies close to the inner part of the intercondylar fossa, and is known as the patellar jacet. In extreme flexion of the kneejoint, as in the position assumed by the miner when at work, the patella by its inner vertical zone articulates witl this facet, which may be called the miner's facet.

The outer surface of the external condyle towards the back part presents the external tuberosity, which gives attachment to the long external lateral ligament of the knee-joint. Immediately above and behind the tuberosity is an impression for the outer head of the gastrocnemius, and behind and below it there is a groove, called the poplitcal groove, which is directed downwards and forwards. 
The tendon of the popliteus arises from the front part of the horizontal portion of the groove, and it is lodged in the groove only when the knee is flexed.

The inner surface of the internal condyle presents at its centre a large blunt eminence, called the internal tuberosity, for the attachment of the internal lateral ligament. Posteriorly, where the internal supracondylar ridge joins the internal condyle, the adductor tubercle is situated, and the line of origin of the inner head of the gastrocnemius extends almost transversely outwards from this tubercle above the internal condyle.

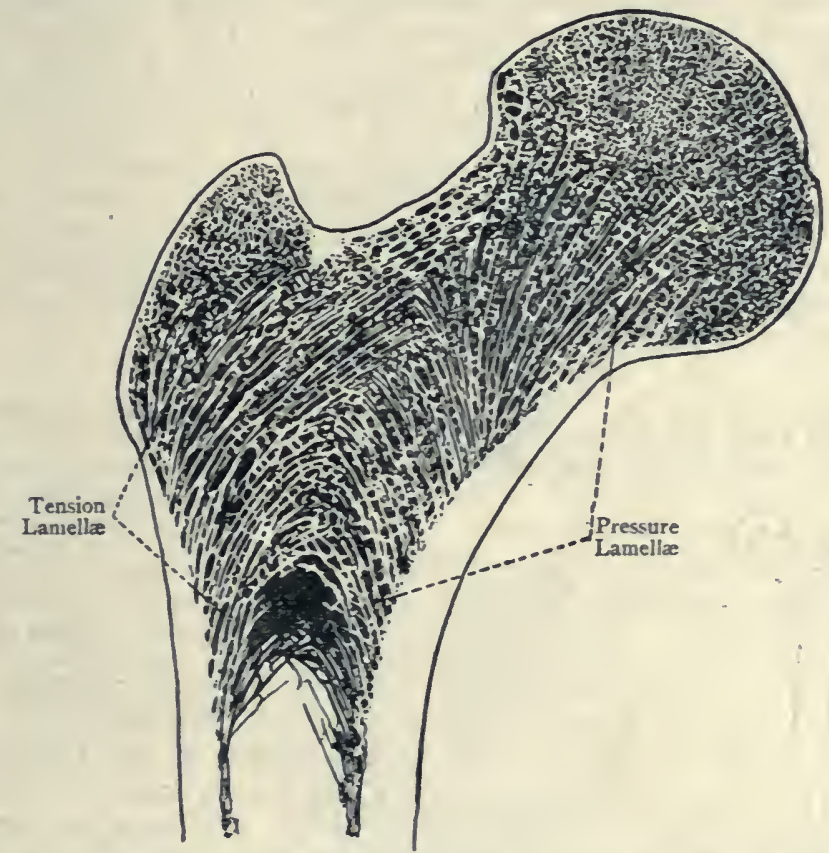

Fig. I40.-Longitudixal Section through the UPPER EÑd of the Femur, Showing the Pressure LaMelle aNd TeNsion Lamelle.

The markings in connection with the intercondylar fossa are for the crucial ligaments. The impression for the anterior crucial ligament is at the back part of the inner surface of the external condyle, whilst that for the posierior crucial ligament is at the front part of the outer surface of the internal condyle, and adjacent portion of the front of the intercondylar fossa. At the front of that fossa in the middle line the ligamentum mucosum is attached.

Articulations.- Superiorly with the acetabulum of the os innominatum, and inferiorly with the head of the tibia below, and the patella in front. 
Structure.-The structure is that of a long bone. The marrow canal extends from a point just below the small trochanter to the level of the apex of the trigonum femoris. Above and below these points the bone is composed of cancellated tissue, except externally, where there is a shell of compact bone. The cancellated tissue at the upper extremity has its lamellæ arranged in a series of curves disposed in two systems, one of which represents the pressure lamellæ, and the other the tension lamellae. The pressure lamella extend from the lower part of the neck and upper part of the shaft internally in a radiating manner, sorne, which are very strong, passing inwards to the head, whilst others pass outwards to the great trochanter. The tension lamellae are disposed almost at right angles to the pressure lamellæ, and arch upwards and inwards from the outer aspect of the shaft, below the great trochanter, to the head and lower part of the neck. Additional strength is afforded by an almost vertically disposed plate of compact bone, called the calcar femorale, which runs upwards and downwards in front of, and above, the small trochanter, and lies in the line in which weight is transmitted. The cancellated tissue at the lower extremity has its lamellæ arranged in obliquely decussating lines which enclose somewhat rectangular meshes.

Varieties.-(1) The gluteal ridge may assume the form of a depression, called the fossa hypotrochanterica. (2) There may be a third trochanter, situated at the upper part of the gluteal ridge. (3) The linea aspera may be

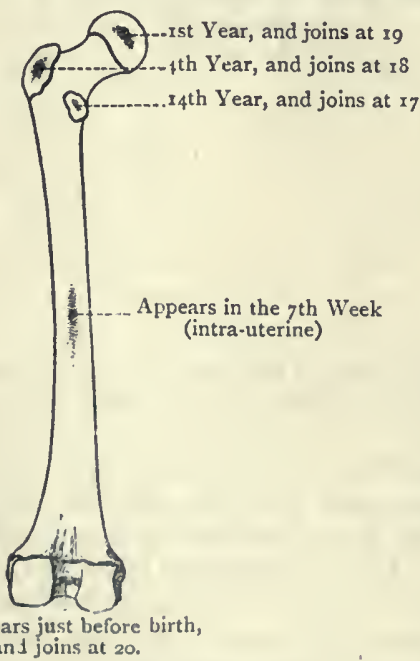

Fig. 141.-OSSification of the FEMUR. unduly prominent owing to muscular action, this condition being known as the pilastered femur. (4) A pressure facet is sometimes met with, as in miners, at the upper end of the front of the neck, close to the cartilage of the head, with which it is continuous, this facet being due to prolonged maintenance of the flexed posture.

The Femur of the Female.(I) The bone is smoother than in the male. (2) The angle formed by the neck with the shaft is about IIo degrees. (3) The bones are farther apart above, more sloped inwards, and nearer to each other below, than in the male.

Ossification.-The femur ossifies in cartilage from one primary, and four secondary, centres. The primary centre appears at the middle of the shaft in the seventh week of intra-uterine life. The centre for the lower extrenity appears just before birth in the botton 
of the intercondylar fossa. At birth the shaft is ossified, and the lower epiphysis is showing signs of ossification, but the three upper epiphyses are cartilaginous. The centre for the head appears in the first year, that for the great trochanter in the jourth year, and that for the small trochanter in the fourteenth year. The small trochanter joins the shaft at seventeen, the great trochanter at eighteen, the head at nineteen, and the lower epiphysis at twenty. The neck is ossified from the centre for the shaft. The line indicating the junction of the lower epiphysis and shaft cuts the adductor tubercle into two, one portion belonging to the lower épiphysis, and the other to the shaft.

\section{The Patella.}

The patella, rotula, or knee-cap, is situated in front of the kneejoint, where it articulates with the patellar surface of the femur. It is originally a sesamoid cartilage developed in the tendon of the quadriceps extensor cruris. The bone is triangular with the apex downwards, and is compressed from before backwards. The
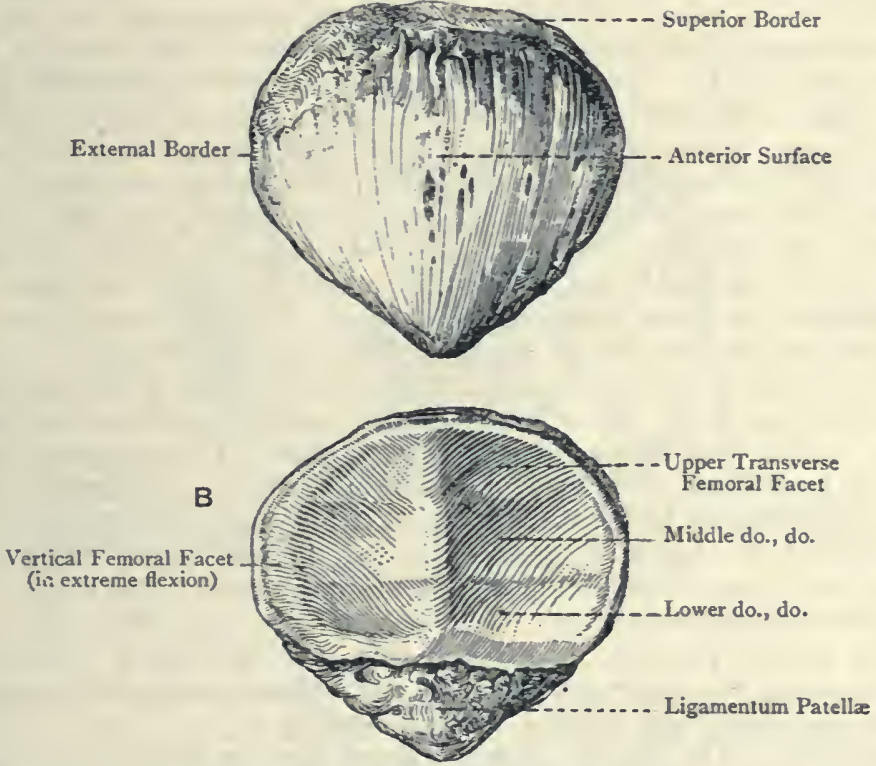

Fig. 142.- The Right Patella.

A, Anterior Surface; B, Posterior Surface.

superior border or base is broad, and its plane is inclined forwards and slightly downwards. It gives insertion anteriorly to the rectus femoris and crureus, in this order from before backwards, and posteriorly it is covered by a portion of the synovial membrane of the knee-joint. The lateral borders are sloped towards the apex, the outer being at first rather more prominent than the inner. The outer border over its upper third gives insertion to a nortion of the vastus externus, and the inner over its upper half to a 
portion of the vastus internus. The apex is blunt, and, together with the adjacent marginal parts, gives attachment to the ligamentum patellæ, by which the bone is connected with the tubercle of the tibia.

The anterior surface, which is slightly convex, is vertically striated and covered by a prolongation of the tendon of the quadriceps extensor cruris. It is perforated by numerous nutrient foramina, and is subcutaneous, being separated from the integument by the prepatellar bursa.

The posterior surface is divided into two parts-articular and non-articular. The non-articular part represents the lower fourth, and is rough and depressed. It lodges a collection of fat covered by synovial membrane. The articular part corresponds with the upper three-fourths, and is divided into two unequal parts by a round vertical ridge, which is received into the groove of the patellar surface of the femur. The external division is broad and concave from side to side, whilst the internal is narrow and convex in the transverse direction. Excluding a narrow vertical zone at the inner part of the inner division, each division is subdivided by two slight transverse ridges into three horizontal zones-upper, middle, and lower, of which the middle is the largest and broadest. These six horizontal facets articulate with the patellar surface of the femur, the lower facets being in contact with the upper part of the patellar surface in extension of the knee-joint, the middle patellar facets with the middle portion of the patellar surface of the femur in semiflexion, and the upper patellar facets with the lower parts of the patellar surface of the femur in flexion of the knee-joint. The vertical zone at the inner part of the inner division of the articular surface (close to the inner border of the bone) constitutes a seventh facet, which may be called the miner's facet. In extreme flexion of the knee-joint this facet articulates with the semilunar facet (miner's facet) on the outer part of the tibial surface of the internal condyle of the femur close to the intercondylar fossa, whilst the upper and outer horizontal facet is in contact with the front part of the external condyle.

The patella receives its arteries from the superficial branch of the anastomotica magna of the femoral, inferior articular branches of the popliteal, and anterior tibial recurrent.

Structure.-The patella, being a short bone, is composed principally of dense cancellated tissue with close meshes, surrounded by compact bone, which is much thicker in front than behind.

Ossification.-The original cartilage is deposited in the tendon of the quadriceps extensor cruris in the third month of in tra-uterine life. In this cartilage a singie centre appears in the third year, and ossification is completed about the age of puberty.

\section{The Tibia.}

The tibia, or shin-bone, is the inner and larger of the two bones of the leg, and alone transmits the weight of the body to the foot. The posterior surfaces of the shafts of the tibia and 
fibula are on the same horizontal plane above and below, but over about the middle three-fifths the fibula projects slightly farther back on account of its curve. Anteriorly the tibia is on a more anterior plane than the fibula, a point to be borne in mind in making flaps by transfixion. The tibia is a long bone, and is divisible into a shaft and two extremities, upper and lower.

The upper extremity, known as the head, is broader from side to side than from before backwards. Antero-laterally it is convex, but posteriorly it is rendered concave by the popliteal notch at its centre. The enlargements of the bone on either side of the head are called the tuberosities, external and internal. The external twberosity is rather smaller than the internal, and at its posterior and under aspect it presents a flat circular facet, directed downwards,

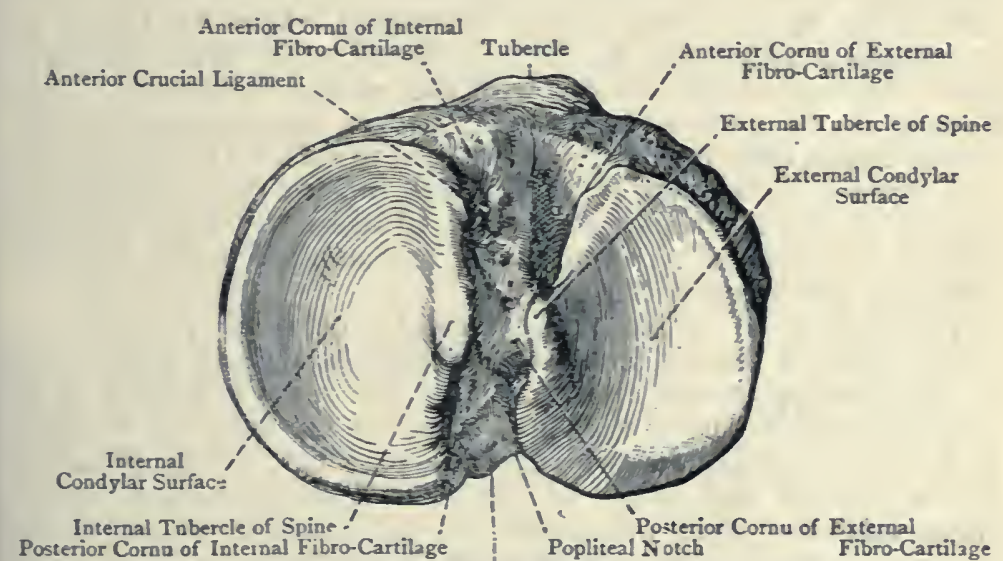

Posterior Crucial Ligament

Fig. 143.-The Head of the Right Tibia (Superior View).

backwards, and outwards, which articulates with the head of the fibula. The cartilage of this facet is occasionally continuous with that of the external condylar surface. At the junction of its anterior and outer surfaces the external tuberosity presents an elevation, which usually assumes the form of a ridge, for the attachment of the ilio-tibial band of the fascia lata. The internal tuberosity is larger than the external, and has a distinct inclination backwards as well as inwards, a point to be noted in setting fractures of this bone. On its posterior aspect it presents a horizontal groove for the insertion of the chief portion of the tendon of the semimembranosus muscle. On the anterior aspect of the superior extremity, at the junction of the head and shaft, there is a well-marked projection, called the tubercle or anterior tuberosity. It is fully I inch in length, and its upper border is about $\frac{3}{4}$ inch 
below the level of the upper surface of the head. It is divisible into two nearly equal parts, upper and lower. The lower division is rough, and is usually strongly ridged in the vertical direction for the attachment of the ligamentum patellæ. The upper division is smooth, and is separated from that ligament by a synovial bursa.

The superior surface of the head presents the two condylar articular surfaces, separated from each other by an irregular interval, which, amongst other markings, presents the bifid tibial spine. Each surface surmounts the corresponding lateral tuberosity. The external condylar surface is broad from side to side, and is almost circular. It is concave from side to side, and concavoconvex from before backwards. Its cartilage rises towards the middle line to coat the external surface of the outer tubercle of the tibial spine, and posteriorly it dips down for a little on the outer part of the back of the external tuberosity, where the tendon of the popliteus glides over it. It is in this situation where the cartilage is occasionally continuous with that of the fibular facet. The internal condylar surface is oval and concave, being elongated from before backwards, but narrow from side to side. The cartilage of this surface rises towards the middle line to coat the internal surface of the inner tubercle of the tibial spine. Each condylar surface is deepened by a semilunar fibro-cartilage, which is placed round its peripheral part.

The interspace between the condylar surfaces presents the spine, which is distant from the posterior border about one-third of the antero-posterior measurement. The spine is formed by an upward rising of the contiguous borders of the condylar surfaces, and is bifid, ending in two tubercles, of which the inner is the better marked and longer of the two. The interspace between these tubercles gives attachment to the posterior cornu of the external semilunar fibro-cartilage, which continues to be attached to a depression behind the outer tubercle. The surfaces of the tubercles which face each other are free from cartilage, but the other surfaces have each a cartilaginous covering.

In front of the spine there is a rough depression where important structures are attached as follows: in front of the outer tubercle of the spine the anterior cornu of the external semilunar fibrocartilage is attached, and in front of the inner tubercle the anterior crucial ligament is attached to the bone. At the extreme anterior and inner part there is an impression for the anterior cornu of the internal semilunar fibro-cartilage. On the outer side of the impression for the anterior crucial ligament, and in front of that for the anterior cornu of the external semilunar fibro-cartilage, there is a depression which is partially occupied by a small collection of fat. At its outer part, however, there is a groove which receives a portion of the external semilunar fibro-cartilage in extension of the knee-joint. The immediately adjacent portion of the external condylar surface is specially facetted for the play of part of the 
external condyle of the femur in extension of the joint. Behind the tibial spine there is a more limited rough depression, which leads backwards to the popliteal notch. The posterior cornu of the internal semilunar fibro-cartilage is attached to the inner part of this depression, and the posterior crucial ligament is attached to its back part, as well as to the popliteal notch.

Order of Structures attached to the Head.-The structures, enumerated as nearly as possible in order from before backwards, are as follows :

I. Anterior cornu of internal semilunar fibro-cartilage.

2. Anterior crucial ligament.

3. Anterior cornu of external semilunar fibro-cartilage.

4. Posterior cornu of external semilunar fibro-cartilage.

5. Posterior cornu of internal semilunar fibro-cartilage.

6. Posterior crucial ligament.

The head is pierced all round by many nutrient foramina for branches of the inferior articular arteries of the popliteal, and of the posterior and anterior tibial recurrents of the anterior tibial.

The shaft is massive and triangular. It diminishes in size from above downwards over its upper two-thirds, and then gradually enlarges towards its lower end. It presents three borders and three surfaces. The anterior border extends from the outer side of the anterior tuberosity above to the anterior margin of the internal malleolus below. Over the upper two-thirds, where it occupies the middle line, it is prominent, and is known as the crest or shinridge. This is doubly curved, the convexity of the upper curve being directed inwards, and that of the lower outwards. Over the lower third the anterior border inclines inwards, and the external surface of the shaft is thus allowed to come forwards. The crest is subcutaneous, and gives attachment to the deep fascia of the leg. The internal border extends from the inner and back part of the internal tuberosity to the posterior margin of the internal malleolus. For 3 or 4 inches superiorly it is rough, and gives attachment to the internal lateral ligament of the knee-joint. Over its middle third it is prominent, and it here gives origin to a portion of the soleus as low as the centre of the bone. The external or interosseous border extends from the front of the fibular facet above to a point about 2 inches from the lower end, where it bifurcates. The two divisions pass to the front and back of the sigmoid cavity, and enclose between them a rough triangular surface for the inferior interosseous ligament. This border is sharp and wiry, and gives attachment to the interosseous membrane.

The internal surface is situated between the crest and internal border. It is for the most part subcutaneous, and slightly convex. Superiorly, where it becomes expanded and flattened, it presents a vertical rough area, behind the tubercle, for the insertion of the sartorius, and behind this two vertical rough impressions in the same line with each other, the upper of which gives insertion 


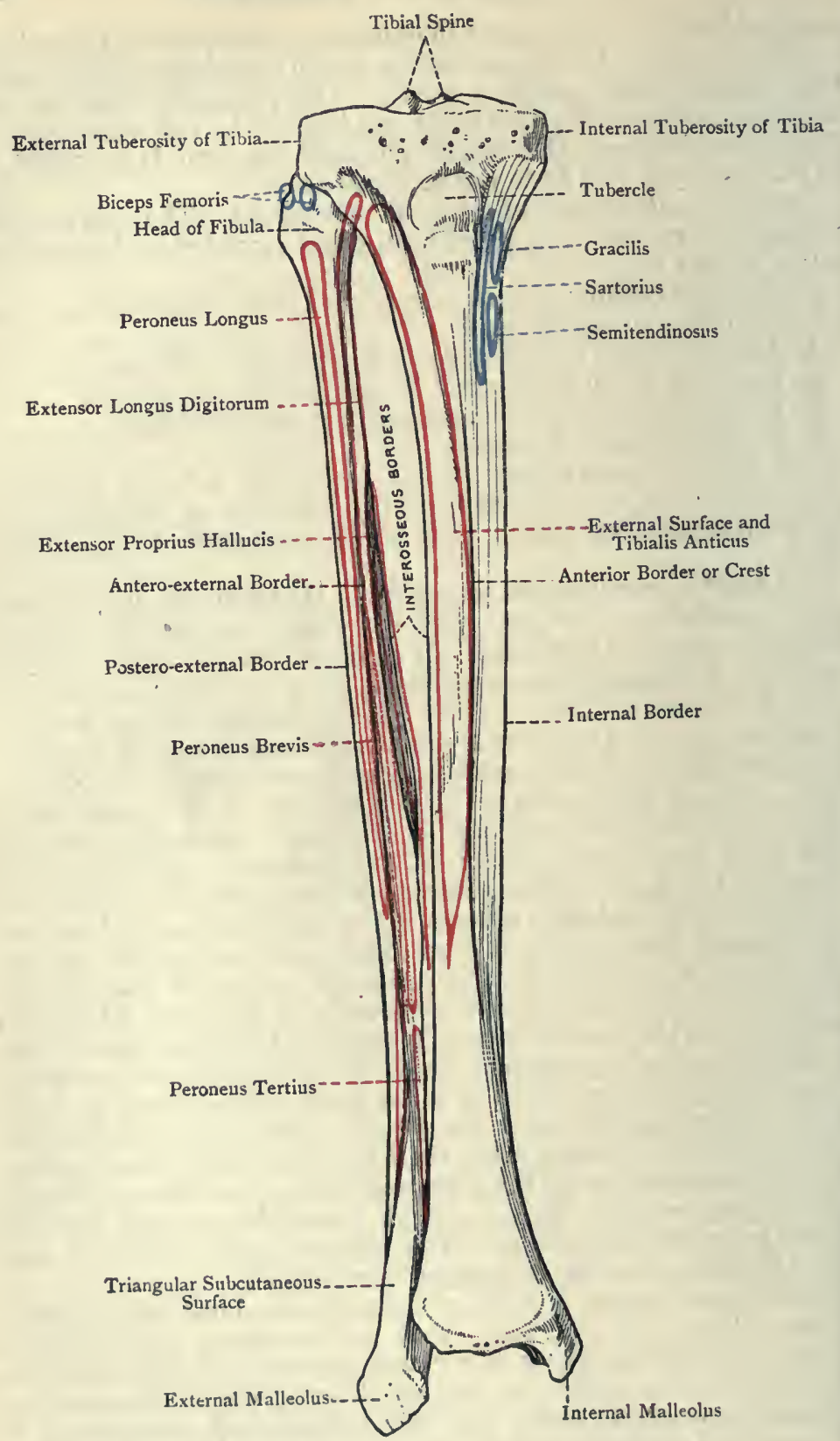

Fig. 144.-The Right Tibia and Fibula (Anterior View). 
to the gracilis, and the lower to the semitendinosus. The external surface is situated between the crest and interosseous border. It is concave over its upper two-thirds, where it gives origin to the tibialis anticus. Over the lower third, where it is convex, it turns to the front and supports the extensor tendons, and anterior tibial vessels and nerve. The posterior surface lies between the interosseous and internal borders. Superiorly it is crossed by the popliteal or oblique line, which is rough, and extends from the fibular facet downwards and inwards to the internal border at about the junction of the upper third and lower two-thirds. This line gives attachment to the popliteal fascia and part of the soleus, whilst the triangular popliteal surface above gives insertion to the popliteus muscle. The posterior surface below the oblique line presents over its middle third a vertical ridge which divides it into two parts. The outer portion is narrow, and gives origin to the tibialis posticus as low as a point just below the centre of the bone. The inner portion is broad, and gives origin to the flexor longus digitorum over the middle two-fourths of the bone. A little below the oblique line, close to the outer side of the vertical ridge, is the medullary foramen for a large branch of the posterior tibial artery. This foramen, which is the largest of its class, and the canal to which it leads are directed downwards. The posterior surface in its lower third supports the flexor tendons, and posterior tibial vessels and nerve.

The lower extremity presents a quadrilateral articular surface, concave from before backwards, and wider in this direction externally than internally. It is broader in front than behind, and articulates with the superior surface of the astragalus. The posterior border projects somewhat lower than the anterior. The anterior surface, immediately above the anterior border, is depressed and rough for the anterior ligament of the ankle-joint. The posterior border gives attachment to the posterior ligament of the ankle-joint as far inwards as the groove behind the internal malleolus. It presents the following grooves: one for the tendon of the flexor longus hallucis near the outer end; one (very faint) near the centre for the posterior tibial vessels and nerve; and one mainly situated on the back of the internal malleolus for the tendons of the tibialis posticus and flexor longus digitorum.

The inner aspect of the lower extremity presents the internal malleolus, which is a strong process having a downward direction. Its internal surface is rough, convex, and subcutaneous. The external surface is covered by cartilage, continuous with that which coats the lower extremity. The plane of this surface is vertical, and the cartilage coats it more deeply in front than behind. It articulates with the internal surface of the astragalus. The anterior border is rough and round for the attachment of the anterior and internal lateral ligaments of the ankle-joint. The lower border is indented by a notch, in front of which is the projection known as the tip, the internal lateral ligament being attached to both of these parts. 


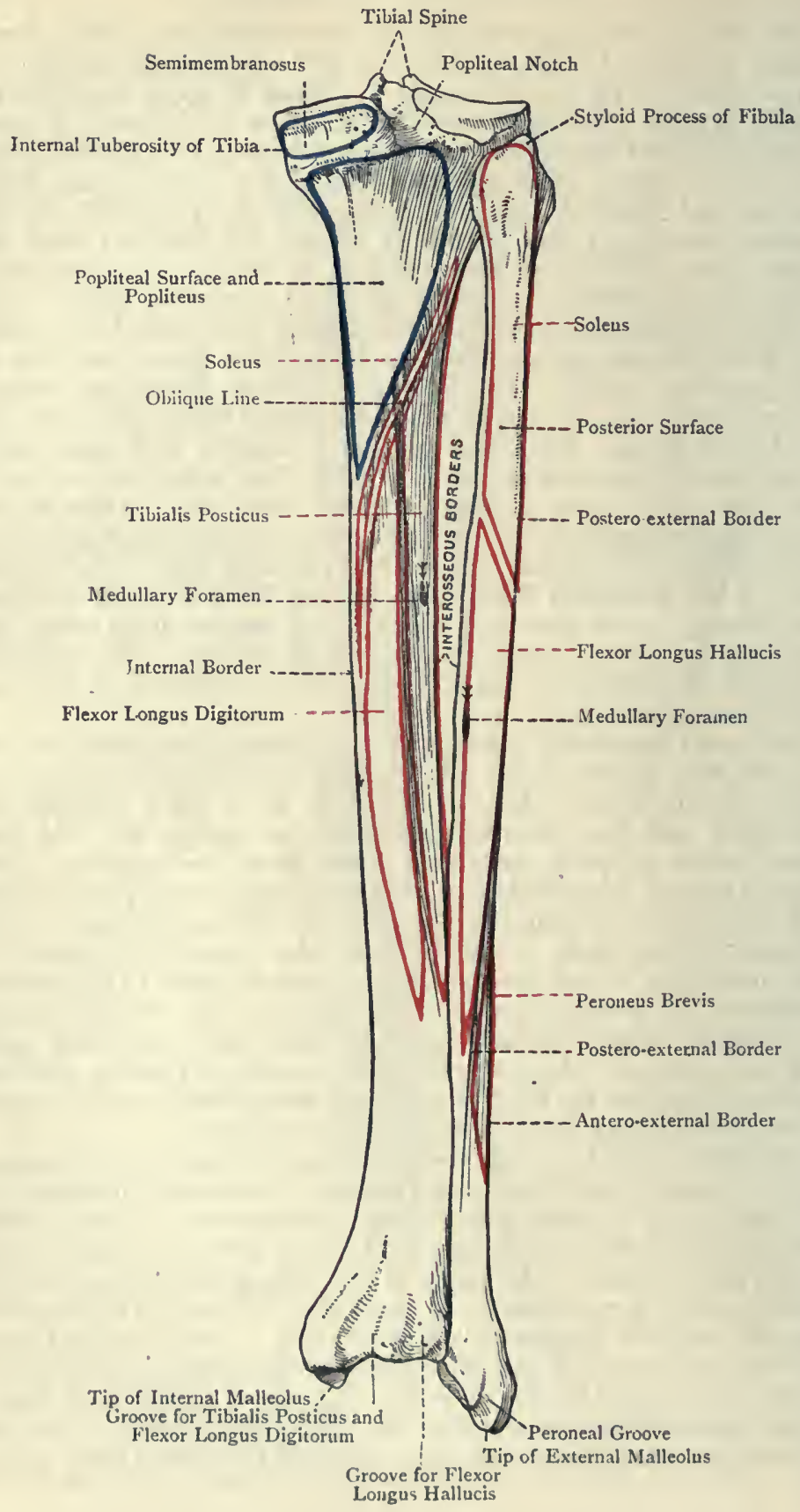

Fig. 145.-Tue Right Tibia and Fibula (Posterior View). 
Posteriorly is the groove for the tendons of the tibialis posticus and flexor longus digitorum. The outer aspect of the lower extremity presents a concave facet for the fibula, and above this a concave triangular rough surface about $\mathrm{I}_{\frac{1}{2}}$ inches long for the inferior interosseous ligament.

The inferior extremity of the tibia presents many nutrient foramina for branches of the anterior and posterior tibial, internal malleolar, and anterior peroneal, arteries.

Articulations.-Superiorly with the condyles of the femur above, and the head of the fibula postero-externally, and inferiorly with the fibula externally, and the superior and internal aspects of the astragalus below.

Structure.-The structure is that of a long bone. The medullary canal extends above to a point about $I_{\frac{1}{2}}$ inches below the lower margin of the anterior tuberosity, and inferiorly to a point about I inch below the lower extremity of the crest. The cancellated tissue of the upper epiphysis has its lamellæ disposed somewhat vertically, whilst that of the upper end of the shaft has its lamellæ arranged in the form of arches. In the lower part of the shaft the cancellous lamelle are disposed vertically, and the cancellated tissue of the lower epiphysis is closely meshed.

Varieties.-(I) The tibia is sometimes much compressed laterally, which leads to an increase in its antero-posterior diameter. In these cases the vertical ridge posteriorly becomes unduly prominent, a condition which is associated with a large development of the tibialis posticus muscle. Such a bone is spoken of as being platycnemic (broad-legged), and the condition is known as platycnemism (broadness of tibia). (2) The anterior aspect of the lower extremity of the bone sometimes presents a pressure facet at its outer part for articulation with the upper surface of the neck of the astragalus in extreme flexion of the ankle-joint.

Ossification.-The tibia is ossified in cartilage fron I primary and 3 secondary centres.

The primary centre appears at the centre of the shaft about the 7 th week. The 3 secondary centres are disposed as follows: 2 are superior. one for the head, and the other for the tubercle; and $\mathrm{I}$ is inferior for the lower extremity and internal malleolus. Upper Extremity. - The centre lor the head appears just before birth, and from it the external and in-

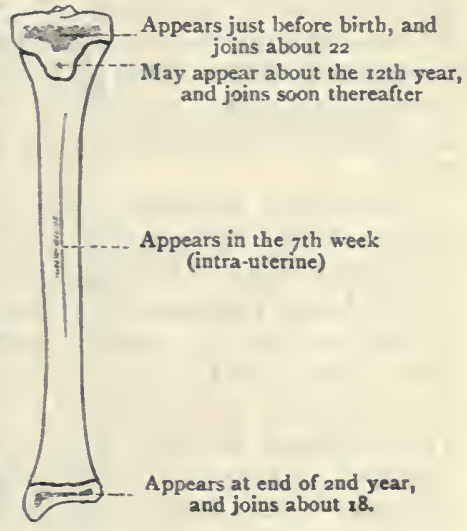

Fig. 146.-Ossification OF THE Tibia. ternal tuberosities are ossified, which constitute the superior epiphysis. The centre for the tubercle appears about the I2th year. Soon afterwards it joins the superior epiphysis, which unites with the shaft about the $22 n d$ year. In some cases the tubercle is entirely ossified from the centre for the head. In other cases the upper smooth part of the tubercle is ossified from the centre for the head, and the lower rough part derives its ossification from the primary centre for the shaft. Lower Extremity. -The centre for the lower extremity and internal malleolus appears towards the end of the $2 n d$ year, and this lower epiphysis joins about the 18 th year. 


\section{The Fibula.}

The fibula, or peroneal bone, is situated on the outer side of the tibia. It is very slender for its length, and is a rudimentary bone. It takes no part in transmitting the weight of the body, but serves chiefly to afford attachment to muscles, though it also forms part of the ankle-joint, and acts as a brace or support to the tibia. It is a long bone, and is divisible into a shaft and two extremities, upper and lower.

The upper extremity, or head, is enlarged and knob-like, its upper surface being somewhat flattened and sloping. It is situated about $\frac{3}{4}$ incl below the level of the head of the tibia. Posteriorly it is prolonged upwards into the styloid process, to the tip of which the short external lateral ligament of the knee-joint is attached. In front of this process the upper surface of the head is sloped downwards and forwards, and is divisible into an articular and a non-articular part. The articular division is internal in position, and takes the form of a flat circular facet, which is directed upwards, inwards, and forwards, to articulate with the facet on the posterior and under aspect of the external tuberosity of the tibia, by which latter it is overhung. The non-articular division is external in position, and takes the form of a rough depression, into which the tendon of the biceps femoris, previously divided into two parts by the long external lateral ligament, takes insertion. Posteriorly the head gives origin to the soleus. Externally it gives origin to the peroneus longus, and at a point nearly $\frac{1}{2}$ an inch anterior to the styloid process its outer margin gives attachment to the long external lateral ligament. Anteriorly it gives origin to the extensor longus digitorum. The constricted part below the head is called the neck.

The upper extremity presents several nutrient foramina for branches of the inferior external articular of the popliteal, and superior fibular of the anterior tibial, arteries.

The lower extremity is prolonged downwards into a massive projection, called the external malleolus, which is not only larger, but lower down and farther back, than the internal malleolus. It is triangular or pyramidal, the base being directed upwards. The external surface is rough, convex, and subcutaneous. The internal surface is divisible into two parts, articular and nonarticular. The articular division is anterior in position, and occupies about two-thirds of the surface. It is triangular and convex, and it mainly articulates with the outer surface of the astragalus. Superiorly, however, for about $\frac{1}{4}$ inch, it assumes a somewhat semilunar outline, and this portion articulates with the outer aspect of the tibia. The non-articular division is posterior in position, and occupies about one-third of the surface. It is rough, depressed, and triangular, and is known as the digital fossa. Superiorly it gives attachment to the transverse ligament of the inferior tibio-fibular joint, and inferiorly to the posterior fasciculus 
of the external lateral ligament of the ankle-joint. Above the external malleolus on the inner aspect there is a rough, convex, triangular surface about $I \frac{1}{2}$ inches long for the inferior interosseous ligament. The anterior border projects at first forwards, and then slopes downwards and backwards to the tip. The projecting part gives attachment to the anterior ligament, and the lower portion of the sloping part to the anterior fasciculus of the external lateral ligament of the anklejoint. The posterior border is shorter than the anterior, and is vertical. It presents the peronea! groove for the tendons of the peroneus longus and peroneus brevis. The tip is the most dependent part, and is situated at the meeting of the posterior border and the lower sloping part of the anterior border. It gives attachment to the middle fasciculus of the external lateral ligament.

The lower extremity presents several nutrient foramina for branches of the anterior and posterior peroneal, and external malleolar, arteries.

The shaft is slightly curved, the convexity being directed backwards in the upper part, and inwards lower down. It is quadrilateral in its upper three-fourths, where it presents four borders and four surfaces, but it is somewhat triangular in the lower fourth. The anteroexternal border, which is the most prominent, commences in front of the head, and passes straight downwards until it reaches the lower fifth, whore it bifurcates. One division passes to the anterior margin of the external malleolus, and the other to

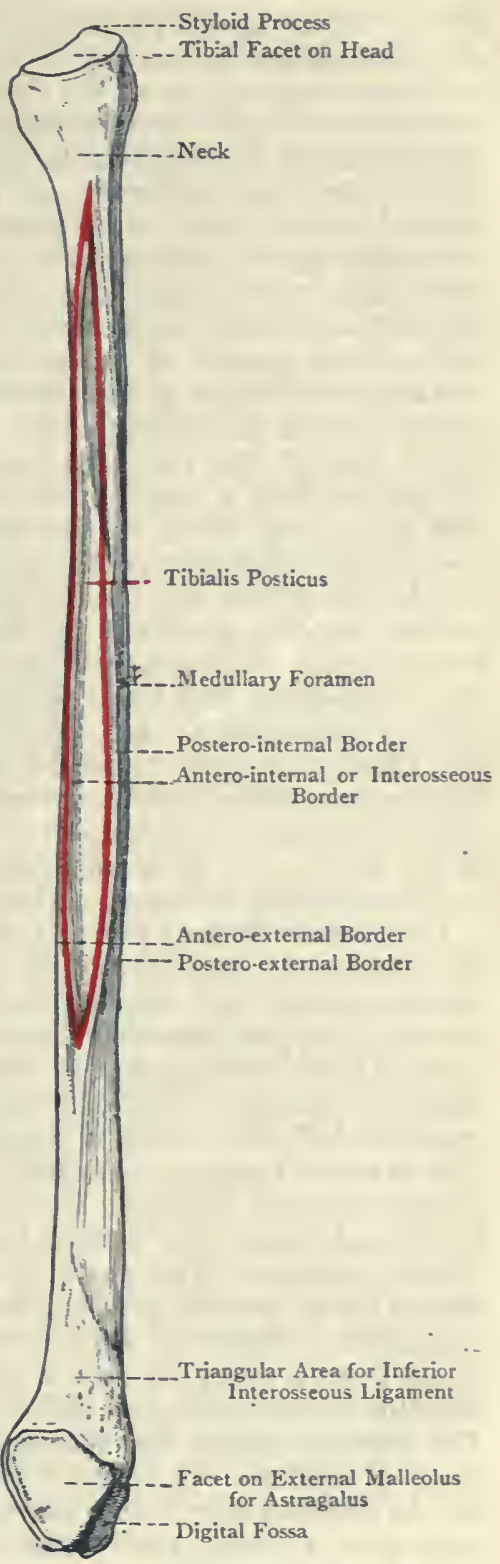

Fig. 147.-The Right Fibula (INTERNal VIEW). 
the posterior margin, external to the peroneal groove. These two divisions enclose between them a triangular area which is continuous with the outer surface of the external malleolus. This border gives attachment to the antero-external intermuscular septum. The antero-internal or interosseous border, which gives attachment to the interosseous membrane, also commences in front of the head, where it is very near to the antero-external border. As it descends it keeps near to that border at first, but beyond the upper third it gradually diverges from it, and on reaching a point about 2 inches above the external malleolus it bifurcates. One division passes to the anterior margin of the malleolus, becoming incorporated with one of the divisions of the antero-external border, whilst the other passes to the posterior margin of the malleolus, internal to the upper end of the peroneal groove. The two divisions, as they diverge, enclose a rough triangular area, which is slightly convex and gives attachment to the inferior interosseous ligament. The postero-internal border commences on the inner side of the head, not far from the antero-internal. It descends in a backwardlycurved manner, gradually leaving the antero-internal border, but subsequently approaching it, until on reaching the junction of the upper two-thirds and lower third it ends by joining it. This border gives attachment to an intermuscular septum, which separates the tibialis posticus from the soleus and flexor longus hallucis. The postero-external border extends from the back of the head to the back of the external malleolus, internal to the peroneal groove, and in its lower part it turns inwards. It gives attachment to the postero-external intermuscular septum.

The anterior surface is situated between the antero-external and antero-internal or interosseous borders. It is very narrow over about its upper half, but becomes wider below. It gives origin over about its upper three-fourths to the extensor longus digitorum, over its lower fourth (except about I inch below) to the peroneus tertius, and over about its middle two-fourths to the extensor proprius hallucis, which is nearest to the antero-internal border. The internal surface is situated between the antero-internal and postero-internal borders. It is concave and fusiform, being narrow above and below, but wide at the centre, and it gives origin to the tibialis posticus. The posterior surface is limited by the posterointernal and postero-external borders, and in its lower fourth it undergoes a twist, and turns round to become internal. Over its upper third it gives origin to a part of the soleus, and over its lower two-thirds, except the last inch or more, to the flexor longus hallucis. The external surface lies between the antero-external and posteroexternal borders. It is the broadest, and in muscular subjects is deeply grooved over rather more than its upper half. Inferiorly it undergoes a twist, and turns round to become posterior, where it leads directly to the peroneal groove on the back of the external malleolus. In this manner the two peroneal tendons are guided to this groove. The upper two-thirds of this surface give origin to 
the peroneus longus, and the lower two-thirds, except the last 2 inches, to the peroneus brevis, these two muscles overlapping towards the centre of the bone.

The medullary foramen, which is small, is usually situated on the posterior surface, but may be on the internal, a little above the centre, and there may be an additional one a little higher up. It is for a branch of the peroneal artery, and the direction of the foramen and the canal to which it leads is dorenwards towards the ankle.

Articulations.-Superiorly with the external tuberosity of the tibia, and inferiorly with the outer aspect of the tibia, and the external surface of the astragalus.

Structure.-The structure is that of a long bone, and the marrow canal is limited to about the middle three-fifths of the shaft.

Ossification.-The fibula ossifies in cartilage from one primary, and two secondary, centres. The primary centre for the shaft appears in the eighth week of intra-uterine life. At birth the shaft is ossified, but the extremities are cartilaginous. The centre for the lower extremity appears in the second year, and that for the upper extremity about the fourth year. The lower epiphysis joins the shaft about twenty, and the upper about twenty-three. The fibula forms an exception to the general law of ossification applicable to long bones with ar epiphysis, or epiphyses, at either end, which may be here restated as follows: 'The epiphysis or epiphyses, at the end towards which the medullary foramen and the canal to which it leads are directed, are the last to show signs of ossification, but they are the first to join the shaft.' In the fibula the lower epiphysis not only joins the shaft first, but it is the first to show signs of ossification-due to the fact that this extremity is the least rudimentary part of the bone.

At about the seventh month of intrauterine life the tibial and fibular malleoli are of nearly equal proportions, but by the second year. previous to the appearance of its centre of ossification, the fibular malleolus has attained

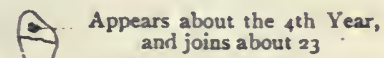
and joins about 23

Appears in the 8th Week (intra-uterine)

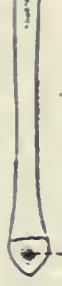

Appears in the and Year, and joins about 20

Fig. 148.-Ossification OF the Fibula. the large relative size which characterizes it throughout life.

\section{The Tarsus.}

The tarsus is composed of seven short bones, namely, the astragalus, os calcis, navicular or scaphoid, three cuneiforms, and cuboid.

The first two constitute the proximal row, the astragalus lying above the os calcis, and the last four comprise the distal row, the order from the inner or tibial to the outer or fibular side being internal, middle, and external cuneiform bones, and cuboid. The navicular occupies an intermediate position. 


\section{The Asiragalus.}

The astragalus, or talus, is characterized by having a head, neck, and body. 'It is situated between the tibia above and the os calcis below, is grasped laterally by the tibial and fibular malleoli, and has the navicular in front. It is the only tarsal bone which receives directly the weight of the body, and it lies with its long axis directed forwards and inwards. In point of size it comes next to the os calcis.

The head forms the anterior part of the bone, and presents an extensive convex articular surface, which looks forwards and also downwards. It is divided into three facets, called navicular, sustentacular, and 'spring.' The navicular facet, which is placed on the anterior surface, is pyriform, and its long axis is directed downwards and inwards. The sustentacular facet, continuous with the foregoing, is situated on the inferior surface. It is convex and elliptical, and its long axis is directed forwards and outwards. It is often crossed by an elevated ridge a little anterior to the centre, and it articulates with the sustentacular facet on the upper surface of the os calcis. The spring facet is situated on the inner aspect of the inferior surface, and is in contact with the superior surface of the inferior calcaneo-navicular or 'spring' ligament.

The neck is the constricted part behind the head. It is conspicuous superiorly, and passes inferiorly into the interosseous groove. This groove is directed forwards and outwards, its inner part being narrow and deep, and the outer wide and shallow. It gives attachment to the strong interosseous ligament which binds the astragalus to the os calcis. The neck is perforated all round with numerous nutrient foramina for offsets of the dorsalis pedis artery and its tarsal branch.

The body is quadrilateral, and presents four surfaces and a posterior border. The superior surface presents an extensive trochlear facet, which is concave from side to side, and convex from before backwards. Posteriorly it slopes downwards, and in this situation it usually presents a transverse groove for the play of the transverse ligament. The inner border is straight and slightly depressed, and as a rule it extends rather farther back than the outer, which latter is somewhat sinuous. The surface is broader in front than behind. The external surface is deep, and presents a large triangular facet for the external malleolus, the apex being downwards. It is concave from above downwards, and, immediately in front of it, the anterior fasciculus of the external lateral ligament of the ankle-joint takes attachment. The internal surface presents superiorly a falciform facet, broad in front and pointed behind, for the internal malleolus. This facet in the fœtus encroaches on the inner side of the neck, a condition which is associated with the inversion of the foot at that period of life. This sometimes occurs in the adult, and, if it does so to any marked extent, it usually accompanies the condition known as talipes varus. 
The inferior surface presents a large oval facet, concave from within forwards and outwards, for articulation with the os calcis. The posterior border is short, stout, and oblique, its direction being inwards and forwards. It presents a groove, which is directed down-
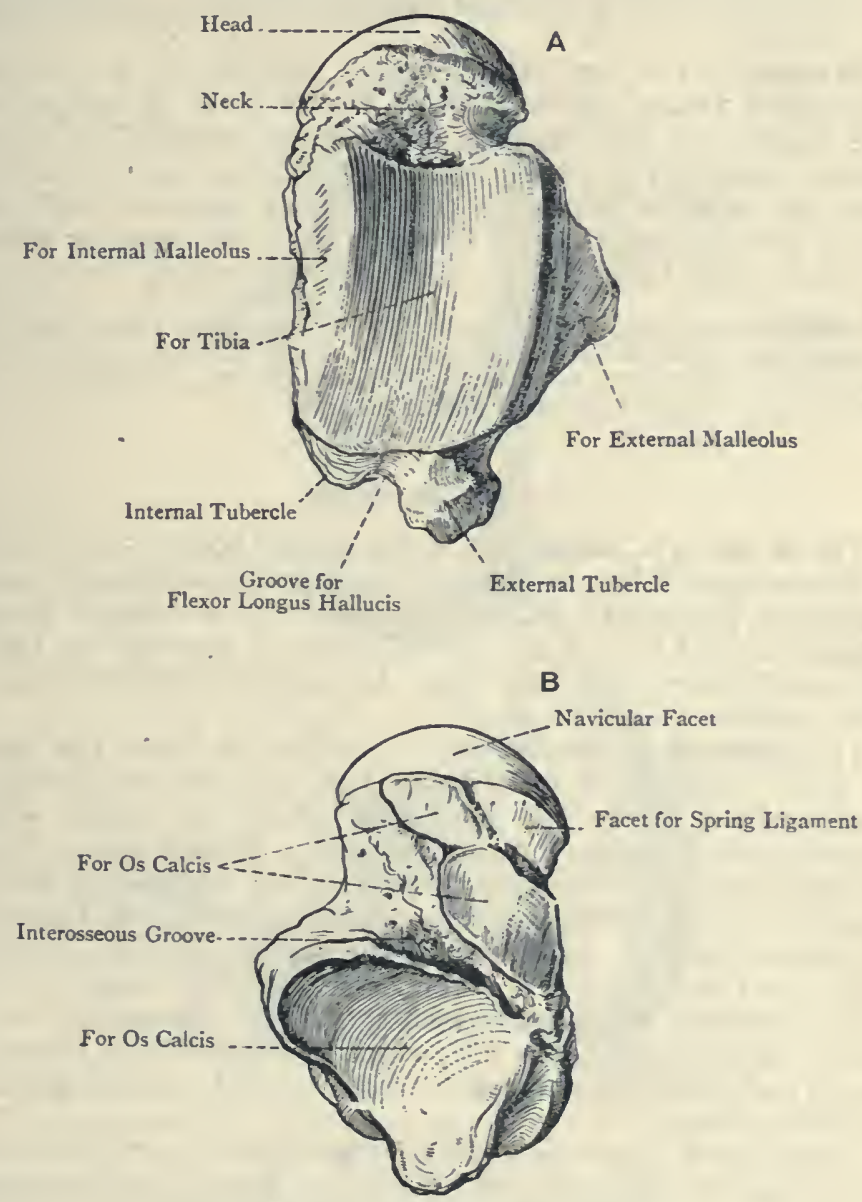

Fig. 149.-The Right Astragalus.

A, Superior View ; B, Inferior View.

wards and inwards, for the tendon of the flexor longus hallucis. On either side of this groove there is a tubercle, the internal being rudimentary, whilst the external is well developed and gives attachment superiorly to the posterior fasciculus of the external lateral ligament of the ankle-joint. 
The astragalus derives its blood-supply from branches of the dorsalis pedis artery.

Articulations.-Superiorly with the shaft, and internally with the internal malleolus, of the tibia; externally with the external malleolus of the fibula; inferiorly with the os calcis; and anteriorly with the navicular, and occasionally with the lower and inner angle of the cuboid.

Structure.-The astragalus, being a short bone, is composed of cancellated tissue, surrounded by a thin shell of compact bone. The lamellæ of the cancellated tissue are arranged in a curved manner, and in two sets. Some pass downwards and backwards from the superior surface to the posterior calcaneal facet, whilst others arch downwards and forwards from the superior surface to the neck, these being the directions in which weight is transmitted.

Varieties.-(I) The external tubercle on the posterior border may form a separate ossicle, called the os trigonum. (2) There may be a pressure facet on the upper surface of the neck at its outer part, due to prolonged contact with the anterior margin of the lower end of the tibia.

\section{The Os Calcis.}

The os calcis, or calcaneum, is the largest bone of the tarsus, and is characterized by its elongation, lateral compression, and enlargement posteriorly into a tuberosity. It is situated below the astragalus, and behind the cuboid, where it lies with its long axis directed forwards and outwards. It presents two extremities and four surfaces.

The posterior extremity, which is enlarged, forms the tuberosity or tuber calcis, and constitutes the prominence of the heel. Posteriorly it is divided into three zones-an upper, which is smooth and separated from the tendo Achillis by a bursa; a middle, rough and vertically ridged, for the insertion of the tendo Achillis; and a lower, which is continuous with the tubercles on the plantar aspect, and supports the fat of the heel. In front of the tuber calcis there is a constriction, called the neck.

The anterior extremity presents a large, somewhat triangular facet, narrow towards the sole, which is concave from above downwards and outwards, and convex from side to side, for articulation with the cuboid.

The superior surface presents over its anterior part two facets for the astragalus, separated by an oblique groove, and posteriorly a non-articular surface. The antero-internal or sustentacular facet surmounts the sustentaculum tali. It is concave and somewhat elliptical, its long axis being directed forwards and outwards. It is constricted in front of the centre, and is sometimes broken up into two facets by a rough groove. The postero-external facet is large, oval, and convex from behind forwards and outwards. The intervening groove, which is directed forwards and outwards, becomes wide and shallow externally, and in front of the outer 
part of the groove the upper surface gives origin to a portion of the extensor brevis digitorum, and the fundiform ligament of Retzius. When the astragalus is in position this groove is converted into a short tunnel, called the sinus tarsi, which is occupied by the interosseous ligament. The superior surface behind the articular portion is rough, and supports a collection of fat.
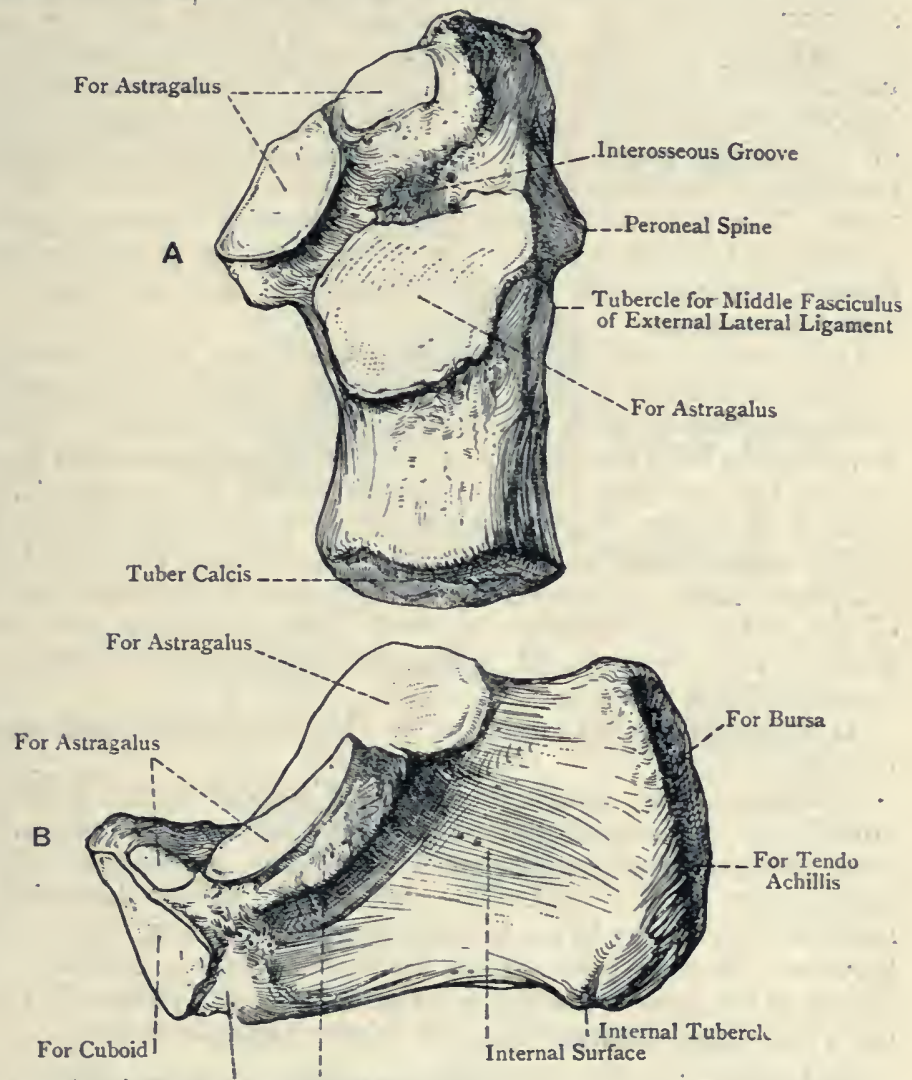

Anterior Tubercle Sustentaculum Tali, with Groove below it for Flexor Longus Hallucis

Fig. 150.-The Right Os Calcis.

A, Superior View; B, Internal View.

The inferior surface is narrow and rough. Posteriorly it presents two tubercles, the outer of which is small but prominent, whilst the inner is large and blunt. The outer tubercle gives attachment to the external division of the plantar fascia, and a portion of the abductor minimi digiti, whilst the inner gives attachment to the central and internal divisions of the plantar fascia, 
the outer head of the abductor hallucis, the flexor brevis digitorum, and a portion of the abductor minimi digiti. The greater part of the inferior surface gives attachment to the long plantar ligament, and anteriorly it presents a small round eminence, called the anterior tubercle, to which the short plantar ligament is attached.

The internal surface is concave, and is overhung at its anterosuperior part by the sustentaculum tali. This latter is concave and articular above for the astragalus, and below it presents a groove for the flexor longus hallucis. Anteriorly it gives attachment to the inferior calcaneo-navicular or ' spring' ligament, below which a slip of the tibialis posticus takes insertion, and its inner margin gives attachment to fibres of the internal lateral ligament of the anklejoint. The general concavity of the internal surface supports the tendon of the flexor longus digitorum and the plantar vessels and nerves, and anteriorly it affords origin to the inner head of the flexor accessorius.

The external surface is for the most part flat. Towards its anterior and lower part it presents a short oblique ridge, called the peroneal spine or ridge, which separates two grooves. The upper groove transmits the tendon of the peroneus brevis, and the lower that of the peroneus longus. Behind and a little above this spine there is a small tubercle, about the centre of the surface, for the middle fasciculus of the external lateral ligament of the ankle-joint.

The os calcis is pierced by many nutrient foramina for offsets of the calcaneal branches of the posterior tibial and external plantar, and the internal and external malleolar branches of the anterior tibial, arteries.

Articulations.-Superiorly with the astragalus, and anteriorly with the cuboid.

Structure. - The structure is that of a short bone. Some of the lamellæ of the cancellated tissue arch downwards and backwards from the large postero-external facet on the superior surface to the prominence of the heel. In addition to these, there are other lamellæ which pass in an antero-posterior direction just above the layer of compact bone which forms the plantar surface. In the region of the groove for the sinus pedis, especially towards the outer part, the upper compact layer is thicker than elsewhere.

Ossiflcation.- The os calcis ossifies in cartilage from one primary, and one secondary, centre. The primary centre appears in the sixth month of intrauterine life. The secondary centre appears in the tenth year, and forms a thin epiphysial scale over the posterior surface of the tuber calcis, which joins in the sixteenth year. This epiphysis includes the outer, and a large part of the inver, tubercle on the under surface, and it may include the whole of the posterior surface, or only the lower two-thirds.

\section{The Navicular Bone.}

The navicular or scaphoid bone is distinguished by its resemblance to a boat. It is situated on the inner side of the foot, where it is placed in front of the astragalus, and belind the three cuneiform 
bones. It is compressed from before backwards, and its long axis is directed inwards and downwards. The anterior surface presents a large convex articular surface, divided into three facets by two ridges which converge inferiorly. The inner facet, for the internal cuneiform, is pyriform, with the narrow end upwards. The middle facet, for the middle cuneiform, is triangular, with the truncated apex downwards. The outer facet, for the external cuneiform, resembles the middle, except that it is rather shorter and has a rounder apex. The posterior surface is characterized by a large concave, pyriform facet for the front of the head of the astragalus, its narrow end being directed downwards and inwards. The

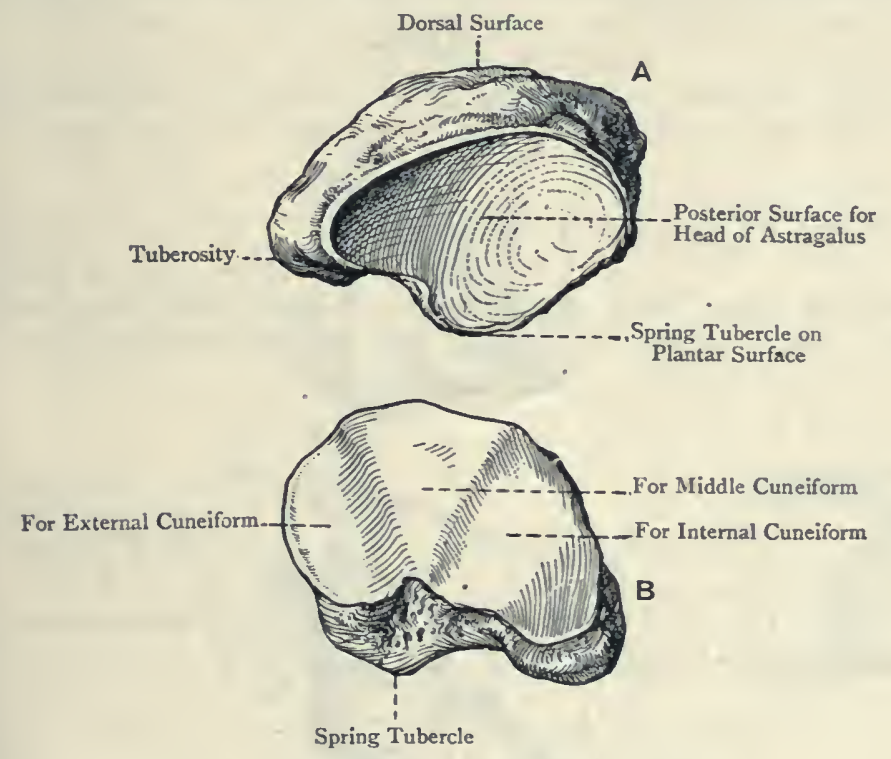

Fig. 151.-The Right Navicular Bone.

A, Postero-superior View; B, Antero-inferior View.

dorsal surface, extensive and rough, is sloped downwards and inwards. The plantar surface, narrow and rough, gives attachment to the inferior calcaneo-navicular or 'spring' ligament, and about its centre there is usually a knob-like projection, called the spring tubercle. The external surface is broad and rough, and it sometimes presents a small facet for the cuboid, contiguous to the outer facet on the anterior surface. The inner extremity (prow of the boat) is inclined downwards, and forms a stout, round projection on the inner side of the sole, called the tuberosity, which gives insertion to the principal portion of the tendon of the tibialis posticus. 
Articulations.-Posteriorly with the astragalus, anteriorly with the three cuneiform bones, and sometimes with the cuboid externally.

Structure.-The structure is that of a short bone.

Variety. - The tuberosity sometimes forms a separate ossicle.

\section{The Cuneiform Bones.}

The cuneiform bones are three in number, namely, internal, middle, and external. They are situated between the navicular and the inner three metatarsal bones, and are characterized by their
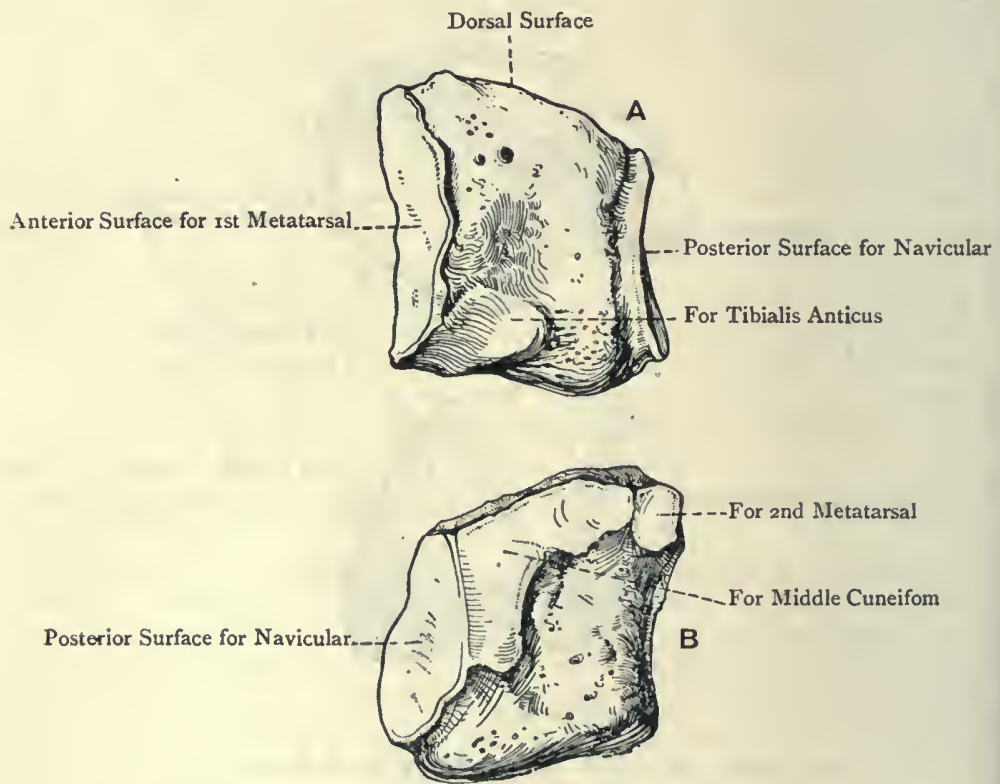

Fig. 152.-The Right Internal Cuneiform Bone.

A, Internal View ; B, External View.

wedge shape. The internal cuneiform is the largest, and the middle is the smallest.

The internal cuneiform bone is situated on the inner side of the foot, where it lies with the narrow end of the wedge upwards, and it supports the first metatarsal. The dorsal surface is narrow and rough. The plantar surface is thick and convex, and posteriorly it presents an eminence for a slip of the tendon of the-tibialis posticus. The internal siorface is traversed by an oblique groore, directed downwards and forwards, for the tendon of the tibialis 
anticus, the principal portion of which is inserted into an impression situated at the lower part of the groove. The external surface presents, close to its superior and posterior borders, an L-shaped facet for the middle cuneiform, at the anterior extremity of which there is a small facet for the inner side of the base of the second metatarsal. When the bone is held in its proper position, the $L$ is placed thus $\Gamma$ in a right bone, and thus 7 in a left. The rest of the surface is concave and rough for strong ligaments, except at the lower and anterior part, where it gives insertion to a slip of the tendon of the peroneus longus. The anterior surface is deep, and presents a convex reniform facet for the first metatarsal, the concave border being directed outwards. The posterior surface, much smaller than the anterior, is characterized by a concave pyriform facet for the navicular, the narrow end being upwards.

Articulations.-Posteriorly with the navicular, anteriorly with the first metatarsal, and externally with the middle cuneiform and second metatarsal.

Variety.-The internal cuneiform may be divided into two parts, dorsal and plantar.

The middle cuneiform bone lies with the broad end of the wedge upwards, and it supports the second. metatarsal. The dorsal

A

Dorsal Surface

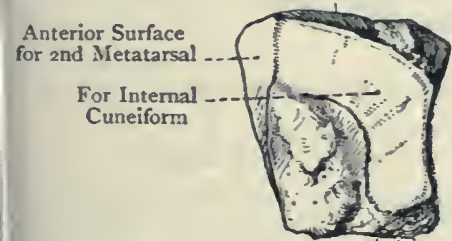

B

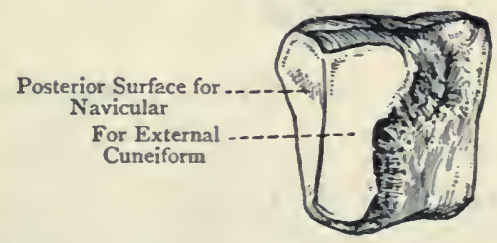

Fig. I53.-The Right Midde Cuneiform Bone.

A, Internal View ; B, External View.

surface is rough and nearly square. The plantar surface, also rough, is narrow, and gives insertion to a slip of the tendon of the tibialis posticus. The internal surface presents, close to its superior and posterior borders, an L-shaped facet for the internal cuneiform, placed thus 7 for a right bone, and thus $\Gamma$ for a left, the remainder of the surface being rough and ligamentous. The external surface has a rertical facet posteriorly for the external cuneiform, and elsewhere it is rough and ligamentous. The anterior and posterior surfaces are triangular and covered by cartilage, the former articulating with the second metatarsal, and the latter with the navicular. They are distinguished from each other in the following manner: the anterior surface is convex, whilst the posterior is concave; the apex of the anterior surface is more pointed than that of the pos- 
terior ; and the posterior surface is rather broader than the anterior, and has one of the limbs of the $L$ facet close to it.

Articulations.-Posteriorly with the navicular, anteriorly with the second metatarsal, internally with the internal cuneiform, and externally with the external cuneiform.

The external cuneiform bone, like the middle, lies with the broad end of the wedge upwards, and it supports the third metatarsal. The dorsal surface is rough, quadrilateral, and elongated from before backwards. The plantar surface, also rough, is narrow, and gives insertion to a slip of the tendon of the tibialis posticus. The internal surface presents a vertical facet posteriorly for the middle cuneiform, and two semi-oval facets anteriorly for articulation with the proximal pair of facets on the outer side of the base of the second metatarsal. The remainder of the surface is rough and ligamentous. The external surface has a large, almost circular, facet near the postero-superior angle for the cuboid, and there may be a

A

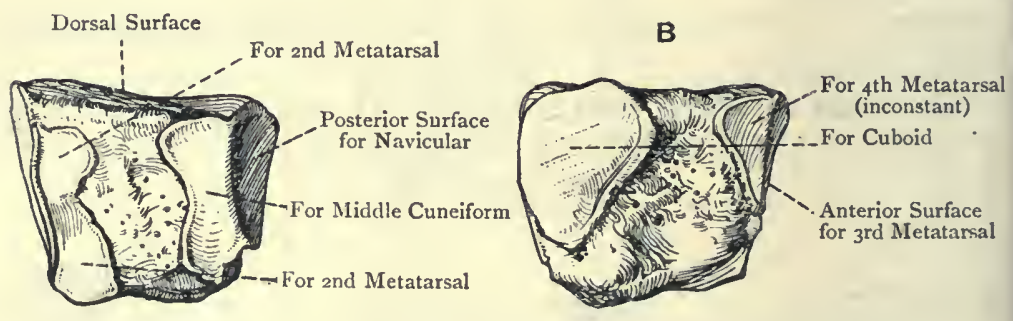

Fig. I 54.-The Right External Cuneiform Bone.

A, Internal View ; B, External View.

small semi-oval facet at the antero-superior angle for the inner side of the base of the fourth metatarsal, but this facet is not constant. Elsewhere the surface is rough and ligamentous. The anterior and posterior surfaces are triangular, and covered by cartilage, the former articulating with the third metatarsal, and the latter with the navicular. They are distinguished from each other in the following manne: : the anterior facet is deeper than the posterior, and its apex is more pointed; the cartilage of the anterior surface extends over its entire length, but the lower part of the posterior surface is non-articular; the anterior facet is slightly concavoconvex from below upwards, but the posterior is concave, and it has the large, almost circular, facet on the external surface contiguous to it.

Articulations.-Posteriorly with the navicular, anteriorly with the third metatarsal, internally with the middle cuneiform and outer side of the base of the second metatarsal, and externally with the cuboid, and, it may be, with the inner side of the base of the fourth metatarsal. 
Structure of the Cuneiform Bones.-The structure of each is that of a short bone.

When the cuneiform bones are in position their posterior surfaces are on the same transverse plane, but the anterior surfaces of the

\section{DORSAL ASPECT}

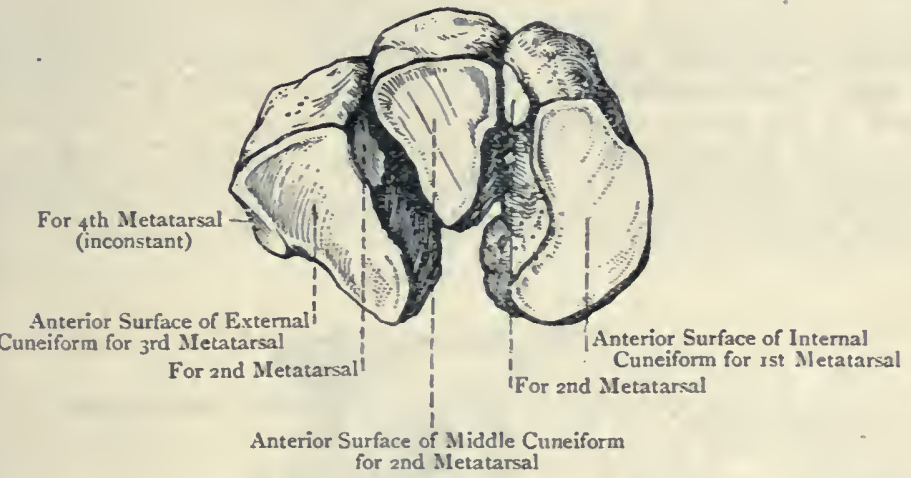

Fig. I55.-The Right.Cuneiform Gones (ANtero-superior View).

internal and external project farther forwards than that of the middle. In this manner a recess is formed, into which the base of the second metatarsal bone is received.

\section{The Cuboid Bone.}

The cuboid bone is characterized by its irregularly cubical shape, and by the groove and ridge on its plantar aspect. It is situated on the outer border of the foot, where it lies between the os calcis and the fourth and fifth metatarsal bones. The anterior surface has its cartilage divided by a vertical ridge into two facets-an inner quadrilateral for the fourth metatarsal, and an outer triangular for the fifth metatarsal. The posterior surface presents a large, somewhat triangular, facet, narrow towards the sole and deep internally, which is convex from above downwards and outwards, and concave from side to side. It articulates with the os calcis, and its internal and inferior angle, called the calcaneal process, projects backwards for a little beneath that bone. Below and inside the calcaneal process there may be a facet for the head of the astragalus. The internal surface, which is extensive and vertically disposed, presents a large, almost circular, facet for the external cuneiform, near the centre and extending to the dorsal surface. Behind this, and usually continuous with it, there may be a small facet for the navicular, the remainder of the surface being rough and ligamentous. The external surface, which is really a 
border, is very short and narrow, and presents a notch leading to the peroneal groove on the plantar surface. The dorsal surface is rough, and is directed upwards and outwards. The plantar surface presents in front the deep peroneal groove, which is directed inwards and forwards, and lodges the tendon of the peroneus longus. Behind the groove is a stout, oblique ridge for the long plantar ligament. This ridge becomes enlarged externally into a tubercle, which is covered by cartilage on its anterior and outer aspects for the play of the sesamoid cartilage, or bone, usually present in the tendon of the peroneus longus. The surface behind the ridge gives attachment to the short plantar ligament, a slip of the tendon of the tibialis posticus, and some fibres of the flexor brevis hallucis, but the latter may spring from the internal surface.
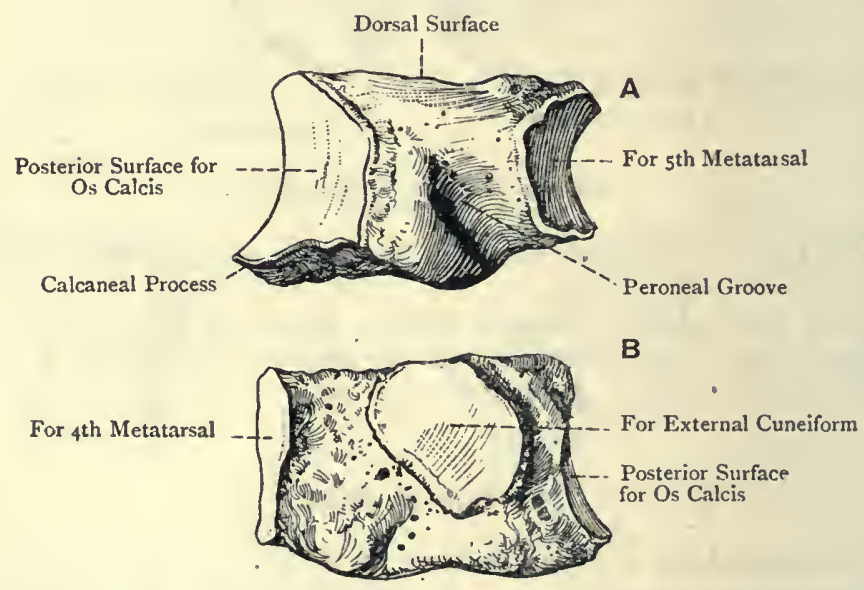

Fig. i.56. - The Right Cuboid Bone.

A, External View ; B, Internal View.

Articulations.-Posteriorly with the os çalcis, anteriorly with the fourth and fifth metatarsal bones, internally with the external cuneiform, and sometimes with the navicular, and at the lower and inner angle occasionally with the astragalus.

Structure.-The structure is that of a short bone.

The tarsus as a whole is convex superiorly, and concave inferiorly, from before backwards as well as from side to side. The part in front of the astragalus and os calcis constitutes the instep, and the entire tarsus forms two columns - an inner, comprising the astragalus, navicular, and three cuneiform bones, and an outer, representing the os calcis and cuboid.

Varieties. - The number of tarsal bones is sometimes increased to eight, which is brought about in one or other of the following ways: (I) the external tubercle on the posterior border of the astragalus may form a separate ossicle. called the os trigonum: (2) the tuberosity of the navicular may form a 
ssparate ossicle: (3) the internal cuneiform may be divided into two parts, dorsal and plantar; or (4) there may be an additional ossicle in the space at the antero-internal part of the os calcis, or between the internal cuneiform and the second metatarsal.

Ossification.-The tarsal bones ossify in cartilage, each from one centre, (except the os calcis, which has one primary, and one secondary, centre), and at the following periods approximately:

Os calcis, 6 th month (intra-uterine).

Astragalus, 7 th month (intra-uterine).

Cuboid, 9 th month (intra-uterine).

\section{Navicuiar, 4 th year.}

For the secondary centre of the os calcis, see page 258 . The external tubercle on the posterior border of the astragalus has sometimes a secondary centre, and then it remains separate as the os trigonum.

\section{The Metatarsus.}

The metatarsus is composed of five long bones, which are named numerically from within outwards, that of the great toe being the first. Each bone is divisible into a shaft and two extremities, proximal and distal. The shaft, which is triangular, is massive in the first, slender and much compressed laterally in the second, third, and fourth, and compressed from above downwards in the fifth. Each shaft, except that of the first, is longitudinally convex on its dorsal aspect, and they are all longitudinally concave on their plantar aspects. The shaft presents three borders and three surfaces. In the outer four bones the borders are two lateral, and a plantar. The lateral borders, external and internal, extend from the sides of the proximal end or base, close to the dorsal aspect, to the dorsal tubercle on either side of the distal end or head, and their outline is sharp. The plantar border, round behind, but sharp in front, extends from the centre of the plantar aspect of the base forwards in the middle line to near the head, where it bifurcates, the divisions passing to the cornua on the plantar aspect of the head. The dorsal surface lies between the external and internal borders, and is narrow. Each lateral surface is situated between the lateral and plantar borders. The lateral surfaces, which are extensive and sloped, bound the interosseous spaces, and give attachment to the interosseous muscles. The shaft of the first metatarsal has its borders disposed as supero-external, inferoexternal, and internal. The dorsal surface is convex, and is directed upwards and inwards. The plantar surface is concave, and supports the tendon of the flexor longus, and the flexor brevis hallucis. The external surface, which is practically vertical, is narrow in front, but wide behind.

The heads of the four outer metatarsal bones are much compressed laterally. The cartilage is prolonged more on the plantar than on the dorsal aspect, and in the former situation it ends in a concave 
border, surmounted at either side by a prominent cormu. On either side the head presents a dorsal tubercle and plantar depression for the lateral metatarso-phalangeal ligament. The head of the first metatarsal is of large size, and elongated transversely. On its plantar aspect it presents two well-marked grooves, separated by a median antero-posterior ridge, for the sesamoid bones in the heads of insertion of the flexor brevis hallucis.

The bases of the metatarsal bones articulate with the tarsus and with each other, except, as a rule, in the case of the first, and they present distinctive characters in each case.

First Metatarsal Bone.-This supports the great toe, and is the thickest and most massive of the series. The base is of large size, and presents a concave reniform surface, with the concavity out-

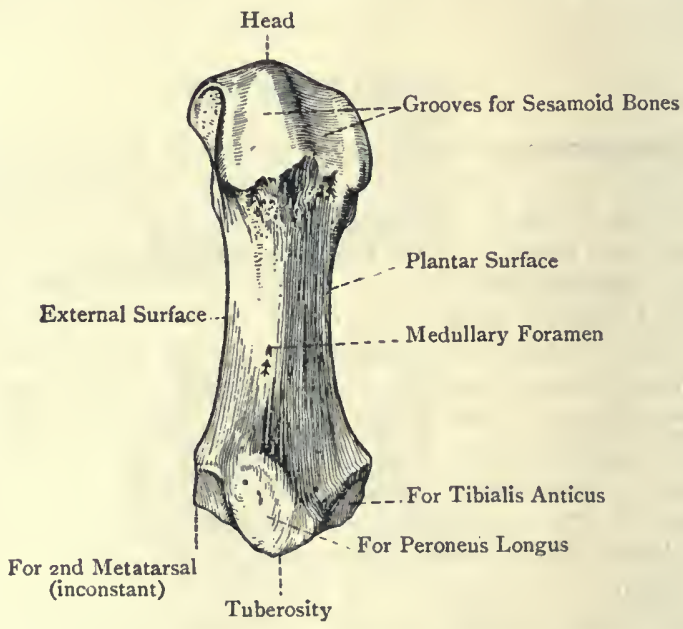

Fig. 157.-The First Right Metatarsal Bone (Plantar View).

wards, for the internal cuneiform. Inferiorly it presents a projection, called the tuberosity, which gives insertion, by its outer aspect, to the principal part of the tendon of the peroneus longus, and by its inner aspect to a slip of the tendon of the tibialis anticus. There is usually no facet on its outer surface, but sometimes it presents one for the second metatarsal, and it always gives origin to the inner head of the first dorsal interosseous.

Articulations.-Posteriorly with the internal cuneiform, and sometimes externally with the second metatarsal; anteriorly with the first phalanx of the great toe; and inferiorly with the two sesamoid bones.

Second Metatarsal Bone.-This supports the second toe. Its base is wedge-shaped, with the broad end upwards. It recedes between the internal and external cuneiform bones, and posteriorly 
presents a concave triangular facet for the middle cuneiform. On the inner side, close to the dorsal aspect, there is a small facet for the internal cuneiform, and sometimes there is an additional facet, below and in front of this, for the first metatarsal. The outer side presents two facets, dorsal and plantar, separated by a rough anteroposterior groove, each of these being subdivided by a vertical ridge into two semi-oval facets. There are thus four.facets in all-

A

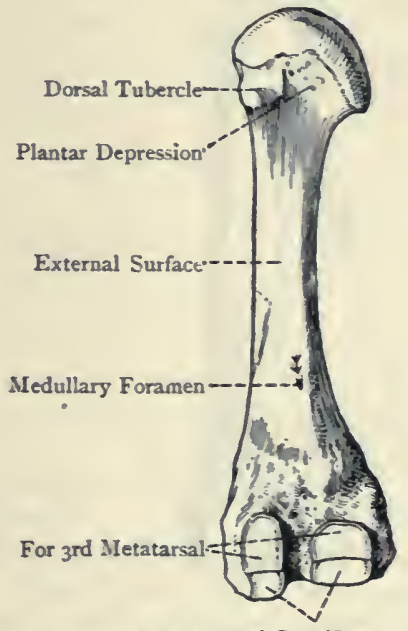

For External Cuneiform
B

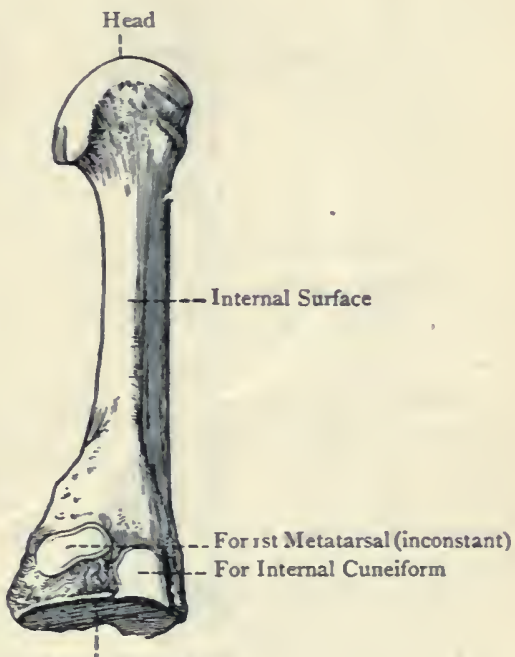

For Middle Cuneiform

Fig 158.-The Second Right Metatarsal Bone.

A, Externål View ; B, Internal View.

a posterior pair for the inner side of the external cuneiform, and an anterior pair for the inner side of the base of the third metatarsal. The plantar surface of the base gives insertion to a slip of the tendon of the tibialis posticus, and origin to a portion of the adductor obliquus hallucis. The shaft gives partial origin to the first and second dorsal interossei.

Articulations.-Posteriorly with the middle cuneiform, internally with the internal cuneiform, and sometimes with the first metatarsal, externally with the external cuneiform and third metatarsal, and anteriorly with the first phalanx of the second toe.

Third Metatarsal Bone.- This supports the third toe. The base resembles in shape that of the second, the broad end being upwards. Posteriorly it presents a triangular facet, concavo-convex from above downwards, for the external cuneiform. The inner side of 
the base presents two semi-oval facets, dorsal and plantar, separated by a rough antero-posterior groove, for the anterior pair of facets on the outer side of the base of the second metatarsal. On the outer side there is a large semi-oval facet, dorsally placed, for the inner side of the base of the fourth metatarsal. The plantar surface of the base gives insertion to a slip of the tendon of the tibialis posticus, and origin to a portion of the adductor

A

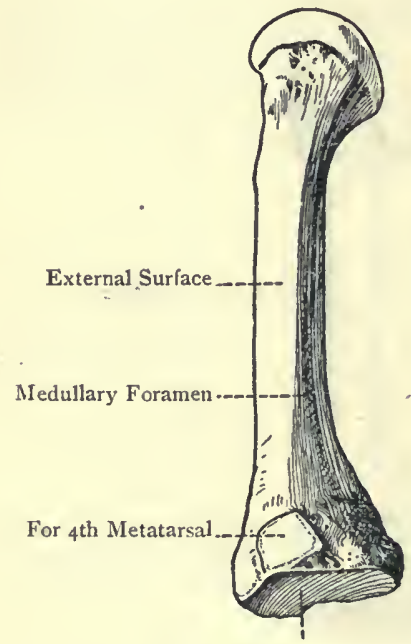

For External Cuneiform

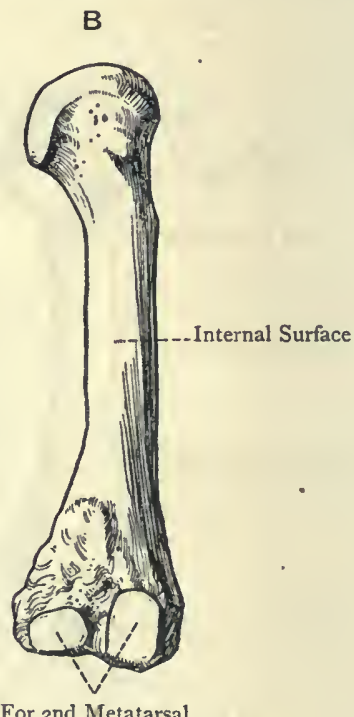

For 2nd Metatarsal

Fig. i59.-The Third Right Metatarsal Bone.

A, External View; B, Internal View.

obliquus hallucis. The shaft gives origin to the first plantar interosseous, and partial origin to the second and third dorsal interossei.

Articulations.-Posteriorly with the external cuneiform, internally with the second metatarsal, externally with the fourth metatarsal, and anteriorly with the first phalanx of the third toe.

Fourth Metatarsal Bone.-This supports the fourth toe. The base is quadrilateral, and is somewhat broader above than kelow. Posteriorly it presents a quadrilateral facet for the cuboid. On the inner side there is a large semi-oval facet for the third metatarsal, and this is sometimes prolonged to the extremity of the base, thus forming an additional facet for the outer side of the external cuneiform. On the outer side there is a large semi-oval facet, dorsally placed, for the inner side of the base of the fiftl metatarsal, and below this there is a deep rough groove. The plantar surface of the base gives insertion to a slip of the tendon 
of the tibialis posticus, and origin to a portion of the adductor obliquus hallucis. The shaft gives origin to the second plantar

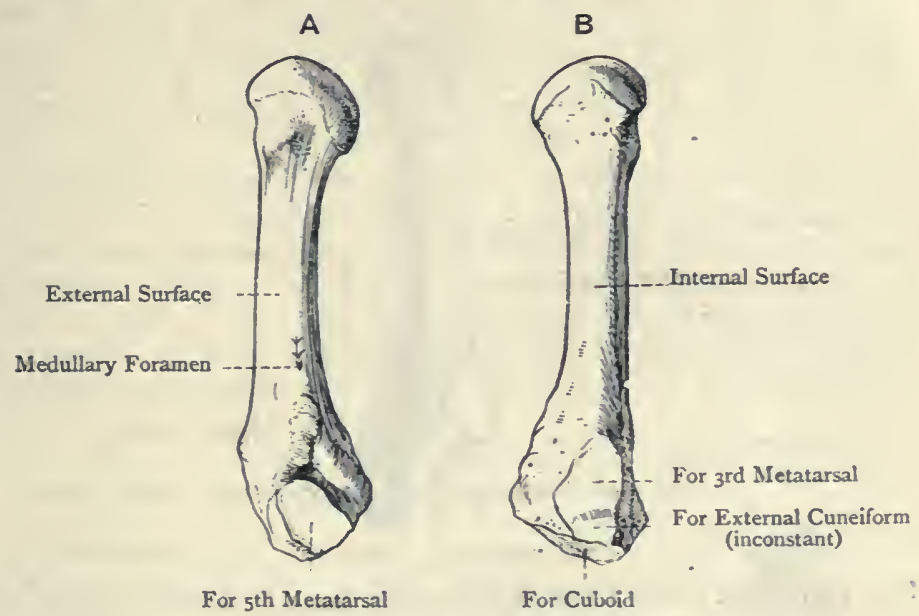

Fig. 160.-The Fourth Right Metatarsal Bone.

A, External view; B, Internal view.

interosseous, and partial origin to the third and fourth dorsal interossei.

Articulations.-Posteriorly with the cuboid, internally with the third metatarsal, and sometimes with the external cuneiform, externally with the fifth metatarsal, and anteriorly with the first phalanx of the fourth toe.

Fifth Metatarsal Bone.-This supports the little toe. The base is elongated from side to side, and compressed from above downwards. Its leading characteristic is a stout, mammillary process, situated on its outer aspect, called the tuberosity, which is directed outwards and backwards, and gives insertion to the tendon of the peroneus brevis. The posterior surface presents a triangular facet for the cuboid, the plane of which is inclined inwards and forwards. This facet does not encroach upon the tuberosity. The inner surface presents a large semi-oval facet for the outer side of the base of the fourth metatarsal. The dorsal surface, which is rough and slightly convex, gives insertion, as a rule, to the tendon of the peroneus tertius. The plantar surface, which is rough and concave, gives origin to the flexor brevis minimi digiti. The shaft gives origin to the third plantar interosseous, and partial origin to the fourth dorsal interosseous.

Articulations.-Posteriorly with the cuboid, internally with the fourth metatarsal, and anteriorly with the first phalanx of the little toe.

Each metatarsal bone presents a medullary foramen, that of 
the first and second, and usually that of the third and fourth, being situated on the outer side of each shaft, whilst that of

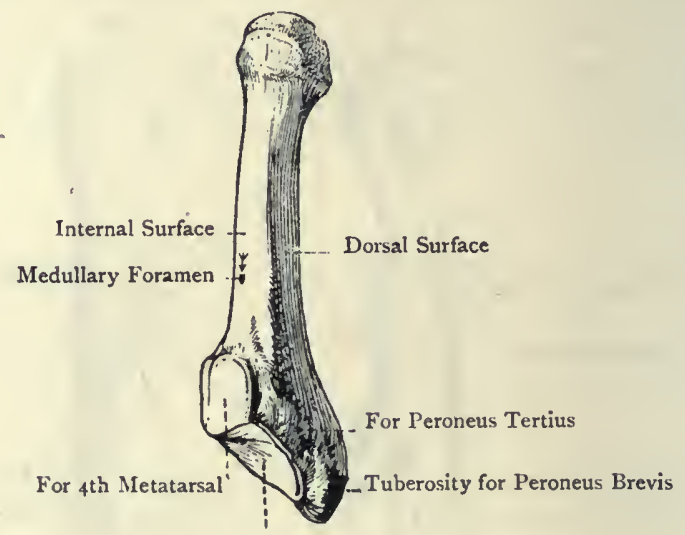

For Cuboid

Fig. i6i.-The Fifth Right Metatarsal Bone (Supero-internal View).

the fifth is situated on the inner side.* The foramen of the first and the canal to which it leads are directed dorenwards towards the head or distal end, but that of each of the other four is directed

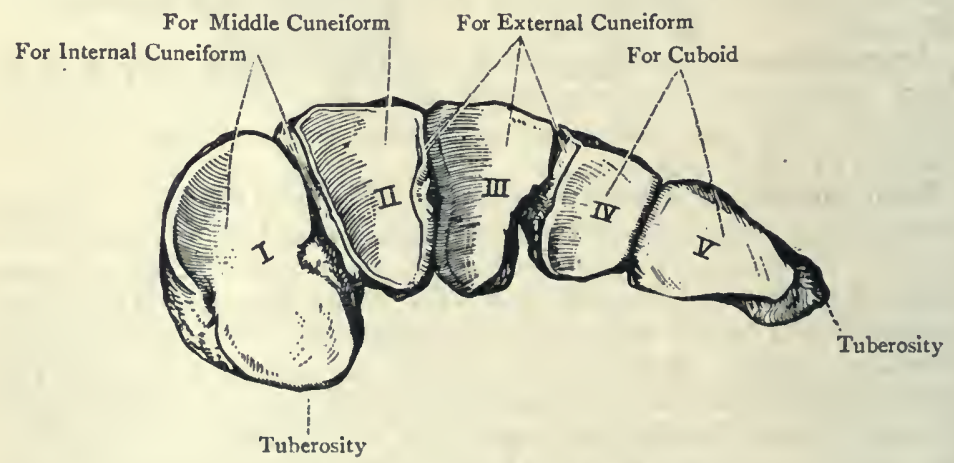

Fig. I62.-The Bases of the Right Metatarsal Bones (Posterior View).

upwards towards the base or proximal end. The first metatarsal receives its nutrient artery from the arteria magna or princeps hallucis, the second from the fourth digital artery, the third usually

* Of roo third, and an equal number of fourth, metatarsal bones examined, 73 third metatarsals had the medullary foramen on the outer side, and 27 on the inner side; and 60 fourth metatarsals had it on the outer side, and 40 on the inner side. 
from the third digital, and the fourth and fifth from the serond digital artery.

Structure. - The structure is that of a long bone.

Varieties.-(1) The tuberosity on the outer side of the base of the fifth metatarsal, or the tuberosity on the plantar surface of the base of the first, may form a separate ossicle. (2) An additional ossicle is sometimes met with between the bases of the first and second metatarsals.

The metatarsus as a whole is convex on its dorsal aspect from side to side, and also longitudinally. The transverse convexity is due to the broad ends of the bases of the second, third, and fourth metatarsals being directed upwards. On its plantar aspect it is concave from side to side, and also longitudinally. All five bones are nearly parallel with each other, being slightly divergent in front. The interosseous spaces are as in the hand, the first being the innermost.

\section{The Phalanges.}

The phalanges are fourteen in numberthree to each of the four outer toes, and two to the great toe. The toes, from within outwards, are called great toe or hallux, second, third, fourth, and fifth or little toe. In their general characters the phalanges so closely resemble those of the hand that a detailed description is unnecessary. The phalanges of the great toe, called proximal and distal, are characterized by their large size and great length, which, with the length of the first metatarsal bone, places. the great toe on a level with those next it. The first phalanx of the four outer toes is characterized by being slender, and much compressed from side to side over its shaft, the proximal end being of large size, and almost triangular. The second phalanx is short, and compressed from above downwards. The ungual phalanx is very small.

Special Muscular Attachments.-The base of the proximal phalanx of the great toe, which presents a fubercular enlargement at either side, gives insertion

internally to the abductor hallucis and inner head of the flexor brevis hallucis ; externally, to the outer head of the flexor brevis hallucis, adductor obliquus hallucis, and adductor transversus hallucis; and on its dorsal surface there is a rough transverse ridge for the innermost tendon of the extensor brevis digitorum. The base of the ungual phalanx of the great toe gives insertion, on its dorsal surface, to the extensor proprius hallucis, and, on its plantar surface, to the flexor longus hallucis. The base of the first phalanx of the second toe gives partial insertion internally to the first dorsal interosseous, and externally to the second dorsal interosseous. The base of the first phalanx of the third toe gives partial insertion internally to the first plantar interosseous, and externally to the third dorsal interosseous. The base of the first phalanx of 
the fourth toc gives partial insertion internally to the second plantar interosseous, and externally to the fourth dorsal interosseous. The base of the first phalanx of the fifth toe gives partial insertion internally to the third plantar interosseous, and externally insertion to the abductor minimi digiti and flexor brevis minimi digiti. The second and ungual phalanges of each of the four outer toes give insertion to extensor and flexor tendons, as in the case of the corresponding bones of the four inner fingers.

The medullary foramen and the canal to which it leads are, in each phalanx, directed towards the distal end. The nutrient arteries are derived from the corresponding plantar digital arteries.

Structure.-The structure of each phalanx resembles that of the corresponding bone in the hand.

Varieties.-Ankylosis of the ungual and second phalanges of the fifth toe is of frequent occurrence, and may even involve those of other toes, up to and including the second.

Sesamoid Bones.-These are two in number, and are of large size. They are associated with the two heads of insertion of the flexor brevis hallucis, and lie on the plantar aspect of the head of the first metatarsal bone.

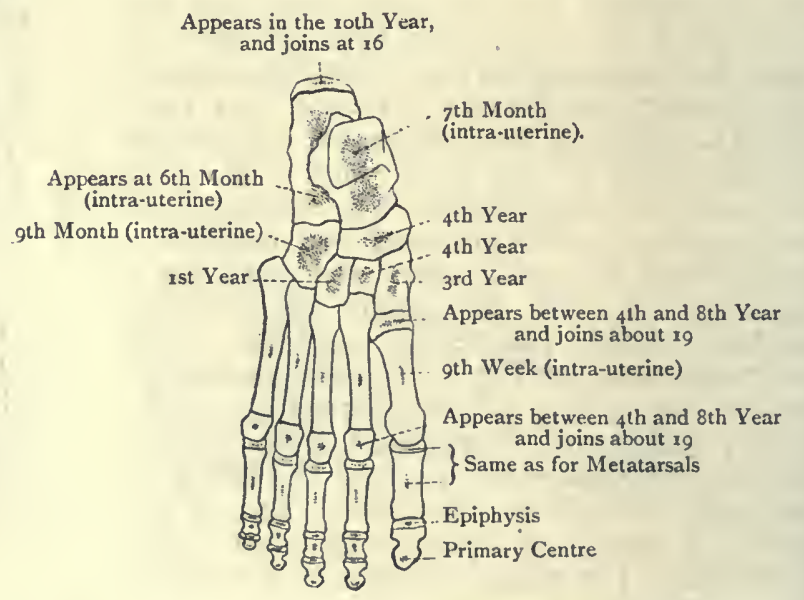

Fig. I64.-OSSIFICATION OF THE BONES OF THE FOOT.

Ossification of Metatarsal Bones and Phalanges.-Each bone ossifies in cartilage from one primary, and one secondary, centre, which closely agree with those of the corresponding bones of the hand in their disposition. The primary centres for the shafts appear about the ninth week of intra-uterine life, whilst the secondary centres appear between the fourth and eighth year. Each epiphysis joins its shaft about the ninetcenth year. 


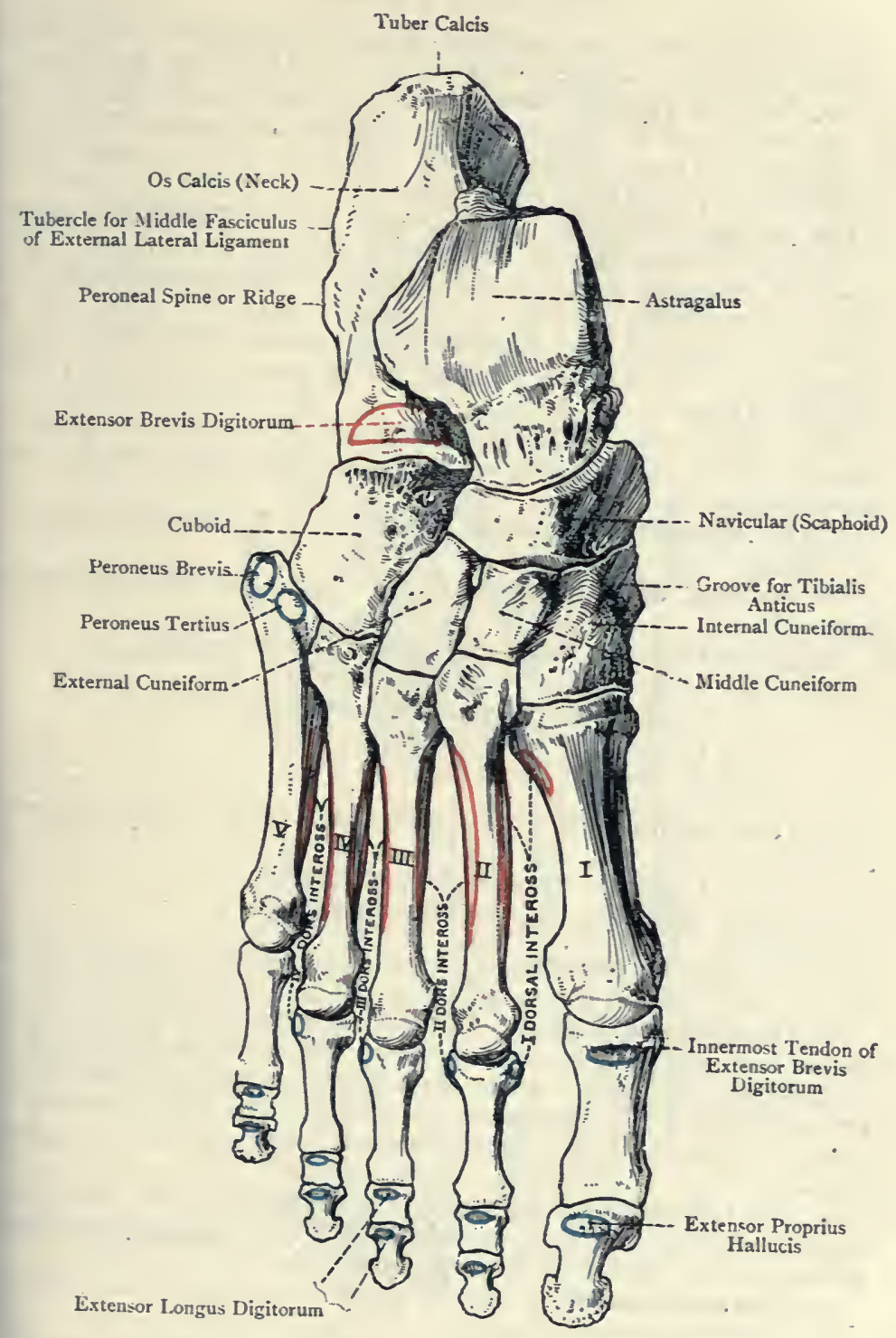

Fig. 16.5. -The Right Foot (Dorsal Surface). 


\section{The Foot as a Whole.}

The foot presents two surfaces--dorsal and plantar-two borders -inner and outer-and two extremities-anterior and posterior.

The dorsal or superior surface is arched, both longitudinally and transversely, and the superior surface of the astragalus constitutes its summit. The astragalus is the only bone of the tarsus which articulates with the tibia and fibula.

The plantar surface is concave, both longitudinally and transversely, in conformity with the longitudinal and transverse arches. When an articulated foot is placed upon a table, with the plantar surface downwards, the parts in contact with the table are as follows: Posteriorly, the internal and external tubercles on the plantar aspect of the tuber calcis, and anteriorly the heads of the metatarsal bones.

The plantar surface presents important projections and grooves, which will be enumerated, as nearly as possible, in order from behind forwards.

I. The internal and external tubercles on the plantar aspect of the tuber calcis.

The internal tubercle gives attachment to the following structures:

(I) Internal division of plantar fascia (part of).

(2) Central division of plantar fascia.

(3) Outer head of abductor hallucis (part of).

(4) Flexor brevis digitorum (part of).

(5) Abductor minimi digiti (part of):

The external tuberele gives attachment to the following structures:

(I) Outer division of plantar fascia.

(2) Abductor minimi digiti (part of).

2. The anterior tubercle of the os calcis, which gives attachment to the short plantar ligament.

3. The sustentaculum tali of the os calcis, close to the inner border of the foot, which is grooved inferiorly for the tendon of the flexor longus hallucis, the groove being continuous with that on the posterior border of the astragalus. Anteriorly, the sustentaculum tali gives attachment to the inferior calcaneo-navicular or spring ligament.

4. The tuberosity of the scaphoid or navicular bone, close to the inner border of the foot, which gives insertion to the principal portion of the tendon of the tibialis posticus.

5. The spring tuberele on the plantar surface of the navicular bone, for the spring ligament.

6. The eminence on the plantar surface of the internal cuneiform. bone, close to the inner border of the foot, for a slip of the tendor of the tibialis posticus. 


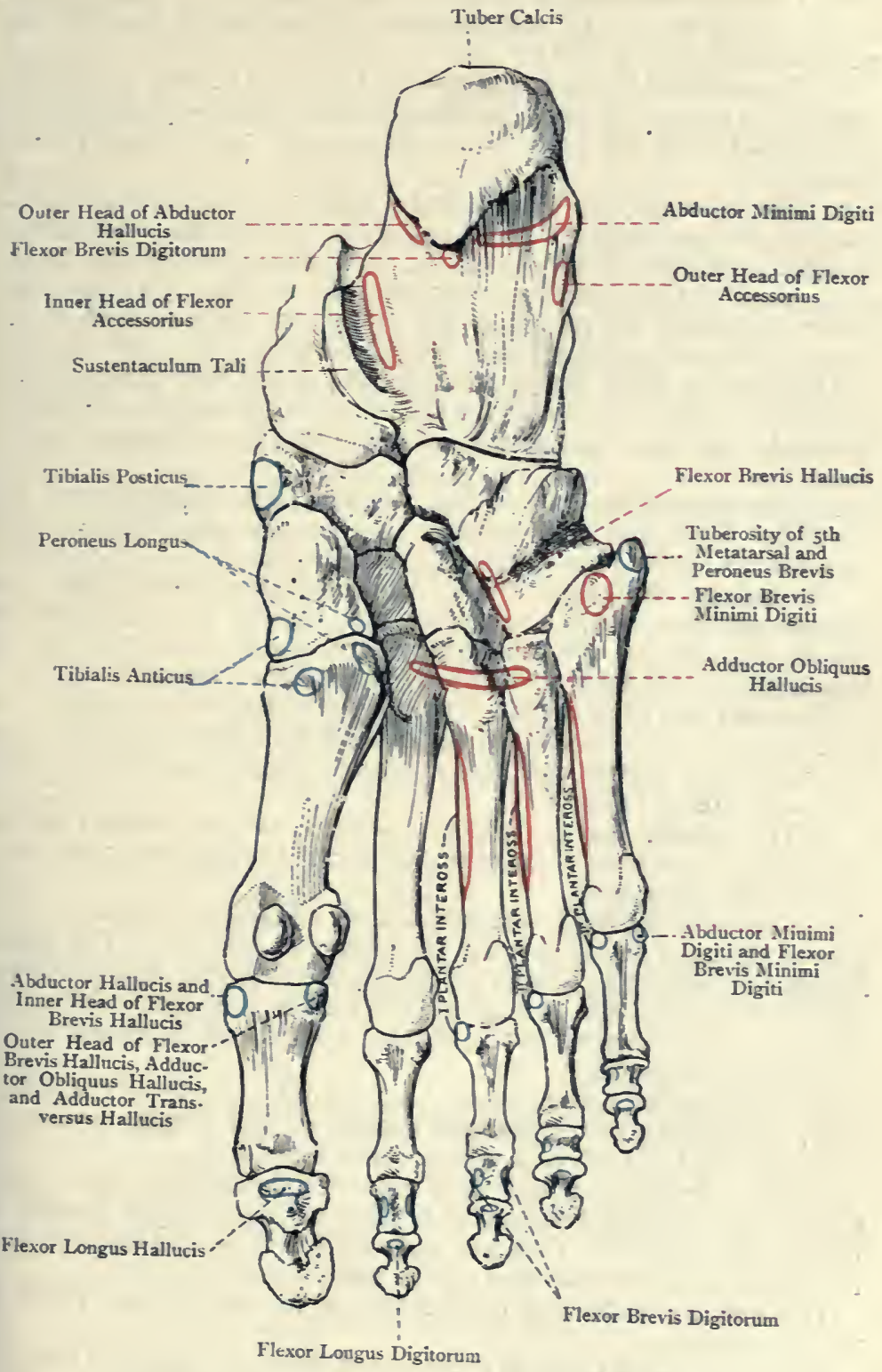

Fig. I66.-The Right Foot (Plastar Surface). 
7. The peroneal ridge on the outer surface of the os calcis, above which is the groove for the peroneus brevis, whilst that for the peroneus longus is below it.

8. The peroneal notch and groove on the outer border and plantar surface of the cuboid bone for the tendon of the peroneus longus. The ridge behind the groove gives attachment to the long plantar ligament.

9. The tuberosity on the plantar aspect of the proximal end or base of the first metatarsal bone, which gives insertion internally to a slip of the tendon of the tibialis anticus, and externally to the main part of the tendon of the peroneus longus. (A slip of the latter tendon is inserted into the lower and anterior part of the external surface of the internal cuneiform bone.)

The inner or tibial border of the foot is in line with the great toe, or hallux. It is constructed by the os calcis, astragalus, navicular, internal cuneiform, the first metatarsal, and the phalanges of the great toe.

The sustentaculum tali of the os calcis, the tuberosity of the navicular bone, and the eminence on the plantar surface of the internal cuneiform bone, pertain to this border.

The internal surface of the internal cuneiform bone presents an oblique groove, directed downwards and forwards, for the tendon of the tibialis anticus, the principal part of which is inserted into an impression at the lower and posterior part of the groove.

The outer or fibular border of the foot is in line with the little toe. It is constructed by the os calcis, cuboid, fifth metatarsal, and the phalanges of the little toe. 'Its markings, enumerated from behind forwards, are as follows:

I. The tubercle for the middle fasciculus of the external lateral ligament of the ankle-joint, situated about the centre of the outer surface of the os calcis.

2. The peroneal ridge, situated a little below and anterior to the preceding tubercle, and lying between two grooves. The upper groove transmits the tendon of the peraneus brevis, and the lower groove transmits the tendon of the peroneus longus, whilst the ridge gives attachment to the fibrous septum, which separates the two peroneal sheaths.

3. The peroneal notch, situated on the outer border of the cuboid bone, and leading to the peroneal groove, on the plantar surface of the bone, for the tendon of the peroneus longus.

4. The tuberosity on the outer side of the proximal end or base of the fifth metatarsal bone, which gives insertion to the tendon of the peroneus brevis. (The peroneus tertius is usually inserted into the dorsal surface of the base of this metatarsal bone.)

The anterior extremity of the foot is formed by the third or ungual plialanges.

It is to be noted that the great toe or hallux is almost as long as, and parallel to, the second toc, in which respects it presents a striking contrast to the thumb or pollex. 
The posterior extremity of the foot is formed by the tuber calcis. Posteriorly this tuberosity presents three transverse zones-upper, for a synovial bursa; middle, for the insertion of the tendo Achillis; and lower, for the fat of the heel.

The posterior border of the astragalus, though it stops short of the posterior extremity of the foot, may be referred to. The markings which it presents are a groove and two tubercles. The groove, which is directed downwards and inwards, transmits the tendon of the flexor longus hallucis, and leads to the groove on the under aspect of the sustentaculum tali of the os calcis. The external tubercle, of large size, gives attachment superiorly to the posterior fasciculus of the external lateral ligament of the ankle-joint. The internal tubercle, which is small, gives attachment internally to a few fibres of the internal lateral or deltoid ligament of the anklejoint.

The sinus tarsi or astragalo-calcaneal canal is the oblique tunnel which lies between the astragalus and the os calcis. Its direction is outwards and forwards, and it is occupied by a strong interosseous ligament, within which is the bursa simus tarsi.

For the Arches of the Foot, see p. 577.

\section{APPROXIMATE HOMOLOGIES OF THE BONES OF THE LIMBS.}

UPPER LIMB.

LOWER LIMB.

\section{Shoulder Girdle and Pelvic Girdle.}

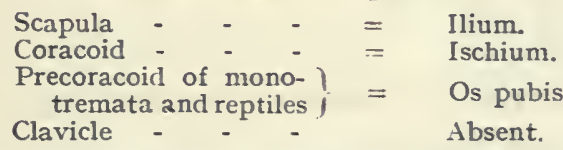

(If, however, the clavicle is the morphological representative of the precoracoid of monotremata and reptiles, then it is homologous with the os pubis.)

UPPER LIMB.

LOWER LIMB.

Special Homelogies of Scapula and Ilium (Flower).

Scapula.

Inferior angle

Superior angle

Axillary border

Superior border

Supraspinous fossa

Infraspinous fossa

Spine and acromion

Subscapular fossa

Glenoid cavity

\section{Ilium.}

Anterior superior spine.

Posterior superior spine.

Crest.

Anterior border.

Posterior border.

Sacral surface.

Iliac fossa.

Ilio-pectineal line.

Gluteal surface (dorsum ilii).

Cotyloid cavity. 
I1. Arm, Forearm, Thigh, and Leg.

$\begin{array}{lll}\text { Humerus - } & = & \text { Femur. } \\ \text { Absent - } & = & \text { Patella. } \\ \text { Radius - } & = & \text { Tibia. } \\ \text { Ulna - } & = & \text { Fibula. }\end{array}$

Special Homologies of Humerus and Femur.

Humerus.

$\begin{array}{lll}\text { Great tuberosity - - } & = & \text { Small trochanter. } \\ \begin{array}{l}\text { Small tuberosity - } \\ \text { External epicondyle and } \\ \text { capitellum - }\end{array} & =\text { Great trochanter. } \\ \begin{array}{l}\text { Internal epicondyle and } \\ \text { trochlea }\end{array} & = & \text { Internal condyle. }\end{array}$

III. Hand and Foot.

Hand.

$\begin{array}{lll}\text { Carpus - }- & = & \text { Tarsus. } \\ \text { Metacarpus - } & = & \text { Metatarsus. } \\ \text { Digital phalanges - } & = & \text { Digital phalanges. } \\ \text { Pollex - } & = & \text { Hallux. }\end{array}$

\section{Homologies of Carpus and Tarsus.}

Carpus.

Scaphoid (except the part represented by the embryonic supernumerary cartilage),

Cuneiform or pyramidal - $\quad=\quad=$ Os calcis.

(The pisiform is generally regarded as the rudiment of a suppressed digit.)

The part of the scaphoid

formed by the embryonic $=$ Navicular or scaphoid.

supernumerary cartilage -

(In apes and water-tortoises there is an additional carpal bone, called the os centrale, which represents the navicular of the tarsus.)

\begin{tabular}{|c|c|c|c|c|}
\hline Trapezium & - & - & $=$ & Internal cuneiform. \\
\hline Trapezoid & - & - & $=$ & Middle cuneiform. \\
\hline Os magnum & - & - & $=$ & External cuneiform. \\
\hline Unciform & - & - & $=$ & Cuboid. \\
\hline
\end{tabular}




\section{ARTHROLOGY}

Ax articulation or joint is the connection between two or more parts of the skeleton in the recent condition. In most joints the parts are osseous, but in certain cases cartilage forms the basis. The manner in which the parts are connected and the amount of movement allowed between them vary, and joints are accordingly divided into three classes, namely, synarthrosis, amphiarthrosis, and diarthrosis.

Synarthrosis.-This is direct union, there being only a small amount of intervening tissue without any joint cavity, and the joint is immovable. There are two varieties of this class-synchondrosis and suture.

I. Synchondrosis.-In this joint a thin plate of liyaline cartilage intervenes between the component parts, as, for example, between the diaphysis or shaft and epiphyses of a long bone. It is essentially a temporary joint.

2. Suture.-In this variety the bones are separated by a small amount of fibrous tissue, which is continuous with the periosteum. Such joints are only met with in the skull. There are three forms of suture, called true, false, and grooved. When the margins of the bones present a number of projections with intervening depressions, so that they become closely interlocked, the suture is called true. When the opposed margins are more or less flat, so that there is merely apposition without interlocking, the suture is spoken of as false. When one margin presents a ridge and the other a cleft into which the ridge is received, it is known as a grooved suture.

True sutures are of three kinds-serrated, dentated, and limbous. In the serrated suture the margins of the bones are saw-like, as in the frontal suture; in the dentated suture the margins present projections like teeth, as in the interparietal suture; and in the limbous suture the margins of the bones are ridged and bevelled so that they overlap, as in the lower and mesial parts of the frontoparietal suture.

False sutures are of two kinds-squamous and harmonic. In the squamous suture the margins are bevelled so that one overlaps the other, as in the squamo-parietal suture. In the harmonic suture the surfaces, which are rough, are in direct apposition, as between 
the superior maxillæ. In the grooved suture a ridge on one bone is received into a cleft on another. Such a suture is known as schindylesis, and it is exemplified in the articulation between the rostrum of the sphenoid and the vomer.

Amphiarthrosis.- - In this class the opposed surfaces are connected either by a disc of fibro-cartilage, or by an interosseous ligament, and the joint is partially movable. When the connecting medium is a disc of fibro-cartilage the name of symphysis is given to the joint, as between the bodies of the vertebræ, the bodies of the pubic bones, and between the presternum and mesosternum. When the connecting medium is an interosseous ligament the joint is called syndesmosis, as in the inferior tibio-fibular articulation. Union by means of an interosseous membrane is also a form of syndesmosis.

Diarthrosis.-While there is more or less continuity at a synarthrosis and an amphiarthrosis, there is none at a diarthrosis. The articular ends of the bones are free, and the joint is freely movable. The ends of the bones are smooth and polished, being covered by articular cartilage; they are connected by ligaments; and the interior of the joint is lined with a synovial membrane, except over the articular cartilages. This membrane secretes the synovia, which lubricates all parts of the interior. There are five varieties of diarthrosis.

I. Enarthrosis or Ball-and-socket Joint.-In this variety one articular end is spherical, and the other is hollowed into a socket for its reception, as in the shoulder- and hip-joints.

2. Condylarthrosis or Condylar Joint.-This is akin to enarthrosis. One articular end has the form of a condyle, and the other presents a shallow cavity, as in the metacarpo- and metatarso-phalangeal joints.

3. Trochlearthrosis, Ginglymus, or Hinge Joint.-In this variety one articular end has the form of a trochlea or pulley, and the other is so shaped as to adapt itself to it, as in the elbow- and ankle-joints.

4. Arthrodia or Gliding Joint.-In this joint the surfaces are almost plane, or in some cases concavo-convex, as in the joints between the articular processes of vertebræ, and the carpal and tarsal joints Under this variety is to be included the reciprocal or saddle joint, where the articular surfaces are saddle-shaped and mutually adapt themselves to each other, as between the trapezium and the first metacarpal bone.

5. Trochoides or Pivot Joint.-In this joint one articular end forms a pivot, and the other is so arranged as to rotate around it, as between the atlas and the odontoid process of the axis.

Ligaments.-These are composed of white fibrous tissue, and, as their name implies, they bind the bones together. At a diarthrosis their chief use is to control movement, the bones being maintained in position by the muscles and atmospheric pressure. At their attachments they are intimately associated with the periosteum. When the fibrous tissue is arranged continuously round the joint the ligament is called a capsular ligament. In other cases the tissuc is disposed as round cords, and in a third variety it forms flattened bands. 
Synovial Membranes.-These membranes are so named because they secrete a fluid, viscid like white of egg, which is called syrovia. They serve to diminish friction and so facilitate movement. Their situations are therefore associated with movable structures, such as joints, gliding tendons, and the integument over bony projections. Accordingly there are three kinds of synovial membrane-namely, articular, tendon or vaginal, and bursal.

The articular synovial membranes line the interior of diarthrodial joints, except where there is articular cartilage, and they stop at the margin of this cartilage. In some joints they give rise to folds, some of which contain adipose tissue. Such folds are known as Haversian mucilaginous glands.

The tendon or vaginal synovial membranes, also known as synovial sheaths, invest those tendons which have to glide within fibrous sheaths. They are met with around the ankle, particularly behind the external and internal malleoli, and upon the palmar aspect of the fingers.

The bursal synovial membranes, commonly called synovial bursæ, are synovial sacs which are situated between the integument or a muscle and some bony projection. They may be deepseated or subcutaneous. The deep-seated burse are situated between a muscle, or its tendon, and the contiguous bone,-e.g., the tendon of the biceps brachii and the anterior part of the bicipital tuberosity of the radius. The subcutaneous burse are placed beneath the integument, which they separate from some bony projection-e.g., the prepatellar bursa.

Structure.-A synovial membrane consists of connective tissue, which is destitute of an endothelial lining properly so called. At intervals it may present scattered groups of branched cells, but these are not endothelial. There are many capillary bloodvessels, and lymphatic vessels are described as being present. The nerves are described as terminating in corpuscles of the nature of end-balls, in a plexiform manner, and in Pacinian corpuscles.

Development. - Synovial membranes are developed from the axial mesenchyme of the limb-buds, in the intervals between contiguous bones, which are being formed from that mesenchyme.

Interarticular Fibro-cartilages.--These are met with in certain joints either in the form of plates separating the articular ends, or as bands placed around cavities, which they serve to deepen, or around flat surfaces, which they render concave. They are known as menisci. When the fibro-cartilage takes the form of a plate it is spoken of as an interarticular meniscus. Such occur at the temporo-mandibular, sterno-clavicular, and radio-carpal joints. They act as buffers to break shock, and they compensate for rregularities of the opposed surfaces. When the fibro-cartilage is limited to the margin of a cavity, or the circumference of a surface, it is called a marginal meniscus, such as the glenoid ligament of the shoulder-joint, the cotyloid ligament of the hip-joint, and the semilunar fibro-cartilages of the knee-joint.

Movements. - The different kinds of movement at diarthrodial oints are angular, circumduction, rotation, and gliding. 
Angular Movement.-This increases or diminishes the angle between two or more bones. When it takes place in a forward and backward direction, so as to bend or straighten a joint, it is spoken of as flexion and extension. When it takes place laterally, away from or towards the median plane of the body, it is called abduction and adduction. In the case of the hand the median line from or towards which abduction and adduction take place is a line passing through the centre of the middle finger, and in the case of the foot, through the centre of the second toe.

Circumduction.- This consists of the four forms of angular movement, occurring successively in such sequence as flexion, abduction, extension, and adduction. It occurs at ball-and-socket and condylar joints, and during the movement a part of the limb describes a cone, the apex of which is formed by the proximal end at the moving joint, whilst the base is described by the distal end.

Rotation.- This is movement of a bone round its axis without much disturbance of its position. It occurs at enarthrodial and trochoidal joints, and also at the knee-joint, which is a ginglymus.

Gliding Movement.-This consists of a simple to-and-fro or sliding movement of two articular surfaces, as between the articular surfaces of vertebræ, and at the carpal and tarsal joints. When the gliding is combined with a certain amount of turning or rolling, so as to bring different parts of the articular surfaces successively into contact in different positions of the joint, the movement is known as coaptation, as at the femoro-patellar joint.

A description of the different articulations will be found in connection with the regions to which they belong. 


\section{THE UPPER LIMB}

\section{THE BACK.}

Landmarks. - The middle line of the neck presents a median furrow, called the nuchal groove, which lies over the line of the ligamentum nuchæ. It is due to the shortness of most of the cervical spinous processes and the prominence at either side, caused principally by the complexus muscle. At the upper end of this groove the strong bifid spine of the axis may be felt by sinking the finger deeply, but the spines of the third, fourth, and fifth cervical vertebræ cannot be detected, as these fall short of the surface so as to allow of dorsal flexion of the neck. At the lower end of the groove the spine of the seventh cervical or vertebra prominens can readily be felt, and usually also that of the sixth, and succeeding to these are the thoracic and lumbar spines. The spine of the first thoracic vertebra is, as a rule, the most conspicuous of the series. Along the middle line of the thoracic and lumbar regions there is an elongated furrow, called the spinal groove, which is produced by the prominence formed by the erector spinæ on either side. This groove is best marked in the lower thoracic and upper lumbar regions, and it subsides about the level of the third sacral spine. The outline of the scapula may be made out, unless in very muscular persons. It will be found to extend from the second to the seventh rib, and the spine and acromion process are usually readily felt. The root of the spine is on a level with the third thoracic spine, and the inferior angle coincides with the seventh rib. The crest of the ilium can be felt at the lower part of the back, its greatest prominence being on a level with the fourth lumbar spine.

Fascia.-The superficial fascia is thick, granular, and fatty, and in it the cutaneous nerves and vessels are met with. The deep fascia is thin and membranous, but firm. It contains no fat, and forms sheaths for the muscles.

Cutaneous Nerves.-These are most readily met with at the level of the deep part of the superficial fascia, and the cutaneous vessels serve as the best guide to them. They are derived from the posterior primary divisions of the spinal nerves, which, with a few exceptions, divide each into an external and internal branch. 
In the thoracic region the internal branches of the upper six nerves become cutaneous near the spines of the vertebræ, and then turn outwards in the integument. The branch of the second is characterized by its great length, and it can be traced well outwards over the scapula. The external branches of the upper six thoracic nerves end in the muscles. The internal branches of the lower six thoracic nerves are muscular, and the external branches become

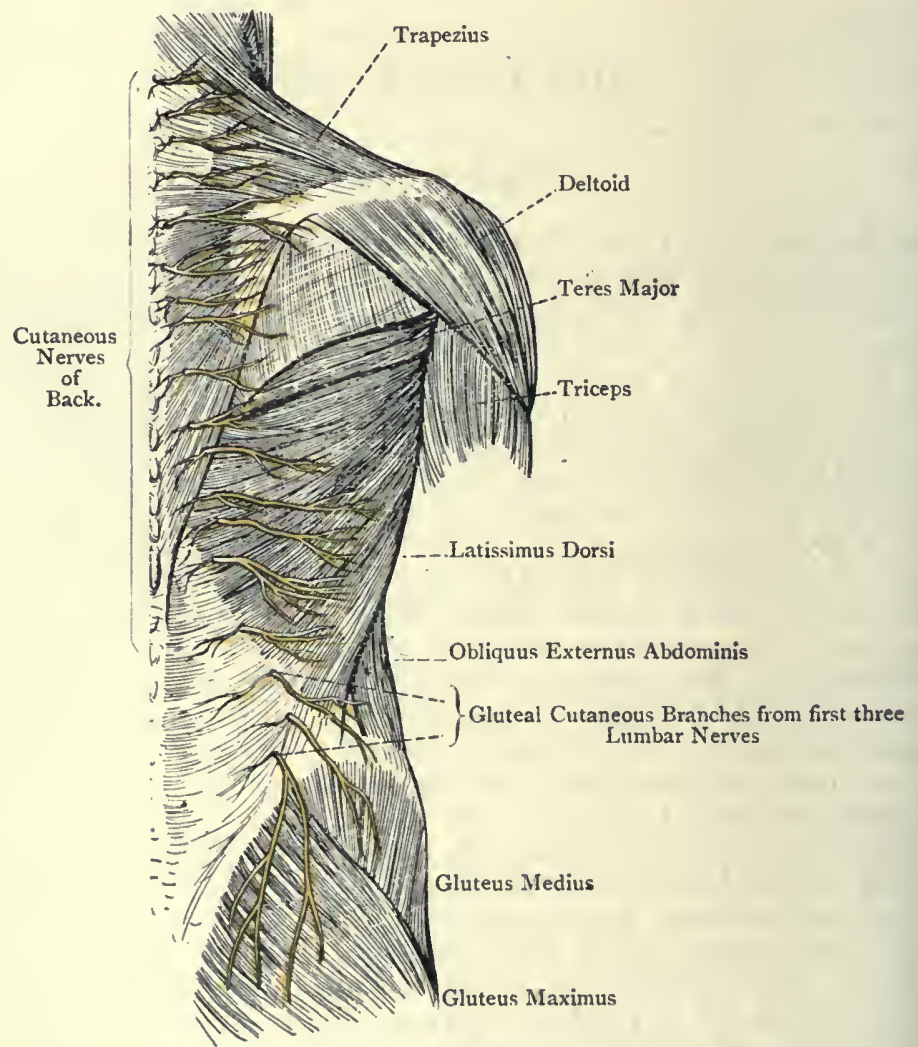

Fig. 167. -The Superficial Dissection of the Back.

cutaneous along the course of the angles of the ribs. In the lumbar region the internal branches end in the muscles. The external branches of the first three nerves furnish cutaneous offsets which descend over the iliac crest in front of the outer border of the erector spinæ to the gluteal integument, supplying in their course the integument of the lumbar region. The external branches of the lower two nerves end in the deep muscles. In all cases the 
distribution of the cutaneous nerves is at a lower level than their origin.

The cutaneous arteries which accompany the cutaneous nerves of the thoracic and lumbar regions are derived from the dorsal branches of the intercostal and lumbar arteries.

Muscles. Trapezius.-This muscle is so named because, along with its fellow, it presents a four-sided appearance like a table. The two muscles have also a resemblance to a monk's hood or cowl; hence the name cucullaris.

Origin.--(I) The inner third of the superior curved line of the occipital bone, and the external occipital protuberance; (2) the ligamentum nuchæ; and (3) the spinous processes and supraspinous ligaments of the last cervical, and, as a rule, all the thoracic vertebræ. The origins of the two muscles are markedly tendinous between the second cervical and third thoracic spines, where they give rise to an elliptical area, widest opposite the sixth cervical spine.

Insertion.-(I) The posterior border of the outer third of the clavicle; (2) the inner border of the acromion process, and upper lip of the posterior border of the spine, of the scapula; and (3) the tubercle which projects at the inner end of the upper lip of the spine near the root. The lower fibres end in a special tendon which glides over the triangular surface at the root of the spine, being separated from it by a synovial bursa.

Nerve-supply.-(I) The spinal accessory nerve (spinal portion), and (2) branches from the cervical plexus, which are derived from the anterior primary divisions of the third and fourth cervical nerves. The nerves enter the deep surface of the muscle after passing beneath its anterior border a little above the clavicle, and the spinal accessory, having become connected with the spinal nerves, can be traced over nearly the whole extent of its deep surface, where it lies about 2 inches from the spines of the vertebræ.

Blood-supply.-There is one specially-named artery, called the superficial cervical, which is a branch of the transverse cervical, and, like the nerves, enters the deep surface of the muscle.

The upper fibres of the muscle are directed downwards, outwards, and forwards; the middle fibres pass more or less horizontally outwards ; and the lower fibres pass upwards and outwards.

Action.-The upper fibres, acting from their origin, elevate the outer end of the clavicle and the point of the shoulder. Acting from their insertion they extend the head and incline the neck towards the same side, the face being directed towards the opposite side. The middle fibres approximate the scapula to the spine, and the lower fibres draw it downwards and inwards, both of these sets of fibres also producing rotation of the bone, so as to elevate the point of the shoulder. The entire muscle draws the scapula to the spine, and produces a certain amount of rotation of the bone, the point of the shoulder being raised, as in the act of shrugging the shoulders. 
Ligamentum Nuchæ.-This is a fibrous band, or intermuscular septum, which occupies the median line of the neck. Its superficial fibres are attached above to the external occipital protuberance, and below to the spine of the seventh cervical vertebra. Its deep fibres are attached to the external occipital crest, and to the spines of cervical vertebræ from the second to the sixth inclusive. They also extend into the interspinous intervals between the interspinales muscles, where they represent interspinous ligaments.

Latissimus Dorsi-Origin.-(I) The spinous processes and supraspinous ligaments of the lower six thoracic vertebræ; $(2)$ the posterior lamina of the lumbar aponeurosis, through means of which it is attached to the lumbar and sacral spines, and the posterior fourth of the outer lip of the iliac crest; (3) the outer lip of the iliac crest a little behind the centre for about 2 inches; $(4)$ the outer surfaces of the last three or four ribs external to their angles by fleshy slips, which interdigitate with slips of the obliquus externus abdominis; and (5) the back of the inferior angle of the scapula, the last origin being inconstant.

Insertion.-The floor of the bicipital groove of the humerus about its middle third for about $\mathrm{I}_{\frac{1}{2}}$ inches.

Nerve-supply.-The middle or long subscapular nerve from the posterior cord of the brachial plexus, its fibres being derived chiefly from the seventh cervical nerve. The nerve is only seen during the dissection of the axillary space, and it enters the muscle on its deep aspect.

Blood-supply.-Branches of the subscapular artery.

The upper fibres of the muscle pass horizontally outwards, and cross the inferior angle of the scapula, which they bind to the chest wall ; the succeeding fibres pass obliquely upwards and outwards ; and those from the iliac crest and lower ribs pass almost vertically upwards.

Action.-The arm being raised, the muscle draws it downwards and backwards, producing at the same time internal rotation, as when the hands are crossed behind the back. When the muscle acts from its insertion, it raises the pelvis and trunk after the outstretched arms, as in the act of climbing a pole. It also elevates the last three or four ribs, as in forced inspiration.

At the inferior angle of the scapula the muscle is behind the teres major; it then winds round the lower border of that muscle; and eventually it is placed in front of it. The lower borders of the tendons of these muscles near their insertions are closely connected, but ultimately they become separated, a synovial bursa intervening between the two. Between the upper border of the latissimus dorsi, the lower border of the trapezius, and the base of the scapula, there is a triangular area in which are exposed a portion of the rhomboideus major, the sixth rib, and the sixth intercostal space.

Triangle of Petit.-The anterior border of the latissimus dorsi, between the iliac crest and last rib, may or may not overlap the 
posterior border of the obliquus externus abdominis. If it does not do so, a small space is left, called the triangle of Petit, which is situated immediately above the centre of the iliac crest. It is bounded in front by the posterior border of the obliquus externus abdominis, behind by the anterior border of the latissimus dorsi, and below by the iliac crest near the centre. It is covered only by skin and fascia, and its floor is formed by a part of the obliquus internus abdominis. In this situation a lumbar hernia may occur, or a lumbar abscess may find its way to the surface.

Levator Anguli Scapulæ-Origin.-By four tendinous slips from the posterior tubercles of the transverse processes of the first four cervical vertebræ.

Insertion.-The base of the scapula from the superior angle to the triangular surface at the root of the spine.

Nerve-supply.-Branches of the cervical plexus, which are derived from the third and fourth cervical nerves, and a branch from the nerve to the rhomboids.

The muscle is directed downwards, backwards, and slightly outwards.

Action.-Acting from its origin the muscle elevates the superior angle of the scapula, thereby rotating the bone and causing the point of the shoulder to be depressed. Acting from its insertion it is a lateral flexor of the neck.

Rhomboideus Minor-Origin.-The lower part of the ligamentum nuchæe, and the spines and supraspinous ligament of the seventh cervical and first thoracic vertebræ.

Insertion.-The base of the scapula opposite the triangular surface at the root of the spine.

Rhomboideus Major-Origin.-The spines and supraspinous ligaments of thoracic vertebræ from the second to the fifth inclusive.

Insertion.-The base of the scapula from the triangular surface at the root of the spine to the inferior angle. The insertion takes place by means of a tendinous expansion, which is firmly attached near the inferior angle. Elsewhere the expansion is connected with the base of the bone by connective tissue, so that the muscle can be detached to a large extent without injury.

Nerve-supply of the Rhomboids.-The nerve to the rhomboids, which is a branch of the fifth cervical. This nerve, having pierced the scalenus medius, gives a branch to the levator anguli scapulæ, and passes beneath the upper border of the rhomboideus minor about I inch from the base of the scapula. Having given a branch to the deep surface of that muscle, it passes beneath the rhomboideus major and enters its upper part.

The direction of the rhomboid muscles is downwards and outwards.

Action. - The muscles draw the scapula backwards and upwards, and rotate the bone so as to depress the point of the shoulder.

Posterior Scapular Artery.-This vessel is usually a branch of the transverse cervical, but it may arise from the third part of the sub- 
clavian. It passes backwards beneath the levator anguli scapulæ, and then downwards beneath the rhomboid muscles, lying close to the base of the scapula. It gives off branches to the adjacent muscles, and several offsets to the front and back of the scapula, which latter take part in the scapular anastomoses of arteries.

At the upper border of the scapula a limited view is obtained of the posterior belly of the omo-hyoid muscle, and the suprascapular artery and nerve. The former arises from the upper border of the bone inside the suprascapular notch, as well as from the suprascapular or transverse ligament. The suprascapular artery passes to the supraspinous fossa over the ligament, and the suprascapular nerve to the same region beneath it. The artery in this part of its course furnishes its supra-acromial branch to the upper surface of the acromion process. The transverse cervical artery is seen, at a higher level than the suprascapular, dividing into its superficial cervical and posterior scapular branches. The former passes superficial to the levator anguli scapulæ, and the latter beneath that muscle.

It will now be convenient to continue the description of the back to its termination, except the structures more immediately involved in the head and neck.

Serratus Posticus Superior-Origin.-The lower part of the ligamentum nuchæ, and the spines and supraspinous ligaments of the last cervical and first two thoracic vertebræ.

Insertion.-By fleshy and tendinous slips into the upper borders and outer surfaces of the second, third, fourth, and fifth ribs, external to their angles.

Nerve-supply.-The second, third, and fourth intercostal nerves.

The origin of the muscle is aponeurotic, and continues so over about half its length. The direction of the fibres is downwards and outwards.

Action.-The muscle elevates the ribs into which it is inserted, and is therefore a muscle of inspiration.

Serratus Posticus Inferior-Origin.-The posterior lamina of the lumbar aponeurosis, through means of which the muscle is attached to the spines and supraspinous ligaments of the lower two thoracic and upper two or three lumbar vertebræ, under cover of the latissimus dorsi.

Insertion.-By four fleshy slips into the lower borders of the last four ribs.

Nerve-supply. - The ninth, tenth, and eleventh intercostal nerves.

The serrations of insertion overlap each other from above downwards; the second is the broadest, and the third to a large extent conceals the fourth. The direction of the fibres is upwards and outwards.

Action.-(I) To draw backwards and slightly depress the lower four ribs, the effect of which is to increase the capacity of the lower part of the thoracic cavity; and (2) to steady the lower four ribs, and thus to act as an auxiliary to the diaphragm. In both cases it acts as a muscle of inspiration. 
Origin.-(I) The lower two-thirds of the ligamentum nuchæ, and (2) the spines of the last cervical and first six thoracic vertebræ.

Insertion-(a) Splenius Capitis.-(I) The lower part of the outer surface of the mastoid process of the temporal bone, and (2) the occipital bone below the outer third of the superior curved line, in each case under cover of the sterno-cleido-mastoid. (b) Splenius Colli.-The posterior tubercles of the transverse processes of the first two or three cervical vertebræ, internal to the levator anguli scapulæ, and in line with the cervicalis ascendens.

Nerve-supply. - The posterior primary divisions of the cervical nerves below the third, and of the upper five thoracic.

The direction of the fibres is upwards and outwards.

Action.-The muscle extends the head and flexes the neck to its own side, the face being turned to the same side.

Erector Spinæ.-This composite muscle is single below in the region between the last rib and iliac crest, where it is strongly tendinous internally, and fleshy externally. It subsequently, however, resolves itself into three columns-outer, middle, and inner, there being three muscles in each of the outer and middle columns, and one in the inner column, thus making seven muscles in all.

Origin.-(I) The spines of the last two thoracic, all the lumbar, and the upper four sacral vertebræ; (2) the back of the fourth sacral vertebra; $(3)$ the posterior sacro-iliac ligament; and (4) the posterior fifth of the iliac crest. The insertion of the muscle is represented by the columns into which it divides. On approaching the last rib it presents a groove on its surface, which indicates its division at this stage into an outer and inner column, the latter representing the combined middle and inner columns.

Outer Column.-This column is composed of three muscles, which, from below upwards, are named ilio-costalis, musculus accessorius ad ilio-costalem, and cervicalis ascendens.

Ilio-costalis.-This is the direct continuation of the outer part of the erector spinæ, and it is inserted by fleshy and tendinous bundles into the angles of ribs from the seventh to the eleventh, and into the lower border of the twelfth rib.

Musculus Accessorius ad Ilio-costalem.-This muscle prolongs the ilio-costalis from the lower six to the upper six ribs. It arises by tendons, internal to the slips of insertion of the ilio-costalis, from the angles of ribs from the seventh to the eleventh, and from the outer surface of the twelfth rib, and it is inserted by tendons into the angles of the upper ribs from the second to the sixth, and into the outer border of the first rib external to the tubercle.

Cervicalis Ascendens.-This continues the musculus accessorius from the upper ribs to the neck. It arises by tendinous slips from the third, fourth, fifth, and sixth ribs, internal to the tendons of insertion of the musculus accessorius, and it is inserted into the posterior tubercles of the transverse processes of the fourth, fifth, and sixth cervical vertebræ, where it is in line with the splenius colli. 
Action.-The muscles of the outer column act as extensors and lateral flexors of the vertebral column, and as depressors of the ribs, as in expiration.

Middle Column.- This column, like the outer, is composed of three muscles, which, from below upwards, are named longissimus

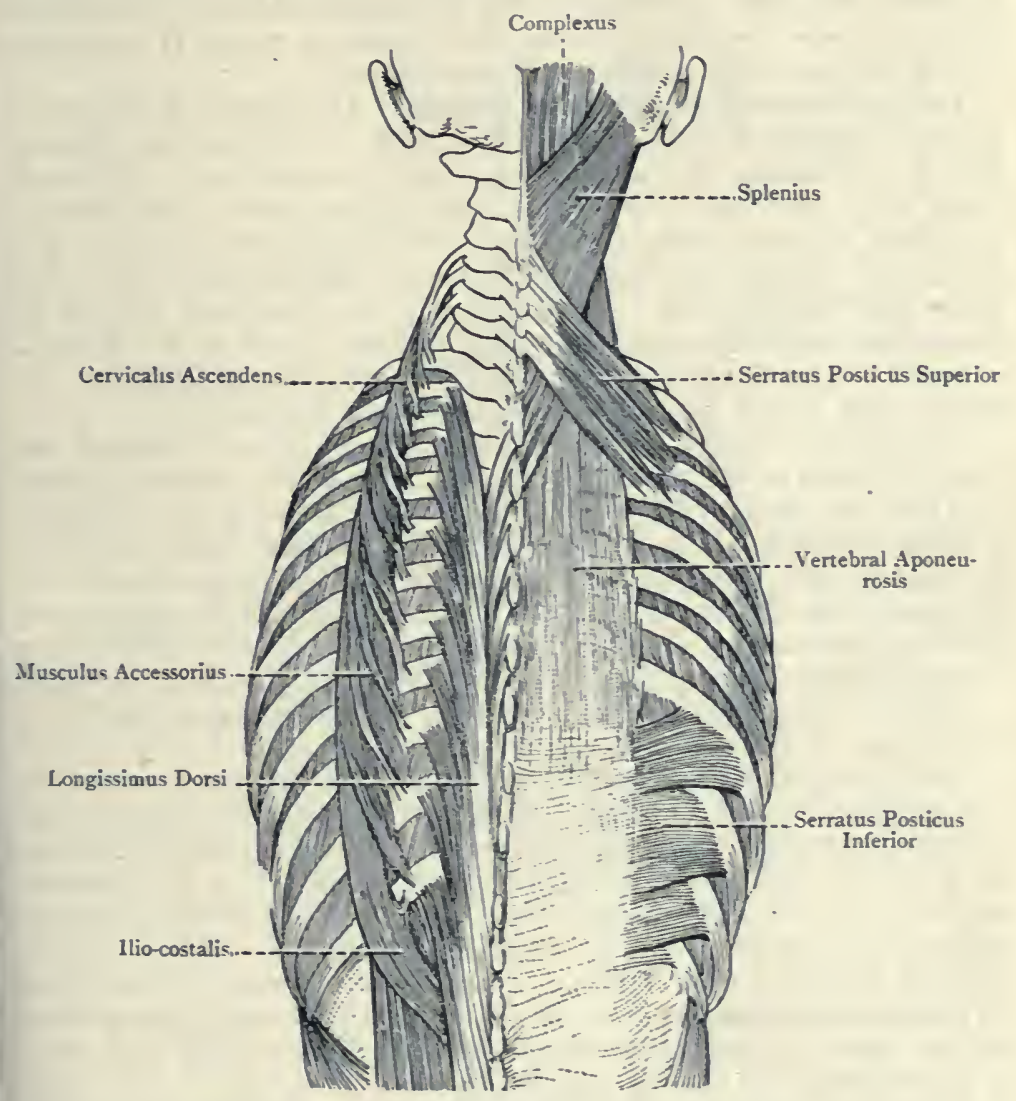

Fig. 169.-The Deep Muscles of the Back.

dorsi, longissimus cervicis (transversalis cervicis), and trachelomastoid (longissimus capitis).

Longissimus Dorsi.- This is the direct continuation of the inner part of the erector spinæ, and it is inserted in a twofold manner. The inner insertions take the form of round, tapering tendons, which are attached to the accessory processes of the lumbar vertebræ and the extremities of the transverse processes of the thoracic vertebræ. 
The outer insertions are fleshy, and are attached to the backs of the transverse processes of the lumbar vertebræ, and the outer surfaces of the lower ten ribs external to the tubercles.

Transversalis Cervicis (longissimus cervicis). -This continues the longissimus dorsi to the neck. It arises from the transverse processes of the upper five or six thoracic vertebræ, and is inserted into the posterior tubercles of the transverse processes of cervical vertebræ from the second to the sixth inclusive, where it is internal to the splenius colli and cervicalis ascendens.

Trachelo-mastoid (longissimus capitis).-This muscle has been so named because it extends from the neck to the mastoid process, and it continues the longissimus dorsi to the head. It arises from the transverse processes of the upper five or six thoracic vertebræ in close connection with the longissimus cervicis, and from the articular processes and capsular ligaments of the lower three cervical vertebræ. Its insertion is into the lower part of the outer surface of the mastoid process under cover of the splenius capitis. Towards its insertion this muscle is very narrow and ribbon-like, and it is marked by a tendinous intersection.

Action.-The muscles of the middle column act as extensors and lateral flexors of the vertebral column. They also extend the head and flex the neck to one side, the face being turned to the same side.

Inner Column.-This consists of the spinalis dorsi, which is intimately associated with the inner part of the longissimus dorsi. It arises from the lower two thoracic and upper two lumbar spines, and it is inserted by tendinous slips into the upper thoracic spines -sometimes the upper four, sometimes as many as the upper eight.

Action.-This muscle is an extensor of the thoracic portion of the vertebral column.

Nerve-supply.-The erector spinæ and its component muscles are supplied by the posterior primary divisions of the spinal nerves.

Between the iliac crest and the last rib the erector spinæ is enclosed in a sheath, the posterior wall of which is formed by the posterior lamina of the lumbar aponeurosis, affording origin to the latissimus dorsi and serratus posticus inferior, whilst the anterior wall is formed by the middle lamina of that aponeurosis. On the back of the sacrum its tendon gives origin deeply to fibres of the multifidus spinæ, whil $t$ superficially, between the sacrum and the iliac crest, a few fibres of the gluteus maximus arise from it.

Complexus-Origin.-(I) The extremities of the transverse processes of the upper six thoracic and last cervical vertebræ; and (2) the backs of the articular processes and capsular ligaments of three or four cervical vertebræ above the seventh. The muscle may receive a fleshy slip from the spine of the seventh cervical.

Insertion. - The occipital bone between the superior and inferior curved lines, commencing at the crest and extending outwards for about 2 inches. The inner fibres of the muscle are interrupted about the level of the sixth cervical vertebra by an elongated tendinous intersection, having the appearance of a tendon, with a fleshy belly 
at either extremity, from which circumstance this part is known as the biventer cervicis. About the level of the axis the muscle usually presents another faint transverse intersection.

Nerve-supply.-(I) The posterior primary division of the first cervical or suboccipital nerve; (2) the great occipital; and (3) the posterior primary divisions of cervical nerves below the second.

The innermost and longest fibres pass almost vertically upwards,

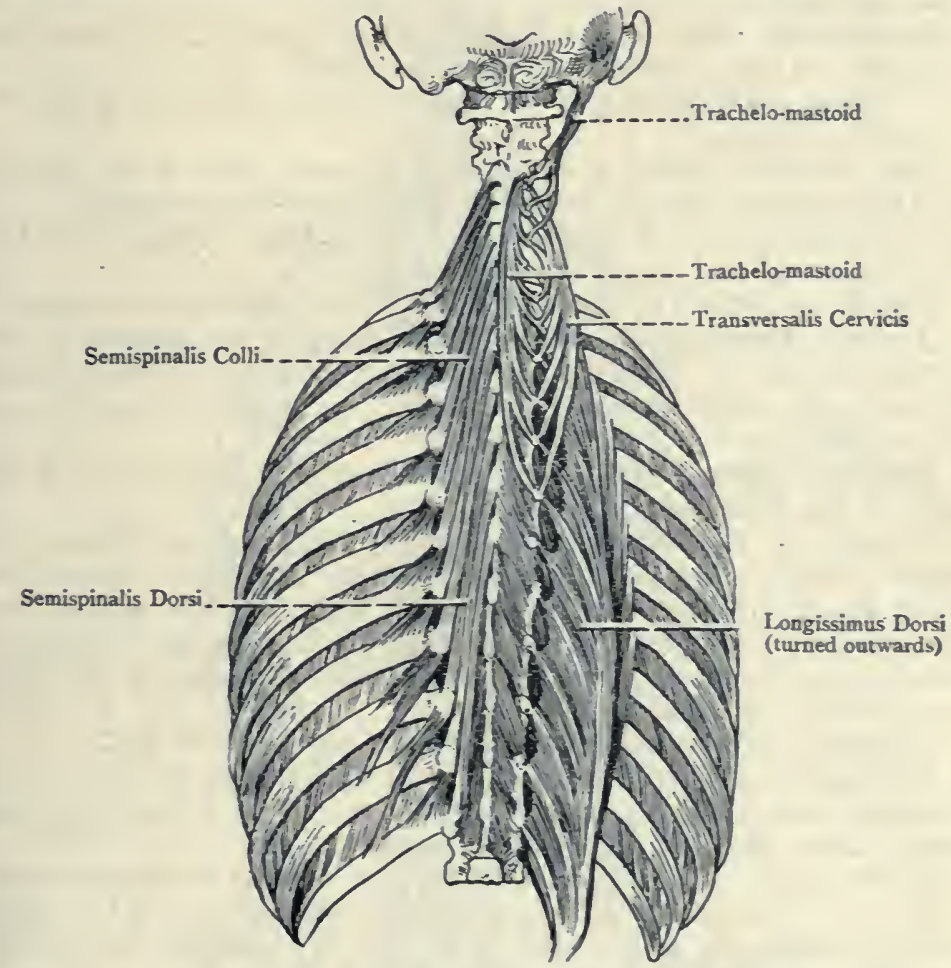

Fig. 170.-The Middle Columin of the Erector Spine, and the Semispinales Muscles.

whilst the outer and upper fibres, which are short, pass upwards and inwards.

Action.-To extend the head and incline it to one side, the face being turned to the opposite side.

A small part of the complexus may appear superficially in the upper part of the posterior triangle of the neck, this portion being pierced by the great occipital nerve. As high as the axis spine the muscle rests upon the semispinalis colli, and in this situation an arterial anastomosis takes place between the deep cervical of the superior intercostal from the second part of the subclavian and the 
deep branch of the ramus cervicalis princeps of the occipital from the external carotid. Above the level of the axis spine it covers the recti capitis postici and obliqui capitis muscles, and forms the roof of the suboccipital triangle.

Semispinalis Dorsi-Origin.-The extremities of the transverse processes of thoracic vertebræ from the sixth to the tenth inclusive.

Insertion.-The spines of the last two cervical and upper four thoracic vertebræ.

Semispinalis Colli-Origin.-The extremities of the transverse processes of the upper five thoracic vertebræ.

Insertion.-The spines of cervical vertebræ from the second to the fifth inclusive.

The bundle of fibres inserted into the axis spine is the largest, and the bundles overlap one another from above downwards.

Nerve-supply of the Semispinales.-The posterior primary divisions of the cervical and upper thoracic nerves.

The fibres of the two muscles are directed upwards and inwards.

Action.-The muscles are extensors and lateral flexors of the vertebral column. The semispinalis colli and the cervical portion of the semispinalis dorsi also rotate the cervical vertebræ towards the opposite side.

Multifldus Spinæ.-This muscle lies deeply in the groove by the sides of the spines of the vertebræ.

Origin.-(I) The sacral groove, and the deep surface of the tendon of the erector spinæ; (2) the posterior sacro-iliac ligament, and the inner lip of the iliac crest at its back part; (3) the mammillary tubercles of the lumbar vertebræ; (4) the transverse processes of the thoracic vertebræ; and (5) the articular processes of the lower four cervical vertebræ.

Insertion.-The spines of the vertebræ from root to tip.

The superficial fibres from any given origin pass over three or four vertebræ before taking insertion, the succeeding fibres pass over two or three, and the deeper fibres pass over one.

Nerve-supply.-The posterior primary divisions of the spinal nerves. Action.-The muscle is an extensor and lateral flexor of the spinal column, producing at the same time rotation towards the opposite side in the cervical and thoracic regions.

Rotatores Spinæ.-These muscles are really the deepest fibres of the multifidus spinæ in the thoracic region. They are eleven in number.

Origin.-The upper and back part of a transverse process.

Insertion.-The lower border of the lamina of the vertebra immediately above.

Nerve-supply.-The posterior primary divisions of the spinal nerves. Action.-To rotate the vertebræ towards the opposite side.

Interspinales.- These muscles are usually confined to the cervical and lumbar regions, where they are arranged in pairs between the spines, one on either side of the middle line. In the neck they are limited to the region of the apices of the spines, but in the 
lumbar region they extend very nearly over their whole length. In the neck the muscles of each pair are separated by a deep process of the ligamentum nuchæ, representing an interspinous ligament, and in the lumbar region they are separated by the interspinous ligaments themselves.

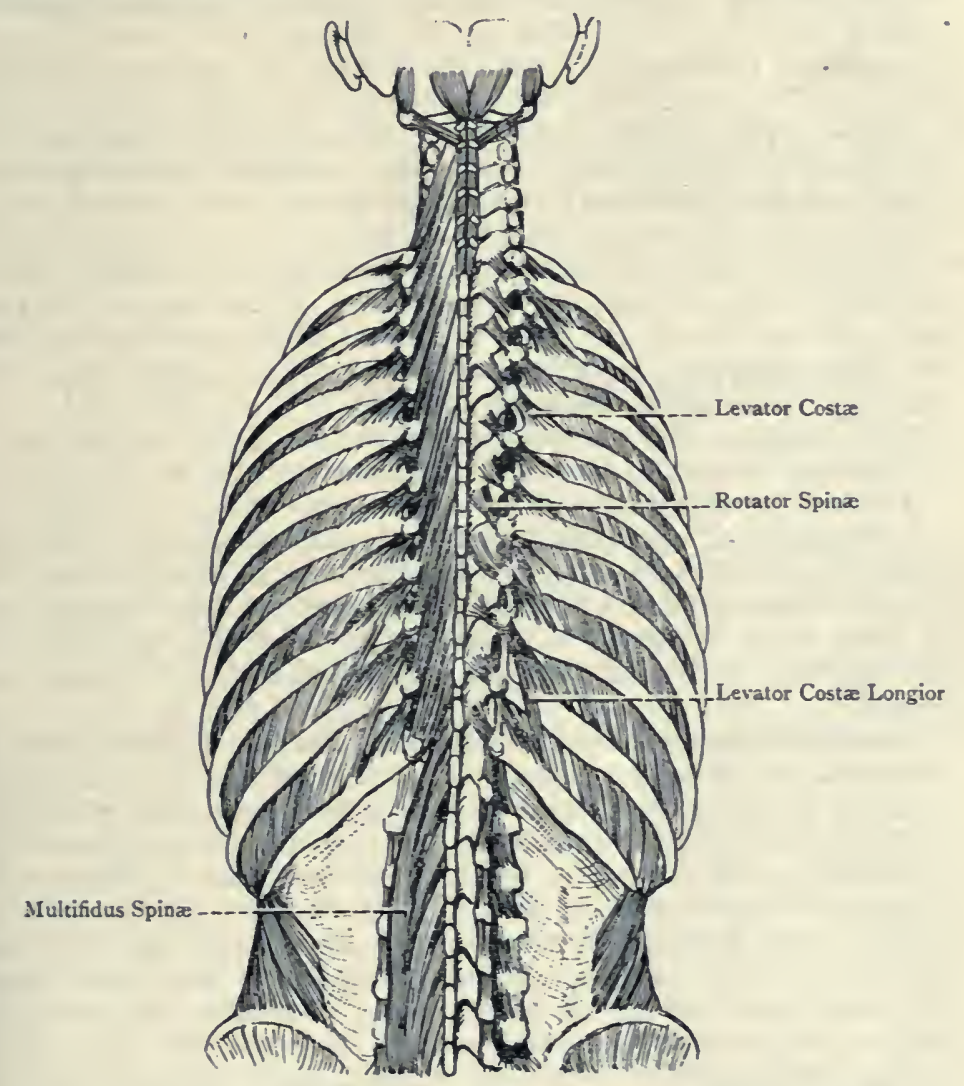

Fig. 171.-The Multifidus Spinfe and Levatores Costarum Muscles.

Nerve-supply.-Posterior primary divisions of spinal nerves. Action.- To extend the vertebræ.

Intertransversales. - These muscles occur chiefly in the cervical and lumbar regions, where they are arranged in pairs in each space. In the neck they extend between the anterior and posterior tubercles of adjacent vertebræ. In the lumbar region the muscles of each pair are arranged as lateral and mesial. The intertransversales laterales extend between two given transverse processes, and the 
intertransversales mediales extend from the accessory process of one vertebra to the mammillary process of the vertebra below. Sometimes intertransversales muscles are met with in the lower thoracic region.

Nerve-supply.-Posterior primary divisions of spinal nerves.

Action.-The muscles act as lateral flexors of the vertebral column.

Levatores Costarum.-These are twelve in number on either side.

Origin.-The highest muscle arises from the tip of the transverse process of the seventh cervical vertebra, and the succeeding eleven arise from the tips of the transverse processes of the thoracic vertebræ from the first to the eleventh inclusive.

Insertion.-Each muscle is inserted into the outer surface of the rib below, from the tubercle to the angle. In the case of the lower two or three muscles the more superficial fibres pass over the first rib below and take insertion into the next rib, these fibres constituting the levatores costarum longiores.

Nerve-supply.-The intercostal nerves.

The muscles are directed downwards and outwards.

Action.-To elevate the ribs, as in inspiration.

Each muscle is somewhat fan-shaped, and contains an admixture of aponeurotic fibres. In direction the muscles coincide with the external intercostals, with which they are closely incorporated by their outer borders. They are covered by the erector spinæe, and by their deep surfaces they are related to the external intercostals.

Posterior Primary Divisions of Spinal Nerves.-These nerves in the thoracic region pass backwards, each through a four-sided space bounded below by the neck of a rib, above by the transverse process of the upper vertebra, externally by the superior costo-transverse ligament, and internally by the body of a vertebra. Between the transverse processes each divides into an internal and external branch. The internal branches incline inwards on the superficial surface of the multifidus spinæ, and the upper six become cutaneous near the spines of the vertebræ, whilst the lower six end in the deep muscles. The external branches pass outwards beneath the middle column of the erector spinæ, and, on reaching the interval between the middle and outer columns of that muscle, they end differently in the upper and lower parts of the back. The upper six end in the deep muscles, but the lower six become cutaneous along the line of the angles of the ribs. In the lumbar region the posterior primary divisions pass backwards, each through a space bounded externally by the quadratus lumborum muscle, internally by the intertransversalis lateralis muscle, and above and below by a transverse process. Their further disposition is as in the thoracic region, but the external branches of the first three only furnish cutaneous nerves, and, as already stated, these are principally gluteal in their distribution. 
The arteries of the thoracic region are the dorsal branches of the intercostal arteries. Each dorsal branch passes backwards in company with the corresponding posterior primary division of a spinal rerve. Before passing through the quadrangular space it gives off a spinal branch, which enters the spinal canal through the intervertebral foramen. After passing backwards it divides into an internal and external branch, which have a distribution similar to those of the nerve.

The veins of the thoracic region terminate in the intercostal veins.

The arteries of the lumbar region are the dorsal branches of the lumbar arteries. Each accompanies the corresponding posterior primary division of a spinal nerve, and its distribution is as in the thoracic region.

The veins of the lumbar region terminate in the inferior vena cava.

\section{THE PECTORAL REGION AND AXILLARY SPACE.}

Landmarks - The outline of the clavicle and acromion process of the scapula are readily made out, and, in connection with the acromion, it is to be borne in mind that the acromial epiphysis may remain permanently detached from the spine, and so simulate a fracture. The roundness of the shoulder is to be noted, this being due largely to the deltoid muscle, but also in part to the head of the humerus. Below the clavicle, at a point external to its centre, is a depression, called the infraclavicular fossa, which indicates the separation between the deltoid and pectoralis major. When the arm is abducted and the finger pressed into this hollow, the inner border of the coracoid process will be felt, and, if the finger be carried outwards under the deltoid, the tip of that process can be made out. In this region it is possible to compress the axillary artery against the second rib, but this requires deep pressure. The course of this artery may be indicated by a line drawn from the centre of the clavicle to the inner border of the coraco-brachialis, the arm having been raised to a right angle with the trunk, so as to define clearly the outline of that muscle. The anterior and posterior folds of the axilla are to be observed, the anterior being formed by the lower border of the pectoralis major, which practically coincides with the line of the fifth rib, and the posterior by the latissimus dorsi winding round the lower border of the teres major. The hollow between these folds indicates the position of the axillary space, and it will be seen to be narrow towards the arm, but wide towards the thoracic wall. In the female the prominence formed by the mammary gland is usually apparent on the surface of the pectoralis major. Its vertical extent is from the second to the sixth rib, and it reaches transversely from the side of the sternum to the anterior fold of the axilla, except at the lower and outer part, where it extends beyond this fold upon the serratus magnus. A little below and internal to the centre of the mammary prominence is the nipple, which is sur- 
rounded by a coloured circle, called the areola. The exact position of the nipple is subject to variation. As a rule it may be said to lie over the fourth intercostal space about 4 inches from the midsternal line, but in corpulent persons, and in females with pendulous mammæ, it is lower in position. Along the middle line the sternum can readily be made out, and about 2 inches below its upper border the transverse ridge, called the sternal angle, may be felt, which is the guide to the second costal cartilage at. either side. Above the centre of the upper border of the sternum is a depression, known as the jugular fossa. Below the lower border of the mesosternum there is the infrasternal depression, which is situated between the seventh pair of costal cartilages, where it lies over the metasternum.

Cutaneous Nerves.-These nerves are arranged in three groupsdescending, anterior, and lateral.

The descending nerves are branches of the cervical plexus, and are derived from the third and fourth cervical nerves. They are three in number - suprasternal or internal, supraclavicular or middle, and supra-acromial or external-and, as they descend, they lie beneath the platysma myoides. The suprasternal nerve crosses the inner end of the clavicle, and its branches are distributed to the subjacent integument, as well as to that over the upper part of the sternum. The supraclavicular nerve crosses the centre of the bone, and its branches have a fairly wide distribution to the pectoral integument. The supra-acromial nerve crosses the outer end of the clavicle, and its branches are distributed to the integument over the upper half of the deltoid.

The anterior cutaneous nerves are the terminal branches of the upper six intercostal nerves, and they pierce the pectoralis major close to the sternum. They furnish small twigs to the integument over that bone, but the principal part of each nerve takes an outward course and supplies the pectoral integument. The branch of the first nerve is sometimes absent.

The lateral cutaneous nerves are branches of the intercostal nerves from the third to the sixth inclusive. The first intercostal nerve gives no lateral cutaneous branch. The lateral cutaneous of the second intercostal nerve is undivided, and crosses the axillary space to the brachial region, under the name of intercosto-humeral. The succeeding lateral cutaneous nerves emerge from their intercostal spaces by piercing the external intercostal muscles not far from the lower border of the pectoralis major. Each then divides into an anterior and a posterior branch, which pass out between the digitations of the serratus magnus, being separated from each other by a slight interval. The anterior branches wind round the lower border of the pectoralis major to supply the pectoral integument, whilst the posterior branches pass backwards to supply the integument over the anterior border of the latissimus dorsi.

The anterior and lateral cutaneous nerves are accompanied by 
corresponding arteries, the anterior being the perforating branches of the internal mammary, and the lateral being branches of the intercostal arteries.

Fascia.-The superficial fascia is continuous over the clavicle with the superficial fascia of the neck, and it is noteworthy in two respects:

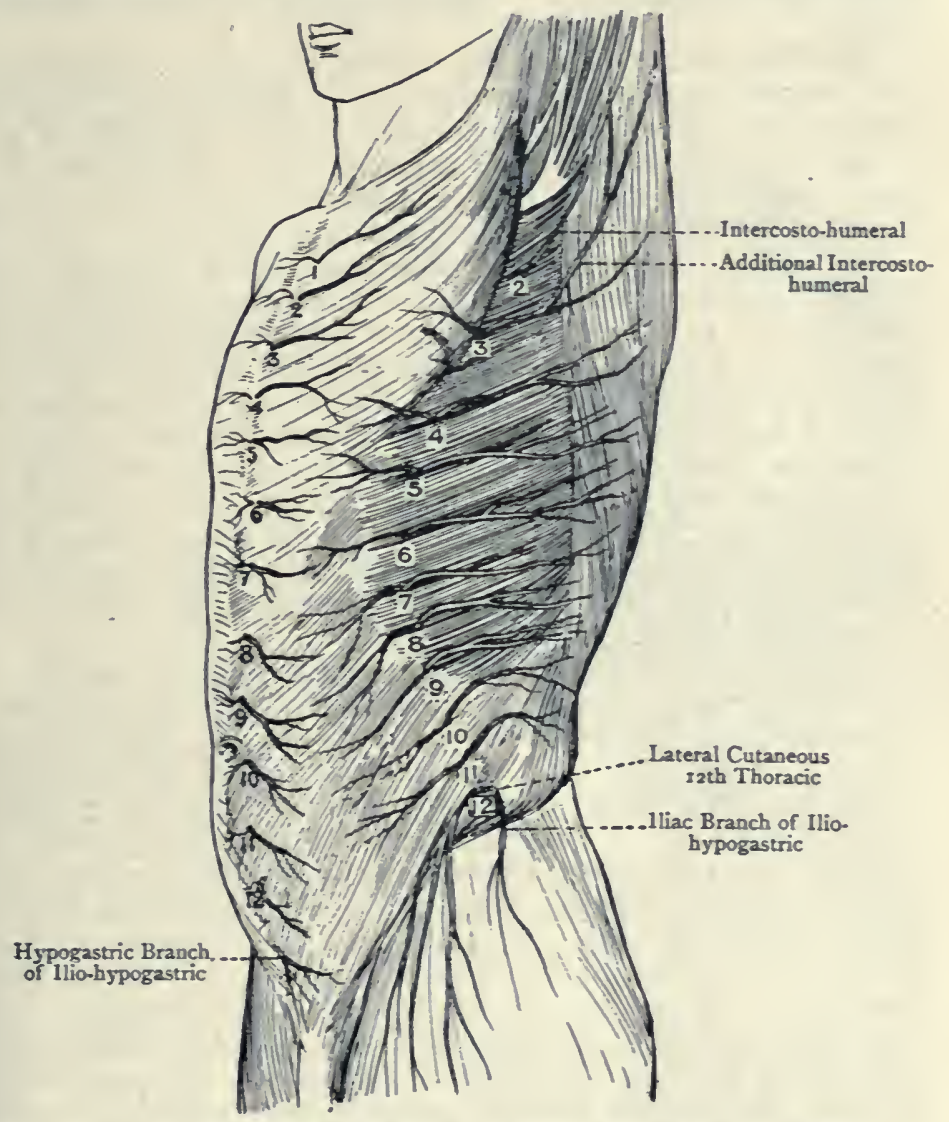

Fig. 172.-Cutaneous Nerves of the TruNk (ANTero-lateral VIEW) (AFTER HENLE).

1-12, Anterior Cutaneous; 2-12, Lateral Cutaneous.

In the first place, just below the clavicle it has a faint reddish colour, due to the fact that over the clavicular portions of the pectoralis major and deltoid it gives origin to fibres of the platysma myoides, which can be seen on incising it. In the second place, in $\checkmark$ the region of the mammary gland it divides into two laminæ, which ensheathe that gland. These laminæ send processes into the sub- 
stance of the gland which support its lobes, and from the anterior lamina fibrous bands pass to the integument, these being known as the ligamenta suspensoria of Cooper. Inferiorly the superficial fascia is continuous with that over the anterior abdominal wall, and externally it is prolonged over the floor of the axillary space to become continuous with the superficial fascia of the brachial region.

The deep fascia is thin and membranous, and it closely invests the pectoralis major. Superiorly it is attached to the front of the clavicle above the origin of that muscle, internally it is fixed to the front of the sternum, externally it is continuous with the deep fascia over the deltoid, and inferiorly it joins the axillary fascia at the lower border of the pectoralis major.

The axillary fascia is a firm membrane which stretches from the anterior to the posterior fold of the axilla, and forms the floor of that space. It is continuous in front with the deep pectoral fascia, and behind it blends with the deep fascia which ensheathes the latissimus dorsi. Externally it is continuous with the deep fascia of the brachial region, and internally with the fascia covering the serratus magnus. Its upper surface is convex, due to the fact that it receives, near the pectoralis major, the clavi-pectoral fascia, to be afterwards described. In this way the axillary fascia is drawn upwards into the space. It is of considerable strength, and is capable of affording much resistance to the pointing of an axillary abscess in this direction.

Mammary Gland.-This gland is situated on the surface of the pectoralis major. It is somewhat hemispherical, and extends vertically from the second to the sixth rib, and transversely from the side of the sternum to the anterior fold of the axilla, except below and externally, where it passes over that fold upon the serratus magnus. A little below, and internal to, the centre of the gland is the corrugated nipple, lying in the centre of a coloured circle, called the areola. There is no fat beneath the nipple and areola, and the skin of these parts is provided with plain muscular tissue disposed circularly, which, by its contraction, contributes to erection of the nipple by compressing its veins. The summit of the nipple is perforated by the openings of the lactiferous ducts. The skin of the areola presents a number of small projections due to sebaceous glands, which are known as the glands of Montgomery. The mamma is a compound racemose gland, which is composed of about twenty lobes, these in turn consisting of lobules, and all being connected by a fibrous stroma. The entire gland is ensheathed by the splitting of the superficial pectoral fascia into two laminæ, and these send processes into its interior which support its component lobes. The posterior layer of the sheath is loosely connected to the deep pectoral fascia covering the pectoralis major, and sometimes deep processes of the gland penetrate into the substance of the muscle. The lobes are pyramidal, and their apices converge toward the nipple. Each of 
them is distinct, and has its own duct. A lobe is made up of lobules, and each lobule is composed of a cluster of tubes, called alveoli or acini, which represent the secreting parts of the gland, and are lodged in spaces known as loculi. The alveolar tubes are lined with cubical epithelium, the cells of which contain fat globules during the period of the functional activity of the organ. The ducts of the lobes are about twenty in number, and are called the lactiferous or galactophorous ducts. They are lined with columnar epithelium, and, as they approach the nipple, each presents a dilatation, called the sinus or ampulla. Thereafter each duct becomes narrow, and enters the nipple to terminate by a minute pore on its sum m it . The mammary glands are present in both sexes, but in the male their development, as a rule, is arrested, so that they are in a rudimentary condition.

Bloodvessels of the Mamma. - The arteries are as follows: long

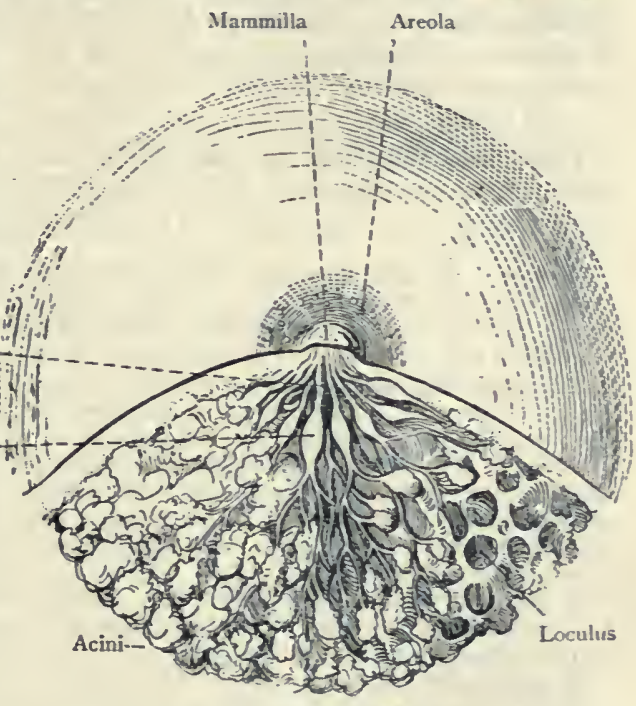

Fig. r73.-The Female Mamma durixg LActation (AFTER LUSchKa).

thoracic (external mammary) of the second part of the axillary; anterior cutaneous or perforating of the internal mammary; and branches from the intercostal arteries of the spaces over which the gland lies.

The veins pass to the axillary and internal mammary veins.

Lymphatics.-The principal lymphatic vessels of the mammary gland arise in the fibrous stroma, and pass towards the nipple, lying in their course between the galactophorous ducts. In the region around the nipple they terminate beneath the areola in a plexus, called the subareolar plexus. This plexus also receives the cutaneous lymphatics of the nipple and areola. The efferent vessels from this plexus pass to the antero-internal or pectoral group of axillary glands, more particularly to those on a level with the third rib.

There are, however, other paths by which lymph is conveyed away from the mammary gland. (I) Some lymphatics emerge from about the outer two-thirds of the gland, and pass to the pectoral group of axillary glands, either directly, or after having joined the principal lymphatics. (2) Other lymphatics (one or 
more) emerge from the deep part of the gland. These pierce the pectoralis major muscle, and terminate in one of the infra-clavicular glands. (3) A third set of lymphatics emerge from about the inner third of the gland. These pierce the pectoralis major muscle near the lateral border of the sternum, the anterior intercostal aponeurosis, and the interchondral portions of the internal intercostal muscles, after which they terminate in the internal mammary glands, otherwise known as the anterior intercostal or sternal glands.

Nerves.-These are derived from the supraclavicular branch of the cervical plexus, and the anterior and lateral cutaneous branches of the upper intercostal nerves.

Accessory mammæ are sometimes met with on the upper, lower, and inner outskirts of the main gland, and more rarely in the axilla, or on the upper part of the anterior abdominal wall.

Structure of the Mamma.-The mammary gland belongs to the class of acino-tubular or compound racemose glands. It consists of-(I) glandular tissue, and (2) a fibrous connective-tissue stroma, which together constitute a firm light red mass, known as the corpus mamma.

The glandular element is composed of large lobes, varying in number from fifteen to twenty. There is no firm fibrous capsule, such as that possessed by the parotid salivary gland, but the superficial pectoral fascia, within which the mammary gland is embedded, sends inwards fatty processes between the lobes, the supporting fibrous tissue of these processes being continuous with the sustentacular tissue of the gland. Each lobe is tapering in outline, and is provided with its ovgn duct, the various lobes being disposed in a radiating manner from the nipple.

A mammary lobe, being large, is made up of smaller lobes, and each small lobe consists of a group of lobules, the structure of which furnishes the key to the structure of the entire gland.

A mammary lobule consists of from one to three acini, and these have one duct in common. This duct is one of the radicles of the main duct of a given mammary lobe. The radicle duct breaks up into two or three somewhat dilated excretory passages, the walls of each of which are convoluted. This convoluted character is due to the fact that the walls are distended into a series of pouches, called alveoli, which beset all parts of the wall of the acinus, including its cæcal end, thus giving it somewhat of the appearance of a grape -hence the name acinus. The wall of each alveolus consists of a delicate basement membrane, which is invested by capillary bloodvessels, and lined with cubical or short columnar epithelium. From one to three acini, as stated, constitute a lobule, and they are consequently appendages of a lobular duct. Contiguous lobular ducts unite and form the duct of a small lobe, and the contiguous ducts of small lobes unite, and form the duct of a large lobe. The number of main ducts corresponds to the number of main lobes-namely, from fifteen to twenty. They are referred to as the lactiferous or galactophorous ducts. The main lactiferous ducts converge towards the nipple. As they lie beneath the areola, each.duct expands into a somewhat spindle-shaped dilatation, called the ampulla or lactiferous sinus, which serves as a reservoir for the milk during lactation. Thereafter the duct becomes narrow, and having traversed the nipple, it opens upon its summit at the bottom of a small depression or foveola. The orifice of the duct is of smaller size than the excretory tube leading to it. The arrangement of orifices at the summit of the nipple may be compared to the arrangement of the orifices of the excretory tubes of the kidney at the apex of a given Malpighian pyramid.

The walls of the ducts consist of areolar and clastic tissue, and they are lined with columnar epithelium. The elastic tissue is disposed both longitudinally and in a circular manner. 
Development of the Mammary Glands.-The mammary glands are of ectodermic origin. In the course of the second month of intra-uterine life a circular thickening of the epidermis (ectoderm) makes its appearance in the region of the future gland. This thickening projects into the subjacent mesodermic corium, and the superficial central cells of the thickening becoming shed, the thickened area is now depressed. From the deep surface of the thickened ectodermic area solid columns of cells grow into the subjacent mesodermic corium, which give off lateral offshoots. These cell columns become hollow, and form the lactiferous or galactophorous ducts, which open upon the area corresponding to the circular thickening of epidermis. The lateral offshoots of the cell columns give rise to the lobes, lobules, and alveoli or acini of the gland. Indeed, each lateral offshoot gives rise to a mammary gland in miniature.

The nipple and areola are developed from the glandular area-that is to say, the area of the original thickening of epidermis. The depression of this area disappears, and its central portion becomes elevated to form the nipple. This consists of epidermis, and contains the terminal parts of the lactiferous ducts, bloodvessels, and connective tissue. The remainder of the glandular area forms the areola. The corium corresponding to the nipple and areola is provided with unstriped muscular itssue.

The connective-tissue stroma of the gland is developed from the mesoderm of the corium.

Pectoralis Major-Origin.-(r) The anterior surface of the clavicle over its inner half, and the anterior sterno-clavicular ligament; (2) one-half of the anterior surface of the sternum as low as the metasternum; (3) the anterior surfaces of the upper six costal cartilages, and slightly from the bony part of the sixth rib; and (4) the upper portion of the external oblique aponeurosis.

Insertion.-The outer bicipital ridge of the humerus over its lower three-fourths.

The muscle is divisible into two portions, clavicular and sternocostal, which are separated by a slight interval extending downwards and outwards from the sterno-clavicular joint.

The tendon of insertion is folded upon itself so as to be composed of two layers, anterior and posterior, the former of which is the horter, and both being continuous below. The anterior layer eceives the sterno-costal fibres above the third costal cartilage, and it also receives superficially the clavicular fibres, which latter lescend lowest at their insertion, where they are intimately conlected with the tendon of the deltoid. The posterior layer receives il the fibres from the third costal cartilage downwards, and the pwest of these fibres, as they pass outwards and upwards, become uccessively folded underneath the fibres above. The result is hat the fibres arising lowest reach the highest part of the posterior tyer of the tendon, whilst the fibres above these reach its lowest art. The posterior layer ascends higher on the outer bicipital idge than the anterior, and from its upper border a tendinous xpansion passes to the great tuberosity of the humerus and the apsular ligament of the shoulder-joint, which conceals the long endon of the biceps brachii. From the lower border of the tendon $\mathrm{n}$ expansion is given to the deep fascia of the arm.

Nerve-supply.-(I) The external anterior thoracic from the outer ord of the brachial plexus, (its fibres coming from the fifth, sixth, id seventh cervical nerves), the branches of which nerve enter 
the deep surface of the clavicular and upper sterno-costal portions ; and (2) the internal anterior thoracic, the branches of which enter the deep surface of the sterno-costal portion, after having pierced the pectoralis minor.

Blood-supply.-Branches of the thoracic axis.

The upper fibres pass downwards and outwards, the middle transversely outwards, and the lower upwards and outwards.

Action.-Acting from its origin the muscle adducts the arm, draws it forwards-that is to say, flexes it at the-shoulder-joint

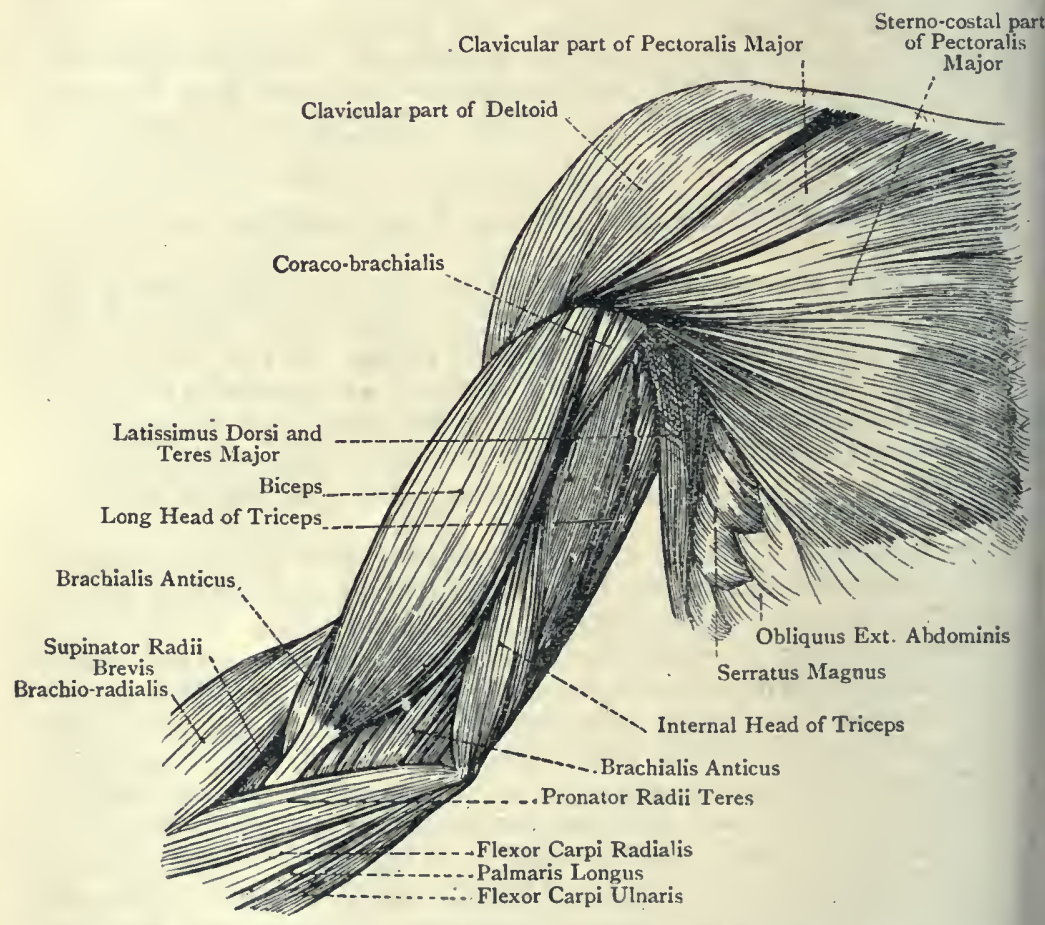

Fig. 174.-The Superficial Muscles of the Front and Inner Side of the Pectoral and Brachial Regions.

(by its clavicular portion), and rotates it inwards: Acting from its insertion it raises the trunk after the outstretched arm, as in climbing a pole, and it elevates the upper ribs in forced inspiration.

The upper border of the muscle is related to the deltoid, from which it is separated by a triangular interval for a short distance below the clavicle, and the cephalic vein and humeral artery intervene between the two. The lower border forms the anterior fold of the axilla. Sometimes there is a muscle, called the sternalis, 
present, on one or both sides. It lies over the sternal fibres of the pectoralis major, and is connected below with the external oblique aponeurosis, whilst above it may terminate in the sternal head of the sterno-cleido-mastoid, or on the presternum.

Clavi-pectoral Fascia.-This is situated beneath the pectoralis major. Superiorly it is disposed in two laminæ, which are attached to the anterior and posterior lips of the subclavian groove of the clavicle, and embrace the subclavius muscle. At the lower border of that muscle they unite to form the costo-coracoid membrane, which, on reaching the upper border of the pectoralis minor, divides to ensheathe it. At the lower border of the muscle the two layers unite to form a single sheet, which joins the axillary fascia, and acts as a suspensory ligament.

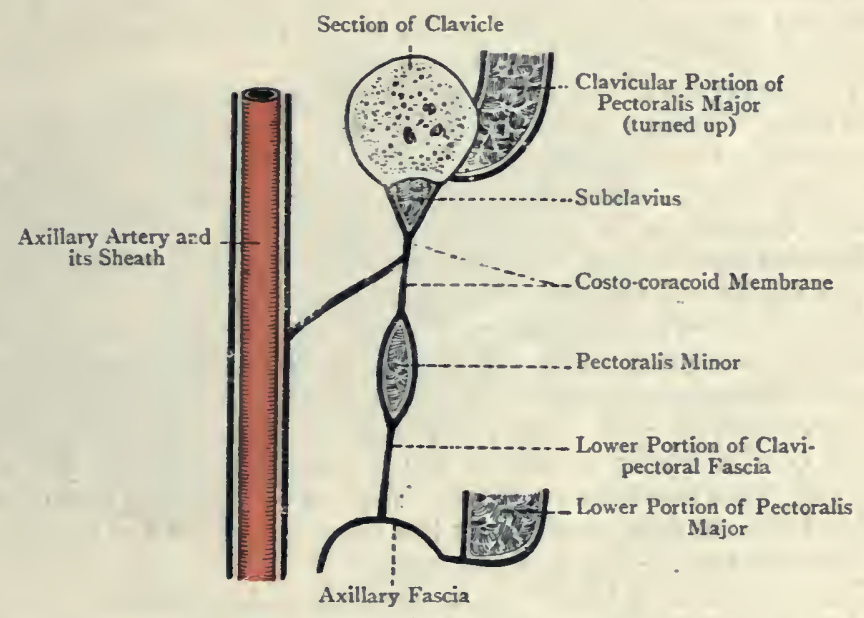

Fig. 175.-Diagram of the Clavi-pectoral Fascia and the Costo-coracoid Membrane.

Costo-coracoid Membrane.-This name is given to that portion of the clavi-pectoral fascia which extends from the lower border of the subclavius to the upper border of the pectoralis minor. The lower portion of the membrane is tense and cord-like, and is known as the costo-coraccid ligament. It is attached internally to the ipper surface of the first rib at its sternal extremity in connection vith the tendon of the subclavius, and externally it is attached to he posterior part of the antero-internal border and adjacent portion ff the upper surface of the coracoid process. This membrane is onnected by its deep surface with the axillary sheath ; it is covered y the clavicular portion of the pectoralis major; and it is pierced $y$ the cephalic vein, the thoracic axis, and the external anterior horacic nerve. 
The costo-coracoid ligament represents the ventral end of the coracoid bar of cartilage, the dorsal end of which forms the.coracoid process.

Pectoralis Minor-Origin.-The upper borders and outer surfaces of the third, fourth, and fifth ribs near their anterior extremities, as well as from the fascia covering the adjacent external intercostal muscles.

Insertion.-The anterior half of the antero-internal border of the coracoid process of the scapula and the adjacent portion of its upper surface, where it is intimately connected with the common origin of the coraco-brachialis and short head of the biceps.

Nerve-supply.-The internal anterior thoracic nerve, which is a branch of the inner cord of the brachial plexus, its fibres being derived from the eighth cervical and first thoracic. The branches of the nerve enter the muscle on its deep surface, and a few of them pierce it to enter the deep surface of the pectoralis major.

Blood-supply.-The thoracic axis.

The direction of the fibres is upwards and outwards.

Action.-Acting from its origin the muscle draws the scapula downwards and forwards, the point of the shoulder being at the same time depressed. Acting from its insertion it elevates the ribs from which it arises, as in forced inspiration.

Subclavius-Origin.-By a rounded, tapering tendon from the upper surface of the first rib and its cartilage.

Insertion.- The subclavian groove on the under surface of the clavicle, extending from the rhomboid impression internally to the interval between the conoid tubercle and trapezoid ridge externally.

Nevve-supply. - The nerve to the subclavius, which arises from the front of the upper trunk of the brachial plexus, its fibres being derived from the fifth cervical. The nerve descends from the neck behind the clavicle, and enters the deep surface of the muscle.

Blood-supply.-The thoracic axis.

The direction of the fibres is upwards and outwards.

Action.-(I) To depress the clavicle and draw it slightly forwards, and (2) to support the sterno-clavicular joint by bracing the clavicle in an inward direction.

Axillary Space.--The axillary space is situated between the upper part of the arm and upper part of the thoracic wall. It has the form of a four-sided pyramid, and presents an apex, a base or floor, and four walls-anterior, posterior, inner, and outer. It is of much greater extent towards the thoracic wall than towards the arm, on account of the convergence in the latter direction of the structures forming the anterior and posterior walls. The apex is the narrowest part of the space, and is directed upwards towards the root of the neck. It is somewhat triangular, and lies between the clavicle, first rib, and upper border of the scapula. The base or floor is of considerable extent, and is formed directly by the axillary fascia, which, as stated, is drawn upwards into the space by the clavipectoral fascia. The anterior wall is formed over its whole extent by the pectoralis major, and, under cover of this, by the pectoralis 
ainor over about its middle third. Above the latter muscle the osto-coracoid membrane enters into the anterior wall. The lower order of the anterior wall, formed by the pectoralis major, contitutes the anterior fold of the axilla. The posterior wall is formed rom above downwards by the subscapularis, teres major, and latisimus dorsi. Towards the arm the tendon of the latissimus dorsi ies in front of the teres major, the latter muscle extending a little ower down. The posterior wall is longer than the anterior, and its ower border, formed by the folding of the latissimus dorsi round the eres major to get in front of it, forms the posterior fold of the axilla. the inner wall is formed by the upper four or five ribs, with their ntercostal muscles, and the corresponding serrations of the serratus

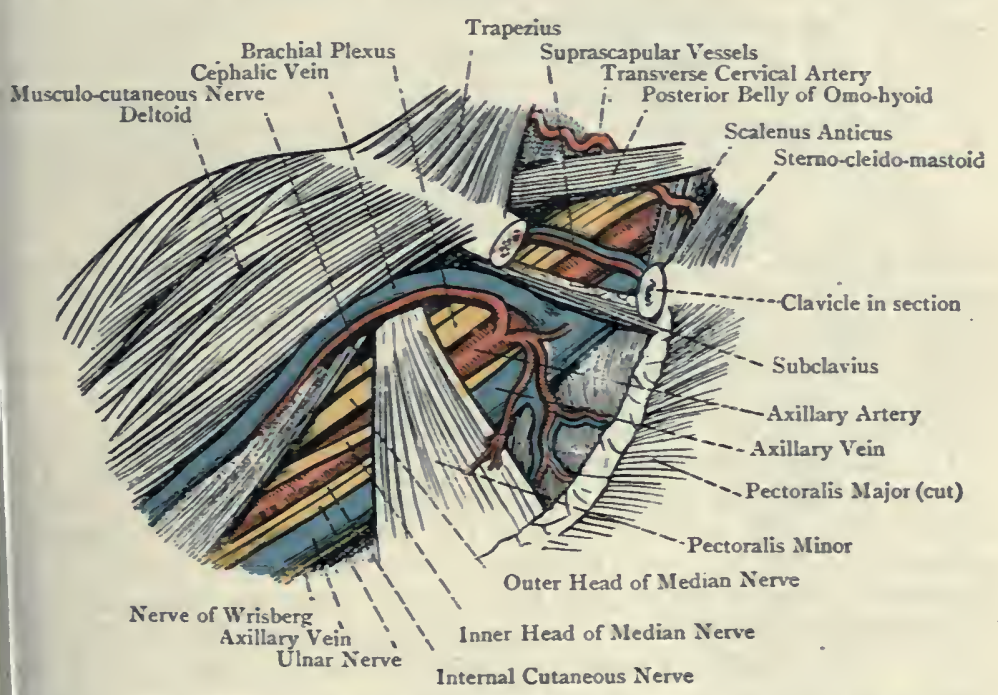

G. I76.-The Axillary Space, After Reflection of the Pectoralis Major, AND the Subclavian Triangle.

agnus. The outer wall, which is very circumscribed, is formed by le upper part of the humerus and the common origin of the coracorachialis and short head of the biceps.

Contents and their Position. - The axillary vessels and the nerves bich arise from the brachial plexus lie for the most part along the iter wall. The thoracic axis and the long thoracic artery pass to e anterior wall, the former above the pectoralis ininor, where it erces the costo-coracoid membrane, the latter along the lower rder of that muscle. The subscapular artery lies on the posterior all, where it passes inwards on the lower border of the subscapuris. The posterior circumflex artery lies for a short distance on the sterior wall, but soon passes backwards between the subscapularis d teres major. The anterior circumflex artery passes outwards 
in front of the humerus beneath the coraco-brachialis and biceps. The superior thoracic artery is above the thoracic axis, where it takes a course inwards to the thoracic wall.

The external anterior thoracic nerve pierces the costo-coracoid membrane to enter the deep surface of the pectoralis major. The internal anterior thoracic nerve enters the deep surface of the pectoralis minor, and sends branches through it to the major. The posterior thoracic nerve descends upon the inner wall, resting on the serratus magnus. The intercosto-humeral nerve pierces the second intercostal space, and crosses the axilla to the inner side of the arm. Below this nerve, on the thoracic wall, the succeeding lateral cutaneous branches of intercostal nerves pierce the intercostal spaces, and the posterior branch of the lateral cutancous of the third intercostal gives a branch which crosses the axilla to the inner side of the arm, communicating in its course with the intercosto-humeral.' The three subscapular nerves lie upon the posterior wall, and supply the muscles which form it. The circumflex nerve lies with the posterior circumflex artery, which it accompanies in its backward course. The musculo-spiral nerve descends behind the axillary artery, and eventually turns to the back of the humerus, but, before doing so, it gives off its internal cutaneous branch. The median nerve is upon the outer side of the main artery, and the ulnar nerve is internal to it, between it and the vein. The lesser internal cutaneous nerve is on the inner side of the vein, and the internal cutaneous nerve is usually met with partly over the line of the main artery and partly on its inner side.

The axillary lymphatic glands are disposed in four groups. The external or humeral glands lie on the outer wall, where they form a chain along the axillary vein. The antero-internal or pectoral glands lie along the long thoracic artery at the lower border of the pectoralis minor, resting upon the serratus magnus. . The posterior or subseapular glands lie on the posterior wall along the subscapular artery. The central glands are situated towards the base of the space.

Axillary Artery.-The axillary artery is the continuation of the subclavian, and it extends from the outer border of the first rib to the lower border of the teres major, where it becomes the brachial. When the arm is by the side of the trunk the vessel describes a curve with the convexity upwards, but, when the limb is abducted to the position of a right angle to the trunk, its course is almost straight. In the latter position of the limb its course may be indicated by a line drawn from the centre of the clavicle to the inner border of the coraco-brachialis. The artery is crossed by the pectoralis minor and is thus divided into three parts-first, second, and third.

First Part. - The first part extends from the outer border of th first rib to the upper border of the pectoralis minor, and it is abou I inch long.

Relations.-Anteriorly it is covered by the skin, superficial fascia 
of the brachial plexus, behind which is a quantity of fat, separating the vessel from the subscapularis. Externally is the outer cord of the brachial plexus. Internally are the inner cord of the brachial plexus, the internal anterior thoracic nerve, and the axillary vein.

Third Part.-The third part extends from the lower border of the pectoralis minor to the lower border of the teres major, and its length is about 3 inches. The upper half of this part is under cover of the pectoralis major, but the lower half is free from muscular covering, which is due to the posterior wall of the axilla being longer than the anterior.

Relations.-Anteriorly over its upper half it is covered by the integument and pectoralis major, and over its lower half only by the integument of the arm. The inner root of the median nerve crosses it obliquely from within outwards, and the external vena comes of the brachial artery crosses it from without inwards. Posteriorly it rests, from above downwards, upon the subscapularis, tendon of the latissimus dorsi, and lower part of the teres major, and the circumflex and musculo-spiral nerves descend behind it. Externally is the coraco-brachialis, which sometimes slightly overlaps it. The outer root of the median nerve lies on its outer side, as does also the musculo-cutaneous. Internally is the axillary vein, and, for a short distance below, the internal vena comes of the brachial artery. Between the axillary vein and the artery are the inner root of the median nerve and the ulnar nerve; internal to the vein is the lesser internal cutaneous nerve; and the internal cutaneous nerve is partly on the artery and partly to its inner side.

Branches.--The first part gives off the superior or short thoracic, and the thoracic axis or acromio-thoracic artery. The second part furnishes the inferior or long thoracic, and the alar thoracic, which is very inconstant as a separate branch. The third part gives off the subscapular, the anterior circumflex, and the posterior circumflex.

Branches of the First Part.-The superior or short thoracic artery is a small branch which arises just below the subclavius. Its course is inwards and downwards behind the axillary vein to the first two intercostal spaces, where it anastomoses with branches of the first and second intercostal arteries. It supplies the adjacent intercostal muscles, upper part of the serratus magnus, and occasionally the pectoral muscles. The thoracic axis or acromio-thoracic artery is a short, but large, trunk which arises immediately above the upper border of the pectoralis minor. Passing straight forwards it pierces the costo-coracoid membrane, and then divides into radiating branches, called thoracic, acromial, humeral, and clavicular. The thoracic branches descend between the two pectoral muscles which they supply. The acromial branches pass outwards over the coracoid process to the deltoid, in which some of them end, whilst others pierce that muscle, and so reach the upper surface of the acromion process, where they anastomose with branches of the suprascapular and posterior circumflex. The humeral (descending) branch passes 
downwards with the cephalic vein between the pectoralis major and deltoid, to the contiguous parts of which muscles it is distributed. The clavicular branch passes upwards to end in the subclavius.

Branches of the Second Part.-The inferior or long thoracic artery, also called the external mammary, is directed downwards and inwards along the lower border of the pectoralis minor to the thoracic wall. It is distributed to the pectoral muscles, serratus magnus, and intercostal muscles, and it sends branches round the lower border of the pectoralis major to the mammary gland. It also gives branches to the pectoral group of axillary glands, and it anastomoses with branches of the aortic intercostals and internal mammary. The alar thoracic artery is distributed to the axillary glands. It is seldom a special branch, its place being usually taken by branches of the long thoracic and subscapular.

Branches of the Third Part.-The subscapular artery, which is the largest of all the branches of the vessel, arises opposite the lower border of the subscapularis, along which it courses downwards and inwards to the lower angle of the scapula, in company with the long subscapular nerve, where it anastomoses with the posterior scapular and long thoracic. Besides giving branches to the muscles on the posterior wall, the serratus magnus, and the posterior group of axillary glands, it gives off near its origin a large branch, called the dorsalis scapulc. This vessel at once passes backwards through the triangular space bounded above by the subscapularis, below by the teres major, and externally by the long head of the triceps. Thereafter it winds round the back of the axillary border of the scapula, piercing the origin of the teres minor and grooving the bone, and so it reaches the infraspinous fossa beneath the infraspinatus. Here it breaks up into numerous branches, which supply that muscle and the bone, and which anastomose with the suprascapular and dorsal branches of the posterior scapular. As it passes through the triangular space it furnishes a ventral branch (infrascapular), which ramifies in the venter of the scapula beneath the subscapularis, and anastomoses with the ventral branches of the suprascapular and posterior scapular. Before piercing the teres minor it gives off a descending or teres branch, which passes downwards between the teres major and minor as far as the lower angle of the scapula,.where it anastomoses with the terminal part of the posterior scapular. The dorsalis scapulæ sometimes arises directly from the axillary.

The anterior circumflex artery, which is of small size, arises from the outer side of the vessel a little below the subscapular and opposite the posterior circumflex. It passes outwards in front of the surgical neck of the humerus, and beneath the coraco-brachialis and biceps. On reaching the bicipital groove it gives off an ascending or bicipital branch, which passes upwards in the groove with the long head of the biceps, to be distributed to the shoulder-joint and head of the humerus. Thereafter it continues its winding course to the outer side of the bone, where it anastomoses with the posterior circumflex. 
The posterior circumflex artery, which is of large size, arises from the back of the vessel a little below the subscapular. Its course is backwards in company with the circumflex nerve through the quadrangular space, which is bounded above by the teres minor (subscapularis in front), below by the teres major, internally by the long head of the triceps, and externally by the surgical neck of the humerus. In its course it winds round the inner and posterior aspects of the neck of the bone, and it furnishes many branches, most of which enter the deep surface of the deltoid. A special acromial branch reaches the upper surface of the acromion process, and there anastomoses with the suprascapular and acromial branches of the thoracic axis. On the outer side of the neck of the bone the artery anastomoses with the anterior circumflex, and in this way an arterial circle is formed which closely embraces the surgical neck. Some of the branches supply the muscles bounding the quadrangular space, and one or two pass downwards between the long and outer heads of the triceps, where they anastomose with the superior profunda of the brachial. The posterior circumflex artery is subject to certain variations. (I) It may arise in common with the subscapular. (2) Its origin may be transferred to the superior profunda of the brachial, in which case it ascends behind the teres major. (3) It may give off the anterior circumflex, superior profunda, or dorsalis scapulæ.

Varieties of the Axillary Artery.-(I) The subscapular, circumflex, and profundæ of the brachial may arise by a common trunk. (2) The artery may give origin to a large branch, which may be the radial, ulnar, vas aberrans, or the in terosseous trunk of the forearm.

The part of the axillary artery most accessible for surgical interference is the lower half of the third part, which is covered only by the integument of the arm.

Axillary Vein.-The axillary vein is the continuation of the basilic vein of the arm. It extends from the lower border of the teres major to the outer border of the first rib, where it becomes the subclavian vein. It is of large size, and throughout its entire course it lies to the inner side of the artery. Most of its tributaries correspond with the arterial branches, but the following two are specially noteworthy: (I) a little above its commencement it receives the trunk formed by the union of the venæ comites of the brachial artery, and (2) below the clavicle it is joined by the cephalic vein.

Axillary Sheath.-The axillary vessels and the brachial plexus of nerves are enclosed in a loose, infundibuliform sheath, which is called the axillary sheath. It is a downward prolongation of the deep cervical fascia, and it blends with the deep surface of the costo-coracoid membrane. It has been compared to the crural sheath of the femoral vessels, but it is in no sense such a defined structure.

Brachial Plexus.- The brachial plexus is situated in the lower part of the posterior triangle of the neck, behind the clavicle, and in the upper part of the axilla. Its complex formation is rendered simple 
by arranging it into four stages, namely, (I) nerve roots, (2) nerve trunks, (3) divisions of nerve trunks, and (4) nerve cords.

First Stage. - The nerves which form the plexus are the anterior primary divisions of the fifth, sixth, seventh, and eighth cervica!, and the greater part of that of the first thoracic. Superiorly the plexus is reinforced by a small descending branch from the fourth cervical, which joins the fifth, and inferiorly it is occasionally reinforced by a branch from the second thoracic, which joins the first. As regards the first thoracic nerve, the part of it which does not join the plexus, and which is of small size, enters the first intercostal space to become the first intercostal nerve. The nerves, as they emerge at the side of the neck, are placed between the scalenus anticus and scalenus medius.

Second Stage.-The fifth and sixth cervical nerves join at the outer border of the scalenus anticus to form the upper trunk; the seventh cervical remains meanwhile single, and forms the middle trunk; and the eighth cervical and greater part of the first thoracic unite between the scalene muscles to form the lower trunk. There are thus three trunks - upper, middle, and lower.

Third Stage.-A little above the clavicle each of the three trunks breaks up into an anterior and a posterior division.

Fourth Stage.--The anterior divisions of the upper and middle trunks unite to form the outer cord of the plexus; the anterior division of the lower trunk, which is of large size, forms the inner cord ; and all three posterior divisions (that of the lower trunk being of small size) unite to form the posterior cord. As a variety, the anterior division of the middle trunk may subdivide into two branches, one entering the outer cord and the other the inier. There are thus three cords-outer, inner, and posterior.

Branches of the Plexus.-The branches are conveniently divided into two groups--supraclavicular, arising above the clavicle and coming from nerve roots and nerve trunks; and infraclavicular, arising below the clavicle and coming from nerve cords.

Supraclavicular Branches.-These are as follows: Muscular branches from the four cervical nerves to the scalene muscles and longus colli.

One root of the phrenic nerve (inconstant) from the front of the fifth cervical.

The Nerve to the Rhomboids. - This branch arises from the back of the fifth cervical close to, or along with, the highest root of the posterior thoracic, and it takes a backward course through the scalenus medius.

The Posterior Thoracic Nerve or External Respiratory Nerve of Bell. - This branch arises by three roots from the back of the fifth, sixth, and seventh cervical nerves. The upper two roots pierce the scalenus medius below the nerve to the rhomboids, either conjointly or separately, whilst the lowest ront passes in front of the scalenus medius, and joins the trunk formed by the others near the first rib. The nerve then courses behind the first part of the axillary artery, 
and subsequently descends upon the axillary surface of the serratus magnus, supplying branches to all its serrations.

The Nerve to the Subclavius. - This small branch arises from the front of the upper trunk, its fibres being derived from the fifth cervical. It descends over the third part of the subclavian artery, and, passing behind the clavicle, it enters the subclavius muscle on its deep aspect. This nerve sometimes communicates with the phrenic nerve.

The Suprascapular Nerve.-This is a large nerve which arises

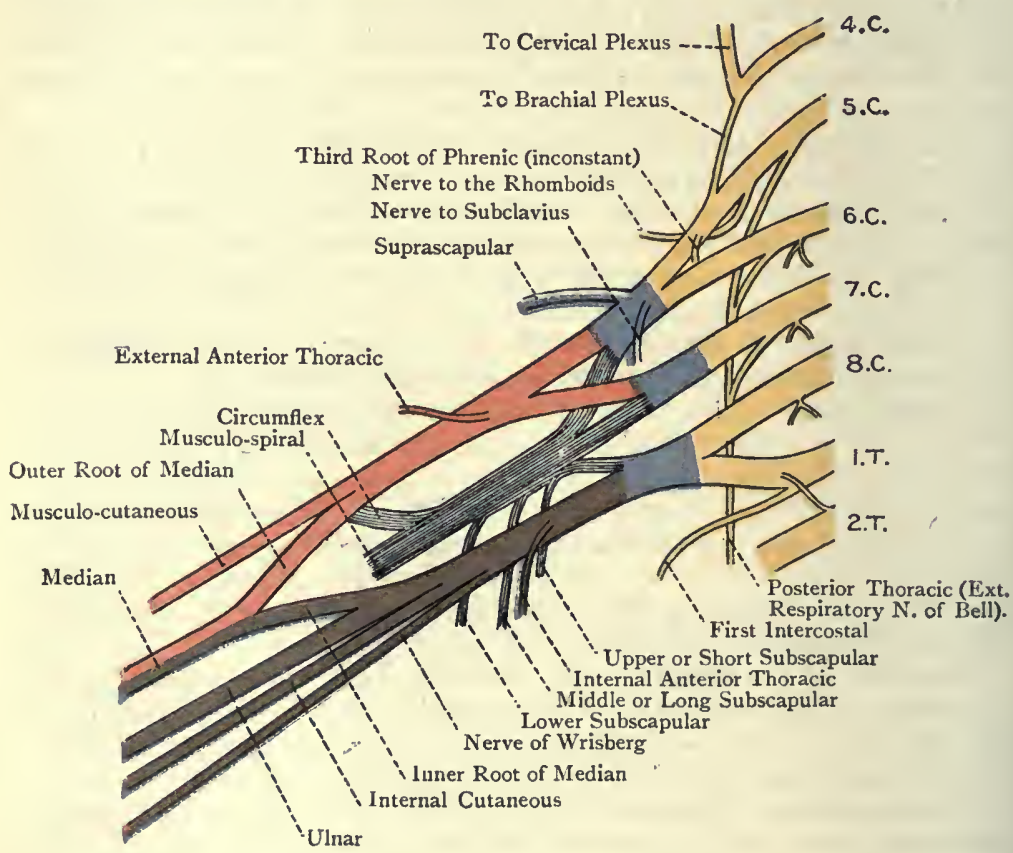

Fig. 178. -The Brachial Plexus.

Yellow $=$ Spinal Nerves and their Branches; Blue $=$ Trunks $;$ Red $=$ Outer Cord : Purple $=$ Inner Cord ; Grey $=$ Posterior Cord.

from the back of the upper trunk, its fibres being derived from the fifth and sixth cervical. It is directed downwards, outwards, and backwards beneath the trapezius and posterior belly of the omohyoid to the upper border of the scapula, on approaching which it gets in company with the suprascapular artery. It is distributed to the supraspinatus, infraspinatus, and shoulder-joint.

Infraclavicular Branches.-Outer Cord.-The branches of this cord are the external anterior thoracic, musculo-cutaneous, and outer root of the median.

The external anterior thoracic, which derives its fibres from the 
fifth, sixth, and seventh cervical, crosses over the first part of the axillary artery from without inwards, and gives a branch to the internal anterior thoracic. Thereafter it pierces the costo-coracoid membrane, and is distributed to the pectoralis major.

The musculo-cutaneous or perforating nerve of Casserius, which derives its fibres from the fifth, sixth, and seventh cervical, arises about the level of the pectoralis minor. For a short distance it lies on the outside of the axillary artery, but it soon leaves the vessel, and pierces the coraco-brachialis in a direction downwards and outwards. Before reaching the muscle it parts with a branch to it, which usually enters it in two divisions. The fibres of this branch are derived from the seventh cervical. The subsequent course of the musculo-cutaneous will be described later on.

The outer root of the median passes slightly on to the axillary artery, where it is joined by the inner root.

Inner Cord. - The branches of this cord are the internal anterior thoracic, the lesser internal cutaneous or nerve of Wrisberg, the internal cutaneous, inner root of the median, and ulnar.

The internal anterior thoracic nerve, which derives its fibres from the eighth cervical and first thoracic, passes behind the first part of the axillary artery, and then comes forwards between the artery and the axillary vein. Having received a branch from the external anterior thoracic nerve, which forms a loop over the first part of the artery, it breaks up into branches which enter the deep surface of the pectoralis minor, a few of them piercing that muscle to enter the deep surface of the pectoralis major.

The lesser internal cutaneous or nerve of Wrisberg, which derives its fibies from the first thoracic, is at first placed behind the axillary vein, but subsequently descends on its inner side, where it communicates with the intercosto-humeral nerve. This nerve is sometimes absent.

The internal cutaneous, which derives its fibres from the eighth cervical and first thoracic, descends partly on the axillary artery and partly to its inner side.

The inner root of the median, which is of smaller size than the outer, passes obliquely over the axillary artery, and joins the outer root a little below the lower border of the pectoralis minor. The trunk of the nerve, which derives its fibres from all the nerves of the plexus, then descends on the outer side of the third part of the artery.

The ulnar nerve is the largest branch of the inner cord, of which it is the continuation, and it derives its fibres from the eighth cervical and first thoracic. It appears at the lower border of the pectoralis minor, and then descends, lying deeply between the third part of the axillary artery and the vein.

Posterior Cord.-The branches of this cord are the three subscapular nerves, circumflex, and musculo-spiral.

The subscapular nerves are distinguished as upper or short, middle or long, and lower. The upper or short subscapular nerve, 
which is of small size, derives its fibres from the fifth and sixth cervical. It is situated high up on the posterior wall of the axilla, and after a short course it enters the upper part of the subscapularis. The middle or long subscapular nerve derives its fibres chiefly from the seventh cervical, but to a certain extent also from the sixth and eighth. It descends along with the subscapular artery to the latissimus dorsi, which it supplies. The lower subscapular nerve derives its fibres from the fifth and sixth cervical. On approaching the lower border of the subscapularis it breaks up into branches, some of which enter the lower part of that muscle, whilst others pass to the teres major.

The circumflex nerve derives its fibres from the fifth and sixth cervical. For a short distance it lies behind the axillary artery, resting on the subscapularis, but, at the lower border of that muscle, it passes backwards through the quadrangular muscular space in company with the posterior circumflex artery. In doing so it furnishes an articular branch to the shoulder-joint, and then breaks up into an upper or anterior and a lower or posterior division. The upper or anterior division accompanies the posterior circumflex artery; and divides into a number of branches which enter the deep surface of the deltoid over its anterior part. Some of the branches, piercing the muscle, supply the integument over about its middle third. The lower or posterior division gives branches to the posterior part of the deltoid, a branch to the teres minor, and a cutaneous branch, which latter turns round the posterior border of the deltoid to be distributed to the integument over its lower third. The nerve to the teres minor presents a small reddish swelling, which has the appearance of a ganglion, but is really a fibrous thickening.

In connection with the distribution of the circumflex nerve Hilton's law may be here stated as follows: a nerve trunk, supply. ing a given joint, also supplies the muscles moving that joint, and the integument covering their insertions. For example, the circumflex nerve supplies the shoulder-joint, the deltoid, and the integument covering its insertion. This law, however, is not universally applicable.

The musculo-spiral nerve is the largest of all the branches of the plexus, and is the continuation of the posterior cord. It derives its fibres from the last four cervical nerves, and sometimes from the first thoracic. It descends behind the third part of the axillary artery, resting upon the subscapularis, latissimus dorsi, and teres major. Whilst in the axillary space it gives off-muscular and cutaneous branches. The muscular branches are destined for the long and inner heads of the triceps, those for the long head entering it high up, whilst those for the inner head enter it at different levels. One of the latter, which is remarkable for its length, descends in company with the ulnar nerve to enter the inner head low down, this branch being known as the ulnar collateral nerve (Krause). The internal cutaneous branch usually arises in common 
with one of the muscular branches, and it takes a backward course behind the intercosto-humeral nerve to be distributed to the integument of the back of the arm, reaching nearly as low as the elbow.

Intercosto-humeral Nerve.-The intercosto-humeral nerve is the undivided lateral cutaneous branch of the second intercostal, and it represents the posterior branch of the other lateral cutaneous nerves. Emerging from the second intercostal space it appears between two serrations of the serratus magnus, and then crosses the axillary space. In its course it usually communicates with a branch of the posterior division of the lateral cutaneous of the third intercostal, and towards the arm it also communicates with the nerve of Wrisberg and the internal cutaneous of the musculo-spiral. On reaching the arm it is distributed to the integument of the inner and back part over the upper half. Sometimes there is another intercosto-humeral nerve, which is the undivided lateral cutaneous branch of the first intercostal nerve. When this is so the nerve of Wrisberg is absent.

The lateral cutaneous nerves have been previously described in connection with the cutaneous nerves of the pectoral region on page 279. It may be stated, in addition, that the posterior branch of the lateral cutaneous of the third intercostal, as a rule, gives a branch across the axilla to the inner side of the arm, which communicates in its course with the intercosto-humeral, and may largely replace it, if it is small.

Axillary Lymphatic Glands.-The axillary glands are very numerous, and lie embedded in the loose adipose tissue of the axillary space. They are disposed in four groups - external, or humeral; antero-internal, or pectoral; posterior, or subscapular; and central.

The external or humeral glands are about six in number, and lie upon the outer wall of the space, where they form a chain along the axillary vein. Their afferent vessels represent (I) most of the superficial and deep lymphatics of the upper limb, (2) some of the efferent vessels of the pectoral axillary glands, and (3) some of the efferent vessels of the subscapular glands. Their efferent vessels pass to (I) the central axillary glands, (2) the infraclavicular glands, and (3) the supraclavicular glands.

The antero-internal or pectoral glands are about six in number. and are situated along the inferior or long thoracic artery at the lower border of the pectoralis minor muscle, and therefore within the anterior fold of the axilla. They lie upon the serratus magnus, in the angle between it and the pectoral muscles, and they extend from the level of the second to the level of the fifth intercostal space. They receive their afferent vessels from $(I)$ the anterior and lateral parts of the thoracic wall, and the supra-umbilical portion of the abdominal wall, and (2) the outer two-thirds of the mammary gland. Their efferent vessels pass to (I) the external axillary glands, (2) the central axillary glands, and (3) the infra-clavicular glands.

The posterior or subscapular glands are about five in number, and are situated upon the posterior wall of the axilla. They lie along 
the lower border of the subscapularis muscle, in contact with the subscapular artery. Their afferent vessels are derived from (I) the superficial lymphatics of the lower and back part of the neck, (2) the superficial lymphatics of the posterior part of the trunk, and (3) some of the superficial lymphatics of the supra-umbilical portion of the lateral wall of the abdomen. Their efferent lymphatics pass to (I) the external axillary glands, (2) the central axillary glands, and (3) the infra-clavicular glands.

The central glands are about five in number, and are situated at the base of the axilla. They lie upon the axillary fascia, and between the external, pectoral, and subscapular groups. They receive as afferent vessels some of the efferent vessels of the external, pectoral;

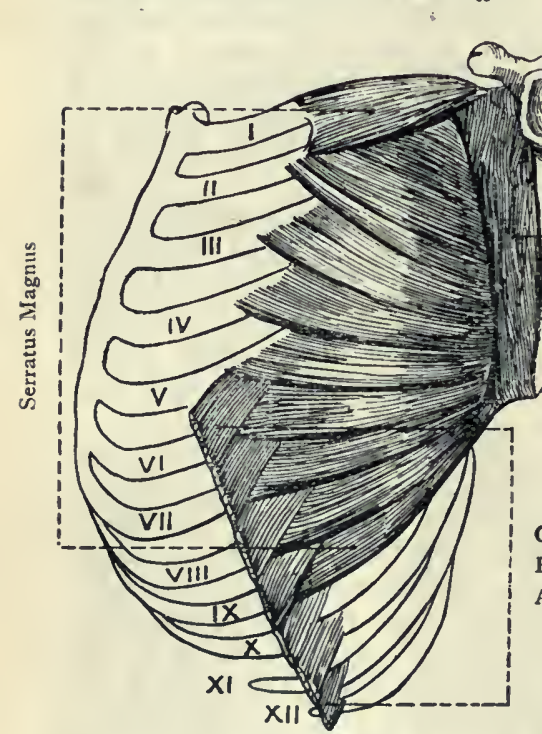

Fig. I79.-The Serratus Magnus MUSCle. and subscapular glands. Their efferent vessels pass to the infra-clavicular glands.

Serratus Magnus-Origin.The outer surfaces of the first eight or nine ribs about midway between the angles Subscapularis and costal cartilages, by means of fleshy serrations which are curved with their convexities forwards. Each serration arises from one rib, except the first, which arises from the first and second ribs and a fibrous arch between them.

Insertion.-The anterior Obliquus surface of the base of the Externus scapula from the superior Abdominis to the inferior angle.

Nerve-supply. - The posterior thoracic nerve, which arises by three roots from the fifth, sixth, and seventh cervical. The nerve descends on the axillary surface of the muscle.

The muscle is arranged in three parts. The upper part is formed by the first serration, which is of large size, and it is inserted into the triangular area on the front of the superior angle of the scapula. The middle part forms a thin, expanded sheet, which is formed by the broad second and the third serrations, and it is inserted into the long, linear impression on the front of the base, reaching to near the superior angle and extending down to near the inferior angle. The lower part, which is formed by the lower five or six serrations, is fan-shaped, and towards the scapula is thick and stout, its insertion being into the expanded area in front of the inferior angle. The lower four or five serrations interdigitate with the obliquus externus abdominis.

Action.-The muscle draws the base of the scapula forwards, as 
in pushing, and the strong lower fibres, acting upon the lower angle, rotate the bone so as to elevate the point of the shoulder." In this latter action the muscle is auxiliary to the trapezius. An important use of the muscle is to steady the scapula, more particularly the glenoid cavity, this condition being necessary before the deltoid can elevate the humerus. The muscle by its contraction serves to keep the lower angle of the scapula in contact with the chest wall. When the shoulder is fixed, the lower part of the muscle may elevate the ribs from which it arises.

When the serratus magnus muscle is paralyzed (in association with the rhomboid muscles), the condition known as winged scapula is produced. The lower angle and vertebral border of the scapula are displaced backwards when an effort is made to abduct the arm or to thrust it forwards.

\section{THE SCAPULAR REGION.}

Cutaneous Nerves.-The cutaneous nerves of the shoulder are derived from the supra-acromial of the cervical plexus and the circumflex. The supra-acromial nerve, having descended over the outer part of the clavicle and trapezius, divides into numerous branches, which supply the integument over the upper third of the deltoid. The circumflex nerve sends branches through the muscle which supply the integument over about its middle third. It also furnishes a special cutaneous branch which turns round the posterior border of the muscle a little below the centre, and supplies the integument over its lower third.

Deep Fascia.-The deep fascia is well marked over the infraspinatus, where it is strongly aponeurotic. It sends septa between that muscle and the teres muscles, and then passes forwards over them to the posterior border of the deltoid, where it splits into two layers which encase that muscle.

Deltoid-Origin.-(I) The anterior border of the outer third of the clavicle; (2) the outer border of the acromion process; and (3) the lower lip of the posterior border of the spine of the scapula.

Insertion.-The deltoid impression on the outer aspect of the humerus, commencing at the centre, and extending upwards for 2 inches or more.

Nerve-supply. - The circumflex nerve, which is a branch of the posterior cord of the brachial plexus, its fibres being derived from the fifth and sixth cervical.

Blood-supply. - The posterior circumflex and acromio-thoracic arteries.

The muscle, which has very coarse fasciculi, is triangular, the base being upwards. The clavicular portion passes downwards and outwards, the acromial downwards, and the spinal downwards and forwards.

Action.-The acromial portion abducts the arm to the position of a right angle with the trunk; the clavicular portion draws it forwards; and the spinal portion draws it backwards.

The acromial portion of the muscle, besides arising from the bone, 
springs to a large extent from the sides of four fibrous septa, which descend into that part, and the fibres arising in this manner are inserted into the sides of three fibrous septa, which ascend from below.

The origin of the muscle corresponds with the insertion of the trapezius. The anterior border is related to the pectoralis major, the cephalic vein and humeral artery lying between the two. The posterior border is to a large extent bound down by the splitting of the deep fascia over the infraspinatus. The muscle covers the shoulder-joint, subacromial bursa, coracoid process, coraco-brachialis, biceps, tendons of insertion of the supraspinatus, infraspinatus, and teres minor, parts of the long and outer heads of the triceps, teres major, tendon of insertion of the pectoralis major, circumflex nerve, and posterior circumflex artery.

Subacromial Bursa. - This is a large bursa which intervenes between the acromion process and deltoid, on the one hand, and the upper part of the capsular ligament and the tendons inserted into the great tuberosity of the humerus, on the other.

Supraspinatus-Origin.- The inner two-thirds of the supraspinous fossa of the scapula, and the aponeurosis covering the muscle.

Insertion.-The upper impression on the great tuberosity of the humerus, its tendon being closely connected with the upper part of the capsular ligament, and with that of the infraspinatus.

Nerve-supply. - The suprascapular nerve, which arises from the back of the upper trunk of the brachial plexus, its fibres being derived from the fifth and sixth cervical.

The direction of the muscle is outwards and downwards.

Action.- To abduct the arm in association with the deltoid.

Posterior Belly of the Omo-hyoid-Origin.-The upper border of the scapula internal to the suprascapular notch, and, as a rule, the adjacent portion of the suprascapular or transverse ligament.

Infraspinatus-Origin.-(I) The infraspinous fossa of the scapula over about its inner two-thirds, the parts excepted being those for the teres muscles, and a portion at the upper and outer part of the fossa ; and (2) the deep fascia covering the muscle.

Insertion.-The middle impression on the great tuberosity of the humerus, its tendon being closely connected with the, back part of the capsular ligament, from which it is sometimes separated by a bursa. The tendon, which is fan-shaped, is at first concealed within the muscle.

Nerve-supply.-The suprascapular nerve.

The direction of the muscle is outwards.

Action.-When the arm is by the side of the trunk the muscle is an external rotator. When the arm is raised the muscle carries it backwards in association with the deltoid.

Suprascapular Artery.-The suprascapular artery (transversalis humeri) is a branch of the thyroid axis of the first part of the sub- 
clavian. Having coursed transversely outwards behind the clavicle, it reaches the upper border of the scapula in company with the suprascapular nerve. The artery then passes backwards over the suprascapular ligament, whilst the nerve passes beneath it, and it descends into the supraspinous fossa beneath the supraspinatus, where it parts with several branches. Thereafter it passes behind the neck of the scapula through the great scapular notch, beneath the spino-glenoid ligament, into the upper and outer part of the infraspinous fossa under cover of the infraspinatus, where it anastomoses with the dorsalis scapulæ and posterior scapular.

Branches.-These are as follows: muscular, in the neck, to the sterno-cleido-mastoid and subclavius; suprasternal, over the inner end of the clavicle, to the integument over the presternum; medullary to the clavicle; supra-acromial, which pierces the trapezius to reach the upper surface of the acromion process, where it anastomoses with branches of the acromio-thoracic and posterior circumflex; articular to the acromio-clavicular and shoulderjoints; ventral to the belly of the scapula, given off as it passes backwards over the suprascapular ligament, this branch anastomosing with the ventral branches of the dorsalis scapulæ and posterior scapular; supraspinous to the fossa and its muscle; and infraspinous to the fossa and its muscle.

Suprascapular Nerve.-This nerve passes backwards beneath the suprascapular ligament into the supraspinous fossa, where it gives branches to the supraspinatus, and articular branches to the acromioclavicular and shoulder-joints. It then accompanies the artery through the great scapular notch, beneath the spino-glenoid ligament, to the infraspinous fossa, where it ends in branches to the infraspinatus.

Teres Minor-Origin.-(I) The dorsum of the scapula close to the axillary border for its upper two-thirds, and (2) the septa between it and the infraspinatus and teres major.

Insertion.-The lower impression on the great tuberosity of the humerus, and the surgical neck of the bone for a short distance below, its tendon being closely connected with the back part of the capsular ligament.

Nerve-supply.-The circumflex nerve, the branch of which has a reddish enlargement presenting the appearance of a ganglion, but being in reality a fibrous thickening.

The direction of the muscle is outwards and slightly upwards.

Action.-The muscle is an external rotator of the arm when it is abducted, and it also helps in depressing the arm.

The muscle is pierced by the dorsalis scapulæ artery.

Teres Major-Origin.-(I) From an oval impression at the lower and outer part of the infraspinous fossa, which extends on to the lower angle, and reaches upwards on the axillary border for about its lower third; and (2) the septa between it and the teres minor, infraspinatus, and subscapularis. 
Insertion.-The inner lip of the bicipital groove of the humerus for 2 inches over about its lower two-thirds.

Nerve-supply. - The lower subscapular nerve, which is a branch of the posterior cord of the brachial plexus, its fibres being derived from the fifth and sixth cervical.

The direction of the muscle is outwards.

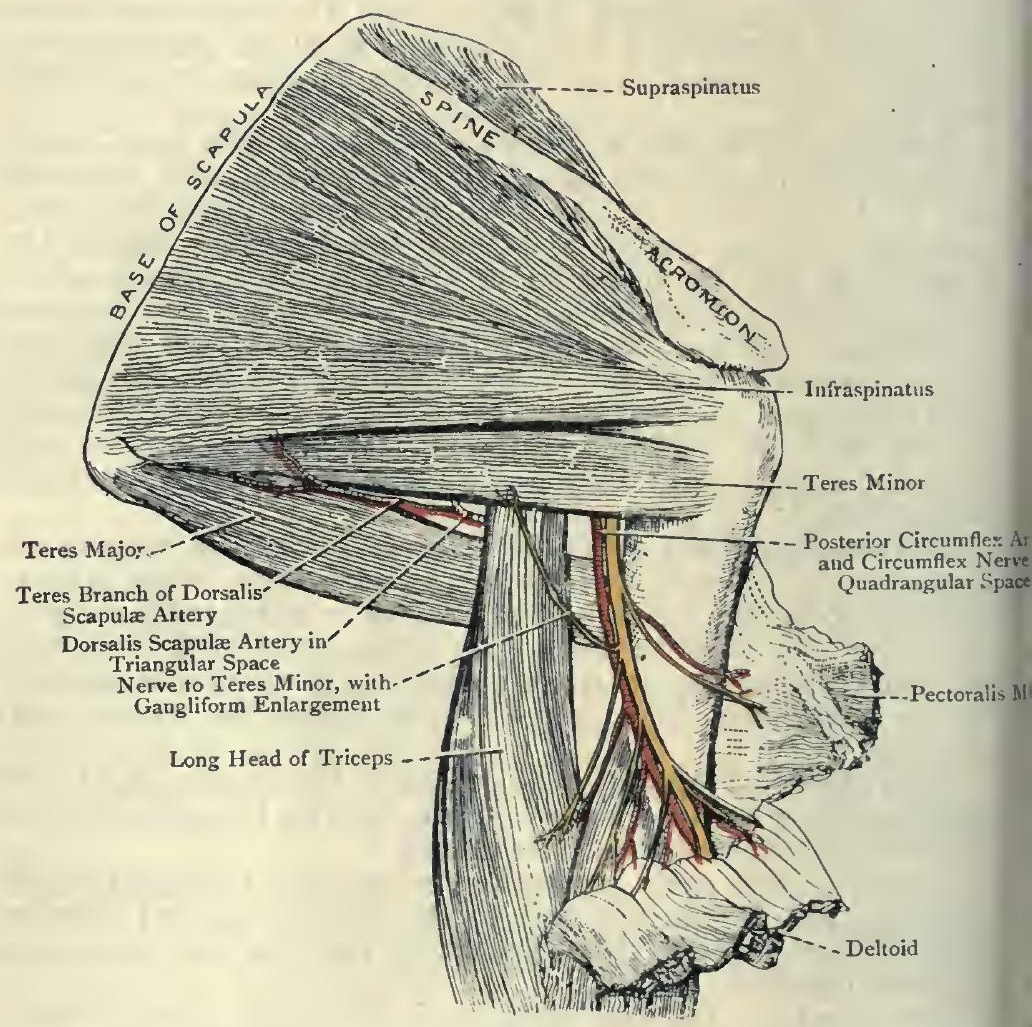

Fig. 180.-Dissection of the Scapular and Upper Brachial Regions FROM BEHIND.

(The Deltoid has been turned down, and the Triangular and Quadrangular Muscular Spaces, with their contents, are shown.)

Action.-The muscle adducts the arm. When the arm is abducted it acts as an internal rotator.

The latissimus dorsi winds round the lower border of the muscle, and is subsequently placed in front of it. The two tendons are at first closely connected by their lower borders, but are after- 
wards sepąrated by a bursa, and there is usually a bursa behind the teres major at its insertion.

Subscapularis-Origin.-(I) The venter of the scapula, except near the neck, and along the front of the base where the serratus magnus is inserted; and (2) the sides of tendinous septa which intersect the muscle and are connected with the ridges on the venter.

Insertion.-The small tuberosity of the humerus, and the surgical neck of the bone for a short distance below.

Nerve-supply.-The upper or short, and part of the lower, subscapular nerves, which are branches of the posterior cord of the brachial plexus, their fibres being derived from the fifth and sixth cervical.

The direction of the muscle is outwards.

Action.-When the arm is by the side of the trunk the muscle is an internal rotator, and, when it is raised, it carries it forwards and downwards.

The muscle is closely connected with the front of the capsular ligament. Between its upper border and the coracoid process and neck of the scapula there is a bursa, which usually communicates with the synovial membrane of the shoulder-joint through an opening in the capsule.

Triangular and Quadrangular Spaces.-When the long head of the triceps is cut and displaced a large triangular space is seen, which, as viewed from behind, is bounded above by the teres minor, below by the teres major, and externally by the surgical neck of the humerus. As viewed from before, the subscapularis replaces the teres minor. When the long head of the triceps is in position it descends in front of the teres minor, and behind the teres major. It therefore passes through the triangular space and divides it into two, one triangular and the other quadrangular.

Triangular Space.-This, as viewed from behind, is bounded aboic by the teres minor, below by the teres major, and externally by the long head of the triceps. As viewed from before, the subscapularis replaces the teres minor. The dorsalis scapulæ artery passes backwards through this space, so long as the subscapularis forms one of its boundaries, but thereafter, and as seen from behind, it only lies in it preparatory to piercing the teres minor and winding round the axillary border.

Quadrangular Space.-This space, as viewed from behind, is bounded above by the teres minor, below by the teres major, internally by the long head of the triceps, and externally by the surgical neck of the humerus, the subscapularis replacing the teres minor in front. The structures which pass through it are the circumflex nerve and posterior circumflex vessels. It is covered by the deltoid.

Scapular Anastomoses of Arteries.-The anastomoses of arteries upon the scapula are divided into two sets-scapular proper, and acromial. 
Scapular Anastomoses Proper.-The arteries which take part in these anastomoses are (I) the suprascapular and posterior scapular, representing the subclavian; and (2) the dorsalis scapulæ of the subscapular, representing the third part of the axillary. The suprascapular is a branch of the thyroid axis of the first part of the subclavian, and the posterior scapular arises from the transverse cervical, which is also a branch of the thyroid axis. The suprascapular is distributed to the supraspinous and infraspinous fossæ and venter, and so also is the posterior scapular. The subscapular is distributed by its dorsalis scapulæ branch to the infraspinous fossa and venter. In the supraspinous fossa the suprascapular anastomoses with the posterior scapular. In the infraspinous fossa the suprascapular anastomoses with the dorsalis scapulæ, as does also the posterior scapular. In the venter of the bone the ventral branches of the suprascapular and posterior scapular anastomose with the ventral branch of the dorsalis scapulæ. At the lower angle of the bone the posterior scapular anastomoses with the descending or teres branch of the dorsalis scapulæ.

Acromial Anastomosis.-The arteries which take part in the acromial anastomosis or rete on the upper surface of the acromion process are as follows : (I) the supra-acromial branch of the suprascapular; (2) branches of the acromio-thoracic artery of the first part of the axillary ; and (3) a branch of the posterior circumflex of the third part of the axillary.

The importance of the scapular anastomoses comes into play after ligature of the subclavian artery in the third part of its course.

\section{THE ARTICULATIONS OF THE CLAVICLE.}

Sterno-clavicular Joint.-This joint belongs to the class diarthrosis, and to the subdivision arthrodia. The articular surfaces are the inner end of the clavicle and the clavicular impression on the upper border of the presternum. The articular surface of the clavicle is of larger size than that on the presternum, and the two are separated by an interarticular fibro-cartilage. The joint is surrounded by a complete capsule, which is weak above and below, but strong in front and behind, where it constitutes the anterior and posterior sterno-clavicular ligaments. Besides these there are the interclavicular and costo-clavicular or rhomboid ligaments.

The anterior sterno-clavicular ligament is broad, and its fibres extend obliquely downwards and inwards from the front of the clavicle to the front of the presternum. The sternal head of origin of the sterno-cleido-mastoid is in contact with it. The posterior sterno-clavicular ligament resembles the anterior, and is similarly disposed behind the joint. The sterno-hyoid muscle 


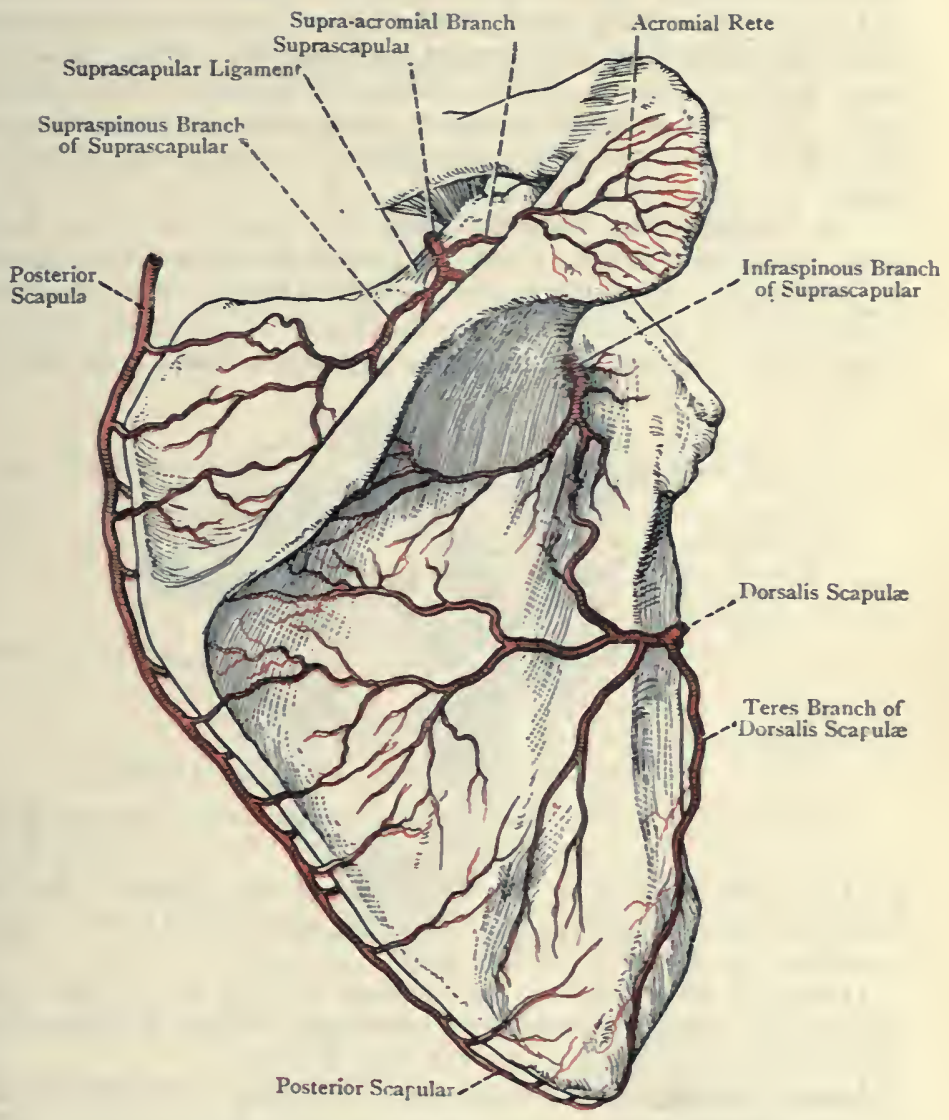

Fig. i8r.-The ANastomoses of Arteries on the Dorscm AND Acromion Process of the Scaplla. 
arises in part from it. The interclavicular ligament is a wellmarked, curved bundle of fibres, which is attached at either side to the upper and back part of the inner end of the clavicle. In crossing between the two bones it curves downwards to be attached to the interclavicular notch on the upper border of the presternum. The costo-clavicular or rhomboid ligament is a strong, quadrilateral band of fibres, which extends from the upper surface of the first costal cartilage to the rhomboid impression on the under surface of the clavicle, its direction being upwards, backwards, and outwards.

The interarticular fibro-cartilage is a nearly circular, flattened plate, which is thinner at the centre and lower part than elsewhere. It is attached superiorly to the upper and back part of the inner end of the clavicle, and inferiorly to the inner end of the first costal cartilage, where it inclines slightly outwards to form part of a socket

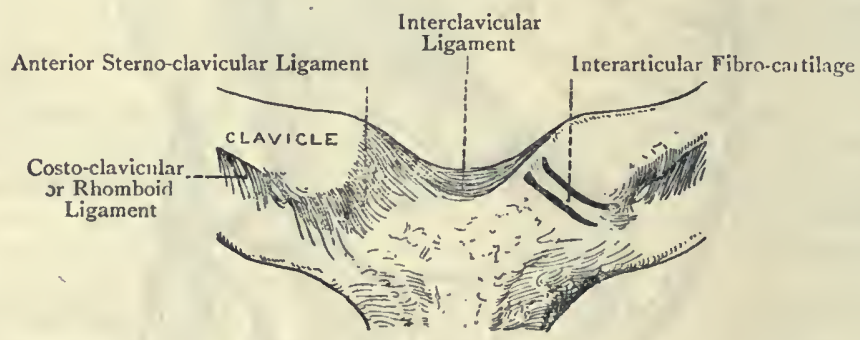

Fig. I82.-The Sterno-Clavicular Joints. .

(The Left Anterior Sterno-clavicular Ligament has been removed.)

for the lower portion of the inner end of the clavicle. Its circumference is connected with the fibrous capsule of the joint. The plate sometimes presents a perforation at its centre.

There are two synovial membranes at this joint, one on either side of the interarticular fibro-cartilage. When the latter is perforated these are continuous with each other.

Arterial Supply.- The suprasternal branch of the suprascapular, and the internal mammary.

Nerve-supply. - The suprasternal branch of the cervical plexus.

Movements. - These take place in an upward, downward, forward, and backward direction. There is also circumduction. In the upward and downward movements the clavicle moves on the fibro-cartilage, and the forward and backward movements take place between the fibro-cartilage and the presternum. In the downward movement of the bone the interarticular ligament is put upon the stretch, and the upward movement is limited by the costo. clavicular ligament.

Acromio-clavicular Joint.--This belongs to the class diarthrosis, and to the subdivision arthrodia. The articular surfaces are the outer end of the clavicle and the facet on the acromion process. 
These surfaces are sometimes partially separated by an interarticular fibro-cartilage, and the joint is surrounded by a complete capsule, which forms the superior and inferior ligaments.

The superior and inferior acromio-clavicular ligaments extend between the contiguous margins of the bones on their upper and under surfaces, the former being strengthened by aponeurotic fibres from the trapezius and deltoid. The interarticular fibrocartilage, when present, is limited to the upper part of the joint, where it is attached to the superior ligament. It may divide the joint into two synovial compartments, but there is usually only one.

The coraco-clavicular ligament, which is to be regarded as accessory to this joint, connects the clavicle with the coracoid process,

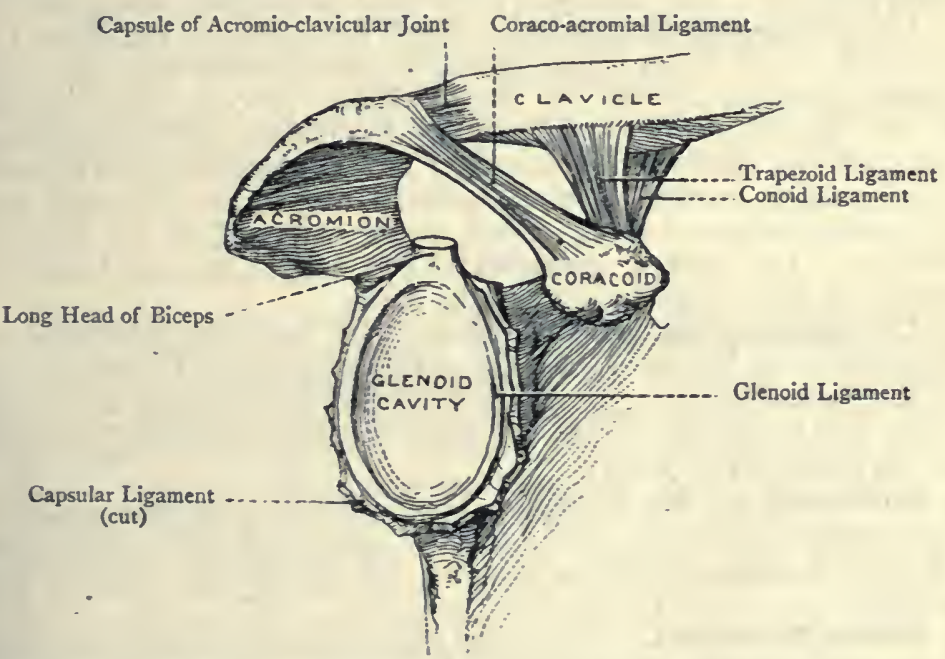

Fig. -r83.-The Rigi.t Glenoid Cavity, and the Adjacent Ligaments.

and is composed of two parts-conoid and trapezoid. The conoid ligament, internal and posterior in position, is attached below by its apex to an impression at the back part of the antero-internal border of the coracoid process, and above by its base to the conoid tubercle of the clavicle, its direction being upwards and backwards. The trapezoid ligament, external and anterior in position, is somewhat quadrilateral. It is attached below to the trapezoid ridge on the back part of the upper surface of the coracoid process, and above to the trapezoid ridge on the under surface of the clavicle, its direction being upwards; backwards, and outwards. Between the two ligaments there is a slight interval, in which there may be a bursa.

Arterial Supply.-The suprascapular and acromio-thoracic arteries:

Nerve-supply. - The suprascapular and circumflex nerves. 
Movements.-The movements at this joint are limited, and are principally of a gliding nature in an upward and downward, and forward and backward, direction.

\section{The Ligaments of the Scapula.}

These are three in number-suprascapular, coraco-acromial, and spino-glenoid.

The suprascapular or transverse ligament extends from the upper border of the scapula, internal to the suprascapular notch, to the root of the coracoid process. It is thin and flat, and it bridges over the notch, which it converts into a foramen. It usually gives origin to some fibres of the posterior belly of the omo-hyoid, and the suprascapular nerve passes backwards beneath it and the suprascapular artery over it. This ligament sometimes undergoes ossification.

The coraco-acromial ligament, which is triangular, is attached by its apex to the tip of the acromion process, and by its base to the postero-external border of the coracoid process. Its superior surface is covered by the deltoid, and the inferior surface overhangs the shoulder-joint, the subacromial bursa intervening. The acromion process, coraco-acromial ligament, and coracoid process form the coraco-acromial arch, within which the head of the humerus fits when the arm is abducted. The arch therefore forms an auxiliary socket for the head of the bone.

The spino-glenoid ligament consists of a few fibres which extend from the outer border of the spine to the adjacent part of the margin of the glenoid cavity. It arches over the suprnscapular artery and nerve as they pass through the great scapular notch on their way to the infraspinous fossa.

Movements of the Scapula.-These movements take place at the acromio-clavicular joint, and are associated with movements of the clavicle. They are of two kinds-namely, gliding and rotation.

Gliding Movements.-These take place upwards, downwards, outwards or forwards, and inwards or backwards. During their occurrence the scapula moves over the dorsal wall of the thorax in such a manner as to describe the arc of a circle. The centre of this circle corresponds to the sterno-clavicular joint, and the clavicle represents a ray of the circle. In the inward or backward movement the base of the scapula is drawn towards the vertebral column, and in the outward or forward movement the base is drawn away from the vertebral column.

Rotatory Movements.- Rotation takes place inwards and outwards. During internal rotation the acromion is elevated, the superior angle is depressed, and the inferior angle moves slightly outwards. In internal rotation the conoid ligament is tightened. During external rotation the superior angle is elevated, the acromion is depressed, and the inferior angle moves slightly inueards. In external rotation the trapezoid ligament is tightened. 
On account of the existence of the acromio-clavicular joint, the direction of the glenoid cavity of the scapula remains unaltered during the movements of the bone.

Chief Muscles concerned in the Movements.-Elevation of Entire Scapula: Cervical part of the trapezius, and the levator anguli scapulæ. Depression of Entire Scapula: The lower fibres of the trapezius, which end upon the somewhat triangular tendon, and the pectoralis minor. Outward or Forward Movement of Entire Scapula: The serratus magnus. Inward or Backward Movement of the Entire Scapula: The middle and lower parts of the trapezius, and the rhomboid muscles.

Special Movements.-Elevation of Acromion: Cervical part of the trapezius. Elevation of Superior Angle: Levator anguli scapulæ, aided by the rhomboid muscles.

The inferior angle of the scapula is kept in contact with the dorsal wall of the thorax by the upper horizontal fibres of the latissimus dorsi muscle, which pass over its dorsal aspect.

The base of the scapula is kept in contact with the dorsal wall of the thorax by the serratus magnus and rhomboid muscles.

\section{THE ARM.}

Landmarks.-The front of the brachial region presents a wellmarked elongated prominence, due to the biceps, which reaches from the anterior fold of the axilla to near the elbow. Internal to this prominence, above, is another swelling caused by the coracobrachialis. On either side of the bicipital prominence is a groove, that on the outer side indicating the position of the cephalic vein, and that on the inner side the position of the basilic vein, brachial artery, and median nerve. External to the bicipital prominence, for a short distance above the elbow, is the prominence formed by the brachio-radialis and extensor carpi radialis longior. At the elbow the internal and external epicondyles of the humerus and the olecranon process of the ulna are to be noted. The internal epicondyle forms a very distinct projection, having an inclination backwards, and behind it, close to the olecranon, is the ulnar nerve. The external epicondyle, which is not well marked, may be felt in zemiflexion of the joint. The olecranon process can easily be felt at the back. In extension of the joint the summit of the olecranon is on the same line with the two epicondyles. When the arm and forearm are placed in the position of a right angle, the summit of the olecranon falls below a line connecting the epicondyles. In extreme flexion of the elbow the summit of the olecranon is anterior to a line connecting the epicondyles. The posterior surface of the olecranon is covered by a subcutaneous bursa. The head of the radius can be felt, in extension of the joint, at the bottom of a depression situated at the outer and back part, where it lies just below 
the external epicondyle. It is most readily felt when the forearm is alternately pronated and supinated. In front of the elbow there is a slight hollow indicating the position of the anticubital fossa, and in this region the outlines of the median basilic and median cephalic veins may be visible, especially the former. On the back of the

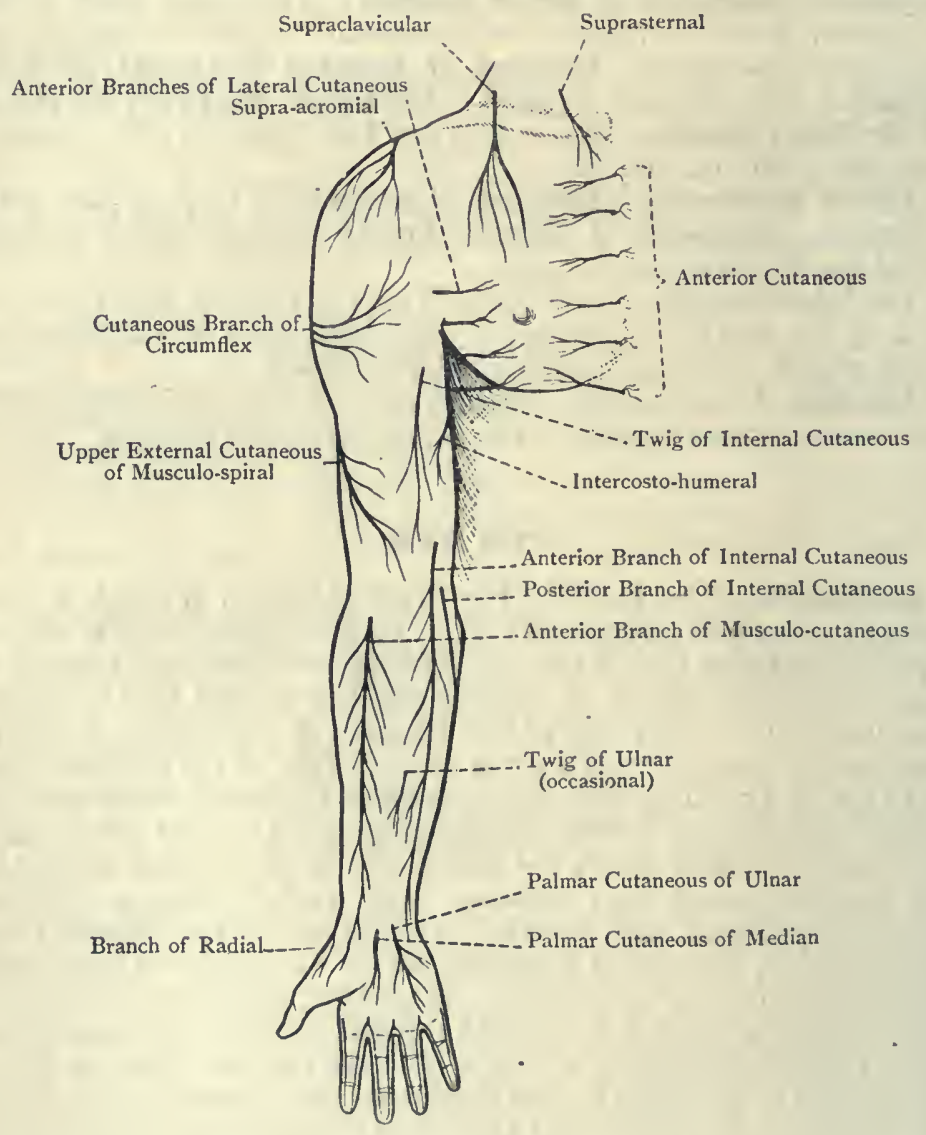

Fig. 184.-Diagram of the Cutaneous Nerves of the Upper Limb (ANTERIOR ASPECT).

forearm the posterior border of the ulna can readily be felt. It leads superiorly to the subcutaneous bursa on the back of the olecranon, and inferiorly it conducts to the styloid process of the bone, which is situated mainly on its posterior aspect. On the outer side of the lower end of the radius its styloid process can easily be felt, 
which projects lower down than that of the ulna, and in front of it is the radial artery. On the back of the radius, about its centre, is the radial tubercle, which bounds externally the groove for the tendon of the extensor longus pollicis.

Olecranon Bursa.-This bursa is situated subcutaneously over the posterior triangular surface of the olecranon process of the ulna.

Cutaneous Nerves. - The intercosto - humeral nerve, having crossed the axillary space, ramifies in the integument of the inner and back part of the arm over its upper half. It may be accompanied by an offset of the posterior branch of the lateral cutaneous of the third intercostal.

The lesser internal cutaneous or nerve of Wrisberg is distributed to the integument of the inner side of the arm, as low as the interval between the internal epicondyle and olecranon.

The internal cutaneous nerve furnishes one or more branches, which, piercing the deep fascia close to the axilla, are distributed to the integument over the biceps. The nerve itself pierces the deep fascia a little below the centre of the arm, and then divides into an anterior and a posterior branch. The anterior branch descends behind the median basilic vein, giving one or two twigs over it, and it is distributed to the integument of the anterior aspect of the inner side of the forearm.

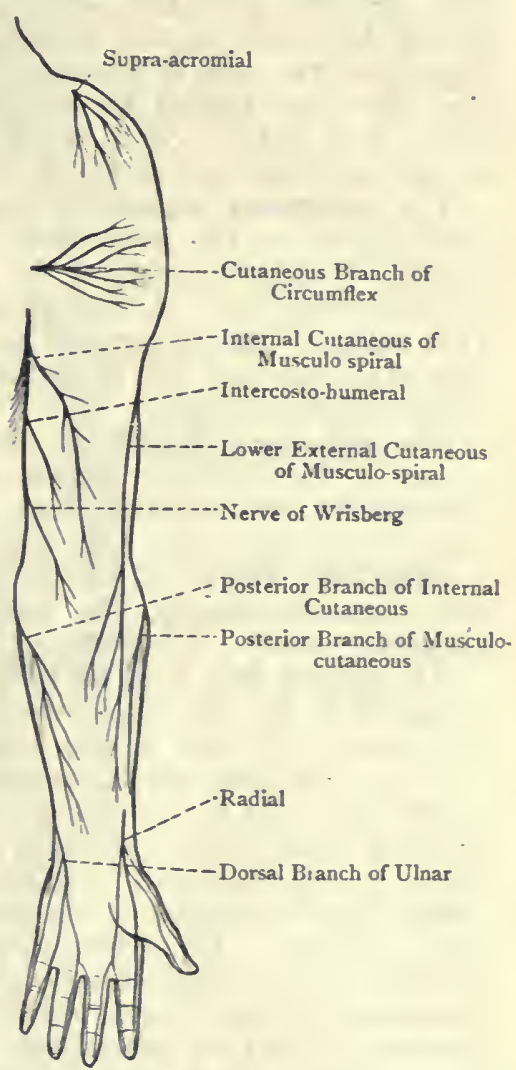

Fig. 185.-Diagram of the Cutaneous NERVES OF THE UPPER LIMB (Posterior ASPECT).

The posterior branch passes downwards and inwards on the inner side of the basilic vein, and over the internal epicondyle, after which it turns backwards to supply the integument over the back of the inner side of the forearm.

The infernal cutaneous of the musculo-spiral is distributed to the integument of the back of the arm, almost as low as the olecranon. 
The external cutaneous branches of the musculo-spiral are two in number-upper and lower. They leave the main trunk towards the lower end of the spiral groove, just before the nerve passes through the external intermuscular septum, and the two branches pierce the deep fascia about $\frac{1}{2}$ inch apart. The upper branch, of small size, descends with the cephalic vein to the front of the elbow, giving branches to the integument of the outer and anterior aspects of the arm in its lower half. The lower branch, of larger size, descends behind the external epicondyle into the forearm, where it is distributed to the integument on the posterior aspect of the outer side as low as the wrist.

The cutaneous branch of the musculo-cutaneous pierces the deep fascia on the outer side of the biceps a little above the elbow. It descends behind the median cephalic vein, giving one or two twigs over it, and then it divides into two branches. One supplies the integument on the anterior aspect of the outer side of the forearm, and the other gives branches to the integument on the posterior aspect.

The cutaneous branch of the ulnar, which arises about the centre of the forearm, pierces the deep fascia, and has a limited distribution to the integument just below the centre, internal to the median line. This branch is inconstant.

Superficial Veins.-There are four principal superficial veins in the forearm, namely, the median, radial, anterior ulnar, and posterior ulnar.

The median vein is formed by the union of a few radicles which originate in the venous plexus in front of the wrist, and its course is upwards in front of the forearm. As it ascends it takes up several veins, and often receives a large tributary from the back of the limb. It is also in free communication with the radial and anterior ulnar veins. On arriving at the hollow in front of the elbow it receives a short but large branch, called the deep median vein, which establishes a communication between it and the deep venæ comites. Thereafter it divides into median cephalic and median basilic, which diverge from each other as they ascend, somewhat like the capital letter $V$. The median cephalic vein, the smaller of the two, passes upwards and outwards in the interval between the biceps and brachio-radialis, having the cutaneous part of the musculo-cutaneous nerve behind, and a few of its twigs over it. A little above the external epicondyle it receives the radial vein, the resulting trunk being called the cephalic vein. The median basilic vein, the larger of the two, passes inwards and upwards, crossing the bicipital fascia, which separates it from the brachial artery, and the anterior branch of the internal cutaneous nerve descends behind it, a few of its twigs passing over it. Just above the internal epicondyle it receives the anterior and posterior ulnar veins, either separately or as a common trunk, and the resulting vessel is called the basilic vein. 


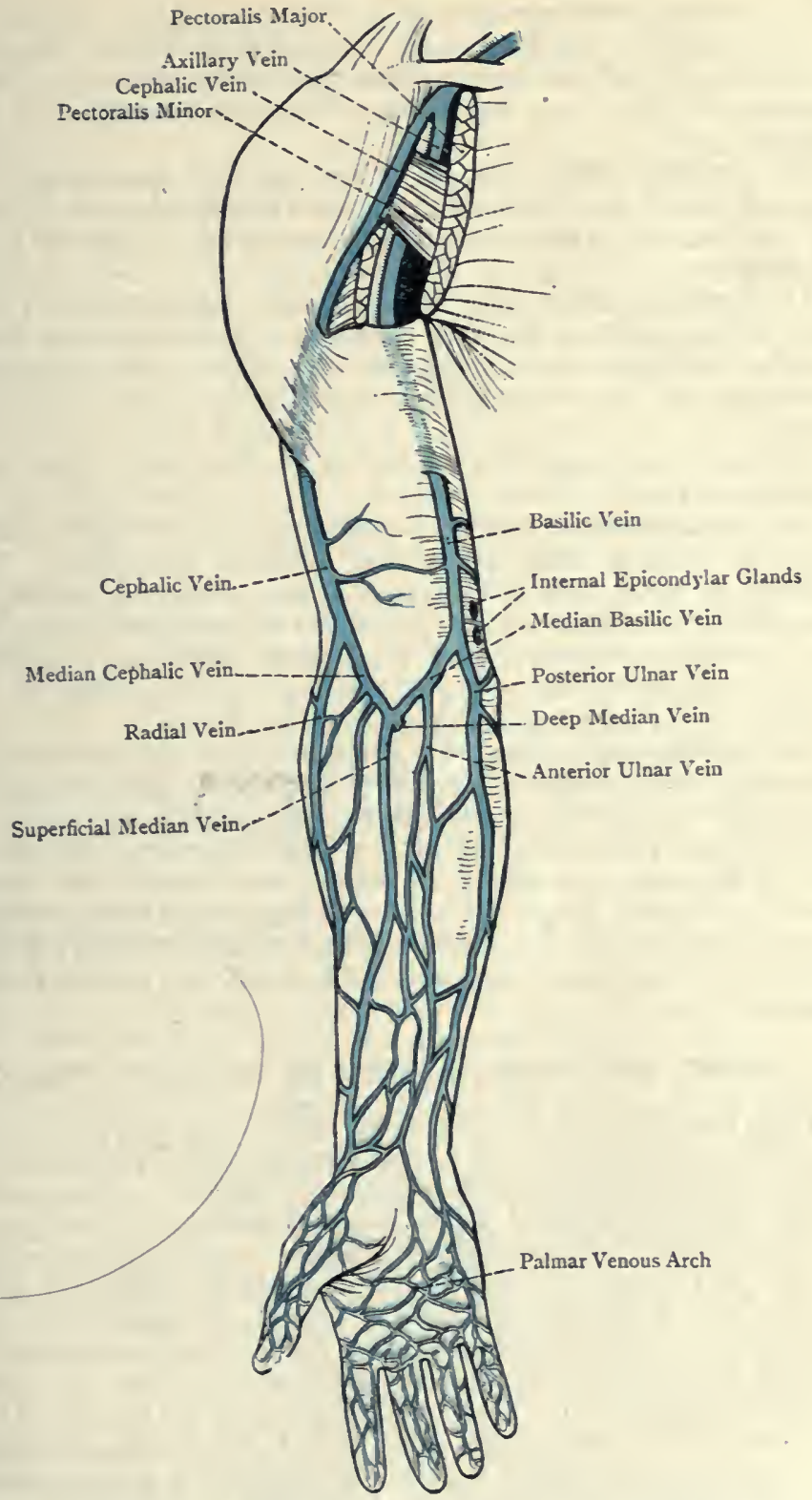

Fig. 186.-The Superficial Veins of the Upper Limb (ANTERIOR VIEW). 
The radial vein commences in the outer part of the plexus on the back of the hand, and it ascends at first on the back of the outer side of the forearm, but gradually inclines to its outer aspect. . A little above the external epicondyle it joins the median cephalic vein.

The anterior ulnar voin commences on the inner aspect of the front of the wrist, and it ascends in front of the inner side of the forearm, to end either in the median basilic or by joining the posterior ulnar vein.

The posterior ulnar vein, of large size, commences in the inner part of the plexus on the back of the hand, and it ascends along the back of the inner side of the forearm to join the median basilic, either separately or having previously taken up the anterior ulnar vein.

The principal superficial veins of the brachial region are the cephalic and the basilic.

The cephalic vein is formed by the union of the median cephalic and the radial a little above the external epicondyle. It then ascends, lying at first in the groove along the outer border of the biceps, and then between the pectoralis major and deltoid. Thereafter it crosses the first part of the axillary artery, and, piercing the costo-coracoid membrane and axillary sheath, opens into the axillary vein above the pectoralis minor.

The basilic vein is formed by the union of the median basilic, anterior ulnar, and posterior ulnar, just above the internal epicondyle. It then ascends in the groove along the inner border of the biceps, lying inside the line of the brachial artery. In the lower half of the arm it is superficial to the deep fascia, but about the centre it pierces it, and becomes the axillary vein at the lower border of the tendon of the teres major. In contact with the basilic vein, just above the internal epicondyle, there are one or two lymphatic glands.

\section{FRONT AND INNER ASPECT OF BRACHIAL REGION.}

Deep Fascia.-The deep fascia or aponeurosis forms a continuous investment to the arm, its fibres being principally disposed transversely, but others run more or less longitudinally. It is continuous above with the axillary fascia and the fascial investments of the pectoralis major and deltoid, the tendons of which give expansions to it. It is thin over the biceps, and somewhat thicker over the triceps, but it becomes specially strong in the region of the elbow, where it is attached to the epicondyles of the humerus and olecranon process of the ulna. In front of the elbow it receives a considerable accession of fibres from the bicipital or semilunar fascia. At about the centre of the arm, on its inner aspect, it presents an opening for the passage of the basilic vein. The deep fascia is connected witl the lower part of the humerus on either side by two deep processes, called intermuscular septa. The external septum is attached to the 
external epicondyle, and external supracondylar ridge as high as a point posterior to the lower part of the tendon of insertion of the deltoid, with which it is connected. It gives origin posteriorly to fibres of the inner head of the triceps, below where it is pierced by the musculo-spiral nerve, and above that point to fibres of the external head. Anteriorly, from above downwards, it gives origin to a small part of the brachialis anticus, brachio-radialis, and extensor carpi radialis longior. It is pierced from behind forwards by the musculospiral nerve and the anterior terminal branch of the superior profunda artery, the posterior terminal branch descending behind it. The internal septum is stronger than the external, and is attached to the internal epicondyle, and internal supracondylar ridge as high as a point behind the insertion of the coraco-brachialis. It gives origin anteriorly to the brachialis anticus, and posteriorly to the inner head of the triceps. It is pierced, at its upper part, from before backwards by the ulnar nerve and inferior profunda artery, and, a little above the elbow, by the posterior branch of the anastomotica magna artery. Connected with the internal septum there is a fibrous band, known as the internal brachial ligament (Struthers), which extends from the humerus below the tendon of insertion of the teres major to the internal epicondyle. The two intermuscular septa divide the lower half of the arm into two compartments-anterior and posterior. The anterior compartment contains the biceps, brachialis anticus, brachio-radialis, extensor carpi radialis longior, basilic vein, brachial vessels, median nerve, ulnar nerve for a short distance above, internal cutaneous nerve, nerve of Wrisberg, and musculo-spiral nerve after it has pierced the external septum. The posterior compartment contains the triceps and a small part of the musculo-spiral nerve.

Internal Epicondylar Lymphatic Glands.--These glands, usually two in number, are situated about $I_{\frac{1}{2}}$ inches above the internal epicondyle of the humerus. They lie superficial to the deep fascia, and in close proximity to the commencement of the basilic vein. Their afferent vessels are derived from (I) the inner three fingers, (2) the inner part of the palm, and (3) the ulnar side of the forearm. Their efferent vessels ascend with the basilic vein, and, about the centre of the arm, they pass with the basilic vein through an opening in the deep fascia. Thereafter they join the deep lymphatics along the brachial artery, which terminate in the external axillary glands.

Anticubital Lymphatic Glands.-These are very inconstant. When present they are about two in number, and lie opposite the bend of the elbow, superficial to the deep fascia. They receive their afferent vessels from the centre of the palm and the front of the forearm. Their efferent vessels ascend superficially along the inner border of the biceps brachii, and, piercing the deep fascia, they terminate in the external axillary glands.

Coraco-brachialis-Origin.-(I) The tip of the coracoid process of the scapula; and (2) the inner aspect of the tendon of the short head of the biceps for 3 inches or more. 
Insertion.-The inner side of the humerus at its centre for about an inch and a half. Some of the upper fibres are inserted into a fibrous band, which ascends in front of the tendons of the latissimus dorsi and teres major to be attached to the humerus below the small tuberosity.

Nerve-supply.-The musculo-cutaneous nerve, by a branch which derives its fibres from the seventh cervical.

The muscle is directed downwards, outwards, and slightly backwards.

Action.-To adduct and flex the humerus. It also braces the head of the bone against the glenoid cavity.

The muscle is pierced by the musculo-cutaneous nerve.

Biceps Flexor Cubiti-Origin.-(x) The short head arises from the tip of the coracoid process of the scapula in association with the coraco-brachialis; (2) the long head arises from the supraglenoid tubercle of the scapula by a rounded tendon, which lies within the capsular ligament of the shoulder-joint, and is continuous on either side with the glenoid ligament.

Insertion.-(I) The posterior rough portion of the bicipital tuberosity of the radius, being separated from the anterior smooth portion by a bursa; and (2) the deep fascia covering the muscles arising from the internal epicondyle of the humerus by means of the bicipital or semilunar fascia.

The short head arises by a short tendon, and the tendon of the long head is about 4 inches in length. This latter tendon arches over the head of the humerus, and leaves the interior of the joint by entering the bicipital groove, beneath the transverse humeral ligament. Within the joint it is invested by a tubular sheath formed by the synovial membrane, which accompanies it for a short distance in the bicipital groove, and is then reflected upwards to become continuous with the synovial lining of the capsular ligament. After leaving the bicipital groove the tendon is replaced by a conical bundle of fleshy fibres, and these join the fibres derived from the short head about the centre of the arm, giving rise to an elongated, oval, fleshy belly. At the level of the epicondyles of the humerus the belly gives place to the strong tendon of insertion, which sinks into the anticubital space, and undergoes a quarter of a turn before reaching its insertion. From the inner side of the tendon, towards its upper part, a strong band of fibres is given off, which passes to the deep fascia covering the muscles arising from the internal epicondyle. It is called the bicipital or semilunar fascia, and it passes over the brachial artery, whilst the median basilic vein lies upon it.

Nerve-supply.-The musculo-cutaneous nerve, which is a branch of the outer cord of the brachial plexus, its fibres being derived from the fifth, sixth, and seventh cervical.

Action.-(I) To flex the elbow-joint ; (2) to supinate the forearm ; (3) by its short head to adduct and flex the arm, and (4) by its long head to raise the arm at the shoulder.

Internal to the muscle in the upper half is the coraco-brachialis, 
and in the lower half the brachial artery and median nerve. External to it is the cephalic vein. The biceps sometimes has a third head, which usually arises from the inner side of the humerus at or near the insertion of the coraco-brachialis. As a rule, it

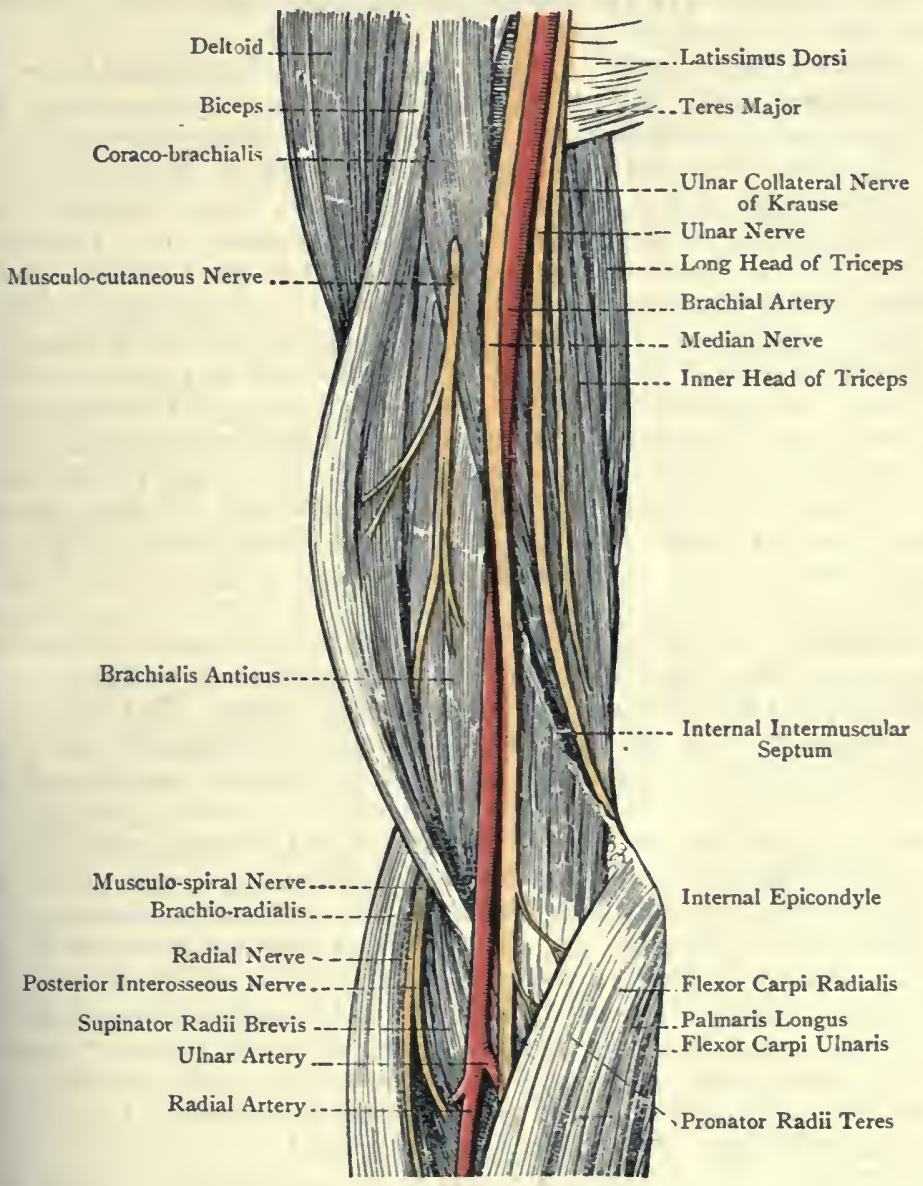

Fig. 187.-Dissection of the Right Brachial Region, AND BeNd of the Elbow.

s external to the brachial artery, but sometimes it crosses the vessel.

Brachialis Anticus-Origin.-(I) The lower half of the front of he humerus ; $(2)$ the front of the internal intermuscular septum over he whole of its extent ; and (3) the front of the external intermusular septum for a short distance above. 
Superiorly the muscle sends a pointed projection upwards on either side of the lower part of the insertion of the deltoid.

Insertion.-The inner part of the rough triangular surface on and below the coronoid process of the ulna.

Nerve-supply.-(I) The musculo-cutaneous nerve, and (2) a twig from the musculo-spiral nerve.

Action.-The muscle is a direct flexor of the elbow-joint.

Brachial Artery.- - The brachial artery is the continuation of the axillary, and it extends from the lower border of the teres major to a point just below the bend of the elbow, where it divides opposite the upper part of the neck of the radius into the radial and ulnar arteries. It is at first internal to the humerus, but gradually inclines to the front of the bone, and at the elbow it is equally distant from the two epicondyles. The course of the vessel is indicated by a line drawn from a point midway between the anterior and posterior folds of the axilla at the humerus to a point midway between the epicondyles of the bone. The artery is accompanied by two venæ comites, one on either side, which communicate with each other over the vessel at frequent intervals. It is for the most part superficial, being only slightly overlapped by the coracobrachialis and biceps. At the bend of the elbow, however, it sinks deeply under cover of the semilunar fascia, and lies in the anticubital space.

Relations-Superficial.-Skin, superficial and deep fasciæ, median nerve about the centre of the arm, semilunar fascia of the biceps, and, superficial to this, the median basilic vein. Deep.-The long head of the triceps, with the intervention of the musculo-spiral nerve and superior profunda artery, inner head of the triceps, insertion of the coraco-brachialis, and brachialis anticus. External.-The coracobrachialis and biceps, both of which slightly overlap the vessel, external vena comes, and the median nerve in the upper half of the arm. Internal.- The internal vena comes, internal cutaneous nerve (which may be slightly over the vessel) as low as the centre of the arm, ulnar nerve also as low as the centre, median nerve in the lower third, and basilic vein, which is superficial to the deep fascia in the lower half, but beneath it in the upper half. The nerve most intimately related to the artery is the median, which lies on its outer side in the upper half of the arm, in front of it for a little at the centre, and on its inner side in the lower third.

Branches.-The vessel gives off from its outer side a series of branches which are distributed to the muscles and integument of the front of the arm. The named branches arise from the inner and back part of the trunk. They are called superior profunda, inferior profunda, nutrient, and anastomotica magna.

The superior profunda artery is a large vessel which arises from the back of the brachial near its commencement. It passes downwards and backwards with the musculo-spiral nerve between the long and inner heads of the triceps, and it then winds round the back of the humerus, lying with the nerve in the spiral groove, 
between the outer and inner heads of that muscle. Towards the lower end of the groove it divides into two terminal branchesanterior and posterior. The anterior branch accompanies the musculo-spiral nerve through the external intermuscular septum, and then descends between the brachio-radialis and brachialis anticus to anastomose with the radial recurrent artery. The poslerior branch descends behind the external intermuscular septum, and anastomoses behind the external epicondyle with the posterior interosseous recurrent, and across the back of the humerus above the olecranon fossa with the anastomotica magna. Besides the two terminal branches the superior profunda gives off the following offsets: muscular to the triceps; an ascending branch, which passes upwards between the long and outer heads of the triceps to anastomose with a branch of the posterior circumflex; and a nutrient branch, which enters a foramen on the back of the humerus. The superior profunda may arise from the third part of the axillary, and it may give off the posterior circumflex.

The inferior profunda artery arises from the brachial about the centre of the arm, or sometimes from the superior profunda. It accompanies the ulnar nerve through the internal intermuscular septum, and then descends with it on the inner head of the triceps to the back of the internal epicondyle, where it anastomoses with the anastomotica magna and posterior ulnar recurrent arteries. In its course it gives muscular offsets to the triceps.

The nutrient or medullary artery arises from the brachial opposite the lower border of the insertion of the coraco-brachialis, or it may come off from the inferior profunda. Its course is downwards, and it enters the medullary foramen of the bone to be distributed to its interior.

The anastomotica magna artery arises about 2 inches above the elbow. It passes inwards on the brachialis anticus, and divides into two branches-a small anterior and large posterior. The anterior pranch descends beneath the pronator radii teres, and anastomoses with the anterior ulnar recurrent artery. The posterior branch pierces the internal intermuscular septum, and then passes outwards beneath the triceps, resting upon the back of the humerus above the blecranon fossa, where it forms an arch with the posterior branch of he superior profunda artery. It gives a branch to the back of the hternal epicondyle, which anastomoses with the inferior profunda ind posterior ulnar recurrent arteries.

Varieties.-1. The brachial artery may divide above the normal level. In tost cases the vessel given off earlier than usual is the radial; more rarely it the ulnar, and in these cases the interosseous trunk arises from the radial; iill more rarely the premature branch is the interosseous trunk, or a large vas berrans. The level at which a high division takes place is most frequently 2 the upper third of the arm, less so in the lower third, and it is of rarest ccurrence in the middle third. When two arteries are present they usually e side by side in the position of the normal vessel. When a vas abesrans present it usually arises from the upper part of the brachial artery, and rminates below by joining, most commonly, the radial artery. 
2. In rare cases the brachial artery divides high up into two vessels of equa size, which become reunited into one trunk a little above the elbow.

3. When a supracondylar process is present the brachial artery, along with the median nerve, may descend towards the internal epicondyle until it gets below the level of the process, round which it turns forward to the front of the elbow. This is the normal course taken by the artery in the Felida, in which there is a supracondylar foramen.

Collateral Circulation.-When the brachial artery has been ligatured above the centre of the arm, the collateral circulation is carried on by the superior profunda artery, which anastomoses below with (I) the radial recurrent, (2) the posterior interosseous recurrent, and (3) the anastomotica magna. When the artery has been ligatured in the vicinity of the elbow, the inferior profunda and anastomotica magna assist the superior profunda by anastomosing with the anterior and posterior ulnar recurrent.

Brachial Venæ Comites.-These are two in number, and they closely accompany the artery, one being placed on either side of it. Along the course of the vessel they communicate with each other across it at frequent intervals. Superiorly the external vena comes crosses inwards over the lower portion of the third part of the axillary artery to join the internal vena comes, and the resulting trunk opens into the axillary vein near the lower border of the subscapularis.

The internal cutaneous nerve and the nerve of Wrisberg are situated on the inner side of the brachial artery, the former slightly encroaching upon it. The median nerve lies on the outer side of the artery as low as the centre of the arm, where it passes over it, and then descends on its inner side in the lower third. Instead of crossing over the vessel it may pass behind it. It gives off no branch in the arm, but it sometimes receives 'a branch from the musculo-cutaneous. The ulnar nerve lies on the inner side of the artery as low as the insertion of the coraco-brachialis. Here it meets with the inferior profunda artery, and with it pierces the internal intermuscular septum from before backwards. It then descends on the inner head of the triceps to the interval between the olecranon and internal epicondyle. It gives off no branch in the arm. The musculo-cutaneous nerve, having pierced the coracobrachialis, passes downwards and outwards between the biceps and brachialis anticus. A little above the elbow it appears at the outer border of the biceps, whence it descends to its cutaneous distribution, already described. Before piercing the coracobrachialis, it gives off the branch to that muscle, and, as it courses between the biceps and brachialis anticus, it furnishes branches tc them. It sometimes gives a communicating branch to the mediar nerve.

Anticubital Space.-This is the name given to the triangulal hollow in front of the elbow-joint. The roof of the space is former by the integument, median basilic and median cephalic veins anterior division of the internal cutaneous nerve, cutaneous par of the musculo-cutaneous nerve, deep fascia, and semilunar fascia 
The floor is formed by the brachialis anticus and a small part of the upinator radii brevis. The base is represented by an imaginary ine connecting the epicondyles of the humerus. The outer boundary $\mathrm{s}$ formed by the brachio-radialis, and the inner by the pronator radii eres, the apex being constructed by the former muscle overlapping

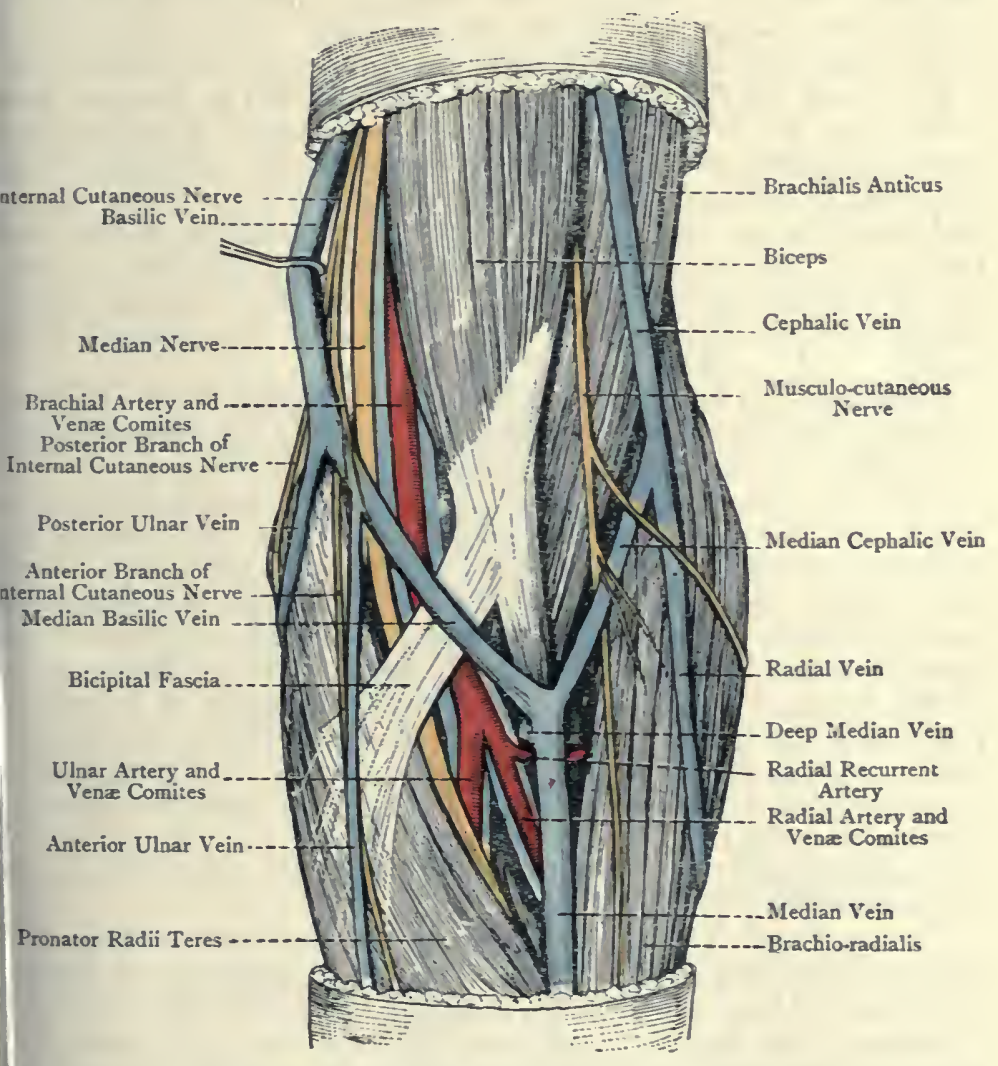

iig. 188.-Superficial Dissection of the Front of the Left Elbow.

he latter. The space contains the terminal part of the brachial, nd the commencement of the radial and ulnar, arteries, with their spective venæ comites. On the outer side of the brachial artery : the tendon of the biceps, and on its inner side is the median erve. Under cover of the brachio-radialis are the radial and osterior interosseous branches of the musculo-spiral nerve. 


\section{BACK OF THE BRACHIAL REGION.}

Triceps Extensor Cubiti-Origin.-(I) The long head arises fron the infraglenoid ridge of the scapula, where it is superficially ten dinous. (2) The external head arises from $(a)$ the outer part of th posterior surface of the humerus, reaching as high as the insertio of the teres minor, and as low as the spiral groove; and $(b)$ th back of the external intermuscular septum above the point wher it is pierced by the musculo-spiral nerve. (3) The internal hea arises from $(a)$ the whole of the posterior surface of the humeru below the spiral groove, reaching upwards on the inner side of th groove, in a tapering manner, as high as a point about $\frac{1}{4}$ inch above and posterior to, the lower border of the insertion of the tere major; (b) the back of the internal intermuscular septum ove its whole extent; and (c) the back of the external inter muscular septum below where it is pierced by the musculo-spira nerve.

Insertion.-(I) The back part of the upper surface of the olecrano process of the ulna; (2) the deep fascia covering the anconeus; an (3) slightly into the posterior ligament of the elbow-joint.

The long and external heads terminate in a broad flat tendon which occupies about the lower half of the arm, the fibres of th long head ending on its inner side, and those of the external hea on its upper and outer parts. Most of the fibres of the interna head terminate on the deep surface of the tendon, but some ar inserted directly into the olecranon. The deepest and lowest fibre of this head are inserted into the posterior ligament of the elbow joint, and form the so-called subanconeus. There is usually bursa over the front part of the upper surface of the olecranon separating the tendon of the muscle from the posterior ligamen of the elbow-joint.

The long head is related to the lower part of the capsular ligamen of the shoulder-joint.

Nerve-supply.-The musculo-spiral nerve, which is a branch o the posterior cord of the brachial plexus, its fibres being deriver from the last four cervical, and sometimes from the first thoracic.

Action.-The external and internal heads are simple extensor: of the elbow-joint. The long head also extends the elbow-joint and from its relation to the shoulder-joint it depresses the arm upon the scapula.

Musculo-spiral Nerve.-This nerve at first lies behind the thir part of the axillary artery, and then behind the upper part of the brachial. Thereafter it passes downwards and backwards, with the superior profunda artery, between the long and internal heads of the triceps. It next winds round the back of the humerus in the spiral groove, between the external and internal heads of the triceps. Having pierced the upper part of the external intermusculat septum, it descends in the groove between the brachio-radiali and brachialis anticus to a point a little above the external epi- 
condyle, where it terminates by dividing into the radial and posterior nterosseous nerves.

Branches-Internal Branches.-These, which arise on the inner

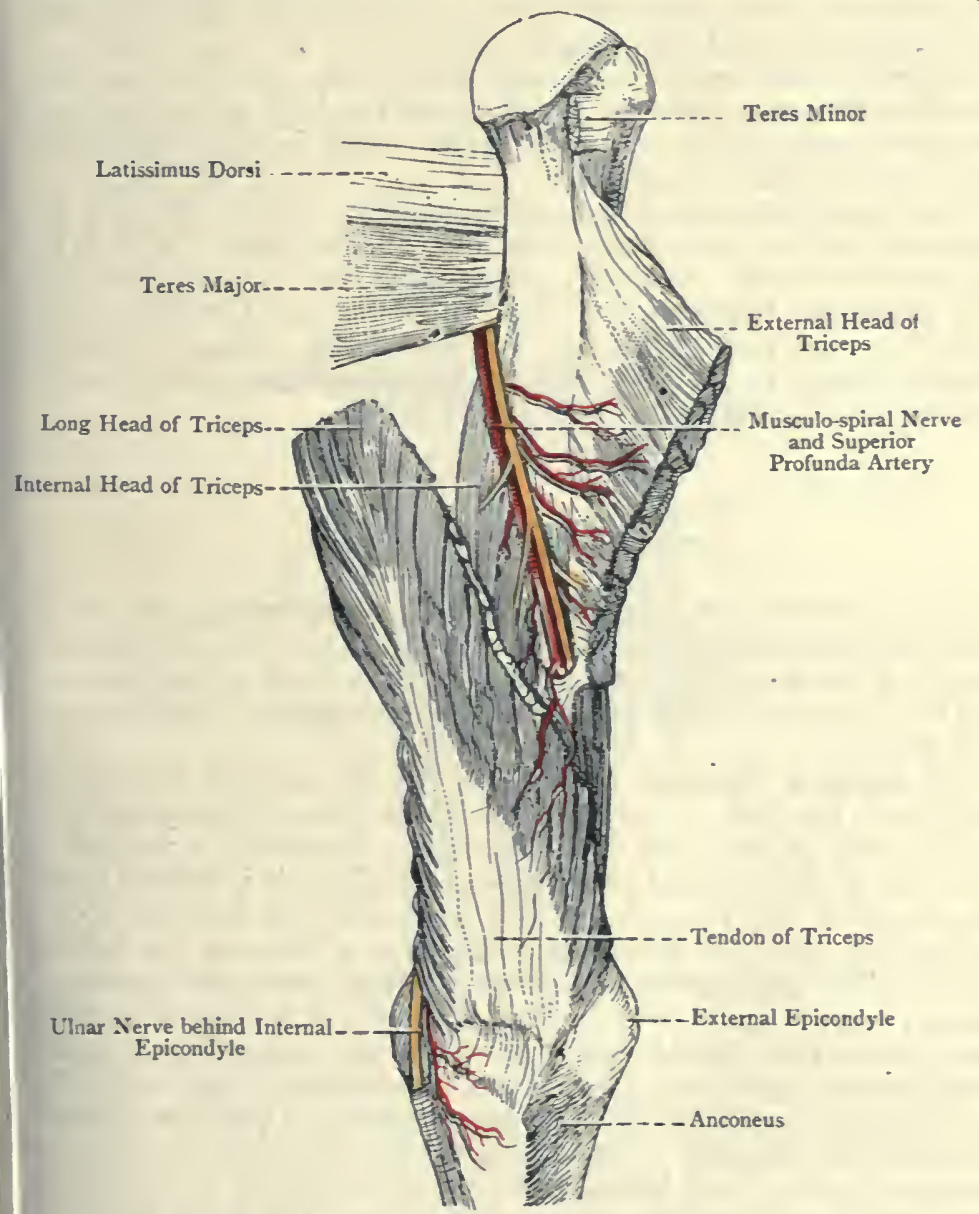

Fig. I E9.-Dissection of the Back of the Brachial Region.

The External Head of the Triceps has been divided and reflected outwards to show the contents of the Spiral Groove.)

ide of the humerus, are muscular and cutaneous. The muscular ranches supply the long and internal heads of the triceps, hose for the long head entering it high up, whilst those for the nternal head enter it at different levels. One of the latter, which 
is remarkable for its length, descends, along with the ulnar nerve, to enter the internal head low down, this branch being known as the ulnar collateral nerve (Krause). The internal cutaneous branch usually arises in common with one of the muscular branches, and is distributed to the integument of the back of the arm, reaching nearly as low as the back of the elbow.

Posterior Branches.-These arise behind the humerus, and are distributed to the external and internal heads of the muscle and to the anconeus, the nerve to the latter, which is long and slender, descending in the internal head.

External Branches.-These arise on the outer side of the humerus, and are cutaneous, muscular, and articular. The upper and lower external cutaneous nerves have been already described. The muscular branches are distributed to the brachio-radialis, extensor carpi radialis longior, and brachialis anticus, the latter branch being a small twig. The articular branches, one or two in number, are distributed to the elbow-joint.

\section{THE SHOULDER-JOINT.}

The shoulder-joint belongs to the class diarthrosis, and to the subdivision enarthrosis (ball-and-socket). The articular surfaces are the glenoid cavity of the scapula and the head of the humerus; and the ligaments are the capsular, coraco-humeral, gleno-humeral, and glenoid.

The capsular ligament is attached to the scapula around the margin of the glenoid cavity close to the glenoid ligament, with which many of its fibres are connected. Superiorly it extends to the root of the coracoid process, and inferiorly it is related to the long head of the triceps. At the humerus it is attached to the anatomical neck, its fibres descending for a little on the inferior aspect. The ligament is very loose, so that, when the muscles in contact with it have been divided, the head of the humerus drops away from the glenoid cavity for over an inch. The ligament presents two openings. One, called the foramen ovale, is situated on its anterior aspect, behind the upper border of the subscapularis. Through this opening a protrusion of the synovial membrane of the joint takes place beneath the upper border of the subscapularis, where it forms the subscapular bursa. The other opening is placed between the great and small tuberosities of the humerus at the commencement of the bicipital groove, and it allows the long tendon of the biceps, with its synovial investment, to leave the interior of the joint, beneath the transverse humeral ligament. There is sometimes a third opening of small size on the posterior aspect of the capsule, which allows the synovial membrane to protrude and form a bursa beneath the infraspinatus.

The coraco-humeral ligament is a strong band which extends 
from the outer border of the coracoid process, near the root, across the upper part of the capsule, with which it is closely connected, to the great tuberosity of the humerus.

The gleno-humeral bands are three in number, and are thickened parts of the capsule which project in an inward direction. The superior band is known as the gleno-humeral ligament, and is regarded as representing the ligamentum teres of the hip-joint. It extends from the apex of the glenoid cavity, and the root of the coracoid process, of the scapula to the small tuberosity of the humerus, where it lies on the inner side of the bicipital groove. The middle band, called Flood's ligament, extends from the anterior

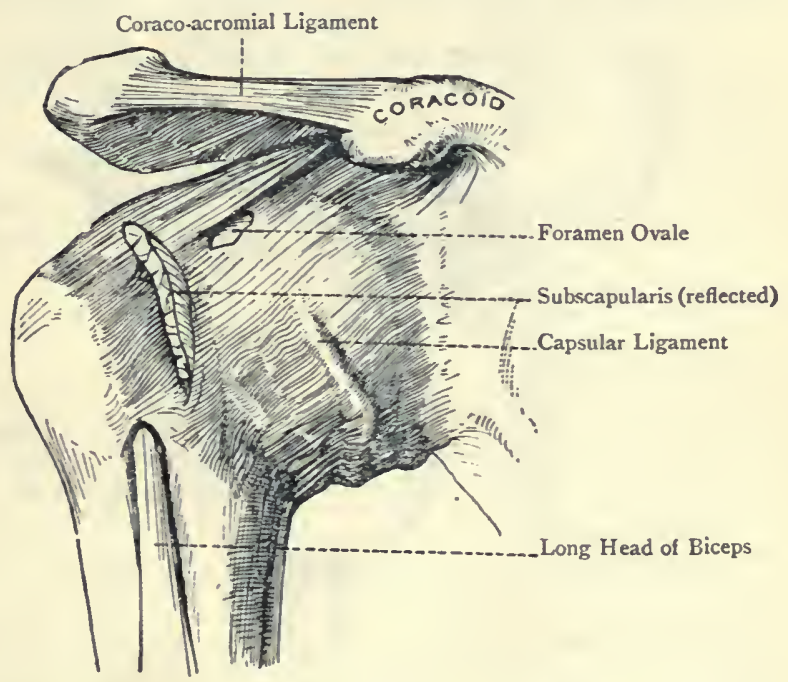

Fig. 190.-The Right Shoulder-Joint (ANterior View).

margin of the glenoid cavity to the lower part of the small tuberosity of the humerus. The inferior band, called Schlemm's ligament, extends from the lower part of the glenoid cavity to the lower part of the neck of the humerus. The gleno-humeral bands are best seen when the joint is opened from behind. Associated with the capsule there is also the transverse humeral ligament, which bridges over the upper end of the bicipital groove between the great and small tuberosities.

The glenoid ligament is a dense fibro-cartilaginous band which is implanted on the edge of the glenoid cavity, and so deepens it for the head of the humerus. Externally it is connected with the capsular ligament, and superiorly each lateral division blends with the long head of the biceps.

The long head of the biceps is to be regarded in the light of a 
ligament, inasmuch as it arches over the head of the humerus, and tends to prevent upward displacement of the bone.

The synovial membrane lines the inner surface of the glenoid ligament, and is reflected from it over the inner surface of the capsule. From this it passes to the anatomical neck of the humerus, which it covers as far as the margin of the articular cartilage of the head. It protrudes through the opening in the front of the capsule to form the subscapular bursa, and it sometimes protrudes through an opening behind to form a bursa beneath the infraspinatus. The long head of the biceps, in passing from the supraglenoid tubercle to the bicipital groove, receives a tubular investment from it. This accompanies the tendon for a short distance in

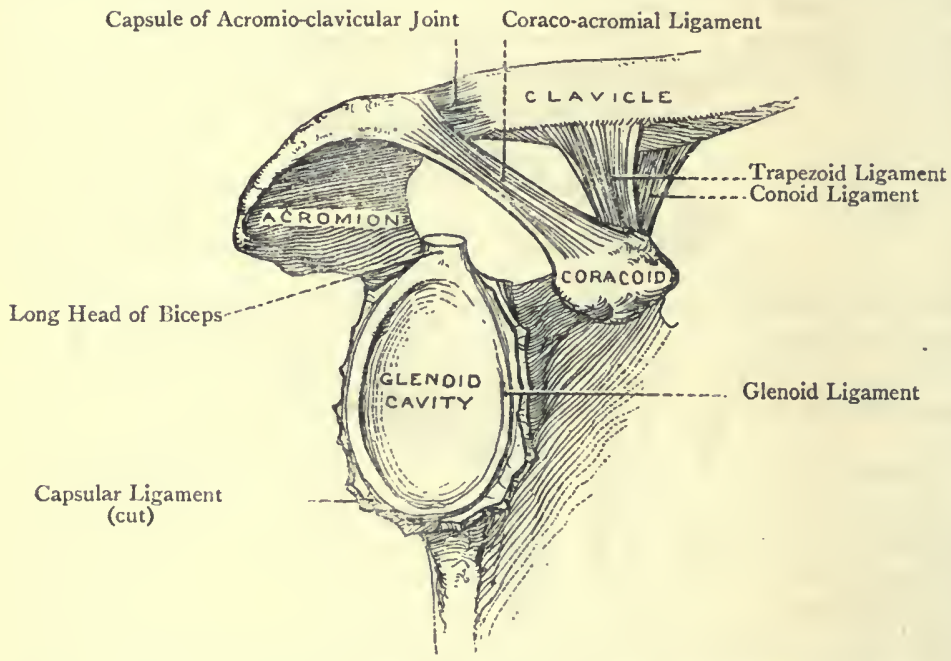

Fig. i9i,-The Right Glenoid Cavity, and the Adjacent Ligaments,

the bicipital groove, after which it is reflected upwards to become continuous with the synovial membrane lining the capsule.

Muscular Relations.-The capsular ligament is closely related to the following muscles: superiorly, the supraspinatus; posteriorly, from above downwards, infraspinatus and teres minor; inferiorly, long head of the triceps ; and anteriorly, subscapularis.

Arterial Supply.--The suprascapular, and anterior and posterior circumflex, arteries.

Nerve-supply.-The suprascapular and circumflex nerves.

Movements.-Seven movements are allowed at the shoulder-joint, namely forward flexion, as in shaking hands, extension, backward flexion, abduction, adduction, rotation, and circumduction. 
Museles concerned in the Movements-Forward Flexion.-The clavicular parts of the pectoralis major and deltoid, short head of the biceps, and coracobrachialis. Extension.- The spinal portion of the deltoid, teres major, and latissimus dorsi. Backward Flexion.-This movement being a continua. tion backwards of extension, the muscles concerned are the same as for that movement. Abduction.-The acromial portion of the deltoid and supraspinatus. Adduction.-The sterno-costal portion of the pectoralis major, short head of the'biceps, coraco-brachialis, teres major, and latissimus dorsi.

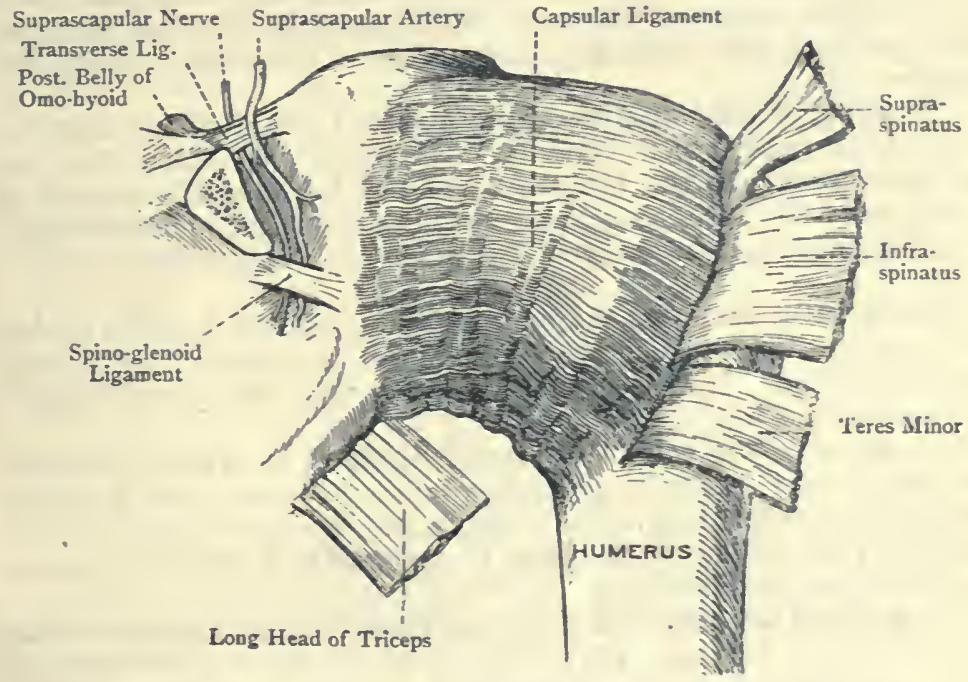

Fig. 192.-The Right Shoulder-Joint (Posterior View).

External Rotation.-Infraspinatus and teres minor. Internal Rotation.Subscapularis, pectoralis major, teres major, and latissimus dorsi. Circumduction.-This movement, being a combination of flexion, abduction, extension, and adduction, is effected by the various muscles concerned in these movements.

Bursæ at Shoulder-Joint.-The bursæ in the vicinity of the shoulder-joint are as follows:

\section{Subscapular. \\ Subacromial. \\ Infraspinatus. \\ Latissimus dorsi.}

\author{
Teres major. \\ Cor aco-clavicular ligament. \\ Bicipital intertubercular.
}

The subscapular bursa lies between the upper border of the tendon of the subscapularis muscle and the upper part of the front of the capsular ligament. It is continuous with the synovial membrane of the joint, which protrudes through the foramen ovale in the capsule.

The subacromial or subdeltoid bursa, which is of large size, is 
situated beneath the acromion process of the scapula, the coracoacromial ligament, and the acromial portion of the deltoid muscle. It covers the upper part of the capsular ligament, and the tendons inserted into the great tuberosity of the humerus. Occasionally it is multilocular. It has no communication with the synovial cavity of the shoulder-joint.

The bursa of the infraspinatus muscle, which is not constant, is situated between the tendon of that muscle and the back part of the capsule. It may communicate with the synovial cavity of the joint through an opening in the back part of the capsule.

The bursa of the latissimus dorsi muscle is situated between the back of the tendon of insertion of that muscle into the floor of the bicipital groove of the humerus and the front of the tendon of insertion of the teres major muscle into the inner bicipital ridge. It has no communication with the synovial cavity of the shoulderjoint.

The bursa of the teres major muscle is situated directly behind the tendon of insertion of that muscle into the inner bicipital ridge of the humerus. It has no communication with the synovial cavity of the shoulder-joint.

The bursa of the coraco-clavicular ligament is situated between the conoid and trapezoid portions of that ligament, which extend between the outer part of the clavicle and the root of the coracoid process of the scapula. It has no communication with the synovial cavity of the shoulder-joint.

The bicipital intertubercular synovial sheath invests the long tendon of the biceps in the upper part of the bicipital groove of the humerus. It is a prolongation of the synovial membrane of the shoulderjoint.

\section{THE FOREARM AND HAND.}

Cutaneous Nerves.-Along the front of the outer side of the forearm is the anterior branch of the cutaneous part of the musculocutaneous, and along the back are its posterior branch and the lower external cutaneous branch of the musculo-spiral. Along the front and back of the inner side are the anterior and posterior divisions of the internal cutaneous. All these nerves have been already described. A small cutaneous branch of the ulnar nerve is given off from it about the centre of the forearm, which, after piercing the deep fascia and communicating with the anterior branch of the internal cutaneous, has a limited distribution to the integument just below the centre, internal to the median line. This branch is not constant.

The integument of the palm of the hand is supplied by the palmar cutaneous branches of the ulnar, median, and radial. The 
palmar cutaneous branch of the ulnar arises in common with the branch to the integument of the forearm and descends on the ulnar artery, keeping beneath the deep fascia until it approaches the wrist, where it becomes cutaneous on the outer side of the tendon of the flexor carpi ulnaris. It then passes over the anterior annular ligament, and is distributed to the integument of the inner part of the palm. The palmar cutaneous branch of the median arises a little above the wrist, and pierces the deep fascia just above the anterior annular ligament, in the interval between the tendons of the flexor carpi radialis and palmaris longus. It then descends

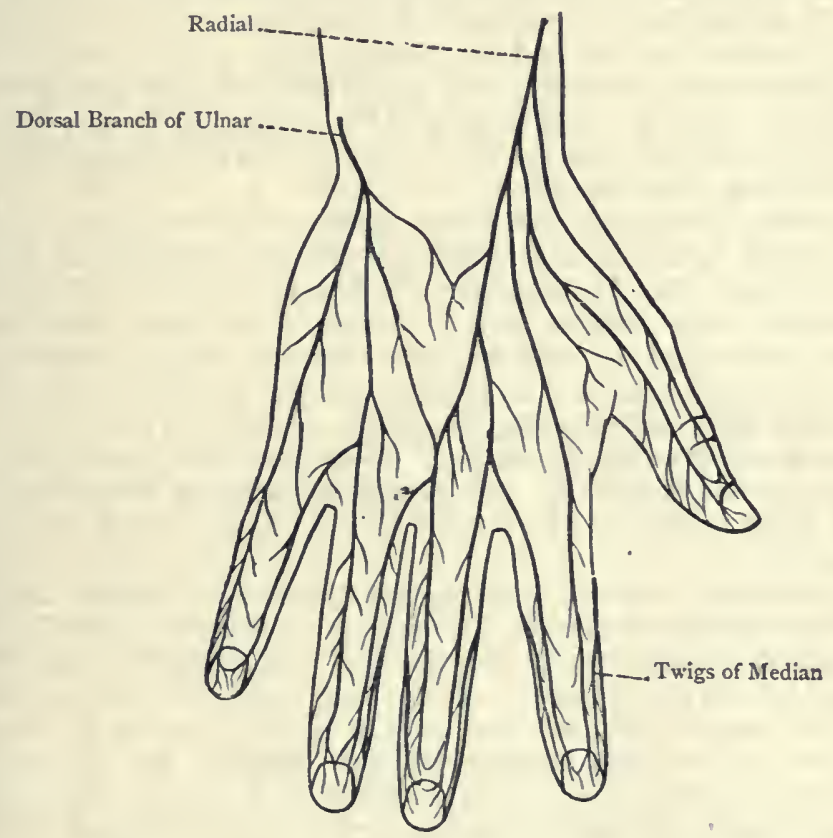

Fig. 193.-Diagram of the Nerves of the HaNd

(DORSAL ASPECT).

over the anterior annular ligament, and is distributed to the integument of the outer part of the palm, and slightly to that of the thenar eminence. It communicates internally with the palmar cutaneous of the ulnar, and externally with that of the radial. The palmar cutaneous branch of the radial arises from the external division of the nerve which passes to the outer side of the thumb. It is joined by a twig from the anterior branch of the musculocutaneous, and is distributed to the integument of the thenar eminence.

The integument of the dorsum of the hand and fingers is supplied 
by the radial nerve and the dorsal branch of the ulnar. The radial nerve winds backwards beneath the tendon of the brachioradialis about 3 inches above the wrist, and then divides into two branches-external and internal. The external branch passes to supply the outer side of the thumb, giving off in its course the palmar cutaneous branch. The internal branch, having communicated with the posterior branch of the musculo-cutaneous, and having given a branch to the back of the wrist which communicates with the dorsal branch of the ulnar, divides into four digital nerves. The first supplies the inner side of the thumb, the second the outer side of the index finger, the third bifurcates at the cleft between the index and middle fingers into two collateral branches for the supply of their contiguous sides, and the fourth passes to the cleft between the middle and ring fingers. The latter in its course is reinforced by an offset from the dorsal branch of the ulnar, and then it divides into two collateral nerves for the supply of the contiguous sides of the middle and ring fingers.

The dorsal branch of the ulnar nerve arises about $2 \frac{1}{2}$ inches above the wrist, and winds backwards beneath the tendon of the flexor carpi ulnaris. It then gives a branch to the back of the wrist, which communicates with a branch of the radial, and thereafter is distributed as follows: one branch supplies the inner side of the little finger; a second branch passes to the cleft between the little and ring fingers, giving an offset to the innermost digital branch of the radial, and it divides into two collateral branches for the supply of the contiguous sides of these fingers; whilst a third branch is distributed to the integument of the back of the hand.

The distribution of the dorsal digital nerves is as follows : on the thumb they extend as far as the nail, on the index finger to the distal end of the second phalanx, on the middle finger to near the distal end of the first phalanx, on the ring-finger to near the distal end of the second phalanx, and on the little finger as far as the nail. The portions of integument not supplied by the dorsal digital nerves derive their innervation from branches which pass backwards from the palmar digital branches' of the median and ulnar nerves.

Veins.-The superficial veins of the forearm, already described, are the radial, median, anterior ulnar, and posterior ulnar. On the back of the hand is the dorsal venous plexus, and in front of the anterior annular ligament is the anterior plexus, of small size. The dorsal venous plexus receives the superficial digital veins, which commence in plexuses in the region of the nails. They are two in number to each finger, one being placed on each side towards the dorsal aspect. These veins form cross arches above and below the interphalangeal joints on the backs of the fingers. At the clefts the collateral veins unite to form in each case a single trunk, and these trunks end in the dorsal venous plexus. The superficial digital vein from the inner side of the little funger passes to the commence- 
ment of the posterior ulnar vein, and is known as the vena salvatella or 'saving vein.' The blood is conveyed away from the outer side of the dorsal venous plexus by the radial vein, and from the inner side by the posterior ulnar vein. The radial vein receives a communicating branch from the venæ comites of the deep palmar arch at the proximal end of the first interosseous space. The posterior ulnar vein receives a communicating branch from the venæ comites of the profunda branch of the ulnar artery, which passes beneath the abductor minimi digiti muscle.

The anterior venous plexus receives its radicles from the palm, and the blood is conveyed away from it by the median vein. The anterior ulnar vein, as stated, commences on the inner aspect of the front of the wrist.

Deep Fascia of the Forearm.-The deep fascia is of considerable strength, its fibres being principally transverse, though some are disposed longitudinally and obliquely. Superiorly, below the internal epicondyle, it receives the semilunar fascia of the biceps, and behind it gets an accession of fibres from the tendon of the triceps. In front of the elbow it presents an opening for the passage of the deep median vein. In the region of the epicondyles it serves as a common tendon of origin to the muscles arising from these prominences, and it sends between them strong intermuscular septa which afford additional origin to them, and are readily recognised on the surface by white lines. It is attached above to the epicondyles of the humerus and the margins of the triangular surface on the back of the olecranon process of the ulna, and along the forearm to the posterior border of the latter bone. Anteriorly it sends an expansion between the superficial and deep muscles, and it acts in a similar manner on the posterior aspect, where the fascia is stronger than in front. At the wrist it blends with the anterior annular ligament, and posteriorly it forms the posterior annular ligament.

\section{FRONT OF THE FOREARM.}

Muscles.-The muscles of the front of the forearm are arranged in three layers-first or superficial, second or intermediate, and third or deep.

First Layer.-The muscles comprising this layer are, in order from without inwards, the pronator radii teres, flexor carpi radialis, palmaris longus (inconstant), and flexor carpi ulnaris.

I. Pronator Radii Teres. - This muscle arises by two headssuperficial or humeral, and deep or coronoid. The superficial head, which is of large size, arises from (I) the front and upper part of the internal epicondyle, and lower part of the internal supracondylar ridge, of the humerus; (2) the common tendon; and (3) the inter. muscular septa between it and the flexor carpi radialis externally, and flexor sublimis digitorum deeply. The deep head, which is of small size, arises from the inner margin of the coronoid process of the 
ulna, and, after a short course, it joins the deep surface of the superficial head at an acute angle.

Insertion.-By means of a flat tendon, about $\mathrm{I}_{\frac{1}{2}}$ inches broad, in to the rough impression on the outer surface of the radius at its centre, where the curve of the bone is most prominent.

Nerve-supply. - The median nerve, which arises from the outer and inner cords of the brachial plexus, its fibres being derived from the last four cervical and first thoracic nerves. The branches are given off just before the median nerve passes between the two heads of origin of the muscle.

The muscle is directed downwards and outwards.

Action.-(I) To pronate the forearm, and (2) to assist in flexion of the elbow-joint.

The median nerve passes between the two heads of the muscle, and the ulnar vessels beneath its deep head.

The muscle may have a third head, arising from the internal intermuscular septum of the arm, or from a supracondylar process, and, when this is so, it bridges over the brachial artery and median nerve.

2. Flexor Carpi Radialis-Origin.-(I) The front of the internal epicondyle by means of the common tendon; (2) the deep fascia covering the muscle; and (3) the intermuscular septa separating it from the pronator radii teres externally, palmaris longus internally, and flexor sublimis digitorum deeply.

Insertion.-The front of the base of the second metacarpal bone, and by a small slip into the front of the base of the third.

The muscle presents a fusiform, fleshy belly in the upper half of the forearm, which is replaced in the lower half by a strong, flat tendon.

Nerve-supply. - The median nerve.

The direction of the muscle is downwards and outwards.

Action.-(I) To flex the wrist-joint, and (2) to assist in flexion of the elbow-joint.

The tendon of the muscle passes through a special compartment of the anterior annular ligament, where it traverses the groove on the palmar surface of the trapezium, and the radial vessels lie upon the outer side of the tendon in the lower half of the forearm.

3. Palmaris Longus-Origin.-(I) The front of the internal epicondyle by means of the common tendon ; (2) the deep fascia covering the muscle; and (3) the intermuscular septa separating it from the flexor carpi radialis externally, flexor carpi ulnaris internally, and flexor sublimis digitorum deeply.

Insertion.-(I) The upper part of the central division of the palmar fascia, and (2) the front of the anterior annular ligament at its lower part.

Nerve-supply. - The median nerve.

Action.-(I) To render tense the central division of the palmar fascia; (2) to assist in flexing the wrist-joint; and (3) to assist feebly in flexing the elbow-joint. 
The palmaris longus is the representative of an original superficial flexor of the fingers, the expanded portion of the tendon of which remains as the palmar fascia.

4. Flexor Carpi Ulnaris. - This muscle arises by two heads. One

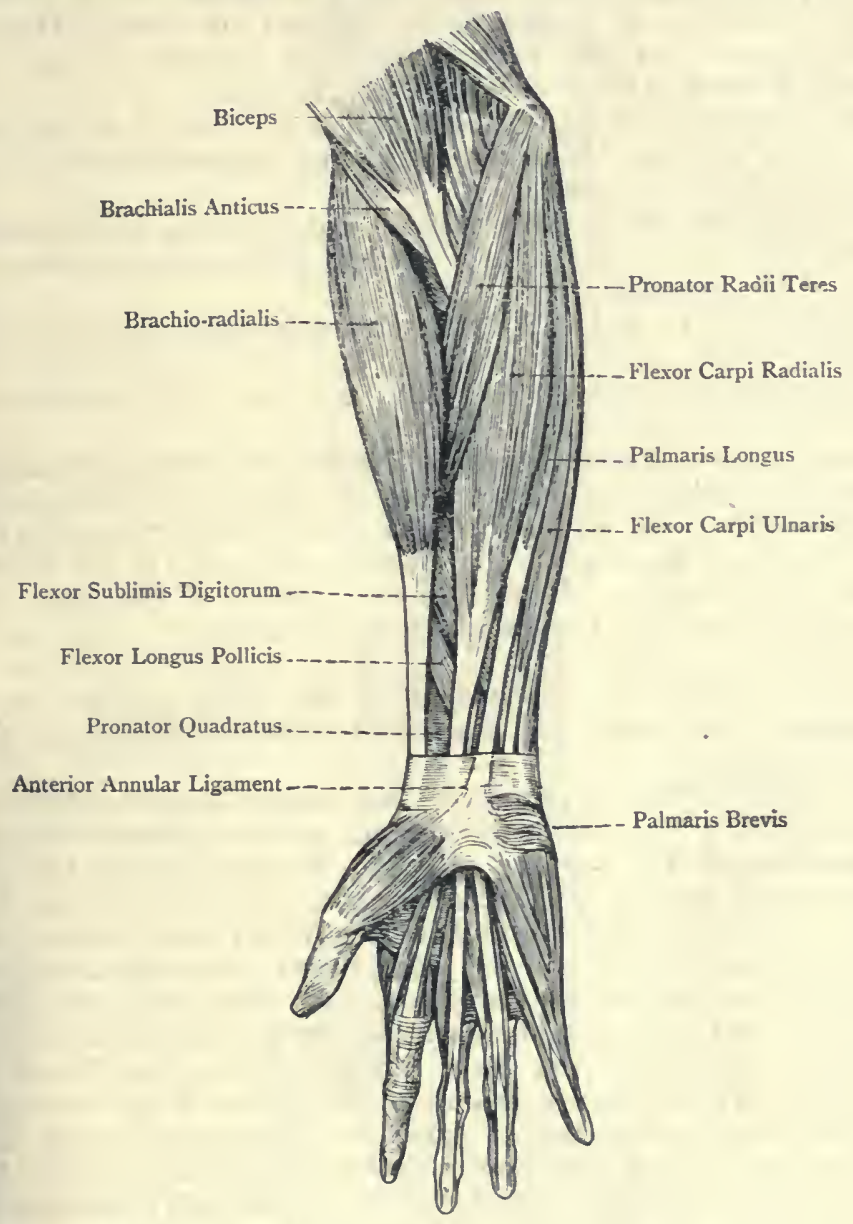

Fig. I94. - The Superficial Muscles of the Front of THE FOREARM.

head arises from (I) the front of the internal epicondyle by means of the common tendon; (2) the deep fascia; and (3) the intermuscular septa between it and the palmaris longus and flexor sublimis digitorum. The other head arises from (I) the inner aspect of the olecranon process, and (2) the upper two-thirds of the posterior 
border, of the ulna, by an aponeurosis common to it, the flexor profundus digitorum, and extensor carpi ulnaris.

Insertion.-The pisiform bone. From the tendon of insertion two prolongations are given off, known as the pisi-uncinate and pisimetacarpal ligaments, the former being attached to the anterior border of the hook of the unciform bone, and the latter to the front of the base of the fifth metacarpal. The tendon also gives off laterally a band to the anterior annular ligament.

Nerve-supply. - The ulnar nerve, which is a branch of the inner cord of the brachial plexus, its fibres being derived from the eighth cervical and first thoracic.

The fibres are directed downwards and forwards, and terminate on the posterior aspect of the tendon which appears about the centre of the forearm.

Action.-(x) To flex and adduct the wrist-joint, and (2) to flex feebly the elbow-joint.

The ulnar nerve and posterior ulnar recurrent artery pass between the two heads of the muscle.

Second Layer.-Flexor Sublimis Digitorum (flexor perforatus)Origin.-The upper part arises from (I) the internal epicondyle; (2) the intermuscular septum between it and the first layer; (3) the internal lateral ligament of the elbow-joint; and (4) the tubercle on the inner margin of the coronoid process of the ulna. The lower part, broad and thin, arises from the anterior oblique line of the radius.

Insertion.-By' four tendons into the sides of the second phalanges of the four inner fingers, at their centre and on their anterior aspect.

The muscle in the lower part of the forearm is replaced by four tendons which pass beneath the anterior annular ligament in pairs. The tendons of the anterior pair are for the middle and ring fingers, whilst those of the posterior pair are for the index and little fingers. In this situation, as well as for a little above the wrist, and as low as about the centre of the palm, they are invested, along with the deep flexor tendons, by the great palmar bursa, to be afterwards described. In the palm the four tendons diverge, and each is accompanied by a tendon of the flexor profundus digitorum, which lies beneath it. At the commencements of the digits each pair of tendons enters the sheath on the palmar aspect of a finger, which binds them to the first and second phalanges. Towards the distal end of the first phalanx the flexor sublimis tendon splits into two parts to allow the flexor profundus tendon to pass through. The two divisions of the superficial tendon are folded round the deep tendon, and unite beneath it at the proximal end of the second phalanx. Beyond this point the superficial tendon is grooved to support the deep tendon, and then divides into its two parts of insertion. The sheath and its accessories will be afterwards described.

Nirve-supply. - The median nerve.

Action.-(I) To flex the second phalanges of the four inner fingers ; 
(2) to flex their metacarpo-phalangeal joints ; (3) to flex the wrist. joint ; and (4) to assist in flexion of the elbow-joint.

Radial Artery.-The radial artery is one of the terminal branches of the brachial, from which it arises in the anticubital space, opposite the upper part of the neck of the radius. It is smaller than the ulnar, which is the other terminal branch, and in point of direction the vessel is the continuation of the brachial. Its destination is the palm, to reach which it passes at first downwards and slightly outwards, as low as the styloid process of the radius. Here the vessel passes to the back of the wrist beneath the styloid process and upon the external lateral ligament, after which it sinks between the two heads of the abductor indicis muscle. - In this way it reaches the palm, where it anastomoses with the profunda branch of the ulnar artery, and so forms the deep palmar arch. The vessel is divided into three parts. The first part lies in front of the forearm, the second on the back of the wrist, and the third in the palm.

First Part.-This part extends from the origin to the styloid process of the radius. Its direction is downwards and slightly outwards, and its course may be indicated by a line drawn from a point just below the bend of the elbow, midway between the epicondyles of the humerus, to a point about $\frac{1}{2}$ inch internal to the styloid process of the radius. In the upper third the vessel lies between the brachio-radialis externally and pronator radii teres internally, being overlapped by the fleshy belly of the former. Thereafter it is placed between the brachio-radialis externally and flexor carpi radialis internally, and in the lower half of the forearm, where these muscles are replaced by their tendons, the vessel is quite superficial.

Relations-Superficial.-The integument, and margin of the brachio-radialis in the upper third, or more. Branches of the musculo-cutaneous nerve are distributed over the line of the vessel. Deep.-From above downwards it lies upon (I) the tendon of insertion of the biceps ; (2) the supinator radii brevis ; $(3)$ the tendon of insertion of the pronator radii teres; $(4)$ the radial origin of the flexor sublimis digitorum; $(5)$ the flexor longus pollicis; (6) the pronator quadratus; and (7) the lower end of the radius. External.-The brachio-radialis throughout the whole of the forearm, and the external vena comes. The radial nerve in the upper part lies a little to the outer side of the vessel; at the centre it is closer to it ; and in the lower part the nerve leaves the artery by turning backwards beneath the tendon of the brachio-radialis. Internal.-The internal vena comes, pronator radii teres in the upper third, and thereafter the flexor carpi radialis.

Branches of the First Part.-The first part gives off the following branches, namely, radial recurrent, muscular, anterior radial carpal, and superficial volar.

The radial recurrent artery, usually of large size, arises from the outer side of the radial close to its commencement, and passes outwards beneath the brachio-radialis, where it rests on the supinator 
radii brevis. Here it divides into branches which come into relation with the musculo-spiral nerve and its terminal divisions. Most of these are distributed to the muscles arising from the external epicondyle, and they anastomose with the posterior interosseous recurrent. One branch, however, ascends with the musculospiral nerve between the brachio-radialis and brachialis anticus, and anastomoses with the anterior terminal branch of the superior profunda of the brachial. The radial recurrent also gives articular twigs to the elbow-joint.

The muscular branches arise at frequent intervals along theforearm.

The anterior radial carpal artery, of small size, arises from the inner side of the radial at the level of the lower border of the pronator quadratus, along which it passes inwards, lying deeply beneath the flexor tendons. At the middle line it anastomoses with the anterior ulnar carpal artery to form the anterior carpal arch. This arch is reinforced from above by the anterior branch of the anterior interosseous artery, and from below by the recurrent branches of the deep palmar arch. In this manner the anterior carpal arch is converted into a rete, the branches of which are distributed to the wristjoint, and the carpal articulations and bones.

The superficial volar artery arises either below the preceding, o sometimes above it. It passes downwards over, or through, the thenar muscles, in which, if of small size, it terminates. When large, it reaches the palm, and anastomoses with the ulnar artery to complete the superficial palmar arch.

Varieties of the First Part.-(I) The artery may arise high up from the brachial, or axillary. (2) When of high origin, it may descend superficial to the semilunar fascia of the biceps and deep fascia of the forearm. (3) The artery may turn backwards over the brachio-radialis just below the centre of the forearm. (4) It may be joined by a vas aberrans from the brachial, or axillary. (5) It may terminate at the lower part of the forearm, its distribution being taken up by the ulnar, median, or anterior interosseous.

The second and third parts of the radial artery will be afterwards described.

Radial Venæ Comites.-The radial artery is accompanied by two venæ comites, which are placed one on either side of the vessel, and they communicate at frequent intervals by transverse branches ying upon it. They terminate above by uniting to form the external brachial vena comes.

Radial Nerve.-The radial nerve is one of the terminal branches of the musculo-spiral, its fibres being derived from the sixth cervical, and sometimes from the fifth and seventh. It passes straight downvards under cover of the brachio-radialis, lying at first a little to the outer side of the radial artery, then getting closer to it at the centre of the forearm, and finally leaving the vessel in the lower art by turning backwards beneath the tendon of the brachioadialis on its way to its cutaneous distribution, which has been lready described. It is a sensory nerve, and gives off no branches intil it reaches the back of the limb. 
Posterior Interosseous Nerve.-This is the other and larger terminal branch of the musculo-spiral, its fibres being derived from the sixth, seventh, and sometimes the eighth, cervical. After a short downward course under cover of the brachio-radialis, it winds round the outer side of the radius, passing through the supinator radii brevis. Having reached the back of the limb near the lower border of that muscle, it descends, in company with the posterior interosseous artery, between the superficial and deep groups of muscles. At the upper border of the extensor longus pollicis it leaves the artery and passes beneath that muscle, where it meets the posterior branch of the anterior interosseous artery on the back of the interosseous membrane. Thereafter it passes through the groove on the back of the radius for the extensor communis digitorum and extensor indicis, lying beneath the tendons and the posterior annular ligament. It terminates on the back of the wrist in a gangliform enlargement, from which branches are given off to the wrist-joint and carpal articulations.

Branches.- The branches are muscular and articular.

Muscular Branches.-Before piercing the supinator radii brevis the nerve gives branches to that muscle and to the extensor carpi radialis brevior. After reaching the back of the forearm, it supplies the extensor communis digitorum, extensor minimi digiti, extensor carpi ulnaris, the three extensors of the thumb, and the extensor indicis.

Articular Branches.-These arise from the terminal gangliform enlargement, and are distributed to the wrist-joint and carpal articulations.

Ulnar Artery.-The ulnar artery is the larger of the two terminal branches of the brachial, and it arises in the anticubital space opposite the upper part of the neck of the radius. Its destination is the palm, which it reaches by passing over the anterior annular ligament, and there it forms the superficial palmar arch. It is at first directed downwards and inwards beneath both heads of the pronator radii teres, flexor carpi radialis, palmaris longus, and flexor sublimis digitorum, and it here describes a slight curve, the convexity of which is directed inwards. Having gained the front of the ulna it meets the ulnar nerve a little above the centre of the forearm, and it then descends on the outer side of the nerve, both structures resting on the flexor profundus digitorum, and being overlapped by the flexor carpi ulnaris. A little above the wrist the artery lies superficially on the outer side of the tendon of that muscle. It then crosses over the anterior annular ligament, lying close to the outer side of the pisiform bone, with the intervention of the ulnar nerve, and shortly thereafter it turns outwards in the palm towards the thenar muscles. The vessel is divided into three parts-first, second, and third.

First Part.-This part extends from the origin to the upper border of the anterior annular ligament. On account of the curve of the vessel at first no definite line can be given to indicate its 


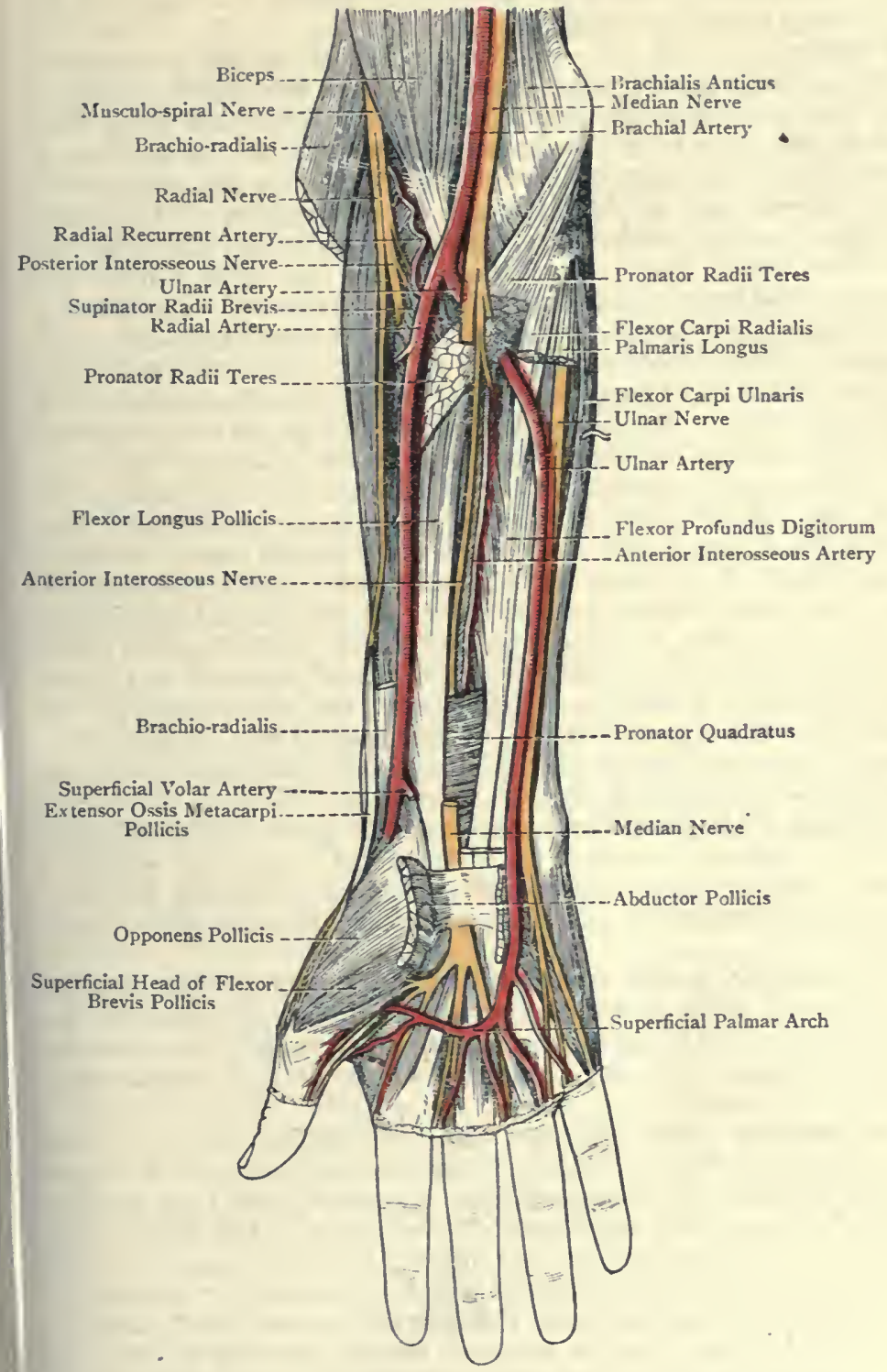

Fig. 196.-Deep Dissection of Front of Right Forearm, and Superficial Dissection of Palm. 
entire course, but in the lower half of the forearm the border of the flexor carpi ulnaris is the guide.

Relations-Superficial.-In the upper half of the forearm the artery is deeply placed, being covered by both heads of the pronator radii teres, flexor carpi radialis, palmaris longus, and flexor sublimis digitorum. In the lower half it is overlapped by the tendon of the flexor carpi ulnaris, except for a little above the wrist, where it lies superficially on the outer side of that tendon. The palmar cutaneous branch of the ulnar nerve descends over the artery in the lower half. Deep.--Brachialis anticus for about $I$ inch, and thereafter the flexor profundus digitorum. External.-The external vena comes, and, in the lower half, the flexor sublimis digitorum. Internal.-The internal vena comes, ulnar nerve for rather more than the lower half, and the tendon of the flexor carpi ulnaris for a little above the wrist. The nerves related to the first part of the artery are the median, ulnar, and palmar cutaneous branch of the ulnar. The median nerve lies for a little at first on the inner side of the vessel, but, at the point where the vessel passes beneath the deep head of the pronator radii teres, the nerve crosses it, being separated from it by the deep head of that muscle, and so gains its outer side. The ulnar nerve, having come from behind the internal epicondyle, is widely separated from the artery for rather more than the upper third of the forearm. A little above the centre the nerve and artery meet, and then descend in close contact, the nerve being on the inner side, with the intervention of the internal vena comes. The palmar cutaneous branch of the ulnar nerve, as stated, descends over the lower half of the vessel.

Branches of the First Part.-The first part gives off the following branches, namely, anterior ulnar recurrent, posterior ulnar recurrent, common interosseous (giving off anterior and posterior interosseous), muscular, posterior ulnar carpal, and anterior ulnar carpal.

The anterior ulnar recurrent artery, of small size, passes upwards and inwards on the brachialis anticus and beneath the superficial head of the pronator radii teres, to supply these muscles and anastomose with the anterior branch of the anastomotica magna of the brachial.

The posterior ulnar recurrent artery, of large size, arises just below the preceding, or sometimes in common with it. It passes inwards beneath the flexor sublimis digitorum, and then ascends with the ulnar nerve between the two heads of the flexor carpi ulnaris to the interval between the internal epicondyle and olecranon process. It supplies the adjacent muscles, ulnar nerve, and elbowjoint, and it anastomoses with the inferior profunda and posterior branch of the anastomotica magna. It also gives twigs over the back of the olecranon, which anastomose with the posterior interosseous recurrent, thus forming the olecranon rete.

The common interosseous artery is a short, thick trunk, which arises, below the preceding, about I inch from the commencement 
of the ulnar. It is directed backwards to the upper border of the interosseous membrane, where it divides into the anterior and posterior interosseous arteries.

(I) The anterior interosseous artery descends on the front of the interosseous membrane, having a vena comes on either side of it, and the anterior interosseous nerve on its outer side. It lies between the flexor longus pollicis externally and the flexor profundus digitorum internally, both of which overlap it, and at the upper border of the pronator quadratus it divides into two terminal branches, anterior and posterior.

Branches.-These are as follows: the median artery (comes nervi mediani) is a long, slender branch which arises from the commencement of the vessel. It at once gets in contact with the median nerve, which it accompanies beneath the flexor sublimis digitorum, supplying the nerve and that muscle. If of large size, it passes beneath, or over, the anterior annular ligament into the palm, where it may join the superficial palmar arch, or if that should be abnormal, it furnishes certain digital arteries. Muscular branches are given off to the deep layer of muscles, and to the extensor muscles of the thumb on the back of the interosseous membrane. The branches to the latter muscles pierce the membrane. The nutrient or medullary arteries enter the radius and ulna. The anterior terminal branch descends beneath the pronator quadratus and joins the anterior carpal arch. The posterior terminal branch, which, passing backwards through the interosseous membrane, and having anastomosed with the posterior interosseous, descends beneath the extensor tendons and the posterior annular ligament to the back of the wrist, where it joins the posterior carpal arch.

(2) The posterior interosseous artery passes backwards between the upper border of the interosseous membrane and the oblique ligament. At the back of the forearm it appears between the supinator radii brevis and extensor ossis metacarpi pollicis, where it is joined by the posterior interosseous nerve. The artery, with the nerve, then descends between the superficial and deep group of muscles until it reaches the upper border of the extensor longus pollicis. Here the artery leaves the nerve, and passes over that muscle and the extensor indicis. At the lower border of the latter muscle it anastomoses with the posterior terminal branch of the anterior interosseous, and then ends in articular branches to the wrist-joint, though it may pass to join the posterior carpal arch.

Branches.-These are as follows: the posterior interosseous recurrent arises as soon as the vessel reaches the back of the forearm. It passes upwards beneath the anconeus to the back of the external epicondyle, where it anastomoses with the posterior branch of the superior profunda of the brachial. It also gives twigs over the back of the olecranon process which anastomose with branches of the posterior ulnar recurrent, and so form the olecranon rete. 
In addition, it anastomoses with the muscular branches of the radial recurrent. Muscular branches supply the adjacent muscles. Articular branches are given off to the wrist-joint.

The muscular branches of the first part of the ulnar artery arise at frequent intervals along the forearm.

The posterior uinar carpal artery arises a little above the pisiform bone, and passes backwards beneath the tendon of the flexor carpi ulnaris to the back of the wrist. Here it turns outwards beneath

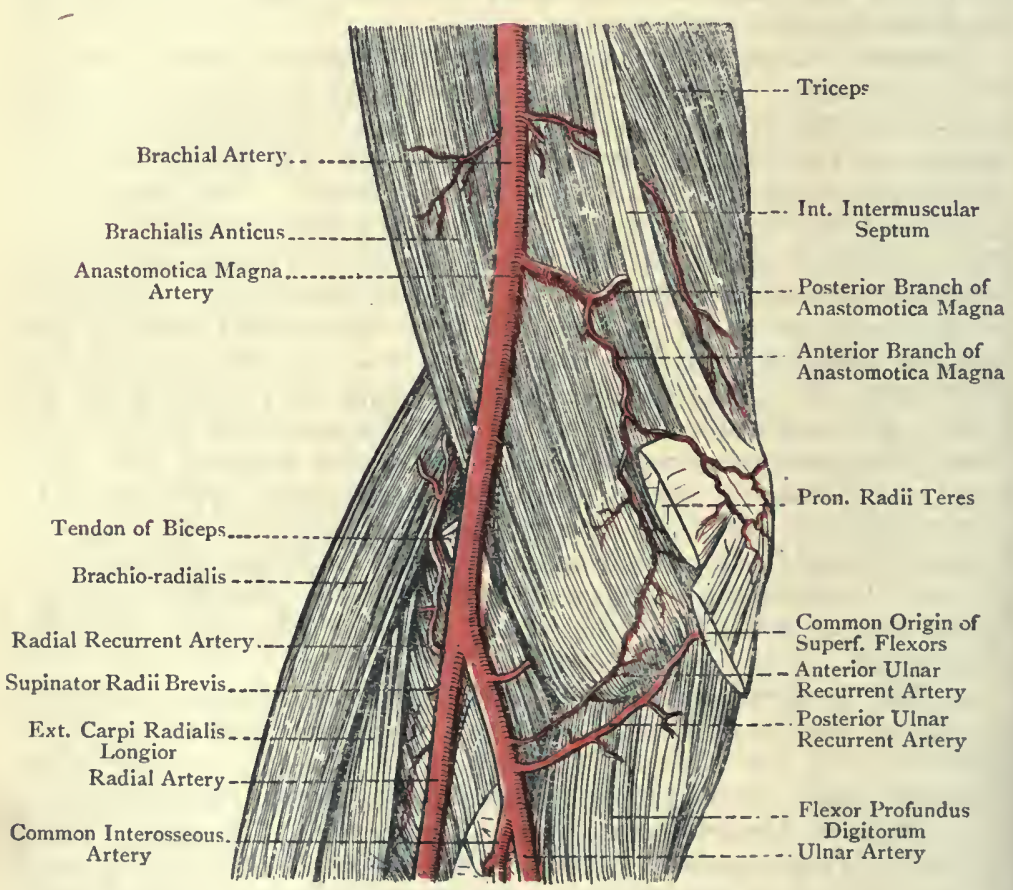

Fig. I97:-Deep Dissection of the Front of the Right Elbow (AFter Tiedemann).

the extensor tendons, and anastomoses with the posterior radial carpal and posterior branch of the anterior interosseous to form the posterior carpal arch. It may give off the dorsal digital artery of the inner side of the little finger.

The anterior ulnar carpal artery arises opposite the lower border of the pronator quadratus. It passes outwards along the lower border of that muscle beneath the flexor profundus digitorum, and anastomoses with the anterior radial carpal, the anterior branch of the anterior interosseous, and the recurrent branches of the deep palmar arch, to form the anterior carpal arch or rete.

Anastomoses round the Elbow-Joint.-The anastomoses of 
arteries round the elbow-joint are very free. In front of the internal epicondyle of the humerus the anterior branch of the anastomotica magna of the brachial anastomoses with the anterior ulnar recurrent. Behind the internal epicondyle the inferior profunda and the posterior branch of the anastomotica magna (both of the brachial) anastomose with the posterior ulnar recurrent. In front of the external epicondyle the anterior terminal branch of the superior profunda of the brachial anastomoses with the radial recurrent. Behind the external epicondyle the

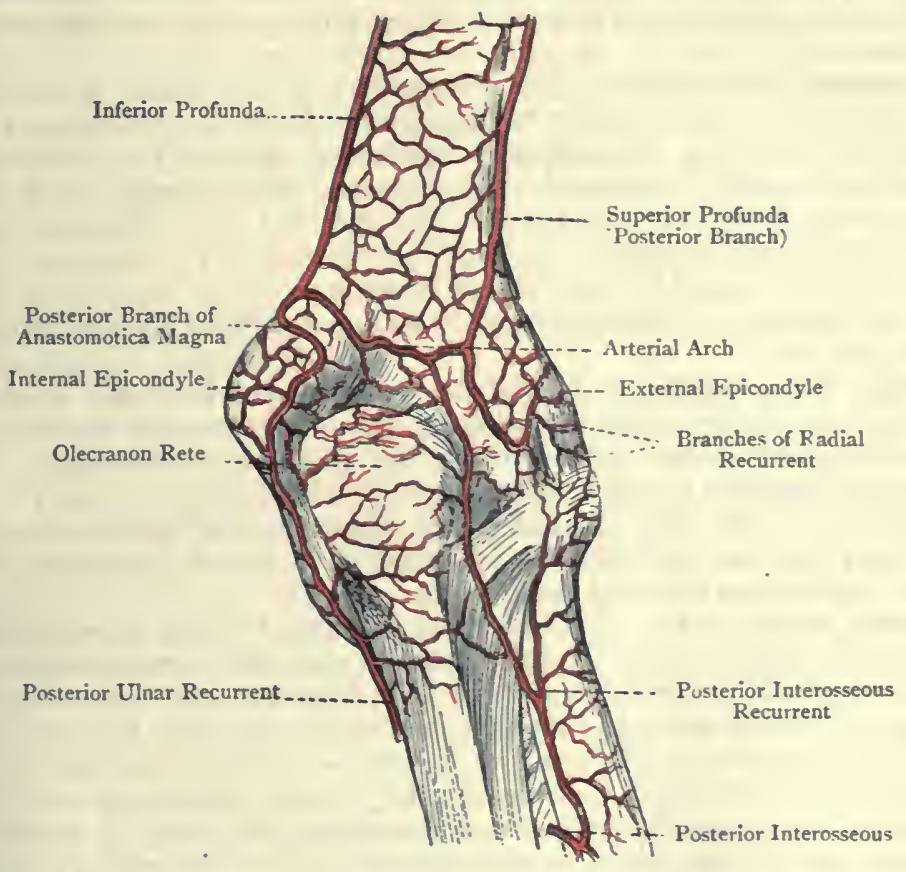

Fig. 198.-The Anastomoses Round the Right Elbow-Joint (Posterior View) (AFTER TIEDEMANN).

posterior terminal branch of the superior profunda anastomoses with the posterior interosseous recurrent. Upon the back of the shaft of the humerus, immediately above the olecranon fossa, a transverse anastomosis takes place between the posterior branches of the superior profunda and anastomotica magna, both of the brachial. Upon the back of the olecranon process is the olecranon arterial rete, which is formed by branches of the posterior interosseous recurrent and posterior ulnar recurrent.

Varieties.-(I) The ulnar artery may arise high up from the brachial, or axillary. In cases of high origin the vessel usually descends over the muscles 
arising from the internal epicondyle of the humerus, and beneath the deep fascia, though it may be superficial to it. In such cases the common interosseous is a branch of the main trunk, and it furnishes the anterior and posterior ulnar recurrent arteries. (2) The artery, though normal in origin, may pass superficial to the muscles arising from the internal epicondyle.

Second Part of the Ulnar Artery.-The second part lies upon the anterior annular ligament, and extends from its upper to its lower border. It has a vena comes on either side of it, and the ulnar nerve on its inner side. It lies on the outer side of the pisiform bone, by which it is overhung, and on the inner side of the hook of the unciform bone, where it is under cover of the pisi-uncinate ligament.

Relations--Superficial.-The integument, the expansion from the flexor carpi ulnaris tendon to the front of the anterior annular ligament, and the pisi-uncinate ligament. Deep.-The anterior annular ligament. External.-The external vena comes, and hook of the unciform bone. Internal. - The internal vena comes, ulnar nerve, and pisiform bone.

The branches of this part are unimportant.

The third part of the ulnar artery will be described in connection with the palm.

Ulnar Venæ Comites.-The ulnar artery in the first and second parts of its course is accompanied by two venæ comites, one being placed on either side of the vessel, and the two communicate at frequent intervals by transverse branches which lie upon it. They commence in the venæ comites of the inner parts of the superficial and deep palmar arches, and they terminate above by uniting to form the internal brachial vena comes.

Ulnar Nerve.-The ulnar nerve enters the forearm by passing through the interval between the internal epicondyle and olecranon process, where it lies between the two heads of the flexor carpi ulnaris. It then descends under cover of that muscle, lying upon the flexor profundus digitorum. A little above the centre of the forearm it meets with the ulnar artery, upon the inner side of which it subsequently lies. On approaching the wrist it escapes from beneath the flexor carpi ulnaris, and lies, with the artery, close to the outer side of its tendon. It then passes over the anterior annular ligament close to the outer side of the pisiform bone, by which it is overhung, and subsequently on the inner side of the hook of the unciform bone. Thereafter it enters the palm, where it divides into its two terminal branches, superficial and deep.

Branches.-These are articular, muscular, and cutaneous.

The articular branches, two or three in number, are given off to the elbow-joint as the nerve passes between the internal epicondyle and olecranon process.

The muscular branches arise in the upper part of the forearm, and are distributed to the flexor carpi ulnaris and the inner portion of the flexor profundis digitorum. 
The cutaneous branches are three in number, and have been already described.

The ulnar nerve in the palm will be afterwards described.

Median Nerve.- The median nerve lies at first in the anticubital space, where it is placed on the inner side of the brachial and ulnar arteries. On leaving the space it passes between the two heads of the pronator radii teres, where it crosses the ulnar artery, the deep head of that muscle intervening between the two. It then passes beneath the radial origin of the flexor sublimis digitorum, and descends under cover of that muscle in the middle line, until it approaches the wrist. Here it escapes from beneath the muscle, and lies between its tendons and that of the flexor carpi radialis, where it parts with its palmar cutaneous branch. Thereafter it passes under the anterior annular ligament into the palm, where it will be afterwards described. The nerve is accompanied by the median artery, which is a branch of the anterior interosseous near its origin.

Branches.-The branches of the nerve in the forearm are articular, muscular, and cutaneous.

The articular branches, one or two in number, enter the elbowjoint on its anterior aspect.

The muscular branches supply all the muscles on the front of the forearm, except the flexor carpi ulnaris and the inner portion of the flexor profundus digitorum. The branch, or branches, to the pronator radii teres arise from the nerve before it passes between the two heads of that muscle. The branches for the flexor carpi radialis, palmaris longus, and flexor sublimis digitorum arise lower down. The flexor longus pollicis, outer portion of the flexor profundus digitorum, and pronator quadratus are supplied by the anterior interosseous branch. This long branch arises from the median just below the neck of the radius, and it descends on the front of the interosseous membrane, lying on the outer side of the anterior interosseous artery, both being overlapped by the contiguous borders of the flexor profundus digitorum and flexor longus pollicis. On reaching the upper border of the pronator quadratus the nerve passes beneath that muscle, and ends in two branches, one of which enters the deep surface of the muscle, whilst the other passes to supply the wrist-joint. The branch to the outer portion of the flexor profundus digitorum arises high up, and it communicates in the muscle with the branch of the ulnar nerve to its inner portion. The anterior interosseous nerve in its course furnishes an interosseous branch, which is distributed to the interosseous membrane, and gives off medullary filaments which accompany the medullary arteries of the radius and ulna.

Third Layer of Muscles.-The muscles comprising the third or deep layer are three in number, namely, the flexor profundus digitorum, flexor longus pollicis, and pronator quadratus.

I. Flexor Profundus Digitorum (flexor perforans)-Origin.(I) The upper three-fourths of the anterior surface of the ulna; 
(2) the inner half of the front of the interosseous membrane; (3) the upper two-thirds of the inner surface of the ulna; and (4) the posterior border of the bone for a similar extent, by an aponeurosis common to it, the flexor carpi ulnaris, and extensor carpi ulnaris.

Insertion.-The front of the bases of the distal phalanges of the four inner fingers.

The tendinous part of the muscle makes its appearance about the centre of the forearm, and the index-finger tendon is usually separate from the rest of the tendinous mass over the greater part of its extent. The other three tendons become separate beneath the anterior annular ligament, so that in the palm there are four diverging tendons, connected with which are the lumbricales. Each deep flexor tendon accompanies a superficial flexor tendon, beneath which it lies, and both enter the sheath on the palmar aspect of a finger. Opposite the distal end of the first phalanx the deep tendon passes through the cleft in the superficial tendon, and so reaches its more distant point of insertion.

Nerve-supply.-(I) The anterior interosseous branch of the median supplies that portion of the muscle which acts upon the index finger, and in part the portion acting upon the middle and ring fingers; and (2) the ulnar nerve supplies that portion which acts upon the little finger, and in part the portion acting upon the ring and middle fingers. Sometimes the anterior interosseous nerve supplies the whole of the portion acting upon the middle finger.

Action.-(I) To flex the distal phalanges of the four inner fingers ; (2) to assist in flexing the second phalanx and metacarpo-phalangeal joint ; and (3) to assist in flexing the wist-joint.

2. Flexor Longus Pollicis-Origin.-(I) The anterior surface of the radius, from the anterior oblique line above to the upper border of the pronator quadratus below; (2) the outer half of the front of the interosseous membrane; and (3) as a rule by a tendinous slip from the inner margin of the coronoid process of the ulna, or from the internal epicondyle of the humerus.

Insertion.-The front of the base of the distal phalanx of the thumb.

The tendon appears on the front of the muscle about the centre of the forearm, and receives fleshy fibres until it is near the wrist.

Nerve-supply. - The anterior interosseous nerve.

Action.-(I) To flex the distal phalanx of the thumb; (2) to assist in flexing its metacarpo-phalangeal joint; and (3) to act as an auxiliary flexor of the wrist-joint.

3. Pronator Quadratus-Origin.-The front of the ulna over its lower fourth.

Insertion.-The front of the radius for about 2 inches at its lower end, and the anterior part of the inner surface.

Nerve-supply. - The anterior interosseous nerve.

The fibres are disposed, for the most part, transversely, and the muscle is covered by a firm aponeurosis over about its inner third. Action.-To pronate the radius upon the ulna. 


\section{FRONT OF THE WRIST AND PALM.}

Landmarks.-Below, and internal to, the styloid process of the radius the tuberosity of the scaphoid can be felt, and below this the tuberosity of the trapezium. On the inner aspect of the front of the wrist the pisiform bone can easily be felt, and below, and external to, it, the hook of the unciform bone. The interval between these two projections indicates the position of the ulnar vessels and nerve. The centre of the palm presents a triangular hollow, the apex of which is directed upwards and inwards towards the wrist, and the base downwards towards the ruots of the fingers, in which latter situation there is a transverse prominence, broken up by grooves leading to the four inner digits. The palmar hollow is bounded above and externally by the thenar eminence, and internally by the hypothenar eminence.

The integument of the palm presents four furrows, two being disposed transversely, and two more or less longitudinally. The lower transverse furrow is about $I$ inch above the roots of the inner three digits, and is most conspicuous when the fingers are flexed. It commences at the inner border of the palm, and, passing outwards in a slightly arched manner, it terminates at the cleft between the index and middle fingers. It is produced by the flexion of the metacarpo-phalangeal joints of the inner three fingers. These joints are situated about midway between this line and the roots of the fingers when these are extended. The upper transverse furrow commences at the outer border of the palm about $\frac{3}{4}$ inch above the root of the index finger, and it passes inwards and slightly upwards to the inner border of the palm, lying about $\frac{1}{2}$ inch above the lower furrow. The outer part of this furrow is due to flexion of the metacarpo-phalangeal joint of the index finger, and the remainder to complete flexion of the metacarpo-phalangeal joints of the inner three fingers. One of the longitudinal furrows commences about the centre of the wrist, and curves downwards and outwards to meet the upper transverse furrow. It is produced by flexion of the joint between the trapezium and metacarpal bone of the thumb during the movement of opposition. The other longitudinal furrow runs downwards from the wrist internal to the preceding, and meets the lower transverse furrow. It is due to the movement of opposition of the little finger. The front of each of the four inner fingers presents three transverse furrows. The distal pair correspond with the interphalangeal joints, but the proximal furrow is about $\frac{1}{2}$ inch beyond the metacarpo-phalangeal joint. The front of the thumb presents only two transverse furrows.

The position of the superficial palmar arch corresponds with a line drawn across the palm from the web between the thumb and index finger (the thumb being abducted) to the outer side of the pisiform bone. This line is slightly arched, with the convexity towards the fingers. From the convexity of the arch the three compound 
digital arteries pass forwards in line with the webs of the fingers, and they lie over the interosseous spaces. An incision, therefore, may be made with safety in the palm in the direction of the middle line of a finger, but it should not be carried nearer the wrist than the line indicating the position of the superficial palmar arch. The deep palmar arch lies about $\frac{1}{2}$ inch nearer the wrist than the superficial. The digital arteries are placed on the lateral aspects of the fingers.

On the back of the hand the radial tubercle may be felt about the centre of the lower end of the bone, and the heads of the metacarpal bones, which form the knuckles, are conspicuous when the fingers are flexed. Below and behind the styloid process of the radius there is a well-marked triangular hollow, the base of which is directed upwards, in which, by sinking the finger deeply, pulsation may be felt in the second part of the radial artery in the living subject. At the upper part the tendons of the radial extensors of the wrist may also be felt. The hollow is bounded internally by a prominence formed by the tendon of the extensor longus pollicis, externally by a prominence due to the tendons of the extensor ossis metacarpi pollicis and extensor brevis pollicis, and above by the lower end of the radius. The scaphoid and trapezium lie in its floor, and the radial vein and branches of the radial nerve are in its roof. This hollow is known as the anatomical smuff-box (Cloquet).

The middle line of the hand, from and towards which abduction and adduction take place, represents a longitudinal line drawn through the centre of the middle finger.

Superficial Fascia.--The superficial fascia, especially over the hollow of the palm, is lobulated, the lobules being separated by fibrous processes which pass between the skin and the central division of the palmar fascia.

Palmaris Brevis.-This is a thin, flat, subcutaneous muscle, of quadrilateral outline, and usually arranged in two or three bundles, separated by fat.

Origin.-(I) The front of the anterior annular ligament at its lower and inner part, and (2) the inner margin of the central division of the palmar fascia at its upper part.

Insertion.--The integument of the inner border of the hand at its inner part.

Nerve-supply.-The superficial division of the ulnar nerve.

Action.- To draw the integument into which it is inserted towards the middle line of the hand, thus giving rise to a wrinkled depression on the inner border at its upper part.

The muscle lies in the superficial fascia over the upper part of the hypothenar eminence, and covers the ulnar vessels and nerve, which it guards against pressure.

Superficial Transverse Ligament.-This ligament is composed of a bundle of transverse fibres, contained within the folds of skin which form the clefts of the four inner fingers. 
Palmar Fascia.-The palmar fascia is arranged in three divisions - central and two lateral.

The central division is triangular, the apex being towards the wrist, where it is continuous with the tendon of the palmaris longus, and attached to the front of the anterior annular ligament close to its lower border. The superficial fibres of this division are longitudinal, and the deep fibres transverse, the latter being most conspicuous between the diverging digital processes. The base is directed towards the roots of the four inner fingers, on approaching which the fascia divides into four digital processes. These diverge and pass to join the sheaths of the flexor tendons on the fronts of the fingers. Each process gives fibres to the superficial transverse liganient and skin of the clefts of the fingers. Each also gives off a deep expansion at either side, which joins the transverse metacarpal ligament at either lateral margin of the head of a metacarpal bone. In this way short canals are formed for the superficial and deep flexor tendons on their way to the fronts of the fingers. Between the diverging digital processes three intervals are left, in which the digital arteries and nerves and lumbricales muscles make their appearance; and, as stated, the transverse fibres are here conspicuous, where they lie superficial to these structures. The central division is bound to the skin by fibrous processes which enclose spaces containing the lobules of the superficial fascia. Its deep surface is related to the great palmar bursa. At either side it gives off a deep septum which joins the interosseous fascia. The outer septum is placed between the thenar muscles and the centre of the palm, where the flexor tendons and digital vessels and nerves lie, and it is.pierced by the digital nerves for the sides of the thumb and index finger. The inner septum is placed between the hypothenar muscles and the centre of the palm, and it is pierced by the digital branches of the ulnar nerve, and by a portion of the superficial palmar arch. In this way a large central fascial canal is formed, which contains the superficial palmar arch and its digital branches, the digital nerves, the superficial and deep flexor tendons, and the lumbricales. On either side of this central canal are the thenar and hypothenar canals, containing the short muscles of the thumb and of the little finger. The central division of the palmar fascia serves as an important protection to the superficial palmar arch and the digital arteries and nerves. From its great strength and capability of resistance it has an important bearing on the course taken by pus in a palmar abscess.

The central division represents the expanded portion of the tendon of an original superficial flexor muscle of the fingers.

The external or thenar division is thin, and covers the thenar muscles. Superiorly it is connected with the tendon of the palmaris longus and anterior annular ligament, and it receives an accession of fibres from the tendon of the extensor ossis metacarpi pollicis. Inferiorly it blends with the sheath of the tendon of the flexor longus pollicis on the front of the thumb. 
The internal or hypothenar division, also thin, is connected above with the anterior annular ligament, and terminates below over the muscles inserted into the inner side of the base of the first phalanx of the little finger.

Third Part of the Ulnar Artery. - The third part of the vessel forms the superficial palmar arch. It descends for a little under cover of the palmaris brevis, and then turns outwards across the palm in an arched manner. About the middle of the thenar eminence it is joined by the superficial volar of the radial, or by a branch of the arteria radialis indicis, or, failing these, by a branch of the arteria princeps pollicis, and so the arch is coinpleted externally. Its convexity is directed towards the fingers, and its course may be indicated by a line drawn across the palm from the web between the thumb and index finger (the thumb being abducted) to the outer side of the pisiform bone, the line being slightly arched, with the convexity towards the fingers.

Relations-Superficial.-The integument, the palmaris brevis for a short distance internally, and the central division of the palmar fascia. Deep.-From within outwards it rests upon the opponens minimi digiti, the digital branches of the ulnar nerve, the flexor. tendons, and the digital branches of the median nerve. The arch lies within the great palmar bursa.

Branches. - The branches of the arch are cutaneous to the integument of the.palm, muscular to the adjacent superficial muscles, the profunda branch, and the digital'branches.

The profunda artery is given off from the commencement of the arch, opposite the lower border of the anterior annular ligament. It at once passes deeply, in company with the deep division of the ulnar nerve, between the abductor and flexor brevis minimi digiti, then through the opponens minimi digiti, and it terminates by anastomosing with the third part of the radial artery, thereby completing the deep palmar arch. In its short course it gives branches to the hypothenar muscles.

The digital arteries arise from the convexity of the arch, and are four in number. They are destined for the sides of the inner three and a half fingers, and are called first, second, third, and fourth, from within outwards. The first is a single artery, but the other three are compound, each ultimately dividing into two. The first digital artery, of small size, passes downwards and inwards over the hypothenar muscles, to which it gives twigs, and then it passes along the inner side of the little finger.

The second, third, and fourth digital arteries, which are compound, pass downwards over the fourth, third, and second interosseous spaces to near the clefts between the little and ring, ring and middle, and middle and index fingers, where each divides into two collateral digital arteries, which pass along the contiguous sides of these fingers. These four digital arteries account for the blood-supply of the sides of the inner three and a half fingers. The outer side of the index finger and both sides of the thumb are supplied by the arteria 


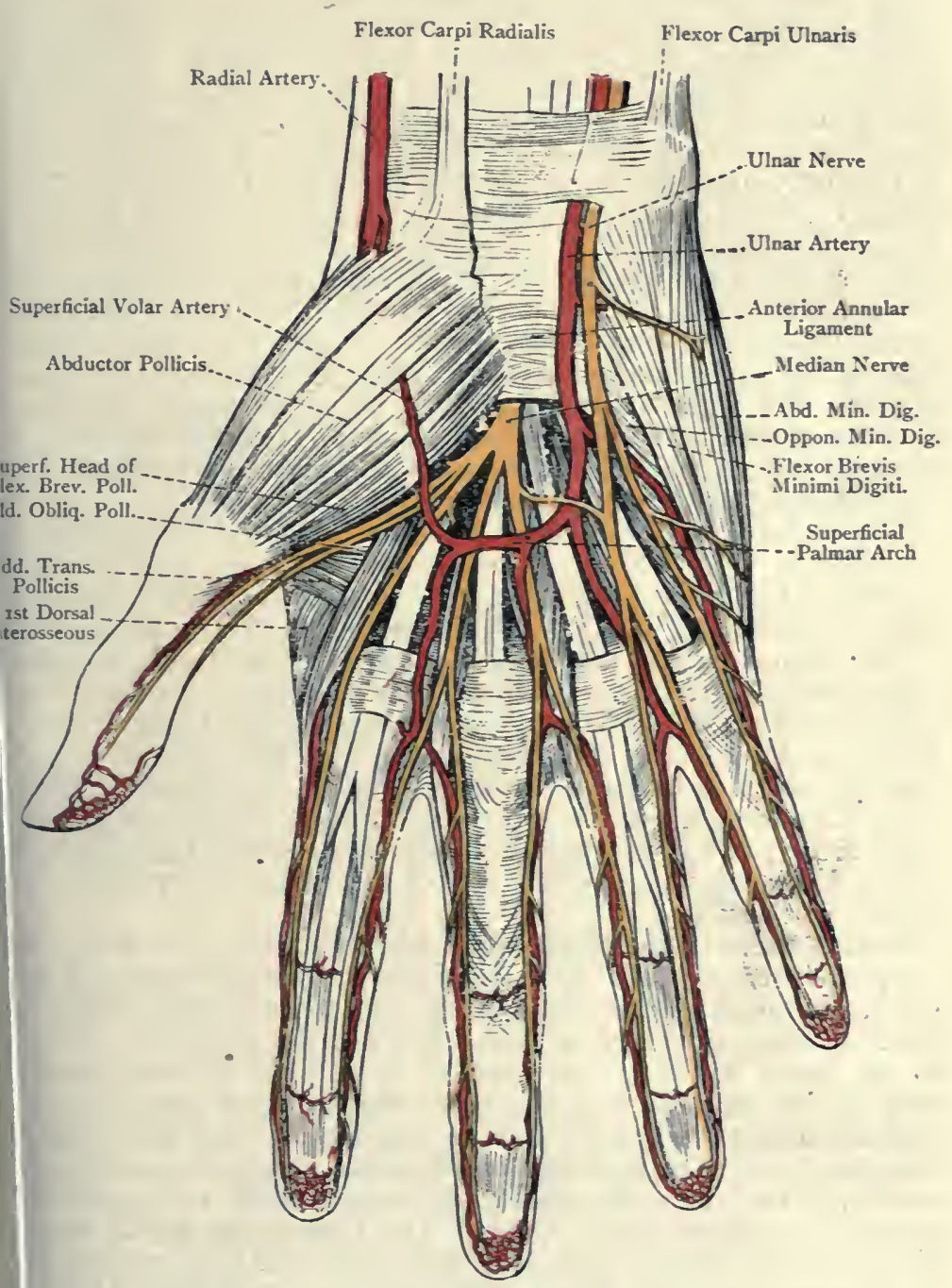

Fig. I99.-The Palmar Aspect of the HaNd。

(The Palmar Fascia has been removed.) 
radialis indicis and arteria princeps pollicis of the radial. As the digital arteries pass towards the fingers they lie between the flexor tendons, and superficial to the digital nerves and lumbricales muscles. Along the sides of the fingers, however, the digital nerves are superficial to the arteries. Just before the outer three digital arteries divide into their collateral branches each is joined by a palmar interosseous artery from the deep palmar arch, and, it may be, by the inferior perforating branches of the dorsal interosseous arteries. The innermost digital artery receives its communicating branch from the innermost palmar interosseous about the centre of the hand. As the arteries pass along the sides of the fingers they anastomose with each other across the front of the phalanges to form arches, which are placed on the proximal side of the interphalangeal joints. Each also supplies the flexor tendons with their sheaths, and gives off a few dorsal branches. In front of each terminal phalanx the arteries of each finger unite to form an arch, from which many twigs are given off to supply the pulp of the finger and matrix of the nail.

The varieties of the superficial palmar arch will be afterwards Jescribed.

Veins.-The superficial palmar arch is accompanied by two venæ comites, and so are the digital arteries. The venæ comites of the digital arteries unite at the clefts of the fingers to form single vessels, and these end in the venæ comites of the superficial palmar arch. The venæ comites of the inner part of the arch unite, and so do those of the inner part of the deep palmar arch, and in this way the venæ comites of the ulnar artery are formed. Those from the outer side of the superficial arch go with the superficial volar artery, and, with those from the outer side of the deep arch which accompany the second part of the radial artery, form the radial venæ comites.

Median Nerve in the Hand.-The median nerve enters the paim by passing beneath the anterior annular ligament, where it lies within the great palmar bursa along with the superficial and deep flexor tendons, giving off articular twigs to the wrist-joint. Close to the lower border of the ligament it presents an enlargement, and breaks up into two divisions-external and internal. The external division gives off a muscular branch and three single digital nerves. The muscular branch passes outwards, and divides to supply the abductor pollicis, opponens pollicis, and superficial head of the flexor brevis pollicis. The three single digital nerves are distributed to the outer and inner sides of the thumb and the outer side of the index finger, and the latter nerve gives a branch to the first, or most external, lumbricalis. The internal division breaks up into two compound digital nerves. The outer of these passes to near the cleft between the index and middle fingers, where it divides into two collateral digital nerves for the supply of the contiguous sides of these fingers. In its course it gives a branch to the second lumbricalis. The inner passes to near the cleft 
between the middle and ring fingers, where it also divides into two collateral digital nerves, which supply their contiguous sides. In its course it communicates with the outer digital branch of the ulnar nerve. In the palm the digital nerves lie beneath the superficial palmar arch and its digital branches, but along the sides of the fingers the nervès are superficial to the arteries. Occasionally a digital artery may pass through a digital nerve. On the sides of the fingers the nerves, which present small swellings, called Pacinian bodies, give branches to the integument of the palmar aspects of the finger;, and the metacarpo-phalangeal and interphalangeal joints. At the extremities of the fingers each nerve

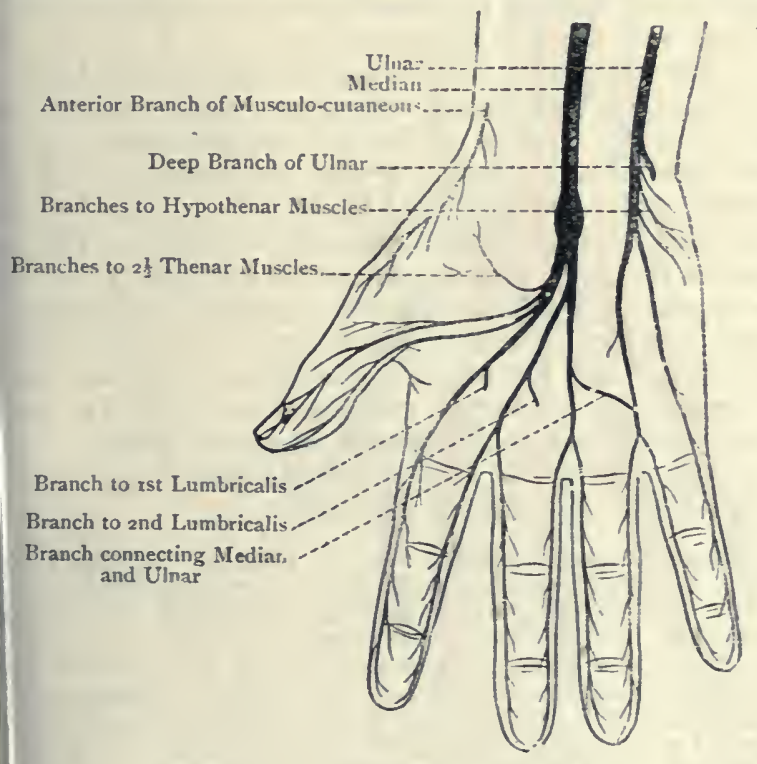

Fig. 200.-Diagram of the Nerves of the PalM.

nds in branches for the pulp and matrix of the nail. The digital erves also give branches which turn backwards to the dorsal aspects f the fingers. These, for the most part, supply the integument s follows: (I) the matrix of the thumb-nail; (2) the back of the erminal phalanx of the index finger; (3) the back of the second ad terminal phalanges of the middle finger; and (4) the back of the rminal, and distal end of the second, phalanges of the ring finger.

Summary of the Median Nerve.-Museular.-It supplies (I) all the muscles $i$ the front of the forearm, except the flexor carpi ulnaris and inner portion the flexor profundus digitorum; (2) two and a half muscles of the thenar ninence, namely, the abductor, opponen;, and superficial head of the xor brevis, pollicis; and (3) the outer two lumbricales. Cutsneous.-It pplies the outer part of the palm, and the outer three and a half digits. rticular.-It supplies branches to the elbow- and wrist-joints, as well as to veral joints of the hand. 
Ulnar Nerve in the Hand.-The ulnar nerve enters the hand by passing over the anterior annular ligament, where it lies on the inner side of the ulnar vessels, and is overhung by the pisiform bone. It then breaks up into two divisions-superficial and deep. The superficial division passes downwards beneath the palmaris brevis, which it supplies, and it ends in two digital nerves. The inner is a single nerve, and is distributed to the inner side of the little finger. The outer, which is compound, passes to near the cleft between the ring and little fingers, where it divides in to two collateral digital nerves for the supply of their contiguous sides. This nerve communicates with the innermost digital branch of the median. The distribution of the nerves on the fingers corresponds with that of the median, and the branches which turn to the backs of the fingers are for the most part distributed as follows : (I) to the back of the terminal, and distal end of the second, phalanges of the ring finger, and (2) to the matrix of the nail of the little finger. The deep division of the ulnar nerve, along with the profunda branch of the ulnar artery, passes between-the abductor and flexor brevis minimi digiti, and then through the opponens minimi digiti. Thereafter it accompanies the deep palmar arch, above which it lies, to the thenar region, and it has an extensive muscular distribution, as follows : it supplies the abductor, flexor brevis, and opponens, minimi digiti, the inner two lumbricales, the seven interossei, and two and a half muscles of the thenar eminence-namely, the deep head of the flexor brevis pollicis, adductor obliquus pollicis, and adductor transversus pollicis. It also gives articular twigs to the wrist-joint, and several of the joints of the hand.

Summary of the Ulnar Nerve.-Muscular.-In the forearm it supplies the flexor carpi ulnaris and inner portion of the flexor profundus digitorum; and in the hand it supplies (I) the palmaris brevis, (2) the three hypothenat muscles, (3) the inner two lumbricales, (4) the seven interossei, and (5) two and a half muscles of the thenar eminence, namely, the deep head of the flexor brevis, adductor obliquus, and adductor transversus, pollicis. Cutaneous.It supplies the integument of (I) the front of the forearm for a little below the centre, and internal to the middle line; $(2)$ the inner portion of the palm ; and (3) the inner one and a half fingers. Articular.-It supplies branches to the elbow- and wrist-joints, and several of the joints of the hand.

Great Palmar Bursa.-This bursa has two compartments, inner and outer.

The inner compartment, which is large and loose, invests the superficial and deep flexor tendons and median nerve as they pass beneath the anterior annular ligament. It extends upwards into the forearm for rather more than an inch above the ligament, and downwards to about the centre of the palm. It is shut off from the synovial sheaths of the flexor tendons of the index, middle, and ring fingers, but internally it is prolonged downwards io be, as a rule, continuous with the synovial sheath of the flexor tendons of the little finger.

The outer compartment, which is long and narrow, invests the tendon of the flexor longus pollicis beneath the anterior annular. 
ligament, and reaches upwards to the same height as the inner compartment. Inferiorly it is prolonged uninterruptedly along the tendon to its insertion, so that it is continuous with its synovial sheath on the thumb. The disposition of the great palmar bursa in relation to the thumb and little finger is to be carefully noted in connection with whitlow of these fingers. It will be evident that pus could readily burrow upwards from the thumb and little finger into the lower part of the forearm, passing in its course beneath the anterior annular ligament.

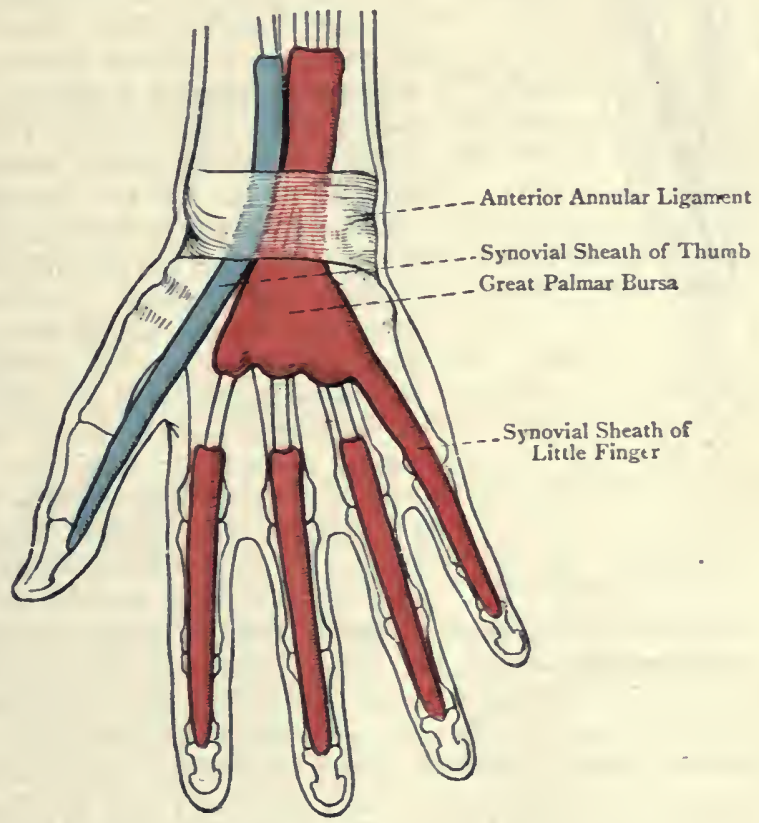

Fig. 201.-The Great Palmar Bursa, and the Synovialo Sheaths OF the Flexor Tendons.

Sheaths of the Flexor Tendons.-As the superficial and deep flexor tendons pass along the fronts of the four inner fingers each pair is contained in a fibro-osseous canal. The osseous wall is formed by the palmar aspects of the first and second phalanges, and the fibrous yall by a sheath. This sheath, over the greater parts of the first and second phalanges, is thick and strong, and these parts of it ire known as the vaginal ligaments. The fibres of these ligaments un transversely, and are attached to the rough lateral margins of he palmar surfaces of the phalangeal shafts. Opposite the joints, $\mathrm{n}$ order not to interfere with flexion, the vaginal ligaments are eplaced by thin membranes, superadded to which are obliquely 
decussating fibres. Each fibro-osseous canal is lined by synovial membrane, which is reflected over the contained tendons in such a manner as to furnish a separate investment for each. The synovial

Flexor Profundus

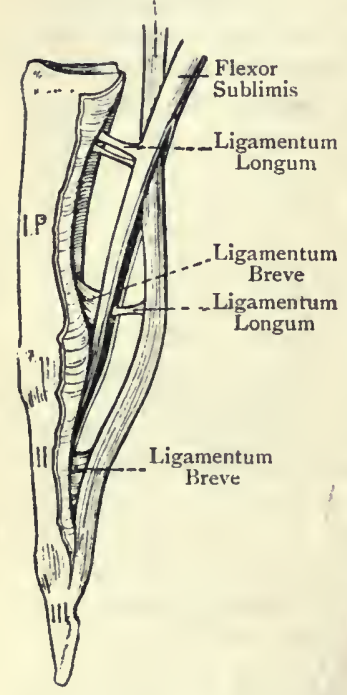

FIG. 202.-THE FLEXOR TENDONS OF A Finger. sheaths of the index, middle, and ring fingers extend into the palm as far as the heads of the metacarpal bones, and they have no connection with the great palmar bursa. The synovial sheath of the little finger, however, is as a rule continuous with the large inner compartment of that bursa. The synovial membranes of the sheaths form ce.tain bands, called vincula accessoria tendinum, which are of two kinds-ligamenta brevia and ligamenta longa. The ligamenta brevia are two in number-one for the superficial and one for the deep flexor tendon. They are broad, laterally compressed, triangular bands which connect each tendon, close to its insertion, with the distal end of the phalanx above that into which it is inserted. The ligamenta longa, few and inconstant, are narrow cords, which pass between the tendons and the phalanges, or from one tendon to the other. The fibro-osseous canal for the tendon of the flexor longus pollicis is similar to those of the other flexor tendons, and its synovial membrane is continuous with the outer compartment of the great palmar bursa.

Lumbricales.--These are four tapering muscles which are connected with the deep flexor tendons in the palm, and they receive numerical names, the most external being the first. The first and second arise each from the outer side of the deep flexor tendon for the index and middle fingers, and the third and fourth arise from the adjacent sides of the two deep flexor tendons between which each lies, the tendons involved being those for the middle, ring, and little fingers. Each muscle tapers off, and ends in a tendon which, turning round the outer side of a metacarpo-phalangeal joint, expands, and is inserted into the outer side of the broad expansion of the extensor tendon on the back of the first phalanx.

Nerve-supply-First. - The digital branch of the median to the outer side of the index finger. Second.-The digital branch of the median, which divides to supply the contiguous sides of the index and middle fingers. Third and Fourth.-The deep division of the ulnar nerve.

Action.-(I) To flex the metacarpo-phalangeal joint, and (2) to extend the interphalangeal joints.

Anterior Annular Ligament.-This is a strong fibrous band which bridges over the concavity on the palmar aspect of the carpus, 
and converts it into a fibro-osseous canal. Externally it is attached to the tuberosities of the scaphoid and trapezium, and internally to the pisiform and hook of the unciform. Its upper border is continuous with the deep fascia of the front of the forearm; its lower border is connected with the palmar fascia ; at its upper and inner part it receives an expansion from the tendon of the flexor carpi ulnaris ; and near its lower border it gives partial insertion to the tendon of the palmaris longus. The ligament is crossed by the lastmentioned tendon and the ulnar vessels and nerve, the latter structures lying close to the pisiform bone, where they are overhung by it, and overlapped by a slip from the flexor carpi ulnaris. At either side the ligament affords origin to muscles of the thenar and hypothenar groups. The fibro-osseous canal formed by the ligament and front of the carpus gives passage to the tendons of the flexor sublimis and flexor profundus digitorum, the tendon of the flexor longus pollicis, and the median nerve. The tendon of the flexor carpi radialis does not pass through this canal, but traverses a special compartment in the outer part of the ligament as it passes through the groove on the front of the trapezium, where it is invested by a special synovial sheath.

Thenar Muscles.-The short muscles of the thumb are five in number, namely, the abductor, opponens, and flexor brevis, pollicis, the adductor obliquus pollicis, and the adductor transversus pollicis.

I. Abductor Pollicis-Origin.-(I) The front of the anterior annular ligament; (2) the tuberosity of the scaphoid; and (3) the tuberosity of the trapezium.

Insertion.-(I) The outer side of the base of the first phalanx of the thumb, in association with the superficial head of the flexor brevis pollicis; and (2) the outer margin of the tendon of the extensor longus pollicis on the back of the first phalanx.

Nerve-supply. - The median nerve.

The muscle, which is triangular, is directed downwards and outwards.

Action.-(I) To abduct the thumb, and (2) to assist in flexing its first phalanx, the result being that the thumb is drawn forwards and a little inwards. It also assists in extending the distal phalanx.

The muscle rests upon the opponens pollicis, and the superficial head of the flexor brevis pollicis lies on its inner side.

2. Opponens Pollicis-Origin.-(I) The front of the anterior annular ligament, and (2) the tuberosity of the trapezium.

Insertion.-The outer border of the shaft of the metacarpal bone of the thumb, and the adjacent part of its palmar surface.

Nerve-supply. - The median nerve.

The muscle, which is triangular, is directed downwards and outwards.

Action.-To flex the first metacarpal bone, the result being that the thumb is drawn forwards and inwards across the palm, so as to oppose its tip to the tips of the four inner fingers, 
The muscle supports the abductor pollicis, and has the superficial head of the flexor brevis pollicis along its inner border.

3. Flexor Brevis Pollicis. - This muscle arises by two heads-a large superficial or outer, and a small deep or inner. The superficial head arises from the outer two-thirds of the lower border of the anterior annular ligament, and the tuberosity of the trapezium. The deep head (interosseus primus volaris) arises from the proximal extremity of the first metacarpal bone on its inner aspect.

Insertion.-The superficial head, along with the abductor pollicis, is inserted into the outer side of the base of the first phalanx of the thumb, a sesamoid bone being contained within it. The deep head joins the adductor obliquus pollicis, and, along with it, is inserted into the inner side of the base of the first phalanx of the thumb.

Nerve-supply.-The superficial head is supplied by the median nerve, and the deep head by the deep division of the ulnar nerve.

Action.-To flex the metacarpo-phalangeal joint of the thumb, and so to assist in opposition.

4. Adductor Obliquus Pollicis-Origin.-By several bundles from (I) the fronts of the bases of the second and third metacarpal bones; (2) the fronts of the trapezoid and os magnum; and (3) the sheath of the tendon of the flexor carpi radialis.

Insertion.-The muscle, having received the small deep head of the flexor brevis pollicis, is inserted, along with the adductor transversus pollicis, into the inner side of the base of the first phalanx of the thumb, a sesamoid bone being contained within it.

In its course the muscle detaches, from its outer side, a large fleshy bundle, which passes outwards behind the tendon of the flexor longus pollicis and joins the superficial head of the flexor brevis pollicis.

Nerve-supply. - The deep division of the ulnar nerve.

The muscle is directed downwards and outwards.

Action.-To adduct the thumb and assist in opposition.

The muscle has the superficial head of the flexor brevis pollicis on its outer side, the tendon of the flexor longus pollicis lying between the two, and the adductor transversus pollicis along its inner and lower aspect, the radial artery passing between the two.

5. Adductor Transversus Pollicis-Origin.-The distal two-thirds of the anterior border of the shaft of the third metacarpal bone.

Insertion.-(I) The inner side of the base of the first phalanx of the thumb, along with the adductor obliquus pollicis and deep head of the flexor brevis pollicis ; and (2) the inner margin of the tendon of the extensor longus pollicis on the back of that phalanx.

Nerve-supply.-The deep division of the ulnar nerve.

The muscle, which is triangular, is directed outwards.

Action.-To adduct the thumb and assist in opposition. It also assists in extending the distal phalanx.

The tendon of the flexor longus pollicis on its way to its insertion 
has on its outer side the superficial head of the flexor brevis pollicis, and on its inner side the adductor obliquus pollicis.

Hypothenar Muscles. - The short muscles of the little finger are three in number, namely, the abductor, flexor brevis, and opponens, minimi digiti.

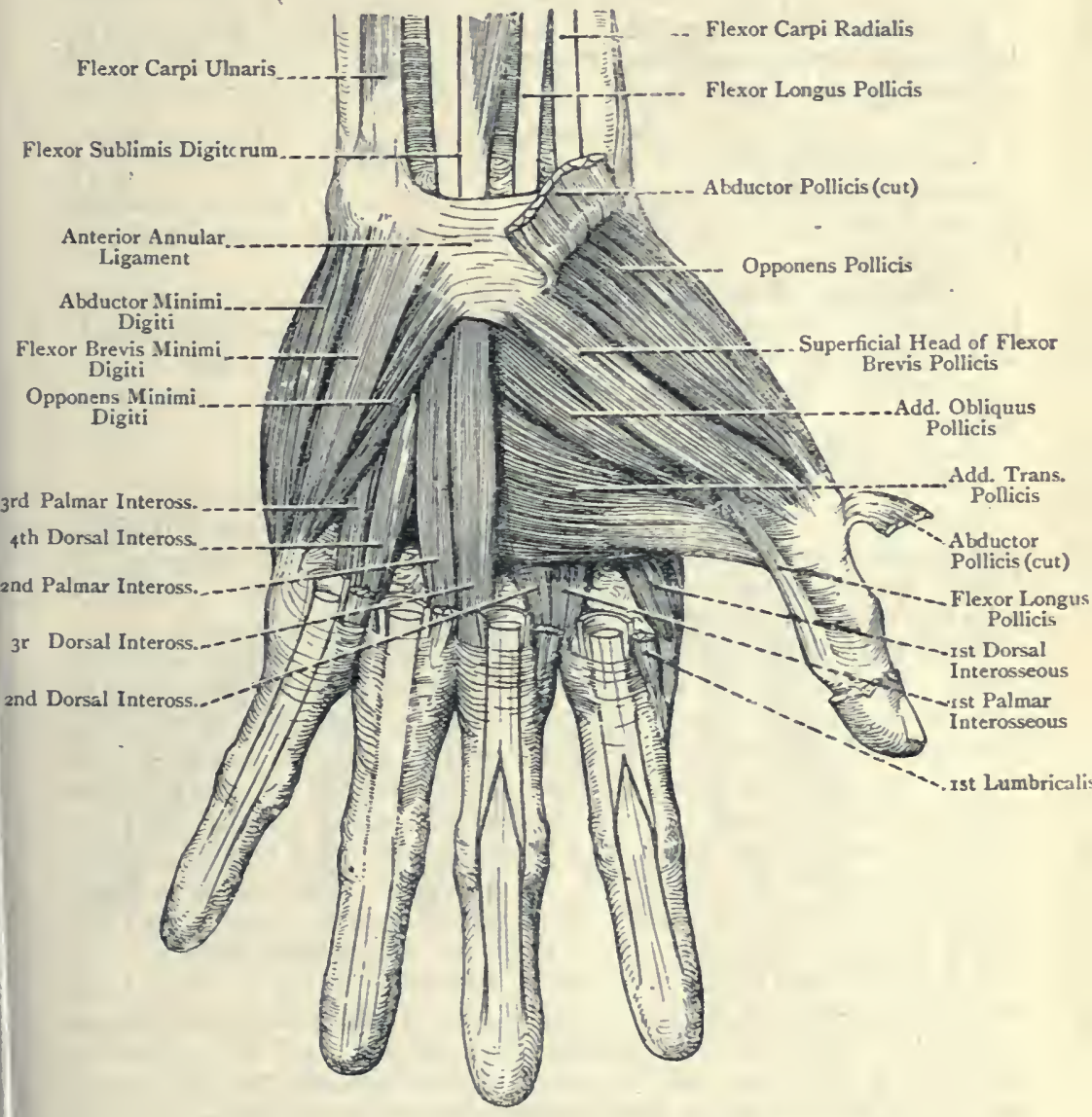

Fig. 203,-The Thenar and Hypothenar Muscles.

I. Abductor Minimi Digiti-Origin.-The lower part of the pisiform bone.

Insertion.-(I) The inner side of the base of the first phalanx of the little finger, in common with the flexor brevis minimi digiti; and (2) the inner margin of the tendon of the extensor minimi digiti on the back of the phalanx.

Nerve-supply.-The deep division of the ulnar nerve. 
Action.-(I) To abduct the little finger, and flex its metacarpophalangeal joint; and (2) to assist in extending the second and third phalanges.

2. Flexor Brevis Minimi Digiti-Origin.-(I) The inner surface of the hook of the unciform bone close to its tip, and (2) the front of the adjacent portion of the anterior annular ligament.

Insertion.-The inner side of the base of the first phalanx of the little finger, in common with the abductor minimi digiti.

Nerve-supply.-The deep division of the ulnar nerve.

Action.-To flex the metacarpo-phalangeal joint of the little finger.

This muscle is of small size, and lies on the outer side of the abductor minimi digiti, from which it is separated, close to its origin, by the profunda branch of the ulnar artery and deep division of the ulnar nerve.

3. Opponens Minimi Digiti-Origin.-(I) The inner surface of the hook of the unciform bone underneath the preceding muscle, and (2) the adjacent part of the anterior annular ligament.

Insertion.-The inner margin of the shaft of the fifth metacarpal bone.

Nerve-supply. - The deep division of the ulnar nerve.

Action.- To flex and adduct the fifth metacarpal bone.

The muscle supports the abductor and flexor brevis minimi digiti, and its deep surface is related to the interosseous muscles of the fourth space. The deep branches of the ulnar artery and nerve pass through it on their way to the deep part of the palm.

Third Part of the Radial Artery. - The third part, which forms the greater portion of the deep palmar arch, extends from the upper part of the first interosseous space to about the base of the fifth metacarpal bone, where it is joined by the profunda branch of the ulnar. It enters the palm by passing between the two heads of the abductor indicis, and then it crosses the palm, resting upon the bases of the second, third, and fourth metacarpal bones, and the adjacent interosseous muscles. In its course it describes a slight arch, the convexity of which is directed towards the fingers, and it is about $\frac{1}{2}$ inch nearer the wrist than the superficial palmar arch. The deep division of the ulnar nerve lies close above it. The third part of the vessel is at first under cover of the adductor obliquus pollicis; it then passes between that muscle and the adductor transversus pollicis; and thereafter-it lies beneath the superficial and deep flexor tendons and lumbricales, where it forms the deep palmar arch.

Branches.-These are the arteria princeps pollicis, arteria radialis indicis, three palmar interosseous, recurrent, and superior perforating, the last three coming from the deep palmar arch.

The arteria princeps pollicis arises from the vessel immediately after it emerges from between the two heads of the abductor indicis, and it passes downwards along the first metacarpal bone, lying upon the abductor indicis and under cover of the adductor obliquus pollicis. On reaching the head of the bone it divides, under cover of the tendon 
of the flexor longus pollicis, into two collateral digital arteries for the sides of the thumb, which appear on either side of the long flexor tendon, between the superficial head of the flexor brevis pollicis and adductor obliquus pollicis. Their distribution is similar to that of the other digital arteries. The arteria princeps pollicis may give a branch to complete the superficial palmar arch.

The arteria radialis indicis passes downwards along the outer side of the second metacarpal bone, lying upon the abductor indicis and under cover of the adductor obliquus, and adductor transversus,

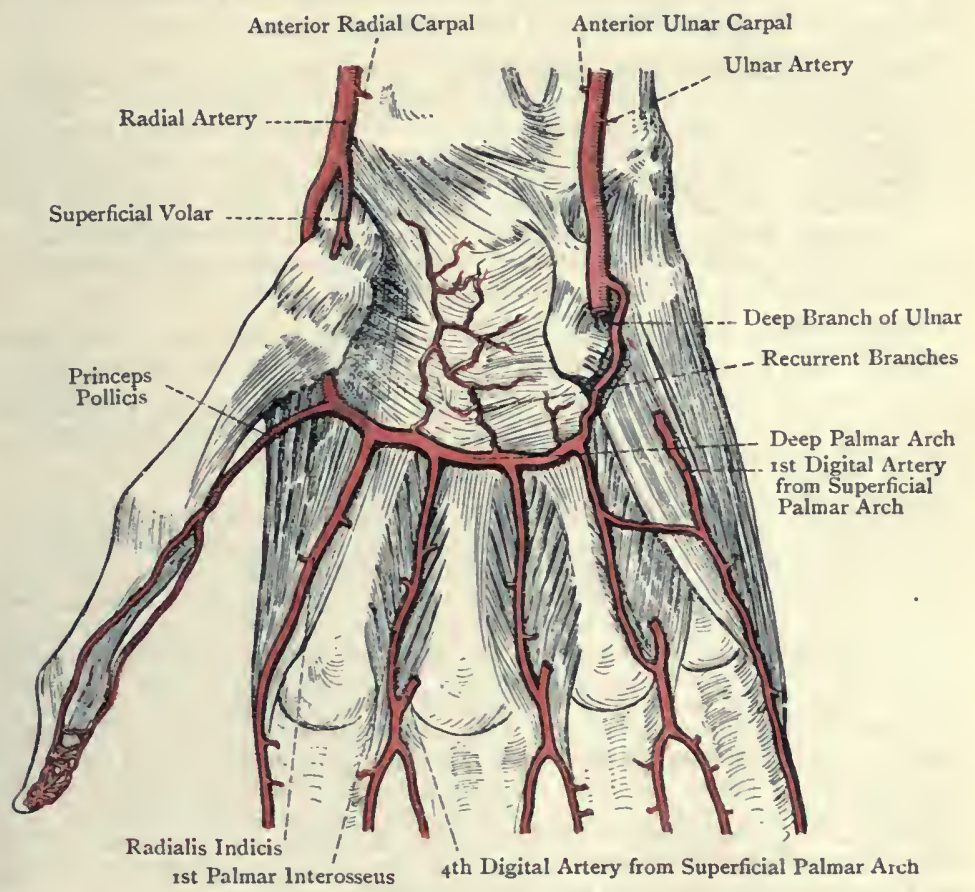

Fig. 204.-The Radial Artery in the Palm (Deep Palmar Arch) (after Spalteholz).

pollicis, and it becomes the digital artery of the outer side of the index finger. It may give a branch to complete the superficial palmar arch.

Branches of the Deep Palmar Arch.-The palmar interosseous arteries, three in number, arise from the convexity of the arch, and pass downwards over the second, third, and fourth interosseous spaces. Near the clefts of the corresponding fingers, they terminate by joining the three compound digital arteries of the superficial palmar arch. The innermost palmar interosseous, as a rule, gives off a communicating branch to join the single digital 
artery from the superficial arch to the inner side of the little finger, the junction taking place about the centre of the palm. In those cases where the digital arteries from the superficial arch are awanting, the palmar interosseous arteries may take their place.

The recurrent branches, few and small in size, arise from the concavity of the arch, and pass upwards to take part in the anterior carpal rete.

The superior perforating arteries, three in number, pass through the upper ends of the inner three interosseous spaces between the two heads of the corresponding dorsal interosseous muscles, and on the back of the hand they join the dorsal interosseous arteries.

Varieties of the UInar and Radial Arteries in the Hand.-I. The ulnar artery may be deficient in the number of digital branches furnished by the superficial palmar arch, or the vessel may end as the profunda artery, in which latter case there would be no superficial arch. Under these circumstances the deficiencies are usually supplied by the palmar interosseous branches of the deep arch, but sometimes by a large median and large superficial volar artery.

2. The radial artery may be deficient in its normal branches, and the arteria princeps pollicis and arteria radialis indicis may arise from the superficial arch, from a median, or from a superficial volar, artery.

Veins.-The deep palmar arch is accompanied by two venæ comites, which receive tributaries corresponding to the branches of the arch. The destination of these venæ comites on either side has been already described in connection with the superficial palmar arch.

Summary of the Palmar Arches.-The superficial palmar arch is formed mainly by the ulnar artery, being completed by the superficial volar of the radial, or, if this fails, by a branch from the arteria radialis indicis, or arteria princeps pollicis. Its digital branches lie over the interosseous spaces, so that, to avoid them, incisions in the palm should be made in line with the centre of a finger, and should not be prolonged farther upwards than the line indicating the position of the superficial palmar arch. On the fingers the digital arteries are placed laterally, so that in cases of whitlow an incision should be made along the middle line of a finger. The superficial palmar arch is sonetimes joined by a large median artery, which is a branch of the anterior interosseous high up in the forearm. In such cases, if the arch were to be punctured, and if the hæmorrhage could not be arrested by the graduated compress, it is evident that ligature neither of the ulnar nor radial artery would suffice. In order to arrest the circulation through a large median artery the ligature would require to be placed on the brachial artery.

The deop palmar arch is formed mainly by the radial artery, and is completed by the profunda branch of the ulnar. It lies about $\frac{1}{2}$ inch above the superficial arch.

\section{BACK OF THE FOREARM AND HAND.}

The cutaneous nerves, already described, are as follows: the posterior branch of the musculo-cutaneous and the lower external cutaneous branch of the musculo-spiral to the outer part, and the posterior division of the internal cutaneous to the inner part.

The deep fascia of the back of the forearm has been described in connection with the anterior aspect, and the posterior annular ligament will be presently referred to. 
Muscles of the Outer Side of the Forearm.-These are three in number, namely, the brachio-radialis, extensor carpi radialis longior, and extensor carpi radialis brevior.

I. Brachio-radialis (supinator radii longus)-Origin.-(I) The upper two-thirds of the external supracondylar ridge of the humerus, and (2) the front of the external intermuscular septum over a corresponding extent.

Insertion.- The outer side of the radius close above the base of the styloid process.

Nerve-supply.-The musculo-spiral nerve.

Action.- Though called a supinator, the muscle is a flexor of the forearm, acting most directly when the limb is in a state of semipronation. An important use of the muscle is to maintain the forearm in the flexed position, as in holding a book. When the forearm is fully pronated the muscle is a feeble supinator, but only to the extent of semisupination. When the forearm is fully supinated it produces semipronation.

The muscle presents a fleshy belly to near the middle of the forearm. Thereafter it is replaced by a flat tendon, which first appears on the deep surface of the muscle. Superiorly the surfaces are directed outwards and inwards, but afterwards they look forwards and backwards.

2. Extensor Carpi Radialis Longior-Origin.-(I) The lower third of the external supracondylar ridge of the humerus, and (2) the front of the external intermuscular septum for a corresponding extent.

Insertion.-The back of the base of the second metacarpal bone over its outer half, a small bursa lying beneath the tendon.

Nerve-supply.-The musculo-spiral nerve.

Action.-(I) To extend the wrist-joint, and (2) to assist in external lateral flexion (abduction) of that joint.

The muscle presents a fleshy belly to near the middle of the forearm, and thereafter a tendon which descends at first upon, and then on the outer side of, the tendon of the extensor carpi radialis brevior. These two tendons pass beneath the posterior annular ligament, and occupy the outermost groove on the back of the lower end of the radius. The surfaces of the muscle are at first directed outwards and inwards, and then forwards and backwards.

3. Extensor Carpi Radialis Brevior-Origin.-(I) The external epicondyle of the humerus by the common tendon ; $(2)$ the external lateral ligament of the elbow-joint; and (3) the intermuscular septa between it and adjacent muscles.

Insertion.-The back of the base of the third metacarpal bone over its outer half, and often by a small slip into the back of the base of the second metacarpal over its inner part. A small bursa is placed beneath the tendon.

Nerve-supply. - The posterior interosseous nerve.

Action.-(I) To extend the wrist-joint, and

(2) to assist in external lateral flexion (abduction) of that joint. 
The tendon appears about the centre of the forearm, and becomes free from fleshy fibres in the lower third. It descends beneath the tendon of the long radial extensor, except near its insertion, where it lies internal to that tendon. It accompanies the long tendon beneath the posterior annular ligament, and passes with it through the outermost groove on the back of the lower end of the radius.

Muscles of the Back of the Forearm.-These are arranged in two layers-superficial and deep.

Superficial Layer. - The muscles of this layer, which are serially continuous with those of the outer side of the forearm, are four in number, namely, the extensor communis digitorum, extensor minimi digiti, extensor carpi ulnaris, and anconeus. The first three have a common tendon of origin, which they share with the extensor carpi radialis brevior.

I. Extensor Communis Digitorum-Origin.-(I) The external epicondyle of the humerus by the common tendon; (2) the deep fascia; and (3) the intermuscular septa between it and adjacent muscles.

Insertion.-The muscle ends in four tendons, which pass beneath the posterior annular ligament to the dorsum of the hand. Here they diverge and pass to the four inner fingers, that for the index finger being accompanied internally by the tendon of the extensor indicis. Having crossed the metacarpo-phalangeal joints, where they give fibres to their lateral ligaments, the tendons form broad expansions covering the backs of the first phalanges, which give insertion to the lumbricales and interossei. Towards the distal end of the first phalanx the expansion divides into three parts-central and two lateral. The central part is inserted into the back of the base of the second phalanx. The two lateral parts unite on the back of the second phalanx, and are inserted into the back of the base of the distal phalanx. The index and middle finger tendons are connected by a weak band of fibres. The ringfinger tendon is connected by a strong band of fibres with the tendon on either side of it, which explains the very limited amount of extension of which the ring-finger is capable, unless the middle and little fingers are extended along with it. The little finger tendon may divide into two parts, one joining the ring-finger tendon and the other the tendon of the extensor minimi digiti.

Nerve-supply.-The posterior interosseous nerve.

Action.-(I) To extend the first phalanges of the four inner fingers, and also the second and third phalanges slightly, these being extended principally by the interossei and lumbricales; and $(2)$ to assist in extension of the wrist-joint.

The common extensor tendons, along with that of the extensor indicis, in passing beneath the posterior annular ligament, occupy the broad innermost groove on the back of the radius.

2. Extensor Minimi Digiti-Origin.-(I) The external epicondyle of the humerus by the common tenclon; (2) the deep fascia; and (3) the intermuscular septum on either side.

Insertion.-Having passed beneath the posterior annular liga- 
ment, where it occupies the groove between the radius and ulna, the tendon divides into two parts, the outer part being joined by the common extensor tendon to the little finger, or by its inner division. Both parts end in a broad expansion on the back of the first phalanx,

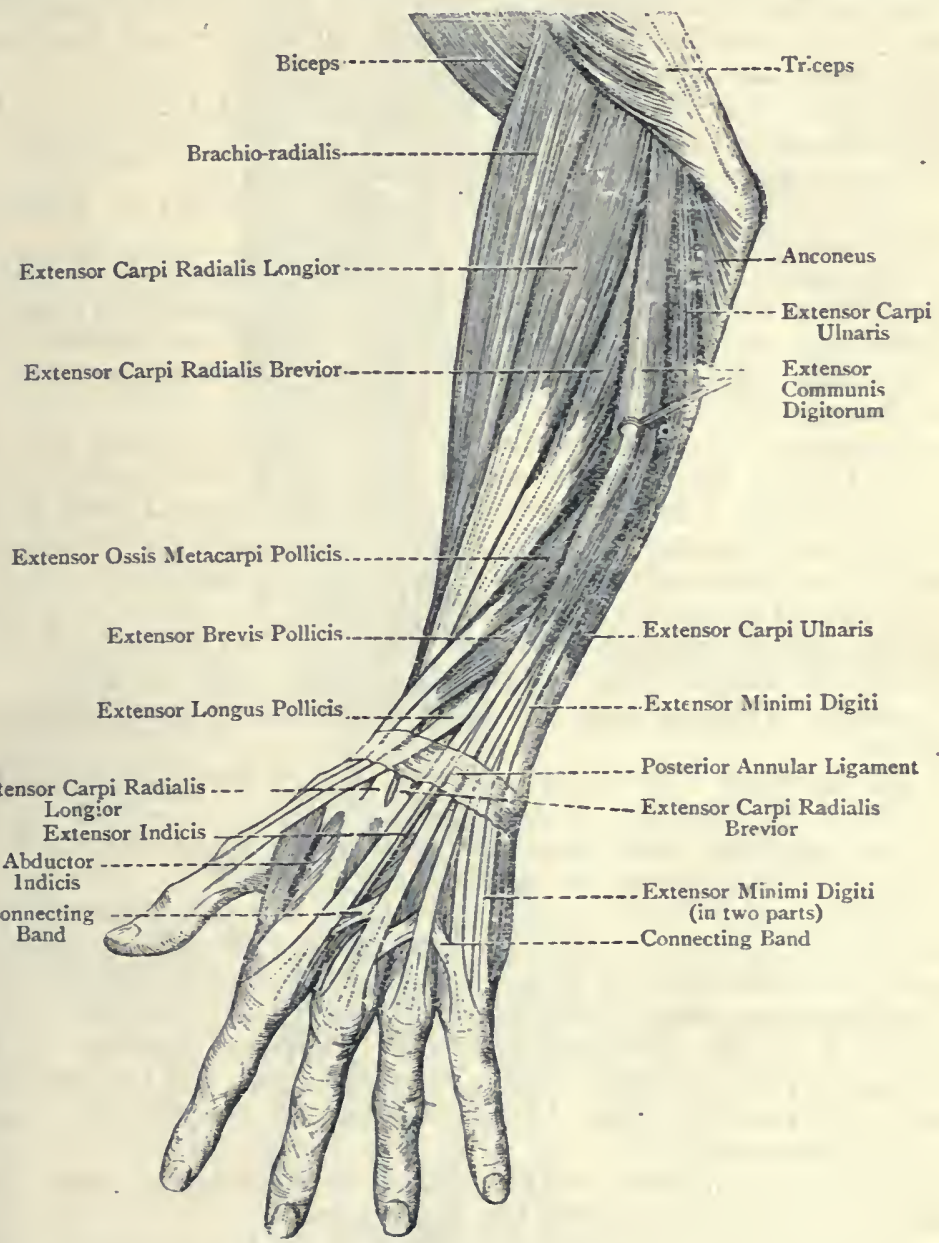

Fig. 205. - The Muscles of the Radial Side and Back of the FOREARM.

and the ultimate insertion is as in the case of the common extensor tendons.

Nerve-supply.-The posterior interosseous nerve.

Action.-The muscle is the special extensor of the little finger, 
as in inserting that finger into the ear canal. It also acts as a feeble auxiliary extensor of the wrist-joint.

3. Extensor Carpi Ulnaris-Origin.-(I) The external epicondyle of the humerus by the common tendon; (2) the deep fascia; (3) the intermuscular septum on either side; and (4) the upper two-thirds of the posterior border of the ulna by an aponeurosis which is common to it, the flexor carpi ulnaris, and the flexor profundus digitorum.

Insertion.-The tubercle on the inner side of the base of the fifth metacarpal bone.

Nerve-supply.-The posterior interosseous nerve.

Action.-(I) To extend the wrist-joint, and (2) to assist in internal lateral flexion (adduction) of that joint.

The muscle rests upon the inner half of the posterior surface of the shaft of the ulna, and its tendon, in passing beneath the posterior annular ligament, occupies the groove on the back of the ulna.

4. Anconeus-Origin.-The back of the external epicondyle of the humerus at its lower part.

Insertion.-The outer surface of the olecranon process, and the upper third of the posterior surface of the shaft, of the ulna.

The muscle, which is continuous with the internal head of the triceps, is triangular, the upper fibres being short and transverse, whilst the others pass obliquely downwards and inwards.

Nerve-supply.-The musculo-spiral nerve, by means of a long branch which descends in the internal head of the triceps, and enters the muscle on its deep surface near the upper border.

Action.-To assist the inner head of the triceps in extending the elbow.

The posterior interosseous recurrent artery ascends beneath the muscle to the back of the external epicondyle.

The posterior interosseous nerve will be found described on p. 358 , and the corresponding artery on p. $3^{6 \mathrm{r}}$.

Deep Layer.-There are five muscles in this layer, namely, the supinator radii brevis, extensor ossis metacarpi pollicis, extensor brevis pollicis, extensor longus pollicis, and extensor indicis.

I. Supinator Radii Brevis-Origin.-(I) The external lateral ligament of the elbow-joint; (2) the orbicular ligament of the radius; (3) the back part of the bicipital hollow, and the supinator ridge, of the ulna; and (4) the aponeurosis covering the upper part of the muscle.

Insertion.-The shaft of the radius on its anterior, outer, and posterior aspects over about the upper third.

Anteriorly the muscle descends as low as the anterior oblique line, externally as low as the insertion of the pronator radii teres, and posteriorly as low as the posterior oblique line. It closely invests the radius over about its upper third, except on its inner aspect.

Nerve-supply. - The posterior interosseous nerve.

Action.-To supinate the radius upon the ulna.

The muscle is pierced by the posterior interosseous nerve, and, 


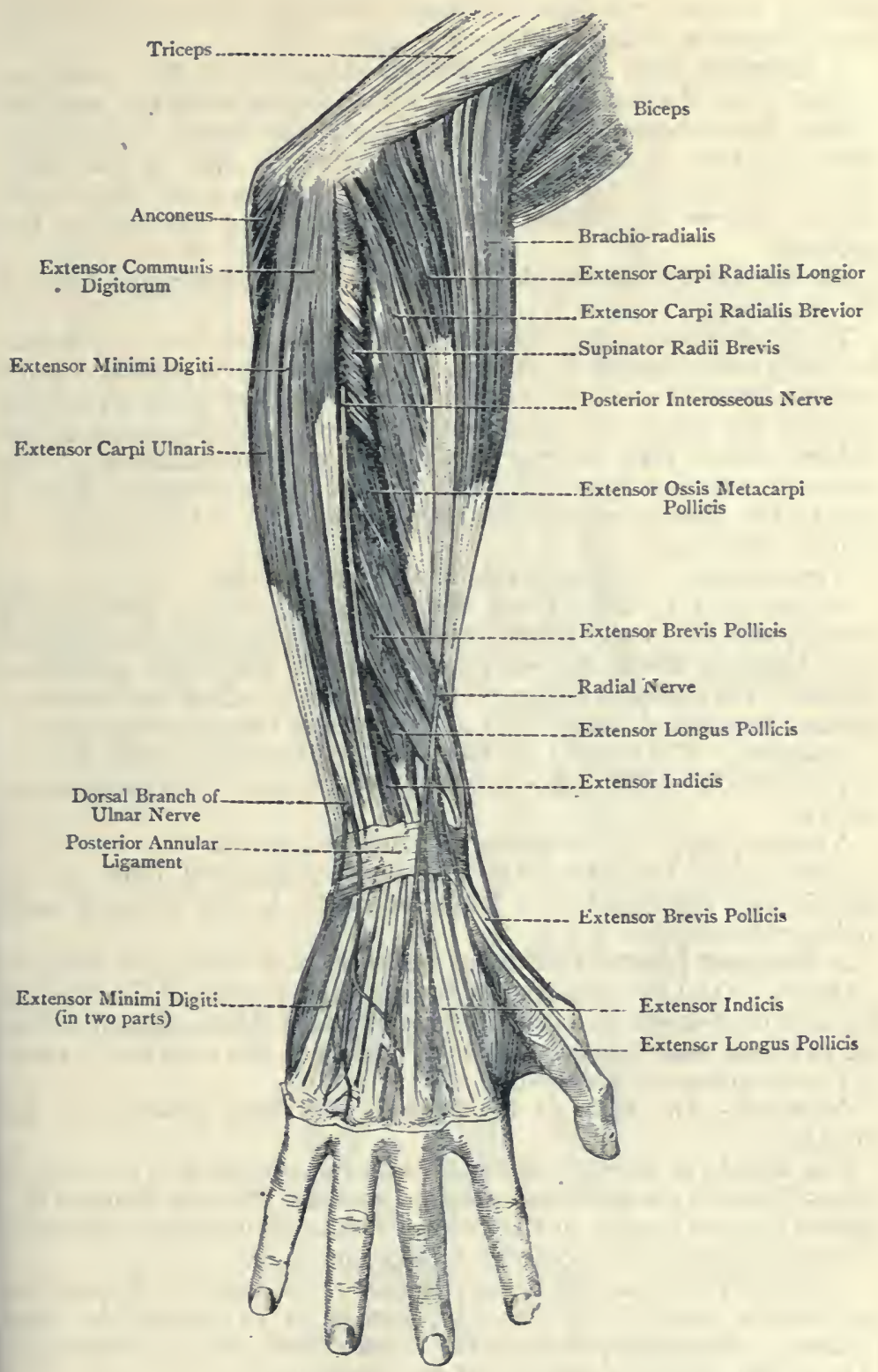

Fig. 206.-Deep Dissection of the Back of the Forearm

(The Radial Nerve and the Dorsal Branch of the Ulnar Nerve are also shown). 
in doing so, the nerve passes between the small superficial and large deep lamina, of which the muscle is composed.

2. Extensor Ossis Metacarpi Pollicis-Origin.-(I) The posterior surface of the shaft of the radius for fully 2 inches below the posterior oblique line, where it meets the supinator radii brevis ; (2) the adjacent portion of the interosseous membrane; and (3) the outer part of the posterior surface of the shaft of the ulna for a short distance below the oblique line, which limits the insertion of the anconeus.

Insertion.-The outer side of the base of the metacarpal bone of the thumb.

The muscle passes downwards and outwards, and its strong tendon is closely accompanied by that of the extensor brevis pollicis. Both of these tendons cross those of the radial extensors of the wrist, and traverse the groove on the outer side of the styloid process of the radius, where they occupy the outermost compartment of the posterior annular ligament. The tendon at its insertion gives a slip to the thenar portion of the palmar fascia, which gives it a double appearance.

Nerve-supply.-The posterior interosseous nerve:

Action.-(I) To extend and abduct the metacarpal bone of the thumb, and (2) to abduct the wrist-joint (external lateral flexion).

3. Extensor Brevis Pollicis (extensor primi internodii pollicis)Origin.-The posterior surface of the shaft of the radius, and the interosseous membrane, for a short distance below the preceding muscle.

Insertion.-The back of the base of the first phalanx of the thumb.

The muscle closely accompanies the extensor ossis metacarpi pollicis.

Nerve-supply. - The posterior interosseous nerve.

Action.-(I) To extend the metacarpo-phalangeal joint of the thumb, and (2) to act as a feeble auxiliary to the extensor ossis metacarpi pollicis.

4. Extensor Longus Pollicis (extensor secundi internodii pollicis) -Origin.-(I) The outer part of the posterior surface of the shaft of the ulna over about its middle third, commencing immediately below the extensor ossis metacarpi pollicis; and (2) the adjacent portion of the interosseous membrane.

Insertion. - The back of the base of the distal phalanx of the thumb.

The muscle is directed downwards and outwards, and its tendon passes beneath the posterior annular ligament, where it occupies the narrow oblique groove on the radius, internal to the radial tubercle.

Nerve-supply.-The posterior interosseous nerve.

Action.-To extend the distal phalanx of the thumb. Thereafter the muscle, which acts strongly, assists in extending the first phalanx and metacarpal bone, the thumb being drawn backwards. It is also an auxiliary extensor of the wrist-joint.

5. Extensor Indicis-Origin.-(I) The outer part of the posterior surface of the shaft of the ulna, commencing just below the middle 


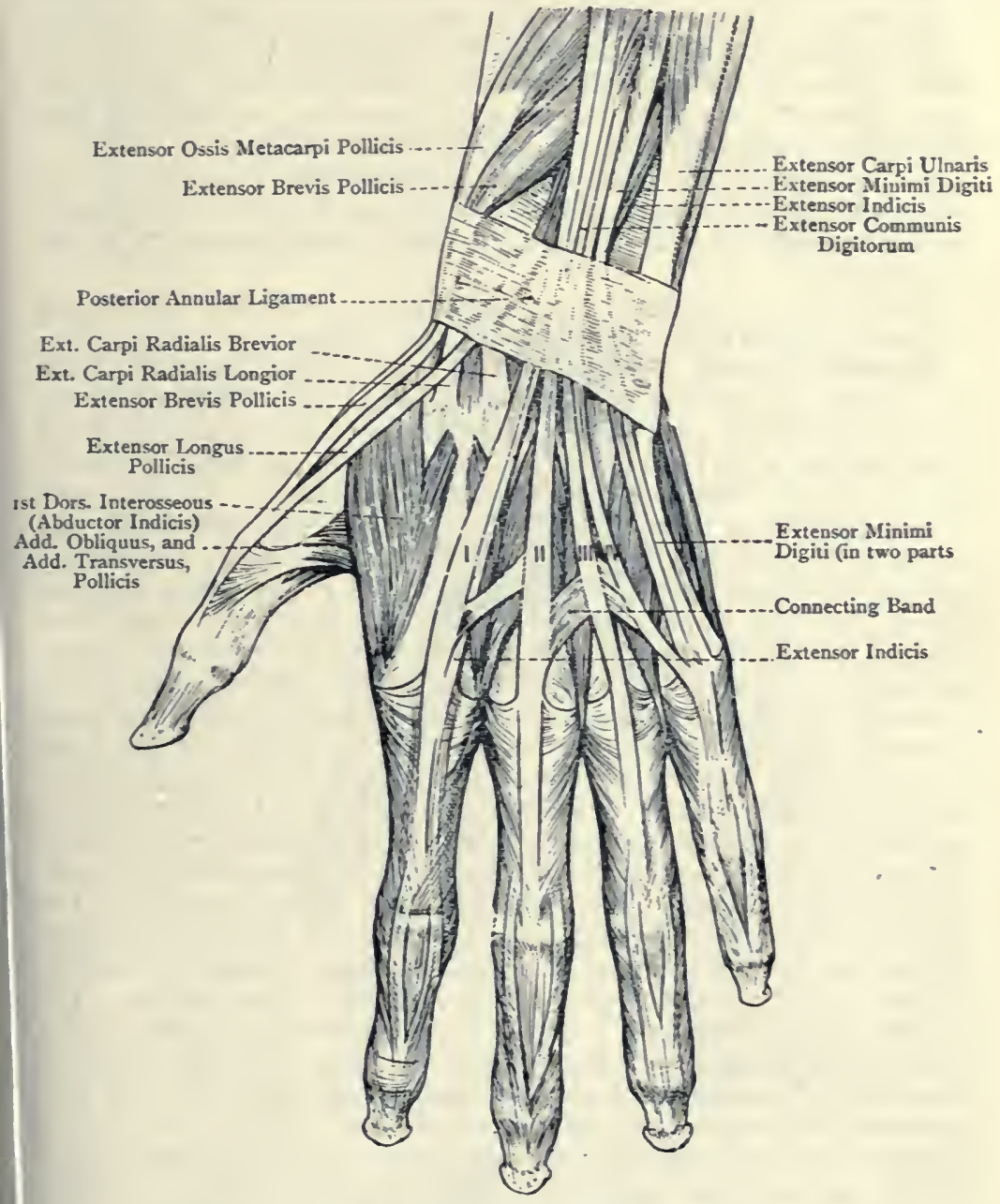

Fig. 207.-The Muscles and Tendoxs of the Back of THE WRIST AND HAND.

I., II., III., IV., Tendons of Extensor Communis Digitorum. 
third, and reaching to near the lower end of the bone; and (2) the lower part of the interosseous membrane.

Insertion.-The inner side of the common extensor tendon of the index finger, which it joins at the metacarpo-phalangeal joint.

The muscle is directed downwards and outwards, and its tendon passes beneath the posterior annular ligament, where it occupies the innermost groove on the back of the radius, in company with the common extensor tendons, beneath which it lies.

Nerve-supply.-The posterior interosseous nerve.

Action.-(I) The muscle is the special extensor of the index finger, as in pointing; and (2) it acts as a feeble auxiliary extensor of the wrist-joint.

The muscle is covered by the extensor communis digitorum, extensor minimi digiti, and extensor carpi ulnaris.

Posterior Annular Ligament.-This is a thickened portion of the deep fascia of the back of the forearm, and it lies obliquely on the back of the wrist. It is attached externally to the lower part of the anterior border of the radius, and initernally to the inner and back part of the cuneiform and pisiform bones, as well as to the hypothenar portion of the palmar fascia. Its direction is inwards and downwards, and it bridges over the various grooves in this region, sending deep processes to be attached to the ridges which separate them. In this manner these grooves are converted into fibroosseous canals for the passage of the extensor tendons. These canals are six in number, four being on the radius, one between that bone and the ulna, and one on the ulna. Proceeding from without inwards, the first canal corresponds with the groove on the outer ....... surface of the siytenu ic metacarpi pollicis and extensor brevis tendons of the extensor oseewu-suds with the outer broad groove pollicis. The second canal correspu.... he tendons of the extensor on the back of the radius, and it contains tn tadialis brevior. The carpi radialis longior and extensor carpi rlaoblique groove on third canal corresponds with the narrow, deep, , which overhangs the back of the radius, internal to the radial tubercle ${ }^{i}$, pollicis. The it, and it contains the tendon of the extensor longus roove on the fourth canal corresponds with the broad, innermost ghe extensor back of the radius, and it transmits the tendons of $t_{2}$, the poscommunis digitorum and extensor indicis, together witior interterior interosseous nerve and posterior branch of the with between osseous artery. The fifth canal corresponds with the groove $x$ tensor
the radius and ulna, and it contains the tendon of the $e^{\text {ve }}$ minimi digiti. minimi digiti. The sixth canal corresponds with the grof the the back of the ulna, between the styloid process and groc naris. bone, and it transmits the tendon of the extensor carpi ul eath,
Each of the foregoing canals is lined by a separate synovi and these sheaths extend for lined by a separate synovial sh annular ligament.

Deep Fascia of the Back of the Hand.-The deep fascia in region is continuous above with the posterior annular ligament, 
at either side with the thenar and hypothenar portions of the palmar fascia. It covers the extensor tendons, with which it is intimately connected. Besides this fascia there is another fascial investment, which is placed beneath the extensor tendons. This deeper layer covers the dorsal interosseous muscles, and, in the intervals between them, it is attached to the dorsal aspects of the shafts of the metacarpal bones.

Second Part of the Radial Artery.-The second part of the vessel winds backwards below the styloid process of the radius to the back of the wrist. It extends from a point on the front of the radius about $\frac{1}{2}$ inch internal to the styloid process to the upper end of the

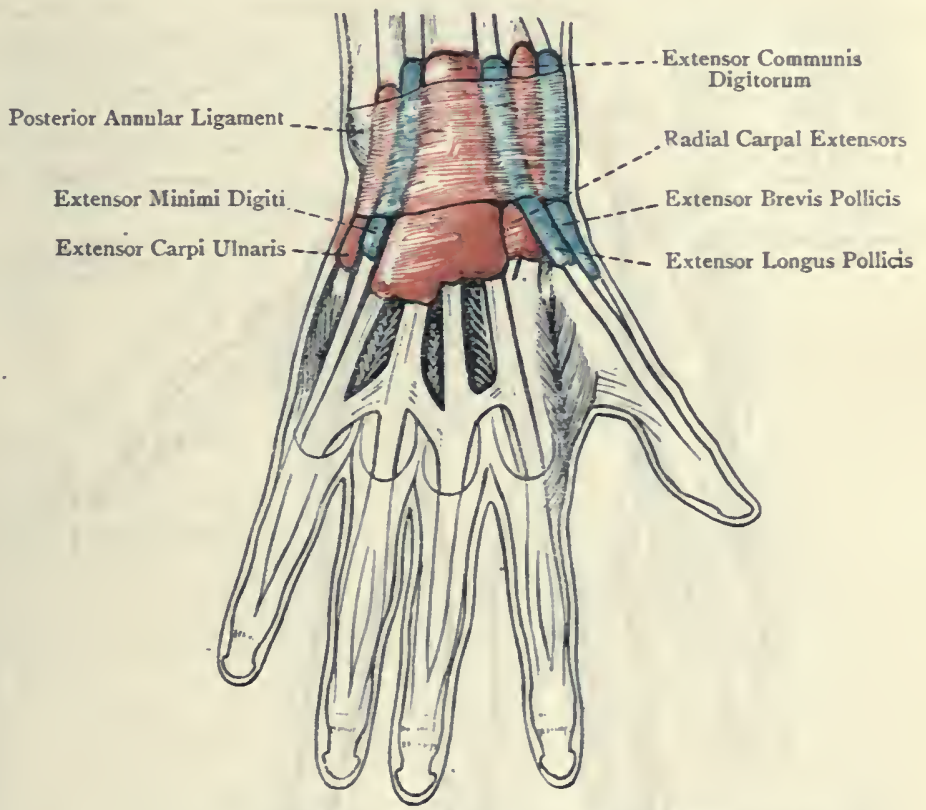

Fig. 208.-The Synovial Sheaths of the Extensor Tendons (afrer L. Testut's 'Anatomie Humaine').

first interosseous space on its posterior aspect, where it dips between the two heads of the abductor indicis muscle, and ends in the third part. Its course is at first backwards and then downwards.

Relations-Superficial. - The integument, radial vein, branches of the radial nerve, tendons of the extensor ossis metacarpi pollicis, extensor brevis pollicis, and extensor longus pollicis, the latter crossing the vessel just before it disappears between the two heads of the abductor indicis. At first it is deeply placed, but afterwards it is superficial, and, in the living subject, pulsation may be felt in it in the triangular hollow below and behind the radial styloid 
process. Deep.-The external lateral ligament of the wrist-joint, and the scaphoid and semilunar bones. It is accompanied by two venæ comites.

Branches.- The branches are the posterior radial carpal, first dorsal interosseous, two arteriæ dorsales pollicis, and arteria dorsalis indicis.

The posterior radial carpal artery, of small size, arises from the radial as it lies upon the external lateral ligament. It passes in-

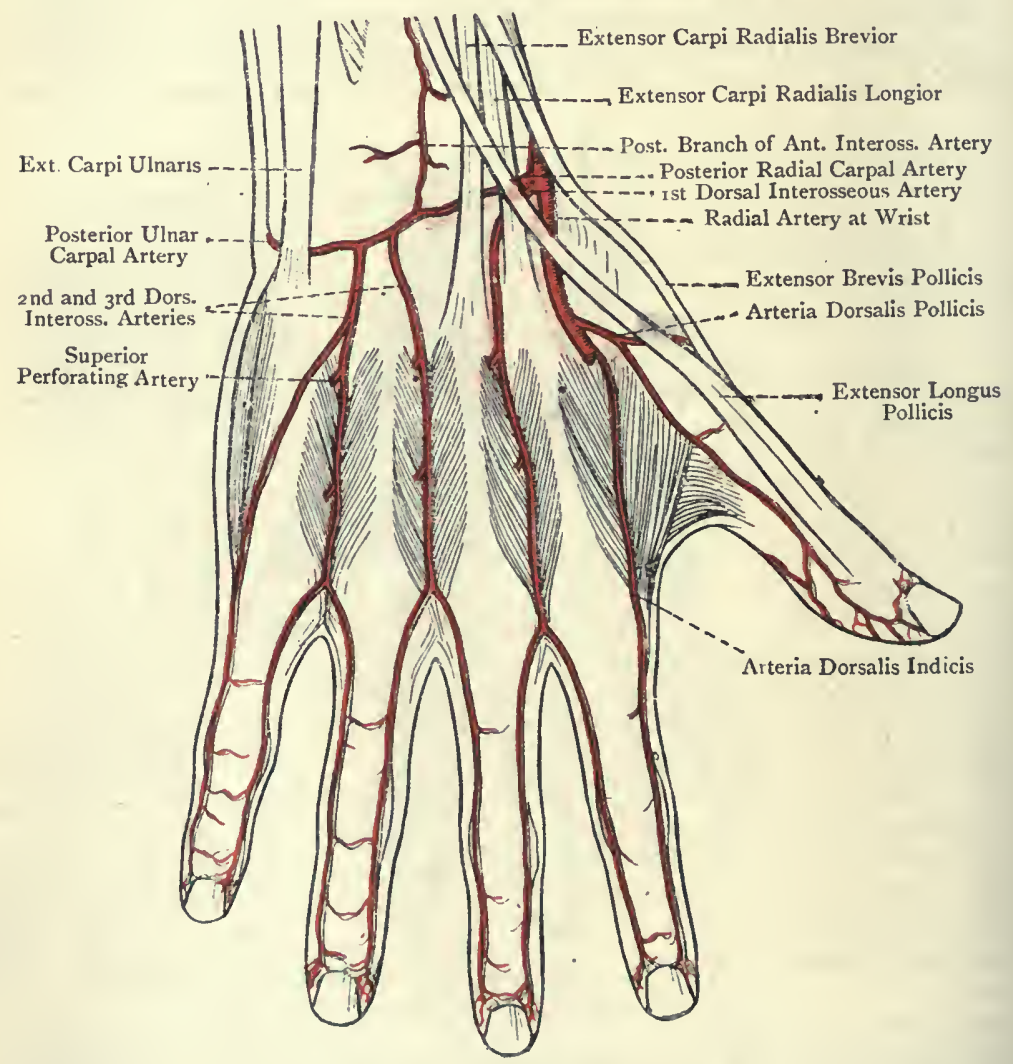

Fig. 209, - The Arteries of the Back of the Wrist and Hand (after L. Testut's 'Anatomie Humaine').

wards on the back of the wrist beneath the extensor tendons, and anastomoses with the posterior ulnar carpal and posterior branch of the anterior interosseous to form the posterior carpal arch.

The first dorsal interosseous artery arises from the preceding, or from the radial a little lower down. It passes to the upper end of the second interosscous space, where it receives a superior 
perforating branch from the deep palmar arch. It then descends over that space towards the cleft between the index and middle fingers, where it may send forward an inferior perforating branch to join the fourth or outermost digital artery from the superficial palmar arch. Thereafter it divides into two dorsal collateral digital arteries for the contiguous sides of the index and middle fingers.

The second and third dorsal interosseous arteries are branches of the posterior carpal arch. They descend over the backs of the third and fourth interosseous spaces, at the upper ends of which each receives a superior perforating branch from the deep palmar arch. Near the clefts between the middle and ring, and ring and little fingers, they may send forwards inferior perforating branches to join the second and third digital arteries from the superficial palmar arch, and thereafter each divides into two dorsal collateral digital arteries for the contiguous sides of the middle and ring, and ring and little fingers. The dorsal digital artery of the inner side of the little finger arises from the third (innermost) dorsal interosseous, or from the posterior ulnar carpal.

The arteriæ dorsales pollicis, two in number, arise separately or by a common trunk, from the radial opposite the base of the first metacarpal bone, and are distributed to the sides of the thumb.

The arteria dorsalis indicis arises below the preceding, just before the radial dips between the two heads of the abductor indicis. It descends on the outer side of the second metacarpal bone, and supplies the outer side of the index finger.

The distribution of the dorsal digitalarteriesceases, as a rule, about the level of the first interphalangeal joint.

\section{The Interosseous Muscles.}

The interosseous muscles re seven in number, and tre arranged in two groups -three palmar and four dorsal. The palmar interJssei are seen only on the palmar aspect, but the lorsal interossei are seen on poth aspects of the hand.

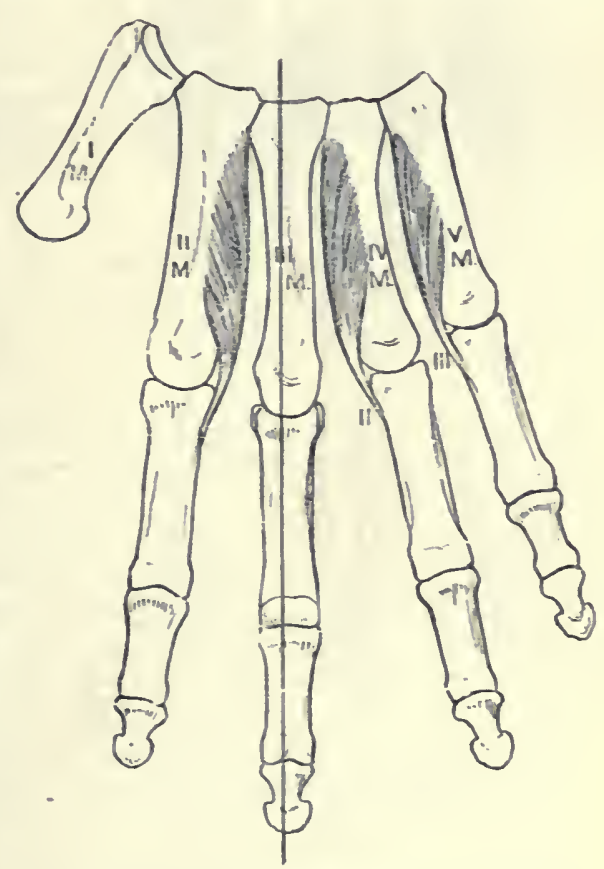

Fig. 21o.-The Palmar Interosseocs Mescles of the Right Hand. 
The palmar interossei belong to the index, ring, and little fingers, and they are named from without inwards-first, second, and third. Each is connected only with one metacarpal bone. The first arises from the inner aspect of the shaft of the second metacarpal bone, and the second and third from the outer aspects of the shafts of the fourth and fifth metacarpals. The first is inserted partly into the inner side of the base of the first phalanx of the index finger, and partly into the-expansion of the extensor tendon on the dorsal aspect of that phalanx. The second and third are inserted in a similar manner in the case of the ring and little fingers, except that each is inserted into the outer side of the base of the first phalanx.

The palmar interossei are covered by a thin fascia, called the interosseous fascia. At either side it joins the thenar and hypothenar portions of the palmar fascia, and inferiorly it is connected with the transverse metacarpal and anterior metacarpo-phalangeal

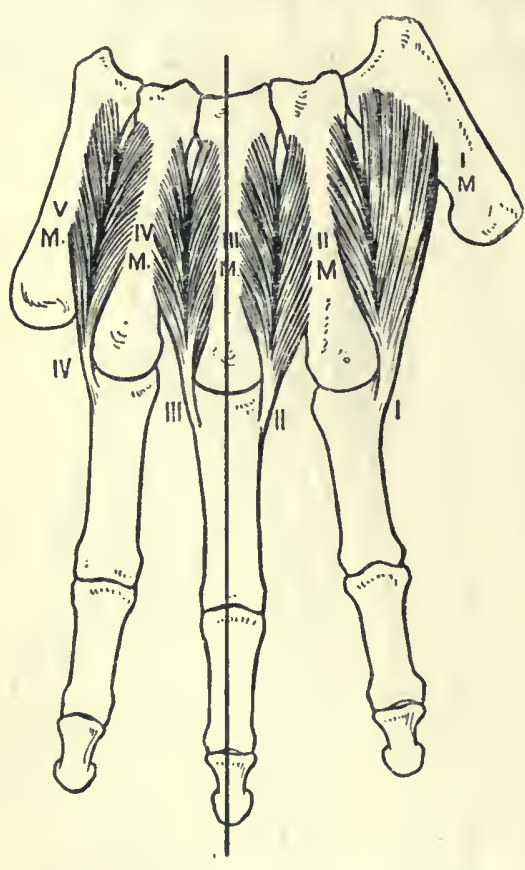

Fig. 2i1.-The Dorsal Interosseous Muscles of the Right HaNd. ligaments. Two septa pass between it and the central portion of the palmar fascia. The dorsal interossei, which are four in number, belong to the index, middle, and ring fingers, the middle finger having two. They are named from without inwardsfirst, second, third, and fourth, the first being specially called the abductor indicis. Each muscle arises by two heads from the contiguous sides of the shafts of the metacarpal bones between which it is placed, and always more extensively from the bone belonging to the finger upon which the muscle acts. The fibres of each muscle end upon a tendon which is placed in the centre, and this tendon is inserted partly into the side of the base of the first phalanx of a finger, and partly into the expansion of the extensor tendon on the back of that phalanx. The first or abductor indieis is of large size. Its outer large head arises from the proximal half of the inner margin of the first metacarpal bone, and its inner small head from the whole length of the outer aspect of the shaft of the second metacarpal. It is inserted 
into the outer side of the base of the first phalanx of the index finger, and the expansion of the extensor tendon on the back of that phalanx. The second is inserted into the outer side, and the third into the inner side, of the base of the first phalanx of the middle finger, and both into the expansion of the extensor tendon on the back of that phalanx. The fourth is inserted into the inner side of the base of the first phalanx of the ring-finger, and into the expansion of the extensor tendon on the back of that phalanx.

Nerve-supply.-All the interossei are supplied by the deep division of the ulnar nerve.

Action.-(I) The palmar interossei are adductors, the fingers which they adduct being the index, ring, and little. (2) The dorsal interossei are abductors, the fingers which they abduct being the index, middle, and ring, the middle finger having two abductors, inasmuch as it can be drawn to either side of the middle line which passes through its centre.

In speaking of adduction and abduction reference is made to an imaginary line passing through the centre of the middle finger, adduction being movement torvards that line, and abduction from it. There is another very important action of all seven interossei, in the performance of which they are assisted by the lumbricales. This action is as follows: (I) to flex the metacarpo-phalangeal joints, in virtue of their insertion into the first phalanges of the four inner fingers, and (2) to extend their interphalangeal joints, in virtue of their insertion into the expansions of the extensor tendons. This action well illustrated in making the hair- or upstroke in writing.

Between the two heads of the abductor indicis superiorly is an interval for the passage of the radial vessels, and between the two heads of each of the other dorsal interossei superiorly there is an interval for the passage of a superior perforating artery from the deep palmar arch.

\section{Lymphatic Vessels of the Upper Limb.}

The lymphatic vessels of the upper limb are arranged in two groups-superficial and deep. The superficial lymphatics lie in the subcutaneous tissue, and the deep lymphatics accompany the deep bloodvessels.

Superficial Lymphatics.-(I) Digital.-There are very rich networks of lymphatics in the fingers, especially over their palmar aspects. The efferent lymphatics from the digital networks form two digital vessels, on either side which lie on each side of each finger, in contact with the corresponding digital artery. At the roots of the fingers these vessels pass to the dorsal aspect of the hand, and, after having communicated with each other and formed a dorsal network, they pass to the back of the wrist.

(2) Palmar.-There is a very rich network of lymphatics in the palm, from which several vessels emerge and pass in various directions-outwards, inwards, downwards, and upwards. The external efferent vessels pass upwards and outwards over the thenar eminence, and join the lymphatics from the thumb. The internal efferent vessels pass inwards, and, having turned round the inner border of the hand, they join the lymphatics from the little finger. The inferior efferent lymphatics pass downwards to the clefts between the fingers, where they turn backwards and join the digital efferent lymphatics. The superior efferent vessels ascend to the front of the wrist. 
(3) Carpal.-The carpal lymphatics form two groups-palmar and dorsal, which derive their vessels from the palmar and dorsal networks of the hand.

(4) Antibrachial.- The superficial lymphatics of the front of the forearm

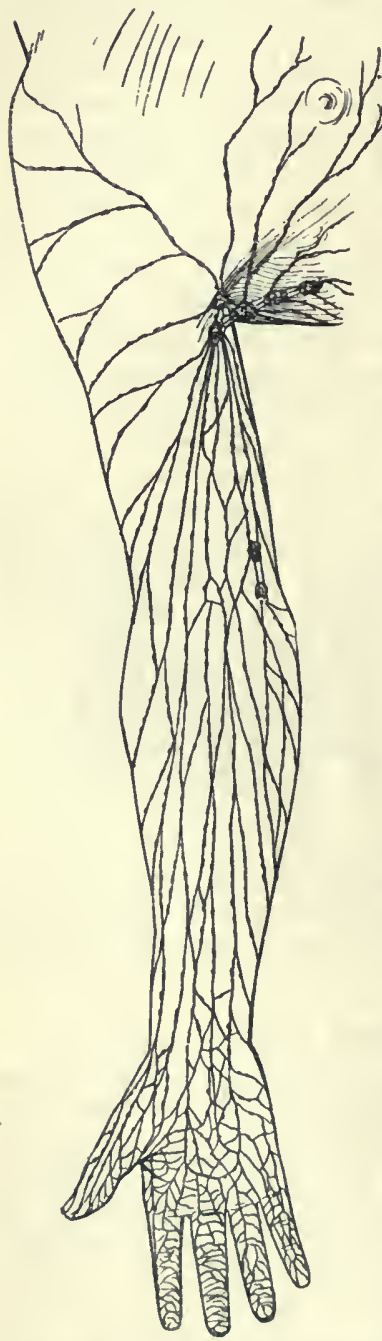

Fig. 2I2.-THE SUPERFICIAL LYMPHATICS OF THE UPPER LIMB (ANTERIOR VIEW). are arranged in three groups-radial, median, and ulnar, which accompany the corresponding veins. As the radial and ulnar lymphatics ascend they are joined at intervals by efferent vessels from the dorsal plexus of the hand, which wind round the radial and ulnar borders of the forearm from its dorsal aspect. The radial lymphatics in part ascend into the arm and accompany the cephalic vein, and in part join the median lymphatics. The median lymphatics may terminate in the anticubital glands, if these are present. When these are absent, they join the ulnar lymphatics, which terminate in the supra-trochlear gland or glands.

(5) Brachial.-The superficial lymphatics of the brachial region form twe groups-inner and outer. The inner lymphatics, which are very numerous, lie along the inner border of the biceps brachii. They largely represent the efferent vessels of the supra-trochlear gland or glands, and anticubital glands, if present, and they lie at first superficial to the deep fascia. Subsequently, however, they pierce that fascia and pass to the external or humeral axillary glands, in association with the deep brachial lymphatics. The outer lymphatics, which are reinforced by some of the radial lymphatics, lie on the outer side of the biceps brachii, along the course of the cephalic vein. They then ascend in the groove between the deltoid and pectoralis major muscles, where they may traverse one or two very minute deep lymphatic glands. Ultimately they pass to the infra-clavicular glands, but one or two of them may ascend over the clavicle, and terminate in one of the supra-clavicular glands.

Deep Lymphatics. - These, as stated, accompany the deep bloodvessels, and they form five groups-radial, ulnar, anterior interosseous, posterior interosseous, and brachial. In connection with each artery there are usually two lymphatic vessels, and those accompanying the radial, ulnar, anterior interosseous, and brachial arteries occasionally traverse one or two deep lymphatic glands, which are sometimes met with along the course of each of these arteries. The brachial lymphatics are formed by the union of the radial, ulnar, anterior interosseous, and posterior interosseous lymphatic vessels. About the centre of the arm they are reinforced by the efferent vessels of the supra-trochlear gland or glands, and they terminate in the external or humeral axillary glands.

Summary of the Lymphatic Glands of the Upper Limb.-The lymphatic glands of the upfer limb form two groups-superficial and deep.

The superficial glands cmbrace the internal epicondylar or supra-trochlear. 
and, it may be, the anticubital glands; but the latter are inconstant. There are no superficial lymphatic glands in the hand or forearm.

The deep glands principally represent the axillary and infra-clavicular glands. A few deep lymphatic glands, however, of very small size are sometimes met with in connection with the deep lymphatic vessels which accompany the arteries of the forearm and the brachial artery. One or two deep lymphatic glands are also sometimes met with in the groove between the deltoid and pectoralis major muscles, along the cephalic vein.

Superficial Glands.

1. Internal epicondylar.

2. Anticubital (ineonstant).

\section{Deep Glands.}

\author{
1. Axillary. \\ 2. Infra-clavicular. \\ 3. Radial (inconstant). \\ 4. Ulnar (inconstant). \\ 5. Anterior interosseous (inconstant). \\ 6. Brachial (inconstant). \\ 7. Cephalic (inconstant).
}

\section{Development of Arteries of Upper Limbs.}

The arterial stem of the left upper limb is developed entirely from the seventh left cervical segmental artery, in association with the left vertebral artery. The component arteries of this stem, from above downwards, are as follows: (I) The subclavian artery; (2) the axillary artery; (3) the brachial artery; and (4) The anterior interosseous artery. The arterial stem of the right upper limb is developed from the fourth right arterial arch, a portion of the right dorsal aorta, and the seventh right cervical segmental artery, in association with the right vertebral artery. The component arteries of this stem are as on the left side.

Before the adult condition of the arteries is reached, several stages of development have to be passed through.

(I) As the anterior interosseous artery becomes diminished, another artery springs from the brachial a short distance below the elbow-joint. This new" vessel accompanies the median nerve, and is called the median artery.

(2) The median artery superiorly furnishes a branch, which becomes the ulnar artery.

(3) As the median artery becomes diminished, a vessel springs from the brachial artery about the centre of the brachial region. This vessel is called the primary radial artery, and it accompanies the radial nerve.

(4) As the upper part of the primary radial artery atrophies, the brachial artery gives off another branch just below the elbow-joint, which soon joins the primary radial, and so the permanent radial artery is formed.

\section{Development of Veins of Upper Limbs.}

The veins of each upper limb form two groups-superficial and deep. The superficial veins are developed prior to the deep, the latter accompanying the arteries.

The primitive vein is the primary ulnar or postaxial vein, which receives distally the digital veins, and opens proximally into the anterior cardinal vein. At a later period the cephalic vein is formed as an outgrowth from the external jugular vein, but it subsequently acquires its permanent connection with the upper part of the axillary vein.

The distal portion of the primary ulnar vein disappears. Its proximal portion persists and gives rise to the basilic, axillary, and subclavian veins.

From the distal end of the basilic vein the anterior and posterior ulnar veins and the median basilic vein are developed.

The radial and median cephalic veins grow distally from the cephalic vein.

The median vein is developed from the median basilic and median cephalic veins. 


\section{THE ELBOW-JOINT.}

The elbow-joint belongs to the class diarthrosis and to the subdivision ginglymus. The articular surfaces are the trochlea and capitellum of the humerus above, and the great sigmoid cavity of the ulna and the cup-shaped depression on the head of the radius below. The trochlea articulates with the great sigmoid cavity, and the humeral capitellum with the head of the radius. The joint is surrounded by a capsule, which is made up of four ligaments-anterior, posterior, external lateral, and internal lateral.

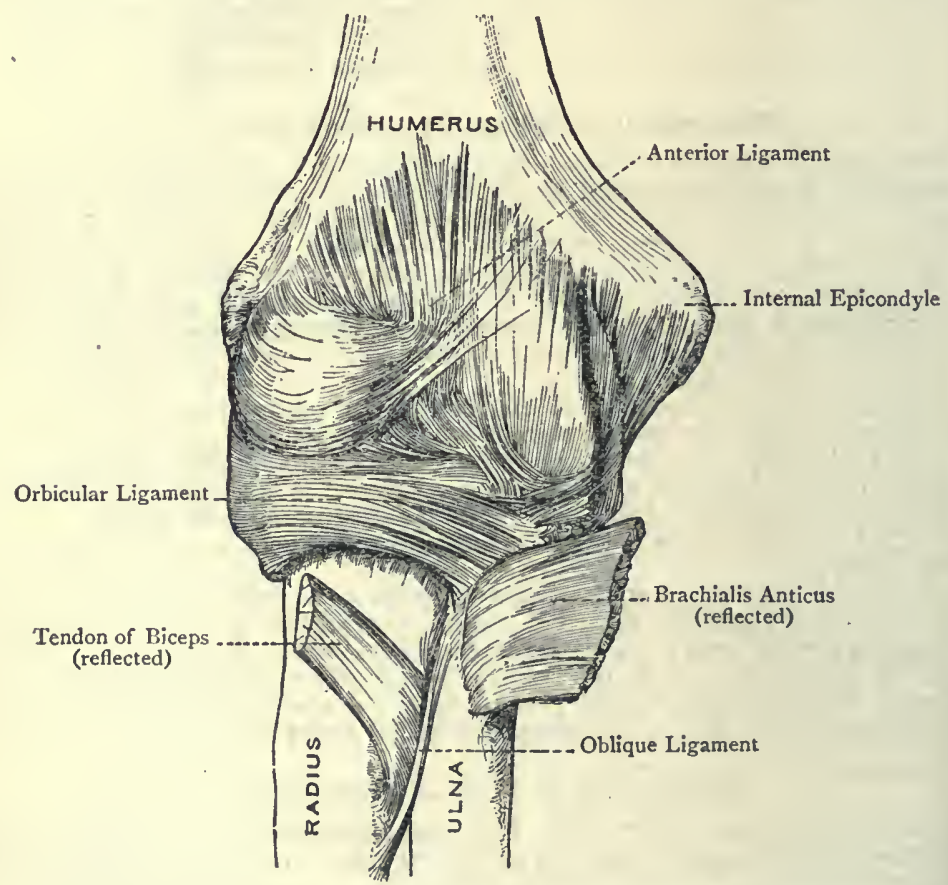

Fig. 2r3.-The Right Elbow-Joint (Anterior View).

The anterior ligament is broad and thin, the central portion being the strongest. Its fibres are attached superiorly to the front of the humerus above the coronoid and radial fossæ, and inferiorly to (I) the front of the coronoid process of the ulna, and (2) the orbicular ligament of the radius. Some of its fibres run vertically and others obliquely, the latter chiefly passing downwards and outwards superficial to the former. The ligament is closely covered by the brachialis anticus muscle. 
The posterior ligament is thin and membranous. Superiorly it is attached to the upper part and sides of the olecranon fossa of the humerus, and inferiorly to (I) the front part of the summit of

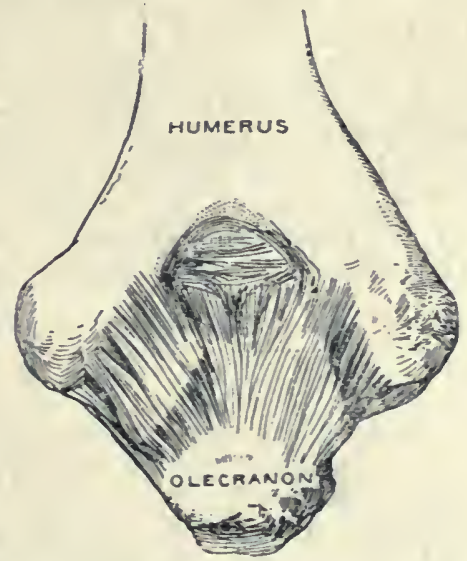

Fig. 214.-The Posterior Ligament of the Elbow-Joint.

the olecranon process of the ulna, and (2) the orbicular ligament of the radius. The most of its fibres are disposed vertically, but a few pass transversely between the margins of the olecranon fossa.

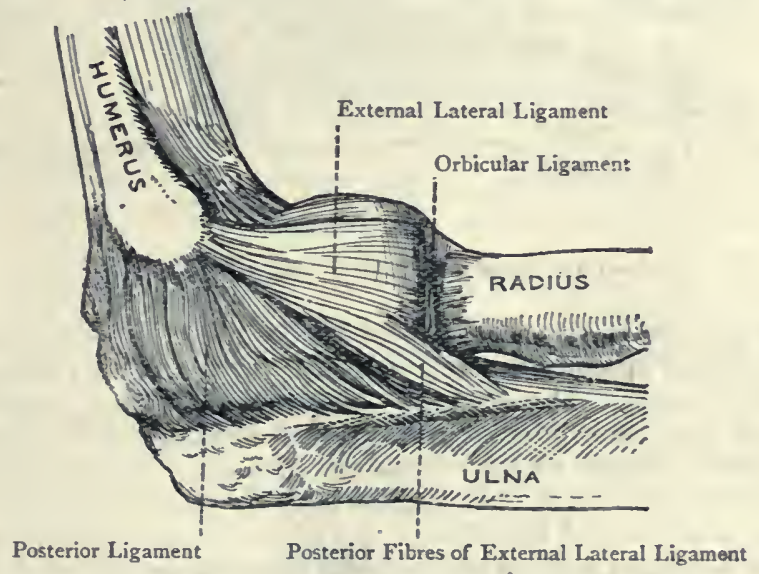

Fig. 215- The Right Elbow-Joint (External View).

The ligament is related to the tendon of insertion of the triceps, some of the fibres of the internal head being inserted into it, under the name of the subanconeus. 
The external lateral ligament is a short, stout, flat band, which is attached superiorly to the lower part of the external epicondyle of the humerus, and inferiorly to the outer part of the orbicular ligament of the radius. A few of its posterior fibres pass over the orbicular ligament, and are attached to the outer margin of the ulna. It is intimately related to the origins of the extensor carpi radialis brevior and supinator radii brevis muscles.

The internal lateral ligament is triangular, with the apex upwards. It is attached superiorly to the anterior, inferior, and posterior parts of the internal epicondyle of the humerus, and inferiorly to the inner margin of the great sigmoid cavity of the ulna. It is divisible into three portions-anterior, posterior, and middle. The

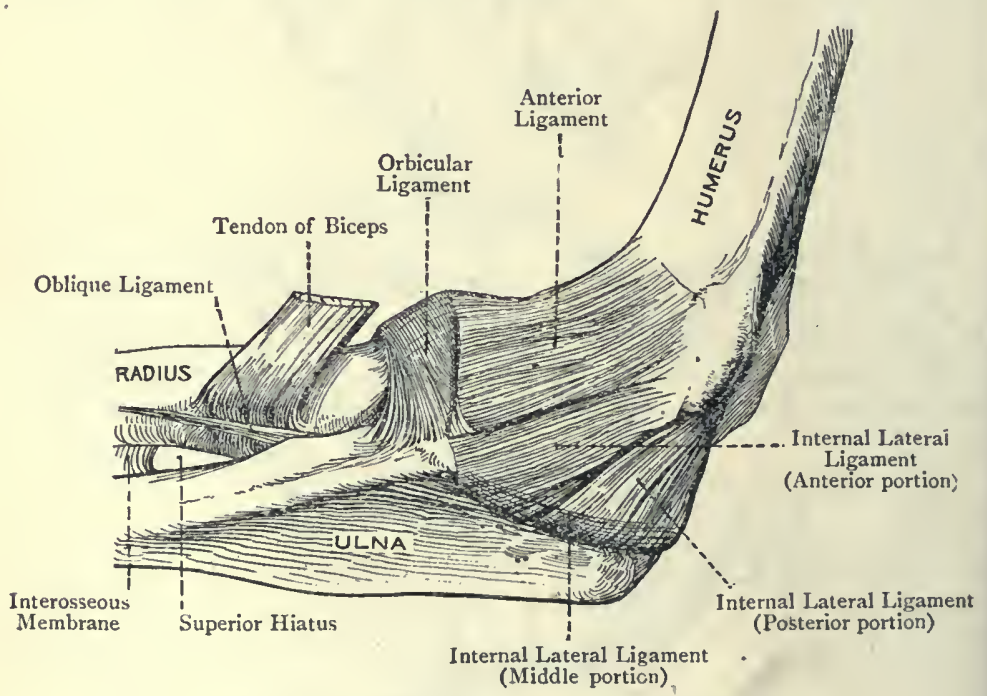

Fig. 216.-The Right Elbow-Joint (Internal View).

anterior portion is attached superiorly to the front and lower part of the internal epicondyle, and inferiorly to the inner margin of the coronoid process. The posterior portion is attached superiorly to the lower and back part of the internal epicondyle, and inferiorly to the inner margin of the olecranon process. The middle portion consists of fibres which pass transversely from the olecranon to the coronoid process, and blend with the fibrous band bridging over the notch on the inner margin of the great sigmoid cavity between these two processes. The ligament is intimately related to the origin of the flexor sublimis digitorum.

The synovial membrane lines the internal surface of the capsule, and inferiorly is continued into the superior radio-ulnar joint, where it lines the internal surface of the orbicular ligament and 
upper part of the neck of the radius. It also lines the coronoid, radial, and olecranon fossæ of the humerus, which contain small cushions of fat.

Muscular Relations.-The capsule is closely related to the following muscles: anteriorly, the brachialis anticus; posteriorly, the triceps; externally, the extensor carpi radialis brevior and supinator radii brevis; and internally, the flexor sublimis digitorum.

Arterial Supply.-The superior and inferior profundæ, anastomotica magna, anterior and posterior ulnar recurrent, radial recurrent, and posterior interosseous recurrent, arteries.

Nerve-supply.-The musculo-spiral, musculo-cutaneous, median, and ulnar nerves.

Movements.-Two movements are allowed, namely, flexion and extension, these being limited by the tension of the structures related to the joint, and flexion also by the coming into contact of the fleshy parts of the forearm and arm.

Muscles concerned in the Movements-Flexion.-This is produced by the biceps, brachialis anticus, brachio-radialis, and, as auxiliaries, the muscles arising from the internal epicondyle of the humerus. Extension.-This is produced by the triceps and anconeus, and, as auxiliaries, the muscles arising from the external epicondyle.

Bursa at Elbow-Joint.-The bursæ in the vicinity of the elbowjoint are four in number, as follows:

\section{Subcutaneous olecranon. Bicipito-radial. \\ Deep olecranon. Epicondylar.}

The subcutaneous olecranon bursa is situated between the integument and the posterior triangular surface of the olecranon process of the ulna. When this bursa becomes inflamed, it constitutes the affection known as miner's elbow. It has no communication with the synovial cavity of the elbow-joint.

The deep or subtendinous olecranon bursa, which is of small size, is situated upon the front part of the upper surface of the olecranon. It separates the tendon of insertion of the triceps muscle from the posterior ligament of the elbow-joint, and it has no communication with the synovial cavity of the joint.

The bicipito-radial bursa is situated between the tendon of insertion of the biceps muscle and the anterior smooth portion of the bicipital tuberosity of the radius. It has no communication with the synovial cavity of the elbow-joint.

The epicondylar bursæ are not constant. There may be two mesial over the internal or mesial epicondyle of the humerus, and lateral over the external epicondyle.

\section{THE RADIO-CARPAL OR WRIST-JOINT.}

The wrist-joint belongs to the class diarthrosis, and to the subdivision condylarthrosis. - The articular surfaces are the carpal surface of the radius and the triangular fibro-cartilage above, and the superior facets of the scaphoid, semilunar, and cuneiform, as well as the interosseous ligaments on either side of the semilunar, 
below. The carpal surface of the radius, which presents an outer or scaphoid and an inner or semilunar division, and the triangular fibro-cartilage form a socket,. which is concave from side to side and from before backwards. The superior facets of the carpal bones extend more upon the dorsal than palmar aspects, and they present a convexity. The ulna is entirely excluded from this joint by the triangular fibro-cartilage. The joint is surrounded by a capsule, which is made up of four ligaments, namely, anterior, posterior, external, and internal.

The anterior ligament is a broad, strong membrane, the fibres of which are attached posteriorly to the front of the lower end of the radius, close above the anterior border, and tc the anterior margin

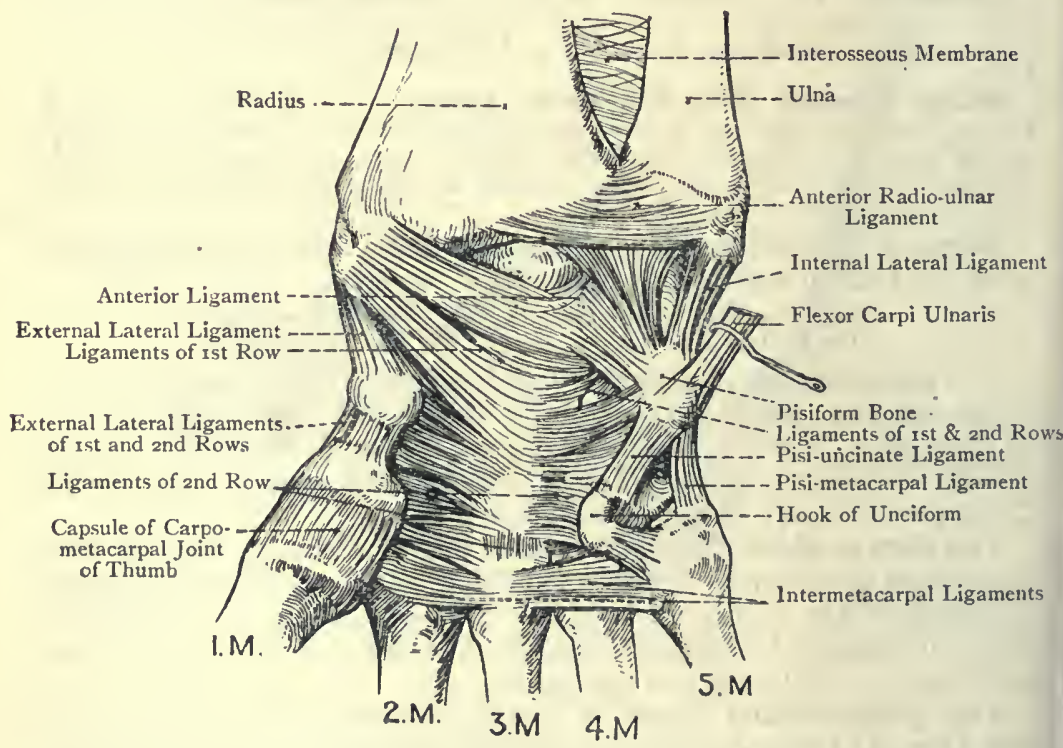

Fig. 217.-The Ligaments of the Radio-Carpal, Carpal, CarioMETACARPal, AND INTERMETACARPAL JOINTS (ANTERIOR VIEW).

of the triangular fibro-cartilage. Inferiorly they are attached to the palmar surfaces of the scaphoid, semilunar, and cuneiform bones. The fibres for the most part are directed downwards and inwards.

The posterior ligament is a broad, weak membrane, the fibres of which are attached superiorly to the posterior border of the radius and triangular fibro-cartilage, and inferiorly to the dorsal surfaces of the scaphoid, semilunar, and cuneiform bones, especially the latter. Its fibres are directed downwards and inwards.

The external lateral ligament is a strong, flattened band, which extends from the tip of the styloid process of the radius to the outer aspects of the scaphoid and trapezium. It supports a portion of the second part of the radial artery. 
The internal lateral ligament is a strong, round cord, which extends from the tip of the styloid process of the ulna to the cuneiform and pisiform bones.

The synovial membrane lines the internal surface of the capsule and the upper surfaces of the interosseous ligaments on either side of the semilunar. In cases where the triangular fibro-cartilage is perforated it is continuous with the synovial membrane of the inferior radio-ulnar joint, but it is distinct from that of the carpal articulations.

Muscular Relations - Anterior.-From within outwards, the flexor carpi ulnaris, flexor profundus digitorum, flexor longus pollicis, and flexor carpi radialis. Posterior. -From within outwards, the extensor carpi ulnaris, extensor minimi digiti, extensor communis digitorum with the extensor indicis, extensor longus pollicis, extensor carpi radialis brevior, and extensor carpi radialis longior. External. - The extensor ossis metacarpi pollicis and extensor

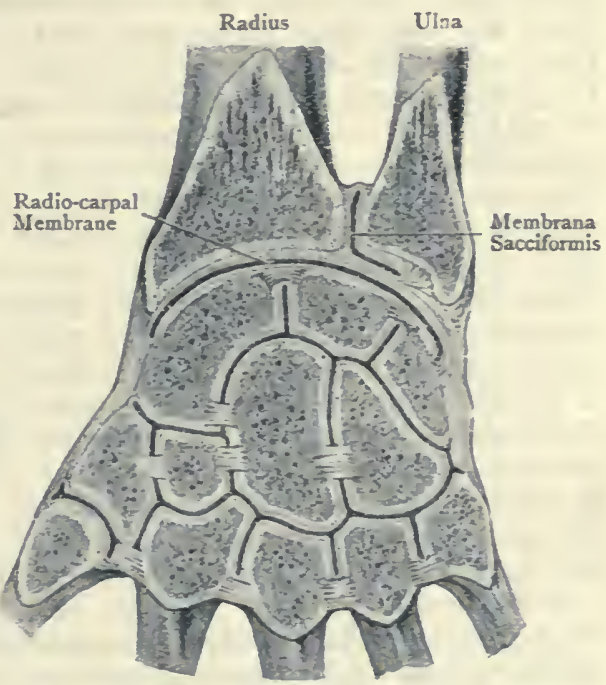

FIG. 2I7A.-RADIO-CARPAL AND CARPAL Sysovial MeMbraxes. brevis pollicis.

Arterial Supply.-The anterior and posterior carpal retia. Nerve-supply.-The median, ulnar, and posterior interosseous, nerves.

Movements.-Six movements are allowed, namely, palmar flexion, dorsal flexion, ullnar flexion or adduction, radial flexion or abduction, extension, and circumduction. In all forms of flexion an angle is produced between the hand and forearm. Extension is straightening, so as to efface the angle produced by flexion. Circumduction is a combination of all the foregoing movements occurring in alternate succession. Adduction is more extensive than abduction, the styloid process of the radius limiting the latter movement. When the hand is in line with the forearm, the scaphoid division of the carpal articular surface of the radius articulates with the scaphoid bone, and the semilunar division of the carpal articular surface of the radius, together with the triangular fibro-cartilage, articulates with the semilunar bone, the ficeted part of the upper surface of the cuneiform being in contact with the inner portion of the capsule. When, however, the hand is adducted-that is to say, flexed in an inward direction-the faceted part of the upper surface of the cuneiform articulates with the triangular fibro-cartilage, the semilunar bone partially articulates with the scaphoid surface of the radius, and the scaphoid bone partially articulates with the outer portion of the capsule. 


\section{THE RADIO-ULNAR JOINTS.}

The radius and ulna are united by a superior and an inferior radio-ulnar joint, and there is an intermediate connection between their shafts.

Superior Radio-ulnar Joint.-This joint belongs to the class diarthrosis, and to the subdivision trochoides. The articular surfaces are the deep inner part of the head of the radius, and the small sigmoid cavity of the ulna. There is one ligament at this joint, namely, the orbicular or annular ligament. This is a strong fibrous band, which forms about four-fifths of a circle, and surrounds the circumference of the head of the radius, which it retains in contact with the small sigmoid cavity. Its extremities are attached to the anterior and posterior margins of the small sigmoid cavity. The ligament is smaller below than above, and so it maintains the head of the radius in position. Its lower border is attached to the neck of the radius by looselydisposed fibres. It gives attachment to $(\mathrm{I})$ the external lateral ligament of the elbow-joint externally, (2) the outer fibres of the anterior ligament anteriorly, and (3) a portion of the posterior ligament posteriorly.

The synovial membrane is continuous with that of the elbow-joint, and lines the inner surface of the orbicular ligament and upper part of the neck of the radius.

Muscular Relations.-Extensor carpi radialis brevior and supinator radii brevis.

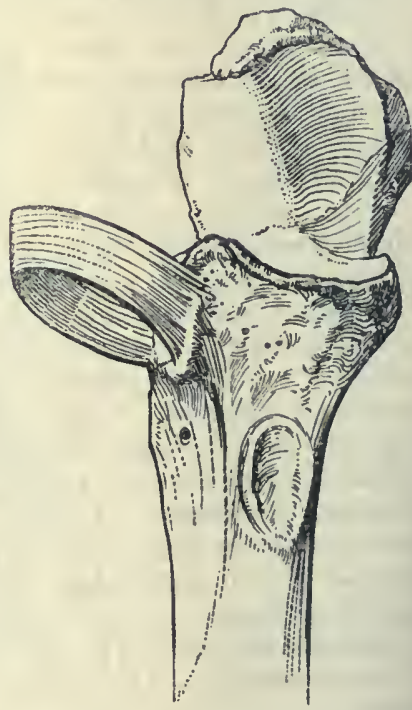

FIG. 218.-THE UPPER END OF THE ULNA, AND THE ORBICUlar Ligament.

Arterial Supply.-The radial recurrent and posterior interosseous recurrent arteries.

Nerve-supply.-The musculo-cutaneous and musculo-spiral nerves.

Inferior Radio-ulnar Joint.-This joint belongs to the class diarthrosis, and to the subdivision trochoides. The articular surfaces are the sigmoid cavity of the radius, and the outer side of the head of the ulna. The ligaments are anterior and posterior. and the triangular fibro-cartilage.

The anterior and posterior radio-ulnar ligaments consist of scattered fibres in front of and behind the joint, which are attached to the adjacent portions of the radius and ulna. The bones, however, 
are chiefly connected by the triangular fibro-cartilage, which is a strong plate. It is attached by its base to the ridge on the radius which separates the sigmoid cavity from the carpal articular surface, and by its apex to the pit on the lower end of the ulna at the root of the styloid process. It is thicker at the circumference than at the centre, and its anterior and posterior borders are connected with the anterior and posterior ligaments of the wrist-joint. Its upper surface is related to the lower end of the ulna, and its lower surface enters into the wrist-joint, articulating with the semilunar bone when the hand is in line with the forearm, and with the faceted part of the upper surface of the cuneiform bone when the hand is adducted. It sometimes presents a perforation.

The synovial membrane is very loose, and is known as the membrana sacciformis. It consists of two parts, vertical and horizontal, which are continuous with each other. The vertical part extends upwards between the head of the ulna and the sigmoid cavity of the radius, and the horizontal part lies between the lower end of the ulna and the upper surface of the triangular fibro-cartilage. When the fibro-cartilage is perforated the membrana sacciformis is continuous with the radio-carpal synovial membrane.

Arterial Supply.-The anterior and posterior ulnar carpal arteries.

Nerve-supply. - The anterior and posterior interosseous nerves.

Intermediate Connection between the Radius and Ulna.-This is a variety of syndesmosis. The shafts of the two bones are connected by two ligaments, namely, the interosseous membrane and the oblique ligament.

The interosseous membrane is a strong expansion which extends between the interosseous borders of the shafts of the radius and ulna. Its fibres pass principally downwards and outwards from the radius to the ulna, but some pass in the opposite direction. Superiorly it reaches to a point about I inch below the bicipital tuberosity of the radius, and inferiorly to the upper part of the sigmoid cavity of that bone. The posterior interosseous artery passes backwards between the two bones over its upper border, and the posterior branch of the anterior interosseous artery pierces it a little above its lower end. The membrane, besides connecting the shafts of the two bones, serves to give origin to muscles of the front and back of the forearm.

Relations-Anterior.-The flexor profundus digitorum and flexor longus pollicis, with the anterior interosseous vessels and nerve lying between the two, as low as the lower fourth, where the pronator quadratus forms the anterior relation. Posterior.-From above downwards, the supinator radii brevis, extensor ossis metacarpi pollicis, extensor brevis pollicis, extensor longus pollicis, extensor indicis, and, for a short distance below, the posterior interosseous nerve and posterior branch of the anterior interosseous artery.

The oblique ligament is a narrow band which extends from the tuberosity of the ulna to the radius, close below the lower and back part of the bicipital tuberosity. Its fibres are directed downwards 
and outwards. The interval between it and the upper border of the interosseous membrane gives passage to the posterior interosseous vessels.

Movements.-The movements allowed are pronation and-supination, the latter being the stronger. In pronation the lower part of the radius, carrying with it the hand, crosses over the lower part of the ulna so as to lie on its inner side, the dorsum of the hand being directed upwards. In supination the radius and hand retrace their steps, so that the lower part of the radius lies on the outer side of the ulna, the palm of the hand being directed upwards. At the superior radio-nlnar joint the head of the radius rotates upon the capitellum of the humerus within the orbicular ligament, and comes into closest relation with the capitellum in semiflexion of the elbow-joint and semipronation of the forearm. At the inferior radio-ulnar joint the radius, carrying with it the triangular fibro-cartilage, rotates upon the head of the ulna, the movement being one of limited circumduction around an axis passing from the centre of the head of the radius to the styloid process of the ulna. There is also a limited amount of circumduction on the part of the ulna, the lower end of that bone moving outwards and backwards in pronation, and inwards and forwards in supination.

Muscles concerned in the Movements-(I) Pronation.-The principal pronator muscles are the pronator radii teres and pronator quadratus. (2) Supination. -The principal supinator muscles are the biceps and supinator radii brevis. The brachio-radialis is also a feeble supinator, but it only acts as such to the extent of semisupination. The biceps is enabled to act as a supinator in virtue of its insertion in to the back part of the bicipital tuberosity of the radius.

\section{THE ARTICULATIONS OF THE HAND.}

Carpal Joints.-The carpal joints are divided into those of the first row, those of the second row, and the transverse carpal joint.

Joints of the First Row.-These belong to the class diarthrosis, and to the subdivision arthrodia. The bones of the first row, with the exception of the pisiform, are united by two dorsal, two palmar, and two interosseous ligaments. The dorsal and palmar ligaments extend transversely from the front and back of the semilunar to the corresponding surfaces of the scaphoid and cuneiform. The twc interosseous ligaments are placed one on either side of the semilunar. and they connect it with the scaphoid and cuneiform. They art situated high up on the lateral surfaces of the bones, and thei upper surfaces form part of the lower wall of the radio-carpal joint

The synovial membrane is continuous with that of the transvers carpal joint.

Pisiform Joint.-The pisiform bone is united to the front of th cuneiform by a capsular ligament, which surrounds the joint and $j$ attached to the bones at the circumference of their opposed articula surfaces. Superiorly the capsule is connected with the tendon c insertion of the flexor carpi ulnaris, and inforiorly with two prolong: tions of that tendon, called the pisi-uncinate and pisi-metacarp, ligaments. The former is attached to the hook of the unciform bon. and the latter to the base of the fifth metacarpal. These ligamen 
act by keeping the pisiform bone fixed during the action of the flexor carpi ulnaris.

The pisiform joint has a special synovial membrane.

Joints of the Second Row. - These belong to the class diarthrosis, and to the subdivision arthrodia. The four bones of the second row are connected by three dorsal, three palmar, and three interosseous ligaments, which are disposed similarly to those of the first row.

The synovial membrane is continuous with that of the transverse carpal joint.

Transverse Carpal Joint.-This is the joint between the first and second rows. It belongs to the class diarthrosis, and partly to

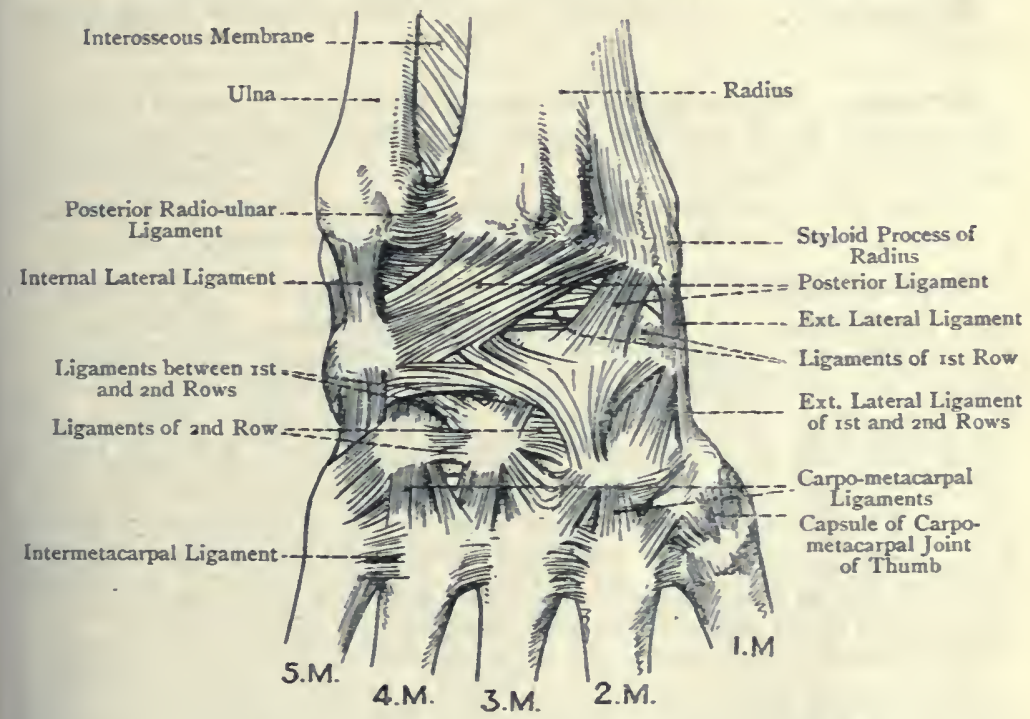

Fig. 219.-Ligaments of the Radio-carpal, Carpal, Carpo-metacarpal, AND INTERMETACARPAL JOINTS (POSTERIOR VIEW).

the subdivision arthrodia and partly to the subdivision condylarthrosis. The opposed surfaces of the two rows are each concavoconvex, the first in a direction from within outwards, and the second from without inwards. The convexity of the first row is formed by part of the scaphoid, and the concavity by part of the scaphoid, semilunar, and cuneiform. The concavity of the second row is formed by the trapezium and trapezoid, and the convexity by the head of the os magnum and part of the unciform. The two rows are connected by dorsal, palmar, and lateral ligaments. The dorsal ligaments are very indefinite and pass in different directions. The palmar ligaments for the most part radiate from the os magnum, 
though a few pass from the other bones of the second row. The external lateral ligament connects the scaphoid with the trapezium, and the internal lateral connects the cuneiform with the unciform.

Carpal Synovial Membrane.-This membrane lines the transverse carpal joint, and gives off vertically-disposed processes. Two of these pass upwards, one on either side of the semilunar as far as its interosseous ligaments. Three pass downwards-one between the trapezium and trapezoid, another between the trapezoid and os magnum, and a third between the os magnum and unciform. Thereafter the synovial membrane is continued into the inner four carpometacarpal joints, and this latter portion of it sends processes into the joints between the bases of the inner four metacarpal bones.

Arterial Supply.-The anterior and posterior carpal retia.

Nerve-supply.-The median, ulnar, and posterior interosseous nerves.

Movements.-The movements between the bones of each row are extremely limited, and are of a gliding nature. The movements at the transverse carpal joint are more free, and take the form of flexion and extension in association with the corresponding movements at the radio-carpal joint. The number of the carpal articulations imparts to this part of the hand considerable strength and elasticity, and so enables it to disperse shock.

Carpo-metacarpal Joints.-Inner Four Joints.-These belong to class diarthrosis, and to the subdivision arthrodia. The bones concerned are the trapezium, trapezoid, os magnum, and unciform above, and the bases of the inner four metacarpal bones below. The ligaments are dorsal, palmar, and interosseous. The second, third, and fourth metacarpal bones receive each two dorsal ligaments as a rule, and the fifth receives one, namely, from the unciform. The palmar ligaments are usually one to each bone. There is only one interosseous ligament, which connects the adjacent parts of the os magnum and unciform with the inner aspect of the base of the third metacarpal bone towards its palmar aspect.

The synovial membrane is a continuation of that of the carpus.

These joints derive their arterial supply from the radial and ulnar arteries, and their nerve-supply from the deep division of the ulnar, and posterior interosseous, nerves.

Movements. - The movements are those of flexion and extension, the former being most free in the case of the fifth metacarpal, as in the formation of the 'palmar cup.'

Carpo-metacarpal Joint of the Thumb. - This belongs to the class diarthrosis, and to that variety of the subdivision arthrodia which is called the reciprocal or sadale-joint. The bones which enter into this important joint are the trapezium and the base of the first metacarpal. The articular surfaces are saddle-shaped, and are connected by a capsular ligament, which is attached round the margin of each articular surface, being strongest on the dorsal and outer aspects. This ligamen $\mathrm{t}$ is sufficiently loose to allow of considerable movement. 
The synovial membrane is peculiar to the joint.

Arterial Supply. - The arteria princeps pollicis of the radial. Nerve-supply.-The median nerve.

Movements. - The movements allowed are flexion, extension, abduction, adduction, and circumduction. It is at this joint where the important movement of opposition'takes place, whereby the tip of the thumb can be opposed in succession to the tip of each of the four inner fingers.

Intermetacarpal Joints. - The basal intermetacarpal joints belong to the class diarthrosis, and to the subdivision arthrodia. The bones concerned are the inner four metacarpals, the first metacarpal bone standing off from the second, with which it has no articulation. The ligaments are dorsal, palmar, and interosseous. The dorsal ligaments are composed of stout fibres, which pass transversely between the contiguous aspects of the bases. The palmar ligaments are similarly disposed in front. The interosseous ligaments are strong bundles which pass between the opposed surfaces of the bases to which they are attached on the distal sides of the articular facets.

Nerve-supply. - The deep division of the ulnar nerve.

Arterial Supply.-The palmar and dorsal interosseous arteries.

The synovial membrane is a downward prolongation from that of the carpus.

The heads of the inner four metacarpal bones are connected by the transverse metacarpal (deep transverse) ligament. It extends transversely across the palmar aspects of the heads, and its fibres are attached to the fibrous plates on the palmar surfaces of the metacarpo-phalangeal joints. It receives the deep expansions of the digital processes of the central division of the palmar fascia, and the digital arteries and nerves pass in front of it, and the interosseous muscles behind it. It is to be noted that the transverse metacarpal ligament excludes the head of the first metacarpal bone, whereas the corresponding ligament of the foot includes the head of the first metatarsal.

The synovial membranes from the inferior radio-ulnar joint down to the intermetacarpal joints are five in number, as follows :

I. Inferior radio-ulnar or membrana sacciformis.

2. Radio-carpal.

3. Pisiform.

4. Carpal, composed of carpal proper, carpo-metacarpal, and intermetacarpal portions, all continuous with one another.

5. Carpo-metacarpal of the thumb.

Metacarpo-phalangeal Joints.-These belong to the class diarthrosis, and to the subdivision condylarthrosis, which is a modification of enarthrosis or ball-and-socket joint. In each joint the round head of a metacarpal bone articulates with the cup-shaped depression on the proximal end of a first phalanx.

Inner Four Metacarpo-phalangeal Joints.-Each of these joints has three ligaments - two lateral, and a palmar. The lateral 
metacarpo-phalangeal ligaments are strong bands which are attached above to the dorsal tubercle and palmar depression on either side of the head of a metacarpal bone, and below to each side of the base of a first phalanx, and also to the lateral margin of the palmar fibrous plate. Each is connected with a deep expansion of a digital process of the central division of the palmar fascia. The palmar ligament takes the form of a fibrous plate. It is closely attached at either side to the lateral ligaments, inferiorly to the palmar aspect of the base of the first phalanx, and superiorly, where it is connected with the transverse metacarpal ligament, it is slightly attached to the palmar aspect of the head of a metacarpal bone, close above the articular cartilage. It increases the extent of the phalangeal socket for the head of a metacarpal bone, and it forms part of the tunnel for the passage of a pair of flexor tendons. Its deep surface is lined by the synovial membrane of the joint. There is no dorsal ligament, its place being taken by the extensor tendon.

Each joint is provided with a synovial membrane, which is looser in front than behind.

Arterial Supply. - The digital arteries from the superficial palmar arch, or the palmar interosseous from the deep palmar arch.

Nerve-supply. - The deep division of the ulnar nerve.

Movements. - The movements are flexion, extension, abduction, adduction, and circumduction. Flexion is particularly free, and is combined with adduction. Extension is associated with abduction, and dorsal flexion is prevented by the palmar fibrous plates and flexor tendons.

Metacarpo-phalangeal Joint of the Thumb.-The lateral ligaments of this joint are similar to those of the other joints, but there is no palmar fibrous plate, its place being taken by two sesamoid bones. Each of these bones resembles a split pea. The palmar surface is convex, whilst the deep surface is almost flat and covered by cartilage, to play upon a groove on the palmar surface of the head of the first metacarpal bone. The two bones are united by fibres which pass between their contiguous surfaces, and are related superficially to the tendon of the flexor longus pollicis. Inferiorly they are connected by fibres with the palmar aspect of the base of the first phalanx. At either side they are connected with the lateral ligaments, and posteriorly they are slightly connected with the head of the first metacarpal above the articular cartilage. The outer sesamoid bone is closely associated with the superficial head of the flexor brevis pollicis, and the inner with the adductor obliquus pollicis. The place of a dorsal ligament is taken by the tendons of the extensor brevis, and extensor longus, pollicis.

Arterial Supply.- The arteria princeps pollicis of the radial.

Nerve-supply. - The median nerve.

Movements.-Flexion, extension, and lateral movement, the latter only when the joint is partially flexed.

Interphalangeal Joints. - These belong to the class diarthrosis and to the subdivision ginglymus. The ligaments are similar t 
those of the inner four metacarpo-phalangeal joints, namely, two lateral, and an anterior fibrous plate, the extensor tendon taking the place of a dorsal ligament. Each joint is provided with a synovial membrane, and the arterial and nerve supply are derived from the digital arteries and nerves. Each of the inner four fingers has two interphalangeal joints, but the thumb, being destitute of a middle phalanx, has only one, and its dorsal ligament is represented by the tendon of the extensor longus pollicis, whilst its fibrous plate is grooved by the tendon of the flexor longus pollicis.

Movements.-The only movements allowed are flexion and extension, the former being very free.

Joints with Interarticular Fibro-cartilages. - The joints of the upper limb which have interarticular fibro-cartilages are as follows:

I. The sterno-clavicular joint.

2. The acromio-clavicular joint (inconstant).

3. The triangular fibro-cartilage of the inferior radio-ulnar articulation is to be regarded as an interarticular fibro-cartilage between the ulna and cuneiform bone at the radio-carpal joint.

The shoulder-joint has a circumferential fibro-cartilage, namely, the glenoid ligament.

\section{GUIDE TO THE UPPER LIMB.}

The Back.-To remove the skin, the following incisions may be made: one along the middle line from the seventh cervical spine to the second sacral spine ; another from the upper end of this incision outwards to the acromion process, and thence downwards over the back of the shoulder in a curved manner to meet the posterior fold of the axilla; and a third from the lower end of the mesial incision outwards along the iliac crest. The cutaneous nerves will be found near the spines of the upper six thoracic vertebræ and near the angles of the lower six ribs. Three branches from the first three lumbar nerves are to be shown descending over the iliac crest to the gluteal region.

The trapezius muscle is to be cleaned, and, in doing so, as well as in the deep stages of this dissection, it is advisable that the dissector of the upper limb should work in concert with the dissector of the head. The spinal accessory nerve will be found passing beneath the anterior border of the trapezius. the latissimus dorsi is next to be cleaned, and turning backwards round its outer border will be found the posterior offsets of the lateral cutaneous branches of the intercostal nerves. Between the contiguous borders of the latissimus dorsi and obliquus externus abdominis, just above the centre of the iliac crest, the triangle of Petit is to be shown. The region between the trapezius, latissimus dorsi, and base of the scapula, and the parts contained therein, are to be studied. The trapezius is to be divided about I inch from the spines of the vertebræ, and the spinal accessory nerve, with branches of the third and fourth cervical nerves, and the superficial cervical artery, are to be dissected on its deep surface. The latissimus dorsi is to be divided by an incision carried downwards from its upper border about 3 inches from the spines of the vertebra to the back part of the iliac crest internal to the iliac origin of the muscle, so as to leave undisturbed its slips of origin from the lower three or four ribs, as well as its iliac origin. In reflecting inwards the inner portion of the muscle care is to be taken not to injure the serratus posticus inferior, and, in turning outwards the outer portion, the serratus magnus is not to be interfered with.

The levator anguli scapulæ and rhombaid muscles are to be dissected, and the nerve to the rhomboids is to be looked for deeply between these two 
muscles about 1 inch from the base of the scapula. The disposition of the rhomboideus major at its insertion is to be noted, and the fibrous band or arch shown. The levator anguli scapulæ is to be divided about its centre, and the rhomboids about $I$ inch from the spines of the vertebræ, which will bring into view the posterior scapular artery lying close to the base of the scapula. A branch to the levator anguli scapulæ from the nerve to the rhomboids is to be looked for, and a very limited view will be obtained of the posterior belly of the omo-hyoid at the upper border of the scapula. In association with it the suprascapular nerve and artery may just be seen, but this dissection is not to be pushed.

The serratus posticus superior is next to be dissected, followed by that of the serratus posticus inferior, and before reflecting these muscles the vertebral aponeurosis is to be studied, and the posterior lamina of the lumbar aponeurosis, which gives origin to the latissimus dorsi and serratus posticus inferior. At this stage the internal or deep surface of the serratus magnus is to be examined. The serratus posticus superior is to be cut about I inch from the spines of the vertebræ, and each serration of the serratus posticus inferior is to be divided just below the rib into which it is inserted. The vertebral aponeurosis is also to be carefully removed. In this manner the erector spinæ muscle is exposed. Before dissecting this muscle, however, the splenius is to be studied, in concert with the dissector of the head, and divided about 1 inch from its origin. Underneath the splenius will be found the complexus, the inner portion of which is known as the biventer cervicis, and external to the complexus is the narrow, ribbon-like trachelo-mastoid or transversalis capitis. The dissector should now replace over the lower part of the erector spinæ the posterior lamina of the lumbar aponeurosis, having at tached to it the latissimus dorsi and serratus posticus inferior. At the outer border of the erector spinæ he should notice a portion of the middle lamina of the lumbar aponeurosis, of which more will come into view by raising the outer border of the muscle. He will thus see that the lower part of the erector spinæ is enclosed in a sheath, the posterior wall of which is formed by the posterior lamina, and the anterior wall by the middle lamina, of the lumbar aponeurosis. The anterior lamina is not visible at this stage. The obliquus internus abdominis may be seen to arise from the lumbar aponeurosis between the iliac crest and the last rib.

The dissection of the erector spinæ is now to be proceeded with. The separation between the outer and middle columns of the muscle will be apparent a little below the twelfth rib as a cellular interval, through which nerves emerge, and in line with which, higher up, nerves, accompanied by arteries and veins, also appear. Dealing first with the outer column, and dissecting it from below upwards, the slips of insertion of the ilio-costalis into the angles of the lower six ribs are to be shown. These are then to be turned outwards, and the slips of origin of the musculus accessorius lying internal to them are to be displayed, followed by the slips of insertion of that muscle in to the upper ribs. The latter having been turned outwards, the slips of origin of the cervicalis ascendens will be found, and this muscle is to be followed up into the neck, where its three slips of insertion are to be brought out. In dealing with the middle column the dissector should first artificially separate from it the inner column, the connection between the two being very close, and he may at once dispose of the inner column or spinalis dorsi. Its four fleshy and telldinous origins below should be shown, and its tendons of insertion above, varying from four to eight in number. The series of arches formed by the tendons of this muscle should be noted. The middle column is next to be dissected, and the two series of insertions of the longissimus dorsi shown, the outer, fleshy, passing to the lumbar transverse processes and ribs, and the inner, round and tendinous, passing to the lumbar accessory processes and thoracic transverse processes. At the upper part of the back the dissector will find that the longissimus dorsi is prolonged in to the neck by the transversalis cervicis, and to the head by the trachelo-mastoid. It is at this stage that the complexis is most advantageously studied. This muscle having been cut high up by the dissector of the head, the semispinalis dorsi and semispinalis colli are to be 
dissected. The semispinales and longissimus dorsi having been removed, the multifidus spinæ is to be studied, and an effort made to show its deepest fibres, called the rotatores spinæ. The levatores costarum are to be carefully dissected, and the intertransversales and interspinales are to be looked to, chiefly in the cervical and lumbar regions.

Pectoral Region and Axillary Space.-To remove the skin, the following incisions should be made : a mesial incision along the sternum; another from the upper end of this along the clavicle to the acromion process, and thence downwards over the front of the shoulder to the inner side of the arm close to the anterior fold of the axilla; and a third transversely outwards from the lower end of the sternum. In the case of a female subject, a circular incision should be made around the inargin of the areola. In removing the skin from over the mammary gland the fibrous processes, known as the ligamenta suspensoria of Cooper, which pass between the superficial fascia in front of the gland and the skin, are to be noted. The following cutaneous nerves are to be displayed: (I) the suprasternal, supraclavicular, and supra-acromial branches of the cervical plexus, which descend over the clavicle beneath the platysma myoides; (2) the anterior cutaneous branches of the intercostal nerves, which emerge through the upper six intercostal spaces close to the sternum; and (3) the anterior offsets of the lateral cutaneous branches of intercostal nerves below the second, which turn round the anterior fold of the axilla.

If the subject is a female, the mammary gland should now receive careful attention. Its relation to the superficial fascia, which ensheathes it, is to be studied, and also its relation to the deep fascia covering the pectoralis major. The thin skin of the areola is to be carefully raised towards the nipple, and the galactophorous ducts, each presenting a dilatation or ampulla, are to be shown. An endeavour should be made to display the glandular structure and loculi.

The pectoralis major and anterior portion of the deltoid are to be dissected, and in the groove between the two the cephalic vein and humeral branch of the acromio-thoracic artery are to be shown. Lying deeply in the upper part of this groove, just below the clavicle, the infraclavicular glands, two or three in number, are to be looked for. The clavicular part of the pectoralis major should now be divided and reflected. In doing so, the cephalic vein, external anterior thoracic nerve, and branches of the acromio-thoracic artery are to be preserved. The region now being dissected (infraclavicular) lies between the clavicle, pectoralis minor, and upper border of the sterno-costal portion of the pectoralis major. The costo-coracoid membrane, which is a part of the clavi-pectoral fascia, is to be shown, and the stout portion of it, called the costo-coracoid ligament, is to be noted.

Having studied the connections of the costo-coracoid membrane and the various structures piercing it, the membrane, along with the axillary sheath beneath it, is to be carefully removed, and the first part of the axillary artery, with its branches, the axillary vein, and the trunks of the brachial plexus are to be displayed. The internal anterior thoracic nerve will be found coming forwards between the artery and the vein, and a communication between it and the external anterior thoracic nerve is to be looked for over the artery. The posterior thoracic nerve, which lies behind the artery, is to be carefully preserved. The removal of the costo-coracoid membrane will also expose the subclavius muscle. Without further disturbing the pectoralis major meanwhile, the axillary space is now to be dissected from below. The axillary fascia forming the floor of the space, and its relations to the fascial investments of the pectoralis major and latissimus dorsi and fascia of the arm are to be noted. The fascia will be seen to be drawn up towards the space, this being due to the insertion of the clavi-pectoral fascia into its upper surface. The axillary fascia having been dissected, the adipose tissue in the space is to be removed with the greatest care. The lateral cutaneous branches of the intercostal nerves (except the first) will be found on the inner wall between the serrations of the serratus magnus. The lateral cutaneous branch of the second intercostal is to be followed as the intercosto-humeral nerve across the space 
to the inner and back part of the arm. The lateral cutaneous branches of the succeeding intercostal nerves are to be shown in two divisious-anterior and posterior. The axillary glands are to be carefully looked for. They will be found in three groups, namely, pectoral, within the anterior fold and on the adjacent part of the inner wall; subscapular, on the posterior wall; and external, on the outer wall. The posterior thoracic nerve is to be followed out upon the serratus magnus, the long thoracic artery will be found along the lower border of the pectoralis minor, and the subscapular artery on the posterior wall. The dorsalis scapulæ branch of the subscapular artery is to be shown. The alar thoracic is seldom a special artery. The middle or long and lower subscapular nerves will be found on the posterior wall, the former, with the subscapular artery, going to the latissimus dorsi, the latter, farther out, supplying the teres major and adjacent part of the subscapularis. The upper or short subscapular nerve should not be looked for at present, as it lies too high up. The structures along the outer wall are next to be shown, namely, the axillary artery, giving off its subscapular, anterior circumflex, and posterior circumflex branches; the axillary vein; and the nerves arising from the cords of the brachial plexus. The internal cutaneous branch of the musculo-spiral nerve should be shown at this stage, and preserved. When the tributaries of the axillary vein have been noted in the course of the foregoing dissection they should be removed.

The sterno-costal portion of the pectoralis major is now to be divided and reflected, in doing which twigs of the internal anterior thoracic nerve will be seen entering its deep surface, after having pierced the pectoralis minor. The latter muscle is now to be dissected, the fascia, removed in preparing it, being continuous above with the costo-coracoid membrane, and being inserted below into the upper surface of the axillary fascia. The internal anterior thoracic nerve is to be shown entering the deep surface of the muscle. The pectoralis minor having been cut, the axillary space will be fully exposed. The second part of the axillary artery is to be dissected, and it will be found, as a rule, to give off the long thoracic, but the alar thoracic is very inconstant. The cords of the brachial plexus and their relation to the second part of the artery are to be noted, and the npper or short subscapular nerve may now be seen at the upper part of the posterior wall of the space, where it immediately enters the upper part of the subscapularis. The origins of the branches of the cords of the brachial plexus are to be shown as follows: external anterior thoracic, musculo-cutaneous, and outer. root of the median from the outer cord ; internal anterior thoracic, lesser internal cutaneous or nerve of Wrisberg, the internal cutaneous, inner root of the median, and ulnar from the inner cord; and the three subscapular nerves, circumflex, and musculo-spiral from the posterior cord.

The next duty of the dissector is to study the sterno-clavicular joint. Thereafter the clavicle is to be sawn through at its centre, and the subclavius muscle divided. At this stage the dissectors of the upper limb and head should work in concert. A full view will be obtained of the continuity between the subclavian and axillary arteries, and the nerve trunks and individual nerves of the brachial plexus, as well as the supra-clavicular branches of that plexus. The suprascapular artery and nerve, and the posterior belly of the omo-hyoid will also be seen. The vessels and nerves are then all to be included in two ligatures placed $\mathrm{I}$ inch apart, and divided between them, and the lower ligature is to be firmly secured to the ou ter cut end of the clavicle. The serratus magnus is to be studied, and it will be put upon the stretch if the shoulder is pressed outwards. The limb is then to be removed by dividing the serratus magnus, posterior belly of the omo-hyoid, and levator anguli scapulæ, if the latter has not been already cut.

Scapular Region.-After removal of the limb, the dissector is to trim the various scapular muscles already dissected, and the arrangement of the tenclon of insertion of the pectoralis major is to be shown. The shoulder being supported on a block, the skin is to be removed from over the deltoid, and the cutaneous nerves shown, namely, the supra-acromial over the upper third, and a large cutaneous branch of the circumflex turning round the posterion 
border of the muscle near its centre. In cleaning this border the relation to it of the deep fascia over the infraspinatus is to be observed. The deltoid having been fully dissected, and twigs of the circumflex nerve which pierce it having been noted, the muscle is to be divided about I inch below its origin. In turning it down the subacromial bursa and the circumflex nerve and posterior circumflex artery are to be shown, the latter two entering its deep surface.

The infraspinatus', teres minor, teres major, and long head of the triceps are then to be dissected, and the gangliform enlargement on the branch of the circumflex nerve to the teres minor is to be observed. The quadrangular and triangular muscular spaces, with their contents, are to be exposed, and the substitution of the subscapularis as a boundary in front for the teres minor behind is to be noted. The dorsalis scapulæ artery is to be shown winding round the axillary border of the scapula through the origin of the teres minor. The supraspinatus is next to be dissected, and, to follow the muscle to its insertion, the acromion process may be sawn through. At the upper border of the muscle will be found the posterior belly of the omo-hyoid and the suprascapular nerve and artery. The subscapularis is to be cleaned, and at this stage the upper or short subscapular nerve will be fully seen.

The supraspinatus, infraspinatus, and teres minor are to be stripped carefully from the bone and dissected up to their insertions, in doing which the intimate connection between their tendons and the upper and back parts of the capsule of the shoulder-joint will arrest attention. Between the infraspinatus tendon and the capsule a small bursa may be found. The careful removal of these muscles will allow the dissector to follow out the suprascapular artery and nerve, and the dorsalis scapulæ artery. The subscapularis is also to be stripped from the bone and dissected to its insertion, to do which the muscle must be raised from beneath the coraco-brachialis and short head of the biceps. The close connection between its tendon and the front of the capsule is to be noted, as well as the opening in the capsule through which the synovial membrane protrudes to form the subscapular bursa. The teres major and latissimus dorsi are to be followed to their insertions, the varying relations between the two are to be made clear, the intervening bursa shown, and in some cases a small bursa behind the tendon of the teres major close to the bone. Before leaving this region the dissector should carefully study the scapular anastomoses of arteries, the muscular relations of the capsule of the shoulder-joint, and the actions of the scapular muscles, with their nerve-supply.

Cutaneous Nerves and Veins of the Arm and Forearm.-The skin should be at once reflected as low as the wrist by a median incision down the front of the limb, and transverse incisions at the elbow and wrist. In reflecting it from over the back of the olecranon process the subcutaneous bursa, there situated, is to be attended to. The following cutaneous nerves are to be followed out to their distribution, care being taken to preserve the cutaneous veins: (I) the intercosto-humeral to the inner and back part of the arm in its upper half ; $(2)$ the internal cutaneous of the mnsculo-spiral to the back of the arm; (3) the nerve of Wrisberg to the lower half of the arm on its inner aspect ; (4) twigs of the internal cutaneous to the front of the arm in its upper part ; and (5) the upper external cutaneous branch of the musculo-spiral (which appears a little below the centre of the outer side of the arm) to the outer side and front of the arm over about its lower half. At the junction of the upper two-thirds and lower third of the arm on its inner side the internal cutaneous nerve will be found piercing the deep fascia in two divisions, separately or conjointly, and from this point the two divisions, anterior and posterior, are to be followed downwards along the inner part of the forearm, as low as the wrist in the case of the anterior. The lower external cutaneous branch of the musculo-spiral is to be followed from the outer side of the arm, a little below the centre, down the back of the outer side of the forearm as low as the wrist. The cutaneous part of the musculo-cutaneous will be found at the outer border of the biceps a little above the elbow, and it is to be traced down the outer side of the forearm as low as the wrist in two divisions, anterior and posterior.

In front of the forearm, just below the centre and internal to the middle line, 
a cutaneous branch of the ulnar nerve may be met with, and towards the wrist the palmar cutaneous branches of the median and ulnar are to be shown. In the lower third of the forearm, posteriorly and on its outer aspect, the radial nerve is to be shown, as well as the dorsal branch of the ulnar nerve on its inner aspect. The cutaneous veins to be dissected are the radial, median, and anterior and posterior ulnar veins in the forearm; the median basilic and median cephalic at the bend of the elbow, with the deep median joining the superficial median close to its termination; and the basilic and cephalic in the arm, the basilic being superficial to the deep fascia in the lower half, but subsequently piercing it, and the cephalic being superficial throughout. In connection with the median basilic vein the semilunar fascia of the biceps is to be shown, and one or two supracondylar glands are to be carefully looked for a little above the internal epicondyle, close to the basilic vein.

Front and Inner Side of the Brachial Region.-The deep fascia of the arm is to be studied, and, as the dissection proceeds, the external and internal intermuscular septa, with the structures related to them, are to be noted. The biceps and coraco-brachialis are to be dissected, and, in connection with the tendon of insertion of the latter muscle, a fibrous band should be looked for passing upwards to the humerus below the sinall tuberosity, and forming an arch over the latissimus dorsi and teres major. The in ternal brachial ligament of Struthers is to be looked for, extending from the humerus near the insertion of the teres major to the internal epicondyle. The musculo-cutaneous nerve is to be shown piercing the coraco-brachialis, and, by raising the biceps, the nerve is to be followed between that muscle and the brachialis anticus to the outer border of the biceps, where it becomes cutaneous, and its muscular branches are to be shown. It may be found to give a communicating branch to the median. The semilunar fascia of the biceps is to be carefully dissected, and its relation to the brachial artery and median basilic vein is to be observed.

The brachial artery is next to be dissected, and the venæ comites on either side of it shown. These will be found to join above, and terminate in the lower part of the axillary vein. The median nerve is to be shown lying at first on the outer side of the artery, then crossing it just below the centre of the arm, and thereafter lying on its inner side. It gives off no branches in the arm, but it may receive one from the musculo-cutaneous. The branches of the brachial artery are to be shown as follows: (I) the superior profunda arises high up from the inner and back part of the vessel, and accompanies the musculo-spiral nerve to the back of the arm; (2) the inferior profunda arises from the inner side a little lower down than the preceding, and accompanies the ulnar nerve through the internal intermuscular septum to the interval between the internal epicondyle and the olecranon ; (3) the nutrient or medullary artery arises from the inner side about the lower border of the tendon of insertion of the coraco-brachialis, and passes downwards to enter the medullary foramen of the humerus: (4) the anastomotica magna arises from the inner side about 2 inches above the elbow, and divides into two branches-anterior, passing downwards in front of the internal epicondyle beneath the pronator radii teres, and posterior, piercing the internal intermuscular septum to reach the interval between the internal epicondyle and the olecranon; and (5) several muscular branches arising from the outer side of the vessel.

The inusculo-spiral nerve is to be shown lying, for a little, behind the upper part of the brachial artery, and the branches which it here gives off, namely, internal cutaneous to the back of the arm, and muscular to the long and internal heads of the triceps, are to be noted. One of the latter descends with the ulnar nerve, as the ulnar collateral nerve of Krause, to enter the internal head of the muscle low down. The ulnar nerve is to be dissected as low as the internal epicondyle and the olecranon, and it is to be slown piercing the internal intermuscular septum. It gives off no branches in the arm. The internal cutaneous nerve and the nerve of Wrisberg are also to be noted. The brachialis anticus is to be dissected by displacing the biceps outwards, and it i: to be separated from the brachio-radialis. In this way a part of the musculo spiral nerve will be exposed, and it is to be followed carefully downwards to near the external epicondyle, where its two terminal branches, radial anc 
posterior interosseous, are to be shown. In this part of its course the nerve will be found to furnish branches to the brachio-radialis and extensor carpi radialis longior, and a twig to the brachialis anticus, the latter arising high up in the spiral groove. Descending with the musculo-spiral nerve in this groove will be found the anterior terminal branch of the superior profunda artery, and coming up from below to anastomose with it a branch of the radial recurrent.

The anticubital space or triangular hollow in front of the elbow is next to be dissected, and its roof, floor, boundaries, and contents carefully studied. If the brachio-radialis is held aside, the posterior interosseous nerve will be seen piercing the supinator radii brevis; and, if the superficial head of the pronator radii teres is raised, the anterior ulnar recurrent artery, of small size, will be found ascending to the front of the internal epicondyle, where it anastomoses with the anterior branch of the anastomotica magna. The mode of insertion of the tendon of the biceps is to be carefully noted, and its action in this connection studied.

Back of the Brachial Region.- The triceps is to be dissected, and its three heads clearly shown. The long head is obvious, but the external and internal heads require careful dissection. The dissector should pull upon the musculospiral nerve to make evident its winding course round the back of the humerus, and he should then make an incision through the muscle over the course of the nerve, avoiding its long head. When the cut parts of the muscle are separated, the spiral groove of the humerus is laid bare, with the musculospiral nerve and superior profunda artery lying in it. The portion of the muscle above and external to the groove is the external head, and the small, peaked portion inside the groove and all the fibres arising from the back of the humerus below the groove represent the internal head.

The branches of the musculo-spiral nerve behind the humerus are to be shown, and one long branch to the anconeus, which descends in the internal head of the triceps, is to be followed out. The nerve, on leaving the spiral groove, will be seen to pierce the external intermuscular septum from behind forwards, along with the anterior branch of the superior profunda artery. This artery is also to be dissected, and its posterior terminal branch is to be shown descending behind the external intermuscular septum to the back of the external epicondyle, where it anastomoses with the posterior interosseous recurrent. The internal head of the triceps may now be cut into inferiorly, in order to show its deepest fibres, under the name of the subanconeus, taking insertion into the back part of the capsule of the elbow-joint.

Acromio-clavicular Joint. - In connection with this joint the coracoclavicular ligament is to be displayed in two parts-conoid and trapezoidand between these will be found a little fat and a small bursa. The ligaments of the joint itself are next to be dissected, and, when the joint is opened, an incomplete interarticular fibro-cartilage may be found within it at the upper part.

Special Ligaments of the Scapula.-The suprascapular or transverse ligament is to be made evident, with the suprascapular nerve-passing backwards beneath it, the suprascapular artery over it, and fibres of the posterior belly of the omohyoid arising from it. The coraco-acromial or deltoid ligament is next to be dissected, and its relation to the capsule of the shoulder-joint carefully noted. The spino-glenoid ligament is to be shown passing between the outer border of the spine and the adjacent part of the margin of the glenoid cavity. It will be seen to bridge over the suprascapular artery and nerve on their way to the infraspinous fossa.

Shoulder-Joint.-The dissector should first revise the subacromial bursa and the muscular relations of the capsule of this joint. The capsular ligament is then to be studied, and the coraco-humeral ligament is to be noted incorporated with its upper aspect. Two openings in the capsule are to be observed. One is situated in front, behind the upper border of the tendon of the subscapularis, and through this opening the synovial membrane will be found protruding to form the subscapular bursa. The other opening is situated between the tuberosities of the humerus. at the entrance to the bi- 
cipital groove, this being for the passage of the long tendon of the biceps. The transverse humeral ligament will be found bridging over this part of the bicipital groove. The joint should now be opened by removing the posterior part of the capsule, and the three gleno-humeral ligaments are to be looked for. They are situated on the inner and anterior aspect of the capsule. The front part of the capsule should now be removed and the glenoid ligament examined. The tendon of the long head of the biceps, as it arches over the head of the humerus, is to be noted, and its relation to the glenoid ligament at the a pex of the glenoid cavity is to be shown. The synovial membrane and movements are then to be studied.

Front of the Forearm.-The deep fascia is to be examined and removed, except the part covering the muscles arising from the internal epicondyle. These muscles are to be dissected, from without inwards, in the following order : pronator radii teres, flexor carpi radialis, palmaris longus, flexor carpi ulnaris, and flexor sublimis digitorum. Having noted the common tendon of origin of these muscles, what is left of the deep fascia is to be removed, and the muscles carefully separated from each other up to their origins. In doing so, the strong intermuscular septa are to be noted. The small, deep head of the pronator radii teres is to be displayed, with the median nerve passing between the two heads of the muscle, and the ulnar artery beneath its deep head, and it should be noted that the median nerve, which is at first internal to the ulnar artery, crosses it to get to its outer side.

The muscular branclies of the median nerve are to be caught high up, as well as its anterior interosseous branch, in connection with which latter care is to be taken to preserve the median branch of the anterior interosseous artery, which is usually of small size. The ulnar nerve is to be shown entering the forearm between the two heads of the flexor carpi ulnaris, where it lies between the internal epicondyle and olecranon, and gives off articular twigs to the elbow-joint. Thereafter its branches to the flexor carpi ulnaris and inner portion of the flexor profundus digitorum should be displayed high up.

The radial artery is next to be dissected as low as the wrist, its venæ comites being noted. The branches to be shown are the radial recurrent, close to the origin, muscular down the forearm, and anterior radial carpal and superficial volar near the wrist. The radial nerve is also to be dissected until it turns to the back of the forearm beneath the tendon of the brachioradialis. The ulnar artery is then to be dissected as low as the wrist, and, descending over its lower half, the palmar cutaneous branch of the ulnar nerve is to be preserved. The branches of the vessel to be shown are the anterior and posterior ulnar recurrents, the interosseous trunk, soon divicling into anterior and posterior interosseous, the former of which gives off the median artery (all of these branches arising high up), muscular down the forearm, and posterior and anterior ulnar carpals near the wrist. The posterior interosseous artery is to be shown passing backwards between the radius and ulna, and the median branch of the anterior interosseous artery is to be carefully studied, as it is sometimes of large size and may be continued into the palm to join the superficial palmar arcli. The ulnar nerve is to be dissected as low as the wrist. Its occasional branch to the front of the forearm below the centre is to be looked for, and the palmar cutaneous and dorsal branches are to be shown, the former arising a little below the centre of the forearm and the latter about 2 inches above the wrist, after which it turns backwards beneath the tendon of the flexor carpi ulnaris.

The flexor sublimis digitorum is then to be raised and held aside, to show the median nerve, with the median artery, descending in close contact with its deep surface as far as a little above the wrist, where the nerve comes to lie on the outer side of the muscle, and gives off its palmar cutancous branch. At this stage the dissector should carefully note that the great palmar bursa is prolonged upwards round the nexor tendons for fully an inch above the anterior annular ligament, and the arrangement of the superficial flexor tendons in pairs before they pass beneath that ligament is to be shown. By pulling upon the individual tendons, those of the anterior pair will be seen 
to belong to the middle and ring fingers, and those of the posterior pair to the index and little fingers.

The deep muscles of the front of the forearm are next to be dissected, namely, the flexor profundus digitorum, flexor longus pollicis, and pronator quadratus. The anterior interosseous nerve and artery, the latter having two venæcomites, are to be followed down the front of the interosseous membrane beneath the pronator quadratus. In this latter situation the nerve should be shown to supply the pronator quadratus, and give an articular branch to the wristjoint. The artery should be here shown to divide into anterior and posterior branches, the former descending to take part in the anterior carpal rete, and the latter piercing the interosseous membrane to reach the posterior carpal rete.

Front of the Hand.-The various landmarks are to be carefully studied. Thereafter the skin is to be removed by a median incision from the centre of the wrist to the cleft between the middle and ring fingers, and a transverse incision across the roots of the fingers. Median incisions are also to be made down the centre of the thumb and each finger, and the skin removed from these parts. In removing the skin from the palm fibrous processes will come into view, which connect it with the central division of the palmar fascia. The lobulated condition of the superficial fascia of the palm is to be noted, and the superficial transverse ligament is to be looked for as a few scattered transverse fibres lying within the skin at the roots of the fingers. The palmar cutaneous branches of the median and ulnar nerves are to be followed out, and twigs of the radial nerve, reinforced by twigs from the musculo-cutaneous, are to be shown over the thenar eminence. The palmaris brevis muscle is to be carefully dissected, as it is subcutaneous and arranged in bundles. The expansion from the tendon of the flexor carpi ulnaris to the anterior annular ligament is to be noted, as well as the pisi-uncinate and pisi-metacarpal expansions, which are known as ligaments. The ulnar artery and nerve are then to be followed over the anterior annular ligament till they disappear beneath the palmaris brevis, and their relation to the pisiform bone and hook of the unciform are to be noted.

The palmar fascia should now receive careful attention. Its thenar and hypothenar divisions, which are thin, are to be noted, but attention is to be concentrated on the central division. Its triangular shape, great strength, and the longitudinal direction of most of its fibres are to be observed. Some of the fibres, however, will be seen to be disposed transversely, especially towards the roots of the fingers. In this latter situation it should be shown dividing into four digital processes, which pass to become incorporated with the sheaths of the flexor tendons of the inner four fingers. The thenar and hypothenar divisions of the fascia are removable with ordinary care in order to expose the muscles, and no important structure is in danger. The central division, however, must be removed with the greatest care. In doing so the dissector should particularly note its great strength, which will show him how it would resist the pointing of a palmar abscess, and the necessity of early incision to give vent to the pus. As this division is being removed a deep lateral expansion is to be looked for at either side of it, these expansions passing to join the more deeply placed interosseous fascia. The result is that a great central fibrous tunnel is formed in the palm, which contains the superficial palmar arch, digital arteries and nerves, and flexor tendons, all enclosed in the great palmar bursa. In reflecting the digital processes of the central division deep lateral expansions should be looked for, which, along with them and the anterior fibrous plates of the metacarpo-phalangeal joints, form short tunnels for each pair of flexor tendons.

On removal of the central division of the palmar fascia the great palmar bursa is to be thoroughly mastered. The superficial palmar arch is then to be dissected, and its position and branches are to be carefully studied. The profunda branch is to be shown coming off from the commencement (inner part) of the arch, and, along with the deep division of the ulnar nerve, it will be seen to disappear between the abductor, and flexor brevis, minimi digiti. Four digital arteries are to be followed from the convexity of the 
arch, the inner one being single and the other three compound, for the supply of the inner three and a half fingers. It should be noted that the digita arteries, in passing to the fingers, lie over the interosseous spaces and super. ficial to the digital nerves. About the centre of the palm the inner artery will be found to be reinforced by a communicating branch from the deeply placed innermost palmar interosseous, and the outer three arteries, close to their points of bifurcation, will be found to be joined each by a palmar inter osseous artery. The possible presence of a large median artery joining th superficial palmar arch is to be borne in mind.

The median and ulnar nerves are next to be dissected in the palm as far a: the roots of the fingers. The median nerve, after emerging from beneath th anterior annular ligament, will be found to present an enlargement, and thei to break up into outer and inner divisions. The outer division is to be show giving off a muscular branch which supplies the abductor pollicis, opponen pollicis, and superficial head of the flexor brevis pollicis, and then to arrang itself into three single digital nerves to both sides of the thumb and outer sid of the index finger, the last branch giving a twig to the first or outermos lumbricalis muscle. The inner division furnishes two compound digital nerves each of which divides near the clefts of the fingers into two branches for th supply of the contiguous sides of the index and middle, and middle and rin fingers. The compound digital nerve to the cleft between the index anc middle fingers will be found to give a twig to the second lumbricalis, and tha to the cleft between the middle and ring fingers communicates by a cros branch with the compound digital branch of the ulnar nerve to the clef between the ring and little fingers.

The ulnar nerve is next to be dissected as far as the fingers. Its division into a superficial and deep branch is to be shown, the latter accompanyin the profunda artery between the abductor, and flexor brevis, minimi digiti The superficial division will be found to supply the palmaris brevis an then to divide into two digital nerves-an inner single to the inner side o the little finger, and an outer compound for the supply of the contiguous side: of the little and ring fingers. The digital arteries and nerves are now to be followed along the sides of the fingers, where the nerves will be seen to be superficial to the arteries, and to present minute swellings, called Paciniar bodies. In dissecting the digital nerves on the outer side of the index finge and both sides of the thumb the arteria radialis indicis and the two brancher of the arteria princeps pollicis are to be shown.

The sheaths of the flexor tendons are now to be examined. The strong almost cartilaginous, vaginal ligaments over the first and second phalanges are to be noted, and the weak membrane opposite the interphalangeal joints, with its obliquely-decussating fibres, is to be shown. The sheath being opened, the synovial lining is to be studied, and the insertions of the superficial and deep flexor tendons are to be examined. In connection with the synovial lining the vincula accessoria tendinum are to be displayed, namely, the ligamenta longa and ligamenta brevia. The sheath of the tendon of the flexor longus pollicis is to be examined in a similar manner. The relations of the synovia? sheaths of the flexor tendons on the digits to the great palmar bursa are to be carefully studied.

The thenar muscles are now to be dissected. The most superficial is the abductor pollicis, and beneath this is the opponens pollicis. The super ficial head of the flexor brevis pollicis lies internal to the opponens pollicis and close to the outer side of the tendon of the flexor longus pollicis, anc the adductor obliquus pollicis is close to the inner side of that tendon. Thi adductor transversus pollicis lies beyond the obliquus, from which it is separ ated by the radial artery. The deep head of the flexor brevis pollicis i clifficult to show. It lies deeply, and arises from the inner side of the base o the first metacarpal bone, and it joins the adductor obliquus pollicis. A larg bundle of fibres should be looked for passing from the adductor obliquu: pollicis to the superficial head of the flexor brevis pollicis, and in each of thes: muscles at its insertion a small sesamoid bone will be met with. The medias nerve will be found to supply the abductor, opponens, and superficial head o 
the flexor brevis pollicis, the others being supplied by the deep division of the ulnar nerve. The three hypothenar muscles are then to be dissected. The profunda branch of the ulnar artery and deep division of the ulnar nerve pass between the abductor, and flexor brevis, minimi digiti, and then pierce the opponens minimi digiti, these three muscles being supplied by the deep division of the nerve.

The anterior annular ligament is now to be dissected, the structures related to it superficially are to be revised, and the ligament is then to be divided in order to examine the contents of the fibro-osseous canal which it forms with the front of the carpus. The great palmar bursa is to be carefully noted passing upwards beneath it, and descending will be found the superficial and deep flexor tendons, tendon of the flexor longus pollicis, and median nerve. Lying in a special compartment of the ligament, at its outer part, will be found the tendon of the flexor carpi radialis, as it traverses the groove on the palmar aspect of the trapezium. The superficial flexor tendons may then be divided in the palm, to enable the deep flexor tendons to be raised and placed over the handle of a scalpel. The lumbricales, arising from these deep tendons, are then to be dissected, and their nervesupply noted, namely, the deep division of the ulnar for the inner two, and the median for the outer two. The deep flexor tendons are to be cut and turned downwards with the lumbricales, in order to expose the radial artery in the palm, where it forms the deep palmar arch, which is completed by the profunda branch of the ulnar. The arteria princeps pollicis and arteria radialis indicis are to be shown arising from the radial as it passes to the front of the hand, and the palmar interosseous, recurrent, and superior perforating branches of the deep palmar arch are to be dissected. The deep division of the ulnar nerve is next to be followed out, and its extensive muscular distribution is to be carefully studied.

Back of the Forearm and Hand.-The skin having been carefully removed, the dorsal venous arch, with its tributaries, is to be shown on the back of the hand, and the radial nerve and the dorsal branch of the ulnar nerve are to be followed to their digital distributions. In removing the deep fascia from the back of the forearm, care should be taken to leave intact the thickened portion of it on the back of the wrist which forms the posterior annular ligament. The muscles on the outer side of the forearm are to be dissected first, in the following order: brachio-radialis, extensor carpi radialis longior, and extensor carpi radialis brevior. The superficial layer of muscles on the back of the forearm are then to be dissected as follows : extensor communis digitorum, extensor minimi digiti, extensor carpi ulnaris, and anconeus. Without disturbing meanwhile the posterior annular ligament, the extensor tendons are to be followed over the back of the hand to their insertions. In doing this, the thin, deep fascia on the dorsum, continuous above with the posterior annular ligament, is to be noted, and care is to be taken to preserve the dorsal arteries. The dissector will find the tendon of the extensor indicis lying inside the common extensor tendon to the index finger, and the tendon of the extensor minimi digiti will usually be found to be double. The flat bands which connect the ring-finger tendon with that on either side of it are to be shown, as well as a band connecting the middle-finger tendon with the common extensor tendon to the index finger. The expansions of the common. extensor tendons over the backs of the first phalanges are to be shown, and it will be seen that these receive the insertions of the lumbricales and interossei. The mode of insertion of the extensor tendons is then to be examined.

Returning to the back of the forearm, the superficial muscles are to be held well aside, and the posterior interosseous nerve and artery dissected. The nerve, having wound round the outer side of the radius in the supinator radii brevis, will be found emerging from that muscle behind near its lower border, and a little below this the artery meets it, after having passed backwards between the radius and ulna. The nerve is now to be followed downwards between the superficial and deep muscles, and then beneath the extensor longus pollicis, but no lower in the meantime, and its muscular distribution 
is to be noted. The posterior interosseous artery will be found to give of its posterior interosseous recurrent branch as soon as it reaches the back o the forearm. This branch is to be followed upwards beneath the anconen to the back of the external epicondyle, where it anastomoses with the posterio. terminal branch of the superior profunda. If well injected, it will be founc to give branches to the back of the olecranon process, which anastomos with branches of the posterior ulnar recurrent to form the olecranon rete The posterior interosseous artery, as it descends with the nerve, gets very small, and usually ceases before reaching the wrist, where its place is taker by the posterior branch of the anterior interosseons, with which it anas tomoses.

The muscles of the deep layer are then to be dissected in the following order supinator radii brevis, extensor ossis metacarpi pollicis, extensor brevi pollicis, extensor longus pollicis, and extensor indicis. The posterior annula ligament is next to be carefully dissected, and, by opening it up, the fibro osseous canals which it forms with the grooves on the lower ends of the radiu and ulna are to be studied. The localization of the various tendons in thes canals is to be mastered, and careful attention is to be given to their synovia sheaths. The mode of termination of the posterior interosseous nerve is also to be shown at this stage. It will be found to end beneath the tendons o the extensor communis digitorum in a gangliform enlargement, from whicl articular twigs are given off to the adjacent joints. The radial artery or the back of the wrist and its branches are next to be dissected, and the dissector is to note that the artery, just before it dips between the two head: of the abductor indicis, is crossed by the tendon of the extensor longus pollicis In connection with the posterior radial carpal artery, the posterior ulnar carpa is to be shown, as well as the posterior carpal arch, beneath the extensoi tendons. The inner two dorsal interosseous arteries are to be shown arising from this arch, and they are to be followed downwards over the inner twe interosseous spaces to near the clefts between the middle and ring, and ring and little fingers, where each divides into two dorsal collateral digital branches The inner of the two arteries may give a branch to the inner side of the little finger, or this may arise from the posterior ulnar carpal. The first dorsa interosseous artery is to be followed from the radial over the second inter. osseous space to near the cleft between the index and middle fingers, where it also divides in to two dorsal collateral digital ar teries. The superior perforating branches of the deep palmar arch are to be shown after they have pierced the upper ends of the inner three interosseous spaces between the two heads of the dorsal interosseous muscles, where each joins a dorsal interosseons artery. Each of these dorsal interosseous arteries may give off an inferior perforating branch, before it bifurcates, to join a digital artery from the superficial palmar arch. The arteria dorsalis pollicis is next to be shown, and it will be found to divide in to two branches (which sometimes arise separately) for the sides of the thumb. Lastly, the arteria dorsalis indicis, which arises from the radial just before it leaves the back of the wrist, is to be followed along the outer side of the second metacarpal bone to the outer side of the index finger.

The interosseous muscles are next to be dissected. In cleaning the palinar interossei the interosseous fascia is to be noted, and its relation to the central division of the palmar fascia is to be revised. In cleaning the dorsal interossei a thin, deep dorsal fascia will be removed. It will also be necessary to dissect the transverse metacarpal (deep transverse) ligament, which stretches across the heads of the four inner metacarpal bones on their palmar aspects. This having been cut, the muscles are now to be fully dissected, and their nerve-supply and actions carefully studied.

Elbow-Joint. - The muscles related to this joint are to be replaced, and theis relation to it noted. The adjacent nerves are also to be observed, and the anastomoses of arteries around the joint are to be thoronghly inastered. The ligaments which compose the capsule, namely, anterior, posterior, external lateral, and internal lateral, are to be dissected, and the joint is then to be opened. The articular surfaces are to be examined, the pads of fat noted and the synovial membranc and movements studied. 
Wrist-Joint.-This joint is to be studied in the same way as the elbow-joint.

Radio-ulnar Joints. - The superior radio-ulnar joint has only the orbicular or annular ligament, and its synovial membrane is continuous with that of the elbow-joint. The chief bond of union at the inferior radio-ulnar joint is the triangular fibro-cartilage, which is to be carefully examined, along with the synovial membrane, which is called the membrana sacciformis. As regards the intermediate connection between the radius and ulna, the interosseous membrane is to be dissected, and its anterior and posterior relations studied. The opening in it near its lower part for the posterior branch of the anterior interosseous artery is to be shown. The oblique ligament at the upper end is to be examined, and between it and the upper border of the interosseous membrane will be seen the interval for the passage of the posterior interosseous vessels. The movements of pronation and supination are to receive close attention, and the dissector is to make himself thoroughly familiar with the muscles concerned in these important movements.

Carpal Joints. - The transverse carpal joint is to be dissected first, and the ligaments and movements are to be studied. The pisiform joint is next to be examined, and its capsular ligament shown. The dorsal, palmar, and interosseous ligaments of the other three bones of the first row are next to be examined, followed by those of the second row. The carpo-metacarpal joints are to be studied, and special attention should be directed to the joint between the trapezium and first metacarpal bone. The intermetacarpal (basal) joints follow next. The deep transverse ligament which connects the palmar aspects of the heads of the four inner metacarpal bones has been already dissected. The metacarpo-phalangeal joints, followed by the interphalangeal, are next to be dissected. The strong anterior fibrous plate is to be noted, and the absence of a dorsal ligament, its place beirg taken by the extensor tendon. In the metacarpo-phalangeal joint of the thumb no anterior fibrous plate will be found, its place being taken by two sesamoid bones. Special attention is to be given to the arrangement of the synovial membranes, from the inferior radio-ulnar joint above, with its membrana sacciformis, to the intermetacarpal (basal) joints below. The number of these synovial membranes and the complexity of that of the carpus are to be noted. 


\section{THE LOWER LIMB}

\section{THE GLUTEAL REGION.}

Landmarks. - The crest of the ilium is almost entirely obscured by the prominence of the abdominal muscles above it and the gluteus medius below it, so that as a rule its position is indicated by a groove. This groove conducts the finger to the posterior superior iliac spine, which is on a level with the second sacral spine and the centre of the sacro-iliac synchondrosis. The spinous processes of the sacral vertebræ, usually four in number, may be distinguished as separate projections, or they may be fused into one median ridge. The fifth sacral spine being undeveloped, no median projection can be felt, but at either side of the middle line the sacral cornua may be made out. The coccyx is felt in the natal cleft between the buttocks. The tuber ischii is obscured by the lower border of the gluteus maximus when the hip-joint is extended, but, when that joint is flexed, the prominence is easily made out. The great trochanter is felt at the lower and outer part of the gluteal region, and behind it there is a natural depression. The prominence of the natis or buttock is formed by the gluteus maximus, covered by a large amount of adipose tissue. The fold of the natis is produced by a fold of the skin and fascia, and is very perceptible when the hip-joint is extended. It takes the form of a transverse furrow, which commences internally a little below the lower border of the gluteus maximus, and terminates externally on the surface of that muscle, having crossed its lower border. The fold is horizontal in direction, whilst the lower border of the gluteus maximus is oblique. It is possible to feel the great sciatic nerve in this fold, but that nerve lies deeply at a point very nearly midway between the great trochanter and tuber ischii, being rather nearer the latter than the former.

Cutaneous Nerves.-These are met with in the following situations : (I) line of origin of gluteus maximus; (2) iliac crest ; (3) outer and lower part of gluteus maximus; and (4) lower border of gluteus maximus.

I. Line of Origin of Gluteus Maximus. - The cutancous nerves met with in this situation are divisible in to three sets. 
(a) Two or three twigs from the external branches of the posterior primary divisions of the first three sacral nerves. These external branches form two sets of loops, one set being situated deeply on the back of the sacrum, and the other set more superficially on the posterior surface of the great sacro-sciatic ligament. It is from this latter set of loops that the two or three cutaneous twigs are derived, and their direction is chiefly outwards.

(b) One or two twigs from the undivided posterior primary divisions of the last two sacral nerves and the coccygeal nerve. These divisions form a loop on the back of the sacrum, from which the cutaneous twigs are derived, their distribution being confined to the coccygeal integument.

(c) Twigs from the so-called coccygeal plexus, which is situated on the pelvic surface of the coccygeus muscle. These twigs pierce that muscle and the small sacro-sciatic ligament, as well as the gluteus maximus, and are distributed to the coccygeal integument.

2. Iliac Crest.-The cutaneous nerves in this situation from behind forwards are also divisible into three sets.

(a) Three offsets from the external branches of the posterior primary divisions of the first three lumbar nerves, which descend over the iliac crest, in front of the outer border of the erector spinæe muscle.

(b) Iliac branch of the ilio-hypogastric nerve, which crosses the iliac crest at the junction of the middle and anterior thirds.

(c) Undivided lateral cutaneous or iliac branch of the anterior primary division of the twelfth thoracic (subcostal) nerve. This is a large and long nerve which descends over the anterior part of the iliac crest, I inch behind the anterior superior iliac spine, and is distributed to the integument of the anterior part of the gluteal region as low as the great trochanter.

3. Outer and Lower Part of Gluteus Maximus.-The cutaneous nerves appearing in this situation are branches of the posterior division of the external cutaneous nerve, which is derived from the lumbar plexus.

4. Lower Border of Gluteus Maximus.-The cutaneous nerves met with in this locality are as follows :

(a) Three or four recurrent branches from the small sciatic nerve, which supply the integument ove- the lower and outer part of the gluteus maximus.

(b) Perforating cutaneous branch of the sacral plexus, more particularly from the back of the second and third sacral nerves, which supplies the integument over the lower and inner part of the gluteus maximus.

Fasciæ.-The superficial fascia is very thick, and loaded with adipose tissue. It is continuous over the iliac crest with the superficial fascia of the back of the trunk, and it contributes to the prominence of the natal region. Between the iliac crest and the upper border of the gluteus maximus there is a considerable accession of adipose tissue to the deep surface of the superficial 
fascia, and so the depression in that region is filled up. The deer fascia forms a part of the fascia lata. Over the fleshy part o the gluteus maximus it is thin, but, at the insertion of the muscl and over the anterior two-thirds of the gluteus medius, it is dense In these latter situations it gives insertion to a considerable par of the gluteus maximus, and origin to the superficial fibres of the gluteus medius in its anterior two-thirds. In passing fron th gluteus medius on to the gluteus maximus it straps down the uppe border of the latter muscle.

Muscles. Gluteus Maximus-Origin.-(I) The posterior 2 inche: of the outer lip of the iliac crest; (2) the upper part of the rougl surface on the dorsum ilii between this part of the crest and the superior gluteal line; (3) the posterior lamina of the lumba aponeurosis; (4) the lateral part of the back of the fourth anc fifth sacral vertebræ; (5) the back of the upper three coccygea vertebræ close to the lateral border ; and (6) the superficial surfac of the great sacro-sciatic ligament.

Insertion.-(I) Rather more than the upper half of the muscle i inserted between two laminæ of the fascia lata on the upper anc outer part of the thigh; (2) the superficial fibres of rather less thar the lower half are also inserted into the fascia lata, but the deef fibres of this portion are inserted into the gluteal ridge of the femur The part of the fascia lata which gives insertion to the gluteu maximus is known as the ilio-tibial band.

Nerve-supply.-The inferior gluteal nerve from the sacral plexus which enters the muscle on its deep or anterior surface in its lowe third.

The direction of the coarse fasciculi of the muscle is downward and outwards.

Action.-Acting from its origin the muscle extends the thigh upor the pelvis, as in rising from the sitting posture, or ascending a stair The upper part, acting alone, would abduct the thigh, and the lowe part would adduct it and rotate it outwards. The muscle also takes part in the completion of extension of the knee-joint, and in the maintenance of extension, as in standing, through means of the ilio-tibial band of the fascia lata. Acting from its insertion the muscle extends the pelvis upon the thigh, as in raising the trunk from the stooping posture.

The gluteus maximus, which is quadrilateral, has a short uppe border which is bound down to the gluteus medius by the fascia lata, and a long lower border which is free.

Deep Relations.-These, from above downwards, are as follows the posterior fleshy third of gluteus medius; superficial division of gluteal artery; extrapelvic part of pyriformis; great and smal sciatic nerves; inferior gluteal nerve; sciatic and internal pudi arteries; pudic nerve; nerve to obturator internus, supplying brancl to gemellus superior; common nerve to gemellus inferior an quadratus femoris; gemellus superior ; extrapelvic part of obturato internus; gemellus inferior; quadratus femoris ; crucial anastomosi 
of arteries; upper horizontal part of adductor magnus; origins of hamstring muscles from tuber ischii; portion of vastus externus, just below the great trochanter; and great sacro-sciatic ligament,

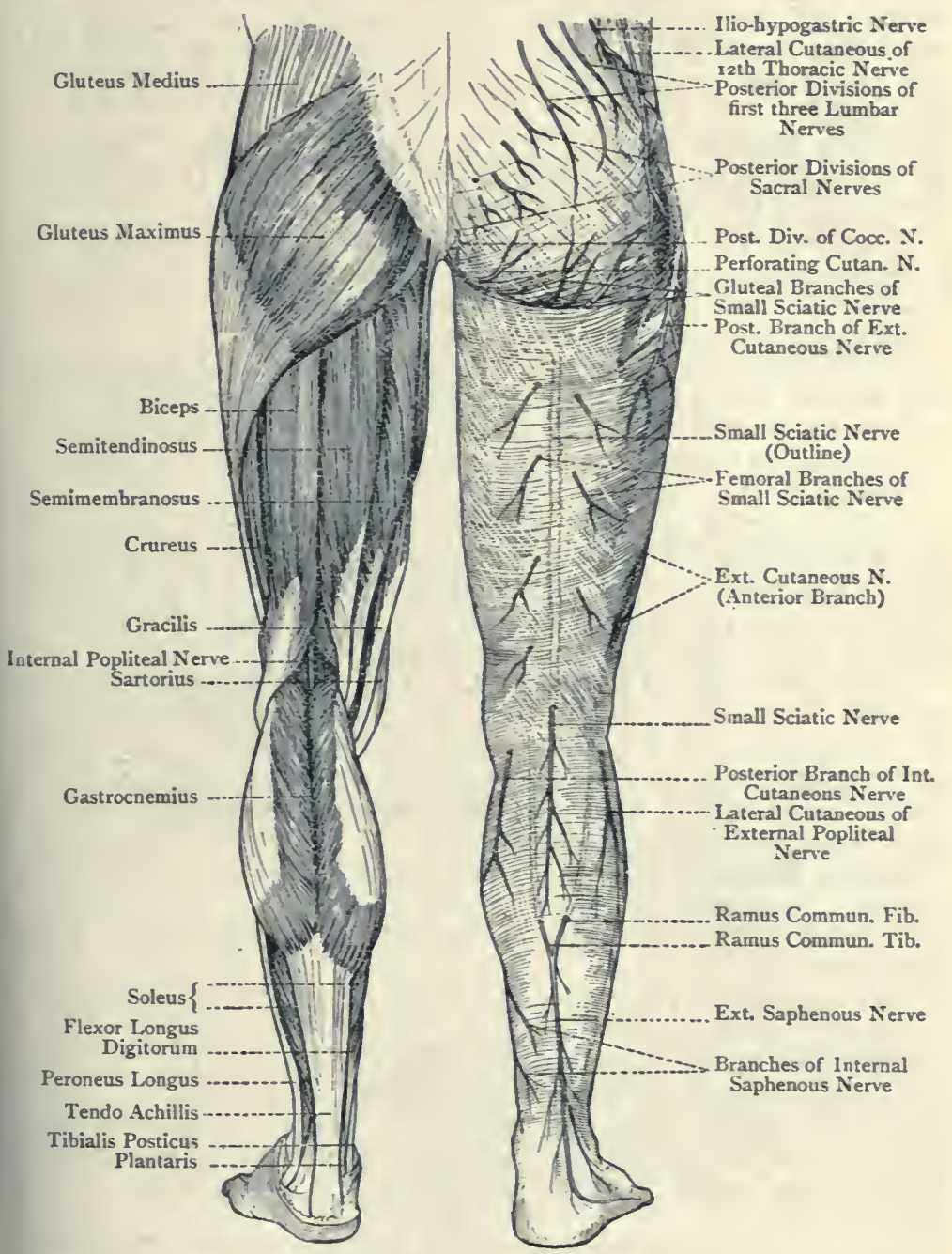

Fig 2:0.-Muscles and Cutaneous Nerves of the lower Limb (POSTERIOR ASPECT).

pierced by the coccygeal branch of the sciatic artery, the sacral branch of the internal pudic artery, and the perforating cutaneous branch of the sacral plexus. 
Three synovial bursæe are found underneath the muscle. One, which is single, is situated between the lower border of the muscle and the tuber ischii. Its importance consists in the fact that it is concerned in the condition known as 'lighterman's bottom.' A second lies between the muscle and the great trochanter, this one being multilocular. The third is found between the muscle and the upper part of the vastus externus, just below the great trochanter.

Gluteus Medius-Origin.-(I) The dorsum ilii between the crest, superior gluteal line, and middle gluteal line; and (2) the fascia lata covering the anterior two-thirds of the muscle.

Insertion.-The oblique impression on the outer surface of the great trochanter, extending from the postero-superior angle downwards and forwards to the antero-inferior angle.

Nerve-supply.-The superior gluteal nerve.

The direction of the anterior fibres of the muscle is downwards and slightly backwards, and of the posterior fibres downwards and forwards. The fibres of the muscle converge from their origin to the fan-shaped tendon of insertion, and they correspond in direction with those of the gluteus minimus.

Action.-Acting from its origin the muscle abducts the thigh. The anterior fibres also act as an internal rotator, and the posterior fibres as an external rotator, of the thigh. Acting from its insertion it balances the pelvis upon the thigh, as in standing upon one leg, and it is also a lateral flexor of the pelvis.

The gluteus medius is triangular. The posterior fleshy third is covered by the gluteus maximus, and the anterior two-thirds by the fascia lata. The muscle covers the gluteus minimus except posteriorly, the deep division of gluteal artery, and the superior gluteal nerve, and a synovial bursa intervenes between it and the upper part of the great trochanter.

Gluteus Minimus-Origin.--The dorsum ilii between the middle and inferior gluteal lines.

Insertion.-The lower part of the anterior surface of the great trochanter.

Nerve-supply. - The lower division of the superior gluteal nerve.

The fibres of the muscle correspond in direction, for the most part, with those of the gluteus medius.

Action.-Acting from its origin the muscle is an abductor and internal rotator of the thigh. Acting from its insertion it balances the pelvis upon the thigh, and is a lateral flexor of the pelvis.

The gluteus minimus is fan-shaped, and the tendon of insertion spreads out into an aponeurotic expansion over its lower part. It is covered by the gluteus medius except at the posterior part, where the pyriformis rests upon it. Its deep surface is related to the capsular ligament of the hip-joint and the posterior or reflected head of the rectus femoris. The tendon of insertion of the muscle 
is separated from the great trochanter by a synovial bursa, and is connected with the upper part of the capsular ligament of the hipjoint by a strong arched band of fibres.

The anterior portion of the gluteus minimus is sometimes detached from the rest of the muscle, and, when this occurs, the separated part represents the gluieus quartus or musculus scansorius (climbing muscle) of certain animals.

Pyriformis-Origin.-(I) By three fleshy slips from the anterior surfaces of the second, third; and fourth sacral vertebræ, which are interposed between, and lie external to, the adjacent anterior sacral foramina; (2) the deep surface of the great sacro-sciatic ligament; and (3) the posterior border of the ilium immediately below the posterior inferior iliac spine.

Insertion.-The upper border of the great trochanter near its centre.

Nerve-supply.-Two branches from the sacral plexus, which enter the intrapelvic part of the muscle. The branches come from the dorsal divisions of the first and second sacral nerves.

Action.-External rotator of the thigh.

The pyriformis, in emerging from the pelvis through the great sacro-sciatic foramen, divides that foramen into a small upper and a large lower compartment. Through the upper compartment the gluteal vessels and superior gluteal nerve pass. Through the lower compartment the following structures are transmitted: the sciatic and internal pudic.vessels, great and small sciatic nerves, inferior gluteal nerve, pudic nerve, nerve to obturator internus, and common nerve to gemellus inferior and quadratus femoris. The extrapelvic part of the pyriformis is sometimes pierced by the external popliteal nerve.

Gemellus Superior-Origin.-The lower border and adjacent portion of the external surface of the spine of the ischium.

Insertion.-The upper border of the tendon of the obturator internus.

Nerve-supply.-Branch from the nerve to the obturator internus, which enters the muscle on its anterior or deep surface near the upper border, close to the origin.

The gemellus superior is sometimes absent.

Obturator Internus-Origin.-(I) The internal surface of the obturator membrane; (2) the posterior surface of the body of the os pubis, descending pubic ramus, and ascending ramus of the ischium; (3) the inclined plane of the ischium, extending as far back as the great sacro-sciatic foramen, and nearly as high as the iliac portion of the ilio-pectineal line; and (4) the parietal pelvic fascia covering the muscle.

Insertion.-The inner surface of the great trochanter, above and in front of the digital fossa.

Nerve-supply.-The nerve to the obturator internus from the sacral plexus.

Action.-External rotator of the thigh. 
The intrapelvic and extrapelvic parts of the muscle form very nearly a right angle with each other. It emerges from the pelvis through the small sacro-sciatic foramen, and the deep surface of its tendon is here broken up into from three to five columns, separated from each other by grooves. The small sciatic notch is covered by cartilage, which presents as many grooves as there are columns on the deep surface of the tendon, these grooves being separated by slight ridges. The grooves lodge the columns of the tendon, and the ridges are received into the grooves between the tendinous columns. A bursa intervenes between the tendon and the cartilage covering the notch.

Gemellus Inferior-Origin.-The upper part of the tuber ischii, and the lower margin of the small sciatic notch.

Insertion.-The lower border of the tendon of the obturator internus.

Nerve-supply.-Branch from the nerve to the quadratus femoris, which enters the muscle on its deep or anterior surface near the upper border, close to the origin.

The gemelli muscles are merely extrapelvic origins of the obturator internus, of which they form accessory parts. As they take insertion into the upper and lower borders of the obturator tendon they are folded over it, so as to overlap and partially conceal the tendon on its superficial aspect. They are auxiliary to the obturator internus in action.

Small Sacro-sciatic Foramen.-The structures which pass through this foramen are as follows: (I) obturator internus; (2) nerve to the obturator internus ; (3) pudic nerve ; and (4) internal pudic artery with its venæ comites.

Quadratus Femoris-Origin.-The outer border of the tuber ischii.

Insertion.-The linea quadrati of the femur, extending as low as the small trochanter.

Nerve-supply.-The nerve to the quadratus femoris from the sacral plexus, which enters the muscle on its deep or anterior surface near the upper border, close to the origin.

Action.-External rotator of the thigh.

The muscle conceals part of the obturator externus and the small trochanter of the femur, from which latter it is separated by a small bursa. When the gemellus inferior and quadratus femoris are separated, part of the obturator externus comes into view. Between the lower border of the muscle and the upper border of the adductor magnus the transverse branch of the internal circumflex artery appears, which here takes part in the crucial anastomosis. When the lower border of the muscle is raised, the small trochanter comes into view with the tendon of insertion of the ilio-psoas.

Arteries.-The chief arteries of the gluteal region are the gluteal, sciatic, and internal pudic.

Gluteal Artery.-This vessel arises from the posterior division of the internal iliac artery piercing the parietal pelvic fascia, it 


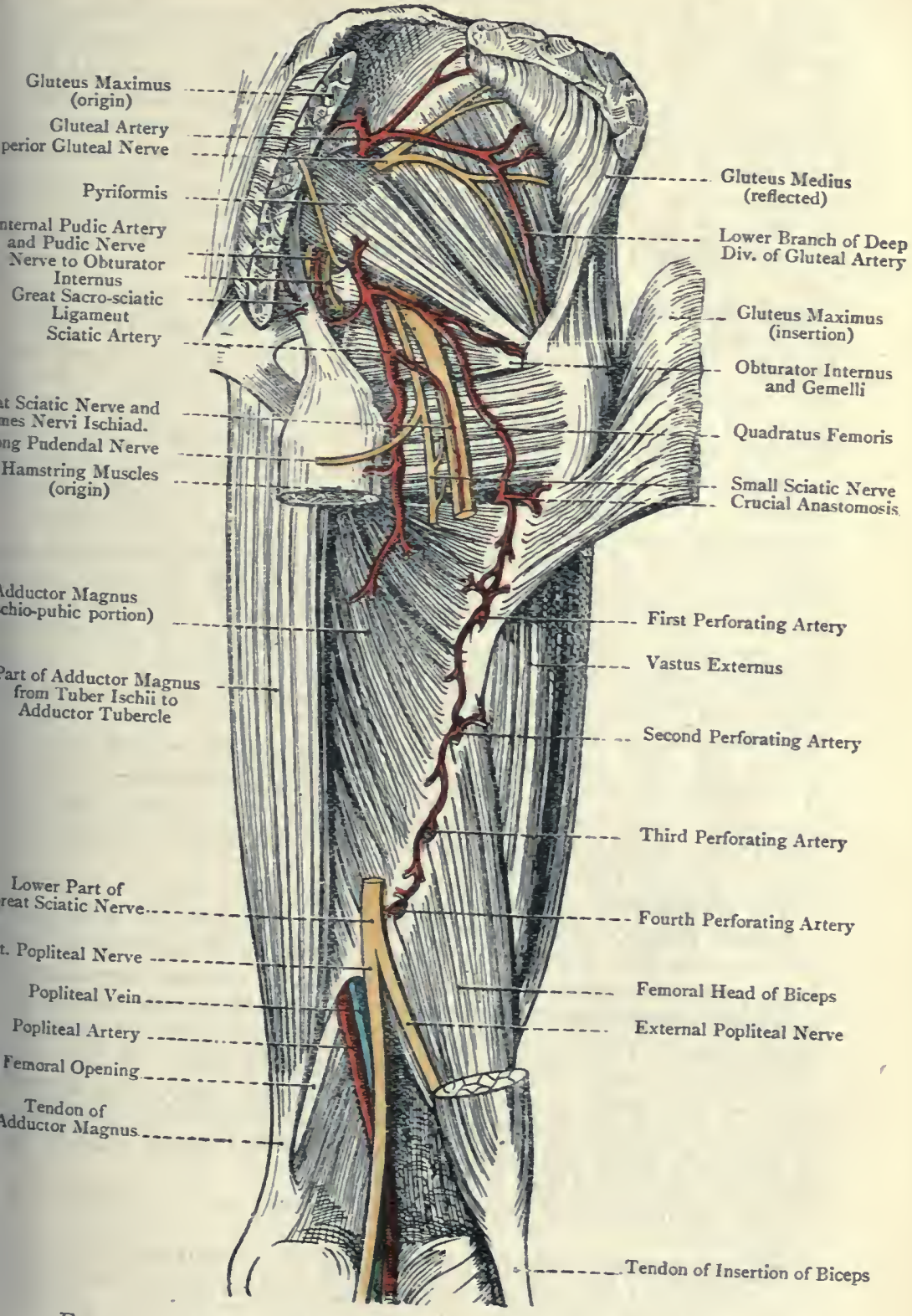

Nerve and
Ischiad.
ind Nerve
rigin) niernal Pudic Artery and Pudic Nerve

Lower Branch of Deep Div. of Gluteal Artery

Gluteus Maximus Quadratus Femoris Small Sciatic Nerve

chio-puhic portion

art of Adductor Magnu from Tuber Ischii to

Fig. 221.-The Gluteal Region AND Back of the Thigh
(Deep Dissection) 
passes between the lumbo-sacral cord and the anterior primary division of the first sacral nerve, after which it emerges through the upper compartment of the great sacro-sciatic foramen above the pyriformis, and breaks up into two divisions-superficial ano deep. The superficial division passes backwards between the posterior border of the gluteus medius and the pyriformis, and then enters the deep or anterior surface of the gluteus maximus near its origin. Some of its branches become cutaneous by piercing the muscle, and they anastomose with the posterior branches of the lateral sacral arteries from the posterior division of the internal iliac.

The deep division passes beneath the gluteus medius, where it subdivides into an upper and a lower branch. The upper branch courses along the upper border of the gluteus minimus, in company with the upper division of the superior gluteal nerve. It supplies the ilium and adjacent muscles, and anasto. moses with the deep circumflex iliac of the external iliac and the ascending branch of the external circumflex of the arteria profunda femoris. The lower branch passes forwards over the centre of the gluteus minimus, in company with the lower division of the superior gluteal nerve. It supplies the gluteus medius and gluteus minimus muscles. It also gives an articular branch to the hip-joint, and a branch to the digital fossa which anastomoses with the sciatic, the ascending branch of the internal circumflex, and a branch of the first perforating of the arteria profunda femoris. The lower branch of the deep division also anastomoses with the ascending branch of the external circumflex.

The place of emergence of the gluteal artery from the pelvis is indicated as follows: the thigh being rotated inwards, draw : line from the top of the great trochanter to the posterior superios iliac spine, and take a point in this line at the junction of the innes third and outer two-thirds.

The gluteal vein terminates in the internal iliac vein.

Sciatic Artery.-This vessel arises from the anterior division o the internal iliac artery. It descends, usually behind the interna pudic, upon the pyriformis and sacral nerves, and emerges throug the lower compartment of the great sacro-sciatic foramen below th pyriformis. It then passes between the great trochanter and tube ischii, lying under cover of the gluteus maximus, on the inne side of the great sciatic nerve, and resting upon the gemell obturator internus, and quadratus femoris. On leaving this hollov it descends upon the posterior surface of the upper part of tl adductor magnus, where it terminates.

For the intrapelvic portion of the artery, see dissection of $\mathrm{tl}$ pelvis.

Branches.-The extrapelvic branches are as follows: coccyge: inferior gluteal, muscular, anastomotic, articular, gluteal cutaneor and comes nervi ischiadici.

The coccygeal branch pierces the great sacro-sciatic ligame 
and gluteus maximus, and is distributed over the back of the coccyx. The inferior gluteal branch enters the deep surface of the gluteus maximus with the inferior gluteal nerve, and it anastomoses in the muscle with the superficial division of the gluteal artery. The muscular branches are distributed to the adjacent external rotator muscles and the origins of the hamstrings. In the latter muscles an anastomosis takes place with the external terminal branch of the obturator artery.

The anastomotic branches are two in number. One passes to the digital fossa, where it anastomoses with the gluteal, ascending branch of the internal circumflex, and first perforating arteries. The other passes to the interval between the quadratus femoris and adductor magnus, where it anastomoses with the transverse branch of the internal circumflex, the transverse branch of the external circumflex, and the first perforating arteries. This fourfold anastomosis is called the crucial anastomosis.

The articular branches are two or three in number. They pass beneath the gemelli and obturator internus with the nerve to the quadratus femoris, and pierce the back part of the capsular ligament of the hip-joint.

The gluteal cutaneous branches pass round the lower border of the gluteus maximus with the gluteal cutaneous branches of the small sciatic nerve, and are distributed to the integument over the lower part of the muscle.

The comes nervi ischiadici is a long branch which descends for some distance with the great sciatic nerve, to which it is distributed, and in which it anastomoses with the perforating branches of the arteria profunda femoris. After ligature of the femoral artery in the upper third of the thigh this branch becomes much enlarged.

The place of emergence of the sciatic artery from the pelvis is indicated as follows: draw a line from the posterior superior iliac spine to the outer border of the tuber ischii, and take a point in this line at the junction of the middle with the lower third.

The sciatic vein terminates in the internal iliac vein.

The sciatic artery in the early embryo is the leading arterial trunk of the lower limb. As the femoral artery is developed it forms a junction with the sciatic in the neighbourhood of the knee, and so becomes the chief artery, and the part of the primitive sciatic between the knee and the gluteal region for the most part disappears.

Internal Pudic Artery.-This vessel arises from the anterior division of the internal iliac, and at first lies within the pelvic cavity. It is only the second part of the vessel which is seen in the gluteal region, and which will be described here. Having emerged from the pelvis through the lower compartment of the great sacrosciatic foramen below the pyriformis, the artery passes downwards for a short distance, and crosses over the back of the spine of the ischium. It then courses through the small sacro-sciatic foramen, and is lost to view. 
Relations-Superficial or Posterior.-Gluteus maximus. Deep or Anterior.-Posterior surface of spine of ischium.

On either side of the artery is a vena comes. The pudic nerve lies on the inner side, and the nerve to the obturator internus on the outer side.

Branches.-Muscular, to gluteus maximus, and sacral, which pierces the great sacro-sciatic ligament, and ramifies over the back of the lower end of the sacrum, where it anastomoses with the coccygeal branch of the sciatic artery.

The position of the second part of the internal pudic artery upon the back of the spine of the ischium, which spine is about 4 inches below the posterior superior iliac spine, is ascertained as follows the thigh being rotated inwards, draw a line from the upper border of the great trochanter to the junction of the sacrum with the coccyx, and take a point in this line at the junction of the inner third and outer two-thirds.

Relation of Structures on Back of Spine of Ischium.-The relation from within outwards is as follows: (I) pudic nerve, (2) internal vena comes, (3) second part of internal pudic artery, (4) external vena comes, and (5) nerve to obturator internus muscle.

Deep Nerves. Superior Gluteal Nerve.-This nerve arises from the sacral plexus, more particularly from the dorsal divisions of the descending branch of the fourth lumbar, the fifth lumbar, and the first sacral nerves. It passes through the upper compartment of the great sacro-sciatic foramen, above the pyriformis, with the gluteal artery, and then beneath the gluteus medius, where it divides into a small upper and large lower brancl. The upper branch accompanies the corresponding division of the deep part of the gluteal artery along the upper border of the gluteus minimus, and it supplies the gluteus medius. The lower branch passes outwards over the centre of the gluteus minimus with the lower division of the deep part of the gluteal artery. It supplies the gluteus medius and gluteus minimus, and terminates by supplying the tensor fasciæ femoris.

Inferior Gluteal Nerve.-This nerve arises from the sacral plexus more particularly from the dorsal divisions of the fifth lumbar, and first and second sacral nerves. It passes through the lower compartment of the great sacro-sciatic foramen, below the pyriformis in close contact with the small sciatic nerve. It then divide into several branches which enter the deep surface of the gluteu: maximus in its lower third.

Nerve to Obturator Internus.-This nerve arises from the sacra plexus, more particularly from the ventral divisions of the fiftl lumbar, and first and second sacral nerves. It passes through th lower compartment of the great sacro-sciatic foramen, below th pyriformis, internal to the great sciatic nerve; over the posterior sur face of the spine of the ischium, where it lies to the outer side of th internal pudic vessels; and through the small sacro-sciatic forame to the intrapelvic part of the obturator internus. At the lowe 
border of the pyriformis it gives a branch to the gemellus superior, which enters that muscle on its deep surface near the upper border, close to the origin.

Nerve to Quadratus Femoris. - This nerve arises from the sacral plexus, more particularly from the ventral divisions of the descending branch of the fourth lumbar, the fifth lumbar, and the first sacral nerves. It passes through the lower compartment of the great sacro-sciatic foramen, below the pyriformis, where it lies in close contact with the deep surface of the great sciatic nerve. It then passes, in succession, beneath the gemellus superior, obturator internus, gemellus inferior, and quadratus femoris, entering the last muscle on its anterior or deep surface near the upper border, close to the origin. As it passes beneath the gemellus inferior, it parts with its branch to that muscle, which enters its deep surface near the upper border, close to the origin.

The nerve to the quadratus femoris also supplies, as a rule, an articular branch to the back of the hip-joint.

Lymphatics. - The superficial lymphatics of the gluteal region terminate in the inguinal glands (superior or oblique superficial inguinal glands). Tile deep lymphatics enter the pelvis, and terminate in the internal iliac glands.

For the great and small sacro-sciatic ligaments see the description of the pelvis in Abdomen Section.

\section{THE THIGH}

\section{Back of the Thigh and Popliteal Space.}

Landmarks. - The hamstring muscles give rise to a prominence along the back of the thigh, but they cannot be individually recognised until they reach the popliteal space. The great sciatic nerve is deeply placed, being under cover of the long or ischial head of the biceps femoris, but its course may be indicated by drawing a line from the centre of the back of the knee-joint to a point between the great trochanter and the tuber ischii, rather nearer the latter than the former. The upper two-thirds of this line correspond with the great sciatic nerve, and the lower third with the internal popliteal nerve.

The situation of the popliteal space behind the knee-joint is indicated by a depression when the joint is flexed. The strong tendon of the biceps femoris can be felt on the outer side, as it descends to reach the head of the fibula, and in front of it the long external lateral ligament of the knee-joint can be distinguished by its tense, cord-like feel. Anterior to this ligament the lower part of the ilio-tibial band of the fascia lata may be felt. The external popliteal nerve is close to the inner side of the biceps tendon. Lower down it can be felt just below the head of the fibula. On the inner side of the popliteal space, over the back of the inner 
condyle, three tendons may be felt: The most superficial one is that of the semitendinosus, which, though firm, is narrow, and is traceable for some distance above the knee-joint. Underneath it is the tendon of the semimembranosus, and, internal to this, is the slender tendon of the gracilis. The course of the popliteal artery practically coincides with the middle line of the popliteal space, but the vessel can only be felt when the joint is well flexed. . During this manipulation the popliteal lymphatic glands, if enlarged, may be detected.

Back of the Thigh.-There is nothing noteworthy in the superficial fascia. The deep fascia will be described in connection with the fascia lata.

Small Sciatic Nerve.-This nerve arises from the sacral plexus, more particularly from the back of the first, second, and third sacral nerves. It emerges through the lower compartment of the great sacro-sciatic foramen, below the pyriformis. It then passes downwards between the great trochanter and tuber ischii, resting upon the superficial surface of the great sciatic nerve, and being under cover of the lower part of the gluteus maximus. After escaping from beneath this muscle, it descends in the middle line superficial to the hamstring muscles, and beneath the deep fascia covering them, and, passing over the popliteal space, it pierces the deep fascia at the back of the knee-joint. It then enters the back of the leg, and descends as low as about the centre of the calf, in company with the external or short saphenous vein. The nerve is entirely sensory.

Branches.- The branches are gluteal cutaneous, long pudendal nerve of Soemmering, femoral cutaneous, and sural cutaneous.

The gluteal cutaneous branches are three or four in number, and take a recurrent course, winding round the lower border of the gluteus maximus, and supplying the integument over its lower and outer part. The long pudendal nerve of Soemmering arises at the lower border of the gluteus maximus, and winds inwards towards the anterior part of the perineum, lying a little below the tuber ischii and crossing behind the origins of the hamstring muscles from that prominence. It then pierces the fascia lata fully $I$ inch in front of the tuber ischii, and, passing over the ischio-pubic ramus and through Colles' fascia, it courses forwards and inwards, in company with the two superficial perineal nerves and the superficial perineal artery, to the scrotum in the male and the labium majus in the female. In the anterior part of the perineum it communicates with the two superficial perineal nerves, and with them forms the three long scrotal (or labial) nerves. Its branches are femoral cutaneous, to the upper and inner part of the thigh on its posterior aspect ; and scrotal or labial, to the scrotum or labium majus, according to the sex. The femoral cutaneous branches of the small sciatic supply the integument of the back of the thigh as low as the knee-joint. The sural cutaneous branches are the terminal branches of the nerve. 
They supply the integument of the back of the leg as low as about the centre of the calf, and communicate with branches of the ramus communicans tibialis from the internal popliteal.

Muscles.-The muscles of the back of the thigh are called the hamstring muscles, and are three in number, namely, biceps femoris, semitendinosus, and semimembranosus.

Biceps Femoris or Biceps Flexor Cruris-Origin.-The muscle arises by two heads--long or ischial, and short or femoral. (I) Long or Ischial Head.- This arises, in conjunction with the semitendinosus, from the lower and inner impression on the posterior surface of the tuber ischii. (2) Short or Femoral Head.-This arises from (I) the outer lip of the linea aspera, (2) the upper two-thirds of the external supracondylar ridge, and (3) the external intermuscular septum.

Insertion. - The upper surface of the head of the fibula in front of the styloid process, by means of a round tendon which sends an expansion downwards to the deep fascia covering the peroneus longus. A few fibres are also inserted into the external tuberosity of the tibia.

The long external lateral ligament of the knee-joint passes through the tendon of insertion, and divides it into two parts, from which the ligament is separated by the bursa wrapped around it. The two divisions of the tendon are anterior and posterior, the anterior, which is the stronger, being inserted into the external tuberosity of the tibia, as well as into the head of the fibula. The expansion to the deep fascia of the leg is derived from the posterior division of the tendon.

Nerve-supply.-Great sciatic nerve. The long or ischial head receives branches from the internal popliteal part of the nerve, and the short or femoral head from the external popliteal part.

Action.-Acting from its origin the muscle is an extensor of the hip-joint and a flexor of the leg upon the thigh, and, having flexed the knee-joint, it acts as an external rotator of the leg. The long or ischial head alone extends the hip-joint. Acting from its insertion the muscle, by means of its long or ischial head, is an extensor of the pelvis upon the thigh. In virtue of its double insertion into the head of the fibula and external tuberosity of the tibia, the biceps femoris contributes materially to the strength of the superior tibiofibular joint by bracing the bones together.

In rare cases the biceps femoris has a third head of origin, which may arise from the tuber ischii, linea aspera, or internal supracondylar ridge.

Semitendinosus-Origin.-The lower and inner impression on the posterior surface of the tuber ischii, in conjunction with the long or ischial head of the biceps femoris.

Insertion.-The upper part of the internal surface of the shaft of the tibia, behind the sartorius and below the gracilis. From the tendon of insertion an expansion is given to the deep fascia of the leg.

Nerve-supply. - Great sciatic nerve: The branches come from the internal popliteal part of the nerve. 
Action.-Acting from its origin the muscle is an extensor of the hip-joint and a flexor of the leg upon the thigh, and, having flexed the knee-joint, it acts as an internal rotator of the leg. Acting from its insertion it is an extensor of the pelvis upon the thigh. The semitendinosus is intimately connected with the long or ischial head of the biceps femoris in the upper fifth of the thigh, and its belly is crossed at its centre by an oblique tendinous intersection. In the lower third of the thigh the muscle has a long, narrow, round tendon, which broadens out at its insertion, and crosses the internal lateral ligament of the knee-joint. A bursa intervenes between it and that ligament, and also between it and the tendon of the sartorius.

Semimembranosus-Origin.-By means of a broad, flat tendon from the upper and outer impression on the posterior surface of the tuber ischii.

Insertion.-The insertion is threefold, as follows: (I) the chief insertion is by means of a strong tendon into the horizontal groove on the posterior surface of the internal tuberosity of the tibia; (2) by an expansion which passes upwards and outwards to the upper and back part of the external condyle of the femur, and which forms a large part of the posterior ligament of the knee-joint, known as the ligamentum posticum Winslowii; (3) by a broad expansion, which passes downwards and outwards to the oblique or popliteal line on the posterior surface of the shaft of the tibia. This expansion covers the popliteus muscle, and is called the popliteal fascia.

Nerve-supply.-Great sciatic nerve. The branches come from the internal popliteal part of the nerve.

Action.-Acting from its origin the muscle is an extensor of the hip-joint and a flexor of the leg upon the thigh, and, having flexed the knee-joint, it acts as an internal rotator of the leg. Acting from its insertion it is an extensor of the pelvis upon the thigh.

The strong tendon of origin is prolonged downwards for some distance upon the outer side of the muscle, and the chief tendon of insertion is prolonged upwards for some distance upon its inner side The belly of the muscle is composed of short, oblique fasciculi, an arrangement which gives it great power of action, but a limited range of movement. The chief tendon of insertion is under cover of the posterior border of the internal lateral ligament of the kneejoint. Previous to this it is separated from the internal head of the gastrocnemius by the popliteal bursa, which frequently communicates with the synovial membrane of the knee-joint through an opening often present in the posterior ligament. The chief tendon of insertion is also separated by a bursa from the upper lip of the groove on the posterior surface of the internal tuberosity of the tibia.

The hamstring muscles descend in close contact through the upper three-fourths of the thigh, being held together by the fascia lata. When, however, they reach the lower fourth, they part 
company, the biceps femoris passing downwards and outwards, and the semitendinosus and semimembranosus downwards and inwards; and so the popliteal space begins to open out.

Great Sciatic Nerve.-This nerve arises from the sacral plexus, more particularly from the descending branch of the fourth lumbar, the fifth lumbar, and the first, second, and upper branch of the third sacral nerves. It emerges from the pelvis through the lower compartment of the great sacro-sciatic foramen, below the pyriformis, and descends between the great trochanter and tuber ischii, being somewhat nearer the latter than the former. It then passes down the middle line of the back of the thigh, under cover of the long or ischial head of the biceps femoris, and, about the junction of the middle and lower thirds, it terminates by dividing into internal and external popliteal nerves.

The great sciatic nerve is about $\frac{3}{4}$ inch broad. Its course may be indicated as follows : draw a line from the centre of the back of the knee-joint to a point between the great trochanter and tuber ischii, rather nearer the latter than the former. The upper two-thirds of this line correspond with the great sciatic nerve, and the lower third with the internal popliteal nerve:

Chief Relations. Superficial or Posterior.-Gluteus maximus, small sciatic nerve, and long or ischial head of the biceps femoris. Deep or Anterior.-From above downwards the nerve is in contact with the following structures: the ischium, the nerve to the quadratus femoris, gemellus superior, obturator internus, gemellus inferior, quadratus femoris, and posterior surface of adductor magnus. Internal.-Semimembranosus.

Branches.-The branches are muscular and terminal.

The muscular branches arise in the upper part of the thigh, with the exception of the branch to the short head of the biceps femoris, which arises about the centre. They supply the hamstring muscles, and also that part of the adductor magnus which descends from the tuber ischii to the adductor tubercle of the femur. The branch to this part of the adductor magnus arises in common with the nerve to the semimembranosus. The branch to the short head of the biceps femoris is derived from the external popliteal part of the great sciatic nerve, but all the other muscular branches come from the internal popliteal part.

The terminal branches are the internal and external popliteal nerves. They arise about the junction of the middle and lower thirds of the thigh, and will be described in connection with the popliteal space.

The great sciatic nerve supplies an articular branch to the hipjoint in those cases where the nerve to the quadratus femoris fails to do so.

Blood-supply. - The nerve is supplied by the arteria comes nervi ischiadici from the sciatic, and the perforating branches of the arteria profunda femoris.

The great sciatic nerve is not infrequently replaced by the external 
and internal popliteal nerves. Under these circumstances the external popliteal nerve often pierces the pyriformis.

Popliteal Space.-The popliteal space or ham* is situated behind the knee-joint, whence it extends upwards to the junction of the upper three-fourths and lower fourth of the thigh, and downwards to the junction of the upper sixth and lower five-sixths of the leg. In shape the space resembles a diamond.

Boundaries. External-(a) Above the Knee-joint.-Biceps femoris. (b) Below the Knee-joint.-External head of the gastrocnemius, and plantaris.

Internal-(a) Above the Knee-joint.--Semitendinosus, semimembranosus, gracilis, sartorius, and tendon of the adductor magnus, in the order named from behind forwards. (b) Below the Kneejoint.-Internal head of the gastrocnemius.

The superior median angle of the space corresponds with the divergence of the hamstring muscles, the inferior median angle by the approximation of the external and internal heads of the gastrocnemius, the external angle by the meeting between the biceps femoris and external head of the gastrocnemius, and the internal angle by the meeting between the semimembranosus and internal head of the gastrocnemius.

The roof is formed by the integument. It contains the small sciatic nerve, and, in its lower part, the terminal portion of the external or short saphenous vein.

The floor is formed, from above downwards, by the popliteal surface or trigone of the femur, the posterior ligament of the kneejoint, and the popliteus muscle, covered by the popliteal fascia.

Contents.-The contents are the popliteal artery and its branches, the popliteal vein and its tributaries (including the termination of the external or short saphenous vein), the internal popliteal nerve and its branches, the external popliteal nerve and some of its branches, the geniculate branch of the obturator nerve (inconstant), lymphatic glands, and a large amount of fat.

Popliteal Artery.--This vessel is the continuation of the femoral artery. It extends from the posterior margin of the femoral opening, which is in connection with the adductor magnus, to the lower border of the popliteus muscle, where it divides in to anterior and posterior tibial arteries. The division takes place on a level with the lower border of the tubercle of the tibia, and fully I $\frac{3}{4}$ inches below the level of the upper surface of the head of that bone. The vessel at first passes downwards and outwards until it reaches the middle line of the limb. It then takes a straight course downwards between the condyles of the femur, and finally it sinks

* The word ham is derived from a Teutonic verb signifying " to be curved or crooked,' and it has been applied to the popliteal space because that space is situated behind the 'crook,' 'curve,' or bend of the knee. By a later extension of the word ham it came to be applied to the whole of the back of the thigh-hence the name hamstring, which is given to the muscles of that region. 
beneath the approximation of the external and internal heads of the gastrocnemius.

General Relations. Superficial or Posterior.-Skin, superficial and deep fasciæ, small sciatic nerve, terminal part of the external or short saphenous vein, fat, semimembranosus for about I inch after the artery enters the space, approximation of the external and internal heads of the gastrocnemius, and the plantaris, which crosses the artery from without inwards. Deep or Anterior.-Fat, popliteal surface or trigone of the femur, posterior ligament of the knee-joint, and popliteus muscle, covered by the popliteal fascia.

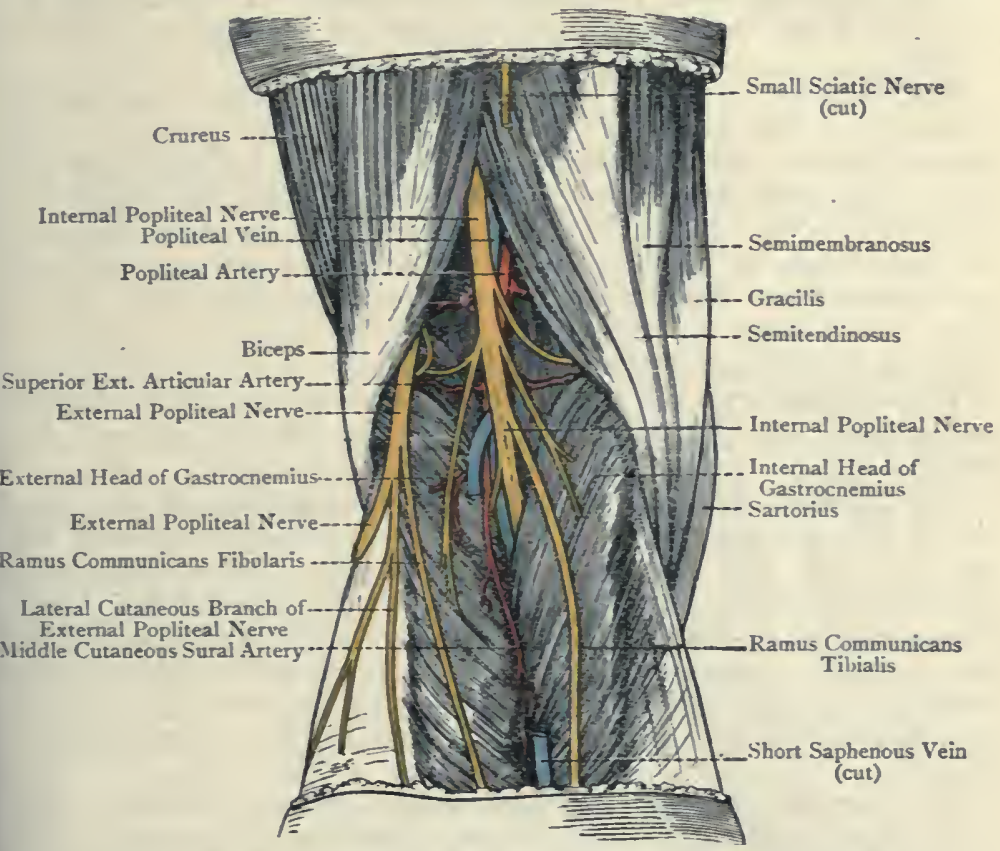

Fig. 222.-The Popliteal Space (Superficial Dissectios).

Special Relations.-In the upper part of the space the popliteal vein is close behind, and on the outer side of, the artery, and the internal popliteal nerve is close behind, and on the outer side of, the vein, so that they overlap one another. In the middle of the space the popliteal vein is entirely behind the artery, and the internal popliteal nerve is directly behind the vein. In the lower part of the space the popliteal vein is close behind, and on the inner side of, the artery, and the internal popliteal nerve is close behind, and on the inner side of, the vein, a relation which is the reverse of that in the upper part of the space. The geniculate branch of the obturator nerve (when present), having pierced the adductor magnus 
close above the femoral opening, descends at first upon the inner side of the popliteal artery, and then in front of it as low as the origin of the central or azygos artery, where the nerve leaves the main vessel and passes, with that branch, through the posterior ligament of the knee-joint to the interior of the articulation.

Branches.-The branches are (I) muscular and cutaneous, and (2) articular.

The muscular and cutaneous branches are divided into a superior and an inferior set.

The superior set are purely muscular, and are distributed to the lower parts of the hamstring muscles. They anastomose with the lower two perforating branches of the arteria profunda femoris.

The inferior or sural set are partly muscular and partly cutaneous. The muscular branches supply both heads of the gastrocnemius and the plantaris. The cutaneous branches are three in number, and supply the integument over the upper half of the calf. The middle cutaneous branch lies in the groove between the two heads of the gastrocnemius with the external or short saphenous vein. This branch is sometimes of large size, and in these cases, under the name of the external saphenous artery, it descends to the back of the external malleolus.

The articular branches are five in number-two superior, external and internal, one central or azygos, and two inferior, external and internal.

The superior external articular artery courses outwards close above the external condyle of the femur, passing beneath the biceps femoris and through the external intermuscular septum into the crureus muscle, where it breaks up into branches. These anastomose with the following arteries: (I) descending branch of the external circumflex; (2) inferior external articular; (3) deep branch of the anastomotica magna; (4) superior internal articular; and $(5)$ fourth or lowest perforating branch of the arteria profunda femoris.

The superior internal articular artery courses inwards close above the internal condyle of the femur, and, having passed beneath the tendon of the adductor magnus, it enters the vastus internus, where it divides into branches. These anastomose with the following arteries: (I) inferior internal articular; (2) deep branch of the anastomotica magna; and (3) superior external articular.

The central or azygos articular artery, if a separate branch, arises from the front of the main artery, but it is often a branch of the superior external articular. It passes through the posterior ligament of the knee-joint to supply the synovial membrane and ligaments within the joint.

The inferior external articular artery courses horizontally outwards beneath the tendon of the biceps femoris and the long external lateral ligament of the knee-joint to the outer side of the 
articulation, where it divides into branches. These anastomose with the following arteries: (I) superior external articular; (2) inferior internal articular; (3) anterior tibial recurrent; and (4) posterior tibial recurrent (inconstant).

The inferior internal articular artery courses at first obliquely downwards and inwards along the upper border of the popliteus muscle, and then passes inwards below the level of the internal tuberosity of the tibia, where it lies beneath the internal lateral ligament of the knee-joint. On reaching the inner side of the articulation, it divides into branches which anastomose with the following arteries: (I) superficial branch of the anastomotica magna; (2) superior internal articular; (3) inferior external articular ; (4) anterior tibial recurrent; and (5) posterior tibial recurrent (inconstant).

Varieties of Popliteal Artery.-I. The vessel may divide into its anterior and posterior tibial branches at the upper border of the popliteus muscle.

2. The terminal branches may be three in number instead of two, the additional branch being the peroneal artery.

3. In very rare cases the popliteal artery divides high up in to two branches of equal size, which subsequently unite prior to the normal termination of the ressel.

Popliteal Vein:-This vessel commences at the lower border of the popliteus muscle, where it is formed by the union of the venæ comites of the anterior and posterior tibial arteries, and it terminates at the posterior margin of the femoral opening, which is in connection with the adductor magnus, where it becomes the femoral vein. The relations of the vessel have already been described. Its tributaries correspond with the branches of the popliteal artery, with the acidition of the external or short saphenous vein.

Internal Popliteal (Tibial) Nerve.-This nerve is one of the terminal branches of the great sciatic, and it derives its fibres from the ventral divisions of the descending branch of the fourth lumbar, the fifth lumbar, the first and second sacral, and the upper branch of the third sacral nerves. It extends from the superior angle of the popliteal space to the lower border of the popliteus muscle, where it becomes the posterior tibial nerve. The relations of this nerve have been given in connection with the popliteal artery.

Branches.-The branches are articular, cutaneous, and muscular. The articular branches are three in number (sometimes two), as follows: superior (inconstant), accompanying the superior internal articular artery ; central or azygos, going with the corresponding artery; and inferior, accompanying the inferior internal articular artery.

The cutaneous branch is called the ramus communicans tibialis. It descends in the middle line beneath the deep fascia as far as the centre of the calf. Here it pierces the deep fascia, and shortly afterwards it is joined by the ramus communicans fibularis from the external popliteal. In this manner the external or short saphenous nerve is formed. 


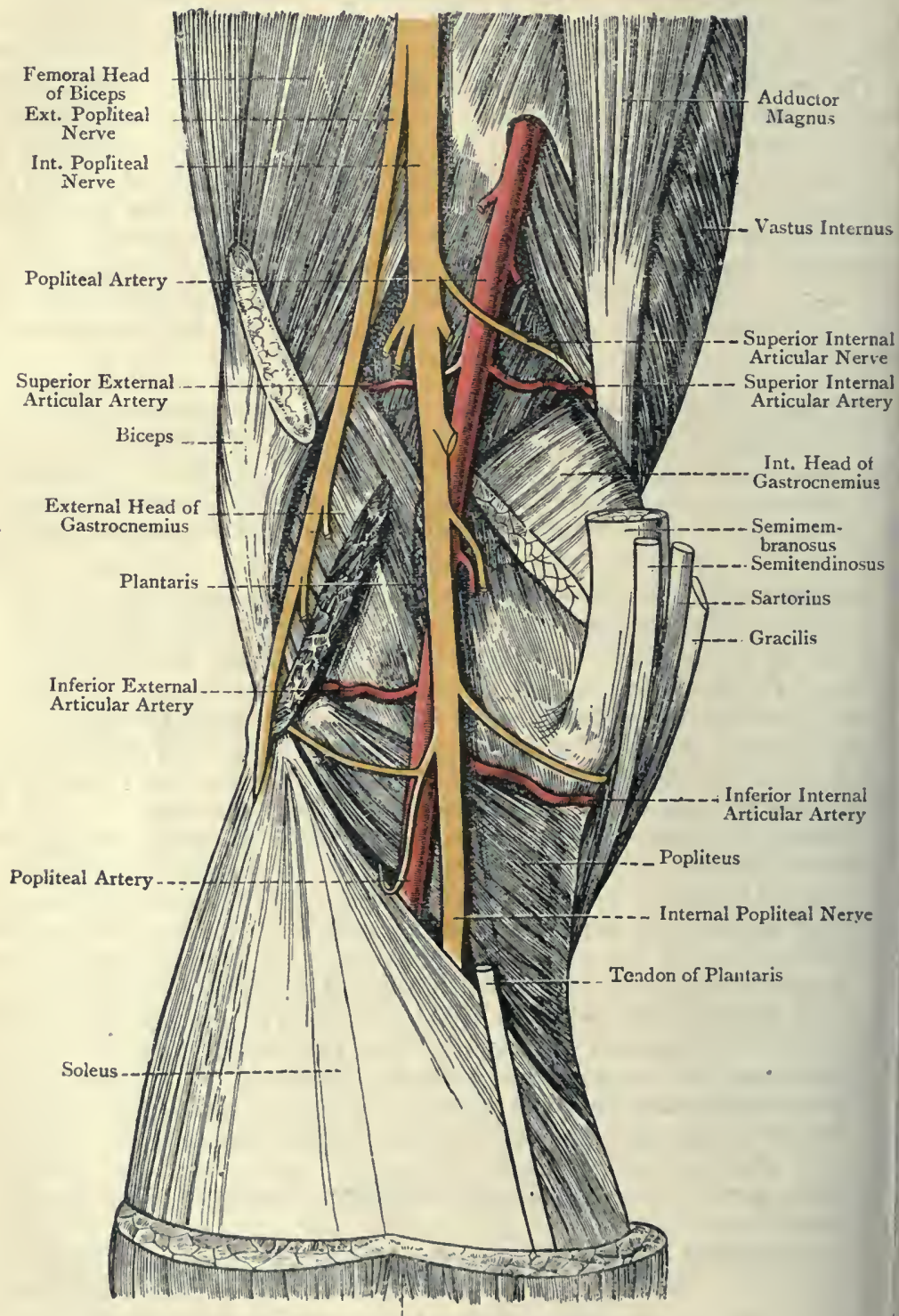

Fig. 223. -The Popliteal Space (Deep Dissection). 
The muscular (sural) branches are usually five in number, which are distributed as follows: one to the external head of the gastrocnemius, one to the plantaris (which sometimes comes from the preceding), one to the internal head of the gastrocnemius, one to the soleus, and one to the popliteus. - The nerve to the popliteus descends over the popliteal fascia, lying to the outer side of the popliteal vessels. On arriving at the lower border of the popliteus, it turns round that border and enters the deep surface of the muscle. This nerve, besides supplying the popliteus, furnishes the following branches: articular to the superior tibio-fibular joint; medullary, which enters the large nutrient or medullary foramen on the back of the tibia; vascular to the anterior and posterior tibial arteries; and interosseous. The interosseous branch is a long nerve which descends in the interosseous membrane to terminate in the inferior tibio-fibular joint.

External Popliteal or Peroneal (Fibular) Nerve.-This nerve is the other terminal branch of the great sciatic, and it derives its fibres from the dorsal divisions of the descending branch of the fourth lumbar, the fifth lumbar, and the first and second sacral nerves. It commences at the superior angle of the popliteal space, and descends obliquely downwards and outwards as low as the back of the neck of the fibula, where it passes forwards between the bone and the peroneus longus, to end on the outer side of the fibular neck in its terminal branches. The nerve lies at first close to the inner border of the biceps femoris, and then it rests upon the back of the external head of the gastrocnemius, between which and the biceps tendon it may be regarded as lying.

Branches.- The branches are articular, cutaneous, and terminal.

The articular branches are three in number, as follows : superior, accompanying the superior external articular artery; inferior, going with the inferior external articular artery, which it reaches as the vessel is about to pass beneath the biceps tendon; and recurrent articular, accompanying the anterior tibial recurrent artery.

The cutaneous branches are two in number, as follows: lateral cutaneous branch, which supplies the integument of the outer side of the leg over about its upper two-thirds, as well as the adjacent integument of the sural region; and ramus communicans Qbularis, which passes downwards and inwards over the external head of the gastrocnemius and beneath the deep fascia to the centre of the calf, where it pierces the deep fascia and shortly afterwards joins the ramus communicans tibialis, to form the external or short saphenous nerve.

The terminal branches are three in number, as follows : recurrent articular, already referred to; anterior tibial (see p. 509); and musculo-cutaneous (see p. 503).

For the geniculate branch of the obturator nerve, see p. 474 .

Popliteal Lymphatic Glands.-These are usually four in number, and lie in close contact with the popliteal artery, one being 
superficial to the vessel, one beneath it, and the remaining two being disposed laterally. They receive their afferent lymphatics from the following sources: (I) sole of the foot; (2) deep part of the back of the leg; (3) some of the superficial lymphatics accompanying the ex. ternal or short saphenous vein ; and (4) the two efferent lymphatics which proceed from the anterior tibial lymphatic gland on the front of the interosseous membrane at its upper part. The course of these two efferent lymphatics is backwards above the interosseous membrane, or through the superior hiatus in it.

The efferent lymphatics of the popliteal glands ascend to become the afferent lymphatics of the deep femoral glands, which lie in contact with the femoral vein near Poupart's ligament.

\section{Front and Inner Side of the Thigh.}

Landmarks.--Thigh.--The anterior superior spinous process of the ilium is situated at the anterior extremity of the iliac crest, and can readily be felt. It is the point from which the measurement of the lower limb is taken. It is also a guide to the great trochanter of the femur, that prominence being situated about 4 inches below the spine, and about 4 inches behind a line let fall vertically from it: The crest of the ilium extends backwards in a curved manner from the anterior superior spinous process. Poupart's ligament passes between the anterior superior iliac spine and the pubic spine. It can be felt as a tense, firm band, especially when the thigh is extended, abducted, and rotated outwards. Immediately below it the inguinal lymphatic glands may be felt. Poupart's ligament, when traced inwards, conducts the finger to the pubic spine, which is situated at the lower and inner part of the anterior abdominal wall about $\mathrm{I}_{4}^{\frac{1}{4}}$ inches outside the upper part of the sym. physis pubis. It is sometimes a sharp-pointed process, and then $\mathrm{i}$ can readily be felt beneath the integument. In most bodies, how ever, it takes the form of a more or less indistinct tubercle, and especially in corpulent bodies, it cannot be felt. In such cases th scrotal integument may be invaginated with the finger so as t raise the adipose tissue from over the spine. If it cannot be felt is this way, the thigh should be well abducted to render prominent th adductor longus muscle, the tendon of origin of which will serve as guide to the spine, which lies above and to the outer side of it. Th pubic spine is the guide to the following openings: the extern abdominal ring, which lies immediately above and to the outer sid of it; the crural or femoral ring, which is situated fully I inc external to the spine in a line drawn outwards from it across th front of the thigh; and the saphenous opening, which is situate below, and a little external to, it.

The pubic crest extends inwards for about I inch from $t]$ pubic spine, and terminates in the pubic angle above the syn physial surface of the os pubis. The crest may be felt if the scrot integument is invaginated and the little finger passed into the $\mathrm{e}$ 
ternal abdominal ring, of which the crest forms the base. The angle cannot usually be felt. The ischio-pubic ramus can be felt extending from the tuber ischii at the back of the limb to the lower part of the symphysis pubis. The saphenous opening is situated below and external to the pubic spine, and it extends downwards for about $\mathrm{I} \frac{1}{2}$ inches below the inner third of Poupart's ligament. At the lower extremity of the opening the superficial femoral or saphenous lymphatic glands may be felt. The most prominent part of the head of the femur will be felt immediately below Poupart's ligament, at a point just external to its centre.

The great trochanter is situated about 4 inches below the anterior superior iliac spine, and about 4 inches behind a line let fall vertically from it. Its outline is more or less obscured by the muscles which cover it, but the following guides may serve to localize the prominence :

Holden's Guide.-The top of the great trochanter is pretty nearly on a level with the pubic spine in the recumbent posture.

Nélaton's Line.-This is a line drawn from the anterior superior iliac spine to the most prominent part of the tuber ischii. It coincides with the top of the great trochanter, and-runs through the centre of the acetabulum.

Bryant's Test-Line. - The subject being in the horizontal position, a triangle (ilio-femoral) is constructed in the following manner: draw two lines from the anterior superior spine of the ilium, one being vertical and traversing the outside of the hip to the horizontal plane of the body, and the other impinging on the tip of the great trochanter. Then construct the base by drawing a line from, and at right angles to, the vertical line to the trochanter. The base represents Bryant's test-line for shortening of the neck of the femur. 'Any shortening of this line, on comparing it with the same taken on the uninjured side, indicates with precision a shortening of the neck of the thigh-bone.'

The position of Scarpa's triangle is indicated by a slight depression which exists below Poupart's ligament. The outline of the adductor longus muscle is easily seen when the limb is firmly abducted. If the prominence thereby produced is followed upwards, the narrow round tendon of origin of the muscle is readily felt at a point on the body of the os pubis below and internal to the pubic spine. The rectus femoris gives rise to a median vertical prominence on the front of the thigh. The well-marked prominence over the lower fourth of the thigh on its inner aspect is due to the vastus internus muscle. When the knee is flexed, the narrow round tendon of the adductor magnus can be felt as it descends, posterior to the vastus internus, to reach the adductor tubercle of the femur.

Knee.-The outline of the patella is readily felt along with that of the ligamentum patellæ, which passes from the lower pointed end of the patella to the tubercle of the tibia.

The internal condyle of the femur forms a large prominence looking inwards. On its inner surface the blunt internal tuberosity is easily felt. At its upper and back part the adductor tuberele can be felt, when the knee-joint is flexed, with the narrow round tendon of the adductor magnus taking insertion into it: The upper border of the 
patellar surface of the femur may be felt when the knee-joint is flexed, but it is somewhat obscured by the tendon of the quadriceps extensor cruris. It is oblique in direction, and rises higher externally than internally. The adductor tubercle is on the same level as the outer part of the upper border of the patellar surface. A line connecting these two points, and at the same time cutting the adductor tubercle into two, indicates the line of junction of the lower epiphysis of the femur with the shaft at the twentieth year. The external condyle of the femur; though not very prominent, may be felt. The external tuberosity of the tibia forms a marked prominence at the outer and anterior part of the knee-joint, and it here presents a tubercle, or ridge, for the ilio-tibial band of the fascia lata. The head of the fibula is easily felt below and behind the external tuberosity of the tibia. The tubercle of the tibia is felt at the upper end of the sharp anterior border or crest of the bone. Its upper border is on the same level as the upper part of the head of the fibula. It is to be noted that the internal tuberosity of the tibia has a slight inclination backwards as well as inwards.

The lower limit of the synovial membrane of the knee-joint corresponds with the level of the tubercle, or ridge, on the anterior and outer part of the external tuberosity of the tibia for the attachment of the ilio-tibial band of the fascia lata. If this tubercle cannot be felt, a transverse line just above the head of the fibula. indicates the lower limit of the membrane. In this direction the membrane clothes the deep or posterior surface of the ligamentum patellæ in its upper half. Behind the lower end of this ligament there is a bursa separating it from the upper smooth part of the tubercle of the tibia. Superiorly the synovial membrane extends upwards above the patella in the form of a large pouch upon the front of the femur for about 2 inches above the upper border of the patellar surface of the bone. This pouch lies beneath the tendon of the quadriceps extensor cruris, and communicates with a bursa which is situated immediately above it, and which extends upwards for about another inch beneath the tendon.

In extension of the knee-joint the patella is situated above the level of the condyles of the femur. In flexion it lies over the intercondylar fossa. In extreme flexion the patella articulates chiefly with the semilunar impression on the outer part of the tibial surface of the internal condyle of the femur, close to the intercondylar fossa. The particular part of the patella which so articulates is the inner vertical zone on its posterior surface, immediately adjoining its internal border. In flexion of the joint there is a depression on either side of the ligamentum patellæ, and also on either side of the patella itself, the latter depression being greater on the inner than on the outer side. In this position of the joint the anterior margin of each tibial tuberosity is readily felt, and, above each, there is a hollow which separates it from the corresponding femoral condyle. In extension of the joint the depression on either side of the patella is also manifest, being, as in flexion, greater on its inner side. The 
depression on either side of the ligamentum patellæ, however, is not present, this being due to the lateral dispersion of the fat which normally lies underneath the ligamentum patellæ.

Cutaneous Nerves. Femoral Branch of Genito-femoral Nerve.The genito-femoral nerve is a branch of the lumbar plexus, its fibres being derived from the first lumbar and the ventral division of the second lumbar nerves. Its femoral branch emerges beneath Poupart's ligament immediately to the outer side of the femoral artery, and within the femoral sheath. Having supplied a twig to the coats of that vessel, it pierces the sheath and the fascia lata, to be distributed to the integument over Scarpa's triangle.

Inguinal Nerve (Ilio-inguinal). - This is a branch of the lumbar plexus, its fibres being derived from the first lumbar nerve. It emerges from the inguinal canal through the external abdominal ring, where it lies directly to the outer side of the spermatic cord, or round ligament of the uterus, according to the sex. It then pierces the intercolumnar fascia, and is distributed to the integument of the inner side of the thigh in the upper third, and to the adjacent integument of the scrotum in the male and the labium majus in the female.

External Cutaneous Nerve.-This is a branch of the lumbar plexus, its fibres being derived from the dorsal divisions of the second and third lumbar nerves. It emerges beneath the outer end of Poupart's ligament, and shortly afterwards divides into a small posterior and a large anterior division. The posterior division is distributed to the integument of the outer side of the thigh in the upper fourth, and also to the integument of the outer and lower part of the gluteal region. The anterior division is at first contained in a tube of the fascia lata for about 4 inches, and then it enters the integument of the outer side of the thigh which it supplies, reaching in some cases as low as the patella, and taking part in the patellar plexus.

Middle Cutaneous Nerve. - This is a branch of the anterior division of the anterior crural nerve. It pierces the fascia lata in two divisions, outer and inner, about 4 inches below Poupart's ligament. The outer division usually passes through the upper part of the sartorius, and then descends with the inner division to supply the integument of the front of the thigh in the lower two-thirds. The two divisions extend as low as the patella, where they take part in the patellar plexus.

Internal Cutaneous Nerve.-This is a branch of the anterior division of the anterior crural nerve. It crosses over the femoral artery from without inwards near the apex of Scarpa's triangle, either as one nerve, or in its two final divisions, anterior and posterior. These two divisions now descend on the inner side of the thigh beneath the fascia lata, and along the line of the long saphenous vein. At the junction of the middle and lower thirds of the thigh the anterior division pierces the fascia lata, and descends near'the tendon of the adductor magnus to the inner side of the knee-joint, 
supplying the integument of the inner side of the thigh in the lower third. It finally turns outwards over the patella, and enters into the patellar plexus. The posterior division descends along the posterior border of the sartorius, and keeps beneath the fascia lata until it reaches the level of the internal condyle of the femur. Here it pierces the fascia lata, and descends to the inner side of the leg, lying posterior to the long saphenous nerve, and supplying the integument as low as the centre. The internal cutaneous nerve, whilst in Scarpa's triangle, gives off two or three cutaneous branches, which, having pierced the fascia lata, cross the femoral artery and are distributed to the integument of the inner side of the thigh in the middle third, along the course of the long saphenous vein. The posterior division of the nerve gives off a branch to the subsartorial plexus about the centre of the thigh on its inner aspect.

Internal or Long Saphenous Nerve.-This is a branch of the posterior division of the anterior crural nerve, and is deeply placed as far as the knee-joint. In the lower two-thirds of Scarpa's triangle it lies close to the outer side of the femoral artery, and in Hunter's canal it gets in front of that vessel. It leaves Hunter's canal at its lower end by piercing the aponeurotic roof, in company with the superficial branch of the anastomotica magna artery, and then it descends beneath the sartorius to the inner side of the kneejoint. Here it pierces the fascia lata on a level with the lower border of the sartorius, and enters the inner side of the leg, along which it courses, usually in two divisions, in company with the long saphenous vein, the larger division being behind that vessel, and the smaller in front. At the ankle-joint the larger division passes in front of the internal malleolus, and then along the inner border of the foot as far as the centre. The long saphenous nerve, in passing through Hunter's canal, gives off a branch which pierces the aponeurotic roof, and enters into the subsartorial plexus. At the level of the internal femoral condyle it furnishes its patellar branch. This passes through the sartorius and fascia lata, and is distributed to the integument over the front of the knee, where it takes part in the patellar plexus.

In its further course beyond the knee-joint the nerve supplies branches to the integument of the inner side of the leg, back of the leg in its lower and inner part, internal malleolus, and inner side of the foot as far as the centre. In the upper part of the leg it communicates with the posterior division of the internal cutaneous nerve, and on the inner side of the foot it is connected with the musculo-cutaneous.

Obturator Nerve.-The anterior division of the obturator nerve usually furnishes a branch which pierces the fascia lata about the centre of the thigh, between the posterior border of the sartorius and the anterior border of the gracilis, to be distributed to the integument for a variable distance.

Patellar Plexus.-This free interlacement of nerves is so named because it is situated over the anterior surface of the patella. 


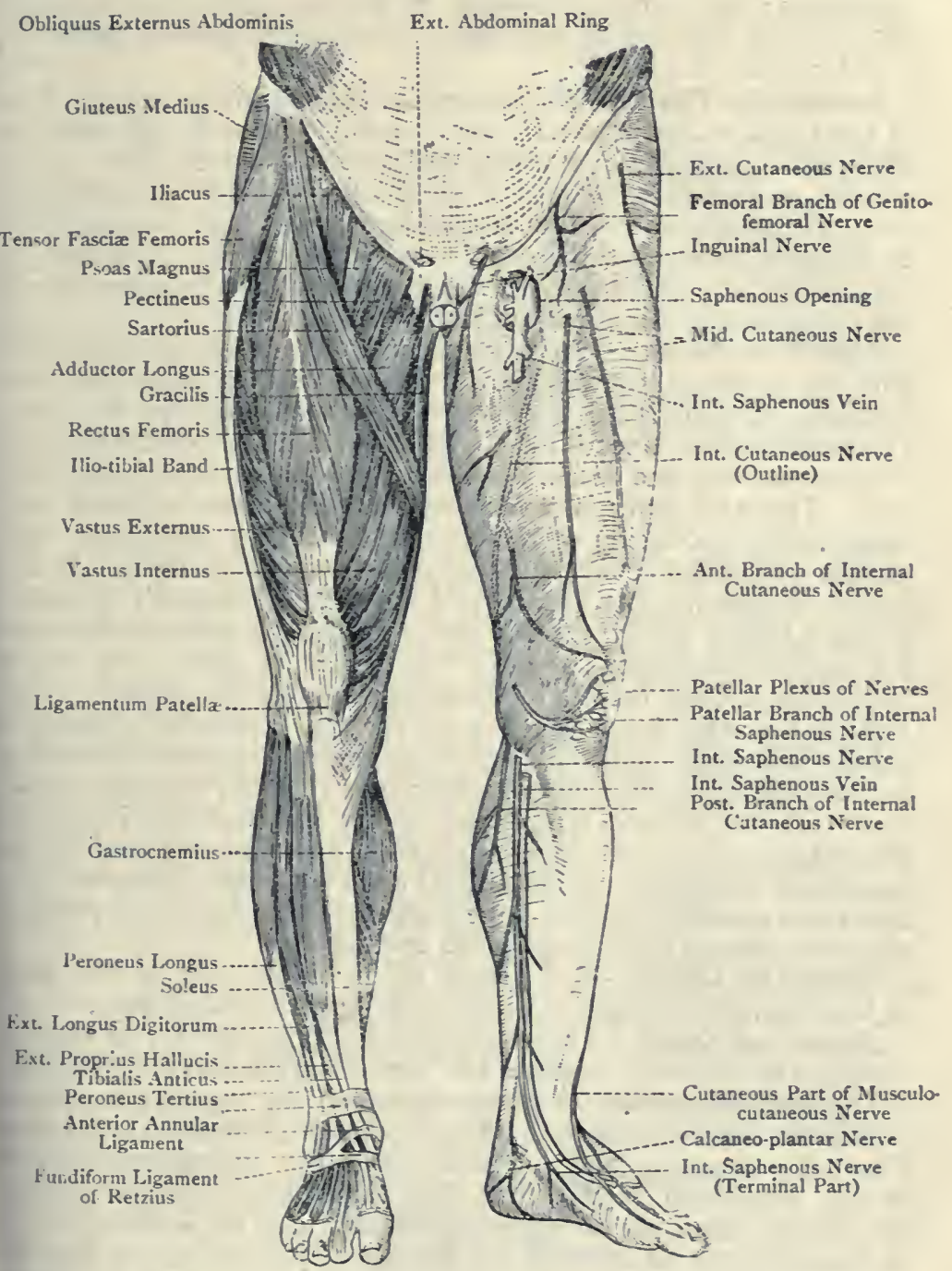

Fig. 224.-Muscles and Cutaneous Nerves of the Lower Limb (ANTERIOR ASPECT). 
The nerves which take part in it are as follows: the patellar branch of the long saphenous; both divisions of the middle cutaneous; the anterior division of the internal cutaneous; and the anterior division of the external cutaneous (which, however, is not constant).

Subsartorial Plexus. - This plexus is situated in the middle third of the thigh on its internal aspect, and lies beneath the sartorius upon the aponeurotic covering of Hunter's canal. The nerves which take part in its formation are as follows: the long saphenous; the posterior division of the internal cutaneous ; and the superficial or anterior division of the obturator. The branches furnished by this plexus are distributed to the integument of the inner side of the thigh for about its middle third.

Superficial Fascia.-The only point to be noted in connection with the superficial fascia is that, for about 3 inches below Poupart's ligament, it is divisible into two layers-subcutaneous and deep. The subcutaneous layer is fatty, and, when traced upwards, becomes continuous with the fascia of Camper on the anterior abdominal wall. The deep layer is a very delicate membrane, which is best seen on the inner side of and beneath the long saphenous vein, as that vessel lies between it and the subcutaneous layer. It can be traced upwards to a line about $\frac{1}{4}$ inch below Poupart's ligament, where it blends with the fascia lata. In passing upwards it covers the saphenous opening, to the outer margin of which it is closely bound by fibrous bands, more especially over the middle third. The part of this layer which lies over the saphenous opening is called the cribriform fascia, because it presents numerous foramina for the passage of the following strùctures: (I) the long saphenous vein ; (2) the efferent lymphatics of the inguinal and superficial femoral glands ; $(3)$ the superior external pudic artery; and (4) the superficial epigastric and superficial circumflex iliac arteries. The latter two vessels, however, sometimes pierce the fascia lata forming the outer margin of the saphenous opening.

Lymphatic Glands. - The glands of the upper part of the front of the thigh are arranged in two groups-superficial and deep.

Superficial Group.- The glands which comprise this group are arranged in two sets-inguinal and superficial femoral or saphenous.

The inguinal glands are otherwise called the superior or oblique superficial inguinal glands. They are situated immediately below Poupart's ligament, and lie underneath the subcutaneous layer of the superficial fascia, their long axes being oblique. They are usually from six to eight in number, and the innermost two or three, which lie internal to the upper part of the saphenous opening in the vicinity of the pubic spine, are spoken of as the pubic glands. The inguinal glands receive their afferent lymphatics from the following sources:

I. The superficial lymphatics from the infra-umbilical portion of the anterior wall of the abdomen, except those from the suprapubic region.

2. The superficial lymphatics from the outer two-thirds of the gluteal region. 
3. The superficial lymphatics from the outer and back parts of the thigh superiorly.

The pubic glands receive their afferent vessels from the following sources:

I. The superficial lymphatics from the suprapubic region of the anterior wall of the abdomen.

2. The superficial lymphatics from the upper and inner parts of the thigh.

3. Some of the superficial lymphatics from the inner one-third of the gluteal region.

4. Most of the lymphatics from the scrotum.

5. The lymphatics from (a) the integument of the penis, including the prepuce and most of the penile portion of the urethra, $(b)$ the prepuce of the clitoris, (c) the labium majus, and $(d)$ the labium minus or nympha. (A few of these lymphatics may pass to the superficial femoral glands.)

6. The superficial lymphatics from the perineum (a few of which may pass to the superficial femoral glands).

7. The lymphatics of the lower part of the anal canal below the anal valves, and of the anus (a few of which occasionally pass to the superficial femoral glands).

8. Some of the lymphatics from the lower one-third of the vagina.

9. A few lymphatics from the body of the uterus.

The efferent lymphatics of both the inguinal and pubic glands pierce the cribriform fascia and anterior wall of the femoral sheath. Thereafter most of them end in the deep femoral glands, most of which lie within the middle compartment of the femoral sheath. A few of them, however, may ascend into the abdomen, and end in the lower external iliac glands.

Blood-Supply.-The inguinal glands are supplied by the superficial circumflex iliac and superficial epigastric branches of the common femoral artery; and the pubic glands are supplied by the superior external pudic branch of that vessel.

The superficial femoral or saphenous glands are otherwise called the inferior or vertical superficial inguinal glands. They are situated at the lower end of the saphenous opening, where they lie, with their long axes vertical, on either side of the terminal part of the internal or long saphenous vein, underneath the subcutaneous layer of the superficial fascia. They are usually from four to six in number, and they reçeive their affereat lymphatics from the following sources:

I. The superficial lymphatics of the foot and leg (internal saphenous lymphatics), except the external saphenous lymphatics which pass to the popliteal glands.

2. The superficial efferent lymphatics of the popliteal glands.

3. The superficial femoral lymphatics, except (1) those from the outer and back parts of the thigh superiorly, which pass to the inguinal glands; and (2) those from the upper and inner parts of the thigh, which pass to the pubic glands.

4. Some of the lymphatics of the knee-joint.

5. Some of the superficial lymphatics from the inner one-third of the gluteal region.

5. Occasionally some of the lymphatics fron $(a)$ the perineum, $(b)$ the scrotum, (c) the integument of the external genital organs (see Pubic Glands), and $(d)$ the lower part of the anal canal and the anus. 


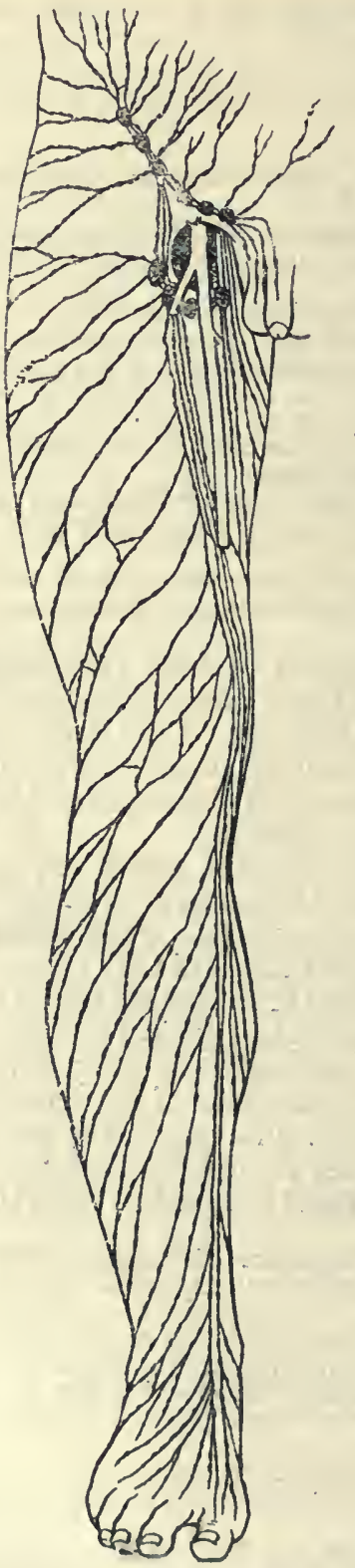

Fig. 225.-The Superficial Lymphatics of the Lower Limb. 
The efferent lymphatics of the superficial femoral or saphenous glands pierce the cribriform fascia, and terminate in a manner similar to those of the inguinal glands.

Blood-supply.-These glands derive their arterial supply from two or three saphenous branches of the superficial femoral artery.

Deep Group.-These are called the deep femoral glands (deep inguinal glands). They are from three to four in number, and are situated within the femoral sheath. One of them (the gland of Cloquet) lies in the femoral canal at its upper end, being attached to the inferior surface of the septum femorale. The other glands are situated in the middle compartment of the femoral sheath on the inner side of the femoral vein. The glands receive their afferent lymphatic vessels from the following sources:

I. The inguinal glands.

2. The superficial femoral or saphenous glands.

3. The deep lymphatics of the knee and thigh.

4. The popliteal glands.

The efferent lymphatics of the deep femoral glands enter the abdominal cavity, where they become the afferent lymphatics of the external iliac glands.

Blood-supply. - The deep femoral glands derive their arterial supply from the superior or superficial external pudic branch of the common femoral artery.

Internal or Long Saphenous Vein.-This vessel arises from the inner end of the venous arch on the dorsum of the foot. It passes in front of the internal malleolus and along the inner side of the leg, where it lies about a finger's breadth from the internal border of the tibia. It then courses along the inner side of the knee-joint, being situated behind the most prominent part of the internal condyle of the femur, and so it enters the thigh. Thereafter it ascends along the inner side of the thigh superficial to the sartorius, until it reaches the upper third of that division of the limb. Here it turns to the front of the thigh, and in its subsequent course lies at the junction of the inner fourth and outer three-fourths. On reaching a point $I_{\frac{1}{2}}$ inches below Poupart's ligament it pierces the cribriform fascia and anterior wall of the crural sheath, and terminates in the femoral vein. Throughout its whole course the vein is superficial to the deep fascia. It receives many tributaries from the front and back of the leg and thigh, and it communicates at frequent intervals with the venæ comites of the anterior and posterior tibial arteries, these communicating branches being intermuscular. Two tributaries in the upper part of the thigh are specially noteworthy from their size and constancy. One, collecting the blood from the front of the thigh, is called the external femoral cutaneous, or anterior saphenous, vein. The other returns the blood from the inner and back parts of the thigh, and is called the internal femoral cutaneous, or posterior saphenous, vein. These two tributaries terminate in the long saphenous vein near the 
saphenous opening. It is to be noted that the external femoral cutaneous or anterior saphenous vein lies over the sartorius muscle at the apex of Scarpa's triangle, where it is liable to be cut in operating in this situation. Before piercing the cribriform fascia the internal or long saphenous vein receives as final tributaries the following veins: the superficial circumflex iliac; the superficial epigastric; the superior or superficial external pudic; and the inferior or deep external pudic. The long saphenous vein has usually about fifteen valves. One of these is found in the vein just before it pierces the cribriform fascia, and another at its opening into the femoral vein.

The guide to the vein in the femoral part of its course is a line drawn from a point immediately behind the most prominent part of the internal condyle of the femur to a point on the front of the thigh $I_{\frac{1}{2}}$ inches below Poupart's ligament, at the junction of the inner fourth and outer three-fourths of the limb.

The vein is occasionally double in the femoral part of its course. This condition is brought about by the vein dividing into two branches shortly after it enters the thigh, which ascend close together and unite to form one trunk close to the saphenous opening.

Deep Fascia or Fascia Lata. - The fascia lata is a very strong fibrous membrane which forms a continuous tubular sheath swathing the powerful muscles in this region. Superiorly it is attached as follows: (I) to Poupart's ligament as far inwards as the pubic spine; (2) to the outer lip of the iliac crest, where it covers the anterior two-thirds of the gluteus medius; (3) to the posterior lamina of the lumbar aponeurosis ; $(4)$ to the back of the lower end of the sacrum and coccyx; (5) to the lower border of the great sacro-sciatic ligament; (6) to the tuber ischii; (7) to the ischiopubic ramus; $(8)$ to the anterior or femoral surface of the body of the os pubis close to the symphysis; and (9) to the anterior lip of the pubic crest as far outwards as the pubic spine. Inferiorly, in the region of the knee, the fascia lata is disposed as follows: (I) on the outer side it is attached to the head of the fibula and external tuberosity of the tibia; (2) anteriorly it is attached to the lateral borders of the patella, in which latter situations it forms the larger portions of the so-called lateral patellar ligaments; from its attachments to the lateral borders of the patella an expansion is sent over that bone and downwards to the head of the tibia, which confines the prepatellar bursa; (3) posteriorly it is continued uninterruptedly into the back of the leg, covering the popliteal space as it descends, and becoming continuous with the deep fascia; (4) internally it is continued into the inner side of the leg, taking a limited attachment to the internal tuberosity of the tibia, and becoming continuous with the deep fascia.

The fibres of the fascia lata interlace freely, some of them being circular and others longitudinal. It is pierced by a large 
number of minute foramina for the passage of bloodvessels and nerves. The fascia is strongest upon the outer side of the thigh, where it gives insertion superiorly to a large part of the gluteus maximus, and lower down to the tensor fasciæ femoris. It is also strong over the popliteal space and prepatellar region, receiving in the former situation accessions of fibres from the hamstring tendons, and, in the latter, similar accessions from the tendons of the muscles which form the quadriceps extensor cruris. Upon the inner side of the thigh the fascia is very thin.

The portion upon the outer side of the thigh, between the fore part of the iliac crest and the anterior part of the external tuberosity of the tibia and outer border of the patella, is spoken of as the ilio-tibial band. At the insertion of the tensor fascix femoris it gives off a deep lamina which passes upwards on the deep surface of the muscle to be attached to the bottom of the groove on the dorsum of the ilium above the acetabulum, where the posterior or reflected head of the rectus femoris takes its origin. This deep lamina is intimately connected with the capsular ligament of the hip-joint and the tendon of the gluteus minimus.

The fascia lata on the front of the thigh divides at a point $\mathrm{I}_{\frac{1}{2}}$ inches below the inner third of Poupart's ligament into two laminæ, iliac and pubic. The iliac lamina passes upwards to be attached to Poupart's ligament, lying superficial to the outer portion of the anterior wall of the crural sheath. The pubic lamina is on a deeper or more posterior plane than the iliac, and passes upwards upon the flat pectineus muscle to be attached to the pectineal portion of the ilio-pectineal line and anterior

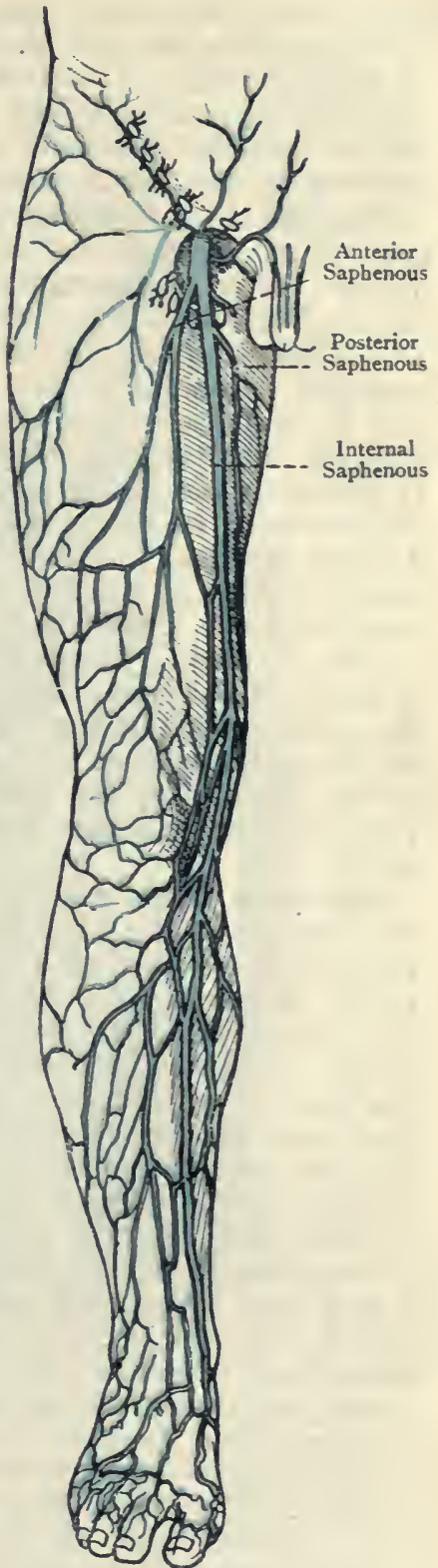

Fig. 226. -The INTERNAL SAPHENOUS VEIN AND ITS Tributaries. 
lip of the pubic crest. This pubic lamina, when followed outwards, passes behind the crural sheath and ends by blending with its posterior wall. It will thus be seen that the iliac lamina is superficial or anterior to the femoral vessels enclosed in the crural sheath, and that the pubic lamina is on a plane deeper than, or posterior to, the femoral vessels.

Processes of the Fascia Lata.-These processes, which for the most part are indicated on the surface by white lines, pass between the muscles, forming intermuscular septa, and taking part in the muscular sheaths. Three of them are specially noteworthy, and are called external, internal, and posterior. The external intermuscular septum is a deep expansion from the ilio-tibial band, and it is attached to the outer lip of the linea aspera and external supracondylar ridge of the femur in its whole extent. It separates the vastus externus and crureus in front from the femoral head of the biceps behind, and it gives partial origin to these muscles. The internal intermuscular septum is a delicate process, which is attached to the inner lip of the linea aspera, especially in its lower part. Over the internal supracondylar ridge its place is taken by an expansion from the tendon of the adductor magnus. This septum separates the vastus internus in front from the adductor longus and adductor magnus behind. The posterior intermuscular septum is a very delicate process which is attached to the summit of the linea aspera. It is situated behind the adductor magnus, which it separates from the femoral head of the biceps.

Saphenous Opening.-This is an opening in the fascia lata for the passage of the long saphenous vein, and it is of importance surgically inasmuch as a femoral hernia may protrude through it. It is situated on the front of the thigh below and outside the pubic spine, just below the inner third of Poupart's ligament. It is not so much an opening as an oval depression in the fascia lata, formed by the division of that fascia into its iliac and pubic laminæ, the iliac lamina passing upwards superficial to the outer part of the anterior wall of the crural sheath, and the pubic lamina passing upwards on a plane posterior to the posterior wall of the crural sheath, with which it becomes continuous. The average length of the opening is $\mathrm{I}_{\frac{1}{2}}$ inches, and its breadth is from $\frac{1}{2}$ to $\frac{3}{4}$ inch. The lower border of the opening is called the inferior cornu, and over this the long saphenous vein passes. It is firm, sharp, and crescentic, with the concavity directed upwards. The inner part, formed by the pubic lamina of the fascia lata, is for the most part flat, the fascia being here spread out upon the pectineus muscle, whence it passes outwards to blend with the posterior wall of the crural sheath. Over the lower fourth, however, the opening has a distinct inner border, which is continuous with the inferior cornu. The outer border, formed by the iliac lamina of the fascia lata, is on a plane superficial to the outer part of the anterior wall of the crural sheath, to which it is kound by fibrous bands. In- 
feriorly this border is continuous with the inferior cornu. Superiorly it curves inwards over the anterior wall of the crural sheath. The whole border is concave or semilunar, the concavity being directed inwards and slightly downwards. The upper border is the portion in front of the anterior wall of the crural sheath, and it lies immediately below Poupart's ligament. It is called the superior cornu.

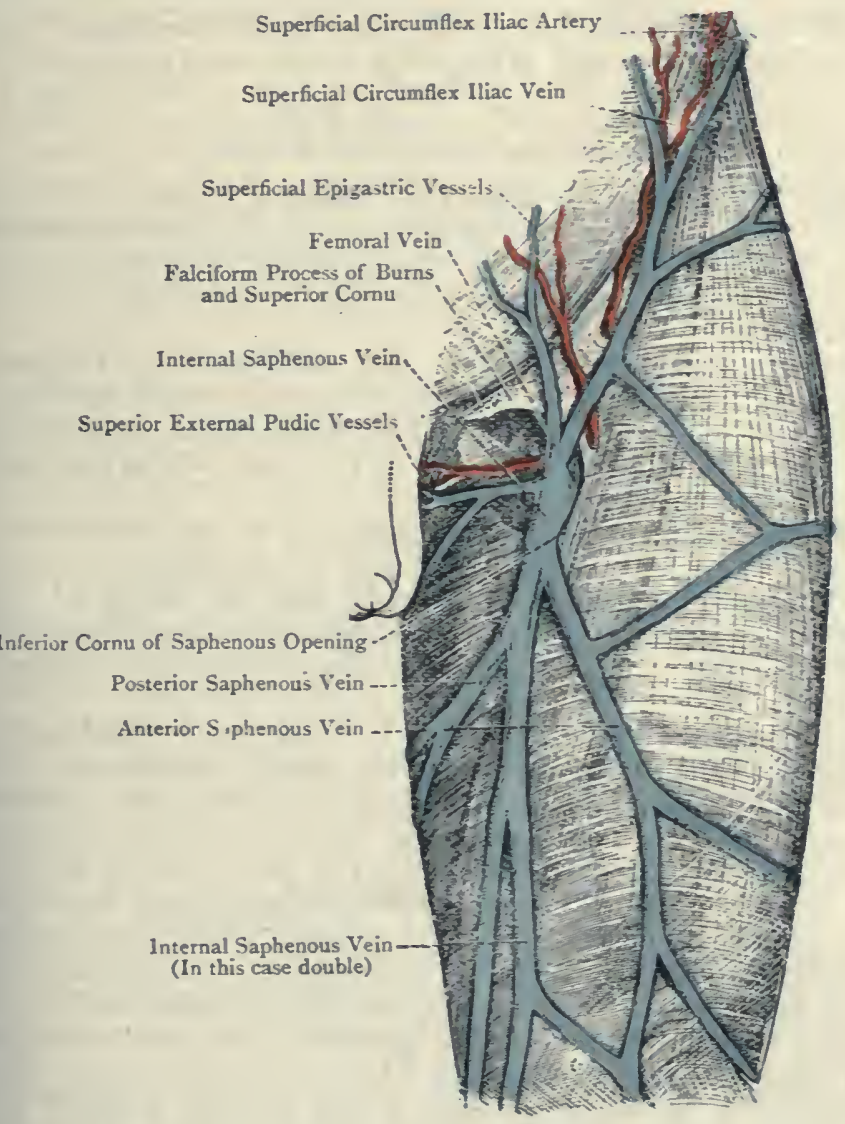

Fig. 227.-The Left Ixtersal Saphexous Vein in the Thigh, with its TRIBUtaries, AND THE SAPHENOUS OPENING.

It is triangular, and is continuous with the outer border. Superiorly it is attached to the inner part of Poupart's ligament, extending inwards as far as the pubic spine, and a few of its fibres join the antero-inferior or femoral surface of Gimbernat's ligament. The inner portion of the superior cornu is internal to the femoral vein, and lies in front of the crural canal. It is this portion, inside the 
femoral vein, which has been called the falciform process of Burns (John), ${ }^{*}$ or the femoral ligament of Hey. The importance of this process or ligament is that it impresses upon a femoral hernia its final change in direction, inasmuch as it causes the protrusion to turn upwards over Poupart's ligament upon the anterior abdominal wall, or upwards and outwards towards the anterior superior iliac spine.

The saphenous opening is covered by the cribriform fascia, which is more intimately attached to the outer border than elsewhere.

\section{Parts concerned in Femoral Hernia.}

Poupart's Ligament or the Superficial Femoral Arch.-This is the thickened lower border of the aponeurosis of the external oblique muscle. It is folded upon itself in a backward direction, and is attached externally to the anterior superior iliac spine and internally to the pubic spine.

Gimbernat's Ligament.-This is the reflection of Poupart's ligament from the pubic spine along the ilio-pectineal line for I inch. It is triangular, and presents a free base which is sharp, wiry, and concave, and is situated immediately to the inner side of the crural or femoral ring.

Deep Femoral Arch.-This is a thickening of the fascia transversalis, due to an accession of fibres as it is prolonged downwards beneath Poupart's ligament. It extends from the centre of that ligament on its deep aspect to the pectineal portion of the iliopectineal line, where it is attached behind Gimbernat's ligament near its base.

Falciform Process of Burns.-This has just been described in connection with the superior cornu of the saphenous opening.

Femoral Sheath.-This sheath extends from Poupart's ligament to a point about $I_{\frac{1}{2}}$ inches below it, where it blends with the special sheath of the femoral vessels. The outer wall is straight, but the inner is oblique, being sloped downwards and outwards. In shape the sheath resembles a funnel, from which circumstance the term infundibuliform has been applied to it. The anterior wall is formed by the fascia transversalis, and the posterior wall by the fascia iliaca, both of which fasciæ are prolonged downwards beneath Poupart's ligament. The outer wall is formed by the union of these two fascix close to the outer side of the femoral artery. The inner wall is formed by their union at a point $\frac{1}{2}$ inch to the inner side of the femoral vein.

Within the sheath there are two septa, external and internal, both of which extend from the anterior to the posterior wall, the external septum being close to the outer side of the femoral vein, and the internal septum close to its inner side. By means of these two septa the interior of the sheath is divided into three distinct compart-

* Edinburgh Medical and Surgical Journal, July 1, 1806, p. 269. 
ments-outer, middle, and inner. The outer compartment contains the common femoral artery and the femoral branch of the genitofemoral nerve for a short distance at its upper part, where the nerve lies immediately external to the artery. The middle compartment contains the femoral vein and three of the deep femoral glands. The inner compartment is called the crural or femoral canal, and it contains one of the deep femoral glands and a certain amount of adipose tissue, the gland lying at the upper end of the canal.

Femoral Canal.-This is the inner compartment of the femoral sheath, and it is $\frac{1}{2}$ inch in length. Its upper end forms the crural or femoral ring, and is on a level with the base of Gimbernat's ligament. Its lower end is just below the superior cornu of the saphenous opening. The boundaries of the canal are as follows: the anterior wall is formed by the fascia transversalis; the posterior wall by the fascia iliaca; the inner wall by the junction of the fascia transversalis and fascia iliaca; and the outer wall by the internal septum within the sheath.

Femoral Ring.-This is by no means a patent opening, but is bridged over by the subperitoneal areolar tissue of the abdominal wall. The septum thus formed between the crural canal and abdominal cavity is called the septum femorale (fascia of Cloquet). It protrudes slightly into the canal, and the lymphatic gland which lies at the upper end of the canal is attached to its under convex surface. When the septum crurale has been removed from the femoral ring, the aperture presents an oval shape, with the long measurement lying transversely, in which direction it measures $\frac{1}{2}$ inch. It readily admits the little finger, and it is somewhat larger in the female than in the male. The situation of the ring is close to the outer side of the base of Gimbernat's ligament, and its position may be ascertained in one of two ways.

(I) Draw a line from the pubic spine horizontally outwards across the front of the thigh, and take a point in this line fully $I$ inch to the outer side of the pubic spine.

(2) Find the central point between the anterior superior iliac spine and the symphysis pubis; then take a point on Poupart's ligament rather more than $\frac{1}{2}$ inch to the inner side of this central point.

Boundaries-Anterior.-The inner part of Poupart's ligament, and the deep femoral arch. Posterior.-The pubic lamina of the fascia lata, joined by Cooper's ligament;* the pectineus muscle; and the superior pubic ramus. Internal.-The base of Gimbernat's

* The pubic ligament of Cooper is a strong bundle of fibres extending between the ilio-pectineal eminence and the pubic spine, between which points it is attached to the pectineal portion of the ilio-pectineal line. It has the pectineal border of Gimbernat's ligament immediately behind it, and is closely incorporated with the pubic lamina of the fascia lata, where that is attached to the pectineal portion of the ilio-pectineal line. 
ligainent. External.-The upper extremity of the internal septum within the femoral sheath.

Normal Relation of Bloodvessels to the Femoral Ring-Anterior.The pubic branch of the deep epigastric artery, as it courses inwards behind the inner half of Poupart's ligament. Superoanterior. - The vessels of the spermatic cord in the male, or of the round ligament of the uterus in the female. Supero-external.-The deep epigastric vessels. External.-The femoral vein, becoming the external iliac vein, and separated from the ring by the upper extremity of the internal septum within the femoral sheath.

There are normally no vessels behind the ring, nor internal to it. Under no circumstances are there ever any vessels behind it, but in certain cases there may be a vessel on its inner side, namely, an abnormal obturator artery.

Abnormal or Aberrant Obturator Artery.-The obturator artery normally arises from the anterior division of the internal iliac, and under these circumstances it has no relation to the femoral ring. In about 30 per cent. of cases, however, the obturator arises from the deep epigastric artery near its commencement. This origin is more common in the female than in the male, and is rarely bilateral. In most of these cases the aberrant obturator passes backwards close to the inner side of the commencement of the external iliac vein, and therefore upon the outer side of the femoral ring. In a few cases the aberrant vessel passes backwards across the centre of the femoral ring. In very rare cases it passes inwards behind Poupart's. ligament, and then arches backwards either close to the base of Gimbernat's ligament, or a line or two inwards from it, and therefore upon the inner side of the femoral ring. If a femoral hernia should occur under these latter circumstances, the aberrant obturator artery would lie upon the inner side of the neck of the sac, and it would thus be endangered in the operation for the relief of the strangulation. This very rare position of an aberrant obturator artery is more frequent in the male than in the female, and, according to Lawrence, it occurred once in a hundred cases.

The origin of an aberrant obturator artery from the deep epigastric is duc to a more or less complete obliteration of a normal obturator at its origin. and an enlargement of the normal anastomosis which takes place between the pubic branches of the deep epigastric and obturator arteries behind the body of the os pubis.

Femoral Hernia. - This is a protrusion of an abdominal viscus, or part of a viscus, through the crural or femoral ring. The course of the hernia is as follows: entering the crural ring by elongating the septum crurale, it descends through the femoral canal, on reaching the lower end of which it is placed in the upper part of the saphenous opening, immediately below the falciform process of Burns or femoral ligament of Hey. This structure now impresses upon the hernia the following change in its course: the front part of the hernia being arrested in its downward course by that structure, the posterior part, which is free, comes down, and, passing forwards, turns upwards upon the anterior abdominal wall, or upwards and outwards. along Poupart's ligament. The course of a femoral hernia, when complete, is thus at first downwards through the femoral ring and femoral canal, then forwards through the upper part of the saphenous opening, and finally either upwards or upwards and outwards.

Coverlngs of a Femoral Hernia.-The coverings, from within outwards, are as follows:

1. Peritoneum, which forms the sac.

2. Septum femorale. 
A

Deep Circumflex Iliac Artery

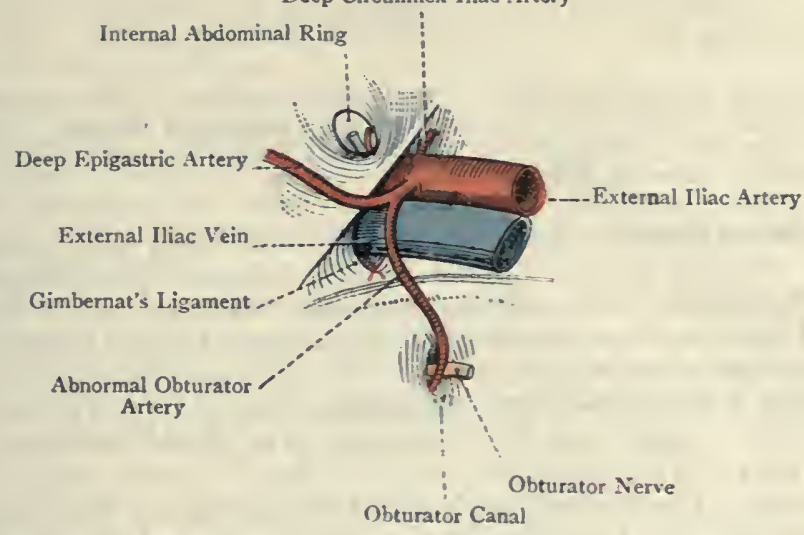

B

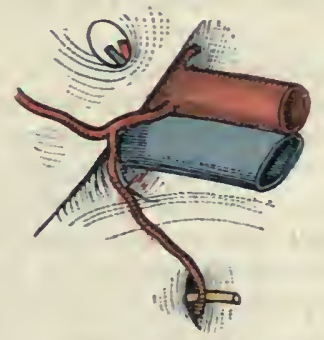

C

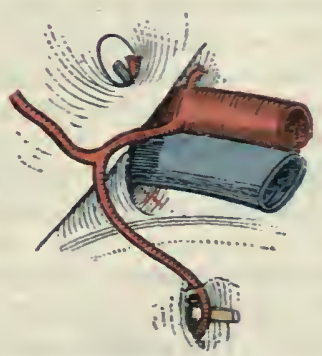

Fig. 228.-Diagrams of Abiormal Obturator Artery (The red $X$ indicates the position of the Femoral Ring).

[A and B after Gray].

A. Artery external to Femoral Ring; B, Artery internal to Ring, and close to Base of Gimbernat's Ligament; C, Artery internal to Ring, and one or two lines from Base of Gimbernat's Ligament. 
3. Anterior wall of the femoral sheath, or fascia transversalis.

4. Cribriform fascia.

5. Subcutaneous layer of superficial fascia.

6. Skin.

The septum crurale is usually inseparably united with the anterior wall of the femoral sheath, thus forming the fascia propria of Cooper. The neck of the sac is on a level with the base of Gimbernat's ligament, which is the most common cause of strangulation.

Prepatellar Bursa.--This bursa, which is of large size, is situated on the anterior surface of the patella beneath the integument. When it becomes inflamed the condition is known as housemaid's knee. There may be another subfascial bursa, between the fascia lata over the patella and the bone.

Anterior Femoral Museles. Sartorius-Origin--(I) The anterior superior iliac spine, and (2) the upper part of the anterior interspinous notch of the ilium.

Insertion.-The internal surface of the shatt of the tibia immediately behind the tubercle. From the tendon of insertion two expansions are given off-one from the upper border to the capsule of the knee-joint, and another from the lower border to the deep fascia of the leg.

Nerve-supply.-The anterior division of the anterior femoral nerve, the branches from which, arising in common with the middle cutaneous nerve, enter the muscle near the apex of Scarpa's triangle.

Action.-Acting from its origin the muscle is (I) a flexor of the knee-joint, and an internal rotator of the leg; and (2) a flexor of the hip-joint, and an external rotator and abductor of the thigh. Acting from its insertion it is a flexor of the pelvis upon the thigh.

The sartorius is a long ribbon-like muscle, the fasciculi of which are the longest of any muscle in the body. The muscle, therefore, has a wide range of movement, but comparatively little power. It is at first directed downwards and inwards over the front of the thigh, where it forms, by its inner border, the outer boundary of Scarpa's triangle. Having crossed the superficial femoral artery at a point about $3 \frac{1}{2}$ inches below Poupart's ligament, the muscle passes vertically downwards as far as the internal condyle of the femur. Beyond this point, it curves forwards to its insertion, where it covers the subjacent tendons of the gracilis and semitendinosus, from which it is separated by a bursa. This bursa is a prolongation of that which lies between the internal lateral ligament of the knee-joint and the more superficially placed tendons of the gracilis and semitendinosus. The muscle, in its lower part, is usually pierced by the patellar branch of the long saphenous nerve.

The two most important relations of the sartorius are as follows : (I) it crosses the superficial femoral artery from without inwards at a point about $3 \frac{1}{2}$ inches below Poupart's ligament; and (2) it covers the superficial femoral artery in Hunter's canal.

Tensor Fasciæ Femoris.-Although this muscle is on the same plane as the gluteus maximus, "it may be described in this place. 
Origin.-(I) The outer lip of the crest of the ilium for $I \frac{1}{2}$ inches at its anterior part; (2) the dorsum ilii for $\frac{1}{4}$ inch close to the upper part of the anterior interspinous notch; and (3) the fascia lata covering the muscle.

Insertion. - The angle of division of the ilio-tibial band of the fascia lata into a superficial and deep lamina, at the junction of the upper fourth and lower three-fourths of the thigh.

Nerve-supply.-The lower division of the superior gluteal nerve from the sacral plexus, the branch of which enters the muscle on its deep surface.

Action.-(I) Abductor and internal rotator of the thign; and (2) extensor of the knee-joint. The muscle, in association with. the gluteus maximus, takes part in the completion of extension of the knee-joint, and in the maintenance of extension through means of the ilio-tibial band, as in standing.

The tensor fasciæ femoris is a flat, strap-like muscle, the direction of which is downwards, with a slight inclination outwards and backwards.

Ilio-Psoas.-The femoral portion of this muscle is alone described in this place. For a full description of the psoas magnus and iliacus, see Abdomen Section. The ilio-psoas is inserted into the small trochanter of the femur, the outer fibres of the iliacus taking insertion into the triangular surface which is situated below and in front of the small trochanter, between it and the spiral line.

The outer portion of the muscle, which is fleshy, represents the iliacus, and the inner portion, which is tendinous, represents the psoas magnus. Lying deeply between the two is the anterior crural nerve; the femoral artery, enclosed within the femoral sheath, rests upon the psoas magnus; and the femoral vein, similarly enclosed, lies between the psoas magnus and pectineus. The iliopsoas covers the front of the capsular ligament of the hip-jcint, a bursa intervening between the psoas portion and the ligament.

Pectineus-Origin.-(I) The pectineal portion of the ilio-pectineal line of the os pubis; (2) the adjacent portion of the upper or pectineal surface of the superior pubic ramus; and (3) the pubic lamina of the fascia lata, as it covers the muscle in this situation.

Insertion.-The upper third of the line which leads from the back of the small troshanter of the femur to the inner lip of the linea aspera.

Nerve-supply.-The anterior division of the anterior femoral nerve, the branch from which passes inwards behind the femoral sheath, and enters the superficial surface of the muscle close to its outer border. The pectineus sometimes receives an additional nerve from the anterior division of the obturator, or from the accessory obturator (when present), which enters the deep surface of the muscle.

Action.-(I) Flexion of the hip-joint, and (2) adduction and external rotation of the thigh, as, for example, in crossing one leg over the other.

The pectineus is a flat, four-sided muscle, which is directed down- 
wards, outwards, and backwards. The surfaces at first look forwards and backwards, but, towards its insertion, the muscle undergoes a slight twist, and its surfaces are then external and internal. Its deep surface is related to the capsular ligament of the hip-joint, the anterior division of the obturator nerve, and the adductor brevis.

Quadriceps Extensor Cruris.-This is a composite muscle, which consists of the rectus femoris, vastus externus, crureus, and vastus internus. The rectus femoris arises from the os innominatum,

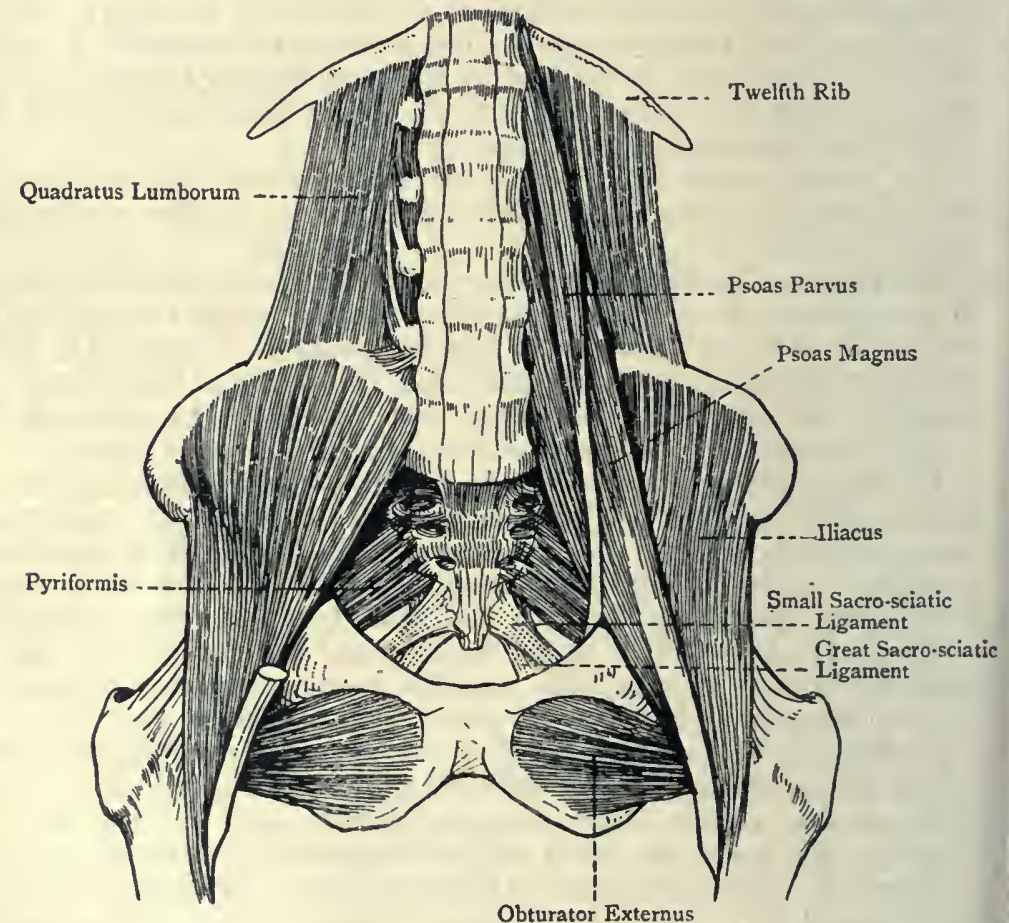

Fig. 229.-The Psoas, Iliacus, and Quadratus I.umborum Muscles.

whilst the other three muscles take origin from the femur, the crureus being situated between the vastus externus and vastus internus.

I. Rectus Femoris-Origin.-By two strong tendinous headsanterior or straight, and posterior or reflected. The anterior or straight head arises from the anterior inferior iliac spine. The posterior or reflected head arises from the groove on the anterior part of the dorsum ilii immediately above the brim of the acetabulum, where it is under cover of the gluteus minimus. The two heads unite at an angle of about 60 degrees. 
Insertion.-The anterior part of the upper border of the patella by a flat tendon, which is free from fleshy fibres in its lower 3 inches.

Nerve-supply.-The posterior division of the anterior femoral nerve, the branch from which furnishes an offset to the hip-joint.

Action.-(I) The muscle is a powerful extensor of the knee-joint; and (2) it is a flexor of the hip-joint. In extending the knee-joint the rectus femoris acts entirely from its anterior or straight head when the hip-joint is extended; but, when the hip-joint is flexed, the muscle in extending the knee-joint acts from its posterior or reflected head.

The fibres which form the fleshy belly are very short, and, springing in a bipenniform manner from the common tendon of origin, they pass obliquely to end upon the tendon of insertion as low as a point 3 inches above the patella. This arrangement gives the muscle great power of action, but limited range of movement. Superiorly the muscle is related to the capsular ligament of the hip-joint.

2. Vastus Externus-Origin.-(I) The upper third of the anterior intertrochanteric line of the femur; (2) the anterior and inferior borders of the great trochanter; (3) the outer side of the gluteal ridge; $(4)$ the outer lip of the linea aspera in its upper half ; $(5)$ the adjacent portion of the shaft of the femur; and (6) the upper part of the external intermuscular septum.

Insertion.-(I) The outer half of the upper border of the patella posterior to the rectus femoris; (2) the upper third of the outer border of the patella; and (3) very slightly into the outer border of the tendon of the rectus femoris.

Nerve-supply. - The posterior division of the anterior femoral nerve, the branch from which furnishes an offset to the knee-joint.

The vastus externus at its origin is aponeurotic, and the aponeurosis spreads downwards upon the superficial surface of the muscle for a considerable distance. The fibres are directed downwards and forwårds or inwards. The anterior border is free.

3. Crureus-Origin.-(I) The lower two-thirds of the anterior intertrochanteric line; (2) the anterior and external surfaces of the shaft of the femur over about their upper three-fourths; (3) the outer lip of the linea aspera in its lower half ; (4) the upper twothirds of the external supracondylar ridge, as well as the immediately adjacent portion of the bone; and (5) the contiguous part of the external intermuscular septum.

Insertion.-The inner half of the upper border of the patella behind the rectus femoris, where it has many of the fibres of the vastus internus associated with it.

Nerve-supply.-The posterior division of the anterior femoral nerve, the branches from which are two or three in number. The most internal of these branches furnishes an offset to the kneejoint, which in its course supplies the subcrureus muscle.

The direction of the fibres of the crureus is chiefly downwards, 
except in the lower and outer part of the muscle, where they pass forwards.

4. Vastus Internus-Origin.--(I) The part of the spiral line of the femur which extends from the inferior cervical tubercle to the inner

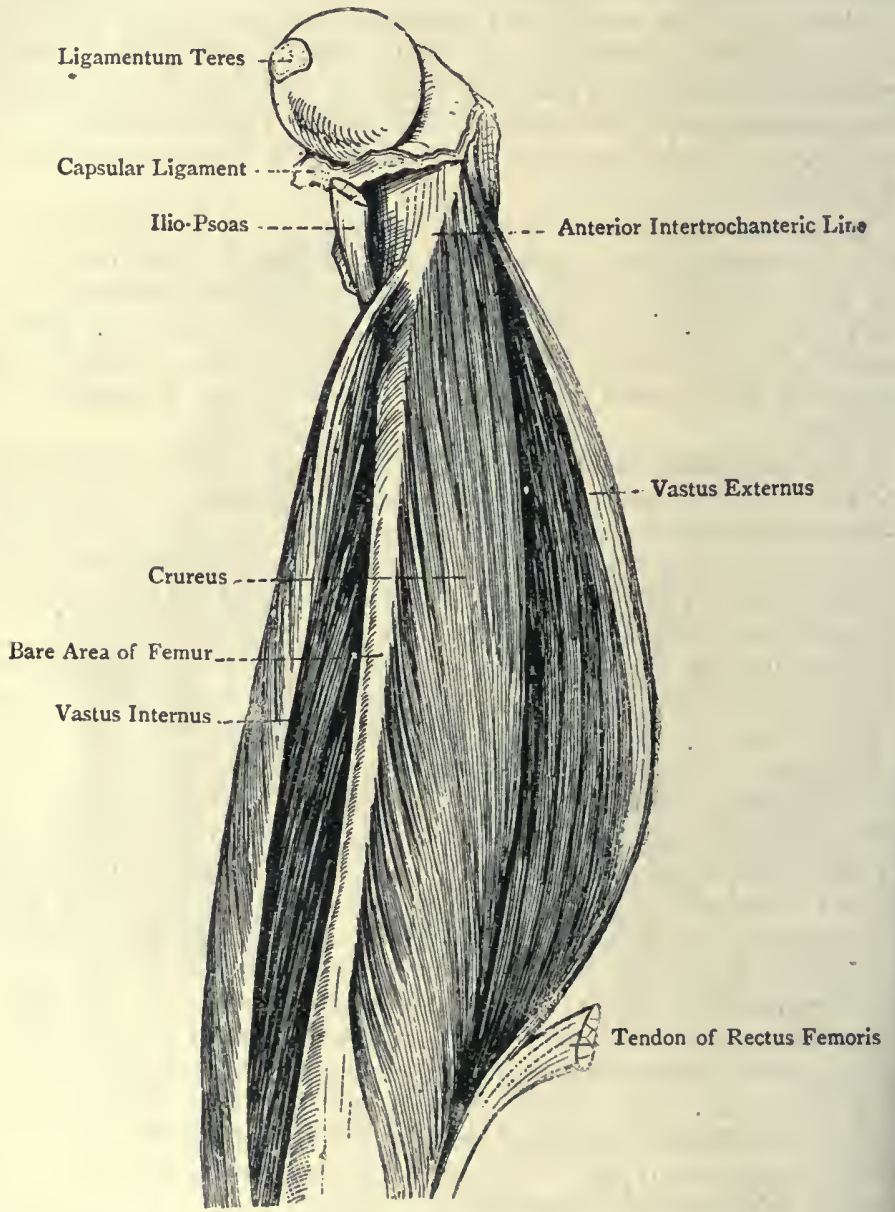

Fig. 230.-Dissection of the Quadriceps Extensor Cruris Muscle.

lip of the linea aspera; (2) the inner lip of the linea aspera in it whole extent; (3) the internal intermuscular septum; and (4) th. front of the tendon of the adductor magnus almost as low as th adductor tubercle.

Insertion.--(I) The inner half of the upper border of the pateila 
in close association with the crureus; (2) the upper half of the inner border of the patella; and (3) very slightly into the inner border of the tendon of the rectus femoris.

Nerve-supply.-The posterior division of the anterior femoral nerve by a branch called the nerve, to the vastus internus. This nerve descends in close contact with, and on the outer side of, the long saphenous nerve, and with that nerve it traverses the upper half of Hunter's canal. It furnishes a large offset to the knee-joint.

The direction of the fibres of the muscle is downwards and forwards or outwards.

Whilst the vastus externus is easily separable from the crureus, the vastus internus and crureus appear at first sight to be one muscle. A cellular interval, howerer, can be traced directly upwards from the inner border of the patella to the inferior cervical tubercle of the femur, along the course of which the two muscles can be distinctly separated. When this separation has been effected, a characteristic elongated strip of the femur is laid bare upon the inner side of the shaft, which is free from muscular fibres.

Suprapatellar Tendon.-This is the common tendon in which the four muscles constituting the quadriceps extensor cruris terminate. The part of it which belongs to the rectus femoris is inserted into the anterior part of the upper border of the patella, whence an expansion of fibres descends over the front of that bone to blend with the fibres of the ligamentum patellæ. The tendon of the crureus is behind that of the rectus femoris, where it has many of the fibres of the vastus internus associated with it, the rest of the last-named muscle terminating partly upon the upper half of the inner border of the patella, and partly upon the inner border of the tendon of the rectus femoris. The vastus externus is partly behind the rectus tendon, and in part it joins the outer border of that tendon, and terminates upon tise upper third of the outer border of the patella. Beneath the suprapatellar tendon there is a bursa, called the suprapatellar bursa. It is continuous with the pouch which the synovial membrane of the knee-joint sends upwards above the patellar surface of the femur.

Action of the Vasti and Crureus.-These three muscles act as powerful extensors of the knee-joint, the lowest fibres of the vastus internus at the same time drawing the patella inwards.

Subcrureus.-This muscle is in reality the lowest and deepest portion of the crureus. Viewing it as an independent muscle, it arises in two bundles from the front of the femur about 4 inches above the patellar surface, and it is inserted into the suprapatellar bursa, which is continuous with the upward prolongation of the synovial membrane of the knee-joint above the patellar surface of the femur.

Nerie-supply.-The articular branch to the knee-joint which comes from the innermost muscular branch to the crureus.

Action.-The subcrureus is a tensor of the synovial membrane of the knee-joint. 
Ligamentum Patellæ (infrapatellar tendon).-This ligament is really a continuation of the common tendon of the quadriceps extensor cruris, the patella being originally a sesamoid cartilage developed in that tendon. It is a very strong, flat, broad ligament, about 2 inches in length. It is attached superiorly to the blunt apex and adjacent margins of the lower part of the patella, and inferiorly to the lower rough half of the tubercle of the tibia, a bursa intervening between the tendon and the upper smooth half of that tubercle.

Anterior Femoral Nerve.-This is the largest branch of the lumbar plexus, its fibres being derived from the dorsal divisions of the second, third, and fourth lumbar nerves. In the abdomen the nerve lies deeply between the psoas magnus and iliacus muscles, and in this position it passes into the thigh beneath Poupart's ligament. In the upper part of Scarpa's triangle it continues to lie deeply between these two muscles, being outside the femoral sheath and about $\frac{1}{4}$ inch to the outer side of the common femoral artery. In this situation the nerve becomes broad and flat, and, at a point about $\frac{3}{4}$ inch below Poupart's ligament, it breaks up into two divisicns. One of these is called the superficial or anterior division, and it is partly muscular and partly cutaneous; the other is called the deep or posterior division, and it is principally muscular and articular, only one cutaneous nerve, namely, the long saphenous, arising from it.

Branches.--These are conveniently divided into intra-abdomiual and extra-abdominal or femoral.

The intra-abdominal branches arise from the trunk of the nerve, and are as follows: muscular, three or four in number, to the iliacus; and an arterial branch to the coats of the femoral artery.

The extra-abdominal or femoral branches arise from the two terminal divisions of the nerve in Scarpa's triangle.

The branches of the superfieial or anterior division are muscular to the sartorius and pectineus, and cutaneous, namely, middle cutaneous and internal cutaneous, to the integument of the front and inner side of the thigh, and of the inner side of the leg in its upper half.

The branches of the deep or posterior division are muscular to the rectus femoris, vastus externus, vastus internus, crureus, and subcrureus; articular to the hip-joint and knee-joint (see nervesupply of quadriceps extensor cruris); and the long saphenous nerve, already described.

Internal Femoral Muscles. Gracilis (adductor gracilis)-Origin.(I) The anterior or femoral surface of the body of the os pubis in its lower half, close to the symphysis; and (2) the front of the descending pubic ramus close to its inner border.

Insertion.-The upper part of the inner surface of the shaft of the tibia, above the semitendinosus, and under cover of the sartorius. 
Nerve-supply.-The anterior or superficial division of the obturator nerve.

Action.-The muscle is an adductor of the thigh, and a flexor of the knee-joint. Having flexed the knee-joint, it acts as an internal rotator of the leg.

The gracilis is flat and strap-like in the upper third of the thigh. In the middle third it becomes thick and narrow, and it gradually tapers into a long, narrow, round tendon which expands towards its insertion. The surfaces are directed inwards and outwards, the borders looking forwards and backwards. In the lower third of the thigh the tendon has the sartorius in front of it, and the semitendinosus behind it, and it is separated from the internal lateral ligament of the knee-joint by a bursa.

Adductor Longus-Origin.-The upper and inner part of the anterior or femoral surface of the body of the os pubis, immediately below and external to the pubic angle.

Insertion.-The inner lip of the linea aspera of the femur.

Nerve-supply. - The anterior or superficial division of the obturator nerve.

Action. - The muscle is an adductor and external rotator of the thigh, and a flexor of the hip-joint.

The adductor longus is a flat, triangular muscle, the direction of which is downwards, outwards, and backwards. It lies to the inner side of the pectineus, and rests upon the adductor brevis, the anterior or superficial division of the obturator nerve, and the adductor magnus. The superficial femoral artery is superficial to it, and the arteria profunda femoris beneath it.

Adductor Brevis-Origin.--(I) The anterior or femoral surface of the body of the os pubis for rather less than its lower half, immedi. ately external to the gracilis; and (2) the front of the descending pubic ramus, where it is likewise external to the gracilis.

Insertion.-The line leading from the back of the small trochanter to the inner lip of the linea aspera.

Nerve-supply.-The anterior or superficial division of the obturator nerve (occasionally the posterior division).

Action.-The muscle is an adductor and external rotator of the thigh, and a flexor of the hip-joint.

The adductor brevis is quadrilateral, and is directed downwards, outwards, and backwards. It lies behind the pectineus and adductor longus, and upon the adductor magnus. The anterior or superficial division of the obturator nerve is in front of it, and the posterior or deep division behind it. It is pierced by the first two perforating branches of the arteria profunda femoris.

Adductor Magnus-Origin.-(I) The anterior or femoral surface of the body of the os pubis in its lower fourth, between the adductor brevis internally and the obturator externus externally; (2) the front of the ischio-pubic ramus; and (3) the inferior portion of the postero-inferior surface of the tuber ischii. 
Insertion.-(I) The inner side of the gluteal ridge of the femur; (2) the inner lip of the linea aspera, and the upper part of the internal supracondylar ridge for fully I inch; and (3) the adductor tubercle, by means of a narrow, round tendon which gives a fibrous expansion to the internal supracondylar ridge below the femoral groove.

Nerve-supply.-The posterior or deep division of the obturator nerve. The postero-inferior part of the muscle, representing the fibres which arise from the tuber ischii, is supplied by the branch of the great sciatic nerve to the semimembranosus.

Action.-The muscle is a powerful adductor of the thigh. The part inserted into the shaft of the femur also acts as an external rotator of the thigh, and the part extending from the tuber ischii to the adductor tubercle as an extensor of the hip-joint.

The adductor magnus is triangular or fan-shaped. The uppermost fibres are horizontal; the succeeding fibres are oblique; and those which arise from the tuber ischii are almost vertical. The part of the muscle which is inserted into the inner side of the gluteal ridge vastus usually forms a distinct portion, which is triangular in shape, and is sometimes called the adductor minimus. The insertion of the muscle into the shaft of the femur is interrupted by four tendinous arches, which give passage to the perforating branches of the arteria profunda femoris. Between the tendon which descends to the adductor tubercle

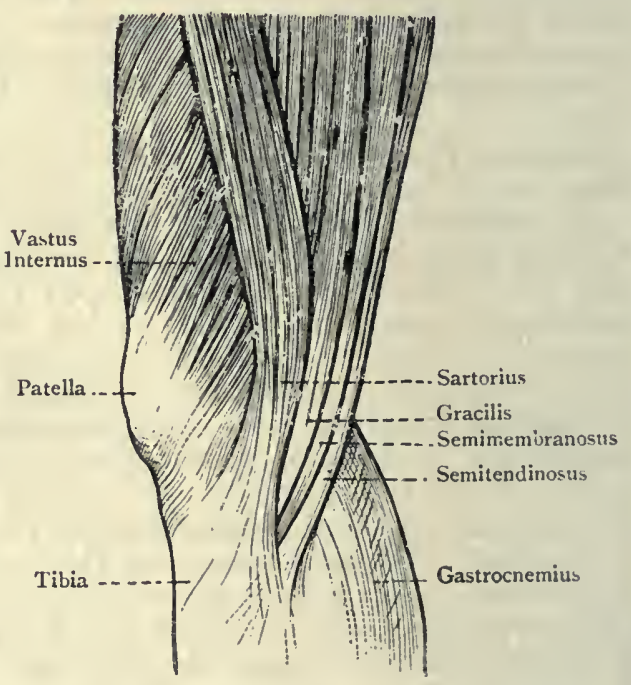

Fig. 231,-The Tendons of The INNER Side OF THE KNEE.

and the part of the muscle which is inserted into the upper end of the internal supracondylar ridge there is an aperture, called the femoral opening, for the passage of the superficial femoral vessels. It is triangular, with the apex rounded off, and is tendinous in front, but fleshy behind. It is of larger size than is necessary for the passage of the vessels, being so adapted that the vessels are not in any way interfered with during the action of the muscle. The muscle supports the adductor brevis, adductor longus, and posterior or deep division of the obturator nerve. Its posterior surface is related to the hamstring muscles and the great sciatic nerve. 
The adductor muscles come into action, in riding upon horseback, enabling the rider to grasp the saddle with his thighs, and they are hence called ' the rider's muscles.' In this act the adductor longus is specially subjected to strain, and its narrow round tendon of origin is occasionally ruptured. At the seat of rupture ossification sometimes takes place, thus giving rise to the so-called ' rider's bone.'

Obturator Externus-Origin.-(I) The external surface of the obturator membrane over its inner half ; (2) the adjacent portion of

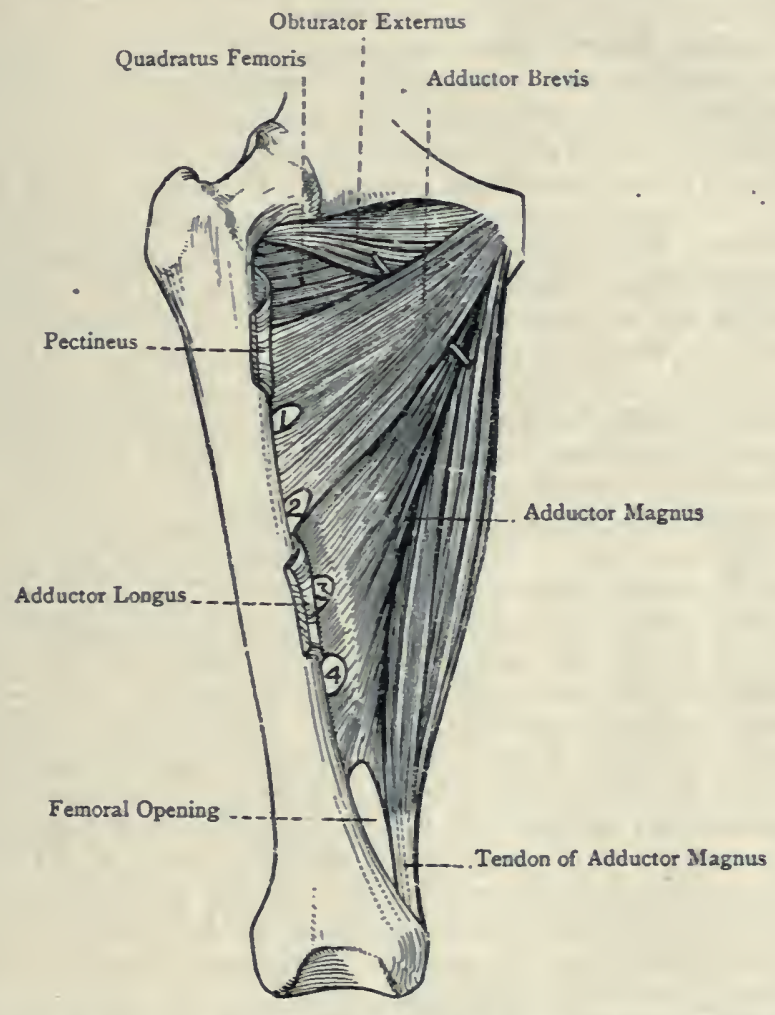

Fig. 232.-The Right Isternal Femoral Muscles (ANterior View). I, 2, 3, 4, Openings for the Perforating Arteries.

the anterior or femoral surface of the body of the os pubis; and (3) the front of the ischio-pubic ramus close to the obturator foramen.

Insertion.-The digital or trochanteric fossa of the femur.

Nerve-supply.-The deep or posterior division of the obturator nerve as it passes through the upper part of the muscle.

Action.--The muscle is an external rotator and adductor of the thigh. 
The obturator oxternus is somewhat triangular, and is directed at first outwards, and then backwards and upwards. Its round tendon of insertion is closely applied to the back of the neck of the femur, which it slightly grooves in its lower or extracapsular part. The superficial or anterior division of the obturator nerve passes over the upper border of the muscle, and the deep or posterior division pierces the upper part of it. The muscle is in close contact with the lower and back part of the capsular ligament of the hipjoint, a bursa being usually interposed.

Obturator Nerve.-This nerve is a branch of the lumbar plexus, and generally arises by three roots from the ventral divisions of the second, third, and fourth lumbar nerves, but the root from the second may be absent. For a description of the nerve within the abdomen and pelvis, see these sections. It escapes from the pelvic cavity by passing through the obturator canal, in which it breaks up into two divisions-superficial or anterior, and deep or posterior. The superficial or anterior division passes over the upper border of the obturator externus, and then descends in front of the adductor brevis, but behind the pectineus and adductor longus. This division communicates with the accessory obturator nerve (when present). The deep or posterior division pierces the upper part of the obturator externus, and then passes behind the adductor brevis and in front of the adductor magnus.

Branches-Superficial or Anterior Division.-The branches of this division are as follows: articular to the hip-joint, which arises in the obturator canal, and enters the joint through the cotyloid foramen; muscular to the gracilis, adductor longus, adductor brevis as a rule, and occasionally to the pectineus; arterial to the coats of the femoral artery; and cutaneous. This last branch commences at the lower border of the adductor longus, and, after a short course downwards, it pierces the fascia lata between the posterior border of the sartorius and the anterior border of the gracilis, to be distributed to the integument of the inner side of the thigh for a variable extent about the centre. Before this branch pierces the fascia lata it gives one or two twigs which communicate with branches of the long saphenous and the posterior division of the internal cutaneous beneath the sartorius, to form the subsartorial plexus.

Deep or Posterior Division.-This division furnishes the following branches: muscular to the obturator externus, adductor magnus, and adductor brevis (provided the latter is not supplicc by the superficial or anterior division); and articular to the kneejoint, called the geniculate branch. This latter branch descends upor the adductor magnus, which it subsequently picrees close abov the femoral opening, and so reaches the popliteal artery. It accom panies that artery, lying at first on its inner side and then in front 0 it, as low as the origin of the central or azygos branch. Here th nerve leaves the main artery, and passes with its central or azygo branch through the posterior ligament of the knee-joint to thi 
interior of the articulation. The geniculate nerve is frequently absent.

Accessory Obturator Nerve.-This nerve is present in about 30 per cent. of bodies. It is comparatively small in size, and arises by two roots from the anterior primary divisions of the third and fourth lumbar nerves, the roots being interposed between those of the anterior crural and obturator nerves. It descends along the inner border of the psoas magnus, close to the brim of the pelvis, underneath the external iliac vessels. It then passes over the superior pubic ramus, where it passes beneath the pectineus, and so emerges on to the thigh beneath Poupart's ligament. Under cover of the pectineus it divides into the following three branches: (I) articular to the hip-joint; (2) muscular to the deep surface of the pectineus; and (3) a reinforcing branch to join the superficial or anterior division of the main obturator nerve.

Scarpa's Triangle. - This triangle is situated on the front of the thigh below Poupart's ligament. The base, which is directed upwards, is formed by Poupart's ligament. The outer boundary is formed by the inner border of the sartorius in its upper fourth, and the inner boundary is constructed by the internal border of the adductor longus in its upper part. The apex is about $3 \frac{1}{2}$ inches below Poupart's ligament, and is formed by the overlapping which there takes place between the superficially placed sartorius and the more deeply placed adductor longus. The roof is formed by the skin, two layers of the superficial fascia, and fascia lata. The following structures also lie in the roof; the femoral branch of the genito-femoral nerve; the inguinal and superficial femoral or saphenous glands; the cutaneous branches of the common femoral artery (except the inferior external pudic), with their corresponding veins; the saphenous opening; and the terminal part of the long saphenous vein, with the anterior and posterior saphenous veins joining it. The floor contains the following muscles, in order from within outwards : adductor longus; pectineus; psoas magnus; and iliacus (the latter two being here united to form one muscle, called the ilio-psoas). Occasionally a small part of the adductor brevis is seen between the adductor longus and pectineus, and, when this is so, the superficial or anterior division of the obturator nerve may be seen in part.

Contents.-The contents are as follows: the common femoral artery and its branches; the first part of the superficial femoral artery and its branches; the upper part of the femoral vein and its tributaries; and the anterior femoral nerve, with its divisions and their branches.

Hunter's Canal.*-This is a somewhat triangular, musculoaponeurotic canal, which occupies the middle two-fourths of the thigh on its inner aspect.

- The canal has received this complimentary name because John Hunter was the first surgeon who tied the superficial femoral artery in this part of its course for popliteal aneurism in the year 1785 . 
Femo-al Branch of Genito-femoral Nerve

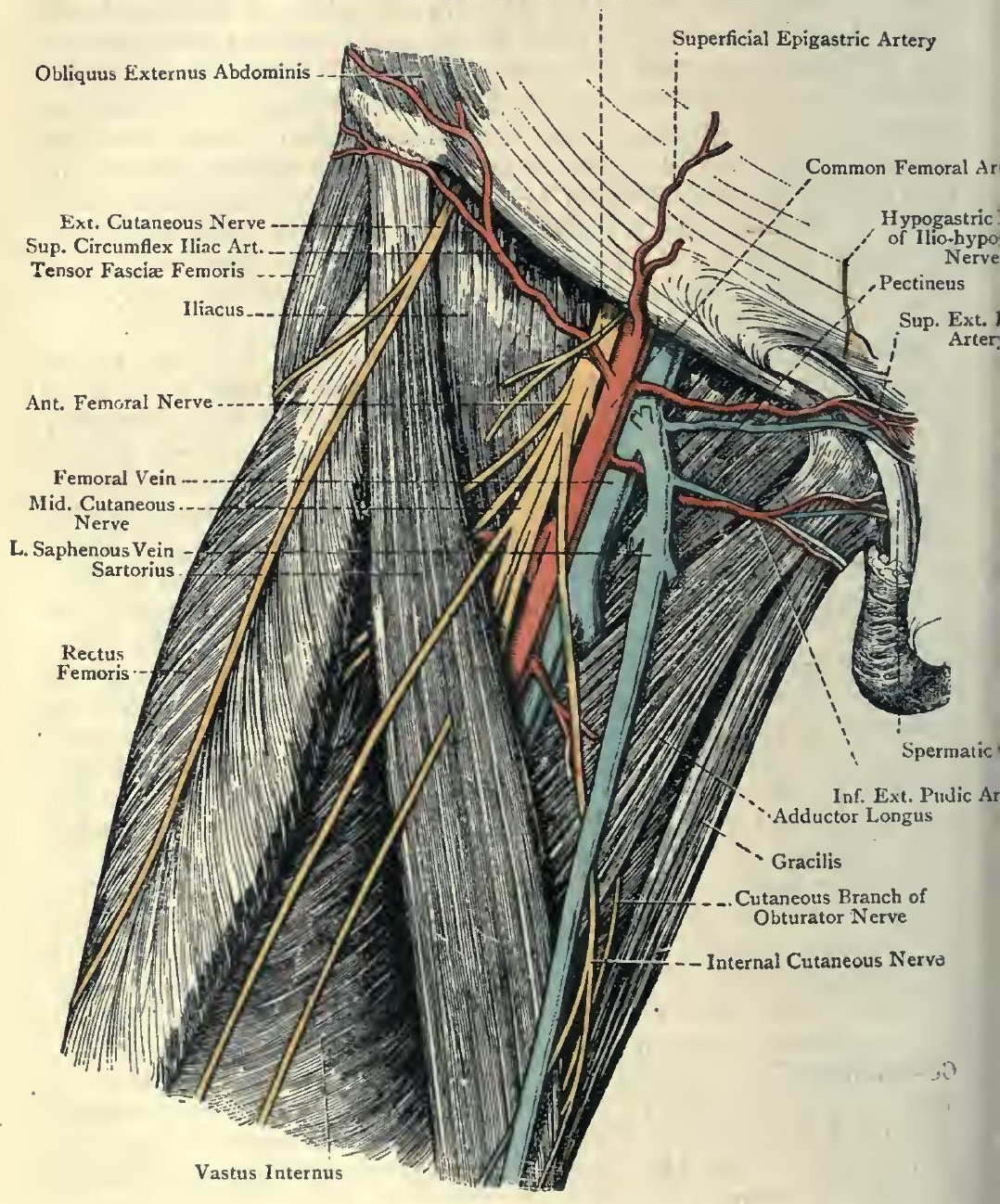

Fig. 233.-The Front of the Thigh (Scarpa's Triangle); 
Boundaries-External. - The vastus internus, closely covering the inner surface of the femur as far back as the inner lip of the linea aspera. Internal and Posterior. - The adductor longus and subsequently the adductor magnus, both of which extend back to the inner lip of the linea aspera, where they meet the vastus internus and so form the apex of the triangle. Anterior or Superficial.-This boundary is commonly called the roof of the canal. It represents the base of the triangle, and is formed by an aponeurotic expansion which extends from the adductor longus and adductor magnus on the inner side to the vastus internus on the outer side. Superficial to this expansion in its whole length is the middle portion of the sartorius, and the subsartorial plexus of nerves lies between the two in the middle third of the thigh. The aponeurosis is com. paratively weak over the upper half of the canal, but over the lower half it is strong, and, below, it is continuous with the anterior margin of the femoral opening in connection with the adductor magnus.

Contents.-These are as follows: the superficial femoral artery in the second part of its course, and its branches; the first portion of the femoral vein, and its tributaries; the long saphenous nerve; and the nerve to the vastus internus muscle in the upper half of the canal.

Femoral Artery.-This vessel extends from the lower border of Poupart's ligament to the posterior margin of the femoral opening, which is in connection with the adductor magnus. It is the direct continuation of the external iliac artery, and at its termination becomes the popliteal. It occupies the upper three-fourths of the thigh, and its direction is downwards and inwards. The course of the vessel is indicated in the following manner: the thigh being partially flexed upon the abdomen, and at the same time slightly abducted and rotated outwards, draw a line from a point midway between the anterior superior iliac spine and the symphysis pubis to the adductor tubercle of the femur, or, if this tubercle cannot be felt, to the internal tuberosity on the inner surface of the internal condyle. This line over its upper three-fourths represents the course of the vessel.

At a point about $3 \frac{1}{2}$ inches below Poupart's ligament the sartorius takes up a position over the artery, and from this point onwards that muscle continues to lie over the vessel. For at least the first $I_{\frac{1}{2}}$ inches of its course the vessel corresponds in size with the external iliac artery. When, however, it reaches a point from $I_{\frac{1}{2}}$ inches to 2 inches below Poupart's ligament it gives off a large branch, called the arteria profunda femoris, after which it undergoes a sudden diminution in size. The part of the ressel between Poupart's ligament and the origin of the arteria profunda femoris is known as the common femoral ; the part beyond the origin of that large branch is called the superficial femoral ; and the arteria profunda femoris is spoken of as the deep femoral ; that is to say, the common femoral artery is regarded as dividing into superficial 
femoral and deep femoral at a point from $I_{2} \frac{1}{2}$ inches to 2 inches below Poupart's ligament. For convenience of description, the femoral artery (meaning thereby the whole of the leading artery of the thigh) is divided into two parts-first and second - the first part lying in Scarpa's triangle, and the second part occupying Hunter's canal.

First Part.--The first part of the femoral artery extends from the lower border of Poupart's ligament to the apex of Scarpa's triangle, within which triangle it lies.

Relations-Superficial or Anterior.-The skin; superficial fascia in two layers; fascia lata; anterior wall of the femoral sheath for the first $\mathrm{I}_{\frac{1}{2}}$ inches, and subsequently the anterior wall of the special sheath; internal cutaneous nerve crossing the vessel from without inwards, either as one nerve or in its two divisions, just above the apex of Scarpa's triangle and outside the special sheath; two or three cutaneous branches of the internal cutaneous nerve on their way to the inner side of the thigh; superficial circumflex iliac vein near Poupart's ligament; and external cutaneous femoral or anterior saphenous vein just above the apex of Scarpa's triangle.

Deep or Posterior. - The posterior wall of the femoral sheath for the first $I_{2}^{\frac{1}{2}}$ inches, and subsequently the femoral vein; branch of the anterior femoral nerve passing inwards to the pectineus muscle, and lying close behind the femoral sheath; psoas magnus in the upper part and pectineus in the lower part, the artery being separated from the latter muscle by the femoral vein and profunda femoris vessels, the femoral vein being nearest to the artery.

External.-The femoral branch of the genito-femoral nerve for a short distance below Poupart's ligament, where the nerve is within the crural sheath; anterior femoral nerve for about $\frac{3}{4}$ inch below Poupart's ligament, and separated from the artery by an interval of about $\frac{1}{4}$ inch; long saphenous nerve and nerve to the vastus internus muscle, the former being nearest to the artery, and both being outside the special sheath; and the arteria profunda femoris for about $\frac{1}{2}$ inch at its commencement.

Internal.-For about 2 inches below Poupart's ligament the femoral vein lies to the inner side of the artery, with the intervention of the external septum of the femoral sheath. Thereafter the vein gradually leaves the inner side of the artery to take up its position behind it, towards the apex of Scarpa's triangle.

The femoral artery immediately below Poupart's ligament is anterior to the inner part of the head of the femur, but in the rest of its course the vessel is situated on the inner side of that bone.

Second Part.-The sccond part of the artery (superficial femoral) extends from the apex of Scarpa's triangle to the posterior margin of the femoral opening in connection with the adductor magnus, where it becomes the popliteal. In this part of its course the superficial femoral lies in Hunter's canal, and passes over the adductor longus, whilst the deep femoral is behind that muscle. 
Relations-Superficial or Anterior.-The skin ; superficial fascia; long saphenous vein ; fascia lata ; sartorius ; subsartorial plexus of nerves ; the aponeurotic covering of Hunter's canal ; and the long saphenous nerve in the act of crossing the vessel from its outer to its inner side. External.--The vastus internus, covering the inner surface of the femur; and the long saphenous nerve and the nerve to the vastus internus, both in the upper half of the canal. Internal.-At first the adductor longus, and lower down the adductor magnus, both of which muscles also lie behind the artery. In Hunter's canal the femoral vein still maintains its position close behind the artery until the lower end of the canal is reached, when the vein inclines a little to the outer side of the artery.

The superficial femoral artery in Scarpa's triangle, as well as in Hunter's canal, is surrounded by a sheath, which also contains the femoral vein.

Branches of the Femoral Artery.-The branches are as follows: superficial epigastric; superficial circumflex iliac; superior or superficial external pudic ; inferior or deep external pudic ; arteria profunda femoris or deep femoral (all from the common femoral); muscular (from the superficial femoral in Scarpa's triangle and in Hunter's canal); saphenous (from the superficial femoral in Scarpa's triangle); and anastomotica magna (from the superficial (emoral in Hunter's canal).

The superficial epigastric artery arises from the common femoral about $\frac{1}{2}$ inch below Poupart's ligament. It then pierces the femoral sheath and the cribriform fascia, or the outer border of the saphenous opening, after which it turns upwards over Poupart's ligament a little to the inner side of the centre, and ramifies in the integument of the anterior abdominal wall as high as the umbilicus. In its course it supplies branches to the inguinal glands, and anastomoses with branches of the deep epigastric of the external iliac.

The superficial circumflex iliac artery frequently arises in common with the superficial epigastric. Having pierced the femoral sheath and the outer border of the saphenous opening, it passes outwards below the outer half of Poupart's ligament to the anterior part of the iliac crest, where it ramifies in the integument of that neighbourhood. In its course it supplies branches to the iliacus, sartorius, and outer inguinal glands, and anastomoses with the deep circumflex iliac of the external iliac, and the gluteal of the internal iliac.

The superior or superficial external pudic artery arises from the common femoral about $\frac{3}{4}$ inch below Poupart's ligament. Having pierced the femoral sleath and cribriform fascia, it emerges through the saphenous opening, after which it passes inwards and upwards over the spermatic cord, or round ligament of the uterus, according to the sex, to be distributed to the integument of the suprapubic region, the adjacent portion of the scrotum in the male, and the labium najus in the female, and the dorsum of the 


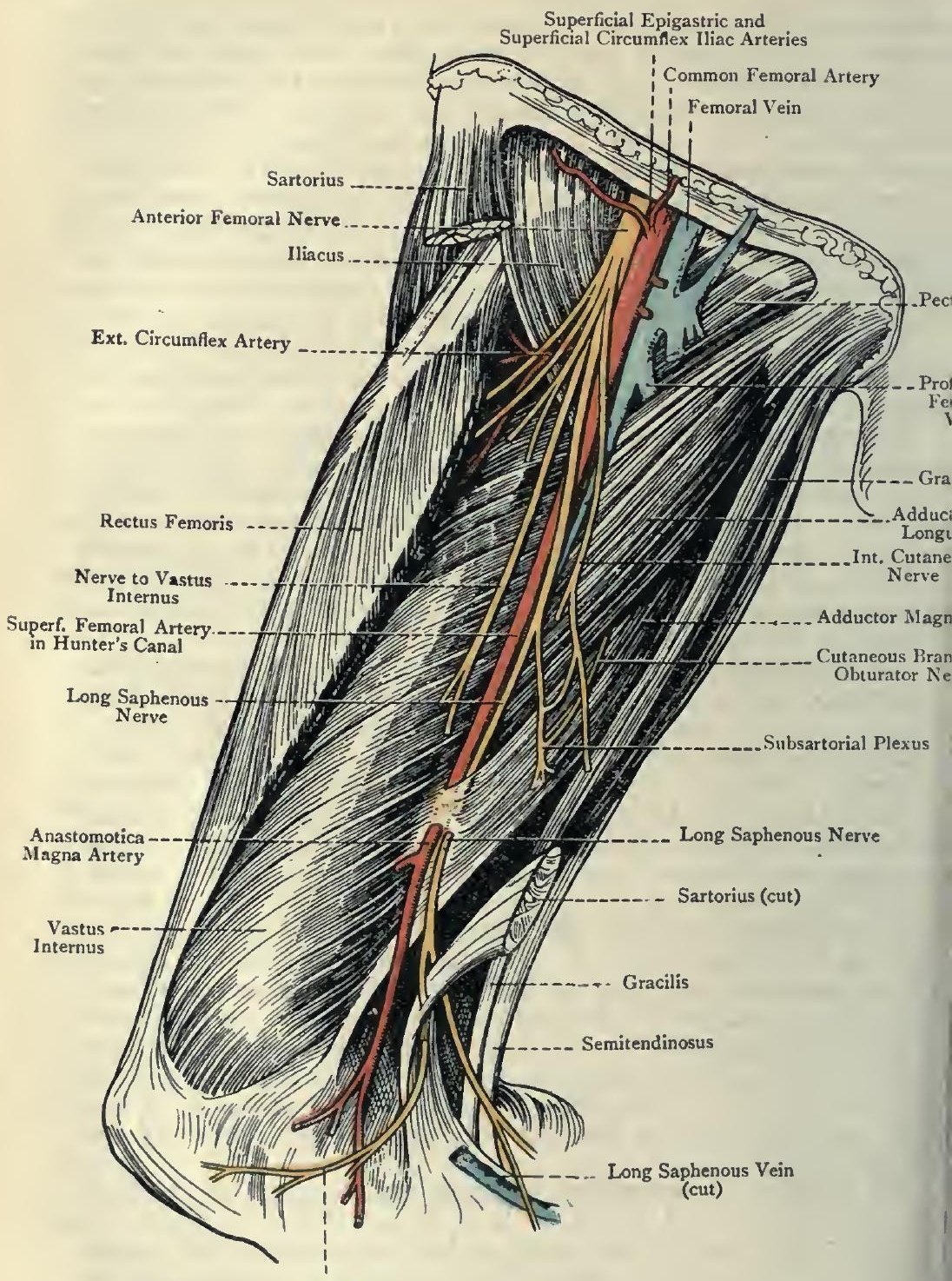

Patellar Brauch of Long Saphenous Nerve

Fig. 234.-The Front and InNer Side of the Thigh (The Sartorius has been in great part removed). 
penis by a branch which extends as far as the prepuce in the vicinity of the corona glandis, lying in its course external to the dorsalis penis artery. The vessel in its course gives branches to the inner inguinal or pubic glands, the deep femoral glands, and the coverings of the spermatic cord or of the round ligament of the uterus. It anastomoses with (I) the cremasteric branch of the deep epigastric, in crossing the spermatic cord, that branch being represented in the female by the artery of the round ligament of the uterus: (2) its fellow of the opposite side; (3) the inferior or deep external pudic; and (4) the dorsalis penis artery, which is a branch of the internal pudic from the internal iliac.

The inferior or deep external pudic artery arises from the common femoral a little lower down than the preceding. Unlike the superior branch, it does not pass through the saphenous opening, but, after piercing the femoral sheath, it passes inwards on the pectineus and adductor longus, under cover of the fascia lata. Having reached the inner side of the thigh, it pierces the fascia lata to be distributed to the side of the scrotum in the male and the labium majus in the female. In its course it gives branches to the muscles upon which it rests, and anastomoses with (I) the superior or superficial external pudic, (2) the cremasteric, and (3) the superficial perineal, which is a branch of the internal pudic.

The four arteries just described are spoken of as the cutarioous arteries of the groin.

Arteria Profunda Femoris or Deep Femoral Artery.-This important vessel is one of the terminal branches of the common femoral, arising from the outer and back part of that artery at a point from $I \frac{1}{2}$ inches to 2 inches below Poupart's ligament. At first it lies for about $\frac{1}{2}$ inch on the outer side of the superficial femoral, where it rests upon the iliacus. It then bends sharply, and passes inwards over the pectineus, where it lies immediately behind the superficial femoral vessels and its own vein, the latter being nearest to it. Thereafter it descends behind the adductor longus, resting, in succession, upon the adductor brevis and adductor magnus, and, having become comparatively small, it terminates in the fourth or last perforating artery. As the profunda vessel passes behind the adductor longus, it is separated by that muscle from the superficial femoral artery.

Branches.-The branches are as follows: external circumflex; internal circumflex ; and perforating, which are four in number.

The external circumflex artery, which is of large size, arises from the arteria profunda femoris close to its origin. Its direction is outwards beneath the sartorius and rectus femoris, and between the nerves arising from the posterior division of the anterior femoral, supplying in this part of its course branches to the surrounding muscles, and resting upon the crureus. It terminates by dividing into three sets of branches-ascending, transverse, and dersending. 


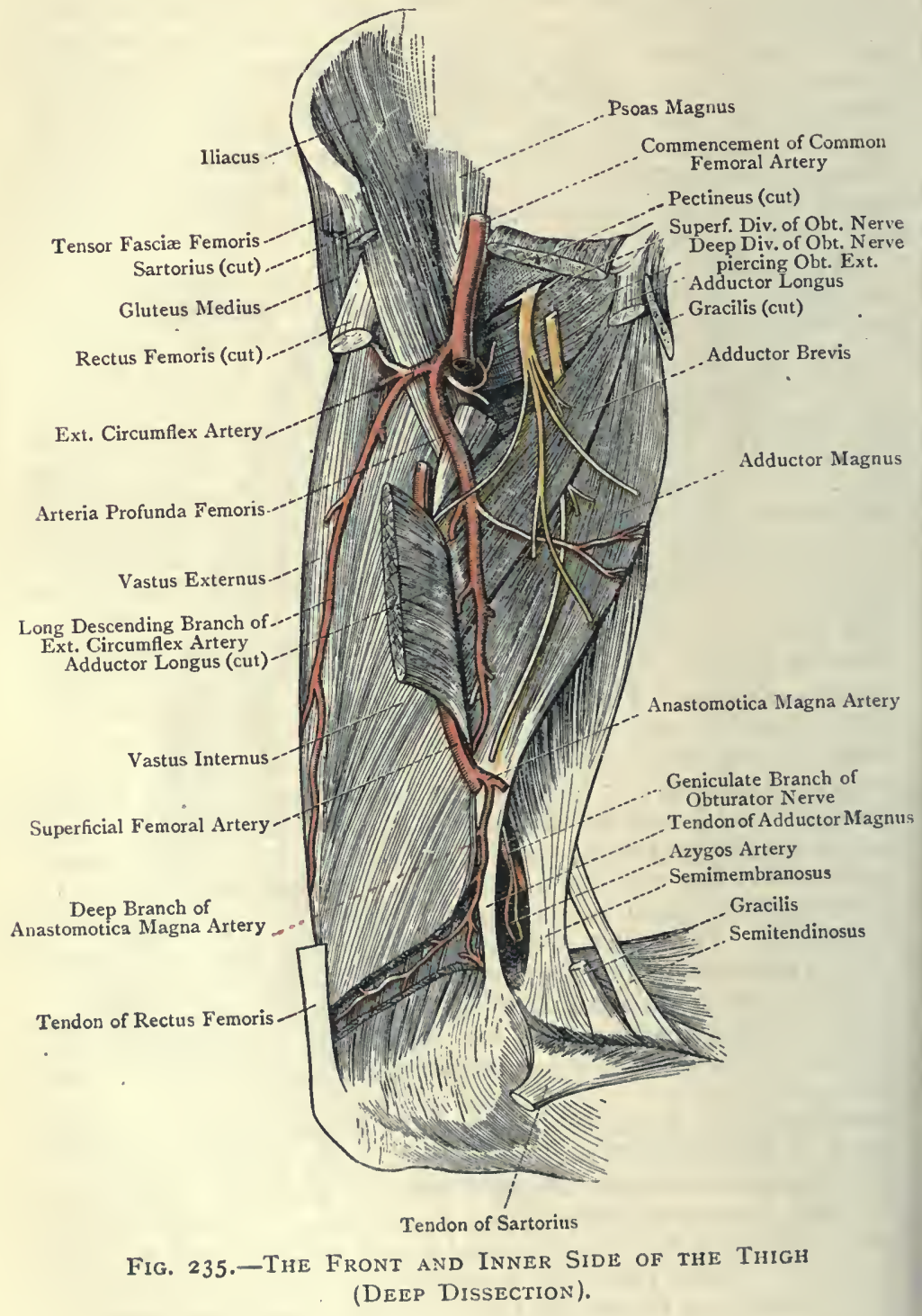


The ascending branch passes upwards beneath the tensor fasciæ femoris to the fore part of the gluteal region. Its branches supply the tensor fasciæ femoris and the anterior portions of the gluteus medius and gluteus minimus. It also supplies an articular branch to the hip-joint, which reaches the articulation under cover of the rectus femoris. The ascending branch anastomoses with the gluteal of the posterior division of the internal iliac, and the deep circumflex iliac of the external iliac.

The transverse branches are at least two in number. They enter the vastus externus, in which they wind round the outer part of the femur, supplying that muscle in their course. One of them, piercing the femoral insertion of the gluteus maximus, reaches the back of the thigh, where it anastomoses with the first perforating artery, the sciatic, and the transverse branch of the internal circumflex, thus forming the crucial anastomosis.

The descending branches are three or four in number. They pass downwards with the nerve to the vastus externus, lying along the anterior border of that muscle, and supplying in their course the rectus femoris, vastus externus, and crureus. One of them is long, and descends upon the vastus externus as low as the knee-joint, where, piercing that muscle, it anastomoses with the superior external articular of the popliteal, the fourth or lowest perforating of the arteria profunda femoris, the deep branch of the anastomotica magna of the superficial femoral, and the lowest muscular branch of the superficial femoral. The other descending branches anastomose in the crureus with the lower two perforating arteries.

The internal circumflex artery is of smaller size than the external. Arising from the inner and back part of the arteria profunda femoris, opposite the origin of the external circumflex, it passes backwards round the inner side of the femur towards the small trochanter. In this course it passes, in succession, between the pectineus and psoas magnus, and between the adductor brevis and obturator externus, giving off branches to the obturator externus and adductor muscles. On reaching the interval between the adductor brevis and obturator externus, it terminates in two branches - ascending or anterior, and transverse or posterior.

The ascending or anterior branch passes with the obturator externus to the digital fossa of the femur, where it anastomoses with the gluteal and sciatic of the internal iliac, and the first perforating, thus forming the digital anastomosis.

The transverse or posterior branch continues the course of the main artery backwards, passing between the upper border of the adductor magnus and lower border of the quadratus femoris, in which situation it anastomoses with one of the transverse branches of the external circumflex, the first perforating, and the sciatic of the internal iliac, thus forming the crucial anastomosis. The branches which the iniernal circumflex gives to the 
obturator externus anastomose in that muscle with the obturator artery, which is a branch of the internal iliac.

The perforating arteries are four in number-first, second, third, and fourth. They pass backwards round the inner side of the femur, and under the tendinous arches of the adductor magnus. The first and second arteries pierce the adductor brevis and adductor magnus, whilst the third and fourth pierce the adductor magnus only. On reaching the back of the thigh, all four arteries give off the following branches: muscular, to the hamstring muscles; branches to the great sciatic nerve; and cutaneous, to the integument of the outer and back parts of the thigh. The first perforating also gives branches to the lower part of the gluteus maximus, and the second (or third) furnishes the chief nutrient or medullary artery of the femur. The perforating arteries, now much reduced in size, wind round the back and outer side of the femur. In this course the first pierces the femoral insertion of the gluteus maximus, and the second, third, and fourth pierce the femoral head of the biceps and the external intermuscular septum. The first and second terminate in the vastus externus, and the third and fourth in the crureus, all four anastomosing with branches of the external circumflex, the fourth also taking part in the deep geniculate arterial rete. At the back of the thigh the perforating arteries anastomose freely with one another. The first perforating also anastomoses with the gluteal, sciatic, and ascending branch of the internal circumflex in the digital fossa, thus forming the digital anastomosis, and with the sciatic and transverse branches of the internal and external circumflex between the adductor magnus and quadratus femoris to form the crucial anastomosis. The lower two perforating arteries anastomose at the back of the thigh with the superior muscular branches of the popliteal. The branches of the perforating arteries to the great sciatic nerve anastomose with the comes nervi ischiadici branch of the sciatic.

The muscular branches of the superficial femoral artery arise partly in Scarpa's triangle and partly in Hunter's canal. The lowest of these branches, which is somewhat large and very constant, is given off at the lower end of Hunter's canal. It passes outwards behind the femur, piercing the external intermuscular septum and the femoral head of the biceps, and it terminates in the crureus, in which it anastomoses with the long. descending branch of the external circumflex, the fourth perforating, and the superior external articular of the popliteal.

The saphenous branches are two or three in number, and arise from the superficial femoral in Scarpa's triangle. They supply the superficial femoral or saphenous glands and the structures around the terminal part of the long saphenous vein.

The arteria anastomotica magna arises from the superficial femoral at the lower end of Hunter's canal, and at once divides into a superficial and deep branch. In miany cases these branches arise independently from the main vessel. "The superficial bran:h 
pierces the aponeurotic covering of Hunter's canal, and descends in company with the long saphenous nerve between the sartorius and gracilis, where it lies beneath the fascia lata. It subsequently pierces that fascia, and enters the leg on its inner aspect, to be distributed to the integument over the upper third. This branch anastomoses with the inferior internal articular of the popliteal. The deep branch at once enters the vastus internus, in which it descends anterior to the tendon of the adductor magnus. It gives branches to the vastus internus and crureus, and anastomoses with the superior internal articular of the popliteal. It also furnishes a cross branch, which passes outwards in front of the femur above the patellar surface to form an arch with a branch of the superior external articular of the popliteal. Additional anastomoses are formed with the long descending branch of the external circumflex, and the lowest perforating branch of the arteria profunda femoris.

Varieties of the Femoral Artery-1. The Trunk.-(I) In rare cases the femoral artery may be found on the back of the thigh in company with the great sciatic nerve. In such cases the vessel is derived from the internal iliac, and may be regarded as a large sciatic artery. Under these conditions the external iliac artery ends as the arteria profunda femoris.

(2) Occasionally the superficial femoral artery divides just below the origin of the arteria profunda femoris into two branches of equal size, which descend in close contact, and subsequently unite to form one trunk before reaching the femoral opening in connection with the adductor magnus.

II. The Branches-(I) Arteria Profunda Femoris.-In normal cases this artery arises from the common femoral from $1 \frac{1}{2}$ to 2 inches below Poupart's ligament. The common femoral artery, however, may divide into superficial femoral and deep femoral at any point between this and Poupart's ligament. The superficial and deep femoral arteries may even spring from the lower part of the external iliac a little above Poupart's ligament, in which case two large arteries would pass out beneath that ligament, instead of one. More rarely, the division of the common femoral into superficial and deep femoral may take place lower down than the normal level, even as low as 4 inches below Poupart's ligament.

(2) External Cireumflex Artery.-This vessel is very liable to variation. It may arise from the common femoral instead of the deep femoral, as in cases of low origin of the latter vessel, or it may arise in two branches, one from the deep femoral and one from the common femoral, or both from the deep femoral, or both from the common femoral.

(3) Internal Circumflex Artery. - This vessel is not so liable to variation as the external circumflex. It may arise along with the external circumflex : it may spring from the common femoral; it may be a branch of the deep epigastric ; or it may be derived from the external iliac.

In cases of low origin of the deep femoral, the external and the internal circumflex arteries usually spring from the common femoral.

(4) Arteria Anastomotica Magna.-In very rare cases the superficial branch of this artery assumes considerable length, and, under the name of the internal saphenous ariery, accompanies the internal saphenous vein down the inner side of the leg as far as the internal malleolus.

Unusual Branches. - The deep epigastric, the deep circumflex iliac, or an abnormal obturator artery, may arise from the common femoral near Poupart's ligament.

Collateral Circulation after Occlusion of the Femoral Artery.-I. The Common Femoral Artery. - (a) The superficial perineal and dorsalis penis arteries, both from the internal pudic of the internal iliac, anastomose with the superior and inferior external pudics of the common femoral. 
(b) The cremasteric from the deep epigastric of the external iliac anastomoses with the superior external pudic of the common femoral.

(c) The obturator of the internal iliac anastomoses with the internal circumflex of the arteria profunda femoris.

(d) The deep circumflex iliac from the external iliac and the gluteal from the internal iliac anastomose with the ascending branch of the external circumflex from the arteria profunda femoris.

(e) The sciatic of the internal iliac anastomoses with the transverse branch of the internal circumflex, the transverse branch of the external circumflex, and the first perforating artery, all branches of the arteria profunda femoris.

$(f)$ The sciatic and gluteal, both branches of the in ternal iliac, anastomose in the digital fossa with the ascending branch of the internal circumflex of the arteria profunda femoris.

2. The Superficial Femoral Artery below the Origin of the Arteria Prolunda Femoris.- (a) The descending branch of the external circumflex of the arteria profunda femoris takes part in the deep geniculate arterial rete.

(b) The third and fourth perforating branches of the arteria profunda femoris anastomose at the back of the thigh with the superior muscular branches of the popliteal.

(c) The fourth perforating artery and the descending branch of the external circumflex, both from the arteria profunda femoris, anastomose with the lowest muscular branch of the superficial femoral.

A continuous anastomotic chain of arteries extends from the gluteal region down the back of the thigh to the region of the knee, and it is formed in the following manner: the gluteal anastomoses with the sciatic, the sciatic with the external and internal circumflex, the gluteal, sciatic, external circumflex, and internal circumflex with the perforating branches of the arteria profunda femoris, and the perforating branches of the arteria profunda femoris with the upper muscular and articular branches of the popliteal. This chain is of special importance after occlusion of the common femoral artery, and the comes nervi ischiadici branch of the sciatic is particularly liable to enlargement.

Femoral Vein.-This vessel extends from the posterior margin of the femoral opening, which is in connection with the adductor magnus, to the lower border of Poupart's ligament, where it becomes the external iliac vein. In the lower part of Hunter's canal it lies close behind the superficial femoral artery, with a slight inclination to the outer side. In ascending, however, it soon takes up a position behind the artery, which it maintains until it has entered Scarpa's triangle. The vein now gradually inclines to the inner side of the artery, and for about 2 inches below Poupart's ligament it is quite to its inner side, but on the same plane, being separated from the artery by the external septum of the femoral sheath, and lying here between the pectineus and psoas magnus.

Tributaries.-These are as follows: the venæ comites of the anastomotica magna artery at the lower end of Hunter's canal; the venæ comites of each of the muscular branches of the superficial femoral artery; the profunda femoris vein at a point about $\mathrm{I}_{4}^{3}$ inches below Poupart's ligament; and the long saphenous vein at a point about $I_{2}^{\frac{1}{2}}$ inches below Poupart's ligament, and above the point where the profunda vein terminates. The long saphenous vein has been previously reinforced by the anterior saphenous or external cutancous femoral, the posterior saphenous or internal cutaneous femoral, the superficial circumflex iliac, the 
superficial epigastric, and the superior and inferior external pudic veins.

The profunda femoris vein is formed by the venæ comites which accompany each of the branches of the arteria profunda femoris. It is a large vessel, and is provided with several valves.

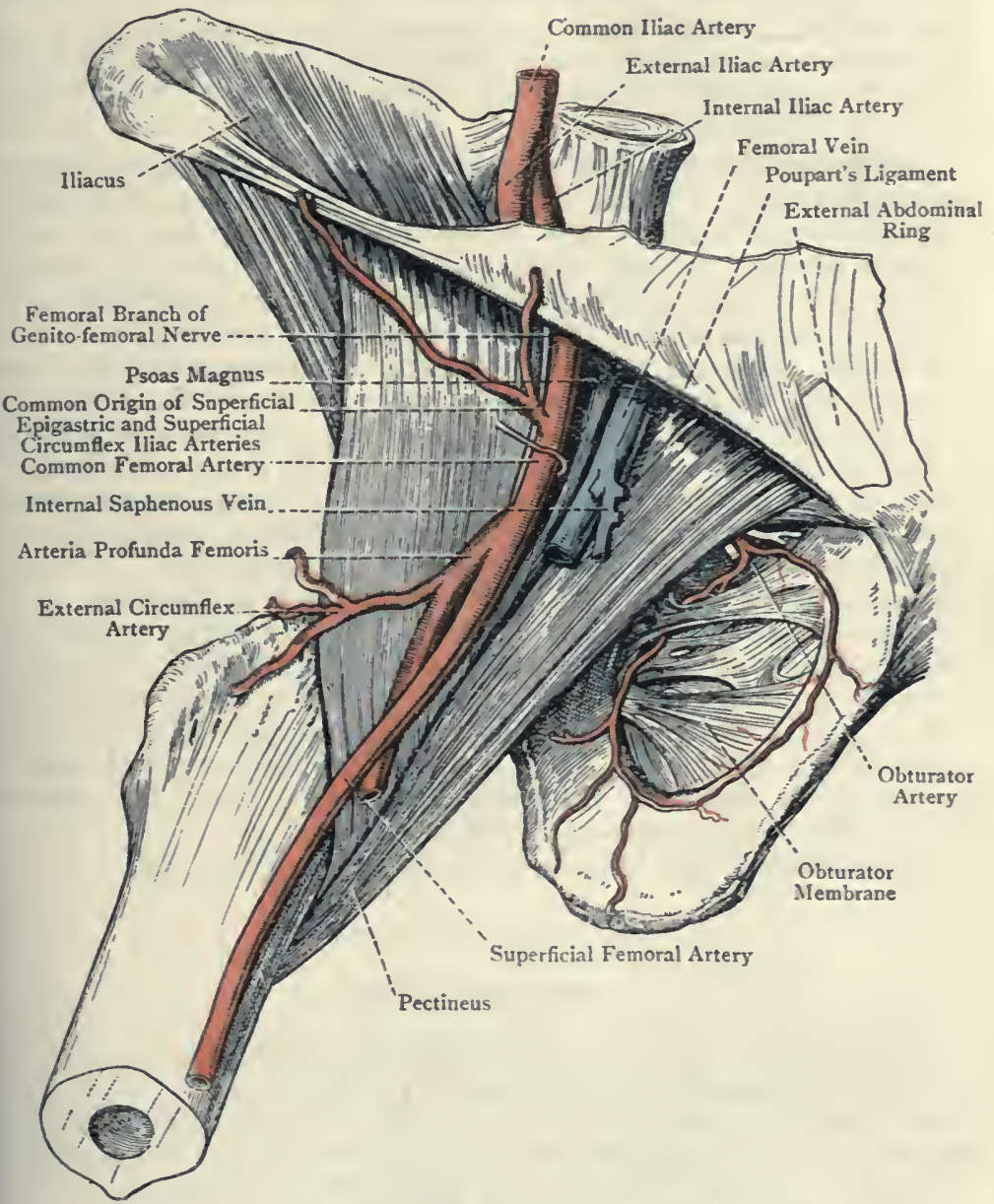

Fig. 236.-Deep Dksection of Scarpa's Triangle.

The femoral vein is provided with five pairs of valves. Three of these are situated in the part of the vessel below the point where it receives the profunda femoris vein. Of the other two, one is placed immediately above the termination of the profunda femoris vein, and the other at the point where the femoral vein ends in the 
external iliac. This last valve is known as the ilio-femoral valve of Bennett.

Varieties of the Femoral Vein.-I. The vein is sometimes absent from Hunter's canal. In such cases, instead of passing through the femoral opening in connection with the adductor magnus, it ascends for some distance upon the posterior surface of that muscle, which it subsequently pierces, and so enters Scarpa's triangle.

2. The vein may be double. simulating the arrangement known as venæ comites, in which case the femoral artery would have a vein on either side of it, with communicating branches passing between the two at frequent intervals over the vessel.

Obturator Artery. -This vessel normally arises from the anterior division of the internal iliac, and for the most part is placed on the outer wall of the pelvic cavity. The artery, having passed through the obturator canal, divides at once into its two terminal branches, internal and external. These descend upon the anterior surface of the obturator membrane under cover of the obturator externus, the internal branch coursing along the inner margin, and the external along the outer margin, of the membrane. The internal branch supplies the obturator externus and adjacent adductor muscles. The external branch divides into two at the lower part of the obturator membrane. One of these divisions passes inwards and anastomoses with the internal branch, thus completing an arterial loop at the circumference of the membrane. The other division takes an outward course below the acetabulum to the region of the tuber ischii, where it supplies the origins of the hamstring muscles and anastomoses with the sciatic artery. This latter division supplies an articular branch to the hip-joint, which passes beneath the transverse ligament. The external branch, in addition to the foregoing offsets, supplies the obturator externus, in which both terminal branches anastomose with the internal circumflex of the arteria profunda femoris.

The obturator vein terminates in the internal iliac vein.

\section{THE HIP-JOINT.}

The hip-joint belongs to the class diarthrosis, and to the subdivision enarthrosis. The articular surfaces are the head of the femur and the acetabulum of the os innominatum. The ligaments are the capsular ligament, with its accessory parts, the ligamentum teres, the cotyloid ligament, and the transverse ligament.

The capsular ligament is of great strength, and completely surrounds the joint. It is tight-fitting, and hardly admits of separation taking place between the articular surfaces. Its superior attachments are as follows: above and behind it is attached to the os innominatum immediately external to the cotyloid ligament and about three lines from the brim of the acetabulum. In front it is attached to the base of the anterior inferior iliac 
spine; the outer surface of the cotyloid ligament; the iliopectineal eminence; the outer extremity of the obturator crest of the superior pubic ramus; and the adjacent portion of the obturator membrane. Antero-inferiorly (opposite the cotyloid notch) it is attached to the outer surface of the transverse ligament. Inferiorly it is attached to the upper part of the groove between the acetabulum and the tuber ischii. Its inferior attachments are as follows : superiorly it is attached to the anterior part of the upper border of the great trochanter of the femur and the superior cervical tubercle. Anteriorly it descends as low as the anterior intertrochanteric line, into which it is firmly implanted as low as the inferior cervical tubercle. Inferiorly and internally it is attached to the femur about $\frac{3}{4}$ inch in front of the small trochanter.

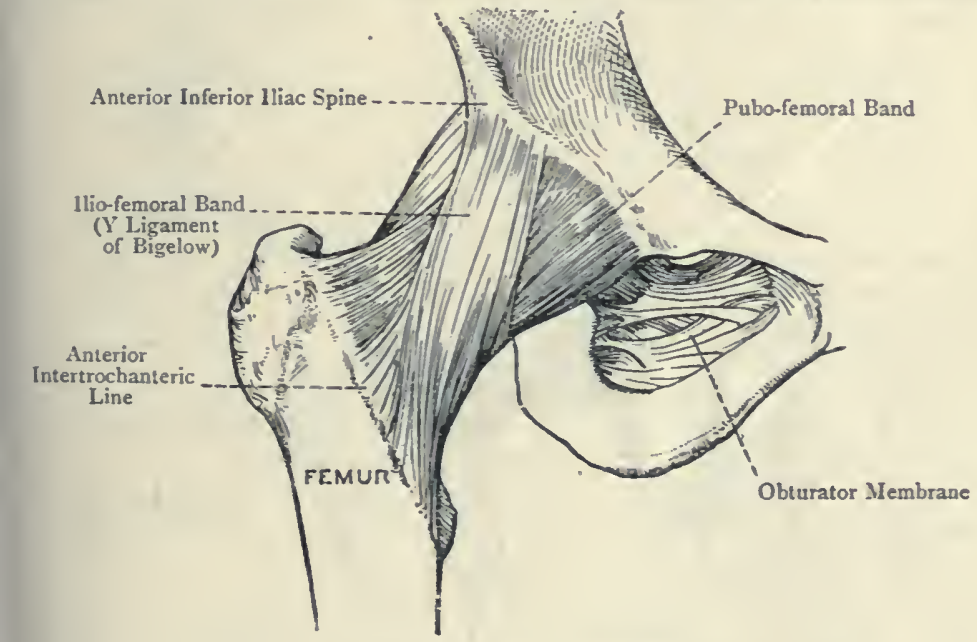

Fig. 237.-The Right Hip-Joint (ANTERIor View).

Posteriorly it is attached very loosely to the neck of the femur at the junction of the middle and lower thirds.

The majority of the fibres of the ligament are disposed longitudinally, but some are circular. The circular fibres are best marked at the posterior and inferior parts of the capsule, where they form a band, nearly $\frac{1}{2}$ inch in depth, called the zona orbicularis. In certain situations the longitudinal fibres form thickened bands which constitute the accessory portions of the capsule. These accessory bands, usually designated as special ligaments, are as follows : ilio-femoral, ilio-trochanteric, ischio-capsular, and pubofemoral.

The ilio-femoral ligament has been compared to an inverted $\mathbf{Y}$, and is known as the Y-shaped ligament of Bigelow. It is situated on the anterior aspect of the capsule, and is attached superiorly 
to the inferior aspect of the anterior inferior iliac spine below the origin of the straight head of the rectus femoris. The lateral fibres as they descend form two strong bands, outer and inner, which diverge very slightly from each other. The inner band passes almost vertically to be attached to the lower end of the anterior intertrochanteric line of the femur and the inferior cervical tubercle. The outer band, shorter than the inner, passes obliquely downwards and outwards to be attached to the upper end of the anterior intertrochanteric line and the superior cervical tubercle. The central fibres are attached to the middle portion of the anterior intertrochanteric line, and the part of the capsule covered by them is comparatively weak.

The ilio-trochanteric ligament is situated immediately external to the outer part of the ilio-femoral ligament. It is attached superiorly

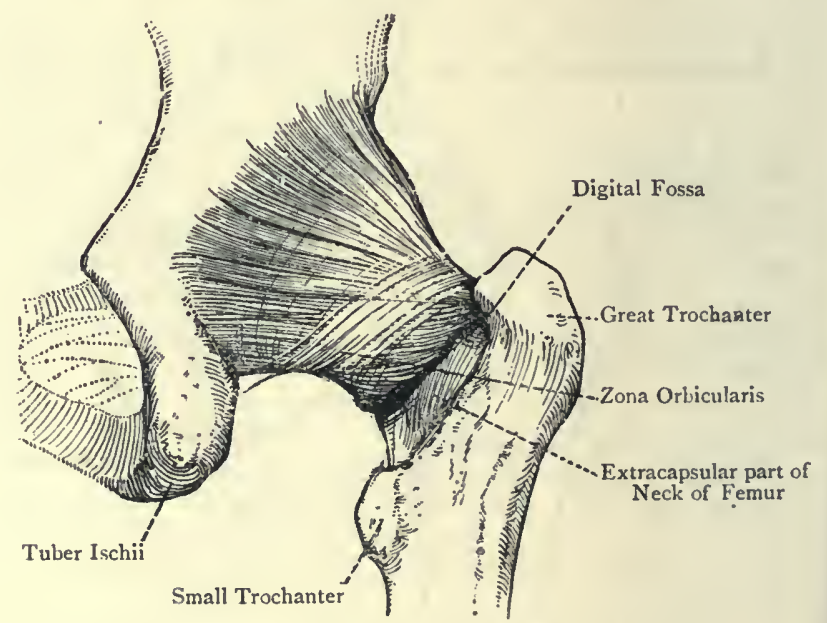

Fig. 238.-The Right Hip-Joint (Posterior View).

to the lower and anterior part of the dorsum ilii immediately above the acetabulum, close to the upper attachment of the ilio-femoral ligament, and inferiorly to the great trochanter in the region of the superior cervical tubercle.

The ischio-capsular ligament is' a thickened band which extends from the upper part of the obturator groove (just below the acetabulum) to the zona orbicularis.

The pubo-femoral ligament is the weakest of the three accessory ligaments. Its fibres are attached in a somewhat scatterec manner to the ilio-pectincal eminence, the outer part of thi obturator crest, and the adjacent portion of the obturator membrane From these points the fibres converge to be attached to the anterior inner, and lower parts of the capsular ligament, where they lie clos to the inner band of the ilio-femoral ligament. 
Two views may be stated regarding the morphology of the ligamentum teres.

I. It may be regarded as a part of the capsule of the hip-joint which has been cut off by the outgrowth of the articular surface of the head of the femur (Keith).

2. It may be regarded as the separated tendon of the pectineus muscle (Bland-Sutton).

The cotyloid ligament is a firm annular band of fibro-cartilage which is implanted upon the brim of the acetabulum, thereby deepening that cavity and bridging over the cotyloid notch. Its fibres are oblique in direction, and are for the most part attached to the outer and inner surfaces of the brim, but opposite the cotyloid notch they are attached to the transverse ligament. The outer surface of the cotyloid ligament is convex, whilst the inner surface is concave and is closely applied to the head of the femur. Both surfaces are covered by the synovial meinbrane. The ligament is triangular, the base being implanted on the brim of the acetabulum, and the apex representing the free margin, which is incurved so as to grasp the head of the femur.

The transverse ligament bridges over the cotyloid notch. It is somewhat complex in structure, being composed of three bundles of fibres intimately blended with one another. The superficial bundle is formed by that part of the cotyloid ligament which stretches over the notch. The other two bundles are more deeply placed, and are arranged as two decussating bands extending between the margins of the notch, and blending closely with the superficial bundle. The transverse ligament does not completely fill up the cotyloid notch, a space being left between the ligament and the bottom of the notch for the passage of articular vessels and nerves.

The synovial membrane covers the anterior surface of the neck of the femur and the upper two-thirds of the posterior surface, passing over the retinacula and extending as high as the margin of the cartilage covering the head. From the neck it is reflected over the inner surface of the capsular ligament, which it completely invests. After leaving the capsular ligament at its acetabular attachments it passes over the cotyloid ligament, and covers both of its surfaces. Thereafter it is reflected over the Haversian gland in the bottom of the acetabulum, from which it is prolonged as a tubular sheath around the ligamentum teres as far as the pit on the head of the femur. In those cases where the capsular ligament presents an opening between the ilio-femoral and pubofemoral ligaments, the synovial membrane is in communication through that opening with the bursa beneath the ilio-psoas. It is to be noted that the ligamentum teres, though intracapsular, is extrasynovial. Further, the synovial membrane is visible from the exterior of the joint at the lower part of its posterior aspect, on account of the very slight attachment of the capsular ligament to the back of the neck of the femur.

The so-called synovial or Haversian gland occupies the rough 
pit in the bottom of the acetabulum, and is simply a collection of adipose tissue covered by the synovial membrane. The ligamentum teres rests upon it, and the synovial membrane which covers it is thicker and more vascular than elsewhere. It serves as a cushion for the head of the femur.

Museular Relations. - Commencing at the inner aspect of the capsular ligament and passing over its anterior, outer, and posterior surfaces to its lower part, the muscles in contact with the ligament are as follows: ( $\mathrm{I}$ ) pectineus on the inner aspect and adjacent part of the anterior surface; (2) ilio-psoas in front; (3) the two heads of the rectus femoris, with the tendon formed by their union, and the gluteus minimus on the outer and upper aspects; (4) pyriformis, gemellus superior, obturator internus, and gemellus inferior on the upper and posterior aspects; and (5) obturator externus on the posterior and inferior aspects. Between the ilio-psoas and the ligament there is a bursa, which sometimes communicates with the synovial membrane of the joint through an opening in the capsule.

Arterial Supply.-The hip-joint receives its arterial supply from the following sources:

I. The transverse branch of the internal circumflex.

2. The ascending branch of the external circumflex.

3. The external terminal branch of the obturator.

4. The inferior branch of the deep division of the gluteal.

5. The sciatic.

Nerve-supply. - The hip-joint receives its nerves from the following sources :

I. The nerve to the rectus femoris, which is a branch of the posterior division of the anterior femoral.

2. The anterior or superficial division of the obturator nerve, or the accessory obturator when it is present.

3. The nerve to the quadratus femoris, or, if that nerve does not supply an articular branch, the great sciatic.

The first two nerves are branches of the lumbar plexus, and the others are derived from the sacral plexus.

Movements.-These are as follows : flexion, extension, abduction, adduction, rotation, and circumduction.

Flexion.-This is of two kinds-ventral and dorsal. In ventral flexion the thigh is drawn upwards towards the anterior abdominal wall. In dorsal flexion the thigh is carried backwards beyond the perpendicular. The extent of ventral flexion depends upon the condition of the knee-joint, being greater when that joint is flexed and more limited when it is kept extended. Assuming the knee-joint to be flexed, ventral flexion is limited by the coming in to contact of the fleshy parts of the front of the thigh and anterior abdominal wall. When the knee-joint is kept extended, ventral flexion is checked earlier by the tension of the hamstring muscles. Dorsal flexion is in most persons soon checked by the tightening of the froct part of the capsular ligament.

Extension.-In extending the hip-joint from the position of ventral flexion the movement is limited by the tightening of the front part of the capsular ligament. When a person stands at rest the hip-joint is in a state of extension. The vertical line passing through the centre of gravity then falls behind the centre of rotation at the hir-joint, and there is thus a tendency on the 
part of the pelvis to fall backwards. This tendency, however, is counteracted by the tightening of the capsular ligament in front, and so the erect attitude is maintained without any muscular effort.

Abduction.-This movement is controlled by the tightening of the pubofemoral ligament and the lower part of the capsule; the locking which takes place between the upper border of the neck of the femur and the adjacent margin of the acetabulum; and the tension of the adductor muscles.

Adduction.-This movement is controlled by the outer band of the iliofemoral ligament and upper part of the capsule, and the loaking which takes place between the inner part of the neck of the femur and the anterior margin of the acetabulum. If the hip-joint is flexed adduction is also controlled by the tightening of the ligamentum teres.

Rotation. - This consists in movement of the femur round its longitudinal axis without much change of position. The axis of rotation is represented by a line passing through the centre of the head of the femur to meet the vertical line of the centre of gravity of the lower limb at a point corresponding with the middle of the intercondylar notch when the knee-joint is extended.

Rotation may take place outwards or inwards. External rotation is controlled by the tightening of the outer band of the ilio-femoral ligament. Internal rotation is checked by the tightening of the ischio-capsular ligament ; by the zona orbicularis; and by the muscles in contact with the back of the joint. The range of rotation is about 60 degrees.

Circumduction.- This is a composite movement, consisting of flexion, abduction, extension, and adduction, following each other in rapid succession, the result being that the femur describes a cone, the head of the bone forming the apex and the lower extremity describing the circumference of the base.

Ligamentum Teres.-This ligament is loose in the state of extension of the hip-joint, but becomes tightened in fexion and adduction.

Muscles concerned in the Movements-Flexion.-Ilio-psoas, rectus femoris, sartorius, pectineus, adductor longus, and adductor brevis, the ilio-psoas being the most powerful flexor. Extension.-Gluteus maximus, biceps femoris by its ischial head, semitendinosus, semimembranosus, and adductor magnus by the fibres which extend from the tuber ischii to the adductor tubercle. Abduction.-Gluteus medius, gluteus minimus, gluteus maximus by its upper fibres, tensor fasciæ femoris, and sartorius. Adduction.-Adductores gracilis, longus, brevis, et magnus, pectineus, gluteus maximus by its lower fibres, and obturator externus. Internal Rotation.-Gluteus medius by its anterior fibres, gluteus minimus, tensor fasciæ femoris, and adductor magnus by the fibres which extend from the tuber ischii to the adductor tubercle. External Rotation.-Gluteus maximus by its lower fibres, gluteus medius by its posterior fibres, pyriformis, obturator internus and gemelli, quadratus femoris, pectineus, adductores longus, brevis, et magnus (the latter by the fibres which are inserted into the shaft of the femur), obturator externus, and sartorius.

\section{Relation of Muscles on the Femoral Aspect of the Body of the Os Pubis.}

Commencing at the symphysis pubis, and passing outwards as far as the inner margin of the obturator foramen, the relation of muscles is as follows : (1) gracilis, (2) adductor brevis, (3) a small portion of the adductor magnus, and (4) obturator externus.

The adductor longus does not take part in this relation, its origin being at the upper and inner part of the anterior or femoral surface of the body of the os pubis, from an impression which can be covered with the point of the little finger.

\section{Relation of Muscles at the Back of the Upper End of the Shaft of the Femur.}

Commencing at the small trochanter, and passing outwards over the back of the femur as far as the outer margin of the gluteal ridge, the relation of muscles is as follows: (1) ilio-psoas, (2) pectineus, (3) adductor bruvis, 
(4) lower fibres of the quadratus femoris, (5) adductor magnus, (6) gluteus maximus, and $(7)$ vastus externus.

Relation of Muscles along the Linea Aspera of the Femur.-Commencing at the outer lip of the linea aspera, and passing inwards as far as the inner lip, the relation of muscles is as follows: (I) vastus externus and crureus in one line; (2) short head of biceps femoris; (3) adductor magnus; (4) adductor longus; and (5) vastus internus.

Bursæ at Hip-Joint.-The bursæ in the vicinity of the hip-joint are as follows:

\section{Psoas. Trochanteric (3). Subtrochanteric.}

The psoas bursa is situated between the tendon of the psoas muscle and the anterior part of the capsule of the hip-joint, between the ilio-femoral and pubo-capsular accessory ligaments, this portion of the capsule being weak. The bursa may be continuous with the synovial membrane of the hip-joint through an opening in the capsule which is sometimes present (one in ten).

The trochanteric bursæ are three in number, and are associated with the great trochanter and the three gluteal muscles. The bursa of the gluteus maximus, which is of large size and multilocular, is situated between the fascial insertion of that muscle and the outer surface of the great trochanter of the femur. The bursa of the gluteus medius is situated between the tendon of insertion of that muscle and the outer surface of the great trochanter above, and in front of, the insertion. The bursa of the gluteus minimus is situated between the tendon of insertion of that muscle and the anterior surface of the great trochanter above the insertion.

The subtrochanteric bursa is situated directly below the bursa of the gluteus maximus, and is also associated with that muscle. It lies between the insertion of the muscle into the gluteal ridge of the femur and the tendinous vastus externus muscle as it skirts the base of the great trochanter.

The trochanteric and subtrochanteric bursæ have no communication with the synovial cavity of the hip-joint. 


\section{THE LEG.}

Landmarks. - The sharp sinuous anterior border of the tibia, being entirely subcutaneous, can readily be felt, and will guide the finger to the tubercle, which is situated at its upper end, where it gives attachment to the ligamentum patellæ. The lower border of the tubercle corresponds with the division of the popliteal artery into anterior and posterior tibial vessels. The head of the fibula forms a prominent landmark on the outer side of the leg, and is situated about.I inch below the level of the upper surface of the external tuberosity of the tibia. The shaft of the fibula is for the most part obscured by muscles. It can be felt, however, over its lower fourth. The bone occupies a more posterior plane than the tibia. The prominence along the front of the leg in its upper two-thirds is mainly due to the fleshy belly of the tibialis anticus.

The internal and external malleoli form bold projections at the lower end of the leg, the internal being formed by the tibia, and the external by the fibula. The malleoli are upon the same plane posteriorly, but the internal malleolus projects further forwards than, and does not descend so low as, the external malleolus; that is to say, the internal malleolus is a little higher up and further forwards than the external, but the two project equally far back. This has to be borne in mind in Syme's amputation at the ankle-joint. It should be carefully noted that, whilst the internal malleolus looks directly inwards, the internal tibial tuberosity has a slight inclination backwards as well as inwards. The tendons of the tibialis posticus and flexor longus digitorum may be felt behind the internal malleolus, the former being the larger and in close contact with the bone. If the inner border of the foot is raised so as to invert the sole, the tendon of the tibialis posticus will be more readily felt. The tendons of the peroneus longus and peroneus brevis are situated behind the external malleolus, where they lie one upon the other, the brevis tendon being in close contact with the bone. Several tendons can readily be felt in front of the ankle-joint. From within outwards they are as follows: tibialis anticus; extensor proprius hallucis; extensor longus digitorum; and peroneus tertius. All these tendons are best felt in the living subject when the foot is flexed upon the leg-that is to say, when the foot is raised.

The back of the leg is characterized by the prominence of the calf, which is produced by the gastrocnemius and soleus muscles. This prominence gives place inferiorly to the tendo 
Achillis, which stands out boldly beneath the integument, and subsides at the tuber calcis. Upon either side of the projection formed by the tendo Achillis there is an elongated furrow. The furrow on the outer side indicates the situation of the short saphenous vein and nerve and the peroneal artery, whilst that on the inner side corresponds with the position of the posterior tibial vessels and nerve.

The course of the anterior tibial artery on the front of the leg is indicated by a line drawn from the superior tibio-fibular articulation to the centre of the front of the ankle-joint, midway between the two malleoli. This practically coincides with the outer border of the tibialis anticus. The course of the posterior tibial artery corresponds with a line drawn from the centre of the popliteal space to a point midway between the tip of the internal malleolus and the inner part of the point of the heel.

It is to be noted that another large artery is situated upon the back of the leg. This is the peroneal branch of the posterior tibial, and in operations it will be caught upon the back of the fibula, the posterior tibial being between the tibia and fibula, except in the lower part of the leg, where it lies on the back of the tibia.

The anterior and posterior tibial arteries can readily be compressed with one hand by placing the thumb in front of the anklejoint midway between the two malleoli, and the middle finger midway between the tip of the internal malleolus and the inner part of the point of the heel.

The long saphenous vein may be visible in front of the internal malleolus, and from this point it may be traceable upwards along the internal subcutaneous surface of the tibia.

The short saphenous vein is rarely perceptible. It lies behind the external malleolus, then in the hollow upon the outer side of the tendo Achillis, after emerging from which it soon gains the middle line, where it lies in the groove between the two heads of the gastrocnemius.

Deep Fascia.-The deep fascia is in part a prolongation of the fascia lata of the thigh, the continuity being established at the inner and posterior aspects of the knee-joint. Elsewhere it is attached superiorly to the head of the fibula, external tuberosity of the tibia, lower part of the patella, ligamentum patellæ, and tubercle, and front of the internal tuberosity, of the tibia. In the leg it is attached to the crest of the tibia, from which it is prolonged backwards over the inner surface to be attached to the internal border. It is also attached to the antero-external and postero-external borders of the fibula. At the ankle the deep fascia is attached to the internal and external malleoli. Over the inner surface of the tibia and at its malleolar attachments it becomes closely incorporated with the periosteum. The chief direction of the fibres of the fascia is vertical. There are, however, superadded transverse fibres behind the knee-joint and in the vicinity of the ankle-joint, in which latter region important annular ligaments are formed. 
The part of the fascia behind the knee-joint presents an opening for the passage of the short saphenous vein. In the region of the gastrocnemius and over the inner surface of the tibia the deep fascia is very thin. Over the front and outer parts of the leg, however, more especially in the upper half, it attains considerable strength and density, and there it gives origin by its deep surface to the superficial fibres of the muscles which it covers. In the region of the knee-joint it receives many strong accessions of fibres from the tendons of the quadriceps extensor cruris, biceps femoris, sartorius, gracilis, and semitendinosus.

Four expansions are given off from its deep surface, which are called intermuscular septa-anterior, antero-external, posteroexternal, and posterior or transverse. The anterior intermuscular septum is limited to the upper third of the leg, where it extends between the tibialis anticus and extensor longus digitorum, to both of which muscles it gives partial origin. The antero-external intermuscular septum is attached to the antero-external border of the fibula, and extends between the extensor longus digitorum and peroneus tertius on the anterior surface, and the peroneus longus and peroneus brevis on the external surface. The postero-external intermuscular septum is attached to the postero-external border of the fibula, where it extends between the peroneus longus and peroneus brevis on the external surface, and the soleus and flexor longus hallucis on the posterior surface. The peroneus longus and peroneus brevis are thus completely shut off from the adjacent muscles by the two external intermuscular septa. These septa, along with the deep fascia at the surface and the external surface of the shaft of the fibula, form a long fibro-osseous canal which contains the peroneus longus and peroneus brevis. The posterior or transverse intermuscular septum is a broad expansion which passes transversely between the postero-external border of the fibula and the internal border of the tibia. It lies beneath the soleus and upon the deep muscles of the back of the leg, as well as upon the posterior tibial vessels and nerve, binding down the structures which it covers.

Annular Ligaments.-These are situated in the region of the ankle-joint, and are thickened portions of the deep fascia. They serve to confine and maintain in position the strong tendons in that vicinity, and are three in number-external, internal, and anterior.

The external annular ligament extends between the posterior border of the external malleolus and the outer border of the tuber calcis and adjacent portion of its outer surface. Its direction is downwards and backwards over the peroneal groove behind the external malleolus. The tendons of the peroneus longus and peroneus brevis pass beneath it, and are thereby kept in position. The tendon of the peroneus brevis is close to the bone, and that of the peroneus longus is directly behind the brevis tendon, both being invested by one common synovial sheath. The external annular ligament at its anterior border is continued forwards to 
form two distinct fibrous sheaths for the peroneal tendons, which are here lying one above the other (the peroneus brevis tendon being the upper one) as they traverse the outer surface of the os calcis. Each tendon has now its own synovial investment, the common synovial sheath beneath the external annular ligament having sent forward two prolongations.

The internal annular ligament is chiefly formed by a thickening of the posterior or transverse intermuscular septum, but superficial to it there is also the proper deep fascia, though that is here very thin. This annular ligament extends between the posterior border of the internal malleolus and the internal border of the tuber calcis, and is directed downwards and backwards. Its upper border is continuous principally with the posterior intermuscular

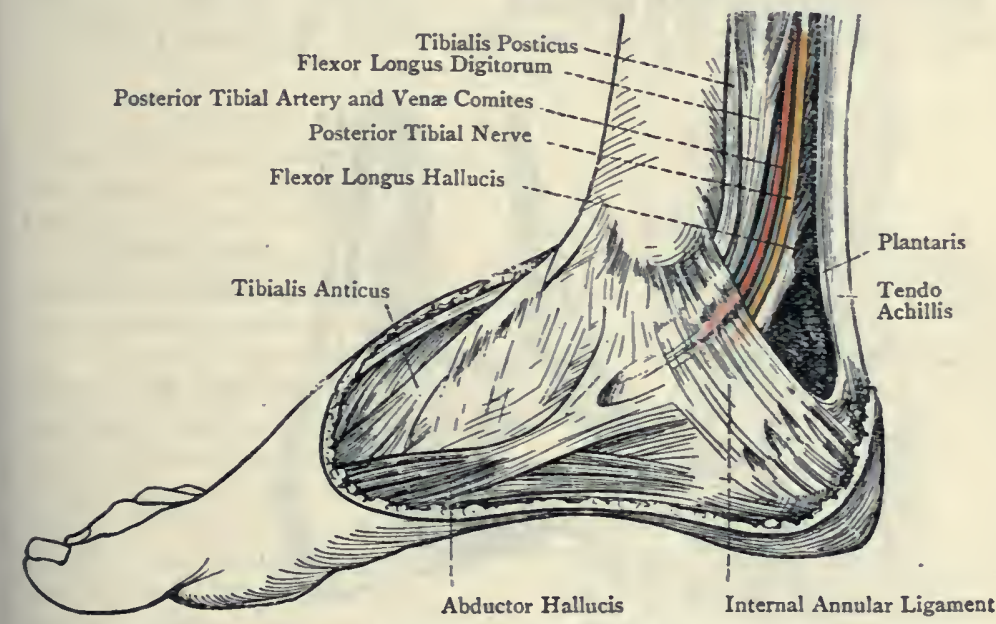

Fig. 240.-The Structures between the Internal Malleolus AND THE HEEL.

septum. Its lower border gives origin on its deep aspect to the inner head of the abductor hallucis, and on its superficial aspect it is in part continuous with the lower limb of the $Y$ division of the anterior annular ligament. The internal annular ligament passes over the grooves on the back of the lower end of the tibia, and converts these into canals for the flexor tendons and posterior tibial vessels and nerve. There are in all four canals, three of them being fibro-osseous and one fibrous. The relation of structures beneath this ligament, from the internal malleolus outwards to the tuber calcis, is as follows: (I) the tendon of the tibialis posticus, lying in the groove behind the internal malleolus close to the bone, and occupying one of the fibro-osseous canals ; (2) the tendon of the flexor longus digitorum, lying behind, and slightly external to, that of the tibialis posticus, and occupying the 
fibrous canal ; (3) the internal vena comes, posterior tibial artery, external vena comes, and posterior tibial nerve, all occupying a second fibro-osseous canal; (in cases of an early division of the posterior tibial nerve into external and internal plantar nerves these two nerves are found in this canal, the internal on the inner side of the internal vena comes, and the external on the outer side of the external vena comes); (4) the tendon of the flexor longus hallucis, occupying the third fibro-osseous canal, and lying in the groove at the outer part of the posterior border of the lower end of the tibia. Each of the three tendons mentioned is invested by a special synovial sheath. The internal annular ligament is pierced by the calcaneo-plantar branch of the posterior tibial nerve and the internal calcaneal branch of the posterior tibial artery.

The anterior annular ligament is composed of two divisions. One division is situated on the front of the leg above the ankle-

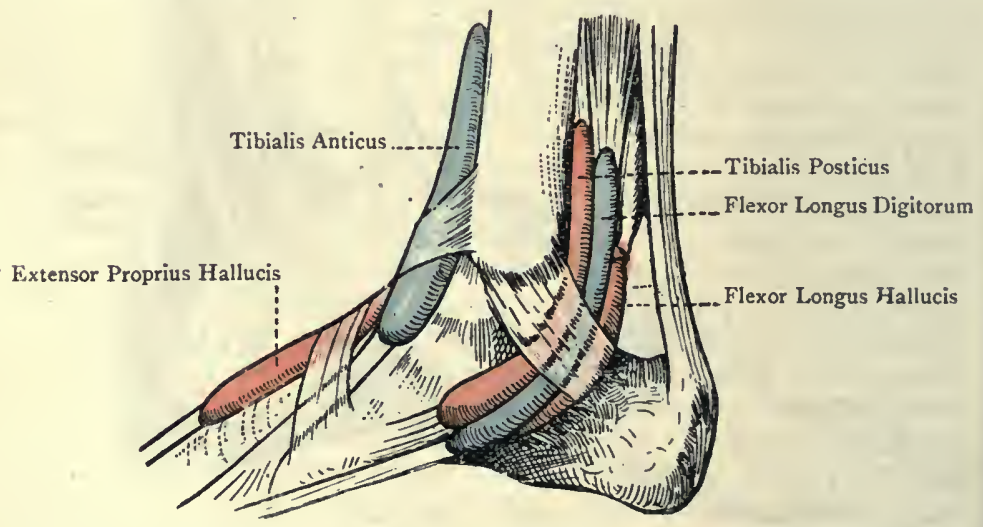

Fig. 24I.-The Synovial Sheaths at the Ankle (Internal View) (After L. Testut's 'Anatomie Humaine').

joint, and is called the superior anterior annular ligament. The other division lies in front of the bend of the ankle, and is called the inferior anterior annular ligament, anterior annular ligament proper, or lambdoid ligament.

The superior anterior annular ligament is a broad band extending transversely between the anterior borders of the tibia and fibula, and measuring about 2 inches from above downwards. Immediately beyond it the deep fascia is exceedingly thin. The structures passing beneath it, in order from within outwards, are (I) tibialis anticus, (2) extensor proprius hallucis, (3) internal vena zomes, (4) anterior tibial artery, (5) external vena comes, (6) anterior tibial nerve, (7) extensor longus digitorum, and (8) peroneus tertius. These structures occupy one common compartment, the tibialis anticus tendon alone being surrounded by a synovial sheath. 
which is continuous with that investing it as it passes through the lambdoid ligament.

The inferior anterior annular or lambdoid ligament is a more defined structure than the superior. Being placed in front of the ankle-joint, it serves to strap down the extensor tendons as they are entering upon their horizontal course on the dorsum of the foot. It may be likened either to the capital letter $\prec$ placed upon its side as indicated, or to the Greek letter $\prec$ (lambda), also laid upon its side, as shown. From the former resemblance it may be called the $Y$ annular ligament. The outer part, which is a single narrow band, is attached to a depression on the upper surface of the os calcis at its anterior and outer part, immediately in front of the interosseous groove. This part forms a loop through which the tendons of the extensor longus digitorum and peroneus tertius pass, invested by one synovial sheath. From the fact that it forms a loop it has been called by Retzius the fundiform ligament. It gives partial origin to the extensor brevis digitorum, and at its inner end it is continued into two diverging bands, upper and lower. The upper band is attached to the internal mallealus, and the tendon of the extensor proprius hallucis passes underneath it, being

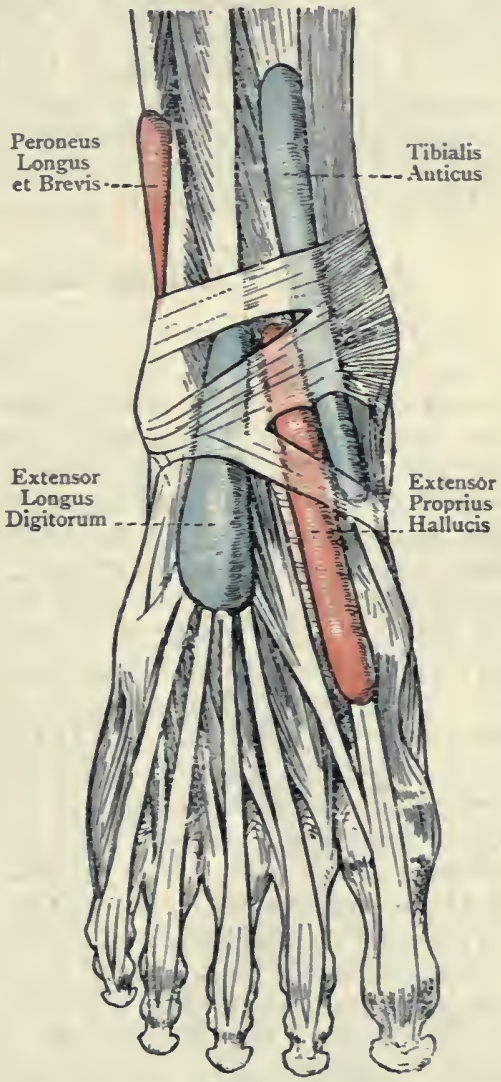

Fig. 242.-The Syxovial Sheaths at ThE ANKLE (ANTERIOR VIEW) (AFTER L. Testut's 'Anatomie Humaine').

surrounded by a synovial sheath. The tendon of the tibialis anticus passes through it, the main portion of the band being underneath that tendon, and a small expansion from it passing superficial to the tendon. The tendon of the tibialis anticus has its own synovial sheath, which is prolonged upwards from this point along the tendon, to be continuous with the synovial sheath investing it as it passes behind the superior anterior 
annular ligament. The lower band of the lambdoid ligament is a comparatively weak structure which passes to the inner border of the foot, where it joins partly the plantar fascia and partly the lower border of the internal annular ligament. The tendons of the extensor proprius hallucis and tibialis anticus both pass underneath it. There are thus three distinct synovial sheaths in connection with the lambdoid ligament-one for the extensor longus digitorum and peroneus tertius, one for the extensor proprius hallucis, and one for the tibialis anticus, the latter being continuous with that which invests the tendon beneath the superior annular ligament. The dorsalis pedis artery, with its venæ comites, and the dorsalis pedis nerve pass underneath or behind both bands of the fundiform ligament.

\section{Internal Aspect of the Leg.}

The internal surface of the tibia, except at its upper end, is subcutaneous. The deep fascia is here very thin, and is intimately incorporated with the periosteum. The structures met with on this aspect are the long saphenous vein and nerve, the posterior division of the internal cutaneous nerve, and the superficial branch of the anastomotica magna artery. The long saphenous vein lies about a finger's breadth from the internal border of the tibia, and it receives many tributaries from the anterior and posterior aspects of the leg. The long saphenous nerve lies immediately behind it. The posterior division of the internal cutaneous nerve is confined to the upper half of the inner aspect of the leg, and the superficial branch of the anastomotica magna artery ramifies in the upper third. At the upper end of the internal surface of the tibia the tendons of insertion of the sartorius, gracilis, and semitendinosus are met with, as well as the internal lateral ligament of the knee-joint. Proceeding in a direction backwards from the tubercle of the tibia to the internal border of the bone, the relation of these structures is as follows: ( $\mathrm{I}$ ) tendon of the sartorius; (2) tendons of the gracilis and semitendinosus in the same vertical line, the gracilis being the higher of the two, and both being under cover of the sartorius ; and (3) the internal lateral ligament of the knee-joint. The tendons of the gracilis and semitendinosus cross the internal lateral ligament in a forward direction, and are separated from it and the subjacent bone by a bursa, which furnishes an expansion to separate them from the more superficially placed sartorius. The portion of the internal lateral ligament met with in this region is a long, flat, expanded band, attached to the internal border, and adjacent portion of the internal surface, of the tibia, upon which it descends for fully 3 inches. The inferior internal articular artery passes transversely forwards underneath it, below the internal tuberosity of the tibia; and beneath the posterior border of the ligament the chief tendon of the semimembranosus passes to be inserted into the horizontal groove on the posterior surface of the internal tuberosity. 


\section{Anterior Aspect of the Leg and Dorsum of the Foot.}

Musculo-cutaneous Nerve (Superficial Peroneal). - This nerve is one of the terminal branches of the external popliteal, arising from that nerve on the outer side of the neck of the fibula, where it lies between the bone and the peroneus longus. It then descends in the antero-external intermuscular septum, lying between the extensor longus digitorum in front, and the peroneus longus and peroneus brevis behind. When it reaches the junction of the upper twothirds and lower third of the leg, it becomes cutaneous by piercing the deep fascia, and almost immediately afterwards it divides into its two terminal branches, internal and external.

Branches. - The branches of the nerve are as follows: muscular to the peroneus longus and peroneus brevis; and cutaneous to the integument of the front of the leg in its lower third, which arises as soon as the nerve pierces the deep fascia.

The internal terminal branch descends to the dorsum of the foot, lying superficial to both divisions of the anterior annular ligament. It then divides into two branches, inner and outer. The inner branch supplies twigs to the integument of the internal malleolus and inner side of the foot, which communicate with the terminal part of the long saphenous nerve, and it then becomes the dorsal digital nerve of the inner side of the great toe. It gives a communicating branch to the dorsalis pedis nerve as that lies upon the first dorsal interosseous

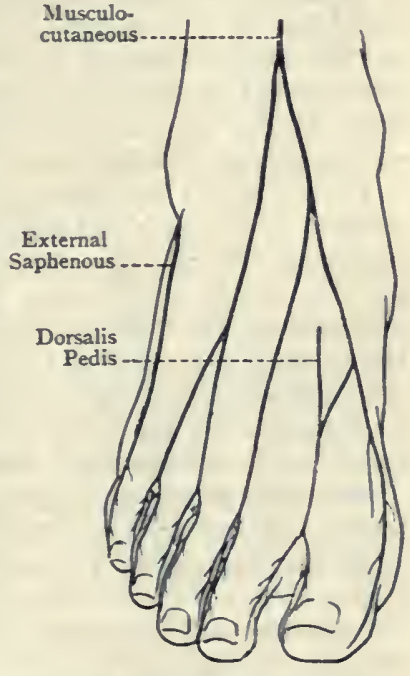

Fig. 243,-Diagram of ThE Nerves of the Foot

(Dorsal AsPECT).

muscle. The outer branch passes to the cleft between the second and third toes, where it divides into two dorsal collateral digital nerves for the supply of the contiguous sides of these toes.

The external terminal branch, like the internal, descends to the dorsum of the foot, and also lies superficial to both divisions of the anterior annular ligament. On the dorsum of the foot it divides into two branches, inner and outer. The inner branch passes to the cleft between the third and fourth toes, and the outer to the cleft between the fourth and fifth toes. At these clefts the inner and outer branches divide each into two dorsal collateral digital nerves for the supply of the contiguous sides of the third and fourth, and fourth and fifth toes. The outer branch in its course supplies twigs to the integument of the 
external malleolus and outer border of the foot which communicate with the terminal part of the short saphenous nerve.

Summary of the Distribution of the Musculo-cutaneous Nerve.-The 11erve is distributed to the peroneus longus, peroneus brevis, integument over the front of the leg in its lower third, integument of the malleolar regions and outer and inner borders of the foot, and the integument of (I) the inner side of the great toe, and (2) the contiguous sides of the second and third, third and fourth, and fourth and fifth toes, all on their dorsal aspects. The dorsal digital nerve of the outer side of the fifth toe is the terminal part of the short saphenous nerve.

The dorsal collateral digital nerves for the supply of the contiguous sides of the great toe and second toe are derived from the dorsalis pedis nerve, which is the continuation of the anterior tibial nerve.

Muscles. Tibialis Anticus-Origin.-(I) The external tuberosity of the tibia at its lower part ; (2) the upper two-thirds of the external surface of the shaft of the tibia and adjacent portion of the anterior surface of the interosseous membrane ; and (3) the deep fascia, and the anterior intermuscular septum which lies between it and the extensor longus digitorum in the upper third of the leg.

Insertion.- The tendon, having traversed the groove on the inner surface of the internal cuneiform bone, divides into two slips. The posterior slip is inserted into an oval impression at the lower part of the inner surface of the internal cuneiform bone, and the anterior slip is inserted into the inner surface of the tuberosity on the plantar aspect of the base of the first metatarsal bone.

Nerve-supply.-The anterior tibial nerve.

Action.-(I) To flex the foot upon the leg, and (2) to raise the inner border of the foot, thereby inverting the sole.

Extensor Proprius or Longus Hallucis-Origin.-The middle twofourths of the anterior surface of the shaft of the fibula, and the adjacent portion of the anterior surface of the interosseous membrane.

Insertion.-The dorsal surface of the base of the distal phalanx of the great toe.

Nerve-supply.--The anterior tibial nerve.

Action.-(I) To extend the distal phalanx of the great toe, and (2) to flex the foot upon the leg:

The extensor proprius hallucis is a very narrow muscle which lies deeply between the tibialis anticus and extensor longus digitorum, both of which conceal it until its tendon appears on the front of the lower part of the tibia.

Extensor Longus Digitorum.-Origin.-(I) The external tuberosity of the tibia; (2) the head and upper three-fourths of the anterior surface of the shaft of the fibula; (3) the anterior surface of the interosseous membrane in its upper fourth ; $(4)$ the intermuscular septa between it and adjacent muscles; and (5) the deep fascia.

Insertion.- The second and distal phalanges of the four outer toes. The four tendons pass to the dorsal aspects of the metatarsophalangeal joints of the four outer toes. At this point they broaden 
out into expansions which receive the tendons of the lumbricales and interosseous muscles. In the case of the tendons destined for the second, third, and fourth toes, their expansions are also joined by the outer three tendons of the extensor brevis digitorum. Each tendinous expansion passes forwards over the dorsum of the first phalanx, and at its distal end divides into three bands. The middle band is inserted into the dorsal aspect of the base of the second phalanx. The two lateral bands, having united, are inserted into the dorsal aspect of the base of the distal phalanx.

Nerve-supply.-The anterior tibial nerve:

Action.-(I) To extend the second and distal phalanges of the four outer toes, and (2) to flex the foot upon the leg.

Peroneus Tertius-Origin.-(I) The anterior surface of the shaft of the fibula in its lower fourth, except for an inch or more below, and (2) the adjacent portion of the anterior surface of the interosseous membrane, and the antero-external intermuscular septum between the muscle and the peroneus brevis.

Insertion. - The dorsal surface of the base of the fifth metatarsal bone.

Nerve-supply.-The anterior tibial nerve.

Action.-(I) To flex the foot upon the leg, and (2) to raise the outer border of the foot slightly.

The peroneus tertius is to be regarded as a detached portion of the extensor longus digitorum.

Anterior Tibial Artery.- This vessel is one of the terminal branches of the popliteal artery. It commences at the lower border of the popliteus muscle on a level with the lower border of the tubercle of the tibia (fully $1 \frac{3}{4}$ inches below the level of the upper surface of the head of that bone), and it terminates on the anterior ligament of the ankle-joint midway between the two malleoli by becoming the dorsalis pedis artery. Being placed at its commencement on the back of the leg, the vessel passes at first. forwards between the two heads of the tibialis posticus, and over the upper border of the interosseous membrane, where it lies below the superior tibio-fibular articulation. (The artery sometimes passes through an aperture in the upper part of the interosseous membrane, called the superior hiatus.) Having reached the front of the leg, the vessel changes its course somewhat abruptly, and then descends in close contact with the anterior surface of the interosseous membrane, until it reaches the junction of the upper two-thirds and lower third of the leg. Beyond this point it gradually inclines towards the anterior aspect of the tibia, and in the lower fourth it lies upon that surface, and finally upon the anterior ligament ofthe ankle-joint. The course of the vessel on the anterior aspect of the leg corresponds with a line drawn from the superior tibiofibular articulation to the centre of the front of the ankle-joint midway between the two malleoli.

Relations-On the Back of the Leg.-The artery lies here between the two heads of the tibialis posticus, having the lower border of the 
popliteus muscle above it, and being under cover of the gastrocnemius.

On the Front of the Leg-Superficial.--In the upper three-fourths the artery is deeply placed, and is covered by the integument, and the meeting between the tibialis anticus on the inner side and

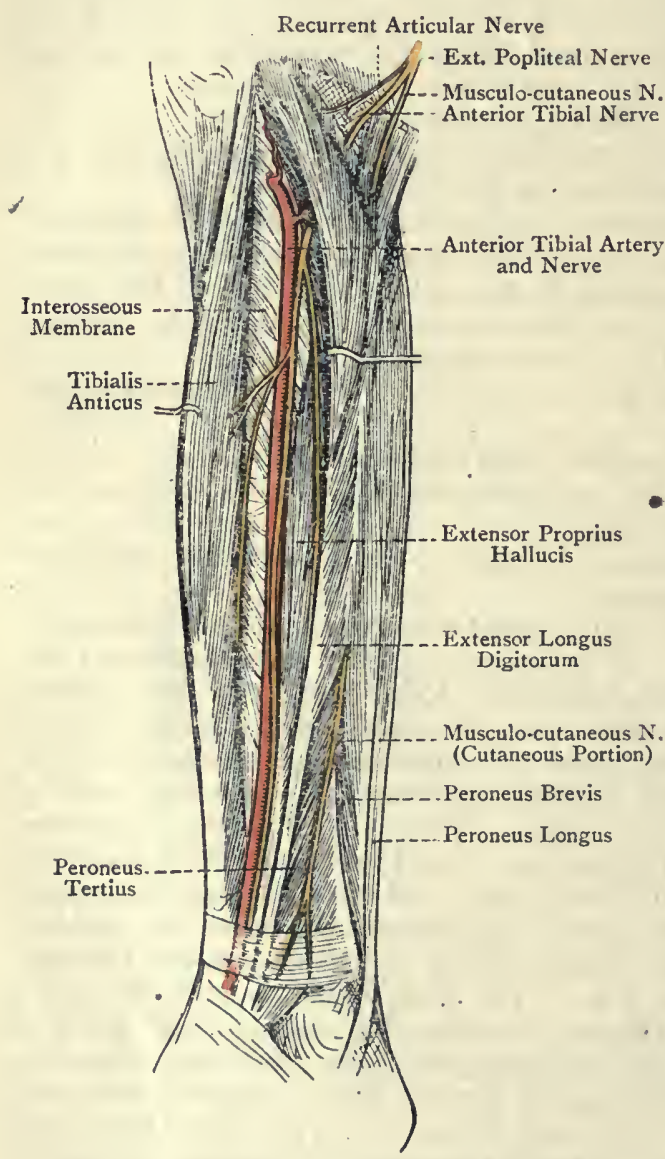

Fig. 244.-The Front of the Left Leg (DEep Dissection).

the extensor longus digitorum and extensor proprius hallucis on the outer side. In the lower fourth the vessel is compara- tively superficial, the muscles having now ended in their tendons, and it is covered by the integument, upper division of the anterior annular ligament, and extensor proprius hallucis, the

- tendon of the latter muscle crossing it from without inwards about $3 \frac{1}{2}$ inches above the ankle-joint. Deep. - Anterior surface of the interosseous membrane (to which it is bound by fibrous tissue), anterior surface of the tibia in lower fourth, and the anterior ligament of the ankle-joint. External.-Extensor longus digitorum in the upper fourth o the leg, extensor pro prius hallucis in the middle two-fourths and extensor longu digitorum again is the lower fourth. In

ternal.-Tibialis anticus in the upper three-fourths, and extenso proprius hallucis in the lower fourth.

The artery is accompanied by two venæ comites, which closel embrace its sides and communicate with each other at frequer intervals by transverse branches, crossing superficial to the vesse These venæe comites, having passed backwards over the uppe 
border of the interosseous membrane (or through a superior hiatus in it), join to form one trunk, which unites with the trunk formed by the junction of the venæ comites of the posterior tibial artery to form the popliteal vein.

The anterior tibial artery in the upper fourth of the leg is separated from the anterior tibial nerve by the fibula and the extensor longus digitorum, the artery having passed forwards between the tibia and fibula, and the nerve having wound obliquely round the outer side of the bone. At the junction of the upper fourth and lower three-fourths of the leg the nerve, having pierced the antero-external intermuscular septum and extensor longus digitorum, comes into contact with the artery, along the outer side of which it descends for a short distance. It then lies on the artery for a little, and finally it again takes up a position upon the outer side of the vessel in the lower fourth of the leg.

Branches.-The branches from above downwards are as follows: posterior tibial recurrent; superior fibular; anterior tibial recurrent; muscular ; internal malleolar; and external malleolar.

The posterior tibial recurrent artery is an inconstant branch. When present it arises from the anterior tibial artery whilst on the back of the leg. Its course is upwards beneath the popliteus muscle, and it is distributed to that muscle, the posterior ligament of the knee-joint, and the superior tibio-fibular articulation. It anastomoses with the external and internal inferior articular arteries on the back of the leg.

The superior fibular artery also arises from the anterior tibial whilst on the back of the leg. Its course is outwards behind the neck of the fibula, where it pierces the soleus, and it is distributed to that muscle, the peroneus longus, and the adjacent integument.

The anterior tibial recurrent artery arises from the anterior tibial whenever it reaches the front of the leg. Entering the upper part of the tibialis anticus, to which, as well as to the superior tibio-fibular joint, it gives branches, it ascends to the external tuberosity of the tibia, in company with the recurrent articular branch of the external popliteal nerve. Its terminal branches anastomose with the external and internal inferjor articular arteries.

The muscular branches are very numerous, and are distributed chiefly to the muscles on the anterior aspect of the leg. Some offsets, however, reach the integument, and others, piercing the interosseous membrane, terminate in the tibialis posticus, in which they anastomose with branches of the posterior tibial artery.

The internal malleolar artery is a small branch which arises from the inner side of the anterior tibial near the lower end of the tibia. Its course is inwards beneath the tendon of the tibialis anticus, and it is distributed over the internal malleolus, where it anastomoses with (I) the internal malleolar branches of the pos- 
terior tibial, (2) the superior internal tarsal branch of the dorsalis pedis, and (3) branches of the internal plantar, thus forming the internal malleolar anastomosis.

The external malleolar artery arises from the outer side of the anterior tibial at a slightly lower level than the internal malleolar. Its course is outwards beneath the tendons of the extensor longus digitorum and peroneus tertius, and it is distributed over the external malleolus, where it anastomoses with (I) the anterior peroneal, (2) the posterior peroneal, and (3) the external tarsal branch of the dorsalis pedis, thus forming the external malleolar anastomosis.

The veins which accompany the branches of the anterior tibial artery are, in each case, arranged as venæ comites, and they terminate as tributaries of the anterior tibial venæ comites.

Varieties-I. Origin.-The vessel may arise from the popliteal at the upper border of the popliteus muscle. In these cases it may descend on the posterior surface of that muscle (this being the more frequent position), or it may pass deeply in front of it.

2. Course. - The vessel in the lower fourth of the leg may be found upon the fibula instead of the tibia, in which cases it makes a sudden bend at the ankle-joint to become the dorsalis pedis artery. In very rare cases the vessel may become superficial at the centre of the leg instead of in the lower fourth.

3. Size.-The vessel is occasionally very small, and, if the diminution in size is very pronounced, it may fail to furnish the dorsalis pedis artery, in which cases that vessel is supplied by the anterior peroneal.

Anastomoses round the Knee-Joint.-The arteries which take part in these anastomoses are as follows : (I). the two superior, and the two inferior, external and internal articular branches of the popliteal; (2) the long descending branch of the external circumflex of the profunda femoris; (3) the anastomotica magna of the superficial femoral; (4) the fourth perforating of the profunda femoris; and (5) the posterior tibial recurrent (inconstant), and the anterior tibial recurrent, both of which are branches of the anterior tibial artery. For the special anastomoses of the foregoing arteries see the descriptions of the individual vessels, and Fig. 25I.

The anastomoses are divided into superficial and deep, the former being placed superficial to the patella, and the latter being in contact with the lower end of the femur and the head of the tibia.

Three transverse arches are to be noted anteriorly as follows: one lies in the substance of the crureus, just above the patellar surface of the femur, and is formed by branches of the superior external articular of the popliteal, and the deep branch of the anastomotica magna of the superficial femoral. A second arch lies in front of the head of the tibia, near the superior surface, and is formed by branches of the inferior external articular, and the superior internal articular. A third arch lies in front of the tibia just above the tubercle, and is formed by branches of the anterior tibial recurrent, and the inferior internal articular artery. 
The terminal part of the anterior peroneal artery, having reached the front of the leg by passing through the inferior hiatus in the interosseous membrane, descends in front of the inferior tibiofibular articulation under cover of the peroneus tertius, to both of which it furnishes branches. It finally takes part in the external malleolar anastomosis, along with (I) the external malleolar of the anterior tibial, (2) the external tarsal of the dorsalis pedis, and (3) the posterior peroneal.

Anterior Tibial Nerve (Deep Peroneal).--This nerve is one of the terminal branches of the external popliteal. It commences upon the outer side of the neck of the fibula, where it lies between the bone and the peroneus longus, and it terminates at the anterior ligament of the ankle-joint, where it becomes the dorsalis pedis nerve. At first it is directed downwards, forwards, and inwards through the antero-external intermuscular septum and extensor longus digitorum, and it comes into contact with the anterior tibial artery at the junction of the upper fourth and lower threefourths of the leg. It then descends in close contact with that artery as far as its termination, lying at first external to the vessel, then in front of it, and finally again on its outer side. The general relations of the nerve closely correspond with those of the artery on the front of the leg.

Branches.-These are muscular and articular. The muscular branches supply the tibialis anticus, extensor longus digitorum, extensor proprius hallucis, and peroneus tertius. The articular branches arise from the lower part of the nerve, and are distributed to the ankle-joint and inferior tibio-fibular articulation.

Anterior Tibial Gland.-This lymphatic gland is situated in front of the interosseous membrane at its upper part in close proximity to the anterior tibial artery. Its afferent vessels take up lymph from the dorsum of the foot and anterior aspect of the leg in its more deeply-placed parts. The efferent vessels, which are usually two in number, pass backwards either above the interosseous membrane or through a superior hiatus in it, lying along the anterior tibial artery, and they become afferent vessels to the popliteal glands.

Dorsum of the Foot. Deep Fascia. - This is a delicate membrane which is prolonged forwards from the lambdoid ligament over the long extensor tendons. Two other thin layers of deep fascia are met with, one covering the extensor brevis digitorum, and the other covering the dorsal interosseous muscles and the dorsal surfaces of the metatarsal bones.

Extensor Brevis Digitorum-Origin.-(I) The anterior part of the upper surface, and adjacent part of the external surface, of the os calcis; and (2) the outer single portion of the lower division of the anterior annular ligament-that is to say, the fundiform ligament of Retzius.

Insertion.-Into the four inner toes, namely, the great toe, second, third, and fourth, by means of four tendons in the following 
manner: the innermost tendon has a special insertion into the dorsal surface of the first phalanx of the great toe near its base. The other three tendons join the outer borders of the long extensor tendons which go to the second, third, and fourth toes, the union taking place at the metatarso-phalangeal joints.

Nerve-supply. - The external branch of the dorsalis pedis nerve.

Action.-(I) Innermost tendon.-This tendon extends the great toe at the metatarso-phalangeal joint, and it also acts as an adductor of that toe. (2) Second, third, and fourth tendons.-These extend the corresponding toes in conjunction with the long extensor tendons. In doing so they tend, by the obliquity of their direction, to draw the toes outwards, and in this way they counteract the opposite tendency of the long extensor tendons, which of themselves would incline the toes inwards.

The muscle lies obliquely upon the dorsum of the foot, and is directed forwards and inwards. The innermost fleshy bundle is always the largest, and separates from the parent muscle sooner than the others. It has accordingly been regarded as a separate muscle, and as such is called the extensor brevis hallucis.

Dorsalis Pedis Artery.-This vessel is the continuation of the anterior tibial artery. It commences at the anterior ligament of the ankle-joint, midway between the two malleoli, and terminates at the proximal end of the first interosseous space, where it divides into two branches, plantar or perforating, and arteria dorsalis hallucis or first dorsal interosseous artery. The course of the vessel is along the inner part of the dorsum of the foot, and is indicated by a line drawn from the centre of the front of the ankle-joint, midway between the two malleoli, to the proximal end of the first interosseous space.

Relations-Superficial.-The skin, superficial fascia, lower division of anterior annular ligament, deep fascia, and innermost tendon of the extensor brevis digitorum, which crosses it from without inwards near its termination. Deep.- -Upper border of the head of the astragalus, navicular, middle cuneiform, base of second metatarsal, and the corresponding ligaments. External.-External vena comes, dorsalis pedis nerve, innermost tendon of the extensor longus digitorum, and, near its termination, the innermost tendon of the extensor brevis digitorum as it is about to cross the artery. Internal.-Internal vena comes, tendon of the extensor proprius hallucis, and, near its termination, the innermost tendon of the extensor brevis digitorum after it has crossed the vessel.

The artery is firmly bound down by connective tissue to the subjacent bones and ligaments. It is accompanied by two venæ comites, one on either side, which communicate with each other by transverse branches lying superficial to the vessel, and ultimately become the venæ comites of the anterior tibial artery.

Branches.--The branches are as follows: internal tarsal, external tarsal, metatarsal, arteria dorsalis hallucis or first dorsal 
interosseous, and plantar or perforating, which latter divides in the sole of the foot into arteria magna or princeps hallucis, or first plantar interosseous, and plantar communicating, which latter completes the plantar arch.

The internal tarsal arteries are two or three in number, and supply the adjacent structures on the inner border of the foot, where they anastomose with branches of the internal plantar

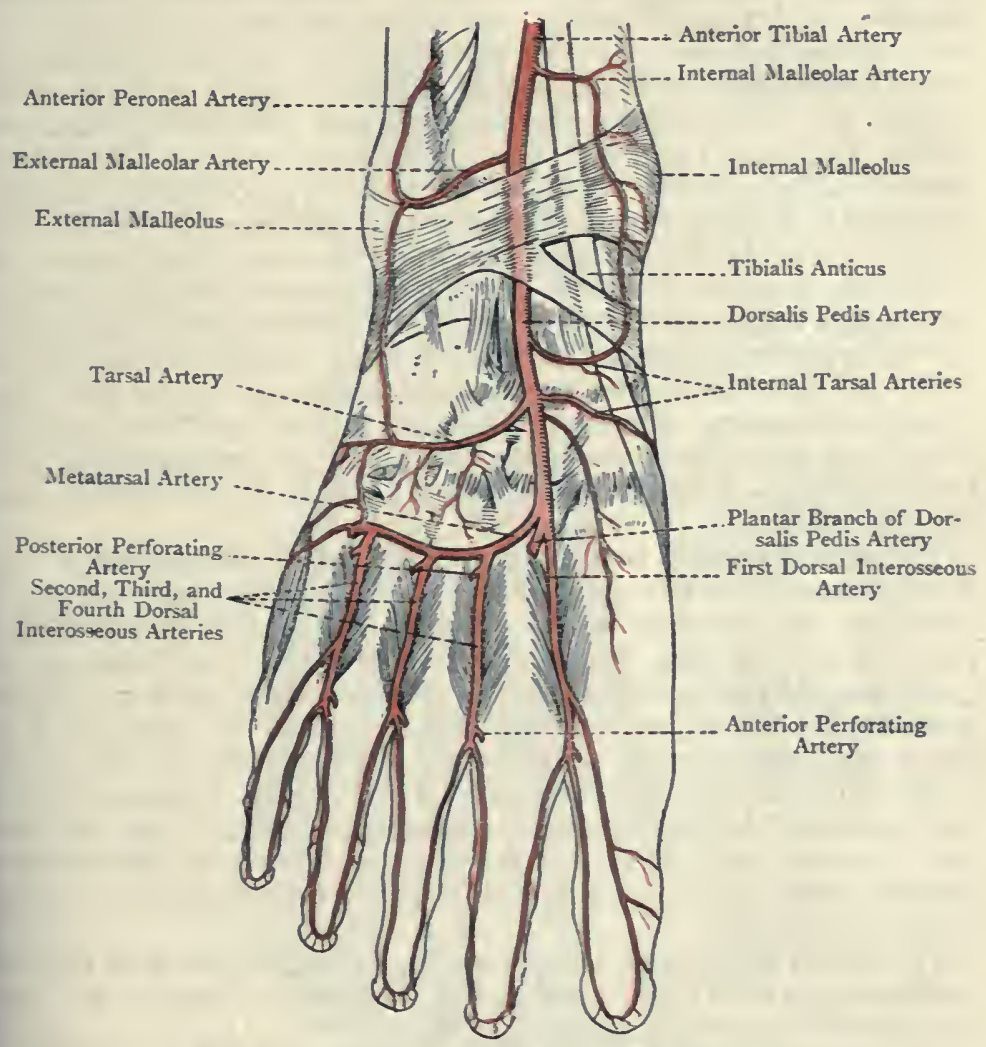

Fig. 245.-The Arteries on the Dorsum of the Right Foot (after L. Testut's 'Anatomie Humaine').

artery. The highest of them also takes part in the internal malleolar anastomosis.

The external tarsal artery passes outwards beneath the extensor brevis digitorum. It supplies the adjacent structures, and at the outer border of the foot it anastomoses with the metatarsal and external plantar arteries. It also takes part in the external malleolar inastomosis. 
The metatarsal artery arises on a level with the bases of the metatarsal bones, over which it passes on its way to the outer border of the foot, being under cover of the extensor brevis digitorum. At the outer border it anastomoses with the tarsal and external plantar arteries. The vessel forms a slight arch with the convexity directed forwards. From the concavity of the arch a few recurrent branches are given off to the tarsal articulations. From the convexity three dorsal interosseous arteries - are given off, which pass forwards over the outer three interosseous spaces. At the level of the metatarso-phalangeal joints each divides into two dorsal collateral digital arteries, which supply the contiguous sides of the second and third, third and fourth, and fourth and fifth toes. The most external dorsal interosseous artery, before it divides into its two collateral branches, furnishes a single dorsal digital artery to the outer side of the fifth or little toe. At the proximal end of the second, third, and fourth interosseous spaces the three dorsal interosseous arteries (in themselves small) are reinforced each by a posterior perforating artery, the three posterior perforating arteries being branches of the plantar arch. At the distal end of these interosseous spaces the three dorsal interosseous arteries are usually further reinforced each by an anterior perforating artery, the three anterior perforating arteries being branches of the three compound plantar digital arteries from the plantar arch.

The arteria dorsalis hallucis is the first dorsal interosseous artery. Arising from the terminal part of the dorsalis pedis artery, it continues the direction of that vessel, and passes forwards over the first interosseous space. On reaching the cleft between the great toe and the second it divides into two dorsal collateral digital branches for the supply of the contiguous sides of these two toes, and it furnishes a small single dorsal digital artery for the supply of the inner side of the great toe. The dorsalis hallucis artery is not reinforced by any posterior perforating branch. Before, however, dividing into its digital branches it receives an anterior perforating artery, which is derived from the arteria magna or princeps hallucis in the sole.

The dorsal digital arteries as they pass along the sides of the toes communicate with each other across their dorsal aspects, and also with the corresponding plantar digital arteries.

The plantar or perforating artery will be described in connection with the sole of the foot.

Varieties of Dorsalis Pedis Artery - r. Origin. - The vessel may be the continuation of the anterior peroneal artery.

2. Course. - The vessel often describes a considerable curve outwards before it reaches the proximal end of the first interosseous space. In these cases the artery may terminate at the back of the second interosseous space instead of the first.

3. Branches.-There is considerable deviation from the normal in respect of branclies. The branch most affected is the metatarsal artery. This may arise in common with the tarsal branch. The metatarsal arch is often very 
indefinite. The metatarsal artery is sometimes absent, and when this occurs the dorsal interosseous arteries of the outer three interosseous spaces are usually furnished by the three posterior perforating arteries from the plantar arch, or, it may be, by the tarsal artery.

Dorsalis Pedis Nerve.-This nerve is the continuation of the anterior tibial. 'It commences at the anterior ligament of the ankle-joint, and terminates at the distal end of the first interosseous space by dividing into two dorsal collateral digital nerves

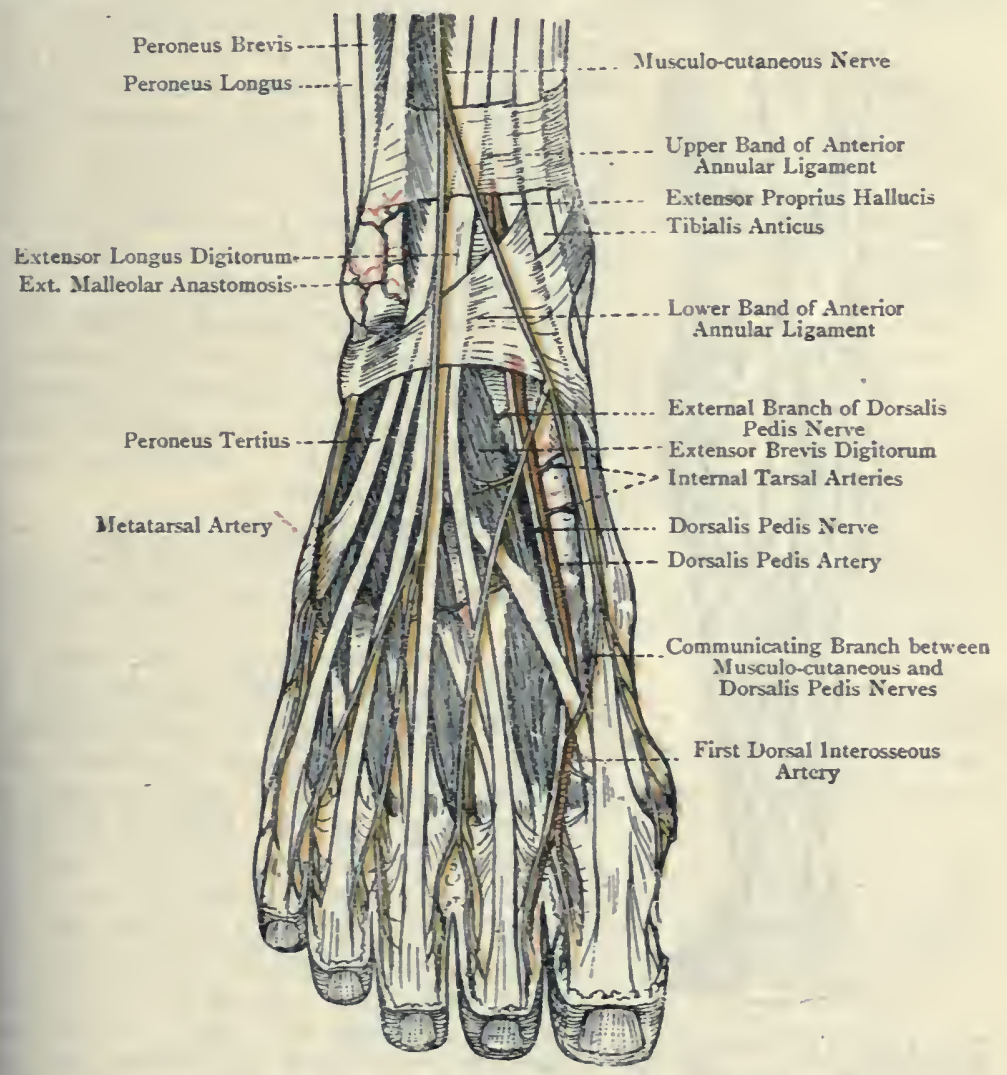

Fig. $24^{6}$.-Dissection of the Dorsum or the Right Foot.

for the supply of the contiguous sides of the great toe and the second. Its relations correspond with those of the dorsalis pedis artery, on the outer side of which it lies, with the intervention of the external vena comes. As the nerve passes over the dorsal aspect of the first interosseous space it is reinforced by an offset from the inner branch of the internal division of the musculo-cutaneous nerve. 
Branches.-The branches are as follows: external or tarsal, interosseous, and terminal.

The external or tarsal branch arises from the dorsalis pedis nerve as soon as it emerges from beneath the lower division of the anterior annular ligament. It passes outwards beneath the extensor brevis digitorum, and in that situation it presents a gangliform enlargement from which branches are furnished to the extensor brevis digitorum and the tarsal articulations. Three

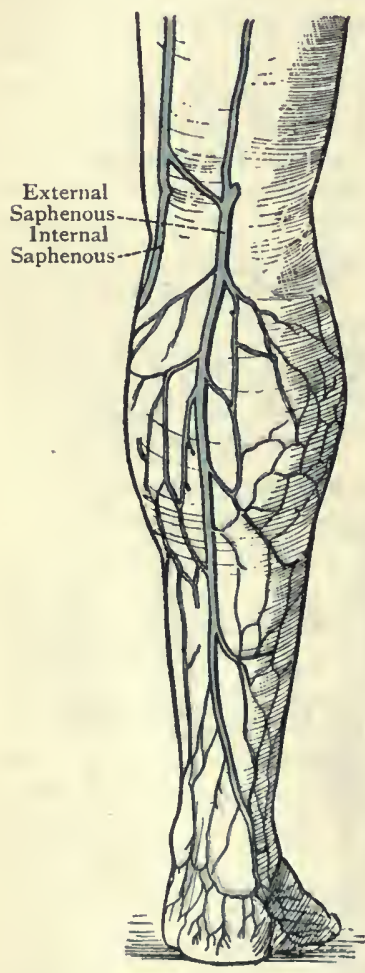

FIG. 247.-The External Saphenous Vein and its Tributaries. interosseous branches also arise from it, which pass over the dorsal aspects of the three outer interosseous spaces, supplying the adjacent tarso-metatarsal and metatarso-phalangeal articulations. The interosseous branch, which lies over the second interosseous space, usually supplies a small twig to the dorsal interosseous muscle of that space.

The interosseous branch passes over the dorsal aspect of the first interosseous space, supplying the adjacent tarso-metatarsal and metatarso-phalangeal articulations. It also gives a twig to the first dorsal interosseous muscle.

The terminal branches are the dorsal collateral digital nerves for the contiguous sides of the great toe and the second.

Dorsal Venous Arch.-This arch is superficial to the deep fascia, and is situated well forward upon the dorsum of the foot, being about 2 inches from the webs of the toes. The convexity of the arch, which is but slight, is directed forwards. It receives (I) the dorsal digital veins, (2) small veins from the dorsum of the foot, and (3) the efferent interdigital veins which come from the plantar transverse venous arch situated near the clefts of the toes. The blood is carried away from the dorsal venous arch by the two saphenous veins. The internal or long saphenous vein arises from the inner end of the arch, and, having received branches from the superficial plantar venous plexus which turn round the inner border of the foot, it passes in front of the internal malleolus, and thus reaches the inner side of the leg. Its further course has been previously described.

The external or short saphenous vein arises from the outer end of the arch, and, having received branches from the superficia 
plantar venous plexus which turn round the outer border of the foot, it passes below and behind the external malleolus, and thus reaches the back of the leg. It then passes upwards and inwards, lying at first to the outer side of the tendo Achillis. On reaching the middle line of the calf, it ascends in the groove between the two heads of the gastrocnemius until it arrives at the interval between the condyles of the femur. Up to this point the vein is superficial to the deep fascia. It now, however, passes through an opening in that fascia, and terminates in the popliteal vein. As high as the centre of the calf the vein is accompanied by the short saphenous nerve, which lies on its outer side. From the centre of the calf up to where the vein pierces the deep fascia it is accompanied by the terminal part of the small sciatic nerve and the middle superficial or cutaneous sural artery. It receives many tributaries from the calcaneal region, and from the outer and back parts of the leg, and it communicates at intervals with the venæ comites of the posterior tibial and peroneal arteries. Just before it pierces the deep fascia a communicating branch passes upwards and inwards from it to join the long saphenous vein. The external or short saphenous vein is provided with about ten valves.

\section{External Aspect of the Leg.}

In this region the lateral cutaneous branch of the external popliteal nerve is met with. It supplies the integument of the outer side of the leg over about its upper two-thirds, as well as the adjacent integument of the sural region.

Muscles. Peroneus Longus-Origin.-(I) The external aspect of the head of the fibula; (2) the adjacent part of the external tuberosity of the tibia; (3) the upper two-thirds of the external surface of the shaft of the fibula; (4) the antero-external and postero-external intermuscular septa; and (5) the deep fascia.

Insertion.-The tendon of insertion divides into two parts. The main part is inserted into the outer side of the tuberosity on the plantar surface of the base of the first metatarsal bone, and the other into the lower and anterior part of the outer surface of the internal cuneiform bone.

Nerve-supply.-The musculo-cutaneous nerve.

Action.-(I) To extend the foot upon the leg; (2) to abduct or turn out the fore part of the foot; (3) to depress the inner border of the foot, the effect of which is to raise the outer border and produce eversion of the sole; and (4) to support and strengthen the transverse arch of the foot.

The tendon descends behind that of the peroneus brevis, and, along with it, passes through the groove behind the external malleolus and beneath the external annular ligament, the two tendons having one synovial sheath in common. After leaving the malleolar groove, the tendon passes forwards on the outer surface of the os 
calcis, where it occupies the groove below the peroneal spine or ridge. In this part of its course the tendon is surrounded by a fibrous sheath, which is derived from the lower border of the external annular ligament, and it is here invested by a special synovial sheath, which is a prolongation of that beneath the external annular ligament. It then turns round the peroneal notch on the

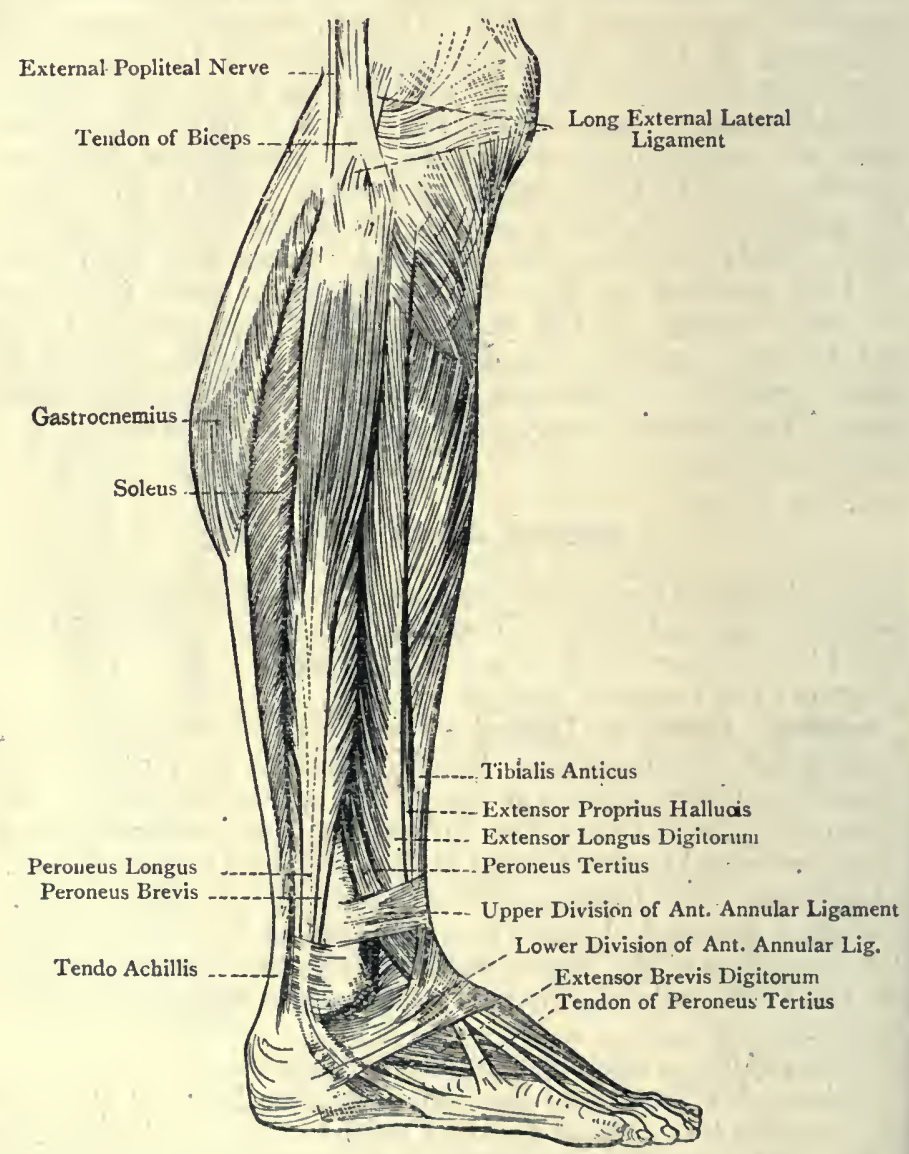

Fig. 248. - Muscles of the Leg (External View).

outer border of the cuboid, and traverses the peroneal groove on the plantar surface of that bone, which is converted into a fibroosseous canal by an expansion derived from the long plantar ligament. Finally, after leaving this fibro-osseous canal, in which it is invested by a special synovial sheath, the tendon crosses the sole of the foot to its twofold insertion, taking a direction forwards 
and inwards. In that part of the tendon which turns round the outer border of the cuboid a sesamoid fibro-cartilage is found.

Peroneus Brevis-Origin.-(I) The lower two-thirds of the external surface of the shaft of the fibula, except the last 2 inches; (2) the antero-external and postero-external intermuscular septa; and (3) the deep fascia.

Insertion.-The tuberosity on the outer side of the base of the fifth metatarsal bone. From the tendon of insertion a slip is often given to the long extensor tendon of the little toe.

Nerve-supply. - The musculo-cutaneous nerve.

Action.-(I) To extend the foot upon the leg, but in a feeble manner ; (2) to abduct or turn out the fore part of the foot; and (3) to raise directly the outer border of the foot, thus producing. eversion of the sole.

The middle third of the external surface of the fibula is occupied by both peroneus longus and peroneus brevis, the former arising from the posterior half, and the latter from the anterior half, so that the two muscles overlap. The tendon of the peroneus brevis passes through the groove behind the external malleolus with the tendon of the peroneus longus, which is directly behind it. Both tendons lie beneath the external annular ligament, where they have one synovial sheath in common. After leaving the malleolar groove the tendon of the peroneus brevis passes forwards on the outer surface of the os calcis, where it occupies the groove above the peroneal spine or ridge. In this part of its course the tendon is surrounded by a fibrous sheath, which is derived from the lower border of the external annular ligament, and it is here invested by a special synovial sheath, which is a prolongation of that beneath the external annular ligament. After leaving the outer surface of the os calcis the tendon passes over the cubcid bone, and so reaches its insertion.

On the outer surface of the os calcis the two peroneal tendons are completely separated from each other, that of the brevis being above that of the longus. The separation is effected partly by a strong fibrous septum, and partly by the peroneal spine or ridge. The peroneus longus and peroneus brevis are contained in a fibroosseous canal formed by the deep fascia, the intermuscular septur on each side, and the external surface of the shaft of the fibula.

\section{Posterior Aspect of the Leg.}

Superficial Structures.-The following superficial structures are met with in this region: the external or short saphenous vein, the cutanecus sural branches of the popliteal artery, the terminal part of the small sciatic nerve, the ramus communicans tibialis nerve, the ramus communicans fibularis nerve, and the external or short saphenous nerve. These, except the last named, have been already described. 
External or Short Saphenous Nerve.-This nerve is formed by the union of the ramus communicans tibialis of the internal popliteal and the ramus communicans fibularis of the external popliteal. The union takes place at, or just below, the centre of the calf, and superficial to the deep fascia. The nerve descends on the outer side of the short saphenous vein, and external to the tendo Achillis. It then passes behind and below the external malleolus, and so reaches the outer border of the foot, along which it passes to the outer side of the little toe, of which it is the dorsal digital nerve. The nerve supplies the integument of the back of the leg in its lower half, the external malleolar region, the outer side of the calcaneum, and the outer border of the foot and outer side of the little toe. It also furnishes articular branches to the anklejoint, and the astragalo-calcaneal articulation.

Muscles.-The muscles of the back of the leg are divided into two groups-superficial and deep.

Superficial Group.-This group comprises the gastrocnemius, soleus, and plantaris, which are collectively called the sural (calf) muscles.

Gastrocnemius.-This muscle is so named because it forms the 'belly of the leg,' that is to say, the fleshy enlargement at the back of the leg which is called the calf.

Origin.-The external head arises from (I) the outer surface of the external condyle of the femur immediately above the commencement of the popliteal groove, and close behind and above the external tuberosity; and (2) the adjacent part of the posterior surface of the femur, vertically, for at least an inch immediately external to the lower part of the external supracondylar ridge. The internal head arises from (I) an oblique impression on the posterior surface of the femur immediately above the internal condyle, and extending inwards for fully an inch as far as the adductor tubercle; and (2) the lower extremity of the internal supracondylar ridge for a short distance.

Insertion. - The fleshy part of the muscle gives place to a flat tendon, which joins the subjacent tendon of the soleus at the centre of tho leg to form the tendo Achillis. Through this latter the gastrocnemius is inserted into the middle zone on the posterior surface of the tuber calcis, a bursa intervening between the tendon and the upper zone.

Nerve-supply.-The internal popliteal nerve, which furnishes two branches, one for each head.

Action.-Acting from its origin, the muscle is a powerful extensor of the foot upon the leg, thus raising the heel. Acting from its insertion, as when the ankle-joint is fixed by the muscles of the anterior aspect of the leg, the gastrocnemius is a flexor of the knee-joint, flexing the femur upon the tibia.

The two heads come into contact at the junction of the upper sixth and lower five-sixths of the leg, and so they limit the lower part of the popliteal space. After coming together they are sepa- 
rated superficially by a longitudinal groove, and when the lips of this groove are held apart a tendinous band or raphé is seen to lie between them. The fleshy fasciculi of both heads are short and oblique, and they terminate upon the lower tendon, which extends upwards upon the deep or anterior surface of the muscle in the form of an expanded aponeurosis. The shortness of the fleshy bundles gives the muscle great power of action, but the range of movement is limited.

The internal head is separated from the tendon of the semimembranosus by a large bursa, called the popliteal bursa, which frequently (one in five) communicates with the synovial membrane of the knee-joint. Another small bursa is situated a little higher, between the internal head and the posterior surface of the femur. In the external head a sesamoid fibro-cartilage is occasionally met with.

Soleus.-This flat muscle has been so named from its resemblance to a sole-fish.

Origin.-(I) The posterior surface of the head, and the upper third of the posterior surface of the shaft, of the fibula; (2) the postero-external intermuscular septum; (3) the fibrous arch thrown over the popliteal vessels and internal popliteal nerve; (4) the oblique or popliteal line of the tibia ; and (5) the internal border of the tibia over its middle third.

Insertion.-The tendon joins that of the gastrocnemius at the centre of the leg to form the tendo Achillis, which is inserted into the middle zone on the posterior surface of the tuber calcis.

Nerve-supply.-(I) The internal popliteal nerve, the branch from which enters the muscle on its superficial or posterior surface near the upper border; and (2) the posterior tibial nerve, the branch from which enters the muscle on its deep or anterior surface near the centre of the leg.

Action.-Acting from its origin the muscle is a powerful extensor of the foot upon the leg, thus raising the heel. Acting from its insertion, as when the ankle-joint is fixed by the muscles of the anterior aspect of the leg, the soleus steadies the leg upon the foot.

The fasciculi of this muscle are short and oblique, like those of the gastrocnemius. It has therefore great power of action, but its range of movement is limited. Only a limited number of the fibres of the soleus spring directly from the bony surfaces. The majority arise from two aponeurotic laminæ, which are almost entirely concealed within the muscle, and which descend, one from the fibula, and the other from the oblique line of the tibia and the fibrous arch over the popliteal vessels. The fibres which arise from the anterior surfaces of these two laminæ terminate upon a median tendinous band or raphé, which is deeply placed, and those arising from their posterior surfaces end upon a broad expanded aponeurosis, which covers the superficial or posterior surface of the muscle, giving it an appearance similar to that of the deep or anterior surface of the gastrocnemius. This aponeurosis is 
simply an upward expansion of the tendon of insertion of the muscle.

Tendo Achillis.* - This very strong tendon is formed by the union of the tendons of the gastrocnemius and soleus. It extends from the centre of the leg to the prominence of the heel, and is inserted into the middle zone on the posterior surface of the tuber calcis, a bursa intervening between it and the upper zone. The tendo Achillis is at first broad and comparatively thin. As it descends, it becomes narrower and thicker until it reaches a point about $I_{2}^{1}$ inches above the tuber calcis, beyond which it again broadens to its insertion. The short saphenous vein and nerve lie on its outer side, the tendon of the plantaris and the posterior tibial vessels and nerve on the inner side, and a large quantity of fat beneath it.

Plantaris-Origin.-(I) The lower 2 inches of the external supracondylar ridge of the femur, internal to the external head of the gastrocnemius; and (2) the adjacent part of the posterior ligament of the knee-joint.

Insertion.-The middle zone on the posterior surface of the tuber calcis immediately to the inner side of the tendo Achillis, with which it is closely connected. It may, however, terminate upon the inner border of the tendo Achillis, in the deep fascia of the leg, upon the internal annular ligament, or in the plantar fascia.

Nerve-supply. - The internal popliteal nerve.

Action.-(I) The plantaris acts as a very weak auxiliary to the gastrocnemius. (2) From its connection with the posterior ligament of the knee-joint the muscle has a slight action as a retractor of that ligament during flexion of the joint, and thus it guards the ligament against being nipped or pressed upon by the articular surfaces of the femur and tibia.

The fleshy belly of the muscle is limited to the upper sixth. The tendon is very narrow and descends inwards between the gastrocnemius and soleus. It is also very extensible, so that when grasped by the fingers and stretched laterally it can be drawn out into a ribbon of about 2 inches in breadth.

The plantaris, which is sometimes absent, is a vestigial muscle, being the crural remains of a superficial flexor of the toes, the plantar portion of the tendon of which, having been divorced, remains persistent as the central division of the plantar fascia. The homologue of the plantaris in the upper limb is the palmaris longus.

Deep Group.-The muscles which comprise this group are four in number, namely, the popliteus, flexor longus digitorum, tibialis posticus, and flexor longus hallucis. The popliteus muscle is covered by the popliteal fascia, which is one of the modes of insertion of the semimembranosus. The other muscles, together with

* Being inserted into the heel, it has been called the tendo Achillis, because it was believed that the heel was the only part in which the Greek hero, Acliilles, could be wounded. 
the posterior tibial vessels and nerve, are covered by the posterior or transverse intermuscular septum.

Popliteus-Origin.-(I) By a narrow round tendon from the front part of the horizontal portion of the popliteal groove on the outer surface of the external condyle of the femur, and (2) slightly from the posterior ligament of the knee-joint.

Insertion.-(I) The popliteal surface of the tibia, and (2) the popliteal fascia which covers the muscle.

Nerve-supply.-The internal popliteal nerve. The branch from this nerve descends over the posterior surface of the muscle, and, turning round its lower border, enters the deep or anterior surface in its lower part.

Action. - The muscle is a feeble flexor of the leg upon the thigh, and, when the knee-joint has been flexed, it acts as an internal rotator of the leg:

The tendon of origin is within the capsular ligament, and beneath the long external lateral ligament, of the knee-joint. Whilst within the joint it is in contact with the posterior and outer aspect of the external semilunar fibro-cartilage, which it grooves. It then pierces the capsule, and the fleshy ibres spread out in the manner of a fan as they diverge downwards and inwards. The popliteal fascia, which covers the muscle, represents part of the insertion of the semimembranosus muscle, and it is attached to the oblique or popliteal line of the tibia.

Flexor Longus Digitorum (flexor perforans)-Origin.-(I) The inner division of the posterior surface of the shaft of the tibia, commencing at the oblique or popliteal line, and extending downwards over the middle two-fourths of the bone; (2) the posterior intermuscular septum which covers the muscle; and (3) the intermuscular septum between it and the tibialis posticus on its outer side.

Insertion.-By means of four tendons which go to the four outer toes, where each is inserted into the plantar surface of the base or proximal end of the distal phalanx.

Nerve-supply.-The posterior tibial nerve.

Action.-(I) The muscle acts as a flexor of the distal phalanges of the four outer toes, and (2) when these have been flexed, it acts as an extensor of the foot upon the leg.

The muscle crosses behind the tibialis posticus obliquely from within outwards in the lower third of the leg. The tendon passes behind the internal malleolus and beneath the internal annular ligament, lying behind and slightly external to the tendon of the tibialis posticus, and occupying a special, purely fibrous compartment, in which it is invested by a distinct synovial sheath. It then enters the sole of the foot, where it passes forwards and outwards to the middle line, crossing beneath the tendon of the flexor longus hallucis, from which it receives a slip. On reaching the middle line it gives insertion to the flexor or musculus accessorius, and immediately afterwards it divides about the centre into four 
tendons which, after having given origin to the four lumbricales muscles, go to the four outer toes. Each tendon, as it passes along the plantar surface of a toe, has a tendon of the flexor brevis digitorum lying close beneath it as far as the second phalanx, the two tendons occupying a fibro-osseous canal lined by a synovial membrane, which furnishes a separate investment to each tendon. Opposite the first phalanx the brevis tendon divides into two parts, and the longus tendon passes through the cleft thus formed (hence the name flexor perforans) on its way to the distal phalanx. The fibro-osseous canals and their synovial membranes, with the vincula accessoria tendinum of the latter, namely, ligamenta brevia and ligamenta longa, correspond with those of the fingers.

In the leg the muscle occupies a fibro-osseous canal formed by intermuscular septa and the posterior surface of the shaft of the tibia.

Tibialis Posticus-Origin.-(I) The outer division of the posterior surface of the shaft of the tibia, extending as high as the commencement of the oblique or popliteal line, and ceasing just below the centre of the bone; (2) the posterior surface of the interosseous membrane, except the lower 2 inches ; (3) the internal surface of the shaft of the fibula ; $(4)$ the posterior or transverse intermuscular septum, as it covers the muscle; and (5) the intermuscular septa on either side, separating it from the flexor longus digitorum internally and the flexor longus hallucis externally.

Insertion.-The tuberosity of the navicular or scaphoid bone. From this insertion eight expansions are given off. One passes backwards to the anterior part of the under surface of the sustentaculum tali of the os calcis. The other seven pass forwards, and are attached as follows : three to the internal, middle, and external cuneiform bones, one to the cuboid bone, and three to the bases of the second, third, and fourth metatarsal bones, all on their plantar aspects.

Nerve-supply.-The posterior tibial nerve.

Action.-(I) The muscle inverts the sole of the foot; (2) it is an extensor of the foot upon the leg; and (3) it contributes in a material degree to the support of the longitudinal arch of the foot, and so helps to guard against the condition known as flat foot, being in this respect auxiliary to the inferior or internal calcaneo-navicular or spring ligament.

In the lower third of the leg the muscle passes obliquely inwards in front of the flexor longus digitorum. The tendon passes through the groove behind the internal malleolus, where it is beneath the internal annular ligament, occupying one of the fibroosseous canals, and having the tendon of the flexor longus digitorum (enclosed within its fibrous canal) lying behind and slightly external to it. In this part of its course the tendon has a special synovial investment, which is also prolonged forwards around it as far as the navicular bone. As it passes over the head of the astragalus and spring ligament it contains a sesamoid fibrocartilage. 
The greater part of the muscle in the leg is contained in a fibroosseous canal formed by the posterior or transverse intermuscular septum, the interosseous membrane, the intermuscular septa on either side, and the surfaces of the tibia and fibula from which it arises.

Flexor Longus Hallucis-Origin.-(I) The lower two-thirds of the posterior surface of the shaft of the fibula, except the last inch or more; (2) the postero-external intermuscular septum, which separates the muscle from the peroneus longus and peroneus brevis; (3) the intermuscular septum between it and the tibialis posticus; and (4) the posterior or transverse intermuscular septum, as it covers the muscle.

Insertion.-The plantar surface of the base of the distal phalanx of the great toe.

Nerve-supply.-The posterior tibial nerve.

Action.-(I) The muscle acts as a flexor of the distal phalanx of the great toe; (2) it is an extensor of the foot upon the leg; (3) it contributes to the support of the longitudinal arch of the foot; and (4) by means of the slip which it gives to the tendon of the flexor longus digitorum in the sole it is an auxiliary of that muscle, more especially in flexing the second and third toes.

The tendon passes through the groove on the back of the lower end of the tibia at its outer part, where it lies beneath the internal annular ligament, occupying one of the fibro-osseous canals, and having a special synovial investment. Beyond this point it passes in succession through the groove on the posterior border of the astragalus, and through that on the under surface of the sustentaculum tali of the os calcis. Each of these grooves is converted into a fibro-osseous canal by a fibrous expansion, and in each case the tendon has a synovial investment. In the sole the tendon is crossed superficially or inferiorly, and from within outwards, by that of the flexor longus digitorum, to which it gives a slip. In most cases this slip is incorporated with the long flexor tendons of the second and third toes (Turner). As it passes along the first phalanx it is contained in a fibro-osseous canal lined by synovial membrane, which also invests the tendon and gives rise to a ligamentum breve. In the leg the flexor longus hallucis is contained within a fibro-osseous canal formed by intermuscular septa and the posterior surface of the shaft of the fibula.

Posterior Tibial Artery.-This vessel is the larger of the two terminal branches of the popliteal, the other being the anterior tibial. It commences at the lower border of the popliteus muscle on a level wi h the lower border of the tubercle of the tibia, fully $\mathrm{I}_{\frac{3}{4}}$ inches below the upper surface of the head of that bone, and it terminates at the lower border of the internal annular ligament by dividing into the internal and external plantar arteries. The termination of the vessel is situated midway between the inner part of the point of the heel and the tip of the internal malleolus. In the upper two-thirds of the leg it is deeply 


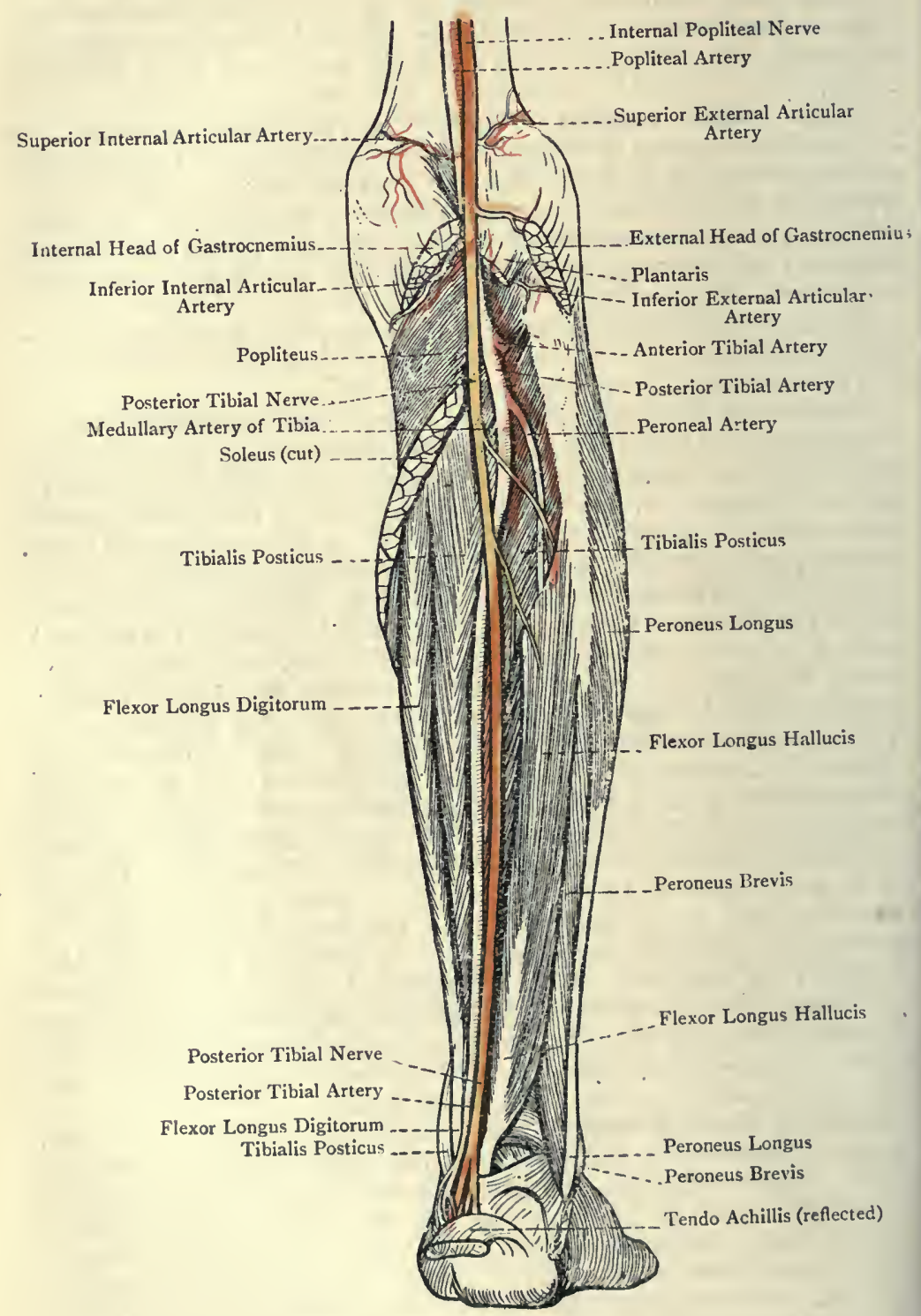

Fig. 249.-The Back of the Right Leg (Deep Dissection). 
placed, being situated between the superficial and deep muscles, and over the interval between the tibia and fibula. It then inclines inwards, and in the lower third of the leg it lies over the back of the tibia and to the inner side of the tendo Achillis, where it is comparatively superficial. The course of the vessel may be indicated by drawing a line from the centre of the lower part of the popliteal space to a point midway between the inner part of the point of the heel and the tip of the internal malleolus.

Relations-Superficial or Posterior.-In the upper two-thirds the vessel lies beneath the gastrocnemius, soleus, and posterior or transverse intermuscular septum; and, in the lower third, it is covered by the skin, superficial fascia, deep fascia, and the internal annular ligament. Anterior or Deep.-The tibialis posticus (to which it is bound by the posterior or transverse intermuscular septum), flexor longus digitorum, posterior surface of the tibia, and internal lateral ligament of the ankle-joint (in this order from above downwards). External.- The tendo Achillis in the lower part of the leg. The artery in this part of its course lies about midway between the inner border of the tendo Achillis and the internal border of the tibia. As the ressel passes beneath the internal annular ligament it occupies a special fibro-osseous canal along with its venæ comites and the posterior tibial nerve, the latter being on the outer side. In this situation the tendons of the tibialis posticus and flexor longus digitorum lie on the inner side of these structures, and the tendon of the flexor longus hallucis on their outer side.

The artery is accompanied by two venæ comites, which closely embrace its sides, and communicate with each other at frequent intervals by transverse branches crossing superficial to the vessel. These venæ comites ultimately join to form one trunk, which unites with that formed by the junction of the venæ comites of the anterior tibial artery to form the popliteal vein, the union taking place at the lower border of the popliteus muscle. The posterior tibial nerve is at first internal to the artery for about $\mathrm{I}_{2}^{\frac{1}{2}}$ inches. It then crosses over the vessel just below the origin of the peroneal branch, and from this point onwards it lies on its outer side. Beneath the internal annular ligament the artery has frequently a nerve upon either side of it, an arrangement which is brought about by an early division of the posterior tibial nerve into its internal and external plantar branches.

Branches.-These are as follows: peroneal, muscular, medullary or nutrient, cutaneous, communicating, internal malleolar, internal calcaneal, and terminal.

The description of the peroneal artery, from its large size and numerous branches, will be deferred to the last.

The muscular branches are numerous, and are distributed to the deep group of muscles and to the soleus.

The medullary or nutrient artery is the largest of all the arteries bearing that name. Arising from the upper part of the posterior 
tibial, it pierces the tibialis posticus and enters the medullary foramen of the tibia, accompanied by the medullary branch of the nerve to the popliteus.

The cutaneous branches are distributed to the integument of the inner and back part of the leg.

The communicating branch arises about an inch above the anklejoint. It passes transversely outwards between the tibia and flexor longus hallucis, and anastomoses with the communicating branch of the peroneal artery.

The internal malleolar branches are usually two in number. Passing inwards beneath the tendons of the flexor longus digitorum and tibialis posticus, they reach the front of the internal malleolus, where they take part in the internal malleolar anastomosis.

The internal calcaneal branch arises beneath the internal annular ligament, which it pierces near the lower border to be distributed over the inner surface of the os calcis, where it anastomoses with the internal calcaneal branches of the external plantar artery.

The terminal branches are the internal and external plantar arteries, which will be afterwards described.

Peroneal Artery.-This vessel, which is of large size, arises from the posterior tibial fully I inch below its commencement. It is at first directed downwards and outwards over the tibialis posticus to the back of the fibula. Having reached that bone, it descends along its posterior surface close to the postero-internal border, lying between the bone and the flexor longus hallucis, or within that muscle, or in a fibrous canal between that muscle and the tibialis posticus. About 2 inches above the ankle-joint it escapes from beneath the flexor longus hallucis, and divides into anterior and posterior peroneal arteries.

Branches.-The branches are as follows : muscular, medullary or nutrient, cutaneous, communicating, and terminal.

The muscular branches are distributed to the adjacent muscles.

The medullary or mutrient artery enters the medullary foramen of the fibula.

The cutaneous branches are distributed to the integument on the outer and back part of the leg.

The communicating branch arises about I inch above the anklejoint. It passes transversely inwards and anastomoses with the communicating branch of the posterior tibial artery.

The terminal branches are the anterior peroneal and posterior peroneal.

The anterior peroneal artery arises from the peroneal artery near the lower part of the interosseous membrane, and passes forwards through the inferior hiatus in it. Having reached the front of the leg, it descends under cover of the peroneus tertius, and takes part in the external malleolar anastomosis, along with the external malleolar of the anterior tibial, the external tarsal of the dorsalis pedis, and the posterior peroneal. 
The posterior peroneal artery is the continuation of the peroneal artery. It passes behind the external malleolus, and along the outer border of the foot for a variable distance. Its branches are chiefly distributed over the outer surface of the os calcis, and these external calcaneal branches take part in the external malleolar anastomosis. Over the prominence of the heel they anastomose freely with the internal calcaneal branches of the external plantar, and on the outer border of the foot the posterior peroneal again mastomoses with branches of the external plantar.

The peroneal artery is accompanied by two venæ comites, which ultimately join the posterior tibial venæ comites:

Varieties of the Posterior Tibial Artery.-The vessel is sometimes very small, in which cases the peroneal artery is of large size, as well as the communicating branch normally given off about $I$ inch above the ankle-joint, and so the small posterior tibial artery is reinforced. In rare cases the vessel does not reach the ankle, and it may even be entirely absent, in which cases there is always a large peroneal artery to make good the deficiency,

Varieties of the Peroneal Artery.-The level at which this vessel arises from the posterior tibial is subject to variety. Its normal origin is fully I inch below the commencement of the posterior tibial, but it may arise lower down or higher up, or it may even spring from the popliteal, or from the anterior tibial. In some cases it is of large size, in order to compensate for a small posterior tibial. In other cases the peroneal artery may stop short of the ankle, its place being taken by a branch of the posterior tibial. The anterior peroneal branch is sometimes of large size, in order that it may reinforce a small anterior tibial, or even furnish the dorsalis pedis artery.

Posterior Tibial Nerve.-This nerve is the continuation of the internal popliteal. It commences at the lower border of the popliteus muscle, and its normal termination corresponds with the lower border of the internal annular ligament, where it divides into internal and external plantar nerves. The division, however, frequently takes place at the upper border of the internal annular ligament, or as the nerve passes beneath it. The nerve closely accompanies the posterior tibial artery throughout the whole of its course. It lies at first on the inner side of the artery, with the intervention of the internal vena comes, but it only maintains this position for about $\mathrm{I}_{\frac{1}{2}}$ inches. It then crosses over the vessel, and descends upon its outer side, the external vena comes intervening. The general relations of the nerve are similar to those of the corresponding artery.

Branches. - These are muscular, fibular, calcaneo-plantar, articular, and terminal.

The muscular branches are given off from the upper part of the nerve, and supply the flexor longus digitorum, tibialis posticus, flexor longus hallucis, and soleus, the branch to the last muscle entering it on its deep or anterior surface near the centre of the leg.

The fibular branch is a long nerve which is usually associated at its origin with the branch to the flexor longus hallucis. It accompanies the peroneal artery, and furnishes (I) a medullary 
branch, which goes with the medullary artery into the interior of the fibula ; (2) periosteal branches to the fibular periosteum; and (3) twigs to the coats of the peroneal artery.

- The calcaneo-plantar branch arises from the posterior tibial whilst it is beneath the internal annular ligament. Having pierced the ligament, it divides into internal calcaneal and plantar branches. The former supply the integument of the inner side of the heel, and the latter are the cutaneous nerves of the inner and posterior part of the sole.

The articular branches, two or three in number, arise from the posterior tibial close to its termination, and enter the ankle-joint on its inner aspect by piercing the internal lateral ligament.

The terminal branches are the internal plantar and external plantar nerves, which will be afterwards described.

\section{THE KNEE-JOINT.}

The knee-joint belongs to the class diarthrosis, and to the subdivision ginglymus. Though the chief movements are flexion and extension, there is also a certain amount of gliding or to and fro movement, as well as rotation. The joint, therefore, partakes of the nature of an arthrodial joint. It is really made up of three joints, namely, one into which the patella and the patellar surface of the femur enter (femoro-patellar), and other two into each of which a femoral condyle and a tibial condylar surface enter (femorotibial). These three joints in man communicate freely with one another.

The articular surfaces are (I) the condyles and patellar surface of the femur, (2) the upper three-fourths of the posterior surface of the patella, and (3) the condylar articular surfaces of the tibia.

The ligaments are divided into two groups, external and internal.

I. External Ligaments.--These are as follows : anterior or ligamentum patellæ, posterior, external lateral, internal lateral, and capsular.

The anterior ligament or ligamentum patellæ is attached superiorly to the apex and adjacent margins of the lower part of the patella, and inferiorly to the lower rough portion of the tubercle of the tibia, a bursa intervening between it and the upper smooth portion of the tubercle. It is a very strong, flat, broad ligament, the lateral margins of which are connected with the lateral patellar ligaments.

The posterior ligament is really a part of the capsular ligament. It covers the back parts of the femoral condyles, and extends between the external and internal lateral ligaments. Superiorly it is attached to the upper margin of the intercondylar 
fossa, and at either side of this to the back of the femur immediately above each condyle. Inferiorly it is attached to the posterior border of the head of the tibia. It constitutes a broad membranous ligament, which in itself is thin, but it receives a considerable accession of fibres from the tendon of the semimembranosus. These fibres form a strong prominent bundle, which represents one mode of insertion of that muscle, and is known as the ligamentum

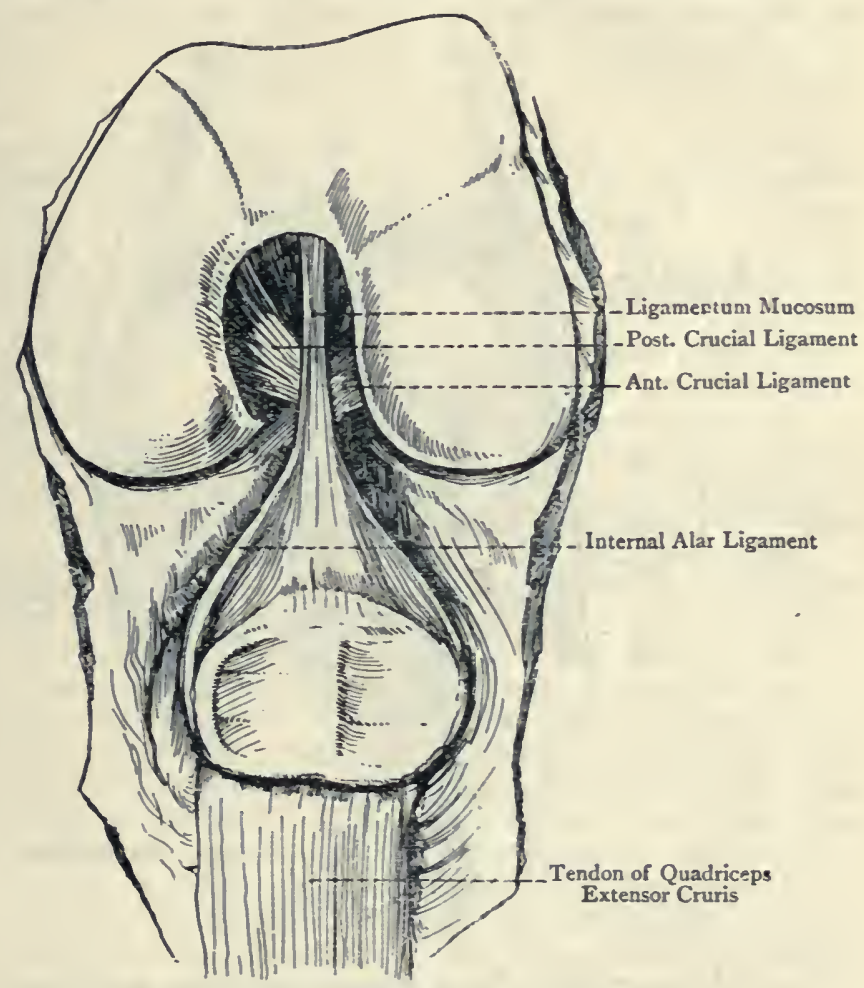

Fig. 250.-INTERIOR OF THE LEFT KNEE-JOINT (ANTERIOR VIEW).

osticum Winslowii. It is directed upwards and outwards from te tendon of the semimembranosus towards the outer condyle of ie femur' and outer head of the gastrocnemius. Internally the osterior ligament blends with the internal lateral ligament, and iternally (regarding it as a part of the capsular ligament) it sends 1 expansion over the long external lateral ligament. The posterior gament presents a number of openings for the passage of nutrient ssels and nerves, the largest of these being at its centre for the 
azygos artery, accompanied by a branch of the internal popliteal nerve and, it may be, the geniculate branch of the obturator nerve. At the outer part of the ligament there is a-special opening for the tendon of the popliteus. Another opening is frequently present over the upper and back part of the inner condyle, and, when this is so, the popliteal bursa between the semimembranosus and the inner head of the gastrocnemius communicates with the synovial membrane of the joint. The upper and outer part of

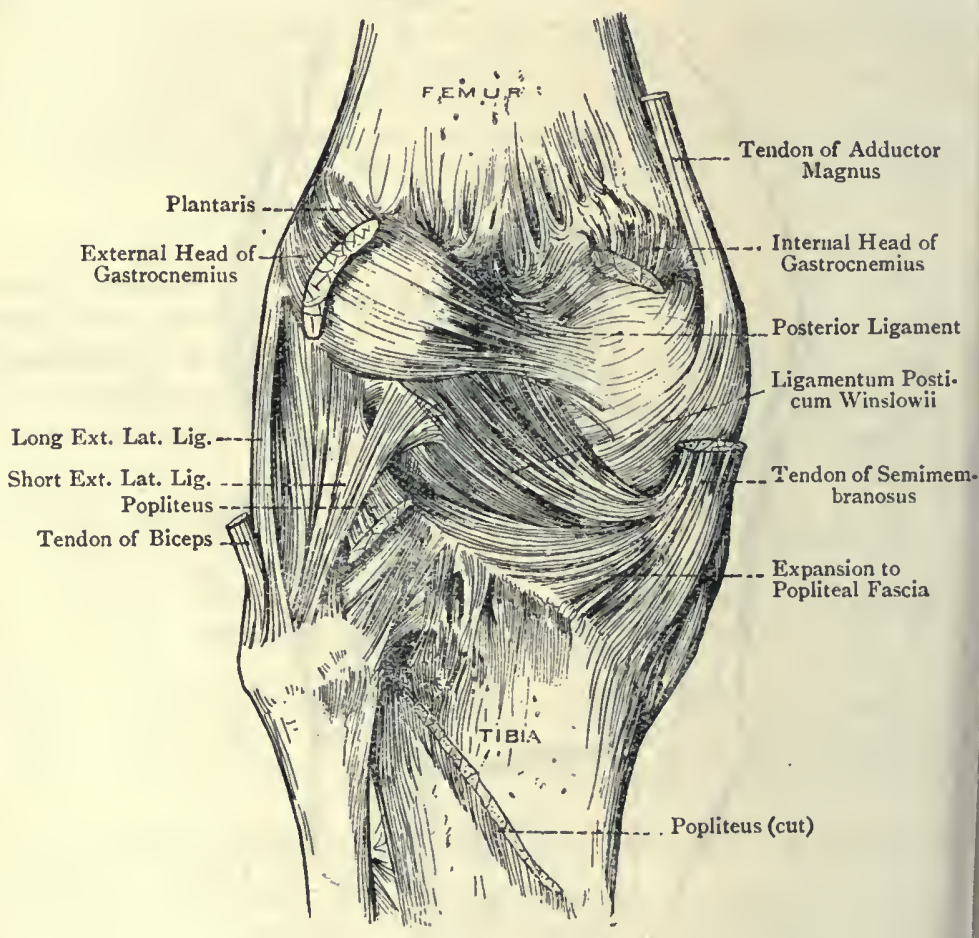

Fig. 25J.-The Left Knee-Joint (Posterior View).

the posterior ligament gives origin to some of the fibres of th plantaris.

The external lateral ligament consists of two divisions-anterio and posterior. The anterior division is the proper external latera ligament, and is known as the long external lateral ligament. It $\mathrm{i}$ firm and cord-like, and passes vertically downwards, lying clear c the joint. It is attached superiorly to the external tuberosity c the femur, and inferiorly to the outer margin of the head of tl: fibula about $\frac{1}{2}$ inch anterior to the styloid process. The liganer crosses the tendon of the popliteus, and, near the head of the fibul. 
it pierces the tendon of the biceps femoris, being there provided with a synovial investment. The inferior external articular vessels and nerve pass beneath it. The long external lateral ligament represents the original tendon of origin of the peroneus longus from the femur. The posterior division of the ligament, which is known as the short external lateralligament, lies a little behind the long, and is not well defined. If distinct, it is attached above to the external condyle close to the outer head of the gastrocnemius, where it blends with the posterior ligament, its inferior attachment being to the styloid process of the fibula. Its direction is downwards and forwards, and it is to be regarded as an expansion in that direction of the posterior ligament.

The internal lateral ligament is a long, flat, strong band of unequal width, being broader at the centre than at either end. It is attached superiorly to the internal tuberosity of the femur, and inferiorly to the internal border and adjacent part of the internal surface of the shaft of -the tibia, extending from the internal tuberosity downwards for fully 3 inches. Its posterior border covers the chief part of the tendon of the semimembranosus, and blends with the posterior ligament. As the ligament passes over the inner side of the knee-joint it is closely adherent to the internal semilunar fibro-cartilage, and, lower down, the inferior articular vessels and nerve pass beneath it. The internal lateral ligament represents the original insertion of the adductor magnus into the tibia.

The capsular ligament is formed to a large extent by expansions derived from the tendons of the vastus externus, vastus internus, sartorius, and semimembranosus, and from the fascia lata. It surrounds the joint except above the patella, where its place is taken by the suprapatellar tendon. The posterior part of the capsule, which forms the posterior ligament, is specially strong, being reinforced by the ligamentum posticum Winslowii. In other situations it is comparatively thin. It furnishes a thin expansion over the ligamentum patellæ, and on either side of the patella it forms the lateral patellar ligaments. Externally, where it is closely incorporated with the ilio-tibial band of the fascia lata, it covers the long external lateral ligament. Internally, where it receives accessions of fibres from the sartorius and semimembranosus, it blends with the internal lateral ligament.

The lateral patellar ligaments are two in number, external and internal, and they take the form of flat membranous bands, sometimes called the retinacula. The external extends between the puter border of the patella and the anterior margin of the outer tuberosity of the tibia. It is intimately connected with, and in part formed by, the ilio-tibial band of the fascia lata. The internal extends between the inner border of the patella and the anterior nargin of the inner tuberosity of the tibia.

2. Internal Ligaments. - The internal ligaments, properly so alled, are as follows: the two crucial ligaments; the two 
semilunar fibro-cartilages, with their coronary ligaments; and the transverse ligament. There are other structures, within the joint, designated as ligaments, namely, the ligamentum mucosum and ligamenta alaria ; but, as these are merely folds of the synovial membrane, they will be described in connection with it.

Crucial Ligaments. - These are two in number, anterior or external, and posterior or internal, and they bind the condyles of the femur to the head of the tibia. They are very powerful, somewhat round bundles situated in the centre of the joint, and so disposed as to form a cross.

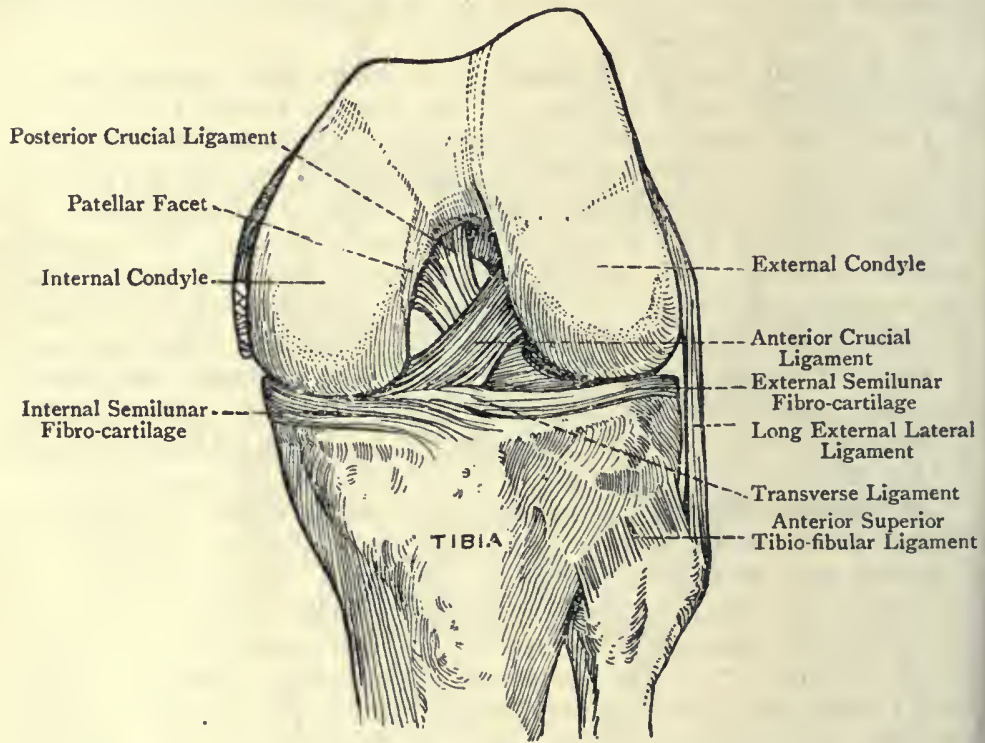

Fig. 252.-The Crucial Ligaments of the Left Knee-Joint (ANTERTOR VIEW).

The anterior or external crucial ligament is attached inferiorly tc the inner part of the rough depression on the upper surface of the head of the tibia in front of the inner tubercle of the spine Superiorly it is attached to the depression on the posterior par of the internal surface of the external condyle of the femur. It direction is upwards, backwards, and outwards. At its tibial attach ment it has the anterior cornu of the internal semilunar fibro-cartilag in front of it, and the anterior cornu of the external semilunar fibro cartilage behind and to its outer side. The anterior crucia ligament is tense in extension of the knee-joint, and prevents ove1 extension. It is also tense in internal rotation of the leg. Th ligament is at one period the internal lateral ligament of the extern: femoro-tibial joint. 
The posterior or internal crucial ligament is attached inferiorly to the back part of the rough depression on the head of the tibia behind the spine, and to the popliteal notch. Superiorly it is attached to the anterior part of the external surface of the internal condyle of the femur, close to the front part of the intercondylar fossa, upon which it slightly encroaches. Its direction is upwards, forwards, and slightly inwards. It is stronger, but shorter, than the anterior crucial ligament, and is not so oblique. At its tibial

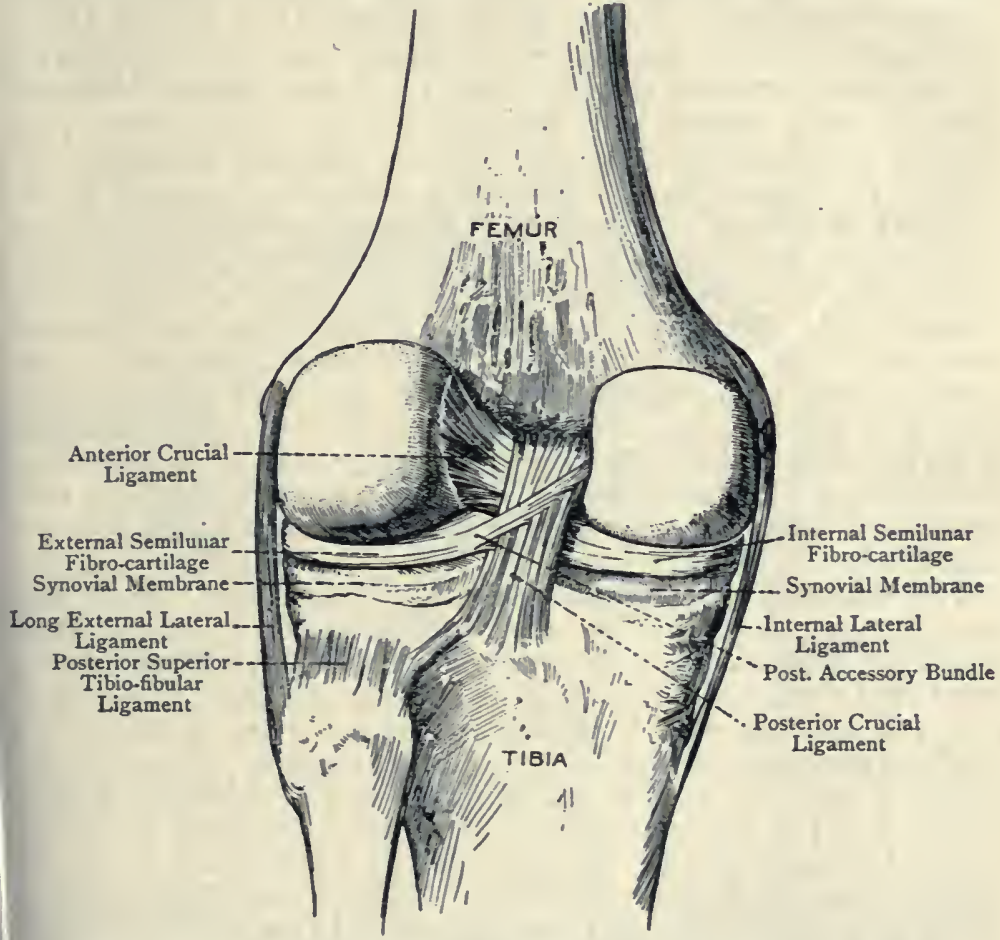

Fig. 253.-The Crucial Ligaments of the Left ínee-Joint (Posterior View) (AFter Spalteholz).

attachment it is behind the posterior cornua of both semilunar Abro-cartilages, the posterior cornu of the internal one being more immediately in front of it and to its inner side.

The posterior crucial ligament is tense in flexion of the kneejoint, and checks over-flexion. It is at one period the external lateral ligament of the internal femoro-tibial joint:

Two views may be stated as to the origin of the crucial ligaments. 1) According to Keith, they are originally parts of the capsule of the kneeoint, but have become separated from that structure as the result of the 
outgrowth of the condyles of the femur. (2) According to Bland-Sutton, they are modifications of muscles, but there are no certain facts as to which muscles they originally belonged.

The semilunar fibro-cartilages are two in number-internal and external. They are lunated plates, of very firm consistence, which are placed upon the peripheral parts of the condylar articular surfaces of the tibia so as to deepen them for the reception of the condyles of the femur. They also serve as cushions, which mitigate pressure and diminish shock. Each presents two surfaces, superior and inferior; two borders, outer and inner; and two extremities or cornua, anterior and posterior. The superior surface is slightly concave to adapt itself to a femoral condyle, being sloped from the outer to the inner border. The inferior surface is flat, and rests upon a condylar surface of the tibia at its circumference. Both these surfaces are covered by the synovial membrane of the joint. The outer border is convex and thick, and it is attached to the margin of the corresponding tuberosity of the tibia by fibres which are really a part of the capsular ligament, but which have received the name of coronary ligament (one for each fibro-cartilage). The external semilunar fibrocartilage is more loosely attached in this manner than the internal. Partly on this account, and partly by reason of the close proximity of its two cornua, the external fibro-cartilage is more movable than the internal. The inner border of each fibro-cartilage is very thin, sharp, and concave. It is quite free, and covered by the synovial membrane.

The extremities or cornua are thin fibrous bands, which have the following attachments: the anterior cornu of the internal semilunar fibro-cartilage is attached to the rough depression in front of the spine of the tibia at its anterior and inner part; or, to put it in another way, it is attached to the upper surface of the head of the tibia in front, and to the outer side, of the internal condylar surface. It has the anterior crucial ligament directly behind it. The posterior cornu is attached to the inner part of the rough depression behind the spine of the tibia, where it has the posterior crucial ligament behind it. The internal semilunar fibrocartilage is semicircular, and may be likened to the capital letter $\mathbf{C}$, its cornua being wide apart, and embracing between them the cornua of the external semilunar fibro-cartilage. It is intimately connected with the internal lateral ligament.

The anterior cornu of the external semilunar fibro-cartilage is attached to the head of the tibia immediately in front of the outer tubercle of the spine, where it is placed on the outer side of and behind the anterior crucial ligament. The posterior cornu is attached to the rough depression between the two tubercles of the spine, and also to a depression immediately behind the outer tubercle. The external semilunar fibro-cartilage is almost circular, thus $\boldsymbol{O}$, its cornua being very close together, and being embraced by the cornua of the internal semilunar fibro-cartilage. The pos- 
front of the internal. It prevents the front part of the external semilunar fibro-cartilage from moving too far backwards during flexion of the joint.

The synovial membrane invests the inner surface of the capsular ligament. On reaching the semilunar fibro-cartilages it is reflected over their upper and under surfaces, and is then conducted by the coronary ligaments to the margins of the tuberosities of the tibia. Between the front of the head of the tibia and the patella the membrane covers a collection of fat, known as the infrapatellar

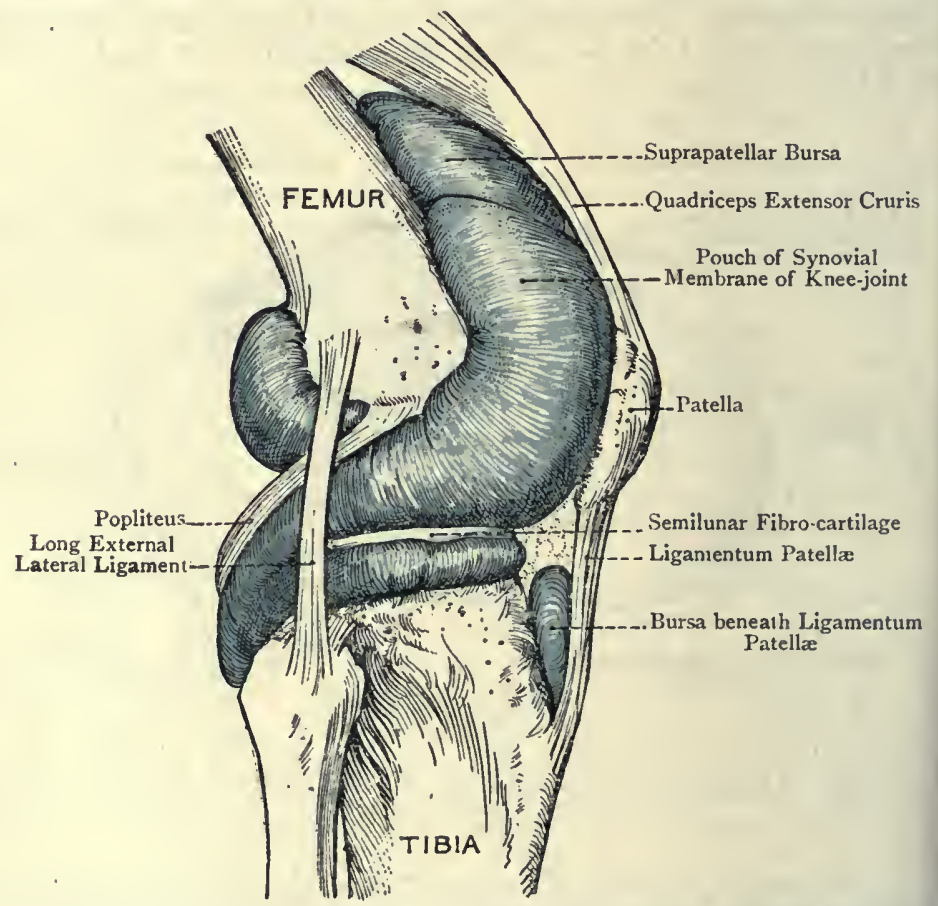

Fig. 255.-The Synovial Membrane of the Right Knee-Joint (External View) (AFter Spalteholz).

pad, and in this situation it is raised into certain folds, called ligaments, namely, the ligamentum mucosum and ligamenta alaria, to be presently described. Above the patella the membrane extends upwards in the form of a large pouch upon the front of the shaft of the femur for about 2 inches above the upper border of the patellar surface of the bone. This pouch lies beneath the suprapatellar tendon of the quadriceps extensor cruris, and communicates with the suprapatellar bursa, which is situated immediately above it, and which extends upwards for about I inch beneath the suprapatellar tendon. 
From the posterior ligament the synovial membrane is reflected forwards in the form of two laminæ, one of which passes over the outer surface of the anterior crucial ligament, and the other over the inner surface of the posterior crucial ligament. After having done so, these two laminæ become continuous with each other in front of the crucial ligaments. It will thus be evident that the following parts of these ligaments are destitute of synovial membrane: (I) the posterior surface of each ligament, and (2) that part of the anterior surface of the posterior ligament which is in contact with the anterior ligament.

In connection with the posterior ligament of the knee-joint it is to be noted that there is a narrow median vertical zone which is destitute of synovial membrane, on account of the forward reflection of that membrane in two laminæ. This interlaminar zone is covered by a certain amount of fat.

The synovial membrane furnishes an investment to the tendon of the popliteus, which separates that tendon from the long external lateral ligament. This prolongation also extends in a downward direction between the posterior part of the external semilunar fibro-cartilage and the outer tuberosity of the tibia, so as to facilitate the movement of the tendon of the popliteus at that point. It may extend so far down as to become continuous with the synovial membrane of the superior tibio-fibular joint. At the back of the joint the synovial membrane frequently communicates with the popliteal bursa between the semimembranosus and inner head of the gastrocnemius.

The synovial membrane, as it covers the infrapatellar pad of fat, forms three folds-the ligamentum mucosum and ligamenta alaria. The ligamentum mucosum, which contains a little fat, extends backwards to be attached to the front part of the interconaylar fossa of the femur. It is narrow and pointed at its femoral attachment, but over the infrapatellar pad of fat it widens out and becomes triangular. The lateral borders of this latter part of the ligamentum mucosum constitute the so-called ligamenta alaria, which extend for a short distance in an upward direction along the lateral margins of the patella.

The ligamentum mucosum, which serves to adjust the infrapatellar pad of fat to the different positions of the joint, is the remains of the double synovial partition which originally separates the femoro-tibial articulations.

The articular fat forms two chief collections, called infrapatellar and suprapatellar, which represent the Haversian glands of the knee-joint. The infrapatellar pad is the larger of the two, and fills up the space between the lower end of the femur, the head of the tibia, and the patella with its ligament. It adapts itself to the different positions of the joint, and the ligamentum mucosum acts as its regulator. The suprapatellar pad is situated around the suprapatellar bursa and the pouch which the synovial membrane sends upwards above the patellar surface of the femur. 
Muscular Relations.--The muscular relations are as follows : Anterior.-The quadriceps extensor cruris.

External.-The biceps femoris.

Posterior.-The popliteus, plantaris, and outer head of the gastrocnemius externally, and the inner head of the gastrocnemius and semimembranosus internally.

Internal.--The sartorius, gracilis, semitendinosus, and semimembranosus.

Arterial Supply.-The joint receives its arterial supply from the following sources: (I) the long descending branch of the external circumflex of the arteria profunda femoris; $(2)$ the deep branch of

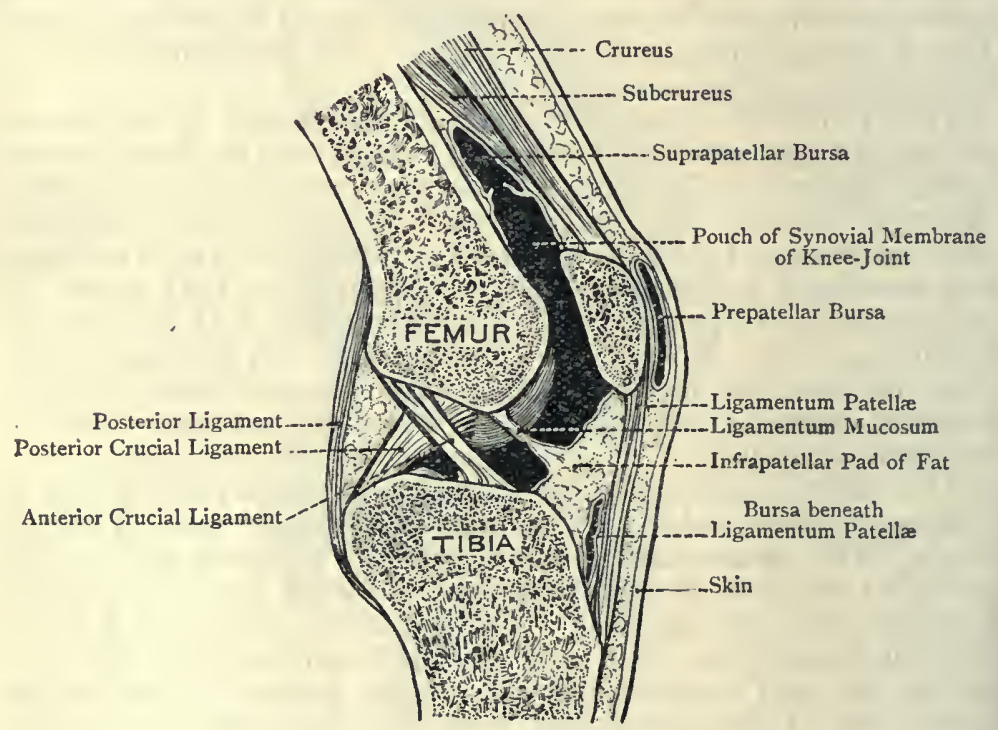

Fig. 256.-Sagittal Section of the Right Knee-Joint, viewed FROM THE OUTER SIDE.

the anastomotica magna of the superficial femoral; (3) the superior and inferior external and internal articular and the central or azygos articular branches of the popliteal; and (4) the anterior tibial recurrent and posterior tibial recurrent (inconstant) branches of the anterior tibial.

Nerve-supply.-The joint receives its nerves from the following sources: (I) the external and internal popliteal, both from the great sciatic; (2) the anterior femoral; and (3) the obturator.

The external popliteal nerve furnishes three articular branches, one with the superior external articular artery, one with the inferior external articular, and a recurrent branch with the anterior tibial rccurrent. The internal popliteal nerve furnishes tliree (sometimes 
two) articular branches, one with the superior internal articular artery (inconstant), one with the central or azygos articular, and one with the inferior internal articular.

The anterior femoral nerve furnishes articular branches as follows: (I) the nerve to the vastus externus furnishes one articular branch; (2) the most internal of the branches to the crureus furnishes another articular branch, which in its course supplies the subcrureus; and (3) the nerve to the vastus internus furnishes a third articular branch, which is of large size, and ul timately accompanies the deep branch of the anastomotica magna artery.

The obturator nerve, by its deep or posterior division, furnishes an articular branch called the geniculate nerve. This branch, however, may be absent.

If the nerve-supply of the knee-joint is compared with that of the hip-joint, it will be evident that there is a nervous sympathy between these two articulations.

\section{Movements-Femoro-} tibial Joints.-The movements between the condyles of the femur and the condylar articular surfaces of the tibia are of two kinds,

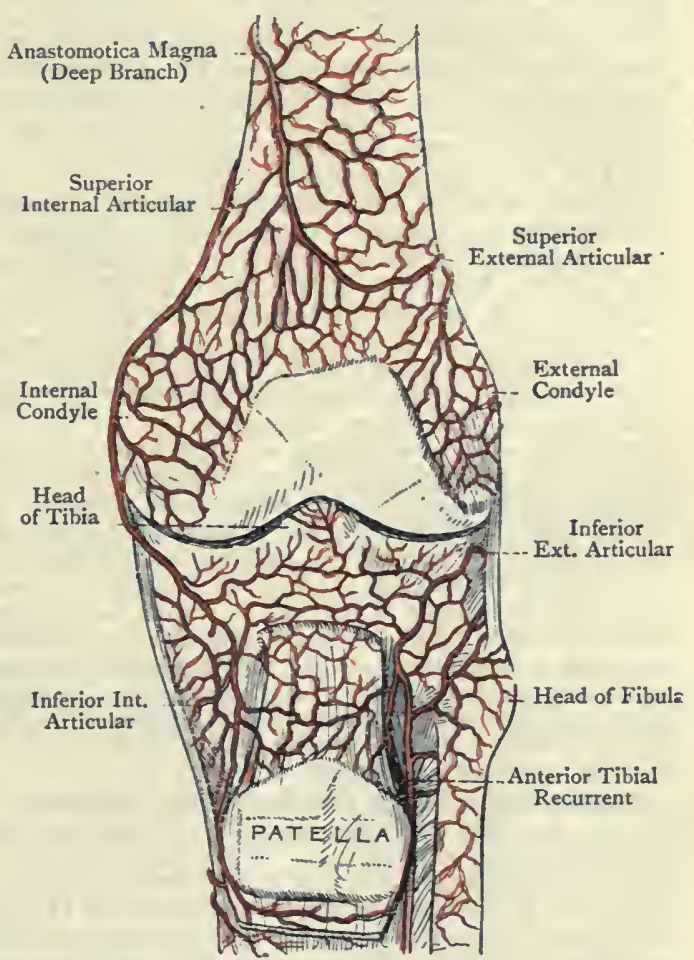

Fig. 257.-The DeEp Anastomoses round the KNEE-JOINT (ANTERIOR VIEW) (TIEDEMANN). namely, flexion and extension, and rotation, the latter movement being only possible when the knee-joint is flexed.

Flexion and Extension.-These movements are complicated by the spiral outline of the femoral condyles, and they partake partly of gliding and partly of rolling. During extension the two condyles move parallel to each other up to a certain stage, namely, towards the end of extension. When this stage has been reached, the anterior part of the outer condyle is in contact with the outer tibial articular surface. The inner condyle, however, being longer than the outer, continues to glide backwards so as to bring its oblique anterior part into contact with the inner tibial articular surface. The result is that the femur is rotated inwards on the tibia. Hence, at the very com- 
mencement of flexion, the femur is rotated outwards on the tibia. When the joint is completely extended, the following ligaments are rendered tense : (I) anterior crucial, (2) posterior ligament, and (3) long external lateral and internal lateral ligaments. Over-extension is checked $(\mathrm{I})$ by the tightening of the anterior crucial, the posterior ligament, and the two lateral ligaments ; (2) by the anterior portions of the semilunar fibro-cartilages being pressed into the depressions of the femoral condyles; (3) by the locking which takes place between the anterior crucial ligament and the front part of the intercondylar fossa ; and (4) by the locking which takes place between the inner depression of the outer femoral condyle and the front of the outer tubercle of the tibial spine (Bruce Young). At the end of extension the outer condyle pushes the anterior and inner part of the external semilunar fibro-cartilage over the anterior and inner border of the onter condylar surface of the tibia. When this has been done, the depression at the inner end of the groove on the outer condyle comes into contact with the front of the outer tubercle of the tibial spine, and with a special facet in front of that tubercle (at the anterior and inner part of the outer condylar surface of the tibia), and so the locking takes place.

In flexion of the knee-joint the external ligaments are relaxed, except the ligamentum patellæ. Over-flexion is checked by ( 1 ) the stretching of the quadriceps extensor cruris; (2) the meeting of the fleshy parts of the calf and back of the thigh; (3) the tightening of the posterior crucial ligament; and (4) the extreme posterior and outer part of the inner condyle (which here presents a special facet) being pressed against the back part of the inner tubercle of the tibial spine (Cleland).

In extension of the joint the semilunar fibro-cartilages move forwards, and in flexion they move backwards.

Rotation.-This movement is impossible in extension of the knee-joint, owing to the tightening of the anterior crucial and the external ligaments, except the ligamentum patellæ. In semiflexion, however, a fair amount of rotation is. allowed. Internal rotation is checked by the anterior crucial ligament, and external rotation by the internal lateral ligament.

The range of rotation is about 40 degrees. Assuming that all the muscles and external ligaments are cut, in which case the femur and tibia would be connected only by the crucial ligaments, if the femur is grasped firmly and the tibia rotated inwards the movement will be seen to be arrested by the tightening of the anterior crucial ligament. If, however, the tibia is rotated outwards neither crucial ligament interferes with the movement, so that the tibia can be made to describe half a circle, thus directing the front of the head of the bone backwards.

Muscles concerned in the Movements-Flexion.-The muscles which produce flexion are the biceps femoris, semitendinosus, semimembranosus, sartorius, gracilis, and popliteus. If the ankle-joint is fixed, the gastrocnemius, with the plantaris as a feeble auxiliary, acts as a flexor. Extension.The chief extensor is the quadriceps extensor cruris. The gluteus maximus and tensor fasciæ femoris, however, take part in the completion of extension by means of the ilio-tibial band of the fascia lata. Internal Rotation. - This movement is produced by the popliteus, semitendinosus, semimembranosus, gracilis, and sartorius, in each case after flexion has been effected. External Rotation.-This movement is produced by the biceps femoris after it has flexed the joint.

Femoro-patellar Joint. - The movement at this joint is of a gliding or to-and-fro nature, associated with a certain amount of rolling of the patella, the result of which is to bring different parts of the opposed articular surfaces into contact in different positions of the joint. This combination of gliding and rolling constitutes the movement called coaptation. In extension of the knee, when the patella is drawn up by the quadriceps extensor cruris, the inferior pair of patellar facets are in contact with the upper part of the patellar surface of the femur. In semiflexion the middle pair of patellar facets are in contact with the middle part of the patellar surface of the femur. In ordinary bexion the upper pair of patellar facets are in contact with the 
lower part of the patellar surface of the femur. In extreme flexion, as in the position assumed by the miner when at work, the patella is entirely off the patellar surface of the fermur, and, being turned outwards by the internal condyle, the inner vertical facet of the patella articulates with the semilunar facet on the outer part of the tibial surface of the internal condyle close to the intercondylar fossa, and the upper and outer horizontal patellar facet is in contact with the front part of the external condyle of the femur. When the patella is turned inwards in extension of the joint, the outer lateral patellar ligament is put upon the stretch, and so prevents over-displacement inwards. When the patella is turned outwards in flexion by the internal condyle, the inner lateral patellar ligament is put upon the stretch, and so prevents overdisplacement outwards.

When a person stands erect with both feet upon the ground, the vertical line of the centre of gravity falls in front of the axis of movement at the knee-joint. There is thus a tendency to over-extension, which, however, is resisted by the tension of the ligaments. In the erect attitnde the knee-joint is, therefore, maintained in a state of extension to a large extent without muscular effort. This is proved by the fact that each patella is freely movable. When, however, one limb is raised from the ground, muscular effort now becomes necessary to maintain extension, and the patella of each limb becomes fixed. The gluteus maximus and tensor fascix femoris muscles, acting through the ilio-tibial band of the fascia lata, contribute to the maintenance of extension at the knee-joint, as in standing.

Bursæ at Knee-Joint.-The region of the knee-joint abounds in bursæ, and in this respect surpasses other articular regions. The bursæ are as follows:

\section{Suprapatellar. Subcrureal. Prepatellar. Infrapatellar. Anserine.}

\section{Popliteal. \\ of popliteal tendon. \\ Of semimembranosus tendon. \\ Gastrocnemial, mesial. \\ Gastrocnemial, lateral. Bicipital.}

The suprapatellar bursa is a large pouch of the synovial membrane of the knee-joint, which extends upwards upon the front of the shaft of the femur for about 2 inches above the upper border of the patellar articular surface of the bone. It lies beneath the suprapatellar tendon of the quadriceps extensor muscle, and its cavity is necessarily continuous with that of the synovial membrane of the knee-joint. In cases of extensive effusion into that joint this bursa becomes distended with fluid, and forms a large swelling, which readily admits of palpation. Superiorly it usually communicates with the subcrureal bursa.

The subcrureal bursa lies upon the front of the femur above, or higher up than, the suprapatellar bursa. It is situated beneath the subcrureus muscle, and usually communicates with the suprapatellar bursa, of which it may be regarded as a differentiated part.

The prepatellar bursæ are situated in front of the patella. There may be three of these-namely, subcutaneous, subfascial, and subtendinous-or there may only be one-namely, subcutaneous.

The subcutaneous prepatellar bursa is situated beneath the integument, in front of the lower part of the patella and upper part of the ligamentum patellæ. It may be more or less multilocular, and it is the seat of the condition known as housemaid's knee. This bursa is always present. 
The subfascial and subtendinous prepatellar bursæ are inconstant. When present, they may be regarded as deep differentiations of the subcutaneous bursa, with which they usually communicate more or less freely, and might therefore be involved in housemaid's knee. They may, however, form distinct bursæ.

The subfascial prepatellar bursa is situated between the fascia lata, which covers the patella, and the tendinous expansion which the suprapatellar tendon of the quadriceps extensor muscle sends downwards over the patella. It may extend downwards over the upper part of the ligamentum patellæ, and it may be multilocular.

The subtendinous prepatellar bursa is the most deeply placed. It is situated between the tendinous expansion of the suprapatellar tendon over the patella and the periosteum of the bone, and it is limited to the front of the bone.

The prepatellar bursæ have no communication with the synovial cavity of the knee-joint, and it is therefore necessary to discriminate between prepatellar bursitis and synovitis of the knee-joint.

The infrapatellar bursæ are two in number-subcutaneous and deep.

The infrapatellar subcutaneous bursa is situated beneath the integument, and lies over the lower rough half of the tubercle or anterior tuberosity of the tibia, which half gives attachment to the lower end of the ligamentum patellæ.

The deep infrapatellar bursa is situated between the lower part of the ligamentum patellæ and the upper smooth half of the tubercle or anterior tuberosity of the tibia. It is on a slightly higher level than the subcutaneous infrapatellar bursa, and neither the subcutaneous nor the deep bursa has any communication with the synovial cavity of the knee-joint.

The bursa anserina is connected with the expanded tendons of insertion of the sartorius, adductor gracilis, and semitendinosus muscles into the upper portion of the inner surface of the shaft of the tibia, on a level with the tubercle or anterior tuberosity of the bone. It is also related superficially to the internal lateral ligament of the knee-joint, and it has no communication with the synovial cavity of that joint. It is called the anserine bursa, because the tendons of the muscles to which it is related are disposed somewhat in the form of a goose's foot, known as the pes anserinus cruris, as distinguished from the pes anserinus faciei, formed by the facial nerve on emerging from the parotid gland.

The popliteal bursa, which is of large size, is situated at the posterior and inner aspect of the knee-joint. It lies between the inner head of the gastrocnemius and the semimembranosus muscle, and frequently (one in five) communicates with the synovial cavity of the joint. Under these circumstances, if effusion should take place into the knee-joint, this bursa might form a fluctuating swelling, which might be palpated at the posterior and inner part of the joint.

The synovial sheath of the popliteal tendon is derived from the 
synovial membrane of the knee-joint, as the tendon lies within the joint. It lies over the lower part of the outer aspect of the external condyle of the femur, and it accompanies the tendon as the latter passes beneath the long external lateral ligament. This bursa may communicate with the superior tibio-fibular joint.

The bursa of the semimembranosus tendon lies between the chief tendon of insertion of that muscle and the upper lip of the horizontal groove on the posterior surface of the internal tuberosity of the tibia. This bursa has no communication with the synorial cavity of the knee-joint.

The mesial gastrocnemial bursa, of small size, is situated between the inner head of the gastrocnemius and the posterior surface of the femur, just above the inner condyle.

The lateral gastrocnemial bursa is situated between the outer head of the gastrocnemius and the adjacent part of the capsule of the knee-joint at its posterior and outer part, just above the external condyle. The two gastrocnemial bursæ, being so close to the kneejoint, may communicate with its synovial cavity.

The bicipital bursa invests the long external lateral ligament of the knee-joint as that ligament passes through the tendon of insertion of the biceps femoris muscle. It lies close above the outer aspect of the head of the fibula, and it has no communication with the synovial cavity of the knee-joint.

\section{SOLE OF THE FOOT.}

Landmarks.-Along the inner border of the foot there are several important bony landmarks which can be made out without much difficulty. The internal malleolus is a good starting-point. One inch below this projection the sustentaculum tali of the os calcis can be felt, and a little in front of it is the tuberosity of the navicular or scaphoid bone. The navicular tuberosity is situated about $I_{\frac{1}{2}}$ inches in front of the internal malleolus, and on a lower level. The recess between the sustentaculum tali and the navicular tuberosity is bridged over by the spring ligament and the tendon of the tibialis posticus, the latter being the more superficial. Immediately in front of the navicular tuberosity the eminence on the plantar surface of the internal cuneiform bone may be felt, and anterior to it is the tuberosity on the plantar aspect of the proximal end of the first metatarsal bone. Along the outer border two projections can be felt, namely, the external malleolus and the tuberosity on the outer side of the proximal end of the fifth metatarsal bone.

The guide to the astragalo-navicular joint on the inner side of the foot is the tuberosity of the navicular bone, the joint being situated immediately behind that tuberosity. The guide to the calcaneo-cuboid joint on the outer side of the foot is a point midway 
between the external malleolus and the tuberosity on the outer side of the proximal end of the fifth metatarsal bone. The astragalonavicular and calcaneo-cuboid joints lie in the same transverse plane, and are the joints at which disarticulation is performed in Chopart's operation. The guide to the joint between the internal cuneiform and first metatarsal bones is a point $I_{2} \frac{1}{2}$ inches in front of the navicular tuberosity. This is to be remembered in connection with the tarso-metatarsal operations of Lisfranc and Hey. The joint between the cuboid and fifth metatarsal bones is situated immediately behind the tuberosity on the outer side of the proximal end of the fifth metatarsal bone.

The course of the external plantar artery is complicated by the fact that it first crosses the sole of the foot from within outwards, and afterwards from without inwards. The former course is indicated by a line drawn from a point midway between the inner part of the point of the heel and the tip of the internal malleolus to a point about $\mathrm{I}$ inch internal to the tuberosity on the outer side of the base of the fifth metatarsal bone. A line drawn from the latter point to the proximal part of the first interosseous space indicates the position of the artery as it recrosses the foot. To indicate the course of the internal plantar artery start from the same point as for the commencement of the external plantar vessel, and draw a line to the centre of the metatarso-phalangeal joint of the great toe.

The skin of the sole is characterized by great thickness over the bony prominences of the heel and balls of the toes, in which situations bursæ are frequently devcloped. The superficial fascia is coarse and granular. It forms a thick pad composed of lobules of 'fat, which lie between, and are supported by, processes of fibrous tissue passing between the skin and the plantar fascia.

Plantar Bursæ.- These bursæ are as follows:

Calcaneal.

First metatarsal.

\section{Fifth metatarsal. \\ Navicular. \\ Peroneal.}

- The calcaneal bursa is placed over the plantar aspect of the tuber calcis, which has two tubercles-inner and outer. This bursa is but little developed, unless in those persons who have to stand for long periods of time.

The first metatarsal bursa is situated over the plantar aspect of the head of the first metatarsal bone, which supports the two phalanges of the great toe or hallux. The head of this bone is of large size, and its plantar surface is rendered prominent by two large sesamoid bones.

Another first metatarsal bursa is sometimes formed over the internal or medial aspect of the metatarso-phalangeal joint of the great toe. This occurs in cases of hallux valgus, which is an external displacement or subluxation of the great toe or hallux at the metatarso-phalangeal joint. The hallux may be displaced outwards to 
such an extent as to be overlapped by $i$ he second toe. Under these circumstances the inner part of the head of the first metatarsal bone and the inner part of the proximal end of the first phalanx give rise to a prominence on the inner aspect of the metatarsophalangeal joint of the great toe. Over this prominence a synovial bursa is formed, and, when this bursa becomes enlarged, the most common form of bunion is the result. If suppuration should ensue, the metatarso-phalangeal joint of the great toe may become involved.

The fifth metatarsal bursa is situated over the plantar aspect of the head of the fifth metatarsal bone, which supports the phalanges of the little toe. This bursa is of small size, and is due to the fact that the external pillar of the longitudinal arch of the foot is nearer the ground than the internal pillar.

Another fifth metatarsal bursa is sometimes formed over the prominent tuberosity on the outer surface of the base or proximal end of the fifth metatarsal bone. This occurs in cases of talipes varus, in which the inner border of the foot is raised.

The navicular bursa is not common. It may be formed over the tuberosity of the navicular or scaphoid bone, about $\mathrm{I}_{2} \frac{1}{2}$ inches in front of the internal malleolus-e.g., in cases of talipes valgus, in which the outer border of the foot is raised.

The tendon-sheath of the peroneus longus invests the tendon of that muscle as it traverses the fibro-osseous canal formed by the long plantar ligament and the peroneal groove on the plantar surface of the cuboid bone. It is deeply placed.

Plantar Fascia or Aponeurosis.- The plantar fascia is connected to the skin by fibrous processes, which enclose and support the lobules of the superficial fascia, and it presents two longitudinal grooves, along the course of which intermuscular septa pass deeply into the sole. These grooves indicate the division of the plantar fascia into three portions-central and two lateral.

The central division covers the flexor brevis digitorum, and is of considerable strength. It is triangular, being somewhat pointed behind and expanded in front. Posteriorly, where it is narrow and thick, it is attached to the inner tubercle on the plantar aspect of the tuber calcis. From this point the fibres pass forwards towards the toes, the central division meanwhile widening out and becoming thinner. It the heads of the metatarsal bones it divides into five digital processes, one for each toe. The direction of its fibres is longitudinal-that is to say, they run from heel to toes, but towards the balls of the toes transverse fibres make their appearance. Moreover, in the webs of the toes there are other superadded transverse fibres, which constitute the superficial transverse ligament. This ligament arches over the digital vessels, nerves, lumbricales muscles, and digital processes. Each of the five digital processes forms an arch over the flexor tendons as these are about to pass to the plantar aspects of the toes. The final disposition of each process is as follows: (I) it is attached superficially to the skin; (2) it joins the vaginal ligament of the 


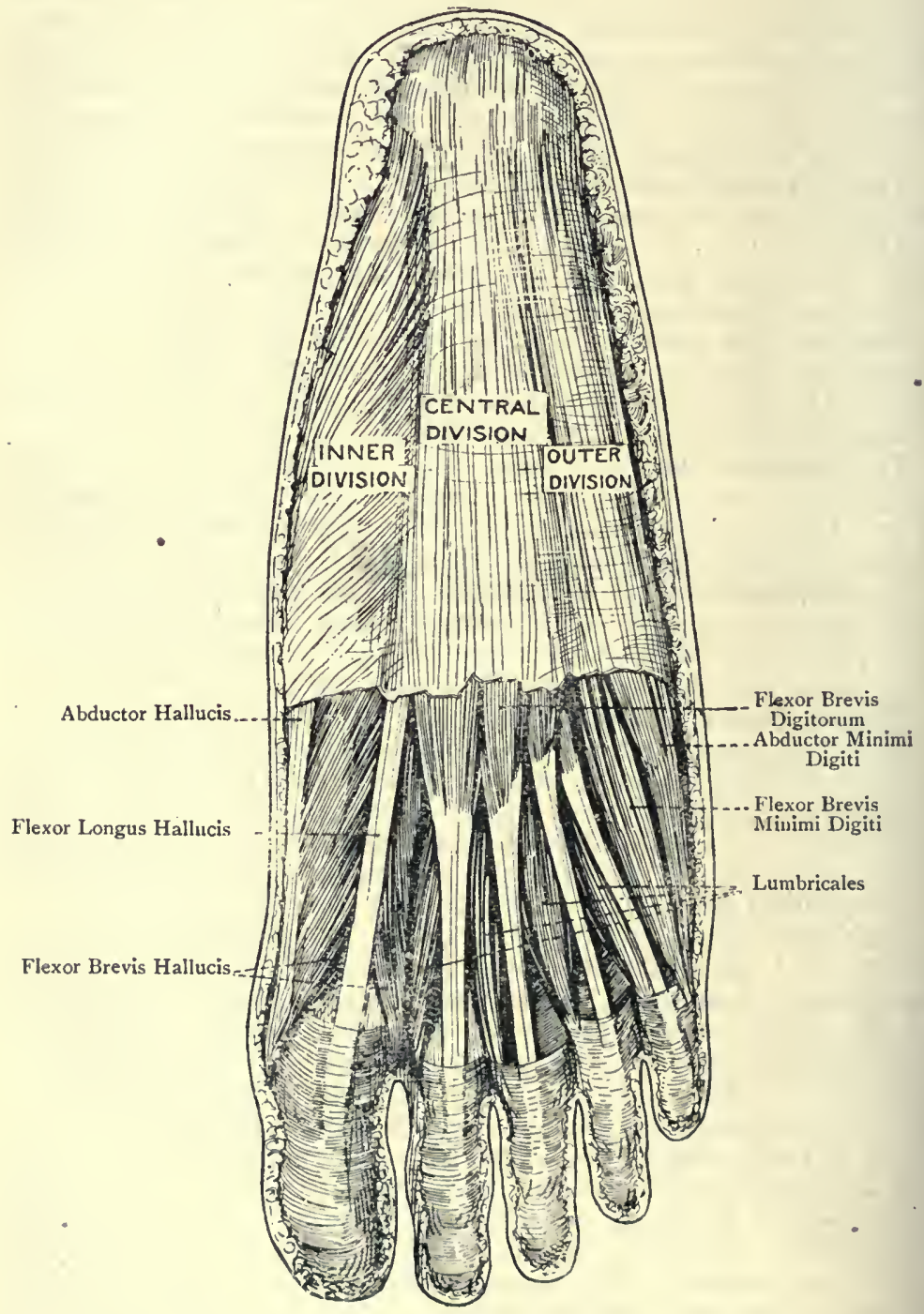

Fig. 258.-The Plantar Fascia. and First layer of Muscles (in part) 
flexor sheath anteriorly; and (3) at each side of the flexor tendons it sends a deep process upwards, which joins the transverse metatarsal (deep transverse) ligament connecting the heads of the metatarsal bones, and is also attached to the corresponding lateral metatarso-phalangeal ligament. There is thus formed a tunnel for the passage of the flexor tendons. The lumbricales muscles and the digital vessels and nerves make their appearance between the diverging digital processes. The central division contributes to the maintenance of the longitudinal arch of the foot by its tendency to approximate the toes to the heel. When it becomes contracted it gives rise to an exaggeration of the longitudinal arch, a condition known as pes cavis. Morphologically it represents the divorced plantar portion of the tendon of the plantaris.

The external division is weaker than the central. It is attached posteriorly to the outer tubercle on the plantar aspect of the tuber calcis, whence it passes forwards over the abductor minimi digiti muscle. Internally it is continuous with the central division along the line of attachment of the external intermuscular septum; externally it is continuous round the outer border of the foot with the deep fascia of the dorsum; and anteriorly, having become very thin, it is attached to the proximal end of the first phalanx of the little toe on its outer aspect. This division forms a specially strong band between the outer tubercle of the os calcis and the tuberosity on the outer side of the base of the fifth metatarsal bone, which is the remains of the abductor ossis metatarsi quinti, or Wood's muscle.

The internal division is the weakest of the three. It is attached posteriorly to the internal aspect of the inner tubercle on the plantar surface of the tuber calcis, and to the lower border of the internal annular ligament, whence it passes forwards over the abductor hallucis muscle. Externally it is continuous with the central division along the line of attachment of the internal intermuscular septum; internally it is continuous round the inner border of the foot with the deep fascia of the dorsum; and anteriorly, where it becomes very thin, it is attached to the proximal end of the first phalanx of the great toe on its inner aspect.

The intermuscular septa are two in number, external and internal, and they extend upwards into the sole at either side of the central division along the course of the longitudinal grooves. They are situated on either side of the flexor brevis digitorum, the internal septum lying between that muscle and the abductor hallucis, and the external septum intervening between it and the abductor minimi digiti. Each septum gives partial origin to the muscles between which it lies. The plantar fascia and the two intermuscular septa form three muscular compartments-inner, middle, and outer.

Cutaneous Nerves. - The cutaneous nerves are as follows: 
calcaneo-plantar, branches of the internal plantar, and branches of the external plantar.

The calcaneo-plantar nerve is a branch of the posterior tibial whilst that nerve is beneath the internal annular ligament. Having pierced that ligament, the nerve divides into internal calcaneal and plantar branches. The former supplies the integument of the inner side of the heel, and the latter the inner and posterior part of the sole.

The plantar cutaneous branches of the internal plantar nerve appear along the groove between the abductor hallucis and flexor brevis digitorum, and, having pierced the plantar fascia, are distributed to the integument of the inner half of the sole.

The plantar cutaneous branches of the external plantar nerve appear along the groove between the flexor brevis digitorum and abductor minimi digiti, and, having pierced the plantar fascia, are distributed to the integument of the outer half of the sole.

It is to be noted that the integument of the outer side of the heel and outer border of the foot, including the outer side of the little toe, is supplied by the short saphenous nerve, the external branch of the musculo-cutaneous nerve also taking part in the supply of the outer border, whilst the integument of the inner border of the foot is supplied by the long saphenous, and by the internal branch of the musculo-cutaneous nerve.

The cutaneous arteries of the sole are branches of the internal and external plantar vessels, and in their course and distribution they accompany the corresponding cutaneous nerves. There is a very copious supply of arteries to the integument of the heel, represented by the internal calcaneal branches of the external plantar and posterior tibial arteries on the inner side, and the external calcaneal branches of the posterior peroneal artery on the outer side.

Superficial Veins.-The superficial veins are very numerous, and are for.the most part arranged in the form of a plexus, which lies immediately beneath the skin. Besides this plexus, there is a transverse venous arch situated near the clefts of the toes. The destination of the venous blood of the plexus and transverse arch is the dorsum of the foot. The blood is conveyed away from the plantar subcutaneous plexus by anterior efferent and lateral efferent vessels. The anterior efferent vessels terminate in the transverse venous arch near the clefts of the toes. The lateral efferent vessels turn round the outer and inner borders of the foot, the external set terminating in the short saphenous vein, and the internal set in the long saphenous vein, just where these vessels are springing from the extremities of the dorsal venous arch. The transverse venous arch near the clefts of the toes receives the anterior efferent vessels of the plantar subcutaneous plexus, and the plantar digital veins. The blood is conveyed away from it by means of efferent veins, called interdigital, which pass upwards to the dorsum of the 
foot, where they terminate in the dorsal venous arch. The superficial veins of the sole are all furnished with valves, which are so placed as to direct the flow of blood to the dorsum of the foot.

Muscles. - The muscles of the sole are divided into four layersfirst, second, third, and fourth.

First Layer.-The first layer consists of the following three muscles, named in order from within outwards : abductor hallucis, flexor brevis digitorum, and abductor minimi digiti. All three lie immediately beneath the corresponding divisions of the plantar fascia, each muscle having a compartment to itself.

Abductor Hallucis - Origin. - The outer head arises from (I) the internal aspect of the inner tubercle on the plantar surface of the tuber calcis; (2) the deep surface of the internal division of the plantar fascia; and (3) the internal intermuscular septum, which separates it from the flexor brevis digitorum. The inner head arises from (I) the lower border of the internal annular ligament on its deep aspect; and (2) the fibrous structures along the adjacent part of the inner side of the sole, such as the tendon of the tibialis posticus and some of its expansions.

Insertion.-The tubercular enlargement on the inner side of the base of the first phalanx of the great toe.

Nerve-supply. - The internal plantar nerve.

Action.-(I) To abduct the great toe from the imaginary middle line of the foot, which passes through the centre of the second toe; and (2) to flex the metatarso-phalangeal joint of the great toe. The tendon of the muscle receives on its outer and deep aspect the fleshy inner head of the flexor brevis hallucis.

Flexor Brevis Digitorum (flexor perforatus)-Origin.-(I) The front of the inner tubercle on the plantar surface of the tuber calcis; (2) the deep surface of the central division of the plantar fascia; and (3) the intermuscular septum on either side.

Insertion.-By means of four tendons which go to the four outer toes, where each is inserted into the sides of the shaft of the second phalanx at its centre and on its plantar aspect.

Nerve-supply. - The internal plantar nerve.

Action.-(I) To flex the second phalanges of the four outer toes, and (2) to flex the metatarso-phalangeal joints.

Each tendon, as it passes along the plantar surface of a toe, has a tendon of the flexor longus digitorum above it as far as the second phalanx, the two tendons occupying a fibro-osseous canal lined by a synovial membrane which furnishes a separate investment for each tendon. Opposite the first phalanx the brevis tendon divides into two parts, and the longus tendon passes through the cleft thus formed; hence the name flexor perforatus for the brevis muscle. Thereafter the two divisions of the brevis tendon unite by their adjacent margins on the upper or dêep surface of the longus tendon, but they soon separate and take insertion into the sides of the shaft of the second phalanx at its centre and on its plantar aspect. Each brevis tendon has a ligamentum breve 


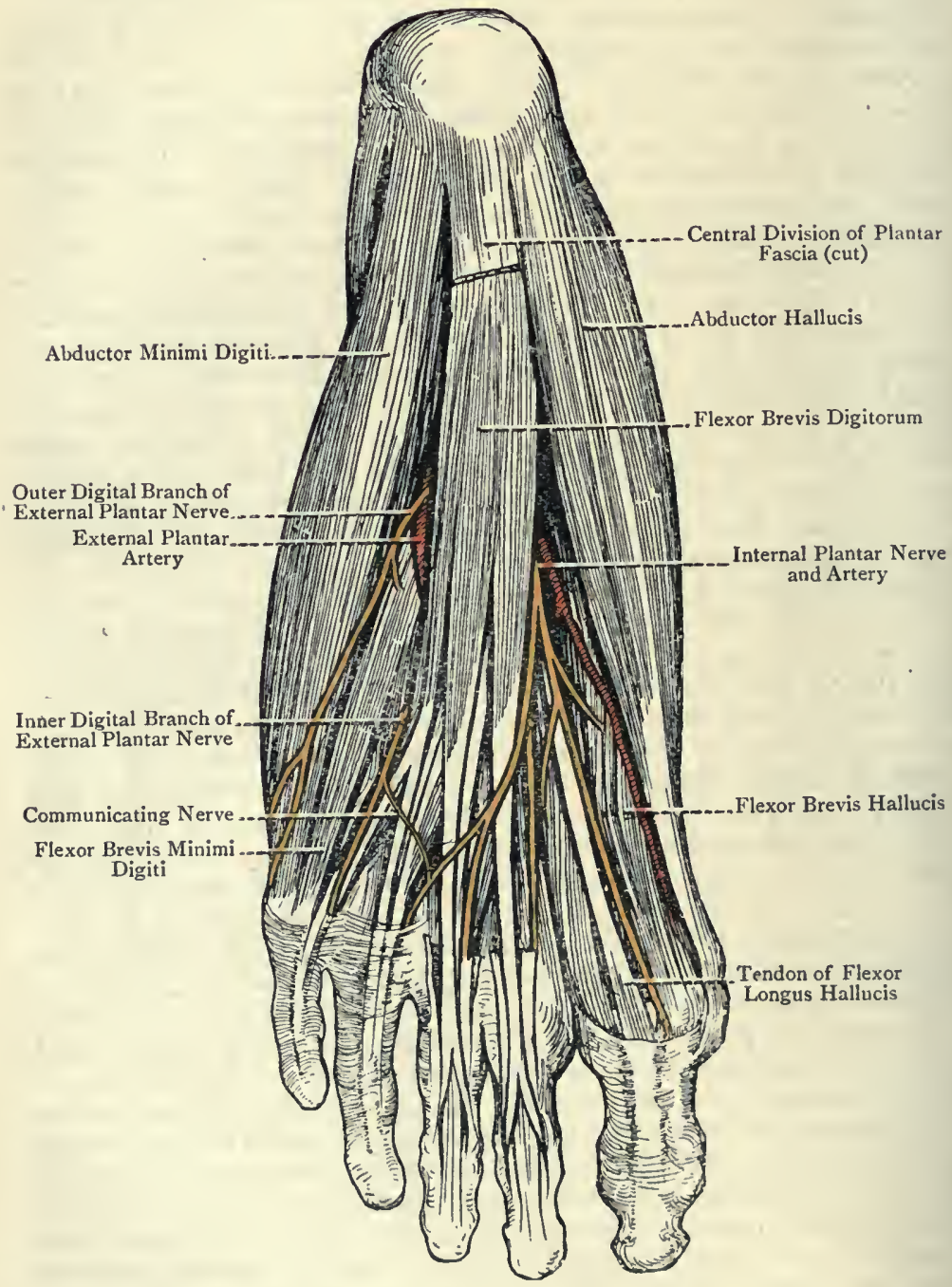

Fig. 259.-The First layer of Plantar Moscles (Left Foot). 
which passes between its upper surface near its final division and the distal end of the first phalanx. The fibrous sheaths of the tendons, as they pass along the plantar aspects of the toes, as well as the accessories of these sheaths, correspond with those of the fingers." As the long and short flexor tendons are about to pass to the plantar aspects of the respective toes, and before entering the fibro-osseous canals, each pair of tendons passes through a short fibrous tunnel, corresponding in position with the balls of the toes, and constructed in the following manner: superiorly or deeply it is formed by a portion of the transverse metatarsal ligament; inferiorly or superficially by a digital process of the central division of the plantar fascia; and on either side by the deep expansion of the digital process which joins the transverse metatarsal ligament and lateral metatarso-phalangeal ligament.

Abductor Minimi Digiti-Origin.--(I) The outer side and front of the outer tubercle on the plantar aspect of the tuber calcis; (2) the front of the inner tubercle; (3) the external intermuscular septum; and (4) the external division of the plantar fascia, more particularly the strong band which extends between the outer tubercle of the os calcis and the tuberosity on the outer side of the base of the fifth metatarsal bone.

Insertion.-The outer side of the base of the first phalanx of the little toe, in conjunction with the flexor brevis minimi digiti. It often takes attachment also to the base of the fifth metatarsal bone on the outer aspect of its plantar surface.

Nerve-supply.-The external plantar nerve.

Action.-(I) To abduct the little toe, and (2) to flex its metatarso. phalangeal joint.

Occasionally a fourth muscle is met with in the first layer, called the abductor ossis metatarsi quinti or Wood's muscle. It arises from the outer tubercle on the plantar aspect of the tuber calcis, and from the adjacent portion of the plantar fascia, and it is inserted into the tuberosity on the outer side of the base of the fifth metatarsal bone. In most cases, however, this muscle has undergone degeneration, and is only represented by the strong band of the external division of the plantar fascia, which extends between the outer calcaneal tubercle and the tuberosity on the outer side of the base of the fifth metatarsal bone.

Second Layer. - This layer consists partly of tendons and partly of muscles. They are as follows: the tendon of the flexor longus hallucis; the tendon of the flexor longus digitorum; the flexor or musculus accessorius ; and the lumbricales.

Tendons of Flexor Longus Hallucis and Flexor Longus Digitorum. -The tendon of the flexor longus hallucis, after leaving the groove on the under surface of the sustentaculum tali of the os calcis, is directed forwards and inwards, lying in its course between the two heads of the flexor brevis hallucis, and the tendon of the flexor longus digitorum is directed forwards and outwards towards the middle line of the sole. The two tendons, 


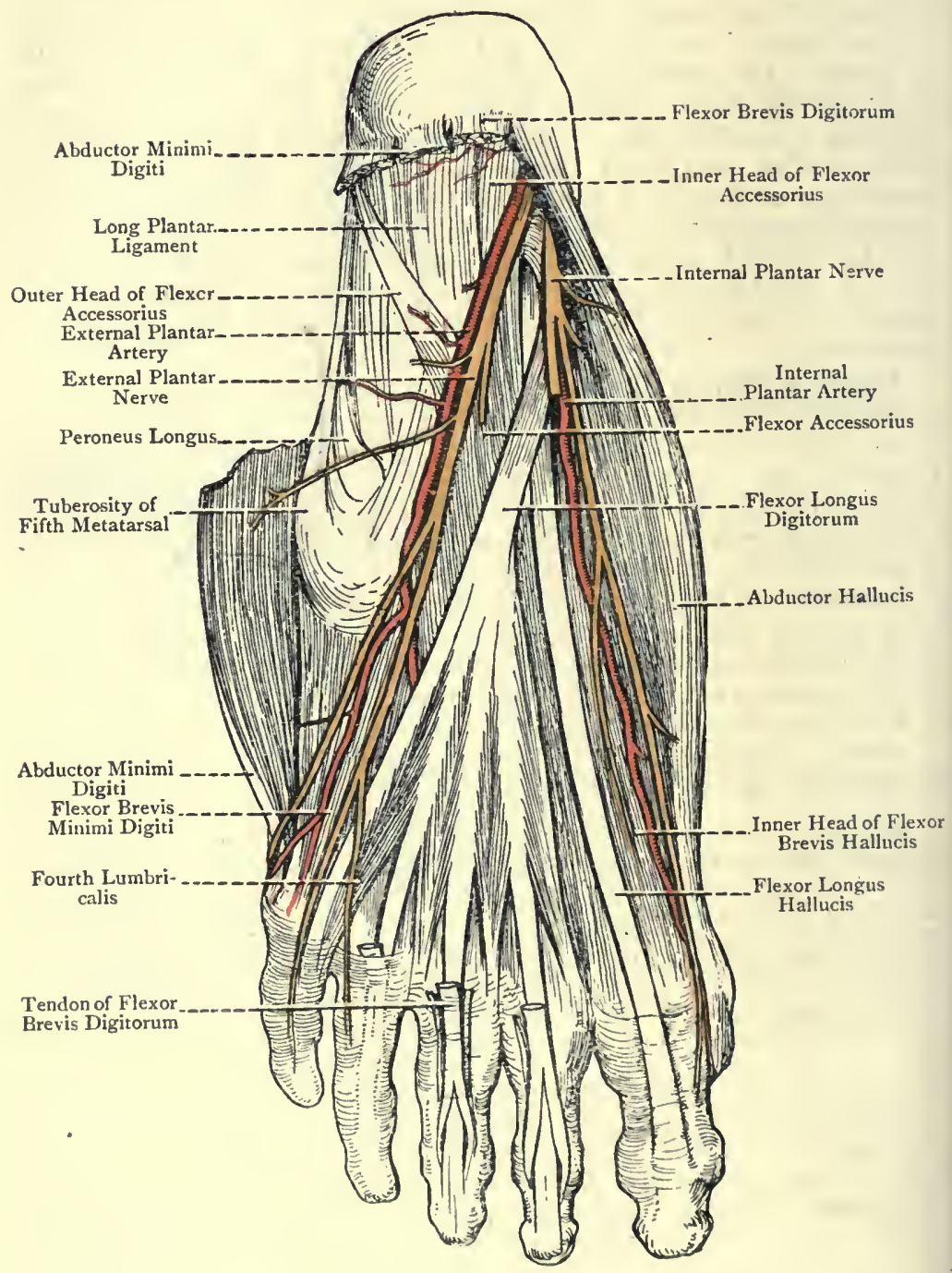

Fig. 260.-The Second Layer of Plantar Muscles (Left Foot). 
therefore, cross each other, that of the flexor longus hallucis being above, or on the deep surface of, the other, and giving a slip to it. The tendon of the flexor longus digitorum, on reaching the middle line of the sole, receives the flexor or musculus accessorius, and thereafter terminates in four tendons for the four outer toes, with which tendons the lumbricales are associated.

Flexor or Musculus Accessorius. - This muscle is so named because it is accessory to the long flexor. It arises, by two heads, inner and outer, which embrace between them the os calcis and the long plantar ligament.

Origin.-The inner head arises from the internal concave surface of the os calcis below the groove on the under surface of the sustentaculum tali. The outer head arises from (I) the junction of the external and plantar surfaces of the os calcis in front of the outer tubercle; and (2) the adjacent portion of the long plantar ligament. Insertion.-The outer border and upper surface of the tendon of the flexor longus digitorum about the centre of the sole.

Nerve-supply. - The external plantar nerve.

Action.-(I) To flex the terminal phalanges of the four outer toes, and (2) to counteract the tendency of the long flexor tendons to draw the toes inwards during flexion. In performing this latter action, the muscle keeps the long flexor tendon in the middle line, so that its four divisions act in a straight line upon the toes.

The inner head of the muscle is broad and fleshy, whilst the outer head is narrow, pointed, and tendinous.

This muscle is to be regarded as a detached portion of the flexor longus digitorum, forming an additional tarsal origin for the purpose of counteracting the obliquity of its tendons.

Lumbricales. - These muscles are four in number.

Origin.-From the tendons of the flexor longus digitorum where these begin to diverge, the outer three taking origin each from the contiguous sides of the tendons between which it lies, and the most internal springing only from the inner side of the long flexor tendon destined for the second toe.

Insertion.-The tendons, having passed round the inner sides of the metatarso-phalangeal joints of the four outer toes, take insertion each into the expansion formed by the extensor tendon on the dorsal aspect of the first phalanx.

Nerve-supply. - The most internal or first lumbricalis is supplied by the internal plantar nerve, more particularly by its second digital branch on its way to the cleft between the great toe and the second. The outer three lumbricales, or the second, third, and fourth, are supplied by the deep division of the external plantar nerve.

Action.-(I) To flex the metatarso-phalangeal joint, and (2) to extend the interphalangeal joints.

The muscles are to be regarded as detached portions of the flexor longus digitorum.

Third Layer.-The third layer consists of the following four 


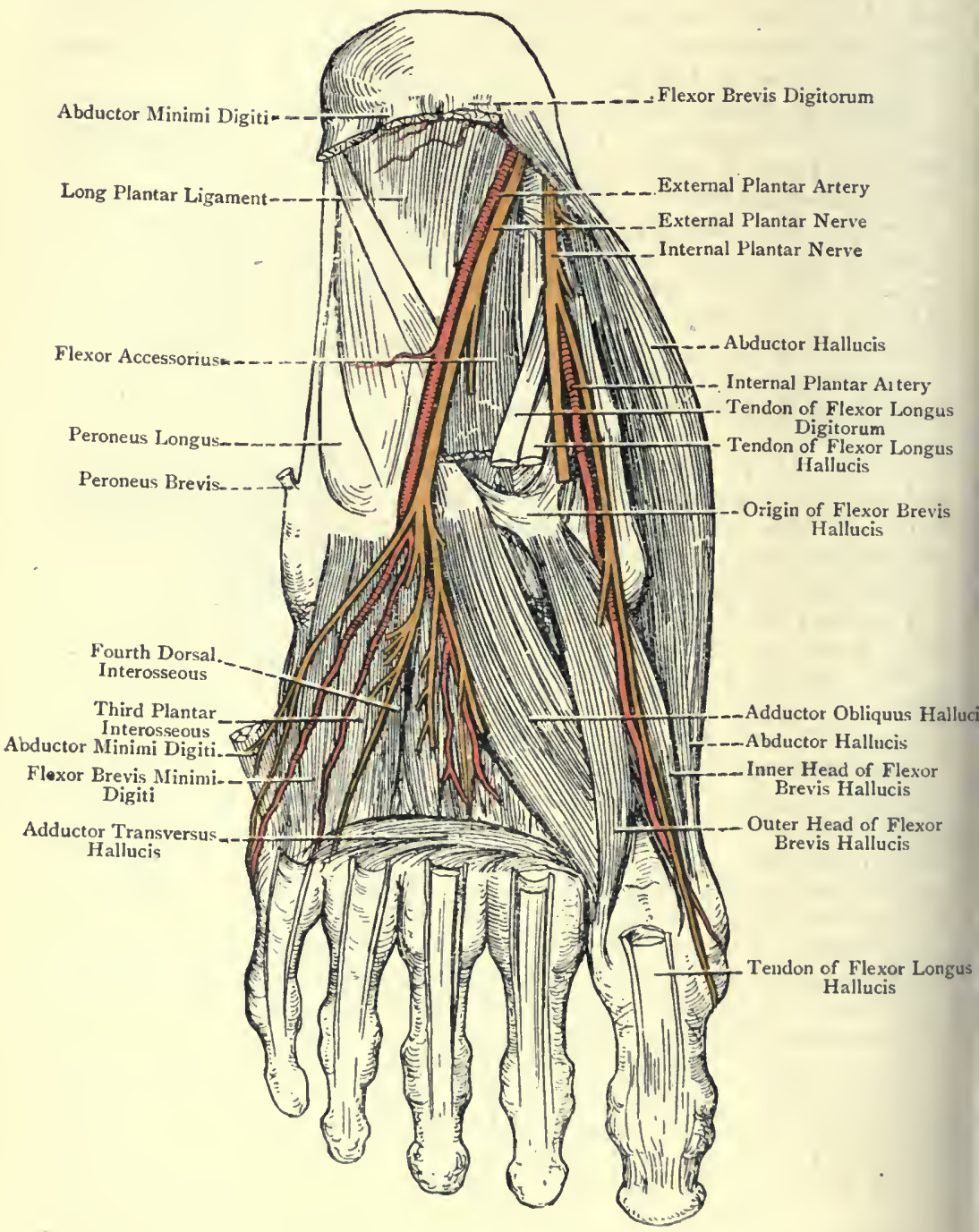

Fig. 26r, - The Third Layer of Plantar Muscles (Left Foot). 
nuscles: flexor brevis hallucis; adductor obliquus hallucis; adductor ransversus hallucis; and flexor brevis minimi digiti.

Flexor Brevis Hallucis-Origin.-(I) The inner part of the plantar urface of the cuboid bone (sometimes the internal surface), and 2) the expansions of the tibialis posticus tendon to the middle and xternal cuneiform bones.

Insertion.-By means of two heads, outer and inner. The inner lead is inserted into the tubercular enlargement on the inner side of the base of the first phalanx of the great toe, in conjunction with he abductor hallucis. The outer head is inserted into the tuber:ular enlargement on the outer side of the base of the first phalanx of the great toe, in conjunction with the adductor obliquus hallucis and adductor transversus hallucis.

Nerve-supply.-The internal plantar nerve, more particularly its irst or most internal digital branch.

Action.-To flex the metatarso-phalangeal joint of the great toe. It may at the same time act as a very slight adductor of that toe.

The flexor brevis hallucis lies along the outer side of the tendon of the abductor hallucis. It is tendinous and narrow behind, but soon becomes fleshy and divides into an inner and outer head of insertion, between which is the tendon of the flexor longus hallucis. In each head there is a sesamoid bone.

Adductor Obliquus Hallucis - Origin.-(I) The sheath of the tendon of the peroneus longus, and (2) the plantar surfaces of the bases of the second, third, and fourth metatarsal bones.

Insertion.-The tubercular enlargement on the outer side of the base of the first phalanx of the great toe, in conjunction with the outer head of the flexor brevis hallucis on the inner side and the adductor transversus hallucis on the outer side.

Nerve-supply.-The deep division of the external plantar nerve. Action.-(I) To adduct the great toe, and (2) to flex the metatarso-phalangeal joint.

The muscle lies obliquely on the outer side of the flexor brevis hallucis.

Adductor Transversus Hallucis (transversalis pedis)-Origin.(I) The inferior metatarso-phalangeal ligaments of the third, fourth, and fifth toes, and (2) the transverse metatarsal ligament.

Insertion.-The tubercular enlargement on the outer side of the base of the first phalanx of the great toe, in conjunction with the dductor obliquus hallucis.

Nerve-supply. - The deep division of the external plantar nerve. Action.-(I) To adduct the great toe, and (2) to approximate the toes to each other.

The muscle consists of three fleshy bundles which join into one, and it lies transversely upon the heads of the four outer netatarsal bones. It is to be regarded as a detached portion of he adductor obliquus hallucis, which has become shifted forwards o the balls of the toes. 
Flexor Brevis Minimi Digiti-Origin.-(I) The plantar surface of the base of the fifth metatarsal bone, and (2) the sheath of the tendon of the peroneus longus.

Insertion.-The outer side of the base of the first phalanx of the little toe, in conjunction with the abductor minimi digiti. A few of the fibres also take attachment to the plantar surface of the shaft of the fifth metatarsal bone over its anterior part. These fibres represent the muscle called opponens minimi digiti, the origin of which corresponds with those fibres of the flexor brevis minimi digiti which spring from the sheath of the tendon of the peroneus longus.

Nerve-supply.-The superficial division of the external plantar nerve, and usually the outer digital branch of that division to the outer side of the little toe.

Action.-To flex the metatarso-phalangeal joint of the little toe. The muscle is a small fleshy slip which lies upon the plantar surface of the fifth metatarsal bone, under cover of the abductor minimi digiti. It is liable to be taken for a plantar interosseous muscle, lying, as it does, in close contact with the most external plantar interosseous.

Plantar Triangle.-This is a muscular triangle in connection with the third layer of muscles, the boundaries of which are as follows : Anterior or Base. - Adductor transversus hallucis. Internal.Adductor obliquus hallucis. External. - Flexor brevis minimi digiti. Floor.- Portions of the plantar and dorsal interosseous muscles, covered by the interosseous fascia. Roof.- The long flexor tendons and the lumbricales. Contents.-(I) A limited portion of the deep part of the external plantar artery; (2) some of the digital branches of the plantar arch, especially the second and third, and the corresponding veins; and (3) a limited portion of the deep division of the external plantar nerve.

Fourth Layer.-The fourth layer consists of the interosseous muscles; the tendon of the peroneus longus; and the tendon of the tibialis posticus.

Interosseous Muscles.-The interosseous muscles are seven in number, and are arranged in two groups-plantar, of which there are three, and dorsal, of which there are four. The plantar muscles are seen only in the sole, but the dorsal muscles are seen on the dorsum of the foot as well as in the sole. The plantar aspects of the muscles are covered by the thin interosseous fascia which joins anteriorly the transverse metatarsal ligament.

Plantar Interossei.--These belong to the three outer toes, and are named first, second, and third from within outwards.

Origin.-(I) The inner surfaces of the shafts of the third, fourth, and fifth metatarsal bones, and (2) the sheath of the tendon of the peroneus longus.

Insertion.-(I) The inner sides of the bases of the first phalanges of the third, fourth, and fifth toes, and (2) the expansions formed by the long and short extensor tendons (in the case of the fifth toe 
long extensor only) on the dorsal aspects of the first phalanges of the same toes.

Dorsal Interossei.- These belong to the second, third, and fourth toes, the second toe having two. They are named first, second, third, and fourth, from within outwards.

Origin.-Each muscle arises by two heads from the adjacent lateral surfaces of the shafts of the metatarsal bones between which it is situated, but more extensively from the metatarsal bone of the toe upon which the muscle acts than from the other bone. In the case of the first (most internal) muscle its inner head is comparatively small, and arises from the outer side of the base of the first metatarsal bone, as well as from the adjacent portion of the internal cuneiform.
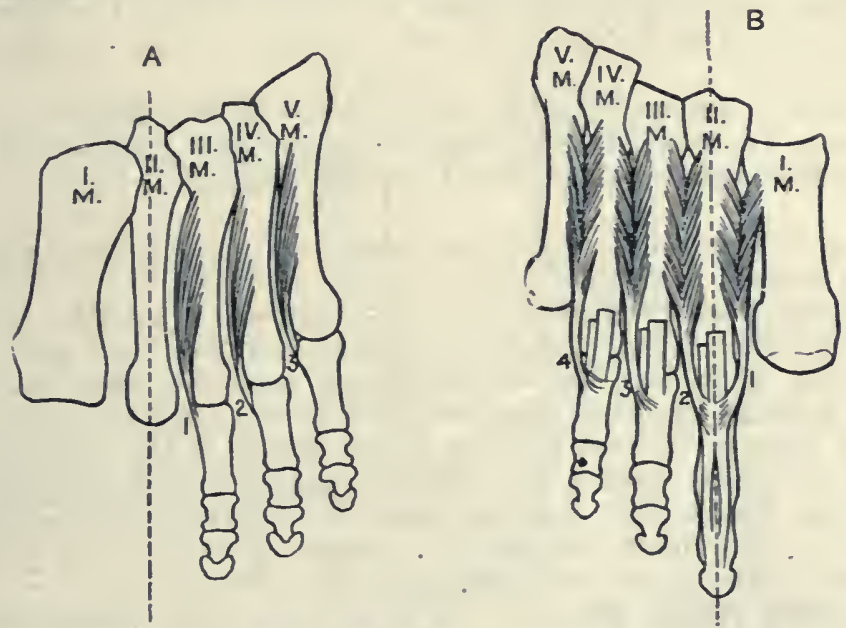

Fig. 262.-The Interosseous Muscles of the Right Foot.

A, Plantar; B, Dorsal.

Insertion.-The dorsal interossei are inserted in a manner precisely similar to the plantar interossei. The first and second dorsal interossei belong to the second toe, one on either side; the third belongs to the outer side of the third toe; and the fourth to the outer side of the fourth toe. In the case of all the interosseous muscles the insertion into a first phalanx is comparatively slight, the chief insertion being into the expansion of the extensor tendon on the dorsal aspect of the first phalanx.

Nerve-supply.-The external plantar nerve in the following manner: the deep division of the nerve usually supplies the interosseous muscles, with the exception of the two which occupy the fourth (most external) interosseous space, namely, the fourth dorsal and third plantar. These two are usually supplied by the outer digital branch of the superficial division of the external plantar 
nerve to the outer side of the little toe. Sometimes, however, the deep division of the nerve supplies all seven interosseous muscles. In all cases the nerves enter the muscles on their plantar aspects.

Action-Plantar Interossei.-These are adductors, the toes which they adduct being the third, fourth, and fifth. Dorsal Interossei.These are abductors, the toes which they abduct being the second, third, and fourth.

In speaking of adduction and abduction in the case of the foot, reference is made to an imaginary line passing through the centre of the second toe, adduction being movement towards that line, and abduction movement from it.

The first and second dorsal interossei, acting both upon the second toe, abduct it from the imaginary line passing through its centre, the first abducting it to the inner side and the second to the outer side. The third and fourth dorsal interossei abduct the third and fourth toes.

The interosseous muscles, aided by the lumbricales, also act as follows: (I) they flex the metatarso-phalangeal joints of the four outer toes, and (2) they extend the interphalangeal joints of these toes.

The tendons of the peroneus longus and tibialis posticus have been already described.

Plantar Nerves.- The plantar nerves are two in number, internal and external. They are the terminal branches into which the posterior tibial nerve divides on a level with the lower border of the internal annular ligament, though the division frequently takes place on a level with the upper border of that ligament, or at some point beneath it. The internal plantar is the larger of the two nerves, and it accompanies the internal plantar artery, which is the smaller of the two plantar arteries. The external plantar nerve accompanies the external plantar artery.

Internal Plantar Nerve.-This nerve has a wider cutaneous, but a more limited muscular, distribution than the external plantar. From its origin it passes forwards on the outer side of the internal plantar artery, being at first under cover of the abductor hallucis, and subsequently lying between that muscle and the flexor brevis digitorum. About the middle (in length) of the foot it divides into its terminal branches.

Branches.-These are muscular, articular, plantar cutaneous, and digital.

The muscular branches supply the abductor hallucis and flexor brevis digitorum.

The articular branches are distributed to the astragalo-navicular and naviculo-cuneiform articulations.

The plantar cutaneous branches are distributed to the integument of the inner half of the sole.

The digital branches are four in number, and are named first, second, third, and fourth from within outwards. The first is a single nerve, but the other three are compound. The first digital 
nerve is distributed to the inner side of the great toe, and it furnishes a branch to the flexor brevis hallucis muscle. The second digital nerve gives off a twig to the first lumbricalis, and near the cleft between the great toe and the second it divides into two collateral plantar digital nerves, which supply the contiguous sides of these two toes. The third digital nerve divides near the cleft between the second and third toes into two collateral plantar digital nerves, which supply the contiguous sides of these two toes. The fourth digital nerve also divides near the cleft between the third and fourth toes into two collateral plantar digital nerves, which supply the contiguous sides of these two toes. Before dividing, it communicates by a twig with that digital branch of the superficial division of the external plantar which supplies the con-

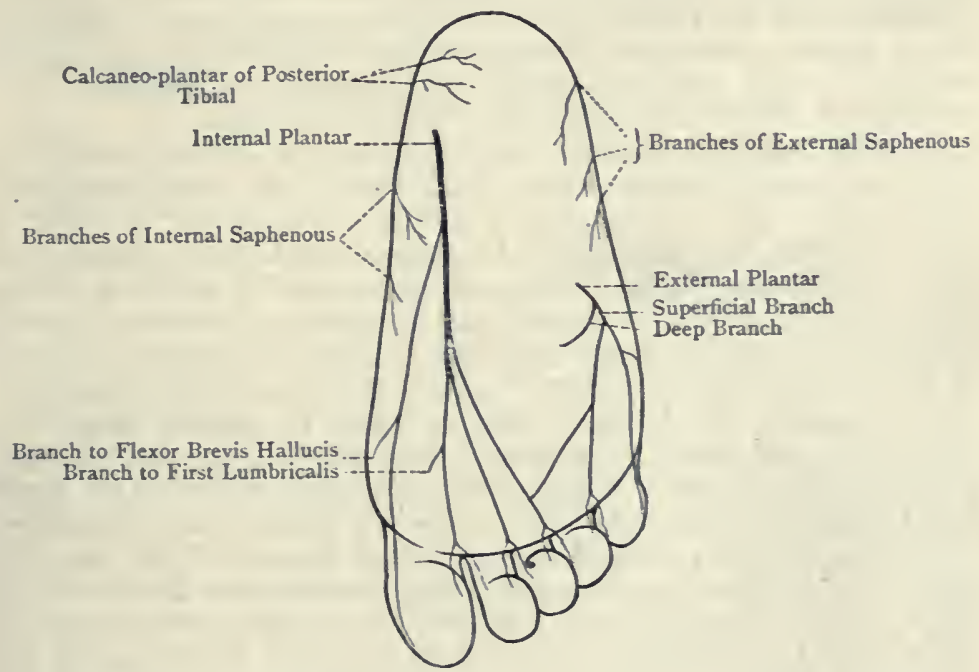

Fig. 263.-Diagram of the Nerves of the Foot (Plantar Aspect).

tizuous sides of the fourth and fifth toes. The nerves on the sides of the toes are placed below the digital arteries. They furnish articular branches to the joints of the toes and cutaneous branches to the plantar and dorsal surfaces of the toes. Finally each terminates in two branches-one to the matrix of the nail, and the other to the pulp of the toe. The branches of the digital nerves are beset with numerous Pacinian bodies.

Summary of the Internal Plantar Nerve.-Muscular branches to abductor hallucis, flexor brevis digitorum, flexor brevis hallucis, and first lumbricalis. Cutaneous branches to the integument of the inner half of the sole and the inner three and a half toes. Articular branches to the astragalo-navicular, naviculo-cuneiform, and digital articulations.

The internal plantar nerve in its digital distribution corresponds closely 
with the median nerve in the hand. There is this difference, however, between these two nerves: the internal plantar has one single digital nerve and three compound digital nerves, whereas the median has three single digital nerves and two which are compound.

External Plantar Nerve.-This nerve has a more limited cutaneous, but a wider muscular, distribution than the internal plantar. From its origin it is directed forwards and outwards to the base of the fifth metatarsal bone, where it breaks up into two divisions, superficial and deep. In this course it lies at first between the flexor brevis digitorum and flexor or musculus accessorius, and subsequently in the groove between the former muscle and the abductor minimi digiti. It is close to the inner side of the external plantar artery, and occupies the concavity of the curve described by that vessel. This part of the nerve is spoken of as the trunk.

Branches of the Trunk.-The branches are as follows: muscular to the flexor or musculus accessorius and abductor minimi digiti ; articular to the calcaneo-cuboid articulation; cutaneous to the integument of the outer half of the sole; and terminal.

The terminal branches are two in number, superficial and deep, and they spring from the trunk at the base of the fifth metatarsal bone.

The superficial division furnishes two digital nerves, outer and inner. The outer digital nerve is single, and is distributed to the outer side of the little toe, supplying branches to the flexor brevis minimi digiti and, as a general rule, to the interosseous muscles of the fourth interosseous space, namely, the fourth dorsal and the third plantar. It may, however, fail to supply these two interosseous muscles, in which case they derive their nerve-supply from the deep division of the external plantar nerve. The inner digital nerve is compound. In its forward course it communicates by a twig with the most external digital branch of the internal plantar nerve which supplies the contiguous sides of the third and fourth toes, and near the cleft between the fourth and fifth toes it divides into two collateral plantar digital nerves, which supply the contiguous sides of these two toes. The digital branches of the superficial division of the external plantar nerve resemble in all respects those of the internal plantar.

The deep division is muscular and articular in its distribution. It sinks deeply into the sole with the external plantar artery as that vessel forms the plantar arch. Its direction is inwards and forwards on the deep or superior surface of the musculus accessorius, long flexor tendons and lumbricales, and adductor obliquus hallucis, and lying upon the bases of the second, third, and fourth metatarsal bones.

Branches.-The branches of the deep division are muscular, articular, and perforating.

The muscular branches supply (I) the interosseous muscles, plantar and dorsal, with the exception, as a general rule, of the two which occupy the fourth interosseous space, namely, the 
fourth dorsal and third plantar-though these two may be included; (2) the outer three lumbricales; (3) the adductor transversus hallucis ; and (4) the adductor obliquus hallucis.

The articular branches supply the tarsal and tarso-metatarsal articulations. They sometimes also supply the metatarso-phalangeal articulations.

The perforating branches pass upwards through the proximal parts of the interosseous spaces, and join the interosseous branches of the dorsalis pedis nerve.

Summary of External Plantar Nerve. - Muscular branches to the flexor or musculus accessorius, abductor minimi digiti, flexor brevis minimi digiti, all seven interossei, outer three lumbricales, adductor transversus hallucis, and adductor obliquus hallucis. Cutaneous branches to the integument of the outer half of the sole and the outer one and a half toes. Articular branches to the tarsal, tarso-metatarsal, and, in some cases, metatarso-phalangeal articulations. Perforating branches to join the interosseous nerves on the. dorsum of the foot.

The external plantar nerve corresponds with the ulnar nerve in the hand.

Plantar Arteries. - The arteries of the sole of the foot are three in number, namely, the internal plantar, the external plantar, and the plantar branch of the arteria dorsalis pedis. The internal and external plantar arteries are the terminal branches into which the posterior tibial divides on a level with the lower border of the internal annular ligament. The internal plantar is much smaller than the external, and each vessel is accompanied by the corresponding plantar nerve.

Internal Plantar Artery. - This vessel passes forwards along the inner side of the sole with the internal plantar nerve, which lies on its outer side, and it usually terminates on the inner aspect of the metatarso-phalangeal joint of the great toe by anastomosing with the digital branch of the arteria magna or princeps hallucis to the inner side of that toe. It is at first under cover of the abductor hallucis, and subsequently lies between that muscle and the flexor brevis digitorum. It is accompanied by two venæ comites.

Branches.-These are as follows :

Muscular to the muscles in its immediate vicinity.

Cutaneous to the integument of the inner half of the sole.

Articular to the articulations along the inner side of the foot.

The internal tarsal branches pass inwards beneath the abductor hallucis to the inner border of the foot, where they anastomose with the internal tarsal branches of the arteria dorsalis pedis.

The superficial digital arteries are three in number, and are usually very small. They accompany the three compound digital branches of the internal plantar nerve to the clefts where these nerves divide into their collateral branches, and there they terminate by joining the inner two digital arteries from the plantar arch and the arteria magna or princeps hallucis. 
External Plantar Artery. - This vessel, which is much larger than the internal plantar, arises from the posterior tibial artery on a level with the lower border of the internal annular ligament,

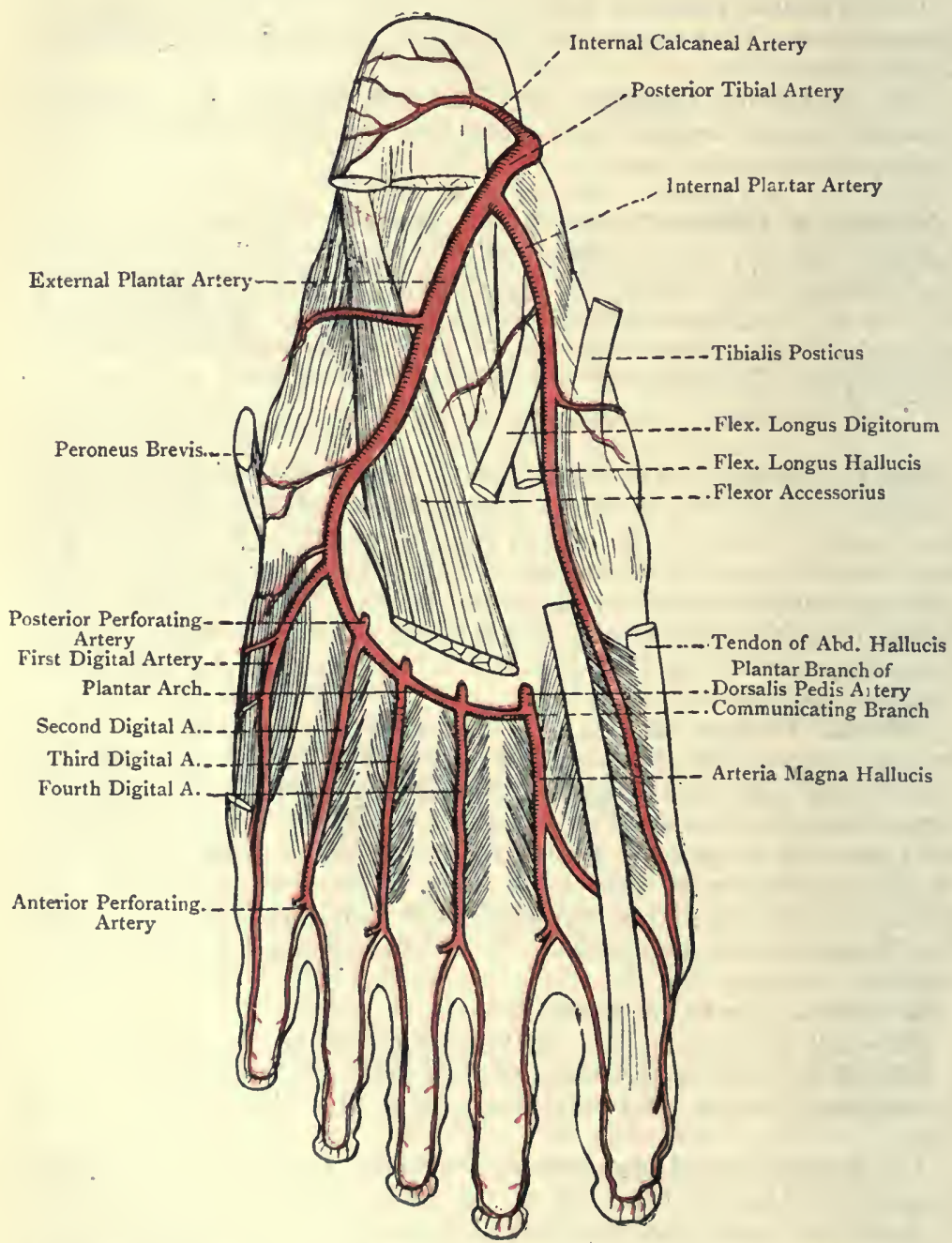

Fig. 264,-The Plantar arteries (Left Foot) (after L. Thstut's 'Anatomie Humaine').

and terminates at the proximal part of the first interosseous space by anastomosing with the communicating branch of the plantar division of the arteria dorsalis pedis. 
The artery is accompanied by the external plantar nerve throughout its whole course, and by two venæ comites. At its origin it is situated on the inner surface of the os calcis, from which point it is directed outwards and forwards across the sole to the base of the fifth metatarsal bone. It here describes a sharp bend, and, sinking deeply, it recrosses the sole on its way to the proximal part of the first interosseous space. The vessel is divided into two parts, first and second. These two parts together describe one great curve, the concavity of which looks inwards and is occupied by the external plantar nerve.

The first part extends from the lower border of the internal annular ligament to the base of the fifth metatarsal bone. Its course may be indicated by drawing a line from a point midway between the tip of the internal malleolus and the inner tubercle on the plantar aspect of the tuber calcis to the base of the fifth metatarsal bone. The direction of this part is outwards and forwards.

Relations. - It is covered in succession by the abductor hallucis, flexor brevis digitorum, and finally only by the skin; superficial fascia, and plantar fascia. It rests upon the os calcis and the musculus accessorius.

It is to be noted that this part of the vessel is very superficial for a short distance close to the base of the fifth metatarsal bone, where it lies between the flexor brevis digitorum and abductor minimi digiti.

The second part extends from the base of the fifth metatarsal bone to the proximal part of the first interosseous space. Its course may be indicated by a line connecting the limits of this part of the vessel. It is directed inwards and forwards in a slightly curved manner, with the convexity of the curve forwards, and thus it forms the plantar arch, which is completed by the communicating branch of the plantar division of the arteria dorsalis pedis. The second part is accompanied by the deep division of the external plantar nerve, and is very deeply placed.

Relations.-It is covered by the skin, superficial fascia, central division of the plantar fascia, flexor brevis digitorum, flexor longus digitorum, lumbricales, and adductor obliquus hallucis. It rests upon the bases of the second, third, and fourth metatarsal bones and the corresponding interosseous muscles.

Branches of the First Part.-The branches are as follows :

Muscular to the muscles in its immediate vicinity.

The internal calcaneal branches are two or three in number. After piercing the origin of the abductor hallucis, they reach the inner surface of the os calcis, where they anastomose with the internal calcaneal branch of the posterior tibial artery. Over the prominence of the heel they also anastomose freely with the external calcaneal branches of the posterior peroneal artery.

Cutaneous to the integument of the outer half of the foot.

Branches also turn round the outer border of the foot, where they anastomose with the tarsal and metatarsal branches of the arteria dorsalis pedis and with the posterior peroneal artery. 
Branches of the Second Part.--The branches of the second part, or plantar arch, are as follows: articular, posterior perforating, and digital.

The articular branches arise from the concavity of the arch, and pass backwards to supply the tarsal articulations.

The posterior perforating arteries, which are three in number, arise from the upper aspect of the arch. They passs upwards through the proximal parts of the three outer interosseous spaces, and between the two heads of the corresponding dorsal interosseous muscles. On reaching the dorsum of the foot they anastomose with the dorsal interosseous branches of the metatarsal artery, which is a branch of the arteria dorsalis pedis.

The digital arteries are four in number-first, second, third, and fourth, from without inwards. They arise from the front or convexity of the arch and pass forwards. The first, which is a single artery, lies over the fifth metatarsal bone and flexor brevis minimi digiti. The second, third, and fourth, which are compound arteries, are placed over the fourth, third, and second interosseous spaces respectively, where they lie upon the corresponding interosseous muscles. The first is distributed to the outer side of the little toe, of which it is the plantar digital artery. The second, third, and fourth pass over the deep surface of the adductor transversus hallucis, and bifurcate near the clefts between the four outer toes, each dividing into two collateral plantar digital arteries. Those of the second supply the contiguous sides of the fourth and fifth toes, those of the third the contiguous sides of the third and fourth toes, and those of the fourth the contiguous sides of the second and third toes. Each of the inner three digital arteries at its point of bifurcation gives off an anterior perforating artery. These anterior perforating arteries pass upwards through the distal ends of the outer three interosseous spaces, and, on reaching the dorsum of the foot, anastomose with the dorsal interosseous arteries. The inner two (third and fourth) digital arteries are joined near the clefts of the toes by the outer two superficial digital branches of the internal plantar artery.

On the sides of the toes the plantar digital arteries furnish branches. to the flexor tendons and their sheaths, and anastomose freely with the dorsal digital arteries. Near the distal end of the first and second phalanx, and on the plantar aspect of each, the plantar digital arteries of opposite sides form arches from which articular twigs are given to the interphalangeal articulations, and on the plantar aspect of the terminal phalanx they end by forming another arch. From this latter arch branches are furnished to the pulp of the toe and matrix of the nail. Each digital artery is accompanied by two vence comites. The external plantar artery corresponds with the deep branch of the ulnar artery in the palm.

It has been seen that the plantar arch, by means of its four digital branches, supplies the outer three and a half tocs. There 
thus remain one and a half to be accounted for, namely, both sides of the great toc and the inner side of the second toe.

Plantar or Perforating Branch of Arteria Dorsalis Pedis.-This artery is one of the terminal branches of the arteria dorsalis pedis. Having entered the sole through the proximal part of the first interosseous space, between the two heads of the first dorsal interosseous muscle, it immediately divides into two branches-communicating and arteria magna or princeps hallucis.

The communicating branch is a short vessel, which ends by joining the second part of the external plantar artery to complete the plantar arch.

The arteria magna or princeps hallucis is the fifth plantar digital artery. Commencing at the proximal end of the first interosseous space it passes forwards over the plantar aspect of that space and the corresponding dorsal interosseous muscle towards the cleft between the great toe and second toe. In this part of its course it furnishes a single plantar digital artery, which, having crossed the first metatarsal bone, beneath the tendon of the flexor longus hallucis, is distributed to the inner side of the great toe. Near the cleft between the great toe and second toe it receives the innermost superficial digital branch of the internal plantar artery, and then divides into two collateral plantar digital arteries for the supply of the contiguous sides of these two toes. Before dividing, it communicates with the arteria dorsalis hallucis by an anterior perforating branch, which passes through the distal end of the first interosseous space.

Varieties-I. Internal Plantar Artery.-This vessel is sometimes very small, and it may then terminate in the flexor brevis hallucis. In other cases it is of fairly large size, and then it may replace the arteria magna- or princeps hallucis, and furnish the plantar digital branches for both sides of the great toe and the inner side of the second toe. In very rare cases the internal plantar artery communicates with the external plantar, and so forms a superficial plantar arch. In such cases the superficial digital arteries arise from this arch.

2. External Plantar Artery.-This artery is liable to be diminished in size, and this may occur to such an extent as to exclude it from any share in the plantar arch. Such deficiencies are compensated for by an enlargement of the arteria dorsalis pedis and its plantar branch.

The posterior perforating branches of the plantar arch are sometimes of comparatively large size, and then they furnish the dorsal interosseous arteries after they reach the dorsum of the foot.

For the tendon of the peroneus longus in the sole see p. 5I6.

\section{Tendons Involved in Club-loot.}

The chief varieties of club-foot are as follows: talipes equinus, talipes varus, talipes equino-varus, talipes valgus, talipes calcaneus, and talipes calcaneo-valgus.

Talipes Equinus.-In this variety the foot is extended upon the leg, the heel being raised from the ground, so that the person walks upon the toes. The chief tendons involved are (I) the tendo Achillis, and (2) the plantaris tendon. The latter, however, is insignificant.

Talipes Varus.-In this variety the inner border of the foot is raised so as to invert the sole, and the foot is at the same time slightly extended upon 
the leg, so that the person walks upon the outer border of the foot. The chief tendons involved are those of the tibialis anticus and tibialis posticus.

Talipes Equino-varus. - This is a combination of talipes equinus and talipes varus, the heel being raised as well as the inner border of the foot. The chief tendons involved are as follows: (I) tibialis posticus, (2) tibialis anticus, (3) tendo Achillis, and (4) plantaris. In addition to these tendons the abductor hallucis and the plantar fascia are usually implicated.

Talipes Valgus. - In this variety the outer border of the foot is raised so as to evert the sole, and the person walks upon the inner border of the foot. The tendons involved are those of the peroneus longus and peroneus brevis.

Talipes Calcaneus.--In this variety the foot is flexed upon the leg, the toes being raised, so that the person walks upon the heel. The tendons involved are as follows: (1) extensor longus digitorum, (2) peroneus tertius, (3) extensor proprius hallucis, and (4) tibialis anticus.

Talipes Calcaneo-valgus.-This is a combination of talipes calcaneus and talipes valgus, the foot being flexed and the outer border of the foot raised. The tendons involved are those which are implicated in talipes calcaneus and talipes valgus.

\section{Summary of the Veins of the Lower Limb.}

The veins of the lower limb are divided into two groups-superficial and deep.

Superficial Veins.-In the sole of the foot there are (1) a plantar subcutaneous plexus, and (2) a transverse venous arch situated near the clefts of the toes, which receives the plantar digital veins. The blood is carried away from the plantar subcutaneous plexus by anterior and lateral efferent vessels. The anterior efferent vessels terminate in the transverse venous arch near the clefts of the toes. The lateral efferent vessels turn round the outer and inner borders of the foot, the external set terminating in the short saphenous vein, and the internal set in the long saphenous vein, where these vessels spring from the dorsal venous arch. The blood is conveyed away from the transverse venous arch by means of efferent veins, called interdigital, which pass upwards to the dorsum of the foot, where they terminate in the dorsal venous arch.

The dorsal venous arch is situated well forward upon the dorsum of the foot, being about 2 inches from the webs of the toes. It receives (I) the dorsal digital veins, (2) small veins from the dorsum of the foot, and (3) the efferent interdigital veins from the plantar transverse venous arch. The blood is carried away from the dorsal venous arch by the long and short saphenous veins. The long saphenous vein arises from the inner end of the arch, and, having received branches from the plantar subcutaneous venous plexus, passes in front of the internal malleolus, and thus reaches the inner side of the leg. Its subsequent course is upwards along the inner side of the leg, knee, and thigh, and finally, having reached the front of the thigh, it passes through the saphenous opening $I \frac{1}{2}$ inches below Poupart's ligament, and terminates in the femoral vein. It receives many tributaries in its course; in the leg it communicates at frequent intervals with the venæ comites of the anterior and posterior tibial arteries by intermuscular branches; and near its termination it is reinforced by the posterior saphenous, anterior saphenous, superficial circumfiex iliac, superficial epigastric, and superior and inferior external pudic veins. The short saphenous veln arises from the outer end of the dorsal venous arch, and, having reccived branches from the plantar subcutaneous venous plexus, it passes below and behind the external mallcolus, and thus reaches the back of the leg. It then passes upwards and inwards, and subsequently straight upwards until it arrives at the interval between the condyles of the femur. Here it passes through an aperture in the fascia lata, and terminates in the popliteal vein. It receives many tributarics from the calcaneal region and the outer and back parts of the leg: it communicates at intervals with the vence comites of the posterior tibial 
and peroneal arteries; and near its termination it communicates with the long saphenous vein.

Deep Veins.-The deep veins accompany the various arteries and their branches. Below the level of the popliteus muscle they are arranged in pairs along the arteries which they accompany, this arrangement being known as venæ comites. Opposite the lower border of the popliteus muscle the posterior tibial venæ comites, having previously received the peroneal venæ comites, unite with the anterior tibial venæ comites, and so the popliteal vein is formed, which is continued into the fermoral vein.

\section{Lymphatic Vessels of the Lower Limb.}

The lymphatic vessels of the lower limb are arranged in two groups - superficial and deep.

Superflcial Lymphatics.- These form two sets, which are conveniently designated as internal saphenous and external saphenous from the veins which they accompany.

1. Internal Saphenous Lymphatics. - On the plantar aspect of each toe there is a digital lymphatic plexus, from which four lymphatic vessels emerge, these being arranged in pairs on either side of the toe. They pass to a plexus on the dorsal aspect of the foot, and this plexus is reinforced by lymphatics from the plantar region which reach it at the clefts between the toes. From the dorsal plexus two sets of vessels issue -internal and external. The intsrnal vessels receive their lymph from about the inner onethird of the dorsal plexus and from the inner two toes. They also receive the internal plantar and internal calcaneal lymphatic vessels, and thereafter they accompany the internal saphenous vein and finally empty into the superficial femoral glands. The externa! vessels of the dorsal plexus receive their lymph from about the outer twothirds of the plexus and from the outer three toes. They also receive some lymphatic vessels from the external plantar region. Thereafter they pass in front of the external malleolus, and, as they ascend in front of the leg, they soon incline inwards and join the internal vessels of the dorsal plexus, which by this time form the internal saphenous lymphatics.

2. External Saphenous Lymphatics.-These ves. sels come from the outer border of the foot, over about its posterior half, and from the outer side of the heel. They accompany the external saphenous vein, and, as they ascend, they take up lymphatics from the superficial structures of the back of the leg. Finally they pierce the deep fascia over the popliteal space, and terminate in the popliteal glands, very often in that gland which lies close to the termination of the external saphenous vein.

The superficial femoral lymphatics from (I) the lower and outer parts, (2) the front, (3) the lower and inner parts, and (4) the posterior parts of the thigh pass to the superficial femoral glands. The

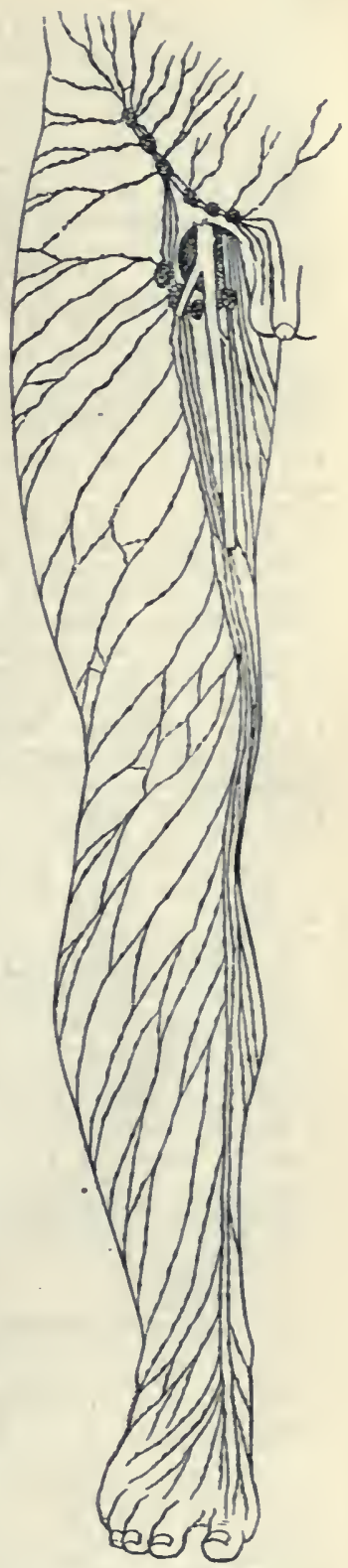

FIG. 265.-SUPERFICIAL LYMPHATICS OF THE LOWER I,IMB. 
lymphatic vessels from the knee-joint pass, for the most part, to the popliteal glands, but some ascend to the superficial femoral glands.

The superficial lymphatics from the outer and back parts of the thigh supeviorly pass to the inguinal glands, and those from the upper and inner parts of the thigh terminate in the pubic glands.

The superficial gluteal lymphatics from the outer two-thirds of the gluteal region pass to the inguinal glands; and those from the inner one-third terminate partly in the pubic glands and partly in the superficial femoral glands.

Deep Lymphaties of Lower Limb. -The deep lymphatic vessels accompany the main arteries. They form groups as follows: ( 1 ) Dorsalis pedis and anterior tibial; (2) plantar and posterior tibial; (3) peroneal; (4) femoral; (5) sciatic; (6) gluteal; and (7) obturator.

The dorsalis pedis and anterior tibial lymphatics arise in the deep structures of the sole of the foot, and they reach the dorsum of the foot along the plantar or perforating branch of the dorsalis pedis artery. They then accompany the dorsalis pedis and anterior tibial arteries, taking up the deep lymphatics of the dorsum of the foot and front of the leg. Thereafter they terminate in the anterior tibial gland, the efferent vessels of which pass to the popliteal glands.

The plantar and posterior tibial lymphatics arise, like the preceding group, in the deep structures of the sole of the foot, and they accompany the two plantar arteries, especially the external-plantar artery.. Thereafter they pass along the posterior tibial artery and terminate in the popliteal glands.

The peroneal lymphatics arise in (1) the back part of the outer border of the foot, (2) the outer surface of the heel, and (3) the front of the external malleolus. They accompany the peroneal artery, taking up lymphatics from the deep structures on the back of the fibula, and in the upper part of the leg they terminate in the posterior tibial lymphatics.

The deep femoral lymphatics represent (I) the efferent vessels of the popliteal glands, which accompany the superficial femoral artery, and (2) the lymphatic vessels which accompany the arteria profunda femoris and its branches. They take up lymphatics from the deep structures on the inner and front aspects of the thigh, and they terminate in the deep femoral glands.

The sciatic lymphatics return lymph from the structures supplied by the sciatic artery, which they accompany. Their destination is the internal iliac glands within the pelvis.

The deep gluteal lymphatics arise in the gluteal muscles and other deep structures of the gluteal region. They accompany the gluteal artery, and, within the pelvis, they terminate in the internal iliac glands.

The obturator lymphatics arise in the obturator membrane, obturator externus muscle, and upper portions of the adductor muscles. They accompany the obturator artery as that vessel passes through the obturator canal, and they terminate in the obturator gland, which, when present, lies in the obturator canal. If this gland is absent, they pass to the middle gland of the internal set of the external iliac glands.

Summary of the Lymphatic Glands.-The lymphatic glands of the lower limb form the following groups:

I. Inguinal.

2. Pubic.

3. Superficial Femoral, or Saphenous.
4. Deep Femoral.

5. Popliteal.

6. Anterior Tibial.

\section{Development of Arteries of Lower Limbs.}

The primary arterial stem of each lower limb is formed by the following arteries, in order from above downwards: (1) The sciatic artery, which accompanies the great sciatic nerve; (2) the popliteal artery; and (3) the peroneal artery. A little below the knce-joint tlie popliteal artery gives off the anterior tibial artery, which is reinforced inferiorly by a branch of the peroneal artery.

The arterial stem, as in the upper limb, is segmental in origin. The common lilac arteries represent a fifth pair of abdominal segmental arteries, and cach 
sciatic artery is developed from the hypogastric portion (subsequently internal iliac portion) of a common iliac artery.

The external iliac and femoral arteries are formed at a later period, the external iliac being a secondary outgrowth from the common iliac at a higher level than the origin of the sciatic artery. The external iliac artery is continued into the femoral, and the femoral artery joins the popliteal artery at the place where the sciatic artery is continued into that vessel. The sciatic artery now undergoes retrogression, and the part of it between the popliteal artery and the gluteal region for the most part disappears. The upper part of the primitive vessel, however, persists, and forms the sciatic artery (arteria glutea inferior) of adult life.

Before the femoral artery joins the popliteal it gives off a fairly large branch, which accompanies the internal saphenous vein, and is called the internal saphenous artery. This vessel for the most part atrophies, but its upper portion persists and represents the superficial branch of the arteria anastomotica magna. The posterior tibial artery is developed from the upper part of the peroneal artery.

\section{Development of Veins of Lower Limbs.}

The veins of the lower limb form two groups-superficial and deep. The superficial veins are developed prior to the deep, the latter accompanying the arteries.

The primitive vein of each lower limb is the primary fibular, or post-axial, vein, which opens proximally into the posterior cardinal vein. From the primary fibular vein a temporary anterior tibial vein is developed. The long saphenous vein grows distally from the posterior cardinal vein. A temporary posterior tibial vein is developed from the long saphenous vein.

The primary fibular vein, which is originally continuous with the sciatic vein, loses its connection with that vessel, and persists as the short saphenous vein.

\section{THE ANKLE-JOINT.}

The ankle-joint belongs to the class diarthrosis, and to the subdivision ginglymus. The articular surfaces are the lower extremity of the shaft and the outer surface of the internal malleolus of the tibia, the inner surface of the external malleolus of the fibula, and the superior and both lateral surfaces of the astragalus. The ligaments are anterior, posterior, internal lateral, and external lateral.

The anterior ligament is a thin membrane which covers the joint in front. Superiorly it is attached from within outwards to the anterior border of the internal malleolus, anterior surface of the lower end of the tibia two or three lines above the anterior border, anterior inferior tibio-fibular ligament, and anterior border of the external malleolus. Inferiorly it is attached to a groove on the upper aspect of the head of the astragalus, immediately behind the cartilaginous surface and in front of the neck. The fibres of the ligament are chiefly disposed in a transverse direction.

In addition to the tendons which lie upon it, this ligament is related to the anterior tibial vessels and nerve. Its deep surface is covered by the synovial membrane of the joint, and inferiorly is in contact with a collection of fat which lies in the hollow on the upper surface of the neck of the astragalus. 
The posterior ligament is weaker and less defined than the anterior. Superiorly it is attached from without inwards to the posterior aspect of the external malleolus internal to the peroneal groove, posterior inferior tibio-fibular ligament, and posterior border of the tibia as far inwards as the groove behind the internal malleolus. Inferiorly it is attached to the upper aspect of the posterior border of the astragalus immediately behind the superior articular surface, where it extends between the posterior fasciculus of the external lateral ligament and the internal lateral ligament. Its fibres are disposed obliquely, and radiate in an inward direction from the external malleolus.

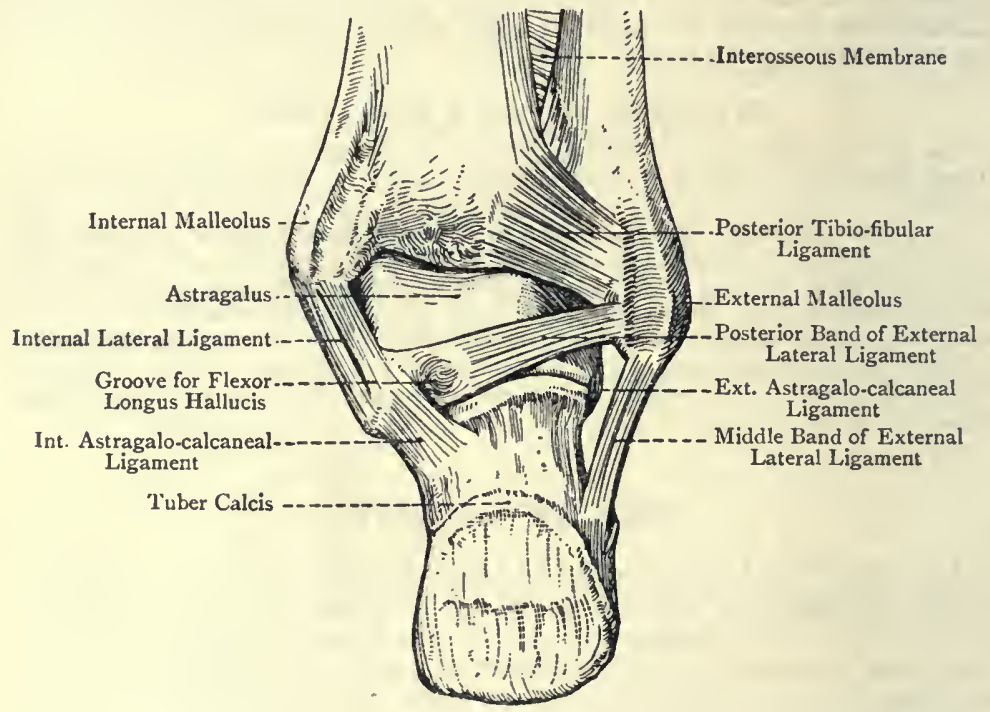

Fig. 266.-The Right Ankle-Joint (Posterior View).

(The Posterior Ligament has been removed.)

The internal lateral ligament is also known as the deltoid ligament. It is a strong, flat, triangular structure, which is attached superiorly to the lower border, tip, and anterior border of the internal malleolus, in which latter situation it is superficial to the fibres of the anterior ligament. A strong bundle of fibres springs from the notch which indents the lower border of the internal malleolus. From the superior attachment the fibres diverge in a radiating manner. The posterior fibres, strong and short, descend with an inclination backwards to be attached to the rough, depressed inner surface of the astragalus below the internal facet, where they extend as far back as the inner tubercle on the posterior border of the bone. The anterior part of the ligament, somewhat thinner and more radiating than the posterior, is attached to 
the inner border of the sustentaculum tali of the os calcis, inner border of the internal calcaneo-navicular or spring ligament, and inner part of the dorsal surface of the navicular bone.

The external lateral ligament is composed of three distinct fasciculi-anterior, middle, and posterior.

The anterior fasciculus (astragalo-fibular) is flat, and extends from the lower part of the anterior border of the external malleolus to the outer surface of the astragalus immediately in front of the external facet. Its direction is forwards and inwards, and it is the shortest of the three fasciculi.

The middle fasciculus (calcaneo-fibular), which is round, is attached superiorly to the tip of the external malleolus, and inferiorly to a tubercle on the outer surface of the os calcis situated about the centre, behind and above the peroneal spine. Its direction is down-

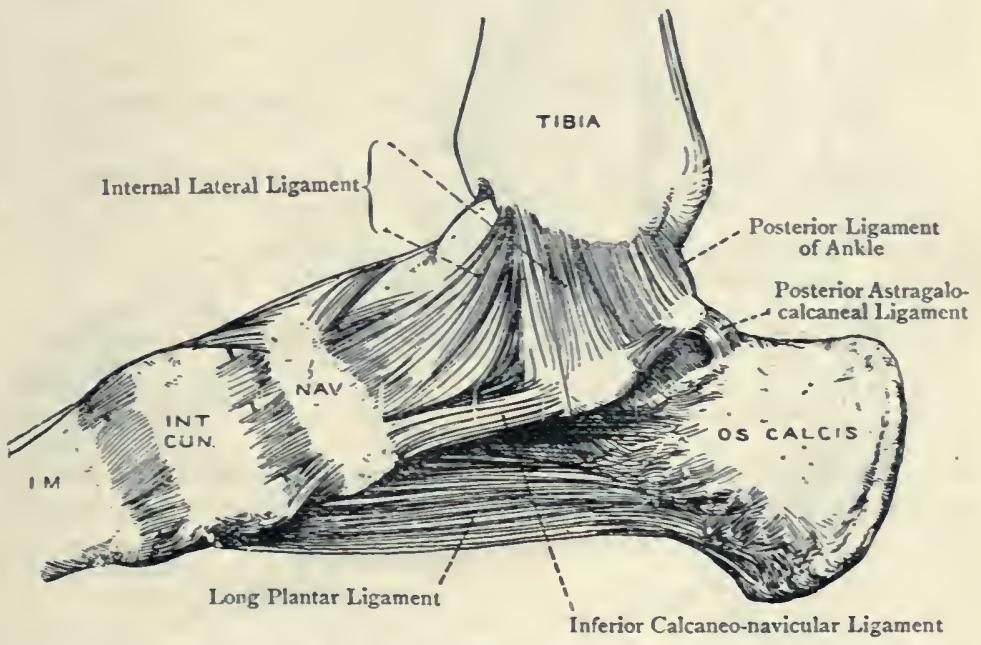

Fig. 267.-Ligaments of the Right Foot (Internal View).

wards and backwards, and it is related to the tendons of the peroneus longus and peroneus brevis.

The posterior fasciculus (astragalo-fibular) is the strongest bundle of the three. It is attached by one extremity to the lower part of the digital fossa on the inner surface of the external malleolus behind the articular facet. The other extremity is attached to the upper surface of the outer tubercle on the posterior border of the astragalus. Its direction is inwards and slightly backwards.

The synovial membrane lines the inner surfaces of the ligaments in a loose manner, and it covers collections of fat (Haversian glands) at the front and back of the joint, where it forms folds 
which project between the astragalus and the tibia. It is also prolonged into the inferior tibio-fibular articulation so as to line the anterior and posterior ligaments of that joint.

Tendinous Relations of the Ankle-Joint-Anterior.-From within outwards these are the tibialis anticus, extensor proprius hallucis, extensor longus digitorum, and peroneus tertius. External.Peroneus longus and peroneus brevis. Posterior.-From without inwards these are the peroneus longus and peroneus brevis, tendo Achillis and plantaris, with the intervention of a large amount of fat, flexor longus hallucis, and flexor longus digitorum and tibialis posticus. Internal.-Tibialis posticus.

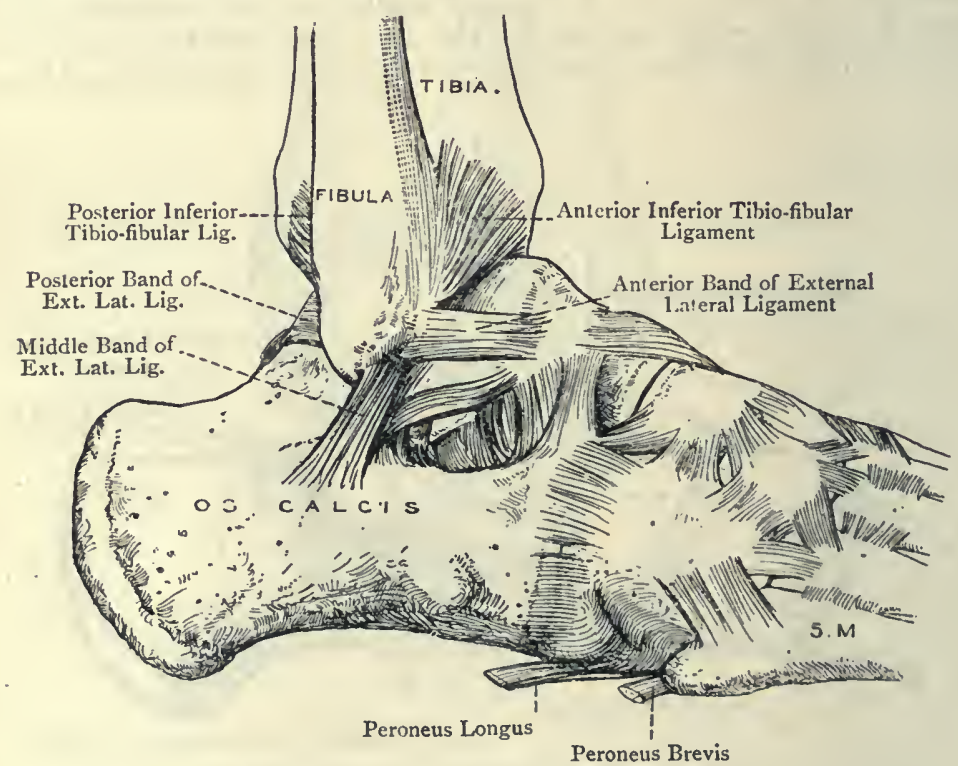

Fig. 268.-Ligaments of the Right Inferior Tibio-fibular, ANkle, TARSAL, and Tä̈SO-Metatarsal Joints (External View).

Arterial Supply.-The anterior tibial, external and internal malleolar, anterior peroneal, posterior tibial, and posterior peroneal arteries.

Nerve-supply. - The posterior tibial, short saphenous, and external division of the dorsalis pedis nerve, or the anterior tibial nerve itself.

Movements.-The chief movements at the ankle-joint are flexion and extension. When, however, the foot is extended a certain amount of lateral movement is allowed. The foot is said to be flexd when it is raised from the ground towards the front of the leg, as in standing upon the heels, and it is said to be extended when the heel is raised towards the back of the leg. 
as in standing upon the toes. In flexion of the foot the broad anterior part of the superior articular surface of the astragalus is carried backwards in to the narrow posterior part of the tibial socket, and lateral movement is then impossible. Flexion is limited by (I) the tension of the posterior and middle portions of the internal lateral ligament; $(2)$ the tension of the posterior and middle fasciculi of the external lateral ligament; and (3) the locking which takes place between the upper surface of the neck of the astragalus and the anterior border of the lower end of the tibia. In extension of the foot the narrow posterior part of the superior articular surface of the astragalus is carried forwards into contact with the broad anterior part of the tibial socket, and a certain amount of lateral movement can now take place. Extension is limited by (I) the tension of the anterior part of the internal lateral ligament; (2) the tension of the anterior and middle fasciculi of the external lateral ligament; (3) the tension of the anterior ligament, particularly of its inner part; and (4) the locking of the posterior part of the astragalus against the posteror border of the lower end of the tibia. The range of movement in the direction of flexion and extension is about 90 degrees, and it takes place round a transverse axis passing through the body of the astragalus in a direction forwards and outwards. At the end of extension there is a tendency to abduction or turning in of the foot, due to the following factors: (I) the greater length posteriorly of the inner border of the superior articular surface of the astragalus; (2) the greater depression of the corresponding part of the outer border of the astragalus; and (3) adduction at the astragalo-calcaneal joint.

The vertical line of the centre of gravity falls in front of the axis of movement at the ankle-joint. There is thus a tendency to over-flexion, which, however, is counteracted by a certain amount of muscular effort on the part of the sural muscles.

Muscles concerned in the Movements-Flexion.-This is produced by the tibialis anticus, peroneus tertius, extensor longus digitorum, and extensor proprius hallucis.

Extension.-This is produced by the gastrocnemius and soleus by means of the tendo Achillis, plantaris, tibialis posticus, flexor longus digitorum, flexor longus hallucis, peroneus longus, and peroneus brevis.

Abduction.-The foot is everted by the peroneus longus and peroneus brevis.

Adduction.-The foot is inverted by the tibialis anticus and tibialis posticus.

Bursæ and Tendon-Sheaths at Ankle-Joint.-The synovial bursæ in the vicinity of the ankle-joint are as follows:

\section{Internal malleolar. \\ External malleolar.}

\section{Tendo Achillis.}

Sinus tarsi.

The malleolar bursæ are not constant. There may be two mesial over the internal malleolus of the tibia, and lateral over the external malleolus of the fibula.

The bursa of the tendo Achillis, which is constant, is situated between the lower part of this tendon and the upper smooth horizontal zone on the posterior surface of the tuber calcis. It lies directly above the insertion of the tendon into the middle rough horizontal zone.

The bursa sinus tarsi is situated within the sinus tarsi, between the inferior surface of the astragalus and the superior surface of the os calcis. It lies within the strong interosseous ligament which binds these two bones together, and it partially separates the anterior and posterior laminæ of which the interosseous ligament is composed.

None of the foregoing bursæ communicate with the synovial cavity of the ankle-joint. 
The synovial or tendon-sheaths around the ankle-joint are very numerous and of great importance. In all there are nine sheaths, three of which are in front of the ankle-joint, three behind the internal malleolus between it and the tuber calcis, one behind the external malleolus, and two on the outer surface of the os calcis.

The anterior tendon-sheaths, from within outwards, are as follows:

Sheath of tendon of tibialis anticus.

Sheath of tendon of extensor longus hallucis.

Sheath of tendons of extensor longus digitorum and peroneus tertius.

These sheaths, which have no communication with the anklejoint, frequently become the seat of teno-vaginitis or teno-synovitis.

The sheath of the tendon of the tibialis anticus like the tendon which it invests, passes through a special compartment in the superior division of the anterior annular ligament, which lies just above the ankle-joint, through a special compartment in the upper band of the inferior division of that ligament, and beneath its lower band, the inferior division of the ligament being placed just in front of the ankle-joint. It is the only synovial sheath within the superior division, and it ceases beneath the lower band of the inferior division, on a level with the scaphoid or navicular bone.

The sheath of the tendon of the extensor longus hallucis only invests that tendon as it passes through a special compartment in the upper band of the inferior division of the anterior annular ligament. It is not present beneath the superior division of the ligament. It commences just above the upper band of the inferior division of the ligament, and it terminates about the level of the joint between the internal cuneiform and first metatarsal bone.

The common sheath of the tendons of the extensor longus digitorum and peroneus tertius only invests these tendons as they pass through a special compartment in the outer single portion (fundiform ligament of Retzius) of the inferior division of the anterior annular ligament. It is not present beneath the superior division of the ligament. It commences a little above the fundiform ligament of Retzius, and it terminates about the level of the front part of the cuboid bone.

The internal malleolar tendon-sheaths are situated between the posterior part of the internal malleolus and the tuber calcis. They are three in number, and are quite independent of one another. The tendons to which they belong are (I) the tendon of the tibialis posticus, (2) the tendon of the flexor longus digitorum, and (3) the tendon of the flexor longus hallucis. All three sheaths lie beneath the internal annular ligament, which extends between the posterior border of the internal malleolus and the internal border of the tuber calcis. They commence about 2 inches above that ligament, and extend forwards beyond it for about 2 inches in advance of the internal malleolus. These three sheaths are frequently the seat of teno-synovitis. 
The external malleolar tendon-sheath is situated behind the external malleolus. It is a common sheath for the tendons of the peroneus longus and peroneus brevis, as they lie one upon the other in the peroneal groove. It is situated beneath the external annular ligament, which extends between the posterior border of the external malleolus and the outer border of the tuber calcis, and it commences about 2 inches above that ligament. After escaping from beneath the ligament the single sheath now forms two independent sheaths, one for the peroneus longus tendon, and the other for the peroneus brevis tendon, as these two tendons pass forwards over the outer surface of the os calcis. The two sheaths terminate about I inch behind the level of the tuberosity on the outer side of the base of the fifth metatarsal bone.

Teno-synovitis is not uncommon in the sheath behind the external malleolus.

All the synovial bursæ and tendon-sheaths in the vicinity of the ankle-joint are quite distinct from the synovial cavity of that joint.

\section{THE TIBIO-FIBULAR JOINTS.}

The superior and inferior extremities of the tibia and fibula form direct articulations, and the shafts are connected by means of an interosscous membrane and inferior interosseous ligament.

Superior Tibio-fibular Joint.-This joint belongs to the class diarthrosis, and to the subdivision arthrodia. The articular surfaces are the facets on the head of the fibula and on the external tuberosity of the tibia, and the ligaments are two in number, namely, anterior and posterior. Their fibres pass downwards and outwards from the outer tuberosity of the tibia to the head of the fibula, and they completely cover the joint in front and behind. Superiorly and inferiorly they meet, and thus construct a capsule for the joint. The anterior division of the tendon of the biceps femoris is closely related to the anterior ligament, and contributes materially to the strength of the joint, the more so because that division has an insertion into the outer tuberosity of the tibia as well as into the upper surface of the head of the fibula.

The synovial membrane is usually distinct from that of the kneejoint. Occasionally, however, it is in communication with it posteriorly by means of the synovial investment which surrounds the tendon of the popliteus.

Arterial Supply.- The arterial supply is derived from the inferior external articular, posterior tibial recurrent (inconstant), and anterior tibial recurrent arteries.

Nerve-supply.-The inferior external articular and recurrent articular, both branches of the external popliteal, and the nerve to the popliteus muscle, which is a branch of the internal popliteal.

Movements.-The movements are extremely limited, and are of a gliding or to-and-fro nature in an upward and downward direction. The knee being 
almost fully extended, if the fingers are placed over the head of the fibula whilst the foot is alternately flexed and extended, the head of the bone will be felt to glide upwards during flexion and downwards during extension of the foot.

Inferior Tibio-fibular Joint.-This joint belongs to the class diarthrosis, and to the subdivision arthrodia. The articular surfaces are the upper part of the facet on the inner surface of the external malleolus of the fibula and the sigmoid cavity on the outer aspect of the lower end of the tibia. The ligaments are anterior, posterior, inferior interosseous, and transverse.

The anterior ligament is a strong, flat band, the fibres of which pass obliquely downwards and outwards from the tibia to the fibula. It is related anteriorly to the peroneus tertius, and posteriorly is in contact with the inferior interosseous ligament.

The posterior ligament, which is disposed like the anterior, is in contact with the transverse ligament inferiorly, and with the inferior interosseous ligament anteriorly.

The inferior interosseous ligament is an important ligament, s and is of considerable strength. It consists of short fibres which pass directly between the opposed rough triangular surfaces at the lower ends of the shafts of the tibia and fibula. It is continuous above with the interosseous membrane, and its extent is about $\mathrm{I} \frac{1}{2}$ inches. Anteriorly and posteriorly it is in part related to the anterior and posterior inferior tibio-fibular ligaments. The part of the inferior tibio-fibular joint occupied by the inferior. interosseous ligament belongs to the class amphiarthrosis, and to the subdivision syndesmosis.

The transverse ligament, which is strong, narrow, and somewhat round, extends almost horizontally from the posterior border of the lower end of the tibia to the upper part of the digital fossa of the external malleolus. Externally it lies along the lower border of the posterior inferior tibio-fibular ligament, by which it is slightly overlapped. It fills up a slight hollow between the tibia and fibula, and it plays across the back part of the upper articular surface of the astragalus, where it usually gives rise to a transverse groove. The transverse ligament completes the back part of the tibial socket for the upper surface of the astragalus.

The synovial membrane is continuous with that of the anklejoint.

Arterial Supply.-The posterior and anterior peroneal arteries, and the external malleolar of the anterior tibial.

Nerve-supply. - The interosseous branch of the nerve to the popliteus muscle, and the external division of the dorsalis pedis nerve.

Movements.-These are very limited, and are of a gliding nature, chiefly upwards and downwards, the fibula moving on the tibia. Though the bones are firmly bound together by the inferior interosseous ligament, there is yet a certain amount of lateral separation allowed during flexion of the foot.

Intermediate Connection between the Tibia and Fibula. - The union between the shafts of the tibia and fibula is effecteu by 
means of an interosseous membrane. This kind of union is a form of syndesmosis (union by an interosseous ligament). The interosseous membrane extends from the external border or interosseous ridge of the tibia to the antero-internal border or interosseous ridge of the fibula. The chief direction of the fibres is downwards and outwards from the tibia to the fibula, but a few pass in the opposite direction. Superiorly it terminates about I inch below the superior tibio-fibular joint in a sharp concave margin, the concavity of which is directed upwards. An interval is thus left above the membrane for the passage of the anterior tibial vessels and the efferent lymphatics of the anterior tibial gland. Sometimes these structures pass through a distinct aperture in the membrane, called the superior hiatus. Inferiorly it becomes continuous with the inferior interosseous ligament, and in this neighbourhood it presents a small opening or inferior hiatus for the passage of the anterior peroneal vessels. The interosseous membrane serves as a surface of origin to muscles.

Relations-Anterior.-Tibialis anticus, over the upper two-thirds of the inner half; extensor longus digitorum, over the upper fourth of the outer half; extensor proprius hallucis, over the middle twofourths of the outer half; peroneus tertius, over the lower fourth of the outer half; anterior tibial vessels, over the upper two-thirds in the middle line; and anterior tibial nerve, over the middle third. Posterior.-Tibialis posticus:

Arterial Supply.-The anterior tibial and peroneal arteries.

Nerve-supply. - The interosseous branch of the nerve to the popliteus muscle, which descends within it to terminate in the inferior tibio-fibular joint.

\section{THE ARCHES OF THE FOOT.}

The foot presents two arches-antero-posterior or longitudinal and transverse (see Figs. I65, I66).

The longitudinal arch has two piers-posterior or calcaneal and anterior or metatarsal. The calcaneal pier is formed by the internal and external tubercles on the plantar aspect of the tuber calcis, and the metatarsal pier is formed by the heads of the metatarsal bones.

The longitudinal arch consists of two pillars-internal and external.

The internal pillar is constructed by the os calcis, astragalus, navicular, three cuneiforms, and inner three metatarsal bones. Its calcaneal pier is formed by the internal and external tubercles on the plantar aspect of the tuber calcis, and its metatarsal pier is formed by the heads of the inner three metatarsal bones. The summit of the internal pillar corresponds to the upper articular surface of the astragalus, and through the medium of that bone the greater part of the superincumbent weight is transmitted to the internal pillar. This pillar is much arched, and is characterized by great strength and elasticity. The part of it most sub- 
jected to pressure is the astragalo-navicular joint. This joint, however, is powerfully braced inferiorly by (I) the elastic inferior calcaneo-navicular or spring ligament, which blends internally with the anterior part of the internal lateral, or deltoid, ligament of the ankle-joint; and (2) the strong tendon of the tibialis posticus muscle, which lies on the inferior or plantar aspect of the spring ligament, and acts as a strong auxiliary to it.

The external pillar of the longitudinal arch is constructed by the os calcis, cuboid, and outer two metatarsal bones. Its calcaneal pier is formed by the internal and external tubercles on the plantar aspect of the tuber calcis, and its metatarsal pier is formed by the heads of the outer two metatarsal bones. The summit of the external pillar corresponds to the highest part of the postero-external facet on the superior surface of the os calcis. Through the medium of the os calcis the external pillar receives from the astragalus a small part of the superincumbent weight. This pillar is only slightly arched, and is characterized by its stability. The part of it most subjected to pressure is the calcaneo-cuboid joint. This joint, however, is powerfully braced inferiorly by the inferior calcaneocuboid ligaments-namely, the long and short plantar ligaments.

The two pillars of the longitudinal arch receive material strength from the plantar fascia.

The transverse arch of the foot is most conspicuous at the tarsometatarsal joints. In this situation it is due to (I) the broad aspects of the middle and external cuneiform bones being dorsally placed, and (2) the broad aspects of the wedge-shaped bases of the second, third, and fourth metatarsal bones being also dorsally placed. The arch is braced inferiorly by plantar and interosseous ligaments, and by the tendon of the peroneus longus muscle.

The arches of the foot serve the following purposes: (I) They impart strength and elasticity to the foot; (2) they protect the important structures which occupy the sole or plantar region; and (3) they allow of the heel-to-toe movement in walking.

\section{THE ARTICULATIONS OF THE FOOT.}

The articulations of the foot are divided into tarsal, tarso-metatarsal, intermetatarsal, metatarso-phalangeal, and interphalangeal.

\section{The Tarsal Articulations.}

I. Astragalo-calcaneal Joints.--The astragalus is connected with the os calcis by two synovial joints, anterior and posterior, both of which belong to the class diarthrosis, and to the subdivision arthrodia.

Posteriar Astragalo-calcaneal. Joint. - The ligaments are interosseous, posterior, internal, and external.

The interosseous ligament passes between the oblique grooves separating the two articular surfaces of each bone, and forming 
by their apposition the tunnel called the simus tarsi. It is very strong, and is to be regarded as the anterior ligament of the posterior astragalo-calcaneal joint.

The posterior ligament extends from the posterior aspect of the outer tubercle on the posterior border of the astragalus to the adjacent upper and inner surfaces of the os calcis. It is thin and membranous, and its fibres are arranged in a radiating manner.

The internal ligament is a narrow band which passes from the inner tubercle on the posterior border of the astragalus to the back of the sustentaculum tali of the os calcis. It is related to the tendon of the flexor longus hallucis.

The external ligament extends from the lower part of the outer surface of the astragalus, below the facet, to be attached to the adjacent part of the outer surface of the os calcis. It is under cover of, and parallel with, the middle fasciculus of the external lateral ligament, which, along witl the internal lateral ligament of the ankle-joint, contributes to the strength of this articulation.

The synovial membrane of this joint is peculiar to it.

Arterial Supply.- The arterial supply is derived from the posterior tibial, external malleolar of the anterior tibial, posterior peroneal, and external tarsal of the dorsalis pedis artery.

Nerve-supply.--The short saphenous and the posterior tibial, or it may be the external plantar, nerves.

Anterior Astragalo-calcaneal Joint.-This joint has a lateral ligament at either side, and a posterior, but it is continuous anteriorly with the astragalo-navicular joint. The ligaments are interosseous or posterior, internal astragalo-calcaneal, and external or superior calcaneo-navicular.

The interosseous or posterior ligament has already been described in connection with the posterior astragalo-calcaneal joint.

The internal astragalo-calcaneal ligament extends from the inner surface of the neck of the astragalus to the upper margin of the inner border of the sustentaculum tali of the os calcis. It blends posteriorly with the inner end of the interosseous ligament, and anteriorly with the upper border of the internal calcaneo-navicular or spring ligament. It is strengthened by the internal lateral ligament of the ankle-joint. The internal or inferior calcaneo-navicular or spring ligament is also an internal ligament of this joint, but, inasmuch as it ranks as a ligament of the astragalo-navicular joint, it will be described in connection with that articulation.

The external or superior calcaneo-navicular ligament is placed on the outer side of the joint. It also ranks as an external lateral ligament of the astragalo-navicular joint. It is strong, and extends from the anterior part of the upper surface of the os calcis, external to the anterior facet, to a depression on the outer surface of the navicular bone near its posterior margin. Inferiorly it blends with the internal calcaneo-navicular ligament, and superiorly with the superior astragalo-navicular ligament. 
The synovial membrane, though distinct from that of the posterior astragalo-calcaneal joint, is continuous in front with that of the astragalo-navicular articulation.

The arterial supply and nerve-supply are the same as for the astragalo-navicular articulation.

Movements between the Astragalus and Os Calcis.-The movements at the astragalo-calcaneal joints are abduction, adduction, and rotation. In abduction the foot and toes are turned outwards, and in adduction they are turned inwards, these movements being associated with a certain amount of rotation round an axis passing from the inner side of the neck of the astragalus downwards, backwards, and outwards to the lower and outer part of the tuber calcis. In adduction or inversion the posterior facet of the os calcis moves forwards and downwards upon the astragalus, and the front part of the os calcis is carried slightly inwards. During the movements the navicular bone rotates on the head of the astragalus, and the cuboid bone moves along with the os calcis.

II. Astragalo-navicular Joint. - This belongs to the class diarthrosis, and to the subdivision enarthrosis. It is in direct continuity behind with the anterior astragalo-calcaneal joint, with which it shares its synovial membrane, and the two articulations are sometimes described as one composite joint under the name of the astragalo-calcaneo-navicular joint. The ligaments are astragalonavicular, external or superior calcaneo-navicular, and internal or inferior calcaneo-navicular, or spring, ligament.

The astragalo-navicular ligament is a thin membrane which covers the joint on its dorsal aspect. It is attached posteriorly to the upper margin of the head of the astragalus close behind the cartilage, and also to its outer and inner surfaces. Anteriorly it is attached to the dorsal surface of the navicular bone. Its fibres are arranged in a radiating manner, and converge towards the navicular bone. At the attachment to the astragalus they frequently form three bands, outer, dorsal, and inner, which, however, are continuous with one another.

The external or superior calcaneo-navicular ligament has been described in connection with the anterior astragalo-calcaneal joint, of which it ranks as the external ligament.

The internal or inferior calcaneo-navicular ligament is one of the most important ligaments of the foot, and is known as the spring ligament. It is a broad, thick, strong band of the consistence of fibro-cartilage, and is composed of fibrous and elastic tissues. Posteriorly it is attached to the front of the sustentaculum tali and the adjacent portion of the plantar surface of the os calcis. Anteriorly it is attached to (I) the plantar surface of the navicular bone, (2) the back of its tuberosity, and (3) the inner part of its dorsal surface. It is directed forwards and inwards, and covers the joint on its inner and lower aspects. Internally it blends with the anterior part of the internal lateral ligament of the ankle-joint and the astragalo-navicular ligament, and externally it blends with the external calcaneo-navicular ligament. The superior or deep surface of the ligament is in contact with a special 
facet on the internal aspect of the inferior surface of the head of the astragalus. This surface of the ligament is covered by synovial membrane, and forms part of the articular socket for the head of the astragalus. It has a smooth, polished appearance, and presents no indication of its fibrous structure. The inferior or superficial surface, on the other hand, has the ordinary fibrous appearance of a ligament, and is in close contact with the tendon of the tibialis posticus, which is a powerful auxiliary to the ligament in supporting the antero-posterior arch of the foot. The spring ligament sometimes contains a sesamoid fibro-cartilage, which occasionally becomes ossified.

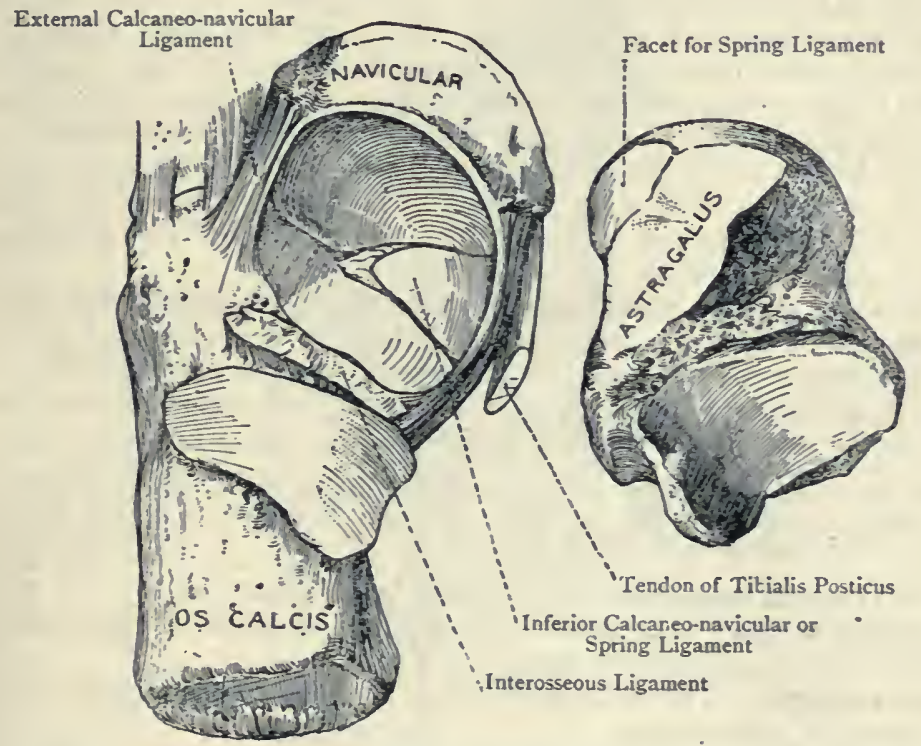

Fig. 269.-- I he INFerior Calcaneo-navicular or Spring Ligament OF THE LEFT FOOT (SUPERIOR VIEW).

The astragalo-navicular, external or superior calcaneo-navicular, and internal or inferior calcaneo-navicular ligaments form together a capsule for the astragalo-navicular joint.

The synovial membrane is continuous with the synovial membrane of the anterior astragalo-calcaneal joint.

Arterial Supply.-The anterior tibial, external tarsal branch of the dorsalis pedis, and internal plantar arteries.

Nerve-supply.-The internal plantar and the external division of the dorsalis pedis nerve.

The movements at this joint will be described with those at the calcaneo-cuboid joint. 
III. Calcaneo-cuboid Joint.-This belongs to the class diarthrosis, and to the subdivision reciprocal or saddle-joint. The ligaments are dorsal, internal or interosseous, long plantar, and short plantar.

The dorsal ligament is a broad, flat band which passes between the superior surfaces of the os calcis and cuboid.

The internal or interosseous ligament is a strong band which lies deeply in the hollow between the fore parts of the astragalus and os calcis, where it is connected with the external calcaneonavicular ligament. Its attachments are to the adjacent inner portions of the os calcis and cuboid.

The long plantar (long inferior calcaneo-cuboid) ligament is very strong, and is attached posteriorly to the plantar surface of the os calcis, upon which it extends from the inner and outer tubercles to the anterior tubercle. Anteriorly most of its fibres are attached to the ridge on the plantar surface of the cuboid bone behind the peroneal groove. Some of them, however, are continued forwards to be attached to the plantar aspects of the bases of the second, third, fourth, and fifth metatarsal bones. These latter fibres, as they pass over the peroneal groove, cover the tendon of the peroneus longus, and so complete its fibro-osseous canal.

The short plantar (short inferior calcaneo-cuboid) ligament is more deeply placed than the preceding. Posteriorly it is attached to the plantar surface of the os calcis in front of the anterior tubercle, as well as to the fore part of that tubercle, and anteriorly to the plantar surface of the cuboid bone behind the ridge.

The long and short plantar ligaments are in early life continuous with the tendo Achillis, but they become subsequently separated from it as the result of the backward growth of the heel.

The synovial membrane of this joint is peculiar to it.

Arterial Supply.- The external tarsal branch of the dorsalis pedis and the external plantar artery.

Nerve-supply.--The external plantar and the external division of the dorsalis pedis nerve.

Movements at the Astragalo-navicular and Calcaneo-cuboid Joints.- The movements at these joints are flexion and extension, combined with abduction, adduction, and rotation. Flexion occurs during extension of the ankle-joint, and extension during flexion of that joint. The axis of these movements passes from the inner side of the neck of the astragalus obliquely downwards, outwards, and backwards to the lower and outer part of the tuber calcis. During flexion and extension of the astragalo-navicular joint rotation of the navicular bone on the head of the astragalus takes place. The most free movement at this joint is downwards and inwards, or upwards and outwards. In adduction and abduction of the foot movement takes place at this joint as well as at the astragalo-calcaneal articulations. In adduction or inversion the navicular bone moves downwards and inwards, and so also does the cuboid at the calcaneo-cuboid joint, the result being that the antero-posterior arch of the foot is diminished. In abduction or eversion the navicular and cuboid bones move npwards and outwards, and the antero-posterior arch is increased.

In walking the head of the astragalus tends to sink upon the spring ligament, 
and a certain amount of abduction or eversion of the foot takes place. When a person stands erect with both feet upon the ground, abduction or eversion is prevented by the astragalo-calcaneal ligaments. If, however, the spring ligament is in a weak condition, the tendon of the tibialis posticus is not of itself sufficient to prevent displacement of the head of the astragalus downwards and inwards. Displacement accordingly takes place in that direction, and so the condition known as flat foot (pes planus) is produced.

At the calcaneo-cuboid joint, besides the movements of flexion and extension combined with adduction and abduction, movement takes place downwards and inwards, or upwards and outwards. The astragalo-navicular and

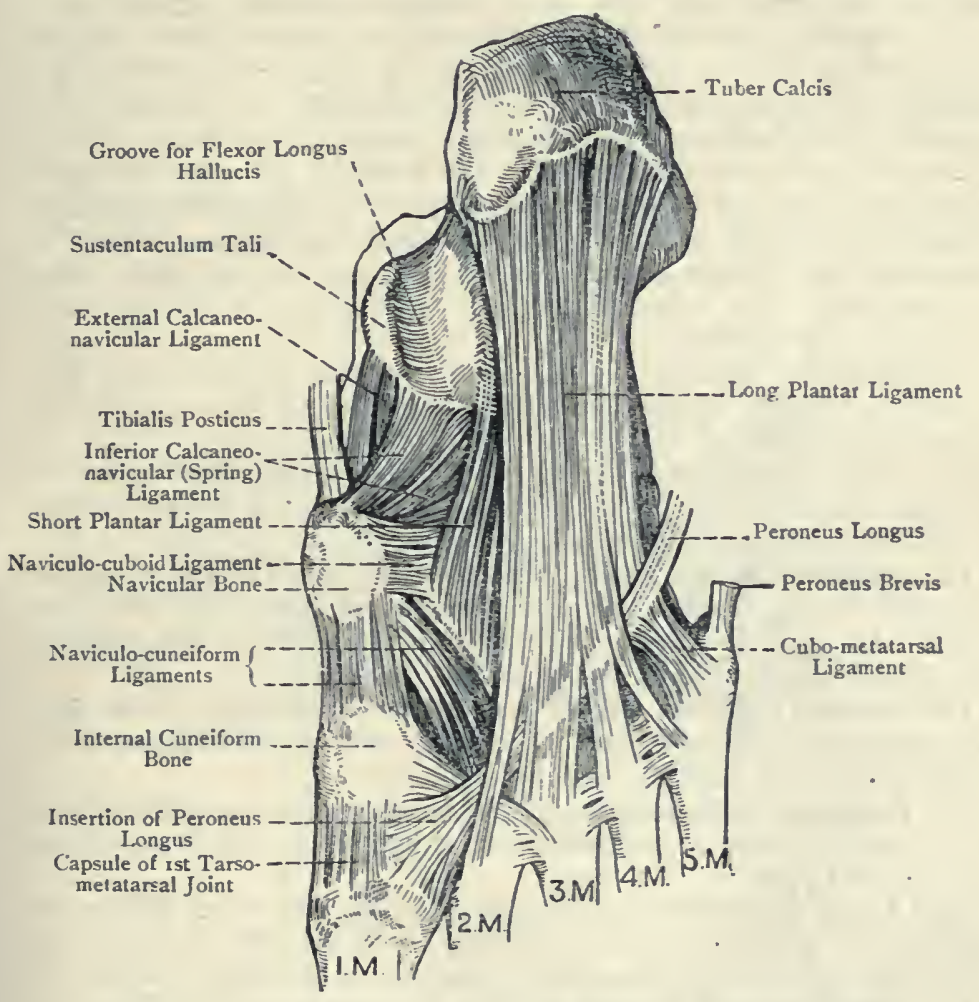

Fig. 270.-Ligaments of the Right Foot (Plantar Aspect).

calcaneo-cuboid joints together constitute the transverse tarsal articulation, the direction of which is in a straight line across the foot. It is at this transverse tarsal joint where disarticulation is performed in Chopart's operation.

IV. Naviculo-cuboid Joint.--This belongs to the class diarthrosis, and to the subdivision arthrodia, but only in those cases where the two bones articulate directly with each other by faceted surfaces. The ligaments are dorsal, plantar, and interosseous.

The dorsal ligament passes from the outer part of the dorsal 
surface of the navicular bone to the middle third of the inner border of the dorsal surface of the cuboid.

The plantar ligament is a strong band which extends from the outer part of the plantar surface of the navicular bone to the internal part of the plantar surface of the cuboid. Its direction is forwards and outwards.

The interosseous ligament is also a strong band which extends between the contiguous surfaces of the two bones. The navicular and cuboid bones do not, as a rule, articulate directly with each other by cartilaginous surfaces, and under these circumstances there is no synovial membrane at this joint. Sometimes, however, the two bones come into actual contact, and then each has a special articular facet, that on the navicular bone being situated on its outer extremity, adjacent to the facet for the external cuneiform, and that on the cuboid being situated on its internal surface, behind the normal facet for the external cuneiform. When the two bones are thus in actual contact the joint is provided with a synovial membrane which is continuous with that of the naviculocuneiform articulation.

V. Naviculo-cuneiform Joint.-This belongs to the class diarthrosis, and to the subdivision arthrodia. The ligaments are dorsal, plantar, and internal.

The dorsal ligament is a strong, continuous sheet of fibres passing from the dorsal surface of the navicular bone to the dorsal surfaces of the three cuneiform bones.

The plantar ligament extends between the plantar surface of the navicular bone and the plantar surfaces of the three cuneiform bones. To a large extent it derives its fibres from the adjacent expansions of the tendon of the tibialis posticus.

The internal ligament passes between the tuberosity of the navicular bone and the inner surface of the internal cuneiform, and it blends with the dorsal and plantar ligaments.

VI. Intercuneiform Joints.-These belong to the class diarthrosis, and to the subdivision arthrodia. The ligaments are dorsal, interosseous, and plantar.

The dorsal ligaments are two in number, and their fibres pass transversely between the dorsal aspect of the middle cuneiform and the dorsal aspects of the internal and external cuneiform bones.

The interosseous ligaments are also two in number, and are very strong. They are deeply placed, and pass between the sides of the middle cuneiform and the contiguous sides of the internal and external cuneiform bones in front of the articular facets. They constitute the chief bond of union between the three bones.

The ligament between the middle and external cuneiform is attached to the entire vertical extent of the contiguous surfaces, whilst that between the middle and internal cuneiform is generally limited to the inferior and anterior portions of the contiguous surfaces. 
The plantar ligament passes between the inner aspect of the eminence on the plantar surface of the internal cuneiform and the plantar aspect of the middle cuneiform.

VII. Cubo-cuneiform Joint.-This belongs to the class diarthrosis, and to the subdivision arthrodia. The ligaments are dorsal, plantar, and interosseous.

The dorsal ligament passes between the dorsal surface of the external cuneiform bone and the dorsal surface of the cuboid.

The plantar ligament passes from the plantar aspect of the external cuneiform bone to the internal surface of the cuboid over its anterior half, close to the plantar surface.

The interosseous ligament, which is strong and deeply placed, passes between the entire vertical extent of the contiguous surfaces of the two bones in front of the articular facets.

Synovial Membrane of the Naviculo-cuneiform, Intercuneiforn, and Cubo-cuneiform Joints.-The naviculo-cuneiform synovial membrane is usually prolonged into the cubo-cuneiform joint, though

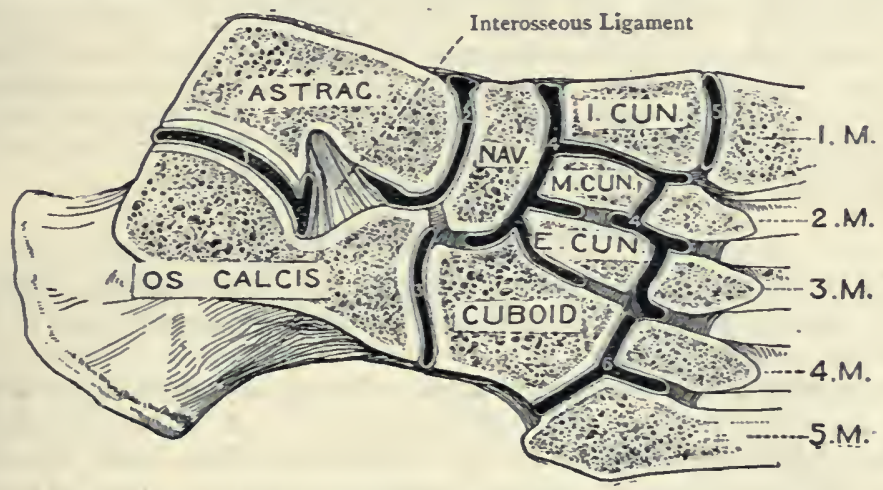

Fig. 271.-The Synovial Cavities of the Tarsal and TARSO-METATARSAL JoINTS.

occasionally the latter has a synovial sac peculiar to it. It is also continued into the naviculo-cuboid joint in those cases where these two bones articulate by facets: Further, it sends forwards two prolongations, one on either side of the middle cuneiform. The prolongation between the middle and external cuneiform and that between the external cuneiform and cuboid are entirely shut off from the synovial cavity of the middle tarso-metatarsal joint (the joint between the cuneiform bones and the second and third metatarsal bones). The explanation of this is that the interosseous ligaments between the middle and external cuneiform and between the external cuneiform and cuboid, which are placed in front of the articular surfaces, are attached over the entire vertical extent of the contiguous surfaces from the dorsal ligaments above to the plantar below. The prolongation, however, between the middle 
and internal cuneiform is usually continuous in front with the synovial membrane of the middle tarso-metatarsal joint, because the interosseous ligament between these two bones is generally limited to the inferior and anterior portions of the contiguous surfaces. If this ligament should reach the dorsal surface, the naviculo-cuneiform synovial membrane would be entirely isolated from that of the middle tarso-metatarsal joint.

Arterial Supply.-The arterial supply of the naviculo-cuboid, naviculo-cuneiform, intercuneiform, and cubo-cuneiform joints is derived from the metatarsal branch of the dorsalis pedis and the internal and external plantar arteries.

Nerve-supply. - The dorsalis pedis, internal plantar, and external plantar nerves.

Movements at the Naviculo-cuneiform, Intercuneiform, and Cubo-cuneiform Joints. - The movements at these joints are very limited, and are of the gliding or to-and-fro variety. They do not affect the position of the foot as regards flexion and extension, or inversion and eversion, but they influence the transverse arch by increasing or diminishing its span. Downward gliding would increase the span, and upward gliding would diminish it.

The portion of the transverse arch which is formed by the three cuneiform and cuboid bones has the following muscles at tached to its various parts-tibialis posticus, flexor brevis hallucis, adductor obliquus hallucis, peroneus longus, flexor brevis minimi digiti, and tibialis anticus. The first four muscles diminish the span, and so deepen the arch, whilst the tibialis anticus increases the span, and so renders the arch wider.

\section{The Tarso-metatarsal Joints.}

The tarso-metatarsal joints are divided into internal, middle, and external. They all belong to the class diarthrosis, and to the subdivision arthrodia.

I. Internal Tarso-metatarsal Joint.--This is the joint between the internal cuneiform and the first metatarsal bone. The dorsal and plantar ligaments of this joint are so disposed as to meet upon its inner and outer aspects, and thus a complete capsule is formed round the articulation, which capsule is stronger inferiorly and internally than elsewhere.

The synovial membrane of this joint is peculiar to it.

II. Middle Tarso-metatarsal Joint.-The bones which enter into this joint are the three cuneiforms and the bases of the second and third metatarsals (sometimes a part of the base of the fourth also). The ligaments are dorsal, plantar, and interosseous.

The dorsal ligaments. - The dorsal surface of the base of the second metatarsal bone receives three dorsal ligaments, one from each cuneiform. The dorsal surface of the base of the third metatarsal bone receives a dorsal ligament from the external cuneiform. Moreover, the external cuneiform is connected by a dorsal ligament with the dorsal surface of the base of the fourth metatarsal bone.

The plantar ligaments are three in number. One passes between the base of the internal cuneiform and the plantar aspects of the 
bases of the second and third metatarsals, having the slips of the tendon of the tibialis posticus behind it; a second passes between the middle cuneiform and the base of the second metatarsal; and a third passes between the external cuneiform and the base of the third metatarsal, the latter two also having the slips of the tendon of the tibialis posticus behind them.

The interosseous ligaments are three in number-internal, middle, and external.

The internal interosseous ligament* extends from the anterior and upper part of the outer surface of the internal cuneiform to the inner surface of the base of the second metatarsal. In each case it is attached below and in front of the articular facet, and it separates the internal from the middle tarso-metatarsal joint. It is a very strong ligament, and offers considerable resistance to the knife in the performance of Lisfranc's operation. The middle interosseous ligament extends from the anterior part of the inner surface of the external cuneiform, between the two semi-oval facets, to the groove on the outer surface of the base of the second metatarsal which separates the two facets. The external interosseous ligament extends from the anterior part of the outer surface of the external cuneiform, below the inconstant antero-superior facet, to the outer side of the base of the third metatarsal below the facet. It is also attached to the inner side of the base of the fourth metatarsal, and it ranks as the interosseous ligament of the cubo-metatarsal joint.

The synovial membrane of the middle tarso-metatarsal joint is usually continuous with that of the naviculo-cuneiform joint; the continuity taking place between the internal and middle cuneiform bones. Sometimes, however, they are quite distinct from each other. It sends prolongations forward between the bases of the second and third and third and fourth metatarsals.

III. External Tarso-metatarsal or Cubo-metatarsal Joint.-The bones which enter into this joint are the cuboid and the fourth and fifth metatarsals. The ligaments are dorsal, plantar, and interosseous.

The dorsal ligament passes from the dorsal surface of the cuboid to the dorsal surfaces of the bases of the fourth and fifth metatarsals.

The plantar ligament extends between the plantar surface of the cuboid in front of the peroneal groove and the plantar aspects of the bases of the fourth and fifth metatarsals. It is closely associated with the forward expansion of the long plantar ligament and with one of the slips of the tendon of the tibialis posticus.

The interosseous ligament is the same as the external interosseous ligament of the middle tarso-metatarsal joint.

The dorsal, plantar, and interosseous ligaments construct a capsule round the cubo-metatarsal joint.

The synovial membrane is peculiar to this joint, and sends a prolongation between the bases of the fourth and fifth metatarsals.

Arterial Supply.-The tarso-metartarsal joints derive their arterial

* Lisfranc's ligament. 
supply from the dorsalis pedis artery and its metatarsal branch, the internal plantar artery, and the plantar arch.

Nerve-supply. - The dorsalis pedis, internal plantar, and external plantar nerves.

Movements at the Tarso-metatarsal Joints. - The movements allowed are flexion and extension. At the internal tarso-metatarsal joint flexion is associated with a certain amount of abduction, whilst extension is accompanied by slight adduction. At the middle tarso-metatarsal joint flexion and extension are allowed, but no lateral movement. At the cubo-metatarsal joint flexion and extension, combined with abduction and adduction, are allowed, lateral movement being tolerably free in the case of the fifth metatarsal. A certain amount of gliding or to-and-fro movement is also permissible at the tarso-metatarsal joints in the following manner: the third metatarsal base can glide upwards, whilst the pair on either side of it can glide downwards, the effect being to diminish the span of the transverse arch of the foot. When the reverse movement takes place the span of the arch is increased.

The portion of the transverse arch which is formed by the bases of the metatarsal bones has the following muscles attached to its various parts: tibialis posticus, adductor obliquus hallucis, peroneus longus, flexor brevis minimi digiti, and tibialis anticus. The first three muscles diminish the span, and so deepen the arch, whilst the tibialis anticus increases the span, and so renders the arch wider.

Surgical Anatomy of the Tarso-metatarsal Joints.-It is in this situation where Lisfranc's and Hey's amputations are performed. The guide to the line of articulation on the outer border of the foot is the tuberosity on the outer side of the base of the fifth metatarsal, which can always be felt without difficulty. The joint between the cuboid and the fifth metatarsal is situated immediately behind this tuberosity. The guide to the line of articulation on the inner border of the foot is a point $1 \frac{1}{2}$ inches in front of the tuberosity of the navicular bone. The line of articulation is in no sense transverse, its inner part being about $I$ inch further forwards than the outer part. The tarso-metatarsal joints are remarkable for their irregularity, which is due to two causes. In the first place, the anterior surfaces of the three cuneiform bones do not present an even frontage. The internal and external cuneiform project further forwards than the middle cuneiform, and so a recess is formed which receives the base of the second metatarsal as that extends backwards to articulate with the middle cuneiform. The base of the second metatarsal thus becomes locked between the internal and external cuneiform. In the second place, the external cuneiform extends a little further forwards than the cuboid. The line of the tarso-metatarsal articulations, from the inner border of the foot to the outer border, is as follows: (1) outwards, between the internal cuneiform and first metatarsal; (2) backwards, for about $\frac{1}{2}$ inch (at this stage a very strong interosseous ligament has to be divided, as it passes between the internal cuneiform and the inner side of the base of the second metatarsal; (3) outwards, between the middle cuneiform and second metatarsal ; (4) forwards, for about $\frac{1}{4}$ inch ; (5) outwards, between the external cuneiform and third metatarsal; (6) backwards, for about $\frac{1}{8}$ inch; and (7) outwards and backwards, between the cuboid and the fourth and fifth metatarsals.

\section{The Intermetatarsal Joints.}

The basal intermetatarsal joints belong to the class diarthrosis, and to the subdivision arthrodia. The bones concerned are the outer four metatarsals. The first metatarsal bone does not usually articulate with the second, but sometimes it does. The ligaments are dorsal, plantar, and interosseous. 
The dorsal ligaments are short transverse bands, three in number, which pass between the dorsal aspects of the bases of the second and third, third and fourth, and fourth and fifth metatarsals.

The plantar ligaments are disposed in a similar manner to the preceding on the plantar aspects of the bases of the four outer metatarsals.

The interosseous ligaments, which are three in number, are deeply placed and of considerable strength. They constitute a very firm bond of union between the contiguous sides of the bases of the outer four metatarsals, to the non-articular parts of which they are attached.

Synovial Membrane.-In the case of the joints between the bases of the second and third and third and fourth metatarsals the synovial membrane is a forward extension of that of the middle tarso-metatarsal joint, which sends forward two prolongations.

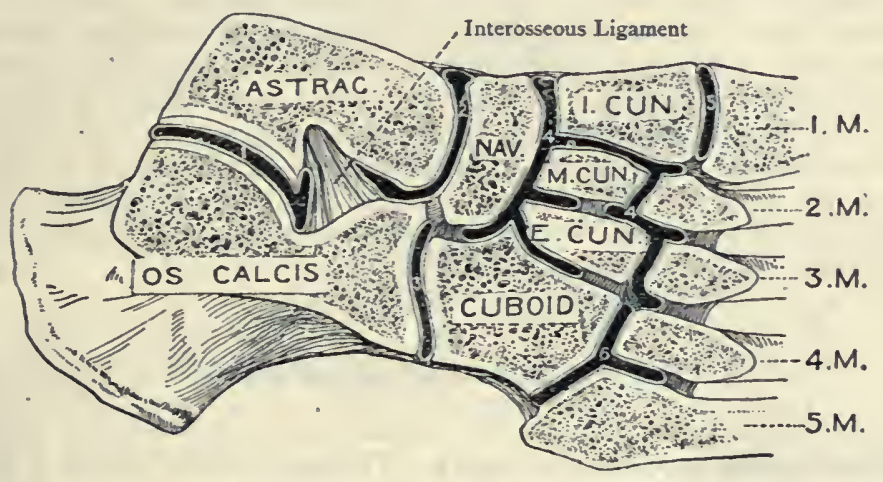

Fig. 272.-The Synovial Cavities of the Tarsal and TARSO-METATARSAL JoINTS.

In the case of the joint between the bases of the fourth and fifth metatarsals the synovial membrane is a forward prolongation of that of the cubo-metatarsal joint. As regards the contiguous sides of the bases of the first and second metatarsals, if they should articulate, a special synovial bursa is usually provided which sometimes communicates with the synovial membrane of the internal tarso-metatarsal joint.

The arterial supply and nerve-supply are derived from the same sources as in the case of the tarso-metatarsal joints.

Movements. - These are of a purely gliding or to-and-fro nature, so as to increase or diminish the span of the transverse arch of the foot.

The heads of all the metatarsal bones are connected by the transverse metatarsal (deep transverse) ligament, which extends across their plantar aspects. Its fibres are attached to the fibrous plates on the plantar surfaces of the metatarso-phalangeal joints, 
and it receives the deep expansions of the digital processes of the central division of the plantar fascia. It is to be noted that the transverse metatarsal ligament includes the head of the first metatarsal bone, whereas the corresponding ligament in the hand (transverse metacarpal) excludes the head of the first metacarpal bone.

Summary of the Tarsal and Tarso-metatarsal Synovial Membranes. - These are usually six in number.

I. Posterior astragalo-calcaneal.

2. Astragalo-calcaneo-navicular.

3. Calcaneo-cuboid.

4. Naviculo-cuneiform, cubo-cuneiform, cubo-navicular (inconstant), intercuneiform, and middle tarso-metatarsal.

5. Internal tarso-metatarsal.

6. External tarso-metatarsal or cubo-metatarsal.

Sometimes the middle tarso-metatarsal synovial membrane is distinct from the naviculo-cuneiform and intercuneiform synovial membrane, in which case the number would be increased to seven. Occasionally the cubo-cuneiform synovial membrane is distinct from the naviculo-cuneiform and intercuneiform, in which case there would be eight synovial membranes. In addition to these, there may be a synovial bursa between the contiguous sides of the bases of the first and second metatarsals if these articulate with each other, as they sometimes do.

\section{The Metatarso-phalangeal Joints.}

These joints belong to the class diarthrosis, and to the subdivision condylarthrosis or condyloid joint. They are formed by the heads of the metatarsal bones and the proximal ends of the first phalanges. The ligaments of the four outer joints are three in number, two lateral and an inferior or plantar fibrous plate. In the metatarso-phalangeal joint of the great toe the plantar fibrous plate of the other joints is replaced by two sesamoid bones, which are closely associated with the heads of insertion of the flexor brevis hallucis. In the case of each joint the expansion of an extensor tendon serves the purpose of a dorsal ligament. Each joint is provided with a synovial membrane. The ligaments, synovial membranes, and movements of these joints closely correspond with those of the metacarpo-phalangeal joints. Abduction, adduction, and circumduction, however, are much more limited.

Arterial Supply.--The plantar digital and dorsal interosseous arteries.

Nerve-supply. - The deep division of the external plantar nerve (either directly or through its branches to the interosseous muscles), or the adjacent digital nerves. 


\section{The Interphalangeal Joints.}

These joints belong to the class diarthrosis, and to the subdivision ginglymus. They are formed by the distal end of one phalanx and the proximal end of the adjoining phalanx. The ligaments are three in number, two lateral and an inferior or plantar fibrous plate, the extensor tendon taking the place of a dorsal ligament. Each joint is provided with a synovial membrane. The ligaments,

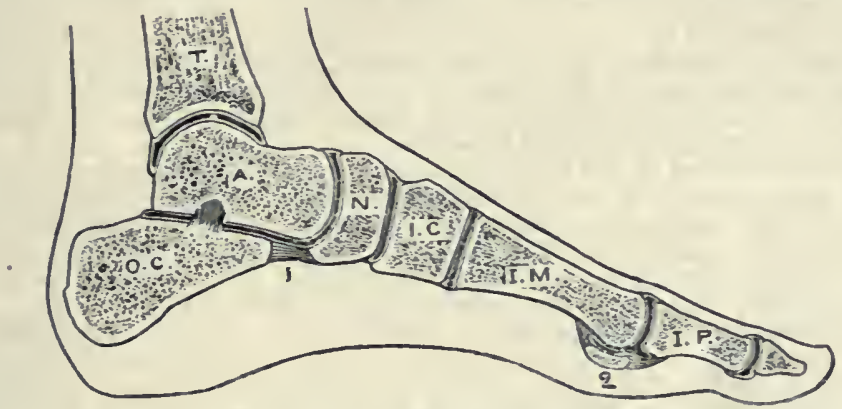

Fig. 273.-Sagittal Section of ANKLE ANd Foot, PASSing through THE GREAT TOE.

I, Spring Ligament; 2, Inner Sesamoid Bone.

synovial membranes, and movements of these joints closely correspond with those of the interphalangeal joints of the hand.

The cnly joint in the lower limb which has interarticular fibro-cartilages is the knee-joint. The hip-joint has a circumferential fibro-cartilage, namely. the cotyloid ligament.

\section{Development of the Limbs.}

In the third week of embryonic life two longitudinal ridges make their appearance, one on either side, external to the mesodermic somites. The rudiments of the fore- and hind-limbs (exclusive of the pectoral and pelvic girdles) appear as processes of these ridges. These prominences assume the form of distinct outgrowths, which are called the limb-buds. The limb-buds undergo elongation, and each bud becomes divided into two segments by means of a transverse groove. The distal segment represents the manus or pes, as the case may be. The proximal segment soon becomes differentiated by means of another groove into two parts-namely, antibrachium and brachium for the fore-limb, and leg and thigh for the hind-limb.

Structure of a Limb-Bud.-Each limb-bud consists of the following elements: (1) A core of mesenchyme (covered with ectoderm), derived from several of the contiguous somites; (2) muscular tissue, derived from the muscle-plates of several contiguous somites: and (3) prolongations (anterior primary divisions) from the contiguous spinal nerve-segments.

The bones are developed from the core of mesenchyme, which first undergoes chondrification and subsequently ossification. The mesenchyme in the intervals between the osseous frameworks assumes a ho!low condition, and 
so gives rise to the joint-cavities, the synovial membranes and ligaments being formed by the surrounding mesenchyme.

The muscular tissue, originally derived from the muscle-plates of the somites, or perhaps from the mesenchyme which forms the core of the limbbud, becomes differentiated in to the limb-muscles.

The prolongations from the spinal nerve-segments become differentiated into the limb-nerves.

Position of the Limbs.-At first the fore- and hind-limbs are disposed with their longitudinal axes parallel with the longitudinal axis of the trunk. As the limbs undergo elongation and division into their ultimate component parts, a change in their position takes place. They become bent in a ventral direction, so that their longitudinal axes are now at right angles to the longitudinal axis of the trunk. The radial or preaxial side of the antibrachium and the pollex, on the one hand, and the tibial or preaxial side of the leg, and the hallux, on the other, are directed towards the cephalic region; the palmar and plantar surfaces of the hands and feet are directed towards the trunk; the elbows are directed outwards and slightly backwards; and the knees are directed outwards and slightly forwards.

Towards the end of the sixth week a second change in the position of the limbs takes place, and their permanent condition is thereby brought about, in which their longitudinal axes coincide with the longitudinal axis of the trunk. This change consists in a rotation of each limb round its longitudinal axis. In the case of the fore-limbs the rotation takes place in an outward direction, but in the case of the hind-limbs the rotation is inwards.

The permanent results of these rotations are as follows: Fore-Limbs ( $E x$ ternal Rotation): (I) The radial or preaxial side of the antibrachium and the pollex are directed outwards; (2) the elbow becomes entirely posterior; and (3) the ventral or flexor aspect of the limb is now anterior, the extensor aspect being posterior. Hind-Limbs (Internal Rotation): (I) The tibial or preaxial side of the leg and the hallux are directed inwards; (2) the knee becomes entirely anterior; and (3) the ventral or flexor aspect of the limb is now posterior, the extensor aspect being anterior.

From the preceding changes it will be evident that, in the adult, the anterior aspect of the upper limb corresponds to the posterior aspect of the lower limb; the outer aspect of the upper limb to the inner aspect of the lower limb; and so on.

\section{GUIDE TO THE LOWER LIMB.}

Gluteal Region.-The landmarks having been studied, the skin is to be removed by making the following incisions : one extending from the level of the spine of the fifth lumbar vertebra outwards along the iliac crest as far as the position of the body will allow, and another extending from the fifth lumbar spine downwards in the middle line as low as the coccyx, after which it is prolonged outwards and downwards to the outer side of the thigh at a point about 5 inches below the great trochanter. The skin having been reflected, the gluteal cutaneous nerves are to be exposed in the following situations: (1) line of origin of gluteus maximus; (2) iliac crest ; (3) outer and lower part of gluteus maximus; and (4) lower border of gluteus maximus. The superficial fascia should now be removed, and the deep fascia examined. It will be seen that, in passing from the gluteus medius to the gluteus maximus, it firmly straps down the upper border of the latter muscle to the former. When the gluteus maximus has been reflected, the fascia will be seen to divide into two laminx, which embrace and give insertion to rather more than the upper half of that muscle. 
The gluteus maximus should be cleaned in the direction of its coarse fasciculi, and in connection with its lower border the bursa between it and the tuber ischii should be displayed. The muscle is to be divided about $1 \frac{1}{2}$ inches from its origin. As the greater part of it is being reflected outwards, the following arteries should be exposed entering its deep surface: the superficial branch of the gluteal, the inferior gluteal branch of the sciatic, and branches of the first perforating of the arteria profunda femoris. The inferior gluteal nerve should also be shown entering the deep surface of the muscle in its lower third. Between the great trochanter and the insertion of the muscle into the fascia lata a large multilocular bursa will be found, and another single bnrsa should be noticed between its insertion and the vastus externus just below the great trochanter. The twofold insertion of the muscle should be noted. The origin of the muscle is now to be removed so as to expose the great sacro-sciatic ligament and the structures piercing it, namely, the coccygeal branch of the sciatic artery, the sacral branch of the internal pudic artery, and the perforating cutaneous branch of the sacral plexus of nerves. On the superficial surface of the ligament will be found the plexiform loops formed by the external branches of the posterior primary divisions of the first three sacral nerves.

The fascia lata should next be removed from the anterior part of the gluteus medius, and in doing so it should be noted that the superficial fibres of the muscle arise from the deep surface of the fascia. The various structures underneath the gluteus maximus are to be cleaned from above downwards, as follows: the posterior fleshy part of the gluteus medius, gluteal vessels, pyriformis, great and small sciatic nerves, sciatic vessels, pudic nerve and internal pudic vessels, nerve to the obturator internus, furnishing a branch to the gemellus superior, common nerve to the gemellus inferior and quadratus femoris, gemellus superior, tendon of the obturator internus, gemellus inferior, quadratus femoris, upper horizontal part of the adductor magnus, origins of the hamstring muscles from the tuber ischii, and the upper tendinous fibres of the vastus externus. The digital and crucial anastomoses should be looked for, the former in the digital or trochanteric fossa, and the latter between the quadratus femoris and adductor magnus.

The small sciatic nerve should be displayed so as to show its long pudendal branch, and the great sciatic nerve is to be exposed in the hollow between the tuber ischii and the great trochanter. The sciatic artery and its branches are to be followed out. The pudic nerve, the internal pudic vessels, and the nerve to the obturator internus (with its branch to the gemellus superior) are to be shown as they cross the back of the spine of the ischium. The tendon of the obturator internus should be divided to show the columns on its under surface, the synovial bursa between the tendon and the small sciatic notch, and the cartilage facing that notch, with its grooves and ridges for the columns of the tendon. The sacro-sciatic ligaments, great and small, should be studied. The mode of formation of the great and small sacro-sciatic foramina should be made out, and the various structures passing through each should be noted. The upper border of the quadratus femoris is to be displaced downwards so as to show the obturator externus and the ascending branch of the internal circumflex artery, both on their way to the digital fossa. The lower border of the quadratus femoris should be raised to show the small trochanter and the insertion of the ilio-psoas in part. The common nerve to the gemellus inferior and quadratus femoris will be found at first underneath the great sciatic nerve, and subsequently passing beneath the gemelli, obturator internus, and quadratus femoris. Its articular branch to the hip-joint should be looked for. If absent, its place will be taken by a branch from the great sciatic nerve.

The gluteus medius (except its anterior fibres) sloould now be stripped from the dorsum ilii, and the bone cleaned by scraping, so as to show the exact bony origin of the muscle. The pyriformis being also divided, the gluteus minimus is to be cleaned, along with the upper and lower branches of the deep divisions of the gluteal artery and superior gluteal nerve. One of each 
of these structures will be found coursing along the upper border of the gluteus minimus, and crossing outwards over its centre. The latter branch (lower) of the artery should be shown to give a branch to the digital fossa to take part in the digital anastomosis, and the corresponding branch of the superior gluteal nerve should be followed outwards to the outer aspect of the thigh as far as possible on its way to the tensor fasciæ femoris, which it supplies. The gluteus minimus (except its anterior fibres) is to be stripped from the bone, after which the surface thereby exposed should be cleaned. The posterior or reflected head of the rectus femoris is to be shown arising from the dorsum ilii immediately above the brim of the acetabulum. The muscular relations of the capsular ligament of the hip-joint should be carefully attended to in so far as the position of the body will allow.

Popliteal Space.- This space should be dissected before the structures on the back of the thigh are disturbed. The landmarks having been studied, the skin is to be removed by the following incisions: (I) a median vertical incision extending from the junction of the upper two-thirds and lower third of the thigh to the junction of the upper fourth and lower three-fourths of the leg; and (2) two transverse incisions, one at either end of the median incision.

The small sciatic nerve is to be displayed after it pierces the fascia lata at the back of the knee-joint, and it should be followed to its terminal distribution over the upper part of the back of the leg. The upper part of the external or short saphenous vein is to be dissected, and in connection with it the following two veins should be noticed: (I) a fairly large tributary vessel, which descends from the lower part of the back of the thigh; and (2) a communicating branch, which passes upwards and inwards to join the long saphenous vein. Three cutaneous sural arteries (branches of the popliteal) may be looked for-an external, over the outer head of the gastrocnemius; an internal, over the inner head; and a middle, accompanying the short saphenous vein. The fascia lata should next be cleaned, and the accession of strength which it here receives from superadded transverse fibres should be observed. Above the level of the knee-joint the small sciatic nerve will be found in the middle line beneath the fascia lata. This fascia is now to be removed, and the boundaries of the popliteal space are to be cleaned.

The principal contents of the space are to be displayed by the removal of a large amount of fat. The great sciatic nerve should be exposed close to the upper median angle, and its external and internal popliteal branches cleaned downwards froin that point. The external popliteal nerve is to be followed along the inner border of the biceps femoris as far as a point just below the head of the fibula. In cleaning this nerve, the dissector should look for the following branches, namely, (I) superior articular, to accompany the superior external articular artery ; (2) inferior articular, to accompany the inferior external articular artery; (3) lateral cutaneous, to the integument of the outer side of the leg over about its upper two-thirds; and (4) ramus communicans fibularis, which passes downwards and inwards to the middle line of the calf on its way to join the ramus communicans tibialis, and so form the external or short saphenous nerve.

The internal popliteal nerve is to be followed through the centre of the space as low as the interval between the heads of the gastrocnemius. In cleaning it, the following branches should be looked for: (I) superior articular (inconstant), to accompany the superior internal articular artery; (2) central or azygos articular, to accompany the corresponding artery; (3) inferior articular, to accompany the inferior internal articular artery; (4) ramus communicans tibialis, which takes a straight course downwards to the calf, where it forms the chief part of the external or short saphenous uerve; and (5) four or five muscular (sural) branches, as follows: (a) one to the outer head of the gastrocnemius; $(b)$ one to the plantaris (which sometimes comes from the preceding branch); (c) one to the inner head of the gastrocnemius; (d) one to the soleus; and (e) one to the popliteus. The nerve to the popliteus should be preserved with great care.

The internal popliteal nerve should be looked to one side, and the popliteal 
vein exposed, its varying relation to the more deeply-placed popliteal artery being noted. The tributaries of the popliteal vein, when dissected, should be cut. These correspond with the branches of the popliteal artery, the short saphenous vein being a special tributary. The vein should next be hooked to one side, which will prepare the popliteal artery for dissection. In order to expose the vessel fully to its termination, the inner head of the gastrocnemius may be divided. In cleaning the artery, the geniculate branch of the obturator nerve should be looked for. If present, it will be found piercing the adductor magnus close above the femoral opening, after which it usually descends at first upon the inner side of the artery and then in front of it, until it comes into contact with the central or azygos branch, which it accompanies through the posterior ligament of the knee-joint to the interior of the articulation. During this dissection the popliteal lymphatic glands may come into view in the interval between the femoral condyles, one gland being superficial to the artery, one beneath it, and one on either side. The branches of the artery are to be carefully cleaned, as follows: ( $I$ ) muscular, to the hamstring and sural muscles; (2) cutaneous, to the upper part of the back of the leg; and (3) the following five articular arteries: (a) two superior, external and internal, above the knee-joint, usually close above the femoral condyles and lying very deeply; $(b)$ central or azygos, at the back of the joint, and coming either from the front (deep) surface of the main vessel, or in many cases from the superior external articular; and (c) two inferior, external and internal, below the level of the joint. The floor of the popliteal space should next be cleaned and examined, as follows: (I) the popliteal surface or trigone of the femur; (2) the posterior ligament of the knee-joint, with the ligamentum posticum Winslowii; and (3) the popliteal fascia covering the popliteus muscle.

Back of the Thigh. - The landmarks having been studied, the skin is to be removed by making a single vertical incision in the middle line. In the superficial fascia the femoral cutaneous branches of the small sciatic nerve are to be looked for, along the inner and outer aspects. At the upper and inner part of the back of the thigh twigs may be met with from the long pudendal branch of the small sciatic nerve.

The superficial fascia having been removed, the deep fascia or fascia lata should be studied. The deep fascia having been removed, the small sciatic nerve should be shown, and the hamstring muscles, namely, the biceps femoris, semitendinosus, and semimembranosus, should be cleaned and studied. The relation of the long head of the biceps to the great sacrosciatic ligament should be noted.

The great sciatic nerve is also to be cleaned to its division in to external and internal popliteal nerves, which latter nerves sometimes take the place of the great sciatic. The muscular branches of the great sciatic should be dissected, and it should be noted that, with one exception, they are derived from the internal popliteal part of the nerve. The exception is the branch to the femoral head of the biceps, which is derived from the external popliteal part of the nerve. It should also be noted that the nerve to the semimembranosus gives a branch to that part of the adductor magnus which extends from the tuber ischii to the adductor tubercle of the femur. The whole course and relations of the great sciatic nerve should be thoroughly mastered. The posterior surface of the adductor magnus should next be cleaned, showing the four arches with the four perforating arteries passing backwards under them, and the femoral opening where the femoral artery becomes the popliteal.

The distribution and anastomoses of the perforating arteries on the back of the thigh should be carefully studied, and it should be noted that they communicate above with the sciatic and gluteal, and below with the popliteal. It should further be noted that the perforating arteries do not terminate on the back of the thigh, and for this purpose an effort should be made to show all four passing outwards preparatory to their winding round the outer side of the femur to the outer side of the thigh, in which situation they end. The relation of muscles at the gluteal ridge of the femur should be studied, as well as the relation of muscles along the linea aspera. 
Front of the Thigh.--The dissector should make himself thoroughly familiar with the landmarks of the front of the thigh and of the knee. The first dissection should be limited to the upper 4 inches in connection with the parts involved in femoral hernia. For this purpose three incisions are required for the removal of the skin, as follows: one along the line of the groin from the anterior superior iliac spine to the pubic angle, a second extending from the pubic angle vertically downwards along the inner side of the thigh for 4 inches, and a third extending from the lower end of the second incision transversely across the front of the thigh as far as its outer aspect.

It is to be desired that the subsequent dissection, which has to do with the superficial fascia and cutaneous vessels, should be undertaken in concert with the dissector of the abdomen. The superficial fascia in this region should be shown to be divisible into a subcutaneous fatty layer and a deep thin membranous layer, which correspond with Camper's and Scarpa's fascix of the lower part of the anterior abdominal wall. In order to reflect the subcutaneous layer, a transverse incision should be carefully made across the thigh, the depth of this incision coinciding internally with the long saphenous vein, which lies between the two layers of the superficial fascia. Another incision should be made vertically upwards on the inner side of the thigh, and extending only through the subcutaneous layer. Acting simultaneously with the dissector of the abdomen, the dissector of the thigh can now reflect outwards the subcutaneous layer, and both dissectors will see that it is continuous over Poupart's ligament with Camper's fascia of the anterior abdominal wall. Care should be taken not to disturb the lymphatic glands of this region. The inguinal glands (including the pubic glands) and the superficial femoral or saphenous glands are next to be dissected. The former will be found lying with their long axes oblique just below Poupart's ligament, and the latter with their long axes vertical along the terminal part of the long saphenous vein.

The cutaneous arteries of the groin, with their corresponding veins, are to be dissected, namely, the superficial epigastric, superficial circumflex iliac, and superior external pudic. The inferior external pudic, being beneath the fascia lata, is not to be dissected at present. The terminal part of the long saphenous vein should be shown, up to the level of the saphenous opening, and the following tributaries should be displayed joining it, namely, the external femoral cutaneous or anterior saphenous from the front of the thigh, the internal femoral cutaneous or posterior saphenous from the inner and back parts of the thigh, and the cutaneous veins of the groin, namely, the superficial epigastric, superficial circumflex iliac, and superior and inferior external pudic.

The deep layer of the superficial fascia is next to be raised towards Poupart's ligament. It lies immediately beneath the long saphenous vein, and upon the deep fascia or fascia lata. When raised towards the groin it will be seen to cover the saphenous epening, and thereafter to be firmly bound down to the fascia lata about $\frac{1}{4}$ inch below Poupart's ligament. The portion of it which covers the saphenous opening should be carefully studied. It is called the cribriform fascia, and it should be shown to be closely attached to the outer border of the saphenous opening, but only loosely to the inner part. It will be obvious that a hernia in passing through the saphenous opening must receive a covering from the cribriform fascia.

The following nerves should now be dissected, namely, (I) branches of the inguinal nerve (so-called ilio-inguinal), which will be found on the inner aspect of the thigh; (2) the femoral branch of the genito-femoral, appearing immediately external to the femoral artery just below Poupart's ligament, after having pierced the outer part of the femoral sheath; and (3) the external cutaneous nerve, which will be found emerging bencath the outer end of Poupart's ligament.

The fascia lata is next to be cleaned, and the saphenous opening exposed, which should be carefully dissected. The best starting-point is the inferior cornu, which will readily come into view by raising the long saphenous vein. The cribriform fascia, is to be carefully removed, and the various 
parts of the opening displayed. Before disturbing the opening, the dissector should note that a part of the anterior wall of the femoral sheath is seen lying within it. Having studied the saphenous opening, the dissector should now separate the outer border of the opening from the anterior wall of the femoral sheath by dividing the fibrous processes which connect them. The superior cornu is next to be detached from Poupart's ligament, and turned downwards and outwards along with the outer border of the opening. This will bring fully into view the anterior wall of the femoral sheath. The deep femoral arch should be shown at this stage as a bundle of fibres extending from the centre of Poupart's ligament on its deep aspect inwards over the anterior wall of the femoral sheath to the pectineal portion of the ilio-pectineal line, where it is attached behind Gimbernat's ligament.

Having studied the sheath as it now appears, and having observed that the femoral branch of the genito-femoral nerve pierces the outer part of the sheath just below Poupart's ligament, three vertical incisions are to be made in its anterior wall-one over the femoral artery, another over the femoral vein, and a third a line or two internal to the vein. The interior of the sheath should then be shown to be divided into three compartments by means of two septa, which pass backwards on either side of the femoral vein. The femoral artery and, for a very limited distance, the femoral branch of the genito-femoral nerve should be shown in the outer compartment, whilst the femoral vein, having on its inner side two or three of the deep femoral (deep inguinal) glands will be found in the middle compartment. The inner compartment is called the femoral canal. It is of considerable surgical importance, and should be studied with the closest attention. It will be seen to contain a little fat, and at its upper end one of the deep femoral glands will be found. The dissectors of the thigh and abdomen, who should be working in concert at this stage, should now thoroughly explore the canal. The little finger should be inserted into it, and carried upwards to a point beneath Poupart's ligament. At the upper end of the canal the finger will detect the septum femorale, which shuts off the canal from the abdominal cavity. By breaking down this septum, the point of the finger will lie in the femoral ring, and the sharp wiry base of Gimbernat's ligament will readily be felt on the inner side of the ring, this being the usual cause of stricture in femoral hernia. Behind the finger as it lies in the femoral ring is Cooper's ligament. This structure, however, can only be shown by making a deep dissection of the parts beneath Poupart's ligament, in conjunction with the dissector of the abdomen. The parts around the femoral ring should be studied with the greatest care, as it is through this ring and the femoral canal that femoral hernia may occur.

The remainder of the skin of the thigh is now to be removed by making a vertical incision down the inner side of the thigh to a point just below the level of the patella, and carrying this incision transversely across the front of the leg immediately below the patella on to the outer aspect of the limb. This will enable the dissector to reflect the skin from the patella as well as from the front of the thigh. The prepatellar bursa will be found lying in front of the bone, where it is confined by an expansion which the fascia lata sends over the bone from its lateral borders.

A complete dissection of the cutaneous nerves and the femoral part of the long saphenous vein is now to be made. The external cutaneous nerve, which has been already caught, should now be followed out. Its small posterior division and large anterior division should be shown, and it should be noted that the latter is contained in a tube of fascia lata for about 4 inches before it enters the integument. The middle cutaneous nerve will be found piercing the fascia lata (usually in two divisions) about 4 inches below Poupart's ligament, and both should be followed down to the patellar plexus. It has been seen that the inguinal nerve is distributed to the integument of the inner aspect of the thigh in its upper third. In dissecting the long saphenous vein in the middle third of the thigh, twigs of the internal cutaneous nerve will be met with along the course of that vein. There may also be found branches of the subsartorial plexus ramifying in this region (middle third). Another nerve which should be looked for at this level is the cutaneous 
branch of the superficial or anterior division of the obturator nerve. When present it will usually be found piercing the fascia lata at about the centre of the inner side of the thigh, between the posterior border of the sartorius and the anterior border of the gracilis. The two divisions of the internal cutaneous nerve, anterior and posterior, should next be dissected. The anterior division will be found piercing the fascia lata at the junction of the middle and lower thirds of the thigh. It should then be followed to the inner side of the knee and patellar plexus. The posterior division will be found piercing the fascia lata at the level of the inner condyle, after which it descends to the inner side of the leg, where it will be afterwards dissected. The patellar branch of the internal or long saphenous nerve is to be shown. It pierces the sartorius and the fascia lata at the level of the inner condyle, and should be followed to the integument over the patella and patellar plexus. The patellar plexus itself is to be carefully dissected.

The fascia lata is now to be cleaned and examined. Its deep processes will only come into view as it is being removed to expose the muscles. The muscles of the front and inner side of the thigh are to be cleaned by removing the fascia lata. A strip of this fascia, the so-called ilio-tibial band, about 2 inches broad, and extending from the fore part of the iliac crest to the front of the external tuberosity of the tibia, should be preserved. In removing the fascia lata from the pectineus and adductor longus the inferior external pudic artery is to be dissected.

The boundaries of Scarpa's triangle should be defined. In the triangle the following muscles should be cleaned from without inwards, namely, the iliacus, psoas magnus, pectineus, and adductor longus. The contents of the triangle are now to be cleaned and studied, namely, (I) the anterior femoral nerve; (2) the common femoral and superficial femoral arteries; (3) the origins of the superficial epigastric, superficial circumflex iliac, superior external pudic, and inferior external pudic arteries; (4) the origin of the arteria profunda femoris, and its external circumflex and internal circumflex branches, and (5) the femoral vein and its tributaries. In cleaning the anterior femoral nerve its branch to the pectineus should be noted as it passes inwards behind the femoral sheath.

Having completed the superficial dissection of Scarpa's triangle, the remainder of the sartorius should be cleaned. The following muscles should also be cleaned in the order named: tensor fasciæ femoris, rectus femoris, and gracilis. In cleaning the tensor fasciæ femoris the branch of the superior gluteal nerve entering its deep surface should be shown by dissecting between the muscle and the gluteus medius, the ilio-tibial band should be studied, and a strong lamina of the fascia lata should be shown passing upwards on the deep surface of the muscle to the dorsum of the ilium. The two heads of the rectus femoris should be exposed.

The sartorius should now be held aside in the middle two-fourths of the thigh, in order to study Hunter's canal and its contents. The aponeurotic covering or roof of the canal should be shown as an upward expansion from the tendinous anterior margin of the femoral opening in connection with the adductor magnus. It should be observed that this tendinous roof is strong over the lower part of the canal, where it is pierced by a branch of the long. saphenous nerve to the subsartorial plexus, and by the arteria anastomotica magna. An cndeavour should be made at this stage to expose the subsartorial plexus as it lies upon the aponeurotic covering of Hunter's canal. This covering should now be removed, and the contents and boundaries of the canal exposed. It should be observed that the nerve to the vastus internus is only contained in the upper half of the canal, and that the arteria anastomotica magna arises from the superficial femoral at the extreme lower end of the canal.

The deep dissection of Scarpa's triangle should now be undertaken. A slight interval may be noted between the lower part of the pectineus and adductor longus, in which a partial view may be obtained of the adductor brevis and the superficial or anterior division of the obturator nerve. The superficial femoral artery should be divided just below the origin of the arteria profunda femoris, as well as the femoral vein. The arteria profunda femoris 
should now be studied as far as the upper border of the adductor longus, and this will be facilitated by dividing the corresponding vein. Its external circumflex branch should be followed out in its ascending, transverse, and descending branches, and the anastomoses of these branches should be carefully studied. An articular offset to the hip-joint from the ascending branch should be looked for. The long descending branch of this artery to the geniculate arterial rete should be noted.

The branches of the anterior or superficial and posterior or deep divisions of the anterior femoral nerve should be dissected, and the following articular nerves should be looked for, namely, one to the hip-joint from the nerve to the rectus femoris, and three to the knee-joint, as follows: one from the nerve to the vastus externus; one from the most internal of the branches to the crureus, supplying in its course the subcrureus; and one from the nerve to the vastus internus, which ultimately accompanies the deep branch of the arteria anastomotica magna.

The vastus externus, crureus, and vastus internus are next to be dissected. The vastus internus and crureus are to be separated by dissecting along the course of a cellular interspace, which extends upwards in the direction of a line drawn from the inner border of the patella to the inferior cervical tubercle of the femur. When the vastus internus is reflected inwards, a bare strip along the inner aspect of the shaft of the femur, devoid of muscular fibres, is to be noted. The crureus should next be turned aside in two halves by making a vertical incision down the centre of the muscle. This will show the lower limit of origin of the muscle, and it will bring into view the subcrureus. The bursa beneath the suprapatellar tendon and the prolongation of the synovial membrane of the knee-joint, which is continuous with it, should be shown. In dissecting the lower part of the vastus internus the deep branch of the arteria anastomotica magna and an articular branch to the knee-joint from the nerve to the vastus internus should be followed out.

The pectineus and adductor longus should be divided and reflected. When the pectineus has been reflected it should be noted that a portion of the capsular ligament of the hip-joint (including the pubo-femoral ligament) is exposed. This will bring fully into view the adductor brevis, with the anterior or superficial division of the obturator nerve resting upon it, and the obturator externus lying above it. The continuation of the arteria profunda femoris is also exposed. The four perforating branches of this vessel should be shown, the first and second piercing the adductor brevis and adductor magnus, whilst the third and fourth pierce only the adductor magnus.

The internal circumflex artery should be studied at this stage. Replacing the pectineus, the artery should be traced backwards between that muscle and the psoas magnus, and then between the adductor brevis and obturator externus. In this latter situation the artery should be shown to divide into its two terminal branches, namely, $(a)$ ascending, or anterior, passing with the obturator externus to the digital fossa; and (b) transverse or posterior, passing backwards between the quadratus femoris and upper border of the adductor magnus, and furnishing in its course an articular branch to the hip-joint which enters beneath the transverse ligament. It should be observed that the internal circumflex artery gives branches to the obturator externus, which anastomose in that muscle with branches of the obturator artery.

The adductor brevis should be divided without injuring the anterior division of the obturator nerve, and the obturator externus should be dissected. Its relation to the neck of the femur and the capsular ligament should be noted, as also its relation to the obturator nerve. This nerve is now to be dissected. An articular branch to the hip-joint from the anterior division should be looked for, and the termination of this division in cutaneous and vascular branches should be noted. The posterior division should be followed out in the expectation of finding it terminating in the inconstant geniculate branch. An accessory obturator nerve may be found emerging over the superior pubic ramus beneath the pectineus, after which it will be seen to end in three 
branches, as follows: one to the hip-joint, one to the pectineus, and one to reinforce the anterior division of the normal obturator nerve.

The obturator externus is next to be cut and reflected, in order to expose the obturator membrane and obturator artery. The artery should be shown to divide into an internal and external branch (the latter furnishing an articular branch to the hip-joint), and the arterial loop formed by these two branches at the circumference of the obturator. membrane should be made out. The anastomosis in the obturator externus between the obturator and internal circumflex arteries will now be readily understood. The adductor magnus is next to be fully studied. Its division into three parts is to be shown, as well as the four tendinous arches under which the four perforating arteries pass. The relation of the muscle to Hunter's canal is to be noted, and the femoral opening for the passage of the superficial femoral artery and corresponding vein is to be dissected. The dissector should now replace the adductor brevis, adductor longus, and pectineus, and the relative positions of these muscles should receive attention. The anterior portions of the gluteus medius and gluteus minimus should be cleaned by removing the fascia lata, and when they are reflected the bursæ between them and the great trochanter should be shown, as well as the strong arched band of fibres which connects the tendon of the gluteus minimus with the upper part of the capsule of the hip-joint.

Hip-Joint. - A careful study of the hip-joint should now be made, preparatory to the removal of the limb, and this should be done in the following order: (I) The muscular relations of the capsular ligament should be carefully noted, the muscles not yet cut should be divided, the bursa beneath the ilio-psoas should be observed, and the two heads of the rectus femoris should be again studied. The actions of the various muscles upon the joint should also be studied. (2) The capsular ligament should be cleaned, and an occasional opening in it should be looked for underneath the ilio-psoas bursa. The various accessory bands should be attended to, and their influence over the movements of the joint should be observed. The attachments of the capsule to the femur in front and behind are to be noted, and the difference in direction of the anterior and posterior fibres, as well as their relative strength, observed. The effect of different positions of the limb upon the head of the femur should be attended to. (3) The capsular ligament should be divided, and the ligaments within the joint studied, namely, $(a)$ the cotyloid ligament, (b) the transverse ligament, and $(c)$ the ligamentum teres. The synovial or Haversian gland is also to be noted. As regards the ligamentum teres, a good plan to adopt is as follows: the dissector of one limb should examine the hip-joint in the usual manner, namely, from the front, and the dissector of the other limb should avail himself of this examination. The dissector of the other limb should saw through the upper part of the shaft of the femur, and leave the hip-joint undisturbed in the meantime. Subsequently, when the pelvis has been sufficiently dissected, the dissectors of the-abdomen, in conjunction with the dissectors of the lower limbs, should open in to the hip-joint on the side on which it has been left undisturbed by operating upon the smooth inclined pelvic aspect of the ischial portion of the hip-bone. This dissection will enable the dissectors to study the action of the ligamentum teres with the capsular ligament left undisturbed. (4) The synovial membrane of the joint is to be studied. (5) The bony articular surfaces are to be examined. (6) The movements at the joint, and the muscles by which these are effected, are to be thoroughly mastered, and the arterial supply and nerve-supply are to be reviewed.

The relations of structures to the anterior intertrochanteric line should next receive attention, and the obturator canal and its contents should be examined. The relative position of the muscles from the symphysis pubis outwards to the obturator foramen should be noted. Finally, the relative positions of the tendons of insertion of the sartorius, gracilis, and semitendinosus, and the bursa in connection with them, slould be examined.

The dissector is now prepared to remove the limb. Any muscles passing between the pelvis and the thigh are to be cut, and the ligamentum teres is 
to be divided, after which the limb can be separated. The first duty of the dissector is to trim the femoral muscles, and revise their attachments and relations. On the inner side of the knee-joint, if not previously displayed, he should show the long saphenous vein and nerve, the posterior division of the internal cutaneous nerve, and the superficial branch of the arteria anastomotica magna. The deep branch of the last-named artery, if not previously dissected, should now be followed out. The relation of the long external lateral ligament of the knee-joint to the tendon of insertion of the biceps femoris, as well as the bursa in this situation, and the expansion from the tendon to the deep fascia of the leg, should be shown.

Front and Sides of the Leg and Dorsum of the Foot. - The dissector is to make himself thoroughly familiar with the landmarks of the leg and foot. The skin is then to be removed by making the following incisions: a vertical incision along the anterior border of the tibia, and along the dorsum of the foot as far as the web between the second and third toes; a transverse incision at the anklejoint, and another at the webs of the toes; a median incision along the dorsum of each toe ; and a transverse incision across the dorsum of each toe close to the nail. The skin is to be reflected to either side, and the following structures should be exposed on the inner aspect of the tibia: (I) the long saphenous vein, which should be shown passing in front of the internal malleolus, and traced as far as the inner end of the dorsal venous arch; (2) the long saphenous nerve, accompanying the foregoing vein as far as the centre of the inner border of the foot ; $(3)$ the posterior division of the internal cutaneous nerve, ramify. ing in the integument of the upper half of the leg; and (4) the superficial branch of the arteria anastomotica magna, ramifying in the upper third. Communications are to be sought for between the long and short saphenous veins, and also between the former and the deep-seated veins on the front of the leg.

The venous arch on the dorsum of the foot should next be displayed, care being taken to preserve the cutaneous nerves. The long saphenous vein will conduct to it, and the small tributary veins from the superficial plantar venous plexus may be shown turning round the inner border of the foot to join the long saphenous vein. The short saphenous vein should be traced from the outer end of the arch to a point below and then behind the external malleolus ; the small tributary veins from the superficial plantar venous plexus may be shown turning round the outer border of the foot to join it ; and an endeavour may be made to show the following tributaries of the dorsal venous arch, namely, (1) the dorsal digital veins; (2) small veins from the dorsum of the foot; and (3) the efferent or interdigital veins from the plantar transverse venous arch.

The dissector should now display the musculo-cutaneous nerve. He will find it piercing the deep fascia on the outer side of the leg about the junction of the upper two-thirds and lower third. Branches should be traced to the integument of the front of the leg in its lower third, and the main nerve should be followed on in two divisions, to be distributed to the inner side of the great toe and the contiguous sides of the second and third, third and fourth, and fourth and fifth toes, as well as to the integument of the malleoli and dorsum of the foot.

The external or short saphenous nerve should next be dissected along the outer border of the foot as far as the outer side of the little toe. Upon the outer side of the leg the dissector will find the lateral cutaneous branch of the external popliteal nerve, which ramifies in the integument of about the upper two-thirds. The deep fascia of the front and sides of the leg should next be studied. Three intermuscular septa are to be noted, namely, anterior, antero-external, and postero-external. In the region of the anklejoint the following parts of the deep fascia are to be specially dissected, namely, (1) the external annular ligament, strapping down the tendons of the peroneus longus and peroneus brevis; and (2) the anterior annular ligament, which will be found in two divisions-one above the ankle-joint and the other in front of it. The following points are to be specially noted in connection with the two divisions of the anterior annular ligament, namely, 
(I) the number of compartments in each division; (z) the tendons passing beneath each, and their relative position; and (3) the number of synovial sheaths.

The anterior tibial muscles are next to be dissected to their insertions in the following order, namely, the tibialis anticus, extensor longus digitorum, peroneus tertius, and extensor proprius hallucis. In connection with the expansions formed by the long extensor tendons on the dorsal aspects of the metatarso-phalangeal joints, the tendons of insertion of the lumbricales and interossei are to be carefully preserved. The anterior tibial nerve will be found coming in to con tact with the outer side of the anterior tibial artery about the junction of the upper fourth and lower three-fourths of the leg, and it should be followed from this point as far as the front of the ankle-joint. The anterior tibial artery is now to be shown lying deeply upon the interosseous membrane over about the upper two-thirds, but upon the anterior surface of the tibia in the lower fourth. In dissecting the upper part of the artery, the anterior tibial lymphatic gland should be looked for, and the venæ comites accompanying the artery should be noted. The following branches of the artery should be shown, namely, anterior tibial recurrent, muscular, internal malleolar, and external malleolar. In dissecting the anterior tibial recurrent artery, the recurrent articular branch of the external popliteal nerve should be looked for. The terminal part of the anterior peroneal artery should be exposed as it appears through the inferior hiatus in the interosseous membrane, and it should be followed downwards under cover of the peroneus tertius and in front of the inferior tibio-fibular articulation, to take part in the external malleolar anastomosis.

The dissector should now turn his attention to the dorsum of the foot. The deep fascia should be examined. The extensor brevis digitorum is to be dissected, and the insertion of its innermost tendon and its relation to the arteria dorsalis pedis are to be noted. The arteria dorsalis pedis and its venæe comites are to be dissected, and the following branches of the artery are to be followed out, namely, internal tarsal, external tarsal, metatarsal, and arteria dorsalis hallucis. The arch formed by the metatarsal branch is to be noted, and three dorsal interosseous arteries are to be dissected forwards from it. The dorsalis pedis nerve (the continuation of the anterior tibial nerve) is next to be dissected as far as the cleft between the great toe and second, where it will be found to divide into two collateral dorsal digital branches for the supply of the contiguous sides of these two toes. The offset which it receives from the branch of the musculo-cutaneous nerve to the inner side of the great toe is to be shown, and the external branch of the nerve is to be followed beneath the extensor brevis digitorum. In this situation its gangliform enlargement is to be looked for, and the offsets arising therefrom may be followed out.

The four dorsal interosseous muscles should now be dissected. In connection with the first dorsal interosseous the plantar or perforating branch of the arteria dorsalis pedis is to be noted, and in connection with the second, third, and fourth, the posterior and anterior perforating arteries should be looked for.

The dissector should now turn his attention to the external aspect of the leg, where the peroneus longus and peroneus brevis are to be dissected. The latter muscle should be dissected throughout its entire course, but the former should only be followed meanwhile as far as the groove on the outer border of the cuboid bone. In dissecting these muscles, the fibro-osseous tunnel in which they lie is to be noted. The musculo-cutaneous and anterior tibial nerves are now to be traced upwards to their origin from the external popliteal at the level of the neck of the fibula. In doing so, in the case of the musculo-cutaneous nerve, both peroneal muscles are to be cut in so far as may be necessary, and in the case of the anterior tibial nerve the extensor longus digitorum and peroneus longus are to be cut. The mode of ending of the external popliteal nerve will now be made manifest, its terminal branches bcing the anterior tibial, musculo-cutaneous, and recurrent articular.

Back of the Leg. - A transverse incision having been made over the heel, the 
skin is to be reflected from the back of the leg. The terminal distribution of the small sciatic nerve having been revised, the dissector should trace the ramus communicans tibialis and ramus communicans fibularis nerves to the centre of the calf, the former lying in the median groove of the gastrocnemius, and the latter descending obliquely inwards over the outer head of that muscle. At or about the centre of the calf these two nerves will usually be found to unite, and so form the external or short saphenous nerve. This nerve, along with the short saphenous vein, should be followed superficial to the deep fascia downwards and outwards along the outer side of the tendo Achillis (the nerve meanwhile giving branches to the integument of the lower half of the back of the leg), then behind and below the external malleolus (in which situation the nerve supplies calcaneal and malleolar branches), and subsequently along the outer border of the foot. Communications between the short saphenous vein and the long saphenous vein are to be sought for, as well as communications between the former and the deeply-seated veins which accompany the posterior tibial and peroneal arteries.

The deep fascia of the back of the leg is next to be dissected, and thereafter the gastrocnemius muscle is to be studied. The inner head of the muscle having been previously cut in connection with the dissection of the lower part of the popliteal space, the outer head is now to be divided, and both heads are to be reflected upwards in order to show the exact origin of each, that of the inner head lying obliquely, whilst that of the outer head lies vertically. The popliteal bursa, which separates the inner head from the semimembranosus, should be sought for, and its relation to the synovial membrane of the knee-joint examined. In the outer head of the muscle will be found a sesamoid fibro-cartilage (sometimes ossified). The belly of the gastrocnemius and its tendon is to be reflected downwards as far as the commencement of the tendo Achillis. The plantaris is then to be dissected, and its long, narrow tendon should be taken between the index-finger and thumb of each hand and stretched laterally to demonstrate its distensibility. The soleus is next to be dissected, and the fibrous arch over the posterior tibial vessels is to be shown, with fibres of the muscle springing from it. A branch from the internal popliteal nerve is to be found entering the superficial surface of the muscle near its upper bcrder. The other nerve-supply from the posterior tibial will only come into view as the muscle is being reflected. The tendon of the soleus is to be shown joining that of the gastrocnemius to form the tendo Achillis. The aponeurotic appearance presented by the deep surface of the gastrocnemius and the superficial surface of the soleus is to be noted. The tendo Achillis is to receive careful attention.

Before the soleus is disturbed, the hollow on either side of the tendo Achillis is to be noted. In the outer hollow the short saphenous vein and nerve have been already dissected, their position being here superficial. Lying deeply in the hollow on the inner side of the tendon are the posterior tibial vessels and nerve. A limited opening should be made in the deep fascia to show the exact position and relations of these structures. The soleus is now to be stripped from its tibial and fibular origins and turned downwards. In the course of this dissection a branch from the posterior tibial nerve is to be shown entering the deep surface of the muscle about the centre of the leg. The tendo Achillis having been tur:ed downwards, the large quantity of fat beneath it is to be observed, and the bursa between the tendon and the upper zone of the posterior surface of the tuber calcis is to be shown. By dissecting into the deep surface of the soleus, the arrangement of its very short fibres will be brought into view.

The lower part of the popliteal vessels and internal popliteal nerve, though previously dissected, should now be revised. The division of the artery into anterior tibial and posterior tibial at the lower border of the popliteus muscle should be noted. The commencement of the anterior tibial artery is to be dissected, showing the vessel passing forwards between the two heads of the tibialis posticus, and between the tibia and fibula. Its branches in this situation are to be shown, namely, posterior tibial recurrent (inconstant), and superior fibular. The nerve to the popliteus from the internal popliteal, if 
not previously dissected, is to be followed out. The inferior external and inferior internal articular arteries are to be studied. The popliteal fascia covering the popliteus muscle is to be inspected, noting that it is one of the modes of insertion of the semimembranosus. The fascia is now to be removed, in doing which it should be noted that the superficial fibres of the popliteus muscle take insertion into its deep surface. In the meantime, the exact origin of that muscle cannot be shown, so that the dissector should content himself with showing the tendon after its escape from the interior of the knee-joint, when it is almost immediately replaced by fleshy fibres.

The sural muscles having been completely turned aside, the expansion of the deep fascia, called the posterior or transverse intermuscular septum, is to be studied. In connection with it the internal annular ligament is to be displayed (but not opened up as yet) between the internal malleolus and the tuber calcis. In doing so, care must be taken to preserve the calcaneo-plantar branch of the posterior tibial nerve and the internal calcaneal branch of the posterior tibial artery, both of which pierce the ligament. The deep muscles of the back of the leg are to be cleaned, and at the same time the posterior tibial vessels and nerve are to be dissected. The muscles from within outwards are the flexor longus digitorum, tibialis posticus, and flexor lnngus hallucis. It should be observed that the tibialis posticus is covered superficially by a strong aponeurotic expansion, which stretches across between the muscles on either side of it. In dissecting the lower portions of these muscles in the neighbourbood of the ankle-joint, the internal annular ligament is now to be opened, when it will be seen to contain four canals, three of which are fibroosseous, and one (for the flexor longus digitorum) purely fibrous. The relation of the tendons, posterior tibial vessels, and posterior tibial nerve as they traverse these canals is to be studied, and the synovial sheaths are to be noted. The branches of the posterior tibial artery and nerve are to be displayed. As regards the branches of the artery, they should be dissected in the following order from above downwards, namely, (I) peroneal, of large size, and arising about $I$ inch below the commencement of the posterior tibial ; (2) medullary, for the tibia (of large size); (3) muscular ; (4) communicating or transverse, near the ankle, and passing transversely outwards to join a similar branch of the peroneal; (5) internal malleolar, usually two in number, and passing beneath the tendons behind the internal malleolus to take part in the internal malleolar anastomosis : (6) internal calcaneal; and (7) the two terminal branches, internal and external plantar arteries. Two venæ comites are to be dissected with the posterior tibial artery, which communicate at frequent intervals by transverse branches placed superficial to the vessel. These venæ comites should be shown to join those of the anterior tibial artery at the lower border of the popliteus muscle, and so form the popliteal vein. The peroneal branch of the posterior tibial artery requires special dissection. After reaching the back of the fibula, it sinks deeply and disappears from view, its course being either between the fibula and the flexor longus hallucis, or in that muscle, or in a fibrous canal between that muscle and the tibialis posticus. It will next be found becoming superficial about 2 inches above the ankle, where it should be shown to end by dividing into an anterior and a posterior peroneal branch. Two venæ comites are to be dissected with the peroneal artery, which end by joining those of the posterior tibial. The following branches of the peroneal artery are to be dissected from above downwards, namely, (1) muscular; (2) medullary to fibila; (3) communicating or transverse ; (4) anterior peroneal, disappearing through the inferior hiatus in the interosseous membrane; and (5) posterior peroneal. The lastnamed artery is to be shown passing behind the external malleolus on to the outer border of the foot for a variable distance. It will be seen to give off external calcaneal branches, which take part in the external malleolar anastomosis, and anastomose across the heel with the internal calcaneal branches of the external plantar. On the outer border of the foot it will be seen again to anastomose with branclies of the external plantar.

Knee-Jolnt. - The knee-joint is to be dissected before proceeding with the sole of the foot. The muscular and tendinous relations of the joint are to be 
studied, and the threefold insertion of the semimembranosus is to be displayed. The arteries entering into the geniculate arterial rete are to be studied as fully as possible. All the external ligaments of the joint are now to be dissected, namely, (1) the ligamentum patellæ; (2) the lateral patellar ligaments; (3) the internal lateral ligament, with the inferior internal articular artery passing beneath it, and the main division of the tendon of the semimembranosus overlapped by it posteriorly ; (4) the long and short external lateral ligaments, with the inferior external articular artery passing beneath the long ligament; (5) the posterior ligament, with its thickened part, known as the ligamentum posticum Winslowii; and (6) the capsular ligament. In connection with the ligamentum patellæ, the bursa between it and the upper part of the tubercle of the tibia should be shown. The exact origin of the tendon of the popliteus is to be displayed, and its relation to the joint and the long external lateral ligament noted. The expansion of the synovial membrane of the joint upwards above the patella and beneath the suprapatellar tendon is to be again studied. The articulation is now to be opened from before by cutting transversely down through the synovial membrane just above the patella. This bone, along with the ligamentum patellæ, having been turned downwards, underneath them will be seen a collection of fat covered by the synovial membrane, and representing the Haversian gland of the joint. In connection with this portion of the synovial membrane, the following so-called ligaments are to be studied, namely, the ligamentum mucosum and the ligamenta alaria. The crucial ligaments are next to be examined in situ, as well as the semilunar fibro-cartilages.

The synovial membrane is to be examined, and thereafter the following external ligaments, are to be cut, namely, the capsular and the posterior, thus leaving only the two lateral ligaments intact. The joint is now to be placed in different positions-e.g., flexion, extension, internal rotation, and external rotation-and the conditions of the crucial and lateral ligaments, as well as of the semilunar fibro-cartilages, are to be carefully attended to.

The la teral ligaments are then to be cut, when the femur will be bound to the tibia only by the crucial ligaments, which should be completely mastered. The femur should be firmly grasped and the tibia rotated inwards in order to see the effect of this upon the anterior crucial ligament. The tibia should next be rotated outwards, and it will then be made to describe half a circle, neither crucial ligament interfering with this movement. In connection with the posterior cornu of the external semilunar fibro-cartilage, the posterior accessory bundle is to be observed, and thereafter the crucial ligaments are to be divided. The upper surface of the head of the tibia boing now fully exposed, the semilunar fibro-cartilages are to be studied, and in connection with their anterior aspects the transverse ligament is to be examined. Lastly, the bony articular surfaces are to be examined.

Sole of the Foot. - The skin is to be removed from the sole and plantar aspects of the toes by making the following incisions: a median incision from the heel to the roots of the toes, a transverse incision across the roots of the toes, a median incision along the plantar aspect of each toe, and a transverse incision across the tip of each toe. Within the skin of the webs of the toes a bundle of transverse fibres, called the superficial transverse ligament, is to be dissected. The superficial fascia should be examined, and the calcaneal and plantar divisions of the calcaneo-plantar branch of the posterior tibial nerve are to be followed out to their distribution.

The superficial fascia is now to be removed so as to expose the dense deep fascia, known as the plantar fascia. In dissecting the three divisions of this important fascia, care is to be taken to display the following structures: ( $I$ ) cutaneous branches of the internal plantar artery and nerve, which appear in the groove between the inner and middle divisions; and (2) cutaneous branches of the external plantar artery and nerve, which appear in the groove between the middle and outer divisions. The three divisions of the fascia are then to be carefully studied, and the grooves between them, indicating intermuscular septa, are to be noted. In the outer groove, near the base of the fifth metatarsal bone, the external plantar artery should be exposed, 
and its superficial position noted. Special attention is to be directed to the middle division of the fascia. On being followed towards the toes, it should be shown to divide into five digital processes, one for each toe. The longitudinal direction of the fibres (from heel to toes) should be observed, but towards the toes superadded transverse fibres are to be displayed. In the interval between the diverging digital processes the plantar digital vessels and nerves are to be sought for and followed along the sides of the toes. The manner in which the digital processes are disposed is to be studied, and the bearing of the middle division of the plantar fascia upon the deformity known as pes cavus is to be carefully noted. In connection with the outer division of the fascia attention should be paid to the strong band which passes between the outer tubercle of the os calcis and the tuberosity on the outer side of the base of the fifth metatarsal bone, and which may be represented by a muscle known as the abductor ossis metatarsi quinti or Wood's muscle.

The plantar fascia is now to be removed in the following manner: (I) the thin inner division is to be removed in the ordinary way so as to expose the abductor hallucis muscle, which it covers; $(2)$ the stronger outer division is also to be removed in the usual way so as to expose the abductor minimi digiti, which lies underneath it; (3) the very strong middle division, which closely covers the flexor brevis digitorum, is to be carefully divided transversely about I inch in front of the heel, the posterior narrow part is to be dissected backwards, and the expanding anterior part is to be reflected forwards. This dissection will bring into view the flexor brevis digitorum, and it will show the extent to which the superficial fibres of that muscle take origin from the deep surface of the middle division of the fascia. In dissecting the tendon of insertion of the abductor hallucis, the inner head of the flexor brevis hallucis is to be shown joining it, and a branch from the internal plantar nerve is to be looked for entering the abductor hallucis on its deep aspect about the centre. In dissecting the flexor brevis digitorum, it should be shown to end in four tendons for the four outer toes. Each tendon will be found to lie superficial to a tendon of the long flexor of the toes, and the canal in which each pair of tendons lies before passing along the toes should be studied.

The sheaths which confine the flexor tendons as they pass along the plantar aspects of the toes are next to be dissected. Upon one or more toes the sheaths are to be laid open, the insertions of the tendons made out, and the vincula accessoria tendinum (ligamenta longa and ligamenta brevia) shown. A similar dissection is to be made in connection with the tendon of the flexor longus hallucis. The intermuscular septum on either side of the short flexor is to be noted, and twigs from the internal plantar nerve are to be looked for entering the muscle on its deep surface. In dissecting the abductor minimi digiti, twigs from the external plantar nerve are to be looked for entering the deep surface of the muscle at its back part.

The muscles of the first layer are to be divided near their origins and thrown forwards. The tendons of the flexor longus digitorum and flexor longus hallucis are now to be dissected, the former passing forwards and outwards, and the latter forwards and inwards. The crossing between these two tendons should be noted, that of the flexor longus hallucis being uppermost, and the slip which this tendon furnishes to that of the long flexor of the toes should be displayed. The tendon of the flexor longus hallucis is to be followed out to its insertion, and the vincula accessoria tendinum shown within its sheath on the great toe. The flexor or musculus accessorius is to be dissected in connection with the tendon of the long flexor of the toes previous to its division. In dissecting the musculus accessorius the external plantar vessels and nerve are to be carefully preserved as they cross the sole, and twigs of the nerve are to be looked for entering the inferior surface of the muscle at its back part. The four lumbricales muscles are to be dissected in connection with the four tendons of the long flexor of the toes. It should be noted that the innermost lumbricalis arises only from one tendon, whilst the other three arise each from the contiguous sides of two tendons. A branch from the second digital nerve of the internal plantar should be shown 
entering the innermost lumbricalis, whilst the other three will be found to receive their nerve-supply from the deep part of the external plantar nerve.

The internal plantar nerve is now to be dissected, as well as the internal plantar artery. The small size of the artery and the large size of the nerve are to be noted, and each is to be followed out to its distribution. The external plantar artery and nerve are next to be dissected in the first part of their course as they cross the sole from within outwards. The large size of the artery is to be noted, and the nerve is to be shown supplying the musculus accessorius and abductor minimi digiti, besides giving off articular and cutaneous branches. The superficial terminal branch of the external plantar nerve is also to be followed out, showing its two digital nerves, the outermost of which supplies the flexor brevis minimi digiti, and, as a rule, the interosseous muscles of the fourth space. The tendons of the flexor longus hallucis and flexor longus digitorum and the musculus accessorius are now to be divided near the ankle and turned forwards, but the plantar vessels and nerves are to be left undisturbed.

The dissector is then to display the third layer of muscles in the following order from within outwards : flexor brevis hallucis, adductor obliquus hallucis, and flexor brevis minimi digiti. Lying across the heads of the four outer metatarsal bones will be found the adductor transversus hallucis (transversalis pedis). In dissecting the flexor brevis hallucis its single origin and double insertion are to be noted, and a sesamoid bone is to be looked for in each head of insertion. Its nerve is to be found coming from the first or most internal digital branch of the internal plantar. The nerve of the adductor obliquus hallucis is to be found coming from the deep part of the external plantar nerve. In dissecting the flexor brevis minimi digiti care must be taken to separate it from the most external plantar interosseous, close to which it lies, and with which it is apt to be confounded. Its nerve is usually to be found coming from the outer digital branch of the superficial part of the external plantar. In dissecting the adductor transversus hallucis it should be noted that the digital nerves on their way to the toes lie superficial to the muscle, whilst the digital vessels lie on its deep surface. The nerve-supply of this muscle is to be found coming from the deep part of the external plantar. The dissector should now show the relation of muscles which take insertion into the base of the first phalanx of the great toe, as follows: inner side-abductor hallucis and inner head of flexor brevis hallucis; outer side-outer head of flexor brevis hallucis, adductor obliquus hallucis, and adductor transversus hallucis. The plantar triangle and its contents should next be examined.

The further stage of the dissection of the sole consists in dividing the flexor brevis hallucis and adductor obliquus hallucis, in order to expose (1) the deep parts of the external plantar artery and nerve, (2) the plantar (and dorsal) interossei muscles, and (3) the plantar or perforating branch of the arteria dorsalis pedis. In the case of the deep part of the external plantar nerve, muscular, articular, and perforating branches are to be looked for. It is to be noted that the deep part of the external plantar nerve gives branches to the outer three lumbricales. In the case of the deep part of the external plantar artery the plantar arch and its branches are to be dissected. The plantar or perforating branch of the arteria dorsalis pedis is to be found appearing at the proximal part of the first interosseous space, and the following branches are to be dissected, namely, arteria magna or princeps hallucis, and communicating to the plantar arch.

The external plantar artery and nerve may now be cut to enable them to be laid fully aside, and the plantar interosseous muscles are to be dissected. Care must be taken not to confound the most external plantar interosseous with the flexor brevis minimi digiti. At this stage of the dissection the insertion of the lumbricales is to be studied. The innervation of the interossei will be found to come from the deep part of the external plantar nerve, except in the case of the interossei of the fourth space, which usually get their nervesupply from the outer digital branch of the superficial part of the external plantar. The dissector should now revise the insertion of all the tendons connected with the foot, and, if any have not yet been followed out to their 
insertions, this should now be done. The actions of the muscles to which these tendons give insertion should be mastered, with special reference to the different varieties of club-foot, and an articulated foot should be before the dissector to enable him to illustrate these varieties and the actions of the muscles involved in producing them.

Attention is now to be directed to the remaining articulations, namely, the tibio-fibular joints, the ankle-joint, and the joints of the foot. Before removing the muscles of the front of the leg and the deep muscles of the back, the anterior and posterior relations of the interosseous membrane should be studied, and the muscular relations of the ankle-joint mastered. Thereafter the muscles are to be removed, and attention is to be directed first to the superior tibio-fibular joint. The relation of the tendon of insertion of the biceps femoris to the joint is to be noted, as well as the relation of the tendon of the popliteus. More especially, the relation of the anterior division of the biceps tendon to the anterior ligament of the joint is to be studied. Having dissected the ligaments (anterior and posterior), the joint is to be opened and the synovial membrane examined. The bony articular surfaces are also to be inspected, and the movements of which the joint is capable are to be studied. The interosseous membrane is next to be dissected. The large deficiency left at its upper part for the passage of the anterior tibial vessels and.lymphatics is to be noted, and the anterior tibial lymphatic gland, if not previously dissected, is now to be shown. In the lower part of the interosseous membrane a small opening is to be displayed for the passage of the anterior peroneal vessels. The inferior tibio-fibular joint is next to be examined, pursuing the same order as in the examination of the superior. The ligaments to be dissected are anterior, posterior, inferior interosseous, and transverse, special attention being directed to the inferior interosseous ligament. The joint having been opened, the synovial membrane (which is continuous with that of the ankle-joint) is to be examined. Attention should next be directed to the bony articular surfaces and the movements of which the joint is capable.

The ankle-joint should now be carefully examined. The dissector should first revise the muscular relations of the joint, and thereafter he should dissect the following ligaments: anterior, posterior, internal lateral or deltoid, and external lateral in three fasciculi, anterior, middle, and posterior. The anterior and posterior ligaments are then to be cut and the synovial membrane examined. Its continuity with the synovial membrane of the inferior tibio-fibular joint is to be noted, and collections of fat covered by it (Haversian glands) are to be shown at the front and back of the joint. The bony articular surfaces are to be examined, and special attention is to be given to the movements of which the joint is capable, and the muscles by which these are effected.

The longitudinal and transverse arches of the foot should now receive careful attention, and a review of the different varieties of club-foot and the tendons involved will prove advantageous. Thereafter the articulations of the foot are to be dissected, and in doing so it is advisable that the dissector should have before him an articulated foot and the individual bones, all belonging to the same side as that which he is dissecting. The tarsal articulations are to be studied first, in the following order: (1) astragalo-calcaneal ; (2) astragalo-navicular; (3) calcaneo-cuboid ; (4) naviculo-cuboid ; (5) naviculocuneiform; (6) intercuneiform; and (7) cubo-cuneiform. Before pursuing this order the dissector should make himself familiar with the transverse tarsal articulation-that is to say, the conjoined astragalo-navicular and calcaneo-cuboid joints, where disarticulation is performed in Chopart's operation. He should also study the expansions from the tendon of insertion of the tibialis posticus, and he should at this stage revise the tendon of the peroneus longus as it crosses the sole of the foot. In connection with the astragalo-calcaneal articulation two joints are to be recognised-posterior and anterior. The following ligaments are to be studied in connection with the posterior joint: interosscous, posterior, internal, and external. Only a lateral vicw of the interosseous ligament will be obtained at this stage, as it lics in the sinus pedis. It is to be noted that this joint has a synovial. membrane peculiar to it. The anterior astragalo-calcaneal joint is next to 
be dissected, and the following ligaments studied : interosseous, internal astragalo-calcaneal, and external or superior calcaneo-navicular. It is to be noted that the synovial membrane of this joint is continuous with that of the astragalo-navicular articulation. The bony articular surfaces of the astragalo-calcaneal joints cannot be studied until the astragalus is removed, which should presently be done. Meanwhile, by removing all the ligaments, except the interosseous, and dividing the synovial membranes, the movements between the astragalus and os calcis are to be studied.

The astragalo-navicular joint should now be examined. Inasmuch as this is one of the most important joints of the foot, it should receive careful study. The ligaments to be dissected are as follows: astragalo-navicular, dorsally placed ; external or superior calcaneo-navicular; and internal or inferior calcaneo-navicular, a most important ligament, commonly spoken of as the spring ligament. The last-named ligament is to be studied from two points of view, namely, from below, by removing the tendon of the tibialis posticus, and from above, by removing the astragalus. In performing the latter dissection the interosseous astragalo-calcaneal ligament is to be studied, and it is to be observed that, whilst it is the anterior ligament of the posterior astragalo-calcaneal joint, it is the posterior ligament of the anterior astragalocalcaneal joint. An excellent view is obtained from above of how the spring ligament supports and forms a part of the socket for the under aspect of the nead of the astragalus, and from below it can be well seen how the tendon of the tibialis posticus serves as an important strengthening adjunct to the ligament. It is to be noted that the synovial membrane of the astragalonavicular joint is continuous behind with that of the anterior astragalocalcaneal. The movements at the astragalo-navicular articulation are to be carefully studied, and the manner in which pes planus may be brought about is to be observed. Attention should also be directed to the support which the spring ligament, aided by the tendon of the tibialis posticus, gives to the inner longitudinal arch of the foot. The bony articular surfaces are also to receive attention.

The calcaneo-cuboid joint is next to be studied. The ligaments to be dissected are dorsal, internal or interosseous, long plantar, and short plantar. On opening the joint the synovial membrane will be found to be peculiar to it, and the movements of which the articulation is capable are to be studied. Attention is also to be given to the bony articular surfaces. The naviculocuboid joint is next to be attended to, the ligaments being dorsal, plantar, and interosseous. The remaining tarsal articulations are to be studied in the following order : naviculo-cuneiform, intercuneiform, and cubo-cuneiform, and the complicated synovial membrane of these articulations should be examined. The bony articular surfaces and movements are also to be studied.

The dissector should next direct his attention to the tarso-metatarsal articulations, which should be studied in the following order: (I) internal tarso-metatarsal, (2) middle tarso-metatarsal, and (3) external tarso-metatarsal. Special notice should be taken of the internal interosseous ligament of the middle tarso-metatarsal joint. It will be found extending between the outer surface of the internal cuneiform and the inner surface of the base of the second metatarsal bone. The synovial membranes, bony articular surfaces, and movements of these articulations are to be attended to, and their surgery is to be carefully studied in connection with Lisfranc's operation.

The intermetatarsal articulations are to be studied next, attention being directed to the basal intermetatarsal joints, and the union between the heads of the metatarsal bones. The tarsal and tarso-metatarsal synovial membranes should be reviewed at this stage. The metatarso-phalangeal articulations are now to be dissected, the ligaments being two lateral and an inferior or plantar fibrous plate. The synovial membranes, bony articular surfaces, and movements are to be studied. Lastly, the interphalangeal articulations are to be dissected in a similar manner and to a like extent. 



\section{THE ABDOMEN}

\section{MALE PERINEUM.}

Landmarks. - The tuber ischii can be felt on either side, as well as the ischio-pubic ramus; but the great sacro-sciatic ligament cannot be felt, on account of the thickness of the lower border of the gluteus maximus. The tip of the coccyx is very accessible to the finger.

The perineum practically corresponds with the outlet of the pelvis, and is somewhat lozenge-shaped, having the subpubic angle in front, the tip of the coccyx behind, and the tuber ischii on either side. It is conveniently divided into two parts by a line connecting the ischial tuberosities, each division being triangular. The anterior represents the uro-genital or urethral division, this being the perineum proper, and the posterior the ischio-rectal or anal division. The skin of the uro-genital division presents an antero-posterior-median elevation, called the perineal raphé, which extends over the posterior and anterior surfaces of the scrotum, and along the under surface of the penis. The position of the deeplyplaced central tendinous point of the perineum is indicated by taking a point in this raphé very nearly $I$ inch in front of the anus.

The anus is the external opening of the anal canal. It is situated posterior to a line connecting the front parts of the ischial tuberosities, and about $I \frac{1}{2}$.inches from the tip of the coccyx. A delicate white line surrounds the anus where the skin and mucous membrane meet, which indicates the separation between the external and internal sphincter muscles (Hilton). The position of each ischio-rectal fossa is indicated by a slight depression between the anus and tuber ischii.

Deep Boundaries of the Perineum-Anterior.-The subpubic angle and subpubic ligament. Posterior.-The coccyx. Lateral. -From behind forwards, the inferior border of the great sacrosciatic ligament, tuber ischii, and ischio-pubic ramus.

\section{Ischio-Rectal Division.}

Cutaneous Nerves.-These are (I) the hemorrhoidal or perineal branch of the lower part of the anterior primary division of the 
fourth sacral, and (2) the inferior hemorrhoidal nerve, which is a branch of the pudic. The former supplies the integument between the tip of the coccyx and the back of the anus, and the latter the integument between the anus and tuber ischii.

Fascia.-The fascia of this division is very thick, and is prolonged into the ischio-rectal fossa, where it forms an elastic pad for the lower part of the rectum and the anal canal. There is no deep fascia.

Ano-coccygeal Body. - This is a collection of fibrous and muscular tissues situated between the coccyx and anal canal, the muscular element being connected with the levatores ani and sphincter ani muscles (Symington). Its importance lies in the fact that it gives support to the anal canal.

Muscles.-The muscles in this division are the sphincter ani externus, corrugator cutis ani, levator ani, and coccygeus.

Sphincter Ani Externus-Origin.-The tip of the coccyx and the integument over it.

Insertion.-The greater part of the muscle is inserted into the central tendinous point of the perineum. The more superficial fibres, however, are inserted into the skin, and a certain amount of decussation takes place across the middle line.

Nerve-supply. - The part of the muscle between the coccyx and the anus is supplied by the hemorrhoidal or perineal branch of the fourth sacral, and the remainder by the inferior hemorrhoidal and the deep branch of the perineal nerve, both of which are derived from the pudic.

Action.-To keep the anal aperture closed, at the same time producing a wrinkled condition of the skin.

The muscle is situated immediately beneath the skin, and is elliptical. Behind and in front of the anus it is single, but around that aperture it is arranged in two symmetrical halves, which are intimately connected with the middle portions of the levatores ani.

Corrugator Cutis Ani (Ellis).-This muscle is represented by a very delicate sheet of involuntary muscular fibres, which pass in a radiating manner from the submucous tissue of the anal aperture to be inserted into the surrounding skin.

Action.-(I) To throw the integument around the anus into wrinkles; and (2) to invert the mucous membrane of the lower end of the anal canal after it has been everted during defæcation.

For the levator ani and coccygeus, see pp. 862 and 863 .

Ischio-rectal Fossa.-This is a deep fossa which is situated on either side between the ischium and rectum. It measures nearly $2 \frac{1}{2}$ inches in depth, 2 inches from before backwards, and I inch from side to side. In transverse section it is triangular, the base being directed downwards and the apex upwards. The outer wall, which is vertical, is formed by the lower part of the obturator internus muscle covered by the obturator fascia. The inner wall, which is oblique and longer than the outer, is formed by the lower part of the rectum, the anal canal, the levator ani muscle covered by the anal fascia, and the sphincter ani externus. The base is 
formed by the skin and fascia which extend between the tuber ischii and anus. The roof is just below the white line, where the anal fascia springs from the obturator fascia. Anteriorly the fossa is partially limited by the reflection of the fascia of Colles round the posterior border of the superficial transverse perineal muscle to join the base of the triangular ligament of the urethra. Above this junction there is a forward prolongation of the fossa, called the anterior diverticulum, which extends almost to the symphysis pubis. It lies between the postero-superior layer of the triangular ligament and the inferior surface of the anterior part of the levator ani muscle,

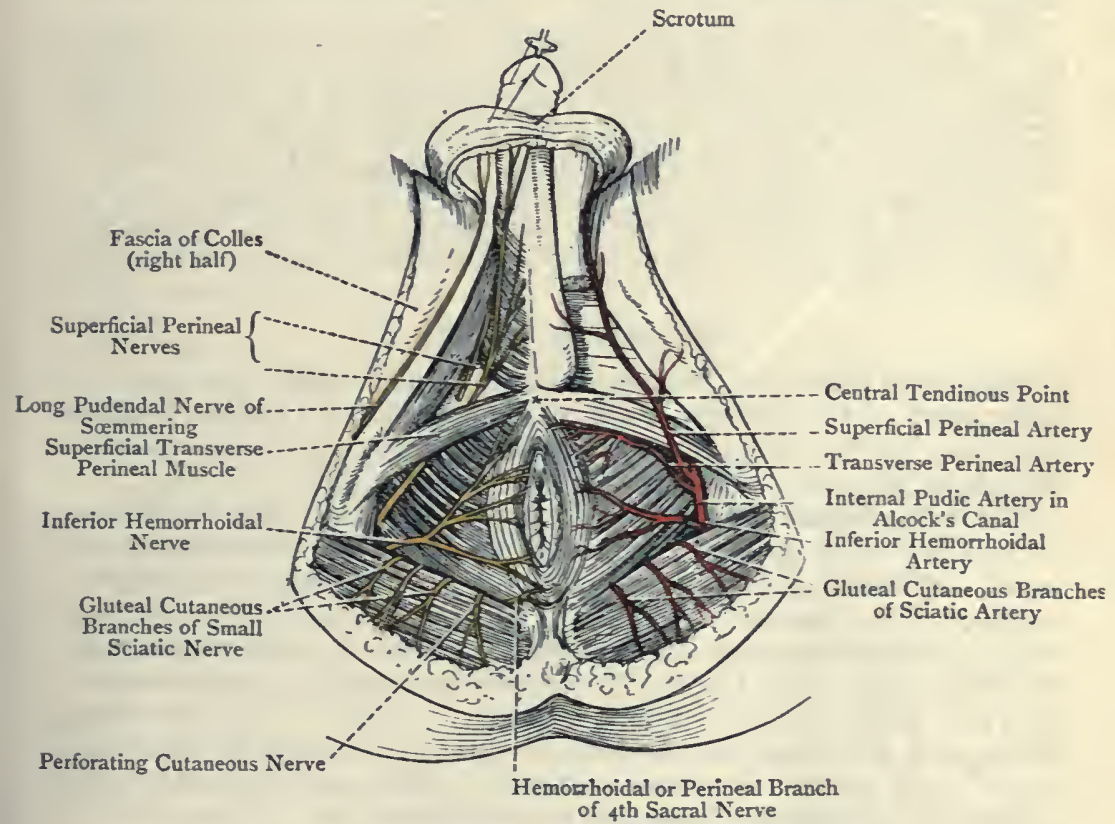

Fig. 274.-Dissection of the Male Perineum

(On the left side the bulbo-cavernosus has been removed, and the crus penis cut).

and its lateral limits are the prostate gland and pubo-prostatic ligament internally, and the ischio-pubic ramus externally. Posteriorly the fossa is partially limited by the margin of the great sacro-sciatic ligament and the lower border of the gluteus maximus muscle. Between these two structures it is prolonged backwards for a short distance in the form of a posterior diverticulum, which extends outwards towards the tuber ischii, and inwards towards the upper part of the coccyx.

Position of Contents.- The internal pudic vessels and the perineal and dorsalis penis branches of the pudic nerve lie upon the outer 
wall about $I_{\frac{1}{2}}$ inches above the lower part of the tuber ischii, and they are contained in Alcock's canal. The inferior hemorrhoidal vessels and nerve cross the fossa transversely from the outer wall to the anal canal. The superficial perineal vessels and nerves on their way forwards lie for a short distance in the front part. At the back part of the fossa, winding round the lower border of the gluteus maximus, the gluteal cutaneous branches of the small sciatic nerve and sciatic artery, as well as the perforating cutaneous branch of the sacral plexus, are met with.

The fossa is filled with loose fat, which also extends into the anterior and posterior diverticula.

This fat is badly supplied with bloodvessels, and its vitality is therefore low. As a consequence, an ischio-rectal abscess is of not infrequent occurrence. When an abscess forms, the pus has a tendency to discharge itself in two directions, namely, through the skin forming the floor of the fossa, and through the wall of the anal canal about half an inch above the anus. When this happens a complete fistula in ano is the result.

The severe pain which accompanies an ischio-rectal abscess is due to pressure upon the following nerves: (1) the inferior hemorrhoidal ; (2) the two superficial perineal nerves; and (3) the gluteal cutaneous branches of the small sciatic.

\section{Uro-Genital Division.}

Superficial Fascia.-The superficial fascia resembles that over the lower part of the anterior wall of the abdomen in being divisible into two layers, which are called the subcutaneous layer and the deep layer or fascia of Colles.

The subcutaneous layer is fatty and granular. When followed backwards on either side it forms the floor of the ischio-rectal fossa. At the middle line it is continuous with the corresponding layer of the opposite side. Externally it passes freely over the ischio-pubic ramus, and is continuous with the superficial fascia of the inner side of the thigh. Anteriorly it passes into the scrotum, where it joins the deep layer or fascia of Colles. The resultant fascia, now free from adipose tissue, contains involuntary muscular tissue, and forms the tunica dartos. The subcutaneous layer represents the fascia of Camper of the lower part of the anterior abdominal wall.

The deep layer constitutes the faseia of Colles. It is membranous and strong. Externally, it is attached to the anterior everted lip of the inner border of the ischio-pubic ramus, as far back as the tuber ischii, immediately outside the attachment of the crus penis. Internally, the fascia of one side is continuous with that of the other along the middle line. Posteriorly, the fascia turns round the posterior border of each superficial transverse perineal muscle, and joins the base of the triangular ligament. Anteriorly, it passes into the scrotum, where it joins the subcutaneous layer, the two forming the tunica dartos.

The fascia of Colles represents the fascia of Scarpa of the anterior abdominal wall. It encloses a space over the anterior division of the perineum, called the perineal pouch. This pouch is partially 
subdivided into two compartments by an incomplete septum, which extends upwards from the deep or superior surface of the fascia of Colles to be attached to the median raphé above. This septum is only complete for a short distance at the back part; elsewhere it is very imperfect, and is continued forwards into the scrotum.

When air is blown beneath the back part of the fascia of Colles on one side of the middle line, the fascia of that side and the corresponding half of the scrotum become distended. As more air is blown in, the fascia of the opposite side and the corresponding half of the scrotum also become distended.

It is into the perineal pouch that urine is extravasated in rupture of the urethra in the perineum. In such cases the urine cannot pass into either ischio-rectal fossa, its backward course being arrested at the posterior borders of the superficial transverse perineal muscles, where the fascia of Colles, as a whole, turns round to join the base of the triangular ligament. Neither can the urine make its way down the inner side of the thigh, its passage in this direction being stopped at the ischio-pubic ramus to which the fascia of Colles is attached. The only course, therefore, which is impressed upon the extravasated urine is forwards into the scrotal wall and on to the penis, in each case beneath the dartos tunic, whence it passes upwards along the spermatic cord to the anterior wall of the abdomen, in which situation it lies beneath the fascia of Scarpa.

The fascia of Colles covers the following structures: the crura penis, covered by the ischio-cavernosi muscles; the bulb of the urethra, covered by the bulbo-cavernosi muscles; the superficial transverse perineal muscles; the superficial perineal vessels of each side; the three long scrotal nerves of each side; and the deep perineal triangle of each side, in the area of which is one half of the antero-inferior layer of the triangular ligament.

Muscles-Transversus Perinæi Superficialis-Origin.-The inner aspect of the tuber ischii above the origin of the ischio-cavernosus.

Insertion.-The central tendinous point of the perineum.

Nerve-supply. - The deep division of the perineal branch of the pudic nerve.

Action.-To draw back and fix the central tendinous point, and so to aid the action of the bulbo-cavernosus.

The muscle is directed obliquely inwards and forwards, being accompanied by the transverse perineal artery, and it forms the base of the deep perineal triangle.

Ischio-cavernosus (erector penis)-Origin.-(I) The inner aspect of the tuber ischii close below the origin of the superficial transverse perineal muscle; and (2) the inner border of the ramus of the ischium on either side of the crus penis.

Insertion.- The under surface of the fibrous sheath of the crus penis in its front part, and the outer and upper surfaces of the fibrous sheath of the corpus cavernosum penis, in which latter situation it is continuous with the fascial investment of the penis and with the insertion of the suspensory ligament of that organ.

Nerve-supply.-The deep division of the perineal branch of the pudic nerve. 
Action.-The muscle compresses the crus penis against the ischiopubic ramus, and, by retarding the return of venous blood, it helps to maintain the penis in a state of erection. It also contributes to the maintenance of erection by compressing the dorsalis penis vein.

The anterior and outer part of the muscle is sometimes detached, and forms a separate muscle, called the compressor venæ dorsalis penis. This arises from the descending pubic ramus, anterior to the origin of the ischiocavernosus, and it terminates in an aponeurotic expansion which joins its fellow of the opposite side over the dorsal vein of the penis.

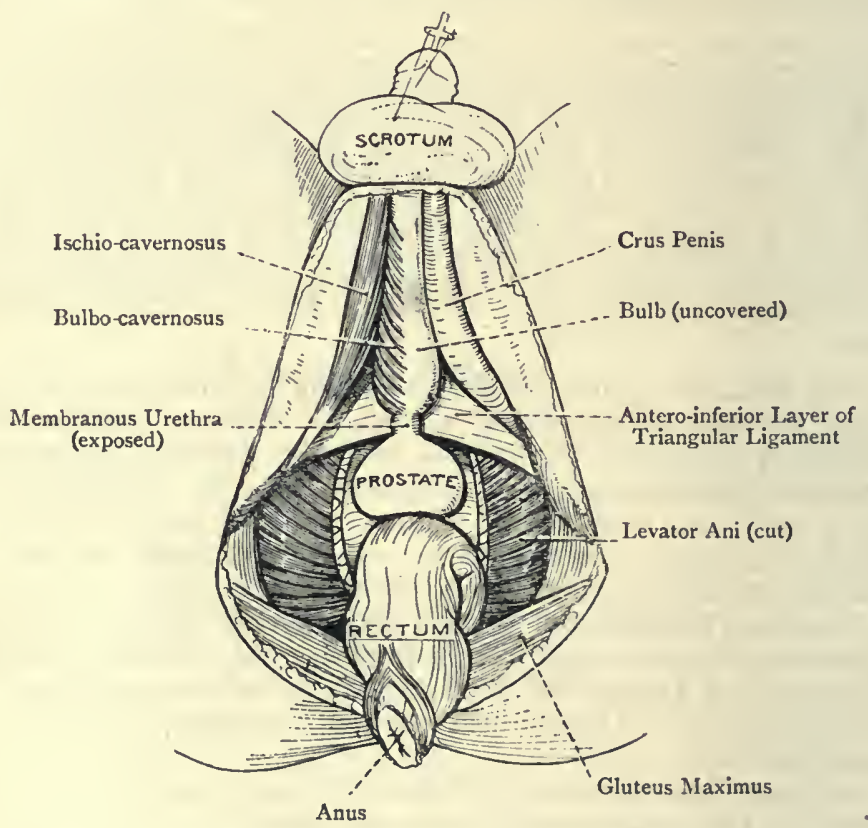

Fig. 275.-Deep Dissection of the Male Perineum

(The rectum has been turned back).

Bulbo-cavernosus (accelerator or ejaculator urinæ)-Origin.(I) The central tendinous point of the perineum ; and (2) the median raphé, which is continued forwards from that point towards the symphysis pubis.

Insertion.-The muscle, as regards its insertion, is conveniently divided into three parts, namely, the chief part, the anterior part and the posterior part.

Chief Part.-The fibres of this part ascend between the crus penis and the side of the bulb to be inserted into the median raphe on the upper surface of the bulb, where they meet the fibres of the corresponding part of the opposite muscle. 
Anterior Part.-The fibres of this part become somewhat detached and form the muscle of Houston. They are inserted partly into the outer surface of the fibrous sheath of the corpus cavernosum penis in front of the ischio-cavernosus, and partly by means of a tendinous expansion into the fascial investment of the penis beneath which the dorsal vein of the penis lies.

Posterior Part.- The fibres of this part arch outwards and backwards to be inserted into the antero-inferior layer of the triangular ligament near its base.

Nerve-supply. - The deep division of the perineal branch of the pudic nerve.

Action.--(I) The chief part of the muscle, acting with its fellow, compresses the bulb. These portions of the two muscles, therefore, come into play at the end of the act of micturition, when they expel the last drops of urine from this part of the urethra. A further action is to contribute to the maintenance of erection of the penis by compressing the veins of the bulb. (2) The anterior part compresses the dorsal vein of the penis, and so contributes to the maintenance of erection. (3) The posterior part tends to fix the bulb and maintain it in position.

The chief portions of the bulbo-cavernosi muscles completely surround the bulb, and may be regarded as forming a sphincter muscle to it.

Compressor or Constrictor Urethræ.-This muscle is also called the muscle of Guthrie. It extends transversely from the inner border of the ischio-pubic ramus of one side to the inner border of the opposite ischio-pubic ramus, lying in each case behind the attachment of the antero-inferior layer of the triangular ligament of the urethra. At the middle line the muscle divides into two layers, which, passing above and below the membranous part of the urethra, form a sphincter muscle to it, the lower layer also ensheathing the glands of Cowper. The posterior fibres of the muscle, which lie close to the base of the triangular ligament, are known as the transversus perinæi profundus (Henle). The highest fibres are connected on either side with the pelvic surface of the descending pubic ramus at its upper part, and they join each other below the membranous portion of the urethra. These highest fibres, which are oblique in direction, constitute the levatores urethræ muscles, or muscles of Wilson.

Nerve-supply. - The dorsal nerve of the penis.

Action.-(I) To constrict the membranous part of the urethra. The muscle comes into play at the end of micturition, and it assists the bulbo-cavernosus in emptying the urethral canal. (2) To contribute to the maintenance of erection of the penis by compressing the plexus of veins, in the misscle, which receives its tributaries from the corpora cavernosa and bulb. (3) To compress Cowper's glands, and so aid in the expulsion of their secretion.

The compressor urethræ muscle lies between the two layers of the triangular ligament of the urethra. Close to its attachment to 
the ischio-pubic ramus it contains within its substance the internal pudic vessels and the dorsal nerve of the penis.

Central Tendinous Point.-Nearly I inch in front of the anus there is a short transverse tendinous septum, about $\frac{1}{2}$ inch long. At its centre it presents a thickening, to which the name of central tendinous point is given. The muscles which meet at this point are as follows: (I) the sphincter ani externus, coming from behind; (2) the bulbo-cavernosi, coming from before; (3) the superficial transverse perineal muscles, coming from either side; and (4) the anterior portions of the levatores ani, which here lie above the posterior portions of the bulbo-cavernosi. The pointed process at the centre of the base of the antero-inferior layer of the triangular ligament of the urethra is also attached to the central tendinous point.

Bulb of the Urethra.-This is the first part of the corpus spongiosum penis, and is so named from its presenting a bulbous enlargement. It measures about $I \frac{1}{2}$ inches in length, and about $\frac{3}{4}$ inch in breadth at its posterior part. Its posterior extremity rests upon the antero-inferior layer of the triangular ligament, and extends as far back as the central tendinous point, where it lies nearly $I$ inch in front of the anus. This part extends fully $\frac{1}{4}$ inch farther back than the bulbous part of the urethra. It here presents on its under surface, in the middle line, a faint groove indicative of a tendency to a bipartite division at this point. The bulb is invested by a fibrous sheath derived from the circumference of the urethral opening in the antero-inferior layer of the triangular ligament, superficial to which lie the fibres of the chief parts of the bulbo-cavernosi muscles. Each lateral wall of the bulb is pierced by Cowper's duct, which, with its fellow of the opposite side, opens upon the floor of the bulbous part of the urethra fully $I$ inch in front of the antero-inferior layer of the triangular ligament.

Crus Penis (crus corporis cavernosi penis).-This is the posterior attached portion of the corpus cavernosum penis. It is attached to the anterior everted lip of the inner border of the ischio-pubic ramus, superficial to the antero-inferior layer of the triangular ligament. Inferiorly and laterally it is covered by the ischiocavernosus, and it lies beneath the fascia of Colles. The artery of the corpus cavernosum, having pierced the antero-inferior layer of the triangular ligament, passes between the attached surface of the crus and the ischio-pubic ramus, and enters the deep surface of the crus, to be continued forwards in the centre of the corpus cavernosum.

Deep Perineal Triangle-Boundaries-External.-The crus penis, covered by the ischio-cavernosus muscle. Internal.-The bulb of the urethra, covered by the bulbo-cavernosus muscle. Posterior, or Base.-The superficial transverse perineal muscle. The floor is formed by the fascia of Colles, with the superficial perineal vessels and the three long scrotal nerves. In the undisturbed position of 
the parts the area of the triangle is concealed by the approximation of the bulbo-cavernosus and ischio-cavernosus muscles. When, however, these muscles are held apart there is seen lying deeply in the area one half of the antero-inferior layer of the triangular ligament of the urethra.

Subpubic Ligament (ligamentum arcuatum).-This is a thick band which lies at the antero-superior part of the subpubic arch, where it fills up the subpubic angle. It is attached superiorly to the lower part of the interpubic disc, and laterally to the adjacent parts of the inner lips of the descending pubic rami. It is about $\frac{1}{4}$ inch in depth, and is slightly arched.

Transverse Pelvic or Perineal Ligament.-This band extends transversely between the descending pubic rami two or three lines below the subpubic ligament. Inferiorly it is closely connected with the truncated apex of the antero-inferior layer of the triangular ligament. Between its upper border and the subpubic ligament there is the opening for the backward passage of the dorsalis penis vein.

Triangular Ligament of the Urethra.-This ligament occupies the subpubic arch, which it fills, except at its antero-superior part where it is replaced by the subpubic and transverse pelvic or perineal ligaments. It is composed of two distinct layers, called antero-inferior (perineal) and postero-superior (pelvic). These two layers are united by their bases, but elsewhere they are separated by an interval of about $\frac{1}{2}$ inch, in which the membranous part of the urethra in the male, and the vagina and urethra in the female, along with other structures to be presently enumerated, lie.

The antero-inferior layer is also called the subpubic, or deep perineal, fascia. It is triangular, the apex being truncated. The apex is closely connected with the transverse pelvic or perineal ligament, which may be regarded as a part of its fibres. Each lateral margin is attached to the inner border of the ischio-pubic ramus, behind its everted lip, on which it extends as far back as the tuber ischii, lying between the attachments of the crus penis and ischio-cavernosus anteriorly and the compressor urethræ posteriorly. In this direction it measures fully 2 inches. The base is directed downwards and backwards, and is joined by the base of the postero-superior layer and the fascia of Colles. In the middle line the base is projected into a slight process, which is connected with the central tendinous point of the perineum. On either side of this median process the base presents a concave margin where it sweeps downwards and outwards to the tuber ischii. The length of the anteroinferior layer in the middle line is about $I \frac{1}{2}$ inches. Its fibres are chiefly disposed transversely. The structures which pierce this layer are as follows: the urethra, the arteries of the bulb, the arteries of the corpora cavernosa penis, the dorsal arteries and the dorsal nerves of the penis, the superficial perineal vessels and nerves, and the ducts of Cowper's glands.

Urethral Opening.-This aperture is situated in the middle line 
fully I inch below the subpubic angle, and it gives exit to the membranous part of the urethra. From the circumference of the opening an expansion is given off, which forms a fascial investment for the bulb.

Openings for the Arteries of the Bulb.-These are two in number, right and left, and are situated one on either side of the upper part of the urethral opening.

Openings for the Arteries of the Corpora Cavernosa Penis.-These are two in number, right and left, and each is situated in the lateral attached border, under cover of the crus penis, about I inch below the level of the subpubic angle.

Openings for the Dorsal Arteries and Nerves of the Penis.-These are two in number on either side, and are situated high up,

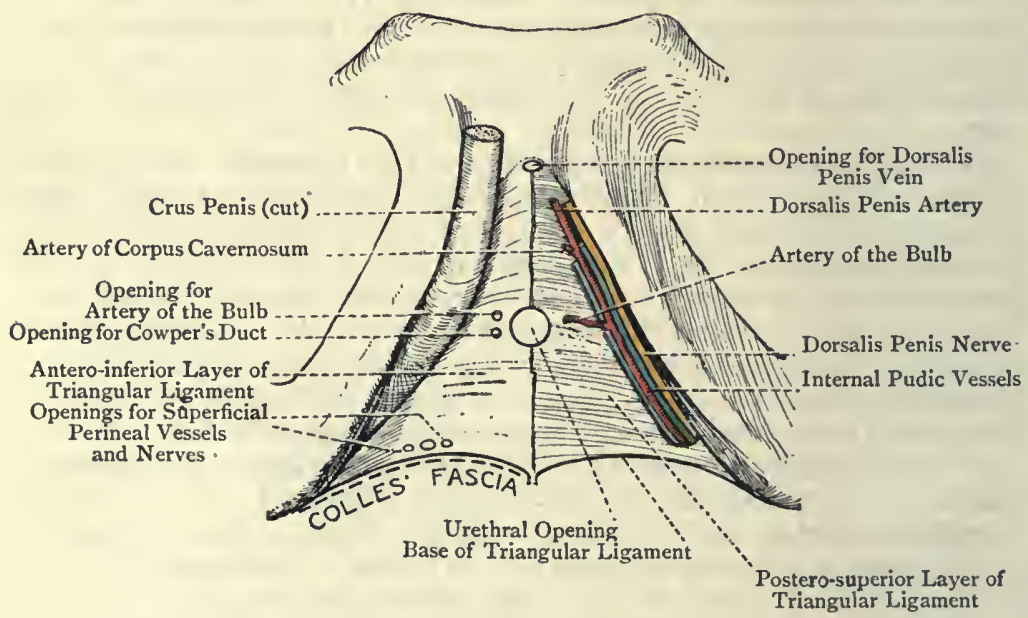

Fig. 276.-The Triangular Ligament of the Urethra

(The antero-inferior layer has been removed on the left side).

being near the subpubic angle, and close to the descending pubic ramus, the opening for the artery being internal to that for the nerve. It is to be noted that the dorsal vein of the penis has a special opening, which is situated in the median line between the subpubic and transverse pelvic or perineal ligaments.

Openings for the Superficial Perineal Vessels and Nerves.-These are situated, on either side, in the base at the line of junction with the fascia of Colles.

Openings for the Ducts of Coroper's Glands.-Each of these is situated on either side of the urethral aperture a little behind and below the opening for the artery of the bulb.

Chief Relations-Antero-inferior.-The bulb of the urethra, and the crura penis, covered by their respective muscles, the superficial transverse perineal muscles, and the fascia of Colles. Postero- 
superior.-The membranous part of the urethra, the glands of Cowper, and the compressor urethræ muscle.

The postero-superior layer is weak, and is formed by the parietal pelvic fascia. It lies about $\frac{1}{2}$ inch above and behind that layer, and extends inwards to the urethra from the inner aspect of each ischio-pubic ramus near the posterior lip, where it is behind the compressor urethræ muscle. Superiorly it is attached to the transverse pelvic or perineal ligament, and its base joins that $\mathrm{cf}$ the antero-inferior layer. At each ischio-pubic ramus it is continuous with the parietal pelvic fascia. When it arrives at the urethra it changes its course, and passes backwards over the anterior border of the levator ani muscle to blend with that portion of the visceral pelvic fascia which ensheathes the prostate gland. The postero-superior layer of the triangular ligament is to be regarded as a part of the parietal pelvic fascia. Antero-inferiorly it is in contact with the membranous part of the urethra and compressor urethræ muscle, whilst postero-superiorly it is related to the anterior fibres of the levator ani of each side, and forms the floor of the anterior diverticulum of the ischio-rectal fossa. The structures which pierce this layer are as follows: the membranous part of the urethra in the male, and the vagina and urethra in the female; and the internal pudic vessels and dorsal nerves of the penis.

Urethral Opening.- This is often a mere cleft, in which cases the postero-superior layer may be described as being arranged in two symmetrical halves. At this opening or cleft it becomes continuous superiorly with the capsule of the prostate gland.

Openings for the Internal Pudic Vessels and Dorsal Nerves of the Penis.-These are situated close to the base, on either side, near the ischial ramus.

\section{Structures between the Layers of the Triangular Ligament.-} These are as follows:

I. The membranous portion of the urethra in great part.

2. The glands of Cowper.

3. The compressor urethræ muscle.

4. The internal pudic arteries, each lying close to the ischiopubic ramus in the compressor urethræ muscle, and each giving off the following branches : $(a)$ the artery of the bulb, which in turn gives off the artery of a Cowper's gland; $(b)$ the artery of the corpus cavernosum; and $(c)$ the dorsal artery of the penis.

5. A plexus of veins which receives its tributaries from the crus (corpus cavernosum) and bulb, and in which the internal pudic venæ comites take their origin.

6. The deep lymphatics of the penis.

7. The dorsal nerves of the penis, each of which lies external to the corresponding internal pudic artery.

Glands of Cowper. - These glands are two in number, right and left. They are situated between the two layers of the triangular ligament, where they lie above the bulb and behind the membranous portion of the urethra, one on either side of the median 
line. Each gland is a firm, round, and lobulated mass about the size of a small pea. Both glands are ensheathed by the lower layer of the compressor urethræ muscle, and within this there is the special fibrous capsule which has an admixture of plain muscular tissue. The glands belong to the class of racemose or acino-tubular glands, and each is composed of several lobules. The alveoli or acini are lined with columnar cells. The ducts are two in number, right and left. They are lined with cubical epithelium, and their walls contain plain muscular tissue. Each duct pierces the anteroinferior layer of the triangular ligament on either side of the urethral opening, a little behind and below the artery of the bulb. The duct then pierces the side of the bulb, and opens, with its fellow, upon the floor of the bulbous part of the urethra, fully I inch in front of the antero-inferior layer of the triangular ligament. Each gland receives a branch from the artery of the bulb.

Cowper's glands are developed from the epithelial lining of the uro-genital sinus or canal.

Internal Pudic Artery.-This vessel is one of the terminal branches of the anterior division of the internal iliac, the other and larger terminal branch being the sciatic. Lying at first within the pelvis, the artery passes downwards over the pyriformis muscle and sacral nerves, having the sciatic artery usually behind it, and it emerges from the cavity through the lower compartment of the great sacrosciatic foramen. It then crosses the back of the spine of the ischium, after which it passes through the small sacro-sciatic foramen, and so enters the ischio-rectal division of the perineum. The vessel now courses along the outer wall of the ischio-rectal fossa, where, contained in Alcock's canal, it lies about $I_{2}^{\frac{1}{2}}$ inches above the lower part of the tuber ischii. On approaching the anterior part of the fossa the artery gradually becomes more superficial, and, after leaving the fossa, it enters the interspace between the two layers of the triangular ligament of the urethra by piercing the postero-superior layer close to its base and near the ischial ramus. It now passes forwards and upwards, embedded in the compressor urethræ muscle, and lying close to the ischio-pubic ramus, where it is comparatively superficial. Having given off the artery of the bulb about $\frac{1}{2}$ inch above the base of the triangular ligament, the vessel finally divides, about I inch below the subpubic angle, into its two terminal branches, the artery of the corpus cavernosum and the dorsal artery of the penis.

In considering the relations and branches of the internal pudic artery it is convenient to divide the vessel into four parts-first, second, third, and fourth.

The first part represents the intrapelvic portion of the vessel, and will be found described on p. 843 .

The second part is the portion of the vessel which lies upon the back of the spine of the ischium. For a description of it see p. 433 . 
The third part is the part of the vessel which lies on the outer wall of the ischio-rectal fossa. It is here contained in Alcock's canal, and is situated about $I_{\frac{1}{2}}$ inches above the lower part of the tuber ischii. For its relations see Alcock's canal.

Branches.-These are as follows : the inferior hemorrhoidal, the superficial perineal, and the transverse perineal.

The inferior hemorrhoidal artery arises, either singly or in two or three branches, from the internal pudic immediately after it has taken up its position in Alcock's canal. The branches pass inwards to the anal canal through the loose fat which fills the ischio-rectal fossa. They are distributed to the external sphincter, levator ani, wall of the anal canal, and superficial structures of one half of the ischio-rectal division of the perineum, and they anastomose with the middle and superior hemorrhoidal arteries, and the inferior hemorrhoidal branches of the opposite side.

The superficial perineal artery arises from the internal pudic at the anterior part of the ischio-rectal fossa. It pierces the base of the antero-inferior layer of the triangular ligament, and passes superficial to (sometimes on the deep surface of) the superficial transverse perineal muscle. Its subsequent course is forwards under cover of the fascia of Colles, where it lies in the floor of the deep perineal triangle, in company with the three long scrotal nerves. On approaching the scrotum it divides into several long slender branches, which supply the back of the scrotum and anastomose with the external pudic branches of the common femoral artery.

The transverse perineal artery, as a rule, arises in common with the superficial perineal, of which it is sometimes regarded as a branch. It may, however, arise directly from the internal pudic immediately in front of the origin of the superficial perineal. It is directed inwards and forwards to the central tendinous point lying superficial to the superficial transverse perineal muscle, and beneath the fascia of Colles. It supplies the muscles which meet at the central tendinous point, and anastomoses with its fellow of the opposite side.

Alcock's Canal.- This canal is situated in the outer wall of the ischio-rectal fossa, and is formed by the obturator fascia. Its contents from below upwards are as follows: (I) the perineal division of the pudic nerve; (2) the lower vena comes; $(3)$ the third part of the internal pudic artery; $(4)$ the upper vena comes; and $(5)$ the dorsal nerve of the penis.

The fourth part of the internal pudic artery lies between the two layers of the triangular ligament. It enters this interspace by piercing the postero-superior layer of that ligament close to its base and near the ischial ramus. It is embedded in the compressor urethræ muscle, and is comparatively superficial. As it lies near the ischio-pubic ramus it has a vena comes on either side of it, and the dorsal nerve of the penis is external to it.

Branches.-These are as follows : the artery of the corpus 
spongiosum, the artery of the corpus cavernosum, and the dorsal artery of the penis.

The artery of the corpus spongiosum (artery of the bulb) arises from the internal pudic about $\frac{1}{2}$ inch above the base of the triangular ligament, and passes transversely inwards in the substance of the compressor urethræ muscle. On approaching the urethra it turns forwards, and, having pierced the compressor urethræ, it passes through an opening in the antero-inferior layer of the triangular ligament at the side of the urethral aperture. It then enters the bulb, and is continued onwards in the corpus spongiosum as far as the glans penis, the erectile tissue of which parts it supplies. It anastomoses with its fellow of the opposite side and with the dorsal arteries of the penis. Whilst between the two layers of the triangular ligament the artery furnishes a branch to Cowper's gland of the corresponding side.

The artery of the corpus cavernosum is one of the two terminal branches of the internal pudic, and is somewhat larger than the dorsal artery of the penis, which is the other terminal branch. It arises about $I$ inch below the subpubic angle, and immediately thereafter pierces the compressor urethræ muscle and the antero-inferior layer of the triangular ligament close to the ischio-pubic ramus. It then passes between the bone and the crus, and, entering the crus on its deep surface, it is continued onwards in the centre of the corpus cavernosum as far as the distal end of that body, the erectile tissue of which it supplies.

The dorsal artery of the penis is the continuation of the internal pudic. For a very short distance it lies between the two layers of the triangular ligament embedded in the compressor urethræ muscle, its course here being upwards. It then turns forwards, and, having pierced that muscle and the antero-inferior layer of the ligament very near its upper part, it ascends between the crus and the symphysis pubis. Its subsequent course is between the two layers of the suspensory ligament of the penis, and then along the dorsum of the organ, where it has the centrally-placed dorsal vein on its inner side and the dorsal nerve of the penis on its outer. On arriving at the neck of the penis it ends in branches for the supply of the glans and prepuce, where it anastomoses with its fellow of the opposite side and the arteries of the corpus spongiosum. In its course along the dorsum of the penis the artery gives off many branches, some of which supply the integument and anastomose with the superior external pudic of the common femoral, whilst others pierce the fibrous sheath of the corpus cavernosum to supply its erectile tissue, these latter anastomosing with the artery of the corpus cavernosum.

Varieties of the Internal Pudlc Artery-1. Trunk.-The vessel is occasionally of small size, and may terminate in the artery of the bulb, or in the superficial perineal artery. In these cases an accessory pudic artery is present, which supplies the deficiencies. This vessel usually arises from the first or intrapelvic part of the internal pudic, though it may spring from an inferior 
vesical artery. Its course is forwards along the side of the bladder, then along the side of the prostate gland to the triangular ligament of the urethra, which it pieices above the membranous part of the canal, and so reaches the root of the penis. The accessory pudic furnishes the artery of the corpus cavernosum and the dorsal artery of the penis, and in some cases the aitery of the bulb.

2. Artery of the Corpus Spongiosum (Artery of the Bulb).-Dometimes two arteries are present on one side; sometimes the artery is absent on one side ; and sometimes it is of very small size. A much more important variety of this artery affects its origin. It may arise from the fourth part of the internal pudic close to the base of the triangular ligament, and even from the third part at the front of the ischio-rectal fossa. In these cases the artery cannot escape division in the operation of lateral lithotomy. In other cases it may arise from. an accessory pudic artery, when it will lie higher up and further forwards than usual.

3. Dorsal Artery of the Penis. - This vessel may arise from the obturator artery in the obturator canal, or from one of the external pudic branches of the common femoral artery.

Veins.-Lying in each compressor urethræ muscle there is a plexus of veins, which receives its tributaries from the corresponding corpus cavernosum and one half of the corpus spongiosum and bulb. The internal pudic venæ comites arise on either side from this plexus, and accompany the internal pudic artery as far back as the upper border of the spine of the ischium, one lying on either side of the vessel. Here they join to form one trunk, which enters the pelvis through the lower compartment of the great sacro-sciatic foramen, and terminates in the internal iliac vein. They receive as tributaries the transverse perineal, superficial perineal, and inferior hemorrhoidal veins, as well as a few veins from the gluteus maximus and external rotator muscles. The inferior hemorrhoidal veins take their origin in a plexus of veins which is situated on the outer surface of the external sphincter muscle. Having crossed the ischio-rectal fossa through its loose fat, and being ultimately reduced to two or three in number, they join the internal pudic venæ comites.

It is to be noted that, though there are two dorsal arteries, there is only one dorsal vein, which takes the following course: after leaving the dorsum of the penis it passes through an opening between the subpubic and transverse pelvic or perineal ligaments, where it communicates on either side with the venous plexus in each half of the compressor urethræ muscle. Having entered the cavity of the pelvis, it divides into two branches, right and left, which join the prostatic plexus of veins.

Lymphatics.-The superficial lymphatics of the perineum, as well as those of the anus and lower part of the anal canal, pass to the inguinal glands, which lie immediately below Poupart's ligament, whilst the deep lymphatics pass to the internal iliac glands.

Pudic Nerve.-The pudic nerve is one of the terminal branches of the sacral plexus, and derives its fibres from the ventral division of the second, the lower branch of the third, and the upper branch of the fourth sacral nerves, the majority of its fibres being derived from the lower branch of the third. Leaving the pelvis through the lower compartment of the great sacro-sciatic foramen, the nerve 
crosses the back of the spine of the ischium, where it lies under cover of the gluteus maximus and on the inner side of the internal pudic vessels. It then passes through the small sacro-sciatic foramen, and so enters Alcock's canal in the outer wall of the ischiorectal fossa. Immediately after doing so, it divides into three branches, namely, inferior hemorrhoidal, perineal, and dorsal nerve of the penis.

The inferior hemorrhoidal nerve passes inwards acioss the ischiorectal fossa to the region of the anus, and is distributed to the external sphincter muscle and the integument which covers the ischio-rectal fossa.

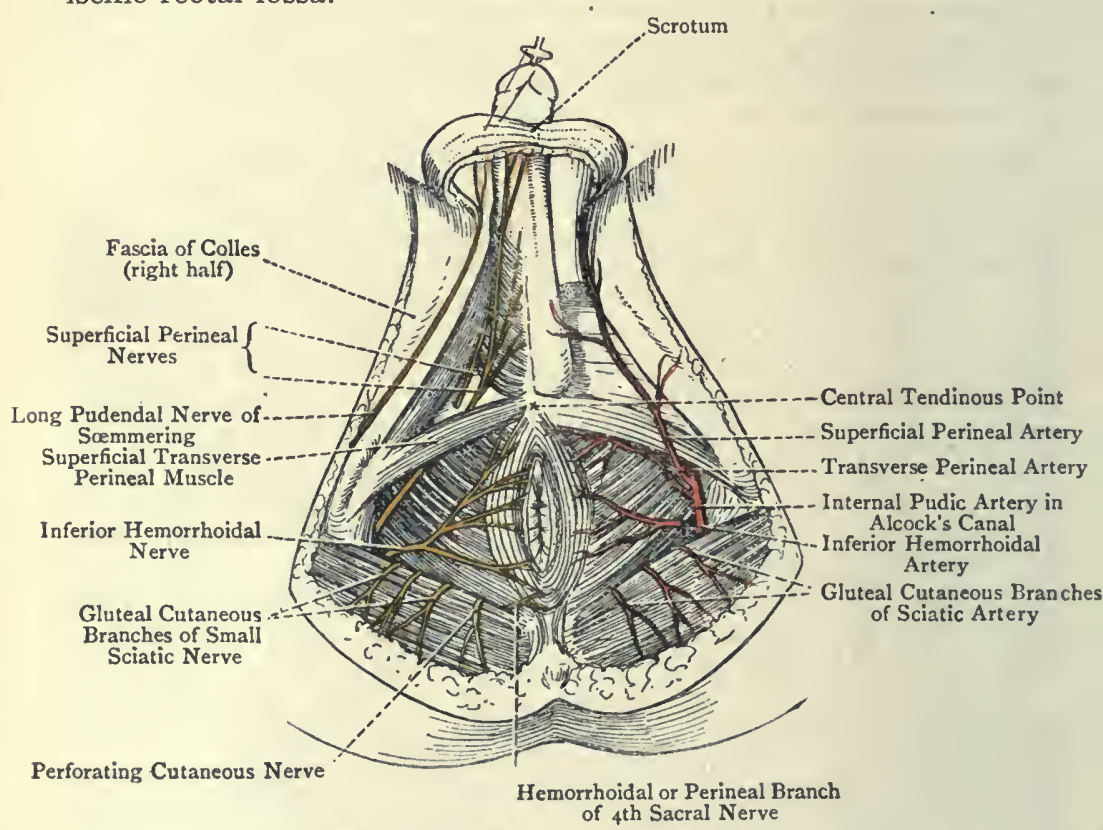

Fig. 277.-Dissection of the Male Perineum

(On the left side the bulbo-cavernosus has been removed, and the crus penis cut).

The perineal nerve is a large branch which passes forwards in Alcock's canal, being the lowest of its contents. It ultimately divides into superficial and deep branches.

The superficial branches are two in number, and are called the external or posterior, and internal or anterior, superficial perineal nerves. Both nerves, having emerged from Alcock's canal, pass forwards and pierce the base of the antero-inferior layer of the triangular ligament. They then run forwards with the superficial perineal artery under cover of the fascia of Colles, and on approach- 
ing the back of the scrotum they divide into long slender branches which supply the scrotal integument.

In the anterior division of the perineum the two superficial perineal nerves communicate freely, and are accompanied by the long pudendal nerve of Sœmmering, which is a branch of the small sciatic. This nerve, having pierced the fascia lata about I inch in front of the tuber ischii, passes inwards over the ischia-pubic ramus and through the fascia of Colles. It then runs forwards and inwards beneath that fascia to the scrotum, to take part in the supply of its integument, and it communicates with the external or posterior superficial perineal nerve.

The two superficial perineal nerves and the long pudendal nerve are known as the long scrotal nerves.

The deep branch of the perineal nerve furnishes offsets which, with one exception, are muscular in their distribution, and supply the anterior part of the external sphincter, the anterior part of the levator ani, the superficial transverse perineal, the ischio-cavernosus, and the bulbo-cavernosus. The non-muscular branch, called the nerve of the bulb, pierces the bulbo-cavernosus muscle and the wall of the bulb, to be distributed to the erectile tissue of the corpus spongiosum and the mucous membrane of the spongy part of the urethra.

The dorsal nerve of the penis is at first contained in Alcock's canal, where it lies above the internal pudic vessels. Having emerged from that canal, it pierces the postero-superior layer of the triangular ligament near its base. It then passes forwards and upwards, with the fourth part of the internal pudic artery, between the two layers of the triangular ligament, in which situation it lies on the outer side of the internal pudic vessels, and close to the ischio-pubic ramus, being embedded in the compressor urethræ muscle. Its subsequent course is similar to that of the dorsal artery of the penis, which it accompanies. On the dorsum of the penis, where it. lies external to the dorsal artery, it is continued as far as the glans, where it ends in branches for the glans and prepuce. As the nerve lies between the two layers of the triangular ligament, it gives branches to the compressor urethræ muscle, and it also furnishes the nerve of the corpus cavernosum. This latter nerve, having pierced the compressor urethræ and antero-inferior layer of the triangular ligament, enters the crus, and is continued forwards in the corpus cavernosum to supply the erectile tissue of these parts. As the nerve passes along the dorsum of the penis, it supplies numerous branches to the integument of that organ.

Structures divided in Left Lateral Lithotomy. - The structures divided in this operation are as follows: (I) the skin: (2) the subcutaneous layer of the superficial fascia; (3) the deep layer of the superficial fascia or the fascia of Colles; (4) the transverse perineal vessels; (5) the superficial transverse perineal muscle; (6) the inferior hemorrhoidal vessels and nerve; (7) the basal part of the antero-inferior layer of the triangular ligament; (8) the compressor urethræ muscle and the plexus of veins embedded in it; (9) the 
membranous part of the urethra; (Io) the postero-superior layer of the triangular ligament; (II) the anterior fibres of the levator ani muscle; (12) a portion of the left lateral lobe of the prostate gland, with its capsule, and some of the veins of the prostatic plexus; and (13) the neck of the bladder.

Structures to be avoided. -The structures to be avoided are as follows: (I) the rectum; (2) the internal pudic vessels as they lie in Alcock's canal; (3) the artery of the bulb; and (4) the common ejaculatory duct.

\section{FEMALE PERINEUM.}

The female perineum is divided into three regions-uro-genital, perineum proper, and ischio-rectal. The uro-genital division is - situated at the anterior part, and comprises the pudendum and urogenital cleft. The perineum proper is situated between the posterior part of the uro-genital cleft and the anus. The ischio-rectal division is situated as in the male.

\section{Uro-genital Division.}

The uro-genital division contains the external uro-genital organs. These collectively constitute the pudendum, and comprise the following parts : the mons Veneris ; labia majora; labia minora, or nymphæ; clitoris; vestibule; meatus urinarius; vaginal orifice, including the hymen, or the carunculæ myrtiformes; frænulum pudendi; fossa navicularis; bulbi vestibuli; and glands of Bartholin or of Duverney.

The mons Veneris is an eminence situated in front of and above the upper part of the symphysis pubis. It is produced by a collection of adipose tissue, the skin over which is more or less freely provided with hair after the age of puberty.

The labia majora are two thick, round folds of integument, which are directed from before backwards, with a slight inclination downwards. The length of each is about 3 inches. Posteriorly they become thin, and join each other at the anterior part of the perineum proper, about $I$ inch in front of the anus. The junction receives the name of the posterior commissure, and is somewhat pointed. Anteriorly they retain their thick, round character, and here also they join and become continuous with the mons Veneris. This latter junction is called the anterior commissure. Each labium majus has two surfaces, outer and inner. The skin covering the outer convex surface is somewhat dark in colour, like that of the scrotum, and contains numerous sebaceous glands of large size. It is also more or less freely provided with hair after the age of puberty, except towards the posterior part. The inner flat surface forms the lateral boundary of the uro-genital cleft, and touches that of the opposite side. The skin covering this surface is smooth and free from hair, and presents the openings of the ducts of sebaceous glands. Each labium majus contains adipose and areolar tissues, and a small amount of dartos tissue. The round ligament of the 
uterus of each side loses itself in this labium, and the fasciæ of Camper and of Scarpa of the lower part of the anterior abdominal wall of each side also enter it. The fissure between these labia is called the uro-genital cleft or rima pudendi, and is almost horizontal, its direction being antero-posterior. The blood-supply and nervesupply of the labia majora correspond with those of the scrotum.

The lymphatics arise from a rich network in each labium majus, these networks being connected with each other across the median line. The most of the efferent vessels pass to the pubic group of inguinal glands, but some may terminate in the superficial femoral or saphenous glands. Some vessels are said to cross the median line and terminate in the glands of the opposite side.
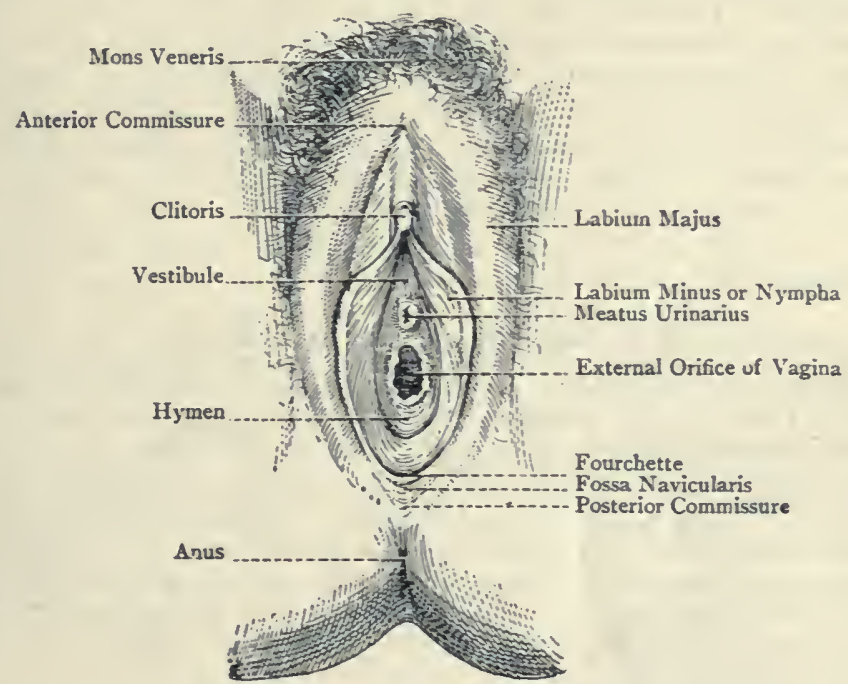

Fig. 278.-The External Genital Organs of the Female.

The labia minora, or nymphæ, are two narrow, more or less pendulous folds of integument, which are situated in the uro-genital space, each lying internal to the corresponding labium majus. They become continuous with each other anteriorly, in the region of the clitoris, a short distance from the anterior commissure, in a manner to be presently described. From this point they diverge as they pass backwards, and so form the lateral boundaries of the space called the vestibule. Each terminates posteriorly by blending with the inner surface of the corresponding labium majus, or in some cases by becoming continuous with the frænulum pudendi. Each labium minus has two surfaces, outer and inner, and two borders, superior and inferior. The outer surface is in contact with the inner surface of the labium majus of the same side, and the inner surface touches that of the opposite nympha. Each surface is covered by a modified form of skin, that on the inner 
surface being extremely delicate, and being somewhat like mucous membrane. The true mucous membrane, however, only commences at the inner side of the base or superior attached border of the labium minus. The labia minora, previous to their union anteriorly, divide each into two laminæ. The upper and larger lamina passes over the clitoris, and becomes continuous with that of the opposite side, thus forming a cap for that organ, called the præputium clitoridis. The lower and smaller lamina passes below the clitoris, where it also becomes continuous with that of the opposite side. At the line of junction of the two lower laminæ they are attached to the under surface of the clitoris, thus forming the frænulum clitoridis. The labia minora are destitute of both hair and fat, but they contain sebaceous glands. They sometimes attain a large degree of development, in which cases they project through the uro-genital fissure. In some African women they become so much developed as to reach down to the knees. When this occurs they form what has been called the apron of Hottentots. The labia minora are homologous with the floor of the spongy part of the urethra and the integument of the under surface of the penis and prepuce in the male.

Development of the Labia Majora and Labia Minora.-The anterior part of the cloacal fossa in the female forms the uro-genital space, and the margins or lips of this space become prominent and give rise to the outer genital folds. At the posterior (lower) part of the uro-genital space there is the uro-genital cleft, which receives the external orifices of the urethra and vagina. The lips of this cleft form two other folds, called the inner genital folds. The outer genital folds form the labia majora, and the inner genital folds give rise to the labia minora. In the male the anterior part of the cloacal fossa (uro-genital furrow) is closed over by the meeting and union of its lips to form the spongy portion of the urethra. The genital folds, which correspond with the outer genital folds in the female, coalesce in a median raphé, and so give rise to the scrotum, which, therefore, corresponds to the labia majora in the female.

The clitoris is situated in the uro-genital space a little behind the anterior commissure, and is composed of two corpora cavernosa and a glans. Each corpus cavernosum is attached by means of a crus to the anterior everted lip of the inner border of the ischiopubic ramus, the crus being covered by the ischio-cavernosus or erector clitoridis muscle, and lying superficial to the antero-inferior layer of the triangular ligament. The two corpora cavernosa unite by their inner flattened surfaces, and so form the body of the clitoris, which is about $I \frac{1}{2}$ inches long. The septum, which is interposed at the line of junction of the corpora cavernosa, is incomplete, and is called the septum pectiniforme. The dorsal surface of the clitoris at its upper end is attached to the front of the symphysis pubis by a small suspensory ligament, and the distal end of the organ is capped by an imperforate glans. The glans, which caps the corpora cavernosa, is composed of erectile tissue, and is extremcly sensitive. It is provided with a prepuce and a frænulum, both of which are continuous with the labia minora. The organ is composed of erectile tissue. 
The clitoris is the homologue of the penis, from which it differs in the following respects: (I) the only part of a corpus spongiosum which it possesses is the glans (the part of the corpus spongiosum of the male which lies between the bulb and the glans penis being represented in the female by the pars intermedia of the bulbi vestibuli); (2). it does not contain the female urethra; and (3) its component parts are much smaller than those of the penis. In reality the clitoris is a diminutive penis, minus the corpus spongiosum and the urethra. It is developed from the genital eminence.

Lymphatics. - The lymphatics of the prepuce of the clitoris accompany those of the labia majora, and pass to the pubic group of inguinal glands, and, it may be, to the superficial femoral or saphenous glands.

The lymphatics of the glans clitoridis run on the dorsum of the clitoris towards the front of the symphysis pubis, where they form a network. The vessels which emerge from either side of this network have the following destinations: (I) Some pass to the deep femoral glands, which lie within the femoral canal; and (2) one or two traverse the inguinal canal, and terminate in the lowest or retro-femoral gland of the outer chain of the external iliac glands.

The lymphatics of the corpora cavernosa probably pass to the internal iliac glands on either side.

The vestibule is the space which is enclosed by the labia minora, and is so called because it is the 'porch' of the vagina. It is triangular, the apex, which is in front, being formed by the glans clitoridis, the lateral boundaries by the labia minora, and the base, which is directed backwards, by an imaginary line passing transversely in front of the external orifice of the vagina and behind the meatus urinarius. It is about I inch in length, and presents a smooth surface formed by mucous membrane, which is covered by stratified squamous epithelium. At its posterior extremity, in the middle line, and immediately in front of the external orifice of the vagina, there is a slight prominence, with somewhat irregular margins. Upon this prominence the meatus urinarius is situated at a point $I$ inch behind the clitoris. The irregular prominence serves as a guide to this opening.

The vestibule represents the remains of the uro-genital sinus.

The external orifice of the vagina is situated immediately behind the vestibule. It is an antero-posterior cleft, having an elliptical shape when partially dilated. The portion of the vagina close above it is the narrowest part of the passage. For the description of the vagina, see Female Pelvis.

The hymen in its normal condition is a thin semilunar fold of mucous membrane which is stretched across the posterior third, or half, of the external orifice of the vagina. Its concave border, which is free, is directed forwards and upwards. Sometimes the hymen completely surrounds the circumference of the orifice, an aperture being left in its centre. In other cases it stretches over the entire opening, but is perforated by apertures which give it a cribriform appearance. In rare cases it is an entire membrane, completely shutting off the vaginal canal from the uro-genital space, and it is then spoken of as an imperforate hymen. In some cases, even in the virgin, it is entirely absent. 
The hymen begins to appear about the fifth month of intra-uterine life as a fold of the mucous membrane at the point where the vagina opens into the uro-genital sinus.

The carunculæ myrtiformes are small elevations which represent the remains of the hymen after its disappearance. Though called caruncula (fleshy), they are really mucous excrescences.

The fourchette, or frænulum pudendi, is a crescentic fold of the uro-genital lining membrane which is situated a little in front of the posterior commissure, and it is best marked in early life.

The fossa navicularis is a small depression which lies between the posterior commissure and the fourchette.

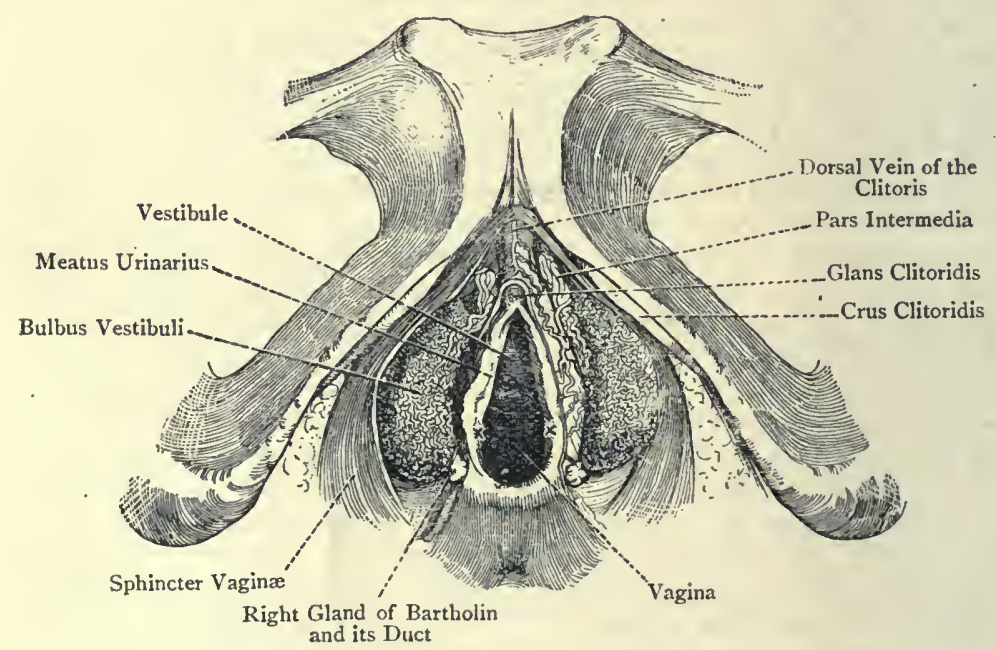

Fig. 279.-Dissection showing the Bulbi Vestibuli and Glands OF BARTHOLIN (MODIFIEd FROM KOBELT)

(The cross on either side of the Vaginal Orifice shows the position of the opening of the Duct of Bartholin's Gland).

The bulbi vestibuli are two ovoid masses of erectile tissue I inch in length, which are situated on either side of the external orifice of the vagina and vestibule beneath the mucous membrane. Each bulb is covered by a delicate fibrous capsule derived from the antero-inferior layer of the triangular ligament, superficial to which the bulb of either side lies. The outer surface is convex, and is covered by one half of the sphincter vaginæ muscle. The inner surface is slightly concave, and is covered by the vaginal mucous membrane. Posteriorly the bulbs diverge, and anteriorly, having become narrow, they pass upwards and forwards, and ultimately meet in the middle line, where they are attached to the antero- 
inferior layer of the triangular ligament. In front of the bulbs there is a plexus of veins which is continuous behind with their erectile tissue, and in front with that of the glans clitoridis. This plexus of veins is known as the pars intermedia of Kobelt. It receives its veins from the labia minora, and its blood is conveyed into the vaginal plexus.

The bulbi vestibuli, together, represent the bulb of the male urethra, which latter presents on its under surface a faint groove in the middle line, indicating a tendency to a bipartite division. The pars intermedia of Kobelt is regarded as representing that part of the male corpus spongiosum which extends from the bulb to the glans penis.

The glands of Bartholin or of Duverney belong to the class of racemose or acino-tubular glands. They are two in number, right and left, and each resembles a small bean. They lie on either side of the external orifice of the vagina towards its back part, immediately behind the posterior extremities of the bulbi vestibuli, and superficial to the base of the triangular ligament. The duct of each gland is about $\frac{3}{4}$ inch long, and opens in the angle between the attached border of the nympha posteriorly and the hymen or its remains, close to the centre of the margin of the vaginal orifice.

These glands are homologous with the glands of Cowper, and their structure is similar.

The glands of Bartholin are developed from the lining epithelium of the uro-genital sinus.

The external uro-genital organs of the female have received the name of vulva. As this word, however, literally signifies ' a covering,' it is strictly applicable only to the labia majora, which by their approximation form a covering for the uro-genital space and its contents.

\section{Development of the External Genital Organs.}

In the early stages no sexual differences are apparent in the development of the external genital organs. The chief parts concerned are-(I) the genital eminence, (2) the genital groove, (3) the internal genital folds, and (4) the external genital folds.

Female External Organs. - The surface-depression corresponding to the cloacal membrane, which bounds the cloaca postero-inferiorly, is known as the cloacal depression. As the cloaca becomes divided into two compartments-dorsal or intestinal, and ventral or urogenital-by the cloacal or uro-rectal septum, the cloacal membrane is also divided into two parts-dorsal or anal, and ventral or urogenital. Moreover, the superficial cloacal depression is likewise divided into two parts-dorsal, which is called the anal depression or proctodaum; and ventral, which is known as the uro-genital depression, and is somewhat cleft-like. When the uro-genital portion of the cloacal membrane ruptures the uro-genital sinus or canal communicates with the exterior by means of the uro-genital opening or cleft. 
In the female the urinary bladder is developed from the cephalic part of the uro-genital sinus. The entire urethra of the female is developed from the cephalic part of the uro-genital sinus, in succession to the urinary bladder. The caudal part of the uro-genital sinus, becoming expanded, gives rise to the vestibule, into the cephalic part of which the urethra opens, whilst the external orifice of the vagina is caudal, or posterior, to it.

The female external genital organs are developed around the urogenital opening. At the cephalic part of the primitive vestibule a small tubercle, called the genital eminence, makes its appearance in the median line. On the lower, or vestibular, surface of this eminence a furrow, called the genital grove, is formed. The lips of this groove, which are laterally disposed, are called the internal genital folds. On either side of the genital eminence, external to the corresponding internal genital fold, a prominent ridge makes its appearance. These ridges are known as the external genital

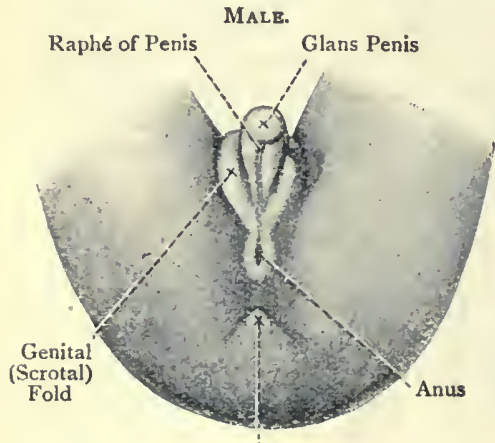

Coccyx

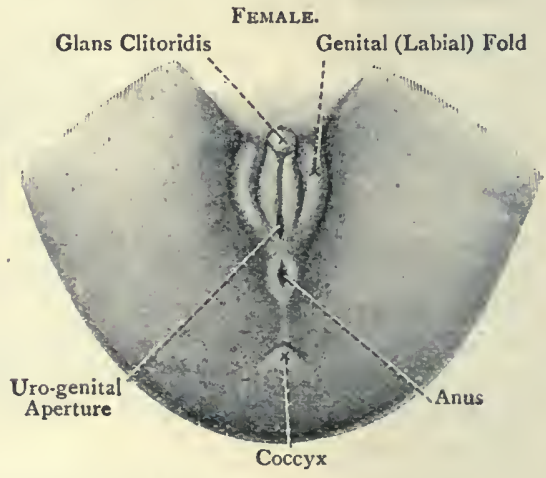

Coccyx

Fig. 280.-Development of the External Genital Organs.

folds. They are continuous with each other on the ventral aspect of the genital eminence, and they extend dorsalwards, lying on either side of the vestibule, and finally meeting behind in the perineum.

The genital eminence undergoes lengthening, and gives rise to the clitoris. The terminal extremity of the eminence becomes enlarged, and forms the glans clitoridis, whilst the remainder gives rise to the corpora cavernosa clitoridis.

The internal genital folds, which in the malc fuse, enclosing the spongy part of the urethra, and forming the corpus spongiosum penis, remain separate in the female, and form the labia minora, or nympha. The external genital folds, which in the male fuse and form the scrotum, remain separate in the female, and give rise to the labia majora. The ventral portions of the external genital folds, which are continuous with each other on the ventral aspect of the genital eminence, form the mons veneris. 
The hymen appears as a semilunar fold of mucous membrane, extending as a rule over the dorsal part of the external orifice of the vagina.

The glands of Bartholin are developed laterally as evaginations of the epithelial lining of the caudal part of the uro-genital sinus, which part, when expanded, forms the vestibule.

The bulbi vestibuli and the pars intermedia of Kobelt are developed as masses of erectile tissue close to the labia minora or nymphæ and clitoris.

Male External Organs.-In the male the genital eminence undergoes considerable lengthening, and gives rise to the corpora cavernosa penis, the terminal enlargement of the eminence forming the glans penis. As the genital eminence elongates, the genital groove on its lower surface undergoes proportionate elongation, and at the same time deepens. The internal genital folds, which form the lateral lips of this groove, and which in the female remain separate from each other as the labia minora, come together in the male, and fuse over the genital groove. This groove is thus transformed into a canal, which constitutes the spongy part of the male urethra. The line of fusion of the internal genital folds is indicated, in adult life, by the median raphé, which traverses the under, or scrotal, aspect of the penis. The portion of the urethra within the glans penis is developed from a collection of ectodermic cells, forming the so-called urethral septum or plate. These cells become tunnelled into a canal, which is continuous with the spongy urethra, developed as stated from the genital groove.

The spongy part of the urethra extends as-far as the uro-genital sinus, from the lower part of which the prostatic and membranous parts of the canal are developed.

In rare cases the internal genital folds fail to unite over some portion of the genital groove. In such cases the spongy urethra opens externally on the under, or scrotal, aspect of the penis, and the condition is known as hypospadias.

The internal genital folds, which enclose the spongy part of the urethra, acquire erectile tissue and constitute the corpus spongiosum penis. The genital eminence, having lengthened considerably, and having acquired erectile tissue, gives rise by its greater part to the corpora cavernosa penis, whilst its terminal enlargement forms the glans penis. As just stated, the corpus spongiosum penis is formed by the fusion of the two internal genital folds, this fusion converting the genital groove into the spongy part of the urethra.

The external genital folds, which in the female remain separate and form the.labia majora, unite in the male and give rise to the scrotum. The line of fusion is indicated, in adult life, by the scrotal raphé.

It is to be noted that, whilst the prostatic and membranous portions of the male urethra are developed from the lower part of the urogenital sinus, and are therefore non-penile, the spongy portion of the canal is developed from (I) the genital groove on the 
lower surface of the genital eminence, and (2) the internal genital folds. The spongy part of the urethra is therefore penile.

The bulb of the corpus spongiosum penis represents the bulbi vestibuli of the female, and the portion of the corpus spongiosum penis between the bulb and the glans penis represents the pars intermedia of Kobelt in the female.

\section{Metamorphoses of the Structures concerned in the Development of the External Genital Organs.}

\section{Structures.}

Genital eminence.

Internal genital folds.

External genital folds. Uro-genital sinus.

\section{Female.}

Clitoris.

Labia minora and bulbi vestibuli.

Labia majora.

Bladder, urethrá, vestibule, and glands of Bartholin.
Male.

Corpora cavernosa penis and glans penis.

Corpus sponglosum and spongy urethra.

Scrotum.

Bladder, prostate gland, prostatic and membranous urethra, and glands of Cowper.

\section{Perineum Proper.}

The perineum proper is the region which lies between the anus and the posterior commissure, and it presents a raphe in the middle line. It is in this division that the perineal body is situated, as well as the central tendinous point. The latter, being similar to that of the male, requires no special description.

Perineal Body.-This body is peculiar to the female perineum. It is situated between the anus and the posterior commissure, and it may be regarded as a deep expansion of the central tendinous point. It is triangular, and is about $I_{\frac{1}{2}}$ inches in breadth. It is bounded in front by the posterior wall of the vagina, behind by the anterior wall of the anal canal, and inferiorly by the integument. The perineal body is produced by a thickening of connective tissue, with a free admixture of elastic tissue, and a few muscular fibres derived from the external sphincter, levatores ani, and sphincter vaginæ muscles. It serves as a support to the posterior wall of the vagina. During parturition it becomes greatly stretched, but its elastic tissue usually guards it against rupture.

Triangular Ligament.-The triangular ligament resembles that of the male in being composed of two layers, antero-inferior and postero-superior.

The antero-inferior layer, on account of the greater width of the subpubic arch in the female, is broader than in the male, though it is more indefinite on account of its being pierced by the vagina. It is attached at either side to the inner border of the ischio-pubic ramus, and superiorly to the transverse pelvic or perineal ligament. In the middle line, where it is pierced by the vagina, it blends with the wall of that canal. Its base is joined by a somewhat indefinite layer of fascia representing the fascia of 
Colles in the male, and by the postero-superior layer. The openings in the antero-inferior layer are similar to those in the male, with this exception, that the ducts of the glands of Bartholin do not pierce it as the ducts of the glands of Cowper do in the male, the glands of Bartholin being situated superficial to this layer. The urethral opening is situated I inch below the symphysis pubis. The opening for the vagina, which is of large size, lies below the urethral orifice, from which it is separated by a few fibres. The openings for the arteries of the bulbi vestibuli are situated one on either side of the vaginal opening. The openings for the artery of the corpus cavernosum clitoridis, for the dorsal artery and nerve of the clitoris, and for the superficial perineal vessels and nerves, all of each side, are situated as in the male.

It is to be noted that the dorsal vein of the clitoris, like the corresponding vessel in the male, passes between the subpubic and transverse pelvic or perineal ligaments.

The postero-superior layer is similar to the corresponding layer in the male, and it presents openings for the urethra, vagina, and internal pudic vessels and pudic nerves of each side.

\section{Ischio-rectal Division.}

The chief characters of the ischio-rectal division in the female are as follows: the aperture of the anus is somewhat nearer the coccyx than in the male, the distance between the ischial tuberosities is greater than in the male, and the ischio-rectal fossæ are wider and shallower than in the male.

Muscles.-The muscles of the fernale perineum, as compared with those of the male, present certain differences.

Levatores Ani.-The anterior fibres of these muscles embrace the vagina instead of the prostate gland, as in the male.

Ischio-cavernosus (erector clitoridis).-This muscle replaces the erector penis, and is of small size.

Bulbo-cavernosus or Sphineter Vaginæ.-This muscle arises from the central tendinous point, where it meets the external sphincter and superficial transverse perineal muscles. It then passes forwards and divides into two symmetrical parts which surround the vaginal orifice and vestibule, each part closely embracing the outer surface of the corresponding bulbus vestibuli. Anteriorly the two parts become very narrow, and each is inserted into the fibrous sheath of the corpus cavernosum. A few fibres are here detached to be inserted into a tendinous expansion on the dorsum of the clitoris covering the dorsal vein, which vessel would be thereby compressed when the muscle is in action. Some of the inner fibres of the sphincter vaginæ are inserted into the mucous membrane of the vestibule.

The sphincter vaginæe is homologous with the bulbo-cavernosi in the male.

Compressor or Constrictor Urethræ.-This muscle, as in the male, lies between the two layers of the triangular ligament. It is 
attached, on either side, to the inner margin of the ischio-pubic ramus, and in the middle line it is almost completely divided into two parts by the vagina. The anterior part passes transversely across the subpubic arch in front of the urethra, whilst the posterior and larger part passes inwards, partly transversely and partly obliquely, to blend with the vaginal wall.

The external sphincter and superficial transverse perineal muscles are similar to those in the male.

Internal Pudic Artery. - This vessel is of smaller size than in the male, but it takes a similar course. The difference, therefore, in the two sexes affects chiefly the branches of the artery.

The superficial perineal artery is larger than in the male, and is distributed to the labium majus.

The artery of the bulb is of comparatively small size, and is distributed to the bulbus vestibuli.

The artery of the corpus cavernosum clitoridis, having pierced the antero-inferior layer of the triangular ligament, enters the crus clitoridis, and is then continued onwards in the centre of the corpus cavernosum.

The dorsal artery of the clitoris, like the preceding, is comparatively small in size. Having pierced the antero-inferior layer of the triangular ligament, it passes between the crura clitoridis, and also between the two layers of the suspensory ligament of the clitoris. It is then continued along the dorsum of that organ as far as the glans, having the dorsal vein of the clitoris on its inner side, and the dorsal nerve of the clitoris on its outer side. On reaching the glans, it divides into branches for the supply of the glans and its prepuce. As it passes along the dorsum of the clitoris it gives off several branches, which enter the corpus cavernosum by piercing its fibrous sheath.

The veins of the female perineum are so similar to those in the male as not to require any special description. An exception, however, has to be made in the case of the dorsal vein of the clitoris. This vein is formed by branches which return the blood from the glans and prepuce, and also to a certain extent from the corpora cavernosa.' It passes backwards in the groove between the corpora cavernosa, where it has on either side of it the dorsal artery, and external to this the dorsal nerve, of the clitoris. In this part of its course it receives tributaries from the corpora cavernosa. On reaching the root of the organ it passes between the two layers of the suspensory ligament of the clitoris, and then between the subpubic and transverse pelvic or perineal ligaments, and so it enters the pelvic cavity, where it terminates in the plexus of veins at the neck of the bladder.

Lymphatics.-The lymphatics of the labia majora, the superficial lymphatics of the clitoris, including those of its glans, and the lymphatics of the lower third of the vagina and of the urethra terminate in the inguinal glands, which lie immediately below Poupart's ligament. The lymphatics of the upper two-thirds of 
the vagina terminate in the internal iliac glands, and the deep lymphatics of the corpora cavernosa clitoridis, having accompanied the dorsal vein of the clitoris, also terminate in the internal iliac glands.

The pudic nerve and its branches are similar to those in the male, the superficial perineal nerves being distributed to the labia majora.

\section{ABDOMIXAL WALL.}

Landmarks. - The position of the linea alba is indicated by the mid-abdominal groove; which extends from the ensiform process of the sternum to the umbilicus, and the mid-abdominal line, which extends from the umbilicus to the upper part of the symphysis pubis. After removal of the integument the linea alba presents a dense white appearance, and is slightly depressed below the level of the adjacent surfaces. It is produced by the decussation of the aponeuroses of the abdominal muscles of opposite sides, except the recti, and it is divided into two parts, supra-umbilical and infraumbilical. The supra-umbilical part is about $\frac{1}{4}$ inch broad, the recti being here separated to that extent. The infra-umbilical part is only about $\frac{1}{8}$ inch wide on account of the approximation of the recti in this situation. Over the whole extent of its posterior or abdominal surface it is invested by the parietal peritoneum, unless in cases of abnormal distension of the bladder, when the peritoneum is stripped from the lower part to an extent corresponding with the height to which the distended bladder ascends. The anterior abdominal wall is thinner and less vascular along the linea alba than at any other part. This line is therefore selected for such operations as suprapubic lithotomy, tapping a distended bladder above the symphysis pubis, and ovariotomy.

The structures which are divided in opening the abdominal cavity along the linea alba are as follows: the integument, the decussating fibres of the aponeuroses of opposite sides, fascia transversalis, subperitoneal areolar tissue, and parietal peritoneum. There are no bloodvessels of any importance in this situation.

The posterior aspect of the linea alba has important visceral relations. The left lobe of the liver lies behind it for about 2 inches below the ensiform process of the sternum. The relation of the stomach to it is variable. When the viscus is moderately distended it lies behind the linea alba below the margin of the liver. In the empty condition, however, it recedes from the linea alba, and this gives rise superficially to the epigastric depression, or scrobiculus cordis ("small trench of the heart'). The transverse colon, covered by the great omentum, as it crosses from right to left, usually lies behind the linea alba just above the umbilicus. The coils of the jejunum and ileum, also covered by the great omentum, lie behind it below the umbilicus. 
In young persons the upper part of the bladder, being extrapelvic, lies behind the lowest part of the linea alba. In adults the upper part of that viscus, when abnormally distended, also lies behind the lowest part of this line.

The umbilicus takes the form of a cicatricial depression which is situated in the linea alba at the junction of the upper threefifths and lower two-fifths. As seen from before it is irregularly circular, the skin being more or less puckered according to the state of distension of the abdomen. When viewed from behind it is smaller in size, and its long measurement lies transversely. Besides cicatricial tissue and fat, the lower part of it contains the upper ends of the urachus and obliterated hypogastric arteries, whilst the upper part is occupied by part of the obliterated umbilical vein. The upper part is weaker than the lower. The umbilicus is on the same horizonal plane as the disc between the bodies of the third and fourth lumbar vertebræ.

During intra-uterine life the umbilicus is an opening by which the hypogastric arteries leave the abdomen of the fœtus on their way to the placenta, and by which the umbilical vein, on its way from the placenta, enters the abdomen. A few days after birth, however, the opening becomes permanently closed.

The lineæ semilunares coincide with the outer borders of the recti abdominis. The position of each is indicated by a line drawn from the lowest part of the eighth costal cartilage to the pubic spine. This line is curved, with the convexity outwards, and at the level of the umbilicus it is about 3 inches from it. Over the upper three-fourths of the rectus abdominis it indicates the splitting of the aponeurosis of the internal oblique into two laminæ, which encase that extent of the muscle in a sheath. Over the lower fourth it indicates where the aponeuroses of the external oblique and internal oblique and the anterior aponeurosis of the transversalis abdominis pass in front of the rectus.

The lineæ transversæ are usually three in number, on either side. They are tendinous intersections or inscriptions which cross the rectus in the following situations: one at the level of the umbilicus, one at the level of the lower part of the ensiform process of the sternum, and one about midway between these two. The positions of the lineæ transversæ are indicated by three faint grooves. The anterior wall of the sheath of the rectus is closely bound down to the tendinous intersections, and so each muscle above the level of the umbilicus is mapped out into two quadrangular areas, and the interior of the sheath, anterior to the muscle, is also divided into two distinct compartments. An abscess may form in one or other of these compartments, or there may be a spasmodic contraction of one or other of these quadrangular areas of the muscle, a condition in each case which would necessarily have a circumscribed limit, and might lead to error in diagnosing affections really having their seat within the abdominal cavity.

The spino-umbilical lines are two in number, right and left, 
and each extends from the anterior superior iliac spine to the umbilicus.

The anterior superior spinous process of the ilium is situated at the anterior extremity of the iliac crest, and, being very superficial, it can be readily felt. It is on the same level with that of the opposite side, and therefore a line connecting the two should be quite horizontal. The plane of this interspinous line is rather lower than the promontory of the sacrum. The anterior superior iliac spine is the point from which the measurement of the lower limb is taken. the other point being the internal malleolus. This spine is also a good ready guide to the position of the great trochanter, which is situated about 4 inches below it, and about $4 \frac{1}{2}$ inches behind a line let fall vertically from it.

The pubic spine is situated at the lower and inner part of the anterior abdominal wall about $I \frac{1}{2}$ inches outside the upper part of the symphysis pubis. It is sometimes a sharp-pointed process, and then it can readily be felt beneath the integument. In most persons, however, it takes the form of a more or less indistinct tubercle, and cannot readily be made out. In such cases the scrotal integument may be invaginated with the finger, and so the adipose tissue raised from over the spine. If it cannot be felt in this way the thigh should be well abducted to render prominent the adductor longus muscle, the tendon of origin of which will serve as a guide to the spine, which lies above and to the outer side of it. The pubic spine is the guide to the external abdominal ring, the crural or femoral ring, and the saphenous opening. The external abdominal ring is situated immediately above, and to the outer side of, the pubic spine. In exploring the ring the best way to proceed is to invaginate the scrotal integument, and carry the examining finger up the inner side of the spermatic cord, when the ring will be reached. In normal circumstances it should admit the point of the little finger. In making this examination the spermatic cord is readily felt, and the vas deferens can be distinguished as a firm cord-like structure lying at its back part, and easily separable from the other constituents of the cord. In the female the round ligament of the uterus takes the place of the spermatic cord, but, being a very ill-defined structure, it usually escapes detection. The femoral ring is situated fully I inch external to the pubic spine in a line drawn transversely outwards from that spine across the front of the thigh. The saphenous opening is situated below, and a little external to, the pubic spine.

The pubic crest extends transversely inwards for about I I inches from the pubic spine, and it terminates in the pubic angle which surmounts the internal surface of the pubic body, and is usually a rudimentary tubercle. The crest may be felt with the finger as the external abdominal ring, of which it forms the base, is being explored.

Poupart's ligament can be felt as a tense band, especially when the thigh is extended, abducted, and rotated outwards, 
passing between the anterior superior iliac spine and the pubic spine.

The internal abdominal ring is situated midway between the symphysis pubis and the anterior superior iliac spine, and $\frac{1}{2}$ inch above Poupart's ligament.

The inguinal canal extends obliquely downwards, forwards, and inwards from the internal to the external abdominal ring, and is situated immediately above the inner half of Poupart's ligament.

Topography of Arteries, Triangles, and Iliac Fossæ.-The bifurcation of the aorta into right and left common iliac arteries usually takes place opposite the centre of the body of the fourth lumbar vertebra, a finger's breadth to the left of the middle line. The position of the bifurcation is indicated in one of two ways: (I) a point fully I inch below the umbilicus and a finger's breadth to the left of the linea alba pretty nearly corresponds with the point of bifurcation; (2) a more definite guide is a point in the line which connects the highest parts of the iliac crests a finger's breadth to the left of where it intersects the linea alba.

The common and external iliac arteries are indicated by a line drawn from a point midway between the anterior superior iliac spine and the symphysis pubis to the point corresponding to the bifurcation of the aorta. This line should be slightly curved, with the convexity directed outwards. Its upper 2 inches indicate the course of the common iliac artery, and the remainder represents the external iliac.

The deep epigastric artery, in its first or oblique part, is indicated by a line drawn from the inner border of the internal abdominal ring to the outer border of the rectus abdominis at a point midway between the upper border of the symphysis pubis and the umbilicus. The subsequent course of the vessel is represented by a line corresponding to the centre of the rectus abdominis, and reaching to a point about 2 inches above the umbilicus. This latter line is about $x_{2} \frac{1}{2}$ inches distant from the linea alba.

The triangle of Hesselbach is situated on the inner side of the first or oblique part of the deep epigastric artery, and above the inner half of Poupart's ligament.

The triangle of Petit is situated immediately above the centre of the iliac crest. In this region a lumbar hernia may protrude, and a lumbar abscess may come to the surface at this point.

The right iliac fossa contains the terminal part of the ileum, the cæcum, the ileo-cæcal valve, and the vermiform appendix in part.

The left iliac fossa contains the iliac colon.

McBurney's point is situated in the right spino-umbilical line between $I \frac{1}{2}$ and 2 inches from the anterior superior iliac spine. According to McBurney it corresponds very accurately in the living subject to the base of the vermiform appendix. Practically it coincides with the centre of the right iliac fossa.

Monro's point is situated at the centre of the spino-unibilical line. Its distance from the anterior superior iliac spine is 2.6 inches. 
Upon the right side McBurney's point and Monro's point very nearly coincide. Both of these points are now regarded as indicating approximately the situation of the ileo-cæcal valve, and not the base of the vermiform appendix. The guide to the base or opening of the appendix is a point on an average rather more than I inch below the ileo-cæcal valve-that is to say, the base or opening of the vermiform appendix is rather more than I inch below McBurney's point.*

Anterior Abdominal Fasciæ.-The superficial fascia of the anterior wall of the abdomen, from Poupart's ligament to a line drawn transversely from the anterior superior iliac spine to the linea alba, resembles the superficial fascia of the uro-genital division of the perineam in being divisible into two layers. The layer immediately beneath the skin is called the subcutaneous layer or fascia of Camper, and the other layer is called the deep layer or fascia of Scarpa.

The subcutaneous layer or fascia of Camper is composed of areolar tissue containing adipose tissue in its meshes. When traced upwards it blends with the deep layer above the level of the line connecting the anterior superior iliac spine with the linea alba. When followed inwards it is continuous with the corresponding layer of the opposite side. In a downward direction it is freely continuous over Poupart's ligament with the subcutaneous layer of the superficial fascia of the front of the thigh. When traced downwards and imwards it passes along the spermatic cord into the scrotal wall and over the penis, in which situations, more especially the former, it contains involuntary muscular fibres, which replace its adipose tissue, and so, with the deep layer which it here joins, it forms the tunica dartos. In the female Camper's fascia passes along the round ligament of the uterus into the labium majus.

The deep layer or fascia of Scarpa is a strong membrane which contains yellow elastic tissue. It is separated from Camper's fascia by the superficial epigastric vessels, and, in the region of Poupart's ligament, by the inguinal glands. Its deep aspect is loosely connected by areolar tissue to the subjacent aponeurosis of the external oblique muscle. Superiorly, above the line connecting the anterior superior iliac spine with the linea alba, it blends with Camper's fascia. At the middle line it is firmly bound down by fibrous bands to the linea alba. Inferiorly it blends with the inner part of Poupart's ligament, but external to this it passes over that ligament, to which it is bound by fibrous bands, and immediately thereafter it becomes incorporated with the fascia lata of the thigh. When traced downwards and inwards it passes along the spermatic cord into the scrotal wall, and over the penis. In the former situation it forms, along with the subcutaneous layer, the tunica dartos. In the female Scarpa's

* Sir Frederick Treves, based upon observations made by Dr. C. Addison and Dr. Arthur Keith. 
fascia passes along the round ligament of the uterus into the labium majus.

The fascia of Camper is continuous, through the tunica dartos, with the subcutaneous layer of the superficial fascia of the uro-

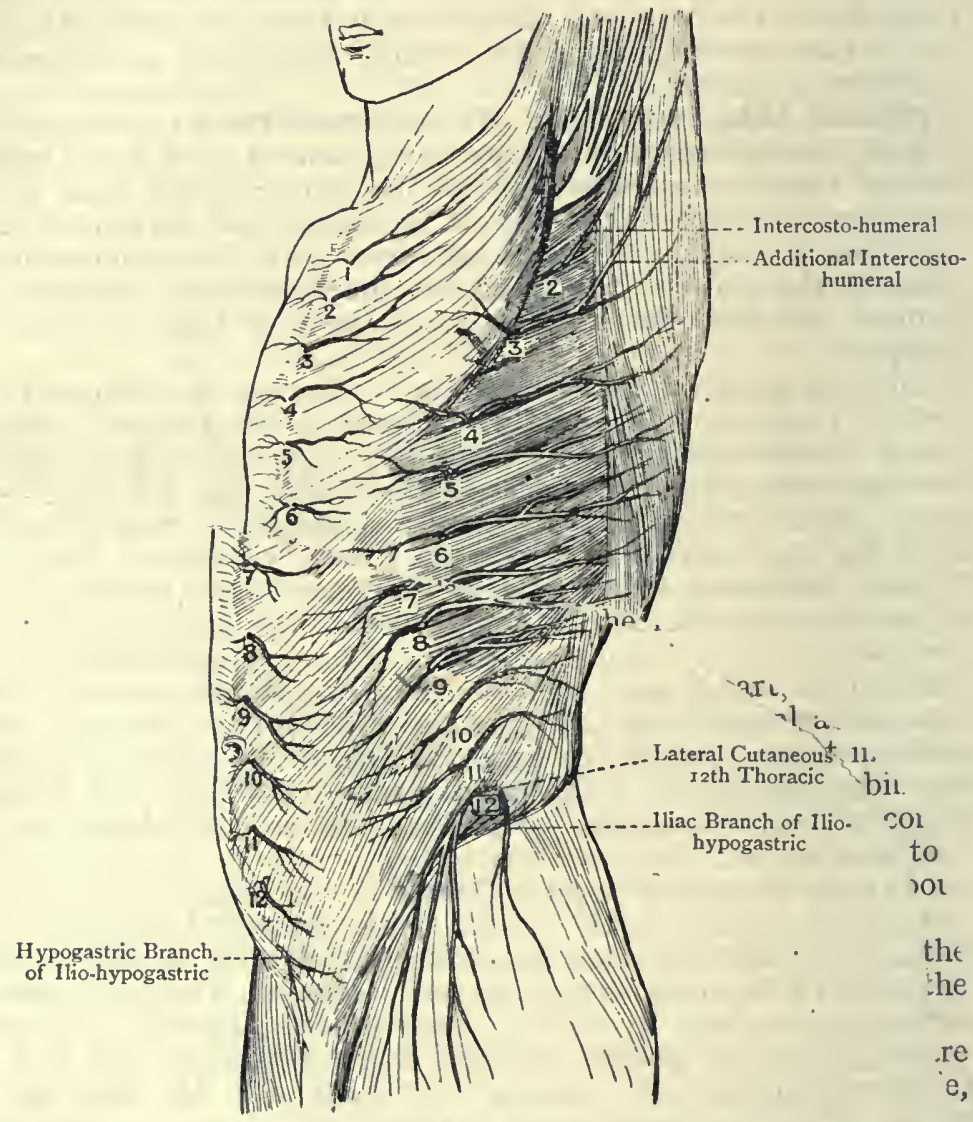

Fig. 281,-Cutaneous Nerves or the Trunk (Antero-lateral le VIEW) (AFTER HENLE).

I-12, Anterior Cutaneous; 2-12, Lateral Cutaneous.

genital division of the perineum, and the fascia of Scarpa is similarly continuous with the deep layer of the superficial fascia, or the fascia of Colles, of that division.

The disposition of Scarpa's fascia at the line of the groin explains why, in cases of extravasation of urine beneath Colles's fascia, tricaic urine, when it reaches the anterior abdominal wall, does not pahess 
downwards to the front of the thigh, but takes an upward course.

The fascia of Scarpa represents the tunica abdominalis of quadrupeds, which is composed almost entirely of elastic tissue, and serves as an important adjunct to the abdominal parietes.

The deep fascia resembles that in other parts of the body in being membranous. Laterally, where the external oblique is fleshy, this fascia is well marked, but, as it approaches the confines of the aponeurosis of that muscle, it becomes very delicate, and ultimately disappears. Over the external oblique aponeurosis, therefore, there is really no deep fascia properly so called, so that the deep layer of the superficial fascia or fascia of Scarpa lies directly upon the external oblique aponeurosis, to which it is loosely connected by areolar tissue.

Cutaneous Nerves. - The anterior cutaneous nerves are the terminal branches of the lower five intercostal nerves and of the anterior primary division of the twelfth thoracic nerve (subcostal nerve). Having emerged through the anterior wall of the sheath of the rectus abdominis in a straggling manner, they give a few twigs inwards, and then turn outwards to supply the anterior abdominal integument, in which they communicate with the anterior branches of the lateral cutaneous nerves.

The hypogastric branch of ilio-hypogastric nerve pierces the external oblique aponeurosis about $I$ inch above the external abdominal ring, and is distributed to the integument of the suprapubic region. It is serially continuous with the anterior cutaneous nerves.

The skin below the ensiform process is supplied by the seventh thoracic nerve; that on a level with the umbilicus by the tenth thoracic; and that over the lower half of the infra-nmbilical region by the twelfth thoracic, and the ilio-hypogastric.

The lateral cutaneous nerves are branches of the lower five intercostal nerves. Having emerged between the serrations of the external oblique muscle in the mid-axillary line, each divides into an anterior and a posterior branch. The posterior branch turns backwards to supply the integument over the lower part of the back. The anterior branch passes forwards to supply the anterior abdominal integument, in which it communicates with an anterior cutaneous nerve.

Cutaneous Arteries. - The superior or superficial external pudic artery arises from the common femoral about $\frac{3}{4}$ inch below Poupart's ligament, after which it pierces the crural sheath and cribriform fascia. Having emerged through the saphenous opening, it passes inwards and upwards over the spermatic cord, or round ligament of the uterus, according to the sex, to be distributed to the integument of the suprapubic region, the adjacent portion of the scrotum in the male and the labium majus in the female, and the dorsum of the penis by a branch which extends as far as the prepuce, lying external to the dorsalis penis artery. The vessel in its course gives branches to the pubic glands, the deep femoral glands, and the coverings of the spermatic cord, or of the round ligament of the uterus. It anasto- 
moses with the following arteries: $(a)$ the cremasteric branch of the deep epigastric, in crossing the spermatic cord, that branch being represented in the female by the artery of the round ligament of the uterus; $(b)$ its fellow of the opposite side; $(c)$ the inferior or deep external pudic; and (d) the dorsalis penis.

The venæ comites of this artery terminate in one vessel which joins the long saphenous vein.

The superficial epigastric artery arises from the common femoral about $\frac{1}{2}$ inch below Poupart's ligament. Having pierced the crural sheath and cribriform fascia, or the outer border of the saphenous opening, it turns upwards over Poupart's ligament a little to the inner side of the centre, and then ascends in the abdominal integument as high as the level of the umbilicus. As it turns upwards it supplies branches to the inguinal glands, and on the abdominal wall it anastomoses with branches of the deep epigastric artery.

There are at first two venæ comites with this artery, but these eventually join to form one vessel which terminates in the long saphenous vein. The radicles of these venæ comites communicate with the following vessels: the parumbilical veins in the region of the umbilicus, which lie on the surface of the round ligament of the liver, and communicate with the branches of the vena portæ; the inferior or long thoracic and subscapular veins, upon the side of the thorax, which are tributaries of the axillary vein; and the superior epigastric.veins, which are tributaries of the internal mammary veins. In the superficial epigastric vein and its tributaries the blood can flow in either direction. The anastomoses of the tributaries of the vein explain why they become engorged in cases of portal obstruction and obstruction of the inferior vena cava.

The superficial circumflex iliac artery often arises in common with the superficial epigastric from the common femoral about $\frac{1}{2}$ inch below Poupart's ligament. Having pierced the crural sheath and the outer border of the saphenous opening, it passes outwards below the outer part of Poupart's ligament to the anterior part of the iliac crest, where it is distributed to the adjacent abdominal integument. In its course it gives branches to the iliacus and sartorius muscles and the outer inguinal glands, and it anastomoses with $(a)$ the deep circumflex iliac of the external iliac, and (b) the gluteal of the internal iliac.

The vein corresponding to this artery terminates in the long saphenous vein.

The anterior cutaneous arteries are derived from the deep and superior epigastric arteries. They emerge through the anterior wall of the sheath of the rectus abdominis in an irregular manner, and accompany more or less closely the anterior cutaneous nerves.

The veins corresponding to these arteries terminate in the deep and superior epigastric veins.

The lateral cutaneous arteries are branches of the lower five aortic intercostal and subcostal arteries, and they emerge with the lateral cutaneous nerves between the serrations of the external oblique muscle in the midaxillary line.

The veins corresponding to these arteries are tributaries of the lower five intercostal and subcostal veins.

Superficial Lymphatics. - The superficial lymphatics below the level of the umbilicus accompany the superficial epigastric vessels, and terminate in the inguinal glands. Those above the level of the umbilicus pass to the axillary glands. The superficial lymphatics of the lateral abdominal wall terminate in two ways. Some accompany the superficial circumflex iliac vessels, and terminate in tlie inguinal glands; others accompany the abdominal branches of the lumbar arteries, and terminate in the deeply-placed lateral group 
of lumbar glands. For the deep lymphatics of the antero-lateral abdominal wall, see p. 664 .

Penis. Coverings. - The skin at the free extremity of the glans, being doubled upon itself, passes backwards until it reaches the constriction behind the corona glandis called the cervix. Here it is reflected forwards, closely investing the cervix, corona glandis, and body of the glans. On reaching the lips of the meatus urinarius it becomes continuous with the mucous membrane of the urethra. The skin covering the glans is provided with papillæ, but these do not appear on the surface. The duplicature, which the skin forms in the region of the glans, is called the praputium penis, or prepuce. The under part of the prepuce is connected to the under surface of the glans by a median, laterally compressed, triangular fold, called the frenum praputii, which extends as far as the lower pait of the meatus urinarius. The skin of the cervix, corona glandis, and adjacent part of the inner surface of the prepuce contains large sebaceous glands, called the glands of Tyson. Their secretion is a whitish curdy substance, and is strongly odorous, from.which character the glands have been called the glandula odorifera, the secretion being called the smegma praputii (unguent of the prepuce). The dartos tunic is situated immediately beneath the skin, and is destitute of adipose tissue. It is continuous with Camper's and Scarpa's fasciæ of the anterior abdominal wall, and with the tunica dartos of the scrotum, like which latter it contains involuntary muscular tissue. The fascial investment or sheath covers the penis, with the exception of the glans. It is situated beneath the dartos tunic, and at the cervix blends with the skin of the glans. Towards the root of the organ it receives expansions from the ischio-cavernosi and bulbo-cavernosi, and it covers the dorsal vessels and nerves. In this way the dorsal vein is compressed during the action of the ischio-cavernosi and bulbocavernosi. At the root of the organ the fascial investment blends with the two layers of the suspensory ligament.

Suspensory Ligament.-This ligament, which is strong and triangular, is composed of fibrous and elastic tissues. It is attached superiorly to the front of the symphysis pubis, where it is single, and inferiorly it divides into two laterally-disposed, diverging laminx, which blend with the fascial sheath of the penis. The interval between the two laminæ is occupied by the dorsal vessels and nerves.

The dorsal arteries.-For the description of these arteries, see p.624.

In addition to the dorsal artery, the integument of the organ is supplied by the superior or superficial external pudic arteries, the branch from each of these vessels lying external to the dorsalis penis artery.

Deep Dorsal Vein.- The tributaries which give rise to this vein come from the glans and corpora cavernosa. They form at first two dorsal veins, but these soon unite into one vessel, which passes backwards in the middle line, occupying the groove between the corpora cavernosa, where it is under cover of the fascial sheath 
of the penis. At the root of the organ it passes between the two laminæ of the suspensory ligament, and then between the subpubic and transverse perineal or pelvic ligaments. In this part of its course it communicates with the venous plexus in the compressor urethræ muscle in which the internal pudic venæ comites arise. On entering the pelvis it divides into two branches, which terminate in the right and left portions of the prostatic plexus of veins.

In addition to the deep dorsal vein, there are two superficial dorsal veins which take up blood from the glans and integument. Each accompanies a branch of the superiot external pudic artery, and opens into the superior external pudic vein.

For a description of the dorsal nerves of the penis, see p. 627.

The relation of the structures on the dorsum of the penis, from the middle line outwards, is as follows: deep dorsal vein, dorsal artery, and dorsal nerve.

Composition of the Penis.-The penis has two surfaces, upper or dorsal and under or scrotal. Viewed as a whole the organ is composed of three cylindrical bodies, namely, two corpora cavernosa and a corpus spongiosum, closely applied to each other. It is divisible into a root, body, cervix, and glans. The root is formed by the crura of the corpora cavernosa, which are attached to the inner margins of the ischio-pubic rami. The upper surface of the root is also connected to the symphysis pubis by the suspensory ligament: The upper or dorsal surface of the body is formed by the corpora cavernosa, the under or scrotal surface being formed by the corpus spongiosum in the middle line and the corpora cavernosa at either side. The corpora cavernosa and corpus spongiosum at their meeting become flattened, and so the shape of the body is subcylindrical. The corpora cavernosa terminate in round extremities, in front of which the corpus spongiosum turns upwards, and, becoming much enlarged, forms the glans penis. The cervix is the constriction between the body and the glans. The glans penis is formed by the corpus spongiosum. It is somewhat conical, and has been likened to an acorn, from which circumstance it has been called the balanus. At the base there is a wheel-like rim, called the corona glandis. The part anterior to the corona is called the body of the glans, and this terminates in a small round tip, which presents a vertical fissure called the meatus urinarius. For the structure of the penis, see p. 856 .

Lymphatics.-These are divided into a superficial and deep set.

The superficial lymphatics of the prepuce, having formed a plexus, pass to the cervix, which they surround in the form of a ring. Those of the glans, having also formed a plexus, pass along with those of the spongy portion of the urethra to the foregoing ring. From this ring three lymphatic trunks proceed towards the symphysis pubis. One of these is median and dorsally placed, whilst the other two are disposed one on either side. The median trunk, when it reaches the suspensory ligament of the penis, divides into two branches, right and left. Each of these passes with the corresponding lateral trunk to the groin of the same 
side, where both terminate in the pubic group of the inguinal glands. The position of the foregoing three lymphatic trunks accounts for the red lines which are often seen along the dorsum and sides of the penis when septic matter is absorbed from a venereal sore on the prepuce or glans. Their mode of termination also explains the occurrence of induration, or of suppuration, in the inguinal glands as the result of such sores, or of gonorrhœea.

The deep lymphaties return the lymph from the substance of the penis, and accompany the dorsalis penis vein into the pelvis, where they terminate in the internal iliac glands.

The lymphatics of the clitoris correspond with those of the penis.

Scrotum.-The wall of the scrotum is complex, and its constituent parts will be made more evident if they are enumerated in the order in which the testes receive them in their original descent from the abdominal cavity. It may be premised that the descent of each testis is preceded by a process of peritoneum, called the processus vaginalis, the lower part of which remains permanent as the tunica vaginalis scroti. The constituent parts of the scrotal wall, enumerated from within outwards, are as follows: tunica vaginalis scroti, subperitoneal areolar tissue, internal spermatic fascia, cremasteric or middle spermatic fascia, intercolumnar or external spermatic fascia, tunica dartos, and skin.

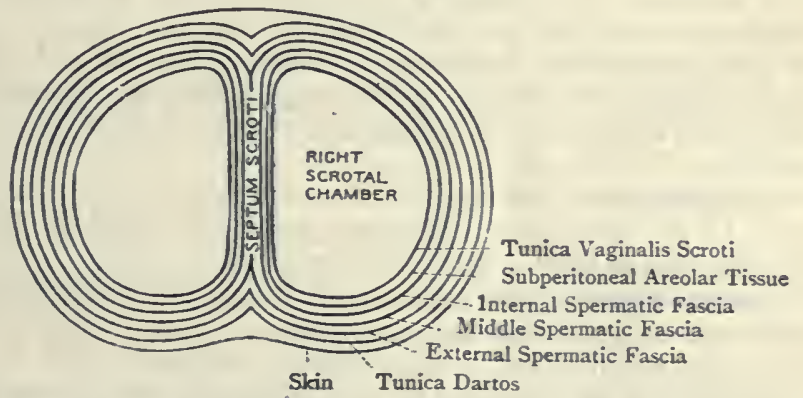

Fig. 282, - Scheme OF the Structures Forming the WALl of the Scrotum (Transverse Section).

The tunica vaginalis scroti is the parietal layer of the tunica vaginalis.

The subperitoneal areolar tissue is composed of areolar and adipose tissues, and a certain amount of plain muscular tissue, its muscular element being known as the middle cremaster.

The internal spermatic fascia is derived from the fascia transversalis at the margins of the internal abdominal ring, in which vicinity it is called the infundibuliform fascia. In association with the subperitoneal areolar tissue it forms the fascia propria of Cooper.

The cremasteric, or middle spermatic, fascia is composed of striated muscular tissue, forming the cremaster muscle (external cremaster), and of areolar and elastic tissues. It is traceable superiorly to the lower border of the internal obliqne and the centre of Poupart's ligament on its deep aspect.

The intercolumnar, or external spermatic, fascia is composed of connec. tive tissue, and is derived from the intercolumnar fibres which extend between the columns of the external abdominal ring. The internal, middle, and external spermatic fascix are closely incorporated with one another. 
The tunica dartos lies immediately within the skin. It is derived from the fascize of Camper and of Scarpa of the anterior abdominal wall. It is chiefly composed of plain muscular tissue, but it also contains fibro-areolar and elastic tissues, and it has a brick-red colour.

The skin is thin, very extensible, somewhat dark in colour, and pro. vided with hairs, sweat-glands, and sebaceous glands. It is more dependent on the left side on account of the lower position of the left testis. In the middle line it presents a raphé, which is continuous behind with that of the anterior part of the perineum, and in front with that on the under surface of the penis. This raphe is an external indication of the bilateral symmetry of the scrotum. On either side of the raphe the skin is thrown into a number of transverse rugæ, the corrugated condition being due to the plain muscular tissue of the tunica dartos.

The interior of the scrotum is divided into two compartments, each of which lodges the corresponding testis. This division is effected by the septum scroti, formed by the fusion of the contiguous walls of the two scrotal chambers, except the skin, which forms one continuous investment to both. Inferiorly the septum is attached to the bottom of the scrotal chamber, and superiorly to the under surface of the root of the penis.

Blood-supply.-The scrotum receives its chief arterial supply from the superior and inferior external pudic branches of the common femoral of each side, and the superficial perineal branches of the internal pudic arteries.

The veins corresponding to these arteries terminate in the long saphenous and internal pudic veins.

The cremasteric branch of the deep epigastric of each side also takes part in the supply of the scrotal wall, the venous blood being returned into the deep epigastric vein, which at its termination is single.

Lymphaties.-The most of the lymphatics of the scrotum terminate in the pubic inguinal glands, but those from the back part pass to the internal iliac glands.

Nerve-supply.- The nerves of the scrotum are as follows: (I) the external or posterior and internal or anterior superficial perineal branches of the pudic; (2) the long pudenal nerve of Sommering from the small sciatic; (3) the inguinal nerve (ilio-inguinal) from the lumbar plexus; and (4) twigs of the genital branch of the genito-crural nerve from the lumbar plexus. The two superficial perineal nerves and the long pudendal nerve are known as the long scrotal nerves.

Development. - The external genital folds.

Muscles of Abdominal Wall. Obliquus Externus AbdominisOrigin.-The outer surfaces of the lower eight ribs by means of eight fleshy slips, the upper five of which interdigitate with slips of the serratus magnus, and the lower thrce with slips of the latissimus dorsi.

Insertion.-(I) The anterior half of the outer lip of the iliac crest by fleshy fibres. (2) By means of an aponeurosis into $(a)$ the linea alba; $(b)$ the pubic spine and crest of the opposite side through means of the triangular fascia; $(c)$ the front of the symphysis pubis; (d) the pubic spine of the same side; (e) the first incl 
of the ilio-pectineal line; and $(f)$ the anterior superior iliac spine.

Nerve-supply.-The lower five intercostal nerves and the subcostal nerve.

Action.-(1) The two muscles, acting conjointly, diminish the size of the abdominal cavity, and so compress the viscera, as in defæcation. (2) The two muscles, acting conjointly from their origins, raise the front part of the pelvis and flex it upon the thorax. (3) Acting conjointly from their insertions, they flex the thorax upon the pelvis, the vertebral column being also flexed in the lower thoracic and lumbar regions ; but, if the column is fixed, the two muscles depress the lower eight ribs. (4) One muscle, acting from its origin, is a lateral flexor of the pelvis. When it acts from its insertion it flexes the thorax towards its own side and rotates it to the opposite side.

The most of the fibres of the external oblique pass downwards and forwards, coinciding in direction with those of the external intercostal muscles. The only parts of the muscle which are free are the part between the last rib and the iliac crest, and the part opposite the pubic crest. The aponeurosis attains its greatest width and strength at, and below, the level of the anterior superior iliac spine, and it is narrowest opposite the umbilicus. Superiorly it gives origin to fibres of the pectoralis major. Crossing the fibres of the aponeurosis in an upward and inward direction there are several superadded fibres. In the lower part of the abdominal wall these are specially well marked, and are here spoken of as the intercolumnar fibres. Under this name they spring from the outer third of Poupart's ligament, whence they pass upwards and inwards in a curved manner, the convexity being downwards. On arriving at the upper and outer part of the external abdominal ring they extend from one column to the other. They round off and close the ring, and are prolonged upon the spermatic cord to form the intercolumnar or external spermatic fascia.

The following parts of the external oblique aponeurosis require special description: Poupart's ligament, Gimbernat's ligament, the triangular fascia, the external abdominal ring, and the linea alba.

Poupart's ligament, also known as the superficial femoral arch, is the thickened lower border of the external oblique aponeurosis, which is folded backwards. Externally it is attached to the anterior superior iliac spine, and internally to the pubic spine. from which it is reflected outwards and backwards upon the ilio-pectineal line for fully I inch to form Gimbernat's ligament. The ligament is curved, the convexity being directed downwards and outwards, which is due to the attachment of the iliac lamina of the fascia lata to its lower border. The convexity is greatest when the thigh is extended, abducted, and rotated outwards, in which position the fascia lata on the front of the thigh is tightened.

Relations-Superficial.-Skin; Camper's and Scarpa's fasciæ; superficial epigastric vessels, a little to the inner side of the centre; superficial circumflex iliac vessels just below its outer part; and the inguinal 
glands along its lower border. Deep.-Internal oblique along the outer half, and transversalis abdominis along the outer third. Along the outer half the fascia transversalis and fascia iliaca are attached, and form a canal containing the deep circumflex iliac vessels. The fascia transversalis continues to be attached to the inner part, but loosely and only as far inwards as the base of Gimbernat's ligament. The deep crural arch is also a deep

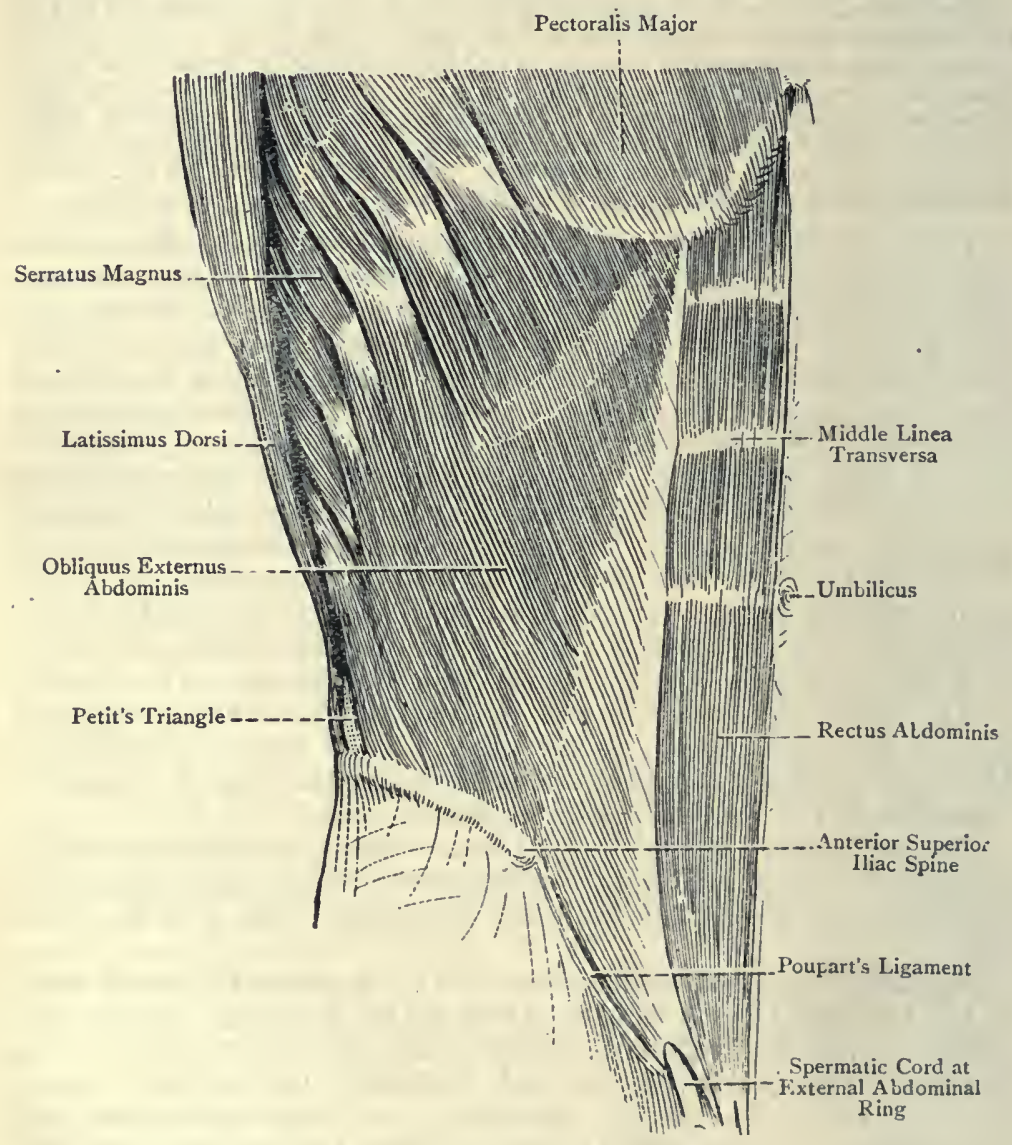

Fig. 283.-The External Obligue Muscle.

relation. Poupart's ligament, along with the irregularly concave border of the os innominatum, forms a large fibro-osseous canal. This region is known as the lacunar region.

The deep femoral arch is a thickening of the fascia transversalis as it passes downwards beneath Poupart's ligament to form the anterior wall of the crural sheath. The thickening is due to superadded fibres which extend inwards from the centre of Poupart's ligament on its deep aspect to the ilio-pectineal line, where they are attached behind Gimbernat's ligament near its base. 
Gimbernat's ligament is the reflection of Poupart's ligament from the pubic spine along the ilio-pectineal line. Its. length is fully I inch, and it is triangular, the apex being at the pubic spine. It presents three borders and two surfaces. Two of the borders are fixed, one, called the Poupart border, being continuous with the inner end of Poupart's ligament, and the other, called the pectineal border, being implanted on the pectineal portion of the ilio-pectineal line. The third border is free, and is called the base. It is sharp, wiry, and concave, and is situated immediately to the inner side of the crural or femoral ring. The surfaces of the ligament are femoral and abdominal. They occupy an oblique plane, the jemoral surface looking downwards, forwards, and slightly outwards, whilst the abdominal surface looks upwards, backwards, and slightly inwards. This latter surface, along its line of junction with Poupart's ligament, forms the floor of the inguinal canal in its lower third, where it supports the spermatic cord in the male, and the round ligament of the uterus in the female. The fascia transversalis takes attachment to the base of the ligament on its abdominal aspect, and the conjoined tendon and fascia transversalis are behind it at the ilio-pectineal line. A few of the fibres of the superior cornu of the saphenous opening terminate on the femoral surface of the ligament, and the pectineus muscle and pubic lamina of the fascia lata are in front of its pectineal attachment.

The triangular fascia, also known as the ligament of Colles, is situated behind the spermatic cord and internal pillar of the external abdominal ring. Its fibres are derived from the external oblique aponeurosis of the opposite side, and, having crossed the linea alba, they take insertion into the pubic spine and crest. A portion of the fascia is seen lying in the lower and inner part of the external abdominal ring.

The external abdominal ring is an opening in the aponeurosis of the external oblique, and it is situated immediately above, and to the outer side of, the pubic spine. It serves for the passage of the spermatic cord in the male, and the round ligament of the uterus in the female. In the natural condition no opening is perceptible, the intercolumnar fibres rounding it off, and being prolonged downwards upon the transmitted structure. When the intercolumnar fibres and fascia have been removed, the ring is seen to be formed in the following manner: the fibres of the external oblique aponeurosis, as they approach the os pubis, are disposed in two diverging bundles. One bundle, which represents the inner end of Poupart's ligament, is fixed to the pubic spine. The other bundle passes to be attached to the front of the symphysis pubis, where its fibres decussate with those of its fellow of the opposite side, the fibres from the right side being superficially placed. An interval is thus left between these diverging bundles, which is widest at the lower and inner part, where it corresponds with a portion of the pubic crest. This interval is the external abdominal ring. Its direction is upwards and outwards, and 
it is parallel with the inner part of Poupart's ligament. Its length is from I inch to $I \frac{1}{2}$ inches, and its breadth at the base about $\frac{1}{2}$ inch. It is triangular, the base being formed by part of the pubic crest, and the apex being directed upwards and outwards. The margins of the ring are called pillars or columns. From the oblique position of the ring one pillar is external and inferior, the other being internal and superior. The internal or superior pillar, which is thin and straight, passes to be attached to the front of the symphysis pubis. The external or inferior pillar is at first thin, but it soon becomes thick and prismatic, and is fixed to the pubic spine. It presents superiorly a concavity which lodges the spermatic cord in the male and the round ligament of the uterus in the female. The intercolumnar or external spermatic fascia is attached to both pillars, and it here lies upon the spermatic cord. Underneath the cord this fascia is attached along the pubic crest between the pillars of the ring. These attachments of the fascia explain why urine, extravasated into the perineum, does not find its way into the inguinal canal. Within the lower and inner part of the ring is the triangular fascia, and directly behind the ring is the conjoined tendon, which strergthens what would othęrwise be a weak part of the abdominal wall. The ring is smaller in the female than in the male.

The linea alba has been already described (see p. 639).

The triangle of Petit is only present when the latissimus dorsi and external oblique do not meet. It is situated immediately above the centre of the iliac crest, and is bounded in front by the posterior border of the external oblique, behind by the anterior border of the latissimus dorsi, and below by the central portion of the iliac crest. It is covered only by skin and fascia, and its floor is formed by a part of the internal oblique. In this situation a lumbar hernia may occur, or a lumbar abscess may find its way to the surface.

Obliquus Internus Abdominis-Origin.-(I) The deep or abdominal surface of Poupart's ligament over its outer half; (2) the summit of the iliac crest over its anterior two-thirds; and (3) the posterior aponeurosis of the transversalis abdominis.

Insertion.-(I) The lower borders of the cartilages of the lower three or four ribs; (2) the lower borders of the cartilages of the seventh and eighth ribs, and the side of the ensiform process of the sternum; (3) the linea alba in its whole length ; $(4)$ the front of the pubic crest; and (5) the pectineal portion of the ilio-pectineal line for $\frac{1}{2}$ inch.

Nerve-supply.-This is similar to that of the external oblique, with the addition of twigs from the inguinal nerve and hypogastric branch of the ilio-hypogastric, both of which are derived from the lumbar plexus, more particularly the first lumbar nerve.

Action.-This is similar to that of the external oblique. "When the right internal oblique acts simultaneously with the left external oblique, the trunk is rotated to the right side, and vice versâ. To 
undcrstand this action, it is to be noted that the fibres of the internal oblique of one side coincide in direction with those of the external oblique of the opposite side.

The most of the fibres pass upwards and forwards across those of the external oblique, and coinciding with those of the internal intercostal muscles. The Poupart fibres, however, pass downwards and inwards, and join the corresponding fibres of the transversalis

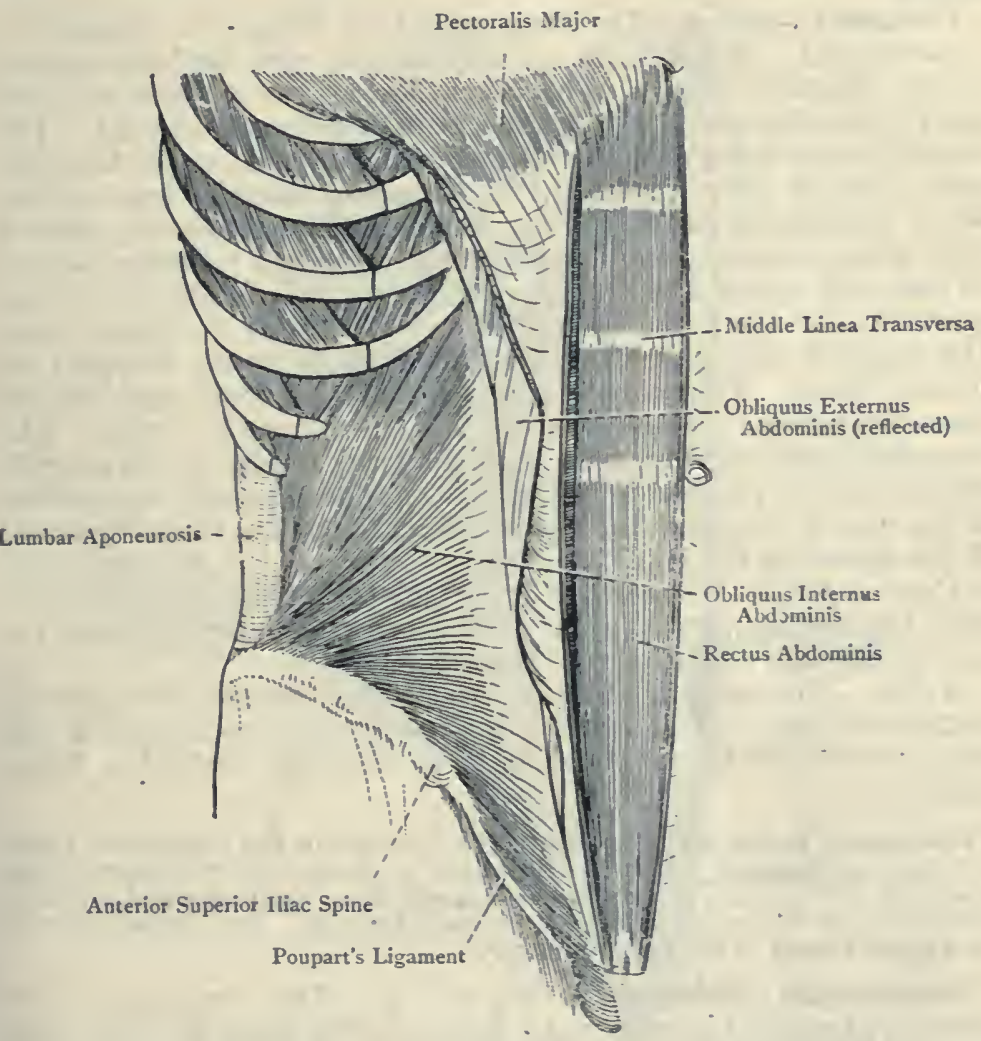

Fig. 284. -The Internal Oblique Míuscle.

abdominis to form the conjoined tendon. The aponeurosis is broader above than below. Along the outer border of the rectus abdominis, over about its upper three-fourths, it divides into two laminæ, one of which passes in front of the muscle and the other behind it. The anterior lamina joins the aponeurosis of the external oblique, and the posterior lamina joins the ariterior aponeurosis of the transversalis abdominis. It is this latter lamina which has an insertion into the seventh and eighth costal cartilages and ensiform 
process. Over about the lower fourth of the rectus the aponeurosis remains single, and passes in front of the muscle. The Poupart fibres of the muscle, as they leave the ligament, are in front of the spermatic cord as it lies in the upper third of the inguinal canal. They then arch over it, and finally descend behind it as it lies in the lower two-thirds of the inguinal canal. In this latter situation the fibres have terminated in the conjoined tendon.

Cremaster-Origin.-The deep aspect of Poupart's ligament in its inner part. A few fibres are also derived from the lower border of the internal oblique. The fibres descend in a series of loops upon the outer and anterior aspects of the spermatic cord. The lowest loops reach the tunica vaginalis of the testis, upon the upper part of which they spread out in an arched manner, some of the fibres terminating on it. The other loops are successively shorter from below upwards, and ascend on the inner and posterior aspects of the cord, where they become tendinous and indefinite, to take insertion into the pubic spine and anterior lip of the pubic crest. The loops of the muscle are separated by intervals occupied by areolar tissue. This combination of muscular loops and areolar tissue forms the cremasteric or middle spermatic fascia. The cremaster muscle lies in series with the lower border of the internal oblique, and is peculiar to the male. It is occasionally represented in the female by a few fibres which descend on the round ligament of the uterus in the inguinal canal.

Nerve-supply. - The genital branch of the genito-femoral nerve from the lumbar plexus, more particularly from the first, and the ventral division of the second, lumbar nerves.

Action.-To support and raise the testis towards the external abdominal ring. The fibres composing the muscle are of the striated variety, but the action is involuntary, and of a reflex character.

Cremasteric Reflex.-The affevent nerve involved in the cremasteric reflex is either the inguinal, which is distributed to the side of the scrotum and inner side of the thigh, or the femoral branch of the genito-femoral, which is distributed to the integument over Scarpa's triangle. The efferent nerve is the genital branch of the genito-femoral.

Transversalis Abdominis-Origin.-(I) The deep aspect of Poupart's ligament over its outer third; (2) the inner lip of the iliac crest over its anterior two-thirds; (3) the lumbar aponeurosis, which, by its posterior lamina, is connected with the spinous processes of the lumbar and sacral vertebræ, and the posterior fourth of the outer lip of the iliac crest, by its middle lamina with the tips of the lumbar transverse processes, and, by its anterior lamina, with the fronts of the lumbar transverse processes near their tips; and (4) the inner surfaces of the cartilages of the lower six ribs by six slips which interdigitate with slips of the diaphragm.

Insertion.-(I) The linea alba; (2) the front of the pubic crest; and $(3)$ the pectineal portion of the ilio-pectineal line for I inch. 
Nerve-supply.-This is similar to that of the internal oblique.

Action.-To diminish the capacity of the abdominal cavity, and so compress the viscera. To a limited extent the two muscles aid the external and internal oblique muscles in elevating the pelvis, flexing the thorax, and depressing the ribs.

The most of the fibres pass transversely forwards, and terminate in the -anterior aponeurosis. The Poupart fibres, however, pass

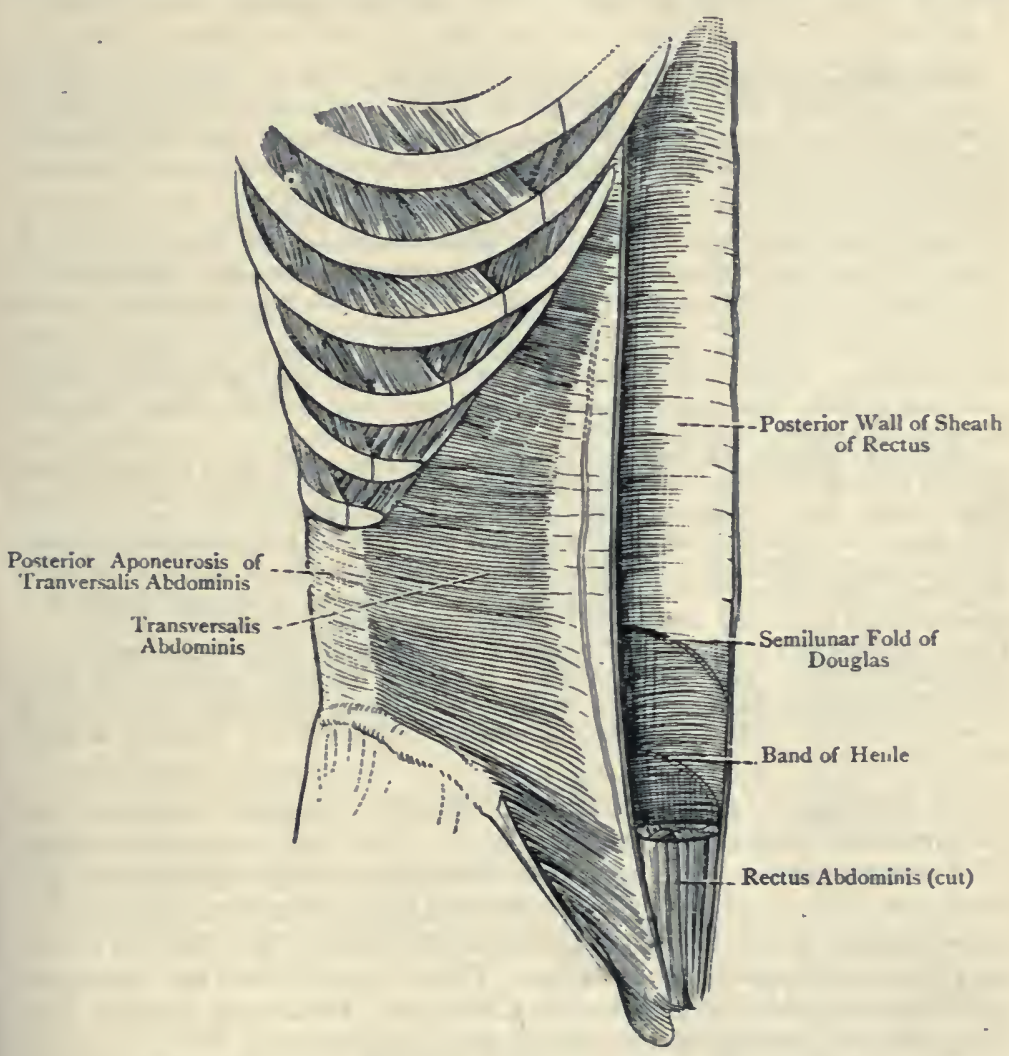

Fig. 285.-The Transversalis Abdominis Muscle.

lownwards and inwards, and join the corresponding fibres of the internal oblique to form the conjoined tendon. The anterior aponeurosis is narrow above, but broad below. Over about the upper three-fourths of the rectus abdominis it passes behind the muscle, but over about the lower fourth it passes in front of it. The transversalis ablominis is continuous superiorly with the triangularis sterni muscle, and its lower border is free where it arches over the spermatic cord, or the round ligament of the uterus. 
For the posterior aponeurosis of the muscle, see p. 765 .

Conjoined Tendon.-This is the tendon which gives a common insertion to the Poupart fibres of the internal oblique and transversalis abdominis muscles. It is inserted into (I) the front of the pubic crest, and (2) the pectineal portion of the ilio-pectineal line for I inch. The tendon is formed principally by the transversalis abdominis, which extends for I inch along the ilio-pectineal line, whilst the internal oblique only extends along that line for $\frac{1}{2}$ inch.

Relations-Superficial.-The structures in front of the conjoined tendon, from the middle line outwards, are as follows: the lower part of the internal pillar of the external abdominal ring; the triangular fascia ; the insertion of the cremaster ; Gimbernat's ligament ; the inner part of Poupart's ligament ; and the spermatic cord. Deep.-The fascia transversalis.

The conjoined tendon varies greatly in development, and in many cases is hardly distinguishable. As a rule, it covers the inner two-thirds of the floor of Hesselbach's triangle, where it lies behind the inner two-thirds of the inguinal canal and has the spermatic cord, or the round ligament of the uterus, as a superficial relation. It also lies directly behind the external abdominal ring, and so strengthens what would otherwise be a weak part of the abdoninal wall. In some cases the conjoined tendon is divisible into two parts, the outer being known as the ligament of Hesselbach, and the inner as the ligament of Henle.

Reflected Tendon of Cooper. - In normal circumstances the conjoined tendon is limited to the first inch of the pectineal portion of the ilio-pectineal line. Sometimes, however, the anterior aponeurosis of the transversalis abdominis is prolonged outwards beyond the normal limit of the conjoined tendon, in the form of a thin semilunar expansion which extends as far as the inner and lower parts of the internal abdominal ring, and is attached inferiorly to the deep crural arch. This semilunar expansion of the conjoined tendon is known as the reflected tendon of Cooper. Its concavity is directed outwards, and, when present, it covers the outer third of the finor of Hesselbach's triangle, where it lies behind the spernatic cord, and is closely connected with the subjacent fascia transversalis, to which it imparts strength.

An inspection of the external oblique, internal oblique, and transversalis abdominis muscles shows that they cross each other at different angles, the external oblique passing downwards and forwards, the internal oblique upwards and forwards (these two muscles thus crossing like the limbs of the letter X), and the transversalis horizontally forwards. They thus form an intricate lattice-work which renders the abdominal wall very strong, and is a powerful safeguard against hernial protrusions.

Rectus Abdominis-Origin.-This muscle arises by two heads, both of which are tendinous. The external head, broad and flat, arises from the pubic crest, and the internal head, narrow and somewhat round, from the fibrous structures in front of the symphysis pubis, where it is closely connected with its fellow of the opposite side. The two heads join about I inch above the symphysis pubis.

Insertion.-By means of three flat slips, which are at first fleshy and subsequently tendinous, into the anterior surfaces of the fifth, sixth, and seventh costal cartilages. The most internal slip is 
sometimes partially inserted into the side of the ensiform process of the sternum.

Nerve-supply.-The lower five intercostal nerves and the subcostal nerve.

Action.-The action is similar to that of the two oblique and transversalis muscles, with the exception that it does not produce lateral rotation of the thorax.

The muscle is long, flat, and strap-like, its fibres being directed vertically upwards by the side of the linea alba. It is narrow beiow and broad above, its greatest breadth being about 3 inches. Below the umibilcus the two muscles are very near each other, the interval between them being not more than about $\frac{1}{8}$ inch. Above the umbilicus, however, the muscles are separated by an interval of about $\frac{1}{4}$ inch. Each muscle is marked by tendinous intersections or inscriptions, which cross it in a somewnat irregular manner at certain intervals. They are usually three in number, and are situated as follows : one at the level of the umbilicus, a second opposite the tip of the ensiform process, and a third about midway between these two. Sometimes there is a fourth inscription, which crosses the muscle a little below the umbilicus, but this one is faint, and does not usually extend the whole width. These inscriptions, which do not usually penetrate to the posterior surface, map out the upper part of the muscle into two quadrangular areas, and are firmly bound to the anterior wall of its sheath. Above the level of the umbilicus, therefore, the interior of the sheath, anterior to the muscle, is divided into two distinct compartments.

The tendinous inscriptions are the remains of the septa which separated the primitive myotomes or muscle-segments, and by some they are regarded as indicating the abdominal ribs of certain animals, such as the crocodile.

Pyramidalis Abdominis-Origin.-The front of the pubic crest.

Insertion.- The linea alba for 2 inches or more above the symphysis pubis.

Nerve-supply.-The subcostal nerve.

Action.- To render tense the linea alba.

The muscle is sometimes present only on one side ; sometimes it is absent on both sides; and sometimes it is double on one or both sides. In man it is a small muscle, and vestigial like the plantaris; but it attains a large size in marsupials, and especially in ornithorhynchus.

Sheath of Rectus Abdominis.-Over about the upper three-fourths of the muscle the anterior wall is formed by the anterior lamina of the internal oblique aponeurosis and the external oblique aponeurosis, the two being closely connected; and the posterior wall is formed by the posterior lamina of the internal oblique aponeurosis and the anterior aponeurosis of the transversalis abdominis, these two being likewise closely connected. Over about the lower fourth the anterior wall is formed by the external oblique aponeurosis, the undivided internal oblique aponeurosis, and the 
anterior aponeurosis of the transversalis abdominis, in this order from before backwards, all three being closely connected. Over this extent the sheath is deficient posteriorly, and the muscle rests upon the fascia transversalis, with an exception to be presently stated. The lower border of the posterior wall presents a free curved margin, with the concavity downwards, called the semilunar fold of Douglas, over which the deep epigastric artery passes. This fold is usually situated about the junction of the upper fourth and lower three-fourths of the infra-umbilical portion of the linea alba. Sometimes the anterior aponeurosis of the transversalis abdominis, though passing in front of the lower fourth of the rectus, sends scattered fibres behind it, which would thus intervene between the muscle and the fascia transversalis for a variable distance below the fold of Douglas. In such cases the posterior wall of the sheath presents another curved semilunar fold, situated from I to 2 inches below the semilunar fold of Douglas, and known as the band of
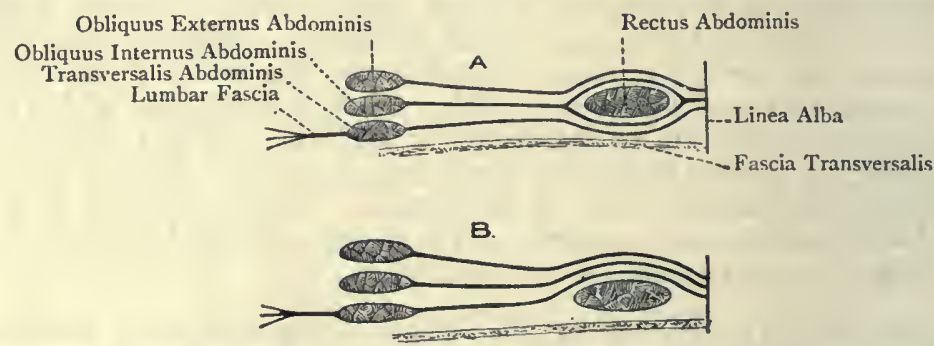

Fig. 286.-Diagram showing the Sheath of the Rectus Abdominis.

$A$, Over the upper three-fourths; B, over the lower fourth.

Henle, over which the deep epigastric artery passes. The contents of the sheath are as follows: the rectus abdominis, pyramidalis abdominis, deep epigastric vessels, superior epigastric vessels, and terminal parts of the lower five intercostal, and subcostal, nerves.

Relation of Structures at the Pubic Crest.-The relation of structures from before backwards is as follows :

1. The triangular fascia.

2. The conjoined tendon.

3. The pyramidalis.

4. The outer head of the rectus abdominis.

5. The fascia transversalis.

A few fibres of the cremaster ale inserted into the pubic crest close to the pubic spine.

Relation of Structures at the Pectineal Portion of the Ilio-pectineal Line.The relation of structures from before backwards is as follows :

I. The pubic lamina of the fascia lata, incorporated with which is the pubic ligament of Cooper.

2. The pectineus.

3. Gimbernat's ligament.

4. The conjoined tendon.

5. The fascia transversalis and the deep fenoral arch. 
Deep Nerves of the Abdominal Wall.-The lower five intercostal nerves, after leaving the intercostal spaces, lie between the internal oblique and transversalis abdominis, and pass downwards and forwards to the outer border of the rectus. Here they pierce, in succession, the posterior wall of the sheath, the rectus, and the anterior wall of the sheath, after which they terminate in the anterior cutaneous nerves. In their course they supply the two oblique, the transversalis, and the rectus muscles. Each nerve gives off a lateral cutaneous branch in the mid-axillary line. These lateral cutaneous branches, having pierced the internal oblique, appear between the slips of the external oblique and divide into anterior and posterior branches.

The subcostal nerve is the anterior primary division of the twelfth thoracic nerve. It is in series with the eleventh intercostal, but is not ranked as an intercostal nerve, inasmuch as it lies along the lower border of the twelfth rib. It is commonly spoken of as the last dorsal nerve. Its abdominal relations, course, and distribution are similar to the preceding nerves, with an additional distribution to the pyramidalis abdominis. Its lateral cutaneous or iliac branch, which is undivided, having pierced the internal and external oblique muscles, descends over the anterior part of the iliac crest, I inch behind the anterior superior iliac spine, and is distributed to the integument of the anterior part of the gluteal region as low as the great trochanter of the femur.

The ilio-hypogastric nerve, having pierced the posterior part of the transversalis abdominis a little above the iliac crest, furnishes its iliac branch (lateral cutaneous), which perforates the internal and external oblique, and, having crossed the iliac crest at the junction of its middle and anterior thirds, is distributed to the integument of the adjacent part of the gluteal region. The nerve then continues its course forwards between the internal oblique and transversalis, supplying branches to these muscles and communicating with the inguinal nerve. About $I$ inch in front of the anterior superior iliac spine it pierces the internal oblique, and runs forwards between the Poupart fibres of that muscle and the external oblique aponeurosis. Finally, it pierces that aponeurosis about I inch above the external abdominal ring, and is distributed to the integument of the suprapubic region, where it is in series with the anterior cutaneous nerves.

The inguinal nerve (ilio-inguinal), as it passes forwards, lies internal to the inner lip of the iliac crest, beneath the transversalis and upon the iliac fascia and iliacus muscle. Near the anterior part of the iliac crest it pierces the transversalis, and here communicates with the hypogastric branch of the ilio-hypogastric. It subsequently perforates the Poupart fibres of the internal oblique, after which it descends through the lower two-thirds of the inguinal canal, and emerges through the external abdominal ring where it lies external to the spermatic cord. Finally, it is distributed to the integument of the inner side of the thigh in its upper third, 
and to the integument of the scrotum, or labium majus, according to the sex. The nerve in its course supplies branches to the internal oblique and transversalis muscles.

Deep Arteries of the Abdominal Wall.-The deep epigastric artery arises from the inner side of the external iliac, about $\frac{1}{4}$ inch above Poupart's ligament. For a very short distance at first it passes inwards between Poupart's ligament and the lower border of the internal abdominal ring. It then changes its course, and passes upwards and inwards, lying close to the inner side of the internal abdominal ring, only the external vena comes intervening. On arriving at the outer border of the rectus abdominis, at a point about midway between the upper border of the symphysis pubis and the umbilicus, the artery passes beneath the muscle and enters the sheath over the semilunar fold of Douglas. It then changes its course, and ascends vertically between the muscle and the posterior wall of the sheath, as high as the umbilicus. Thereafter it enters the muscle, and, about 2 inches above the umbilicus, it ends in its terminal branches, which anastomose with branches of the superior epigastric artery. The deep epigastric lies at first in the subperitoneal areolar tissue, having the parietal peritoneum on its deep surface, and the fascia transversalis superficial to it. Shortly after passing the internal abdominal ring the vessel pierces the fascia transversalis, and in its course to the outer border of the rectus it forms the outer boundary of Hesselbach's triangle. As the vessel turns from the lower border to the inner side of the internal abdominal ring it has the spermatic cord in front of it, and the vas deferens here hooks round its outer side. The course of the vessel in its first or oblique part is indicated by a line drawn from the inner border of the internal abdominal ring to the outer border of the rectus abdominis at a point about midway between the umbilicus and the upper border of the symphysis pubis. The course of the second or vertical part of the vessel is represented by a vertical line corresponding with the centre of the rectus, and distant from the linea alba about $\mathrm{I} \frac{1}{2}$ inches.

Branches.-These are as follows : cremasteric, pubic, muscular, cutaneous, peritoneal, and terminal or anastomotic.

The cremasteric artery enters the spermatic cord and supplies the cremaster muscle and the other coverings of the cord. It anastomoses with the spermatic artery, the superior and inferior external pudic arteries, and the superficial perineal artery. The cremasteric artery is replaced in the female by the artery of the round ligament of the uterus.

The pubic artery passes inwards behind the inner half of Poupart's ligament to the back of the body of the os pubis, where it anastomoses with the pubic branch of the obturator artery and its fellow of the opposite side.

The muscular branches arise chiefly from the outer side of the deep epigastric, and supply the two oblique, transversalis, and 
rectus muscles. They anastomose with the lower two intercostal arteries, the subcostal artery, the abdominal branches of the lumbar arteries, and the lateral or intermuscular epigastric (so called ' ascending branch ') of the deep circumflex iliac artery.

The cutaneous branches perforate the rectus and the anterior wall of its sheath, to be distributed to the integument in which they anastomose with branches of the superficial epigastric.

The peritoneal branches pierce the posterior wall of the sheath of the rectus, to be distributed to the adjacent parietal peritoneum.

The terminal or anastomotic branches enter the rectus above the level of the umbilicus and anastomose with the superior epigastric of the internal mammary.

For an abnormal or aberrant obturator artery, see p. 462 .

There are two venæ comites with the deep epigastric artery, one on either side. These ultimately join to form one vessel, which terminates in the external iliac vein.

The superior epigastric artery is one of the terminal branches of the internal mammary from the first part of the subclavian. It descends behind the seventh costal cartilage, passing between the sternal and costal portions of the diaphragm, and enters the sheath of the rectus, where it lies at first between the muscle and the posterior wall of its sheath. It then enters the muscle and anastomoses with the deep epigastric. Its branches are as follows: metasternal, which crosses in front of the metasternum and anastomoses with its fellow of the opposite side; phrenic, to the diaphragm; muscular to the muscles of the abdominal wall; anterior cutaneous to the integument; falciform or hepatic (present only on the right side), which passes to the liver in the falciform ligament and anastomoses with the hepatic artery; and peritoneal, which pierce the posterior wall of the sheath of the rectus, to be distributed to the adjacent parietal peritoneum. The vessel is accompanied by two venæ comites, which terminate in those of the internal mammary artery.

The deep circumflex iliac artery arises from the outer side of the external iliac nearly opposite the origin of the deep epigastric. It passes outwards and upwards behind the outer half of Poupart's ligament, where it is contained in a canal formed at the junction of the fascia transversalis and fascia iliaca. Having arrived at the anterior superior iliac spine, it pierces the fascia transversalis, and courses outwards and backwards along the iliac crest on its inner aspect. At its termination it anastomoses with the lumbar branch - of the ilio-lumbar from the internal iliac. Over about the anterior half of the iliac crest the artery lies beneath the transversalis muscle, but about the centre of the crest it pierces that muscle, and subsequently lies between it and the internal oblique.

Branches. - In the first part of its course the vessel gives branches to the ilio-psoas, sartorius, and tensor fasciæ femoris, in which latter muscle it anastomoses with the ascending branch of the external circumflex from the arteria profunda femoris. 
As it courses along the iliac crest it furnishes branches to the muscles of the abdominal wall, some of which reach the gluteal region, where they anastomose with the gluteal of the internal iliac and the superficial circumflex iliac of the common femoral. It also gives branches to the iliacus muscle, which anastomose with the iliac branch of the ilio-lumbar from the internal iliac. One of the muscular branches of the artery is very constant and of large size. It springs from the vessel near the anterior superior iliac spine, and, having pierced the fascia transversalis and transversalis muscle, it ascends vertically between that muscle and the internal oblique, where it anastomoses with the abdominal branches of the lumbar arteries. This branch is usually spoken of as the ascending branch, but it may be called the lateral or intermuscular epigastric.

The deep circumflex iliac artery is accompanied by two venæ comites. These ultimately join to form one vessel, which crosses the external iliac artery from without inwards about $\frac{1}{2}$ inch above Poupart's ligament, and terminates in the external iliac vein.

The lower two intercostal arteries ultimately leave the tenth and eleventh intercostal spaces and pass towards the rectus muscle, lying in their course between the internal oblique and transversalis. They anastomose with the superior epigastric, deep epigastric, and subcostal arteries.

The subcostal artery lies below the last rib, and is in series with the aortic intercostal arteries above and the lumbar arteries below. On the abdominal wall it anastomoses with the last intercostal, the abdominal branch of the first lumbar, and the superior epigastric, artèries.

The abdominal branches of the lumbar arteries pass forwards towards the rectus abdominis. They anastomose with the deep epigastric, subcostal, and lateral or intermuscular epigastric of the deep circumflex iliac.

The veins corresponding to the lower two intercostal arteries terminate in the corresponding intercostal veins. The subcostal vein of the right side terminates in the right azygos vein, and that of the left side in the lower left azygos vein. The veins accompanying the abdominal branches of the lumbar arteries terminate in the lumbar veins, which are tributaries of the inferior vena cava.

Deep Lymphatics. - The deep lymphatics of the lower part of the anterior abdominal wall accompany the deep epigastric and deep circumflex iliac vessels, and terminate in the external iliac glands ; those of the upper part accompany the superior epigastric artery, and terminate in the sternal glands.

The deep lymphatics of the lower part. of the lateral abdominal wall accompany the abdominal branches of the lumbar arteries and the ilio-lumbar artery, and terminate in the lateral group of lumbar glands; those of the upper part accompany the adjacent anterior intercostal and musculo-phrenic arteries, and terminate in the sternal glands. 
For the superficial lymphatics of the antero-lateral abdominal wall, see p. 646.

Fascia Transversalis.-The fascia transversalis is situated underneath the transversalis abdominis muscle. It is of greatest strength over the lower part of the abdominal wall, particularly between the lower free border of the transversalis and Poupart's ligament. When traced upwards to the costal margin it becomes very thin, and is there continuous with the fascia which covers the abdominal surface of the diaphragm. Along the linea alba it is continuous with the fascia of the opposite side. In the lumbar region it is continuous with the anterior wall of the sheath of the quadratus lumborum. In the region of the iliac crest it is attached to the anterior two-thirds of the crest immediately internal to its inner lip, where it meets and becomes continuous with the fascia iliaca, both of these fasciæ being here interposed between the transversalis and iliacus muscles. The most important disposition of the fascia is along the line of the groin, where its attachments are as follows: along the outer half of Poupart's ligament it is firmly attached to that ligament on its deep aspect, and over that extent it meets and is continuous with the fascia iliaca, the two fasciæ here forming a canal, which contains the deep circumflex iliac vessels. In the situation of the external iliac vessels, and as far inwards as the base of Gimbernat's ligament, it is only loosely attached to Poupart's ligament, and is here prolonged downwards beneath the ligament to the thigh, where it lies in front of the common femoral vessels, and forms the anterior wall of the crural sheath. As the fascia is prolonged beneath Poupart's ligament it is strengthened by superadded fibres, which are known as the decp crural arch. In the region of Gimbernat's ligament the fascia is attached in succession to the pectineal portion of the ilio-pectineal line behind the conjoined tendon, and to the pubic crest. Behind the symphysis pubis the fascia descends into the pelvis, and becomes continuous with the pubo-prostatic, or anterior true, ligaments of the bladder. The strongest part of the fascia transversalis, as before stated, is between the lower free border of the transversalis muscle and Poupart's ligament, this part of the abdominal wall being uncovered by muscular structures. It is in this situation where the fascia is pierced by the spermatic cord in the male and the round ligament of the uterus in the female.

Internal Abdominal Ping.-This ring is situated in the fascia transversalis at a point midway between the symphysis pubis and the anterior superior iliac spine, and $\frac{1}{2}$ inch above Poupart's ligament. It serves for the passage of the spermatic cord in the male and the round ligament of the uterus in the female. As viewed from before no opening is visible in the undissected state, because the fascia transversalis is prolonged from the margins of the aperture round the spermatic cord as the infundibuliform or internal spermatic fascia. When fully dissected the ring is oval, with the long axis lying vertically, in which direction it measures $\frac{3}{4}$ inch, 
the transverse measurement being $\frac{1}{2}$ inch. The deep epigastric artery lies at first below, and then on the inner side of, the ring, the vessel being here beneath the fascia transversalis. The reflected. tendon of Cooper may be upon the inner side of, and below, the ring. On the outer side of the ring are the Poupart fibres of the internal oblique, and above is the lower free border of the transversalis. The fascia at the outer and lower parts of the ring is stronger than elsewhere.

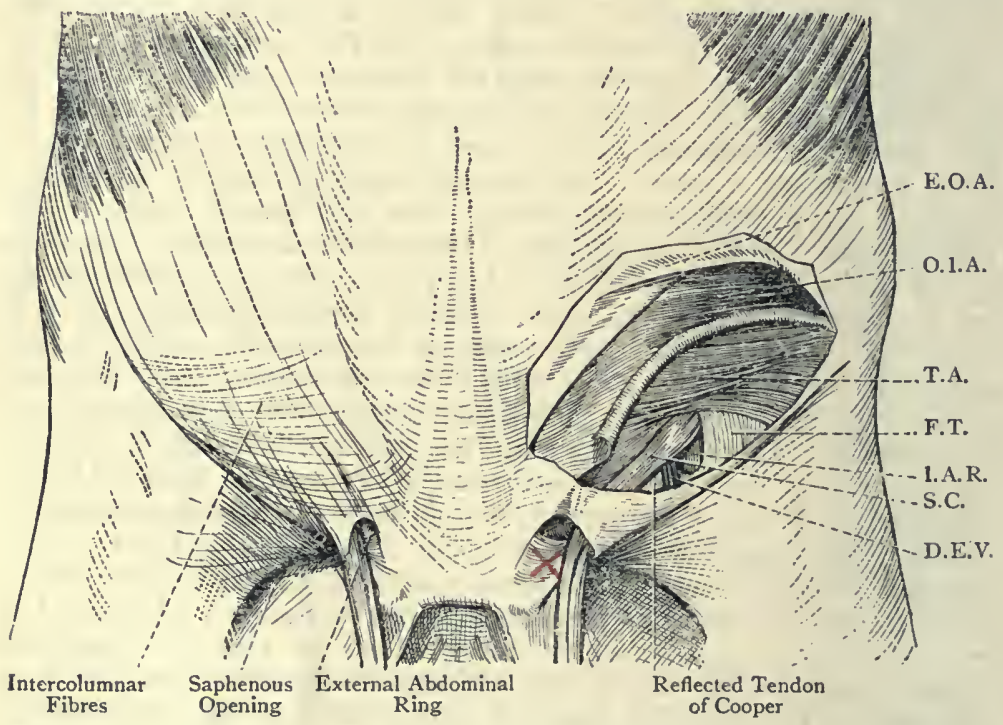

Fig. 287.-Superfictal and Dhep Dissection of the Lower Part of the Anterior Abdominal Wall (after Cooper).

E.O.A. External Oblique Aponeurosis O.1.A. Obliquus Internus Abdominis

T.A. Transversalis Abdominis

F.T. Fascia Transversalis
I.A.R. Internal Abdominal Ring S.C. Spermatic Cord

D.E.V. Deep Epigastric Vessels $\mathrm{X}$. Triangular Fascia

Inguinal Canal.--The inguinal canal is the oblique passage in the lower part of the anterior abdominal wall, which transmits the spermatic cord, or round ligament of the uterus, according to the sex, and also the inguinal nerve in its lower two-thirds. It is situated immediately above the inner half of Poupart's ligament, its direction being downwards, forwards, and inwards, and it is $I_{2}^{\frac{1}{2}}$ inches in length. The inlet to the canal is the internal abdominal ring, the outlet being the external abdominal ring. Its component parts are a floor, a roof, an anterior wall, and a posterior wall. The floor in its upper two-thirds is formed by the meeting between the fascia transversalis and Poupart's ligament. This portion of it presents a groove, which lodges the spermatic cord. In the lower third the floor is formed by the 
abdominal surface of Gimbernat's ligament along its line of junction with Poupart's ligament. The roof is formed by the apposition of the external and internal oblique muscles, and also by the lower borders of the internal oblique and transversalis. The anterior wall is formed by the following structures from before backwards : (I) the skin; (2) fascia of Camper ; (3) fascia of Scarpa ; (4) external oblique aponeurosis (all of these four structures extending over the whole length of the anterior wall); and (5) the Poupart portion of the internal oblique over the outer third. The posterior wall is formed by the following structures, in order from behind forwards : (I) the parietal peritoneum; (2) subperitoneal fat; (3) fascia

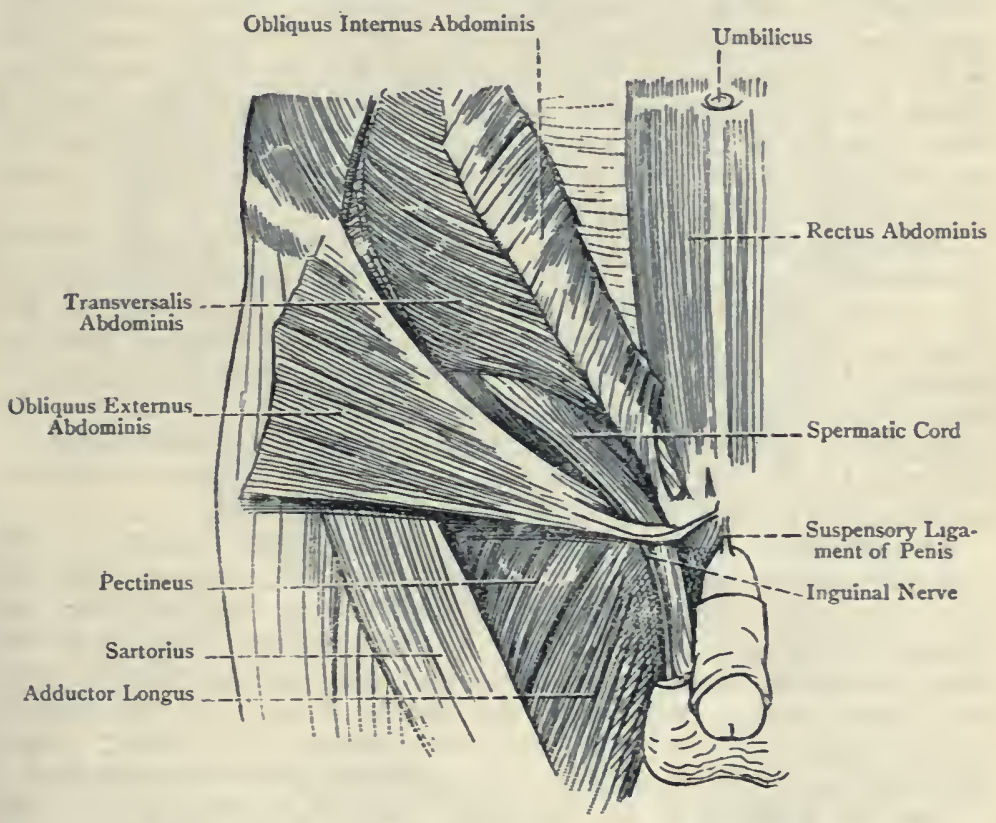

Fig. 288.-Dissection of the Inguinal Region.

transversalis; (4) conjoined tendon over the inner two-thirds, and it may be, the reflected tendon of Cooper over the outer third; and (5) the outer portion of the triangular fascia (provided that fascia is well developed), which lies over the extreme inner part of this wall.

In early life the inguinal canal is very short. Indeed, at one period of life it is non-existent, inasmuch as the internal abdominal ring in very early life lies directly behind the external ring. As the pelvis, however, increases in breadth, the internal ring is gradually shifted outwards, and so the inguinal canal becomes formed.

The inguinal canal in the female differs from that in the male in 
being of smaller size, and in containing the round ligament of the uterus.

Triangle of Hesselbach.-This triangle is situated at the lower part of the anterior abdominal wall above the inner half of Poupart's ligament.

Boundaries-Internal.-The outer border of the rectus abdominis over about its lower 2 inches. External.-The deep epigastric vessels. Inferior (base).-The inner half of Poupart's ligament. The apex corresponds with the point where the deep epigastric vessels pass beneath the outer border of the rectus. The floor is covered over its whole extent by the fascia transversalis, superficial to which, over the inner two-thirds, is the conjoined tendon, and over the outer third, sometimes the reflected tendon of Cooper. The floor is, therefore, conveniently divided into an inner two-thirds, where the conjoined tendon lies, and an outer third, where the reflected tendon of Cooper may be situated. The triangle is covered superficially by the skin, the fasciæ of Camper and of Scarpa, and the external oblique aponeurosis.

Spermatic Cord.- The spermatic cord extends from the internal abdominal ring to the upper part of the posterior border of the testis. For the first $I \frac{1}{2}$ inches of its course it lies in the inguinal canal, and is directed downwards, forwards, and inwards. After passing through the external abdominal ring it enters the scrotum, in which it descends almost vertically. The relations of the cord in the inguinal canal will be understood on referring to the description of that canal. In the lower two-thirds of the canal it is accompanied by the inguinal nerve.

The spermatic cord is composed of the following structures: the vas deferens or excretory duct of the testis; three arteries, namely, the spermatic artery, the artery of the vas deferens, and the cremasteric artery; the spermatic or pampiniform plexus of veins; the lymphatics of the testis and epididymis; the spermatic plexus of sympathetic nerves; and the genital branch of the genitocrural nerve. These structures are connected by areolar tissue, and the cord receives certain coverings to be presently described.

The vas deferens is the excretory duct of the testis. It commences at the lower cnd or globus minor of the epididymis, and terminates at the base of the prostate gland by joining the duct of the vesicula seminalis to form the common ejaculatory duct. This latter duct, having passed through the prostate gland for about I inch, opens upon (sometimes within) the margin of the opening of the sinus pocularis or prostatic vesicle on the floor of the prostatic portion of the urethra. The vas deferens at its commencement is slightly tortuous, but it soon becomes straight. It lies at first on the inner side of the epididymis, and along the posterior border of the testis. After entering the cord it is placed behind all the other elements, where it can be readily felt. It maintains this position until it arrives at the internal abdominal ring, where it lies on the inner side of the other elements of the cord. 
After passing through the internal abdominal ring it hooks round the outer side of the deep epigastric artery, and, having crossed the external iliac vessels from without inwards, it dips down on the inner side of the external iliac vein, and so enters the pelvis under cover of the peritoneum, where it will be afterwards described. The vas deferens in its natural state measures about I foot in length, but when straightened it attains a length of from $I_{2}^{\frac{1}{2}}$ to 2 feet.

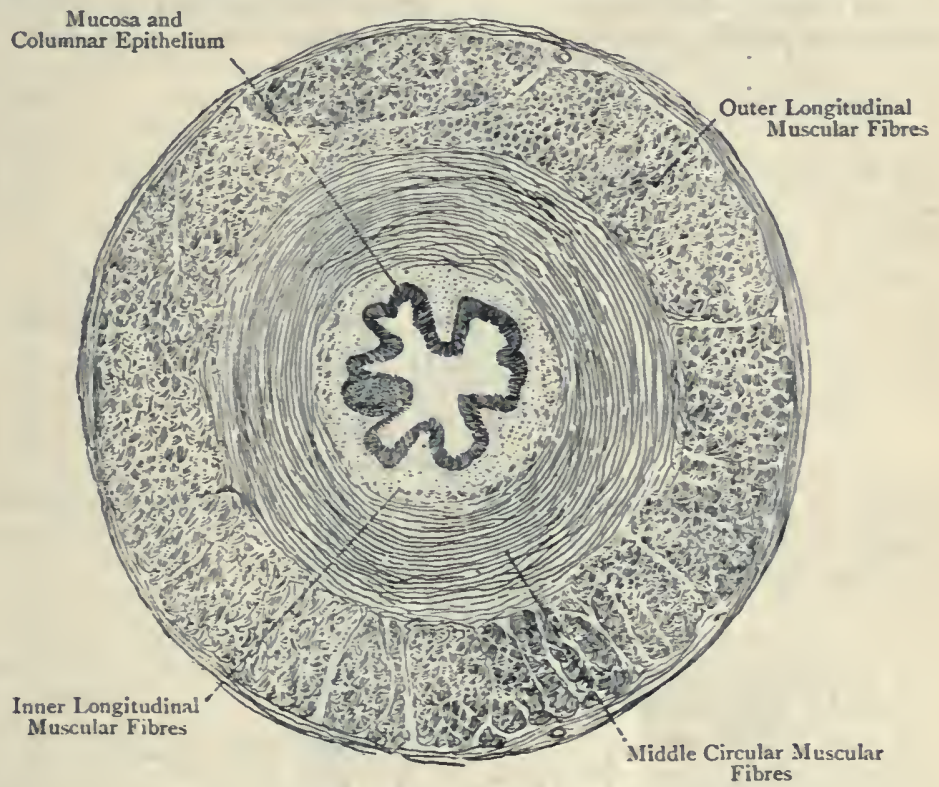

Fig. 289. -Transverse Section of the Vas Deferens, showing its Minute Structure.

Structure of the Vas Deferens.-The vas deferens has a very thick wall, and feels like a piece of whip cord. Its outer coat consists of fibrous tissue. Within this there is a thick nuscular coat composed of plain muscular tissue, which is arranged in three layers-an outer longitudinal, a middle circular (both of which are thick), and an inner thin longitudinal layer. Within the muscular coat is the mucosa, which is covered by non-ciliated columnar epithelium.

Development.-From the lower part of the Wolffian duct.

The artery of the vas deferens, or deferential artery, is usually a branch of the superior vesical of the internal iliac, though it may arise from the inferior vesical, and it divides into a descending and an ascending branch. The descending branch passes downwards to supply the lower part of the vas deferens and the vesicula seminalis. The ascending branch accompanies the vas deferens through the inguinal canal to the testis, supplying the vas deferens, and giving a few twigs to the globus minor of the epididymis, in which latter situation it anastomoses with the epididymal branch 
of the spermatic artery. The deferential artery is sometimes of large size, and then it takes the place of the spermatic artery when that vessel is absent.

The deferential vein opens into the vesical plexus of veins, and thence into the internal iliac vein.

The spermatic artery arises from the abdominal aorta about I inch below the renal artery. On approaching the upper part of the testis it divides into testicular and epididymal branches, the former supplying the testis and the latter the epididymis. As

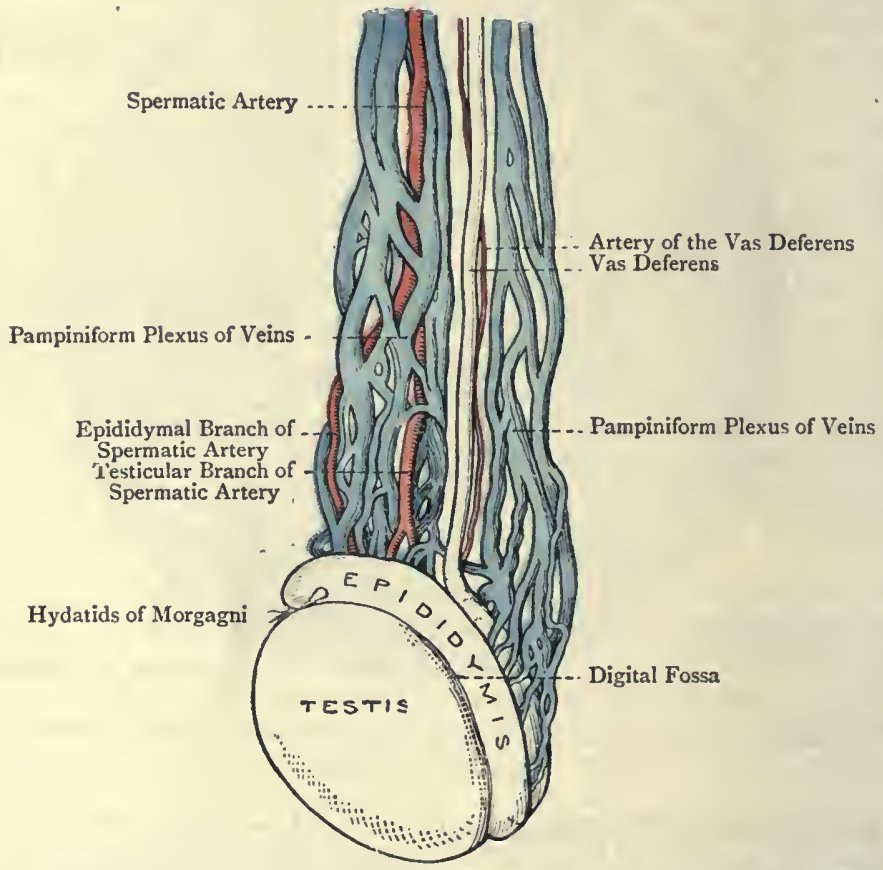

Fig. 290.-Dissection of the Spermatic Cord, showing the Blood. vessels and Duct of the Testis (after Sappey).

the artery descends in the spermatic cord it supplies branches to its coverings which anastomose with the cremasteric artery, and its epididymal branches anastomose with the deferential artery.

For the cremasteric artery, see p. 662.

The spermatic veins issue from the testis along its posterior border. In the cord they form a copious plexus, called the pampiniform plexus. At the internal abdominal ring two veins emerge from this plexus, which range themselves on either side of the spermatic artery. These, as they ascend, soon join to form one vessel, that of the right side opening into the inferior vena cava, and that of the left side into the left renal vein. The veins of 
the spermatic plexus have valves, but they are not competent. There is, however, a competent valve as a rule at the termination of each spermatic vein.

The lymphatics of the testis and spermatic cord, the spermatic plexus of sympathetic nerves, and the genital branch of the genitocrural nerve will be afterwards described.

Coverings of the Spermatic Cord.-The coverings, enumerated from within outwards, are as follows :

I. The subperitoneal areolar tissue, which is continuous with that of the abdominal wall through the internal abdominal ring. It contains a certain amount of muscular tissue, which is known as the middle cremaster.

2. The fascia transversalis, prolonged from the margins of the internal abdominal ring, and known as the internal spermatic fascia. This covering near the ring is funnel-shaped, and is thence called the infundibuliform fascia, but lower down it becomes incorporated with the subperitoneal areolar tissue, and forms the fascia propria of Cooper.

3. The cremasteric fascia, or middle spermatic fascia.

4. The intercolumnar fascia, or external spermatic fascia.

5. The tunica dartos.

6. The skin.

Within the innermost of these coverings there are a few scattered muscular fibres, which constitute the internal cremaster of Henle, and which are regarded as representing the inverted lateral bands of the gubernaculum testis.

It is to be noted that the middle cremaster is the muscular tissue contained in the subperitoneal areolar tissue on the surface of the cord, whilst the external cremaster is the more distinct muscular structure which is derived from the lower border of the internal oblique. The fibres of the external cremaster are of the striated variety, but those of the middle and internal are of the plain variety.

Descent of Testis and Formation of Inguinal Canal.- The testis originally lies in the lumbar region of the abdomen upon the psoas magnus muscle, on a level with the last thoracic and first lumbar vertebræ. It is situated on the mesial side of the WVolffian body or mesonephros and behind the peritoneum, the epithelial cells of the peritoneum being the superficial cells of the germinal epithelium.

In order to understand the descent of the sexual organ (testis or ovary) it is necessary to refer to certain embryological structures.

As stated, the testis (as well as the ovary) is situated on the mesial side of the Wolffian body. The sexual organ is attached to the mesial part of the IVolffian body by a short peritoneal fold, called the meso-testis or mesorchium in the male, and the mesovarium in the female. The Wolffian body is attached to the posterior abdominal wall by a peritoneal fold, the so-called Wolfian mesentery (more strictly Wolfian ligament). External to the Wolffian body there are (I) the Wolffian duct, which gives origin to the epididymis, vas deferens, and common ejaculatory duct, and (2) the Müllerian duct, from which the uterus and vagina are developed. These two ductsWolffian and Müllerian-lie within a fold of peritoneum, called the urogenital Iold, by which they are attached to the outer part of the Wolffian body, the Müllerian duct, at this level lying upon the outer side of the Wolffian duct. 
In addition to the mesorchium or mesovarium, according to the sex, the testis and ovary are each provided with two other peritoneal folds--superior and inferior. The superior testicular fold, which ascends towards the diaphragm, is called the plica vascularis, and it contains the bloodvessels of the spermatic cord. In the female it forms the ovario-pelvic ligament. The inferior testicular (or ovarian) fold joins the urogenital fold at the lower part of the Wolffian body, and will be presently referred to.

After atrophy (to a large extent) of the Wolffian body the mesorchium, or mesovarium, and the urogenital fold become incorporated with the Wolffian mesentery (ligament) and form the urogenital mesentery (ligament), which may be regarded as an augmented secondary mesorchium. A fold of the urogenital mesentery ascends to the diaphragm, and is known as the diaphragmatic ligament of the mesonephros or Wolffian body. It is not concerned in the migration of the sexual organ, and may therefore be disregarded.

Gubernaculum Testis. - At the lower part of the Wolffian body the urogenital fold is joined by the inferior testicular fold, as stated. After this junction has taken place the urogenital fold furnishes a peritoreal fold which extends to the inguinal region at a point corresponding to the situation of the
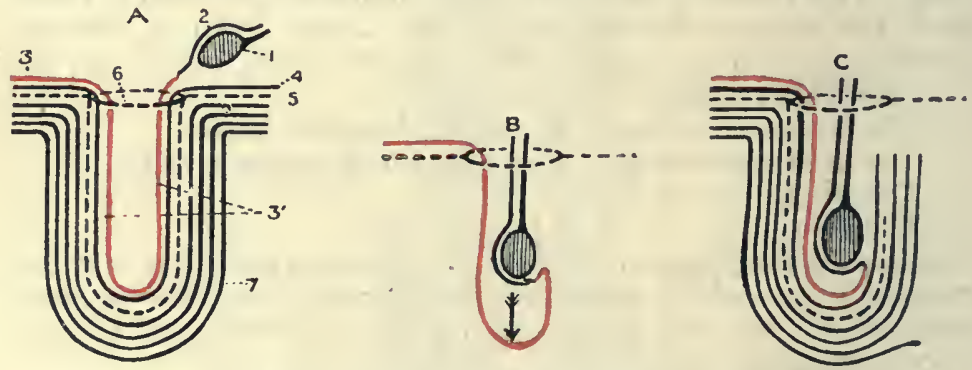

Fig. 291 -Diagram showing the Descent of the Teśtis.

A, First Stage : B, Second Stage ; C, Final Stage.

I. Testis (in Abdomen)

2. Primitive Peritoneum (Tunica Adnata)

3. Parietal Peritoneım

3'. Processus Vaginalis

\author{
4. Subperitoneal Areolar Tissue \\ 5. Fascia Transversalis \\ 6. Internal Abdominal Ring \\ 7. Integument
}

future internal abdominal ring. This offshoot of the urogenital fold is called the plica gubernatrix or plica inguinalis. In the female this fold, as it descends, becomes connected with the corresponding Müllerian duct at the level where this duct fuses with its fellow to form the rudiment of the uterus and vagina.

IVithin the plica gubernatix fold a fibro-muscular cord is developed, consisting of connective tissue and plain muscular tissue. This cord is called the gubernaculum testis. Inferiorly it is attached, at first, to the posterior surface of the anterior abdominal wall in the inguinal region, at a point corresponding to the situation of the future internal abdominal ring, whence it, or the greater part of it, ultimately extends to the bottom of the corresponding scrotal chamber. Superiorly its attachment is twofold: (I) It is principally attached to the lower part of the epididymis, at the junction of the globus minor and vas deferens. (2) A portion of it ascends within the inferior testicular fold to be attached to the caudal end of the testis. According to some authors the lower part of the gubernaculum testis is reinforced by striated muscular fibres derived from the internal oblique and transversalis abdoninis muscles. These fibres, which form the so-called inguinal cone, are superadded to the fibro-muscular cord, just referred to, which constituter the core of the gubernaculum. Superiorly these superadded fibres from the 
inguinal cone are described as being attached to the testis and epididymis. Inferiorly, thrce attachments are ascribed to them, as follows: (1) The outer - undle is attached to the deep aspect of Poupart's ligament near its centre; (2) the middle or principal bundle accompanies the gubernaculum testis to the bottom of the scrotal chamber; and (3) the inner bundle is attached to the pubic crest.

Two views are thus entertained regarding the structure of the gubernaculum testis. - According to one view it consists solely of plain muscular tissue and connective tissue arranged as a cord within the peritoneal fold, called the plica gubernatrix or plica inguinalis. According to the other view, in addition to these fibro-muscular elements, there are superadded striated muscular fibres derived from the internal oblique and transversalis abdominis muscles in the form of the inguinal cone. However constituted, the gubernaculum testis soon becomes a stout thick cord, the final destination of which will be presently referred to.

Descent of the Testis.-As stated, the testis originally lies in the abdomen on a level with the last thoracic and first lumbar vertebra, being placed behind the peritoneum which forms its mesorchium. As it descends it takes

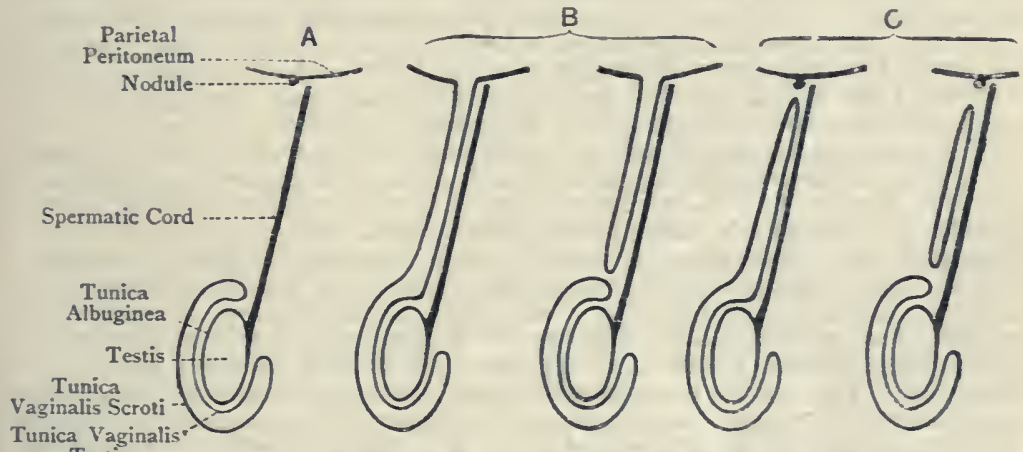

Fig. 292.-VARIeties of the Tunica V:aginalis.

\section{A, Normal Type ; B, Ccngenital Type; C, Infantile Type.}

along with it the superficial cells of its germinal epithclium, and the mesorchium also accompanies it.

The descent, or more properly the migration, of the organ commences about the third month of intra-uterine life, and its usual destination is the corresponding scrotal chamber. The migration is accomplished in four stages-pelvic, inguinal, intraparietal, and scrotal-and throughout all these stages it follows the lead of the gubernaculum, which necessarily undergoes shortening.

The pelvic stage soon brings the testis into the iliac fossa, where it lies near the brim of the true pelvis, having the epididymis externally and the vas deferens internally, the latter dipping into the peltic cavity.

The inguinal stage takes the testis to the posterior aspect of the inguinal portion of the anterior abdominal wall, at a point corresponding to the future internal abdominal ring, where it arrives about the sixth month.

Inasmuch as there are no abdominal rings and no inguinal canal at this period, a pause now takes place in the migration of the testis, in order to permit of a path being cleared for its progress. In the immediate vicinity of the lower or inguinal end of the gubernaculum testis a peritoneal depression is formed, and the principal part of the inguinal end of the gubernaculum now slowly penetrates the compact anterior abdoninal wall in the inguinal region, thus giving rise to the inguinal canal and abdominal rings. In pre- 
paring this path for the testis, its gubernaculum takes with it the parietal peritoneum forming the peritoneal depression, just alluded to, which accordingly constitutes a peritoneal process, called the processus vaginalis. This process may be likened to the finger of a glove, being open towards the peritoneal or abdominal cavity, but closed at its distal end. The principal part of the gubernaculum lies behind the processus vaginalis, which latter aids the stout gubernaculum in the formation of the inguinal canal.

As the processus vaginalis penetrates the inguinal portion of the abdominal wall, it elongates before it the several elements which compose the wall, in the following order from within outwards:

I. Subperitoneal areolar tissue.

2. Fascia transversalis.

3. Lowermost Poupart fibres of internal oblique muscle (cremaster).

4. External oblique aponeurosis.

5. Scarpa's and Camper's fasciæ (tunica dartos).

6. Skin of scrotum.

In the foregoing manner the internal abdominal ring, inguinal canal, and cxternal abdominal ring are formed by the gubernaculum testis and processus vaginalis. Beyond the external abdominal ring these two structures enter the corresponding scrotal chamber, the lower part of the processus vaginalis forming a serous lining for it. The lower part of the gubernaculum extends lower down than the lower end of the processus vaginalis, and this portion is attached to the fundus of the scrotal chamber.

The intraparietal stage in the migration of the testis consists in the passage of the organ through the inguinal canal. This stage commences about the seventh month of intra-uterine life, and the testis follows the lead of the middle or principal bundle of the gubernaculum testis, gliding along the posterior wall of the processus vaginalis, and being post-vaginal in position.

The scrotal stage consists in the entrance of the testis into the corresponding scrotal chamber, which usually takes place about the end of the eighth month of intra-uterine life. The organ still lies behind the lower part of the processus vaginalis, which it invaginates from behind, to form the tunica vaginalis. The middle or principal bundle of the gubernaculum testis has now become very short, and is ultimately represented by an indefinite fibrous bundle, spoken of as the remnant of the gubsnaculum, which connects the lower parts of the epididymis and testis to the fundus of the scrotal chamber.

As the testis descends into the scrotum, the outer or Poupart and inner or pubic bundles of the gubernaculum testis, according to the view that there is a conus inguinalis, are drawn downwards on the sides of the spermatic cord.

There are two theories regarding the migration of the testis, which may be called developmental and muscular. According to the developmental theory the migration is not an active process, due to muscular contraction, but is brought about by developmental changes, of the nature of disproportionate or unequal growth, which take place in the lumbar, iliac, and inguinal regions of the trunk. According to the muscular theory, maintained by those who favour the existence of a conus inguinalis, the migration is brought about by muscular contraction, as follows: All three bundles of the muscular inguinal cone would draw the testis down to the inguino-pubic region, and thereafter the middle bundle would draw it down into the scrotal chamber, the outer and inner bundles being elongated downwards. The non-striated muscular fibres in the core of the gubernaculum may also take part in the descent, and the descent may be aided by the 'retraction' of the connective tissue of the gubernacular core.

Metamorphoses of the foregolng Perltoneal Folds-Male.-The plica gubernatrix or plica inguinalis in the male contains the gubernaculum testis. The inferior testicular fold forms part of the plica gubernatrix. The superior testicular fold or plica vascularis contains the clements of the-spermatic cord. The mesorchium, derived from the greatly reduced urogenital mesentery, connects the testis and epididynis. 
Female.-The portion of the plica gubernatrix or plica inguinalis (of which plica the embryonic inferior ovarian fold forms a part) between the caudal end of the ovary and the fusion of the Mullerian duct with its fellow to form the rudiment of the uterus, represents the ligament of the ovary; and the part which extends from the Müllerian fusion through the inguinal canal to the labium majus contains the round ligament of the uterus, which is the homologue of part of the gubernaculum testis. In other words, the entire plica gubernatrix in the male contains the gubernaculum testis; whereas in the female it pertains to (I) the ligament of the ovary, and (2) the round ligament of the uterus. The embryonic superior ovarian fold represents the ovario-pelvic ligament. The urogenital mesentery of either side, which is formed by the mesovarium, Wolffian mesentery, and urogenital fold (within which latter the Müllerian duct lies along with the Wolffian duct) becomes the corresponding broad ligament of the uterus.

Metamorphosis of the Processus Vaginalis.-As stated, the processus vaginalis is originally a tubular process or diverticulum of the parietal peritoneum of the inguinal region, resembling the finger of a glove, which precedes the descent of the testis, and behind which the testis descends, following the lead of the middle bundle of the gubernaculum testis. After the testis has reached the scrotal chamber, it invaginates the processus vaginalis from behind, to form the tunica vaginalis, and that process now undergoes certain changes. To understand these changes familiarity with the following facts is necessary: (1) The processus vaginalis is closed below and open above; (2) the lumen of the process is simply a diverticulum of the cavity of the peritoneum; (3) the part of the process related to the testis is called the testicular portion; and (4) the part in front of the spermatic cord is referred to as the funicular portion.

The changes are as follows: (1) About the period of birth the processus vaginalis usually becomes constricted and closed superiorly at the internal abdominal ring, and then the process is an elongated tube, closed at each end, its lumen being now shut off from the cavity of the peritoneum. (2) A few days after birth the process usually becomes constricted and closed a little above the testis. (3) The funicular portion of the process usually becomes impervious and converted into a fibrous thread, which as a rule disappears in the course of the first month after birth. (4) The testicular portion of the process persists as a shut serous sac, called the tunica vaginalis. Normally the persistent remains of the embryonic processus vaginalis in the adult are (I) the testicular portion, which forms the tunica vaginalis, and (2) a small nodule on the parietal peritoneum immediately behind the internal abdominal ring. Instead of a mere nodule, however, a slender fibrous thread, called the ligamentum vaginale, may extend downwards from this part of the parietal peritoneum in front of the spermatic cord as low as the external abdominal ring, and sometimes as low as the tunica vaginalis.

In the female the processus vaginalis is represented by a tubular process of the peritoneum which lies in front of the round ligament of the uterus for a short distance in the inguinal canal. When this process remains open superiorly, it is known as the canal of Nuck.

Abnormal Conditions of the Processus Vaginalis-Congenital Type.-(I) The processus vaginalis may remain permanently open throughout, under which circumstances the cavity of the tunica vaginalis is in direct communication with the general peritoneal cavity. (2) The processus vaginalis may be closed just above the tunica vaginalis, but it may remain as a permanently open tube above this point.

Infantile or Funicular Type.--(I) The processus vaginalis may be closed only at its upper end near the internal abdominal ring. In these cases the tunica vaginalis, instead of being limited to the region of the testis, is prolonged upwards as an elongated tube in front of the spermatic cord in to the inguinal canal. (2) The processus vaginalis may be closed above, near the internal abdominal ring, and also below, just above the tunica vaginalis, the portion of it intervening between these two points remaining as an elongated tube, closed at either end, and lying in front of the spermatic cord. 
The funicular portion of the processus vaginalis is sometimes closed at intervals along the course of the spermatic cord, and, when scrous fluid accumulates in the intervening patent portions, the condition is known as encysted hydrocele of the cord.

Abnormal Positions of Testis.-(I) The testis may remain permanently in the abdominal cavity. (2) Its descent may be arrested in the inguinal canal, or at the external abdominal ring. Such conditions constitute the condition known as cryplorchismus.

Ectopia Testis.- The testis may occupy unusual situations. (1) It may be found in the anterior part of the perineum. (2) It may be found on the front of the thigh in the region of the saphenous opening, in which cases it might simulate a femoral hernia.

For the structure and development of the testis, see pp. 683,634 .

Subperitoneal Fat or Areolar Tissue.-This is situated between the fascia transversalis and the parietal peritoneum. Its condition is subject to much variety, being fairly well marked in some bodies, and in others hardly perceptible. Internal to the external iliac vein at Poupart's ligament it forms the septum crurale, which closes the upper end of the crural canal, and at the internal abdominal ring it is carried downwards round the spermatic cord underneath the infundibuliform fascia.

Parietal Peritoneum.-This is the innermost covering of the abdominal wall, and it is connected with the fascia transversalis by the subperitoneal areolar tissue. Behind the internal abdominal ring it forms a slight projection, which in some cases enters the ring, the corresponding depression on its abdominal aspect at this point being known as the digital fossa. The projecting part of the parietal peritoneum corresponds with. the upper end of the original processus vaginalis, and presents a slight nodule, or it may be a slender thread-like process. It is here in the female where a diverticular process may extend for a short distance into the inguinal canal in front of the round ligament of the uterus, forming the canal of Nuck.

Peritoneal Plicæ and Inguinal Fossæ.-The peritoneum lining the posterior surface of the anterior abdominal wall below the umbilicus presents plicæ or folds and fossæ or pouches.

The plicæ are five in number, one being situated in the middle line, and two on either side. "The median plica extends from the apex of the bladder to the umbilicus, and contains the urachus. It is called the plica urachi. Of the two lateral plice the more internal one contains the obliterated hypogastric artery, and is cailed the plica hypogastrica. It is oblique in direction, and meets the plica urachi and $\mathrm{its}$ fellow of the opposite side at the umbilicus. It corresponds to the junction of the inner two-thirds and outer third of Hesselbach's triangle. The external of the two lateral plice is produced by the deep epigastric artery, and is called the plica epigastrica.

The inguinal fossæ are six in number, three right and three left, and are called internal, middle, and external. The internal inguinal fossa is situated between the plica urachi and plica hypogastrica, and lies behind the inner two-thirds of Hesselbach's 
before it the peritoneum forming that fossa. It then passes through the internal abdominal ring, and traverses the entire length of the inguinal canal, from which it emerges through the external abdominal ring into the scrotum, thus forming a complete external oblique inguinal hernia. Throughout its entire course the hernia lies in front of the spermatic cord, and its descent is arrested at the upper part of the testis, which can be felt at its lower and back part. The bowel may be arrested at any part of the inguinal canal, the hernia being then called incomplete, and forming what is known as a bubono. cele. In its descent the bowel elongates and carries before it certain investments from the structures to which it is related, these investments being called the coverings of the hernia. The first covering of this form of hernia, as of the other varieties, is derived from the parietal peritoneum, the particular part being that which forms the external inguinal fossa, and this constitutes the sac. The other coverings are simply those of the spermatic cord, which are all superadded to the sac.

The coverings, enumerated in order from within outwards, are as follows:

1. Parietal peritoneum, which forms the sac.

2. Subperitoneal fat or areolar tissue.

3. Fascia transversalis, from the margins of the internal abdominal ring, forming the infundibuliform or internal spermatic fascia.

4. Cremasteric or middle spermatic fascia, at the lower border of the internal oblique muscle.

5. Intercolumnar or external spermatic fascia, from the pillars of the external abdominal ring.

6. Tunica dartos.

7. Skin.

A short distance below the internal abdominal ring the subperitoneal fat and infundibuliform fascia become united, and are known as the lascia propria.

The sac is composed of the following parts: (I) the mouth, which is the opening by which its interior communicates with the general peritoneal cavity; (2) the neck, which is the constricted part immediately beyond the mouth; and (3) the body. The neck of the sac is on a level with the margins of the internal abdominal ring, and the deep epigastric vessels lie immediately on its inner side.

The seat of stricture may be ( 1 ) at the external abdominal ring ; $(2)$ at the lower border of the internal oblique muscle; or (3) at the neck of the sac, the last being the most common situation.

Internal Direct Inguinal Hernia.-This variety is called internal because the hernia, as it leaves the abdominal cavity, is internal to the deep epigastric artery, and divect, from its straight course through the abdominal parieties. The course of this variety is as follows : the hernia enters the internal inguinal fossa, elongating before it the peritoneum forming that fossa. It then passes through the inner two-thirds of Hesselbach's triangle, and so reaches directly the external abdominal ring without traversing the inguinal canal. Having emerged through the external ring it descends into the scrotum, thus forming a complete internal direct inguinal hernia, which is in front of, and internal to, the spermatic cord. It is to be noted ( $I$ ) that there is no natural opening in the fascia transversalis over the inner two-thirds of Hesselbach's triangle, as there is external to the deep epigastric artery, and (2) that the conjoined tendon covers tlie inner two-thirds of Hesselbach's triangle. The coverings of this variety, enunerated in order from within outwards, are as follows:

1. Parietal peritoneum.

2. Subperitoneal fat or areolar tissue.

3. Fascia transversalis.

4. Conjoined tendon.

5. Intercolumnar or external spermatic fascia.

6. Tunica dartos.

7. Skin.

If the liernia occurs suddenly, rupture of the conjoined tendon may take place, in which cases the bowel would pass through the fissure. The seat of 
stricture in an internal direct inguinal hernia may be (I) at the external abdominal ring, (2) at the fissure in the conjoined tendon, if that structure should be ruptured, or (3) at the neck of the sac. The latter situation is the most common, and it is to be noted that the deep epigastric vessels lie on the outer side of the neck of the sac.

Internal Oblique Inguinal Hernia.-This variety is called internal because the hernia, as it leaves the abdominal cavity, is internal to the deep epigastric artery, and oblique, because it has to descend through the lower two-thirds of the inguinal canal. The course of this variety is as follows: the hernia enters the middle inguinal fossa, elongating before it the peritoneum forming that fossa. It then passes through the outer third of Hesselbach's triangle and descends through the lower two-thirds of the inguinal canal, from which it emerges through the external abdominal ring into the scrotum, thus forming a complete internal oblique inguinal hernia. Practically the only difference between the course of an internal oblique and an external oblique inguinal hernia is that the external oblique variety enters the inguinal canal by its natural inlet, namely, the internal abdominal ring, whereas the internal oblique variety obtrudes itself into the upper part of the inguinal canal through its posterior wall. It is to be noted (I) that there is no natural opening in the fascia transversalis over the outer third of Hesselbach's triangle, as there is external to the deep epigastric artery, and (2) that there is no conjoined tendon over the outer third of Hesselbach's triangle. The coverings of this variety, enumerated in order from within outwards, are as follows :

I. Parietal peritoneum.

2. Subperitoneal fat or areolar tissue.

3. Fascia transversalis.

4. Cremasteric or middle spermatic fascia.

5. Intercolumnar or external spermatic fascia

6. Tunica dartos.

7. Skin.

If the coverings of an external oblique, and an internal oblique, inguinal hernia are compared with each other, it will be seen that the former has a tube of fascia transversalis already prepared for it, namely, the infundibuliform fascia, whereas the latter has to elongate before it a fresh portion of fascia transversalis. In some cases the fascia transversalis over the outer third of Hesselbach's triangle is covered by an expansion from the conjoined tendon, which is known as the reflected tendon of Cooper. In such cases that reflected tendon must be added as a covering of internal oblique inguinal hernia, its position being immediately superficial to the covering formed by the fascia transversalis. The relation of an internal oblique inguinal hernia to the spermatic cord is similar to that of an external oblique, and the possible seats of stricture are also similar. The neck of the sac is the most common situation, and the deep epigastric vessels lie immediately on its outer side. The extreme difficulty which must be experienced in diagnosing between an external oblique and an internal oblique hernia is explained by the fact that the former leaves the abdominal cavity immediately external to the deep epigastric vessels, and the latter immediately internal to them. Hence, the practical rule followed in operating is to cut upwards and not transversely. The propriety of this rule is further enhanced if it be remembered that an external oblique inguinal hernia of old standing may so drag upon the internal abdominal ring as to displace it downwards and inwards to a point behind the external ring, and thus a hernia which is really external oblique may simulate one of the internal direct variety.

Varieties of External Oblique Inguinal Hernia.--There are two varieties of this form of hernia, the special features of which depend upon abnormal conditions of the processus vaginalis (see p. 673). These varieties are named congenital and infantile:

Congenital Hernia.- There are two forms of congenital hernia. (I) The processus vaginalis may remain permanently open throughout, in which case the bowel descends within that process into the cavity of the tunica vaginalis at its lower extremity. The tunica vaginalis thus represents the 
sac of the hernia, and this form is therefore spoken of as a hernia into the tunica vaginalis. In such cases the bowel more or less completely envelops the testis. (2) The processus vaginalis may be shut off only just above the testis, the part above this remaining as a funicular process communicating above with the general peritoneal cavity. In such cases the bowel descends into the funicular process, which thus forms the sac of the hernia. This form is therefore spoken of as a hernia into the funicular process.

Infantile Hernia.-There are two forms of infantile hernia, infantile hernia proper and encysted hernia. In both there is a funicular process which is closed above, towards the internal abdominal ring. It may also be closed below just above the testis, being thus distinct from the tunica vaginalis, or it may simply be an upward extension of the tunica vaginalis. In either case it is situated in front of the spermatic cord. In infantile hernia proper the bowel, having elongated the parietal peritoneum to form a sac, descends between the spermatic cord and the funicular process. Its importance consists in the fact that, before the bowel can be exposed in operating, three serous layers must be divided, two of these belonging to the funicular process and the other representing the wall of the hernial sac. In this form the descent of the bowel is arrested at the upper part of the testis. In encysted hernia the bowel, having elongated the parietal peritoneum to form a sac, pushes against the upper part of the funicular process so as to invaginate it in a downward direction in the form of a cup, in which the bowel, enclosed in its sac, lies. The condition of matters is therefore very much like an egg set in its cup, assuming that the top of the shell is removed, and that the wall of the cup is formed of two layers. To bring out this simile, the contents of the egg may be taken as representing the bowel, the shell of the egg being the sac of the hernia, and the assumed two layers of the wall of the cup representing the two serous layers of the doubled down or invaginated funicular process, the cavity thus formed representing the inside of the cup. In this form, as in infantile hernia proper, three serous layers must be divided before the bowel is exposed in operating, two of these belonging to the invaginated funicular process and the other representing the wall of the hernial sac.

Umbilical Hernia (Exomphalos).-By an umbilical hernia is meant a protrusion of bowel or of great omentum from the abdominal cavity through the umbilicus. The protrusion occurs through the upper part of the umbilicus, which, containing only the obliterated umbilical vein, is weaker than the lower part where the obliterated hypogastric arteries and the urachus lie. The coverings of an umbilical hernia are as follows :

I. Parietal peritoneum.

2. Subperitoneal fat or arealar tissue.

3. Fascia transversalis.

4. An expansion from the decussating fibres of the aponeuroses of the abdominal muscles of opposite sides.

5. Superficial fascia.

6. The cicatricial cutaneous umbilical tiss'se.

There is no vessel liable to be injured in operating on this form of lernia, the deep epigastric artery being about $\mathrm{I} \frac{1}{2}$ inches from the linea alba.

The congenital form of umbilical hernia consists in a protrusion of bowel or omentum through the centre of the umbilicus into the umbilical cord, in which it may descend for some distance. Its possible presence will show the propriety of carefully examining the cord before ligaturing it after birth.

The anterior abdominal wall above the umbilicus presents pos: teriorly a median fold of parietal peritoneum, which represents a part of the upper border of the falciform ligament of the liver. This fold contains a portion of the obliterated umbilical vein, which is now spoken of as the round ligament of the liver. It extends upwards from the umbilicus to the interlobar or umbilical 
notch on the anterior border of the liver, through which it passes to enter the umbilical fissure on the under surface of the viscus. As the round ligament ascends to the liver the peritoneum, within which it lies, is being gradually elongated in the form of two closely applied laminæ, which thus project to form a part of the falciform ligament. The apex of this ligament is therefore at the umbilicus.

\section{The Tunica Vaginalis and Testis.}

Tunica Vaginalis.-This is a closed serous sac, behind which the testis lies. It is formed by the lower part of the processus vaginalis, or peritoneal diverticulum, which precedes the descent of the testis from the abdomen. Like all serous membranes, it is composed of two layers, parietal and visceral. The parietal layer is known as the tunica vaginalis scroti, from the circumstance that it lines the scrotal chamber of its own side. It is much larger and looser than the visceral layer, with which it is continuous along the posterior border of the testis and on the spermatic cord about $:_{2}^{1}$ inch above the organ. The visceral layer is known as the tunica vaginalis testis. It closely invests the tunica albuginea of the testis, to

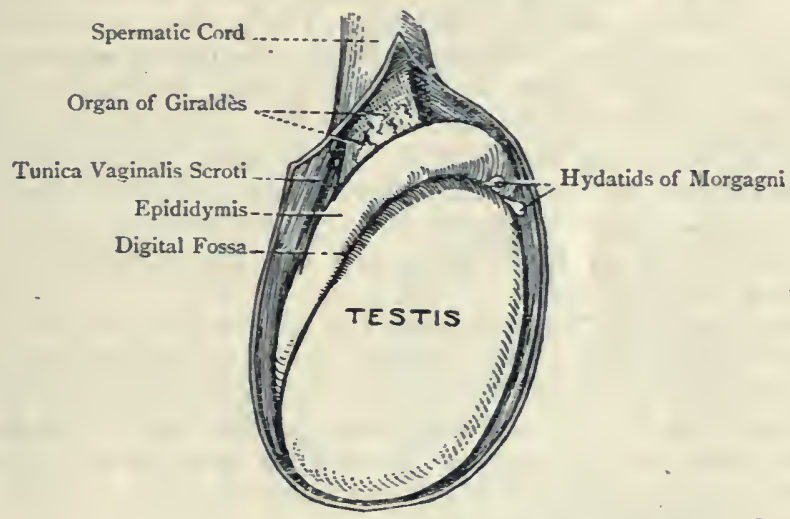

Fig. 294.-The Testis ANd its Coverings.

which it is inseparably united. It also invests the epididymis except at its posterior border, where the constituents of the spermatic cord enter or leave the testis. Between the epididymis and the testis it forms a recess, called the digital fossa, and it extends upwards on the spermatic cord for about $\frac{1}{2}$ inch above the testis. Along the posterior border of the organ, where this layer becomes continuous with the parietal layer, there is a narrow strip which is free from serous investment. The portion of the tunica vaginalis which is related to the spermatic cord is called the funicular par. When fluid accumulates between the parietal and visceral layers, the condition is known as hydrocele of the tunica vaginalis. 
Testis. - The testis is suspended obliquely by the spermatic cord in its scrotal compartment, to the bottom of which it is loosely attached by the fibrous remains of the gubernaculum testis. The left testis is a little lower than the right. The organ is oval, and compressed from side to side. Its exterior, which is smooth, is closely invested by the visceral layer of the tunica vaginalis, except where the constituents of the cord enter or leave the organ. The surfaces are external and internal, the former looking slightly backwards, and the latter forwards. The extremities are superior and inferior, the former being inclined forwards, and the latter backwalds. The borders are anterior and posterior. The anterior border looks slightly downwards and outwards, and is free. The posterior border looks upwards and inwards, and is attached. The average weight of the testis is about 7 drachm:

Epididymis. - This is an elongated narrow body, composed of the convolutions of the excretory duct, and lying along the posterior border and adjacent portion of the external surface of the testis. Its upper extremity, which is above the upper end of the testis, is enlarged, and is called the caput or globus major; the lower and smaller end is called the tail or globus minor; and the intervening narrow portion represents the body. The globus major and globus minor are connected to the testis by fibrous tissue, and by a reflection of the tunica vaginalis, the former being further connected to the organ by the efferent ducts. The body, except at its posterior border, is free, being separated from the testis by the digital fossa. The epididymis is almost completely invested by the visceral layer of the tunica vaginalis except at its posterior border, where there is a duplicature of that membrane containing bloodvessels and attaching it to the testis.

Hydatids of Morgagni.- These are two small pyramidal bodies which are situated on the anterior aspect of the globus major at its lower part, or on the front of the upper end of the testis below the globus major, one of them being pedunculated and the other sessile. Each is composed of connective tissue and bloodvessels, covered by the visceral layer of the tunica vaginalis. The hydatids are vestiges of Müller's duct.

These small bodies are two in number. One is pedunculated or stalked, and is situated on the front of the caput epididymis at its lower part. The other hydatid is sessile, and is placed on the upper end of the testis below the caput epididymis.

Organ of Giraldès or Paradidymis. -This organ is situated on the front of the spermatic cord, immediately above the globus major, and under cover of the funicular part of the tunica vaginalis. It consists of a few irregular nodules of convoluted tubules, which are lined with ciliated columnar epithelium. These nodules are remains of the Wolffian body.

Development.-The organ of Giraldès represents a portion of the caudal part of the Wolffian body. 
Arterial Supply of the Testis and Epididymis.-The testis derives its arterial supply from the testicular branch of the spermatic (which enters the posterior border of the organ), and the epididymis derives its supply from the epididymal branch of the spermatic, which vessel arises from the abdominal aorta about I inch below the renal artery. The globus minor of the epididymis also receives a few twigs from the deferential artery, which is usually a branch of the superior vesical from the internal iliac.

The veins of the testis issue at the upper part of the posterior border, and, along with those of the epididymis, enter the spermatic cord, where they form the pampiniform plexus. The right spermatic vein, in which the right plexus ultimately ends, opens directly into the inferior vena cava, and the left into the left renal vein.

Lymphatics.- These ascend in the spermatic cord, and accompany the spermatic vessels as high as the lateral and median groups of lumbar glands in which they terminate.

Nerve-supply.-The spermatic plexus of the sympathetic system which derives its fibres from the aortic and renal plexuses.

The testis is homologous to the ovary of the female (testis muliebris).

General Structure of the Testis and Epididymis.-The testis is an aggregation of seminiferous tubules collected into Iobules, which are encased within a capsule called the tunica albugrnea. This tunic is a dense, bluish white, inelastic membrane, composed of bundles of fibrous tissue. Its onter surface is closely covered by the tunica vaginalis testis. Its inner surface is invested by a copious vascular network, known as the tunica vasculosa of Cooper. At the posterior border of the testis the tunica albuginea passes for a certain distance into the interior, this inflection being called the corpus Highmorianum, or mediastinum testis. This mediastinum extends into the organ for one-fourth of its antero-posterior measurement, and from its sides and anterior border a number of trabecula, containing plain muscular tissue. pass off, which extend in various directions as far as the inner surface of the tunica albuginea, to which they are attached. By means of these the interior of the testis is

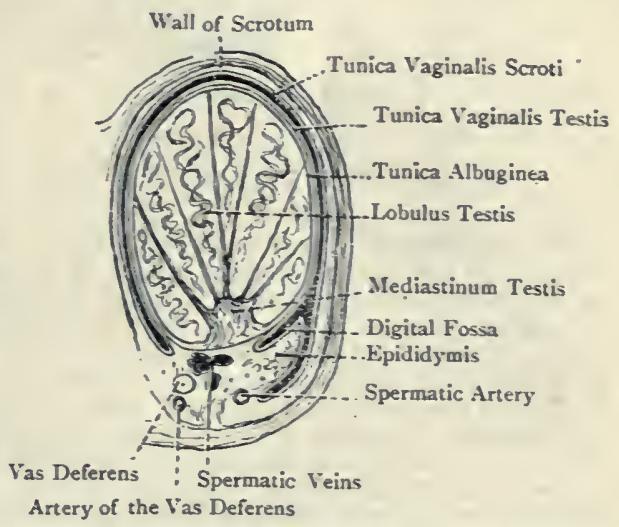

FIg. 295.-DiagraM SHowing a Transverse Section of the Testis axd Scrotum.

mapped out into a number of loculi, the trabecula which enclose them containing the branches of the spermatic artery as they make their way to the tunica vasculosa. These compartments contain the seminiferous tubules collected into bundles, called the lobuli testis, which vary in number from 300 to 400 . Each lobule contains from two to four tubules, and is conical, the base being directed towards the circumference of the testis and the apex 
towards the mediastinum. Each tubule is about $\frac{1}{20}^{\frac{1}{20}}$ inch in diameter and is convoluted. When the coils are undone the tubule measures about 2 feet in length. The tubules of each lobule unite into one, and the tubules of adjacent lobules unite in turn, and so give rise to the tubuli recti, each of which is about $\frac{1}{40}$ inch in diameter, and about inch in length. These tubuli recti enter the mediastinum, where they form by their division a net. work, called the rete testis. From this rete tubules, called vasa efferentia, proceed, which are about $\frac{\pi}{3}$ inch in diameter, their number varying from twelve to twenty. These leave the testis at the upper part of its posterior border. For a short distance thereafter they remain straight, but they are soon thrown into convolutions, which form conical masses, called coni vasculosi. The length of each conus vasculosus is about $\frac{1}{3}$ inch, and its apex is directed towards the testis. When the convolutions are undone, the tube assumes a length of about 8 inches, its diameter gradually diminishing from about $\frac{1}{20}$ inch at its commencement to about $\frac{1}{60}$ inch at its termination The coni vasculosi open by separate orifices into the canal of the epididymis.

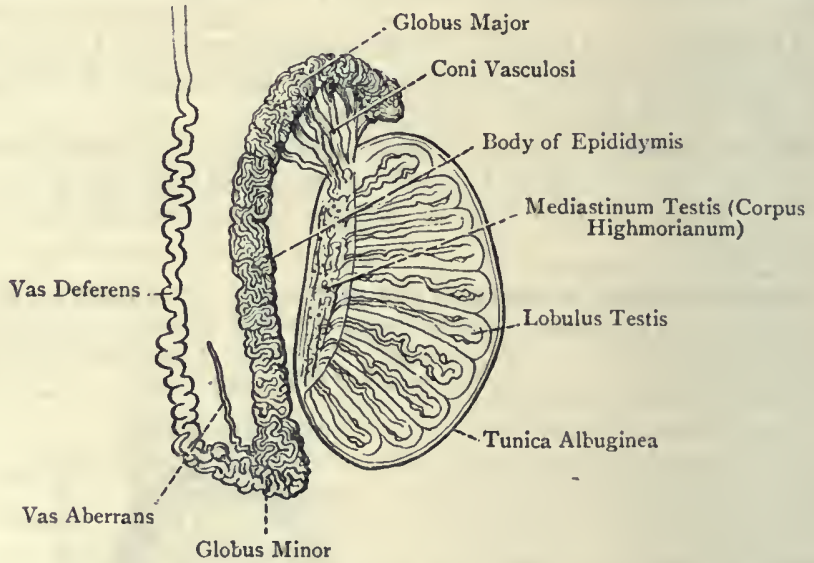

Fig. 296.-The Structure of the Testis and Epididymis.

The epididymis consists of one tube, liaving a diameter of about ${ }_{11}^{1}$ inch in the globus major, where it commences in a blind extremity. In the body it diminishes a little in diameter, and in the globus minor it again enlarges. The tube presents a great number of convolutions, which, being folded upon themselves and connected together by loose tissue, give rise to a series of lobules. When the convolutions are undone the length of the epididymis has been variously estimated at from 12 to 20 feet. At its upper extremity it receives the coni vasculosi, and beyond the globus minor it terminates in the vas deferens. At the point where it terminates in the vas deferens there is a diverticulum connected with it, called the vas aberrans of Haller, which extends upwards in a convoluted manner between the epididymis and the adjacent part of the vas deferens. The vas aberrans, when the coils are undone, is from 8 to 12 inches in length.

Minute Structure. - The tubuli seminiferi are composed of a liyaline basement membrane, lining which there are several layers of epithelial cells. (I) The most external layer consists of cubical cells, known as the parietal cells. They line the basement nembrane of the tubule, and are of two kinds. The majority of them give rise to the spermatozoa, and these are called the spermatogenic cells, or spermatogonla. Others are of a supporting nature. and are called the sustentacular cells, or cells of Sertoli, which subsequently 
form the columns of Sertoli. (2) The spermatogonia undergo mitotic division, and give rise to a second layer of cells, called the primary spermatocytes, or mother-cells. (3) The primary spermatocytes also undergo mitotic division, and give rise to a third layer of cells, called the secondary spermatocytes, or daughter-cells. (4) The secondary spermatocytes also undergo mitotic division, and give rise to a fourth layer of cells, called the spermatoblasts, or spermatids. These spermatids, having undergone considerable modifications, give rise to the spermatozoa. These spermatozoa lie with their heads buried between the more deeply placed cells, and their long tails projecting free into the lumen of the tubule. The enlarged ends or heads of the spermatozoa, whilst they lie buried between the deeper cells, are connected with the sustentacular cells of the lining epithelium. The tubuli recti are composed of a basement membrane lined with a single layer of cubical epithelium. The tubules of the rete testis are destitute of a basement membrane, its place being taken by the connective tissue of the mediastinum. The lining membrane of the tubules consists of a single layer of cubical cells. The vasa efferentia and coni vaseulosi are composed of a basement membrane, external to which there is a layer of plain muscular fibres arranged in a circular manner. The lining epithelium is of the ciliated columnar variety.

The structure of the epididymis is similar to that of the vasa efferentia and coni vasculosi.

\section{Development of the Internal Sexual Organs.}

The testis and the ovary do not present any distinctive sexual characters in the earliest stage of their development, and this stage, in each case, is therefore spoken of as the indifferent stage.

Indifferent Stage.- The first indication of a sexual organ is the appearance of a thick longitudinal fold on the inner surface of the Wolffian body. This fold is called the genital ridge, and it is formed by (1) a thickening of the cœlomic epithelium which covers the inner surface of the Wolffian body, and (2) the subjacent mesodermic tissue.

The genital ridge presents three regions-namely, anterior or rete region. middle or sex-gland region, and posterior or mesenteric region, the last-named taking no part in the development of the sexual organ.

The colomic epithelium of the genital ridge constitutes the germinal epithelium, and the cells of this epithelium over the sex-gland region of the genital ridge invade the subjacent mesoderm in the form of strands called the sex-cords. The cells of these strands are of two kinds, some being small epithelial cells, whilst others are large primitive sexual cells. As the stage of differentiation proceeds, these sexual cells give rise to spermatozoa, or seminal cells, in the case of the testis, and to ova in the case of the ovary.

The indifferent sexual organ is thus represented by the genital ridge, the mesodermic basis of which is invaded by sex-cords, the cells of which are partly epithelial and partly sexual. Moreover, the indifferent organ lies on the inner side of the Wolffian body.

The stage of differentiation now commences. 
Development of the Testis and its Excretory Apparatus.-The embryonic rudiments pertaining to the development of the testis are as follows:

\author{
Sex-cords. \\ Rete-cords. \\ Genital ridge. \\ Anterior tubules of the meso- \\ nephros, or Wolffian body. \\ Wolffian duct.
}

The cells of the germinal epithelium of the sex-gland region of the genital ridge which invade the mesoderm are, as stated, small epithelial cells and large sexual cells, and they are arranged as cylindrical columns, which, as stated, are known as the sex-cords. These cords become separated from the epithelium of the genital ridge when the tunica albuginea of the testis is formed from connective tissue derived from 'the basal nuclei of the sexcords.' They are broken up into spherical masses which, like the sex-cords, consist of small epithelial cells and large sexual cells. These masses become hollow, and, after undergoing elongation, they give rise to the secreting seminiferous tubules. The sexual cells of the masses become spermatogonia, or primordial sperm-cells, from which the spermatozoa are derived, whilst the small epithelial cells are probably transformed into the cells of Sertoli. For spermatogenesis, or the formation of spermatozoa, see Index.

The excretory tubules (tubuli recti and rete testis) are usually regarded as being developed from the anterior or sexual tubules of the mesonephros, or Wolffian body. From the epithelium of the Malpighian corpuscles of these tubules solid cords of cells, called the rete-cords, grow into the developing testis. These cords become tubular, and they give off branches which acquire connections with the secreting seminiferous tubules. In this manner the tubuli recti and rete testis are formed. The vasa efferentia are developed from the anterior or sexual tubules of the Wolffian body.

Another view is that the rete-cords arise from the cells of the germinal epithelium over the anterior or rete-region of the genital ridge, being serially homologous with the sex-cords. The rete-cords are at first solid, but subsequently acquire a lumen. They extend posteriorly into the proper glandular substance, formed by the sex-cords. As they do so, they give off branches, each of which passes to one of the spherical masses into which the sex-cords are broken up. Within this mass it becomes continuous with a secreting seminiferous tubule.

The proximal ends of the rete-cords become detached from the epithelium of the rete-region of the genital ridge. They then come into close contact with the capsules of Bowman of the Malpighian corpuscles belonging to the anterior or sexual tubules of the mesonephros or Wolffian body, which tubules give rise to the vasa efferentia of the testis. The contact may be direct, or through means of evaginations of the walls of Bowman's capsules. In either case fusion takes place, and the proximal ends of the rete-cords (rete-tubules open into the capsules of Bowman, and become continuous with the anterior or sexual tubules (vasa efferentia of the testis) of the mesonephros. These mesonephric tubules open into the Wolffian duct, which gives rise to the epididymis, vas deferens, and common ejaculatory duct.

The convoluted canal of the epididymis, the vas deferens, and the common ejaculatory duct are developed from the Wolman duct. The vesicula seminalis is formed as a blind diverticulum of the caudal part of the Wolffian duct; and the vas aberrans is a slender diverticulum of that portion of the Volffian duct which forms the globus minor, or tail, of the epididymis.

Summary. - The testis, genetically considered, consists of (I) secreting scminiferous tubules and seminal cells, and (2) excretory tubules. The secreting seminiferous tubules and their seminal cells are developed from the sex-cords of that portion of the germinal epithelium which covers the sex-gland region of the genital ridge. The tubuli recti and rete testis are developed from the epithelium of the Malpighian corpuscles of the anterior or sexual tubules of the mesonephros, or Wolffian body, which covers the anterior or sexual gland rcgion, or from the germinal cpithelium of the rete-rcgion of the genital ridge. The vasa efferentia are developed from the anterior or sexual tubules of the mesonepliros or Wolffian body. The epididynis, vas deferens, 
vas aberrans, vesicula seminalis, and common ejaculatory ducts are all developed from the Wolffian duct.

The interstitial stroma of the testis is developed from the mesoderm of the genital ridge. The tunica albuginea is formed from connective tissue derived from 'the basal nuclei of the sex-cords.'

Development of the Ovary. - The embryonic rudiments pertaining to the development of the ovary are as follows:

$$
\begin{aligned}
& \text { Genital ridge and germinal epithelium. } \\
& \text { Egg-columns or egg-tubes of Medullary cords. } \\
& \text { Pfluger (sex-cords). }
\end{aligned}
$$

Rete-cords.

The ovary, like the testis, is developed from the indifferent sexual organ. Whilst in the case of the testis the anterior or sexual tubules of the mesonephros or Wolffian body and the Wolffian duct pertain to its development, inasmuch as the excretory tubules of the male sexual gland are associated with the epithelium of their Malpighian corpuscles; in the case of the ovary the Wolffian tubules and the Wolffian duct become vestigial.

The ovary is developed from the sexual cords and the mesoderm of the genital ridge. The epithelial cells of the genital ridge, as in the male, constitute the germinal epithelium. The deep cells of the epithelium of the sex-gland region of the genital ridge invade the mesoderm of the ridge, and are disposed as cellular cords, which are separated by ingrowths of connective tissue, derived from the mesoderm of the genital ridge. These cellular cords are called the egg-columns, or egg-tubes of Pflüger (sex-cords). These egg-columns consist of small epithelial cells (follicular cells) and large sexual cells or primitive ova. They become separated from the epithelium of the genital ridge when the somewhat indefinite tunica albuginea of the ovary is formed from the connective tissue derived from 'the basal nuclei of the medullary cords."

The sexual cells of the egg-columns increase in size, and each column, being invaded by connective tissue, is thereby broken up into groups of cells. known as cell-nests, which form the primary Graafian follicles. Each of these follicles consists of one sexual cell, or primitive ovum, and numerous small epithelial cells, with an external investment of connective tissue. These small cells are disposed as a single layer around the ovum, which layer represents the nembrana granulosa, and the investing connective tissue forms the theca folliculi. At a later period the cells of the membrana granulosa undergo proliferation, and so give rise to several layers. A fluid, called liquor folliculi, is then formed by these cells; the ovum is thereby displaced to one side of the follicle; and the mass of cells, within which it is embedded, forms the discus proligerus.

The egg-columns (egg-tubes of Pfluger) form the cortical part of the ovary.

The medullary cords, which contribute to the medullary part of the ovary, like the sex-cords of the testis, are probably developed from the germinal epithelium of the sex-gland region of the genital ridge. They bear a resemblance to the egg-columns, by which they are succeeded, and contain follicular cells and primitive ova. They become broken up into a number of primary Graafian follicles, but these soon degenerate. In the medullary part of the ovary they give rise to groups of follicular cells, and these subsequently become converted into connective tissue.

The rete-cords are developed, as in the male, from the germinal epithelium of the rete-region of the genital ridge. They differ from the rete-cords of the male inasmuch as they do not become hollow, so as to form tubules, but remain solid. They resemble the medullary cords, and, along with the remains of these cords, they form the medullary part of the ovary, giving rise to groups of follicular cells, which subsequently become converted into connective tissue. This arrangement is known as the rete otarii.

The epithelium, which covers the surface of the ovary, in adult life represents the superficial cells of the germinal epithelium, which take no part in the formation of the egg-columns. The cells of this epithelium differ in a marked manrier from those of the adjacent peritoneum inasmuch as they are columnar, whilst the adjacent cells are the usual endothelial cells of a serous membrane. 
Summary:-The cortical part of the ovary, with its Graafian follicles and their contents, is developed from the cell-nests formed by the ovarian ends of the egg-columns or egg-tubes of Pfluger, which arise from the germinal epithelium of the sex-gland region of the genital ridge.

The medullary part of the ovary is formed by the ovarian ends of the medullary cords and rete-cords, which are now transformed into a connectivetissue stroma, the latter cords constituting the so-called rete ovarii. The medullary cords have a similar origin to that of the egg-tubes of Pflüger; and the rete-cords arise from the germinal epithelium of the rete-region of the sex-gland.

The tunica albuginea of the ovary is developed from the connective tissue derived from ' the basal nuclei of the medullary cords.'

The columnar epithelium, which covers the surface of the adult ovary, represents the superficial cells of the embryonic germinal epithelium.

Mesorchium and Mesovarium.-The Wolffian body is attached to the dorsal wall of the body-cavity by a fold of peritoneum, which is spoken of as its 'mesentery,' but is really its ligament. 'This ligament is prolonged to the diaphragm, under the name of the diaphragmatic ligament, and externally it blends with the uro-genital fold, which contains the Wolffian and Müllerian ducts. From the caudal part of the WVolffian body another fold of peritoneum, containing connective tissue and plain muscular tissue, extends to the inguinal region. This fold is called the inguinal ligament.

As the genital ridge, or indifferent sexual organ, which lies on the inner side of the Wolffian body, becomes differentiated into the testis and the ovary, it retains its connection with the Wolffian body, and is attached to the dorsal wall of the body-cavity by a fold of peritoneum, which acts as a suspensory ligament. This fold forms the mesorchium and mesovarium, respectively, and at the level of the caudal part of the Wolffian body it blends with the uro-genital fold, the latter having previously fused with the ligament ('mesentery') of the Wolffian body. The sexual gland is retro-peritoneal: that is to say, it lies behind the peritoneum.

As the caudal tubules of the Wolffian body become atrophied, the inguinal ligament acquires a connection with the caudal end of the sexual organ. In the male it constitutes the gubernaculum testis, which is referred to in connection with the descent of the testis. In the female the inguinal ligament, as it descends, becomes connected with the Müllerian duct at the level where this duct fuses with its fellow to form the uterus and vagina, and thereafter it continues its course to the inguinal region, traversing the inguinal canal, and terminating within the labium majus. The part of the inguinal ligament between the ovary and the uterus forms the ligament of the ovary, and the part which extends from the uterus through the inguinal canal to the labium majus constitutes the round ligament of the uterus.

The descent of the inguinal ligament through the inguinal canal is preceded, or accompanied, by a small diverticulum of the peritoneum, which lies in front of the inguinal ligament. This diverticulum generally becomes obliterated. In rare cases, however, it remains pervious as a blind diverticulum, communicating only with the peritoneal cavity, and known as the canal of Nuck.

Development of the Epoöphoron. - The longitudinal tube, which lics parallel to the Fallopian tube, is a persistent part of the Wolffian duct, and represents the canal of the epididymis in the male. In some animals, e.g., the sow, the Wolffian duct remains persistent, and, under the name of the duct or canal of Gärner, can be traced from the broad ligament of the uterus along the side of that organ to the lateral wall of the vagina in its upper part, where it disappears. In the human female it sometimes takes a similar course, and the portion of it on the uterine and vaginal walls is to be regarded as representing the vas deferens in the malc. The transverse tubules of the cpoöphoron extending from the region of the ovary to the longitudinal tube (so-called duct of Gärtner), into which they open at right angles, are vestiges of the anterior segmental tubes of the Wolftian body, and represent the tubuli recti, rete testis, vasa efferentia, and coni vasculosi of the testis in the male.

Development of the Paroöphoron.-These vestigial tubules are derived from the more posterior segmental tubes of the Wolffian body, and they represent the paradidymis or organ of Girald es in the male. 


\section{ABDOMINAL CAVITY.}

The abdominal cavity is somewhat ovoid, the vertical measurement greatly exceeding the transverse. Its superior boundary is formed by the diaphragm, which here presents a concave surface. The inferior boundary is formed by the levatores ani and coccygei muscles, covered superiorly by the visceral pelvic fascia and inferiorly by the anal fascia. This boundary is concave on its upper aspect. The superior and inferior boundaries, being fleshy, are capable of contracting and relaxing alternately. During contraction the diaphragm descends on each side, and the levatores ani ascend, thus diminishing the vertical measurement of the cavity. During relaxation the reverse takes place, the diaphragm ascending and the levatores ani descending, and so the cavity is increased in its vertical measurement. The anterior and lateral boundaries are partly osseous and partly musculo-aponeurotic. The osseous boundaries are formed by the lower ribs above, and the pelvis below. Elsewhere these boundaries are formed by the musculo-aponeurotic planes of the abdominal muscles. The posterior boundary is formed by the bodies and discs of the lumbar vertebræ, psoas magnus and quadratus lumborum muscles with their fascial investments, sacrum, and coccyx. The cavity is divided into two regions, the abdomen proper and the pelvis. The abdomen proper is limited below by the brim of the pelvis, and its visceral contents are the abdominal portion of the alimentary canal, with the exception of the pelvic colon and rectum, the liver, pancreas, spleen, kidneys, and suprarenal bodies. The pelvis is situated below the level of the brim, and contains the pelvic colon, rectum, and internal uro-genital organs.

\section{Abdomen Proper.}

Division into Regions.-The abdomen proper is divided into nine regions by means of two horizontal and two vertical lines, with their corresponding planes. The horizontal lines are called subcostal and intertubercular. The subcostal line encircles the abdomen proper on a level with the lowest parts of the tenth costal cartilages, and the plane corresponding to it is called the subcostal plane. The intertubercular line connects the bighest points of the iliac crests which can be felt from the front, where there is usually a tubercle on the outer lip about 3 inches from the anterior superior iliac spine. The plane corresponding to this line is called the intertubercular plane. The vertical lines are called the Poupart lines, right and left, and each extends vertically upwards from the centre of Poupart's ligament. The planes corresponding to these lines are known as the Poupart planes. The subcostal and intertubercular lines, with their corresponding planes, map out the abdomen proper into three horizontal zones, called subcostal, umbilical, and hypogastric. The two Poupart lines, with their 
corresponding planes, subdivide each of these zones into three regions, two lateral, right and left, and a central. The abdomen proper is thus eventually divided into nine regions, three in each of the three horizontal zones, as follows: the subcostal zone is subdivided into right hypochondriac, epigastric, and left hypochondriac regions; the umbilical zone is subdivided into right lumbar, umbilical, and left lumbar regions; and the hypogastric zone is subdivided into right iliac, hypogastric, and left iliac regions.

Superficial View of the Contents.-On taking a superficial view of the contents of the abdomen proper the sharp anterior border of the liver is seen on the right side projecting beyond the right costal margin, and also beyond the ensiform process. The great bulk of the organ, however, lies concealed in the right hypochondrium, and the extent to. which it passes into the left hypochondrium usually corresponds to the left mammary line. In the middle line it projects beyond the ensiform process for about 2 inches, but along the right costal margin it does not usually project more than about $\frac{1}{4}$ inch. The anterior border presents two notches. One, which is well defined, is called the interlobar or umbilical notch. It is situated fully I inch to the right of the middle line, and transmits the obliterated umbilical vein or round ligament. The other, which is situated about 2 inches to the right of the interlobar notch, is usually somewhat indefinite, and is called the eystic notch. It allows the fundus of the gall-bladder to come forward opposite the ninth right costal cartilage at a point coinciding with the outer border of the right rectus muscle. The falciform or suspensory ligament is conspicuous as it takes attachment to the supero-anterior surface of the liver, which it divides into two lobes, right and left.

On the left side a portion of the stomach is visible, though a large part of the viscus lies deeply in the left hypochondrium. The portion which is seen in the epigastrium is partially covered by the left lobe of the liver, but a part of it is in contact with the anterior abdominal wall, provided the viscus is not empty. Descending from the great curvature of the stomach there is an extensive fold of peritoneum, which hangs down in the form of a curtain, and so conceals the jejunum and ileum. This fold is called the great or gastro-colic omentum. In normal circumstances it descends as low as the level of the sacral promontory upon the left side, but it stops a little short of that level on the right side. The condition of the great omentum is subject to much variety. In some bodies it is very narrow, and much puckered in the vertical direction, so as to leave exposed the viscera which are normally covered by it. In other cases it is displaced to one or other side, or it may even be raised into the left hypochondrium. In normal circumstances a few coils of the ileum are visible beyond the great omentum on the right side, and, more especially in the female, one or two loops of the ileum may descend into the pelvic cavity to occupy the pouch of 
Douglas. In the right iliac fossa the cæcum is in part seen, and in the left iliac fossa the iliac colon is partially visible.

When the great omentum is raised and laid over the costal margin, the coils of the jejunum and ileum come into view, occupying the umbilical and hypogastric regions, and extending into the right and left lumbar, and iliac, regions. The transverse colon is also seen crossing in an arched manner from the right to the left hypochondriac regions.

When moderately distended, the stomach is pyriform and curved. It presents for consideration the following parts: Two extremities, two surfaces, two curvatures, and two orifices.

Extremities.-The extremities are left and right. The left extremity is known as the cardiac or splenic end or fundus. It is large

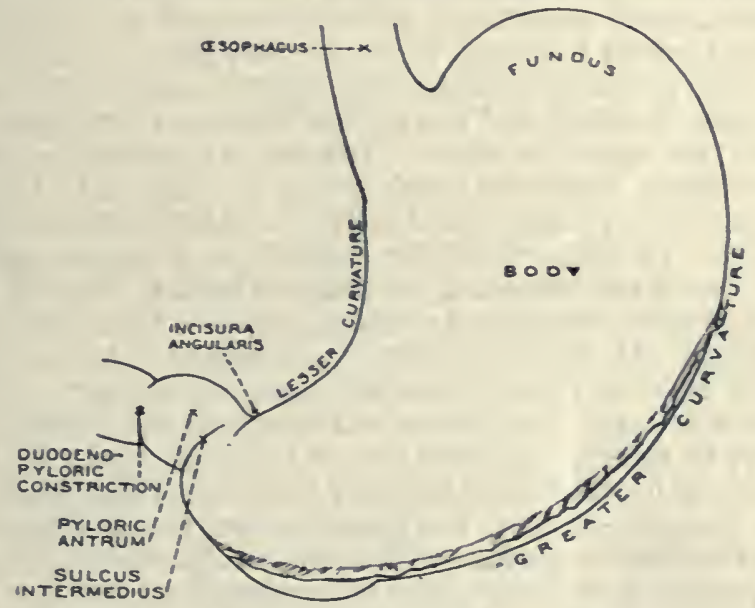

Fig. 29S.-The Stomach (Extersal View).

and round, and forms a cul-de-sac. Its direction is upwards, backwards, and to the left, and it is related to the left half of the diaphragm posteriorly and the spleen. The right extremity is known as the pyloric end. It lies beneath the quadrate lobe of the liver, and is directed backwards. It is narrow, and is continuous with the first part of the duodenum. Its position is indicated superficially by a well-marked, circular constriction, called the duodeno-pyloric constriction.

Surfaces.-These are antero-superior and postero-inferior. The antero-superior surface is convex, and, though mainly directed upwards, it has a slight inclination forwards. It is closely related to (I) the under surface of the left lobe of the liver, (2) the left half of the diaphragm, and (3) the anterior abdominal wall. The postero- 
inferior surface is somewhat flat, and has a slight inclination backwards. Its relations are as follows:

I. The diaphragm.

2. The gastric surface of the spleen.

3. The left suprarenal capsule.

4. The gastric area at the upper part of the front of the left kidney.

5. The antero-superior surface of the pancreas.

6. The transverse colon.

7. The upper surface of the transverse mesocolon.

Curvatures.-The curvatures, also known. as borders, are small and great. The small curvature, or posterior border, extends downwards and to the right from the right side of the lower end of the œsophagus to the duodeno-pyloric constriction. It is concave, and is directed backwards and towards the right. The small or gastro-hepatic omentum connects the small curvature with the lips of the portal fissure of the liver, and between the two layers of the gastro-hepatic omentum, along the small curvature, there are the gastric artery and the pyloric branch of the hepatic artery, with the corresponding veins. Towards its pyloric extremity the small curvature presents a notch, which is produced by the stomach being bent upon itself. This notch is called the incisura angularis. It indicates the division of the stomach into cardiac and pyloric parts. The great curvature, or anterior border, extends from the left side of the lower end of the œsophagus to the duodeno-jejunal constriction. It is convex, and much arched. At first it arches over the fundus, passing upwards, backwards, and to the left. Thereafter it passes downwards and forwards, and finally it extends from left to right. The direction of the greater part of the great curvature is forwards and towards the left. It gives attachment to two omenta-namely, the great or gastro-colic omentum, and the gastro-splenic omentum. The gastro-colic omentum is attached to the greater part of the great curvature, from which it depends. Between its two layers there are the right gastro-epiploic artery and the left gastro-epiploic artery, together with the gastro-epiploic veins, right and left. The gastro-splenic omentum is attached to the great curvature to the left of the attachment of the gastro-colic omentum. The transverse colon lies immediately below the great curvature, under cover of the great omentum. About $\mathrm{I} \frac{1}{\mathrm{c}}$ inches from the pyloric end the great curvature presents a notch, called the sulcus intermedius, which indicates the subdivision of the pyloric part of the stomach into a pyloric canal and a pyloric vestibule.

Orifices.-These are two in number-namely, œsophageal and pyloric. The œsophageal orifice is also known as the cardiac oriflce, or cardia, and through it the œsophagus opens into the stomach. It is situated at the upper and left extremity of the small curvature, fully 2 inches to the right of the highest part of the fundus. The pyloric or duodenal orifice, through which the stomach communicates with the duodenum, is narrow, and is 
situated at the right extremity of the stomach. It is directed backwards, and is guarded by the pyloric valve, which will be described in connection with the structure of the stomach. Its position is indicated superficially by the duodeno-pyloric constriction already referred to.

Divisions of the Stomach.-The stomach is divided into two parts - cardiac and pyloric-by means of a line connecting the incisura angularis on the small curvature with the opposite point on the great curvature. The cardiac part lies to the left of this line, and is of large size. It consists of the fundus and body of the stomach, the separation between these two parts being indicated by a line connecting the cardia with the opposite point on the great curvature. The pyloric part, which is short, is subdivided into two portions-namely, the pyloric canal and the pyloric vestibule-by means of the sulcus intermedius on the great curvature. The pyloric canal adjoins the duodeno-pyloric constriction. It is about $\mathrm{I} \frac{1}{4}$ inches in length, and is narrow and cylindrical in outline, like a portion of the small intestine. Its walls are thick, and it is directed backwards. The pyloric vestibule, or antrum pylori, is a dilatation, situated to the left of the pyloric canal, from which it is separated by the sulcus intermedius. Its direction on the great curvature is downwards.

Position of the Stomach. - When the stomach is empty it is comparatively small, which is due to the contracted state of its walls during life. It is situated horizontally in the left hypochondrium and the left part of the epigastrium, and is falciform in outline. The fundus is directed backwards; the cardiac portion, somewhat saccular, is directed forwards and slightly to the right; the pyloric portion, tubular in outline, passes to the right; the pylorus lies about $\frac{1}{2}$ inch to the right of the median line; the surfaces look upwards and downwards; and the great curvature looks forwards, and the small curvature backwards.

When the stomach becomes distended, it usually assumes an oblique position, its long axis being directed downwards, forwards, and to the right. The organ increases in length; the pylorus is carried towards the right side, assuming a position from $I_{\frac{1}{2}}$ to 2 inches on the right side of the median plane; the pyloric canal is bent backwards; the fundus becomes enlarged and directed upwards and towards the left; and the upper surface acquires an inclination forwards, and the under surface an inclination backwards. The position of the cardia is practically unaltered. The stomach still occupies the left hypochondrium and the epigastrium. but, when much distended, part of it may enter the umbilical and left lumbar regions. Occasionally the stomach, when distended, retains the horizontal position occupied by it when empty.

Topography of the Stomach.-The cardiac orifice, or cardia, is situated to the left of the median line about I inch below the sternal attachment of the sevcnth left costal cartilage. It is about 4 inches distant from the anterior abdominal wall, and is on a level with the upper part of the body of the eleventh thoracic vertebra. 
The pyloric orifice, or pylorus, is on a lower level and more anterior plane than the cardia, and, moreover, it usually lies to the right of the median line. Relatively to the vertebral column it is on a level with the upper part of the body of the first lumbar vertebra, and it is opposite the tip of the ninth right costal cartilage. When the stomach is empty, the pylorus usually lies about $\frac{1}{2}$ inch to the right of the median line, but this distance is increased during distension to $\mathrm{I}_{\frac{1}{2}}$ or 2 inches, or even more. The pylorus may be said to lie about 4 inches below the junction of the seventh right costal cartilage with the sternum. Its level may also be stated as being midway between the interclavicular notch on the upper border of the manubrium sterni and the upper border of the symphysis pubis.

Peritoneal Relations.-The stomach is almost completely invested by peritoneum, the anterior surface deriving its covering from the peritoneum of the general cavity, and the posterior surface from that of the small sac. The parts uncovered by peritoneum are as follows: a narrow line along the small curvature between the two layers of the gastro-hepatic omentum for the passage of the gastric and pyloric arteries; a narrow line along the great curvature between the two layers of the gastro-colic omentum for the passage of the right and left gastro-epiploic arteries; and the uncovered area or trigone. This latter area is situated on the posterior surface, balow, and a little to the left of, the cardiac orifice. It is about 2 inches in breadth, and rather less from above downwards, its shape being triangular. This part of the stomach is in contact with the left crus of the diaphragm, and sometimes with the left suprarenal capsule. The reflection of the peritoneum around this area from the stomach to the diaphragm is known as the gastro-phrenic ligament, which lies on the left side of the lower end of the osophagus. 793.

For the structure and development of the stomach, see pp. $78 \mathrm{I}$,

\section{Position, Connections, and Component Parts of the Intestinal} Canal.-The intestinal canal commences at the pyloric end of the stomach and terminates at the anus. It is divided into small intestine and large intestine.

The small intestine commences at the pyloric extremity of the stomach, and terminates in the right iliac fossa by opening obliquely into the large intestine. It measures fully 23 feet in length, and is divided into three parts, which, from above alownwards, are called the duodenum (twelve fingers' breadth), jejunum ('empty'), and ileum (" coiled'). The duodenum is from ro to II inches in length, and its limits are the pyloric extremity of the stomach, and the left side of the body of the second lumbar vertebra. Being very leeply placed, its position and connections will be described further on (p. 73r). Of the remainder of the small intestine the upper two-fifths represent the jejunum; and the lower three-fifths the ileum. There is no evident external mark of separation between the three divisions of the small intestine, so that they merge imperceptibly into each other; but there are internal characters which serve to distinguish these divisions. On the left side of the body of the second lumbar vertebra, where the duodenum terminates in the jejunum, the bowel describes a bend in a downward and for- 
ward direction, called the duodeno-jejunal flexure, which is suspended from the left crus of the diaphragm by a fibro-muscular band, called the musculus suspensorius duodeni. The jejunum and ileum are very much convoluted, their coils being covered to a greater or less extent by the great omentum. They lie below the transverse colon, and occupy the umbilical, hypogastric, right and left lumbar, and right and left iliac regions. A few coils of the ileum sometimes dip into the pelvis, and when this occurs they occupy, in the female, the pouch of Douglas. The jejunum and ileum are attached to the vertebral column by a fold of peritoneum, called the mesentery proper, which contains their bloodvessels, nerves, and lymphatics, and which is of such a nature as to permit of great mobility in this part of the intestinal tube. They are surrounded by peritoneum except along a narrow interval corresponding with the attachment of the mesentery proper, this border of the bowel being called the attached or mesenteric border, as distinguished from the free or anti-mesenteric border. The small intestine is a smooth cylindrical tube, which gradually diminishes in size from above downwards. The terminal portion of the ileum, as it is about to join the large intestine, is directed upwards and to the right, with a slight inclination backwards.

Meckel's Diverticulum.-This is a protrusion which is sometimes found connected with the free or anti-mesenteric border of the ileum about 3 feet above the ileo-creal valve. It represents the persistent proximal part of the vitelline or vitello-intestinal duct, which connects the yolk-sac with that portion of the primitive alimentary canal from which the lower part of the ileum is formed. It usually measures from 2 to 3 inches in length, and its calibre generally corresponds with that of the tube from which it springs. In most cases it resembles the finger of a glove, but occasionally it is reduced to the condition of a cord. It is rarely provided with a mesentery.

The large intestine commences in the right iliac fossa, and terminates at the anus. It measures about 6 feet in length, and gradually diminishes in size from its commencement to its ternination. It is composed of the colon and the rectum. The colon is subdivided into the crcum (with the vermiform appendix), ascending or right colon, hepatic flexure, transverse or middle colon, splenic flexure, descending or left colon, iliac colon, and pelvic colon.

Cæcum.-The cæcum (caput cæcum coli) is the commencement of the large intestine. It represents that part of the gut which extends below the ileo-creal orifice, and is situated in the right iliac fossa, where it rests upon the ilio-psoas muscle with the intervention of the fascia iliaca. Its lower end or fundus has an inclination inwards towards the pelvic brim, close to which it usually lies. The average length of the cæcum is about $2 \frac{1}{2}$ inches, its breadth being about 3 inches. When empty it is more or less covered by coils of the ileum. In the distended state it comes into contact with the anterior abdominal wall, and at the same time it descends as low as the outer half of Poupart's ligament. At its inner and back part, at a point about $2 \frac{1}{2}$ inches from its 
lower end, it receives the termination of the ileum, the opening being guarded by the ileo-cæcal valve. The position of this valve corresponds with a point in the right spino-umbilical line between $I \frac{1}{2}$ and 2 inches from the anterior superior iliac spine ( $M c B u r n e y$ 's point). In normal cases the cæcum is very movable, being completely covered by peritoneum. The line of reflection of the peritoneum posteriorly may correspond with the level of the ileocæcal orifice. In some cases, however, the peritoneum, after having invested the posterior aspect of the cæcum, gives a covering to the posterior wall of the ascending colon for $I_{\frac{3}{4}}$ inches (Treves), after which the reflection takes place. The line of reflection may be transverse or oblique, and the peritoneum so reflected is continuous with the left or inferior layer of the mesentery proper.

In a few cases (about 6 per cent.) the upper part of the posterior surface of the cæcum is destitute of peritoneum, and is bound down by connective tissue to the subjacent fascia iliaca. Under these circumstances its mobility is more or less curtailed.

Varieties of Cæcum. -The cæcum is characterized by extreme variableness as regards form, but the variations may be grouped into the following four principal types (Treves):

First Type.--In the fœetus the cæcum is conical, the vermiform appendix springing from the apex of the cone. The three longitudinal muscular tæniæ are disposed as follows : one is situated on the postero-internal aspect; a second lies along the postero-external aspect; and the third is placed on the anterior aspect. They are nearly equally distant from each other, and meet at the apex of the cæcum.

Second Type.-The cæcum is quadrilateral. The lower end presents two sacculi, and the vermiform appendix springs from the interval between them. The tæniæ are situated as in the preceding type.

Third Type.-In this variety the part of the cxcum to the right of the anterior tænia becomes more developed, and consequently more prominent, than the part to the left of that band, and the anterior wall undergoes greater growth than the posterior wall. During these changes the apex is being gradually shifted backwards and to the left, until finally it takes up a position near the ileo-cxcal junction, where it corresponds with the origin of the vermiform appendix. The part to the right of the anterior trenia becomes so much developed, especially in a downward direction, as to give rise to a false apex. This is the most common form of cxcum, the origin of the appendix being transferred to the left and posterior aspect.

Fourth Type.-In this variety the part to the right of the anterior tænia undergoes excessive development, and the part to the left becomes much atrophied, and in many cases practically disappears. This causes the anterior tænia to terminate at the lower part of the ileo-cæeal junction, and the vermiform appendix arises immediately posterior to that point.

Vermiform Appendix.-The vermiform appendix is a small diverticulum of the cæcum, which opens into its inner and back part rather more than I inch below the ileo-crecal orifice. The crecal end of the appendix is called its base, and the guide to it is rather more than I inch below McBurney's point. Its diameter corresponds with that of an ordinary goose-quill, and its length varies from 2 to 6 inches, or more. Its outline is serpentine, and the lumen is usually confined to its crecal half, though it may extend throughout its entire length. The opening by which the appendix 
communicates with the cæcum is occasionally guarded by a fold of mucous membrane, which is known as the valve of Gerlach. The appendix is provided with a mesentery, called the appendicular mesentery or meso-appendix. It seldom reaches more than half or two-thirds along the appendix, which latter is thus rendered more or less convoluted or serpentine. The meso-appendix and its variations will be found described on $\mathrm{p} .725$.

The position occupied by the appendix is extremely variable. According to Lockwood and Rolleston, the normal positions may be tabulated as follows :

I. The vermiform appendix often lies under the left or inferior layer of the mesentery, where it takes a course upwards and to the left in the direction of the spleen. (According to Treves this is its usual position.)

2. It may lie on the brim of the pelvis, or it may project into the pelvic cavity.

3. If the appendicular mesentery is long, the appendix may lie to the right of the cæcum and ascending colon, in which situation it may ascend over the right kidney towards the right lobe of the liver.

4. It may lie free over the cæcum and ascending colon.

5. It may lie free underneath the cæcum. (This is the second most common situation according to Treves.)

As regards abnormal positions of the vermiform appendix, it may, amongst other situations, be found lying in the retro-cæcal, or in the ileo-cæcal, fossa. It has been found extending horizontally across the vertebral column at the level of the lumbo-sacral angle, with its tip resting on the left psoas magnus muscle (Treves).

Ascending or Right Colon.-This extends from the cæcum, on a level with the ileo-cæcal orifice, to the under surface of the right lobe of the liver at a point to the right of the gall-bladder. Here it describes a bend, called the hepatic flexure, which indents the liver, and so gives rise to the impressio colica. The ascending colon occupies a part of the right iliac, right lumbar, and right hypochondriac regions, in which it lies deeply, being in contact with the posterior abdominal wall. Posteriorly it rests upon a portion of the right iliacus muscle covered by the fascia iliaca, the right quadratus lumborum invested by its sheath, and the front of the right kidney in its lower and outer part. Anteriorly it is more or less covered by the coils of the jejunum and ileum. Internally, it has the coils of the jejunum and ileum, and the right psoas magnus muscle, covered by its fascia. The ascending colon in most cases is covered by peritoneum in front and at the sides, but not behind. Sometimes, however, it is completely invested by the serous membrane, which then forms behind it a mesentery, called the ascending meso-colon. Occasionally a peritoneal fold is met with, which extends from the right side of the ascending colon to the abdominal wall at, or a little above, the-level of the iliac crest. It is called the sustentaculum hepatis, and occurs in about I8 per cent. of cases (Treves). It presents anteriorly a free concave border, and it measures about $I_{\frac{1}{2}}$ inches in width, and about 2 inches from before backwards.

Hepatic Flexure.-This is the bend formed by the gut between 
the termination of the ascending colon and the commencement of the transverse colon. The bend takes place in a direction forwards and to the left, and so brings the bowel in front of the second or vertical part of the duodenum. The hepatic flexure has the impressio colica on the inferior surface of the right lobe of the liver above it, the sharp anterior margin of the liver on its outer side, and the second part of the duodenum on its inner side. Posteriorly it is in contact with the right kidney in the same locality as the upper part of the ascending colon, and it is here uncovered by peritoneum.

Transverse or Middle Colon.-This, which is comparatively long and very arched, commences in the right hypochondrium in front of the second part of the duodenum, and terminates in the left hypochondrium in the splenic flexure. Its extremities are deeply placed, the right being a little lower and more superficial than the left, and both being comparatively fixed on account of the shortness of the transverse meso-colon at these points. The greater part of it descends into the umbilical region, where it usually lies just above the umbilicus. The transverse colon is completely invested by peritoneum, except occasionally for I inch or more posteriorly at its right extremity. The serous membrane forms an extensive fold behind it, called the transverse meso-colon, which passes backwards to the anterior border of the pancreas, and is of very limited extent at its right and left extremities. The transverse colon is covered in front by the great omentum. Above it, from right to left, are the liver, gall-bladder, great curvature of the stomach, inferior surface of the pancreas, and basal surface of the spleen; behind it are the second part of the duodenum, head of the pancreas, and transverse meso-colon; and below it are the coils of the jejunum.

Splenic Flexure.-This is situated in the left hypochondrium in contact with the basal surface of the spleen, and behind the cardiac end of the stomach. It occupies a higher and deeper position than the hepatic flexure, and its posterior surface is uncovered by peritoneum. Connected with its left aspect there is a triangular fold of the serous membrane, which attaches it to the diaphragm opposite the tenth or eleventh left rib. This fold is called the phreno-colic or costo-colic ligament, and it forms a platform upon which the basal surface of the spleen rests: hence the name sustentaculum lienis ('support of the spleen '). It will be found described on p. 720 .

Descending or Left Colon.-This, which is of comparatively small calibre, commences in the left hypochondrium at the splenic flexure, and terminates in the lower part of the left lumbar region on a level with the back part of the iliac crest, where it passes into the iliac colon. It lies deeply in the left hypochondriac and left lumbar regions, being directed at first downwards and slightly inwards, and subsequently vertically downwards. Posteriorly it is in contact, from above downwards, with the front of the left kidney at its lower and outer part, and the left quadratus lumborum muscle invested by its sheath. Anteriorly it is covered by coils 
of the jejunum and ileum. Internally coils of the jejunum and ileum form a superficial relation, whilst more deeply there are the lower part of the left kidney and the left psoas magnus muscle covered by its fascia. The descending colon in most cases is covered by peritoneum in front and at the sides, but not behind. Sometimes, however, it is completely invested by the serous membrane, which then forms behind it a mesentery, called the descending meso-colon.

The ascending, transverse, and descending parts of the colon form an arch, within the concavity of which the coils of the jejunum and ileum are disposed.

Iliac Colon.-This commences at the lower extremity of the descending colon on a level with the back part of the iliac crest, and terminates at the inner border of the left psoas magnus, anterior to the left sacro-iliac articulation. At this point it enters the pelvic cavity and becomes the pelvic colon. It is situated in the left iliac fossa, where it lies upon the ilio-psoas muscle with the intervention of the fascia iliaca, its direction being downwards and inwards. Anteriorly it is covered, when empty, by coils of the ileum, but when distended it lies in contact with the anterior abdominal wall. The iliac colon in most cases is covered by peritoneum in front and at the sides, but not behind. Sometimes, however, it is completely invested by the serous membrane, which then forms behind it a mesentery, called the iliac meso-colon.

For the pelvic colon and rectum, see p. 859 .

The large intestine, with the exception of the vermiform appendix and rectum, is characterized by well-marked sacculations, which present a striking contrast to the smooth cylindrical contour of the wall of the small intestine. These sacculations are due to the longitudinal muscular fibres being gathered into three longitudinal bands, called tania coli (ligaments of the colon), which are shorter than the portion of bowel to which they are applied. The sacculations are separated from each other by constrictions filled with fat. Another characteristic of the greater part of the large intestine is the presence, at frequent intervals, of small projections of the peritoneal coat containing fat, called appendices epiploica. These characteristics will be found described in connection with the structure of the large intestine on p. 795.

For the structure and development of the intestinal canal, see Index.

Position, Connections, and Component Parts of the Spleen.-The spleen (lien) is a ductless gland which lies deeply in the left hypochondrium opposite the ninth, tenth, and eleventh ribs, and extending from about the level of the ninth thoracic spine to that of the eleventh. The organ can only be seen when the stomach is drawn out from the left hypochondrium. It is soft, spongy, easily torn, and exceedingly vascular, and it has a dark red colour. It is so liable to become enlarged that it is subject to much variety as regards dimensions and shape. The following statement, therefore, 
of its dimensions is only to be accepted as approximately accurate. The average length of the spleen may be said to be about 5 inches, its breadth, at the widest part, being about 3 inches, and its thickness about $I \frac{1}{2}$ inches. The weight, which is very variable, is about 6 ounces. The organ occupies an oblique position, its long axis being directed downwards, outwards, and forwards. Its lower twothirds are situated in the left hypochondrium, and the upper third in the epigastrium. When it has been hardened in situ its shape resembles that of an irregular tetrahedron (Cunningham) - that is to say, it resembles a solid figure enclosed by four equilateral triangles. The following description is based upon this view of its shape.

Apex.-This corresponds with the upper end, and lies in the epigastrium, about 2 inches from the median line. It is directed upwards, inwards, and slightly forwards.

Surfaces.-One aspect of the organ is directed towards the diaphragm, the other looking towards the abdominal cavity and its viscera. The former aspect represents the parietal or ex. ternal surface, which is convex, and adapts itself to the concavity of the diaphragm. It is called the phrenic surface, and its direction is outwards, backwards, and upwards. It is in contact with the diaphragm opposite the

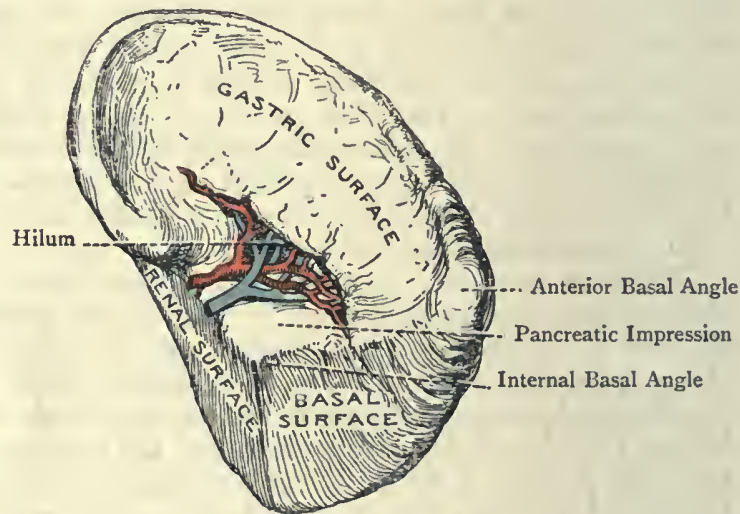

Fig. 299.-The Spleen (Visceral Are ninth, tenth, and eleventh ribs, the left pleural sac descending for some distance between that part of the diaphragm and the adjacent ribs. A part of the base of the left lung, very thin and covered by pleura, also descends for a short distance so as to intervene between the upper part of this surface of the spleen and the exterior. The abdominal or visceral aspect is complex, and presents three surfaces, which are separated from each other by more or less well-marked ridges, radiating from the internal basal angle (Cunningham). These surfaces are called gastric, renal, and basal.

The gastric surface is large, concave, and somewhat semilunar. Its direction is forwards, inwards, and downwards, and it accurately adapts itself to the fundus of the stomach on its posterior aspect. It is limited anteriorly by the sharp anterior border of the spleen, which separates it from the phrenic surface, and 
posteriorly it is separated from the renal surface by the ridge which extends from the internal basal angle upwards to the apex. A little in front of this ridge, and therefore situated on the gastric surface, there is a fissure, called the hilum, for the passage of the splenic vessels, lymphatics, and nerves. Instead of a hilum there is sometimes a row of foramina. The narrow portion of the gastric surface behind the hilum is, at its lower end, in contact with the tail of the pancreas.

The renal surface, which is posterior to the gastric surface, is narrow. It is directed inwards and downwards, and is in contact with the front of the left kidney at its upper and outer part, close to the external border. It is separated from the gastric surface by the ridge already referred to, and is limited behind by the posterior border, which separates it from the phrenic surface. Inferiorly it is separated from the basal surface by the ridge which extends from the internal basal angle to the posterior angle.

The basal surface is the small triangular surface which looks. downwards and inwards. It rests upon the splenic flexure of the colon and upper surface of the sustentaculum lienis or peritoneal platform formed by the costo-colic ligament. It is separated from the renal surface by the ridge already referred to, and from the gastric surface by the ridge which passes between the internal and anterior basal angles. The angles of this surface are called internal, posterior, and anterior, the last being the most prominent.

Borders. - These are anterior, posterior, and inferior. The anterior border is situated between the phrenic and gastric surfaces. It is sharp, and usually presents several notches. The posterior border is situated between the phrenic and renal surfaces. It is blunt, and its position and direction practically coincide with the lowest left intercostal space. The inferior border separates the phrenic and basal surfaces, and is somewhat sharp.

Peritoneal Relations.-The spleen is surrounded by peritoneum, except at the hilum, and where the gastro-splenic omentum and phreno-splenic ligament are connected with it. The serous membrane forms three folds, called gastro-splenic omentum, lieno-renal ligament, and phreno-splenic ligament. The gastro-splenic omentum is attached by one extremity to the gastric surface of the spleen just in front of the hilum, the other extremity being connected with the cardiac end of the stomach on its posterior aspect and the left border of the great omentum. The lieno-renal ligament is attached by one extremity to the gastric surface along the line of the hilum, the other extremity being attached to the front of the left kidney at its upper and outer part. The phreno-splenic or lieno-phrenic ligament extends between the spleen near its upper extremity and the contiguous part of the diaphragm.

Occasionally small accessory spleens, varying in number from one to twenty, are found in the gastro-splenic omentum in the neighbourhood of the hilum, or more rarely in the great omentum. 
Area of Splenic Dulness.-This area is limited posteriorly by the mid-scapular line between the ninth and eleventh left ribs, and anteriorly by the mid-axillary line as it crosses the ninth, tenth, and eleventh ribs, or by a line connecting the left sterno-clavicular joint with the tip of the eleventh left rib. The length of the area is about 3 inches, and its breadth from 2 to $2 \frac{1}{2}$ inches.

For the structure and development of the spleen, see Index.

Position, Connections, and Component Parts of the Liver.-The liver (hepar), which is the largest gland in the body, occupies almost all the right hypochondrium, a great part of the epigastrium, and a small part of the left hypochondrium, in which latter direction it usually extends as far as the left Poupart plane. It is maintained in position by the following peritoneal ligaments : the posterior or coronary ligament, the right and left lateral or triangular ligaments, and the falciform or suspensory ligament.

Topography.-The size of the liver is such a variable factor that the following statement of its limits is only to be regarded as approximately accurate. In the right mammary line it extends from a point about $\frac{1}{2}$ inch below the right nipple to a point about $\frac{1}{2}$ inch below the tip of the bony part of the tenth right rib, being covered by the right ribs from the fifth to the tenth inclusive. In the mid-axillary line the right aspect of the organ extends from the seventh to the eleventh rib, and in [the scapular line its superior and inferior limits are on a level with the ninth and eleventh thoracic spinous processes respectively, the ribs to which it is here related being the ninth, tenth, and eleventh. The left limit of the organ usually corresponds to the left Poupart plane. In mapping out the upper limit the middle line may be taken as the starting. point. In this situation the limit is indicated by a line crossing the sternum at the level of the sixth costal cartilages, this line being slightly arched downwards. The line should then be prolonged to the left, with a slight curve upwards, to a point about 2 inches to the left of the left border of the sternum. In continuing the line to the right it should be carried upwards so as to reach a point about $\frac{1}{2}$ inch below the right nipple. The line, on being prolonged from this point towards the right side, must be carried slightly downwards so as to reach the mid-axillary line at the level of the seventh right rib. The lower limit of the organ extends from a point about $\frac{1}{2}$ inch below the tip of the bony part of the tenth right rib to the left extremity of the line indicating the upper limit. The direction of the line indicating the lower limit is upwards and to the left.

Summary of Outline of Liver.-Commencing 2 inches from the left border of the sternum and 1 inch below the nipple, draw a line downwards and to the right to the left border of the sternum at the level of the sixth costal cartilage, next across the sternum with a slight curve downwards, then upwards and to the right to a point about $\frac{1}{2}$ inch below the right nipple. From this point carry the line downwards and to the right as far as the seventh rib in the right mid-axillary line, then downwards and backwards to the ninth rib on a level with the ninth thoracic spine, whence the line is carried downwards to the 
eleventh rib on a level with the eleventh thoracic spine. From this latter point the line should proceed forwards and slightly upwards to a point about $\frac{1}{2}$ inch below the tip of the bony part of the tenth right rib, and finally upwards and to the left to reach the starting-point.

The liver is to a very large extent under cover of the lower ribs and costal cartilages of the right side, the ensiform process, and the sixth, seventh, and eighth, costal cartilages of the left side. It is accurately moulded on the under surface of the diaphragm, which separates it from the base of the right lung covered by pleura, and the heart enclosed in the pericardium. The thin marginal part of the base of the right lung, with its pleural investment, descends in the angular interval between the diaphragm and the thoracic wall, and so partially covers the liver, a relation which has to be borne in mind in percussing the organ. In the right mammary line the lung descends as low as the sixth rib, whilst the liver ascends to the upper border of the fifth. In the right mid-axillary line the lung descends as low as the eighth rib, whilst the liver ascends as high as the seventh. In the right scapular line (inferior angle of scapula) the lung descends as low as the tenth rib, whilst the liver ascends as high as the ninth. The liver comes nearest to the surface beyond the right costal margin and ensiform process. In the former situation it projects about $\frac{1}{4}$ inch, and in the latter about 2 inches, and in each situation is in contact with the anterior abdominal parietes.

Various circumstances affect the position of the liver. "During respiration, the liver descends in inspiration and ascends in expiration. In the horizontal posture it ascends, and in the sitting or upright posture it descends. In distension of the stomach and intestines, as well as in ascites, it ascends. In right hydro-thorax, hypertrophy of the heart, and hydro-pericardium it descends. Long-continued pressure, as in tight lacing, causes the liver to be displaced downwards. Finally, when the abdomen proper is encroached upon by the gravid uterus or by an ovarian tumour the liver is displaced upwards.

The liver has a reddish-brown colour, and presents for the most part a smooth surface. It is firm to the touch, but under pressure is friable, that is to say, easily crumbled. The dimensions of the organ can only be stated approximately. In the transverse direction (from right to left) it measures from 7 to ro inches, the measurement from before backwards at its right extremity being about 6 inches, which also represents its vertical measurement at the thickest part of the right lobe. Its weight in the adult ranges from 45 to 60 ounces, or from 3 to 4 pounds, the weight in the female being rather less, and its relation to the body weight being in the proportion of one to forty in the adult. In early life the liver is proportionately larger than in the adult, and in a child at the period of birth its relation to the body weight is as one to twenty.

Surfaces.-These are superior, anterior, inferior, right lateral, and posterior. It is not to be supposed, however, that these surfaces are all clearly separated from one another by well-defined borders, only one margin being in reality distinct, namely, the anterior border, which has a very sharp outline. 
The superior surface is markedly convex in its right portion, and accurately adapts itself to the concavity of the diaphragm with which it is in contact. To the left of this convex part there is a depression, called the impressio cardica, produced by the heart with the intervention of the diaphragm. To the left of this impression the superior-surface again becomes convex, and adapts itself to the concavity of the left half of the diaphragm. The superior surface is separated from the posterior, anterior, and right lateral surfaces by round, somewhat indistinct, borders.

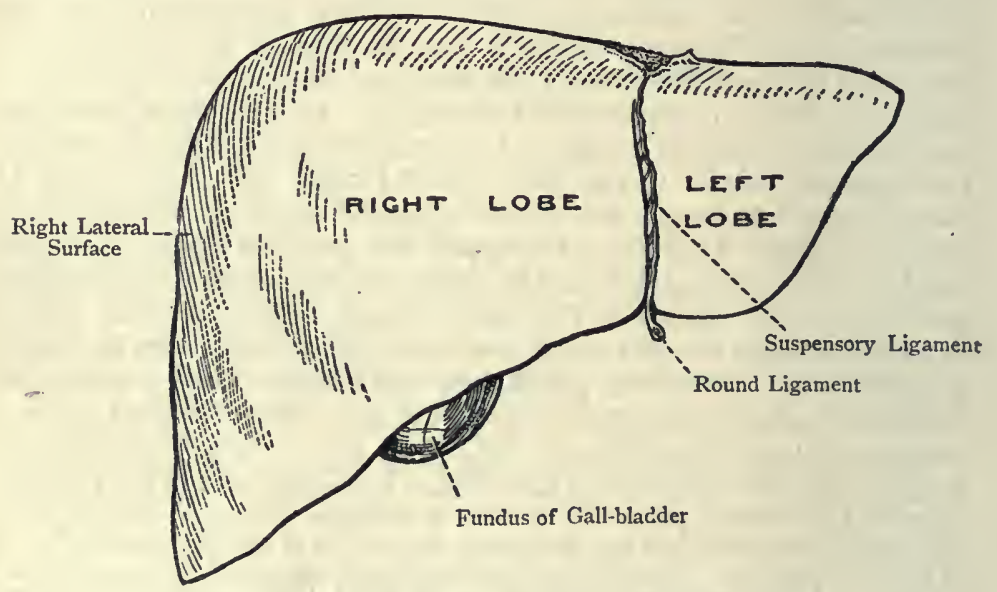

Fig. 300.-The Liver (Antero-superior View).

The anterior surface looks forwards and is triangular. The apex is directed towards the left extremity of the liver, whilst the base is towards the right extremity. One side of the triangle corresponds to the anterior border, the other side being formed by the round border which separates the anterior from the superior surface. The anterior surface is mainly in contact with the diaphragm and the right and left costal margins, but at the middle line it is in relation with the ensiform process, and, for about 2 inches below that process, it is in contact with the anterior abdominal wall. The superior and anterior surfaces are divided into two lateral parts by the falciform ligament, the part to the right of this ligament being called the right lobe, and the part to the left the left lobe. The right lobe forms about four-fifths of the entire supero-anterior surface in the adult, but in early life the two lobes are very nearly of equal size.

The inferior surface looks downwards with an inclination to the left. It is divided into two parts, right and left, by the umbilical fissure. The part to the left of this fissure represents a portion of the left lobe. It lies in front of the cardiac orifice 
The portal or transverse fissure forms a right angle with the back part of the umbilical fissure, from which it extends over the inferior surface of the right lobe for a distance of about 2 inches. It is bounded in front by the quadrate lobe, and behind by the caudate lobe and the lower margin of Spigel's lobe. The two layers of the gastro-hepatic omentum are attached to its anterior and posterior lips. It serves for the passage of the following structures, in order from before backwards : (I) the hepatic duct ; (2) the hepatic artery, accompanied by the hepatic sympathetic plexus of nerves and lymphatic vessels; and (3) the vena portæ, all surrounded by the capsule of Glisson.

The caudate lobe is the narrow portion of liver substance which connects the right extremity of the lower margin of Spigel's lobe with the adjacent part of the inferior surface of the right lobe. It lies behind the portal fissure, and has the vena portæ in front of it, and the inferior vena cava behind it. It forms the upper boundary of the foramen of Winslow.

The lower margin of Spigel's lobe, like the caudate lobe, is situated belind the portal fissure. It is divided by a notch into two eminences of unequal size. The right eminence, which is the smaller of the two, is continuous with the caudate lobe. The left eminence is of large size, and is known as the tuber papillare. The part of the inferior surface of the right lobe which lies to the right of the gall-bladder is of large extent, and presents three impressions, namely, impressio colica, impressio renalis, and impressio duodenalis. The impressio colica, which looks downwards, is situated in front, where it lies to the right side of the body of the gall-bladder. It is in contact with the hepatic flexure of the colon. The impressio renalis, which is of large size, looks backwards as well as downwards, is posterior to the impressio colica, and is in contact with a large part of the front of the right kidney. The impressio duodenalis is situated on the inner side of the impressio renalis, just external to the neck of the gall-bladder. It is in contact with the commencement of the second part of the duodenum.

The umbilical fissure is so named because it contains the remains of the umbilical vein, now known as the round ligament of the liver. It commences at the anterior border of the organ in the umbilical or interlobar notch, and extends as far back as the left extremity of the portal fissure, with which it forms a right angle. It separates the quadrate lobe from the inferior surface of the left lobe. Sometimes the fissure is more or less completely bridged over by a portion of hepatic substance, which thus forms a pons hepatis. The obliterated umbilical vein terminates by joining the left division of the vena portæ, and posteriorly the left division of the vena portæ is joined by the obliterated ductus venosus.

The right lateral surface is convex, and is in contact with the diaphragm and right ribs from the scventh to the cleventh, the margin of the base of the right lung and pleura here descending between the ribs and diaphragm as low as the eighth rib. There is 
no well-marked line of demarcation between this surface and the posterior, superior, and anterior surfaces, but it is distinctly separated from the inferior surface by the right portion of the anterior border.

The posterior surface presents a concavity corresponding with the convexity of the bodies of the tenth and eleventh thoracic vertebræ. It is related to the diaphragm, and its component parts from left to right are as follows: the posterior part of the left lobe; the venosal fissure for the ductus venosus; Spigel's lobe, except its lower margin; the caval fossa for the inferior vena cava; and the uncovered area of the right lobe.

The posterior part of the left lobe at its left extremity is a mere margin, but elsewhere it presents a distinct surface marked by

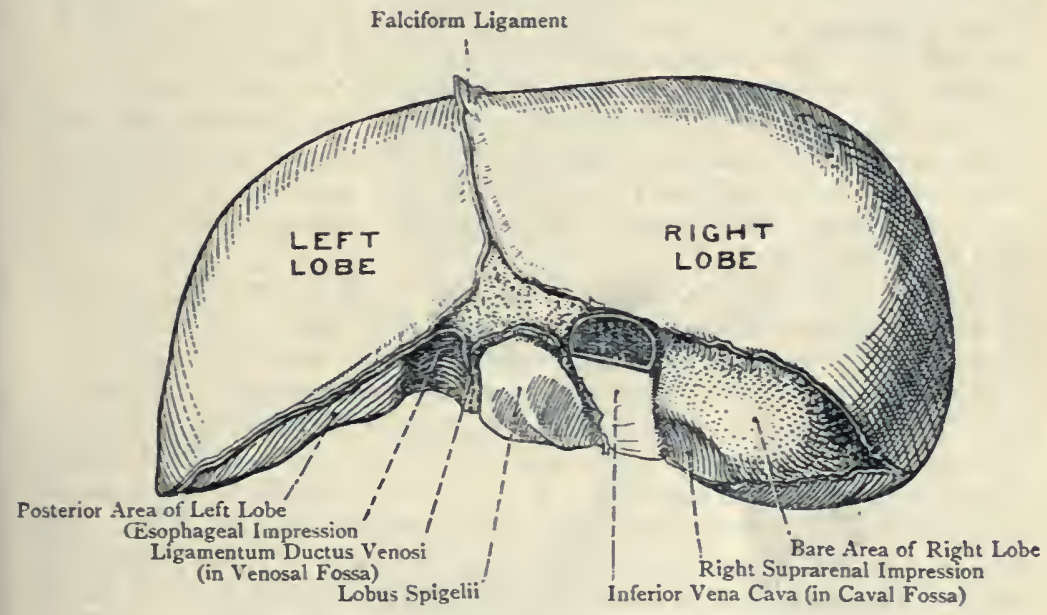

Fig. 302.-The Posteriok Surface of the Liver.

the asophageal groove, which is in contact with the right side of the lower end of the oesophagus.

The venosal fissure lies vertically on the posterior surface, having Spigel's lobe on its right, and the cesophageal groove of the left lobe on its left. Inferiorly it meets the left extremity of the portal fissure and the posterior extremity of the umbilical fissure, and superiorly it meets the caval fossa. It lodges the obliterated ductus venosus, which is connected below with the left division of the vena portæ, and above with the inferior vena cava. The venosal and umbilical fissures separate the right and left lobes on the posterior and inferior surfaces respectively.

Spigel's lobe, with the exception of its lower margin, lies vertically on the posterior surface. It is bounded on the right side by the caval fossa, on the left by the venosal fissure, and inferiorly by the portal or transverse fissure. It looks backwards and a little 
inwards, and is in contact with the right crus of the diaphragm opposite the tenth and eleventh thoracic vertebræ. Its lower margin has been already described (p. 706). The lobe lies at the upper end of the small cavity of the peritoneum.

The caval fossa lodges a part of the inferior vena cava. It lies vertically, and somewhat deeply, on the posterior surface, having the uncovered area of the right lobe on its right side, Spigel's lobe on its left side, and the caudate lobe below. This fossa is sometimes bridged over by a portion of liver substance, called a pons hepatis. At the upper part of this fossa the hepatic veins open into the inferior vena cava.

The uncovered area of the right lobe represents its back part. It measures from $2 \frac{1}{2}$ to 3 inches in the transverse direction, and fully 2 inches from above downwards, except at the extreme right, where it tapers to a point. It is destitute of peritoneum, and is enclosed between the two serous layers which form the coronary ligament, being attached to the diaphragm by areolar tissue. Its direction is backwards and a little inwards. The extreme left end of this area, at a point immediately to the right of the lower end of the caval fossa and near the caudate lobe, presents a somewhat triangular impression, called the impressio suprarenalis, for the right suprarenal body.

Borders.- The chief borders are three in number, namely, posterosuperior, postero-inferior, and anterior. The postero-superior and postero-inferior borders give attachment to the two layers of peritoneum which form the coronary ligament, and they enclose between them the posterior surface. The anterior border is sharp. At its right extremity it passes backwards so as to separate the inferior from the right lateral surface. At its left extremity it also passes backwards, and so forms the thin left margin of the left lobe. Its anterior portion presents two notches, umbilical or interlobar and cystic. The umbilical or interlobar notch is situated fully I inch to the right of the middle line, and transmits the obliterated umbilical vein or round ligament. The cystic notch, often hardly perceptible, is situated about 2 inches to the right of the umbilical notch, and allows the fundus of the gall-bladder to come into contact with the anterior abdominal wall.

Peritoneal Relations.-The liver is covered by peritoneum except in the following regions: the uncovered area of the right lobe; a small triangular area at the posterior extremity of the hepatic attachment of the falciform ligament; the portal fissure; and the cystic fossa, unless in those cases where the gall-bladder is completely invested by peritoneum.

For the ligaments of the liver, see Index.

Excretory Apparatus of the Liver.-This consists of the hepatic duct, the gall-bladder, the cystic duct, and the common bile-duct.

The hepatic duct is formed by the union of a right and left branch which issue from the respective lobes at the portal fissure. It is the most anterior of the structures in that fissure, and, after 
a course of rather more than I inch downwards and to the right, it joins the cystic duct, and so gives rise to the common bile-duct. The diameter of the hepatic duct is about $\frac{1}{6}$ inch.

The gall - bladder is a reservoir for the bile. It is pyriform, and is situated obliquely on the inferior surface of the right lobe, where it occupies the cystic fossa, having the quadrate lobe on its left side, and a large part of the right lobe on its right side. It extends from the anterior border of the liver to near the portal fissure, and presents a fundus, body, and neck. The fundus, which is round, looks downwards, forwards, and to the right. It occupies the cystic notch on the anterior border, and is in contact

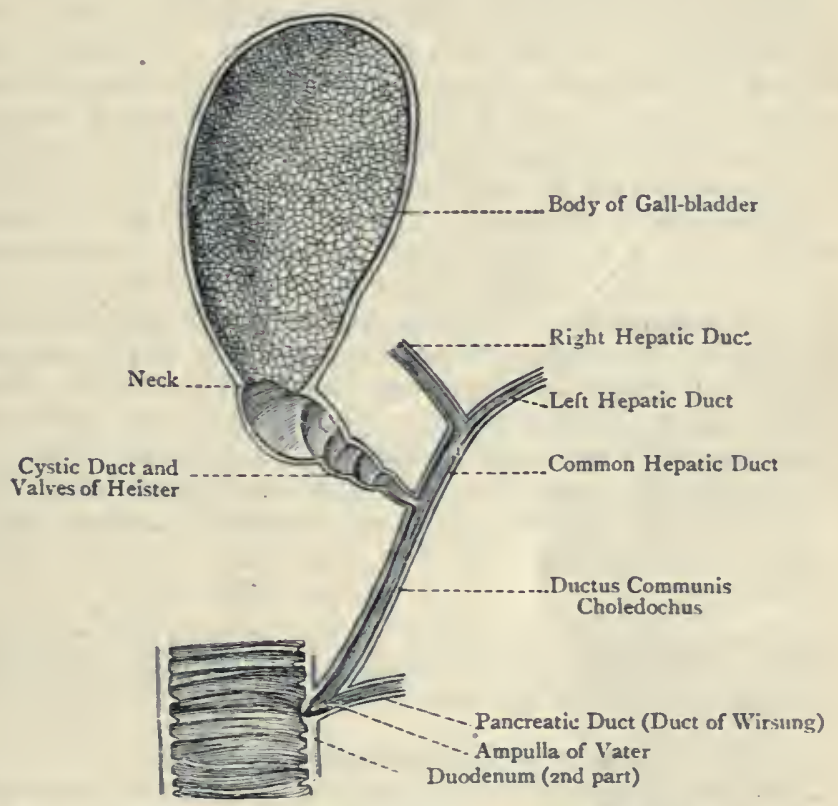

Fig. 303.-The Excretory Apparatus of the Liver.

with the anterior abdominal wall opposite the $n$ inth right costal cartilage, at the outer border of the right rectus abdominis muscle. The body is directed upwards, backwards, and to the left. Its inferior and lateral surfaces are free, but superiorly it is attached by areolar tissue to the cystic fossa. It rests upon the right end of the transverse colon and the first part of the duodenum. The neck describes two curves like the letter $\mathrm{S}$, after which it is continued into the cystic duct. The gall-bladder is usually covered by peritoneum, except on its upper surface. Sometimes, however, the serous membrane entirely surrounds it and forms a ligamentous fold above it, by which it is loosely and movably suspended 
from the cystic fossa. The gall-bladder measures about 3 inches in length, its breadth at the widest part being $I_{\frac{1}{2}}$ inches. Its capacity is from I to $I \frac{1}{2}$ ounces. For the structure of the gallbladder, see Index.

The cystic duct is about $I_{2} \frac{1}{2}$ inches long, its diameter being about $\frac{1}{1^{2}}$ inch. Its course is backwards, downwards, and to the left, and it ends by joining the hepatic duct near the portal fissure to form the common bile-duct.

The common bile-duct (ductus communis choledochus) is formed by the union of the hepatic and cystic ducts near the portal fissure. Its length is fully 3 inches, its diameter being about $\frac{1}{4}$ inch. It passes downwards between the two layers of the gastro-hepatic omentum in front of the foramen of Winslow, where it has the hepatic artery on its left side, and the vena portæ behind. It afterwards descends behind the first part of the duodenum, and subsequently between the second part of the duodenum and the head of the pancreas on their posterior aspect. It then enters the wall of the second part of the duodenum in company with the duct of the pancreas, and runs obliquely in the wall for $\frac{3}{4}$ inch. Thereafter it joins the pancreatic duct, and the resulting duct forms a dilatation, called the ampulla of Vater, which, having become constricted, pierces the mucous membrane and opens on the tip of the bile-papilla at the junction of the inner and posterior walls of the second part of the duodenum, where the upper two-thirds and lower third of that part meet. The distance of this opening from the pylorus is about 4 inches. The common bile-duct sometimes opens into the duodenum independently of the pancreatic duct, but close to it.

It is in the ampulla of Vater where a gall-stone may become lodged and delayed in its downward progress towards the duodenum.

Liver in Early Life.-The size of the liver in early life is much greater than in the adult, the left lobe in particular assuming large dimensions. As age advances, however, the left lobe undergoes a marked diminution in size.

For the structure and development of the liver, see Index.

Peritoneum.-The peritoneum is the serous membrane which lines the abdominal parietes, and invests more or less completely most of the viscera. It is composed of two layers, parietal and visceral, the contiguous surfaces of which are smooth and moist. In the male the interval between the two layers forms a shut sac, but in the female, at the fimbriated extremity of each Fallopian tube, the sac communicates with the lumen of that tube, and through it with the cavity of the uterus and the vagina. It is at the margin of the fimbriated extremity of each Fallopian tube where the endothelium of the peritoneum undergoes a sudden transition into the columnar ciliated epithelium of the Fallopian tube.

The peritoneum forms certain reflections or folds which are of three kinds, namely, omenta, mesenteries, and ligaments. 
An omentum is a double fold of peritoneum passing between two abdominal viscera. The omenta are three in number, namely, the great or gastro-colic omentum (epiploön), which passes between the great curvature of the stomach and the transverse colon, the small or gastro-hepatic omentum, which passes between the small curvature of the stomach and the portal fissure of the liver, and the gastro-splenic omentum, which passes between the posterior surface of the cardiac end of the stomach and the gastric surface of the spleen.

A mesentery is a double fold of peritoneum passing between a portion of intestine and the abdominal wall. The mesenteries in the adult are normally as follows: the mesentery proper, which connects the jcjunum and ileum to the vertebral column; the appendicular micscntery or meso-appendix, which is connected with the vermiform appendix; the transverse meso-colon, which cxtends between the transverse colon and the posterior wall of the abdomen at the anterior border of the pancreas; and the pelvic meso-colon, which connects the pelvic colon to the anterior surface of the sacrum as low as the third sacral vertebra. Occasionally the ascending colon, descending colon, and iliac colon are each provided with a mesentery, called respectively the ascending meso-colon, descending meso-colon, and iliac meso-colon.

A ligament is a fold of peritoneum which connects a viscus not intestine to the abdominal or pelvic parietes. The peritoneal ligaments are as follows: (I) the ligaments of the liver, namely, the falciform ligament, the coronary ligament, and the right and the left lateral or triangular ligaments; (2) the gastro-phrenic ligament; (3) the lieno-phrenic ligament; (4) the false ligaments of the urinary bladder, namely, two posterior, two lateral, and superior ; and $(5)$ the broad ligaments of the uterus.

Course of the Peritoneum.- The parietal and visceral layers of the peritoneum are in unbroken continuity with each other, and this continuity is shown by tracing the membrane in the vertical and transverse directions.

Vertical Course.-Commencing at the portal fissure of the liver two layers of peritoneum descend to the small curvature of the stomach, forming the gastro-hepatic omentum. On reaching the small curvature these two layers separate, one passing over the anterior surface, and the other over the posterior surface, of the stomach. At the great curvature they come together, and descend in the form of a curtain over the coils of the jejunum and ileum to the lower part of the abdomen, forming the two anterior or descending layers of the gastro-colic omentum. These two layers are then folded backwards and ascend to the transverse colon, thus forming the two posterior or ascending layers of the gastrocolic omentum. On reaching the transverse colon they separate, one layer passing in front of, and above, the bowel, and the other layer below and behind it. Having enclosed the transverse colon, the two layers meet, and are prolonged backwards to the posterior 
wall of the abdomen at the anterior border of the pancreas, thus forming the transverse meso-colon. On reaching the anterior border of the pancreas the two layers of the transverse meso-colon take leave of each other, and form an ascending and a descending layer. The ascending layer passes upwards over the anterior surface of the pancreas, the second part of the duodenum, and the posterior portion of the under surface of the diaphragm.

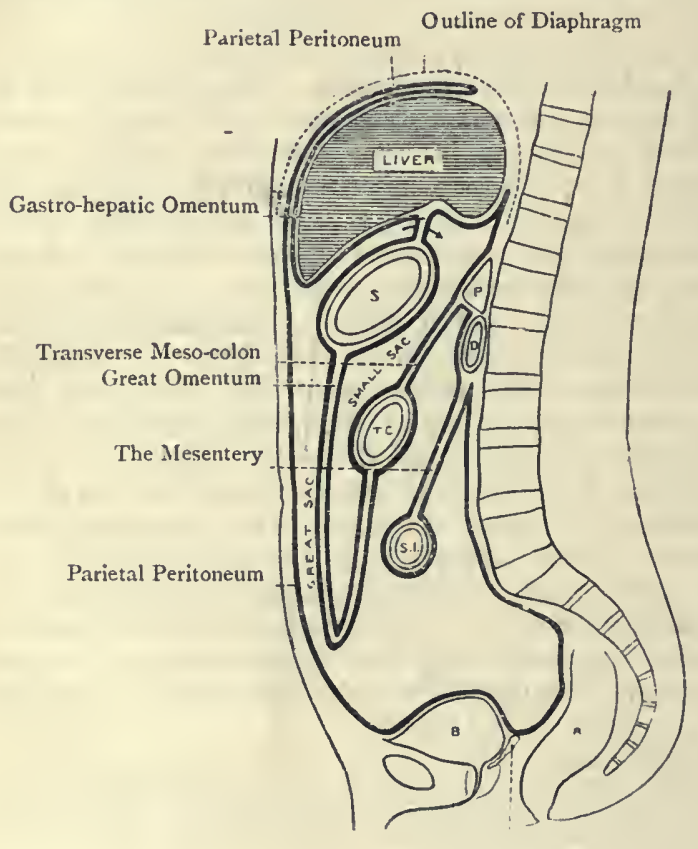

Vesicula Seminalis

Fig. 304.-Diagram of the Peritoneum in the Adult Malf. (Vertical Section).
S. Stomach
P. Pancreas
D. Duodenum
'I.C. Transverse Colon
S.J. Small Intestine
B. Urinary Bladder
R. Rectum

The arrow is through the Foramen of Winslow.

from which it passes to the postero-inferior border of the liver, thus forming the inferior layer of the coronary ligament. Having covered Spigel's lobe; it arrives at the posterior lip of the portal fissure, where it is continuous with the posterior layer of the gastrohepatic omentum.

The descending layer of the transverse meso-colon passes at first backwards upon the inferior surface of the pancreas, and then downwards over the third part of the duodenum, at the lower border of which it is conducted off to the jejunum and ileum by the 
superior mesenteric vessels. Having surrounded these portions of the small intestine it passes to the posterior abdominal wall upon the other aspect of the superior mesenteric vessels, and so forms the mesentery proper. It then descends over the abdominal aorta and inferior vena cava into the pelvis, where its course will be subsequently traced. From the apex of the urinary bladder this layer of the peritoneum is reflected on to the posterior surface of the anterior abdominal wall, after lining which it passes to the anterior portion of the under surface of the diaphragm, whence it is reflected on to the postero-superior border of the liver, thus forming the superior layer of the coronary ligament. It then passes over the superior and anterior surfaces of the liver, and, turning round its anterior border, it arrives at the anterior lip of the portal fissure, where it is continuous with the anterior layer of the gastro-hepatic omentum.

Transverse Course. - The peritoneum may be traced in the transverse direction at two levels, namely, above the transverse colon, or at the level of the foramen of Winslow, which is situated behind the right or free border of the gastro-hepatic omentum, and below the transverse colon, or at the level of the umbilicus. •

Above the Transverse Colon, or at the Level of the Foramen of Winslow.-In front of the foramen of Winslow there are the two layers of peritoneum, anterior and posterior, which form the right or free border of the gastro-hepatic omentum, and which contain between them the common bile-duct, the hepatic artery, and the vena portæ. Tracing the gastro-hepatic omentum from this point to the left, its two layers separate to enclose the stomach, after which they pass to the gastric surface of the spleen as the gastro-splenic omentum. The two layers of this omentum are anterior and posterior, and they contain between them the vasa brevia of the splenic artery. At the spleen the two layers are immediately in front of the hilum. The anterior layer now takes temporary leave of the posterior layer, and turns completely round the spleen, covering its gastric, bașal, phrenic, and renal surfaces in succession. On leaving the renal surface of the organ it again passes to the gastric surface, but it is now behind the hilum. Here it meets the posterior layer of the gastro-splenic omentum, which had remained meanwhile stationary immediately in front of the hilum. These two layers now pass backwards to the anterior surface of the left kidney at its upper extremity, and close to its external border, thus forming the lieno-renal ligament. The two layers of this ligament are disposed as right and left, and between them are the splenic branches of the splenic artery. The right layer corresponds with the posterior layer of the gastro-splenic omentum, and the left layer with the anterior layer of that omentum. The right layer of the lieno-renal ligament, after leaving the left kidney, passes to the right over the aorta and inferior vena cava. As it covers the latter vessel it is placed behind the foramen of Winslow. 
It then continues its course to the right, and, having given a partial covering to the front of the right kidney, it passes over the right lateral and anterior wall of the abdomen as far as the middle line. The left layer of the lieno-renal ligament, after leaving the left kidney, passes over the left lateral and anterior wall of the abdomen, and, on arriving at the middle line, it becomes continuous with the right layer, which has just been traced as far as the middle line. Along the posterior surface of the anterior wall of the abdomen in the middle line, above the level of the umbilicus, the peritoneum meets with the obliterated umbilical vein or round ligament

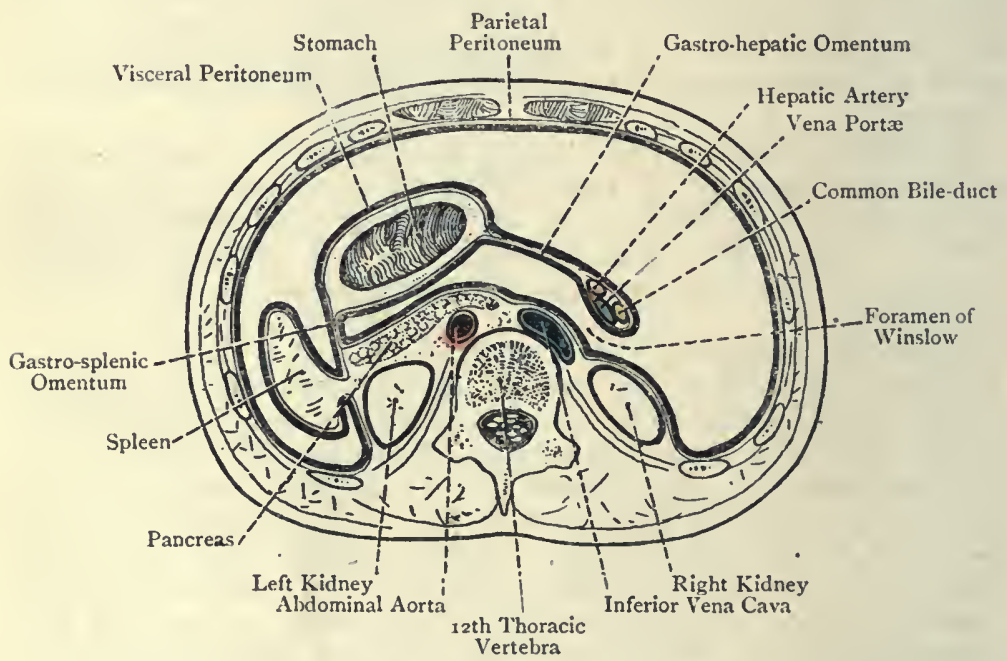

Fig. 305.-Diagram of the Peritoneum at the Level of the Foramen of Winslow ('Transverse Section).

of the liver, around which it is reflected, and here it is carried off from the abdominal wall to form part of the falciform or suspensory ligament.

Another way to trace the peritoneum transversely at the level of the foramen of Winslow is to commence just behind that foramen. Beginning at this point, the peritoneum, which covers the inferior vena cava, passes to the right, and, having given a partial covering to the front of the right kidney, it is continued over the right lateral wall of the abdomen, and thence over the right half of the anterior wall. At the middle line, above the level of the umbilicus, it meets with the obliterated umbilical vein or round ligament of the liver, around which it is reflected, and here it is carried off from the abdominal wall to form part of the falciform or suspensory ligament. It then continues its course over the left 
half of the anterior abdominal wall and the left lateral wall, whence it passes on to the front of the left kidney at the upper part, close to the external border. From this point it passes to the right over the posterior wall of the abdomen, covering the aorta and inferior vena cava, and so it arrives at a point behind the foramen of Winslow. Between the left kidney and the hilum of the spleen the peritoneum forms the lieno-renal ligament, and between the gastric surface of the spleen, just in front of the hilum, and the posterior aspect of the cardiac end of the stomach it forms the gastro-splenic omentum. The two layers of this omentum, having separated, enclose the stomach. They then meet at the small curvature to form the gastro-hepatic omentum, the layers of which, when followed to the right, arrive at the front of the foramen of Winslow.

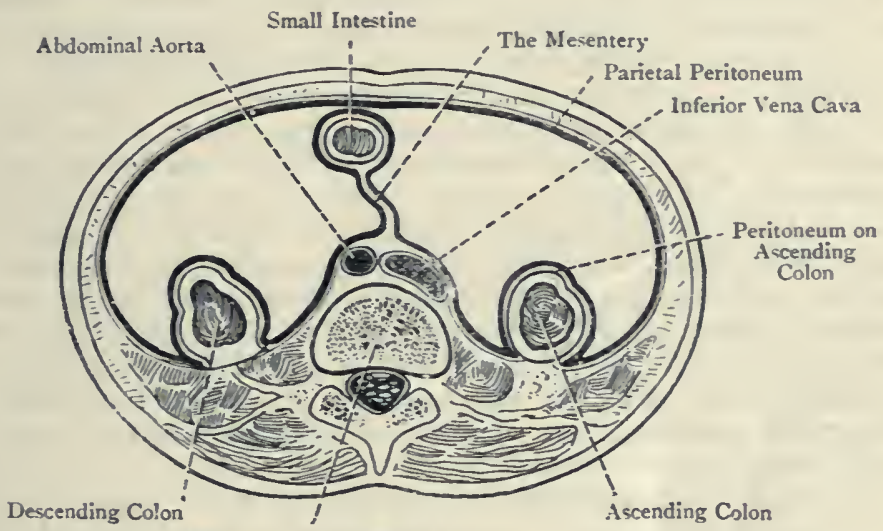

Section of Lumbar Vertebra

Fig. 306.-Diagram of the Peritoneus at the Level of the Urbilicus (Traxsverse Sectiox).

Below the Transverse Colon, or at the Level of the Umbilicus.Commencing at the middle line and passing to the right, the peritoneum covers the right half of the anterior abdominal wall and the right lateral wall as far as the lumbar region. It then covers the right side, anterior surface, and left side of the ascending colon, whence it passes over the front of the right kidney at its lower and inner part. Thereafter it is reflected over the inferior vena cava, and, meeting with the superior mesenteric vessels, it is carried off by them to the jejunum and ileum, both of which it invests. It is then conducted back again to the vertebral column by the superior mesenteric vessels, thus forming the mesentery proper. It next passes to the left over the aorta, and, having partially covered the front of the left kidney at its lower and inner part, it meets with the descending colon in the left lumbar region, which it covers on the right side, anterior surface, and left side. Thereafter it is 
reflected over the left lateral and left half of the anterior wall of the abdomen as far as the middle line.

The relations of the peritoneum to the duodenum, pancreas, and kidneys will be described when these viscera fall to be considered.

Omenta.-The great or gastro-colic omentum (epiploön) extends from the great curvature of the stomach and first inch of the first part of the duodenum to the transverse colon, descending in its course usually as low as the pelvic brim, and lower on the left side than on the right, which accounts for the greater frequency of an omental hernia on the left side. It covers the coils of the jejunum and ileum. At the great curvature of the stomach it contains between its two layers the right and left gastro-epiploic arteries, and the epiploic branches of these vessels, which are long and slender, descend into it. The great omentum is often of small size, thus leaving many of the coils of the small intestine uncovered, or it may even be displaced into the left hypochondrium. It is composed of four layers of peritoneum, two of which descend from the great curvature of the stomach, inseparably united, to the region of the pelvic brim, these being called the anterior or descending layers. The other two layers, also inseparably united, ascend from the region of the pelvic brim to the transverse colon, these being called the posterior or ascending layers. Between the two anterior and the two posterior layers there is usually, in healthy persons, a space which represents a part of the small cavity of the peritoneum, and is known as the bag or sac of the great omentum. In many cases, however, this space is scarcely demonstrable on account of adhesions. It is most conspicuous a little below the great curvature of the stomach.

The small or gastro-hepatic omentum extends from the small curvature of the stomach and first inch of the first part of the duodenum to the portal fissure of the liver, and also to the venosal fissure. It is composed of two layers of peritoneum which, at the small curvature of the stomach, contain between them the anterior and posterior branches of the gastric and pyloric arteries. For the most part its two layers are inseparably united, but at its right border, which is free, it contains between them the following structures: (I) the common bile-duct to the right side; (2) the hepatic artery, invested by the hepatic plexus of sympathetic nerves, to the left side; (3) the vena portæ, which lies between these two, and on a plane posterior to both; and (4) lymphatic vessels. This right or free border, with the foregoing contents, lies in front of the foramen of Winslow. The left border of the gastro-hepatic omentum is short on account of the oblique position of the stomach, and is attached to the diaphragm between the caval and œsophageal openings. The anterior layer of the small omentum is formed by peritoneum belonging to the great sac, and the posterior layer by that belonging to the small sac, these two layers becoming continuous with each other round the right or free border of the omental fold in front of the foramen of Winslow. 
The gastro-splenic omentum extends between the posterior surface of the cardiac end of the stomach and the gastric surface of the spleen just in front of the hilum. It is composed of two layers, anterior and posterior, which are continuous with the two anterior layers of the great omentum. The anterior layer is formed by peritoneum belonging to the great sac, and the posterior layer by that belonging to the small sac. The fold contains between its two layers the vasa brevia of the splenic artery.

Mesenteries.-The mesentery proper is the fold of peritoneum which attaches the jejunum and ileum to the vertebral column. Its vertebral border is called the root, and is comparatively short, measuring from 5 to 6 inches in length. Its line of attachment extends from the commencement of the jejunum on the left side of the body of the second lumbar vertebra, at the anterior border of the pancreas, to the termination of the ileum in the right iliac fossa, near the right sacro-iliac articulation. This line of attachment passes obliquely from left to right, and in this course the root of the mesentery proper crosses in succession the third part of the duodenum, aorta, inferior vena cava, and right psoas magnus. The other border of the mesentery proper is called the intestinal border, and is attached to the jejunum and ileum throughout their whole length. This border is of considerable length, and equals that of the jejunum and ileum. The widening of the mesentery proper takes place gradually, so that it is thrown into a number of folds, an arrangement which accounts for the coils of the jejunum and ileum. It: average breadth, from the root to the intestinal border, is about 8 inches. The fold is composed of two layers of peritoneum, right or superior, and left or inferior. The right or superior layer is continuous with the inferior layer of the transverse meso-colon, and with the peritoneum which covers the ascending colon, whilst the left or inferior layer is continuous with the peritoneum which covers the descending colon. Both layers are formed by peritoneum belonging to the great sac. The two layers contain between them the following structures: (I). the superior mesenteric vessels, and the rami intestini tenuis; (2) the superior mesenteric plexus of sympathetic nerves, and its secondary offshoots; (3) the lacteal vessels; and (4) the mesenteric lymphatic glands.

In some cases the mesentery proper presents one or more openings, known as mesenteric holes, which may be congenital or traumatic. If a portion of intestine slipped through one of these holes a mesenteric hernia would result, and, if the hole is situated in one layer only, the herniated portion of intestine would take up a position in the space between the two layers. These openings are liable to be met with in that portion of the mesentery proper which is attached to the lower part of the ileum, within the arch formed by the ileo-colic branch of the superior mesenteric artery and the last ramus intestini tenuis (Treves).

The appendicular mesentery or meso-appendix is a fold of 
peritoneum which is derived from the left or inferior layer of the mesentery proper near the terminal part of the ileum. It is triangular, and usually lies obliquely. Its right end reaches in a pointed manner to the ileo-cæcal junction, and its left end forms a concave free border which transmits the appendicular vessels and sympathetic plexus of nerves. It seldom extends for more than half or two-thirds along the appendix, which is thus rendered more or less convoluted or serpentine. It may, however, extend along its entire length. In some cases it lies vertically, and then it loses its hold upon the mesentery proper, its attachment being transferred to the cæcum, or right fascia iliaca, or even to the back of the ascending colon. The base of the appendix is sometimes destitute of a mesentery, in which cases that portion of it is closely connected to the posterior aspect of the cæecum. Occasionally the entire meso-appendix is awanting, and then the appendix is found adhering to the back of the cæcum. The meso-appendix may present a small opening through which a portion of bowel may pass and become strangulated. In very rare cases the meso-appendix is disposed in such a manner as to divide the ileo-cæcal fossa into an upper and a lower compartment.

The transverse meso-colon is a broad fold of peritoneum which extends between the transverse colon and the posterior abdominal wall at the anterior border of the pancreas. Its layers contain between them the middle colic vessels, sympathetic nerves, and the lymphatics of the transverse colon. Mesenteric holes may be present in the transverse meso-colon, under which circumstances a meso-colic hernia may occur.

The pelvic meso-colon is a fold of peritoneum which attaches the pelvic colon to the front of the sacrum. It extends from the inner border of the left psoas magnus (covered by the left fascia iliaca) near the left sacro-iliac articulation to the front of the third sacral vertebra, and its length is such as to render the pelvic colon freely movable. Its line of attachment posteriorly extends obliquely from left to right, and it is composed of two layers disposed laterally, and containing between them the superior hemorrhoidal vessels, sympathetic nerves, lymphatic vessels, and a certain amount of fat.

The ascending colon, descending colon, and iliac colon are each, in normal circumstances, devoid of a mesentery. Occasionally, however, an ascending meso-colon, a descending meso-colon, and an iliac meso-colon are present.

Peritoneal Ligaments-Ligaments of the Liver.-The peritoneal ligaments of the liver are four in number (the round ligament not being regarded as of a peritoneal nature), and they are as follows : the falciform ligament, the coronary ligament, the right lateral ligament, and the left lateral ligament.

The falciform ligarnent is also known as the ligamentum latum and suspensory ligament. It extends between the inferior surface of the diaphragm and the posterior surface of the anterior abdominal 
wall on the one hand and the superior and anterior surfaces of the liver on the other. Its base, which is free, extends from the umbilicus to the interlobar notch of the liver, and contains between its two layers the obliterated umbilical vein or round ligament. The line of attachment of the ligament to the anterior and superior surfaces of the liver map the organ out into a right and left lobe, and along this line the two layers of the ligament separate from each other, the right layer extending over the right lobe and the left qver the left lobe. Near the postero-superior border of the liver the two layers of the ligament diverge somewhat abruptly, and leave between them a small triangular area which is destitute of peritoneum. Thereafter they become continuous, on either side, with the superior layer of the coronary ligament.

The coronary ligament is also known as the posterior ligament. It is composed of two layers of peritoneum, superior and inferior, which are attached to the postero-superior and postero-inferior borders of the liver on the one hand, and the diaphragm on the other. These layers are separated from each other by an interval, which corresponds with the uncovered area of the right lobe of the liver. The superior layer is continuous with the falciform ligament, and the inferior layer is continuous with the peritoneum which covers the inferior vena cava and the fiont of the right kidney.

The right and left lateral or triangular ligaments are situated at the extreme right and left ends of the coronary ligament, and are formed by the meeting, at these points, of the two layers of that ligament.

The round ligament of the liver, though not a peritoneal ligament, may here be described. It is a fibrous cord formed by the obliterated umbilical vein, and is contained within the base of the falciform ligament between the umbilicus and the interlobar notch of the liver, its course between these points being upwards, backwards, and to the right. At the interlobar notch it enters the umbilical fissure on the inferior surface of the liver, and terminates by joining the left branch of the vena portæ.

A minute portion of the lumen of the left umbilical vein remains pervious within the round ligament of the liver. This pervious portion communicates at the portal fissure of the liver with the left division of the portal vein, and at the umbilicus it is connected with the epigastric veins of the anterior abdominal wall. It thus forms a channel of communication between the left division of the portal vein and the systemic veins of the anterior abdominal wall. Through this pervious portion of the left umbilical vein the blood can flow towards the umbilicus. The anastomosis thus established between the left umbilical vein and the epigastric veins of the anterior abdominal wall explains the enlargement of the veins of the anterior abdominal wall in cases of portal obstruction within the liver.

A small portion of the right primitive umbilical vein near the umbi'icus also remains pervious, and this portion likewise forms an anastomosis with the epigastric veins of the anterior abdominal wall.

On the surface of, or within, the round ligament of the liver there are a few very small veins, called parumbilical veins. These anastomose at the umbilicus with the epigastric veins of the anterior abdominal wall, and supcriorly they are connected with the left division of the portal vein. They are also concerned in the enlargement of the veins of the anterior abdominal wall in cases of portal obstruction within the liver. 
The ligament of the stomach is called the gastro-phrenic ligament. It is of small size, and extends between the region of the uncovered trigone of the stomach and the inferior surface of the diaphragm, lying immediately to the left of the lower end of the œsophagus.

The ligaments of the spleen are two in number, namely, phrenosplenic or lieno-phrenic, and lieno-renal.

The phreno-splenic or lieno-phrenic ligament is also called the suspensory ligament. It extends between the spleen, near its upper extremity, and the contiguous part of the diaphragm. The lienorenal ligament extends from the hilum of the spleen to the front of the left kidney at its upper and outer part. Its direction is backwards, and it is composed of two layers of peritoneum, right and left, which contain between them the splenic branches of the splenic artery. The right layer corresponds with the posterior layer of the gastro-splenic omentum, and the left with the anterior layer of that omentum.

The phreno-colic or costo-colic ligament (sustentaculum lienis) extends between the splenic flexure of the colon and the diaphragm opposite the tenth or eleventh left rib. It is triangular, and its surfaces are superior and inferior, its anterior border being free. It forms a platform upon which the basal surface of the spleen rests.

The sustentaculum hepatis is a fold of peritoneum which is sometimes met with in connection with the ascending colon. (Treves found it in eighteen out of one hundred bodies.) When present it extends from the right side of the ascending colon to the abdominal wall at a point a little above the level of the iliac crest. Its free border looks forwards, and it forms a shelf which supports the right margin of the liver.

Cavity of the Peritoneum.-The peritoneal cavity is divided into two compartments, great and small, which communicate with each other through the foramen of Winslow behind the right or free border of the gastro-hepatic omentum.

The great cavity or sac is the space which is exposed to view after opening the abdominal cavity. It is separated from the small sac by the liver, gastro-hepatic omentum, stomach, gastro-colic omentum, gastro-splenic omentum, lieno-renal ligament, and transverse meso-colon.

The small cavity or sac is an offshoot from the great sac, the introversion taking place at the foramen of Winslow. It extends upwards behind the stomach to the posterior part of the inferior surface of the diaphragm and the Spigelian lobe of the liver, and downwards into the gastro-colic omentum, the part of it within that omentum being known as the bag or sac of the great omentum.

Boundaries of the Small Sac-Anterior.-From below upwards, the two anterior or descending layers of the gastro-colic omentum, the posterior surface of the stomach, the gastro-hepatic omentum, and the posterior surface of the Spigelian lobe of the liver. Posterior. -From below upwards, the two posterior or ascending layers of the gastro-colic omentum, the transverse colon, the transverse mesocolon, and the ascending layer of the transverse meso-colon, 
Superior.-The posterior part of the inferior surface of the diaphragm. Inferior.-The bend of the gastro-colic omentum, where the two anterior or descending layers are folded backwards to become the two posterior or ascending layers. Left.-The spleen; the gastro-splenic omentum; the lieno-renal ligament; and the left border of the gastro-colic omentum.

In a great many cases that part of the small sac which is contained within the gastro-colic omentum is very limited in its downward extent, on account of adhesions having formed between the layers of the great omentum.

Foramen of Winslow.-This is the opening by which the great and small sacs of the peritoneum communicate with each other. It is situated behind the right or free border of the gastro-hepatic omentum, on a level with the body of the I2th thoracic vertebra. Its direction is forwards and to the right, and it admits of the passage of one finger, and in some cases of two fingers. It is, however, often blocked by inflammatory products.

Boundaries-Anterior.- The right or free border of the gastrohepatic omentum, containing between its two layers (I) the common bile-duct, to the right side; (2) the hepatic artery, invested by the hepatic plexus of sympathetic nerves, to the left side ; $(3)$ the vena portæ, which lies between these two, on a plane posterior to both; and (4) lymphatic vessels. Posterior.-The inferior vena cava covered by peritoneum. Superior.-The caudate lobe of the liver. Inferior.-The first part of the duodenum, and the hepatic artery in the first part of its course, as it curves forwards and upwards from the coeliac axis. When the foramen is blocked by inflammatory products, if fluid should be effused into the small sac the condition known as hydrops saccatus would result. It is possible for a loop of bowel to pass through the foramen, thus forming one variety of internal hernia.

Peritoneal Fossæ or Pouches.-The peritoneum presents in certain situations small pockets, which are known as peritoneal fossa or pouches. Their importance consists in the fact that a small portion of intestine may enter one or other of them and become strangulated, thus constituting an internal hernia, which, except in the cases of the inguinal pouches, is called a retro-peritoneal hernia. The fossæ, according to their situation, are called duodenal, duodeno-jejunal, peri-cæcal, and intersigmoid.

Duodenal Fossæ.- Four varieties of duodenal fossæ are met with in connection with the terminal part of the duodenum, namely, inferior duodenal, superior duodenal, para-duodenal, and retro-duodenal (Jonnesco).

The inferior duodenal fossa is the most common and largest. It is situated on the left side of the terminal part of the duodenum ind opens upwards. It is bounded in front by a thin triangular portion of -itoneum, called the inferior duodenal fold, which presents a free cresceniic border or base superiorly. The fossa may admit the thumb, and may be nearly an inch deep. It is said to be present in 75 per cent. of cases (Jonnesco).

The superior duodenal lossa is less constant, and of smaller size, than the inferior, and lies about an inch above it. It opens downwards, and its orifice faces that of the inferior duodenal fossa. It may admit the tip of a finger. It is bounded in front by a thin triangular portion of peritoneum, called the superior duodenal fold, which presents a free crescentic border or base inferiorly. The fossa is said to be present in 50 per cent. of cases (Jonnesco). 
The para-duodenal possa is situated a little to the left of the terminal part of the duodenum. It is bounded on the left side by a faint fold of peritoneum, called the inferior mesenteric fold, which is produced by the inferior mesenteric vein.

The retro-duodenal lossa is si uated behind the terminal part of the duodenum.

Duodeno-jejunal Fossa.--This fossa is situated on the left side of the duodeno-jejunal flexure, and leads upwards and towards the left side. It is bounded by two free portions of peritoneum, called the duodeno-mesocolic folds, and it has the pancreas above, the left kidncy on the left, and the aorta on the right. It is of small size, and may admit the tip of the little finger. It is said to be present in from 15 to 20 per cent. of cases.

Perl-Cæcal Fossæ.-These fossæ are three in number, namely, ileo-colic, ileo-cæcal, and retro-cæcal.

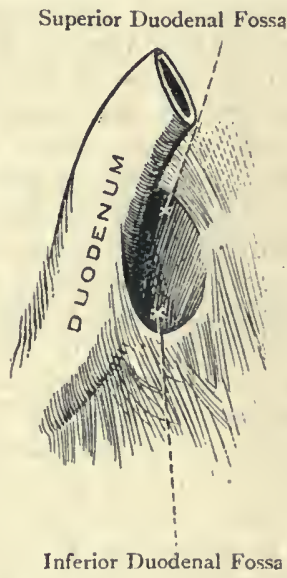

Fig. 307.-Duodenal Fossze.

The ileo-colic lossa is situated in the angle between the termination of the ileum and the commencement of the ascending colon, in front of the adjacent part of the mesentery proper. It opens inwards, and is bounded in front by a portion of the peritoneum, called the ileo-colic pold, which is produced by the anterior cæcal artery; behind, by the mesentery proper; below, by the ileum; and, on the right side, by the commencement of the ascending colon. In size and depth the fossa is small.

The ileo-cæeal Possa is situated in the angle of junction of the ileum and cæcum, and opens downwards and inwards. It may extend upviards for a variable distance behind the ascending colon, and sometimes is capable of admitting two fingers. It is bounded anteriorly and inferiorly by a portion of peritoneum, called the lleo-cæcal fold (bloodless fold of Treves); posteviorly. by the meso-appendix; externally, by the cxcum; and superiorly, by the posterior aspect of the terminal part of the ileum and the inferior layer of the mesentery proper. The importance of this fossa consists in the fact that it often contains the vermiform appendix, or a portion of it.

The retro-cæcal lossa is situated behind the cacum, on the outer side of the meso-appendix. It may extend upwards for a variable distance behind the ascending colon, and is sometimes divided into an external and internal compartment. It occasionally contains the vermiform appendix, or a portion of it.

Intersigmoid Fossa. - This fossa is of rare occurrence in the adult, but is frequently present in early infancy. It is situated on the left side of the polvic meso-colon near the bifurcation of the left common iliac artery, and 


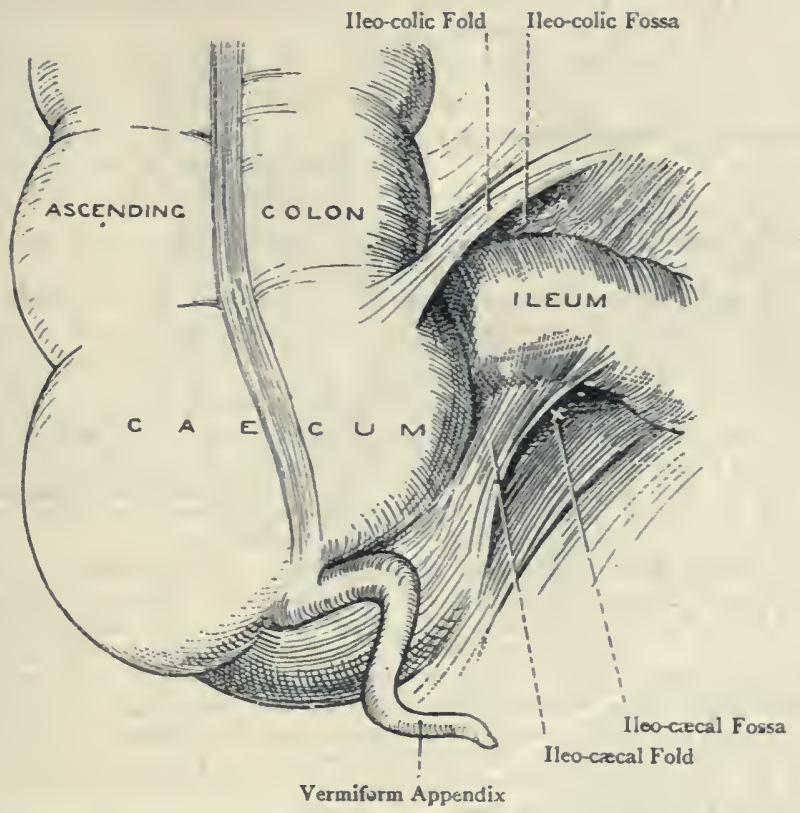

Fig. 308. - The Ileo-colic and Ileo-cacal Foss.x.

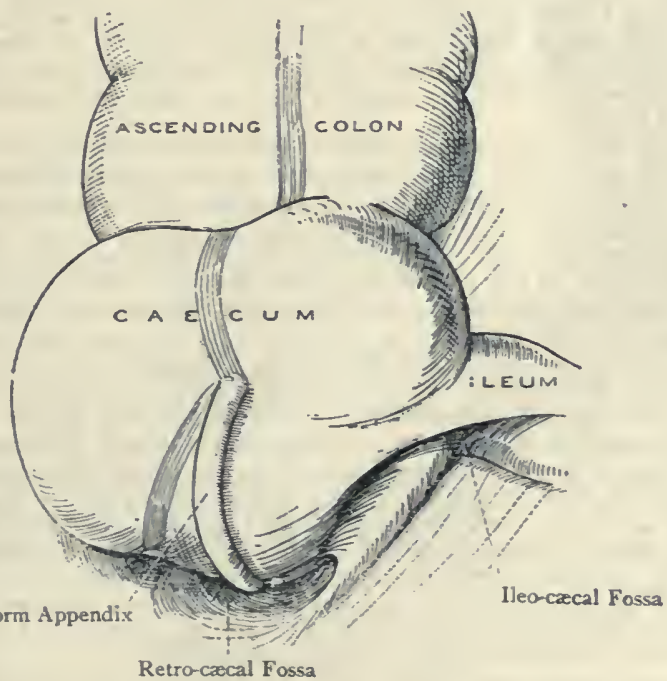

Fig. 309.-The Retro-cecal and Ileo-cecal Fosse. 
is produced by a fold of peritoneum which contains the sigmoid artery. It opens downwards and towards the left side.

In early life the alimentary tube is very short and straight. Its ventral and dorsal aspects are attached to the ventral and dorsal body-walls by mesodermic folds, which constitute the primitive ventral and dorsal mesenteries.

The primitive ventral mesentery extends from the pharynx to the duodenum. Its cephalic or cardiac portion gives rise to the ventral and dorsal cardiac mesenteries or mesocardia. Its caudal or posterior part forms the ventral or anterior gastro-duodenal mesentery.

The primitive dorsal mesentery connects the dorsal aspect of the entire primitive alimentary tube to the dorsal body-wall.

The ventral gastro-duodenal mesentery extends from the ventral aspect of the primitive stomach and upper part of the duodenum to the ventral bodywall on the cephalic side of the umbilicus. It is closely associated with the lower layer of the septum transversum, within which the liver undergoes development. As the liver descends, it carries with it the ventral gastroduodenal mesentery, which it divides in to two parts. The part between the liver and (1) the ventral portion of the diaphragm, and (2) the ventral wall of the abdomen as low as the umbilicus, forms the falciform ligament of the liver; and the part between the liver (portal fissure) and stomach (small curvature) forms the small or gastro-hepatic omentum.

The primitive dorsal mesentery receives names corresponding to the parts of the alimentary tube with which it is connected. Thus, in the abdomen, there are the mesogastrium, mesoduodenum, mesojejunum, meso-ileum, mesoappendix, mesocolon (ascending, descending, iliac and pelvic), and meso-rectum.

As development proceeds, these mesenteric folds undergo important changes.

Mesogastrium. - This is the primitive posterior mesentery of the stomach, and, as stated, is a portion of the primitive dorsal mesentery. It consists of two layers of peritoneum, which extend between the great curvature of the stomach (at this period directed backwards) and the dorsal body-wall, where the two layers enclose the pancreas.

As the stomach changes its position, the pyloric end being tilted forwards, and the whole organ along with the duodenum turning round upon its right side, the great curvature, which originally looked backwards, is now directed downwards and towards the left side. During this change the mesogastrium becomes stretched. Thereafter a gradual extension of the mesogastrium takes place in a downward divection from the great curvature of the stomach over the transverse colon, jejunum, and ileum; and in this man wer the great omentum is formed.

At first the two posterior or ascending layers of the great omentum pass over the front and upper part of the trans verse colon without separating to enclose it, and then pass backwards to the posterior abdominal wall, where they enclose the pancreas. The lower layer is now reflected ventralwards from the posterior abdominal wall until it meets with the transverse colon. Having invested this part of the gut, it retraces its steps to the posterior abdominal wall, thus forming the two layers which constitute the primitive transverse meso-colon. On referring to Fig. 3 Io, it will be seen that, in this region, there are four layers of peritoneum at this stage. The upper two layers are continuous with the two posterior or ascending layers of the great omentum, and represent the original mesogastrium. The lower two layers belong to the primitive transverse mesocolon. Subsequently the lower of the upper two layers and the upper of the lower two layers unite and disappear. There are thus left only two layers of peritoneum, which constitutc the transverse mesocolon of the adult, the lower layer of which is part of the primitive transverse mesocolon, whilst the upper layer is part of the great omentum. In fact, botlı layers are ultimately derived from the two posterior or ascending layers of the grcat omentum. As the result of these changes, the pancreas comes eventually to lie behind the peritoncum, whercas it was originally contained between the two layers of the mesogastrium.

The remaining portions of the primitive dorsal mesentery are disposed as follows: 
The mesoduodenum disappears.

The mesojejunum and meso-ileum persist and represent the mesentery proper.

The meso-appendix persists as the appendicular mesentery.

The ascending and descending mesocolon and the iliac mesocolon, as a rule, disappear.

The pelvic mesocolon

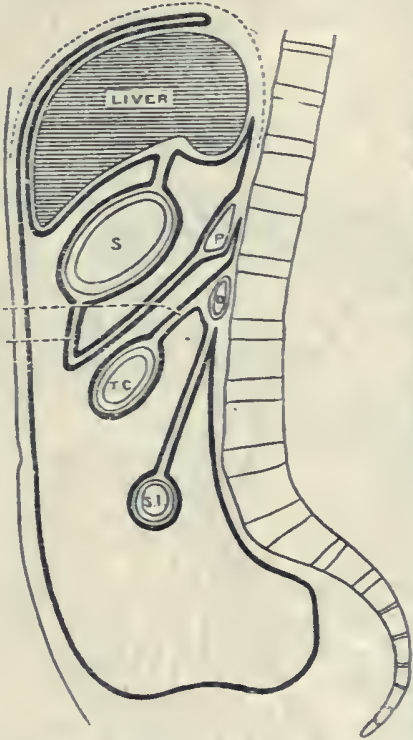
persists and the mesorectum disappears.

Structure of the Peritoneum.- The peritoneum is a typical serous membrane like the pleura, the serous portion of the pericardium, and the tunica vaginalis. Briefly stated, it consists of a homogeneous conriective-tissue basement membrane, containing elastic tissue, and lined with endothelium. Its free surface presents many stomata (especially over the diaphragm), which communicate with the cavity of the peritoneum and with the lymph-canalicular system.

Devel opment.-The parietal peritoneum is developed from the somatic mesoderm of the somatopleure of the body-wall. The visceral peritoneum is Fig. 3IO.-Diagram OF the PERITONEUM IN developed from the Early Life (Vertical Section).

splanchnic mesoderm of the splanchnopleure of the

S. Stomach; P. Pancreas; D. Duodenum; primitiv? intestinal tube. T.C. Transverse Colon; S.I. Small Intestine.

Blood-supply of the Intestinal Canal.-The intestinal canal receives its blood-supply from the superior and inferior mesenteric arteries, with the exception of the upper portion of the duodenum, and a portion of the rectum.

Superior Mesenteric Artery.-This vessel springs from the front of the abdominal aorta about $\frac{1}{4}$ inch below the cœliac axis. It is at first directed downwards behind the body of the pancreas and the splenic vein. It then passes downwards and forwards in front of the uncinate process of the pancreas and third part of the duodenum, at the lower border of which latter it takes up its position between the two layers of the mesentery proper. The vessel then passes downwards near the root of the mesentery, its course being slightly curved with the convexity towards the left

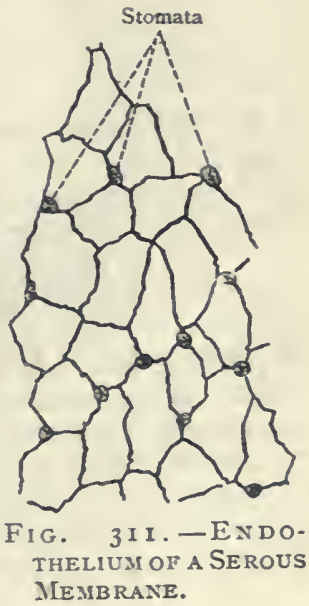


side, and it terminates near the ileo-cæcal junction in the last ramus intestini tenuis, which anastomoses with the ileal branch of the ileo-colic artery. The vessel is surrounded by a tough sheath formed by the superior mesenteric sympathetic plexus.

Branches-Left Branches.-These are called the rami intestini tenuis, and are at least twelve in number. They pass downwards

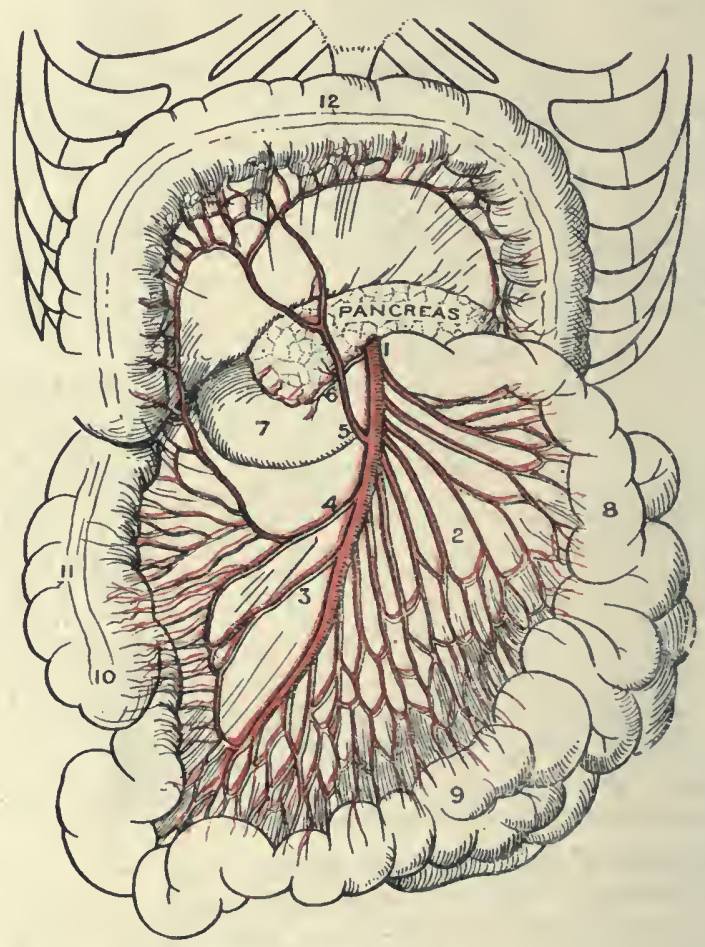

Fig. 3I2.-The SUperior Mesenteric Artery and its Branches (AFTER TiedemanN).

1. Superior Mesenteric Artery

2. Rami Intestini Tenuis

3. Ileo-colic

4. Right Colic

5. Middle Colic

6. Inferior Pancreatico-duodenal
7. Duodenum
8. Jejunum
9. Ileum
10. Crecum
11. Ascending Colon
12. Transverse Colon

and to the left between the two layers of the mesentery proper, and supply the jejunum and ileum. After a course of about 2 inches each divides into two branches, which by their junction with contiguous branches give rise to primary arches. From the convexities of these arches small branches are given off, which act in a similar manner, and give rise to secondary arches. This disposition of arteries goes on so as to form tertiary, quaternary, and even 
quinary arches. The minute vessels arising from the arches of the last tier enter the wall of the jejunum and ileum along the mesenteric border, where each divides into two branches, which encircle the bowel beneath its serous covering. From the rings thus formed branches penetrate deeply to reach the mucous coat. Each ramus intestini tenuis, as well as its various branches, conducts to the bowel an offshoot of the superior mesenteric sympathetic plexus.

Right Branches-Ileo-colic Artery.-This vessel is the lowest of the right branches, and in many cases it arises in common with the right colic. Its course is downwards and outwards towards the right iliac fossa behind the peritoneum, and it divides into two branches, colic and ileo-cæcal. The colic branch passes upwards and forms an arch with the descending branch of the right colic, from which branches proceed to the lower part of the ascending colon. The ileo-crecal branch passes to the upper part of the ileo-creal junction, where it furnishes the following branches: ileal, to the terminal part of the ileum, where it anastomoses with the last ramus intestini tenuis; appendicular, which, descending behind the terminal part of the ileum, passes between the two layers of the meso-appendix, and so reaches the vermiform appendix; anterior cacal, to the front of the crecum; and posterior cacal, to its posterior aspect.

Right Colic Artery.-This is the second branch in order from below upwards, and in many cases it arises in common with the ileo-colic. Its course is transversely to the right behind the peritoneum, and it divides into two branches, descending and ascending. The descending branch anastomoses with the colic branch of the ileo-colic, and the ascending brancle with the right branch of the middle colic. The arches thus formed furnish branches to the ascending colon, which in their course form secondary and tertiary arches.

Middle Colic Artery.-This vessel arises from the right side and front of the main trunk about 2 inches above the right colic, on a level with the lower border of the third part of the duodenum. Its course is forwards between the two layers of the transverse mesocolon, and it divides into a short right and a long left branch. The right branch anastomoses with the ascending branch of the right colic, and the left branch with the ascending branch of the left colic from the inferior mesenteric. The arches thus formed furnish branches to the middle colon, which in their course form secondary and tertiary arches.

Inferior Pancreatico-duodenal Artery.-This small vessel usually arises from the right side of the main trunk opposite the upper border of the third part of the duodenum, but it may spring from the first ramus intestini tenuis. Its course is to the right behind the superior mesenteric vein, and between the head of the pancreas and the third part of the duodenum. Thereafter it ascends for a little between the head of the pancreas and the second part of the duodenum, and anastomoses with the superior pancreaticoduodenal of the gastro-duodenal from the hepatic. It supplies the 
adjacent portions of the pancreas and duodenum. The vessel is accompanied by an offshoot from the superior mesenteric sympathetic plexus, and, when it arises from the first ramus intestini tenuis, it passes behind the superior mesenteric artery.

Superior Mesenteric Vein.-This vein is formed by tributaries which return the blood from the parts of the intestinal canal supplied by the superior mesenteric artery, and it receives in addition the right gastro-epiploic vein. It ascends on the right side of the superior mesenteric artery. After leaving the mesentery it passes over the third part of the duodenum and uncinate process of the pancreas, and finally behind the neck of the latter organ, where it joins the splenic vein to form the vena portæ. The vessel and its tributaries are destitute of valves, so that the blood can regurgitate in cases of portal obstruction.

Superior Mesenteric Sympathetic Plexus.-This plexus is derived from the solar plexus. It closely surrounds the superior mesenteric

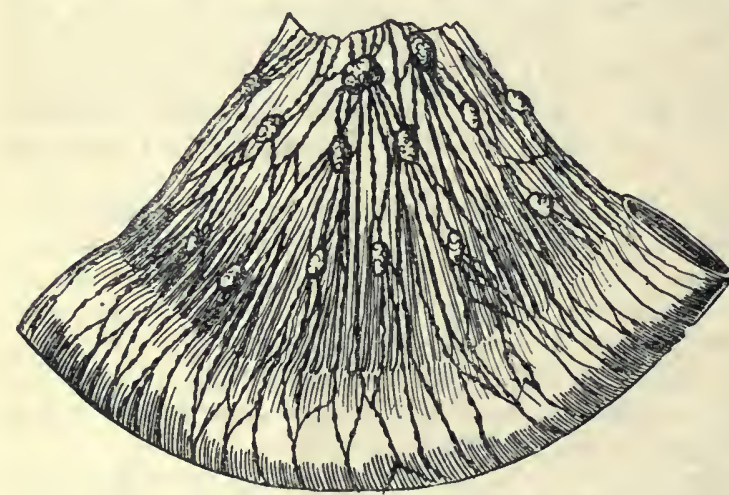

Fig. 3r 3.-Portion of Jejunum with its Mesentery, Showing Lacteal Vessels and Mesenteric Glands. artery in the form of a tough sheath, and it furnishes offshoots which accompany all the branches of that vessel.

$\mathrm{L} \mathrm{y} \mathrm{m} \mathrm{phatic}$ Vessels of Small Intestine.-These which are called lacteals, originate in the villi of the mucous membrane of the small intestine (see p. 790). They leave the wall of the bowel at the mesenteric border, those of the jejunum exceeding in number those of the ileum. Within the mesentery they take a course inwards and upwards, becoming, in succession, the afferent and efferent vessels of the groups of mesenteric glands. At the root of the superior mesenteric artery the lacteals, which have now emerged from the innermost mesenteric glands, terminate in from one to four lymphatic trunks, which open into the receptaculum chyli.

Superior Mesenteric Glands.-These are about I5O in number, and are situated within the mesentery proper and along the course of the trunk of the superior mesenteric artery. In health their average size is about that of a small pea, except along the course of the main artery, where they are somewhat larger, and they are more numerous in the jejunal than in the ileal mesentery. They receive the lacteals from the lower part of the duodenum, the jejunum, and the ileum, and also the lymphatics from the ascending and transverse colon. There are no glands in the portion of mesentery close to the intestine 
for about 2 inches. In the ileo-colic angle there is a special group of glands, called the ileo-colic glands. These receive afferent vessels from the terminal part of the ileum, the cacum, and the vermiform appendix, and their efferent vessels pass to the innermost group of superior mesenteric glands.

Lymphatic Vessels of Ascending and Transverse Colon.-The lymphatics of the ascending colon terminate in two ways, as follows: those from the lower part pass to the innermost group of superior $\mathrm{m}$ es e $\mathrm{nt}$ eric glands, whilst those from the upper part go to the mesocolic glands. The lymphatics of the transverse colon become afferent vessels of the meso-colic glands, the efferent vessels of which join the terminal intestinal lymphatic trunks from the superior mesenteric glands.

\section{Inferior Mes-} enteric Artery.This vessel arises from the front of the abdominal aorta towards its left side, about $I_{2} \frac{1}{2}$ inches above the bifurcation. Its course is downwards and to the left towards the left iliac fossa. It is

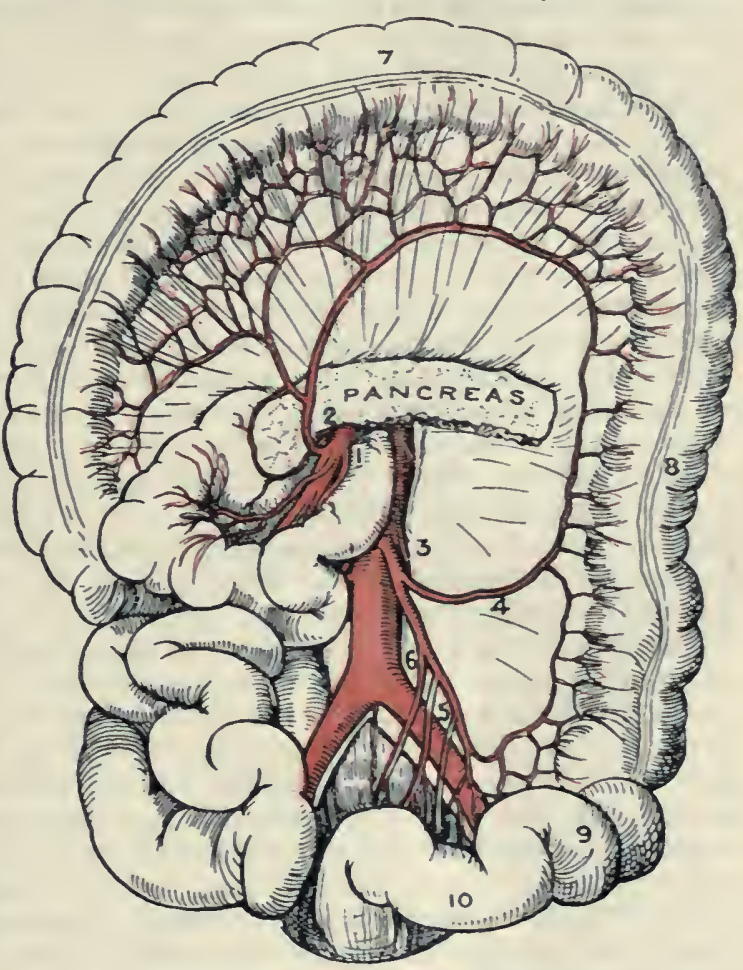

Fig. 3I 4.-The Ivferior Mesenteric Artery AND its BRANCHES (AFter TIEDEMANN).
6. Superior Hemorrhoidal

7. Transverse Colon

8. Descending Colon

g. 1liac Colon

ro. Pelvic Colon

be'int the peritoneum, and lies first upon the aorta, and then on its left side, where it is supported by the psoas magnus. Subsequently it is continued as the superior hemorrhoidal artery over the left common iliac vessels. The artery is surrounded by the inferior mesenteric sympathetic plexus.

Branches-Left Colic Artery.-This vessel passes transversely to the left, behind the peritoneum and over the lower part of th: 
left kidney, and it divides into two branches, ascending and descending. The ascending branch anastomoses with the left branch of the middle colic, and the descending branch with the ascending branch of the superior sigmoid artery. The arches thus formed supply branches to the left extremity of the transverse colon and the descending colon, which in their course form secondary and tertiary arches.

Sigmoid Arteries.-These are usually three in number, superior, middle, and inferior, but they frequently arise as a single trunk. They pass downwards and to the left over the psoas magnus, and supply the lower part of the descending colon, the iliac colon, and the pelvic colon. The superior sigmoid artery, which lies, as a rule, behind the peritoneum, divides into two branches, ascending and descending. The ascending branch forms an arch with the descending branch of the left colic, and the descending branch passes between the two layers of the pelvic meso-colon, where it anastomoses with the middle sigmoid artery. The middle and inferior sigmoid arteries pass between the two layers of the pelvic meso-colon, where they form arches with the descending branch of the superior sigmoid, with one another, and with the superior hemorrhoidal artery. The branches of these arches form secondary, or even tertiary, arches before the terminal branches are given off.

The superior hemorrhoidal artery is the continuation of the inferior mesenteric, and will be found described on p. 875 .

Inferior Mesenteric Vein.-This vein is formed by tributaries which return the blood from the parts of the large intestine supplied by the inferior mesenteric artery. It lies at first near the left side of its artery, but soon leaves it and ascends on the left psoas magnus, where it lies on the left side of the aorta behind the peritoneum. In this course it crosses the left spermatic artery and left renal vein. It finally passes behind the pancreas with an inclination to the right, and usually terminates in the splenic vein. It may, however, open into the angle of junction of the splenic and superior mesenteric veins, or into the superior mesenteric vein, near its termination. The inferior mesenteric vein and its tributaries are destitute of valves, so that the blood can regurgitate in cases of portal obstruction.

Inferior Mesenteric Sympathetic Plexus.-This plexus is derived from the left half of the aortic plexus. It forms a tough sheath round the artery, and furnishes offshoots with its branches.

Inferior Mesenteric Glands.--These glands are situated around the root, and along the trunk and branches, of the inferior mesenteric artery. Those around the root of the vessel constitute the inferior mesentcric group of the pre-aortic glands.

Their afferent vessels are derived from (I) the lower part of the descending colon, (2) the iliac colon, (3) the pelvic colon, and (4) some of the lymphatics of the rectum.

Their efferent vessels pass to the inferior mesenteric group of preaortic glands. 
Lymphatic Vessels of Descending, Iliac, and Pelvi Colon.-The lymphatics of the descending colon terminate in two ways, as follows: those of the upper part pass to the meso-colic glands, whilst those of the lower part join the left lymphatic trunk from the median lumbar glands. The lymphatics of the iliac and pelvic colon also join the left lymphatic trunk from the median lumbar glands.

Lymphatic Glands of Large Intestine (colic glands). - These glands are arranged in groups, named according to the portion of intestine to which they are related, and they are situated behind the respective parts, except those belonging to the transverse colon which lie between the two layers of the transverse meso-colon, and are known as the meso-colic glands.

Position and Connections of the Duodenum.-The duodenum is the first part of the small intestine. It measures from Io to II inches in length, and is the widest and least movable part. It extends from the pylorus to the left side of the body of the second lumbar vertebra, where it ends in the jejunum. It describes a somewhat $\mathbf{U}$-shaped curve with the concavity directed upwards and to the left in close adaptation to the head of the pancreas. It is devoid of a mesentery, and is divided into three parts, first, second, and third.

First or Superior Part.- The first part extends from the pylorus to the right side of the neck of the gall-bladder. It lies in the epigastric region, and is about 2 inches in length, its direction being upwards, backwards, and to the right when the stomach is empty, but directly backwards when that organ is distended. The gastrohepatic omentum furnishes a complete covering to about the first inch, and the remainder is covered by peritoneum only in front. The first part is therefore comparatively movable.

Relations-Superior and Anterior.-The quadrate lobe of the liver and the gall-bladder. Poslerior.-The vena portæ, gastroduodenal artery, common bile-duct, and neck of the pancreas. Inferior. - The head of the pancreas. The first part lies below the foramen of Winslow.

Second or Descending Part.-This part extends from the right side of the neck of the gall-bladder to the right side of the body of the third (sometimes fourth) lumbar vertebra. It lies at first in the epigastric, and subsequently in the umbilical, region; its length is from 3 to 4 inches, and its direction is almost vertically downwards behind the right extremity of the transverse colon. The anterior surface is covered by peritoneum, except opposite the transverse colon. If there is no transverse meso-colon at this point, there is a distinct strip near the centre left uncovered and connected to the colon by areolar tissue. If, however, there is a transverse meso-colon present at this point, the bare area is trifling. The posterior surface is destitute of peritoneum. The second part is therefore very immovable.

Relations-Anterior.-From above downwards the gall-bladder near its neck, the right extremity of the transverse colon, and some coils of the small intestine. Posterior.-The anterior surface of the right kidney near the hilum, and the inferior vena cava. Right.- 
The hepatic flexure of the colon, and the right lobe of the liver. Left.-The head of the pancreas, with the common bile-duct behind, and the superior pancreatico-duodenal artery in front. The common bile-duct and pancreatic duct enter the wall of this part at the junction of the inner and posterior aspects, a little below the centre.

Third or Inferior Part.-This part extends from the right side of the body of the third (sometimes fourth) lumbar vertebra to the left side of the body of the second on a level with its upper border. At this point it makes a sharp bend forwards, and terminates in the jejunum, thus forming the duodeno-jejunal flexure. It lies at first in the umbilical, and subsequently in the epigastric, region; its length is about 5 inches, and its direction is at first obliquely to the left and upwards, and afterwards vertically upwards. Its anterior surface is covered by peritoneum derived from the descenciing layer of the transverse meso-colon, except where it has the superior mesenteric vessels in front of it. There is no peritoneum behind it, and consequently it is fixed in position.

Relations-Anterior. - The superior mesenteric vessels and the upper part of the root of the mesentery, with portions of the small intestine on either side of these. Posterior.-The inferior vena cava, aorta (below the origin of the superior mesenteric artery), left renal vein, left psoas magnus, and left crus of the diaphragm. Superior.The lower part of the head of the pancreas (including its uncinate process), and the inferior pancreatico-duodenal artery.

The terminal portion of the third part is sometimes spoken of as the fourth or ascending part of Treves. It is covered by peritoneum on the left side, as well as in front, and in cases where a retro-duodenal fossa is present it is partially covered by peritoneum behind, towards the left side. The duodenal fossæ of the peritoneum are met with on the left side of this portion. The duodeno-jejunal flexure is suspended from the left crus of the diaphragm by a fibro-muscular bundle, called the musculus suspensorius duodeni.

$\bar{B}$ lood and Nerve Supply of the Duodenum. - The arteries of the duodenum are as follows: (I) The pyloric branch of the hepatic; (2) the superior pancreatico-duodenal branch of the gastro-duodenal of the hepatic; and (3) the inferior pancreatico-duodenal branch of the superior mesenteric.

The veins terminate in the superior mesenteric and splenic veins.

The nerves are derived from the hepatic and superior mesenteric sympathetic plexuses.

The lymphatic vessels of the first part of the duodenum pass to the retro-pyloric glands, and those of the second and third parts pass to the pre-pancreatico-duodenal and retro-pancreatico-duodenal glands.

Position and Connections of the Pancreas.-The pancreas is a long narrow gland which is situated behind the stomach, on a level with the first and second lumbar vertebræ. Its right extremity occupies the duodenal curve, and its somewhat pointed left ex- 
Posterior.-The inferior vena cava, right crus of the diaphragm, aorta, and left renal vein. Superior.-The first part of the duodenum. Inferior. - The third part of the duodenum, and the inferior pancreatico-duodenal artery. Right. - The second part of the duodenum, with the common bile-duct behind, as low as a little below the centre, and the superior pancreatico-duodenal artery in front.

The neck may be defined as the part in front of the origin of the vena portæ and the termination of the superior mesenteric vein. It springs from the anterior surface of the head near its upper part, and is about I inch in length. Its direction is upwards and to the left, and it forms the connecting link between the head and body. The gastro-duodenal and superior pancreatico-duodenal arteries occupy a groove on its right side, and the commencement of the first part of the duodenum is in front of it, whilst the origin of the vena portæ and the termination of the superior mesenteric vein are behind it.

The body passes to the left, with a slight inclination backwards after it has crossed the aorta. It is triangular, and presents three surfaces-antero-superior, posterior, and inferior, and three borders -superior, anterior, and posterior.

The antero-superior surface, which is covered by peritoneum, is in relation with the posterior surface of the stomach. At its right extremity, just below the cœliac axis, it presents a prominence, called the omental tuberosity from its relation to the gastro-hepatic omentum. The posterior surface, which is destitute of peritoneum, is related to the following structures : the aorta below the cœliac axis, with a portion of the solar plexus; the origin of the superior mesenteric artery; the left suprarenal capsule; and the left kidney with its vessels. The splenic vein passes from left to right in contact with this surface near the superior border. The inferior surface, which is covered by peritoneum, is moulded on the duodeno-jejunal flexure, some coils of the jejunum, and the left extremity of the transverse colon. The cœliac axis projects forwards over the superior border above the omental tuberosity. To the left of this axis the splenic artery pursues its tortuous course to the spleen, and to the right of it the hepatic artery lies for a short distance. The transverse meso- $\bullet$ colon is attached to the anterior border along which its separation into ascending and-descending layers takes place, the former covering the antero-superior surface of the organ, and the latter, on its way backwards, investing the inferior surface. The posterior border presents nothing noteworthy.

The tail corresponds with the left extremity where the pancreas is narrowest, and is in contact with the lower end of the gastric. surface of the spleen belind the hilum.

For the structure and development of the pancreas, see pp. 809, 812 .

Solar or Epigastric Plexus of the Sympathetic System.-The solar plexus is of large size, and is situated deeply in the epigastric region, behind the stomach and in front of the crura of the diaphragm and the aorta close to the origins of the cœliac axis and 


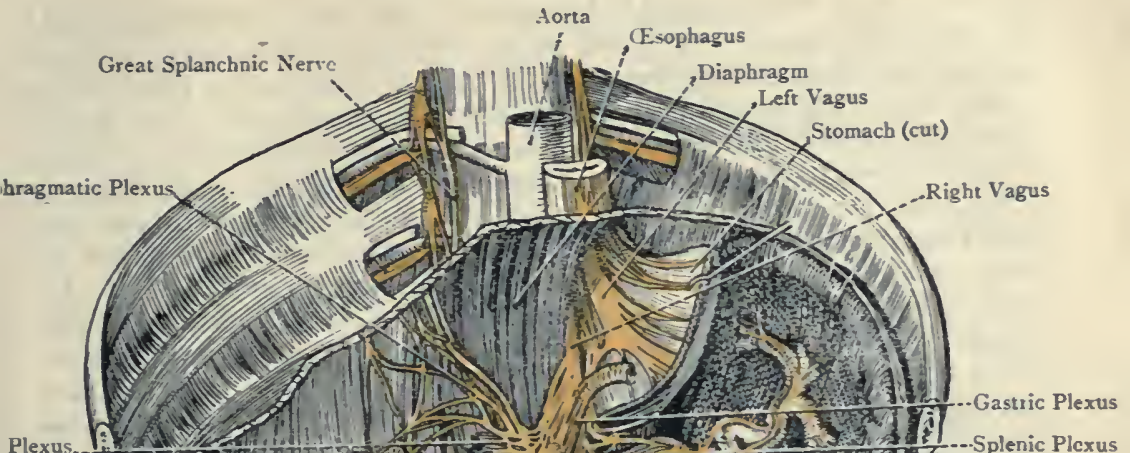

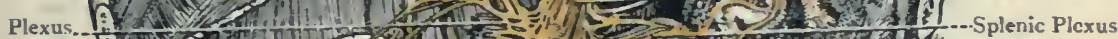

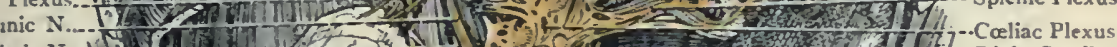

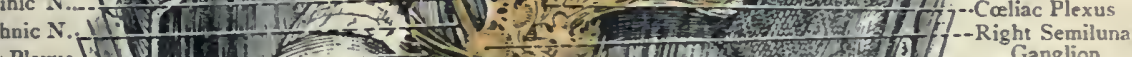

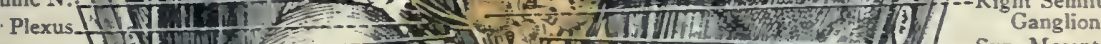

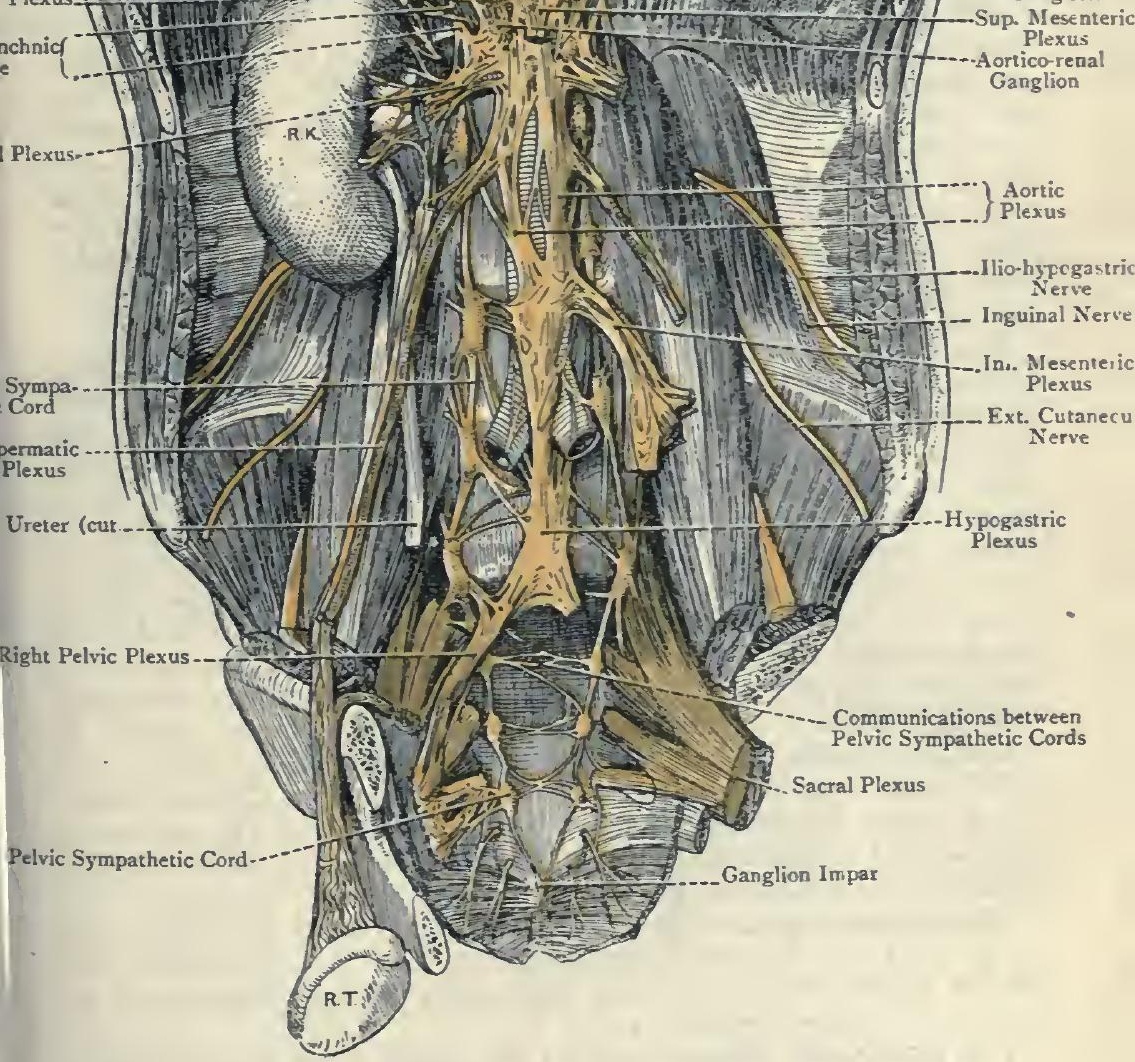

Fig. 316. -The Sympathetic System in the Abdomen and Pezvis (Hirschfeld axd Leveillé).

R.K. Right Kidney; R.T. Right Testis. 
superior mesenteric artery. It extends from one suprarenal capsule to the other, and is composed of nerve-fibres and ganglia, with an admixture of adipose tissue. The plexus receives its chief fibres from the great and small splanchnic nerves of each side, which contain a large number of spinal fibres. The great splanchnic nerve is formed by roots derived usually from the fifth to the ninth thoracic sympathetic ganglia inclusive, and it enters the abdomen by piercing the crus of the diaphragm. The small splanchnic nerve arises by two roots from the tenth and eleventh thoracic ganglia, and it also enters the abdomen by piercing the crus of the diaphragm. The plexus also receives fibres from the right pneumogastric nerve. Two of the ganglia of the solar plexus are of large size, and are situated one at either lateral pole. They are called the semilunar ganglia, right and left. Each lies over the corresponding crus of the diaphragm close to the suprarenal body, that of the right side being under cover of the inferior vena cava, and each receives at its upper part the great splanchnic nerve. The lower part of each ganglion is more or less detached, and is known as the aortico-renal ganglion, which lies over the root of the renal artery, and in which the small splanchnic nerve terminates. From each semilunar ganglion branches proceed in a radiating manner upwards, outwards, downwards, and inwards. The inner group of fibres extend from one ganglion to the other, embracing the coeliac axis as they cross the aorta, and forming the cœliac plexus, which receives fibres from the right pneumogastric nerve, and contains numerous small ganglia.

The cœliac plexus furnishes three secondary plexuses-gastric, splenic, and hepatic. The gastric plexus accompanies the corresponding artery to the small curvature of the stomach, and supplies branches to the adjacent portions of the anterior and posterior surfaces of that organ. The splenic plexus goes with the splenic artery, and receives branches from the right pneumogastric nerve. It is distributed, with the branches of the artery, to the pancreas, cardiac extremity of the stomach, left half of its great curvature and adjacent portions of its surfaces, and the spleen. The hepatic plexus accompanies the artery of that name, and receives branches from the left pneumogastric nerve. Its distribution corresponds with that of the artery, and its offshoots are as follows : pyloric to the small curvature of the stomach; gastro-duodenal, dividing into right gastro-epiploic to the great curvature of the stomach, and superior pancreatico-duodenal to the head of the pancreas, and the first and second parts of the duodenum; cystic to the gallbladder ; and hepatic to the liver.

The diaphragmatic or phrenic plexus receives its fibres from the upper part of the semilunar ganglion, and it accompanies the inferior phrenic artery to the diaphragm, giving branches in its course to the suprarenal plexus.

The suprarenal plexus receives its fibres from the semilunar ganglion and coliac plexus. It contains small ganglia, and is 


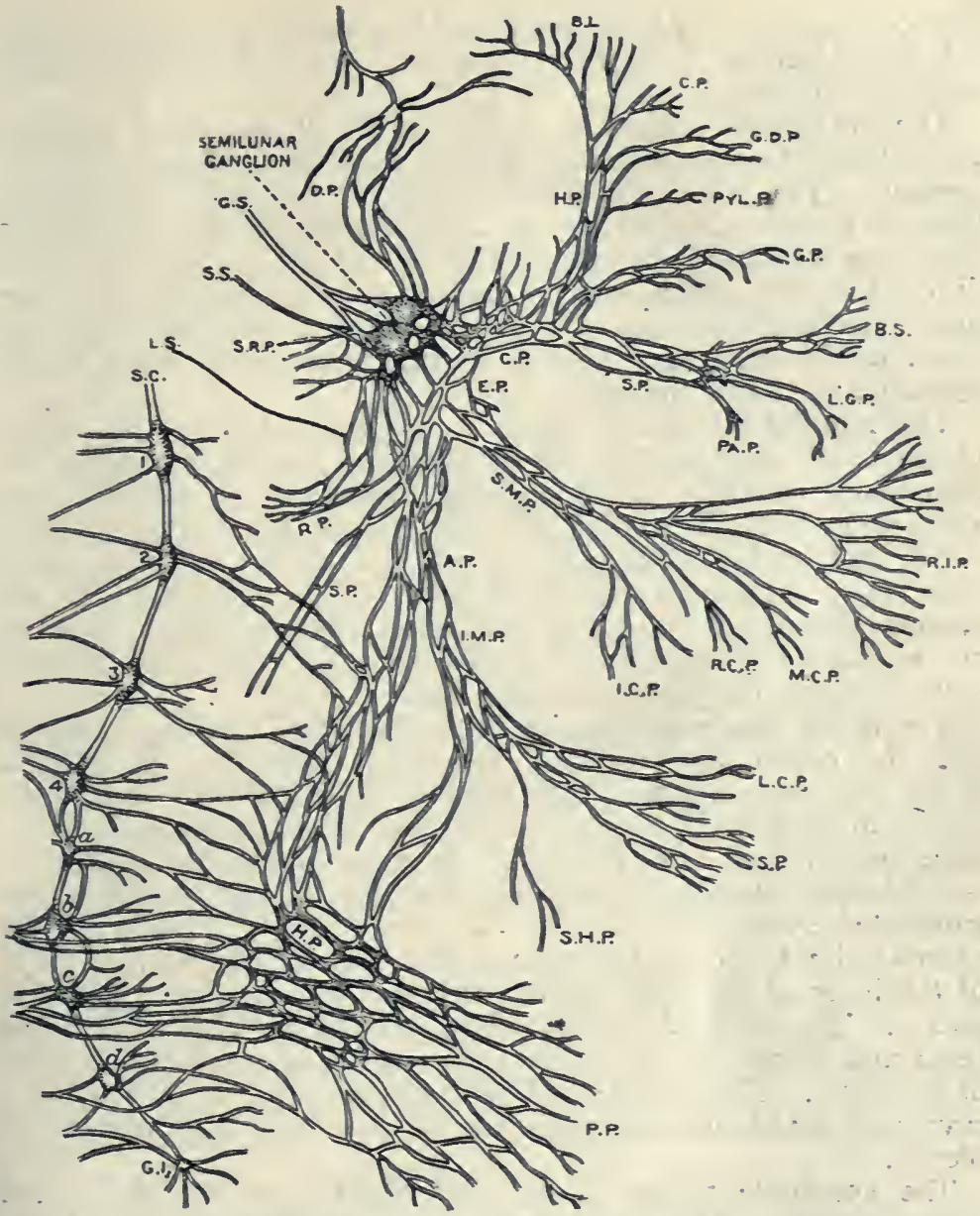

Fig. 317.-Scheme of the Sympathetic Nerve in the Abdomen and Pelvis (Flower).

S.C. Sympathetic Cord $\mathbf{x}, 2,3,4$. Lumbar Ganglia $a, b, c, d$. Pelvic Ganglia

G.I. Ganglion Impar

G.S. Great Splanchnic

S.S. Small Splanchnic

L.S. Least Splanchnic

D.P. Diaphragmatic Plexus

S.R.P. Suprarenal Plexus

R.P. Renal Plexus

S.P. Spermatic Plexus

A.P. Aortic Plexus

I.M.P. Inferior Mesenteric Plexus

L.C. P. I Jeft Colic Plexus

S.P. Sigmoid Plexus

S.H.P. Superior Hemorrhoidal Plexus

H.P. Hypogastric Plexus
P.P. Pelvic Plexus

E.P. Epigastric Plexus

C.P. Coeliac Plexus

S.P. Splenic Plexus

Pa.P. Pancreatic Plexus

L.G.P. Left Gastro-epiploic Plexus

B.S. Branches to Spleen

G.P. Gastric Plexus

H.P. Hepatic Plexus

Pyl.P. Pyloric Plexus

G.D.P. Gastro-duodenal Plexus

C.P. Cystic Plexus

b. L. Branches to Liver

S.M.P. Superior Mesenteric Plexus

1.C.P. Ilev-colic Plexus

R.C.P. Right Colic Plexus

M.C. P. Midulle Colic Plexus

R.I.P. Rami Intestini Tenuis Plexuses 
joined from above by branches from the diaphragmatic plexus, and below by branches from the renal plexus. It is distributed to the suprarenal capsule.

The renal plexus derives its fibres from the aortico-renal ganglion, the coliac and aortic plexuses, and the least splanchnic nerve when present. (The least splanchnic nerve arises from the twelfth thoracic ganglion, and enters the abdomen behind the ligamentum arcuatum internum, or through the crus of the diaphragm). The fibres of the renal plexus, which contain ganglia here and there, are distributed with the renal artery to the kidney, branches being also given to the suprarenal plexus, spermatic plexus (ovarian in the female), and to the ureter.

The superior mesenteric plexus is a continuation of the cœliac plexus, and also receives fibres from the semilunar ganglia. It contains a ganglion, called superior mesenteric, in contact with the origin of the artery of that name, and it accompanies that vessel and its branches to be distributed to the intestinal canal from (and including) the third part of the duodenum to the commencement of the descending colon. Its secondary plexuses are as follows: rami intestini tenuis, ileo-colic, right colic, middle colic, and inferior pançreatico-duodenal.

The aortic plexus derives its fibres from the semilunar ganglia, and the cœliac and superior mesenteric plexuses. It extends along the aorta, beyond the origin of the superior mesenteric artery, in the form of two lateral strands which communicate freely with one another over the vessel by many interlacing fibres. It is reinforced laterally by branches from the lumbar portion of the gangliated sympathetic cord. The two lateral strands of the plexus ultimately cross the common iliac arteries, and unite in front of the body of the fifth lumbar vertebra to form the hypogastric plexus. The aortic plexus furnishes, on either side, branches to the renal and spermatic (or ovarian) plexuses, and supplies the coats of the aorta. The right strand gives branches to the inferior vena cava, and the left furnishes the chicf fibres of the inferior mesenteric plexus.

The spermatic plexus derives its fibres from the renal and aortic plexuses, and accompanies the spermatic artery to the testis. In the female it is called the ovarian plexus, which goes with the artery of that name to the ovary.

The inferior mesenteric plexus is derived chiefly from the left strand of the aortic plexus, and contains a ganglion, called inferior mesenteric, which lies below the root of the inferior mesenteric artery. The plexus accompanies the inferior mesenteric artery, and furnishes left colic, sigmoid, and superior hemorrhoidal plexuses, which supply the descending colon, iliac colon, pelvic colon, and rectum.

The hypogastric plexus is formed by the fusion of the two halves of the aortic plexus after these have crossed the common iliac arteries. It is reinforced by branches from the lumbar ganglia, and is 
situated in front of the body of the fifth lumbar vertebra betweer the common iliac vessels. It is a large flat plexus, measuring about $\mathrm{I} \frac{1}{2}$ inches in breadth, and it ends in two divisions, which become the right and left pelvic plexuses.

Cœliac Axis.-The coeliac axis is a short thick trunk which arises from the front of the aorta between the crura of the diaphragm just below the aortic opening. Its direction is straight forwards over the superior border of the body of the pancreas, and, after a course of about $\frac{1}{2}$ inch, it divides into three radiating branchesgastric, splenic, and hepatic. Of these the splenic is the largest, except during fotal life when it is exceeded by the hepatic. The branches of the axis supply the stomach, duodenum, pancreas, spleen, liver, and gall-bladder.

Relations.-The right lobe of the liver above, the superior border of the body of the pancreas below, the gastro-hepatic omentum in front, and a semilunar ganglion on either side, with the Spigelian lobe of the liver as an additional right relation. The axis is closely surrounded by the cœliac sympathetic plexus.

The gastric (coronary) artery is directed upwards and to the left as far as the small curvature of the stomach on the right side of the œesophagus. It then bends sharply forwards and downwards, and descends in two divisions from left to right along the small curvature towards the pylorus, where it anastomoses with the two divisions of the pyloric branch of the hepatic. The artery is at first behind the small sac of the peritoneum, but along the small curvature it lies between the two layers of the gastro-hepatic omentum, and is surrounded by the gastric sympathetic plexus.

Branches.-These are œsophageal, cardiac, and gastric. The cesophageal branches arise when the artery reaches the small curvature, and they ascend through the cesophageal opening of the diaphragm to anastomose on the gullet with the lower œsophageal branches of the thoracic aorta. The cardiac branches are distributed to the cardiac end of the stomach, where they anastomose with the vasa brevia of the splenic. The gastric branches arise from the two divisions of the artery on the small curvature, and pass to the front and back of the stomach, where they anastomose with branches of the gastro-epiploic arteries.

The gastric vein ascends from right to left along the small curvature of the stomach as far as the œsophagus, where it receives a few osophageal tributaries, after which it turns to the right and opens into the vena portæ.

The splenic artery takes a tortuous course to the left along the superior border of the body of the pancreas behind the small sac. On reaching the front of the left kidney it breaks up into several splenic branches which enter the spleen through the hilum. The artery is invested by the splenic sympathetic plexus, and the splenic vein lies below it, and behind the pancreas.

Branches.-These are pancreatic, left gastro-epiploic, vasa brevia, 
and splenic. The pancreatic branches arise at intervals along the superior border of the pancreas, which they enter. One of them, known as the arteria pancreatica magna, enters the organ towards its left end, and passes from left to right, lying a little above the pancreatic duct. The left gastro-epiploic artery arises near the spleen, and passes within the gastro-splenic omentum to the great curvature of the stomach, along which it descends from left to right

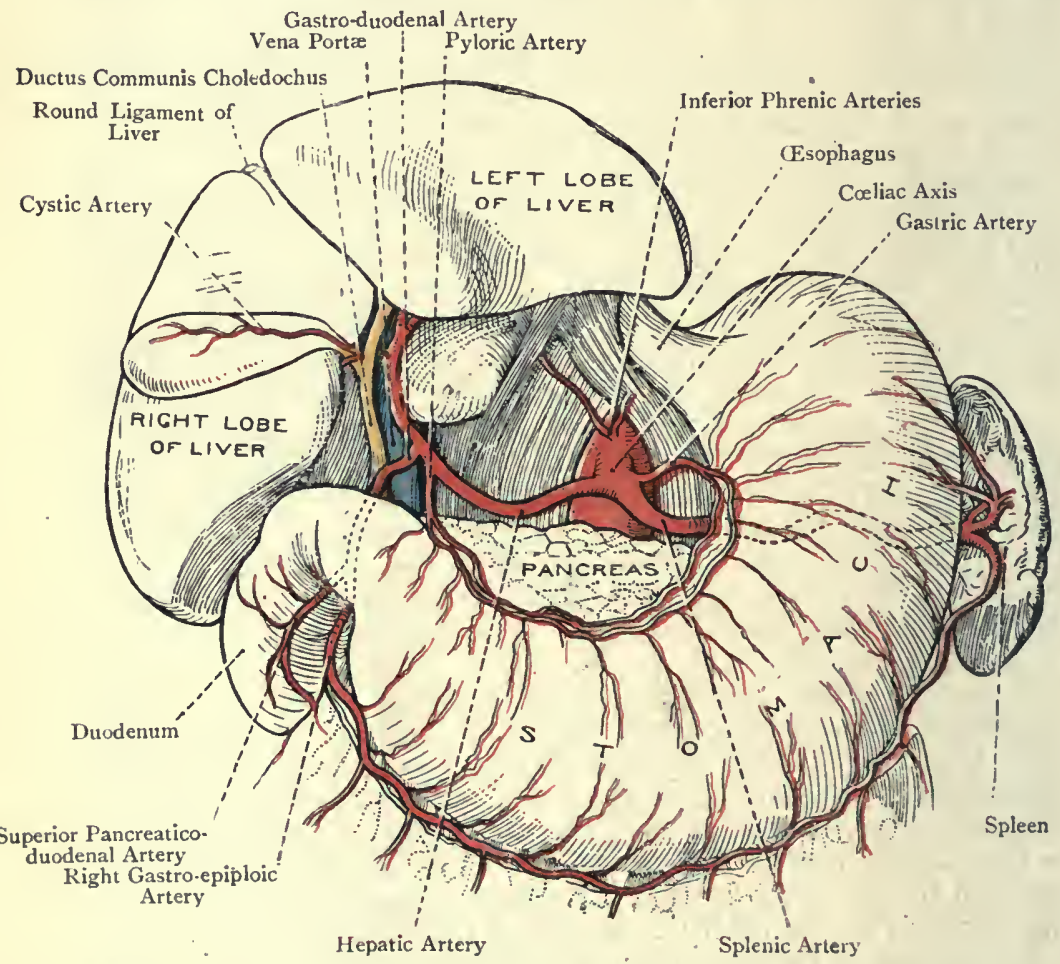

Fig. 318.-The Arteries of the Stomach, Liver, and Spleen (AFTER MERKEL).

between the two layers of the gastro-colic omentum as far as the centre, where it anastomoses with the right gastro-epiploic. It furnishes gastric branches to the front and back of the stomach, which anastomose with branches of the gastric artery, and epiploic branches, which descend into the gastro-colic omentum, these latter being long and slender. The vasa brevia arise from the terminal part of the splenic and from its splenic branches. They are about five in number, and, having passed within the gastrosplenic omentum to the cardiac extremity of the stomach, they 
anastomose with branches of the left gastro-epiploic and gastric arteries. The splenic branches are about five in number, and pass to the spleen within the lieno-renal ligament.

The splenic vein is formed by the union of about five veins which emerge from the spleen. It is of large size, and passes from left to right behind the pancreas near its superior border, where it lies below the splenic artery. Having crossed the aorta, it joins the superior mesenteric vein to form the vena portæ behind the neck of the pancreas. The vein takes up the following tributaries: the vasa brevia, the left gastro-epiploic, many pancreatic veins, and the inferior mesenteric (as a rule).

The hepatic artery passes at first to the right along the superior border of the pancreas for a short distance, where it lies behind the small sac. It then turns forwards below the foramen of Winslow to the upper border of the first part of the duodenum near the pylorus, and it subsequently ascends between the two layers of the gastro-hepatic omentum in front of the foramen of Winslow towards the portal fissure of the liver, on approaching which it divides into a right and left hepatic branch. The vessel is accompanied by the hepatic sympathetic plexus. As it ascends between the two layers of the gastro-hepatic omentum it has the common bile-duct on its right side, the vena portæ being behind both.

Branches.-These are pyloric, gastro-duodenal, and right and left hepatic. The pyloric artery, of small size, arises near the pylorus, and passes to the small curvature of the stomach, where it divides into two branches. These lie between the two layers of the gastro-hepatic omentum, and supply offsets to the front and back of the stomach. They anastomose with the two divisions of the gastric artery. The gastro-duodenal artery also arises near the pylorus, and descends behind the first part of the duodenum, having the common bile-duct on its right, and the vena portæ behind it. Having reached the lower border of the first part of the duodenum, it occupies a groove on the right of the neck of the pancreas, and here divides into its two terminal branches-right gastro-epiploic and superior pancreatico-duodenal. The right gastro-epiploic artery passes from right to left along the great curvature of the stomach as far as its centre between the two layers of the gastro-colic omentum, and its distribution and anastomoses are similar to those of the left gastro-epiploic artery. The superior pancreatico-duodenal artery descends anteriorly between the head of the pancreas and the second part of the duodenum, towards the lower end of which latter it anastomoses with the inferior pancreaticoduodenal of the superior mesenteric. It supplies the first and second parts of the duodenum, and furnishes branches to the adjacent portion of the pancreas. The hepatic branches are the terminal divisions of the trunk. The right, which is the larger, enters the portal fissure at its right end, whilst the left, small in size, enters that fissure at its left end, having previously furnished a branch to 
the Spigelian lobe. The right branch gives off the cystic artery, and this divides into two branches, superior and inferior, which ramify on the upper and under surfaces of the gall-bladder.

The pyloric vein passes from left to right, and opens into the vena portæ near the pylorus.

The right gastro-epiploic vein passes from left to right, and opens in to the superior mesenteric vein near its termination.

The pancreatico-duodenal vein takes up blood from the right end of the pancreas and from the duodenum, and opens into the superior mesenteric vein near its termination.

The cystic vein usually, ends in the right division of the vena portæ.

All the veins which return the blood from the stomach, duodenum, pancreas, and spleen are destitute of valves, so that the blood can regurgitate in cases of portal obstruction.

Cœliac Glands.-The glands of this group are numerous. They surround the cœliac axis, and extend over the aorta as low as the origin of the superior mesenteric artery. They receive their afferent vessels from the gastric, pancreatic, splenic, and hepatic glands, and their efferent vessels either join the in testinal lymphatic trunk (or trunks) of the superior mesenteric glands, or open independently into the receptaculum chyli.

Gastric Lymphatic Glands.-These are arranged in two groups, superior and inferior, the former lying along the small curvature of the stomach, and the latter along the great curvature. They receive their afferent vessels from the stomach, and their efferent vessels pass to the cœliac glands.

Pancreatic Glands.-These lie along the superior border of the pancreas. They receive their afferent vessels from that organ, and their efferent vessels pass to the cœliac glands.

Splenic Glands.- These are numerous, and are situated near the hilum of the spleen in contact with the tail of the pancreas. They receive their afferent vessels from the spleen, and their efferent vessels, having been joined by some of those from the left half of the great curvature of the stomach, pass to the cœliac glands.

Hepatic Glands.-These are situated between the two layers of the gastro-hepatic omentum near the portal fissure. They receive as afferent vessels those of the deep lymphatics of the liver which accompany the branches of the portal vein, and also some of the superficial lymphatics of the inferior surface of the liver, and their efferent vessels pass to the coeliac glands.

Vena Portæ.-This vein is formed by the union of the superior mesenteric and splenic veins, and is about 3 inches in length. It commences on a level with the body of the first lumbar vertebra a little to the right of the middle line, where it lies behind the neck of the pancreas. It ascends behind the first part of the duodenum, and then between the two layers of the gastro-hepatic omentum in front of the foramen of Winslow, where it has anterior to it the hepatic artery and common bile-duct, the artery being 
on the left of the duct. When the vessel arrives at the right extremity of the portal fissure of the liver it presents a slight enlargement, called the portal sinus, and then divides into two branches, right and left, the former being the larger and shorter of the two. The right branch, having received the cystic vein, enters the right lobe of the liver. The left branch, having traversed the portal fissure from right to left, and furnished branches to the quadrate and Spigelian lobes, crosses the longitudinal fissure, and

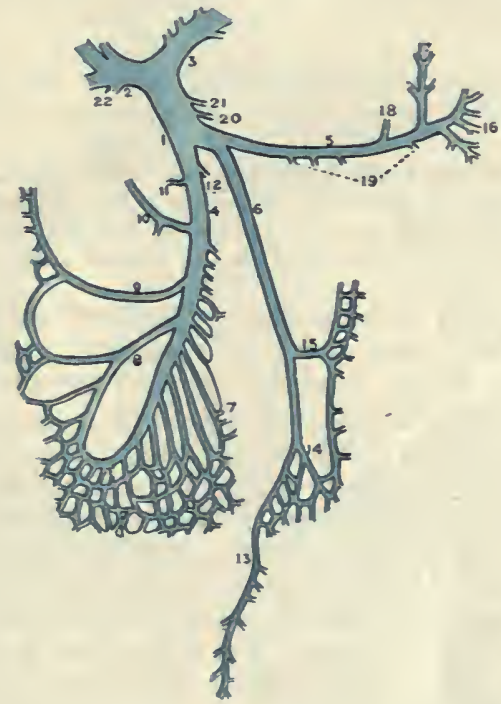

Fig. 3ig.- Vena Porta and its Tributaries.

2. Right Division

3. Left Division

4. Superior Mesenteric

5. Splenic

6. Infericr Mesenteric

7. Rami Intestini Tenuis

8. Ileo-Colic

9. Right Colic

ra Middle Colic

11. Right Gastro-epiploic
1. Vena Portae

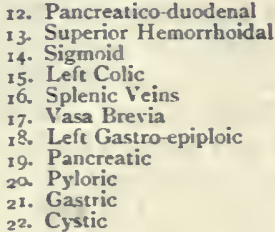

enters the left lobe. As it crosses this fissure it is joined in front by the round ligament of the liver, which is the remains of the umbilical vein of foetal life. Posteriorly, and slightly to the right of this point, it is connected with the fibrous cord which represents the fœtal ductus venosus. The vena portæ near the pylorus receives the pyloric and gastric veins. The distinctive character of the vessel is that it behaves like an artery, its blood ultimately entering the intralobular plexuses in the liver.

The sources from which the vein receives its blood are as follows: 
(I) the stomach, (2) the small and large intestine, except a portion of the anal canal, (3) the pancreas, (4) the spleen, and (5) the gallbladder.

Summary of the Tributaries of the Vena Portæ.-(1) The superior mesenteric vein, which takes up $(a)$ the right gastro-epiploic, $(b)$ the pancreatico-duodenal, $(c)$ the rami intestini tenuis, $(d)$ the ileo-colic, $(e)$ the right colic, and $(f)$ the middle colic. (2) The splenic vein, which takes up $(a)$ the gastric vasa brevia, (b) the left gastro-epiploic, (c) many pancreatic veins, and (d) the inferior mesenteric (as a rule), which in turn takes up the superior hemorrhoidal, sigmoid, and left colic veins. (3) The pyloric vein. (4) The gastric vein. (5) The cystic vein.

The portal vein and its tributaries are destitute of valves, so that the blood can regurgitate in cases of portal obstruction.

Development of the Vena Portæ. - The lower portion of the vein results from the union of the two vitelline veins. The upper portion is developed from the left half of the lower venous ring and the right half of the upper venous ring, formed by the vitelline veins around the primitive duodenum.

For a description of the common bile-duct, see p. 7 Io.

Kidneys. - The kidneys are two in number, right and left, and are situated deeply at the posterior part of the abdomen, where they lie behind the peritoneum. They chiefly occupy portions of the epigastric and hypochondriac regions, but also extend slightly into the umbilical and lumbar regions. Relatively to the vertebral column they extend from the level of the upper border of the last thoracic vertebra to about the centre of the body of the third lumbar, the right kidney being usually somewhat lower than the left. The long axis of each organ is directed downwards and slightly outwards, so that their upper ends are rather nearer the middle line than the lower. The superior limit of the right kidney is usually the lower border of the eleventh rib, whilst that of the left is usually the upper border of the eleventh rib. Inferiorly the organs approach the iliac crests, from which they are distant about 2 inches, the right being the nearer of the two. Each kidney is surrounded by a quantity of areolar and adipose tissues, constituting the adipose capsule, which, along with the adjacent viscera, anchors the organ. The length of a kidney is about 4 inches, the breadth about $2 \frac{1}{2}$ inches, and the thickness about $I_{\frac{1}{4}}$ inches. The right kidney is usually shorter and broader than the left. The weight of the organ is about $5 \frac{1}{4}$ ounces. In form the kidney is bean-shaped. It presents two smooth surfaces, two extremities, and two borders: The anterior surface looks outwards as well as forwards, and presents important visceral impressions, whilst the posterior surface looks inwards as well as backwards, and presents muscular impressions. The extremities are enlarged and round, the superior more so than the inferior, the latter often assuming a somewhat pointed appearance. The external border has an inclination backwards, and is convex and free. The internal border has an inclination forwards, is concave, and is connected with the renal vessels and the pelvis of the kidney. 
Reiations-Anterior Surface of the Right Kidney.-This surface is overlapped by the right suprarenal capsule for a very short distance at its upper and inner part. It presents three visceral areas-hepatic, duodenal, and colic. The hepatic area lies somewhat obliquely, and occupies about the upper two-thirds, being in contact with the impressio renalis on the under surface of the right lobe of the liver.

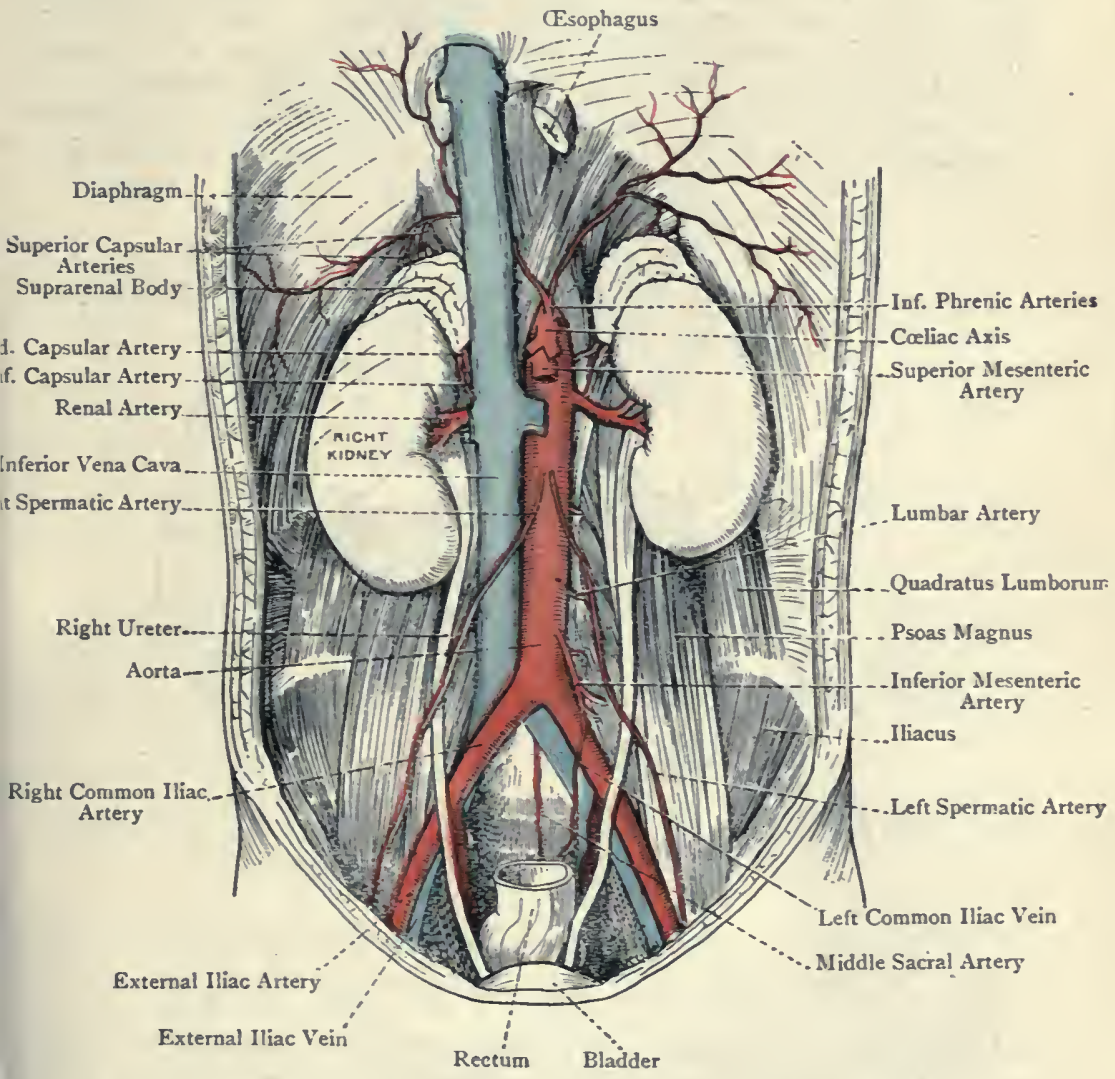

Fig. 320.-Dissection of the Posterior Abdoninal Wall.

It is covered by peritoneum. The duodenal area corresponds with an elongated narrow strip lying close to the hilum, and reaching a little above and below it. It is in contact with the posterior wall of the second part of the duodenum, both being destitute of peritoneum. The colic area lies below the hepatic, and, like it, is oblique. It is in contact with the upper end of the ascending colon and the hepatic flexure without the intervention of peritoneum. Between the lower part of the duodenal and the colic impression, 
that is, at the lower and inner part of the anterior surface, there is a small area covered by peritoneum which is in contact with a portion of the small intestine.

Anterior Surface of the Left Kidney.-This surface is overlapped by the left suprarenal capsule for a very short distance at its upper and inner part. It presents five visceral areas-splenic, gastric, pancreatic, colic, and jejunal. The splenic area is situated at the upper and outer part, close to the external border, and is in contact with the renal surface of the spleen, the peritoneum of the great cavity intervening. The gastric area, somewhat triangular, lies at the upper end, between the splenic and capsular areas, and above the pancreatic area. It is in contact with the postero-inferior surface

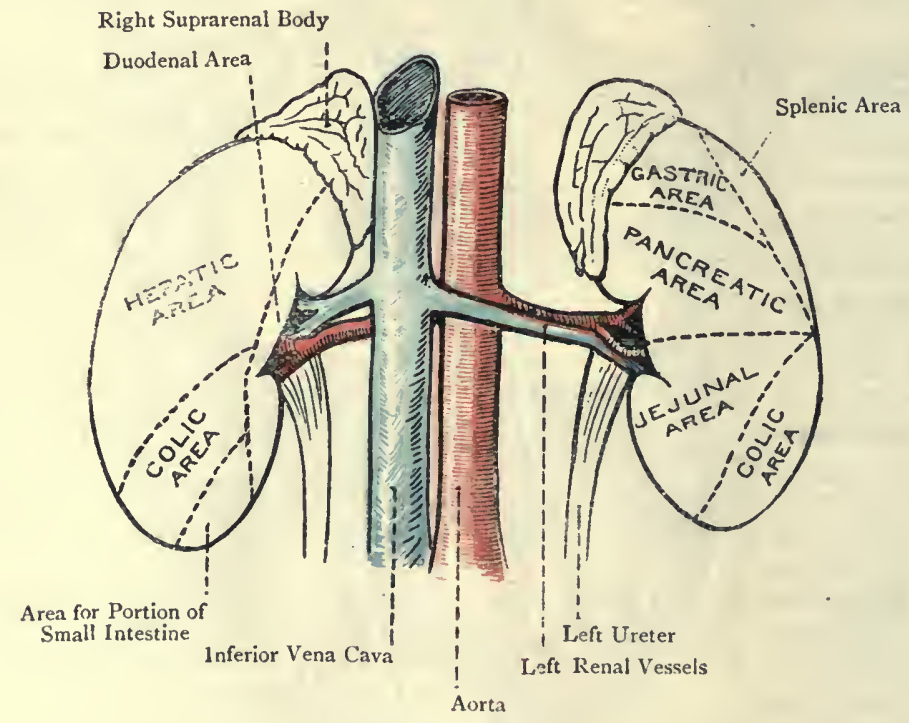

Fig. 321. -The Visceral Areas of the Kidneys

(In this case the right renal vein was higher than usual).

of the stomach with the intervention of the peritoneum of the small sac. The pancreatic area lies transversely below the gastric area, and extends as low as about the centre of the hilum. It is in relation with the posterior surface of the body of the pancreas and the splenic vessels, without peritoneum. The colic area is situated at the lower and outer part, and is in contact with the splenic flexure and the commencement of the descending colon, without peritoneum. At the lower and inner part there is a small area covered by peritoneum, which is related to a part of the jejunum.

Posterior Surface.-This surface is readily recognised by observing the posterior position of the pelvis of the kidney at the 
hilum, with which it coincides. The inner portion of it rests upon $(a)$ the psoas magnus and its sheath, and $(b)$ the crus of the diaphragm. The outer portion rests, from above downwards, upon (a) the twelfth rib (in the case of the left kidney the eleventh rib also), (b) the diaphragm, and (c) the quadratus lumborum, covered

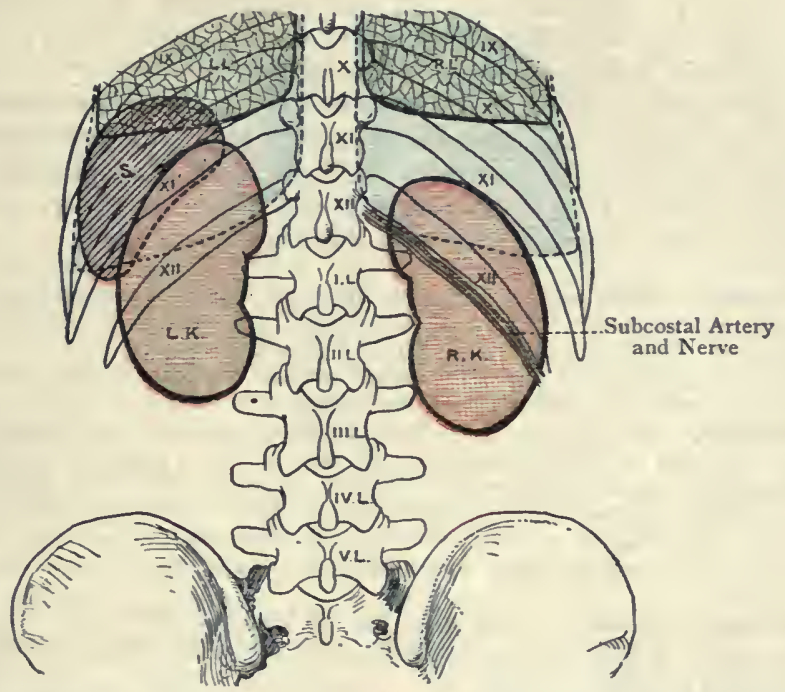

Fig. 322.-Diagram showing the Relations of the Kidneys from Behind.

\author{
R.L. Right Lung \\ L.I. Left Lung \\ S. Spleen \\ R.K. Right Kidney \\ L.K. Left Kidney \\ IX. Ninth Rib \\ X. Tenth Rib
}

\author{
XI. Eleventh Rib \\ X11. Twelfth Rib \\ 1. L. First Lumbar Vertebra \\ I1.I. Second Lumbar Vertebra \\ 11I. L. Third Lumbar Vertebra \\ 1V.L. Fourth Lumbar Vertebra \\ V.I. Fifth Lumbar Vertebra
}

by the anterior lamina of the lumbar aponeurosis. An important surgical relation of the upper part of this division of the posterior surface is that the pleura, in descending between the diaphragm and the twelfth rib, lies behind the kidney. Three nerves pass downwards and outwards behind the organ, namely, the subcostal, ilio-hypogastric; and inguinal.

The superior extremity is capped by the suprarenal capsule, which also extends for a little over the anterior surface and adjacent portion of the internal border.

The external border, which is convex, rests on the posterior aponeurosis of the transversalis abdominis. The external border of the right kidney, over about its upper two-thirds, is in contact with the liver, whilst the external border of the left kidney at its upper end is in contact with the spleen.

The internal border of the right kidney lies very near the 
inferior vena cava, whilst that of the left is situated fully I inch from the aorta. This border is concave, and presents a longitudinal fissure, called the hilum, which extends over about its middle third. It presents two somewhat thick lips, anterior and posterior, and it leads to a cavity within the organ, called the sinus. It transmits the following structures in order from before backwards : the branches of the renal vein, the branches of the renal artery, with branches of the renal sympathetic plexus and lymphatics, and the pelvis of the kidney.

The side to which a kidney belongs may be ascertained in the following way : if the organ is held with that end downwards towards which the ureter is naturally inclined, and that surface backwards which coincides with the pelvis at the hilum, the convex border will then indicate the side to which the organ belongs.

Varieties-Form.-The kidneys may be much elongated, or somewhat round, or triangular. The lobulated condition, which is characteristic of the kidney in early life, may persist in the adult.

Size.-One kidney may be diminished in size, in which cases there is a proportionate increase in the other organ.

Position.-It is very rare to find the kidneys higher than usual, but one or both not infrequently extend into the iliac fossa, or over the pelvic brim.

Number-Diminution.-One kidney (usually the left) may be entirely suppressed, in which cases the solitary kidney usually occupies its normal position, and may, or may not, be of large size. Increase.-The number may be increased to three, the additional organ being lateral, or median, in position.

Horseshoe Kidney. - This condition is brought about by the fusion of the lower parts of the organs, the connecting band of renal substance extending across the vertebral column.

Preternatural Mobility. - The kidney is usually anchored in its normal position by its capsule and the adjacent viscera, but it is sometimes movable, which may be due to one of two causes: (I) the capsule may be very loose, giving rise to the condition known as movable kidney; or (2) the organ may be attached to the posterior abdominal wall by a peritoneal fold, called the meso-nephron, in which case the condition known as floating kidney occurs, this being said to be more frequent on the right side.

For the structure and development of the kidney, see Index.

Ureter.-The ureter is the excretory duct of the kidney, and it conveys the urine to the bladder. It is a cylindrical, thick-walled tube, like a goose-quill, its average length being about I2 inches, and its diameter about $\frac{1}{6}$ inch. The ureter proper commences towards the lower end of the kidney, where it is the continuation of the pelvis, and it terminates in the bladder. The pelvis is funnelshaped and flattened from before backwards. It lies partly in the sinus, where it receives the calices, and partly outside the hilum, where it lies behind the other transmitted structures. Its direction is downwards and inwards; and, having become narrow, it passes in to the ureter proper towards the lower end of the kidney.

The ureter proper passes downwards and inwards behind the peritoneum, in contact with the posterior abdominal wall. I rests at first upon the psoas magnus and its sheath, being here crossed superficially by the spermatic (or ovarian) vessels, whicl 
are taking a course downwards and outwards, and deeply by the genito-crural nerve, which is taking a similar course. In this part of its course the right duct has the inferior vena cava near it on its inner side, whilst the left duct has the aorta on its inner side, but at some little distance. The ureter next crosses the terminal part of the common iliac artery (or the commencement of the external iliac), after which it enters the pelvic cavity, the right ureter being crossed by the lower part of the root of the mesentery proper, and the left by a portion of the pelvic meso-colon. In the pelvis the ureter passes downwards and forwards in front of the internal iliac artery to the outer wall of the pelvis, lying beneath the peritoneum, and crossing internally the obturator vessels and nerve, and the obliterated hypogastric artery. It subsequently passes inwards to the bladder, being crossed internally by the vas deferens. Having arrived at the lateral or ureteric angle of the bladder, it commences to pierce the vesical wall anterior to the upper free end of the vesicula seminalis, being here about 2 inches distant from its fellow, and about $I_{\frac{1}{2}}$ inches from the base of the prostate gland. It now pursues an oblique course through the wall of the bladder, lying in it for about $\frac{3}{4}$ inch, and it finally opens into the interior by a very small slit-like aperture placed obliquely at one angle of the base of the internal trigone, where it is distant from its fellow and from the urethral orifice by about $\mathrm{I} \frac{1}{4}$ inches. In the female the ureter, in its pelvic course, passes along the side of the cervix uteri and upper part of the vagina.

Varieties.-(1) The pelvis may be absent, its place being taken by two, or three, tubes. (2) Double Ureter.-The foregoing tubes may remain separate for some distance beyond the hilum, or even as low as the bladder, thus giving rise to a double ureter. A triple ureter is extremely rare. (3) Dilated Ureter.-This condition is liable to result in such cases as urethral stricture, enlarged prostate, and vesical calculus.

For the structure of the ureter, see Index.

Suprarenal Capsules or Bodies.-The suprarenal capsules (adrenals) are two in number, right and left, and are situated in the epigastric region. Each is compressed from before backwards, and broad from side to side. It is set upon the superior extremity of the corresponding kidney, to which it is bound by connective tissue. Each capsule encroaches upon the adjacent parts of the anterior surface and internal border of the kidney, the left being mainly situated upon the internal border. The dimensions of the organ are so variable that they can only be stated approximately as follows: the height is about $I \frac{1}{4}$ inches, and the breadth rather less than 2 inches. The weight, on an average, is about 2 drachms.

The right capsule is rather smaller than the left, and is triangular. It is wedged in between the diaphragm and the posterior surface of the right lobe of the liver, and its surfaces are anterior and posterior, the former having an inclination outwards, and the latter inwards. The anterior surface, close to the inner border, is in contact with the inferior vena cava, and elsewhere it is related to the pos- 
terior surface of the right lobe of the liver. At its upper and inner part there is a small fissure, called the hilum, through which the right suprarenal vein emerges. The peritoneum gives a partial covering to this surface at its lower part. The posterior surface is in contact with the diaphragm and the upper part of the anterior surface of the right kidney. The right semilunar ganglion lies on the inner side of the right capsule.

The left capsule is somewhat semilunar. The anterior surface is related above to the postero-inferior surface of the stomach, with the intervention of the small sac, which furnishes. it with a peritoneal covering. Below this it is related to the posterior surface of the body of the pancreas and the splenic vessels; without peritoneum. The hilum, through which the left suprarenal vein emerges, is situated at the lower and inner part of this surface. The posterior surface at its upper part is in contact with the left crus of the diaphragm, and, below, it rests upon the upper and inner part of the front of the left kidney, as well as upon its internal border.

For the structure and development of the suprarenal capsule see Index.

Abdominal Aorta. - The abdominal aorta commences at the aortic opening of the diaphragm on a level with the lower border of the body of the twelfth thoracic vertebra, where it lies in the middle line, and it usually terminates opposite the centre of the body of the fourth lumbar vertebra, a finger's breadth to the left of the middle line, by dividing into the right and left common iliac arteries. The position of the bifurcation may be indicated in one of two ways, as follows : (I) by taking a point fully I inch below the umbilicus and a finger's breadth to the left of the linea alba; or (2) by taking a point in the line which connects the highest parts of the iliac crests a finger's breadth to the left of where it intersects the linea alba. The bifurcation may take place a little lower down or higher up than tlie normal level. The length of the vessel is about 5 inches. It occupies. the epigastric and umbilical regions, where it lies very deeply behind the peritoneum, and its direction is downwards with a slight inclination to the left.

Relations-Anterior.-The direct anterior relations, from above downwards, are as follows: the origins of the inferior phrenic arteries, the cœliac axis, cœliac glands, and solar plexus, the ascending layer of the transverse meso-colon, the pancreas and splenic vein, the root of the superior mesenteric artery, the third part of the duodenum and left renal vein, the origins of the spermatic (or ovarian) arteries, the aortic plexus, the median group of lumbar glands, the peritoneum of the general cavity, and the origin of the inferior mesenteric artery. The more remote anterior relations are the gastro-hepatic omentum, stomach, transverse colon with its meso-colon, coils of the small intestine, root of the mesentery proper, and gastro-colic omentum. Posterior.-The disc between the twelfth thoracic and first lumbar, vertebra, the bodies and discs of the upper four lumbar vertebra, and 
the anterior common ligament, the left lumbar veins, and the origins of the lumbar and middle sacral arteries. Right.-The right crus of the diaphragm, with the receptaculum chyli and right rena azygos lying deeply between the vessel and the right crus, the former being nearest the aorta, and slightly covered by it; and the inferior vena cava. Left.-The left crus of the diaphragm, the left semi-

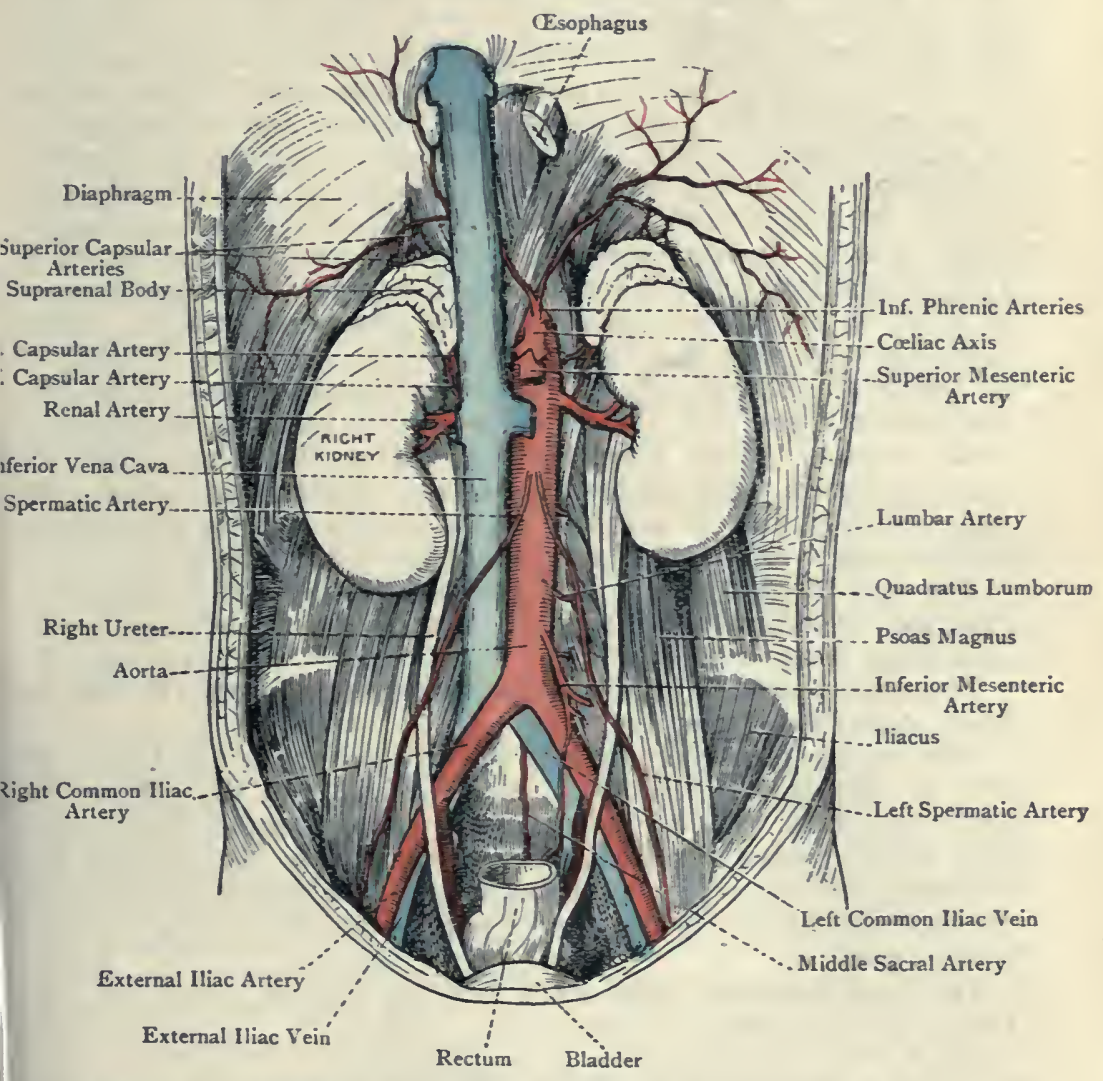

Fig. 323,-Dissection of the Posterior Abdominal Wal.L.

lunar ganglion, the terminal portion of the third part of the duodenum (ascending part of Treves), and the left sympathetic gangliated cord.

Branches.-These are nine in number, and are arranged in two groups, visceral and parietal, four of them being single, and five arranged in pairs. The four single branches are the coliac axis, superior mesenteric, inferior mesenteric, and middle sacral. The five pairs are the inferior phrenic, middle capsular, renal, spermatic 
(ovarian in the female), and the lumbar. Excluding the lumbar arteries, the order of origin of the branches is as follows : (I) inferior phrenic, (2) cœliac axis, (3) superior mesenteric and (4) middle capsular, both on same level, (5) renal, (6) spermatic (or ovarian), (7) inferior mesenteric, and (8) middle sacral. In what follows the letter $\mathrm{P}$ after an artery signifies parietal, and $\mathrm{V}$ visceral.

The inferior phrenic arteries (P) are two in number, right and left, and they are called inferior to distinguish them from the superior phrenic of the internal mammary, right and left. They arise, either separately or by a common trunk, from the front of the aorta, whenever the vessel has passed through the aortic opening of the diaphragm. They at once diverge, each passing outwards and upwards over the crus of the diaphragm, the right vessel lying behind the inferior vena cava, and the left behind the œsophagus. Each ends by dividing into two branches, anterior and external. The anterior branch passes forwards and inwards in a curved manner in front of the central tendon, and anastomoses with its fellow of the opposite side, and the superior phrenic and musculo-phrenic of the internal mammary. The external branch passes outwards, and anastomoses with the musculo-phrenic and the lower intercostal arteries. Each inferior phrenic artery furnishes a superior capsular branch (or branches) to the suprarenal capsule, the right vessel also giving off a few branches to the inferior vena cava, whilst the left supplies a few branches to the œsophagus.

The right inferior phrenic vein opens into the inferior vena cava, and the left terminates in the left suprasenal vein, left renal vein, or inferior vena cava.

The cœliac axis (V) and superior mesenteric artery (V) will be found described on pp. 725,739 .

The middle capsular arteries (V) are of small size, and are two in number, right and left, each arising from the side of the aorta on a level with the origin of the superior mesenteric artery. The vessel passes outwards and upwards over the crus of the diaphragm to the suprarenal capsule, in which it anastomoses with the superior capsular of the inferior phrenic and the inferior capsular of the renal.

The right suprarenal vein opens into the inferior vena cava, and the left in to the left renal vein.

The suprarenal veins originally open into the cardinal veins. The right suprarenal vein becomes transferred to the upper segment of the inferior vena cava (above the right renal vein). The left suprarenal vein, after the obliteration of the adjacent portion of the left cardinal vein, becomes transferred to the left renal vein.

The renal arteries (V) are remarkable for their large size, and are two in number, right and left. They arise from the side of the aorta about $\frac{1}{2}$ inch below the superior mesenteric on a level with the body of the first lumbar vertebra, the right artery being usually a little lower than the left. They form right angles with 
the aorta, and cross the crura of the diaphragm on their way to the hila of the kidneys, the right vessel passing behind the inferior vena cava, second part of the duodenum, and head of the pancreas, whilst the left passes behind the body of the pancreas. Each vessel has its own vein in front of it, and the aortico-renal ganglion lies over its root. On approaching the renal hilum each vessel divides into three or four branches, one of which usually passes behind the pelvis of the kidney, whilst the others lie between the renal vein in front and the pelvis behind. For the subsequent distribution of the branches in the kidney, see p. 822 .

Before breaking up into its proper renal branches the vessel gives off an inferior capsular artery to the suprarenal capsule, peri-renal branches to the capsule, and ureteric to the upper part of the ureter.

Diferences between the Renal Arteries.-(I) The right artery is rather lower than the left. (2) The right is rather longer than the left, on account of the inclination of the aorta to the left of the middle line. (3) The right passes behind the inferior vena cava, second part of the duodenum, and head of the pancreas, whilst the left passes behind the body of the pancreas.

Varieties.-1. Very often there is an accessory renal artery present, arising close to the main vessel, and usually above it. 2. The renal artery may divide into its renal branches close to its origin. 3. There may be an aberrant renal artery, which may arise from the inferior phrenic, spermatic (or ovarian), inferior mesenteric, common or external iliac, or middle sacral. 4. In cases of variations, or even in the normal condition, one or more of the renal branches may fail to enter the hilum, and may pierce the kidney on its anterior surface, or near the upper or lower end.

The renal veins, like the arteries, are of large size. Each lies in front of its artery, and receives tributaries from the adipose tunic and the upper part of the ureter. The vessels are tributaries of the inferior vena cava, which they join almost at a right angle, the left a little higher than the right. The left vein crosses in front of the aorta, taking up in its course the left suprarenal and left spermatic (or ovarian) veins. In some cases there is a single semilunar valve at the lower part of the opening of each renal vein into the inferior vena cava.

Difference between the Renal Veins.--(I) The left vein is longer than the right, on account of the position of the inferior vena cava on the right side. (2) The left is rather higher than the right. (3) The left crosses in front of the aorta. (4) The left receives the left suprarenal and left spermatic (or ovarian) veins.

The renal veins originally open into the cardinal veins. Subsequently the right renal vein opens into the junction of the upper and lower segments of the inferior vena cava. When the left cardinal vein becomes obliterated, in great part the left renal vein becomes continuous with the left division of the primitive inferior vena cava. The mesial portion of the left renal vein is developed from the left division of the primitive inferior vena cava. 
The spermatic arteries (V) are two in number, right and left, and they arise from the front of the aorta about I inch below the renal arteries. If they arise separately they are close to each other, but they sometimes spring by a common trunk. They are long, slender, somewhat tortuous vessels, which at once diverge, each passing obliquely downwards and outwards behind the peritoneum. In this course the vessel rests upon the aorta for a short distance, and then upon the psoas magnus and its sheath, where it crosses the ureter. The right artery passes in front of the inferior vena cava and behind the terminal part of the ileum; whilst the left passes behind the iliac colon. Subsequently the artery, on its way to the internal abdominal ring, lies upon the terminal part of the external iliac. At the internal ring it approaches the vas deferens to form, with other structures, the spermatic cord. The vessel then passes through the internal abdominal ring, along the inguinal canal, and through the external abdominal ring into the scrotum, where it divides into testicular and epididymal branches. In the abdomen the spermatic artery furnishes ureteric branches to the ureter, and in the scrotum it gives off cremasteric branches to the coverings of the spermatic cord, which anastomose with the cremasteric branch of the deep epigastric. During fotal life the vessel is very short, and takes a transverse course to the testis, which is then lying below and behind the kidney. As the testis, however, descends into the scrotum the vessel gradually becomes much elongated.

Varieties.-(I) One or both spermatic arteries may be absent, in which cases the testis is supplied chiefly by the deferential artery. (2) A spermatic artery may arise from a renal artery.

The spermatic veins spring from the pampiniform plexus of the spermatic cord at the internal abdominal ring, and are at first two in number on each side, which lie one on either side of the corresponding artery. They subsequently unite to form a single vessel, which on the right side opens at an acute angle into the inferior vena cava, and on the left at a right angle in to the left renal vein. There is usually a valve at the point of termination of each vein, though this may be absent. In the left spermatic vein, where it joins the left renal vein, the valve directs the current of blood entering by the spermatic vein in the direction of the inferior vena cava. It also prevents the blood in the left renal vein from entering the spermatic vein by directing the current over the mouth of the latter vessel. The left spermatic vein is rather longer than the right.

The spermatic veins originally open into the lower cardinal veins. The right spermatic vein opens into that portion of the right cardinal vein which persists as the lower segment of the permanent inferior vena cava. The upper part of the left spermatic vein is formed from that portion of the left cardinal vein which lies immediately below the left renal vein. In this manner the difference in the mode of termination of the two spermatic veins in the adult is accounted for. 
The ovarian arteries (V), in the female, take the place of the spermatic arteries in the male, and their course and relations in the abdomen correspond with those of the spermatics. The ovarian arteries are, however, shorter than the spermatics, and they do not pass out through the inguinal canal, but enter the pelvis by crossing the commencement of the external iliac artery. In the pelvis each vessel becomes very tortuous, and passes between the two layers of the broad ligament of the uterus to be distributed to the ovary. In the abdomen the artery supplies branches to the ureter, and in the pelvis it furnishes the following offsets : tubal to the Fallopian tube; a uterine branch to the side of the uterus; and a ligamentous branch to the round ligament of the uterus, which it accompanies as far as the inguinal canal. The ovarian arteries, like the spermatic, are very short and transverse in direction during fotal life, when the ovary occupies a position similar to that of the testis. They, however, gradually become elongated as the ovary descends to its future abode in the pelvis.

The ovarian veins spring from the ovarian or pampiniform plexus between the two layers of the broad ligament, close to the ovary. After emerging therefrom their subsequent course and mode of termination resemble those of the spermatic veins.

For the inferior mesenteric artery (V) and vein, see p. 729; for the middle sacral artery (P) and vein, see p. 86I; and for the lumbar arteries $(\mathrm{P})$ and veins, see $\mathrm{p} .772$.

Inferior Vena Cava.- The inferior vena cava commences opposite the upper border of the body of the fifth lumbar vertebra a little to the right of the middle line, where it is formed by the union of the right and left common iliac veins, and it terminates at the postero-inferior angle of the right auricle of the heart. It ascends along the right side of the aorta, resting upon the anterior and right aspects of the lumbar vertebræ as high as the level of the second. Beyond this point it diverges from the aorta, and is supported by the right crus of the diaphragm. It then occupies the caval fossa on the posterior surface of the right lobe of the liver. On leaving this fossa it passes through the caval opening in the central tendon of the diaphragm, and, almost immediately thereafter, it opens into the postero-inferior angle of the right auricle of the heart. As the vein passes through the caval opening its walls are connected with the margins of that opening, and so the patency of the vessel is maintained.

Relations-Anterior.-From below upwards, the right common iliac artery, lower part of the root of the mesentery proper, right spermatic (or ovarian) vessels, third part of the duodenum. head of the pancreas, origin of the vena portæ, first part of the duodenum, foramen of Winslow, and posterior surface of the liver. Posterior.-The bodies, discs, and anterior common ligament of the lower thiee lumbar vertebræ, the corresponding right lumbar vessels, inner border of the' right psoas magnus' and its sheath, 
right sympathetic gangliated chain, right renal artery, right semilunar ganglion, right crus of the diaphragm, right suprarenal capsule, and right infesior phrenic artery. Right.-From below upwards, the right ureter and the internal border of the right kidney.

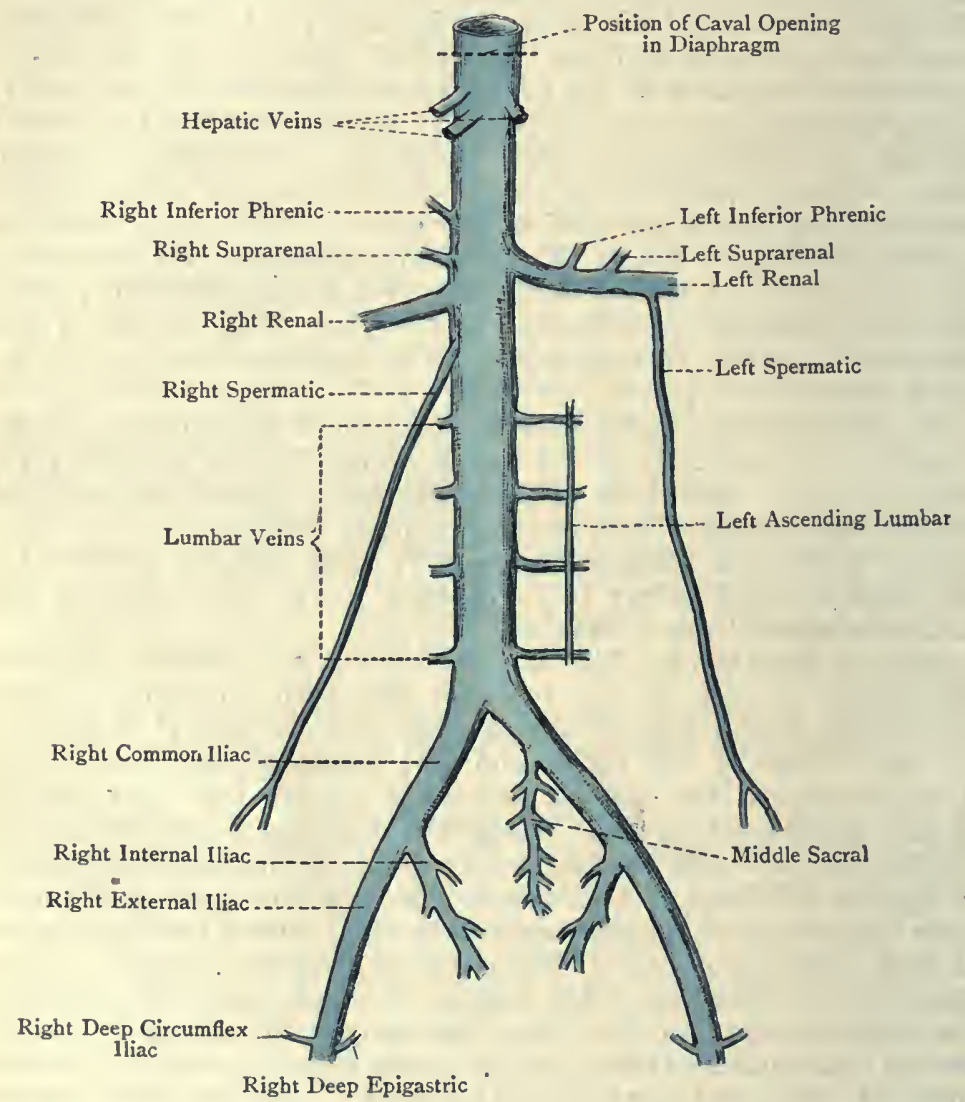

Fig. 324.-Schieme of the Inferior Vena Cava and its Tributaries.

Left.-From below upwards, the aorta and the right crus of the diaphragm.

Tributaries.-These are as follows : the right and left common iliac veins, right and left lumbar veins, the right spermatic (or ovarian) vein, the right and left renal veins, the right suprarenal vein, the right inferior phrenic vein (and sometimes the left), and the hepatic veins at the caval fossa of the liver.

Chief Varieties. - (1) The vessel may be found on the left side of the aorta in its lower part, in which cases it subsequently crosses that vessel to take up 
its usual position on its right side. This may be due to a transposition of viscera, or to a permanent patency of the lower part of the left cardinal vein oi fœtal life. (2) The inferior vena cava, in very rare cases, may terminate in a large right azygos vein, and, through means of that, in the superior vena cava. Under these circumstances the hepatic veins open into the right auricle of the heart.

Development.-The postrenal segment of the inferior vena cava is usually regarded as being developed from the lower portion of the right posterior cardinal vein. The prerenal segment is developed from (I) the upper portion of the right subcardinal vein; (2) the hepatic sinusoids, and (3) the vena hepatica communis (Lewis).

Aortic Lymphatic Glands.-These glands are very numerous, and are arranged in four groups, which lie around the abdominal aorta. The groups are spoken of as pre-aortic, lateral aortic, right and left, and retro-aortic, respectively.

Pre-aortic Glands.-These are usually disposed in three sets, namely, (I) cœliac glands, which constitute a very distinct set around the origin of the coliac axis; (2) superior mesenteric glands, in the region of the origin of the superior mesenteric artery, and along the trunk of the vessel; and (3) inferior mesenteric glands, in the region of the origin of the inferior mesenteric artery.

The afferent vessels of the pre-aortic glands are derived from the following sources:

I. Rectum.

2. Pelvic colon.

3. Iliac colon.

4. Descending colon.

5. Transverse colon.

6. Ascending colon.

7. Cæcum.

8. Vermiform appendix.

9. Small intestine.

I0. Stomach.

II. Liver.

I2. Pancreas.

I3. Spleen.

I4. Lateral aortic glands.

The cœliac glands, more particularly, receive their chief afferent vesseils from the following glands:

I. Coronary or gastric glands.

2. Retro-pyloric glands.

5. Pancreatic glands.

3. Hepatic glands.

4. Splenic glands.

The efferent vessels of the pre-aortic glands usually unite to form one trunk, called the intestinal lymphatic trunk, which opens into the receptaculum chyli, a few of them having traversed the retroaortic glands.

Lateral Aortic Glands (Lateral Lumbar Glands).--These are disposed in two groups-right and left. The right lateral aortic glands lie both in front of, and behind, the inferior vena cava; whilst the glands of the left side form a single chain on the left side of the abdominal aorta.

The lateral aortic glands, of either side, receive their afferent vessels from the following sources:

I. Common iliac glands.

2. Lumbar (lateral lumbar)

3. Testis.
4. Ovary, Fallopian tube, and adjacent half of body of uterus.

5. Suprarenal body.

6. Kidney. 
Their efferent vessels, for the most part, unite, on either side, to form a lateral aortic lymphatic trunk, right and left, which opens into the receptaculum chyli. Some pass to the pre-aortic glands, and others to the retro-aortic glands.

Retro-aortic Glands.-These glands are about four in number, and they lie behind the abdominal aorta. Their afferent vessels are derived from the pre-aortic and lateral aortic glands, as well as from the vertebral bodies and ligaments to which they are related. Their efferent vessels unite to form a single trunk, called the retroaortic lymphatic trunk, which opens into the receptaculum chyli.

Diaphragm-Origin-Sternal Portion.-By two fleshy slips from the back of the ensiform process close to its lower end. Costal Portion.-By six fleshy slips, at either side, from the inner surfaces of the lower six costal cartilages, which interdigitate with slips of the transversalis abdominis. Vertebral Portion.-From the external and internal arcuate ligaments, and the fronts of the bodies of lumbar vertebræ, as well as the intervertebral discs and anterior common ligament, by two crura, the right crus reaching usually as low as the disc between the third and fourth lumbar bodies, and the left as low as the disc between the second and third.

Insertion. - The central tendon on all sides.

Nerve-supply. - The right and left phrenic nerves, each of which springs chiefly from the anterior primary division of the fourth cervical nerve, and usually receives a branch from the third, and sometimes from the fifth. Each phrenic nerve, on approaching the diaphragm, divides into branches which pierce its muscular part separately in front of the central tendon, and are distributed on the abdominal surface of the muscle. In the right half of the muscle a communication takes place between the right phrenic nerve and the right inferior phrenic sympathetic plexus, a small ganglion, called the ganglion diaphragmaticum, being situated at the place of communication. A similar connection is established on the left side, but no ganglion is present.

Arterial Supply. - (r) The inferior phrenic branches of the abdominal aorta; (2) the superior phrenic (comes nervi phrenici) and musculo-phrenic, both branches of the internal mammary of each side; (3) the phrenic branch of the superior epigastric of the internal mammary of each side; and (4) branches from the lower intercostal arteries.

Lymphatics.-These pass to the sternal, anterior mediastinal, posterior mediastinal, intercostal, and lumbar glands.

Action. - The diaphragm by its contraction increases the vertical diameter of each half of the thorax, and is therefore a muscle of inspiration. The middle portion of the central tendon is fixed by reason of the fibrous portion of the pericardium, which is implanted into it, being connected above with the deep cervical fascia. The fleshy portion, however, becomes flattened, and descends towards the abdomen, displacing the viscera, and so increasing the vertical 
diameter of each half of the thorax. .The diaphragm also elevates the lower ribs, except the last.

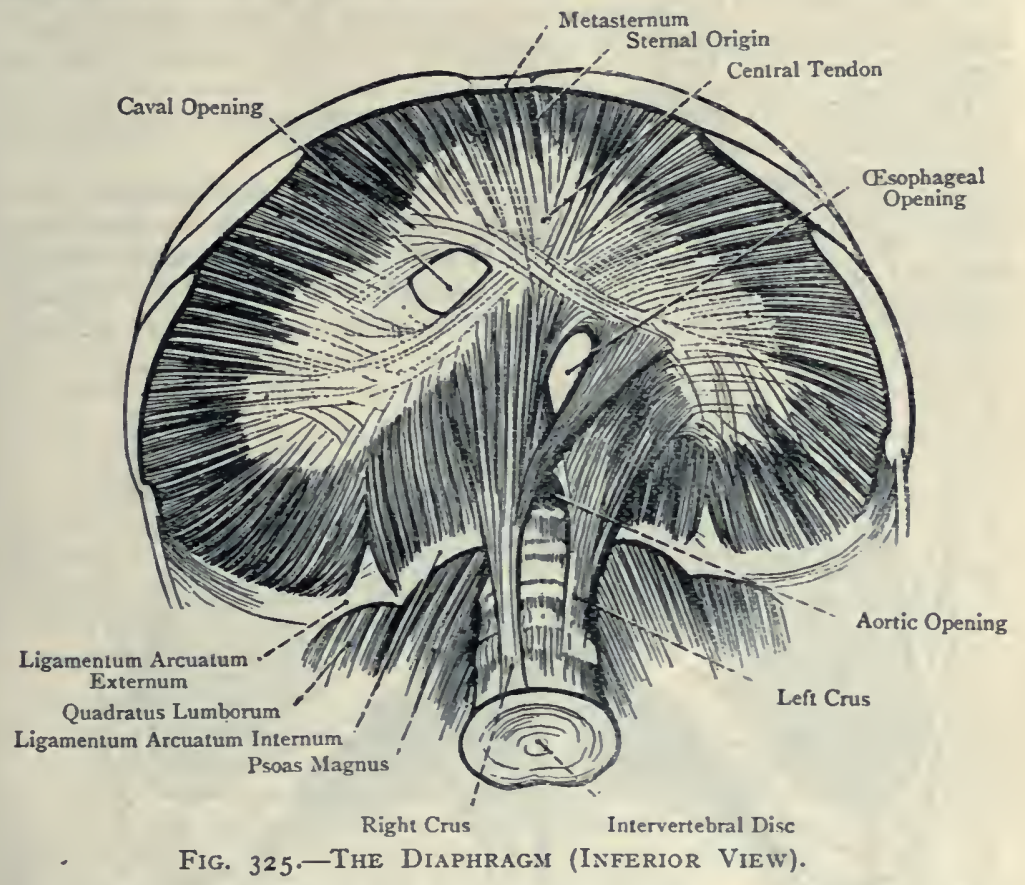

The muscular fibres pass in an arched manner upwards and inwards to the central tendon, upon which they converge from all points. The sternal portion is separated on either side from the costal portion by a small interval occupied by areolar tissue, through which the superior epigastric vessels and some of the superficial lymphatics of the upper surface of the liver pass. Above and below this interval are the pleura and peritoneum respectively. In this situation a diaphragmatic hernia may take place, involving one or other of the abdominal viscera. Between the lowest costal fibres and the corresponding external arcuate ligament there is sometimes another areolar interval of small size. The crura are strong, thick musculo-tendinous bundles disposed vertically, the left being the smaller, shorter, and more posterior of the two. Each crus is fleshy externally, and strongly tendinous internally, the lower extremity of each being entirely tendinous. On a level with the lower border of the body of the twelfth thoracic vertebra the inner tendinous fibres of the crura are connected by a fibrous band, called the middle arcuate ligament, which lies in front of the aorta. The muscular fibres of the crura pass upwards in a diverging manner to be inserted in to the posterior border of the central tendon. The innermost muscular fibres on either side, re:nforced by fibres springing from the middle arcuate ligament, decussate before reaching the central tendon and enclose between them the œsophageal opening. In the decussation the bundle derived from the right crus passes in front of th $t$ from the left, which latter is of small size.

The central tendon is also called the cordiform or trefoil tendon. It is much elongated from side to side, convex in front, and con- 
cave behind. It is divided into three lobes or alæ, right, middle, and left, of which the right is the largest, and the left the smallest and narrowest. The separation into lobes is indicated by two small notches situated in the course of the anterior convex border.

The diaphragm presents three foramina, namely, aortic, caval, and œesophageal.

The aortic opening is situated in the middle line between the upper portions of the crura, and in front of the disc between the bodies of the twelfth thoracic and first lumbar vertebræ. It is bounded on either side by a crus, in front by the middle arcuate ligament, and behind by the anterior common ligament of the

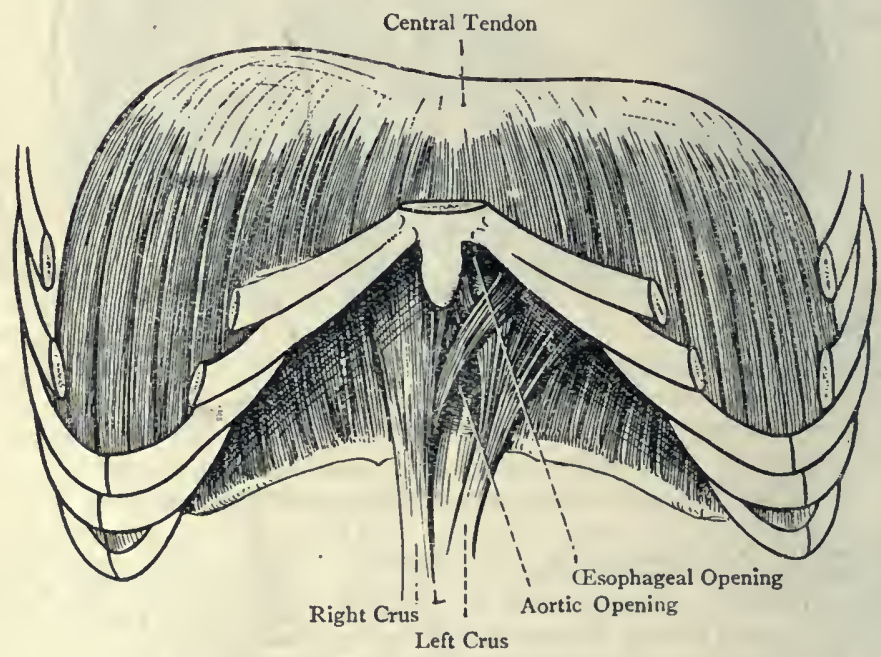

Fig. 326,-The Diaphragm (Superior View)

(After L. Testut's "Anatomie Humaine ").

vertebral column. It is therefore not really an opening in the diaphragm, but is situated behind it. It transmits the aorta, thoracic duct, and right azygos vein, in this order from left to right.

The caval opening (foramen quadratum) is situated in the central tendon close to its posterior border, and at the junction of the right and middle lobes. It is somewhat four-sided with rounded angles, and transmits the inferior vena cava and some of the deep lymphatics of the liver.

The cesophageal opening is situated in the fleshy part of the muscle. It is elliptical, and lies in front, and a little to the left, of the aortic opening, being separated from it behind by the inner decussating fibres of the crura. It transmits the œsophagus, the 
right and left pneumogastric nerves, and the œsophageal branches of the gastric artery.

In addition to the foregoing foramina, the diaphragm presents certain small fissures, as follows : each crus is pierced by the great and small splanchnic nerves, and sometimes by the least. The left crus is also pierced by the lower left azygos vein. The musculo-phrenic artery pierces the costal portion, and the branches of the phrenic nerve are also transmitted through the muscle.

The arcuate ligaments are five in number, as follows : external, right and left ; internal, right and left ; and middle. The external arcuate ligament is a thickening of the upper part of the anterior wall of the sheath of the quadratus lumborum, and extends from the last rib to the tip of the transverse process of the first lumbar vertebra. The subcostal artery and nerve pass downwards and outwards behind it. The internal arcuate ligament is a thickening of the upper part of the sheath of the psoas magnus, and extends from the tip of the transverse process of the first lumbar vertebra to the side of its body. The gangliated cord of the sympathetic passes into the abdomen behind it, and sometimes the least splanchnic nerve. The middle arcuate ligament is a fibrous band which connects the innermost tendinous fibres of the crura on a level with the lower border of the body of the twelfth thoracic vertebra, and it arches over the aorta.

Relations of the Diaphragm-Superior.-The right and left pleuræ with the lungs, and the pericardium with the heart. Inferior.-The peritoneum, except opposite the uncovered area of the posterior surface of the liver ; the liver with its suspensory, coronary, and right and left lateral or triangular ligaments; the stomach; spleen; pancreas; kidneys; and suprarenal capsules.

Development. - The diaphragm is developed in two parts-ventral and dorsal. The ventral part, which is the first to appear, is developed from the upper layer of the septum transversum. It shuts off the pericardial cavity from the peritoneal cavity, leaving two passages, one on each side of the primitive alimentary tube, which are called the thoracic prolongations of the peritoneal cavity, and which are destined to form the pleural cavities.

The dorsal part of the diaphragm is developed from the two pleuro-peritoneal membranes, formed by the pleuro-peritoneal folds, which grow from the dorsal and lateral body-walls, and which close the primitive pleuro-peritoneal openings on either side. The fusion between these pleuro-peritoneal membranes and the ventral part of the diaphragm may be incomplete on one or other side, in which cases a communication is left between the thoracic and abdominal cavities, through which a diaphragmatic hernia may occur.

Gangliated Cord of the Sympathetic.-This cord enters the abdomen behind the inner portion of the internal arcuate ligament. It then descends along the inner border of the psoas magnus, where it lies in front of the bodies of the lumbar vertebræ, and, having passed behind the common iliac artery, it enters the pelvis. The right lumbar cord is under cover of the inferior vena cava, whilst the left lies a little to the left of the aorta, and the lumbar vessels of each side pass beneath the corresponding cord. Each cord usually contains four ganglia.

Branches.-These are arranged in two sets, namely, rami communicantes, and branches of distribution. The rami communicantes, which are long, are of two kinds, white and grey, the latter being 
the more numerous. The white rami are composed of medullated nerve-fibres, and are not more than three in number. They are derived from the anterior primary divisions of the first three lumbar nerves, and proceed to the ganglia. The grey rami pass from all four ganglia to join the anterior primary divisions of the five lumbar nerves. Both white and grey rami pass together beneath the fibrous arches of the psoas magnus in company with the lumbar vessels, and the communications with the lumbar nerves are established close to the intervertebral foramina. The branches of distribution proceed partly from the ganglia and partly from the connecting cord, and are distributed to the coats of the aorta, the bodies and ligaments of the Iumbar vertebræ, and the hypogastric plexus, the last branches crossing the common iliac artery.

Receptaculum Chyli.-This is the dilated commencement of the thoracic duct. It is situated deeply at the upper. part of the posterior abdominal wall in front of the bodies of the first and second lumbar vertebræ, where it has the aorta on its left side and slightly in front, and the right azygos vein on its right side. It is overlapped by the right crus of the diaphragm. It is somewhat elliptical, being about $\frac{1}{6}$ inch broad at its widest part, and about 2 inches in length. Superiorly it becomes narrow, and is continued into the thoracic duct, which enters the thorax through the aortic opening of the diaphragm. It receives the following afferent vessels from below upwards: the right and left lumbar lymphatic trunks from the median lumbar glands, which join its lower narrow end; some of the efferent vessels from the lateral lumbar glands; and the intestinal lymphatic trunk (or trunks) from the mesenteric glands, which sometimes takes up the efferent vessels of the cœliac glands, though these may enter the receptaculum chyli independently.

Azygos Veins.-These are three in number, namely, the vena azygos major, or right azygos vein, the vena azygos minor or lower left azygos vein, and the upper left, or accessory, azygos vein.

The vena azygos major or right azygos vein usually commences in the right ascending lumbar vein, which is formed by longitudinal anastomosing branches passing between the lumbar veins in front of the lumbar transverse processes, and so disposed as to form one vein which communicates with the inferior vena cava and with one or other of the following veins of the right side : the common iliac, the internal iliac, the ilio-lumbar, or the lateral sacral. Sometimes, however, the right azygos vein springs from the posterior aspect of the inferior vena cava close to the right renal vein, or from that renal vein itself, or from the first right lumbar vein. It ascends upon the body of the first lumbar vertebra, where it lies on the right side of the receptaculum chyli under cover of the right crus of the diaphragm, and it enters the thorax through the aortic opening of that muscle. For its subsequent course in the thorax, where it terminates in the superior vena cava, see the section of the thorax.

The vena azygos minor or lower left azygos vein commences in 
the leit ascending lumbar vein, through which it has communications similar to those of the right azygos vein. It may, however, spring from the left renal vein, or the first left lumbar vein. It enters

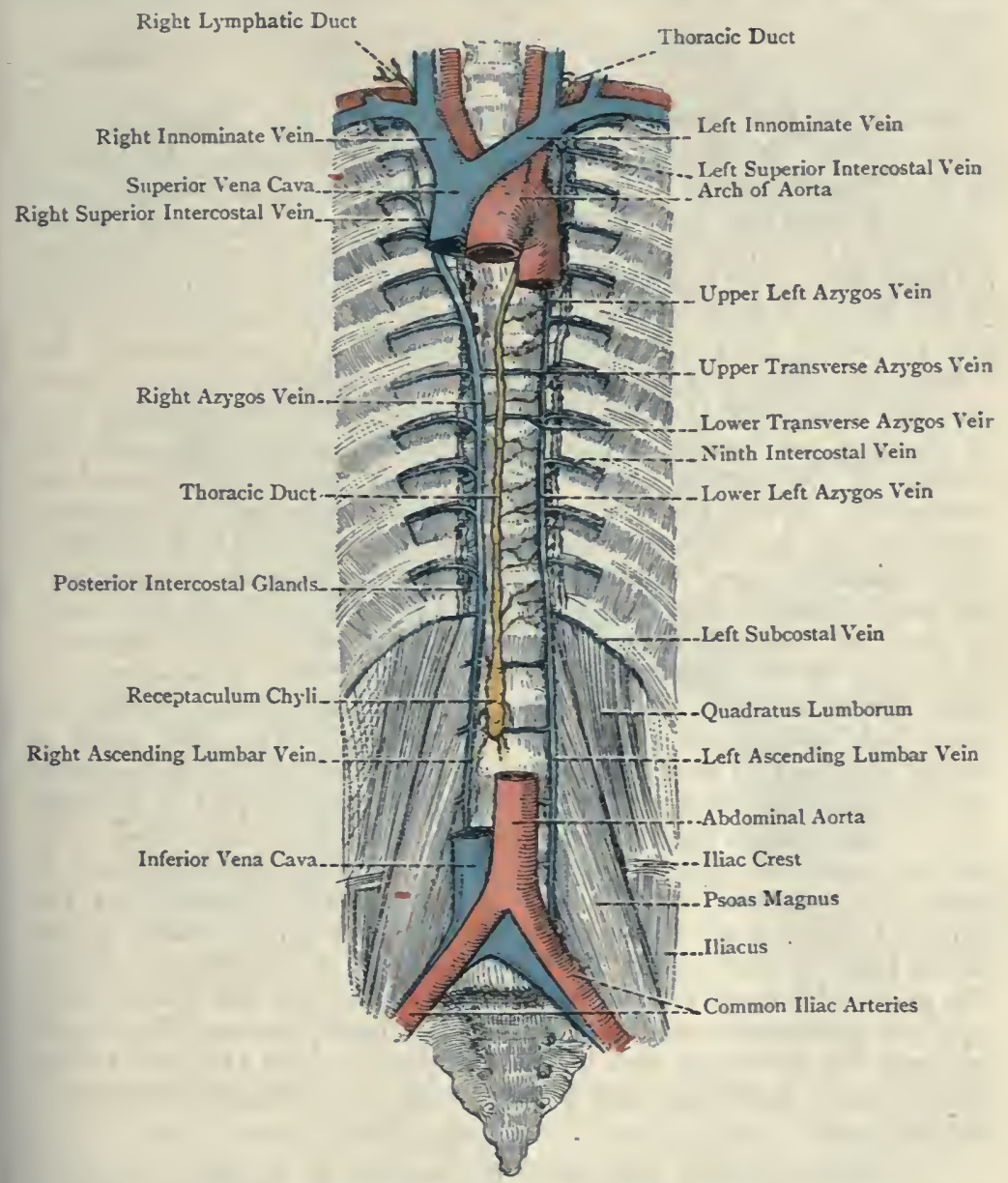

Fig. 327.-The Thoracic Duct, Azygos Veins, and Posterior Intercostal Glands.

the thorax through the left crus of the diaphragm, and subsequently terminates in the right azygos vein.

The right and lower left azygos veins are persistent portions of the right and left cardinal veins of fotal life.

The right and lower left azygos veins, through their connections with the ascending lumbar veins, establish communications with 
the inferior vena cava, and with the common iliac veins or some of their tributaries. They therefore form important channels by which a considerable quantity of blood is returned from the lower limbs and abdominal wall in cases of obstruction of the inferior vena cava.

The upper left, or accessory, azygos vein will be found described in connection with the thorax.

Fasciæ of the Posterior Abdominal Wall-Iliae Fascia.--This fascia covers the iliacus and psoas magnus muscles. Above the level of the iliac crest it is related only to the psoas magnus, and the part covering that muscle is spoken of as the psoas sheath. Superiorly it forms the internal arcuate ligament, which extends between the tip of the first lumbar transverse process and the side of the body of that vertebra. Internally it is attached to $(a)$ the intervertebral discs and contiguous margins of the bodies of the lumbar vertebræ, and $(b)$ the fibrous arches over the lumbar vessels opposite the centre of each lumbar body. Externally, near the tips of the lumbar transverse processes, it blends with the anterior lamina of the lumbar aponeurosis which covers the quadratus lumborum. Below the level of the iliac crest the iliac fascia covers the iliacus as well as the psoas magnus. This part of it is known as the fascia iliaca, and it passes uninterruptedly from the iliacus on to the psoas magnus. Externally it is attached to the anterior two-thirds of the iliac crest immediately within its inner lip, and internally to the ala of the sacrum and the iliac portion of the ilio-pectineal line. Inferiorly it is disposed in the following manner : along the outer half of Poupart's ligament on its deep aspect the fascia is firmly attached to that ligament, and joins the fascia transversalis, the two constructing a canal which contains the first part of the deep circumflex iliac artery. Opposite the external iliac vessels the fascia passes downwards behind them and Poupart's ligament to form the posterior wall of the crural sheath. Internal to the external iliac vessels it is continuous with the pubic lamina of the fascia lata, as that covers the upper part of the pectineus. From the point of junction between the iliac fascia and the pubic fascia lata an intermuscular septum (ilio-pectineal) passes backwards between the pectineus and the psoas magnus to be attached to the ilio-pectineal eminence and the front of the capsular ligament of the hip-joint.

The importance of the iliac fascia has reference to the course taken by pus in cases of lumbar (psoas) abscess. The pus cannot pass outivards over the quadratus lumborum withouit bursting through the psoas sheath, because the psoas sheath is bound down to the anterior wall of the sheath of the quadratus lumborum at the outer border of the psoas muscle. It cannot pass across the vertebral column on account of the attachments of the psoas sheath in that situation. The usual course, therefore, taken by the pus is to diffuse itself downwards within the psoas sheath. On reaching the iliac fossa it may diffuse itself outwards beneath the iliac fascia as that covers the iliacus muscle, but it cannot enter the pelvic cavity on account of the attachment of the iliac fascia to the pelvic brim, unless it bursts through the psons sheath. Subsequently the pus usually treks along the psoas magnus 
witlin its sheath, and, passing behind Poupart's ligament and the femoral vessels encased in the crural sheath, it usually points over the saphenous opening on a level with the small trochanter, where it may simulate a femoral hernia.

Lumbar Aponeurosis.-This is situated between the last rib and the iliac crest, and is to be regarded as the posterior aponeurosis of the transversalis abdominis muscle. When followed backwards it divides into three laminæ, anterior, middle, and posterior. The anterior lamina, which is thin, covers the quadratus lumborum, and is attached internally to the fronts of the transverse processes of the lumbar vertebræ near their tips. In this situation it is interposed between the quadratus lumborum and psoas magnus, and receives the iliac fascia which forms the psoas sheath. At the outer

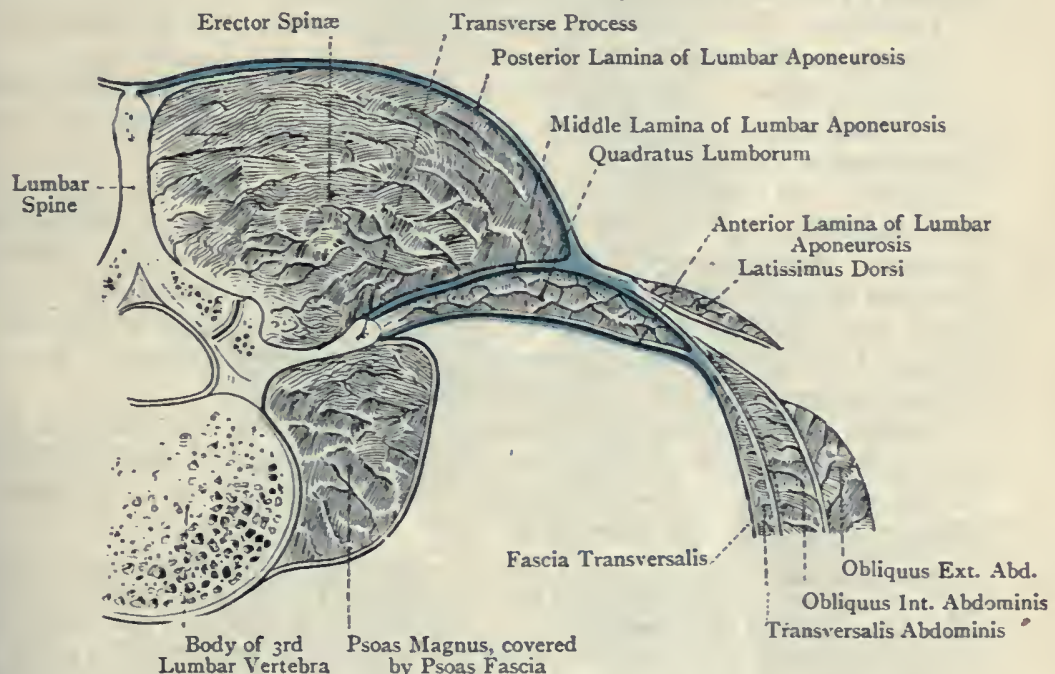

Fig. 328.-Diagram of the Lumbar Aponeurosis.

border of the quadratus lumborum it joins the middle lamina, and is here also continuous with the fascia transversalis. Superiorly it forms the external arcuate ligament, which extends between the last rib and the tip of the first lumbar transverse process. Inferiorly it is attached to the ilio-lumbar ligament and the contiguous part of the inner lip of the iliac crest. The middle lamina, which is of considerable strength, is attached internally to the tips of the lumbar transverse processes, and externally, at the outer border of the quadratus lumborum, it is joined by the anterior lamina, whilst at the outer border of the erector spinæ it receives the posterior lamina. Superiorly it is attached to the lower border of the last rib, and inferiorly to the back part of the summit of the iliac crest. The middle lamina lies between the quadratus lumborum and the 
erector spinæ. The posterior lamina, which is also very strong, is attached to the spinous processes of the lumbar and sacral vertebræ, and the posterior fourth of the outer lip of the iliac crest. It lies behind the erector spinæ, at the outer border of which it blends with the middle lamina, and so the muscle is enclosed in a sheath. The posterior lamina is joined by the vertebral aponeurosis, and affords attachment to the latissimus dorsi and serratus posticus inferior.

\section{Muscles of the Posterior Abdominal Wall-Psoas Magnus-} Origin.-(I) The fronts of the transverse processes of the lumbar vertebræ; (2) the lateral aspects of the intervertebral discs, and of the adjacent borders of the twelfth thoracic, and all the lumbar, vertebræ; and (3) a series of fibrous arches which cross the lumbar vessels at the centres of the bodies of the lumbar vertebræ.

Insertion.- The small trochanter of the femur, by a tendon which receives, on its outer side, the greater part of the iliacus.

Nerve-supply. - The lumbar plexus. The branches come more particularly from the anterior primary divisions of the second and third lumbar nerves.

Action.-Acting from its origin the muscle is a powerful flexor of the thigh upon the pelvis, coming into play in walking, or ascending a stair. Acting from its insertion it is a flexor of the lumbar portion of the vertebral column upon the pelvis, and of the pelvis upon the thigh, as in the act of stooping. The muscle of one side, acting from its insertion, is capable of producing lateral flexion of the lumbar portion of the vertebral column.

As the muscle descends close to the pelvic brim the fibres of the iliacus begin to join the outer side of its tendon, and they continue to do so as far as the insertion, thus giving rise to a conjoined muscle known as the ilio-psoas.

Psoas Parvus.-This muscle is present in man on one or both sides in about 45 per cent. of bodies. It arises from the lateral aspect of the intervertebral disc between the twelfth thoracic and first lumbar vertebrx, and from the tiouous borders of their bodies, by means of a small fleshy belly, which is concin $\downarrow$ u $1+2$ inches long. It is then replaced by a long, narrow, flat usually abou 2 rsonds as it is about to take insertion into the middle of the tendon, which expu the ilio-pectineal eminence, in which latter situation ilio-pectineal line and the ilio-pectineal eminence, in

it blends with the ilio-pectimarior primary division of the first lumbar nerve.

The nerve-supply is the antc. - tends to flex the pelvis upon the vertebral Acting from its origin the muscle sheath. Acting from its insertion it column, and is a tensor of the psoas reswart of the thoracic portion, of the tends to flex the lumbar portion, and lower 1 "is teristic action is seen in the vertebral column upon the pelvis. Its charcertic to the act of leaping, that position assumed by saltatory animals preparatory' is and vertebral column. position consisting in a drawing forwards of the pelvis and vertebral column.

The psoas parvus lies along the anterior aspect of the psoas magnus closed tendon turn to its inner border, except at the pelvic brim, where its expan, to the inner side of that muscle.

Iliacus-Origin.-(I) The upper surface of the ala of the sacrum (2) the anterior sacro-iliac, ilio-lumbar, and lumbo-sacral lis raments and (3) the upper half of the iliac fossa, reaching anteriorly, is lov as the anterior inferior iliac spine. 
Insertion.-(I) The outer aspect of the tendon of the psoas magnus; (2) the triangular surface which is situated below, and in front of, the small trochanter of the femur (between it and the spiral line); and (3) the ilio-femoral ligament. The fibres inserted into the ilio-femoral ligament are those which arise in the region of the anterior inferior iliac spine. They are sometimes separated from the rest of the muscle, and are then known as the iliacus miror or ilio-capsularis.

Nerve-supply. - The anterior femoral nerve.

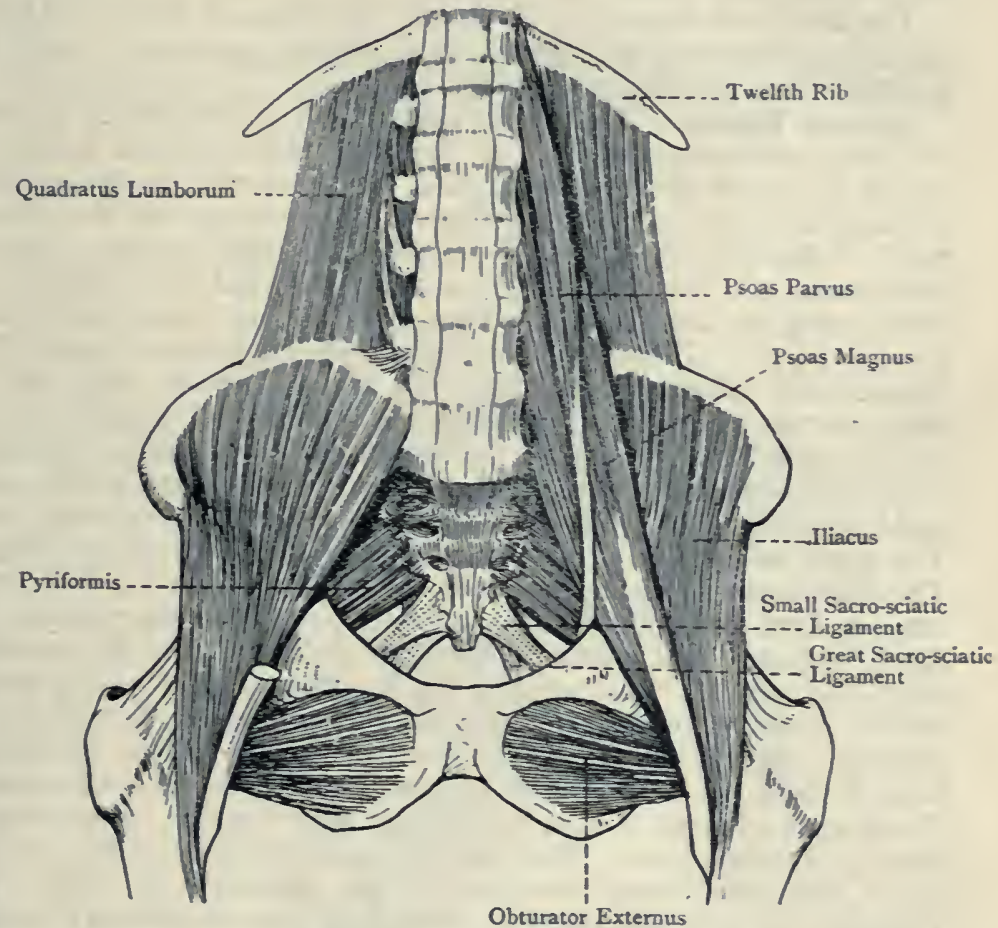

Fig. 329.-The Psoas, Iliacus, and Quadratus Lumborum Muscles.

Action.-Acting from its origin the muscle is a flexor of the thigh upon the pelvis. Acting from its insertion it is a flexor of the pelvis upon the thigh.

Quadratus Lumborum-Origin.-(I) The ilio-lumbar ligament; (2) the inner lip of the crest of the ilium for about 2 inches behind and outside the ilio-lumbar ligament; and (3) the tips of the transverse processes of the lower three or four lumbar vertebræ.

Insertion.-(I) The lower border of the last rib along its inner half, and (2) the tips of the transverse processes of the upper three or 
four lumbar vertebræ, by tendinous slips which lie behind the slips of origin.

Nerve-supply. - The subcostal nerve and the anterior primary divisions of the first two lumbar nerves.

Action.-Acting from its origin the muscle depresses and fixes the last rib, and is therefore a muscle of inspiration, inasmuch as it is auxiliary to the diaphragm. In depressing the last rib the muscle is also capable of producing lateral flexion of the vertebral column. Acting from the last rib it will produce lateral flexion of the pelvis.

The muscle is encased in a sheath, the anterior wall of which is formed by the anterior lamina of the lumbar aponeurosis, and the posterior wall by the middle lamina.

Lumbar Plexus.- The lumbar plexus is situated deeply in front of the transverse processes of the first three lumbar vertebræ, where it lies in the psoas magnus. It is formed by the anterior primary divisions of the first three lumbar nerves and the greater part of that of the fourth. In addition, the anterior primary division of the first lumbar is usually reinforced by a small communicating branch from the subcostal nerve, called the dorsolumbar nerve. The nerves concerned in the lumbar plexus first furnish the following branches: (I) the first gives twigs to the psoas parvus when present; (2) the first and second supply branches to the quadratus lumborum; (3) the second and third give branches to the psoas magnus; and (4) the upper two or three furnish white rami communicantes to the lumbar sympathetic gangliated cord. The mode of formation and branches of the plexus are as follows: The first lumbar, having been, as a rule, reinforced by the dorso"umbar from the subcostal, furnishes, from above downwards, the A. musclengastric and inguinal, and then it descends to join a branch ilio-hypus - .... The second, third, and that part of the fourth from the second. This mitu arises break up into a small anterior or which enters into the plexinracic ardorsal division. The descending ventral, and a large posterior or ${ }^{\varsigma}$ by mio from the ventral division of branch from the first joins a branch then ${ }^{1}$ ?erve, which arises next in the second to form the genito-femoral iaminenous nerve arises by two order to the inguinal. The external cutanir sepcond and third. The roots from the dorsal divisions of the $\sec ^{\text {rision }} v$ - the dorsal divisions anterior femoral nerve arises by three roots from cting? third being the of the second, third, and fourth, the root from the the roots from the largest. The obturator nerve arises usually by thre acit the root from ventral divisions of the second, third, and fourth, whe is nerve, when the second may be absent. The accessory obturati-n pith, which are present, arises by two roots from the third and fous ${ }^{\circ}$ al and main interposed between the roots of the anterior crui of obturator nerves.

The branches of the lumbar plexus are accordingly nteri. lumbar. $^{2}$. Muscular to

I. Muscular to psoas parvus (when present), from firs ${ }^{\star}$ and second lumbar. 
3. Muscular to psoas magnus, from second and third lumbar.

4. Two or three white rami communicantes, to the lumbar sympathetic gangliated cord, from the upper two or three lumbar.

5. Ilio-hypogastric and inguinal, from first lumbar.

6. Genito-femoral, from first and ventral division of second lumbar.

7. External cutaneous, from dorsal divisions of second and third lumbar.

8. Anterior femoral, from dorsal divisions of second, third, and fourth lumbar. lumbar.

9. Obturator, from ventrul divisions of second, third, and fourth

I0. Accessory obturator (when present), from third and fourth lumbar, between the roots of the anterior crural and main obturator.

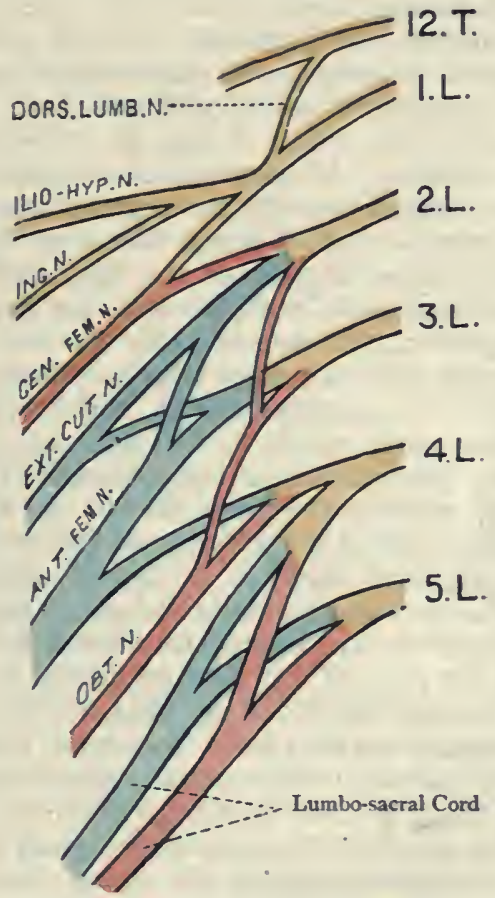

Fig. 330.-Diagrail of the Right Lumbar Plexus.

The ilio-hypogastric nerve, having pierced the outer border of the psoas magnus near its upper part, passes outwards and downwards over the quadratus lumborum, lying below the subcostal nerve and behind the kidney. It then pierces the posterior part of the transversalis abdominis a little above the iliac crest, and furnishes its iliac or lateral cutaneous branch. which, perforating 
the internal and external oblique, crosses the iliac crest at the junction of its middle and anterior thirds to be distributed to the integument of the adjacent part of the gluteal region. The nerve continues its course forwards between the internal oblique and transversalis abdominis, supplying branches to these muscles and communicating with the inguinal nerve. About $I$ inch in front of the anterior superior iliac spine it pierces the internal oblique, and then runs forwards between the Poupart fibres of that muscle and the external oblique aponeurosis. - Finally it pierces that aponeurosis $\mathbf{I}$ inch above the external abdominal ring, and is distributed to the integument of the suprapubic region, where it is in series with the anterior cutaneous nerves. The ilio-hypogastric nerve is serially continuous with the intercostal nerves, like which it gives off a lateral cutaneous branch, called iliac, and then ends as an anterior cutaneous nerve.

The inguinal nerve (ilio-inguinal), having pierced the outer border of the psoas magnus lower down than, but close to, the ilio-hypogastric, passes obliquely outwards and downwards over the quadratus lumborum, where it may lie below the lower end of the kidney or behind it. It then passes forwards internal to the inner lip of the iliac crest beneath the transversalis abdominis. In this part of its course it rests upon the iliac fascia and iliacus muscle. Near the anterior part of the iliac crest it pierces the transversalis, and here communicates with the hypogastric branch of the ilio-hypogastric. It subsequently perforates the Poupart fibres of the internal oblique, after which it descends through the lower two-thirds of the inguinal canal, and emerges through the external abdominal ring, where it lies external to the spermatic cord. Finally, having pierced the intercolumnar fascia, it is distributed to the integument of the inner side of the thigh in its upper third, and the integument of the scrotum, or labium majus, according to the sex. The inguinal nerve in its course supplies branches to the internal oblique and transversalis abdominis muscles. It differs from the ilio-hypogastric and intercostal nerves in the following two respects : it does not give off any iliac or lateral cutaneous branch, and it is not distributed to the integument of the abdominal wall.

The genito-femoral nerve passes forwards through the psoas magnus, and appears on its superficial surface about the level of the body of the third lumbar vertebra, where it lies close to the inner border of the muscle. It sometimes pierces the muscle in two parts, due to an early division of the nerve into its genital and crural branches. It then descends upon the psoas sheath, passing slightly outwards, and crossing behind the ureter. At a variable distance above Poupart's ligament (sometimes in the psoas magnus) the nerve divides into two branches, genital and crural. The genital branch lies upon the external iliac artery close above. Poupart's ligament, and enters the inguinal canal through the internal abdominal ring to be distributed to the cremaster muscle. The crural branch descends on the outer side of the external iliac artery 
and passes out behind Poupart's ligament, having, just prior to this, crossed the deep circumflex iliac artery. On entering the thigh it lies for about $\frac{1}{2}$ inch within the crural sheath, and subsequently, piercing the outer wall of that sheath, it is distributed to the integument over Scarpa's triangle.

The external cutaneous nerve pierces the outer border of the psoas magnus near its centre, and takes a direction downwards and outwards over the back part of the iliac crest into the iliac fossa. It then crosses the iliacus under cover of the fascia iliaca towards the anterior superior iliac spine, where it emerges on to the thigh behind the outer end of Poupart's ligament. For the distribution of the nerve in the thigh, see p. 449.

The anterior femoral nerve pierces the outer border of the psoas magnus about the level of the back part of the pelvic brim. It then passes forwards, lying deeply between the psoas magnus and iliacus, and emerges on to the thigh behind Poupart's ligament. Whilst in the abdominal cavity it gives branches to the iliacus muscle. The course and distribution of the nerve in the thigh will be found on p. 470 .

The obturator nerve pierces the inner border of the psoas magnus at the back part of the pelvic brim, and it lies upon the ala of the sacrum, having the lumbo-sacral cord on its inner side. Passing deeply behind the common iliac artery it enters the pelvic cavity, and passes along the outer wall a little below the pelvic brim, where it lies above the obturator artery. It then emerges on to the thigh through the obturator canal. For the course and distribution of the nerve in the thigh, see P. 474.

The accessory obturator nerve (when present) pierces the inner border of the psoas magnus close to the main obturator nerve, but, unlike it, does not enter the pelvic cavity. Its course is forwards along the inner border of the psoas magnus underneath the external iliac vessels, and it emerges on to the thigh by passing over the superior pubic ramus beneath the pectineus muscle. Under cover of that muscle it divides into the following three branches: articular to the hip-joint; muscular to the deep surface of the pectineus; and a reinforcing branch to join the superficial or anterior division of the main obturator nerve.

Varieties of the Lumbar Plexus.-These assume the form of two types, high or prefixed, and low or postfixed. In the prefixed type the anterior primary division of the third lumbar is a nervus furcalis, and takes part in the sacral plexus, whilst in the postfixed type the anterior primary division of the fifth lumbar is a nervus furcalis, and takes part in the lumbar plexus.

Lumbo-sacral Cord.-This is formed by the union of the ventral and dorsal divisions of the descending branch of the fourth lumbar nerve with the ventral and dorsal divisions of the anterior primary division of the fifth lumbar. It is a large double trunk, which rests upon the ala of the sacrum, being at first under cover of the psoas magnus, and subsequently lying on its inner side, where it has the obturator nerve external to it. In its course it passes behind the common and internal iliac vessels, and in the pelvis, its two divisions having joined those of the anterior primary division of the first sacral nerve, it takes part in the sacral plexus, entering 
more particularly the upper or outer band of that plexus which is continued into the great sciatic nerve.

The anterior primary division of the fourth lumbar nerve is known as a nervus furcalis from the fact that it is distributed partly to the lumbar and partly to the sacral plexus.

Lumbar Arteries.- These are branches of the abdominal aorta, being parietal in their distribution, and they are serially continuous with the aortic intercostal and subcostal arteries. They are eight in number, four right and four left, and they arise in pairs, separately or conjointly, from the posterior aspect of the parent trunk. They occupy the grooves at the centres of the bodies of the first four lumbar vertebræ. As each artery winds round a vertebral body it passes beneath one of the fibrous arches of the psoas magnus and the lumbar sympathetic gangliated cord. It then passes behind the psoas magnus and lumbar plexus, and, on reaching the interval between two adjacent lumbar transverse processes, it divides into two branches, dorsal and abdominal. The upper two arteries pass beneath the corresponding crus of the diaphragm, and those of the right side also pass beneath the receptaculum chyli and right azygos vein. All four arteries on the right side pass beneath the inferior vena cava. The trunk of each lumbar artery gives off a few vertebral branches to the body and ligaments of the adjacent vertebra, and muscular branches to the psoas magnus. The dorsal branch passes backwards between the adjacent transverse processes, in company with the posterior primary division of a spinal nerve, and it divides into an internal and external branch. The internal branch supplies the multifidus spinæ, and the external branch supplies the erector spinæ, giving also cutaneous branches which accompany the cutaneous nerves to the integument. Opposite an intervertebral foramen the dorsal branch furnishes a spinal branch, which enters the spinal canal through the foramen, to be distributed to the spinal cord and its coverings, as well as to the wall of the canal.

The abdominal branch is to be regarded as the continuation of a lumbar artery. These branches usually pass behind the quadratus lumborum, with the exception, as a rule, of that of the fourth. At the outer border of that muscle they pierce the posterior aponeurosis of the transversalis abdominis, and pass forwards between that muscle and the internal oblique, as far as the lower part of the rectus abdominis, which they enter. They furnish the following offsets, in order : muscular to the quadratus lumborum; capsular to the capsule of the kidney, which anastomose with branches of the renal artery; subperitoneal to the subperitoneal areolar tissue, which anastomose therein with branches of the ilio-lumbar, the inferior phrenic, and the hepatic, colic, and renal arteries, thus forming the subperitoneal arterial plexus of Turner; muscular to the abdominal muscles, which anastomose above with the lower two intercostal and subcostal arteries, below with the lateral or intermuscular epigastric of the deep circumflex iliac, and ilio-lumbar, 
and in front with the deep epigastric. Sometimes there are five lumbar arteries on each side, the fifth pair coming usually from the middle sacral artery. Each of these passes beneath the corresponding common iliac vessels, and, having furnished a dorsal branch, usually to the gluteus maximus, it is distributed over the lateral mass of the sacrum and ends in the iliacus, where it anastomoses with the deep circumflex iliac artery.

The lumbar veins open into the inferior vena cava, those of the left side passing behind the abdominal aorta. The vessels of each side are connected by a series of longitudinal anastomosing veins in front of the lumbar transverse processes, and the longitudinal vessel thus formed is called the ascending lumbar vein.

Subcostal Artery.-This vessel is the last parietal branch of the thoracic aorta. It lies below the last rib, and is in series with the aortic intercostals above and the lumbar arteries below. It winds round the side of the body of the twelfth thoracic vertebra, and courses along the lower border of the twelfth rib with the subcostal nerve, passing behind the external arcuate ligament of the diaphragm and in front of the quadratus lumborum. This part of the vessel is behind the kidney and the ascending or descending colon, according to the side. Its subsequent course corresponds with that of the abdominal branches of the lumbar arteries. It anastomoses with the lower two intercostal arteries, the abdominal branches of the lumbar arteries, the lateral or intermuscular epigastric of the deep circumflex iliac, and the deep epigastric artery. This vessel has to be borne in mind in such operations as nephrotomy, nephrorrhaphy, and nephrectomy.

The subcostal vein of each side enters the thorax behind the external arcuate ligament of the diaphragm, the right opening into the right azygos vein, and the left into the lower left azygos vein.

Subcostal Nerve.-This is the anterior primary division of the twelfth thoracic nerve, and is in series with the eleventh or last intercostal. It accompanies the subcostal artery, and ultimately enters the sheath of the rectus abdominis, which muscle it pierces from behind forwards to become an anterior cutaneous nerve. In its course it gives off an undivided lateral cutaneous or iliac branch, which pierces the internal and external oblique muscles, and descends over the iliac crest to be distributed to the integument of the anterior part of the gluteal region. Besides this branch it furnishes the following offsets: (I) dorso-lumbar to the anterior primary division of the first lumbar nerve; and (2) branches to the quadratus lumborum, transversalis abdominis, internal oblique, and pyramidalis abdominis.

Lumbar Glands.- These are very numerous, and are divided into three groups - median, and two lateral, right and left.

The median lumbar glands are about twelve in number, and lie behind the parietal peritoneum, in front of, behind, and along the sides of the aorta (as high as the root of the superior mesenteric artery) and inferior vena cava.

A few of the lower glands lie along 
the common iliac arteries, and these are sometimes spoken of as the common iliac glands. The median lumbar glands receive their afferent vessels from the following sources:(I) the external iliac glands; (2) the internal iliac glands; (3) the sacral glands; (4) the efferent lymphatics of the testes in the male, and of the ovaries, Fallopian tubes, and upper end of the uterus in the female; $(5)$ the kidneys; (6) the suprarenal capsules; $(7)$ the vertebral part of the diaphragm; and (8) some of the efferent vessels of the lateral lumbar glands. Their efferent vessels unite to form two lumbar lymphatic trunks, right and left, which open into the lower end of

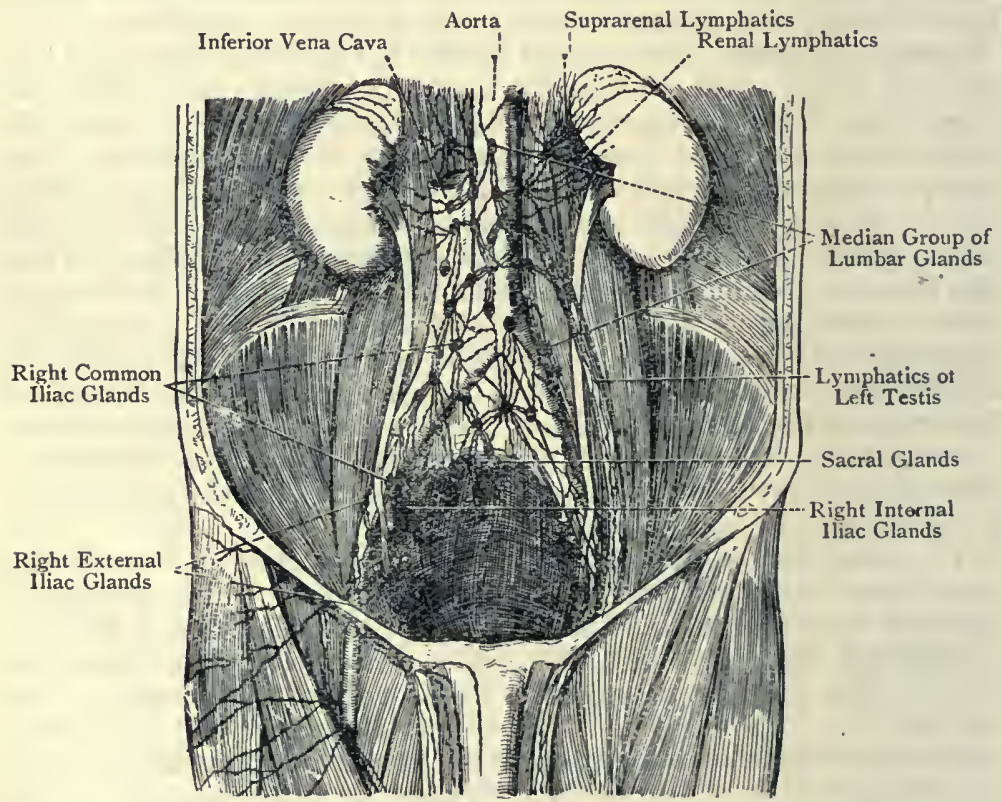

Fig. 33I.-Lymphatics of the Abdomen (After Mascagni).

the receptaculum chyli on a level with the body of the second lumbar vertebra. The left trunk receives the lymphatics of the lower part of the left colon, iliac colon, and pelvic colon.

The lateral lumbar glands are small but very numerous, being about twenty in number. They are situated behind the psoas magnus, where they lie between the lumbar transverse processes, and they receive their afferent vessels from the following sources: (I) the posterior abdominal wall and deep muscles of the back, and (2) the spinal canal. Some of their efferent vessels join the right and left lumbar lymphatic trunks, whilst others open independently into the lower end of the receptaculum chyli.

Common Iliac Arteries.-These vessels are the terminal 
branches of the abdominal aorta. They arise from that vessel opposite the centre of the body of the fourth lumbar vertebra, a finger's breadth to the left of the middle line, and they at once diverge from each other. Their course is obliquely downwards and outwards over the lower portion of the body of the fourth and the whole of that of the fifth lumbar vertebra, as well as the disc between the two. Each artery, on arriving opposite the lumbosacral articulation, ends by dividing into external and internal iliac arteries. The length of the right common iliac is about 2 inches, and that of the left about $I_{\frac{3}{4}}$ inches. The left vessel is less oblique in direction than the right, and the course of each may be indicated in the following manner: draw a line from a point fully I inch below the umbilicus, a finger's breadth to the left of the middle line, to a point at the groin midway between the anterior superior iliac spine and the symphysis pubis, and let this line be slightly curved with the convexity directed outwards. About the upper 2 inches of this line indicate the course of the common iliac artery, and the remainder that of the external iliac vessel.

Relations-Anterior.-The peritoneum, coils of the small intestine, one half of the aortic sympathetic plexus, and the ureter, which latter crosses the artery close to its termination, though it may be transferred to the commencement of the external iliac vessel. An additional superficial relation of the left common iliac artery is that it is crossed by the superior hemorrhoidal vessels.

Posterior.-Each artery rests upon the lower half of the body of the fourth and the whole of that of the fifth lumbar vertebra, as well as the disc above and below the latter, and the gangliated sympathetic chain. The right vessel is separated from the foregoing structures by the commencement of the inferior vena cava, the terminal part of the left common iliac vein, and the right common iliac vein, whilst the left vessel is free from posterior venous relations. Lying deeply behind each artery there are the obturator nerve, lumbo-sacral cord, and ilio-lumbar artery.

External.-On the outer side of the right vessel there are, from above downwards, the inferior vena cava, right common iliac vein, and psoas magnus. On the outer side of the left vessel is the psoas magnus.

Internal.-On the inner side of the right vessel, from below upwards, there are the right common iliac vein, the left common iliac vein, and the hypogastric sympathetic plexus. On the inner side of the left vessel there are the left common iliac vein, and the hypogastric plexus.

It is to be noted that the left artery is related only to its own vein, which lies on its inner side. The right artery, on the other hand, is related to three veins as follows: the inferior vena cava, which lies partly behind its upper end and partly on its outer side; the terminal part of the left common iliac vein, which lies partly on its inner side and partly behind it; and the right common iliac 
vein, which, from below upwards, lies first on its inner side, then behind it, and finally on its outer side.

Branches.-These are as follows: peritoneal to the peritoneum and subperitoneal areolar tissue; muscular to the psoas magnus; ureteric to the ureter (all of small size and unimportant); external iliac; and internal iliac. In some cases the common iliac gives off one or other of the following vessels : ilio-lumbar, middle sacral, lateral sacral, lumbar, or an aberrant renal artery.

Varieties. - The chief variety affects the length of the vessel. It may be very short, which is due either to a low bifurcation of the aorta or a high bifurcation of the artery itself ; or it may be very long, which is due to exactly opposite causes. When abnormally long, the vessel is usually more or less tortuous.

Collateral Circulation.-After ligature of a common iliac artery, the chief channels by which the circulation is carried on are as follows : (I) the superior epigastric of the internal manmary from the first part of the subclavian anastomoses with the deep epigastric of the external iliac; (2) the lumbar branches of the aorta anastomose with $(a)$ the lateral or intermuscular epigastric of the deep circumflex iliac from the external iliac, and $(b)$ the ilio-lumbar of the internal iliac; (3) the superior hemorrhoidal of the inferior mesenteric from the aorta anastomoses with $(a)$ the middle hemorrhoidal of the internal iliac, and $(b)$ the inferior hemorrhoidal of the internal pudic from the internal iliac; (4) the middle sacral from the aorta anastomoses with the lateral sacral branches of the internal iliac; and (5) the pubic branches of the obturator from the internal iliac and of the deep epigastric from the external iliac, both of one side, anastomose across the middle line with the corresponding branches of the opposite side. The vesical, and middle and inferior hemorrhoidal arteries of one side anastomose in a similar manner with those of the opposite side.

Common Iliac Veins.-Each vein is formed by the union of the external and internal iliac veins opposite the corresponding sacroiliac articulation, on a level with the brim of the pelvis. They unite to form the inferior vena cava opposite the upper border of the body of the fifth lumbar vertebra a little to the right of the middle line, behind and on the right side of the right common iliac artery. The right vein is necessarily shorter than the left, and it ascends almost vertically, lying at first internal to, then behind, and finally on the outer side of its own artery. The left vein ascends very obliquely from left to right, lying internal to its own artery, and then behind that of the right side. It crosses the middle sacral artery, and is crossed by the superior hemorrhoidal vessels and the left half of the aortic plexus. The common iliac veins are usually destitute of valves.

Tributaries.- These are chiefly the external iliac, internal iliac, and ilio-lumbar. In addition, the left vein receives the middle sacral vein.

The left common iliac vein is mainly the persistent and enlarged transverse branch (transverse iliac) which connects the right and left cardinal veins of the embryo above the back part of the pelvic brim. Its commencement, however, is developed from a portion of the left cardinal vein. The right common iliac vein is developed from the part of the right cardinal vein which intervenes between the termination of the right external iliac vein and the right extremity of the transverse iliac vein. 
Common Iliac Lymphatic Glands.-These glands are about nine in number, and are arranged in three groups-external, posterior, and internal-which lie along the common iliac artery. The afferent vessels of the external group are derived from the external iliac glands; those of the posterior group come from the internal iliac glands; and the afferent vessels of the internal group proceed from (I) the prostate gland, (2) the base of the bladder, (3) the lower part of the vagina, and (4) the cervix uteri.

The efferent vessels of all the common iliac glands of one side pass to the lateral aortic glands of the same side.

External Iliac Artery.-This vessel is the larger of the two terminal divisions of the common iliac in the adult. It extends from the lumbo-sacral articulation to the lower margin of Poupart's ligament, where it is continued into the common femoral artery. Its course is along the pelvic brim, and at the groin it passes through the vascular lacuna at a point midway between the anterior superior iliac spine and the symphysis pubis. The course of the vessel may be indicated in the following manner: Draw a line from a point fully I inch below the umbilicus, a finger's breadth to the left of the middle line, to a point at the groin midway between the anterior superior iliac spine and the symphysis pubis, and let this line be slightly curved with the convexity directed outwards. About the upper 2 inches of this line indicate the course of the common iliac artery, and the remainder that of the external iliac vessel. The line indicating the course of the vessel corresponds to the lower part of that which has been given as indicating the course of the common iliac. The vessel is from $3 \frac{1}{2}$ to 4 inches long, and its direction is downwards, outwards, and forwards.

Relations-Anterior.-The artery is covered by the parietal peritoneum and subperitoneal areolar tissue, the portion of the latter which is related to it being known as Abernethy's fascia. The right vessel at its commencement is crossed by the terminal part of the ileum, and sometimes by the vermiform appendix, whilst the left at its commencement is crossed by the pelvic colon, and each may be crossed by the ureter. In the female both arteries are crossed superiorly by the ovarian vessels. Near Poupart's ligament each vessel is crossed by the deep circumflex iliac vein, and the genital branch of the genito-crural nerve lies upon it. The spermatic vessels in the male also lie for a short distance upon it in this situation, and the vas deferens (or round ligament of the uterus) arches over it from without inwards. The external iliac glands lie along the artery. Posterior.-The artery rests upon the iliac fascia at the pelvic brim, except for a little above Poupart's ligament, where it lies upon the psoas muscle with the intervention of the iliac fascia, as that forms its sheath. The right artery at its commencement has its own vein behind it for a short distance, and each vessel may have the accessory obturator nerve as a deep posterior relation. External.- The psoas magnus covered by the iliac fascia, the genito. crural nerve, and its crural branch Internal.-The peritoneum, 
the subperitoneal areolar tissue (Abernethy's fascia), which binds the artery with its vein to the iliac fascia, the external iliac vein (except for a short distance above on the right side, where the vein is behind its artery), and the vas deferens near Poupart's ligament.

Branches.--These are as follows: muscular to the psoas magnus; glandular to the external iliac glands (both unimportant); deep epigastric; and deep circumflex iliac. For the latter two, see pp. 662,663 .

Varieties of the Branches.-(1) The origin of the deep epigastric may be transferred to the common femoral, or to the arteria profunda femoris, and the deep circumflex iliac may be transferred to the common femoral. (2) The internal circumflex, obturator, or arteria profunda femoris may arise from the external iliac, in which latter case two large arteries would emerge on to the thigh beneath Poupart's ligament.

The external iliac vein is the continuation of the femoral vein. It extends from the lower border of Poupart's ligament to the sacro-iliac articulation on a level with the brim of the pelvis, where it joins the internal iliac, and so forms the common iliac vein. The right vein lies at first internal to its artery, and then behind it. The left vein lies internal to its artery throughout. Its chief tributaries are the deep epigastric and deep circumflex iliac veins.

The external iliac vein of adult life is preceded in function by the sciatic vein of the embryo, which is the primitive vein of the lower limb. In the process of development the upper part of the femoral and the whole of the external iliac vein of the adult are continued upwards from the long saphenous vein to the cardinal portion of each common iliac vein, and the sciatic vein is now a tributary of the internal iliac.

Collateral Circulation. - When the external iliac artery is ligatured, the collateral circulation is carried on through the following channels: (I) the superior epigastric of the internal mammary from the first part of the subclavian anastomoses with the deep epigastric of the external iliac; (2) the pubic branch of the obturator from the internal iliac anastomoses with the pubic branch of the deep epigastric ; (3) the ilio-lumbar and gluteal, both from the internal iliac, and the abdominal branches of the lumbar arteries from the aorta anastomose with the deep circumfiex iliac of the external iliac; $(4)$ the obturator from the internal iliac anastomoses with the internal circumflex of the arteria profunda femoris; (5) the sciatic from the internal iliac anastomoses with the internal and external circumflex, and the first perforating of the arteria profunda femoris; (6) the gluteal anastomoses with the external circumflex and the ascending branch of the internal circumflex from the arteria profunda femoris ; (7) the arteria comes nervi ischiadici of the sciatic anastomoses with the perforating branches of the arteria profunda femoris; and (8) the superficial perineal and dorsalis penis of the internal pudic from the internal iliac anastomose with the superior and inferior external pudics of the common femoral.

External Iliac Lymphatic Glands.-These glands are related to the external iliac vessels, and are about twelve in number. They are usually arranged in three chains-outer, middle, and inner-there being about four glands in each chain. The outer chain lies on the 
outer side of the external iliac artery, between it and the psoas magnus muscle, except the lowest gland, which lies upon that muscle. The middle chain lies in front of the external iliac vessels. The inner chain lies below the level of the external iliac vein, upon the upper part of the lateral wall of the pelvis, above the obturator nerve. One of the glands of this chain may lie within the pelvic entrance to the obturator canal, and is spoken of as the obturator gland, but it is not constant. The lowest gland of each chain lies close to the deep aspect of Poupart's ligament, and these are known as the retro-femoral glands-external, middle, and internal respectively.

The afferent vessels of the external iliac glands convey lymph from the following sources:

I. The deep femoral glands.

2. Some of the inguinal and superficial femoral glands.

3. The deep structures of the antero-lateral abdominal wall below the umbilicus.

4. To a certain extent the glans penis, or glans clitoridis, these lymphatics passing along the inguinal canal.

5. The adductor muscles.

6 . The prostate gland and prostatic urethra, in part.

7. The bladder.

8. Part of the membranous and the bulbar portions of the urethra.

9. The upper part of the vagina.

Io. The body and cervix of the uterus.

The efferent vessels of all the external iliac glands pass to the common iliac glands.

Lacunar Region.-The lacunar region is situated between Poupart's ligament and the anterior margin of the os innominatum, and is divided into two compartments-muscular and vascular.

The muscular lacuna is subdivided into two portions, external or iliac, and internal or pectineal, by the ilio-pectineal septum, which separaces the psoas magnus from the pectineus. This septum passes between the ilio-pectineal eminence and the fascia iliaca at its point of junction with the upper part of the pubic portion of the fascia lata. The iliac compartment, which is of large size, is bounded in front by the outer part of Poupart's ligament and the iliac fascia, behind by the anterior margin of the ilium, and internally by the ilio-pectineal septum. It transmits (I) the iliopsoas muscle, (2) the external cutaneous nerve, and (3) the anterior femoral nerve. The pectineal compartment is situated between the superior pubic ramus behind, and the upper part of the pubic lamina of the fascia lata in front, the ilio-pectineal septum being external to it. It contains the origin of the pectineus muscle, and is shut off from the abdominal cavity by the attachment of the pubic lamina of the fascia lata to the pectineal portion of the iliopectineal line. In connection with this portion of the fascia lata 
there is a bundle of fibres, known as the pubic ligament of Cooper. This ligament extends between the ilio-pectineal eminence and the pubic spine, between which points it is attached to the pectineal portion of the ilio-pectineal line in front of Gimbernat's ligament, being closely incorporated with the pubic lamina of the fascia lata.

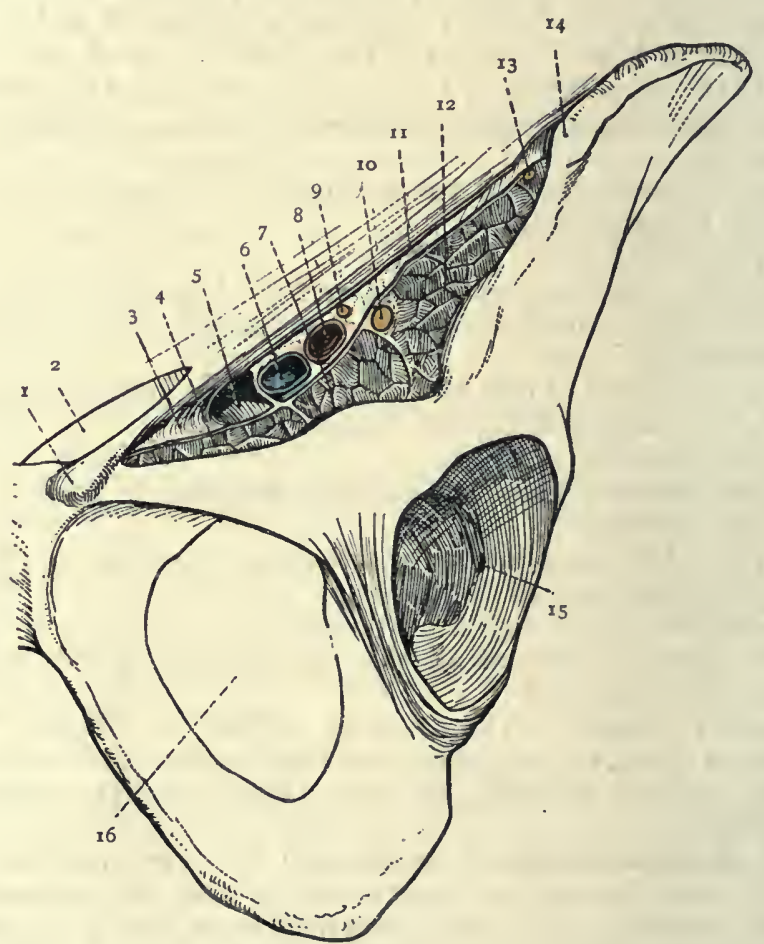

r. Pubic Spine

2. External Abdominal Ring

3. Gimbernat's Ligament

4. Pectineus

5. Crural or Femoral Ring

6. Femoral Vein
7. Psoas Magnus
8. Femoral Artery
9. Femoral Branch of Genito- femoral Nerve
10. Anterior Femoral Nerve
rx. Poupart's Ligament

12. Iliacus

13. External Cutaneous Nerve

14. Ant. Superior lliac Spine

15. Acetabuluin

16. Obturator Foramen

Fig. 332.-Poupart's Ligament and the Lacunar Region.

The vascular lacuna is situated anterior to the other two. It is bounded posteriorly by the connection between the iliac fascia and the pubic lamina of the fascia lata, whilst anteriorly it is bounded by the central portion of Poupart's ligament and the downward prolongation of the fascia transversalis to form the anterior wall of the femoral sheath, that fascia being here strengthened by the decp femoral arch. It gives passage to (I) the external iliac vessels, the 
vein being internal to the artery, and (2) the femoral branch of the genito-femoral nerve, which lies close to the outer side of the artery. . The part of this lacuna internal to the external iliac vein forms the crural or femoral ring, which is closed by the septum femorale.

\section{STRUCTURE AND DEVELOPMENT OF THE ABDOMINAL VISCERA}

\section{Structure of the Stomach.}

The wall of the stomach is composed of four coats-serous, muscular, submucous, and mucous.

The serous coat is formed by the peritoneum, which covers every part of the organ except (I) along the great and small

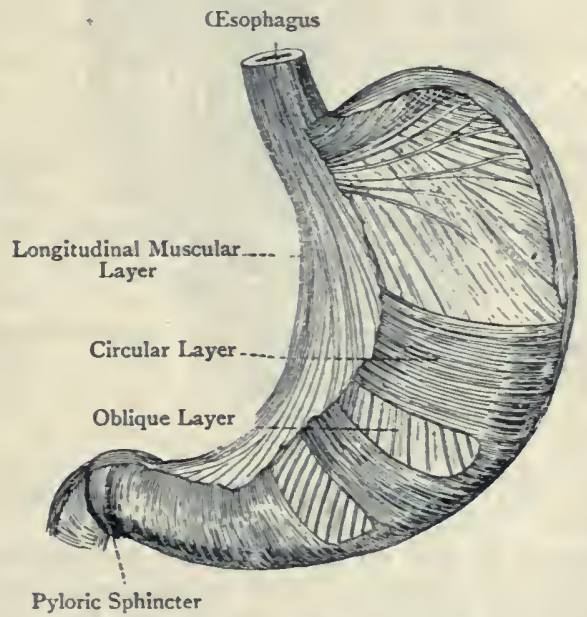

Fig. 333.-Dissection siowing the Muscular Layers of the Stomach.

curvatures, and (2) the uncovered trigone, situated on the posterior surface, below and a little to the left of the cardiac orifice.

The muscular coat (muscularis extcrna) is composed of plain muscular tissue disposed in three layers external or longitudinal, middle or circular, and internal or oblique. The external or longiIndinal fibres are continuous with the longitudinal fibres of the cesophagus, and at the pyloric end of the stomach they are continuous with the longitudinal fibres of the duodenum. They are most abundant along the small curvature. The middle 
or circular fibres completely surround the stomach from the fundus to the pyloric end. At first they are thin and irregular in position, but over the pyloric canal they are thick. At the pylorus they become augmented, and are gathered together into a thick muscular ring, called the sphincter pylori, which lies within a circular fold of the mucous membrane. The outermost fibres of this ring become continuous with the circular fibres of the duodenum. Some of the circular fibres appear to be continuous with the superficial circular fibres of the right side of the lower end of the œesophagus. The internal or oblique fibres are continuous with the circular fibres of the left side of the lower end of the œsophagus. They loop over the stomach immediately to the left of the cardia, and run very

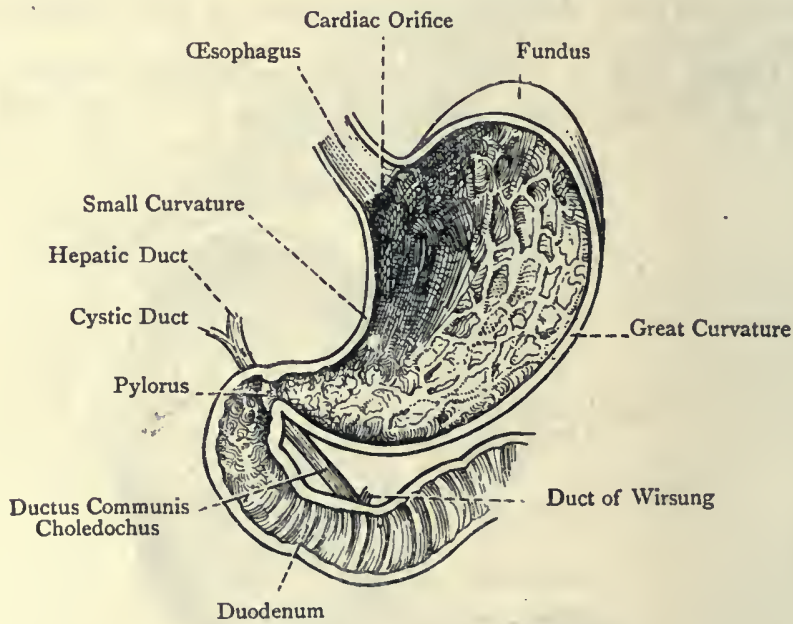

Fig. 334.-The Stomach and Duodenum opened.

obliquely downwards and to the right for a considerable distance on both surfaces of the organ. They cannot be traced as far as the pylorus, but end by inclining downwards to the great curvature, where they blend with the circular fibres.

The submucous coat is situated between the muscular and mucous coats. It is composed of loose areolar tissue, and serves partly as a connecting medium, and partly as a bed in which the arteries subdivide before entering the mucous coat.

The mucous coat is covered by a single layer of columnar epithelium, many of the cells being mucus-secreting goblet cells. It is sott and pulpy, and in the empty state of the viscus is thrown into rugæ, which are for the most part longitudinal, and are due to the loose connection between the muscular and mucous coats. These, however, are readily effaced when the stomach becomes distended. 
It is thickest towards the pyloric end, and in healthy adults it has a light crimson colour, while in early life this is heightened into a bright rosy tint. After death, however, it presents a mottled appearance, being marked with grey-brown patches. When examined with a lens, it presents a great number of polygonal depressions, varying in diameter from $\frac{1}{100}$ to $\frac{1}{350}$ inch, the largest being near the pylorus. These impart to it a honeycomb appearance. The mucous membrane surrounding them is elevated into ridges by subjacent capillary networks, and in the region of the pylorus these ridges present processes, called plice villosa. The polygonal depressions are beset with minute pores, which are the orifices of the gastric glands. These glands, which belong to the tubular variety, are placed perpendicularly in the mucous coat, and are closely packed together like upright stakes. They are of two kinds, cardiac and pyloric, between which there are certain differences.

The cardiac glands are situated in the cardiac two-thirds. The duct of each forms about one-third of the entire length of the gland. It is lined with a single layer of columnar epithelium. The deep end of the duct is connected with two or three glandtubes, which represent

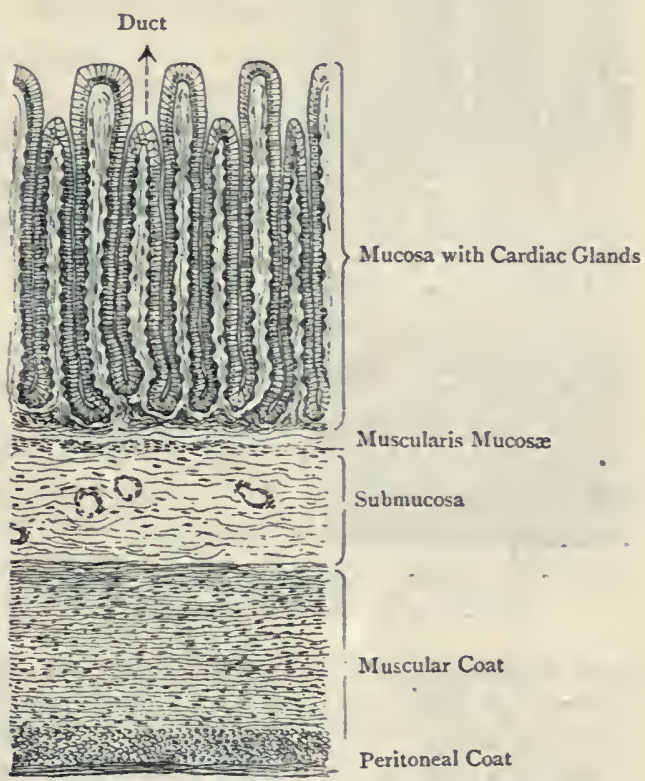

Fig. 335.-The Cardiac Glands of the STOMACH (HIGHLY MAGNIFIED). two-thirds of the entire gland. Each gland-tube is divisible into a neck, body, and fundus. The neck is the part connected with the duct, and it forms one-third of the length of the glandtube. It is narrower than the body, and is lined with coarsely granular polyhedral cells, which almost completely fill it, thus leaving a very small lumen. These are called the central or chief cells. Between these and the basement membrane there are interposed large oval or spherical granular cells, each having a clear nucleus. These, which are called the parietal or oxyntic ("acidforming ') cells, do not form a continuous layer, but are placed at intervals, and they give rise to small swellings on the wall of the neck. The body is wider than the neck, and forms two-thirds of 
the length of the gland-tube. It is lined by a prolongation of the central or ehief cells of the neck, which almost completely fill it, and which have now become somewhat columnar and transparent. Between these cells and the basement membrane there are a few

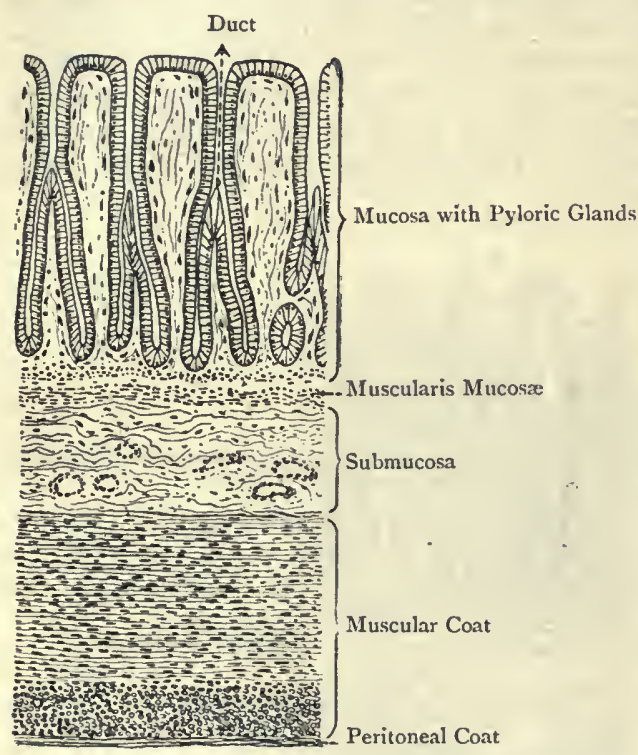

Fig. 336.-The Pyloric Glands of the STOMACH (HIGHLY MAGNified). parietal or oxyntic cells here and there. The parietal cells of the neck and body impart the characteristic beaded appearance to the glandtube. The fundus is the blind deep end of the gland-tube.

The pyloric glands are situated in the pyloric third. The duct of each forms one half of the entire length of the gland. It is lined with a single layer of columnar epithelium. The deep end of the duct is connected with two or three gland-tubes, which represent one half of the entire gland. The neck of each tube is comparatively short, and the body is branched at its deep extremity. The neck and body are lined with cubical granular cells, representing the central or chief cells of the cardiac glands, and they are not so crowded as in the cardiac glands, so that there is a very distinct lumen. There is an entire absence of parietal or oxyntic cells, and the body of each gland-tube has an undulating, convoluted outline. The pyloric glands are serially continuous with Brunner's glands of the small intestine.

\section{Summary of the Cardiac and Pyloric Glands.}

\section{Cardlac Glands.}

I. Ducts short.

2. Gland-tubes long.

3. Gland-tubes almost filled with coarsely granular polyhedral cells, called central or chief cells.

4. Lumen very small.

5. Gland - tubes have parietal or oxyntic cells between the central cells and the basement membrane.

\section{Pyloric Glands.}

1. Ducts long.

2. Gland-tubes short.

3. Gland-tubes lined with cubical granular cells.

4. Lumen distinct.

5. Gland-tubes destitute of parietal or oxyntic cells. 
At the deepest part of the mucous coat, and forming a part of it, there is a stratum of plain muscular tissue, called the muscularis mucose (muscularis interna), which is disposed in two layers-outer longitudinal, and inner circular. The mucous membrane is also provided with lymphoid tissue in the interspaces between the deep ends of the gastric glands. In the cardiac part of the stomach this lymphoid tissue occurs in the form of isolated collections, called lymph follicles, which bear a resemblance to the solitary glands of the intestinal mucous membrane. In the neighbourhood of the pylorus these lymph follicles become aggregated, and so resemble somewhat the agminated glanas or Peyer's patches of the small intestine.

Blood-supply-Arteries.-Along the small curvature there are (I) the gastric branch of the cœliac axis in two divisions, and (2) the pyloric branch of the hepatic, also in two divisions. Along the great curvature there are (I) the right gastro-epiploic of the gastroduodenal of the hepatic from the cœliac axis, and (2) the left gastroepiploic of the splenic irom the cœliac axis. At the fundus there are the vasa brevia of the splenic artery.

The branches arising from all these arteries enter the muscular coat without piercing the peritoneum. They subsequently make their way inwards to the submucous coat, where they break up into branches which freely anastomose with one another. Fine branches then enter the mucous coat, which run upwards between the closely-packed gastric glands, round which they form by their anastomoses a delicate capillary network with its meshes elongated in the direction of the gland-tubes. From this network somewhat larger vessels proceed upwards, which by their anastomoses form a coarser and more superficial network around the orifices of the ducts of the glands.

Veins.-These arise from the superficial network of capillaries round the orifices of the ducts of the glands. They take a downward course between the gland-tubes, and on reaching their deep ends they form a plexus. From this plexus branches proceed outwards to the submucous coat, in which they form another plexus. The branches arising from this latter plexus, having passed through the muscular coat, terminate: in the following veins : (I) the right gastro-epiploic, which opens into the superior mesenteric; (2) the left gastro-epiploic and vasa brevia, which open in to the splenic; (3) the gastric; and (4) the pyloric, the latter two opening directly into the vena portæ. The veins of the stomach contain numerous valves, which are sufficiently competent in early life to oppose the return of venous blood, but in the adult they are incompetent.

Lymphatics. - These commence near the free surface of the mucous membrane either in loops or in enlargements, and they take a downward course between the gland-tubes, where they open into a network of lacunar spaces. The branches which proceed from this network on reaching the deep ends of the glands form a plexus, 
and the vessels issuing from this plexus on entering the subinucous coat form another plexus, the lymphatics of which are furnished with valves. The vessels which emerge from this latter plexus accompany the bloodvessels, and pass to the small and great curvatures, and the vicinity of the hilum of the spleen. At the curvatures they are connected with the superior and inferior gastric lymphatic glands, whilst those which accompany the vasa brevia arteries pass through the splenic glands, the efferent vessels of all these glands ultimately passing to the cœliac glands. In addition to the lymphatic vessels just described there is a subperitoneal Jymphatic plexus.

Nerves.-These are derived from (I) the two pneumogastric nerves, and (2) sympathetic plexuses from the solar plexus. The right pneumogastric nerve descends upon the posterior surface of the stomach, whilst the left nerve descends upon the anterior surface. The sympathetic .plexuses closely accompany the arteries. The nerves form two gangliated plexuses composed of non-medullated nerve-fibres. One of these is situated between the longitudinal and circular fibres of the external muscular coat, and corresponds to the : plexus myentericus of Auerbach of the intestine. The other plexus 'is situated in the submucous coat, and corresponds to the plexus of Meissner of the intestine.

The explanation of the right pneumogastric nerve descending upon the posterior surface, and the left upon the anterior surface, of the stomach is found in the position assumed by the stomach in the early embryo. Briefly stated, at that period of life the stomach is a straight tube, and its surfaces are right and left. The two pneumogastric nerves, therefore, right and left, naturally descend on the right and left surfaces of the viscus. When, however, the stomach turns over on its right side, the surface which was originally right becomes posterior, and the surface which was originally left becomes anterior. Thus the right nerve eventually descends on the posterior (originally the right) surface, and the left nerve descends on the anterior (originally the left) surface.

Pylorus.-The opening between the pyloric end of the stomach and the duodenum is provided with a sphincter muscle, called the sphincter pylori. This is formed by an aggregation of the circular muscular fibres, which causes the mucous membrane to project in the form of an annular fold, thus giving rise to the pyloric valve. The sphincter pylori is only relaxed when the contents of the stomach are being passed into the duodenum. At all other times it is in a condition of firm contraction, and the pyloric orifice then takes the form of a cleft.

The average length of the stomach is about 12 inches, and its average width at the widest part about 5 inches. I $t$ weighs about $4 \frac{1}{2}$ ounces, and its capacity, which is very variable, ranges from 3 to 5 pints. 


\section{Structure of the Intestinal Canal.}

Small Intestine.-The wall of the small intestine, which is cylindrical, is composed of four coats-serous, muscular, submucous, and mucous.

The serous coat is formed by peritoneum derived from the mesentery proper. In the case of the duodenum it is incomplete, but it forms a complete investment to the jejunum and ileum, except along a narrow interval corresponding to the mesenteric border of the bowel, where the peritoneal investment becomes continuous with the two layers of the mesentery proper.

The muscular coat (muscularis externa) is composed of plain muscular tissue, disposed in two layers, external or longitudinal, and internal or circular. The external or longitudinal fibres are continuous with the corresponding fibres of the stomach, and they are best marked along the anti-mesenteric border. The internal or circular fibres are continuous with the outermost fibres of the sphincter pylori, and form a much thicker layer than the longitudinal. The muscular coat attains its greatest thickness in the duodenum, whence it gradually diminishes. Between the two muscular layers there is a gangliated plexus of non-medullated nerve-fibres, called the plexus myentericus of Auerbach, and also a plexus of lymphatic vessels.

The submucous coat is situated between the muscular and mucous coats. It is composed of lnose areolar tissue, and serves partly as a connecting medium and partly as a bed in which the arteries subdivide. It contains a gangliated plexus of non-medullated nerve-fibres, called the plexus of Meissner, and a plexus of lymphatic vessels. In the duodenum this coat lodges Brunner's glands, and the deep ends of the solitary glands project into it throughout.

The mucous coat is red and thick in the upper part of the small intestine, but pale and thin in the lower part. It is covered by a single layer of columnar epithelium, many of the cells being mucussecreting goblet cells. The protoplasm of the cells is longitudinally fibrillated, and their free ends are covered by a delicate striated basilar border, the striations being vertically disposed. Underneath the epithelium there is a basement membrane, known as the subepithelial endothelium, and underneath this is the main part of the mucous coat, which is essentially composed of adenoid tissue, that is to say, retiform tissue containing in its meshes lymph corpuscles. At the deepest part of the mucous coat, and forming a part of it, there is a stratum of plain muscular tissue, called the muscularis mucose (muscularis interna), which in many situations is disposed in two layers-outer longitudinal, and inner circular. 
In some places, however, only the outer longitudinal layer is present. The mucous coat is beset all over with minute projections, called villi, and is sometimes called the villous coat. These villi impart to it a woolly appearance like the nap of velvet. It diminishes in thickness from above downwards, and is characterized by the following structures: (I) valvulæ conniventes, (2) villi, (3) Brunner's glands, (4) crypts or follicles of Lieberkühn, (5) solitary glands, and (6) agminated glands or Peyer's patches.

Of the foregoing structures the valvulæ conniventes, villi, and Peyer's patches constitute the macroscopical (naked-eye) characters of the mucous membrane, the others forming its microscopical characters.

The valvulæ conniventes (valves of Kerkring) are permanent folds of the mucous membrane, which cannot be effaced. They are absent from the first part of the duodenum for a distance of from I to 2 inches from the pylorus. Commencing about the upper end of the second part of the duodenum as small straggling folds, they become large and distinct at the place of entrance of the ductus communis choledochus and pancreatic duct (about

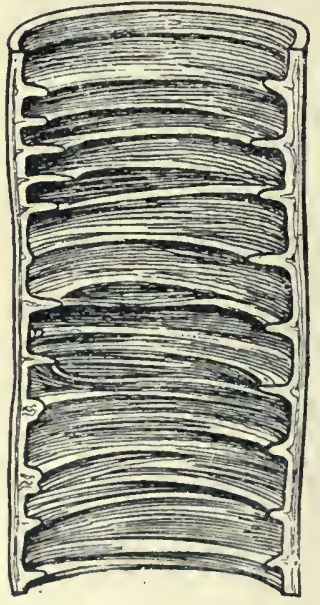

FIG. 337.-THE VALvula Conniventes. 4 inches from the pylorus). Throughout the rest of the duodenum and in the upper part of the jejunum they are still prominent, and are placed close to each other. In the lower part of the jejunum they become smaller, and are placed farther apart. In the upper part of the ileum they become still smaller and more irregular, and they finally disappear. just beyond the centre of the ileum. They are crescentic folds placed across the bowel, and each consists of two layers of mucous membrane applied back to back, with a little submucous areolar tissue intervening. Their average length is about $2 \frac{1}{2}$ inches, and the average breadth of each is about $\frac{1}{3}$ inch. The majority of them extend round the bowel for from one-half to two-thirds of its circumference. Some, however, describe complete circles, whilst a few are arranged in a spiral manner so as to describe from one to three turns round the tube. Some of them begin and terminate in bifurcated extremities, whilst others present abrupt single extremities. The purpose served by the valvulæ conniventes is a twofold one. In the first place they increase the extent of the absorbing and secreting surface of the mucous membrane, and in the second place they delay the passage of the intestinal contents, and so afford time for digestion and absorption.

In connection with the valvula conniventes of the duodenum the common orifice of the ductus communis choledochus and 
pancreatic duct has to be noted. At the junction of the inner and posterior aspects of the second part of the duodenum where the upper two-thirds and lower third of that part meet, there is a small eminence of the mucous membrane, called the bile papilla. It lies at the lower end of a vertically-placed valvula connivens, which bifurcates so as to form a kind of cap for it. From the lower part of the papilla a fold extends downwards for some distance, which acts as a bridle, and gives the apex a downward direction. On the summit of this papilla there is an opening which represents the common orifice of the two ducts. These ducts, having traversed the wall of the second part of the duodenum obliquely for $\frac{3}{4}$ inch, unite to form one duct which, before piercing the mucous coat, presents an enlargement called the ampulla of Vater, but subsequently narrows at its final ending. In the ampulla of Vater a gallstone may become lodged and delayed in its downward progress toward the duodenum. About I inch above the bile papilla there is another small papilla upon which there is another minute opening. This represents the orifice of the accessory pancreatic duct, or duct of Santorini.

The villi commence at the beginning of the duodenum on the outer side of the pylorus, and extend as far as the margins of the segments of the ileo-cæcal valve. They are minute projections of the mucous membrane, to which they impart a woolly appearance, and may be visible to the naked eye, but are more readily seen with the aid of an ordinary lens if a portion of bowel is floated in water. They are closely set upon the mucous membrane (valvulæ conniventes included), except over the solitary glands. Their total number is said to be about four millions (Krause). The villi are conical, cylindrical, leaf-like or finger-like processes, varying in length from $\frac{1}{30}$ to $\frac{1}{10}$ inch. They are larger and more numerous in the lower part of the duodenum and in the jejunum, especially at its upper part, than in the ileum, and they diminish both

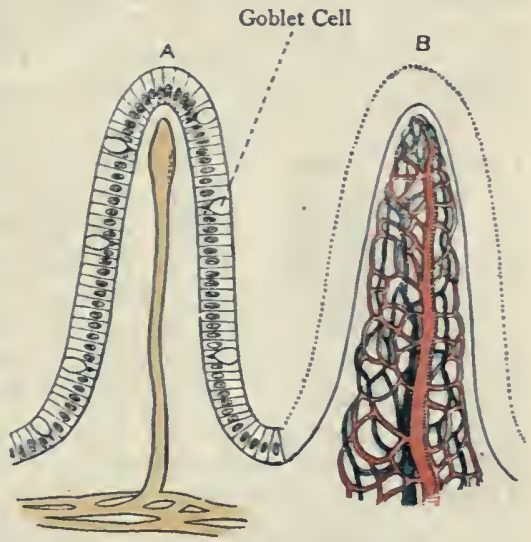

Fig. 338.-Two Villi.

A. Villus, showing Striated Basilar Border, Columnar Epithelium, Goblet Cells, and Lacteal Vessel. B. Villus, showing the Capillary Bloodvessels.

in size and number from above downwards, becoming somewhat filiform in the ileum. Each villus is an elevation of the mucous membrane covered by a single layer of columnar epithelium. It is composed of (I) adenoid tissue, (2) a capillary network of 
bloodvessels, (3) one or more lacteal vessels ensheathed by plain muscular tissue; and (4) arborisations of nerve-fibrils derived from the plexus of Meissner.

Between the columnar epithelial cells of the free surface, many of which are mucus-secreting goblet cells, there are amœboid lymph corpuscles. Beneath the epithelium is a basement membrane composed of flattened cells, and known as the subepithelial endothelium. The cells of this basement membrane send processes outwards between the columnar cells of the free surface, and they also send processes inwards which are connected with the branched cells of the retiform tissue of the adenoid tissue. One artery (sometimes two) enters the base of the villus and ascends to near the centre. Here it breaks up into a number of branches, which form a copious capillary network. From this plexus the blood is returned by one or two venous radicles, which leave the villus at its base, where they open into the venous plexus of the mucous membrane. In the centre of the villus there is a lacteal vessel, which commences near the tip in a blind bulbous extremity, or, if there should be two lacteals, they originate in the form of a loop. The wall of the.lacteal vessel is formed by a single layer of endothelial plates, which are connected by processes with the branched cells of the retiform tissue of the adenoid tissue. The vessel is ensheathed by longitudinal plain muscular fibres derived from the muscularis mucosæ, their fibre-cells being connected with the basement membrane of the villus. The villus is pervaded by adenoid tissue-that is to say, retiform tissue with its meshes filled with amœboid lymph corpuscles. The branched cells of this retiform tissue are connected by processes on the one hand with the endothelial plates which compose the wall of the lacteal vessel, and on the other hand with the cells of the basement membrane near the surface, and these latter in turn send out processes between the columnar epithelial cells of the free surface.

The villi play a most important part in absorption, partly through their copious capillary networks, and partly through their lacteals. The lacteals serve specially for the absorption of fats, which is probably effected in the following manner : the columnar epithelial cells at the free surface take up the saponified and emulsified fats, which they transfer to the amoboid lymph corpuscles between them. These corpuscles then carry the fats inwards through the adenoid tissue into the lacteal vessel.

Brunner's glands are confined to the duodenum, and are serially continuous with the pyloric glands of the stomach. They are very numerous in the commencement of the duodenum, where they form a continuous layer of gland tissue extending as low as the entrance of the common bile-duct and pancreatic duct. Beyond this point they gradually diminisb in number, and ultimately disappear near the duodeno-jejunal flexure. They belong to the class of racemose or acino-tubular glands, and they differ from the pyloric glands of the stomach in having their tubules 
more branched, and in having longer ducts. Otherwise the structure of the two kinds of glands is similar. Brunner's glands lie embedded in the submucous coat, and their long ducts pass through the whole thickness of the mucous coat, upon the surface of which they open between the crypts of Lieberkühn. Some of them, however, open into these crypts. The glands can easily be displayed by removing the peritoneal and muscular coats of the duodenum and a little of the submucous areolar tissue, when they appear as small, round, grey-coloured masses like millet seeds, varying in diameter from $\frac{1}{12}$ to $\frac{1}{50}$ inch.

The crypts or follicles of Lieberkühn are found in large numbers over the whole of the mucous membrane of the small intestine, as well as that of the large bowel. They belong to the class of simple tubular glands, and are to be regarded as small diverticula o the mucous membrane. Each crypt takes the form of a simple tube, which is closed and slightly enlarged at its deep extremity, and opens by its other end on the surface between the villi. The crypts are present on the valvulæe

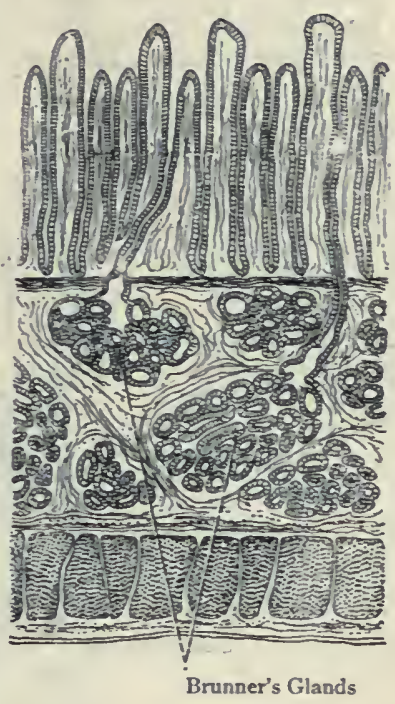

FIG. 339.-SEction OF THE DUODENUM, SHOWING BRUNNER'S GIANDS (HIGHLY MAGNIFIED). conniventes as well as in the intervening parts. They are placed vertically and close together, and are confined entirely to the mucous coat, in which they extend from the free surface to the muscularis mucosæ. In length they vary from $\frac{1}{60}$ to $\frac{1}{180}$ inch. Each crypt is composed of a basement membrane lined with columnar epithelium, many of the cells being mucus-secreting goblet cells, and the lumen is of large size.

The solitary glands are present over the whole extent of the mucous membrane of the small intestine. They assume the form of small white round or oval nodules, which project by their deep ends into the submucous coat, whilst their superficial ends give rise to slight elevations of the free surface, where they have the openings of the crypts of Lieberkühn placed around them. They are found upon, as well as between, the valvulæ conniventes. In structure each solitary gland is composed of adenoid tissue containing large numbers of lymph corpuscles, and permeated by capillary networks. Each gland is surrounded at its deep part by a copious plexus of lymphatic vessels, or by lymphatic sinuses. The solitary glands are simply lymphoid nodules.

The agminated glands or Peyer's patches are peculiar to the 
small intestine, and average about thirty in number in the adult, being more numerous in early life. They are situated along the free or anti-mesenteric border of the bowel, which must, therefore, be opened along its attached or mesenteric border in order to preserve them. They are largest, best marked, and most plentiful in the lower half of the ileum. In the upper part of the ileum and

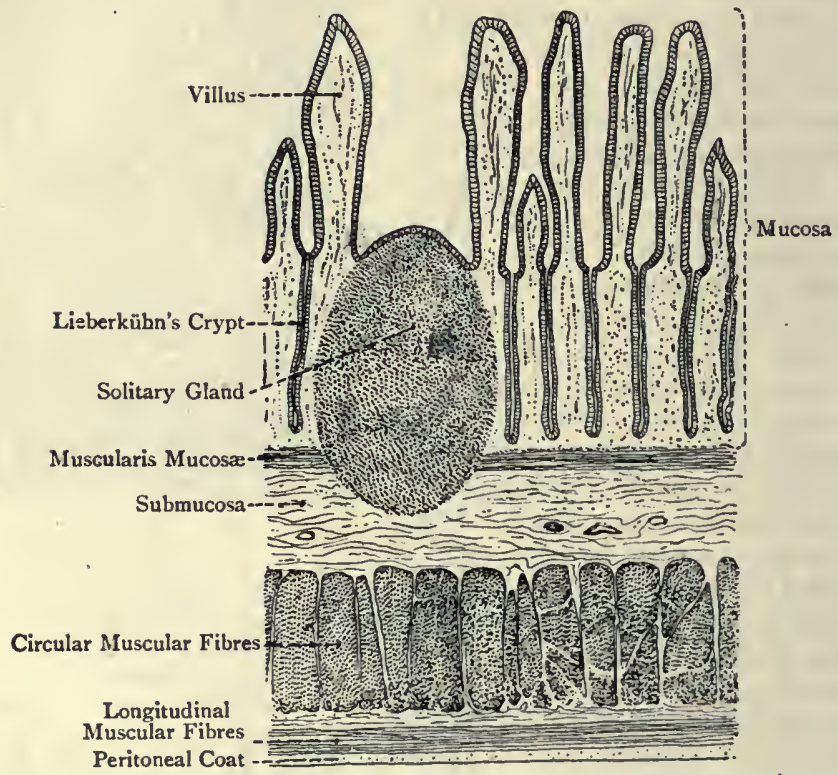

Fig. 340.-Vertical Transverse Section of the Small Intestine (HIGHLY MAGNIFIED).

- lower part of the jejunum they become smaller and more scarce, and they disappear as a rule above the centre of the jejunum. They vary in length from $\frac{1}{2}$ inch to 4 inches, their breadth ranging from $\frac{1}{2}$ inch to I inch. They are for the most part oblong, their long axis coinciding with that of the bowel. In the upper part of the ileum and lower part of the jejunum, however, they are somewhat circular. Each patch is composed of a group of solitary glands or lymphoid nodules, surrounded by lymphatic plexuses or lymphatic sinuses. The area of each patch is slightly elevated, and there are no villi over the lymphoid nodules, whilst the openings of the crypts of Lieberkühn are arranged in a circular manner round each nodule. There are no valvulæ conniventes over Peyer's patches. The patches are best marked in young persons. Towards middle life they fade away, and in old persons they are usually only distinguishable as discoloured portions of the mucons membrane. 
Bloodosupply--Arteries.- The duodenum receives its arteries from the superior pancreatico-duodenal of the gastro-duodenal from the hepatic, and the inferior pancreaticoduodenal of the superior mesenteric. The jejunum receives its arteries from the rami intestini tenuis of the superior mesenteric. The ileum also receives its arteries from the rami intestini tenuis, and its terminal part in addition receives its arterial supply from the ileal branch of the ileo-colic from the superior mesenteric.

Veins.-The destination of the venous blood of the small intestine is the superior mesenteric vein, and thereafter the vena portæ. The veins are provided with valves which are competent in early life, but in the adult they are incompetent, and therefore allow regurgitation of blood to talie place towards the small intestine, as happens in cases of portal obstruction.

Lymphatics.-These form a copious plexus of valved vessels, which is situated in the submucous coat. This plexus receives the lymphatics of the mucous membrane and the

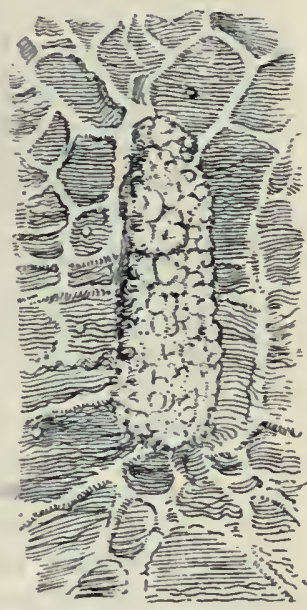

Fig. 34I.-A PEYER'S Patch. lacteals of the villi, and it surrounds the deep ends of the solitary glands. Its efferent vessels pierce the muscular coat, and in doing so they take up the lymph from an intramuscular plexus of lymphatics, which lies between the longitudinal and circular layers. They then leave the bowel at the mesenteric border, where they pass between the two layers of the mesentery, and on their way to the receptaculum chyli they traverse the superior mesenteric glands.

Nerves.-These are derived from the superior mesenteric sympathetic plexus, and they form two gangliated plexuses of nonmedullated nerve-fibres. One of these is situated in the muscular coat between the longitudinal and circular layers, and is known as the plexus myentericus of Auerbach (plexus of the muscular coat of the intestine). The other is situated in the submucous coat, and is called the plexus of Meissner. The branches of this latter plexus are distributed to the muscularis mucosæ and the mucous membrane with its villi.

Characters of Different Parts of the Small Intestine-Duodenum-Peritoneum. -There is no mesentery and only a partial investment of peritoneum. Muscular Coat.-This is very thick. Submucous Coat.- This contains the tubular portions of Brunner's glands. Mucous Coat.- The characters of this coat are as follows: (I) valvulæ conniventes, except in the first I or 2 inches, (2) villi, (3) Lieberkühn's crypts, (4) solitary glands. (5) ducts of Brunner's glands, (6) common orifice of the ductus communis choledochus and pancreatic duct, and (7) orifice of the accessory pancreatic duct, or duct of Santorini. 
The diameter of the duodenum is from $I \frac{1}{2}$ to 2 inches.

Jejunum-Peritoneum.-There is a mesentery, and the bowel is surrounded by peritoneum except along its mesenteric border. Muscular Coat.This is comparatively thin. Mucous Coat.-This has the following characters : (1) valvulæ conniventes, (2) villi in abundance, (3) Lieberkühn's crypts, (4) solitary glands, and (5) Peyer's patches in its lower half.

The diameter of the jejunum is about $I \frac{1}{2}$ inches.

Ileum-Peritoneum. - In this respect the ileum resembles the jejunum. Muscular Coat. - This is very thin. Mucous Coat. - The characters of this coat are as follows: (I) valvulæ conniventes in upper half, but small and sparse, there being none in the lower half; (2) villi, but in fewer numbers; (3) Lieberkühn's crypts ; (4) solitary glands; and (5) Peyer's patches.

The diameter of the ileum is about $\mathrm{I}_{4}$ inches.

Large Intestine.-The wall of the large intestine, which is sacculated, is composed of four coats-serous, muscular, submucous, and mucous.

The serous coat forms a complete investment to the vermiform appendix, cæcum, transverse colon, and pelvic colon. As regards the ascending colon, descending colon, and iliac colon, it is incomplete, being absent behind.

The muscular coat (muscularis externa) is composed of plain muscular tissue, disposed in two layers-external or longitudinal, and internal or circular.

The longitudinal muscular fibres are for the most part collected into three flat bands, called tæniæ coli, or ligaments of the colon, except upon the rectum. In the intervals between these bands there are some longitudinal fibres, but they are very few and scattered. The tæniæ, which are about $\frac{1}{4}$ inch in breadth, commence upon the cæcum at the base of the vermiform appendix, and they extend along the several parts of the large intestine as far as the rectum, where they spread out and form a continuous covering, which completely surrounds that part of the bowel. Upon the cæcum, ascending colon, descending colon, and iliac colon the tæniæe from their disposition are called anterior, postero-internal, and posteroexternal. Upon the transverse colon they are so placed as to be called anterior or omental (great omentum), postero-inferior, and superior or meso-colic (transverse meso-colon). They are shorter than the bowel to which they are applied, with the result that the tube is drawn together or puckered, and thus thrown into sacculi. There being three tæniæ, there are three rows of sacculi between them, and, inasmuch as the tæniæ are placed at nearly equal distances from each other, the sacculi are pretty much of equal dimensions. Between the successive sacculi there are constrictions, usually containing fat. The sacculi give rise internally to large pouches, and the constrictions between the sacculi produce internally sharp crescentic rugæ, which separate the pouches from cach other. When the tæniæ are divided, the sacculi and constrictions entirely disappear, and the large bowel becomes elongated into a smooth cylindrical tube. Along the course of the tæniæ there are a number of small processes of peritoneum containing fat, called appendices epiploicæ. 
Except in the case of the transverse colon, these are chiefly found along the postero-internal tænia, but in the case of the transverse colon they are principally met with along the postero-inferior tænia.

The circular fibres are thin and scattered over the sacculi, but in the constrictions between them they become aggregated. Upon the rectum and anal canal they form a thick layer, which in the latter situation is known as the sphincter ani internus.

The submucous coat is in all respects similar to that of the small intestine.

The mucous coat is pale and greyish in colour, except in the rectum, where it is red. Its epithelium is.similar to that of the small intestine. It is destitute of valvulæ conniventes and villi, and consequently presents a smooth surface. It contains large numbers of the crypts or follicles of Lieberküh, which abound in mucus-secreting goblet cells. It also contains solitary glands or lymphoid nodules, which are especially prevalent in the vermiform appendix and crcum. The deepest part of the mucous coat is formed by the muscularis mucose (muscularis interna).

Blood-supply-Arteries.-These are as follows: (I) appendicular, for the vermiform appendix; (2) anterior and posterior cæcal, for the cæcum; (3) colic of ileo-colic, and right colic, for the ascending colon ; (4) middle colic, for the transverse colon (all branches of the superior mesenteric) ; (5) left colic, for the descending colon; and (6) sigmoid arteries, for the iliac colon and pelvic colon (the latter two being branches of the inferior mesenteric).

Veins. - The destination of the venous blood of the vermiform appendix, cæcum, ascending colon, and transverse colon is the superior mesenteric vein, whilst the blood of the descending colon, iliac colon, and pelvic colon is carried into the inferior mesenteric vein. In both cases the further destination of the blood is the vena portæ. As in the small intestine, the veins have valves which are competent in early life, but not so in the adult.

The lymphaties will be found described on pp. 729 and 730.

Nerves.-These are derived from the superior mesenteric sympathetic plexus, and the inferior mesenteric plexus, which latter is an offshoot from the aortic plexus. The disposition of the nerves corresponds with that in the small intestine.

The large intestine diminishes gradually in size from its commencement to its termination. Its diameter varies in different parts, the extremes being $2 \frac{1}{2}$ inches and $I$ inch.

Characters of the Large Intestine-Peritoneal Coat.-This presents at frequent intervals small projections called appendices epiploicæ. Muscular Coat. -The longitudinal fibres are for the most part arranged in three tæniæ. Mucous Coat. - This is destitute of (a) valvulæ conniventes, (b) villi, (c) Brunner's glands, and (d) Peyer's patches, but it is provided with (I) Lieberkühn's crypts, and (2) solitary glands or lymphoid nodules. Outline of Tabe.The bowel presents three rows of sacculi, except in the vermiform appendix and rectum. 
Structure of the Vermiform Appendix.-The vermiform appendix is entirely covered by peritoneum, which forms a more or less complete meso-appendix or appendicular mesentery. It has a muscular coat, composed of an external longitudinal and internal circular layer, both of which completely surround it. The

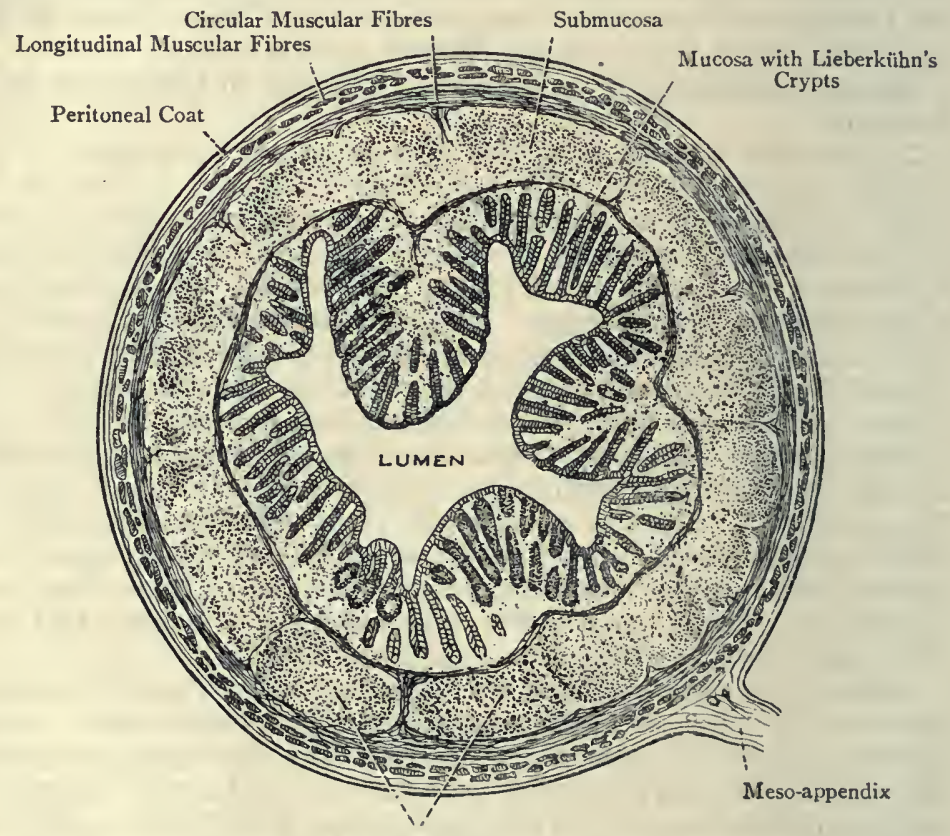

Lymphoid Nodules

Fig. 342.-Transverse Section of the Vermiform Appendix (HIGHLY MAGNIFIED).

submucous coat contains, in great abundance, lymphoid nodules (solitary glands) of large size, and the mucous coat contains a few lymphoid nodules, as well as a few crypts of Lieberkühn. The base of the appendix is situated at a point on an average rather more than I inch below the ileo-cæcal valve according to Treves. The opening by which the appendix communicates with the crecum is occasionally guarded by a fold of mucous membrane, which is known as the valve of Gerlach.

The vermiform appendix is usually regarded as the remains of the herbivorous crecum. It is also looked upon as an appendage of the lymphoid system, and as such it would belong to the class of structures represented by Peyer's patches, namely, lymphoid organs. In its typical form it is peculiar to man, the higher apes, and the marsupial wombat. In certain rodents, however-e.g., the rabbit and hare-the very large capacious crecun shows an approach to a vermiform appendix at its lower extremity. 
Ileo-cæcal or Ileo-colic Valve (valve of Tulpius).-This valve is situated at the point where the terminal part of the ileum opens into the junction between the cæcum and ascending colon. The orifice, as seen from the interior of the large intestine, has the form of a slightly oblique cleft about $\frac{1}{2}$ inch long, and running in an anteroposterior direction. It is bounded above and below by the two segments which form the valve, and which project into the large intestine. The upper or ileo-colic segment is prominent, and occupies an almost horizontal plane. The lower or ileo-cacal segment, longer than the upper, is concave superiorly, and occupies an oblique plane. The anterior part of the cleft is rounder off, whilst the

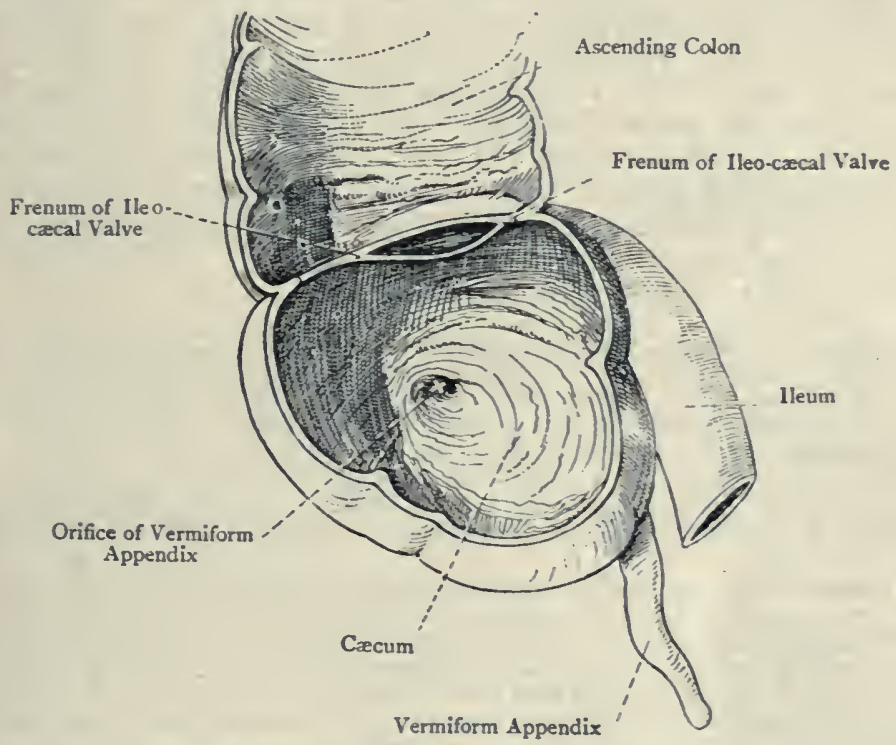

Fig. 343.-The Intertor of the Cacum and the Ileo-ceccal Valve.

posterior part tapers to a point. The segments meet in front of and behind the cleft, and form two prominent folds, which are prolonged round the wall of the bowel for some distance. These folds are known as the frena or retinacula of Morgagni. Each segment is composed of two layers of mucous membrane, one of which belongs to the ileum, and the other to the cæcum or colon as the case may be. These two layers are continuous with each other at the free margin of the segment, and they contain between them submucous areolar tissue and circular muscular fibres, both of which are derived partly from the ileum and partly from the large intestine. The longitudinal muscular fibres and the serous or peritoneal coat take no part in the formation of the segments, being continued uninterruptedly from the ileum to the large 
intestine. The mucous membrane which covers the opposed surfaces of the segments belongs to the ileum, and is, therefore, provided with villi. The mucous membrane of the other surfaces, that is to say, the surfaces which look away from each other (downwards and upwards respectively), belong to the cæcum and colon, and are destitute of villi. The villi thus disappear at the margins of the segments. The valve may be described as being formed by an inversion of the terminal part of the ileum into the large intestine. That part of the ileum, having passed upwards and to the right with a slight inclination backwards, enters the large intestine. As it does so, it leaves behind its serous and longitudinal muscular coats, and takes with it its circular muscular, submucous, and mucous coats, the corresponding coats of the large intestine accompanying it in the inversion. The ileo-cæcal valve prevents the regurgitation of the contents of the cæcum into the ileum. It is generally believed that the mode of action of the valve is as follows: when the cæcum becomes distended the fræna or retinacula of the valve are stretched, and exercise traction upon its segments, which are thereby brought together, very much as an old-fashioned purse is closed by pulling upon the two strings.

For the structure of the rectum, see Index.

Development of the Stomach and Intestinal Canal.-The primitive gut, or archenteron, consists of splanchnopleure-that is to say, entoderm covered externally by splanchnic mesoderm-and it extends from the head-fold to the tail-fold, lying on the ventral aspect of the notochord. It is divided into three parts-namely, the fore-gut, mid-gut, and hind-gut.

The fore-gut gives rise to (I) the tongue and back part of the oral cavity, (2) the pharynx, (3) the œsophagus, (4) the stomach, (5) the greater part of the duodenum, and (6) the organs developed as outgrowths from the foregoing parts.

The mid-gut gives rise to (1) a small part of the duodenum, (2) the jejunum, (3) the ileum, and (4) the colon, except a small portion at its lower end.

The hind gut gives rise to (I) a small portion of the colon at its lower end, (2) the rectum, and (3) the portion of the anal canal above the anal valves. The anus and the portion of the anal canal below the anal valves are developed from the proctodaum, which is an invagination of the ectoderm at the caudal end of the trunk to meet the intestinal entoderm.

The cephalic end of the primitive gut is closed, and is separated from the stomodæum, or primitive mouth (which is developed from an invagination of the ectoderm), by a septum, known as the pharyngeal membrane. This septum consists of two layers, one of which is formed by the ectoderm of the stomodæum, whilst the other consists of entoderm belonging to the cephalic or anterior end of the primitive gut (pharyngeal portion).

The primitive gut is also closed near its caudal end by a septum, called the cloacal membrane. This membrane, from its mode of formation, bears a close resemblance to the pharyngeal membrane. It consists of two layers, one of which is formed by the ectoderm of the exterior, whilst the other is formed by the entoderm of the hind-gut.

The mid-gut remains for some time in free communication with the yolksac. This communication is at first wide, but a constriction takes place on a level with the umbilical aperture, so that the mid-gut and yolk-sac now communicate by a narrow neck, called the vitelline, vitello-intestinal, or umbllical duct. "The ventral body-wall being now formed, and the yolk-sac being extraembryonic, this duct extends inwards, along the umbilical cord, through the umbilical aperture, and, after entering the embryonic colon or body-cavity, 
it opens into the mid-gut. The part of the mid-gut which is connected with this duct gives rise to the lower portion of the ileum. The duct, as a rule, becomes closed and disappears. In some cases, however, its intestinal portion retains its communication with the gut, and gives rise to a blind evagination of the gut, which is known as Meckel's divericulum. In very rare cases the intestinal portion of the vitelline duct undergoes no closure, under which circumstances it is pervious throughout, opening internally into the gut, and externally into the cavity of the umbilical cord. Such a condition of matters gives rise to a congenital umbilical facal fistula.

The primitive gut is lined throughout with entoderm, from which the future intestinal epithelium is developed, as well as the epithelium of the gland-ducts which open into the gut. This entoderm is covered externally by splanchnic mesoderm, these two combined layers forming the splanchnopleure. The splanchnic mesoderm gives origin to the tissues which form the other coats of the gut.

The primitive gut, thus constituted, is separated from the body-wall, which is composed of somatopleure (ectoderm and somatic mesoderm combined) by the cœlom or body-cavity, which becomes partitioned off into pericardial, pleural, and peritoneal cavities.

Stomach. - The primitive gut is a straight tube up to about the fourth week of intra-uterine life. About that time the first indication of the stomach shows itself as a spindle-shaped dilatation of the primitive gut in the neighbourhood of the embryonic heart, this dilatation being at first straight. After the stomach descends into the abdominal cavity its dorsal aspect undergoes greater development than the ventral, and the organ becomes curved. The convexity of this curve is, at this period, directed dorsalwards, and the concavity ventralwards. The surfaces of the organ are right and $l e f t$, and they thus naturally receive, at this period, the corresponding pneumogastric nerves. The pyloric end, which is directed caudalwards, is now tilted forwards towards the ventral body-wall, and it carries the duodenal portion of the gut

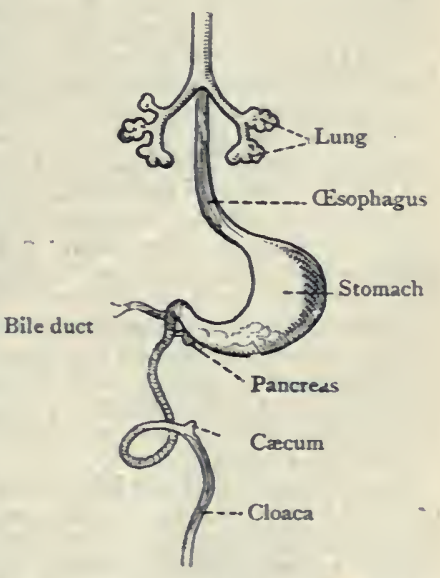

Fig. 344. - The Almentary CANAL OF THE EMBRYO (HIS). along with it, the duodenal loop being thereby produced. Thereafter the stomach undergoes partial rotation round its long axis towards the right side, and the cephalic or cardiac end is carried to the left of the median line. The stomach, as it rotates, carries with it the lower part of the œsophagus and the duodenal loop, which latter thus takes up a position on the right side of the median line.

The original right surface of the stomach now becomes dorsal or posterior, and the original left surface becomes ventral or anterior. This change in the direction of the surfaces explains why the right pneumogastric nerve is dislributed to the posterior surface of the stomach in the adult, and the left pneumogastric nerie to the anterior surface of the organ. The partial rotation of the lower part of the esophagus towards the right also explains why, in the adult; the right pnewmogastric nerve lies behind the lower part of the cesophagus, and the left in front of the lower part of that tube.

The great curvature of the stomach, which was originally directed dorsalwards, is now directed caudalwards and towards the left: whilst the small curvature, which originally looked ventralwards, is now directed cephalicwards or upwards, and towards the right. 
Intestinal Canal. - This canal is at first very short and almost straight, and, as has been stated, it communicates freely with the yolk-sac. At this stage there is no indication of a division into small and large intestine. When the wide opening leading to the yolk-sac becomes constricted and converted into the vitelline duct, the intestinal canal undergoes lengthening, and a conspicuous loop is formed, which projects into the cavity of the proximal part of the umbilical cord, this cavity being a direct prolongation of the cœlom or body-cavity. This loop is spoken of as the U-loop. The convexity of the bend of the loop is directed ventralwards, and the vitelline duct is connected with the convexity. The loop has two limbs, which lie at first parallel to each other. One limb is cephalic, upper, or proximal, and leads from the duodenal loop of the gut; the other limb is caudal, lower, or distal, and leads to the caudal end of the gut. Upon the distal limb a bud makes its appearance. This assumes the form of a blind diverticulum, or cul-de-sac, which is the rudiment of the cæcum. The appearance of the cæcal evagination is the first indication of the division of the intestinal canal into small and large intestine. The primitive small intestne is the part on the proximal side of the rudimentary cæcum, and the primitive large intestine is the part extending from the rudimentary cæcum to the caudal end of the gut.

The U-loop soon undergoes rotation in such a manner that the distal limb bearing the cæcal diverticulum crosses obliquely over, or ventral to, the proximal limb, so that the original distal or lower limb now becomes proximal or upper. The part of the gut which is crossed ventrally by a portion of the distal limb represents the future descending part of the duodenum. This crossing accounts for the fact that, in the adult, the commencement of the transverse colon lies in front of the descending part of the duodenum.

The $U$-loop is occupied by a mesentery, which represents the mesentery proper of the adult. Close to the point of crossing of the two limbs of the loop, its mesentery contains the superior mesenteric artery. The loop itself, as stated, lies in the cavity of the proximal part of the umbilical cord. At a later period (about the ninth week of embryonic life) it is withdrawn from its original extra-embryonic position into the body-cavity (abdomen). In rare cases the prolongation of the cœlom into the proximal part of the umbilical cord may persist at birth, and so predispose to the occurrence of a congenital umbilical hernia.

The elongation of the jejunal and ileal portions progresses after withdrawal of the U-loop, and takes place more rapidly than that of the large intestine. Moreover, the small intestine outgrows in length the body-cavity, in consequence of which the jejunum and ileum are thrown into coils. These coils are placed, at first, on the right side of the median line.

The primitive large intestine is represented by (1) the cxcum, which is its initial part, (2) a short transverse colon, (3) a descending colon, and (4) a terminal part, which subsequently forms an iliac colon, pelvic colon, rectum, and upper two-thirds of the anal canal. There is no ascending colon at this stage. These initial parts (cæcum and colon) lie, at first, on the left side of the median line, the cæcum being underneath the left part of the liver. The cæcum gradually shifts to the median line, and then to the right side, still lying beneath the liver, and, as it thus alters its position, the transverse colon undergoes elongation. Finally, the cæcum, as a rule, descends slowly to its permanent abode in the right iliac fossa. During this descent the ascending colon becomes formed.

The large intestine having, like the small, outgrown the body-cavity, now has the form of an arch or loop, in the concavity of which the coiled jejunum and ileum lie. In the course of these changes in the position of the crecum, the jejunum and ileum are also shifting their position. Originally, as stated, they lie on the right side of the median line, but they, along with the lower part of the duodenum, are gradually shifted (but not entirely) towards the left side, so as to be brought within the loop of the large intestine.

The foregoing transposition of parts is brought about by the twist which has been referred to as occurring in the $U$-loop. This twist, or rotation, involves not only the loop itself, but also its mesentery, and it takes place 
from left to right round an axis, represented by the superior mesenteric artery. Inasmuch as the mesentery of the $U$-loop is involved in this rotation, the original right side of the mesentery becomes, after rotation, the left side, and vice versa.

Cæcum and Vermiform Appendix.-The cæcum appears about the fifth week of embryonic life as a bud in connection with the distal limb of the $\mathrm{U}$-loop of intestine. It assumes the form of a blind diverticulum, which is at first of uniform dimensions. It soon, however, becomes conical. The proximal part undergoes enlargement, and represents the permanent eæcum. The distal part remains long and narrow, and represents the vermiform appendix, which ultimately becomes connected with the left and posterior part of the cæcum. The various positions occupied by the cæcom and its migration to the right iliac fossa have just been referred to. Suffice it to say that it lies, at first, on the left side of the median line, then it crosses to the right side, being at this stage high up below tlse liver, and finally it sinks into its usual permanent abode. The descent of the cæcum, however, may be arrested at any stage.

For the development of the rectum and anal canal, see Index.

Development of the Peritoneal Cavity and Peritoneum.-The peritoneal cavity is one of the four divisions of the coelom or body-cavity. The other three divisions-namely, the pericardial cavity and the two pleural cavities, are contained within the thorax, and are separated from the peritoneal cavity by the diaphragm.

The parietal peritoneum is formed by the somatic mesoderm of the somatopleure of the body-wall, and the visceral periloneum is derived from the splanchnic mesoderm of the splanchnopleure of the primitive intestinal tube. The opposed surfaces of these two layers are lined with mesothelium, the flattened cells of which give rise to the lining endothelium of the peritoneal cavity.

In early life the entire alimentary tube is characterized by its shortness and straightness. Its ventral and dorsal aspects are attached to the ventral and dorsal body-walls by mesodermic folds, called primitive mesenteries. These mesenteries are two in number-namely, ventral and dorsal-and they establish a continuity between the splanchnic mesoderm of the alimentary tube and the somatic mesoderm of the body-wall.

The primitive ventral mesentery extends from the pharynx to the upper part of the duodenum, and consists of splanchnic mesoderm. The cephalic part is known as the cardiac portion. The heart and pericardium are developed within it, and it gives rise to the ventral and dorsal mesocardia. The caudal or posterior part of the ventral mesentery forms the ventral, or anterior, gastro-duodenal mesentery. The primitive dorsal mesentery is the fold which attaches the dorsal aspect of the primitive alimentary tube, over its entire length, to the dorsal body-wall. Like the ventral mesentery, it consists of splanchnic mesoderm.

The primitive mesenteries are thus two in number-namely, ventral and dorsal.

The ventral gastro-duodenal mesentery extends from the ventral aspects of the primitive stomach and upper part of the duodenum to the ventral bodywall on the cephalic side of the umbilicus. As the liver, which is undergoing development within the caudal or hepatic layer of the septum transversum, descends, it separates from the niddle or diaphragmatic layer of that septum, which gives rise to the ventral portion of the diaphragm; and as it descends it passes between the two layers of the gastro-duodenal mesentery. This mesentery is now divided into two parts by the developing liver. The part between the liver and (1) the ventral portion of the diaphragm and (2) the ventral wall of the abdomen as low as the umbilicus becomes the falciform ligament of the liver; and the part between the liver (future portal fissure) and (I) the stomach (future small curvature); and (2) the duodenum (future first part for about an inch) becomes the small or gastro-hepatic omentum.

The dorsal mesentery receives names corresponding to the parts of the alimentary tube with which it is connected. Thus, in the abdomen, there are 
the mesogastrium, mesoduodenum, mesojejunum, meso-ileum, mesocolon, and mesorectum.

In the course of development these various parts of the primitive dorsal mesentery undergo marked changes.

I. Mesogastrium.-The mesogastrium is that portion of the primitive dorsal mesentery which extends vertically between the great curvature of the stomach, which at this period is directed dorsalwards, and the dorsal bodywall. It is at first comparatively short, but it undergoes lengthening as the stomach turns round on its right side. During this partial rotation of the stomach the mesogastrium is somewhat doubled upon itself, and forms a pouch, which is directed downwards and to the left. This pouch is the initial stage in the formation of the small sac of the peritoneum. The pouch is bounded ventvally by (1) the small or gastro-hepatic omentum, and (2) the dorsal surface of the stomach (originally the right surface); dorsally by the dorsal body-wall; and caudalwards by the somewhat doubled mesogastrium. During the rotatory movement of the stomach, the small or gastro-hepatic omentum, the direction of which was originally sagittal-that is, dorsoventral-now assumes a coronal or transverse position. In this manner, combined with a slight doubling of the mesogastrium, a small part of the peritoneal cavity is incompletely partitioned off, and this is the portion which constitutes the upper part of the small sac, lying, as just stated, dorsal to the small omentum and dorsal surface of the stomach. This upper part of the small sac communicates with the general peritoneal cavity (from which it has been partitioned off) by an opening, called the foramen of Winslow, which is situated behind the right or free border of the small or gastro-hepatic omentum. This opening is at first large, but, as the liver grows, it becomes considerably curtailed.

The great, or gastro-colic, omentum is developed from the mesogastrium, which increases slowly in size downwards. When fully developed, it forms the extensive sheet which descends from the great curvature of the stomach, and lies ventral to the transverse colon and the coils of the jejunum and ileum. As the great omentum grows downwards, the pouch, which represents the upper part of the small sac, increases in size and also grows downwards. This downward extension of the small sac occupies the interval between the two anterior, or descending and the two posterior, or ascending, layers of the great omentum, this part of the small sac being known as the bag or sac of the great omentum.

For the changes which this curtain undergoes, prior to assuming its condition in the adult, see the Peritoneum in the Fœtus.

The gastro-splenic omentum and lieno-renal ligament are associated with the development of the spleen (lien) in the mesogastrium. The portion of the mesogastrium between the stomach and spleen gives rise to the gastrosplenic omentum, whilst the portion between the spleen and the left kidney forms the lieno-renal ligament.

It is to be noted that the great or gastro-colic omentum is developed from the mesogastrium, which is a portion of the primitive dorsal mesentery, whilst the small or gastro-hepatic omentum is formed, as stated, by that portion of the ventral gastro-duodenal mesentrry which extends between the liver (the future portal fissure) and the stomach (the future small curvature).

As the development of the abdominal portion of the alimentary tube proceeds, it undergoes considerable changes, which impress modifications upon the peritoneum, and impart to it a remarkably complicated character.

The stomach appears at first as a spindle-shaped dilatation of the alimentary tube, which, together with the duodenal portion of the intestinal tube, is at first straight. For the subsequent changes in form and position of the stomach and intestinal canal, see their development. It is sufficient in this place to state that the stomach becomes curved, and turns round on its right side, carrying the duodenum along with it, whilst the intestinal tube undergoes elongation and becomes very tortuous.

The primitive ventral gastro-duodenal mesentery, as stated, gives rise to(1) the falciform ligament of the liver, and (2) the small or gastro-hepatic omentum. 
The mesogastrium (part of the primitive dorsal mesentery), as stated, gives rise to-(1) the great or gastro-colic omentum; (2) the gastro-splenic omentum; and $(3)$ the lieno-renal ligament.

2. Mesoduolenum. - This fold disappears, so that the duodenum, except for about an inch at its commencement, is retroperitoneal.

3. Mesojejunum and Meso-ileum.-These folds persist and constitute the mesentery proper. Its dorsal end contains the superior mesenteric artery, and is of limited extent. As the jejunum and ileum increase in length, the intestinal end of the mesentery proper acquires a much greater length than the vertebral end. Moreover, as these parts of the intestinal tube become coiled, the mesentery proper towards its intestinal end becomes curved, and presents a number of segments of circles, which impart to it an undulating outline.

4. Cæcum.-The cxcum, being originally a bud or outgrowth of the gut, has no mesentery, and is entirely surrounded by peritoneum.

5. Vermiform Appendix.-The vermiform appendix, being originally the blind narrowed end of the cacum, or, in other words, a diverticulum of the cæcum, is also destitute of a mesentery, properly so called, and is invested by an extension of the peritoneal envelope of the cæcum. There is, however, in most cases a fold of peritoneum pertaining to the vermiform appendix, which extends along it for about $\frac{1}{2}$ or $\frac{2}{3}$ of its length. This fold is called the mesoappendix or appendicular mesentery, and it is derived from the left or inferior layer of the mesentery proper, close to the ileo-colic junction.

6. Ascending Mesocolon.-This fold, in most cases, disappears.

7. Transverse Mesocolon.-This fold is persistent.

8. Descending Mesocolon.--This fold, like the ascending mesocolon, in most cases disappears.

9. Iliac Mesocolon.-This fold in most cases disappears.

ro. Pelvic Mesocolon. - This fold is persistent.

11. Mesorectum.-This fold disappears.

\section{Structure of the Liver.}

The liver has two coats, external and internal. The external or serous coat is formed by the peritoneum, and is incomplete (see p. 703). Within the serous coat is the fibrous or areolar coat, which is known as the fibrous capsule of the organ. It is for the most part thin, except where the peritoneal coat is deficient. It is continuous all over the surface with the scanty amount of areolar tissue which pervades the interior of the organ and connects the hepatic lobules. At the portal fissure it surrounds the hepatic duct, hepatic artery, and vena portæ, under the name of the capsule of Glisson. The subdivisions of this capsule accompany the various branches of the duct, artery, and vein as these ramify throughout the liver in the portal canals. The liver-substance is composed of a great number of small polyhedral masses, called hepatic lobules, which are closely packed together, and are set, each by one surface, on the walls of the sublobular veins, to which they are attached by means of intralobular or central veins. In man there is very little areolar tissue between the lobules, which are therefore for the most part confluent. In some animals, however, notably the pig and camel, there is a very distinct amount of areolar tissue between the lobules, which, therefore, stand clearly apart from each other. 
The average diameter of a hepatic lobule is $\frac{1}{20}$ inch. One of its surfaces is called the buse. It is by this surface that the lobule is set upon the wall of a sublobulal vein, and the intralobular or central vein, having emerged from the lobule through the centre of its base, opens at once into the sublobular vein. The lobules, therefore, relatively to the sublobular veins, on which they are ranged, are sessile. When a sublobular vein is opened and viewed from within, an appearance something like mosaic work presents itself, the closely-set bases of the lobules being visible through the thin wall of the vein, and the minute openings of the intralobular or central veins appearing in the centre of each base. Each lobule is composed of hepatic cells, permeated by capillary networks of bloodvessels and bile capillaries.

Rloodvessels.-The liver derives its blood from two sources,

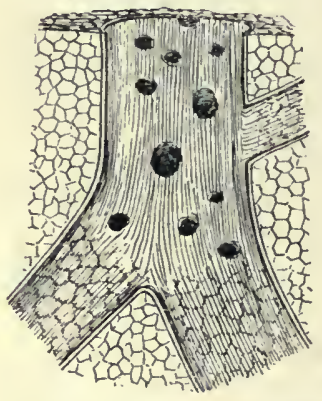

Fig. 345.-SEction of THE LIVER, SHOWING a Large HEPATIC VEIN AND ITS TRIBUTARIES (AFTER KIERNAN). namely, the vena portæ, and the hepatic artery. These two vessels, together with the hepatic duct, are invested by the capsule of Glisson at the portal fissure. Their several branches, ensheathed by prolongations of Glisson's capsule, ramify from this point throughout the liver, being contained in the system of canals, known as portal canals. Each of these canals contains (I) a branch of the vena portæ, (2) a branch of the hepatic artery, accompanied by a plexus of nerves, (3) lymphatic vessels, and (4) a minute duct, all these being loosely surrounded by a prolongation of Glisson's capsule.

The vena portæ ramifies within the liver like an artery. In the portal canals its branches receive as tributaries small capsular and vaginal veins, and they go on ramifying until they arrive at the inter. lobular areas. Here they anastomose freely with one another around the lobules, and so form the interlobular plexuses. The branches which arise from these plexuses enter the lobules on all sides except their bases, and form in the interior of each lobule an intralobular plexus. From this plexus a few radicles converge towards the centre of the lobule, where they form by their union the intralobular or central vein. The portal blood, though dark in colour, is very rich, being derived from, amongst other sources, the stomach and small intestine.

The hepatic artery is a branch of the cœliac axis. As its branches traverse the portal canals they give off capsular branches to the fibrous capsule of the liver, and vaginal branches, which supply the walls of the vessels in the portal canals, as well as their Glis. sonian sheaths. The branches of the artery finally end as minute interlobular arteries, which supply the walls of the interlobular 
veins and bile-ducts. According to some authorities they send minute capillary branches into the interior of each lobule to join the intralobular plexus of the venæ portæ, but this view is not held by others.

The function of the hepatic artery and its branches is to nourish the tissues of the liver, whilst the portal blood supplies the materials which are elaborated by the hepatic cells.

The hepatic veins commence in the centre of each lobule as an intralobular or central vein. The intralobular veins open into the sublobular veins, which are closely adherent to the bases of the lobules. The sublobular veins join to form larger sub. lobular veins, and these in turn terminate in the hepatic veins, which are not in direct contact with the lobules. The hepatic veins pass to the caval fossa on the posterior surface of the liver, towards which they converge, taking up sublobular veins in their course. The passages which contain the veins are known as the hepatic canals. On arriving at the upper part of the caval fossa they are reduced to two or three in number, which pour their contents directly into the inferior vena cava. It is to be noted that the hepatic veins converge to the caval fossa on the posterior surface, whilst the

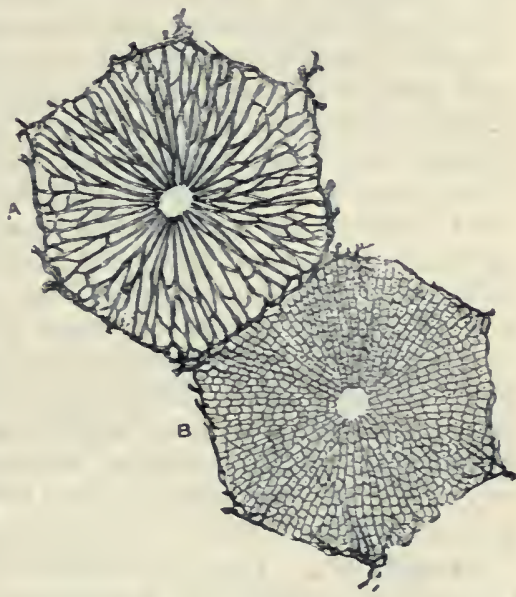

Fig. 346.-Two Hepatic Lobules (HIGHLY MAGNIFIED).

A, Lobule showing the Intralobular Plexus; B, Lobule showing the Hepatic Cells.

branches of the vena portæ radiate in all directions from the portal fissure. The hepatic veins are accompanied only by lymphatic vessels, and are surrounded by a very scanty amount of areolar tissue, which explains why they present open mouths when cut across.

Distinguishing Characters of the Hepatic and Portal Veins-Hepatic Veins. -(I) These present open mouths when cut across. (2) They are accompanied only by lymphatic vessels. (3) Their walls are very thin, and are practically in direct contact with the substance of the liver. (4) They converge towards the caval fossa on the posterior surface.

Portal Veins. - (I) The mouths of these veins are practically closed or collapsed when cut across. (2) The veins are accompanied by branches of the hepatic artery, and by ducts. (3) Their walls are fairly thick, and are separated from the substance of the liver by the sheaths derived from Glisson's capsule. (4) They radiate in all directions from the portal fissure in portal canals.

Hepatic Cells.-These are situated within the lobules. They are polyhedral, granular cells, having a diameter of $\frac{1}{1000}$ inch, and each contains a round nucleus. They have no cell-wall, properly 
so called, and they are connected by a delicate supporting tissue. The cells, which are capable of amoeboid movement, lie between, and around, the capillary vessels, where they are arranged in radiating rows converging towards the centre of the lobule.

Bile-ducts.-These commence within the lobules as bile-capillaries or bile-canaliculi, which are in reality intercellular passages. They have a very delicate wall, and are bounded on all sides by the hepatic cells-hence the name intercellular passages. According to Pflüger and Kupffer, the cells contain vacuoles, which communicate by intracellular passages with the bile-capillaries. The hepatic cells intervene between the bile-capillaries and the capillary bloodvessels. At the circumference of the lobule the bile-capillaries pass into the small interlobular bile-ducts. These join to form larger ducts, and these in turn go on joining until, on arriving at the portal fissure, only two ducts result, which emerge one from each lobe. These now join to form the hepatic duct, and this joins the cystic duct to form the common bile-duct. The walls of the interlobular ducts are very thin, being composed of a basement membrane lined with polygonal epithelium. The larger ducts in the portal canals have thicker walls, which, from without inwards, are composed of a fibro-elastic coat, containing plain muscular tissue, arranged longitudinally and circularly, and a mucous coat lined with columnar epithelium. The mucous membrane of the larger ducts presents numerous openings, which lead into blind mucus-secreting recesses. The structure of the hepatic and common bile-ducts is similar to the preceding, though on an increased scale.

Lymphatics. - The lymphatic vessels of the liver are arranged in two groups-superficial and deep.

Superficial Lymphatics.-These form plexuses beneath the peritoneal coat, and have different destinations.

Supero-anterior Surface.-(I) The lymphatics from the vicinity of the falciform ligament enter that ligament, and, passing through the diaphragm into the thorax, they terminate in the anterior mediastinal glands. (2) The lymphatics from the right part of the superior surface and those from the right lateral surface enter the right lateral ligament, and, passing through the diaphragm, they terminate in the middle diaphragmatic glands of the right side. (3) The lymphatics from the left part of the superior surface and those from the left extremity enter the left lateral ligament, and pass to the peri-cesophageal glands in relation to the lower end of the œsophagus, the efferent vessels of which terminate in the celiac glands. (4) The lymphatics from the anterior part of the supero-anterior surface, except those from the vicinity of the falciform ligament, turn round the anterior border of the liver, and end in the hepatic glands within the small omentum. (5) The lymphatics from the posterior surface of the right lobe pass between the two layers of the coronary ligament, and, having pierced the diaphragm, they terminate in the caval glands within the thorax.

Inferior Surface.-(I) The lymphatics from the greater part of the inferior surface of the right lobe, including those of the quadrate 
lobe, pass to the hepatic glands. (2) The lymphatics from the posterior part of the inferior surface of the right lobe pierce the diaphragm, and end in the caval glands. (3) The lymphatics from the greater part of Spigel's lobe pierce the diaphragm, and end in the caval glands; whilst those from the lower part of this lobe pass to the hepatic glands. (4) The lymphatics from the inferior surface of the left lobe pass to the hepatic glands.

Deep Lymphatics.-These vessels form two distinct sets. Some of them accompany the branches of the portal vein, and, having emerged through the portal fissure, they terminate in the hepatic glands. Others pass with the hepatic veins to the caval fossa of the liver, and thereafter they enter the thorax along with the inferior vena cava, their destination being the caval glands.

Nerves.-The nerves of the liver are derived from the hepatic plexus, which is an offshoot of the cœliac plexus, that in turn being derived from the solar plexus of the sympathetic system. The cœliac plexus, though principally composed of sympathetic fibres, is reinforced by a few twigs from the right pneumogastric nerve. The hepatic plexus accompanies the hepatic artery to the portal fissure, where it receives branches from the left pneumogastric nerve, which have ascended from the antero-superior surface of the stomach between the two layers of the gastro-hepatic omentum. In the liver the nerves, which are chiefly non-medullated, are distributed to the walls of the bloodvessels and ducts, penetrating as far as the interspaces between the hepatic cells.

\section{Development of the Liver.}

Liver.-The hepatic cells and the epithelium of the bile-ducts are of entodermic origin, whilst the connective tissue of the gland and its vascular constituents are developed from the mesoderm.

The rudiment of the liver appears as a longitudinal groove on the inner aspect of the ventral wall of the duodenal portion of the primitive gut. This groove gives origin to a diverticulum or evagination of the entoderm of the ventral duodenal wall, called the hepatic diverticulum or liver-bud, which has at first a wide communication with the gut. At a later period this communication becomes constricted, and forms a pedicle, which, after undergoing elongation, gives rise to the common bile-duct. From this pedicle, close to the duodenal wall, the ventral diverticulum of the pancreas arises.

The hepatic diverticulum or liver-bud invades the lower or caudal layer of the septum transversum, composed of mesoderm, within which it bifurcates, the two divisions representing the right and left hepatic ducts.

The cells of these two divisions of the hepatic diverticulum undergo proliferation within the mesoderm of the lower layer of the septum transversum, and thereafter each division breaks up into a number of solid trabeculæ, which are known as the hepatic eylinders. These cylinders give off secondary solid trabeculæ, and these again ramify extensively. The subdivisions of the various trabeculæ anastomose freely, and in this manner intricate networks are formed around the vitelline and umbilical veins, which veins traverse the septum transversum as they pass to the sinus venosus. The hepatic cylinders invade these veins, carrying the endothelial walls of the vessels before them. The veins are thus freely subdivided into blood-channels, which are known as sinusoids. These sinusoids form capillary networks, which occupy the meshes of the networks formed by the hepatic cylinders.

Many of the solid trabeculæ become tubular, and give rise to the tileeapillaries or bile-canaliculi, and bile-ducts. Others give rise to the hepatic 
cells. The liver thus consists originally of intricate reticula of solid cellular trabeculx, or hepatic cylinders, disposed around the vitelline and umbilical veins.

As the liver increases in size it projects downwards, carrying with it the lower layer of the septum transversum, which forms its capsule and connective tissue. The organ now lies between the two layers of the ventral or gastroduodenal mesentery, which gives rise to-(1) the falciform ligament; (2) the posterior or coronary ligament; and (3) the gastro-hepatic or small omentum.

A bout the middle of intra-uterine life the liver occupies a large part of the abdominal cavity, and the right and left lobes are of equal size. In the latter half of intra-uterine life, however, the right lobe gradually attains greater size than the left. About the period of birth the liver extends almost as low as the umbilicus. After birth it undergoes diminution in size, the circulation of placental blood through it, by the umbilical vein, having been permanently arrested at birth.

At an early period in its development the liver presents four lobes-two vitelline and two umbilical. The vitelline lobes represent the parts which are developed around the vitelline veins, whilst the umbilical lobes are developed around the umbilical veins. As, however, development proceeds the lobar condition characteristic of the full-grown liver is gradually. assumed.

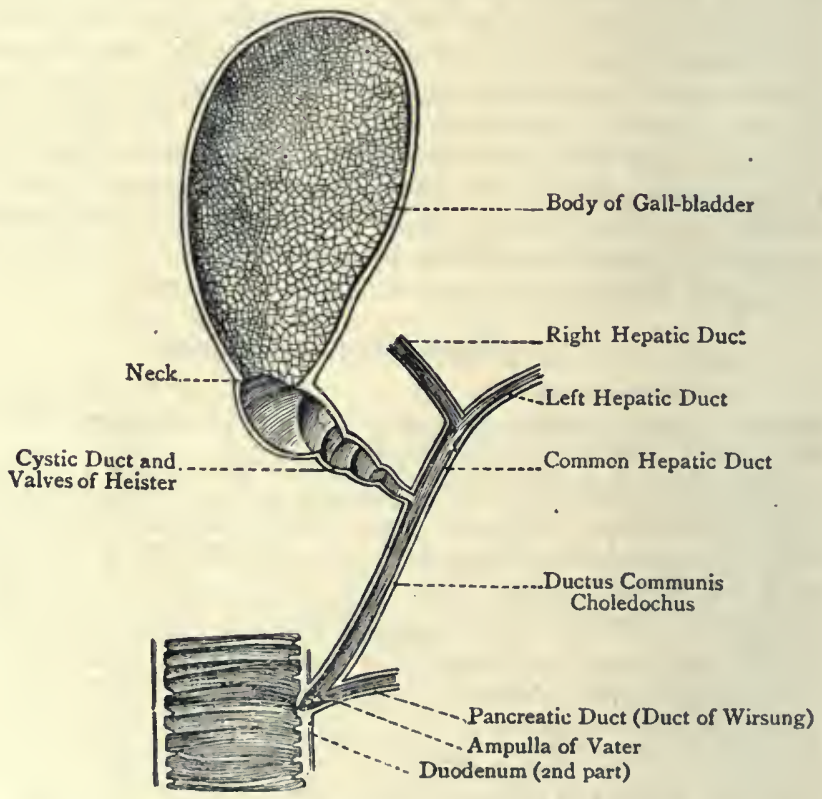

Fig. 347.-The Excretory Apparatus of the Liver.

Structure of the Gall-bladder.-The wall of the gall-bladder is composed of three coats-serous, fibro-muscular, and mucous.

The serous coat is formed by the peritoneum, and is usually incomplete, being confined to the inferior and lateral surfaces. Sometimes, however, the peritoneum completely surrounds the organ, and attaches its superior surface to the cystic fossa of the liver by a ligamentous fold, 
The fibro-muscular coat is composed of fibrous and plain muscular tissues. The fibrous bands are disposed in all directions, and interlace freely with one another. The muscular fibres, which intermingle with the fibrous bands, are arranged both longitudinally and transversely.

The mucous coat is covered by columnar epithelium. Its surface presents a great number of small ridges, which interlace in all directions and enclose polygonal depressions or alveoli of various sizes. This pitted appearance bears a resemblance to honeycomb, and the mucous membrane of the vesiculæ seminales in the male is similarly arranged. The mucous coat is richly provided with mucous glands. In the neck of the gall-bladder, which describes two curves like the letter $\mathbf{S}$, there are two fibro-mucous folds, which project into the interior, one opposite each curve, and serve as valves.

Structure of the Cystic Duct.-The wall of the duct is composed of a fibro-elastic and muscular coat, and a mucous coat. The former resembles that of the gall-bladder. The mucous coat, which is covered by columnar epithelium, presents several crescentic folds, disposed obliquely round the wall in a spiral or somewhat corkscrew manner, and succeeding each other at frequent intervals. When the duct is distended, its exterior presents a series of spiral constrictions with intervening swellings. The spiral folds in the interior serve as valves, which are known as the valves of Heister, or of Amussat.

Blood-supply.-The gall-bladder is supplied by the cystic artery, which is a branch of the right division of the hepatic. Its course is forwards between the hepatic and cystic ducts to the neck of the organ, where it divides into two branches, superior and inferior. The superior branch ramifies on the upper surface of the organ, between it and the cystic fossa of the liver, whilst the inferior branch is distributed over the inferior surface beneath the peritoneum. The cystic vein, which is formed by the union of superior and inferior branches, usually opens into the right division of the vena portæ.

Lymphatics.-These pass between the two layers of the gastrohepatic omentum to the hepatic glands.

Nerves.-These are derived from the hepatic sympathetic plexus.

Development of the Gall-bladder and Cystic Duct.-These are formed in the course of the second month as a diverticulum from the common bile-duct.

\section{Structure of the Pancreas.}

The pancreas belongs to the class of compound racemose or acinotubular glands, and bears a close resemblance to a serous or true salivary gland, e.g., the parotid. It has a greyish-pink colour, and is somewhat soft in consistence. It is from 6 to 8 inches long, from I to $I \frac{1}{2}$ inches deep, except at the right and left extremities, and from $\frac{1}{2}$ to $\frac{3}{4}$ inch thick. Its weight is about $3 \frac{1}{2}$ ounces. It is 
destitute of a fibrous capsule, properly so called, and is invested merely by a thin connective-tissue covering, which readily allows the outline of the lobules to be seen.

It is composed of a number of lobules, which are loosely held together by ducts and areolar tissue. Each lobule consists of a group of alveoli or acini which are long, tubular, and convoluted, wherein they differ from the saccular alveoli of such a gland as the parotid. From each lobule a duct passes off, which unites with the ducts of adjacent lobules in the left extremity of the gland to form the commencement of the principal duct. Within the lobule the lobular duct divides into junctional ducts, each of which belongs to an acinus, and is lined with flattened cells.

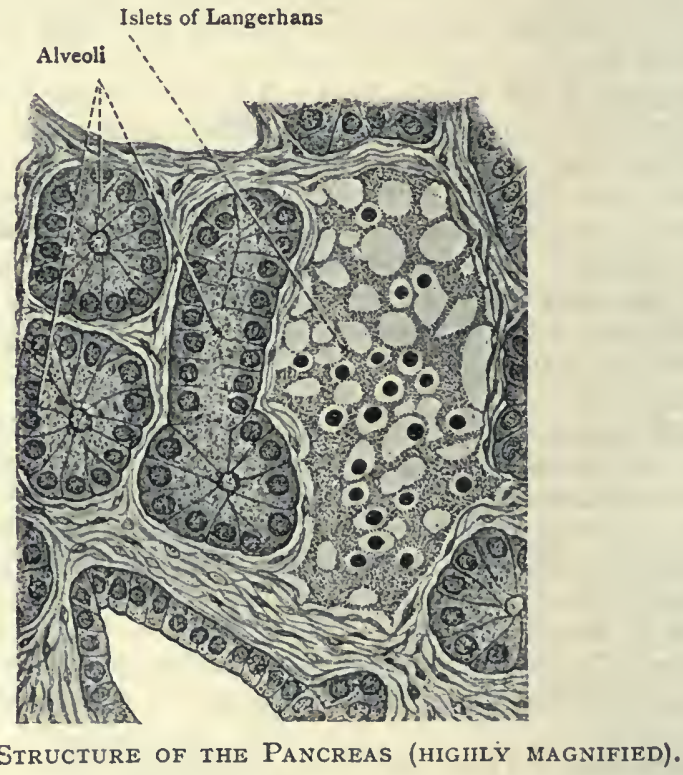

The alveoli or acini are each composed of a basement membrane, reticular in structure, and lined with secreting columnar epithelium, the basement membrane being continuous with the wall of the junctional duct. The lumen of each acinus is small, and is usually occupied by spindle-shaped cells, known as the centro-acinar cells of Langerhans, which are continuous with the cells of the junctional duct. The connective tissue which covers the pancreas sends expansions inwards between the lobules, along which the bloodvessels are conducted. Further, the interlobular connective tissue penetrates into the lobules, and so conducts the bloodvessels into their interior. The interalveolar connective tissue has a loose disposition, and in cartain parts contains small groups of epithelium-like cells, 
surrounded by large convoluted capillary vessels. These groups constitute the islets of Langerhans, which are characteristic of the pancreas.

Excretory Apparatus of the Pancreas.-There is one principal duct, called the pancreatic duct or duct of Wirsung. It is buried in the substance of the gland, and is readily recognised by its white colour. It lies nearer the posterior than the anterior surface, and rather nearer the lower than the upper part of the gland. It commences in the tail, whence it runs through the body as far as the neck, receiving in its course a great many tributaries. On arriving at the neck it gets a branch from the accessory pancreatic duct, and then it describes a bend, and passes into the head in a direction downwards, backwards, and to the right. Finally, on ieaving the pancreas it meets with the common bile-duct, and the two, entering the wall of the second part of the duodenum, terminate in the manner already described (see p. 7Io).

The accessory pancreatic duct or duct of Santorini is comparatively small, and varies much in size. If well developed, it commences in the lower part of the head, where it takes up the ducts of the lobules of that part. It then passes upwards with an inclination to the right, and divides into two branches. One of

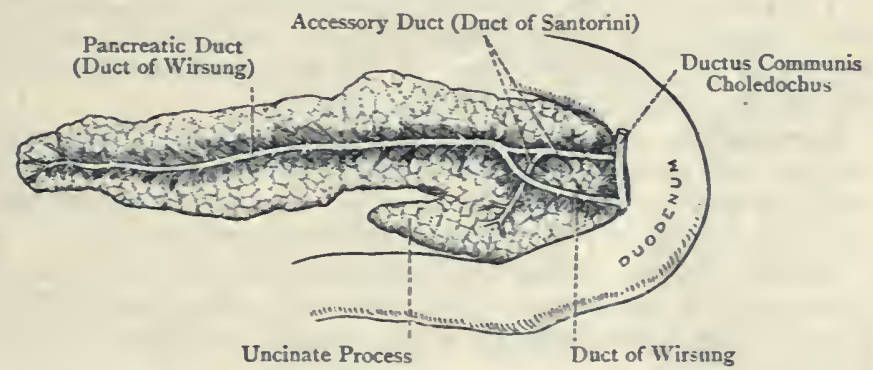

Fig. 349. -The Pancreas and its Ducts (Posterior View).

these joins the duct of Wirsung in the neck, whilst the other opens into the second part of the duodenum at a point about I inch above the common opening of the common bile-duct and duct of Wirsung. The secretion conveyed by the duct of Santorini in early life is usually regarded as flowing into the duodenum, whilst in the adult it is diverted into the duct of Wirsung.

The tributaries of the principal duct, as well as of the accessory duct, when followed into the pancreas, become in succession interlobular and intralobular ducts. The intralobular ducts pass within the lobules, and end in intermediary, junctional, or intercalary* ducts, with which the alveoli or acini are directly connected. The part of the duct between the intermediary and the intralobular duct is called the neck. The walls of the intermediary ducts are thin, and are formed of a basement membrane covered by flattened

* Interposed or inserted between the alveoli and the intralobular ducts. 
epithelial cells, the neck being lined by polyhedral cells. In the larger ducts a connective-tissue coat is superadded to the basement membrane, which is now covered by columnar epithelium. The duct of Wirsung, though of comparatively large size, has a thin wall destitute of muscular fibres, and composed of two coatsexternal fibrous and internal mucous. The mucous coat is smooth and covered by columnar epithelium.

Varieties-(I) Small Pancreas.-This consists in a permanent detachment of the unciform process, or that part of the pancreas which extends along the upper aspect of the third part of the duodenum and has the superior mesenteric vessels in front of it. (2) The head of the pancreas may surround the second part of the duodenum more or less completely. (3) Accessory Panereas. When an accessory pancreas is present it is usually met with in the wall of the upper part of the duodenum, but it may be found in the wall of the stomach. (4) The duct of Wirsung sometimes opens into the duodenum independently of, but close to, the common bile-duct.

Blood-supply.-The pancreas derives its arteries from (I) the pancreatic branches of the splenic artery, one of which accompanies the principal duct from left to right, and is known as the arteria pancreatic magna; (2) the superior pancreatico-duodenal of the gastro-duodenal from the hepatic; and (3) the inferior pancreatico-duodenal of the superior mesenteric.

The veins are (I) the pancreatic veins, which open into the splenic vein, and (2) the pancreatico-duodenal vein, which terminates in the superior mesenteric vein. All the pancreatic venous blood eventually passes into the portal vein.

Lymphatics. - These commence as lymphatic clefts around the alveoli, and pass chiefly to the cceliac glands. Some of them, however, terminate first in the superior mesenteric glands in contact with the upper part of the superior mesenteric artery.

Nerves.-These are derived from offshoots of the hepatic, splenic, and superior mesenteric plexuses, and accompany the arteries. They are chiefly composed of non-medullated fibres.

Development of the Pancreas.-The tubular portion of the pancreas is of entodermic origin, but its connective tissue and vascular elements are developed from mesoderm.

The pancreas is developed from two entodermic diverticula-dorsal and ventral. The dorsal diverticulum is an evagination of the entoderm of the dorsal wall of the duodenal portion of the primitive gut. The ventral diverticulum springs from the primitive hepatic diverticulum close to the ventral wall of the duodenum, which diverticulum subsequently forms the common bile-duct. The dorsal diverticulum extends between the two layers of the mesogastrium, where it comes into relation with the developing spleen. This diverticulum gives off lateral epithelial tubes, which ramify freely, and so build up an acinotubular gland. The acini appear as enlargements of the walls of the terminal tubes. By means of the ramifications of the dorsal diverticulum, the greater part of the head, body, and tail of the adult pancreas are formed.

The ventral diverticulum, which is small, gives rise to ramifying epithelial tubes, like the dorsal diverticulum, and these form the lower part of the head of the adult pancreas. The ventral diverticulum, an evagination, as stated, from the future common bile-duct, gives rise to the principal duct of the pancreas, or duct of Wirsung, and the dorsal diverticulum forms the accessory pancreatic duct, or duct of Santorini, which consequently opens directly into the duodenum. 
The ventral and dorsal parts of the pancreas join, and the dorsal diverticulum (duct of Santorini) and ventral diverticulum now communicate by freely anastomosing branches. Thereafter the duodenal portion of the duct of Santorini usually atrophies and disappears. It may, however, persist, and open, as an independent duct, into the second part of the duodenum. The duct of Wirsung is persistent, and constitutes the principal duct of the fully developed pancreas. From its mode of development it necessarily opens into the common bile-duct.

The islands of Langerhans, which are characteristic of the pancreas, are formed of cells derived from the walls of the original epithelial tubes. These cells become differentiated, and by their multiplication, they give rise to isolated cell-groups, lying in the mesodermic connective tissue between the lobules of the gland, where they are soon permeated by bloodvessels.

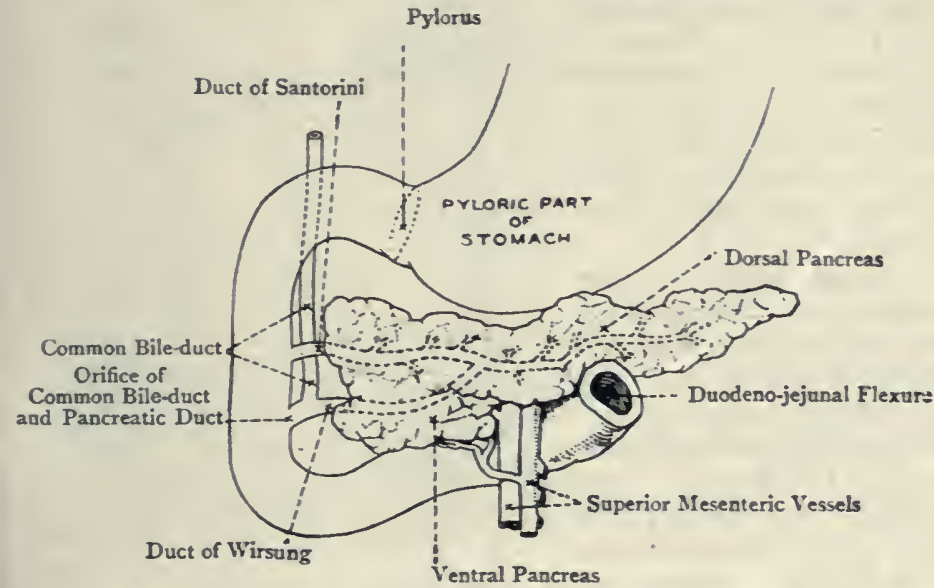

Fig. 350.-The Pancreas in Early Life.

The development of the pancreas, as stated, proceeds within the messgastrium. It occupies a mesial position at this stage, and is completely invested by the mesogastrium. When, however, the stomach and duodenum undergo partial rotation towards the right, the pancreas also turns on its right side. The head of the gland inclines towards the right, and occupies the U-shaped curve described by the duodenum. The body and tail pass to the left, the body coming to lie in front of the left kidney, and the tail applying itself to the spleen. The entire gland, therefore, now lies across the dorsal body-wall.

The dorsal layer of the mesogastrium, covering the dorsal surface of the pancreas, becomes absorbed, and consequently the pancreas of adult life is situated behind the peritoneum, and is therefore spoken of as being retroperitoneal.

\section{Structure of the Spleen.}

The spleen is the largest ductless gland in the body. It has two coats, serous and fibrous, inseparably connected together. The serous coat is formed by the peritoneum. The fibrous coat or tunica propria is composed of fibrous tissue, with a considerable admixture of elastic tissue, and a certain amount of 
plain muscular tissue, all of which build up a strong distensible tunic.

The organ is composed of a trabecular network, the spleen pulp, bloodvessels, and Malpighian corpuscles.

The tunica propria sends into the organ a number of trabeculæ which, like the tunica propria, are composed of fibrous, elastic, and plain muscular tissues. These divide and subdivide, and unite with one another, as well as with processes de. rived from the sheaths which the bloodvessels carry in with them at the hilum. There is thus formed a trabecular network which pervades the interior, and contains in its meshes the spleen pulp, capillary tufts, and Malpighian corpuscles.

The spleen pulp occupies the meshes of the network formed by the trabeculæ. It is soft, and has a dark red colour, which, however, becomes brighter on exposure to the air. The matrix of the spleen pulp is reticular, and is formed by branched connective-tissue corpuscles, which constitute the sustentacular cells of the organ.

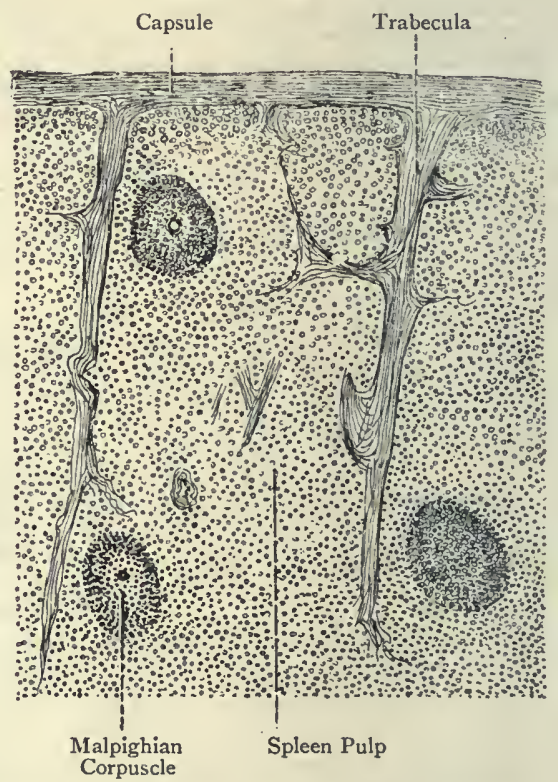

Fig. 35I.-Section of the Spleen. The matrix is, therefore, in reality retiform tissue. The interstices of the reticulum contain blood, in which there is a large number of white corpuscles, and also special cells characteristic of the spleen, and called the splenic cells. These latter are of large size, and are amœboid. They contain pigment, and red blood-corpuscles in various stages of disintegration.

Bloodvessels and Malpighian Corpuscles--Arteries.-The splenic artery furnishes five or six large branches which enter the organ at the hilum, and carry in with them trabecular sheaths from the tunica propria. In the interior they divide and subdivide, and finally terminate in pencil-like clusters of capillary vessels, by which time they have laid aside all their coats except the endothelial lining. The endothelial cells then become separated from each other by spaces, and, being continuous with the sustentacular cells of the spleen pulp, the blood flows directly into the interstices of the reticulum of the pulp. The arteries, which are at first accompanied by trabecular sheaths, ultimately lose these sheaths, and 
enter the spleen pulp to end in clusters of capillaries. Before they terminate in these clusters their external coat undergoes an important modification, which consists in its transformation into lymphoid or adenoid tissue. This lymphoid tissue forms at intervals small round or oval enlargements, called the Malpighian corpuscles, which on section appear as minute white specks in the dark red spleen pulp. These corpuscles are simply localized expansions of the lymphoid tissue which forms the external coat of the small arteries. The expansion may be confined to one side of the artery, or it may include the whole of its circumference. Each corpuscle receives minute twigs from the artery on which it is set, and contains large numbers of lymph corpuscles, as well as capillary bloodvessels.

Veins.-The arterial blood, on leaving the capillary vessels, flows directly into the interstices of the reticular matrix of the spleen pulp, and from these it is taken up by radicle veins which commence in a manner similar to that in which the capillaries end. Endothelial cells, continuous with the sustentacular cells of the pulp, come together and cohere, so as to form very delicate tubular vessels, having closed walls. As these radicle veins unite and become larger the usual other coats are superadded to the endothelial lining. Ultimately five or six veins leave the spleen at the hilum, which unite to form the splenic vein, this in turn uniting with the superior mesenteric vein to form the vena portæ.

It is to be noted that, in the circulation through the spleen, the arterial blood leaves tubular vessels and flows through the interstices of the reticulum of the spleen pulp, where it bathes the Malpighian corpuscles, after which it enters tubular vessels of the nature of veins.

The cells of the spleen are of three kinds as follows: (I) the sustentacular cells of the retiform tissue of the spleen pulp; (2) the splenic cells; and (3) lymphoid corpuscles.

Lymphatics. - These are arranged in two groups - trabecular and perivascular. The trabecular lymphatics are contained in the trabeculæ, and communicate with a lymphatic network in the tunica propria underneath the peritoneal coat. The perivascular lymphatics commence in the lymphoid tissue which forms the external coat of the smaller arteries. At the hilum both sets of lymphatics meet and pass to the splenic glinds, and thence to the coliac glinds.

Nerves.-These are derived from the splenic plexus, which is an offshoot from the cœliac plexus of the solar plexus. The fibres, which are mostly non-medullated, are derived partly from the sympathetic system and partly from the right pneumogastric nerve.

Development of the Spleen.-The spleen is usually regarded as being developed from the mesoderm between the two layers of the mesogastrium. It is in close proximity to the developing pancreas, which, however, is of entodermic origin. Towards the end of the second month the mesenchyme becomes thickened by an accumulation of lymphoid cells. This mass is increased by the addition of cells which migrate from the surface-cells of the mesogastrium, and it becomes permeated by bloodvessels. The cells give off processes, and these interlace, thus giving rise to the trabecular network. 
At a later period the Malpighian corpuscles make their appearance. These are formed from lymphoid cells, which are grouped around the small branches of the splenic artery, before these branches terminate in their clusters of capillaries.

As the spleen grows towards the left side it carries with it the left layer of the mesogastrium, and from this are formed the gastro-splenic omentum, the lieno-renal omentum, and the lieno-phrenic, or phreno-splenic ligament.

\section{Structure of the Suprarenal Capsules.}

The suprarenal capsules or bodies (adrenals) belong to the so-called ductless glands. Each capsule is enclosed in a thin sheath of connective tissue, from the deep part of which processes are given off into the interior, where they form a supporting stroma. The proper substance of the capsule is divided into an external or cortical, and internal or medullary part.

The cortical part lias a somewhat yellowish tint, and is com. posed of cells supported by a fibrous stroma. The cells being variously arranged in different parts, the cortex is divisibleinto three $z$ ones, named, from without inwards, zona glomerulosa, zona fasciculata, and zona reticularis. The zona glomerulosa, which is narrow, lies immediately within the external capsule, and is so named because its component cells are grouped in such a manner as to form glomeruli, which are embedded in a fibrous stroma. The cells are polyhedral, and each contains a clear round nucleus. The zona fasciculata forms the chief part of the cortex, and is so named because its component cells are arranged in columns or

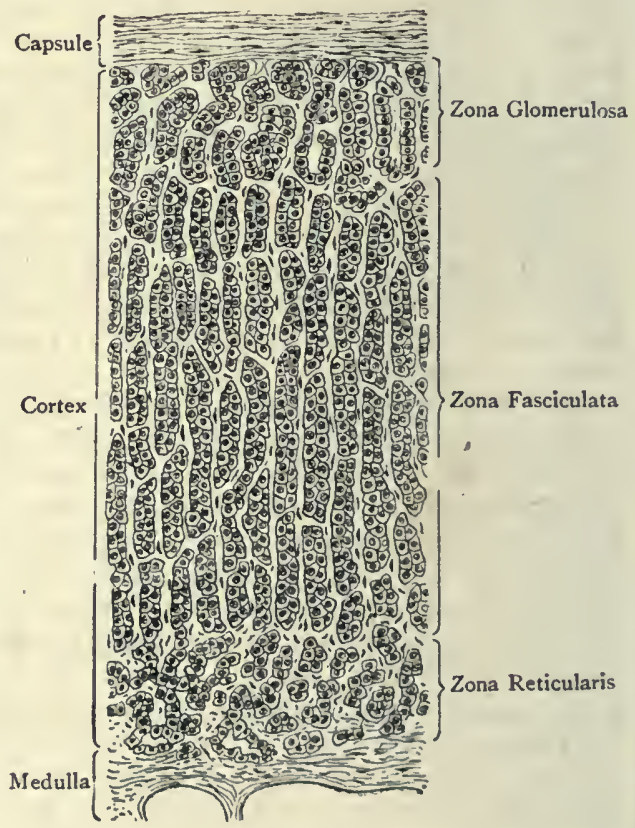

Fig. 352.-Section of the Suprarenal Capsule. fasciculi. The cells are similar to those of the zona glomerulosa, and the cell-columns are separated from one another by fibrous trabeculæ, which act as carriers of the bloodvessels, nerves, and lymphatics. The zona reticularis, which is narrow, lies within the zona fasciculata, and is so named because its component groups of polyhedral cells are connected with one another in such a manner as to form a reticulum. 
The medullary part is confined to the centre of the capsule in the adult, is soft in consistence, and has a reddish-brown colour, due to the contained venous blood. The supporting fibrous stroma of the cortex pervades it, and is arranged in a reticular manner. The meshes of this reticulum are occupied by cells, resembling somewhat epithelial cells, and larger and more irregular than those of the cortex, some of them being branched.

Blood-supply-Arteries.-These are (I) the superior capsular of the inferior phrenic or diaphragmatic from the abdominal aorta, (2) the middle capsular from the abdominal aorta, and (3) the inferior capsular from the renal.

The veins of each capsule eventually unite to form one suprarenal vein. This vessel emerges through the hilum, and on the right side it opens directly into the inferior vena cava, whilst on the left side it terminates in the left renal vein.

The lymphatics terminate in the lateral lumbar glands.

Nerves.-The suprarenal capsules are very richly supplied with nerves, which are derived from (I) the inferior or diaphragmatic plexus from the semilunar ganglion of the solar plexus, (2) the suprarenal plexus from the semilunar ganglion and cœliac plexus, and (3) offshoots from the renal plexus. According to Bergmann, the suprarenal capsules also receive fibres from the phrenic and pneumogastric nerves. The fibres, which are chiefly non-medullated, form rich plexuses in the medullary part, where they have numerous ganglion cells connected with them.

Development. - The suprarenal capsule makes its appearance internal to the Wolffian body. The cortex is of mesodermic origin, and the medulla is developed from the ganglia of the primitive abdominal sympathetic chain.

The cortex is developed from cellular outgrowths of the mesothelium of the cœlom or body-cavity, on the mesial aspect of the mesonephros, or Wolffian body. These outgrowths soon become separated from the cœlomic mesothelium, and unite to form a solid mass, which constitutes the cortex.

The mequlla is derived from the ganglia of the primitive abdominal sympathetic chain. Groups of cells grow out from the ganglia (these cells being consequently of ectodermic origin), and they invade the cortex. They lose their connection with the sympathetic ganglia, and become differentiated into two groups. The cells of ope group are chromaffin cells, and stain a dark yellow colour with chromic acid salts. The cells of the other group are ganglion cells. All the cells developed from the sympathetic ganglia gradually pass to the centre, where they constitute the medulla.

The suprarenal body thus develops in two parts-cortex, derived from the cœlomic mesothelium (mesoderm), and medulla, derived from the abdominal sympathetic ganglia, and therefore of ectodermic origin.

\section{Structure of the Kidneys.}

The kidneys are compound tubular glands. Each organ is invested by a capsule, composed of fibrous tissue with a certain amount of elastic fibres. The deep surface of this capsule is attached to the peripheral portion of the renal substance by fine fibrous processes and small bloodvessels. Underneath it there is a certain amount of plain muscular fibre-cells, arranged in a somewhat plexiform manner. At the hilum on the inner border it 
is prolonged inwards to line a cavity to which the hilum leads, called the sinus, where it becomes continuous with the fibrous coats of the calices.

When a kidney is cut into two halves by a longitudinal incision carried from the outer to the inner border, it is seen to be composed of two parts-cortical and medullary.

The medullary part, which is internal in position, is arranged in conical bundles, termed the pyramids of Malpighi, which vary in number from eight to eighteen, the average number being about twelve. The bases of these pyramids are directed towards the circumference, whilst their apical parts, called papilla, project into the sinus, where they are grasped by the calices. Each Malpighian

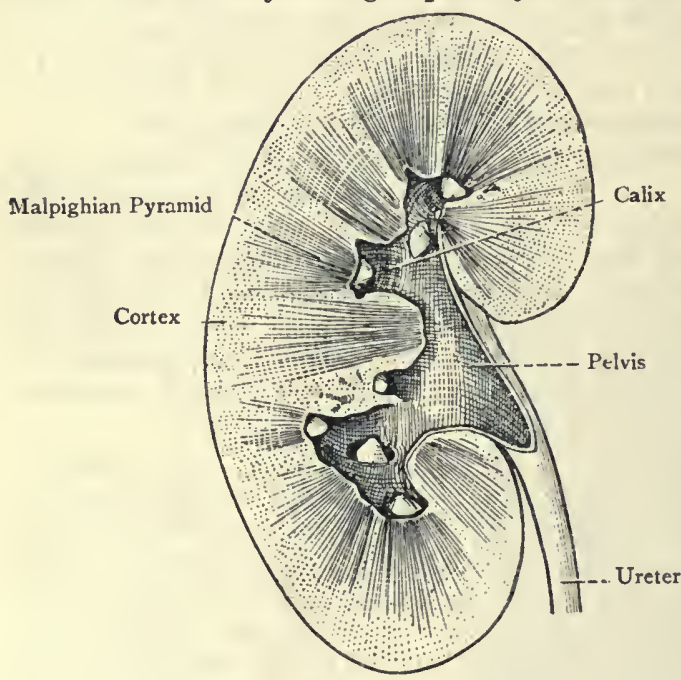

Fig. 353.-Diagram OF THE KIDNEy IN Longitudinal Section. pyramid is divided into three parts, namely, the boundary zone or basal part, the papillary zone, and the apex. The boundary zones of the pyramids form collectively the boundary or intermediate layer of the kidney. They abut against the cortical substance, which sends prolongations between them, called septula renum or columna Bertini. These prolongations extend as far as the commencement of the apical parts of the papillary zones, where they cease, so that these apical parts project into the calices free from cortical investments. Each Malpighian pyramid is composed of straight, slightly diverging uriniferous tubules (tubuli recti) and straight bloodvessels, the number of tubules being very much greater in the boundary zone or basal part than elsewhere. This straight arrangement of tubules and bloodvessels imparts to each pyramid a longitudinally striated appearance from apex to base. The boundary zones or basal parts have a dark reddish-brown colour, which becomes brighter in the papillary zones.

The cortical part of the kidney is mainly situated within the fibrous capsule. It has a reddish-brown colour and, as already stated, sends prolongations between the Malpighian pyranids. Like tlie medullary part, it is composed of uriniferous tubules and bloodvessels, but the tubules are principally convoluted (tubuli 
contorti), though there are also bundles of straight tubules (tubuli recti) which have issued from the Malpighian pyramids, and form the medullary rays or pyramids of Ferrein.

Cortex. - The cortical part is composed of the labyrinth and the medullary rays or pyramids of Ferrein.

Labyrinth.- This portion of the cortex is so named from the very complicated arrangement of its tubules. It is situated in the interspaces between the medullary rays, and is composed of convoluted uriniferous tubules, bloodvessels, and Malpighian corpuscles, each of which corpuscles is enclosed within a capsule of Bowman.

Uriniferous Tubules,-Each tubule commences in the labyrinth of the cortex in a spherical dilatation, called the capsule of Borman, within which there is a tuft of convoluted capillary bloodvessels, known as a glomerulus (Ruysch) or Malpighian corpuscle. Bowman's capsule presents two poles. One of these is formed by two bloodvessels, afferent and efferent, which pierce the capsule at separate points, but close to each other. At the other pole Bowman's capsule becomes constricted, and forms the neck of a cylindrical tubule. After the tubule has passed the neck it becomes convoluted, and forms the first or proximal convoluted tubule. The basement membrane and lining epithelium of Bowman's capsule are continuous with the basement membrane and lining epithelium of the proximal convoluted tubule, and the space between Bowman's capsule and the glomerulus is continuous with the lumen of the tubule. The proximal convoluted tubule soon becomes straight, though slightly wavy, and, entering a medullary ray, it forms the spiral twbule. This tubule, on reaching the junction of the cortex and boundary layer, becomes suddenly very narrow, and traverses the boundary zone of a Malpighian pyramid. It is known as the descending tubule of Henle. On leaving the boundary zone it enters the papillary zone, and after a short course it describes a very sharp bend, called the loop of Hevile's tubule. It now retraces its steps from the papillary zone into the boundary zone of a Malpighian pyramid, in which latter situation it becomes suddenly enlarged. This part, which retraces its steps, is called the ascending tubule of Henle. It re-enters the cortex, where it becomes narrower, and passes into a medullary ray, in which it lies for a short distance. It subsequently, however, leaves the ray, and, entering the labyrinth, winds between the convoluted tubules as the irregular or zigzag tubule, which has become slightly enlarged. This zigzag tubule then passes into the second or distal convoluted (intercalary) tubule. This tubule, becoming narrow, passes in to the junctional or collecting tubule, which is slightly wavy. The junctional tubule leads into the straight collecting tubule which is slightly enlarged, and is situated in a medullary ray. This straight collecting tubule now passes to the boundary zone of a Malpighian pyramid, taking up in its course other junctional tubules from the labyrinth. In passing through the various parts of a Malpighian pyramid the straight collecting tubules unite at 
acute angles, and so become less numerous and at the same time larger. They run in straight, slightly converging lines towards the apex of a papilla, where they are very much reduced in numbers,

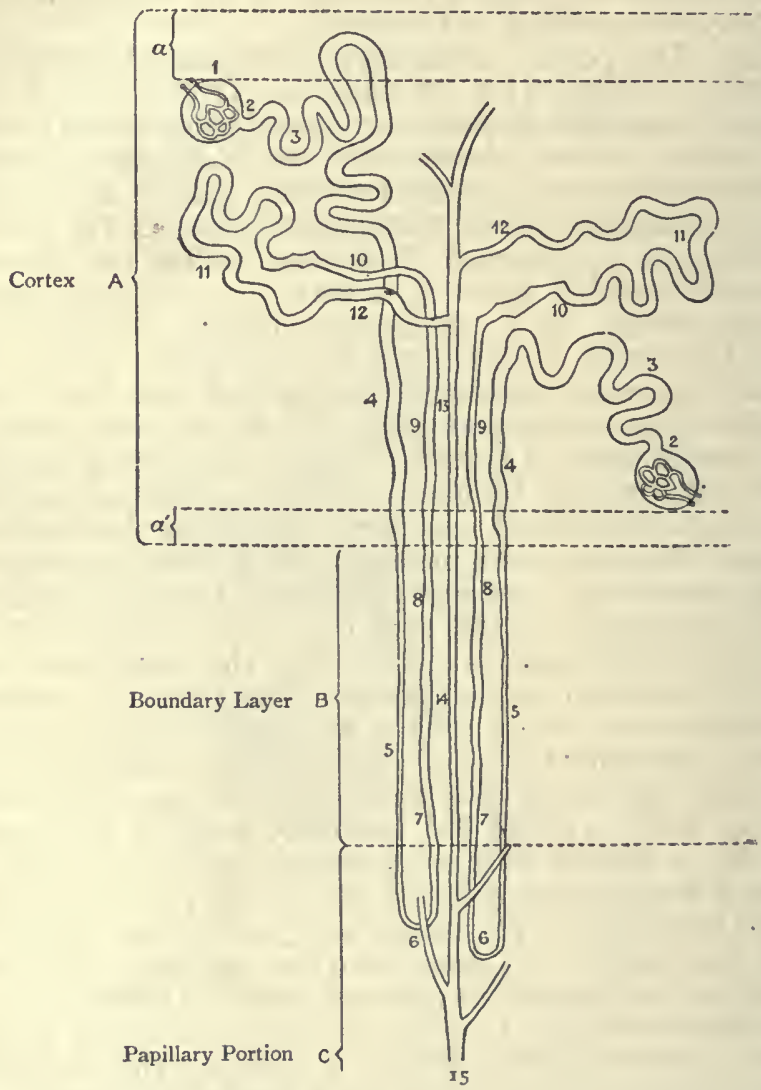

Fig. 354.-Diagram of the Uriniferous Tubules of the Kidney (Klein).

$a$ and $a^{\prime}$, Outer and Inner Zones of Cortex, which are free from Malpighian Corpuscles.
x. x. Capsule of Bowman
2. 2. Neck of Capsule
4. 4. Spiral Tubule
3. 3. Proximal Convoluted Tubule
5. 5. Descending Limb of Henle's Loop
6. 6. Loop of Henle

\section{7., 8. 8., 9. 9., Ascending Limb of Henle's Loop}

хo. хо. Irregular Tubulc

11. xх. Distal Convoluted Tubule

x2. 12. Junctional Tubule
13., r4. Straight Collecting Tubule

15. Excretory Tube or Duct of Bellini

but of large size. In this final part of their course each straight collecting tubule forms the excretory tube or duct of Bellini. These excretory tubes open on the apex of a papilla, where there is often 
a foveola or small depression, and through these openings the urine escapes into a calix.

The diameter of the excretory tubes or ducts of Bellini is about the $\frac{1}{2} \frac{1}{00}$ inch.

\section{Summary of a Tubule from Beginning to End.}

1. The capsule of Bowman, containing a glomerulus or Malpighian corpuscle, in the labyrinth.

2. The neck, in the labyrinth.

3. The first or proximal convoluted tubule, in the labyrinth.

4. The spiral tubule, in a medullary ray.

5. The descending tubule of Henle (small), in a boundary zone, and in part of a papillary zone, of a Malpighian pyramid.

6. The loop of Henle's tubule, in a papillary zone.

7. The ascending tubule of Henle (large), in part of a papillary zore, a boundary zone, and the cortex, in which latter it is in a medullary ray.

8. The irregular or zigzag tubule, in the labyrinth.

9. The second or distal convoluted (intercalary) tubule, in the labyrinth.

10. The junctional tubule, in the labyrinth on its way to a medullary ray.

I I. The straight collecting tubule, in a medullary ray of the cortex, and in a boundary zone of a Malpighian pyramid.

12. The excretory tube, or duct of Bellini, in a papillary zone of a Malpighian pyramid.

Structure of the Uriniferous Tubules.-The tubules are composed of a basement membrane lined with a single layer of epithelial cells. The basement membrane itself consists of flattened epithelial cells. The tubules vary in size, but their average diameter may be stated as z of the lining epithelial cells present differences in the different tubules, which will now be considered in order.

1. The capsule of Bowman is lined with a single layer of flattened epithelium, which is reflected over the glomerulus or Malpighian corpuscle, where it is more distinct in early life than in the adult.

2. The neck is lined with cubical epithelium.

3. The first or proximal convoluted tubule is also lined with cubical epithelium, but the cells, which are thick, are peculiar. The inner part of each cell-that is the part next the lumen of the tubule, including the spherical nucleus, has granular protoplasm, whilst the outer part-that is, the part next the basement membrane-has its protoplasm striated or fibrillated, owing to the presence of rod-shaped fibrils disposed vertically to the basement membrane (Heidenhain). These fibrillated cells are provided laterally with processes, by which they embrace one another. The lumen of the tubule is distinct.

4. The spiral tubule is lined with epithelium similar to that of the proximal convoluted tubule, and its lumen is distinct.

5. The descending tubule of Henle is lined with clear, thin, flattened cells. The tubule, though narrow, has a distinct lumen.

6. The loop of Henle's tubule is lined with epithelium which resembles that of the descending tubule.

7. The ascending tubule of Henle is lined with fibrillated, cubical epithelial cells like those of the proximal convoluted and spiral tubules. Though it is of comparatively large size, its lumen is small.

8. The irregular or zigzag tubule is lined with cubical epithelial cells, which are markedly fibrillated, and its lumen is minute.

9. The second or distal convoluted tubule is lined with epithelium which resembles that of the proximal convoluted tubule, with the following differences: $(a)$ the cells are longer; and $(b)$ they are highly refractive. In size it corresponds with the proxinal convoluted tubule.

10. The junctional tubule is lined with clear flattened, cubical epithclial cells, and its lumen is large. 
I I. The straight collecting tubule is lined with epithelial cells, which, in its earlier part, are clear and cubical, but in its later part they are columnar, and the lumen is very distinct.

I2. The excretory tube or duct of Bellinl is lined with epithelial cells which are clear and columnar.

Medullary Rays or Pyramids of Ferrein.-These take the form of pyramidal bundles of uriniferous tubules, which are separated from each other by portions of the labyrinth. The tubules are straight, and emerge from the boundary zones of the Malpighian pyramids. As these medullary rays are followed from the boundary zones to the periphery, each gradually diminishes in breadth, and ultimately tapers to a point, which stops a short distance from the fibrous capsule, being separated from it by a portion of the labyrinth. The rays are thus conical, their bases being at the boundary zones of the Malpighian pyramids, and their apices near the fibrous capsule-hence the name 'pyramids.' The explanation of this shape is that the tubules at the circumference of a medullary ray enter the labyrinth sooner than those in the centre, which latter form the apex of the ray.

Summary of a Medullary Ray.-Each contains the following tubules: (I) spiral tubules; (2) ascending tubules of Henle; and (3) straight collecting tubules.

Medulla of the Kidney. - The medullary portion has been already generally described. It is composed of Malpighian pyramids, consisting of uriniferous tubules and bloodvessels (true and false).

The tubules in the various parts of the Malpighian pyramids are as follows :

I. Boundary Zone.-This contains the following tubules :

Portions of the descending tubules of Henle.

Portions of the ascending tubules of Henle.

Straight collecting tubules.

2. Papillary Zone.-This contains the following tubules:

Portions of the descending tubules of Henle.

The loops of Henle's tubules.

Portions of the ascending tubules of Henle.

The excretory tubes or ducts of Bellini.

3. Apex of the Papillary Zone.-This contains only the excretory tubes or ducts of Bellini.

Bloodvessels of the Kidneys-Arteries.-Each kidney receives a large amount of blood from the renal artery, which is a branch of the abdominal aorta. This vessel, as it approaches the hilum, divides into four or five branches, which enter the sinus, where they are embedded in fat along with the calices. They then subdivide into interlobar branches, which pass between the Malpighian pyramids, where they continue to subdivide. On arriving at the junction of the cortex and boundary layer they form a series of arches, which are independent of one another, and are called the cortico-medullary arches. Alongside of these there are venous arches, which, unlike the arterial arches, anastomose freely with 
one another. The convexities of the incomplete arterial arches are directed towards the cortex, and the concavities towards the Malpighian pyramids. The

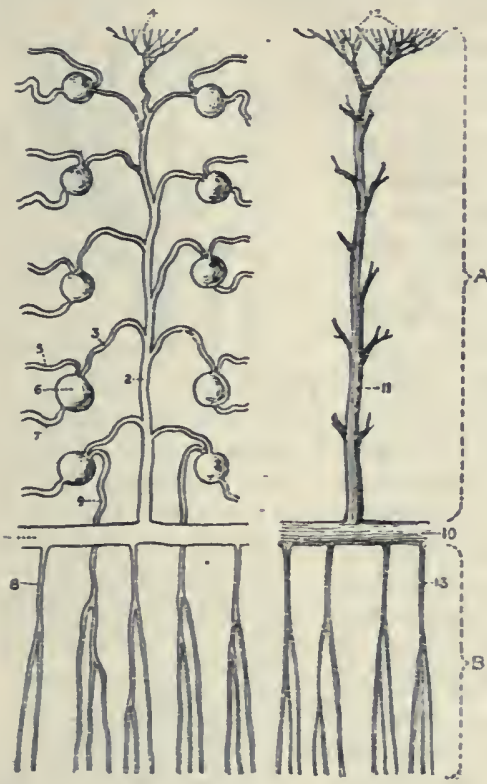

Fig. 355.-Diagram of THE BloodVESSELS OF THE KIDNEY.

A, Cortex; B, Medulla.

I. Arterial Arch

2. Interlobular Artery

3. Afferent Artery of Glomerulus

4. Capsular Branches

5. Efferent Vessel of Glomerulus

6. Malpighian Corpuscle

7. Proximal Convoluted Tubule

8. True Arteria Recta

9. False Arteria Recta

ra. Venous Arch

II. Interlobular Vein

12. Venæ Stellatze

13. Venæ Rectæ

branches of the arches are interlobular and arteriæ rectæ.

The interlobular arteries (cortical) arise from the convexities of the arches and enter the labyrinth of the cortex, in which they pass outwards between the medullary rays. They give off afferent and capsular branches.

The afferent branches are so named because they carry blood to the glomeruli or Malpighian corpuscles. They arise from the interlobular arteries at frequent intervals, and each passes to a capsule of Bowman without giving off any branch. Having pierced the capsule at one pole, the afferent vessel breaks up into a number of convoluted capillary vessels, which form a small vascular ball, called a glomerulus or Malpighian corpuscle. The blood is conveyed away from the glomerulus by an efferent vessel, which is variously regarded as an artery and a vein. This efferent vessel is smaller than the afferent artery. It pierces Bowman's capsule at the same pole as the afferent artery, but separate from, though close to, it. Thereafter it breaks up into a network of capillary vessels, which invest the corresponding proximal convoluted uriniferous tubule, the meshes of the network being polygonal. The efferent vessels of those glomeruli which lie nearest to the Malpighian pyramids are disposed in a different manner. They break up into bundles of straight vessels, called false vasa recta, which enter the boundary zones or basal parts of the pyramids, where they supply to a large extent the uriniferous tubules, upon the walls of which they form capillary networks with elongated meshes. It is, however, to be noted that the Malpighian pyramids also receive true arteriæ rectæ from the cortico-medullary arterial arches. 
Malpighian Corpuseles.-A Malpighian corpuscle of the kidney is a glomerulus or small ball of convoluted capillary bloodvessels, having two vessels connected with it, one of which is an afferent artery and the other an efferent vessel. The vessels of many of the corpuscles are collected into bundles, an arrangement which renders these corpuscles lobulated, the number of lobules varying from two to five. Each corpuscle lies within a capsule of Bowman, and the flattened epithelium of the capsule is reflected over the corpuscle from the points of entrance and exit of the afferent and efferent vessels. The epithelial cells covering the corpuscle are thicker and less flattened than those lining the capsule of Bowman, and are better marked in early life than in the adult. In the case of the lobulated corpuscles the epithelial investment dips in between the component lobules. The epithelium of Bowman's capsule and the epithelium of the corpuscle, which in each case forms a single layer, are separated from one another by a slight interspace. The diameter of a Malpighian corpuscle is about $\frac{1}{20} 0$ inch. The corpuscles are confined to the cortex of the kidney, where they lie in rows in the labyrinth between the medullary rays. There is a narrow zone immediately within the external capsule, and another narrow zone

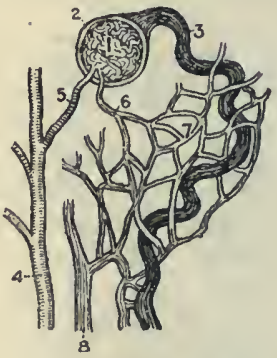

FIG. 356 . DIAGRAM SHOWING A MALPIGHIAN CORPUSCLE OF THE KIDNEY WITH ITS AFFERENT AND EFFERENT VESSELS, AND A Próximal Convo. LUTED Tubule With its Venous Plexus (BOWMAN).
r. Glomerulus
2. Bowman's Capsule
3. Uriniferous Tubule
4. Interlobular Artery
5. Afferent Vessel
6. Efferent Vessel
7. Venous Plexus around Tubule
8. Interlobular Vein close to the Malpighian pyramids, from both of which Malpighian corpuscles are absent.

The capsular branches of the interlobular arteries supply the external fibrous capsule of the kidney, in which they anastomose with branches of the lumbar arteries from the abdominal aorta.

The arteriæ rectæ (medullary), or vasa recta (true), arise from the concavities of the cortico-medullary arterial arches, and at once enter the boundary zones or basal parts of the Malpighian pyramids. Here they break up into bundles of straight, slightly diverging arterioles, which run between the bundles of straight, slightly converging uriniferous tubules, a mutual arrangement which imparts to the Malpighian pyramids a longitudinally-striated appearance. The capillary networks formed by these arterioles have necessarily elongated meshes. It is to be noted that the Malpighian pyramids derive their blood-supply from two sources, namely, (I) the arteriæ rectæ, or vasa recta (true), from the corticomedullary arterial arches, and (2) the false vasa recta from the efferent vessels of those glomeruli which lie nearest the Malpighian pyramids. It is also to be noted that a Malpighian corpuscle of 
the kidney is a ball of convoluted capillary bloodvessels, which is enclosed within a capsule of Bowman, whereas a Malpighian corpuscle of the spleen is a collection of lymphoid or adenoid tissue which is a localized expansion of the external or lymphoid coat of the small arteries in the interior of that organ.

Veins-Veins of the Cortex.-These are called the interlobular veins. Some of them commence on the surface of the kidney beneath the capsule, from which they return blood, and these are called vence stellate, because the venous radicles which give rise to them converge to a point, and so present a star-like appearance. Others originate in the plexuses around the uriniferous tubules. The interlobular veins terminate by joining the convexities of the venous arches, which are situated between the cortex and the Malpighian pyramids.

Veins of the Medulla.-The veins of the Malpighian pyramids coinmence in plexuses which surround the excretory tubes or ducts of Bellini in the apical parts of the papillary zones, and they are called vene recta. As these traverse the pyramids they are collected into bundles of straight vessels, which open into the concavities of the cortico-medullary venous arches.

Cortico-medullary Venous Arches.-These arches, which are complete, are situated between the cortex and the Malpighian pyramids, where they lie alongside of the incomplete arterial arches. They derive their blood from the interlobular veins of the cortex, and the venæ rectæ of the Malpighian pyramids. The veins which proceed from these arches pass between the pyramids to the sinus of the kidney, where they unite to form the renal vein, which terminates in the inferior vena cava.

Lymphatics.-These consist of two groups, superficial and deep. The superficial lymphatics, which are few in number, form a plexus in the fibrous capsule, and communicate with lymph spaces between the uriniferous tubules in the outer part of the cortex. They terminate by joining the deep lymphatics at the hilum. The deep lymphaties accompany the bloodvessels, and communicate with lymph spaces between the uriniferous tubules of the cortex and boundary layer. On emerging at the hilum they receive the superficial lymphatics, and then pass to the lateral group of lumbar glands.

Nerves.-The kidney receives numerous nerves from the renal plexus, which dorives its fibres from (I) the aortico-renal ganglion, in which the small splanchnic nerve terminates; (2) the coliac plexus; and (3) the aortic plexus. If there is a least splanchnic nerve present it reinforces the renal plexus. The nerve-fibres are partly sympathetic, partly spinal through the small and least splanchnic nerves, and in part derived from the right pneumo-gastric through the coliac plexus. They accompany the arterial branches, and ramify upon the walls of the vessels and uriniferous tubules.

Excretury Apparatus of the Kidney.-This consists of the calices or infundibula, pelvis, and ureter. 
Ureter.-This is the excretory duct of the kidney, and it has the form of a cylindrical tube, like a goose-quill, its colour being a dull white. Its length varies from I2 to 16 inches, and its diameter is about $\frac{1}{5}$ inch. At its lower extremity it opens into the bladder, and at the hilum of the kidney it presents a dilatation called the pelvis of the kidney, which is funnel-shaped, being wide above and narrow below. The pelvis is flattened from before backwards, and lies partly in the sinus and partly outside the hilum, its direction being downwards and inwards. It breaks up into two or three primary divisions within the sinus, and these again form secondary divisions, the resulting tubes being called calices or infundibula, which vary in number from 7 to 13 . The number of calices thus falls short of the number of Malpighian pyramids ( 8 to I 8 ), the explanation being that one calix grasps two papillæ or even three. The calices embrace the papillæ of the Malpighian pyramids, which thus project into them, and they receive the urine as it oozes through the pores on the apices of the papillæ, these pores being the openings of the excretory tubes or ducts of Bellini. 'The interspaces between the calices in the sinus are occupied by the branches of the renal bloodvessels and by fat.

Structure of the Ureter.-The ureter is a thick-walled muscular tube, lined with mucous membrane, and it consists of three coatsexternal or fibrous, middle or muscular, and internal or mucous. The fibrous coat is composed of fibrous tissue, very compactly arranged, and it contains the bloodvessels and nerves, which here subdivide. The muscular coat is composed of plain muscular tissue, which is disposed in three layers-outer longitudinal, middle circular, and internal longitudinal. The outer longitudinal layer is best marked in the lower half of the ureter, where it forms a continuous investment. In the upper half of the tube its bundles are somewhat scattered. The mucous coat superiorly is continued over the papillæ of the Malpighian pyramids, and inferiorly becomes continuous with the mucous membrane of the bladder. It is covered by stratified transitional epithelium, the cells being arranged in four layers. In the most superficial layer (nearest the lumen of the tube) the cells are cubical, and present depressions on their deep surfaces, which receive the round ends of the pyriform cells of the layer beneath. In the second layer the cells are pyriform, the round superficial ends being capped by the cubical cells of the first layer, and the narrow deep ends projecting between the deeper cells. In the third and fourth layers the cells are round or oval. The mucous membrane is thrown into longitudinal folds, and the lumen of the tube, which is of small size, presents under the microscope a branched appearance in cross section.

Blood-supply-Arteries.--The ureter receives branches from the renal, spermatic (ovarian in the female), common iliac, and superior vesical, arteries.

The veins terminate in the vessels corresponding to these arteries. 
Lymphaties.-These pass to the lateral group of lumbar glands, and to the internal iliac glands.

Nerves.-These accompany the arteries, and are derived from the renal, spermatic (ovarian in the female), and pelvic plexuses. The fibres form plexuses in the fibrous and muscular coats, which contain small ganglia.

\section{Middle Circular Muscular Fibres}

Outer Longitudinal Muscular Fibres

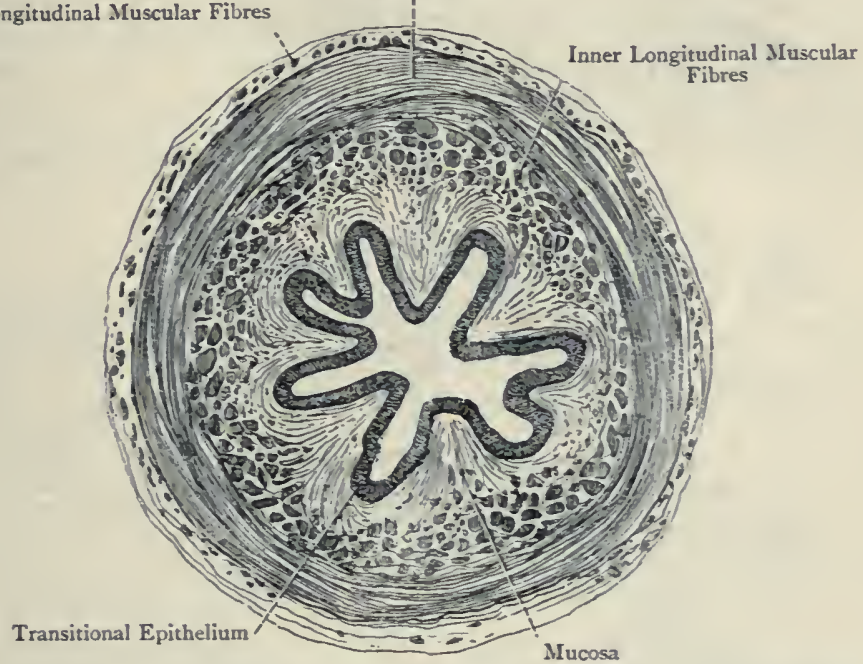

Fig. 357.-Transverse Section of the Lower Part of the Ureter, SHOWiNg ITS MiNute StructuRE.

Structure of the Pelvis and Calices. -The structure of these parts of the excretory apparatus resembles that of the ureter, with the exception that they have only two layers of plain muscular tissue, instead of three as in the ureter. The layer which is wanting is the outer longitudinal layer. The calices are attached to the bases of the papillæ, and in these situations their fibrous coats become continuous with that part of the fibrous capsule of the kidney which is prolonged inwards through the hilum to line the sinus. The internal longitudinal muscular fibres disappear towards the papilla, but the circular muscular fibres accompany a calix to its termination, where they are arranged in the form of a circular band, which surrounds the base of a papilla at the line of attachment of a calix. The stratified transitional epithelium of the mucous coat of a calix is prolonged over the apex of each papilla.

Stroma of the Kidneys.-Between the uriniferous tubules and bloodvessels there is a certain amount of connective tissue, which is present in greatest abundance in the region of the papillary zones. 


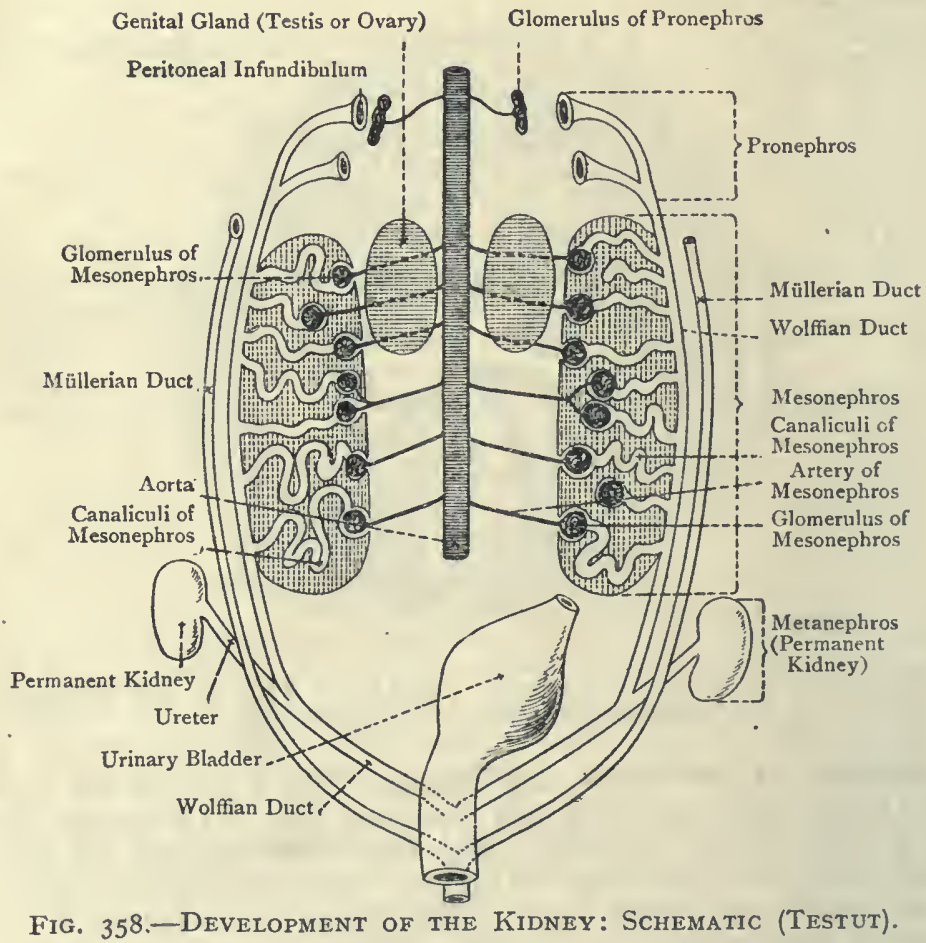


This constitutes what is known as the fibrous or interstitial stroma, of which there is a very small amount.

Early Condition of the Kidneys. - The kidneys of a child at the period of birth are lobulated, each lobule representing a Malpighian pyramid surrounded by cortical substance. In this respect they resemble the permanently lobulated kidneys of certain animals, e.g., the ox. The lobules first become apparent towards the end of the third month of intra-uterine life, and all traces of them have usually disappeared by the eighth or tenth year. They may, however, remain persistent to a greater or less extent thronghout life, though this condition is of very rare occurrence. It is, however, not uncommon to find the surface of an adult kidney marked by faint grooves indicative of its original lobulated condition. The disappearance of the lobulated condition is brought about by the adjacent lobules coalescing, this being accompanied by an increased development of cortical substance at the surface. The portions of cortical substance which remain in the spaces between the medullary pyramids (except their apical parts) represent the septula renum or columnæ Bertini.

\section{Development of the Kidney and Ureter.}

The kidneys are of mesodermic origin, and are developed in connection with the intermediate cell-mass, which appears along the groove separating the mesodermic somites of the paraxial mesoderm from the lateral plate of mesoderm. The intermediate cell-mass, like the paraxial mesoderm, undergoes segmentation, its segments being known as nephrotomes, each of which is a solid cord of cells.

In the course of the development of the urinary system in vertebrates, three sets of organs have to be considered-namely: (I) the pronephros, (2) the mesonephros, and (3) the metanephros.

The pronephros, fore-kidney, or head-kidney, which is functional in lower vertebrates, originates on either side as a thickening of the lateral part of the corresponding intermediate cell-mass, called the Wolffian ridge, which projects into the cœlom or body-cavity. The Wolffian ridge consists of solid cords of cells, which soon become tubular and give rise to a longitudinal canal, called the pronephric or segmental duct. This duct extends from the region of the heart anteriorly to the cloaca posteriorly, into the ventral compartment, of which latter it opens. Associated with the cephalic end of this duct there are two epithelial tubales, known as the pronephric tubules, which lie at right angles to it. By one end these tubules open into the pronephric duct in those vertebrates in which the pronephros is functional, but in the human embryo they do not communicate with the pronephric duct. By their other extremities they open into the colom or body-cavity (peritoneal cavity), the openings being known as nephrostomata. In the neighbourhood of the nephrostomata, but independent of them, there are tufts of capillary bloodvessels. covered by epithelium. These are called the pronephric glomeruli.

In those vertebrates in which the pronephros is well developed-e.g., certain Fishes, and Amphibia during the larval stage, the urinary secretion makes its way from the pronephric glomeruli into the colomic cavity, from which it passes through the nephrostomata into the pronephric tubules, and thence into the pronephric duct. The latter duct conveys it to the cloaca.

In most vertebrates the pronephros is a rudimentary structure, and its tubules soon atrophy. The pronephric duct, however, persists as the Wolffian duct, which belongs to the mesonephros.

The mesonephros, mid-kidney, or Wolffian body consists of (I) a series of transverse tubules, called the mesonephric or Wolffian tubules, and (2) the Wolffian duct.

The Wolffian tubules are developed from the intermediate cell-mass, which. as stated, is broken up into segments, called nephrotomes. Each solid 
nephrotome becomes hollow and gives rise to a mesonephric tubule, otherwise known as a segmental tubule. These tubules open by one end into the pronephric duct, now called the mesonephric or Wolfian duct. Their other extremities open into the cœlom by nephrostomata, but these soon become closed.

The Wolffian duct extends from the region of the heart to the cloaca, into the ventral compartment of which it opens-that is to say, the uro-genital sinus or canal. The Wolffian duct on either side lies external to the Wolffian body, and the Müllerian duct is on the outer side of the Wolffian duct. These two ducts lie within a fold of mesoderm, called the uro-genital fold, by which they are connected with the outer part of the Wolffian body. Anteriorly the uro-genital folds, right and left, are wide apart, but posteriorly they converge and fuse. By this fusion a dense fold of mesoderm is formed, called the genital cord, which contains the four ducts-namely, the right and left Wolffian ducts and the right and left Müllerian ducts. The Müllerian duct of either side having crossed over the Wolffian duct, the two Müllerian ducts lie close together at the median line within the genital cord, and in this situation they blend to form the rudiment of the uterus and vagina (utricle or uterus masculinus in the male).

Each Wolffian tubule presents a saccular dilatation towards its cœlomic end, which dilatation is invaginated by a tuft of capillary bloodvessels.

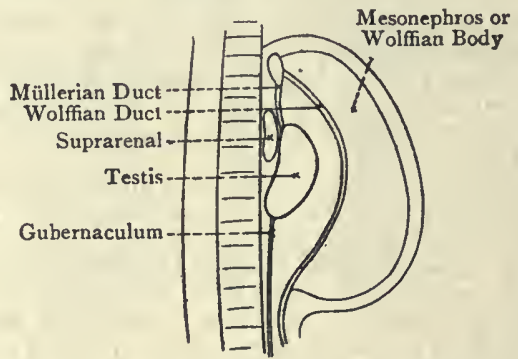

Fig. 359.-Mesonephros AND Contiguous Structures of THE EMBRyo Pig: Schematic (B. M. Allen).

This tuft represents a glomerulus, and the saccular part of the tube invaginated by it represents a capsule of Bowman, the glomerulus and capsule of Bowman corresponding to a Malpighian corpuscle of the permanent kidney. The glomerular part of the tube is the secreting part; and the portion opening into the Wolffian duct is the excretory part, which becomes convoluted. The Wolffian tubules just described are known as the primary tubules. Subsequently secondary and tertiary tubules are formed. These tubules have no communication with the cœlomic cavity. At one end, which is blind, they acquire Malpighian corpuscles, and their other ends join the excretory parts of the primary tubules, and so form collecting tubes.

The mesonephros or Wolffian body persists in Fishes and Amplibia, and constitutes the functional kidney. In Mammals, Birds, and Reptiles it soon atrophies, and is largely replaced by the metanephros or permanent kidney. The fate of the Wolffian body will be aftcrwards referred to.

The metanephros (after-kidney), or permanent kidney, is developed from (1) the renal diverticulum, which springs from thc dorsal aspect of the caudal or posterior end of the Wolffian duct close to the uro-genital sinus of the cloaca, and probably (2) the metanephric blastema. The renal divertlculum elongates into a tube which grows ceplialicwards, lying dorso-mesial to the Wolffian duct. Its cephalic end cxtends into the intermediate cell-mass, ancl is surrounded by mesodermic tissue, known as the metanephrio blastema, where it undergoes enlargement. The caudal part of the diverticulum repre-

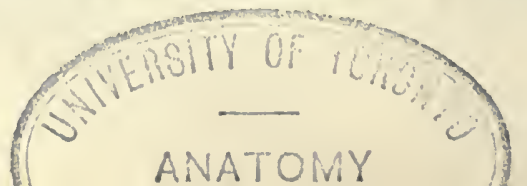


sents the primitive ureter, and its cephalic enlargement corresponds to the primitive renal pelvis. The pelvic enlargement furnishes secondary diverticula, which grow into the metanephric blastema, and represent the calices. These, in turn, give off tubular branches, which, becoming extended and subdivided, give rise to the excretory twbules of the medullary part of the kidney.

The complicated system of tubules in the cortical part, beyond the collecting or excretory tubules-namely, the secretory tubules-are developed from the metanephric blastema, which surrounds the enlarged cephalic extremity of the renal diverticulum. The tissue of this blastema becomes differentiated into two zones-outer and inner. The mesodermic tissue of the outer zone furnishes the connective-tissue stroma and capsule of the kidney as well as the columns of Bertini.

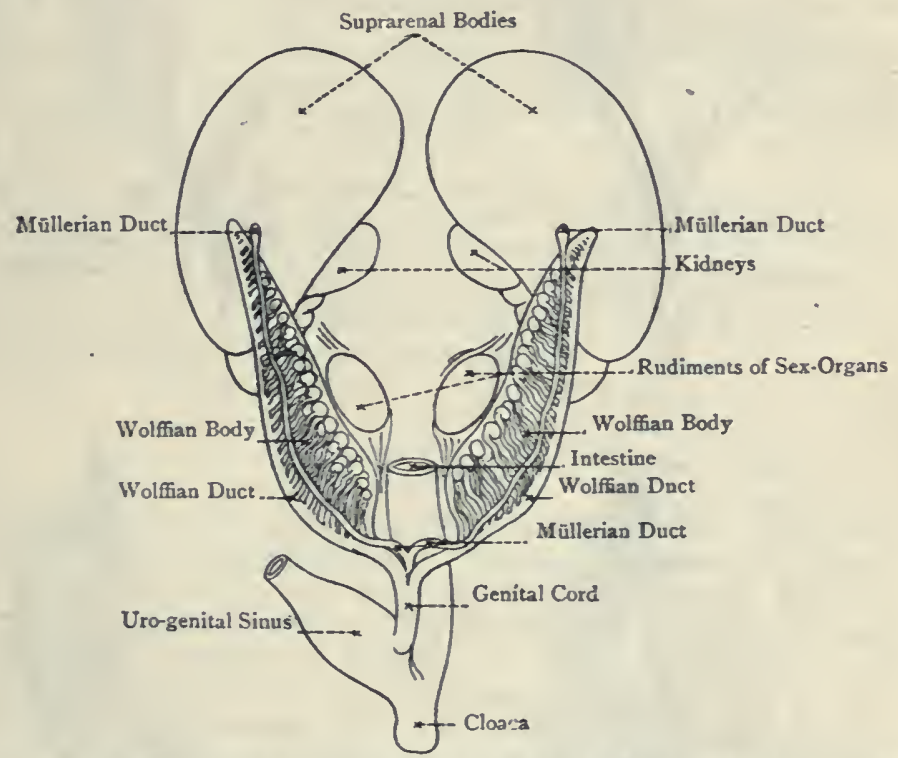

Fig. 360.-The Wolffian Bodies: Wolffian and Müllerian Ducts, axd Contiguous Parts. (Allen Thomson).

The mesodermic tissue of the inner zone gives rise to solid outgrowths, which at a later period become hollow and separated from the inner zone. These outgrowths are called the renal vesicles. Each vesicle becomes elongated. and forms an S-shaped tubule. One end of the S becomes connected with the contiguous collecting tubule, into which it opens. The other end pertains to a Malpighian corpuscle.

Within the lower loop of the $S$ a collection of mesenchyme appears, and within this a glomerulus is developed. This glomerulus invaginates a portion of the upper wall of the lower limb of the $S$, and the part thus invaginated forms Bowman's capsule. The remainder of the lower limb represents the commensement of the proximal convoluted tubule. As the $\mathrm{S}$ tubule elongates it forms several curves, and thus gives rise to (1) the remainder of the proximal convoluted tubule, (2) the spiral tubule, (3) Henle's looped tubule, (4) the irregular, or zigzag tubule, (5) the second convoluted tubule, and (6) the junctional tubule. 

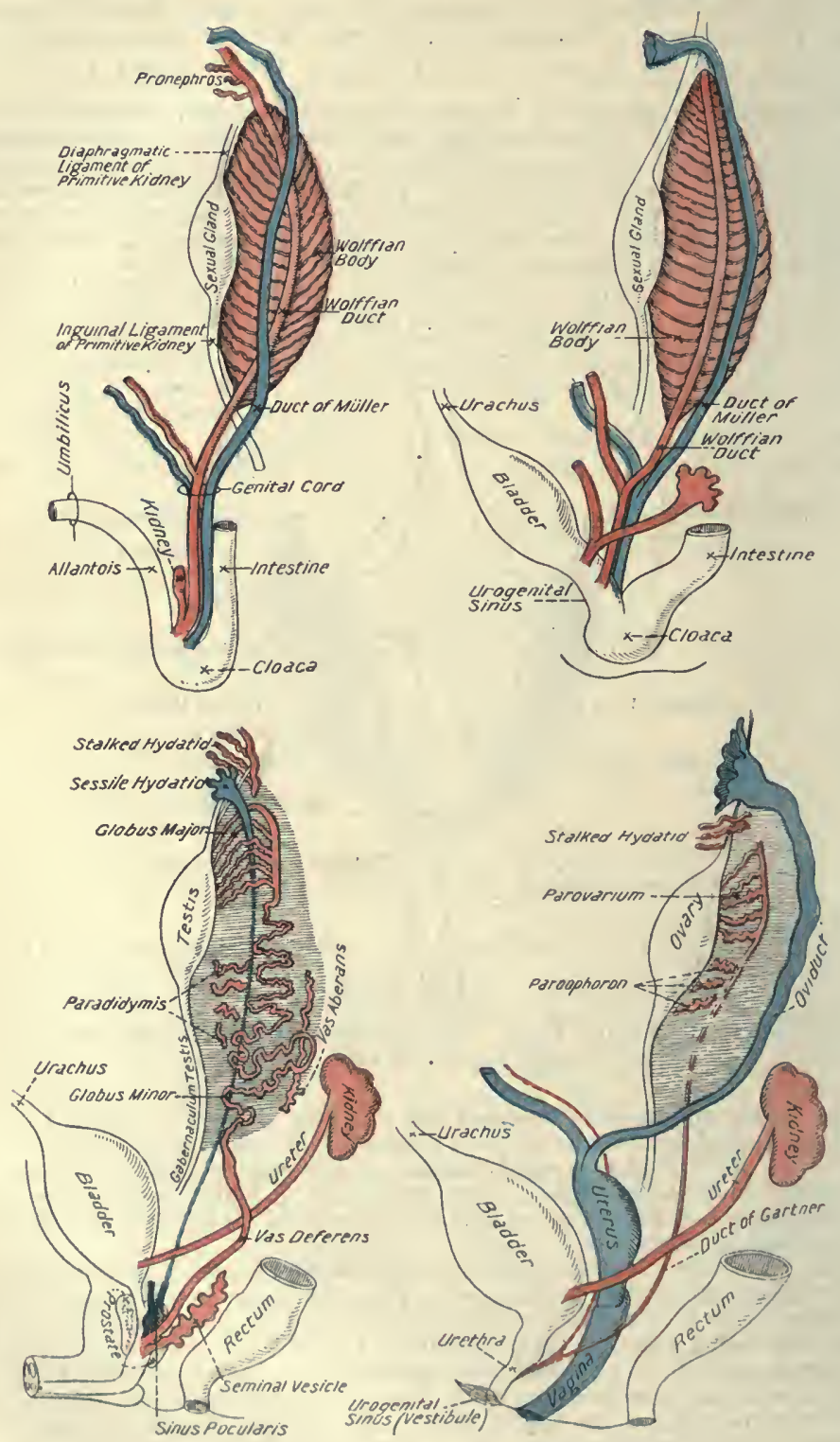

Fig. 361.-Development of Genito-Urinary, system (alter. Heisle 
Another view is that the complicated system of convoluted tubules, just referred to as lying beyond the collecting or excretory tubules, is developed as a ramification of these tubules, just as the collecting tubules are ramifications of the calices. In other words, according to this view the entire system of ariniferous tubules-secretory and excretory-the calices, the pelvis, and the ureter, spring from one common stem-namely, the renal diverticulum from the caudal end of the Wolffian duct. The blind extremity of a proximal convoluted tubule becomes enlarged, and is invaginated by a tuft of capillary bloodvessels, called a glomerulus; the invaginated blind end of the proximal convoluted tubule forms the capsule of Bowman; and the glomerulus and the capsule of Bowman form a Malpighian corpuscle of the kidney.

The vascular constituents, the interstitial connective-tissue stroma, the columns of Bertini, and the renal capsule are developed from the metanephric blastema.

The ureter, of either side, which originally opens into the urogenital sinus in common with the Wolffian duct, becomes detached from that duct and opens by an independent orifice into the urogenital sinus on its dorsal aspect. It may here be stated that the urinary bladder is developed from the urogenital sinus.

Summary. - The pronephros is functional in lower vertebrates - e.g., certain Fishes, and Amphibia during the larval stage. In Man it is rudimentary. The pronephric duct persists as the mesonephric or Wolffian duct.

The mesonephros or Wolfian body succeeds to the pronephros, and persists as the functional kidney in Fishes and Amphibia. In Man it atrophies to a large extent, and is replaced by the metanephros.

The metanephros is the permanent human kidney.

\section{THE PELVIS.}

The pelvis is the lower division of the abdomen which lies below the level of the ilio-pectineal lines and sacral promontory. Unlike the abdomen proper the walls of the pelvis are chiefly osseous. The posterior wall is constructed by the sacrum and coccyx, with the origins of the pyriformes muscles. Each lateral wall is formed by the body and spine of the ischium, and is covered by the obturator internus muscle. The anterior wall is formed by the bodies of the pubic bones which, in the median line, construct the symphysis pubis. Between the posterior wall and each lateral wall is the large sacro-sciatic space, which is partly closed by the powerful great and small sacro-sciatic ligaments. This space is divided into two parts by the small sacro-sciatic ligament and the spine of the ischium. The upper part forms the great sacro-sciatic foramen, which transmits the pyriformis muscle, the gluteal vessels and nerves, the sciatic vessels and nerves, the internal pudic vessels and pudic nerve, the nerve to the obturator internus and gemellus superior, and the nerve to the quadratus femoris and gemellus inferior. The lower part forms the small sacro-sciatic foramen, which transmits the obturator internus muscle and its nerve, the internal pudic vessels, and the pudic nerve. At each lateral part of the anterior wall is the obturator foramen, which is closed, except superiorly, by the obturator membrane, covered by the obturator internus muscle. Below the symphysis pubis is the subpubic arch, which is occupied by the triangular ligament of the urethra. The 
pelvic wall, thus constructed, is clothed by the pelvic fascia. Superiorly the pelvic cavity is quite open and in free communication with the abdomen proper. Inferiorly it is for the most part closed, a complex septum separating it from the perineum underneath. This septum is partly muscular and partly fascial. The muscles entering into it are the levatores ani and the coccygei, which construct the pelvic diaphragm. The fascial element is formed on either side by the visceral pelvic fascia, which clothes the superior or pelvic surface of the muscular diaphragm, and the anal fascia, which covers its inferior or perineal surface. The floor of the pelvis is consequently movable, being capable of ascending and descending. It affords passage to the rectum, and, in addition, to the vagina in the female.

Contents of the Pelvis.-The contents of the male pelvis are as follows : the pelvic colon and rectum; the bladder, with the lower portions of the ureters and the prostate gland, the latter containing the prostatic part of the urethra; the vesiculæ seminales and the lower portions of the vasa deferentia; the internal iliac vessels and their branches; portions of the superior hemorrhoidal and middle sacral vessels; the hemorrhoidal, vesical, and prostatic venous plexuses, the latter receiving the dorsal vein of the penis in two divisions; the sacral and sacro-coccygeal plexuses and their branches; the pelvic portions of the gangliated sympathetic chains ; and the obturator nerves in part of their course.

The differences in the contents of the female pelvis, as compared with those of the male, are as follows :

For the prostate gland and prostatic portion of the urethra substitute the female urethra, uterus, and vagina. For the vesiculæ seminales and portions of the vasa deferentia substitute the broad ligaments of the uterus and their contents, namely, the Fallopian tubes, the ovaries with their ligaments, and the round ligaments of the uterus. Add to portions of the superior hemorrhoidal and middle sacral vessels portions of the ovarian vessels. For the prostatic venous plexus substitute the pudendal plexus, and add the uterine and ovarian venous plexuses.

\section{The Male Pelvis.}

General Position of the Viscera.-The pelvic colon and rectum are situated upon the posterior wall, the pelvic colon reaching as low as the third sacral vertebra, and the rectum extending thence downwards and forwards to the interval between the levatores ani muscles, where it is succeeded by the anal canal. The bladder is anterior in position, being situated behind the bodies of the pubic bones, and resting by its base upon the rectum. The vesiculie seminales and the lower portions of the vasa deferentia lie in contact with the base of the bladder, between it and the rectum. The prostate gland surrounds the neck of the bladder and the prostatic part of the urethra.

Peritoneum.-The disposition of the peritoneum will be simplified 
by defining the limits of the pelvic colon and rectum, and the different parts of the bladder. The pelvic colon extends from the inner border of the left psoas magnus, just anterior to the left sacro-iliac articulation, to the level of the third sacral vertebra. The rectum extends from the third sacral vertebra to a point $I \frac{1}{2}$ inches in front of and below the tip of the coccyx, where it pierces the pelvic diaphragm to terminate in the anal canal, which is the part of the large gut surrounded by the sphincter muscles. The bladder, when empty, or only moderately distended, presents the following parts: (I) an apex, which is directed forwards, and lies behind the upper part of the symphysis pubis, where it has connected with it the fibrous cord representing the urachus; (2) a

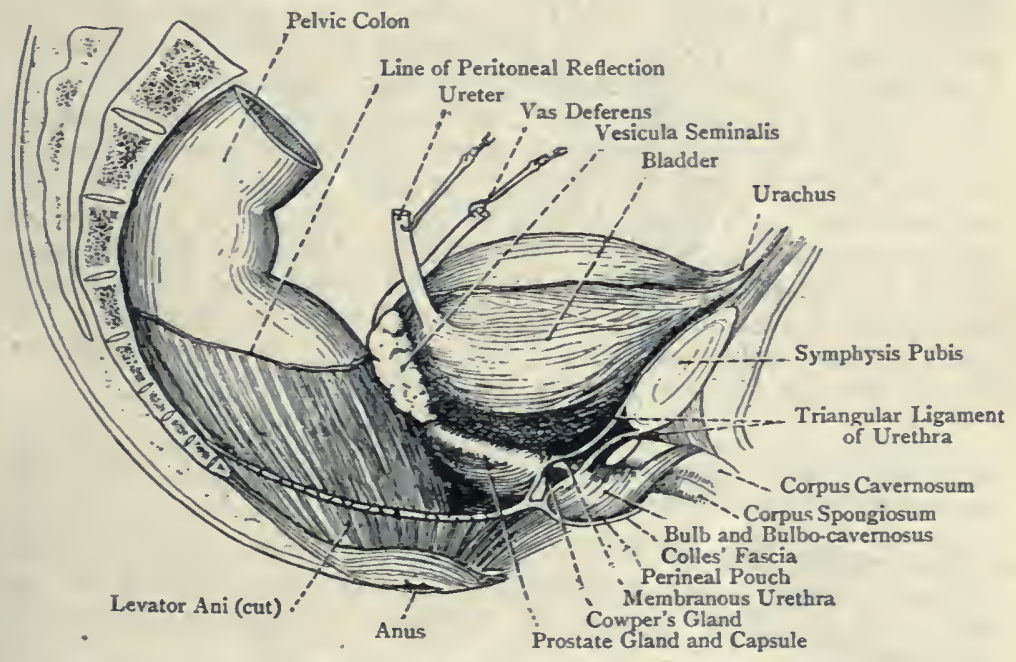

Fig. 362. - The Viscera of the Male Pelvis (Lateral View).

base or fundus, directed backwards and downwards towards the rectum, from which it is separated by the vesiculæ seminales and vasa deferentia ; (3) a body, which has a superior surface, an inferior surface, and three borders, two being lateral and one posterior ; and (4) a neck.

The peritoneum, having descended from the posterior wall of the abdomen over the common iliac vessels, enters the back part of the pelvic cavity, where it invests the pelvic colon, forming behind it an expanded, wavy mesentery, called the pelvic meso-colon, which attaches it to the front of the sacrum as low as the third sacral vertebra. The peritoneum is then prolonged upon the rectum, the upper third of which it covers anteriorly and laterally, but not posteriorly, the middle third being covered by it only anteriorly, whilst the lower third is destitute of peritoneal covering. 
The point at which the peritoneum leaves the rectum is fully 3 inches above the anus. The membrane is now carried forwards to the upper ends of the vesiculæ seminales, and the adjacent portions of the vasa deferentia, which it covers. Thereafter it passes forwards over the superior surface of the bladder, which it completely covers as far as the apex. Here it meets with the urachus, and by this it is conducted from the bladder to the posterior surface of the anterior abdominal wall. Along each lateral border of the bladder the peritoneum is reflected over the corresponding lateral wall of the pelvis. In passing from the rectum to the upper part of the base of the bladder, the peritoneum forms the rectovesical pouch. The bottom of this pouch is, as a rule, fully I inch distant from the base of the prostate gland, thus leaving the part of the base of the bladder, called the external trigone, quite free from peritoneum. The peritoneum, as it passes to and from the bladder, forms certain folds, which constitute the false ligaments of the viscus. These are five in number, as follows : two posterior, which represent the laterally-disposed lips of the mouth of the recto-vesical pouch; two lateral, right and left, which represent the reflection of peritoneum from each lateral border of the bladder to the corresponding lateral wall of the pelvis; and superior, which is the reflection of the peritoneum from the apex of the bladder to the posterior surface of the anterior abdominal wall along the urachus. The parts of the bladder which are left uncovered by peritoneum are (I) the inferior surface, and (2) the external trigone.

The pelvic peritoneum on either side of the bladder and rectum presents three fossæ when these viscera are empty, which are named, from before backwards, paravesical, paravesicular, and pararectal.

Retro-pubic Pad of Fat.-This is a collection of areolar and adipose tissues which lies between the posterior aspect of the bodies of the pubic bones, the bladder, and the pubo-prostatic ligaments.

Pelvic Fascia.-This fascia clothes the inner wall of the pelvis, and furnishes inward expansions, which have an intricate connection with, and serve to support, the contained viscera. It is divisible into two portions-parietal and visceral.

Parietal Portion.-Over the posterior wall of the pelvic cavity the parietal portion of the pelvic fascia, which is here very thin, covers the intrapelvic portion of each pyriformis muscle and the corresponding sacral plexus, this portion being known as the fascia of the pyriformis. Over the lateral wall the fascia attains considerable strength, and is attached superiorly to the back part of the iliac portion of the ilio-pectineal line for a short distance, where it becomes continuous with the iliac fascia. In front of this the fascia falls short of the ilio-pectineal line, and is fixed to the lateral wall of the pelvis a little below the line, its attachment being oblique, and accurately following the upper border of the obturator internus muscle. When it arrives at the upper part of 
the obturator foramen, there is a break in the osseous attachment of the fascia, which here joins the upper border of the obturator membrane over the upper border of the obturator internus, and so converts the obturator groove into a canal for the passage of the obturator vessels and nerve. At the upper and inner part of the obturator foramen the fascia resumes its osseous attachment, but falls more and more short of the pelvic margin, its connection with the back of the body of the os pubis being in a direction downwards and inwards till it reaches a point just below the lower part of the symphysis pubis. The fascia descends from the foregoing line of attachment, closely covering the obturator internus, to be

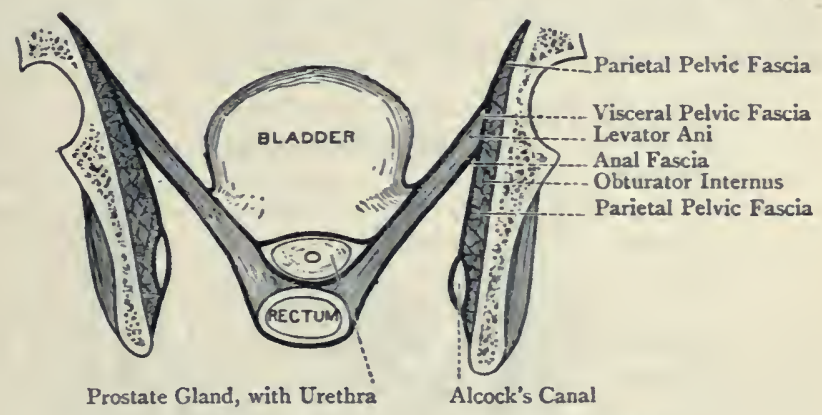

Fig. 363.-The Pelvic Fascia (Anterior View).

attached as follows from before backwards : (I) to the pelvic aspect of the ischio-pubic ramus near the subpubic arch, where it lies on the mesial side of the obturator internus ; $(2)$ to the inner margin of the falciform process of the great sacro-sciatic ligament at the lower part of the ischial ramus, and also to the great sacro-sciatic ligament itself, where that is attached to the inner margin of the tuber ischii ; and (3) to a portion of the great sacro-sciatic ligament near its ischial attachment, and to the anterior margin of the great sciatic notch. The lateral portion of the parietal pelvic fascia, from its relation to the obturator internus muscle, is sometimes called the obturator fascia. From its lower attachment the fascia is continued backwards and inwards over the great sacro-sciatic foramen, pyriformis, and sacral plexus to the sacrum, as the fascia of the pyriformis. As it covers the foramen it is perforated by the gluteal, sciatic, and internal pudic vessels.

At the anterior part of the pelvic cavity the fascia, after taking attachment to the pelvic surface of the ischio-pubic ramus near the subpubic arch, is continued inwards over that arch, where it lies behind the .compressor urethræ muscle, and forms one half of the postero-superior or deep layer of the triangular ligament, the other half being formed by the corresponding portion of the fascia of the opposite side. When it arrives at the urethra, it changes its course, and passes backwards over the anterior border of the 
levator ani to blend with that portion of the visceral pelvic fascia which ensheathes the prostate gland.

In the direction of a line passing from the inner aspect of the ischial spine to the back of the body of the os pubis, near its lower end and close to the symphysis, the lateral pelvic fascia is strengthened by fibres which impart to it a white colour. This part is called the white line, and it serves to divide the fascia into two portions, upper or pelvic proper, and lower or perineal. The pelvic portion looks into the pelvic cavity, and covers the upper part of the obturator internus, whilst it is itself covered by the peritoneum. The perineal portion covers the lower part of the obturator internus, and lies on the outer wall of the ischio-rectal fossa, where it constructs Alcock's canal. The under aspect of the white line affords extensive origin to the levator ani muscle.

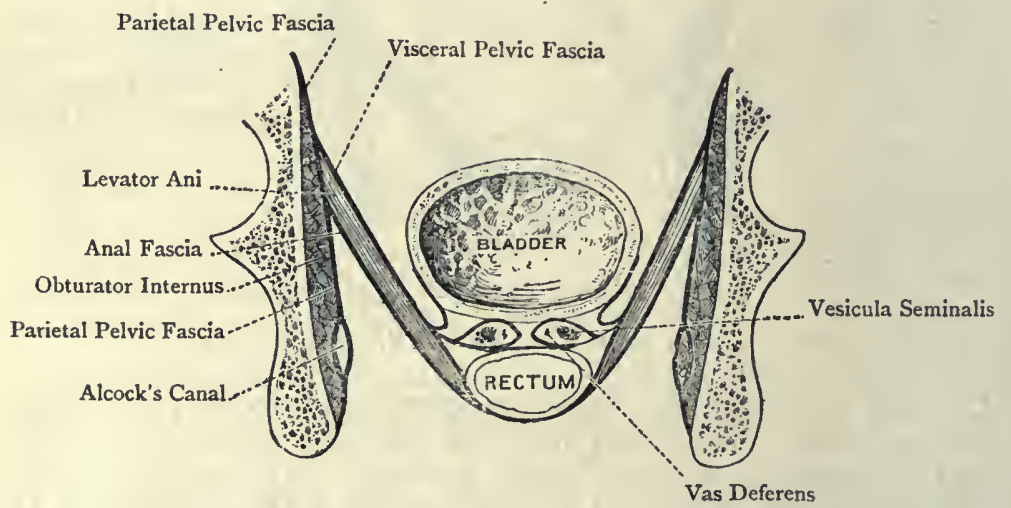

Fig. 364.-Diagram of the Pelvic Fascia from Behind.

Visceral Portion of the Pelvic Fascia.-The visceral portion is destined to support the lower part of the bladder, vesiculæ seminales, terminal portions of the vasa deferentia, prostate gland, and rectum, It is a laminar offshoot of the parietal portion, from which it springs along the white line. Though, however, this is its principal source, it has an independent origin anteriorly on either side of the middle line from the posterior aspect of the body of the os pubis near the lower part of the symphysis. In this latter situation it lies a little above the attachment of the parietal portion, the anterior fibres of the levator ani taking origin from the portion of bone between the two fasciæ. Though the visceral portion forms one continuous sheet along its pelvic attachment, its ultimate disposition is so intricate that it is convenient to consider its arrangement under three divisions.

I. Opposite the bladder, vesicula seminalis, and rectum. In this region the visceral portion of the fascia passes inwards as far as the outer border of the vesicula seminalis. In doing so it covers 
the upper surfaces of the levator ani and coccygeus, and is in turn covered by the peritoneum. At the outer border of the vesicula seminalis it divides into three laminæ-upper, middle, and lower. The upper or vesical lamina ascends upon the lateral aspect of the bladder for a short distance, and ultimately loses itself in the muscular tissue of the vesical wall. This portion at each side forms the lateral true ligament of the bladder. The middle or rectovesical lamina passes inwards between the base of the bladder and the rectum, and is continuous with the corresponding lamina of the opposite side. As it passes inwards it furnishes a sheath to the vesicula seminalis and adjacent portion of the vas deferens, closely strapping these structures to the base of the bladder. The lower or rectal lamina descends upon the side of the rectum, and passes to its posterior surface, where it is continuous with the rectal lamina of the opposite side.

2. Opposite the neck of the bladder and prostate gland. In this situation the visceral portion of the fascia also divides into three laminæ-upper, middle, and lower. The upper or prostatic lamina passes inwards over the anterior surface of the prostate gland, and is continuous with the prostatic lamina of the opposite side. The middle or recto-prostatic lamina passes inwards between the posterior surface of the prostate gland and the rectum, and is continuous with the corresponding lamina of the opposite side. The lower or rectal lamina is unaltered in its disposition. The upper or prostatic and middle or recto-prostatic laminæ furnish a stout sheath to the prostate gland.

3. Between the pubic bodies and the upper aspect of the neck of the bladder. The portion of the visceral fascia in this region represents the upper lamina in the other regions, and may be called the pubo-prostatico-vesical lamina. It is arranged in the form of two stout cords, which are known as the pubo-prostatic ligaments or the anterior true ligaments of the bladder. Each is attached anteriorly to the posterior aspect of the body of the os pubis, near the lower part of the symphysis, and a little above the attachment of the parietal portion of the pelvic fascia in this region, the anterior fibres of the levator ani taking origin from the portion of bone between the two fasciæ. As the cord-like fascial bands pass backwards they are connected by a portion of the visceral fascia which covers the dorsal vein of the penis and lies deeply, thus giving rise to a small pouch, which is capable of admitting the point of a finger. This pouch is filled with fat, which is continuous with the retro-pubic pad of fat. The anterior portion of the visceral fascia lies upon the anterior surface of the prostate gland, and on reaching the neck of the bladder it passes forwards to its inferior surface, where it soon disappears in the muscular tissue of the vesical wall. The pubo-prostatic ligaments are largely composed of plain muscular tissue, which is derived from the longitudinal fibres on the inferior surface of the bladder as they pass to take attachment to the back of the bodies of the pubic bones on either 
side of the middle line. These fibres constitute the so-called pubo-vesical muscles.

Anal Fascia.-This very thin sheet of fascia is an offshoot from the parietal pelvic fascia just below the white line and the origin of the levator ani. It closely covers the ischio-rectal surface of that muscle and of the coccygeus, upon which it descends to the region of the anus, where it blends with the aponeurotic investment of the sphincter ani externus.

Sympethetic Plexuses in the Pelvis.-These are derived from the hypogastric plexus, which is formed by the fusion of the two lateral strands of the aortic plexus after they have crossed the common iliac arteries. It is reinforced by branches from the ganglia of the lumbar sympathetic cords, and is situated in front of the body of the fifth lumbar vertebra between the common iliac vessels. It is a large, flattened plexus, measuring about $\mathrm{I}_{\frac{1}{2}}$ inches in breadth, and it breaks up into two divisions, which form the right and left pelvic plexuses. Each of these enters the pelvis on the inner side of the internal iliac artery, and takes up a position on the side of the rectum. The pelvic plexus of each side is reinforced by branches from the upper one or two ganglia of the pelvic sympathetic chain, and by spinal fibres from the anterior primary divisions of the third and fourth sacral nerves (sometimes also the second), therekbeing very small ganglia at the places of junction. From each plexus the following secondary plexuses are given off, which accompany the corresponding branches of the internal iliac artery : hemorrhoidal, vesical, prostatic, vesicular, and deferential, the latter three being replaced in the female by the vaginal and uterine plexuses.

Internal Iliac Artery.-This vessel arises from the common iliac opposite the lumbo-sacral articulation, and terminates opposite the upper border of the great sciatic notch by dividing into an anterior and a posterior division. The length of the vessel is about $\mathbf{I} \frac{1}{2}$ inches, and its direction is downwards and backwards.

Relations-Anterior.--The artery is covered by the peritoneum, and the ureter descends over it. The terminal part of the ileum forms an anterior relation of the right vessel, whilst the pelvic colon is similarly related to the left. Posterior. - The vessel rests chiefly upon its own vein, but near its origin it is placed over the commencement of the common iliac vein. Behind the veins there are the lumbo-sacral cord and ala of the sacrum. External.-The psoas magnus, with the intervention of the external iliac vein, and subsequently the lateral wall of the pelvis, with the intervention of the obturator nerve. Internal.--The peritoneum.

Varioties.--The chief variety affects the length of the vessel. It may be shorter or longer than usual, according as the common iliac is longer or shorter than normal, or according to the height at which the internal iliac ends in its two divisions.

Fotal Condition.-During fotal life the internal iliac is represented by the hypogastric and umbilical arteries, the size of which greatly exceeds that of the external iliac. The function of these arteries is to carry the impure blood 
from the fœtus to the placenta of the mother. The hypogastric artery passes forwards to the posterior surface of the anterior wall of the abdomen, being crossed by the vas deferens. It then ascends to the lower part of the umbilicus, where it leaves the abdomen with its fellow. Beyond the umbilicus the hypogastric is known as the umbilical artery, and the two umbilical arteries, together with the umbilical vein, form the umbilical cord, in which the arteries describe spiral coils around the vein. The arteries convey the impure blood to the placenta, where it is purified, after which it is returned to the abdomen of the fœtus by the umbilical vein. After birth, there being no further use for the placental circulation, the umbilical cord is tied, and the child separated from the mother. Thereafter the hypogastric arteries become impervions, and each is converted into a fibrous cord. The obliteration, however, does not involve the first $I \frac{1}{2}$ inches of the vessel, which persist as the internal iliac artery of the adult. Moreover, the proximal end of the fibrous cord representing the obliterated hypogastric remains pervious also, and, being connected with the anterior division of the internal iliac, it furnishes the superior vesical artery or arteries. This pervious portion lies along the side of the pelvis beneath the peritoneum, where it is crossed by the vas deferens in the male, and the round ligament of the uterus in the female. The fœtal hypogastric artery gives off the sciatic, which is the primitive main artery of the lower limb, until the external iliac, which becomes the femoral, is developed.

Branches.--These are subject to much variation. In normal cases they arise from the two terminal divisions, anterior and posterior, some of them being parietal in their distribution, which will be indicated by the letter $\mathrm{P}$, whilst others are visceral, which will be indicated by the letter $\mathrm{V}$. The branches are as follows :

\section{Anterior Division.}

\section{Visceral.}

Superior vesical.

Inferior vesical.

Middle hemorrhoidal.
Parietal.

Obturator.

Internal pudic. Sciatic.

\section{Posterior Division.}

\section{Parietal.}

Ilio-Iumbar.

Lateral sacral.

Gluteal.

In the female the inferior vesical artery may be replaced by the vaginal, or the vaginal may be an independent branch, and the uterine artery is always a special branch.

Anterior Division.- The superior vesical artery (V) arises from the pervious portion of the fibrous cord which represents the fœtal hypogastric artery, and at once breaks up into several twigs which frequently have independent origins. They are distributed to the upper portion of the bladder, and anastomose with the vesical of the obturator, and the inferior vesical of the same side, and with the superior vesical of the opposite side. The superior vesical furnishes the following branches: urachal to the urachus; ureteric to the lower end of the ureter; and deferential (as a rule) to the vas deferens, though this branch may arise from the inferior vesical. The deferential artery, which is usually of small size, divides into a descending and an ascending branch. The descending branch passes downwards to supply the ampulla of the vas deferens, and the vesicula seminalis. The ascending branch accompanies the vas deferens through the internal abdominal ring and inguinal canal in to the scrotum, supplying the vas deferens, and giving a few twigs 
to the globus minor of the epididymis, in which latter situation it anastomoses with the epididymal branch of the spermatic artery. In the spermatic cord it also anastomoses with the cremasteric branch of the deep epigastric. The deferential artery is sometimes of large size, and then it takes the place of the spermatic artery, if that vessel should be absent. One of the branches of the superior vesical artery is sometimes spoken of as the middle vesical.

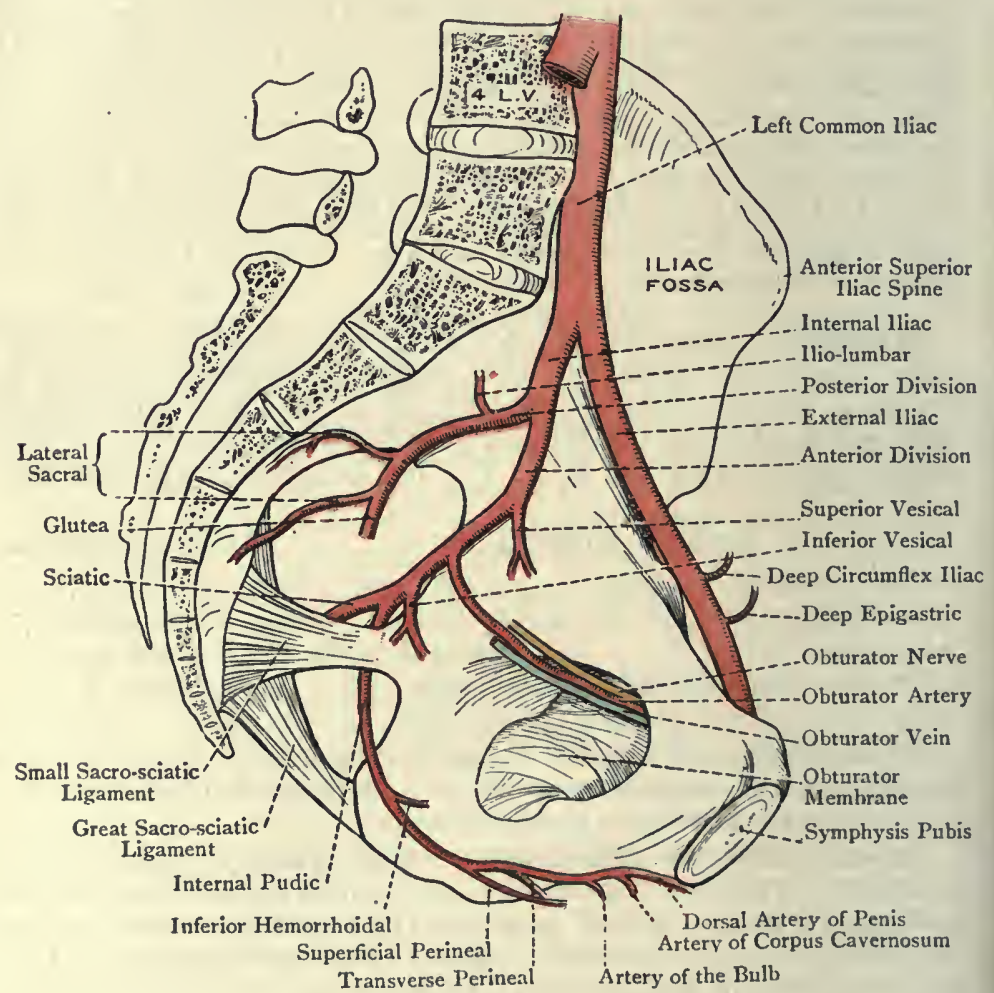

Fig. 365.-The Left Internal Iliac Artery and its Branches.

The inferior vesical artery (V) takes an inward course to the lower portion of the bladder, which it supplies, giving branches to the vesicula seminalis, ampulla of the vas deferens, and prostate gland, and in the female to the vagina. It sometimes gives off the deferential artery, and it may give origin to an accessory pudic. It anastomoses with the superior vesical and middle hemorrhoidal of its own side, and with its fellow of the opposite side.

The middle hemorrhoidal artery (V) often arises in common with 
the inferior vesical. It is distributed to the rectum, and gives branches to the vesicula seminalis, ampulla of the vas deferens, and prostate gland. The anastomoses which it establishes are with the superior hemorrhoidal of the inferior mesenteric, the inferior hemorrhoidal of the internal pudic, the inferior vesical of its own side, and its fellow of the opposite side.

The obturator artery $(\mathrm{P})$ passes along the outer wall of the pelvic cavity on its way to the obturator canal, lying between the parietal pelvic fascia and the peritoneum. It has the obturator nerve above it, and its own vein below it. In entering the obturator canal, by which it emerges from the pelvis, the artery does not pierce the parietal pelvic fascia, but passes over its upper border, where that joins the upper part of the obturator membrane. As the artery passes along the pelvic wall it furnishes the following branches: muscular to the obturator internus; iliac to the iliac fossa, which supply the bone, psoas magnus, and iliacus, and anastomose with the iliac branch of the ilio-lumbar; vesical, which reaches the side of the bladder within the lateral false ligament; and pubic to the back of the body of the os pubis, where it anastomoses with the pubic branch of the deep epigastric from the external iliac, and its fellow of the opposite side.

For the distribution of the obturator artery outside the pelvis, see p. 488 .

The obturator artery sometimes arises from the deep epigastric; see p. 426 .

The internal pudic artery $(\mathrm{P})$ is one of the terminal branches of the anterior division. It descends with the sciatic artery upon the pyriformis and sacral nerves, and emerges from the pelvis through the lower compartment of the great sacro-sciatic foramen, having previously pierced the parietal pelvic fascia. The intrapelvic branches of the vessel are unimportant, and are distributed to the pyriformis, coccygeus, obturator internus, and pelvic fascia. For the further course and distribution of the artery, see the gluteal region and the perineum, pp. 433 and 622.

The sciatic artery $(\mathrm{P})$ is the other and larger terminal branch of the anterior division. It descends, usually behind the internal pudic, upon the pyriformis and sacral nerves, and emerges from the pelvis through the lower compartment of the great sacrosciatic foramen, having previously pierced the parietal pelvic fascia. Within the pelvis the artery gives off branches to the pyriformis, levator ani, coccygeus, rectum, bladder, vesicula seminalis, and prostate gland.

For the extrapelvic course and distribution of the vessel, see p. 432 .

Posterior Division.-The ilio-lumbar artery $(\mathrm{P})$ passes upwards and outwards in front of the sacro-iliac articulation, between the lumbosacral cord and obturator nerve. In its course it passes behind the external iliac, or, it may be, the common iliac, vessels, and also behind the psoas magnus and iliacus. On reaching the back part 
of the iliac fossa, it divides into two branches, iliac and lumbar, The iliac branch, passing transversely, ramifies in the iliacus and ilium, and anastomoses with the iliac branch of the obturator and branches of the deep circumflex iliac from the external iliac. The lumbar branch ascends beneath the psoas magnus on to the quadratus lumborum, where it anastomoses with the terminal part of the deep circumflex iliac, and the last lumbar of the abdominal aorta. In its course it furnishes a spinal branch, which enters the spinal canal through the intervertebral foramen between the fifth lumbar and first sacral vertebræ, to be distributed in a manner similar to the other spinal arteries.

The lateral sacral arteries $(\mathrm{P})$ are usually two in number, superior and inferior. They course downwards and inwards to the front of the lateral mass of the sacrum, passing in front of the pyriformis and sacral nerves. Upon the sacrum both arteries lie external to the anterior sacral foramina, the superior being confined to the region of the first two foramina, whilst the inferior descends as low as the coccyx, where it anastomoses with the middle sacral artery. They are distributed to the pyriformis and sacral nerves, and furnish spinal branches, which enter the anterior sacral foramina, and so reach the sacral canal, where they supply its contents. Each spinal branch, before entering the sacral canal, gives off a posterior branch, which emerges through the posterior sacral foramen, and anastomoses with branches of the gluteal, sciatic, and internal pudic. The lateral sacral arteries anastomose with each other and with the middle sacral.

The gluteal artery $(\mathrm{P})$ is a large vessel, which is the continuation of the posterior division. It is destined for the supply of the gluteal region, and lies within the pelvis for a very short distance. Its direction is backwards through the parietal pelvic fascia, and between the lumbo-sacral cord and the anterior primary division of the first sacral nerve, its escape from the pelvis being through the upper compartment of the great sacrosciatic foramen. The branches of the artery within the pelvis are unimportant.

For the extrapelvic course and distribution of the vessel, see p. 430 .

The branches of the internal iliac, with one exception, have to pierce the pelvic fascia. The exception is the oblurator artery, which, as stated, passes over the upper border of the parietal pelvic fascia at the obturator canal.

Internal Iliac Vein.- This vessel results from the union of branches which correspond, for the most part, with branches of the internal iliac artery. The ilio-lumbar vein, however, is an exception, inasmuch as it is a tributary of the common iliac vein. Moreover, during fotal life the umbilical vein, which corresponds to the hypogastric (subsequently umbilical) artery, passes to the liver after entering the abdomen of the foetus. The internal iliac vein extends from the upper part of the great sciatic notch to the sacro- 
iliac articulation on a level with the pelvic brim, where it joins the external iliac, and so the common iliac vein is formed. In its course it lies behind the corresponding artery. There are no valves in the vein itself, but its branches are freely provided with them.

The internal iliac vein is developed from the lower part of the cardinal vein.

Internal Iliac Lymphatic Glands.-These glands are about ten in number, and are associated with the origins of the branches of the internal iliac artery. One of them lies near the side of the rectum, in relation with the middle hæmorrhoidal artery, and is spoken of as the middle hemorrhoidal gland.

The afferent vessels of the internal iliac glands return lymph from the parts supplied by the branches of the internal iliac artery. Thus, they receive afferent vessels from the following parts:

I. Upper part of the anal canal, above the anal valves.

2. Lower part of the rectum.

3. Bladder.

4. Vesicular seminalis and vas deferens.

5. Prostate gland.

6. Prostatic, membranous, and bulbar portions of the urethra, in part.

7. Uterus (cervix).

8. Vagina (central part).

9. Deep structures of gluteal region, supplied by gluteal artery.

Io. Deep structures of upper part of back of thigh, supplied by sciatic artery.

II. Deep structures of perineum.

The efferent vessels of the internal iliac glands pass to the posterior group of common iliac glands.

Anterior Primary Divisions of the Sacral and Coccygeal Nerves. -There are five sacral nerves and one coccygeal nerve on either side. The anterior primary divisions of the first four sacral nerves emerge into the pelvis through the anterior sacral foramina, and that of the fifth sacral passes between the inferior lateral angle of the sacrum and the transverse process of the first coccygeal vertebra, through the coccygeus muscle. The anterior primary division of the coccygeal nerve enters the pelvis below the transverse process of the first coccygeal vertebra, through the coccygeus muscle. The first and second sacral nerves are of large size, and their course is obliquely downwards and outwards. Beyond the second they diminish rapidly in size, and pass more horizontally. They receive grey rami communicantes froin the adjacent ganglia of the pelvic sympathetic cord, and the third and fourth nerves (sometimes also the second) furnish white rami communicantes to the corresponding pelvic plexus. The anterior primary divisions of the first and second sacral nerves divide each into an anterior or ventral and a posterior or dorsal branch. The anterior primary division of the third sacral nerve divides into an upper and a lower 
branch, and is known as the nervus bigeminus. The anterior primary division of the fourth sacral nerve also divides into an upper and a lower branch, and it is known as a nervus furcalis. The lumbo-sacral cord in two divisions, ventral and dorsal, the anterior primary divisions of the first two sacral nerves, the upper and lower branches of the third sacral, and the small upper branch of the fourth sacral form the sacral plexus, whilst the large lower branch of the fourth sacral, the fifth sacral, and the coccygeal form the sacro-coccygeal plexus.

Sacral Plexus.-This plexus, formed as stated, is a large flattened

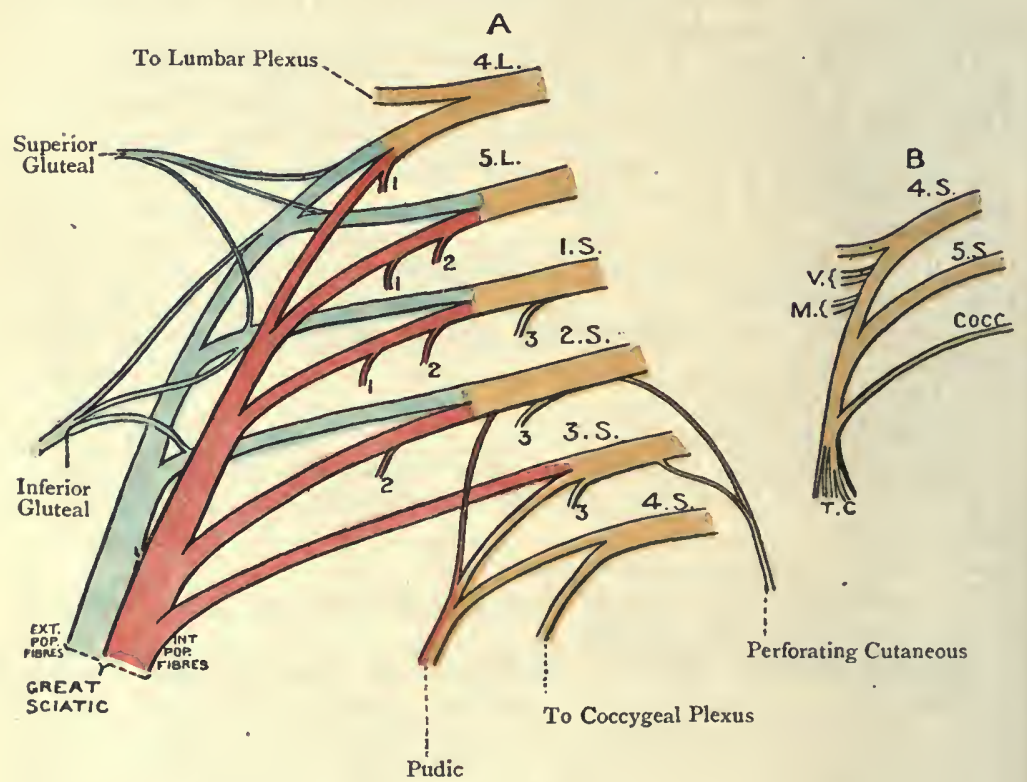

Fig. 366.-A. The Sacral Plexus; B, The Sacro-Coccygeal Plexus.

A

r, $x$, x. Nerve to Quadratus Femoris

2, 2, 2. Nerve to Obturator Internus

3, 3, 3. Small Sciatic Nerve

\section{B}

4.S. Fourth Sacral, giving a Branch to Sacral Plexus

V. Visceral Branches

M. Muscular Branches

T.C. Terminal Cutaneous Branches

mass, lying upon the pyriformis muscle, and behind the parietal pelvic fascia, which separates it from the sciatic and pudic vessels. The mass ultimately forms two bands, upper and lower. The upper or sciatic band, which is the larger of the two, receives the lumbo-sacral cord, first sacral, larger portion of the second sacral, and upper branch of the third sacral nerves. It is flat and somewhat triangular, and is continued into the great sciatic nerve, which leaves the pelvis through the lower part of the great sacro-sciatic 
foramen below the pyriformis, without piercing the parietal pelvic fascia. The upper band and its contributory nerves are sometimes spoken of as the sciatic plexus. The lower or pudic band, the smaller of the two, receives fibres from the second, lower branch of the third, and upper branch of the fourth sacral nerves, and is continued into the pudic nerve, which leaves the pelvis, like the great sciatic, through the lower compartment of the great sacro-sciatic foramen without piercing the parietal pelvic fascia. The lower band and its contributory nerves are sometimes spoken of as the pudic plexus. The great sciatic and pudic nerves are thus the terminal branches of the sacral plexus. The reason of the name nervus bigeminus, as applied to the third sacral nerve, is because it enters into the sciatic band by its upper branch, and in to the pudic band by its lower branch.

Branches.-The branches of the sacral plexus are arranged in two groups-collateral and terminal.

Collateral Group.-The branches of this group form three sets - visceral, muscular, and cutaneous.

Visceral Branches.-These are derived from the third sacral and that part of the fourth which enters into the sacral plexus (sometimes also from the second). They are white rami communicantes, which reinforce the pelvic plexus of the sympathetic, a few of them being traceable independently to the pelvic viscera. They are known as the pelvic splanchnics.

Muscular Branches.-The superior gluteal nerve arises by three roots from the dorsal divisions of the descending branch of the fourth lumbar, fifth lumbar, and first sacral nerves. It passes outwards and backwards, and leaves the pelvis with the gluteal artery through the upper compartment of the great sacro-sciatic foramen, to be distributed to the gluteus medius, gluteus minimus, and tensor fasciæ femoris muscles.

The inferior gluteal nerve arises by three roots from the dorsal divisions of the fifth lumbar and first and second sacral nerves. It leaves the pelvis through the lower compartment of the great sacro-sciatic foramen, below the pyriformis and superficial to the great sciatic nerve, to be distributed to the gluteus maximus. It is usually intimately associated with the small sciatic nerve.

The nerves to the pyriformis, which are usually two in number, spring from the dorsal divisions of the first and second sacral nerves.

The nerve to the obturator internus and gemellus superior arises by three roots from the ventral divisions of the fifth lumbar and first and second sacral nerves. It leaves the pelvis through the lower compartment of the great sacro-sciatic foramen, crosses the back of the ischial spine, where it lies external to the internal pudic vessels, and then passes through the small sacro-sciatic foramen to the outer wall of the ischio-rectal fossa, where it enters the inner or pelvic surface of the obturator internus. In the gluteal region it gives a branch to the gemellus superior when that muscle is present. 
The nerve to the quadratus femoris and gemellus inferior arises by three roots from the ventral divisions of the descending branch of the fourth lumbar, fifth lumbar, and first sacral nerves. It leaves the pelvis through the lower compartment of the great sacro-sciatic foramen, and then lies between the ischium and the great sciatic nerve. It subsequently descends over the back of the capsular ligament of the hip-joint, to which it usually gives a branch, and beneath the gemelli and obturator internus to the deep surface of the quadratus femoris, in which it ends, having previously given a branch to the gemellus inferior.

Cutaneous Branches. - The small sciatic nerve arises by three roots from the posterior aspects of the first, second, and third sacral nerves. It leaves the pelvis through the lower compartment of the great sacro-sciatic foramen, and is distributed to (I) the lower and outer part of the gluteal region by its gluteal cutaneous branches, (2) the integument of the scrotum by the long pudendal nerve of Soemmering, (3) the integument of the back of the thigh, and (4) the integument of the back of the leg as low as about the centre of the calf.

The perforating cutaneous nerve arises by two roots from the posterior aspects of the second and third sacral nerves. It passes backwards through the great sacro-sciatic ligament, after which it turns round the lower border of the gluteus maximus near the coccyx to be distributed to the integument over the lower and inner part of that muscle.

Terminal Group.-This group is comprised of two nerves, namely, the great sciatic and the pudic.

The great sciatic nerve is the continuation of the upper or sciatic band of the sacral plexus. In reality it is made up of the two divisions in which it ultimately ends, namely, the external and internal popliteal, which lie close together within the same sheath. Sometimes, however, these two divisions arise separately from the sacral plexus, in which cases the external popliteal nerve may pass through the pyriformis. Again, though the great sciatic nerve may be apparently single, a careful dissection of it, after the removal of its sheath, will reveal the external and internal popliteal nerves. which can be shown to have independent origins. The external popliteal nerve derives its fibres from the dorsal divisions of the descending branch of the fourth lumbar, fifth Iumbar, and first and second sacral nerves; and the internal popliteal nerve derives its fibres from the ventral divisions of the foregoing nerves, and in addition from the upper branch of the third sacral.

The great sciatic nerve leaves the pelvis through the lower compartment of the great sacro-sciatic foramen below the pyriformis.

The pudic nerve is the continuation of the lower or pudic band of the sacral plexus. It arises by three roots from the ventral division of the second, the lower branch of the third, and the upper branch of the fourth sacral, the root from the third being the largest. The nerve leaves the pelvis through the lower compart- 
ment of the great sacro-sciatic foramen, crosses the back of the ischial spine, where it lies internal to the internal pudic vessels, passes through the small sacro-sciatic foramen, and then traverses the outer wall of the ischio-rectal fossa. Having given off the inferior hemorrhoidal nerve, it divides into the perineal and dorsalis penis nerves, which with the internal pudic vessels are contained in Alcock's canal.

The sacral plexus and its branches being situated behind the parietal pelvic fascia, the branches do not pierce that fascia as they leave the pelvis. The branches of the internal iliac artery, on the other hand, being placed on the superficial surface of the parietal pelvic fascia, have to pierce it, with the single exception of the obturator artery.

Sacro-coccygeal Plexus.--The nerves which form this plexus are the lower branch of the anterior primary division of the fourth sacral, the anterior primary division of the fifth sacral, and the anterior primary division of the coccygeal nerve. The upper branch of the fourth sacral enters the pudic band of the sacral plexus, and the fourth sacral is therefore a nervus furcalis, inasmuch as it gives a branch to the sacral plexus and one to the sacro-coccygeal plexus. Before entering the sacro-coccygeal plexus the lower branch of the fourth sacral nerve gives off visceral and muscular branches. The visceral branches for the most part reinforce the pelvic plexus of the sympathetic as white rami communicantes, but a few of them pass independently to the pelvic viscera. The muscular branches supply the levator ani, coccygeus, and sphincter ani externus. The branch to the latter muscle reaches the perineum either by piercing the coccygeus, or by passing between it and the levator ani, and, besides supplying the external sphincter, it gives branches to the integument between the coccyx and the anus. The nerve is known as the hemorrhoidal or perineal branch of the fourth sacral. The lower branch of the fourth sacral nerve, having parted with the foregoing offsets, descends upon the coccygeus where it joins the fifth sacral, which has just entered the pelvis through that muscle. The conjoined nerve now descends, and is soon reinforced by the coccygeal nerve, which also enters the pelvis by piercing the coccygeus. In this manner the sacro-coccygeal plexus is formed. It lies at the lower part of the posterior wall of the pelvis upon the coccygeus, and the nervous loop takes a downward course. Subsequently it divides into several twigs, which leave the pelvis by piercing the coccygeus, the small sacro-sciatic ligament, and the adjacent portion of the gluteus maximus to be distributed to the integument over the coccyx.

Ureters.-The ureter of each side, having crossed the termination of the common iliac, or the commencement of the external iliac, artery, enters the pelvis. It then passes downwards and forwards in front of the internal iliac artery to the outer wall of the pelvis, lying beneath the peritoneum, and crossing internally the obturator vessels and nerve, and the obliterated hypogastric 
artery. It subsequently passes inwards to the bladder, being crossed internally by the vas defereins. Having arrived at the lateral or ureteric angle of the bladder, it passes obliquely through the vesical wall anterior to the upper free end of the vesicula seminalis, being here about 2 inches distant from its fellow, and about $I \frac{1}{2}$ inches from the base of the prostate gland.

Bladder.-The bladder, when empty, or only moderately distended, lies entirely within the cavity of the pelvis. When, however, it is fully distended, the apical part of the viscus rises above the level of the symphysis pubis into the hypogastric region of the abdomen for at least 2 inches, and, in cases of marked over-distension, it may reach to the umbilicus, or even higher. The bladder, therefore, has to be considered under two aspects, namely, when empty, or only moderately distended, and when fully distended.

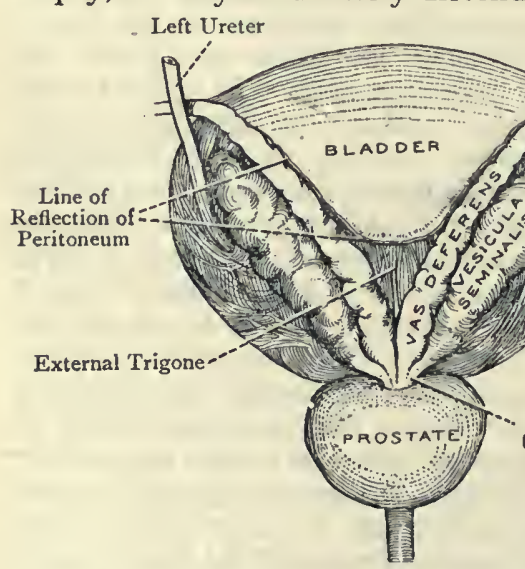

Fig. 367.-Dissection OF THE BASE

OF THE BLADDER, SHOWING . THE

Vesiculat Seminales, VAsa DeferENTIA, AND External Trigone.
The bladder, when empty, or moderately distended, is triangular, and presents the following component parts: an apex, a base, and a body, the latter presenting a superior surface, an inferior surface, two lateral borders, a posterior border, two lateral angles, and a neck.

The apex is directed forwards and lies Common Kjaculatory forwards and lies
Duct of Right Side behind the upper part of the symphysis pubis. It has connected with it the fibrous cord representing the urachus, which ascends on the posterior surface of the anterior abdominal wall to the lower part of the umbilicus. The base or fundus is directed backwards and downwards towards the rectum, from which it is separated by the vesiculæ seminales and terminal portions of the vasa deferentia, and the recto-vesical lamina of the visceral portion of the pelvic fascia. The base is separated from the superior surface by the posterior border, and from each rounded half of the inferior surface by a slight elevation, which extends from the lateral or ureteric angle of either side to the urethra! opening. The superior surface is three-sided and slightly convex. It is usually related to a few coils of the small intestine, and a loop or two of the pelvic colon. The inferior surface, which is convex, rests upon (I) the symphysis pubis and the adjacent portions of the posterior surfaces of the bodies of the pubic bones, (2) the retro-pubic pad of fat, (3) the pubo-prostatic ligaments, and (4) the prostate gland. This surface, being rounded, is somewhat indefinitely divided into two lateral 
portions. The lateral borders start from the apex, whence they pass backwards in a diverging manner, and each marks the separation between the superior surface and the corresponding half of the inferior surface. Posteriorly each lateral border meets the posterior border. The posterior border extends transversely between the superior surface and the base. At either end it is joined by the lateral border. The lateral or ureteric angles are situated on either side at the junction of the lateral with the posterior border. At these angles the ureters pass through the vesical wall. The neck is situated at the meeting of the base and inferior surface. It is surrounded by the base of the prostate gland, and it is in this situation where the vesical cavity becomes continuous with the urethra. The neck lies fully 2 inches behind the symphysis pubis, being a little lower than its upper border, and it is practically fixed in position by the prostate gland and pubo-prostatic ligaments.

The external trigone is the name given to a limited triangular space upon the exterior of the base, which is bounded as follows: above, by the bottom of the recto-vesical pouch of peritoneum; laterally, by the ampulla of the vas deferens, external to which is the vesicula seminalis; and below, by the approximation of the common ejaculatory ducts at the base of the prostate gland. The length of the space from the apex below to the base above is, as a rule, fully I inch, but its breadth is limited by the encroachment upon it of the deferential ampullæ. There is no peritoneum over this region.

The bladder during distension, and when fully distended. As the bladder becomes distended the neck alters its position only to a very slight extent in a downward direction. The demarcations between the component parts of the viscus gradually disappear, and it becomes ovoid. The apex appears above the symphysis pubis, and, as the organ increases in size, the apex and the superior portion of the body ascend into the hypogastrium in such a manner as to strip off the parietal peritoneum from the back of the linea alba. In this way a portion of the original inferior surface, devoid of peritoneum, is brought into direct contact with the back of the linea alba, which is now also free from peritoneal covering. A distended bladder may, therefore, be punctured or opened through the linea alba above the symphysis pubis without doing any injury to the peritoneum. The bladder is now becoming spheroidal, and its long axis is directed downwards and backwards. The part which rises highest is not the original urachal apex, but that part of the superior surface which is immediately adjacent to, and behind, the apex.

Peritoneal Relations.-The only part of the bladder which is covered by peritoneum is the superior surface. The peritoneum is reflected from the apex along the urachus, and at either side it leaves the organ along the lateral border. When the bladder is distended the lateral reflection of peritoneum appears to take place along the course of the obliterated hypogastric artery, but this 
is due to the lateral distension and elevation of the viscus, the obliterated hypogastric itself lying along the outer-wall of the pelvis. For the structure and development of the bladder, see Index.

Vas Deferens in the Pelvis.-The vas deferens enters the abdomen through the internal abdominal ring, where it lies on the inner side of the other constituents of the spermatic cord. It then hooks round the outer side of the deep epigastric artery, and, having crossed the external iliac vessels from without inwards, it dips down on the inner side of the external iliac vein. In this way it enters the pelvis under cover of the peritoneum. It now passes backwards and downwards upon the lateral wall, crossing the obliterated hypogastric artery and ureter, both on the inner side. Thereafter it is directed inwards to the inner aspect of the upper end of the vesicula seminalis, whence it passes downwards and inwards, and then vertically downwards upon the base of the bladder, where it lies close to the inner side of the seminal vesicle. This part of the vas deferens, which comes very near its fellow, is dilated and sacculated, like the adjacent vesicula seminalis, and is known as the ampulla. Close to the base of the prostate gland the sacculations disappear, and the duct, having become very narrow, is joined on the outer side at an acute angle by the duct of the seminal vesicle, and so the common ejaculatory duct is formed, which will be presently described. For the structure and development of the vas deferens, see Index.

Vesiculæ Seminales.-These are two in number, right and left, and lie between the base of the bladder and the rectum. They are sacculated reservoirs for the seminal fluid, and each is conical, being about 2 inches long, and about $\frac{1}{2}$ inch broad at the widest part. The broad end is free, and looks upwards and outwards. It is covered posteriorly by the peritoneum which forms the rectovesical pouch, and anterior to it is the ureter, as that is about to pass through the bladder. The upper ends of the two seminal vesicles are wide apart, and the bottom of the recto-vesical pouch descends between them for a short distance. The lower end of each, which is narrow and free from sacculations, approaches its fellow, the vasa deferentia intervening. This lower end represents the duct, which, as stated, joins the vas deferens at an acute angle close to the base of the prostate gland, and so gives rise to the common ejaculatory duct. For the structure and development of the vesiculæ seminales, see Index.

Common Ejaculatory Ducts.-These are two in number, right and left. Each is formed by the union of the duct of the vesicula seminalis with the vas deferens close to the base of the prostate gland, and it is about I inch in length. The two ducts pass downwards and inwards through the prostate gland, betwcen the middle and lateral lobes, and each enters the lateral wall of the sinus pocularis. Thereafter each duct terminates in a minute aperture, which is situated either on the lateral margin of the opening of the 
sinus pocularis, or immediately within the lateral margin. For the structure and development of the common ejaculatory ducts, see Index.

Prostate Gland.-This gland surrounds the neck of the bladder, as well as the first $x \frac{1}{4}$ inches of the urethra. It is firm in consistence, and in shape and colour resembles a chestnut - that is to say, it is conical and of a reddish-brown colour. It is subject to much variety in size, but its average measurements may be stated as follows: the transverse diameter at the base is about $I_{\frac{1}{2}}$ inches; the vertical diameter from base to apex is about I $\frac{1}{4}$ inches; and the antero-posterior diameter is about $\frac{3}{4}$ inch. The average weight of the organ is about $4 \frac{1}{2}$ drachms.

The gland is situated fully $I \frac{1}{2}$ inches from the anus, and its anterior surface is about $\frac{1}{2}$ inch behind the lower part of the symphysis pubis. It presents a base, an apex, a posterior or rectal surface, an anterior or pubic surface, and two lateral surfaces. The base, which is directed upwards, surrounds the neck of the bladder, and is intimately connected with the vesical wall. Notwithstanding this, however, there is a superficial line of demarcation between the two, which takes the form of an annular groove, occupied by a portion of the prostatic venous plexus. The common ejaculatory ducts enter the base close to the upper part of the posterior or rectal surface, where there is a small transverse cleft, called the prostatic fissure. The apex is directed downwards, and is the most dependent part of the organ. It is in contact with the postero-superior layer of the triangular ligament of the urethra. The posterior or rectal surface is flat and triangular. It is directed backwards and slightly downwards, and is set upon the anterior wall of the rectum, from which it is separated by the recto-prostatic lamina of the visceral portion of the pelvic fascia. This surface is accessible to manipulation on introducing the finger into the anal canal, and carrying it up for fully $I_{2} \frac{1}{2}$ inches. The anterior or pubic surface, which is convex, is so much projected as to be really an anterior round border, which separates the two lateral surfaces. It lies about $\frac{1}{2}$ inch behind the lower part of the symphysis pubis, from which it is separated by a portion of the prostatic venous plexus, the retro-pubic pad of fat, and the pubo-prostatic ligaments. The anterior surface projects between the anterior borders of the levatores ani muscles. The lateral surfaces are convex, and stand out in full relief. Each is embraced by the anterior fibres of the corresponding levator ani, which constitute the so-called levator prostatæ muscle. When an accessory pudic artery is present it passes over the lateral surface, and might be endangered in lateral lithotomy.

The gland is usually regarded as composed of three lobes, two lateral and a middle, but, though the middle lobe can usually be demarcated without much difficulty, there is no external indication of any separation between the lateral lobes. The lateral lobes form the chief bulk of the gland, and they meet and become con- 
tinuous in front of and behind the prostatic portion of the urethra. The middle lobe represents that part of the basal portion of the gland which is wedged in between the common ejaculatory ducts and the adjacent portion of the neck of the bladder, and which lies just below the apex of the internal trigone. It is important to note that the middle lobe lies behind the commencement of the urethra. It is liable to become hypertrophied in old age, and then it may produce undue elevation of the uvula vesicæe in the interior of the bladder, which is a natural product of it. In this manner, by blocking the urethral orifice, it may not only give rise to difficulty in micturition, but may also obstruct catheterization.

The prostate gland is traversed by the first $I_{\frac{1}{4}}^{\frac{1}{4}}$ inches of the urethra. The common ejaculatory ducts are also contained within the gland, in which they pass downwards and inwards between the middle and lateral lobes. For the structure and development of the prostate gland, see Index.

Urethra.- The male urethra commences at the urethral orifice in the neck of the bladder, and terminates at the extremity of the

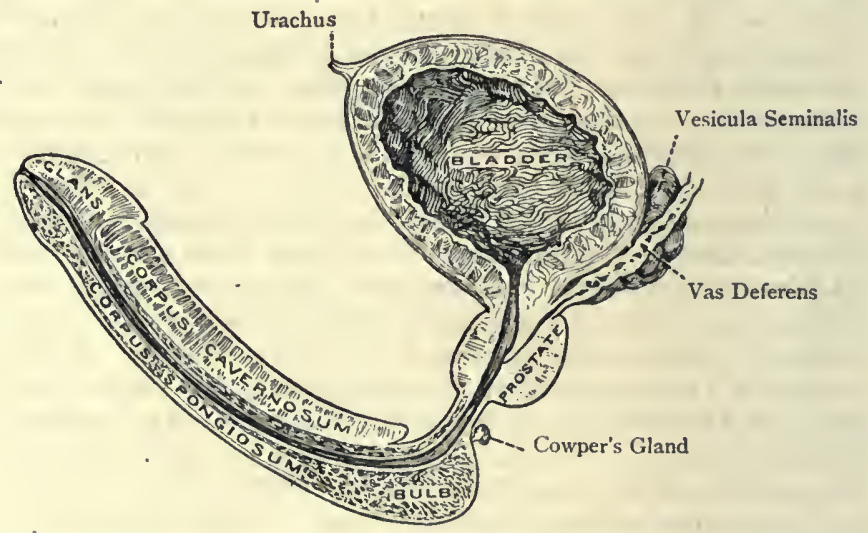

Fig. 368.-Section of the Bladder and Penis to show the Urethra.

glans penis in a vertical fissure, called the meatus urinarius. It is about 8 inches in length, and is divided into three portions, which, from the bladder outwards, are called prostatic, membranous, and spongy. The prostatic and membranous portions constitute the non-penile part of the canal, and the spongy portion, being contained within the corpus spongiosum of the penis, represents the penile part.

The prostatic portion of the urethra is the part of the canal which is contained within the prostate gland. It is $I_{4}^{1}$ inches in length, and its course is almost vertical, there being a slight curve with the concavity directed forwards. It is spindle-shaped, being wider at the centre than at either end, and at its upper or vesical 
end it is rather wider than at the lower end. Its diameter at the centre, which is the widest part of the entire canal, is rather more than $\frac{1}{3}$ inch; at the upper or vesical end it is rather less than this; and at the lower end it is still less. This portion of the urethra, though surrounded by the prostate gland, is the most dilatable part of the whole canal. Close to the neck of the bladder, however, it usually offers some resistance to the passage of an instrument. Its walls are anterior and posterior, the latter being often spoken of as the floor. These walls are in contact with each other, except during the passage of fluid, and the mucous membrane is thrown into longitudinal folds. The posterior wall presents along the middle line a prominent narrow elevation of the mucous membrane, called the erest. The other names by which it is known are verumontanum, caput gallinaginis, and colliculus seminalis. It is about $\frac{3}{4}$ inch long, and commences either a little below the urethral orifice of the bladder or at the lower end of the uvula vesicæ. As it descends it gradually becomes more prominent, assuming a height of about $\frac{1}{8}$ inch, and then rapidly subsides. It is due to a thickening of the submucous tissue, which causes an elevation of the mucous membrane. On account of this projection a transverse section of this portion of the urethra is curved or crescentic, with the convexity directed forwards. On either side of the crest there is a longitudinal groove, called the prostatic sinus, into which the majority of the prostatic ducts open. A few of these ducts, however, from the middle lobe open in the median line above the crest, or, if the crest commences at the lower end of the uvula vesicæ, upon its upper part. Immediately below the most prominent part of the crest there is the opening of a small blind recess, called the utricle. The other names by which it is known are sinus pocularis, vesicula prestatica, and uteris masculimus. Its direction is upwards and backwards behind the middle lobe of the prostate, and it is from $\frac{1}{4}$ to $\frac{1}{2}$ inch in length. It is somewhat flask-shaped, being narrow at its urethral orifice, but expanded at its deep caecal end. Upon the lateral margins of its orifice, or it may be just within them, there are the minute openings of the common ejaculatory ducts. The utricle represents the uterus and vagina in the female, being developed from the fusion of the posterior or caudal ends of the Miillerian ducts.

When the middle lobe of the prostate becomes hypertrophied, it blocks the urethral orifice of the bladder, as has bcen stated, by pressing the uvula vesicæ over it from behind, and so gives rise to difficulty in micturition, and obstructs catheterization. When both lateral lobes become uniformly hypertrophied, the prostatic portion of the urethra undergoes increase in length. When only one lateral lobe is involved in the hypertrophy it presses against the urethra, and, producing distortion of the canal, gives rise to difficulty in micturition, and often considerable obstruction in catheterization.

Structure.-The mucous membrane of the prostatic urethra is 
surrounded externally by the inner circular muscular fibres of the prostate, and is covered internally by transitional epithelium.

The membranous portion of the urethra succeeds to the prostatic portion, and extends from the apex of the prostate gland to the commencement of the spongy portion. It is contained, for the most part, between the two layers of the triangular ligament, but it also extends for a short distance ( $\frac{1}{4}$ inch) beyond the anteroinferior layer of that structure. It is the shortest and narrowest part of the canal, with the exception of the meatus urinarins. Its length is $\frac{3}{4}$ inch along the anterior wall, and $\frac{1}{2}$ inch along the posterior, the difference being due to the fact that the membranous urethra passes into the spongy part in a slanting manner at a point $\frac{1}{4}$ inch in front of the posterior extremity of the bulb. The back part of the bulb projects backwards for $\frac{1}{4}$ inch over the posterior wall of the membranous urethra, and is here lying in front of the antero-inferior layer of the triangular ligament. It is in this situation where a false passage is liable to be made in catheterization, partly because the walls are here very thin, and partly by reason of the backward extension of the posterior extremity of the bulb. The diameter of the membranous urethra is $\frac{1}{5}$ inch. It lies about I inch behind and below the subpubic ligament, and its direction is downwards and slightly forwards. It describes a gentle curve, the concavity of which looks forwards and upwards towards the lower part of the symphysis pubis. As the membranous urethra passes through the postero-superior layer of the triangular ligament, which is formed by the parietal pelvic fascia, the fascia is prolonged upwards to form part of the capsule of the prostate gland. Moreover, as it pierces the antero-inferior layer of the triangular ligament about I inch below the symphysis pubis, it carries with it a prolongation from the margins of the urethral opening, which forms a fascial investment for the bulb. Between the two layers of the triangular ligament the membranous urethra is surrounded by the fibres of the compressor urethræ muscle, and Cowper's glands lie behind it, one on either side of the middle line.

Structure.-External to the mucous membrane there is a layer of erectile tissue, and outside this there is a layer of circularlydisposed plain muscular fibres, which are continuous above with the circular muscular fibres of the prostate around the prostatic urethra. External to this, again, there are the sphincter fibres of the compressor urethra. The mucous membrane is covered by columnar epithelium. The membranous portion of the urethra in transverse section presents the appearance of a circular opening, the lumen of which is branched, this being due to the longitudinal folds into which the mucous membrane is thrown.

The spongy portion of the urethra succeeds to the membranous portion. It is contained within the corpus spongiosum of the penis, and extends from a point $\frac{1}{4}$ inch in front of the posterior extremity of the bulb and of the antero-inferior layer of the triangular ligament to the meatus urinarius on the extremity of 
the glans penis. It is about 6 inches in length, and its calibre is unequal throughout. It presents two dilatations, one situated in the bulb (which represents about the first $I_{2}^{\frac{1}{2}}$ inches of the corpus spongiosum), and the other in the glans penis, the latter being

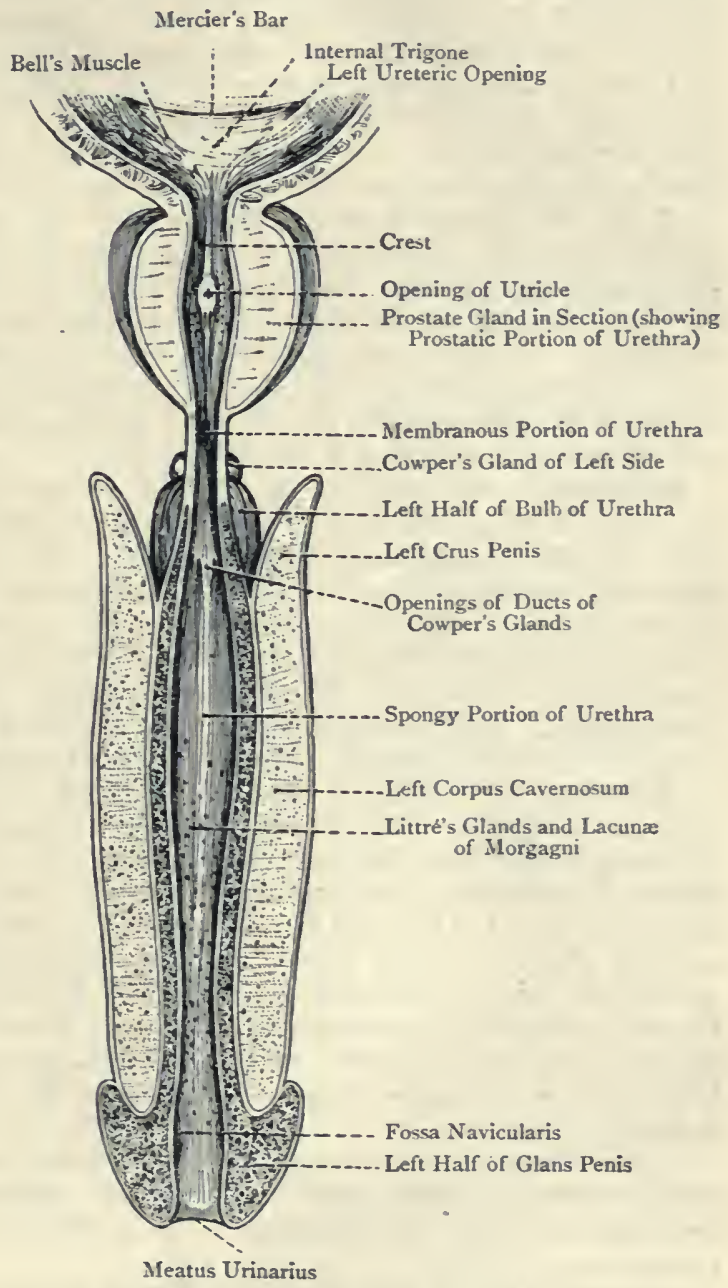

Fig. 369.-The Interior of the Male Urethra.

called the fossa navicularis. The bulbous enlargement is about $I_{\frac{1}{4}}$ inches in length, whilst the fossa navicularis is about $\frac{1}{2}$ inch long. The diameter of that part of the spongy urethra which intervenes between the bulbous enlargement and the fossa navi- 
cularis is about $\frac{1}{4}$ inch. At the meatus urinarius the calibre of the canal is diminished, this being the narrowest part of the entire canal. The spongy portion at its commencement is directed forwards for a short distance, and then bends downwards. It thus describes a curve, which is situated about 2 inches from its commencement, the concavity being directed downwards. This curve is fixed, and corresponds with the angle of the penis, the situation of which is immediately in front of the penile attachment of the suspensory ligament, where drooping of the organ takes place. Another curve, but of a temporary nature, can be produced by raising the penis towards the abdomen, as in catheterization. The walls of the spongy part of the urethra are in contact, except during the passage of fluid. A transverse section of it, except in the glans, presents a transverse slit with anterior and posterior lips. In the glans, however, the slit is vertical with its lips laterally disposed. At the upper end of the fossa navicularis-that is, the end most remote from the meatus urinarius - the vertical slit is modified by the addition of a short transverse slit, and so presents the shape of an inverted T, as follows, $\perp$.

Structure.-The mucous membrane of the spongy portion is provided with elastic tissue, and is very vascular. It is covered by columnar epithelium, except in the glans, where it is of the stratified squamous variety. Outside the mucous membrane is the submucous coat, which contains two layers of plain muscular tissue -inner longitudinal and outer circular. External to the submucous coat there is a plexus of veins, which forms part of the corpus spongiosum. The mucous membrane contains both simple and compound mucous glands, called the glands of Littré, the openings of the ducts of which are studded over the surface. In addition to these there are other openings, which lead into small blind recesses, called the lacunæ of Morgagni, these openings being directed forwards. One lacuna, of large size, called the lacuna magna, or sinus of Guerin, is situated on the roof of the fossa navicularis, about I inch from the meatus urinarius. The mucous membrane which surrounds this lacuna is known as the valvule of Guerin. Besides the foregoing openings, the ducts of Cowper's glands open upon the floor of the bulbous portion of the urethra about $I$ inch in front of the antero-inferior layer of the triangular ligament.

There is sometimes a congenital deficiency in the floor of the spongy urethra, constituting the condition known as hypospadias. In other cases the deficiency may be on the roof, and it is then known as epispadias.

Lymphaties of the Male Urethra-Penile Portion.-The lymphatic vessels of the penile portion, excluding those of the bulbar part, run from behind forwards, and open into the ring formed by the lymphatics of the glans penis behind the corona glandis. They ultimately pass to (I) the pubic group of the inguinal glands, (2) the decp femoral glands, which lie within the crural canal, and (3) the lowest 
or retro-femoral gland of the outer chain of the external iliac glands, by means of one or two vessels which traverse the inguinal canal.

Bulbar and Memtranous Portions.-The lymphatics of these portions pass to (I) the internaliliac glands, and (2) the inner chain of the externaliliac glands.

Prostatic Portion.- The lymphatics of this portion join those of the substance of the prostate gland, and pass to ( 1 ) the middle chain of the external iliac glands, (2) the internal iliac glands, (3) the lateval sacral glands, and (4) the inner group of the common iliac glands.

Pelvic Colon.-The pelvic colon succeeds to the iliac colon. It commences at the inner border of the left psoas magnus, just anterior to the left sacro-iliac articulation, and terminates in front of the third sacral vertebra, where it becomes continuous with the rectum. It measures on an average about $\mathrm{I}_{5}$ inches in length, and usually lies in the pelvic cavity over the upper part of the posterior wall, where it rests below upon the bladder and rectum, and may descend on either side of these viscera. It is.surrounded by peritoneum, which forms behind it an expanded wavy mesentery, called the pelvic meso-colon, which contains between its two layers the lower two sigmoid and superior hemorrhoidal arteries, with the corresponding veins. The root of this mesentery is composed of two limbs, which become continuous at an acute angle. One limb takes an upward course along the inner border of the psoas magnus, and the other passes downwards over the sacral promontory to be attached to the anterior surfaces of the first three sacral vertebræ. At its extremities, which are near each other, the mesentery is short, and the extremities of the pelvic colon are nocessarily fixed. The intervening portion, however, is long, and so allows of considerable mobility on the part of the greater portion of the attached gut. A very common course for the pelvic colon to take is to pass to the right wall of the pelvis, where it bends and crosses to the left wall, and then bending again it passes to the right as far as the middle line. In such cases, therefore, it describes the letter S. A portion of it is sometimes met with in the abdominal cavity.

Structure.-The chief difference in the structure of the pelvic colon, as compared with the other divisions of the colon, affects the arrangement of the longitudinal muscular fibres. Though arranged at first in three tæniæ, these gradually become disposed as two broad tæniæ, anterior and posterior. IThen this has taken place, the longitudinal fibres almost surround the gut, except along the sides, in which situations the usual sacculations are formed. Appendices epiploicæ are met with over the pelvic colon.

Blood-supply.-The pelvic colon receives its arteries from the lower two sigmoid arteries, which are branches of the inferior mesenteric. The veins pass to the inferior mesenteric vein, and ultimately into the vena portre.

The lymphaties pass to the left lumbar lymphatic trunk.

The nerves are derived from the inferior mesenteric sympathetic plexus. 
Rectum.-The rectum succeeds to the pelvic colon, and extends from the front of the third sacral vertebra to a point $I \frac{1}{2}$ inche: in front of and below the tip of the coccyx, where it pierces the pelvic diaphragm, and terminates in the anal canal. Its direction is at first downwards and slightly backwards, then vertically downwards, and finally downwards and forwards. It is fully 5 inches in length, and its diameter, for the most part, is about $I_{2}^{\frac{1}{2}}$ inches in the empty state, but it becomes enlarged above the anal canal, this dilated part being called the ampulla. When empty, its anterior and posterior walls are in contact, and in transverse section it appears as a transverse slit. The rectum is destitute of a mesentery. The peritoneum covers the upper third laterally and anteriorly, but not posteriorly, the middle third being covered by peritoneum only anteriorly, whilst the lower third is free from serous covering. The point at which the peritoneum leaves the bowel is usually about 3 inches above the anus.

The rectum, so far from being straight in man, presents both antero-posterior and lateral curves. The antero-posterior curves are two in number, upper and lower. The upper curve extends from the third sacral vertebra to the posterior or rectal surface of the prostate gland, and its concavity is directed forwards. The lower curve, which is abrupt, corresponds with the rectal surface of the prostate, its concavity looking backwards, and containing the anococcygeal body. The lateral curves are of importance, because they tend to obstruct the passage of instruments by giving rise to the valves of Houston. These curves are usually three in numberupper, middle, and lower. The upper and lower curves have their convexities directed towards the right, whilst the middle curve has its convexity directed towards the left. In the concavities of these curves the rectal wall becomes inflected, and so gives rise to more or less prominent shelves of mucous membrane, which are known as the rectal valves of Houston. The lateral inflections and resultant valves are associated with the erect posture of man, which posture throws considerable pressure upon the anal aperture and its sphincter muscles. They are best marked in the distended rectum, and the lateral curves are brought about in the following manner: The longitudinal muscular fibres of the rectum are rather shorter than the gut to which they are applied. Moreover, they are principally disposed in two stout broad sheets, one on the anterior and the other on the posterior wall, there being very few longitudinal fibres along the lateral walls. The shortness of these longitudinal fibres, therefore, throws the rectum into lateral curves on account of the sparseness of the longitudinal fibres at the sides. These lateral curves may be taken as representing the sacculations of the other parts of the colon.

Relations-Anterior. - The recto-vesical pouch of peritoneum for a short distance; the base of the bladder, vesiculæ seminales, and vasa deferentia, with the intervention of the recto-vesical lamina of the visceral pelvic fascia ; and the posterior or rectal surface of the prostate gland, with the intervention of the recto-prostatic 
lamina of the visceral pelvic fascia. In the female the anterior relations are (I) the recto-uterine pouch of peritoneum, or pouch of Douglas, with a few coils of small intestine, in front of which are the posterior surface of the body of the uterus and the upper part of the posterior wall of the vagina; and (2) the greater portion of the posterior wall of the vagina. Posterior.-The lower three sacral vertebræ, coccyx, levatores ani, and ano-coccygeal body. Behind the rectum there is a large amount of areolar tissue. Lateral.-The pelvic sympathetic plexuses, levatores ani, and coccygei muscles, the lateral divisions of the superior hemorrhoidal artery, and the corresponding veins.

Anal Canal.-This is the terminal portion of the large intestine. It extends from the lower end of the rectum, where that has pierced the pelvic diaphragm at a point corresponding to the apex of the prostate gland, to the anus, and it is the part which is surrounded by the sphincter muscles. It is about $I \frac{1}{2}$ inches in length, and its lateral walls are in contact, so that in transverse section it appears as an antero-posterior slit, in which respect it differs from the rectum proper. Its direction is downwards and backwards, and its antero-posterior measurement is from $\frac{1}{2}$ to $\frac{3}{4}$ inch.

Relations-Anterior. - The bulb of the corpus spongiosum, the base of the triangular ligament, and the membranous part of the urethra. In the female the perineal body is anterior to it, and separates it from the lower end of the vagina. Posterior.-The ano-coccygeal body. Lateral. - The fat of the ischio-rectal fossæ. The anal canal is closely guarded by muscles in the following manner, and to the following extent: the sphincter ani externus surrounds its termination in the anal aperture, the levatores ani grasp it at its commencement, and the intervening part is surrounded by the sphincter ani internus. For the structure and development of the rectum and anal canal, see Index.

Parts felt per Rectum in Catheterization.-A catheter having been passed into the bladder, the following parts, in order from before backwards, may be felt through the anterior wall of the bowel on the introduction of the index finger: (I) the membranous portion of the urethra; (2) the posterior or rectal surface of the prostate gland; and (3) the apical part of the external trigone of the bladder, destitute of peritoneum, with a vas deferens on either side, and very near each other-indeed, almost touching. During this examination the finger may come in contact with the lower left, and perhaps the right, valve of Houston.

Middle Sacral Artery.-This vessel arises from the posterior aspect of the abdominal aorta just above the bifurcation. Its course is downwards in the middle line, behind the left common iliac vein and the hypogastric sympathetic plexus, and it rests in succession upon the following parts : the lower half of the body of the fourth lumbar vertebra and the disc between it and the fifth, the body of the fifth lumbar and the disc between it and the first sacral vertebra, and the pelvic surfaces of the sacrum and coccyx. In the pelvis it lies within the root of the pelvic meso-colon, being covered by a portion of the pelvic colon, as low as the third sacral vertebra, 
and beyond that it is placed behind the rectum. On reaching the tip of the coccyx it terminates in the coccygeal body. The branches of the artery are as follows: anterior or rectal to the posterior wall of the rectum, where they anastomose with the superior and middle hemorrhoidal arteries; lateral to the front of the sacrum and coccyx, which anastomose with the lateral sacral arteries; and terminal to the coccygeal gland. The vessel occasionally furnishes a fifth pair of lumbar arteries, which wind round the sides of the body of the fifth lumbar vertebra:

The middle sacral artery represents the caudal aorta of animals, and its lateral branches are serially homologous with the lumbar branches of the abdominal aorta.

The middle sacral vein is at first arranged as two venæ comites, but these subsequently unite to form a single vessel, which usually terminates in the left common iliac vein.

Coceygeal Gland (Body) of Luschka.-This so-called gland is situated in front of the tip of the coccyx. It is about the size of a small pea, and is composed of a few nodules which are held together and invested by connective tissue. It receives the terminal twigs of the middle sacral artery. In structure it consists of groups of polyhedral cells, united by connective tissue, and permeated by blood-capillaries and sympathetic nerve-filaments. It is similar to the carotid body, which is situated behind the common carotid artery of each side close to its bifurcation.

Sacral Glands.-This group comprises a few lymphatic glands which lie in front of the sacrum along the middle line, behind the rectum. They receive their afferent vessels from the rectum and upper part of the anal canal, and from the posterior wall of the pelvis, and their efferent vessels pass to the median lumbar glands.

'Gangliated Cord of the Sympathetic.-This is situated close to the inner side of the anterior sacral foramina. The two cords, right and left, converge as they descend, and in front of the coccyx they are connected by a loop which sometimes presents a single median ganglion, called the ganglion impar or coccygeal ganglion. The number of ganglia on each cord is usually four.

Branches.-(I) Grey rami communicantes, which spring from the ganglia and pass to the anterior primary divisions of the sacral and coccygeal nerves. These rami are very short. (2) Visceral branches, which pass from the upper one or two ganglia to the pelvic plexus. (3) Parietal branches, which are distributed over the front of the sacrum, and which communicate with those of the opposite side, so as to form a plexus upon the middle sacral artery. From the terminal loop and ganglion impar (when present) branches proceed to the front of the coccyx and coccygeal body. The ganglia of the sacral chain do not receive any white rami communicantes from the sacral nerves, these, under the name of the pelvic splanchnics, going chiefly to the pelvic plexus.

Levator Ani-Origin.-(I) The posterior surface of the body of the os pubis in its lower part, below the attachment of the pubo- 
prostatic or anterior true ligaments of the bladder, and above the attachment of the parietal pelvic fascia; (2) the internal surface of the parietal pelvic fascia along the white line ; and (3) the pelvic surface of the spine of the ischium in its lower part, below the coccygeus.

Insertion.-(I) The side of the lower part of the coccyx; (2) the post-anal or ano-coccygeal raphé, where it meets its fellow of the opposite side ; (3) the wall of the anal canal, where the fibres blend with the longitudinal fibres of the gut, and ultimately pass with them between the external and internal sphincter muscles to be attached to the anal integument; and (4) the pre-anal raphé, and the contral tendinous point of the perineum, where the muscle again meets its fellow of the opposite side.

The levator ani is a broad, flat, and thin fleshy muscle, the direction of which is downwards, inwards, and backwards, and with its fellow of the opposite side it forms an incomplete muscular floor to the pelvic cavity. It is covered on its pelvic aspect by the visceral portion of the pelvic fascia, and on its perineal aspect by the anal fascia, and in this manner a separation is formed between one half of the pelvic cavity and the ischio-rectal fossa of the same side.

The anterior border is free close to the body of the os pubis, but, after it passes downwards and backwards upon the side of the prostate gland in the male, or vagina in the female, it meets its fellow of the opposite side, between the prostate gland and the commencement of the membranous part of the urethra in the male on the one hand, and the rectum on the other, or between the vagina in the female and the rectum, the meeting taking place in the pre-anal raphé. Between the anterior borders of the two muscles the membranous part of the urethra in the male, and the vagina in the female, emerge from the pelvic cavity. The anterior or pubo-prostatic fibres are sometimes spoken of as the levator or compressor prostatæ. The posterior border adjoins the coccygeus muscle.

Nerve-supply.-(I) The anterior primary division of the fourth sacral nerve, and (2) the perineal branch of the pudic nerve (deep division).

Action.-(I) To elevate and support the floor of the pelvis, thus diminishing the vertical measurement of the abdominal cavity; (2) to compress the anal canal, and so assist in expelling its contents ; (3) to elevate the anus; (4) to elevate and compress the prostate gland in the male, and the vagina in the female; and (5) to flex the coccyx.

Coceygeus (ischio-coccygeus)-Origin.-(I) The pelvic surface of the spine of the ischium, above the origin of the posterior fibres of the levator ani, and (2) slightly from the inner surface of the parietal pelvic fascia above the ischial spine.

Insertion.-The side of the upper two coccygeal and lower two sacral vertebræ.

Nerve-supply.-The anterior primary divisions of the fourth and fifth sacral nerves. 
Action.-To flex the coccyx.

The coccygeus is a thin, flat, triangular muscle, the fleshy fibres having a large admixture of tendinous fibres. The internal or pelvic surface is related to the visceral pelvic fascia, sacro-coccygeal plexus and rectum, and the external surface to the small sacro-sciatic ligament. The superior border is adjacent to the pyriformis, with the intervention of the structures which leave the pelvis below that muscle. The inferior border adjoins the posterior border of the levator ani.

The coccygeus muscle is to be regarded as a detached portion of the levator ani.

The levatores ani and coccygei muscles form the pelvic diaphragm, which presents a superior concave, and an inferior convex, surface. The greater part of this diaphragm is formed by the levatores ani, and this part of it gives passage in the middle line to the rectum.

Pyriformis-Origin.--(I) By three fleshy slips from the anterior surfaces of the second, third, and fourth sacral vertebræ, which are interposed between, and lie external to, the adjacent anterior sacral foramina; (2) the deep surface of the great sacro-sciatic ligament; and (3) the posterior border of the ilium immediately below the posterior inferior spine.

Insertion.-The upper border of the great trochanter of the femur near its centre.

Nerve-supply.--Two branches from the sacral plexus, more particularly from the dorsal divisions of the first and second sacral nerves.

Action.-External rotator of the thigh.

The intrapelvic portion of the muscle is covered by a prolongation of the parietal pelvic fascia, called the fascia of the pyriformis, and it supports the nerves of the sacral plexus, branches of the anterior division of the internal iliac artery, and pelvic colon. For the extrapelvic portion, see Gluteal Region.

Obturator Internus-Origin.-(I) The internal surface of the obturator membrane; (2) the posterior surface of the body and descending ramus of the os pubis, and ramus of the ischium ; $(3)$ the inclined plane of the ischium, extending as far back as the great sacro-sciatic foramen, and nearly as high as the iliac portion of the ilio-pectineal line; and (4) the parietal pelvic fascia covering the muste.

Insertion.-The inner surface of the great trochanter above and in front of the digital fossa.

Nerve-supply.-The nerve to the obturator internus, from the sacral plexus.

Action.-External rotator of the thigh.

Relations of Intrapelvic Part-Internal.-As low as the rehite line the internal surface of the muscle is covered by the parietal pelvic fascia and peritoneum with the subperitoneal areolar tissue, and it is directed towards the pelvic cavity. The obturator vessels and nerve here lie between the subperitoneal arcolar tissue and the parietal pelvic fascia. Below the level of the white line the internal 
surface is still covered by the parietal pelvic fascia, which constructs Alcock's canal. This portion of the muscle lies upon the outer wall of the ischio-rectal fossa. External.-The internal surface of the obturator membrane, and the surrounding bone. For the extrapelvic part of the muscle, see p. 429 .

\section{STRUCTURE OF THE VISCERA OF THE MALE PELVIS.}

\section{The Bladder.}

The wall of the bladder is composed of four coats-serous, muscular, submucous, and mucous.

The serous coat is formed by the peritoneum, and is confined to the superior surface and upper part of the base. It forms the false ligaments of the viscus.

The muscular coat consists of plain muscular tissue, which is arranged in three layers - external longitudinal, middle circular, and internal longitudinal.

The external longitudinal fibres are most apparent on the superior and inferior surfaces. Laterally they are scarce, and are disposed in an interlacing manner. They have an independent bony attachment to the posterior aspects of the bodies of the pubic bones near the lower part of the symphysis, where they constitute the so-called pubo-vesical muscles. They then pass witbin the pubo-prostatic ligaments to the neck of the bladder, where they are connected with the base of the prostate gland. Having covered the inferior surface as far forwards as the apex, some of them are there prolonged along the urachus for a short distance, whilst others turn to the superior surface ove- which they pass to the base. Having descended upon the base, they enter the prostate gland, where they blend with its muscular tissue. The longitudinal stratum has been called the detrusor urinæ muscle from its supposed function in expelling the urine from the bladder.

The middle circular fibres are somewhat indistinct, and more or less reticular over the greater part of the viscus. In the region where the fundus and inferior surface meet they become more distinct, ard around the neck of the bladder, where the urethral opening is situated, they form 2 tolerably well-marked annular bundle, known as the sphincter vesicæ, beyond which they are continuous with the muscular tissue of the prostate gland.

The internal longitudinal fibres are somewhat indefinite, and are chiefly recognisable on the inferior surface.

When portions of the mucous membrane project between the scattered muscular bundles, the bladder is said to be sacculated. When, on the other hand, the muscular bundles become hypertrophied from any cause, such as enlarged prostate or stricture, they give rise to inward projections of the mucous coat, which are 
arranged in a coarsely reticular manner, a condition known as the fasciculated bladder.

The submucous coat is situated between the muscular and mucous coats, which it connects in a loose manner for the most part, and it is composed of areolar tissue with an admixture of elastic tissue. It serves as a bed in which the arteries and nerves subdivide before entering the mucous coat.

The mucous coat is soft in consistence, and of a pinkish colour in health." It is continuous with the mucous membrane of the ureters and urethra, and over the greater part of the empty bladder is thrown into folds, which, however, disappear as the viscus becomes distended. This rugose condition is explained by the loose connection which exists between the mucous and muscular coats through means of the submucous coat. Over the internal trigone, however, to be presently described, the mucous membrane is quite smooth, and also very sensitive. The mucous coat is covered by stratified transitional epithelium, similar to that of the ureters. In the most superficial layer the cells are cubical, and they present depressions on their deep surfaces, which receive the round ends of the pyriform cells of the layer beneath. In the second layer the cells are pyriform, the round ends being capped by the cubical cells of the first layer, and the narrow ends being deeply placed amongst the deeper cells. In the third and fourth layers the cells are round or oval.

Orifices of the Bladder.-These are three in number, namely, urethral, and two ureteric. The urethral orifice is the opening by which the urine leaves the bladder, and it is situated at the posterior extremity of the inferior surface, where it meets the base. This is the most dependent part of the viscus, and corresponds with the neck, which is surrounded by the base of the prostate gland. Immediately above the urethral orifice the mucous membrane presents a short median vertical ridge, which projects forwards over the orifice. This ridge is called the uvula vesica. It is produced by the middle lobe of the prostate gland, and is much more conspicuous when that lobe is enlarged. The ureteric openings assume the form of small slit-like, somewhat elliptical, apertures, which are about $I_{4}^{1}$ inches apart, and an equal distance from the urethral orifice, their direction being obliquely downwards and inwards. The ureters, before so.terminating, have pierced the vesical wall obliquely: lying in it for about inch, and in this manner reflux of urine is prevented.

Internal Trigone of the Bladder.--This is situated at the lower part of the basal surface, and the mucous membrane over it is so closely connected to the muscular coat that it is always smooth, and so presents a marked contrast to the rugose condition of the mucous membrane over the rest of the empty bladder. The trigone assumes the form of an equilateral triangle, the angles of which correspond with the urethral and ureteric openings, whilst the sides áre constructed by ridges connecting these openings. The inter-ureteric ridge, called Mercier's bar, is produced by a 
bundle of muscular fibres, and is slightly convex, with the convexity directed downwards towards the urethral orifice. The urethro-ureteric ridge on either side is also produced by a bundle of muscular fibres, these bundles being known as the muscles of Bell. The uvula vesicæ is situated at the lower part of the trigone in the middle line, above the urethral orifice.

Lumen of the Empty Bladder.-This is very small, and is composed of two limbs, anterior and posterior. The anterior limb, which is long, lies almost horizontally in front of the urethral orifice, and is formed by the juxtaposition of the superior and inferior walls. The posterior limb, which is short, is directed upwards and backwards, and is formed by the juxtaposition of the back part of the superior wall and the base. The anterior and posterior limbs join each other at the urethral orifice, and are there continued onwards into the lumen of the prostatic urethra. A triradiate appearance is thus imparted to the lumen of the empty bladder and of the prostatic urethra, the three rays diverging from a central point, namely, the urethral orifice, one ray being urethral, and two vesical, of which latter one is the anterior limb of the vesical lumen, and the other the posterior limb.

The Infantile Bladder.-The bladder in early life is pyriform. The narrow end is directed downwards, and is on a level with the upper border of the symphysis pubis, from which point it gradually descends as age advances. The broad end is directed upwards, and lies in the hypogastric region of the abdomen. The base is absent at this period, and the anterior surface of the viscus, devoid of peritoneum, is in contact with the posterior surface of the anterior wall of the abdomen, which is likewise destitute of peritoneum. There being no base, the bottom of the recto-vesical pouch of peritoneum lies close to the base of the prostate gland.

Ligaments of the Bladder.-These are arranged as false and true ligaments. The false ligaments are formed by the peritoneum, and are five in number, as follows : two posterior, which represent the laterally-disposed lips of the mouth of the recto-vesical pouch ; two lateral, right and left, which represent the reflection of the peritoneum from each lateral border of the bladder to the corresponding lateral wall of the pelvis; and superior, which is the refiection of the peritoneum from the apex of the bladder to. the posterior surface of the anterior abdominal wall along the urachus. The true ligaments are also five in number, as follows: two lateral, right and left, which are formed by the vesical layers of the visceral portion of the pelvic fascia on the sides of the bladder; two anterior or pubo-prostatic, which are the reflections of the visceral pelvic fascia from the back of the bodies of the pubic bones near the lower part of the symphysis to the neck of the bladder, and which pass in their course over the anterior surface of the prostate gland; and superior, which is the urachus. Each anterior true ligament contains some of the external or longitudinal muscular fibres of the bladder. 
Blood-supply-Arteries.-These are as follows : (I) superior vesical, from the pervious portion of the obliterated hypogastric; (2) inferior vesical, from the anterior division of the internal iliac; (3) vesical, from the intrapelvic portion of the obturator; (4) vesical, from the intrapelvic portion of the sciatic; and (5) branches from the uterine and vaginal arteries in the female.

Veins.-These are very copious, and are arranged in two plexuses -superior vesical and prostatico-vesical. The vesical plexus is situated over the fundus and sides of the bladder, and its blood is conveyed into the prostatico-vesical plexus. The prostatico-vesical plexus is composed of two parts, continuous with each other, namely, vesical and prostatic. The vesical portion surrounds the neck of the bladder, and its blood is conveyed by one or more vesical veins into the internal iliac vein.

Lymphatics.-These pass to the external and internal iliac glands.

Nerves.--Each half of the bladder receives sympathetic and spinal fibres from the following sources: (I) the pelvic plexus of the sympathetic, which contains spinal fibres from the third and fourth sacral nerves (sometimes also from the second), these being known as the pelvic splanchnics; and (2) special twigs from the pelvic splanchnics, which can be traced independently to the bladder. It is to be noted that the bladder also receives spinal fibres from the upper two or three lumbar nerves through the intervention of (a) the ganglia of the lumbar sympathetic cord, (b) the aortic plexus, $(c)$ the hypogastric plexus, and $(d)$ the pelvic plexus.

\section{Structure of the Penis.}

Corpora Cavernosa.-Each corpus cavernosum has a strong capsule, called the tunica albuginea. This tunic is composed of fibrous, plain muscular, and elastic tissues. It is disposed in two laminæ-external and internal. The fibres of the external lamina are arranged longitudinally, and are common to both corpora cavernosa. The fibres of the internal lamina run circularly round each corpus cavernosum, and in the middle line those of each side meet and are prolonged inwards as a septum, which is imperfect except near the roots of the penis, being traversed by vertical clefts. This partition is called the septum pectiniforme. From the inner surface of the tunica albuginea strong trabeculæ are given off, which penetrate into the interior of the corpus cavernosum. These trabeculæ, which are fibro-muscular and elastic in character, are arranged in a reticular manner, and enclose the cavernous spaces of the erectile tissue. The artery of the corpus cavernosum lies in the centre, and, in addition to this chief vessel, several other arteries, derived from the dorsal artery of the penis, enter it from the surface. The branches of these arteries run in the trabeculie, and terminate in capillaries, which pour their blood directly into the cavernous or intertrabecular spaces. Some of the small arteries in the trabeculæ are thrown into spiral loovs, which project into the intertra- 
becular spaces. Such vessels are called the helicine (spiral) arteries (Müller). From the intertrabecular spaces the radicle veins proceed, and by them the blood is returned from the organ.

Corpus Spongiosum.-This differs from the corpora cavernosa in being destitute of any bony attachments. Its length is about 6 inches, and it is expanded at either end. It is divided into a bulb, body, and glans. The glans has been already described (see p. 648). The bulb represents about the first $I \frac{1}{2}$ inches of the corpus spongiosum, and at its widest part it measures $\frac{3}{4}$ inch. It

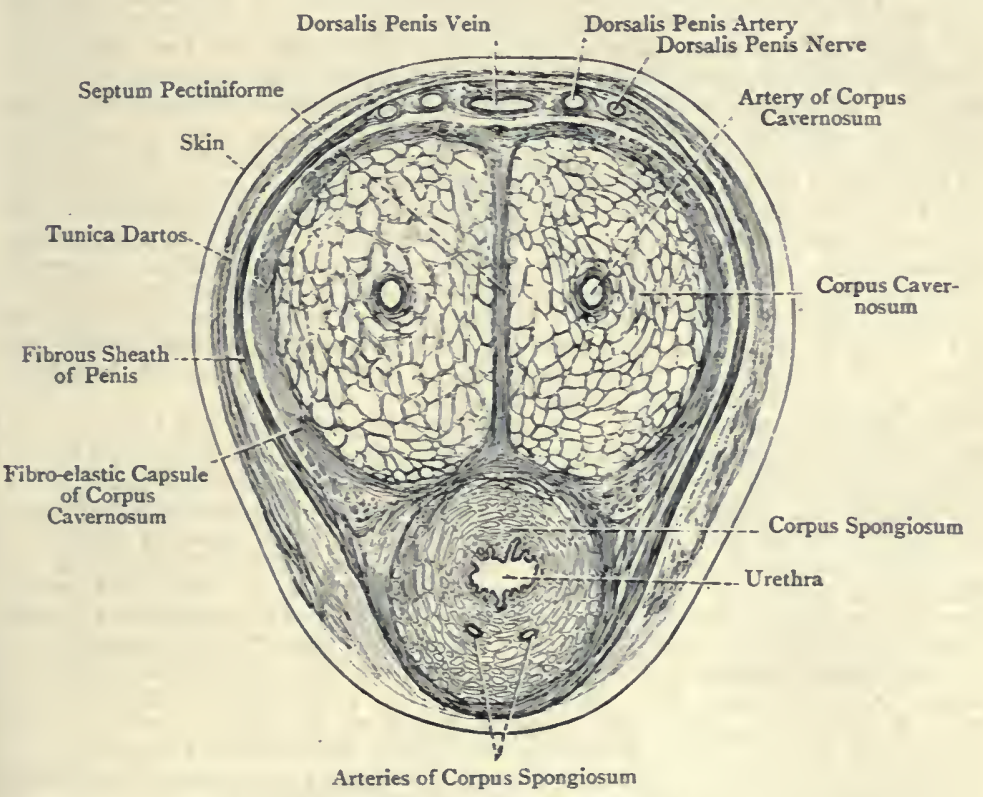

Fig. 370.-Transverse Section of the Penis of a Child as seen UNDER A LOW POWER.

is surrounded by the bulbo-cavernosi muscles, and its enlarged posterior extremity rests upon the front of the antero-inferior layer of the triangular ligament of the urethra, where it is about I inch in front of the anus. The body is cylindrical. It has a capsule or tunica albuginea, but this is very thin. Within the capsule there is the erectile tissue, which resembles that of the corpora cavernosa, though not so well developed, and in the centre is the spongy portion of the urethra. The corpus spongiosum is traversed by the arteries of the corpus spongiosum, right and left, which are branches of the internal pudic arteries. They are situated underneath the urethral canal, one on either side of the middle line.

The venous blood of the corpora cavernosa and corpus spongiosum 
is returned by the dorsal vein of the penis, and by the internal pudic venæ comites.

For the structure of the different parts of the urethra, see Index.

The glans penis and corpora cavernosa are developed from the genital eminence and the corpus spongiosum is developed from the internal genital folds.

Development of the Bladder and Urethra-The Allantois. - The allantois is a hypoblastic diverticulum from the ventral wall of the hind-gut, which, with an investment of mesoblast, extends into the space between the true and false amnion. There are, therefore, two constitucnt parts in the allantois, namely, the hypoblastic diverticulum and its mesoblastic investment. This latter receives the embryonic allantoic or umbilical arteries, and, being very vascular, it carries bloodvessels to the villi of the chorion. The allantois thus establishes a communication between the hind-gut, which is intra-embryonic, and the chorion, which is extra-embryonic, and functionally it is associated with the nutritive and respiratory processes of embryonic life. The extra-embryonic and intra embryonic parts are in continuity with each other through the umbilical spening. The former is of interest on account of its mesoblastic constituent, which, as has been stated, carries blood to the chorionic villi, and gives nse to the fœtal element of the placenta. The intra-embryonic part of the allantois is at first directly continuous with the upper part of the bladder, but in the course of the second month its lumen usually disappears, and then this portion is transtormed into a fibrous cord, which is called the urachus. It 1s, however, to l,e noted that the lumen of the intra-embryonic part of the allantois may remait persistent for some time, thus giving rise to the condition which is known, after birth, as an umbilical urinary fistula.

The cloaca, which is the common blind chamber of the hind-gut and uro-genital ducts, becomes divided into two compartments-dorsal and ventral -by the cloacal or uro-rectal septum. The dorsal compartment gives rise to the rectum and upper two-thirds of the anal canal, or portion above the anal valves, whilst the ventral compartmenl constitutes the uro-genital sinus.

The proximal end of the allantois is connected with the ceplialic end of the uro-genital sinus, and the two Wolffian ducts, together witl the two Müllerian ducts (now fused), open into it. Further, the ureter of either side is a diverticulum of the corresponding Wolffian duct close to the uro-genital sinus.

The urinary bladder is developed from the cephalic part of the uro-genital sinus. As the vesical dilatation proceeds, the ureters lose their connection with the Wolffian ducts, and are transferred to the dorsal portion of the developing bladder, into which they now open. The terminal portions of the Wolffian ducts, being shifted downwards, give rise to the common ejaculatory ducts, which open into the caudal part of the uro-genital sinus.

Prostatic Urethra.-The upper portion of the prostatic urethra, as low as the sinus pocularis, is, like the bladder, developed from the cephalic part of the uro-genital sinus, and in the female the upper part of the urethra is formed from it. The lower portion of the prostatic urethra is developed from the caudal part of the uro-genital sinus, as is also the lower part of the female urethra.

Membranous Urethra.- The membranous portion of the urethra is developed from the caudal part of the uro-genital sinus.

Development of the Spongy Portion of the Urethra.-The cloaca, which is the common opening of the hind-gut and uro-genital ducts, is bounded behind and below by a membrane called the cloacal membrane. This membrane in very early embryonic life shuts off the cavity of the hind-gut from the exterior. It is composed of two layers, internal or hypoblastic belonging to the hind-gut, and extcrnal or epiblastic, which is an invagination of the epiblast. The cloacal membrane forms the floor of a fossa, called the cloacal lossa. In the course of the sccond month the cloaca itself becomes partitioned off into two compartments by a septum formed of two lateral transverse portions, which fuse in the middle line. The dorsal division is called the rectal compartment, and the ventral is termed the uro-genital compartment. Shortly after- 
wards a transverse fold appears, which crosses the cloacal fossa in such a position as to coincide with the posterior end of the internal partition. This transverse fold is called the perineal fold. In the female it gives rise to the perineum proper, and in the male it forms the tegmental parts of that portion of the perineum which lies between the scrotum and the anus. The part of the cloacal fossa behind the perineal fold forms the anal depression or proctodeum, and the portion of the cloacal membrane which forms it is called the anal membrane. When the anal membrane disappears (about the third month) the anus becomes formed, and the rectal compartment of the cloaca now opens into the back part of the cloacal fossa. The uro-genital sinus, which is the posterior portion of the uro-genital compartment of the cloaca, opens into the cloacal fossa in front of the perineal fold by means of a narrow vertical cleft, called the uro-genital cleft. Shortly before the completion of the division of the cloaca into its two compartments (namely, about the fifth or sixth week), a prominence makes its appearance at the anterior or cephalic part of the cloacal membrane. This prominence receives the name of the genital or sexual eminenee, and its importance consists in the fact that the penis and clitoris are developed from it. Leading backwards from the genital eminence there is a furrow, which ends at the uro-genital cleft. The genital eminence is surrounded at the front and sides by an epiblastic fold, called the genital fold, which, as it extends backwards, encloses between its two epiblastic layers the cloacal fossa and the cloacal membrane. The posterior surface of the genital eminence presents a groove, which is continuous with the uro-genital furrow. The lips of this groove, by their subsequent meeting and fusion, form a canal, which represents the part of the spongy urethra contained in the glans penis. Posterior to this the lips of the uro-genital furrow come together and unite as far back as the uro-genital cleft, and thus convert the front part of the cloacal fossa into a canal, which represents the spongy part of the urethra behind the glans. The spongy part is thus to be regarded as a forward extension of the urogenital sinus, which opens at the uro-genital cleft. When the lips of the uro-genital furrow fail to meet and unite at any part, the condition known as hypospadias is produced, in which the spongy urethra opens externally on the under surface of the body of the penis. It is to be noted that, whilst the prostatic and membranous portions of the urethra are developed from the ventral or uro-genital compartment of the hypoblastic cloaca, the spongy portion is developed from the anterior part of the epiblastic cloacal fossa, the posterior part of that fossa giving rise to the anus and anal canal.

\section{Structure of the Vesiculæ Seminales.}

Each vesicula seminalis consists of a tube which is thrown into a number of coils, these being held together by fibrous tissue. When these coils are undone, and the tube straightened out, it measures from 5 to 6 inches in length. Its upper end is closed, and along its course several diverticula are met with. Each seminal vesicle in its natural sacculated condition is surrounded by a sheath, which is derived from the recto-vesical lamina of the visceral portion of the pelvic fascia. Within this sheath the tube has a fibrous wall composed of delicate fibrous tissue, and within this there is a muscular coat, which is formed of plain muscular tissue arranged in two layers-outer longitudinal and inner circular. Within the muscular coat is the mucous coat, which presenics a number of reticular ridges with intervening alveoli. It thus bears a resemblance to honeycomb, in which respect it corresponds with the mucous membrane of the ampulla of the vas deferens and of the gall-bladder, the last-named having coarser meshes. The 
epithelium which covers the mucous membrane is of the columnar non-ciliated variety.

Blood-supply-Arteries.-These are derived from the inferior vesical, the middle hemorrhoidal, the descending branch of the deferential artery, and the intrapelvic portion of the sciatic.

The veins are fairly large and numerous, and are disposed in a plexiform manner. They communicate with the prostatico-vesical plexus.

Lymphatics.-These go to the internal iliac glands.

Nerves.-These are derived from the pelvic plexus.

Development.-Each vesicula seminalis is developed as a diverticulum from the posterior or caudal part of the Wolffian duct, from which the vas deferens originates.

\section{Structure of the Common Ejaculatory Ducts.}

The wall of each duct is composed of three layers, as follows : an outer fibrous layer, which is very delicate; a middle muscular layer, composed of an outer circular and inner longitudinal stratum ; and an inner mucous layer, lined by columnar non-ciliated epithelium:

Development.-Each duct is developed from the posterior or caudal part of the Wolffian duct.

\section{Structure of the Prostate Gland.}

The prostate gland is encased in a strong capsule, which is formed principally by the visceral portion of the pelvic fascia, and partly by the parietal pelvic fascia, in the following manner : the anterior wall of the capsule is formed by the prostatic lamina, and the posterior wall by the recto-prostatic lamina, of the visceral portion of the pelvic fascia; and on either side the capsule is joined by the corresponding half of the deep or postero-superior layer of the triangular ligament of the urethra (which is formed by the parietal pelvic fascia), after it has passed backwards over the anterior border of the corresponding levator ani muscle. The capsule has a material influence in fixing the prostate gland in its position. Within this capsule there is the prostatic plexus of veins, and within this plexus there is another special capsule, composed of fibrous tissue, which immediately surrounds the glandular substance. The prostatic venous plexus is thus enclosed between the two capsules. The substance of the gland is composed of two elements-muscular and glandular. The muscular tissue, which is of the plain variety, is arranged as (I) an external, partly longitudinal and partly transverse, layer, which lies beneath the special fibrous capsule, and (2) an internal circular layer, which surrounds the prostatic urethra, being continuous above with the fibres of the sphincter vesicæ, and below with those around the membranous portion of the urethra. Between these two layers the muscular fibres pervade the gland in a decussating manner, so as to con. struct a muscular reticulum, the meshes of which contain the 
glandular tissue. The chief part of the gland in front of the urethra is composed of muscular tissue. The glandular element consists of branched tubular alvecli or acini, the walls of which are formed by a basement membrane, covered internally by columnar epithelium. The tubular alveoli lead into the excretory or prostatic ducts, the structure of which is similar to that of the gland-tubes. The ducts average twenty in number, ten on either side, and they open by independent orifices upon the posterior wall of the prostatic portion of the urethra, for the most part into the prostatic sinus on either side of the crest.

Blood-supply-Arteries.-These are derived from the inferior vesical, the middle hemorrhoidal, and the intrapelvic portion of the sciatic.

The veins form a copious prostatic plexus, which is most plentiful over the anterior and lateral surfaces. It receives in front the dorsal vein of the penis in two divisions, and discharges its blood into the plexus which surrounds the neck of the bladder, and thence by one or more vesical veins into the internal iliac vein. The prostatic plexus is liable to become much enlarged in old age.

Lymphatics. - These pass to the external iliac, internal iliac, sacral, and common iliac glands.

Nerves.-These are derived from the pelvic sympathetic plexus.

Development. - The glandular part of the prostate is developed from the epithelium of the uro-genital sinus. The epithelial cells in the course of the third month send out ramifying branches, which are at first solid, but subsequently become tubular, and so form the glandular part.

\section{Structure of the Rectum.}

The wall of the rectum is composed of five coats-serous, fascial, muscular, submucous, and mucous.

The serous coat is formed by the peritoneum, and, as has been stated, is imperfect.

The fascial coat represents a sheath which is derived from the visceral pelvic fascia. In front of the rectum it is composed of the recto-vesical and recto-prostatic laminæ, whilst posteriorly it is formed by the rectal lamina of that fascia. It is best marked over the lower third of the rectum, where the peritoneum is absent. Elsewhere it is comparatively thin, and over the peritoneal area it merges into the subperitoneal areolar tissue.

The muscular coat is well developed, and is composed of plain muscular tissue, arranged as an external longitudinal and internal circular layer. The longitudinal layer is present all round the gut, but attains its greatest development along the anterior and posterior walls, where it forms two broad stout laminæ. Along the sides it is somewhat sparse. The circular fibres form a continuous covering for the rectum, and inferiorly they become increased in amount around the anal canal, and so give rise to the sphincter ani internus.

The submucous coat is composed of areolar tissue, and connects 
the muscular and mucous coats in a very loose manner, so that the latter is thrown into a number of temporary folds in the empty condition of the gut. The internal hemorrhoidal plexus of veins is embedded in this coat.

The mucous coat is characterized by great thickness and vascularity. In health, therefore, it has a reddish colour, and is capable of free movement upon the muscular coat. The mucous membrane is covered by columnar epithelium, and is provided with crypts of Lieberkühn and lymphoid nodules. It is thrown into a number of folds in the empty state, but the majority of these are temporary, and become effaced when the gut is distended. There are, however, at least three permanent folds, which constitute the rectal valves of Houston. The mucous membrane is also studded over with a number of minute tubular depressions, called rectal pits, which are surrounded at their deep ends by lymphoid tissue (Birmingham).

The rectal valves (valves of Hauston) are horizontal infoldings of the wall of the rectum in certain situations. Each valve is crescentic or semilunar, and consists of (I) the rectal mucous membrane, (2) the submucous areolar tissue, and (3) a variable amount of the circular muscular fibres. The valves are usually three in numberfirst, second, and third-from above downwards, and their exact positions are subject to variation. The first or highest valve is usually situated on the right wall of the rectum at the level of the third sacral vertebra, and therefore immediately below the lower end of the pelvic colon. The second or intermediate valve, which is the most indefinite, projects from the left wall about $\Upsilon^{\frac{1}{2}}$ inches below the first valve. The third or lowest valve, which may be regarded as the principal one, usually projects from the anterior wall of the rectum, but may also encroach upon the right wall. Its distance from the anus is about 3 inches, and it is on a level with the bottom of the recto-vesical pouch of the peritoneum.- It is therefore related to the fundus of the bladder. This valve is sometimes referred to as the plica transversalis recti, and it is occasionally annular. The rectal muscular fibres (circular), which it contains, constitute the so-called sphincter ani tertius, or sphincter of Nélaton.

The rectal valves are probably sustentacular in function, serving to support the rectal contents. They may give rise to obstruction during the introduction of instruments.

\section{Structure of the Anal Canal.}

The wall of the anal canal is composed of three coats-muscular, submucous, and mucous. The muscular coat is composed of plain muscular tissue, arranged as an external longitudinal and internal circular layer. The longitudinal fibres are continuous with those of the rectum, and, blending with them, there are fibres of the levator ani. Inferiorly they pass between the internal and external sphincters, to be attached to the anal integument. Associated 
with the plain longitudinal fibres on the posterior wall of the anal canal there are two minute muscles (sometimes united into one), called the recto-coccygei muscles. These spring from the front of the coccyx, and descend upon the posterior wall, where they blend with the longitudinal fibres.

There is nothing specially noteworthy in the submucous coat.

The mucous coat presents the columns of Morgagni, and the anal valves of Morgagni. The columns of Morgagni are six or more in number, and take the form of permanent vertical folds, which are confined to the upper two-thirds of the anal canal, where they are separated from each other by longitudinal grooves. They are composed of inflections of the mucous membrane, which contain plain muscular tissue belonging to the muscularis mucosæ, and two small bloodvessels. They stop short about $\frac{1}{2}$ inch above the anus. The anal valves of Morgagni are situated at the lower ends of the columns of Morgagni, about $\frac{1}{2}$ inch above the anus. They lie in the lower ends of the longitudinal grooves, where they extend between adjacent columns, and they bear a resemblance to the valves which are met with in veins. They are semilunar folds of the mucous membrane, their free margins being directed upwards, and behind each valve there is a small recess or "sinus." The mucous membrane of the anal canal is replaced in the lower third (below the anal valves) by modified skin, continuous with that of the exterior. In the upper two-thirds of the canal the mucous membrane is lined by columnar epithelium, and in the upper third it is provided with a few Lieberkühn's crypts and lymphoid nodules. The cutaneous wall of the lower third of the anal canal is covered by modified epidermic epithelium, which is continuous at the anal margin with the epidermis. It is at the margins of the anal valves where this modified epidermic epithelium gives place to the columnar epithelium of the mucous membrane, and here also the modified skin of the lower third of the canal becomes continuous with the mucous membrane of the upper two-thirds. The point of transition is indicated by a faint line, which is known as the white line of Hilton.

Sphincter Ani Internus.- This so-called muscle is merely a thickening of the circular plain muscular fibres of the gut. It is confined to the region of the anal canal, and, viewing it as an independent muscle, or rather thickening of muscular tissue, it begins very near the upper end of the anal canal, and terminates about $\frac{1}{2}$ inch above the anus. Though auxiliary to the external sphincter, the principal action of the internal sphincter is to expel the contents of the anal canal.

Blood-supply of the Rectum and Anal Canal-Arteries. - The rectum and anal canal receive their arterial supply from (I) the superior hemorrhoidal, which is a single vessel, (2) the middle hemorrhoidals, right and left; $(3)$ the inferior hemorrhoidals, right and left; and (4) branches from the middle sacral and sciatic arteries.

The superior hemorrhoidal artery is of large size, and is the direct continuation of the inferior mesenteric. Having crossed the left common iliac 
vessels, it descends within the root of the pelvic meso-colon as low as the third sacral vertebra, where the rectum commences. Here it divides into two branches, right and left, which descend upon the sides of the rectum. From each of these divisions six or more branches are given off, which enter the rectal wall near its centre. Having pierced the muscular coat, they enter the submucous coat, and there subdivide into small terminal branches, which descend in the submucous coat as low as the anal valves, some of them lying within the columns of Morgagni. As these terminal branches descend they give off twigs, which, by anastomosing with branches of the middle and inferior hemorrhoidals, give rise to an arterial anastomotic network in the submucous coat. The disposition of the two primary divisions of the superior hemorrhoidal artery on the sides of the rectum is to be borne in mind in the performance of Whitehead's operation for the removal of hemorrhoids.

The middle hemorrhoidal arteries are two in number, right and left, and are branches of the anterior divisions of the internal iliacs, often arising in common with the inferior vesicals. Having reached the sides of the rectum about its centre, they divide into branches, some of which supply the muscular wall, whilst others enter the submucous coat to take part, along with the superior and inferior hemorrhoidals, in the anastomotic network.

The inferior hemorrhoidal arteries are two in number, right and left, and each may be a single artery, or there may be two or three on each side. In any case, they are branches of the internal pudic, immediately after that vessel has taken up its position in Alcock's canal on the outer wall of the ischio-rectal fossa. Having pierced the wall of Alcock's canal, and traversed the fat of the ischio-rectal fossa, they approach the wall of the anal canal, where they break up into branches, some of which supply the muscular wall, including the external sphincter and adjacent fibres of the levator ani, whilst others pass into the submucous coat, where they take part, along with the superior and middle hemorrhoidals, in the anastomotic network already referred to. The middle and inferior hemorrhoidal arteries of one side anastomose with their fellows of the opposite side.

The rectum also receives twigs from the middle sacral and sciatic arteries.

Veins.-The veins, all of which are destitute of valves, form two rich plexuses, internal hemorrhoidal, situated in the submucous coat, and external hemorrhoidal, lying along the exterior of the rectum, both being confined to its lower third. The internal hemorrhoidal plexus, situated in the submucous coat, receives its radicles from a set of vessels, termed anal veins, which commence beneath the anal integument. These anal veins ascend in the wall of the anal canal, some of them lying within the columns of Morgagni, and they are reinforced by other veins from the anal wall. Having given rise by their communications to the internal hemorrhoidal plexus over the lower third of the rectum, the blood is conveyed away from the plexus in two channels as follows: (I) the veins from tlie upper part of the plexus pierce the wall of the rectum, and open into the external hemorrhoidal plexus; and (2) the veins from the lower part of the plexus pass through the external sphincter to end in a plexus on the outer surface of that muscle, in which the inferior hemorrhoidal veins originate. The external hemorrhoidal plexus is situated on the outer wall of the rectum over its lower third, and is continuous below with the plexus on the outer surface of the external sphincter. It is from this external hemorrl niclal plexus that the hemorrhoidal veins, which correspond with the hemoirhoidal arteries, pass off. The superior hemorrhoidal vein leaves the plexus in two divisions right and left, which ultimately join to form one vessel, the destination of which is the inferior mesenteric vein. It is, therefore, an indirect tributary of the vena portx. The middle hemorrhoidal veins, right and left, terminate in the internal iliac veins. The inferior hemorrholdal velns, right and left, pass to the internal pudic veins. Through means of the external hemorrhoidal plexus a free communication is established between the systemic and portal venous channels, and, there being no valves, when the portal circulation is obstructed the condition known as hemorrhoids frequently results. 
Lymphatic Vessels of Rectum, Anal Canal, and Anus-(I) Rectum. -The lymphatics of the upper part of the rectum pass partly with the superior hemorrhoidal artery to the inferior mesenteric group of the pre-aortic glands, and partly to the rectal glands. The lymphatics of the lower part of the rectum pass with the middle hemorrhoidal artery to the internal iliac glands.

(2) Anal Canal.-The lymphatic vessels of the upper part of the anal canal, above the anal valves, pass to the internal iliac glands. Those of the lower part of the anal canal, below the anal valves, pass to the pubic, and, it may be, the superficial femoral, or saphenous glands.

(3) Anus.-The lymphatic vessels of the integument of the anus pass to the pubic, and, it may be, the superficial femoral or saphenous glands.

Lymphatic Glands of Rectum.- The rectal glands, which are small and few in number, lie behind the rectum. They receive as afferents some of the lymphatic vessels of the upper part of the rectum, and their eferent vessels pass to the lateral sacral glands.

Nerves.-These are partly sympathetic and partly spinal. The superior hemorrhoidal artery conducts to the rectum and anal canal the superior hemorrhoidal sympathetic plexus, which is an offshoot from the inferior mesenteric plexus, that in turn coming from the aortic plexus. The middle hemorrhoidal arteries conduct the middle hemorrhoidal sympathetic plexuses, which are offshoots from the pelvic plexuses. The spinal fibres are derived from the third and fourth sacral nerves (sometimes also the second), and they belong to the pelvic splanchnics. They are further derived from the upper two or three lumbar nerves, all in the manner described in connection with the innervation of the bladder. The fibres from the pelvic splanchnics carry motor impulses to the longitudinal muscular fibres of the rectum, and inhibitory impulses to the circular fibres; whilst the sympathetic fibres are motor as regards the circular fibres, and inhibitory as regards the longitudinal fibres. The anal canal, close to the external sphincter, receives twigs from the inferior hemorrhoidal branch of the pudic nerve.

\section{Development of the Rectum, Anal Canal, and Anus.}

The terminal portion of the hind-gut presents a blind dilatation, lined with entoderm, which is called the eloaca (entodermal cloaca). This chamber is freely connected with (I) the hind-gut, (2) the uro-genital ducts, and (3) the allantoic diverticulum, all of which open into it in early embryonic life. The ventral wall and terminal extremity of the cloaca are formed by a membrane, derived from the caudal part of the primitive streak, called the cloacal membrane. This membrane consists of two layers, external and internal. The external layer consists of ectoderm, and the internal layer of entoderm, in which respects it is homologous with the bucco-pharyngeal membrane at the cephalic end of the fore-gut.

The cloacal membrane separates the cloaca from a superficial depression called the cloacal fossa, or ectodermal cloaca, which is divided into (I) the anal fossa or proctodxum, and (2) the uro-genital fossa, by means of the pre-anal or perineal fold.

The cloaca is persistent in Amphibia, Reptiles, Birds, and Monotremata; 
and, after the rupture of the cloacal membrane in these animals, a common outlet is formed by which intestinal or fæcal matter, urine, and the products of the genital organs escape.

In Man the cloaca becomes partitioned off into two tubular compartments by a fold, called the cloacal or uro-rectal septum. The two compartments or canals are dorsal and ventral respectively. The dorsal canal gives rise to (I) the rectum, and (2) the upper two-thirds of the anal canal, or the part above the anal valves. The ventral canal leads caudalwards from the opening of the allantoic diverticulum, and is called the uro-genital sinus or canal. The allantoic diverticulum and the uro-genital ducts open into it.

The recto-anal and uro-genital canals are directed towards the cloacal membrane, which closes both of them ventrally and caudalwards. The uro-rectal septum is connected inferiorly with the cloacal membrane, and this membrane is divided into two parts-namely, dorsal or anal, known as the anal membrane, and ventral or uro-genital.

The anal and uro-genital parts of the cloacal membrane are separated externally by a transverse fold, known as the pre-anal, ano-gential, or perineal fold. This fold undergoes considerable thickening, and gives rise to the perineal body.

The anal membrane soon becomes invaginated, and a superficial pit is thereby produced, called the anal depression or proctodæum. The depression consists of invaginated ectoderm which meets the intestinal entoderm, and the two layers construct the anal membrane, or anal part of the cloacal membrane. The condition of matters may be compared to the invagination of ectoderm, which forms the primitive oral cavity or stomodæum, and which, meeting with the entoderm of the pharyngeal part of the fore-gut, forms the bucco-pharyngeal membrane.

About the beginning of the fourth month the cloacal membrane (anal and uro-genital) ruptures and disappears. The rupture of the anal part of the cloacal membrane gives rise to an aperture called the anus, through which the hind-gut and proctodæum become continuous. The lower one-third of the anal canal-that is to say, the limited portion below the anal valves-is formed by the ectoderm of the anal fossa or proctodæum.

In some cases the anal membrane-namely, that part of the cloacal membrane which separates the hind-gut from the anal fossa or proctodæum-is persistent. Such a condition is known as atresia ani or imperforate anus.

The uro-genital part of the cloacal membrane becomes depressed, and so gives rise to the uro-genital fossa. When this part of the membrane ruptures, the uro-genital opening or cleft is formed, by which the uro-genital compartment of the cloaca-namely, the uro-genital sinus or canal-communicates with the exterior.

\section{The Female Pelvis.}

The female pelvis contains the pelvic colon and rectum, with a few coils of the small intestine; the bladder and urethra; the uterus and vagina; and the uterine appendages connected with the broad ligaments, namely, the Fallopian tubes, the ovaries, with the parovarium or epoöphoron, and paroöphoron (the latter in early life); and the round ligaments of the uterus. The arteries are the same as in the male, with the addition of portions of the ovarian arteries, and the uterine arteries, and the substitution of the vaginal arteries for the inferior vesical arteries in the male. The venous plexuses are hemorrhoidal, vesical, pudendal, ovarian, uterine, and vaginal. The nerve-plexuses peculiar to the female are the uterine, ovarian, and vaginal.

General Position of the Viscera.-The pelvic colon and rectum are situated as in the male pelvis. The bladder lies anteriorly, being situated immediately behind the bodies of the pubic bones, 
and the urethra is very closely related to the anterior wall of the vagina. The virgin uterus lies upon the superior surface of the bladder, and the broad ligaments extend from either side of it to the lateral wall of the pelvis. The vagina passes off from the lower end of the uterus, and in its outward course it lies between the base of the bladder and the rectum.

Peritoneum.-The peritoneum is related to the pelvic colon and rectum as in the male. On leaving the rectum at a point fully 3 inches above the anus it passes to the posterior wall of the vagina, which it covers for about its upper fourth. It then mounts upwards over the posterior surface of the supravaginal portion of the cervix uteri and the body of the uterus. On reaching the fundus it turns round to the anterior surface, which it invests as low as the junction of the body and cervix. On leaving the viscus it passes to the upper part of the base of the bladder, whence it extends forwards over the superior surface, which it covers as far as the apex. Its course thereafter is as in the male. Along each lateral border of the bladder the peritoneum is reflected on to the lateral wall of the pelvis. Along either side of the uterus it is reflected on to the lateral wall of the pelvis, and in this manner the broad ligaments are formed. Between the rectum and the upper portion of the posterior wall of the vagina and supravaginal portion of the cervix uteri the peritoneum forms a recess, called the pouch of Douglas, which corresponds to the recto-vesical pouch in the male. The mouth of this pouch is bounded on either side by a semilunar peritoneal fold, which extends from the front of the sacrum over the side of the rectum to the cervix uteri. These folds are known as the sacro-uterine folds, or recto-uterine folds of Douglas. Each fold contains a collection of fibrous and plain muscular tissues, connected on the one hand with the fibrous structures in front of the lower part of the sacrum, and on the other with the cervix uteri. They become continuous with each other over the back of the isthmus uteri (junction of body and cervix), and there they give rise to a transverse ridge, called the torus uterinus. The pouch of Douglas is bounded in front by the upper part of the posterior wall of the vagina and the supravaginal portion of the cervix uteri, and behind by the rectum. In front of the uterus the peritoneum gives rise to a small recess, called the utero-vesical pouch, the entrance to which is bounded laterally by two folds, called the utero-vesical folds. As regards the bladder, the peritoneum forms for it false ligaments as in the male, namely, one superior, two lateral, and two posterior. The latter are simply the utero-vesical folds, and may be regarded not only as posterior ligaments of the bladder, but also as anterior ligaments of the uterus.

Pelvic Fascia.- The parietal pelvic fascia in the female is similar to the corresponding fascia in the male. The visceral pelvic fascia, however, is complicated by the interpolation of the vagina. On either side of the pelvic viscera it divides into four laminæ, as follows: vesical, which forms, on either side, the lateral true liga- 
ment of the bladder; vesico-vaginal, which passes between the bladder and the vagina; recto-vaginal, which passes between the vagina and the rectum; and rectal, which passes over the sides and posterior wall of the rectum.

Broad Ligaments of the Uterus.-These are also called the alce vespertilionis, from their supposed resemblance to a bat's wings. Each is an extensive fold of peritoneum composed of two layers,

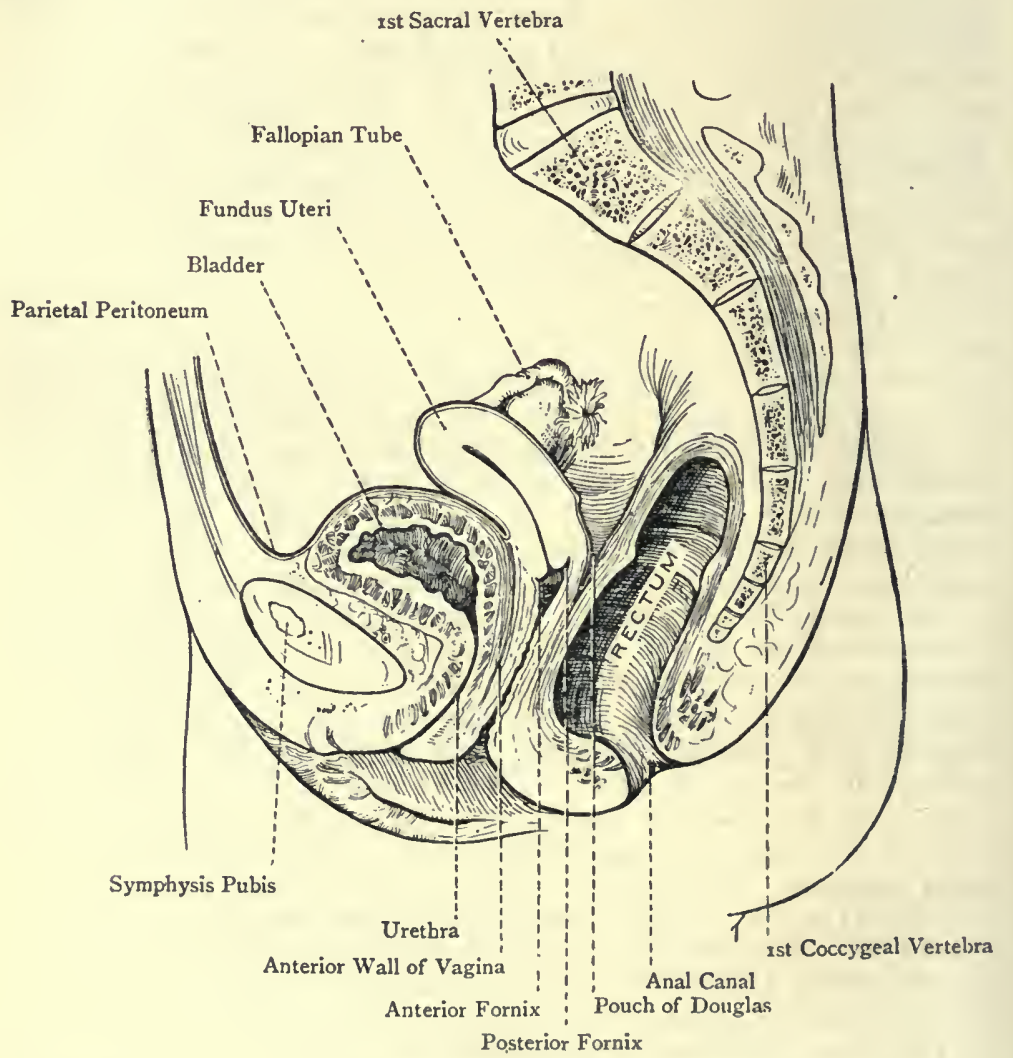

Fig. 37I.-Vertical Sagittal. Section of the Female Pelvis.

anterior and pusterior, which pass between the side of the uterus and the lateral wall of the pelvis. The broad ligament has associated with it the following important structures : (I) the Fallopian tube or oviduct, which lies within the superior border of the ligament; (2) the ovary and its ligament, which lie within a backward extension of the posterior layer of the broad ligament at a lower level than the Fallopian tube; (3) the round ligament of the uterus, 
which lies within a forward projection of the anterior layer of the broad ligament, also at a lower level than the Fallopian tube; (4) the epoöphoron, which lies within the ligament between the ovary and the Fallopian tube ; and ( $\xi$ ) the paroöphoron (in early life), which also lies within the ligament, internal to the epoöphoron and near the uterus. The double fold of peritoneum attaching the anterior border of the ovary over its whole length to the back of the broad ligament forms a short mesentery for it, called the mesovarium, which contains between its two layers the bloodvessels and nerves of the ovary. The portion of the broad ligament which lies between the Fallopian tube and the ovary with its ligament is called the mesosalpinx. It is somewhat falciform, and is narrow internally, but broad externally. It is bounded above by the Fallopian tube, below by the ovary and its ligament, internally by the uterus, and externally by the ovarian fimbria and the suspensory ligament of the ovary.

Round Ligament of the Uterus.-This is a narrow, cord-like band, about 5 inches long, which is attached to the upper part of the side of the uterus in front of, and a little below, the uterine end of the Fallopian tube. It is composed of fibrous connective tissue, which, near the uterus, has an admixture of plain muscular fibres continuous with those of the uterus. It lies within the anterior layer of the broad ligament, where it gives rise to a slight prominence. Its direction is outwards, upwards, and forwards over the obliterated hypogastric artery and pelvic brim to the internal abdominal ring, close to which it hooks round the outer side of the deep epigastric artery, and crosses the external iliac vessels from within outwards. Escaping by the internal abdominal ring, it traverses the inguinal canal, and, emerging through the external abdominal ring, it ends in the subcutaneous tissue of the labium majus. For a short distance after entering the inguinal canal it is covered by a process of the peritoneum, which represents the processus vaginalis in the male fotus. This process is at first tubular, and receives the name of the canal of Nuck. It is usually, however, obliterated in the adult, though it may remain pervious, under which circumstances the condition known as hydrocele of the round ligament may occur. A few muscular fibres may be found in front of the round ligament in the inguinal canal, which are continuous with the lower fibres of the internal oblique, and represent the cremaster in the male.

The round ligament is supplied by two arteries as follows: the ligamentous branch of the ovarian artery, which supplies it as far as the inguinal canal; and the ligamentous branch of the deep epigastric, which supplies it beyond the internal abdominal ring, and corresponds to the cremasteric artery in the male. The principal venous blood is returned by a ligamentous vein, which is a tributary of the ovarian plexus.

The round ligament represents the gubernaculum testis in the male.

Ovaries.-The ovaries are two small flattened bodies, each of 
which lies within a backward extension of the posterior layer of the broad ligament, with which it is connected by the mesovarium. The ovary is laterally compressed, and usually lies, with its long axis vertical, igainst the lateral wall of the pelvis in a peritoneal depression, called the ovarian fossa. It may, however, lie obliquely, and may even be shifted near to the uterus. In size it may be likened to the half of a small walnut, its average length being from $I$ inch to $I_{2}^{\frac{1}{2}}$ inches, its breadth about $\frac{3}{4}$ inch, and its thickness (from side to side) from $\frac{1}{4}$ to $\frac{1}{2}$ inch. Its average
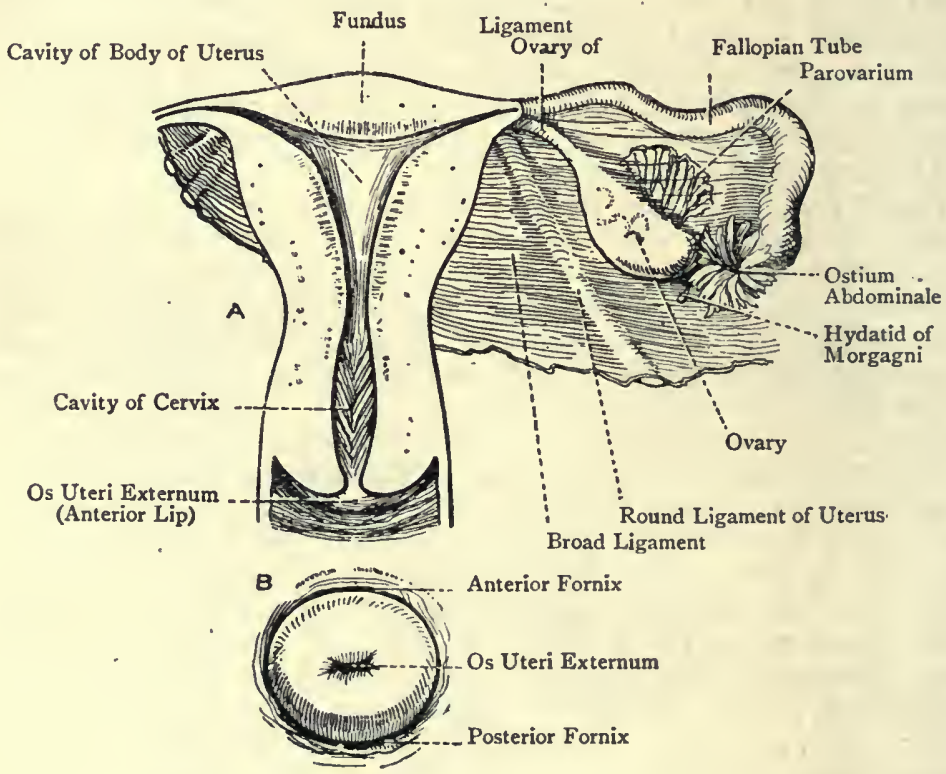

Fig. 372.-The Uterus AND ITS ApPendages.

A, The Uterus opened, and the Right Broad Ligament (Posterior View); B, The Os Uteri Externum.

weight is about 2 drachms. It presents two surfaces, two borders, and two extremities.

The surfaces are laterally disposed, and are called internal or mesial, which is related to the Fallopian tube, and external or. lateral, which is related to the ovarian fossa. The borders are anterior and posterior. The anterior or attached border is straight. Along it are attached the two layers of the mesovarium, and between these it presents a hilum for the passage of the bloodvessels, nerves, and lymphatics. The posterior border is convex and free. The extremities are named poles, superior and inferior respectively. The superior is named the tubal pole, because the 
ovarian fimbria is attached to it. Connected with it there is also a fold of peritoneum, which passed upwards to join the peritoneum over the psoas magnus near the upper part of the external iliac vessels. This fold, which is termed the suspensory ligament of the ovary, is continuous with the outer part of the broad ligament, and contains the ovarian vessels and nerves. The inferior pole is known as the uterine pole, and is connected with the superior angle of the uterus by a round cord, called the ligament of the ovary. The attachment of this ligament to the uterus is behind and a little bel w w the uterine end of the Fallopian tube.

The surface of the ovary is covered by peritoneum, continuous with the mesovarium, the only exception being along the anterior border, where the hilum exists. The connective-tissue element of this peritoneum is inseparably blended with the so-called tunica albuginea of the ovary, and it is covered by an epithelium the cells of which present a striking contrast to those of the endothelial covering elsewhere. It is composed of short columnar cells, and imparts a somewhat dull appearance to the surface, which contrasts with the polished appearance elsewhere. The ovarian epithelium is the remains of the germinal epithelium, from which the ovary is developed, and at the circumference of the organ it passes abruptly into the endothelial cells of the adjacent peritoneum. Prior to puberty the surface of the ovary is smooth, but after that period it gradually assumes a pitted and scarred appearance, which is due to the periodical escape of the ova from the Graafian follicles.

Descent of the Ovary. - The ovary, like the testis, originally lies in the lumbar region of the body-cavity, by the side of the vertebral column. At this period the inguinal ligament, as stated, extends from the caudal end of the ovary to the inguinal region, where it traverses the inguinal canal and terminates within the labium majus. As it descends it becomes connected, as stated, with the Mullerian duct at the level where this duct fuses with its fellow to form the uterus. About the third month of intra-uterine life the ovary begins to descend towards the brim of the pelvis. Having arrived at the pelvic brim, it remains there for some time, and is still at that level at the period of birth. At a later period it descends into the pelvic cavity, as a rule, and assumes its normal position within a backward extension of the broad ligament of the uterus.

Two factors are probably concerned in the descent of the ovary, namely, (I) the shortening of that part of the inguinal ligament which extends from the ovary to the side of the uterus, close to the uterine end of the Fallopian tube; and (2) the traction exercised by the fusion of the two Müllerian ducts to form the uterus and vagina.

As each ovary descends, its mesovarium is taken along with it. This mesovarium is intimately connected with the uro-genital fold, which contains the Wolffian and Müllerian ducts; and the uro-genital fold, in turn, is combined with the Wolffian 'mesentery' or ligament. The vestigial portions of the Wolffian body in the female are therefore carried down along with the descending ovary, these vestigial portions representing (I) the so-called duct of Gärtner, (2) the epoöphoron or parovarium, and (3) the paroopphoron. The combined mesovarium, uro-genital fold, and Wolffian 'mesentery' or ligament, of each side become continuous mesially, and form one continuous sheet. Within this sheet the uterus is formed by the fusion of the two Müllerian ducts, and each lateral part of the sheet constitutes the broad ligament of the uterus. 
Abnormal Positions of the Ovary.-(I) The ovary, in its original descent, may pass into the inguinal canal, and even into the labium majus ; (2) it may pass through the crural ring into the crural canal, and lie over the saphenous opening, where it may simulate a femoral hernia ; (3) an ovary, when enlarged, may become prolapsed, and pass downwards and inwards behind the uterus into the pouch of Douglas, where it may be palpated through the posterior fornix of the vagina.

For the structure and development of the ovary, see p. 892 et seq.

Epoöphoron (Parovarium of Kobelt), or Organ of Rosenmuiller.The epoöphoron is situated in that portion of the mesosalpinx which lies between the ovary and the Fallopian tube. It is composed of a number of small blind tubules, lined with epithelium, which converge towards the ovary, but do not meet. Their tubal ends are united by a longitudinal tube, which 1 s parallel with, and a little below, the Fallopian tube.

Paroöphoron of Waldeyer.-The paroöphoron is situated in that part of the mesosalpinx which lies between the ligament of the ovary and the Fallopian tube, where it is placed near the uterus. It is composed of a few minute blind tubules, which can only be discerned at or shortly after the period of birth.

For the development of the epoöphoron and paroöphoron, see Index.

Fallopian Tubes.-The Fallopian tubes, right and left, serve to convey the ova, after their escape from the Graafian follicles, into the cavity of the uterus. They are, therefore, functionally the ducts of the ovaries, and are hence spoken of as the oviducts. Each tube is contained within the superior border of the broad ligament, except at its extreme inner end, where the tube is embedded in the uterine wall. It is fully 4 inches in length. Proceeding from the uterus, it passes at first horizontally outwards for about I inch towards the lower or uterine pole of the ovary. It then ascends vertically for a short distance upon the lateral wall of the pelvis, where it lies internal to the anterior or attached border of the ovary. Having arrived at the upper or tubal pole, it arches backwards and descends along the posterior convex border, and adjacent portion of the internal surface of the ovary. Each Fallopian tube is divided into the following parts : pars uterina, isthmus, ampulla, neck, and corpus fimbriatum; and each has two openings, ostium uterinum, and ostium abdominale.

The pars uterina is the limited portion which is contained within the uterine wall at the superior angle, and it presents the ostium uterinum, which is about I millimetre in diameter. The isthmus succeeds to the pars uterina, and represents about one-third of the tube. It is straight, round, and firm to the touch, due to the predominance of circular muscular fibres in this portion. Its diameter is about $2 \frac{1}{2}$ millimetres. The ampulla succeeds to the isthmus, 
and forms rather more than half of the tube. It is larger than the isthmus, less resistant (being chiefly mucous in structure), and tortuous. Its diameter gradually increases in the distal direction, the average being about 7 millimetres. The ampulla at its outer end becomes constricted to form the neck, which presents the ostium abdominale, opening into the pelvic cavity, its diameter being about 2 millimetres. It is in this situation where the general cavity of the peritoneum in the female is continuous with the lumen of the Fallopian tube, and through it with the cavity of the uterus and vagina. Beyond the neck the tube expands in the form of a funnel, called the infundibulum or pavilion, in the body of which the ostium abdominale is situated. The circumference of the infundibulum is broken up into a number of irregular fringes, called fimbria, and the outer end of the tube is hence called the corpus fumbriatum. The larger fimbriæ are broken up into smaller filiform processes. The outer surfaces of the fimbriæ, which look into the pelvic cavity, are covered by peritoneum, but the inner surfaces, which look into the infundibulum, are covered by mucous membrane continuous with that of the Fallopian tube. At the free margins of the fimbriæ the peritoneum, with its endothelial cells, becomes continuous with the mucous membrane, which is covered by ciliated columnar epithelium. One of the fimbriæ, which is larger and longer than the others, is called the ovarian fimbria, and is connected with the upper or tubal pole of the ovary usually directly, but sometimes by a delicate fibrous band. This fimbria presents a longitudinal furrow, which serves as a channel of communication between the ostium abdominale and the ovary.

For the structure and development of the Fallopian tubes, see Index.

Uterus.-The uterus is a hollow muscular organ, which receives at its superior angles the Fallopian tubes, and opens below into the upper part of the anterior wall of the vagina. Through the Fallopian tubes it receives the ova at periodical intervals, and, when an ovum becomes impregnated, the uterus retains it during development, and thereafter expels the fœtus through the vagina. The virgin uterus lies upon the superior surface of the bladder, and is usually inclined to the right of the middle line. Above it there are a portion of the pelvic colon and usually a few coils of the ileum. At its lower end is the vagina, and the broad ligament stretches from either side. It is somewhat pyriform, the wide end being directed upwards and forwards, and is flattened from before backwards. It is firm and resistant to the touch, on account of its very thick muscuiar walls. Its average length is 3 inches, the breadth at the upper part being 2 inches, and the thickness $I$ inch. The organ is divided into a fundus, body, and cervix.

The fundus is that portion which lies above the level of a line connecting the superior angles, where the Fallopian tubes pass through the uterine wall. It is convex from side to side, and also 
from before backwards. The lateral borders of the uterus are sloped downwards and inwards, and at the junction of the upper two-thirds and lower third of the organ there is a slight constriction or concavity, called the isthmus, which is also present in front and behind, and is most conspicuous in early life. The part between the fundus and the isthmus is the body, and the part below the isthmus is the cervix.

The body, which is 2 inches long, is triangular, and presents two smooth surfaces, anterior and posterior, and two lateral borders. The anterior or vesical surface, which has an inclination downwards, is slightly convex. The posterior or rectal surface, which has an inclination upwards, is more markedly convex. Each lateral border extends from the superior angle to the isthmus, and is sloped downwards and inwards. The superior angles are situated at the points of entrance of the Fallopian tubes, and correspond with the portions of the uterus which are elongated into cornua in someanimals.

The cervix, which measures I inch in length, is cylindrical, and narrower than the body. It is received into the upper part of the anterior wall of the vagina, the walls of which are attached to it in such a manner as to divide it into two portionssupravaginal and vaginal. The posterior wall of the vagina extends higher upon the cervix behind than the anterior wall does in front. The anterior surface of the supravaginal portion, which is about $\frac{1}{2}$ inch in extent, is related to the base of the bladder, with the intervention of some loose cellular tissue. The posterior surface of the supravaginal portion, which is about $\frac{1}{4}$ inch in extent, forms a part of the anterior wall of the pouch of Douglas, and is crossed transversely by the torus uterinus. At the lower end of the vaginal portion, where the cervix is slightly protuberant, there is an opening, called the os uteri externum, through which the cavity of the cervix communicates with that of the vagina. This opening is also known as the os tince, because it is supposed to resemble the mouth of the tench fish, on account of its lips being of unequal size. In early life this opening is circular, but later, in the virgin, it assumes the form of a transverse slit about $\frac{1}{12}$ inch long. It is bounded by two lips, anterior and posterior, which in the virgin are smooth, but in multiparæ they are often more or less fissured, especially the posterior lip. The anterior lip is thick, round, and short, whilst the posterior is thin, sharp, and long. The anterior lip descends lower into the vagina than the posterior, by reason of the oblique manner in which the cervix uteri passes into the canal. It is the anterior lip which first meets the finger in making vaginal examinations. The greater length of the posterior lip is due to the fact that the posterior wall of the vagina extends higher on the back of the cervix than the anterior wall does in front. The os uteri externum is directed downwards and backwards towards the posterior wall of the vagina, this being due to the oblique position of tle cervix. 
Surrounding the vaginal portion of the cervix there is a vaulted recess, which is divided into anterior, posterior, and lateral fornices.

General Relations of the Uterus. - The anterior surface of the body rests upon the superior surface of the bladder, and the anterior surface of the supravaginal portion of the cervix is related to the base of the bladder. The lateral relations are the Fallopian tubes, round ligaments, ligaments of the ovaries, broad ligaments, and a certain amount of adipose tissue, containing large bloodvessels, which lies upon either side of the cervix, and extends upwards over the lateral border between the layers of the broad ligament. This collection is known as the parametrium. The posterior surface of the body is related to the rectum, and the posterior surface of the supravaginal portion of the cervix forms a part of the anterior boundary of the pouch of Douglas.

Peritoneal Relations.-The following parts of the uterus are covered by peritoneum: the supravaginal portion of the cervix posteriorly, the posterior surface of the body, the fundus, and the anterior surface of the body as low as the front of the cervix. The following parts are free from peritoneal covering: the vaginal portion of. the cervix, the supravaginal portion of the cervix anteriorly, and a narrow strip along each lateral border where the two layers of peritoneum pass off to form the broad ligament. The uterine peritoneal folds are as follows: two utero-vesical, sometimes called the anterior uterine ligaments, but they may also be regarded as the posterior false ligaments of the bladder; the sacro-uterine folds, with the torus uterinus; and the broad ligaments.

Position of the Uterus. - The virgin uterus occupies a position of anteflexion and anteversion, assuming the bladder and rectum to be empty. In speaking of the uterus as being anteflexed it is to be understood that the body of the organ is bent forwards at the isthmus in such a manner that it forms with the cervix an angle, which is open anteriorly. This is brought about in the following manner: the cervix uteri is more fixed than the body, from its connection with the vaginal walls and base of the bladder; and the cervix is less yielding than the body. In speaking of anteversion of the uterus it is to be understood that the entire uterus is inclined forwards, so that its long axis forms an angle with the longitudinal axis of the trunk. The anterior surface has therefore a downward inclination towards the superior surface of the bladder, and the posterior surface has an upward inclination, and so supports a portion of the pelvic colon and a few coils of the ileum. When the bladder is distended, the position. of the uterus becomes altered. The organ is raised along with the distended bladder, the anteflexion and anteversion become less, and the uterus may even assume a vertical position. Any coils of the ileum lying in contact with its posterior surface, as well as the pelvic colon, would be displaced, and the organ would come to be closely related to the rectum. 
Interior of the Uterus.-The interior is divided into two portions - the cavity of the body and the cavity of the cervix. The cavity of the body is very small compared with the thickness of the uterine walls, and is triangular, with the base directed upwards towards the fundus. Its three sides are convex towards the cavity, and its anterior and posterior walls are in contact. In the vicinity of each superior angle it narrows, and gradually tapers to the ostium uterinum of the Fallopian tube, with the lumen of which it is continuous. Inferiorly the cavity also becomes narrow, and, at the junction of the body and cervix, it ends in a circular opening,

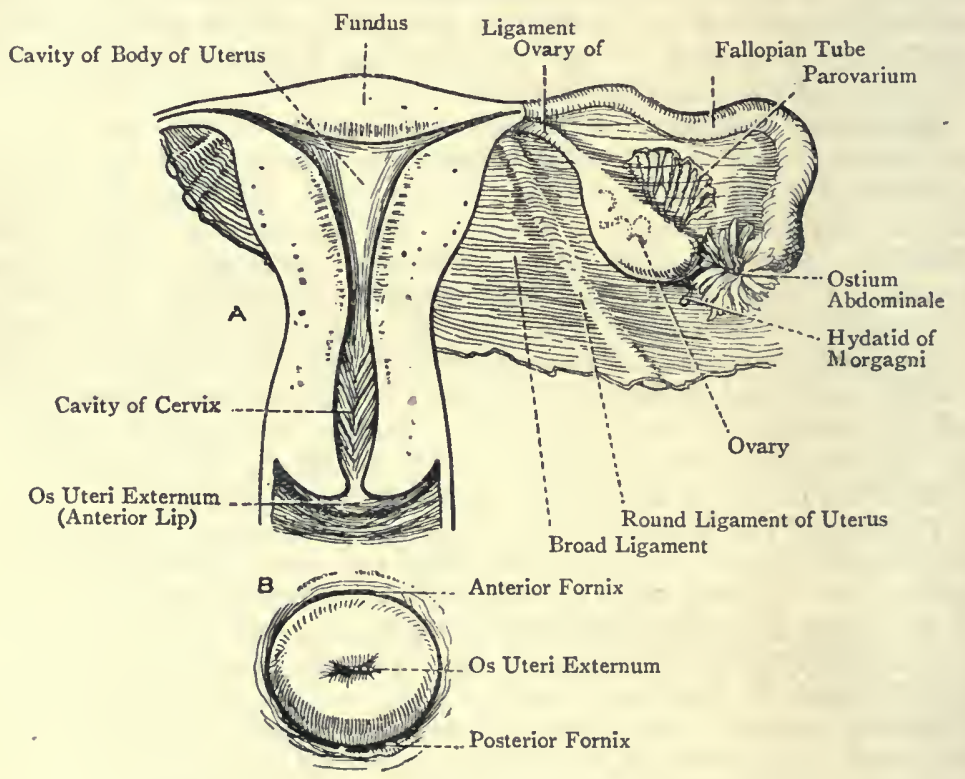

Fig. 373. - The Uterus and its Appendages.

A, The Uterus opened, and the Right Broad Ligament (Posterior View); B, The Os Uteri Externum.

called the os uteri internum, which is smaller than the os externum. Through this opening it becomes continuous with the cavity of the cervix. The cavity of the cervix is spindle-shaped, being wider at the centre than at either end. It is somewliat flattened from before backwards, and is continuous above with the cavity of the body through the os internum, and below with the cavity of the vagina through the os externum. Its mucous membrane presents two longitudinal ridges, anterior and posterior, from each of which a number of rugæ extend in an upward and outward direction, the appearance thus produced being known as the arbor vite uterina, or plica palmata. 
Uterus at Birth.-At birth the neck of the uterus is larger than the body, and there is no fundus. At each superior angle it tapers very much, and resembles somewhat a uterus bicornis. The arbor vitæ uterina extends all along the interior.

Varieties.-These are as follows: (I) uterus bicornis; (2) uterus unicornis ; and $(3)$ double uterus.

For the structure and development of the uterus, see p. $\$_{9} 6$ et seq.

Vagina.-This is a musculo-membranous passage which extends from the cervix uteri to the vulva. It is from 3 to $3 \frac{1}{2}$ inches long, being 3 inches along the anterior wall, and $3 \frac{1}{2}$ inches along the posterior. It is slightly curved, and its direction is downwards and forwards. Its axis forms an obtuse angle with that of the uterus $\left(100^{\circ}\right.$ to $\left.110^{\circ}\right)$. In the erect posture it forms with the horizontal an angle of about $60^{\circ}$. Superiorly it is closely connected with the cervix uteri, the posterior wall rising higher than the anterior. It is rather narrower at either end than at the centre, the lower end being the narrowest part of the passage. The walls are anterior and posterior, and they are in contact. In transverse section the vagina appears as an $H$-shaped fissure at its lower end, as a transverse fissure at the centre, and at its upper end it presents a lumen which is almost circular.

Relations-Anterior.-The base of the bladder, and the urethra. Posterior.-From above downwards there are the pouch of Douglas for a short distance, the rectum, with the intervention of the recto-vaginal lamina of the visceral pelvic fascia, and the anal canal, from which it is separated by the perineal body. The posterior wall is covered by peritoneum over about its upper fourth. Lateral.-The ureter at the upper end for a short distance, and the levatores ani muscles.

The vagina passes through the triangular ligament, and its lower end has a bulbus vestibuli on either side, with the sphincter vaginæ surrounding the external orifice. When the finger is passed into the posterior fornix, which is the recess between the posterior lip of the os uteri externum and the posterior wall of the vagina, the pouch of Douglas can be palpated, and a few coils of the ileum, or a prolapsed ovary, may be felt in it. The base of the bladder may be palpated through the anterior fornix, and the urethra through the anterior wall of the vagina lower down.

For the structure and development of the vagina, see Index.

Bladder.-The base is directed backwards, and is related to the front of the supravaginal portion of the cervix uteri and a portion of the anterior wall of the vagina, the utero-vesical pouch of the peritoneum intervening. The superior surface is in contact with the anterior surface of the body of the uterus. The false ligaments, formed by the peritoneum, are at least three in number, namely, one superior and two lateral. The peritoneal folds on either side 
of the utero-vesical pouch are usually regarded as the anterior uterine ligaments, but they may also be looked upon as the posterior false ligaments of the bladder. The true ligaments are similar in both sexes.

Ureters.-Each ureter lies for a short distance on the side of the cervix uteri and upper part of the wall of the vagina, being crossed near the cervix by the uterine artery.

Urethra.-The urethra is very closely related to the anterior wall of the vagina. Its length is $I_{2}^{\frac{1}{2}}$ inches, and its transverse diameter is about $\frac{1}{4}$ inch, the narrowest part being at the meatus urinarius. The direction of the canal is downwards and forwards, and its walls are anterior and posterior, these being in contact except during micturition. In its course it passes between the two layers of the triangular ligament, where it is embraced by the compressor urethræ muscle. The canal is capable of considerable distension, and may, under anæsthetics, admit the index-finger. The meatus urinarius is situated at the posterior extremity of the vestibule in the middle line, immediately in front of the external orifice of the vagina, and is placed on a slight prominence, the margins of which are somewhat irregular.

For the structure and development of the urethra, see p. 870 .

Rectum-Relations-Anterior.-The uterus and vagina, the recto-vaginal pouch of Douglas intervening for a short distance in the vicinity of the cervix uteri. Posterior. - As in the male.

Anal Canal.- This is separated from the vagina by the perineal body.

Ovarian Artery in the Pelvis. - This vessel, which arises from the abdominal aorta about I inch below the renal artery, enters the pelvis by crossing the commencement of the external iliac. It then passes within the broad ligament of the uterus, where it is very tortuous, and its branches to the ovary enter that organ through the hilum on the anterior border without piercing the peritoneum. Besides supplying the ovary the vessel furnishes the following branches: uterine to the uterus, near the superior angle, where it anastomoses with branches of the uterine artery from the internal iliac; tubal to the Fallopian tube; and ligamentous to the round ligament of the uterus, which accompanies it as far as the inguinal canal.

The ovarian vein of each side originates as two vessels in the ovarian or pampiniform plexus, which lies within the broad ligament. This plexus receives the veins which emerge through the hilum of the ovary, as well as tributaries from the Fallopian tube and round ligament of the uterus, and it communicates freely with the uterine plexus. The two ovarian veins, having emerged from the ovarian plexus, leave the pelvis, and soon join to form a single vein, that of the right side opening into the inferior vena cava, and that of the left side into the left renal vein. 
Uterine Artery. - This vessel is derived from the anterior division of the internal iliac artery. It is directed downwards and inwards to the side of the cervix uteri, near which it crosses the ureter. On reaching the cervix it turns upwards along the lateral border of the body in a very tortuous manner, lying between the two layers of the broad ligament. As it ascends it gives off tortuous branches to the front and back of the body, and near the inner end of the Fallopian tube it anastomoses with the uterine branch of the ovarian artery. Along the side of the body it also gives offsets to the round ligament, ligament of the ovary, and Fallopian tube. At the cervix the uterine artery furnishes two branches-

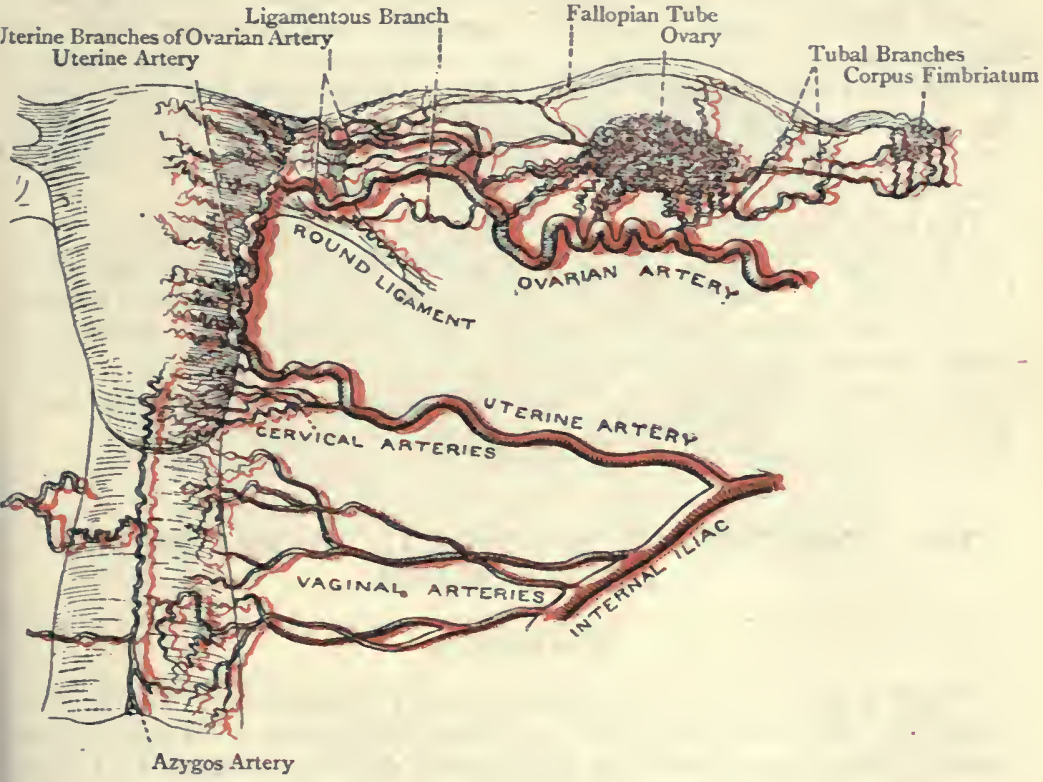

Fig. 374-The Ovarian, Uterine, and Vaginal Arteries. (Posterior View) (AFTER HYrtL).

cervical and vaginal. The cervical branch supplies offsets to the cervix. One of these, called the coronary artery, divides into two branches, which with their fellows of the opposite side form an arterial circle around the cervix. The vaginal branch divides into two, anterior and posterior, which descend in the middle line of the anterior and posterior walls of the vagina, where they anastomose with branches of the vaginal arteries.

The uterine veins, which are destitute of valves, form a copious plexus within the broad ligament close to the uterus, where it is embedded in the parametrium. The blood from the lower part 
of this plexus is conveyed away by two uterine veins, which are tributaries of the internal iliac vein. A large proportion of the blood, however, passes from the upper part of the plexus into the ovarian plexus. The uterine plexus communicates below with the vaginal plexus.

Vaginal Artery.--This vessel, which usually replaces the inferior vesical of the male, arises from the anterior division of the internal iliac, occasionally in common with the uterine, or the middle hemorrhoidal, artery. It passes downwards and inwards to the wall of the vagina, where it divides into branches which anastomose with their fellows of the opposite side, the vaginal branches of the uterine arteries, and, towards the lower end of the vagina, with branches of the internal pudic. Along the anterior and posterior walls, in the median line, an arterial chain is constructed by the vaginal arteries and the vaginal branches of the uterine arteries, thus forming the vessels known as the vaginal azygos arteries. The vaginal artery also furnishes branches to the bladder, rectum, and bulbus vestibuli.

The veins of the vagina form a rich plexus in the muscular coat, which is more copious towards the lower end. They communicate above with the uterine plexus, in front with the pudendal plexus around the urethra, behind with the hemorrhoidal plexus, and below -with the veins of the bulbus vestibuli. The vaginal vein leaves the upper part of the vaginal plexus and opens into the internal iliac vein.

\section{THE STRUCTURE OF THE SPECIAL VISCERA OF THE FEMALE PELVİS.}

\section{The Ovaries.}

The ovary is covered by a layer of short columnar epithelial cells. These are the remains of the germinal eptihelium from which the organ is developed, and they rest upon a delicate, connective-tissue membrane, which is blended with the so-called tunica albuginea. Interposed between the columnar cells there are a few spheroidal cells of larger size, which are primordial ova. The connectivetissue membrane is continuous with the peritoneum which forms the mesovarium along the margins of the hilum, and which is covered by endothelium. Within this membrane, and blended with it, there is a continous covering, consisting of fibrous connective tissue, which is called the tunica albuginea ovarii, from its supposed resemblance to the tunica albuginea testis. It is, however, really a condensation of the ovarian stroma at the surface. This stroma pervades the interior of the ovary, and is composed of fibrous connective tissue, which is richly provided with spindle-shaped cells and elastic tissue. There are also a few plain muscular fibres in the deeper part of the ovary close to the hilum. The spindle- 
shaped cells are regarded by some authorities as muscular fibrecells, but they probably belong to the connective tissue of the stroma. The stroma is freely permeated by bloodvessels, and contains the Graafian follicles. Immediately within the surface there is a layer of the stroma which presents a granular appearance, especially in young persons, due to the presence of an immense number of Graafian follicles, with their contained ova, in an early stage. This part of the ovary is called the cortex, the remainder being known as the medulla. Lying more deeply in the stroma there is another set of Graafian follicles, less numerous, but of larger size, these being in a more advanced stage. Still more deeply there is

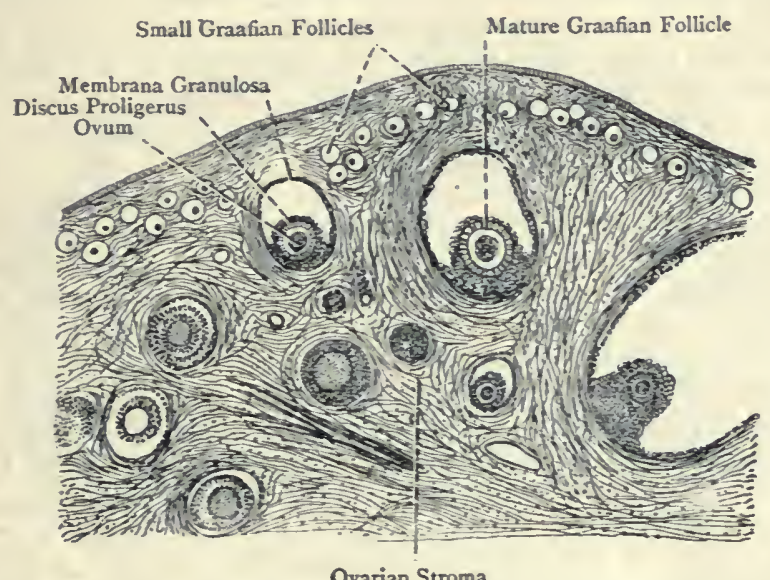

Ovarian Stroma

Fig. 375--Section of the Ovary, showing its Minute Structure.

another and less numerous set of follicles, of still larger size, which are almost in a state of maturity. When these latter have attained full development, they pass towards the surface, where they may sometimes be seen as clear follicles causing slight projections. When fully developed, they attain a diameter of about $\frac{1}{12}$ inch. At periodical intervals one or more of these mature follicles rupture, this being accompanied by the discharge of a fluid-the liquor folliculi-and the simultaneous escape of the contained ovum or ova. After the discharge of its contents the follicle becomes filled with blood and cellular tissue, and assumes a yellow colour. It is then known as a corpus luteum. This undergoes atrophy in the virgin, and, assuming a white colour, is known as a corpus albicans.

Structure of the Graafian Follicles.-The smallest follicles near the surface, which are about $\frac{1}{100}$ inch in diameter, consist of a single investing layer of f.rttened cells, closely embracing the contained ovum. It is computed that the ovaries of a child at birth 
contain as many as 70,000 of these follicles. In follicles a little more advanced the investing epithelium becomes columnar, and is arranged in two layers-outer and inner, the latter surrounding the ovum. In more mature follicles fluid, called the liquor folliculi, accumulates between the outer and inner cellular layers, except at the point where the ovum lies. The outer layer is then known as the membrana granulosa, and the inner as the discus proligerus. The cells of these two layers become continuous at the part where the liquor folliculi is absent, so that in this manner the ovum is anchored to one point of the wall of the follicle. In the most mature follicles the liquor folliculi has increased in amount, and the cells of the membrana granulosa and discus proligerus have multiplied so as to form several strata. Each of these follicles has a distinct wall, called the theca folliculi, which is formed by a condensation of the surrounding stroma, and in which two layers can be recognised-an outer fibrous and an inner vascular. There is usually only one ovum in each follicle.

Structure of the Ovum. - The ovum is a spherical cell, having a diameter of about $\frac{1}{125}$ inch. It has two membranes-an external, called the zona radiata (zona pellucida), and an internal, called the vitelline membrane, the latter representing the cell-wall. Within this is the cell-body, called the yolk, or vitellus, which contains a nucleus, known as the germinal vesicle, and that in turn contains a nucleolus, called the germinal spot. The zona radiata is fairly thick, and is perforated by pores or canals, arranged in a radiating manner, hence the name 'radiata.' These canals serve for the passage of nutritive material, and also for spermatozoa preparatory to impregnation. The vitelline membrane is merely a condensation of the yolk at the surface. The yolk, or vitellus, is semifluid, very refracting, and contains many yolk granules, which are fatty and albuminoid in their nature. These granules form the nutritive yolk, or deutoplasm, the remainder of the yolk being called the formative yolk, or cytoplasm. The nucleus or germinal vessel is about $\frac{1}{500}$ inch in diameter. It is eccentric in position in the cytoplasm, and has the usual characters of a cell-nucleus. The nucleolus, or germinal spot, is granular in character.

Blood-supply of the Ovary. - The ovary receives its blood from the ovarian artery.

Nerves.-These come from the ovarian sympathetic plexus, which derives its fibres from the renal and aortic plexuses, and accompanies the ovarian artery.

Lymphatics.-The lymphatic vessels of the ovary accompany the ovarian bloodvessels, and terminate, for the most part, in the lateral aortic glands, on either side. A lew, however, terminate in the pre-aortic glands. They are joined by most of the lymphatics of the body of the uterus and by those of the Fallopian tube.

The ovaries represent the testes in the male, and they have been called the testes mulicbres. 
Ligament of the Ovary.-This is composed of plain muscular and fibrous tissues, the former being continuous with the muscular tissue of the uterus. It derives its blood-supply from the ovarian artery, and represents the upper part of the gubernaculum testis in the male fœtus.

\section{Structure of the Fallopian Tubes.}

The wall of the Fallopian tube is composed of four coats-serous, muscular, submucous, and mucous. The serous coat is formed by the peritoneum. The muscular coat is composed of plain muscular tissue, arranged as an outer longitudinal and inner circular layer,

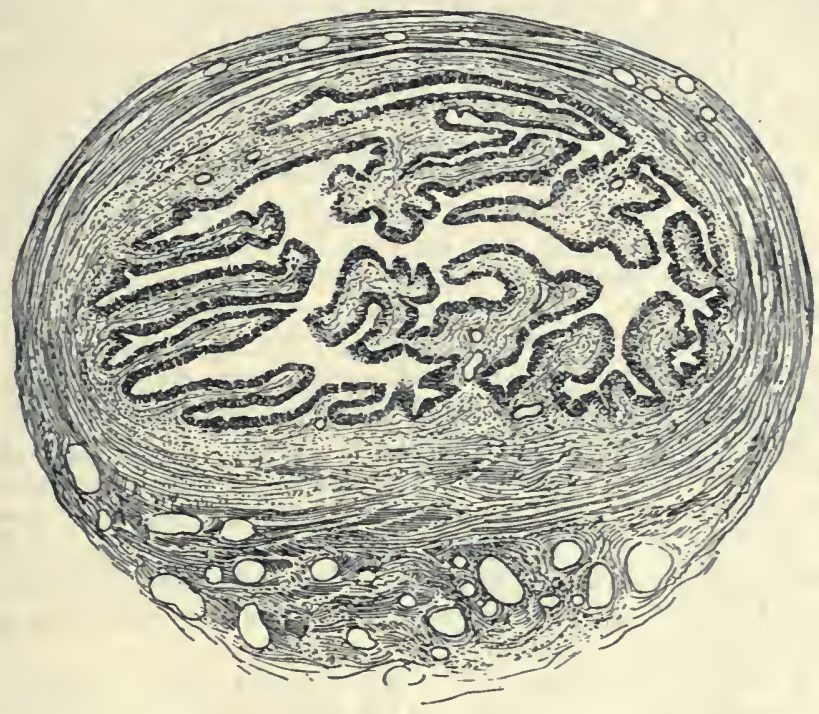

Fig. 376.-Transverse Section of the Fallopian Tube (highly MAGNIFIED).

the latter being the thicker. The submucous coat is areolar in structure. The mucous coat is continuous with that of the uterus on the one hand, and with the peritoneum $n$ the other, at the margins of the fimbriæ. It is thrown into longitudinal folds, which are simple in the isthmus, but complex in the ampulla, where they are beset with secondary folds, and communicate in such a manner as to give rise to alveolar spaces, thus imparting an almost glandular appearance to the coat. In transverse section the tube presents a branched lumen, which is nearly filled by the leaf-like processes formed by the mucous folds. The mucous membrane is covered by ciliated columnar epithelium, which, at the free margins of the fimbrix, passes into the endothelium of the peritoneum.

Blood-supply.-The ovarian and uterine arteries. 
Nerves.-These are derived from the ovarian and uterine sympathetic plexuses.

Lymphatics.- These pass to the median lumbar glands along with those of the ovary and upper part of the body of the uterus.

\section{Structure of the Uterus.}

The wall of the uterus consists of three coats-serous, muscular, and mucous - there being no submucous coat.

The serous coat is formed by the peritoneum, already described.

The muscular coat is composed of plain muscular tissue, with an admixture of areolar tissue, and it imparts great thickness to the wall. The muscular tissue is disposed in three strata-outer, middle, and inner. The outer stratum is thin, and its fibres are disposed longitudinally over the front and back of the organ, becoming continuous with one another by turning over the fundus. Those nearest the lateral borders incline outwards, and are prolonged into the round ligaments, Fallopian tubes, and ligaments of the ovaries. Some from the back of the supravaginal portion of the cervix are prolonged into the sacro-uterine folds. The middle stratum is very thick, and is composed of fibres which interlace in a complex manner over the body, but in the neck they are arranged circularly. The bloodvessels and nerves are freely interspersed throughout this layer. The inner stratum, which is also very thick, has its fibres disposed longitudinally in the cervix. As they ascend over the body they become oblique, and at the superior angles they run circularly. The uterine glands project into this stratum, and it contains a free admixture of areolar tissue. It is right to mention that the inner stratum is regarded as a very much thickened muscularis mucosæ, according to which view it would form a part of the mucous coat (Williams).

The mucous membrane of the cavity of the body is smooth, and soft in consistence, and is covered by ciliated columnar epithelium. It is beset with a number of openings, which are the mouths of the uterine or utricular glands. These are simple tubular glands, which extend in a somewhat convoluted manner through the entire thickness of the mucous coat, and project by their deep blind ends into the inner muscular stratum, there being no submucous coat. Each gland is composed of a basement membrane, which is lined with ciliated columnar epithelium, continuous with that of the cavity of the body. Each has a distinct lumen, except at its deep end, where it is filled with cells. The mucous membrane of the cavity of the cervix is of firmer consistence than that of the cavity of the body, and, as has been stated, it presents the appearance known as the arbor vitæ uterina. It is provided with papillae, and is covered by columnar epithelium, except near the os externum, where the epithelium is of the stratified squanous variety, as it is over the exterior of the vaginal portion of the cervix 
and vagina. On the summits of the rugæ the columnar cells are ciliated, but in the furrows between them they are devoid of cilia. The mucous membrane is freely provided with racemose glands, which in the upper part of the cervix are lined with columnar cells, and in the lower part with cubical cells. In both regions the cells are non-ciliated. The glands in the lower part of the cervix have each a large lumen, and they secrete a very tenacious mucus during pregnarcy, which, in the later stages of that period, plugs the os externum.

In addition to these glands clear vesicles of a yellowish colour, called the ovula Nabothi, may be seen in the mucous membrane between the rugæ of the arbor vitæ uterina, which are supposed to result from the closure of some of the racemose glands.

Blood-supply.- The uterus is supplied with blood by the uterine arteries, and the uterine branches of the ovarian arteries.

Nerves.-The chief nerves are derived from the uterine sympathetic plexus, which is an offshoot from the pelvic plexus, and accompanies the uterine artery. It is to be noted that the pelvic plexus contains spinal fibres derived from the third and fourth sacral nerves (sometimes also the second), and from the upper two or three lumbar nerves, as in the case of the bladder and rectum. The uterus also receives sympathetic fibres from the ovarian plexus, which is derived from the renal and aortic plexuses.

Lymphatics-Cervix Uteri.-The lymphatic vessels of the cervix have a threefold destination, namely, (I) the middle chain of the external iliac glands, (2) the internal iliac glands, and (3) the inner group of the common iliac glands.

Body.-(I) The most of the lymphatics of the body of the uterus join the lymphatics of the ovary, and pass chiefly to the lateral aortic glands, but a few terminate in the pre-aortic glands. (2) A few lymphatics pass to the middle chain of the external iliac glands. (3) Some lymphatics accompany the round ligament of the uterus, and terminate in the pubic group of the inguinal glands.

The lymphatics of the cervix communicate freely with those of the body and with those of the upper part of the vagina.

\section{Structure of the Vagina.}

The wall of the vagina is composed of three coats-fibrous, muscular, and mucous.

The fibrous coat is composed of dense connective tissue.

The muscular coat consists of plain muscular tissue, arranged as an onter longitudinal and inner circular layer, both being closely connected. Embedded in this coat there is a network of anastomosing veins, representing erectile tissue, which is well developed round the lower part of the passage. The plain muscular coat is replaced at the external orifice by the striated sphincter vaginæ muscle.

The mucous membrane is covered by stratified squamous epithelium, and is provided with papillæ. In the upper part of 
the passage it contains mucous glands. Along the middle line of the anterior and posterior walls it presents a ridge, these ridges being called the columns of the vagina, or columna rugarum. Passing off from them at right angles there are, in the virgin, numerous transverse rugæ, these appearances being well inarked in the lower part of the passage, and absent in the upper part.

Blood-supply. - The vagina is supplied with blood by the vaginal arteries, the vaginal branches of the uterine arteries, and branches of the internal pudic arteries.

Nerves.-These are derived from the vaginal sympathetic plexus of each side, which is an offshoot from the pelvic plexus.

Lymphaties.-The lymphatic vessels are disposed in two setssuperior and inferior. The superior lymphatics come from about the upper two-thirds of the vagina, and they pass to (I) the middle chain of the external iliac glands, and (2) the internal iliac glands, on either side. The inferior lymphatics come from about the lower third, and they pass to (I) the inner group of the common iliac glands, and (2) the pubic groups of the inguinal glands.

The superior and inferior vaginal lymphatics communicate freely with one another; the superior lymphatics communicate with those of the cervix uteri; and the inferior set communicate with those of the labia majora.

\section{Development of the Fallopian Tubes, Uterus, and Vagina.}

The Fallopian tubes, uterus, and vagina are developed from the Müllerian ducts.

As the mesonephros, or Wolffian body, approaches its full development, a longitudinal tube makes its appearance on the outer side of the Wolffian

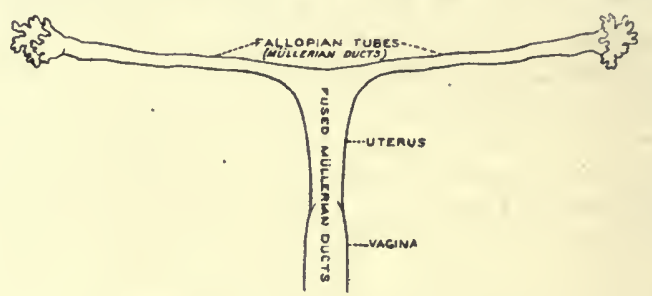

Fig. 376a.-Development of Fallopian Tubes, Uterus, and Vagina.

duct. This tube is called the Muillerian duct, and it is developed from an invagination of the peritoneum, which covers the anterior end of the genital ridge. The duct extends caudalwards, lying within the uro-genital fold along with the Wolffian duct, upon the outer side of which it is placed. Posteriorly, where the two uro-genital folds fuse to form the genital cord, the Miillerian duct of either side crosses over the corresponding Wolffian duct, and then lies on its inner side. The two Müllerian ducts and the two Wolffian ducts are contained within the genital cord, and the Müllerian ducts are now in close contact at the median line.

In this part of their course the Miillerian ducts unite, and form a single mesial canal, which, together with the Wolffian ducts, opens into the ventral compartment of the cloaca-that is to say, the uro-genital sinus or canal. The single nilesial canal, formed by the fusion of the Müllerian ducts with the genital cord, gives rise to the uterus and the vagina. In the male it forms 
the sinus pocularis, or uterus masculinus, which is appended to the prostatic portion of the urethra.

The Müllerian ducts on the cephalic side of their fusion form the Fallopian tubes, and the cephalic extremity of each Müllerian duct, which remains in communication with the cœlom, or body-cavity, constitutes the infundibulum, or pavilion, of the corresponding Fallopian tube, in the centre of which infundibulum the ostium abdominale of the tube is situated.

The abnormal condition of the uterus known as uterus bicornis is brought about by the fact that the two Müllerian ducts have united at a more posterior (caudal) level than they usually do. The condition known as uterus unicornis is due to imperfect development of one or other Müllerian duct. In extremely rare cases the Müllerian ducts fail to unite, and, by opening independently into the uro-genital sinus, they give rise to a double uterus and a double vagina:

\section{Structure of the Urethra.}

The wall of the urethra is composed of three coats-muscular, vascular, and mucous. The muscular coat, which is continuous with that of the bladder, is composed of plain muscular tissue, arranged as an outer circular and an inner longitudinal layer. The circular fibres are well developed, especially at the upper end, where they partake somewhat of the nature of a sphincter muscle. Superficial to the circular fibres the urethra, as it lies between the two layers of the triangular ligament, is embraced by the striated fibres of the compressor urethræ. The vascular coat is composed of a rich plexus of veins, supported and pervaded by areolar and elastic tissues. This plexus is continuous above with that around the neck of the bladder. The mucous coat is covered by transitional epithelium in its upper part, and stratified squamous epithelium in its lower part. It is provided with papillæ, and is thrown into longitudinal folds, which are temporary above, but permanent below. One fold, situated on the posterior wall, is larger than the others, and is known as the crest. The mucous membrane. is furnished with tubular mucous glands, and between the permanent folds in the lower part there are crypts or lacunæ.

Lymphatics.-The lymphatic vessels of the female urethra join those of the bladder, which pass to the external iliac, internal iliac, and common iliac glands.

Development of the Urethra.-The female urethra is developed from the anterior or cephalic part of the ventral or uro-genital compartment of the cloaca, which also gives rise to the bladder. It represents the prostatic portion of the male urethra as low as the sinus pocularis or uterus masculinus.

\section{The Articulations of the Pelvis.}

Lumbo-sacral Articulation.-The union between the fifth lumbar vertebra and the base of the sacrum is effected by means of the following ligaments: an intervertebral disc, prolongations of the anterior and posterior common ligaments of the bodies of the vertebræ above, capsular ligaments and synovial membranes for the articular processes, ligamenta subflava for the laminæ, and interspinous and supraspinous ligaments for the spinous processes. These are similar to the corresponding ligaments above the level 
of the fifth lumbar vertebra. The articulation between the bodies of the fifth lumbar and first sacral vertebræ belongs to the class amphiarthrosis, and the joints between the articular processes belong to the class diarthrosis, and the subdivision arthrodia. In addition to the foregoing ligaments there are two special ligaments, called lateral lumbo-sacral and ilio-lumbar. The lateral lumbosacral ligament, at either side, extends from the lower aspect of the transverse process of the fifth lumbar vertebra anteriorly to the upper surface of the ala of the sacrum at its anterior and outer part close to the sacro-iliac articulation. It is somewhat fan-shaped, and corresponds to the intertransverse ligaments of the lumbar vertebræ, and the superior costo-transverse ligaments of the thoracic region. The ilio-lumbar ligament extends from the tip of the transverse process of the fifth lumbar vertebra to the inner lip of the iliac crest, where it is attached for about 2 inches above the back part of the iliac fossa. It is triangular, and its direction is outwards and slightly backwards. It is closely associated with the lower part of the anterior lamina of the lumbar aponeurosis.

Arterial Supply.-Ilio-lumbar and superior lateral sacral arteries.

Nerve-supply. - Fourth and fifth lumbar nerves, and sympathetic filaments.

Movements.-Flexion, extension, and lateral movements between the opposed bodies, and gliding and rotation between the articular processes.

Sacro-coceygeal Articulation.-This belongs to the class amphiarthrosis. The bony elements are the fifth sacral and first coccygeal vertebræ. The opposed surfaces are transversely oval, and are separated by an intervertebral disc, unless in advanced life, when ankylosis takes place, this occurrence being earlier and more frequent in the male than in the female. The ligaments are as follows : anterior and posterior sacro-coccygeal, which are continuations of the anterior and posterior common ligaments of the bodies of the vertebræ; supracornual, which pass between the sacral and coccygeal cornua; and intertransverse or lateral, which pass between the inferior lateral angles of the sacrum and the transverse processes of the first coccygeal vertebra. The latter ligament is liable to become ossified.

Arterial Supply.-Inferior lateral and middle sacral arteries.

Nerve-supply.-Lower two sacral, and coccygeal, nerves.

Movements.-Forward and backward movements are allowed.

Intereoceygeal Articulations.-These only exist prior to middle life. The union between the coccygeal segments is effected by intervertebral discs, and anterior and posterior ligaments. The adjacent fibres of the great and small sacro-sciatic ligaments serve as lateral ligaments.

Sacro-iliac Articulation.-This belongs to the class amphiarthrosis. The bony elements are the auricular and ligamentous surfaces of the sacrum and ilium. The cartilage of the auricular surface is about $\frac{1}{12}$ inch thick, and it may be a single plate for both surfaces, 
or it may exist as two plates, one for each surface, in which latter case a small synovial cavity exists between the two plates. IWhen the bones are forcibly separated, a portion of the cartilage is usually left adherent to each auricular surface. The ligaments at the joint are anterior, posterior, and oblique. The anterior sacro-iliac ligament is composed of short fibres which are placed in front of the joint. The posterior sacro-iliac ligament, which is very strong, extends from the ligamentous surface of the ilium to the ligamentous surface of the sacrum and the transverse tubercles on the dorsum of the bone, the direction of the fibres being downwards and inwards. The oblique sacro-iliac ligament lies superficial to the posterior, and extends from the posterior superior iliac spine and the adjacent part of the iliac crest to the third and fourth transverse tubercles on the dorsum of the sacrum. It is really a detached part of the posterior sacro-iliac ligament. The great and small sacro-sciatic ligaments are accessory to this joint.

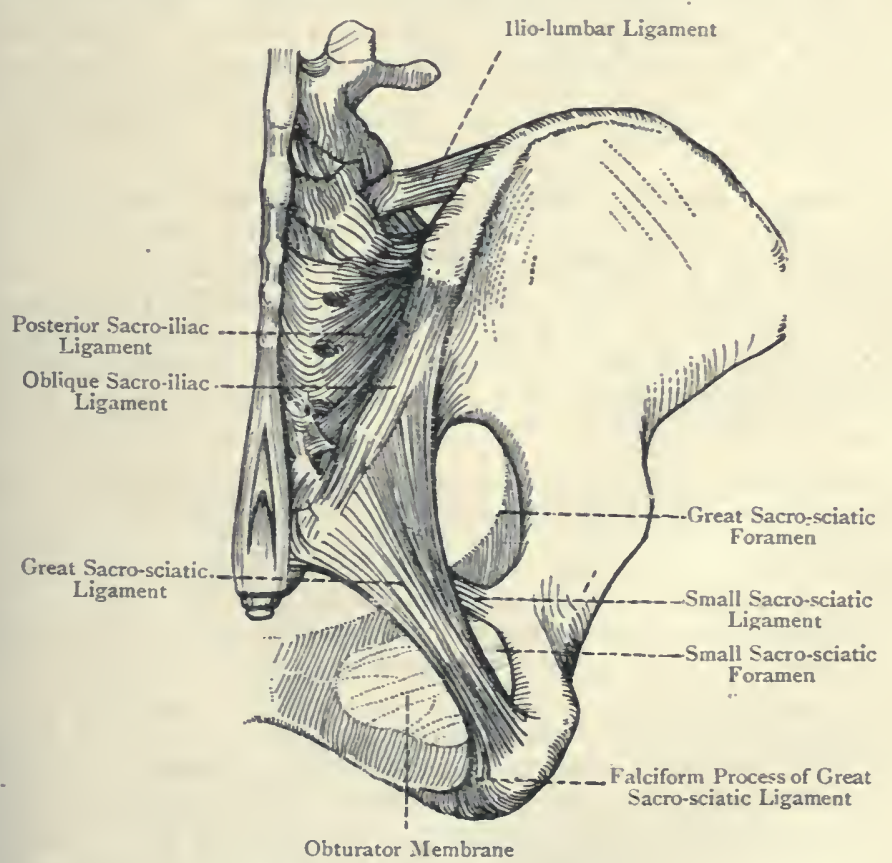

Fig. 377.-Ligamexts of the Right Haif of the Pelvis (Posterior VIEW).

The great or posterior sacro-sciatic ligament is attached by one extremity to the posterior inferior iliac spine, and the sides of the last three sacral and first coccygeal vertebræ, and by the other extremity to the inner border of the tuber ischii. From 
this latter point it sends forwards an expansion, called the falciform process, which is attached to a sharp ridge on the lower part of the inner surface of the ramus of the ischium, close to its internal border. The ligament is broad at its attached ends, especially the upper, but in crossing towards the tuber ischii it becomes narrow. Its direction is downwards and forwards, and its ischial fibres are continuous with the tendinous origin of the long head of the biceps femoris. By its superficial surface it gives origin to part of the gluteus maximus, and resting on this surface are the plexiform loops formed by the external branches of the posterior primary divisions of the first three sacral nerves. Its deep surface gives origin to some fibres of the pyriformis, and lower down is intimately connected with the small sacro-sciatic ligament. The falciform process affords attachment to the lower part of the parietal pelvic fascia. The great sacrosciatic ligament is pierced by the coccygeal branch of the sciatic artery, the sacral branch of the internal pudic artery, and the perforating cutaneous nerve from the sacral plexus. The ligament assists in the formation of the great and small sacro-sciatic foramina.

The great sacro-sciatic ligament is to be regarded as the separated tendon of the long head of the biceps femoris muscle.

The small or anterior sacro-sciatic ligament, which is triangular, is attached by its base to the sides of the last.two sacral and first coccygeal vertebræ, where it is intimately connected with the more superficially placed great sacro-sciatic ligament. Its apex is attached to the tip of the spine of the ischium. Its deep surface is incorporated with the coccygeus muscle, and, along with the spine of the ischium, it forms the separation between the great and. small sciatic foramina.

The small sacro-sciatic ligament is to be regarded as resulting from the fibrous degeneration of the superficial part of the coccygeus muscle.

Arterial Supply of the Sacro-iliac Articulation.-Ilio-lumbar, superior lateral sacral, and gluteal arteries.

Nerve-supply.-Superior gluteal, and anterior primary divisions, and external branches of the posterior primary divisions, of the first two sacral nerves.

Movements.-Stability being required at this joint, it is almost immovable. The two ossa innominata by their union at the pubic articulation form an arch, the convexity of which is directed downwards and forwards. The piers of this arch are separated by a wide interval, into which the sacrum fits in the form of a wedge. The sacrum being narrower behind than in front, the superincumbent weight of the trunk has a tendency to displace it downwards into the pelvic cavity, but this is resisted partly by the powerful posterior sacro-iliac ligaments, which suspend the bone, and partly by the strong hold which the sacrum has upon the ilium, in virtue of the irregularities of the opposed surfaces. Under the influence 
of the superincumbent weight there is a tendency on the part of the sacrum to rotate round an axis passing transversely through the sacro-iliac joints. This tendency, however, is checked by the great and small sacro-sciatic ligaments. The ilio-lumbar ligaments prevent displacement of the fifth lumbar vertebra over the base of the sacrum.

Symphysis Pubis.-This belongs to the class amphiarthrosis. The articular surfaces are the symphysial aspects of the pubic bones. The ligaments are anterior, posterior, superior, inferior, and interpubic disc. The anterior pubic ligament is strong, and is composed of superficial and deep fibres. The superficial fibres are arranged in an obliquely decussating manner, and are chiefly constructed by the aponeurotic fibres of the external oblique and inner heads of the recti abdominis mu cles. The deep fibres are disposed transversely. The posterior and superior pubic ligaments are weak, and consist of scattered fibres. The inferior or subpubic ligament is a strong, thick band of fibres, which lies at the antero-superior part of

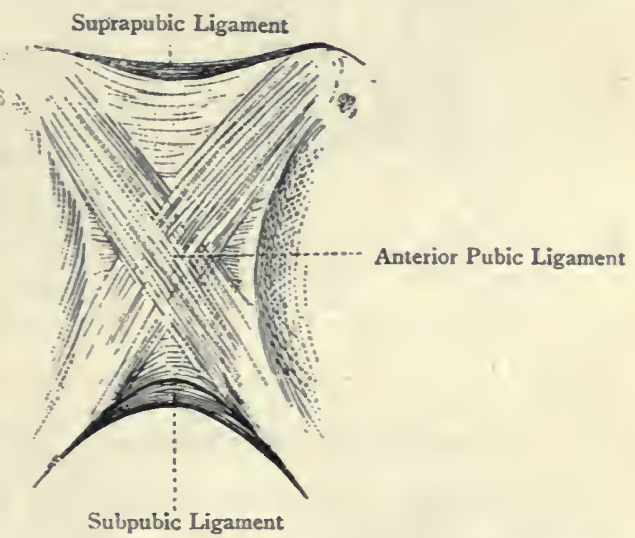

Sulpubic Ligament

Fig. 378.-The Ligaments of the Symphysis Pubis (ANTERIOR Aspect). the subpubic arch, where it fills up and rounds off the subpubic angle. It is attached superiorly to the lower part of the interpubic disc, and laterally to the adjacent parts of the inner lips of the descending pubic rami. It is about $\frac{1}{4}$ inch in depth, and is slightly arched, from which latter circumstance it is known as the ligamentum arcuatum. The interpubic dise lies between the plates of cartilage which cover the bony articular surfaces. It is composed of fibro-cartilage, is thicker in front than behind, and usually contains a fissure at its upper and back part, which may extend for one half, or even the whole length, of the disc as an oblique cleft, parallel to the plane of the bony surfaces. This fissure is brought about by absorption of the tissues in that situation, and it does not appear until about the tenth year of life. It is larger in the female than in the male.

The depth of the symphysis pubis is less in the female than in the male.

Arterial Supply.-Pubic branches of the deep epigastric and obturator, and superior or superficial external pudic arteries. 
Nerve-supply.-Probably the hypogastric branch of the iliohypogastric, inguinal, and pudic nerves.

Movements.-Very slight separation is allowed at this joint, due

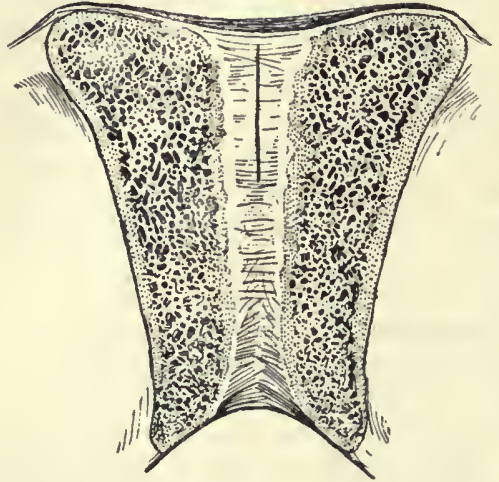

Fig. 379.-Vertical Section of the Symphysis PUBis.

small sacro-sciatic ligament. through it, see Gluteal Region. to yielding of the connecting structures. This is most apparent during pregnancy and parturition.

Great Sacro-sciatic Foramen. - This foramen is formed by the great sciatic notch, the spine of the ischium, the great sacrosciatic ligament, and the small sacro-sciatic ligament. For its compartments, and the structures which pass through them, see p. 429.

Small Sacro-sciatic Foramen. -This foramen is formed by the small sciatic notch, the spine of the ischium, the great sacro-sciatic ligament, and the For the structures which pass

\section{Obturator Membrane and Ob.urator Canal.}

The obturator membrane, also called the thyroid membrane, is attached to the circumference of the obturator foramen, except superiorly opposite the obturator groove, which it converts into a fibro-osseous canal for the passage of the obturator vessels and nerve. In this situation it is joined posteriorly by the parietal pelvic fascia. Its fibres are arranged in an irregular, decussating manner. Its posterior or pelvic surface is closely covered by the obturator internus muscle. The anterior or femoral surface is in like manner covered by the obturator externus muscle, and at its circumference there is an arterial loop, formed by the external and internal terminal branches of the obturator artery.

The obturator canal is a fibro-osseous canal, which is situated above the upper border of the obturator membrane. Its upper boundary, which represents the osseous element, is formed by the obturator groove on the inferior surface of the superior pubic ramus, the direction of the groove being downwards, forwards, and inwards. The lower boundary, which represents the fibrous element, is formed by the junction of the parietal pelvic fascia with the upper border of the obturator membrane over the upper border of the obturator internus. The canal transmits the obturator vessels and obturator nerve, the nerve being above the artery. 


\section{GUIDE TO THE DISSECTION OF THE ABDOMEN.}

\section{Male Perineum.}

Before the subject is placed in the lithotomy positon the dissectors should practise passing the catheter, which should then be left in the urethra and bladder. The subject having been placed in the lithotomy position, the penis and scrotum are to be tied up to the end of the catheter. The first duty of the dissector is to make himself familiar with the landmarks, boundaries, and arbitrary divisions of the perineum, and he should have before him a ligamentous pelvis. He should also introduce his finger well into the rectum, when he will feel through its upper wall the following structures in order from without inwards: (I) the membranous part of the urethra, with the catheter lying in it; (2) the posterior or rectal surface of the prostate gland, easily recognised by its firmness and resistance ; and (3) the external trigone of the bladder, which is destitute of peritoneum. The rectum is then to be lightly stuffed with tow, and the margins of the anal aperture are to be brought together with a few stitches. Before removing the skin the dissector should note the antero-posterior median raphe in the uro-genital division of the perineum, and he should follow this raphé over the posterior and anterior surfaces of the scrotum to the under surface of the penis. He should note the position of the central tendinous point, which is about an inch in front of the anus. He should also observe that the anus is situated posterior to a line connecting the front parts of the ischial tuberosities. A slight hollow on the surface between the anurs and the tuber ischii indicates the situation of the ischio-rectal fossa.

To remove the skin the following incisions should be made: a median incision, extending from the root of the scrotum to the tip of the coccyx, and surrounding the anus; a transverse incision, extending outwards from the tip of the coccyx for fully 3 inches; another transverse incision, extending outwards from the central tendinous point for fully 3 inches; and a third transverse incision, extending outwards from the anterior extremity of the median incision (at the root of the scrotum) for a similar distance. The sphincter ani externus is to be first displayed. An effort should be made to show the corrugator cutis ani, but this is not an easy dissection. Between the tip of the coccyx and the anus the dissector will meet with the hemorrhoidal or perineal branch of the fourth sacral nerve, and, in the region of the anus and over the ischio-rectal fossa, will be found twigs of the inferior hemorrhoidal nerve. The lower border of the gluteus maximus is next to be dissected, taking care to preserve the gluteal cutaneous branches of the small sciatic nerve and sciatic artery, and the perforating cutaneous branch of the sacral plexus, the latter making its appearance near the coccyx. The dissectors should conjointly make an effort to show the ano-coccygeal body. which lies in the middle line between the tip of the coccyx and the anal canal.

The fat is to be removed from the ischio-rectal fossa, in doing which its loose character is to be noted, as well as the fact that it fills up the fossa, and also extends into the anterior and posterior diverticula connected witl it. In removing the fat the dissector should cut from the anus outwards, taking care to preserve the inferior hemorrhoidal vessels and nerve, which cross the fossa transversely. In removing the fat from the front part of the fossa the superficial perineal artery and the two superficial perineal nerves, external and internal, are to be preserved. The outer wall of the fossa is to be dissected, showing the obturator fascia covering the obturator internus muscle, and containing Alcock's canal. Upon one side Alcock's canal should be opened and its contents displayed, in the following order from below upwards: the perineal division of the pudic nerve, vena comes, internal pudic artery, vena comes, and dorsal nerve of the penis. The position of the artery, its distance from the surface, and its relation to the tuber ischii are to be noted. An opening is also to be made in the obturator fascia to 
show the fibres of the obturator internus muscle. In dissecting the inner wall of the fossa the anal or ischio-rectal fascia is to be studied, and thereafter removed to show the levator ani muscle descending upon the side of the rectum and anal canal. Posterior to the levator ani is the coccygeus, and emerging between the two will be found the hemorrhoidal or perineal branch of the fourth sacral nerve. Under cover of the lower border of the gluteus maximus there should be shown the inferior border of the great sacro-sciatic ligament. The anterior limit of the fossa is not to be disturbed in the meantime, but the dissector should make himself aware that it is partially limited by the reflection of Colles's fascia round the superficial transverse perineal muscle to join the base of the triangular ligament.

The ischio-rectal fossa having now been displayed, the following points in connection with it are to be noted : its measurements, including its depth; its boundaries, and, in connection with the outer boundary, the position of the internal pudic vessels, and in connection with the inner boundary, the rectum and anal canal ; the loose fat which fills the fossa ; the inferior hemorrhoidal vessels and nerve crossing the fossa from side to side ; and the superficial perineal artery and two superficial perineal nerves in the anterior part of the fossa, passing in a forward direction. It is further to be observed that there are two prolongations or diverticula connected with the fossa, anterior and posterior. The anterior diverticulum is to be found at the front part of the fossa above the junction of Colles's fascia with the base of the triangular ligament. It is of large size, and reaches almost as far forwards as the symphysis pubis, but it will be better understood in the deep dissection of the anterior part of the perineum. The posterior diverticulum will be found between the great sacro-sciatic ligament and the lower border of the gluteus maximus, its direction being outwards and inwards.

The dissector is now to turn his attention to the anterior or uro-genital division of the perineum. The arrangement of the superficial fascia is to receive careful attention. It can easily be shown to be divisible into a subcutaneous fatty layer, having no special name, and a deep membranous layer, known as the fascia of Colles. The subcutaneous fatty layer is traceable backwards over and into the ischio-rectal fossa, outwards to the inner aspect of the thigh, and forwards into the scrotum, where it will be again noticed. The fascia of Colles has an important bearing upon the course taken by urine when extravasated into the anterior part of the perineum. The disposition of this fascia is best studied by inserting the end of a blow-pipe underneath it at its back part, on one side of the middle line. Air having been blown in, the fascia will be seen to be attached externally to each ischiopubic ramus; posteriorly it turns round the posterior border of each superficial transverse perineal muscle to join the base of the triangular ligament; internally (towards the median line) it is continuous with the corresponding fascia of the opposite side ; and anteriorly it passes into the scrotum, where it joins the subcutaneous fatty layer, and forms the tunica dartos. Inflation with air reveals the fact that beneath the fascia there is a space known as the perineal pouch, which is divided posteriorly into two compartments by an incomplete septum. This is demonstrated by the air at first passing forwards on its own side, then crossing the median line, and travelling backwards on the opposite side. Further, the air passes forwards into the scrotal wall beneath the dartos tunic. The attachments of the fascia of Colles clearly explain in what direction extravasated urine can only pass, and from what courses it is debarred.

Beneath Colles's fascia the following structures are to be dissected on each side, namely, the superficial perineal vessels and the three long scrotal nerves. The two superficial perineal nerves will be found entering the perineal pouch at its back part, and the long pudendal nerve of Soemmering will be found appearing through the fascia lata of the thigh about an inch in front of the tuber ischii, after which it passes inwards and forwards, entering the perineal pouch from the outer side. All these structures are to be dissected forwards to the scrotum. The following structures are next to be dissected : the bulbocavernosus, covering the bulb in the middle line; the ischio-cavernosus, 
covering the crus penis along the line of the ischio-pubic ramus; and the superficial transverse perineal muscle, in the direction of a line passing inwards and forwards from the tuber ischii to the central tendinous point. This last muscle is often very indistinct, and in its dissection care is to be taken to preserve the transverse perineal artery, which lies close to the muscle.

The dissector should now separate the ischio-cavernosus from the bulbocavernosus, and the deep perineal triangle will be thereby exposed. In the area of each triangle a membrane will be seen, which represents the anteroinferior layer of the triangular ligament of the urethra. Through it the fibres of the compressor urethræ muscle may be visible. At this stage the dissector should study the central tendinous point of the perineum, and note the various muscles which are connected with it. The bulbo-cavernosus muscle of one side is now to be carefully removed from the bulb of the urethra, by cutting along the median raphe between the two muscles. The different modes of insertion of the muscle are to be observed, and the bulb of the urethra is to be studied, its relation to the triangular ligament and central tendinous point being noted. The ischio-cavernosus should next be reflected, and attention given to the crus penis.

The dissector is now ready to study the triangular ligament of the urethra. Its antero-inferior layer, also called the subpubic fascia or deep perineal fascia, has been already laid bare in the area of each deep perineal triangle. The several structures piercing it are to be noted, and it should be observed that the base of this layer is joined by the fascia of Colles. This layer having been studied in all its bearings, attention should be directed to the subpubic ligament, which will be found close below the symphysis pubis. The anteroinferior layer of the triangular ligament is now to be carefully removed, and the following structures are to be dissected: the compressor urethræ, the posterior fibres of which are known as the transversus perinxi profundus; the internal pudic vessels and dorsal nerve of the penis, lying close to the ischiopubic ramus; the following branches of the internal pudic artery, namely, the artery of the bulb or of the corpus spongiosum, lying usually about half an inch from the base of the triangular ligament, the artery of the crus or of the corpus cavernosum, and the dorsal artery of the penis; a plexus of veins lying in the compressor urethræ muscle, and receiving its tributaries from the corresponding corpus cavernosum, and one half of the corpus spongiosum and bulb; and Cowper's glands, two in number, situated one at either side of the middle line, underneath the membranous part of the urethra. The postero-superior or deep layer of the triangular ligament is next to be dissected. It is very ill-defined, and will be found about half an inch above and behind the antero-inferior layer. The structures which pierce it are to be noted, and the anterior diverticulum of the ischio-rectal fossa should now be studied, its floor being formed by the postero-superior layer. The membranous part of the urethra, with the catheter lying in it, is to be examined. The fibres of the postero-superior layer of the triangular ligament should be cut through in order to show the anterior fibres of the levator ani. The various structures which lie between the two layers of the triangular ligament are now to he carefully reviewed.

The dissector is next to direct his attention to the internal pudic artery and pudic nerve, making whatever dissection may now be necessary. The course, relations, and branches of these structures are to be studied. The structures which are divided in the operation of left lateral lithotomy are to be mastered, and, by way of concluding the dissection of the perineum, the dissector should cut transversely downwards at the central tendinous point so as to enable him to turn the anal canal and rectum well backwards. This will expose the rectal surface of the prostate gland.

\section{Female Perineum.}

The dissector should practise passing the female catheter before the subject is placed in the lithotomy position. When placed in position, he should make himself familiar with the landmarks of the perineum. Its three divisions are to be noted, and each constituent part of the pudendum 
or vulva is to be carefully inspected. The guide already given to the dissection of the ischio-rectal or anal division of the imale perineum is equally applicable to that of the female. An effort should be made to show the perineal body, which is peculiar to the female. The guide to it is the central tendinous point, of which it may be regarded as a deep expansion. In connection with the vaginal orifice the following structures are to be dissected: the bulbo-cavernosus or sphincter vaginæ; the bulbi vestibuli, one on either side of the orifice, and the pars intermedia of Kobelt, which is situated in front of the bulbi vestibuli ; and the glands of Bartholin, which are also situated on either side of the vaginal orifice, just posterior to the bulbi vestibuli. If these glands should be displayed, an effort should be made to dissect their ducts, each of which is about three-quarters of an inch in length. The muscles requiring special attention are the following: the bulbo-cavernosus or sphincter vaginæ already dissected; and the ischio-cavernosus, or erector clitoridis. The other muscles are practically similar in both sexes. The internal pudic artery takes a similar course in the female to what it does in the male. The pudic nerve is practically the same in both sexes.

\section{Antero-lateral Abdominal Wall.}

The first duty of the dissector is to make himself familiar with the surfacemarkings of the anterior wall of the abdomen. The abdomen is to be inflated with air, by introducing a blow-pipe through the umbilicus. On withdrawing the blow-pipe, the opening is to be secured by a ligature, a circular cut through the skin, around and close to the umbilicus, having been previously made in order to give the string a hold. The skin is to be removed by making the following incisions : a median incision extending from the ensiform process of the sternum to the upper border of the symphysis pubis ; an incision along the line of Poupart's ligament from the anterior superior iliac spine to the lower end of the median incision; a transverse incision from the ensiform process to a point as far outwards as the position of the body will allow; and another transverse incision from the anterior superior iliac spine inwards as far as the median line. The skin having been removed, the arrangement of the superficial fascia over the lower part of the anterior abdominal wall is to be studied. The region referred to extends from the level of Poupart's ligament upwards to the level of a line extending horizontally inwards from the anterior superior iliac spine to the linea alba.

A dissection of this region will show that the superficial fascia is divisible into a subcutaneous fatty layer, called Camper's fascia, and a deep membranous and elastic layer, known as Scarpa's fascia. This arrangement of the superficial fascia will at once recall to the dissector a similar arrangement of the superficial fascia of the anterior division of the perineum, the deep layer being there known as the fascia of Colles. A good guide to the separation between Camper's fascia and Scarpa's fascia is the superficial epigastric vessels (especially the vein), Camper's fascia being superficial to them and Scarpa's fascia underneath them. The difference in the naked-eye appearance of the two fasciæ is usually a sufficient indication of the existence of two layers, Camper's fascia being fatty, and Scarpa's fascia membranous. Along the line of the groin the separation is quite distinct, being here effected not only by the superficial epigastric vessels, but also by the inguinal glands (superior or oblique superficial inguinal glands). The dispositions of Camper's fascia and Scarpa's fascia are to be very carefully studied, especially the disposition of Scarpa's fascia, beneath which extravasated urine ultimately comes to lie in cases of rupture of the bulb of the urethra in the perineum. A careful incision is to be made through Camper's fascia transversely inwards from the anterior superior iliac spine. Before dividing it along the linea alba its contiuuity with the corresponding fascia of the opposite side is to be noted, and, in turning it downwards to the front of the thigh, this should be done in concert with the dissector of the lower limb. A similar transverse incision is next to be made through Scarpa's fascia as deep as the external oblique aponeurosis, and another vertical incision along the linea alba. The fascia 
is then to be reflected downwards, partly by using the handle of the scalpel, and partly by using the fingers. Its connection with the external oblique aponeurosis is to be noted; its attachments along the line of the groin are to be demonstrated; and the manner in which this fascia, along with the fascia of Camper, is conducted by the spermatic cord into the scrotum to form the tunica dartos (or by the round ligament of the uterus into the labium majus). is to be studied. In the region of the symphysis pubis the suspensory ligament of the penis is to be dissected. The deep fascia is next to be examined. It will be found to be present over the lateral wall of the abdomen, where the external oblique muscle is fleshy, but, on being traced forwards, it will be seen to fade away, and ultimately to disappear, in the region of the aponeurosis of that muscle.

In the course of the foregoing dissection the superficial arteries, with their accompanying veins, are to be displayed, namely, the superior or superficial external pudic, crossing inwards and upwards over the spermatic cord (or round ligament of the uterus) to the suprapubic region; the superficial epigastric, turning upwards over Poupart's ligament a little to the inner side of the centre; the superficial circumflex iliac, lying below the outer part of Poupart's ligament; the anterior cutaneous arteries, emerging through the anterior wall of the sheath of the rectus abdominis, in a somewhat irregular manner, and at variable distances from the linea alba; and the lateral cutaneous arteries, appearing between the serrations of the external oblique in the mid-axillary line. Simultaneously with the dissection of the foregoing bloodvessels, the following cutaneous nerves should be dissected; the hypogastric branch of the ilio-hypogastric, piercing the external oblique aponeurosis about an inch above the external abdominal ring; the anterior cutaneous nerves, emerging through the anterior wall of the sheath of the rectus abdominis in a straggling manner, some appearing near the linea alba, and others a little farther from it; the lateral cutaneous nerves, which will be found emerging between the serrations of the external oblique in the mid-axillary line, and each of which (with one exception, which immediately follows) divides into an anterior and $\mathrm{a}$ posterior branch; the undivided lateral cutaneous or iliac branch of the anterior primary division of the twelfth thoracic nerve, which should be shown descending over the iliac crest, about an inch behind the anterior superior iliac spine, to the anterior part of the gluteal region ; and the iliac branch of the ilio-hypogastric, descending to the gluteal region over the iliac crest at the junction of the middle and anterior thirds.

The superficial dissection of the penis and the dissection of the scrotum are next to receive attention. The prepuce and frenum praputii are to be examined, and the glans penis is to be studied, attention being paid to the meatus urinarius, corona glandis, and cervix. The median raphé of the scrotum is to be noted, and it may be traceable along the under surface of the penis. A catheter is then to be introduced into the bladder, and the penis is to be made tense by fixing it with string to the end of the instrument. The thin skin of the penis is to be carefully removed, and the reddish-coloured underlying fascia, continuous with the tunica dartos of the scrotum, is to be noted. If not previously dissected, the suspensory ligament is to be displayed, and its bilaminar disposition is to be shown. The fascial investment or sheath of the penis is to be studied, and thereafter the following structures are to be dissected: the dorsal vein, occupying a median position between the corpora cavernosa; the two dorsal arteries, one on either side of the vein; the dorsal nerves, each external to the corresponding artery; and the dorsal penile branches of the superior or superficial external pudic arteries, each lying external to the corresponding main dorsal artery. The dissection of the penis as regards structure is to be postponed until the pelvic cavity is dissected. The scrotum is now to be attended to. Having observed the dark colour and puckered condition of the skin, to the lateral aspect of which twigs of the inguinal nerve are distributed, the dissector is to remove it carefully, and thereafter study the brick-red scrotal fascia, known as the tunica dartos. The relation of the 
dartos tunic to Camper's and Scarpa's fasciæ of the anterior abdominal wall, and to the two layers of the superficial fascia of the anterior division of the perineum, is to be carefully noted. The testis. is next to be withdrawn from the scrotum, by making forcible traction upon the spermatic cord, and, when this has been done upon both sides, a view will be obtained of the interior of the scrotum. The dissector will notice that there are two scrotal chambers, each lodging a testis, and separated from one another by a partition, known as the septum scroti. The constituent parts of the scrotal wall and scrotal septum are next to receive attention, and the blood-supply and nerve-supply are to be fully reviewed.

The dissector should now replace the testis in the scrotum, and return to the dissection of the antero-lateral wall of the abdomen. The external oblique is to be dissected and studied. In dissecting the posterior border of the muscle its relation to the latissimus dorsi is to be noted. If it is not overlapped by the last-named muscle, the interval between them, called the triangle of Petit, is to be examined. The interdigitations with the latissimus dorsi and serratus magnus are to be shown, and, in connection with the external oblique aponeurosis, the intercolumnar fibres are to be displayed. In the region of the external abdominal ring these fibres are to be shown folding themselves around the spermatic cord in the form of the finger of a glove, thus forming the intercolumnar or external spermatic fascia. The attachment of this fascia along the pubic crest is to be noted, and, partly on this account, and partly by reason of the attachment of the intercolumnar fibres to the pillars of the external abdominal ring, it will be evident that extravasated urine, in passing upwards along the spermatic cord, cannot enter the inguinal canal. The following parts of the external oblique aponeurosis require special attention : Poupart's ligament; Gimbernat's ligament ; the triangular fascia or ligament of Colles, which will be found lying within the external abdominal ring behind the spermatic cord and internal pillar; the external abdominal ring; and the linea alba. In connection with the external abdominal ring, its condition is to be carefully studied ( 1 ) in its natural state, (2) after partial dissection, a circular cut having been made in the intercolumnar fascia at the mouth of the ring, and (3) after complete dissection, the intercolumnar fibres having been completely removed. The following structures should be observed through the ring, namely, the triangular fascia or ligament of Colles, and the conjoined tendon of the internal oblique and transversalis abdominis. The position of the spermatic cord as it passes through the ring is to be noted.

The removal of the external oblique is to be effected in the following manner : an incision is to be carried inwards through the aponeurosis from the anterior superior iliac spine to the linea alba, and another is to be made vertically downwards in the median line to the symphysis pubis. The triangular flap of the aponeurosis so included, containing the external ring, is to be turned downwards, and left intact, so that it can be replaced when studying the inguinal region in connection with hernia. Beneath this portion of the aponeurosis the dissector is to preserve the hypogastric branch of the iliohypogastric nerve as far outwards as about an inch in front of the anterior superior iliac spine. The external oblique is next to be detached from the iliac crest and lower eight ribs, preserving the lateral cutaneous nerves, and the muscle, with the remainder of its aponeurosis, is to be reflected as far forwards as possible. At no point is it possible to reflect it quite up to the linea alba, but it can be reflected more completely below the level of the umbilicus than above it. The reason why the external oblique aponeurosis cannot be separately raised quite up to the linea alba will be seen to be because it blends inseparably with the subjacent anterior lamina of the aponeurosis of the internal oblique. This latter muscle is now to be cleaned, preserving the hypogastric branch of the ilio-hypogastric nerve, and the latcral cutaneous nerves, all of which pierce it. In cleaning the Poupart fibres of the muscle, the origin of the cremaster is to be brought into view. The complex relation of the Poupart fibres to the spermatic cord is to reccive attention, and the cremaster is to be dissected. In connection with this muscle the genital 
branch of the genito-crural nerve and the cremasteric branch of the deep epigastric artery are to be noted, and the cremasteric fascia is to be studied.

The internal oblique is to be removed in the following manner : make an incision carefully through the muscle just in front of the anterior superior iliac spine, and expose the lateral or intermuscular epigastric branch (so-called ascending branch) of the deep circumflex iliac artery. This is the best guide to the separation between the internal oblique and transversalis abdominis. Now carry an incision inwards through the internal oblique towards the linea alba, and leave the Poupart fibres undisturbed in the meantime. The internal oblique is next to be detached from the iliac crest, from the posterior aponeurosis of the transversalis abdominis, and from the lower three or four costal cartilages, and the muscle, with its aponeurosis, is to be thrown forwards as far only as the outer border of the rectus abdominis. During the reflection of the muscle care must be taken to preserve the following structures, namely, the lower five intercostal nerves; the anterior primary division of the twelfth thoracic nerve (subcostal nerve); the hypogastric branch of the ilio-hypogastric nerve; the inguinal (so-called ilio-inguinal) nerve; the lateral or intermuscular epigastric artery; the abdominal branches of the lumbar arteries; and the lower two intercostal arteries. The transversalis abdominis is next to be cleaned and studied. The lower five intercostal nerves and the anterior primary division of the twelfth thoracic nerve are to be followed forwards as far as the outer border of the rectus abdominis, where they disappear by piercing the sheath to get behind the muscle. The posterior aponeurosis of the muscle (which forms the lumbar aponeurosis) cannot be displayed in the present position of the body, but the anterior aponeurosis should be dissected as far as the outer border of the rectus abdominis, where it will be further studied in connection with the sheath of that muscle.

The dissector should now direct his attention to the Poupart fibres of the internal oblique and transversalis abdominis. He will find that the Poupart fibres of the two muscles are intimately connected together, but a careful dissection will show that, whilst the internal oblique arises from the outer half of Poupart's ligament on its abdominal aspect, the transversalis abdominis arises only from the outer third. The former muscle, then, reaches lower down along Poupart's ligament than the latter. An incision having been made through the transversalis abdominis in a transverse direction inwards from the anterior superior iliac spine, the Poupart fibres of the two muscles are to be detached from the ligament, and, in the course of reflecting them inwards without injuring the fascia transversalis, the dissector should observe their varying relations to the spermatic cord and internal abdominal ring, and also the lower border of each muscle. These fibres of the two muscles will be found to end in the conjoined tendon, and this structure should be followed to its insertion and studied. It will be found that the anterior aponeurosis of the transversalis abdominis contributes more to the conjoined tendon than the aponeurosis of the internal oblique. The relation of the conjoined tendon to Hesselbach's triangle will be presently seen. Mleanwhile the dissector is to observe that the tendon lies behind the spermatic cord (or round ligament of the uterus), and that it also lies behind the external abdominal ring, thereby strengthening an otherwise weak part of the abdominal wall. The dissector should look for the reflected tendon of Cooper. If present, it will be found as a thin semilunar expansion, extending outwards from the outer border of the conjoined tendon to the inner and lower part of the internal abdominal ring, where it is attached to the deep crural arch. Its relation to Hesselbach's triangle, as well as to the inguinal canal, will be seen further on in the dissection. At this stage the dissector of the abdomen should associate himself with the dissector of the lower limb in order to make a dissection of the deep crural arch.

The anterior wall of the sheath of the rectus abdominis is now to be opened and reflected by making a vertical incision through it about an inch and a half from the linea alba, and turning one half inwards and the other outwarls, preserving at the same time the transmitted nerves. In doing so the close 
connection between the anterior wall of the sheath and the tendinous inscriptions of the muscle is to be noted at the following levels, namely, at the umbilicus, opposite the tip of the ensiform process, and about midway between these two points. Occasionally a fourth connection is established a little below the umbilicus. The anterior wall of the sheath having been reflected, the pyramidalis muscle should be dissected. If present, it will be found in front of the lower part of the rectus abdominis. The rectus itself is then to be fully dissected. Its two heads of origin and three slips of insertion are to be shown, and the three (sometimes four) tendinous inscriptions are to be studied. The varying relation of the inner border of the muscle to its fellow along the linea alba is to be observed, and both borders of the muscle are to be freed from their connections. In freeing the outer border, the lower five intercostal nerves and the anterior primary division of the twelfth thoracic nerve are to be shown on their way to the back of the nuscle (within the sheath). The deep epigastric artery is to be displayed, disappearing beneath the outer border of the muscle at a point about midway between the upper border of the symphysis pubis and the umbilicus. Before further disturbing the muscle, the triangle of Hesselbach is to be studied. It will be found between the lower fourth of the rectus abdominis and the deep epigastric vessels. The relation of the conjoined tendon to the triangle, and the manner in which it divides the triangle into two compartments are to be noted. If the reflected tendon of Cooper should be present, its relation to the triangle is also to be noted.

The rectus abdominis is now to be raised from its sheath, but not yet divided. As this is being done, the lower five intercostal nerves and the anterior primary division of the twelfth thoracic nerve will be seen making their way from behind forwards through the muscle to become anterior cutaneous nerves. At this stage also the superior epigastric artery is to be shown entering the deep surface of the upper part of the muscle from behind the seventh costal cartilage. The continuation of the deep epigastric artery in the substance of the muscle is to be dissected from behind to a point about 2 inches above the umbilicus, and the anastomosis between it and the superior epigastric at that level may be shown. The sheath of the rectus is next to be studied, the muscle itself having been previously divided a little above the umbilicus. The splitting of the internal oblique aponeurosis into two laminæ anterior and posterior, along the outer border of the muscle is to be shown. This, however, will be found to cease at a point a little above the level of the anterior superior iliac spine, below which point the undivided aponeurosis will be found to pass in front of the muscle. The anterior aponeurosis of the transversalis abdominis will be seen to pass behind the rectus for a similar extent to that over which the splitting of the internal oblique aporieurosis takes place ; but, over the lower part of the muscle (below a point a little above the level of the anterior superior iliac spine), it passes in front of it. The deficiency of the sheath posteriorly over its lower fourth is to be noted, and the semilunar fold of Douglas, with the deep epigastric artery passing over it, is to be studied. Sometimes the anterior aponeurosis of the transversalis abdominis will be found to send scattered fibres behind the rectus for a variable distance below the semilunar fold of Douglas. In such cases another curved semilunar fold, known as the band of Henle, is to be looked for from I to 2 inches below the semilunar fold of Douglas. The composition of the sheath in front and behind is to be mastered; the different attachments superiorly of the anterior and posterior walls are to be noted; the contents of the sheath are to be studied; and the linea alba should be thoroughly revised. The dissector should next direct attention to that part of the deep epigastric artery which forms the outer boundary of Hesselbach's triangle, showing its relations to the internal abdominal ring and fascia transversalis, and also its two venæ comites. The cremasteric and pubic branches of the artery are to be shown, but the artery itself is not to be followed, at this stage, to its origin from the external iliac. The deep epi. gastric artery should now be studied throughout its course, its branclies should be niastered, and the anastomoses of the vessel should be carefully attended to. 
The next stage of the dissection consists in ( $\mathrm{I}$ ) detaching the transversalis abdominis from the iliac crest, (2) cutting through posterior aponeurosis in the region between the iliac crest and the last rib, and (3) dividing the muscle as it escapes from beneath the costal margin. When the muscle has been reflected forwards to the outer border of the rectus, the various nerves on its surface having been divided in front and turned outwards, and the ilio-hypogastric and inguinal nerves being carefully preserved in the region of the iliac crest, a good view will be obtained of the fascia transversalis. Before proceeding to study this fascia, however, it will well repay the dissector to replace for a moinent the external oblique, internal oblique, and transversalis abdominis, in order to show the intricate piece of lattice-work which these muscles construct by the varying direction of their fibres, a circumstance which adds materially to the strength of the abdominal wall. The fascia transversalis should be followed upwards beneath the costal margin, where, now very thin, it becomes continuous with the areolar investment of the diaphragm. Towards the lower part of the abdominal wall it becomes better marked. Its disposition along the linea alba and along the iliac crest is to be studied ; its arrangement along the line of Poupart's ligament should be carefully attended to ; the deep crural arch is to be revised in connection with it ; its relation to Hesselbach's triangle is to be noted; and the internal abdominal ring, with the infundibuliform fascia prolonged from its margins, is to receive the closest attention. To see the ring the spermatic cord (or round ligament of the uterus) should be pulled upon, and a circular incision made through the infundibuliform fascia close to the ring. In performing this dissection, it will be seen that the infundibuliform fascia is prolonged upon the cord as the internal spermatic fascia. If the cord be pulled upon it will be seen to receive a covering from the subperitoneal fat or areolar tissue through the internal ring. The internal abdominal ring in all its bearings is to be fully studied, and the relation of the deep epigastric vessels to it carefully noted. At this stage the deep circumflex iliac artery should be dissected in the first part of its course. It will be found in a canal behind the outer half of Poupart's ligament formed by the meeting of the fascia transversalis and fascia iliaca, but it should not be dissected at this stage beyond the anterior superior iliac spine, in which region it will be found to pierce the fascia transversalis.

The inguinal canal is next to be attended to, and its boundaries and relations studied. The spermatic cord, or round ligament of the uterus, according to the sex, is to be observed traversing it throughout, and the inguinal nerve will be found in the lower two-thirds of the canal, after which it will be seen to emerge through the external abdominal ring external to the spermatic cord. Hesselbach's triangle should be revised at this stage, and the relation of the conjoined tendon to the inguinal canal is to be noted. Further, if the reflected tendon of Cooper should be present, its relation to the canal is to be observed. The spermatic cord is next to be dissected. Lying in front of its upper part for a variable distance may be found a thread-like prolongation of the peritoneum. The various coverings of the cord are to be studied, and the dissector is to note that there are three spermatic fascix. By teasing and separation the various constituents of the cord are to be shown. Certain of these will not be easily made out, such as the spermatic plexus of nerves and the lymphatics of the testis, but the vas deferens can be separated without difficulty. The spermatic artery (in the centre of the cord), the pampiniform plexus of veins, and the deferential artery are to be looked for. The round ligament of the uterus (a very variable structure in this part of its course) requires very little dissection, and may be followed into the labium majus. A few fibres, representing the cremaster muscle in the male, should be looked for in front of the round ligament. The fascia transversalis is now to be removed as carefully as possible, and in doing so the dissector should observe that it is connected to the underlying peritoneum by the subperitoneal fat or areolar tissue. In connection with this tissue two points have to be noted, namely, (1) internal to the external iliac vein it forms the septum crurale, closing the crural or femoral ring, which should be studied iu conjunction with the 
dissector of the lower limb ; and (2) at the internal ring it is carried downwards with the spermatic cord.

The parietal peritoneum is now to be examined. It will be readily recognised by its bluish-white colour, and upon it a small nodule is to be looked for just behind the internal abdominal ring, representing the upper end of the original processus vaginalis. Instead of a mere nodule the dissector may find a fibrous thread descending for a variable distance on the spermatic cord, and sometimes containing a canal in the female, known as the canal of Nuck. The disposition of the fascia transversalis and fascia iliaca along the outer half of Poupart's ligament, and in the region of the external iliac vessels at the groin is to be attended to, the deep crural arch being at the same time revised. Gimbernat's ligannent may be looked to at this stage, but its complete examination should be postponed till a later period.

The abdominal cavity is now to be opened. This is to be done only partially at first by means of the following incisions : commencing immediately below the umbilicus, an incision is to be made downwards and outwards, on either side, to Poupart's ligament at the junction of its inner two-thirds and outer third. In this manner a triangular portion of the anterior abdominal wall below the umbilicus can be thrown down. The parietal peritoneum lining this portion having been sponged, the following plicæ and fossæ are to be studied : (I) Folds. - (a) plica urachi, in the middle line; (b) plica hypogastrica; and $(c)$ plica epigastrica, which is usually a little external to the plica hypogastrica, though sometimes the two correspond. (2) Fossæ.-These are the internal, middle, and external inguinal fossæ. The relations of the internal and middle fossæ to the two divisions of Hesselbach's triangle are to be noted, as well as the relation of the external fossa to the internal abdominal ring. At this stage the dissector should study the descent of the testis, and the abnormal conditions of the processus vaginalis. A complete revisal of all the structures of the abdominal wall in the region of Poupart's ligament should now be made, and thereafter inguinal hernia is to receive careful attention in the following order: external oblique hernia; internal direct hernia : internal oblique hernia; congenital hernia; and infantile hernia. Having mastered these varieties of hernia, the dissector should now turn his attention to umbilical hernia, or exomphalos. The relation of structures at the pubic crest is next to be mastered, as well as the relation of parts at the pectineal portion of the ilio-pectineal line.

It will be proper to dissect the testis at this stage, if it is in proper condition. If not, it should be delayed until the organ has been sufficiently hardened by immersion in spirit. The testis, along with the lower part of the spermatic cord, is to be removed and laid upon a board, and the parts are to be kept in position by pins. The tunica vaginalis is to be studied, and the dissector should note that the lower fibres of the cremaster spread out in a scattered manner over its upper part. An attempt should be made to inflate it, by making an aperture in it, just large enough to admit the small end of a blow-pipe. If this is successfully done, an instructive view of the membrane will be obtained. The tunica vaginalis is then to be divided by a vertical incision, when its two parts will come readily into view, namely, tunica vaginalis scroti and tunica vaginalis testis. These two parts having been studied, attention is next to be directed to the following structures: (I) the body of the testis, closely invested by the dense tunica albuginea, which, in turn, is closely covered by the tunica vaginalis testis; $(2)$ the epididymis, which will be seen lying along the posterior border and adjacent portion of the external surface of the testis; and (3) the digital fossa, which is a recess formed by the tunica vaginalis testis between the testis and the epididymis. The epididymis will be seen to be composed of a head or globus major, situated at the upper end; a body, succeeding to the head, long and narrow ; and a tail or globus minor, situated at the lower end of the body. Before proceeding further, the dissector should look carefully for the following vestiges of embryonic structures, namely (I) the hydatids of Morgagni, whicl will usually be seen on the front of the globus major, or on the front of the testis just below the globus major; (2) the organ of Giraldés or para- 
didymis, situated on the front of the spermatic cord immediately above the globus major, beneath the visceral layer of the funicular portion of the tunica vaginalis ; and (3) the vas aberrans of Haller, situated at the point where the globus minor passes into the vas deferens. The tissue between the globus major and the testis should be carefully unravelled, and an effort made to show the coni vasculosi entering the globus major after leaving the testis. The vas deferens should be shown as it passes off from the globus minor, and the tortuosity which it here presents is to be noted. The tunica albuginea is next to be opened by making a vertical, median, anterior incision. On separating the two halves from the substance of the testis the dissector is to observe the fibrous processes, thereby torn, which pass into the interior of the organ between the lobuli testis. An attempt should now be made to show the lobuli testis by teasing out the glandular tissue, which may bring into view the tubuli seminiferi. A transverse incision should next be made through the testis, in order to show the forward projection of the tunica albuginea from the back part of the testis into the interior for a short distance, known as the corpus Highmorianum, or mediastinum testis.

The abdominal wall above the umbilicus is to be removed by making a vertical incision through it at either side of the middle line, thus preserving meanwhile a median strip. The lateral parts of the wall are then to be divided along each costal margin as far back as the position of the body will allow. Reverting to the median vertical strip, the dissector should study on its posterior surface a median fold of parietal peritoneum, representing a part of the falciform ligament of the liver, which contains the obliterated umbilical vein. The umbilicus and this peritoneal fold are to be preserved.

Without in any way disturbing the contents of the abdominal cavity, the dissector should remove with a sponge any fluid which may have accumulated. He should then make himself acquainted with the various regions into which the cavity is divided, and the lines and planes by which these divisions are effected. He should also study the boundaries of the cavity. The succeeding stages of the dissection being very complicated, it is ad"usable that the dissector should exercise considerable method in his mode of procedure. His first duty is to take a superficial view of the contents of the abdomen in their undisturbed position, and this should be done in the following order : the liver, the stomach, and the great omentum, depending from the great curvature of the stomach. It should be noted that the great omentum descends lower on the left side than on the right, and that a few coils of the ileum are visible beyond it on the right side. The crecum will be partly seen in the right iliac fossa, and the iliac colon is partly visible in the left iliac fossa.

The great omentum is next to be raised and laid over the costal margin, when the coils of the jejunum and ileum will come into view. The transverse colon will also be seen crossing from the right to the left hypochondrium. If the urinary bladder should be distended, a part of it would be seen in the hypogastrium. The foregoing represent the contents which are seen on taking a superficial view. The coils of the jejunum and ileum should now be carried over to the left side, and the ascending colon examined. Superiorly it will lead to the hepatic flexure, and, without disturbing the parts, the right kidney should be felt. The cæecum will now be fully exposed at the lower end of the ascending colon, and the vermiform appendix, and the mode of ending of the ileum in the large intestine, should be carefully examined. The coils of the jejunum and ileum are then to be carried over to the right side, and the descending colon, commencing above at the splenic flexure and terminating below in the iliac colon, is to be examined. The left kidney is to be felt for, and, if the jejunum is followed upwards, the duodeno jejunal flexure will be exposed.

The dissector should now study (1) the position and connections of the stomach, and (2) the position and connections of the intestinal canal (except the duodenum, pelvic colon, and re:tum). In connection with the sma!l intestine the possible presence of Meckel's diverticulum, about 3 feet from the ileo-creal valve, is to be borne in mind. In studying the laige intestin? 
special attention should be paid to the cæcum, including its varieties, and the vermiform appendix. The ascending, transverse, descending, and iliac colon should next be studied in the order named. The position and connections of the spleen are next to be studied, and for this purpose the stomach is to be well drawn out from the left hypochondrium. It is to be remembered that occasionally accessory spleens are met witl in the gastro-splenic omentum, or in the great omentum. The dissector is next to turn his attention to the position, connections, ligaments, and component parts of the liver, in so far as they can at present be made out. The gall-bladder is to be included in this examination.

A study of the foregoing structures will have prepared the dissector for the examination of the peritoneum. The description given of this mem. brane and its various folds should be read, and it should be compared with, and verified from, the dissection. In a great number of bodies, however, verification is rendered impossible by the existence of adhesions, but the dissector should do his best. The continuity between the parietal and visceral peritoneum is to be shown in two ways, namely, in the vertical direction, and in the transverse direction. The continuity can be demonstrated in the vertical direction without much difficulty, and the best starting point is the portal fissure of the liver. The peritoneum is to be traced in the transverse direction at two levels, namely, above the transverse colon, or at the level of the foramen of Winslow, and below the transverse colon, or at the level of the umbilicus. The former is difficult of accomplishment, but the dissector should try his best. The latter will not cause much trouble. The various folds formed by the peritoneum, namely, omenta, mesenteries, and ligaments, are next to be studied in the following order: (I) the great omentum ; (2) the small omentum; $(3)$ the gastro-splenic ornentum; (4) the mesentery proper; (5) the appendicular mesentery or meso-appendix; $(6)$ the transverse meso-colon; $(7)$ the pelvic meso-colon (which, however, belongs to the pelvis); (8) the peritoneal ligaments of the liver; (9) the peritoneal ligament of the stomach, namely, the gastrophrenic ligament; (Io) the peritoneal ligaments of the spleen, namely, the lieno-phrenic and lieno-renal ligaments; and (II) the peritoneal ligament of the splenic flexure of the colon, namely, the phreno-colic or costo-colic ligament (sustentaculum lienis). In connection with the ascending colon, a peritoneal fold, known as the sustentaculum hepatis, may be met with.

The cavity of the peritoneum is next to be studied, attention being given first to the great cavity, and then to the small cavity. The boundaries of the small cavity are to be studied, which will be facilitated by making an incision through the descending layers of the great omentum about an inch below the great curvature of the stomach. The hand should then be introduced through the incision into the small cavity, and, unless there are adhesions, the dissector will be able to insinuate it downwards between the descending and ascen ling layers of the great omentum until it is arrested where that omentum describes its bend. In this way the bag or sac of the omentum (great) is demonstrated He should also pass his hand upwards behind the stomach and gastro-hepatic omentum, and he will feel Spigel's lobe of the liver at the upper end of the small cavity. Thereafter the foramen of Winslow should be mastered. It will be found behind the right or free border of the gastrohepatic omentum. In order to familiarize himself with this opening, the dissector should again pass his hand into the small cavity throngh the incision already made below the stomach, keeping the thumb outside in the general cavity. If the hand (except the thumb) is now carried upwards behind the stomach and gastro-hepatic omentum, the index finger can easily be pushed through the foramen of Winslow (unless it is blocked by inflammatory pro$\bar{a}=$ ts) into the great cavity, where it can be made to meet the thumb. Again, the index finger of one hand should be passed through the foramen into the small cavity, and the thumb of the same hand should be brought down upon the right or free border of the gastro-hepatic omentum. This having been done, the dissector will have two layers of peritoneum between the index finger and thumb, one of which belongs to the small cavity, and the other 
to the great cavity. The structures contained between these two layers are to be noted. As the finger lies in the foramen of Winslow the caudate lobe of the liver will be felt above it. It is possible for a hernia to occur through this foramen.

The relation of the duodenum to the peritoneum should next be attended to. Certain peritoneal fossæ are to be studied in the following order: (I) the duodenal and duodeno-jejunal fossa; (2) the peri-creal fossæ, namely, ileo-colic, ileo-cæcal, and retro-cæcal ; and (3) the intersigmoid. The study of the peritoneum in the adult having been concluded, the dissector should attend to the condition of the peritoneum in the fotus.

The dissection of the mesentery proper should now be proceeded with. The great omentum, with the transverse colon, is to be raised and laid over the costal nargin, and the jejunum and ileum are to be carried over to the left side. The dissector is to place portions of the mesentery successively upon a platter, and remove the right or upper layer. The trunk of the superior mesenteric artery is to be dissected beyond the point where it escapes from under cover of the pancreas, and the superior mesenteric sympathetic plexus around it is to be noted, as well as the superior mesenteric vein on its right side. About tweive rami intestini tenuis are to be shown arising from the left side of the parent trunk, and these are to be followed out to their primary, secondary, and at least tertiary arches, attention being given at the same time to the accompanying sympathetic plexuses and veins. During this dissection the mesenteric glands are to be shown, and an effort should be made to display the lacteals, which enter the glands as afferent vessels, and emerge from them as efferent vessels.

The right side of the superior mesenteric artery will be found to give off, in order from above downwards, the inferior pancreatico-duodenal, middle colic, right colic, and ilco-colic branches, but they should be followed out from below upwards. The ileo-colic is to be shown dividing into a colic and an ileo-cæcal branch, the latter of which will be found to give off appendicular. anterior cæcal, posterior cæcal, and ileal branches. The right colic and middle colic are to be shown, each dividing into two branches, the latter lying between the two layers of the transverse meso-colon. Throughout this dissection the tributaries of the superior mesenteric vein are to be noted, as well as the offshoots from the superior mesenteric sympathetic plexus. The inferior pancreatico-duodenal artery should not be followed out at this stage.

The jejunum and ileum are now to be carried over to the right side, and the left branch of the middle colic artery is to be followed to its termination. This will guide the dissector to the descending branch of the left colic artery, and, as he follows this down, he will readily expose the trunk of the inferior mesenteric artery, arising from the aorta about $1 \frac{1}{2}$ inches above the bifurcation. In doing so, he is to note that the artery is behind the parietal peritoneum. It will be found to give off a left colic branch, one, two, or three sigmoid branches, and a superior hemorrhoidal branch. The inferior mesenteric sympathetic plexus and ganglion, and the offshoots from the plexus should be noted. The left colic and superior sigmoid arteries are to be followed to their distribution, and the superior hemorrhoidal is to be shown crossing the left common iliac vessels on its way to the pelvis, where it disappears between the two layers of the pelvic meso-colon. The superior hemorrhoidal, sigmoid, and left colic veins will be found to form the inferior mesenteric vein, which is to be followed upwards on the left psoas magnus as high as the pancreas, care being taken to show that it crosses the left spermatic artery and left renal vein.

The intestines are now to be removed in the following manner: a double ligature is to be placed upon the jejunum at its commencement, and another double ligature at the point where the iliac colon passes into the pelvic colon. The gut is then to be divided between each of these double ligatures, and the jejunum and ileum are to be separated from the mesentery proper by dividing the latter with a knife, or clipping it with scissors. When this has been done, the large intestine should be forcibly drawn out from its deep position, any adhesions which it has formed being severed with a knife. The parts of intestine left behind are ( 1 ) the duodenum, and (2) the pelvic colon and rectum. 
The portion of gut removed is to be cleansed by allowing water to flow through it, and small portions are to be separated, inflated, and dried for future inspection. These portions are to be taken from the following regions : (1) the upper and lower ends of the jejunum ; (2) the upper end, and near the lower end, of the ileum; and (3) two pieces from the ascending colon. One of these pieces, from the small intestine, is to be dissected in the inflated and moist condition, in order to show the longitudinal and circular muscular fibres. One of the pieces from the ascending colon, when inflated, should also be dissected in the moist condition.

The sacculated appearance and the three muscular tæniæ having been noted, the peritoneum is to be carefully removed, and the arrangement of the muscular fibres studied. Thereafter the three treniæ are to be divided, and the elongation which then takes place in the wall of the gut is to be observed.

The small intestine is to be opened along the mesenteric border by means of a pair of scissors, one of the points of which has been guarded with a piece of cork. The gut is then to be spread out upon a board, and the characters of the mucous nembrane are to be studied. A small portion should be examined under water with the aid of an ordinary lens, which will make clear the villi. With the exception of the crcum and the first 2 inches of the ascending colon, the large intestine is to be treated in a similar manner. The cæcum and 2 inches of the ascending colon, along with the last 2 inches of the ileum, are to be treated in a different manner. The bowel is to be filled with water from the colic end, the ileal end being left free. It will then be seen that, in distension of the cæcum, no water escapes into the ileum, thus demonstrating the action of the ileo-cæcal valve. The exact attachment of the base of the vermiform appendix should be noted, and the meso-appendix should receive attention. The cæcum and colon are then to be laid open along the right wall, or that opposite the ileum, and the ileo-crcal valve is to be studied. The opening of the vermiform appendix should be examined, and the valve of Gerlach, guarding it, noted. The extent to which the appendix is pervious should also be attended to.

It is advisable that the dissectors should associate themselves with the dissectors of another abdomen, in order that the cæcum and parts of the ascending colon and ileum from one abdomen may be inflated and dried, so as to allow of the ileo-cæcal valve being examined in the dried state.

The intestines having been studied, the dissector is to cut away the remainder of the mesentery proper, leaving only small portions of the superior mesenteric vessels, as well as of the inferior mesenteric vein, for future reference. A blow-pipe having been introduced into the small portion of the jejunum which has been left, the duodenum and stomach are to be moderately inflated, and the stomach is to be turned upwards. When this has been done, the position and connections of the duodenum are to be studied, the musculus suspensorius duodeni is to be shown passing between the duodeno-jejunal flexure and the left crus of the diaphragm, and the peritoneal fossa in this region are to be revised. The position and connections of the pancreas are next to receive attention, followed by the spleen and the liver.

The blood-supply of the foregoing viscera should be shown by displaying the cœliac axis without injuring the important plexus of nerves which surrounds it. The axis will be found appearing at the superior border of the pancreas, where it breaks up into gastric, splenic, and hepatic branches, which are to be followed out in the order named. The gastric artery will be seen to pass upwards and to the left behind the small cavity of the peritoneum, and it is to be followed out. The splenic artery is recognised by taking a tortuous course to the left along the superior border of the pancreas, and its pancreatic, left gastro-epiploic, vasa brevia, and proper splenic branches are all to be shown. The hepatic artery is next to be dissected as far as the portal fissure of the liver. In displaying the vessel in front of the foramen of Winslow, opportunity is to be taken to show the common bile-duct descending on its right side, and the vena portæ ascending behind the two. The following branches are to be shown arising from the hepatic artery, all of which are to be followed out: (I) pyloric; (2) gastro-duodenal, dividing into superior 
pancreatico-duodenal aud right gastro-epiploic; and (3) right and left hepatic. The right hepatic branch will be found to give off the cystic artery, which divides into superior and inferior branches. It is at this stage that the inferior pancreatico-duodenal artery is to be studied.

In dissecting the foregoing arteries, the following groups of lymphatic glands are to be noted: (i) the cneliac glands surrounding the coliac axis; (2) the gastric glands arranged in two sets-superior, along the small curvature of the stomach, and inferior, along the great curvature: (3) the pancreatic glands along the superior border of the pancreas; (4) the splenic glands near the hilum of the spleen; and $(5)$ the hepatic glands within the gastro-hepatic omentum. The veins corresponding to the arteries are to be preserved, if possible, in connection with the portal system.

The vena portx is to receive careful attention. Its mode of formation behind the neck of the pancreas is to be noted, and its mode of tcrmination at the right extremity of the portal fissure of the liver, where it presents the portal sinus, is to be shown, with the cystic vein joining its right branch. The sources from which the vena portæ receives its blood should be thoroughly mastered. The common bile-duct is next to be studied. Its formation by the union of the hepatic duct with the cystic duct is to be dissected, and the hepatic duct itselt is to be shown as formed by the union of two ducts, one issuing from the right. and the other from the left, lobe of the liver. The duct is to be followed down in front of the foramen of Winslow, behind the first part of the duodenum. and between the head of the pancreas and the second part of the duodenum on their posterior aspect, to about as low as the junction of the upper twothirds and lower third of the second part. Its mode of termination in the interior of the second part will fall to be noted when the duodenum is opened for inspection.

The liver is now to be removed, in doing which the inferior vena cava should be ligatured below it, and then divided. It must also be divided above the liver, close to the diaphragm. The viscus is then to be thoroughly studied in all its aspects, and so also is the gall-bladder, the interior of which is to be examined to display the reticulated mucous membrane. An effort should be made at this stage to display the right and left pneumogastric nerves as they enter the abdomen through the cesophageal opening of the diaphragm. The right nerve may be followed to the posterior surface of the stomach, and the left to the anterior surface. The stomach, duodenum, pancreas, and spleen are then to be removed en masse, the osophagus having been previously tied below the seat of division. These organs are now to be carefully studied. After washing out the stomach and duodenum, they are to be opened and their interior examined. The main pancreatic duct, or duct of Wirsung, is to be shown by dissecting the pancreas from behind, and an effort should be made to find the small pancreatic duct, or duct of Santorini, in the head of the gland. The junction of the duct of Wirsung with the common bile-duct in the duodenal wall is to be displayed, and the common internal opening of the two ducts is to be examined. The spleen is to be studied, and sections of it should be made and washed, with pressure, under a stream of water, in order to remove the pulp, and show the trabecular framework.

\section{Posterior Wall of the Abdomen.}

The prevertebral sympathetic plexuses, namely, the epigastric or solar and hypogastric, with their secondary offshoots, are to be carefully dissected. The dissector will find the great splanchnic nerve piercing the crus of the diaphragm, and it will guide him to the semi-lunar ganglion. These ganglia, two in number, lie at either lateral pole of the solar plexus, close to each suprarenal capsule, that of the right side being under cover of the inferior vena cava. The lower part of each ganglion will be found to be semi-detached as the aortico-renal ganglion, which lies over the root of the renal artery, and terminating in this ganglion will be found the small splanchnic nerve, after it has pierced the crus of the diaphragm. The least splanchnic nerve, if present, may pierce the crus, or it may pass behind the ligamentum 
arcuatum internum, to terminate in the renal plexus. Passing inwards from each semilunar ganglion a matted leash of nerve-fibres, mixed with minute ganglia, is to be dissected. These fibres mect in front of the aorta, and invest the cœliac axis and root of the superior mesenteric artery, in which latter situation is the superior mesenteric ganglion. The offshoots from the solar plexus to be shown are as follows: inferior phrenic; cœliac, furnishing gastric, splenic, and hepatic; superior mesenteric; capsular; renal; aortic; spermatic (ovarian in the female); and inferior mesenteric. The spermatic plexus will be seen to come from the renal and aortic plexuses, and the aortic plexus is to be shown in two halves, right and left, which comnunicate freely with each other over the front of the aorta. The inferior mesenteric plexus will be found to arise chiefly from the left half of the aortic plexus, and the inferior mesenteric ganglion is to be looked for in it, near the root of the artery of that name. The various secondary plexuses will be found to accompany the corresponding branches of the abdominal aorta. The two halves of the aortic plexus, reinforced by branches from the ganglia of the lumbar sympathetic chains, are to be followed over the common iliac arteries, after which they will be found to blend, in the angle between these vessels, to form the hypogastric plexus. This latter is to be shown dividing into the right and left pelvic plexuses.

Attention is next to be directed to the kidneys and suprarenal capsules. The adipose tunic of the kidney is to be carefully removed, and the suprarenal capsule preserved on the upper part of the organ. The great vascularity and copious nerve-supply of the suprarenal capsule are to be noted, and only one vein is to be looked for, emerging from each through an anterior hilum, that of the right side opening into the inferior vena cava, and that of the left into the renal vein of that side. The structures at the hilum of the kidney are to be shown, and the relations of the organ carefully studied, the visceral areas on the anterior surface of each being noted. The inferior vena cava is next to be dissected, and its tributaries shown, one of these on the right side being the spermatic, or ovarian, vein, whilst the vein of the left side, in each sex, is a tributary of the left renal. The abdominal aorta and its branches are to be dissected, followed by the common iliac vessels. Along the front of the aorta the median lumbar glands should be looked for, and along each common iliac artery the so-called common iliac glands. By drawing aside the right crus of the diaphragm, the receptaculum chyli will be found on the right of the aorta, in front of the first and second lumbar vertebræ, and, on the right of it, the right azygos vein. The ureter is to be dissected as low as the pelvic brim, and the pelvis at its upper end is to be shown. The renal vessels are to be studied, and the kidney is then to be removed. The organ is to be divided into two halves by a vertical incision carried from the outer to the inner border. This will show the fibrous capsule (which should be stripped off), sinus, calices, Malpighian pyramids, and cortex.

The diaphragm is next to be dissected. Its central tendon, crura, ligamenta arcuata, and openings are to be studied. The iliac fascia is next to be dissected, with its upward prolongation on the psoas magnus, known as the psoas fascia. Its mode of attachment to the vertebral column and pelvic brim are to be noted, and its disposition at the outer border of the psoas magnus above the iliac crest is to be carefully studied. The nerves on the posterior wall arc now to be shown. Emerging from beneath the ligamentum arcuatum externum, and passing along the lower border of the twelfth rib, will be found the anterior primary division of the twelfth thoracic nerve (subcostal nerve), and with it the subcostal vessels. The genito-crural nerve pierces the psoas magnus close to its inner border, singly or in two branches, genital and crural, and these are to be followed, the former to the cremasteric muscular covering of the spermatic cord, and the latter to Poupart's ligament, beneath which it escapes on to the front of the thigh, close to the outer side of the external iliac artery. The ilio-hypogastric and inguinal nerves appear at the outer border of the psoas magnus, and cross the quadratus lumborum close together, the inguinal being the lower of the two. The external cutaneous nerve also appears at the outer border of the psoas magnus below 
the preceding, and is readily recognised by crossing the iliac fossa to a point beneath the outer end of Poupart's ligament, where it passes out to the thigh. The anterior crural nerve will be found lying deeply in the groove between the psoas magnus and iliacus, and the obturator nerve will be found lying deeply on the inner side of the psoas magnus, on its way to the lateral wall of the pelvis. If an accessory obturator nerve is present, it will usually be found passing deeply along the inner side of the psoas magnus, close to the pelvic brim, and then coursing over the superior pubic ramus, where it passes beneath the pectineus.

The psoas magnus is now to be dissected, and a psoas parvus is to be looked for. The nerves are tlien to be followed upwards and inwards to their origins, by cutting through the psoas magnus. In this manner the lumbar plexus will be exposed, which should be carefully dissected, the lumbo-sacral cord being shown, at the same time, descending to take part in the sacral plexus. The gangliated cord of the sympathetic is to be displayed in front of the bodies of the lumbar vertebra, and the length of its rami communicantes, which accompany the lumbar arteries, is to be noted. The lumbar branches of the aorta are now to be dissected as far back as the intervals between the lumbar transverse processes, where the lateral lumbar glands should be looked for. If the lumbar veins have not been previously dissected, this should now be done. In connection with these veins, at either side, the ascending lumbar vein is to be shown, that of the right side ending as the right azygos vein, and that of the left side ending as the lower left azygos vein. The posterior aponeurosis of the transversalis abdominis and the quadratus lumborum are next to be dissected, and, if that muscle is cut, it will be seen to be encased in a sheath which is formed by the anterior and middle laminx of the aponeurosis. The iliacus muscle and the external iliac vessels are now to be dissected, and the relation of the vessels to the peritoneum, subperitoneal tissue, and iliac fascia is to be carefully noted, as well as their relation to the genito-crural nerve and constituents of the spermatic cord. The principal branches of the artery, namely, the deep epigastric and deep circumflex iliac, are to be shown, and related to the artery will be found the external iliac glands, three of them being placed just above Poupart's ligament, one on the outer side, another on the inner side, and a third in front of the vessel. The deep circumflex iliac artery is to be followed behind the outer half of Poupart's ligament, and along the iliac crest, and its anastomoses are to be studied. The lacunar region, which lies between Poupart's ligament and the front of the hip-bone, is to be examined, and its two muscular lacuna, outer and inner, with the vascular lacuna in front of them, are to be s.lown, with the structures lying in each.

\section{Pelvis.}

The pelvis is to be separated by cutting through the disc between the third and fourth lumbar bodies, and then applying the saw. The various muscles connected with the exterior having been trimmed, the bladder is to be partially inflated, and the general position of the viscera and their relations are to be studied, as far as possible, in situ. The extent of the pelvic culon is to be noted, and its extensive mesentery, with the superior hemorrhoidal vessels between its two layers, observed.

The reflections of the peritoneum are then to be examined. If a male subject, the relation of the peritoneum to the bladder is to be thoroughly mastered, and the false ligaments, which it forms for the viscus, noted. The recto-vesical pouch of the peritoneum is also to be observed. If a female subject, the relation of the peritoneum to the uterus is to be studied, and the recto-uterine pouch, or pouch of Douglas, is to be carefully examined, which usually contains a few coils of the ileum, and, it may be, a prolapsed ovary. The broad ligament of the uterus, formed by two layers of peritoneum, is to be studied. The position of the Fallopian tube along its upper border, of the ovary with its ligament in a backward extension of it, and of the round ligament of the uterus in a forward extension of it, are to be observed. 
The exact position of the ovary should receive special attention, and the corpus fimbriatum of the Fallopian tube, with its ovarian fimbria, is to be noted.

The peritoneum is now to be stripped from the walls of the pelvis to expose the pelvic fascia. On the lateral wall of the cavity the obturator vessels and nerve are to be shown on their way to the obturator canal, through which they pass. The pelvic fascia is then to be carefully examinea. This fascia will be seen to line the lateral wall, where it covers the obturator internus, and the posterior wall, where it forms the fascia of the pyriformis. To see it in front, the bladder must be drawn well backwards. The lining portion of the fascia is called the parietal pelvic fascia. To study it more fully the dissector should remove a large part of the os innominatum by means of the following cut : place the saw over the back part of the iliac crest and over the pubic bone, keeping as near as possible to the pelvic brin, but external to it. The bone is now to be sawn downwards in such a manner as to remove a large part of the tuber ischii, but the great and small sciatic notches are to be avoided. The obturator externus muscle is then to be carefully removed from the outer surface of the obturator membrane, and the obturator artery is to be shown in two branches, which form a loop upon the membrane round its circumference. The obturator membrane is then to be removed, which will expose a portion of the obturator internus.

When this part of that muscle is taken away, a good view of the parietal pelvic fascia from the outside will be obtained through the obturator foramen. The thickened portion of the fascia, known as the white line, is to be observed extending from near the lower part of the symphysis pubis to the spine of the ischium. On looking deeply into the pelvic cavity, the bladder having been well displaced to one side, the parietal pelvic fascia, along the white line, will be seen to send inwards its visceral layer, and the portion of the parietal fascia below the white line will be seen, from the perineal point of view, to line the outer wall of the ischio-rectal fossa, where it contains Alcock's canal. From the under aspect of the white line the levator ani muscle arises, and immediately below this the parietal pelvic fascia gives off the anal fascia, which covers the perineal surface of the levator ani. The dissector will now understand what structures separate the pelvic cavity, on either side of the bladder, from the ischio-rectal fossa, this partition being formed, from above downwards, by the visceral pelvic fascia, the levator ani, and the anal fascia.

At the ipper part of the obturator foramen the parietal pelvic fascia should be shown, on the side not yet dissected, to join the upper border of the obturator membrane over the obturator internus muscle, thus converting the obturator groove on the under surface of the superior pubic ramus into a fitro-osseous tunnel, called the obturator canal. Through this canal the obturator vessels and nerve will be seen to pass, and it will be evident that the obturator artery escapes from the pelvic cavity without piercing the parietal pelvic fascia. As the parietal fascia is attached along the ischio-pubic ramus, at either side, it is continued inwards until it meets with the urethra. This portion of it, with its fellow, forms the postero-superior layer of the triangular ligament. From the urethra the fascia is continued backwards over the anterior border of the levator ani to the prostate gland, where it becomes continuous with the visceral layer of the fascia, which ensheathes that gland.

The visceral layer of the fascia is now to be followed inwards to the bladder, rectum, and prostate gland. Opposite the vesicula seminalis it divides into an upper or vesical, a middle or recto-vesical, and a lower or rectal layer, the vesical layer forming the lateral true ligament of the viscus. Opposite the prostate the visceral fascia divides into prostatic, recto-prostatic, and rectal layers. In the female the visceral fascia divides into four layers, namely, vesical, vesico-vaginal, recto-vaginal, and rectal. The bladder being pulled well backwards, two stout bands of the visceral pelvic fascia will be seen to extend from the lower part of the body of each pubic bone over the anterior surface of the prostate gland to the bladder. These constitute the anterior true ligaments of the bladder, and each will be found to contain some of its external longitudinal muscular fibres. Between these two ligaments a well- 
marked depression, occupied by fat, is to be shown. To facilitate this dissection, the pelvic brim may be removed on one side.

The prostate gland is now to be exposed, and the anterior fibres of the levator ani shown on its side. Within its fascial sheath will be found the prostatic venous plexus, which receives the dorsal vein of the penis in two divisions. If the subject is a female, a portion of the vagina is to be shown. The ureter, vas deferens, and obliterated hypogastric artery are to be displayed, and their relations noted. The ureter will be found on the lateral wall of the pelvis in front of the internal iliac artery, and, as it curves inwards to the lateral angle of the bladder, it will be seen to be crossed by the vas deferens. In the female, before reaching the bladder, it should be shown to lie on the side of the extravaginal portion of the cervix uteri, and for a short distance on the lateral wall of the vagina at its upper part. The obliterated hypogastric artery will be found passing forwards from the anterior division of the internal iliac artery over the lateral wall of the pelvis towards the apex of the bladder. Thereafter the internal iliac artery and its branches are to be dissected, as follows: from the anterior division, the pervious portion of the obliterated hypogastric, giving off two or three superior vesical arteries; inferior vesical, usually giving off middle hemorrhoidal; obturator; internal pudic; and sciatic; and, from the posterior division, the ilio-lumbar; two or three lateral sacral; and gluteal. The special branches in the female are the uterine and vaginal arteries. The arteries are accompanied by offshoots from the pelvic sympathetic plexus.

In dissecting the branches of the artery, care is to be taken of the nerves which arise from the sacral plexus. By displacing the viscera, this plexus and its branches are to be dissected, including the lumbo-sacral cord. In dissecting the plexus, the nerves which form the lumbo-sacral cord, the cord itself, and the first two sacral nerves are to be shown, by careful dissection, in two divisions, ventral and dorsal, and the third sacral (nervus bigeminus) is to be shown dividing into an upper and a lower brancin. The fourth sacral is a nervus furcalis. The sciatic and pudic bands of the plexus are to be displayed. The branches will be found to arise as follows : superior gluteal, by three roots from the dorsal divisions of the descending branch of the fourth lumbar, the fifth lumbar, and the first sacral; inferior gluteal, by three roots from the dorsal divisions of the fifth lumbar, and first and second sacral; muscular branches to the pyriformis, from the dorsal divisions of the first and second sacral ; nerve to the obturator internus and gemellus superior, by three roots from the ventral divisions of the fifth lumbar, and first and second sacral ; nerve to quadratus femoris and gemellus inferior. from the ventral divisions of the descending branch of the fourth lumbar, fifth lumbar, and first sacral ; visceral branches to the bladder, from the third and part of the fourth sacral ; small sciatic, by three roots from the posterior aspect of the first, second, and third sacral ; perforating cutaneous, by two roots from the posterior aspect of the second and third sacral; great sciatic, which is the continuation of the upper or sciatic band of the plexus; and pudic, which is the continuation of the lower or pudic band. The sacro-coccygeal plexus, formed by part of the fourth sacral, fifth sacral, and coccygeal nerves, is to be dissected, and the pelvic sympathetic cord is to be displayed with the ganglion impar on the first coccygeal vertebra. The middle and lateral sacral arteries are to be followed out, and an effort should be made to show the coccygeal gland of Luschka near the tip of the coccyx. Branches of the middle sacral artery may help to guide the dissector to it. The pyriformis. levator ani, and coccygeus muscles are to be dissected, and the relation of the latter two to the pelvic floor is to be noted. The obturator internus is also to be studied.

Viscera of the Pelvis.-The position of the rectum, and its relations to the bladder and prostate gland in the male, and uterus and vagina in the female, having been studied as far as possible in situ, the viscera are to be removed en masse along with the superior hemorrhoidal vessels. The bladder, if empty, should be partially inflated, and the rectum and anal canal slightly distended with tow, as well as the vagina, if the subject is a female. The relations of the 
rectum are now to be more fully examined, after which the gut is to be separated from the other structures, and subjected to a special dissection. The superior hemorrhoidal artery is to be followed out in two divisions, laterally disposer. The tow having been removed, and one end securely tied, the gut is to be inflated from the other end, which is then to be ligatured. The arrangement of the longitudinal and circular muscular fibres are to be shown, and the thickening of the latter along the anal canal, which gives rise to the internal sphincter, is to be noted. The gut is now to be laid open from end to end, and its interior examined. The valves of Houston, three in number, are to be looked for as transverse crescentic folds. One will be found on the right side fully 3 inches above the anus, and two on the left side about I inch above and below the right valve. The part of the bowel surrounded by the internal sphincter muscle is to be studied as the anal canal. The longitudinal folds seen here, called the columns of Morgagni, are to be cxamined, and a little above the anus these columns will be seen to be connected by circularlydisposed semilunar folds, known as the anal valves. The entire blood-supply of the rectum should be carefully revised.

The bladder is next to be dissected, showing the ureters at the lateral angles, and the vesiculæ seminales and vasa deferentia at the fundus. The commencement of each common ejaculatory duct is also to be shown, this being formed by the union of a vesicula seminalis and vas deferens. The limited area on the fundus, bounded on either side by these structures, and called the external trigone, is to be studied. The peritoneum having been removed from the upper surface of the bladder, the muscular fibres of the viscus are to be displayed as well as possible. They will be found to be arranged as an external longitudinal, a middle circular, and an internal longitudinal, layer, the last being very indefinite. The fibrous cord, representing the urachus, is to be shown passing from the apex of the bladder. The prostate gland is now to be fully examined, and, if not previously dissected, its recto-vesical capsule and the prostatic venous plexus are to be shown. At the base of the gland the dissector should look for the middle lobe, which is represented by the sniall part lying between the common ejaculatory ducts and the wall of the bladder, the rest of the organ being disposed as two lateral lobes. The membranous part of the urethra is to be shown succeeding to the apex of the prostate gland. A vesicula seminalis may now be cut into, in order to show the reticulated arrangement of its mucous lining. The bladder is to be opened, and its interior carefully examined. The openings of the ureters and the urethral opening are to be noted, and the smooth internal trigone examined, which lies within ridges connecting these openings. The prostate gland is also to be laid open along its anterior surface, and the whole penis is to be laid open, so as to expose the interior of the corpora cavernosa, and the urethra contained in the corpus spongiosum. In the prostatic urethra the central dilatation and the constriction at either end are to be observed, as well as the following markings: the crest, along the posterior wall; the sinus pocularis, having on the lateral margins of its opening, or just within them, the opcnings of the common ejaculatory ducts ; and the prostatic sinus, on either side of the crest, in which the prostatic ducts open. The spongy urethra is next to be examined, and the dilatation towards its centre, and also in the glans, are to be observed. the latter being called the fossa navicularis. The openings of the glands of Littré and the lacunæ of Morgagni are to be noted. The septum pectiniforme of the corpora cavernosa, and their trabecular tissue, which supports the erectile tissue, are to be observed.

In the female the bladder is to be examined as in the male. The absence of a prostate gland is to be noted, and the urethra is to be laid open, its shortness and distensibility being observed. The vagina is to be opened from end to end by an incision along the lateral wall. Its inucous rugxe are to be examined, as well as the carunculæe myrtiformes at its external orifice, and the lower part of the cervix uteri, which is received into its anterior wall at the upper end. The transverse slit of the os uteri externum is to be studied, along with its anterior and posterior lips. The recesses formed by the vagina around the lower part of the cervix uteri, called the fornices, are to be 
explored, and, on introducing the finger into the posterior fornix, the dissector will find that he can palpate the recto-uterine pouch of peritoneum or pouch of Douglas. The uterus is next to be carefully examined, and its relations, peritoneal included, are to be revised. The fundus, body, isthmus, and cervix are to be attended to. On each side of the upper part of the vagina and cervix uteri a collection of fat, called the parametrium, is to be shown. The body of the uterus is now to be laid open from before by a triangular incision, the apex of which is on a level with the isthmus, whence the two linbs diverge upwards and outwards to each lateral angle. The thick, triangular flap of the anterior wall thus formed having been raised, the cavity of the body is to be examined. The smoothness of the mucous lining is to be observed, and the uterine openings of the Fallopian tubes at each lateral angle, as well as the os uteri internum at the isthmus, are to be studied. The cervix is then to be opened along its anterior wall on one side of the middle line, and the arrangement of the mucous membrane, known as the arbor vitæ uterina, is to be noted. The Fallopian tube, with the corpus fimbriatum at its outer end, is to be studied, followed by the round ligament of the uterus. The ovary is next to be fully examined, and the scarred condition of its surface noted. The part of the broad ligament between the ovary and Fallopian tube, called the meso-salpinx, on being held up to the light, may be seen to contain the epoopphoron (parovarium, or organ of Rosenmüller). In a young subject a few other tubules may be seen nearer the uterus, which are known as the paroōphoron. The ovarian and uterine arteries, if injected, are to be followed to their distribution.

Articulations of the Pelvis. - The sacro-vertebral joint is to be examined, and the sacro-vertebral and ilio-lumbar ligaments dissected. The symphysis pubis is to receive careful attention, and the oblique direction of the joint is to be noted. Its ligaments are to be shown, and the interarticular fibro-cartilage is to be examined. Occasionally an interspace will be found in it towards the upper and back part, in which there may be a little fluid. The movement at this joint is only of a very slight vielding nature, except in the female during parturition. The sacro-iliac joint or synchondrosis is next to be examined. Its anterior ligament is weak. The posterior ligament, however, which is to be studied by partially separating the bones, is of considerable strength, and the part which it takes in supporting weight is to be carefully studied. The great and small sacro-sciatic ligaments are to be studied, and the manner in which they convert the sciatic notches on the posterior border of the hipbone into foramina is to be noted. The dissector is to observe the cartilaginous facing of the sinall sciatic notch, and the grooves, with intervening ridges, which it presents, for the columns on the under surface of the tendon of the obturator internus. Finally, the sacro-coccygeal and intercoccygeal joints are to be examined. 


\section{THE THORAX}

\section{Thoracic Wall.}

Muscles-Intercostal Muscles.-Thèse are two in number in each space, external and internal, and they are arranged as thin sheets of obliquely-disposed muscular fibres, with a large admixture of tendinous fibres.

External Intercostal Muscles-Origin.-The lower border of the upper rib.

Insertion.--The outer margin of the upper border of the lower rib.

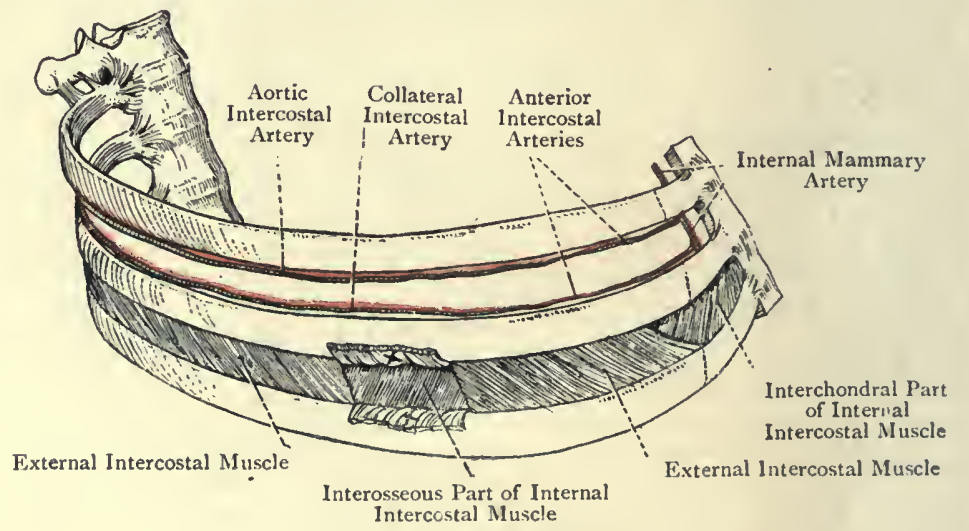

Fig. 380. Diagram of the Intercostal Muscles and Intercostal Arteries

(A small portion of the external intercostal muscle has been divided and reflected).

Nerve-supply.--The intercostal nerve of the corresponding space. The fibres of each muscle are directed downwards and forwards, and in this respect coincide with those of the obliquus externus abdominis. The muscles, as a rule, extend as far forwards as the junction of the ribs with their cartilages, but sometimes they stop short of this point, especially in the upper spaces. From the 
point where the fibres cease the upper six muscles are continued inwards to the side of the sternum by a thin delicate membrane, called the anterior intercostal aponeurosis. This occupies the spaces between the true costal cartilages. Posteriorly the muscles extend as far as the tubercles of the ribs.

Internal Intercostal Muscles-Origin.-The upper margin of the subcostal groove of the upper rib.

Insertion.-The inner margin of the upper border of the lower rib.

Nerve-supply. - The intercostal nerve of the corresponding space.

The fibres of each muscle are directed downwards and backivards, and in this respect coincide with those of the obliquus internus abdominis. The upper six muscles extend as far inwards as the side of the sternum, and the lower two are continuous anteriorly with the obliquus internus abdominis. Posteriorly the muscles extend as far back as the angles of the ribs. In this situation each is replaced by a delicate membrane, called the posterior intercostal aponeurosis, which lines the adjacent portion of the external intercostal muscle, and blends behind with the anterior or external superior costo-transverse ligament. The internal intercostal muscles are covered internally by the parietal pleura.

Action of the Intercostal Muscles.-This subject has given rise to much discussion and difference of opinion. Three views are entertained. (I) According to Haller, the external and internal intercostal muscles both act as elevators of the ribs, and are therefore muscles of inspiration. Inasmuch as the fibres of the two muscles decussate, they must, according to this view, act on the principle of the parallelogram of forces. The common nerve-supply of the two muscles tends to favour this view. (2) According to Hamberger, the external intercostal muscles are elevators of the ribs, and therefore muscles of inspiration, whilst the internal intercostal muscles are depressors of the ribs, and therefore muscles of expiration. (3) According to Hutchinson, the external intercostal muscles and the interchondral portions of the internal intercostal muscles act as elevators of the ribs, and are therefore muscles of inspiration, whilst the interosseous portions of the internal intercostal muscles act as depressors of the ribs, and are therefore muscles of expiration.

When a rib is elevated, its lower border is at the same time everted.

For the levatores costarum muscles, see Index.

Intercostal Nerves.-These are eleven in number on each side, and are the anterior primary divisions of the upper eleven thoracic spinal nerves. The anterior primary division of the twelfth thoracic spinal nerve is not an intercostal nerve, but lies along the lower border of the twelfth rib, and is known as the subcostal nerve ('last dorsal nerve').

The lower five intercostal nerves ultimately leave the intercostal spaces and pass on to the anterior abdominal wall.

Upper Six Intercostal Nerves.-Each of these nerves, as it enters the back part of an intercostal space, lies between the posterior 
intercostal aponeurosis and the parietal pleura. About the level of the angle of the rib it pierces the posterior intercostal aponeurosis, and passes forwards in the subcostal groove of the upper rib, lying between the intercostal muscles. It continues its forward course in this position, in company with the aortic intercostal artery and intercostal vein, the order of these from above downwards being intercostal vein, aortic intercostal artery, and intercostal nerve. The nerve is concealed by the overlapping lower border of the upper rib. Having reached a point about midway between the vertebral column and the side of the sternum, the nerve gives off

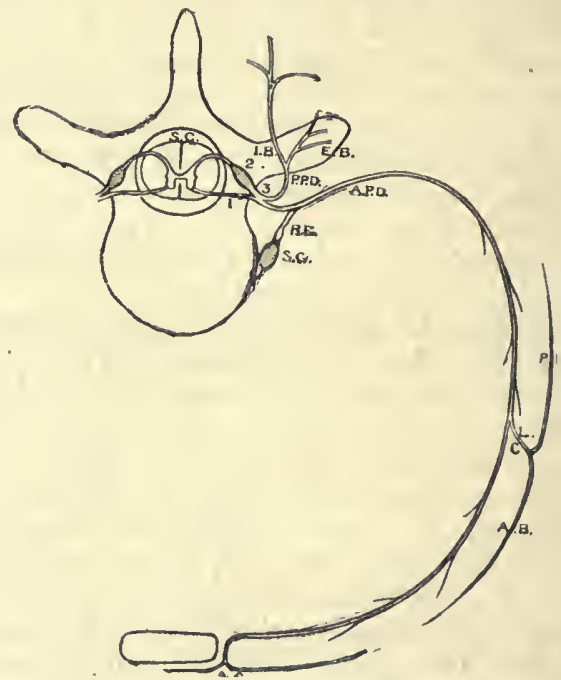

Fig. 381.-Scheme of an Intercostal Nerve.

S.C. Spinal Cord

I. Anterior Root of Spinal Nerve

2. Posterior Root, with Ganglion

3. Spinal Nerve

A.P. U. Anterior Primary Division

(Intercostal Nerve)

S.G. Sympathetic Ganglion

R.C. Rami Communicantes
L.C. Lateral Cutaneous Nerve

A. B. Anterior Branch

P.B. Posterior Branch

A.C. Anterior Cutaneous Nerve

P.P.D. Posterior Primary Division

E. B. External Branch

1.B. Internal Branch

a large branch, called the lateral cutaneous nerve. The intercostal nerve itself, now reduced in size, leaves the intermuscular space hitherto occupied by it, and enters the internal intercostal muscle, in the substance of which it continues its forward course.

Having arrived at the anterior extremity of the osseous rib, the nerve cmreges from the internal intercostal muscle and passes inwards on its deep surface, lying at first upon the parietal pleura, and subsequently upon the triangularis sterni muscle, crossing in its course the internal mammary vessels. On reaching the side of the sternum it passes straight forwards, to become an anterior 
cutaneous nerve, piercing in succession the following structures : the interchondral portion of the internal intercostal muscle; the anterior intercostal aponeurosis ; the pectoralis major muscle; and the deep fascia.

Branches.-These are muscular, lateral cutaneous, and anterior cutaneous.

The muscular branches supply the following muscles : the levatores costarum, serratus posticus superior, subcostal muscles, intercostal muscles, and triangularis sterni.

The lateral cutaneous nerves arise about midway between the vertebral column and the side of the sternum. They pierce the external intercostal muscles, and make their appearance between the digitations of the serratus magnus. The first intercostal nerve, as a rule, gives off no lateral cutaneous branch, and that of the second is known as the intercosto-humeral nerve.

The anterior cutaneous nerves are the terminal branches of the upper six intercostals, that of the first being sometimes absent. For their description, see Index.

Intereostal Arteries.-These vessels are arranged in two setsposterior and anterior.

Posterior Series.- The intercostal arteries of the first two spaces are derived from the superior intercostal artery (see Index). The intercostal arteries of the lower nine spaces are branches of the descending thoracic aorta, and are called the aortic intercostal arteries. Each at first lies between the posterior intercostal aponeurosis and the parietal pleura. About the level of the angle of the rib it pierces the posterior intercostal aponeurosis, and gives off its collateral intercostal branch, which inclines downwards. These two arteries now pass forwards between the two intercostal muscles, the main aortic intercostal lying in the subcostal groove of the upper rib, where it has the intercostal vein above it and the intercostal nerve below it, and the collateral intercostal lying along the upper border of the lower rib. These two vessels, in the case of the upper nine spaces, terminate by anastomosing with the anterior intercostal arteries, which are two in number in each of these spaces, and will be presently described. These anastomoses take place a little in front of the mid-point between the vertebral columin and the side of the sternum.

Branches.-These are dorsal, collateral intercostal, and lateral cutaneous.

The dorsal branch passes backwards to the muscles and integument of the back, giving off in its course a spinal branch, which enters the spinal canal through the intervertebral foramen.

The collateral intercostal branch, as stated, arises about the level of the angle of the rib, and inclines downwards to the upper border of the lower rib, along which it passes between the intercostal muscles.

The lateral cutaneous branch accompanies the corresponding lateral cutaneous nerve. 
The aortic intercostal and collateral intercostal arteries give branches to the intercostal muscles and ribs. Those of the third, fourth, and fifth spaces, at least, furnish branches to the mammary gland and pectoral muscles, which anastomose with the thoracic branches of the acromio-thoracic artery from the first part of the axillary, and with the long thoracic from the second part of that vessel. The first aortic intercostal artery, as it enters the third intercostal space, gives off a branch which ascends to the back part of the second intercostal space, and anastomoses with the branch of the superior intercostal artery to that space.

Anterior Series.-The internal mammary artery furnishes two anterior intercostal arteries to each of the upper six intercostal spaces, and the musculo-phrenic branch of the internal mammary furnishes two anterior intercostal arteries to each of the seventh, eighth, and ninth intercostal spaces. The arteries of the upper six spaces lie at first upon the triangularis sterni muscle, and then upon the parietal pleura, being under cover of the internal intercostal muscle. Afterwards all the anterior intercostal arteries pass between the external and internal intercostal muscles. In each space they anastomose with the aortic intercostal and collateral intercostal arteries, and also with the corresponding intercostal arteries of the upper two spaces.

Branches.- These are distributed to the intercostal muscles, ribs, mammary gland, and pectoral muscles.

The lower two intercostal spaces are not furnished with anterior intercostal arteries. The aortic intercostal arteries of these spaces, after leaving them, enter the abdominal wall, and pass forwards between the internal oblique and transversalis muscles to the rectus abdominis, in which they anastomose with the superior epigastric and deep epigastric arteries.

Intercostal Veins.-These veins accompany the corresponding arteries.

The chief intercostal vein passes backwards in the subcostal groove of the upper rib, in company with the aortic intercostal artery, above which it lies. In the region of the angle of the rib it is joined by the collateral intercostal vein, which accompanies the corresponding artery. Close to the vertebral column the chief intercostal vein receives a large dorsal branch, which returns blood from the muscles and integument of the back, the dorsal spinal venous plexus, and the spinal canal. The mode of termination of the chief intercostal veins differ on each side, and will be described in connection with the dissection of the posterior wall of the thorax.

The anterior intercostal veins accompany the anterior intercostal arteries. Those of the upper six intercostal spaces pass to the internal mammary, and those of the succeeding three spaces pass to the musculo-phrenic, veins.

Intercostal Lymphaties. - The lymphatic vessels of the intercostal spaces pass partly to the posterior intercostal glands, which lie at the back parts of the intercostal spaces, and partly to the anterior 
intercostal or sternal glands, which lie along the course of the internal mammary artery.

Internal Mammary Artery.-This vessel arises from the lower portion of the first part of the subclavian artery, and it passes downwards, forwards, and inwards behind the inner part of the clavicle and the first costal cartilage. It then descends vertically behind the succeeding costal cartilages as low as the sixth intercostal space, where it terminates by dividing into two branches, namely, the superior epigastric and the musculo-phrenic. The artery lies about $\frac{1}{2}$ inch from the margin of the sternum.

Cervical Relations-Anterior.-The clavicular portion of the sterno-cleido-mastoid muscle, and the internal jugular and subclavian veins. The phrenic nerve crosses it superficially from without inwards. Posterior.-The dome of the pleura.

Thoracic Relations-Anterior.-The pectoralis major; upper six costal cartilages; anterior intercostal aponeuroses ; internal intercostal muscles; and upper six intercostal nerves. Posterior.-The pleura above, and subsequently the triangularis sterni muscle. Lateral.--The artery has a vena comes on either side of it. The anterior intercostal or sternal glands lie along the course of the vessel.

Branches.-The artery gives off no branches in the neck. In the thoracic part of its course it furnishes the following branches:

$\begin{array}{ll}\text { Superior phrenic. } & \text { Anterior intercostal. } \\ \text { Mediastinal. } & \text { Perforating. } \\ \text { Pericardial. } & \text { Musculo-phrenic. } \\ \text { Sternal. } & \text { Superior epigastric. }\end{array}$

The superior phrenic artery, long and slender, arises high up, and accompanies the phrenic nerve to the diaphragm, from which circumstance it is known as the comes nervi phrenici. It gives twigs to the pericardium and pleura, and in the diaphragm it anastomoses with the inferior phrenic branch of the abdominal aorta and with the musculo-phrenic branch of the internal mammary. The mediastinal branches are distributed to the contents of the anterior mediastinum, namely, the remains of the thymus body, anterior mediastinal glands, and areolar tissue. The pericardial branches supply the front part of the pericardium. The sternal branches are distributed to the sternum and triangularis sterni muscle. The anterior intercostal arteries are two in number to each of the upper six intercostal-spaces. For their description, see Index. The perforating branches are six in number, one arising opposite each of the upper six intercostal spaces. Each vessel pierces the internal intercostal muscle, anterior intercostal aponeurosis, and pectoralis major. Thereafter it gives a few twigs to the front-of the sternum, and then turns outwards to supply the pectoral integument. The second, third, fourth, and fifth perforating branches give offsets to the inner portion of the mammary gland.

The musculo-phrenic artery is one of the terminal branches of the 
internal mammary. It arises from that vessel in the sixth intercostal space, and passes obliquely downwards and outwards behind the seventh, eighth, and ninth costal cartilages. About the level of the tenth rib it perforates the diaphragm and terminates in the lateral wall of the abdomen, where it anastomoses with the lateral epigastric branch (ascending branch) of the deep circumflex iliac artery. It gives off anterior intercostal and muscular branches. The anterior intercostal arteries are two in number to each of the seventh, eighth, and ninth intercostal spaces, in which they are

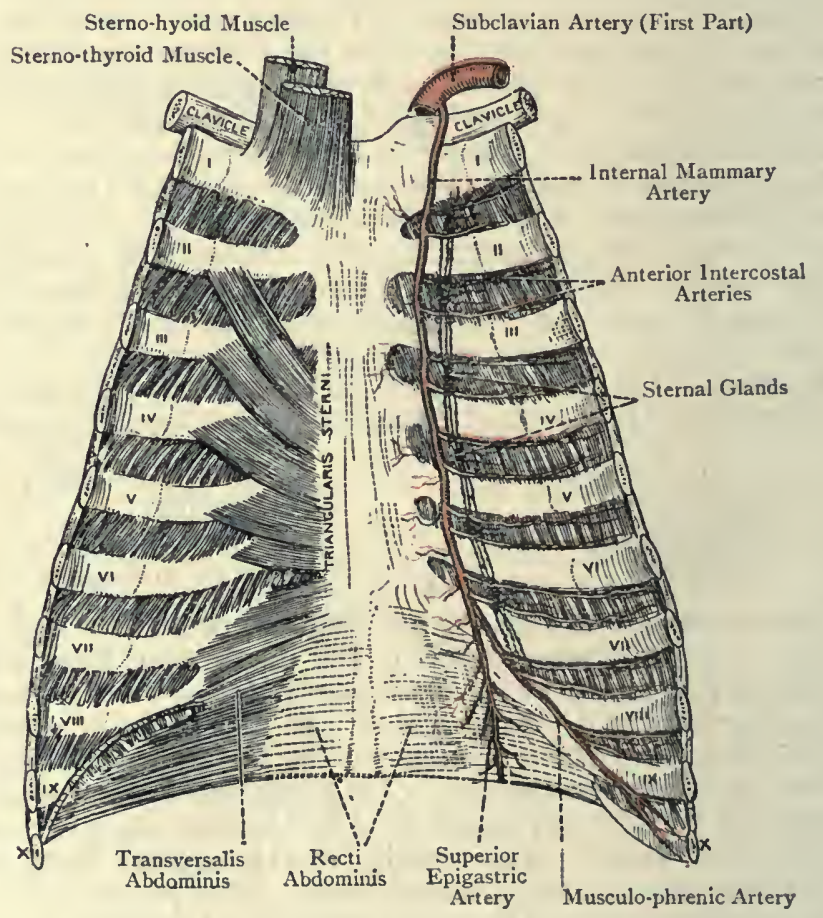

Fig. 382.-Dissection of the ANTERIor Wall of the THorax (POSTERIOR VIEW).

disposed in a manner similar to the anterior intercostal branches of the internal mammary. The muscular branches are distributed to the diaphragm and lateral wall of the abdomen. In the diaphragm they anastomose with the inferior phrenic branch of the abdominal aorta and the superior phrenic branch of the internal mammary.

The superior epigastric artery is the other terminal branch of the internal mammary, of which it is the continuation. It descends behind the seventh costal cartilage, and passes through the areolar interval between the sternal and costal portions of the diaphragm 
In this manner it enters the sheath of the rectus abdominis, lying at first behind the muscle, but afterwards entering it. In the muscle it anastomoses with the deep epigastric artery, which is a branch of the external iliac.

The branches of the superior epigastric artery will be found described on p. 663 .

The internal mammary veins (vence comites) are two in number, and lie one on each side of the artery. They are formed, respectively, by the union of the venæ comites of the musculo-phrenic and superior epigastric arteries. In their course they receive tributaries corresponding to the branches of the artery. About the level of the first intercostal space the outer vein crosses over the artery and joins the inner vein to form a single vessel, which opens into the corresponding innominate vein. The internal mammary veins are provided with valves at intervals.

Sternal or Internal Mammary Lymphatic Glands.-These glands form a chain along the course of the internal mammary vessels on cither side, there beiug one, or it may be two, in the anterior extremity of each of the upper six intercostal spaces.

They receive their afferent vessels from (I) the inner third of the mammary gland, (2) the anterior half of the costal pleura, (3) the anterior halves of the external and internal intercostal muscles, (4) the lymphatics which accompany the superior epigastric artery, and (5) the anterior diaphragmatic glands. Their efferent vessels pass to the thoracic duct, or to the right lymphatic duct, according to the side on which the glands lie.

Triangularis Sterni-Origin.-(I) The deep surface of the ensiform process and body of the sternum, close to the lateral border, and extending as high as the level of the third costal cartilage; and (z) the deep surfaces of the lower two or three true costal cartilages at their sternal ends.

Insertion.-The deep surfaces and lower borders of the costal cartilages from the sixth to the second. The insertion takes place by separate slips, and one or two of the upper slips may be partially attached to the rib itself.

Nerve-supply.-The intercostal nerves of the adjacent spaces.

The lowest fibres of the muscle are horizontal; the succeeding fibres pass obliquely upwards and outwards; and the upper fibres are almost vertical.

Action.-To depress the anterior extremities of the ribs, and so take part in expiration.

The muscle forms a thin musculo-tendinous sheet, which is situated on the deep surfaces of the costal cartilages and side of the sternum, and it is serially continuous with the transversalis abdominis muscle. It supports the internal mammary vessels, sternal glands, and certain of the intercostal nerves, whilst its deep surface rests upon the parietal pleura. 


\section{Thoracic Cavity.}

Contents and their General Position.-The thoracic cavity is chiefly occupied by the lungs and heart. The lungs are situated one in each half of the cavity, and each lung is provided with a serous membrane, called the pleura. The heart lies obliquely between the lungs, projecting more to the left of the sternum than to the right, and it is enclosed within a fibro-serous sac, called the pericardium. Each lung is free to expand except at the hilum pulmonis, which is situated on its inner surface. Through this hilum the bronchus, pulmonary artery, and pulmonary veins pass, along with other structures, and the pedicle so formed is called the root of the lung. The upper part, or apex, of the lung rises into the root of the neck, where it is covered by the dome of the pleura, that in turn being covered by Sibson's fascia. The lower part, or base, of the lung rests upon the corresponding half of the diaphragm, the heart lying upon the central tendon of that muscle.

The pulmonary artery springs from the base of the right ventricle of the heart, and the aorta from the base of the left ventricle, being at first covered by the pulmonary artery. The left innominate vein courses along the upper aspect of the arch of the aorta in front of the origins of the innominate, left common carotid, and left subclavian arteries, and it unites with the right innominate vein behind the sternal end of the first right costal cartilage to form the superior vena cava. This latter vessel opens into the postero-superior angle of the right auricle of the heart, and, just before piercing the pericardium, it receives the right azygos vein, which arches forwards over the right bronchus. The inferior vena cava, having entered the thorax through the foramen quadratum in the central tendon of the diaphragm, almost immediately opens into the posteroinferior angle of the right auricle of the heart.

The phrenic nerve, on each side, descends in front of the root of the lung, and is intimately related to the pericardium, especially on the left side. The small anterior pulmonary plexus of nerves lies in front of the root of each lung. The pneumogastric nerve, on each side, descends behind the root of the lung, and forms in that part of its course the much larger posterior pulmonary plexus. The following important nerves descend in front of the arch of the aorta : the left phrenic, the left pneumogastric, the upper cervical cardiac branch of the left sympathetic, and the lower cervical cardiac branch of the left pneumogastric. The left superior intercostal vein lies in front of the back part of the aortic arch. Thic superficial cardiac plexus of nerves lies within the concavity of the arch of the aorta, and the deep cardiac plexus lies behind the arch and in front of the trachea, close to its bifurcation into the two bronchi. The left recurrent laryngeal nerve passes backwards under 
the arch, and then ascends behind it. Within the concavity of the aortic arch the trunk of the pulmonary artery breaks up into its right and left divisions, and the ligamentum ductûs arteriosi extends from the root of the left pulmonary artery to the back part of the concavity of the aortic arch, immediately beyond the origin of the left subclavian artery. The gangliated cord of the sympatlietic lies very deeply, and descends over the heads of the ribs close behind the parietal pleura, and the two, or three, splanchnic nerves lie obliquely on the sides of the bodies of the lower thoracic vertebræ.

The œesophagus lies in contact with the front of the vertebral column, and the trachea is anterior to it. The descending thoracic aorta lies very deeply, being situated at first on the left side of the vertebral column, but subsequently in front of it. The thoracic duct ascends on its right side, and the right azygos vein ascends on the right side of the thoracic duct, both structures being under cover of the œsophagus. The two left azygos veins, upper and lower, as well as the two transverse azygos veins, upper and lower, are closely related to the vertebral column.

The thoracic cavity contains the following sets of lymphatic glands : the anterior mediastinal, in front of the pericardium; the superior mediastinal, above the pericardium, along the arch of the aorta and innominate veins; the posterior mediastinal, behind the pericardium; the posterior intercostal, in the back parts of the intercostal spaces; and the bronchial, in the interval between the diverging bronchi, and also at the root of each lung. In early life a portion of the thymus body, which is then of large size, lies behind the upper part of the sternum, whence it extends into the lower part of the neck.

Pleuræ.-The pleuræ are the two serous sacs which invest the lungs, and line the adjacent parietes. Each forms a shut sac, which is quite distinct from its fellow. Like other serous membranes, the pleura consists of two portions-parietal and visceral-which, however, are continuous with one another.

The parietal pleura lines the parietes, and is divisible into five portions, namely, costal, diaphragmatic, pericardial, cervical, and mediastinal. The costal pleura lines the inner surfaces of the ribs and internal intercostal muscles. The diaphragmatic pleura covers the upper surface of one-half of the diaphragm. The pericardial pleura is in intimate contact with the pericardium. The cervical pleura forms what is known as the dome, and rises into the neck for about I inch above the clavicle, being a little higher on the right side than on the left, on account of the projection formed by the liver. The mediastinal pleura of each side bounds the mediastinum thoracis.

The visceral pleura closely invests the lung, and is known as the pulmonary pleura. It is intimately connected with the lung substance, and extends into the fissure, or fissures, which map out the lung into lobes. Below the root of each lung it forms a fold, called the ligamentum latum pulmonis, which descends to the diaphragm, and is connected with the pericardium. 
Continuity and Reflections of the Pleura.-(I) In the transverse direction.-Commencing at the deep surface of the sternum, the parietal pleura of each side passes backwards to the pericardium, the two being in contact except for a little inferiorly. They form the lateral boundaries of the space which is called the anterior mediastinum. When they reach the pericardium the two pleuræ separate, each keeping to its own side of that sac, and so forming the pericardial pleura, which bounds laterally the space known as the middle mediastinum. Each pleura in this manner reaches the anterior aspect of the root of the corresponding lung, where it becomes the visceral pleura. This covers the front of the root of the lung, and then invests the entire organ, dipping into its fissure or fissures, as the case may be, and giving a covering to the posterior

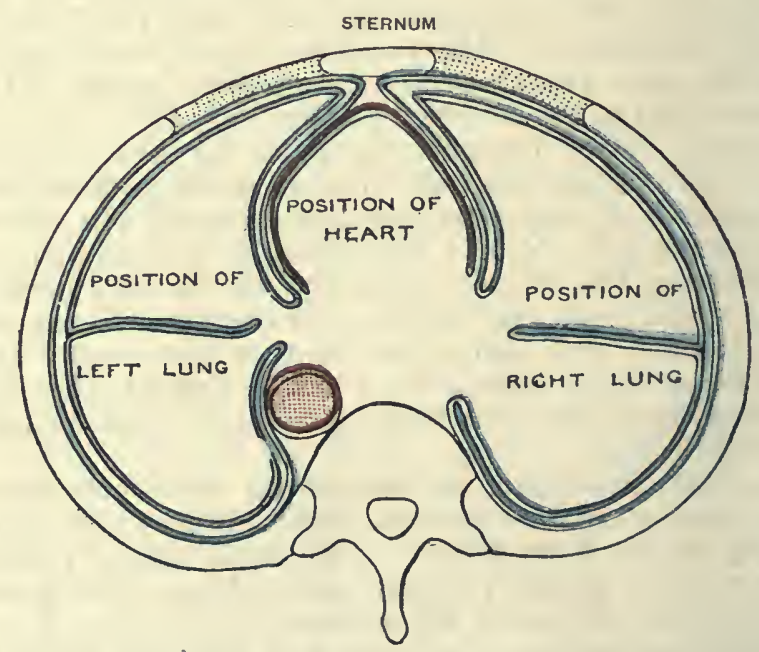

Fig. 383.-Diagram showing the Reflections of the Pleura (Transverse Section).

aspect of the root. On leaving the back of the root of each lung, the two pleuræ pass backwards, slightly over the pericardium, trachea, and osophagus, and over the descending thoracic aorta, to the lateral aspects of the bodies of the thoracic vertcbræ. These portions form the lateral boundaries of the space which is called the posterior meaiastinum. From the vertebral column each pleura passes outwards over the gangliated cord of the sympathetic, and then lines the inner surfaces of the ribs, as well as the internal intercostal muscles. In this manner it reaches the deep surface of the sternum.

From the foregoing description it will be evident that the pleura, in passing from the dcep surface of the sternum to the vertebral column, meets with, and is reflected over, the lung and its root 
At the level of the manubrium sterni the pleura passes uninterruptedly backwards to the vertebral column. That of the right side passes over the side of the superior vena cava, innominate artery, right innominate vein, and trachea ; whilst that of the left side passes over the side of the left common carotid and left subclavian arteries, œsophagus, and thoracic duct. The two pleuræ, as they pass backwards at this level, form the lateral boundaries of the space which is called the superior mediastinum.

Below the level of the root of the lung the antero-posterior reflection of the pleura is complicated by a triangular fold, called the ligamentum latum pulmonis. This fold consists of two layers of pleura, in close apposition, which are continuous superiorly with the anterior and posterior pleural investments of the root of the lung. It extends, on the one hand, between the lower border of the root of the lung and the diaphragm, to which latter it is atached, and, on the other hand, between the pericardium and the inner surface of the lung below the level of the root. In the last-named situation its two layers separate, to encase the lung at that level. Its lower border is free and concave.

(2) In the vertical direction.-Superiorly the parietal pleura of each side rises, in the form of a dome, into the root of the neck for about I inch above the clavicle, where it is covered by Sibson's fascia. This fascia is attached, on the one hand, to the internal border of the first rib, and, on the other, to the front of the transverse process of the seventh cervical vertebra. The subclavian artery and innominate vein are intimately related to the dome of the pleura internally and anteriorly, the artery being the higher of the two. Inferiorly the parietal pleura of each side is reflected from the thoracic wall on to the upper surface of the corresponding portion of the diaphragm, which it covers where the base of the lung rests upon it. The intervening portion of the diaphragm (central tendon) is covered by the serous portion of the pericardium. Internally the diaphragmatic pleura becomes continuous with the mediastinal pleura.

Lines of Reflection of the Pleuræ-Sternal Reflection.-Behind the manubrium sterni the right and left pleuræ are separated from each other by an interspace which represents the superior mediastinum. At the level of the junction of the manubrium and body of the sternum they meet each other, and descend behind the body of the bone, in close contact and inclining slightly to the left of the median line. At the level of the lower border of the fourth costal cartilage the left pleura parts company with the right, and passes outwards and downwards close to the left border of the sternum, and in intimate relation with the pericardium. There is, however, a small triangular area of that sac which is uncovered by the pleura, and lies in direct relation to the anterior thoracic wall. The left pleura continues its downward course as far as the inner surface of the sixth costal cartilage, lying not far from the left border of the sternum. At this level it again passes outwards and downwards, 
to be reflected on to the diaphragm. The right pleura pursues an undeviating course downwards behind the sternum as far as the junction of the body and ensiform process. At this level it leaves the bone, and passes obliquely outwards, downwards, and backwards over the inner surface of the seventh costal cartilage, from which it is reflected on to the diaphragm.

Costo-diaphragmatic Reflection.-The level of this reflection may be considered in the first place as it affects certain definite vertical lines. In the left sternal line it takes place at the level of the sixth costal cartilage, and in the right sternal line at the level of the seventh costal cartilage. In the left mammary line it takes place at the level of the eighth costal cartilage, where it joins the osseous part of the rib, and on the right side at a similar level. In the left mid-axillary line it takes place on a level with the lower border of the tenth rib, this being the lowest point to which the pleura descends. In the right mid-axillary line it takes place on a level with the upper border of the tenth $\mathrm{rtb}$, or, it may be, the lower border. Posteriorly, in the scapular line (inferior angle of the scapula), on each side, it takes place at the level of the lower border of the twelfth rib. From this point inwards to the vertebral column the line of pleural reflection is a little below the adjacent border of the twelfth rib, and is very nearly midway between the vertebral end of that rib and the first lumbar transverse process.

From the foregoing description it will be evident that the pleura descends lowest in the mid-axillary line, and that posteriorly, close to the vertebral column, it actually descends below the level of the lower border of the twelfth rib. The direction of the line of costo-diaphragmatic reflection, from before backwards, is at first obliquely downwards and outwards, until the level of the tenth rib is reached. Thereafter the line passes backwards and upwards to the vertebral column. The costo-diaphragmatic pleural line is on a distinctly lower level than the margin of the base of the lung, but it is a little above the line of the costal attachment of the diaphragm. The portion of the diaphragm below the line is therefore in direct contact with the thoracic wall and adjacent internal intercostal muscles, without the intervention of the pleura, and the costo-diaphragmatic reflection is connected to these structures by a fascial expansion.

The free surfaces of the parietal and visceral pleuræ are, in health, in close contact. They are polished and moistened by a slight amount of serous fluid, so that they glide smoothly upon each other. In cases of pleurisy, however, certain changes take place. The free surfaces are, in the first place, rendered dry, and thereafter they become roughened at intervals by the deposit of lymph. Under these circumstances the movement of the lung is accompanied by the sound known as pleuritic friction. Adhesions are also frequently formed, and a serous exudation takes place into the pleural sac, which tends to compress the lung injuriously, the condition being known as hydro-thorax. In cases of serous exudation the cavity of the pleura, which is a shut sac, is clearly demonstrable, but in 
health no such cavity exists, the parietal and visceral pleuræ being at all times in intimate contact with each other. The attached surfaces of the parietal and visceral pleuræ are roughened by fibrous processes, by means of which they are connected to the parts which they cover.

The costal pleura is the thickest, and can readily be stripped fiom the inner surfaces of the ribs and internal intercostal muscles. There is a fairly thick layer of subserous areolar tissue on its attached surface. The diaphragmatic and pericardial pleuræ are thinner than the costal pleura, and are more adherent to the subjacent structures. The pulmonary pleura is the thinnest and most adherent. Beneath it there is a layer of subserous areolar tissue, containing much elastic tissue, and this is in continuity with the areolar tissue which pervades the lung.

Differences between the Two Pleural Sacs.-The right pleural sac rises higher into the root of the neck, and is shorter and wider, than the left. These differences are due partly to the projection formed by the liver on the right side, and partly to the greater inclination of the heart to the left of the sternum than to the right.

Blood-supply. - The parietal pleura receives its arteries from (I) the aortic intercostals, and (2) the superior phrenic, anterior intercostal, anterior mediastinal, pericardial, and musculo-phrenic branches of the internal mammary.

The veins pursue courses corresponding to the arteries.

Nerve-supply.-The nerves are derived from the sympathetic and phrenic nerves.

Lymphatics-Visceral or Pulmonary Pleura.-The lymphatic vessels of this part of the pleura open into the superficial lymphatics of the lungs. Parietal Pleura.-The lymphatics of the costal pleura open into the lymphatics of the internal intercostal muscles, which terminate in (I) the sternal or internal mammary glands, and (2) the intercostal glands. The lymphatics of the diapliragmatic pleura open into the lymphatics of the diaphragm. The lymphatics of the mediastinal pleura open into (I) the anterior mediastinal glands, and (2) the posterior mediastinal glands.

Structure.-The pleura is a typical serous membrane like the serous portion of the pericardium, the peritoneum, and the tunica vaginalis. Such membranes are called serous because their free surfaces are moistened by a small quantity of serous fluid. Briefly stated, the pleura consists of a homogeneous, connective-tissue basement membrane, containing elastic tissue, and lined with endothelium. It presents many slomata, which communicate with the pleural cavity and with the pleural lymphatic vessels.

Development. - The pleura is developed from the walls of the cœlom, or body-cavity, which is the cleft in the mesoblast separating the splanchno. pleure and somatopleure.

Mediastinum Thoracis.-The mediastinum thoracis is formed by the approximation of the two pleural sacs in the region of the median antero-posterior line of the thorax. The interval between the two sacs is called the mediastinal space, and its boundaries are 
as follows: in front, the sternum; behind, the bodies of the thoracic vertebræ; and, on either side, the corresponding pleural sac. The mediastinal space contains structures so numerous as to necessitate its subdivision into four parts-superior, anterior, middle, and posterior.

The superior mediastinum is situated above the pericardium, and its boundaries are as follows : in front, the deep surface of the manubrium sterni, with the origins of the sterno-hyoid and sterno-thyroid muscles; behind, the bodies of the upper four thoracic vertebræ and the lower portions of the longus colli muscles; above, an imaginary plane corresponding to the superior aperture of the thorax; below, an imaginary plane passing from the lower border of the manubrium sterni to the lower border of the body of the fourth thoracic vertebra; and, laterally, the mediastinal pleura of each side as it extends from the deep surface of the manubrium sterni to the vertebral column.

Contents.-These are as follows: (I) the arch of the aorta; (2) the innominate artery, and the thoracic portions of the left common carotid and left subclavian arteries ; $(3)$ the right and left innominate veins, and the upper half, or extrapericardial portion, of the superior vena cava ; 4 ) the phrenic, pneumogastric, left recurrent laryngeal, and cardiac nerves ; $(5)$ the trachea, œesophagus, and thoracic duct ; (6) the superior mediastinal glands; and (7) the remains of the thymus body.

The anterior mediastinum is situated behind the body of the sternum, and its boundaries are as follows : in front, the deep surface of the body of the sternum, and the left triangularis sterni muscle ; behind, the pericardium ; and, laterally, the mediastinal pleura of each side as it extends from the deep surface of the body of the sternum to the lateral aspects of the pericardium. Its direction is downwards and to the left. At its upper part there is no interspace, because the two pleural sacs come into contact behind the first piece of the body of the sternum. Below this level, however, the left pleural sac diverges from its fellow, so as to leave an interspace.

Contents.-These are the anterior mediastinal glands, some lymphatic vessels, the anterior mediastinal branches of the internal mammary artery, and areolar tissue.

Anterior Mediastinal Glands.-These glands are situated within the areolar tissue of the lower part of the anterior mediastinum, between the lower part of the body of the sternum and the front of the pericardium.

They receive their afferent vessels from (I) the antero-mesial portion of the diapliragm, corresponding to the ensiform process of the sternum, (2) the supero-anterior surface of the liver, on either side of the falciform ligament, (3) the anterior part of the pericardium, (4) the anterior part of the mediastinal pleura, and (5) the lower sternal, or internal mammary, glands. Their efferent. vessels ascend and open into the superior mediastinal or cardiac glands. 
The middle mediastinum lies behind the anterior, and below the superior, mediastinum, and is the widest part of the interpleural space.

Contents.-These are as follows: (I) the heart, enclosed in the pericardium; (2) the ascending aorta; (3) the trunk of the pulmonary artery; (4) the lower half, or intrapericardial portion, of the superior vena cava; $(5)$ the phrenic nerves and the superior phrenic

\section{Parietal Pleura Internal Mammary Vessels}

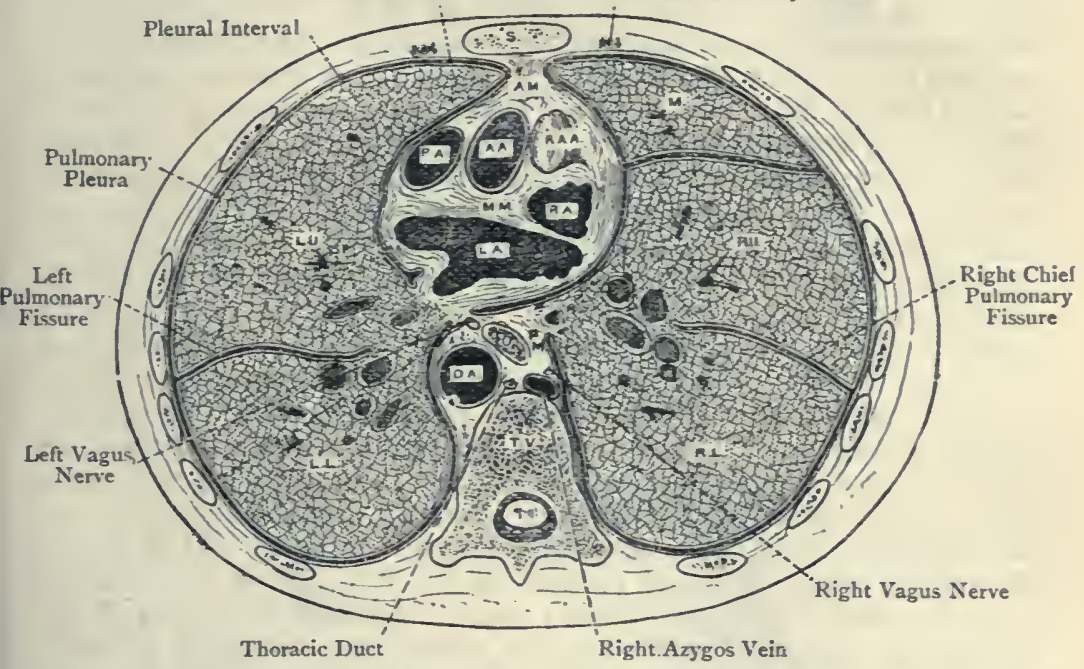

Fig. 384.-Transverse Section of the Thorax through the Second Sternebra in Front and the Body of the Ninth Thoracic Vertebra Behind, Showing the Reflections of the Pleura and the Position OF THE VISCERA.

S. Sternum

A.M. Anterior Mediastinum

A.A. Ascending Aorta

P.A. Pulmonary Artery

R.A.A. Right Auricular Appendix

R.A. Right Auricle

M.M. Middle Mediastinum

L.A. Left Auricle
P.M. Posterior Mediastinum

O. Esophagus

D.A. Descending Thoracic Aorta

T.V. Thoracic Vertebra

M. Middle Lobe of Right Lung

R.U. Right Upper Lobe

R. L Right Lower Lobe

L.U. Left Upper Lobe

L. L Left Lower J obe

vessels; (6) the bifurcation of the trachea; and (7) the roots of the lungs.

The posterior mediastinum is situated behind the pericardium, and below the posterior part of the superior mediastinum, with which latter it is continuous. Its boundaries are as follows: in front, the pericardium, and below this the posterior part of the diaphragm; behind, the bodies of the thoracic vertebræ below the level of the lower border of the body of the fourth; and laterally, the mediastinal pleura of each side as it extends from the back of the root of the lung to the side of the vertebral column. 
Contents.-These are as follows: (I) the descending thoracic aorta; (2) the œsophagus, and the two pneumogastric nerves; (3) the thoracic duct; (4) the right azygos vein; (5) the upper and lower left azygos veins, with the corresponding transverse azygos veins; (6) the great splanchnic nerves, right and left; and (7) the posterior mediastinal glands.

Phrenic Nerve.-This nerve arises chiefly from the anterior primary division of the fourth cervical nerve. It usually receives a small root from the anterior primary division of the third cervical, and, as a rule, an additional root from that of the fifth cervical. Having descended on the superficial surface of the scalenus anticus muscle to the root of the neck, it crosses the internal mammary artery superficially from without inwards. Thereafter it enters the thorax and descends in front of the root of the lung, in close contact with the side of the pericardium, and under cover of the mediastinal pleura. Having reached the diaphragm, the nerve divides into several branches, which pierce that muscle to be distributed to its abdominal surface. The terminal branches of each nerve are connected, in the diaphragm, with filaments of the corresponding inferior phrenic plexus of the sympathetic, which is an offshoot from the solar plexus. At the place of junction of the two sets of fibres on the right side there is a small ganglion, called the ganglion diaphragmaticum. In the thorax the phrenic nerve occupies the superior and middle mediastinal spaces. The right phrenic nerve lies, in succession, on the outer side of the right innominate vein and superior vena cava, and then descends in front of the root of the right lung. The left phrenic nerve descends in the interval between the left common carotid and left subclavian arteries, where it crosses the left pneumogastric nerve from without inwards. Thereafter it passes behind the left innominate vein, and crosses over the arch of the aorta, after which it descends in front of the root of the left lung. Each nerve is accompanied by the superior phrenic artery, which is a branch of the internal mammary artery.

Branches. - The nerve of each side furnishes twigs to the pericardium and the mediastinal pleura, its principal branches being distributed to the diaphragm.

Differences between the two phrenic nerves-(I) The right nerve is shorter than the left, because the right half of the diaphragm, having the bulk of the liver below it, is higher than the left half. (2) The right nerve is straighter than the left, because the heart, enclosed in the pericardium, projects less to the right side than to the left. (3) The right nerve occupies a deeper position in the upper part of the thorax than the left.

Pericardium.-The pericardium is the fibro-serous sac which loosely surrounds the heart in the middle mediastinum. It is somewhat conical, being wide below, where it is in contact with the diaphragm, and narrow above, where it surrounds the great vessels connected with the base of the heart. On each side it is intimately related to the mediastinal pleura, and is embraced by the inner 
surfaces of the lungs anterior to their roots. The phrenic nerve, on each side, descends in very close contact with it. In front of it are the body of the sternum and the sternal ends of the corresponding costal cartilages. Its anterior surface is, to a greater or less extent, encroached upon by the adjacent portions of the anterior margins of the lungs and by the two pleuræ. There is, however, a small portion of this surface which is immediately related to the sternum below the level of the lower border of the fourth left costal cartilage at its sternal end. Posteriorly, the pericardium lies in front of the posterior mediastinum, and the œsophagus is here related to it opposite the posterior aspect of the left auricle of the heart.

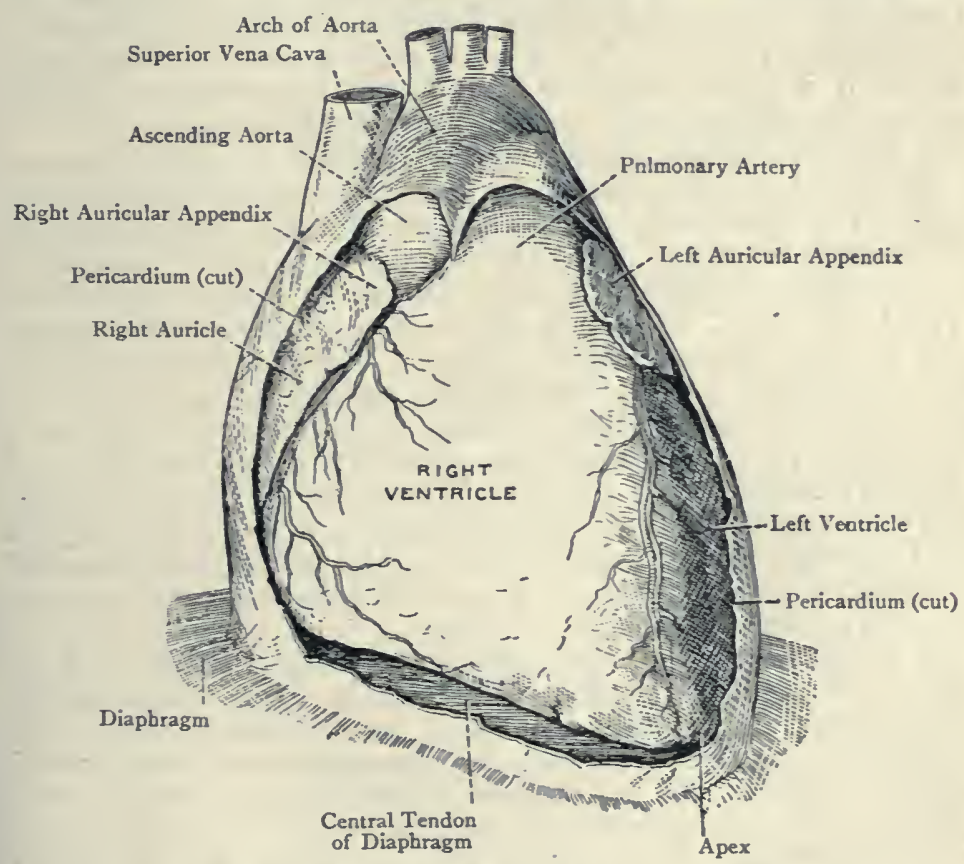

Fig. 385.-The Heart (Anterior View) and Pericardium

(The anterior part of the pericardium has been removed).

The pericardium consists of two portions-an external or fibrous, and an internal or serous. The fibrous portion is strong and dense. Inferiorly it is attached to the central tendon of the diaphragm, and slightly to its muscular part on the left side. Superiorly it ensheathes the great vessels connected with the base of the-heart, with the single exception of the inferior vena cava. These sheaths are eight in number. One, which is the strongest, invests the whole of the ascending aorta; two invest the right and left pulmonary arteries; one surrounds the lower half of the 
superior vena cava; and the remaining four invest the pulmonary veins. The inferior vena cava receives no sheath from the fibrous portion, because that vessel opens into the right auricle of the heart almost immediately after it passes through the foramen quadratum in the central tendon of the diaphragm.

The fibrous layer is attached to the sternum by two fibrous bands, which are known as the superior and inferior sterno-pericardial ligaments of Luschka, the former being attached to the deep surface of the manubrium, and the latter to the deep surface of the ensiform process.

The serous portion is a typical serous membrane, and consists of two layers, parietal and visceral, which together form a shut sac.

The parietal portion lines the inner surface of the fibrous part, to which it is closely adherent, and it also intimately covers the upper surface of the central tendon of the diaphragm.

The visceral portion, known as the epicardium, closely invests the heart, and also the great vessels at its base more or less completely. The continuity between the parietal and serous portions is established inferiorly along the inferior vena cava. Superiorly the continuity is established along the great vessels at the base of the heart about $I_{\frac{1}{2}}$ inches above it. In this situation the serous portion forms an arterial sheath which encloses within it the ascending aorta and trunk of the pulmonary artery, for, as stated, about $I_{\frac{1}{2}}$ inches, this being the only complete sheath formed by the serous portion. Behind this sheath, with its contents, and in front of the auricular portion of the heart, there is a passage, called the transverse sinus of the pericardium, which leads from the right to the left side of the serous sac. The serous portion is also related to the superior vena cava and the pulmonary veins, but it only covers them in front and at the sides.

The serous portion of the pericardium forms a triangular fold, called the vestigial fold of Marshall, which is situated between the left pulmonary artery and the upper left pulmonary vein. Its base is directed towards the left, and its surfaces are anterior and posterior. Between its two delicate layers there is a small fibrous cord, which is a vestige of the left duct of Cuvier.

The free surfaces of the parietal and visceral layers of the serous portion are smooth, polished, and lubricated by serous fluid to allow of free movement on the part of the heart. In the course of pericarditis they become at first dry, and then roughened by deposits of lymph, thus giving rise to the pericarditic friction murmur, and, it may be, to adhesions. They may also become separated from each other by effusion into the pericardial sac, thus giving rise to the condition known as hydro-pericardium.

Blood-supply. - The fibrous portion of the pericardium and the parietal layer of the serous portion receive their arteries from (I) the pericardial and superior phrenic branches of the internal mammary, and (2) the descending thoracic aorta. The visceral layer of the serous portion reccives arterial twigs from the coronary arteries of the heart. 
The veins pass to the internal mammary, superior phrenic, and azygos veins.

Nerve-supply. - The phrenic, pneumogastric, and sympathetic nerves.

Lymphatics.-These pass to the anterior, superior, and posterior mediastinal glands.

Structure. - The fibrous portion of the pericardium is composed of fibrous tissue, and is very dense, but not very extensible. The serous portion consists of a homogeneous, connective-tissue basement membrane, containing some elastic fibres, and lined with endothelium. The parietal layer is much thicker than the visceral, and the latter is intimately connected with the cardiac muscular tissue, except along the grooves which are occupied by adipose tissue and bloodvessels.

Development. - The serous portion of the pericardium is developed from the walls of the cœlom, or body-cavity, which is the cleft in the mesoblast separating the splanchnopleure and somatopleure.

Thymus Body (Gland).- This body is present only in the fotus and young child. It attains its greatest size at the period of birth, and forms a very conspicuous object in the dissection of a child during the first year or two of life. It soon, however, begins to diminish. In its fully-developed condition it is situated partly in the thorax, and partly in the neck. In the former situation it occupies the superior and anterior mediastinal spaces, extending as low as about the level of the fourth costal cartilages, and lying in front of the great bloodvessels and upper part of the pericardium, the upper part of the sternum being in front of it. In the neck it extends as high as the lower part of the thyroid body, being under cover of the sterno-hyoid and sterno-thyroid muscles. In this situation it embraces the front and sides of the trachea, completely concealing it from view, and it encroaches upon the carotid sheath on either side. Its length is about $2 \frac{1}{2}$ inches, and its breadth, which is greatest inferiorly, is about $I_{2} \frac{1}{2}$ inches. Its colour is pinkish ; it is soft in consistence ; and its surface shows indications of lobulation. It consists of two asymmetrical lateral lobes, each of which is pyramidal. In some cases the left lobe is the larger, and in other cases the right. These lobes are in close contact, but are still quite distinct. Sometimes a third lobe is present, occupying an intermediate position between the other two.

Blood-supply. - The arteries are chiefly derived from the internal mammary, inferior thyroid, and superior thyroid.

The veins open into the right and left innominate and thyroid veins.

Nerve-supply.-The nerves are derived from the sympathetic and pneumogastric.

Structure.-Each lobe has a capsule of fibrous tissue, from which trabeculæ pass into the interior. These trabeculæ map out each lobe into large and small lobules. Each lobule consists of an outer cortical, and an inner medullary, portion. The cortex is composed of lymphoid tissue, the lymphoid element predominating over the retiform, and it is surrounded by a capillary network of bloodvessels, which contains many lymph corpuscles. The lymphoid tissue of the cortex is incompletely subdivided into nodules by means of trabeculæ. 
The medulla is more transparent than the cortex, the retiform element of the lymphoid tissue is more conspicuous, and the lymph corpuscles are less numerous. In addition, the medulla contains small groups of cells, more or less concentrically arranged, which are known as the concentric corpuseles of Hassall. According to one view these cells are remains of the diverticula from which the thymus body is developed; but another view is that they are concerned with the formation of bloodvessels and connective tissue.

Development. - The thymus body is developed in two lateral parts from the entodermic epithelium of the pharyngeal portion of the primitive gut. The epithelium of the third visceral cleft, on either side, becomes evaginated, and gives rise to a hollow diverticulum. This diverticulum has a thick epithelial wall, but a small lumen, and it grows in a downward direction. Its distal end, which lies ventral to the pericardium, forms a solid enlargement, and the proximal end loses its connection with the third visceral cleft. The enlarged distal end gives off numerous solid epithelial buds, which are invested with mesoderm. This budding gradually extends to the proximal part of the diverticulum. The solid buds ramify freely and give rise to the corresponding lobe of the thymus. The original diverticulum thus assumes a lobulated appearance, and resembles a racemose gland. The buds or acini, however, are solid, and not hollow, as in racemose or acinous glands. The acini are separated by connective tissue and bloodvessels, which are developed from the surrounding mesoderm. Lymphoid tissue is also developed around the acini, and this tissue forms the greater part of the adult thymus. The epithelial elements of each lobe are subordinate to the lymphoid tissue, and are ultimately represented by the concentric corpuscles of Hassall.

Thymus Body in the Adult.--Only the remains of the thymus body are present in the form of a collection of adipose and areolar tissues, which lies in the superior mediastinum in front of the innominate and left common carotid arteries, above the arch of the aorta. This mass receives a few twigs from the mediastinal branches of the internal mammary artery, and it usually contains a certain amount of the original lymphoid tissue.

Lungs.-The lungs are two in number-right and left. They are spongy in consistence, float in water, and are readily compressed. When pressed between the fingers crepitation is elicited, this being due to the displacement of air. When the lung is incised, similar crepitation is heard, and a muco-serous fluid, mixed with air, exudes. They possess considerable elasticity, their colour is that of a dark slate, and they are usually mottled, this being duc to carbonaceous matter. In early life, however, the colour is rosepink.

The lungs occupy the grcater part of the thoracic cavity. Normally they are at all times in close contact with the thoracic walls, the pleuræ intervening. Unless adhesions have formed during life between the visceral and parietal pleura, the surface of each lung is quite free except in two situations, namcly, at the root, which occupies a limited area of the inner surface, and at the attachment of the ligamentum latum. Each lung is conical, the base being directed downwards. It presents for consideration an apex, a base, two surfaces, outer and inner, and two borders, anterior and posterior.

The apex is blunt, and rises out of the thoracic cavity into the 
root of the neck for about $I_{\frac{1}{2}}$ inches. It is here covered by the dome of the pleura, and a little below its highest point it presents a groove on its internal and anterior aspects. In the case of the right lung this groove is produced by the innominate and right subclavian arteries, and, in the case of the left lung, by the subclavian artery of that side. Below this groove there is another groove, produced on either side by the innominate and subclavian veins.

The base is extensive, semilunar in outline, and concave in adaptation to the upper arched surface of the diaphragm, upon which it

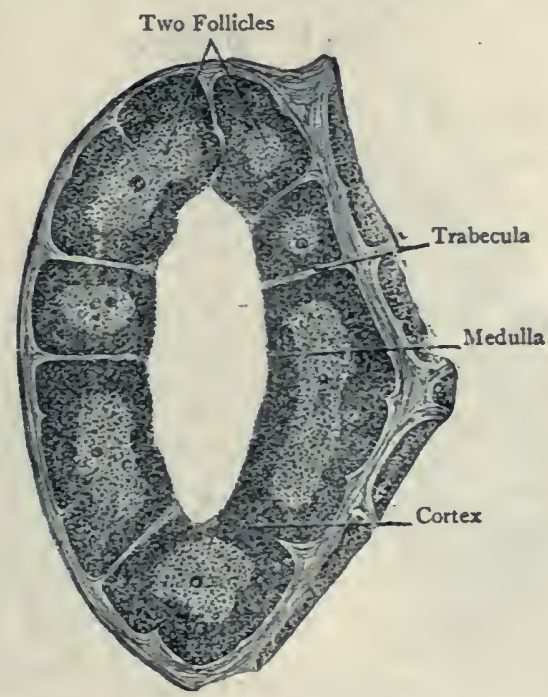

Fig. 386.-Transverse Section of a Lobule of the Thymus Gland of A KITTEN

(The concentric corpuscles of Hassall are shown in the medulla).

rests, with the intervention of the pleura. The base of the right lung is related to the right lobe of the liver, and that of the left lung to the left lobe of the liver, the stomach, and the spleen, the diaphragm intervening in each case. The margin of the base is thin and sharp, and it extends into the costo-diaphragmatic space, reaching lowest behind, but nowhere as low as the line of the costodiaphragmatic reflection of the pleura.

The outer surface is extensive and convex, and in health it is closely applied to the inner surfaces of the ribs and of the internal intercostal muscles.

The inner surface is of much more limited extent than the outer. 
The greater part of it is concave in adaptation to the heart, enclosed in the pericardium, the concavity being greater in the case of the left lung on account of the projection of the heart to the left side. About the junction of the anterior two-thirds and posterior third this surface presents a vertical fissure, called the hilum pulmonis, at which the root of the lung is situated. The inner surface of the right lung, behind the hilum, is related to the œsophagus, and the corresponding portion of the inner surface of the left lung presents part of the aortic groove, vertically placed and produced by the descending thoracic aorta. Anterior to the lower part of this groove the left lung is related to the osophagus, but less

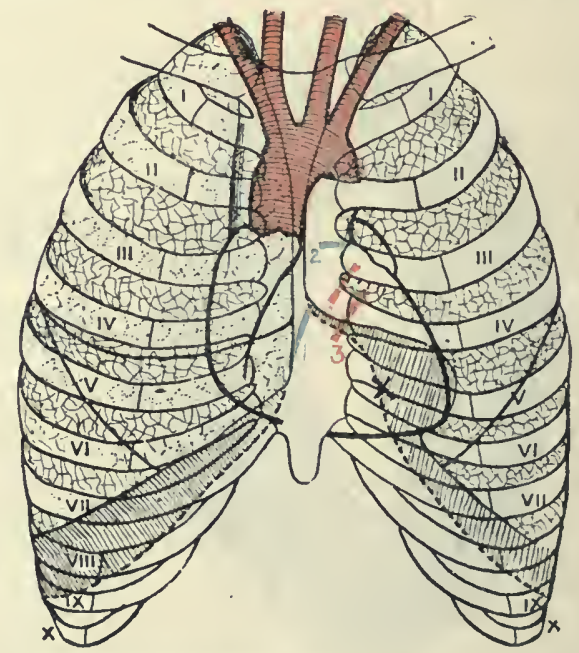

Fig. 387 -Diagram showing the Heart and Lungs in Situ.
I. Tricuspid Orifice
2. Pulmonary Urifice.
3. Mitral Orifice 4. Aortic Orifice

$X$, Region of Latham's Circle

intimately than the right lung. The inner surface of the right lung presents the following grooves above the level of the hilum, named in order from below upwards: (I) azygos, for the right azygos vein; (2) caval and innominate, for the superior vena cava and right innominate vein; and (3) arterial, for the innominate artery. The inner surface of the left lung presents the following grooves above the level of the hilum: (I) the commencement of the aortic groove, transversely disposed, for the arch of the aorta ; (2) the subclavian groove, for the left subclavian artery, leading upwards from the preceding; and (3) an indefinite groove, anterior to the subclavian groove, for the left innominate vein.

The borders are anterior and posterior. The anterior border is thin and short, and overlaps the pericardium, more so during 
inspiration than expiration, but leaving an area of the pericardium uncovered, which is known as the area of precordial dulness. The anterior border of the right lung keeps behind the sternum as low as the sixth right costal cartilage. The corresponding border of the left lung, beyond the lower border of the fourth left costal cartilage, presents a deep V-shaped notch, called the cardiac notch, for the reception of the apical portion of the heart, enclosed in the pericardium. The posterior border is elongated, thick; and round, and it occupies the pulmonary groove of tile thorax, by tine side of the thoracic portion of the vertebral column.

Each lung is divided into two lobes, upper and lower, by ani extensive, oblique, and deep fissure, which penetrates almost to

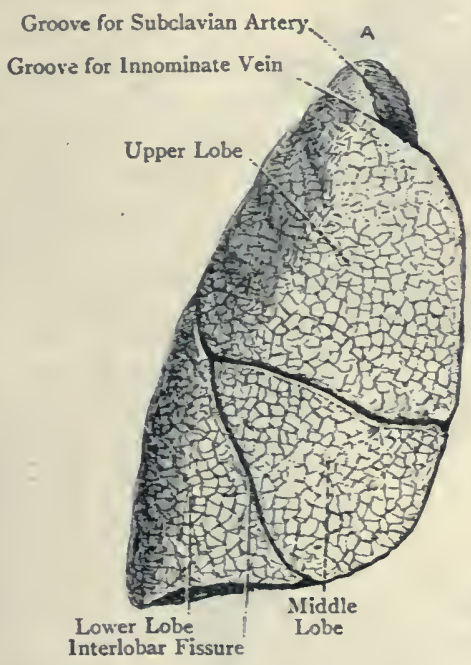

Fig. 388.-The Luxgs (Axterior View).

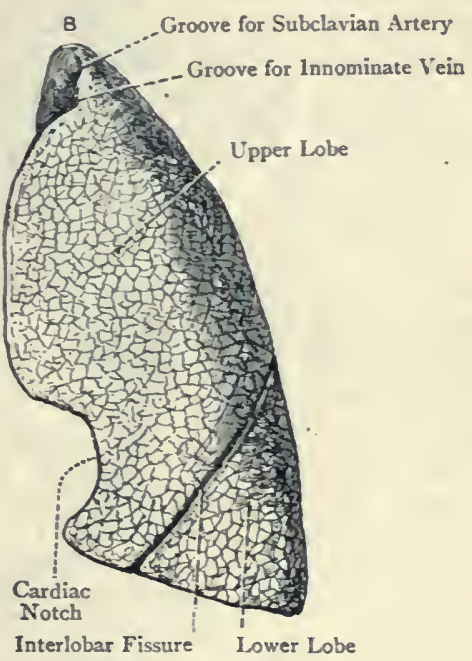

A, the Right Lung; B, the Left Lung.

the hilum. So deep is this-fissure that, unless adhesions have formed, the lung appears to consist of two halves. The fissure commences on the inner surface, near the posterior border, about 3 inches below the apex. It then turns round the posterior border, and passes obliquely downwards and forwards over the outer surface to the basal margin. In the case of the right lung the fissure joins the basal margin some little distance from the lower end of the anterior border ; but, in the case of the left lung, the fissure joins the basal margin distinctly farther forwards. A good ready guide to this fissure is the lower border of the pectoralis major muscle, as it forms the anterior fold of the axilla. 
The upper lobe is comparatively small, and includes the apex, about the upper 3 inches of the posterior border, the anterior border, and, in the case of the left lung, practically all that can be auscultated anteriorly. The lower lobe is of large size, and lies behind and below the great pulmonary fissure. It includes the base, the posterior border except the upper 3 inches, and practically all that can be auscultated posteriorly.

By means of the great pulmonary fissure each lung, as stated, is divided into two iones. In the case of the left lung the division proceeds no further. In the case of the right lung, however, there is an additional fissure, which extends from the chief fissure, at

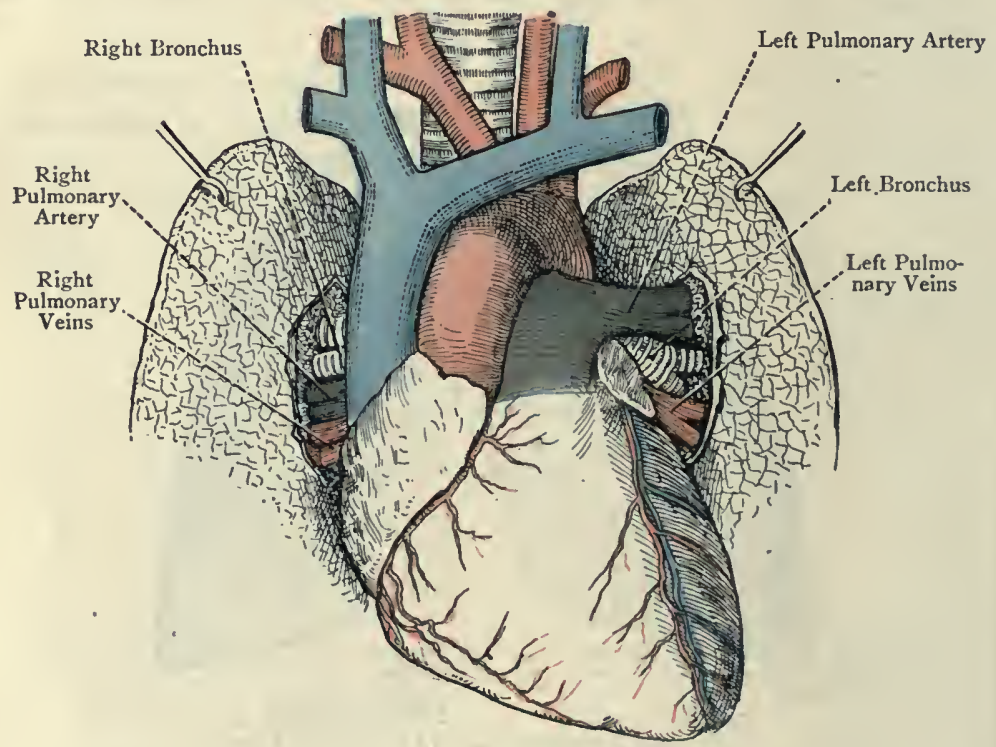

Fig. 389.-The Roots of the Lungs (Anterior View).

the posterior border, horizantally forwards over the outer surface to the anterior border at about the level of the lower margin of the fourth right costal cartilage, which corresponds to the commencement of the cardiac notch on the anterior border of the left lung. This additional fissure cuts off from the upper lobe a triangular or wedge-shaped portion, which is called the middle lobe.

Differences between the two lungs-(I) The right lung has two fissures and three lobes, whilst the left lung has only one fissure and two lobes. (2) The anterior border of the right lung is uninterrupted, whilst that of the left lung presents inferiorly the cardiac. 
notch. (3) The right lung is larger and heavier than the left, the weight of the right being about 20 ounces and that of the left about IS ounces. (4) The right lung is shorter than the left, this being due to the fact that the liver causes the right half of the diaphragm to rise higher than the left half. $(5 ;$. The right lung is broader than the left, because the heart projects more to the left side than to the right.

Vertical Extent of the Lungs. - In the mammary line the right lung descends as low as the sixth rib; in the mid-axillary line, as low as the eighth rib; and; in the scapular line (inferisi angle of the scapula), as low as the tenth rib. The lower limits of the lieft. lung exceed those of the right by about the depth of a rib.

Root of the Lung.-The root is situated at the hilum pulmonis on the inner surface. Its chief constituents are as follows: (I) the bronchus or air-tube; (2) the pulmonary artery, which conveys venous blood to the lung; and (3) the two pulmonary veins, which convey the arterial or oxygenated blood from the lung to the left auricle of the heart. In addition to these constituents there are (a) the bronchial arteries and veins, (b) the pulmonary lymphatic ressels, (c) the pulmonary nerves, and $(d)$ the bronchial lymphatic glands. All these constituents are connected by areolar tissue, and the entire root is invested by the pleura.

Relations. -The following relations are common to both roots: in front, the phrenic nerve, with the superior phrenic artery, and the anterior pulmonary plexus of nerves; behind, the pneumogastric nerve and the posterior pulmonary plexus of nerves; and, below, the ligamentum latum pulmonis.

Special Relations - Right Root-Anterior. - The - superior vena cava and the upper part of the right auricle of the heart. Superior.-The right azygos vein, as it arches forwards over the right bronchus to open into the superior vena cava. Posterior.The right azygos vein. Left Root-Superior.-The arch of the aorta. Posterior.-The descending thoracic aorta.

Relative Position of the Chief Constituents.-The relation from before backwards is the same on each side, and is as follows: (I) the upper of the two pulmonary veins; (2) the pulmonary artery; and (3) the bronchus. The relation from above dowmeards differs on the two sides. On the right side the relation is bronchus, pulmonary artery, and pulmonary veins; and, on the left side, pulmonary artery, bronchus, and pulmonary veins.

The difference in the position of the bronchus on each side is due to the fact that the right bronchus gives off a branch known as the eparterial bronchus, which lies above the right pulmonary artery, the larger portion of the right bronchus and the whole of the left bronchus being hyparterial.

Structure of the Lungs. - The trachea divides into two bronchi, right and left, the structure of which is similar to that of the trachea. The right bronchus, at about inch from its origin, gives off superiorly a branch, 
called the eparterial bronchus, for the upper lobe of the right lung, and beyond this point, where it is now hyparterial, it divides into two branches, one for the middle, and the other for the lower, lobe. The left bronchus is entirely hyparterial, and divides into two branches, one for the upper, and the other for the lower, lobe of the left lung. The structure of these primary divisions of the bron ini is similar to that of the bronchi themselves. Within each lung these primary divisions undergo subdivision, to a certain extent dichotomously, iut the ramifications are chiefly due to branches being given off laterally, and these never anastomose. The ramifications of the airtubes within the lungs are called the bronchial tubes, and their ultimate subdivisions within the lobules are known as the bronchioles. Each bron. chiole transmits air to and from a group of infundibuk, or ultimate lobules.

Each lobular bronchial tube, after entering a lobule, divides into as many bronchioles as there are groups of infundibula or ultimate lobules. Each

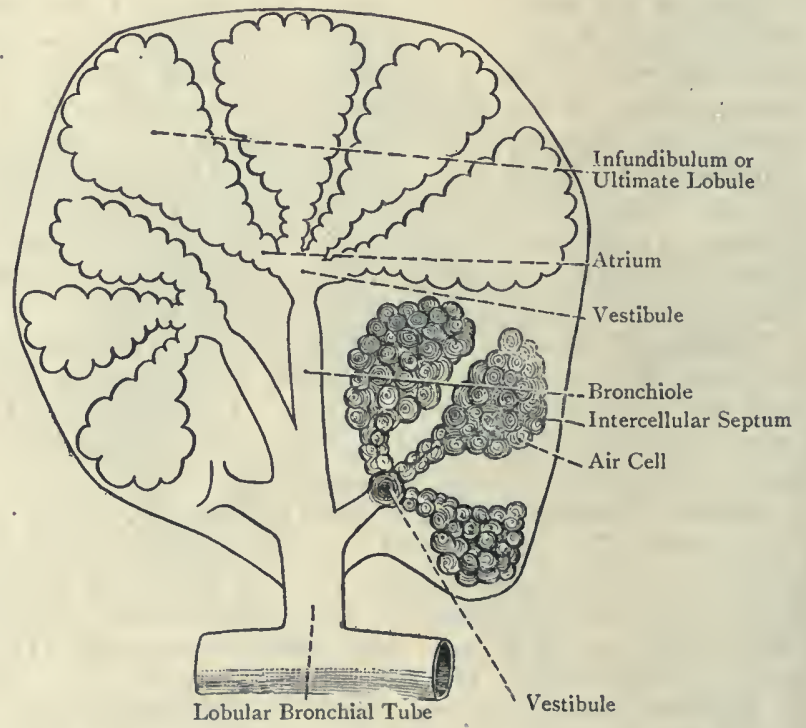

Fig. 390.-Scheme of a Pulmonary Lobule (after L. Testut's 'Anatomie Humaine').

bronchiole, on approaching a cluster of, say, two or three infundibula, presents a dilatation, called the vestibule, and from this vestibule receptionchambers, known as the atria, proceed to the infundibula.

An infundibulum or ultimate lobule is an irregular, funnel-shaped passage, closed at one end, and having its walls and closed extremity beset with air-cells or pulmonary alveoli, which also beset, though more sparsely, the walls of the atria.

The bronchial tubes within the lungs are destitute of membranous walls posteriorly, and are cylindrical. This is due to the fact that their irregular plates of cartilage are disposed round the circumference of the wall. The muscular fibres are arranged in complete rings round the tubes, and the elastic tissue forms longitudinal bundles. Tlie mucous membrane is freely provided with racemose mucous glands, and is covered with stratified ciliated columnar epithelium. When the tubes, by division, have attained a diameter of about a inch, the cartilaginous plates disappear, and the walls consist of a 
fibro-elastic membrane and circularly-disposed muscular fibres, with a thin mucous coat, destitute of mucous glands, and covered with simple ciliated columnar epithelium, there being here and there patches of squamous, nonciliated cells. The walls of the vestibule, atria, and air-cells are very thin and consist of areolar, elastic, and muscular tissues, the elastic element being specially developed at the margins of the orifices of the cells. This elastic tissue enables the cells to recoil after distension. The interior of the vestibule, atria, and air-cells is lined with a single layer of squamous, non-ciliated epithelium, which is of extreme delicacy in the air-cells. Upon the outer walls of the cells there are dense networks of capillary bloodvessels, which also per-

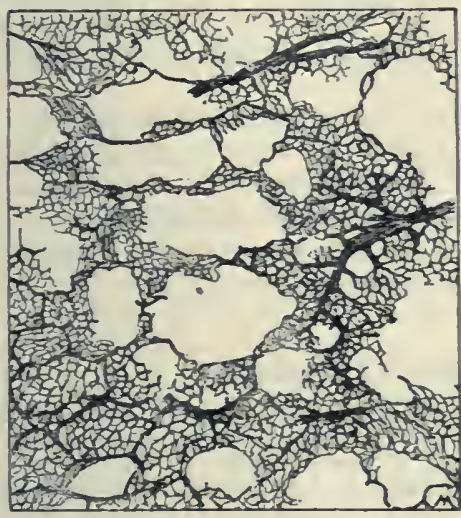

Fig. 391.-Section of Lung (INJECTED). vade the septa between the cells, these septa being formed by infoldings of the contiguous cell-walls. Each septum contains only one capillary layer. The venous blood is thus brought into the most intimate relation with the air, all that separates the two being the very thin walls of the cells and the very delicate walls of the capillary bloodvessels. Moreover, there being only one capillary layer in each intercellular septum, the blood in the septal capillaries is exposed to the air on each side.

Summary of the Structure of the Lung.-The lung consists of an immense number of lobules, irregularly polygonal in outline, and each of, these is provided with its own bronchial tube. A lobule is composed of groups of infundibula or ultimate lobules, and the lobular bronchial tube, on entering the lobule, divides into as many bronchioles as there are groups of infundibula. Each bronchiole, on approaching a cluster of infundibula, presents a dilatation, called the vestibule, from which atria lead to the infundibula. The walls of the infundibula are freely beset with air-cells, which are also present, though more sparsely, on the walls of the atria. Upon the walls of the air-cells are dense networks of capillary bloodvessels, and each intercellular septum contains a single capillary layer.

Bloodvessels of the Lungs.-Two sets of arteries are associated with each lung, namely, pulmonary and bronchial, the former having to do with the respiratory function of the organ, and the latter with the nutrition of its component tissues. The pulmonary arteries are two in number, right and left. They result from the bifurcation of the pulmonary trunk, and convey venous blood to the lungs. Each artery ramifies freely within the lung, its branches accompanying the bronchial tubes, but they never anastomose with one another. Ultimately they terminate in dense capillary networks which lie upon the walls of the air-cells, and also in the septa between adjacent cells.

The pulmonary veins commence as radicles in the capillary networks already referred to, and they pass to the root of each lung, where they give rise to two pulmonary veins, which proceed to the left auricle of the heart and convey to it arterial or oxygenated blood. The pulmonary veins and their tributaries are destitute of 
valves. It is to be noted that the pulmonary arteries carry venous blood, whilst the pulmonary veins carry arterial blood.

The bronchial arteries convey arterial blood to the lungs for the nutrition of their component tissues. They will be described in connection with the descending thoracic aorta, with which they are associated.

The bronchial veins return their blood chiefly into the right azygos and upper left azygos veins respectively.

Lymphatics. - The lymphatic vessels of each lung are arranged in two sets-superficial and deep. At the hilum these two sets open into the interbronchial glands. The superficial set receives the lymphatics of the visceral or pulmonary pleura.

Nerves.-These are derived from the anterior and posterior pulmonary plexuses, which are formed by the pneumogastric nerves, aided by branches from the sympathetic. The nerves penetrate as far as the air-cells, upon the walls of which they are regarded as terminating in arborizations. The anterior and posterior pulmonary plexuses will be found described on p. 967 .

\section{Development of the Respiratory Apparatus.}

The respiratory apparatus consists of the larynx, trachea, and lungs. The earliest indication of it is a median longitudinal groove on the inner aspect of the ventral wall of the csophageal part of the fore-gut. This groove is called the pulmonary or laryngo-tracheal groove, and it produces an evagination of the ventral wall of the œsophagus. It consists of entoderm, derived from that of the fore-gut, and it is covered by splanclinic mesoderm. This groove gradually deepens, and by the fusion of its lips a longitudinal diverticulum of the ventral wall of the osophageal part of the fore-gut is formed, called the pulmonary or laryngo-tracheal diverticulum. It lies on the ventral aspect of the œsophagus, from which it becomes gradually separated in a caudo-cephalic direction. At the cephalic end, however, the separation is arrested, and a communication (superior laryngeal aperture) persists between this part of the pulmonary diverticulum and the pharyngo-œesopliageal tube. The pulmonary diverticulum consists of (I) entoderm, derived from that of the fore-gut, and (2) mesoderm, which invests it.

The condition of matters now is that there are two tubes, dorsal or pharyngocesophageal, and ventral or pulmonary (laryngo-tracheal), which communicate freely cephalicwards.

Larynx.-The larynx is developed from the cephalic or proximal part of the pulmonary or laryngo-tracheal diverticulum. (see the Larynx).

Trachea.-The trachea is developed from the caudal or distal part of the pulmonary or laryngo-tracheal diverticulum, the cartilaginous rings, connective tissue and muscular tissue of the trachea being developed from the mesodermic investment of the primitive diverticulum.

Lungs. - The rudiments of the lungs appear as two lung-buds, right and left, which are formed by the bifurcation of the pulmonary diverticulum at its caudal end. Like the diverticulum itself, these buds consist of entoderm, invested by mesoderm. The entoderm of each lung-bud and of its various ramifications furnishes all the epithelial elements, bronchial and alveolar, of the corresponding lung. The mesoderm of the bud and of its various ramifications gives rise to the bloodvessels, connective and muscular tissues, and cartilages of the bronchlal tubes, as well as to the visceral pleura. The pedicles of the lung-buds give rise to the bronchi. The right lung-bud gives off thrce processes or vesicles, and the left lung-bud gives off two processes, and in this 
manner the three-lobed condition of the adult right lung and the two-lobed condition of the adult left lung are indicated.

Each of these processes gives rise, by budding, to secondary processes, and these in turn give rise, successively, to other processes. This budding goes on very freely, and the ramifications constitute the pulmonary lobes. All the buds or processes, which carry along with them an investment of mesoderm, give rise to the ramifying system of bronchial tubes. The terminal ramifications form the bronchioles and infundibula. The air-cells or pulmonary alveoli are formed as hollow sessile buds or evaginations of the walls of the infundibula, with the cavities of which they communicate freely.

As stated, the epithelial cells of the lung-buds and of all their ramifications, as well as the epithelial cells of the pulmonary diverticulum, are developed from the entoderm of the fore-gut.

Lungs of the Fœtus.- The lungs prior to birth, having been impervious to air, feel solid, like liver, and they would at once sink if placed in a vessel containing water.

Superior Mediastinal or Cardiac Glands.-These glands, which are numerous and important, are situated in the superior mediastinum, along the upper aspect of the arch of the aorta, in front of the lower end of the trachea, and along the right and left innominate veins.

They receive their afferent vessels from (I) the anterior mediastinal glands, (2) the upper part of the pericardium, (3) the heart, and (4) the thymus body, in early life. Their efferent vessels terminate in the thoracic duct and in the right lymphatic duct.

Innominate Veins.-These vessels are two in number, right and left, and each is formed by the junction of the internal jugular and subclavian veins behind the inner end of the clavicle. They both lie in the superior mediastinum.

The right innominate vein is about an inch in length, and passes downwards with a slight inclination inwards. At the level of the lower border of the first right costal cartilage, close to the sternum, it unites with the left innominate vein to form the superior vena cava. Externally it is closely related to the right phrenic nerve and right pleura; its internal relation is the upper part of the innominate artery; and, behind it, is the right pneumogastric nerve.

The left innominate vein is about 3 inches in length, and passes obliquely inwards and downwards from left to right. As stated, it joins its fellow of the right side to form the superior vena cava. In front of it there are the upper part of the manubrium sterni, the origins of the sterno-hyoid and sterno-thyroid muscles, and the remains of the thymus body. Behind it are the origins of the innominate, left common carotid, and left subclavian arteries, the left pneumogastric and left phrenic nerves, and two superficial cardiac nerves from the cervical portions of the left pneumogastric and left sympathetic. Below it there is the arch of the aorta.

There are no valves in the innominate veins.

Tributaries. - Each vein receives the following tributaries : (I) the vertebral vein; (2) the inferior thyroid vein : (3) the internal 
mammary vein; and (4) the first intercostal vein. The last-named vessel, however, sometimes opens into the vertebral vein, and occasionally the right inferior thyroid vein opens into the left innominate vein. The left innominate vein receives, as an additional tributary, the left superior intercostal vein.

Development.-The right innominate vein is developed from that portion of the right anterior cardinal vein which intervenes between the place where it receives the right subclavian vein and the place where the transverse jugular vein joins it.

The left innominate vein is developed from the transverse jugular vein.

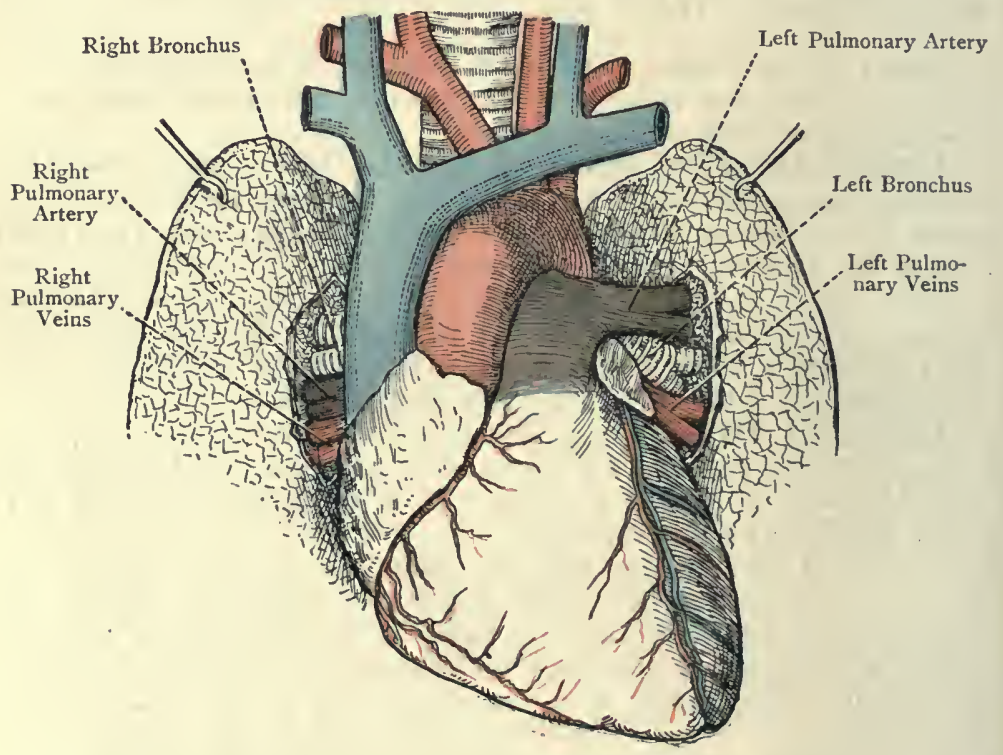

Fig. 392.-The Roots of the Lungs (ANTerior View).

Superior Vena Cava. - This vessel is formed by the union of the right and left innominate veins behind the lower border of the first right costal cartilage, close to the sternum. It is about 3 inches in length, and descends almost vertically to the level of the upper border of the third right costal cartilage, where it opens into the postero-superior angle of the right auricle of the heart. In its course it pierces the fibrous portion of the pericardium. The upper half of the vessel is extrapericardial, and lies in the superior mediastinum ; but the lower half is intrapericardial, and lies in the middle mediastinum.

Relations - Upper Half-External. - The right phrenic nerve and the right pleura. Internal.- The lower part of the innominate artery. Lower Half-Internal.--The ascending aorta. Postcrior.The root of the right lung. The serous portion of the pericardium 
covers the lower part of the vessel except over about its posterior fourth.

The superior vena cava is destitute of valves.

Tributaries.-The chief tributary is the right azygos vein, after it has arched forwards over the right bronchus. It opens into the superior vena cava immediately before that vessel pierces the fibrous portion of the pericardium. Other minute tributaries are pericardial and mediastinal veins.

Development.-The portion of the superior vena cava above the right azygos vein is developed from that part of the right primitive jugular vein which lies below the point where it is joined by the transverse jugular vein : and the portion below the right azygos vein is developed from the right duct of Cuvier.

Inferior Vena Cava.-This vessel enters the thorax through the foramen quadratum in the central tendon of the diaphragm, and immediately thereafter it is received within the fibrous portion of the pericardium. Its course in the thorax is practically nil, as it may be said to open at once into the postero-inferior angle of the right auricle of the heart.

Thoracic Aorta. - The thoracic aorta extends from the base of the left ventricle of the heart to the level of the lower border of the body of the twelfth thoracic vertebra. At this point it passes through the aortic opening of the diaphragm, and thereafter enters upon the abdominal part of its course. It passes at first upwards and to the right; thereafter it sweeps, in an arched manner, upwards, backwards, and to the left, over the root of the left lung; and finally it descends in close contact with the vertebral column, lying at first upon its left side, but subsequently in front of it. It is therefore conveniently divided into three parts, namely, the ascending aorta, the arch of the aorta, and the descending aorta.

Ascending Aorta.-The ascending aorta commences at the base of the left ventricle of the heart, behind the left border of the sternum; on a level with the lower margin of the third left costal cartilage, and it terminates at a point behind the right border of the sternum on a level with the upper margin of the second right costal cartilage. Its course is upwards and to the right, with an inclination forwards. It lies in the middle mediastinum, and within the fibrous pericardium, which ensheathes it, and, for about the first $I_{\frac{1}{2}}$ inches of its course it is enclosed, along with the adjacent portion of the pulmonary artery, in a sheath formed by the serous part of the pericardium. The ascending aorta measures about 2 inches in length. At its commencement it presents three bulgings, which correspond to the sinuses of Valsalva in the interior and lie opposite the segments of the aortic valve. The bulgings are disposed as right antero-lateral, left antero-lateral, and posterior respectively. Along the right side of the vessel there is another sornewhat extensive dilatation, called the great sinus of the aorta.

Relations-Anterior.-The root of the pulmonary artery and the right auricular appendix, at first, and subsequently the first piece 
of the body of the sternum, from which it is separated by the pericardium, right pleura, and anterior margin of the right lung. Posterior.- The right pulmonary artery and the left auricle. Right.The superior vena cava, and the right auricle. Left.-The trunk of the pulmonary artery.

Branches.- These are the two coronary arteries, right and left.

The right coronary artery arises from the right antero-lateral sinus of Valsalva. Passing forwards between the right auricular: appendix and the root of the pulmonary artery, it enters the right auriculo-ventricular groove, which it traverses from front to back as far as the commencement of the inferior interventricular groove. At this point it divides into two branches, transverse and descending. The transverse branch enters the posterior part of the left auriculo-ventricular groove, in which it anastomoses with the transverse branch of the left coronary artery. The descending branch traverses the inferior interventricular groove as far as the region of the apex, where it anastomoses with the descending branch of the left coronary artery.

The right coronary artery furnishes branches to the right auricle and to both ventricles. One, of large size, called the right marginal branch, passes along the right border, or margo acutus, towards the apex.

The left coronary artery arises from the left antero-lateral sinus of Valsalva, and is at first concealed by the root of the pulmonary artery. It then passes forwards between the pulmonary artery and the left auricular appendix, and divides into two branches, transverse and descending. The transverse branch enters the left auriculo-ventricular groove, which it traverses from front to back, anastomosing posteriorly with the transverse branch of the right coronary artery. The descending branch traverses the anterosuperior interventricular groove as far as the region of the apex, where it anastomoses with the descending branch of the right coronary artery.

The left coronary artery furnishes branches to the left auricle and to both ventricles. One, of large size, called the left marginal branch, springs from its transverse branch and passes along the left border, or margo obtusus, towards the apex.

Development.-The ascending aorta, along with the trunk of the pulmonary artery, is developed from the aortic bulb.

Arch of the Aorta. - The arch of the aorta commences behind the right border of the sternum, on a level with the upper margin of the second right costal cartilage, and it terminates on the left side of the body of the fourth thoracic vertebra, at the lower-border of which it becomes the descending aorta. It passes upwards, backwards, and to the left, sweeping over the root of the left lung, and, when it reaches the left side of the body of the fourth thoracic vertebra, it descends. The height to which the arch reaches corresponds to the centre of the manubrium sterni, which is about 
ficial cardiac plexus, which they form. All these nerves are overlaid by the left pleura. Farther back than these nerves is the left superior intercostal vein. The remains of the. thymus body constitute an additional anterior relation. Posterior.-The trachea, deep cardiac plexus of nerves, œsophagus, thoracic duct, and left recurrent laryngeal nerve. Superior.-The left innominate vein, and the origins of the following three great arteries, named in order from right to left, and also from before backwards: the innominate, the left common carotid, and the left subclavian. Inferior.-The left bronchus, the bifurcation of the pulmonary artery, the superficial cardiac plexus of nerves, the left recurrent laryngeal nerve, and the ligamentum ductûs arteriosi. The lastnamed fibrous cord is attached to the back part of the concavity of the arch, immediately beyond the level of the origin of the left subclavian artery.

The arch presents a constriction immediately beyond the origin of the left subclavian artery, called the aortic isthmus, and this is succeeded by a short fusiform dilatation, known as the aortic spindle of $H i$ s. These conditions are best marked in the fotus.

Branches.-These are three in number, namely, the innominate, left common carotid, and left subclavian arteries. They arise in the order named, proceeding from before backwards, and also from right to left.

Innominate Artery.-This vessel is the first and largest of the three branches which arise from the arch of the aorta. It springs from the upper aspect of the arch rather above the level of the upper border of the second right costal cartilage, and it terminates behind the upper border of the right sterno-clavicular joint by dividing into the right common carotid and right subclavian arteries. It is from $\mathrm{I} \frac{1}{2}$ to 2 inches in length, its direction is upwards and outwards, and it lies in the superior mediastinum.

Relations-Anterior.--The right half of the manubrium sterni, with the origins of the right sterno-hyoid and sterno-thyroid muscles ; the left innominate and the right inferior thyroid veins ; the right sterno-clavicular joint; and some remains of the thymus body. Posterior. - The trachea at first, but, as the artery ascends obliquely to the right, it leaves the front of the trachea and is placed on its right side. The three cervical cardiac branches of the right sympathetic also lie behind the artery on their way to the deep cardiac plexus. Right.-The right pleura ; the innominate vein, with the right pneumogastric nerve behind it ; the superior .vena cava; and the right phrenic nerve. Left.-The left common carotid artery, and the trachea, in this order from below upwards.

Branches.- These are terminal, and are two in number, namely, the right common carotid and the right subclavian. The artery, as a rule, gives off no branches in its course. Occasionally, however, it gives origin to a vessel, of variable size, callẹd the arteria thyroidea ima (lowest thyroid artery). The interest attached to this occasional branch is that, in ascending to the isthmus of the 
thyroid body in the neck, it lies in front of the trachea, and would be endangered in performing the low operation of tracheotomy.

Varieties.-(I) The innominate artery may be shorter or longer than usual. (2) In cases of high bifurcation the artery may so encroach upon the trachea as to be endangered in performing the low operation of tracheotomy.

Left Common Carotid Artery in the Thorax.-This vessel arises from the upper aspect of the arch of the aorta, just to the left of, and posterior to, the origin of the innominate artery. It lies in the superior mediastinum, and its direction is upwards and to the left. Having reached the posterior aspect of the left sternoclavicular joint, it enters upon the cervical part of its course.

Relations-Anterior.-The left half of the manubrium sterni, with the origins of the left sterno-hyoid and sterno-thyroid muscles, but these structures lie at a little distance from the vessel ; the left innominate vein; and some remains of the thymus body. Posterior.-The trachea at first, and subsequently the œsophagus (which here deviates slightly to the left of the trachea), and the thoracic duct. Right.- The innominate artery at first, and thereafter the trachea. Left.-The left pneumogastric and left phrenic nerves, with the superior cervical cardiac branch of the left sympathetic and the inferior cervical cardiac branch of the left pneumogastric; and the left pleura and lung.

The thoracic portion of the left subclavian artery is on the left of, and posterior to, the vessel, but at a little distance from it.

The thoracic portion of the left common carotid artery gives off no branches.

First Part of the Left Subclavian Artery in the Thorax.-This vessel arises from the upper aspect of the arch of the aorta, a little to the left of, and posterior to, the origin of the left common carotid artery. It lies deeply in the superior mediastinum, and is almost parallel to the thoracic portion of the left common carotid, its course being nearly vertical.

Relations-Anterior.- The left common carotid artery; the left pneumogastric and left phrenic nerves, with the superior cervical cardiac branch of the left sympathetic and the inferior cervical cardiac branch of the left pneumogastric, all these nerves lying between it and the left common carotid artery; and the left innominate vein. Posterior.-The osophagus, and the thoracic duct. Right.-The trachea, and the left recurrent laryngeal nerve. Left.The left pleura, and the inner aspect of the left lung, the latter being grooved by the vessel.

The thoracic portion of the left subclavian artery gives off no branches.

Varieties of the Aorta-1. Position.-(a) The arch of the aorta may rise ts high as the upper border of the manubrium sterni, or it may stop short of the level of the centre of the manubrium. (b) It may be a right aortic arch, instead of a left, in which cases there may, or may not, be a transposition of the viscera. The presence of a right aortic arch is due to its formation from the fourth right arterial arch, and the persistence of the right primitive dorsal aorta. 


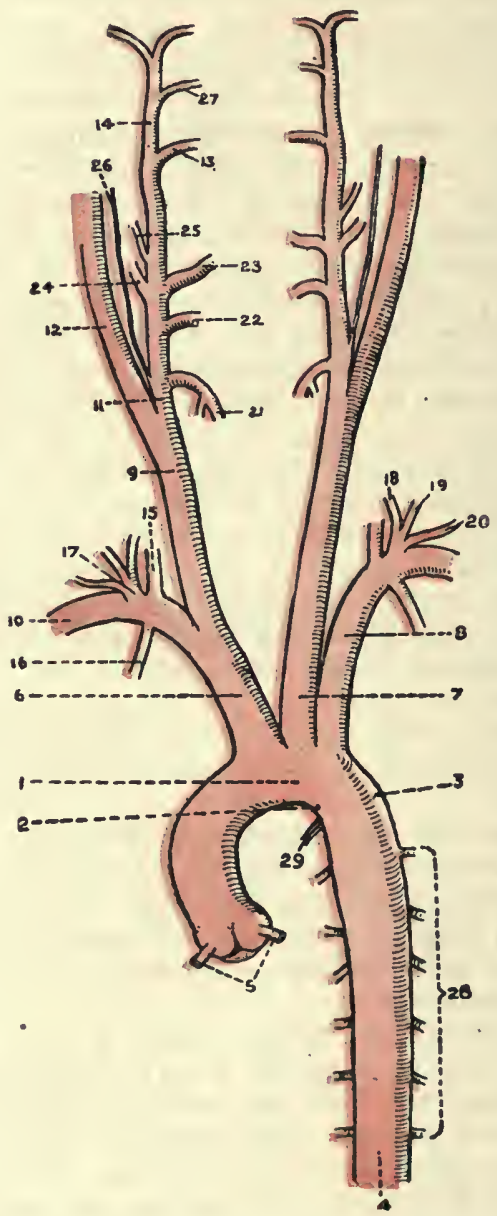

Fig. 394.-The Aorta in the Thorax, and the Principal Arteries of THE HEAd AND NECK.
3. Arch of the Aorta
2. Aortic Isthmus
3 Aortic Spindle
4. Descending Aorta
5. Coronary Arteries (from Ascending Aorta)
6. Innominate Artery
7. I.eft Common Carotid
8. Left Suliclavian
9. Right Common Carotid

10. Right Subclavian

11. External Carotid

12. Internal Carotid

13. Internal Maxillary

24. Superficial Temporal

15. Vertebral

16. Internal Mamnary

17. Thyroid Axis

18. Inferior Thyroid

19. Transverse Cervical
20. Suprascapular

21. Superior Thyroid

22. Lingual

23. Facial

24. Occipital

25. Posterior Auricular

26. Ascending Pharyngeal

27. Trancverse Facial

28. Aortic Intercosials

29. Lig. Ductûs Arteriosi 
2. Branches of the Areh and their Positions.-Varieties in these respects are very numerous. The normal number of branches arising from the arch is three. There may, however, be four. The most common additional branch is the left vertebral artery, its place of origin being between the left common carotid and left subclavian arteries. The right vertebral artery sometimes arises from the arch, but this is somewhat rare. An arteria thyroidea ima may arise from the arch between the innominate and left common carotid arteries. In rare cases the internal mammary artery, or the inferior thyroid, may spring from the arch. The innominate artery may be absent, in which cases the right subclavian and right common carotid have independent origins. Under these circumstances the right subclavian artery may be the last of the branches from the upper part of the arch, and, when this is so, in order to reach the right side of the neck, it crosses in front of the vertebral column, lying behind the œsophagus, or between the trachea and the oesophagus.

Development of the Arch of the Aorta and its Branches.-As already stated, the ascending aorta is developed, along with the pulmonary artery, from the aortic bulb. The arch of the aorta is developed chiefly from the fourth left arterial arch, but slightly also from the root of the left primitive ventral aorta, that is, the part as high as the fourth left arterial arch.

The innominate artery is developed from the root of the right primitive ventral aorta - that is, the part as high as the fourth right arterial arch.

The left common carotid artery is developed from that portion of the left primitive ventral aorta which lies between the fourth and third left arterial arches.

The left subclavian artery is developed from a segmental artery.

For the description of the descending aorta, see p. I023.

Pulmonary Artery.-This is the great vessel which, by means of its right and left divisions, carries the venous blood from the right ventricle of the heart to the lungs. It is therefore an example of an artery which conveys venous blood, and in this respect resembles the hypogastric and umbilical arteries of the fœtus. It arises from the infundibulum, or conus arteriosus, of the right ventricle of the heart, on a level with the upper margin of the third left costal cartilage at its junction with the sternum. It is directed upwards and backwards, and, after a course of about 2 inches, breaks up into two divisions, right and left, within the concavity of the arch of the aorta." The vessel lies in the middle mediastinum, and, along with the ascending aorta, it is contained within the pericardium, the serous portion of which forms one common sheath for the two arteries over about the first $\mathrm{I} \frac{1}{2}$ inches of their course.

Relations-Anterior.-The sternal extremity of the second left intercostal space and second left costal cartilage; and the left pleura and left lung. Posterior.-The root of the ascending aorta ; the commencement of the left coronary artery ; and the left auricle of the heart. Right.-The right coronary artery; the right auricular appendix ; and the ascending aorta. Left.-The left coronary artery ; and the left auricular appendix.

The only branches of the artery are the two terminal divisions.

The right pulmonary artery passes outwards to the right, behind the ascending aorta and superior vena cava, to the root of the right lung, where it divides into two branches, upper and lower. The upper branch is distributed to the upper lobe, and the lower branch, which is the larger of the two, is distributed to the middle and lower lobes. 
The left pulmonary artery passes outwards to the left, in front of the left bronchus and descending aorta, to the root of the left lung, where it divides into two branches, one for the upper, and the other for the lower, lobe. The ligamentum ductûs arteriosi is attached to the upper aspect of its root.

The right pulmonary artery is larger and longer than the left.

Development.- The trunk of the pulmonary artery, along with the ascending aorta, is chiefly developed from the aortic bulb, but a small portion of it is formed by the commencement of the sixth left arterial arch, which remains connected with that portion of the aortic bulb which becomes partitioned off to form the pulmonary trunk.

The right and left pulmonary arteries are developed as branches from the sixth left arterial arch near its commencement, the remainder of that arch giving rise to the ductus arteriosus of the fœtus.

Ligamentum Ductûs Arteriosi.--This is a fibrous cord which is the remains of an important vessel, peculiar to foetal life, called the ductus arteriosus. It extends from the upper aspect of the root of the left pulmonary artery to the under surface of the arch of the aorta, immediately beyond the level of the origin of the left subclavian artery. Its direction is upwards, backwards, and slightly to the left.

During fœtal life the right and left pulmonary arteries are of small size, and the ductus arteriosus conveys the greater part of the venous blood from the right ventricle of the heart into the aorta at a point beyond the origin of the left subclavian artery. None of this blood, therefore, can pass into the great vessels which spring from the upper aspect of the arch of the aorta.

Development.-The ductus arteriosus is developed from the dorsal part of the sixth left arterial arch.

Pulmonary Veins.-These vessels carry the arterial or oxygenated blood from the lungs to the left auricle of the heart. Though they are called veins, they contain arterial blood, and in this respect resemble the umbilical vein of the fœtus. They are four in number, two right and two left, and, at the root of each lung, the upper of the two is on a more anterior plane than the lower. The right veins pass bchind the superior vena cava and the right auricle, and the left veins pass in front of the descending aorta. All four vessels open into the left auricle on its posterior aspect. On leaving the roots of the lungs the veins are said to receive small bronchial tributaries from the adjacent bronchial tubes and glands.

The right pulmonary veins are larger and longer than those of the left side.

Pneumogastric Nerves in the Thorax.-These nerves, right and left, differ so much from each other in their course and relations as to require separate descriptions.

The right pneumogastric, or vagus, nerve, having descended in front of the first part of the. right subclavian artery; and having given off its inferior or recurrent laryngeal branch at the lower border of that vessel, enters the thoracic cavity. It then descends in the superior mediastinum behind the innominate vein, and, inclining backwards, it reaches the right side of the trachea, along which it courses to the posterior aspect of the root of the right lung. 
Behind the root of the right lung the nerve becomes flattened out and breaks up into numerous branches, which are disposed in a plexiform manner, and constitute the right bosterior pulmonary plexus, from which branches are given off to the right lung. From the lower part of this plexus the nerve issues in the form of two cords, which descend in the posterior mediastinum upon the right side of the œsophagus, or gullet, and communicate freely with the corresponding cords of the left side. In this manner a plexus is formed, which is called the plexus gula. Subsequently the two

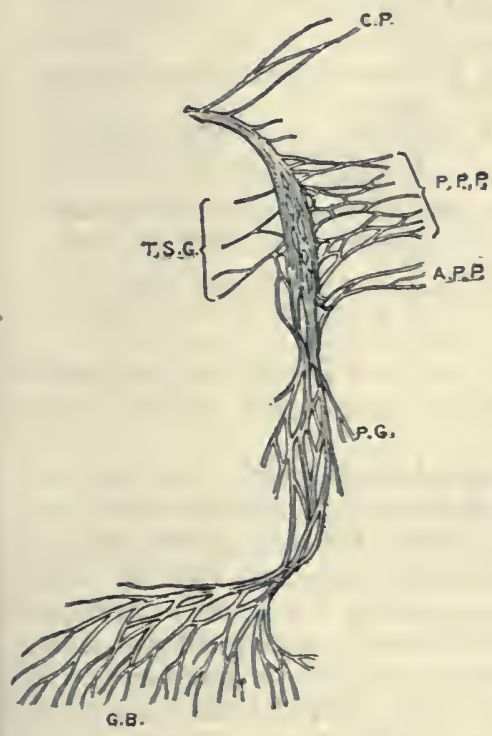

Tig. 395.- Scheme of PNeumogasTRIC NerVe in Thorax AND ABDOMEN (Flower).

C.P. Branches to Cardiac Plexus

P.P.P. Branches to Posterior Pulmonary Plexus

A.P.P. Branches to Anterior Pul. monary Plexus

T.S.G. Branches from Upper Thoracic Ganglia of Sympathetic

P.G. Plexus Fula

G.B. Gastric Branches cords of the right side unite to form a single nerve, which descends on the posterior surface of the œsophagus, and enters the abdomen through the cesophageal opening of the diaphragm to be distributed to the posterior surface of the stomach.

The left pneumogastric, or vagus, nerve enters the thoracic cavity between the left common carotid and left subclavian arteries, and descends in the superior mediastinum behind the left innominate vein. It then passes in front of the arch of the aorta, having the left phrenic nerve on its right side and anterior to it, with the intervention of the superior cervical cardiac branch of the left sympathetic and its own inferior cervical cardiac branch. At the lower border of the arch it gives off its inferior or recurrent laryngeal branch, and then passes to the posterior aspect of the root of the left lung. Behind the root of the left lung the nerve, as on the right side, becomes flattened out and breaks up into numerous branches, which are disposed in a plexiform manner, and constitute the left posterior pulmonary plexus, from which branches are given off to the left lung. From the lower part of this plexus the nerve, as on the right side, issues in the form of two cords, which descerid in the posterior mediastinum upon the left side of the œsophagus or gullet, and communicate freely with the corresponding cords of the right side. In this manner, as stated, a plexus is formed, which is called the plexus gulce. Subsequently the two cords of the left side unite to form a single nerve, which descends on the anterior 
surface of the œsophagus, and enters the abdomen through the œsophageal opening of the diaphragm, to be distributed to the anterior surface of the stomach.

\section{Differences between the Right and Left Pneumogastric Nerves.}

\section{Right Pneumogastric Nerve.}

1. Descends in front of first part of right subclavian artery.

2. Gives off its recurrent laryngeal branch at lower border of right subclavian artery.

3. Lies behind right innominate vein.

4. Lies on right side of trachea.

5. Lies at first on right side of cesophagus, and then behind it.

6. Ramifies on posterior surface of stomach.

\section{Left Pneumogastric Nerve.}

I. Descends between left common carotid and left subclavian arteries.

2. Passes belind left innominate vein.

3. Crosses in front of arch of aorta.

4. Gives off its recurrent laryngeal branch at lower border of arch.

5. Lies at first on left side of œsophagus, and then in front of it.

6. Ramifies on anterior surface of stomach.

Branches.-These are as follows : the left inferior or recurrent laryngeal ; cardiac, from the right nerve ; pulmonary ; œsophageal ; and pericardial.

The left inferior or recurrent laryngeal nerve arises from the left pneumogastric in front of the arch of the aorta, on a level with its lower border. It passes backwards within the arch at the place of attachment of the ligamentum ductûs arteriosi, and then it turns upwards behind the arch. Having reached the groove between the trachea and the œsophagus, it ascends therein to the neck, where its subsequent course and distribution will be described. In the thorax the nerve, which contains fibres derived from the bulbar or accessory portion of the spinal accessory nerve, furnishes a few cardiac branches to the deep cardiac plexus as it winds round the arch of the aorta.

The right inferior or recurrent laryngeal nerve is extra-thoracic, inasmuch as it arises from the right pneumogastric at the root of the neck, and it winds round the first part of the right subclavian artery.

The recurrent course of the right and left recurrent laryngeal nerves is brought about by the change in position which the heart and great vessels undergo in the course of development. In early embryonic life each recurrent laryngeal nerve passes inwards to the larynx below the corresponding sixth arterial arch. This arch on the right side, together with the fifth, disappears, and the right recurrent laryngeal nerve then becomes related to the fourth right arterial arch, from which the first part of the right subclavian artery is developed. The sixth left arterial arch gives rise to the ductus arteriosus, which, however, becomes obliterated, and then the left recurrent laryngeal nerve becomes related to the fourth left arterial arch (the fifth having disappeared) at the place where it receives the ductus arteriosus, and from this fourth left arch the arch of the aorta is developed. As the heart and great vessels descend from the neck into the thorax each recurrent laryngeal 
nerve is necessarily drawn downwards, and the right nerve becomes recurrent round the first part of the right subclavian artery (the original fourth right arterial arch), whilst the left nerve becomes recurrent round the arch of the aorta (the original fourth left arterial arch) at the place of attachment of the ligamentum ductûs arteriosi.

The cardiac branches of the right pneumogastric are two or three in number, and they descend upon the trachea to the deep cardiac plexus. (The cardiac branches on the left side are derived, as stated, from the left recurrent laryngeal nerve as it winds round the arch of the aorta.)

The pulmonary branches are arranged in two sets, anterior and posterior. The anterior pulmonary branches are two or three in number, and arise from the parent trunk before it disappears behind the root of the lung. They pass to the anterior aspect of the root, and, being joined by sympathetic twigs, they form the anterior pulmonary plexus, which is reinforced by twigs from the deep cardiac plexus, and, in the case of the left anterior pulmonary plexus, by twigs from the superficial cardiac plexus. The branches of the anterior pulmonary plexus enter the lung, and accompany the ramifications of the bronchial tubes.

The posterior pulmonary branches arise from the pneumogastric nerve behind the root of the lung. They are larger and more numerous than the anterior branches, and, being joined by twigs from the second, third, and fourth thoracic sympathetic ganglia, they form the posterior pulmonary plexus. The branches of this plexus, like those of the anterior, enter the lung, and accompany the ramifications of the bronchial tubes.

The osophageal branches arise chiefly from the plexus gulæ, below the level of the roots of the lungs, and they are distributed to the portion of the œsophagus which occupies the posterior mediastinum. Other œesophageal branches, however, arise above the level of the roots of the lungs, and are distributed to the portion of the œsophagus which occupies the superior mediastinum.

The pericardialbranches arise from the plexus gulæ, and are dis. tributed to the pericardium, which they enter from behind.

\section{Summary of Branches.}

Right Pneumogastric Nerve.

Cardiac.

Anterior Pulmonary.

Posterior Pulmonary.

Esophageal.

Pericardial.

\section{Left Pneumogastric Nerve.}

Recurrent Laryngeal (which gives off Cardiac branches).

Anterior Pulmonary.

Posterior Pulmonary.

Esophageal.

Pericardial.

Cardiac Plexus.-The cardiac plexus is one of three large prevertebral plexuses associated with the sympathetic system, the other two, namely, the epigastric or solar and the hypogastric, being situated in the abdominal cavity. The plexus is situated partly in the concavity of the arch of the aorta, and partly upon 
the trachea above the bifurcation and behind the aortic arch. It is formed by branches of the pneumogastric and sympathetic nerves, and consists of two portions, superficial and deep, which communicate with each other.

The superficial cardiac plexus, which is comparatively small, is situated in the concavity of the arch of the aorta, between the ligamentum ductûs arteriosi and the right pulmonary artery. It is formed by (I) the superior cervical cardiac branch of the left sympathetic, and (2) the inferior cervical cardiac branch of the left pneumogastric, nerves. These two nerves descend over the arch of the aorta, lying between the left phrenic and left pneumogastric nerves. At the place where the two nerves join there may be a small ganglion, which is known as the ganglion of Wrisberg.

Branches.-The plexus gives branches to the left anterior pulmonary plexus, and, having received a considerable accession of fibres from the right half of the deep cardiac plexus, it is prolonged into the right coronary plexus.

The deep cardiac plexus, of larger size than the superficial, is situated upon the trachea immediately above the bifurcation and behind the arch of the aorta. It is formed by (I) all the cervical cardiac branches of the right sympathetic, namely, superior, middle, and inferior, and of the right pneumogastric, namely, superior and inferior; (2) one or two cardiac branches from the right recurrent laryngeal nerve; (3) one or two thoracic cardiac branches from the right pneumogastric; (4) the middle and inferior cervical cardiac branches of the left sympathetic; (5) the superior cervical cardiac branch of the left pneumogastric; and (6) the thoracic cardiac branches of the left recurrent laryngeal nerve. It is arranged in two halves, right and left, which communicate with each other; the right half also gives a considerable accession of fibres to the superficial cardiac plexus. Each half receives the following branches :

\section{Right Halt.}

I. The three cervical cardiac branches of the right sympathetic.

2. The two cervical cardiac branches of the right pneumogastric.

3. The cardiac branches of the right recurrent laryngeal.

4. The thoracic cardiac branches of the right pneumogastric.

\section{Left Hall.}

I. The middle and inferior cervical cardiac branches of the left sympathetic.

2. The superior cervical cardiac branch of the left pneumogastric.

3. The cardiac branches of the left recurrent laryngeal.

Branches.-The right half of the deep cardiac plexus gives off (I) branches to the right anterior pulmonary plexus ; (2) branches to the right auricle of the heart; $(3)$ branches to reinforce the right coronary plexus, which is derived, as stated, from the superficial cardiac plexus; and (4) a few branches to the left coronary plexus. The left half of the deep cardiac plexus gives off (I) branches to the 


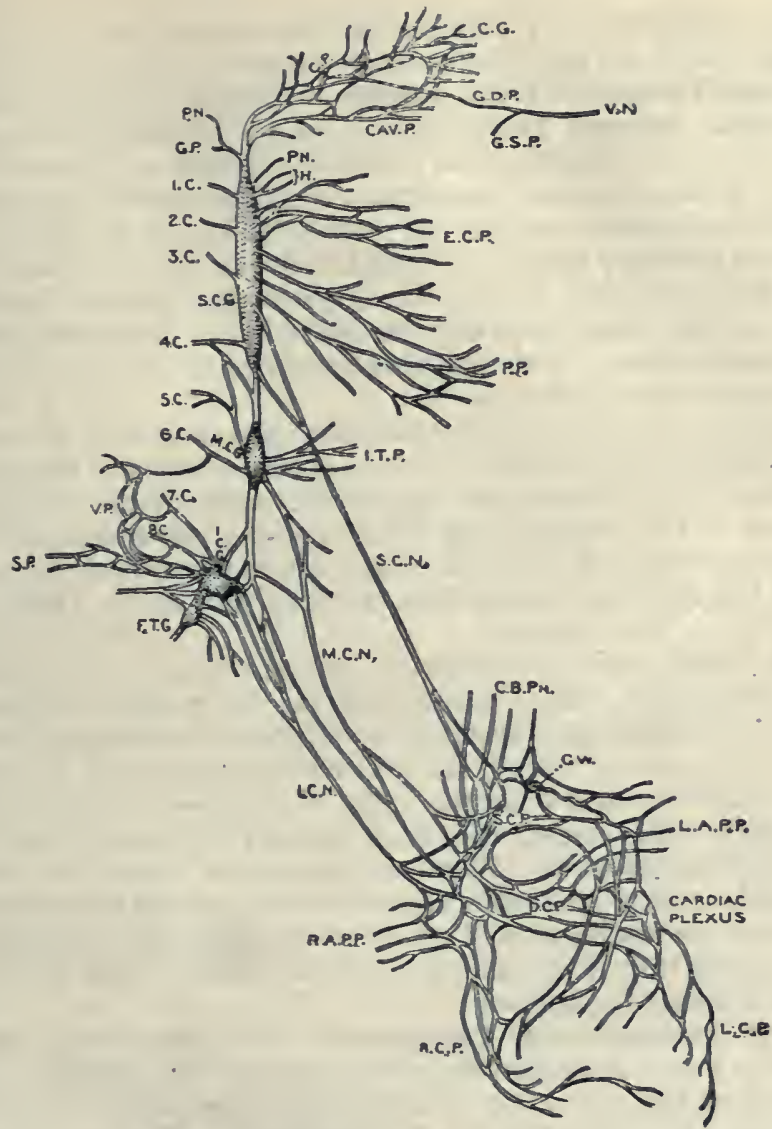

Fig. 396.-Scheme of the Sympathetic Nerve in the Neck, and of the Cardiac Plexus (Flower).

\section{S.C.G. Superioz Cervical Ganglion.}

P.N. and G.P. Branches to Pneumogastric and Glosso-pharyngeal

Ic, $2 c, 3 c$, 4 c. Branches to Upper Four Cervical Nerves

C P. Carotid Plexus

Cav. P. Cavernous Plexus

C.G. Branch to Ciliary Ganglion

G.D.P. Great Deep Petrosal

G.S.P. Great Superficial Petrosal

V.N. Vidian Nerve

P.N. To Ganglion of Trunk of Pneumogastric

H. To Hypoglossal

E.C.P. To External Carotid Plexus

P.P. Tn Pharyngeal Plexus

S.C.N. Superior Cardiac Nerve

M.C.G. Middle Cervical Gangllon.

5c, 5c. To Fifth and Sixth Cervical Nerves

I.T.P. To laferinr Thyroid Plexus

M.C.N Middle Cardiac Nerve

\section{L.C.G. Inferior Cervical Gangilon.}

$7 c, 8 c$. To Seventh and Eighth Cervical Nerves V.P. To Vertebral Plexus

S.P. To Subclavian Plexus

I.C.N. Inferior Cardiac Nerve

\section{F.T.G. First Thoracic Ganglion}

\section{Cardiac Plexus}

C. B.PN. Cardiac Branches of Pneumogastric S.C.P. Superficial Cardiac Plexus

G.IV. Ganglion of Wrisherg

D.C.P. Deep Cardiac Plexus

R.A.P.P. Right Anterior Pulmonary Plexus

L. A.P P. Left Anterior Pulmonary Plexus

R.C.P. Right Coronary Plexus

L.C.P. Left Coronary Plexus 
left anterior pulmonary plexus; (2) branches to the left auricle of the heart ; and (3) the left coronary plexus.

Coronary Plexuses.- - These are two in number, right and left.

The right coronary plexus is formed by branches from (I) the superficial cardiac plexus, and (2) the right half of the deep cardiac plexus. It accompanies the right coronary artery, and furnishes branches to the right auricle and right ventricle of the heart.

The left coronary plexus is formed by branches which are derived chiefly from the left half of the deep cardiac plexus, but it also receives a few branches from the right half. It accompanies the left coronary artery, and furnishes branches to the left auricle and left ventricle of the heart.

Ganglia are met with in the coronary plexuses, and in the course of the fibres which supply the walls of the auricles. They are also present on the fibres which supply the walls of the ventricles in the region of the auriculo-ventricular groove, but nowhere else. In the heart of the calf the nerves are easily recognised beneath the visceral layer of the pericardium, as they pass across the muscular fibres in an oblique manner.

Heart.-The heart is a hollow, nuuscular organ, which, enclosed within the pericardium, is situated in the middle mediastinum, where it lies obliquely between the two lungs. It is conical in shape, and is free to move within its pericardial sac, except at the base, where it is connected with the great bloodvessels. Its relation to the thoracic wall, during life, is influenced by posture and by the respiratory movements. When a person lies upon the left side, or when the prone position is assumed, the organ is more intimately related to the thoracic wall than in the opposite postures; and during inspiration it is less intimately connected with the thoracic wall than during expiration.

General Relations and Topography.-The heart lies obliquely behind the lower three-fourths of the body of the sternum. About two-thirds of the organ are contained in the left half of the thoracic cavity, and about one-third in the right half. The base is directed upwards, backwards, and to the right, and lies opposite the bodies of the middle four thoracic vertebræ, namely, the fifth, sixth, seventh, and eighth. The apex is directed downwards, forwards, and to the left, and, during life, it strikes the thoracic wall in the fifth left intercostal space, $\mathrm{I}_{2}^{\frac{1}{2}}$ inches below the left nipple, and about $\frac{3}{4}$ inch within the left mammillary line. This point represents the apex-beat, and is about $3 \frac{1}{2}$ inches from the median line of the sternum. 'The antero-superior surface, which is convex, lies behind the lower three-fourths of the body of the sternum and the corresponding costal cartilages, right and left, namely, the third, fourth, fifth, and sixth, more particularly those of the left side. This surface is encroached upon by the pleuræ and the thin anterior margins of the lungs. Opposite the cardiac notch on the anterior margin of the left lung there is a small portion which is uncovered by lung, unless during deep inspiration, and this cor- 
responds with the area of precordial dulness. Latham's circle is taken as defining this area, and the directions for describing the circle are as follows: "Make a circle of 2 inches in diameter round a point midway between the left nipple and the end of the sternum.' Strictly speaking, the area of precordial dulness is triangular in conformity to the $\mathbf{V}$-shaped cardiac notch on the anterior margin of the left lung, and it may be mapped out by the following lines: one drawn from the position of the apex-beat to the median line of the sternum on a level with the fourth left costal cartilage ; another drawn from the position of the apex-beat to the median line of the sternum at the junction of the body and ensiform process; and a third connecting the mesial ends of these two lines, and extending along the middle of the sternum. The inferior or diaphragmatic surface, which is flat, is directed downwards, and rests upon the upper surface of the central tendon of the diaphragm, covered by the serous portion of the pericardium.

The outline of the heart, in reference to the anterior wall of the thorax, may be indicated, with approximate accuracy, in the following manner :

Base.-Draw a line across the sternum on a level with the upper border of the third right, and the lower border of the second left, costal cartilages, and prolong this line for $\frac{1}{2}$ inch to the right of the sternum, and I inch to the left of it.

Inferior (Right) Border, or Margo Acutus.--Draw a line from the sternal end of the sixth right costal cartilage to the position of the apex-beat. This line corresponds to the lower limit of the heart.

Right Limit.-Draw a line from the upper border of the third right costal cartilage, $\frac{1}{2}$ inch from the sternum, to the sternal end of the sixth right costal cartilage. This line should be curved outwards to such an extent that its greatest convexity will be $I \frac{1}{2}$ inches distant from the median line of the sternum. It corresponds with the right limit of the right auricle.

Left Border, or Margo Obtusus vel Rotundus.-Draw a line from the lower border of the second left costal cartilage, I inch from the sternum, to the position of the apex-beat. This line should be slightly curved outwards, but it must not include the left nipple. It corresponds to the left limit of the heart.

Course of the Circulation.-The interior of the heart is divided by two septa (interauricular and interventricular) into two halves, right and left, and each half is subdivided by a transverse constriction into two chambers, an upper or auricle, and a lower or ventricle, right and left respectively. The auricles, except in the fœtus, are completely separated from each other by a septum, and so also are the ventricles; but the auricle and ventricle of each side communicate freely with each other by the auriculo-ventricular orifice. The right auricle receives the venous blood chiefly from the superior and inferior venæ cavæ and the coronary sinus. From the right auricle the blood passes into the right ventricle, and thence into the trunk of the pulmonary artery. The right and 
left pulmonary arteries convey it to the lungs, and in passing through the pulmonary capillaries it is oxygenated and becomes arterial blood. It is then taken up by the pulmonary venous radicles, and conveyed to the pulmonary veins, which carry it to the left auricle of the heart. From the left auricle it passes into the left ventricle, whence it is driven into the aorta. The aorta and its various ramifications convey the arterial blood to the different parts of the body, and thereafter it is returned as venous blood to the right auricle of the heart.

Exterior of the Heart.-The exterior of the heart presents distinct indications of its division internally into four chambers.

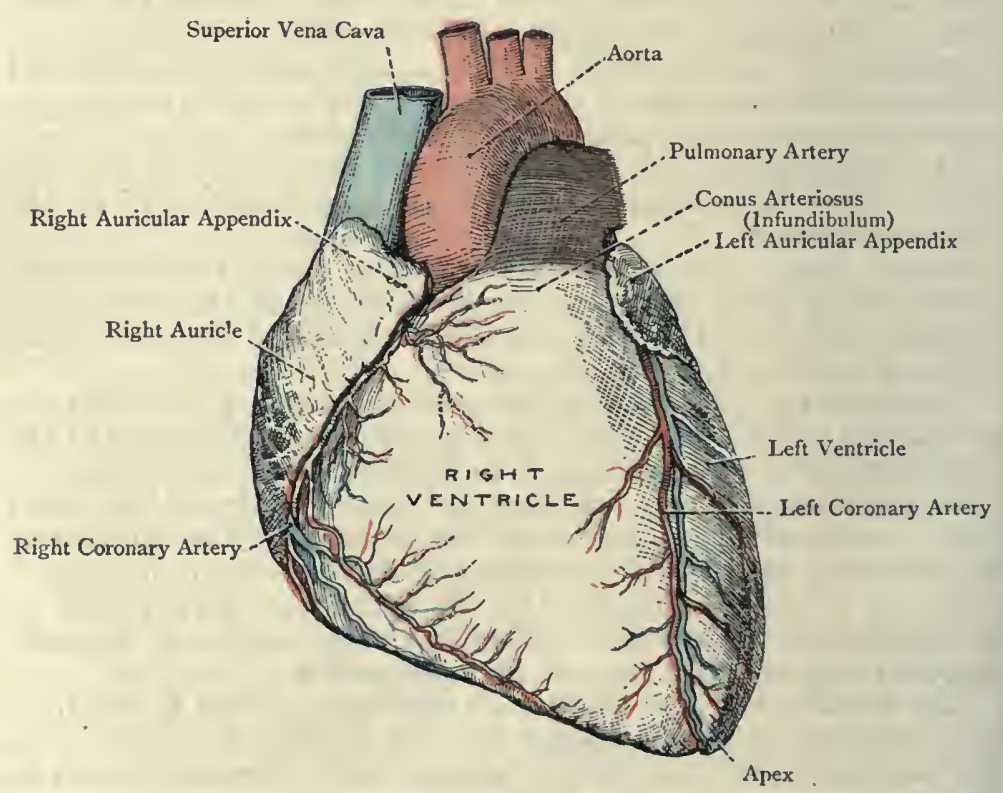

Fig. 397.-The Heart (ANTerior View).

These take the form of grooves, namely, auriculo-ventricular, interauricular, and interventricular.

The auriculo-ventricular groove, which is deep; divides the heart into auricular and ventricular portions, and it surrounds the organ except in front, where the roots of the aorta and pulmonary artery are situated. The auricular portion is posterior and superior in position, whilst the ventricular portion is anterior and inferior. The right half of the auriculo-ventricular groove contains (I) the right coronary artery, and (2) the right or small cardiac vein, which latter lies chiefly in its posterior part. The left half of the auriculoventricular groove contains (I) the transverse branch of the left coronary artery, (2) the transverse branch of the right coronary 
artery, which lies in its posterior part; (3) a portion of the great cardiac or coronary vein, which lies in its anterior part, and also slightly in its posterior part ; and (4) the coronary sinus, which lies in its posterior part.

The auricular portion of the heart has the form of a crescent, the horns of which represent the auricular appendices, and are directed forwards. The concavity of the crescent is also directed forwards, and it lodges the roots of the aorta and pulmonary artery, the latter being the more anterior of the two. The walls of the auricular portion are thin, and it is divided into two auricles, right and left. The external indication of this division is the interauricular groove, which is situated vertically on the posterior surface, to the left of the openings of the superior and inferior venæ cavæ. This groove corresponds to the posterior attachment of the interauricular septum in the interior. The greater part of the posterior surface of the auricular portion is formed by the left auricle. The large posterior part of each auricle is called the atrium ("receptionchamber '), and projecting forwards from the anterior and upper part of each atrium there is the auricular appendix. The two auricular appendices embrace between them the roots of the aorta and pulmonary artery.

The right auricle forms the anterior and right part of the base of the heart, and is quadrangular. The superior vena cava enters its atrium at the postero-superior angle, and the inferior vena cava at the postero-inferior angle. Near the latter vein the coronary sirus also opens into the atrium. The right auricular appendix is prolonged forwards from the antero-superior angle of the atrium. and inclines to the left in front of the root of the ascending aorta. It is shorter, broader, and less curved than the left auricular appendix, and its margin is notched, but not so much so as in the left appendix. The right auricle is traversed by a groove, called the sulcus terminalis, which extends from the front of the termination of the superior vena cava to the right side of the termination of the inferior vena cava. This groove indicates where the saccus reuniens of embryonic life meets the primitive auricle.

The left auricle forms the posterior and left part of the base of the heart. It is quadrilateral, and the greater part of it lies flattened behind the ascending aorta and trunk of the pulmonary artery. The pulmonary veins, two right and two left, open into the posterior part of its atrium. The left auricular appendix is prolonged forwards from its left aspect, and inclines to the right over the left side of the root of the pulmonary artery. It is longer, narrower, and more curved than the right auricular appendix, and its margin is more deeply notched. The back of the left auricle is related to the asophagus, with the intervention of the pericardium, and the small oblique vein of Marshall passes downwards and inwards upon it, to open into the coronary sinus.

The ventricular portion of the heart is conical, its walls are thick, and it is divisiole into two ventricles, right and left. The ex- 
ternal indication of this division is the interventricular groove, which corresponds to the attachment of the interventricular septum in the interior. This groove consists of two parts, anterosuperior and inferior. The antero-superior interventricular groove extends over the antero-superior surface of the heart from the left side of the root of the pulmonary artery to the inferior border, or margo acutus, to the right of, and near, the apex. At this point it becomes continuous with the inferior interventricular groove. It contains, besides fat, ( $I$ ) the descending branch of the left coronary artery, and (2) a part of the great cardiac or coronary vein (more

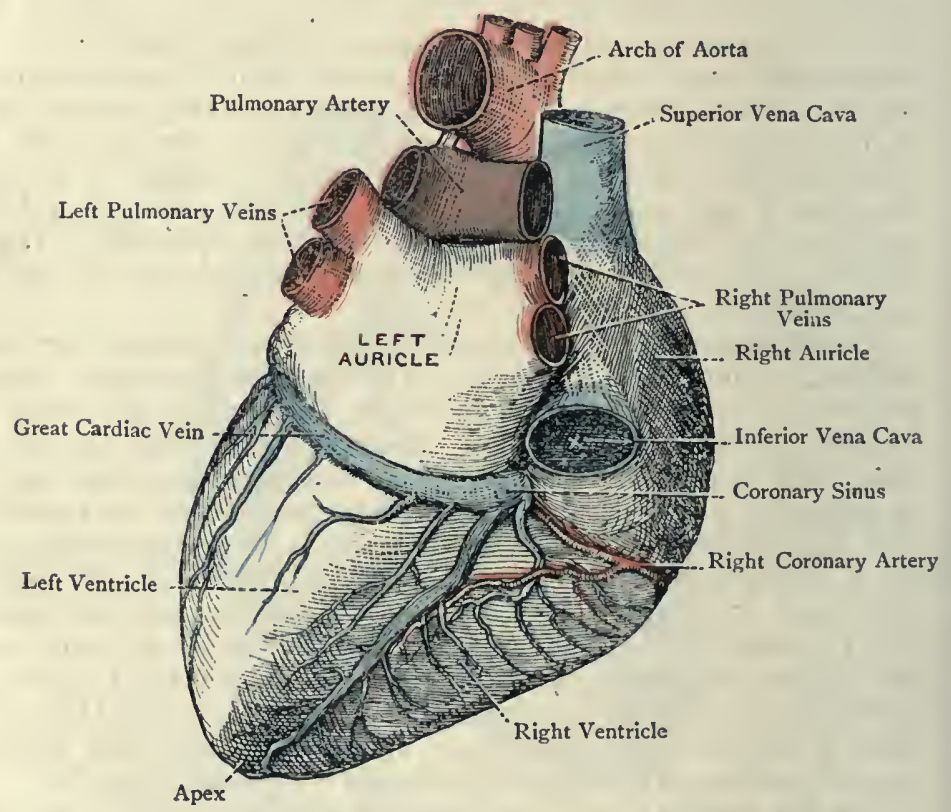

Fig. 398.-The Heart (Posterior View).

properly called the antero-superior interventricular vein in this region). The inferior interventricular groove is situated on the inferior surface of the heart, and, as stated, is continuous with the preceding. It contains, besides fat, ( $\mathrm{I}$ ) the descending (inferior) branch of the right coronary artery, and (2) the middle cardiac vein (more properly called the inferior interventricular vein).

The ventricular portion of the heart presents an apex, a base, two surfaces, and two Dóruers. " The apex is directed downwards, forwards, and to the left, and forms the apex of the heart. The left ventricle alone enters into its formation. The base is directed upwards, backwards, and to the right, and is connected with the 
atria of the auricles and the origins of the aorta and pulmonary artery, the former being behind, and the latter in front.

The surfaces are antero-superior and inferior. The anterosuperior surface is convex, and is traversed by the antero-superior interventricular groove. As this groove lies near the left border, the greater part of this surface (about two-thirds) is formed by the right ventricle, and the remainder by the left ventricle. In postmortem examinations, therefore, when the pericardium is opened, the right ventricle is chiefly exposed for inspection. On this aspect of the right ventricle there are the anterior cardiac veins. Its upper and left part is somewhat conical, and is called the infundibulum or conus arteriosus. It gives origin superiorly to the trunk of the pulmonary artery. The inferior surface is flat, and is traversed by the inferior interventricular groove. As this groove lies near the inferior border, the greater part of this surface (about two-thirds) is formed by the left ventricle, and the remainder by the right ventricle. On this aspect of the left ventricle there are the posterior cardiac veins.

The borders are inferior (right) and left. The inferior border is comparatively long, and extends from right to left. It is formed by the right ventricle, and is sharp in outline, from which circumstance it is known as the margo acutus. The marginal branch of the right soronary artery and one of the anterior cardiac veins lie along it. The left border is shorter than the inferior, and is blunt and round, from which circumstances it is known as the margo obtusus vel rotundus. The marginal branch of the left coronary artery lies along it.

Interior of the Heart-Right Auricle.-The wall of the auricular appendix is marked by a number of muscular elevations, arranged as closely-set, vertical, parallel bands, like the teeth of a comb, from which circumstance they are called the musculi pectinati. These bands, relatively to each other, are more or less reticular. They are also present on the right wall of the atrium, being more comb-like here than in the appendix, and they terminate posteriorly at a vertical ridge, called the crista terminalis. This crest corresponds in position to the sulcus terminalis externally, and it has the same significance. Internally and posteriorly the walls of the auricular atrium are destitute of musculi pectinati, and present a smooth appearance. The atrium of the right auricle presents the following openings: the opening of the superior vena cava; the opening of the inferior vena cava; the opening of the coronary sinus; the foramina Thebesii ; and the auriculo-ventricular opening.

The orifice of the superior vena cava, which is destitute of a valve, is situated at the postero-superior angle of the atrium. It is directed downwards and forwards, and the upper part of the crista terminalis is continuous with its anterior margin.

The orifice of the inferior vena cava is situated at the posteroinferior angle of the atrium, and is directed upwards and inwards. In front of the orifice, and to a certain extent overlapping it, there 
is a crescentic fold of endocardium, which is the remains of the Eustachian valve of fotal life. The convexity of the crescent is continuous with the anterior margin of the orifice of the vein, and the inner horn of the crescent is continuous with the anterior limb of the annulus ovalis, to be presently described. The fold is a

- somewhat indefinite structure in the adult, being subject to much variety as regards size, and sometimes presenting several small openings. During fœtal life, however, the Eustachian valve is of the utmost importance, inasmuch as it directs the blood entering by the inferior vena cava through the foramen ovale into the left auricle.

The orifice of the coronary sinus is situated between the vestigial Eustachian valve and the auriculo-ventricular orifice. It is

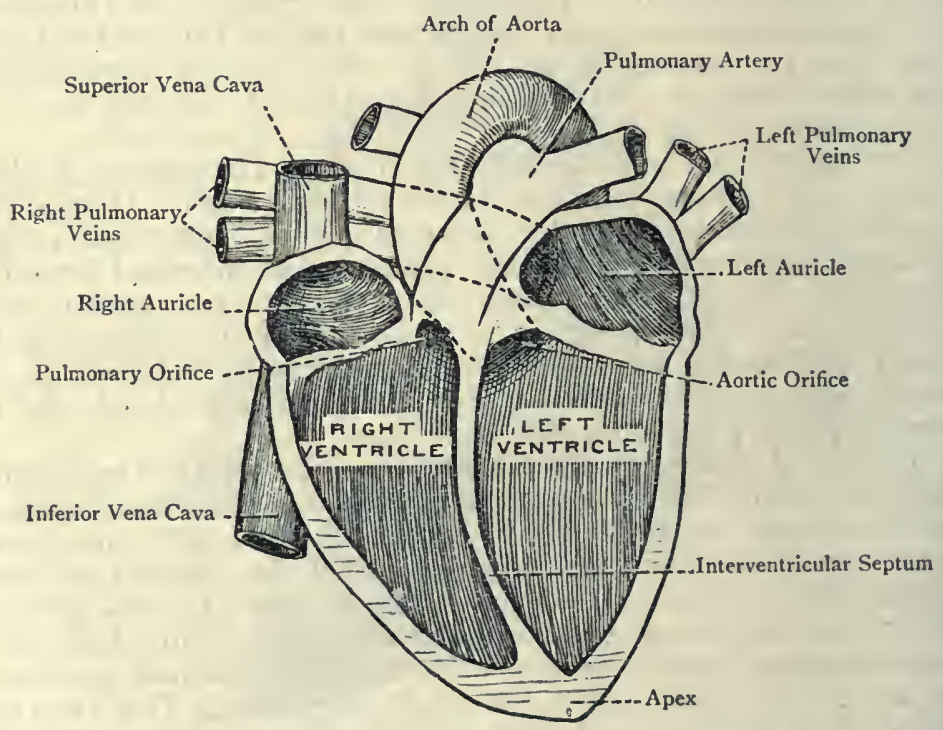

Fig. 399,-Diagrammatic View of the Interior of the Heart (after Milne EDWards).

guarded by a delicate semicircular fold of the endocardium, called the coronary valve, or valve of Thebesius, which, however, is functionally incompetent.

The foramina Thebesii represent several minute openings on the wall of the atrium. Some of these are simply blind recesses, whilst others are the orifices of minute veins, called the vena minima cordis, which return the blood from the wall of the auricle.

The auriculo-ventricular, or tricuspid, orifice is situated in the lower and anterior part of the atrium, in front of the orifice of the inferior vena cava, with the intervention of that of the coronary sinus. It is oval, and, in health, the index, middle, and ring fingers, 
held close together and side by side, can be passed through it without difficulty. Through this opening the blood passes from the right auricle into the right ventricle, its return being prevented by the tricuspid valve, which will be described in connection with the right ventricle.

The so-called twbercle of Lower is an unimportant slight projection, which is situated on the interauricular septum, just below the opening of the superior vena cava and behind the upper part of the fossa ovalis.

The posterior wall of the atrium corresponds to the intercuricular septum, and it presents for consideration the fossa ovalis and the annulus ovalis.

The fossa ovalis is an oval depression which is situated upon the lower part of the interauricular septum, a little above and to the left of the orifice of the inferior vena cava. It indicates the position of the foramen ovale of the fœtal heart, which is a communication between the two auricles through which the blood entering the right auricle by the inferior vena cava passes into the left auricle. The floor of the fossa ovalis is very thin, and is bounded above and at the sides by a prominent, crescentic margin, called the annulus ovalis. The annulus is deficient below, and the concavity of the crescent is directed downwards. Its anterior limb is continuous with the vestigial Eustachian valve. In some cases a minute oblique communication between the two auricles persists in the adult, being situated under cover of the upper portion of the annulus ovalis.

Interior of the Right Ventricle.-When exposed to view in the ordinary way the interior of the right ventricle is triangular, the base being directed backwards and upwards, and the apex forwards and downwards. It is completely separated from the left ventricle by the interventricular septum, which forms the posterior wall of the ventricle, and bulges into it so as to be convex towards it. In transverse section, therefore, the right ventricle is semilunar. Its wall, which is about three times thinner than that of the left ventricle, is thickest at the base, and becomes thinner towards the apex. The capacity of the ventricle is about 4 ounces. Its upper and left part forms the infundibulum or comus arteriosus, from the upper part of which the trunk of the pulmonary artery springs. The walls of the infundibulum are smooth, but elsewhere the walls of the ventricle are elevated into muscular bands, called columna carnece. These project into the cavity, and, from their reticular arrangement, they render the wall-very irregular. According to the manner in which the columnæ cornex are attached to the wall, they are arranged in three sets: (I) some are simple elevations, which are attached to the wall by their entire length, as well as by their extremities; (2) some are attached to the wall only by their extremities, being free elsewhere; and (3) others are attached only by one extremity. These latter are called musculi pupillares. They are conical, and their bases are attached to the 
wall of the ventricle. Their free extremities are connected with a number of filiform processes, called chorda tendinea, which pass to the margins and ventricular surfaces of the segments of the auriculo-ventricular valve. When the ventricle contracts, the musculi papillares also contract, and, by tightening the chordæ tendineæ, they prevent the segments of the auriculo-ventricular valve from being driven back into the auricle. The segments are therefore maintained in contact during the ventricular systole, and no regurgitation of blood from the ventricle into the auricle is allowed, in health. The musculi papillares are arranged in two groups, anterior and posterior, and their bases are attached to the

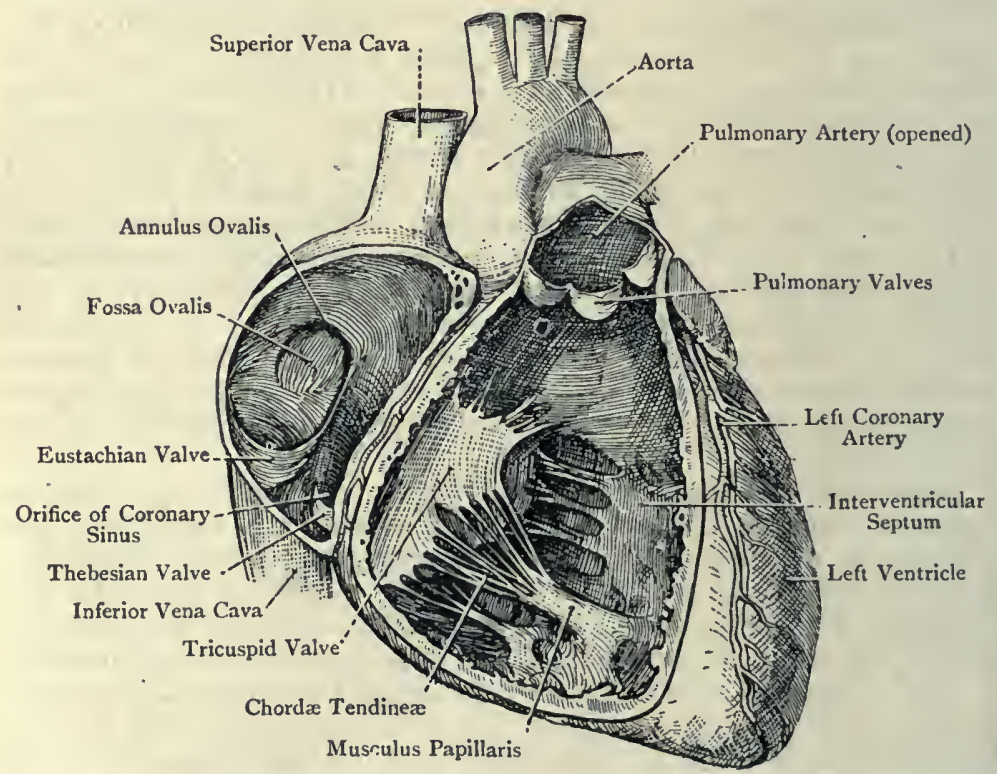

Fig. 400.-The Interior of the Right Auricle and Right Ventricle.

wall of the ventricle in the region of the apex of the cavity. The anterior musculus papillaris is of large size, and the posterior is usually broken up into two or more secondary papillary muscles.

In most hearts a fleshy column, called the moderator band, is met with in the right ventricle, which extends from the interventricular septum to the base of the anterior papillary muscle. It is regarded as controlling distension of the right ventricle, which it effects by the influence it exerts upon the anterior wall.

The openings connected with the right, ventricle are two in number, namely, the auriculo-ventricular and pulmonary. They are situated at the base of the cavity, and are guarded by most important valves. The auriculo-ventricular orifice is situated on the 
right and posteriorly, whilst the pulmonary orifice is situated on the left and anteriorly, being also on a higher level than the other. Vence minime cordis are said to open into the right ventricle.

The auriculo-ventricular, or tricuspid, orifice is, as already stated in connection with the right auricle, oval, and it readily admits the index, middle, and ring fingers held close together. It allows the venous blood to flow from the right auricle into the right ventricle, and, in order to prevent regurgitation of blood from the ventricle into the auricle during the ventricular systole, it is guarded by an important valve, called the right auriculo-ventricular or tricuspid valve. This valve is composed of three segments or cusps, which are covered with endocardium. These project into the cavity of the ventricle, and are triangular. The bases of the cusps are continuous with one another, and form a ring, which is attached to the margin of the auriculo-ventricular orifice. The largest cusp is situated in front, and to the left, of the auriculo-ventricular orifice. It intervenes between that orifice and the infundibulum, and is known as the left or infundibular cusp. Another cus pis situated behind the auriculo-ventricular orifice, and is in contact with the interventricular septum. It is called the posterior or septal cusp. The third cusp is situated to the right, near the margo acutus, and is called the right or marginal cusp. In the angular intervals between the basal parts of the three large cusps there are usually three small cusps.

Each cusp consists of two layers of endocardium, with fibrous tissue between them, especially at their central parts. The middle portion of each cusp is therefore thicker than the marginal portions, these latter being thin and transparent. The margins themselves are notched. The auricular surfaces of the cusps are smooth, but their ventricular surfaces are roughened by the chordæ tendineæ, which are also attached to the margins.

The chordæ tendineæ are filiform, fibrous processes which are connected, on the one hand, with the wall of the ventricle, and, on the other, with the cusps of thë auriculo-rentricular valve. Most of them spring from the two papillary muscles, but a few of them arise from the interventricular septum and from small papillary eminences upon it. Those which are connected with the anterior papillary muscle pass to the interval between the infundibular and marginal cusps; those which are connected with the posterior papillary muscle and its subdivisions pass to the interval between the septal and marginal cusps; and those which are connected with the interventricular septum pass to the interval between the infundibular and septal cusps. The connection of the chordæe tendineæ with the cusps of the valve is of a threefold nature, as follows: (I) the most numerous are connected with the marginal parts of the cusps; (2) others are connected with the thickened central portion of each cusp ; and (3) a few pass to the basal portion of each cusp, where they are connected with the fibrous ring around the auriculo-ventricular orifice. 
The pulmonary valve guards the orifice of the pulmonary artery, and prevents regurgitation of blood from the trunk of the pulmonary artery into the right ventricle during the elastic recoil of the arterial wall. It is composed of three semilunar or sigmoid segments, and the wall of the artery, opposite each segment, presents a recess, these recesses being called the sinuses of Valsalva. The valve and the sinuses of Valsalva are similar to corresponding structures in connection with the aortic orifice, and will be fully described along with that orifice.

Interior of the Left Auricle.-The musculi pectinati are present only in the auricular appendix, whereas in the right auricle they are

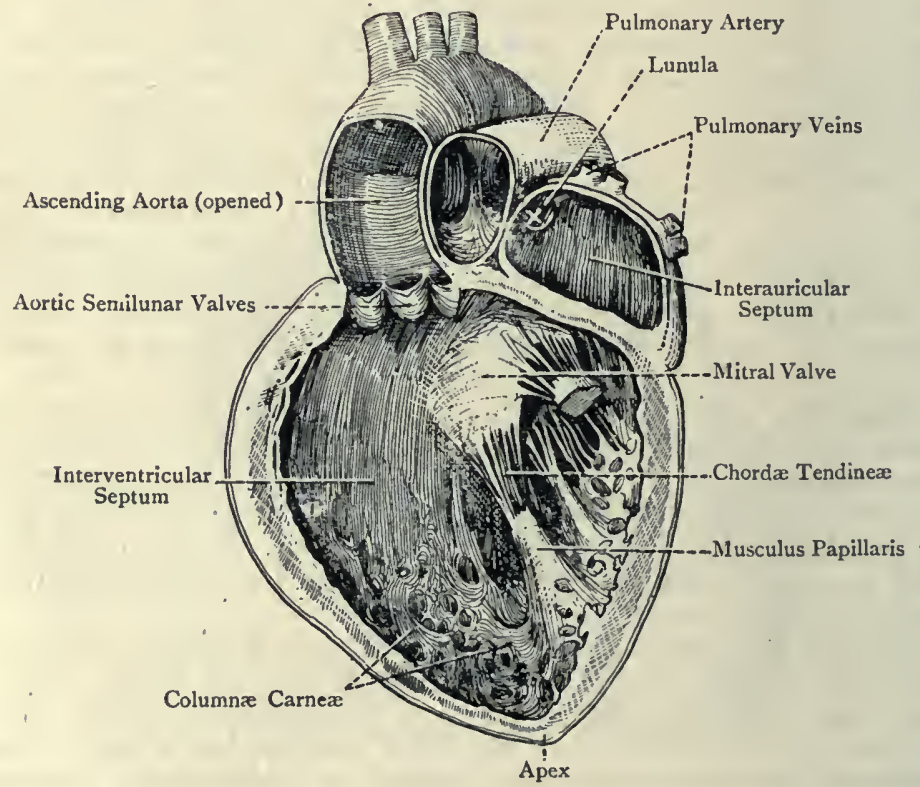

Fig. 40I.-The Interior of the Left Auricle and Left Ventricle.

present both in the auricular appendix and on the right wall of the atrium. The wall of the left atrium is entirely smooth. The atrium presents five openings, nam ly, the openings of the four pulmonary veins, and the auriculo-ventricular opening.

The orifles of the four pulmonary veins are situated on the posterior wall at either side, two right and two left, and they are destitute of valves.

The auriculo-ventricular, or mitral, orifice is situated in the anterior part of the floor of the atrium. It is oval, and, in health, it admits two fingers held side by side, and in close contact.

Vene minima cordis are said to open into the left auricle. 
The interauricular septum presents a slight depression, limited inferiorly by a faint crescentic ridge, the concavity of which is directed upwards. These indicate the position of the foramen ovale of the fotal heart.

Interior of the Left Ventricle.-The cavity of the left ventricle extends quite to the apex of the heart, and is longer and narrower than that of the right ventricle. It is somewhat conical, the base being directed backwards and upwards. The interventricular septum recedes from the cavity, and is concave towards it. In transverse section, therefore, the left ventricle is oval, or nearly circular. The wall of the left ventricle is about three times thicker than that of the right, the difference being readily accounted for
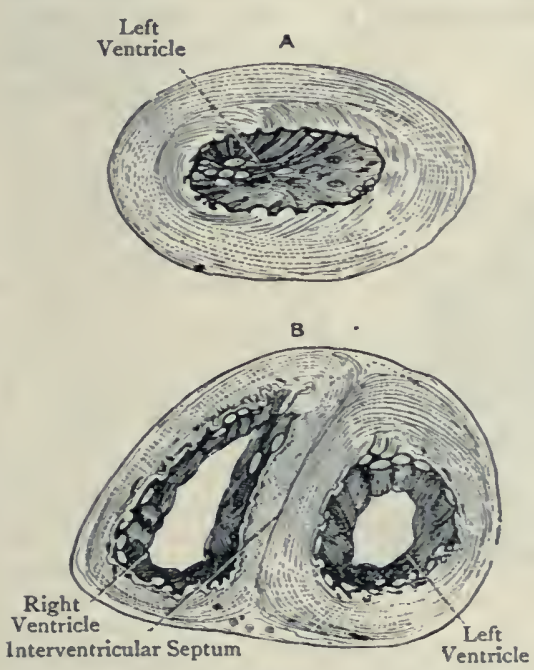

Fig. 402,-Transverse Sections of THE HEART.

A, Through Apex (Superior View):

B, Through Ventricles (Inferior View). by the fact that the left ventricle is concerned with the systemic circulation, whilst the right ventricle has to do with the pulmonary circulation, the latter involving a much shorter circuit. The wall of the left ventricle attains its maximum thickness about the junction of the upper fourth and lower three-fourths, and it is thinnest in the region of the apex. The capacity of the ventricle is about 4 ounces. The portion of the cavity immediately below the aortic orifice is known as the aortic vestibule, the walls of which are fibrous.

The left ventricle, like the right, is provided with columnæ carneæ. They are arranged in a very intricate manner, more particularly in the region of the apex and over the posterior wall. The aortic vestibule, and the interventricular septum, at least over its upper part, are destitute of columnæ carneæ, and present a smooth. appearance. The musculi papillares are much larger than those in the right ventricle ; they are two in number, anterior and posterior; and they are attached by their bases to the respective walls of the ventricle, whilst their free ends are connected with the chordæ tendineæ.

The openings connected with the left ventricle are two in number, namely, auriculo-ventricular and aortic. They are situated at the base of the ventricle in close proximity to each other, and are guarded by important valves. The auriculo-ventricular orifice is 
situated on the left and posteriorly, whilst the aortic orifice is situated on the right and anteriorly. The aortic orifice is also the higher of the two. Vence minimae cordis are said to open into the left ventricle.

The auriculo-ventricular, or mitral, orifice is oval, and, in health, it admits two fingers held side by side. It allows the arterial blood to flow from the left auricle into the left ventricle, and, in order to prevent regurgitation of blood from the ventricle into the auricle during the ventricular systole, it is guarded by an important valve. This valve is called the left auriculo-ventricular, mitral, or bicuspid valve. It is composed of two large segments or cusps, with two small cusps in the angular intervals between their basal parts. The cusps are similar in shape and structure to those of the tricuspid valve, but, on account of the nature of their.work, they are thicker and stronger. They are disposed obliquely, and are of unequal

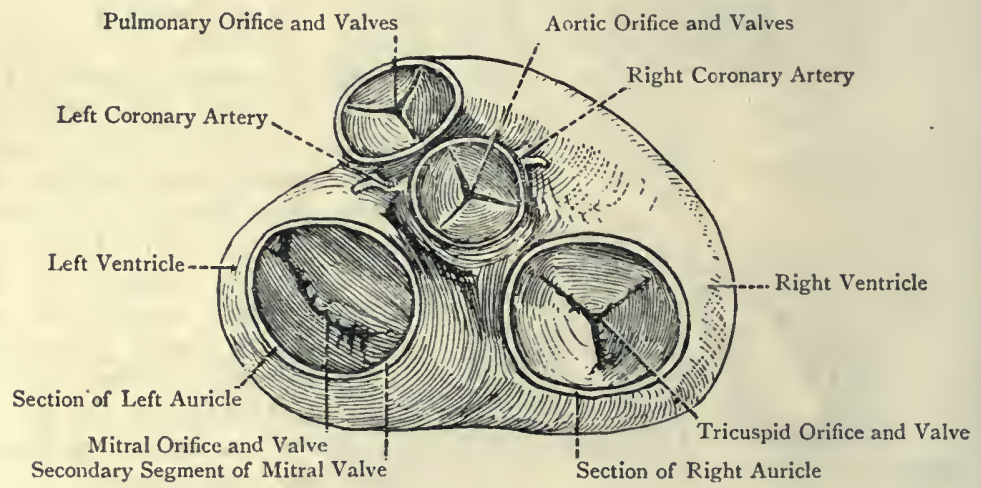

Fig. 403.-The Auriculo-Ventricular and Arterial Orifices of the Heart, with their Valves (SUPerior View).

(The Auricles have been removed.)

size. The larger of the two is placed in front, and to the right, of the orifice. It intervenes between the auriculo-ventricular and aortic orifices, and is known as the anterior or aortic cusp. The smaller cusp is placed behind, and to the left, of the orifice, and is known as the posterior or marginal cusp.

The chordæ tendineæ are attached to the cusps, as in the case of the tricuspid valve. They are, however, fewer in number, and of greater thickness and strength, than on the right side.

Function of the Tricuspid and Mitral Valves.-These valves serve to prevent regurgitation of blood from the ventricles into the auricles during the ventricular systole. Whilst the ventricle is being filled, some of the blood gets behind the segments of the auriculo-ventricular valve, that is to say, between each segment and the wall of the ventricle, and the segments are thus carried towards the auriculo-ventricular orifice. When the ventricle is 
filled with blood, the segments are in contact, and the ventricular systole now takes place. At the same time the musculi papillares contract. Blood is forced against the segments of the valve, but it cannot, in health, enter the auricle, because the segments are maintained in close contact, and are prevented from being driven back into the auricle, by the chorda tendineæ, which are under the control of the musculi papillares. If there were no musculi papillares, in which case the chordæ tendinea would spring directly from the wall of the ventricle, then the segments of the valve would not be held tight, but would, under the pressure of the blood, be driven back into the auricle, and regurgitation of blood would of necessity occur. The explanation of this lies in the fact that when the ventricle contracts a kind of screwing up or wringing movement takes place in its wall, as, so to speak, in wringing a wet towel. The effect of this peculiar action is to approximate successive parts of the ventricular wall to the auriculo-ventricular orifice, and this would have the effect of relaxing the chordr tendineæ, and so allowing the segments of the valve to be driven back into the auricle. The chordæ tendineæ, however, spring from musculi papillares, and these are elevations of the wall of the ventricle, directed towards the auriculo-ventricular orifice. During the wringing movement, therefore, of the ventricular wall in systole the musculi papillares, as stated, contract, and so maintain the chordæ tendineæ taut, or tightly drawn.

When the mitral valve opposes the entrance of blood into the left ventricle the cardiac affection is known as mitral obstruction (stenosis). When the mitral valve is incompetent, and allows regurgitation of blood to take place from the left ventricle into the left auricle, the cardiac affection is known as mitral regurgitation (incompetence).

The aortic orifice is circular, and is separated from the auriculoventricular orifice by the anterior or aortic cusp of the mitral valve. It is guarded by the aortic valve, which, by means of its segments, prevents regurgitation of blood from the aorta into the left ventricle during the elastic recoil of the arterial wall. It is composed of three semilunar or sigmoid segments, consisting of fibrous tissue, covered on their ventricular surfaces by endocardium, and on their

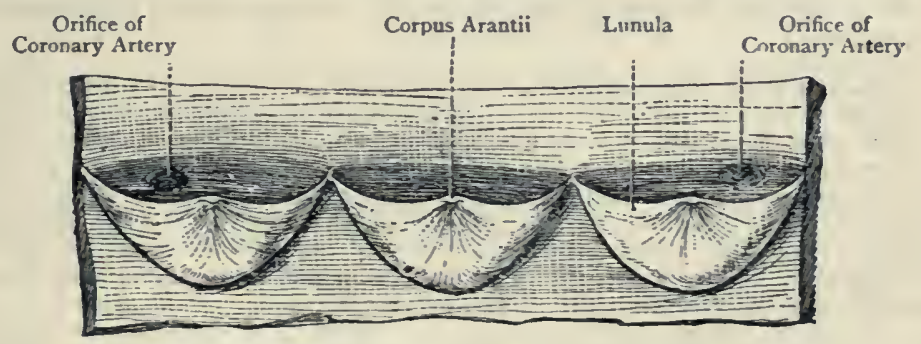

Fig. 404.-The Aortic Semilunar Valves.

arterial surfaces by the endothelial lining of the artery. Each segment is attached by its convex border to the wall of the artery at the place where it springs from the ventricle. The other border of the segment is free, and is directed away from the ventricle. Each segment, therefore, is so disposed as to allow the blood to pass freely into the aorta from the left ventricle. The free border of each segment is strengthened by a band of fibrous tissue, and at 
the centre of the border there is a small sweiling, called the nodule or corpus Arantii. This gives rise to a slight projection, and on either side of it the border is concave. The attached convex border of each segment is also strengthened by fibrous tissue. In addition to these fibrous thickenings, fibrous tissue pervades each segment from the nodule to the attached border, with the exception of the portions immediately below the lateral concave parts of the free border. These portions, in each segment, are semilunar, and are called the lumula. They are the thinnest parts of the segment, and are transparent, consisting practically of endocardium and the endothelial lining of the artery.

The interior of the wall of the aorta presents three well-marked recesses, each of which is placed opposite a segment of the valve. These recesses are called the sinuses of Valsalva, and they are disposed as right antero-lateral, left anterolateral, and posterior, respectively. The right antero-lateral sinus of Valsalva presents the orifice of the right coronary artery, and the left antero-lateral sinus of Valsalva presents the orifice of the left coronary artery. Each sinus of Valsalva, together with the corresponding segment of the aortic valve, forms a small pocket, and the three pockets open away from the left ventricle, that is to say, in a direction corresponding to the normal blood-flow.

Function of the Aortic Valve.-This valve serves to prevent regurgitation of blood from the aorta into the left ventricle during the elastic recoil of the arterial wall close to the heart. During the ventricular systole, when the blood is being driven through the aortic orifice, the aortic valve is passive, and its three segments are applied to the arterial wall. During the elastic recoil of the arterial wall, however, the valve is in action. The first effect of the elastic recoil is to force sufficient blood backwards towards the left ventricle to close the aortic valve. This blood enters the pockets formed by the sinuses of Valsalva and the segments of the valve. The segments are pressed towards the centre of the aortic orifice, and they come into contact in the following manner: the three nodules or corpora Arantii come into close contact at the centre of the aortic orifice, and so close what would otherwise be a small space, and the respective lunula are closely pressed against one another. In this manner the aortic orifice is completely closed, and regurgitation of blood into the left ventricle is, in health, effectually guarded against. It will be evident that the strain of the backward pressure of blool must be borne by those portions of the segments which are strengthened by fibrous tissue extending from each isodule to the attached border of each segment. Though the lunulæ are thin and weak, nevertheless, being, so to speak, doubled up against one another, the more they are pressed upon the more closely they fit together.

When the aortic valve offers opposition to the entrance of blood into the aorta, the cardiac affection is called aortic obstruction (stenosis). On the 
i)ther hand, when the aortic valve is incompetent, and allows regurgitation of blood to take place into the left ventricle, the cardiac affection is called aortic regurgitation (incompetence).

The pulmonary valve, which guards the orifice of the pulmonary artery, is similar to the aortic valve, and the preceding description is, for the most part, applicable to it. The segments of the pulmonary valve are, however, weaker than those of the aortic valve, and the sinuses of Valsalva are destitute of any arterial orifices. These sinuses are disposed as anterior, right postero-lateral, and left postero-lateral, respectively.

The function of the pulmonary valve is similar to that of the aortic valve.

Interventricular Septum.-This septum completely separates the right and left ventricles. The external indications of its attachments are the antero-superior and inferior interventricular grooves. It is thick and, for the most part, fleshy. It is thickest in the region of the apex, and becomes thinner towards the base of the ventricles. It bulges into the right ventricle, so as to be convex on that aspect, whilst it recedes from the left ventricle, so as to be concave towards it. The upper or basal part of the septum presents anteriorly a small portion which is destitute of muscular fibres. This portion is thin, and consists of fibrous tissue, covered on either side by endocardium. It is known as the pars membranacea septi, and is developed from the lower part of the septum which divides the aortic bulb into the ascending aorta and trunk of the pulmonary artery. It forms part of the aortic vestibule of the left ventricle. In rare cases the interventricular septum is deficient in the region of the pars membranacea, and a communication thus exists between the right and left ventricles.

Topography of the Orifices of the Heart.-The pulmonary orifice is situated on a level with the upper margin of the third left costal cartilage at its junction with the sternum. The aortic orifice, which is more deeply placed than the pulmonary, is situated behind the left border of the sternum on a level with the lower margin of the third leit costal cartilage.

The tricuspid orifice lies behind the body of the sternum opposite the fourth intercostal spaces and fourth and fifth costal cartilages. The mitral orifice, which is placed very deeply, is situated behind the left border of the sternum on a level with the fourth left costal cartilage.

Cardiac Bloodvessels-Arteries.-The nutrient vessels of the heart are the coronary arteries, right and left, which arise from the root of the ascending aorta. For a description of these vessels, see p. 958 .

Veins.-The cardiac veins are as follows : the great or left cardiac or coronary vein ; the coronary sinus; the posterior cardiac veins; the middle cardiac vein; the right or small cardiac or coronary vein; the anterior cardiac veins; the oblique vein of Marshall; and the venæ minimæ cordis or smallest cardiac veins. With the 
exception of the last-named cardiac veins (venæ minimæ cordis), all the others are seen upon the exterior of the heart.

The great or left cardiac or coronary vein commences at the apex of the heart, and ascends in the antero-superior interventricular groove alongside of the descending branch of the left coronary artery. In this part of its course it is more properly called the antero-superior interventricular vein. It is of large size, and receives tributaries from both ventricles and from the inter-

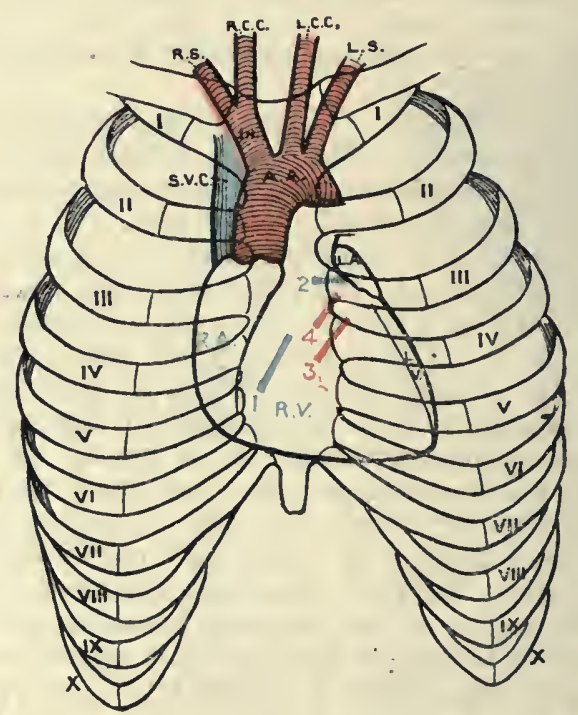

Fig. 406.-Diagram showing the Position of the Heart and its Valvular Orifices in Relation to the Anterior Wall of the Thorax.

R.A. Right Auricle
R.V. Right Ventricle
L.A. Left Auricle
L.V. Left Ventricle
A.A. Arch of Aorta
IN. Innominate Artery
R.S. Right Subclavian Artery

R.C.C. Right Common Carotid Artery

L.C.C. Left Common-Carotid Artery

L.S. Left Subclavian Artery

S.V.C. Superior Vena Cava

Numbers ON STERnum.
I. Tricuspid Orifice
2. Pulmonary Orifice
3. Mitral Orifice
4. Aortic Orifice

ventricular septum. On reaching the auriculo-ventricular groove, it enters the left division of that groove, in which it courses along with the transverse branch of the left coronary artery. Having turned round the left margin of the heart, it joins the left extremity of the coronary sinus, with which it is continuous. The name coronary is strictly applicable to this vessel only where it lies in the left auriculo-ventricular groove.

The coronary sinus (see Fig. $39^{8}$, p. 974) is the dilated terminal 
part of the great cardiac vein. It is about $I$ inch in length, and occupies a portion of the posterior part of the left auriculo-ventricular groove. Its left extremity is continuous with the great cardiac vein, and its right extremity opens into the right auricle between the vestigial Eustachian valve and the auriculo-ventricular orifice, the opening being guarded by the Thebesian valve. At the place where the coronary sinus is continuous with the great cardiac vein there is a valve, which is composed of two segments.

The coronary sinus is the persistent terminal part of the left duct of Cwier and left lateral cornu of the sinus venosus.

The posterior cardiac veins ascend upon the inferior surface of the left ventricle, and open partly into the coronary sinus, and partly into the contiguous portion of the great cardiac vein. One of them is known as the left marginal vein.

The middle cardiac vein (more properly called the inferior interventricular vein!, which is of large size, commences at the apex of the heart, and passes along the inferior interventricular groove with the descending branch of the right coronary artery. It receives tributaries from the adjacent surface of the right ventricle and from the interventricular septum, as well as a few from the left ventricle, and it opens into the right extremity of the coronary sinus.

The right or small cardiac or coronary vein occupies the right auriculo-ventricular groove, in company with the right coronary artery, and it opens into the right extremity of the coronary sinus. It is subject to some variation, but it usually receives tributaries from the contiguous parts of the right auricle and right ventricle, including the anterior cardiac veins.

The anterior cardiac veins ascend on the front of the right ventricle, and open into the right cardiac vein. One of them is known as the right marginal vein.

The oblique vein of Marshall, which is a persistent part of the left duct of Cuvier, passes downwards and inwards over the posterior aspect of the left auricle, and opens into the left extremity of the coronary sinus, its orifice being destitute of a valve.

Development.-The oblique vein of Marshall represents the portion of the left duct of Cuvier which adjoins the terminal part, this latter part giving rise to the coronary sinus.

Tributaries of the Coronary Sinus.-These are as follows : (I) the great or left cardiac vein; (2) some of the posterior cardiac veins, others of these veins opening into the great cardiac vein; $(3)$ the middle cardiac vein ; (4) the right cardiac vein ; and (5) the oblique vein of Marshall. With the exception of the last-named tributary, all the others are provided with valves at their terminal orifices, but elsewhere they are destitute of valves.

The smallest cardiac veins, or vence minima cordis, also known as the vence Thebesii, are not visible upon the exterior of the heart. They are very minute, and they open into the right auricle, their orifices constituting some of the foramina Thebesii. Similar 
minute veins are said to open into the left auricle, and also into both ventricles.

Lymphaties.-The lymphatic vessels of the heart form two networks-subendocardial and subpericardial. The vessels are ultimately collected into two trunks, right or posterior, and left or anterior. The right trunk receives the lymphatics of the right side of the heart, and the left trunk takes up those of the left side. Each trunk accompanies the corresponding coronary artery, and both pass backwards on either side of the common pulmonary artery. Having pierced the pericardium, they terminate in the superior mediastinal or cardiac glands.

Nerves.- The nerves of the heart are derived from the superficial and deep cardiac plexuses, and, through these, from the pneumogastric and sympathetic nerves. The branches to the auricles are derived partly from the deep cardiac plexus, and partly from the coronary plexuses. They form a gangliated plexus on the surface of each auricle beneath the epicardium, and from this plexus branches proceed to the muscular wall. The branches to the ventricles are derived frorn the right and left coronary plexuses. These branches lie upon the surface of each ventricle beneath the epicardium, and in the heart of the calf they are readily recognisable as delicate, thread-like streaks. Minute ganglia are met with, at intervals, on these nerves in the region of the base of the ventricles, but none on those nerves which lie over the lower two-thirds of each ventricle. The branches which enter the muscular, substance of the ventricular walls form plexuses, but are destitute of ganglia.

Structure of the Heart.-The cardiac wall is composed of muscular tissue, which is known. as the myocardium. This is intimately covered by the visceral layer of the serous portion of the pericardium, this layer being called the epicardium, and the cavities of the heart are lined with a membrane which is known as the endocardium.

The epicardium resembles a serous membrane in structure, and is covered by endothelium. It is provided with stomata, which establish a communication between its lymph-canalicular system and the cavity of the pericardium. Beneath the epicardium there is a variable amount of fat, which is chiefly met with in the auriculo-ventricular and interventricular grooves.

The endocardium is a smooth, delicate membrane, destitute of bloodvessels, and covered on its internal surface by endothelium. It consists of a connective-tissue basement membrane, containing elastic fibres, and is continuous, through the arterial and venous orifices, with the tunica intima of the vessels. It enters into the formation of the segments of the auriculoventricular and semilunar valves.

Fibrous Tissue.-The auriculo-ventricular and arterial orifices of the heart are each surrounded by a rịng of fibrous tissue, and these rings, in the case of the auricles and ventricles, connect them together. The fibrous tissue of these rings furnishes that which is met with in the segments of the various valves. In the triangular interval between the aortic and the two auriculoventricular orifices there is a collection of dense fibrous tissue, of the consistence of fibro-cartilage, which is connected with the fibrous rings just referred to. When a heart is boiled, the fibrous tissue is dissolved, and the auricular portion can be separated from the ventricular portion.

Myocardium. - The muscular tissue of the auricles is mostly distinct from 
that of the ventricles, the fibrous rings at the auriculo-ventricular orifices intervening between the two, and serving to connect them.

Auricles. - The muscular fasciculi of the auricles are arranged in a superficial set, common to both auricles, and a deep set, confined to each auricle. The superficial fasciculi are disposed transversely, and some of them enter the interauricular septum. They are best marked in front. The deep fasciculi are arranged in two sets, looped and circular. The looped fasciculi arch vertically over the auricle, their extremities being attached to the ring of fibrous tissue which surrounds the auriculo-ventricular orifice. The circular fasciculi are present in the auricular appendix, around the venous orifices, and around the fossa ovalis. The walls of the auricles, though muscular, are thin.

Ventricles.-The muscular fasciculi of the ventricles are, as stated, mostly distinct from those of the auricles, and are disposed in a very complicated manner. They spring, for the most part, from the rings of fibrous tissue which surround the auriculo-ventricular orifices. The superficial fasciculi descend obliquely towards the apex, where they are disposed in a twisted or whorled manner, after which they pass on to the inner surface of the left ventricle. Some of them become continuous with the musculi papillares of that ventricle, whilst others ascend as a vertica: layer on the inner surface of the ventricle to be attached to the left auriculo-ventricular fibrous ring. The superficial fasciculi are common to both ventricles.

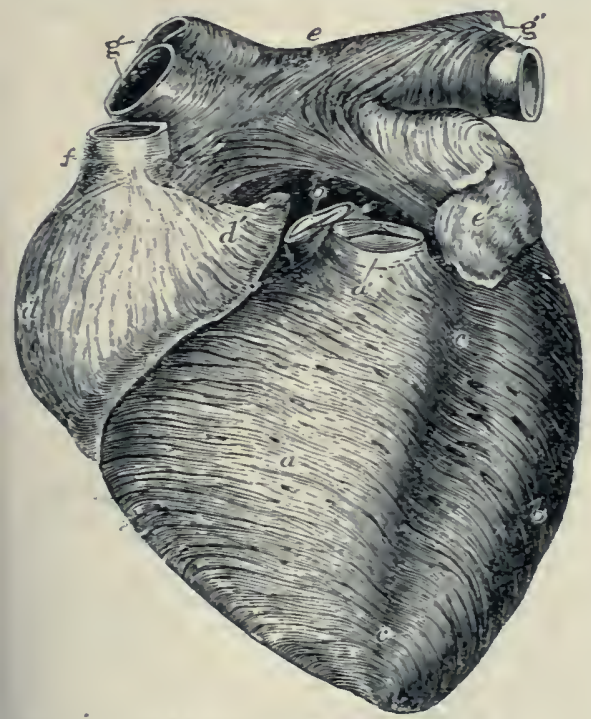

This figure is planned after one of Luschka's, but its details were chiefly taken from an original preparation. The aorta, $b^{\prime}$, and pulmonary artery, $a^{\prime}$, have been cut short close to the semilunar valves, so as to show the anterior fibres of the auricles. $a$, superficial layer of the fibres of the right ventricle: $b$, that of the left; $c, c$, anterior interventricular groove; $d$, right auricle; $d^{\prime}$, its appendix, both showing chiefly perpendicular fibres; $e$, upper part of the left auricle ; between $e$ and $b^{\prime}$, the transverse fibres which behind the aorta pass across both auricles; $e^{\prime}$. appendix of left auricle: $f$. superior vena cava, around which, near the auricie, circular fibres are seen; $g, g^{\prime}$, right and left pulmonary veins with circular bands of fibres sur. rounding them.

Fig. 407.-ANTERIor View OF HeArt OF A Young SubJect dissected AFter Lovg Bolliyg, to show the Superficial Muscular Fibres (Allex Thomsox). $\frac{2}{3}$. (FROM Quar's 'ANATOMY.')

The remaining fasciculi are very numerous, and must be described separately for each ventricle. The principal fasciculi of the left ventricle spring from the left auriculo-venticular fibrous ring, and they pass more or less obliquely towards the apex. In their course they turn inwards, and enter the front part of the interventricular septum inferiorly. Some of them now pass upwards to the base of the ventricle to be attached to the collection of dense tibrous tissue, of the consistence of fibro-cartilage, already described; 
others pass across to the postero-inferior wall of the right ventricle, where they partly end in a musculus papilaris, and partly pass to be attached to the right auriculo-ventricular fibrous ring; whilst a third set pass to the postero-inferior wall of the left ventricle, where they become circular in direction. The principal fasciculi of the right ventricle are, like the superficial fasciculi, continuous with those of the left ventricle, and they spring from the fibrous rings around the auriculo-ventricular and pulmonary orifices. Those on the postero-inferior surface of the right ventricle pass into the interventricular septum, and, having traversed it obliquely forwards and upwards, they issue from it, and become continuous with the deep fibres of the left ventricle. Those on the antero-superior surface of the right ventricle also pass into the interventricular septum, and having traversed it obliquely backwards and downwards, they pass on to the postero-inferior wall of the left ventricle. Those from the inferior aspect of the right ventricle enter the lower part of the interventricular septum, and ascend in the septum to the collection of dense fibrous tissue of the consistence of fibro-cartilage at the base. Although the ventricular muscular fasciculi are disposed for the most part obliquely, there are some circular fasciculi, but these are confined to the thickest part of each ventricular wall.

Fibres of Purkinje.-These fibres are situated between the endocardium and the cardiac muscular tissue. - They consist of elongated cells, united at their ends so as to form chains. The central part of each cell contains granular protoplasm, within which there are two nuclei; and the peripheral part presents transverse striations. These cells are probably vestiges of an early condition of cardiac muscular fibres.

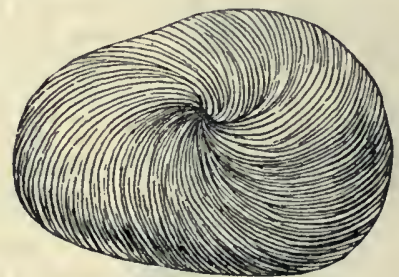

Fig. 408.-The Superficial Muscular Fibres of the Heart in the Region of the Apex (C. Gegenbaur).

The whorled arrangement is well shown.

Auriculo-Ventricular Bundle.--This bundle constitutes the only auriculoventricular muscular connection, and functionally it is of a conducting nature. It may be described in three sections-auricular (atrial), nodal, and ventricular.

The auricular section consists of fibres which are derived from (1) the circular fibres of the right auricle, just above the base of the septal cusp of the tricuspid valve, and (2) the muscular tissue of the interauricular septum at a point situated at the lower and anterior part of the septum on its right side, just above the anterior end of the base of the septal cusp of the tricuspid valve. These auricular fibres (circular and septal) enter the central fibrous body of the heart, which is situated between the aortic and the two auriculo-ventricular orifices.

The nodal section is situated within the central fibrous body. It is a small knot, consisting of an intricate network of inuscular fibres, intermingled with, and embedded in, fibrous tissue.

The ventricular section emerges from the nodal section as a single bundle. which represents the main stem of the auriculo-ventricular bundle. This stem passes forwards along the upper inargin of the muscular portion of the interventricular septum, lying imnediately below the pars membranace. septi, and beneath the anterior part of the base of the septal cusp of the tricuspid valve. In this situation the main stem breaks up into two divisions -right and left. Eacls of these divisions enters the septal wall of the corre- 
sponding ventricle, and divides into branches which ramify beneath the endocardium, where they form part of the system of Purkinje's fibres. Ultimately the terminal ramifications blend with the muscular fibres of the ventricles and musculi papillares.

The fibres of the auriculo-ventricular bundle are pale, and are furnished with nerve-fibres and ganglia. The clinical significance of the bundle has reference to Stokes-Adams disease, or heart-block. (Kent, His junior, Tawara, and Keith and Flack.)

Structure of the Cardiac Valves.-Each segment of the tricuspid and mitral valves consists of two layers of endocardium, enclosing between them fibrous tissue which is derived from the fibrous ring around the corresponding auriculoventricular orifice. Each segment of the aortic and pulmonary valves also consists of two layers of endocardium, enclosing between them fibrous tissue which is derived from the fibrous ring around the corresponding arterial orifice. The segments of all the valves are destitute of bloodvessels.

Minute Structure of the Myocardium.-Cardiac muscle is composed of transversely striated fibres, but these differ so much from ordinary striated

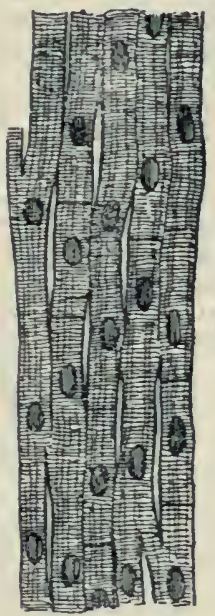

Fig. 409.-Cardiac Muscular Fibres showing their Transverse Stria, i. Divisions, Junctions, and Position of the Nuclei.

voluntary muscular fibres as to require a special description. They possess no sarcolemma; they branch freely, and adjacent branches unite, so that an intricate network is formed by them; and the transverse strix are fainter and less regular than those of voluntary muscle. Each fibre and its branches are broken up into a series of short quadrangular muscle-corpuscles, arranged in rows or chains, and separated from one another by cross-marks. Each muscle-corpuscle contains one nucleus, which occupies the centre of the corpuscle and the corpuscle presents longitudinal strixe as well as transverse. A cardiac muscular fibre, as well as its branches, is therefore a chain of musclecorpuscles, each of which has a nucleus at its centre, and there is an absence of sarcolemma.

Weight of the Heart.-The average weight of the heart of the adult male is $10 \frac{1}{2}$ ounces, and of the adult female $8 \frac{1}{2}$ ounces.

Size.-The size of a normal heart has been compared to the closed hand of the individual person.

Development of the Heart.-The heart makes its first appearance as two tubes, which are hollowed out in the splanchnic mesoderm of the pericardial region (cardiac portion of the ventral mesentery). These tubes are 
at first separated from each other, but subsequently they come together and fuse, giving rise to a single longitudinal tube, composed of splanchnic meso$d \in v_{m}$, which represents the primitive heart, this tube being situated mesially along the ventral aspect of the pharyngeal portion of the fore-gut. It thus occupies the region which subscquently becomes the neck.

The lumen of each of the two primitive tubes is lined with mesothelium, which becomes part of the cardiac endothelium. The mesenchyme, which lies outside the lining mesothelium, gives rise to the following cardiac tissues: (1) the myocardium, or cardiac muscular tissue; (2) the epicardiuin, or vasceral layer of the serous portion of the pericardium; and (3) the connective-tissue element of the endocardium.

After the fusion of the two tubes, which represent the cardiac rudiments, the primitive heart has the form of a longitudinal tube. At the caudal end of the tube is the sinus venosus.

Mesocardial Folds. - At this stage the primitive heart is connected dorsally with the ventral wall of the pharyngeal portion of the fore-gut, and ventrally with the somatopleuric body-wall, by means of splanchnic mesoderm, disposed in two layers. These bilaminar mesodermic folds form the dorsal mesocardium and ventral mesocardium, which are of the nature of mesenteries. They soon disappear, the ventral folds entirely and the dorsal folds to a large extent.

After the disappearance of these folds the cardiac tube is for the most part free, its ends being more or less fixed.
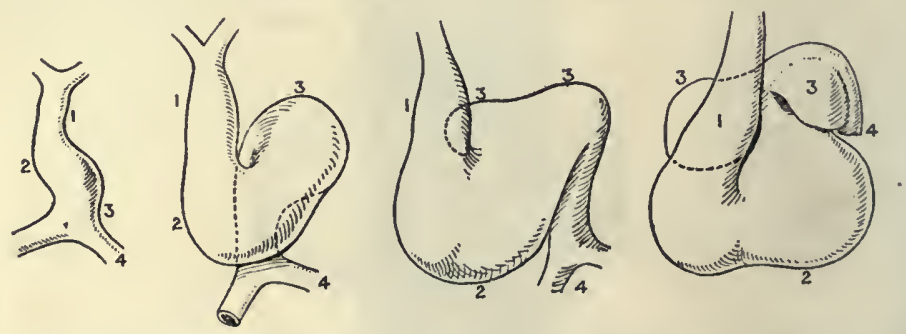

Fig. 410.--Scheme showing Four Successive Stages in the Develop. ment of the Heart (L. Testut's 'Anatomie Humaine').

I. Arterial Buib. 2. Ventricle. 3. Auricle.

4. Veins opening into the Sinus Venosus.

Further Development of the Heart. - The cardiac tube soon presents two constrictions, which indicate its division into three parts-namely, auricular (atrial), ventricular, and bulbar. The auricular portion pertains to the venous end of the doubly-bent cardiac tube; and the ventricular and bulbar portions belong to the arterial end, the bulbar portion being differentiated from the primitive heart as the aortic bulb or bulbus arteriosus (bulbus cordis). The ventricular portion is therefore intermediate in position between the auricular and bulbar parts. The auricular division is closely associated with the sinus venosus, which will be described presently, and which, though not a division of the primitive cardiac tube, becomes ultimately merged into its auricular portion. The constriction between the auricular and ventricular portions gives rise internally to a passage called the auricular, or atrial canal ; and the constriction betwcen the ventricular and bulbar portions gives rise internally to a passage known as the fretum Halleri or strait of Haller.

The primitive sinuous cardiac tube thus becomes differentiated into three chambers-namely: (1) the primitive auricle, (2) the primitive ventricle, and (3) the aortic bulb, or bulbus artcriosus.

Whilst these differentiations are in progress, the tube undergoes two bends, and now rescmbles a capital $S$, laid somewhat thus- $S$. The venous loop 
is directed dorsalwards, and migrates cephalicwards, whilst the arterial loop is directed ventralwards, and migrates caudalwards.

Transformation of the Single Heart into the Double Heart.-The double function of the permanent heart is concerned in (1) the pulmonary circulation, and (2) the systemic circulation. In order to prepare the organ for the performance of this two fold function, the primitive single heart undergoes a series of changes, which result in the division of its auricular and ventricular portions each into two halves, and of the aortic bulb into the ascending aorta and trunk of the pulmonary artery. The permanent heart, therefore, is a double organ, consisting of a right or pulmonary half, and a left or systemic half.

The transformation of the single auricle into two halves, right and left, is of a complicated nature. Prior to the commencement of the transformation the single auricle presents a pocket-like dilatation or diverticulum on either side of the median line. These two diverticula represent the right and leIt auricular appendices. A groove then makes its appearance on the upper and dorsal walls of the single auricle. This groove indicates the position internally of a falciform fold, which grows downwards and forwards within the auricular cavity. The lamina is called the septum primum (interauricular septum) or septum superius, and it eventually joins the upper part of the septum intermedium, to be presently described. It may here be stated, however, that the septum intermedium divides the auricular canal in to two parts, right and left, which represent the permanent auriculo-ventricular orifices. In the foregoing manner the primitive single auricle and the single auricular canal are divided each into two-right and left. The two auricles, right and left, are for the most part separated by the septum primum, and the two auriculo-ventricular orifices are separated by the septum intermedium. The septum primum is incomplete, and the two auricles communicate with each other by an opening, called the ostium interauriculare primum, which is situated between the septum primum and septum intermedium: This ostium, however, is soon closed by the fusion of the septum primum with the septum intermedium.

At the dorsal attachment of the septum primum a new opening, called the loramen ovale or ostium interauriculare secundum, makes its appearance. which establishes a communication between the two auricles.

Another septum, called the septum secundum, is also developed. It grows downwards from the roof of the right auricle, and lies on the right side of, and parallel with, the septum primum. This secondary septum ends in a semilunar or falciform border, which bounds the foramen ovale above, in front, and below, and forms the annulus ovalis or limbus Vieussenii. The portion of the septum primum within the free semilunar border of the septum secundum projects slightly into the cavity of the left auricle, and is so disposed as to allow blood to pass freely from the right auricle into the left, but it guards against regurgitation. This portion of the septum primum is consequently called the valve of the foramen ovale.

About the period of birth the ventral margin of this valve becomes as a rule adherent to the left side of the annulus ovalis, and the foramen ovale is thereby closed. Thereafter the valve (a portion of the septum primum) forms the floor of the depression known as the fossa ovalis.

In some cases the union of the valve with the left side of the annulus ovalis is incomplete, and in these circumstances a patent foramen ovale persists, giving rise to the condition known as morbus ceruleus.

Transformation of tine Auricular Canal.-The auricular canal, which is the narrow communication between the auricular and ventricular divisions of the primitive cardiac tube, undergoes invagination in to the ventricular chamber. Internally it presents two thickenings of endocardium, called the endocardial cushions, one of which is situated ventrally and the other dorsally. As these cushions grow they come together and fuse, thus giving rise to the septum intermedium. This septum divides the auricular canal into two compartments, which represent the right and leit auriculo-ventricular orifices. The upper part of the septum intermedium, as stated, joins the lower part of the septum primum, a temporary opening, known 
as the ostium interauriculare primum, being situated between the two, prior to complete fusion. The right auriculo-ventricular orifice now pertains to the right auricle, and the left auriculo-ventricular orifice to the left auricle.

Transformation of the Single Ventricle.-The division of the single ventricular chamber into two ventricles, right and left, is effected by the formation of a partition, called the interventricular septum, or septum inferius. The position of this septum internally coincides with a groove externally. called the interventricular groove, which traverses the ventral and dorsal surfaces of the ventricular division. The interventricular septum appears first in the lower part of the ventricular chamber, and grows in an upward and backward direction. The dorsal part of its upper border blends with the dorsal part of the lower border of the septum intermedium. The ventral part of its upper border falls short of the root of the aortic bulb, so that the interventricular septum is incomplete in this region. The two ventricles therefore communicate with each other at this stage by a somewhat oval aperture, called the ostium interventriculare, which is situated between the root of the aortic bulb and the ventral part of the upper border of the interventricular septum. This aperture, however, becomes closed by the downward growth of the membranous aortic septum, which divides the aortic bulb into the ascending aorta and trunk of the pulmonary artery. As the membranous aortic septum grows downwards, it closes the ostium interventriculare by meeting, and fusing with, the ventral part of the upper border of the interventricular septum. The partition between the two ventricles is then complete. The interventricular septum is thus partly muscular and partly membranous. The membranous portion, comparatively small, is formed by the downward growth of the aortic septum, and this part of the interventricular septum is known as the pars membranacea septi. The superior connections of the interventricular septum, as stated, are twofold, as follows: ventrally, it blends with the downward growth of the aortic septum, and, dorsally, it joins the dorsal part of the lower border of the septum intermedium.

Cases have been recorded in which the pars membranacea septi has been found perforated, or, in other words, in which the original ostium interventriculare has not been completely closed by the downward growth of the aortic septum. Wherever such a condition exists, an intercommunication between the venous blood in the right ventricle and the arterial blood in the left ventricle is allowed.

The ostium interventriculare represents the normal communication which exists between the two ventricles in the hearts of some Reptilia-e.g., Lizards and Chelonia (Tortoises and Turtles).

Transformation of the Aortic Bulb.-The aortic bulb or bulbus arteriosus is divided into the ascending aorta and the trunk of the pulmonary artery by a partition, which is called the aortic septum. This septum is formed by the meeting and fusion of two Iongitudinal endocardial ridges which appear on opposite walls of the aortic bulb internally. It is first developed in the upper part of the bulb, and in this situation the formative ridges appear on the ventral and dorsal walls of the bulb. The septum is therefore disposed at first antero-posteriorly.

As the endocardial ridges grow downwards in a spiral manner, they become disposed laterally, as right and left ridges. This accounts for the ascending aorta and the trunk of the pulmonary artery lying side by side superiorly (aorta to the right, and trunk of the pulmonary artery to the left), whilst inferiorly, close to the heart, the trunk of the pulmonary artery is anterior, and the aorta lies behind it. The aortic membranous septum, as it grows downwards, meets, and fuses with, the ventral part of the upper border of the interventricular septum. In so doing, as stated, it closes the ostium interventriculare, and forms that part of the interventricular septum which is known as the pars membranacea septi.

Two grooves appear on the exterior of the aortic bulb, which coincide with the attached borders of the aortic septum. $\Lambda \mathrm{s}$ these grooves decpen, the aortic bulb becomes divided into (1) the ascending aorta, and (2) the trunk of the pulmonary artery. 
Sinus Venosus. - The sinus venosus (saccus reuniens) is at first an appendage of the auricular division of the primitive heart, below and behind which it lies. Subsequently, however, it becomes merged into the right auricle, and forms the posterior part of that chamber of the adult heart. Into it the following veins discharge their blood: (I) The two ducts of Cuvier, from the body of the embryo; (2) the two vitelline veins from the yolk-sac; and (3) the two umbilical or allantoic veins from the chorion. It is elongated transversely, and its lateral portions, where the ducts of Cuvier open into it, are spoken of as the lateral cornua.

The communication between the sinus venosus and the auricular chamber soon becomes small, and it shifts towards the right side, to a point between the right lateral cornu and the right half of the auricular chamber. Ultimately, when the auricular chamber has become divided into two auricles, the sinus venosus opens entirely into the right auricle. The narrow opening is guarded by two endocardial folds, right and left, which are known as the venous valves. These valves are continuous superiorly with a ridge called the septum spurium, on the roof of the atrium. The left venous valve disappears, but the right venous valve persists, and gives rise to (I) the Eustachian valve, and (2) the Thebesian valve.

The venous valves, right and left, are situated at the opening of the sinus venosus into the right auricular cavity, and they are formed by the margins of the opening which project slightly into the right auricular cavity. The upper end of the right venous valve is continuous with a fold, called the septum spurium, situated on the roof of the right auricular cavity on the right side of the septum primum. The right venous valve gives rise to the Eustachian and Thebesian valves. The left venous valve is continuous with the septum secundum, which bounds the foramen ovale above, in front, and below. The semilunar border of the septum secundum, together with the left venous valve, forms the annulus ovalis.

The right lateral cornu, which receives the right duct of Cuvier, undergoes enlargernent, and, along with the central portion of the sinus venosus, it forms the atrium of the right auricle. The right lateral cornu carries with it the right duct of Cuvier, or right superior vena cava. The left lateral cornu which receives the left duct of Cuvier, is not merged in to the right auricle, but remains connected with it, forming the coronary sinus, which is the persistent and pervious cardiac end of the left duct of Cuvier, or left superior vena cava. Whilst the right auricle receives the sinus venosus, the left auricle receives the trunk formed by the union of the pulmonary veins, but these latter vessels are, at this stage, of little importance, the pulmonary circulation bing merely of a nutritive nature.

The common orifice of the pulmonary veins becomes enlarged and forms the atrium of the left auricle; and subsequently the veins open into the atrium by four orifices, two right and two left.

Formation of the Valves of the Heart.-The muscular tissue of the ventricular walls of the heart is at first loosely arranged in the form of trabecula, which are disposed in a reticular manner. This muscular reticulum, including its meshes, is lined with endocardium. The superficial trabeculre soon come together, and form a compact muscular wall, but towards the interior the reticular arrangement is retained. Muscular bundles, of different kinds, are therefore formed-e.g., columne carnec, musculi papillares, and moderator bands.

In the portions of the ventricular walls around the auriculo-ventricular orifices the muscular tissue is replaced by fibrous tissue. This fibrous tissue gives rise to thin, somewhat triangular lamellæ, and these become the segments of the auriculo-ventricular valves.

The chorde tendine represent muscular trabeculx, which, at their valvular ends, have been replaced by fibrous tissue, whilst their ventricular ends retain their muscular character, and form the musculi papillares.

The aortic and pulmonary semilunar valves are developed in connection with the ventricular end of the aortic bulb, prior to its division into the ascending aorta and trunk of the pulmonary artery. They appear as four fibrous cushions on the inner wall of the ventricular end of the aortic bulb, and are anterior, posterior, and right and left lateral respectively. When the 
aortic bulb is divided into the ascending aorta and trunk of the pulmonary artery, each lateral cushion is divided into two equal segments-anterior and posterior-by the aortic septum as it descends. The aortic and pulmonary orifices are thus provided each with three valves. The pulmonary valves are disposed as follows: One is anterior in position, and it represents the original anterior cushion of the lower end of the undivided aortic bulb; whilst two are postero-lateral, right and left, and they represent the anterior segments of the lateral cushions of the lower end of the undivided aortic bulb. The aortic valves are disposed as follows: Two are antero-lateral, right and left, and they represent the posterior segments of the lateral cushions of the lower end of the undivided aortic bulb; whilst one is postevior in position, and it represents the posterior cushion of the lower end of the undivided aortic bulb.

At this stage the root of the pulmonary artery is in front of the root of the aorta, and the segments of the pulmonary and aortic valves occupy the

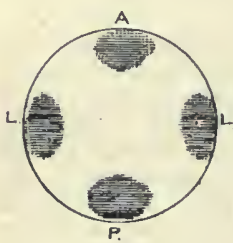

Endocardial Cushions of Primilive Aortic Bulb

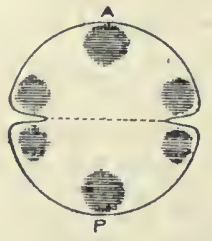

Aortic Bulb undergoing Division : Segmentation of Lateral Cushions

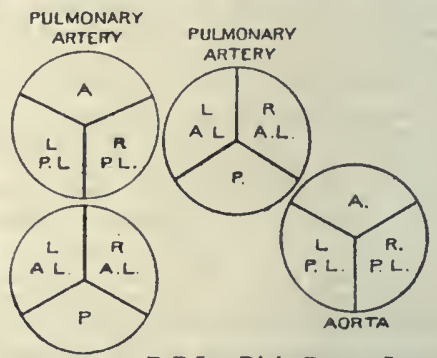

AORTA R.P.L. = Right Postero-Lateral R.A.L. = Right Antero-Lateral

Fig. 4II.-Development of Aortic and Pulmonary Valves.

positions which have just been assigned to them. Towards the end of intrauterine life, however, the heart undergoes a rotation from right to left to such an extent as to place the root of the pulmonary artery in front, and to the left, of the root of the aorta. The aorta, as well as the pulmonary artery, is involved in this rotation, and the segments of the pulmonary and aortic valves are now permanently altered in position. The relations of the various segments in the adult are as follows: The pulmonary valves are disposed as two antero-lateval, right and left, and one posterior; whilst the aortic valves are one anterior and two postero-lateral, right and left.

The rotation, just referred to, also explains why the left ventricle of the adult heart is chiefly postero-inferior in position, comparatively little of it appearing on the antero-superior surface of the adult heart.

Peculiarities of the Fœtal Circulation. - The structures peculiar to the fœtus in connection with the circulation of the blood are as follows :

r. Foramen ovale.

2. Eustachian valve.

3. Ductus arteriosus.
4. Hypogastric and umbilical arteries. 5. Umbilical vein.

6. Ductus venosus.

The foramen ovale is an oval opening in the interauricular septum. Up to the fourth month of intra-uterine life it is quite free, but after that period the valve of the foramen ovale gradually extends upwards on the left side of, and beyond, the annulus ovalis, and acts as a valve during the latter months of fatal life. The blood can then 
only flow from the right auricle into the left, its return being prevented by this valve.

The Eustachian valve is of large size during fotal life, and is associated with the orifice of the inferior vena cava at the posteroinferior angle of the right auricle. It is a crescentic fold of the endocardium, which extends between the anterior margin of the orifice of the inferior vena cava and the anterior horn of the annulus ovalis. Its situation is such as to direct the blood entering the right auricle by the inferior vena cava to the foramen ovale.

The ductus arteriosus connects the trunk of the pulmonary artery to the arch of the aorta at a point immediately beyond the origin of the left subclavian artery, and it conveys the most of the blood from the right ventricle to the descending thoracic aorta.

The hypogastric arteries, right and left, are prolonged from the common iliac arteries to the umbilicus, through which they leave the body of the foetus, and, under the name of the umbilical arteries, they pass along the umbilical cord to be distributed to the fotal part of the placenta. They convey the impure blood of the foetus to the placenta.

The umbilical vein extends from the fotal part of the placenta through the umbilicus to the inferior surface of the liver, where it traverses the umbilical fissure, and gives off right and left branches to the right and left lobes, which join the branches of the vena portæ. After parting with its right and left branches the umbilical vein is prolonged, under the name of the ductus venosus, to the inferior vena cava, which it joins at the caval fossa of the liver. The umbilical vein conveys pure blood from the placenta into the body of the fœtus.

Fœtal Circulation.-The right auricle receives blood from the superior and inferior venæ cavæ, and from the coronary sinus. The superior vena cava returns the renous blood from the head and neck, the upper limbs, and the thoracic portion of the trunk; and the inferior vena cava returns the blood from the lower limbs, the abdominal portion of the trunk, and the placenta. The placental blood is pure, and reaches the inferior vena cava in two ways: (I) a large quantity of it is conveyed directly into the inferior vena cava by the ductus venosus; and (2) some of it circulates through the liver in the branches of the vena portæe, and is thereafter conveyed to the inferior vena cava by the hepatic reins. The blood entering the right auricle by the inferior vena cava is necessarily of a mixed nature, being partly placental and partly venous.

The impure blood which enters the right auricle by the superior vena cava, having received a small quantity of the mixed blood which enters by the inferior vena cava, passes through the right auriculo-ventricular orifice into the right ventricle, and from thence is driven into the trunk of the pulmonary artery. A small quantity of it is conveyed to the lungs by the right and left pulmonary arteries, which at this period are of small size, and it is 
returned from the lungs to the left auricle by the pulmonary veins. By far the greater part of the right ventricular blood, however, enters the ductus arteriosus, and is conveyed by it to the aorta immediately beyond the origin of the left subclavian artery. None of this blood, therefore, enters the three great vessels which spring from the arch of the aorta. The whole of it descends in the descending thoracic and abdominal portions of the aorta, and has a

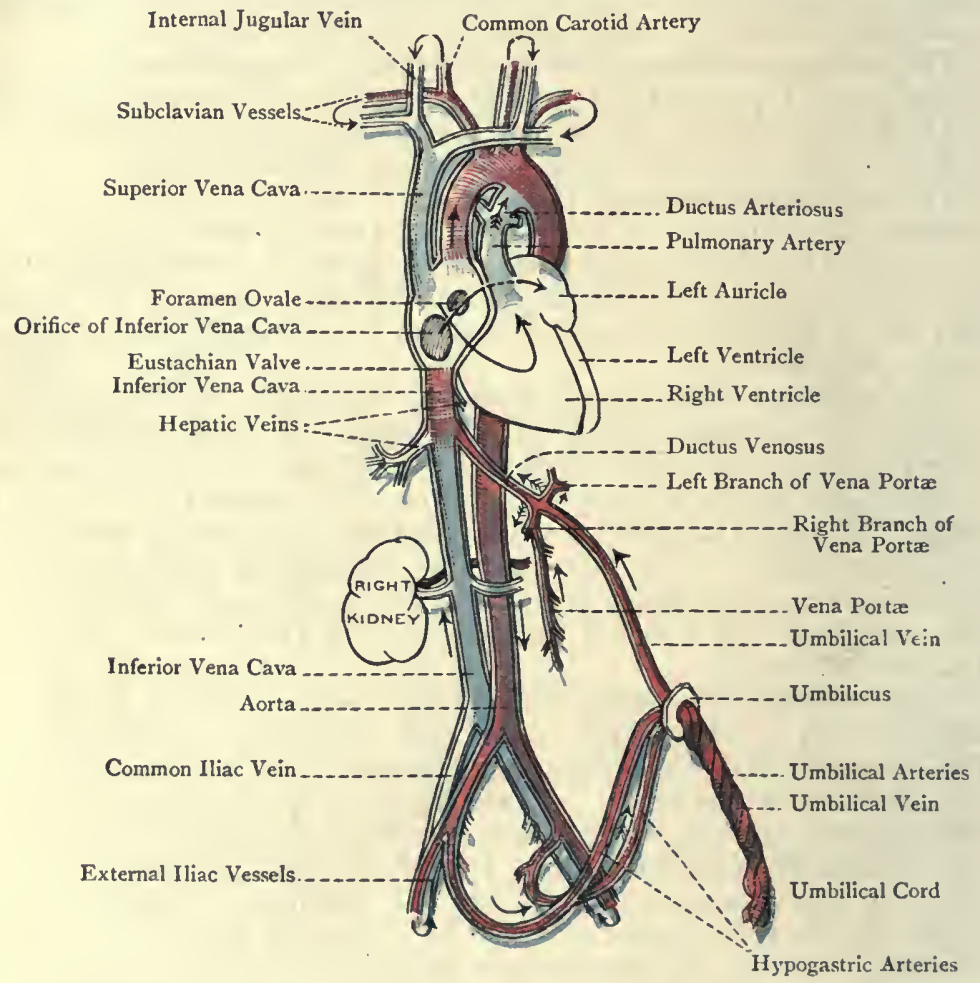

Fig. 412.-Diagram of the Organs of Circulation in the Fotus (The arrows indicate the course of the Circulation).

twofold destination. Part of it is distributed to the abdominal and pelvic viscera, and the lower limls, whence it is returned by the vena portæ and inferior vena cava; but the greater part of it is conveyed out of the body of the foetus to the placenta by means of the hypogastric and umbilical arteries. Having been purified in the placenta, it is returned thence by the umbilical vein into the body of the fotus. This placental blood, as stated, reaches the inferior vena cava in two ways, partly directly by means of the 
ductus venosus, and partly indirectly by means of the hepatic veins after having circulated through the liver.

A small quantity of the mixed blood which enters the right auricle by the inferior vena cava mingles with the venous blood which enters that cavity by the superior vena cava ; but by far the greater part of it is directed by the Eustachian valve to the foramen ovale, through which it passes into the left ventricle. Here it receives the blood which has been returned from the lungs by the pulmonary veins, and then it passes through the left auriculoventricular orifice into the left ventricle. From the left ventricle it is driven into the aorta, and the greater part of it passes into the innominate, left common carotid, and left subclavian arteries to be distributed to the brain, head and neck, and upper limbs, as well as to the substance of the heart by the coronary arteries. A small quantity, however, of this mixed blood passes into the descending thoracic aorta, and mixes with the venous blood entering by the ductus arteriosus. The blood is returned to the right auricle of the heart from the brain, head and neck, and upper limbs, by the superior vena cava, and from the substance of the heart by the coronary sinus.

Changes at Birth.-At birth respiration is established, and the lungs perform their respiratory function; the right and left pulmonary arteries undergo rapid enlargement; and the placental circulation is entirely arrested. The hypogastric arteries become obliterated and transformed into fibrous cords, except at their roots, where they persist as the internal iliac arteries. The umbilical vein becomes obliterated, and is now known as the round ligament of the liver. The ductus venosus becomes transformed into a fibrous cord, called the ligamentum ductûs venosi, which occupies the venosal fissure of the liver. The foramen ovale is closed by the valve of the foramen ovale becoming adherent to the margin of the anmulus ovalis or limbus Vieussenii on its left side. In sorne cases, however, this union is incomplete, and a minute opening persists, through which a small probe may be passed. In very rare cases a fairly large opening may remain, as in the condition known as morbus caruleus. As viewed from the interior of the left auricle, the upper crescentic border of the valve of the foramen ovale is visible upon the interauricular septum, and above this border there is a slight depression. The ductus arteriosus becomes obliterated, and persists as a fibrous cord, called the liganentum ductus arteriosi, which passes from the root of the left pulmonary artery to the arch of the aorta immediately beyond the origin of the left subclavian artery.

The closure of all the peculiar structures associated with the fotal circulation is usually complete from the eighth to the tenth Jay after"birth.

Development of the Principal Arteries.-In the embryo the aortic bulb or bulbus arteriosus divides into two vessels, called the primitive ventral aortæ, right and left, which pass cephalicwards on the ventral aspect of the fore-gut. 
From each of these vessels two arterial arches are given off, and from the aortic bulb there spring four arterial arches on either side; the bulb representing their common stem. Subsequently two of the bulbar arterial arches, on either side, are transferred to the ventral aortæ, whilst the other two maintain their connection with the bulb. There are thus six arterial arches on either side, four of which spring directly from the corresponding ventral aorta, these being the cephalic four, whilst the caudal pair, on either side, arise directly from the aortic bulb. These six arches are called first, second, third, fourth, fifth, and sixth, in this order from the cephalic region caudalwards. The fifth arch, on either side, is rudimentary and of temporary duration, and it takes no part in the development of permanent bloodvessels. The arterial arches traverse the visceral arches, and, in doing so, they sweep from before backwards on either side of the fore-gut. They are sometimes spoken of as the visceral arch arteries.

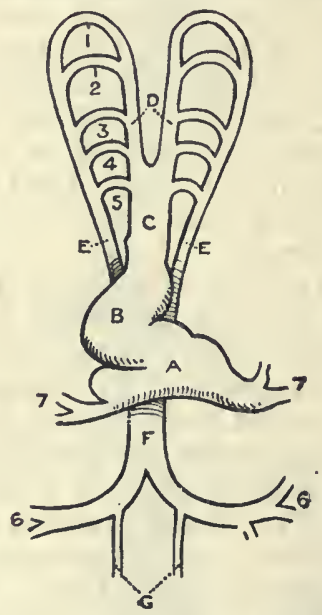

Fig. 4I3.-Diagram of the Heart and Primitive Vessels of the Embryo CHICK, AS SEEN FROM BELOW AND ENLARged (AFTER Allen Thomson, in QUain's 'EMbryology ').

A, Auricle; B, Ventricle; C, Bulbus Arteriosus; D, Primitive Ventral Aortæ; E, E, Primitive Dorsal Aortæ ; F, Single Dorsal Aorta ; G, Continuations of the Dorsal Aortæ beyond the origins of the Vitelline Arteries; i to 5, Aortic Arches; 6,6 , Vitelline Arteries; 7, 7, Vitelline Veins.

Each arterial arch, after having swept dorsalwards, opens into the corresponding primitive dorsal aorta. These primitive dorsal aortæ are two in number-right and left, and each is continuous with the corresponding primitive ventral aorta through means of the first arterial arch of its own sicle. The series of arterial arches therefore establish direct communications between the ventral and dorsal aorta, and the extremities of each arterial arch are referred to as ventral and dorsal roots respectively.

The primitive dorsal aortx pass caudalwards on either side of the notochord, and each gives off a series of vessels, which are known as segmental arteries. In the cervical region there are seven segmental arteries; and in the thoracic and lumbar regions there are the intercostal and lumbar arteries respectively, all of which belong to the segmental type. The upper two or three thoracic segmental arteries disappear, and the anastomotic loop between them forms, on either side, the superior intercostal artery. 
Caudalwards each primitive dorsal aorta furnishes the vitelline or omphalomesenteric artery, which is its principal mode of termination. The two vitelline arteries pass to the yolk-sac. Another branch of each primitive dorsal aorta, however, passes caudalwards, which, though small, is really the continuation of the corresponding dorsal aorta. After retrogression of the yolk-sac, the vitelline arteries are replaced by the allantoic arteries.

At a subsequent period the two primitive dorsal aortæ unite at about the level of the future fourth thoracic vertebra, and so give rise to one vessel, namely, the dorsal aorta. This vessel is continued onwards as the median saudal aorta. which is ultimately known as the middle sacral artery.

The arterial arches, as stated, are called first, second, third, fourth, fifth

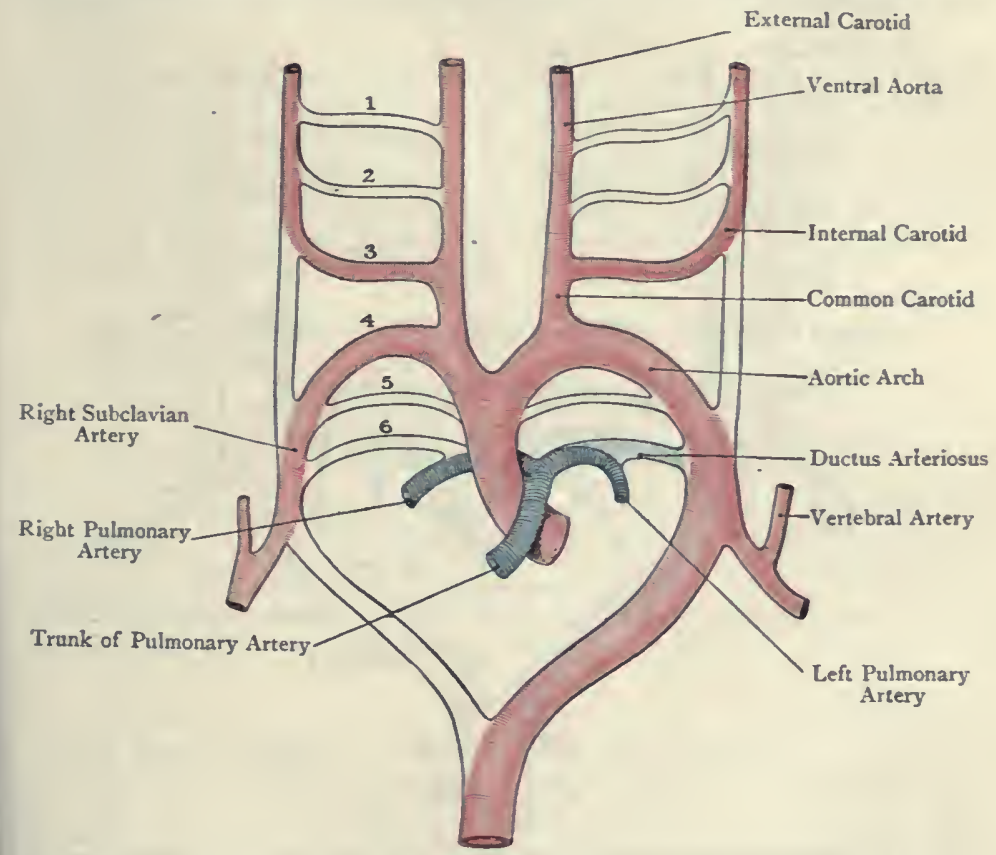

Fig. 4I 4--Scheme of the Aortic Arches and their Destination (MODIFIED FROM KOLLMANN) (FROM GRAY'S 'ANATOMY').

(rudimentary and temporary), and sixth. As the development of the principal arteries proceeds, important changes take place in these arches. Keeping in view the fact that, according to some authorities, the arterial arches are five in number, and not six, the following description will be equally applicable to each view, provided the sixth arch of the view adopted is regarded as being equivalent to the fifth arch of the other view.

The first, second, and fifth arehes disappear, on either side.

The sixth right arch disappears.

The part of the left dorsal aorta between the dorsal roots of the third and fourth left arches disappears.

The part of the right dorsal aorta, which extends from the dorsal root of the third right arch to the place of junction of the two dorsal aorta, disap- 
pears, except the small portion which contributes to the formation of the right subclavian artery.

Permanent Vessels. - The development of these vessels is here described, in a caudo-cephalic order, or from behind forwards.

The ventral portion of the sixth left arch gives rise to the right and left pulmonary arteries respectively, which pass backwards to the lungs.

The dorsal part of the sixth left arch-that is to say, the part beyond the origin of the right and left pulmonary arteries-persists up to birth, and forms the important vessel called the ductus arteriosus (duct of Botalli), which joins the left dorsal aorta. After birth the ductus arteriosus undergoes obliteration, and is converted into a fibrous cord, known as the ligamentum ductûs arteriosi.

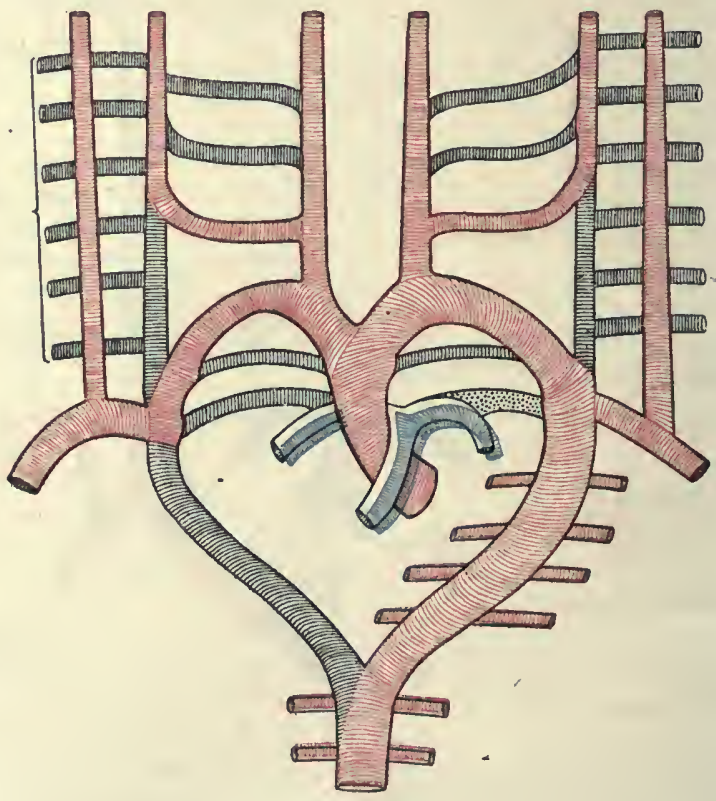

Fig. 4I 5.-Development of Cervical, Segmental, and Vertebral ARTERIES.

The root, or proximal part, of the left ventral aorta-that is to say, the part between the aortic bulb and the ventral root of the fourth left arch-forms the part of the arch of the aorta between the innominate and left common carotid arteries.

The fourth left arch forms the part of the arch of the aorta between the left common carotid artery and the point of entrance of the ductus arteriosus. As development proceeds, the fourth left arch gradually descends, along with the lieart, into the cavity of the future thorax, and so comes to occupy a lower plane than the fourth riglit arch.

The root, or proximal part, of the right ventral aorta - that is to say, the part between the aortic bulb and the ventral root of the fourth right archgives rise to the innominate artery.

The lourth right arch contributes to the formation of the right subciavian 
artery. Two other factors, however, are to be taken into account in connection with this vessel-namely: (I) the part of the right dorsal aorta between the dorsal roots of the fourth and sixth right arches, and (2) the seventh right cervical segmental artery, which arises from the right dorsal aorta at the level of the dorsal root of the sixth right arch. This segmental artery furnishes the root of the right vertebral artery. The vertebral artery is at first larger than the subclavian, which latter appears then to be a branch of the vertebral, but as the right subclavian artery grows out into the corresponding upper limb-bud to become the right axillary artery, it becomes larger than the vertebral artery.

The orgin of the left subclavian artery is much more simple than that of the right vessel. The left subclavian artery is a lateral branch of the seventh left cervical segmental artery, which arises from the left dorsal aorta at the level of the dorsal root of the sixth left arch. This segmental artery furnishes the root of the left vertebral artery, which latter vessel is, as on the right side, at first larger than the subclavian. As the left subclavian vessel, however, grows out in to the left upper limb-bud to become the left axillary, it becomes larger than the vertebral artery. Another subsequent factor in the formation of the left subclavian is the root of the seventh left cervical segmental artery, which thus brings the left subclavian vessel on to the arch of the aorta, and makes the left vertebral now a branch of it, though originally the vertebral artery was really its parent.

\section{Developmental Differences between the two Subclavian Arteries.}

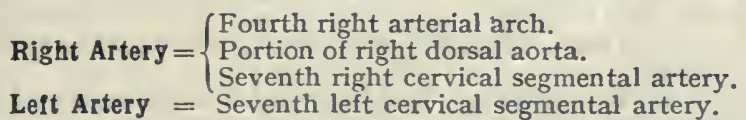

The part of each ventral aorta between the ventral roots of the third and fourth arches forms the corresponding common carotid artery.

The part of each ventral aorta above the ventral root of the third arch gives rise to the corresponding external carotid artery.

The third arch and the part of the dorsal aorta above its dorsal root give rise, on either side, to the internal carotid artery.

\section{Summary.}

Right Ventral Aorta.-(I) The root, or part between the aortic bulb and the ventral root of the fourth right arch, gives rise to the innominate artery. (2) The part between the ventral roots of the third and fourth right arches forms the right common carotid artery. (3) The part above the ventral root of the third right arch gives rise to the right external carotid artery.

Left Ventral Aorta.-(I) The root, or part between the aortic bulb and the ventral root of the fourth left arch forms the part of the arch of the aorta between the innominate and left common carotid arteries. (2) The part between the ventral roots of the third and fourth left arches gives rise to the left common carotid artery. (3) The part above the ventral root of the third left arch forms the left external carotid artery.

Rignt Dorsal Aorta.-(I) The part between the dorsal root of the third right arch and the place of junction of the two dorsal aortæ disappears, except the small portion which contributes to the formation of the right subclavian artery. (2) The part above the dorsal root of the third right arch persists. and forms part of the right internal carotid artery.

Left Dorsal Aorta.- (1) The part between the dorsal roots of the third and fourth left arches disappears. (2) The part above the dorsal root of the third left arch persists and forms part of the left internal carotid artery. (3) The part between the dorsal root of the sixth left arch and the place of junction of the two dorsal aorte forms the commencement of the descending aorta. 
Arterial Arches.-(I) The first, second, and fifth arches, on either side, disappear; and the sixth right arch also disappears. (2) The third arch, on each side, persists and forms part of the corresponding internal carotid artery. (3) The fourth right arch forms part of the right subclavian artery. (4) The fourth left arch forms the part of the arch of the aorta between the left common carotid artery and the point of entrance of the ductus arteriosus. (5) The ventral portion of the sixth left or pulmonic arch gives rise to the right and left pulmonary arteries respectively. (According to some authorities, the right pulmonary artery arises from the ventral part of the sixth right arch, which would then be a pulmonic arch like the sixth left.) (6) The dorsal part of the sixth left arch-that is to say, the part beyond the origin of the right and left pulmonary arteries-persists up to birth, and forms the ductus arteriosus.

Summary of the Permanent Aorta.-(I) The ascending aorta is developed from the aortic bulb or bulbus arteriosus, along with the trunk of the pulmonary artery. (2) The part of the arch of the aorta between the innomInate and left common carotid arteries is developed from the root of the left ventral aorta - that is to say, the part between the aortic bulb and the ventral root of the fourth left arch. (3) The part of the arch of the aorta between the left common carotid artery and the point of entrance of the ductus arteriosus is developed from the fourth left arch.. (4) The descending aorta is formed from above downwards by (a) a portion of the left dorsal aorta, and (b) the fusion of the right and left dorsal aortx.

Summary of the Pulmonary Artery.-(I) The trunk of the pulmonary artery, otherwise spoken of as the common pulmonary artery, is developed from the aortic bulb or bulbus arteriosus, along with the ascending aorta. (2) The right and left pulmonary arteries are developed from the ventral portion of the sixth left arch.

Development of the Segmental, Vertebral, and Left Subclavian Arteries.Each primitive dorsal aorta in the cervical part of its course gives off seven arteries, which are called the cevvical segmental arteries. There are thus seven pairs of these arteries, serially arranged. The first or highest lies between the occiput and the atlas, and is the refore suboccipital; and the seventh or lowest corresponds to the interval between the sixth and seventh cervical vertebræ. These seven vessels, on either side, are connected by intersegmental anastomotic vessels, which, by their continuity, establish a longitudinal anastomotic chain. Superiorly this chain is prolonged through the foramen magnum into the cranial cavity, where it terminates by anastomosing with the corresponding internal carotid artery.

The upper six cervical segmental arteries soon disappear, but the seventh segmental artery and the longitudinal anastomotic chain persist, on either side.

The first part of the vertebral artery is developed from the seventh cervical segmental artery. The second part of the vertebral artery, which traverses the upper six costo-transverse, or vertebrarterial, foranina, represents the persistent longitudinal anastomotic chain, except its cephalic part. The third, or suboccipital, and fourth, or intracranial, parts of the artery are developed from the cephalic part of the longitudinal anastomotic chain, which, as stated, is prolonged through the foramen magnum into the cranial cavity.

The seventh cervical segmental artery, on either side, is of special importance for two reasons. In the first place it gives origin to the vertebral artery, which takes a course cephalicwards. In the second place, it gives origin, on the left side, to the left subclavian artery, and on the right side it contributes to the formation of the right subclavian artery. Eacl subclavian artery takes an outward course to the corresponding upper limb-bud. The seventh left cervical segmented artery thus constitutes a common stem for the left vertebral and left subclavian arteries, whilst the seventh right cervical segmental artery forms a common stem for the right vertebral artery and a part of the right subclavian artery.

The origin of a vertebral artery precedes that of the corresponding sub- 
clavian artery, so that the subclavian artery is originally a lateral branch of the vertebral artery. As development proceeds, however, the subclavian artery increases in size, and greatly exceeds the vertebral artery, the latter vessel being now regarded as a branch of the subclavian, whereas originally it is the parent trunk.

The thoracic and abdominal segmental arteries have been already referred to in connection with the dorsal aorte.

\section{Development of the Principal Veins.}

The primitive veins form two giroups. One group retums the blood from the yolk-sac and the placenta; and the other group returns the blood from the head and neck, anterior limbs, body-wall, Wolffian bodies, and posterior limbs. The first group comprises: (I) The vitelline veins, in connection with which the portal vein is developed; and (2) the umbilical or allantoic veins. The second group consists of (1) the anterior cardinal veins; (2) the posterior cardinal veins; and (3) the subcardinal or supracardinal veins. The veins of each group are arranged in pairs, right and left, as follows:

Two Vitelline Veins.

Two Umbilical A llantoic Veins. Two Subcardinal or Supracardinal Veins.
Two Anterior Cardinal Veins.

Two Posterior Cardinal Veins.

Vitelline or Omphalo-Mesenteric Veins and Vena Portæ.-These veins are developed first, and they return the blood from the yolk-sac. They enter the body of the embryo along the vitelline duct, and finally open into the sinus venosus, after traversing the septum transversum. Within the body they ascend, parallel with each other, at first in front of, and subsequently on either side of, the duodenal portion of the primitive intestinal tube. In the latter region, on the caudal side of the hepatic bud, they become connected by three transverse anastomotic vessels, two of which lie across the ventral aspect of the gut, and one being placed on its dorsal aspect. The first or lowest anastomotic vessel lies on the ventral aspect of the gut; the second or middle vessel is dorsal to the gut; and the third or highest, like the first or lowest, is ventral to the gut.

By means of these three anastomotic vessels two venous rings-lower, or caudal, and upper, or cephalic-are formed around the duodenal portion of the primitive intestinal tube, these rings constituting the sinus anuularis. During their formation the two divisions of the liver-bud are breaking up into hepatic cylinders, and these are giving off secondary cylinders. Owing to these hepatic developments the vitelline veins cease to communicate directly with the sinus venosus. The portions of the vitelline veins above the upper duodenal venous ring (shown in Fig. 4I 7 to proceed from its sides) become surrounded by the hepatic cylinders, and invaded by the secondary cylinders. In this manner these portions of the vitelline veins are freely subdivided into blood-channels, which are known as sinusoids (Minot). These sinusoids form a network which occupies the meshes of the network formed by the branches of the hepatic cylinders. The veins which convey blood from the upper duodenal ring to the hepatic sinusoids are now known as the vene hepatice advehentes, and they become the right and left divisions of the portal vein. The veins which carry the blood from the hepatic sinusoids to the sinus venosus are know $\mathrm{n}$ as the vence hepatica revehentes, and they become the hepatic veins.

Trunk of the Portal Vein.-The portions of the two vitelline veins which ascend in front of the primitive duodenum lie close together and parallel with each other. These portions fuse for a short distance, and form a single venous stem, which opens into the first, or lowest, ventral anastomotic vessel, or, in other words, into the lower part of the lower duodenal venous ring. This short stem receives the veins of the primitive intestinal tube, and it forms the root of the portal vein. The primitive portal vein, therefore, receives 

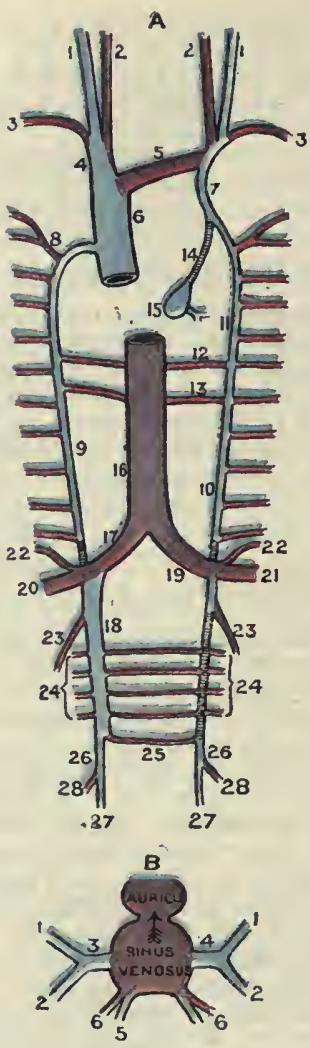

FIG. $4^{16}$.

A, Scheme of the Development of the Principal Veins. B, Scheme of the Primitive Veins Which open into the Primitive Heart.

A

x. External Jugular Vein (Primitive Jugular)

2. Internal Jugular Vein

3. Subclavian Vein

4. Right Inmominate Vein

5. Left Innominate Vein

6. Superior Vena Cava

7. Left Superior Intercostal Vein

8. Right Superior Intercostal Vein

9. Right Azygos Vein

10. Lower Left Azygos Vein

11. Upper Left Azygos Vein

12, 13. Transverse Azygos Veins

34. Left Duct of Cuvier (Remains of)

15. Coronary Sinus

16. Inferior Vena Cava

17, 18. Inferior Vena Cava

19. Mesial Part of Left Renal Vein

20. Right Renal Vein

21. Lateral Part of Left Renal Vein

22. Suprarenal Vein

23. Spermatic Vein (Ovarian Vein)

24. Lumbar Veins

25. Transverse Iliac Vein

26. Common Iliac Vein

27. Internal Iliac Vein

28. External Iliac Vein

B

x, x. Primitive Jugular Veins

2, 2. Cardinal Veins

4. Left Duct of Cuvier

5, 5. Vitelline V'cins

3. Right Duct of Cuvier

6, 6. Umbilical Veins 
its blood from (1) the yolk-sac, and (2) the primitive intestinal tube, within the abdomen.

As the yolk-sac atrophies the portions of the vitelline veins between it and the commencement of the portal vein also atrophy, and the tributaries of the portal vein gradually assume their condition in adult life.

The following parts of the sinus anmularis, or double duodenal venous ring, undergo atrophy:

I. Right half of lower ventral anastomotic vessel.

2. Right half of lower duodenal ring.

3. Left half of upper duodenal ring.

The following parts of the sinus annularis persist:

I. Left half of lower ventral anastomotic vessel.

2. Left half of lower duodenal ring.

3. Middle or dorsal anastomotic vessel.

4. Right half of upper duodenal ring.

5. Upper ventral anastomotic vessel.

These persistent portions, with the exception of the upper ventrai anastomotic vessel, form the greater part of the trunk of the portal vein. The upper ventral anastomotic vessel represents a part of the left division of the portal vein.

The portal vein has originally a spiral relation to the duodenal portion of the primitive intestinal tube-that is to say, it winds round the left side and dorsal aspect of the duodenum, and then appears on its right side.

Divisions of the Portal Vein.-These are connected with the upper duodenal venous ring. As previously stated, the veins which convey the blood from this ring to the hepatic sinusoids are known as the vence hepatice advehentes, right and left. The right division of the portal vein is formed by the right vena hepatica advehens, which springs from the right half of the upper duodenal venous ring. The left division is formed by (1) the upper ventral anastomotic vessel, and (2) the left vena hepatica advehens.

\section{Summary of the Development of the Portal Vein.}

Root $=$ Fusion of vitelline veins ventral to the primitive duodenum.

(I. Left half of lower ventral anastomotic vessel.

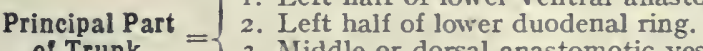

of Trunk $=\left\{\begin{array}{l}\text { 3. Middle or dorsal anastomotic vessel. } \\ \text {. }\end{array}\right.$

Right Division $=\quad$ 4. Right half of upper duodenal ring

Lelt Division $=\left\{\begin{array}{l}\text { 1. Upper ventral anastomotic vessel. } \\ \text { 2. Left vena hepatica advehens. }\end{array}\right.$

According to some authorities the common stem formed by the fusion of the vitelline veins in front of the duodenal portion of the intestinal tube, together with the left half of the lower duodenal ring, gives rise to the superior mesenteric vein, which is joined by the splenic vein. According to this view, the portal vein is formed by (I) the middle or dorsal transverse anastomotic vessel, and (2) the right half of the upper venous ring.

\section{Summary of the Vitelline Veins.}

The vitelline veins, which are developed first, return the blood from the yolk-sac to the sinus venosus. They enter the body of the embryo along the vitelline duct. At first they ascend in front of the duodenal portion of the primitive intestinal tube, lying close to, and parallel with, each other. Subsequently they ascend on either side of the duodenal gut. The portions of the veins in front of the duodenal gut fuse and form the root or lower part of the portal vein, which receives the veins of the primitive intestinal tube. The portions on either side of the duodenal gut become connected by three 
transverse anastomotic vessels, two of which, lower and upper, lie across the ventral aspect of the duodenal gut, and one-middle-dorsal to it. A double venous ring (sinus annularis) is now formed, the rings being known as lower or caudal and upper or cephalic, and these embrace the duodenal gut. The portions of the two vitelline veins above the upper venous ring cease to be connected directly with the sinus venosus, owing to the developmental changes which are now taking place in the hepatic diverticulum or liver-bud. They are invaded by the hepatic cylinders, and are freely subdivided into sinusoids. The veins which carry the blood from the upper venous ring to these sinusoids are called the vene hepatica advehentes, and the veins which carry the blood from the sinusoids to the sinus venosus are

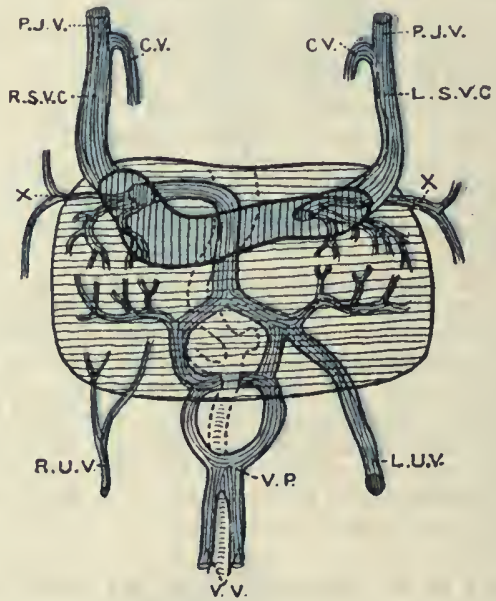

Fig. 4I7.-The Venous Trunks of the Human Embryo (His). P.J.V. Primitive Jugular Vein

R.S.V.C. Right Superior Vena Cava

L. S.V.C. Left Superior Vena Cava
X.X. Upper separated portions of Umbilical Veins R.U.V. Right Umbilical Vein L.U.V. Left Umbilical Vein V.P. Vena Portx

\section{V.V. Vitelline Veins}

called the vene hepatica revehentes, the latter giving rise to the hepatic veins of adult life.

A summary of the portal vein has just been given. The initial portions of the vitelline veins atrophy.

The vitelline veins thus give rise to (I) the portal vein and its ramifications, and (2) the hepatic veins.

Umbilical or Allantoie Veins, - The two umbilical veins return the blood from the placenta to the sinus venosus. They are of small size during the period of the vitelline circulation, but become enlarged as the yolk-sac atrophies and as the allantois undergoes increase in size. The two veins unite and form a single trunk within the umbilical cord. At the umbilicus this trunk enters the body of the embryo, and immediately divides into two umbilical veins, right and left, which traverse the septum transversum and open into the sinus venosus. As they traverse the septum transversum they are intimately related to the developing liver.

The two umbilical veins soon lose their connection with the sinus venosus, and they are now subdivided, like the vitelline veins, in to hepatic sinusoids 
by the hepatic cylinders. At this stage the blood of the umbilical veins flows through the hepatic sinusoids, from which it is taken up by the venæ hepatica revehentes, or hepatic veins, and these veins convey it to the sinus venosus.

At a later period the right umbilical vein, for the most part, atrophies. A portion of it, however, near the umbilicus, persists, and establishes a connection with the epigastric veins of the abdominal wall. In this persistent portion the blood can flow towards the umbilicus. As the right umbilical vein undergoes atrophy, the left umbilical vein becomes enlarged. Immediately below the developing liver it joins the upper duodenal venous ring, formed by the vitelline veins. Meanwhile a vein is being developed, which passes from the convexity of the upper duodenal venous ring-that is to say, from the left division of the portal vein-to the right vena hepatica revehens, or right hepatic vein, near the sinus venosus. This new vein constitutes the ductus venosus, or duct of Arantius, and through this channel the greater part of the pure placental blood passes directly to the heart, without circulating through the liver. A certain amonnt of pure placental blood, however, reaches the liver through the left vena hepatica advehens, which, as previously stated, forms a part of the left division of the portal vein.

The left vena hepatica revehens, or left hepatic vein, loses its connection with the sinus venosus, and now opens into the right vena hepatica revehens, or right hepatic vein, at the level where the latter receives the ductus venosus. A common venous stem is now formed, representing (I) the right hepatic vein, (2) the left hepatic vein, and (3) the ductus venosus. This stem is known as the vena hepatica communis, and it gives rise to the terminal or cardiac portion of the prerenal division of the inferior vena cava.

The left umbilical vein and the ductus venosus remain pervious, and are important vessels, until the period of birth, being concerned in the placental circulation. After the cessation of this circulation both vessels atrophy, the ductus venosus entirely, and the left umbilical vein almost entirely. The obliterated ductus venosus is known as the ligamentum ductus venosi, and the obliterated left umbilical vein forms the round ligament of the liver. A minute portion of the lumen of the left umbilical vein remains pervious within the round ligament of the liver. This pervious portion communicates at the liver with the left division of the portal vein, and at the umbilicus it is connected with the epigastric veins of the abdominal wall. It thus forms a channel of communication between the left division of the portal vein and the systemic veins of the anterior abdominal wall. In this pervious portion the blood can flow towards the umbilicus. This anastomosis between the portal and systemic circulations accounts for the enlargement of the veins of the anterior abdominal wall in cases of portal obstruction within the liver.

\section{Summary of the Umbilical Veins.}

The umbilical veins return the blood from the placenta to the sinus venosus. The two veins unite and form a single trunk within the umbilical cord. At the umbilicus this trunk enters the body of the embryo, and immediately divides into two umbilical veins, right and left, which traverse the septum transversum and open into the sinus venosus. They soon lose their connection with this sinus, and become broken up into sinusoids by the hepatic cylinders. At a later period the right umbilical vein, for the most part, atrophies; but a portion of it, near the umbilicus, persists and conveys blood towards the umbilicus, where it communicates with the epigastric veins of the abdominal wall. The left umbilical vein now enlarges, and, immediately below the developing liver, it joins the upper duodenal venons ring. Meanwhile a vein is being developed, which passes from the convexity of the upper duodenal ring-that is to say, from the left division of the portal vein-to the right hepatic vein. This new vessel is the ductus venosus, and through it the greater part of the pure placental blood passes directly to the heart. The 
left hepatic vein loses its connection with the sinus venosus, and opens into the right hepatic vein at the level where that vein receives the ductus venosus. The common stem, thus formed, is known as the vena hepatica communis, which forms the terminal part of the inferior vena cava.

The left umbilical vein and the ductus venosus persist throughout fotal life. Thereafter the ductus venosus atrophies and forms the ligamentum ductus venosi. The left umbilical vein atrophies almost entirely, and gives rise to the round ligament of the liver. A minute portion of its lumen, however, remains pervious at the centre of the round ligament of the liver, and this portion forms an anastomotic channel between the left division of the portail vein and the epigastric veins of the abdominal wall. In this pervious portion the blood can flow towards the umbilicus.

Anterior Cardinal Veins.-These veins are two in number-right and leftand they return the blood from the head and fore-limbs. Each vein consists of two parts-intracranial and extracranial. The intracranial part gives rise, directly and indirectly, to the intracranial sinuses, whilst the extracranial part, which is the true anterior cardinal vein, becomes the internal jugular vein. The intracranial part is known as the vena capitis medialis. This vein lies at first on the mesial side of the primitive cranial nerve-roots. In this position it is connected with venous loops which embrace the nerveroots. The mesial portions of these loops disappear, and their lateral portions become connected by an anastomotic vessel, which forms another temporary vein, known as the vena capitis lateralis. This vein commences just beyond the primitive Gasserian ganglion, and it leaves the cranial cavity in company with the primitive facial nerve, becoming thereafter continuous with the extracranial part of the anterior cardinal vein.

Vena Capitis Medialis. - This primitive vessel extends as far as the Gasserian ganglion, lying, as stated, on the mesial side of the primitive cranial nerve-roots. The portion of it on the mesial side of the Gasserian ganglion gives rise to the cavernous sinus, and the remainder disappears.

Vena Capitis Lateralis.-This temporary vein receives three chief intracranial tributaries-anterior, middle, and posterior cerebral. The primitive anterior cerebral vein derives its blood from the optic vesicle (eye), fore-brain, and mid-brain. Certain of the tributaries of the anterior cerebral vein, of each side, become connected longitudinally by an anastomotic vessel, lying close to the median line. These two anastomotic vessels, right and left, constitute the rudiment of the superior longitudinal sinus, which sinus is consequently in two halves originally. Subsequently, however, the two anastomotic vessels blend, and so give rise to one superior longitudinal sinus. The primitive middle cevebral vein derives its blood from the cerebellum, and the primitive posterior cerebral vein derives its blood from the medulla oblongata. The posterior cerebral vein issues from the cranial cavity through the primitive jugular foramen, prior to joining the vena capitis lateralis, whilst the anterior and middle cerebral veins, having joined the vena capitis lateralis, necessarily issue from the cranial cavity along with the facial nerve through a temporary foramen.

Subsequently anastomotic connections are formed between the three primitive cerebral veins. The vena capitis lateralis, which, as just stated, leaves the cranial cavity along with the facial nerve, undergoes atrophy, and the venous blood of the entire encephalon issues from the cranial cavity through the jugular foramen.

Subsequent Metamorphoses.-The superior longitudinal sinus, originally in two halves and developed from certain of the tributaries of the anterior cerebral veins, acquires two anastomotic connections, first with the cephalic end of the middle cerebral vein, and afterwards with the posterior cerebral vein. The latter anastomosis gives rise to the greater or transverse part of the lateral sinus, the posterior cerebral vein forming the sigmoid part of that sinus. The middle cerebral vein becomes the superior petrosal sinus. The Inferior petrosal sinus is developed from an anastomotic connection between the dorsal part of the cavernous sinus and the distal part of the lateral sinus. 
The extracranial part of the anterior cardinal vein, after the obliteration of the vena capitis lateralis, commences at the jugular foramen, whence it extends caudalwards, receiving in its course the cervical segmental veins. In the vicinity of the sinus venosus it meets the posterior cardinal vein of its own side, with which it unites. The venous trunk thus formed is called the duct of Cuvier, of which they are two-right and left. These two ducts pass transversely, one on either side, to the sinus venosus, into which they open. At this stage the sinus venosus also receives the vitelline and umbilical veins of each side. The ducts of Cuvier form the primitive right and left superior venæ cavæ.

As the heart descends from the region of the fore-gut, the ducts of Cuvier become vertical, and are in line with the anterior cardinal veins. IVhen the sinus venosus becomes merged into the right auricle, and the heart undergoes a slight rotation from right to left, the left duct of Cuvier is placed dorsal to the left auricle, before opening into the right auricle.

Each anterior cardinal vein is joined, near its caudal extremity, by the corresponding subclavian vein.

Close to the junction the subclavian vein receives the external jugular vein. This vein is a secondary formation, and is probably derived from a posterior auricular vein, being subsequently reinforced by a pre-auricular vein.

A transverse anastomotic vessel is now formed, called the transverse jugular vein. It extends from the junction of the left anterior cardinal and left subclavian veins to the right anterior cardinal vein at a point a little below the place where it receives the right subclavian vein. This transverse jugular vein, which extends obliquely from left to right, with a downward inclination, gives rise to the left innominate vein. The venous blood from (I) the left side of the head and neck and (2) the left fore-limb now passes through the left innominate vein into the permanent superior vena cava. The right innominate vein is formed by the short portion of the right anterior cardinal vein which intervenes between the place where it receives the right subclavian vein and the place where the transverse jugular vein joins it. The portion of the right anterior cardinal vein, which lies immediately below the place where the transverse jugular vein joins it, forms the upper or extrapericardial part of the permanent superior vena cava-that is to say, the part above the point where the right azygos vein opens into it. The lower or intrapericardial part of the superior vena cava is developed from the right duct of Cuvier. The permanent superior vena cava therefore represents (I) the lower part of the right anterior cardinal vein, and (2) the right duct of Cuvier.

The portion of the left anterior cardinal vein immediately below the left extremity of the transverse jugular vein forms the upper part of the left superior intercostal vein, thus accounting for the ending of that vein in the left innominate vein, which, as stated, is formed by the transverse jugular vein.

The left duct of Cuvier undergoes partial obliteration. Its terminal part, along with the left lateral cornu of the sinus venosus, gives rise to the coronary sinus. The portion next the terminal part also persists in the form of a very minute vein, called the oblique vein of Marshall, which lies over the posterior aspect of the left auricle. The obliterated portion is represented by the vestigial fold of Marshall, which is contained within a small triangular fold of the serous portion of the pericardium, in front of the root of the left lung.

Posterior Cardinal Veins. - These are two in number-right and left-and they return the blood from the Wolffian bodies, body-wall, and hind-limbs. They lie on either side of the aorta, dorsal to the mesial part of the Wolffian bodies. The caudal end of each vein receives the sciatic vein, which is the primitive vein of the hind-limb. A little above this point it is joined, at a later period, by the external iliac vein, which, along with the femoral vein, has now been developed.

The cephalic end of each posterior cardinal vein joins the corresponding 
anterior cardinal vein, and the trunk so formed is called the duct of Cuvier (right and left), which opens in to the sinus venosus.

Amongst other tributaries, the posterior cardinal veins receive segmental veins, such as the lumbar and intercostal veins, and veins from the intermediate cell-mass, and they are connected with each other by transverse retro-aortic vessels, which constitute the transverse retro-aortic posterior cardinal anastomoses. One of these, situated in the lower lumbar region, enlarges and forms the transverse iliac vein. This vessel extends somewhat obliquely from the left posterior cardinal vein, at a point a little above the place where it receives the left external iliac vein, to the right posterior cardinal vein, its inclination in crossing being upwards. The transverse iliac vein gives rise to the greater part of the left common iliac vein, the commencement of the latter vessel being formed by that portion of the left posterior cardinal vein which intervenes between the place where it receives the left external iliac vein and the left extremity of the transverse iliac vein. After the formation of the transverse iliac vein, the blood from the left half of the pelvis and left hind-limb is now diverted through the transverse iliac vein in to the right posterior cardinal vein.

In the thoracic region, two (sometimes one) of the retro-aortic posterior cardinal anastomoses persist as the lower and upper transverse azygos veins.

Between the anterior cardinal veins, and also between the posterior cardinal veins, there are, as stated, several transverse anastomotic vessels. Four (sometimes three) of these are of special importance, and may be here summed up as follows, in order from above downwards:

I. Transverse jugular between anterior cardinals, forming the left innominate vein.

2. Upper retro-aortic posterior cardinal anastomosis in thorax, forming the upper transverse azygos vein.

3. Lower retro-aortic posterior cardinal anastomosis in thorax, forming the lower transverse azygos vein.

4. Transverse iliac, between posterior cardinals in lower lumbar region, forming greater part of left common iliac vein.

The posterior cardinal veins constitute a somewhat circuitous route to the heart. Moreover, in the region of the Wolffian bodies they become, to a certain extent, subdivided into sinusoids. New and more direct channels are therefore formed.

New Secondary Venous Channels.-As the development of the hind-limbs proceeds, two new secondary venous channels are formed, which are auxiliaries of the posterior cardinal veins, and relieve the obstruction caused by the developing Wolffian bodies. They are called the subcardinal veins (F. T. Lewis). Each subcardinal vein is formed by a longitudinal anastomotic vessel, which connects the small veins passing from the mesentery to the posterior cardinal veins, and it lies ventral to the mesial part of the Wolffian body. The subcardinal veins run parallel with the posterior cardinal veins, on their ventral aspect, and each extends from the caudal end of a posterior cardinal vein to near its cephalic end, being connected with the posterior cardinal at either extremity. Between these points the two veins (posterior cardinal and subcardinal) are connected, at frequent intervals, by dorsoventral anastomoses. Each vein is also connected with its fellow of the opposite side by transverse anastomoses, those between the posterior cardinal veins being retro-aortic, whilst those between the subcardinal veins are preaortic. The subcardinal anastomotic vessels, for the most part, disappear, but one of them becomes large, and lies below the root of the superior mesenteric artery, in front of the aorta and opposite the future renal veins. It is known as the great pre-aortic transverse subcardinal anastomotic vessel. It also forms an indirect anastomosis between the adjacent parts of the posterior cardinal veins. At the level of this anastomotic vessel each renal vein opens into the corresponding posterior cardinal vein.

One of the dorso-ventral anastomotic vessels on the right side connects 
the subcardina vein with the posterior cardinal vein, opposite the future right renal vein.

The right subcardinal vein becomes connected superiorly, behind the liver and within the caval mesentery, with hepatic veins by means of a fusion between hepatic and subcardinal sinusoids. In this manner a venous channel is formed, which leads to the common hepatic vein and thence to the heart. The right subcardinal and posterior cardinal veins being in free communication with each other, much of the venous blood from the caudal part of the body can flow through the new channel, formed in the caval mesentery by the fusion of the hepatic and subcardinal sinusoids, into the common hepatic

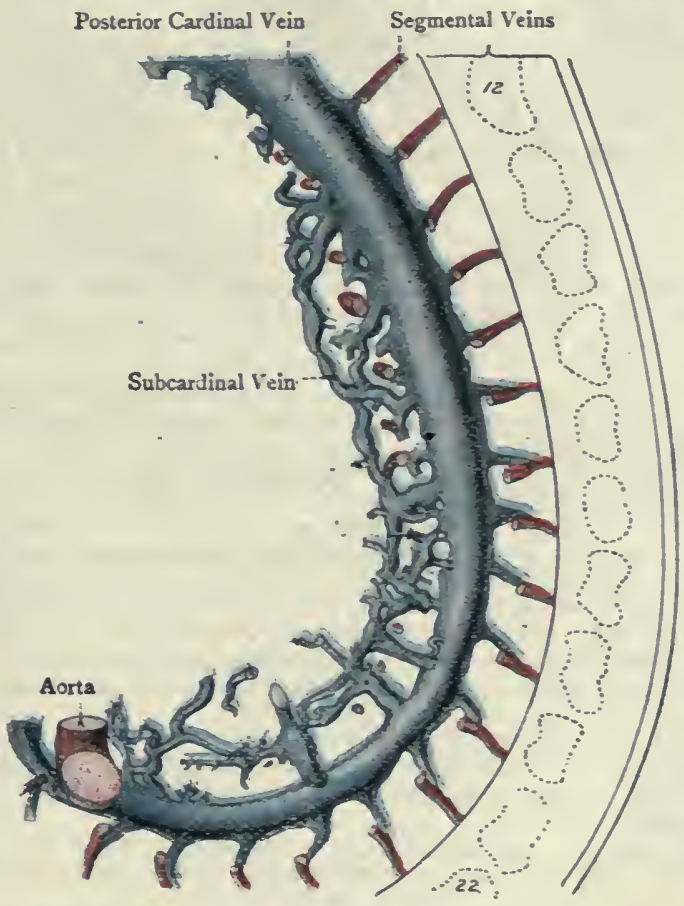

Fig. 4i 8.-The Development of the Vena Cava Inferior (Early Stages). Cardinal and Subcardinal Veins, etc.

(Frederick T. Lewis, in the American Journal of Anatomy.)

vein, and thence to the heart. The blood of the posterior cardinal veins can therefore now reach the heart through two channels-namely: (1) the upper part of the right subcardinal vein, the new path within the caval mesentery, and the common hepatic vein; and (2) the duct of Cuvier. The first channel is taken by the blood of the lower parts of the posterior cardinal veins, and the second channel by the blood from the upper parts of the posterior cardinal veins, above the Wolffian bodies.

The subcardinal and posterior cardinal veins of each side are divided into two parts-lower and upper-by the great transverse pre-aortic anastomotic vessel. The lower parts of the two subcardinal veins atrophy. The upper part of the right subcardinal vein persists, and forms a portion of the 
inferior vena cava of adult life, and the part of the left subcardinal vein above the great transverse pre-aortic anastomotic vessel forms the terminal portion of the left suprarenal vein. The great transverse pre-aortic anastomotic vessel persists, and forms the mesial part of the left renal vein of adult life.

Additional important changes take place in the course of development, which will now be stated.

Metamorphoses of Leit Posterior Cardinal Vein.-(1) The part below the left extremity of the transverse iliac vein persists, and forms from above downwards: (a) the commencement of the left common iliac vein, and $(b)$ the left Internal iliac vein, into which latter the left external iliac vein subsequently opens.

2. The part between the left extremity of the transverse iliac vein and the place of entrance of the left spermatic vein undergoes atrophy.

3. The part between the place of entrance of the left spermatic (or ovarian) vein and the future left renal vein persists, and forms the upper, or terminal, part of the left spermatic (or ovarian) vein. This circumstance explains why the left spermatic (or ovarian) vein opens into the left renal vein, whereas the right spermatic vein opens into the inferior vena cava.

4. The part immediately above the left renal vein disappears.

5. The remainder of the left posterior cardinal vein persists, and gives rise to $(a)$ the lower left azygos vein, (b) the upper left azygos vein, and $(c)$ the lower portion of the left superior intercostal vein, the upper portion of which is formed by that part of the left anterior cardinal vein which lies immediately below the left extremity of the transverse jugular vein.

6. The part between the lower and upper left azygos veins, and the part between the upper left azygos and left superior intercostal veins may, or may not, undergo obliteration.

Metamorphoses of the Right Posterior Cardinal Vein.-(1) The part below the right extremity of the transverse iliac vein persists, and forms from above downwards (a) the right common iliac vein, and (b) the right internal iliac vein, into which latter the right external iliac vein subsequently opens.

2. The part between the right extremity of the transverse iliac vein and the future right renal vein persists, and forms the lower, or postrenal, division of the inferior vena cava. Into this part, superiorly, the right spermatic vein opens, which explains the mode of ending of that vein in the adult, as compared with the mode of ending of the left spermatic vein, which, as stated, opens into the left renal vein.

3. The part immediately above the right renal vein disappears.

4. The remainder of the right posterior cardinal vein persists, and gives rise to the right azygos vein, which, superiorly, takes up the right superior intercostal vein.

The right superior intercostal vein is developed from the anastomotic channels which connect the upper three thoracic segmental veins of the right side.

The right and left posterior cardinal veins, in the thoracic region, are connected by two transverse retro-aortic anastomotic vessels. The lower of these cross-branches persists, and forms the lower transverse azygos vein, which diverts the blood from the lower left azygos vein into the right azygos vein. The upper cross-branch also persists, and gives rise to the upper transverse azygos vein, which diverts the blood from the upper left azygos vein likewise into the right azygos vein. There may only be one transverse azygos vein.

Metamorphoses of the Subcardinal Veins-Right Subcardinal.-(i) The right subcardinal vein, as stated, becomes connected with the hepatic veins by a fusion between hepatic and subcardinal capillaries dorsal to the liver and within the caval mesentery. (2) The portion of it which lies above the large transverse anastomotic vessel, connecting the subcardinal and posterior cardinal veins below the level of the root of the superior mesenteric artery, persists, and forms part of the prevenal division of the inferior vena cava. (3) 'The portion of it on the caudal side of the large transverse anastomotic vessel disappears. 
Left Subcardinal.-The left subcardinal vein, in the cephalic direction, has no connection with the liver. The part above the level of the great transverse pre-aortic subcardinal anastomosis persists as the left suprarenal vein; and its lower part, below the level of the anastomosis just named, atrophies.

\section{Development of the Inferior Vena Cava.}

The inferior vena cava, as regards its development, consists of two divisions -lower, or postrenal, and upper, or prerenal.

As previously stated, the posterior cardinal and subcardinal veins of the right side are connected by the great transverse pre-aortic anastomotic vessel below the root of the superior mesenteric artery, and at the level where the right renal vein opens into the right posterior cardinal vein. The part of the right posterior cardinal below this anastomotic vessel comes into line with the part of the right subcardinal above it, and so one channel is formed.

The postrenal division of the inferior vena cava is formed by the lower portion of the right posterior cardinal vein-that is to say, the portion below the great transverse pre-aortic anastomotic vessel, which forms a part of the left renal vein.

The prevenal division is more complicated in its development. As previously stated, the upper part of the right subcardinal vein becomes connected with the hepatic veins by a fusion between the hepatic and subcardinal sinusoids, dorsal to the liver and within the caval mesentery, a new channel being thereby formed to the common hepatic vein and heart. (The common hepatic vein is formed by the union of the right and left hepatic veins and ductus venosus.) The prevenal division of the inferior vena cava is formed, from below upwards, by (1) the upper portion of the right subcardinal veinthat is to say, the portion above the great transverse pre-aortic anastomotic vessel, (2) the fusion of hepatic and right subcardinal sinusoids within the caval mesentery, (3) the right hepatic vein, and (4) the common hepatic vein, which is connected with the heart.

\section{Summary of Development of Inferior Vena Cava.}

Postrenal Division $=$ Portion of right posterior cardinal between right renal and transverse iliac veins.

(1. Anastomotic connection between right posterior cardinal and right subcardinal veins at level of right renal vein. Prerenal Division $=\left\{\begin{array}{l}\text { 2. Upper portion of right subcardinal vein-that is to } \\ \text { say, the portion above the great transverse pre- } \\ \text { aortic anastomotic vessel. }\end{array}\right.$

3. Fusion of hepatic and right subcardinal capillaries within the caval mesentery.

4. Right hepatic vein.

5. Common hepatic vein.

Development of Renal Veins.-Each renal vein is originally a segmental vein of the corresponding intermediate cell-mass, in which the renal organs are developed. The right vessel has no other source, but the terminal part of the left renal vein is formed by the great transverse pre-aortic anastomotic vessel, which lies across the aorta below the root of the superior mesenteric artery.

Development of Suprarenal Veins.-Each vessel probably originates as a segmental vein of the suprarenal area of the corresponding intermediate cellmass. The right vessel has no other source, but the terminal part of the left vein $s$ formed by the part of the left subcardinal vein above the great transverse pre-aortic anastomotic vessel, which persists as the mesial part of the left renal vein.

Development of Lumbar Veins. - The right and left lumbar veins are originally the segmental veins of the lumbar region. The right vessels have no further 
source, but those portions of the left lumbar veins, which cross behind the abdominal aorta to reach the inferior vena cava, represent the persistent transverse retro-aortic posterior cardinal anastomotic vessels.

Development of Azygos System of Veins.-The right vena azygos represents the upper part of the right posterior cardinal vein, above the level of the future right renal vein. The lower and upper left azygos veins are developed from the thoracic portion of the left posterior cardinal vein. The lower and upper transverse azygos veins are persistent transverse retro-aortic posterior cardinal anastomotic vessels.

Development of Spermatic and Ovarian Veins.-Each of these veins is originally a segmental vein of the corresponding intermediate cell mass, in which the sexual glands are developed. The vessels of the right side have no other source, but the terminal part of each left vein is formed by that portion of the left posterior cardinal vein which extends between the point of entrance of the left spermatic (or ovarian) vein and the future left renal vein.

\section{Development of the Lymphatic System.}

The lymphatic system consists of lymphatic vessels and lymphatic glands or nodes. There is also lymphoid or adenoid tissue, as in the thymus body, lymphoid follicles and villi of the intestinal mucous membrane, Malpighian corpuscles of the spleen, palatine tonsils, and pharyngeal tonsil. There are two stages in the development of the lymphatic system-primary and secondary. The primary stage is concerned in the formation of lymph-sacs, and the secondary stage consists in the formation of lymphatic vessels and lymphglands.

Lymph Sacs.--There are two pairs of lymph-sacs and two single sacs, as follows:

1. Cervical or jugular (2).

2. Retro-peritoneal or pre-aortic (I).

3. Receptaculum or cisterna chyli ( 1 ).

4. Caudal or sciatic (2).

Opinions differ as to the development of these lymph-sacs. The view of Florence R. Sabin will be stated first. According to this lady the sacs are developed as sproutings from the endothelial lining of veins, and this constitutes the primary stage in the development of the lymphatic system.

Jugular Sacs.-The jugular lymph-sacs, right and left, are the first to appear. Each is situated on the outer side of the lower part of the primitive anterior cardinal vein, which becomes the internal jugular vein. It is formed from part of a capillary venous plexus, connected in early life with the anterior cardinal vein. A large part of this plexus disappears, whilst the connection of the remainder with the anterior cardinal vein is severed. There thus results a detached collection of capillaries, lined with endothelium and in close proximity to the outer side of the lower part of the anterior cardinal vein. These capillaries become dilated, and subsequently join to form the jugular lymph-sac of each side, which is lined with endothelium, and which establishes a fresh connection with the lower part of the anterior cardinal vein, where it is joined by the subclavian vein, a valve being formed at the venous junction by a protrusion of part of the lymph-sac at the place of junction. The jugular sac of each side is connected externally with peripheral lymphatic vessels which extend to the head, neck, and fore-limb bud of the same side. The caudal end of the left sac becomes connected with the thoracic duct, whilst the caudal end of the right sac becomes connected with the right lymphatic duct. The dorsal part of the sac becomes converted into a plexus of lymphatic vessels, from which chains of lymph-glands are developed.

Retro-Peritoneal Sac.-The retro-peritoneal or pre-aortic lymph-sac is single, and is formed from a capillary venous plexus in the root of the mesentery, which plexus is connected with the great pre-aortic transverse anastomotic vessel which extends between the two subcardinal veins. The capillary 
plexus in the root of the mesentery becomes converted into a lymph-sac, without any venous connection, and this constitutes the retro-peritoneal or pre-aortic sac, which establishes a communication with the-receptaculum or cisterna chyli, and through it with the thoracic dnct. It is ultimately replaced by a plexus of lymphatic vessels, and from this plexus chains of lymphatic glands are developed which lie along the ventral aspect of the abdominal aorta. From the retro-peritoneal sac lymphatic vessels pass within the mesentery along the branches of the superior mesenteric artery. and these form another lymphatic plexus, from which the mesenteric lymphatic (lacteal) glands are developed. Subsequently lymphatic (lacteal) vessels enter the wall of the small intestine.

Sciatic Sacs. - The sciatic lymph-sacs, right and left, are developed from capillary venous plexuses in connection with the two primitive sciatic veins. On either side the sac extends from near the caudal end of the receptaculum chyli along the outer side of the primitive sciatic vein. It ultimately becomes converted in to lymphatic glands.

Reseptaculum Chyli. - The receptaculum or cisterna chyli is a single sac, situated at the caudal end of the thoracic duct. It is developed from, and replaces, a venous plexus. The thoracic duct connects the receptaculum chyli with the left jugular lymph-sac, and the receptaculum chyli communicates with each sciatic sac. From each jugular sac a vessel grows caudalwards. On the right side this vessel constitutes the right lymphatic trunk, aad on the left side it forms the thoracic duct. As the thoracic duct approaches the developing aortic arch it divides and gives rise to two thoracic ducts, which embrace that arch and then pass to join separately the receptaculum chyli opposite the Wolffian bodies. Subsequently these two ducts fuse, and one duct is formed.

The thoracic duct is developed from a series of venous capillaries, originally having a connection with veins.

Lymphatic Vessels. - The formation of these vessels, along with that of lymph-glands, constitutes the second stage in the development of the lymphatic system. According to Sabin, the lymphatic vessels are formed from the endothelial lining of the lymph-sacs, whilst the lymph-glands are developed from plexuses of lymphatic capillaries. The development of the thoracic duct, as stated, takes place from several groups of venous capillaries. It is the largest lymphatic vessel, and it connects the receptaculum chyli with the left jugular sac.

The lymphatics derived from the jugular lymph-sacs pass to the head. neck, and fore-limb buds; those from the sciatic lymph sacs extend to the hind-limb buds; and those from the retro-peritoneal (mesenteric) lymphsac enter the mesentery, within which lymph-glands (mesenteric) are developed in connection with them. From these mesenteric glands, in succession, fresh lymphatic vessels pass to the intestinal tube.

The other view of the development of the lymphatic system, held by Huntington amongst others, is that the connection of the lymphatic vessels with the venous system is secondary, and not primary, as Sabin holds. According to this other view the lymphatic vessels spring from lymph-spaces, which are formed in the mesoderm (mesenchyme), and are lined with meschelium. The endothelial cells of the lymphatic vessels are derived from this mesothelium, and not from the venous endothelium according to Sabin, and the vessels establish a connection with the venous system at a later period.

Lymph-Glands. - Some of these are formed in connection with the lymphsacs, whilst others are formed in connection with peripheral lymphatic vessels. There are three stages in the development of a lymph-gland. The first stage consists in the formation of a plexus of lymphatic capillaries pervaded by connective-tissue septa. The second stage consists in the invasion of these septa by blood-capillaries, surrounded by lymphocytes. The third stage consists in the formation of a lymph-sinus from the original plexus of lymphatic capillaries.

The essential elements of a lymph-gland are thus threefold-namely: 
(1) a plexus of lymph-capillaries, (2) blood-capillaries, surrounded by lymphocytes, within connective-tissue septa, and (3) a lymph-sinus.

Each lymph-gland is connected with several lymphatic vessels. Those which enter the gland are known as afferent vessels, and they open into the peripheral or cortical part of the lymph-sinus. Those which emerge from the gland are termed efferent vessels, and they arise in the central or medullary part of the lymph-sinus. In no case does a lymphatic vessel pass uninterruptedly through a lymph-gland. The gland is a station in the path of a lymphatic vessel, which represents the terminus of that vessel, but not the terminus of the lymph. That fluid, after leaving the afferent vessel, flows

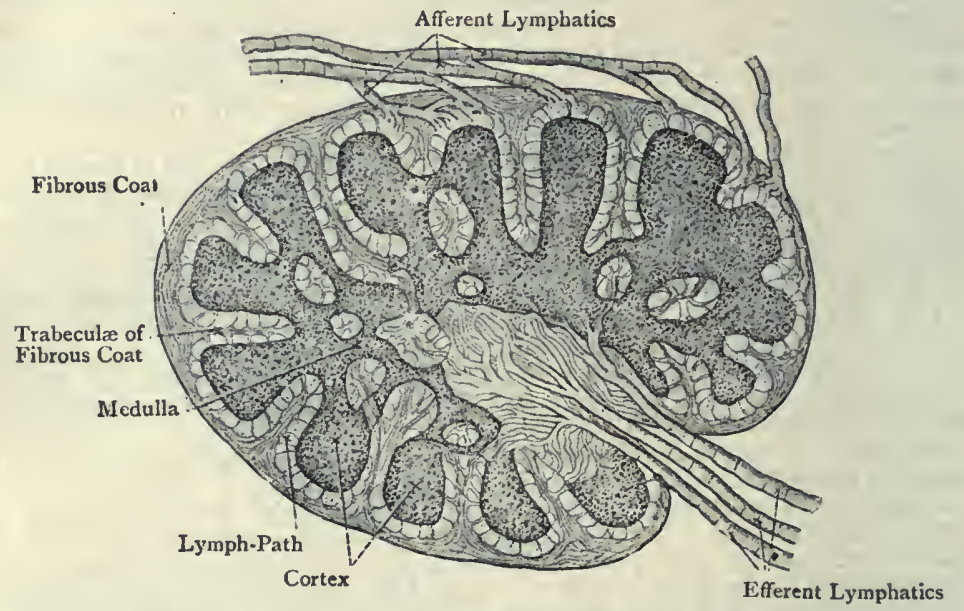

Fig. 4i 9.-Diagrammatic Section of Lymphatic Gland (Sharpey, from QUAIN).

in succession through the cortical and medullary parts of the lymph-sinus, and from the medullary part it flows into the efferent vessels. It is therefore, so far as the lymph is concerned, a case of, so to speak, changing carriages at a glandular station. During this process any injurious matter is taken up by the branched cells (phagocytes) of the lymph-sinus, and the lymph is furnished with a contingent of lymphocytes

\section{Structure of Lymphatic Vessels.}

A lymphatic vessel, of large size, consists of thrce coats-inner, middle, and outer. The inner coat (tunica intima) consists of a longitudinal network of elastic fibres, lined with endothelial cells. The middle coat (tunica media) consists of plain muscular and elastic tissues, disposed for the most part transversely. The outer coat (tunica externa) consists of (I) longitudinal bundles of connective tissue, and (2) plain muscular and elastic fibres, disposed for the most part longitudinally, lined with endothelium.

Lymphatic vessels are furnished with bloodvessels and nerves. Most of them are also provided with valves, similar to those of the veins. Each valve consists of two semilunar segments, facing one another, which are foldings of the inner coat, containing connective and elastic tissues. They project slightly towards each other, and their free edges are directed in the course of the lymph-stream. The valves are situated at short intervals, and they serve to prevent reflux of lymph. When a lymphatic vessel becomes distended it 
presents a beaded appearance, with constrictions between the projections, these constrictions corresponding to the attached margins of the valvular segments.

Development.-According to Sabin the lymphatic vessels are developed from the endothelial lining of the primitive lymph-sacs (see Development of Lymphatic System).

\section{Structure of Lymphatic Glands.}

A lymphatic gland consists of a capsule, which encloses the glandular substance. The capsule is composed of connective tissue, containing elastic fibres.

The glandular substance presents two parts-superficial or cortical, and central or medullary. Each of these parts is permeated by a supporting framework of trabeculæ derived from the capsule. These trabeculæ are composed of connective tissue, with a few plain muscular fibres. They subdivide the cortex into follicles, between which they form incomplete septa. In the medulla the trabeculæe are arranged in a reticular manner.

The glandular substance is formed by lymphoid or adenoid tissue, which consists of retiform tissue, with lymphocytes in its meshes. In the cortex of the gland this lymphoid tissue is disposed as lymph-follicles, and in the medulla it forms lymph-cords. In both parts of the gland there are spaces between the glandular substance and the supporting trabecular framework. These spaces, which are for the passage of lymph, constitute the lymph-sinus. This sinus is broken up at all parts of the gland by retiform tissue, the meshes of which are partially lined with branched cells of the nature of phagocytes. The glandular substance, except the lymph-sinus, are permeated by bloodcapillaries.

Lymphatic glands are furnished with bloodvessels, nerves, and lymphatic vessels. The arterioles pass to the glandular substance, being at first ensheathed by the supporting trabeculæ of connective tissue, and subsequently by the retiform tissue of the glandular substance. The nerves are destined for the plain muscular tissue of the trabeculae and bloodvessels.

The lymphatic vessels are of two kinds-afferent and efferent. The afferent vessels enter the gland over its surface, whilst the efferent vessels emerge at a definite part of the gland, where there is a slight depression, called the hilum. The afferent vessels convey lymph to the cortical part of the lymph-sinus, whilst the efferent vessels convey lymph from the medullary part of that sinus. Inasmuch as the medullary part of the gland extends quite to the surface at the place where the depression, known as the hilum, exists, the efferent vessels emerge from the gland through this hilum, which also gives passage to the arteries and veins.

Development.-Lymphatic glands are developed partly from the primitive lymph-sacs, and partly from peripheral lymphatic vessels (see Development of Lymphatic System).

Trachea in the Thorax.-The thoracic portion of the trachea extends from the level of the upper border of the manubrium sterni to the level of the intervertebral disc between the bodies of the fourth and fifth thoracic vertebræ, where it divides into the two bronchi, right and left. It occupies a median position in the superior mediastinum, and its length is about $2 \frac{1}{2}$ inches. As in the neck, it is cylindrical and firm in front and at the sides, but posteriorly it is flattened and membranous, where it rests upon the œsophagus.

Relations-Anterior.-The manubrium sterni, and the origins of the sterno-hyoid and sterno-thyroid muscles; the remains of the thymus body; the origins of the innominate and left common carotid arteries, and the left innominate vein; and the arch of the aorta, which lies upon it immediately above its bifurcation into the 
two bronchi, with the intervention of the deep cardiac plexus of nerves. Posterior.-The œesophagus, which inclines partially to the left of the trachea. Right.-The right pneumogastric nerve; the innominate artery, after it has left the front of the trachea; and the right pleural sac. Left.-The arch of the aorta and the left common carotid artery, after these have left the front of the trachea ; the left subclavian artery ; and the left recurrent laryngeal nerve.

Bronchi.- The bronchi, right and left, commence at the bifurcation of the trachea, and each passes to the hilum of the corresponding lung, where its ramifications commence. As in the trachea, each bronchus is cylindrical and firm in front and at the sides, but posteriorly it is flattened and membranous. There being certain differences between the bronchi, a separate description is required for each.

The right bronchus is about $x$ inch in iength up to the point where it gives off its first, or eparterial, branch. It has about six cartilaginous rings, and is larger than the left bronchus. It is more vertical than the left bronchus, and therefore coincides with the direction of the trachea to a greater extent than its fellow.

Relations-Superior.-The right azygos vein arches over it to open into the superior vena cava. Posterior.-The right pneumogastric nerve. Inferior.--The right pulmonary artery, which subsequently lies anterior to it.

About I inch from its commencement the right bronchus gives off a branch from its outer side which passes to the upper lobe of the right lung. This branch is known as the eparterial bronchus, because it arises above the right pulmonary artery. The rest of the right bronchus is spoken of as being hyparterial.

The left bronchus is about 2 inches in length. It has about twelve cartilaginous rings, and is smaller than the right bronchus on account of the smaller size of the left lung. Its course is more oblique than that of its fellow, and it is directed downwards and outwards beneath the arch of the aorta to the hilum of the left lung.

Relations-Anterior.-The left pulmonary artery, which subsequently lies above it. Posterior.-The cesophagus and the descending thoracic aorta.

The left bronchus has no eparterial branch.

When the interior of the trachea is viewed from above, as in using the laryngoscope, the openings of the two bronchi are seen to be separated by a ridge which is situated to the left of the median line, and more of the interior of the right bronchus than of the left is visible. Partly on this account, and partly by reason of the more vertical direction of the right bronchus, as well as its larger size, a foreign body getting into the trachea is more apt to descend into the right bronchus than into the left. 
The structure of the trachea will be described in connection with the description of the windpipe in the neck.

Esophagus in the Thorax. - The œsophagus or gullet extends from the pharynx to the stomach. In the first part of its course it lies in the neck, and this portion will be found described in connection with that region. The thoracic part (see Fig. 422) extends from the level of the upper border of the manubrium sterni to the level of the body of the twelfth thoracic vertebra, where it passes through

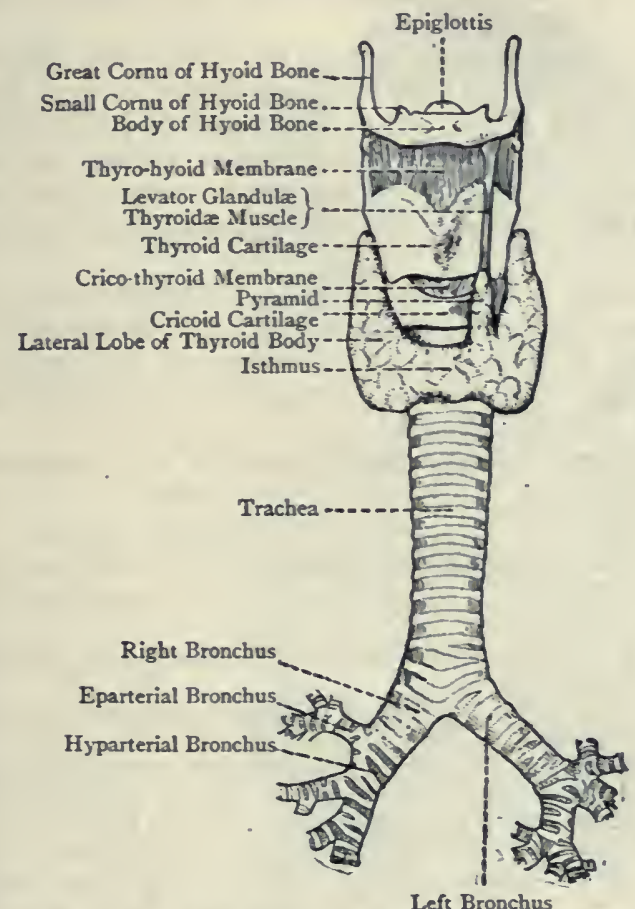

Fig. 420.-The Hyotd Bone, Larynx, Trachea, Broncht, aNd THYROID BODY (ANTERIOR VIEW).

the œesophageal opening of the diaphragm, and terminates at the cardiac orifice of the stomach. Its course is not quite vertical. At its commencement the thoracic portion lies partially to the left of the middle line, but as it descends it comes to occupy a median position about the level of the fifth thoracic vertebra, and inferiorly it again inclines partially to the left. It occupies the superior and posterior mediastinal spaces, and lies in front of the vertebral column, accurately following the thoracic antero-postei ior curve.

Relations-Anterior. - The trachea as low as the intervertebral disc between the bodies of the fourth and fifth thoracic vertebræ; 
the root of the left bronchus; the posterior wall of the pericardium, opposite the back of the left auricle of the heart; and the vertebral portion of the diaphragm. Posterior. -The vertebral column; the longus colli muscles, especially the left; the thoracic duct and right azygos vein; the right aortic intercostal arteries; the upper and lower transverse azygos veins; and, inferiorly, the descending aorta. Right.-The right posterior mediastinal pleura. Left.The thoracic portion of the left subclavian artery; the upper part of the thoracic duct; the left posterior mediastinal pleura; and the descending aorta, except inferiorly.

The right and left pneumogastric nerves are intimately related to the œsophagus. They are at first disposed laterally, and their branches give rise to the plexus gula. Subsequently the right pneumogastric nerve descends on the posterior surface of the œsophagus, and the left on the anterior surface, in which positions they accompany the gullet through the œsophageal opening of the diaphragm.

Blood-supply-Arteries.-These are (I) the œesophageal branches of the descending aorta, (2) the osophageal branches of the gastric artery from the coliac axis, and (3) twigs from the left inferior phrenic artery. In the neck the osophagus receives branches from the right and left inferior thyroid arteries.

The veins accompany the corresponding arteries, and terminate in the right azygos vein and the two left azygos veins.

Lymphatics. - These pass to the posterior mediastinal glands.

Nerves.-These are derived from the pneumogastric and sympathetic nerves.

Structure.-The wall of the nesophagus consists of three coats, namely, muscular, submucous, and mucous.

The muscular coat is thick, and is disposed in two layers, an external longitudinal, and an internal circular.

The longitudinal muscular fibres are attached superiorly to the upper part of the median ridge on the posterior surface of the cricoid cartilage, and from this point they descend as two flattened bands, one on either side of the tube. These expand and soon meet, giving rise to one continuous layer which completely surrounds the gullet. Inferiorly they are continuous with the longitudinal muscular fibres of the stomach. Accessory fleshy slips are described as passing from this layer to the back of the trachea, the back of the root of the left bronchus, the pericardium, and the left pleura.

The circular muscular fibres are continuous superiorly with the lower fibres of the inferior constrictor muscles of the pharynx, and inferiorly with the circular, and also with the oblique, muscular fibres of the stomach.

The muscular tissue of the osophagus is of the striated variety over about the upper third of the tube, but elsewhere it is of the plain or non-striated variety.

The submucous coat consists of loose areolar tissue, which contains the larger bloodvessels and the mucous glands.

The mucous coat is thick, and is thrown into longitudinal folds, on account of the loose disposition of the submucous coat. The portion of it next the submucous coat consists of plain muscular fibres, which are arranged longitudinally. This portion is known as the muscularis mucosa, and it is best marked in the lower part of the gullet. The inner surface of the mucous 
coat is provided with numerous papillæ, and it is covered by stratified squamous epithelium, which is thrown into elevations by the papillx.

The mucous glands are racemose, and are situated in the submucous coat. Their ducts are large and long, and on their way to the free surface some of them traverse small collections of lymphoid tissue.

Development.- The œsophagus is developed from that part of the fore-gut which succeeds to the portion from which the pharynx is developed. At first it is very short, on account of the imperfect development of the neck. As, however, the neck becomes formed, and as the stomach descends, the osophagus becomes elongated.

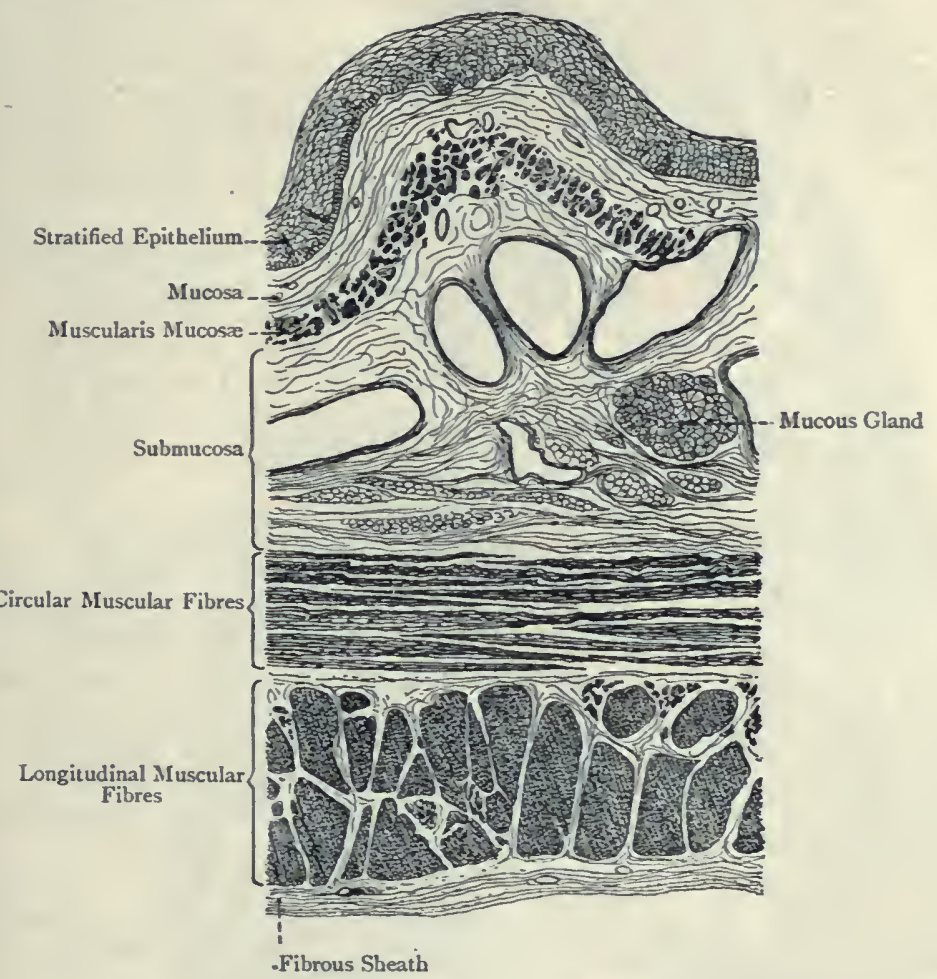

Fig. 421.-Transverse Section of the Wall of the CEsophagus.

Descending Aorta.-The descending aorta, which is the con: tinuation of the aortic arch, commences on the left side of the body of the fourth thoracic vertebra on a level with its lower border, and it terminates at the lower border of the body of the twelfth thoracic vertebra. At the latter level it passes through the aortic opening of the diaphragm, and enters upon the abdominal part of its course. The vessel lies in the posterior mediastinum, and its course is downwards and inwards, so that, though at first on the 
left side of the vertebral column, it subsequently takes up a position in front of it.

Relations-Anterior.-From above downwards, the root of the left lung; the pericardium; the œsophagus; and the vertebral por-

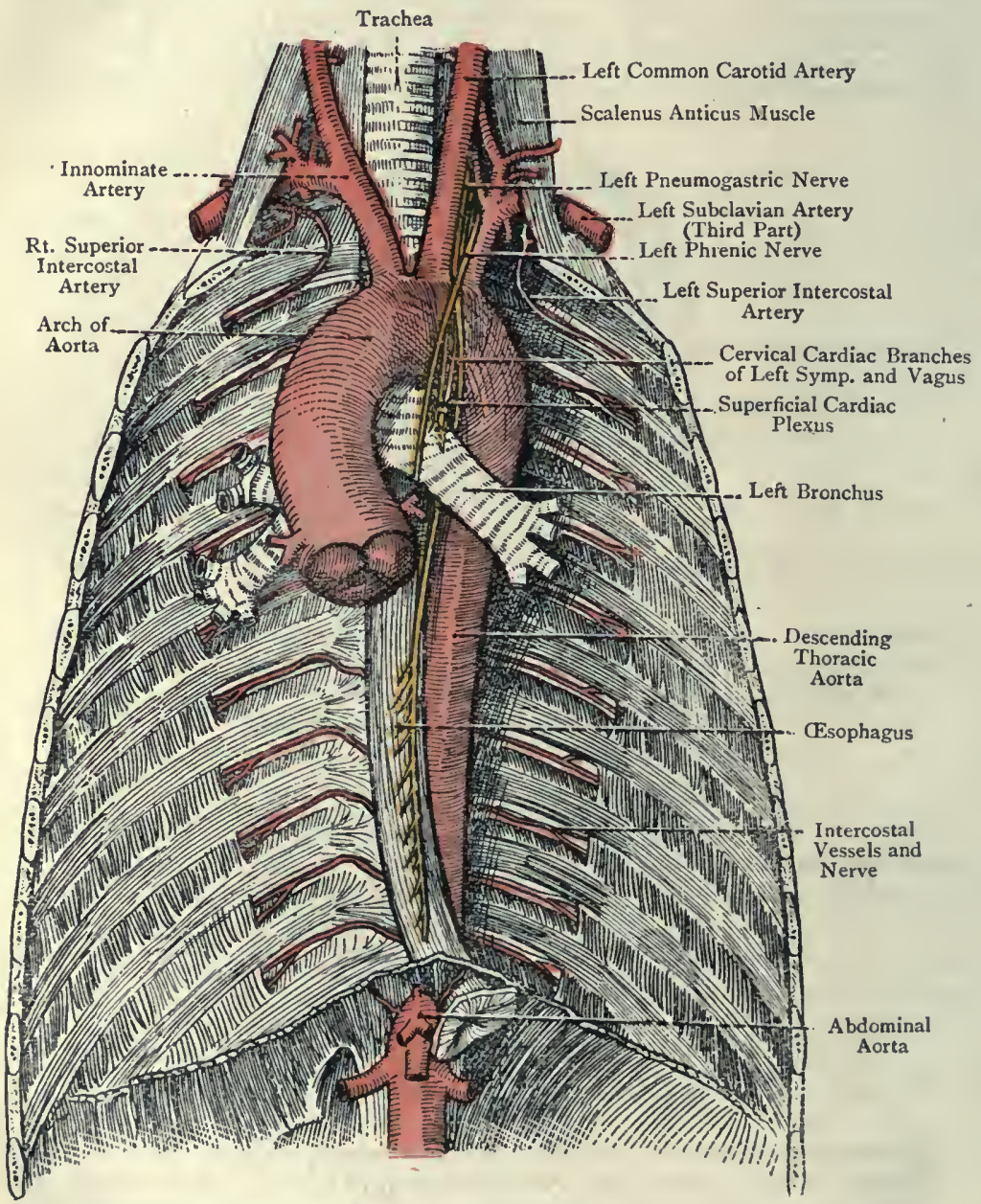

Fig. 422.-Dissection of the Posterior Wall of the Thorax.

tion of the diaphragm. Posterior. - The bodies and intervertebral discs of thoracic vertebra below the fourth; and the upper and lower transverse azygos veins. Right. - The osophagus, superiorly ; the thoracic duct; and the right az;gos vein. Left.-The left pos- 
terior mediastinal pleura; and the upper and lower left azygos veins. Inferiorly the œesophagus inclines slightly to the left of the vessel. It is to be noted that the cesophagus has a threefold relation to the descending aorta. At first it lies upon the right side of the vessel; then directly in front of it; and finally it inclines slightly to its left side.

Branches.-These are as follows: bronchial, pericardial, œsophageal, posterior mediastinal, intercostal, and subcostal.

The bronchial arteries supply the lungs and the bronchial lymphatic glands. They are usually three in number-one right and two left. The right bronchial artery arises from the aorta in common with the upper left bronchial artery, or sometimes from the first right aortic intercostal artery. The two left bronchial arteries, upper and lower, arise directly from the aorta, near each other. The arteries enter the respective lungs behind the bronchi, and in their further course and distribution they follow the ramifications of the bronchial tubes.

The bronchial veins accompany the corresponding arteries. The right vein opens into the right azygos vein; and the left vein opens either into the upper left azygos vein, or into the left superior intercostal vein.

The pericardial branches supply the posterior part of the pericardium.

The oesophageal branches are numerous, and arise at irregular intervals. Superiorly they spring from the right side of the aorta, and inferiorly from its anterior wall. They anastomose freely with one another along the wall of the œsophagus; superiorly with the œsophageal branches of the inferior thyroid arteries; and inferiorly with the œsophageal branches of the gastric artery from the cœliac axis, the latter branches entering the thorax through the œsophageal opening of the diaphragm.

The posterior mediastinal branches are very minute, and supply the lymphatic glands and areolar tissue in the posterior mediastinum.

The intercostal arteries are arranged in pairs, and are nine in number on each side. They are destined for the lower nine intercostal spaces, the first two spaces being supplied by the superior intercostal artery, which is a branch of the second part of the subclavian on the right side, and of the first part on the left side. They arise in pairs from the posterior wall of the descending aorta, and they pass outwards upon the bodies of the vertebræ to the posterior extremities of the intercostal spaces. The arteries of the right side pass behind the osophagus, the thoracic duct, and the right azygos vein ; and those of the left side pass behind the upper and lower left azygos veins, according to their level. The arteries of both sides pass behind the gangliated cord of the sympathetic. The upper right intercostal arteries are longer than those of the left side, on account of the position of the descending aorta on the left side of the vertebral column. All the arteries lie behind the 
parietal pleura. Each artery, on entering an intercostal space, lies at first between the parietal pleura and the posterior intercostal aponeurosis. Its course is outwards and slightly upwards, and partly on this latter account, but chiefly on account of the downward slope of the rib, it soon gains the lower border of the upper rib. At a point corresponding to the angle of the rib the artery pierces the posterior intercostal aponeurosis, and, passing between the external and internal intercostal muscles, it enters the subcostal groove of the upper rib. In this position it courses forwards, and ends by anastomosing with the upper anterior intercostal branch of the internal mammary artery, or of its musculo-phrenic branch, according to the level.

The companion intercostal vein lies above the artery, and the corresponding intercostal nerve lies below it. The order of structures, therefore, in the subcostal groove, from above downwards, is as follows : intercostal vein, intercostal artery, and intercostal nerve. The upper seven aortic intercostal arteries are confined to the intercostal spaces which they occupy; but the lower two, namely, those in the tenth and eleventh intercostal spaces, ultimately leave these spaces, and pass into the abdominal wall, where they have been described in connection with the abdomen.

Branches. - These are dorsal, giving off a spinal branch; collateral ; and lateral cutaneous.

The dorsal branch arises from the aortic intercostal artery as that vessel enters the posterior extremity of an intercostal space. It passes backwards, in company with the posterior primary division of the corresponding spinal nerve, between the adjacent transverse processes, where it lies internal to the superior costo-transverse ligament. Opposite the intervertebral foramen it gives off its spinal branch, which enters the spinal canal through the foramen to be distributed to its osseous and ligamentous walls, as well as to the spinal cord and its membranes. The dorsal branch, continuing its course backwards, divides into an internal and external branch, which supply the muscles and integument of the back.

The collateral intercostal artery arises from the main aortic intercostal opposite the angle of the rib. It passes obliquely downwards and outwards to the upper border of the lower rib, along which it courses, lying between the external and internal intercostal muscles. Anteriorly it ends by anastomosing with the lower anterior intercostal branch of the internal mammary artery, or of its musculophrenic branch, according to the level.

The lateral cutaneous branches accompany the lateral cutaneous branches of the corresponding intercostal nerves to the integument.

The first aortic intercostal artery, namely, that which lies in the third intercostal space, furnishes a branch, of variable size, which ascends over the neck of the third rib to the second intercostal space. This branch anastomoses with the second intercostal 
artery, which is a branch of the superior intercostal, and may even replace it.

The subcostal arteries, right and left, are the last branches of the descending thoracic aorta. They are serially continuous with the aortic intercostal arteries above, and with the lumbar arteries below. Each vessel winds round the side of the body of the twelfth thoracic vertebra, and, passing beneath the external arcuate ligament of the diaphragm, it enters the wall of the abdomen, where it lies along the lower border of the twelfth rib. These vessels will be found described in connection with the abdomen (see p. 773).

The first and second intercostal spaces receive their arteries from the superior intercostal artery, which is a branch of the second part of the subclavian on the right side, and of the first part on the left side. Having descended in front of the neck of the first rib to the posterior extremity of the first intercostal space, the vessel furnishes the first intercostal artery to that space, and it also gives off the second intercostal artery, which descends in front of the neck of the second rib to the second intercostal space: As stated, the second intercostal artery receives a branch from the third intercostal artery, which ascends over the neck of the third rib, the third intercostal being the first aortic intercostal.

Development of the Descending Aorta.-The upper portion of the descending aorta is developed from that part of the left primitive dorsal aorta which lies between the fourth left arterial arch and the place of junction of the two primitive dorsal aortæ. The greater portion of it, however, restilts from the union of the two primitive dorsal aortx. The intercostal arterles are developed from thoracic segmental arteries.

Intercostal Veins.-The intercostal veins are eleven in number on either side, and each lies in the subcostal groove above the corresponding aortic intercostal artery. In the region of the angle of the rib each vein receives the collateral intercostal vein, which accompanies the artery of that name. At the posterior extremity of an intercostal space each. vein receives a large dorsal branch, which returns blood from the muscles and integument of the back, the dorsal spinal venous plexus, and the spinal canal. With the exception of the upper three or four veins, all the other intercostal veins pass inwards, behind the corresponding sympathetic cord, on to the bodies of the thoracic vertebræ, from which they receive small twigs. Their mode of termination differs on the two sides. On the right side the veins, having passed behind the œsophagus, terminate in the right azygos vein. On the left side the lower four veins, namely, the eighth, ninth, tenth, and eleventh, open into the lower left azygos vein; and the succeeding three (or four), namely, the fifth, sixth, and seventh (and, it may be, the fourth also), open into the upper left azygos vein.

The first intercostal vein of each side accompanies the corresponding superior intercostal artery, and terminates in the innominate vein, or, it may be, in the vertebral vein, of its own side.

The second and third intercostal veins (and, it may be, the fourth) 
of each side unite to form the superior intercostal vein. The right superior intercostal vein, after a downward course, joins the upper part of the right azygos vein. The left superior intercostal vein ascends over the back part of the arch of the aorta, and opens into the left innominate vein. It sometimes receives the left bronchial vein as a tributary.

Summary of the Intercostal Veins-Right Intercostal Veins.-The first opens into the right innominate vein, or sometimes into the right vertebral vein. The second and third (and, it may be, the fourth also) unite to form the right superior intercostal vein, which opens into the right azygos vein. The lower eight (sometimes the lower seven) are direct tributaries of the right azygos vein.

Left Intercostal Veins.-The first opens into the left innominate vein, or sometimes into the left vertebral vein. The second and third (and it may be the fourth also) unite to form the left superior intercostal vein, which opens into the lfft innominate vein. The fifth, sixth, and seventh (and, it may be, the fourth also) terminate in the upper left azygos vein. The eighth, ninth, tenth, and eleventh are tributaries of the lower left azgyos vein.

The left superior intercostal vein is developed from two sources. The upper part is formed by the portion of the left primitive jugular vein below, and adjacent to, the commencement of the transverse jugular vein. The lower part is formed by the upper portion of the left cardinal vein.

Intercostal Glands. - These glands form a chain on either side of the vertebral column, in line with the heads of the ribs. The main glands of each chain lie in the posterior parts of the intercostal spaces, and one or two glands accompany each posterior intercostal artery for a very short distance.

The intercostal glands receive their afferent vessels from (1) the posterior half of the costal pleura, (2) the posterior halves of the external and internal intercostal muscles, (3) the deep muscles of the back, and (4) the spinal canal. Their efferent vessels, on either side, pass to the thoracic duct.

The efferents of the upper right intercostal glands sometimes open into a right broncho-mediastinal lymphatic trunk, which terminates in the right lymphatic duct.

The right superior intercostal vein is developed from the anastomotic channels which connect the upper three thoracic segmental veins of the right side. left.

Azygos Veins.-These are three in number, one right and two

The vena azygos major or right azygos vein commences in the abdomen as the right ascending lumbar vein, and enters the thorax through the aortic opening of the diaphragm, lying on the right side of the aorta, the thoracic duct intervening. It then ascends, under cover of the osophagus, upon the bodies of the thoracic vertebræ, crossing in its course the right aortic intercostal arteries. Having reached the level of the fifth thoracic vertebra it leaves the vertebral column, and, arching forwards over the right bronchus, it opens into the superior vena cava, just before that vessel pierces the pericardium. In the thorax the right azygos vein continues to lie on the right side of the aorta, the thoracic duct intervening.

Tributaries.-These are as follows :

I. The right subcostal vein.

2. The lower seven (sometimes the lower eight) right intercostal veins. 
3. The right superior intercostal vein.

4. The right bronchial vein.

5. Some cesophageal veins.

6. Some pericardial veins.

7. The lower and upper transverse azygos veins.

Right Lymphatic Duct

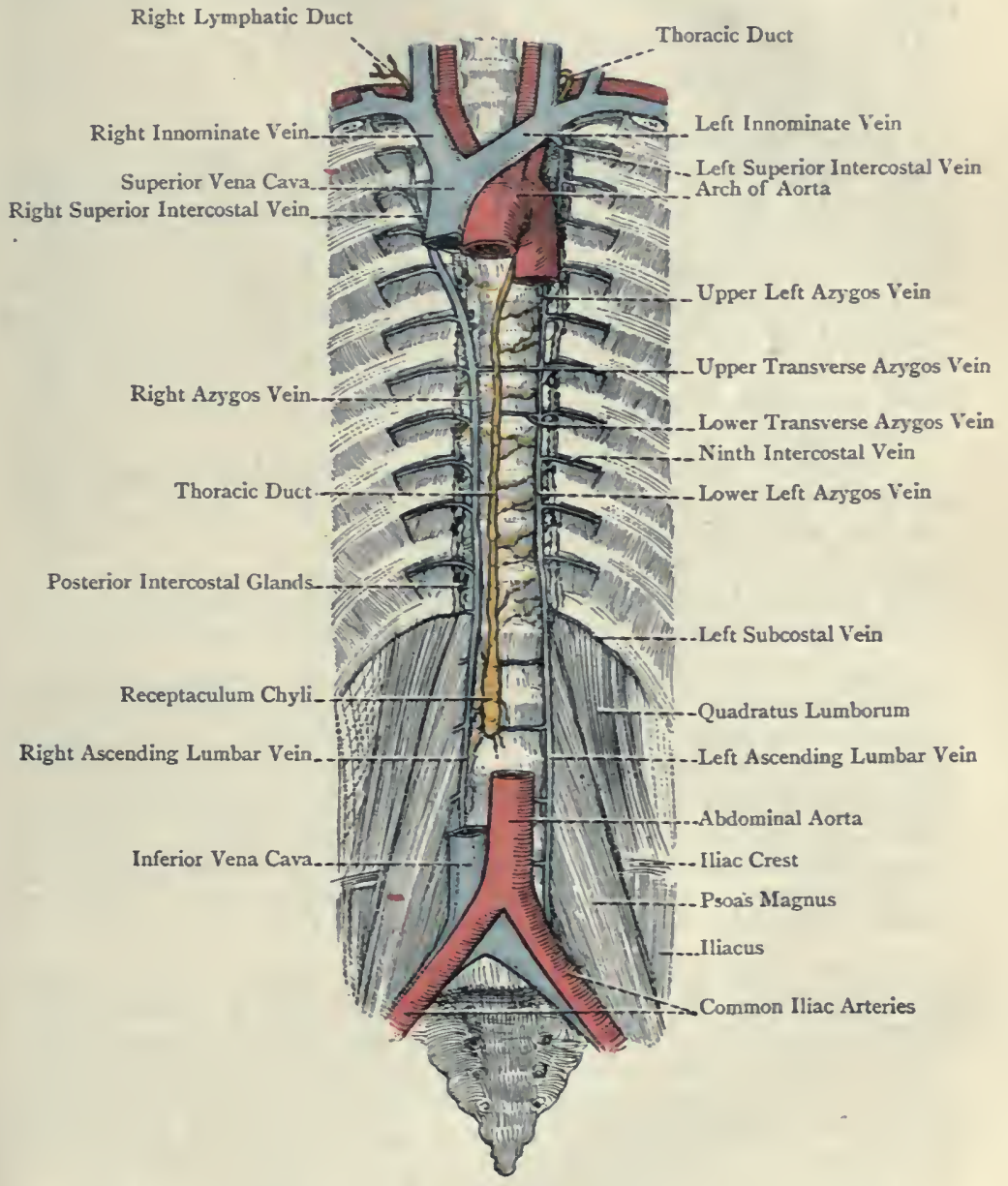

Fig. 423,-The Thoracic Dect, Azygos Veins, and Posterior INTERcostal Glands.

The vena azygos minor or lower left azygos vein commences in the abdomen as the left ascending lumbar vein, and enters the thorax by piercing the left crus of the diaphragm. It ascends upon the vertebral column to the level of the eighth thoracic ver- 
tebra, lying in front of the lower aortic intercostal arteries, and it takes up the lower four left intercostal veins. It then crosses the vertebral column from left to right, under the name of the lower transverse azygos vein, passing behind the descending aorta and thoracic duct, and thereafter it opens into the right azygos vein.

Tributaries.-These are as follows :

I. The left subcostal vein.

2. The lower four left intercostal veins.

3. Some œsophageal veins.

The upper left, or accessory, azygos vein is formed by the union of the fifth, sixth, and seventh left intercostal veins (sometimes also the fourth). At the level of the seventh thoracic vertebra it crosses the vertebral column from left to right, under the name of the upper transverse azygos vein, passing behind the descending aorta and thoracic duct, and thereafter it opens into the right azygos vein. It communicates above with the left superior intercostal vein, and below with the lower left azygos vein.

Tributaries.-These are as follows :

I. The fifth, sixth, and seventh left intercostal veins (sometimes also the fourth).

2. The left bronchial vein, as a rule.

3. Some œsophageal veins.

The lower and upper left azygos veins, which are subject to much variation, sometimes unite to form one transverse azygos vein.

Summary of the Azygos Veins-Right Azygos Vein.-This vessel receives (1) the right subcostal vein; (2) the lower seven (sometimes the lower eight) right intercostal veins; (3) the right superior intercostal vein; (4) the right bronchial vein; (5) some osophageal veins; $(6)$ some pericardial veins; and (7) the lower and upper transverse azygos veins.

Lower Left Azygos Vein.-This vessel receives (1) the left subcostal vein; (2) the lower four left intercostal veins; and (3) some œsophageal veins.

Upper Left Azygos Vein.-This vessel receives (1) the fifth, sixth, and seventh (sometimes also the fourth) left intercostal veins; (2) the left bronchial vein, as a rule; and (3) some œsophageal veins.

The right azygos vein and the lower left azygos vein, through their connections with the ascending lumbar veins, establish communications with the inferior vena cava and with the common iliac veins or some of their tributaries. They therefore form important channcls by which a considerable quantity of blood is returned from the lower limbs and abdominal wall in cases of obstruction of the inferior vena cava.

Development. - The right azygos vein is developed from the thoracic portion of the right cardinal vein. The lower and upper left azygos veins are developed from the thoracic portion of the left cardinal vein.

Subcostal Veins.-These are two in number, right and left, and they are serially continuous with the intercostal veins. Each vessel enters the thorax from the abdomen by passing behind the 
external arcuate ligament of the diaphragm. As stated, the right vein opens into the right azygos vein, and the left into the lower left azygos vein.

\section{Anterior Primary Divisions of the Thoracic Spinal Nerves.-These} are twelve in number on each side. The first eleven enter intercostal spaces, and are called the intercostal nerves. The last, which belongs to the abdomen, lies along the lower border of the twelfth rib, and is called the subcostal nerve ("last dorsal nerve '). At the posterior extremities of the intercostal spaces the intercostal nerves are near the gangliated cord of the sympathetic, and each nerve is connected with the adjacent sympathetic ganglion by two rami communicantes, which are necessarily very short. One of these rami, being composed of spinal fibres, is white, and the other, which consists of sympathetic fibres, is grey. Each intercostal nerve lies below the corresponding artery, and, like it, lies between the parietal pleura and the posterior intercostal aponeurosis, as far as the angle of the rib. Its subsequent course corresponds to that of the artery. The first intercostal nerve is of small size, because the greater part of the anterior primary division of the first thoracic nerve takes part in the formation of the brachial plexus. The second intercostal nerve sometimes gives off a small branch which ascends to join the portion of the anterior primary division of the first thoracic nerve which takes part in the brachial plexus. The further course and distribution of the intercostal nerves belong to the thoracic and abdominal walls, in connection with which they will be found described.

Thoracic Duct.-The thoracic duct commences in the abdomen in a dilatation, called the receptaculum chyli, which is situated in front of the bodies of the first and second lumbar vertebræ, and it terminates by opening into the angle of junction of the internal jugular and subclavian veins of the left side. It is about I 8 inches in length, and enters the thorax from the abdomen by passing through the aortic opening of the diaphragm, where it lies between the aorta on the left and the right azygos vein on the right. In this position it ascends in the thorax, resting upon the vertebral column, the right aortic intercostal arteries, and the lower and upper transverse azygos veins, being under cover of the œsophagus. Up to the level of the fourth or fifth thoracic vertebra it occupies the middle line. At this level it passes behind the arch of the aorta, inclining to the left of the.middle line. Thereafter it ascends in close contact with the left side of the œesophagus, and behind the thoracic portion of the left subclavian artery. In this position it enters the root of the neck on the left side, where it ascends upon the left side of the osophagus, between the left common carotid and left subclavian arteries. At about the level of the seventh cervical vertebra it describes a curve, and passes outwards, forwards, and downwards, in contact with the dome of the left pleura. Thereafter it inclines inwards, and terminates by opening into the angle of junction between the internal jugular and subclavian veins 
of the left side. In the lower part of the thorax the thoracic duct is of smaller calibre than in the upper part. Its course is somewhat undulating, and when distended it presents a beaded appearance, especially in the upper part, due to the number of valves with which it is provided. Sometimes the duct divides into two branches in the lower part of the thorax, which reunite at a higher level. The duct is freely provided with valves, especially in its upper part, and at its termination there is an important valve, consisting of two segments, which are so directed as to prevent effectually the reflux of chyle, or the flow of blood, into the duct.

The thoracic duct receives lymphatic vessels from the following sources: (I) the lower limbs; (2) the abdomen and its viscera, except some of the lymphatics from part of the upper surface of the liver; (3) the left half of the thoracic wall ; (4) the left lung and the left half of the heart; (5) the left upper limb; and (6) the left side of the head and neck. Most of the lymphatics of the right ahalf of the thorax and those of the right lung and right half of the heart see the section dealing with the neck.

Thoracic Lymphatic a flands.-These are arranged - in several groups, as follows : anterior intercostal or sternal, posterior intercostal, superior mediastinal, anterior ... mediastinal, posterior mediastinal, bronchial, and caval.

The anterior intercostal or sternal glands wili be found described on p. 933. They receive their afferent vessels frond (I) the anterior parts of the upper six intercostal spaces; $(2)$ the ininer portion of the mammary gland ; $(3)$ the lymphatics accompanying the superior epigastric artery from the upper part of the anterior abdominal wall; and (4) the lymphatics accompanying the musculo-phrenic artery from the anterior parts of the seventh, eighth, and ninth intercostal spaces, and from a portion of the diaphragm. The efferent vessels of the right glands terminate in the right lymphatic duct, and those of the left glands in the thoracic duct.

The posterior intercostal glands are situated on either side of the vertebral column, where they lie in the intercostal spaces, there being from one to three in each space. They receive their afferen' vessels from (I) the posterior parts of the intercostal spaces; (2) the parietal pleura; (3) the spinal canal; and (4) the deep muscles of the back. The efferent vessels of the left intercostal glands open into the thoracic duct. On the right side the efferent vessels from the lower glands pass to the thoracic duct, but those from the upper glands open into the right lymphatic duct.

The superior mediastinal glands lie in the superior mediastinum in relation to the arch of the aorta and the innominate veins, and they are continuous with the posterior mediastinal and bronchial glands. They receive their afferent vessels from the pericardium, the heart, and the thymus body in early life, and their efferent vessels pass to the thoracic duct and right lymphatic duct.

The anterior mediastinal glands lie in the lower part of the anterior 
mediastinum, in front of the pericardium. They receive their afferent vessels from (I) the mesial portions of the right and left lobes of the liver in the vicinity of the falciform ligament; (2) the adjacent portion of the diaphragm; (3) the anterior part of the pericardium; and (4) the lower anterior intercostal or sternal glands. Their efferent vessels pass to the thoracic duct and right lymphatic duct.

The posterior mediastinal glands are situated in the posterior mediastinum, along the course of the descending aorta and œesophagus. They receive their afferent vessels from the œsophagus, the posterior part of the pericardium, and the vertebral portion of the diaphragm. Their efferent vessels pass, for the most part, to the thoracic duct.

The bronchial glands are very numerous, and are situated partly between the two bronchi, where these spring from the trachea, and partly at the root of each lung. They are very dark in colour, and receive their afferent vessels from the lungs and the visceral pleuræ. Their efferent vessels pass to the thoracic duct and right lymphatic duct.

The caval glands are situated in contact with the limited thoracic portion of the inferior vena cava. They receive their afferent vessels from the uncovered area of the posterior surface of the liver, and also those deep lymphatics of that organ which accompany the hepatic veins to the caval fossa. Their efferent vessels pass to the thoracic duct.

Gangliated Cord of the Sympathetic.-The sympathetic system in the thorax consists of (I) two gangliated cords, right and left, and (2) a prevertebral plexus, namely, the cardiac plexus. The latter plexus has been already described. The gangliated cord lies on each side of the vertebral column behind the parietal pleura, and superficial to the intercostal vessels. It presents, as a rule, twelve ganglia, but the first is frequently united with the inferior cervical ganglion, in which cases the number is reduced to eleven. The upper ten ganglia are placed upon the heads of the corresponding ribs, but the lower two lie upon the sides of the bodies of the eleventh and twelfth thoracic vertebræ. The first thoracic ganglion is the largest of the thoracic series, and is known as the ganglion stellatum. The sympathetic cord leaves the thorax by passing behind the inner part of the internal arcuate ligament of the diaphragm, and so enters the abdomen.

Branches-I. Of Communication.-These are called the rami communicantes. Two of these, one white and the other grey, pass between each ganglion and the adjacent intercostal nerve. The white fibres are of spinal, and the grey of sympathetic, origin. From the proximity of the ganglia to the intercostal nerves the rami communicantes are characterized by their shortness.

2. Of Distribution.-From the upper five ganglia small vascular branches are given off, which are distributed to the coats of the thoracic aorta. From the second, third, and fourth ganglia pul- 
monary branches are given off to the posterior pulmonary plexus. From the fifth ganglion downwards the three splanchnic ('visceral ') nerves-great, small, and least-are given off.

The great splanchnic nerve arises by five separate roots from the fifth, sixth, seventh, eighth, and ninth ganglia, the fibres of the

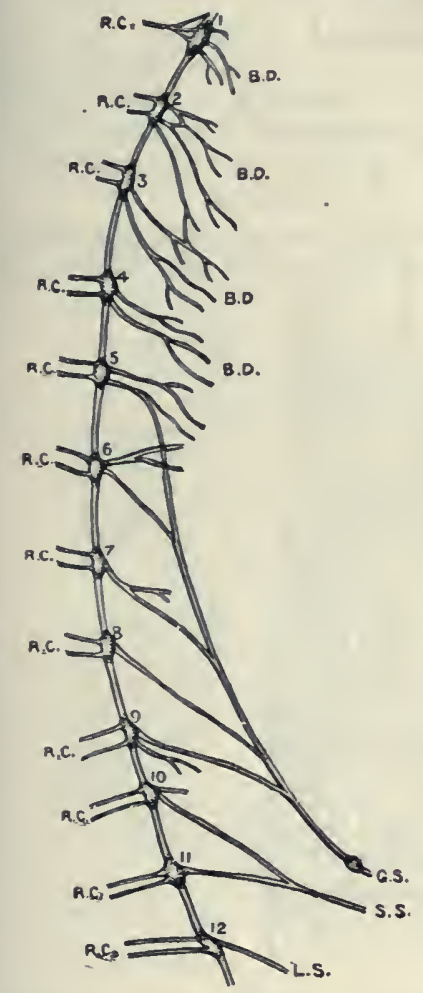

Fig. 425.-Schene of the Gang. LIATED CORD OF THE SYMPATHETIC IN THE THORAX (AFTER FLOWER).

I to 12. Thoracic Ganglia

R.C. Rami Communicantes

B.D. Branches of Distribution from Upper Ganglia

G.S. Great Splanchnic Nerve and

S.S. Small Splanchnic Nerve

L.S. Least Splanchnic Nerve upper roots being traceable in the sympathetic cord as high as the second ganglion. The roots arch obliquely forwards and downwards upon the sides of the bodies of the adjacent vertebræ, and by their union they form a large nerve, which pierces the crus of the diaphragm and terminates in the semilunar ganglion of the epigastric or solar plexus. The great splanchnic nerve contains a large number of spinal fibres, which impart to it a white colour and firm consistence. The right nerve presents a small ganglion just before it leaves the thorax, called the spianchnic ganglion, and there may be one on the left nerve. The great splanchnic nerve gives vascular branches to the lower part of the thoracic aorta.

The small splanchnic nerve arises by two roots from the tenth and eleventh ganglia. It pierces the crus of the diaphragm, and terminates in the aortico-renal ganglion of the epigastric or solar plexus.

The least splanchnic nerve, which is sometimes absent, arises by one root from the twelfth ganglion. It either passes behind the internal arcuate ligament of the diaphragm or through the crus, and it terminates in the renal plexus. When the least splanchnic nerve is absent, its place may be taken by a branch from the small splanchnic nerve.

Subcostal Muscles. - These are small indefinite muscular sheets, which lie on the inner surfaces of the ribs in the region of the angles. They coincide in direction with the internal intercostal muscles, and are best dereloped inferiorly. They are attached to the inner surfaces of the ribs 
near the angles, and each muscle crosses over one, or it may be two, intercostal spaces.

They are supplied by the intercostal nerves.

The diaphragm will be found described in connection with the abdomen.

\section{The Articulations of the Vertebral Column.}

I. Articulations of the Bodies of the Vertebræ.-These joints belong to the class amphiarthrosis and subdivision symphysis. The ligaments are as follows: the anterior common ligament, the posterior common ligament, and the intervertebral discs.

The anterior common ligament is a dense band of white glistening fibres, which extends over the anterior surfaces of the bodies of

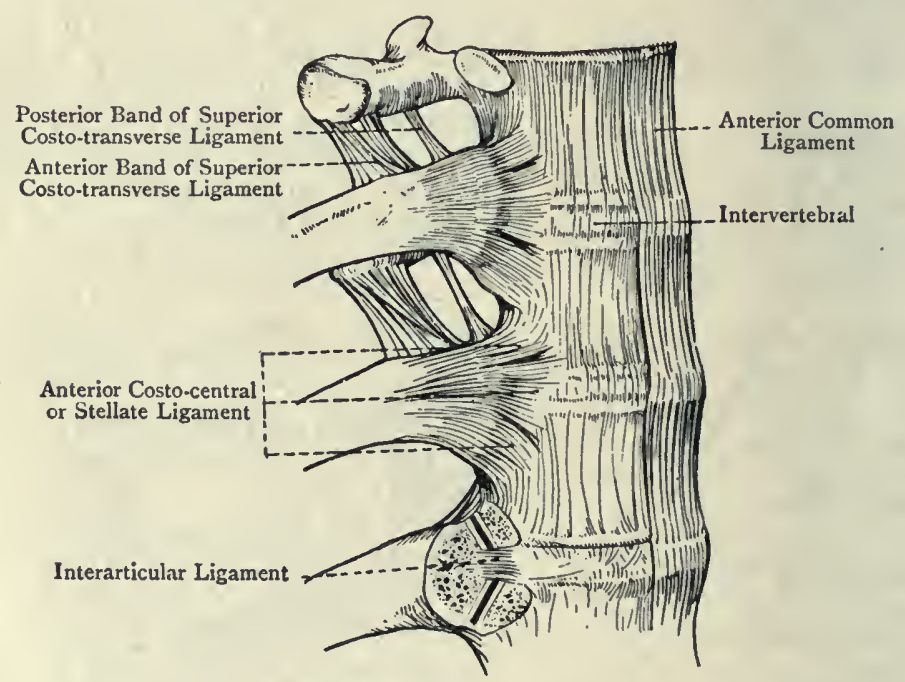

Fig. 426,-Ligaments of the Bodies of the Vertebre and Right COSTO-CENTRAL JOINTS.

the vertebræ and intervertebral discs. It extends from the axis to the first segment of the sacrum, and its fibres are disposed longitudinally. The most superficial fibres extend from a given vertebra to the fifth below it; the intermediate fibres pass from a given vertebra to the third below it; and the deepest fibres pass from a given vertebra to the one immediately below it. The fibres are firmly attached to the intervertebral discs and margins of the vertebral bodies, but very loosely to the centres of the bodies, on account of the presence of bloodvessels. The anterior common ligament is broadest in the lumbar region, and thickest in the 
thoracic region. It is thicker opposite the centres of the bodies than elsewhere, and in these situations it fills up the concavities, and so renders the front of the column less undulating than it otherwise would be. Over the lateral surfaces of the bodies a few scattered fibres are present, which pass from one vertebra to that below. In the sacral region the anterior common ligament is lost in the periosteum of the bone, but it reappears lower down as the anterior sacro-coccygeal ligament. The anterior common ligament is serially continuous superiorly with the anterior atlantoaxial ligament.

The posterior common ligament is situated within the spinal canal, and extends over the posterior surfaces of the bodies of the

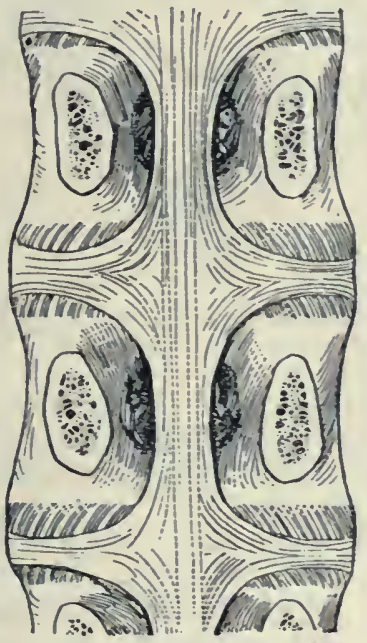

Fig. 427.-Posterior CoMmon LigAMENT OF THE BODIES OF THE VERTEBRE.

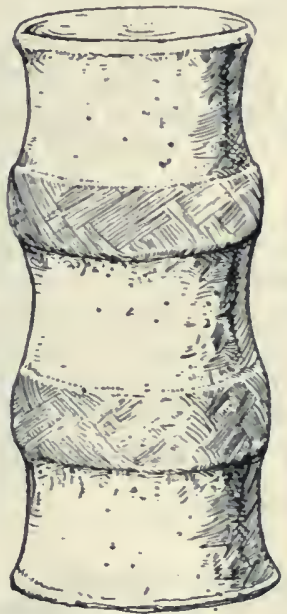

Fig. 428.-INTERvertebral Discs (ANTERIOR VIEW).

vertebræ and intervertebral discs. It is broader above than below, and consists of glistening fibres, which extend from the axis to the first coccygeal vertebra, its sacral part, however, being very narrow and delicate. Its fibres are firmly attached to the intervertebral discs and margins of the vertebral bodies, but they are separated from the centres of the bodies by the transverse venous communications between the anterior intraspinal veins. In the cervical region the ligament is of almost uniform breadth, being expanded over the vertebral bodies, as well as over the intervertebral discs. In the thoracic and lumbar regions, however, it is narrow opposite the vertebral bodies, and broad opposite the intervertebral discs. Its margins, therefore, present dentations, which give it a denticulated appearance. The arrangement of its fibres is similar to the 
arrangement of those of the anterior common ligament. The posterior common ligament is serially continuous superiorly with the posterior occipito-axial ligament.

The intervertebral dises are situated between the adjacent surfaces of the bodies of the vertebræ, and they constitute the chief bond of union between them.

Their outline corresponds to that of the bodies between which they are placed, and they are elastic and compressible. Except in early life, the first or highest disc is situated between the bodies of the axis and the third cervical vertebra, and in the adult the last or lowest disc is situated between the bodies of the fifth lumbar and first sacral vertebræ.

Each disc is composed of a circumferential fibrous part, disposed in the form of superimposed laminæ, and a central portion, which

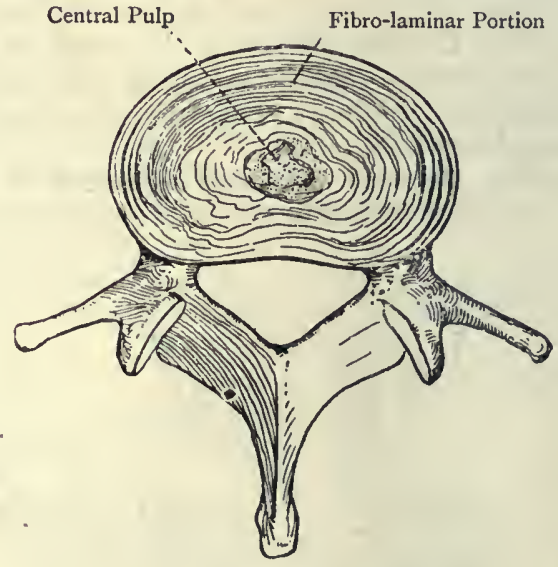

Fig. 429.-INTERVERTEBral Disc (Transverse Section). is soft and pulpy. The external laminar part forms more than half of the disc, and is composed of fibrous tissue and fibro-cartilage. As seen in transverse section the laminæ are arranged concentrically

Fibro-Jaminar Portion

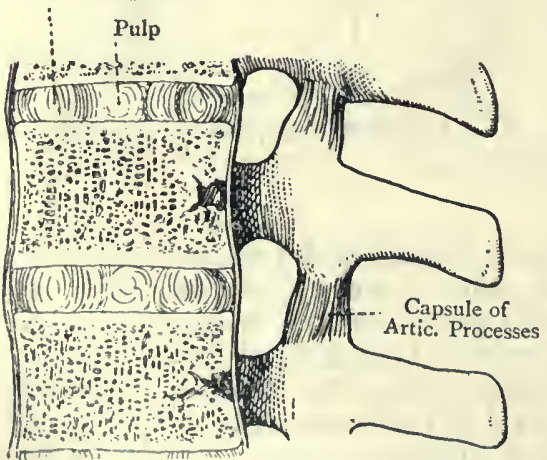

Fig, 430.-Vertical Sagittal Section of Two InTERVERTEBral Discs. around the central pulp, which they closely embrace and compress. The fibres of which they are composed are arranged in parallel bundles, which extend obliquely between the adjacent surfaces of the vertebral bodies, being attached to the layer of hyaline car. tilage which covers them. The fibres of successive laminæ pass obliquely in opposite directions, and are disposed thus $\mathbf{X}$. The outer laminx consist of fibrous tissue, but the majority are composed of white fibro-cartilage. As seen in vertical section the outermost laminæ are bent outwards, and those around the central pulp are bent inwards towards it, this arrangement contributing to 
the elasticity of the vertebral column. The central portion of the disc consists of a soft, elastic, pulpy substance, having a lobate arrangement. Being surrounded and compressed on all sides by the external laminar part, when a section of a disc is made the pulp, being relieved from pressure, projects beyond the level of the cut surface. It is composed of a cellular reticulum, supported by a delicate fibrous stroma.

The pulp is a persistent portion of the notochord.

The intervertebral discs form about one-fourth of the length of the vertebral column, and are thickest in the lumbar region. In the cervical and lumbar regions they are deeper in front than behind, and they give rise to the curve forwards in the cervical region, whilst they increase the forward curve in the lumbar region. In the thoracic region they are of uniform depth. Throughout the

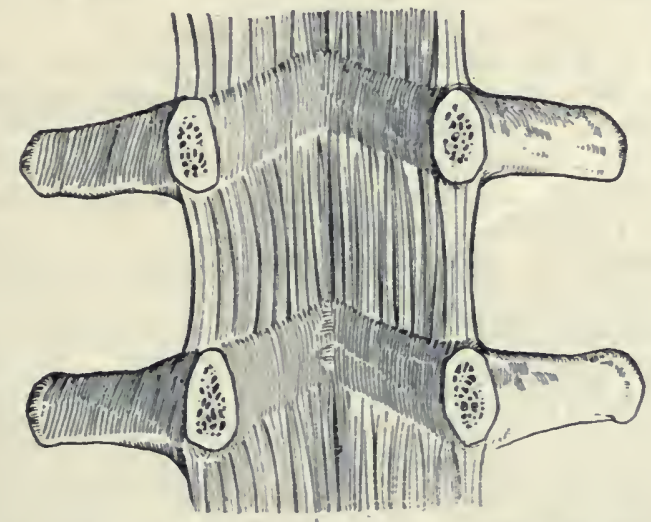

Fig. 43I.-Ligamenta Subflava in the Lumbar Region (ANTERIOR VIEW)

(The Pedicles have been sawn through, and the Vertebral Bodies removed).

column they are intimately connected with the anterior and posterior common ligaments, and, in the thoracic region, with the anterior costo-central or stellate ligaments and the interarticular ligaments of the heads of most of the ribs. In the cervical region the discs are not present at either lateral aspect of the opposed surfaces of the bodies. In these regions there is a synovial space on either side, between the projecting lateral lip of the upper surface of the lower body and the bevelled lateral margin of the lower surface of the upper body. The opposed surfaces are covered by cartilage, and there is an indistinct capsular ligament.

2. Ligaments of the Laminæ.-These are called the ligamenta subflava. They are strong, thick plates of yellow. elastic tissue, which connect the laminæ together, and they extend from the axis to the first sacral segment. They are best seen from the interior 
of the spinal canal, and as they extend between the neural arches they close in the canal in these situations. Each ligamentum subflavum extends from the root of the articular process to the place where the lamina joins its fellow to form the spinous process. At this point it comes into relation with the ligament of the opposite side, a small interval being left between the two for the passage of veins. Superiorly the ligament is attached to the anterior surface of the upper lamina a little above its lower border, and inferiorly it is attached to the upper border, and adjacent part of the posterior surface, of the lower lamina. The ligamenta subflava are wider in the cervical and lumbar regions than in the thoracic region, and over the greater part of the latter region, as viewed from the exterior, they are concealed from view by the imbricated laminæ. Their importance consists in their great elasticity, which enables them to maintain the vertebral column erect, and to restore it to the erect position after it has been bent forwards.

3. Ligaments of the Articular Processes.-The joints between the articular processes belong to the class diarthrosis, and to the subdivision arthrodia. The articular surfaces are covered by cartilage, and the joint is surrounded by a capsular ligament, lined with a synovial membrane. These ligaments are disposed more loosely in the cervical region than elsewhere.

4. Ligaments of the Spinous Processes.-These are supraspinous and interspinous.

The supraspinous ligament consists of longitudinal fibres which connect the extremities of the spinous processes. It extends from the spine of the seventh cervical vertebra to the spine of the fourth sacral segment, and its fibres are arranged in a manner similar to those of the anterior common ligament. In the cervical region the supraspinous ligament is replaced by the ligamentum nuchæ. The interspinous ligaments, which are thin and membranous, are situated between adjacent spinous processes, to the margins of which they are attached from root to tip. They are strongest in the lumbar region, and in the neck they are replaced by deep processes of the ligamentum nuchæ.

5. Ligaments of the Transverse Processes.--These consist of scattered fibres, which pass between the extremities of the transverse processes in the thoracic and lumbar regions. In the neck they are replaced by the intertransversales muscles.

Movements.- The movements allowed in the vertebral column are flexion, extension, lateral movement, rotation, and circumduction.

Flexion and extension are freely allowed in the cervical and lumbar regions. In the thoracic region these movements are very limited on account of (I) the small amount of intervertebral substance, and (2) the imbrication of the laminx. Lateral flexion is allowed in the cervical, thoracic, and lumbar regions, but in the neck it is associated with rotation. During these combined movements of lateral flexion and rotation in the neck one inferior articular process glides upwards and forwards on that which is opposed to it, whilst the other inferior articular process glides downwards and backwards on the one opposed to it. Pure rotation is allowed in the thoracic region round an axis corresponding with the centre of a circle of which the surfaces 
of the articular processes form segments. This centre is necessarily anterior to the articular processes, and corresponds pretty nearly with the centres of the bodies of the vertebræ. In the lumbar region rotation is impossible, for the following two reasons: (I) the centre of the circle of which the articular processes form segments is posterior to these processes; and (2) the articular processes are so disposed as to be locked. In the lumbar region circumduction is allowed, which consists in a combination of flexion, extension, and lateral movements.

Summary of Movements-Cervical Region.-(1) Flexion and extension; and (2) a combination of lateral flexion and rotation. Thoracic Region.(1) Flexion and extension, but only to a limited extent; (2) lateral flexion ; and (3) pure rotation. Lumbar Region.--(1) Flexion and extension ; (2) lateral flexion; and (3) circumduction.

The articulations of the atlas, axis, and occipital bone will be found described in the section dealing with the head and neck.

\section{THE ARTICULATIONS OF THE RIBS, COSTAL CARTILAGES, AND STERNUM.}

I. Ribs-Costo-vertebral Articulations.--These are divided into costo-central and costo-transverse.

Costo-central Articulations.-These unite the heads of the ribs to the centra or bodies of the thoracic vertebra, and they are sometimes spoken of as the capilular joints. They belong to the class diarthrosis, and to the subdivision condylarthrosis. The articular surfaces are the demi-facets on the heads of the ribs and the demifacets on the sides of the bodies of the thoracic vertebræ, that is to say, the lower demi-facet of the vertebra above and the upper demi-facet of the vertebra below, the intervertebral disc intervening between the two. In the case of the Ist, (sometimes the Ioth), the IIth, and the I2th vertebræ there is only one facet, and the corresponding intervertebral discs do not enter into the joints, unless in the case of the Ist joint, into which the disc between the $7^{\text {th }}$ cervical and Ist thoracic vertebræ may enter.

Ligaments.-These are as follows : anterior costo-central, capsular, and interarticular.

The anterior costo-central ligament consists of strong white fibres which are attached to the anterior margin of the head of the rib. From this point the fibres radiate inwards in three bands, one of which passes upwards to be attached to the body of the vertebra above, a second horizontally inwards to be attached to the intervertebral disc, and a third downwards to be attached to the body of the vertebra below. From the radiating disposition of these bands the ligament is known by the name of stellate or radiate. In the case of the Ist, Ioth, IIth, and I2th joints, into each of which only one vertebra enters, the ligament is composed of two bands, instead of three. In the Ist joint the lower band passes to the 
body of the Ist thoracic vertebra, and the upper band to the body of the 7 th cervical. In each of the Ioth, Irth, and I2th joints the lower band passes to the body with which the head of the rib articulates, and the upper band to the body of the vertebra above.

The capsular ligament is incomplete, and consists of thin loose fibres, which cover the posterior, superior, and inferior aspects of the joint.

The interarticular ligament consists of short stout fibres, which extend from the ridge on the head of the rib, separating the two facets, to the intervertebral disc. It divides the joint into two complete synovial cavities, and it is wanting in the Ist, Ioth, IIth, and $\mathrm{I}$ th joints.

There are two distinct synovial membranes in those joints which are provided with an interarticular ligament, namely, from the

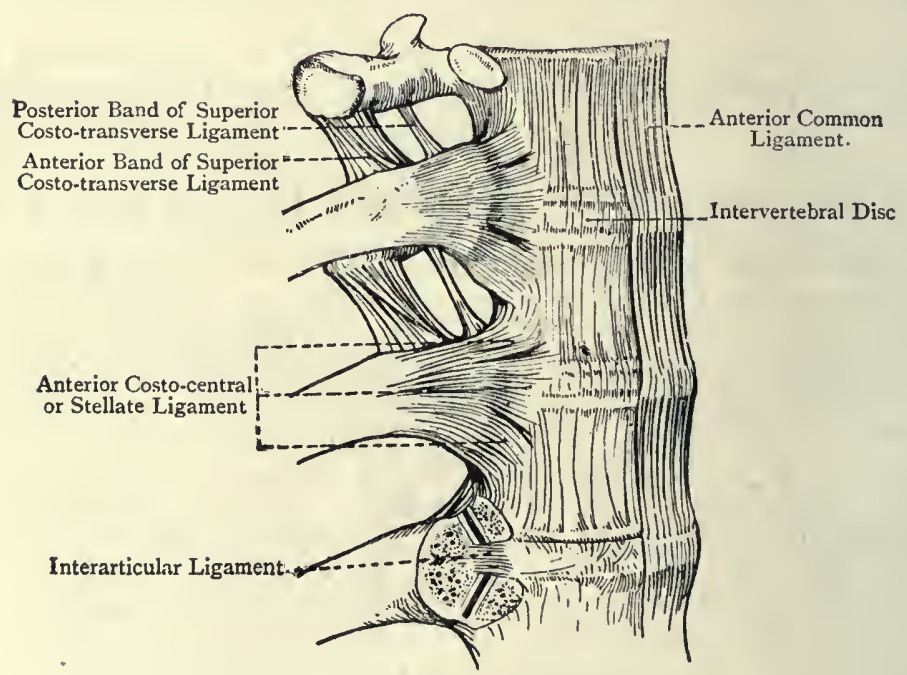

Fig. 432,-Ligaments of the Bodies of the Vertebres and Right Costo-CEnTRAL JoInts.

2nd to the 9th.inclusive, one being above the ligament and the other below it. In those joints in which the interarticular ligament is absent, namely, the Ist, Ioth, Ixth, and I2th, there is only one synovial membrane.

Arterial Supply.-Branches from the intercostal arteries.

Nerve-supply.-Branches from the intercostal nerves.

Costo-transverse Articulations.-These belong to the class diarthrosis, and to the subdivision arthrodia. The articular surfaces are the facet on the tubercle of the rib and the facet on the anterior aspect of the extremity of the transverse process of the thoracic vertebra. 
Ligaments.-These are as follows: posterior costo-transverse, middle costo-transverse, superior costo-transverse, and capsular.

The posterior costo-transverse ligament is a strong flat band, situated on the posterior aspect of the joint, and extending from the extremity of the transverse process to the non-articular part of the tubercle of the rib. The direction of its fibres is upwards and outwards.

The middle costo-transverse or interosseous ligament consists of short strong fibres which pass between the posterior surface of the neck of the rib and the anterior surface of the adjacent transverse process, namely, that with which the tubercle of the rib articulates. Its fibres, which are disposed horizontally, extend from the costocentral to the costo-transverse joint. This ligament is rudimentary in the IIth and I2th ribs.

The superior costo-transverse ligaments are two in number, anterior and posterior. The anterior ligament is a broad flat band, external in position, the fibres of which pass from the crest on the upper border of the neck of the rib to the lower border of the transverse process immediately above, in the vicinity of its tip. Its fibres are directed upwards and outwards, and its outer border is continuous with the posterior intercostal aponeurosis. The posterior ligament is a narrow band, internal to the preceding, and extending from the back of the neck of the rib, below the crest, to the lower aspect of the transverse process immediately above, close to its base.

The superior costo-transverse ligaments are wanting in the case of the Ist rib.

The capsular ligament is formed in part by the posterior costotransverse ligament, and elsewhere by a thin loose membrane, the fibres of which are attached external to the articular processes. It is absent in the case of the IIth and I2th ribs.

The synovial membrane is single and small.

Arterial and Nerve Supply.-Offsets from the posterior branches of the intercostal arteries and nerves.

The costo-transverse articulations are wanting in the case of the IIth and I2th ribs, and sometimes in the case of the Ioth.

Costo-chondral Articulations.-These belong to the class synarthrosis. The outer extremity of the costal cartilage is implanted in the oval pit on the anterior extremity of the rib, and the union is effected by the continuity which takes place between the periosteum of the rib and the perichondrium of the cartilage.

2. Costal Cartilages-Chondro-sternal Articulations.-These belong to the class diarthrosis, and to the subdivision ginglymus, except in the case of the Ist joint, which belongs to the class synarthrosis. The cartilages which take part in these joints are the first seven on either side, these being received into the costal pits or facets on the side of the sternum.

The ligaments are anterior, posterior, superior, and inferior, and, in the case of the 2nd joint at least, interarticular. 
The anterior chondro-sternal ligament is a triangular band, the upper fibres of which ascend upon the sternum, and the lower descend, whilst the intervening fibres pass horizontally forwards. They decussate with those of the opposite side, and blend with the tendinous fibres of origin of the pectoralis major muscle.

The posterior chondro-sternal ligament is disposed in a manner similar to the anterior ligament.

The superior and inferior chondro-sternal ligaments pass from the upper and lower borders of the costal cartilage to the side of the sternum.

The interarticular ligament is present in the and joint, and may be present in some of those succeeding to it. Its fibres extend horizontally between the centre of the sternal end of the 2nd costal cartilage and the plate of fibro-cartilage between the manu-

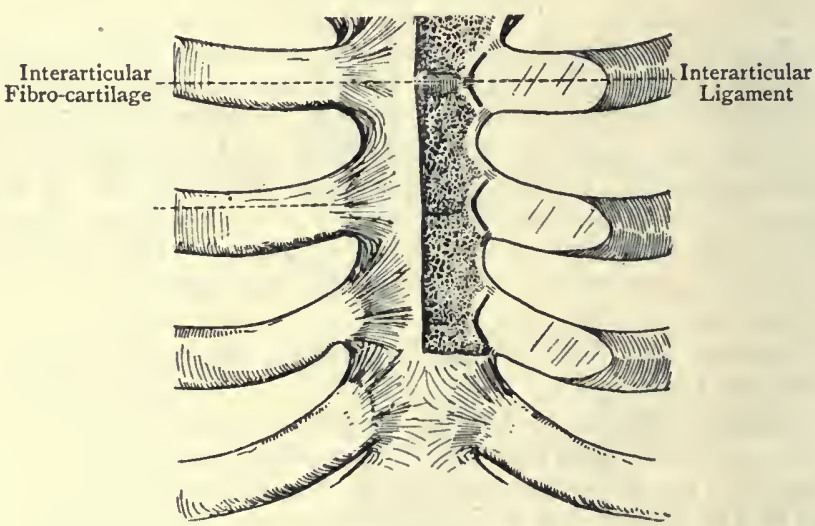

Fig. 433.-The Chondro-sternal Joints (Anterior View).

The Secuss Third, and Fourth Joints of the Left Side are seen in Section.

brium and body of $\cdots$

complete synovial cavitiesternum. It divides this joint into two

The Ist joint has no synov.

being directly united to the niombrane, the first costal cartilage as stated, has two synovial membicrium sterni. The 2nd joint, below the interarticular ligament. ' 1 one above and the other each usually one synovial membrane, bu succeeding joints have of them may have two.

Arterial Supply. - The anterior or perfor metimes one or more

Nerve-supply. - The inter. anterior cutaneous nerves.

Interchondral the subdivision arthrodia. $T$ to the class diarthrosis, and the 6 th, $7^{\text {th }}$, and 8 th, but $\mathrm{so}_{\text {e cartilages involved }}$ retimes also the $9^{\text {th, }}$ 
and it may be the $5^{\text {th }}$, and even the Ioth. The lower border of each cartilage projects downwards, and comes into contact with the upper border of the cartilage below. Each joint is surrounded by fibres disposed in the form of a capsule, and it is provided with a synovial membrane.

Arterial Supply.- The musculo-phrenic branch of the internal mammary artery.

Nerve-supply.--The adjacent intercostal nerves.

3. Sternal Articulations. - The joint between the manubrium and body of the sternum belongs to the class amphiarthrosis, and subdivision symphysis. The opposed surfaces are covered by hyaline cartilage, and a plate or disc of fibro-cartilage is interposed between them, which is connected at either side with the interarticular ligament of the second chondro-sternal joint. This disc may contain a small cavity. In front of and behind the joint there are ligamentous fibres which are disposed longitudinally.

The entire sternum is strengthened by its dense periosteum, by the radiating fibres of the chondro-sternal ligaments, and by the tendinous fibres of origin of the pectoralis major muscles.

Movements of the Ribs. - The movement allowed at the costo-central and costo-transverse joints is of a gliding nature, and takes place in an upward and downward direction. During this movement the rib rotates round the costo-vertebral axis, which corresponds to a line passing obliquely through the costo-central joint, the neck of the rib, and the costo-transverse joint. Owing to the curve and downward inclination of the rib, the result of this rotation is that the anterior and lateral parts of the rib are elevated. Simul. taneously, on account of the obliquity of the axis of rotation, the anterior part of the rib is carried forwards, and along with it the sternum, thus increasing the antero-posterior diameter of the thorax. The lateral part of the rib, and to a certain extent the anterior part also, are carried outwards, thus giving rise to eversion of the lower border of the rib, and at the same time the angle between the rib and its costal cartilage is opened out. In this manner an increase in the transverse diameter of the thorax is produced. During the elevation of the anterior and lateral parts of the rib and the eversion of its lower border the movement takes place round the costo-sternal axis, which corresponds to a line drawn from the costo-central joint of one side to the corresponding chondro-sternal joint. It is usual to liken this movement to the movement of the handle of a backet.

In the case of the first rib elevation and depression are the chief movements allowed, the amount of eversion being trivial, inasmuch as the axis of rotation is almost transverse. In the case of the second, third, fourth, fifth, and sixth ribs elevation and depression, along with eversion, are allowed, the axis of rotation in each case becoming successively more oblique. The seventh, eighth, ninth, and tenth ribs, in which the costo-transverse articular surfaces are almost flat, besides rotating round the costo-vertebral axis, also rotate round the costo-sternal axis. In the case of these ribs elevation is accompanied by a backward movement, and depression by a forward movement. These backward and forward movements take place more freely in the case of the eleventh and twelfth ribs, which have no costo-transverse joints. At the chondro-sternal joints the movement is limited, and consists in elevation and depression, together with forward and backward movement.

At the interchondral joints slight gliding movement is allowed.

Muscles concerned in Respiration.- In ordinary quiet inspiration the muscles concerned are as follows: (1) the diaphragm; (2) the external and internal intercostal muscles, assuming Haller's view to be correct; (3) the 
Ievatores costarum; (4) the serratus posticus superior; (5) the serratus posticus inferior; and (6) the quadratus lumborum, as being auxiliary to the diaphragm, the serratus posticus inferior being also anxiliary. Ordinary quiet expiration is due to (I) the elastic recoil of the lungs, (2) the elastic recoil of the thoracic wall (costal cartilages and sternum), (3) the triangularis sterni muscle, and (4) the muscles of the antero-lateral wall of the abdomen, which press mildly upon the abdominal viscera, and thereby push the diaphragm upwards towards the thorax.

In forced inspiration the following muscles come into play: ( 1 ) the scaleni ; (2) the sterno-cleido-mastoid; (3) the serratus maguus; (4) the pectoralis major and pectoralis minor; and (5) the latissimus dorsi. As auxiliary muscles

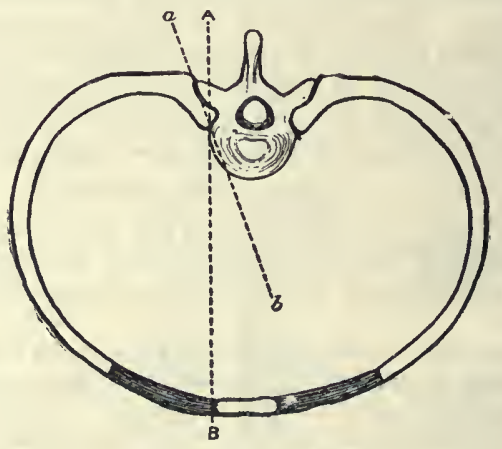

Fig. 434.-Diagram showing the Axes of Rotation of the Ribs (From Halliburton's ' Handbook of Physiology').

A, B, Axis passing from Costo-central to Chondro-sternal Joint ; $a, b$, Axis passing through Costo-transverse and Costo-central Joints. (The movement round the axis $\mathrm{A}, \mathrm{B}$ resembles the raising of the handle of a bucket.)

there are the trapezius, levator anguli scapulæ, and rhomboid muscles, which, by their action upon the scapula, fix the shoulder.

In forced expiration the muscles of the antero-lateral wall of the abdomen act with considerable strength, and now depress those ribs with which they are connected, and necessarily also the sternum. By some authorities the serratus posticus inferior is regarded as being concerned.

In inspiration the thoracic cavity is enlarged in its vertical, antero-posterior, and transverse diameters. The increase in the vertical diameter is due to the contraction and descent of the diaphragm ; the increase in the antero-posterior diameter is caused by the anterior parts of the ribs, and along with them the sternum, being carried forwards; and the increase in the transverse diameter is brought abont by the eversion of the lower borders of the ribs, and the opening ont of the angles between the ribs and their costal cartilages.

\section{GUIDE TO THE THORAX.}

Thoracic Wall.-The upper limb having been separated from the trunk, the dissector should remove the remains of the following muscles: the pectoralis major, pectoralis minor, tendon of origin of the subclavius, serratus magnus, obliquus externus abdominis, latissimus dorsi, and rectus abdominis. In removing the remains of the pectoralis major the anterior cutaneous nerves 
should be preserved, as well as the perforating branches of the internal mam. mary artery. In removing the serrations of the serratus magnus the lateral cutaneous nerves and arteries, which appear between them, should also be preserved.

The insertions of the scalenus anticus and scalenus medius into the first rib, and of the scalenus posticus into the second rib, are to be left intact in the meantime. If the sternum has not been interfered with, for the pnrpose of injecting the subject from the ascending aorta, the dissector should make himself familiar with the sternal angle at the junction of the manubrium and body of the sternum. In this connection he should note that the angle serves as the guide to the second rib, the cartilage of which articulates partly with the manubrium, and partly with the body of the bone.

The external intercostal muscle is to be dissected in at least two intercostal spaces. The direction of its fibres is to be carefully observed, and it is to be noted that the muscle does not extend farther forwards than the junction of the osseous rib with its costal cartilages; indeed, in some cases not so far as this. The anterior intercostal aponeurosis is also to be shown in the intervals between the costal cartilages. In one or more spaces a small portion of the external intercostal muscle is to be carefully divided transversely, and reflected upwards and downwards in two pieces. This dissection will expose a limited portion of the interna! intercostal muscle, and it will enable the dissector to contrast the difference in direction of the two muscles. In another space as much of the external intercostal muscle as possible should be detached from the upper border of the lower rib, and turned upwards with great care, preserving at the same time the lateral cutaneous nerve and artery. The anterior intercostal aponeurosis of the same space should also be carefully removed, care being taken to preserve the anterior cutaneous nerve and perforating artery. This dissection will expose the internal intercostal muscle, the intercostal nerve, and the various intercostal arteries. The direction of the fibres of the internal intercostal muscle is to be observed, and it is to be noted that the muscle extends quite up to the lateral border of the sternum, where it is pierced by the anterior cutaneous nerve.

The intercostal nerve will be found under cover of the lower border of the upper rib, where it lies in the subcostal groove, having the aortic intercostal artery above it, the intercostal vein being in turn above the artery. It is well, therefore, to make slight traction upon the nerve before attempting to display it, and for this purpose the lateral cutaneous nerve will suffice, which is one reason for preserving that nerve. The first intercostal nerve, as a rule, gives off no lateral cutaneous branch, and those of the succeeding nerves will be found piercing the external intercostal muscles about midway between the vertebral column and the sternum. Up to this point the intercostal nerve will be met with between the two intercostal muscles, but subsequently it lies in the substance of the internal intercostal muscle, and finally, between the costal cartilages, it is placed beneath the internal intercostal muscle.

The arteries to be dissected in each space are as follows: (I) the aortic intercostal and its collateral intercostal branch, both passing forwards, the former within the lower border of the upper rib, above the nerve, and the latter along the upper border of the lower rib ; and (2) the anterior intercostal arteries, two in each space, except the lower two spaces, one lying along the lower border of the upper rib, and the other along the upper border of the lower rib. The internal intercostal muscle should now be carefully removed, after which an instructive view of the parietal pleura will be obtained. This, however, must be left intact.

The dissector is next to turn his attention to the internal mammary vessels and anterior intercostal or sternal glands. These structures are to be displayed in a different manner on the two sides.

On one side the costal cartilages are not to be interfered with in the meantime, and on the other side the second, third, fourth, fifth, and sixth costal cartilages are to be cut through at their inner and outer ends, and then lifted 
out. On both sides the anterior intercostal aponeuroses and interchondral fibres of the internal intercostal muscles should be removed. The interna! mammary artery and its brancles, along with its venæ comites, and the anterior intercostal or sternal glands are then to be dissected. The artery will be found about $\frac{1}{2}$ inch from the side of the sternum, and its superior phrenic branch (comes nervi phrenici) is to be preserved until a later period. The venæe comites are also to be subsequently shown uniting superiorly to form one vessel. In the sixth intercostal space the internal mammary artery is to be shown dividing into its two terminal branches, namely, musculo-phrenic and superior epigastric. The triangularis sterni muscle is then to be dissected from before. On the side on which the costal cartilages have been left intact they are now to be cut through from the second to the sixth inclusive, close to the osseous ribs. Thereafter the ribs corresponding to these costal cartilages on each side are to be divided with the bone-forceps, and this should be done as far back as possible by turning the subject round on either side. The divided ribs are then to be bent backwards, the parietal pleura having been carefully separated from them. The sternum, meanwhile, is to be left undisturbed, with whatever costal cartilages are attached to it. The parietal pleura is then to be carefully studied. By way of showing that it does not descend as low as the line of attachment of the diaphragm, the external and internal intercostal muscles of one or two of the lowest spaces should be removed, when it will be seen that the diaphragm comes into view without any trace of parietal pleura.

The manubrium sterni is next to be partially sawn through near its lower border, the seventh costal cartilage on each side is to be cut through, and the great bulk of the sternum is then to be bent upwards in such a manner that it can be replaced when required. If the bone should break, the two parts should be connected by wire sutures. The relations of the anterior borders of the two pleural sacs to the sternum, to each other, and to the pericardium, are now to be carefully studied. The anterior mediastinum and the remains of the thymus body are to be inspected, and the area of the pericardium which is uncovered by the left pleura, and is directly related to the thoracic wall, is to receive special attention.

At this stage an opening should be made in the cervical portion of the trachea, and a blow-pipe, provided with a stop-cock, should be introduced and tied firmly in position. The lungs are now to be inflated through the blow-pipe, the stop-cock is to be turned, and the following points are to be accurately observed: (r) the extent to which the lungs encroach upon the heart, enclosed within the pericardium; and (2) the extent to which they descend between the diaphragm and the ribs. By reversing the stop-cock the lungs will be seen to collapse and recede from the precordial area. During these observations the greater part of the sternum should be alternately raised and lowered.

The chief part of the sternum is now to be removed, aloug with whatever costal cartilages are connected with it. On the side on which the cartilages remain in connection with the bone the triangularis sterni and the internal mammary vessels are to be dissected from behind. The chondro-sternal joints may be dissected at this stage, or it may be convenient to preserve the sternum for their future dissection.

Phrenic Nerves, Pleuræ, Pericardium, and Lungs.-Before the further stages of the dissection are entered upon, it is necessary that the dissector should have some knowledge of the general position of the structures to be exposed. From their complicated nature it is not possible to lay down hard-and-fast directions, and in his mode of procedure he must keep a careful look-out.

The phrenic nerve is to be dissected, and its close relation to the pericardium is to be noted, as well as its course in front of the root of the lung. Possibly the superior phrenic branch (comes nervi phrenici) of the internal mammary artery may be found coming into contact with the nerve high up in the thorax. The reflections of the pleura are to be studied both in the transverse and in the vertical directions. If any adhesions are present, these should be separated with the fingers, and any fluid which has accumulated must be 
removed, and the parts thoroughly cleansed. The lines of the sternal and costo-diaphragmatic refiections of the pleura are to be carefully studied. In examining the antero-posterior reflections of the membrane the ligamentum latum pulmonis, below the root of the lung, is to be noted, and the mediastinum thoracis, with its divisions, is to receive very careful attention, these divisions being anterior, superior, middle, and posterior. The two pleural sacs which form the lateral boundaries of the anterior mediastinum are to be separated, when the middle mediastinum will be exposed, with the pericardium, enclosing the heart, lying in it.

The lungs are next to be studied. They have been already examined in their inflated and collapsed conditions; but attention is now to be directed to their form, component parts, fissure or fissures, and lobes. The differences between the two lungs are also to be noted, which impress corresponding differences upon the pleural sacs. The roots of the lungs are not to be dissected at this stage.

The superior mediastinum should now be examined, without disturbing the pericardium, and the remains of the thymus body, together with the superior mediastinal glands, are to be dissected.

The right and left innominate veins are next to receive attention, and their inferior thyroid and internal mammary tributaries, with, in some cases, the first intercostal vein, are to be noted. The left superior intercostal vein is to be displayed as an additional tributary of the left innominate vein, which it joins not far from its commencement, after having crossed the back part of the arch of the aorta.

The superior vena cava, formed by the junction of the two innominate veins, is to be shown and followed down to where it pierces the pericardium, but no farther in the meantime. The right azygos vein is to be displayed arching over the root of the right lung, and opening in to the superior vena cava just before that vessel pierces the pericardium.

The pericardium is now to be studied from the exterior. Connected with its anterior wall parts of the superior and inferior sterno-pericardial ligaments of Luschka are to be looked for. The relation of the fibrous portion of the pericardium to the diaphragm is to be looked to, and its disposition upon the great vessels at the base of the heart, for which it forms sheaths, is to receive attention.

The intimate relation of the phrenic nerves to the pericardium has been already noted. The pericardium is now to be laid open by means of a vertical median incision, extending over its entire length, and a transverse incision opposite the roots of the lungs. The serous portion of the pericardium is then to receive careful attention. It is to be observed that, being a serous membrane, it is composed of parietal and visceral portions, which enclose between them a shut sac. The close relation of the parietal portion to the fibrous part is to be noted, and the disposition of the visceral portion upon the ascending aorta and trunk of the pulmonary artery is to be carefully studied. It will be seen to form a single sheath for them, and to rise upon the ascending aorta for from $I$ to $1 \frac{1}{2}$ inches. The transverse sinus of the pericardium should be explored with the finger. It will be found lying behind the ascending aorta and trunk of the pulmonary artery, and in front of the auricular portion of the heart. In connection with the serous portion of the pericardium the triangular fold, called the vestigial fold of Marshall, is to be looked for in front of the root of the left lung, between the left pulmonary artery and the subjacent pulmonary vein. When this fold is carefully dissected it will be found to contain a delicate fibrous cord, which is a vestige of the left duct of Cuvier of embryonic life.

The pericardium is now to be removed, which will show the lower part of the superior vena cava. The heart, being now exposed, should receive the most careful attention. Its external form and component parts are to be observed, and the relations of these parts to the thoracic wall must be thoroughly mastered. More especially the relation of the valvular orifices of the heart to the thoracic wall calls for the closest observation. The trunk of the pulmonary artery is to be studied, as it springs from the conus arteriosus 
at the base of the right ventricle, and it is to be noted that it conceals the origin of the aorta from the base of the left ventricle. Further, it will be seen that both of these vessels are embraced by the auricular appendices.

The right and left coronary arteries, each accompanied by the corresponding coronary plexus of nerves, are to be displayed as they come forwards on either side of the root of the pulmonary artery. The superior vena cava is to be shown opening into the postero-superior angle of the right auricle, and, by raising the heart, the inferior vena cava will be seen to open into the posteroinferior angle of the right auricle. The four pulmonary veins, two right and two left, will be found to open into the back part of the left auricle.

The ascending aorta is to be dissected, and in conjunction with it the trunk of the pulmonary artery, the superior vena cava being also kept in view. The arch of the aorta is next to be dissected, along with the innominate artery, and the thoracic portions of the left common carotid and left subclavian arteries.

Crossing in front of the aortic arch will be found the left phrenic and left pneumogastric nerves, and between them the superior cervical cardiac branch of the left sympathetic and the inferior cervical cardiac branch of the left pneumogastric, both of these two small nerves being on their way to the superficial cardiac plexus.

The left recurrent laryngeal nerve is to be shown arising from the left pneumogastric opposite the lower margin of the arch of the aorta, and passing backwards beneath the arch, and then upwards behind it, where it gives off one or two cardiac branches to the deep cardiac plexus. The left superior intercostal vein will be seen crossing over the back part of the arch on its way to the left innominate vein.

Within the concavity of the arch, in addition to the left recurrent laryngeal nerve, the superficial cardiac plexus of nerves and the ligamentum ductûs arteriosi fall to be dissected. The superficial cardiac plexus lies in front of the trunk of the pulmonary artery, close to its bifurcation, and on the right side of the ligamentum ductûs arteriosi, and it requires the most careful dissection. The best way to proceed is to follow downwards from the front of the arch of the aorta the superior cervical cardiac branch of the left sympathetic and the inferior cervical cardiac branch of the left pneumogastric, both of which will conduct to the plexus. In this plexus a small ganglion, called the ganglion of Wrisberg, is to be looked for. The plexus itself will be found to furnish delicate filaments to the left anterior pulmonary plexus, after which it is prolonged into the right coronary plexus, which latter is reinforced by fibres from the right half of the deep cardiac plexus. The ligamentum ductûs arteriosi will be found extending from the root of the left pulmonary artery to the under aspect of the arch of the aorta immediately beyond the place of origin of the left subclavian artery.

The left lung is now to be turned well forwards, and fixed in that position by hooks. The trunk of the left pneumogastric nerve is to be followed downwards behind the root of the left lung. Before reaching that point it is to be shown furnishing a few branches which pass to the front of the root, and there form the small anterior pulmonary plexus. Behind the root of the left lung the nerve will be found to become flattened, and to break up into a number of branches, which form the posterior pulmonary plexus. From this plexus the nerve is to be shown issuing as two cords which pass downwards to the osoplaagus, where they are to be left in the meantine. The right pneumogastric nerve will be found behind the right innominate vein. and on the right side of the right innominate artery and trachea. A little above this point the nerve has crossed over the first part of the right subclavian artery, and parted with the riglit recurrent laryngeal nerve opposite the lower margin of that vessel. The right pneumogastric nerve will be seen to occupy a much deeper position than the left. A branch to the deep cardiac plexus should be looked for coming from it, and the main nerve is to be followed downwards behind the root of the right lung, that organ having been turned well forwards, and kept in that position by hooks. Behind the root of the right lung the right pneumogastric nerve acts in a manner similar 
to the left nerve, and forms the right posterior pulmonary plexus, from which it emerges as two cords, and these, as on the left side, descend to the œsophagus.

The lungs having been replaced in position, the root of each is now to be fully dissected, the bronchus, pulmonary artery, and pulmonary veins being clearly separated from each other all round the root. In performing this dissection the anterior and posterior pulmonary plexuses of nerves, already referred to, are to be studied, and the bronchial lymphatic glands are to be displayed. These glands are usually quite black, this being due to the deposit of carbonaceous matter in them. The general relations of each root, and the special relations of the chief constituents, are to be thoroughly mastered.

The right and left pulmonary arteries should now be examined from their origin from the main trunk to the root of each lung, and their relations are to be noted, as well as the ligamentum ductûs arteriosi in connection with the root of the left pulmonary artery. The pulmonary veins on each side are also to be followed from the root of the lung to the back part of the left auricle of the heart. In the dissection of the roots of the lungs the bronchial arteries, one right and two left, and the bronchial veins are to be kept in view.

Exterior of the Heart. - The dissection of the heart is now to be proceeded with. Its external form is to be carefully examined; the directions of the base, apex, and borders are to be observed; and the grooves by which it is divided into auricular and ventricular portions, and these, in turn, into right and left auricles, and right and left ventricles, are to be noted. The apex will be seen to be formed entirely by the left ventricle.

The right and left coronary arteries are to be fully dissected, and their origins from the root of the ascending aorta clearly shown, the trunk of the pulmonary artery being displaced as may be required. The right coronary artery is to be followed along the front and back of the right auriculo-ventricular groove, and its marginal branch is to be shown passing along the margo acutus. On the back of the heart the main vessel will be found to divide into two terminal branches, transverse and descending. The transverse branch is to be followed into the back part of the left auriculo-ventricular groove, where it anastomoses with the transverse branch of the left coronary artery; and the descending branch is to be followed along the inferior interventricular groove as far as the region of the apex, where it anastomoses with the descending branch of the left coronary artery.

The left coronary artery is to be shown dividing very early into a transverse and descending branch on the left side of the root of the pulmonary artery. The transverse branch is to be followed round the left auriculoventricular groove, and its marginal branch is to be shown passing along the margo obtusus vel rotundus. The descending branch is to be followed along the antero-superior interventricular groove as far as the region of the apex.

In dissecting the coronary arteries the right and left coronary plexuses of nerves are to be kept in view, and the various cardiac veins are to be at the same time displayed.

In the back part of the left auriculo-ventricular groove the coronary sinus, about $\mathrm{r}$ inch leng, will be found, and its dilated condition is to be noted. The great cardiac or coronary vein (anterior interventricular vein) will be found in the antero-superior interventricular groove, along with the descending branch of the left coronary artery. On entering the front part of the left auriculo-ventricular groove (where it is now strictly the great cardiac or coronary vein), it should be followed, in company with the transverse branch of the left coronary artery, to the back part of the groove, where it will be found to become continuous with the coronary sinus. The middle cardiac vein (posterior interventricular vein) will be met with in the inferior interventricular groove, where it accompanies the descending branch of the right coronary artery, and it should be shown to join the right extremity of the coronary sinus. The right or small cardiac or coronary vein will be 
found in the back part of the right auriculo-ventricular grouve, and, it may be, in the front part of it. The anterior cardiac veins will be found on the front of the right ventricle and along the margo acutus, and they are to be traced to the right coronary vein. The posterior cardiac veins will be found on the inferior surface of the left ventricle, and they are to be followed to the lower border of the coronary sinus. The oblique vein of Marshall is to be carefully looked for on the back of the left auricle, along which it passes downwards and inwards to open into the left extremity of the coronary sinus. The tributaries of this sinus are now to be fully reviewed, and thereafter it is to be laid open with scissors. This will bring into view a valve. composed of two segments, which is situated at the place where the great cardiac or coronary vein opens into the sinus, namely, about I inch from the ending of the sinus in the right auricle.

The very delicate nerves derived from the coronary plexuses course upon the surfaces of the ventricles, but in the human heart they do not lend themselves very readily to inspection. The heart of the calf is much more favourably disposed for their examination.

Interior of the Heart.-In this dissection the best way to proceed is to follow the course of the blood through the heart. The right auricle is to be laid open by two incisions as follows: (I) a vertical incision is to be made from the auricular extremity of the superior vena cava to near the point of entrance of the inferior vena cava, keeping clearly in front and to the left of it, in order not to injure the vestigial Eustachian valve which is associated with its orifice; and (2) an oblique incision is to be made from the auricular appendix to about the centre of the vertical incision. Having removed the venous blood and sponged the interior of the auricle, the following points are to receive attention: (I) the musculi pectinati, situated on the wall of the auricular appendix and the adjacent portion of the right wall of the atrium ; (2) the crista terminalis ; (3) the fossa ovalis and annulus ovalis on the interauricular septum; (4) the so-called tubercle of Lower; (5) the vestigial Eustachian valve in connection with the orifice of the inferior vena cava; (6) the opening of the coronary sinus, and the Thebesian valve ; (7) the foramina Thebesii; and (8) the auriculo-ventricular orifice. It is to be noted that no valve guards the opening of the superior vena cava.

In connection with the annulus ovalis a very minute opening may be found under cover of it, leading into the left ventricle. Its possible presence may be determined by exploring with a fine probe. Three fingers, held side by side, should be passed through the auriculo-ventricular orifice, and, if normal, it will be found to admit them readily.

The right ventricle is to be opened by means of two incisions, the left forefinger having previously been passed through the auriculo-ventricular orifice. A transverse incision is to be carried along the upper part of the ventricle, just below and parallel to the auriculo-ventricular groove. This incision is to be prolonged as far as a point below the root of the pulmonary artery. The other incision is to commence at the left end of the foregoing incision, and is to be carried downwards just to the right of the antero-superior interventricular groove. In this manner a triangular flap, representing the front wall of the right ventricle, can be turned down, and the cavity fully exposed. The second incision, just stated, may be replaced by one extending along the margo acutus to the lower end of the antero-superior interventricular groove, and then a triangular flap would be raised towards the left.

After cleansing the ventricle, the dissector is to observe (I) that its cavity is excluded from the apex of the heart, (2) that it is prolonged to the root of the pulmonary artery as the infundibulum or conus arteriosus, and (3) that the interventricular septum bulges into it, so as to be convex on its right aspect. The fleshy elevations, called columnæ carneæ, are to receive attention, their various kinds are to be noted, and special attention is to be given to the variety known as the musculi papillares. These latter will be found to be disposed in two groups anterior and posterior. The absence of columna carnex from the infundibulum is to be noted, and the moderator band is to be looked for extending from the anterior papillary muscle to the interven- 
tricular septum. The tricuspid valve is next to receive attention, and its three main segments are to be carefully studied, the small secondary segments lying between their basal parts being noted. The chordæ tendineæ and their relations to the segments of the tricuspid valve should be closely examined, and the function of that valve is to receive careful consideration. The study of the pulmonary valve, which guards the orifice of the pulmonary artery, is to be postponed till a later period.

On account of the posterior position of the left anricle, and preparatory to opening it, the inferior vena cava may be divided in order to liberate the heart from the diaphragm. The heart is then to be turned over to the right side, and also upwards. A transverse incision may then be made above and parallel to the auriculo-ventricular groove, and any other which may suggest itself to the dissector, who is supposed to know by previous study the internal arrangement of the parts. The interior having been cleansed, it will be at once apparent that the musculi pectinati are confined entirely to the region of the auricular appendix. On examining the left surface of the interauricular septum the position of the foramen ovale of the fœtal heart, and the remains of the valve which originally closed it, will be recognised as a slight depression, which is limited inferiorly by a crescentic border with the concavity directed upwards. It may be compared to the imprint of a fingernail.

The orifices of the pulmonary veins on the posterior wall-two right and two left-are to be examined, and the absence of valves noted. The left auriculo-ventricular orifice is next to receive attention. If two fingers, held side by side, are passed through the orifice, it will be found, if normal, to admit them readily.

The left ventricle is to be opened by two incisions, one carried transversely across the ventricle, just below and parallel to the auriculo-ventricular groove, and the other extending from the right end of the preceding incision to the lower part of the ventricle on the left side of the apex. The ventricle having been cleansed, the great thickness of its wall is to be noted, and the dissector is to observe (I) that its cavity extends quite to the apex of the heart, and (2) that the interventricular septum recedes from it, so as to be concave on this aspect. The portion of the cavity adjacent to the aortic orifice is to receive attention, this part, which is known as the aortic vestibule of Sibson, having fibrous walls. The columnæ carneæ and their varieties are to be studied as on the right side, their very intricate arrangement being noted, and special attention is to be directed to the musculi papillares, which are anterior and posterior. The two large cusps of the mitral valve, with two small cusps placed between their basal parts, are to be carefully studied, and the relation of the anterior or aortic cusp to the aortic orifice is to be noted. The chordæe tendineæ, and their relations to the segments of the valve, should be closely examined, and the function of the valve is to receive careful consideration. The auriculo-ventricular and aortic orifices are to be studied, but the aortic valve is to be left over for future consideration. Before leaving the study of the interior of the heart, the relations of its auriculo-ventricular and arterial valvular orifices to the thoracic wall should be thoroughly mastered.

Deep Cardiac Plexus. - To expose this plexus the ascending acrta should be divided near its upper end, and the superior vena cava is to be divided a little below the point where it receives the right azygos vein. The arch of the aorta is then to be displaced towards the left side, and kept out of the way by hooks. If necessary, the ligamentum ductûs arteriosi should be divided, but the superficial cardiac plexus is to be left intact. This dissection will expose the thoracic portion of the trachea, in front of which the deep cardiac plexus is situated, just above the bifurcation into the two bronchi. By very careful dissection the nerves forming this plexus are to be shown passing downwards and inwards on the sides of the trachea to its anterior aspect, where they end in the plexus. The deep cardiac plexus is arranged in two halves, right and left, which are in free commnnication with each other. The contributory nerves to the right half of the plexus are 
(1) the upper, middle, and lower cervical cardiac branches of the right sympathetic: (2) the upper and lower cervical cardiac branches of the right pneumogastric; (3) one or two branches from the right recurrent laryngeal nerve ; and (4) one or two thoracic cardiac branches from the right pneumogastric nerve. The contributory nerves to the left half of the plexus are (I) the middle and lower cervical cardiac branches of the left sympathetic ; (2) the upper cervical cardiac branch of the left pneumogastric; and (3) branches from the left recurrent laryngeal nerve. As regards the upper cervical cardiac branch of the left sympathetic, and the lower cervical cardiac branch of the left pneumogastric, they have already been followed over the arch of the aorta into its concavity, where they have been seen to form the superficial cardiac plexus. The connection between the two halves of the deep cardiac plexus is to be made out, as well as that between the right half and the superficial cardiac plexus. The right coronary plexus is to be shown coming from the superficial and right half of the deep cardiac plexus. From the right half of the deep cardiac plexus branches are to be looked for passing to the right anterior pulmonary plexus, and also to the right auricle. The left coronary.plexus is to be shown coming from the left half of the deep cardiac plexus, of which it forms the chief prolongation. Branches are also to be looked for passing to the left anterior pulmonary plexus, and to the left auricle.

The heart is now to be removed by dividing the trunk of the pulmonary artery and the pulmonary veins. The aorta and pulmonary artery are, in succession, to be attached to a water-tap, and by turning the stop-cock the flow of water will demonstrate the action of the arterial valves. The three bulgings on the exterior of each vessel close to the corresponding ventricle, and produced by the sinuses of Valsalva in the interior, will be at the same time shown. The auricular portion of the heart should now be separated from the ventricular portion. There is no continuity between the auricular and ventricular muscular fibres, these being only connected through the medium of the fibrous rings round the auriculo-ventricular orifices. A heart usually requires to be boiled in the first place, in order to dissolve the fibrous tissue, but the separation of the auricular from the ventricular portion is possible by careful dissection. After its completion an instructive view will be obtained of the orifices at the base of the ventricular portion. The arriculoventricular orifices will be seen to lie side by side, the aortic orifice being in front of and between them, and the pulmonary orifice being in front of the aortic. In the angle between the auriculo-ventricular and aortic orifices a collection of dense fibrous tissue, of the consistence of cartilage, will be found, and processes are to be shown passing from it to the fibrous rings which surround the auriculo-ventricular and aortic orifices.

The pulmonary artery is next to be laid open by an incision passing between two of the three segments of which the pulmonary valve is composed. The segments of the valve and the sinuses of Valsalva are to be examined, but these admit of much better observation in the case of the aortic valve. The aorta is also to be laid open, observing the same precaution as in opening the pulmonary artery, and the segments of the aortic valve are to receive most careful attention. The two lunulæ and corpus Arantii of each segment are to be noted, the direction of the free margins of the segments is to be observed, and the sinuses of Valsalva are to be looked to. The orifices of the right and left coronary arteries are to be examined, that of the right being connected with the right antero-lateral sinus of Valsalva, and that of the left with the left antero-lateral sinus of Valsalva. Lastly, the function of the aortic and of the pulmonary valves is to be carefully studied.

For the purpose of examining the arrangement of the muscular fasciculi of the auricles and ventricles a sheep's heart may be obtained and boiled for a short time in order to dissolve the fibrous tissue.

Posterior Mediastinum. - The thoracic part of the trachea is now to be dissected, and its bifurcation into two bronchi, right and left, is to be shown, as well as some bronchial glands which lie within the angle of bifurcation. The greater size of the right bronchus and the greater length of the left 
bronchus are to be observed, and it is to be specially noted that the right bronchus is more in line with the trachea than the left. The trachea is then to be carefully divided a little above its bifurcation, and the lower part of it, with the two bronchi and two lungs, is to be removed. The right bronchus is to be dissected, and its eparterial and hyparterial divisions are to be shown. The ramifications of the bronchial tubes within the lungs are then to be followed out.

The difference between the main bronchus and its pulmonary ramifications, as regards the extent of the cartilaginous rings in their walls, should attract attention. The bronchial tubes are to be laid freely open, and their mucous lining is to be inspected. The lung is also to be incised at different parts, and the cut surfaces examined. A portion of healthy lung should be put into a dish of water, and the result should be noted.

The thoracic part of the osophagus is next to be displayed. The dissector is to note that it lies at first on the right side of the descending aorta, then in front of it, and finally slightly to its left side. The right and left pneumogastric nerves will be found in very close contact with the gullet, the right nerve gradually inclining from the right side of the tube to its posterior aspect, and the left nerve gradually inclining from the left side of the tube to its anterior aspect. The plexus gulæ, formed by branches of these two nerves, should be displayed, and the œesophageal branches of the descending aorta are to be borne in mind.

The osophagus is to be displaced, and the thoracic duct is to be dissected. The dissectors should remember that this duct is sometimes double in the thorax. It will be found lying close to the right side of the descending aorta, between it and the right azygos vein. It is to be carefully followed up beneath the arch of the aorta and along the left side of the cesophagus, on its way to the root of the neck on the left side. The dissectors of the thorax should now associate themselves with the dissectors of the head and neck, in order to follow the thoracic duct to its termination in the angle of junction between the left internal jugular and left subclavian veins.

The descending aorta falls to be dissected next, along with its branches. The aortic intercostal arteries are to be shown on either side, and they are to be followed into the back parts of the intercostal spaces. At this stage the dissector should display the delicate posterior intercostal aponeurosis between which and the parietal pleura each intercostal artery passes. The corresponding intercostal vein and nerve are to be shown in position, and near the angle of the rib the aortic intercostal artery will be found to give off its collateral intercostal branch, having previously, on its entrance into the space, given off its dorsal branch. In dissecting the descending aorta the bronchial arteries which nourish the lungs are to be looked for behind the corresponding bronchus. There is usually one right bronchial artery, which arises either from the descending aorta or from the first right aortic intercostal artery, and there are two left bronchial arteries, which spring from the parent trunk.

The azygos veins are next to receive attention. The right azygos vein will be found under cover of the csophagus, where it has the thoracic duct on its left side, and it is to be followed up to its termination in the superior vena cava, a tag of which vessel has been specially preserved. The following tributaries are to be shown entering the right azygos vein: (I) the lower seven, or it may be eight, right intercostal veins; (2) the lower and upper transverse azgyos veins; and (3) the right superior intercostal vein, which takes up the second and third, and it may be the fourth, intercostal veins of the right side. The first right intercostal vein opens into the corresponding innominate vein, or sometimes into the vertebral vein. The lower and upper left azygos veins are then to be dissected, along with the lower and upper transverse azygos veins; and the left superior intercostal vein, if not previously dissected, is to be followed over the back part of the arch of the aorta, and shown entering the left innominate vein. The first left intercostal vein is disposed similarly to its fellow of the right side.

The superior intercostal artery on either side is to be dissected, and its 
distribution to the first and seond intercostal spaces shown. The vessel will be found descending in front of the neck of the first rib. A branch from the first aortic intercostal artery-that, namely, to the third space-should be looked for ascending over the neck of the third rib to join the second intercostal artery, which it sometimes replaces.

The thoracic portion of the gangliated cord of the sympathetic should now be dissected. "It will be found lying over the heads of the ribs under cover of the parietal pleura, and gradually inclining inferiorly on to the side of the vertebral column. Two rami communicantes are to be shown passing between each ganglion and the adjacent intercostal nerve, and it is to be noted that these rami are very short. The vascular branches of distribution of the upper ganglia, as well as the branches to the posterior pulmonary plexus of nerves, are not easy of dissection, but the splanchnic nerves admit of this readily, the great and small splanchnic nerves being constant, but the least being frequently absent.

The upper surface of the diaphragm is next to be dissected, and attention should be directed to (I) its thoracic relations, (2) the central tendon, (3) the foramen quadratum, (4) the aortic and œsophageal openings, and (5) the relation of the sympathetic cords and splanchnic nerves to it. The diaphragm may now be laid open, in association with the dissectors of the abdomen, and the thoracic duct is then to be followed down to its commencement in the receptaculum chyli. Thereafter the subcostal muscles should be dissected. They will be found over the back parts of the ribs in the vicinity of the angles.

Articulations.-I. Vertebral Column.-The part available for the dissection of the ligaments is the portion below the first thoracic vertebra, and probably as low as the tenth or eleventh thoracic vertebra. The ligaments to be dissected are (I) the anterior common ligament, which is easily displayed; (2) the posterior common ligament, to show which the neural arches of the vertebræ must be removed by sawing through the pedicles; $(3)$ the intervertebral discs, which are to be dissected by means of transverse and vertical sections; (4) the ligamenta subflava, which connect the laminæ, and are readily seen after removal of the neural arches; (5) the capsular ligaments, which connect the articular processes; $(6)$ the supraspinous and interspinous ligaments; and (7) the intertransverse ligaments.

The movements of the vertebral column are to be carefully studied.

2. Ribs-Costo-central Joints.-The ligaments to be dissected are (1) the anterior costo-central or stellate, easily recognised; and (2) the interarticular, which is only seen on opening the joint, and which is absent in the first, sometimes the tenth, the eleventh, and twelfth joints. The two synovial cavities are to be noted, except in the case of the joints just enumerated as having no interarticular ligament.

Costo-transverse Joints.-The ligaments to be dissected are the posterior, middle, and superior costo-transverse. Within the posterior costo-transverse ligament the synovial membrane of the joint is to be shown. The middle costo-transverse or interosseous ligament can only be studied properly by sawing horizontally through the neck of the rib and the adjacent trans. verse process. The superior costo-transverse ligaments are to be displayed as two distinct bands-anterior or external, which is large ; and posterior or internal, which is small. The continuity of the outer border of the anterior ligament with the posterior intercostal aponeurosis is to be shown.

Costo-chondral Joints. - Each rib is connected to its cartilage by the continuity which takes placc between the periosteum of the rib and the perichondrium of the cartilage.

Sterno-chondral Joints. - The ligaments to be dissected are anterior, posterior, superior, and inferior. In the case of the second joint, and it may be in some of the succeeding joints, there is an interarticular ligament, which divides the joint into two distinct synovial compartments. The direct connection of the first costal cartilage with the manubrium stcrni is to receive attention.

Interchondral Joints.-These are met with from about the fifth to the 
ninth or tenth costal cartilages. They are provided with capsular ligaments and synovial membranes.

3. Sternum. - The manubrium and body are connected by ligamentous fibres, disposed longitudinally in front and behind, and by a plate of fibrocartilage which is interposed between the articular surfaces. It is to be noted that the entire sternum is strengthened by its dense periosteum, by the radiating fibres of the chondro-sternal ligaments, and by the tendinous fibres of origin of the pectoralis major muscles.

The movements of the ribs are to be studied with the greatest care, and the muscles concerned in these movements are to be specially noted. 


\section{THE HEAD AND NECK}

\section{BACK OF THE SCALP AND NECK}

Landmarks.- The external occipital protuberance can, as a rule, readily be made out, and the superior curved line, if well developed, may be felt extending outwards from it. The mastoid process of the temporal bone can be distinguished without difficulty behind the auricle. The occipital artery, with the great occipital nerve on its inner side, lies a little internal to the centre of a line connecting the external occipital protuberance and the tip of the mastoid process. The occipital lymphatic gland, or glands, may be felt, when enlarged, over the upper part of the trapezius or complexus muscle. The spine of the seventh cervical vertebra, or vertebra prominens, can easily be felt, and that of the sixth may also be made out. Extending from the external occipital protuberance to the seventh cervical spine there is an elongated depression, called the nuchal furrow, which indicates the position of the ligamentum nuchæ. It is possible to feel the strong bifid spine of the axis by sinking the finger deeply into the upper part of the nuchal furrow, near the occipital bone, but the spines of the third, fourth, and fifth cervical vertebræ lie too deeply for detection. The lymphatic glands, known as the glandulæ concatenatæ, may be felt, when enlarged, along the posterior border of the sterno-cleido-mastoid muscle.

Back of the Neck-Fasciæ.-The superficial fascia presents nothing worthy of note. The deep fascia closely invests the cervical portion of the trapezius, and is then prolonged over the posterior triangle of the neck to the posterior border of the sternocleido-mastoid, where it divides to ensheathe that muscle. It will be described in connection with the deep cervical fascia.

Nerves.-The nerves of this region are as follows: the great occipital; the least or third occipital; the internal branches of the posterior primary divisions of the third, fourth, and fifth cervical spinal nerves; the small occipital ; the great auricular; the superficial cervical ; the spinal accessory ; and the descending superficial branches of the cervical plexus, namely, the suprasternal, supraclavicular, and supra-acromial. 
The great occipital nerve is the internal branch of the posterior primary division of the second cervical nerve. It pierces the upper part of the complexus muscle, and sometimes the trapezius, about $\frac{1}{2}$ inch from the middle line. It then turns upwards, with an inclination outwards, and accompanies the occipital artery to the cranial integument, lying on the inner side of that vessel. Its branches are long, and have an extensive distribution, reaching as far as the vertex of the skull. Externally it communicates with the small occipital nerve, and internally with the least occipital.

The least or third occipital nerve is a small offset from the internal branch of the posterior primary division of the third cervical nerve. Having pierced the trapezius, it ascends internal to the great occipital nerve, with which it communicates, and has a limited distribution to the occipital integument inside that nerve.

The internal branches of the posterior primary divisions of the third, fourth, and fifth cervical nerves appear through the trapezius close to the middle line, and turn outwards to supply the skin of the back of the neck, that of the third furnishing, as just stated, the least occipital nerve.

The small occipital nerve is one of the ascending superficial branches of the cervical plexus, and arises from the anterior primary division of the second cervical nerve, sometimes receiving a branch from that of the third. It appears at the posterior border of the sterno-cleido-mastoid muscle, and ascends along that border to the occipital region. Here it divides into mastoid and occipital branches, which supply the skin of the mastoid, outer part of the occipital, and adjacent portion of the parietal, regions. It furnishes an auricular branch to the skin of the inner surface of the auricle, and it communicates with the great occipital, the mastoid and auricular branches of the great auricular, and the posterior auricular branch of the facial. The small occipital nerve is sometimes double, and in these cases one portion of it usually pierces the anterior border of the trapezius.

The great auricular nerve, like the small occipital, is one of the ascending superficial branches of the cervical plexus, and arises by two roots from the anterior primary divisions of the second and third cervical nerves. It turns round the posterior border of the sterno-cleido-mastoid immediately below the small occipital nerve, and passes upwards and forwards upon that muscle towards the lobule of the auricle, on approaching which it divides into mastoid, auricular, and facial branches. The mastoid branch is distributed to the skin over the mastoid region; the auricular branch supplies the skin of the inner surface of the auricle, and sends one or two twigs through the cartilage of the auricle to the skin covering its outer surface ; and the facial branch is distributed to the skin over the parotid gland. The mastoid and auricular branches communicate with the small occipital and posterior auricular nerves, and the facial branch communicates in the parotid gland with the cervico-facial division of the facial nerve. 
The superficial or transverse cervical nerve, like the small occipital and great auricular, is a superficial branch of the cervical plexus, and arises by two roots from the anterior primary divisions of the second and third cervical nerves. It appears at the posterior border of the sterno-cleido-mastoid, close below the great auricular,

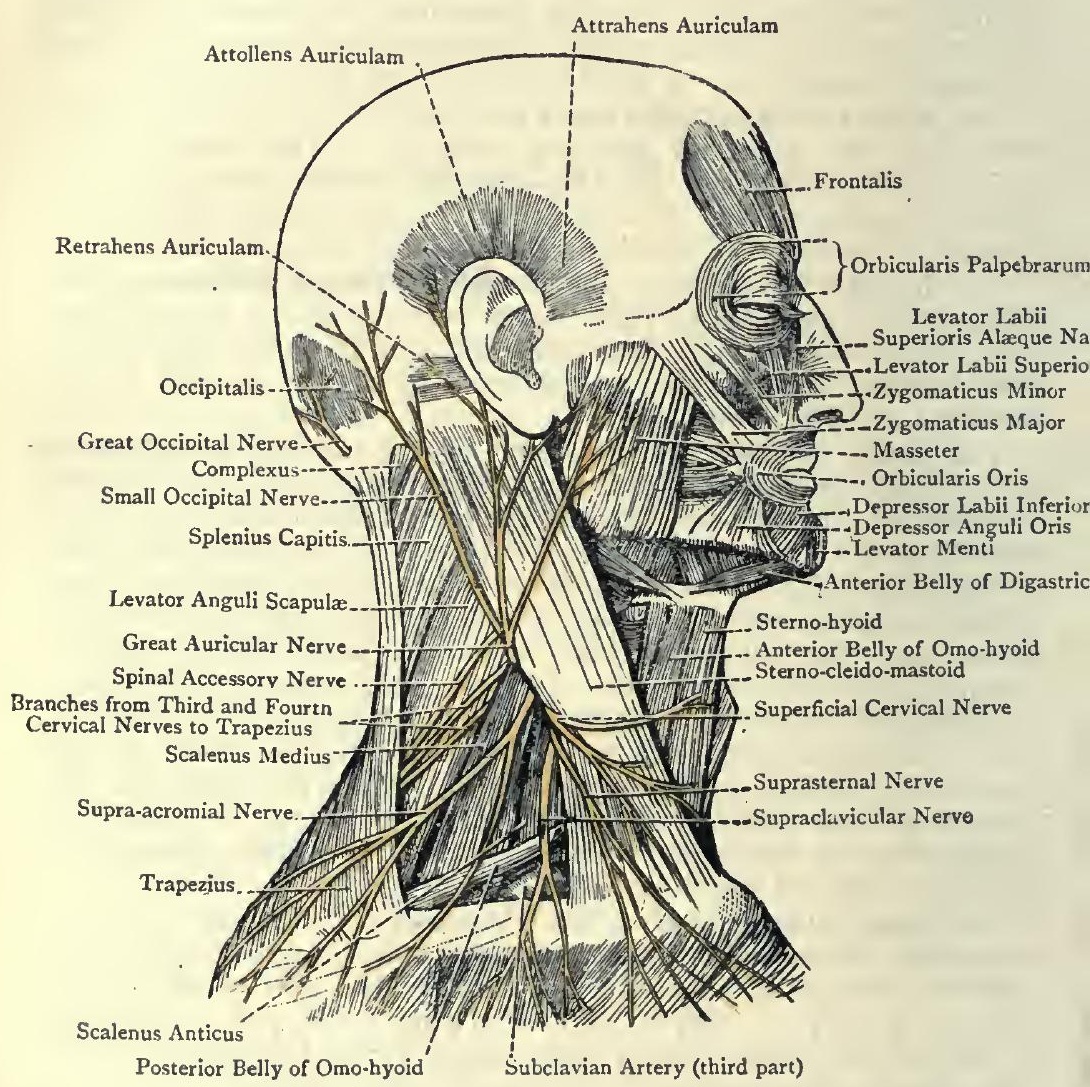

Fig. 435.-The Right Side of the Head and Neck.

(The Platysma Myoides has been removed, and the Nerves are shown).

and turns over that muscle to reach the front of the neck, where it will be afterwards described.

The spinal accessory nerve (spinal portion) appears at the posterior border of the sterno-cleido-mastoid near its centre, below the superficial cervical, and then crosses the posterior triangle of the neck downwards and backwards to the anterior border of the trapezius, beneath which it passes. It is accompanied by two small branches 
of the anterior primary divisions of the third and fourth cervical nerves, which lie below it, and it forms a plexiform communication with them beneath the trapezius, to which muscle it is distributed.

The descending superficial branches of the cervical plexus appear at the posterior border of the sterno-cleido-mastoid, below the spinal accessory, usually as a single trunk which arises by two roots from the anterior primary divisions of the third and fourth cervical nerves. This trunk, as it descends, divides into three nerves, namely, suprasternal, supraclavicular, and supra-acromial, which pass downwards over the clavicle, and will be afterwards referred to.

Muscles-Cervical Portion of the Trapezius-Origin.-(I) The inner third of the superior curved line of the occipital bone, and the external occipital protuberance ; and (2) the ligamentum nuchæ.

Insertion.-(I) The posterior border of the outer third of the clavicle; and (2) the inner border of the acromion process of the scapula.

Nerve-supply.-(I) The spinal accessory nerve (spinal portion); and (2) branches from the cervical plexus, which are derived from the anterior primary divisions of the third and fourth cervical nerves. The nerves enter the deep surface of the muscle after passing beneath its anterior border a little above the clavicle, and they here form a plexiform communication with each other.

Blood-supply.-There is one specially named artery, called the superficial cervical artery, which is a branch of the transverse cervical, and, like the nerves, enters the deep surface of the muscle.

The fibres are directed downwards, forwards, and outwards.

Action.-The cervical fibres, acting from their origin, elevate the outer end of the clavicle and the point of the shoulder. Acting from their insertion tbey extend the head, and incline the neck towards the same side, the face being directed towards the opposite side.

The anterior border of the muscle forms the posterior boundary of the posterior triangle of the neck.

Occasionally a small muscle, called the transversus nuchæ, is met with, extending from the external occipital protuberance to the tendon of insertion of the sterno-cleido-mastoid. It may be fleshy or tendinous:

Ligamentum Nuchæ.-This is a strong fibrous band which occupies the median line of the neck. Its superficial fibres are attached superiorly to the external occipital protuberance, and inferiorly to the spine of the seventh cervical vertebra. Its deep fibres are attached to the external occipital crest, and to the spines of cervical vertebræ, from the second to the sixth inclusive. They also extend into the interspinous intervals, between the interspinales muscles, where they represent interspinous ligaments.

Insertion and Posterior Border of the Sterno-cleido-mastoid.This muscle is inserted into (I) the outer surface of the mastoid process of the temporal bone, and (2) the superior curved line of 
the occipital bone over about its outer half, or more. The posterior border of the muscle forms the anterior boundary of the posterior triangle of the neck, and has the following nerves related to it: (I) the small occipital ascends along it to the head; (2) the great auricular passes upwards and forwards over it towards the lobule of the auricle ; (3) the superficial cervical crosșes it in a forward direction; (4) the spinal accessory appears from behind it, along with the branches of the third and fourth cervical nerves to the trapezius; and (5) the descending superficial branches of the cervical plexus emerge from underneath it.

The glandulæ concatenatæ belong to the deep cervical glands, and are situated along the posterior border of the sterno-cleidomastoid muscle. They will be afterwards described.

The levator anguli scapulæ, rhomboids, serratus posticus superior, splenius, complexus, transversalis cervicis, trachelo-mastoid, semispinalis colli, intertransversales, and interspinales muscles will be found described in connection with the muscles of the back, see p. 287 et seq.

Second and Third Parts of the Occipital Artery.-At the mastoid process this vessel lies very deeply. Having crossed the rectus capitis lateralis muscle, it enters the occipital groove on the inner aspect of the mastoid process, where it is covered by the following structures, in order from the surface downwards : (I) sterno-cleidomastoid; (2) splenius capitis; (3) trachelo-mastoid; (4) mastoid process ; and $(5)$ the posterior belly of the digastric. In its backward course the vessel rests, in succession, upon the insertions of the obliquus capitis superior and complexus, and it escapes from beneath the muscles covering it in the following order: posterior belly of the digastric, trachelo-mastoid, splenius capitis, and sternocleido-mastoid. After emerging from beneath the last-named muscle, it lies for a little in the posterior triangle, close to the apex, and then, piercing the occipital origin of the trapezius, it enters upon the third part of its course. This part ascends, along with the great occipital nerve, to the occipital region, where it divides in to several long tortuous branches.

Branches.-The branches of the second and third parts are as follows : mastoid, posterior meningeal, arteria princeps cervicis, communicating, muscular, and terminal. The mastoid artery passes through the arterial fissure on the outer surface of the mastoid process, and supplies the diploë and mastoid cells, as well as the adjacent dura mater. The posterior meningeal artery enters the cranial cavity through the jugular foramen, and takes part in the supply of the dura mater of the posterior fossa. The arteria princeps cervicis arises under cover of the splenius capitis, and at the outer border of the complexus it divides into a superficial and deep branch. The superficial branch is distributed to the splenius capitis and trapezius, and anastomoses, in the latter muscle, with the superficial cervical artery. The deep branch passes beneath the complexus, and anastomoses, upon the semispinalis 
colli, with (I) the deep cervical of the superior intercostal of the second part of the subclavian, and (2) branches of the vertebral artery. The communicating branches enter the suboccipital triangle, where they anastomose with branches of the vertebral artery. The muscular branches supply the contiguous muscles. The terminal branches are at first two in number, but these soon divide into several long tortuous branches, which supply the occipitalis and the integument as high as the vertex. They anastomose with the posterior auricular, the posterior division of the superficial temporal, and their fellows of the opposite side.

The occipital venous plexus communicates with the posterior auricular, and the posterior branch of the superficial temporal, veins. It receives the parietal emissary vein, which emerges through the parietal foramen, and so a communication is established with the superior longitudinal sinus. Sometimes it receives a small occipital emissary vein, which emerges through an opening at the external occipital protuberance, and then a communication would be established with the torcular Herophili.

Two or three occipital veins emerge from the occipital plexus. The external vessel is known as the posterior jugular vein. It receives the mastoid emissary vein, which emerges through the mastoid foramen, and so an important communication is established with the lateral sinus. Having been reinforced by tributaries from the superficial structures at the upper part of the back of the neck, the posterior jugular vein passes on to the sterno-cleido-mastoid, where it opens into the external jugular. The middle occipital vein (inconstant) accompanies the occipital artery, and usually opens into the internal jugular. The internal occipital vein, having in some cases pierced the trapezius, passes beneath the complexus, and enters the suboccipital triangle. In this situation it joins the suboccipital plexus, from which the deep cervical and vertebral veins emerge.

Occipital Glands.--These glands are usually two in number, and lie over the occipital portion of the trapezius, or over the upper part of the complexus. They receive their afferent lymphatics from the back part of the scalp, and their efferent lymphatics pass to the deep cervical glands.

Deep Cervical Artery.-This vessel is a branch of the superior intercostal of the second part of the subclavian. Having passed backwards between the transverse process of the seventh cervical vertebra and the neck of the first rib, it ascends upon the semispinalis colli, under cover of the complexus, towards the level of the spine of the axis, where it anastomoses with the deep branch of the arteria princeps cervicis of the occipital. In its course it furnishes a spinal branch, which enters the spinal canal through the intervertebral foramen for the eighth cervical nerve, and muscular branches, which anastomose with branches of the vertebral artery.

The deep cervical vein commences, within the suboccipital triangle, in the suboccipital plexus, which receives the internal occipital 
vein. It descends, in company with the deep cervical artery, to the lower part of the neck, where it passes forwards between the transverse process of the seventh cervical vertebra and the neck of the first rib, and ends by joining the vertebral vein just before that vessel terminates in the innominate vein.

In addition to the muscular branches of the occipital and deep cervical arteries, the deep muscles of the back of the neck receive twigs from the second part of the vertebral artery, which traverses the costo-transverse foramina of the upper six cervical vertebræ. These twigs pass backwards through the intertransverse spaces, and anastomose with branches of the arteria princeps cervicis and deep cervical arteries.

Suboccipital Region-Muscles - Rectus Capitis Posticus Major -Origin.- One of the tubercles in which the spine of the axis terminates.

Insertion.-The outer part of the inferior curved line of the occipital bone, and the subjacent area.

Nerve-supply. - The posterior primary division of the suboccipital nerve.

The direction of the fibres is upwards and outwards.

Action.-(I) To rotate the head towards the same side; and (2) to extend the head.

The muscle is triangular, and its insertion is slightly overlapped by the obliquus capitis superior.

Rectus Capitis Posticus Minor-Origin.-The posterior arch of the altas, close to, and encroaching upon, the posterior tubercle.

Insertion.-The inner third of the inferior curved line of the occipital bone, and the subjacent area as low as the foramen magnum.

Nerve-supply.-The posterior primary division of the suboccipital nerve.

The direction of the fibres is upwards in an expanded manner.

Action.-To extend the head.

The muscle is fan-shaped, and lies deeper than, and internal to, the rectus capitis posticus major.

Obliquus Capitis Inferior-Origin.-One of the tubercles in which the spine of the axis terminates.

Insertion.-The lower surface of the extremity of the transverse process of the atlas at its back part.

Nerve-supply.-The posterior primary division of the suboccipital nerve,

The direction of the fibres is outwards and slightly upwards.

Action.-To rotate the atlas, along with the head, towards the same side.

The muscle is thick and fleshy. At its origin it is interposed between the rectus capitis posticus major superiorly and the highest bundle of the semispinalis colli inferiorly. The great occipital nerve winds round its lower border.

Obliquus Capitis Superior-Origin.-The upper surface of the 
extremity of the transverse process of the atlas at its back part.

Insertion.-The occipital bone between the outer parts of the superior and inferior curved lines.

Nerve-supply.-The posterior primary division of the suboccipital nerve.

The direction of the fibres is upwards and inwards.

Action.-(I) To extend the head ; and (2) to act as a slight lateral flexor of the head.

The muscle is triangular.

Suboccipital Triangle.-This triangle is situated under cover of the upper part of the complexus, close below the occipital bone.

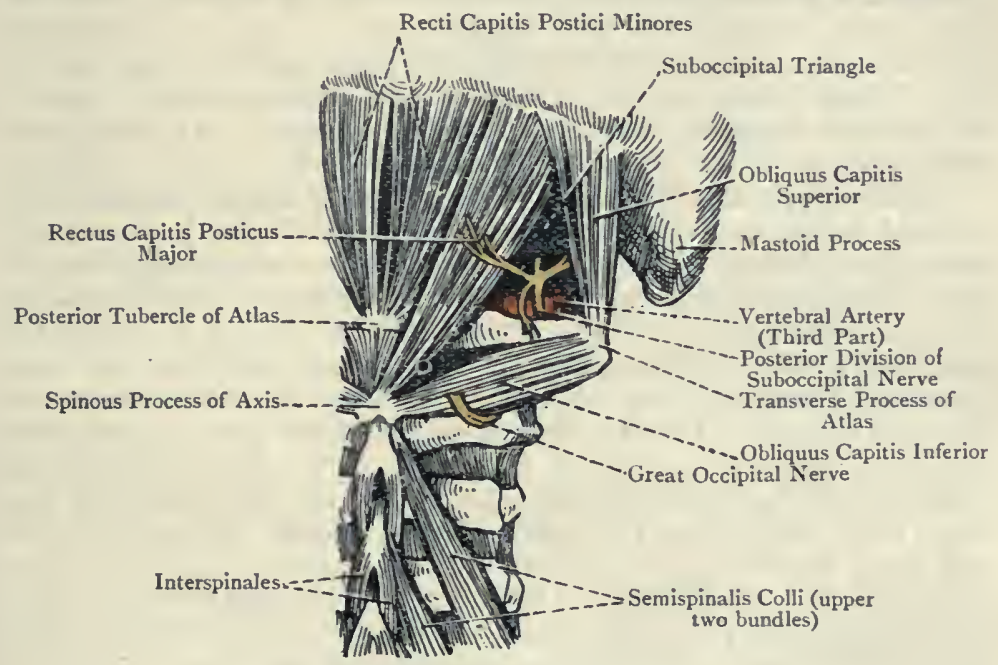

Fig. 436.-The Suboccipital Triangle.

Boundaries-Supero-internal.-The rectus capitis posticus major. Supero-external.-The obliquus capitis superior. Inferior.-The obliquus capitis inferior. The roof is formed by the complexus, and the floor by half of the posterior arch of the atlas, and half of the posterior occipito-atlantal ligament. The contents are (I) the third part of the vertebral artery, (2) the suboccipital plexus of veins, and (3) the posterior primary division of the suboccipital nerve.

Third Part of the Vertebral Artery.-The vertebral artery, having emerged from the costo-transverse foramen of the atlas, enters upon the third part of its course, and takes a winding course backwards and inwards. It lies in the vertebrarterial groove of the atlas, the posterior primary division of the suboccipital nerve being beneath it, and it passes under an arched band of the 
posterior occipito-atlantal ligament. It leaves the suboccipital triangle by piercing the dura mater, and enters the cranial cavity through the foramen magnum. The third part of the vessel furnishes muscular branches to the adjacent muscles which anastomose with the arteria princeps cervicis of the occipital, and the deep cervical of the superior intercostal.

Suboccipital Plexus of Veins.-This plexus is formed by branches which proceed from (I) the intraspinal venous plexus, $(2)$ the adjacent muscles, and (3) the occipital periosteum. It receives the internal occipital vein, and the blood is conveyed away from it by the deep cervical vein, already described, and the vertebral vein, which will be afterwards considered.

Posterior Primary Division of the Suboccipital Nerve.-This division passes backwards, as a single nerve, in the vertebrarterial groove of the atlas, lying between the bone and the third part of the vertebral artery. On entering the suboccipital triangle it divides into branches which supply the obliqui, recti postici, and complexus muscles.

Posterior Primary Divisions of the Cervical Spinal Nerves.-The cervical nerves are eight in number, the first being known as the suboccipital nerve. Each breaks up into an anterior and a posterior primary division. The posterior primary division of the first or suboccipital is single, and has just been described. The posterior primary divisions of the lower seven arise in the intervertebral foramina, and each soon divides into an external and internal branch. The external branches of all are distributed to the deep muscles, and do not become cutaneous. The internal branch of the posterior primary division of the second, which is of large size, constitutes the great occipital nerve. It winds round the lower border of the obliquus capitis inferior, and pierces the complexus, to which it gives branches. Thereafter it may pierce the trapezius to reach its cutaneous distribution, which has been already described. The internal, branches of the third, fourth, and fifth are directed inwards upon the semispinalis colli, to which, as well as to the complexus, they give branches. Close to the cervical spinous processes they pass backwards, internal to the biventer cervicis (part of the complexus), and, having pierced the trapezius, they reach the integument, which they supply. The internal branch of the posterior primary division of the third, before piercing the trapezius, gives off a small ascending branch, known as the least or third occipital. It passes upwards, lying at first under cover of the trapezius, and then passing through it, to reach the occipital integument. The internal branches of the posterior primary divisions of the sixth, seventh, and eighth are directed inwards towards the cervical spinous processes beneath the semispinalis colli, and are distributed to the adjacent muscles. They do not furnish any branches to the integument. 


\section{The Scalp and Temporal Region.}

Scalp-Fascia.-The superficial fascia is firm and granular, and is separated into lobules by fibrous processes which pass between the skin and the epicranial aponeurosis. The deep fascia is represented by the epicranial aponeurosis of the occipito-frontalis muscle, to be presently described.

Nerves of the Scalp Proper.-The great, small, and least or third occipital nerves, and the mastoid branch of the great auricular have been already described. In the fronto-parietal region two sensory nerves are met with, namely, the supra-orbital and supratrochlear, both of which are derived from the frontal nerve, which is one of the branches of the ophthalmic division of the fifth cranial nerve.

The supra-orbital nerve, which is of large size, leaves the orbit through the supra-orbital foramen. It then ascends, lying at first beneath the upper part of the orbicularis palpebrarum and the frontalis muscles, but subsequently piercing the latter muscle in two branches, outer and inner. The outer branch is the larger of the two, and its offsets extend well back over the parietal region. As the supra-orbital nerve passes through the supra-orbital foramen it furnishes one or two twigs to the mucous membrane of the frontal air-sinus and to the diploe of the frontal bone, and, after emerging from the foramen, it sends downwards palpebral branches to the integument of the upper eyelid. Thereafter the nerve supplies the integument of the fronto-parietal region.

The outer and inner branches of the supra-orbital nerve sometimes emerge from the orbit separately, the outer branch passing through the supra-orbital foramen, and the inner branch passing over the frontal notch.

The supratrochlear nerve, which is of small size, is internal to the supra-orbital nerve. It leaves the orbit close to the internal angular process of the frontal bone, where it lies above the trochlea or pulley of the superior oblique muscle of the eyeball. It then ascends beneath the upper part of the orbicularis palpebrarum and the frontalis muscles, and, piercing the latter muscle, it has a limited distribution to the frontal integument close to the median line. As the nerve leaves the orbit it furnishes palpebral twigs to the inner part of the integument of the upper eyelid.

Arteries.-The third part of the occipital artery has been already described. The arteries of the fronto-parietal region are three in number, namely, the supra-orbital, frontal, and anterior branch of the superficial temporal.

The supra-orbital artery is a branch of the ophthalmic, and emerges from the orbit with the supra-orbital nerve. It is distributed to the structures covering the frontal bone, and anastomoses with the frontal, and the anterior division of the superficial temporal, arteries. In the supra-orbital foramen it furnishes one or two branches to the mucous membrane of the frontal air-sinus and to 
the diploë of the bone, and, after emerging from the foramen, it sends downwards palpebral branches to the integument of the upper eyelid.

The supra-orbital vein passes downwards and inwards to a point just above the inner canthus, where it joins the frontal vein. In this manner the angular vein is formed, which is the commencement of the facial vein. The supra-orbital vein receives tributaries from the frontal region and the upper eyelid, and in the region of the inner canthus it communicates with the superior ophthalmic vein. It also communicates with the superior ophthalmic vein through the supra-orbital notch, and this communication receives the frontal diploic vein, which returns the blood from the diploë of the frontal bone and mucous membrane of the frontal air-sinus.

The frontal artery, like the supra-orbital, is a branch of the ophthalmic, and emerges from the orbit with the supratrochlear nerve. It is distributed to the structures over the frontal bone internal to the supra-orbital artery, with which it anastomoses, as well as with its fellow of the opposite side.

The frontal vein, of large size, passes downwards near the median line, and at a point just above the inner canthus is joined by the supra-orbital vein, the resultant vessel being the angular vein. At the root of the nose it communicates with its fellow of the opposite side by a transverse vessel, called the transverse nasal vein.

The supra-orbital and frontal veins communicate with each other, and with the tributaries of the anterior branch of the superficial temporal vein.

The anterior branch of the superficial temporal artery will be described in connection with the temporal region.

Occipito-frontalis.--This muscle consists of the following parts : ( $\mathrm{I}$ ) the occipitalis, (2) the frontalis, and (3) the epicranial aponeurosis.

Occipitalis. - This consists of two thin fleshy bellies, right and left. Origin.-(I) The outer parts of the highest and superior curved lines of the occipital bone; and (2) the outer surface of the mastoid process of the temporal bone, immediately above the insertion of the sterno-cleido-mastoid muscle.

Insertion.-The epicranial aponeurosis.

Each belly forms a thin, broad sheet, the length of the fasciculi being about $I_{\frac{1}{2}}$ inches. The two bellies, right and left, are separated towards the median line by a portion of the epicranial aponeurosis, which here dips down between them.

Frontalis.-This consists of two parts, right and left, which are separated superiorly by a narrow angular portion of the epicranial aponeurosis, but inferiorly, and for the most part, they are in contact. Origin.-(I) The subcutaneous tissue of the eyebrow and root of the nose, where the fibres blend with the upper part of the orbicularis palpebrarum and the corrugator supercilii; and (2) slightly from the external angular process of the frontal bone, and from the nasal bone.

Insertion.-The epicranial aponeurosis, a little below the level of the fronto-parietal suture. 
The fasciculi are about $3 \frac{1}{2}$ inches long, and the innermost fibres inferiorly are regarded by some as being prolonged downwards upon the nasal bone as the pyramidalis nasi muscle.

Epicranial Aponeurosis.-This is the intermediate tendon of the occipito-frontalis muscie. It forms an aponeurotic stratum beneath the superficial fascia, and is connected to the skin by fibrous processes, which separate the superficial fascia into lobules. Its deep surface is loosely connected by areolar tissue to the subjacent

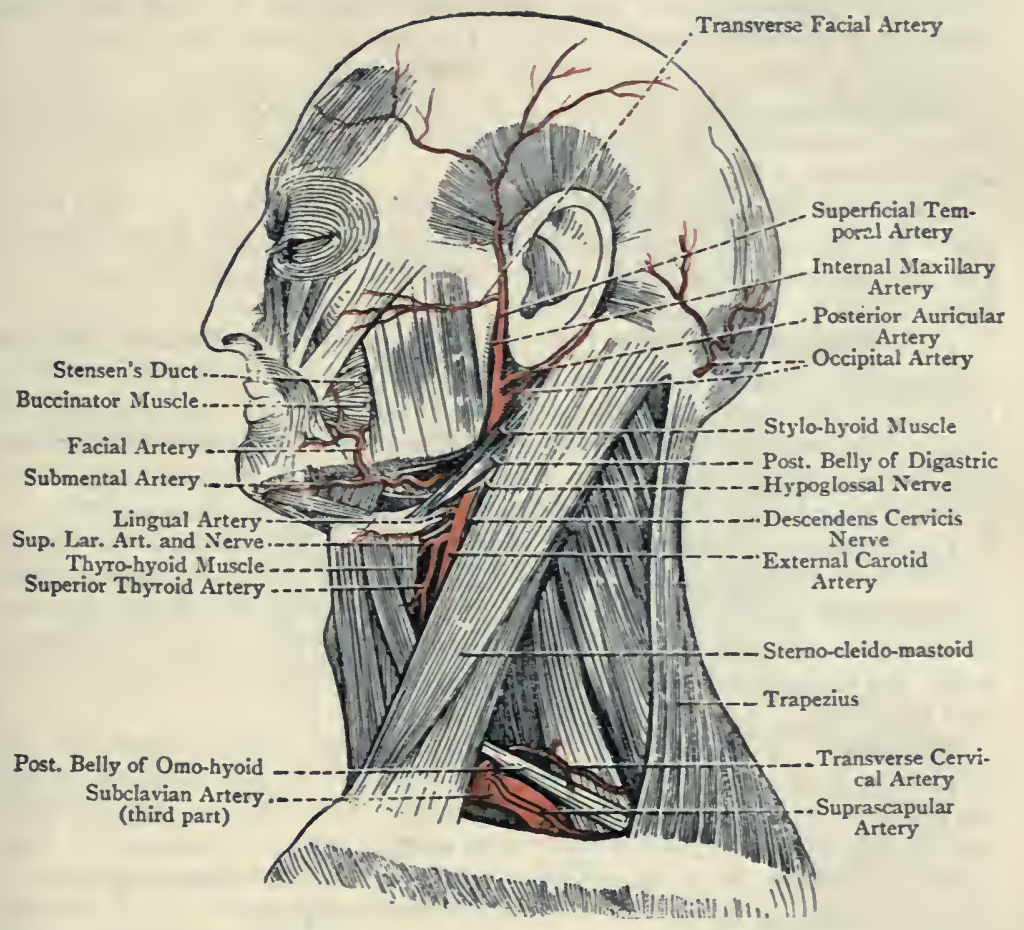

Frg. 43i-The Left Side of the Head and Neck.

(The Platysma Myoides has been removed).

periosteum. Posteriorly it gives insertion to the two bellies of the occipitalis muscle, and, in the interval between these, it dips down to be attached to the inner part of the highest curved line of the occipital bone and the external occipital protuberance. Anteriorly it gives insertion to the two halves of the frontalis muscle. Laterally, below the superior temporal ridge, it is prolonged downwards over the temporal fascia as a delicate expansion, which gives origin to the attollens auriculam and attrahens auriculam muscles.

Nerve-supply.-The occipitalis muscle is supplied by the pos- 
terior auricular branch of the facial nerve, and the frontalis muscle is supplied by the temporal branches of the temporo-facial division of the facial nerve.

Action.-The two bellies of the occipitalis draw backwards the epicranial aponeurosis, and along with it the scalp. The two halves of the frontalis, acting from below, draw forwards the epicranial aponeurosis, and along with it the scalp. Acting from above they elevate the eyebrows, and throw the skin of the frontal region into transverse wrinkles. When the occipitalis and frontalis act simultaneously the scalp is drawn backwards, and the eyebrows are forcibly raised. When the two muscles act alternately the scalp is drawn backwards and forwards.

Pericranium.-This is the periosteum of the cranium, and it is loosely connected by areolar tissue to the superjacent epicranial aponeurosis.

Temporal Region-Nerves.-These are as follows: (I) the temporal branches of the temporo-facial division of the facial nerve ; (2) the auriculo-temporal nerve; and (3) the temporal branch of the orbital or temporo-malar nerve.

The temporal branches of the temporo-facial division of the facial nerve pass upwards a little in front of the auricle, and supply the following muscles: the frontalis, upper part of the orbicularis palpebrarum, corrugator supercilii, attollens auriculam, and attrahens auriculam. They communicate with the auriculo-temporal, temporal branch of the orbital, and supra-orbital nerves.

The auriculo-temporal nerve is a branch of the posterior division of the inferior maxillary nerve, which is the third division of the fifth cranial nerve. It emerges from the upper part of the parotid gland, and ascends immediately in front of the auricle, in company with the superficial temporal artery, lying between that vessel and the auricle.

Branches.-In this part of its course the nerve furnishes the following branches: (I) two meatal branches to the meatus auditorius externus, which enter the canal between its osseous and cartilaginous walls, and supply the integument of the meatus, the upper of the two branches giving an offset to the membrana tympani ; (2) two anterior auricular branches, which supply the skin of the tragus and outer aspect of the auricle over its upper half; and (3) temporal branches, which supply the integument above and in front of the auricle, and of the temporal region generally.

The temporal branch of the orbital or temporo-malar nerve from the superior maxillary is of small size, and pierces the temporal fascia about $I$ inch above the anterior part of the zygoma, and close behind the marginal process of the malar bone. Having communicated with one of the temporal branches of the facial nerve, it has a limited distribution to the integument of the anterior part of the temporal region.

The superficial temporal artery is one of the terminal branches of the extcrnal carotid artery, from which it arises within the parotid gland on a level with the neck of the lower jaw. Emerging from the 
gland it ascends in front of the auricle, in company with the auriculo-temporal nerve, which lies between the vessel and the auricle, and, after a course of about 2 inches, it divides into its two terminal branches.

Branches. - These are as follows: (I) transverse facial ; (2) articular; (3) anterior auricular; (4) middle temporal; (5) orbital; and (6) terminal, namely, anterior temporal and posterior temporal.

The transverse facial branch will be described in connection with the face. It arises within the parotid gland, and in its course

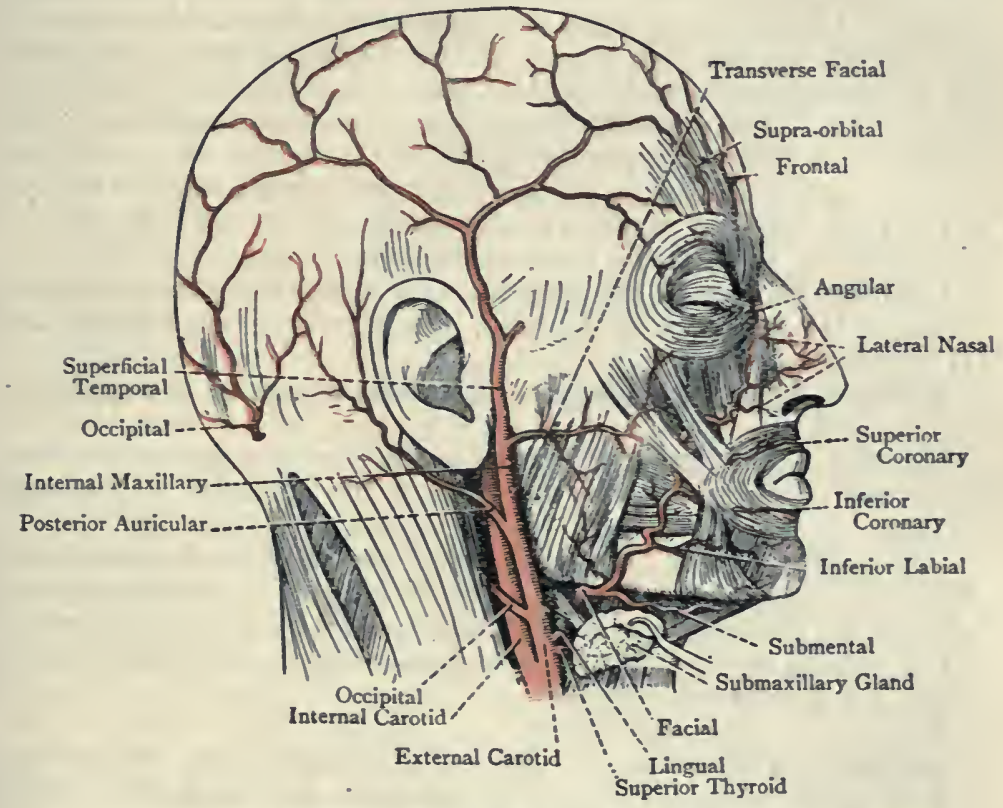

Fig. 438. - The Arteries of the Right Side of the Head (After L. Testut's 'ANatomie Humaine').

furnishes glandular branches. The articular branches supply the temporo-mandibular joint. The anterior auricular branches supply the outer aspect of the auricle, and in part the meatus auditorius externus. The middle temporal branch pierces the temporal fascia and temporal muscle immediately above the zygoma, and then ascends in a groove on the outer surface of the squamous part of the temporal bone. It gives branches to the temporal muscle, and anastomoses with the deep temporal arteries, which are branches of the internal maxillary. The orbital branch passes forwards close above the zygoma, where it lies between the two layers of the temporal fascia. It is distributed to the outer part of the orbicularis palpebrarum muscle. The anterior temporal branch passes forwards and upwards in a tortuous manner, and is distributed 
to the structures covering the frontal bone, where it anastomoses with the supra-orbital and frontal branches of the ophthalmic artery, and with its fellow of the opposite side. The posterior temporal branch takes an arched course upwards and backwards above the auricle, supplying the adjacent structures, and anastomosing with the posterior auricular and occipital arteries, and with its fellow of the opposite side.

The superficial temporal vein is formed in front of the auricle by the union of an anterior and a posterior branch, the former communicating freely with the supra-orbital and frontal veins, and the latter with the posterior auricular vein and the occipital venous plexus. Above the zygoma it is joined by the middle temporal vein, which originates in a plexus in the temporal fossa, and pierces the temporal muscle and temporal fascia, receiving in its course the orbital vein. The superficial temporal vein then descends over the zygoma and enters the parotid gland, within which it receives the anterior auricular, articular, and transverse facial veins. Finally, it joins the internal maxillary vein about the level of the neck of the lower jaw to form the temporo-maxillary vein.

Extrinsic Muscles of the Auricle.-These are three in number, as follows : (I) attollens auriculam; (2) attrahens auriculam; and (3) retrahens auriculam.

Attollens Auriculam-Origin.-The lateral part of the epicranial aponeurosis.

Insertion.-The inner or cranial surface of the auricle over the convexity corresponding to the fossa of the antihelix on its outer surface.

Nerve-supply.-The temporal portion of the temporo-facial division of the facial nerve. The posterior part of the muscle may be supplied by the posterior auricular branch of the facial nerve.

The muscle is very thin, pale, and fan-shaped, and its fibres converge in a downward direction.

Action.-To raise the auricle.

Attrahens Auriculam - Origin.-The lateral part of the epicranial aponeurosis in front of the attollens auriculam, with which muscle it is continuous.

Insertion.-The anterior part of the helix of the auricle.

Nerve-supply. - The temporal portion of the temporo-facial division of the facial nerve.

The muscle is directed backwards.

Action.-To draw forwards the auricle.

Retrahens Auriculam-Origin.-The upper part of the outer surface of the mastoid process of the temporal bone.

Insertion.--The inner or cranial surface of the auricle over the convexity of the concha.

Nerve-supply.-The posterior auricular branch of the facial nerve.

The muscle is directed forwards.

Action.-To draw back the auricle.

The retrahens auriculam is more distinct than the other two muscles, and is usually arranged in two bundles. 
Posterior Auricular Nerve.-This is a branch of the facial nerve immediately after it emerges from the aqueduct of Fallopius through the stylo-mastoid foramen. It passes upwards between the mastoid process and the auricle, where it lies deeply in company with the posterior auricular artery, and it divides into two branches-auricular and occipital. The auricular branch supplies the retrahens auriculam muscle on its deep aspect, and those intrinsic muscles of the auricle which lie on its inner or cranial aspect. It may also send a branch upwards beneath the retrahens auriculam to supply the posterior part of the attollens auriculam. The occipital branch passes backwards, and supplies the corresponding half of the occipitalis muscle. The posterior auricular nerve communicates with the small occipital and great auricular nerves, and also with the auricular branch (Arnold's nerve) of the pneumogastric.

Nerve of Arnold.-This is the auricular branch of the ganglion of the root of the pneumogastric nerve. Having traversed the auricular canaliculus in the petrous part of the temporal bone, it emerges through the auricular or tympano-mastoid fissure, between the mastoid process and tympanic plate, and thereafter divides into two branches-auricular and communicating. The auricular branch takes part in the supply of the inner or cranial surface of the auricle, and also supplies the lower and back part of the meatus auditorius externus ; and the communicating branch joins the posterior auricular nerve.

Posterior Auricular Artery.-This vessel is one of the two posterior branches of the external carotid artery, the other branch being the occipital artery. Having passed backwards and upwards under cover of the parotid gland, it lies deeply between the mastoid process and the auricle, in close relation to the posterior auricular nerve, and divides into two branches-auricular and mastoid. The auricular branch supplies the inner or cranial surface of the auricle and adjacent structures, and it anastomoses with the posterior branch of the superficial temporal artery. The mastoid branch passes backwards to the corresponding half of the occipitalis muscle, and anastomoses with the occipital artery.

The posterior auricular vein is of fairly large size, and returns the blood from the lateral portion of the scalp posteriorly, and the inner surface of the auricle. Its radicles communicate with the occipital plexus and with the posterior branch of the superficial temporal vein. The vessel passes downwards and forwards over the upper part of the sterno-cleido-mastoid muscle, and terminates about the level of the angle of the lower jaw by joining the posterior division of the temporo-maxillary vein to form the external jugular vein.

Posterior Auricular, or Mastoid, Glands.-These glands lie close to the insertion of the sterno-cleido-mastoid muscle. They receive their afferent lymphatics from the lateral part of the back of the scalp and the back of the auricle, and their efferent lymphatics pass to the superficial cervical glands. 
Temporal Fascia.-This is a strong aponeurosis which covers the temporal muscle. Superiorly it is attached from before backwards to the temporal border of the malar bone, the superior temporal ridge of the frontal and parietal bones, and the supramastoid crest of the temporal bone. Inferiorly it divides into two laminæ, which are attached to the outer and inner margins of the upper border of the zygomatic arch. Between these two laminæ there are a small amount of adipose tissue and the orbital branch of the superficial temporal artery. Superficial to the fascia there are the delicate prolongation of the lateral portion of the epicranial aponeurosis and the attollens and attrahens auriculam muscles. Superiorly the fascia gives origin, by its deep surface, to superficial fibres of the temporal muscle, but it is separated from that muscle towards the zygoma by adipose tissue.

Temporal Muscle - Origin.-(I) The temporal fossa, extending as high as the inferior temporal ridge of the frontal and parietal bones, and as low as the infratemporal crest on the external surface of the great wing of the sphenoid ; and (2) the deep surface of the temporal fascia over its upper part.

The muscle is fan-shaped. The anterior fasciculi descend almost vertically; the posterior fasciculi pass almost horizontally forwards; and the intervening fasciculi descend with varying degrees of obliquity.

A complete description of the temporal muscle will be found on p. I200.

Lymphatic Vessels of the Scalp-Frontal Region.-These pass to the intraparotid lymphatic glands. Temporo-parietal Region.The anterior lymphatics pass to the intraparotid glands, and the posterior lymphatics terminate in the mastoid, or posterior auricular, glands.

\section{The Cranial Cavity.}

The meninges of the encephalon, namely (I) the dura mater, with its septa and venous sinuses, (2) the arachnoid membrane, and (3) the pia mater, will be found described in Index.

Cranial Nerves at the Base of the Skull.-The cranial nerves are arranged in twelve pairs, and as they leave the cranial cavity they receive sheaths from the meninges of the encephalon.

The olfactory bulb rests upon one-half of the cribriform plate of the ethmoid bone. Through the foramina of that plate it receives about twenty olfactory flaments, which arisc as the axons of the olfactory cells of the olfactory mucous membrane of the nasal fossa.

The nasal nerve passes forwards and inwards beneatl the dura mater on one-half of the cribriform plate of the ethmoid bone to the nasal slit.

The optic nerve passes forwards and outwards from the optic commissure to the optic foramen, through which it enters the orbit. It is accompanied by the ophthalmic artery, which lics beneath it.

The third or oculo-motor nerve pierces the dura mater, which 
forms the outer wall of the cavernous sinus, a little in front of the posterior clinoid process of the sphenoid bone.

The fourth or trochlear nerve, of small size, pierces the dura mater at a point a little behind the posterior clinoid process of the sphenoid bone, external to the third nerve, and beneath the free margin of

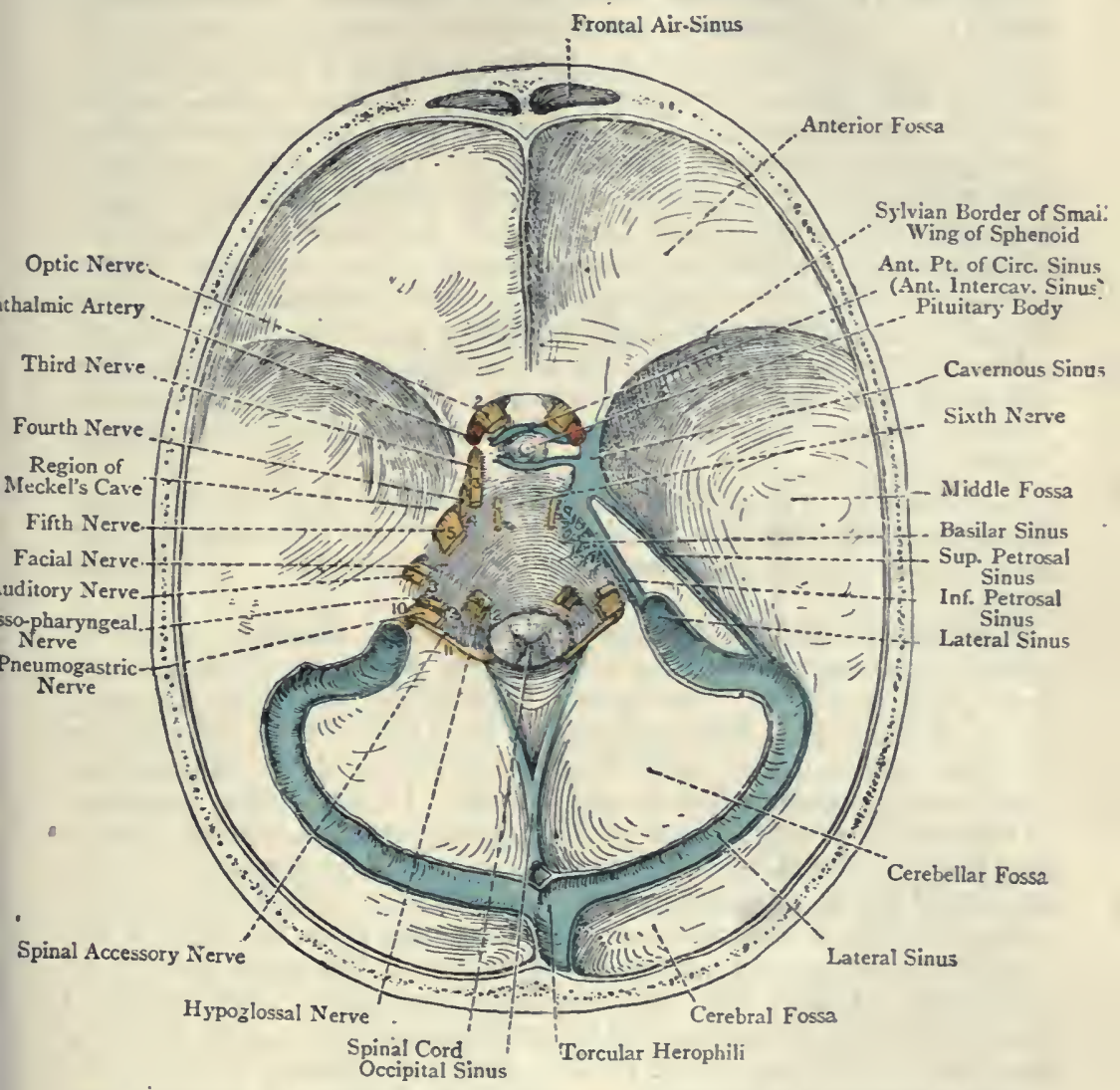

Fig. 439.-The Internal Base of the Skull, Showing the Craniat Nerves and Venous Sinuses.

the tentorium cerebelli. Thereafter it traverses the outer wall of the cavernous sinus.

The fifth or trigeminal nerve consists of two roots-sensory and motor. These two roots pierce the dura mater at the apex of the petrous part of the temporal bone, and enter a recess of the dura mater, called Meckel's cave, where they will be presently described.

The sixth or abducent nerve pierces the dura mater at the outer. border of the dorsum sellæ of the sphenoid bone, a little below the 
posterior clinoid process, where it occupies a notch on the bone. It lies a little to the inner side of the fifth nerve.

The seventh or facial nerve, the eighth or auditory nerve, and the pars intermedia of Wrisberg, accompanied by the internal auditory artery, enter the meatus auditorius internus.

The ninth or glosso-pharyngeal nerve, the tenth or pneumogastric nerve, and the eleventh or spinal accessory nerve pass through the middle compartment of the jugular foramen. This foramen is divided into three compartments, namely, antero-internal, middle, and postero-external. The antero-internal compartment gives passage to the inferior petrosal sinus. The middle compartment transmits the glosso-pharyngeal, pneumogastric, and spinal accessory nerves, in this order from before backwards. The glossopharyngeal nerve pierces the dura mater separately, and receives special sheaths from the dura mater and arachnoid membrane. The pneumogastric and spinal accessory nerves pass through the same aperture in the dura mater, and receive common sheaths from the dura and arachnoid membrane. The postero-external compartment transmits the lateral sinus, which terminates in the internal jugular vein.

The twelfth or hypoglossal nerve pierces the dura mater in two bundles, which pass through separate apertures. As these bundles pass through the anterior condylar foramen they unite to form one nerve.

Structures passing through the Foramen Magnum.-(I) The medulla oblongata, with its membranes, passes out and becomes the spinal cord. (2) The spinal portion of the spinal accessory nerve, of either side, having ascended from the interval between the posterior roots of the cervical spinal nerves and the ligamentum denticulatum, enters the cranial cavity. It then turns outwards to the middle compartment of the jugular foramen, where it meets the bulbar portion of the nerve, with which it becomes closely connected as it passes through the foramen. (3) The vertebral artery of each side enters the cranial cavity, after having left the vertebrarterial groove on the upper aspect of the posterior arch of the atlas. (4) The two anterior spinal branches of the vertebral arteries descend on the front of the medulla oblongata, and, inclining inwards towards each other, they unite in passing through the foramen magnum to form the anterior spinal artery. (5) The two posterior spinal branches of the vertebral arteries pass outwards through the foramen magnum. (6) Parts of the cerebellar amygdalie.

Gasserian Ganglion.-The Gasserian ganglion occupies the Gasserian depression on the superior surface of the petrous part of the temporal bone near the apex, where it lies in a recess of the dura mater, called Meckel's cave. It is somewhat semilunar, being convex in front and concave behind, and measures fully $\frac{1}{2}$ inch from before backwards. It is associated with the sensory root of the fifth nerve, which, before entering the posterior concave aspect of the ganglion, becomes expanded, its fasciculi at the same 
time becoming separated and assuming a plexiform arrangement. Internally the ganglion is intimately related to the back part of the cavernous sinus and the internal carotid artery at the foramen lacerum medium, and on this aspect it receives delicate filaments from the carotid sympathetic plexus. The motor root of the fifth nerve, small in size, lies in close contact with the deep surface of the ganglion; but there is no blending of the two sets of fibres, the motor root passing independently in an outward direction to the foramen ovale. The great superficial petrosal nerve, on its way to the foramen lacerum medium, passes beneath the ganglion.

The ganglion contains cells similar to those of a spinal ganglion, and it receives its blood from the middle and small meningeal arteries.

Branches.-The branches of the ganglion arise from its anterior convex aspect, and are known as divisions. They are as follows : the first or ophthalmic division; the second or superior maxillary division; and the third or inferior maxillary division. The ophthalmic and superior maxillary nerves are entirely sensory, but the inferior maxillary nerve, being joined by the motor root, is both sensory and motor.

The first division of the fifth nerve, or the ophthalmic nerve, is the smallest of the three branches of the ganglion. It passes forwards in the outer wall of the cavernous sinus, where it lies below the fourth nerve. On approaching the sphenoidal fissure it parts with its chief branches, in the following order from behind forwards: (I) nasal, (2) lachrymal, and (3) frontal, the last-named representing the continuation of the nerve. All these three branches enter the orbit through the sphenoidal fissure. As the ophthalmic nerve passes forwards it receives a communicating branch from the cavernous sympathetic plexus, and it furnishes a recurrent meningeal branch to the tentorium cerebelli. It also communicates with each of the third, fourth, and sixth nerves.

The second division of the fifth, or the superior maxillary nerve, passes horizontally forwards for a short distance in the lower part of the outer wall of the cavernous sinus, and it leaves the cranial cavity through the foramen rotundum, which leads to the sphenomaxillary fossa. Before leaving the cranial cavity it furnishes a delicate recurrent meningeal branch to the dura mater of the middle fossa.

The third division of the fifth, or the inferior maxillary nerve, is the largest of the three branches of the ganglion. It passes downwards to the foramen ovale, through which it leaves the cranial cavity, and so enters the zygomatic fossa. It is accompanied by the motor root of the fifth nerve, which joins it as it passes through the foramen ovale. The recurrent meningeal branch of the inferior maxillary nerve is not given off until after the parent trunk has issued through the foramen ovale, and it enters the cranial cavity through the foramen spinosum, in company with the middle meningeal artery. It then divides into two offsets, one of which supplies the dura mater over the great wing of the sphenoid bone, 
and the other passes through the fissure between petrous and squamous parts of the temporal bone to supply the mucous membrane of the mastoid cells.

Great Superficial Petrosal Nerve.-This nerve is a branch of the geniculate ganglion of the facial nerve in the aqueduct of Fallopius. It enters the middle fossa of the base of the skull through the hiatus Fallopii, and passes forwards and inwards in a groove on the superior surface of the petrous part of the temporal bone. Having passed beneath the Gasserian ganglion, it enters the upper part of the foramen lacerum medium, where it is placed on the outer side of the internal carotid artery, and it joins the great deep petrosal nerve from the carotid sympathetic plexus. In this manner the Vidian nerve is formed, which enters the pterygoid or Vidian canal by its opening on the anterior aspect of the foramen lacerum medium, and so reaches Meckel's ganglion in the spheno-maxillary fossa. The great superficial petrosal nerve is accompanied by the petrosal branch of the middle meningeal artery.

Small Superficial Petrosal Nerve.-This nerve represents the continuation, through the tympanic plexus, of the tympanic branch (Jacobson's nerve) of the glosso-pharyngeal nerve. It is reinforced by a small branch from the geniculate ganglion of the facial nerve, which joins it as it traverses a small canal in the petrous part of the temporal bone, beneath the canal for the tensor tympani muscle. The nerve enters the middle fossa through the accessory hiatus on the superior surface of the pars petrosa. It then passes for a little forwards and inwards, and leaves the cranial cavity through the canaliculus innominatus (when present), or through the fissure between the pars petrosa and the great wing of the sphenoid, or, it may be, through the foramen ovale, after which it terminates in the otic ganglion.

External Superficial Petrosal Nerve.-This nerve, which is inconstant, passes from the sympathetic plexus on the middle meningeal artery backwards and outwards on the superior surface of the petrous part of the temporal bone, and it leaves the cranial cavity through a minute aperture situated within the thin margin of the hiatus Fallopii. It terminates in the geniculate ganglion of the facial nerve.

Interior of the Cavernous Sinus.-The interior of this sinus is broken up by a number of delicate trabeculæ, which are arranged in a reticular manner. The outer wall of the sinus contains the following cranial nerves, in order from above downwards : the third, the fourth, the ophthalmic division of the fifth, and the superior maxillary division of the fifth. These nerves, as they pass forwards, are separated from the blood-current by the endothelial lining of the outer wall of the sinus. The cavity of the sinus is traversed by (I) the cavernous part of the internal carotid artery, accompanied by the cavernous sympathetic plexus, and (2) the sixth cranial nerve, which lies in close contact with the outer side of the internal carotid artery. These structures are also separated from the blood-current by the endothelial lining of the sinus.

For the processes and other sinuses of the dura mater see. Index.

Cavernous Part of the Internal Carotid Artery.-This part of the internal carotid artery lies within the cavernous sinus, which occupies the cavernous groove on the lateral aspect of the body of the sphenoid bone. It is separated from the blood-current by the endothelial lining of the sinus. The course of the vessel is at first upwards, between the lingula sphenoidalis and the posterior petrosal 
process of the sphenoid ; then forwards, as far as the anterior clinoid process of the sphenoid ; and, finally, upwards internal to the anterior clinoid process. In the latter situation it pierces the dura mater which forms the roof of the cavernous sinus, and enters upon the cerebral part of its course.

The vessel is accompanied by the cavernous sympathetic plexus, and the sixth cranial nerve lies in close contact with its outer side, all being invested by the endothelial lining of the sinus.

Branches.-These are as follows : pituitary, to the pituitary body ; ganglionic to the Gasserian ganglion; cavernous, to the wall of the cavernous sinus, and to the third, fourth, fifth, and sixth cranial nerves; meningeal, to the dura mater of the middle cranial fossa; and the ophthalmic artery. The latter vessel arises from the cavernous part of the internal carotid on the inner side of the anterior clinoid process, and it enters the orbit, with the optic

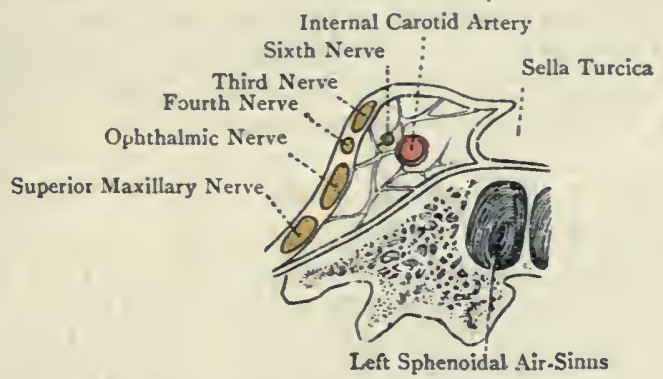

Fig. 4 40.-Diagram of the Left Cavernous Venous Sinus and its Outer Wall, showing the Relative Positions of the Contained StrucTURES (Posterior VIEW).

nerve, through the optic foramen, lying at first beneath the nerve, and then on its outer side.

Cavernous Sympathetic Plexus.-This plexus is situated principally on the inner and lower aspects of - the bend which the cavernous part of the internal carotid artery describes internal to the anterior clinoid process. Its branches are as follows: (x) vascular, to the internal carotid artery and its branches; (2) pituitary, to the pituitary body; (3) communicating, to the third, fourth, ophthalmic division of the fifth, and sixth cranial nerves; and (4) the sympathetic root of the ciliary ganglion. The last-named branch enters the orbit through the sphenoidal fissure.

The pituitary body (hypophysis cerebri) is a small oval mass which is situated in the pituitary fossa, or sella turcica, of the sphenoid bone. It lies under cover of a circular fold of the dura mater, called the diaphragma sella, in the centre of which is an opening for the passage of the infundibulum. Its long measurement extends transversely, and it consists of two lobes-anterior or buccal, and posterior or cerebral. The anterior lobe is the larger of the two, and the posterior 
lobe is connected with the anterior part of the tuber cinereum by means of the infundibulum.

The infundibulum projects downwards from the anterior part of the tuber cinereum to the posterior lobe of the pituitary body. Its upper part is hollow, and contains a funnel-shaped diverticulum of the cavity of the third ventricle.

Structure.-The anterior lobe consists of several tubules, lined with epithelium, and invested by capillary bloodvessels. The posterior lobe, though developed from the brain, is destitute of nervous elements. It is composed of a reticulum of connective tissue, which contains branched cells.

Development. - The anterior lobe is developed from a diverticulum of the buccal or stomatodral epiblast, which is known as the pouch of Rathke. The diverticulum grows upwards, and, when the cranio-pharyngeal canal of early life becomes closed, the connection of the diverticulum with the buccal epiblast is severed. The diverticulum thereafter becomes converted into tubules, which form the anterior lobe. The posterior lobe of the pituitary body and the infundibulum, which is connected with it, are developed as a diverticulum which grows downwards from that part of the thalamencephalon or diencephalon which forms the floor of the third ventricle. The cavity of the diverticulum remains permanent in the upper part of its infundibular portion, but elsewhere it becomes obliterated. The lower part of the diverticulum thereafter becomes converted into a reticulum of connective tissue, with branched cells, which forms the posterior lobe.

\section{Side of the Neck.}

The side of the neck has a quadrilateral outline, the boundaries of which are as follows : superior, one half of the base of the inferior maxilla, and a line prolonged from the angle of that bone over the mastoid process to the inner third of the superior curved line of the occipital bone; inferior, the clavicle, and one half of the upper border of the manubrium sterni; anterior, the middle line of the neck, that is to say, a line connecting the symphysis of the inferior maxilla with the centre of the upper border of the manubrium sterni ; and posterior, a line connecting the inner third of the superior curved line of the occipital bone with the outer third of the clavicle.

Landmarks. - The base and angle of the inferior maxilla can easily be felt, and the clavicle, together with the upper border of the manubrium sterni, is conspicuous. A deep depression, called the suprasternal or jugular fossa, is perceptible above the upper border of the manubrium sterni, lying between the sternal heads of origin of the sterno-cleido-mastoid muscles. The outline of the sternocleido-mastoid muscle is readily discernible when the head is turned so as to direct the face towards the opposite shoulder. The muscle extends in a diagonal direction from the sterno-clavicular joint to the mastoid process and outer half or two-thirds of the superior curved line of the occipital bone, and it divides the side of the neck into two triangles - anterior and posterior. A small triangular interval may be felt between the sternal and clavicular heads of origin of the sterno-cleido-mastoid, just above the inner end of the clavicle, in which the common carotid artery and internal jugular vein lie deeply. The external 
jugular vein crosses the sterno-cleido-mastoid muscle in the direction of a line extending from a point just behind the angle of the inferior maxilla to a point above the centre of the clavicle, and it is accompanied by the superficial cervical glands. The common carotid artery lies under cover of the anterior border of the sternocleido-mastoid muscle, in the direction of a line drawn from the sterno-clavicular joint to a point midway between the angle of the inferior maxilla and the tip of the mastoid process of the temporal bone. The vessel extends along this line as high as the level of the upper border of the thyroid cartilage, and thereafter it is replaced by the external carotid artery. The internal jugular vein is close to the outer side of the common carotid artery, and the pneumogastric nerve lies deeply between the two vessels. If deep pressure is made over the common carotid artery, on a level with the cricoid cartilage of the larynx, the tubercle of the costal process of the sixth cervical vertebra (commonly spoken of as the anterior tubercle of the transverse process) may be felt. It is known as the carotid tubercle of Chassaignac.

The commencement of the right innominate vein and the bifurcation of the innominate artery lie behind the upper border of the right sterno-clavicular joint; and the commencement of the left innominate vein and the left common carotid artery lie behind the left sterno-clavicular joint. Near the middle line of the neck the anterior jugular vein descends vertically. The spinal portion of the spinal accessory nerve passes downwards and outwards beneath the anterior border of the sterno-cleido-mastoid to pierce the deep part of that muscle at about the junction of the upper fourth and lower three-fourths. The nerve is met with fully I inch below the tip of the mastoid process, and in the direction of a line let fall vertically from the mastoid tip. It is very nearly on a level with the body of the hyoid bone. Along the posterior border of the sterno-cleido-mastoid the lymphatic glands, known as the glandulæ concatenatæ, may be felt.

Important structures occupy the middle line of the neck, and can be usually distinguished without difficulty. These are as follows, in order from above downwards: (I) the body of the hyoid bone, lying just below the inferior maxilla, and having the great cornu projecting bachwards and upwards on either side; (2) the thyro-hyoid membrane ; (3) the thyroid cartilage, with its prominent angle or isthmus (pomum Adami) in the middle line, leading up to the $\mathrm{V}$-shaped thyroid notch on the upper border, and its expanded ala on either side, each of which terminates posteriorly in a superior and an inferior cornu ; (4) the crico-thyroid membrane; (5) the narrow, anterior part of the cricoid cartilage, which coincides with the level at which the anterior belly of the omo-hyoid muscle crosses the carotid sheath ; and (6) the rings of the trachea, which, however, are covered superiorly by the isthmus of the thyroid body, and inferiorly by the sterno-hyoid and sterno-thyroid muscles and adipose tissue. 
The lateral lobes of the thyroid body may be felt on either side of the larynx and trachea as low as about the fifth ring; and, in young children, the thymus body extends upwards upon the trachea for some distance above the manubrium sterni.

The great cormu of the hyoid bone is the guide to the lingual artery for operative purposes, the vessel lying just above the great cornu.

The upper border of the thyroid cartilage is on a level with the disc between the bodies of the third and fourth cervical vertebræ.

The narrow anterior part of the cricoid cartilage is on a level with the disc between the bodies of the fifth and sixth cervical vertebræ, at which level the pharynx terminates in the osophagus. In this situation a foreign body, when swallowed, is liable to become impacted.

Above the middle third of the clavicle, between the sternocleido-mastoid and trapezius muscles, there is a depression known as the supraclavicular fossa, which indicates the position of the subclavian or supraclavicular triangle, with the third part of the subclavian artery and the trunks of the brachial plexus of nerves.

Platysma Myoides-Origin.-The superficial fascia which covers the clavicular parts of the pectoralis major and deltoid muscles.

Insertion.-(I) The outer aspect of the base of the inferior maxilla from the symphysis to the anterior border of the masseter muscle; and (2) the angle of the mouth, where the fibres blend with those of the depressor anguli oris and orbicularis oris.

Nerve-supply. - The inframandibular (cervical) branch of the cervico-facial division of the facial nerve, which communicates with branches of the superficial cervical nerve.

The fibres are directed upwards and forwards or inwards over the clavicle and side of the neck.

Action.-(I) To draw the angle of the mouth downwards and outwards; (2) to act as a feeble depressor of the inferior maxilla; (3) to raise the skin of the neck and upper pectoral region, as far out as the acromion process, throwing it into obliquely-disposed folds. By diminishing pressure upon the external jugular vein during forced inspiration the muscle contributes to the return of blood through that vessel.

The muscle forms an extensive, thin, pale sheet, which is embedded within the superficial fascia. In the region of the symphysis of the inferior maxilla the innermost fibres decussate across the middle line with those of the opposite side, the fibres of the right muscle being superficial. The muscle covers the external and anterior jugular veins, the superficial branches of the cervical plexus of nerves, the subclavian triangle, and the sterno-cleidcmastoid, sterno-hyoid, omo-hyoid, and digastric muscles.

The platysma myoides in man is a remnant of a subcutaneous muscular sheet, called the panniculus carnosus, which exists in many animals, and by which the twitching of the skin is produced.

Jugular Veins. - The jugular veins are four in number on either side, namely, anterior, external, posterior, and internal. 
The anterior jugular vein commences in the roof of the submaxillary or digastric triangle, where it is formed by the union of radicles which communicate with the submental vein, and are joined by radicles which have descended from the structures over the body of the inferior maxilla. It descends vertically near the median line, lying at first superficial to the deep cervical fascia, but sub-

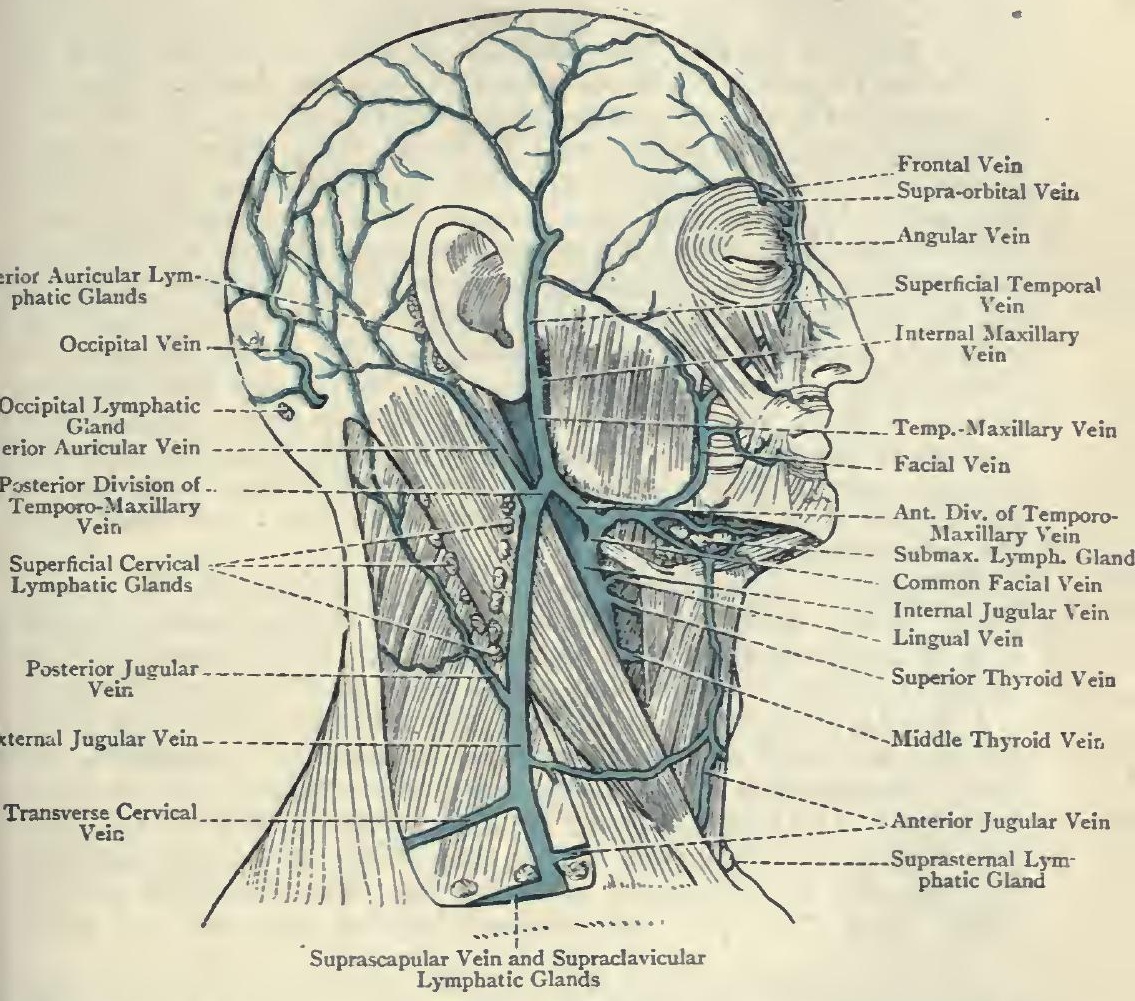

Fig. 44ri - The Superficial Veins and Superficial Lymphatic Glands of the Right Side of the Head and Neck.

(The Platysma Myoides Muscle has been removed).

sequently entering the suprasternal interfascial space, or space of Burns. It then describes a bend, and, passing outwards behind the sterno-cleido-mastoid muscle, and in front of the scalenus anticus, it opens into the lower part of the external jugular vein. It may, however, open into the subclavian vein. It communicates with the external jugular vein by one or more branches, and it usually receives a branch from the facial vein which descends along the anterior 
border of the sterno-cleido-mastoid and joins it towards the lower part of the neck. Further, it communicates with its fellow of the opposite side by a transverse branch which crosses in front of the trachea, and lies in Burns's space.

The anterior jugular veins are usually asymmetrical, one or other being of small size. Occasionally there is only one vein, which is mesially placed, and, under these circumstances, it divides into two vessels inferiorly. The anterior jugular vein is destitute of valves.

The external jugular vein commences close behind the angle of the inferior maxilla in the substance of the parotid gland, where it is formed by the union between the posterior division of the temporomaxillary vein and the posterior auricular vein. Having emerged from the parotid gland it descends almost vertically to a point above the centre of the clavicle. In its course the vessel crosses the sternocleido-mastoid muscle, lying superficial to its sheath, and beneath the superficial fascia containing the fibres of the platysma myoides. At this level the main part of the superficial cervical nerve courses forwards beneath the vein, and offsets of the nerve pass over it. Having crossed the sterno-cleido-mastoid muscle, the vein descends not far from its posterior border, where it lies in the roof of the subclavian or supraclavicular triangle, over the third part of the subclavian artery. Up to this point the vessel is superficial to the deep cervical fascia, but it now pierces that fascia and opens into the subclavian vein.

The course of the external jugular vein is indicated by a line drawn from a point close behind the angle of the inferior maxilla to a point above the centre of the clavicle. Its tributaries are as follows : (I) posterior jugular, which joins it about the middle of the neck ; (2) transverse cervical ; (3) suprascapular ; and (4) anterior jugular, the latter three joining it not far from its termination. It also communicates with the anterior jugular vein by one or more branches. The vessel is provided with valves, both at its termination and about $I \frac{1}{2}$ inches above this point, and the transverse cervical and suprascapular veins are also furnished with valves, where they open into the external jugular vein, or near their orifices.

The external jugular vein is sometimes very small, or even absent ; and it may communicate with the cephalic vein by a vessel which passes over the clavicle.

Development.-The external jugular vein is usually regarded as being developed from the primitive jugular vein; but according to some authorities the primitive jugular vein gives rise to the internal jugular vein, the external jugular being of later development.

The posterior jugular vein represents the most external occipital vein, and is formed by tributaries which issue from the outer part of the occipital.plexus, reinforced by veins from the superficial structures at the back of the neck. Having usually received the mastoid emissary vein, it passes downwards and forwards, and joins the external jugular vein about the middle of the neck. 
The internal jugular vein will be found described on p. III3.

Superficial Cervical Glands.--These are about six in number, and they lie upon the sterno-cleido-mastoid muscle, along the course of the external jugular vein, and beneath the superficial fascia and platysma myoides muscle. They receive their afferent lymphatics from the adjacent superficial structures, the occipital and mastoid glands, and some of those of the parotid and submaxillary lymphatic glands. Their efferent lymphatics pass to the deep cervical and supraclavicular glands.

Deep Cervical Fascia.-The deep cervical fascia is divided into (I) a superficial layer, which completely invests the neck in the form of a collar, and (2) deep processes or laminæ, which invest the muscles, viscera, and chief bloodvessels and nerves.

Superficial Layer.- This layer is attached posteriorly to the ligamentum nuchæ; superiorly to (I) the superior curved line of the occipital bone; (2) the mastoid process of the temporal bone; (3) the zygomatic arch; and (4) the base of the inferior maxilla, under cover of the platysma myoides, as far forwards as the symphysis; and inferiorly to the clavicle and upper border of the manubrium sterni, being pierced above the centre of the clavicle by the external jugular vein. Along the middle line of the neck anteriorly it is continuous with the superficial layer of the opposite side.

The superficial layer invests the cervical portion of the trapezius, and from the anterior border of that muscle it passes forwards over the posterior triangle of the neck to the posterior border of the sterno-cleido-mastoid. In doing so it furnishes a deep process, which ensheathes the posterior belly of the omo-hyoid muscle. This process also embraces the intervening tendon of that muscle, after which it passes downwards and inwards to be attached to the back of the inner end of the clavicle and the first rib. In this manner the horizontal position of the posterior belly of the omohyoid is accounted for. When the superficial layer of the deep cervical fascia reaches the posterior border of the sterno-cleidomastoid it splits into two laminæ which ensheathe that muscle, the superficial lamina being underneath the external jugular vein and platysma myoides. At the anterior border of the sterno-cleidomastoid the two laminæ reunite, and the fascia passes forwards over the anterior triangle of the neck to the median line, where it is continuous with the corresponding layer of the opposite side.

Between the upper part of the anterior border of the sternocleido-mastoid and the angle of the inferior maxilla the superficial layer is of considerable strength, and draws that border of the muscle forwards and upwards, so as to render it convex and keep it over the line of the leading vessels. Between the mastoid process and the angle of the inferior maxilla the superficial layer is prolonged upwards over the parotid gland as the parotid fascia, which is very dense, and is attached superiorly to the lower border of the zygomatic arch. 
Below the level of the thyroid body the superficial layer divides into two laminæ, anterior and posterior, both of which are superficial to the depressor muscles of the hyoid bone. At the middle line these laminæ are continuous with those of the opposite side, and inferiorly they are attached to the anterior and posterior margins of the upper border of the manubrium sterni. Between them there is an interfascial interval, called the suprasternal space, or space of Burns. This interval contains areolar tissue, one or more lymphatic glands, the lower portions of the anterior

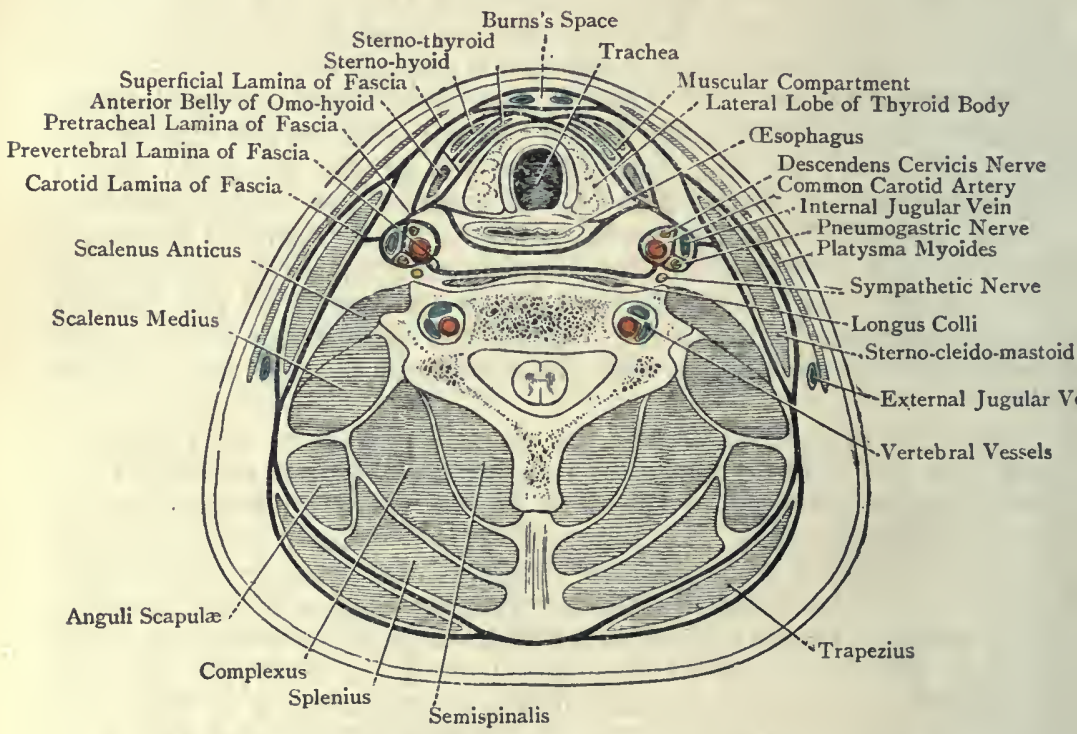

Fig. 442.-Diagram of a Transverse Section of the Neck at the Level of the Sixth Cervical Vertebra, showing the Arrangement of the Deep Cervical Fascia, and the Positions of Other STRUCTURES.

jugular veins, with the transverse vessel which here connects them, and the sternal heads of the sterno-cleido-mastoid muscles.

Deep Processes or Laminæ.-The deep laminæ, as stated, invest the muscles, viscera, and chief bloodvessels and nerves. The most important are derived from that lamina of the superficial layer which forms the posterior wall of the sheath of the sternocleido-mastoid muscle, and they are three in number, namely, carotid, pretracheal, and prevertebral, all of which have an intimate initial connection.

The carotid lamina splits to form the carotid sheath, which contains, in separate compartments ( $I$ ) the common carotid artery and descendens cervicis nerve, (2) the internal jugular vein, and (3) the 
pneumogastric nerve, the latter being contained within the back part of the septum, which separates the artery from the vein.

The pretracheal or visceral lamina, which is at first intimately connected with the anterior wall of the carotid sheath, passes forwards behind the depressor muscles of the hyoid bone, in which situation it splits to ensheathe the thyroid body, trachea, and œsophagus, and then it passes to the median line, where it is continuous with the. pretracheal lamina of the opposite side. The pretracheal lamina is attached superiorly to the body of the hyoid bone, and inferiorly it descends over the trachea and bloodvessels into the superior mediastinum of the thorax, where it blends with the fibrous layer of the pericardium.

The prevertebral lamina, which is at first intimately connected with the posterior wall of the carotid sheath, passes forwards behind the pharynx and œsophagus, and in front of the prevertebral muscles. It is known as the prevertebral fascia. At the middle line it is continuous with the corresponding lamina of the opposite side ; superiorly it is attached to the base of the skull ; and inferiorly it descends over the longus colli muscle into the posterior mediastinum of the thorax. Along a line corresponding to the inner wall of the carotid sheath the pretracheal lamina furnishes a secondary lamina, called the bucco-pharyngeal fascia, which covers the constrictor muscles of the pharynx and the buccinator muscle. Between the bucco-pharyngeal and prevertebral fasciæ there is an interval, called the retro-pharyngeal space, which contains the loosely arranged connective tissue uniting the two fasciæ. This space extends as high as the base of the skull, and inferiorly is continuous with the posterior mediastinum of the thorax. Another process of the prevertebral fascia passes downwards and outwards in front of the scalenus anticus muscle. Thereafter it invests the third part of the subclavian artery and subclavian vein, together with the nerve-trunks of the brachial plexus, and, passing behind the clavicle, it becomes continuous with the axillary sheath, which latter blends with the posterior aspect of the costo-coracoid membrane. In the region of the subclavian triangle there is an interfascial space between this process of the pretracheal fascia and the superficial layer of the deep cervical fascia. This space extends downwards behind the clavicle to the point where the axillary sheath and costo-coracoid membrane join. It contains the posterior belly of the omo-hyoid muscle, the suprascapular and transverse cervical vessels, the lower part of the external jugular vein, and the terminal portion of the anterior jugular vein.

Interfascial Compartments. - It has been stated that the deep cervical fascia reaches the median line of the neck anteriorly in three layers, namely, anterior or superficial in (two divisions), pretracheal, and prevertebral. It. is therefore evident that there are four interfascial compartments, as follows: (I) the suprasternal space, or space of Burns, which is situated between the two divisions of the anterior or superficial layer, and contains the structures 
already enumerated ; (2) the muscular compartment, which is situated between the anterior or superficial layer and the pretracheal layer, and contains the depressor muscles of the hyoid bone ; (3) the visceral compartment, which lies between the pretracheal and prevertebral layers, and contains the larynx, trachea, thyroid body, pharynx, œsophagus, and carotid sheath, the retro-pharyngeal space being in the subdivision of this compartment behind the bucco-pharyngeal fascia; and (4) the vertebral compartment, which lies between the prevertebral layer and the attachment of the fascia to the ligamentum nuchæ posteriorly, and contains the vertebral column, spinal cord, and prevertebral and postvertebral muscles.

The suprasternal and muscular compartments are shut off from the thoracic cavity. The visceral compartment, in front of the trachea, is continuous with the superior mediastinum of the thorax, and behind the œsophagus it, along with the retro-pharyngeal space, is continuous with the posterior mediastinum.

Parotid Process of the Deep Cervical Fascia.-This process is given off a little below the angle of the inferior maxilla, and it passes upwards on the deep surface of the parotid gland to the skull. Along with the parotid fascia superficial to the gland it forms a dense sheath which closely invests the glandular substance. The parotid process furnishes sheaths to the posterior belly of the digastric, styloid, and pterygoid muscles, and it also gives an investment to the superficial part of the submaxillary gland. Connected with the parotid process there are several bands, usually called ligaments, which are as follows: (I) spheno-mandibular; (2) stylo-mandibular; (3) pterygo-mandibular; and (4) pterygospinous.

The spheno-mandibuiar ligament will be described in connection with the temporo-mandibular joint, of which it ranks as the internal lateral ligament.

The stylo-mandibular ligament extends from the styloid process of the temporal bone, near its tip, to the angle and adjacent part of the posterior border of the ramus of the inferior maxilla, where it is placed between the masseter and internal pterygoid muscles.

The pterygo-mandibular ligament is a narrow band which extends from the hamular process of the internal pterygoid plate of the sphenoid bone to the posterior extremity of the mylo-hyoid ridge of the inferior maxilla, close to the last molar alveolus. Anteriorly it gives origin to fibres of the buccinator muscle, and posteriorly to fibres of the superior constrictor muscle of the pharynx.

The pterygo-spinous ligament is a narrow band which extends from a sharp spine on the posterior border of the external pterygoid plate of the sphenoid bone, towards its upper part, to the spinous process of the sphenoid. This ligament is liable to become ossified.

Sterno-cleido-mastoid-Origin.-The sternal head, which is narrow and round, arises from the upper and outer part of the anterior surface of the manubrium sterni. It is tendinous in front, 
and fleshy behind. The clavicular head, which is broad and flat, arises from a rough ridge about $I \frac{1}{2}$ inches long on the upper surface of the clavicle at its inner end, near the postero-superior border.

Insertion.-(I) The outer surface of the mastoid process of the temporal bone, and (2) the superior curved line of the occipital bone over about its outer half or two-thirds.

Nerve-supply.-(I) The spinal portion of the spinal accessory nerve, and (2) a branch from the cervical plexus, more particularly from the anterior primary division of the second cervical nerve.

The spinal portion of the spinal accessory nerve passes beneath the anterior border of the muscle fully $I$ inch below the tip of the mastoid process, and, in passing downwards and backwards, it pierces the deep part of the muscle, giving off, as it does so, its branches to it.

The muscle is directed upwards, outwards, and backwards.

Action.-(I) To flex the head towards the side on which the muscle is placed, the face being turned towards the opposite side. This is the position of the head in torlicollis or wry-neck, a condition which may be due to an organic contraction of the muscle, affecting both heads, or, it may be, the sternal head alone. (2) When both muscles act together from their origin they flex the head towards the thorax. (3) When both muscles act together from their insertion they elevate the upper part of the anterior thoracic wall in forced inspiration.

The sternal and clavicular heads are separated by a triangular cellular interval for a short distance above the sterno-clavicular joint, and before their junction the fibres of the clavicular head to a large extent pass behind those of the sternal head, so that overlapping takes place. The muscle is surrounded by a strong sheath, which is formed by the deep cervical fascia. The platysma myoides covers a large part of it, and the external jugular vein, the superficial cervical and great auricular nerves, and the superficial cervical glands are related to its superficial surface, under cover of the platysma myoides. The principal deep relations are as follows: in the lower part of the neck it covers the first and second parts of the subclavian artery, the sterno-hyoid, sterno-thyroid, omohyoid, and scalenus anticus muscles, with the phrenic nerve lying upon the last-named muscle. In this situation it also covers the anterior jugular vein, and the transverse cervical and suprascapular arteries. Higher up it covers the cervical plexus of nerves, the levator anguli scapulæ, scalenus medius, and scalenus posticus muscles, and the spinal portion of the spinal accessory and the hypoglossal nerves. At its insertion it covers the splenius capitis, trachelo-mastoid, and posterior belly of the digastric muscles, and a portion of the occipital artery, in this order from the surface downwards. The anterior border of the muscle forms the posterior boundary of the anterior triangle of the neck, and covers the carotid sheath, with its contents, as high as the level of the upper border of the thyroid cartilage; and above that level it covers the 
external and internal carotid arteries. This border also overlaps slightly the lateral lobe of the thyroid body. The posterior border forms the anterior boundary of the posterior triangle of the neck, and along it there lie the following structures: the glandulæ concatenatæ, the small occipital nerve, great auricular, superficial cervical, spinal portion of the spinal accessory, and the descending superficial branches of the cervical plexus of nerves, and a portion of the external jugular vein.

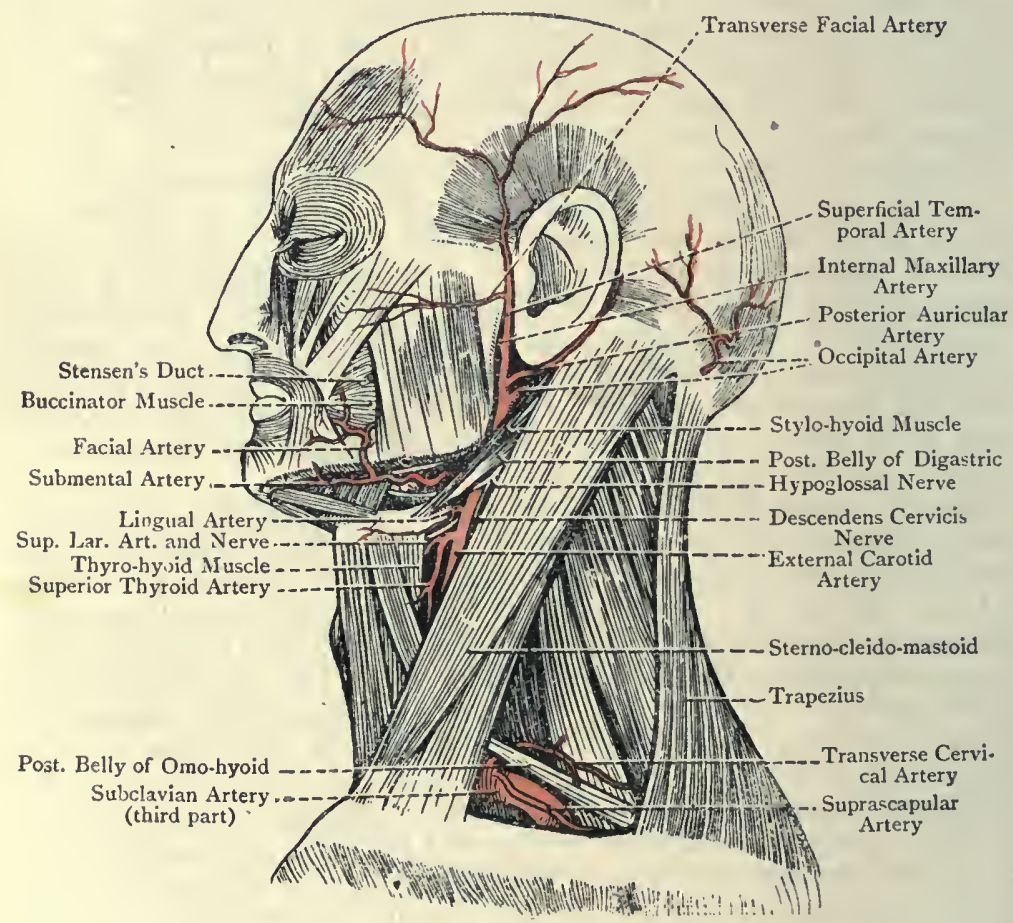

Fig. 443.-The Left Side of the Head and Neck.

(The Platysma Myoides has been removed).

The sterno-cleido-mastoid musclc, from its diagonal position upon the side of the neck, divides the quadrilateral space into two triangles-posterior and anterior.

Posterior Triangle.-This is the region which lies behind the sterno-cleido-mastoid muscle.

Boundaries-Anterior. - The posterior bolder of the sternocleido-mastoid. Posterior.-The anterior horder of the trapezius. Inferior (base).- The middle third of the clavicle. The apex is at 
the superior curved line of the occipital bone, where the sternocleido-mastoid and trapezius may meet, but the apex is usually truncated. The roof is formed by the skin, superficial and deep fasciæ, and, for a short distance inferiorly, by the platysma myoides. The small occipital nerve lies in the upper part of the roof, and the descending superficial branches of the cervical plexus and the external jugular vein lie in the lower part of the roof. The floor is formed by the following muscles, in order from above downwards : (I) a small angle of the complexus, provided the trapezius is not well dereloped at the occiput; $(2)$ the splenius capitis; (3) the levator anguli scapulæ; (4) the scalenus medius and scalenus posticus; (5) the posterior belly of the omo-hyoid; and (6) the scalenus anticus.

The posterior triangle is subdivided by the posterior belly of the omo-hyoid into a large upper portion, called the occipital triangle, and a small lower portion, called the subclavian or supraclavicular triangle.

Occipital Triangle-Boundaries-Anterior.-The posterior border of the sterno-cleido-mastoid. Posterior. - The anterior border of the trapezius. Inferior (base).-The posterior belly of the omohyoid. The muscles in its floor are (I) a small angle of the complexus (inconstant), (2) splenius capitis, (3) levator anguli scapulæ, and (4) scaleni medius et posticus. The contents are the superficial branches of the cervical plexus, the spinal accessory nerve, the branches of the cervical plexus to the levator anguli scapulæ and trapezius, a small part of the occipital artery close to the apex, and the glandulæ concatenatæ.

Cervical Plexus.- The cervical plexus is situated under cover of the upper part of the sterno-cleido-mastoid muscle, and lies immediately in front of the slips of origin of the scalenus medius. It is formed by the anterior primary divisions of the first three cervical nerves and the greater part of that of the fourth, a small branch of the latter descending to join the anterior primary division of the fifth, and so taking part in the brachial plexus. Each of the anterior primary divisions of the first four cervical nerves is connected with the superior cervical ganglion of the sympathetic by a grey ramus communicans.

The anterior primary division of the first cervical nerve lies at first in the vertebrarterial groove of the atlas under cover of the vertebral artery. It then passes forwards in a groove on the outer surface of the superior articular process of the atlas, having the vertebral artery on its outer side. It next emerges between the rectus capitis lateralis muscle (to which it gives a branch) and the rectus capitis anticus minor, and descends in front of the root of the costal process of the atlas to join the ascending branch of the second nerve. From the loop so formed branches are given to the recti antici muscles, major and minor, and one or more branches pass to the hypoglossal nerve, the destination of their fibres being the descendens cervicis and thyro-hyoid nerves. 
The anterior primary divisions of the second, third, and fourth cervical nerves, having emerged between the corresponding intertransversales muscles, divide each into an ascending and a descending branch, which unite with adjacent branches to form loops. The descending branch of the fourth nerve, as stated, joins the fifth, and enters into the brachial plexus. The cervical plexus is therefore a looped plexus, the number of loops being three.

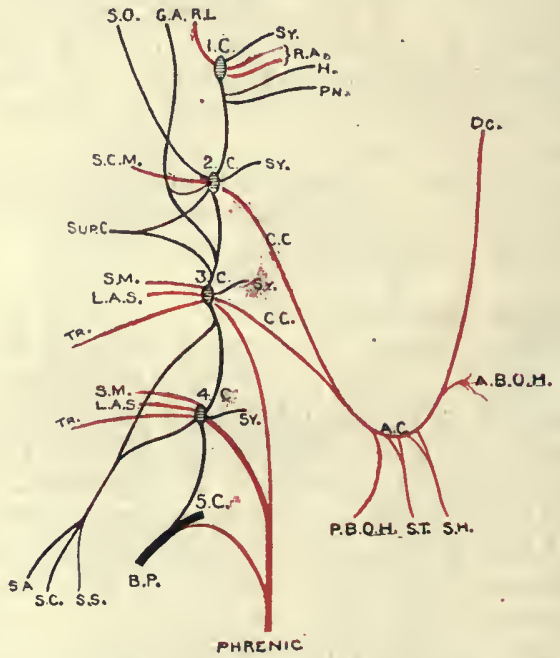

Fig. 444--Scheme of the Cervical Plexus of Nerves.

The Descendens Cervicis Nerve is also shown.

Ic, 2c, 3c, Anterior Primary Divisions of 4c, 5c. Upper Five Cervical Nerves

R.I. To Rectus Lateralis

G.A. Great Auricular

S.O. Small Occipital

S.C.M. To Sterno-cleido-mastoid

Sup.C. Superficial Cervical

S.M., S.M. To Scalenus Medius

L.A.S., To Levator Anguli Scapulæe

$T_{R}$. TR. To Trapezius

S.A. Supra-acromial

S.S. Suprasternal
Sv. Branches to Sympathetic

R.A. To Rectus Capitis Anticus Major et Minor

H. To Hypoglossal

PN. To Pneumogastric (Ganglion of Trunk)

C.C., C.C. Communicantes Cervicis

D.C. Descendens Cervicis

A.B.O.H. To Anterior Belly of Ono-hyoid

A.C. Ansa Cervicis

S.H. To Sterno-hyoid

S.T. To Sterno-thyroid

P.B.O.H. To Posterior Belly of Omo-hyoid

Branches.-These are divided into two groups-superficial and deep.

Superficial Group.-The nerves of this group are ascending, transverse, and descending.

The ascending and transverse nerves arise from the second and third cervical nerves.

The ascending nerves are the small occipital and great auricular (sec p. I059). The transverse nevve is the superficial (transverse) cerviçal. 
The superficial or transverse cervical nerve arises by two roots from the anterior primary divisions of the second and third cervical nerves, and, turning round the posterior border of the sternocleido-mastoid muscle, it passes forwards over the muscle, lying beneath the platysma myoides and the external jugular vein. Having reached the anterior triangle of the neck, it divides into two branches, upper and lower, which are distributed to the integument over the anterior triangle. The offsets of the upper branch communicate freely with the inframandibular branch of the facial nerve beneath the platysma myoides.

The descending nerves are the suprasternal, supraclavicular, and supra-acromial, and they arise in common from the third and fourth cervical nerves. As they descend they form distinct nerves, which lie in the roof of the subclavian triangle under cover of the platysma myoides. For their distribution, see p. 298.

Deep Group.-The nerves of this group are arranged in two setsexternal and internal.

External Set.-The nerves of this set are muscular. (I) The second nerve furnishes a branch to the sterno-cleido-mastoid, which communicates in that muscle with the branch of the spinal portion of the spinal accessory nerve. (2) The third and fourth nerves furnish $(a)$ two branches to the trapezius, which communicate in a plexiform manner with the spinal portion of the spinal accessory nerve beneath the upper part of the muscle; $(b)$ two branches to the levator anguli scapulæ; and (c) branches to the scalenus medius.

Internal Set.-The nerves of this set are communicating and muscular.

The communicating branches are as follows: (I) connecting branches (grey rami communicantes) pass from the upper cervical ganglion of the sympathetic ; (2) communicating branches pass to the pneumogastric and hypoglossal nerves from the highest loop of the plexus; and (3) two rami communicantes cervicis pass from the second and third nerves forwards and downwards, usually over, but sometimes underneath, the internal jugular vein, and terminate by joining the descendens cervicis, conjointly or separately, thus forming the ansa cervicis (ansa hypoglossi).

The muscular branches are distributed to the rectus capitis lateralis, recti antici, major and minor, upper part of the scalenus anticus, longus colli, and the diaphragm. The nerve to the diaphragm is the phrenic nerve (internal respiratory nerve), and, from its importance, it requires a special description.

The phrenic rerve arises, as a rule, by two roots, the larger of which is derived from the anterior primary division of the fourth cervical nerve, and the other from that of the third. In some cases the fifth cervical nerve, which enters into the brachial plexus, furnishes an additional small root. In the neck the nerve descends upon the scalenus anticus muscle, which it crosses obliquely downwards and inwards, passing beneath the tendon of the omo-hyoid muscle, the transverse cervical and supra. 
scapular arteries, and the anterior jugular vein. At the root of the neck the nerve; having left the scalenus anticus, passes behind the terminal part of the subclavian vein, and crosses over the internal mammary artery from without inwards. Having come into contact with the inner surface of the dome of the pleura, it disappears behind the inner end of the clavicle, and enters upon the thoracic part of its course (see p. 942).

The right nerve at the root of the neck is superficial to the second part of the right subclavian artery, with the intervention of the scalenus anticus muscle. The left nerve at the root of the neck is anterior and parallel to the first part of the left subclavian artery, and lies behind the terminal part of the thoracic duct.

The phrenic nerve is sometimes reinforced towards the root of the neck by a branch from the nerve to the subclavius muscle, and, when this takes place, the root from the fifth cervical nerve is usually absent. Before leaving the neck the phrenic nerve receives a twig from the middle, or lower, cervical ganglion of the sympathetic.

No branches arise from the phrenic nerve in the neck.

Supraclavicular Group of Deep Cervical Glands.-These glands lie in the anterior part of the supraclavicular triangle, and are related superficially to the clavicular nerve, and deeply to the upper and middle trunks of the brachial plexus. Superiorly they are continuous with the external chain of the substerno-mastoid glands. They receive their afferent vessels from the following sources:

I. The back of the neck.

2. The external group of axillary glands.

3. The upper part of the pectoral region.

4. Occasionally the lymphatics along the cephalic vein, which may ascend over the clavicle.

Their efferent vessels unite, on either side, with those of the internal chain of substerno-mastoid glands (superior and inferior groups) to form the common jugular lymphatic trunk.

Subclavian or Supraclavicular Triangle.-The subclavian triangle is the lower division of the posterior triangle of the neck, and is separated from the upper division, or occipital triangle, by the posterior belly of the omo-hyoid muscle. It is situated above the middle third of the clavicle, and is of small size until the deep cervical fascia, which ensheathes the posterior belly of the omo-hyoid, has been divided.

Boundaries-Superior.-The posterior belly of the omo-hyoid muscle. Inferior. - The middle third of the clavicle. Anterior.The clavicular part of the sterno-cleido-mastoid muscle. Roof.-The skin; superficial fascia and platysma myoides muscle; suprasternal, supraclavicular, and supra-acromial nerves; a part of the external jugular vein; and the deep cervical fascia. Floor.-The scalenus medius and scalenus posticus muscles.

The extent of the triangle is affected by (I) the height to which the posterior belly of the omo-hyoid ascends above the clavicle, and (2) the extent of the clavicular attachments of the sterno-cleidomastoid and trapezius muscles. The depth of the triangle is in- 
fluenced by the position of the shoulder, being greater when the shoulder is raised and carried forwards, and less when it is depressed and carried backwards.

Contents,-The contents are as follows: (I) the greater portion of the third part of the subclavian artery; (2) small portions of the transverse cervical artery and vein; (3) the lower portion of the external jugular vein; (4) the nerve-trunks of the brachial plexus; and (5) the nerve to the subclavius muscle, and the suprascapular and posterior thoracic nerves.

Third Part of the Subclavian Artery.-This part of the vessel extends from the outer border of the scalenus anticus muscle to the outer border of the first rib, where it becomes the axillary artery. Its course is downwards and outwards, and, for the greater part ot its extent, it lies in the subclavian triangle. Subsequently, however, it passes under cover of the clavicle and subclavius muscle.

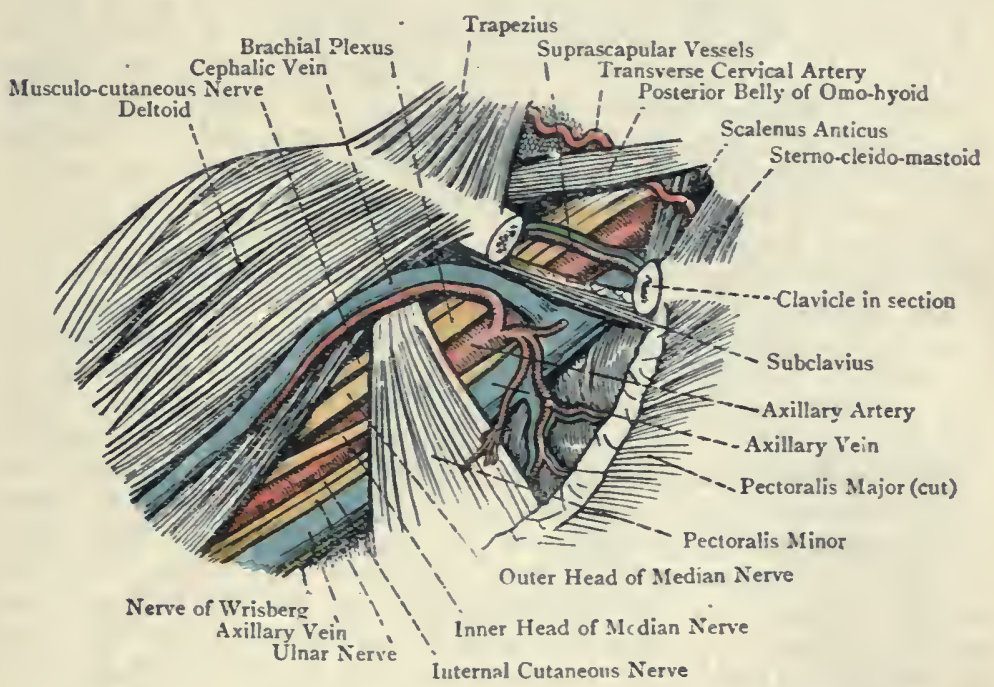

Fig. 445.-The Axillary Space, after Reflection of the Pectoralis Major; aNd the Subclavian Triangle.

Relations-Anterior.-The skin; superficial fascia and platysma myoides ; suprasternal, supraclavicular, and supra-acromial nerves ; deep cervical fascia; clavicle and subclavius muscle; transverse cervical vessels; suprascapular vessels; nerve to the subclavius muscle; and the terminal portion of the external jugular vein. The last-named ressel crosses in front of the artery close to the sterno-cleido-mastoid muscle, and in this situation is joined by the transverse cervical and suprascapular veins. A plexiform arrangement of veins is sometimes met with over the artery, which may be rendered more complex by a branch ascending over the clavicle 
from the cephalic vein. Posterior. - The scalenus medius, with the close intervention of the lower nerve-trunk of the brachial plexus. Superior.-The upper and middle nerve-trunks of the brachial plexus, the latter being nearest the vessel. Inferior.The upper surface of the first rib and the subclavian vein, the vein being on a more anterior plane than the artery, and lying behind the clavicle.

The third part of the subclavian artery does not usually furnish any branch. In some cases, however, the posterior scapular artery arises from it, instead of from the transverse cervical branch of the thyroid axis from the first part of the subclavian. In these cases the posterior scapular artery passes outwards between the nervetrunks of the brachial plexus.

The direction of the third part of the artery is indicated by a line drawn from a point on the posterior border of the sterno-cleidomastoid muscle, about $\frac{1}{2}$ inch above the clavicle, to the centre of that bone. The artery is accessible to compression as it passes over the first rib, the guide to it at this point being the centre of the clavicle. In ligation of the artery the guide to it is the scalene tubercle or spine (tubercle of Lisfranc) on the inner border of the first rib, behind which tubercle the vessel lies; and the scalenus anticus muscle, which is usually covered by the clavicular part of the sterno-cleido-mastoid, leads to this tubercle.

For the manner in which the collateral circulation is carried on after ligation of the third part of the subclavian artery, see p. 324 .

The subclavian vein, in the region of the subclavian triangle, is situated behind the clavicle, where it lies below and anterior to the artery. On the upper surface of the first rib it is anterior to the scalenus anticus, and it receives the external jugular vein, and in some cases the anterior jugular vein.

The transverse cervical artery lies in the subclavian triangle for a short distance only, close to where the posterior belly of the omohyoid passes beneath the sterno-cleido-mastoid. It passes outwards beneath the former muscle into the lower part of the occipital triangle, where it divides into its superficial cervical and posterior scapular branches, the former entering the trapezius, and the latter passing beneath the levator anguli scapulæ, after which it descends along the base of the scapula, under cover of the rhomboid muscles.

The transverse cervical vein opens into the external jugular vein, there being a valve at, or near, its ending.

The suprascapular artery is not in the subclavian triangle, but lies behind the clavicle, close to its upper aspect. It will be described in connection with the first part of the subclavian artery (see p. II43).

The suprascapular vein also lies behind the clavicle, and it opens into the external jugular vein, there being a valve at, or near, its ending.

The external jugular vein will be found described on p. Io8 4 .

Brachial Plexus.- The brachial plexus is situated in the lower part of the posterior triangle of the neck, bchind the clavicle, and in the upper part of the axilla. Its complex formation is rendered 
simple by arranging it into four stages, namely (I) nerve-roots, (2) nerve-trunks, (3) divisions of nerve-trunks, and (4) nervecords.

First Stage.-The nerves which form the plexus are the anterior primary divisions of the fifth, sixth, seventh, and eighth cervical, and the greater part of that of the first thoracic. Superiorly the plexus is reinforced by a small descending branch from the fourth cervical, which joins the fifth, and inferiorly it is occasionally reinforced by a branch from the second thoracic, which joins the first. As regards the first thoracic nerve, the part of it which does not join the plexus, and which is of small size, enters the first intercostal space to become the first intercostal nerve. The nerves, as they

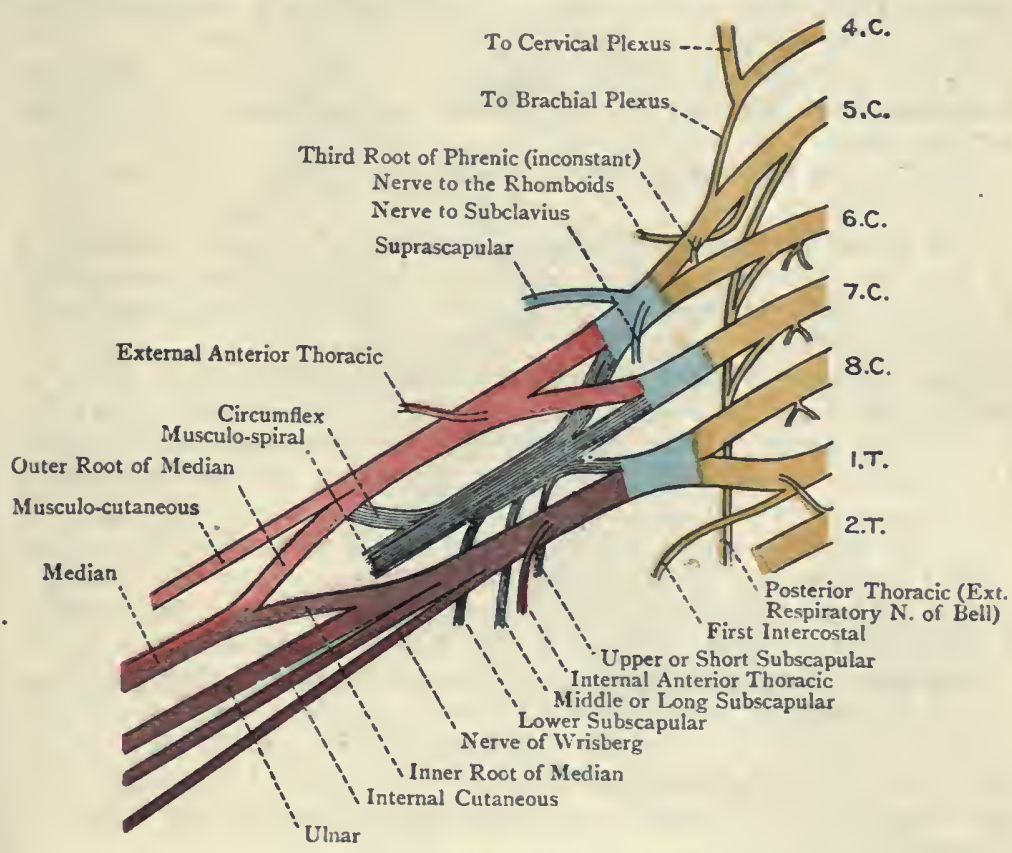

Fig. 446.-The Brachial Plexus.

( Yellow $=$ Spinal Nerves and their Branches; Blue $=$ Trunks; Red =Outer Cord ; Purple $=$ Inner Cord ; Grey $=$ Posterior Cord.)

emerge at the side of the neck, are placed between the scalenus anticus and scalenus medius.

Second Stage.-The fifth and sixth cervical nerves join at the outer border of the scalenus anticus to form the upper trunk; the seventh cervical remains meanwhile single, and forms the middle trunk ; and the eighth cervical and greater part of the first thoracic unite between the scalene muscles to form the lower trunk. There are thus three trunks-upper, middle, and lower. 
Third Stage-A little above the clavicle each of the three trunks breaks up into an anterior and a posterior division.

Fourth Stage.-The anterior divisions of the upper and middle trunks unite to form the outer cord of the plexus; the anterior division of the lower trunk, which is of large size, forms the inner cord ; and all three posterior divisions (that of the lower trunk being of small size) unite to form the posterior cord. As a variety, the anterior division of the middle trunk may subdivide into two branches, one entering the outer cord and the other the inner. There are thus three cords-outer, inner, and posterior.

Branches of the Plexus. - The branches are conveniently divided into two groups-supraclavicular, arising above the clavicle, and coming from nerve-roots and nerve-trunks; and infraclavicular, arising below the clavicle, and coming from nerve-cords.

Supraclavicular Branches.-These are as follows: Muscular branches from the four cervical nerves to the scalene muscles and longus colli.

One root of the phrenic nerve (inconstant) from the front of the fifth cervical.

The Nerve to the Rhomboids.--This branch arises from the back of the fifth cervical, close to or along with the highest root of the posterior thoracic, and it takes a backward course through the scalenus medius.

The Posterior Thoracic Nerve or External Respiratory Nerve of Bell.-This branch arises by three roots from the back of the fifth, sixth, and seventh cervical nerves. The upper two roots pierce the scalenus medius muscle below the nerve to the rhomboids, either conjointly or separately, whilst the lowest root passes in front of the scalenus medius, and joins the trunk formed by the others near the first rib. The nerve then courses behind the brachial plexus and the first part of the axillary artery to the axillary surface of the serratus magnus, which it supplies.

The Nerve to the Subclavius. - This small branch arises from the front of the upper trunk, its fibres being derived from the fifth cervical. It descends over the third part of the subclavian artery, and, passing behind the clavicle, it enters the subclavius muscle on its deep aspect. This nerve sometimes communicates with the phrenic nerve.

The Suprascapular Nerve. - This is a large nerve which arises from the back of the upper trunk, its fibres being derived from the fifth and sixth cervical. It is directed downwards, outwards, and backwards beneath the trapezius and posterior belly of the omo-hyoid to the upper border of the scapula, on approaching which it gets in company with the suprascapular artery. It is distributed to the supraspinatus and infraspinatus muscles and shoulder-joint.

For the infraclavicular branches of the brachial plexus, see p. 3I4.

Anterior Triangle.- This triangle is situated in front of the sternocleido-mastoid muscle, and its base is directed upwards.

Boundaries-Anterior.-The middle line of the neck, that is to say, a line extending from the chin to the upper border of the 
manubrium sterni. Posterior. - The anterior border of the sternocleido-mastoid muscle. Superior.-One half of the base of the inferior maxilla, and a line drawn from the angle of that bone to the mastoid process. The triangle is covered by the skin, superficial cervical fascia, platysma myoides, and deep cervical fascia. Superficial to the deep fascia there are the following structures: the anterior jugular vein, the ramifications of the superficial or

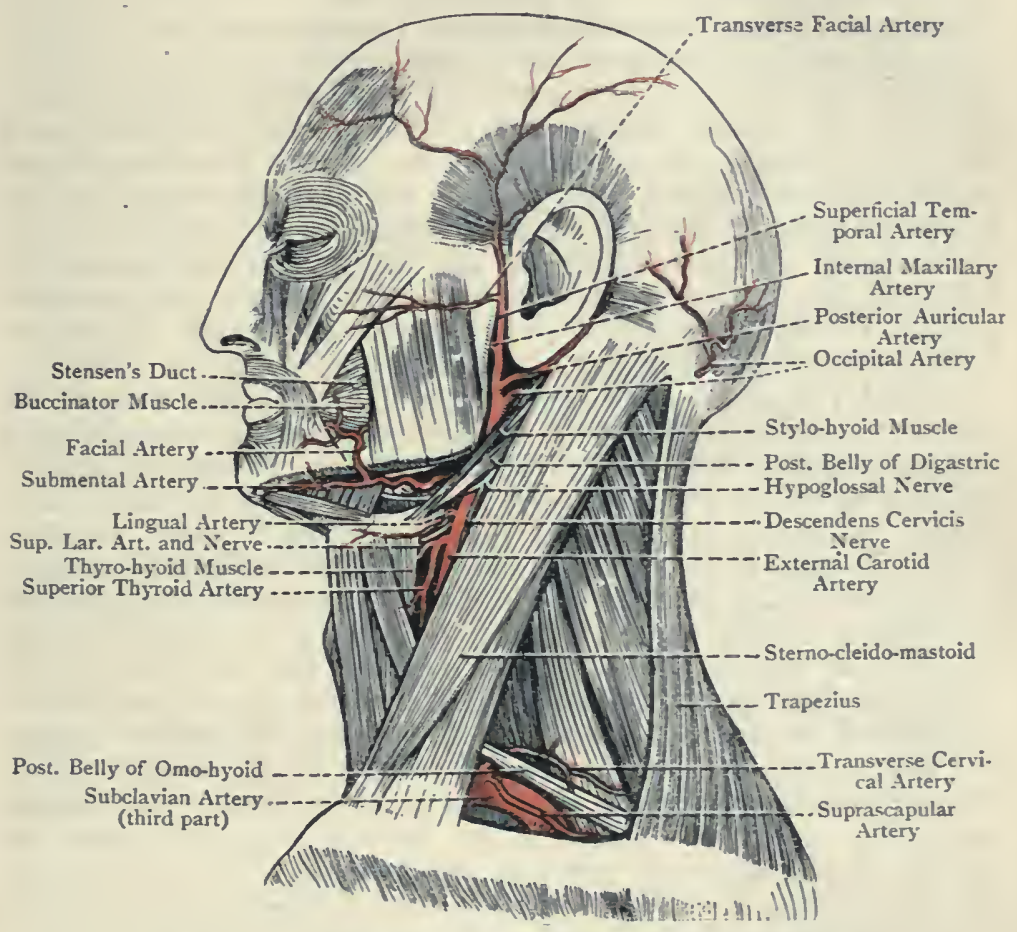

Fig. 447.-The Left Side of the Head and Neck.

(The Platysma Myoides has been removed).

transverse cervical nerve, and the inframandibular branch of the facial nerve.

The anterior triangle is divided into three triangles by the anterior belly of the omo-hyoid muscle inferiorly, and the two bellies of the digastric muscle superiorly. The subdivisions from below upwards are called muscular, carotid, and digastric or submaxillary.

The muscular triangle (inferior carotid) is bounded anteriorly by the middle line of the neck; posteriorly by the anterior border of the sterno-cleido-mastoid; and superiorly by the anterior belly of the omo-hyoid. 
The carotid triangle (superior carotid) is bounded inferiorly by the anterior belly of the omo-hyoid; superiorly by the posterior belly of the digastric and stylo-hyoid; and posteriorly by the anterior border of the sterno-cleido-mastoid.

The digastric or submaxillary triangle is bounded postero-inferiorly by the posterior belly of the digastric and stylo-hyoid; anteroinferiorly by the anterior belly of the digastric; and superiorly by one half of the base of the inferior maxilla, and a line drawn from the angle of that bone to the mastoid process.

Contents of the Triangles-Muscular Triangle.-The area of this triangle is occupied by the sterno-hyoid and sterno-thyroid muscles ; hence the name muscular triangle. Under cover of these muscles there are the carotid sheath with its contents, the lateral lobe of the thyroid body, the trachea, and the larynx. The cesophagus lies behind the trachea, with a slight inclination towards the left side at the root of the neck, and the inferior or recurrent laryngeal nerve lies in the groove between the trachea and the osophagus. The inferior thyroid artery pursues a tortuous course inwards behind the lower part of the carotid sheath, and the gangliated cord of the sympathetic descends behind the sheath.

Carotid Triangle.-This triangle contains the upper part of the common carotid, and the commencements of the external and internal carotid, arteries, all of these being overlapped by the anterior border of the sterno-cleido-mastoid, when the deep fascia which ensheathes that muscle is undisturbed. The common carotid and internal carotid arteries, together with the pneumogastric nerve, are contained within the carotid sheath, and the descendens cervicis nerve lies upon the sheath, or within it, being situated in either case over the common carotid artery. The sterno-cleido-mastoid artery and the superior thyroid vein cross the sheath near the bifurcation of the common carotid artery, and the carotid body lies behind the vessel about the same level. The deep cervical glands lie along the course of the internal jugular vein. The origins of the superior thyroid, lingual, facial, and occipital arteries are contained in this triangle, and the ascending pharyngeal branch of the external carotid lies deeply between that vessel and the internal carotid. The internal jugular vein in this triangle receives the common facial, lingual, and superior thyroid veins. The hypoglossal nerve lies along the lower border of the posterior belly of the digastric muscle, and it here gives off, from behind forwards, the descendens cervicis and thyro-hyoid nerves, the former passing downwards over, or within, the carotid sheath, and the latter passing forwards and downwards at an acute angle with the parent trunk. "The internal branch of the superior laryngeal nerve lies deeply behind the bloodvessels, and the external laryngeal branch of that nerve descends internal to them, and passes beneath the depressor muscles of the hyoid bone. The pneumogastric nerve is within the carotid sheath, and the gangliated cord of the sympathetic is behind it. The spinal portion of the spinal accessory nerve lies deeply, its course being downwards 
and backwards beneath the sterno-cleido-mastoid, the deep portion of which it pierces.

Digastric or Submaxillary Triangle.-This triangle is divided into two parts, anterior and posterior, by the stylo-mandibular ligament. The anterior part contains the superficial part of the submaxillary gland, the facial vein being superficial to it, and the facial artery being embedded in its upper and back part. In this triangle the facial artery gives off its inferior or ascending palatine, tonsillar, glandular, and submental branches. The muscles in the floor of the anterior part of the triangle are the mylo-hyoid, and a part of the hyo-glossus. The superficial part of the submaxillary gland rests upon the mylo-hyoid muscle, and conceals the mylo-hyoid nerve and submental artery, which are in direct contact with the muscle. The hypoglossal nerve lies upon that part of the hyo-glossus muscle which appears in the anterior part of the triangle, but it soon disappears beneath the posterior free border of the mylo-hyoid muscle, in company with the deep part of the submaxillary gland and the lingual nerve. The posterior part of the digastric triangle contains a portion of the parotid gland, and under cover of this part is the external carotid artery, which here furnishes its posterior auricular branch.

The anterior jugular vein and the superficial or transverse cervical nerve have been already described (see pp. I083 and I093). The inframandibular branch of the facial nerve will be found described on P. II75.

Submaxillary Lymphatic Glands.-These glands lie upon the superficial surface of the submaxillary salivary gland, under cover of the deep cervical fascia. They form a chain beneath the corresponding half of the base of the mandible, which extends from near the angle of the bone to near the origin of the anterior belly of the digastric muscle. The central gland of the chain is closely related to the facial artery as that vessel is about to ascend over the base of the mandible. They receive their afferent vessels from the following sources:

I. The side of the nose.

2. A few from the lower eyelid.

3. The lower part of the cheek.

4. Half of the upper lip.

5. The lateral part of the lower lip.

6. The anterior third of the lateral border of the tongue.
7. The subjacent portion of the floor of the mouth.

8. Half of the upper gum.

9. The lateral part of the lower gum.

Io. The superficial facial glands.

II. The submaxillary and sublingual salivary glands.

Their efferent-vessels pass to those glands of the internal group of the substerno-mastoid glands which are on a level with the upper border of the thyroid cartilage of the larynx.

The suorahyoid or submental glands are situated beneath the chin in the submental triangle, and are two or three in number. They receive their afferent lymphatics from the tip of the-tongue, the front of the floor of the mouth, and the inner part of the lower 
lip; and their efferent lymphatics pass to the submaxillary lym. phatic glands.

The prelaryngeal glands, when present, are situated in front of the crico-thyroid membrane, and are one or two in number. They receive their afferent lymphatics from the interior of the larynx, below the rima glottidis, and from the adjacent part of the thyroid body. Their efferent lymphatics pass to the inferior group of deep cervical glands.

The pretracheal glands lie in front of the cervical portion of the trachea, from which, as well as from the adjacent part of the thyroid body, they receive their afferent lymphatics. Their efferent lymphatics pass to the inferior group of deep cervical glands.

Occasionally a few lymphatic glands are met with along the course of the anterior jugular vein.

Deep Cervical Glands.-These glands lie beneath the sternocleido-mastoid muscle, and are about twenty-five in number. They are arranged in two groups, superior and inferior.

The superior group lie along the internal jugular vein above the level of the upper border of the thyroid cartilage. They receive their afferent lymphatics from the cranial cavity, the internal maxillary glands, some of the parotid and submaxillary lymphatic glands, the root of the tongue, the upper part of the thyroid body, the larynx, and the lower part of the pharynx." Their efferent lymphatics pass to the glands of the inferior group.

The inferior group lie along the lower part of the internal jugular vein, and extend outwards and backwards beneath the sternocleido-mastoid, as far as its posterior border, where they give rise to a chain known as the glandula concatenata. The glands of this chain are continuous inferiorly with the supraclavicular glands, and, through these,. with the external axillary glands. They receive their afferent lymphatics from the superior group of deep cervical glands, the upper superficial cervical glands, the lower part of the thyroid body, and the cervical portions of the trachea and oesophagus. Their efferent lymphatics unite to form a single vessel, called the jugular lymphatic trunk, which opens on the left side into the thoracic duct, and on the right side into the right lymphatic duct.

Infrahyoid Muscles.-These are four in number, on either side, as follows : the omo-hyoid, sterno-hyoid, sterno-thyroid, and thyrohyoid.

Omo-hyoid.--This muscle consists of two bellies, anterior and posterior, and an intervening tendon. Origin.-By means of the posterior belly from (I) the upper border of the scapula close to the inner side of the suprascapular notch, and (2) the suprascapular or transverse ligament, which bridges over the suprascapular notch.

Insertion.-By means of the anterior belly into the outer third of the lower border of the body of the hyoid bone, immediately external to the insertion of the sterno-hyoid muscle. 
Nerve-supply.-The anterior belly is supplied by the descendens cervicis nerve, and the posterior belly derives its branches from the ansa cervicis (ansa hypoglossi).

The posterior belly is contained in the posterior triangle of the neck, and separates the occipital from the subclavian or supraclavicular triangle. Its course is forwards and slightly upwards, and it passes beneath the sterno-cleido-mastoid muscle, where its fibres terminate in the intervening tendon. It is ensheathed by a deep process of the deep cervical fascia, as that fascia crosses the posterior triangle, and this process is attached to the back of the inner end of the clavicle and the first rib, which explains the almost horizontal position occupied by the posterior belly.

The anterior belly proceeds from the intervening tendon, and passes upwards and slightly inwards to the body of the hyoid bone. As it emerges from beneath the anterior border of the sternocleido-mastoid muscle the anterior belly crosses the carotid sheath on a level with the narrow anterior part of the cricoid cartilage, and in the anterior triangle of the neck it forms the separation between the muscular (inferior carotid) and carotid (superior carotid) triangles.

Sterno-hyoid-Origin.-(I) The posterior surface of the manubrium sterni at its upper and outer part; $(2)$ the posterior sternoclavicular ligament; and, it may be, (3) the posterior surface of the clavicle at its inner end.

Insertion.-The inner two-thirds of the lower border of the body of the hyoid bone, extending from the middle line to the insertion of the anterior belly of the omo-hyoid.

Nerve-supply.-The ansa cervicis (ansa hypoglossi).

The muscle is flat and ribbon-like, and rests upon the sterno. thyroid and thyro-hyoid.

Sterno-thyroid-Origin.--(I) The posterior surface of the manubrium sterni at its upper and outer part, below the origin of the sterno-hyoid; and (2) the posterior surface of the first costal cartilage.

Insertion.-The oblique line on the outer surface of the ala of the thyroid cartilage.

Nerve-supply.-The ansa cervicis (ansa hypoglossi).

The muscle is broader, but shorter, than the sterno-hyoid, underneath which it lies. Within the thorax the right muscle lies in front of the innominate artery, and the left in front of the left common carotid artery and left innominate vein. In the neck each muscle rests upon the carotid sheath and the corresponding lateral lobe of the thyroid body.

The sterno-hyoid muscles as they emerge from the thorax are separated by an interval, in which situation the sterno-thyroid muscles lie in close contact. As the muscles ascend, the sternolyyoids converge, but the sterno-thyroids diverge.

Thyro-hyoid-Origin.- The oblique line on the outer surface of the ala of the thyroid cartilage.

Insertion.-(I) The outer half of the lower border of the body of 
the hyoid bone; and (2) the basal half of the great cornu of that bone.

Nerve-supply. - The thyro-hyoid nerve, which is a special branch of the hypoglossal. The nerve enters the deep surface of the muscle close to its posterior border.

The muscle is quadrilateral. Its superficial surface supports the anterior belly of the omo-hyoid and the sterno-hyoid muscles, and its deep surface is related to the ala of the thyroid cartilage, the thyro-hyoid membrane, the internal branch of the superior laryngeal nerve, and the superior laryngeal artery.

The nerves which supply the infrahyoid group of muscles are regarded as being of spinal origin.

Action of the Infrahyoid Muscles-Omo-hyoid.-(I) To depress the hyoid bone ; and (2) to render tense the deep cervical fascia in the lower part of the neck.

Sterno-hyoid.-To depress the hyoid bone.

Sterno-thyroid. - To depress the thyroid cartilage.

Thyro-hyoid.-(I) To depress the hyoid bone; and (2) to elevate the thyroid cartilage, as in the production of high notes, or in deglutition.

Structures in the Median Line of the Neck.-The median line of the neck is divisible into two regions, suprahyoid and infrahyoid.

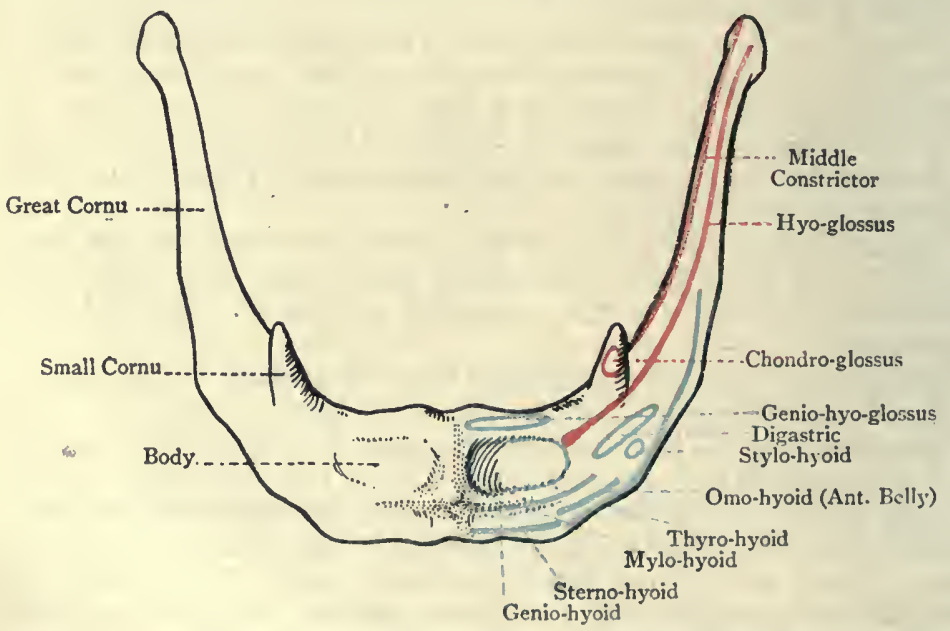

Fig. 448.-The Hyoid Bone, showing its Muscular Attachments,

Suprahyoid Region.-The innermost fibres of the two platysma myoides muscles decussate at the median line for a short distance below the chin. On either side of the median line, under cover of the platysma myoides, is the anterior belly of the digastric muscle. The anterior bellies of opposite sides are near each other at the chin, but, as they descend with an inclination outwards, they diverge 
from each other and leave between them a triangular interval, called the submental triangle. The base of this triangle is formed by the body of the hyoid bone, and each lateral boundary is constructed by the anterior belly of the digastric, the apex being placed at the chin. The area of the triangle is occupied by the anterior portions of the mylo-hyoid muscles, which meet at the median line in a tendinous raphé, and superficial to these muscles there are the suprahyoid or submental glands. Immediately underneath the mesial portions of the mylo-hyoid muscles are the genio-hyoid muscles, these two latter muscles being in very close contact at the median line. Beneath each genio-hyoid muscle is the inferior portion of the genio-hyo-glossus.

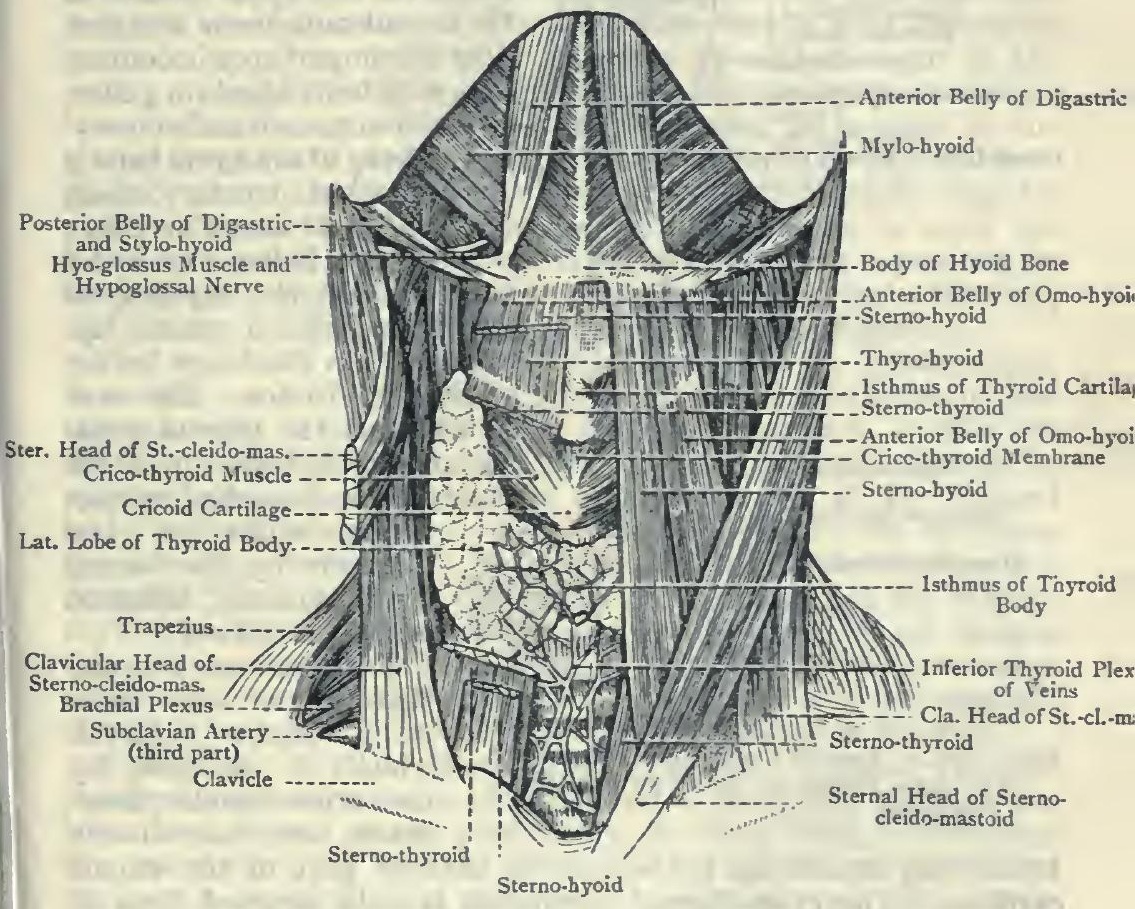

Fig. 449.-Dissection of the Front of the Neck.

(The area bounded on either side bv the Anterior Belly of the Digastric and below by the body of the Hyoid Bone is the Submental Triangle.)

The relation of structures in the suprahyoid region, from the surface downwards (in a deep direction), is as follows:
r. Platysma myoides.
2. Mylo-hyoid.
3. Genio-hyoid.
4. Genio-hyo-glossus.

Submental, or Suprahyoid, Lymphatic Glands.-These glands, usually two in number, lie in the submental triangle, beneath the 
chin and above the body of the hyoid bone, one being on either side of the median line. Each gland receives its afferent vessels from (I) the mesial portions of the lower lip and lower gum; (2) the tip of the tongue and adjacent portion of the floor of the mouth; and (3) the integument of the chin. Their efferent vessels pass to (I) the submaxillary lymphatic glands, and (2) that gland of the internal group of the substerno-mastoid glands which lies on a level with

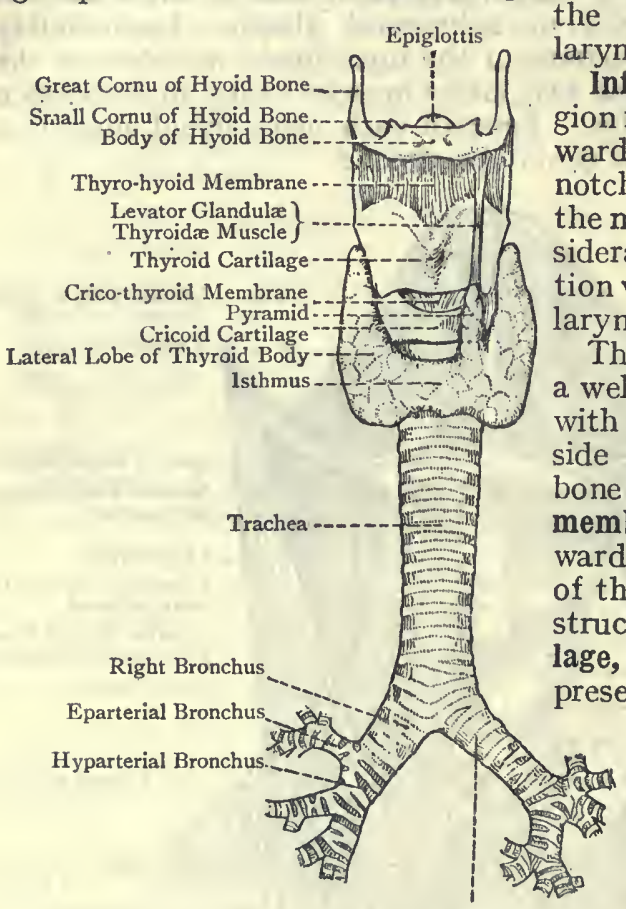

L.eft Bronchus

Fig. 50. - The Hyoid Bane, Larynx, Trachea, Bronchi, and Thyroid Body (ANTERIOR VIEW). below this membrane is the narrow anterior part of the cricoid cartilage. The crico-thyroid membrane is only exposed close to the median line, being elsewhere covered by the two crico-thyroid muscles. The exposed part of the membrane is crossed by the crico-thyroid arterial arch, which is situated midway between the thyroid and cricoid cartilages, and lying upon the membrane there may be one or two prelaryngeal lymphatic glands. Laryngotomy may be performed in the crico-thyroid region, and the crico-thyroid arterial arch has to be borne in mind.

Succeeding to the cricoid cartilage is the trachea, which, as it descends, inclines backwards, and therefore becomes somewhat 
inaccessible. The thyroid body is intimately related to it superiorly. Each latera? lobe closely embraces it laterally as low as about the fifth ring, and the isthmus lies in front of the second and third rings as a rule, but its position is liable to variation. Crossing the upper border of the isthmus there is one of the branches of the superior thyroid artery, known as the artery of the isthmus.

There is nothing of any importance in front of the trachea above the isthmus of the thyroid body. Below the isthmus there is a more or less copious plexus of veins, called the inferior thyroid plexus, from which the right and left inferior thyroid veins descend. Occasionally a small artery, called the arteria thyroidea ima, ascends directly in front of this part of the trachea, in the median line, to reach the isthmus of the thyroid body. The innominate, and even the right common carotid, artery, and the left innominate vein sometimes encroach upon the front of the trachea towards the root of the neck. In early life the upper part of the thymus body covers the front of the trachea. The foregoing structures are covered by the sterno-thyroid and sterno-hyoid muscles in the following manner: the two sterno-thyroid muscles are in contact with each other for a short distance above the manubrium sterni, so as to cover the trachea, but the two sterno-hyoid muscles are here separated by an interval; superiorly the two sterno-thyroid muscles diverge, and the two sterno-hyoid muscles come very nearly together.

The operation performed upon the trachea is tracheotomy. According as it is performed above or below the isthmus of the thyroid body, it is spoken of as the high operation, or the low ope ation. In the high operation there is no anatomical obstacle, unless it be a close attachment of the isthmus of the thyroid body to the tracheal rings which it covers. In the low operation the following obstacles are presented: (I) the trachea is here less accessible, because it recedes from the surface; (2) the inferior thyroid plexus of veins might prove troublesome; $(3)$ an arteria thyroidea ima may be present ; (4) the innominate and right common carotid arteries, and the left innominate vein may be endangered; and (5) in young children the thymus body would be in the way.

Descendens Cervicis (Descendens Hypoglossi) Nerve.-The descendens cervicis nerve arises from the hypoglossal nerve as the latter hooks round the occipital artery, its fibres being derived from the communicating branch which the hypoglossal receives from the loop between the first and second cervical nerves. The nerve, which is long, passes downwards and slightly forwards, lying upon, or within, the carotid sheath, and in either case directly over the line of the common carotid artery. Before reaching the centre of the neck it furnishes a branch to the anterior belly of the omo-hyoid muscle. Thereafter it is joined by a branch which is formed by the union of the two rami communicantes cervicis from the anterior primary divisions of the second and third cervical nerves. These two rami, however, sometimes join it separately. In this manner a loop is formed, which is called the ansa cervicis 
(ansa hypoglossi). The convexity of the loop is directed downwards, and from it branches are given off to (I) the sterno-hyoid, (2) the sterno-thyroid, and (3) the posterior belly of the omo-hyoid, muscles.

The fibres of the descendens cervicis nerve are regarded as being of spinal, not hypoglossal, origin.

For the rami communicantes cervicis, see Cervical Plexus.

Thyro-hyoid Nerve.-This nerve, which is composed of spinal fibres, derived from the loop between the first and second cervical nerves, arises from the hypoglossal at the lower border of the

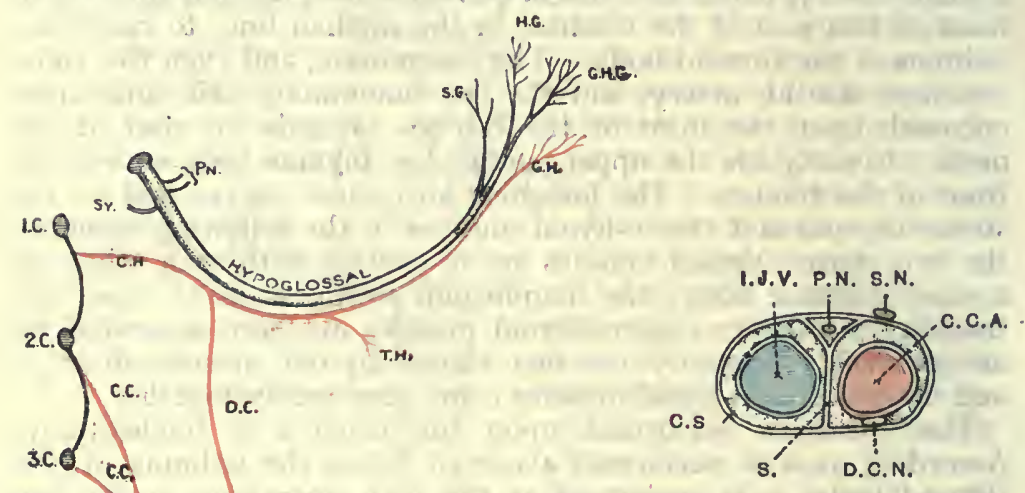

FIG. 45 IB.-CAROTID SHEATH.

I.J.V., internal jugular vein; P.N., pneumogastric nerve; S.N., sympathetic nerve ; C.C.A., common carotid artery; C.S., carotid sheath ; S., septum; D.C.N., descendens

Fig. 45IA.-Scheme of the Hypoglossal. cervicis nerve.

NeRVE, SHOWING ITS CONNECTIONS WITH

Cervical Spinal Nerves.

Sy., twig frem sympathetic; PN., communicating with pneumogastric ; IC, 2C, 3C, first, second, and third cervical ; C.H., communicans hypoglossi ; C.C., communicantes cervicis; D.C., descendens cervicis; A.B.O.H., to anterior belly of omo-hyoid ; A.C., ansa cervicis; S.H., to sterno-hyoid ; S.T., to sterno-thyroid ; P.B.O.H., to posterior belly of omo-hyoid; T.H., to thyro-hyoid; G.H., to genio-hyoid ; G.H.G., to genio-hyo-glossus; H.G.. to hyo-glossus ; S.G., to stylo-glossus.

posterior belly of the digastric. It passes forwards and downwards, forming an acute angle with the parent trunk, and it disappears beneath the posterior border of the thyro-hyoid muscle, immediately after which it enters its deep surface.

Carotid Sheath. - The carotid sheath, as stated in connection with the deep cervical fascia, is derived from the posterior lamina of the sheath of the sterno-cleido-mastoid muscle, and is intimately connected anteriorly with the pretracheal process, and posteriorly with the prevertebral process, of the deep cervical fascia. The interior of the sheath is divided into three compartments-outer, 


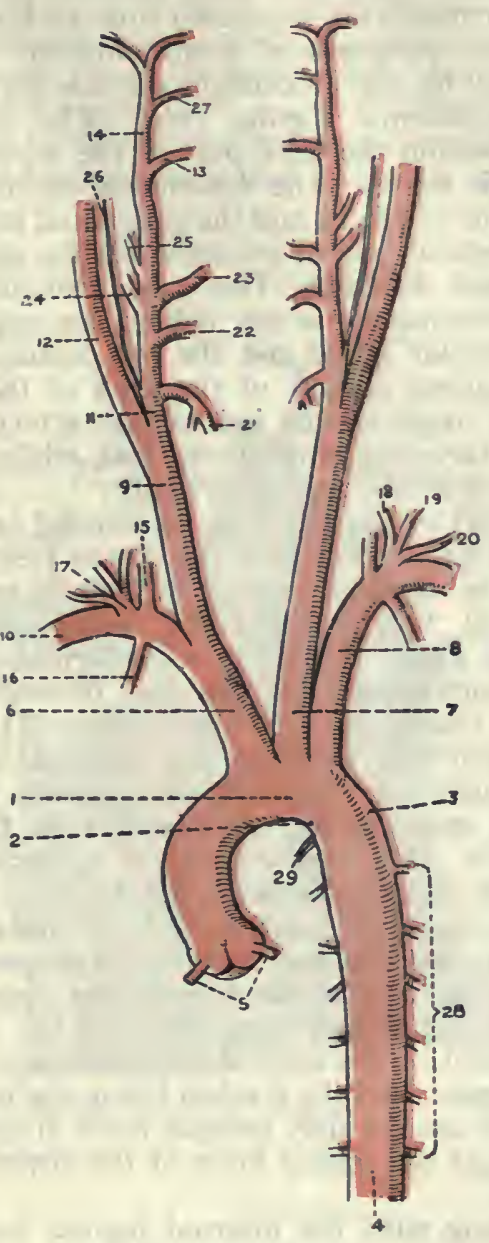

Fig. 452,-The Aorta in the Thorax, ANd the Principal Arteries of the Head and Neck.
x. Arch of the Aorta
2. Aortic Isthmus
3. Aortic Spindle
4. Descendin Aorta
5. Coronary Arteries (from Ascending Aorta)
6. Innominate Artery
7. Left Common Carotid
8. Left Subclavian
9. Right Common Carotid
10. Right Subclavian
11. External Carotid
12. Internal Carotid
13. Internal Maxillary
74. Superficial Temporal
15. Vertebral
16. Internal Mamnary
17. Thyroid Axis
18. Inferior Tbyroid
rg. Transverse Cervical

20. Suprascapular

21. Superior Thyroid

22. Lingual

23. Facial

24. Occipital

25. Posterior Auricular

26. Ascending Pharyngeal

27. Transverse Facial

28. Aortic Intercostals

29. Lig. Ductles Arteriosi 
inner, and posterior, the latter being mesially placed. The outer and inner compartments are separated from each other by a mesial septum, the inner compartment containing the common carotid artery and, it may be, the descendens cervicis nerve, and the outer compartment the internal jugular vein. The posterior compartment is situated within the back part of the septum, and contains the pneumogastric nerve. The descendens cervicis nerve may lie upon the sheath, or within it, and the gangliated cord of the sympathetic descends behind, and in intimate relation with, it.

Common Carotid Arteries.-The right common carotid artery arises from the innominate artery behind the upper border of the right sterno-clavicular joint, and the left common carotid artery arises from the upper surface of the arch of the aorta, in close proximity to the origin of the innominate artery. The vessel of the right side is therefore entirely cervical, whilst that of the left side is partly thoracic and partly cervical.

The thoracic part of the left common carotid artery has already been described in connection with the thorax (see p. 96r).

In the neck the common carotid artery of each side extends from the back of the corresponding sterno-clavicular joint to the level of the upper border of the thyroid cartilage of the larynx, which corresponds to the disc between the bodies of the third and fourth cervical vertebræ. At this level the vessel divides into the external and internal carotid arteries. The place of bifurcation is sometimes opposite the body of the hyoid bone, and, more rarely, on a level with the cricoid cartilage of the larynx. The vessel is about $3 \frac{3}{4}$ inches long, and its course is upwards and outwards in the direction of a line drawn from the sterno-clavicular joint to a point midway between the angle of the inferior maxilla and the mastoid process of the temporal bone. This line, as high as the level of the upper border of the thyroid cartilage, represents the course of the common carotid artery. At the root of the neck the two common carotid arteries are not very far apart, and the trachea lies in the intervening space. As the two vessels ascend they become more divergent, on account of the projection of the lateral lobes of the thyroid body and the thyroid cartilage.

The artery, along with the internal jugular vein and pneumogastric nerve, and, it may be, the descendens cervicis nerve, is contained within the carotid sheath, already described. Opposite the cricoid cartilage it is crossed by the anterior belly of the omohyoid muscle. Below this level it lies deeply in the region of the muscular triangle, being under cover of the sterno-hyoid and sternothyroid muscles, in addition to the platysma myoides and the anterior border of the sterno-cleido-mastoid. Above this level it is situated in the carotid triangle, being here more superficially placed, its only muscular coverings' being the platysma myoides and the anterior border of the sterno-cleido-mastoid.

Relations-Anterior.-The skin; superficial fascia and platysma 
myoides; superficial layer of the deep cervical fascia; anterior border of the sterno-cleido-mastoid ; sterno-hyoid; sterno-thyroid; anterior belly of the omo-hyoid; and the anterior wall of the carotid sheath. Three veins cross the artery from without inwards, as follows : the anterior jugular vein crosses it immediately above the clavicle, superficial to the sterno-hyoid and sterno-thyroid muscles ; the middle thyroid vein crosses it just below the level of the cricoid

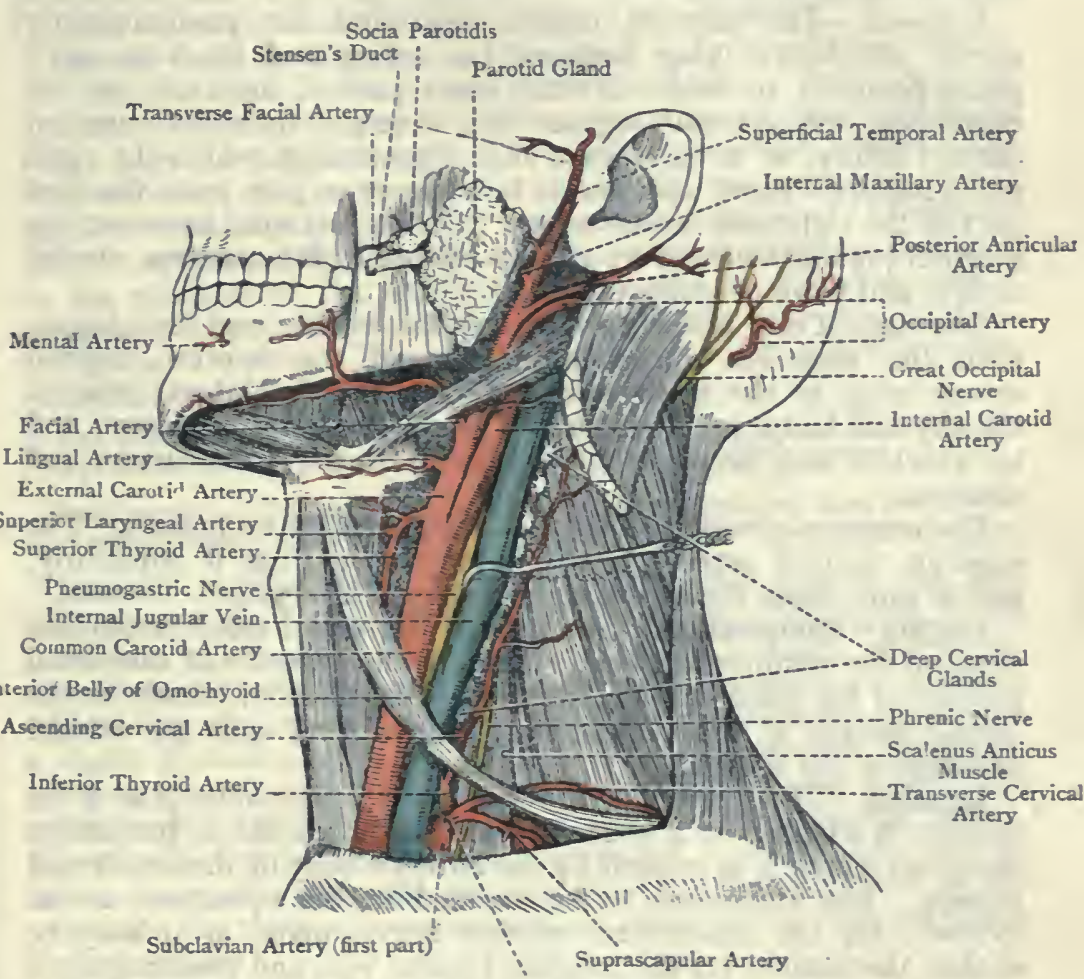

Thyroid Axis

Fig. 453.-Deep Dissection of THE Left Side of The Neck (after Spalteholz).

cartilage ; ard the superior thyroid vein crosses it near its bifurcation. The sterno-cleido-mastoid branch of the superior thyroid artery, which is of small size, passes obliquely downwards and outwards over the carotid sheath in the carotid triangle. The descendens cervicis nerve descends upon, or it may be within, the carotid sheath, lying directly over the artery, and the ansa cervicis (hypoglossi) lies upon the sheath.

Posterior. - The posterior wall of the carotid sheath; the cervical 
transverse processes as high as the level of the fourth ; the longus colli, scalenus anticus, and part of the rectus capitis anticus major, muscles; the gangliated cord of the sympathetic, which is intimately related to the posterior wall of the carotid sheath; the recurrent laryngeal nerve; and the inferior thyroid artery, both of which latter structures pass inwards and upwards behind the lower part of the sheath. The carotid body lies behind the vessel close to its bifurcation.

External.--The internal jugular vein and the pneumogastric nerve, the latter lying between the artery and the vein, on a plane posterior to both. At the lower part of the neck, on the right side, the internal jugular vein diverges from the common carotid artery, so as to leave a slight interval in which the right pneumogastric nerve appears as it is about to pass over the first part of the right subclavian artery. On the left side, however, the internal jugular vein is very closely related to the common carotid artery, and more or less overlaps it.

Internal.-From below upwards (I) the trachea and œsophagus, with the recurrent laryngeal nerve and the inferior thyroid artery lying in the intervening groove; (2) the lateral lobe of the thyroid body, upon which the vessel impresses a groove, and by which it may be slightly overlapped; and (3) the larynx and pharynx.

The common carotid artery, as a rule, gives off no branch. The superior thyroid artery, however, may arise from it superiorly, and in some cases the ascending pharyngeal artery.

Surgery-Compression.-The part of the vessel most favourably situated for compression lies in front of the tubercle of the costal process of the sixth cervical vertebra, this tubercle, known as the carotid tubercle of Chassaignac, being on a level with the cricoid cartilage of the larynx.

Ligation.-The part of the vessel most favourably placed for ligation is situated on a level with the cricoid cartilage, just above the point where it is crossed by the anterior belly of the omo-hyoid muscle. The structures to be avoided in the operation are as follows: (I) the descendens cervicis nerve, upon, or it may be within, the sheath; $(2)$ the internal jugular vein and pneumogastric nerve, both of which are within the sheath, and upon the outer side of the artery; and (3) the gangliated cord of the sympathetic, which lies behind, and in close contact with, the sheath. The small sterno-cleido-mastoid artery will probably be cut, as it passes obliquely downwards and outwards over the sheath in the carotid triangle. Ligation of the artery below the level of the cricoid cartilage is attended with difficulty, the vessel being here covered by the sterno-hyoid and sterno-thyroid muscles, in addition to the platysma myoides and sterno-cleido-mastoid. $C_{n}$ the left side the internal jugular vein would constitute an additional difficulty.

Collateral Circulation after Ligation.-( I $\Rightarrow$ Cross anastomoses take 
place freely between the external and internal carotid arteries of opposite sides. (2) The inferior thyroid artery of the side operated upon anastomoses freely with the superior thyroid of the same side, which is a branch of the external carotid. (3) The deep cervical branch of the superior intercostal, which latter is a branch of the second part of the subclavian artery on the right side, and of the first part on the left side, anastomoses with the ramus cervicalis princeps of the occipital, which is a branch of the external carotid. (4) The vertebral artery undergoes much enlargement.

Carotid Body or Ganglion.-This small body is situated behind the common carotid artery close to its bifurcation. It is composed of a few lobules, united by connective tissue, and it receives minute twigs from the adjacent part of the common carotid artery. The lobules consist of groups of polyhedral cells, permeated by bloodcapillaries and sympathetic nerve-filaments. Some of the cellular constituents are chromaffin cells, similar to those which are met with in the medulla of the suprarenal body and in the sympathetic ganglia. These cells are derived from the contiguous ganglia of the sympathetic system. The carotid body of each side is similar to the coccygeal body and organs of Zuckerkandl.

The carotid body may be regarded as being developed in part from the sympathetic system, and as belonging also to the lymphatic system.

Development.-The common carotid arteries are developed from the parts of the ventral aortæ which are situated between the third and fourth arterial arches.

Internal Jugular Vein.-The internal jugular vein is the continuation of the intracranial lateral sinus. It commences in the postero-external compartment of the jugular foramen, and terminates behind the inner end of the clavicle by joining the subclavian vein to form the innominate vein. At its commencement it presents a slight dilatation, called the bulb. The vein descends vertically, lying at first on the outer side of the internal carotid artery, and subsequently on the outer side of the common carotid artery, the pneumogastric nerve being interposed in each case, and all being enclosed within the carotid sheath. The relations of the vessel for the most part correspond to those of the arteries which it accompanies.

Tributaries.-These are as follows :

Inferior petrosal sinus.

Common facial.

Lingual.
Pharyngeal.

Superior thyroid.

Middle thyroid.

A small vein accompanying the occipital artery may occasionally open into it.

The inferior petrosal sinus leaves the cranial cavity through the antero-internal compartment of the jugular foramen, and opens into the internal jugular vein close to the base of the skull.

Development. - The internal jugular vein is developed from the anterior cardinal or primitive jugular vein. 
crossed by the posterior belly of the digastric and stylo-hyoid muscles, and the hypoglossal nerve. Thereafter the vessel is embedded in the substance of the parotid gland, where it is crossed from behind forwards by the facial nerve.

Relations-Anterior.-The skin; superficial fascia; platysma myoides; deep fascia ; anterior border of the sterno-cleido-mastoid ; the lingual and common facial veins; the hypoglossal nerve (all the foregoing being anterior relations, whilst the artery lies in the superior carotid triangle); the posterior belly of the digastric and stylo-hyoid muscles; the greater part of the parotid gland; the temporo-maxillary vein; and the facial nerve. Posterior.-The

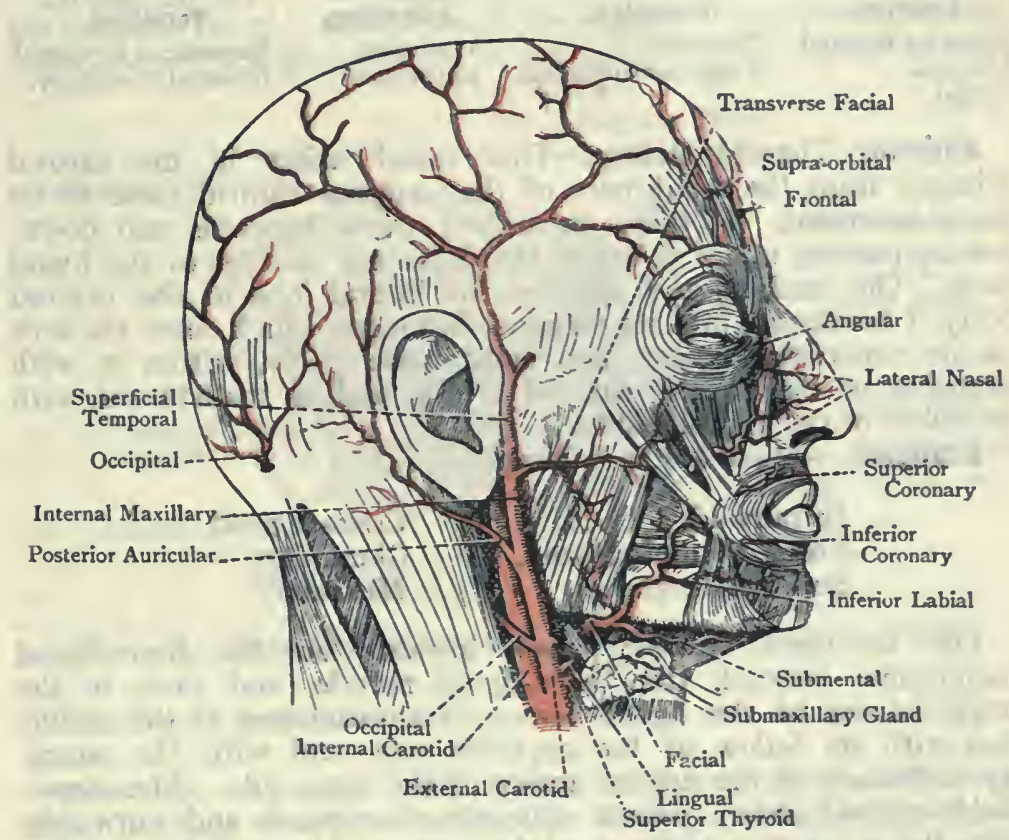

Fig. 455.-ThE Arteries of the Right Side of the Head (After L. Testut's ' Anatomie Humaine'):

chief posterior relations are as follows: (I) the stylo-pharyngeus muscle, glosso-pharyngeal nerve, and styloid process of the temporal bone, all of which intervene between the vessel and the internal carotid (the latter now lying behind the external carotid); and (2) a small portion of the parotid gland. External.-The internal carotid artery for a short distance. Internal.-The pharynx and hyoid bone; the superior laryngeal nerve; a portion of the parotid gland; and the posterior border of the ramus of the inferior maxilla.

The external carotid artery has no vein in the sense of a com- 
panion vessel, but the temporo-maxillary vein descends in front of it, in the parotid gland, to near the angle of the inferior maxilla, beyond which point the artery has no vein.

The course of the vessel may be indicated by a line drawn from the side of the cricoid cartilage of the larynx to the tragus of the auricle.

Development.-The external carotid artery is the persistent part of the ventral aorta above the level of the third arterial arch.

Branches.- These are arranged in four sets, anterior, posterior, ascending, and terminal, and are as follows:

Anterior. Superior thyroid. Lingual. Facial.
Posterior.

Occipital.

Posterior auricular.
Ascending. Ascending pharyngeal.

\section{Terminal.}

Superficial temporal. Internal maxillary.

Superior Thyroid Artery.-This vessel arises in the carotid triangle from the front part of the external carotid close to its commencement. It takes an arched course forwards and downwards, passing under cover of the depressor muscles of the hyoid bone. On reaching the apex of the lateral lobe of the thyroid body it breaks up into its terminal branches, which enter the lobe on its superficial aspect, and anastomose freely, within it, with branches of the inferior thyroid artery, and, in the isthmus, with its fellow of the opposite side.

Branches.-These are as follows :

Infrahyoid.

Sterno-cleido-mastoid.

Superior laryngeal.
Crico-thyroid.

Glandular.

Muscular.

The infrahyoid artery passes inwards on the thyro-hyoid membrane, beneath the thyro-hyoid muscle, and close to the lower border of the hyoid bone. It anastomoses at the middle line with its fellow of the opposite side, and with the suprahyoid branch of the lingual artery of the same side. The sternocleido-mastoid artery passes obliquely downwards and outwards, over the upper part of the carotid sheath, to enter the deep surface of the muscle from which it takes its name. It is liable to be cut in ligation of the common carotid artery. The superior laryngeal artery accompanies the internal laryngeal nerve, and passing beneath the outer border of the thyro-hyoid muscle, it pierces the thyro-hyoid membrane, to be distributed to the interior of the larynx. The crico-thyroid artery passes transversely inwards upon the crico-thyroid membrane, and anastomoses with its fellow of the opposite side to form the crico-thyroid arch. This arch is situated midway between the cricoid and thyroid cartilages, but, notwithstanding this position, it may be in danger in the operation of laryngotomy. The glandular branches are distributed to the lateral lobe of the thyroid body. They anastomose freely with 
branches of the inferior thyroid of the same side, and with branches of its fellow of the opposite side. One very constant branch, known as the artery of the isthmus, courses along the upper border of the isthmus, and anastomoses with its fellow of the opposite side. The muscular branches are distributed to the depressor muscles of the hyoid bone.

The superior thyroid vein issues from the upper part of the lateral lobe of the thyroid body, and crosses in front of the common carotid artery, near its bifurcation, to open into the internal jugular vein. Its tributaries for the most part correspond to the branches of the artery, amongst these being the crico-thyroid and superior laryngeal veins.

Lingual Artery. - The lingual artery arises from the front part of the external carotid, a little above the origin of the superior thyroid, and opposite the great cornu of the hyoid bone. From its com-

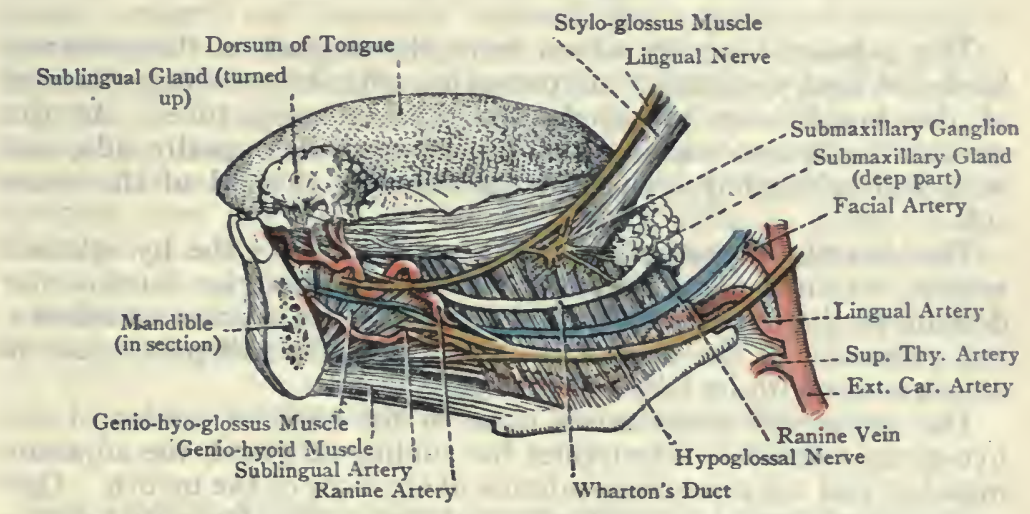

Fig. 456.-Deep Dissection of the Left Submaxillary Region.

plicated course it is convenient to divide the artery into threc parts.

First Part.-The first part of the vessel ascends for a little, and then, bending sharply, descends to the great cornu of the hyoid bone, passing beneath the posterior belly of the digastric and stylohyoid muscles. So far the vessel lies in the carotid triangle, and the bend which it describes is crossed by the hypoglossal nerve. It is for the most part comparatively superficial.

Second Part.-The second part passes horizontally forwards along the upper border of the hyoid bone beneath the hyo-glossus muscle, the hypoglossal nerve and ranine vein being superficial to that muscle. At the anterior border of the hyo-glossus it enters upon the third part of its course. The second part lies deeply in Lesser's triangle, the boundaries of which are as folluws : the base, which is directed upwards, is formed by a portion of the hypo- 
glossal nerve; the two sides are formed by the tendon of the digastric, which is here disposed in the form of a V; and the floor is formed by the hyo-glossus muscle. The artery lies under cover of that muscle, and rests upon the middle constrictor of the pharynx.

Third Part.-Near the anterior border of the hyo-glossus muscle the lingual artery describes another sharp bend in an upward direction, and ascends almost vertically to the under surface of the tongue, resting upon the genio-hyo-glossus, and being under cover of the anterior border of the hyo-glossus. Having reached the tongue, the artery passes forwards on its under surface in a tortuous manner, under the name of the ranine artery.

Branches.-These are as follows :

I. Suprahyoid.

2. Dorsalis linguæ.
3. Sublingual.

4. Ranine.

The suprahyoid artery arises from the lingual at the posterior border of the hyo-glossus, and passes inwards along the upper border of the hyoid bone to supply the adjacent structures. At the median line it anastomoses with its fellow of the opposite side, and with the infrahyoid branch of the superior thyroid of the same side.

The dorsalis linguce artery arises under cover of the hyo-glossus muscle, which it pierces, and so reaches the posterior third of the dorsum of the tongue. It is distributed to the mucous membrane and substance of the tongue, the tonsil, and the soft palate, and it anastomoses with its fellow of the opposite side.

The sublingual artery arises close to the anterior border of the hyo-glossus muscle. It supplies the sublingual gland, the adjacent muscles, and the mucous membrane of the floor of the mouth. One of the lateral branches anastomoses at the median line with a corresponding branch of the opposite artery, and another of them is known as the artery of the frenum lingure.

The ranine artery is the terminal part of the lingual. It passes forwards on the under surface of the tongue, lying immediately. external to the insertion of the genio-hyo-glossus, between it and the inferior lingualis. It is more or less embedded in the substance of the tongue, and its course is tortuous in adaptation to the mobility of the organ to which it is so intimately related. Towards the tip of the tongue the vessel is very superficially placed, lying close by the side of the frenum lingux, and it anastomoses with its fellow of the opposite side near the tip. Elsewhere the cross anastomoses are unimportant. The ranine artery furnishes branches freely to the substance of the tongue. Its close relation to the frenum linguæ is to be carefully noted in connection with the operation for relief of tongue-tied children.

The lingual veins are as follows: (I) the ranine vein, which is of large size, commences under the tip of the tongue, and passes 
backwards, in company with the hypoglossal nerve, over the hyo-glossus muscle, receiving tributaries from the surrounding structures; (2) two vence comites accompanying the lingual artery; and (3) the dorsal lingual veins, which originate in a plexus beneath the mucous membrane over the posterior third of the organ. These three sets of veins may join into a common trunk, called the lingual vein, which opens into the internal jugular vein, or they may terminate independently in that vein.

Lingual Lymphatic Glands.-These glands, which are of small size, lie upon the outer surfaces of the genio-hyo-glossus and hyoglossus muscles, along the ranine vein. They are really small glandstations, lying in the course of the lymphatic vessels of the tongue, as these pass to join the deep cervical glands.

Facial Artery. - The facial artery arises from the front part of the external carotid in the carotid triangle, immediately above the lingual artery, or sometimes in common with that vessel. It passes upwards and forwards, beneath the hypoglossal nerve and the posterior belly of the digastric and stylo-hyoid muscles, into the submaxillary triangle. Thereafter it becomes embedded in a groove on the upper and back part of the submaxillary gland, its course in this situation being horizontally forwards. Having emerged from this groove, it describes a sharp bend and mounts upwards over the base of the inferior maxilla, in front of the masseter muscle. The vessel then enters upon the facial part of its course, for a description of which see p. II78.

Branches.-Four branches arise from the cervical part of the facial artery, as follows :

I. Inferior palatine.
2. Tonsillar.

\section{Glandular.}

4. Submental.

The inferior palatine artery passes upwards between the styloglossus and stylo-pharyngeus muscles, and then over the upper border of the superior constrictor of the pharynx, along with the levator palati muscle. It is distributed to the soft palate, tonsil, and Eustachian tube. The tonsillar artery passes upwards between the stylo-glossus and internal pterygoid muscles, and, after piercing the superior constrictor muscle, it is distributed to the tonsil and the posterior part of the side of the tongue. The glandular branches are distributed to the submaxillary gland. The submental artery arises from the facial as it is about to mount over the base of the inferior maxilla, and it passes forwards upon the mylohyoid muscle. It gives branches to the submaxillary gland and mylo-hyoid muscle, some of the branches piercing that muscle to reach the sublingual gland and anastomose with the sublingual artery. Near the symphysis it ascends over the base of the inferior maxilla, and supplies the muscles of the lower lip and the integument of the chin. The terminal part of the vessel anastomoses with the mental and inferior labial arteries, and with its fellow of the opposite side. 
The cervical part of the facial vein passes downwards and backwards superficial to the submaxillary gland. Having received tributaries corresponding to the branches of the cervical part of the artery, it unites with the anterior division of the temporo-maxillary vein to form the common facial vein, which opens into the internal jugular opposite the body of the hyoid bone.

Occipital Artery.-The occipital artery arises from the posterior aspect of the external carotid opposite the facial artery. It passes at first upwards and slightly backwards beneath the posterior belly of the digastric and stylo-hyoid muscles, and the hypoglossal nerve, having hooked round it, passes forwards over it. Having reached the level of the interval between the transverse process of the atlas and the mastoid process, the artery changes its course, and passes backwards to occupy the occipital groove on the inner aspect of the mastoid process. In this backward course it crosses the internal carotid artery, internal jugular vein, and pneumogastric, spinal accessory, and hypoglossal nerves. As it lies in the occipital groove the vessel is very deeply placed, being covered by the following structures : $(x)$ the origin of the posterior belly of the digastric; (2) the trachelo-mastoid; (3) the splenius capitis; and (4) the sterno-cleido-mastoid. After escaping from beneath the splenius capitis, the vessel takes an upward course over the complexus to the occipital region, where it ramifies in a tortuous manner, along with the branches of the great occipital nerve.

Branches.-These are as follows : muscular ; meningeal ; mastoid ; ramus princeps cervicis ; and terminal.

The muscular branches are distributed to the adjacent muscles. One of them crosses the hypoglossal nerve and enters the deep surface of the sterno-cleido-mastoid muscle, in company with the spinal portion of the spinal accessory nerve. The meningeal branch accompanies the internal jugular vein, and enters the cranial cavity through the jugular foramen to supply the dura mater of the posterior fossa. The mastoid branch passes through the arterial fissure in the mastoid process, and supplies the adjacent dura mater. The ramus princeps cervicis and the terminal branches have been already described (see p. I062).

The occipital veins will be found described on p. 1063.

Posterior Auricular Artery. - This vessel arises from the posterior aspect of the external carotid a little above the origin of the occipital artery, and above the posterior belly of the digastric. It passes upwards and slightly backwards under cover of the lower part of the parotid gland, and over the styloid process of the temporal bone, being crossed by the facial nerve. Having reached the groove between the back of the auricle and the mastoid process, where it meets the posterior auricular nerve, it divides into two branches, auricular and mastoid.

Branches.-These are as follows : muscular; glandular; stylomastoid ; auricular; and mastoid.

The muscular branches supply the adjacent muscles. The glandular branches are distributed to the lower part of the parotid 
gland. The stylo-mastoid artery enters the aqueduct of Fallopius through the stylo-mastoid foramen. It is distributed to the tympanic cavity and the mastoid cells, and anastomoses with the tympanic branch of the first part of the internal maxillary artery. With this latter branch it forms a ring at the circumference of the membrana tympani on its inner aspect. Within the aqueduct of Fallopius the stylo-mastoid artery anastomoses with the petrosal branch of the middle meningeal artery, which branch enters the aqueduct through the hiatus Fallopii. The auricular branch passes upwards beneath the retrahens auriculam muscle, and furnishes branches to the inner aspect of the auricle, some of which reach the outer surface by piercing the cartilage and by turning round its margin. The auricular branch anastomoses with the posterior division of the superficial temporal artery. The mastoid branch passes backwards over the mastoid process to the occipital region, and anastomoses with the occipital artery.

The posterior auricular vein, of fairly large size, unites with the posterior division of the temporo-maxillary vein near the angle of the inferior maxi"a, and by this union the external jugular vein is formed.

Ascending Pharyngeal Artery.-This long, slender vessel arises from the deep surface of the external carotid near its commencement. It passes vertically upwards towards the base of the skull, lying very deeply upon the rectus capitis anticus major muscle, and between the internal carotid artery and the pharynx.

Branches.-These are as follows :
ז. Pharyngeal.
2. Palatine.
3. Prevertebral.
4. Tympanic.

5. Meningeal.

The pharyngeal branches are distributed to the pharynx. The palatine branch passes over the superior constrictor muscle of the pharynx, and is distributed to the soft palate, Eustachian tube, and tonsil. The prevertebral branches supply the prevertebral muscles. The tympanic branch passes with the tympanic branch of the glossopharyngeal nerve through the tympanic canaliculus in the petrous part of the temporal bone, and so reaches the tympanum, to the inner wall of which it is distributed. The meningeal arteries are the terminal branches of the ascending pharyngeal, and are three in number: One passes through the foramen lacerum medium, a second through the jugular foramen, and a third through the anterior condylar foramen, to be distributed to the dura mater in the vicinity of these foramina.

No specially-named vein accompanies the ascending pharyngeal artery.

For the superficial temporal and internal maxillary branches of the external carotid artery, see pp. Io70 and I202.

The internal carotid artery will be found described on p. I222. 
Thyroid Body.-The thyroid body is situated on either side of the upper part of the trachea and the larynx, and a small portion of it lies in front of the upper part of the trachea. Its size is subject to much variation; its weight is rather more than I ounce; and it is larger in the female than in the male. It consists of two lateral lobes and an isthmus.

Each lateral lobe is conical, the rounded base being directed downwards, and it measures about 2 inches in length. It extends from the inferior cornu and adjacent portion of the ala of the thyroid cartilage to about the level of the fifth ring of the trachea. Its superficial surface, which looks forwards and outwards, is somewhat convex, and is covered by the sterno-thyroid, sterno-hyoid, and anterior belly of the omo-hyoid muscles. It is also overlapped by the anterior border of the sterno-cleido-mastoid. Its deep surface is concave in adaptation to the trachea and larynx. The anterior border is thin, and towards its lower part is connected with that of the opposite lateral lobe by means of the isthmus. The posterior border is thick, and is in contact with the pharynx and œsophagus. The lateral lobe slightly overlaps the common carotid artery, enclosed in the carotid sheath, and is frequently grooved by that vessel. Inferiorly it overlaps the recurrent laryngeal nerve and inferior thyroid artery. The apex of the lateral lobe rests upon the inferior constrictor muscle of the pharynx, and the superior thyroid artery enters it superficially.

The isthmus is inconstant as regards size and position. Its depth ranges from $\frac{1}{4}$ to $I$ inch, and its breadth is about $\frac{1}{2}$ inch. It connects the lower parts of the anterior borders of the lateral lobes, but does not reach quite so low as their bases. It lies in front of the trachea, usually upon the second and third rings, sometimes upon the upper three rings, or it may be the second, third, and fourth rings. It fits closely to the rings upon which it rests. Along its upper border there is a branch of the superior thyroid artery, known as the artery of the isthmus, which anastomoses with its fellow of the opposite side. From its lower border several veins issue, which take part in the inferior thyroid plexus of veins in front of the trachea.

In some cases an additional lobe is present, called the pyramidal, or middle, lobe. It takes the form of a long pyramid which is attached by its base to the upper border of the isthmus, usually at its junction with the left lateral lobe. Its apex is attached to the body of the hyoid bone by a fibrous band, which sometimes contains muscular fibres, known as the levator glandula thyroidece muscle.

The thyroid body is invested by a fibrous sheath which is derived from the pretracheal lamina of the deep cervical fascia.

When a portion or portions of the pyramidal lobe, or of a lateral lobe, become detached, the isolated masses are known as accessory thyroids.

Blood-supply-Arterles.-The thyroid body is characterized by great vascularity. The arteries on either side are $(I)$ the superior 
thyroid, which is a branch of the external carotid, and (2) the inferior thyroid, which is a branch of the thyroid axis of the first part of the subclavian. Occasionally there is a third thyroid artery, called the arteria thyroidea ima, which is derived from the innominate artery, or from the arch of the aorta, and is distributed to the isthmus, its position being in front of the trachea at the median line, or close to it.

The veins are three in number on either side, namely, superior,

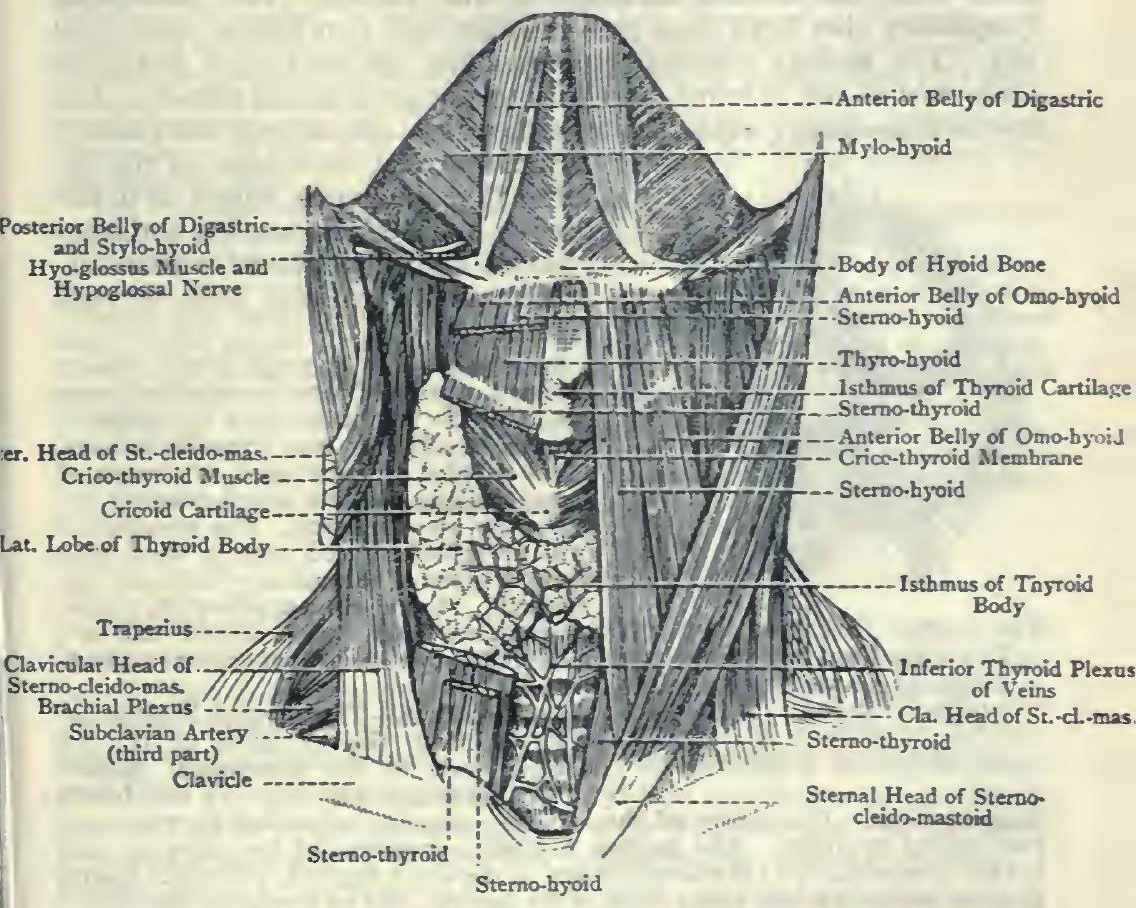

Fig. 457.-Dissection of THE Front of the NEck.

(The area bounded on either side by the Anterior Belly of the Digastric and below by the body of the Hyoid Bone is the Submental Triangle.)

middle, and inferior. The superior and middle thyroid veins open into the internal jugular. The inferior thyroid veins, right and left, issue from a plexus of veins in front of the trachea below the isthmus. The left vein opens into the left innominate vein, whilst the right has a variable mode of termination. It may open into the left innominate vein, or into the angle of junction of the right and left innominate veins, or into the lower part of the right innominate vein. 
Nerves.-These are derived from the sympathetic plexuses which accompany the superior and inferior thyroid arteries.

Lymphatics.- The lymphatic vessels of the thyroid body are disposed in two groups-ascending and descending. The ascending lymphatics form three sets-median and two lateral, right and left. The median ascending lymphatics return lymph from the upper part of the isthmus, and pass to the prelaryngeal glands. The lateral ascending lymphatics, on either side, accompany the superior thyroid artery, and pass to the inferior deep cervical glands on a level with the cricoid cartilage of the larynx. The descending lymphatics also form three sets - median and two lateral, right and left. The median descending lymphatics return lymph from the lcwer part of the isthmus, and pass to the pretracheal glands. The lateral descending lymphatics, on either side, accompany the inferior

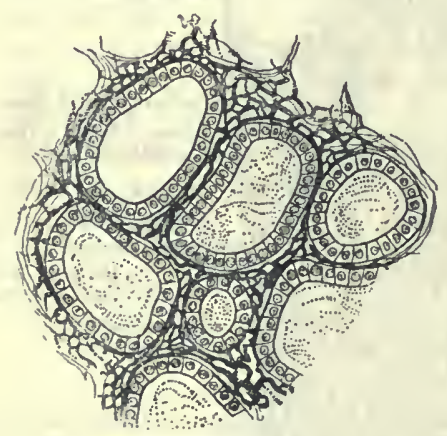

Fig. 458. - SECTION OF THE THYROID BODY, SHOWING THE VESICLES AND THEIR EPITHELIAL LINING.

(The Colloid is indicated.) thyroid artery, and pass to the inferior thyroid glands, which lie in the groove between the trachea and œsophagus, the efferents of which terminate in the inferior deep cervical glands.

Structure.-The thyroid body has an external capsule of dense connective tissue which sends trabeculæ into the interior, thereby dividing it in to irregular lobules. These lobules are composed of groups of closed resicles, which are connected together by areolar tissue. The vesicles are oval or spherical, and each is lined with a single layer of columnar, or cubical, epithelium. They contain a yellowish, viscid, albuminous fluid called colloid, and are surrounded by networks of capillary bloodvessels.

Development. - The thyroid body is developed from the entoderm of ( 1 ) the ventral wall of the pharyngeal portion of the primitive gut, and (2) the fourth visceral clefts. It consists of three parts-namely, a median thyroid, and two lateral thyroids. The median thyroid represents (I) the isthmus, and (2) the greater parts of the lateral lobes, of the adult thyroid, whilst the lateral thyroids represent only small parts of the lateral lobes of the adult thyroid.

The first indication of the median thyroid is an evagination of the ventral pharyngeal entoderm between the ventral ends of the second visceral clefts, immediately behind the tuberculum impar. This evagination is called the median thyroid diverticulum. It forms a thick-walled epithelial vesicle, embedded in mesoderm, which soon becomes solid. As the vesicle grows in a downward direction over the ventral aspect of the primitive larynx, its distal end becomes bilobed. Superiorly it retains for some time its communication with the ventral wall of the pharynx, behind the tuberculum impar, by a hollow pedicle, which constitutes the thyro-glossal duct. This duct usually disappears, its superior orifice being represented on the dorsum of the adult tongue by the blind recess, called the foramen cacum. In very rare cases the lingual portion of the duct may persist for a short distance, in which cases the foramen cacum leads to the lumen of a short tube, known as the ductus lingualis.

The median thyroid, as stated, gives rise to the isthmus and greater parts of the lateral lobes of the adult thyroid body. 
The first indication of each lateral thyroid is an evagination of the ento. derm of the corresponding fourth visceral eleft. This evagination is called the lateral thyroid diverticulum. These lateral diverticula, like the median, form two thick-walled epithelial vesicles, which soon become solid, and lose their connections with the fourth visceral clefts. Thereafter each fuses with the corresponding lobe at the caudal end of the median vesicle, and one solid epithelial mass is thereby formed, which is invested by mesoderm, and which lies across the laryngo-tracheal tube. The lateral thyroids, as stated, form only small parts of the adult thyroid body.

Connective tissue, derived from this mesodermic investment, now invades the solid mass, and it is broken up into numerous solid epithelial cords, which anastomose freely, and so give rise to an intricate reticulum, the meshes of which are occupied by connective tissue and bloodvessels, of mesodermic origin. The solid epithelial cords of the reticulum become hollow, and the lumina, so produced, are broken up at intervals, by constrictions, into closed vesicles, which contain the collold material.

The pyramid of the thyroid body sometimes met with in connection with the isthmus of the adult thyroid, is developed from the caudal part of the thyro-glossal duet.

The epithelial cells of the vesicles of the adult thyroid are derived from the entoderm of the pharyngeal part of the fore-gut.

Parathyroid Bodies. - The parathyrolds are four in number, and are arranged in pairs. The upper pair are related to the dorsal borders of the lateral lobes of the thyroid body, and the lower pair are placed behind the lower ends of the lateral lobes. They are developed as evaginations of the entoderm of the third and fourth visceral elefts, of either side. The parathyroids present no traces of closed vesicles for the colloid material.

Accessory Thyroid Bodies.-These bodies are sometimes met in the neighbourhood of the hyoid bone, and are known as the suprahyoid and prehyoid bodies. They are developed as buds or evaginations of the thyro-glossal duct, and they consist of thyroid tissue.

\section{The Trachea and Esophagus.}

Trachea.-The trachea extends from the cricoid cartilage of the larynx to about the level of the disc between the bodies of the fourth and fifth thoracic vertebræ, where it divides into the two bronchi, right and left. Its average length is about $4 \frac{1}{2}$ inches, and its width about I inch. Anteriorly and laterally it is cylindrical and firm, but posteriorly it is flattened and membranous, so that it does not press upon the œsophagus, over which it lies. It occupies a mesial position, and its direction is downwards with an inclination backwards. It is divisible into two parts, cervical and thoracic.

For the trachea in the thorax, see p. rorg.

The cervical part of the trachea extends from the cricoid cartilage to the level of the upper border of the manubrium sterni, and it measures about $2 \frac{1}{2}$ inches in length. It is freely movable, and is surrounded by areolar tissue, which contains many elastic fibres, and is somewhat loosely arranged.

Relations-Anterior.-The isthmus of the thyroid body lies directly upon the second and third rings as a rule, the first ring usually lying exposed between its upper border and the cricoid cartilage. Superficial to the first ring, on the left of the median line, there may be the lower part of the pyramidal, or middle lobe of the thyroid body. Below the isthmus is the inferior thyroid plexus of veins, from which the right and left inferior thyroid veins 
pass downwards, one on either side of the median line. The arteria thyroidea ima may ascend to the isthmus of the thyroid body, lying in front of the trachea at the median line, or slightly to the right of it. The anterior jugular veins, right and left, are anterior to it, and just above the manubrium sterni it is crossed by the communicating. branch which passes between these two veins. In children under two years of age the cervical portion of the thymus body forms an important anterior relation. Close to the upper border of the manubrium sterni the innominate artery

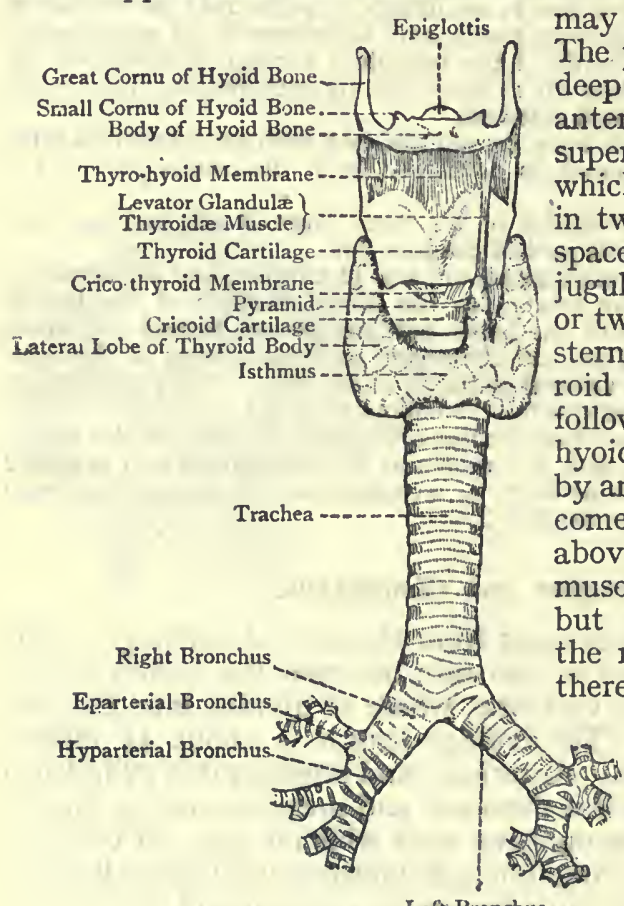

Left Bronchus

Fig. 459.-The Hyoid Bone, LARynX, Trachea, Bronchi, and Thyroid Body (ANTERIOR View). of the fifth ring, and external to this is the carotid sheath with its contents, the common carotid artery being nearest the lateral lobe.

Posierior.-The trachea rests upon the œesophagus, which projects a little to its left side towards the root of the neck. Between the two there is a groove, in which the recurrent laryngeal nerve and inferior laryngeal artery ascend. For a reference to the high and low operations of tracheotomy, see p. Iro7.

Blood-supply.- The arteries of the cervical part of the trachea are derived from the inferior thyroid of each side, which is a branch of the thyroid axis. 
The veins terminate in the inferior thyroid plexus and inferior thyroid veins.

The lymphaties pass to the lower group of deep cervical glands.

Pretracheal Lymphatic Glands.-These glands lie upon the front of the cervical part of the trachea, below the isthmus of the thyroid body. Their afferent vessels are derived from (I) the front of the trachea, and (2) the lower part of the isthmus of the thyroid body. Their efferent vessels pass to the inferior deep cervical glands. The

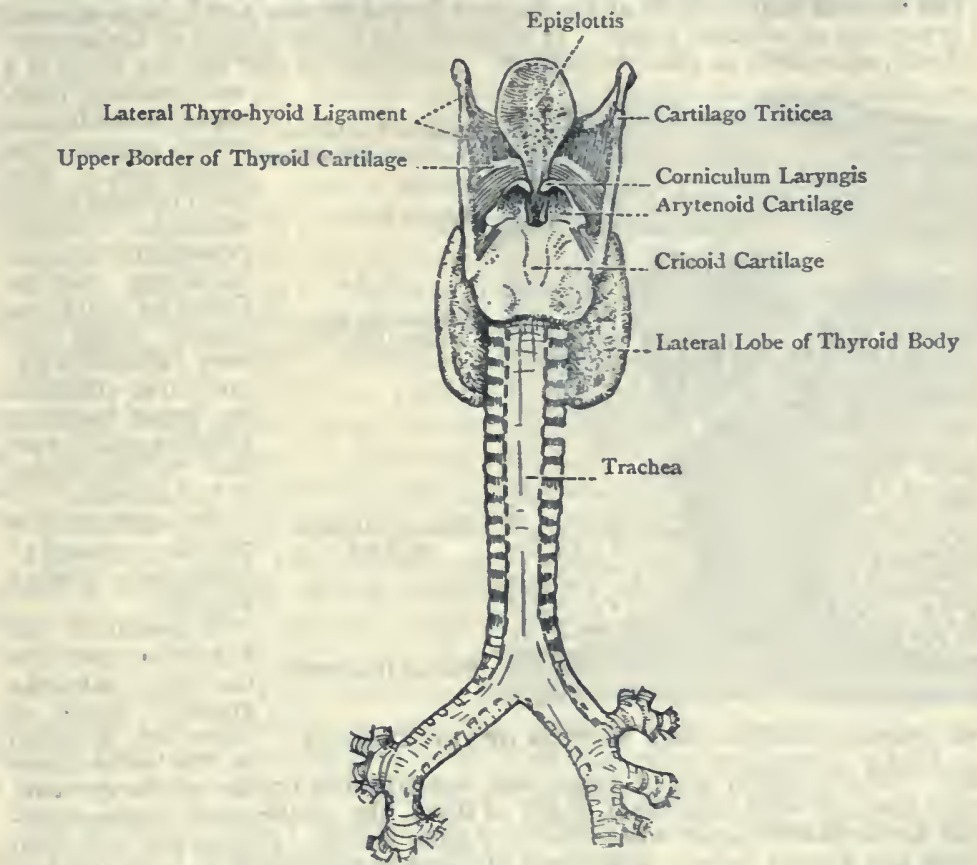

Fig. 460.-The Epiglotits, Larynx, Trachea, Bronchi, and Thyroid BODY (POSTERIOR VIEW).

lymphatic vessels of the cervical part of the trachea pass to the pretracheal, inferior thyroid, and inferior deep cervical glands.

Nerve-supply. - The nerves are derived from the pneumogastric, recurrent laryngeal, and sympathetic.

Structure.-The trachea is composed of about twenty so-called rings of hyaline cartilage, which are incomplete posteriorly. They serve to keep the tube permanently open for the transmission of air, and are embedded in fibrous tissue, which also connects together their contiguous borders. They are shaped thus - $\mathbf{C}$, and each forms rather more than two-thirds of a circle, being flat externally, and convex internally. The deficient portions of the rings are, as stated, placed posteriorly, and here each ring ends in two round extremities. The intervals between these extremities are bridged over by fibrous tissue, continuous with that which connects the borders of the rings and in which they are embedded. In some cases a ring may terminate in a bifurcated extremity, or it may join one of the adjacent rings. The lowest 
ring is deep, and its lower border projects backwards so as to form a ridge between the openings of the two bronchi.

In the posterior wall of the trachea, within the fibrous layer, there is a continuous layer of unstriped muscular tissue, the fibres of which extend transversely between the ends of the rings to which they are attached. In the intervals between the extremities of the rings they are attached to the fibrous coat. These fibres serve to approximate the ends of the rings, and so diminish the calibre of the tube.

The submucous coat consists of loosely-arranged areolar tissue, and contains the larger bloodvessels and nerves, together with the mucous glands.

The mucous coat consists of areolar and elastic tissues, and a large amount of lymphoid or adenoid tissue. It contains the ramifications of the arteries and nerves, as well as the lymphatics. Superficial to the mucosa there is a

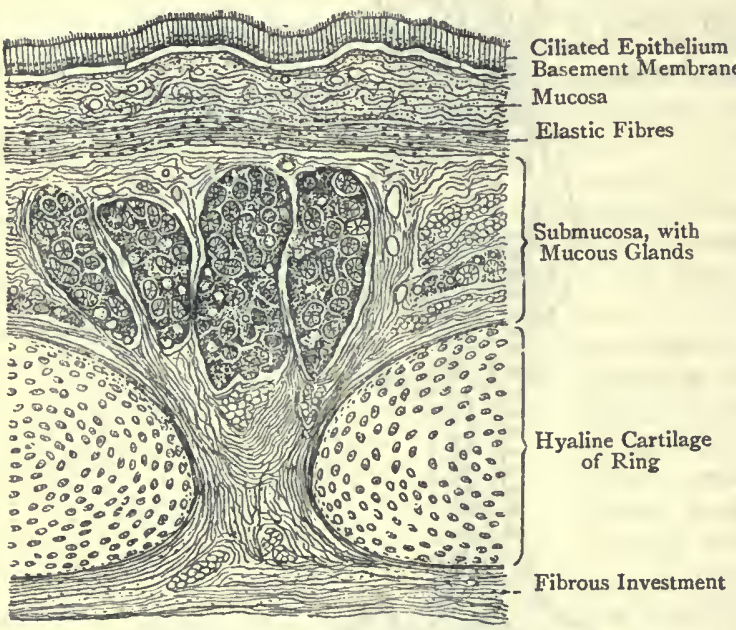

Fig. 46ia.-Longitudinal Section of the Trachea. well-marked basement membrane which supports the epithelium. The deep portion of the mucosa consists principally of elastic fibres. On the posterior wall these elastic fibres are very numerous, and are arranged in longitudinal bundles which give rise to elevations of the mucosa.

The epithelium is of the stratified columnar ciliated variety.

The wall of the trachea contains many mucous glands. Some of these are situated in the submucous coat. Others, which are of large size, are situated on the posterior wall, where they are very superficial, many of them, like small grains, lying superficial to the fibrous layer, where they are quite visible. Others are contained within the fibrous layer. The ducts of these glands have to pass through (1) the muscular layer, (2) the elastic layer (part of the mucosa), and (3) the mucosa, upon the surface of which they open by apparent orifices.

Development. - The trachea is developed from the lower part of a median diverticulum from the ventral aspect of the fore-gut superiorly, the upper part of this diverticulum giving rise to the larynx.

Esophagus.-The œsophagus is that part of the alimentary canal which extends from the pharynx to the stomach. In the neck it commences on a level with the lower border of the cricoid cartilage, and terminates on a level with the upper border of the manubrium sterni. It is compressed from before backwards, and lies between the trachea and the vertebral column covered by the longus colli muscles. At first it occupies the median line, but as it descends it inclines slightly to the left side, so as to be partially visible on the left side of the trachea.

Relations-Anterior.-The trachea; the posterior parts of the lateral lobes of the thyroid body; the recurrent laryngeal nerves; 
and the inferior thyroid arteries. Posterior. - The vertebral column and the longus colli muscles, covered by the prevertebral fascia. Lateral.-On either side there is the carotid sheath with its contents. The right and left recurrent laryngeal nerves are intimately related to the œesophagus, and ascend on each side in the groove between it and the trachea.

The cervical part of the œsophagus receives its blood-supply from the inferior thyroid arteries, right and left, each of which accompanies the corresponding recurrent laryngeal nerve.

Inferior Thyroid Lymphatic Glands.-These glands lie in the groove between the cervical parts of the trachea and œesophagus, along the course of the inferior thyroid artery and recurrent laryngeal nerve. Their afferent vessels are derived from (I) the lower part of the lateral lobe of the thyroid body, and (2) the adjacent parts of the trachea and œsophagus. Their efferent vessels pass to the inferior deep cervical glands.

For a description of the œsophagus in the thorax, including its structure and development, see Index.

Suprahyoid Region-Muscles-Digastric. - The digastric muscle consists of two bellies, posterior and anterior. Origin.-The posterior belly arises from the digastric groove on the inner aspect of the mastoid part of the temporal bone; and the anterior belly arises from the digastric impression on the inner surface of the body of the inferior maxilla, close to the base and symphysis.

Insertion.-The two bellies end upon an intervening tendon, about 2 inches long, which is inserted by means of a broad fibrous band into ( $I$ ) the anterior surface of the body of the hyoid bone at its outer part, and (2) the adjacent portion of the great cornu.

Nerve-supply. - The posterior belly is supplied by the digastric branch of the facial nerve; and the anterior belly derives its nervesupply from the mylo-hyoid branch of the inferior dental, which is a branch of the inferior division of the fifth cranial nerve.

The posterior belly is directed downwards and forwards, and the anterior belly downwards and slightly outwards.

Action.-(I) To elevate the hyoid bone, as in the act of deglutition; and (2) to depress the inferior maxilla.

Relations. - The posterior belly is at first deeply placed, being overhung by the mastoid process, and lying under cover of the trachelo-mastoid, splenius capitis, and sterno-cleido-mastoid muscles. It is also overlapped by the lower part of the parotid gland. It crosses the external and internal carotid arteries, internal jugular vein, and hypoglossal nerve. The stylo-hyoid muscle lies above it, and the hypoglossal nerve below it for a short distance. The intervening tendon is embraced by the fibres of the stylo-hyoid muscle. This tendon crosses the hypoglossal nerve, and forms two sides of a triangle, known as the triangle of Lesser, the base of which is directed upwards, and is formed by the hypoglossal nerve. In the area of this triangle is a portion of the hyo-glossus muscle, and beneath this part is a portion of the lingual artery. The anterior belly, which is shorter than the posterior, is covered by the integu- 
ment, platysma myoides, and deep cervical fascia. It rests upon the mylo-hyoid muscle, and forms the lateral boundary of the submental triangle. Its inner border is connected with that of its fellow by a fascial expansion.

The posterior belly of the digastric, along with the stylo-hyoid muscle, like the stapedius muscle, is associated with the posterior end of the second visceral, or hyoid, arch. The nerve of this arch is the faeial nerve, and this explains the nerve-supply of the posterior belly.

The anterior belly is associated with the anterior or mesial end of the first visceral, or mandibular, arch. The nerve of this arch is the inferior maxillary division of the fifth cranial, or trigeminal, nerve, and this explains the nervesupply of the anterior belly.

Stylo-hyoid-Origin.-The posterior and outer aspect of the styloid process of the temporal bone near its base.

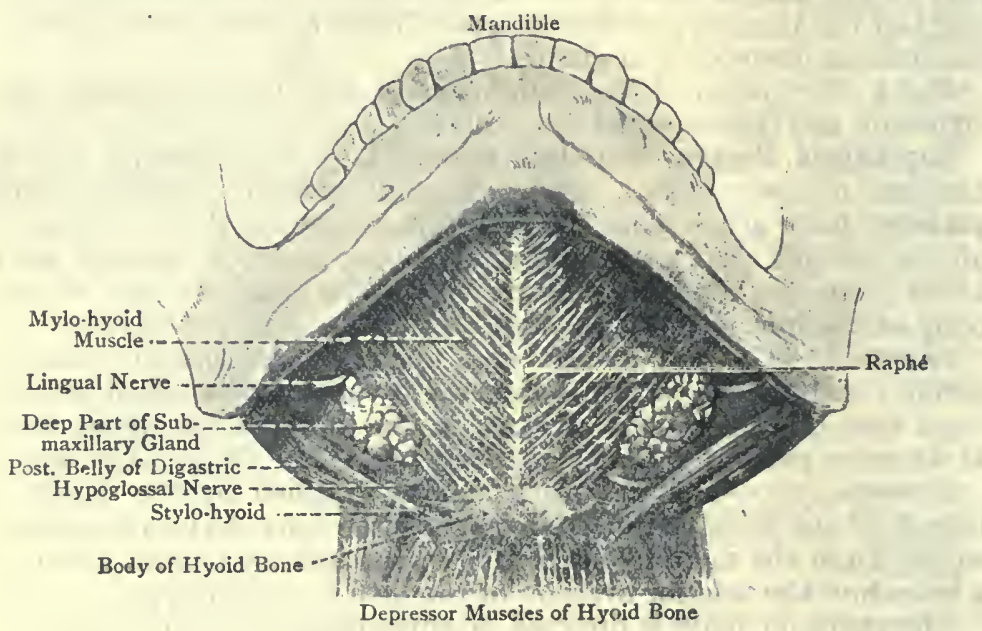

Fig. 46IB.-The MYLO-HYOID REgION.

Insertion.-The anterior surface of the hyoid bone at the junction of the body and great cornu.

Nerve-supply.-The stylo-hyoid branch of the facial nerve, which arises in common with the digastric branch.

The muscle is directed downwards and forwards.

Action.-To draw the hyoid bone upwards and backwards.

The muscle lies close above the posterior belly of the digastric, and, before taking insertion, it usually splits into two bundles, which embrace the intervening tendon of the digastric.

The stylo-hyoid muscle, along with the posterior belly of the digastric, like the stapedius muscle, is associated with the posterior end of the second visceral, or hyoid, arch. The nerve of this arch is the faclal nerve, and this explains the nerve-supply of this muscle.

Mylo-hyoid-Origin.-The mylo-hyoid ridge, or internal oblique line, of the inferiowmaxtlta momo 
Insertion.-The posterior fibres are inserted into the anterior surface of the body of the hyoid bone; and the principal part of the muscle is inserted into a central fibrous raphé, which extends from the symphysis of the inferior maxilla on its deep and lower aspect to the body of the hyoid bone.

Nerve-supply. - The mylo-hyoid branch of the inferior dental nerve, which is a branch of the inferior maxillary division of the fifth cranial nerve.

The muscle is directed downwards and forwards.

Action.-(I) To raise the floor of the mouth, and, in doing so, to press the tongue against the hard palate, as in the first stage of the act of deglutition; (2) to elevate the hyoid bone, and draw it forwards ; and (3) to depress the inferior maxilla.

The two mylo-hyoid muscles form a muscular floor for the buccal cavity, which is known as the diaphragma oris.

Relations-Superficial or Inferior.-The anterior belly of the digastric; the superficial part of the submaxillary gland, lodging a portion of the facial artery; and the mylo-hyoid nerve and submental branch of the facial artery.

Posterior Border.-Passing beneath the posterior free border of the muscle there are the following structures, in order from above downwards : (I) the lingual nerve ; (2) a portion of the submaxillary gland; and (3) the hypoglossal nerve, and the ranine vein.

Deep or Superior.-The hyo-glossus muscle, and, resting upon it, (I) the lingual nerve, (2) the submaxillary ganglion, (3) the deep part of the submaxillary gland and Wharton's duct, (4) the hypoglossal nerve, and (5) the ranine vein. In front of the hyo-glossus is the genio-hyoid muscle, and between it and the hyo-glossus is a portion of the genio-hyo-glossus, with the sublingual gland resting upon it.

Genio-hyoid-Origin.-The lower genial spine of the inferior maxilla, close to the symphysis on its deep aspect.

Insertion. - The inner two-thirds of the anterior surface of the body of the hyoid bone over its upper part. At its insertion the muscle is divided externally into two laminæ, anterior and posterior, the former of which extends furthest out upon the hyoid bone. The innermost fibres of origin of the hyo-glossus pass inwards between these two laminæ.

Nerve-supply.-The hypoglossal nerve, the branch of which is regarded as being composed of spinal fibres.

The muscle is directed downwards and slightly backwards.

Action.-(I) To elevate the hyoid bone and draw it forwards, and $(2)$ to depress the inferior maxilla.

The muscle is in intimate contact with its fellow of the opposite side at the median line. Its inferior or superficial surface is covered by the mylo-hyoid, and its superior or deep surface is related to the lower or posterior border of the genio-hyo-glossus.

Genio-hyo-glossus-Origin.-The upper genial spine of the inferior maxilla, close to the symphysis on its deep aspect. 
Insertion.-(I) The under surface of the tongue close to the median line, and extending from near the tip to the root; (2) the inner part of the anterior surface of the body of the hyoid bone close to its upper margin ; and (3) slightly into the side of the pharynx, where the fibres blend with those of the middle constrictor muscle.

Nerve-supply.-The hypoglossal nerve, the branches of which enter the outer surface of the muscle.

The upper or anterior fibres arch upwards and forwards, and the lower downwards and backwards, whilst the intervening fibres spread out in a diverging manner.

Action.-(I) The entire glossal fibres depress the tongue at the

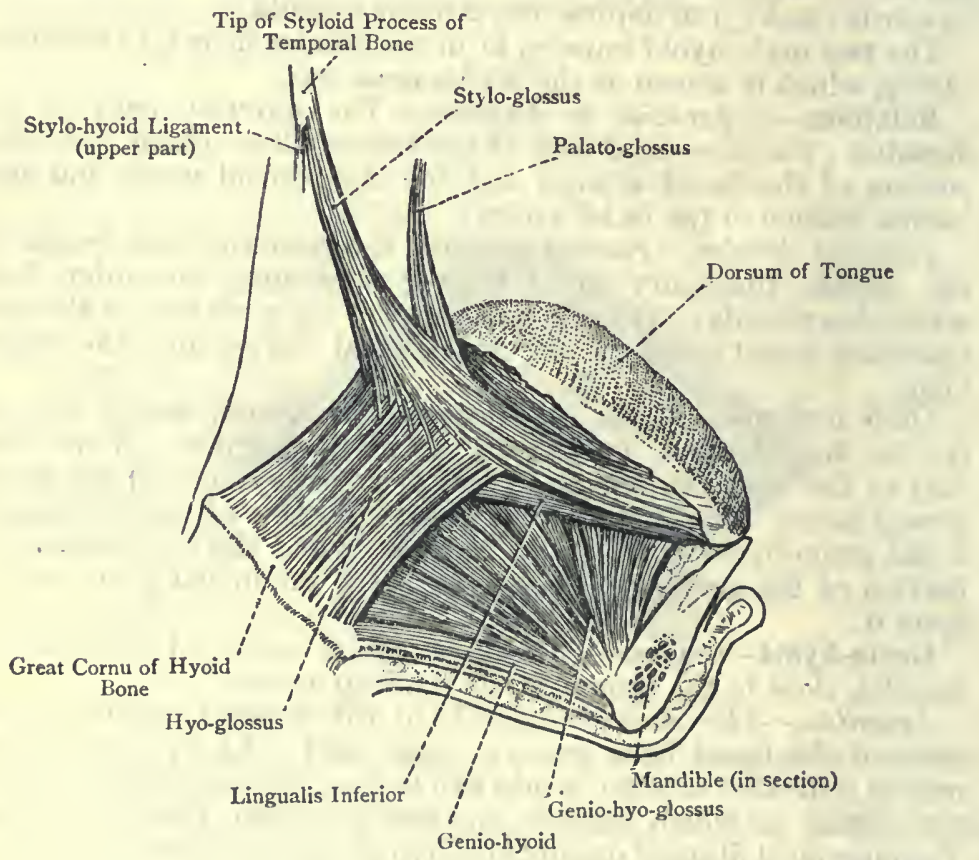

Fig. 462.-The Extrinsic Muscles of the Tongue. (Tine Lingualis INFERIOR IS AN INTRINSIC MUSCLE).

median line, and, along with those of the opposite side, they give rise to an antero-posterior groove, or gutter, on the dorsum of the organ; (2) the posterior glossal fibres draw forwards the tongue, so as to cause its tip to be protruded from the mouth; (3) the anterior glossal fibres retract the tip of the tongue when it has been protruded from the mouth; and (4) the lower or posterior (hyal) fibres elevate the hyoid bone, and draw it forwards.

The muscle is fan-shaped. The internal surface is closely applied to that of its fellow of the opposite side. The external surface is 
related to the inferior lingualis muscle, the ranine artery, the hyoglossus and stylo-glossus muscles, and the sublingual gland. The upper or anterior border is covered by the buccal mucous membrane, and the lower or posterior border by the genio-hyoid muscle.

Hyo-glossus-Origin.-(I) The great cornu of the hyoid bone over its entire length; (2) the anterior surface of the body of the bone over about its outer half ; and (3) the small cornu (inconstant). The innermost fibres of origin from the hyoid bone pass inwards between the two laminæ of the genio-hyoid muscle.

Insertion.-The posterior half of the under surface of the tongue close to its lateral border, the fibres being situated internal to those of the stylo-glossus, and both sets of fibres being intimately intermixed with each other and with the intrinsic lingual muscles.

Nerve-supply. - The hypoglossal nerve, the branches of which enter the superficial surface.

The muscle is for the most part directed upwards, but its anterior fibres have a slight inclination forwards.

Action.-(I) To depress the side of the tongue, and, along with its fellow, to render the dorsum of the organ convex; and (2) to assist in retracting the protruded tongue.

The hyo-glossus is a flat, four-sided muscle.

Relations-Superficial.-The mylo-hyoid muscle; the intervening tendon of the digastric, and stylo-hyoid muscle; the lingual nerve, with the submaxillary ganglion lying a little below it ; the deep part of the submaxillary gland, and Wharton's duct; the hypoglossal nerve; and the ranine vein. Posterior Border.-The following structures pass beneath this border, in order from above downwards : (I) the glosso-pharyngeal nerve; (2) the stylo-hyoid ligament; and (3) the lingual artery. Deep.- The posterior part of the geniohyo-glossus; a portion of the middle constrictor muscle of the pharynx; the lingual artery; the lower end of the stylo-hyoid ligament; and the glosso-pharyngeal nerve.

The fibres of the hyo-glossus which arise from the small cornu of the hyoid bone are separated from the rest of the muscle by those fibres of the genio-hyo-glossus which take insertion into the side of the pharynx, and they are regarded by some as a separate muscle, called the chondro-glossus. These fibres, however, are inconstant.

Stylo-glossus-Origin.-(I) The front of the styloid process of the temporal bone near its tip; and (2) the upper extremity of the stylo-mandibular ligament.

Insertion.- The under surface of the tongue close to its lateral border. The fibres extend as far forwards as the tip, and are situated external to the fibres of the hyo-glossus, both sets of fibres being intimately intermixed with each other, and with the intrinsic lingual muscles.

Nerve-supply. - The hypoglossal nerve.

The muscle is directed downwards, forwards, and inwards.

Action.-(I) To draw the tongue backwards; and (2) to elevate the root of the tongue. 
Stylo-hyoid Ligament.-This is a narrow fibrous cord which is attached superiorly to the tip of the styloid process, and inferiorly to the small cornu of the hyoid bone. Its direction is downwards and forwards, and its lower extremity is covered by the hyo-glossus muscle. It is liable to become ossified, more or less completely.

Submaxillary Gland.-This gland is situated in the anterior part of the submaxillary or digastric triangle. It consists of a large superficial part and a small deep part. The superficial part superiorly occupies the submaxillary fossa on the inner surface of the body of the inferior maxilla, and inferiorly it is covered by the skin, superficial fascia, platysma myoides, and deep fascia. The facial vein descends over it. Its deep surface rests anteriorly upon the mylo-hyoid muscle, with the intervention of the mylo-hyoid nerve and submental branch of the facial artery; and posteriorly upon the hyo-glossus, and slightly upon the posterior belly of the di-

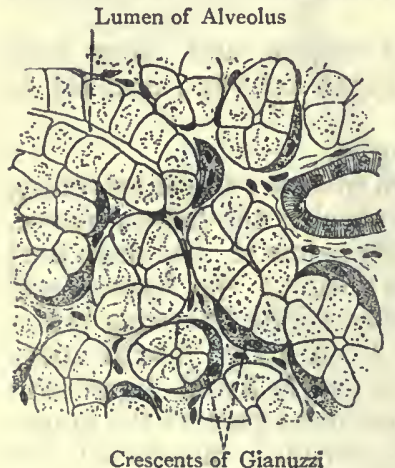

Fig. 463.-Section of THE SubMaxillary Gland oF A Dog. gastric and stylo-hyoid, muscles. The superficial part is grooved at its upper and back part by the facial artery, which lies embedded in the gland. Posteriorly the superficial part is related to the cervical portion of the parotid gland, from which it is separated by the stylo-mandibular (stylo-maxillary) ligament. The deep part of the gland is of small size, and is continuous with the superficial part at the posterior border of the mylo-hyoid muscle. It lies under cover of that muscle upon the hyoglossus, and is related to the sublingual gland.

The duct of the submaxillary gland is known as Wharton's duct, and it emerges from the deep aspect of the superficial part of the gland close to the posterior border of the mylo hyoid muscle. It is about 2 inches in length, and it passes inwards upon the hyo-glossus muscle, lying beneath the deep part of the gland. In this situation it has the submaxillary ganglion and lingual nerve above it, and the hypoglossal nerve below it. After leaving the hyo-glossus muscle, the duct lies upon the genio-hyo-glossus, and, passing slightly upwards, it is crossed from above downwards by the lingual nerve. Having passed beneath the sublingual gland, the duct opens upon the floor of the mouth by a minute orifice, which is situated on the summit of a papilla lying close to the side of the frenum of the tongue.

The submaxillary gland is enclosed in a capsule, which is derived from the deep cervical fascia.

Blood-supply. - The gland derives its blood chiefly from the cervical part of the facial artery. 
Nerve-supply. - The nerves are derived from the submaxillary ganglion, and through this from (I) the chorda tympani, (2) the lingual, and (3) the sympathetic plexus on the facial artery.

Lymphatics. - These pass to the submaxillary lymphatic glands, and thence to the superficial and deep cervical glands.

Structure.-The submaxillary gland is a muco-serous gland, and its general structure is similar to that of the parotid gland. The essential difference between the two has reference to the alveoli or acini, and the nature of their secretion. The cells of the parotid alveoli are serous or albuminous. The submaxillary alveoli contain, around the lumen, mucous cells filled with granular mucigen, which is discharged as mucus. They, however, also contain some albuminous cells, known as the marginal cells, which are situated, external

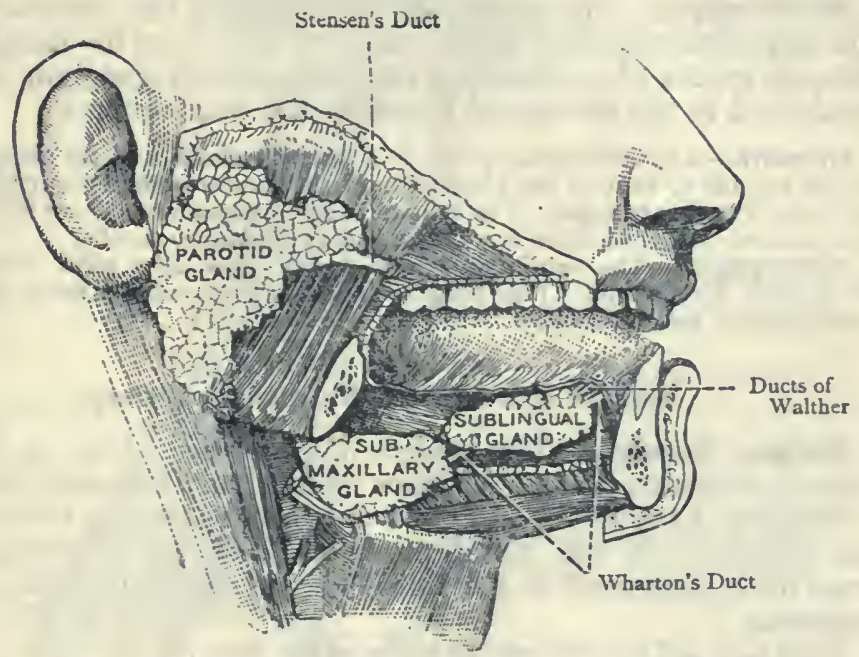

Fig. 464.-The Salivary Glands of the Right Side.

to the mucous cells, but within the membrana propria of the alveolus. These cells usually form groups which, from their crescentic arrangement, are known as the crescents of Gianuzzi.

Development. - The submaxillary gland is developed as a solid outgrowth of the buccal epithelium. This outgrowth undergoes ramifications, and these subsequently become hollow.

Sublingual Gland.-This is the smallest of the salivary glands, and resembles an almond in shape. It measures about $I \frac{1}{2}$ inches in length, and is situated beneath the mucous membrane of the floor of the mouth, where it gives rise to a mucous fold, called the plica sublingualis. Inferiorly it rests upon the mylo-hyoid muscle (under cover of it), and is here related to the deep part of the submaxillary gland, Wharton's duct, and the lingual nerve. Externally it occupies the sublingual fossa on the inner surface of the body of the inferior maxilla above the mylo-hyoid ridge; and internally it is in contact with the genio-hyo-glossus muscle. Its mesial 
extremity comes into contact with its fellow of the opposite side over the anterior border of the genio-hyo-glossus muscle.

The sublingual ducts vary in number from ten to twenty, and are known as the ducts of Walther (ducts of Rivini). A few of them open into Wharton's duct, but the majority open in a linear manner upon the summit of the plica sublingualis. One of the ducts, called the duct of Bartholin, or great duct of Rivini, issues from the external lobule of the gland, and, accompanying Wharton's duct, it opens quite close to the orifice of that duct.

Blood-supply.--The gland derives its blood from the sublingual branch of the lingual, and the submental branch of the facial, arteries.

Nerve-supply.-The nerves are derived from the chorda tympani and lingual nerves, and the sympathetic plexus on the facial artery, through means of a branch of the submaxillary ganglion, which is conducted to the sublingual gland by the lingual nerve.

Structure. - The sublingual gland is a mucous gland, and its general structure is similar to that of the parotid and submaxillary glands, but the lobules are more loosely arranged. The cells of the sublingual alveoli are for the most part mucous cells, but there are also serous or albuminous cells.

Development. - The sublingual gland is developed as a solid outgrowth of the buccal epithelium. This outgrowth undergoes ramifications, and these subsequently become hollow.

\section{Scalene Muscles and Subclavian Artery.}

Scalene Muscles. - The scalene muscles are three in number, namely, scalenus anticus, scalenus medius, and scalenus posticus.

Scalenus Anticus-Origin.-By four short tapering tendons from the tubercles of the costal processes (anterior tubercles of the transverse processes) of the third, fourth, fifth, and sixth cervical vertebræ.

Insertion. - The scalene tubercle of the first rib.

Nerve-supply.-The anterior primary divisions of the fourth, fifth, and sixth cervical nerves.

The muscle is directed downwards, outwards, and forwards.

Action.-(I) To fix the first rib in ordinary inspiration, and to elevate it in forced inspiration ; and (2) to flex the neck to one side and slightly forwards.

Chief Relations-Anterior. - The phrenic nerve, which crosses the muscle obliquely downwards and inwards; the internal jugular vein, which lies over its origin; and the subclavian vein close to its insertion. Posterior.-The scalenus medius, with the intervention of the second part of the subclavian artery, the nerve-roots of the brachial plexus, and the dome of the pleura. Internal.-The ascending cervical artery, and the origin of the rectus capitis anticus major.

Scalenus Medius-Origin.-By six short tapering tendons from the tubercles of the transverse processes (posterior tubercles) of the lower six cervical vertebræ. 
Insertion.-The upper surface of the first rib from the groove for the subclavian artery backwards to the tubercle.

Nerve-supply. - The anterior primary divisions of cervical nerves from the third to the eighth inclusive.

The muscle is directed downwards and outwards.

Action.-(I) To fix the first rib in ordinary inspiration, and (2) to flex the neck to one side.

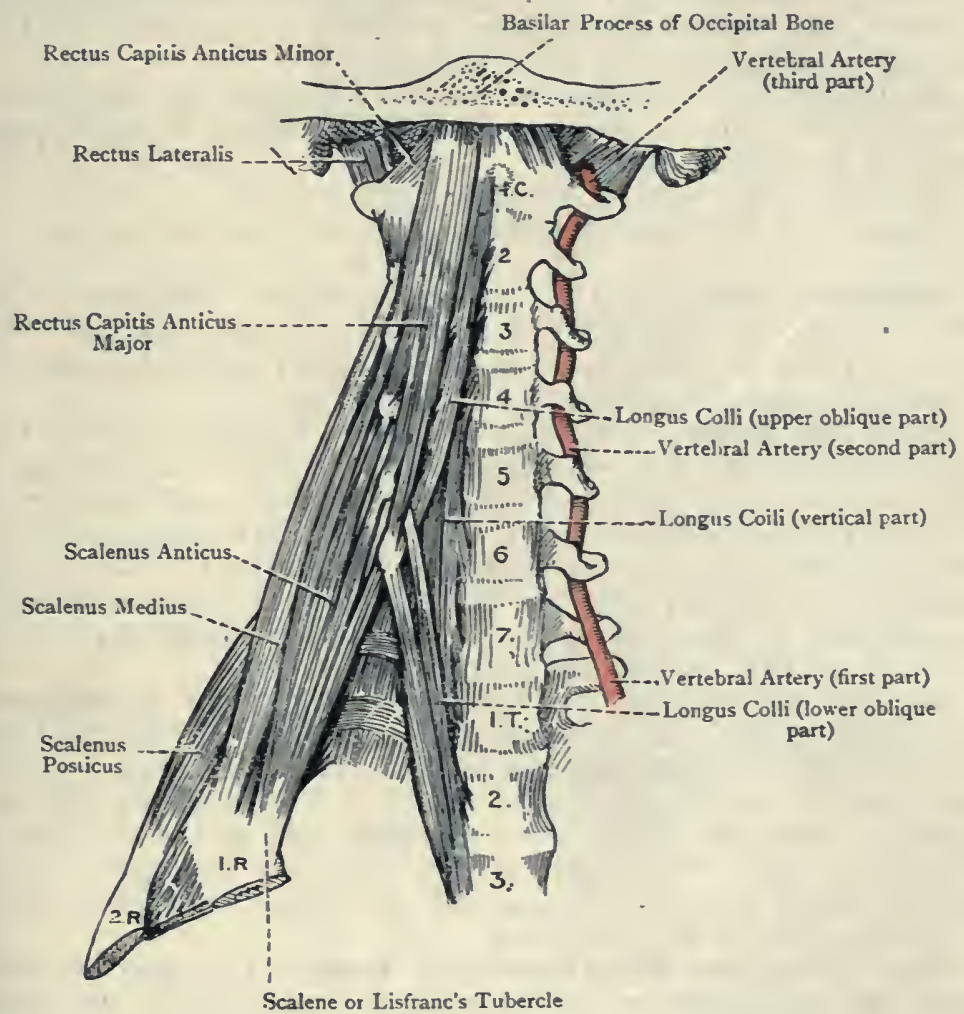

Fig. 465.-The Right Prevertebral Muscles.

(The Vertebral Artery is also shown).

Relations-Anterior. - The cervical plexus, the nerve-roots of the brachial plexus, except the first thoracic, and the second and third parts of the subclavian artery. Posterior.-The levator anguli scapulæ and scalenus posticus muscles.

The scalenus medius is pierced by the following nerves: $(I)$ the nerve to the rhomboids, which passes in a backward direction ; and (2) the upper and middle roots of the posterior thoracic nerve, or external respiratory nerve of Bell. These two roots usually emerge 
from the muscle as a single cord, and the lower or third root of the nerve descends in front of the scalenus medius, and joins the foregoing cord about the level of the first rib.

Scalenus Posticus-Origin.-By two or three short tendons from the tubercles of the transverse processes (posterior tubercles) of the lower two or three cervical vertebræ.

Insertion.-The upper part of the outer surface of the second rib, in front of the insertion of the highest slip of the serratus posticus superior, and behind the origin of a portion of the first, and the second; serrations of the serratus magnus.

Nerve-supply.-The anterior primary divisions of the sixth, seventh, and eighth cervical nerves, the branches of which pass through the scalenus medius.

The muscle is directed downwards and outwards.

Action.-(I) To elevate the second rib, and (2) to flex the neck to one side.

Relations-Anterior.-The scalenus medius. Posterior.-The lower two tendons of origin of the levator anguli scapulæ. The scalenus posticus is intimately connected with the scalenus medius, of which it may be regarded as a detached part.

Subclavian Artery. - The right subclavian vessel arises from the innominate artery behind the right sterno-clavicular joint on a level with its upper part, and the left subclavian vessel arises from the upper aspect of the arch of the aorta towards its back part. On each side the artery terminates at the outer border of the first rib by becoming the axillary artery. In its course the vessel is crossed superficially by the scalenus anticus muscle, which divides it into three parts, first, second, and third.

The first part extends from the origin of the vessel to the inner border of the scalenus anticus; the second part lies behind that muscle; and the third part extends from the outer border of the muscle to the outer border of the first rib. The total length of the vessel on the right side is about 3 inches, and, on the left, about $4 \frac{1}{2}$ inches. The artery describes an arch over the apex of the corresponding lung and pleura, and the height to which it rises above the clavicle is about $\frac{1}{2}$ inch.

First Part of the Right Subclavian Artery.-This part extends from the bifurcation of the innominate artery behind the right sterno-clavicular joint, on a level with its upper part, to the inner border of the scalenus anticus muscle. Its direction is upwards and outwards, and it lies very deeply.

Relations-Anterior.-The skin, superficial fascia and platysma myoides, deep cervical fascia, clavicular origin of the sterno-cleidomastoid, and sterno-hyoid and sterno-thyroid muscles. Three veins are related to it superficially. The internal jugular and vertebral veins cross it from above downwards close to the scalenus anticus, the latter vessel being under cover of the former, and the anterior jugular vein crosses it from within outwards, but superficial to the sterno-hyoid and sterno-thyroid muscles. Important 


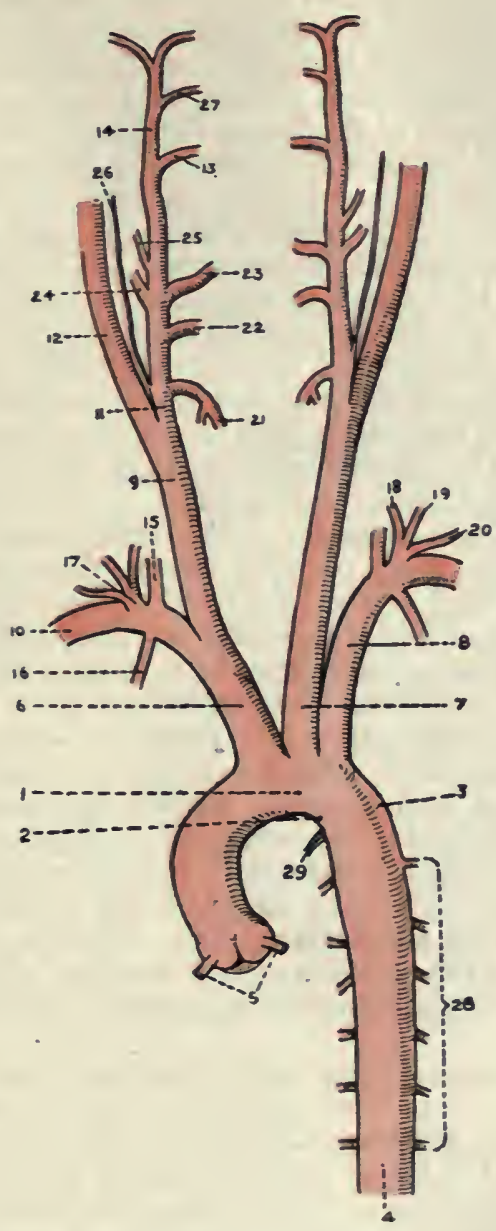

Fig. 466.-The Aorta in the Thorax, and the Principal Arteries op the Head aNd Neck.

1. Arch of the Aorta

2. Aortic Isthmus

3. Aortic Spindle

4. Descending Aorta

5. Coronary Arteries (from Ascending Aorta)

6. Innominate Artery

7. Left Common Carotid

8. Left Subclavian

9. Right Common Carotid 1a. Right Subclavian

11. External Carotid

12. Internal Carotid

13. Internal Maxillary

14. Superficial Temporal

15. Vertebral

16. Internal Mammary

17. Thyroid Axis

13. Inferior Thyroid

Ig. Transverse Cervical
20. Suprascapular

21. Superior Thyroid

22. Lingual

23. Facial

24. Occipital

25. Posterior Auricular

26. Ascending Pharyngeal

27. Transverse Facial

28. Aortic Intercostals

29. Lig. Ductûs Arteriosi 
nerves also cross it superficially, namely, the pneumogastric and its cervical cardiac branches, the cervical cardiac branches of the sympathetic, and the nerve-loop known as the ansa Vieussenii. Posterior.-The recurrent laryngeal nerve, gangliated cord of the sympathetic, fat, longus colli muscle, first thoracic vertebra, dome of the pleura, and apex of the lung. Inferior.--The recurrent laryngeal nerve, part of the ansa Vieussenii, and the pleura.

The right subclavian and right internal jugular veins unite anterior to this part of the vessel to form the right innominate vein.

First Part of the Left Subclavian Artery. - This part extends from the upper aspect of the arch of the aorta, towards its back part, to the inner border of the scalenus anticus muscle. It is therefore placed at first in the thoracic cavity. Its course is almost vertical until it reaches the root of the neck, where it curves sharply outwards upon the dome of the pleura, and so reaches the scalenus anticus. The relations of the intrathoracic portion have been described in connection with the thorax (see p. 96r).

Cervical Relations-Anterior. - The skin, superficial fascia and platysma myoides, deep cervical fascia, clavicular origin of the sterno-cleido-mastoid, sterno-hyoid and sterno-thyroid muscles, and the thoracic duct which arches over it. The left internal jugular and subclavian veins unite in front of it to form the left innominate vein, and the left vertebral vein descends in front of it under cover of the left internal jugular vein. Posterior.-The gangliated cord of the left sympathetic with the inferior cervical ganglion, fat, and the left longus colli muscle. Right.-The trachea, osophagus, left recurrent laryngeal nerve, and thoracic duct.

The varieties in origin of the subclavian artery have been described in connection with the arch of the aorta (see p. 963).

Second Part of the Subclavian Artery.- This portion of the vessel is situated behind the scalenus anticus muscle. It lies about $\frac{1}{2}$ inch above the clavicle, and forms the highest part of the arch described by the vessel.

Relations-Anterior.-The skin, superficial fascia and platysma myoides, deep cervical fascia, clavicular part of the sterno-cleidomastoid, scalenus anticus, phrenic nerve on the right side, and subclavian vein, the latter vessel being on a lower level than the artery, and separated from it by the scalenus anticus. Posterior.The pleura. Superior.-The nerve-roots of the brachial plexus. Injerior. - The pleura.

In some cases the second part of the vessel passes through the scalenus anticus, and in rare cases it passes in front of the muscle.

For the third part of the subclavian artery, see p. 1095 .

Development.-The right subclavian artery, as far as the origin of the internal mammary artery, is developed from the fourth right arterial arch.

The left subclavian artery is developed from the seventh left segmental artery.

Branches of the Subclavian Artery. - These are four in number, namely. the vertebral, internal nammary, thyroid axis, and 
superior intercostal. The first three arise from the first part of the artery towards its termination, and the last arises from the second part on the right side, and from the first part on the left side.

Vertebral Artery.-This is the first branch in order. On the right side it arises from the upper and back part of the artery about an inch from its origin, and on the left side from the ressel just after it enters the root of the neck. It passes for a short distance upwards, backwards, and slightly outwards, and disappears from view by entering through the costo-transverse (vertebrarterial) foramen in the transverse process of the sixth cervical vertebra as a rule. It then ascends vertically through the successive costo-transverse foramina above that of the sixth vertebra, and in passing from that of the axis to that of the atlas it describes a bend outwards. Having traversed the costo-transverse foramen of the atlas, it passes backwards and inwards, lying in the vertebrarterial groove on the upper surface of the posterior arch of the atlas behind the superior articular process. Thereafter it pierces the dura mater and arachnoid, and, having reached the side of the spinal cord, it turns upwards in the subarachnoid space, and enters the cranial cavity through the foramen magnum. Being now related to the medulla oblongata, it gradually inclines from its lateral to its ventral aspect, and at the lower border of the pons Varolii it joins its fellow to form the basilar artery.

On account of its complicated course, the vertebral artery is divided into four parts, namely, first or cervical, second or costotransverse, third or suboccipital, and fourth or intracranial.

The first or cervical part extends from the origin of the vessel to the costo-transverse foramen in the transverse process of the sixth cervical vertebra. It lies between the scalenus anticus and longus colli muscles, having the internal jugular and vertebral veins in front of it ; being crossed by the inferior thyroid artery; and having the sympathetic gangliated cord and the transverse process of the seventh cervical vertebra behind it. The vessel of the left side has the thoracic duct as an additional anterior relation.

The first part gives off no branches.

The second or costo-transverse part traverses the costo-transverse foramina of the cervical vertebra from, as a rule, the sixth upwards. It is surrounded by the vertebral venous plexus, and by the vertebral plexus of the sympathetic, and it lies in front of the cervical spinal nerves as these emerge from the intervertebral foramina.

Branches.-These are as follows: lateral spinal and muscular. The lateral spinal branches enter the spinal canal through the intervertebral foramina. The muscular branches supply the deep muscles of the neck, and anastomose with the deep cervical, ascending cervical, and occipital arteries.

For the third or suboccipital, and the fourth or intracranial, parts of the vertebral artery, see Index.

Varieties.-(I) The left vertebral artery occasionally arises from the arch of the aorta between the origins of the left common carotid and left subrlavian 
arteries. (2) The vessel may pass the costo-transverse foramen of the sixth cervical vertebra, and may enter that of the fifth, or even that of the fourth. (3) The vessel has been found in rare cases to enter the costo-transverse foramen of the seventh cervical vertebra.

Development. - The vertebral artery is developed from ( 1 ) the seventh cervical segmental artery, and (2) the longitudinal anastomotic chain which connects the seven cervical segmental arteries.

Internal Mammary Artery.-This vessel is so named in contradistinction to the external mammary, or long thoracic, which is a branch of the second part of the axillary artery. It arises from the lower aspect of the first part of the subclavian artery very nearly opposite the thyroid axis. Its course is downwards, forwards, and slightly inwards, and it disappears behind the sternal end of the clavicle and first costal cartilage. For its subsequent course and relations, see p. 93 I.

Relations of Cervical Part-Anterior.-The clavicular part of the sterno-cleido-mastoid, and the internal jugular and subclavian veins. The phrenic nerve crosses the vessel superficially from without inwards. Posterior.-The pleura.

No branches arise from this part of the vessel.

Thyroid Axis.- This is a short trunk which springs from the front of the first part of the subclavian artery close to the inner border of the scalenus anticus muscle. It almost immediately divides into three diverging branches, namely, inferior thyroid, transverse cervical, and suprascapular.

The inferior thyroid artery passes upwards and inwards in a tortuous manner in front of the vertebral artery, and behind the carotid sheath and sympathetic nerve, the middle cervical ganglion of which latter rests upon it. Having reached the lower part of the lateral lobe of the thyroid body, the artery breaks up into its terminal branches, which enter the lobe on its deep aspect, and ramify in it, anastomosing with the superior thyroid, and with its fellow of the opposite side in the isthmus. As the vessel ascends it is intimately related to the recurrent laryngeal nerve, which usually lies behind it.

Branches.-These are as follows : muscular, ascending cervical, inferior laryngeal, tracheal, and œsophageal.

The muscular branches supply the scalenus anticus, longus colli, depressor muscles of the hyoid bone, and inferior constrictor of the pharynx. The ascending cervical artery arises from the inferior thyroid as it passes inwards behind the carotid sheath. It ascends in front of the transverse processes of the cervical vertebræ, lying between the scalenus anticus and rectus capitis anticus major, and having the phrenic nerve on its outer side. It anastomoses with branches of the vertebral, occipital, and ascending pharyngeal arteries. It may furnish some spinal branches which enter the spinal canal through the intervertebral foramina, to be distributed in a manner similar to the other spinal arteries. The inferior laryngeal artery accompanies the recurrent laryngeal nerve to the larynx. The tracheal and ascphageal branches are distributed as their names suggest. 
The transverse cervical artery passes transversely outwards in front of the scalenus anticus and phrenic nerve, and behind the clavicular part of the sterno-cleido-mastoid, lying a little above the clavicle, and also above the suprascapular artery. It then enters the subclavian triangle, which it soon leaves by passing beneath the posterior belly of the omo-hyoid. Thereafter it passes in front of, or between, the nerve-trunks of the brachial plexus, and beneath the trapezius muscle. Having reached the anterior border of the levator anguli scapulæ, it divides into its two terminal branches-superficial cervical and posterior scapular.

The superficial cervical artery passes superficial to the levator anguli scapulæ, and is distributed to the trapezius, levator anguli scapulæ, splenius, and the adjacent lymphatic glands. The posterior scapular artery passes backwards beneath the levator anguli scapulæ to the superior angle of the scapula. Thereafter it descends along the base of the bone, under cover of the rhomboid muscles, as far as the lower angle, where it anastomoses with the teres branch of the dorsalis scapulæ of the subscapular from the third part of the axillary artery. The artery gives branches to the adjacent muscles, and ventral and dorsal branches to the scapula, which take part in the scapular anastomoses proper (see p. 325).

The posterior scapular artery is frequently a branch of the third part of the subclavian, in which cases there is no transverse cervical artery, the superficial cervical artery taking its place and arising from the thyroid axis.

The suprascapular artery (transversalis humeri) passes downwards and outwards in front of the scalenus anticus and phrenic nerve, and behind the clavicular part of the sterno-cleido-mastoid. Having reached the back of the clavicle, it courses outwards behind that bone, passing in front of the third part of the subclavian artery and the trunks of the brachial plexus. It then passes to the upper border of the scapula, in company with the suprascapular nerve. For the further course of the vessel see p. 320 ; and for the scapular anastomoses of arteries see Index.

Branches in the Neck. - These are as follows: muscular, suprasternal, and medullary. The muscular branches supply the sternocleido-mastoid and subclavius. The suprasternal branch descends over the inner end of the clavicle to supply the integument over the manubrium sterni. The medullary artery of the clavicle is directed outreards, and enters the medullary foramen of that bone.

Superior Intercostal Artery.-This vessel arises from the second part of the subclavian on the right side, and from the first part on the left side, in each case from the posterior aspect of the parent trunk. On the right side it is therefore behind the scalenus anticus, and on the left side close to the inner border of that muscle. The vessel at first passes backwards for a short distance, and then descends in front of the neck of the first rib. At the posterior extremity of the first intercostal space it furnishes the first intercostal artery to that space, and then descends in front of the neck 
of the second rib to become the second intercostal artery. The superior intercostal artery, therefore, furnishes the first and second intercostal arteries for the first and second intercostal spaces, their distribution being similar to that of the succeeding aortic intercostal arteries. Besides these two branches, the vessel gives off the deep cervical artery. This branch, which is homologous with the dorsal branch of an aortic intercostal artery, arises from the superior intercostal before it descends in front of the neck of the first rib. It passes backwards between the transverse process of the seventh cervical vertebra and the neck of the first rib, and then ascends between the complexus and semispinalis colli muscles to about the level of the axis. In this latter situation it anastomoses with the deep branch of the ramus cervicalis princeps of the occipital from the external carotid. . The deep cervical in its course gives off muscular branches which anastomose with branches of the vertebral and ascending cervical arteries. It also furnishes a spinal branch which enters the spinal canal through the intervertebral foramen between the seventh cervical and first thoracic vertebræ.

The superior intercostal artery on each side is developed from the anastomotic loop between the upper two or three thoracic segmental arteries.

Subclavian Vein.-This vessel, which is the direct continuation of the axillary vein, extends from the outer border of the first rib to the back of the inner end of the clavicle, where it unites with the internal jugular to form the innominate vein. It lies in front of, and on a lower plane than, the subclavian artery, and it passes in front of the scalenus anticus muscle and phrenic nerve. In rare cases the vein has been met with passing behind the scalenus anticus. Its principal tributary is the external jugular vein (sometimes also the anterior jugular). The external jugular vein joins it close to the outer border of the sterno-cleido-mastoid.

The thoracic duct opens at the angle of junction of the left subclavian and left internal jugular veins, and the right lymphatic duct opens into the angle of junction of the corresponding veins on the right side.

Vertebral Vein.-This vein commences in the suboccipital venous plexus within the suboccipital triangle. It passes through the costo-transverse foramen in the atlas, and accompanies the vertebral artery through the succeeding costo-transverse foramina as low as the sixth, forming a plexus around the vessel. This plexus is ultimately replaced by a single vein, which emerges through the costo-transverse foramen in the sixth cervical vertebra (sometimes the seventh). It then descends in front of the first part of the subclavian artery, under cover of the internal jugular vein, and opens into the back part of the innominate vein, the opening being provided with a single or double valve.

The chief tributaries of the vertebral vein are as follows: muscular, spinal, anterior vertebral, and deep cervical. It also, as a rule, receives the first intercostal vein.

No vein accompanies the cervical part of the internal mammary 
artery. The inferior thyroid vein, which does not accompany the corresponding artery, will be found described in connection with the thyroid body.

The transverse cervical and suprascapular veins return the blood from the parts supplied by the corresponding arteries, and are tributaries of the lower part of the external jugular vein.

The deep cervical vein (posterior vertebral vein) commences in the suboccipital venous plexus within the suboccipital triangle. It descends in company with the deep cervical artery, and passes forwards between the transverse process of the seventh cervical vertebra and the neck of the first rib to terminate in the vertebral vein.

The anterior vertebral vein commences in a plexus in front of the upper cervical transverse processes. It descends in company with the ascending cervical branch of the inferior thyroid artery, receiving muscular tributaries in its descent, and it opens in to the lower part of the vertebral vein.

Collateral Circulation after Ligation of the Third Part of the Subclavian Artery.-(I) The suprascapular and the posterior scapular branch of the transverse cervical, both branches of the thyroid axis from the first part of the subclavian, take part in the scapular anastomoses, and anastomose freely with branches of the first and third parts of the axillary artery. (2) The internal mammary from the first part of the subclavian anastomoses with the long thoracic from the second part of the axillary.

Collateral Circulation after Occlusion of the First Part of the Subclavian Artery.-(I) The vertebral artery of one side anastomoses with that of the opposite side.

(2) The internal mammary anastomoses with the deep epigastric from the external iliac, and with the aortic intercostal arteries.

(3) The inferior thyroid anastomoses with the superior thyroid from the external carotid. (4) The superior intercostal anastomoses with the first aortic intercostal. (5) The deep cervical of the superior intercostal anastomoses with the deep branch of the ramus cervicalis princeps of the occipital from the external carotid. (6) The scapular branches of the thyroid axis anastomose with branches of the axillary artery. (7) The thoracic branches of the axillary artery anastomose with the aortic intercostals.

Cervical Part of the Thoracic Duct.-This part of the thoracic duct occupies the root of the neck on the left side, and lies upon the side of the osophagus, between the left common carotid and left subclavian arteries. In this position it ascends to about the level of the seventh cervical vertebra, and then it.describes a curve and passes outwards, forwards, and downwards in contact with the dome of the left pleura. Thereafter it inclines inwards, and, after receiving the left jugular and axillary lymphatic trunks, terminates in the angle of junction of the internal jugular and subclavian veins of the left side. The terminal orifice of the duct is guarded by a valve composed of two segments, which are so 
directed as to effectually prevent the reflux of chyle, and the flow of blood, into it. The duct crosses the left vertebral artery, and subsequently the left subclavian artery.

Right Lymphatic Duct.-The position of this duct corresponds to that of the thoracic duct on the left side. It is about $\frac{1}{2}$ inch in length, and is formed by the union of the right axillary lymphatic trunk and right jugular lymphatic trunk. Besides returning lymph from the right side of the head and neck and the right upper limb, it receives the lymphatic vessels from the right side of the heart, the right lung, the upper part of the right half of the thoracic wall, and some of those from the supero-anterior surface of the liver. It terminates in the angle of junction of the internal jugular and subclavian veins of the corresponding side, its orifice being guarded by a double valve.

Cervical Portion of the Lung - The apex of the lung projects into the root of the neck for from I to $I_{\frac{1}{2}}$ inches. It is covered by the dome of the pleura, on the inner and anterior aspects of which the subclavian artery arches outwards. The dome of the pleura is covered by Sibson's fascia, which extends from the internal border of the first rib to the front of the transverse process of the seventh cervical vertebra. This fascia is probably derived from the adjacent scalene muscles, and it may contain some muscular fibres.

\section{The Orbit.}

The contents of the orbit are as follows : (I) the eyeball and the optic nerve; (2) the capsule of Tenon; (3) seven muscles, six of which belong to the eyeball, namely, the four recti and two obliqui, whilst the seventh belongs to the upper eyelid, and is called the levator palpebræ superioris; (4) the third, fourth, and sixth cranial nerves, the three branches of the ophthalmic division of the fifth cranial nerve, namely, frontal, lachrymal, and nasal, and the orbital or temporo-malar branch of the superior maxillary division of the fifth cranial nerve ; $(5)$ the ciliary, lenticular, or ophthalmic ganglion; (6) the lachrymal gland; and (7) a quantity of soft fat. The foregoing contents are surrounded by a fibrous sheath which is formed by the periosteum.

Periosteum.-The periosteum of the orbit is continuous posteriorly with the dura mater through the sphenoidal fissure. Anteriorly it becomes continuous with the pericranium of the frontal bone, and the periosteum of the superior maxillary and malar bones. Along the supra-orbital and infra-orbital margins the superior and inferior palpebral ligaments blend with it. The periosteum is loosely attached to the bony walls of the orbit, and forms a sheath for the contents of the cavity, which is incomplete in front.

Lachrymal Gland.--This gland lies at the anterior and outer part of the orbit superiorly, where it occupies the lachrymal fossa on the inner side of the external-angular process of the frontal 
bone. It is almond-shaped. Its upper surface is convex, and its under surface is concave in adaptation to the eyeball. The anterior part of the gland is more or less detached, and is known as the palpebral portion, the chief part being known as the orbital portion. The ducts, which issue from both portions, are about twelve in number, and they open in a row into the outer part of the superior fornix conjunctive.

Blood-supply. - The lachrymal artery.

Nerve-supply. - The lachrymal nerve, and sympathetic filaments.

The structure of the gland is similar to that of the parotid gland.

Development.- The lachrymal gland, like the Meibomian glands and glands of Moll, is developed as solid epithelial cords from the conjunctiva. The solid epithelial cords spring from the upper and outer part of the conjunctiva, where it is reflected from the inner surface of the upper eyelid on to the front of the eyeball. The solid cords grow into the contiguous mesoderm, and give off lateral processes. Cords and processes, at first solid, soon become hollow, and so give rise to the alveoli, or aeini, and ducts of the lachrymal gland.

Muscles.-These are seven in number, as follows: levator palpebræ superioris, superior rectus, inferior rectus, internal rectus, external rectus, superior oblique, and inferior oblique.

Levator Palpebræ Superioris-Origin.-The under surface of the roof of the orbit above, and in front of, the optic foramen.

Insertion.-By a broad membranous expansion, which divides into three layers. The middle layer, which represents the chief insertion, and consists largely of involuntary muscular fibres, is attached to the upper margin of the superior tarsal plate. The upper or superficial layer, which is fibrous, passes over, or in front of, the superior tarsal plate, lying between it and the palpebral fibres of the orbicularis palpebrarum. Its fibres pierce the superior palpebral ligament, and, having passed between the bundles of the palpebral portion of the orbicularis palpebrarum, they are attached to the skin of the upper eyelid. The lower or deep layer, which is also fibrous, is attached to the conjunctiva along the superior fornix. The outer and inner margins of the broad membranous expansion are attached to the outer and inner margins of the orbit close to the external and internal tarsal ligaments, and at the inner angle a slip is detached to the pulley of the superior oblique muscle.

Nerve-supply. - The upper division of the third cranial nerve, the branch from which enters the deep or ocular surface of the muscle near its origin.

Action.-To raise the upper eyelid. The muscle is the antagonist of the superior palpebral fibres of the orbicularis palpebrarum.

Relations.-Superiorly, the frontal nerve and the supra-orbital artery. Inferiorly, the superior rectus and the conjunctiva.

Recti Muscles-Superior Rectus-Origin.-The upper part of the fibrous ring in relation to the optic foramen.

Insertion.-By a thin expanded tendon into the upper surface of the sclerotic about three or four lines from the margin of the cornea. 
Nerve-supply. - The upper division of the third cranial nerve, the branch from which enters the deep or ocular surface of the muscle near its origin.

The muscle is flattened from above downwards, and its direction is forwards and slightly outwards.

Action.-To elevate the eyeball, so as to direct the cornea upwards. The muscle imparts a certain amount of inward movement to the eyeball, accompanied by slight rotation.

Relations.-Superiorly, the levator palpebræ superioris, which is the narrower of the two muscles. Inferiorly, the soft fat of the orbit.

Internal Rectus-Origin.-The upper and lower parts of the fibrous ring in relation to the optic foramen, the origin being wide,

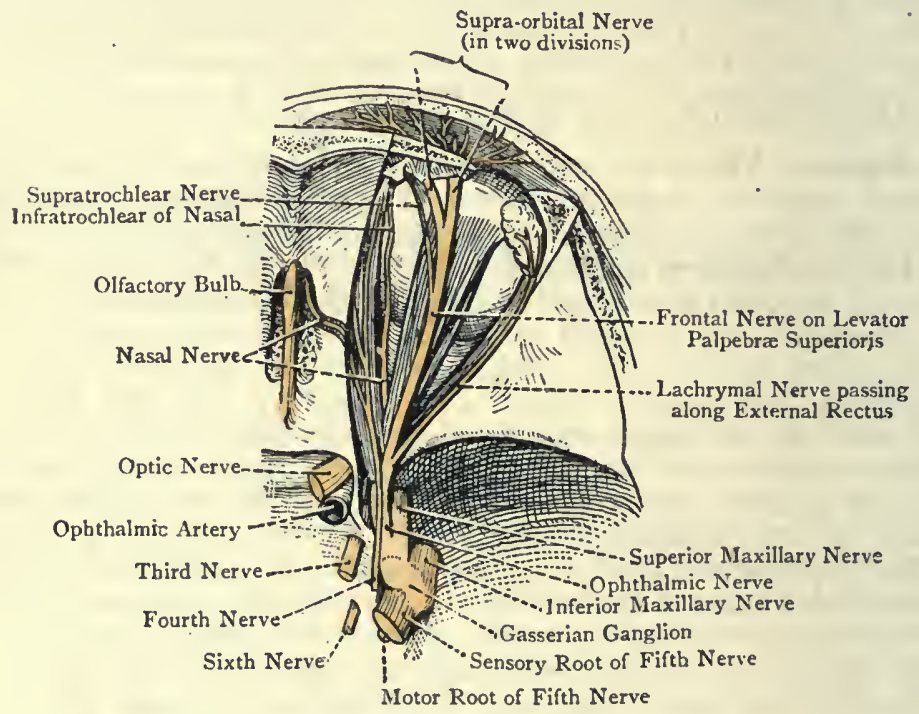

Fig. 467.-Dissection of the ORbit From ABOVE (Hirschfeld AND LEVEILLÉ).

and being situated partly on the inner side of, and partly below, the optic foramen.

Insertion.-By a thin expanded tendon into the inner surface of the sclerotic about three or four lines from the margin of the cornea.

Nerve-supply. - The lower division of the third cranial nerve, the branch from which enters the deep or ocular surface of the muscle.

The muscle is flattened from within outwards, and its borders are superior and inferior. Its direction is almost straight forwards.

Action.-To adduct the eyeball, so as to direct the cornea inwards.

Relations.-Above the upper border of the muscle is the superior oblique, and between the two the nasal nerve and the anterior and posterior ethmoidal vessels pass. 
Inferior Rectus-Origin.-The lower part of the fibrous ring in relation to the optic foramen.

Insertion.-By a thin expanded tendon into the lower surface of the sclerotic about three or four lines from the margin of the cornea.

Nerve-supply.-The lower division of the third cranial nerve, the branch from which enters the deep or ocular surface of the muscle.

The muscle is flattened from above downwards, and its direction is forwards and slightly outwards.

Action.-To depress the eyeball, so as to direct the cornea downwards. The muscle imparts a certain amount of inward movement to the eyeball, accompanied by slight rotation.

Relations-Inferior.-A part of the inferior oblique.

External Rectus-Origin.-This muscle arises by two heads.

Inferior Head.-(I) The lower part of the fibrous ring in relation to the optic foramen, where it is contiguous to the inferior rectus ; and (2) the prominent spine on the lower margin of the sphenoidal fissure near its inner end.

Superior Head.-The outer portion of the upper part of the fibrous ring in relation to the optic foramen, where it is above the sphenoidal fissure, external to the optic foramen, and contiguous to the superior rectus. The two heads are connected by a tendinous band which arches over the sphenoidal fissure, and gives origin to some of the fibres of the inferior head of the muscle.

Insertion.-The outer surface of the sclerotic from three to four lines from the margin of the cornea.

Nerve-supply. - The sixth cranial nerve, the branches of which enter the deep or ocular surface of the muscle.

The muscle is flattened from without inwards, and its direction is forwards and outwards.

Action.-To abduct the eyeball, so as to direct the cornea outwards.

Relations.- The following structures pass between the two heads of the muscle, in order from above downwards: (I) the upper division of the third nerve ; $(2)$ the nasal nerve ; $(3)$ the lower division of the third nerve; (4) the sixth nerve; and (5) the ophthalmic vein, or veins.

Common Tendon of the Recti Muscles.-This tendon takes the form of a fibrous ring, which is attached to the upper, inner, and lower margins of the optic foramen. It then crosses transversely the inner portion of the sphenoidal fissure to a prominent spine on the lower margin of that fissure near its inner end. From this spine it recrosses the sphenoidal fissure in a vertical direction near its centre, and finally reaches the upper part of the optic foramen. This latter portion gives origin to some fibres of the inferior head of the external rectus. The fibrous ring presents an upper and a lower part. The upper part is prolonged forwards into the superior tendon of Lockwood, which gives origin from within outwards to part of the internal rectus, the superior rectus, and the superior head of the external rectus. The lower part is also 
prolonged forwards into the inferior tendon or ligament of Zinn, which gives origin from within outwards to part of the internal rectus, the inferior rectus, and the inferior head of the external rectus.

Obliqui Muscles-Superior Oblique-Origin.-The wall of the orbit directly in front of the upper and inner part of the optic foramen, where it is contiguous to the superior and internal recti.

Insertion.-By an expanded tendon into the upper and outer aspect of the sclerotic, just beyond the outer border of the superior rectus, and midway between the margin of the cornea and the entrance of the optic nerve.

The muscle is situated at the upper and inner part of the orbit, internal to the levator palpebræ superioris. It is directed forwards towards the inner angle of the orbit, on approaching which it ends in a small round tendon. This tendon passes over a fibro-cartilaginous pulley or trochlea, close to the internal angular process of the frontal bone. Thereafter it changes its direction, and passes downwards, outwards, and backwards beneath the tendon of the superior rectus to reach its insertion just external to that tendon.

The fibro-cartilaginous pulley or trochlea is attached by fibrous tissue to the fovea trochlearis on the orbital plate of the frontal bone close to the internal angular process. It is lined with synovial membrane to lubricate the tendon and facilitate its movement at this abrupt change in its course. The tendon receives a fibrous investment from the outer margin of the pulley.

Nerve-supply. - The fourth cranial nerve, which enters the muscle on its superficial or orbital surface near its origin.

Action.- To depress and abduct the eyeball, or turn it outwards, the eyeball being at the same time slightly rotated.

Relations-Superior.-The roof of the orbit and the superior rectus. Inferior. - The upper border of the internal rectus, with the intervention of the nasal nerve, and the anterior and posterior ethmoidal vessels. External.-The levator palpebræ superioris.

Inferior Oblique-Origin.-A small depression at the anterior and inner part of the floor of the orbit, immediately external to the upper opening of the lachrymal canal.

Insertion.-The outer surface of the sclerotic under cover of the external rectus, and slightly posterior to the level of the insertion of the superior oblique.

Nerve-supply.-The lower division of the third cranial nerve, the branch of which is long and enters the posterior border of the muscle.

The muscle at first passes outwards and backwards upon the floor of the orbit beneath the inferior rectus, and then it turns upwards between the sclerotic and the external rectus, where its tendon expands before taking insertion.

Action.-To elevate and abduct the eyeball, which is at the same time slightly rotated.

Relations.-Towards the eyeball the muscle is related to the inferior rectus and the sclerotic, and towards the orbit to the floor 
of the cavity and the external rectus. The borders of the muscle are anterior and posterior, the latter, as stated, receiving the nervesupply.

Movements of the Eyeball.-The movements of the eyeball consist of rotation round a point situated just behind the centre of its antero-posterior axis. When the eyeball rotates round its vertical axis horizontal or lateral movements take place, and the cornea is directed outwards or inwards according to the muscle which acts. The external rectus abducts the eyeball, so as to direct the cornea outwards, and the internal rectus adducts the eyeball, so as to direct the cornea inwards. When the eyeball rotates round its transverse axis vertical or upward and downward movements take place, and the cornea is directed upwards or downwards according to the muscle which acts. The superior rectus elevates the eyeball, and the inferior rectus depresses it. It is to be borne in mind, however, that these two muscles in passing forwards have each a slight inclination outwards. Whilst, then, they respectively elevate and depress the eyeball, each of them also imparts to it a certain amount of inward movement, accompanied by slight rotation. In the case of the superior rectus this inward and rotatory movement is corrected by the inferior oblique acting in association with it, and in the case of the inferior rectus by the superior oblique acting in association with it.

Direct elevation of the eyeball is therefore effected by the superior rectus, aided by the inferior oblique, and direct depression of the eyeball is effected by the inferior rectus, aided by the superior oblique. The superior oblique, acting alone, depresses the eyeball, and abducts it, or turns it outwards, and the inferior oblique, acting alone, elevates the eyeball, and abducts it, these movements in each case being accompanied by slight rotation.

Fascia of the Orbit.-This fascia forms (I) the capsule of Tenon, and (2) sheaths for the ocular muscles.

The capsule of Tenon forms a connective-tissue covering for the sclerotic coat of the eyeball, and extends from the point of entrance of the optic nerve to near the margin of the cornea. Posteriorly it is perforated by the ciliary vessels and nerves, and blends with the dura matral sheath of the optic nerve, and anteriorly it passes beneath the ocular conjunctiva, with which it unites near the margin of the cornea. The outer surface of the capsule is in contact with the orbital fat, and anteriorly with the ocular conjunctiva. The inner surface, which is smooth, is separated from the sclerotic by a narrow space, which is crossed by delicate trabeculæ of connective tissue. This interspace is called the perisclerotic space, or Tenon's space, and is regarded as a lymph space. The capsule of Tenon forms a cavity or socket, within which the eyeball glides with perfect freedom from friction.

The lower part of the capsule of Tenon presents a thickening, which is known as the suspensory ligament of the eye (Lockwood). It is attached externally to the malar bone, and internally to the 
lachrymal bone, in association with the forward expansions from the sheaths of the external and internal recti muscles. It is expanded beneath the eyeball, to the fore part of which it acts as a sling or support.

The muscular sheaths are derived from the capsule of Tenon. The tendons of the six ocular muscles pierce the capsule on their way to their insertions, which latter are under cover of the capsule, each muscle receiving a prolongation from the margin of the cleft in the capsule of Tenon through which its tendon passes. These prolongations extend backwards around the muscles, and ultimately blend with their perimysial sheaths. As regards the superior oblique muscle, the prolongation around its tendon extends upwards, inwards, and forwards as far as the margin of the trochlea, to which it is attached. The prolongation around the tendon of the inferior oblique muscle extends downwards as far as the outer part of the floor of the orbit.

The sheaths of the four recti muscles give off important expansions. The expansion from the sheath of the external rectus is strong, and is attached to the orbital process of the malar bone, and that from the sheath of the internal rectus, also strong, is attached to the lachrymal crest of the lachrymal bone. These two expansions, at their bony attachments, are connected with the lateral extremities of Lockwood's suspensory ligament. The expansion from the sheath of the superior rectus blends with the deep layer of the tendon of the levator palpebræ superioris, and that from the sheath of the inferior rectus is connected with the inferior tarsal plate. These expansions from the sheaths of the recti, especially from those of the external and internal recti, moderate the action of the muscles, and the latter two are hence known as the check ligaments.

Nerves in the Orbit-Optic Nerve.-The optic nerve extends forwards and outwards from the optic commissure, and enters the orbit through the optic foramen, having the ophthalmic artery below it at first, and then on its outer side. It receives sheaths from the dura mater and arachnoid, the former being strong, and at its entrance into the orbit it is surrounded by the origins of the four recti muscles. Its direction is forwards and outwards, with a slight inclination downwards, to the back part of the eyeball, where it pierces the sclerotic coat about $\frac{1}{8}$ inch internal to, and a little below, the level of the centre. Thereafter it pierces the choroid coat, and terminates in the nerve fibre-layer of the retina. The lenticular or ciliary ganglion is in close contact with the outer side of the nerve towards the back part of the orbit, and anterior to this the nerve is surrounded by the ciliary nerves and vessels. It is crossed superiorly by the ophthadmic artery, the superior ophthalmic vein, and the nasal nerve. Inferiorly, about $\frac{1}{2}$ inch behind the eyeball, the arteria centralis retinæe enters it as far as the centre, and thereafter runs forward within it to the retina. 
Third, or Oculo-motor, Nerve.-This nerve, having left the outer wall of the cavernous sinus, breaks up at the sphenoidal fissure into two divisions, upper and lower. These enter the orbit between the two heads of the external rectus muscle, the nasal nerve being situated between the two.

The upper division furnishes two branches, which supply the levator palpebræ superioris and the superior rectus, the twigs entering the muscles on their deep or ocular surfaces.

The lower division, larger than the upper, furnishes three branches, which supply the internal rectus, the inferior rectus, and the inferior oblique. The twigs to the internal and inferior recti enter these muscles on their deep or ocular surfaces. The branch to the inferior oblique is a long nerve which passes forwards between the inferior and external recti, and its twigs enter the inferior oblique

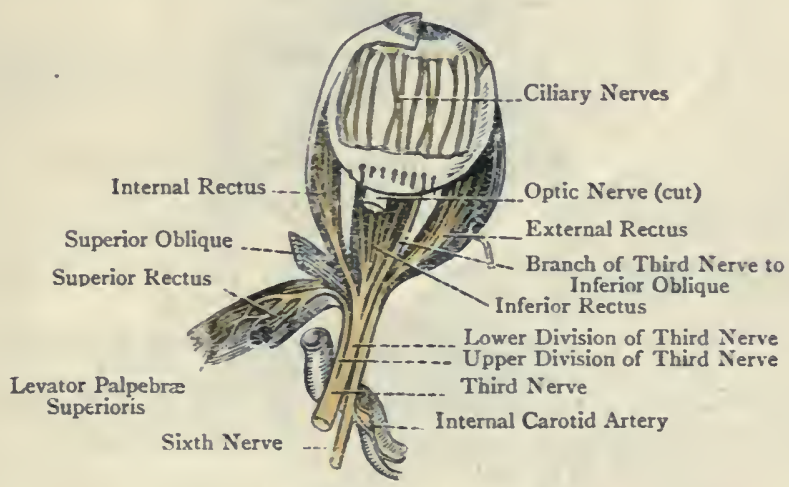

Fig. 468.-The Third axd Sixth Craxial Nerves in the Oreit (Hirschfeld and Leveillê).

muscle at its posterior boraier. Posteriorly it furnishes the ganglionic branch, which forms the short or motor root of the lenticular or ciliary ganglion.

The fourth, trochlear, or pathetic nerve, of small size, having left the wall of the cavernous sinus, enters the orbit through the sphenoidal fissure above the muscles, and internal to, but on a slightly higher plane than, the frontal nerve. Having passed inwards over the levator palpebræ superioris, it enters the posterior part of the superior oblique muscle on its superficial or orbital surface, this being the only muscle which it supplies.

The sixth or abducent nerve, having left the cavernous sinus, enters the orbit through the sphenoidal fissure, and passes between the two heads of the external rectus muscle. In this situation it has the lower division of the third nerve above it, and the ophthalmic veins below it. It supplies the external rectus, its twigs entering the muscle on its deep or ocular surface. 
Branches of the Ophthalmic Division of the Fifth Nerve.-The ophthalmic nerve, having left the outer wall of the cavernous sinus, and before arriving at the sphenoidal fissure, gives off the nasal nerve, and then divides into the frontal and lachrymal nerves.

The frontal nerve, of large size, enters the orbit through the sphenoidal fissure above the muscles. In this situation it has the lachrymal nerve external to, and on the same plane with, it, and the fourth nerve internal to it, but on a slightly higher plane. It then passes forwards on the upper surface of the levator palpebræ superioris, underneath the periosteum of the roof of the orbit, and before arriving at the supra-orbital margin it divides into two branches, supra-orbital and supratrochlear.

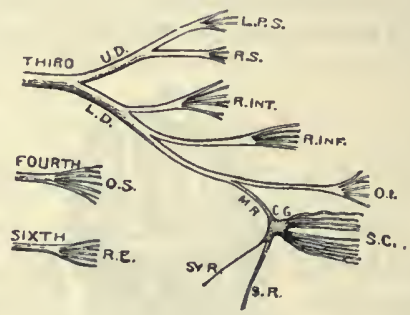

Fig. 469.-Scheme of the Distribution of the Third, Fourth, AND Sixth Cranial Nerves (Flower).

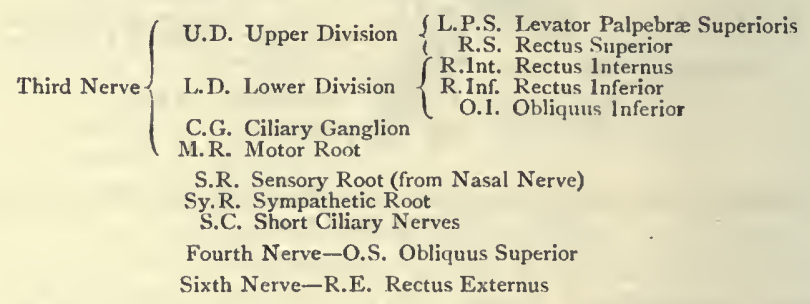

The supra-orbital nerve, which in size and direction is the continuation of the frontal, passes forwards to the supra-orbital notch, or foramen, through which it emerges in company with the supraorbital artery. On the forehead it divides into two branches, outer and inner, which have been already described (see p. ro57). Sometimes this division takes place within the orbit, and in these cases the inner branch emerges through the frontal notch.

The supratrochlear nerve, of small size, passes forwards and inwards to the trochlea or pulley of the superior oblique muscle, above which it emerges on to the forehead, where it has been previously described (see p. I067). Before leaving the orbit it gives off a small twig, which passes downwards, close to the pulley of the superior oblique, to form a loop with the infratrochlear branch of the nasal nerve. 
The lachrymal nerve enters the orbit through the sphenoidal fissure, above the muscles, and external to the frontal nerve. It then passes forwards and outwards along the upper border of the external rectus muscle, in company with the lachrymal artery. On reaching the lachrymal gland it furnishes numerous branches to its deep surface, and is then continued beneath it through the superior palpebral ligament, to be distributed to the skin and conjunctiva of the outer part of the upper eyelid, and the skin in the region of the external angular process of the frontal bone. Near the lachrymal gland it sends downwards a small twig, which joins the temporal branch of the orbital or temporo-malar nerve.

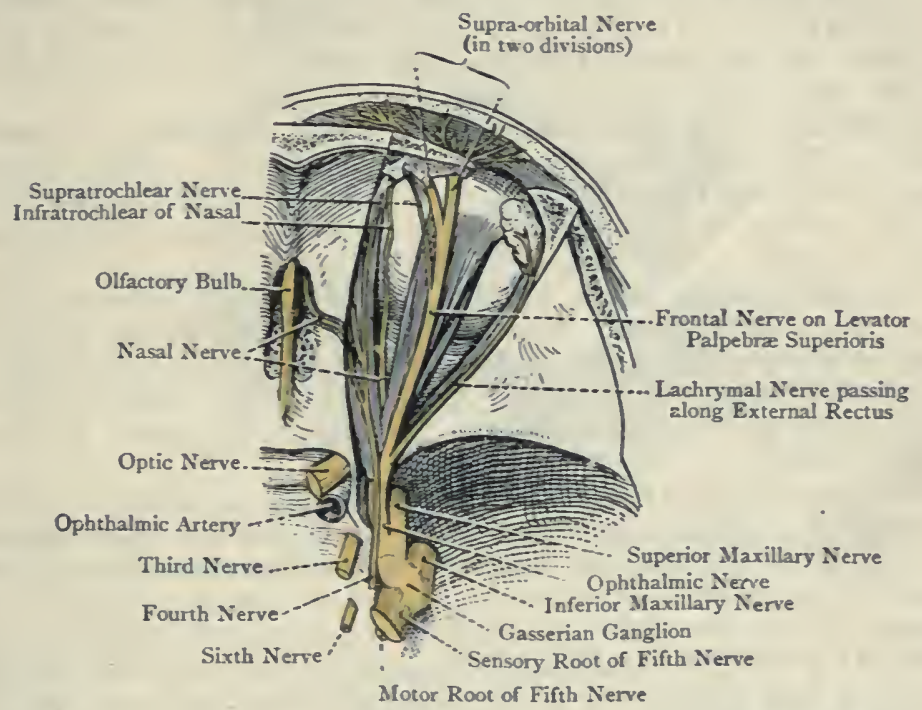

Fig. 470.-Dissection of The ORBIt Froy ABOVE (HIRSCHFEld AND LEVEILLÉ).

The nasal nerve is more deeply placed than the frontal and lachrymal. It enters the orbit through the sphenoidal fissure, and passes between the two heads of the external rectus muscle, lying between the upper and lower divisions of the third nerve. It then passes obliquely inwards and forwards to the inner wall of the orbit, lying beneath the superior rectus and above the optic nerve, and subsequently between the superior oblique and the internal rectus. Having reached the inner wall of the orbit at the anterior ethmoidal, or internal orbital, foramen it parts with its infratrochlear branch, and then passes through that foramen, in company with the anterior ethmoidal artery, and so enters the anterior fossa of the base of the skull. It then passes forwards and inwards in a groove on the upper surface of the cribriform plate of the ethmoid bone, 
lying under cover of the dura mater. Having reached the nasal slit at the side of the fore part of the crista galli, it descends through this slit into the nasal fossa, and immediately gives off a septal branch. Thereafter the nerve descends in a groove on the posterior surface of the nasal bone, furnishing offsets to the mucous membrane of the outer wall of the nasal fossa, in front of the superior and middle conchæ. Finally, the nerve emerges between the lower border of the nasal bone and the upper lateral nasal cartilage, and descends beneath the compressor naris muscle to supply the skin of the tip and lower part of the side of the nose.

In connection with this remarkable course of the nerve it is to be noted that it is to be met with in four different regions, namely, (I) in the orbit; (2) on the cribriform plate of the ethmoid bone in the anterior cranial fossa ; (3) in the nasal fossa ; and (4) on the face, in the region of the tip of the nose.

Branches.-The ganglionic branch forms the long or sensory root of the lenticular ganglion. It is slender, about $\frac{1}{2}$ inch long, and arises from the nasal as it passes between the two heads of the external rectus. It enters the ciliary ganglion at its posterosuperior angle.

The long ciliary nerves, usually two in number, arise from the nasal immediately after it has crossed the optic nerve, and they communicate with the short ciliary nerves from the ciliary ganglion, which they accompany to the back part of the sclerotic. Here they pierce the sclerotic, and are distributed along with the short ciliary nerves.

The infratrochlear nerve arises from the nasal close to the anterior ethmoidal foramen, and passes forwards beneath the superior oblique muscle. Near the pulley of that muscle it receives a twig from the supratrochlear nerve, and then, passing beneath the pulley, it emerges from the orbit at the inner canthus to be distributed to the skin and conjunctiva of the inner parts of the eyelids, the side of the root of the nose, the lachrymal sac, and the caruncula lacrimalis.

As the nerve traverses the anterior ethmoidal canal it furnishes twigs to the mucous membrane of the frontal air-sinus and of the anterior ethmoidal cells.

A spheno-ethmoidal or posterior ethmoidal branch is described as passing through the posterior ethmoidal canal to supply the mucous membrane of the posterior ethmoidal cells and of the sphenoidal air-sinus.

For the branches of the nasal nerve to the exterior of the nose and to the nasal fossa, see p. II76 and Index.

Ciliary, Lenticular, or Ophthalmic Ganglion.-This is a small quadrilateral body, about the size of a moderately large pin-head, which is situated in the posterior part of the orbit, where it lies flattened upon the outer side of the optic nerve. It is usually in close contact with that nerve, and is recognised by its reddish-pink colour. Posteriorly it receives its three roots, and anteriorly the 
short ciliary nerves pass forwards from it. The roots are called long, short, and middle. The long or sensory root is derived from the nasal nerve as it passes between the two heads of the external rectus. It is slender, about $\frac{1}{2}$ inch long, and enters the posterosuperior angle of the ganglion. The short or motor root is derived from the long branch of the lower division of the third nerve which supplies the inferior oblique muscle, and it enters the posteroinferior angle of the ganglion. The middle or sympathetic root

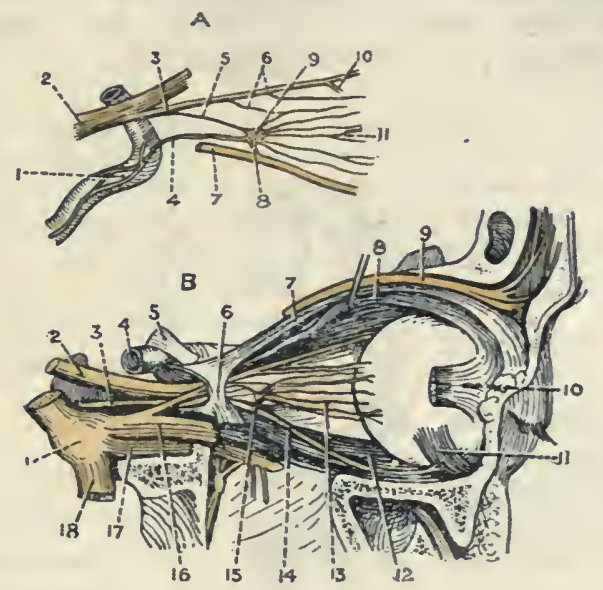

Fig. 471.-A, The Ciliary Ganglion; B, The Nerves of the Orbit (External View) (Hirschfeld ANd LeVEILlê).

A

1. Cavernous Plexus

2. Ophthalmic Nerve

3. Nasal Nerve

4. Sympathetic Root of Ciliary Ganglion
1. Tasserian Ganglion
2. Third Nerve
3. Sixth Nerve
4. Internal Carotid Artery
5. Optic Nerve
6. Fibrous King for Recti

5. Sensory Root of Ciliary Ganglion

6. Long Ciliary Nerves

7. Branch of Third Nerve to Inferior Oblique

\section{B}

7. Levator Palpebra Superioris 8. Superior Rectus

9. Frontal Nerve

Io. External Rectus

11. Inferior Oblique

12. Inferior Rectus

13. Short Ciliary Nerves
8. Motor Root of Ciliary Gang lion

9. Ciliary Ganglion

10. Infratrochlear Branch of Nasal Nerve

11. Short Ciliary Nerves

14. Branch of Third Nerve to Inferior Oblique

15. Ciliary Ganglion

16. Ophthalmic Nerve

17. Superior Maxillary Nerve

8. Inferior Maxillary Nerve

is a very minute nerve which is derived from the cavernous plexus of the sympathetic, and it usually reaches the ganglion in company with the long root. It may, however, join the ganglion separately, and then it does so in close proximity to the entrance of the long root.

The branches of the ciliary ganglion are called the short ciliary nerves. They are from six to eight in number, and come off, in two groups, from the antero-superior and antero-inferior angles. The 
nerves of the lower group usually exceed those of the upper in number. As they pass forward above and below the optic nerve they divide, and give rise to from sixteen to twenty delicate filaments. Having reached the back of the eyeball, they pierce the sclerotic around the entrance of the optic nerve. They then pass forwards between the sclerotic and the choroid, giving twigs to the latter, and they are finally distributed to the ciliary muscle, the iris, and the cornea. The short ciliary nerves thus convey to the eyeball sensory fibres from the ophthalmic division of the fifth nerve, motor fibres from the third nerve, and sympathetic fibres from the cavernous plexus of the sympathetic.

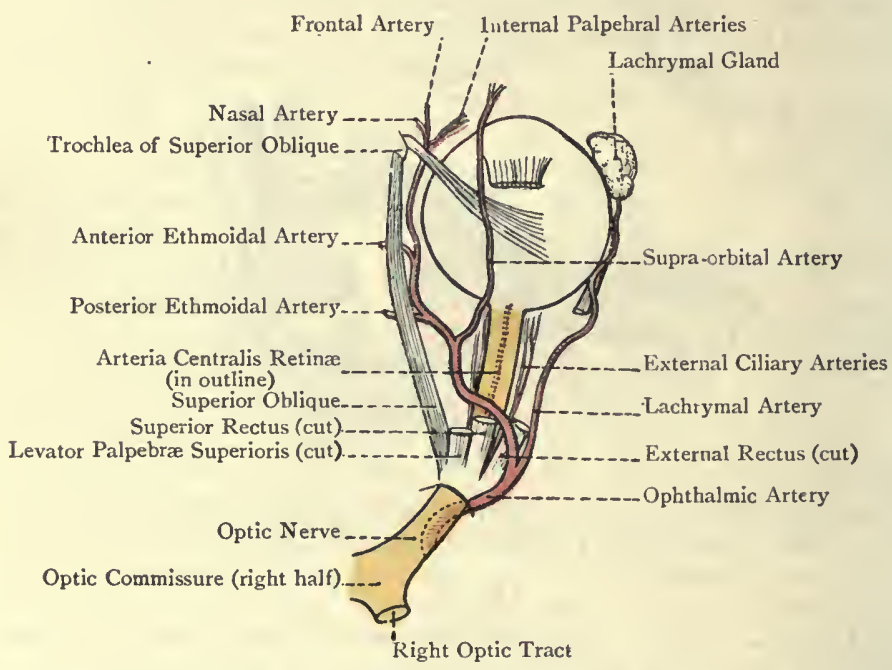

Fig. 472.-Dissection of the Right ORBIT FROM ABOVE, SHOWing the Optic Nerve, Ophthalmic Artery, and Superior Oblique Muscle.

Summary of the Ophthalmic Nerve. - This nerve is entirely sensory. It supplies (I) the skin of $(a)$ the frontal region and top of the skull, $(b)$ the upper eyelid, and $(c)$ the root, tip, and lower part of the side of the nose; (2) the caruncula lacrimalis and lachrymal sac; (3) the mucous membrane of the nasal fossa, and the palpebral conjunctiva; (4) the eyeball; and (5) the lachrymal gland.

Summary of the Ciliary Ganglion.-The branches of this ganglion supply the cornea, iris, and ciliary muscle. The fibres which supply the sphincter pupillæ are derived from the third or oculo-motor nerve, through means of the motor root of the ganglion. The fibres which supply the dilator pupillæ are derived from the sympathetic, through means of the sympathetic root of the ganglion, which is derived from the cavernous sympathetic plexus.

Branch of the Superior Maxillary Division of the Fifth.-The orbital or temporo-malar nerve, of small size, arises from the superior maxillary in the spheno-maxillary fossa. It enters the orbit through the spheno-maxillary fissure, and soon divides into two 
branches, temporal and malar. The temporal branch ascends upon the outer wall of the orbit, and, having received a communicating twig from the lachrymal nerve, it leaves the cavity by passing through the temporal canal in the malar bone. Its destination is the skin of the anterior part of the temporal region.

The malar branch passes forwards in the angle between the outer wall and floor of the orbit, and leaves the cavity by passing through the malar canal, from which it subsequently emerges through the malar foramen. It is distributed to the skin over the malar bone.

Ophthalmic Artery.- This vessel arises from the internal carotid artery on the inner aspect of the anterior clinoid process, and it enters the orbit through the optic foramen, lying beneath the optic nerve. Within the orbit it is situated for a short distance on the outer side of the optic nerve, and then it passes obliquely inwards and forwards in a tortuous manner over the nerve to the inner wall of the orbit. Thereafter it passes forwards to the region of the internal angular process of the frontal bone, near which it divides into its two terminal branches, nasal and frontal.

Branches.- These are as follows :

I. Arteria centralis, retinæ.

2. Posterior ciliary.

3. Lachrymal.

4. Muscular.

5. Supia-orbital.
6. Posterior ethmoidal.

7. Anterior ethmoidal.

8. Palpebral (two).

9. Nasal.

I0. Frontal.

The arteria centralis retinæ arises from the ophthalmic at the back part of the orbit below the optic nerve. Its direction is forwards beneath the nerve, and, at a point about $\frac{1}{2}$ inch behind the eyeball, it enters the nerve, and passes fonwards in its centre to the retina, upon which it ramifies.

The posterior ciliary arteries arise from the ophthalmic whilst it is below the optic nerve. They are arranged in two sets, external and internal, which pass forwards on either side of the optic nerve to the back part of the eyeball. Here they pierce the sclerotic, and the majority of them enter the choroid coat under the name of the short ciliary arteries. Two of them, however, are fairly large, and are known as the long ciliary arteries. They pass forwards between the sclerotic and the choroid, one on each side, and are distributed to the ciliary muscle and iris.

The lachrymal artery arises from the ophthalmic on the outer side of the optic nerve, and passes forwards along the upper border of the external rectus muscle to the lachrymal gland. In its course it furnishes the following branches: (I) a recurrent meningeal branch, which passes through the outer part of the sphenoidal fissure to the middle cranial fossa, where it anastomoses with the middle meningeal artery ; (2) muscular and anterior ciliary branches, the latter piercing the sclerotic very near the corneal margin; 
(3) temporal and malar offsets, which pass through the temporal and malar canals of the malar bone to the face and temporal region, in company with the temporal and malar branches of the-temporomalar or orbital nerve ; (4) two external palpebral arteries, superior and inferior, which pass inwards in the upper and lower eyelids, and anastomose with the internal palpebral branches of the ophthalmic, thus forming arterial arches ; and (5) glandular branches to the lachrymal gland.

The muscular branches arise from the ophthalmic in two sets. outer and inner, and also from some of its branches, e.g., the lachrymal and supra-orbital. They furnish a few of the anterior ciliary arteries.

The supra-orbital artery arises from the ophthalmic as it crosses

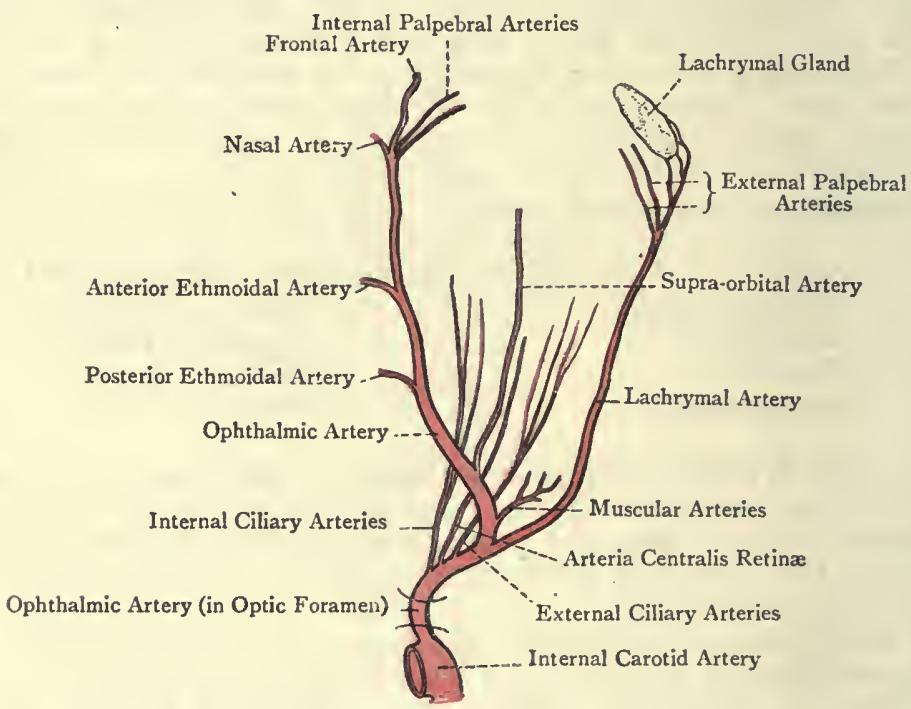

Fig. 473.-Diagram of the Ophthalmic Artery and its Branches.

inwards over the optic nerve. It courses forwards to the supraorbital notch, through which it passes, with the corresponding nerve, to the forehead. It supplies the upper eyelid and the frontal region, and anastomoses with the superficial temporal and frontal arteries.

The posterior ethmoidal artery, a small vessel, passes through the posterior ethmoidal canal on the inner wall of the orbit, and is distributed to the mucous membrane of the posterior ethmoidal cells, and the mucous membrane of the upper and back part of the outer wall of the nasal fossa. The latter branches pass through foramina in the cribriform plate.

The anterior ethmoidal artery is larger than the preceding. It 
passes through the anterior ethmoidal canal along with the nasal nerve, and enters the anterior fossa of the base of the skull. Here it crosses the cribriform plate of the ethmoid bone to the nasal slit by the side of the crista galli. It then descends through this slit, still in company with the nasal nerve, traverses the nasal groove on the posterior surface of the nasal bone, and finally emerges between the lower border of the nasal bone and the upper lateral nasal cartilage to the tip of the nose. Its branches supply the dura mater in the anterior cranial fossa, the mucous membrane of the anterior ethmoidal cells and corresponding frontal sinus, the mucous membrane of the upper and anterior parts of the nasal fossa, and the skin of the tip of the nose.

The palpebral arteries (internal) are two in number, superior and inferior, and are distributed to the eyelids. They arise, separately or conjointly, from the ophthalmic close to the pulley of the superior oblique muscle, and each takes an outward course in the corresponding eyelid. They anastomose with the superior and inferior palpebral branches of the lachrymal artery, thus forming arterial arches.

The nasal artery is one of the terminal branches of the ophthalmic. It emerges from the orbit above the internal tarsal ligament, and is distributed to the upper part of the side of the nose in the region of the root, where it anastomoses with the angular and lateral nasal branches of the facial artery.

The frontal artery is the other terminal branch of the ophthalmic. It emerges from the orbit at the inner angle, and then ascends to the frontal region, along with the supratrochlear nerve, to be distributed to the integument by the side of the median line. It anastomoses with the supra-orbital artery and its fellow of the opposite side.

Ophthalmic Veins.-These are two in number, superior and inferior. The superior ophthalmic vein, of large size, commences at the inner angle of the orbit, where it communicates freely with the angular vein, which is the commencement of the facial vein. It accompanies the ophthalmic artery, passing outwards and backwards over the optic nerve. Having reached the inner end of the sphenoidal fissure, the vessel passes between the two heads of the external rectus muscle, and through the fissure, after which it opens into the anterior part of the cavernous sinus. Its tributaries correspond for the most part to the branches of the ophthalmic artery. It does not, however, receive the frontal vein, nor the supra-orbital vein, but it is in communication with the latter by means of a small branch through the supra-orbital foramen. The inferior ophthalmic vein arises in connection with the lower posterior ciliary and lower muscular veins. It passes backwards along the floor of the orbit, and, as a rule, it joins the superior ophthalmic vein before that vessel passes between the two heads of the external rectus, thus giving rise to what is known as the common ophthalmic vein. It may, however, leave the orbit separately and open independently into the anterior part of the cavernous sinus. It com- 
municates freely with the pterygoid plexus of veins through the spheno-maxillary fissure, and that plexus sometimes represents its mode of termination.

Structures passing through the Sphenoidal Fissure.-These structures are as follows:

I. The third nerve.

2. The fourth nerve.

3. The nasal, lachrymal, and frontal nerves.

4. The sixth nerve.

5. The sympathetic root of the ciliary ganglion.
6. The two ophthalmic veins.

7. The orbital branch of the middle meningeal artery.

8. The recurrent branch of the lachrymal artery.

9. A process of the dura mater.

The lachrymal, frontal, and fourth nerves enter the orbit above the external rectus muscle, in the order named from without inwards.

The third nerve, the nasal nerve, and the sixth nerve enter the orbit between the two heads of the external rectus muscle, the third

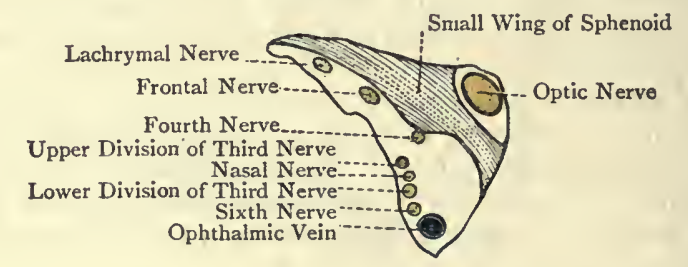

Fig. 474.-Diagram of the Left Sphenoidal Fissure, showing the Transmitted Structures (Posterior View).

nerve having previously broken up into two divisions ; and the two ophthalmic veins leave the orbit between the two heads of the external rectus. The order of parts between the two heads of the external rectus, from above downwards, is as follows :

I. The superior division of the third nerve.

2. The nasal nerve.

3. The inferior division of the third nerve.

4. The sixth nerve.

5. The ophthalmic veins (or vein).

The sympathetic root of the ciliary ganglion passes through the sphenoidal fissure independently, or along with the nasal nerve, or sometimes with the third nerve. 


\section{THE FACE.}

Landmarks. - The nasal eminence or glabella of the frontal bone can be felt just above the root of the nose, and extending outwards from it on either side is the superciliary ridge, behind the inner part of which is the corresponding frontal air-sinus. Below the superciliary ridge the supra-orbital arch can readily be felt, and at the junction of its inner third and outer two-thirds is the supra-orbital notch, or foramen, for the passage of the supra-orbital nerve and artery. A line drawn from the position of the supra-orbital notch to the base of the inferior maxilla, in such a manner as to pass between the lower bicuspid teeth, crosses in succession the infraorbital and mental foramina. The infra-orbital foramen lies in this line at a point about $\frac{1}{4}$ inch below the infra-orbital margin, and it indicates the exit of the infra-orbital nerve and artery from the infra-orbital canal. The mental foramen in the adult lies midway between the alveolar and basilar borders of the inferior maxilla, and it indicates the exit of the mental nerve and artery from the inferior dental canal.

In line with the tragus of the auricle the zygomatic arch can be felt, and leading backwards from it above the orifice of the meatus auditorius externus is the posterior root of the zygoma. This root is continued into the supramastoid crest, which corresponds to the level of the tegmen antri. In connection with the supramastoid crest it is important to note the position of the suprameatal triangle of Macewen. This triangle is bounded above by the supramastoid crest, below by the postero-superior part of the meatus auditorius externus, and behind by a vertical line connecting the upper and lower boundaries, this line being continuous with the posterior part of the meatus auditorius externus.

The superficial temporal artery and auriculo-temporal nerve lie directly in front of the tragus, the division of the artery into its anterior and posterior branches taking place about the level of the upper part of the auricle. The anterior branch of the artery lies nearly an inch above the external angular process of the frontal bone, and the posterior branch about an inch above the upper part of the auricle.

The parotid gland is situated in front of the auricle. It is limited above by the zygoma, behind by the auricle, and inferiorly it extends for a limited distance into the digastric triangle of the neck. Anteriorly it extends for a short distance over the masseter muscle, and Stensen's duct issues from its anterior border. The course of this duct may be indicated by a line drawn from the incisura intertragica, or from the junction of the lobule and cartilage of the auricle, to a point midway between the nostril and the red margin of the upper lip. About the middle third of this line corresponds to the duct. Above it is the transverse facial artery, and below it are the infra-orbital branches of the facial nerve. The course of the 
facial nerve through the parotid gland may be indicated by a line drawn forwards from the lobule of the auricle parallel to the line indicating the course of Stensen's duct.

The posterior border of the ramus of the inferior maxilla can readily be felt. It leads superiorly to the condyle and temporomandibular joint, and inferiorly to the angle of the bone. Extending forwards from the angle is the basilar border, which, about 2 inches from the angle, supports the facial artery, pulsation being readily felt, during life, in this part of the vessel, in front of the masseter muscle, the facial vein intervening between the two. In this situation a lymphatic gland lies in contact with the artery. From this point the facial artery extends, in a tortuous manner, to the inner canthus of the eye, passing close to the angle of the mouth, the facial vein pursuing a comparatively straight course.

In the median line of the face the structures to be noted are the nasal bones, nasal cartilages, dorsum and tip of the nose, alæ nasi; columella nasi, and the philtrum, which latter is the median groove leading from the columella nasi to the upper lip.

For the component parts of the auricle, see p. II92.

The palpebræ or eyelids are to be noted, along with the palpebral fissure between them, and the canthi, outer and inner, at either extremity. The tarsal plates of the upper and lower eyelids can easily be felt, and connected with their inner extremities is the internal tarsal ligament, or tendo oculi. This latter may be made tense by approximating the eyelids, and drawing them outwards. It crosses the lachrymal sac a little above the centre. The eyelids being everted, the outlines of the Meibomian glands may be seen, these being arranged in a linear manner, and being placed perpendicular to the palpebral margins. The eyelashes, or cilia, on the margins of the eyelids are to be noted, and within these are the orifices of the ducts of the Meibomian glands, which are arranged in a row. Along the line of the eyelashes the skin of the eyelid becomes continuous with the palpebral conjunctiva, and along the line of reflection of the palpebral conjunctiva on to the eyeball the recess, known as the fornix, is to be noted. At the inner canthus, where the eyelids do not meet, there is a recess between the lids and the eyeball, which lodges a small red fleshy protuberance, called the caruncula lacrimalis, which is provided with a few delicate hairs. In this situation there is the fold called the plica semilunaris. Upon the eyelids at their inner ends the papillæ lacrimales are visible, and on each papilla is a minute orifice, called the punctum lacrimale. The lower papilla is larger than the upper, and is placed a little farther out. Each punctum leads into the lachrymal canal, and by these canals the tears are conveyed into the lachrymal sac, and thence by the nasal duct into the inferior meatus of the nose. The position of the lachrymal sac may be indicated by drawing the eyelids outwards, so as to render tense the tendo oculi, which, as stated, crosses the sac a little above its centre. 


\section{Muscles of the Face.}

Orbicularis Palpebrarum-Orbital Portion-Origin.-(I) The inner part of the internal tarsal ligament; $(2)$ the outer surface of the nasal process of the superior maxilla; and (3) the internal angular process of the frontal bone.

Insertion.-This corresponds to the origin, there being no attachment to the outer part of the orbit.

Palpebral Portion-Origin.-The upper and lower surfaces of the internal tarsal ligament.

Insertion.-The upper and lower surfaces of the external tarsal ligament.

Nerve-supply.-The facial nerve.

Action-Orbital Portion.-(I) The upper half depresses the eyebrow, and antagonizes the frontalis muscle; (2) the lower half elevates the skin of the infra-orbital region. Palpebral Portion.-This closes the eyelids, as in winking, the upper lid being depressed and the lower raised, the former movement being more free than the latter. Through means of its connection with the internal tarsal ligament the palpebral portion draws forwards the front part of the lachrymal sac, and so contributes to the removal of the tears. When the entire muscle contracts the lids are forcibly closed, and drawn slightly inwards.

The upper part of the muscle is related by its deep surface to the frontalis, the supra-orbital vessels and nerve, and the supratrochlear nerve. The chief deep relations of the lower part are the levator labii superioris, and, beneath that, the infra-orbital nerve.

Internal Tarsal Ligament.-This is also called the tendo oculi, or tendo palpebrarum. It is attached internally to the outer surface of the nasal process of the superior maxilla, immediately in front of the lachrymal groove. Its direction is outwards in front of the lachrymal sac, and it bifurcates at the inner canthus, the divisions diverging, and taking attachment to the inner extremities of the tarsal plates. As the ligament passes in front of the lachrymal sac it is in intimate contact with that sac, and it gives origin to the palpebral portion of the orbicularis palpebrarum, which explains the action of that portion of the muscle upon the lachrymal sac.

External Tarsal Ligament.-This is attached externally to the frontal process of the malar bone. Its direction is inwards, and it bifurcates at the outer canthus, the divisions taking attachment to the outer extremities of the tarsal plates.

Tensor Tarsi (Muscle of Horner)-Origin.-The crest of the lachrymal bone, behind the lachrymal sac.

Insertion.-By means of two slips into the inner extremities of the tarsal plates, where the fibres become continuous with the ciliary fibres of the orbicularis palpebrarum.

Nerve-supply. - The facial nerve.

The direction of the muscle is outwards and forwards, and its two slips pass behind the lachrymal canals. 
Action.-To draw backwards the outer part of the internal tarsal ligament, and in this manner compress the lachrymal sac, the effect of which is to propel the tears into the nasal duct.

Corrugator Supercilii-Origin.-The inner extremity of the superciliary ridge of the frontal bone.

Insertion.-The deep surface of the skin of the eyebrow at the centre.

Nerve-supply.-The facial nerve.

The direction of the muscle is outwards and upwards, and to reach the skin the fibres pass through the upper part of the orbicularis palpebrarum and the adjacent portion of the frontalis.

Action.-To draw the skin of the eyebrow inwards and downwards, giving rise to vertical wrinkles between the eyebrows, as in frowning.

The muscle is under cover of the inner portion of the upper half of the orbicularis palpebrarum, and it conceals the supratrochlear nerve as it emerges from the orbit.

Muscles of the Nose-Pyramidalis Nasi-Origin.-The aponeurosis of the compressor naris over the lower part of the nasal bone.

Insertion.-The deep surface of the skin over the glabella of the frontal bone. Some of the fibres become continuous with the mesial portion of the frontalis.

Nerve-supply. - The facial nerve.

Action.-To draw downwards the skin between the eyebrows, giving rise to transverse wrinkles.

Compressor Naris-Origin.-The superior maxilla between the canine fossa and the nasal notch.

Insertion.-By means of an expanded aponeurosis which blends with its fellow of the opposite side over the cartilaginous portion of the nose, and gives origin superiorly to the pyramidalis nasi.

Nerve-supply.-The facial nerve.

Action.-To depress the cartilaginous portion of the nose.

The muscle is triangular, and at its origin is under cover of the levator labii superioris alæque nasi.

Levator Labii Superioris Alæque Nasi-Origin. - The outer surface ot the nasal process of the superior maxilla by a pointed extremity.

Insertion.-By means of two slips. The inner or nasal slip is inserted into the skin of the ala of the nose. The outer or labial slip is inserted into the skin of the upper lip, some of its fibres blending with the levator labii superioris, and others with the upper part of the orbicularis oris.

Nerve-supply. - The facial nerve.

Action.-(I) To raise the upper lip, and (2) to raise, and dilate, the ala of the nose.

The muscle is triangular, and covers the origin of the compressor naris.

Depressor Alæ Nasi-Origin.-The incisor fossa of the superior maxilla. 
Insertion.-The posterior part of the ala, and the adjacent part of the septum, of the nose.

Nerve-supply. - The facial nerve.

Action.-To depress the ala of the nose.

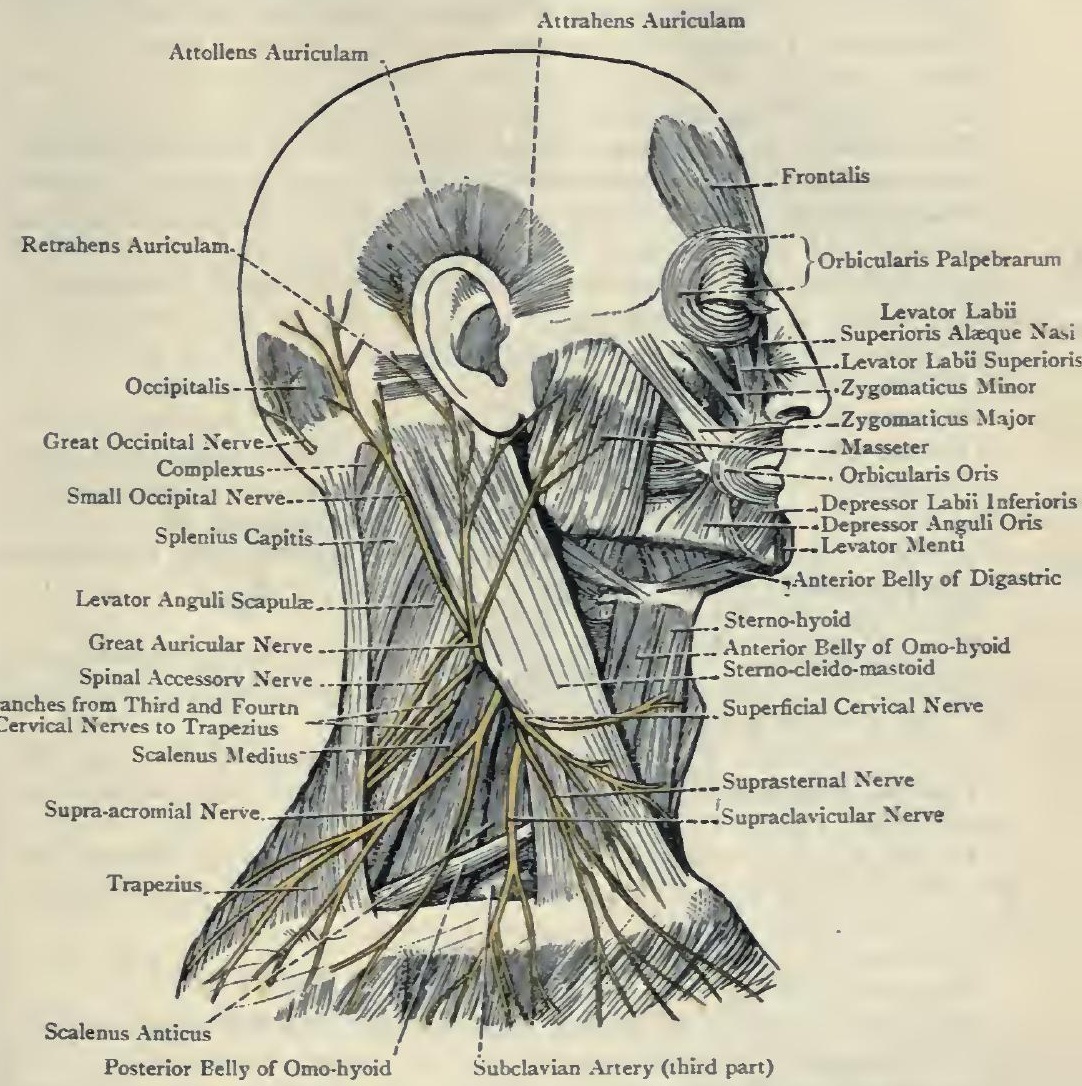

Fig. 475--The Right Side of the Head axd Neck.

(The Platysma Myoides has been removed, and the Nerves are shown).

Dilator Naris Anterior-Origin.-The cartilage of the aperture of the nose.

Insertion.-The deep surface of the skin over the ala of the nose.

Nerve-supply.-The facial nerve.

Action.-To dilate the nostril.

Dilator Naris Posterior-Origin.-(I) The margin of the nasal notch of the superior maxilla; and (2) the accessory or sesamoid cartilages of the nose. 
Insertion.-The skin over the back part of the ala of the nose. Nerve-supply. - The facial nerve. Action.-To dilate the nostril.

Levator Labii Superioris-Origın.-The superior maxilla between the infra-orbital foramen and the lower margin of the orbit.

Insertion.-The skin of the upper lip.

Nerve-supply. - The facial nerve.

Action.-To raise the upper lip.

The muscle is quadrilateral. At its origin it is overlapped by the lower half of the orbicularis palpebrarum, and it covers the infra-orbital nerve and artery as they emerge from the infra-orbital foramen. At its insertion the fibres interlace with those of the upper half of the orbicularis oris. Its inner border is joined by some of the fibres of the outer or labial slip of the levator labii superioris alæque nasi, and its outer border is joined by some fibres of the zygomaticus minor.

Levator Anguli Oris-Origin.-The upper part of the canine fossa of the superior maxilla, under cover of the levator labii superioris.

Insertion.-The angle of the mouth, where some of the fibres are inserted into the skin, whilst others decussate with those of the depressor anguli oris, and enter the lower lip, mingling with those of the lower half of the orbicularis oris.

Nerve-supply. - The facial nerve.

The direction of the muscle is downwards and slightly outwards. Action.-To raise the angle of the mouth, and at the same time to draw it slightly inwards.

The infra-orbital nerve and artery are superficial to the muscle.

Zygomaticus Minor-Origin.-The anterior and lower part of the malar bone, close to the superior maxilla.

Insertion.-The skin of the upper lip immediately external to the levator labii superioris, with the outer border of which muscle some of its fibres blend.

Nerve-supply. - The facial nerve.

The muscle is directed downwards and inwards.

Action.-To raise feebly the upper lip, and at the same time to draw it slightly outwards.

Zygomaticus Major-Origin.-The outer surface of the malar bone near the zygomatic suture.

Insertion. - The skin at the angle of the mouth, where its fibres blend with those of the orbicularis oris.

Nerve-supply. - The facial nerve.

The muscle is directed downwards and inwards.

Action.-To draw the angle of the mouth upwards and outwards.

Risorius of Santorini-Origin.-The deep fascia which covers the masseter muscle and parotid gland.

Insertion.-The skin at the angle of the mouth, where its fibres blend with the orbicularis oris.

Nerve-subply. - The facial nerve. 
The direction of the muscle is inwards, some of its fasciculi also ascending.

Action.-To draw the angle of the mouth outwards and slightly downwards. It is not a muscle of laughter, but is expressive of scorn or pain.

The muscle consists of a few scattered fasciculi, which are embedded in the adipose tissue over the buccinator, and it is to be regarded as a detached portion of the platysma myoides.

Buccinator-Origin.--(I) The outer surfaces of the alveolar borders of the superior and inferior maxillæ, opposite the three molar alveoli ; and (2) the anterior aspect of the pterygo-mandibular ligament.

Insertion. - The orbicularis oris at the angle of the mouth.

Long Buccal Nerve (cut)

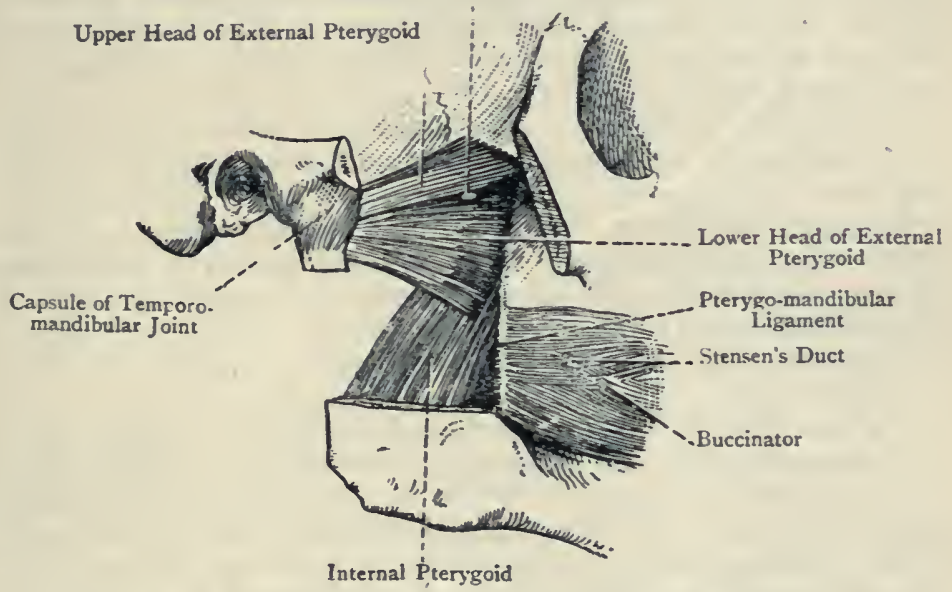

Fig. 476.-The Pterygoid and Buccinator Muscles.

The central fibres decussate, those from above entering the lower lip, and those from below entering the upper lip. The highest and lowest fibres take no part in this decussation, the highest passing directly into the upper lip, and the lowest into the lower lip.

Nerve-supply. - The short buccal nerves, which are derived from (I) the infra-orbital branches of the temporo-facial division of the facial, and (2) the buccal branches of the cervico-facial division of the facial, nerve.

Action.--(I) To draw the angle of the mouth outwards, and press the lips against the teeth, thus preventing the food from accumulating between the lips and the teeth during mastication; and (2) to regulate the expulsion of air from the buccal cavity when the buccal aperture is narrowed, as in playing the flute, or in whistling.

The muscle is exp?nded over the cheek, but towards the angle 
of the mouth it becomes narrow and thick. It is pierced by Stensen's duct opposite the second upper molar tooth, and the long buccal nerve also passes through it on its way to the mucous membrane of the cheek. Externally the muscle is overlapped by the anterior border of the masseter, from which it is separated by the buccal pad of fat.

Buccal Pad of Fat.-This is a well-defined collection of fat which is situated upon the buccinator muscle close to the anterior border of the masseter. It is continuous with the fat of the zygomatic fossa, and is well developed in young children.

Depressor Anguli Oris (triangularis menti) -Origin.-The external oblique line of the inferior maxilla, from about the level of the canine alveolus to about the level of the first molar alveolus.

Insertion.-The angle of the mouth, where some of the fibres are attached to the skin, whilst others decussate with those of the levator anguli oris and enter the upper lip, in which they mingle with the upper part of the orbicularis oris.

Nerve-supply. - The facial nerve.

Action.-To depress the angle of the mouth.

Internally the muscle overlaps a portion of the depressor labii inferioris.

Depressor Labii Inferioris (quadratus menti)-Origin.-The lower part of the outer surface of the body of the inferior maxilla, commencing almost close to the symphysis, and extending outwards to a point just below the mental foramen.

Insertion.-The skin of the lower lip.

Nerve-supply. - The facial nerve.

The muscle is directed upwards and inwards.

Action.--To draw the lower lip downwards and slightly outwards.

The muscle is overlapped externally by a portion of the depressor anguli oris, and internally it is in contact superiorly with its fellow of the opposite side. The deep surface is related externally to the mental foramen and the mental nerve and vessels, and internally to part of the levator menti. In the lower lip its fibres pass amongst those of the lower part of the orbicularis oris.

Levator Menti, or Levator Labii Inferioris-Origin.-The incisor fossa of the inferior maxilla.

Insertion.-The skin of the chin.

Nerve-supply. - The facial nerve.

The muscle is directed downwards and forwards.

Action.-(I) To raise the integument of the chin ; and (2) to elevate and protrude the lower lip. It gives rise to a proud or imperious expression, and has been hence called the musculus superbus.

Superiorly the muscle is under cover of the depressor labii inferioris. Inferiorly it appears along with its fellow, between the two depressor labii inferioris muscles.

Orbicularis Oris.--This is a complex muscle, being composed of three strata. The superficial stratum consists of fibres which are prolonged from the elevators and depressors of the angles of the 
mouth, and extend as far as the centre of the lip, but are not continuous with those of the opposite half. They are inserted into the skin, and are partially reinforced by fibres from the elevators of the upper lip, the zygomatic muscles, the risorius, the posterior fibres of the platysma myoides, and the depressor labii inferioris.

The intermediate stratum consists of fibres which are derived from the buccinator muscles. These fibres are disposed transversely, and those of opposite sides are directly continuous. The fibres of this stratum extend quite to the margins of the lips.

The foregoing fibres are destitute of bony and cartilaginous attachments.

The deepest stratum consists of fibres which are so disposed as to constitute specially-named muscles. These are four in number for the upper lip, two for each half, namely, the musculus incisivus superior and the musculus naso-labialis; and two for the lower lip, one for each half, namely, the musculus incisivus inferior.

The musculus incisivus superior arises from the incisor fossa of the superior maxilla, and turns outwards in the upper lip towards the angle of the mouth, where the fibres blend with the orbicularis oris. The musculus naso-labialis arises from the back part of the nasal septum, and, like the preceding, turns outwards in the upper lip to blend with the orbicularis oris. The musculus incisivus inferior arises from the incisor fossa of the inferior maxilla, and turns outwards in the lower lip towards the angle of the mouth, where the fibres blend with the orbicularis oris. In addition to the foregoing fibres there are oblique fibres which extend between the skin and the mucous membrane.

Nerve-supply.-The facial nerve.

Action.-(I) To close the oral aperture; and (2) to press the lips against the alveolar margins.

The muscle is not a sphincter muscle, properly so called, inasmuch as the upper and lower fibres are not directly continuous at the angles of the mouth. It is separated from the buccal mucous membrane by the coronary arteries and the labial mucous glands.

The labial mucous glands, which are racemose, or acino-tubular, are numerous, and each is about the size of a small pea. They are situated on the deep surface of the mucous membrane of the lips. where they lie in the loose areolar tissue between the mucous membrane and the orbicularis oris. Their ducts open into the vestibule of the mouth.

The buccal mucous glands, also racemose, or acino-tubular, are situated between the mucous membrane of the cheek and the buccinator.

The molar mucous glands, also racemose, or acino-tubular, lie upon the buccinator in the vicinity of the terminal part of Stensen's duct. They are four or five in number, and their ducts, having pierced the buccinator, open into the vestibule of the mouth opposite the molar teeth. 


\section{Nerves of the Face.}

The nerves of the face are thirteen in number on either side. Twelve of these are sensory nerves, and one is motor, namely, the facial nerve.

The facial or seventh cranial nerve (portio dura) will only be described here after its emergence from the aqueduct of Fallopius in the petrous part of the temporal bone. It emerges from that

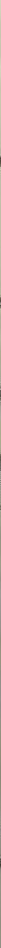

Fig. 477.- Superficial Dissection of the Right Side of the Head and Upper Part of the Neck (Hirschfeld and Levelllé).

I, Sterno-cleido-mastoid ; 2 , Trapezius.

aqueduct through the stylo-mastoid foramen, after which it passes downwards and then forwards into the parotid gland. Near the posterior border of the ramus of the inferior maxilla it breaks up into two terminal parts, called the temporo-facial and cervicofacial divisions.

Branches.-These are as follows : the posterior auricular, digastric, stylo-hyoid, and the two terminal divisions, namely, temporo-facial, and cervico-facial. 
The posterior auricular nerve arises from the facial close to the stylo-mastoid foramen. It ascends between the auricle and the mastoid process, where it lies deeply, and divides into two branches, auricular and occipital. The auricular branch passes upwards behind the auricle and beneath the retrahens auriculam, to be distributed to that muscle, and it may be to the attollens auriculam, as well as to the small intrinsic muscles on the inner aspect of the auricle. The occipital branch passes backwards to supply the occipitalis muscle. The posterior auricular nerve communicates with the great auricular, the small occipital, and the auricular branch (Arnold's nerve) of the pneumogastric.

The digastric nerve is directed downwards, and breaks up into branches which enter the posterior belly of the digastric muscle. One of the digastric branches communicates with the glosso-pharyngeal nerve.

The stylo-hyoid nerve usually arises in common with the preceding. It is of some length, and, passing forwards, it enters the stylo-hyoid muscle about its centre.

The terminal divisions, as stated, are called temporo-facial and cervico-facial. Both divisions continue to pass forwards in the parotid gland, crossing superficially the temporo-maxillary vein and the external carotid artery, the direction of these vessels being vertical. In this part of their course these divisions receive the following branches; the temporo-facial division receives two branches from the auriculo-temporal nerve, and the cervico-facial division receives branches from the great auricular nerve. The temporo-facial division, which is the larger of the two, traverses the upper part of the parotid gland, and the cervico-facial division passes through the gland in a forward and downward direction. Each division breaks up, within the gland, into three branches, and, as these pass in various directions over the face and upper portion of the neck, they ramify freely. The branches frequently communicate with each other in a plexiform manner, both in the parotid gland and on the face, the plexus thus formed being known as the pes anserinus or goose's foot. The ramifications also communicate with the branches of the three divisions of the fifth nerve which appear on the face, these being sensory nerves.

The temporo-facial division furnishes three sets of branches, namely, temporal, malar, and infra-orbital. The temporal branches ascend over the zygomatic arch to the temporal region, and are distributed to the following muscles: the attrahens and attollens auriculam, the intrinsic muscles on the outer surface of the auricle, the frontalis, the upper part of the orbicularis palpebrarum, and the corrugator supercilii. The temporal branches communicate with the supra-orbital and lachrymal branches of the ophthalmic, the temporal branch of the orbital from the superior maxillary, and the auriculo-temporal of the inferior maxillary, all of these being sensory nerves.

The malar branches pass forwards over the malar bone towards 
the outer angle of the orbit, and supply the outer part of the orbicularis palpebrarum. They form communications with the lachrymal branch of the ophthalmic, with the malar of the orbital, and with the infra-orbital, both these latter being branches of the superior maxillary, and all of them being sensory nerves.

The infra-orbital branches, the largest of this group, pass forwards to the region between the lower margin of the orbit and the upper lip. They supply the lower part of the orbicularis palpebrarum,

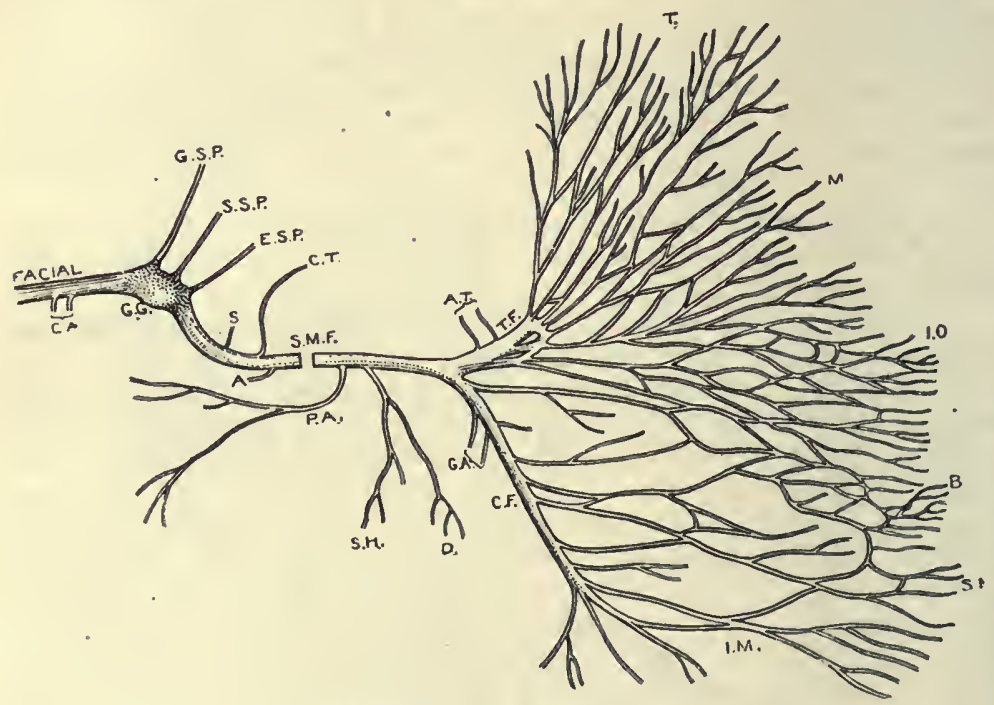

Fig. 478.-Scheme of the Facial Nerve (Flower).

C.A. Communicating Twigs with Auditory

G.G. Geniculate Ganglion

G.S.P. Great Superficial Petrosal

S.S.P. Branch to Small Superficial Petrosal

E.S.P. External Superficial Petrosal

S. Branch to Stapedius Muscle

C.T. Chorda Tympani

A. Auricular Branch (Arnold's Nerve)

S. M.F. Stylo-mastoid Foramen

P.A. Posterior Auricular

S.H. Branch to Stylo-hyoid Muscle

D. Branch to Posterior Belly of Digastric
A.T. Communicating Branches from Auriculo-temporal

T.F. Temporo-facial Division

T. Temporal Branches

M. Malar Branches

I.O. Infra-orbital Branches

G.A. Communicating Branches from Great Auricular

C.F. Cervico-facial Division

B. Buccal Branches

S.M. Supramandibular Branches

I. M. Inframandibular Branches

the muscles of the nose, the elevators of the upper lip, the upper part of the orbicularis oris, and the buccinator. They communicate with the following sensory nerves: the infra-orbital of the superior maxillary, the infratrochlear of the nasal, and the terminal cutaneous branch of the nasal, from the ophthalmic. The communications with the infra-orbital nerve take place under cover of the levator labii superioris muscle, and form a somewhat intricate plexus, called the infra-orbital plexus.

The cervico-facial division likewise furnishes three sets of 
branches, namely, buccal, supramandibular, and inframandibular or cervical.

The buccal branches pass forwards over part of the masseter and the buccinator muscles to the angle of the mouth. They supply the buccinator and the outer part of the orbicularis oris, and are connected with the infra-orbital branches of the temporo-facial division, and with the long buccal nerve, which is a branch of the inferior maxillary division of the fifth nerve. The latter communications take the form of a plexus situated on the superficial surface of the buccinator muscle, called the buccal plexus.

The supramanaibular branches pass forwards over the mandible to the region below the lower lip. They supply the lower part of the orbicularis oris, depressor anguli oris, depressor labii inferioris,

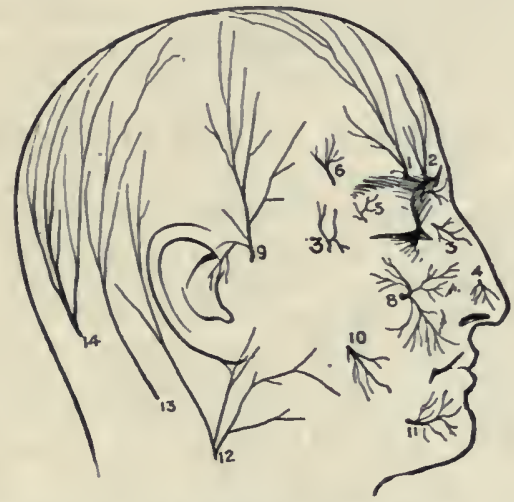

Fig. 479.-Diagram of the Sensory Nerves of the Right SidB OF THE HEAD.

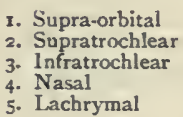

1. Supra-orbital

2. Supratrochlear

4. Nasal

5. Lachrymal
6. Temporal of Orbital
7. Malar of Orbital
8. Infra-orbital
9. Auriculo-temporal
ro. Long. Buccal

rI. Mental

12. Great Auricular

13. Small Occipital

r4. Great Occipital

and levator menti muscles. They communicate with the mental branch of the inferior dental from the inferior maxillary nerve, under cover of the depressor anguli oris.

The inframandibular or cervical branch descends beneath the upper part of the platysma myoides and the deep cervical fascia to the suprahyoid region. Having pierced the deep fascia, it divides into branches which curve forwards and supply the platysma on its deep surface. It communicates freely with the upper branch of the superficial cervical nerve from the cervical plexus.

Summary of the Facial Nerve.-The facial nerve, after its emergence from the aqueduct of Fallopius through the stylo-mastoid foramen, supplies the following muscles: (I) the extrinsic and intrinsic muscles of the auricle; (2) the posterior belly of the digastric and the stylo-hyoid; (3) the muscles of the scalp, namely, the occipitalis and the frontalis; (4) the superficial 
muscles of the face, including the buccinator, but not the masseter, nor the levator palpebræ superioris ; and (5) the platysma myoides. All these muscles, except the posterior belly of the digastric and the stylo-hyoid, are spoken of as muscles of expression. The nerve establishes free communications with all three divisions of the fifth nerve, which are sensory. It also communicates with the small occipital, great auricular, and superficial cervical, which are branches of the cervical plexus.

Sensory Nerves of the Face.-These, as stated, are twelve in number on either side. With one exception they are derived from the fifth cranial (trigeminal or trifacial) nerve, the exception being in the case of the great auricular, which is a branch of the cervical plexus. Most of them reach their distribution as independent nerves, but some sensory fibres reach the skin of the face through their connection with the branches of the facial nerve, as, for example, in the case of the auriculo-temporal and great auricular nerves. The sensory nerves are as follows :

\section{A. Branches of the Fifth Cranial Nerve.}

Distal Sources.

1. Ophthalmic nerve.

2. Superior maxillary nérve.

3. Inferior maxillary nerye.
Proximate Sources.
(a) Frontal division.
(b) Lachrymal division.
(c) Nasal division.

(a) Superior maxillary.

(b) Orbital, or

(Temporo-malar.

(a) Anterior division.

(b) Posterior division.

(c) Inferior dental of posterior division.
Nerves.

$\{$ Supra-orbital.

\{ Supratrochlear.

Superior palpebral.

Infratrochlear.

External terminal branch of nasal. Infra-orbital.

$\{$ Temporal.

\{ Malar.

Long buccal. -

Auriculo-temporal,

\section{B. Facial Branches of the Great Auricular Nerve.}

Branches of the Fifth Cranial Nerve.-The supra-orbital and supratrochlear nerves 'have been already described (see Index). The former is distributed to the skin of the frontal region and the upper part of the scalp; and the latter to the skin of the lower and central portion of the frontal region. Both these nerves furnish twigs to the skin of the upper eyelid.

The superior palpebral branch of the lachrymal nerve pierces the superior palpebral ligament, and is distributed to the skin and conjunctiva of the outer part of the upper eyclid, as well as to the skin in the immediate vicinity of the external angular process of the frontal bone.

The infratrochlear branch of the nasal nerve emerges from the orbit below the pulley of the superior oblique muscle, and furnishes twigs to the skin and conjunctiva of the inner parts of the eyelids, 
the side of the root of the nose, the lachrymal sac, and the caruncula lacrimalis.

The external terminal branch of the nasal nerve emerges between the lower border of the nasal bone and the upper lateral nasal cartilage, and descends beneath the compressor naris muscle to the tip of the nose. Its twigs supply the skin of the tip, and lower part of the side, of the nose.

The infra-orbital nerve is the terminal part of the superior maxillary division of the fifth. It emerges from the infra-orbital canal through the infra-orbital foramen, where it is under cover of the levator labii superioris. In this situation it communicates with the infra-orbital branches of the facial, forming the infra-orbital plexus, and it divides into three sets of branches, namely, inferior palpebral, lateral nasal, and superior labial. The inferior palpebral branches ascend to supply the skin and conjunctiva of the lower eyelid. The nasal branches pass inwards, and are distributed to the skin of the side of the nose. The superior labial branches, long and large, descend to supply the skin and mucous membrane of one half of the upper lip. As they descend they furnish twigs to the skin between the infra-orbital foramen and the upper lip.

The temporal branch of the orbital or temporo-malar nerve is of small size. Having traversed the temporal canal in the malar bone, it pierces the temporal fascia about I inch above the front part of the zygoma, and behind the marginal process on the postero-superior or temporal border of the malar bone, and is distributed to a limited portion of the skin over the anterior part of the temporal region.

The malar branch of the orbital or temporo-malar nerve is of small size, and emerges from the malar canal in the malar bone through the malar foramen. It is distributed to the skin over the malar bone.

The long buceal nerve is a branch of the anterior division of the inferior maxillary nerve. It emerges from beneath the anterior border of the masseter on to the superficial surface of the buccinator, where it divides into branches which communicate freely with the buccal branches of the facial nerve, forming the buccal plexus. Its final distribution is to the skin and mucous membrane of the cheek, the branches to the latter piercing the buccinator.

The auriculo-temporal nerve, which is a branch of the posterior division of the inferior maxillary nerve, has been already described as regards its cutaneous distribution (see p. I070).

The mental nerve is one of the terminal branches of the inferior dental, which, in turn, is a branch of the posterior division of the inferior maxillary nerve. It emerges from the dental canal in the inferior maxilla through the mental foramen, under cover of the depressor anguli oris, and in line with the interval between the two lower bicuspid alveoli. Its branches supply the skin and mucous membrane of one half of the lower lip, and the skin over one half of the body of the inferior maxilla.

The supra-orbital, infra-orbital, and mental nerves, being fre- 
quently the seat of neuralgia, their positions for purposes of operative treatment can readily be ascertained in the following manner: A line drawn upwards from the position of the mental foramen, which is in line with the interval between the two lower bicuspid alveoli, to the supra-orbital notch, which is situated at the junction of the outer two-thirds and the inner third of the supra-orbital arch, will coincide with the infra-orbital foramen. This line, therefore, is over the points of emergence of these three important sensory nerves.

Branches of the Great Auricular Nerve.--The facial branches of the great auricular nerve, which is a branch of the cervical plexus, are distributed to the skin over the parotid gland. They send twigs into the gland which communicate with the cervico-facial division of the facial nerve.

\section{Arteries of the Face.}

The arteries of the face are as follows :

Sources.

Arteries.

$\begin{array}{cl}\begin{array}{c}\text { External } \\ \text { carotid. }\end{array} & \text { Facial. } \\ \begin{array}{c}\text { Ophthalmic } \\ \text { from } \\ \text { internal } \\ \text { carotid. }\end{array} & \left\{\begin{array}{l}\text { Nasal. } \\ \text { Frontal. } \\ \text { Supra-orbital. } \\ \text { Internal palpebral } \\ \text { (superior and inferior). } \\ \text { Terminal branch of an- } \\ \text { terior ethmoidal. }\end{array}\right. \\ \begin{array}{c}\text { Lachrymal, } \\ \text { from } \\ \text { ophthalmic. }\end{array} & \begin{array}{l}\text { External palpebral } \\ \text { (superior and inferior). } \\ \text { Temporal. } \\ \text { Malar. }\end{array}\end{array}$

Sources.

Superficial

temporal.

Internal maxillary.

Inferior dental,

$\left.\begin{array}{c}\text { from } \\ \text { internal maxil- } \\ \text { lary. }\end{array}\right\}$ Mental. Buccal.
Arteries.

Transverse facial. $\left\{\begin{array}{l}\text { Anterior temporal. } \\ \text { Orbital. }\end{array}\right.$

\section{Infra-orbital.}

The chief of these arteries are the facial and the transverse facial.

Facial Artery. - The facial artery (external maxillary) is one of the three anterior branches of the external carotid, being the highest in order. It is situated at first in the upper portion of the anterior triangle of the neck, where it has been previously described (see p. III9). It leaves the neck and enters upon its facial course by mounting over the base and body of the inferior maxilla, in front of the anterior border of the masseter, where it is very superficial, being covered only by the platysma myoides and the integument. From this point it extends to the inner canthus of the eye, where it pierces the levator labii superioris alæque nasi, or becomes embedded in it, and terminates in its angular branch. Its course is upwards and forwards, and it describes many bends, being remarkable for its tortuosity, this being in adaptation to the great mobility of the surrounding parts. Though embedded in the fat, and covered by certain muscles, the facial part of the vessel is in no sense deeply placed.

Relations-Superficial. - The integument and platysma myoides, the risorius, the zygomatic muscles, and branches of the facial 
nerve. Its relation to the levator labii superioris is variable. It may be under cover of, or over, that muscle. Deep.-The body of the inferior maxilla (where pulsation can readily be felt in the vessel), the buccinator, the levator anguli oris, and the infraorbital nerve. External.-The facial vein, which is at a little distance from it, except at the body and base of the mandible, where the vein lies close to the outer side of the vessel.

Facial Branches.-(I) Muscular branches, of small size, arise from the outer side of the vessel, and are distributed to the structures in the masseteric, buccal, and infra-orbital regions, where they anastomose with the buccal, transverse facial, and infra-orbital arteries. (2) The inferior labial artery passes forwards over the body of the mandible, and beneath the depressor anguli oris, to supply the structures between the lower lip and the base of the mandible. It anastomoses with the inferior coronary, mental, and submental arteries. (3) The inferior coronary artery arises just below the angle of the mouth, and passes inwards beneath the depressor anguli oris. In the lower lip it lies near the margin, between the orbicularis oris and the mucous membrane, and it anastomoses with its fellow of the opposite side, and with the inferior labial. (4) The superior coronary artery, of larger size than the inferior, arises just above the angle of the mouth, under cover of the zygomaticus major. Its position in the upper lip is similar to that of the inferior coronary in the lower lip, and it anastomoses with its fellow of the opposite side. Near the middle line it furnishes the artery of the septum nasi, which ramifies on the columella nasi as far as the tip of the nose. The superior and inferior coronary arteries of each side sometimes arise together. The vessels of each side, superior and inferior, form in each lip a tortuous arterial arch, which is necessarily divided in operations upon one or other lip. (5) The lateral nasal arteries arise opposite the nose, upon the side of which they ramify. They anastomose with the nasal branch of the ophthalmic, the artery of the septum, the terminal branch of the anterior ethmoidal artery, and its fellow of the opposite side. (6) The angular artery is the terminal branch of the facial at the inner canthus of the eye. It lies either beneath, or embedded in, the levator labii superioris alæque nasi, and supplies the side of the root of the nose and the adjacent part of the orbicularis palpebrarum. It anastomoses with the nasal branch of the ophthalmic artery.

The facial vein commences above the inner canthus of the eye in the angular vein, which is formed by the union of the frontal and supra-orbital veins. The course of the vessel is downwards and outwards, external to the artery and at a little distance from it, except over the body and base of the inferior maxilla, where it lies close to its outer side and in contact with the anterior border of the masseter. The vein is much less tortuous than the artery, and in the lower part of its course descends almost vertically. The cervical part of the vein, which terminates in the internal jugular, has been already described. The muscular 
relations of the vein correspond pretty much with those of the artery.

Its tributaries are as follows: (I) the frontal vein; (2) the supra-orbital vein, which communicates with the ophthalmic vein; (3 the angular vein, which receives internally the lateral nasal veins, and externally a few superior palpebral veins, whilst posteriorly it communicates with the commencement of the superior ophthalmic vein, and internally with its fellow of the opposite side by means of the transverse nasal vein, which lies over the bridge of

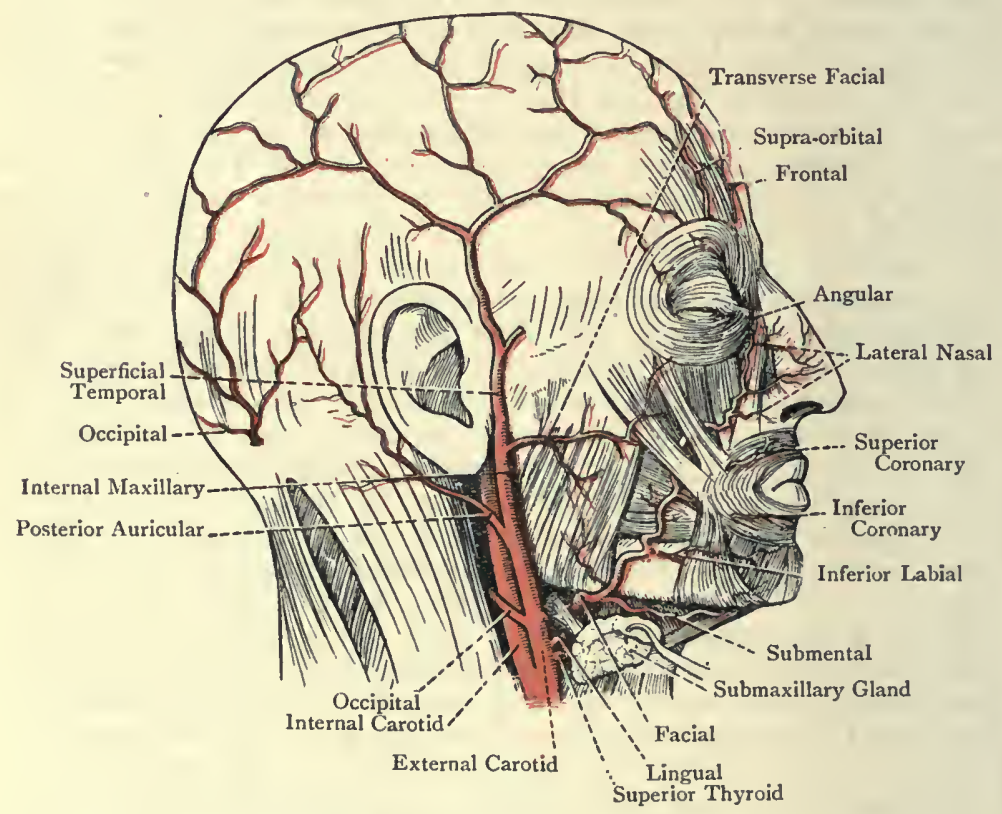

Fig. 480,-The Arteries of the Right Side of the Head (After

L. Testut's 'Anatomie Humaine').

the nose ; (4) a few inferior palpebral veins, which are in communication with the infra-orbital vein; (5) the superior labial vein, which issues from a plexus in the upper lip (the blood from the similar plexus in the lower lip being chiefly conveyed by a few vessels to the roof of the digastric triangle of the neck, where they take part in the formation of the anterior jugular vein); (6) the deep facial vein, of large size, which, emerging from the pterygoid plexus, appears from behind the anterior border of the ramus of the inferior maxilla and of the masseter muscle, and passes forwards over the buccinator ; and (7) parotid, masseteric, and buccal branches. 
Superficial Facial Lymphatic Glands.-These glands lie on the face along the course of the superficial facial artery and vein. Some are situated upon the mandible, beneath the platysma myoides, one of them being placed upon the base of the mandible, close to the facial vessels; others (buccal) rest upon the fascia covering the buccinator muscle; and the remaining glands of this group are met with between the angle of the mouth and the inner canthus. They receive their afferent vessels from the adjacent structures, and their efferent vessels pass to the submaxillary lymphatic glands.

Transverse Facial Artery. - This vessel arises from the superficial temporal in the parotid gland, and passes horizontally forwards. After emerging from beneath the anterior border of the gland it crosses the masseter, having the zygoma above it and Stensen's duct below it, the infra-orbital branches of the facial nerve being, in turn, below Stensen's duct. The order of structures from above downwards is, accordingly, artery, duct, and nerves. The artery is distributed to the parotid gland, the masseter muscle, and the structures on the side of the face, and it anastomoses with the infra-orbital, malar, facial, and buccal arteries. vein.

The transverse facial vein opens into the superficial temporal

The nasal artery is one of the terminal branches of the ophthalmic, and emerges from the orbit at the inner canthus above the internal tarsal ligament. It is distributed to the lachrymal sac and the side of the root of the nose, and anastomoses with the angular and lateral nasal arteries. It gives off a transverse nasal branch, which crosses the root of the nose and anastomoses with its fellow of the opposite side.

The transverse nasal vein connects the two angular veins over the root of the nose, and the other veins corresponding to the nasal artery open into the angular and superior ophthalmic veins.

The supra-orbital and frontal arteries have been already described (see Index).

The internal palpebral arteries, superior and inferior, arise from the ophthalmic, and emerge from the orbit at the inner canthus, one passing above, and the other below, the internal tarsal ligament. Their course is outwards between the palpebral fibres of the orbicularis palpebrarum and the tarsal plates, and they anastomose, and form arches, with the palpebral branches of the lachrymal artery. They are distributed to the palpebral structures, the lachrymal sac, and the caruncula lacrimalis.

The corresponding veins from the upper eyelid open into the angular vein, and those from the lower eyelid in to the facial vein.

The terminal branch of the anterior ethmoidal artery emerges. along with the terminal branch of the nasal nerve, between the lower border of the nasal bone and the upper lateral nasal cartilage. It is distributed to the skin of the tip, and the lower part of the side, of the nose. 
The external palpebral arteries, superior and inferior, are branches of the lachrymal artery within the orbit. Their course is inwards between the palpebral fibres of the orbicularis palpebrarum and the tarsal plates, and they anastomose, and form arches, with the palpebral branches of the ophthalmic.

The external palpebral veins terminate in the orbital vein, which opens into the middle temporal vein, this in turn joining the superficial temporal vein.

The temporal and malar arteries are branches of the lachrymal, and they accompany the temporal and malar branches of the temporo-malar or orbital nerve.

The orbital and anterior temporal arteries have been already described (see Index).

The infra-orbital artery arises from the third part of the internal maxillary in the spheno-maxillary fossa. Having passed horizontally forwards, with the infra-orbital nerve, through the infraorbital canal it emerges with that nerve through the infra-orbital foramen, lying under cover of the levator labii superioris muscle. It then gives branches upwards to the lower eyelid, inwards to the side of the nose, and downwards towards the upper lip. It anastomoses with the inferior palpebral, facial, transverse facial, and buccal arteries.

The infra-orbital vein, having traversed the infra-orbital canal, opens into the pterygoid plexus.

The buccal artery is a branch of the second part of the internal maxillary. It accompanies the long buccal nerve to the superficial surface of the buccinator muscle, and is distributed to that muscle and to the mucous membrane of the cheek. It anastomoses with branches of the facial artery.

The mental artery is one of the terminal branches of the inferior dental from the first part of the internal maxillary, and it emerges from the dental canal of the inferior maxilla, along with the mental nerve, through the mental foramen, lying under cover of the depressor anguli oris muscle. It supplies the structures in this vicinity, and anastomoses with the inferior coronary, inferior labial, and submental arteries.

The mental vein joins the inferior dental vein, which opens into the pterygoid plexus.

Lymphatics. - The lymphatics of the face are arranged in two sets, superficial and deep. The superficial lymphatics for the most part take a course similar to that of the facial vein, and terminate in the submaxillary lymphatic glands. They receive the lymphatics of $(I)$ the inner portion of the frontal region at the inner canthus; (2) the inner portions of the eyelids; $(3)$ the side of the nose; $(4)$ the portion of the face between the lower eyelid and upper lip; and (5) the upper and lower lips. The lymphatics from the temporal and outer part of the frontal regions, from the front of the auricle, from the outer portions of the eye- 
lids, and from the outer part of the cheek terminate in the parotid lymphatic glands. The deep lymphatics, including those of the orbit, anterior part of the nasal cavity, roof of the mouth, and temporal and zygomatic fossæ, terminate in the internal maxillary glands.

Parotid Lymphatic Glands.-These glands are arranged in two groups-superficial parotid and intraparotid.

The superficial parotid or pre-auricular lymphatic glands (lymphoglandulce auriculares anteriores) lie upon the superficial surface of the parotid salivary gland, immediately beneath the parotid fascia, and in front of the tragus of the auricle. They receive their afferent vessels from the following sources:

I. The outer surface of the auricle.

2. The anterior wall of the external auditory meatus.

3. The eyebrow, and upper and lower eyelids.

4. The root of the nose.

5. The upper part of the cheek.

Their efferent vessels pass to (I) the upper superficial cervical, or external jugular, glands, and (2) the superior deep cervical glands.

The intraparotid lymphatic glands (lympho-glandula parotidece) lie within the parotid salivary gland, along the terminal part of the external carotid artery. They receive their afferent vessels from the following sources:

I. The tympanic cavity, in part.

2. The frontal region of the scalp.

3. The anterior temporo-parietal region of the scalp.

4. The parotid salivary gland.

5. The deep facial, or internal maxillary, glands.

Their efferent vessels pass to the superior deep cervical glands.

Buccal Lymphatic Glands. - These glands are situated on the buccinator muscle. They receive their afferent lymphatics from the malar region and the lateral aspect of the face, and their efferent lymphatics pass to the parotid and submaxillary lymphatic glands.

Parotid Gland.-This salivary gland, which is of large size, is situated on the side of the face immediately in front of the auricle, and also below the auricle, where it occupies the space between the ramus of the lower jaw and the sterno-cleido-mastoid muscle. Its limits are as follows: superiorly, the zygoma; inferiorly, the stylo-maxillary ligament, in the direction of a line drawn from near the tip of the styloid process of the temporal bone to the angle of the inferior maxilla, this ligament separating it from the submaxillary gland; and posteriorly, the external auditory meatus, the mastoid process, and the anterior border of the sterno-cleidomastoid muscle. The anterior border rests" upon the posterior part of the masseter, and it is from this border, above its centre, that the parotid duct emerges. The duct is usually accompanied by a 
small portion of the gland, which lies above it, and may take the form of a distinct lobe. It is known as the pars or glandula socia parotidis. The superficial surface of the gland, which presents the usual lobulated appearance of compound racemose or acino-tubular glands, is covered by the parotid fascia, which is an upward extension of the deep cervical fascia. It is very dense, and consequently a parotid abscess is subjected to considerable tension. Lying beneath it, on the surface of the gland, there is the superficial set of parotid lymphatic glands. The deep connections of the gland are very intricate. It extends deeply between the mastoid process and sterno-cleido-mastoid behind, and the posterior border of the ramus and angle of the inferior maxilla in front.

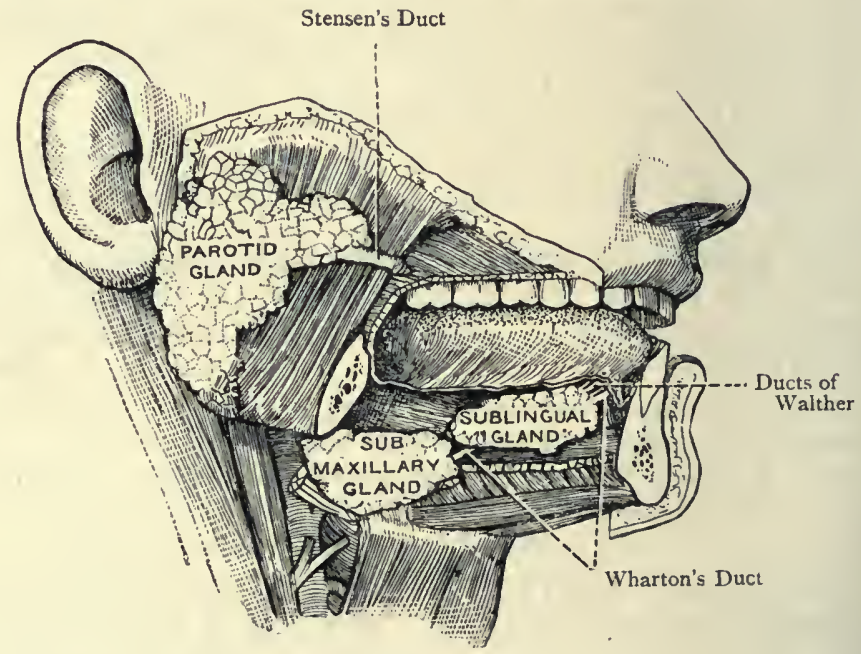

Fig. 48r.-The Salivary Glands of the Right Side.

Superiorly, a deep extension of the gland, known as the glenoid portion, occupies the posterior part of the glenoid fossa, formed by the tympanic plate, and lying behind the fissure of Glaser. Lower down it extends both in front of, and behind, the styloid process and styloid muscles, and is here intimatcly related to the internal carotid artery, internal jugular vcin, and glossopharyngeal, pneumogastric, spinal accessory, and hypoglossal nerves. The deep surface of the gland is invested by an upward extension of the deep cervical fascia, which, with the parotid fascia, forms a capsule for the gland. Anteriorly, the gland extends deeply behind the posterior border of the ramus of the lower jaw, as far at least as the posterior border of the internal pterygoid muscle, or it may be for a short distance between that muscle and the external pterygoid. 
Several important structures traverse the gland. (I) The external carotid artery ascends deeply in it as high as the level of the neck of the inferior maxilla, where it divides into the superficial temporal and internal maxillary arteries, which are at first embedded in the gland. Whilst in the gland the superficial temporal artery gives off its transverse facial and auricular branches. (2) The superficial temporal and internal maxillary veins unite in the gland, at the level of the neck of the inferior maxilla, to form the temporomaxillary vein, which descends from that level within the gland, lying superficial to the external carotid artery, and close to the lower border of the gland it breaks up into an anterior and a posterior division. (3) The external jugular vein is formed in the lower part of the gland by the union of the posterior auricular vein with the posterior division of the temporo-maxillary vein. (4) The facial nerve traverses the gland from behind forwards, and, in doing so, breaks up into its temporo-facial and cervico-facial divisions, the branches of which emerge from the gland anteriorly and superiorly. The nerve is superficial to the external carotid artery and temporo-maxillary vein. (5) Entering the lower part of the gland are branches of the great auricular nerve, which communicate within the gland with the facial nerve. (6) The auriculo-temporal nerve is related to the deep surface of the upper part of the gland, where it gives branches to it which communicate with the facial nerve.

Parotid Duct.-This excretory tube, which feels dense and tough, is usually called the duct of Stensen or Steno. It emerges from the anterior border of the gland, and passes forwards on the masseter muscle, lying fully $\frac{1}{2}$ inch below the zygomatic arch. In this part of its course it is accompanied, for a short distance, by the pars or glandula socia parotidis, which, with the transverse facial artery, lies above it, whilst the infra-orbital branches of the facial nerve are placed below it. After leaving the surface of the masseter muscle the duct dips deeply through the fat covering the buccinator, and pierces that muscle. Thereafter it passes forwards for a very short distance beneath the buccal mucous membrane, which it finally pierces to terminate in a minute opening on a small papilla situated opposite the crown of the second upper molar tooth. The duct is about the size of a crow-quill, its diameter being about $\frac{1}{8}$ inch, except at its buccal orifice, where it becomes narrow. It is about 2 inches in length, and its course may be indicated by a line drawn from the incisura intertragica, or from the junction of the lobule and cartilage of the auricle, to a point midway between the nostril and the red margin of the upper lip. About the middle third of this line corresponds to the duct.

Blood-supply.-The arteries are derived from (I) the external carotid, (2) the superficial temporal, (3) the transverse facial, and (4) the posterior auricular.

Lymphaties. - These pass to the superficial and deep cervical 
glands, having previously traversed the parotid lymphatic glands, and, in the case of some, the submaxillary lymphatic glands.

Nerve-supply.--(I) The auriculo-temporal nerve, which conveys to the gland secretory fibres of the glosso-pharyngeal nerve through its tympanic branch (Jacobson's nerve), the small superficial petrosal nerve, and the otic ganglion; (2) the great auricular nerve; (3) the sympathetic plexus on the external carotid artery; and (4) the facial nerve.

Structure.-The parotid gland is a compound racemose or acino-tubular gland, and is composed of large lobules, which are united by connective tissue. Each of these is made up of smaller lobules, likewise connected by connective tissue. Each small lobule is a gland on a minute scale, and is made up of a group of more or less sacculated tubules, called alveoli or acini. A

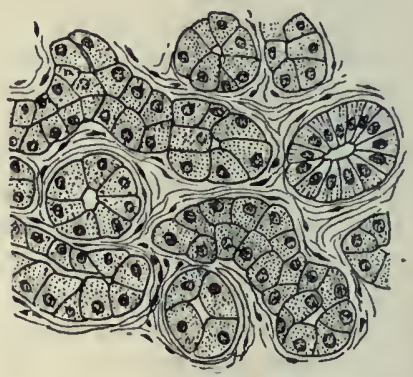

Fif. 482,-StRUCTURE OF THE PAROTID GIAND. duct passes from each small lobule, which unites with adjacent ducts, and these in turn unite, larger and still larger .ducts being formed, the resultant being the principal duct, called Stensen's duct. Each alveolus is serous or albuminous as regards the nature of its secretion, and is composed of a basement membrane continuous with the wall of the duct, and surrounded by a plexus of capillaries. The alveolus is lined with polyhedral cells, which contain albuminous granules, and almost completely fill the tube, leaving only a small lumen. The first portion of the duct, called the intercalary duct, is lined with flattened epithelium. Beyond this the duct becomes constricted into a neck, which is lined with cubical cells, these being replaced in the intralobular duct by columnar cells. These cells are granular towards the lumen of the tube, but striated in the outer part. Each of the larger ducts is composed of a basement membrane, strengthened externally by a layer of connective tissue, superadded to which there is a stratum of plain muscular fibres.

Development of the Salivary Glands. - The salivary glands-parotid, submaxillary, and sublingual-are of ectodermic origin. They appear as solid outgrowths of the epithelium of the stomodæum, or primitive buccal cavity, which grow into the adjacent mesodermic connective tissue. The epithelial constituents of each gland are derived from the stomodaal ectoderm, whilst the capsule and connective-tissue elements are of mesodermic origin.

Each solid epithelial outgrowth ramifies very freely, and these ramifications, as well as the primary outgrowth, become tubular. The hollowing process commences in the primary outgrowth, and extends thence throughout its numerous ramifications. The primary outgrowth represents the principal duct of each gland, and the acini, or alveoli, appear as dilatations of the walls of the terminal ramifications.

The sublingual gland, from its numerous ducts, is to be regarded as a cluster of small alveolo-lingual glands. A few of these glands are developed from diverticula of Wharton's duct, which is the duct of the submaxillary gland. The majority of them, however, like the parotid and submaxillary glands, are developed from solid epithelial outgrowths of the ectoderm of the stomodæum. 


\section{Ocular Appendages.}

The appendages of the eye consist of the eyelids and the lachrymal apparatus.

Eyelids.--The eyelids, or palpebræ, are two movable curtains, placed in front of the eyeball, to which they form an important protection. The inner surface of each is covered by mucous membrane, which constitutes the conjunctiva. The upper eyelid is larger than the lower, and, when closed, covers the transparent part of the eye or cornea. It is also more movable than the lower, being provided with a special elevator muscle, namely, the levator palpebræ superioris. The elliptical interval between the lids is called the fissura palpebrarum, and the lateral extremities of - this fissure are called the outer canthus and inner canthus. The outer canthus is formed by the junction between the two lids. At the inner canthus the lids are separated by a recess, called the lacus lacrimalis, in which there is a small body, called the caruncula lacrimalis. In this region the eyelids are separated from the eyeball by a vertical, semilunar fold of the conjunctiva, called the plica semilunaris. The margin of each eyelid presents, at the commencement of the lacus lacrimalis, a slight conical elevation, called the papilla lacrimalis, the apex of which presents a small orifice, termed the punctum lacrimale, its direction being towards the eyeball. Each punctum is the entrance to a passage, called the canaliculus lacrimalis, by which the tears are conveyed from the surface of the eyeball into the lachrymal sac, and thence, through the nasal duct, into the inferior meatus of the nasal fossa.

The free margins of the eyelids, external to the puncta lacrimslia, are provided with hairs, called the cilia or eyelashes. They are short, stout, and curved, and are arranged in two rows. Those of the upper lid are more numerous and larger than those of the lower. The upper eyelashes are curved upwards, and the lower downwards, and in this manner intermingling is avoided. Within the lines of attachment of the eyelashes there is a row of modified sweat-glands, known as the glands of Moll, the openings of which are associated with the follicles of the eyelashes, and with the condition known as stye.

Strueture of the Eyelids.-Each eyelid is composed of the following structures, in order from without inwards :

I. Skin.

2. Connective tissue.

3. Palpebral fibres of the orbicularis palpebrarum.
4. Tarsal plate.

5. Meibomian glands.

6. Conjunctiva.

In addition to the foregoing structures the upper eyelid contains the tendinous insertion of the levator palpebræ superioris muscle.

The skin is very thin, and at the ciliary margins of the eyelids it becomes continuous with the conjunctiva, which is a mucous membrane. The subcutaneous connective tissue is very loose, and 
devoid of fat. The palpebral fibres of the orbicularis palpebrarum form a very delicate, pale sheet. The tarsal plate, or tarsus, one for each eyelid, is a fibrous plate, and is composed of compact fibrous tissue. The superior tarsal plate is larger than the inferior, and is semilunar, its depth at the centre being about $\frac{1}{4}$ inch. Its upper and anterior part gives insertion to the levator palpebræ superioris. The inferior tarsal plate is narrow, and is almost of the same depth throughout. The ciliary margins of the tarsal plates are free, straight, and comparatively thick. The orbital margins are attached to the circumference of the orbit by membranous expansions, called the superior and inferior palpebral ligaments. The superior palpebral ligament is attached above to the upper part of the circumference of the orbit, where it blends with the periosteum, and below it blends with the tendon of insertion of the levator palpebræ superioris on the superior tarsal plate. The inferior palpebral ligament extends between the lower part of the circumference of the orbit and the lower margin of the inferior tarsal plate. The superior and inferior palpebral ligaments together constitute a partition, called the septum orbitale, which intervenes between the superficial and deep palpebral structures.

Externally and internally the tarsal plates have attachments by means of the tarsal ligaments, which are two in number, external and internal. The external tarsal ligament is formed by the junction of the superior and inferior palpebrel ligaments, and is attached to the malar bone. The internal tarsal ligament, tendo palpebrarum, or tendo oculi, is independent of the palpebral ligaments. Internally it is attached to the external surface of the nasal process of the superior maxilla in front of the lachrymal groove. From this point it passes horizontally outwards for about $\frac{1}{6}$ inch, and then divides into two laminæ, which are attached to the superior and inferior tarsal plates. It passes in front of the lachrymal sac, and furnishes an offset, which passes behind the sac to be attached to the lachrymal crest of the lachrymal bone. The ligament gives origin to a few fibres of the orbicularis palpebrarum.

The Meibomian glands are situated on the internal surface of each tarsal plate, and lie between the plate and the conjunctiva at right angles to the ciliary margin. There are about thirty in the upper eyelid, and about twenty in the lower, and they are arranged in parallel rows, which occupy grooves on the ocular surface of each tarsal plate. Each gland opens by an independent orifice, and these orifices are arranged in a single row, lying a little behind the ciliary margin of the eyelid.

Structure.-The Meibomian glands are sebaceous glands, and their secretion lubricates the margins of the eyelids, and prevents them from adhering. Each consists of a tube, closed at one end, and having its sides beset with diverticula. The wall of the tube is composed of a basement membrane, which is lined witli cubical epithelium throughout the greater part of the gland, but close to the orifice this is replaced by stratified epithelium. 
In the vicinity of the closed ends of the Meibomian glands there are some convoluted tubules, which are known as the posterior tarsal, or accessory lachrymal, glands, the orifices of which are placed close to the fornix conjunctivæ.

Conjunctiva.-This is the mucous membrane which covers the ocular surfaces of the eyelids and the front of the eyeball. It consists of two parts-palpebral and ocular.

The palpebral conjunctiva lines the ocular or inner surfaces of the eyelids, and, at their ciliary margins, it is continuous with the skin on their outer surfaces. It is also continuous, through the puncta lacrimalia, with the lining membrane of the lachrymal canals, lachrymal sac, nasal duct, and inferior meatus of the nose. In the region of the inner canthus it gives rise to the plica semilunaris, and at the outer part of the upper eyelid it is continuous with the lining membrane of the lachrymal ducts. The palpebral conjunctiva is fairly thick and highly vascular, and it presents numerous papillæ.

The ocular conjunctiva consists of two portions-sclerotic and corneal. It is continuous with the palpebral conjunctiva, and the line of reflection from the eyelids is known as the fornix conjunctivæ, superior and inferior respectively. In this vicinity the orifices of the posterior tarsal, or accessory lachrymal, glands are met with. The sclerotic conjunctiva covers the part of the sclerotic which is adjacent to the cornea. It is loosely connected to the sclerotic, is thin, non-papillary, and transparent, and contains only a few bloodvessels, the whiteness of the sclerotic being unaffected by it. The corneal conjunctiva is very thin, and consists only of the conjunctival epithelium.

The palpebral conjunctiva is covered by columnar epithelium, which, at the ciliary margin, passes into the stratified epithelium of the skin; the sclerotic conjunctiva is also covered by columnar epithelium; but the corneal conjunctiva is represented by the stratified epithelium of the cornea.

The conjunctiva is supplied with blood by offsets from the palpebral branches of the ophthalmic artery and its lachrymal branch. The vessels are disposed in a tortuous manner, and are movable upon the eyeball when the conjunctiva is pressed upon and displaced. The nerves are numerous, and form plexuses. The lymphatics commence close to the corneal margin in a network, from which vessels proceed to a network in each eyelid behind the tarsal plate. The efferent vessels ultimately reach the parotid and submaxillary lymphatic glands.

The caruncula lacrimalis occupies the lacus lacrimalis in the region of the inner canthus. It is a small, reddish, spongy body, consisting of a detached portion of skin, containing modified sweat and sebaceous glands. The latter open into the follicles of very delicate hairs with which the surface of the caruncle is provided, and they furnish the white secretion which may accunulate at the inner canthus. 
The plica semilunaris is a vertical, semilunar fold of the conjunctiva which is situated on the outer side of the caruncle, its concave margin being directed outwards. It corresponds to the membrana nictitans, or third eyelid, of some animals.

Development of the Eyelids and Meibomian Glands.-The eyelids make their appearance as two folds of skin, above and below the developing eyeball. Each fold contains some mesodermic tissue which gives rise to the connectivetissue element and tarsal plate of the lid. The ectoderm of the posterior surfaces of the lids acquires the characters of mucous membrane, and forms the conjunctival epithelium. In the course of the third month the eyelids grow together and unite along their margins, a space being thereby enclosed between the lids and the front of the developing eyeball. The union affects the epithelium only, and persists until near the end of intra-uterine life.

During the period of fusion of the eyelids the Meibomian glands and the cilia or eyelashes are formed. The Meibomian glands are developed from the epithelium along the line of fusion of the lids. Solid rods of epithelial cells are formed which grow into the mesodermic tissue of the two lids and give off lateral processes. These solid rods beçome hollow, and so form the Meibomian glands. Some of the epithelial rods give rise to the glands of Moll. A short time before birth the eyelids become separated, and the palpebral fissure is thereby formed.

The plica semilunaris is developed as a vertical fold of conjunctiva near the inner canthus of the eye, external to the caruncle, but it attains little development in man.

The caruncle is developed from that portion of the margin of the lower eyelid which intervenes between the inferior punctum lacrimale and the inner canthus. The Meibomian glands in this region become modified, and the tissue containing these modified glands becomes raised, and forms the reddish, spongy caruncle.

Lachrymal Apparatus.-The constituent parts of the lachrymal apparatus are as follows:

I. The lachrymal gland.

2. The lachrymal canaliculi.
3. The lachrymal sac.

4. The nasal duct.

The lachrymal gland will be found described on p. I 46 .

The lachrymal canaliculi are two in number, superior and inferior. They commence at the puncta lacrimalia on the summit of the papillæ lacrimales, which latter are situated on the ciliary margins of the eyelids, close to the lacus lacrimalis. The superior canaliculus at first ascends vertically for about $\frac{1}{12}$ inch, after which it makes a sudden bend, and passes inwards and downwards to the lachrymal sac. The inferior canaliculus at first descends vertically for about $\frac{1}{12}$ inch, after which it makes a sudden bend and passes almost horizontally inwards to the lachrymal sac. The two canaliculi open into the lachrymal sac, either close together or by a common orifice, and their mucous membrane is lined with stratified squamous epithelium. The two slips of the tensor tarsi muscle are closely related to the horizontal portions of the canaliculi.

The lachrymal sac is the dilated upper part of the passage by which the tears are conveyed from the lachrymal canaliculi to the inferior meatus of the nose. It occupies the lachrymal groove of the lachrymal bone and nasal process of the superior maxilla. 
Superiorly it presents a round, closed extremity, and inferiorly it opens into the nasal duct. Externally it receives the lachrymal canaliculi, separately or conjointly, and anteriorly it is crossed by the tendo palpebrarum or internal tarsal ligament, which furnishes an offset round it. Posteriorly it is related to the tensor tarsi muscle.

The guide to the lachrymal sac is the lachrymal tubercle or spine of the superior maxilla, which is situated at the point of continuity of the infra-orbital margin of the bone and the crista lacrimalis anterior on the outer surface of the nasal process. The lachrymal sac lies immediately internal-to this tubercle. In many cases, however, the tubercle or spine is very rudimentary.

The nasal duct extends from the lower end of the lachrymal sac to the anterior part of the inferior meatus of the nose, under cover of the front part of the inferior concha. Its length is about $\frac{3}{4}$ inch,

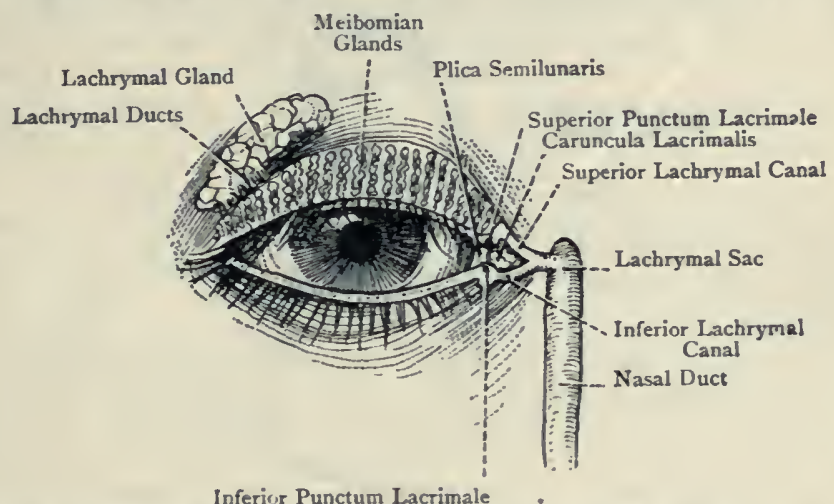

Fig. 483.-The lachrymal Apparatus of the Right Eye.

(The Meibomian Glands of the Upper Eyelid are also shown.)

and its diameter about $\frac{1}{8}$ inch. It is directed downwards, outwards, and backwards, and its opening into the anterior part of the inferior meatus of the nose is usually guarded by an imperfect mucous fold, known as the valve of Hasner. The nasal orifice of the duct is about $I_{4}^{1}$ inches from the anterior nasal aperture.

Strueture of the Lachrymal Sac and Nasal Duct. - The wall is composed of fibro-elastic tissue, which adheres closely to the periosteum of the bones, and is covered by mucous membrane. The epithelial lining is of the columnar variety, and at intervals the cells are furnished with cilia. The mucous membrane is continuous superiorly with the conjunctiva through the lachrymal canaliculi and puncta, and inferiorly it is continuous with the nasal mucous membrane. In the nasal duct it may present one or two folds.

Development of the Nasal Duet and its Appendages. -The efferent lachrymal apparatus consists of (I) the lachrymal canaliculi, (2) the lachrymal sac, and (3) the nasal duct.

In the course of the development of the face, the maxillary process and the tateral nasal process, of either side, are separated by a groove which extends 
from the inner angle of the eye to the olfactory pit. This groove is called the naso-optic, or oculo-nasal, groove, and it indicates, deeply, the position of the lachrymal duct. In the bottom of this groove a solid epithelial cord makes its appearance, which, becoming hollow, forms the nasal duct. The upper extremity of the duct bifurcates, its two divisions becoming connected with the margins of the eyelids near their inner ends, and forming the lachrymal canaliculi. The lower end of the nasal duct, after the completion of development, opens into the lower part of the nasal cavity. The lachrymal sac is the upper expanded extremity of the nasal duct.

\section{Auricle.}

The auricle, or pinna, is that part of the external ear which projects from the side of the head. It is somewhat pyriform, with the broad end upwards, and it presents two surfaces, outer and inner.

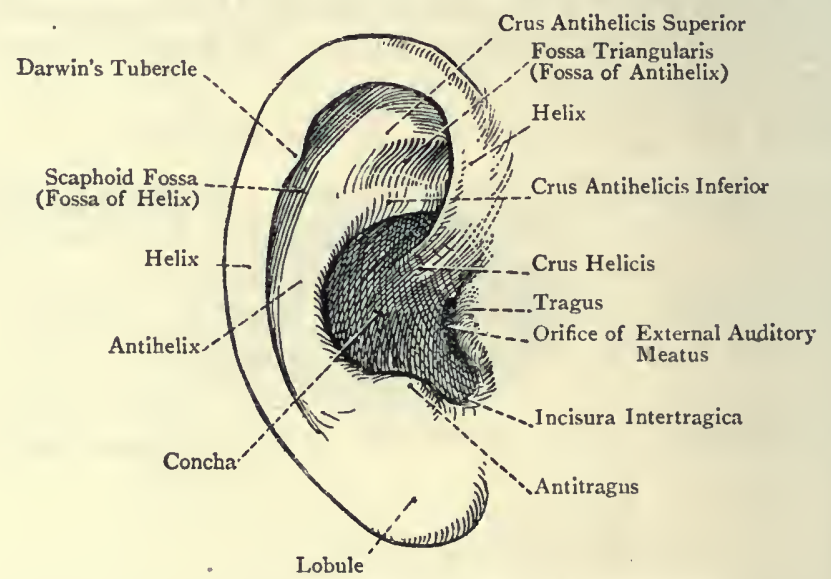

Fig, 484.-The Right Auricle (External View).

The outer surface is irregularly concave, and presents certain elevations and depressions. About its centre there is a large deep fossa, called the concha, which leads to the meatus auditorius externus. Towards the upper and anterior part of the concha there is an elevation, called the crus helicis, which is directed upwards and forwards to the anterior border of the auricle. The concha is thus divided into two parts, upper and lower. In front of the concha there is a small, somewhat conical, prominence, called the tragus, which projects slightly backwards over the orifice of the meatus auditorius externus, and is provided with hairs on its inner aspect. A short distance behind the tragus, and on a lower level than it, there is another prominence, called the antitragus, which is separated from the tragus by a deep notch. called the incisura intertragica. The skin over the antitragus is also provided with hairs. Below the antitragus and incisura intertragica is the most dependent part of the auricle, called the lobule, which is compara- 
tively soft in consistence. The prominent rim of the auricle is called the helix. It is incurved, and commences at the upper and anterior part of the concha in the crus helicis, already referred to. Thereafter it surrounds the upper part of the anterior margin, and the superior and posterior margins, of the auricle, and subsides below in the back part of the lobule. In some cases the helix presents a projection, called the tubercle of Darwin, which is situated on the incurved margin, slightly above the level where the antihelix, to be presently described, bifurcates into its crura. This projection is well developed in the ears of quadrupeds, and forms the point or tip. A short distance within the helix there is another curved elevation, called the antihelix. This commences inferiorly at the back part of the antitragus and ascends behind the concha, above which it divides into two diverging crura, upper and lower. Between the helix and the antihelix is an elongated, narrow fossa, called the fossa of the helix, or the scaphoid fossa, and between the diverging crura of the antihelix there is a depression, called the fossa of the antihelix, or the triangular fossa.

The inner or cranial surface of the auricle presents convexities corresponding to the fossa on the outer surface, the convexity opposite the concha being especially prominent.

Structure of the Auricle.-The auricle, with the exception of the lobule, is composed of a plate of yellow elastic fibro-cartilage covered by skin. This plate imparts firmness and elasticity to it, and is provided with ligaments and intrinsic muscles. The skin is thin, and adheres closely to the fibrocartilaginous plate. It is provided with hairs, which are most plentiful in the regions of the tragus and antitragus. The cartilage of the auricle is rolled upon itself so as to form the outer or cartilaginous part of the meatus auditorius externus. This portion of it is attached internally to the external auditory process of the temporal bone by fibrous tissue. The rolled or tubular portion presents a deficiency at the anterior and upper part, between the tragus and the commencement of the helix, which is occupied by a fibrous membrane. It also presents a variable number of transverse clefts, called the fissures of Santorini, which are filled with fibrous tissue. The lower extremity of the cartilage of the helix is separated from the cartilage of the antihelix by a deep cleft. The part of the cartilage of the helix behind this cleft is known as the processus caudatus helicis. At the upper and anterior part of the auricle, where the helix begins to curve backwards, the cartilage presents a small sharp projection, called the spine of the helix.

Ligaments of the Auricle.-These are two in number, anterior and posterior. The anterior ligament extends from the spine of the helix to the zygoma of the temporal bone close to its root. The posterior ligament extends from the cranial aspect of the concha, under cover of the retrahens auriculam muscle, to the mastoid process.

Intrinsic Muscles.-These muscles, which are very thin and pale, are confined to the auricle and are six in number, four being situated on the outer surface, and two on the inner surface.

Muscles on Outer Surface.-These are as follows: (I) the musculus helicis major; (2) the musculus helicis minor; (3) the musculus tragicus; and (4) the musculus antitragicus. 
The musculus helicis major extends from the spine of the helix along the anterior part of the helix as high as the level at which it curves backwards.

The musculus helicis minor lies upon the crus helicis.

The musculus tragicus lies upon the outer surface of the tragus, its fibres being almost vertical.

The musculus antitragicus extends from the outer surface of the antitragus backwards and slightly upwards, to be attached to the processus caudatus of the helix.

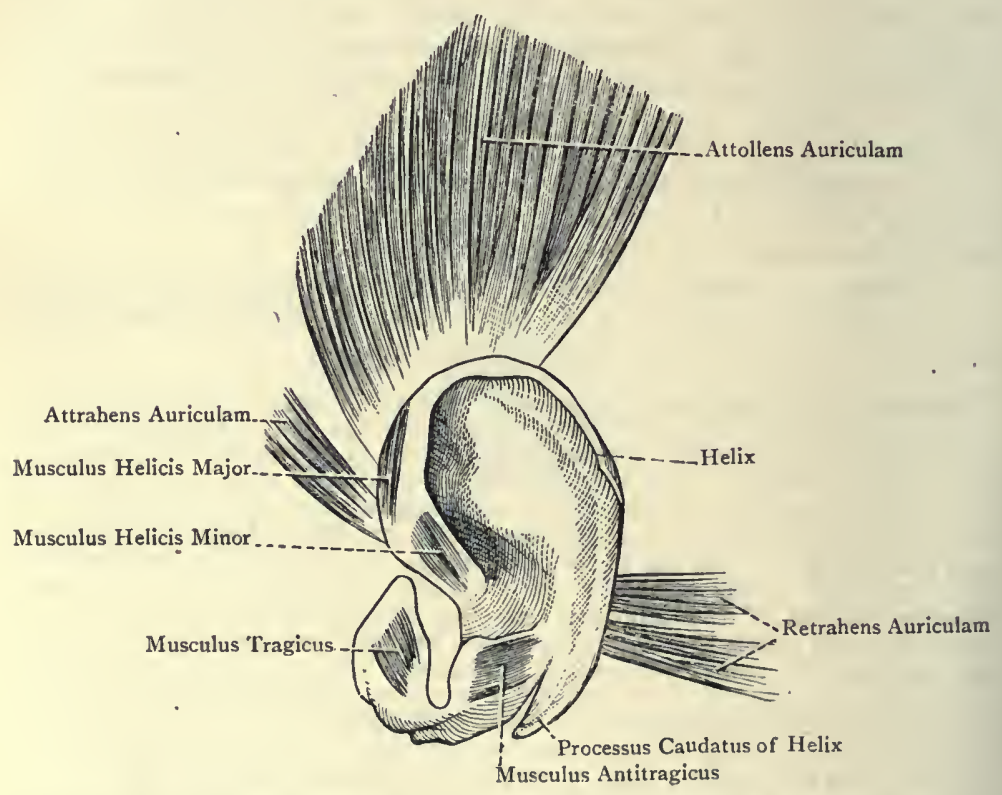

Fig. 485.-The Outer Surface of the Left Auricle and its Muscles (ARNold).

Muscles on Inner Surface.-These are as follows: (I) the musculus transversus, and (2) the musculus obliquus.

The musculus transversus extends over the depression which corresponds to the antihelix on the outer surface, its attachments being to the convexity of the concha on the one hand, and the convexity of the fossa of the helix on the other.

The musculus obliquus extends over the depression corresponding to the lower crus of the antihelix on the outer surface.

Action of the Intrinsic Muscles.-The musculus tragicus and musculus antitragicus diminish the orifice of the meatus auditorius externus, and the muscles of the helix, major and minor, have an opposite effect. 
Nerve-supply.-The facial nerve.

Blood-supply of the Auricle.-The arteries are derived from (I) the posterior auricular branch of the external carotid, (2) the anterior auricular branches of the superficial temporal, and (3) the deep auricular branch of the first part of the internal maxillary, the last named giving offsets to the cartilaginous part of the meatus auditorius externus.

The veins terminate in the posterior auricular, superficial temporal, and internal maxillary veins, and one or two of them may open into the mastoid emissary vein.

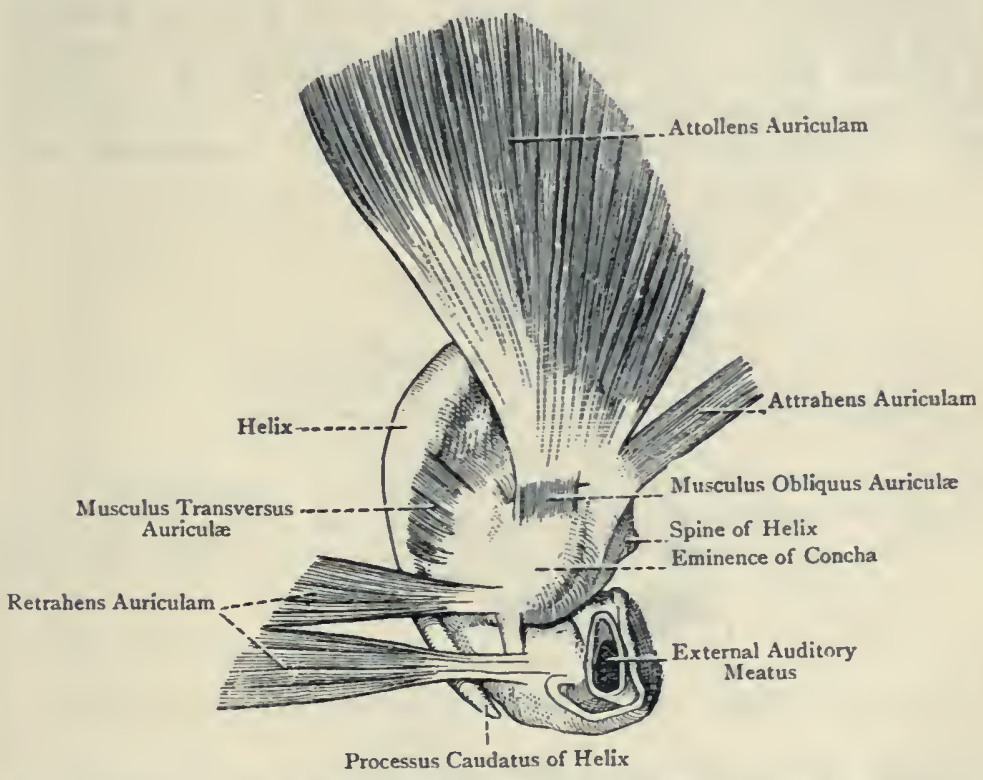

Fig. 486.-The Inner Surface of the Left Auricle and its MUSClES (ARNOLD).

The lymphatic vesse ls of the inner surface of the auricle pass chiefly to the mastoid, or posterior auricular, glands; but a few end in the superior deep cervical glands. The lymphatics of the lobule pass to (I) the superficial cervical, or external jugular, glands, and (2) the superior deep cervical glands. The lymphatics of the outer surface of the auricle pass to the superficial parotid, or anterior auricular, lymphatic glands.

Nerve-supply.-The inner surface receives three cutaneous nerves, as follows: the great auricular supplies about the lower threefourths, the small occipital about the upper fourth, and the auricular branch (Arnold's nerve) of the pneumogastric supplies 
the integument over the convexity of the concha. The outer surface is supplied by two cutaneous nerves, as follows: the auriculo-temporal nerve supplies the upper two-thirds, and the great auricular supplies the lower third. The motor nerve of the intrinsic muscles is, as stated, the facial nerve.

\section{The Nose.}

The nose, as it appears upon the face, presents a root, situated below the glabella of the frontal bone; a tip, or point, situated inferiorly; and a dorsum, which occupies an intermediate position. The upper part of the dorsum is known as the bridge of the nose. At the lower part of the nose there are the openings of the nostrils, or anterior nares. The outer margin of each nostril is slightly prominent and curved, and is called the ala. The nostrils are separated from each other by a septum, called the columella nasi,

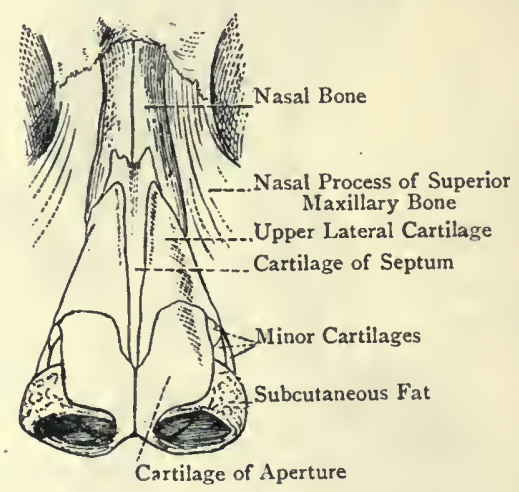

Fig. 487.-The Cartilages of the Nose (Anterior View) (Arnold).

which, as well as the ala, is composed of fibrous tissue and skin. Within the circumference of each nostril there are several stout hairs or vibrissa.

The superficial or facial aspect of the nose derives its arteries from (I) the lateral nasal and angular branches of the facial, and (2) the nasal branch of the ophthalmic.

The nerves are derived from the nasal and infra-orbital nerves, the branches from the nasal being the infratrochlear and the terminal cutaneous offsets.

The cutaneous lymphatics of the root of the nose pass to the superficial parotid, or anterior auricular, lymphatic glands. Those from the greater part of the nasal integument pass to the submaxillary lymphatic glands, the superficial facial glands forming glandstations in their path.

The framework of the nose is both osseous and cartilaginous. 
Cartilages of the Nose.-The nasal cartilages are five in number, as follows :

Upper lateral, right and left.

Lower lateral, right and left.

Septal.

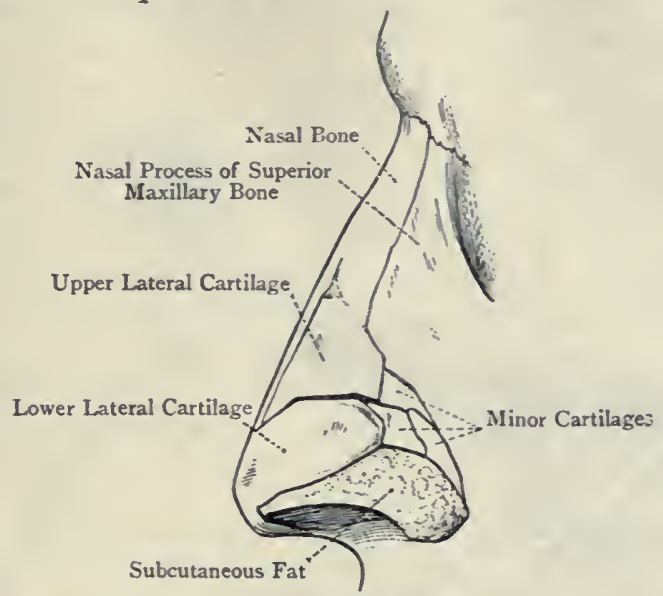

Fig. 488.-The Cartilages of the Nose (Lateral View) (ARnold).

The upper lateral cartilages are situated immediately below the nasal bones. They are triangular, and their anterior borders are continuous with each other, and with the anterior margin of the

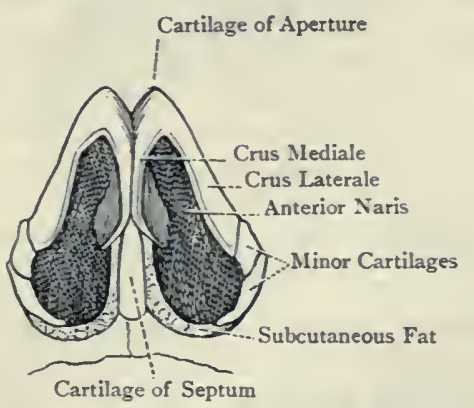

Fig. 489.-The Cartilages of the Nose (Inferior View) (Arnold).

septal cartilage, in each case superiorly. Inferiorly, the anterior borders are separated by a slight interval, in which the anterior margin of the septal cartilage is visible. The posterior border of each cartilage is attached to the lower sloping border of the nasal bone, and also to the upper part of the nasal notch on the mesial 
border of the superior maxilla. The lower border is connected by fibrous tissue to the upper margin of the lower lateral cartilage.

The lower lateral cartilages are also known as the cartilages of the apertures. They are situated below the upper pair, and each is bent so as to lie in front, and on each side, of the nostril, which it keeps open. Its outer portion is called the crus laterale, and its inner portion the crus mediale.

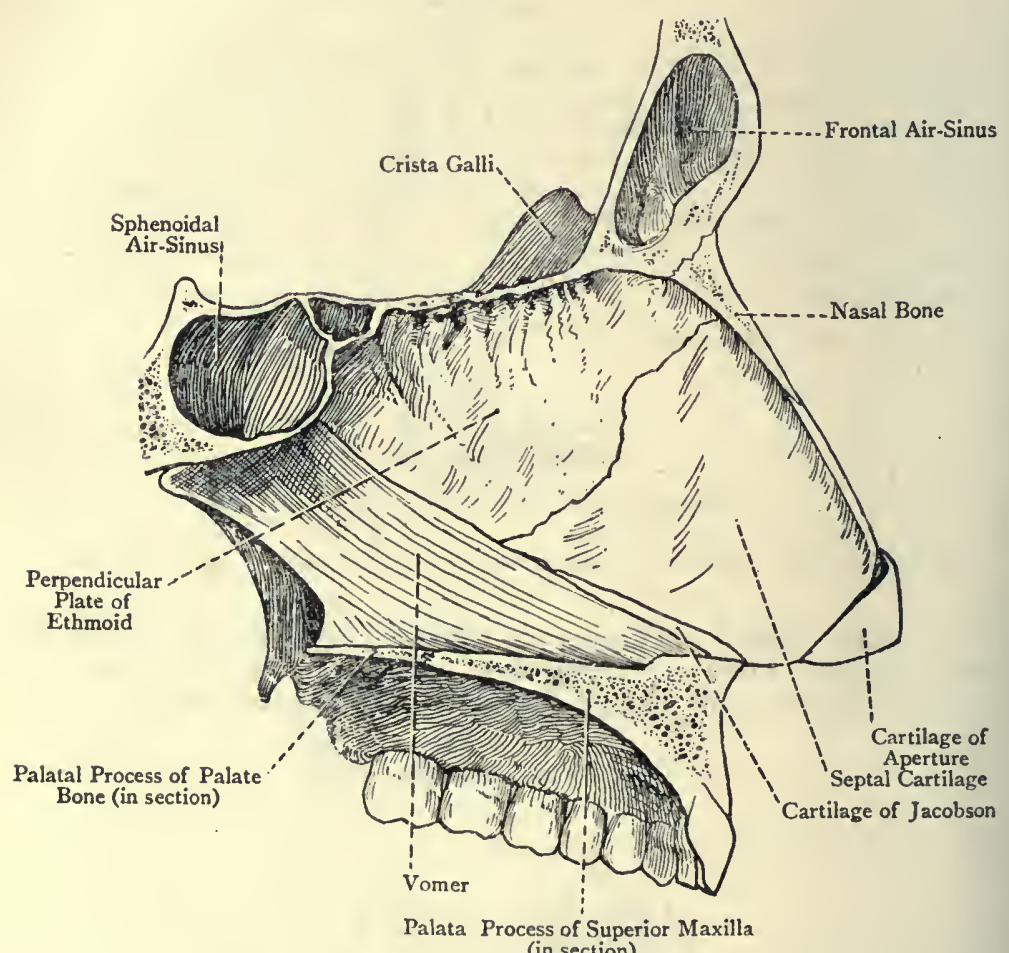
(in section)

Fig. 490.-The Osseous and Cartilaginous Nasal Septum (Right LATERAL View).

The crus laterale is attached by fibrous tissue to the upper lateral cartilage, and to the lower part of the nasal notch on the mesial border of the superior maxilla. The crus mediale is folded backwards, and is contiguous to its fellow of the opposite side. It lies along the upper part of the columella nasi, and along the anteroinferior border of the cartilage of the septum. Anteriorly it is separated from its fellow by a notch.

In the fibrous tissue which connects the crus laterale to the superior maxilla two or more isolated portions of cartilage are met with, called the sesamoid or minor cartilages. 
Development. - The lateral nasal cartilages are derived from the cartilaginous core of the lateral nasal process.

The cartilage of the septum is mesially placed, and is usually inclined slightly to one side, most frequently the left. It forms a large part of the nasal septum anteriorly, and has the form of an irregularly four-sided, laterally-compressed plate. Its anterior border is attached to the back of the nasal bones, along the course of the internasal suture; below this it is connected to the anterior borders of the upper lateral nasal cartilages; and below these it lies between the middle crura of the lower nasal cartilages. Its posterior bordey is accurately applied to the irregular anterior margin of the perpendicular plate of the ethmoid bone. Its inferior border is received into the front part of the groove on the anterior border of the vomer. The antero-inferior border passes upwards and forwards from the front part of the inferior border to the anterior border. In early life the septal cartilage is prolonged backwards to the body of the sphenoid bone in the form of a narrow strip, which intervenes. between the lower border of the perpendicular plate of the ethmoid and the vomerine groove. This portion is known as the sphenoidal or posterior process. Along the inferior border of the septal cartilage, between it and the anterior border of the vomer, there are two narrow, elongated strips of cartilage, right and left, which are called the cartilages of Jacobson, or the vomerine cartilages of Huschke.

Development. - The septal cartilage is derived from the chondrocranium.

\section{The Temporal and Ptergyo-maxillary Regions.}

Muscles of Mastication.-These are four in number, namely, the masseter, temporal, external pterygoid, and internal pterygoid.

Masseter-Origin-(I) Superficial Portion.-The anterior twothirds of the lower border of the zygomatic arch. (2) Deep Portion. -The posterior third of the lower border, and the whole of the deep or internal surface of the zygomatic arch.

Insertion.-The superficial portion is inserted into the lower half, and the deep portion into the upper half, of the outer surface of the ramus of the inferior maxilla. The superficial fibres extend as far as the angle, and the deep fibres encroach on the coronoid process.

Nerve-supply. - The masseteric branch of the anterior portion of the inferior maxillary division of the fifth cranial nerve. This branch emerges from the pterygoid region by passing over the sigmoid notch of the inferior maxilla, below the zygoma, and it therefore enters the muscle on its deep surface, accompanied by the masseteric artery.

The superficial portion of the muscle is directed downwards and slightly backwards, and the deep portion downwards and very slightly forirards. 
Action.--To elevate the inferior maxilla. The superficial portion also draws it slightly forwards.

Relations-Superficial.-The parotid gland and Stensen's duct, branches of the facial nerve, the transverse facial artery, the risorius, and the platysma myoides. Deep.-The ramus of the infarior maxilla, and the masseteric nerve and artery. The anterior border overlaps the buccinator, from which it is separated by the buccal pad of fat.

Temporal Muscle-Origin.--(I) The temporal fossa, extending as high as the inferior temporal ridge of the frontal and parietal bones, and as low as the infratemporal crest on the external surface of the great wing of the sphenoid, but excluding the portion of the fossa formed by the malar bone; and (2) the deep surface of the temporal fascia over its upper part.

Insertion.-(I) The internal surface, summit and anterior border of the coronoid process of the inferior maxilla; and (2) the elongated triangular surface on the internal surface of the ramus of the inferior maxilla, close within the anterior border, and extending as low as a point on the inner side of the last molar alveolus.

Nerve-supply.-The deep temporal nerves, usually three in number, which are branches of the anterior portion of the inferior maxillary division of the fifth cranial nerve, and which enter the deep surface of the muscle.

The muscle is fan-shaped. The anterior fibres descend almost vertically; the middle fibres pass obliquely downwards and forwards; and the posterior fibres pass forwards almost horizontally.

Action.--To raise the inferior maxilla, that is, to draw it upwards into contact with the superior maxilla, as in closing the mouth. The posterior fibres also retract the inferior maxilla, and act in opposition to the external pterygoid, which protracts it.

Relations-Superficial.-The temporal fascia, supporting the attrahens and attolleris auriculam muscles; the superficial temporal artery, auriculo-temporal nerve, and temporal branches of the facial nerve; the zygoma; and fat, which is continuous with the buccal pad. Deep.- The temporal fossa, the deep temporal arteries and nerves, and the external pterygoid muscle.

The long buccal nerve passes downwards and forwards under cover of the musc!e close to the anterior border of the ramus of the inferior maxilla, and the masseteric nerve and artery pass outwards close to the posterior border in the region of the sigmoid notch of the inferior maxilla.

For the temporal fascia, see Index.

External Pterygoid-Origin-(I) Upper Head.-The zygomatic division of the outer surface of the great wing of the sphenoid, below the infratemporal crest. (2) Lower Head.-The outer surface of the external pterygoid plate of the sphenoid. The upper head is small, and the lower head is of large size.

Insertion.-(I) The depression on the front of the neck of the 
inferior maxilla ; and (2) the frontof the interarticular fibro-cartilage of the temporo-mandibular joint.

Nerve-supply.-The external pterygoid nerve, which is a branch of the long buccal nerve, that, in turn, being a branch of the anterior portion of the inferior maxillary division of the fifth cranial nerve.

The direction of the muscle is backwards and slightly outwards.

Action.-(I) To draw forwards the neck and condyle of the inferior maxilla, and also the interarticular fibro-cartilage. When the muscles of opposite sides act in concert the inferior maxilla is protruded, and the lower incisor and canine teeth project beyond the level of those of the superior maxilla. The muscles of opposite sides, however, usually act in reciprocal succession, as in the

Long Buccal Nerve (cut)

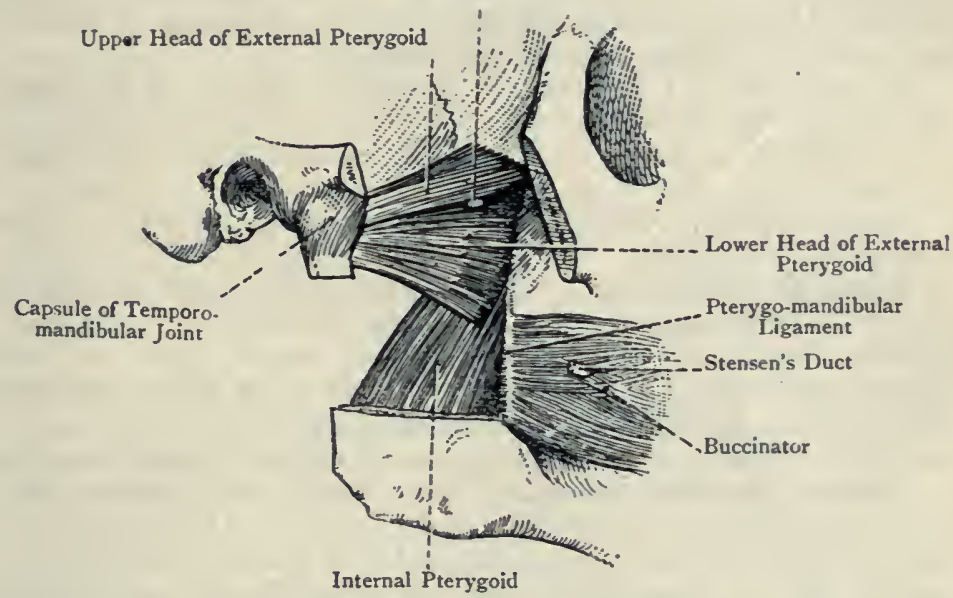

Fig. 491.-The Pterygoid and Buccinator Muscles.

oblique or grinding movement, the lower molars of one side being carried forwards and inwards upon the corresponding upper molars, and vice versâ. At the same time the elevators of the inferior maxilla are in action. (2) To take part in opening the mouth by drawing the condyle of the inferior maxilla and interarticular fibrocartilage forwards on to the eminentia articularis of the temporal bone. The external pterygoid is antagonistic to the posterior portion of the temporal muscle.

Relations-Superficial.-Part of the ramus of the inferior maxilla, the lower part of the temporal muscle, part of the pterygoid plexus of veins, the long buccal nerve, and, it may be, the second part of the internal maxillary artery. Deep.-The upper portion of the internal pterygoid muscle, the spheno-mandibular ligament, in some cases the second part of the internal maxillary artery, the middle meningeal artery, a part of the pterygoid plexus of veins, 
the inferior maxillary nerve, the otic ganglion, and the chorda tympani nerve. Superior.-The masseteric and middle and posterior deep temporal nerves. Injerior.-The inferior dental and lingual nerves, and the spheno-mandibular ligament. The long buccal nerve, with the anterior deep temporal nerve, makes its appearance between the upper and lower heads, and the internal maxillary artery may sink between them.

Internal Pterygoid-Origin.-(I) The inner surface of the external pterygoid plate of the sphenoid, and the portion of the tuberosity of the palate bone which forms the lower part of the pterygoid fossa; and (2) the outer surface of the tuberosity of the palate bone, and the adjacent portion of the tuberosity of the superior maxilla.

Insertion.-(I) The inner aspect of the angle of the inferior maxilla and (2) the back part of the inner surface of the ramus, between the angle and the dental foramen.

Nerve-supply. - The internal pterygoid branch of the anterior portion of the inferior maxillary division of the fifth cranial nerve.

The direction of the muscle is downwards, backwards, and outwards.

Action.-(I) To elevate the inferior maxilla; and (2) to draw it forwards.

Relations-Superficial.-The external pterygoid muscle to a slight extent superiorly, the spheno-mandibular ligament, the internal maxillary and inferior dental vessels, and the inferior dental and lingual nerves. Deep.-The tensor palati muscle, and the superior constrictor muscle of the pharynx.

For a description of the spheno-mandibular ligament, see Index.

Internal Maxillary Artery.-This vessel is the larger of the two terminal branches of the external carotid. It arises from that artery opposite the neck of the inferior maxilla, and within the parotid gland. Its course is at first forwards and inwards behind the neck of the mandible, and superficial to the spheno-mandibular ligament. It then inclines upwards and forwards through the zygomatic fossa, usually passing superficial to the external pterygoid muscle, though in many cases it passes beneath it. Having reached the interval between the two heads of the external pterygoid, it sinks deeply between them, and, passing through the pterygomaxillary fissure, it enters the spheno-maxillary fossa, where it gives off its terminal branches. In those cases where the artery passes beneath the external pterygoid muscle, it forms a projecting curve between the two heads of the muscle. The course of the vessel is very tortuous in adaptation to the mobility of the surrounding structures.

Owing to its complexity it is convenient to divide the artery into three parts, namely, first, second, and third. The first, or mandibular, part is situated between the neck of the inferior maxilla and the spheno-mandibular ligament, and its course is horizontally forwards and inwards. It is accompanied by the internal maxillary vein, and 
lies along the back part of the lower border of the external pterygoid muscle, crossing in front of the inferior dental nerve. The second, or pterygoid, part usually lies upon the lower head of the external pterygoid, and under cover of the insertion of the temporal muscle. Its course through the zygomatic fossa is upwards and forwards, and it sinks between the two heads of the external pterygoid on its way to the pterygo-maxillary fissure. In many cases, however, the second part of the vessel passes beneath the lower head of the external pterygoid, crossing in front of the internal pterygoid muscle and lingual nerve. Under these circumstances it forms a projecting curve between the two heads of the external pterygoid beneath the long buccal nerve. The third, or spheno-maxillary, part is situated in the spheno-maxillary fossa, which it enters by passing through the pterygo-maxillary fissure. In this fossa the vessel and its branches are intimately related to the superior maxillary nerve and Meckel's ganglion, with its branches.

Branches.-These are as follows :

First Part.

Deep auricular.

Tympanic.

Middle meningeal. giving off small meningeal.

Inferior dental.

\section{Second Part.}

Masseteric.

Internal pterygoid.

External pterygoid.

Posterior deep temporal.

Anterior deep temporal.

Buccal.

\section{Third Part.}

Posterior dental.

Infra-orbital.

Descending palatine.

Vidian.

Pterygo-palatine.

Spheno-palatine.

Branches of the First Part.-The deep auricular artery, of small size, ascends within the parotid gland, just behind the temporomandibular joint, and pierces the anterior cartilaginous wall of the meatus auditorius externus. It supplies the cutaneous lining of that passage, and the outer surface of the membrana tympani.

The tympanic artery may be associated with the preceding at its origin. It ascends beneath the external pterygoid, and enters the tympanic cavity by passing through the fissure of Glaser. It is distributed to the structures within the tympanum, and to the inner surface of the membrana tympani. Around the circumference of that membrane it forms an arterial ring with an offset of the stylo-mastoid artery, which is a branch of the posterior auricular.

The middle meningeal artery, of large size, ascends beneath the external pterygoid muscle, and, passing between the two roots of origin of the auriculo-temporal nerve, it enters the cranial cavity through the foramen spinosum in the sphenoid bone. It then passes upwards and forwards to the inner aspect of the anteroinferior angle of the parietal bone, where it divides in to two branches. anterior and posterior, which ramify in the branching grooves on the inner surface of the parietal bone. The artery is accompanied by a plexus of sympathetic nerves, and by two veins. For the distribution of the vessel within the cranium, see Index. Before disappearing through the foramen spinosum the middle meningeal 
artery usually gives off the small meningeal artery, which enters the cranial cavity through the foramen ovale.

The inferior dental artery arises nearly opposite the middle meningeal artery, and descends upon the spheno-mandibular ligament in company with the inferior dental nerve, lying on its posterior and outer side. Having reached the inferior dental foramen, it gives off the small mylo-hyoid artery, and then it passes through the inferior dental foramen and enters the inferior dental canal which it traverses as far as the level of the mental foramen, where

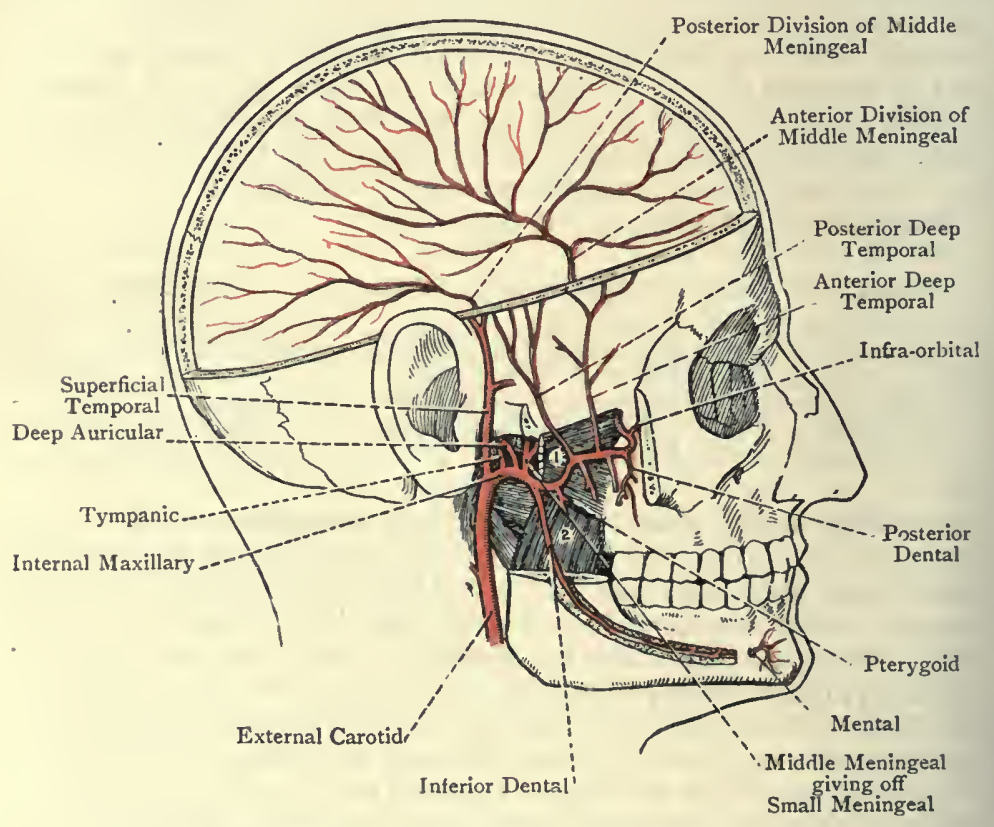

Frg. 492.-The Internal Maxillary Artery and its Branches.

(The Ramus of the Mandible and one half of the Calvaria have been removed).

I. External pterygoid muscle. 2. Internal pterygoid muscle.

it ends by dividing into its mental and incisor branches. Within the inferior dental canal the artery is accompanied by the inferior dental nerve and inferior dental vein.

Branches.-The mylo-hyoid artery, of small size, arises at the level of the inferior dental foramen. In company with the mylohyoid nerve it pierces the spheno-mandibular ligament, and descends in the mylo-hyoid groove to be distributed to the under surface of the mylo-hyoid muscle. The molar, bicuspid, and canine branches arise within the inferior dental canal, and supply the pulps of these teeth, which they reach by passing through the foramina on the extremities of their fangs. The mental artery emerges from the 
inferior dental canal through the mental foramen, and has been already described (see Index). The incisor artery supplies the pulps of the incisor teeth of one side.

Branches of the Second Part.-The branches of this part are muscular in their distribution. The masseteric artery passes outwards, with the corresponding nerve, over the sigmoid notch of the inferior maxilla, and enters the deep surface of the masseter. The internal and external pterygoid branches are distributed to the corresponding muscles. The posterior and anterior deep temporal arteries pass upwards to the posterior and anterior parts of the temporal fossa beneath the temporal muscle. They supply the muscle and the bones forming the fossa, and anastomose with the middle temporal artery, which is a branch of the superficial temporal. The anterior deep temporal artery also anastomoses with the lachrymal artery by twigs which pass through minute foramina in the outer wall of the orbit. The buccal artery passes downwards and forwards in company with the long buccal nerve, and is distributed to the buccinator muscle and the buccal mucous membrane which lines it internally.

Branches of the Third Part.--The posterior dental artery arises from the internal maxillary as it is about to pass through the pterygomaxillary fissure into the spheno-maxillary fossa, and is sometimes associated with the infra-orbital artery at its origin. It descends upon the zygomatic surface of the superior maxilla, posterior to the malar process, and its principal branches traverse the posterior dental canals to supply the pulps of the upper molar teeth of one side. It also furnishes twigs to the mucous lining of the antrum of Highmore, and to the gum.

The infra-orbital artery arises in the spheno-maxillary fossa, sometimes in common with the posterior dental. It passes through the spheno-maxillary fissure in company with the superior maxillary nerve, and traverses the infra-orbital groove and canal on the floor of the orbit, the accompanying nerve being now called the infra-orbital nerve. From this canal it emerges through the infra-orbital foramen on to the face, where it has been already described (see Index). The artery is accompanied by the infraorbital vein. As the artery traverses the infra-orbital canal it furnishes (I) orbital branches to the structures on the floor of the orbit ; and (2) the middle and anterior dental arteries, which descend in the middle and anterior dental canals in the superior maxilla, in company with the corresponding nerves, to supply the pulps of the upper bicuspid, incisor, and canine teeth of one side, and the mucous lining of the antrum of Highmore.

The descending palatine artery passes downwards in the posterior palatine canal, in company with the great or anterior descending palatine nerve, to the hard palate, where it passes forwards and inwards to the anterior palatine fossa. In this situation it furnishes a branch which ascends through the incisor canal, or canal of Stensen, to anastomose with the naso-palatine artery. As the artery 
traverses the posterior palatine canal it gives off a posterior and an external branch, which accompany corresponding nerves in the posterior and external accessory palatine canals, and supply the soft palate and tonsil.

The Vidian artery passes backwards through the Vidian canal in company with the Vidian nerve, and its branches are: (I) pharyngeal, to the upper part of the pharynx; (2) Eustachian, to the Eustachian tube; and (3) tympanic, to the tympanum.

The pterygo-palatine artery, of small size, passes backwards through the pterygo-palatine canal, in company with the pharyngeal nerve, and is distributed to the upper part of the pharynx, the Eustachian tube, and the mucous lining of the corresponding sphenoidal air-sinus.

The spheno-palatine artery enters the superior meatus of the nasal fossa through the spheno-palatine foramen. Its branches are distributed extensively on the outer wall of the nasal fossa, and supply the mucous membrane of the antrum, ethmoidal cells, and frontal air-sinus. One branch, called the naso-palatine artery, or artery of the septum, descends upon the septum to the incisor foramen, where it anastomoses with the ascending branch of the descending palatine artery.

Pterygoid Plexus of Veins. - This is a large plexus which surrounds the external pterygoid muscle. Its tributaries correspond, for the most part, to the branches of the internal maxillary artery, and are chiefly as follows : the deep auricular, tympanic, two middle meningeal, inferior dental, masseteric, pterygoid, deep temporal, buccal, superior dental, infra-orbital, palatine, and spheno-palatine. The blood is conveyed away from the plexus by two veins, namely, the internal maxillary and the deep facial.

The internal maxillary vein is a short vessel which issues from the posterior part of the plexus, and accompanies the first part of the internal maxillary artery. Opposite the neck of the inferior maxilla it joins the superficial temporal vein within the parotid gland to form the temporo-maxillary vein.

The deep facial vein issues from the anterior part of the pterygoid plexus, and, passing downwards and forwards, it emerges from behind the mandibular ramus and masseter muscle, and joins the facial vein on the buccinator muscle. The pterygoid plexus establishes the following important connections superiorly : it communicates with the intracranial cavernous sinus by means of emissary veins, which pass through the foramen ovale, foramen Vesalii, and foramen lacerum medium; and it communicates with the inferior ophthalmic vein at the spheno-maxillary fissure.

Internal Maxillary, Deep Facial, or Zygomatic Lymphatic Gland (Lymphoglandula faciales profunda). - These glands lie upon the external pterygoid muscle. Their afferent vessels are derived from (I) the zygomatic and temporal fossæ; (2) the orbit; (3) the palatal mucous membrane; (4) the nasal fossa, in part; (5) the cerebral dura mater; and (6) the tympanic cavity. Their efferent vessels pass to 
(I) the intraparotid lymphatic glands, and (2) the superior deep cervical glands.

Inferior Maxillary Nerve.-This is the third division of the fifth nerve. It is a mixed nerve, and consists of two roots-sensory and motor. The sensory root, which is of large size, arises from the Gasserian ganglion, and the motor root represents the entire motor root of the fifth nerve. Both roots leave the cranial cavity through the foramen ovale, and immediately after their exit they unite to form a mixed nerve, that is to say, a nerve composed of both sensory and motor fibres. This nerve is very short, and lies deeply in the zygomatic fossa, under cover of the external pterygoid muscle, where it gives off two branches, namely, a recurrent branch, and the internal pterygoid nerve. Thereafter it immediately breaks up into two parts, known as the anterior and posterior divisions. The recurrent branch enters the cranial cavity through the foramen spinosum, along with the middle meningeal artery, and divides into two branches-anterior and posterior. The anterior branch is distributed to the adjacent dura mater, and the posterior branch passes through the fissure between the petrous and squamous parts of the temporal bone, to be distributed to the mucous lining of the mastoid cells. The internal pterygoid nerre arises from the deep surface of the undivided inferior maxillary nerve, and passes downwards to enter the deep surface of the internal pterygoid muscle. Close to its origin it is intimately related to the otic ganglion.

Anterior Division of the Inferior Maxillary Nerve.-This division is smaller than the posterior, and is principally motor in function, the only sensory branch furnished by it being the long buccal nerve. Its branches are as follows: (I) masseteric, furnishing the posterior deep temporal; (2) middle deep temporal; and (3) long buccal, giving off the external pterygoid and anterior deep temporal, after which it is purely sensory.

The masseteric nerve passes upwards beneath the upper head of the external pterygoid muscle, where it furnishes the posterior deep temporal nerve. It then passes outwards over the upper border of the external pterygoid, and over the sigmoid notch of the lower jaw, behind the temporal muscle, to enter the upper part of the masseter on its deep surface.

The deep temporal nerves are three in number-anterior, middle, and posterior. The anterior deep temporal nerve usually arises from the long buccal after it has emerged between the two heads of the external pterygoid. It passes upwards, superficial to the upper head of that muscle, and enters the anterior part of the temporal muscle on its deep surface. The middle deep temporal nerve is a direct branch of the anterior division of the inferior maxillary, and it ascends beneath the external pterygoid to enter the middle part of the temporal muscle on its deep surface. The posterior deep temporal nerve springs from the masseteric nerve beneath the 
upper head of the external pterygoid, and, passing upwards, it enters the posterior part of the temporal muscle on its deep surface.

The long buccal nerve passes outwards between the two heads of the external pterygoid, and then downwards and forwards in contact with the inner surface of the temporal muscle at its insertion. Having emerged from underneath the mandibular ramus and anterior border of the masseter, it is received upon the outer surface

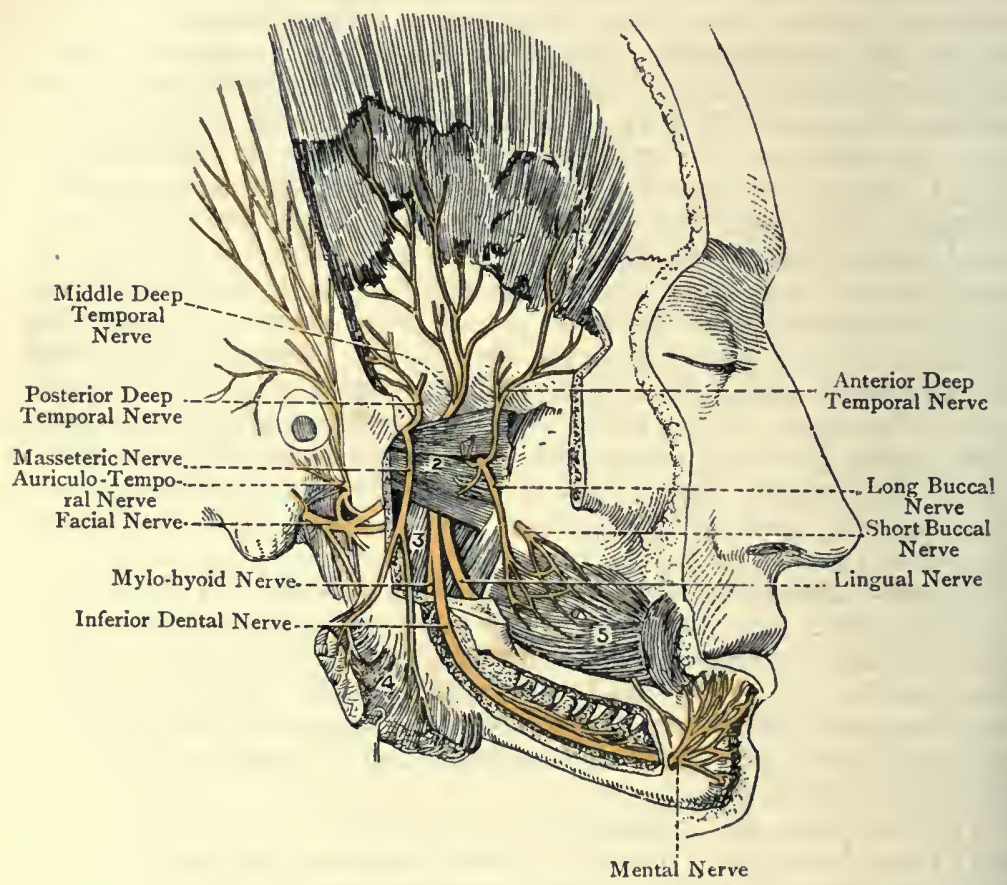

Fig. 493,-The Nerves of the Temporal and Pterygo-maxillary Regions (Hirschfeld AND LeVEillê).

I, Temporal Muscle ; 2, External Pterygoid ; 3, Internal Pterygoid ; 4, Masseter; 5, Buccinator.

of the buccinator, where it communicates with the short buccal branches of the facial nerve to form the buccal plexus. Thereafter its terminal branches are distributed to the skin over the buccinator muscle and the mucous membrane which lines it. The long buccal nerve furnishes two branches, namely, the external pterygoid nerve and the anterior: deep temporal nerve. The external pterygoid nerve is detached from it near its origin, and enters the external pterygoid muscle on its deep surface. The anterior deep temporal nerve arises from it after it has emerged between the two heads of the external 
pterygoid. These two branches conduct away all the motor fibres from the long buccal nerve, which thereafter is entirely sensory.

Posterior Division.-This division is larger than the anterior, and is almost entirely sensory, the only motor fibres which it contains being destined for the mylo-hyoid branch of the inferior dental nerve. Its branches are three in number, namely, (I) auriculotemporal, (2) inferior dental, and (3) lingual (gustatory).

Auriculo-temporal Nerve.-This nerve, which is sensory, arises by two roots, between which the middle meningeal artery ascends to the foramen spinosum. Thereafter the two roots join, and the nerve is directed backwards under cover of the external pterygoid muscle. Having passed close behind the temporo-mandibular joint to the interval between that joint and the auricle, it enters the upper part of the parotid gland. It then changes its course, and passes upwards through the upper part of the gland, after emerging from which it crosses the zygoma and ascends close behind the superficial temporal artery to end in its terminal temporal branches.

Branches of Communication.-(I) Each root of the auriculotemporal nerve receives a small branch from the otic ganglion. These branches contain fibres of the glosso-pharyngeal nerve, through means of (a) its tympanic branch (Jacobson's nerve), (b) the tympanic plexus, and $(c)$ the small superficial petrosal nerve, which latter is reinforced by a branch from the geniculate ganglion of the facial nerve. These glosso-pharyngeal fibres are destined for the parotid gland. (2) Two communicating branches pass to the temporo-facial division of the facial nerve in the parotid gland.

Branches of Distribution.-(I) Articular branches enter the temporo-mandibular joint through the back part of the capsule. (2) Glandular branches are distributed to the parotid gland, to which they conduct fibres of the glosso-pharyngeal nerve. (3) Meatal branches, upper and lower, enter the meatus auditorius externus between its cartilaginous and osseous parts, and supply the skin which lines it, the upper branch also giving twigs to the outer layer of the membrana tympani. (4) Anterior auricular branches are distributed to the skin of the tragus and the upper part of the outer surface of the auricle. The distribution of the meatal and auricular branches explains why pain, due to affections of the lower teeth, may be referred to the ear canal and auricle. (5) The temporal branches are terminal. They accompany the branches of the superficial temporal artery, and supply the skin of the temporal region as high as the vertex of the skull. They communicate with the temporal branches of the facial, and the temporal branch of tie temporo-malar from the superior maxillary nerve.

Inferior Dental Nerve.-This nerve, though chiefly sensory, contains motor fibres, which, however, leave it in its mylo-hyoid branch. It passes downwards, being at fitst under cover of the external pterygoid muscle. After escaping from beneath that muscle, it 
descends upon the spheno-mandibular ligament and internal pterygoid muscle to the inferior dental foramen, through which it passes into the inferior dental canal, after having parted with its mylo-hyoid branch. The lingual nerve is anterior and internal to it, and the inferior dental artery is posterior and external to it. Within the dental canal the nerve is accompanied by the inferior dental artery, and, having arrived at the level of the mental foramen, it terminates by dividing into two branches, mental and incisor.

Branches.-(I) The mylo-hyoid nerve is given off from the parent trunk just before it passes through the inferior dental foramen. It conducts away all the motor fibres from the parent trunk, and in company with the mylo-hyoid artery pierces the lower part of the spheno-mandibular ligament, and then passes downwards and forwards in the mylo-hyoid groove of the inferior maxilla. The nerve and artery are maintained in position within this groove by an expansion from the lower part of the spheno-mandibular ligament, which is attached to the lips of the groove. Thereafter the nerve passes forwards on the inferior surface of the mylo-hyoid muscle, under cover of the superficial part of the submaxillary gland. Having furnished twigs to the mylo-hyoid muscle, it terminates in the anterior belly of the digastric.

(2) The dental branches arise from the parent trunk, whilst it traverses the inferior dental canal. They communicate with each other to form a delicate plexus, from which branches are given off to the pulps of the lower molar and bicuspid teeth, as well as to the adjacent part of the gum. The pulp-branches correspond in number to the fangs of the teeth, and each enters through a minute opening on the extremity of the fang.

(3) The mental nerve is one of the two terminal branches. It emerges from the inferior dental canal through the mental foramen. For its distribution, see p. II77.

(4) The incisor nerve represents the termination of the inferior dental. It commences at the level of the mental foramen, and passes almost as far as the middle line. Its dental branches communicate in a plexiform manner, and supply the pulps of the lower canine and incisor teeth, as well as the adjacent portion of the gum.

Lingual Nerve.-This nerve is sensory. It descends under cover of the external pterygoid muscle, lying anterior and internal to the inferior dental nerve. Whilst under cover of that muscle it receives near its origin the chorda tympani nerve, which joins it from behind at an acute angle, in a direction downwards and forwards, after emerging from the tympanum through the iter chordæ anterius, or canal of Huguier. After issuing from beneath the external pterygoid muscle the lingual nerve passes downwards and forwards between the internal pterygoid muscle and the mandibular ramus, and over the mandibular fibres of the superior constrictor muscle. Below the level of the third lower molar tooth it lies immediately beneath the mucous membrane of the moutl, and is 
here accessible for operative purposes. Thereafter it crosses the stylo-glossus, and passes forwards over the hyo-glossus close to the side of the tongue. Upon the latter muscle it describes a slight curve with the convexity downwards. It then passes beneath the mylo-hyoid muscle, where it lies above the deep part of the submaxillary gland and Wharton's duct, and has the submaxillary ganglion suspended from it. Finally, having crossed Wharton's duct from above downwards, it continues its course as far as the tip of the tongue. As it passes along the side of the tongue the nerve is immediately beneath the mucous membrane.

Branches of Communication.-(I) Chorda tympani (sensory of facial); (2) two branches to the submaxillary ganglion; and (3) one

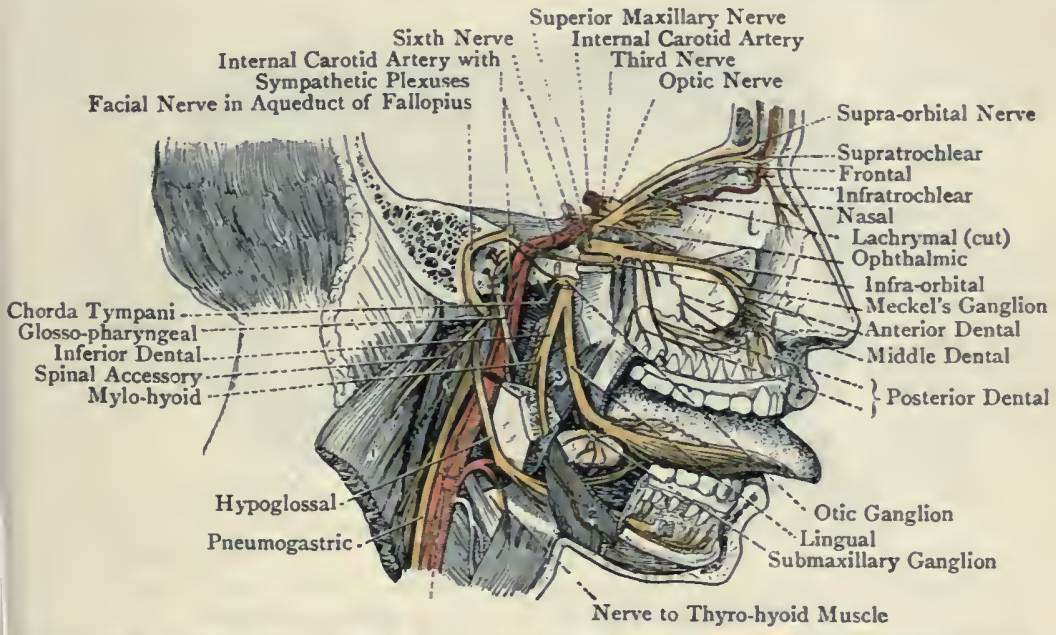

Fig. 494.-General View of the Fifth Cranial Nerve (Hirschfeld aNd LEVEILlÉ).

I, Sterno-cleido-mastoid ; 2, Mylo-hyoid; 3, Internal Pterygoid.

or two filaments to the hypoglossal nerve at the anterior border of the hyo-glossus muscle.

Branches of Distribution.-(I) Buccal, to the mucous membrane of the floor of the mouth and of the gums; (2) glandular, to the sublingual gland; and (3) lingual, to the mucous membrane of the sides and dorsum of the tongue over its anterior two-thirds. These lingual branches pierce the muscular tissue of the tongue, and are destined chiefly for the conical and fungiform papillæ.

Otic Ganglion.-The otic ganglion, or Arnold's ganglion, is a small oval body, of a pinkish colour, which is situated close to the foramen ovale, on the deep surface of the inferior maxillary nerve at the place of origin of the internal pterygoid nerve, with which it is closely connected. It has the middle meningeal artery 
behind it, and the cartilaginous part of the Eustachian tube on its inner side. The ganglion has three roots. One root (motorsensory) is derived from the internal pterygoid nerve, and conveys to the ganglion motor fibres, and probably also sensory fibres. A second root (sensory-motor) is represented by the small superficial petrosal nerve, which joins the back part of the ganglion. This root also conveys to the ganglion sensory and motor fibres. The sensory fibres come from the glosso-pharyngeal, through means of (I) the tympanic nerve or nerve of Jacobson, (2) the tympanic plexus, and (3) the small superficial petrosal. The motor fibres conveyed by this root come from the geniculate ganglion of the facial nerve, through means of the branch which that ganglion gives

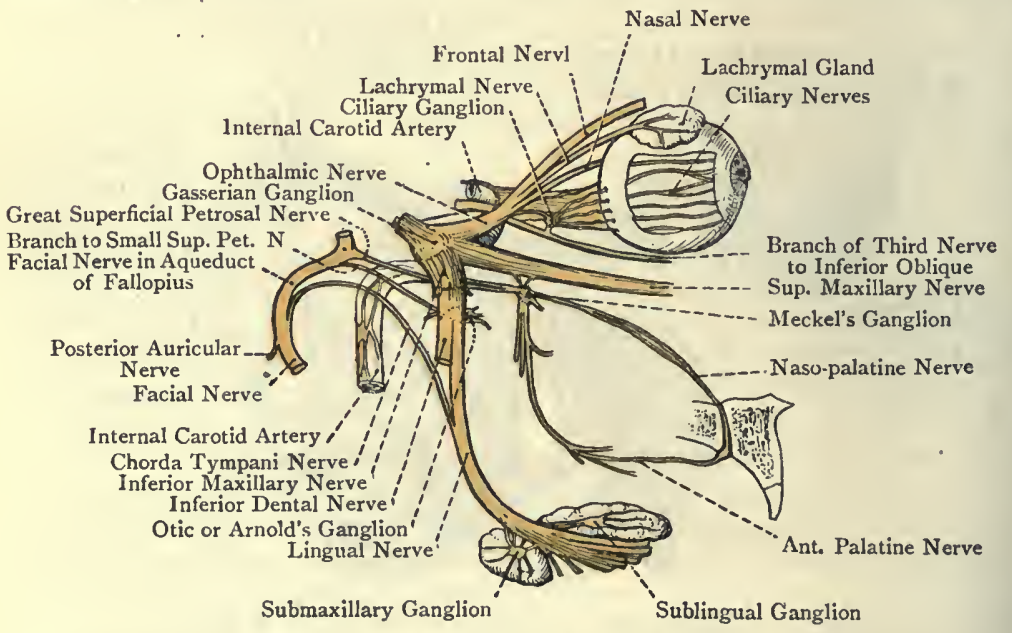

Fig. 495.-Scheme of the Fifth Cranial Nerve and its Ganglia (HiRsChFELd AND LEVEILlÉ).

to join the small superficial petrosal nerve. The third root (sympathetic) comes from the sympathetic plexus around the middle meningeal artery.

Branches-(I) Of Communication.-(a) Two branches pass to the auriculo-temporal nerve, one to each root. These convey to that nerve glosso-pharyngeal fibres which are destined for the parotid gland. (b) A brancl joins the chorda tympani. (c) A branch joins the Vidian nerve.

(2) Of Distribution.-Muscular branches are given to the tensor tympani and tensor palati.

Submaxillary Ganglion.-This ganglion is of small size, and is connected with the lingual nerve in the submaxillary region. It is situated upon the upper part of the hyo-glossus muscle, between the lingual nerve and the deep part of the submaxillary gland, and under cover of the posterior part of the mylo-hyoid muscle. It is 
suspended from the lingual nerve by two connecting branches, posterior and anterior, which stand apart from each other. The posterior connecting branch, sometimes broken up into two or three twigs, conveys to the ganglion sensory fibres from the chorda tympani (sensory portion of the facial) and lingual nerves, the latter being a branch of the inferior maxillary division of the fifth cranial nerve. The anterior connecting branch represents fibres passing from the ganglion to the lingual nerve.

The posterior branch consists of fibres which are derived from the chorda tympani of the facial, and from the lingual nerve. This posterior branch may exist as two twigs. The anterior branch

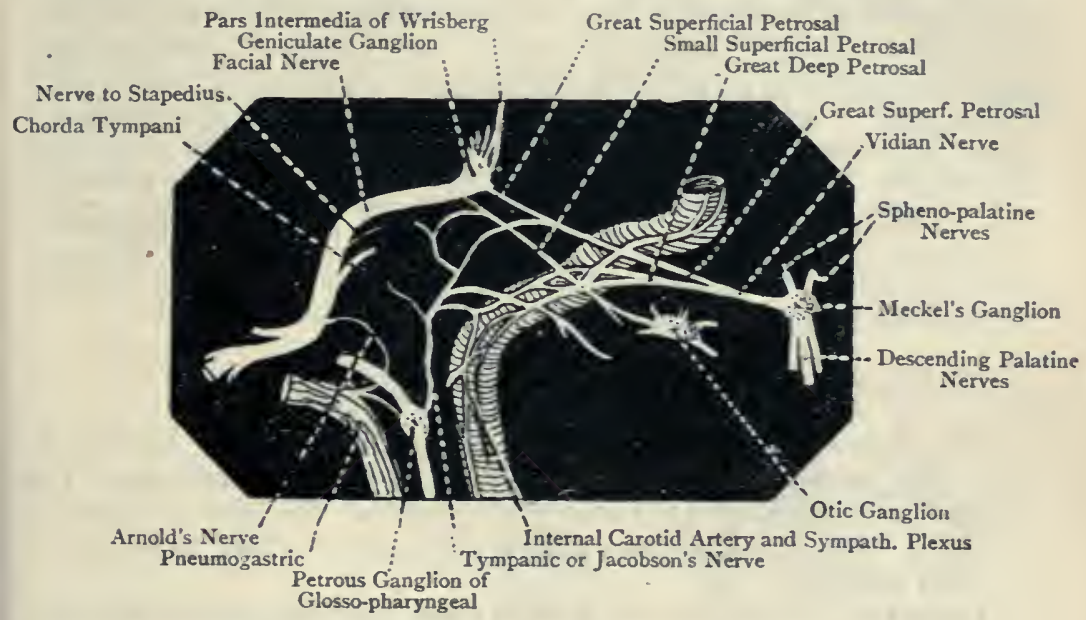

Fig. 496.-The facial Nerve in the AQueduct of Fallopius, eitc.

(HIRSCHFELD AND LEVEILLÉ).

may be regarded as an offset from the ganglion to join the lingual. The sympathetic root of the ganglion is derived from the plexus on the cervical portion of the facial artery.

\section{Roots of the Submaxillary Ganglion.}

\section{Secretory. \\ Sensory. \\ Sympathetic.}

From chorda tympani From lingual. From plexus on cervical portion of facial. of facial artery.

The submaxillary ganglion has three roots-secretory, sensory, and sympathetic. The secretory root comes from the chorda tympani nerve; the sensory root from the lingual nerve; and the sympathetic root from the plexus on the cervical portion of the facial artery. The secretory and sensory roots represent the posterior connecting branch of the ganglion.

Branches.-These proceed from the lower and anterior parts of the ganglion, and are as follow's: (I) glandular to the submaxillary 
gland; (2) branches to Wharton's duct; (3) buccal, to the mucous membrane of the floor of the mouth; and (4) an anterior connecting branch, which passes to the lingual nerve. The anterior connecting branch probably consists of both chorda tympani and lingual fibres, and it accompanies the lingual nerve to the tongue. It furnishes twigs to the sublingual gland, and a small ganglion associated with these twigs has been described under the name of the sublingual ganglion.

Summary of the Inferior Maxillary Nerve- 1 . Cutaneous Distribution.-It supplies ( $\mathrm{I}$ ) a portion of the dura mater, and the mucous membrane of the mastoid cells; (2) the skin over the greater part of the temporal region: (3) the skin of the external auditory meatus, and the outer surface of the membrana tympani ; (4) the skin of the tragus, and of the upper part of the outer surface of the auricle; (5) the skin over the body of the inferior maxilla, including the skin of the chin and lower lip, as well as the mucous membrane of the lower lip; (6) the mucous membrane of the floor of the mouth, and the lower gum ; and (7) the sides and dorsum of the tongue over its anterior two-thirds. 2. Articular Distribution.-It supplies the temporo-mandibular joint. 3. Dental Distribution.-It supplies the pulps of all the lower teeth of one side, and the mucous membrane of the outer surface of the lower gum of one side. 4. Glandular Distribution.-It gives branches to the parotid, submaxillary, and sublingual glands. 5. Muscular Distribution.It supplies (I) the muscles of mastication, namely, the masseter, temporal, external pterygoid and internal pterygoid; (2) the mylo-hyoid and anterior belly of the digastric ; and (3) the tensor palati and tensor tympani.

\section{The Temporo-mandibular Joint.}

The temporo-mandibular (temporo-maxillary) joint belongs to the class diarthrosis, and to the subdivision condylarthrosis. The articular surfaces are (I) the anterior part of the glenoid fossa, in front of the fissure of Glaser, and (2) the condyle of the mandible or inferior maxilla.

Ligaments.-These are as follows : the external lateral, capsular, internal lateral, stylo-mandibular, and interarticular fibro-cartilage.

The external lateral ligament is a short strong bundle of fibres, which is attached above to the tubercle and adjacent portions of the outer surface and lower border of the zygoma, and below to the condylar tubercle and the outer and back part of the neck of the mandible. Its fibres are directed obliquely downwards and backwards. This ligament forms the external portion of the capsular ligament.

The capsular ligament consists of scattered fibres which form a thin loose investment to the joint on its anterior, internal, and posterior aspects, being completed on the outer aspect by the external lateral ligament.

The internal lateral, or spheno-mandibular, ligament is a long flat band, which stands off from the joint, and therefore has no direct relation to it. It is somewhat triangular, being narrow above and broad below. Superiorly it is attached to the spinous process of the sphenoid bone, and inferiorly to the lingula and the inner margin of the inferior dental foramen of the mandible. Its fibres are directed downwards and slightly forwards. Superiorly it is covered by the external pterygoid muscle; subsequently the first 
part of the.internal maxillary artery rests upon it, and separates it from the neck of the mandible; and inferiorly the inferior dental vessels and nerve and the auriculo-temporal nerve intervene between it and the ramus of the mandible. At its lower attachment it is spread over the upper end of the mylo-hyoid groove, and is here pierced by the mylo-hyoid nerve and artery. This ligament is sometimes spoken of as the long internal lateral ligament, in contradistinction to the internal portion of the capsule, which is regarded as the short internal lateral ligament.

The spheno-mandibular ligament represents a portion of the filcrous investment of Meckel's cartilage.

The stylo-mandibular ligament is a stout process of the deep cervical fascia, which extends from the styloid process of the temporal bone near its tip to the angle and adjacent portion of the posterior border of the ramus of the mandible. Superiorly it gives origin to a few fibres of the styloglossus, and inferiorly it is implanted between the masseter and internal pterygoid muscles. It separates the submaxillary gland from the lower portion of the parotid gland.

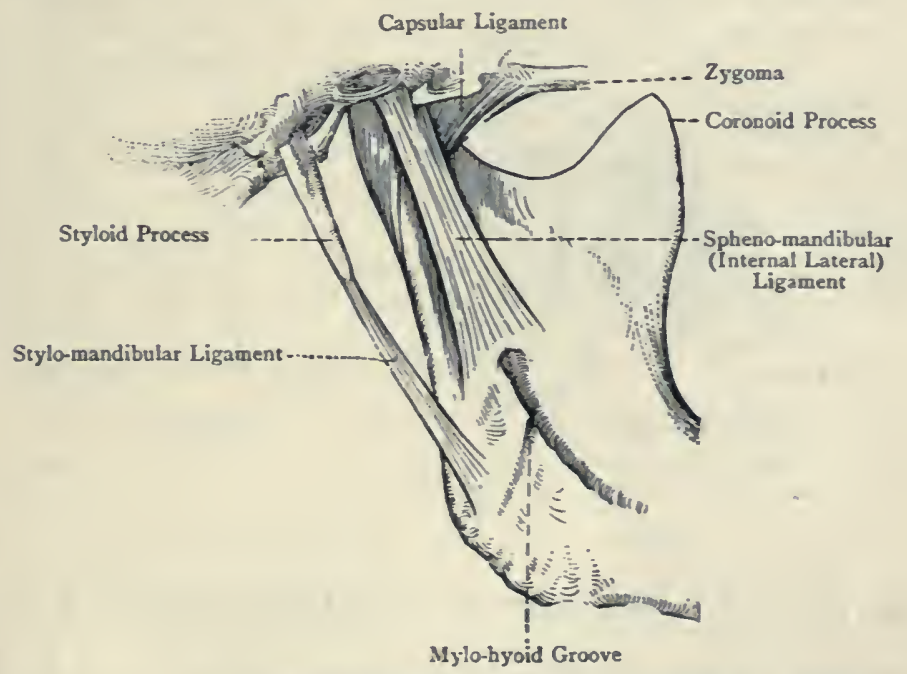

Fig. 497.-The Temporo-Mandibolar Jolit (INternal View).

The interarticular fibro-cartilage is an oval plate which is interposed between the two articular surfaces. It is thinnest at the centre, where it is occasionally perforated, and thickest posteriorly. Its superior surface is concavo-convex from before backwards, in adaptation to the convexity of the eminentia articularis and the concavity of the glenoid fossa. Its inferior surface is concave, and fits upon the condyle of the mandible. Its circumference is connected with the capsular ligament, and anteriorly it gives partial 
insertion to the external pterygoid muscle. It divides the joint into two compartments, upper and lower, which are usually distinct.

The synovial membranes are two in number, upper and lower, one being above and the other below the plate of fibro-cartilage. The upper synovial membrane is larger and looser than the lower, and, when the plate of fibro-cartilage is perforated at the centre, the two synovial membranes are continuous through the perforation.

Arterial Supply. - The superficial temporal artery chiefly.

Nerve-supply. - The auriculo-temporal nerve and offsets from the masseteric nerve.

Movements.-These are as follows : (1) depression, (2) elevation, (3) protraction, (4) retraction, and (5) lateral movements. There being two divisions of the joint, upper and lower, different movements occur in each. The movement in the upper compartment is of a gliding character, whilst in the lower comparlment it is of a ginglymoid or hinge character. When the mandible is depressed, as in opening the mouth, the condyle and the plate of fibrocartilage move forwards on to the eminentia articularis. In cases of overdepression, as in violent yawning, or forcing too large a body into the moutl, the condyle is apt to slip over the eminentia articularis into the zygomatic fossa, and, when this takes place. dislocation of the mandible is the result.

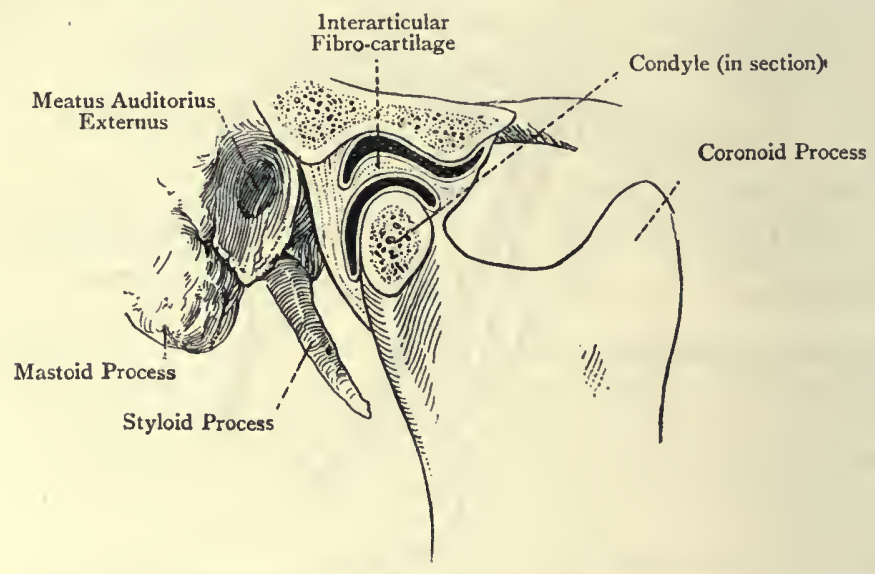

Fig. 498.-The Temporo-Mandibular Joint Opened (External View).

The movement of forward gliding on the part of the condyle and plate of fibro-cartilage during depression takes place in the upper compartment of the joint. Another movement, however, of a ginglymoid or linge character is taking place in the lower compartment of the joint, namely, between the condyle and the interarticular plate. This consists in the condyle rotating on the under surface of the plate round a transverse axis.

When the mandible is elevated, as in shutting the mouth, the clianges which occur in both compartments of the joint are the reverse of those just described as taking place in depression. The condyle and fibro-cartilage glide backwards in to the glenoid cavity, and at the same time the condyle rotates back to its previous position. In protraction, as when the lower incisors are protruder beyond the upper, and in retraction, the movement mainly takes place in the upper compartment of the joint, ancl consists in the condyle and plate of fibrocartilage gliding forwards and backwards. When these movements are per- 
formed alternately on each side the lateral oblique movements, as in grinding or chewing, take place, and the condyle and fibro-cartilage of one side move forwards and backwards, whilst the other condyle and fibro-cartilage move in the opposite direction. During these movements oblique rotation is taking place in the lower compartment of the joint.

Muscles Concerned in the Movements. - Depression is effected by the platysma myoides, mylo-hyoid, anterior belly of the digastric, and geniohyoid muscles; and elevation by the anterior fibres of the temporal, masseter, and internal pterygoid muscles. Protraction is produced by the external pterygoid, the superficial fibres of the masseter, and slightly by the internal pterygoid; and retraction by the posterior fibres of the temporal, and the deep fibres of the masseter. The grinding movement is effected by the external pterygoid muscles acting alternately.

\section{The Superior Maxillary Nerve.}

The superior maxillary nerve is the second division of the fifth cranial nerve, and in size is intermediate between the inferior maxillary and the ophthalmic. It is entirely sensory, and arises from the Gasserian ganglion. Its course is forwards to the foramen rotundum, by which it leaves the cranial cavity. Thereafter it enters the posterior part of the spheno-maxillary fossa, and then crosses the upper part of that fossa to the spheno-maxillary fissure, through which it passes on to the floor of the orbit. It now takes on the name of the infra-orbital nerve, and traverses the infra-orbital groove, and then the infra-orbital canal, on the floor of the orbit. Finally, it emerges from this canal through the infraorbital foramen, under cover of the levator labii superioris, and ends in its terminal branches. The course of the superior maxillary nerve (and its continuation under the name of the infra-orbital nerve) is almost directly forwards, there being a slight inclination outwards.

Branches.-These are as follows :

\section{Intracranial.}

Recurrent meningeal.

In Spheno-maxillary Fossa.

Orbital or temporo-malar.

Spheno-palatine.

Posterior superior dental.

\section{In Infra-orbital Canal.}

Middle superior dental.

Anterior superior dental.

Facial.

Inferior palpebral.

Lateral nasal.

Superior labial.

The recurrent meningeal branch supplies the dura mater of the middle cranial fossa.

The orbital or temporo-malar nerve is the first branch of the superior maxillary in the spheno-maxillary fossa. It arises from its upper surface, and enters the orbit through the spheno-maxillary fissure. For its further course, see p. IoS2.

The spheno-palatine nerves are two in number. They arise from the lower aspect of the parent trunk, and, after a very short descending course, they end for the most part in Meckel's ganglion, to which they convey sensory fibres, and of which they are the 
sensory roots. Many of their fibres, however, pass clear of the grey matter of the ganglion, and are prolonged into the nasal and descending palatine branches of the ganglion.

The posterior superior dental nerve arises from the superior maxillary as it is about to pass through the spheno-maxillary fissure, and soon divides into two branches, which sometimes arise separately. They descend in grooves on the zygomatic surface of the superior maxilla, in company with branches of the posterior dental artery, and give off branches to the gum and contiguous part of the mucous membrane of the cheek. Thereafter they traverse the posterior dental canals, and within the substance of the bone they communicate with each other and with the middle superior dental nerve to form a delicate plexus, from which branches are given off to the pulps of the three upper molar teeth. Slender filaments are also furnished to the mucous membrane lining the antrum of Highmore.

The middle superior dental nerve arises from the main trunk as it lies in the infra-orbital groove near the spheno-maxillary fissure. It descends, in company with the middle dental artery, in the middle dental canal of the superior maxilla. Its branches communicate with each other, and with the posterior superior and anterior superior dental nerves within the substance of the bone, to form a delicate plexus, from which branches are given to the pulps of the upper two bicuspid teeth and to the gum. This nerve also furnishes delicate filaments to the mucous membrane lining the antrum of Highmore. Two enlargements are described in connection with the middle superior dental nerve. One is situated at its communication with the posterior superior dental nerve, and is known as the ganglion of Valentin ; and the other at its communication with the anterior superior dental, this one being known as the ganglion of Bochdalek.

The anterior superior dental nerve arises from the infra-orbital nerve near the front part of the infra-orbital canal, and descends, in company with the anterior dental artery, in the anterior dental canal. Its branches communicate with each other and with the middle superior dental nerve to form a delicate plexus, from which branches are given to the pulps of the upper canine and incisor teeth. This nerve also furnishes delicate filaments to the mucous membrane lining the antrum of Highmore, and a nasal branch, which enters the nasal fossa and supplies the mucous membrane of the anterior part of the inferior meatus and adjacent portion of the inferior concha.

The facial branches of the infra-orbital nerve, namely, inferior palpebral, lateral nasal, and superior labial, have been already described, see p. II 77 .

It is to be noted that there are three superior dental nerves, right and left, the posterior being a branch of the superior maxillary, and the middle and anterior being branches of the infra-orbital: whilst there is only one inferior dental nerve, which is a branch of the inferior maxillary. 
Spheno-palatine Ganglion.-This is also called Meckel's ganglion. It is a small triangular, somewhat reddish body, situated on the upper part of the spheno-maxillary fossa, close to the sphenopalatine foramen, which leads through the inner wall of the fossa to the superior meatus of the nasal fossa. It is suspended from the superior maxillary nerve by some of the fibres of the two spheno-palatine branches of that nerve, many fibres of these two branches, as stated, passing clear of the grey matter of the ganglion into its nasal and descending palatine branches. The fibres conveyed to the grey matter of the ganglion by the two sphenopalatine nerves represent its sensory root or roots.

The ganglion has sensory and sympathetic roots, but no motor root. The sensory roots are derived from two sources-superior

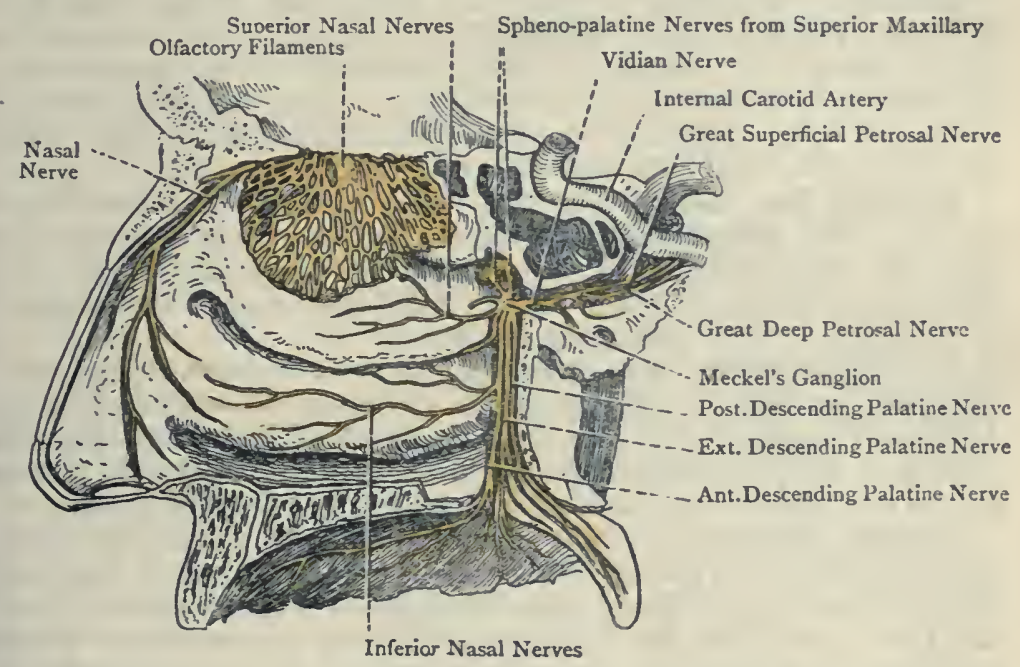

Fig. 499.-Nerves of the Nasal Fossa, and the Spheno-palatine or Meckel's Ganglion, with its Branches (INTERnal View) (Hirsch. FELD AND LEVEILI.é).

maxillary and facial. The superior maxillary sensory root-fibres form two bundles, and represent portions of the two spheno-palatine nerves, by which the ganglion is suspended from the trunk of the superior maxillary nerve in the spheno-maxillary fossa. The facial sensory root-fibres are represented by the great superficial petrosal nerve, which is a branch of the geniculate ganglion of the facial nerve. The sympathetic root is formed by the great deep petrosal nerve from the carotid plexus of the sympathetic.

The facial sensory root (great superficial petrosal) and the sympathetic root (great deep petrosal) join to form the Vidian nerve in the foramen lacerum medium. After traversing the Vidian canal from behind forwards, the Vidian nerve enters the spheno-maxillary fossa and joins the back part of Meckel's ganglion. In the Vidian 
canal the Vidian nerve gives off one or two nasal branches, which pass through the floor of the canal, and are distributed to the mucous membrane of the posterior part of the roof of the nasal fossa and adjacent part of the septum.

Branches of the Spheno-palatine Ganglion.-These are arranged in four sets, and are as follows :

Ascending.

Orbital.
Posterior.

Pharyngeal or Vidian.
Internal.

Superior nasal. Naso-palatine.
Descending.

Great or anterior palatine.

Small or posterior palatine.

External palatine.

Ascending Branches. - These are called the orbital branches. They are two or three in number, and enter the orbit through the spheno-maxillary fissure, to be distributed to the periosteum. They have been described by Luschka as sending filaments through the inner wall of the orbit to supply the mucous membrane of the posterior ethmoidal cells and the sphenoidal air-sinus.

Posterior Branches.-These are the pharyngeal and the Vidian nerves. The pharyngeal nerve passes backwards in the pterygopalatine canal, along with the pterygo-palatine artery, and is distributed to the mucous membrane of the upper part of the pharynx in the region of the Eustachian orifice.

The Vidian nerve (just described), if it is to be regarded as a posterior branch of the ganglion, establishes a communication between it and (I) the geniculate ganglion of the facial, through means of the great superficial petrosal, and (2) the carotid. plexus of the sympathetic, through means of the great deep petrosal.

Internal Branches.-These are the superior nasal and nasopalatine nerves. The superior nasal nerves are of small size, and derive some of their fibres from the spheno-palatine nerves. They are about six in number, and, springing from the inner part of the ganglion, they enter the superior meatus of the nose through the spheno-palatine foramen. They supply the mucous membrane of the superior and middle conchæ, the superior meatus, the posterior ethmoidal cells, the middle meatus, and the upper and back part of the septum.

The naso-palatine nerve (nerve of Cotunnius) enters the nasal fossa along with the superior nasal nerves. It crosses the roof of the fossa, and then passes downwards and forwards upon the septum, lying in the groove on the lateral surface of the vomer. Having arrived at the region of the incisor crest, the left naso-palatine nerve descends through the anterior foramen or canal of Scarpa, and the right descends through the posterior. Having reached the anterior palatine fossa in this manner, the two nerves communicate in a plexiform manner, and delicate filaments are furnished to the mucous membrane of the hard palate behind the incisor teeth. In this situation communications are established with the great or anterior descending palatine nerve of each side. As the naso- 
palatine nerve traverses the groove on the lateral surface of the vomer, it furnishes twigs to the mucous membrane of the nasal septum, and in this part of its course it is accompanied by the nasopalatine artery (artery of the septum); but this artery does not accompany it through the foramen or canal of Scarpa.

Descending Branches.-These are the great or anterior, the small or posterior, and the external palatine nerves. They arise from the lower part of the ganglion, usually by a common trunk, and they derive some of their fibres from the spheno-palatine nerves.

The great or anterior palatine nerve descends in the posterior palatine canal, and escapes from it through the posterior palatine foramen. Thereafter it divides into branches which pass forwards and inwards, occupying grooves on the hard palate, to the mucous membrane and glands of which, as well as to the mucous membrane of the upper gum on its inner aspect, they are distributed. In the region of the anterior palatine fossa this nerve communicates with the naso-palatine nerve. As it descends in the posterior palatine canal, it furnishes two inferior nasal nerves, which enter the nasal fossa, and are distributed to the mucous membrane over the inferior concha, except anteriorly, and to that of the adjacent portion of the inferior meatus. The small or posterior palatine nerve descends in the posterior accessory palatine canal, after emerging from which it is distributed to the mucous membrane of the soft palate and tonsil. It may furnish branches to the levator palati and azygos uvulæ muscles, the fibres of these branches being derived from the geniculate ganglion of the facial through means of the great superficial petrosal, which contributes to the formation of the Vidian nerve, and conveys motor fibres to Meckel's ganglion. The two muscles in question, however, are most probably supplied by the bulbar part of the spinal accessory nerve through the pharyngeal plexus. The external palatine nerve descends through the external accessory palatine canal, after emerging from which it is distributed to the mucous membrane of the outer portion of the soft palate and tonsil.

Summary of the Superior Maxillary Nerve and Meckel's Ganglion1. Cutaneous Distribution. - (I) The skin of the anterior part of the temporal region, and over the malar bone; (2) the skin from the lower eyelid down to the upper lip, including the skin of the side of the nose; (3) the mucous membrane of the naso-pharynx in the vicinity of the Eustachian orifice: (4) the mucous membrane of the nasal fossa; $(5)$ the mucous membrane of the antrum of Highmore; (6) the mucous membrane of the soft palate and tonsil ; and ( 7 ) the mucous membrane of the hard palate, with its glands, and that of the inner surface of the upper gum. 2. Dental Distribution.The pulps of all the upper teeth of one side, as well as the mucous membrane of the outer surface of the upper gum.

In addition to the foregoing, the superior maxillary nerve supplies the periosteum of the orbit, and the dura mater of the middle cranial fossa.

\section{Deep Dissection of the Neck.}

Stylo-pharyngeus-Origin.-The base of the styloid process of the temporal bone on its inner aspect. 
Insertion--(I) The superior and posterior borders of the ala of the thyroid cartilage, and (2) the lateral wall of the pharynx, its fibres blending with those of the palato-pharyngeus and middle constrictor muscles.

Nerve-supply.-The glosso-pharyngeal nerve.

The muscle is directed downwards and inwards.

Action.-To elevate the pharynx and larynx.

The muscle passes between the superior and middle constrictor muscles. The glosso-pharyngeal nerve lies at first along its lower border, and then crosses over it on its way to the posterior part of the tongue.

Internal Carotid Artery.-The internal carotid artery is one of the terminal branches of the common carotid. It commences on a level with the upper border of the thyroid cartilage, and passes vertically upwards to the under surface of the petrous part of the temporal bone. Here it enters the carotid canal within the pars petrosa, and, after traversing this canal, it enters the cranial cavity. The vessel is divided into three parts-extracranial or cervical, petrous, and intracranial or cavernous.

Extracranial or Cervical Part.-This part of the vessel extends from the level of the upper border of the thyroid cartilage to the foramen caroticum on the inferior surface of the petrous part of the temporal bone. Its course is vertically upwards, and at first it lies in the carotid triangle, where it is placed external to, and behind, the external carotid artery.

Relations-Anterior.-This part of the vessel is comparatively superficial, being covered by the integument, platysma myoides, and sterno-cleido-mastoid, the latter overlapping it. After leaving the carotid triangle the artery becomes deeply placed, and passes beneath the posterior belly of the digastric and stylo-hyoid muscles, parotid gland, styloid process, and stylo-pharyngeus muscle. In this part of its course the vessel is crossed by the hypoglossal, glosso-pharyngeal, and pharyngeal branch of the pneumogastric, nerves, and by the occipital and posterior auricular arteries. The relation of the artery to the external carotid here undergoes a change. The level where the change takes place is as the internal carotid passes beneath the styloid process of the temporal bone, and the change consists in the external carotid taking up a position directly anterior to the internal carotid. The two arteries are, however, separated from each other by the following structures: (I) the styloid process; (2) the stylo-pharyngeus muscle; (3) the glosso-pharyngeal nerve; and (4) the pharyngeal branch of the pneumogastric nerve.

Posterior.-The rectus capitis anticus major, the superior cervical ganglion of the sympathetic, and the superior laryngeal branch of the pneumogastric nerve.

External.-The internal jugular vein and pneumogastric nerve, the nerve being on a plane posterior to both artery and vein. These three structures are still surrounded by a sheath which is an upward extension of the carotid sheath, previously described. Close to the 
base of the skull the glosso-pharyngeal, spinal accessory, and hypoglossal nerves lie between the internal carotid artery and the internal jugular vein. The glosso-pharyngeal nerve soon passes forwards superficial to the artery, the spinal accessory nerve passes backwards superficial to the vein, and the hypoglossal nerve passes forwards superficial to the artery at the lower border of the posterior belly of the digastric muscle.

Internal.-The ascending pharyngeal artery, the lateral wall of the pharynx, the tonsil, and the superior laryngeal nerve.

Development.-The internal carotid artery is developed from the third arterial arch and the part of the dorsal aorta above it.

\section{The Glosso-pharyngeal Nerve.}

The glosso-pharyngeal or ninth cranial nerve is a mixed nerve, consisting of both afferent or sensory and efferent or motor fibres. It emerges from the cranial cavity through the middle compartment of the jugular foramen, along with the pneumogastric and spinal accessory nerves, but enclosed in a separate sheath of the dura mater, and lying on the outer side of, and anterior to, these two nerves. In passing through the jugular foramen, the nerve presents two ganglia, the upper one, which is small and situated in the upper part of the foramen, being called the jugular ganglion; and the lower one, which is rather larger, being called the petrous ganglion. The latter is about $\frac{1}{4}$ inch in length, and lies in the lower part of the furamen, where it occupies a groove on the petrous portion of the temporal bone. After emerging from the jugular foramen, the nerve at first descends between the internal carotid artery and internal jugular vein, being external to the line of the pneumogastric nerve. It then passes downwards and forwards in front of the internal carotid artery, and under cover of the styloid process, stylopharyngeus muscle, and external carotid artery. It next curves round the lower border of the stylo-pharyngeus, and crosses its superficial surface in a forward direction. Finally, it passes beneath the hyo-glossus muscle, and so reaches the posterior third of the tongue, where it divides into its terminal branches.

The jugular ganglion, as stated, is very small, and involves only a few of the fibres of the nerve, the other fibres passing clear of, but close to, the ganglion, and being continued into the nerve beyond it. This ganglion neither gives nor receives any branches, and is sometimes absent.

The petrous ganglion, unlike the jugular, involves all the fibres of the nerve, and it furnishes connecting branches and the tympanic nerve.

Branches of the Glosso-pharyngeal Nerve- $I$. From the Petrous Ganglion-(a) Branches of Communication.-These are three in number, as follows: one to the superior cervical ganglion of the sympathetic; one to the auricular branch of the ganglion of the root of the pneumogastric; and one to the ganglion of the root of the pneumogastric. 
(b) Branch of Distribution.-This is called the tympanic nerve, or nerve of Jacobson. After leaving the petrous ganglion, it ascends, in company with the tympanic branch of the ascending pharyngeal artery, through the tympanic canaliculus. In this way it enters the tympanic cavity at the junction of the inner wall and floor, and ascends upon the inner wall. Being joined by the caroticotympanic branch of the carotid plexus of the sympathetic, it breaks up into branches which occupy grooves upon the promontory, and form the tympanic plexus. From this plexus the tympanic nerve emerges under the name of the small superficial petrosal nerve, which leaves the tympanic cavity and traverses a minute canal in the petrous bone, passing in its course beneath the upper portion of the canal which lodges the tensor tympani muscle. Whilst in this canal, it is joined by a communicating branch from the geniculate ganglion of the facial nerve. It emerges from the canal through the accessory hiatus on the superior surface of the petrous bone, which is external to the hiatus Fallopii, and then passes forwards and inwards beneath the dura mater. Thereafter it passes through the canaliculus innominatus, when present, or through the fissure between the pars petrosa and great wing of the sphenoid, or sometimes through the foramen ovale, into the zygomatic fossa, where it joins the otic ganglion, to which it conducts glosso-pharyngeal and facial fibres.

For a description of the tympanic plexus, see Index.

2. From the Trunk of the Nerve.-(I) A communicating branch sometimes passes from the nerve a little below the petrous ganglion to join the digastric branch of the facial nerve. (2) A carotid branch passes to the sympathetic plexus on the internal carotid artery. (3) Pharyngeal Branches.-These are about four in number. Three of them unite, over the middle constrictor of the pharynx, with the subdivisions of the pharyngeal branch of the ganglion of the trunk of the pneumogastric, and with branches of the superior cervical ganglion of the sympathetic, to form the pharyngeal plexus. The other pharyngeal branch pierces the superior constrictor, and is distributed to the mucous membrane of the upper part of the pharynx. (4) A muscular branch to the stylo-pharyngeus muscle, some of the fibres of which pierce the muscle to supply the mucous membrane of the pharynx. (5) Tonsillar branches are given off near the tongue, which are distributed to the tonsil and the anterior and posterior pillars of the fauces. (6) Lingual or Glossal Branches.-Under cover of the hyo-glossus muscle, the terminal part of the nerve divides into two branches, dorsal and lateral. The dorsal branch passes to the dorsum of the tongue, and is distributed to the circumvallate papillæ and the mucous membrane over the posterior third, extending as far back as the front of the epiglottis. The lateral branch is distributed to the mucous membrane of the side of the tongue over about its posterior half. 
Auriculo-temporal Nerve

Facial Nerve

Posterior Auricular Nerve

Glosso-pharyngeal Nirve
Spin il Accessory Nerve

Great Occipital Nerve

Hypoglossal Nerve

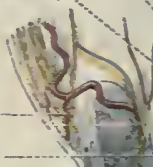

Sup. Cervical Symp. Ganglion

External Carotid Artery

Internal Carotid Artery

Sympathetic Cord

m. Car. Art. and Symp. Plexus

Phrenic Nerve

Right Ansa Vieusseni
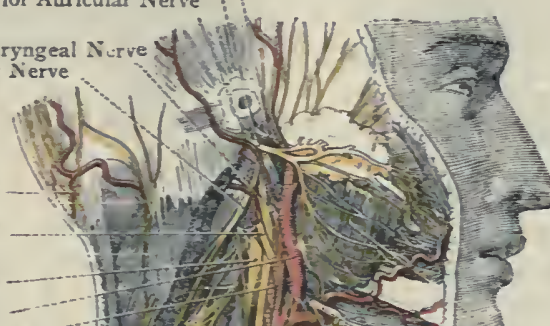
Summary of the Glosso-pharyngeal Nerve-r. Cutaneous Distribution.It supplies $(a)$ the mucous membrane of the tympanum, the posterior third of the tongue, the pillars of the fauces, and the pharynx; and $(b)$ the tonsil. 2. Muscular Distribution.-It supplies the stylo-pharyngeus. 3. Glandular Distribution.-Through means of (I) its tympanic, subsequently small superficial petrosal, branch, (2) the otic ganglion, and (3) the auriculo-temporal nerve, it furnishes secretory branches to the parotid gland.

\section{The Pneumogastric Nerve.}

The pneumogastric (vagus) or tenth cranial nerve is a mixed nerve, consisting of both afferent and efferent fibres. It emerges from the cranial cavity through the middle compartment of the jugular foramen, being enclosed in the same sheath of dura mater as the spinal accessory, and, with that nerve, lying on the inner side of, and slightly posterior to, the glosso-pharyngeal. In the foramen it presents a small enlargement, called the ganglion of the root. After emerging from the foramen, it is joined by the accessory portion of the spinal accessory nerve, and it here presents another enlargement, called the ganglion of the trunk. This ganglion is larger and longer than that of the root, measuring fully $\frac{3}{4}$ inch in length, and presenting a somewhat plexiform appearance. In this part of its course the nerve lies between the internal carotid artery and the internal jugular vein, the glosso-pharyngeal nerve being in front of it, and the spinal accessory behind it. The hypoglossal nerve lies at first deeply behind it, but subsequently turns round the outer side of the ganglion of the trunk. The pneumogastric then descends within the carotid sheath, lying at first between the internal carotid artery and the internal jugular vein, and then between the common carotid artery and the internal jugular vein, being on a plane posterior to both vessels. Within the sheath the nerve occupies a special compartment, situated in the back part of the septum which separates the artery from the vein. Beyond the root of the neck the right and left nerves present important differences in their course and relations (see p. 964).

Branches-A. Ganglion of the Root-I. Branches of Communication.-(a) It receives two filaments from the bulbar or accessory portion of the spinal accessory nerve. (b) It communicates by a small twig with the petrous ganglion of the glosso-pharyngeal nerve. (c) It communicates with the facial, and with the posterior auricular branch of the facial, through means of its auricular branch of distribution (Arnold's nerve). (d) It receives a twig from the superior cervical ganglion of the sympathetic.

2. Branches of Distribution.-(a) The meningeal branch takes a recurrent course, and, after entering the cranial cavity, supplies the dura mater which lines the cerebellar fossa of the occipital bone. (b) The auricular branch, or nerve of Arnold, is of small size, and is soon reinforced by a twig from the petrous ganglion of the glossopharyngeal. It enters the auricular canaliculus through an aperture on the posterior part of the outer wall of the jugular fossa. In this 
canaliculus it traverses the petrous portion of the temporal bone, crossing in its course the inner aspect of the descending portion of the aqueduct of Fallopius a little above the stylo-mastoid foramen, where it forms its first communication with the facial nerve. It then emerges from the pars petrosa through the auricular or tympano-mastoid fissure, between the mastoid process and the tympanic plate, and thereafter divides into two branches. One of these joins the posterior auricular branch of the facial nerve, and the other is distributed to $(a)$ the skin of the inner surface of the auricle, and (b) the skin of the lower and back part of the external auditory meatus.

B. Ganglion of the Trunk-I. Branches of Communication.(a) The most important branch of communication is the bulbar or accessory portion of the spinal accessory nerve, which passes over the surface of the ganglion in intimate contact with it. Most of the bulbar fibres are continued into the pharyngeal and superior laryngeal nerves, but some of them descend in the main trunk of the pneumogastric, and pass into its cardiac and inferior laryngeal branches. The bulbar fibres are to be regarded as of two kinds, namely, motor, for the muscles of the soft palate, pharynx, and larynx, and cardiac inhibitory. (b) Branches pass between the ganglion of the trunk and the hypoglossal nerve. (c) A communicating branch is received from the superior cervical ganglion of the sympathetic.

2. Branches of Distribution.-These are two in number, namely, pharyngeal and superior laryngeal.

The pharyngeal nerve arises from the upper part of the ganglion of the trunk, its fibres being chiefly derived from the bulbar or accessory portion of the spinal accessory nerve. It passes forwards and downwards over the internal carotid artery, and divides into branches opposite the middle constrictor muscle, which join the pharyngeal branches of the glosso-pharyngeal and superior cervical ganglion of the sympathetic to form the pharyngeal plexus.

The pharyngeal plexus is situated upon the middle constrictor muscle of the pharynx. It is formed by (I) the pharyngeal branch of the ganglion of the trunk of the pneumogastric ; $(2)$ the pharyngeal branches of the glosso-pharyngeal; and (3) the pharyngeal branches of the superior cervical ganglion of the sympathetic.

Branches.-These are distributed to (I) the constrictor muscles of the pharynx; (2) the mucous membrane of the pharynx; and (3) the palato-glossus, palato-pharyngeus, levator palati, and azygos uvulæ muscles. Branches of communication pass between the plexus and the superior laryngeal and external laryngeal nerves, and another branch, known as the lingual branch of the vagus, passes to the hypoglossal nerve.

Superior Laryngeal Nerve.-This nerve, which is principally sensory, arises from the ganglion of the trunk of the pneumogastric near its centre, and contains fibres of the bulbar or accessory portion of the spinal accessory nerve. It passes downwards and 
forwards behind the internal carotid artery, having previously received communicating branches from the superior cervical ganglion of the sympathetic and from the pharyngeal plexus. As it passes behind the internal carotid artery, it divides into two branches, internal and external. The internal laryngeal nerve, which is sensory, passes forwards, in company with the superior laryngeal branch of the superior thyroid artery, to the thyro-hyoid membrane, which it pierces under cover of the posterior border of the thyro-hyoid muscle. Having reached the interior of the larynx, it divides into branches which supply the mucous membrane of the epiglottis, with its folds, the mucous membrane of the larynx as low as the true vocal cords, and that covering the lateral and posterior portions of the cricoid cartilage. One branch descends over the inner surface of the ala of the thyroid cartilage, and joins a branch of the inferior or recurrent laryngeal. The external laryngeal nerve, of small size, descends beneath the sterno-thyroid muscle to the crico-thyroid muscle, which it supplies. It furnishes some twigs to the inferior constrictor, and a filament which joins the superior cardiac branch of the sympathetic. It receives a communicating branch from the superior cervical ganglion of the sympathetic, and one or two branches from the pharyngeal plexus.

Inferior or Recurrent Laryngeal Nerve.-This nerve is mainly motor, and is the principal motor nerve of the intrinsic muscles of the larynx. It contains fibres of the bulbar or accessory portion of the spinal accessory nerve. The right nerve arises from the pneumogastric at the root of the neck in front of the first part of the subclavian artery, round the lower border of which it bends, and then ascends behind it. Thereafter it passes upwards and inwards behind the carotid sheath and inferior thyroid artery to the groove between the trachea and the œsophagus. The left nerve arises in the thorax from the pneumogastric in front of the arch of the aorta, on a level with its lower border. It passes backwards within the arch at the place of attachment of the ligamentum ductûs arteriosi, and then it turns upwards behind the arch. Having reached the groove between the trachea and the œsophagus, it ascends therein to the neck. The right and left nerves pass upwards in the groove between the trachea and the œsophagus, on each side, lying behind the corresponding inferior thyroid artery. At the level of the cricoid cartilage each nerve passes beneath the lower border of the inferior constrictor muscle, and enters the larynx behind the crico-thyroid joint.

Extralaryngeal Branches.-(I) Communicating twigs pass between the recurrent laryngeal nerve and the inferior cervical ganglion of the sympathetic ; (2) cardiac branches, which contain bulbar fibres from the spinal accessory, are furnished to the deep cardiac plexus ; (3) tracheal and osophagcal branches are supplied to these tubes; and (4) muscular offsets pass to the lower part of the inferior constrictor.

Intralaryngeal Branches.-These are chiefly muscular, and i apply 
the intrinsic muscles of the larynx, except the crico-thyroid, which is supplied by the external laryngeal nerve. Sensory branches, however, are given to the laryngeal mucous membrane below the true vocal cords, except over the lateral and posterior portions of the cricoid cartilage. Within the larynx the nerve communicates with a branch of the internal laryngeal nerve, under cover of the lower part of the ala of the thyroid cartilage.

For the explanation of the recurrent course of each inferior laryngeal nerve, see p. 966.

Cervical Cardiac Branches.-These are subject to variation as regards numbers. As a rule, there are two, upper and lower, both of which contain bulbar fibres from the spinal accessory. Those of the right side pass behind the first part of the subclavian artery, and end in the deep cardiac plexus. On the left side the upper nerve passes to the deep cardiac plexus, but the lower nerve terminates in the superficial cardiac plexus.

Summary of the Pneumogastric Nerve in the Neck-I. Motor Distribution. -The nerve supplies ( $I$ ) the muscles of the soft palate, except the tensor palati; (2) the constrictor muscles of the pharynx; (3) the intrinsic muscles of the larynx; and (4) the muscular tissue of the cesophagus and trachea. 2. Sensory Distribution.-It supplies (I) the pharynx, csophagus, and trachea; (2) a limited portion of the dura mater; and (3) a small portion of the skin on the inner surface of the auricle, as well as of the lower and back part of the external auditory meatus. 3. Communications.-Each pneumogastric nerve forms connections with the facial, glosso-pharyngeal, spinal accessory, hypoglossal, and sympathetic nerves. It is also connected with the loop between the first and second cervical spinal nerves. Its most important connection is that which is established with the bulbar or accessory portion of the spinal accessory nerve. The bulbar fibres of the nerve are probably chiefly concerned in the innervation of the muscles of the soft palate (except the tensor palati), pharynx, and larynx, but also to a certain extent in the innervation of the heart.

\section{Spinal Accessory Nerve.}

The spinal accessory or eleventh cranial nerve consists of two parts, bulbar or accessory, and spinal. In the jugular foramen these two parts either unite to form one trunk, or freely intermingle, and a communication is established between the accessory part and the ganglion of the root of the pneumogastric by means of two twigs. The nerve emerges through the jugular foramen, being enclosed in the same sheath of dura mater as the pneumogastric, behind which it lies. Immediately beneath the jugular foramen the two parts of the nerve separate from each other.

The bulbar or accessory part passes over, but in intimate contact with, the ganglion of the trunk of the pneumogastric, and its fibres are continued partly into the pharyngeal and superior laryngeal branches of the ganglion of the trunk, and partly into the trunk of the vagus below the ganglion. These latter fibres are ultimately prolonged into the cardiac and recurrent laryngeal branches of the nerve: The bulbar or accessory fibres of the spinal accessory are regarded as (I) the motor nerves of $(a)$ the muscles of the soft 
palate, except the tensor palati, $(b)$ the constrictor muscles of the pharynx, and (c) the intrinsic muscles of the larynx; and (2) the inhibitory fibres of the heart.

The spinal part of the spinal accessory nerve passes backwards and downwards either behind or in front of the internal jugular

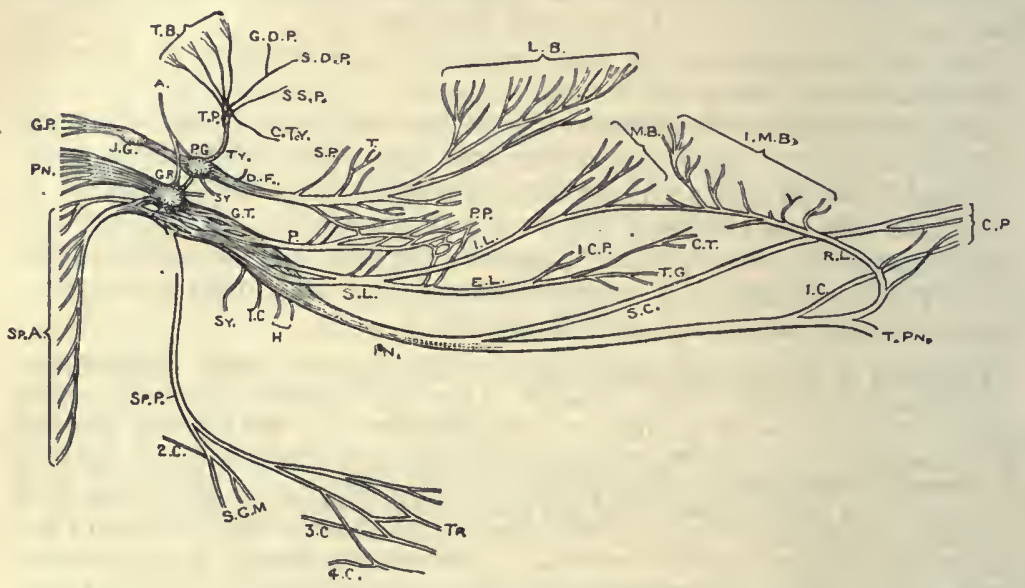

Fig. 50i.-Scheme of the Glosso-Pharyngeal, Pneumogastric, and Spinal Accessory Nerves (Flower).

\section{G.P. Glosso-Pharyngeal.}

J.G. Jugulis Ganglion

P.G. Petrous Ganglion

TY. Tympanic Branch (Jacobson's Nerve)

C.Ty. Carotico-tympanic Nerve

T.P. Tympanic Plexus

T.B. Branches to Tympanum

S.S.P. Small Superficial Petrosal

S.D.P. Small Decp Petrosal

G.D.P. Branch to Great Deep Petrosal

Sx. Communicating from Sympathetic

D.F. Twig from Digastric Branch of Facial

S.P. Branch to Stylo-pharyngeus Muscle

T. Tonsillar Branches

L. B. Lingual Branches

PN. Pneumogastric.

G.R. Ganglion of Root

A. Auricular Branch (Arnold's Nerve)

G.T. Ganglion of Trunk

Sy. Communicating from Sympathetic

Ic. Branch from First Cervical

H. Communicating with Hypoglossa!

P. Pharyngeal Branch
P.P. Pharyngeal Plexus

S L. Superior Laryngeal

I.L. Internal Laryngeal

M.B. Branches to Mlucous Membrane of Larynx

E.L. External Laryngeal

I.C.P. Branch to Inferior Constrictor Muscle

T.G. Branches to Thyroid Body

C.T. Branch to Crico-thyroid Iuscle

S.C. Superior Cardiac Branch

I.C. Inferior Cardiac Branch

R.L. Recurrent Laryngeal

1.M.B. Branches to Intrinsic Laryngeal Mus cles (except Crico thyroid)

C.P. To Cardiac Plexus

T.PN. Thoracic Pneumogastric

SP.A. Spinal Accessory.

SP.P. Spinal Portion of Spinal Accessory

2c. From Second Cervical

S.C.M. To Sterno-cleido-mastoid

3c. From Third Cervical

4c. From Fourth Cervical

TR. To Trapezius

vein. It then pierces the deep surface of the sterno-cleido-mastoid, which it supplies along with a branch of the second cervical nerve. Thereafter it crosses the posterior triangle of the neck obliquely downwards and backwards to the anterior border of the trapezius, beneath which it passes, supplying the muscle, in company with branches from the third and fourth cervical nerves. 
Summary.-The spinal portion of the spinal accessory nerve supplies two muscles, namely, the trapezius and the sterno-cleido-mastoid. The bulbar portion, through its connection with the pneumogastric nerve and its branches, supplies (I) the muscles of the soft palate, except the tensor palati; (2) the constrictor muscles of the pharynx; and (3) the intrinsic muscles of the larynx. It also furnishes the inhibitory fibres of the heart, which reach that organ through the cardiac branches of the pneumogastric.

\section{Hypoglossal Nerve.}

The hypoglossal or twelfth cranial nerve consists at first of two bundles, which pierce the dura mater separately. In passing through the anterior condylar foramen these two bundles unite to form one trunk. After emerging from that foramen the nerve lies deeply under cover of the internal carotid artery and internal jugular vein. It then turns round the outer side of the ganglion of the trunk of the pneumogastric, with which it is closely connected, and passes forwards between the internal carotid artery and internal jugular vein. Thereafter it descends to the lower border of the posterior belly of the digastric, at which level it hooks round the occipital artery from without inwards, and crosses in front of the external carotid artery and its lingual branch. In this part of its course the nerve lies in the superior carotid triangle. It next courses forwards above the hyoid bone, passing beneath the tendon of the digastric and the lower part of the stylo-hyoid muscle, and resting on the hyo-glossus. Thereafter it disappears beneath the mylo-hyoid, still resting upon the hyo-glossus, where it is accompanied by the ranine vein, and lies below the deep part of the submaxillary gland. At the anterior border of the hyo-glossus it is connected with the lingual nerve, and then enters the genio-hyoglossus.

Branches- $I$. Of Communication.-After emerging from the anterior condylar foramen, the hypoglossal nerve communicates with the ganglion of the trunk of the pneumogastric. It then receives a branch from the superior cervical ganglion of the sympathetic, and one from the first cervical nerve, or the loop between the first and second. As it hooks round the occipital artery, it receives the lingual branch of the vagus from the pharyngeal plexus; and at the anterior border of the hyo-glossus it is connected with the lingual nerve.

2. Of Distribution.-One or two meninreal branches arise from the nerve in the anterior condylar foramen, which take a recurrent course, and enter the cranial cavity to supply the dura mater in the vicinity of the foramen.

The descendens cervicis (descendens hypoglossi) nerve has been already described (see Index.

The thyro-hyoid nerve will be found described on p. II08.

The muscular branches are given off from the nerve as it lies upon the hyo-glossus under cover of the mylo-hyoid. These supply the stylo-glossus, hyo-glossus, genio-hyo-glossus, and genio-hyoid. The 
nerve to the genio-hyoid is generally regarded as being composed of spinal fibres, derived from the first, second, and third cervical nerves.

The terminal or glossal branches pierce the under surface of the tongue, and are distributed to its muscular tissue.

The hypoglossal nerve receives many spinal fibres. Those derived from the first cervical nerve, or the loop between the first and second, soon become applied to it, and the most of them pass off to form the descendens cervicis nerve. A few of these, however, still pass along the main trunk. The spinal fibres derived from the second and third cervical nerves in part ascend in the descendens cervicis nerve, and then pass off from it to be applied to the main trunk in its onward course.

Summary-I. Distribution.-The hypoglossal fibres of the nerve supply the stylo-glossus, hyo-glossus, genio-hyo-glossus, and intrinsic muscles of the tongue. The spinal fibres supply both bellies of the omo-hyoid, the sternohyoid, sterno-thyroid, thyro-hyoid, and genio-hyoid muscles. The hypoglossal nerve also supplies the dura mater in the vicinity of the cranial aspect of the anterior condylar foramen. 2. Connections. - It establishes connections with (I) the ganglion of the trunk of the vagus; (2) the superior cervical ganglion of the sympathetic ; (3) the first, second, and third cervical nerves ; (4) the pharyngeal plexus, through means of the lingual branch of the vagus; and (5) the lingual nerve from the inferior maxillary division of the fifth cranial nerve.

\section{Gangliated Cord of the Sympathetic.}

Cervical Portion.-This portion of the sympathetic cord extends from the base of the skull, at the foramen caroticum on the under surface of the petrous part of the temporal bone, to the root of the neck, where it enters the thorax, passing, on the right side, behind the subclavian artery. It lies directly behind the carotid sheath, and in front of the transverse processes of the cervical vertebræ, resting upon the rectus capitis anticus major and longus colli muscles. The cord presents three ganglia-superior, middle, and inferior.

Superior Cervical Ganglion.-This ganglion is elongated and fusiform. It is regarded as representing four ganglia united into one, and corresponding to the first four cervical nerves. It is situated in front of the transverse processes of the second and third cervical vertebræ, and lies upon the rectus capitis anticus major, the pneumogastric nerve being external to it. At either end it tapers, being continued superiorly into its ascending branch, and inferiorly into the descending cord.

Branches.- These are numerous and complicated. They may be conveniently arranged into ascending, external or communicating, internal or pharyngeal, superior cardiac, and anterior or vascular.

The ascending branch enters the carotid canal in the petrous part of the temporal bone, in company with the internal carotid artery, 


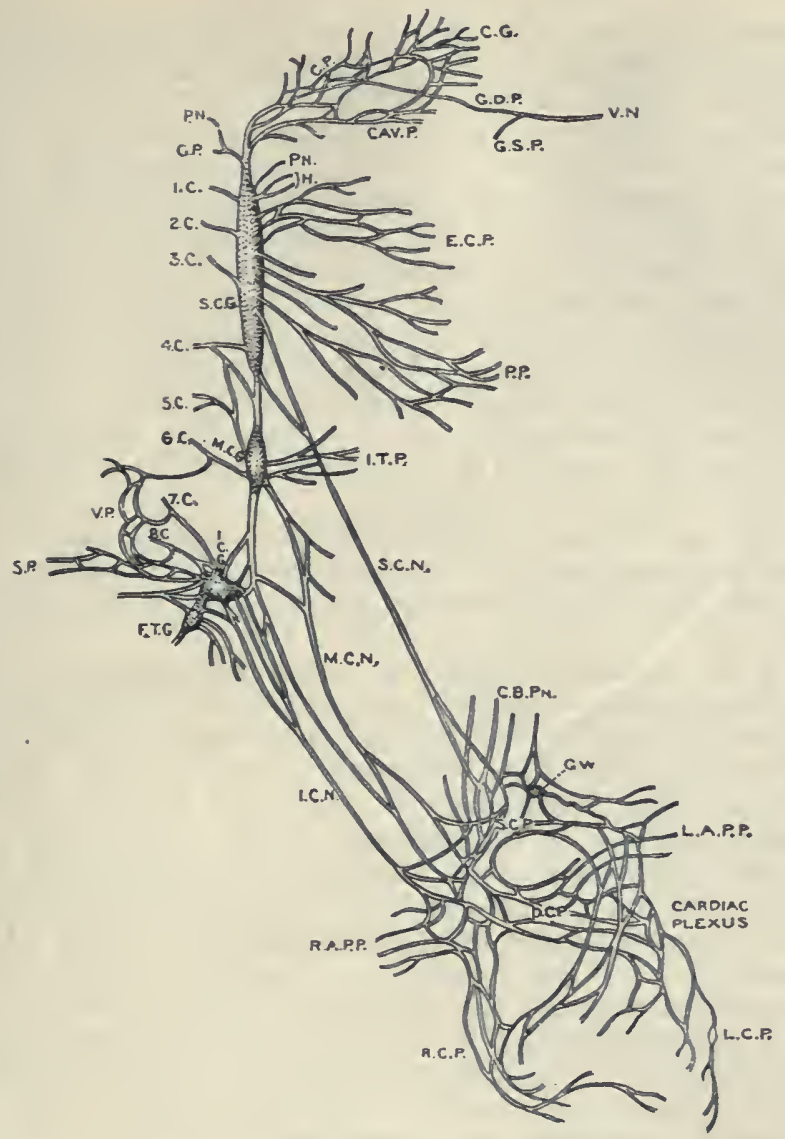

Fig. 502.-Scheme of the Sympathetic Nerve in the Neck, and of the Cardiac Plexus (Flower).

S.C.G. Superior Cervical Ganglion.

P.N. and G.P. Branches to Pneumogastric and Glosso-pharyngeal

xc, 2c, 3C, 4C. Branches to Upper Four Cervical Nerves

C.P. Carotid Plexus

Cav. P. Cavernous Plexus

C. G. Branch to Ciliary Ganglios.

G.D.P. Great Deep Petrosal

G.S.P. Great Superficial Petrosal

V.N. Vidian Nerve

P.N. To Ganglion of Trunk of Pneumogastric

H. To Hypoglossal

E.C.P. To External Carotid Plexus

P.P. To Pharyngeal Plexus

S.C.N. Superior Cardiac Nerve

M.C.G. Middle Cervical Ganglion.

5c, 5c. To Fifth and Sixth Cervical Nerves

I.T.P. To Inferior Thyroid Plexus

Mi.C.N. Middle Cardiac Nerve

\section{I.C.G. Inferior Cervical Ganglion.}

$7 c, 8 c$. To Seventh and Eighth Cervical Nerves V.P. To Vertebral Plexus

S.P. To Subclavian Plexus

1.C.N. Inferior Cardiac Nerve

\section{F.T.G. First Thoracic Ganglion}

\section{Cardiac Plexus.}

C. B.P. Cardiac Branches of Pneumogastric S.C.P. Surerficial Cardiac Plexus

G. W. Ganglion of Wrisberg

D.C.P. Deep Cardiac Plexus

R.A.P.P. Right Anterior Pulmonary Plexus

L.A.P.P. Left Anterior Pulmonary Plexus

R.C.P. Right Cornnary Plexus

L.C.P. Left Coronary Plexus 
and divides into two parts. The external division lies upon the outer side of the artery, and its subdivisions form by their interlacement the carotid plexus. From this plexus several communicating branches are given off. (I) The carotico-tympanic nerve enters the tympanum, and joins the tympanic branch (Jacobson's nerve) of the glosso-pharyngeal, to take part in the tympanic plexus. (2) The great deep petrosal nerve joins the great superficial petrosal from the geniculate ganglion of the facial, in the upper part of the foramen lacerum medium, to form the Vidian nerve, which passes through the Vidian canal to join Meckel's ganglion. (3) A branch (or branches) passes to join the sixth cranial nerve. (4) One or more branches pass to join the Gasserian ganglion. The carotid plexus furnishes filaments to the coats of the internal carotid artery, and it receives the small deep petrosal nerve from the tympanic plexus.

The internal division of the ascending branch of the superior cervical ganglion lies upon the inner side of the internal carotid artery, to which it furnishes twigs, and its subdivisions form by their interlacement the cavernous plexus. This plexus lies in contact with the internal carotid artery as it traverses the cavernous sinus. Its communicating branches pass to join the third, fourth, and ophthalmic division of the fifth cranial nerves, and the ciliary or lenticular ganglion. The last-named branch forms the sympathetic root of the ciliary ganglion, and enters the orbit through the sphenoidal fissure. It is the source of the motor supply of the dilator pupillæ muscle. The cavernous plexus furnishes filaments to the coats of the internal carotid artery, and offsets which accompany the branches of that artery, around which they are disposed as plexuses.

The external branches are communicating, and pass to (I) each of the upper four cervical nerves ; $(2)$ the petrosal ganglion of the glosso-pharyngeal ; (3) the ganglion of the root, and the ganglion of the trunk, of the vagus ; and (4) the hypoglossal nerve.

The internal branches are pharyngeal and cardiac. The pharyngeal branches, two or three in number, unite with the pharyngeal branches of the glosso-pharyngeal and vagus to form the pharyngeal plexus (see p. 1227).

The superior cardiac nerve arises by two roots from the lower part of the superior cervical ganglion, and descends behind the carotid sheath, lying internal to the main sympathetic cord. The nerve of the right side passes either over or beneath the first part of the subclavian artery, and then along the innominate artery, to terminate in the deep cardiac plexus. In its course down the neck it receives twigs from the external laryngeal nerve, and is joined by the upper cervical cardiac branch of the pneumogastric nerve. As it enters the thorax, it receives a filament from the recurrent laryngeal nerve. The nerve of the left side within the thorax accompanies the left common carotid artery, and passes over the arch of the aorta to terminate in the superficial cardiac plexus. 
The anterior branches are vascular, and pass to the external carotid artery, upon which and its branches they form delicate plexuses.

Middle Cervical Ganglion.-This ganglion is situated opposite the sixth cervical vertebra, and usually lies close to the inferior thyroid artery. It is the smallest of the three, and probably represents two ganglia united into one. Occasionally it is absent. It is connected by grey rami with the fifth and sixth cervical nerves, and its branches of distribution are thyroid and middle cardiac. The thyroid branches accompany the inferior thyroid artery to the thyroid body, and they communicate with the external and recurrent laryngeal nerves. The middle cardiac nerve of each side terminates in the deep cardiac plexus. The nerve of the right side passes either in front of, or behind, the first part of the right subclavian artery. It then descends in front of the trachea, and joins the right half of the deep cardiac plexus. The nerve of the left side enters the thorax between the left common carotid and left subclavian arteries, and it joins the left half of the deep cardiac plexus.

Inferior Cervical Ganglion.-This ganglion is situated between the transverse process of the seventh cervical vertebra and the neck of the first rib, where it lies behind the root of the vertebral artery. It is intermediate in size between the superior and middle ganglia, and, like the middle, it probably represents two ganglia united into one. It is frequently fused with the first thoracic ganglion. One or two cords establish a communication between it and the middle cervical ganglion, passing in front of the first part of the subclavian artery. The cord or cords in front of the vessel form a loop around it, called the ansa Vieussenii, or ansa subclavia. The inferior cervical ganglion is connected by grey rami with the seventh and eighth cervical nerves, and its branches of distribution are vascular and inferior cardiac. The vascular branches accompany the vertebral artery in the form of the vertebral plexus, which gives filaments to the vessel and offshoots along its various branches. The plexus is continued along the basilar artery and its branches, and on to the posterior cerebral arteries. The inferior eardiac nerve on each side joins the deep cardiac plexus. The nerve of the right side passes behind the first part of the right subclavian artery, and then in front of the trachea. It is connected with the middle cardiac and recurrent laryngeal nerves. The nerve of the left side in many cases joins the middle cardiac nerve, and so reaches the deep cardiac plexus.

\section{Constitution of the Cervical Sympathetic Cord.}

The sympathetic cord in the neck contains the following important groups of fibres, namely, (I) dilator pupillæ, (2) vaso-constrictor and vaso-dilator, (3) secretory, and (4) accelerator.

The dilator pupillæ fibres are derived from the upper three thoracic nerves, and ascend in the sympathetic cord to the superior cervical ganglion, in which they terminate. From this ganglion they are continued as sympathetic 
fibres to the cavernous plexus, through this plexus to the ciliary ganglion, and thence to the dilator pupillæ muscle.

The vaso-constrictor fibres are derived from a variable number of thoracic nerves, commencing at the second and terminating at the eighth. The origin of the vaso-dilator fibres is not definitely known. The vaso-constrictor fibres ascend to the superior cervical ganglion, in which they terminate. From this ganglion they are continued as sympathetic fibres into the plexus around the external carotid artery, and thence along the branches of that vessel.

The secretory fibres are destined for the submaxillary gland, and are derived chiefly from the second and third thoracic nerves. They also ascend to the superior cervical ganglion, in which they terminate. They are thence continued as sympathetic fibres into the external carotid plexus, from that into the facial plexus, and thence into the submaxillary ganglion, from which they pass into the submaxillary and sublingual glands.

The accelerator fibres of the heart are chiefly derived from the second and third thoracic nerves, but some also spring from the first, fourth, and fifth nerves. The spinal fibres pass to the middle and lower cervical ganglia, from each of which they are continued as sympathetic fibres.

\section{The Mouth.}

The mouth, or buccal cavity, extends from the rima oris in front, where it opens externally on the face, to the isthmus faucium behind, where it opens into the pharynx. It is divided by the upper and lower dental arches into two compartments, the anterior of which is called the vestibule and the posterior the mouth proper.

The vestibule is bounded anteriorly and externally by the lips and cheeks, and internally by the dental arches and gums. Superiorly and inferiorly it is bounded by the reflection of the mucous membrane from the lips and cheeks on to the gums. In the median line, above and below, the mucous membrane forms a small vertical fold, called the frenulum labii. On the inner surface of each cheek the vestibular mucous membrane presents a small papilla opposite the second upper molar tooth, and on this is the minute opening of Stensen's duct.

The mouth proper lies within the dental arches. It communicates with the vestibule by the interval between the upper and lower teeth, and also by an opening at either side situated behind the last molar tooth. Anteriorly and externally it is bounded by the dental arches and gums, and posteriorly it opens into the pharynx by means of the isthmus faucium. The roof, which is arched, is formed by the hard palate and the velum pendulum palati, or soft palate. The floor is formed by the tongue, and the reflection of mucous membrane from the inner surface of the lower dental arch on to its under surface. In the region of the tip of the tongue the lateral reflections of mucous membrane from the two sides of the lower dental arch are continuous across the median line. In the median line the mucous membrane forms a prominent fold, called the frenum lingua, which is connected above with the under surface of the tongue a little distance from the tip. In some children it may extend quite to the tip, impairing the utility of the organ, and giving rise to the condition known as 'tongue-tied.' Close to each side of the frenum there is a small papilla, on which is the 
opening of Wharton's duct, and near to this is the opening of the duct of Rivini or of Bartholin, which comes from the outermost lobule of the sublingual gland. A little posterior to this is a fold of the mucous membrane, at either side, produced by the upper border of the subjacent sublingual gland. This fold is known as the plica sublingualis, and it extends from the side of the tongue to the dental arch. It is upon this plica that the majority of the ducts of Walther (sublingual ducts) open.

The lips are covered by skin externally, and mucous membrane internally. Between these two layers are the muscular fibres

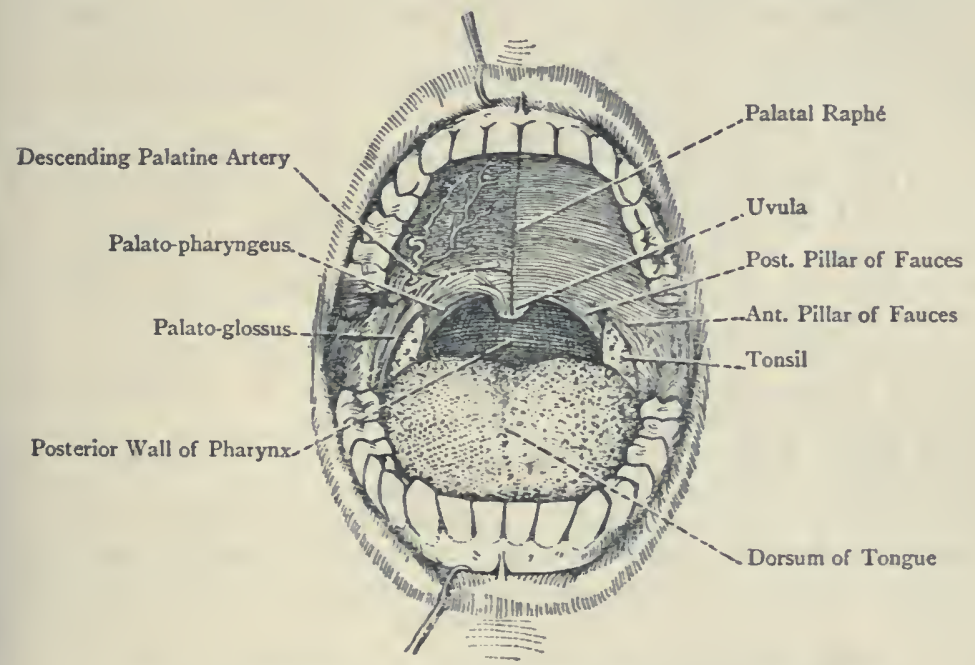

Fig. 503.-The Buccal Cavity and Istmius Faucium.

(The Maxilla are wiclely separated).

of the orbicularis oris, blended with which are the fibres of the buccinator, and, in the case of the upper lip, fibres of the depressor anguli oris, whilst in the case of the lower lip there are the fibres of the levator anguli oris. Between the mucous membrane and the muscular element there are a number of small racemose glands, called the labial glands, the ducts of which open on the inner surface of each lip. The tortuous coronary arteries are embedded in each lip, and those of opposite sides anastomose at the median line.

Between the upper lip and the columella nasi there is a groove, called the philtrum.

The lymphatic vessels of the upper lip pass, on either side, to the submaxillary lymphatic glands. The lymphatic vessels of the lateral portion of the lower lip also pass, on either side, to the submaxillary lymphatic glands; and those of the mesial portion pass to the submental, or suprahyoid, gland of the corresponding side. 
The cheeks are covered externally by skin, and internally are lined with mucous membrane. The muscular element consists of the buccinator, which is covered by the buccal fascia. Posteriorly this fascia is continuous with the pharyngeal fascia, which is derived from the deep cervical fascia, the two being known as the bucco-pharyngeal fascia. Superiorly and inferiorly it is attached to the dental arches.

The lymphatic vessels of the cheek pass to (I) the superficial parotid or pre-auricular lymphatic glands, and (2) the submaxillary lymphatic glands, the buccal lymphatic glands being a gland-station in the path of the latter.

The buccal pad of fat is situated upon the buccal fascia, and is well developed in young children. It is of soft consistence, and is continuous with the fat beneath the temporal muscle under cover of the ramus of the lower jaw.

The buccal fascia, buccinator, and lining mucous membrane are pierced by Stensen's duct. Between the buccinator and the mucous membrane there are several racemose buccal glands. In addition to these there are three or four molar glands, mucous in character, which are situated superficial to the buccal fascia in the angle between the masseter and buccinator.

The masseteric fascia is an upward prolongation of the deep cervical fascia. It is attached superiorly to the zygoma, and externally is continuous with the parotid fascia.

The dental arches are formed by the alveolar borders of the superior and inferior maxillæ, the teeth, and the gums.

The gums (gingivæ) consist of dense fibrous tissue, which is covered by mucous membrane and is closely connected with the periosteum of the alveolar borders of the superior and inferior maxillæ. The mucous membrane, which is very vascular, is continuous with the labial and buccal mucous membrane on the one hand, and with that of the floor of the mouth on the other. Close to the necks of the teeth it is beset with vascular papillæ.

The lymphatic vessels of the upper gum pass, on either side, to the submaxillary lymphatic glands. The lymphatic vessels of the lateral portion of the lower gum also pass, on either side, to the submaxillary lymphatic glands; and those of the mesial portion pass to the submental or suprahyoid gland of the corresponding side.

The mucous membrane of the gums is separated from the subjacent periosteum by dense connective tissue, which connects the two in such a close manner that the mucous membrane is immovable. Close to the necks of the teeth it is beset with papillæ. The mucous membrane of the hard palate, like that of the gums, is separated from the periosteum by a thick layer of dense connective tissue, which binds the two so closely that the mucous membrane is immovable. It presents a median raphé, which is continued over the soft palate, and ends in front at the anterior palatine fossa in a small papillary elevation, known as the incisor papilla. On either side of the raplie anteriorly there are a few transverse ruga. Eacli lateral half of the hard palate is traversed by the ramifications of 
the descending palatine artery, the branches of which extend forwards and inwards from either lateral angle posteriorly. The mucous membrane of the hard palate is provided with racemose palatal glands of a serous character, which are arranged in two symmetrical groups laterally disposed.

The lymphatic vessels of the mucous membrane of the hard palate pass to (I) the deep facial, or internal maxillary, glands, and (2) the superior deep cervical glands.

Nerves.-The great or anterior palatine nerve descends in the posterior palatine canal, from which it emerges through the posterior palatine foramen. Thereafter it divides into branches which pass forwards in grooves on the hard palate, and supply the mucous membrane, glands, and inner aspect of the upper gum. The naso-palatine nerve is distributed to the mucous membrane behind the incisor teeth, where it communicates with twigs of the great or anterior palatine nerve. To reach the hard palate the nerve of the right side passes through the posterior foramen of Scarpa, whilst the left nerve passes through the anterior foramen. The mucous membrane of the cheek is supplied by the long buccal nerve, which is a branch of the inferior maxillary division of the fifth cranial nerve. The mucous membrane of the floor of the mouth derives its nerves from the submaxillary ganglion.

Arteries.-These are the right and left descending palatine branches of the third part of the internal maxillary. Each descending palatine artery takes the same course as, and has a similar distribution to, the great or anterior palatine nerve. At the anterior palatine fossa it terminates in a small branch which ascends through Stensen's canal, and anastomoses at the upper end of that canal with the naso-palatine branch of the spheno-palatine artery.

\section{Development of the Mouth, Lips, and Gums.}

The rudiment of the mouth appears as an invagination of the ectoderm of the ventral aspect of the embryo between the fore-brain (which is folded downwards) and the pericardial region. This invagination gives rise to a depression, called the oral fossa or stomodæum. As the stomodæum deepens, the invaginated ectoderm approaches the pharyngeal end of the fore-gut, with which it unites. There is thus formed a bilaminar stratum, called the bueco-pharyngeal membrane, one layer of which is formed by the stomodical ectoderm, whilst the other layer consists of the entoderm of the fore-gut (pharynx). This membrane forms a septum between the pharyngeal part of the fore-gut and the stomodæum. In the course of the fourth week this septum ruptures and disappears, and thereafter the pharynx communicates freely with the stomodæum.

The formation of the stomodæum and bucco-pharyngeal membrane bears a close resemblance to the formation of the anal fossa or proctodæum and cloacal membrane, at the caudal end of the hind-gut.

The primitive oral cavity, or stomodæum, includes the cephalic part of the pharynx, and from the stomodæum the permanent oral cavity or mouth and the nasal cavities are formed.

The boundaries of the stomodæum are as follows: Dorsally, the buccopharyngeal membrane; superiorly and ventrally, the fronto-nasal process: inferiorly and behind, the two mandibular arches; and, laterally, the corresponding maxillary process. 
Annexed to the stomodæum there are (1) the pouch of Rathke, (2) the tongue (3) the salivary glands, (4) the tonsils, and (5) the teeth.

The pouch of Rathke is an evagination or diverticulum of the roof of the ectodermic stomodæum, ventral to the bucco-pharyngeal membrane. It grows in a cephalic direction, and gives rise to the anterior lobe of the pituitary body, or hypophysis cerebri, the posterior lobe of which is developed from a diverticulum of the floor of the third ventricle of the cerebrum.

The development of the tongue, salivary glands, tonsils, and teeth will be found after the description of each of these organs.

The stomodæum becomes divided into two chambers-upper or nasal and lower or oral-this division being effected by the formation of the hard palate. The nasal chamber becomes transformed into the two nasal fossæe. and the oral chamber constitutes the oral cavity or permanent mouth.

The permanent oral cavity is much more circumscribed than the primitive oral cavity or stomodæum. The latter, as already stated, includes the cephalic part of the pharynx, but that part of the pharynx is, in adult life, shut off from the permanent oral cavity by the velum pendulum palati or soft palate.

The development of the hard palate is described in connection with the visceral arch skeleton of the face, but it may be here briefly restated.

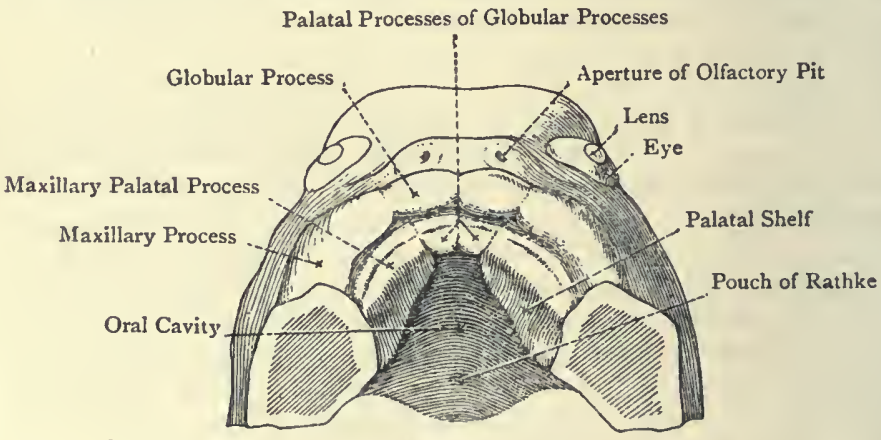

Frg. 504-Roof of Mouth (Marshall, After His).

The horizontal or palatal plate of the palate bone and the greater part of the palatal plate of the superior maxilla, of either side, are developed from the palatal shelf of the corresponding maxlllary process of the first visceral, or mandibular, arch. The incisor or premaxillary portion of the palatal plate of the superior maxilla-that is to say, the portion ventral to the incisor or premaxillary suture on the under aspect-is developed, on either side, from the nasal lamina of the corresponding globular process of the mesial nasal process of the fronto-nasal process. The formation of the hard palate is completed about the end of the third month.

Lips (labia oris) and Gums (gingiva).- Shortly after the fusion of the maxillary and globular processes to form the superior maxilla, a groove, produced by an ingrowth of epithelium, appears along each of these processes, on either side. The margins of this groove, which form parallel ridges, are external and internal, relatively to the oral cavity. The external, or labial, ridge gives rise to the upper lip, the globular portion of the ridge representing the premaxillary part of the lip, and the maxillary portion forming the remainder. The internal or gingival ridge is the rudiment of the upper gum.

The philtrum is probably developed from the mesodermic investment of the united globular processes.

A similar groove and similar ridges (labial and gingival) appear along each mandibular arch, and from the ridges the lower lip and lower gum are developed.

The angles of the mouth correspond to the union between the two lips, on either side, and each originally represents the angle between a mandibular arch and maxillary process. 


\section{The Tongue.}

The tongue is a muscular and very sensitive organ, covered by the buccal mucous membrane, and situated on the floor of the mouth. It is concerned in the sense of taste, mastication, deglutition, and speech, and it consists of a root or base, a body, and a tip. The root is attached to the hyoid bone. The tip is the anterior free extremity, which, in the quiescent state, lies behind the upper incisor teeth. The body presents an upper surface or dorsum, an under surface, and two lateral borders, right and left. The dorsum is convex from before backwards, and presents over its anterior two-thirds a median depression or raphé, on either side of which the surface is convex from side to side. This raphe terminates posteriorly in the foramen cacum. In this region the dorsum presents a $V$-shaped groove, known as the sulcus terminalis. The point of the $V$ is at the foramen crecum, and the two limbs pass outwards and forwards to the sides of the tongue, where the anterior pillars of the fauces are connected with it. The sulcus terminalis marks the union of the two portions, anterior and posterior, from which the tongue is developed. The portion behind it, representing one-third, is the basal, lymphoid, or pharyngeal portion. It occupies the buccal portion of the pharynx, and overhangs the epiglottis. The portion in front of the sulcus terminalis, representing two-thirds, is known as the buccal or papillary portion.

Mucous Membrane.-The mucous membrane covering the basal or pharyngeal portion of the tongue is destitute of proper papillæ, but is freely provided with lymphoid follicles similar to those of the tonsils, and with mucous glands. The follicles are ranged upon the walls of crypts, the mouths of which open upon the surface. The mucous membrane in this region forms the glosso-epiglottidean fold, or frenum epiglottidis, which is mesially placed. On either side of this fold, between it and each pharyngo-epiglottidean fold, there is a pouch or depression, called the vallecula. In front of the sulcus terminalis the mucous membrane of the dorsum, borders, and tip of the tongue is freely covered by characteristic papillæ, which, being visible to the naked eye, impart to this part its distinctive appearance. These papillæ bear secondary papillæ, which, however, are concealed by the epithelial covering. The mucous membrane on the under surface of the tongue in the median line forms, near the tip, a vertical fold, called the frenum linguce. A little external to the frenum, on either side, is an indistinct fringed fold, called the plica fimbriata. The two plicæ converge as they pass forwards towards the tip, and inside each the outline of the ranine vein may be visible. On either side of the tongue, in the region of the limbs of the sulcus termina'is, the mucous membrane presents a few ridges which represent the papille foliate of such animals as the rabbit. 
Papillæ.-These are of three kinds, namely, conical, fungiform, and circumvallate, and they are confined to the anterior twothirds of the organ. The conical papillæ are the most numerous, and are arranged in more or less parallel, closely-set rows, directed forwards and outwards from the median line, except towards the tip, where the rows tend to become transverse. On the sides of the tongue they are arranged in vertical parallel rows. In some

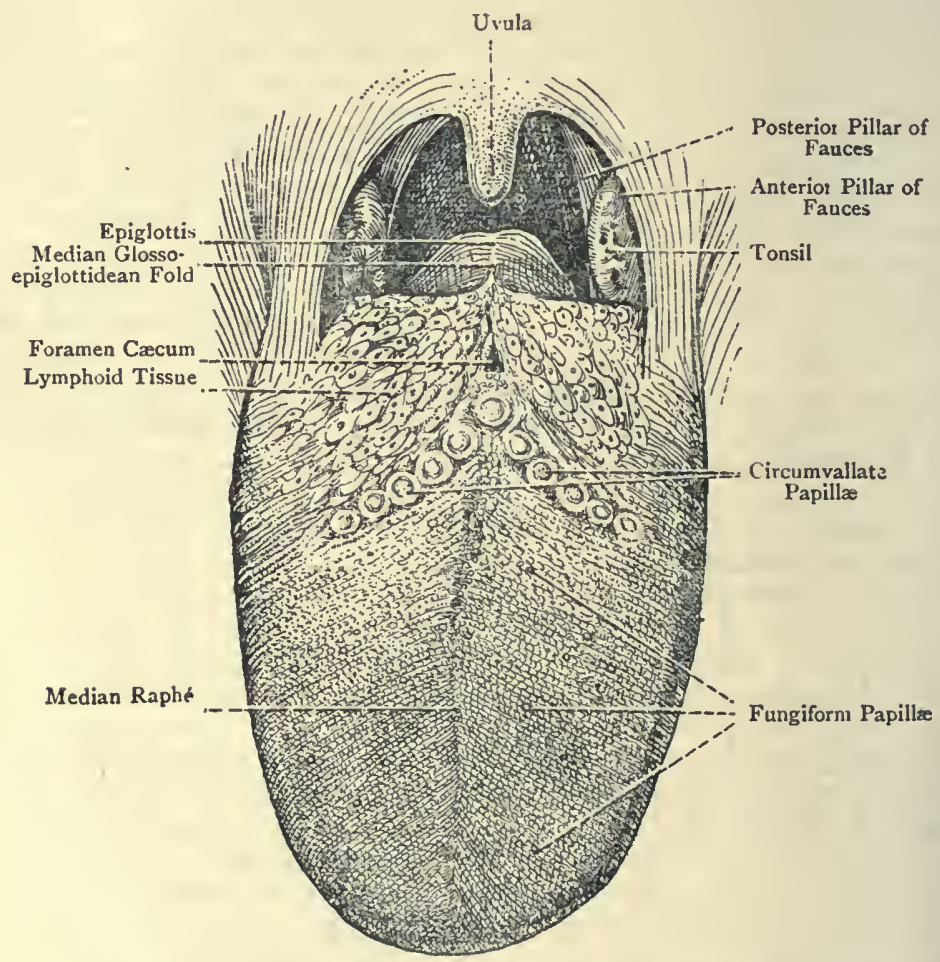

Fig. 505.-The Dorsum of the Tongue, and the Fauces.

conical papillæ the epithelium which caps the secondary papillæ forms thread-like processes. Such are known as filiform papillæ.

The fungiform papillæ are scattered amongst the conical, and are most numerous at the sides and tip, being rather sparse over the dorsum. Each is round and enlarged at the surface, but tapers at its deep end, and it bears secondary papillæ, but there is no filiform arrangement of the epithelial caps.

The circumvallate papillæ are conspicuous, and vary in number from seven to thirteen. They are arranged in two diverging rows 
which lie anterior to, and parallel with, the limbs of the sulcus terminalis. The two rows converge backwards and inwards, and so form a capital $\mathbf{V}$. In the median line, at the point of the $\mathbf{V}$, there is a single circumvallate papilla, behind which is the foramen cæecum. The free surface of each papilla is broad and flat, and bears secondary papillæ, whilst the deep end is somewhat constricted, and is received into a circular pit of the mucosa. In this manner each papilla is surrounded by a space known as the trench. The outer wall of the trench projects slightly beyond the level of the free surface of the papilla, thus forming a circular elevation around it, called the vallum (rampart). It is from this circumstance that the papillæ

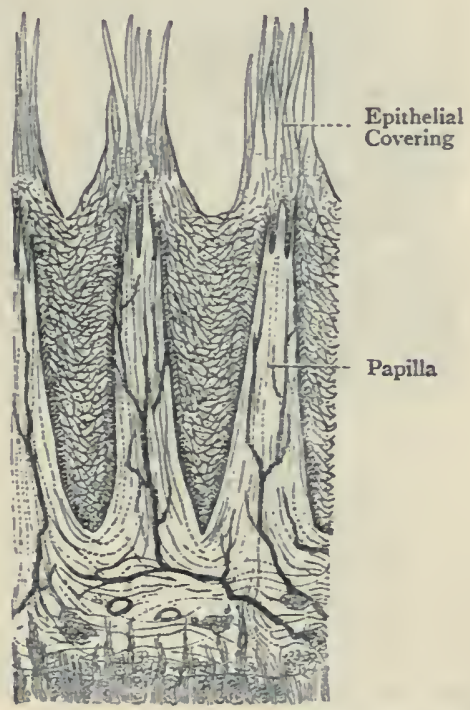

Fig. 506.-The Conical (Filiform) Papille of the Tongue.

have received the name 'circumvallate.' Their sides, as well as the wall of the vallum, contain the taste-buds.

The tongue contains a number of acinous glands. Some of them open into the trenches around the circumvallate papillæ, where tastebuds are present, and are serous in character, these being known as the glands of Ebner. Others open into the foramen cæcum, into the crypts on the posterior third of the dorsum, and along the sides of the organ, these being mucous in character, and being known as the glands of Weber. Beneath the apex of the tongue, on either side of the median line, there is a small group of glands, partly serous and partly mucous. These two groups are known as the glands of Blandin, or of Nuhn. 
The muscular tissue of the tongue is of the striped variety. It forms twc sets of muscles, namely, extrinsic and intrinsic. The extrinsic muscles are those which have their origins outside the tongue, and their insertions into it. They are as follows : (I) the genio-hyo-glossus, (2) the hyo-glossus (including the chondroglossus), (3) the stylo-glossus, and (4) the palato-glossus, all of which have been already described. The intrinsic muscles are those which are contained entirely within the tongue, and they are arranged in pairs on either side of the median fibrous septum,

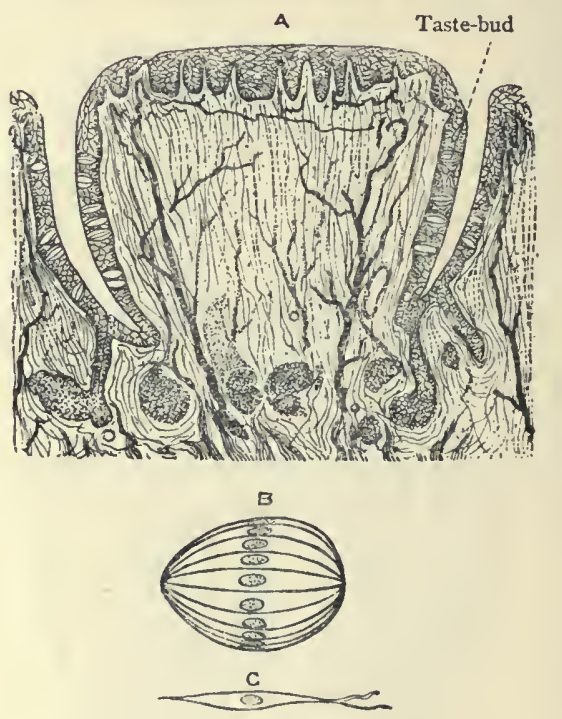

Fig. 507.-Vertical Section of a Circumvallate Papilla of the Tongue.

A, Circumvallate Papilla, beset with Secondary Papillæ ;

B, Taste-bud; C, Gustatory Cell.

which extends from the tip of the tongue as far back as the body of the hyoid bone. The intrinsic muscles are as follows: (I) the lingualis superficialis, (2) the lingualis inferior, (3) the lingualis transversus, and (4) the lingualis verticalis.

The lingualis superficialis is an expanded sheet placed on the dorsum, immediately beneath the mucous membrane. Its fibres are disposed longitudinally, and the muscle extends from the tip backwards to the body of the hyoid bone. Posteriorly it is overlapped by fibres of the hyo-glossus. Its fibres do not extend over the whole length of the tongue, but take attachment, at short intervals, to the mucous membrane. 
The lingualis inferior is disposed as a round bundle on the inferior surface of the tongue. Posteriorly it lies between the insertions of the genio-hyo-glossus internally and the hyo-glossus externally, and anteriorly between the former muscle internally and the stylo-glossus externally. The muscle extends from the tip of the tongue to the body of the hyoid bone, with which it is connected.

The lingualis transversus constitutes a layer of considerable thickness, which is placed between the lingualis superficialis and

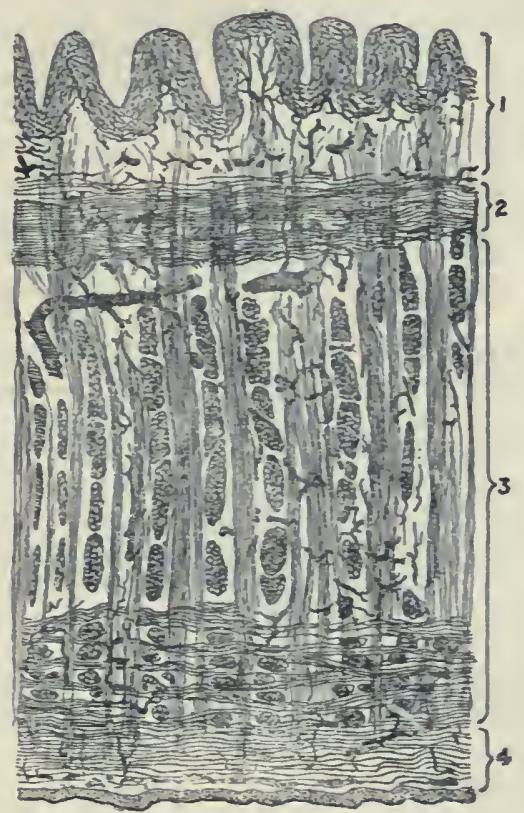

Fig. 508.-Longitudinal Section of the Tosgue of a Cat (injected).

r. Mlucosa

2. Lingualis Superficialis
3. Lingualis Transversus et Verticalis

4. Lingualis Inferior

lingualis inferior. It arises from the median septum, and extends outwards, the upper fibres curving upwards, to be inserted into the mucous membrane of the side of the tongue and adjacent portion of the dorsum. This muscular stratum is interspersed with fat, and is much broken up by fibres of the lingualis verticalis and geniohyo-glossus.

The lingualis verticalis extends from the mucosa of the dorsum to that of the inferior surface. Its fibres describe curves, having the convexities directed inwards, and they decussate freely with the fibres of the lingualis transversus. 
Septum Linguæ.--The septum of the tongue is a fibrous partition, which extends in the median line from the tip of the organ to the body of the hyoid bone. It divides the tongue into two symmetrical halves, and the lingualis transversus muscle arises from it on either side.

Arteries.-These are as follow's: (I) the ranine artery, which is a branch of the lingual, and is situated on the inferior surface, immediately external to the line of insertion of the genio-hyo-glossus muscle. It anastomoses with its fellow beneath the tip. (2) The dorsalis linguæ artery, which is also a branch of the lingual. (3) The tonsillar branch of the cervical portion of the facial artery. (4) The palatine branch of the ascending pharyngeal artery.

The veins pass to the internal jugular vein.

Lymphatics.-The lymphatic vessels of the tongue are disposed in four groups-apical, marginal, basal, and central.

The apical lymphaties issue from the tip, and are two in number, on either side. One passes to the submental, or suprahyoid, gland of the same side; and the other passes to one of the internal chain of substerno-mastoid glands, on a level with the cricoid cartilage of the larynx.

The marginal lymphatics convey lymph from the anterior twothirds of the lateral border, and the marginal portion of the dorsum for a similar extent. The anterior lymphatics of this set pass through the mylo-hyoid muscle, and terminate in the anterior submaxillary lymphatic glands. The posterior lymphatics of this set pass to the superior deep cervical glands, and, more particularly, to those situated under cover of the posterior belly of the digastric muscle. The small lingual glands, which lie upon the outer surface of the hyo-glossus muscle, serve as gland-stations in their path.

The basal lymphaties return lymph from the posterior third of the tongue, and have the same termination as the posterior marginal lymphatics, just stated.

The central lymphaties return the lymph from median part of the tongue, and pass to the deep cervical glands, which extend from the posterior belly of the digastric muscle to the level of the cricoid cartilage of the larynx.

Nerves.-The sensory nerves are: (I) the lingual branch of the inferior maxillary division of the fifth cranial nerve, which is distributed to the mucous membrane over the anterior two-thirds of the tongue, including the conical and fungiform papilla, upon which it confers common sensibility; $(2)$ the chorda tympani nerve, which accompanies the lingual nerve to the anterior twothirds of the tongue, of which it is usually regarded as the nerve of special sense; (3) the lingual branch of the glosso-pharyngeal nerve, which is distributed to the mucous membrane of the posterior third and to the circumvallate papilla, of which it is the nerve of special sense; (4) the internal branch of the superior laryngeal nerve, which furnishes a few twigs to the mucous membrane of the 
root of the tongue, in the region of the epiglottis; and (5) the hypoglossal nerve, which supplies the lingual muscles.

Sympathetic fllaments are also conducted to the tongue by the various arteries.

Taste-buds. - These constitute the gustatory organs. They are modified epithelial cells, and are present in the following situations: (I) The sides of the circumvallate papillæ, and the opposed surface of each vallum; (2) the sides of the anterior two-thirds of the tongue, partly in connection with the fungiform papillæ, and partly embedded in the stratified epithelium; (3) the folds which form the papilla foliate; (4) the buccal surface of the soft palate; and (5) the posterior surface of the epiglottis. They extend vertically through the epithelium from the free surface to the corium of the mucosa.

Each taste-bud is a flask-shaped body. The base rests upon the corium of the mucosa, and gives passage to nerve-fibres. The apex or narrow end lies between the surface-cells of the epithelium, and it is perforated by a minute opening, called the gustatory pore, through which the peripheral processes of the gustatory cells in the interior of the bud project as gustatory hairs.

Structure.-The wall of a taste-bud is composed of flattened, nucleated, epithelial cells, called the sustentacular or tegmental cells. These cells are elongated in the direction of the bud, they taper at either end, and their margins are closely applied to each other. The interior of the bud consists of a bundle of gustatory cells. Each gustatory cell is nucleated and spindle-shaped. The body of the cell is prolonged, at either end, into a process-peripheral and central respectively. The peripheral process passes to the gustatory pore at the apex of the bud, through which it projects as a gustatory hair. The central process passes towards the base of the bud, which rests upon the corium of the mucosa. It is usually branched, and terminates in free extremities. It does not therefore become continuous with nerve-fibres, and therein it differs from the corresponding process of an olfactory cell.

The base of each taste-bud, as stated, gives passage to nerve-fibres. The fibres, as they enter the bud, lose their medullary sheaths, and their axons terminate within the bud in arborizations around the constituent gustatory cells. Nerve-fibrils also enter the epithelial wall of the bud, and ramify between the sustentacular or tegmental cells.

Development.-The tongue is developed in the floor of the pharynx in two parts-buccal and pharyngeal-which are separated from each other by the foramen cæcum and $V$-shaped sulcus terminalis.

The buccal or papillayy part, which represents the anterior two-thirds, is developed from the ventral ends of the first or mandibular visceral arch, on either side of the tuberculum impar. It is therefore originally in two parts, right and left, which subsequently fuse and envelop the tuberculum impar. This eminence may give rise to the portion of the buccal part directly in front of the foramen cæcum.

The pharyngeal or basal part of the tongue, which represents the posterior one-third, behind the foramen cæcum and sulcus terminalis, is developed from 
the ventral ends of the second, or hyoid, and third, or thyro-hyoid, visceral arches. The union between the buccal and pharyngeal parts is indicated by the V-shaped sulcus terminalis, between the two limbs of which is the foramen cacum, or upper end, of the thyro-glossal duct. This foramen represents the persistent upper extremity of the embryonic median diverticulum, called the ductus thyro-glossus, from the lower part of which the isthmus of the thyroid body is developed. The tubereulum impar makes its appearance as a round eminence in the median line, opposite and between the first visceral clefts. Behind it, and separated from it by a groove, is the fureula, which is a forked eminence, mesially placed opposite the second and third visceral arches, and resembling a horseshoe. It gives origin to the epiglottis and aryteno-epiglottidean folds. The ventral ends of the second and third visceral arches of either side coalesce, and the united ends of these arches join each other in the interval between the tuberculum impar and the furcula, forming the copula. It is from the copula that the root of the tongue is formed. It is developed from it as two elevations, which take an upward and forward direction,

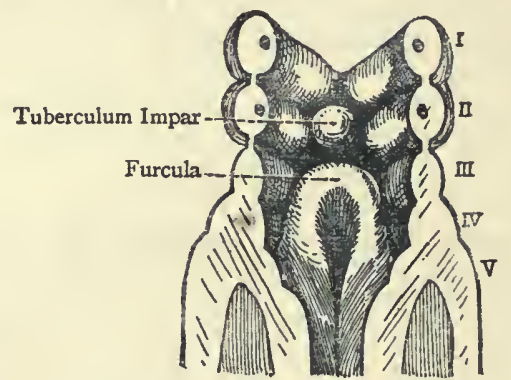

Fig. 509. -The Visceral Arches of the Embryo (Posterior View) (His).

I. Mandibular Arch

11. Hyoid Arch
I1I. Thyro-hyoid Arch

V. Fifth Arch
IV. Fourth Arch

diverging at the same time in a $\mathbf{V}$-shaped manner, so as to embrace the buccal part. After the pharyngeal and buccal portions of the tongue have coalesced, a $V$-shaped groove persists for some time, indicating the line of union of the two parts of the organ; and in adult life this is represented by the sulcus terminalis.

\section{The Soft Palate.}

The soft palate, or velum pendulum palati, is a movable musculoaponeurotic curtain situated at the back part of the hard palate, where it projects downwards and backwards into the pharynx. Anteriorly it is attached to the posterior border of the hard palate, laterally it is connected with the side of the tongue and the wall of the pharynx, and postero-inferiorly it presents a free border. Its surfaces, which occupy an oblique plane, are antero-inferior or buccal, which is concave, and postero-superior or pharyngeal, which is convex and looks towards the naso-pharynx. The posteroinferior border at its centre presents a conical process, called the uvula. On either side of the base of the uvula there are two prominent folds of mucous membrane, which extend outwards and downwards in a diverging manner. These folds constitute the 
anterior and posterior pillars of the fauces. Each posterior pillar belongs to the postero-inferior border of the soft palate, and it sweeps outwards, downwards, and backwards on the lateral wall of the pharynx. It is due to the palato-pharyngeus muscle. Each anterior pillar belongs to the buccal surface of the soft palate, and it sweeps outwards, downwards, and forwards to the back part of the side of the tongue. It is produced by the palato-glossus muscle. Between the diverging anterior and posterior pillars, on either side, there is a triangular interval, which is occupied by the tonsil. The passage which leads from the buccal cavity into the pharynx is called the fauces or isthmus faucium. It is somewhat constricted, and is bounded above by the soft palate, below by the

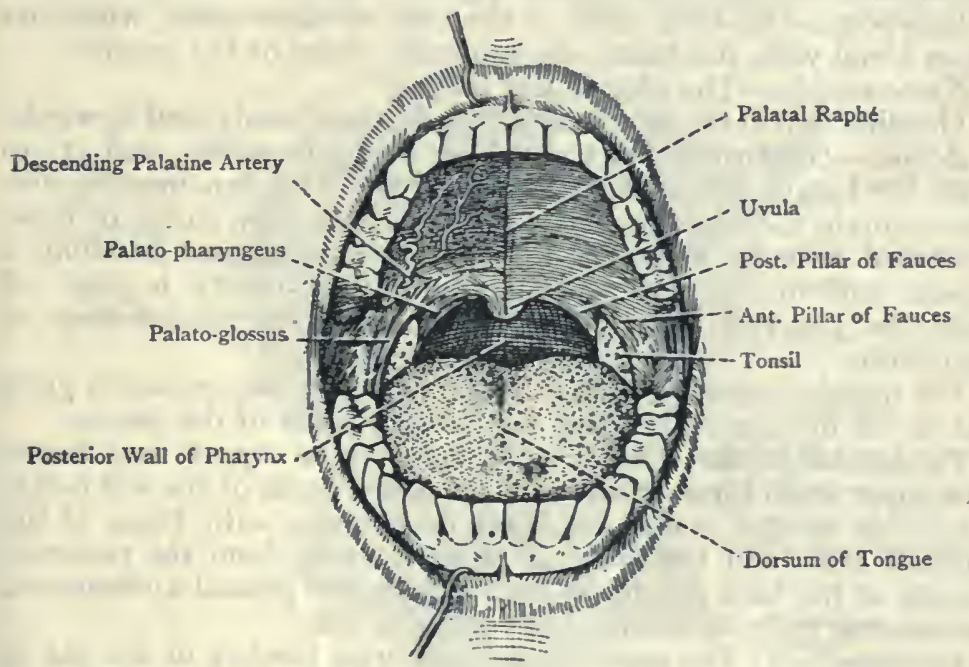

Fig. 5ro,-The Buccal Cavity and Isthuus Faucium.

(The Maxillæ are widely separated).

back part of the dorsum of the tongue, and on either side by the anterior pillar of the fauces.

The buccal surface of the soft palate presents a faint mesial raphé.

Structure.-The soft palate is composed of a double fold of mucous membrane, which contains between its two layers an aponeurosis, muscles, and many racemose glands, with bloodvessels and nerves. The mucous membrane on the buccal surface presents, as stated, a median raphé, which is continuous with that on the mucous membrane of the hard palate, and along which the originally separate halves of the soft palate unite. On the buccal surface and along the postero-inferior border it is covered by stratified squamous 
epithelium, but on the pharyngeal surface by ciliated columnar epithelium. The glands, which are racemose or acinous, are especially abundant on the buccal surface beneath the mucous membrane, where they are closely packed together. The aponeurosis is attached anteriorly to the posterior border of the hard palate, and laterally it becomes continuous with the aponeurosis of the pharynx. It affords attachment to portions of the palatal muscles.

Muscles.-These are arranged in pairs, and are as follows: palato-glossus, palato-pharyngeus, azygos uvulæ, levator palati, and tensor palati.

Palato-glossus (Constrictor Isthmi Faucium)-Origin.-The under surface of the palatal aponeurosis, its fibres being continuous across the niddle line with those of the opposite muscle.

Insertion.-The back part of the side of the tongue, where its fibres blend with the transverse muscular fibres of the tongue.

Nerve-supply.-The pharyngeal plexus.

The direction of the muscle is outwards, downwards, and forwards.

Action.-(I) To depress the side of the soft palate, and (2) to draw the tongue upwards and backwards. The two muscles also approximate the anterior pillars of the fauces. By means of these combined actions, aided by the tongue, the isthmus faucium is closed, and the anterior part of the buccal cavity is shut off from the pharynx at the commencement of the second stage of deglutition.

The muscle forms the lowest layer in the soft palate, and it gives rise to the mucous fold, called the anterior pillar of the fauces.

Palato-pharyngeus-Origin.-By two layers, upper and lower. The upper small layer arises from the aponeurosis of the soft palate above the azygos uvulæ, its fibres decussating with those of the opposite side; and the lower large layer arises from the posterior margin of the hard palate, as well as from the palatal aponeurosis, decussating with its fellow of the opposite side.

Insertion.-(I) The superior and posterior borders of the ala of the thyroid cartilage, and (2) the lateral and posterior wall of the pharynx, its fibres blending with those of the stylo-pharyngeus.

The muscle receives an accessory slip from the lower part of the cartilage of the Eustachian tube, which is known as the salpingopharyngeus muscle.

Nerve-supply. - The pharyngeal plexus.

The muscle is directed downwards and backwards.

Action.-(I) To approximate the posterior pillar of the fauces to its fellow at the commencement of the second act of deglutition, and (2) to elevate the pharynx.

The muscle gives rise to the mucous fold, called the posterior pillar of the fauces.

Azygos Uvulæ-Origin. - The side of the posterior nasal spine and the adjacent aponeurosis.

Insertion.- The submucous tissue of the uvula, having previously united with its fellow of the opposite side. 
Nerve-supply.-The pharyngeal plexus. The direction of the muscle is backwards and downwards.

Action.-To elevate and shorten the uvula.

The muscle is double at its origin, but single at its insertion. It lies above the levator palati, and beneath the upper layer of the palato-pharyngeus.

Levator Palati-Origin.-(I) The rough surface on the inferior aspect of the petrous part of the temporal bone between the apex and the carotid foramen, and (2) the lower and posterior part of the cartilage of the Eustachian tube.

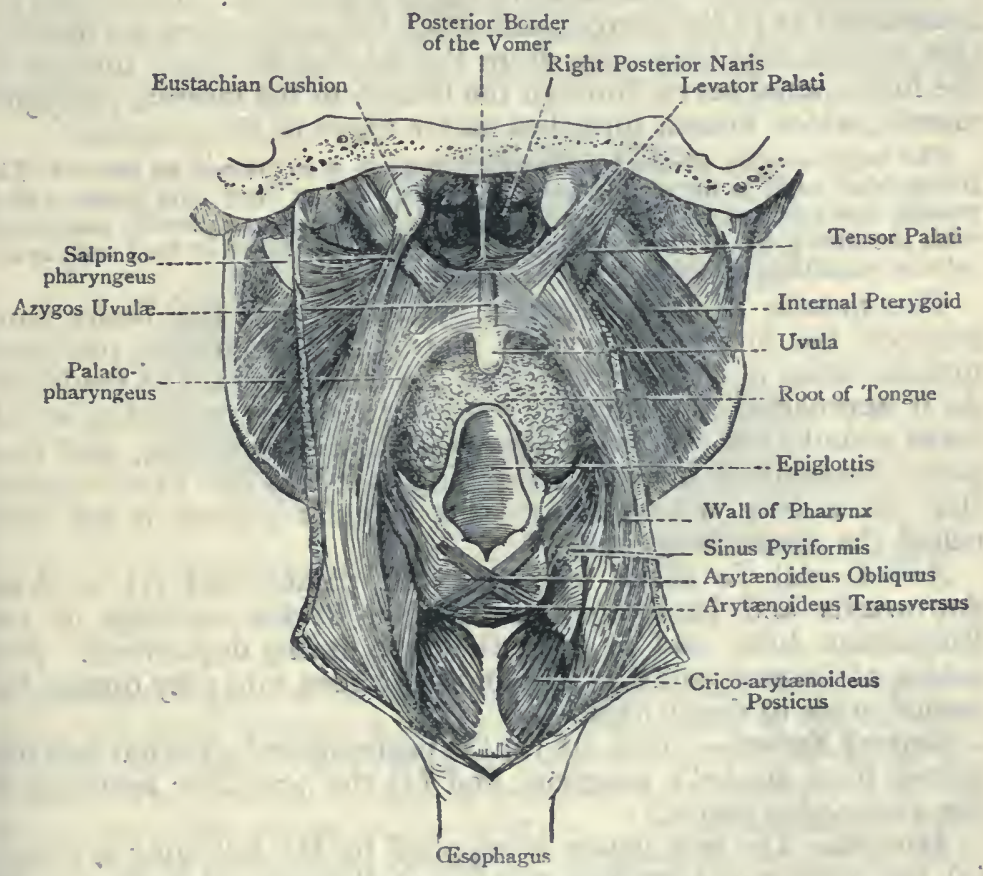

Fig 5II.-The PharynX OPENed From Behind.

Insertion.-The aponeurosis of the soft palate, its posterior fibres hecoming continuous across the middle line with the corresponding fibres of the opposite muscle.

Nerve-supply. - The pharyngeal plexus. Another view, however, is that the muscle is supplied by the posterior descending palatine nerve from Meckel's ganglion, which conveys fibres of the facial nerve through the great superficial petrosal and Vidian nerves.

The muscle is directed downwards, forwards, and inwards.

Action.-(I) To raise the soft palate, and (2) to press upwards the membranous floor of the Eustachian tube, and so lead to closure of the Eustachian orifice during deglutition (Cleland). 
The muscle, which is round and fleshy, passes over the upper border of the superior constrictor and through the pharyngeal aponeurosis. At its origin it is closely related to the membranous portion of the Eustachian tube.

Tensor Palati-Origin.-(I) The scaphoid fossa at the root of the internal pterygoid plate of the sphenoid; (2) the spine of the sphenoid ; and (3) the outer side of the Eustachian tube.

Insertion.-(I) The transverse ridge on the under surface of the horizontal plate of the palate bone, near the posterior border ; and (2) the aponeurosis of the soft palate.

Nerve-supply. - A branch from the otic ganglion. Two views are maintained as to the source from which the nerve-fibres are derived. One view is that they come from the inferior maxillary division of the fifth cranial nerve, through the branch to the internal pterygoid muscle, which branch furnishes motor fibres to the ganglion.

The nerve-supply of the tensor palati muscle is explained as follows: The Eustachian tube is developed from the first visceral cleft; the tensor palati muscle lies in front of the Eustachian tube, and is consequently associated with the first visceral, or mandibular, arch; and the nerve of that arch is the inferior maxillary division of the fifth cranial nerve.

The muscle at first descends vertically as a flat fleshy band between the internal pterygoid plate and the internal pterygoid muscle, being in close contact with the mesial surface of the latter. As it approaches the hamular process it ends in a tendon, which turns round that process, a synovial bursa intervening, and then passes horizontally inwards, expanding as it does so. From the fact that the muscle is bent around the hamular process it has been called the circumflexus palati.

Action.-(I) To make tense the soft palate, and (2) to draw downwards and backwards the margins of the cartilage of the Eustachian tube, and so open the tube during deglutition. (See action of levator palati.) The muscle comes into play during the second stage of deglutition.

Sensory Nerves.-These are (I) the posterior and external palatine nerves from Meckel's ganglion, and (2) the tonsillitic branches of the glosso-pharyngeal.

Arteries.- The soft palate is supplied by the following arteries : (I) the inferior or ascending palatine of the cervical portion of the facial; (2) the palatine branch of the ascending pharyngeal ; and (3) the posterior and external branches of the descending palatine artery from the internal maxillary, which descend in the posterior and external accessory palatine canals.

Relation of Structures in the Soft Palate.-Supposing the soft palate to be transfixed from its buccal to its pharyngeal surface, the following structures would be pierced in the order stated : (I) the mucous membrane covering the buccal surface, (2) the layer of closely-set racemose glands, (3) the palato-glossus, (4) the lower layer of the palato-pharyngeus, (5) the palatal aponeurosis and tensor palati, (6) the levator palati, (7) the azygos uvulæ, (8) the upper layer of the palato-pharyngeus, and (9) the mucous membrane covering the pharyngeal surface. 
Development.-The soft palate is developed from a differentiated portion of the palatal shelf or plate of the maxillary process of either side. This differentiated portion does not undergo ossification, but acquires muscular tissue. Like the hard palate, the soft palate and the uvula are developed in two symmetrical halves.

\section{The Tonsils.}

The tonsils (amygdala) are two in number, right and left. Each is situated in the triangular depression between the anterior and posterior pillars of the fauces on either side, and, above it, is a small recess, known as the supratonsillar fossa, which is the remains of the inner portion of the second visceral cleft. The tonsil stands out as an oval enlargement, covered by mucous membrane, and it lies opposite the angle of the inferior maxilla, being under cover of it and

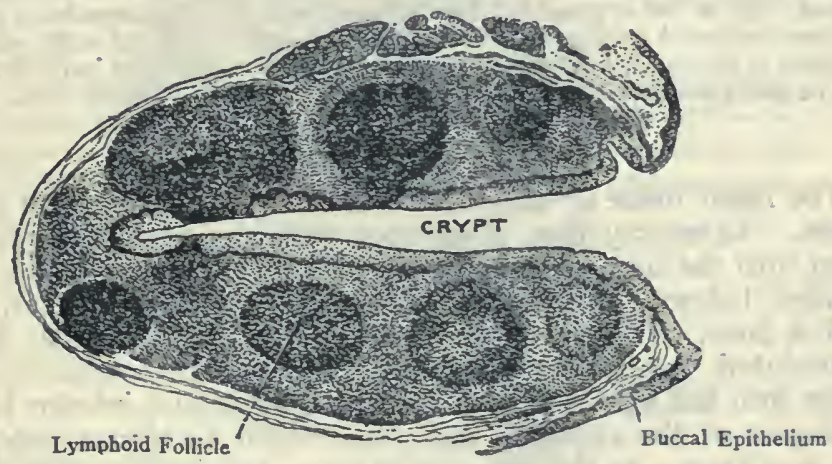

Fig. 5I2.-Section through a CRypt of the Tonsil

the adjacent portion of the ramus. The organ varies much in size, but on an average it measures about $I$ inch in length, about $\frac{3}{4}$ inch from before backwards, and about $\frac{1}{2}$ inch from within outwards. The internal surface is pitted with a number of orifices, which lead into crypts in the interior. The outer surface, which has a fibrous covering, is related to the superior constrictor of the pharynx, some loose tissue intervening, and external to the superior constrictor is the internal pterygoid muscle. Two of the tonsillar arteries, namely, the tonsillar and inferior or ascending palatine, lie between the superior constrictor and internal pterygoid. The cervical portion of the facial artery in its course lies a little below the outer aspect of the tonsil. The internal carotid artery is situated about I inch from it on its outer and posterior aspect.

Arteries.-These are as follows: (I) the tonsillar and inferior or ascending palatine branches of the cervical portion of the facial; (2) the ascending pharyngeal branch from the external carotid; (3) the dorsalis linguæ from the lingual; and (4) the posterior and external palatine offsets of the descending palatine artery from the third part of the internal maxillary. 
The veins form a plexus on the outer surface of the tonsil, from which the blood passes into the pharyngeal plexus.

Lymphaties. - These pass to the upper group of deep cervical glands.

Nerves. - The nerves are derived from (I) the glosso-pharyngeal, (2) the posterior and external palatine branches of Meckel's ganglion, and (3) the sympathetic.

Structure.-The tonsils are composed of lymphoid follicles. The follicles are ranged upon the sides of the crypts which penetrate into the organ, these crypts being lined with mucous membrane, covered by stratified squamous epithelium. Lymph corpuscles migrate from the follicles into the crypts, and become salivary corpuscles.

Development.-The tonsil of either side is developed from the epithelium of the corresponding second visceral cleft. About the fourth month a depression, known as the sinus tonsillaris, makes its appearance. Solid epithelial outgrowths or buds then extend from this sinus into the surrounding mesoderm. 'These buds'subsequently become hollow; the surface of the sinus becomes pitted; and so the crypts of the tonsil are formed. The mesoderm in relation to the buds and crypts becomes pervaded with lymphoid cells, and in this manner is formed the lymphoid tissue which constitutes the bulk of the tonsil.

The supratonsillar fossa is a remnant of the second visceral cleft.

\section{The Nasal Fossæ.}

The nasal fossæ extend from the anterior nares to the posterior nares. Anteriorly they open upon the face, and posteriorly they open into the naso-pharynx. Each fossa is narrow above, but expanded below. It presents two walls, outer and inner, a roof, and a floor. The outer wall is rendered very irregular by three convoluted bony eminences, disposed antero-posteriorly, which bulge into the fossa. These are the superior and inferior turbinate processes of the ethmoid, and the inferior turbinate bone. They are also known as the superior, middle, and inferior spongy bones, or conchæ. They overhang deep channels, which are known as the meatûs-superior, middle, and inferior respectively.

The superior meatus is confined to the back part of the outer wall, and lies between the superior and middle conchæ. It is short and oblique, and opening into it there are (I) the spheno-palatine foramen, which leads from the spheno-maxillary fossa, and (2) the posterior ethmoidal cells, by one or more openings. Above and behind the superior concha is a depression, called the sphenoethmoidal recess, into which the sphenoidal air-sinus opens.

The middle meatus is situated between the middle and inferior conchæ, is directed from behind forwards, and is overhung by the middle concha. Anteriorly it describes a bend, and passes upwards under cover of the front part of the middle concha, to be continued into the infundibulum, which leads from the frontal air-sinus of the corresponding side. The openings into the middle meatus are as follows: (I) the infundibulum, leading from the frontal air-sinus, with the opening of the anterior ethmoidal cells; (2) the opening of the maxillary air-sinus or antrum of Highmore; and (3) one or two openings of the middle ethmoidal cells. These various openings 
are concealed by the middle concha. When this is removed the lateral wall of the middle meatus is seen to present a deep curved groove, called the hiatus semilunaris, which is directed downwards and backwards from the lower end of the infundibulum. Behind and above this hiatus there is a round prominence, called the bulla ethmoidalis. The opening of the anterior ethmoidal cells is situated in the vertical portion of the hiatus semilunaris, and the opening of the antrum of Highmore, which is of small size, is placed

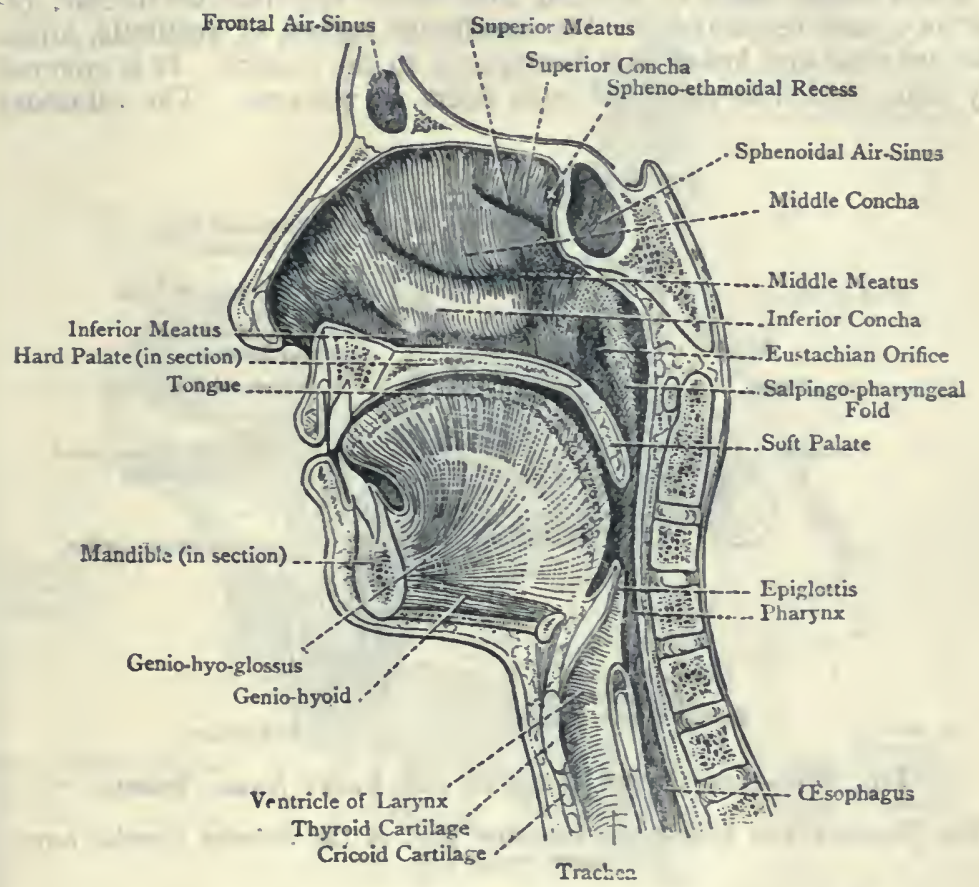

Fig. 513.-Sagittal Section through the Nasal Fossa, Mouth, Pharynx, Csophagus, aNd Larynx.

(The Outer Wall of the Right Nasal Fossa is shown).

in its horizontal portion, whilst the middle ethmoidal cells open above the bulla ethmoidalis. In front of the middle meatus is the region known as the atrium, which communicates anteriorly with the vestibule, the latter being situated just within the ala of the nostril.

The inferior meatus is situated below the inferior concha. Opening into its anterior part, under cover of the inferior concha, is the lower orifice of the nasal duct, which is provided with an imperfect mucous fold, called the valve of Hasner. The orifice is about $\mathrm{I}_{\frac{1}{4}}$ inches from the anterior nasal aperture. 
The inner wall forms the septum nasi.

The roof, which is narrow, is horizontal in its central part, the anterior portion being sloped downwards and forwards, and the posterior portion downwards and backwards.

The floor is smooth, and at its anterior and inner part is the incisor foramen, into which a funnel-shaped portion of the mucous membrane extends. This region represents the wide communication which existed in early life between the nasal and buccal cavities.

Each nasal fossa is divided into three regions-vestibular, olfactory, and respiratory. The vestibular region, or vestibule, forms the anterior and lower part contiguous to the nostril. It is covered by skin, which is provided with hairs or vibrissa. The olfactory

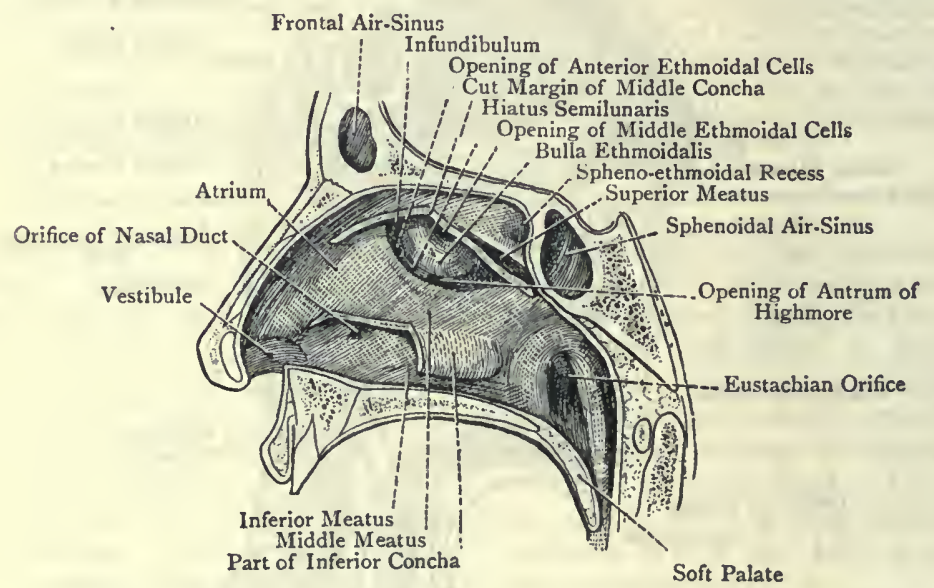

Fig. 514.-The Outer Wall of the Right Nasal Fossa.

(The Superior and Middle Conchæ and part of the Inferior Concha have been removed).

region is situated superiorly, and corresponds to the superior concha and the upper third of the septum nasi. The respiratory region comprises the middle and inferior conchæ, the middle and inferior meatûs, and the corresponding part of the septum.

Mucous Membrane.-With the exception of the vestibule, which is lined with skin, the nasal fossa is provided with a highly vascular and sensitive mucous membrane, called the pituitary or Schnciderian membrane. It is continuous through the posterior nares with the mucous membrane of the naso-pharynx; with that of the nasal duct, lachrymal sac, and lachrymal canaliculi, and thence with the conjunctiva; and with that of the various air-sinuses which communicate with the nasal fossa. It is thick and spongy over the conchæ, especially along the lower borders of the middle 
and inferior conchæ, and also on the septum nasi, but over the floor, atrium, and meatus it is comparatively thin. It is freely provided with acinous glands, and contains a certain amount of lymphoid tissue.

The epithelium varies in different regions. In the vestibular region, where the lining membrane is skin, the epithelium is of the stratified squamous variety ; in the respiratory region, as wcll as in

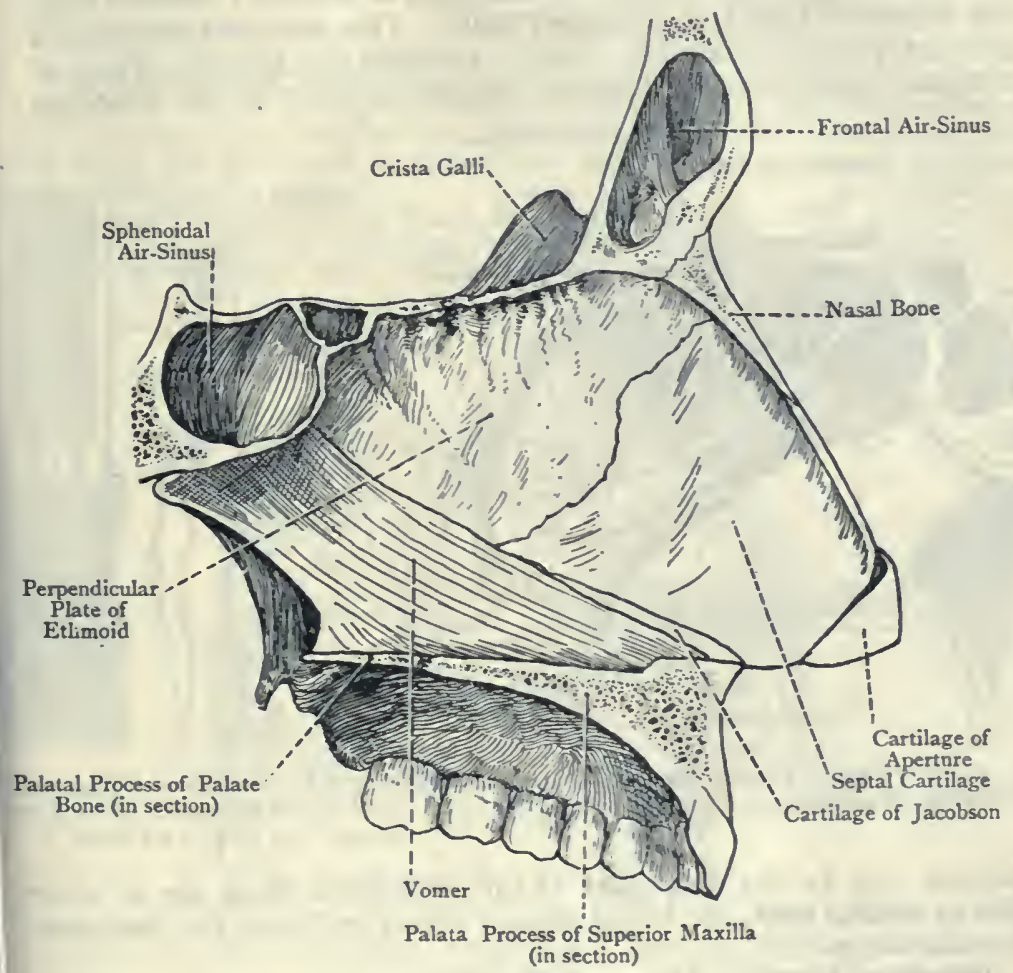

Fig. 515.-The Osseous and Cartilaginous Nasal Septum (Right LATERAL View).

the air-sinuses, it is stratified, columnar, ciliated epithelium; and in the olfactory region it is non-ciliated columnar epithelium.

Olfactory Mucous Membrane.-In the olfactory region the mucous membrane is thick and pulpy, and has a yellowish-brown colour, due to pigment in the epithelial cells. It contains a copious plexus of olfactory nerve-fibres, and many serous glands, which are known as the glands of Bowman. The epithelium is thick, and, as just stated, is of the non-ciliated columnar variety, its free surface being covered by a delicate limiting membrane. The cells 
of which it is composed are of three kinds. (I) Long columnar nucleated cells, called the sustentacular cells, the deep ends of which are prolonged each into a branched process. (2) Between these sustentacular cells there are the olfactory cells, which are elongated and spindle-shaped. Each contains an almost spherical nucleus, and has a superficial and a deep process or pole. The superficial pole extends through the limiting membrane to the free surface, and projects slightly in the form of a tuft of delicate hairlike filaments called the olfactory hairs. The deep pole, which is a delicate varicose filament, extends towards the mucosa, where it becomes continuous with one of the nerve-fibrils of the olfactory
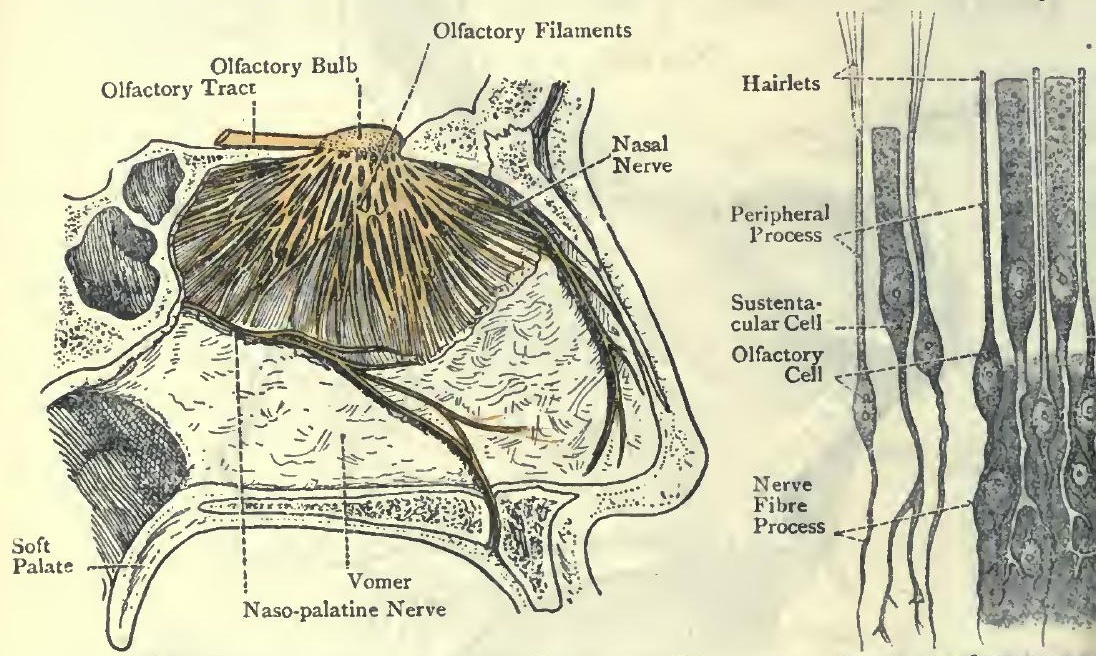

Fig. 516A.The Nerves of The Nasal Septum (Hirsch Feld and LeVeillé).

Fig. 516B.-Cells of Olfactor MuCOUS MEMBRANE (SCHULTZI FROM QUAIN'S 'ANATOMY').

plexus. (3) In the deep part of the epitheliam there are in some places conical cells, the broad enc's of which rest upon the basement membrane.

Olfactory Nerves.-These are from fifteen to twenty in number on each side. After leaving the inferior surface of the olfactory bulb, they pass through the foramina in the corresponding half of the cribriform plate of the ethmoid bone, and so reach the upper part of the nasal fossa, invested by prolongations of the membranes of the brain. Within the nasal fossa they are arranged in two groups, inner and outer. The nerves of the inner group are distributed to the mucous membrane of the septum nasi over about its upper third. The nerves of the outer group are distributed to the mucous membrane in the region of the superior concha and olfactory sulcus. The nerves form a copious plexus in the mucous membrane, and the filaments which issue from this plexus become continuous, as 
stated, with the deep poles of the olfactory cells. The olfactory nerves are destitute of a medullary sheath.

Nerves of Ordinary Sensation.-These are derived from the ophthalmic and superior maxillary divisions of the fifth cranial nerve, and are as follows :

1. Superior nasal nerves.

2. Nasal branches of the Vidian nerve.

3. Inferior nasal nerves.
4. Internal branches of the nasal nerve.

5. Nasal branch of the anterior superior dental nerve.

6. Naso-palatine nerve.

The superior nasal nerves are derived from Meckel's ganglion. They enter the superior meatus through the spheno-palatine foramen, and are distributed to the mucous membrane (1) over the superior and middle conchæ, (2) over the upper and back part of the septum nasi, and (3) within the posterior ethmoidal cells.

The nasal branches of the Vidian nerve pierce the floor of the pterygoid or Vidian canal, and are distributed to the mucous membrane over the back part of the roof of the nasal fossa and the adjacent part of the septum.

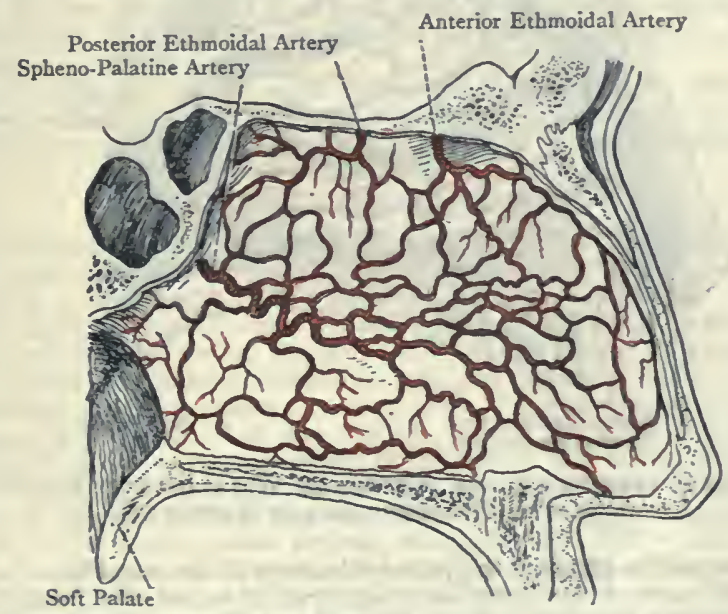

Fig. 517.-The Arteries of the Nasal Septua (Hirschfeld and LEVEILLÉ).

The inferior nasal nerves arise from the great or anterior descending palatine branch of Meckel's ganglion, as that nerve traverses the posterior palatine canal. They enter the nasal fossa through foramina in the vertical plate of the palate bone, and are distributed to the mucous membrane over the greater part of the inferior concha, and the corresponding parts of the middle and inferior meatûs.

The internal branches of the nasal nerve are two in number, septal and external. The septal branch is distributed to the mucous membrane over the anterior and upper part of the septum, and the external branch to that over the anterior portions of the middle and inferior conchæ, and over the outer wall in front of these.

The nasal branch of the anterior superior dental nerve is distributed to the mucous membrane over the anterior part of the inferior meatus and the adjacent part of the floor of the nasal fossa. 
The naso-palatine nerve, or nerve of Cotunnius, arises from Meckel's ganglion, and enters the nasal fossa through the spheno-palatine foramen. It then crosses the roof, and so reaches the septum, upon which it descends, with a forward inclination, occupying the groove on the outer surface of the vomer. Thereafter it passes downwards to the anterior part of the hard palate, the left nerve traversing the anterior foramen of Scarpa, and the right nerve the posterior foramen of Scarpa. As the naso-palatine nerve lies upon the septum nasi it furnishes branches to its mucous membrane.

Arteries of the Nasal Fossa.-These arteries are derived from the following sources:

1. The spheno-palatine.

2. The descending palatine.

3. The Vidian.

4. The anterior ethmoidal.

5. The posterior ethmoidal.

6. The superior coronary.

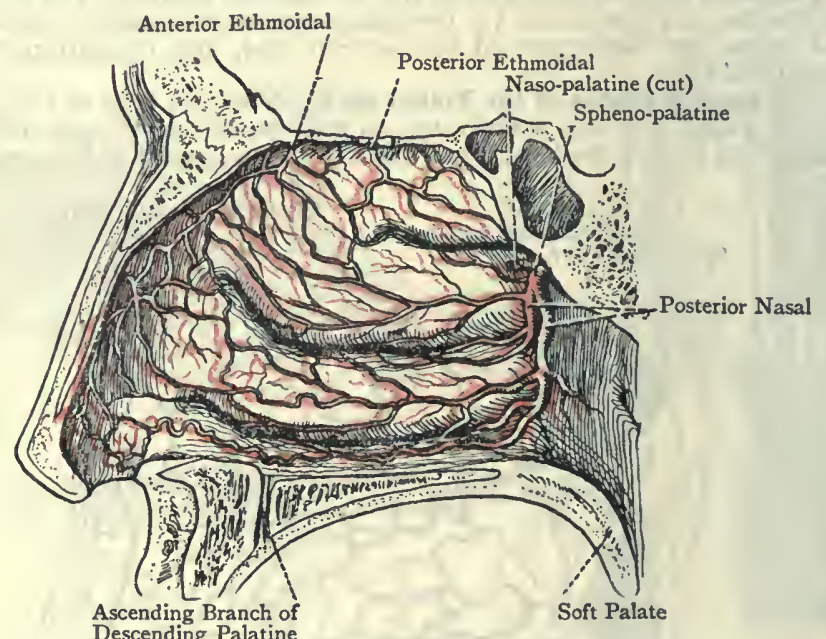

Fig. 518.-The Arteries of the External Wall of the Right Nasal Fossa (after Hirschfeld and Leveillé).

The spheno-palatine artery is the principal artery of the nasal fossa. Arising from the third part of the internal maxillary, it enters the fossa through the spheno-palatine foramen. Most of its branches are distributed to the outer wall; but one, called the naso-palatine branch, accompanies the nasopalatine nerve. This branch gives offsets to the septum, and terminates by entering the incisor foramen, where it anastomoses with a branch of the descending palatine artery, which ascends from the palate in the incisor canal (Stensen's canal).

The descending palatine artery arises from the third part of the internal maxillary. As it traverses the posterior palatine canal it gives off two or three branches which accompany the inferior nasal nerves through foramina in the vertical plate of the palate bone, to be distributed to the back part of the inferior concha and the adjacent parts of the middle and inferior meatûs.

The Vidian artery, arising from the third part of the internal maxillary, traverses the pterygoid or Vidian canal, and furnishes a few twigs which accompany the nasal offsets of the Vidian nerve, and supply the back part of the roof of the nasal fossa and the adjacent part of the septum.

The anterior and posterior ethmoidal arteries are branches of the ophthalmic. The anierior ethmoidal enters the nasal fossa along with the nasal nerve, and furnishes branches to the anterior and upper part of the septum, the front 
part of the outer wall, and the anterior portions of the middle and inferior concha. The posterior ethmoidal furnishes nasal branches which enter the nasal fossa through some of the foramina in one half of the cribriform plate of the ethmoid bone, and are distributed to the roof and upper part of the septum.

The superior coronary artery, a branch of the facial, furnishes the artery of the septum, which supplies the anterior part of the septum and the columella nasi.

The veins form a copious plexus, especially over the inferior concha and the lower margin and back part of the middle concha. The vessels which carry away the blood from the plexus correspond to the various arteries, and their principal destination is threefold, namely, (I) the pterygoid plexus, (2) the superior ophthalmic vein, and (3) the facial vein. The ethmoidal veins, which open into the superior ophthalmic vein, communicate with the intracranial anterior meningeal veins and with the angular vein of the face by means of twigs which pass through minute apertures in the nasal process of the superior maxilla, and, it may be, through a small foramen in the nasal bone. In those cases where the foramen cæcrm is pervious it transmits an emissary vein which passes between the intracranial superior longitudinal sinus and the veins of the roof of the nasal fossa.

The lymphatics of the atrium and vestibule pass to the submaxillary lymphatic glands. The principal lymphatics pass to (I) the retro-pharyngeal glands; (2) the deep facial, or internal maxillary, glands; and (3) the superior deep cervical glands. The lymphatics of the air-sinuses end in a similar manner.

The lymphatics of the nasal fossæ communicate with lymphatic spaces which are related to the olfactory nerve-filaments; and these lymphatic spaces in turn communicate with the intracranial, subdural, and subarachnoid spaces.

\section{Development of the Nose.}

The primitive oral cavity, or stomodæum, becomes divided into two chambers, upper or nasal, and lower or oral, this division being effected by the formation of the hard palate. The oral chamber constitutes the permanent mouth, and the nasal chamber becomes transformed into the two nasal fossa.

The first indications of the olfactory organ are the two olfactory areas. They consist of thickened ectoderm, and are placed on the ventral aspect of the anterior cerebral vesicle, on either side of the mesial nasal process of the fronto-nasal process, and on the cephalic side of the orifice of the stomodrum.

Each olfactory area soon becomes depressed and forms the olfactory pit. The two pits, right and left, are situated one on each side of the mesial nasal process of the fronto-nasal process. Each pit has the corresponding globular process of the mesial nasal process internally, and the lateral nasal process externally, this position corresponding to the situation of the fnture anterior naris on either side. At this stage the olfactory pits communicate with the stomodæum, and the orifice of each pit constitutes the corresponding primitive snterior naris.

The lateral nasal process of the fronto-nasal process is at first separated from the maxillary process by a groove, called the oculo-nasal, naso-optic, or lachrymal sulcus, which extends from the ocular pit on the outer side of the lateral nasal process (which forms the ala of the nostril) to the olfactory pit. This sulcus indicates the position of the future lachrymal sac and nasal duct of the corresponding side. When the maxillary process (previously joined by the lateral nasal process) grows inwards to unite with the globular process, it lies below the olfactory pit. In this manner the orifice of the olfactory pit-namely, the primitive anterior naris-is cut off from communication with the stomodæum.

The blind olfactory pits now grow dorsalwards along the roof of the stomodæum, and so give rise to the nasal sacs which represent the primitive nasal lossæ. As each pit grows dorsalwards it is deepened by the formation of a groove, called the nasal groove, which extends to the roof of the stomodrum. This groove is bridged over by ectoderm, and is thus separated from the corresponding olfactory pit. When the maxillary process (previously joined 
by the lateral nasal process) unites with the globular process, the nasal groove is obliterated, and the olfactory pit is cut off from the stomodæum. By the development of the hard palate, on either side, the ectodermic covering of the nasal groove disappears. The posterior part of this covering constitutes the naso-stomodcal membrane. When this membrane ruptures, on either side, a dorsal aperture, called the primitive posterior naris, is formed, likewise on either side, which establishes a fresh communication between the olfactory pit (blind end) and the stomodæum on the cephalic side of the corresponding palatal shelf.

By the formation of the hard palate the nasal fossæe are almost entirely shut off from the permanent mouth, and by the formation of the septum nasi the fossæ are entirely separated from each other. The communications which originally existed between the olfactory pits and the stomodæum become considerably curtailed as the hard palate is being formed, and they are permanently represented, in man, by the canals of Stensen, in the region of the anterior palatine fossa. In certain animals-e.g., ruminantia-there is a wide passage on either side of the median line, between the ventral part of the hard palate and each nasal fossa, which passage leads to the organ of Jacobson.

Septum Nasi.--The septal cartilage of the nose is derived from the ethmoidal division of the basal sheet of cartilage, called the chondrocranium, and the vomer is developed in the mesoderm which invests the posterior and inferior parts of the septal cartilage. The perpendicular plate of the ethmoid is developed from the ethmoidal division of the chondrocranium, as is also the cribriform plate.

The superior, middle, and inferior spongy bones, or concha, appear as folds of ectoderm which project from the outer wall of the nasal fossa. These folds contain mesoderm, and in this mesoderm cartilage, pertaining to the cartilaginous nasal capsule, is formed. The cartilage undergoes ossification, and gives rise to the three conchæ, the upper two forming part of the lateral mass of the ethmoid, whilst the inferior concha persists as an independent bone, usually spoken of as the inferior turbinate bone.

Air-Sinuses. - The frontal, ethmoidal, sphenoidal, and maxillary air-sinuses are developed as evaginations of the nasal mucous membrane, which extend into spaces formed by absorption within the respective bones. The mastoid cells are developed as evaginations of the tympanic mucous membrane, which is continuous with the nasal mucous membrane through the Eustachian tube and naso-pharynx.

Olfactory Organ.-The true olfactory organ is situated in the upper part, or olfactory region, of each nasal fossa. The olfactory epithelium, which covers the mucous membrane of this region, is developed from the upper part of the corresponding olfactory pit.

External Nose.-The dorsum and tip of the nose, and the columella nasi are developed from the portion of the mesial nasal process which lies between the globular processes. The upper and lower lateral nasal cartilages are derived from the cartilaginous core of the corresponding lateral nasal process. The anterior naris of each side represents, as stated, the external orifice of the corresponding olfactory pit.

Organ of Jacobson.- Jacobson's organ is rudimentary in man, but is well developed in ruminantia. It takes the form of a blind pouch, which is situated in the lower and anterior part of the septum nasi on either side. The minute orifice of the pouch lies above the recess or depression of mucous membrane which projects slightly into the upper end of Stensen's canal. The pouch extends upwards and backwards in the septum nasi for a very short distance, and terminates in a blind extremity. The cartilage of Jacobson lies underneath it. Jacobson's organ does not seem to perform any function in man. In those animals, however, in which it is well developed, e.g., ruminantia, it receives two nerves, one of which 
is olfactory, the other being derived from Meckel's ganglion. Moreover, its epithelial lining is similar to that of the olfactory region of the nasal fossa, inasmuch as it contains olfactory cells, the deep poles of which are continuous with olfactory filaments. In such animals it acts as a supplementary organ of smell.

Development of Organ of Jacobson.- This organ is developed as a diverticulum of a portion of the ectoderm of the olfactory pit. The diverticulum projects upwards and backwards into a recess situated laterally within the lower and anterior part of the septal nasal cartilage, just above the upper end of Stensen's canal (incisor canal).

\section{The Pharyax.}

The pharynx is situated behind the posterior nares, isthmus faucium, and larynx, and it extends from the basilar region of the base of the skull to the level of the lower border of the cricoid cartilage of the larynx, where it becomes continuous with the œsophagus. It is a musculo-aponeurotic tube, about 5 inches long, and it attains its greatest width between the base of the skull and the hyoid bone. Below the latter level it narrow's, and is flattened from before backwards, so as to assume the form of a transverse cleft, except during the act of deglutition.

Relations.-Posteriorly it rests upon the bodies and discs of the cervical vertebræ as low as the sixth, and the prevertebral muscles covered by the prevertebral fascia. Between it and the last-named fascia is the retropharyngeal space, which is occupied by connective tissue, but this is so loosely arranged that no obstacle is offered to the movement of the tube, and a post-pharyngeal abscess can readily diffuse itself. Anteriorly it communicates with (I) the nasal fossæ through the posterior nares, (2) the Eustachian tubes, (3) the buccal cavity through the isthmus faucium, and (4) the larynx. On this aspect its attachments are effected by means of the constrictor muscles in its walls. Laterally it is related to the principal bloodvessels and nerves of the neck, and comes into contact with the styloid muscles and the process from which they arise. Superiorly it is attached to the basilar region of the base of the skull. Inferiorly it is continuous with the œsophagus.

The wall of the pharynx consists of the following four strata, in order from without inwards: (I) the pharyngeal portion of the bucco-pharyngeal fascia, (2) the muscular coat, (3) the pharyngeal aponeurosis, and (4) the mucous coat.

Pharyngeal Fascia.-This is an offshoot of the prevertebral lamina of the deep cervical fascia along the mesial aspect of the carotid sheath. In front it becomes continuous with the fascia covering the buccinator muscle. In association with this fascial stratum there are many veins upon the posterior and lateral walls of the pharynx, which constitute the pharyngeal venous plexus.

Muscular Coat. - This is composed, on either side, of the three constrictor muscles, inferior, middle, and superior, the stylopharyngeus, and the palato-pharyngeus (including the salpingopharyngeus). 
Inferior Constrictor-Origin.-(I) The side of the cricoid cartilage at its posterior part; (2) the inferior cornu and the oblique line of the ala of the thyroid cartilage; and (3) the upper border of the ala behind the superior tubercle.

Insertion.-The median raphé on the posterior wall of the pharynx, where it meets its fellow of the opposite side.

The lower fibres of the muscle are horizontal, but the upper fibres pass upwards and backwards more and more obliquely, and the highest fibres of the two muscles meet in the raphe to form a peak, which is about an inch below the basilar process of the occipital bone. The lower border of the muscle overlaps the upper end of

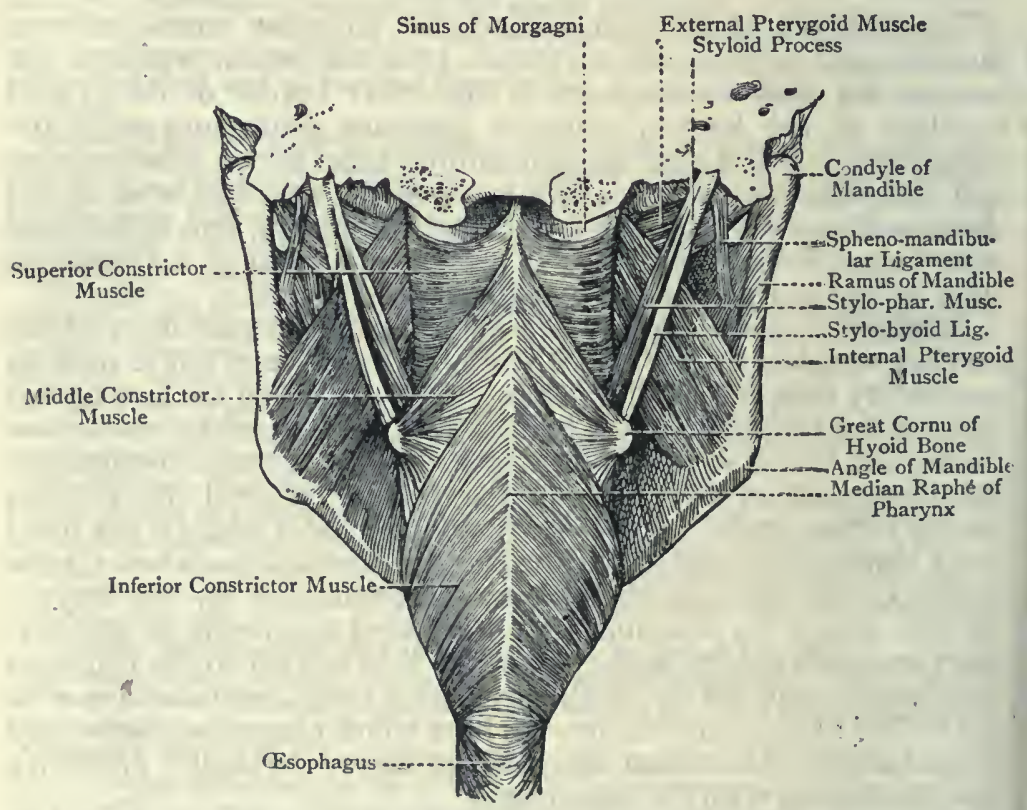

Fig. 519.-The Posterior Wali of the Pharynx and Adjacent STRUCTURES.

the œsophagus, and, to a limited extent, some of the fibres are continuous with the œsophageal muscular fibres. The recurrent laryngeal nerve and inferior laryngeal artery pass upwards beneath the lower border behind the crico-thyroid joint. The upper border, which is very oblique, overlaps the lower portion of the middle constrictor, and the internal laryngeal nerve and the superior laryngeal artery, on their way to pierce the thyro-hyoid membrane, pass between the two muscles anteriorly.

Middle Constrictor-Origin.--(I) The upper surface of the great cornu of the hyoid bone over its entire length, (2) the small cornu, and (3) the hyoid extremity of the stylo-hyoid ligament. 
Insertion--The median raphe on the posterior wall of the pharynx, where it meets its fellow of the opposite side.

The muscle is fan-shaped, and its fibres consequently diverge very much as they pass round to reach the raphé. The inferior fibres descend very obliquely, and are overlapped by the upper fibres of the inferior constrictor, the internal laryngeal nerve and superior laryngeal artery passing to the thyro-hyoid membrane between the two muscles anteriorly. The middle fibres pass more or less transversely. The superior fibres ascend obliquely, and reach the basilar process of the occipital bone. They overlap the lower portion of the superior constrictor, and the stylo-pharyngeus muscle and glosso-pharyngeal nerve pass between the two. The lingual artery rests upon the muscle at the great cornu of the hyoid bone.

Superior Constrictor-Origin.-(I) The lower third of the posterior border of the internal pterygoid plate, and the hamular process of the sphenoid bone; (2) the posterior aspect of the pterygo-mandibular ligament, along which it meets the buccinator muscle; (3) the posterior extremity of the mylo-hyoid ridge of the lower jaw; $(4)$ the mucous membrane of the mouth; and (5) the side of the tongue.

Insertion.-The median raphé on the posterior wall of the pharynx, where it meets its fellow of the opposite side. A few of the highest fibres are inserted into the pharyngeal tubercle on the under surface of the basilar process of the occipital bone.

The muscle is somewhat four-sided. The fibres for the most part pass horizontally, but the lower fibres radiate in a downward direction, whilst the upper fibres curve backwards and upwards. The lower portion of the muscle is overlapped by the upper part of the middle constrictor, the stylo-pharyngeus muscle and glossopharyngeal nerve passing between the two. Between the upper, concave border and the base of the skull there is an interval occupied by the pharyngeal aponeurosis, which is here stronger than elsewhere, and so compensates for the absence of muscular fibres. This interval is semilunar, and is known as the sinus of Morgagni.

Nerve-supply of the Constrictor Muscles.-The nerves are derived from the pharyngeal plexus, which is formed by the pharyngeal branch of the pneumogastric, the pharyngeal branches of the glosso-pharyngeal, and sympathetic filaments. The motor fibres of the plexus are derived from the pharyngeal branch of the pneumogastric, but their ultimate source is the bulbar portion of the spinal accessory nerve. The inferior constrictor muscle also receives twigs from (I) the external laryngeal branch of the superior laryngeal nerve, and (2) the inferior or recurrent laryngeal nerve as it passes beneath the lower border of the muscle.

Action.-The constrictor muscles are concerned in the act of deglutition. The superior constrictor and upper portion of the middle constrictor act upon the naso-pharynx, that is, the compartment of the pharynx which lies above the soft palate, and is in communication with the nasal fossæ through the posterior nares. They narrow the naso-pharynx in the lateral direction, and this 
movement, in conjunction with the action of the posterior pillars of the fauces, shuts off the naso-pharynx, thus preventing regurgitation into and through the nasal fossæ. The lower portion of the middle constrictor and the inferior constrictor diminish the calibre of the buccal compartment of the pharynx. Coming into action during the second stage of deglutition, they grasp the bolus of food and press it downwards into the œsophagus. The constrictor muscles contract rapidly, and in order from above downwards.

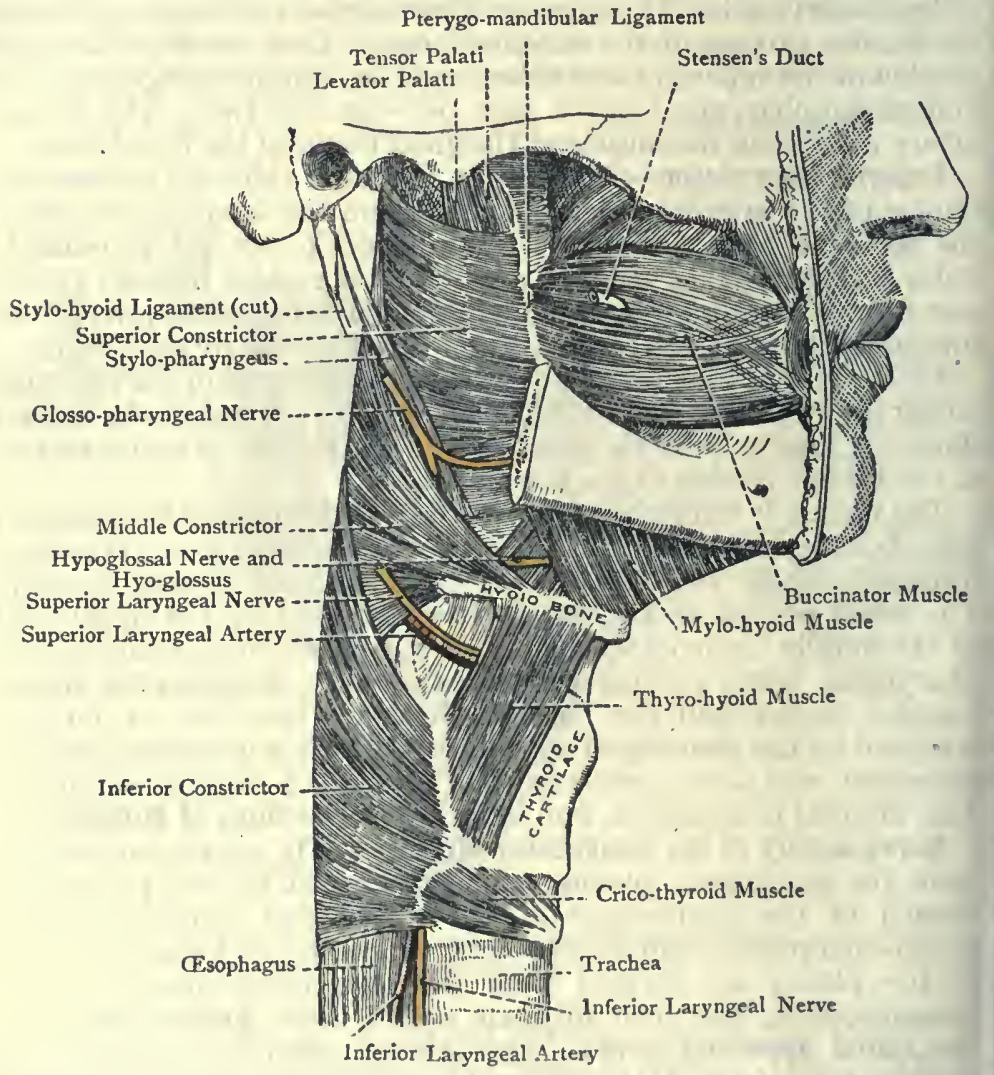

Fig. 520.-Dissection showing the Cheek, Pharynx, Submaxillary REgION, AND LARYNX (RIGHT VIEW).

For a description of the stylo-pharyngeus and palato-pharyngeus muscles (including the salpingo-pharyngeus), see Index.

Pharyngeal Aponeurosis. - This is situated between the muscular coat and the mucosa. Inferiorly it is weak and indistinct, but superiorly it acquires greater firmness and density, expecially where it is attached to the basi-occipital, the apex of the petrous part of the temporal bone, the adjacent portion of the Eustachian tube, 
and the internal pterygoid plate of the sphenoid bone. It receives an accession of strength in the median line from a strong bundle of fibres which descends from the pharyngeal tubercle on the under surface of the basilar process of the occipital bone, and which forms the raphe of the pharynx.

Interior of the Pharynx.-The mucous membrane, which forms the fourth stratum in the pharyngeal wall, is of a papillary character, and has the following important continuations: (I) with the mucous membrane of the Eustachian tube, and thence with that of the

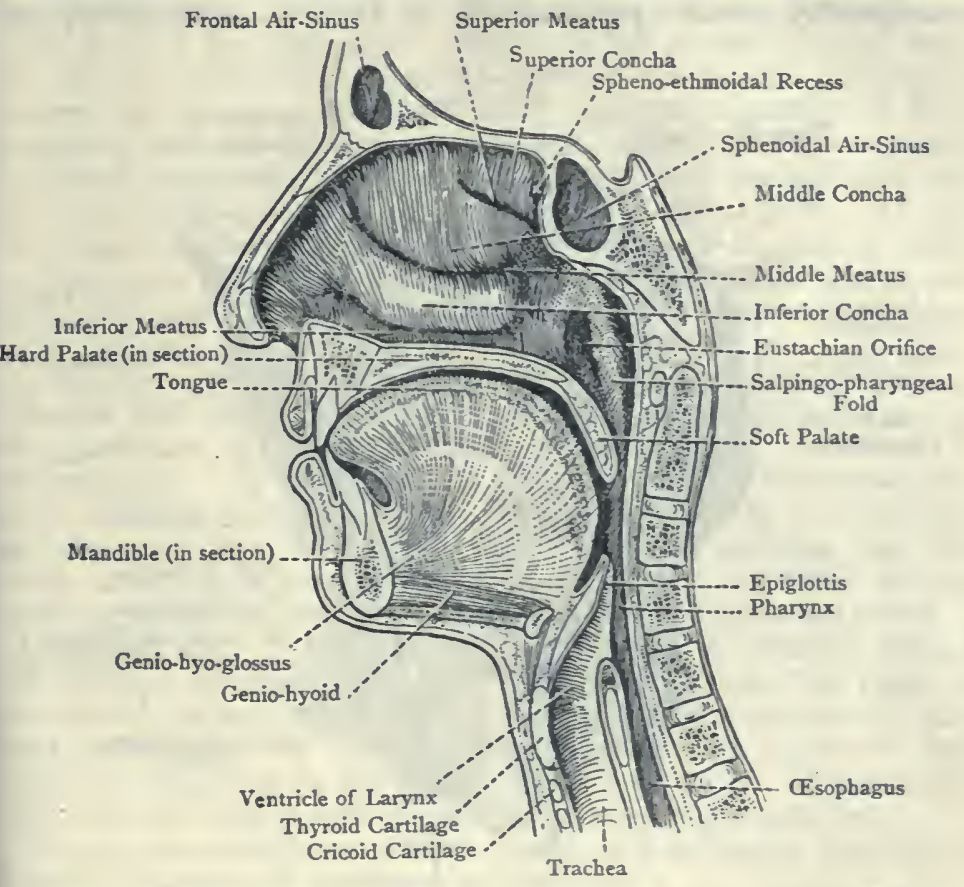

Fig. 521.-Sagittal Section through the Nasal Fossa, Mouth, Pharynx, CEsophagus, and Larynx.

(The Outer Wall of the Right Nasal Fossa is shown)

tympanum or middle ear; (2) with that of the nasal fossæ through the posterior nares; (3) with that of the buccal cavity through the isthmus faucium; (4) with that of the larynx through the superior laryngeal aperture; and (5) with that of the œsophagus. At its upper and back part it is richly provided with lymphoid tissue, to be presently referred to, and in the submucous tissue there are many acinous glands of a mucous character.

The soft palate projects into the cavity of the pharynx in a downward and backward direction, and divides it into two regions, upper and lower. The upper region is known as the naso-pharynx, 
and is in communication with the nasal fossæ and Eustachian tubes. It also communicates with the lower region by means of the pharyngeal isthmus. The lower region is subdivided into two parts, buccal and laryngeal. The buccal part is limited above by the soft palate, and below by the superior aperture of the larynx, and it communicates with the buccal cavity through the isthmus faucium. The laryngeal part is situated behind the larynx, with which it communicates, as well as with the osophagus inferiorly.

Naso-pharynx.-This is entirely respiratory in function, and is consequently always pervious. It is bounded in front by the

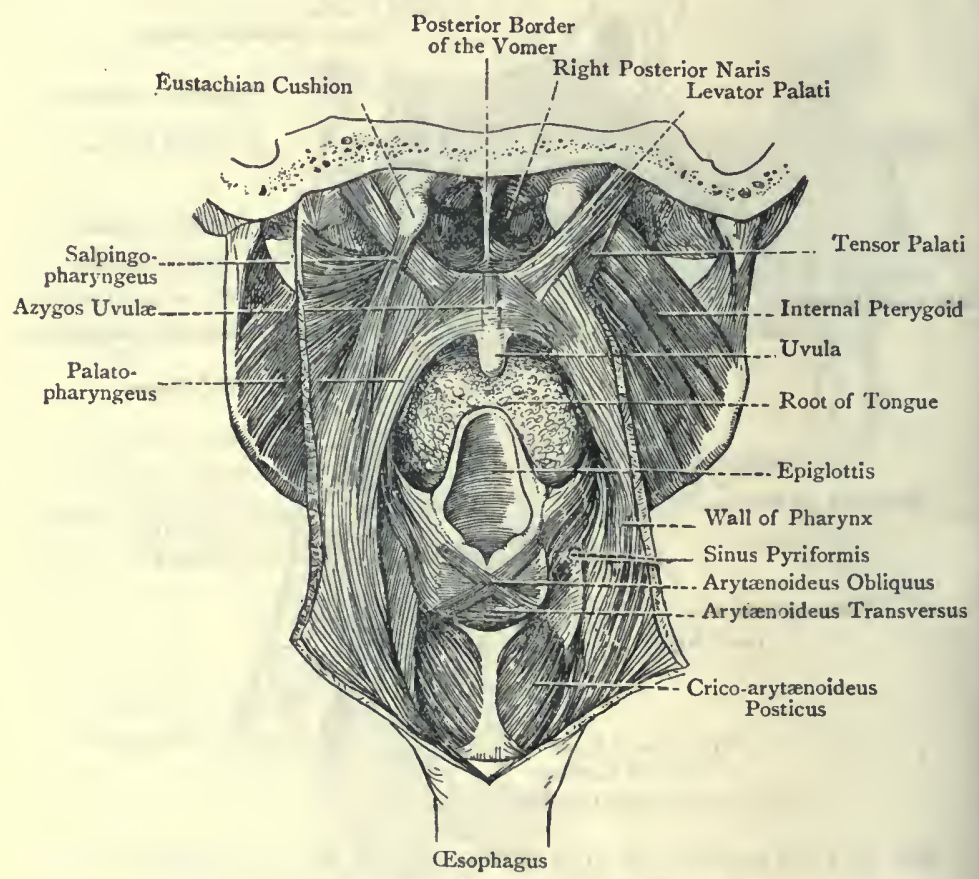

Fig. 522.-The Pharynx opened from behind.

posterior nares and the posterior border of the vomar; behind, by the vertebral column; above, by the basilar process of the occipital and part of the body of the sphenoid; and below by the soft palate, which can be elevated and depressed. Four openings communicate with the naso-pharynx. The anterior wall presents the oval openings of the posterior nares leading from the nasal fossæ, and separated from each other by the posterior border of the vomer. Each opening in the recent state measures about $I$ inch from above downwards, and about $\frac{1}{2}$ inch from side to side. Through it, on the outer wall of the corresponding nasal fossa, are seen the posterior parts of the inferior turbinate bone and the inferior turbinate 
process of the ethmoid bone, covered by mucous membrane; and above and below the inferior turbinate bone are seen the posterior parts of the middle and inferior meatus. The orifice of the Eustachian tube is situated on each lateral wall, behind and external to the corresponding posterior naris, and on the same level as the posterior end of the inferior turbinate bone. It is somewhat oval, and presents, above and behind, a prominent round border, formed of cartilage, called the cushion. Behind the cushion there is a deep pouch on the lateral wall of the naso-pharynx, called the lateral pharyngeal recess, or fossa of Rosenmuller, which represents the upper part of the pharyngeal portion of the second visceral cleft. The mucous membrane extending between the lateral pharyngeal recesses, at the upper and back part, presents a number of folds containing a large amount of lymphoid tissue, which constitute the pharyngeal tonsil. When enlarged this mass is liable to obstruct the Eustachian orifices, and even the posterior nares. At the lower part of the pharyngeal tonsil there may be seen a small median recess, called the pharyngeal bursa, which leads upwards and backwards towards the pharyngeal tubercle on the basilar process of the occipital bone.

The mucous membrane is covered by ciliated columnar epithelium.

Buccal Part of the Pharynx.-This part is situated between the soft palate and the superior aperture of the larynx. Anteriorly it communicates with the buccal cavity through the isthmus faucium, below which is the root of the tongue. Each lateral wall presents the posterior pillar of the fauces, the interval between the two pillars corresponding to the pharyngeal isthmus. In front of each posterior pillar is a triangular depression which lodges the tonsil.

Laryngeal Part of the Pharynx.--This part is situated behind the larynx. Anterior to it there are the epiglottis, the superior aperture of the larynx, on either side of which is a recess called the sinus pyriformis, and the posterior parts of the arytenoid and cricoid cartilages, with the muscles related to them. It communicates with the larynx anteriorly and the œsophagus inferiorly.

The mucous membrane of the buccal and laryngeal portions is covered by stratified squamous epithelium.

Blood-supply. - The arteries of the pharynx are derived from several sources, e.g., the ascending pharyngeal branch of the external carotid, the inferior or ascending palatine and tonsillar branches of the cervical part of the facial artery, and the descending palatine and pterygo-palatine branches of the internal maxillary.

The veins form a copious pharyngeal plexus, which is disposed upon the lateral and posterior walls of the pharynx. It communicates superiorly with the pterygoid venous plexus, and receives tributaries from the soft palate, tonsils, and Eustachian tubes. Inferiorly the blood is conveyed from it into the internal jugular vein directly, or through means of the common facial vein.

Lymphatics.-The lymphatic vessels from the upper part of the pharynx pass to the internal maxillary glands, which are associated with the lateral walls of the tube, and those from the remaining 
part pass to the upper group of deep cervical glands. Some of the lymphatics from the upper part pass to the retropharyngeal gland, two in number, each of which lies upon the upper part of the corresponding rectus capitis anticus major muscle.

\section{Development of the Pharynx, and of the Pharyngeal Pouches or Visceral Clefts and Visceral Arches.}

The pharynx is developed from the anterior or cephalic part of the fore-gut.

On the ventral wall of this part there are two elevations, the anterior of which is called the tuberculum Impar, whilst the posterior is called the furcula. The latter has the form of a horseshoe, the open part of which is placed posteriorly. The furcula is separated from the tuberculum impar in front, and from the lateral wall of the fore-gut on either side, by a groove called the sinus arouatus. The tuberculum impar gives origin to the anterior two-thirds, or buccal portion, of the tongue ; the anterior part or loop of the furcula gives rise to the epiglottis; the posterior extremities of the furcula to the arytenoid cartilages; and the depression within the furcula becomes deepened and developed into the larynx and trachea. Posterior to the furcula the foregut becomes dilated to form the rudimentary stomach, and as this descends the cesophagus is formed.

\section{Pharyngeal Pouches.}

The pharyngeal pouches (visceral clefts) are situated on each lateral wall of the pharyngeal part of the fore-gut, and are arranged in pairs from the cephalic region caudalwards. Their direction is dorso-ventral, or from behind forwards, and they are almost parallel with each other, there being a slight convergence ventralwards. The number of pouches on either side is five in the human embryo. The fifth pouch is rudimentary, and is an appendage of the fourth, from which it projects in a caudal and ventral direction. The pouches are separated from each other by rounded bars, which represent the visceral arches. In order from the cephalic region caudalwards they are designated as first, second, third, fourth, and fifth pouches.

Each pouch is developed as an outward protrusion of the entodermic lining of the pharyngeal part of the fore-gut. To meet each entodermic protrusion from the interior an inward extension of the ectoderm takes place from the exterior. In this manner furrows are formed on the exterior of the pharynx. The bottom of each pharyngeal pouch is closed, and is separated from the corresponding external furrow by an epithelial septum, which constitutes the closing membrane. This septum is bilaminar, its internal layer being entodermic, and its external layer ectodermic, both being in intimate contact.

Metamorphoses of the Pharyngeal Pouches-First Pharyngeal Pouch. - The closing membrane at the bottom of this pouch separates it from the first external furrow which corresponds to it. The pouch itself, being pharyngeal, is entodermic, but the external furrow is ectodermic. From this pouch (entoderm) the tympanitic cavity and the Eustachian tubo are developed. The external furrow (ectoderm) becomes the external auditory meatus, and the lips of this furrow become differentiated into the component elements of the pinna. The closing membrane, originally bilaminar, becomes trilaminar, a third layer, consisting of mesoderm, having been developed between the two original entodermic and ectodermic laminæ. So constituted, the closing membrane forms the membrana tympanl.

Second Pharyngeal Pouch.-The remains of this pouch in the adult are(1) The tonsil; (2) the supratonsillar lossa, a small depression which lies above the tonsil; and (3) the lateral pharyngeal recess or fossa of Rosenmiiller, behind the cushion of the pharyngeal orifice of the Eustachian tube.

Third Pharyngeal Pouch. - This pouch disappears, but it furnishes a diver ticulum from which the corresponding lateral lobe of the thymus body is developed.

Fourth Pharyngeal Pouch.-This pouch likewise disappears, but, like thi third, it furnishes a diverticulum from which a portion of the corresponding 
lateral lobe of the thyroid body is developed. It also gives rise to the sinus pyriformis of the larynx.

Visceral or Branchial Arches.-Between the pharyngeal pouches a thickening of mesoderm takes place in the intervals between the entoderm and ectoderm. These thickenings give rise to elevated, rounded bars, which constitute the visceral or branchial arches. The number of these arches on either side is six. The sixth, however, which lies behind the fifth pharyngeal pouch, remains undifferentiated. Like the pharyngeal pouches, the visceral arches are arranged in pairs. The dorsal extremities of the arches, right and left, stand apart, and are attached to the sides of the head. The ventral extremities, on either side, approach the mesial line of the future embryonic neck, and subsequently they become connected along the mesial line. Each arch projects into the pharyngeal part of the fore-gut, and the first four also form projections on the exterior, which increase the depth of the external

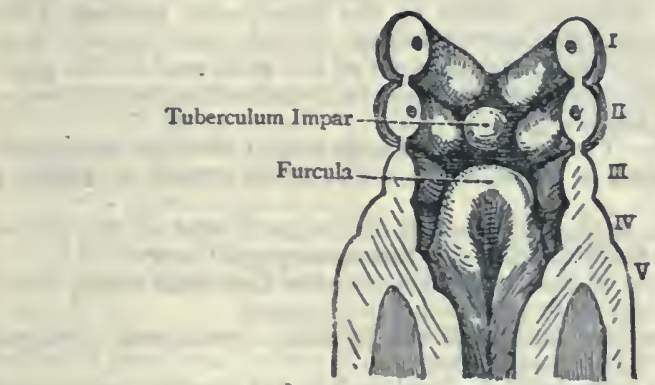

Fig. 523.-The Visceral Arches of the Embryo (Posterior View) (His).

I. Mandibular Arch

II. Hyoid Arch

V. Fifth Arch

III. Thyro-byoid Arch

IV. Fourth Arch

furrows, corresponding to the visceral clefts. The names of the arches, from the cephalic region caudalwards, are as follows:

First, or mandibular, arch.

Second, or hyoid, arch.

Third

Fourth

Fifth Branchial arches proper.

The first arch on either side is situated between the first pharyngeal pouch and the stomodxum or primitive oral cavity. By means of their mandibular and maxillary processes, right and left (the latter being supplemented by the fronto-nasal process), the first arches support the primitive oral cavity, forming its lower or posterior and upper or anterior boundaries. They are therefore known as the oral arches, the succeeding arches being post-oral.

Each visceral arch, in its primitive condition, consists of (I) a central portion, or core, and (2) an epithelial investment. The core consists of mesenchyme (mesoderm), and is peculiar to its own arch. The epithelial investment is derived internally from the entoderm of the pharyngeal part of the fore-gut, and externally from the ectoderm of the exterior.

At a later stage each visceral arch acquires the following four elements: (1) An artery; (2) a cervical myotome or muscle-segment; (3) a nerve (or nerves); and (4) a rod of cartilage.

The artery is known as a visceral-arch artery, and it is one of the primitive aortic arches, which arches, on either side, establish a communication between the corresponding right and left primitive ventral and dorsal aortæ. 
Myotomes of Visceral Arches.-The cervical myotome in each arch gives rise to certain muscles. The myotome of the first arch furnishes (I) the anterior belly of the digastric; (2) the mylo-hyoid ; and (3) the muscles of masticationnamely, $(a)$ masseter, $(b)$ temporal, $(c)$ external pterygoid, and $(d)$ internal pterygoid.

The myotome of the second arch furnishes (I) the posterior belly of the digastric; (2) the stylo-hyoid; and (3) the stapedius. From this myotome are also derived the muscles of expression on the face and epicranial region, as well as the platysma.

The myotome of the third arch furnishes the stylo-pharyngeus, and the middle constrietor of the pharynx may be regarded as derived from it.

The myotome of the fourth arch (and perhaps that of the fifth arch) may be regarded as furnishing the Inferior constrlctor of the pharynx.

Nerves of Visceral Arches-First, or Mandibular (oral), Arch,-The common nerve of this arch is the fifth cranial, or trigeminal, nerve. The inferior maxillary or mandibular division belongs to the mandibular process of the arch, and to its muscles, and the lower teeth of one side; the superior maxillary division belongs to the maxillary process of the arch, and to the upper teeth of one side; and the ophthalmic division belongs to the supplementary fronto-nasal process (one-half).

Second, or Hyoid, Arch.-The nerve of this arch and of the first pharyngeal pouch is the seventh cranial, or faclal, nerve. The eighth cranial, or auditory, nerve may be included.

Third, or Thyro-Hyoid, Arch.-The nerve of this arch and of the second pharyngeal pouch is the ninth eranial, or glosso-pharyngeal, nerve.

Fourth Arch.--The nerve of this arch is the superior laryngeal nerve, which is a branch of the tenth cranial, or pneumogastric (vagus).

Fifth Arch.-The nerve of this arch is the inferior, or recurrent laryngeal, nerve, which is a branch of the pneumogastric.

The bar of cartilage, in each arch, is developed in the mesenchyme, which forms the core of the arch.

\section{Metamorphoses of the Visceral Arches.}

First Visceral, or Mandibular, Arch.-This arch is situated, as stated, between the first pharyngeal pouch and the stomodxum or primitive oral cavity. Its artery is the first primitive aortic arch; its common nerve is the filth cranial or trigeminal nerve; and its cartilaginous bar is known as Meckel's cartilage. The ventral end of this cartilage meets its fellow of the opposite side, and is joined to it by connective tissue. The dorsal end is related to the periotic cartilaginous capsule, and furnishes an offshoot, called the maxillary process. For a full account of the transformation of the first visceral arch see the Development of the Skull. It will be sufficient here to note the following points:

1. The upper or dorsal end of Meckel's cartilage, becoming ossified, gives rise to the malleus, and probably the incus.

2. The lower or ventral end of Meckel's cartilage gives rise to the incisor portion of the mandible.

3. The part of Meckel's cartilage between the upper and lower ends disappears. The membranous investment of the lower or mandibular portion of this part gives rise to the chief part of one-half of the body of the mandible, and the lower half of the ramus as high as the inferior dental foramen. The membranous investment of the upper portion, between the periotic cartilaginous capsule and the inferior dental foramen, forms the spheno-mandibular ligament.

4. The maxillary process, aided by part of the fronto-nasal processnamely, the globular and lateral nasal processes, gives rise to the superior maxilla (see Development of the Skull).

5. The upper end of the first arch gives rise to the tragus, and part of the helix of the pinna.

Second, or Hyoid, Arch.-This arch is situated, as stated, between the first and second pharyngeal pouches. Its artery is the second primitive aortic 
arch ; its nerve is the seventh cranial, or facial, nerve, with the chorda tympani and the auditory nerves, and its cartilaginous bar is called the hyoid bar, or cartilage of Reichert. This bar ventrally is connected with its fellow of the opposite side and with the thyro-hyoid bar of the same side by a transverse piece of cartilage, called the copula, which lies between the tuberculum impar and the loop of the furcula.

The second arch becomes transformed into the following structures:

1. The upper or dorsal segment of the hyoid bar gives rise to the head, neck, and crura of the stapes, the foot-piece of that ossicle being developed from the cartilaginous capsule of the labyrinth, within the fenestra ovalis.

2. The succeeding segment of the hyoid bar gives rise to $(a)$ the tympanohyal, and $(b)$ the stylo-hyal, which collectively constitute the styloid process of the temporal bone.

3. The next portion of the hyoid bar becomes converted into fibrous tissue, and forms the stylo-hyoid ligament, which sometimes exists as an epi-hyal bone.

4. The lower or ventral segment of the hyoid bar gives rise to the ceratohyal, or small cornu of the hyoid bone.

The second arch also gives rise superiorly to the antihelix, antitragus, and lobule of the pinna; and inferiorly, along with the third arch, to one-half of the posterlor third of the tongue.

Third, or Thyro-Hyoid, Arch.-This arch is situated between the second and third pharyngeal pouches. Its artery is the third primitive aortic arch ; its nerve is the ninth cranial, or glosso-pharyngeal nerve; and its cartilaginous bar is known as the thyro-hyold bar. This bar is connected ventrally with its fellow, and with the hyoid bar of the same side, by the copula already referred to.

A large portion of the thyro-hyoid bar disappears, but its lower or ventral segment gives rise to the thyro-hyal or great cornu of the hyold bone. The basi-hyal or body of the hyoid bone is developed from the copula.

Fourth and Fifth Visceral Arches.-The artery of the fourth arch is the lourth primitive aortic arch, and its nerve is the superior laryngeal nerve, which is a branch of the pneumogastric. The artery of the fifth arch is the fifth primitlve aortlc arch, and its nerve is the inferior, or recurrent, laryngeal branch of the pneumogastric. The greater portions of these two arches disappear; but the lower or ventral ends of their cartilaginous bars are usually regarded as giving rise to the corresponding ala of the thyrold cartilage.

Sixth Visceral Arch.-The artery of this arch is the sixth primitive aortic arch. The sixth arch itself, being undifferentiated, leaves no traces behind it.

The metamorphoses of the pharyngeal pouches have been already described in connection with these pouches.

The first external furrow, corresponding to the first internal pharyngeal pouch, gives rise, as stated, to the external auditory meatus, and the walls of this furrow become differentiated into the component parts of the pinna.

Sinus Cervicalis and Cervical Fistula. - The first or mandibular and second or hyoid arches increase more rapidly in all directions than the succeeding branchial arches proper. The third and fourth branchial arches therefore become overlapped by the second or hyoid arch, and now lie at the bottom of a depression. This depression is called the sinus cervicalis. The lining membrane of the sinus is formed by the ectodermic constituents of the overlapped branchial arches. The sinus is, as a rule, transitory, being soon obliterated as the second visceral arch grow's backwards towards the fifth arch, the lips of the sinus fusing. In rare cases, however, the sinus cervicalis may persist as an ectodermal cervical cyst. In other rare cases the ectoderm of the sinus may become ruptured, and then a communication might be established with a mucous canal-namely, the pharyngeal portion of the fore-gut, thus giving rise to the condition known as cervical fistula.

Morphology of the Visceral Arches and Clefts.-In aquatic animals-e.g., Fishes, and Amphibia at an early stage, but only in Perennibranchiata permanently-these are called the branchial or gill-arches and clefts. The clefts range in number from five to eight, and they differ from those of Mammals and Birds inasmuch as they are complete clefts, the closing membrane being 
absent. They therefore establish free communications between the extericr and the fore-part of the alimentary canal, or throat, for the entrance and exit of water.

The functional branchial arches are those which are post-oral, commencing with the second. The second branchial or gill-arch is not, however, a real branchial arch in the functional sense, but is opercular, giving rise, on either side, to the operculum or gill-cover. The real branchial or gill-arches, properly so-called functionally, are those which succeed to the second, of which the third and fourth are conspicuous. The mucous membrane of the real branchial arches is folded into parallel lamellæ, which are placed close together, and are freely furnished with capillary bloodvessels, the blood being derived from the branchial-arch arteries. These lamellæ constitute the branchiæ or gills.

Aquatic respiration consists in the passage of currents of water, containing oxygen, through the complete gill-clefts into the pharyngeal part of the fore-gut. As the water bathes the branchiæ, or gills, its oxygen is taken up into the blood within the branchial capillaries, and the carbon dioxide of the capillary blood is yielded up to the water. Thereafter the water is expelled through the gill-clefts, and is immediately replaced by a fresh current of respiratory water. The branchiæ, or gills, of aquatic animals therefore correspond functionally to the lungs of Mammals and Birds, whose respiration is aerial.

\section{Eustachian Tube.}

The Eustachian tube leads from the tympanum to the nasopharynx, and is about $I \frac{1}{2}$ inches in length. It is directed forwards, inwards, and slightly downwards, and is composed of two parts, osseous and cartilaginous. The osseous or postero-external part is about $\frac{1}{2}$ inch long, and represents the lower or Eustachian compartment of the canal situated in the angle between the petrous and squamous parts of the temporal bone. The cartilaginous or antero-internal part is about $I$ inch in length, and lies in the groove between the great wing of the sphenoid and the apical portion of the petrous part of the temporal bone. It is at first narrow, but gradually enlarges, so as to resemble a trumpet. The narrowest portion of the whole tube is at the junction of the osseous and cartilaginous parts, this being known as the isthmus, and the widest part is at the pharyngeal orifice. The roof, inner wall, and upper part of the outer wall of the cartilaginous part consist of a triangular plate of cartilage, the margins of which are slightly rolled towards each other. The floor-and most of the outer wall are formed of a dense fibrous membrane. The pharyngeal orifice of the tube is expanded, and is situated on the lateral wall of the naso-pharynx behind, and external to, the corresponding posterior naris, and on the same level as the posterior end of the inferior turbinate bone. It is somewhat oval, and presents, above and behind, a prominent border, formed by the thick margin of the cartilage, and called the cushion. Posterior to this is the lateral pharyngeal recess or fossa of Rosenmüller.

The tube is lined with mucous membrane, which is continuous with that of the tympanum on the one hand, and with that of the naso-pharynx on the other. It is thin in the osseous part of the tube, but in the cartilaginous part it is thick, and contains mucous 
glands and lymphoid tissue. It is covered by stratified columnar ciliated epithelium.

Muscles connected with the Eustachian Tube.-The levator palati has an origin from the lower margin of the cartilage of the tube, the tensor palati from the outer side of the cartilage, and the salpingopharyngeus is attached to the lower and front part of the tube. During deglutition the orifice of the tube is usually regarded as being kept open. According to Cleland, however, who had unusual opportunities of examining matters on the living person, 'the anatomical facts make it evident that any dilating influence of the tensor palati is exercised on the hinder and smaller part of the tube, whilst the levator palati closes the anterior (that is, pharyngeal) orifice' by raising the membranous floor of the tube.

Arteries.-These are derived from (I) the Vidian branch of the third part of the internal maxillary, and (2) the ascending pharyngeal branch of the external carotid.

Nerves.-The nerves are derived from (I) the tympanic plexus on the inner wall of the tympanum, and (2) the Vidian nerve.

Development. - The Eustachian tube is developed from the hypoblast of the first visceral cleft.

\section{The Larynx.}

The larynx is the upper part of the respiratory passage, being modified in structure so as to enable it to act as the organ of voice. It is situated in the median line of the neck above the trachea, and it lies opposite the fourth, fifth, and sixth cervical vertebræ. Superiorly it opens into the laryngeal portion of the pharynx, and inferiorly into the trachea. It is covered in front by the integument and the deep cervical fascia, and the laryngeal portion of the pharynx lies behind it. On either side it is in relation with the upper part of the lateral lobe of the thyroid body, the sterno-hyoid, omo-hyoid, sterno-thyroid, and thyro-hyoid muscles, and the common carotid artery.

Structure.-The larynx consists of a framework of cartilages, some of which are connected by joints and ligaments; it is provided with special muscles, spoken of as intrinsic; and it is lined with mucous membrane.

Cartilages.-These are nine in number, three being single and three arranged in pairs. The single cartilages are the epiglottis, the thyroid, and the cricoid, and the three arranged in pairs are the arytenoid, the cornicula laryngis, and the cuneiform.

The epiglottis is a leaf-like plate of yellow elastic fibro-cartilage, which stands bolt upright between the base of the tongue and the superior aperture of the larynx. Its lower part forms a narrow pedicle, which is attached to the receding angle of the thyroid cartilage, just below the median notch on its upper border, by means of a fibro-elastic band, called the thyro-epiglottidean ligament. Superiorly it presents a broad, round, free margin. Each lateral border is free above, but its lower part is contained within 
the aryteno-epiglottidean fold of mucous membrane. The anterior or lingual surface is free over its upper part, where it faces the base of the tongue, and is covered by mucous membrane. This membrane is prolonged on to the base of the tongue as the glossoepiglottidean fold, or frenum epiglottidis, which is mesially placed. It is also prolonged from the sides of the epiglottis on to the lateral walls of the pharynx as the pharyngo-epiglottidean folds. On either side of the frenum epiglottidis, between it and each

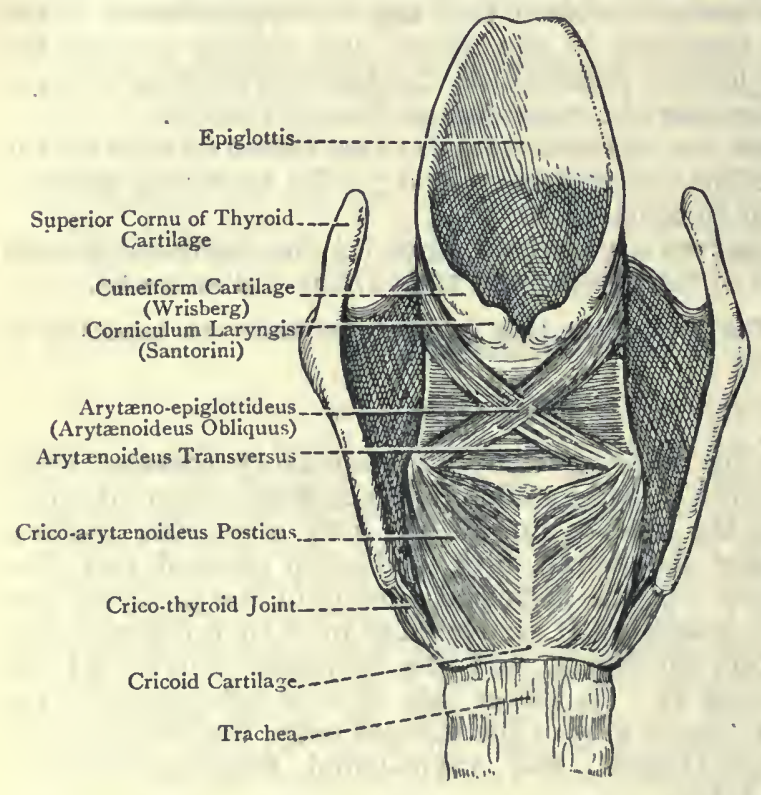

Fig. 524.-The Intrinsic Muscles of the LarynX (Posterior View).

pharyngo-epiglottidean fold, there is a depression or fossa, which is known as the vallecula. Lower down than these folds the anterior surface is connected to the back of the upper border of the body of the hyoid bone by an elastic, semilunar membrane, called the hyo-epiglottidean ligament. Above the upper border of the thyroid cartilage the anterior surface is separated from the posterior aspect of the thyro-hyoid membrane by a collection of adipose and elastic tissues, which is known as the periglottis (epiglottidean gland). The posterior or laryngeal surface is free over its whole extent, and is covered by mucous membrane. It is concave from side to side, and concavo-convex from above downwards. The lower convexity forms the cushion or pulvinar. When the mucous membrane is removed the epiglottis presents a number of small glandular pits.

Development.- The epiglottis is developed from the loop of the furcula. 
Thyroid Cariilage. - This cartilage is composed of two flat quadrilateral alæ, which meet in front by their anterior borders, but diverge widely behind. The angular projection formed by their union is called the isthmus or pomum Adami. The union is confined to about the lower half of each anterior border, and there is left superiorly a deep triangular cleft called the thyroid notch. The posterior border, of greater length than the anterior, is round, and gives attachment to fibres of the palato-pharyngeus and stylo-pharyngeus muscles. At either extremity it is prolonged into a projection, the superior cornu and inferior cornu respectively. The superior cornu gives attachment to the lateral thyro-hyoid ligament, and the inferior cornu, which is slightly incurved, is facetted on its inner aspect to articulate with the cricoid cartilage. The superior border is for

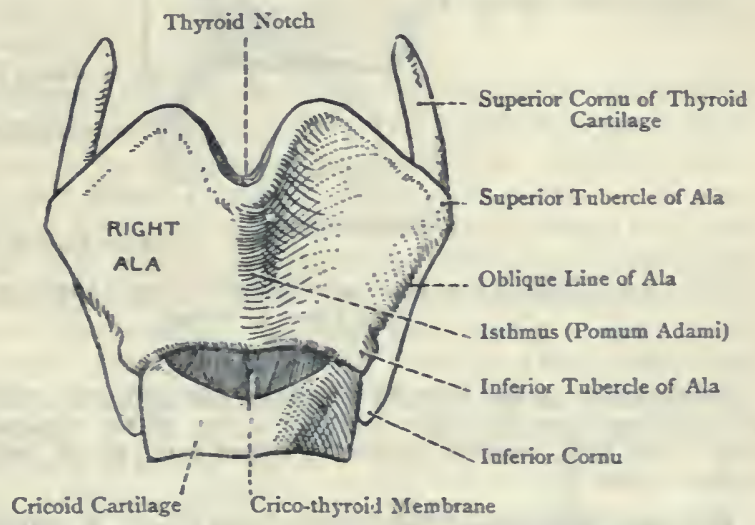

Fig. 525.-The Thyroid and Cricoid Cartilages of the Larynx (ANTERIOR VIEW).

the most part convex. and near its back part it presents a slight eminence, called the superior tubercle. The inferior border is almost horizontal, and presents an eminence about the junction of the posterior third with the anterior two-thirds, called the inferior tuberele. It gives attachment to the median portion of the cricothyroid membrane and the crico-thyroid muscle. The outer surface of the ala is marked by an oblique line, or, it may be, ridge, which extends downwards and forwards from the superior to the inferior tubercle. This line gives insertion to the sterno-thyroid, and origin to the thyro-hyoid, muscles. It divides the outer surface into two unequal parts, an anterior three-fourths and a posterior fourth, the latter giving origin to fibres of the inferior constrictor muscle. The inner surface of the ala is smooth, slightly concave, and covered by mucous membrane. In the median line, behind the pomum Adami, there is a vertical depression known as the receding angle. Commencing just below the thyroid notch on the upper border and passing downwards, this region gives attachment to the 
following structures : (I) the thyro-epiglottidean ligament, (2) the superior thyro-arytenoid ligaments (false vocal cords), and (3) the inferior thyro-arytenoid ligaments (true vocal cords), in association with which are the fibres of the thyro-arytenoid muscles.

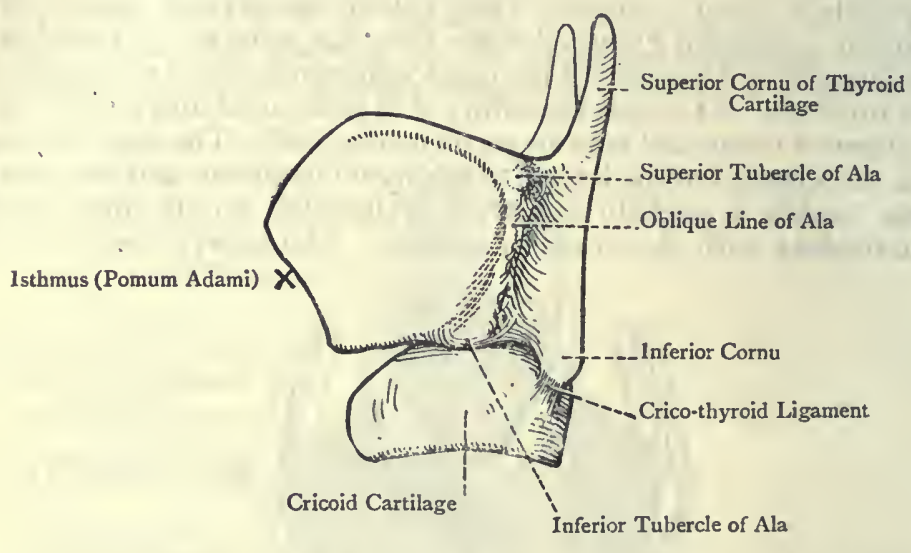

Fig. 526 - The Thyroid and Cricoid Cartilages of the LarynX (LATERAL View).

Development of the Thyroid Cartilage.-The thyroid cartilage is regarded as being developed from the ventral portions of the skeletal cartilages of the fourth and fifth visceral arches of either side, which become united by a median plate.

Cricoid Cartilage.-The cricoid cartilage is situated below the thyroid cartilage, and forms the lower part of the larynx. It bears some resemblance to a signet-ring, and it

Arytenoid Facet

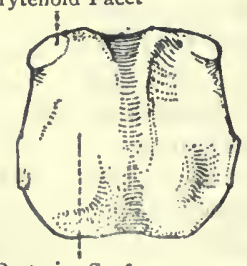

Posterior Surface

FIG. 527.-THE CRICOID CARTILAGE OF THE LARYNX (POSTERIOR VIEW). consists of two parts-an anterior arch and a posterior lamina.

The anterior arch is narrow from above downwards as well as from side to side. The inferior border is horizontal, and is connected with the first ring of the trachea by a fibroelastic membrane. The superior border is connected with the inferior border of thyroid cartilage by the median portion of the cricothyroid membrane.

The posterior lamina is quadrilateral and broad. It attains considerable depth, owing to the rapid elevation of the superior border of the anterior arch as it passes backwards. The inferior border is connected laterally with the first ring of the trachea by a fibro-elastic membrane, and mesially, where the tracheal rings are deficient, to the same membrane. The superior border presents a median notch, and on either side of this an oval 
convex facet for articulation with the base of the arytenoid cartilage. The posterior surface is divided into two depressed areas by a median vertical ridge, which gives attachment to the longitudinal muscular fibres of the œsophagus. The depressed area on either side of this median vertical ridge gives origin to the posterior cricoarytenoid muscle.

The outer surface of the cricoid cartilage presents posteriorly a circular facet for articulation with the inferior cornu of the ala of the thyroid cartilage. The upper sloping border of this part gives attachment, along its inner margin, to the lateral portion of the crico-thyroid membrane.

The internal surface of the cricoid cartilage is lined with the mucous membrane of the larynx.

Development. - The cricoid cartilage, like the rings of the trachea, is developed in the mesoblast of the respiratory tube.

Arytenoid Cartilages.-These cartilages, which are two in number, surmount the superior border of the cricoid cartilage posteriorly. Each has the form of a three-sided pyramid, and measures about $\frac{1}{2}$ inch in height, and about $\frac{1}{4}$ inch in width at the base. The apex looks upwards, and is curved in a backward and inward direction. It is surmounted by the corniculum laryngis. The base looks downwards, and is slightly concave and facetted to articulate with the superior border of the cricoid cartilage posteriorly. Two of the three angles of the base are prominent, namely, the anterior and the external. The anterior angle, somewhat pointed, is directed straight forwards, and is known as the vocal process. It gives attachment to the inferior thyro-arytenoid ligament or true vocal cord. The external angle, thick and somewhat round, has an inclination backwards, as well as outwards, and is known as the mascular process. Anteriorly it gives in-

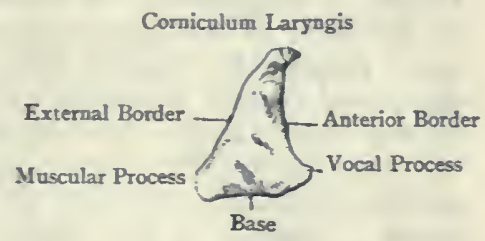

Fig. 528.-The Right ARYTENoId Cartilage a.d CORNiculum LARYNGIS (EXTERNAL VIEW). sertion to the crico-arytænoideus lateralis, and posteriorly to the crico-arytrenoideus posticus. The surfaces are antero-external, posterior, and internal. The anteroexternal surface, a little above the vocal process, gives attachment to the superior thyro-arytenoid ligament or false vocal cord, and, above and external to the vocal process, to the thyro-arytænoideus muscle. The posterior surface is triangular and concave, and gives attachment to a portion of the arytrnoideus muscle. The internal surface faces its fellow of the opposite side, and is covered by mucous membrane. It forms the posterior part of the lateral boundary of the rima glottidis. The borders are anterior, posterior, and external. The anterior border separates the internal from the anteroexternal surface, and terminates below in the vocal process. The posterior border separates the internal from the posterior surface. 
The external border separates the antero-external from the posterior surface, and terminates below in the muscular process.

The thyroid and cricoid cartilages usually retain their cartilaginous condition up to about the twentieth year. In the case of the thyroid cartilage ossification proceeds from the inferior cornu, there being a special osseous nucleus in the region of the pomum Adami.

The anterior part and lower margin of the cricoid cartilage remain cartilaginous for some time, but the remainder undergoes ossification simultaneously with the thyroid cartilage.

Ossification of the arytenoid cartilages takes place at a later date than in the case of the two preceding cartilages.

Cornicula Laryngis or Cartilages of Santorini.-These are two small, somewhat conical nodules of yellow elastic cartilage which cap the apical parts of the arytenoid cartilages, their direction being backwards and inwards. Each lies within the arytenoepiglottidean fold of mucous membrane.

Cuneiform Cartilages or Cartilages of Wrisberg.-These are two nodules of yellow elastic cartilage, which are situated, one on either side, in the aryteno-epiglottidean fold of mucous membrane at its back part, not far from the cornicula laryngis.

Development. - The arytenoid cartilages and the aryteno-epiglottidean folds are developed from the lateral parts of the furcula. The cornicula laryngis are offshoots of the arytenoid cartilages. The cuneiform eartilages are derived from the epiglottis.

Ligaments of the Larynx-Thyro-hyoid Membrane.-This is a broad membranous sheet, which passes between the superior border of the thyroid cartilage and the back of the upper border of the body of the hyoid bone, as well as the deep surface of each great cornu. Its central and lateral portions are strong, and are composed chiefly of elastic tissue. The central portion is known as the middle thyro-hyoid ligament, its lower attachment being to the margins of the thyroid notch. The lateral portions, round and cord-like, are very elastic, and are known as the lateral thyrohyoid ligaments. Each extends from the superior cornu of the thyroid cartilage to the tip of the great cornu of the hyoid bone, and enclosed within it, towards its upper part, there is a small nodule of cartilage called the cartilago triticea. Between the upper median portion of the thyro-hyoid membrane and the concave posterior surface of the body of the hyoid bone there is a synovial bursa. It is to be noted that the superior attachment of the thyrohyoid membrane is such as to enable the upper part of the thyroid cartilage, when raised, to be received within the outline of the hyoid bone. The central portion of the membrane is subcutaneous, but on either side it is covered by the thyro-hyoid muscle. Beneath the posterior border of this muscle the internal laryngeal nerve and superior laryngeal artery pierce this membrane.

Crico-thyroid Membrane.-This membrane is composed of a median and two lateral portions. The median portion is composed chiefly of elastic tissue, and is attached to the adjacent borders of the cricoid and thyroid cartilages. It is subcutaneous in the median 
line, but laterally it is overlapped by fibres of the crico-thyroid muscles. Its centre is crossed by the crico-thyroid arterial arch, formed by the crico-thyroid branches of the superior thyroid arteries, and it presents a few openings for the passage of bloodvessels. Through this portion laryngotomy may be performed. The lateral portion of the crico-thyroid membrane is connected below with the inner margin of the upper border of the lateral part of the cricoid cartilage. Superiorly it is not connected with the thyroid cartilage, but passes within its ala into the larynx, where it expands in an inward direction, and extends from the back of the ala of the thyroid cartilage in its lower part, close to the receding angle, to the under aspect of the vocal process of the arytenoid cartilage. Between these points it becomes continuous with the inferior thyro-arytenoid ligament or true vocal cord. The lateral portion of the crico-thyroid membrane (within the ala of the thyroid cartilage) is covered by the lateral crico-arytenoid and thyro-arytenoid muscles.

Superior Thyro-arytenoid Ligaments.-These form two small fibrous bands, one at either side, which lie within the folds of mucous membrane, called the false vocal cords. Each is attached

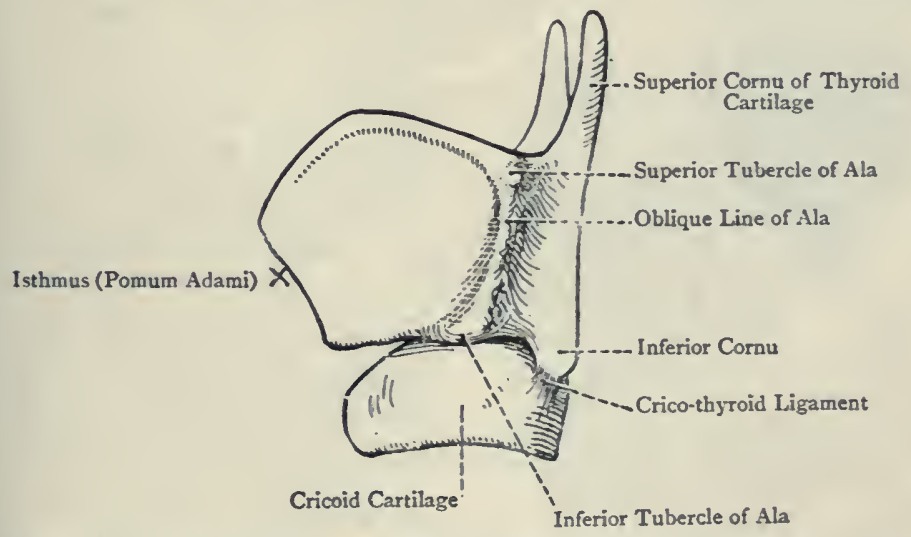

Fig. 529.-The Thyroid and Cricoid Cartilages of the Larynx (Lateral View).

in front to the receding angle of the thyroid cartilage immediately below the attachment of the thyro-epiglottidean ligament, and behind to the antero-external surface of the arytenoid cartilage a little above the vocal process.

Inferior Thyro-arytenoid Ligaments.-These important ligaments, covered by mucous membrane, constitute the true vocal cords, and each is continuous with the upper part of the expanded lateral portion of the crico-thyroid membrane. Each is composed of 
yellow elastic tissue, and, with its fellow, is attached in front to the receding angle of the thyroid cartilage at its centre, and behind to the vocal process of the arytenoid cartilage. Its inner border, which is covered by mucous membrane, is free and clearly defined. In its front part there is a small nodule of elastic cartilage called the cartilage of Luschka (Klein).

Crico-thyroid Joint.- This belongs to the class diarthrosis. The articular surfaces are the facet on the inner surface of the inferior cornu of the thyroid cartilage, and that on the outer surface of the cricoid cartilage posteriorly. The joint is surrounded by a capsular ligament, and this is lined with a synovial membrane. The movements allowed are as follows: (I) rotation of the thyroid cartilage round an axis passing transversely through both joints; and (2) gliding, in which the cricoid moves upwards and backwards, or

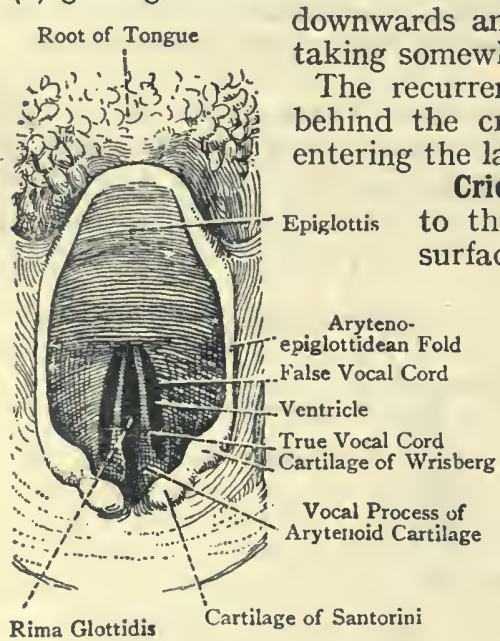

Fig. 530.-The Superior Aperture of THE LARYNX AND ADJACENT Parts.
Crico-arytenoid Joint.-This belongs to the class diarthrosis. The articular surfaces are the convex facet on the superior border of the cricoid cartilage posteriorly, and the concave under surface of the base of the arytenoid cartilage. The joint is surrounded by a capsular ligament, and this is lined with a synovial membrane. The movements allowed are as follows: ( $\mathrm{I}$ ) rotation, in which the arytenoid cartilage rotates on a nearly vertical axis, the effect being to invert, or evert, the vocal process; and (2) gliding in a lateral direction, in which one cartilage moves inwards towards its fellow, or outwards away from its fellow.

The cornicula laryngis are usually connected to the arytenoid cartilages by fibrous tissue, but in some cases there is a synovial articulation.

Prelaryngeal Lymphatic Glands.-These glands are inconstant. When present, there may be one, or there may be two. They lie upon the median portion of the crico-thyroid membrane, in the interval between the crico-thyroid muscles. Their afferent vessels are derived from ( $\mathrm{x}$ ) the infraglottic portion of the larynx, the lymphatics from which pierce the crico-thyroid membrane; $(2)$ the commencement of the trachea; and (3) the upper part of the isthmus of the thyroid body: Their efferent vessels pass either to the inferior deep cervical glands, or to the pretracheal glands as an intermediate gland-station. 
Interior of the Larynx.-The superior aperture of the larynx is situated behind and below the epiglottis. It is triangular, being wide in front and narrow behind, and its plane is sloped obliquely downwards and backwards. Above and in front it is bounded by the epiglottis, and behind by the fold of mucous membrane which stretches between the arytenoid cartilages. On either side are the prominent aryteno-epiglottidean folds, which extend from the tips of the arytenoid cartilages to the sides of the epiglottis and contain muscular fibres. Each of these folds, close to the arytenoid cartilage, contains the corniculum laryngis or cartilage of Santorini, which gives rise to a slight elevation. and a little in front of this it presents another slight elevation produced by the cuneiform cartilage or cartilage of Wrisberg. Between the arytenoid cartilage and the back part of the aryteno-epiglottidean fold internally and the back part of the ala of the thyroid cartilage externally there is a depression, opening upwards, called the sinus pyriformis. In this depression a foreign body may become lodged.

The interior of the larynx commences at the superior aperture and terminates on a level with the lower border of the cricoid cartilage. It is divided into three compartments by means of two antero-posterior folds of mucous membrane, wich project into it from each lateral wall. The upper pair of folds are called the false vocal cords, and the lower pair the true vocal cords. The upper compartment is known as the vestibule, and it extends from the superior aperture down to the level of the false vocal cords. It is wider above than below, and its anterior depth exceeds the posterior. The middle or ventricular compartment is situated between the false vocal cords above and the true vocal cords below. On either side it presents a recess, called the ventricle of the larynx or laryngeal sinus. This is bounded above by a false vocal cord, and below by a true vocal cord. Its outer wall is covered externally by fibres of the

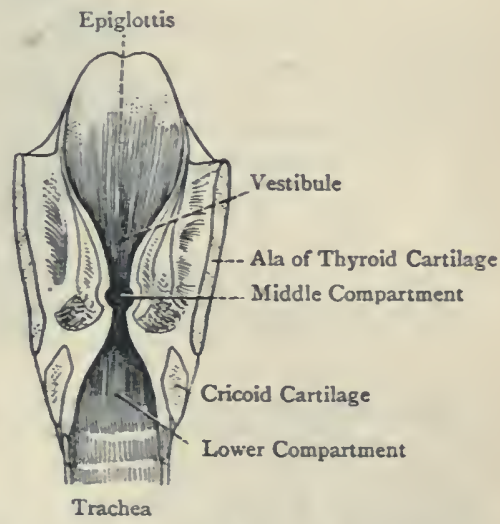

Fig. 531. - Vertical Transverse SECTION OF THE LARYNX, SHOWING THE POSTERIOR SURFAcE OF THE ANTERIor HalF OF the ORgaN (MARShaLL).

thyro-arytenoid muscle. At the anterior part of the ventricle there is a small valvular aperture, which leads to a diverticulum of the ventricle, called the laryngeal saccule or pouch. This extends upwards between the false vocal cord and the ala of the thyroid cartilage, reaching as high as the upper border of the latter. On its inner aspect there are some muscular fibres, 
which are known as the compressor sacculis laryngis or Hilton's muscle.

The lower compartment of the larynx is compressed from side to side above, but becomes circular inferiorly, where it opens into the trachea.

Vocal Cords.-These are arranged in two pairs, laterally disposed, called false and true, or superior and inferior.

The superior or false vocal cords are two folds of mucous membrane, which extend at either side from the receding angle of the thyroid cartilage immediately below the attachment of the thyroepiglottidean ligament to the antero-external surface of the arytenoid cartilage, a little above the vocal process. Each contains

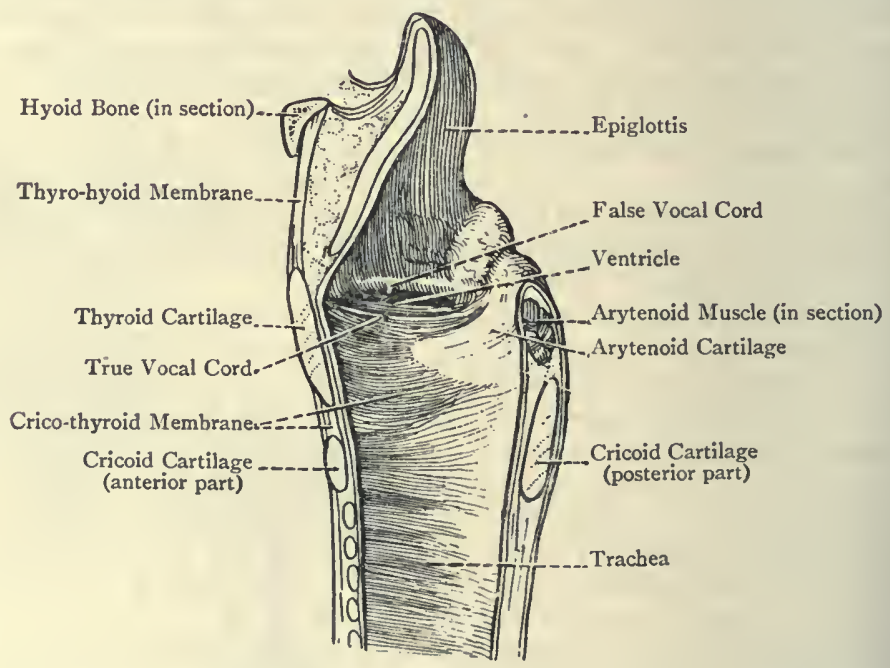

Fig. 532,-Sagittal Section of the Larynx and Trachea, showing the Vocal Cords and Ventricle of the Right Side.

some fibrous tissue forming the superior thyro-arytenoid ligament. The false vocal cords are widely separated from each other by an interval known as the false glottis, so that the true vocal cords are visible on looking into the larynx from above.

The inferior or true vocal cords are concerned in the production of the voice. They are prominent folds at either side, which are rather less than an inch in length, and extend from the receding angle of the thyroid cartilage to the vocal process of each arytenoid cartilage. Each cord consists of the inferior thyro-arytenoid ligament, which is continuous with the upper part of the expanded lateral portion of the crico-thyroid membranc, and is composed of yellow elastic tissue. The mucous membrane covering the cord is thin, and firmly adherent to the elastic tissue of the ligament, 
and it has a characteristic pearly white colour. The true vocal cords are much nearer to each other than the false, so that the latter are not visible when the larynx is viewed from below.

Rima Glottidis.-The rima glottidis, or glottis vera, is the narrow fissure by which the upper and lower compartments of the larynx communicate with each other, and it is the narrowest part of the cavity. It is elongated from before backwards, and is narrow in front at the receding angle of the thyroid cartilage, but wider behind, where it is closed by the interarytenoid fold of mucous membrane. It is divisible into an interchordal portion, called the glottis vocalis, and an interarytenoid portion, called the glottis respiratoria. The glottis vocalis is narrow, and is bounded on either side by the true vocal cord. Its length, as stated, is rather less than an inch, and it forms about two-thirds of the entire glottis.

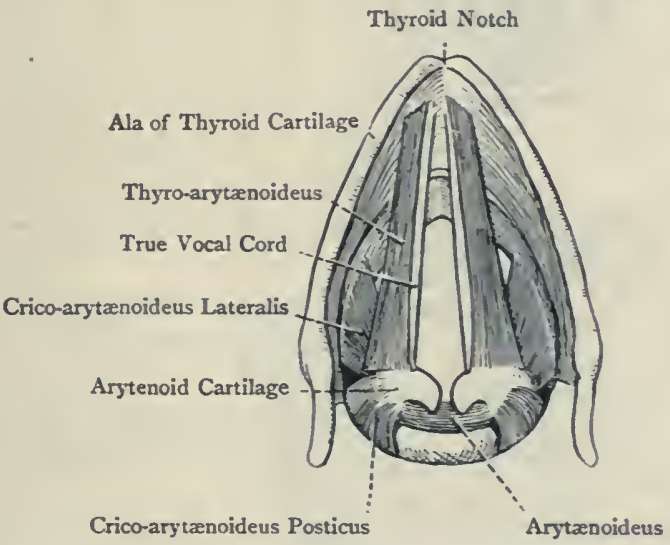

Fig. 533.-Dissection of the Laryxx, showing the Muscles, True Vocal Cords, ANd Rima Glotidis (Superior View).

The glottis respiratoria is wider than the glottis vocalis, and is bounded on either side by the inner aspect of the base of the arytenoid cartilage. It is about $\frac{1}{4}$ inch in length, and forms about one-third of the length of the entire glottis.

The shape of the rima glottidis is subject to alteration, and has to be considered under three conditions.

(I) During quiet respiration it has the form of an elongated triangle, the apex being in front at the thyroid cartilage, and the base behind at the interarytenoid fold of mucous membrane. (2) During a deep inspiration the rima is widely dilated, and assumes a somewhat diamond-shape, the widest part being opposite the tips of the vocal processes of the arytenoid cartilages, where the lateral angles of the diamond are placed, the posterior angle, at the interarytenoid fold of mucous membrane, being truncated. (3) During vocalization, phonation, and especially in singing high 
notes, the vocal cords become so closely approximated as to be practically parallel, and the glottis vocalis assumes the form of a narrow chink.

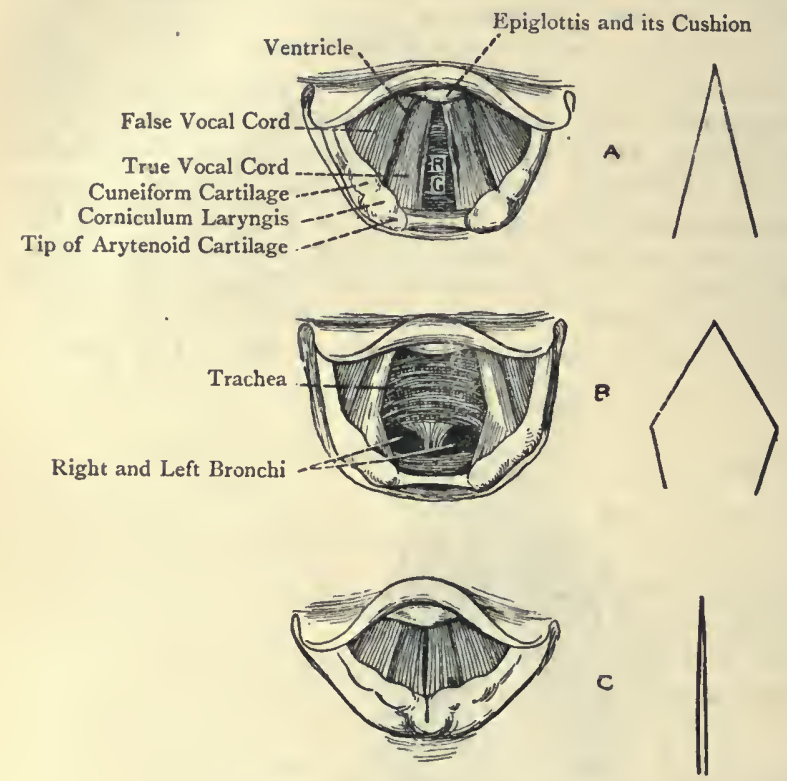

Fig. 534.-The Superior Aperture of the Larynx and the Rima GLOTTIDIS, AS SEEN BY THE AID OF THE LARYNGOSCOPE UNDER DIFFERENT CONDITIONS.

(The Figures on the left side are copied from Czermak).

A, Ordinary quiet inspiration

$B$, Very deep inspiration
C, Vocalization, especially in singing high notes R.G. Rima Glottidis

Mucous Membrane of the Larynx.-This is continuous above with the mucous membrane of the pharynx, and below with that of the trachea. Above the level of the rima glottidis its subjacent attachment is of a loose nature on account of the presence of submucous areolar tissue, particularly in the vicinity of the aryteno-epiglottidean folds, a condition which favours the occurrence of œedema, but over the laryngeal surface of the epiglottis it is firmly attached. Over the true vocal cords it is very thin, and is firmly connected to the inferior thyro-arytenoid ligaments, which they contain. The membrane is covered by ciliated columnar epithelium, except (I) near the margin of the aryteno-epiglottidean folds, where it is of the stratified squamous variety, as in the laryngeal portion of the pharynx and the buccal cavity; and (2) over the true vocal cords, where it is also of the stratified squamous variety. Bodies resembling the taste-buds of the tongue are met with on the 
Nerve-supply.-The external laryngeal branch of the superior laryngeal nerve.

The fibres are directed upwards and backwards in a diverging manner, the posterior being horizontal and the anterior oblique. These two sets of fibres are usually distinct.

Action.-To approximate the front parts of the cricoid and thyroid cartilages in the following manner : the posterior horizontal fibres draw the cricoid cartilage backwards, and the anterior oblique fibres elevate the anterior part of the cricoid cartilage, thus imparting a swinging movement to the cartilage. As a result of this movement the deep posterior part of the cricoid cartilage, with the arytenoid cartilages on its upper border, are depressed. In this manner the vocal cords are put upon the stretch, so that the muscles are tensors of the cords.

Between the two muscles anteriorly there is a triangular interval about $\frac{1}{2}$ inch wide, in which the central portion of the crico-thyroid membrane is visible, this portion being crossed at its centre by the crico-thyroid arch of arteries. In this region, just above the cricoid cartilage, laryngotomy may be performed.

Posterior Crico-arytenoid-Origin.- The posterior surface of the cricoid cartilage on one side of the median vertical ridge.

Insertion.-The posterior aspect of the muscular process or external angle of the base of the arytenoid cartilage.

Nerve-supply.--The recurrent laryngeal nerve.

The fibres of the muscle are directed upwards and outwards, the highest being short and nearly horizontal, the middle being oblique, and the lowest almost vertical.

Action.-To draw the muscular process of the arytenoid cartilage backwards and inwards, thereby swinging the vocal process or anterior angle outwards, the result of which is to open or widen the rima glottidis. The muscle is, therefore, a dilator of the rima glottidis.

The muscle is separated from its fellow by the median vertical ridge on the back of the cricoid cartilage.

Lateral Crico-arytenoid-Origin.-The lateral portion of the cricord cartilage along its upper sloping border, extending as far back as the crico-arytenoid joint.

Insertion.-The anterior aspect of the muscular process of the arytenoid cartilage, and the adjacent portion of the anteroexternal surface.

Nerve-supply.-The recurrent laryngeal nerve.

The fibres of the muscle are directed backwards and upwards.

Action.-To draw the muscular process of the arytenoid cartilage forwards and inwards, thereby swinging the vocal process or interior angle inwards, the result of which is to narrow the rima glottidis. The two muscles, therefore, approximate and relax the true vocal cords, and they come into action in vocalization. When the posterior and lateral crico-arytenoid muscles act together they assist the arytenoid muscle in approximating the arytenoid carti- 
lages, and so the rima glottidis is closed. The lateral crico-aryțenoid muscle is covered externally by the ala of the thyroid cartilage and the upper fibres of the crico-thyroid muscle, and internally by the lateral expanded portion of the crico-thyroid membrane.

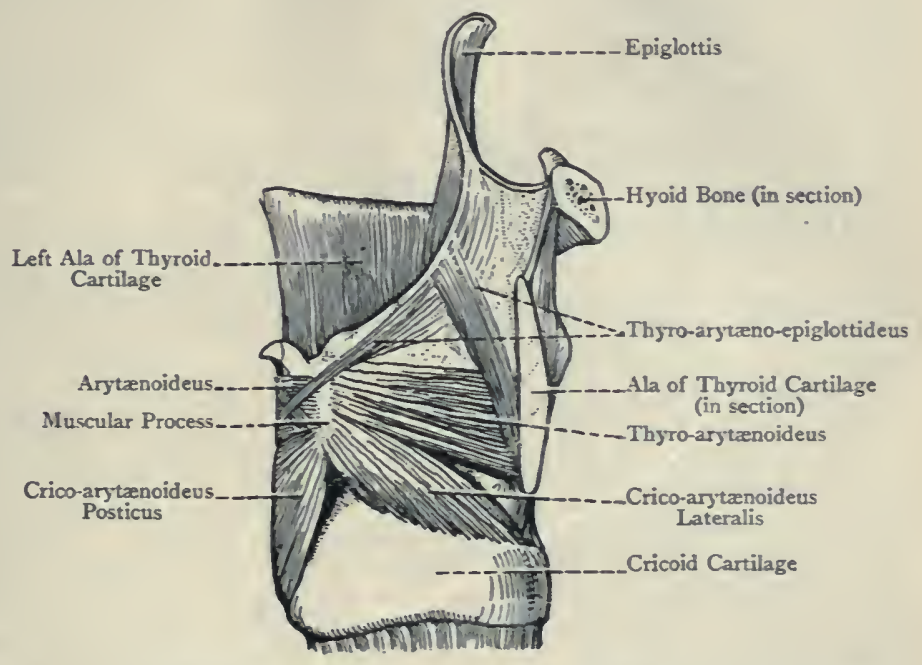

Fig. 536.-The Intrinsic Mescles of the Laryxx (Lateral View). (The greater part of the Right Ala of the Thyroid Cartilage has been removed)

Thyro-arytenoid.-This muscle consists of two portions, external and internal.

External Portion-Origin.-(I) The lower half of the inner surface of the ala of the thyroid cartilage, and (2) the outer surface of the lateral portion of the crico-thyroid membrane.

Insertion - The front of the muscular process and the adjacent part of the outer border of the arytenoid cartilage, a few of the fibres passing round the cartilage to become continuous with the arytænoideus. The uppermost fibres of this portion pass upwards and backwards to the aryteno-epiglottidean fold, and thence to the epiglottis, under the name of the thyro-epiglottideus.

Internal Portion-Origin.-(I) The receding angle of the thyroid cartilage, and (2) the cartilage of Luschka in the anterior part of the true vocal cord. According to Ludwig the deepest fibres of this portion, known as the ary-vocalis, spring from the anterior part of the true vocal cord.

Insertion. - The outer surface of the vocal process of the arytenoid cartilage, and the adjacent portion of the antero-external surface.

The fibres pass from before backwards, some of them having a slight inclination outwards, and the highest backwards. 
Nevve-supply. - The recurrent laryngeal nerve.

Action.-(I) Internal Portion. To draw forwards the arytenoid cartilage, and the posterior part of the cricoid cartilage, swinging the latter in an upward and forward direction. The result of this action is to relax the true vocal cords by approximating the arytenoid cartilage to the thyroid cartilage. This portion of the muscle is, therefore, the antagonist of the crico-thyroid. It is to be borne in mind, however, that one factor in relaxation of the true vocal cords must of necessity be elastic recoil. The fibres representing the ary-vocalis muscle of Ludwig act, according to him, by rendering tense that part of the true vocal cord which is in front of them, and relaxing the part behind them.

(2) External Portion. In virtue of its insertion into the muscular process of the arytenoid cartilage this portion will draw forwards that process, the effect of which is to swing inwards the vocal process. The fibres known as the thyro-epiglottideus have been supposed to assist in depressing the epiglottis.

The outer portion of the muscle lies within the ala of the thyroid cartilage. Its lower border is contiguous to the lateral cricoarytenoid, and its upper fibres lie on the outer wall of the ventricle and saccule. The inner portion of the muscle is in close contact with the outer side of the true vocal cord.

Arytenoid.-The arytenoid muscle lies across the posterior surfaces of the arytenoid cartilages. It consists of two parts-superficial and deep. The superficial part is composed of two decussating bundles, each of which is known as the arytenoideus obliquus; and the deep part constitutes the arytenoideus transversus.

Arytenoideus Obliquus.-Each of these muscles, which has the form of a narrow oblique bundle, arises from the back of the muscular process of the arytenoid cartilage. Its direction is upwards and inwards, and at the median line it decussates with its fellow of the opposite side, thus $x$. Having reached the summit of the opposite arytenoid cartilage, a few of the fibres terminate upon it, but the majority enter the corresponding aryteno-epiglottidean fold. Being reinforced by a few fibres from the summit of the arytenoid cartilage, the fibres now constitute the aryteno-epiglottideus muscle, which passes forwards within the aryteno-epiglottidean fold to be inserted into the side of the epiglottis. Associated with the arytenoepiglottideus there are those fibres of the external portion of the thyro-arytenoid muscle which are known as the thyro-epiglottideus.

Nerve-supply.-The inferior or recurrent laryngeal nerve.

Action. - The two muscles, having the arytenoid cartilages within their embrace, sweep these cartilages together, in which action they are aided by the transverse arytenoid muscle, and the superior aperture of the larynx is narrowed, very much after the manner of a sphincter muscle.

Arytenoideus Transversus,- The fibres of this muscle extend transversely from the posterior surface and outer border of one arytenoid cartilage to the posterior surface and outer border of the other 
cartilage. Some of the fibres are continuous with those of the external portion of the thyro-arytenoid muscle.

Nerve-supply.-The inferior or recurrent laryngeal nerve.

Action.-To draw the arytenoid cartilages together, so as to approximate the true vocal cords, and even close the rima glottidis.

The arytenoideus transversus muscle, clothed with mucous membrane internally, bounds the rima glottidis posteriorly; and its superficial or posterior surface supports the decussating arytenoidei obliqui muscles.

Compressor Sacculus Laryngis, or Hilton's Muscle.-This is the name given to a thin layer of muscular fibres on the inner side and upper end of the sacculus laryngis. The fibres are related superiorly to those of the arytæno-epiglottideus, which are contained within the aryteno-epiglottidean fold.

Summary of the Actions of the Laryngeal Muscles.-The intrinsic muscles of the larynx, by regulating the condition of the rima glottidis, contribute to vocalization, and modify the pitch of notes. In so doing they lengthen, so as to render tense, or shorten, so as to relax, the vocal cords; and they also bring the cords together, or draw them apart. In other words, the intrinsic muscles bring about tension and parallelism of the true vocal cords, or give rise to the opposite conditions, namely, relaxation and divergence.

Tension is effected by the crico-thyroid muscles, which swing the anterior part of the cricoid cartilage upwards and backwards towards the thyroid, the result being to increase the distance between the receding angle of the thyroid cartilage and the vocal processes of the arytenoid cartilages. In this manner the true vocal cords are stretched, and tension of them is produced.

Parallelism of the true vocal cords is effected by (I) the lateral crico-arytenoid muscles, and (2) the arytenoid muscle, the latter also producing narrowing of the glottis respiratoria (interarytenoid portion of glottis). The lateral crico-arytenoid muscles draw forwards the muscular processes of the arytenoid cartilages, the result of which is to swing inwards the vocal processes, and so produce parallelism. The arytenoid muscle draws the arytenoid cartilages together, and so narrows the glottis respiratoria, contributing at the same time to the condition of parallelism.

Relaxation of the true vocal cords is brought about by the thyroarytenoid muscles, which draw the arytenoid cartilages, along with the posterior part of the cricoid cartilage, forwards. In this manner the distance between the receding angle of the thyroid cartilage and the arytenoid cartilages is diminished, and so relaxation of the true rocal cords is produced. They are at the same time shortened in virtue of their elastic recoil. The fibres of the thyro-arytenoid muscle, known as the ary-vocalis, act by rendering tense the part of the true vocal cord in front of them, and relaxing the part behind them (Ludwig).

Divergence of the true vocal cords and opening of the rima glottidis is effected by the posterior crico-arytenoid muscles, which draw the muscular processes of the arytenoid cartilages backwards and 
inwards, the result of which is to swing the vocal processes outwards. The actions of the intrinsic muscles may be tabulated thus:

Tension.

Crico-thyroids.

Posterior crico-arytenoids.

Parallelism and Closure.

Lateral crico-arytenoids.

Arytenoid.

\section{Relaxation.}

Thyro-arytenoids.

Lateral crico-arytenoids.

\section{Divergence and Opening.}

Posterior crico-arytenoids.

Function of the Epiglottis.-Two views are held as regards the function of the epiglottis. (I) According to one view it is folded down, like a lid, over the superior aperture of the larynx. This lid-like action is brought about by $(a)$ the pressure exerted by the base of the tongue, $(b)$ the action of the aryteno-epiglottidei muscles, and (c) the action of the thyro-hyoid muscles, which elevate the thyroid cartilage. (2) According to Anderson Stuart, the epiglottis is never folded down like a lid. The superior aperture of the larynx is, according to this author, closed during deglutition by the action of (I) the transverse arytenoid muscle, and (2) the external portions of the thyro-arytenoid muscles. The transverse arytenoid muscle approximates the arytenoid cartilages, and the external portions of the thyroarytenoid muscles draw the arytenoid cartilages forwards, until their apical parts come into contact with the cushion of the epiglottis.

The sphincter-like action of the aryteno-epiglottidei muscles upon the superior aperture of the larynx must, however, be taken into account.

Prima facie the lid-like action appears to be the most natural one. The results, however, of ulceration of the epiglottis, and consequent loss of function, would tend to support Anderson Stuart's view.

Nerves.-The nerves of the larynx are the superior and inferior, or recurrent, laryngeal, both of which are branches of the pneumo-gastric.

The superior laryngeal nerve divides into two branches, external and internal. The external branch (external laryngeal nerve), which is comparatively small, supplies the crico-thyroid muscle, and also furnishes twigs to the inferior constrictor muscle of the pharynx. The internal branch (internal laryngeal nerve), which is sensory, passes beneath the posterior border of the thyro-hyoid muscle, and enters the larynx by piercing the thyro-hyoid membrane, in company with the superior laryngeal artery, above which it lies. In the interior of the larynx it breaks up into branches, some of which ascend to the aryteno-epiglottidean fold and posterior surface of the epiglottis, a few of them passing through the latter to reach its anterior surface. The other branches descend to supply the laryngeal mucous membrane, and one of them joins a twig from the inferior or recurrent laryngeal nerve.

The inferior or recurrent laryngeal nerve is the principal motor nerve of the larynx. On the right side it arises from the vagus at the root of the neck, and hooks round the first part of the right subclavian artery. On the left side it arises from the vagus in the upper part of the thorax, and hooks round the arch of the aorta. 
Having ascended in the groove between the trachea and œsophagus, it passes beneath the lower border of the inferior constrictor muscle, and ascends upon the cricoid cartilage, lying close behind the crico-thyroid joint. Here it divides into two branches, anterior and posterior. The anterior branch ascends under cover of the thyroid cartilage, and is distributed to the lateral cricoarytenoid, thyro-arytenoid, thyro-epiglottideus, and aryteno-epiglottideus muscles. The posterior branch passes upwards on the back of the cricoid cartilage beneath the posterior crico-arytenoid muscle, which it supplies, and then it goes on to end in the arytenoid muscle.

The recurrent laryngeal nerve furnishes some sensory branches to the mucous membrane of the larynx below the rima glottidis, and it communicates with the internal branch of the superior laryngeal. The motor fibres of the external branch of the superior laryngeal and of the inferior laryngeal are derived from the bulbar portion of the spinal accessory nerve.

Summary of the Laryngeal Nerves.-The superior laryngeal nerve, through its internal branch, is sensory, and its external branch supplies the cricothyroid muscle, and, in part, the inferior constrictor muscle of the pharynx.

The inferior or recurrent laryngeal nerve is chiefly motor, and it supplies the intrinsic muscles of the larynx, with the single exception of the crico-thyroir? muscle.

Arteries.-The arteries of the larynx are the superior and the inferior laryngeal. The superior laryngeal artery is a branch of the superior thyroid. It accompanies the internal laryngeal nerve, below which it lies, and enters the larynx by piercing the thyrohyoid membrane. The inferior laryngeal artery is a branch of the inferior thyroid, and it accompanies the inferior or recurrent laryngeal nerve.

Veins. - The superior laryngeal vein opens into the superior thyroid, and the inferior laryngeal vein into the inferior thyroid vein.

Lymphatics.-These are arranged in two sets-superior and inferior. The superior lymphatics come from the portion of the larynx above the rima glottidis. Having pierced the thyro-hyoid membrane, they pass to the upper group of deep cervical glands. The inferior lymphatics come from the portion of the larynx below the rima glottidis. Having pierced the crico-thyroid membrane, they pass to the lower group of deep cervical glands, having previously traversed the prelaryngeal glands.

In early life the larynx occupies a higher position than it does in the adult, its descent, which is gradual, being completed by puberty. Up to that period the projection known as the pomum Adami is not present. After puberty important changes take place. The cartilages increase in size, the pomum Adami assumes marked development, especially in the male, and the true vocal cords undergo increase in length. These various changes account for the modifications which the voice undergoes at and after puberty. 
Development of the Larynx.- The larynx is developed from the upper part of a median diverticulum from the ventral aspect of the fore-gut, which diverticulum by its lower part gives rise to the trachea. The epiglottis, arytenoid cartilages, and aryteno-epiglottidean folds are developed from the furcula, which is an elevation, like a horseshoe, lying between the second, third, and fourth visceral arches of either side (see Fig. 523). The front part of the furcula gives origin to the epiglottis, the lateral parts to the arytenoepiglottidean folds, and the back part (extremities of the horseshoe bend) to the arytenoid cartilages. The groove within the furcula represents the superior aperture of the larynx.

\section{Prevertebral Muscles.}

Rectus Capitis Anticus Major-Origin.-By four short tendons from the tubercles of the costal processes (anterior tubercles of the transverse processes) of the third, fourth, fifth, and sixth cervical vertebræ, where it coincides with the origin of the scalenus anticus.

Insertion.-The inferior surface of the basilar process of the occipital bone, from the pharyngeal tubercle obliquely outwards and forwards for about $\frac{1}{2}$ inch.

Nerve-supply. - The cervical plexus.

The muscle is directed upwards and inwards.

Action.-To flex the head.

Relations-Anterior.-The upper part of the common carotid, and the internal carotid, arteries, the internal jugular vein, the pneumogastric and sympathetic nerves, and the pharynx. Posterior.-A part of the longus colli, and a large portion of the rectus capitis anticus minor.

Rectus Capitis Anticus Minor-Origin.-The front of the root of the costal process of the atlas.

Insertion. - The inferior surface of the basilar process of the occipital bone, between the foramen magnum and the outer part of the insertion of the rectus capitis anticus major.

Nerve-supply. - The anterior primary division of the first cervical nerve.

The muscle is directed upwards and slightly inwards.

Action.-To flex the head.

Rectus Capitis Lateralis-Origin.-The upper aspect of the extremity of the transverse process of the atlas at its front part.

Insertion.-The inferior surface of the jugular process of the occipital bone.

Nerve-supply. - The anterior primary division of the first cervical nerve.

The muscle passes vertically upwards.

Action.-To flex the head to one side.

Longus Colli.-This complex muscle consists of three partsupper oblique, vertical, and lower oblique.

Upper Oblique Part-Origin.-The fronts of the tubercles of the costal processes (anterior tubercles of the transverse processes) 0 ? the third, fourth, fifth, and sixth cervical vertebræ.

Insertion.-The lateral aspect of the anterior tubercle of the atlas. 
The fibres are directed upwards and inwards.

Vertical Part-Origin.-The fronts of the bodies of the last two cervical and first three thoracic vertebræ.

Insertion.-The fronts of the bodies of the second, third, and fourth cervical vertebræ.

Lower Oblique Part-Origin.-The fronts of the bodies of the first three thoracic vertebræ in common with the lower portion of the vertical part.

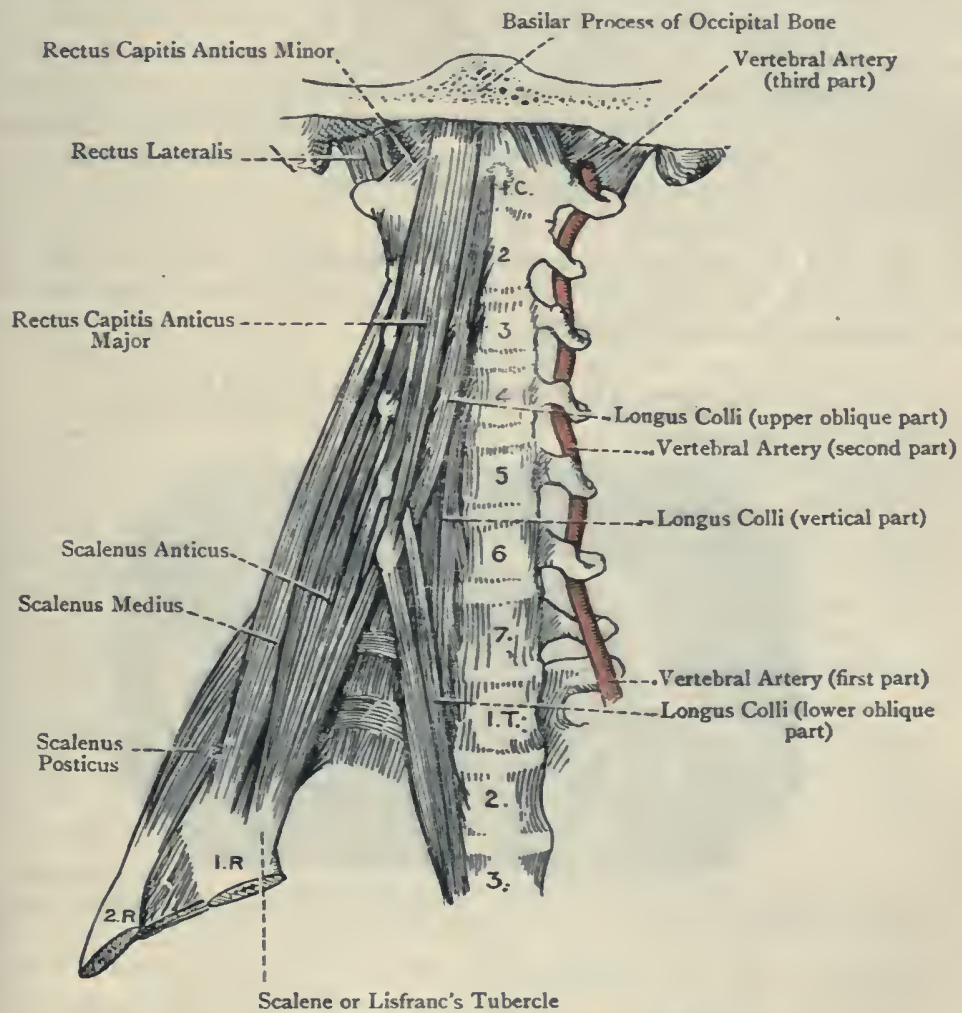

Fig. 537.-The Right Prevertebral Muscles.

(The Vertebral Artery is also shown).

Insertion.-The fronts of the tubercles of the costal processes (anterior tubercles of the transverse processes) of the fifth and sixth cervical vertebræ.

Nerve-supply.-The anterior primary divisions of the adjacent spinal nerves.

Action.-To flex the cervical part of the vertebral column. 
Relations-Anterior. - The pharynx and œsophagus, the common and internal carotid arteries, and internal jugular vein ; the pneumogastric nerve, and the gangliated cord of the sympathetic; the rectus capitis anticus major superiorly ; and the recurrent laryngeal nerve, inferior thyroid artery, and first part of the subclavian artery inferiorly. Posterior.-The bodies and discs of the adjacent vertebræ, and their transverse processes.

Petrous Part of the Internal Carotid Artery.-This part of the internal carotid artery is contained within the carotid canal of the petrous part of the temporal bone. It is at first directed upwards, and then, describing a bend, it passes forwards and inwards to the foramen lacerum medium, where it enters upon the cavernous part of its course. The vessel is surrounded by small tympanic veins, and is accompanied by the ascending branch of the superior cervical ganglion of the sympathetic nerve. This branch breaks up into two divisions. One of these lies on the outer side of the artery, and gives rise to the carotid sympathetic plexus, whilst the other lies on the inner side of the artery, and goes on to form the cavernous sympathetic plexus.

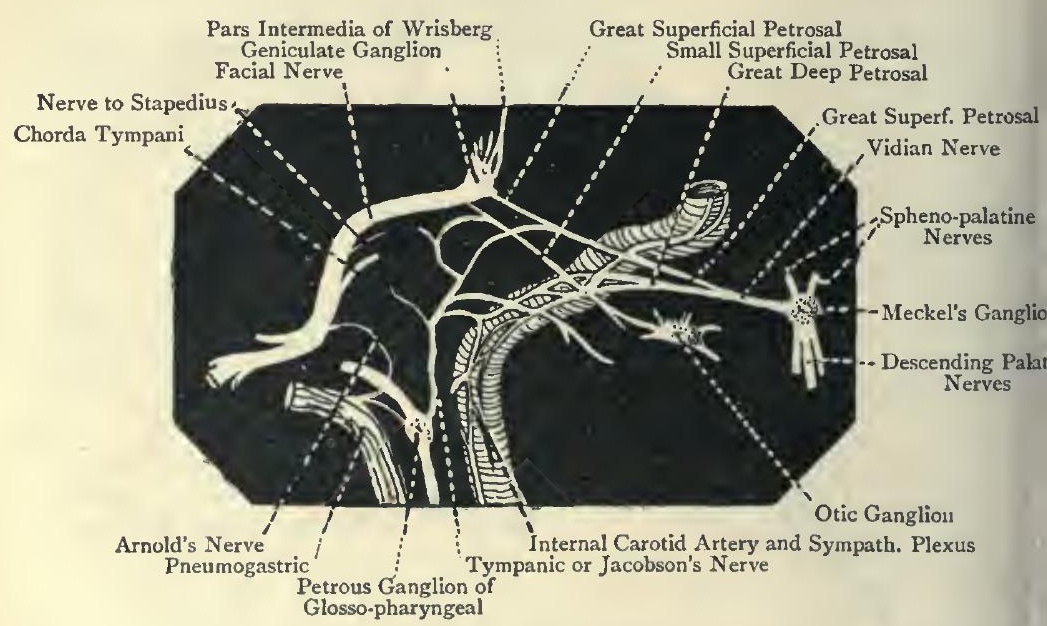

Fig. 538.-The Facial Nerve in the Agueduct of Fallopius, etc. (HIRSCHFELd AND LEVEILlE).

As the artery ascends in the carotid canal it is situated in front of, and below, the tympanum and cochlea; as it bends it has the Eustachian tube on its anterior and outer side; and as it passes forwards and inwards it has the Gasserian ganglion above it, the partition between the two being for the most part membranous.

Branches.-The petrous part gives off a tympanic branch which enters the tympanum through the posterior wall of the carotid 
canal, and it may furnish a petrosal branch to accompany the great deep petrosal nerve.

For the cavernous part of the internal carotid artery, see p. Io 78 .

Petrous Part of the Facial Nerve.-This part of the nerve extends from the orifice of the meatus auditorius internus, on the posterior surface of the petrous part of the temporal bone, to the stylomastoid foramen, and it traverses (I) the meatus auditorius internus, and (2) the aqueduct of Fallopius.

Meatal Portion.-This portion of the nerve is directed outwards, and is accompanied by the pars intermedia of Wrisberg, the auditory nerve, and the internal auditory artery. It is placed upon the upper and anterior aspect of the auditory nerve, and the pars intermedia lies between the two, and here joins the facial nerve. At the deep end of the internal auditory meatus the facial nerve parts company with the auditory nerve, and enters the aqueduct of Fallopius.

Branches.-Two branches connect the facial nerve with the auditory nerve.

Portion in the Aqueduct of Fallopius.-The direction of this portion of the nerve corresponds to that of the aqueduct. It may conveniently be divided into three stages-first, second, and third. In the first stage, which is very short, the nerve passes horizontally outwards, between the cochlea and vestibule, to the inner wall of the tympanum, where it presents an enlargement, called the geniculate ganglion. It then describes a sharp bend, and in the second stage passes backwards, lying above the fenestrz ovalis. Thereafter it describes another abrupt bend, and in the third stage descends behind the posterior wall of the tympanum to the stylomastoid foramen, by which it escapes from the aqueduct. The hiatus Fallopii leads from near the commencement of the aqueduct to the superior surface of the pars petrosa. As the aqueduct descends it communicates with the canal of the pyramid of the tympanum, and below this it presents another opening, called the iter chorde posterius.

Branches.-These are as follows:

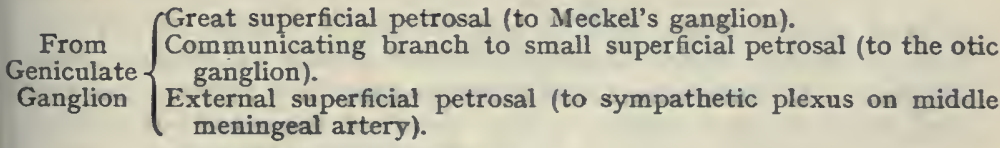
Nerve to the stapedius muscle.

Chorda tympani nerve.

Communicating branch to the auricular branch (Arnold's nerve) of the pneumogastric.
}

The great superficial petrosal nerve arises from the geniculate ganglion and passes forwards through the hiatus Fallopii, by which it emerges from the aqueduct of Fallopius. It then courses inwards and forwards in a groove on the superior surface of the petrous part of the temporal bone, and passes beneath the Gasserian ganglion to the foramen lacerum medium. In the upper 
part of this foramen it joins the great deep petrosal nerve from the carotid plexus to form the Vidian nerve, which passes forwards through the Vidian canal into the spheno-maxillary fossa, and joins the back part of Meckel's ganglion. The great superficial petrosal nerve contains some motor fibres, but to a large extent it consists of sensory fibres. These represent peripheral branches of the unipolar cells of the geniculate ganglion, the central branches of which give rise to the pars intermedia of Wrisberg.

A communicating branch passes from the geniculate ganglion to the small superficial petrosal nerve, which latter represents the tympanic branch (Jacobson's nerve) of the glosso-pharyngeal, after it has emerged from the tympanic plexus on the inner wall of the tympanum. Through means of the small superficial petrosal nerve this communicating branch is conducted to the otic ganglion.

The external superficial petrosal nerve (sometimes absent) emerges at a point just within the orifice of the hiatus Fallopii, and passes to the sympathetic plexus around the middle meningeal artery.

The nerve to the stapedius muscle arises from the facial nerve in the descending part of the aqueduct of Fallopius, opposite the pyramid of the tympanum. It enters a small canal in the pyramid, and so reaches the stapedius as that muscle lies within the canal.

The chorda tympani nerve arises from the geniculate ganglion, and it remains closely associated with the motor portion of the facial nerve in the descending part of the aqueduct of Fallopius. A little above the stylo-mastoid foramen it leaves the motor portion of the nerve, and passes upwards and forwards in a somewhat recurrent manner through a minute canal, called the iter chorde posterius, by which it enters the tympanum. At first it is placed on the posterior wall of the tympanum, close to the posterior margin of the membrana tympani, and on a lower level than the pyramid. The nerve then passes forwards upon the membrana tympani, near its upper margin, lying between its mucous and fibrous layers, so as to be ensheathed by the mucous membrane. In this part of its course it passes over the inner aspect of the handle of the malleus. Having arrived at the inner end of the fissure of Glaser, it leaves the tympanum by passing through the iter chorda anterius or canal of Hugvier. It then enters the pterygo-maxillary region, and passes downwards and forwards under cover of the external pterygoid muscle, where it receives a communicating twig from the otic ganglion, and thereafter joins the lingual nerve at an acute angle, under cover of the external pterygoid muscle. The subsequent distribution of the nerve has been already described (see p. I246).

The chorda tympani is composed of fibres which are the peripheral processes of the unipolar cells of the geniculate ganglion of the facial nerve. These represent the fibres which are distributed to the sides and dorsum of the tongue over its anterior twothirds. They are, therefore, regarded as gustatory, or pertaining to taste, and functionally are afferent. They belong to the same 
group of fibres as compose the pars intermedia of Wrisberg, which represent the central processes of the unipolar cells of the geniculate ganglion of the facial nerve. In other words, the greater portion of the chorda tympani and the pars intermedia are to be regarded as one nerve, with the unipolar cells of the geniculate ganglion interpolated in their course in the form of cell-stations. The chorda tympani nerve, however, also contains purely facial fibres, which are the secretory fibres of the submaxillary and sublingual glands. The nerve is, therefore, a mixed nerve.

A communicating branch is given off from the facial nerve, just above the stylo-mastoid foramen, which connects it with the auricular branch (Arnold's nerve) of the pneumogastric.

Pars Intermedia of Wrisberg. - The fibres of this small nerve arise from the unipolar cells of the geniculate ganglion of the facial, being the central processes of these cells, the peripheral processes representing the principal fibres of origin of the chorda tympani. The pars intermedia is at first closely incorporated with the facial nerve, and passes from the aqueduct of Fallopius into the internal auditory meatus. Here it separates from the facial nerve, and lies between it and the auditory nerve. After emerging through the orifice of the internal auditory meatus the pars intermedia passes to the lower border of the pons Varolii, where it enters the medulla oblongata, and terminates in the fasciculus solitarius and upper part of the glosso-pharyngeal nucleus.

The chorda tympani and the pars intermedia of Wrisberg, with the geniculate ganglion interpolated, may be regarded as representing the afferent or sensory root of the facial nerve.

Geniculate Ganglion.-The geniculate ganglion is situated on the facial nerve in the aqueduct of Fallopius, at the point where the aqueduct, having reached the inner wall of the tympanum, makes a sharp bend before passing backwards. Like a spinal ganglion and the Gasserian ganglion, it consists of unipolar cells, each of which has a central process and a peripheral process. The ganglion is the nucleus of origin of the sensory fibres of the facial nerve. The central processes of the unipolar cells form the pars intermedia of Wrisberg, and the majority of the peripheral processes form the chorda tympani nerve. Some of the peripheral processes, however, give rise to (I) the great superficial petrosal nerve; (2) the communicating branch to the small superficial petrosal nerve; and (3) the external superficial petrosal nerve (inconstant).

The facial nerve is partly motor and partly sensory. The sensory portion, as stated, is represented by $(I)$ the pars intermedia of Wrisberg; (2) the geniculate ganglion; and (3) the chorda tympani nerve. The great superficial petrosal nerve is to be regarded as a branch of the sensory portion of the facial nerve.

Summary of the Petrosal Nerves.-There are three superficial petrosal nerves, namely, great, small, and external; and there are two deep petrosal nerves, namely, great and small. 
Auditory Nerve in the Internal Auditory Meatus.--This nerve passes outwards in the internal auditory meatus in company with the pars intermedia of Wrisberg, the facial nerve, and the internal auditory artery. The facial nerve is placed upon its upper and anterior aspect, and the pars intermedia lies between the two. Two branches connect the auditory nerve with the facial.

At the deep end of the meatus the auditory nerve breaks up into two divisions - an upper, called the vestibular nerve, and a lower, called the cochlear nerve. The vestibular nerve presents a ganglion, called the ganglion of Scarpa, which is situated at the deep end of the meatus, its bipolar cells giving origin to the vestibular fibres. Thereafter it breaks up into two parts, upper and lower. The upper portion furnishes branches which pass to the ampullæ of the superior and external semicircular canals, and to the utricle. These pass through the foramina of the superior vestibular area of the upper fossa of the lamina cribrosa at the deep end of the meatus. The lower portion supplies branches to the ampulla of the posterior

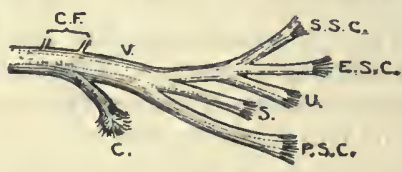

Fig. 539.-Scheme of the Auditory Nerve (Flower).
C.F. Communicating with Facial
C. Cochlear Nerve
V. Vestibular Nerve
S.S.C. To Superior Semicircular Canal
E.S.C. To External Semicircular Canal
U. To Utricle
S. To Saccule
P.S.C. To Posterior Semicircular Canal

semicircular canal and to the saccule. The former pass through the foramen singulare in the lower fossa of the lamina cribrosa, and the latter through the foramina of the inferior vestibular area of the lower fossa of the lamina cribrosa.

The branches of the cochlear nerve pass through the foramina of the cochlear area of the lower fossa of the lamina cribrosa.

Superficial Petrosal Nerves. - The great superficial petrosal nevve is a branch of the geniculate ganglion of the facial in the aqueduct of Fallopius. After emerging from that aqueduct through the hiatus Fallopii, it passes to the upper part of the foramen lacerum medium. Here it joins the great deel petrosal nerve to form the Vidian nerve, which passes forwards through the Vidian canal into the spheno-maxillary fossa, and joins the back part o Meckel's ganglion.

The small superficial petrosal nerve issues from the tympanic plexus on th inner wall of the tympanum, and represents the continuation of the tympani branch (nerve of Jacobson) of the petrous ganglion of the glosso-pharyngea nerve. As it traverses a canal in the petrous portion of the temporal bon it is joined by a small branch from the geniculate ganglion of the facial Emerging from this canal through the accessory hiatus, it passes througl the canaliculus innominatus, when present, or through the fissure betwees the pars petrosa and great wing of the sphenoid, or sometimes through th foramen ovale, into the zygomatic fossa, where it joins the otic ganglion, clos below the foramen ovale.

The external superficial petrosal nerve (inconstant) is a branch of the genicu 
ite ganglion of the facial. It emerges from the petrous part of the temoral bone through a small opening (inconstant) close to the hiatus Fallopii, ad joins the sympathetic plexus on the middle meningeal artery.

Deep Petrosal Nerves. - The great deep petrosal nerve is a branch of the carotid exus of the sympathetic. It joins the great superficial petrosal in the upper art of the foramen lacerum medium to form the Vidian nerve, which, as ated, passes through the Vidian canal into the spheno-maxillary fossa, and ins the back part of Meckel's ganglion.

The small deep petrosal nerve is a branch of the tympanic plexus. It asses forwards within the processus cochleariformis to the upper part of te foramen lacerum medium, where it joins the carotid plexus of the sympaletic, and the great deep petrosal nerve, or, it may be, the great superficial etrosal nerve. In this manner some of its fibres pass in the Vidian nerve Meckel's ganglion.

\section{The Articulations of the Atlas, Axis, and Occipital Bone.}

Atlanto-axial Joints.-These are three in number, and they belong the class diarthrosis. One is mesially placed, the articular surices being the atlantal facet on the anterior surface of the odontoid

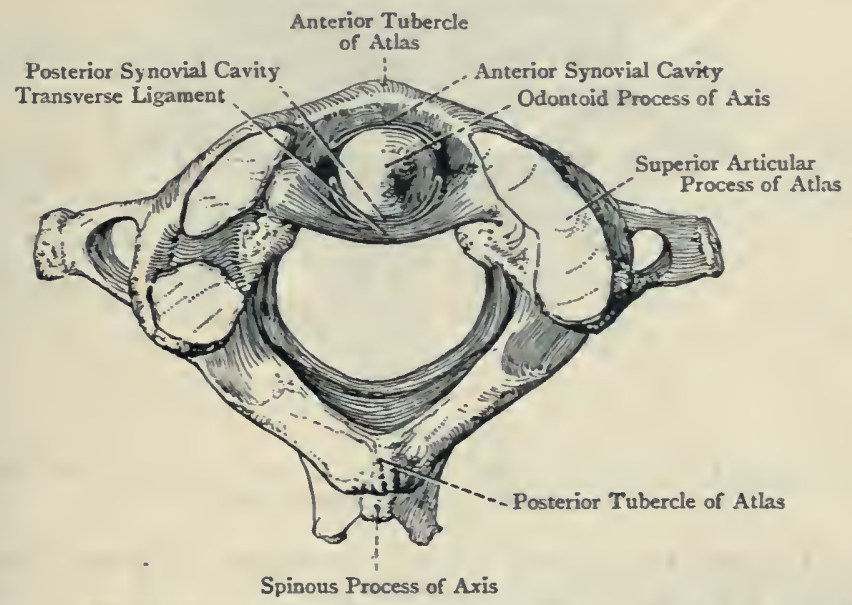

Fig. 540. -The Articulation between the ANterior Arch of the Atlas and the Odontoid Process of the Axis.

rocess of the axis and the odontoid facet on the posterior surface if the anterior arch of the atlas. This joint belongs to the subivision trochoides or pivot-joint. The other two are placed one on ither side, the articular surfaces of each being the inferior articular rocess of the atlas and the corresponding superior articular process f the axis. These two joints belong to the subdivision arthrodia.

Ligaments.-These are as follows: the transverse ligament, the capsular ligaments, and the accessory ligaments. Besides these here are the anterior and posterior atlanto-axial ligaments, which ire not directly related to any of the joints. 
The transverse ligament is usually spoken of as the transverse ligament of the atlas. It represents the transverse portion of the cruciform ligament, to be presently referred to, and it constitutes a strong band, which is attached on either side to the tubercle on the inner aspect of the lateral mass of the atlas. It is arched backwards behind the odontoid process, and at the median line it is connected on the posterior aspect with the crura of the vertical portion of the cruciform ligament. Between the ligament and the odontoid process there is an extensive synovial membrane, which extends well over each lateral aspect of the process, so as to come very near another synovial membrane between the front of the process and the anterior arch of the atlas.

The capsular ligaments are loose sacs which surround the articula-

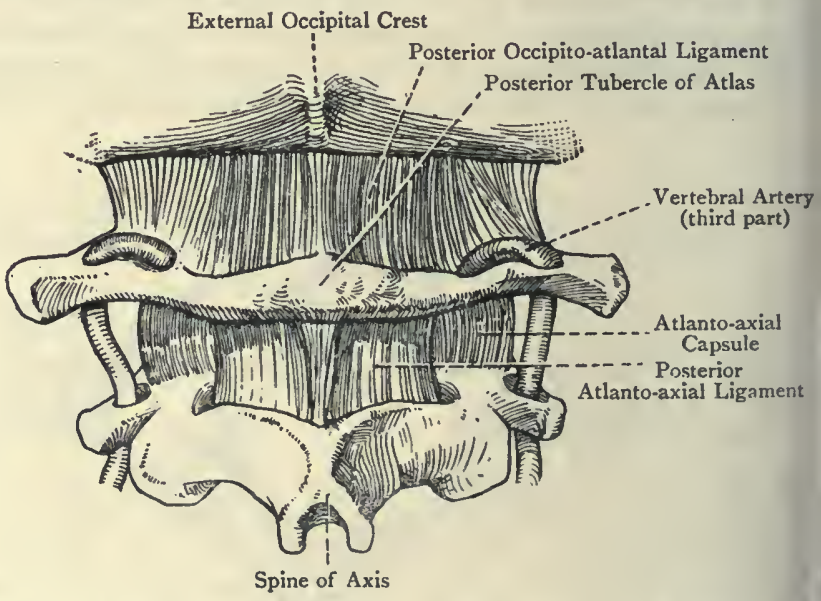

Fig. 54r.-The Occipital, Atlantal, and Axial Ligaments (SUPERfictal, POSTERIOR View).

tions between the inferior articular processes of the atlas and the superior articular processes of the axis.

The accessory ligaments are two in number-right and left. Each extends from the posterior surface of the body of the axis, close to the root of the odontoid process, to the inner and posterior part of the lateral mass of the atlas. The direction of each ligament is upwards and outwards, and it is closely related superiorly to the capsular ligament, which it strengthens internally and posteriorly. The accessory ligaments are auxiliary in function to the check ligaments, and take part in limiting rotation of the atlas upon the axis.

The anterior atlanto-axial ligament, broad, thin, and men1. branous, is attached superiorly along the lower margin of the anterior arch of the atlas, and inferiorly to the anterior aspect 0 the body of the axis. It is serially continuous with the anterio 
common ligament of the bodies of the vertebre, and its central portion is rendered thick by accessory fibres derived from the anterior common ligament.

The posterior atlanto-axial ligament, also broad, thin, and membranous, extends from the under aspect of the posterior arch of the atlas to the upper borders and adjacent portions of the outer surfaces of the laminæ of the axis. It is serially continuous with, and represents, the ligamenta subflava of succeeding vertebræ.

Atlanto-occipital Joints.-These are two in number, right and left. They belong to the class diarthrosis and to the subdivision condylarthrosis. The articular surfaces are the condyles of the occipital bone and the superior articular processes of the atlas.

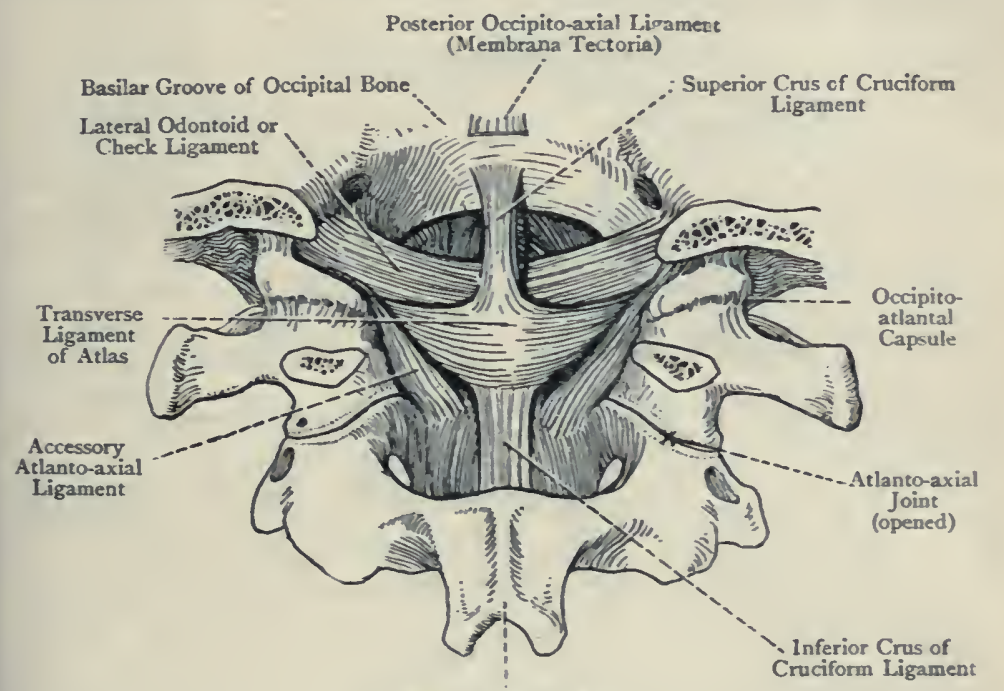

Spinous Process of Axis

Fig. 542.-Occipital, Atlantal, and Axial Ligaments (Posterior View).

Ligaments.-These are as follows: the capsular ligaments and the anterior and posterior occipito-atlantal ligaments, the latter two being indirectly connected with the joints.

The capsular ligaments are loose sacs which directly surround the articulations. Their fibres are attached superiorly around the margins of the occipital condyles, and inferiorly to the lateral masses of the atlas, external to the circumference of the superior articular processes.

The anterior atlanto-occipital ligament, thin and membranous, is attached inferiorly to the upper margin of the anterior arch of the atlas, and superiorly to the under surface of the basilar process of the occipital bone, close to the front part of the foramen magnum 
between the occipital condyles. In the median line it is rendered thick by the addition of a stout, round bundle of fibres, representing the accessory ligamient, the fibres of which for the most part are attached below to the anterior tubercle of the atlas, but some of them are prolonged into the thickened anterior accessory part of the anterior atlanto-axial ligament.

The posterior atlanto-occipital ligament, broad, thin, and membranous, is attached inferiorly to the upper margin of the posterior arch of the atlas, except in the region of the vertebrarterial grooves, and superiorly to the lower margin of the foramen magnum behind the occipital condyles. Over each vertebrarterial groove of the atlas this ligament forms an arch, beneath which the vertebral artery and suboccipital nerve pass.

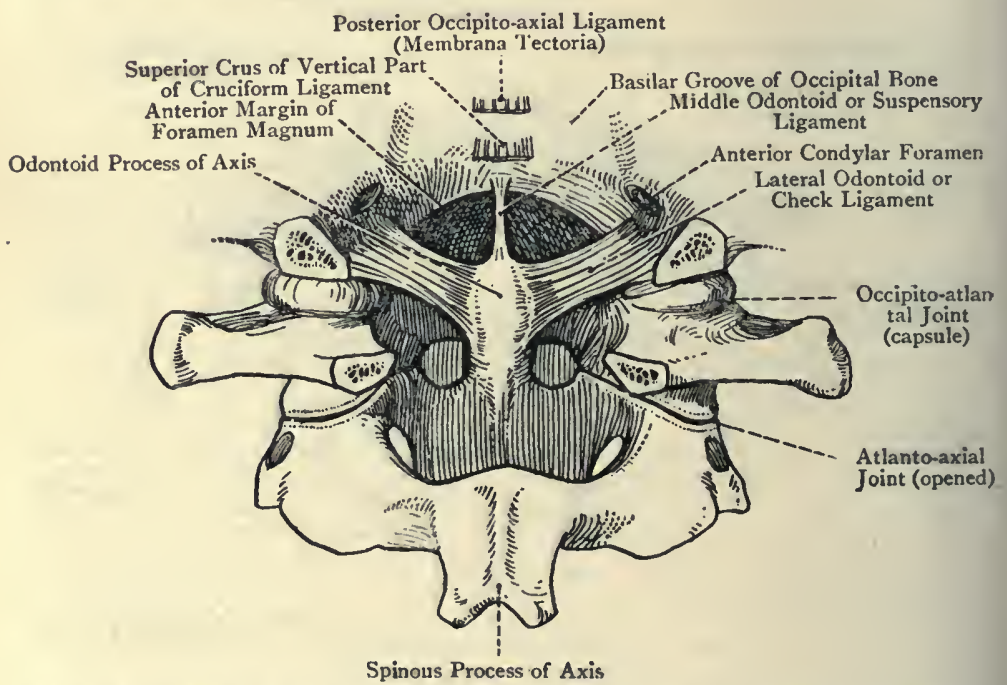

Frg. 543.-The Occipital, Atlantal, and Axial Ligaments (Deep POSTERIOR VIEW).

Occipito-axial Ligaments.-These are as follows: the posterior occipito-axial ligament; the lateral odontoid, or check, ligaments; the middle odontoid, or suspensory, ligament; and the vertical portion of the cruciform ligament.

The posterior occipito-axial ligament, also called the membrana tectoria, is a broad membranous band, which is attached inferiorly to the posterior surface of the body of the axis, where it is continuous with the fibres of the posterior common ligament of the bodies of the vertebræ, and superiorly to the posterior part of the basilar groove of the occipital bone. It covers the odontoid process of the axis and the odontoid and cruciform ligaments, hence the name membrana tectoria. 
The vertical portion of the cruciform ligament consists of two parts, upper and lower, which are spoken of as the superior crus and inferior crus. The superior crus extends from the posterior surface of the transverse ligament at the median line to the posterior part of the basilar groove of the occipital bone, between the anterior margin of the foramen magnum and the superior attachment of the membrana tectoria, under cover of which latter it lies. This crus, as it ascends, is in contact with the posterior surface of the head of the odontoid process. The inferior crus extends from the posterior surface of the transverse ligament at the median line to the posterior surface of the body of the axis above the inferior attachment of the membrana tectoria.

The transverse portion of the cruciform ligament constitutes the transverse ligament of the atlas, already described.

The middle odontoid or suspensory ligament is a narrow round cord which is attached inferiorly to the ridge on the head of the odontoid process, and superiorly to the anterior margin of the foramen magnum in the median line.

The lateral odontoid or alar ligaments, also known as the check ligaments, form two very strong bands which are attached inferiorly to the lateral surfaces on the head of the odontoid process, and superiorly to an impression on the inner surface of each condylar part of the occipital bone. The direction of each ligament is outwards and slightly upwards.

Movements-Atlanto-Axial Joints.-The movements are of a rotatory kind. the atlas, bearing the head, rotating on the axis, the odontoid process of which serves as a pivot. The extent of rotation is about 30 degrees, and it is limited by the lateral odontoid ligaments, aided slightly by the accessory atlanto-axial ligaments. As stated in the section on Osteology, the superior articular processes of the axis are each divided by a slight transverse elevation into two parts-anterior and posterior. When the atlas lies directly over the axis, as when the face is directed straight forwards, the inferior articular processes of the atlas are not in accurate contact with the superior articular processes of the axis. Between the contiguous pairs there is a distinct interval all round. When, however, the atlas is rotated, the anterior division of the axial articular process of one side is brought into accurate contact with the corresponding atlantal articular process, and the posterior division of the opposite axial articular process into accurate contact with the atlantal articular process of that side. Atlanto-oceipitai Joints.--The movements allowed at these joints are as follows: (I) Flexion; (2) extension; and (3) oblique movement. Flexion and extension constitute the forward and backward, or nodding, movements. In overextension (dorsi-flexion) the posterior margins of the superior articular processes of the atlas enter the posterior condylar fossa of the occipital bone. and locking takes place. In complete forward or ventral flexion the anterior margins of the superior articular processes of the atlas come into contact with the occipital bone in front of each condyle.

Oblique movement occurs in the position of semiffexion ventralwards. As stated in the section on Osteology, the condyles of the occipital bone, like the superior articular processes of the axis, are each divided into two parts, anterior and posterior, by a slight elevation, which, in the case of the occipital condyles, is disposed obliquely. During oblique movement the anterior division of one occipital condyle is in contact with the corresponding superior articular process of the atlas, whilst the posterior division of the opposite occipital condyle is in contact with the superior articular process of the atlas on that side. 


\section{THE NERVOUS SYSTEM}

THE nervous system is arranged in two divisions, cerebro-spinal and sympathetic.

The sympathetic nervous system consists of (I) two gangliated cords, right and left, situated on either side of the vertebral column, and (2) three prevertebral plexuses, namely, the cardiac plexus, situated in the thorax; the epigastric or solar plexus; and the hypogastric plexus, the latter two being situated in the abdomen.

The cerebro-spinal nervous system or axis consists of the encephalon and the spinal cord, the former being situated within the cranial cavity, and the latter within the spinal canal. The continuity between these two divisions is established through the foramen magnum.

The cerebro-spinal axis is central in position, and is connected with the various parts of the body by the cranial and spinal nerves. It is composed of two kinds or nervous matter, white and grey. The white matter consists chiefly of nerve-fibres, and the grey matter consists of nerve-cells, with their axis-cylinder processes or axons and dendrites, the pervading supporting tissue in each case being called neuroglia. In the brain the white matter is disposed in the interior as the medulla, and the grey matter forms the exterior or cortex. In the spinal cord the white matter is disposed externally in the form of three columns on either side, whilst the grey matter is situated in the interior.

The cerebro-spinal axis is surrounded by three membranes, or meninges, which, from without inwards, are named the dura mater, arachnoid membrane, and pia mater.

\section{The Spinal Cord.}

Membranes of the Spinal Cord.-The membranes are three in number, namely, the dura mater, the arachnoid membrane, and the pia mater.

Dura Mater.-This is the most external covering of the cord. I 306 


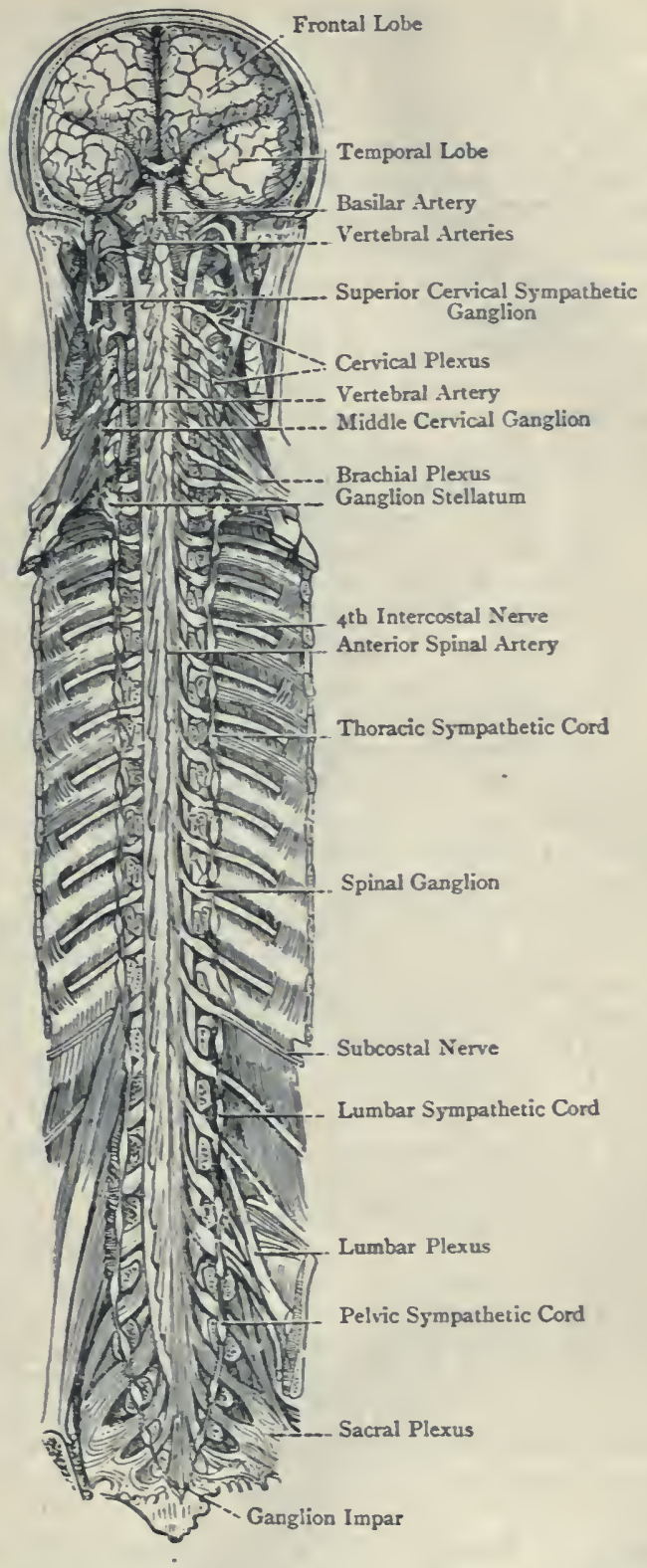

Fig j44.-The Cerebro-Sptval and Sympathetic Systems (ANTerior VIEW) (HIRSCHFELd AND LEVEILLÉ). 
It forms a dense fibrous tube, known as the theca, which extends from the margin of the foramen magnum of the occipital bone to the level of the second sacral vertebra. Inferiorly, where it has become tapering behind the second sacral vertebra, the theca is perforated by the filum terminale, and from this level the spinal dura mater is prolonged downwards around the filum terminale, with which it blends. Finally it is attached, along with the filum terminale, to the back of the first coccygeal vertebra, where it is incorporated with the periosteum. In this situation the spinal dura mater is firmly fixed.

The theca surrounds the spinal cord very loosely, and it is separated from the wall of the spinal canal by an interval, called the epidural space (cavum epidurale B.), which is occupied by venous plexuses and loose areolar tissue.

Opposite the intervertebral foramina of each side the theca presents a series of openings, which are arranged in two parallel rows. The openings constituting each pair are placed side by side, but are distinct from each other, and they transmit the ventral or anterior and dorsal or posterior roots of the spinal nerves. Each of these roots, as it makes its exit, receives a tubular sheath from the margin of the corresponding thecal opening, and these sheaths remain distinct as far as the spinal ganglion of the dorsal root. Thereafter the contiguous sheaths fuse and form one sheath, which blends with the sheath of the corresponding spinal nerve.

The spinal dura mater is maintained in position by several connections. (I) Superiorly it is fixed to the margin of the foramen magnum of the occipital bone. (2) Opposite the body of the axis it is firmly attached, anteriorly, to the posterior occipito-axial ligament. (3) Below the level of the axis it is loosely connected, anteriorly, with the posterior common ligament of the bodies of the vertebræ by fibrous bands. (4) Laterally it is connected with the sheaths of the spinal nerves by means of the tubular sheaths which it gives to the ventral and dorsal nerve-roots. (5) Inferiorly it blends with the periosteum over the back of the first coccygeal vertebra through means of the filum terminale. Posteriorly it is quite free from connections.

The spinal dura mater differs from the cranial dura mater in the following respects: (I) It is destitute of an outer or periosteal layer; (2) it does not send septa into the spinal cord; and (3) it does not contain venous sinuses.

Blood-supply of Spinal Dura Mater.-The arteries are derived from (I) the spinal branches of the vertebral, intercostal, and lumbar arteries; and (2) the lateral sacral arteries, which are branches of the internal iliac trunk.

Nerve-supply.--The nerves are partly spinal and partly sympathetic.

Lymphatic Vessels. - There are no lymphatic vessels, their place being taken by perivascular lymph-spaces in connection with the arteries. 
Structure.-The spinal dura mater consists of fibrous tissue and some elastic tissue disposed in parallel longitudinal bundles. Its internal and external surfaces are covered by endothelial cells.

Subdural Space.-Between the spinal dura mater and the arachnoid there is a narrow cleft-like interval, which is known as the subdural space (cavum subdurale B.). It contains a small amount of fluid, and it communicates freely with the lymph-spaces or clefts in the sheaths of the spinal nerves. It has, however, no communication with the subarachnoid space.

Spinal Arachnoid Membrane.-This is a delicate transparent membrane which loosely surrounds the spinal cord between the theca externally and the pia mater internally. It is separated from the theca by the subdural space, and from the pia mater by the subarachnoid space. Superiorly it is continuous with the cranial arachnoid, and inferiorly it encloses the cauda equina. On either side it forms sheaths for the processes of the ligamentum denticulatum as far as the inner surface of the theca. The ventral and dorsal roots of the spinal nerves also receive sheaths from it, which accompany them through the openings in the theca, but soon cease.

\section{Subarachnoid Space}

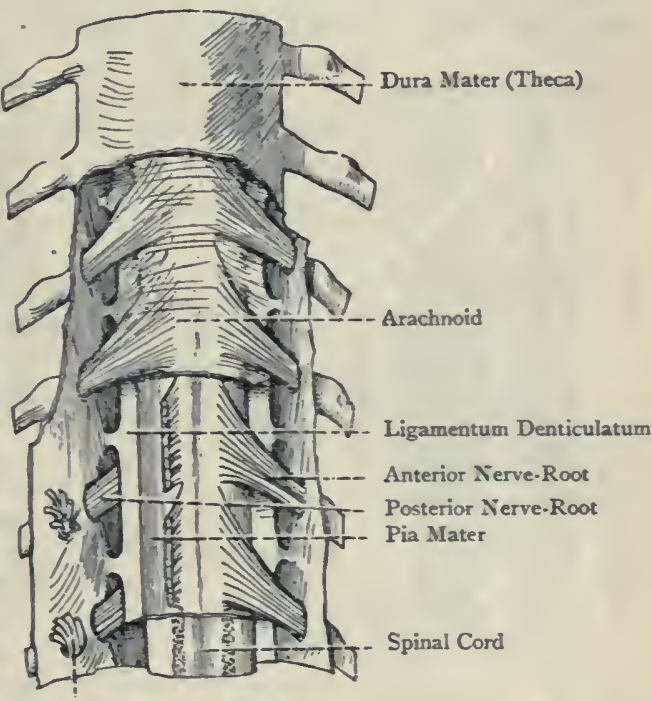

Anterior Nerve-Root (cut)

Fig. 545.-Portion OF the SPINAL CORD, SHowing THE MEMBRANEs, Liganexta Denticulata, AND Roots of the SPINal Nerves. (Cavum Subarachnoidale.B.).-This space, which is wide, is situated, as stated, between the arachnoid and pia mater. It contains cerebro-spinal fluid, and its dorsal part is continuous superiorly with the cisterna magna of the cranial subarachnoid space, which communicates with the fourth ventricle by three openings (foramen of Magendie and foramina of Luschka).

The subarachnoid space is partially divided into two compartments, ventral and dorsal, by ligamenta subflava, which form incomplete lateral septa. The ventral roots of the spinal nerves traverse the ventral compartment, and the dorsal roots traverse the 
dorsal compartment. The dorsal compartment is partially subdivided into two portions, right and left, by means of a third incomplete septum, called the septum posticum. This partition extends from the pia mater, as it crosses the dorsal median fissure of the spinal cord, to the dorsal part of the arachnoid at the median line. All the compartments of the subarachnoid space communicate freely with each other.

The subarachnoid space has no communication with the subdural space.

Structure of the Arachnoid Membrane.- The arachnoid consists of fine fibrous tissue, arranged in interlacing bundles, the intervals between these bundles being occupied by delicate cellular membranes. Several such layers, intimately blended together, form the membrane.

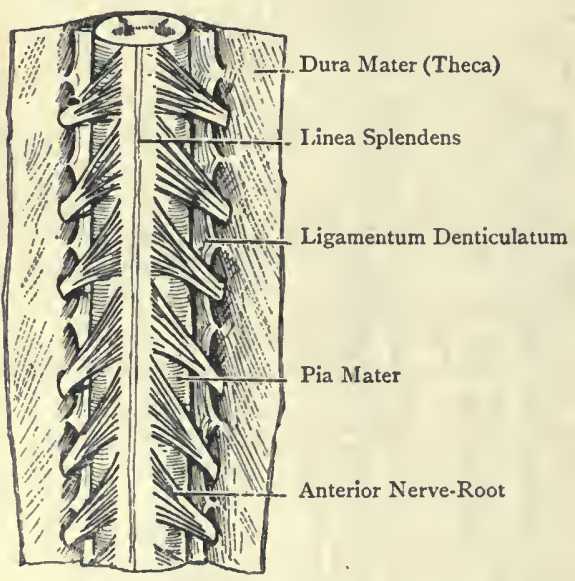

Fig. 546.-A Portion of the Spinal Cord (ANTERIOR VIEW).

(The Theca has been laid open, and the Arachnoid Membrane removed).

Beneath the arachnoid, and constituting a part of it, there is a reticulum of subarachnoid trabecula. These trabeculæ consist, as in the case of the arachnoid proper, of fine fibrous tissue, but the intertrabecular spaces, instead of being occupied by cellular membranes, contain cerebrospinal fluid. The trabecular reticulum connects the arachnoid with the subjacent pia mater.

Spinal Pia Mater.-This is the most internal membrane of the spinal cord. It is very vascular and closely invests the cord, the substantia gelatinosa externa or glial sheath being situated immediately beneath it. Superiorly it is continuous with the cranial pia mater, and inferiorly it is prolonged from the conus medullaris over the upper half of the intrathecal part of the filum terminale. On either side it forms tubular sheaths for the ventral and dorsal roots of the spinal nerves, which blend with the sheaths of the nerves.

Along the course of the ventral median fissure of the cord it sends a vascular fold into that fissure. Along the course of the dorsal median fissure, over which it passes, the neuroglial septum occupying that fissure is attached to it. From the deep surface of the pia mater several septa are prolonged into the cord, which carry with them portions of the glial sheath.

The pia mater is separated from the arachnoid by the subarachnoid space, and opposite the dorsal median fissure of the cord it is connected with the dorsal part of the arachnoid by the septum posticum. 
Blood-supply.-The pia mater derives its arteries from (I) the anterior and posterior spinal arteries, and (2) the neural branches of the lateral spinal arteries.

Nerve-supply. - The nerves are derived from the sympathetic system.

Structure.-The spinal pia mater consists of two layers-outer and inner. The outer layer consists of fibrous tissue, which is disposed for the most part in parallel longitudinal bundles. The inner layer consists of areolar tissue, containing a great many bloodvessels, and its outer and inner surfaces are covered by endothelial cells. Between the two layers there are narrow cleftlike lymphatic spaces, which communicate with the subarachnoid space, and with lymphatic clefts around the arteries of the pia mater.

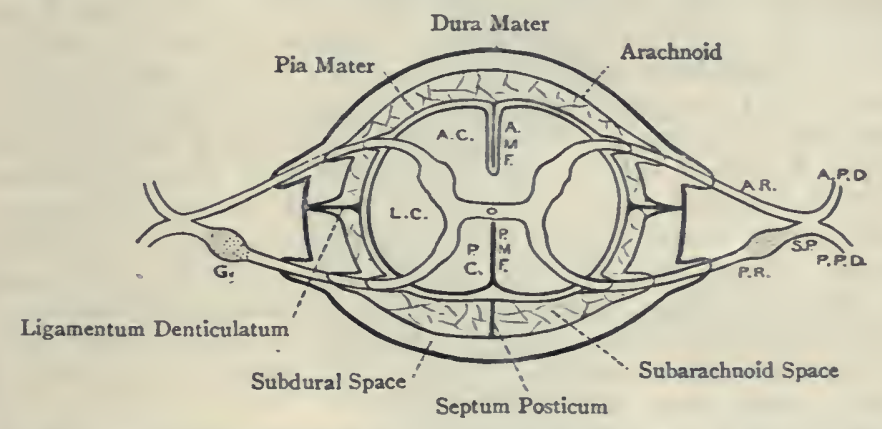
Fig. 547.-Diagram of a Transverse Section of the Spinal Cord
and its Membranes.

A.M.F. Anterior Median Fissure, with Process of Pia Mater

P.M.F. Posterior Median Fissure

A.C. Anterior Column

I. C. Lateral Column

P.C. Posterior Column
A.R. Anterior Nerve-Root

P.R. Posterior Nerve-Root

G. Spinal Ganglion

S.P. Spinal Nerve

A.P.D. Anterior Primary Division

P.P.D. Posterior Primary Division

The spinal pia mater differs from the cranial pia mater in being thicker and more adherent to the nervous matter. The greater thickness is due to the presence of the outer layer, the cranial pia mater representing the inner layer of the spinal pia mater..

Linea Splendens. - The pia mater presents a glistening vertical fibrous band, which lies over the ventral median fissure. This band is known as the linea splendens, and it extends along the entire length of the cord and along the conus medullaris on to the filum terminale.

Ligamentum Denticulatum, - This is a fibrous band which extends along the spinal cord, on each side, opposite the corresponding lateral column. It lies between the ventral and dorsal roots of the spinal nerves, and extends from the margin of the foramen magnum to the lower end of the cord. It lies within the subarachnoid space, and partially divides that space into two compartments-ventral and dorsal. 
Internally it forms an uninterrupted band which is attached to the pia mater along the lateral column of the cord. Externally it is denticulated. The denticulations (about twenty-one in number) carry with them sheaths from the arachnoid, and their pointed outer ends are attached to the inner surface of the theca in the intervals between each pair of openings for the exit of the ventral and dorsal nerve-roots.

The two ligamenta denticulata act as lateral supports to the spinal cord.

Structure.-Each ligamentum denticulatum consists of fibrous tissue, which is continuous with the outer layer of the pia mater.

External Characters of the Spinal Cord.-The spinal cord, or medulla spinalis, which is somewhat cylindrical, is that division of the cerebro-spinal axis which is situated within the spinal canal. It extends from the lower margin of the foramen magnum in the occipital bone to about the level of the disc between the bodies of the first and second lumbar vertebræ, and it is about $\mathrm{r} 8$ inches in length. Superiorly it is continuous with the medulla oblongata, and inferiorly it terminates in a tapering portion, called the conus medullaris. From the lower end of this cone a slender, glistening thread, called the filum terminale, is continued downwards between the bundles of lumbar, sacral, and coccygeal nerves of either side, which constitute the cauda equina, to be attached to the back of the first coccygeal segment.

The spinal cord is of smaller dimensions than the spinal canal, and is therefore relieved from pressure during the ordinary movements of the vertebral column. It is surrounded by three membranes, already described, namely, the dura mater, the arachnoid membrane, and the pia mater, in this order from without inwards. Within its theca of dura mater the cord is suspended by means of the ligamenta denticulata, and the nerveroots as they emerge through the openings in the theca.

The spinal cord varies in shape in different regions. In the cervical region, as seen in transverse section, it is transversely oval, and is slightly flattened from before backwards. In the thoracic. region it is somewhat circular, but the transverse diameter exceeds the antero-posterior. In the lumbar region it is still more circular than in the thoracic region.

The cord presents two swellings, which are known as the cervical and lumbar enlargements, and which are associated with the numerous and large nerve-trunks. destined for the upper and lower limbs. The cervical enlargement extends from near the commencement of the cord to the second thoracic vertebra, and its breadth is greatest opposite the sixth cervical vertebra. The lumbar enlargement, which is less conspicuous than the cervical, extends from the level of the tenth thoracic vertebra to the conus medullaris, and its breadth is greatest opposite the twelfth thoracic vertebra. 
Filum Terminale.-This delicate glistening thread is also known as the central ligament of the cord. It lies in the median line between the lumbar, sacral, and coccygeal nerves of either side, which constitute the cauda equina, and it extends from the apex of the conus medullaris to the back of the first coccygeal segment. It is about 6 . inches in length. As low as the back of the body of the second sacral segment it is situated within the theca, but at that level it pierces the theca, from which it receives an investment, and then passes to be attached to the back of the first coccygeal

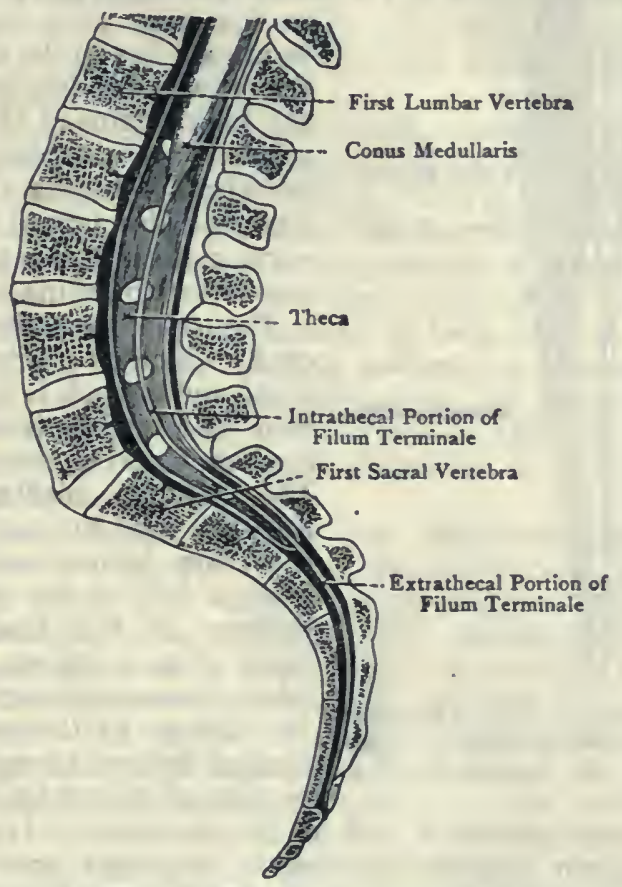

Fig. 548.-Lumbar and Sacral Portions of Spinal Canal, showing Lateral View of Conus Medullaris, Filum Terminale, and Theca (TESTut).

segment, where it blends with the periosteum. The intrathecal portion is known as the filum terminale internum, and the extrathecal portion as the filum terminale externum.

Structure. - The filum terminale internum in its upper half consists of pia mater, prolonged from the conus medullaris of the spinal cord. This encloses grey matter, within which, over about the upper third, there is a continuation of the central canal of the cord. The lover half consists chiefly of connective tissue. The filum terminale externum is a mere fibrous filament, invested by a prolongation of 
the theca which blends with it. It is also composed of pia mater prolonged downwards from the conus medullaris, and reinforced by fibres derived from the lower portions of the ligamenta denticulata

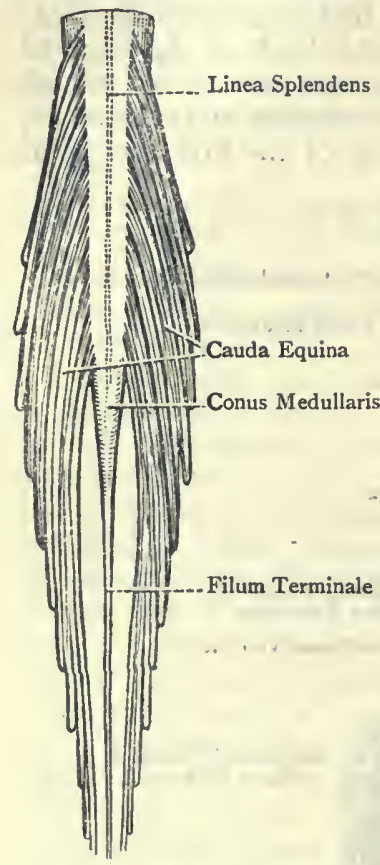

Fig. 549. - The Terminal Part of the Spinal Cord, and the Cauda Equina. and linea splendens. Its lower part is purely fibrous.

Cauda Equina.-This is situated within the lower part of the theca. It consists of the lumbar, sacral, and coccygeal nerves of each side, which are arranged in the form of a leash, and the filum terminale lies in the median line between the two nerve-leashes. On account of the high origins of the individual nerves, relatively to the positions of the intervertebral foramina through which they pass, the direction of the nerves is almost vertical until they reach the level of their respective foramina of exit.

Fissures of the Spinal Cord.-The spinal cord, which is somewhat flattened in front and behind, is incompletely divided into two symmetrical halves by two median fissures, anterior and posterior. The anterior median fissure extends into the cord for one-third of its thickness from before backwards, and it contains a fold of the pia mater. At the bottom of the fissure the transverse band of nerve-fibres, called the anterior white commissure, crosses between the two halves of the cord. The posterior median fissure, though so named, is not an actual fissure like the anterior, and does not contain a fold of the pia mater. It is just wide enough to contain a septum formed by neuroglia, and it extends into the cord for about half of its thickness from before backwards. The posterior grey commissure lies at the bottom of the fissure. The anterior median fissure is more distinct and wider than the posterior, but it is not so deep.

Each half of the cord presents a groove along the line of entrance of the fasciculi of the posterior nerve-roots, called the postero-lateral sulcus, but there is no similar groove along the line of emergence of the fasciculi of the anterior nerve-roots, these being spreald over an area of some breadth. By means of the postero-lateral sulcus on the one hand and the area corresponding to the emergence of the fasciculi of the anterior nerve roots on the other, each half of the spinal cord is divided superficially into three columns, anterior, lateral, and posterior.

The anterior column is situated between the anterior median 
fissure and the most external fasciculi of the anterior nerve-roots; the lateral column is the area between the most external fasciculi of the anterior nerve-roots and the postero-lateral sulcus; and the posterior column lies between the postero-lateral sulcus and the posterior median fissure. Practically the anterior column represents the region in front of the anterior nerve-roots, the lateral column the region between the anterior and posterior nerve-roots, and the posterior column the region behind the posterior nerve-roots. According to some authorities there are only two columns, namely, antero-lateral and posterior, the former extending from the anterior median fissure to the postero-lateral sulcus, and representing the combined anterior and lateral columns.

In the cervical region the surface of each posterior column presents a slight groove which is situated nearer the posterior median fissure than the postero-lateral sulcus. This groove is called the posterior intermediate or paramedian furrow. It contains a septum of pia mater, and in this manner the posterior column of the cord is marked off into two tracts. The inner and smaller tract is called the postero-median column, or column of Goll, and the outer and larger is called the postero-external column, or column of Burdach. These two columns extend throughout the cord, but it is only above the level of the mid-thoracic region that they are separated from each other by a septum of pia mater, known as the posterior intermediate septum.

Origin of the Spinal Nerves. - There are thirty-one pairs of spinal nerves, which arise from the sides of the spinal cord. They are arranged in five groups, on either side, as follows: cervical, eight in number; thoracic, twelve; lumbar, five; sacral, five; and coccygeal, one. Each spinal nerve is attached superficially to the cord by two roots, anterior and posterior, the posterior root being the larger of the two. The portion of the cord from which each pair of spinal nerves arise is spoken of as a segment of the cord. Each root is ensheathed by tubular prolongations of the coverings of the cord, namely, the pia mater, arachnoid, and dura mater, in this order from within outwards, and these sheaths ultimately blend with the perineurium. The roots are separated from each other by the lateral column of the cord and the ligamentum denticulatum, and they pass through separate openings in the theca of dura mater.

The anterior roots are composed of efferent or motor fibres, and their fasciculi emerge from the cord in an irregular manner, being spread over an area corresponding in breadth to the caput of the anterior cornu of the grey matter in the interior. The posterior roots are composed of afferent or sensory fibres, and their fasciculi enter the cord in a straight line along the course of the postero-lateral sulcus. Each posterior root presents an oval swelling, called the spinal ganglion. These ganglia are, for the most part, situated in the intervertebral foramina, and immediately beyond each ganglion the anterior and posterior roots unite to form 
a spinal nerve, which is necessarily a mixed nerve, inasmuch as it is composed of afferent and efferent fibres.

Each spinal nerve breaks up into an anterior and a posterior primary division.

The upper cervical nerve-roots are short, and pass almost horizontally outwards. The succeeding nerve-roots, however, gradually increase in length, and incline downwards as they pass outwards: This downward inclination goes on increasing until it becomes almost vertical in the case of the lumbar, sacral, and

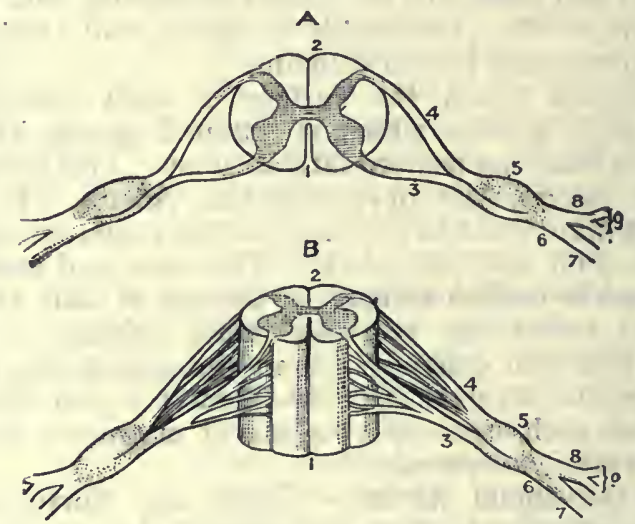

Fig. 550.-Two Segments of the Spinal Cord, showing the AttachMENTS OF THE ANTERIOR AND POSTERIOR NERVE-ROOTS, AND THE Spinal Ganglia.

A, Superior View ; B; Anterior View.

I, I. Anterior Median Fissure.

2, 2. Posterior Median Fissure

3. 3. Anterior or Motor Nerve-Root.

4, 4. Posterior or Sensory Nerve-Root

9, 9. External and Internal Branches of Posterior Primary Division
5, 5. Spinal Ganglion

6, 6. Spinal Nerve

7, 7. Anterior :Primary Division

8, 8. Posterior Primary Division

coccygeal nerves. which constitute the cauda equina. From this disposition it follows that, in the majority of cases; the superficial origins of the spinal nerves are on a higher level than the intervertebral foramina through which they emerge from the spinal canal.

Relation of the Spines of Vertebræ to the Bodies and to the Origins of the Nerves:-In the case of the cervical and the eleventh and twelfth thoracic vertebrx the extremities of the spinous processes correspond to the lower margins of the bodies of the respective vertebræ. In the case of the thoracic vertebræ, from the first to the tenth inclusive; the extremity of each spinous process corresponds to the upper margin of the body immediately below. In the case of the lumbar vertebræ the extremity of each spinous process corresponds to the centre of the body of its own vertebra.

Each cervical spinous process is nearly opposite the lower fasciculi of the roots of the nerve below. The spinous process of the seventh cervical vertebra 
(vertebra prominens) is opposite the roots of the first thoracic nerve. IFrom the third to the tenth thoracic vertebra the spinous processes correspond to the second root below. The eleventh thoracic spine corresponds to the first and second lumbar nerves. The twelfth thoracic spine corresponds to the third, fourth, and fifth lumbar nerves. The first lumbar spine corresponds to the first, second, and third sacral nerves. (Gowers, from an original investigation).

Mode of distinguishing the Anterior and Posterior Surfaces of the Spinal Cord.-These surfaces may be recognised by attending to the following points :

\section{Anterior Surface.}

I. Linea splendens in median line.

2. Anterior spinal artery in median line.

3. Fasciculi of anterior nerveroots spread over a wide area.

\section{Posterior Surface.}

I. Ganglion on each posterior nerveroot.

2. Fasciculi of posterior nerve-roots lie in a straight line, and enter through postero-lateral sulcus.

3. Arterial anastomotic chain behind and in front of the posterior nerve-roots.

Internal Structure of the Spinal Cord.-The spinal cord, as seen in transverse section, consists of a central portion composed of grey matter, and an external portion composed of white matter.

Grey Matter.-This is arranged in the form of two irregular crescents, the concavities of which are directed outwards, and the convexities inwards, the latter being connected across the middle line by the grey commissure. The arrangement has been likened to the letter $\mathbf{M}$. The grey commissure lies at the bottom of the posterior median fissure, and presents about its centre the minute opening of the central canal of the cord. The part of the commis. sure in front of this canal is known as the anterior grey commissure, and the part behind as the posterior grey commissure. In front of the anterior grey commissure there is a transverse band of white matter, called the anterior white commissure, which lies at the bottom of the anterior median fissure. Each crescent of grey matter consists of two cornua, anterior and posterior, the former being in front of, and the latter behind, the grey commissure. The anterior cornu is broad and blunt, and it stops short of the surface of the cord, being separated from the surface by white matter which is traversed by the fasciculi of the anterior nerve-roots. The blunt extremity of the anterior cornu is called the caput cornu, and the portion adjoining the grey commissure, which is slightly constricted, is called the cervix cormu. The posterior cornu is, for the most part, long, narrow, and tapering, and its pointed extremity almost reaches the surface of the cord at the bottom of the postero-lateral sulcus. This pointed extremity is called the apex cornu, and it contains a translucent substance, known as the substantia gelatinosa of Rolando, which forms a cap for the caput 
cornu posterioris. It contains a small amount of neuroghia, and numerous nerve-cells. The portion adjoining the grey commissure, which is slightly constricted, is called the cervix cornu, and the portion contiguous to the cervix, which is slightly enlarged, is called the caput cornu. The part between the two cornua is called the body.

About the centre of the concavity of the body crescent the grey matter projects outwards into the lateral column in the form of processes arranged in a reticular manner and enclosing white matter. This network is called the processus reticularis, and it is most conspicuous in the cervical region. In the thoracic region, more particularly in its upper part, the grey matter of each crescent forms a triangular projection, which extends outwards for a short distance immediately in front of the processus reticularis, and adjacent to the junction of the anterior cornu with the grey com-

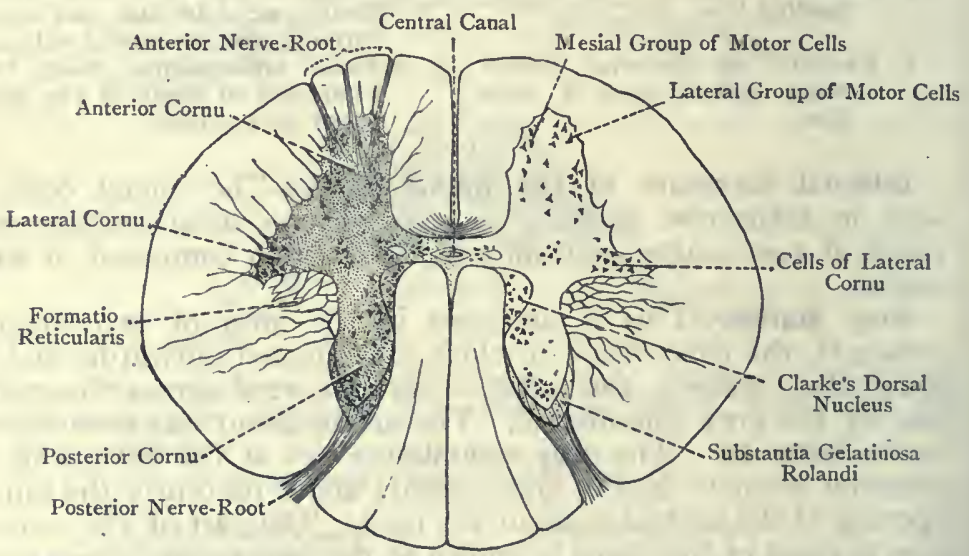

Fig. 551.-Transverse Section of the Spinal Cord in the Upper Thoracic Region, showing the ARrangement of the Grey MatTer and Cells (Semi-diagrammatic) (After Poirier).

missure. This projection is variously known as the lateral cornu, the intermedio-lateral tract of Lockhart Clarke, or the intermediate process of Gowers. When followed into the lower cervical and into the lumbar regions it blends with the anterior cornu, the thickness of which it increases, but it is again present above the level of the fourth cervical vertebra.

The grey matter varies in amount in different parts of the cord. It is present in largest quantity in the lumbar enlargement, where the large nerve-trunks for the lower limbs arise, and next to this in the cervical enlargement, where the large nerve-trunks for the upper limbs arise.

The cornua of the crescents of grey matter vary in shape in different regions. In the cervical region the anterior cornua are short, broad, and blunt, and the posterior cornua are long, narrow, 
and pointed. In the thoracic region both cornua are narrow, though the posterior is more so than the anterior. In the lumbar region both cornua are broad, though the anterior is more so than the posterior. These differences render sections of the spinal cord in the cervical, thoracic, and lumbar regions easily recognisable. As stated, the lateral cornu is also a characteristic of the cord in the thoracic region, more particularly in its upper part.

Central Canal.-This minute canal is situated about the centre of the grey commissure, and extends throughout the entire length of the spinal cord. Superiorly it is continued into the lower half of the medulla oblongata, and it opens into the lower part of the fourth ventricle at the calamus scriptorius. Inferiorly, near the apex of the conus medullaris, it becomes enlarged, and assumes the shape of an inverted $T$, thus $\perp$. This enlargement is known as the ventriculus terminalis of Krause. From this point it is prolonged for some distance into the filum terminale, and it ends in a closed extremity. In the cervical and thoracic regions the central canal
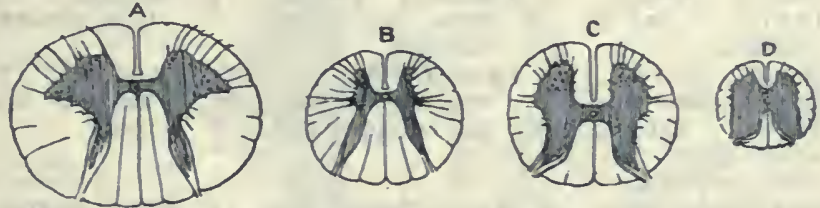

Fig. 552.-Transverse Sections of the Spinal Cord in Diffreent REGIONS.

A, Cervical Region ; B, Mid-thoracic Region; C, Lumbar Region ;

D, Conus Medullaris.

is nearer the anterior surface of the cord than the posterior, but in the lumbar region it occupies the centre. In the conus medullaris it is near the posterior surface.

The canal is lined with ciliated columnar epithelium, the columnar cells being known as ependymal cells.

The central canal represents the lumen of the neural tube of epiblast from which the spinal cord is developed.

White Matter of the Spinal Cord.-The white matter forms the outer part of the cord, and is arranged in three columns, anterior, lateral, and posterior. The anterior column is situated betiveen the anterior median fissure and the anterior cornu of grey matter, and extends as far as the most external fasciculi of the anterior nerve-roots. It therefore includes the superficial coating of the anterior cornu; where it is traversed, over an area of some breadth, by the scattered fasciculi of the anterior nerve-roots. The lateral column is situated between the anterior and posterior cornua of grey matter, in the concavity of the crescent. Its superficial limits are the most external fasciculi of the anterior nerve-roots and the fasciculi of the posterior nerve-roots at the postero-lateral sulcus. 
The posterior column is situated between the posterior median fissure and the posterior cornu of grey matter, its superficial limit being the fasciculi of the posterior nerve-roots at the postero-lateral sulcus.

The white matter increases in quantity from below upwards, and septa of pia mater and neuroglia fibres pass into it at various points.

\section{Chief Distinguishing Characters of the Spinal Cord in Different Regions; as seen in Transverse Sections.}

Cervical Region.

1. Transversely oval.

2. Anterior Cornu, short, broad, and blunt.

Posterior Cornu, long, narrow, and tapering.

3. Formatio Reticularis, well marked.

4. I,ateral Cornu, merged into anterior, excapt above fourth cervical vertebra.

5. White Matter, large in amount.

6. Central Canal, nearer the ventral than the dorsal surface.

7. Postero - intermediate Sulcus and Septum of pia mater, well marked.
Thoracic Region.

I. Approaching to circular.

2. Anterior and Posterior Cornua both narrow, posterior more so than anterior.

3. Formatio Reticularis, not very distinct.

4. Lateral Cornu, conspicuous, especially in upper part.

5. White Matter, less in amount, but large in proportion to Grey Matter.

6. Central Canal, nearer the ventral than the dorsal surface.

7. Postero - intermodiate Sulcus, ab. sent, but Septum of pia mater recognisable.
Lumbar Reglon.

I. Almost circular.

2. Anterior and Posterior Cornua both broad, anterior more so than posterior.

3. Formatio Reticularis, absent.

4. Lateral Cornu, merged into anterior.

5. White Matter, small in amount, and Grey Matter, large.

6. Central Canal, in the centre.

7. Postero-intermediate Sulcus and Septum of pia mater, ab. sent.

Minute Structure of the Spinal Cord-Grey Matter.-The grey matter consists of nerve-cells, nerve-fibres, and neuroglia, and is very vascular.

The nerve-cells are present in great numbers, and are multipolar. Each cell sends off at various points several protoplasmic processes, one of which becomes the axis-cylinder of a nerve-fibre, and is called the axis-cylinder process, or axon. The other processes are known as the protoplasmic processes of Deiters, or dendrites, and, after successive branchings, they terminate in free extremities. There are no anastomoses between the dendrites of the same cell, nor between those of contiguous cells. A multipolar nerve-cell, with its axon and dendrites, constitutes a neuron. The multipolar 
cells form longitudinal columns of various lengths, and, as seen in transverse sections of the cord, they are arranged in groups which occupy particular regions. These cell-columns or groups are three in number, namely, anterior or ventral, in the anterior cornu of grey matter; lateral, in the lateral cornu of grey matter; and posterior, constituting the posterior vesicular column of Lockhart Clarke, and being very conspicuous in the mesial portion of the cervix of the posterior grey cornu in the thoracic region. Besides these main columns or groups, other nerve-cells are present, which are scattered irregularly throughout the other portions of the grey matter.

The anterior or ventral cell-column is situated, as stated, in the anterior cornu of grey matter, and extends throughout the

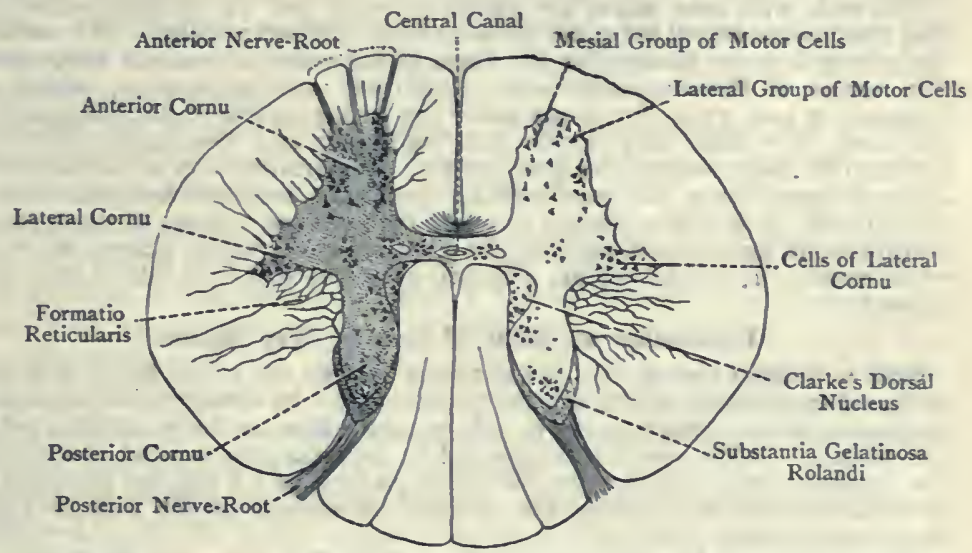

Fig. 553.-Transverse Section of the Spinal Cokd is the Upper Thoracic Region, showing the ARrangement of the Grey Matter and Cells (Semi-diagrammatic) (After Poirier).

whole length of the spinal cord. Its cells are of large size and very conspicuous, and their axons, which are at first non-medullated, become medullated, and then constitute the fasciculi which emerge to form the anterior nerve-roots. These cells are therefore the sources from which the efferent or motor nerve-fibres proceed, and the ventral column is consequently spoken of as the motor or ganglionic column. The ventral or motor cells of this column are arranged in two groups, mesial and lateral. The mesial group occupies the mesial part of the anterior grey cornu, and the lateral group is situated in its outer part. In the cervical and lumbar enlargements of the cord the cells of the lateral group are very numerous, and are arranged in two sub-groups, ventro-lateral and dorso-lateral.

The intermedio-lateral cell-column is situated, as stated, in the 
lateral cornu of grey matter, and the cells constitute a column, known as the intermedio-lateral tract of Lockhart Clarke, or the dorsal nucleus of Stilling. This tract extends throughout the entire thoracic region of the cord, and for a short distance into the cervical and lumbar enlargements.

The posterior vesicular cell-column is situated in the mesial part of the cervix of the dorsal cornu of grey matter. The cells constitute the posterior vesicular column of Lockhart Clarke, and they are of large size. Clarke's column exists chiefly in the thoracic portion of the cord.

The cells of the grey matter differ as regards their axons, some having short, and others long, axons.

The cells with short axons have their axons confined to the grey matter, in which they ramify not far from the parent-cells. They serve to bring contiguous cells into relation with one another.

The cells with long axons are partly root-cells and partly association-cells, and their axons travel for some distance from the parent-cells. "The axons of the root-cells leave the cord in the fibres of the ventral or motor nerve-roots. The axons of the association-cells constitute association-fibres, which are disposed in two ways: (1) Some enter the white matter of the same side of the cord, in which they divide into ascending and descending branches. Eventually they re-enter the grey matter, and terminate in arborizations at some distance from the parent-cells. (2) Other association-fibres cross to the opposite side in the ventral or white commissure. Some of these end in arborizations around the cells of the grey crescent, whilst others enter the white matter, in which they are disposed as on the side from which they have crossed.

\section{Destination of Axons of Cells of Grey Matter.}

Cells of Ventral Cornu.--(I) Many axons become the axis-cylinder processes of the efferent fibres of the ventral nerve-roots. (2) Other axons constitute association-fibres, which cross to the opposite side in the ventral or white commissure. After crossing, some end in arborizations around the cells of the ventral cornu; others enter the white matter; and a few are regarded by some authorities as entering the ventral nerve-roots of the side to which they have crossed.

Cells of Lateral Cornu.-The axons of the cells of the intermedio-lateral tract of Clarke pass to the efferent fibres of the ventral nerve-roots, and they are regarded as furnishing the white rami communicantes of the sympathetic system.

Cells of Dorsal Cornu.-The axons of the cells of the dorsal cornu have various directions: (I) Some pass to the ventral cornu and ventral or white commissure. (2) The axons of the cells of Clarke's column pass to the lateral column, and are usually regarded as entering the dorsal cerebellar and ventrolateral cerebellar tracts. (3) The axons of the cells of the substantia gelatinosa of Rolando pass partly to the lateral column, adjacent to the dorsal horn, where they divide into ascending and descending branches, and partly into the posterior marginal bundle of Lissauer. (4) The axons of other cclls in the lateral part of the cervix of the dorsal cornu pass to the lateral and ventral cornua, the ventral or white commissure, and the lateral column. (5) The axons of the cells of the caput cornu posterioris pass to the lateral column of the same side, and a few are regarded as passing to the opposite side in the ventral or white commissure.

Dorsal or Grey Commissure. - This commissure lies at the bottom of the dorsal median fissure. It consists of (I) grey matter, containing a few small nerve-cells, and (2) medullated nerve-fibres. These fibres pass across from one side to the other, and thereafter 
diverge in each grey crescent. They serve as association-fibres which bring the cells of opposite sides into relation with one another. This commissure contains the central canal of the cord, which for the most part is nearer the ventral portion of the commissure than the dorsal. The part of the commissure surrounding the central canal is called the substantia gelatinosa centralis. It consists of neuroglia, a few nerve-cells, and nerve-fibres; and it is invaded by processes derived from the deep ends of the ciliated columnar epithelial cells which line the central canal.

Summary of the Gelatinous Substances of the Grey Matter.-These are(1) The substantia gelatinosa of Rolando, which forms a cap for the caput cornu posterioris; (2) the substantia gelatinosa centralis, which surrounds the central canal of the cord; and (3) the substantia gelatinosa externa, which forms the glial sheath of the cord beneath the pia mater.

White Matter.-The white matter of the cord consists of longitudinal medullated nerve-fibres, traversed by septa of the pia mater, and embedded in neuroglia. The fibres have no primitive sheath or neurilemma.

Ventral or White Commissure.-This commissure lies at the bottom of the ventral median fissure, and it is separated from the central canal of the cord by a part of the dorsal or grey commissure. It consists of medullated nerve-fibres, destitute of a neurilemma, some of which pass transversely, but most of them decussate, entering the commissure ventrally on one side, and leaving it dorsally on the opposite side. The fibres, after crossing, enter the grey crescent and the ventral column. They are derived from (I) the direct pyramidal tract, or tract of Turck, (2) the processes of root-cells and of association-cells, and (3) the fibres of the spinothalamic tract, to be presently described.

Fibres of Roots of Spinal Nerves-Ventral or Anterior NerveRoots. -The fibres of the ventral nerve-roots arise within the cord from several sources. (I) Many of them are axons of the mesial cells of the ventral horn of grey matter of the same side. (2) Some are axons of the lateral cells of the ventral cornu.

(3) Others are axons of the cells of the intermedio-lateral tract of Clarke of the same side. (4) A few are axons of cells in the dorsal cornu of grey matter of the same side. (5) A few are regarded as being axons of the mesial cells of the ventral cornu of the opposite side, which cross in the ventral or white commissure. All the axons receive their medullary sheaths near the parent-cells, and they form funiculi, which leave the white matter of the ventral column over an area corresponding to the caput of the ventral cornu of grey matter, after which each fibre acquires its primitive sheath or neurilemma.

The most of the axons of the fibres of the ventral nerve-roots belong to the ventral (motor) cells of the ventral cornu of grey matter of the same side.

Dorsal or Posterior Nerve-Roots. - The fibres of the dorsal nerveroots arise from the unipolar (originally bipolar) cells of the spinal ganglia. The single pole or process of each of these cells is T-shaped. 
One half of the horizontal limb of the $\mathrm{T}$-is central, and enters th cord at the dorso-lateral sulcus between the dorsal and latera columns of white matter. The other half of the horizontal limb peripheral, and passes outwards in the course of the nerve.

Within the cord'a few of the fibres enter the marginal bundle o Lissauer, and the dorsal cornu of grey matter, but the most of them

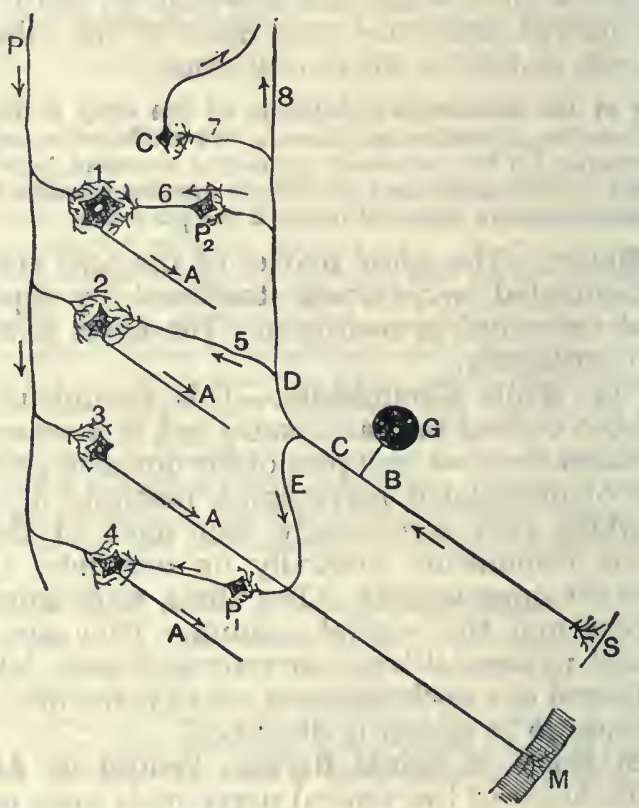

Fig. 554.-Course of Nerve-Fibres in the Spinal Cord (from HallibURTON'S 'HANDbOOK OF PHysiology' (AFter Schafer).

P.'Pyramidal Tract

1, 2, 3, 4. Anterior Cornual Cells

A, A, A, A. Axons of Anterior Cornual Cells

M. Muscular Fibre

G. Unipolar Cell of a Spinal Ganglion, giving Origin to a Fibre of a Posterior Nerve-Root

B. Peripheral Branch of Fibre

S. Skin

C (lower C) Central Branch of Fibre, passing into the Spinal Cord

E. Descending Branch of Fibre in the Spinal Cord
D. Ascending Branch of Fibre in the $\$ p i n$ Cord

Pr, ${ }^{P}$ 2. Posterior Cornual Cells

$\mathrm{C}$ (upper C). Cell of Clarke's Column

5. Collateral, passing directly to arborize arous an Anterior Cornual Cell (2)

6. Collateral, with an Intermediate Cell-Stati in a Posterior Cornual Cell ( $\left.\mathbf{P}_{2}\right)$

7. Collateral, arborizing around a Cell of Clarkı Column (upper C)

8. Continuation of Main Ascending Branch Fibre

pass into the column of Burdach, close to the dorsal cornu of gre: matter. Within this column the fibres divide into two branchesascending and descending. 'The descending branches, after a shor course, enter the dorsal cornu. These descending fibres are usuall regarded as forming the 'comma tract of Schultz.' The ascendini branches are longer than the descending, and, at various levels they also enter the dorsal cornu. The ascending branches of th 
fibres of the dorsal roots of the lower spinal nerves enter the column of Goll.

The ascending and descending branches give off numerous collateral fibrils, which enter the dorsal cornu. These collaterals have the following destinations: (r). The dorsal cornu of the same. side, and that of the opposite side through the dorsal or grey commissure; and (2) the ventral and lateral cornua of the same side. In each case they come into close relation with the corresponding nerve-cells-e.g., the cells of the dorsal cornu, including Clarke's column, the ventral or motor-cells of the ventral cornu, and the cells of the intermedio-lateral tract of Clarke in the lateral cornu.

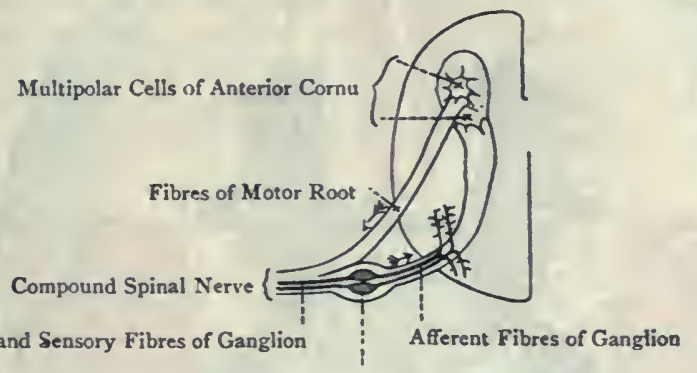

Spinal Ganglion with two Bipolar Cells

Fig. 555-Origin of Motor and Sensory fibres of a Spinal Nerve.

Spinal Ganglia.-These are situated on the posterior roots of the spinal nerves in the intervertebral foramina, and outside the theca, though invested by a prolongation from it. Each ganglion is oval, and consists of unipolar nerve-cells. The single pole of each cell divides into two processes, one of which is centripetal and forms part of the posterior nerve-root, whilst the other is centrifugal and passes into the spinal nerve. The pole and its inward and outward processes resemble the letter $T$. In early life the cells are bipolar.

The fibres of the posterior nerve-roots have their deep origins in the unipolar cells of the spinal ganglia, and they grow into the spinal cord. On the other hand, the fibres of the anterior nerve-roots have their deep origins within the spinal cord, where they arise as the axons of the multipolar nerve-cells of the anterior cornu of grey matter, and they grow outwards.

\section{Tracts of the Spinal Cord.}

Posterior Column.-The tracts of this column are ascending and descending, and they are as follows:

Ascending Tracts.
Tract of Goll.
Tract of Burdach.
Tract of Lissauer.

This tract also belongs to the lateral column. 
Ascending Tracts.-The tract of Goll is situated close to the posterior median sulcus, and superiorly it is known as the funiculus gracilis. Its fibres are derived from the dorsal roots of the coccygeal, sacral, lumbar, and lower thoracic nerves. They are at first contained in the tract of Burdach, but, as they ascend, they are gradually displaced inwards, and so form a special tract. The fibres terminate superiorly in connection with the cells of the nucleus gracilis of the medulla oblongata.

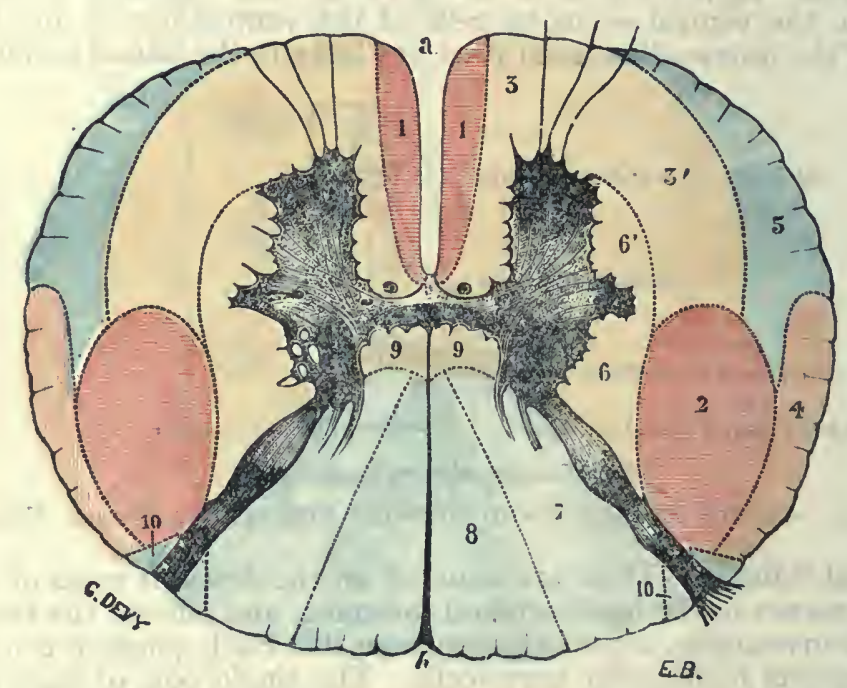

Fig. 556. - The Tracts of the Spinal Cord (L. Testut's 'Anatomie HuMaine').
a. Anterior Median Fissure
b. Posterior Median Fissure
x. Direct Pyramidal Tract
2. Crossed Pyramidal Tract
3. Anterior Ground Bundle
3. Lateral Ground Bundle
ro. Tract of Lissauer

HUMAINE'

The tract of Burdach (known superiorly as the funiculus cuneatus), is situated on the outer side of the tract of Goll, next to the dorsal horn of grey matter.

Above the mid-thoracic region it is separated from Goll's tract by the dorsal intermediate or paramedian furrow and a septum of pia mater. Its fibres are derived from the dorsal nerve-roots. Above the mid-thoracic region they are derived from the dorsal roots of the upper thoracic and cervical spinal nerves, and these fibres terminate superiorly in connection with the cells of the nucleus cuneatus of the medulla oblongata. Below the mid-thoracic region the fibres are derived from the lower dorsal nerve-roots, and 
these, being displaced inwards into the tract of Goll, terminate in connection with the cells of the nucleus gracilis.

The tract of Lissauer, or posterior marginal bundle, is close to the outer surface of the cord. It embraces the contiguous parts of the posterior and lateral columns, and occupies the region of the dorsolateral sulcus where the funiculi of the dorsal nerve-roots enter the cord. It lies between the substantia gelatinosa of Rolando and the surface of the cord. Its fibres are derived from the dorsal nerve-roots, and they ascend close to the substantia gelatinosa of Rolando, in which they terminate at different levels.

Descending Tract.-The comma tract of Sehultz is situated in the outer part of the tract of Burdach. Its fibres are usually regarded as being derived from the descending branches of the fibres of the dorsal nerve-roots, in which case they are exogenous. The other view, however, is that the fibres are intrinsic or endogenous, and spring from the cells of the dorsal cornu of grey matter.

Association Fibres.-These fibres are also known as intrinsic, commissural, or intersegmental fibres. Unlike those just described, they are not derived from the dorsal nerve-roots, but spring from the cells of the dorsal cornu of grey matter. They are therefore intrinsic or endogenous. Thereafter they enter the white matter of the posterior column and divide into ascending and descending branches. Subsequently these branches re-enter the grey matter of the dorsal cornu at different levels, and terminate in connection with its cells. They serve to connect different segments of the cord, and they form ascending and descending bundles.

The ascending bundle lies in the deep or ventral part of the posterior column, close to the dorsal or grey commissure and dorsal median fissure.

The descending intrinsic fibres are disposed in two bundles. One is known as the cornu-commissural bundle. It is met with in the lumbo-sacral region, and is contiguous to the dorsal cornu of grey matter and the dorsal or grey commissure.

The other descending bundle occupies different positions in the cord at different levels. (I) In the sacral region it occupies a small triangular area in the postero-internal part of Goll's column. (2) In the lumbar region it lies contiguous to the centre of the dorsal median fissure and at the corresponding part of the dorsal surface of the cord. This bundle is known as the septo-marginal or oval bundle. (3) In the thoracic and cervical regions it may be represented by the comma tract of Sehultz, if the fibres of that tract are to be regarded as intrinsic.

Antero-Lateral Column.-The tracts of this column are arranged into descending and ascending, and are as follows:

Descending Tracts.

Crossed Pyramidal Tract.

Direct Pyramidal Tract.

Uncrossed Lateral Pyramidal Tract.

Anterior Marginal or Ground-Bnndle.

Prepyramidal or Rubro-spinal Traet.

Bulbo-spinal Tract.
Ascending Tracts.

Dorsal Spino-cerebellar Tract. Ventral Spino-cerebellar Tract. Spino-thalamic Tract. Spino-tectal Tract.

Descending Tracts. - The crossed or lateral pyramidal tract fasciculus cerebro-spinalis lateralis B) is a long descending tract, of large size, which is situated deeply in the dorsal part of the lateral 
column, directly in front of the dorsal cornu of grey matter. It is separated from the outer surface of the cord by the dorsal spinocerebellar tract. It diminishes in size as it descends, and in the lumbar region it becomes superficial. At about the level of the third sacral nerve it terminates. The fibres of this tract have their origin in the pyramidal cells of the Rolandic or motor area of the cortex of the cerebral hemisphere of the opposite side. From this origin they descend through (I) the internal capsule of the corpus striatum, (2) the crus cerebri, and (3) the pons Varolii. On leaving the pons they enter the pyramid of the medulla oblongata on the side from which they have arisen. At the lower part of the pyramid they cross to the opposite side and take up their position deeply in the dorsal part of the lateral column of the spinal cord. The fibres of the crossed pyramidal tract of one side therefore come from the cerebral hemisphere of the opposite side, and they form the inner and larger part of the pyramid of the medulla oblongata, also of the opposite side. As this tract descends, fibres leave it in each segment of the cord. These fibres enter the ventral cornu of grey matter and end in close relation with the ventral or motor cells, the axons of many of which form the axis-cylinder processes of the fibres of the ventral or motor nerve-roots.

The direct pyramidal tract (fasciculus cerebro-spinalis anterior B) is also known as the tract of Turck. It is of small size, and is situated in the anterior column, where it lies close to the ventral median fissure: It diminishes in size as it descends, and usually terminates about the centre of the thoracic region, but fibres have been traced as low as the fourth sacral nerve. The fibres of this tract, like those of the crossed pyramidal tract, have their origin in the pyramidal cells of the Rolandic or motor area of the cortex of the cerebral hemisphere, but in this case of the same side. The fibres of the direct pyramidal tract of one side therefore come from the cerebral hemisphere of the same side. They pursue a similar downward path as low as the pyramid of the medulla oblongata of the same side, of which they form the outer and smaller part. They take no part, however, in the decussation of the pyramids, as do the fibres of the crossed pyramidal tract. Their course is directly downwards into the corresponding half of the spinal cord, where most of them take' up their position in the anterior column, close to the ventral median fissure. The fibres of the direct pyramidal tract, though they take no part in the decussation of the pyramids, cross to the opposite side at regular intervals as they descend in the anterior column of the cord: The crossing takes place in the ventral or white commissure, and, having entered the ventral cornu of grey matter of the opposite side, the fibres end, like those of the crossed pyramidal tract of that side, in close relation with the ventral or motor cells, the axons of many of which pass to the ventral or motor nerve-roots.

Most of the pyramidal fibres therefore cross from the side on which 
they arise to the opposite side. In the case of the crossed pyramidal tract the crossing takes place in the lower part of the medulla oblongata. In the case of the direct pyramidal tract the crossing takes place in the ventral median fissure of the spinal cord along the course of the tract.

The uncrossed lateral pyramidal tract consists of a few fibres which descend from the corresponding pyramid of the medulla oblongata into the lateral column of the same side, where they mix with the fibres of the crossed lateral pyramidal tract. It differs from the crossed lateral, and direct, pyramidal tracts, inasmuch as its fibres never cross to the opposite side. It is therefore a direct motor path from the Rolandic motor area of the cerebral cortex of the same side.

The anterior marginal or ground-bundle of Lowentbal (fasciculus anterior proprius B.) or tractus vestibulo-spinalis is situated in the anterior column, where it lies superficially. Its fibres are derived from the cells of the nuclei of Deiters in the medulla oblongata, chiefly on the same side as the bundle. These nuclei receive fibres of the corresponding vestibular nerve, and are closely related to the vestibular nuiclei. The bundle descends through the medulla oblongata and ventral column of the spinal cord as low as the lumbar region, and its fibres terminate in the anterior cornu of grey matter around the ventral or motor cells. The nucleus of Deiters and the vestibular nucleus, of either side, are connected with small grey nuclei which lie in the white matter of the cerebellum, and in this manner an indirect connection is established between the cerebellum and the ventral or motor-cells of the ventral grey cornu of the spinal cord, as follows: The grey nuclei within the cerebellum are connected with the nuclei of Deiters, and the axons of the cells of Deiters represent the origin of the fibres of the anterior marginal or ground-bundle, which fibres terminate around the ventral or motor-cells of the ventral grey cornu of the spinal cord.

The prepyramidal or rubro-spinal tract, or bundle of Monakow, is situated in the lateral column, on the ventral aspect of the crossed pyramidal tract. Its fibres are chiefly derived from the red nucleus of the tegmentum or dorsal part of the crus cerebri of the opposite side, and they are regarded as terminating in the dorsal part of the ventral cornu of grey matter.

The bulbo-spinal tract, or bundle of Helweg, is confined to the cervical region of the cord, and is triangular. Its fibres are regarded as arising in the medulla oblongata behind the olive, but their mode of termination is not known. They lie near the surface of the cord opposite the lateral cornu of grey matter.

Ascending Tracts.-The dorsal spino-cerebello tract, or direct cerebellar tract of Flechsig (fasciculus cerebello-spinalis B.), is situated in the lateral column. It lies in front of the dorso-lateral sulcus, between the crossed pyramidal tract and the outer surface 
of the cord. It commences in the lower part of the thoracic region, and superiorly it traverses the lower part of the medulla oblongata on its lateral aspect, after which it enters the restiform body, by which it is conducted to the vermis of the cerebellum. Its fibres are usually regarded as being derived from the posterior vesicular column of Clarke.

The ventral spino-cerebellar tract, or tract of Gowers (fasciculus antero-lateralis superficialis B.), is situated chiefly in the lateral column, in front of the dorsal cerebellar tract close to the outer surface of the cord. It is comma-shaped in section, its dorsal part being broad, but, as it extends forwards between the funiculi of the ventral nerve-roots, it tapers and enters the ventral column superficially. It commences near the lumbar region of the cord. Superiorly it extends through the medulla oblongata and pons, and thereafter passes along the superior cerebellar peduncle into the cerebellum, terminating in the vermis. It therefore takes an indirect course as compared with that of the dorsal spino-cerebellar tract. Its fibres are usually regarded as being derived from the posterior vesicular column of Clarke. The ventral spino-cerebellar tract contains the spino-thalamic and spino-tectal tracts.

The spino-thalamic tract consists of fibres which arise as the axons of cells of the dorsal grey cornu, around which cells the fibres of the dorsal nerve-roots have terminated. The spino-thalamic fibres cross to the opposite side in the ventral or white commissure, thus giving rise to a spinal inferior sensory decussation or spinothalamic decussation, as distinguished from the superior sensory decussation in the bulb, called the decussation of the fillets, which is produced by the deep arcuate fibres which arise from the cells of the nucleus gracilis and nucleus cuneatus. The spino-thalamic fibres, having crossed in the ventral white commissure, ascend in the tract of Gowers, and, after traversing the bulb and pons, they terminate in the optic thalamus of the side to which they have crossed as a cell-station. It is important to note that there are two sensory decussations-lower or spinal, and upper or bulbar. In unilateral lesions of the spinal cord there would only be partial anæsthesia on the opposite side; whereas in unilateral lesions of the bulb, involving both the fillet-fibres and the spinothalamic fibres, there would be complete anæsthesia on the opposite side.

The spino-tectal tract is also an ascending tract. Its fibres are connected with the cells of the ventral cornu of grey matter. They ascend in conjunction with the tract of Gowers and pass through the formatio reticularis of the bulb and pons. Thereafter they decussate with those of the opposite side, forming the fountaindecussation of Meynert, which lies between the two red nuclei, to which nuclei the spino-tectal fibres furnish collaterals. After the decussation the fibres of either side pass to the corresponding superior colliculus of the corpora quadrigemina. 
The tracts of the antero-lateral column may be otherwise arranged as follows:

Ventral or Anterior Column.

Direct Pyramidal Tract (descending). Anterior Marginal Bundle (Lowenthal) (descending).

Ventral Part of Ventral Spino-cerebellar Tract (ascending).
Lateral Column.

Crossed Pyramidal Tract (descending). Dorsal Spino-cerebellar Tract (ascending).

Ventral Splno-cerebellar Tract (chiel part of) (ascending).

Prepyramidal Tract (descending).

The Spino-Thalamic and Spino-Tectal Tracts (ascending) are contained in the ventral spino-cerebellar tract. A part of the posterior marginal bundle (Lissauer's tract) lies superficially in the dorsal part of the lateral column, and it has been described in connection with the dorsal column.

Association-Fibres of Antero-Lateral Column.-The part of the anterolateral column which is not occupied by the descending and ascending tracts, is adjacent to the grey matter, and it constitutes the antero-lateral marginal or ground-bundle. It is divided into two parts-anterior and lateral.

The anterior marginal or ground-bundle of Lowenthal is situated in the ventral column, in front of the ventral cornu of grey matter, and has been already described.

The lateral marginal or ground-bundle (fasiculus lateralis proprius B.) occupies the lateral column, ventral and mesial to the crossed pyramidal tract.

The portion of the ventro-lateral ground-bundle adjacent to the grey matter, and almost surrounding it, is known as the limiting zone.

The fibres of the entire antero-lateral ground-bundle are association-or longitudinal commissural fibres, which serve to connect the grey matter of successive segments of the spinal cord. They are derived from the cells of the grey matter of the same side, and also of the opposite side, the latter crossing in the ventral or white commissure.

Arteries of the Spinal Canal and Spinal Cord-Arteries of the Spinal Canal.-These vessels enter the spinal canal through the intervertebral and sacral foramina. In the cervical region they are branches of the vertebral and deep cervical arteries; in the thoracic and lumbar regions they are derived from the dorsal branches of the intercostal and lumbar arteries; and in the sacral region they come from the lateral sacral arteries. Within the spinal canal each spinal artery divides into three branches, namely, neural or central, and two parietal, anterior and posterior. The neural or central branch pierces the theca of the spinal cord. It supplies the coverings of the cord and the nerve-roots, and it anastomoses with the anterior and posterior spinal arteries on the cord. The neural branch is sometimes spoken of as the lateral spinal artery. The anterior parietal branch of each side passes to the back of the body of a vertebra, near the pedicle, and there it divides into two branches, ascending and descending. These anastomose with the adjacent offsets of the contiguous anterior parietal branches, and in this manner a lateral longitudinal anastomotic chain is formed, which communicates, at frequent intervals, with its fellow of the opposite side by transverse branches. From these transverse branches ascending and descending offsets are 
given off, which anastomose with adjacent offsets of contiguous transverse branches, and in this manner a median longitudinal anastomotic chain is formed on the backs of the bodies of the vertebræ. There are thus three longitudinal chains on the posterior surfaces of the bodies of the vertebræ, one being median and two lateral. From these chains branches are furnished to the bodies of the vertebræ and their ligaments. The posterior parietul branch of each side passes to the front of a lamina, where it acts in a manner similar to the anterior parietal branch. Two posterior longitudinal anastomotic chains are thus constructed in front of the laminæ, one at either side, which communicate at frequent intervals by transverse branches, and from these sources offsets are furnished to the laminæ and ligamenta subflava.

Arteries of the Spinal Cord.-These are: (I) the anterior spinal artery; (2) the posterior spinal arteries, right and left; and (3) the lateral spinal arteries, right and left (neural or central branches just described in connection with the spinal canal).

The anterior spinal artery is formed by the union of the anterior spinal branches, right and left, of the vertebral arteries. It descends along the front of the cord in the median line, and is reinforced at regular intervals by the lateral spinal arteries. In this manner an anterior longitudinal anastomotic chain is formed, which descends for some distance on the filum terminale.

The posterior spinal arteries are two in number, right and left, and each is a branch of the corresponding vertebral artery. Each vessel descends on the side of the cord in two branches, one being in front of, and the other behind, the posterior nerve-roots. These are reinforced by branches from the lateral spinal arteries, and the lateral longitudinal anastomotic chains, formed in this manner, extend over the entire length of the cord. There are thus five longitudinal anastomotic arterial chains in connection with the cord, namely, one anterior, and two lateral on each side, of which one is in front of, and the other behind, the posterior nerve-roots. From these chains branches are freely given to the pia mater, and through it to the cord.

Veins of the Spinal Column and Spinal Cord-Veins of the Spinal Column.-These veins, which form plexuses, are disposed in five sets, namely, (I) dorsal, (2) veins of the vertebral bodies, (3) anterior intraspinal, (4) posterior intraspinal, and (5) anterior extraspinal.

The dorsal spinous venous plexus is situated deeply upon the superficial surface of the neural arches of the vertebræ, under cover of the multifidus spinæ muscle. It receives its tributaries from the integument and muscles of the back, and it communicates with the posterior longitudinal intraspinal plexus by branches which pierce the ligamenta subflava. In the neck the blood is conveyed away from the plexus by veins which open into the vertebral venous plexus around the vertebral artery of each side; in the thoracic region by veins which join the dorsal branches of 
the intercostal veins; and in the lumbar region by veins which join the dorsal branches of the lumbar veins.

The veins of the bodies of the vertebræ (vence basis vertebra) are contained within the cancellated tissue of the vertebral bodies. They communicate in front with the anterior extraspinal veins, and posteriorly they terminate in two venous trunks which, emerging through the two foramina on the posterior surface of each vertebral body, open into the transverse communicating branch between the two anterior longitudinal intraspinal veins.

The anterior longitudinal intraspinal veins form two anastomotic chains, which are situated on the posterior surfaces of the bodies of the vertebræ, one on either side. They communicate with each other opposite the centre of each body by transverse branches which receive the terminal trunks of the venæe basis vertebræ. These transverse branches pass between the posterior common ligament and the bodies of the vertebræ. Superiorly the anterior intraspinal veins communicate with the vertebral and the transverse or basilar plexuses of veins, and laterally an offset passes outwards through each intervertebral foramen, which, with that of the posterior intraspinal vein, forms a plexus around the adjacent spinal nerve.

The posterior longitudinal intraspinal veins are situated in front of the laminæ, one on either side, and they are connected at frequent intervals by transverse branches. They communicate with the dorsal spinous venous plexus by branches which pierce the ligamenta subflava. Superiorly they communicate with the marginal sinuses, on either side of the foramen magnum and vermiform fossa, which by their union form the occipital sinus. With the marginal sinuses and the anterior intraspinal veins they form a venous ring at the foramen magnum. Laterally each vein sends outwards through the corresponding intervertebral foramen an offset, which, with that of the anterior intraspinal vein, forms a plexus around the adjacent spinal nerve.

The anterior and posterior intraspinal veins are situated between the theca of dura mater and the wall of the spinal canal.

The anterior extraspinal veins form a plexus along the anterior aspect of the bodies of the vertebræ, which is most copious in the neck. On either side it communicates with the vertebral plexus, around the vertebral artery in the neck, the intercostal veins in the thoracic region, and the lumbar veins in the lumbar region. It is also connected with the venæ basis vertebræ.

Veins of the Spinal Cord.- These vessels lie within the substance of the pia mater, and are disposed as venous chains, one being in front, one behind, and two on either side. The anterior vessel lies over the anterior median fissure beneath the anterior spinal artery; the posterior vessel is also mesially placed; and the two lateral vessels are situated one in front of, and the other behind, the posterior nerve-roots. Besides these principal chains the veins form a plexus on the surface of the cord. Laterally branches 
emerge through the intervertebral foramina, which along with the offsets of the anterior and posterior intraspinal veins, form plexuses around the spinal nerves. From these plexuses the blood is conveyed on either side into the vertebral plexus and deep cervical vein in the neck, and into the intercostal and lumbar veins in the corresponding regions.

Lymphaties.-There are no lymphatic vessels in the spinal cord. Their place is taken by spaces in the outer coat of the arteries, called perivascular spaces, which are in communication with the subarachnoid space.

\section{Development of the Spinal Cord.}

The spinal cord is developed from the wall of the neural tube, and the neural tube is developed from the neural plate. This plate, situated in the anterior part of the embryonic area, consists of ectodermic cells, and it presents

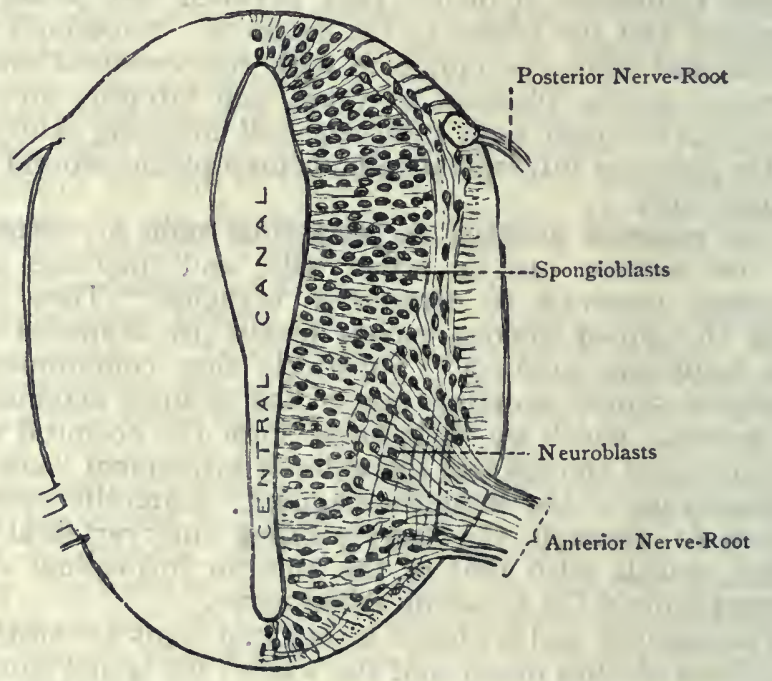

Fyg. 557.-Section of the Spinal Cord of a Human Embryo of FOUR WEERS (HIS).

a median longitudinal groove, called the neural groove. The lateral margins of the plate become elevated into two ridges, the noural folds, and these by their approximation and fusion give rise to the neural tube or canal. At either end the neural tube presents an opening-the anterior and posterior neuropore, but these openings soon become closed.

The ectodermic cells of the wall of the tube undergo proliferation, the wall becomes thickened, and it consists of two kinds of cells-namely, (I) sustentacular or supporting cells, and (2) nerve-cells proper. The former constitute the ependyma and neuroglia of the spinal cord, and the latter give rise to the grey and white matter. The cells are loosely arranged, and form what is known as the myelospongium. The colls of this myelospongiun become condensed internally and externally, and these condensed layers form the internal and external limiting membranes. The wall of the neural 
tube is arranged in three layers or zones-namely, (I) inner or ependymal, (2) intermediate or mantle zone, and (3) outer or marginal zone.

The ependymal zone consists of a single layer of elongated cells, connected with the internal limiting membrane. Their bases are directed towards the lumen of the neural tube, and from their apices delicate radial fibres pass outwards to the external limiting membrane. Amongst these there are some conspicuous cells, called germinal cells. These lie close to the wall of the neural tube, and by their proliferation they give rise to (I) ependymal cells, (2) spongioblasts, and (3) neuroblasts. The latter two migrate outwards into the mantle zone.

The mantle zone consists of branching cellular strands of myelospongium, which radiate from the centre to the periphery. It contains spongioblasts and neuroblasts, and it forms the rudiment of the grey matter of the spinal cord.

The marginal zone consists of reticula of myelospongium, traversed by nerve-tracts. It is the rudiment of the white matter of the spinal cord.

The cells of the wall of the neural tube as they undergo differentiation form two groups-spongioblasts, which form neuroglia, and neuroblasts or ganglionic cells.

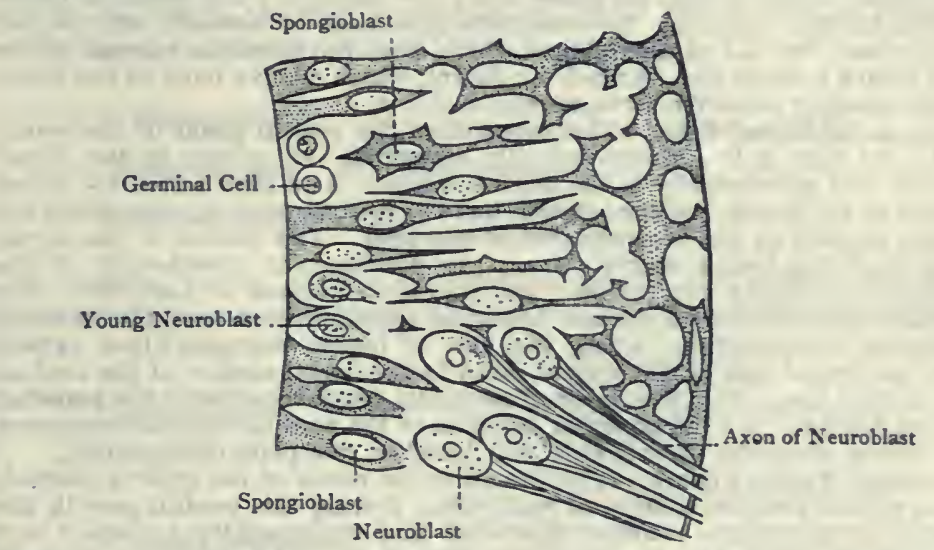

Fig. j58.-The Celis of the Spinal Cord of the Human Embryo (L. Testut's 'Anatomie Humaine ') (After His).

Development of Neuroglia.-This tissue consists of (I) ependymal cells, (2) neuroglia-cells, and (3) neuroglia-fibres, all of which are derived from the spongloblasts. These spongioblasts traverse the entire thickness of the wall of the neural tube, and are disposed in strands which radiate outwards from the lumen of the tube, giving off lateral processes. The spongioblasts adjacent to the lumen of the tube form the ependymal cells, and these form the internal limiting membrane. The remaining spongioblasts form the neuroglia-cells.

Neuroglia-cells have many branches, and are spoken of as glia-cells or spider-cells. The neuroglia-fibres are fibrillations of the peripheral protoplasm of the cells, from which they become differentiated.

The neuroblasts lie in groups within the mantle layer, and they give rise to the nerve-cells of the spinal cord. Each cell is primarily unipolar and pear-shaped. It has a prominent nucleus, and the body is prolonged into a process or pole, whch represents the axon or axis cylinder process of a nervefibre. Subsequently the pear-shape is lost, due to the formation of secondary processes or dendrites, the cell being now multipolar.

The lateral walls of the neural tube become thickened owing to cell-proliferation, but ventrally and dorsally it remains thin, these parts forming the 
Iloor-plate and rool-plate. Each lateral wall is divided into two platesventral and dorsal-by a longitudinal groove, called the sulcus limitans. The ventral plate, which is motor, is spoken of as the basal lamina, and the dorsal plate, which is sensory, is referred to as the alar lamina. The basal lamina is thick, and its mantle zone contains many cells from which the anterior cornu of grey matter is developed. The axons of these cells pass outwards through the marginal zone and form the anterior roots of the spinal nevves. The fibres of the posterior nerve-roots enter from the spinal ganglia at the sulcus limitans, and in the marginal zone of the alar lamina they form by their bifurcations longitudinal strands, which constitute the posterior column of the cord.

Metamorphoses of the Primitive Spinal Cord.-The primitive spinal cord consists of (I) two lateral walls, (2) a ventral or floor-plate, and (3) a dorsal or roof-plate.

Changes In Lateral Walls.-Each lateral wall, as stated, is subdivided into a ventral or basal lamina and a dorsal or alar lamina by the sulcus limitans. The ventral or basal lamina on either side gives rise to (I) the anterior cornu of grey matter, (2) the anterior or motor-nerve roots, and (3) the longitudinal funiculi of nerve-fibres, which form the anterior and lateral ground-bundles. The dorsal or alar lamina on either side gives rise to (1) the posterior cornu of grey matter, including the substantia gelatinosa of Rolando, and (2) the longitudinal funiculi of nerve-fibres which form the posterior column of the cord (Goll's column and Burdach's column), which receive most of the fibres of the entering posterior nerve-roots.

Lumen of Neural Tube.-This constitutes the central canal of the spinal cord. At first it is a dorso-ventrally elongated slit, widest in the dorsal portion, and subsequently in the ventral region. At a later period the dorsal portion of the lumen becomes closed, but the ventral portion, now somewhat round, persists as the central canal of the cord. The closure of the dorsal portion of the lumen occurs during the development dorsalwards of the posterior cornua of grey matter and posterior columns of the cord. The lateral walls of the dorsal portion, becoming approximated, fuse, the dorsal or roof-plate disappearing. Along the line of fusion neuroglia-fibres extend from the dorsal wall of the central canal to the dorsal surface of the cord at the median line. This thin layer of neuroglia-fibres constitutes the posterlor median septum. As the posterior columns of the cord undergo development the septum elongates dorsalwards, the dorsal or roof-plate disappearing.

Anterior Median Fissure.-This fissure is the result of the growth ventralwards of the anterior column of each side. During this ventral growth the ventral or floor-plate becomes depressed, and consequently a distinct cleft is produced, ventral to the depressed floor-plate, and intervening between the protuberant anterior columns. This cleft forms the anterior median flssure of the cord, which is occupied by an inflection of the pia mater. The floor-plate now lies at the bottom of the fissure, and that plate forms the anterior white commissure of the cord. It consists of decussating nervefibres, derived from the neuroblasts of the mantle layer of either side.

For the development of the spinal ganglia and the sensory and motor roots of the spinal nerves, see Neural Crests under Development of the Peripheral Nervous System.

Membranes of Spinal Cord.-The membranes-namely, pia mater, arachnoid, and dura mater (theca)-are developed from the mesoderm which invests the neural tube.

Growth of Spinal Cord.-The cord originally occupies the entire length of the spinal canal of the vertebral column. The vertebral column, how ever, grows more rapidly than the cord, so that at the period of birth the cord does not extend lower than the level of the third or fourth lumbar vertebra. Subsequently its lower limit is the intervertebral disc between the bodies of the first and second lumbar vertebra. This produces a change in the course of the lumbar, sacral, and coccygeal nerves. In order to reach the level of the intervertebral foramina through which they emerge from the spinal canal they descend almost vertically, and constitute the bundles of nerves known as the cauda equina. 


\section{THE ENCEPHALON.}

The encephalon is the part of the cerebro-spinal axis which is contained within the cranial cavity. It is composed of the medulla oblongata, pons Varolii, cerebellum, and cerebrum. Originally it consists of three hollow vesicles, namely, the anterior cerebral vesicle, which represents the prosencephalon or fore-brain; the middle cerebral vesicle, representing the mesencephalon or mid-brain; and the posterior cerebral vesicle, corresponding to the rhombencephalon or hind-brain.

$$
\text { Encephalon }=\left\{\begin{array}{l}
\text { Prosencephalon or Fore-brain. } \\
\text { Mesencephalon or Mid-brain. } \\
\text { Rhombencephalon or Hind-brain. }
\end{array}\right.
$$

The subdivisions of the prosencephalon are the telencephalon and the thalamencephalon or diencephalon; the mesencephalon remains undivided; and the subdivisions of the rhombencephalon are the metencephalon and the myelencephalon.

$$
\begin{aligned}
& \text { Prosencephalon }=\left\{\begin{array}{l}
\text { Telencephalon. } \\
\text { Thalamencephalon or Diencephalon. }
\end{array}\right. \\
& \text { Mesencephalon }=\text { Mesencephalon. } \\
& \text { Rhombencephalon }=\left\{\begin{array}{l}
\text { Metencephalon. } \\
\text { Myelencephalon. }
\end{array}\right.
\end{aligned}
$$

The various parts of the encephalon which are developed from these subdivisions will be made evident from the following table :

$$
\begin{aligned}
& \text { Telencephalon } \quad=\left\{\begin{array}{l}
\text { Cerebral Hemispheres. } \\
\text { Lateral Ventricles. } \\
\text { Anterior part of Third Ventricle. } \\
\text { Foramina of Monro. } \\
\text { Olfactory Lobes. }
\end{array}\right. \\
& \left.\begin{array}{c}
\text { Thalamencephalon } \\
\text { or } \\
\text { Diencephalon }
\end{array}\right\}=\left\{\begin{array}{l}
\text { Posterior Part of Third Ventricle. } \\
\text { Optic Thalami and Corpora Geniculata. } \\
\text { Pineal Body. } \\
\text { Interpeduncular Structures. } \\
\text { Pituitary Body. } \\
\text { Optic Nerve and Retina. }
\end{array}\right. \\
& \text { Mesencephalon } \quad=\left\{\begin{array}{l}
\text { Corpora Quadrigemina. } \\
\text { Crura cerebri. } \\
\text { Aqueduct of Sylvius. }
\end{array}\right. \\
& \text { Metencephalon } \quad=\left\{\begin{array}{l}
\text { Cerebellum. } \\
\text { Pons Varolii. } \\
\text { Pontine Part of the Fourth Ventricle. }
\end{array}\right. \\
& \text { Myelencephalon }=\left\{\begin{array}{l}
\text { Medulla Oblongata or Bulb. } \\
\text { Bulbar Part of Fourth Ventricle. }
\end{array}\right.
\end{aligned}
$$

\section{General Description of the Base and Superior Surface of the Encephalon.}

The inferior aspect of the encephalon is known as the base. In the following general description of the parts which it presents the order pursued is, as nearly as possible, from behind forwards and upwards. 
The medulla oblongata, or bulb, lies on the under aspect of the cerebellum, in the median line, occupying the vallecula which separates the two cerebellar hemispheres. The surface exposed is the ventral surface, which presents (I) the anterior median fissure, crossed at its lower part by the decussation of the pyramids; (2) the pyramid, on either side of this fissure; and (3) the olivary body, external to each pyramid.

The hemispheres of the cerebellum lie one on either side of the medulla oblongata, and they conceal from view the posterior parts of the cerebral hemispheres, and the posterior part of the great longitudinal fissure. They are characterized by the laminated arrangement of their nervous matter, the laminæ being curved and separated from each other by fissures. Posteriorly the hemispheres are separated from each other by the posterior notch. When the medulla oblongata is raised, and the cerebellar hemispheres slightly separated from each other, the vallecula is fully exposed, and the inferior vermis is seen lying deeply in it, with the sulcus valleculce on either side of it.

The pons Varolii forms a prominent elevation above the medulla oblongata, the surface exposed being the ventral surface. In the median line this surface presents a longitudinal groove, which is occupied by the basilar artery. On either side of the pons the middle peduncle of the cerebellum is seen, passing outwards and backwards into the cerebellar hemisphere.

The temporal lobes of the cerebrum are situated in front of the cerebellar hemispheres, and are conspicuous by their prominence. Each terminates anteriorly in a projecting extremity, called the temporal pole.

The stem of the fissure of Sylvius lies immediately in front of the temporal lobe, and is occupied by the middle cerebral artery. At the inner end of the stem of the fissure of Sylvius is the depression known as the vallecula Sylvii.

The frontal lobe of the cerebrum lies in front of the stem of the fissure of Sylvius. The exposed part is the orbital surface, which is separated from its fellow of the opposite side by the great longitudinal fissure. Each orbital surface presents a straight fissure, called the olfactory sulcus, which is situated near the great longitudinal fissure, and is parallel to it. This sulcus is occupied by the olfactory tract and olfactory bulb.

The crura cerebri, right and left, appear at the upper border of the pons Varolii, and soon diverge from each other as they pass forwards and upwards to sink into the cerebral hemispheres.

The optic tract of each side winds round the outer and ventral aspects of the corresponding crus cerebri. Its course is forwards and inwards towards its fellow of the opposite side.

The optic commissure, or chiasma, connects the two optic tracts after their convergence.

The optic nerves, right and left, leave the front of the commissure, and pass forwards and outwards to the optic foramina. 
The interpeduncular space is situated in front of, and above, the pons Varolii. It is somewhat diamond-shaped, and its boundaries are as follows : posteriorly, the upper border of the pons ; anteriorly, the optic commissure; and, laterally, the crus cerebri and optic tract, in this order from behind forwards. The following parts lie within this space, in the order named, from behind forwards:

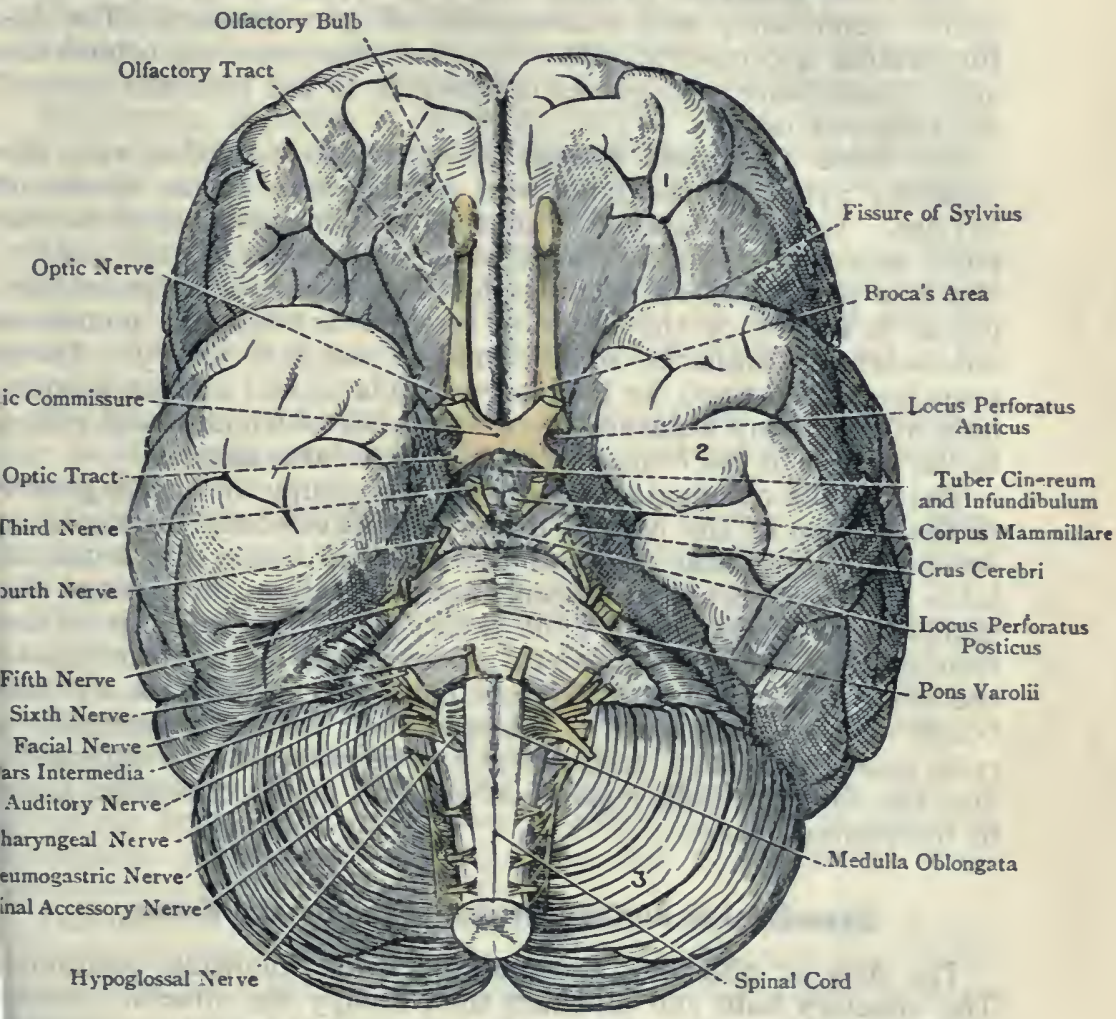

Fig. 559.-The Base of the Encephalon, axd the Cranial Nerves.

I, Frontal Lobe (Orbital Surface) ; 2, Temporal Lobe ; 3, Cerebellum.

I) the locus perforatus posticus; (2) the corpora albicantia or mammillaria; and (3) the tuber cinereum, with the infundibulum. (The region which is directly above the interpeduncular space constitutes the third ventricle.)

The locus perforatus posticus corresponds to the posterior median angle of the diamond-shaped interpeduncular space, and it lies in a deep depression, called the interpeduncular fossa, or fossa Tarini. 
The grey matter which forms it is perforated by openings for the passage of the postero-mesial branches of the posterior cerebral arteries.

The corpora albicantia or mammillaria are situated directly in front of the locus perforatus posticus, and present the appearance of small, white, pea-like bodies, lying close to the median line.

The tuber cinereum extends from the corpora albicantia to the optic commissure, and is composed of grey matter. The infundibulum is connected with the tuber cinereum close behind the optic commissure, and passes downwards to the posterior part of the pituitary body.

The locus perforatus anticus of each side coincides with the vallecula Sylvii at the inner end of the stem of the fissure of Sylvius. It lies outside the interpeduncular space, close to the outer aspect of the optic commissure. It consists of grey matter, which is perforated by openings. for the passage of a few anteromesial branches of the anterior cerebral artery, and numerous antero-lateral branches of the middle cerebral artery. These branches are destined for the nucleus caudatus and nucleus lenticularis of the corpus striatum, the grey matter of which nuclei comes to the surface of the brain at the locus perforatus anticus.

The medulla oblongata and pons Varolii occupy the basilar groove of the interior of the base of the skull; the cerebellar hemispheres occupy the cerebellar fossæ of the occipital bone; the temporal lobes of the cerebrum sink deeply into the lateral divisions of the middle fossa of the base of the skull ; the orbital surfaces of the frontal lobes occupy the lateral divisions of the anterior fossa; the stem of the fissure of Sylvius faces the posterior or Sylvian border of the small wing of the sphenoid; the optic commissure rests upon the olivary eminence and optic groove of the sphenoid; and the olfactory bulb rests upon one half of the cribriform plate of the ethmoid bone.

\section{Superficial or Surface Origins of the Cranial Nerves.}

The first or olfactory nerve occupies the following positions: The olfactory bulb and olfactory tract occupy the olfactory sulcus on the orbital surface of the frontal lobe, near the great longitudinal fissure; and the olfactory filaments pass through the foramina of the cribriform plate of the ethmoid bone on their way from the olfactory cells of the olfactory mucous membrane to the olfactory bulb. Posteriorly the olfactory tract divides into two roots, mesial and lateral. The mesial root curves inwards behind the area of Broca to the callosal gyrus. The lateral root passes backwards and outwards across the outer part of the locus perforatus anticus. The triangular area of grey matter, which is situated between the diverging roots of the olfactory tract, is called the trigomum olfactorium. It is sometimes spoken of as the middle or grey root of the olfactory tract. The area of Broca is situated in front of the mesial 
(inner) root of the olfactory tract, and is continuous with the callosal gyrus.

The optic tract curves round the crus cerebri from without inwards. The two optic tracts, having converged, are connected by the optic commissure, which lies at the anterior part of the interpeduncular space. The optic nerve is connected with the lateral extremity of the front part of the optic commissure.

The third or oculo-motor nerve emerges through the oculo-motor sulcus on the inner aspect of the crus cerebri, just above or in front of the pons Varolii, and close to the locus perforatus posticus.

The fourth, pathetic, or trochlear nerve, having emerged from the upper part of the superior medullary velum, or valve of Vieussens, makes its appearance in the interval between the crus cerebri internally and the temporal lobe externally.

The fifth, trigeminal, or trifacial nerve consists of two roots, which emerge, close together, from the lateral aspect of the ventral surface of the pons Varolii. The sensory root is large, and the motor root, which is small, lies above and slightly internal to the sensory root.

The sixth or abducent nerve emerges at the lower border of the pons Varolii, just external to the pyramid of the medulla oblongata.

The seventh or facial nerve (portio dura) emerges at the lower border of the pons, in front of the restiform body of the medulla oblongata.

The eighth or auditory nerve (portio mollis) likewise emerges at the lower border of the pons, in front of the restiform body of the medulla oblongata. It lies on the outer side of the facial nerve.

The pars intermedia of Wrisberg is a small nerve which appears between the facial and auditory nerves.

The ninth or glosso-pharyngeal nerve emerges, in the form of about six fasciculi, from the postero-lateral sulcus of the medulla oblongata, between the olivary body and the restiform body, immediately below the facial nerve.

The tenth or pneumogastric nerve lies directly below the glossopharyngeal nerve, and emerges by several fasciculi from the posterolateral sulcus of the medulla oblongata in front of the restiform body.

The eleventh or spinal accessory nerve has several roots which lie below the fasciculi of the pneumogastric nerve. These emerge from the medulla oblongata and the upper part of the lateral column of the spinal cord as low as the level of the fifth cervical nerve, and they lie below the fasciculi of the pneumogastric nerve, and external to, or in front of, the posterior roots of the adjacent cervical spinal nerves.

The twelfth or hypoglossal nerve emerges by several fasciculi through the antero-lateral sulcus of the medulla oblongata, between the pyramid and the olivary body. These fasciculi lie in line with the sixth nerve superiorly, 
Arteries at the Base of the Encephalon.-The arteries which supply the brain are the two vertebral and the two internal carotid arteries.

The vertebral arteries incline inwards as they ascend on the ventral aspect of the medulla oblongata, and at the lower border of the pons Varolii they unite to form the basilar artery. The branches of each vertebral artery to be noted are as follows: (I) the posterior spinal branch, which arises from the main vessel immediately after it has pierced the dura mater, and descends upon the side of the medulla oblongata to the spinal cord ; (2) the anterior spinal branch, which arises higher up than the preceding, and passes downwards and inwards on the ventral aspect of the medulla oblongata to unite with its fellow and form the anterior spinal artery ; and (3) the posterior inferior cerebellar branch, of large size, which arises from the main vessel near the pons, and passes backwards round the medulla oblongata to enter the vallecula of the cerebellum.

The basilar artery extends from the lower border of the pons Varolii to the upper border, occupying the basilar groove on its ventral surface. It is formed by the union of the two vertebral arteries, and terminates by dividing into the two posterior cerebral arteries. The branches of the basilar artery to be noted on either side are as follows: (I) the transverse arteries of the pons; (2) the internal auditory artery, which accompanies the auditory nerve through the meatus auditorius internus; (3) the anterior inferior cerebellar artery, which arises from the basilar about its centre, and passes backwards to the inferior surface of the cerebellar hemisphere ; (4) the superior cerebellar artery, which arises from the basilar near its termination, and passes outwards close to the upper border of the pons, and then round the outer side of the crus cerebri to the superior surface of the cerebellar hemisphere; and (5) the posterior cerebral artery, which arises from the termination of the basilar, and passes outwards, parallel to the superior cerebellar artery, and then round the outer side of the crus cerebri to the inferior surface of the occipital lobe. The posterior cerebral and superior cerebellar arteries are separated from each other by the third and fourth cranial nerves, the latter being the external of the two. The branches of the posterior cerebral artery to be noted, amongst others, are as follows : (I) postero-mesial, which pass to the locus perforatus posticus; (2) postero-lateral, which pass round the outer side of the crus cerebri ; and (3) posterior choroidal, which pass to the upper part of the choroidal fissure.

The internal carotid artery of each side appears at the vallecula Sylvii, and there divides into the anterior and middle cerebral arteries. Near its termination it gives off the posterior communicating artery, which passes backwards to join the posterior cerebral artery. It also gives off the anterior choroidal artery, which passes backwards and outwards between the crus cerebri and the uncinate gyrus to the lower and anterior part of the choroidal fissure. 
The anterior cerebral artery passes forwards and inwards, and enters the great longitudinal fissure. As it is about to enter that fissure it is connected with its fellow of the opposite side by the anterior communicating artery, which is short, but of fairly large size. Amongst other branches the following are to be noted arising from the anterior cerebral artery : (I) antero-mesial, few and inconstant, and (2) antero-lateral, both of which pass to the locus perforatus anticus.

The middle cerebral artery, of large size, sinks into the fissure of Sylvius, which it traverses in an outward direction. Before disappearing into the fissure antero-lateral ganglionic branches are to be

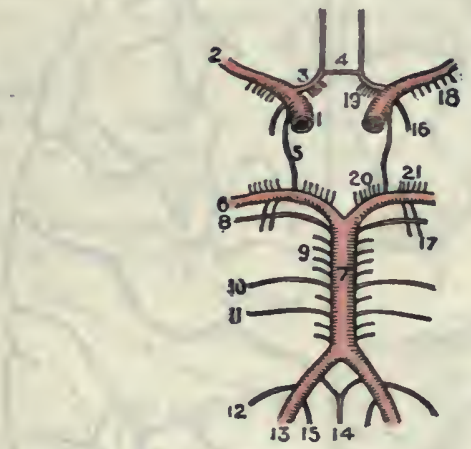

Fig. 560.-The Arteries at the Base of the Brain, and the Circle of Willis.

x. Internal Carotid

2. Middle Cerebral

3. Anterior Cerebral

4. Anterior Communicating

5. Posterior Communicating

. Posterior Cerebral

7. Basilar

Superior Cerebellar
9. Transverse Pontine

10. Internal Auditory

1. Anterior Inferior Cerebellar

12. Posterior Inferior Cerebellar

13. Vertebral

14. Anterior Spinal

15. Posterior Spinal

16. Anterior Choroid x7. Posterior Choroid

18. Central or Ganglionic

19. Central or Ganglionic

20. Central or Ganglionic (Postero-mesial)

2r. Central or Ganglionic (Postero-lateral)

noted arising from it, which are arranged in two sets, internal striate and external striate, which are destined chiefly for the component parts of the corpus striatum.

Circle of Willis.-This is an important communication between the vertebral and internal carotid arterial systems at the base of the brain, which is situated around the interpeduncular space. It is not actually a circle, though so named, but is a heptagon-that is to say, it has seven angles and seven sides.

Commencing at the median line posteriorly, and proceeding forwards on either side to the median line in front, at the great longitudinal fissure, the component arteries of the circle are as follows: (I) the basilar, (2) the posterior cerebral, (3) the posterior communicating, (4) the internal carotid, (5) the anterior cerebral, and (6) the anterior communicating. The connection between the 
two posterior cerebral arteries is effected by means of the basilar artery; the connection between the posterior cerebral and internal carotid arteries is effected by means of the posterior communicating artery; and the connection between the two anterior cerebral arteries is effectcd by means of the anterior communicating artery. These communications serve to insure a regular supply of arterial blood to the brain in cases of obstruction to one or other of the

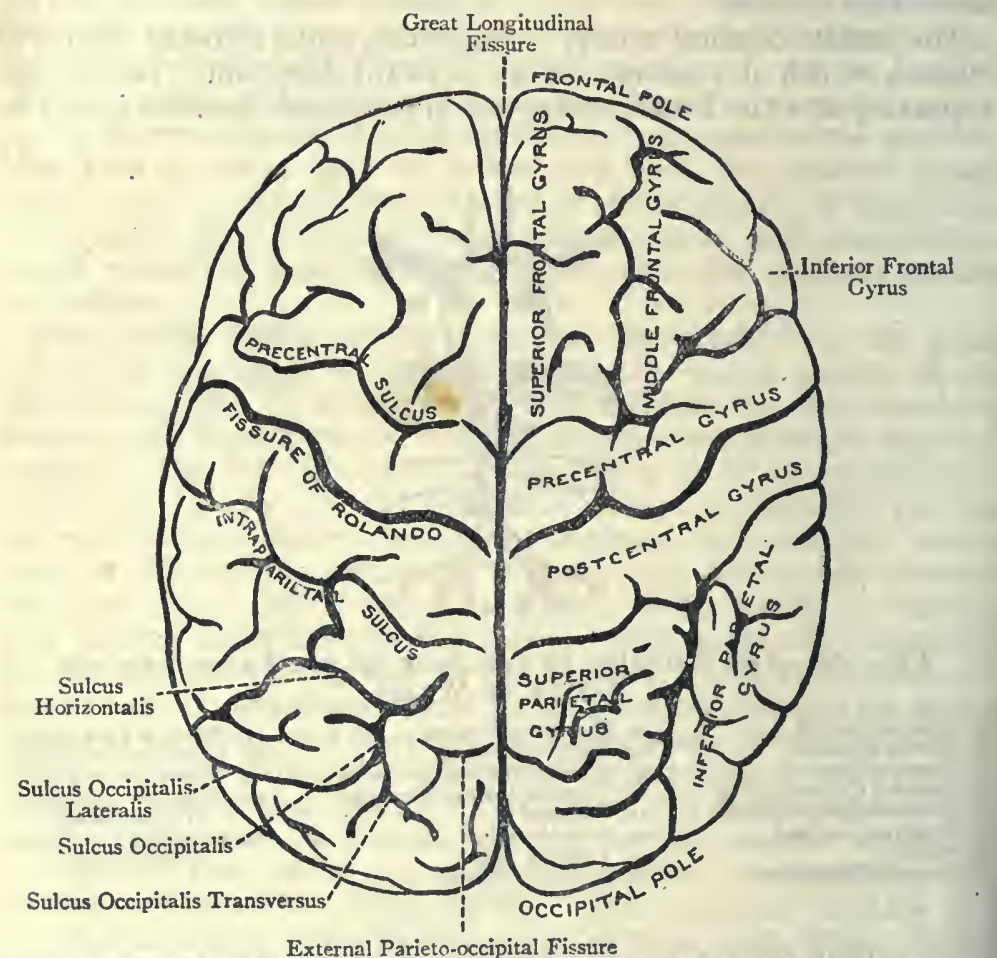

Fig. 56r.-The Cerebrat Hemispheres (Superior View).

principal arterial trunks. The communications also serve to equalize the circulation of blood through the different parts of the brain.

Superior Surface of the Brain.-The brain is ovoid superiorly, its greatest breadth corresponding to the positions of the parietal eminences of the parietal bones. In the median line it presents a deep cleft, called the great longitudinal fissure, which extends from the front to the back, and divides it into two liemispheres, right and left. This fissure is occupied by a process of the dura mater, called the falx cerebri, and the corpus callosum lies at its deep part. In front of the corpus callosum the fissure extends down to, and is 
visible on, the base of the brain, but behind the corpus callosum it only extends to the level of the tentorium cerebelli, which separates the cerebellum from the posterior parts of the cerebral hemispheres. The fissure, therefore, in this situation is not visible inferiorly until the cerebellum and the tentorium cerebelli have been removed.

Each hemisphere is semi-ovoid, its mesial or inner surface being flat. The anterior and posterior extremities are rounded, the former being the thicker of the two. The anterior extremity is known as the frontal pole, and the posterior extremity forms the occipital pole. The surface of each hemisphere consists of grey matter, which is spoken of as the cerebral cortex. Superiorly and externally it is convex in adaptation to the concavity of the vault of the cranium. It is broken up into a number of tortuous eminences, called gyri or convolutions, and these are separated from each other by clefts, called sulci or fissures. The surfaces of the gyri which bound the sulci are covered with grey matter, like their exterior. The pia mater closely covers the gyri, and also dips into the sulci, so as to cover the opposed surfaces of the gyri. The arachnoid membrane, however, does not dip into the sulci, but passes over them. The sulci are of various dèpths, but the average depth may be said to be $\frac{1}{2}$ inch.

\section{RH OMBENCEPHALON.}

\section{The Medulla Oblongata.}

The medulla oblongata or bulb is continuous with the spinal cord and extends from the lower margin of the foramen magnum of the occipital bone to the lower border of the pons Varolii. Its direction is upwards and slightly forwards, and it measures I inch in length, $\frac{3}{4}$ inch in breadth at the widest part, and fully $\frac{1}{2}$ inch in thickness. Inferiorly its girth corresponds with that of the spinal cord, but it widens superiorly, so that it is somewhat pyramidal. Its ventral surface faces the basilar groove of the occipital bone, and its dorsal surface is directed towards the vallecula of the cerebellum.

The bulb is composed of two symmetrical halves, its bilateral symmetry being indicated superficially by upward prolongations of the ventral or anterior and dorsal or posterior median fissures of the spinal cord. The anterior median fissure extends as high as the lower border of the pons Varolii, where it expands slightly and forms a blind recess, called the foramen cacum. In its lower part this fissure is interrupted and crossed by bundles of nerve-fibres, which are derived from the inner three-fourths of each pyramid, the decussation thus formed being known as the decussation of the pyramids, or motor decussation. The posterior median septum only extends along the lower half of the bulb, and it terminates superiorly at the level of the calamus scriptorius. 
Each half of the bulb presents two grooves-ventro-lateral and dorso-lateral. The ventro-lateral sulcus is situated between the pyramid and the olivary body, and along this sulcus the funiculi of the hypoglossal nerve emerge in line with the ventral roots of the spinal nerves. Whilst, however, the latter are spread over a certain area, the hypoglossal funiculi emerge along a straight line corresponding to the ventro-lateral sulcus of the bulb. The ventrolateral sulcus is not represented on the surface of the spinal cord. The dorso-lateral sulcus is serially continuous with the dorso-lateral sulcus of the spinal cord, and it lies on the dorso-lateral aspect of the olivary body. Along this sulcus, in order from above downwards, there are (I) the funiculi of the glosso-pharyngeal nerve,

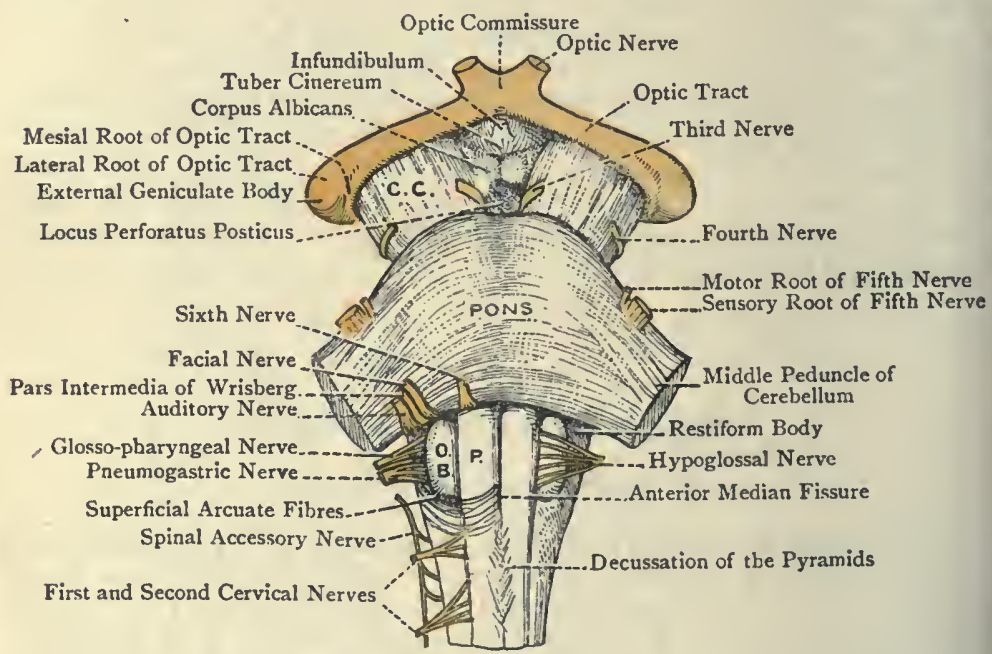

Fig. 562. - The Medulia Oblongata, Pons Varolit, and Interpedunctiak REGION.

C.C., Crus Cerebri ; P., Pyramid : O.B. Olivary Body.

(2) the funiculi of the pneumogastric or vagus nerve, and (3) the funiculi of the bulbar part of the spinal accessory nerve. These nerve-funiculi are in line with the dorsal roots of the spinal nerves.

The bulb in its lower half contains a prolongation of the central canal of the spinal cord. This part of the bulb is spoken of as the closed part, and it extends as high as the level of the calamus scriptorius. In the upper half of the bulb the central canal opens out at the level of the calamus scriptorius into the fourth ventricle, and the dorsal aspect of the bulb forms the lower or bulbar half of the floor of the fourth ventricle. The upper half of the bulb is therefore spoken of as the open part.

The surface of each half of the bulb is divided into three areas by 
the ventro-lateral and dorso-lateral sulci, with the corresponding nerve-funiculi. These surface areas are called ventral, lateral, and dorsal.

Ventral or Anterior Area.-This superficial area is situated between the ventral median fissure and the ventro-lateral sulcus

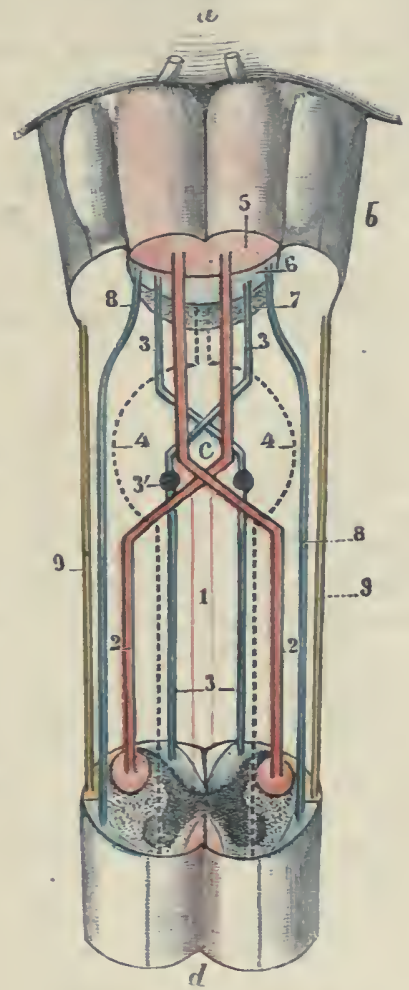

Fig. 563. - The Decussation of the Pyramids. Scheme representing the Passage of the Various Tracts from the Spinal Cord to the Medulla (L. Testut's 'Anatomie Humaine").
a. Pons Varolii
b. Medulla Oblongata (anterior aspect)
c. Decussation of the Pyramids
d. Sectiou of the Cervical Spinal Cord
I. Direct Pyramidal Tract
2. Crossed Pyramidal Tract
3. Sensory Tract
3. Nucleus Gracilis et Nucleus Cuneatus
4. Antero-lateral Ground-Bundle
5. Anterior Pyramid
6. Fillet
7. Posterior Longitudinal Bundle
8. Tract of Gowers
9. Direct Cerebellar Tract

alorg which the funiculi of the hypoglossal nerve emerge. It constitutes the pyramid of the bulb. The two pyramids, right and left, represent the motor tracts of the bulb. As regards position, the pyramid represents the anterior column of the spinal cord, and 
it consists of bundles of nerve-fibres, disposed longitudinally. Inferiorly it is somewhat narrow, but it widens superiorly. At the lower border of the pons Varolii it undergoes a slight constriction, after which it sinks into the pons. As it traverses the pons its funiculi become separated into several strata, and these are gathered together at the upper border of the pons into the crus cerebri of the corresponding side.

Inferiorly each pyramid is disposed in two parts-inner and outer. The inner portion represents as a rule the inner three-fourths, and its fibres cross to the opposite side in the lower part of the ventral median fissure of the bulb. Thereafter they sink deeply into the dorsal part of the lateral column of the spinal cord on the side to which they have crossed, where they constitute the crossed pyramidal tract. The intercrossing of fibres which takes place in the lower part of the ventral median fissure of the bulb is called the decussation of the pyramids, or the motor decussation, and, as stated, it usually involves the fibres of the inner three-fourths of the pyramid.

The outer portion of the pyramid represents as a rule the outer fourth, and its fibres take no part in the decussation. The path of most of them is downwards into the anterior column of the spinal cord of the same side, where they lie close to the anterior median fissure of the cord and constitute the direct pyramidal tract. A few of them, however, descend into the lateral column of the same side, and constitute the uncrossed lateral pyramidal tract.

The pyramid of the bulb, therefore, only corresponds topographically with the anterior column of the spinal cord. The direct pyramidal tract of the anterior column of the cord forms the greater part of the outer fourth of the corresponding pyramid of the bulb; and the crossed pyramidal tract of the lateral column of the cord forms the inner three-fourths of the pyramid of the opposite side. The remainder of the anterior column of the cord sinks deeply into the bulb and lies on the dorsal aspect of the pyramid.

The ventral surface of each pyramid is crossed above the level of the decussation of the pyramids by the anterior superficial arcuate fibres, which emerge from the ventral median fissure and take an arched course outwards and then backwards to the restiform body.

The sixth cranial nerve emerges close to the lower border of the pons Varolii, immediately external to the pyramid, and in line with the funiculi of the hypoglossal nerve as these emerge from the ventro-lateral sulcus.

Lateral Area of the Medulla Oblongata.-This superficial area is situated between the ventro-lateral sulcus, with the funiculi of the hypoglossal nerve, and the dorso-lateral sulcus, with the funiculi of the glosso-pharyngeal, pneumogastric, and bulbar part of the spinal accessory nerves. Superiorly it presents an oval eminence, called the olive (oliva B.). Inferiorly it has the appearance of being a prolongation of the lateral column of the spinal cord, but this is not the case. The crossed pyramidal tract of the lateral 
column of the cord sweeps obliquely across to the opposite side, where it forms the greater part of the pyramid of that side. The parts, therefore, of the lateral column of the cord which form the lateral area of the bulb below the olive are (I) the dorsal or direct spino-cerebellar tract, (2) the ventral spino-cerebellar tract, and (3) the lateral ground-bundle. The dorsal spino-cerebellar tract as it ascends, soon inclines obliquely backwards to join the restiform body. The ventral spino-cerebellar tract and lateral groundbundle ascend until they reach the lower end of the olive. They then for the most part sink deeply and ascend to the pons Varolii on the dorsal or deep aspect of the olive. A few of the fibres, however, remain on the surface and ascend in the small interval

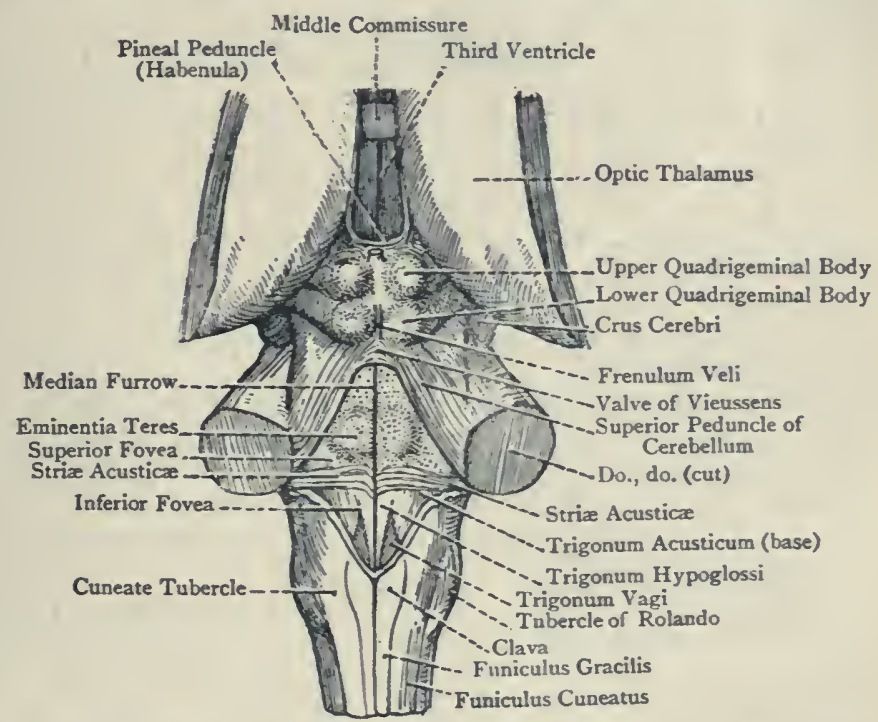

Fig. 564, - The Floor of the Fourth Ventricle and Adjacent Parts.

which lies between the outer part of the olive and the funiculi of the glosso-pharyngeal and pneumogastric nerves.

The lateral area of the bulb below the olive thus represents the dorsal or direct spino-cerebellar tract, ventral spino-cerebellar tract, and lateral ground-bundle of the lateral column of the spinal cord of the same side.

Superiorly, as stated, the lateral area presents an oval eminence, called the olive. It lies between the funiculi of the hypoglossal nerve on the one hand, and the funiculi of the glosso-pharyngeal and pneumogastric nerves on the other, with the intervention of some ascending fibres belonging to the ventral spino-cerebellar tract. Its long axis is placed vertically, and in this direction it 
measures about $\frac{1}{2}$ inch. Superiorly it is separated from the pons Varolii by a deep transverse groove, and inferiorly the anterior superficial arcuate fibres arch over its lower part.

At the lower border of the pons Varolii, external to the upper end of the olive, the facial and auditory nerves make their appearance. The facial nerve is internal in position, and is in line with the funiculi of the glosso-pharyngeal nerve. The auditory nerve appears external to the facial nerve, and between the two is the small pars intermedia of Wrisberg.

Dorsal or Posterior Area of the Medulla Oblongata.-This superficial area is limited in front by the dorso-lateral sulcus, containing the funiculi of the glosso-pharyngeal, pneumogastric, and bulbar part of the spinal accessory nerves. Posteriorly its lower half extends as far as the dorsal median fissure, and its upper half extends only as far as the lateral boundary of the lower or bulbar half of the floor of the fourth ventricle. Inasmuch as this area pertains to both the closed and open parts of the bulb it will be considered in two sections-lower and upper.

Lower Portion of Posterior Area.-This portion, as stated, is limited behind by the dorsal median fissure, and it is in direct continuity with the dorsal column of the spinal cord of the same side, which is composed of the columns of Goll and of Burdach. It presents three longitudinal eminences-namely, the funiculus gracilis, funiculis cuneatus, and funiculus of Rolando.

The funiculus gracilis is a prolongation of Goll's column of the spinal cord, and lies close to the dorsal median fissure. The funiculus cuneatus is a prolongation of Burdach's column of the cord, and lies external to the funiculus gracilis, from which it is separated by an upward continuation of the dorsal intermediate or paramedian furrow of the cord.

At the level of the calamus scriptorius each of these two funiculi becomes enlarged and terminates in a prominence or bulb. The enlargement formed by the funiculus gracilis is called the clava, and that formed by the funiculus cuneatus is termed the cuneate tubercle. The two clavæ, right and left, lie on either side of the calamus scriptorius, and, as the bulb opens out dorsally at this level to form the lower or bulbar half of the floor of the fourth ventricle, each clava is displaced laterally. An angular interval now separates the two clavæ, and the prolongation of the central canal of the spinal cord through the lower or closed part of the bulb opens into the fourth ventricle in the angle between the two clave.

The funiculus gracilis, with its clava, and the funiculus cuneatus, with its cuneate tubercle, are to a large extent produced by the collections of grey matter which they contain-namely, the nucleus funiculi gracilis and nucleus funiculi cuncati.

The funiculus of Rolando is situated on the outer side of the funiculus cuneatus, between it and the funiculi of the bulbar part of the spinal accessory nerve. It is produced by the substantia gelatinosa of Rolando, which comes close to the surface in the 
lower or closed part of the bulb. Inferiorly the funiculus of Rolando is narrow, but it widens as it ascends, and superiorly it terminates in an enlarged extremity, called the tubercle of Rolando.

The funiculus and tubercle of Rolando are covered by a thin layer of longitudinal nerve-fibres which represent the spinal or descending sensory root of the fifth cranial nerve.

Upper Portion of Posterior Area.-This portion, as stated, pertains to the upper or open part of the bulb, and extends as far as the lateral boundary of the lower or bulbar half of the floor of the fourth ventricle. It presents a prominent round tract, called the restiform body (corpus restiforme $\mathrm{B}$.), which is situated between the lower half of the floor of the fourth ventricle and the funiculi of the pneumogastric and glosso-pharyngeal nerves. Its direction is upwards, outwards, and backwards, and it enters the corresponding hemisphere of the cerebellum. It is otherwise known as the inferior cerebellar peduncle.

The restiform body succeeds to the funiculus gracilis and funiculus cuneatus of the lower portion of the posterior area of the bulb, but it is quite distinct from these funiculi, and receives no fibres from them. The sources of its fibres will be stated in connection with the internal structure of the bulb. Meanwhile it may be stated that it constitutes the great tract of connection between the cerebellar hemisphere, the bulb, and the spinal cord.

The restiform body becomes conspicuous above the level of the cuneate tubercle, and forms the lateral boundary of the lower or bulbar half of the floor of the fourth ventricle.

Internal Structure of the Medulla Oblongata.-Each half of the bulb is composed of grey nervous matter and tracts of white nervous matter.

Grey Matter.--The grey matter lies largely in the interior. Over the dorsal aspect of the upper or open part of the bulb, however, it comes to the surface and covers the lower or bulbar half of the floor of the fourth ventricle.

As compared with the grey matter of the spinal cord, it presents important modifications, and its component parts are as follows:

I. Substantia or formatio reticularis.

2. A thick layer of grey matter around the central canal in the lower or closed part of the bulb.

3. A thick layer of grey matter over the floor of the fourth ventricle in the upper or open part of the bulb.

4. Substantia gelatinosa of Rolando.

5. Nuclei of grey matter.

The modifications undergone by the grey matter of the bulb in its lower or closed part are brought about by the decussation of the pyramids. The nerve-funiculi of the crossed pyramidal tract of the spinal cord, on one side as they are traced upwards, pass through the ventral grey cornu of that side, and then cross in the lower part of the ventral median fissure of the bulb to the pyramid 
of the opposite side, of which they form the inner and larger part. The nerve-funiculi of the crossed pyramidal tract of the other side are disposed in a similar manner. The ventral grey cornu of either side is thus broken up by the corresponding crossed pyramidal tract. Its basal part remains on the ventral and lateral aspects of the central canal, but its caput is detached and displaced laterally by the pyramid and olive of the same side.

The dorsal cornu of grey matter is gradually displaced laterally and ventralwards, in the lower or closed part of the bulb, by the funiculus gracilis and funiculus cuneatus. Its basal part remains on the dorsal and lateral aspects of the central canal; its cervix is broken up into a network by intersecting nerve-fibres; and its caput is thereby detached. The caput lies close to the detached caput of the ventral cornu of grey matter, but does not blend with it.

Substantia or Formatio Reticularis.--The grey matter of the detached caput of the ventral grey cornu is broken up into a network

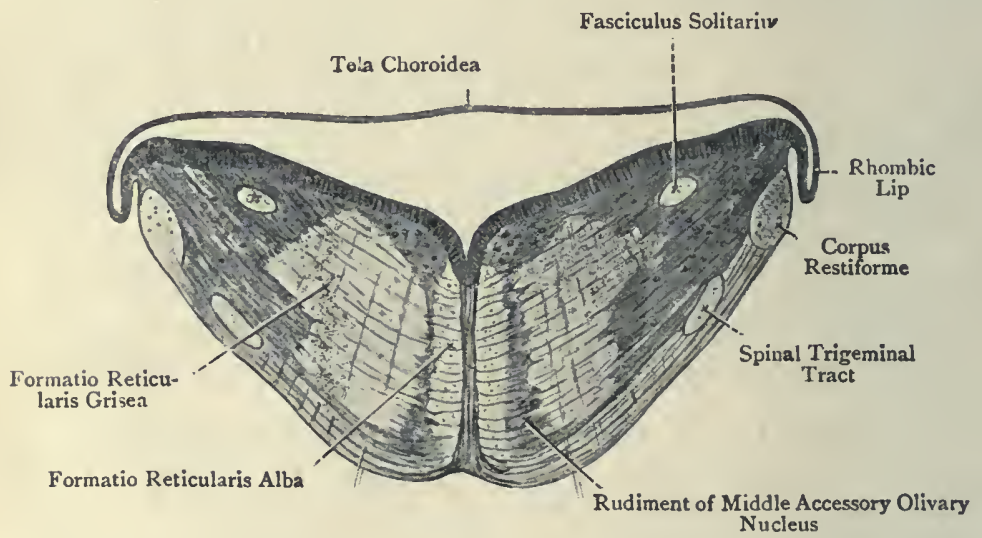

Fig. 565. - Transverse Section of Medulla Oblongata (Keibel and MALL).

by intersecting nerve-fibres, which run longitudinally and transversely. This reticulum, augmented by the network formed in the cervix of the dorsal grey cornu, constitutes the substantia or formatio reticularis of the bulb. It lies deeply within the bulb, dorsal to the olive and pyramid of the same side, and it consists of grey matter, longitudinal and transverse nerve-fibres, and some nerve-cells.

The funiculi of the hypoglossal nerve, as they pass forwards to the ventro-lateral sulcus of the bulb, divide the formatio reticularis into two parts-lateral and mesial. The lateral portion is situated behind the olive, and is called the formatio reticularis grisea, from the large amount of grey matter, with nerve-cells, which it contains. The mesial portion is situated behind the pyramid, and is 
called the formatio reticularis alba. It contains little grey matter and few nerve-cells.

Central Grey Matter.-The grey matter which surrounds the central canal in the lower or closed part of the bulb is derived from the basal portions of the ventral and dorsal grey cornua of the upper part of the spinal cord. In the upper or open part of the bulb this central grey matter spreads out and forms a thick layer over the lower or bulbar part of the floor of the fourth ventricle. The mesial part of this layer represents the basal part of the ventral grey cornu, and it contains the hypoglossal nucleus. The lateral part represents the basal part of the dorsal grey cornu, and it contains pneumogastric, glosso-pharyngeal, and auditory nuclei.

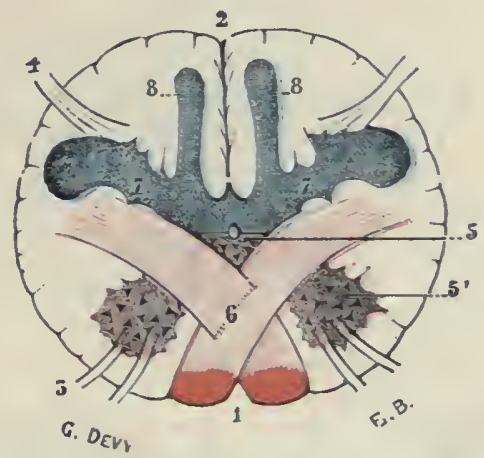

Fig. 566.-Section of the Miedulla Oblongata at the Level of the Decussation of the Pyramids (L. Testut's 'Anatomie Humaine').

x. Anterior Median Fissure

2. Posterior Median Fissure

3. Motor Roots

4. Sensory Roots
5. Base of the Anterior Cornu from which the Caput, 5', has been detached by the passage of the crossed Pyramidal Tract

6. Decussation of the crossed Pyramidal Tracts

7. Posterior Cornu

8. Nucleus Gracilis

Substantia Gelatinosa of Rolando.-This caps the detached and displaced caput of the dorsal horn of grey matter. Having increased in amount and lying close to the surface, it gives rise to the funiculus and tubercle of Rolando.

Nuclei of Grey Matter.-The nuclei, which will be considered in this place, are as follows:

I. Nucleus gracilis.

2. Nucleus cuneatus.

3. Olivary nuclei.

4. Arcuate nucleus.

5. Nucleus lateralis.

The nucleus gracilis is a collection of grey matter within the funiculus gracilis. For the most part it is connected with the grey 
matter on the dorsal and lateral aspects of the central canal, and it may be regarded as being, in large part, an extension from the basal part of the dorsal grey cornu. It is elongated and increases in size as it ascends. It gives rise to the prominence of the funiculus gracilis and to the clava, and the fibres of the funiculus gracilis, as they ascend, terminate at intervals around the cells of the nucleus.

The nucleus cuneatus is a collection of grey matter within the funiculus cuneatus. It is a direct extension from the basal part of the dorsal grey cornu which lies on the dorsal and lateral aspects of the central canal. Like the nucleus gracilis it is elongated, and increases in size as it ascends. It gives rise to the prominence of

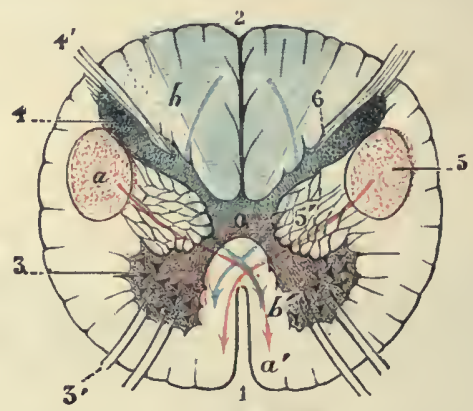

Fig. 567.-Section of the Medulla Oblongata through the Lower Part of the Decussation of the Pyramids (L. Testut's 'Anatomie Humaine').

I. Anterior Median Fissure
2. Posterior Median Fissure
3: Anterior Cornu
3. Anterior Nerve-roots

4. Posterior Cornu

4: Posterior Nerve-roots

5. Crossed Pyramidal Tract

6. Column of Burdach

The red arrows, $a, a^{\prime}$, indicate the paths taken by the crossed Pyramidal Tracts at the level of the decussation of the Pyramids; and the blue arrows, $b, b^{\prime}$, indicate the paths of the Sensory Fibres.

the funiculus cuneatus and to the cuneate tubercle, and the fibres of the funiculus cuneatus, as they ascend, terminate at intervals around the cells of the nucleus.

On the outer side of the nucleus cuneatus there is a small collection of grey matter, which is known as the external or accessory cuneate mucleus. It is on a higher level than the decussation of the pyramids, and it may be regarded as a detached portion of the substantia gelatinosa of Rolando.

The olivary nuclei are associated with the olive, and are three in number-namely, inferior, and two accessory-mesial and dorsal.

The inferior olivary nucleus, which is the chief nucleus, is situated within the olive. As seen in transverse sections through the olive, it appears as a wavy lamina of grey mattcr, curved in such a manner as to form an incomplete capsule, which encloses white matter. The open part of the capsule is called the hilum, and is directed 
towards the median line, but it stops short of either end of the nucleus. A great many nerve-fibres pass through the hilum, some inwards and others outwards, and these form what is known as the olivary peduncle. The wavy lamina is traversed by nerve-fibres.

The superior olivary nucleus is situated in the dorsal or tegmental part of the pons Varolii.

The mesial accessory and dorsal accessory olivary muclei are situated on the mesial and dorsal aspects, respectively, of the inferior or chief olivary nucleus, from which, however, they are distinct. Each consists of a band of grey matter, and the upper part of the mesial accessory nucleus lies opposite the hilum of the chief nucleus.

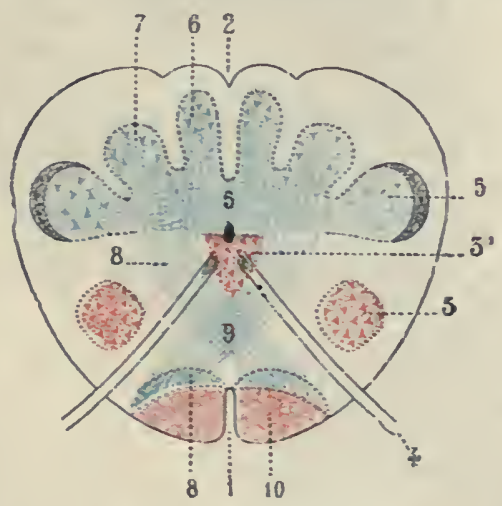

Fig. 568. - Transverse Section passing through the Sensory Decussation (Schematic) (L. Testut's 'Anatomie Humaine').

I. Anterior Median Fissnre

2. Posterior Median Fissure

3. $3^{\mathrm{r}}$. Caput and Base of Anterior Cornu (in red)

4. Hspoglossal Nerve

5. 5. Caput and Base of Posterior Cornu
6. Nucleus Gracilis

7. Nucleus Cuneatus

8, 8. Fillet, or Sensory Tract

9. Sensory Decussation

Ia. Pyramidal Tract

Structure of Inferior Olivary Nucleus. The wavy lamina consists of many small nerve-cells and nerve-fibres which traverse it. The axons of the nerve-cells leave the nucleus as nerve-fibres, and pass to the raphe of the bulb. Some of the nerve-fibres which traverse the wavy grey lamina terminate in connection with its cells, and other fibres pass through it.

The inferior olivary nucleus receives a tract of fibres, called the thalamo-olivary tract, which descends from the optic thalamus through the central part of the tegmentum.

The structure of the two accessory olivary nuclei corresponds to that of the chief or inferior olivary nucleus.

Arcuate Nucleus.-This nucleus consists of a lamina of grey matter which lies upon the ventral aspect of the pyramid of the bulb, above the level of the decussation of the pyramids, and 
beneath the anterior superficial arcuate fibres as they arch outwards over the pyramid after emerging from the ventral median fissure. Superiorly it lies over the mesial aspect of the pyramid, close to the ventral median fissure. It contains small nerve-cells, in connection with which some of the anterior superficial arcuate fibres terminate, whilst others arise as axons of the cells, and many of them pass over the nucleus without entering it.

Nucleus Lateralis. - This is a special collection of nerve-cells in that portion of the formatio reticularis grisea which lies on the dorso-lateral aspect of the olive. It is situated deeply between the olive and the substantia gelatinosa of Rolando.

White Matter of the Medulla Oblongata.-The white matter is situated chiefly on the surface. Over the dorsal aspect of the upper or open part of the bulb, however, the grey matter comes to the surface and covers the lower or bulbar half of the floor of the fourth ventricle. The white matter is disposed in tracts or strands which are chiefly longitudinal, but a few run transversely in an arched manner. The tracts are as follows:

I. Pyramidal tract, or pyramid.

2. Dorsal or direct spino-cerebellar tract.

3. Ventral spino-cerebellar tract.

4. Restiform body.

5. Funiculus cuneatus.

6. Funiculus gracilis.

7. Dorsal or posterior, and ventral or anterior longitudinal, bundles.

8. Superficial arcuate tract.

9. Deep arcuate tract.

ro. Fillet.

II. Olivo-cerebellar tract.

The pyramid of either side, and the decussation of the pyramids, have been already described. It may, however, be again stated that the path of their motor nerve-fibres is downwards into the spinal cord. The fibres comprising about the inner three-quarters of a pyramid, having taken part in the decussation, enter the dorsal portion of the lateral column of the cord on the side to which they have crossed, and here they constitute the deeply placed crossed lateral pyramidal tract. The fibres comprising about the outer quarter of a pyramid take no part in the decussation. Most of them pursue a direct path downwards into the anterior column of the cord on the same side: Having entered the anterior column, they take up a position close to the ventral median fissure and constitute the direct or ventral pyramidal tract (tract of Turck). A few of them, however, enter the lateral column of the same side, and constitute the uncrossed lateral pyramidal tract.

The pyramidal tract has descended from the pons Varolii.

Dorsal or Direct Spino-cerebellar Tract.-This tract extends upwards from the lateral column of the spinal cord. It traverses 
the lower part of the lateral area of the bulb nearly as high as the lower part of the olive, after which it passes backwards and upwards into the restiform body, of which it forms a part.

Ventral Spino-cerebellar Tract.--This tract, like the dorsal or direct spino-cerebellar tract, extends upwards from the lateral column of the spinal cord. It is situated chiefly on the dorsal aspect of the olive, but some of its fibres appear close to the outer

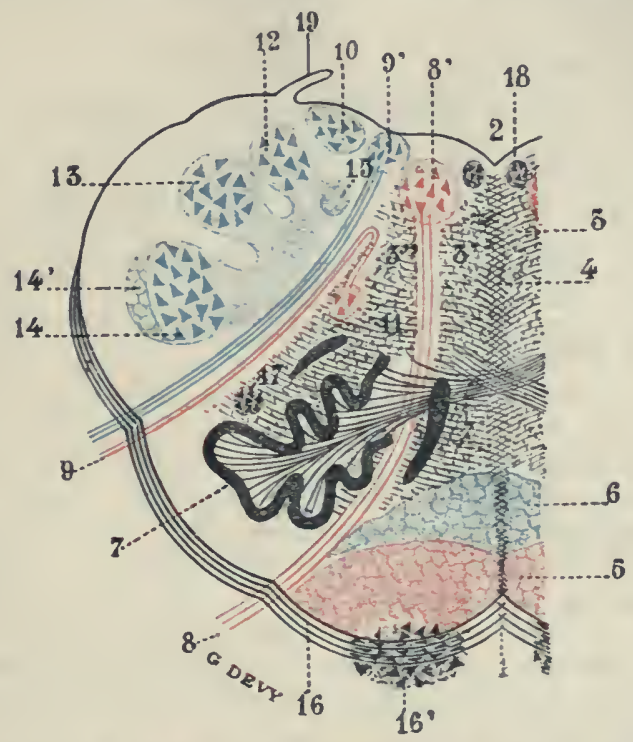

Fig. 569.-The Formatio Reticularis of the Medulla Oblongata, shown by a Horizontal Section passing through the Middle of THE Olivary Body (Demi-Schematic) (L. Testut's ‘Anatomie Humaine').

I. Anterior Median Fissure

2. Fourth Ventricle

3. Formatio Reticularis

3. Reticularis Alba

3". Reticularis Grisea

4. Raphé

5. Anterior Pyramid

6. Fillet

7. Inferior Olive, with the two Accessory Nuclei
7 . Peduncle of Olivary Body
8. Hypoglossal Nerve
8'. Hypoglossal Nucleus
9. Pneumogastric Nerve
9. Terminal Nucleus of Pneu- mogastric Nerve
ro. External Dorsal Auditory Nucleus
II. Nucleus Ambiguus
12. Nucleus Gracilis

13. Nucleus Cuneatus

14. Caput of Posterior Cornu

I 4. Lower Sensory Root of Fifth Nerve

15. Fasciculus Solitarius

r6. Fxternal Anterior Arcuate Fibres

16'. Arcuate Nucleus

I7. Lateral Nucleus

18. Nucleus of Fasciculus Teres

side of that body. Whilst the dorsal spino-cerebellar tract passes into the restiform body and so reaches the cerebellar hemisphere directly, the ventral spino-cerebellar tract is continued upwards into the pons Varolii before reaching the cerebellar hemisphere.

Restiform Body. - The restiform body is situated on the dorsal aspect of the bulb in its upper or open part, the funiculus gracilis and funiculus cuneatus occupying the dorsal aspect in its lower or closed part. It succeeds to the clava and cuneate tubercle in which 
these two funiculi respectively end, but it receives no nerve-fibres from the funiculi. It makes its first appearance in relation to the nucleus cuneatus, and above the cuneate tubercle it is a conspicuous massive bundle, which forms the lateral boundary of the lower or bulbar half of the floor of the fourth ventricle. Its course is upwards, outwards, and backwards. It sinks into the corresponding hemisphere of the cerebellum, and is otherwise known as the inferior cerebellar peduncle.

The restiform body is composed of fibres which are derived from the following sources:

I. The olivo-cerebellar fibres of the inferior olivary nucleus of the opposite side.

2. The direct cerebellar tract of the lateral column of the spinal cord of the same side.

3. The anterior superficial arcuate fibres from the nucleus gracilis and nucleus cuneatus of the opposite side.

4. The posterior superficial arcuate fibres from the nucleus gracilis and nucleus cuneatus of the same side.

5. Vestibular fibres from the vestibular nuclel of the vestibular division of the auditory nerve.

The restiform body, from its composition, serves as an important means of connection between the cerebellar hemisphere superiorly and the medulla oblongata and spinal cord inferiorly.

Funiculus Cuneatus and Funiculus Gracilis.-These tracts are prolonged upwards from the posterior column of the spinal cord. As stated, each contains a grey nucleus, around the cells of which the corresponding sensory nerve-fibres terminate at intervals as they ascend. Towards the clava and cuneate tubercle the fibres become few and are spread over the clava and cuneate tubercle, finally ending in connection with the cells of the grey nuclei which give rise to these prominences.

Dorsal or Posterior Longitudinal Bundle.-The fibres of this bundle (fasciculus longitudinalis medialis B.), when followed downwards into the anterior column of the spinal cord on the same side, represent the fibres of (I) the ventral ground-bundle and (2) the anterior marginal bundle of Lowenthal. As these fibres are followed into the lower part of the bulb they form a bundle, which lies close to the median raphé and directly dorsal to the corresponding pyramid. This strand represents the dorsal longitudinal bundle in the lower part of the bulb. The deep arcuate fibres, to be presently described, pass obliquely through it to the median line, where they decussate with those of the opposite side. This decussation takes place in the interval between the right and left dorsal longitudinal bundles. Having now reached the other side, the deep arcuate fibres take an upward course, close to the median line, as the mesial fillet. The dorsal longitudinal bundle and fillet are therefore now closely related to one another in the lower part of the bulb, both lying dorsal to the pyramid, the fillet lying close to the raphé.

In the upper part of the bulb the two tracts become distinct. 
The dorsal longitudinal bundle is displaced dorsalwards during the formation of the fillet, and it comes into contact with the grey matter on the floor of the fourth ventricle; whilst the fillet lies on the dorsal aspect of the pyramid.

The dorsal longitudinal bundle is prolonged into the ventral column of the spinal cord on the same side, where it is represented, as stated, by the ventral ground-bundle and anterior marginal bundle of Lowenthal.

A ventral or anterior longitudinal bundle (tecto-spinal tract) is described as lying on the ventral aspect of the dorsal or posterior longitudinal bundle. This bundle, however, is not well defined. It descends into the anterior column of the spinal cord, and is accompanied by the ponto-spinal tract, the fibres of which spring from the cells of the formatio reticularis of the pons.

Arcuate Tracts.-These tracts form two groups-superficial and deep.

The superficial areuate fibres are arranged in two sets-anterior and posterior.

The anterior superficial arcuate fibres arise from the nucleus gracilis and nucleus cuneatus of the opposite side, and a few arise from the arcuate nucleus of the same side. At the median line they decussate with those of the opposite side, and emerge at the ventral median fissure, where many of them arch over the mesial and ventral aspects of the pyramid. Others pierce the pyramid, whilst some emerge at the ventro-lateral sulcus between the pyramid and olive. The fibres now pass outwards and dorsalwards, some arching over the lower part of the olive, and they finally enter the restiform body.

The posterior superficial arcuate fibres arise from the nucleus gracilis and nucleus cuneatus of the same side, and they enter the restiform body also of the same side.

The deep arcuate fibres are disposed in two sets-lemniscal and olivo-cerebellar. The lemniscal deep arcuate fibres arise from the nucleus gracilis and nucleus cuneatus of the same side. They sweep forwards and inwards towards the raphé, passing obliquely through the dorsal longitudinal bundle. At the median line they decussate with those of the opposite side above the level of the decussation of the pyramids. Having reached the opposite side, the deep arcuate fibres change their course, and now pass upwards. The ascending tract thus formed constitutes the mesial fillet or lemniscus.

The decussation which takes place between the deep arcuate fibres in the median line, immediately above the decussation of the pyramids, is called the decussation of the fillets (decussatio lemniscor um B.), or the superior sensory decussation, as distinguished from the inferior sensory or spino-thalamic decussation, which takes place in the spinal cord.

The olivo-cerebellar deep arcuate fibres arise from the inferior olivary nucleus of the same side. Emerging through the hilum, they pass across the median line to the opposite side. They then 
pass over, or through, the inferior olivary nucleus of that side, on the dorsal aspect of which they are collected into a distinct tract. This tract, arching backwards, applies itself to the restiform body, on its deep aspect, and is thereby conducted to the cerebellar hemisphere. Its fibres terminate in the cortex of the vermis and cerebellar hemisphere. The olivo-cerebellar arcuate fibres constitute the olivo-cercbellar tract, which connects the inferior olivary nucleus of one side with the cerebellar hemisphere of the opposite side.

Fillet. -The fillet, or lemniscus, as present in the bulb, is a wellmarked tract of fibres which lies on the dorsal aspect of the pyramid close to the raphé. As just stated, its fibres are derived from the lemniscal deep arcuate fibres of the opposite side. In the lower part of the bulb the fillet and dorsal longitudinal bundle are closely related. In the upper part of the bulb, however, as previously stated, the dorsal longitudinal bundle is displaced dorsalwards by the developing fillet, and the fillet, now distinct from the dorsal longitudinal bundle, lies on the ventral aspect of that bundle, and on the dorsal aspect of the pyramid. The ventral region of the bulb is thus traversed by three longitudinal tracts, all of which lie close to the median line. " These tracts are related to each other in the following order from before backwards:

Pyramid.

Fillet.

Dorsal longitudinal bundle.

Olivo-cerebellar Tract.-This tract has already been described in connection with the olivo-cerebellar deep arcuate fibres.

Raphé of the Medulla Oblongata. - The raphé of the bulb occupies the median plane above the decussation of the pyramids, and is composed of fibres which, for the most part, cross obliquely from one side to the other. These fibres represent (I) the anterior superficial arcuate fibres, (2) the lemniscal deep arcuate fibres, and (3) the olivo-cerebellar deep arcuate fibres. A few fibres pass ventro-dorsally, and some are disposed longitudinally. The fibres are therefore arranged in an intersecting manner.

Central Canal of the Medulla Oblongata. - The central canal of the spinal cord is prolonged upwards through the lower or closed part of the bulb. As it ascends it is gradually displaced backwards, first by the decussation of the pyramids, and afterwards by the decussation of the fillets. It is surrounded by a thick layer of grey matter, which is derived from the basal portions of the ventral and dorsal grey cornua of the spinal cord. Superiorly, at the level of the calamus scriptorius, it opens into the lower part of the fourth ventricle in the angle between the two diverging clava. The grey matter which surrounds the canal is now spread out and forms a thick covering over the lower part of the ventricular floor, as previously stated.

Areas of Flechsig. - These areas involve the whole substance of the bulb, and are mapped out by the funiculi of the liypoglossal and pneumogastric 
nerves. As seen in transverse section, these funiculi lie near each other as they arise from their nuclei in the grey matter of the lower part of the floor of the fourth ventricle. As the funiculi of the hypoglossal nerve pass forwards and those of the pneumogastric nerve outwards, they diverge from each other, and the substance of the bulb is thereby divided into three segments, which constitute the areas of Flechsig-ventral, lateral, and dorsal.

The ventral area lies between the raphe of the bulb and the funiculi of the hypoglossal nerve. Throughout its thickness this area contains the following structures:

The pyramid and arcuate nucleus.

The fillet.

The dorsal or posterior longitudinal bundle.

The formatio reticularis alba.

The lateral area lies between the funiculi of the hypoglossal nerve and those of the pneumogastric nerve. Throughout its thickness this area contains the following structures:

The olive and inferior olivary nucleus.

The nucleus lateralis.

The nucleus ambiguus (to be afterwards described).

The formatio reticularis grisea.

The dorsal area is the region behind the funiculi of the pneumogastric nerve. Throughout its thickness this area contains the following structures:

The restiform body.

The upper part of the cuneate nucleus.

The descending root of the vestibular nerve/To be afterwards

The spinal root of the fifth cranial nerve $\int$ described.

The substantia gelatinosa of Rolando.

Course of Chief Nerve-Funiculi of Spinal Cord through Medulla Oblongata.

Spinal Cord. Medulla Oblongata.

Posterior Column.

Column of Goll. Column of Burdach.

Funiculus Gracilis and Nucleus Gracilis. Funiculus Cuneatus and Nucleus Cuneatus.

Lateral Column.

Crossed Lateral Pyramidal Tract.

Uncrossed Lateral Pyramidal Tract.

Dorsal or Direct Spino-cerebellar Tract.

Ventral or Indirect Spino-cerebellar Tract

Prepyramidal or Rubro-spinal Tract

Lateral Ground-Bundle

Inner three-quarters of opposite Pyramid.

Outer one-quarter of Pyramid of same side.

Lateral Area below Olive, and Restiform Body.

Lateral Area below Olive, and Formatio Reticularis.

Anterior Column.

Direct Pyramidal Tract.

Ventral Ground-Bundle Ventral Ground-Bundle
Anterior Marginal Bundle of Lowen-
thal

Outer one-quarter of Pyramid of same side.

Dorsal Longitudinal Bundle.

Development of Medulla Oblongata.-The bulb is developed from the myelencephalon, which is the caudal division of the rhombencephalon. 


\section{The Pons Varolii.}

The pons Varolii is situated above the medulla oblongata, anc between the hemispheres of the cerebellum. With the exceptior of the restiform bodies, all the other parts of the medulla oblongat are prolonged into it. The pons presents two surfaces, ventral anc dorsal, and two borders, upper and lower. The ventral surface rests upon the upper part of the basilar groove of the occipital bon and the dorsum sellæ of the sphenoid. It is convex from side to side, and from above downwards, and has a transversely-striatec appearance, due to the disposition of its superficial fibres. Alon the median line it presents the basilar groove, which extends from the lower to the upper border, and lodges the basilar artery. Or either side of this groove the ventral surface is rendered prominen by the prolongation upwards of the pyramids of the medulla oblongata, and the basilar groove is chiefly due to this circumstance The sensory and motor roots of the fifth nerve, lying close together appear on the lateral aspect of the ventral surface, the small moto root being the upper of the two. The portion external to these two nerve-roots constitutes the middle peduncle of the cerebellum It is composed of the transverse fibres of the pons, these on eithe: side being collected into a large bundle, which passes backward: and outwards into the corresponding cerebellar hemisphere.

The dorsal surface is directed towards the cerebellum. If presents a triangular area which is covered with grey matter. This area is continuous with the dorsal surface of the upper or open par of the medulla oblongata, and it forms the upper or pontine part of the floor of the fourth ventricle. On either side it is boundec by the superior peduncle of the cerebellum as it passes upwards anc inwards.

The upper border is slightly depressed at the centre, and on eithes side of the median depression it slopes outwards and downwards towards the middle peduncle of the cerebellum. The crura cerebri right and left, sink into the pons at the upper border.

Internal Structure of the Pons Varolii.-The pons is composed o. a large ventral and a small dorsal part.

Ventral Part.-This portion consists of: (I) bundles of transverse fibres, (2) bundles of longitudinal fibres, and (3) a large amount 0 : grey matter.

The bundles of transverse fibres intersect the bundles of longi. tudinal fibres, and on either side they are collected into the middle peduncle of the cerebellum, which enters the corresponding cerebellar hemisphere. Some of the transverse fibres arise in the cortex of the cerebellum as the axons of the cells of Purkinje, and these terminate in the pons in arborizations around the cells of the mucleus pontis, mostly on the opposite side to that on which they arise. Other transverse fibres arise in the pons as the axons of the cells of the nucleus pontis on one side. They then cross to the other side, and enter the cerebellar hemisphere of that side, where they 
terminate in arborizations in the cortex. The fibres, therefore, of which the middle peduncle of the cerebellum is composed may be regarded as being of two kinds, namely, efferent and afferent. The efferent fibres arise in the cerebellar cortex and terminate in the pons, whilst the afferent fibres arise in the pons and terminate in the cerebellar cortex.

The bundles of longitudinal fibres in each half of the ventral part of the pons are derived from the breaking up of the crusta or pedal portion of the corresponding crus cerebri, which enters the pons at its upper border. Nost of these bundles are collected together at the lower border of the pons, and form the pyramid of the medulla oblongata on the same side. Certain of the fibres of the crusta of the crus cerebri, however, terminate in the pons, as follows: (I) some end in arborizations around the cells of the motor nucleus of the fifth cranial nerve, the nucleus of the sixth cranial nerve, and the nucleus of the seventh cranial, or facial, nerve; and (2) others end in arborizations around the cells of the nucleus pontis, all of the same side.

The grey matter of the pons, which is large in amount, occupies the intervals between the intersecting transverse and longitudinal bun-

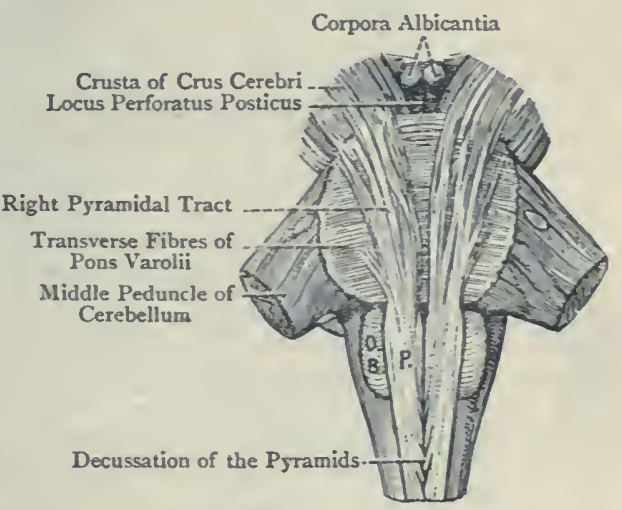

Fig. 570.-Dissection of the Pons VAROLII, SHowiNg the COURSE OF THE PYRAMidal Tracts OF the Medulla ObloNgata (HirschFeld A.ND LeveILlE).

P, Right Pyramid ; O.B., Right Olivary Body. dles, and contains small multipolar nerve-cells. It is known as the mucleus pontis, and is continuous with the arcuate nuclei of the medulla oblongata.

Corpus Trapezoides or Trapezium.-The trapezium represents a group of transverse fibres, on either side, which have no connection with the corresponding middle peduncle of the cerebellum. The fibres are situated in the lower part of the pons, dorsal to the pyramidal bundles. Within the trapezium are large multipolar cells, which constitute the nucleus of the trapezium. The fibres of the trapezium arise chiefly as the axons of the cells of the ventral cochlear nucleus and also of the lateral cochlear nucleus or tuberculum acusticum, in which nuclei the fibres of the cochlear division of the auditory nerve terminate. Some of the fibres arise from the superior olivary nucleus; others are the axons of the cells of the nucleus of the trapezium; whilst a third set (strice acustica) arise 
from the tuberculum acusticum of the opposite side. Certain of the fibres of the trapezium terminate in the superior olivary nucleus, but the majority cross the median plane, where they decussate with those of the opposite side. Having crossed to the opposite side, they become longitudinal, and form a well-marked ascending tract in the dorsal part of the pons Varolii, called the lateral fillet, which lies on the outer side of the main or mesial fillet.

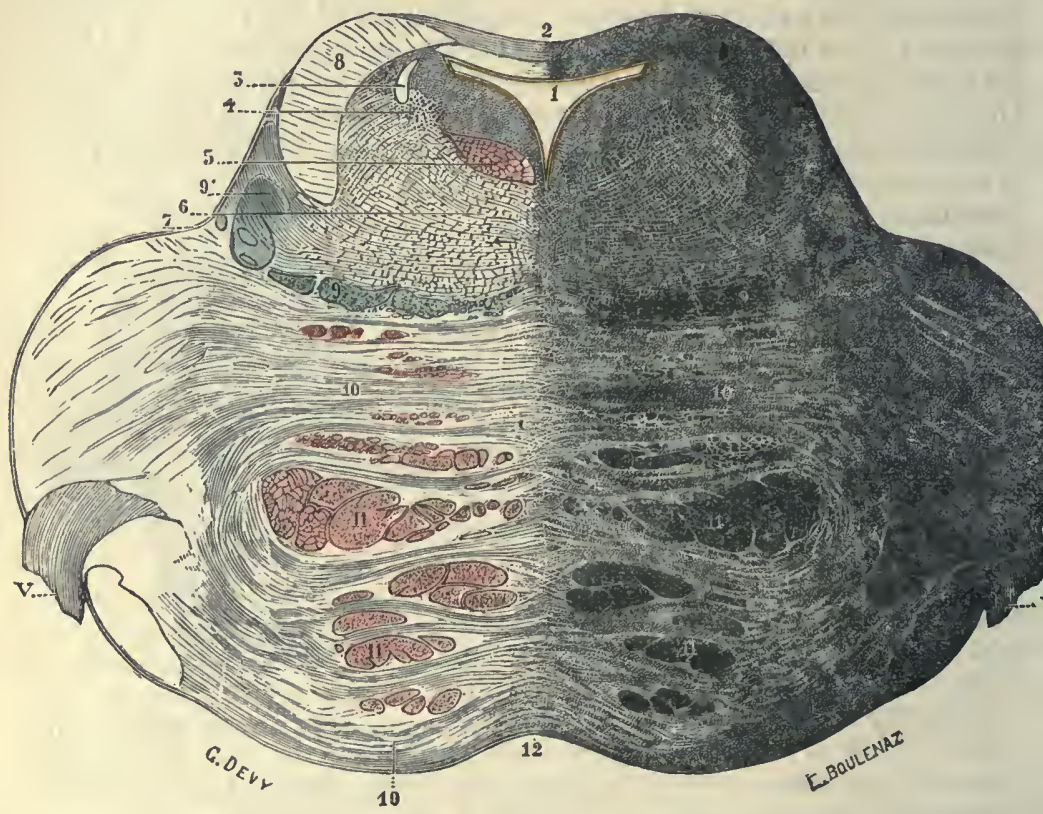

Fig, 571,-Vertical Transverse Section through the Upper Part of the Pons Varolit (From L. Testut's 'Anatomie Humaine,' after Stilling).

1. Fourth Ventricle

2. Valve of Vieussens

3. Superior Root of Fifth Nerve

4. Nerve-cells which accompany this Root

5. Posterior Longitudinal Bundle

6. Formatio Reticularis

7. Lateral Fissure of Isthmus
8. Section of Superior Cerebellar Peduncle 9, 9. Mesial and Lateral Portions of the Fillet

Io, 10. Transverse Fibres of the Pons

I I, II. Longitudinal Fibres of the Pons

12. Raphe

V. Fifth Nerve

Dorsal or Tegmental Part of the Pons.-This portion is divided into two symmetrical halves by a median raphé, which is continuous with that of the upper or open part of the medulla oblongata. It consists of formatio reticularis, which is continued upwards from the formatio reticularis of the bulb. The formatio reticularis of the dorsal part of the pons contains certain tracts of nerve-fibres and nuclei, with which important nerves are connected. These 
are so complicated that it is convenient to divide the dorsal part of the pons into two regions-lower and upper.

Lower Region.-This region corresponds to the level of the trapezium in the ventral part of the pons, and it succeeds to the upper end of the bulb. The restiform body of the bulb lies for a short distance on the outer aspect of this region, but that body soon passes backwards and sinks into the hemisphere of the cerebellum.

The tracts and nuclei of the formatio reticularis of the lower region, which will be described in this place, are as follows:

I. Spinal sensory root of the fifth cranial nerve.

2. Motor nucleus of the facial nerve.

3. Superior olivary nucleus.

4. Nucleus of the sixth cranial nerve.

5. Dorsal longitudinal bundle.

6. Fillet.

The funiculi of the spinal or descending sensory root of the fifth cranial nerve appear on the inner side of the restiform body. Close to the inner side of this root, and accompanying it in its downward course, there is the inferior sensory nucleus of the fifth nerve, around the cells of which the fibres of the spinal or descending root of that nerve terminate at intervals. The inferior sensory nucleus is an upward prolongation of the substantia gelatinosa of Rolando, and inferiorly it extends to about the level of the second cervical spinal nerve.

The motor nucleus of the facial nerve is internal to the funiculi of the spinal root of the fifth nerve. It lies deeply in the lower region of the dorsal part of the pons, on the dorsal aspect of the superior olivary nucleus. The motor fibres of the facial nerve arise as the axons of the cells of this nucleus, and take a course which will be afterwards described.

The superior olivary nueleus is situated on the ventral aspect of the facial nucleus, and is close to the lateral part of the trapezium, the fibres of which arch round its ventro-lateral aspect. Some of these fibres terminate in the superior olivary nucleus, whilst others arise from its cells.

In connection with the superior olivary nucleus three other nuclei are described-namely, (I) an accessory superior olivary nucleus on the mesial side of the principal nucleus; (2) a lateral pre-olivary nucleus on the ventral aspect of the principal nucleus; and (3) a mesial pre-olivary nucleus on the ventral aspect of the nucleus of the trapezium.

The nucleus of the sixth eranial nerve lies immediately beneath the grey matter of the pontine part of the floor of the fourth ventricle, and on the outer side of the dorsal longitudinal bundle which separates the nucleus from the median raphé. It corresponds to that portion of the eminentia teres which lies on the pontine part of the floor of the fourth ventricle directly above the striæ acusticæ.

The posterior longitudinal bundle, or median longitudinal fasciculus, 
lies close to the median raphé, and on the mesial side of the nucleus of the sixth nerve. Like that nucleus, it lies immediately beneath the grey matter of the pontine part of the floor of the fourth ventricle.

The fillet (main fillet) lies, as it does in the bulb, ventral to the dorsal longitudinal bundle, but in the dorsal part of the pons the two strands are separated by a distinct interval. The fillet occupies a broad area in that portion of the lower region of the dorsal part of the pons which is contiguous to the ventral part. The area extends outwards from the median raphé.

Upper Region of the Dorsal Part of the Pons.-This region lies above the level of the trapezium in the ventral part of the pons. The tracts and nuclei of this region, which will be described in this place, are as follows:

I. Superior peduncle of the cerebellum.

2. Nuclei of the fifth cranial nerve.

3. Dorsal longitudinal bundle.

4. Mesial fillet.

5. Lateral fillet.

The superior peduncle of the cerebellum, after emerging from the corresponding cerebellar hemisphere, lies on the lateral aspect of this region, where it forms the lateral boundary of the upper or pontine part of the floor of the fourth ventricle. Its dorsal aspect is connected with that of its fellow of the opposite side by the superior medullary velum, and ventrally it sinks into the upper region of the dorsal part of the pons.

The pontine nuclei of the fifth cranial nerve are two motor and two sensory, upper and lower. The motor roots are pontine and Sylvian.

The pontine motor nucleus is situated close to the superior peduncle of the cerebellum at the lower part of the lateral margin of the upper or pontine part of the fourth ventricle. It lies near the surface, and the axons of its cells form many of the fibres of the motor root of the nerve. The remaining fibres of the motor root represent the descending, mesencephalic, or Sylvian root. These fibres arise from the Sylvian motor nucleus in the grey matter on the lateral aspect of the aqueduct of Sylvius, and as they descend towards the pontine motor nucleus, they lie close to the mesial side of the superior peduncle of the cerebellum.

The upper sensory nucleus is situated deeply on the outer side of the pontine motor nucleus, and on the ventral aspect of the superior peduncle of the cerebellum. Some of the fibres of the sensory root ascend and terminate in arborizations around the cells of this nucleus.

The lower sensory nucleus succeeds to the upper sensory nucleus, and is a continuation upwards of the substantia gelatinosa of Rolando. It is elongated and cxtends into the upper part of the spinal cord to about the level of the second cervical nerve. It lies on the inner side of the spinal or descending scnsory root of the 
fifth nerve, and the fibres of that root terminate at intervals in arborizations around its cells.

The posterior longitudinal bundle has the same position in the upper region as it has in the lower region. It lies close to the median raphé and immediately below the grey matter of the corresponding part of the floor of the fourth ventricle.

The main or mesial fillet, like the main fillet in the lower region, lies in that portion of the upper region of the dorsal part of the pons which is contiguous to the ventral part, and it forms a layer of some breadth, extending outwards from the median raphé.

The lateral fillet represents a strand of fibres which lies on the lateral or outer side of the mesial fillet. Associated with the lateral fillet, and lying between it and the mesial fillet, there is a collection of nerve-cells, called the nucleus of the lateral fillet.

Development of the Pons Varolii.-The pons is developed from the ventral and lateral walls of the metencephalon, which is one of the divisions of the rhombencephalon.

\section{The Cerebellum.}

The cerebellum, or small brain, occupies the inferior occipital or cerebellar fossæ of the occipital bone. It lies beneath the posterior parts of the hemispheres of the cerebrum, from which it is separated by a septum of the dura mater, called the tentorium cerebelli, and it is posterior to the medulla oblongata and pons Varolii. It is composed of white and grey matter, the white matter being situated in the interior, where it constitutes the medullary substance, and the grey matter being spread over the surface as the cortex. In appearance it is laminated or foliated, the laminæ being separated from each other by parallel, slightly curved sulci. It is composed of two large lateral portions, called hemispheres, and a connecting median portion, termed the vermis ('worm') or vermiform process, these parts being much more distinctly demarcated below than above. It presents, in the median line, two notches, anterior and posterior. The anterior notch, which is wide, is known as the incisura semilunaris, and it contains the inferior pair of quadrigeminal bodies and the superior cerebellar peduncles. The posterior notch is narrow, and is occupied by the falx cerebelli. The most conspicuous sulcus of the cerebellum is the great horizontal fissure, which extends round the circumference, and passes for some distance into the interior. By means of this fissure the cerebellum is divided into two parts, upper and lower.

Upper Surface of the Cerebellum.-This surface presents in the median line the upper part of the vermis, known as the superior vermis. It extends from the incisura semilunaris to the posterior notch, and it forms a laminated elevation, which is higher in front than behind, the most prominent part being known as the monticulus cerebelli. On either side of the superior vermis the upper surface of each hemisphere inclines downwards to the circumference, and there is no distinct demarcation between it and the superior vermis. 
The superior vermis is composed of five lobules, named, in order from before backwards, the lingula, central lobule, culmen monticuli, clivus monticuli, and folium cacuminis. The lingula is deeply placed, and consists of about four laminæ or folia, which lie over the superior medullary velum as it extends between the superior cerebellar peduncles. Its laminæ may be continued on either side over the superior cerebellar peduncle, and, when this is so, the prolongation is known as the frenulum lingulæ.

The central lobule is of small size, and lies at the bottom of the incisura semilunaris. It is separated from the lingula by the precentral fissure, and from the culmen monticuli by the postcentral fissure.

The culmen monticuli forms the summit of the superior vermis. It is composed of several laminæ, and posteriorly is separated from the clivus by the preclival fissure.

The clivus monticuli represents the sloping part of the monticulus

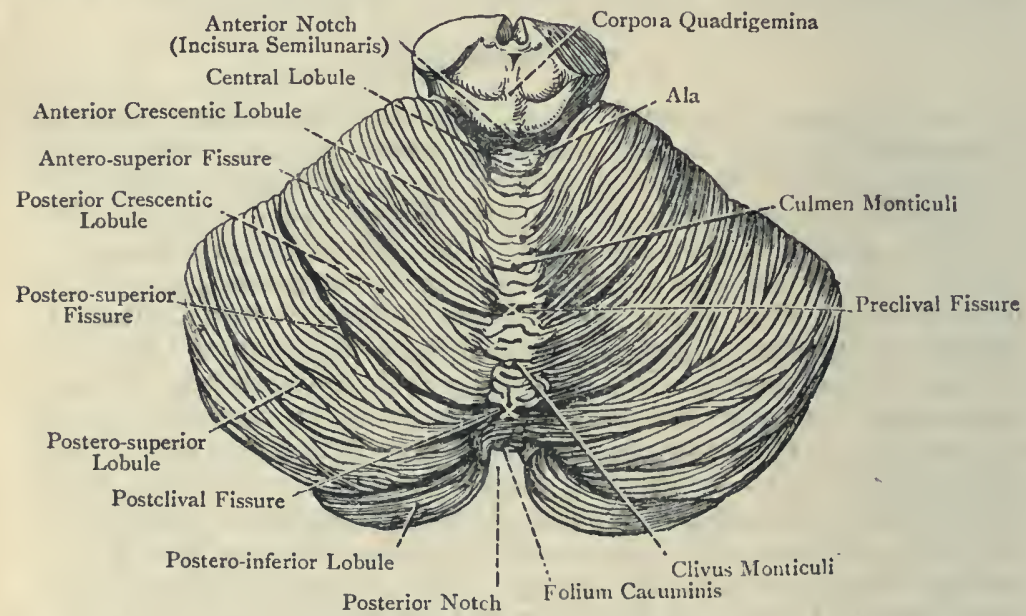

Fig. 572.-The Cerebellum (Superior View).

cerebelli. It is situated behind the culmen monticuli, and is com. posed of several laminæ. Posteriorly it is separated from the folium cacuminis by the postclival fissure.

The folium cacuminis forms the posterior extremity of the superior vermis, and lies at the posterior notch, where it is placed above the great horizontal fissure.

The upper surface of each hemisphere is mapped out into lobules, which are continuous with the subdivisions of the superior vermis, with the exception of the lingula. These are called, in order from before backwards, the ala, anterior crescentic lobule, posterior crescentic lobule, and postero-superior lobule.

The ala is continuous with the central lobule, from which it is 
prolonged for a limited distance round the anterior part of the hemisphere in the region of the incisura semilunaris.

The anterior crescentic lobule is continuous with the culmen monticuli, and represents the anterior subdivision of the upper surface of the cerebellar hemisphere. It is limited posteriorly by a curved sulcus, called the antero-superior fissure, which is continuous with the preclival fissure, and opens at the circumference into the great horizontal fissure. The right and left anterior crescentic lobules, together with the culmen monticuli, form the lobus culminis.

The posterior crescentic lobule is continuous with the clivus monticuli. It is limited in front by the antero-superior fissure, and behind by the postero-superior fissure, the latter being continuous with the postclival fissure, and opening at the circumference into the great horizontal fissure. The right and left posterior

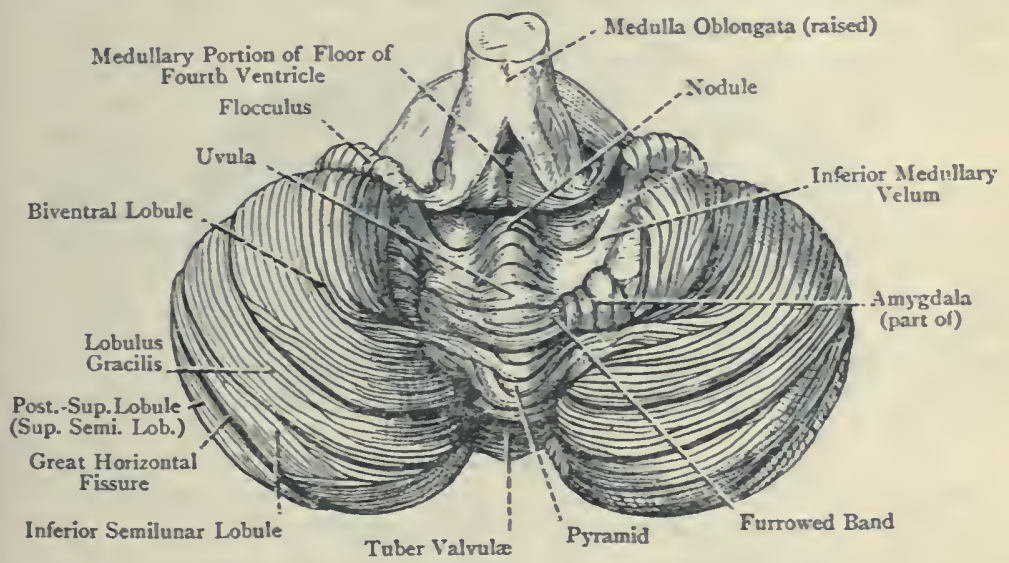

Fig. 573.-The Cerebellum (INferior view).

(The Inferior Semilunar Lobule and the Lobulus Gracilis together constitute the Postero-inferior Lobule).

crescentic lobules, together with the clivus monticuli, form the lobus clivi.

The postero-superior lobule (superior semilunar lobule) corresponds to, but is much more extensive than, the folium cacuminis. It is limited in front by the postero-superior fissure, and behind by the great horizontal fissure. The right and left postero-superior lobules, together with the folium cacuminis, form the lobus cacuminis.

Under Surface of the Cerebellum.-The under surface presents in the median line a deep groove, called the vallecula, which is continuous behind with the posterior notch. Anteriorly it lodges the medulla oblongata, and lying in the bottom of it there is the lower part of the vermis, which is known as the inferior vermis. 
The vallecula separates the two cerebellar hemispheres from each other, and the inferior vermis is separated on either side from the corresponding hemisphere by a furrow called the sulcus vallecula. The under surfaces of the hemispheres are markedly convex, and are received into the inferior occipital or cerebellar fossæ of the occipital bone.

The inferior vermis is composed of four lobules, named, in order from behind forwards, tuber valvulæ, pyramid, uvula, and nodule.

The tuber valvulæ (tuber posticum) forms the posterior part of the inferior vermis, and is composed of several laminæ. On either side it is prolonged into the corresponding hemisphere, and becomes continuous with the postero-inferior lobule. It is the only part of the inferior vermis which is prolonged into the cerebellar hemispheres.

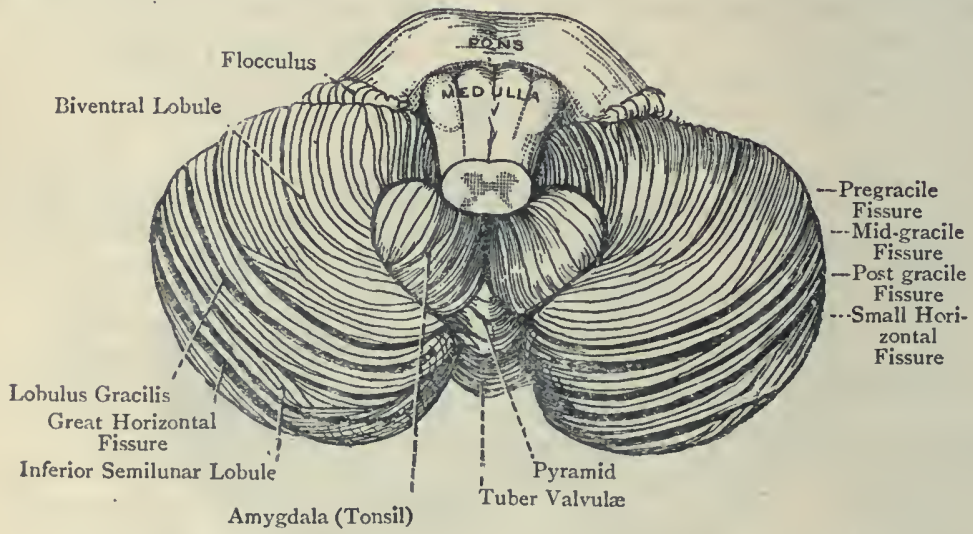

Fig. 574- -The Cerebellum (INFerior View).

(The Inferior Semilunar Lobule and the Lobulus Gracilis constitute the Postero-inferior Lobule).

The pyramid is situated in front of the tuber valvulæ. It presents about four laminæ, and is separated by deep sulci from the tuber valvulæ behind and the uvula in front, whilst on either side it is separated from the cerebellar hemisphere by the sulcus valleculæ. Laterally it is connected with the biventral lobule of the hemisphere by means of a faint ridge, but this lies low in the sulcus valleculæ as it crosses it.

The uvula is situated in front of the pyramid, and between the amygdale or tonsils of the hemispheres. It is triangular, the base being dirceted backwards, and it consists of scveral laminæ. It is separated on either side from the hemisphere by the sulcus valleculae. Laterally its narrow part is connected with the amygdala by a ridge of grey matter, but this lies low in the sulcus 
valleculæ as it crosses it. This ridge is notched at intervals, and is called the furrowed band.

The nodule forms the anterior part of the inferior vermis, and is composed of several laminæ, which are largely concealed by the uvula. It is connected on either side with the flocculus by a thin semilunar band of white matter, which is the lateral portion of the inferior medullary velum.

The fissures of the inferior vermis are three in number, as follows: postpyramidal, between the pyramid and the tuber valvulæ; prepyramidal, between the pyramid and the uvula; and postnodular, between the nodule and the uvula.

The under surface of each hemisphere is mapped out into four lobules, which are called, from behind forwards, the posteroinferior lobule, the biventral lobule, the amygdala, and the flocculus.

The postero-inferior lobule is situated at the back part of the under surface of the hemisphere. It is divided into four curved parts by three curved fissures. The anterior two parts are known as the lobulus gracilis, and the posterior two as the inferior semilunar lobule. The right and left postero-inferior lobules, together with the tuber valvulæ, form the lobus tuberis.

The biventral lobule is composed of curved laminæ, and is somewhat triangular. The pointed end is directed backwards and inwards, and it is connected with the pyramid by a faint ridge, which, as stated, lies low in the sulcus valleculæ. The base is directed forwards towards the flocculus. The lobulus gracilis lies external to it, and the amygdala is on its inner side. The biventral lobule is divided by a sulcus into two portions, outer and inner; hence the name 'biventral.' The right and left biventral lobules, together with the pyramid, form the lobus pyramidis.

The amygdala (tonsil) forms a conspicuous prominence between the uvula and the biventral lobule. It is situated in a depression of the vallecula, which is known as the nidus avis ('bird's nest '), and its direction is almost sagittal. It is connected with the narrow part of the uvula by the furrowed band, which, as stated, lies low in the sulcus valleculæ. The right and left amygdalæ, together with the uvula, form the lobus uvulæ.

The flocculus (subpeduncular lobule) is a small irregular lobule which is situated between the front of the biventral lobule and the middle peduncle of the cerebellum. Internally it is connected with the nodule by the lateral portion of the inferior medullary velum. The right and left flocculi, together with the nodule, form the lobus noduli.

The fissures of the under surface of each hemisphere are as follows: (I) a continuation of the postnodular sulcus, between the biventral lobule and the flocculus; (2) a continuation of the prepyramidal sulcus, between the amygdala and the biventral lobule; (3) the pregracile, or anterior arcuate, sulcus, between the biventral lobule and the lobulus gracilis; (4) the mid-gracile, or middle arcuate 
sulcus, within the lobulus gracilis; (5) the postgracile, or posterior arcuate, sulcus, between the lobulus gracilis and the inferior semilunar lobule; and (6) the small horizontal sulcus within the inferior semilunar lobule.

Peduncles of the Cerebellum.-The peduncles are three in number on either side, namely, superior, middle, and inferior, and they are composed of fibres which enter, or emerge from, the central white medullary substance.

The superior peduncles are composed of efferent fibres, and are at first concealed from view by the upper or anterior portions of the hemispheres. After emerging from the hemispheres they pass upwards on the lateral aspects of the dorsal surface of the pons Varolii in a converging manner towards the quadrigeminal bodies. They form the lateral boundaries of the upper part of the floor of the fourth ventricle, and by their convergence they project slightly over that part of the ventricle, so as to take part in its roof. The superior medullary velum extends between the two peduncles, and closes the interval between them. On reaching the inferior pair of quadrigeminal bodies the two peduncles pass beneath them and enter the mesencephalon, where their course will be subsequently described (see Index). The most of the fibres of each superior peduncle are derived from the corresponding nucleus dentatus, but a few come from the grey matter of the cerebellar cortex.

The middle peduncles are of large size, and are formed by the transverse fibres of the pons Varolii, these being gathered together on either side into a large bundle, which passes backwards and outwards into the white central medullary substance of the corresponding hemisphere. The fibres of each middle peduncle are both afferent and efferent. The afferent fibres arise in the pons from the cells of the nucleus pontis of the opposite side, and terminate in arborizations around the cells of the cerebellar cortex. The efferent fibres arise from the cells of the cerebellar cortex of the same side, and terminate in arborizations around the cells of the nucleus pontis, mostly on the opposite side.

The inferior peduncles are principally composed of afferent fibres, which are derived chiefly from the dorsal or direct cerebellar tract and the olivo-cerebellar tract of either side. The fibres of the dorsal cerebellar tract terminate in the cortex of the superior vermis on both sides of the median line. The fibres of the olivo-cerebellar tract, which are derived from the inferior olivary nucleus of the medulla oblongata on the opposite side, terminate in the cortex of the vermis and cerebellar hemisphere. The superficial arcuate fibres, which form part of the inferior peduncle, are connected with the cortex of the vermis and cerebellar hemisphere.

White and Grey Matter of the Cerebellum.-In the hemispheres and vermis the white matter is situated in the centre as the medulla, and the grey matter is disposed superficially as the cortex. The white matter in the interior of the vermis is known as the corpus trapezoides. When sagittal sections of a liemisphere are made, the 
mass of white matter in the centre is seen to send offshoots into the lobules. From the sides of these offshoots secondary processes are given off, and these in turn furnish tertiary processes, the white matter in all cases being covered by grey matter. When the section is made across the direction of the laminæ or folia the appearance presented is like the trunk and branches of a tree: hence the name arbor vite cerebelli is applied to it.

Nuclei.-The corpus dentatum is a collection of grey matter which is situated within the white matter of each hemisphere, and it is similar to the inferior olivary nucleus in the olivary body of the medulla oblongata. It is composed of a wavy grey lamina, disposed in the form of a capsule, which encloses white matter. The capsule presents an opening or hilum at its upper and inner part, and through this a large number of the fibres of the superior cerebellar peduncle emerge from the interior.

There are three other nuclei on each side, as follows: (I) the nucleus emboliformis, close to the inner side of the hilum of the corpus dentatum; (2) the nucleus globosus, internal to the preceding; and (3) the nucleus fastigii, or roof-nucleus, situated in the corpus trapezoides of the vermis, close to the median line, and contiguous to its fellow of the opposite side.

Commissural and Association Fibres.-The commissural abres pass from the white matter of one hemisphere to that of the opposite hemisphere. They traverse the corpus trapezoides of the vermis in two sets, superior and

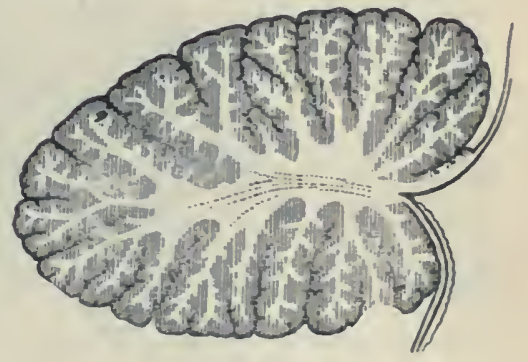

Fig. 575.-Sagittal Section of a HeMisphere of THE CEREBEllum, SHOWING THE ARBOR VITE.

inferior. The association fibres are confined to each side, and they connect adjacent laminæ, passing across the bottom of the fissures which separate them.

Medullary Vela.-These are thin laminæ or curtains of white matter, and are two in number, superior and inferior.

The superior medullary velum, or valve of Vieussens, is continuous with the corpus trapezoides or white matter of the vermis. It extends between the converging superior cerebellar peduncles, bridging over the interval between them, and becoming continuous with their inner margins. Superiorly it extends to the inferior pair of quadrigeminal bodies, and inferiorly it passes into the corpus trapezoides or white matter of the vermis. It forms a large portion of the roof of the upper part of the fourth ventricle, and its dorsal surface supports the lingula of the superior vermis. From the upper part of the superior medullary velum a band of white fibres, called the fremulum veli, passes to the lower part of the median longitudinal groove which separates the lateral pairs of quadri- 
geminal bodies. Immediately below the inferior pair of quadrigeminal bodies the fourth pair of cranial nerves emerge from the superior medullary velum, on either side of the frenulum veli. The tract of Gowers, after having traversed the formatio reticularis of the medulla oblongata and the dorsal part of the pons Varolii, passes into the superior medullary velum, and then desends in the superior cerebellar peduncle to the cerebellum.

The inferior or posterior medullary velum is a thin lamina of white matter, which consists of three parts-median and two lateral, right and left.

The median part supports dorsally the nodule of the inferior vermis. It is a prolongation of the white matter of the corpus trapezoides of the vermis, and lies on the upper or ventral aspect of the nodule, to which it is adherent. As it leaves the white matter

Layer of Purkinje's Cells

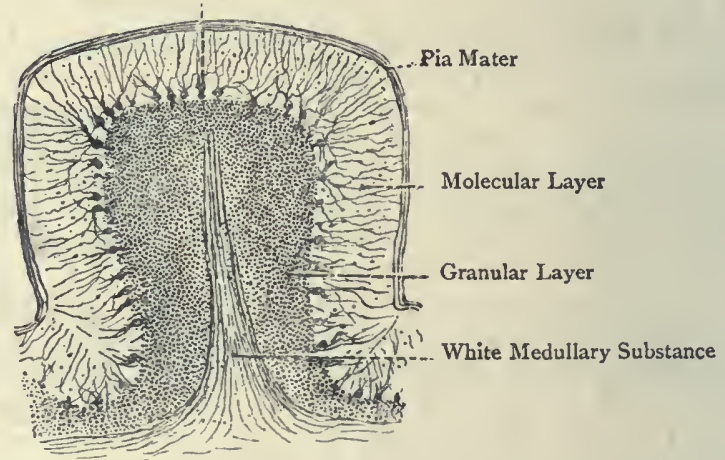

Fig. 576.-Structure of a Lamina of the Cerebellum (highly MAGNIFIED).

it is contiguous to the superior medullary velum, but the two laminæ take different directions. The superior medullary velum passes upwards between the dorsal parts of the superior peduncles of the cerebellum. The median part of the inferior medullary velum passes ventralwards and then downwards. It is succeeded in a downward direction by the ependymal epithelium and pia mater (tela choroidea inferior), which form a large portion of the lower part of the roof of the fourth ventricle. The middle part of the inferior medullary velum forms the upper portion of the lower part of the roof of the fourth ventricle.

Each lateral part of the inferior medullary velum extends outwards to the corresponding flocculus in the form of a semilunar band. The ventral surface of this band is directed towards the fourth ventricle, and the dorsal surface is related to the amygdala or tonsil. One border of the band is free and concave, whilst the other is continuous with the white matter of the corresponding cerebellar hemisphere. 
The inferior medullary velum forms a part of the lobus $n: d u l i$, the other parts, as stated, being the nodule and the two flocculi.

Minute Structure of the Cerebellar Laminæ.-Each lamina or folium of the cerebellum consists of (I) a central part or core of white matter, which is an offshoot from the white medullary substance, and (2) an external part or cortex of grey matter.

Grey Cortex.-The grey cortex is composed of two layers, an outer molecular layer, and an inner granular layer. Between these two layers there is a stratum of characteristic large cells, called the cells or corpuscles of Purkinje.

The cells of Purkinje are pyriform or flask-shaped, and are situated, as just stated, between the molecular and granular layers. The narrow or superficial end of each cell projects into the molecular layer, and the broad or deep end rests on the granular layer. From the broad or deep end of each cell a single axon passes off, which enters the granular layer, where it soon becomes medullated, and then forms a nerve-fibre of the white medullary substance. The axon of Purkinje's cell gives off a few collateral recurrent branches, some of which end in the granular layer, whilst others enter the molecular layer.

From the narrow or superficial end of each cell one or two dendrons are given off. These divide and subdivide at frequent intervals in the molecular layer 'like the antlers of a deer.' The dendritic processes so formed are arranged in an arborescent manner, and are distinct from those of adjacent cells. They permeate the molecular layer as far as the surface.

The molecular layer consists of a few nerve-cells and many nervefibres.

The nerve-cells are situated partly in the inner or deep portion of the molecular layer, and partly in its outer portion.

The inner cells are known as the basket-cells, and they lie in the vicinity of the cells of Purkinje. Each basket-cell has several dendritic processes which ramify in all directions. In addition to these processes there is an axon, which springs from the side of the cell and takes a transverse course. It gives off a number of collaterals which pass. towards the bodies of the cells of Purkinje. These collaterals terminate by ramifying very freely around the cells of Purkinje, as well as around the axons of these cells for a short distance. The minute terminal ramifications form a close basket-work, which encloses the cell of Purkinje and its axon for a short distance.

The outer eells of the molecular layer are small, and each has several dendritic processes and an axon. Each axon springs from the side of the cell, and, taking a transverse course, it ends in numerous ramifications.

The fibres of the molecular layer are derived from the following sources: (I) The dendritic processes, and the recurrent collaterals of the axons, of the cells of Purkinje; (2) the dendritic processes and axons of the outer cells; (3) the dendritic processes of the inner or basket-cells; (4) the axons of the granule-cells of the granula1 
layer; (5) the fibres of Bergmann, which represent the processes of glia-cells in the granular layer; (6) the dendrons of the cells of Golgi; and (7) some fibres from the white medullary substance of the lamina.

The granular layer consists of (I) nerve- and glia-cells, and (2) fibres.

The nerve-cells are of two kinds-namely, granule-cells and cells of Golgi.

The granule-cells are small and very numerous. They are closely packed together and impart to this layer a granular appearance. Each has several dendrons and one axon. The dendrons soon ramify and the dendritic processes terminate in minute clusters within the granular layer, which are closely related to the granule-cells. The axon of each granule-cell passes into the molecular layer, where it ramifies, its branches diverging and being closely related to the dendritic processes of the cells of Purkinje.

The cells of Golgi lie near the cells of Purkinje, and are larger than the granule-cells. They are stellate, and each has several dendrons and an axon. The dendrons enter the molecular layer, in which they ramify. The axon ramifies very freely in the granular layer. The cells of Golgi may be regarded as association-cells.

The glia-cells are situated close to the cells of Purkinje, and lie between the outer granule-cells. Their superficial processes enter the molecular layer and constitute the fibres of Bergmann, which pass as far as the pia-matral covering of the lamina. Their deep processes pass between the granule-cells of the granular layer, and some of them enter the white medullary substance.

The fibres of the granular layer are derived from the following sources: (I) The axons of the cells of Purkinje; (2) the moss-fibres of Cajal; (3) the dendritic processes of the granule-cells; (4) the ramifications of the axons of the cells of Golgi; (5) some of the deep processes of the glia-cells; and (6) some fibres from the white medullary substance.

White Matter.--The white matter of a cerebellar lamina is an offshoot of the principal white medullary substance, and composed of nerve-fibres. (I) Some of these are the axons of the cells of Purkinje, and these enter the white matter. (2) Others pass through the granular layer into the molecular layer, where they divide into branches which are closely related to the more deeply placed dendritic processes of the cells of Purkinje. (3) A third set terminate in the granular layer, where they divide into branches which present moss-like swellings, furnished with short delicatc filaments. These fibres are known as the moss-fibres of Cajal.

Development of the Cerebellum.-The cerebellum is developed from the roof-plate of the metencephalon, which is the cephalic division of the rhombencepalon.

\section{The Fourth Ventricle.}

The fourth ventricle (ventriculus quartus B.) is situated behind (I) the upper or open half of the medulla oblongata or bulb and (2) the pons Varolii. It has two walls-ventral and dorsal. 
Ventral or Anterior Wall.--This wall is usually referred to as the floor. It is formed by (I) the dorsal surface of the upper or open half of the bulb, and (2) the dorsal surface of the pons Varolii, between the converging superior peduncles of the cerebellum. In shape it is rhomboidal, the bulbar and pontine parts being triangular and having their bases applied to each other. The floor is sometimes spoken of as the fossa rhomboidea.

The lower end is tapering and lies between the clavæ of the funiculi graciles. It constitutes the calamus scriptorius. In this situation the cavity of the ventricle is continuous with the central canal of the spinal cord, after that canal has traversed the lower or closed half of the bulb. The upper end is somewhat tapering and lies between the conierging superior peduncles of the cerebellum. In this situation the cavity of the ventricle is continued into the aqueduct of Sylvius, which traverses the mesencephalon and opens superiorly into the third ventricle.

The floor is widest across its centre, which is on a level with the upper ends of the restiform bodies of the bulb. The cavity of the ventricle is here prolonged on either side round the outer aspect of the corresponding restiform body towards the olive. This prolongation is known as the lateral recess.

The floor is covered by a thick layer of grey matter, which is continuous with the central grey matter of the lower or closed half of the bulb. This grey matter is covered by ependyma, the epithelial cells being continuous with those which line the central canal of the spinal cord.

The floor is traversed in the median line by a slight longitudinal groove, which divides it into two symmetrical longitudinal halves. Each half is crossed at its widest part by bundles of white fibres, called strice acustica. They wind round the upper part of the restiform body, and pass transversely across the corresponding half of the floor as far as the median longitudinal groove, into which they sink. They belong to the cochlear division of the auditory nerve, in connection with which they will be described.

The floor is divided into two parts, lower and upper, by the striæ acusticæ of either side.

Lower or Bulbar Part.- The bulbar part is formed by the dorsal surface of the upper or open part of the bulb. It is bounded on either side by (I) the clava of the funiculus gracilis, (2) the cuneate tubercle of the funiculus cuneatus, and (3) the restiform body, in this order from below upwards. The lower tapering end constitutes, as stated, the calamus scriptorius. The bulbar part is traversed in the median line by a longitudinal groove, already referred to, and this groove subdivides it into two symmetrical halves.

Immediately below the striæ acusticæ on either side, and not far from the median longitudinal groove, there is a small triangular depression, known as the fovea inferior. Its apex extends to the striæ acusticæ, and the lateral angles of its base are prolonged downwards as two grooves-inner and outer. The inner groove passes in a somewhat curved manner towards the point of the 
calamus scriptorius, and the outer groove passes downwards and outwards towards the lateral boundary. Between these two diverging grooves there is a triangular area, called the trigonum vagi. Its apex is at the fovea inferior, and its base is directed downwards and outwards. It has a dark colour, and from this circumstance it is known as the ala cinerea. Subjacent to it there is the dorsal nucleus of the vagus and glosso-pharyngeal nerves.

A second triangular area, called the trigonum hypoglossi, is situated between the median longitudinal groove and the inner of the two grooves prolonged from the angles of the base of the fovea inferior. Its base is directed upwards towards the striæ acusticæ, and its apex downwards towards the point of the calamus scriptorius. The area is slightly elevated, and is associated with the lower part of the eminentia teres. Subjacent to the inner part of this area is the upper part of the nucleus of the hypoglossal nerve.

A third triangular area, called the trigonum acusticum, is situated between the lateral boundary of the floor and the outer of the two grooves prolonged from the angles of the base of the fovea inferior. Its base, like that of the trigonum hypoglossi, is directed upwards, and is continued into an eminence, over which the striæ acusticæ pass. This eminence is known as the tuberculum acusticum. Subjacent to the trigonum acusticum and tuberculum acusticum there is the dorsal or chief terminal nucleus of the vestibular division of the auditory nerve.

Upper or Pontine Par. - The pontine part of the floor is formed by the dorsal surface of the pons Varolii between the converging superior peduncles of the cerebellum, which constitute its lateral boundaries. Its upper somewhat tapering end adjoins the lower end of the aqueduct of Sylvius. Like the bulbar part, it is traversed in the median line by a longitudinal groove, already referred to, which subdivides it into two symmetrical halves.

Immediately above the striæ acusticæ, and in line with the fovea inferior, there is a slight depression, called the fovea superior, the two foveæ being separated from each other by the tuberculum acusticum. Between the fovea superior and the median longitudinal groove there is a well-marked prominence, called the eminentia teres or medialis. Subjacent to this eminence, immediately above the striæ acusticæ, is the abducent nucleus, or nucleus of the sixth cranial nerve. The eminence is continued downwards into the trigonum hypoglossi, and superiorly it extends towards the lower end of the aqueduct of Sylvius. Extending upwards from the fovea superior towards the region of the lower end of the aqueduct of Sylvius there is a slight depression, known as the locus cæruleus, which has a dark grey or somewhat blue colour. This colour is due to a subjacent group of deeply pigmented nerve-cells, known as the substantia ferruginea. This group may belong to the chief motor nucleus of the fifth cranial nerve, or it may be a terminal nucleus for some of the sensory fibres of that nerve.

Dorsal or Posterior Wall of Fourth Ventricle.-This wall is 
usually referred to as the roof, and it is divisible into two partsupper and lower.

The upper part is formed chiefly by the superior or anterior medullary velum, or valve of Vieussens, which extends between the inner margins of the dorsal aspects of the superior peduncles of the cerebellum. It is also formed to a certain extent by these peduncles as they converge and slightly overhang the angular space between them. The lower part of the roof is formed, from above downwards, by (I) the inferior or posterior medullary velum, and (2) the ependymal epithelium of the ventricle, covered by pia mater. The inferior medullary velum succeeds to the superior medullary velum and terminates in a free margin. Beyond this

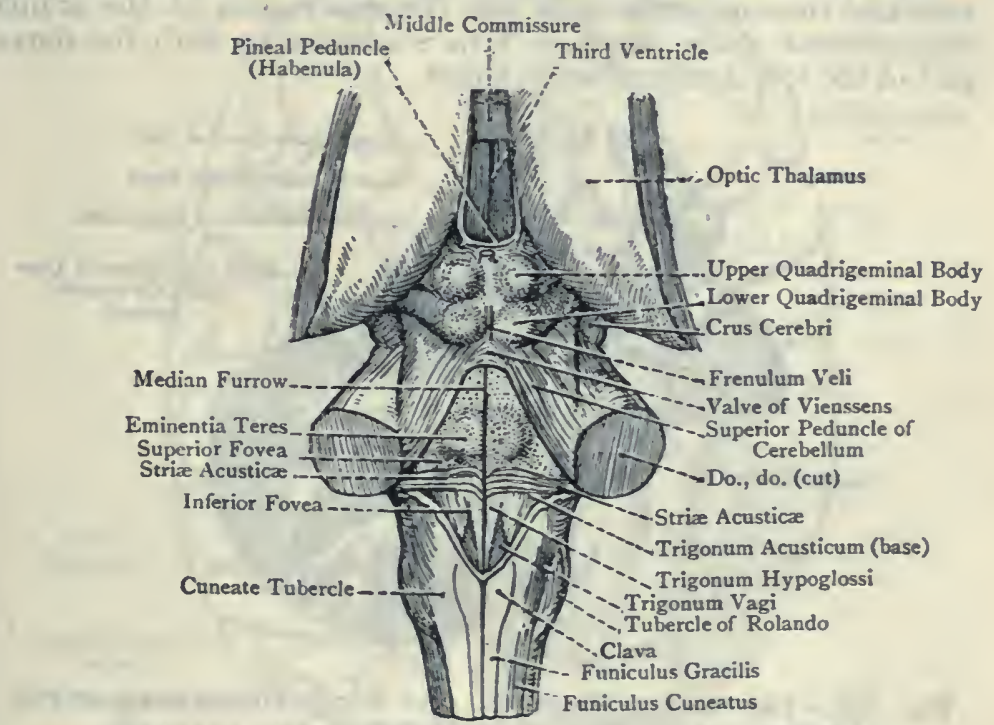

Fig. 577. -The Floor of the Fourth Ventricle and Adjacent Parts.

free margin there is the ependymal epithelium of the ventricle, covered, as stated, by pia mater. This portion of pia mater is called the tela choroidea inferior.

The epithelial part of the roof presents superficially three laminæ of white nervous matter-namely, the obex and the ligulæ. The obex is a thin triangular lamina which is situated at the point of the calamus scriptorius, being attached laterally to the diverging clavæ. The ligula are two in number, right and left. Each ligula is a narrow band which is continuous inferiorly with the obex. It is attached inferiorly to the clava and the cuneate tubercle. It then passes transversely outwards over the dorsal aspect of the restiform body. The transverse part of the ligula forms the lower boundary of the lateral recess of the ventricle. 
Openings in the Roof.-These openings are three in number-one median and two lateral. They are situated in the non-nervous part of the roof-that is to say, the part formed by the ependymal epithelium of the ventricle, covered by pia mater, called the tela choroidea inferior; and they penetrate both of these layers lepithelial and pia-matral). The median opening is known as the foramen of Magendie, and is situated immediately above the obex. The lateral openings, right and left, constitute the foramina of Luschka. Each is situated in the roof of the corresponding lateral recess, where that recess terminates.

By means of these three foramina-foramen of Magendie and foramina of Luschka, right and left-the cavity of the fourth ventricle communicates with the cisterna magna of the cranial subarachnoid space, which in turn is continuous with the dorsal part of the spinal subarachnoid space.

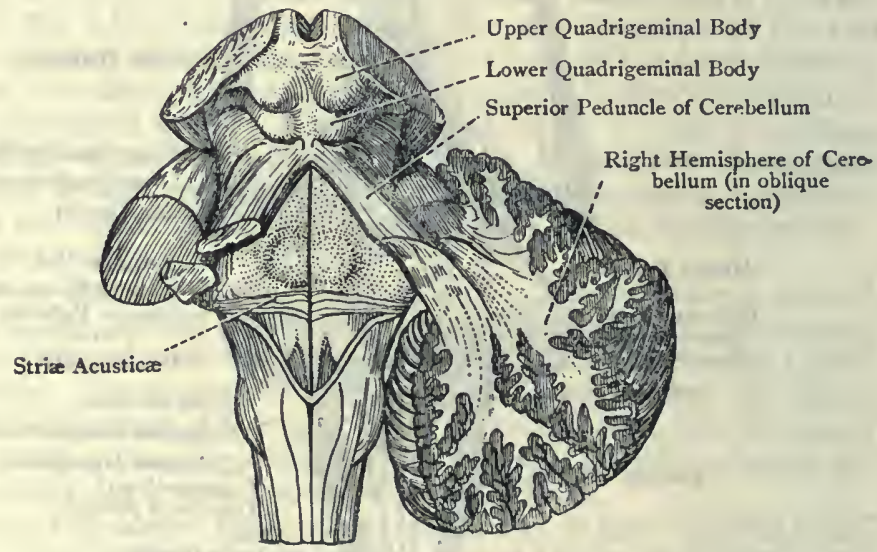

Fig. 578. -The Fourth Ventricle and Right Hemisphere of the Cerebellum (in Section) (Hirschfeld and Leveille).

Choroid Plexuses of Fourth Ventricle.-These are two in number, right and left. Each is a longitudinal inflexion of the pia mater which forms the tela choroidea inferior, and it invaginates the ependymal epithelium of the lower part of the roof of the ventricle, by which it is covered on its ventricular surface. Each choroid plexus consists of two parts-longitudinal and transverse, and the two plexuses are disposed somewhat thus: $7[$. The longitudinal parts lie on either side of the median line, and extend upwards from the region of the foramen of Magendie. Each transverse part extends outwards into the corresponding lateral recess of the ventricle.

Development of Fourth. Ventricle.-The lower or bulbar part is developed from the myelencephalon; and the upper or pontine part is developed from the metencephalon, these being the two divisions of the rhombencephalon. 


\section{TELENCEPHALON.}

\section{Cerebral Hemispheres.}

The cerebral hemispheres are two in number, right and left. Each is semi-ovoid, and presents two extremities and three surfaces.

The extremities are anterior and posterior. The anterior extremity is thick and round, and its most projecting part is called

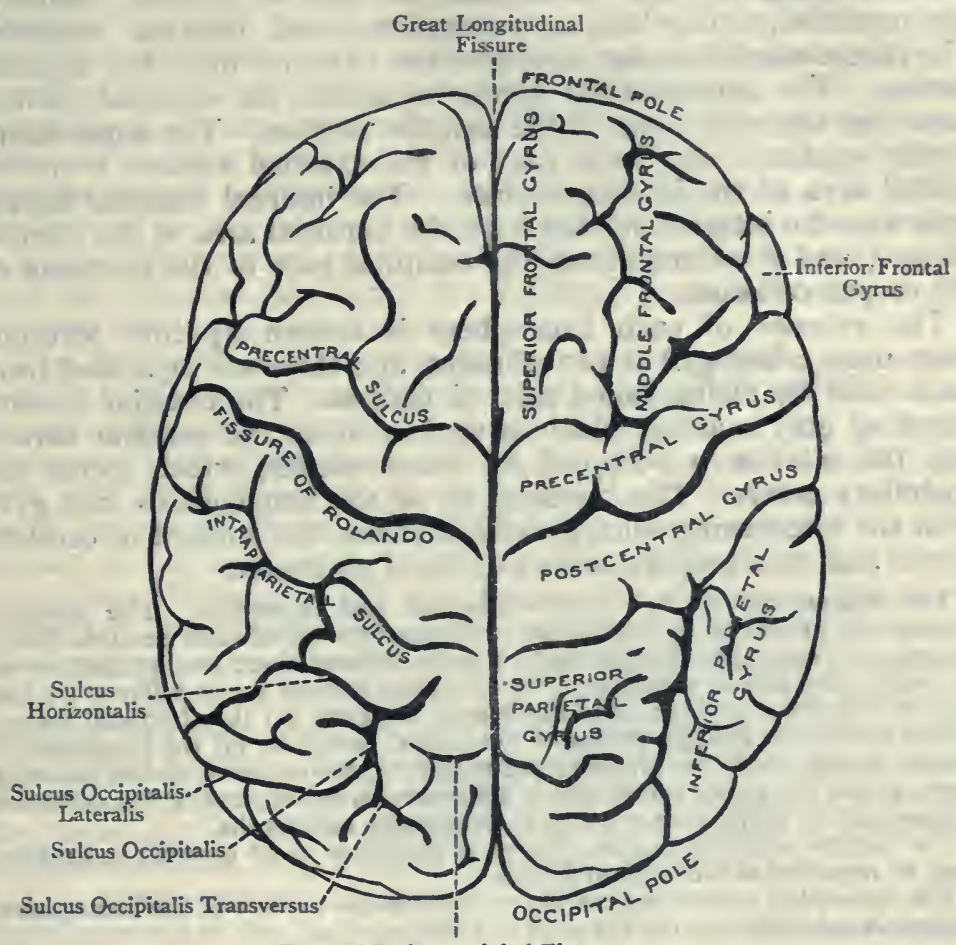

External Parieto-occipital Fissure

Fig. 579,-The Cerebral Hemispheres (Superior View).

the frontal pole. The posterior extremity is narrow and pointed, and its most projecting part is called the occipital pole. The surfaces are external, internal, and inferior. The external surface is convex, in adaptation to the concavity of the cranial vault. The internal surface is flat and vertical, and it forms the lateral boundary of the great longitudinal fissure. For the most part it is in contact with the falx cerebri. The inferior surface is irregular, being adapted to the corresponding lateral divisions of the anterior and middle fossæ of the interior of the base of the skull and the upper surface of the tentorium cerebelli. It is crossed transversely by a deep cleft, representing the stem of the fissure of Sylvius. The 
portion in front of this fissure is known as the orbital area, and is concave, in adaptation to the convexity of the orbital plate of the frontal bone, upon which it rests. The extensive portion behind the stem of the Sylvian fissure is known as the tentorial area, and is prominent and arched. Its anterior portion is received into the lateral division of the middle cranial fossa, and its posterior portion rests upon the tentorium cerebelli.

The borders of each hemisphere are four in number, namely, supero-mesial, infero-lateral, superciliary, and internal occipital. The supero-meslal border separates the external from the internal surface. The infero-lateral border separates the external surface from the tentorial area of the inferior surface. The superciliary border separates the front part of the external surface from the orbital area of the inferior surface. The internal occipital border separates the internal surface from the tentorial area of the inferior surface, and it extends from the occipital pole to the splenium of the corpus callosum.

The exterior of each hemisphere is broken up into tortuous eminences, called gyri or convolutions, and these are separated from each other by clefts, called sulci or fissures. The exterior is composed of grey matter, which is spoken of as the cerebral cortex, and the interior is occupied by white matter, which forms the medullary centre. The breaking up of the hemispheres into gyri, with the intervening sulci, greatly increases the amount of cerebral cortex and, to a proportionate extent, of pia mater.

The fissures are of two kinds-complete and incomplete. The complete fissures are produced by infoldings of the entire wall of each cerebral hemisphere, and corresponding to each of these fissures there is a projection on the wall of the lateral ventricle. The complete fissures are as follows: (I) The fissure of Sylvius, giving rise to the corpus strlatum; (2) the choroidal fissure, giving rise to the chorold plexus of the lateral ventricle; (3) the hippocampal fissure, giving rise to the hippocampus major; (4) the calcarine fissure (anterior part), giving rise to the calcar avis or hippocampus minor; and (5) the collateral fissure (middle part), giving rise to the emlnentia collateralis.

The parieto-occipital fissure, though it gives rise to no internal projection, may be regarded as a complete fissure.

The incomplete fissures are merely furrows on the surface of the hemisphere, formed in association with the growth of the gyri.

Each hemisphere presents six principal fissures, called interlobar fissures, and by means of these it is divided into six lobes. The fissures and lobes are as follows :

\section{Interlobar Fissures.}

I. Fissure of Sylvius.

2. Fissure of Rolando.

3. Parieto-occipital.

4. Calloso-marginal.

5. Collateral.

6. Circular or Limiting.
Lobes.

I. Frontal.

2. Parietal.

3. Occipital.

4. Temporal.

5. Island of Reil or Insula.

6. Limbic.

Interlobar Fissures.-The fissure of Sylvius, which is the first fissure to appear in the course of development, commences on the inferior surface of the hemisphere at the locus perforatus anticus in a depression, called the vallecula Sylvii. From this point it passes 
horizontally outwards to the Sylvian point on the external surface of the hemisphere, where it divides into three diverging branches. It is a deep cleft, which is overhung posteriorly by the front part of the temporal lobe, and it separates the orbital surface of the frontal lobe from the temporal lobe. The posterior or Sylvian border of the small wing of the sphenoid bone faces the fissure, which lodges the middle cerebral artery. The limbs into which the fissure divides at the Sylvian point are, as stated, three in number, namely, anterior horizontal, anterior ascending, and posterior horizontal. The anterior horizontal limb passes forwards into the frontal lobe, its length being about $\frac{3}{4}$ inch. The anterior ascending limb passes upwards and slightly forwards into the frontal lobe for about I inch, but its length is variable. The posterior horizontal limb is the longest and most conspicuous. It passes backwards on the external surface of the hemisphere for at least 2 inches, having portions of the frontal and parietal lobes above it, and the temporal lobe below it. Finally it turns upwards into the parietal lobe for a very short distance.

The fissure of Rolando, also known as the central sulcus, commences at the supero-mesial border of the hemisphere, a little behind its mid-point, and terminates above the centre of the posterior horizontal limb of the fissure of Sylvius. It does not usually open into this limb, but may do so. Superiorly the fissure in most cases intersects the supero-mesial border to reach the internal surface of the hemisphere, upon which it passes backwards for a very short distance. The direction of the fissure of Rolando is irregularly downwards and forwards over the external surface of the hemisphere, and it separates the frontal from the parietal lobe. It describes two bends. The upper genu has its concavity directed forwards, and is situated about the junction of the upper and middle thirds of the fissure. The lower genu has its concavity directed backwards, and is situated on a more anterior plane than the upper genu. Below the lower genu the direction of the fissure is almost vertical, with a slight inclination backwards.

The parieto-occlpital fissure is situated about 2 inches behind the upper end of the fissure of Rolando, and it separates the parietal from the occipital lobe. It is composed of two limbs, external and internal, which are continuous with each other at the supero-mesial border of the hemisphere, where they form a right angle. The external limb is situated on the external surface of the hemisphere, upon which it passes transversely outwards for about $\frac{3}{4}$ inch, when it is arrested by the convolution which connects the parietal and occipital lobes. The internal limb appears as a deep, almost vertical, cleft on the internal surface of the hemisphere, which opens into the calcarine fissure a short distance behind the splenium of the corpus callosum.

The calloso-marginal fissure is situated on the internal surface of the hemisphere. It commences below the rostrum of the corpus callosum, contiguous to the locus perforatus anticus, and, bending round the genu, it passes backwards above the corpus callosum, 
from which it is separated by the callosal gyrus. At a point a little behind the centre of the internal surface of the hemisphere it turns upwards and terminates at the supero-mesial border, a short distance behind the upper end of the fissure of Rolando. The callosomarginal fissure lies between the frontal and limbic lobes, the marginal gyrus being above it and the callosal gyrus below it.

The collateral fissure is situated on the inferior surface of the hemisphere. It commences near the occipital pole, and extends forwards to near the temporal pole. Posteriorly it has the calcarine fissure above, and in line with, it, and anteriorly it has the hippor campal gyrus on its mesial side. It separates the temporal lobe from the hippocampal portion of the limbic lobe. The middle

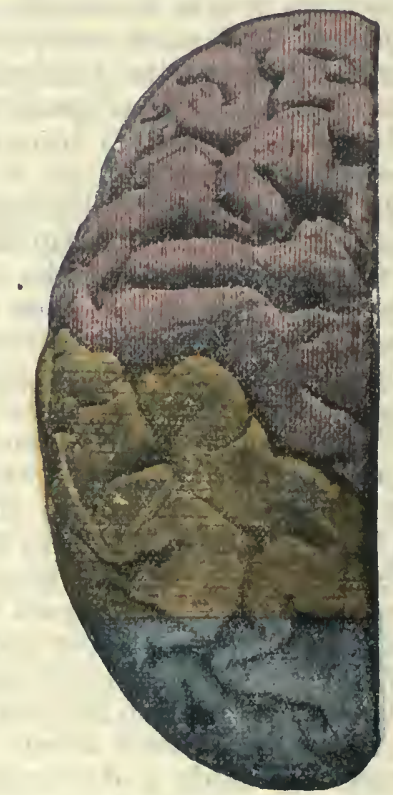

Fig. 580. - The Left Cerebral Hemisphere (Superior Surface).

Red $=$ Frontal Lobe. Orange $=$ Parietal Lobe. Blue $=$ Occipital Lobe.

portion of the collateral fissure gives rise to the eminentia collateralis in the floor of the lateral ventricle.

The circular or limiting fissure is situated deeply in the anterior part of the posterior horizontal limb of the fissure of Sylvius. It almost surrounds the convolutions which constitute the island of Reil or insula, and is composed of three parts-superior, inferior, and anterior. The superior part separates the insula from the frontal and parietal lobes, the inferior part separates it from the temporal lobe, and the anterior pari separates it from the frontal lobe. The circular fissure is deficient in the region of the apex of the insula. 
Lobes of the Cerebral Hemisphere-Frontal Lobe.-This is of large size. On the external surface of the hemisphere it is bounded behind by the fissure of Rolando, and below by the posterior horizontal limb of the fissure of Sylvius. On the inferior surface it is bounded behind by the stem of the fissure of Sylvius. On the internal surface it is bounded by the calloso-marginal fissure. The frontal lobe has three surfaces-external, inferior, and internal.

External Surface.-This surface presents three principal sulci, namely, precentral, superior frontal, and inferior frontal.

The precentral sulcus is more or less parallel to the fissure of Rolando, the ascending frontal or precentral gyrus intervening between the two. It may be a single fissure, but it more frequently consists of two parts, superior and inferior. The superior part is usually joined above by the superior frontal sulcus. The inferior

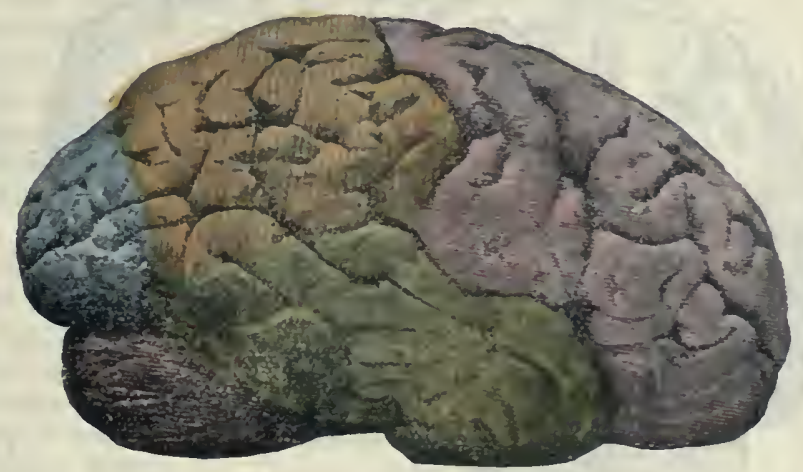

Fig. 58r.-The Right Cerebral and Cerebellar Hemispheres (External SURFACE).

Red = Frontal Lobe.

Orange $=$ Parietal Lobe.

Blue= Occipital Lobe.

Green = Temporal Lobe. Purple $=$ Cerebellar Hemisphere.

part passes superiorly into the middle frontal gyrus for a short distance in a forward and upward direction.

The superior and inferior frontal sulci extend forwards from the precentral sulcus.

The gyri of the external surface are as follows : ascending frontal, superior frontal, middle frontal, and inferior frontal.

The ascending frontal or precentral gyrus is bounded behind by the fissure of Rolando or central sulcus, and in front by the superior and inferior parts of the precentral sulcus. It extends from the supero-mesial border of the hemisphere to a little behind the Sylvian point, which corresponds to the place where the stem of the fissure of Sylvius appears on the external surface of the hemisphere, and divides into its three branches. Below the lower end of the fissure of Rolando it is, as a rule, connected with the ascending parietal or postcentral gyrus by an annectant gyrus. 
The supericr or first, middle or second, and inferior or third frontal gyri are arranged in tiers, which are disposed antero-posteriorly, the first and second being usually each subdivided, so as to make five tiers in all. They are separated from the ascending frontal or precentral gyrus by the superior and inferior parts of the precentral sulcus.

The superior frontal gyrus is narrow, and lies between the superomesial border of the hemisphere and the superior frontal sulcus. It is continuous with the marginal gyrus on the internal surface of

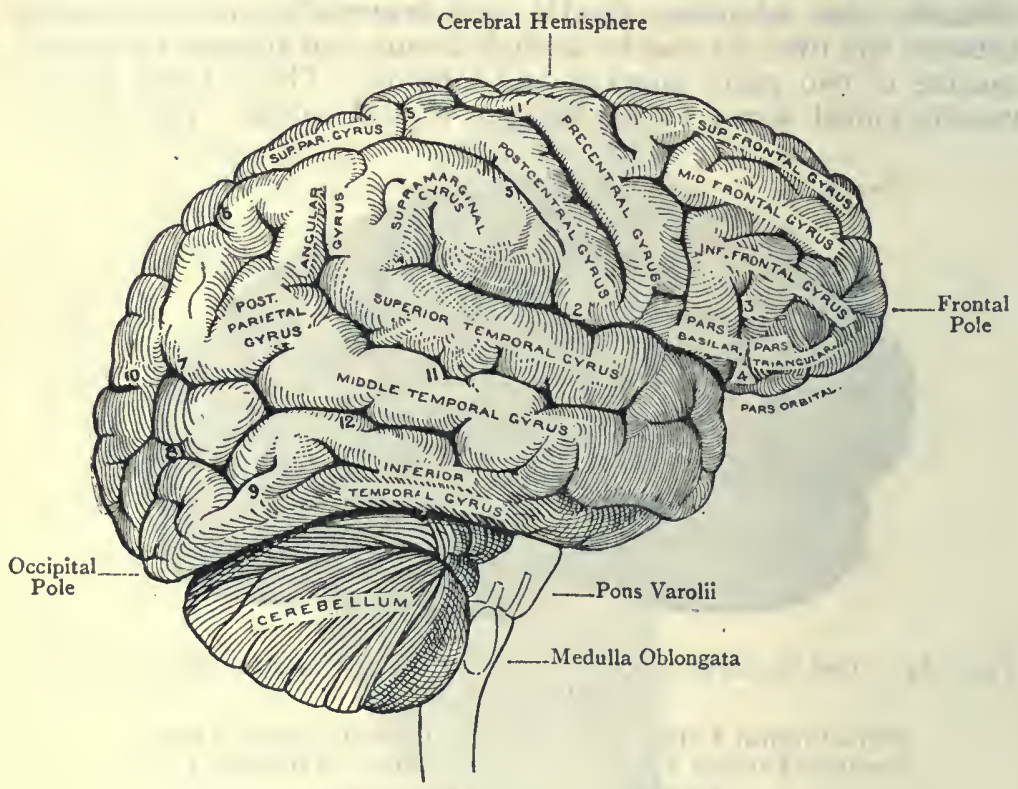

Fig. 582.-The Encephalon (Right Lateral View) (Hirschfeld AND LEVEILlÉ).

1. Fissure of Rolando.

2. Posterior Horizontal Iimb of Fissure of Sylvius.

3. Anterior Ascending Limb of Fissure of Sylvius.

4. Anterior Horizontal Limb of Fissure of Sylvius.

5, 5. Intraparietal Sulcus.
6. Ramus Horizontalis.

7. Ramus Occipitalis.

8. Transverse Occipital Sulcus.

9. Lateral Occipital Sulcus.

10. External Part of Occipito-Parietal Fissure.

x. Superior Temporal, or Parallel, Sulcus:

12. Inferior Temporal Sulcus.

the hemisphere, and is partially broken up into two parts, upper and lower.

The middle frontal gyrus, which is broad, lies between the superior and inferior frontal sulci, and is usually connected with the ascending frontal or precentral gyrus by an annectant gyrus. It is broken up anteriorly into two parts, upper and lower, by an antero-posterior secondary sulcus ; and it is cut into behind by the upper portion of the inferior part of the precentral sulcus. 
The inferior frontal gyrus lies below the inferior frontal sulcus, and in front of the lower part of the precentral sulcus. The anterior horizontal and the ascending limbs of the fissure of Sylvius enter it and subdivide it into three parts, namely, pars orbitalis, pars triangularis, and pars basilaris. The pars orbitalis lies below the anterior horizontal limb of the fissure of Sylvius ; the pars triangularis is situated between the anterior horizontal and the ascending limbs of the fissure of Sylvius; and the pars basilaris is placed between the ascending limb of the fissure of Sylvius and the lower part of the precentral sulcus. The inferior frontal gyrus is connected posteriorly with the lower end of the ascending frontal or precentral gyrus by an annectant gyrus.

The inferior frontal gyrus of the left side is specially important, inasmuch as the speech-centre is assigned to the portion of it which forms the pars triangularis (le cap of Broca).

Inferior or Orbital Surface of the Frontal Lobe.-This surface presents two sulci, olfactory and orbital.

The olfactory sulcus is parallel to the mesial border, from which it is separated by the gyrus rectus. It lodges the olfactory tract and olfactory bulb. The orbital sulcus (triradiate sulcus of Turner) is of very variable form, but, as a rule, bears some resemblance to the letter $\mathbf{M}$. It has therefore three limbs-inner, outer, and transverse. The inner limb is separated from the olfactory sulcus by the internal orbital gyrus. The outer limb is curved, and has external to it the orbital part of the inferior frontal gyrus. The transverse limb passes in a more or less curved manner between the inner and outer limbs.

The gyri of the orbital surface are as follows: gyrus rectus, internal orbital gyrus, anterior orbital gyrus, external orbital gyrus, and posterior orbital gyrus.

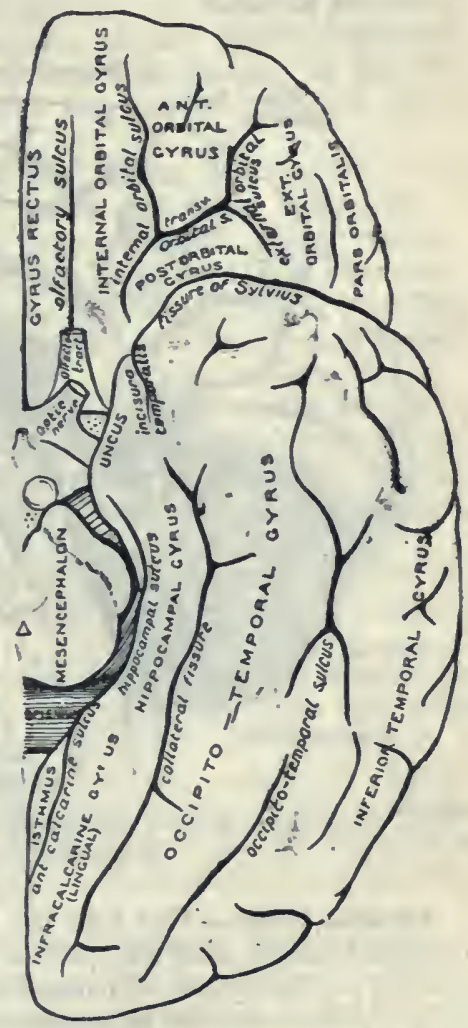

Fig. 583. -The Inferior Surface of the Left Cerebral HemiSPHERE, SHOWING THE GYRI AND Sulci.

The gyrus rectus lies between the olfactory sulcus and the mesial border. The internal orbital gyrus is placed between the olfactory sulcus and the inner limb of the orbital sulcus. The external 
orbital gyrus is external to the outer limb of the orbital sulcus. The anterior orbital gyrus is situated in front of the transverse limb of the orbital sulcus. The posterior orbital gyrus lies behind the transverse limb of the orbital sulcus.

Internal Surface of the Frontal Lobe.-The internal or mesial surface presents only one convolution, namely, the marginal gyrus, which is situated between the supero-mesial border of the hemisphere and the calloso-marginal sulcus. It is continuous with the superior frontal gyrus, and anteriorly is broken up by one or two sulci. Its posterior part is almost completely detached, and forms the paracentral lobule, which contains the upper end of the fissure of Rolando.

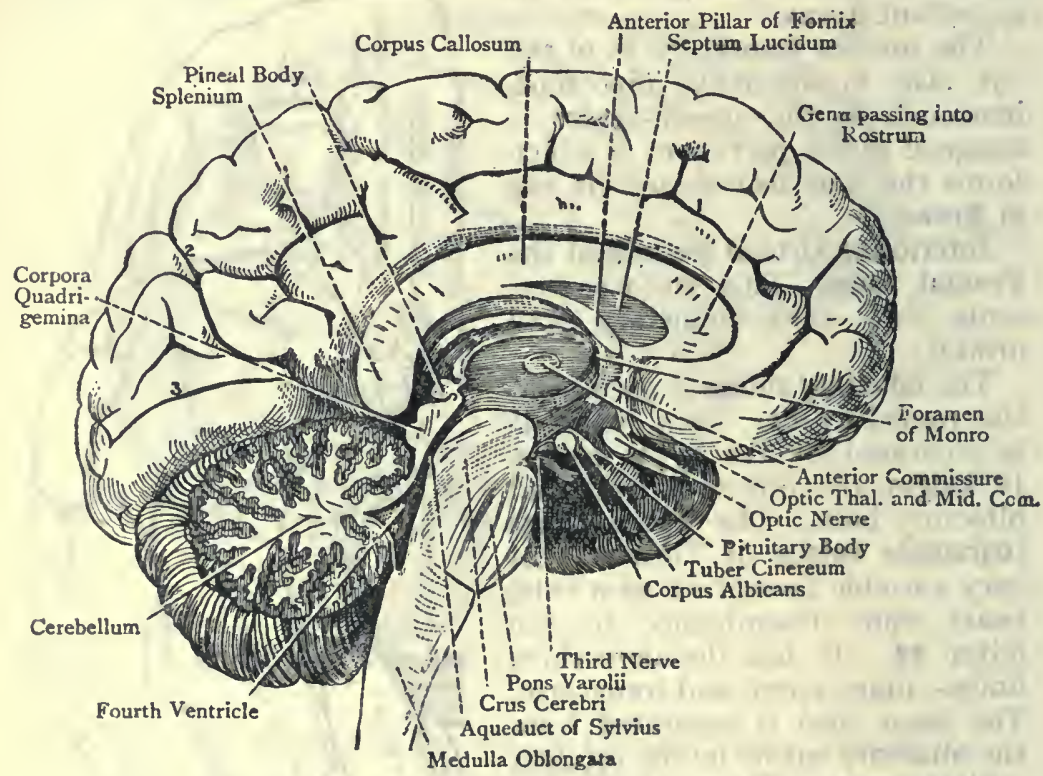

Fig. 584.-The Mestal Surface of the Left Cerebral Hemisphere (HirschFeld AND LEVEILLÉ).

Parietal Lobe.-This lobe lies between the large frontal and small occipital lobes, and above the temporal lobe. It is bounded anteriorly by the fissure of Rolando, which separates it from the frontal lobe. Posteriorly it is 'bounded by (I) the external part of the parieto-occipital fissure, and (2) a line drawn across the external surface of the hemisphere from the extremity of this fissure towards the pre-occipital notch on the infero-lateral border of the hemisphere, from $I_{2}^{1}$ to 2 inches in front of the occipital pole.

The parietal lobe has two surfaces-external and internal. 
External Surface.-This surface presents the following sulci: the intraparietal sulcus, composed of four parts; and the terminal portions of $(a)$ the posterior limb of the fissure of Sylvius, (b) the first temporal or parallel sulcus, and (c) the second temporal sulcus.

The intraparietal sulcus (Turner) is composed of four parts, namely, inferior postcentral, superior postcentral, ramus horizontalis, and ramus occipitalis.

The inferior and superior postcentral sulci may be distinct, or continuous with each other. They lie behind the fissure of Rolando, with which they are parallel, and from which they are separated by the ascending parietal or postcentral gyrus.

The ramus horizontalis passes backwards and slightly upwards from the upper end of the inferior postcentral sulcus. It has the superior parietal gyrus above it, and the inferior parietal gyrus below it.

The ramus occipitalis lies behind the ramus horizontalis, with which it is usually connected. It forms the outer boundary of the gyrus which arches round the external part of the parietooccipital fissure, namely, the arcus parieto-occipitalis (first annectant convolution). Posteriorly the ramus occipitalis divides into two short sulci, which diverge, one ascending, and the other descending, in the occipital lobe.

The terminal portions of $(a)$ the posterior limb of the fissure of Sylvius, $(b)$ the first temporal or parallel sulcus, and $(c)$ the second temporal sulcus, are confined to the lower part of the external surface of the parietal lobe, where they lie in the order named, from before backwards.

The gyri of the external surface are as follows : ascending parietal ; superior parietal; and inferior parietal, with its supramarginal, angular, and postparietal gyri.

The ascending parietal or postcentral gyrus is situated immediately behind the fissure of Rolando, which separates it from the ascending frontal or precentral gyrus in front of that fissure. Posteriorly it is limited by the superior and inferior postcentral sulci. It extends from the supero-mesial border of the hemisphere to the posterior horizontal limb of the fissure of Sylvius, and it lies parallel to the ascending frontal or precentral gyrus, with which it is connected below the fissure of Rolando.

The superior parietal gyrus is situated between the ramus horizontalis and the supero-mesial border of the hemisphere, where it is continuous with the quadrate lobule, or pracuneus, of the interna] surface. Anteriorly it is limited by the superior postcentral sulcus, round the upper end of which it is continuous with the ascending parietal gyrus. Posteriorly it is bounded by the external part of the parieto-occipital fissure, round the extremity of which it is connected with the occipital lobe by the arcus parieto-occipitalis (first annectant convolution).

The inferior parietal gyrus is situated behind the inferior post- 
central sulcus, and below the ramus horizontalis and ramus occipitalis. It is broken up into three gyri, namely, supramarginal, angular, and postparietal, in this order from before backwards. The supramarginal gyrus arches round the ascending extremity of the posterior limb of the fissure of Sylvius. It is continuous in front with the ascending parietal gyrus below the lower end of the inferior postcentral sulcus; posteriorly with the angular gyrus; and postero-inferiorly with the first temporal gyrus. The angular gyrus arches round the ascending extremity of the first temporal or parallel sulcus, and is continuous with the second temporal gyrus. The postparietal gyrus arches round the ascending extremity of the second temporal sulcus, and is continuous with the third temporal gyrus.

Internal Surface of the Parietal Lobe.-The internal or mesial

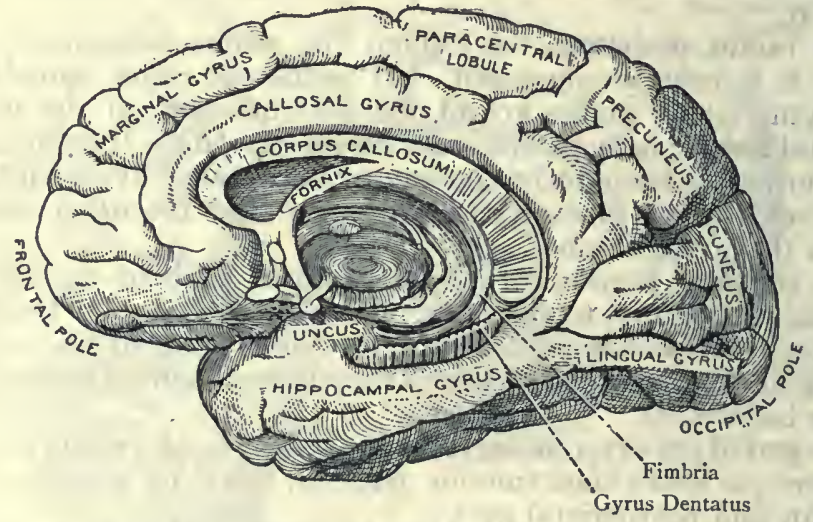

Fig. 585.-The Mesial Surface of the Right Cerebral Hemisphere (HiRsChFELd AND LEVEILlê).

surface is of quadrilateral outline, and constitutes the quadrate lobule or præcuneus. It is bounded in front by the upturned posterior extremity of the calloso-marginal sulcus, behind by the internal part of the parieto-occipital fissure, and below by the postlimbic sulcus and a portion of the limbic lobe.

Occipital Lobe. - This lobe lies behind the parietal and temporal lobes, and forms the posterior part of the cerebral hemisphere.

Externally the lobe is bounded in front by the external part of the parieto-occipital fissure, and a line connecting this fissure with the pre-occipital notch on the infero-lateral border of the hemisphere. Internally it is bounded in front by the internal part of the parieto-occipital fissure, which separates it from the quadrate lobule, or præcuneus, of the parietal lobe. Inferiorly it is continuous with the temporal and limbic lobes, but the separation may be indicated by a line connecting the pre-occipital notch with the 
portion of the limbic lobe which lies below the splenium of the corpus callosum, this portion being known as the isthmus of the limbic lobe.

The occipital lobe is pyramidal, and presents an apex and three surfaces-external, internal, and inferior.

The apex forms the occipital pole of the cerebral hemisphere.

External Surface.-This surface presents two sulci, transverse occipital and lateral occipital. The transverse occipital sulcus is formed by the bifurcation of the posterior end of the ramus occipitalis of the intraparietal sulcus, and it crosses the upper part of the occipital lobe transversely. Its upper limb lies a little behind the external part of the parieto-occipital fissure, from which it is separated by a portion of the arcus parieto-occipitlais; and its lower limb is behind the post-parietal gyrus. The lateral occipital sulcus is situated on the external surface of the occipital lobe, and extends almost horizontally from behind forwards. It divides the external surface of the lobe into two parts, upper and lower, which are connected with the parietal and temporal lobes by annectant gyri.

Internal Surface. - The internal or mesial surface presents the ealcarine fissure. This is a deep cleft which commences on the internal aspect of the occipital pole in a bifurcated manner. It takes a curved course forwards, passing at first upwards and then downwards, and it terminates by entering the limbic lobe beneath the splenium of the corpus callosum. It is joined at a point anterior to its centre by the internal part of the parieto-occipital fissure, and between the two fissures is the cuneus. The caicarine fissure may be regarded as being composed of two parts, anterior, representing the portion in front of the internal part of the parietooccipital fissure, and posterior, representing the portion behind that fissure. The anterior calcarine fissure gives rise to the hippocampus minor, or calcar avis, on the inner wall of the posterior cornu of the lateral ventricle.

The gyri of the internal surface are two in number, namely, the cuneus and the gyrus lingualis.

The cuneus is triangular, and is wedged in between the posterior calcarine fissure and the internal part of the parieto-occipital fissure. The gyrus lingualis (infracalcarine gyrus) is situated between the calcarine fissure above and the posterior part of the collateral fissure below. Anteriorly it becomes narrow, and joins the hippocampal gyrus. The lower portion of this gyrus is visible on the inferior surface of the lobe.

Inferior Surface.-The inferior or tentorial surface presents the posterior part of the occipito-temporal gyrus, internal to which is the posterior part of the collateral fissure, and internal to this again there is the lower portion of the gyrus lingualis.

Temporal Lobe.-The temporal (temporo-sphenoidal) lobe is prominent, and of large size. It is situated below the posterior horizontal limb of the fissure of Sylvius, and behind the stem of that fissure. Superiorly it is bounded by (I) the horizontal portion 
of the posterior limb of the fissure of Sylvius, and (2) a line prolonging this limb backwards to meet the posterior boundary of the parietal lobe. Anteriorly it is bounded by the stem of the fissure of Sylvius, which separates it from the orbital area of the frontal lobe. Posteriorly it is continuous with the occipital lobe, but the separation may be indicated by the following lines: externally by a line connecting the extremity of the external part of the parietooccipital fissure with the pre-occipital notch, and inferiorly by a line connecting the pre-occipital notch with the portion of the limbic lobe which lies below the splenium of the corpus callosum (isthmus of the limbiclobe). Inferiorly it is separated from the hippocampal gyrus by the collateral fissure. The temporal lobe is somewhat pyramidal, the rounded apex being directed forwards. The apical part forms the temporal pole, and it overhangs from behind the stem of the fissure of Sylvius. The uncus of the hippocampal gyrus lies on its inner side, but on a more posterior level, and separated from it by the temporal sulcus.

The lobe presents three surfaces-superior, external, and inferior.

The superior or opercular surface is concealed within the fissure of Sylvius, and is directed towards the island of Reil.

The external surface presents two horizontal sulci and three convolutions, the latter being disposed in horizontal tiers.

The sulci are called first temporal and second temporal, respectively. The first temporal sulcus is parallel to the posterior limb of the fissure of Sylvius, from which circumstance it is called the parallel sulcus. It commences near the temporal pole, and posteriorly it turns upwards into the parietal lobe, where the angular gyrus arches round it. The second temporal sulcus is parallel to the first, below which it lies, and it is usually broken up into two or more parts by annectant gyri. Posteriorly it turns upwards into the parietal lobe, where the postparietal gyrus curves round it.

The gyri of the external surface, as stated, are arranged in horizontal tiers, and are three in number, first, second, and third, in this order from above downwards.

The first temporal gyrus is situated between the posterior limb of the fissure of Sylvius and the parallel sulcus. Posteriorly it is continuous with the supramarginal, and slightly with the angular, gyri of the parietal lobe. The second temporal gyrus lies between the parallel and second temporal sulci, and posteriorly is continuous with the angular and postparietal gyri of the parietal lobe. The third temporal gyrus lies below the second temporal sulcus, and posteriorly is continuous with the lower part of the external surface of the occipital lobe.

The inferior or tentorial surface of the temporal lobe presents the occipito-temporal sulcus and the occipito-temporal gyrus. The occipito-temporal sulcus extends from before backwards, lying near the infero-lateral margin of the hemisphere, and external to the collateral fissure. It is usually broken up into parts by annectant 
convolutions. The occipito-temporal gyrus is situated between the occipito-temporal sulcus and the collateral fissure, and extends from the occipital pole to the temporal pole. External to the occipito-temporal sulcus there is the narrow inferior or tentorial surface of the third temporal gyrus.

Island of Reil, Central Lobe, or Insula.-This lobe is situated deeply within the fissure of Sylvius, and is concealed from view by the opercular gyri, to be presently described. It is triangular, the apex being directed downwards towards the vallecula Sylvii and locus perforatus anticus. The circular or limiting sulcus being here absent, the grey matter of the apex is continuous with that of the locus perforatus anticus, this point being called the limen insula. Elsewhere the island of Reil is surrounded by the circular or limiting sulcus, which has been already described. The insula presents several sulci, which diverge as they pass from the apical region to the base, and these map it out into gyri. One of these sulci is known as the sulcus centralis insulæ. It extends from the apex to the base in an upward and backward direction, almost in line with the fissure of Rolando, and it divides the insula into two lobules, precentral and postcentral.

The precentral lobule is composed of three short gyri, called the gyri breves, which converge as they descend from the base, but they do not reach the apex or pole of the precentral lobule. The postcentral lobule is formed by the gyrus longus, which is usually broken up into two gyri towards the base of the insula.

The direct internal or mesial relation of the insula is the claustrum, internal to which there are, in succession, the external capsule, the nucleus lenticularis, the internal capsule, and the nucleus caudatus.

The insula and the corpus striatum represent the stem of the cerebral hemisphere, whilst the remainder of the hemisphere is known as the pallium or mantle. 
Opercula Insulæ.-The parts of the cerebral hemisphere which bound the three limbs of the fissure of Sylvius and overhang the insula are called the opercula insulæ. They are four in number, namely, fronto-parietal, temporal, frontal, and orbital.

The fronto-parietal operculum forms the upper lip, and the temporal operculum forms the lower lip, of the posterior limb of the fissure of Sylvius. The frontal operculum represents the pars triangularis of the inferior frontal gyrus-that is to say, the part which lies between the ascending and the anterior limbs of the fissure of Sylvius. The orbital operculum represents the pars orbitalis of the inferior frontal gyrus, and lies below the anterior limb of the fissure of Sylvius.

Limbic Lobe.-This lobe is situated on the internal or mesial surface of the cerebral hemisphere. It is composed of two convo-

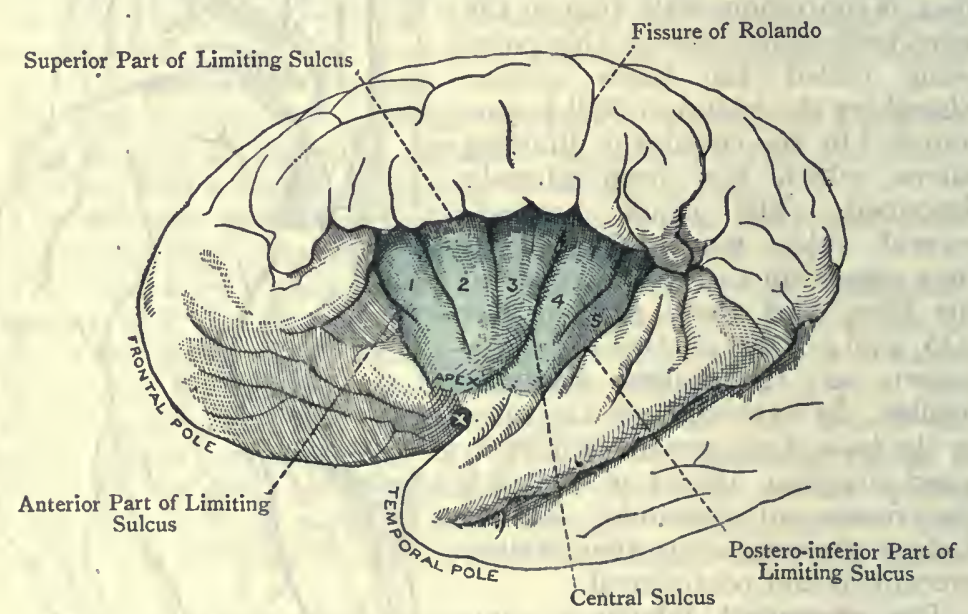

Fig. 587.-The Leff. Island of Reil (Poirier, from Eberstaller).

I, 2, 3, Gyri Breves ; 4, 5, Gyri Longi ; x, Limen Insulæ.

lutions, namely, the callosal gyrus, or gyrus fornicatus, and the hippocampal gyrus, so disposed as to form an almost complete ring round the corpus callosum and subjacent parts. The open part of the ring, which is very small, is situated at the locus perforatus anticus, and is occupied by the roots of the olfactory tract. Around its circumference the limbic lobe is bounded superiorly by the calloso-marginal fissure, posteriorly by the postlimbic sulcus, and inferiorly by the anterior part of the collateral fissure and the incisura temporalis. The callosal and hippocampal gyri become continuous beneath the splenium of the corpus callosum, the connecting portion, which is narrow, being known as the $i$ sthmus of the limbic lobe.

The eallosal gyrus, or gyrus cinguli, arches round the corpus callosum: hence the name gyrus fornicatus. It commences at the 
locus perforatus anticus, below the rostrum of the corpus callosum, and it terminates below the splenium of that body. Between these two points it pursues a sweeping course, passing forwards beneath the rostrum, upwards in front of the genu, backwards above the body of the corpus callosum, and finally curving round the splenium, to be continued into the hippocampal gyrus through the isthmus. It is bounded superiorly by the calloso-marginal fissure, which separates it from the marginal gyrus and paracentral lobule, and, posterior to the latter, it is partially separated from the præcuneus by the postlimbic sulcus. The callosal gyrus is separated from the corpus callosum by the callosal sulcus.

The hippocampal gyrus constitutes the lower part of the limbic lobe, the upper part being formed by the callosal gyrus. Posteriorly, below the splenium of the corpus callosum, it is continuous above with the callosal gyrus through means of the isthmus, and behind and below with the lingual gyrus. As it passes forwards it has the hippocampal or dentate fissure above it, and the anterior part of the collateral fissure below it. Anteriorly, near the apex of the temporal pole and close behind the locus perforatus anticus, it forms an enlargement, known as the caput gyri hippocampi, which is separated from the temporal pole by a slight fissure, called the incisura temporalis. From the caput a hook-like process, known as the uncus, passes backwards fcr a short distance above the anterior part of the hippocampal or dentate fissure. The caput represents the largely-developed lobus pyriformis of many mammals, and it constitutes the olfactory centre of the cerebral cortex. Along with the uncus it forms part of the rhinencephalon or rhinopallium, the rest of the hippocampal gyrus belonging to the neopallium.

The part of the gyrus which lies below the hippocampal fissure is known as the subiculum.

The incisura temporalis, which, as stated, separates the caput gyri hippocampi from the temporal pole, represents the rhinal fissure, which bounds the well-developed rhinencephalon in some animals.

The cingulum is connected with the limbic lobe, which is composed of the callosal and hippocampal gyri. According to Cajal, its fibres arise as the axons of cells of the callosal gyrus, upon the under surface of which gyrus the cingulum lies. On entering the cingulum some fibres pass forwards, and others backwards, whilst a few are described as branching into forward and backward branches. The anterior branches pass as far as the caput of the corpus striatum, where they are described as blending with the fibres which enter the internal capsule. Some may even pass to the cortex of the prefrontal region. The posterior branches turn round the splenium and then lie upon the subiculum, or upper part of the hippocampal gyrus, as far forwards as the caput and uncus. The posterior fibres are described as ending in the cortex of (I) the subiculum hippocampi and (2) occipital lobe. The cingulum belongs to the class of long association fibres. 
Hippocampal or Dentate Fissure.-This fissure commences behind the splenium of the corpus callosum, where it is continuous with the callosal sulcus. It is directed forwards, lying between the gyrus dentatus above and the hippocampal gyrus below, and it terminates within the uncus of the hippocampal gyrus.

The hippocampal fissure is a complete fissure. It appears in the course of the fifth week, and is parallel to the temporal portion of the choroidal fissure, below which it lies. The portion of the vesicular wall between these two fissures represents the gyrus dentatus, and the portion below the hippocampal fissure forms the hippocampal gyrus.

The hippocampal fissure, being complete, gives rise to an internal elevation, namely, the hippocampus major, on the wall of the descending cornu of the lateral ventricle.

Gyrus Dentatus (Fascia Dentata of Tarinus).--The gyrus dentatus is situated above the hippocampal gyrus, and below the fimbria. It is separated from the hippocampal gyrus by the hippocampal fissure, and from the fimbria by a slight groove, called the fimbriodentate sulcus. The dentate gyrus is narrow, and its free margin is indented or notched: hence the name dentatus. It commences behind the splenium of the corpus callosum, and is directed forwards above the hippocampal gyrus into the curve of the uncus. Here it describes a bend, after which it emerges from the curve of the uncus, and, crossing the recurved part, is lost on its outer aspect. This portion of the dentate gyrus is called the frenulum Giacomini.

Posteriorly it is continuous round the splenium with the rudimentary gyrus supracallosus, which contains the mesial and lateral longitudinal strice of one half of the upper surface of the corpus callosum.

Fimbria.-The fimbria is the prolongation of the posterior pillar of the fornix. It is situated above the gyrus dentatus, from which it is separated by the fimbrio-dentate sulcus. Posteriorly it turns upwards round the posterior extremity of the optic thalamus, and so becomes continuous with the posterior pillar of the fornix. Anteriorly it enters the uncus.

Development of the Cerebral Hemispheres.-Each hemisphere is developed from the wall of the cerebral vesicle, which is a hollow protrusion from the upper and lateral part of the telencephalon, one of the divisions of the prosencephalon. The anterior wall of that portion of the telencephalon which lies between the two cerebral vesicles is called the lamina terminalis.

The hemispheres grow out of proportion to the other parts of the encephalon in a forward, upward, and backward direction. Their backward growth is so great that they completely cover the other parts of the encephalon by the seventh month of intra-uterine life.

The sulci and gyri of the hemispheres first appear about the fifth month of intra-uterine life.

Development of the Insula and Fissure of Sylvlus.-The insula, or island of Reil, appears as a depression, called the Sylvian lossa, on the lateral aspect of the cerebral vesicle. The wall of this fossa becomes developed into the opercula insulce, and, as these grow, they cover the insula, and give rise to the limbs of the fissure of Sylvius. 


\section{Olfactory Lobe.}

The olfactory lobe is rudimentary in man. It is composed of two lobules, anterior and posterior (His).

Anterior Lobule.-This consists of the following parts: (I) the olfactory bulb; (2) the olfactory tract, with its two roots, mesial and lateral ; (3) the trigonum olfactorium; and (4) the area of Broca.

The olfactory bulb is the enlarged anterior extremity of the olfactory tract. It is oval, and its upper surface is in contact with the orbital surface of the frontal lobe, whilst its lower surface rests upon one half of the cribriform plate of the ethmoid bone. The lower surface receives the olfactory nerves, which arise from the olfactory cells of the olfactory mucous membrane and pass through the foramina of the cribriform plate.

The olfactory tract is a white band which extends backwards from the olfactory bulb, both of them occupying the olfactory sulcus on the inner part of the orbital surface of the frontal lobe. Posteriorly it divides into two roots, inner and outer, which diverge and enclose between them the trigonum olfactorium.

The inner or mesial root passes inwards, in a curved manner, behind the area of Broca. Some of its fibres pass into this area, and others enter the anterior extremity of the callosal gyrus, or gyrus fornicatus.

The outer or lateral root passes backwards and outwards over the outer part of the posterior lobule (locus perforatus anticus), and enters the anterior part of the hippocampal gyrus.

The trigonum olfactorium is the area of grey matter which lies between the diverging mesial and lateral roots of the olfactory tract. It is sometimes described as the middle or grey root of the olfactory tract.

The area of Broca is situated in front of the inner root of the olfactory tract. It is continuous with the commencement of the callosal gyrus.

Posterior Lobule.-This portion of the olfactory lobe is otherwise known as the locus perforatus anticus. It is bounded anteriorly by the fissura prima of His, which separates it from the trigonum olfactorium; posteriorly by the caput gyri hippocampi; externally by the lateral root of the olfactory tract; and internally by the anterior part of the optic tract and the lateral margin of the optic commissure. Its openings give passage to the central or ganglionic branches of the anterior and middle cerebral arteries.

The grey matter of the locus perforatus anticus is continuous superiorly with the grey matter of the lenticular nucleus and of the large anterior end of the nucleus caudatus.

Development. - The olfactory lobe is developed as a hollow protrusion from the antero-inferior part of the cerebral vesicle. This protrusion becomes solid, and gives rise to the olfactory tract and olfactory bulb. 
Rhinencephalon.-The rhinencephalon consists of the olfactory lobe, uncus, gyrus dentatus, supracallosal and subcallosal gyri, half of the septum lucidum, half of the fornix, and hippocampus major.

\section{Development of the Olfactory Organ.}

The olfactory organ is developed in two parts - the olfactory lobe, and the olfactory epithelium. The olfactory lobe is intracranial, and is an outgrowth from the anterior part of the ventral aspect or floor of the telencephalon,

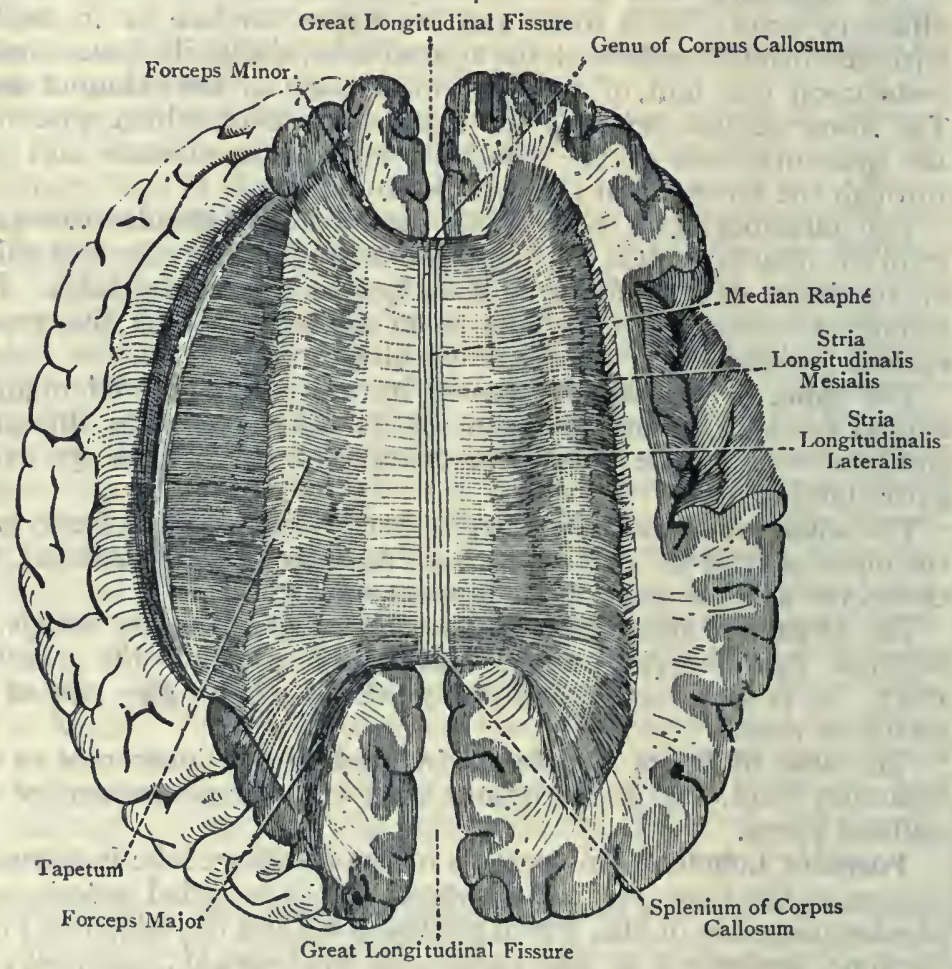

Fig. 588.-The Corpus Callosum (Superior View) (Hirschfeld AND LEVEILlê).

which is the cephalic division of the prosencephalon, or fore-brain. It constitutes the olfactory lobe (rhinencephalon) of the brain, and it becomes transformed into several parts, which will presently be stated, its terminal portion being the olfactory bulb, which rests upon one-half of the cribriform plate of the ethmoid bone.

The olfactory eplthelium is intranasal, and occupics the upper part of the nasal fossa of either side. It represents a neuro-cpithelium, which is derived from an invagination of the surface-ectoderm. The axons of its sensory cells constitute the olfactory nerve-filaments, which pass upwards through the foramina of the cribriform plate, and enter the under surface of the olfactory bulb, 
Olfactory Lobe.-The olfactory lobe appears as a hollow protrusion of the anterior cerebral vesicle on its ventral aspect, and near its anterior part. The cavity of this protrusion, which is continuous with the lateral ventricle, soon undergoes obliteration, and the protrusion becomes solid. Its terminal extremity undergoes enlargement, and the entire protrusion becomes differentiated into the following parts: (1) The olfactory bulb; (2) the olfactory tract; (3) the inner or mesial, and outer or lateral, olfactory roots; (4) the trigonum olfactorium; (5) the area of Broca; and (6) the locus perforatus anticus. Of these parts, the olfaetory bulb is the enlarged terminal extremity of the original protrusion, and, as stated, it rests upon one-half the cribriform plate of the ethmoid bone, through the foramina of which half it receives the olfactory filaments, which are the axons of the sensory cells of the olfactory epithelium of the upper part of the nasal fossa.

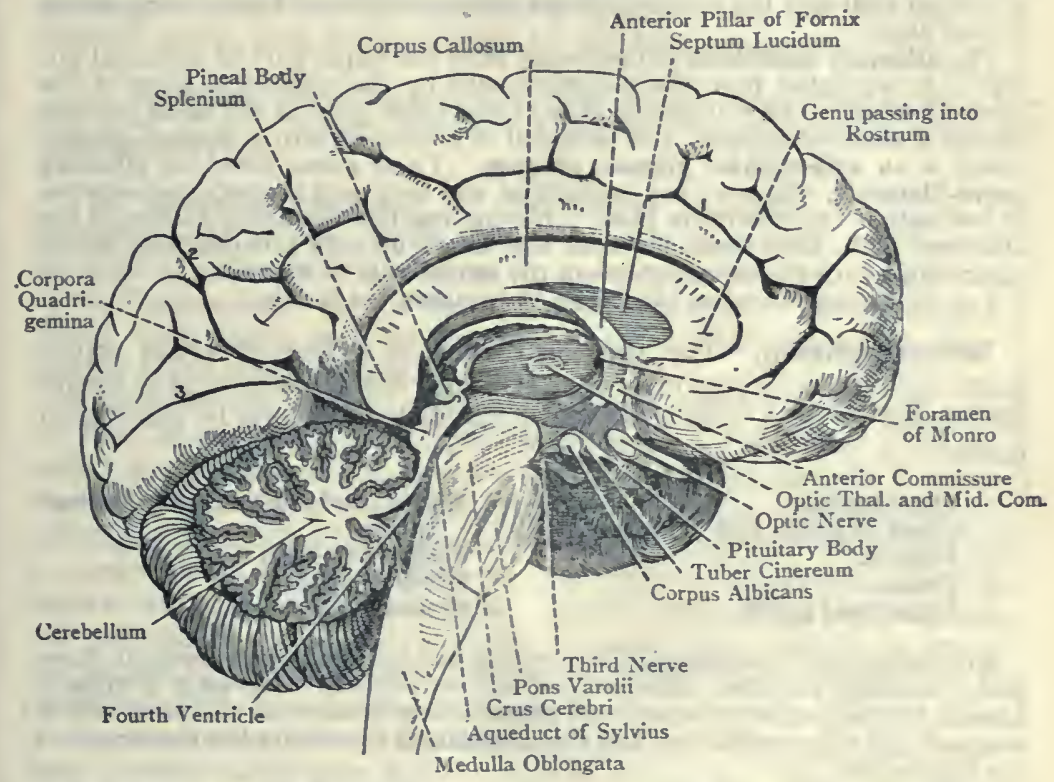

Fig. 589.-The Mesial Surface of the Left Cerebral Hemisphere (HiRschFELD AND LeVEILl E).

Olfactory Epithelium.-The first indications of the olfactory organ are the two olfactory or nasal areas. They consist of thickened ectoderm, and are placed on the ventral aspect of the anterior cerebral vesicle, on either side of the mesial nasal process of the fronto-nasal process, and on the cephalic side of the orifice of the stomodæum. Each olfactory area soon becomes depressed, and forms the olfactory or nasal pit. The two pits, right and left, are situated one on each side of the mesial nasal process of the fronto-nasal process. Each pit has the corresponding globular process of the mesial nasal process internally, and the lateral nasal process externally, this position corresponding to the situation of the future anterior naris on either side. At this period the olfactory pits communicate with the stomodrum, and the orifice of each pit, slightly constricted, constitutes the corresponding primitive anterior naris. This naris, on either side, becomes separated from the stomodæum by the union of the maxillary process (previously joined by the lateral nasal process) with the globular process. 
The blind olfactory pits now grow dorsalwards along the roof of the stomodxum, and so give rise to the nasal sacs, which represent primitive nasal fossæ. As each pit grows dorsalwards it is deepened by the formation of a groove, called the nasal groove, which extends to the roof of the stomodrum. This groove is bridged over by ectoderm, and is thus separated from the corresponding olfactory pit. When the maxillary process (previously joined by the lateral nasal process) unites with the globular process, the nasal groove is obliterated, and the olfactory pit is cut off from the stomodrum. By the development of the hard palate on either side, the ectodermic covering of the nasal groove disappears. The posterior part of this covering constitutes the naso-stomodaal membrane. When this membrane ruptures on either side, a dorsal aperture, called the primitlve posterior naris, is formed, likewise on either side, which establishes a fresh communication between the olfactory pit (blind end) and the stomodæum on the cephalic side of the corresponding palatal shelf.

The olfactory eplthelium is developed from the upper part of the nasal pit, and it is separated from the olfactory bulb by the cribriform plate of the ethmoid bone. The ectodermic cells of the upper part of the nasal pit constitute a neuro-epithelium, and each cell is prolonged into a slender process, which is an axis-cylinder process, or axon. These axons form the olfactory nerve-fllaments, which are non-medullated, and they pass through the foramina in one-half of the cribriform plate. Having reached the under surface of the olfactory bulb, they enter the bulb and break up into arborizations, which intermingle with the arborizations of the mitral cells of the bulb.

For the Development of the Organ of Jacobson see Index.

Rhinencephalon.-The rhinencephalon or olfactory brain represents the olfactory region of each cerebral hemisphere, and is feebly developed in man. It consists of the following closely associated parts:

1. Olfactory lobe.

2. Caput et uncus gyri hippocampl.

3. Fimbria.

4. Fornix.

5. Subcallosal gyrus.
6. Mesial and lateral longitudinal striæ.

7. Fasciola cinerea.

8. Gyrus dentatus.

9. Septum pellucidum.

Morphologically considered, the cerebral hemisphere is composed of three parts-namely, the stem, rhinopallium, and neopallium. The stem or stalk is formed by the corpus striatum; the rhinopallium consists of the parts which compose the rhinencephalon; and the neopallium represents the remainder of the hemisphere.

Corpus Callosum. - The corpus callosum connects the two cerebral hemispheres. It is situated at the bottom of the great longitudinal fissure, and extends nearer to the front than back of the hemispheres.

It is arched and thicker in front and behind than at the centre, its greatest thickness being posteriorly, where more fibres cross in it than elsewhere, on account of there being more of the hemisphere behind it than in front of it.

The superior surface is related to the falx cerebri, but is in contact with it only posteriorly. It is covered by a thin layer of grey matter, and presents a transversely-striated appearance, indicative of the direction of its fibres. In the median line there is a slight antero-posterior furrow or raphé, and on either side of this there is a white longitudinal band, called the stria longitudinalis mesialis. The two strix longitudinales mesiales, right and left, are sometimes 
spoken of as the nerves of Lancisi. External to each mesial stria, and situated under cover of the gyrus fornicatus or callosal gyrus, there is another bard, composed of grey matter, called the stria longitudinalis lateralis. Each lateral stria is sometimes spoken of as the tania tecta ('covered tænia'), from the circumstance that it is covered by the callosal gyrus.

The strix of each side may be traced posteriorly round the splenium into the corresponding gyrus dentatus. Anteriorly each mesial stria, along with the grey matter in which it lies, passes round the genu and backwards on the under surface of the rostrum, under the name of the geniculate gyrus. This enters the subcallosal gyrus, and finally passes to the temporal pole.

The mesial and lateral longitudinal strix of each side, together with the thin layer of gray matter, represent a rudimentary convolution called the supracallosal gyrus.

The posterior extremity of the corpus callosum is called the splenium. It is very thick, which is due, as stated, to the great number of fibres which cross in it, on account of there being more of the hemisphere behind it than in front of it. The splenium is rolled upon itself, so that its lower part is directed forwards. It lies over the mesencephalon.

Anteriorly the corpus callosum is bent upon itself, and passes at first downwards and then backwards. The bent portion is called the genu, and the portion which passes backwards is termed the rostrum. The rostrum terminates in the median line by becoming connected with the lamina terminalis, and on either side it passes into the peduncles of the corpus callosum, otherwise known as the subeallosal gyri. Each subcallosal gyrus, with the contained stria longitudinalis mesialis, now called, as stated, the geniculate gyrus, passes downwards on the internal surface of the cerebral hemisphere to the locus perforatus anticus, lying in front of the lateral portion of the lamina cinerea. The geniculate gyrus then passes backwards and outwards along the posterior margin of the locus perforatus anticus, and so reaches the temporal pole.

The inferior surface of the corpus callosum is divisible into a mesial and two lateral portions. The mesial portion is connected posteriorly with the fornix, and over the remainder of its extent with the septum lucidum. Each lateral portion enters into the roof of the body and anterior cornu of the corresponding lateral ventricle.

Destination of the Callosal Fibres.-The transverse fibres of the corpus callosum, on entering the white medullary substance of each cerebral hemisphere, traverse it in a radiating manner as they pass to the cerebral cortex. They constitute the radiatio corporis callosi, and intersect in their course the fibres which pass between the internal capsule and the cerebral cortex, which form the corona radiata. The fibres from the central portion or body and upper part of the splenium of the corpus callosum constitute the tapetum. This forms the roof of the body of the lateral ventricle, 
the chief part of the roof and the outer wall of the commencement of the middle or descending cornu, and the roof and outer wall of the posterior cornu. The fibres of the tapetum ultimately pass into the temporal and occipital lobes. The fibres from the region of the genu curve forwards into the front part of the frontal lobe, and form the roof of the anterior cornu of the lateral ventricle. They constitute the forceps minor. The fibres from the lower part of the splenium curve backwards into the occipital lobe, and give rise to an eminence on the inner wall of the posterior cornu of the lateral ventricle. They constitute the forceps major.

Development.-The corpus callosum may be regarded as being developed from the lamina terminalis.

Fornix.-This is an arched lamina of white longitudinal fibres, which lies beneath the corpus callosum, with which it is connected posteriorly, but from which it is separated anteriorly by the septum lucidum. It is composed of two lateral halves, which are united together in the median line to form the body of the fornix; but in front and behind they are separated from each other, and form the anterior and posterior pillars. The fornix is thus composed of a body, two anterior pillars, and two posterior pillars.

The body is triangular, being narrow in front, where it is continuous with the anterior pillars, and broad behind, where it is prolonged into the posterior pillars. The superior surface of the body is connected posteriorly with the corpus callosum, and anteriorly with the septum lucidum. Each lateral border is well defined, and projects slightly into the lateral ventricle. The inferior surface rests directly upon the velum interpositum, beneath which, in the median line, is the third ventricle, and on either side the upper surface of the optic thalamus.

The anterior pillars are two round bundles, which are continuous with the anterior part of the body, and are slightly separated from each other. They pass downwards in front of the foramina of Monro, traversing the grey matter on the sides of the third ventricle. On reaching the base of the brain each pillar becomes twisted in the form of a loop, and forms the white portion of the corresponding corpus albicans or mammillare. The fibres of the anterior pillar terminate in the grey nucleus of the corpus albicans, and from this nucleus a bundle of fibres, called the bundle of Vicq d'Azyr, passes upwards and backwards into the optic thalamus.

The anterior commissure lies in front of the anterior pillars.

The posterior pillars are prolongations of the posterior part of the body on either side. They are flattened bands, which at first adhere to the under surface of the corpus callosum. Subsequently however, each curves outwards and downwards round the posterior extremity of the optic thalamus, and enters the descending cornu of the lateral ventricle. Here the posterior pillar comes into contact with the hippocampus major, upon the surface of which some of its fibres become spread out, forming the alveus. The rest of the 
fibres are prolonged as a narrow band of white matter, called the fimbria, or tænia hippocampi, along the inner concave border of the hippocampus major, to which it is attached, as far as the uncus. As the two posterior pillars diverge from each other they enclose between them a small triangular space on the under surface of the corpus callosum posteriorly. This space is crossed by transverse fibres, and is known as the lyra or psalterium, from its supposed resemblance to a lyre. The transverse fibres form a commissure between the two hippocampi majores, and the lyra is therefore known as the hippocampal commissure. Each lateral half of the fornix is to be regarded as establishing a communication between the hippocampus major, in which the majority of its fibres originate, and the optic thalamus of the same side through means of (I) the anterior pillar, (2) the corpus mammillare, and (3) the bundle of Vicq d'Azyr.

Development. - The fornix may be regarded as being developed from the lamoina terminalis.

Anterior Commissure.-This is a round bundle of white fibres which crosses the middle line immediately in front of the anterior pillars of the fornix. Anteriorly its central portion is connected with the lamina terminalis, and posteriorly the central portion appears between the anterior pillars of the fornix, where it forms part of the anterior boundary of the third ventricle, and is covered by the ventricular ependyma. On either side the commissure enters the cerebral hemisphere, and divides into two parts, olfactory and temporal. The olfactory portion is of small size, and enters the corresponding olfactory tract. Some of its fibres serve to connect the olfactory bulb of one side with that of the other side. The other fibres connect the olfactory bulb of one side with the temporal lobe of the opposite side. The temporal portion is of large size, and its fibres disappear in the white matter of the temporal lobe.

The anterior commissure serves to connect the olfactory bulbs and the temporal lobes.

Septum Lucidum.-This is a thin vertical partition which is situated between the anterior cornua of the lateral ventricles, as well as between the front parts of the bodies of these ventricles. It is triangular, being broad in front and narrow behind. Posteriorly it is attached above to the under surface of the corpus callosum, and below to the upper surface of the body of the fornix. Anteriorly it occupies the concavity behind the genu of the corpus callosum, being attached above to the corpus callosum and below to the rostrum of that body. The septum lucidum is composed of two delicate laminæ. The outer surface of each lamina looks into the corresponding lateral ventricle, and is covered by the ventricular ependyma (epithelium). The inner surface faces that of its fellow, a narrow interval, representing the fifth ventricle, intervening between the two. Each lamina consists of white 
matter, which is covered by grey matter on the surface looking towards the fifth ventricle.

The two laminæ are formed from portions of the mesial walls of the two cerebral hemispheres which have become detached in the course of the development of the corpus callosum and fornix.

Fifth Ventricle.--The fifth ventricle, or ventricle of the septum, is the narrow cleft-like interval between the two laminæ of the septum lucidum. It is a closed space, and has therefore no communication with the other ventricles. It is destitute of any ependymal lining, and contains a very little fluid.

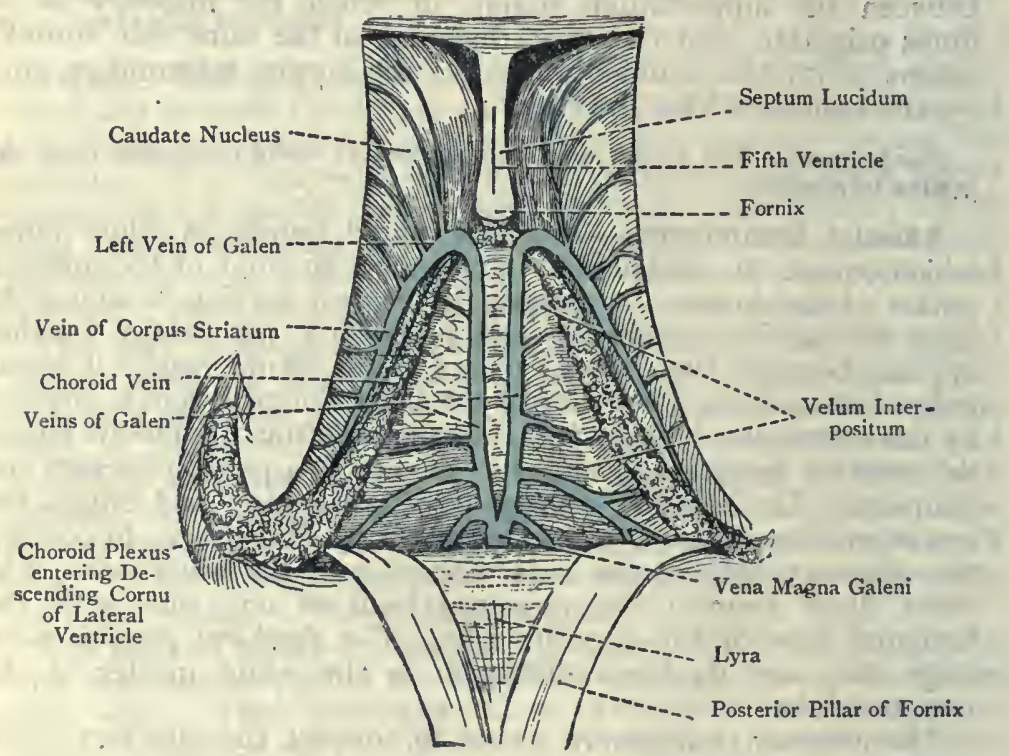

Fig. 590.-The Velum Interpositum and Veins of Galen.

As regards development, it differs from the other ventricles, having been originally a part of the great longitudinal fissure.

Velum Interpositum.-This is also known as the tela choroidea superior, in contradistinction to the tela choroidea inferior, which is the pia mater forming the roof of the lower part of the fourth ventricle. It lies immediately beneath the fornix, and rests upon the ependymal roof of the third ventricle, and also upon the adjacent portions of the optic thalami. It consists of two layers of pia mater, and is triangular, the apex being situated behind the anterior pillars of the fornix at the foramina of Monro and the base lying beneath the splenium of the corpus callosum. In the latter situation the two layers of the velum interpositum become 
continuous with the pia mater, which has entered through the transverse fissure, situated between the splenium of the corpus callosum and the corpora quadrigemina. On either side the velum interpositum projects beyond the lateral border of the fornix, and appears as a vascular fringe in the lateral ventricle, where it is covered by the ventricular ependyma. This fringe is known as the choroid plexus of the lateral ventricle. Posteriorly it is prolonged into the descending cornu. Anteriorly it approaches its fellow of the opposite side, and the two unite in the median line behind the foramina of Monro. From this junction two other choroid plexuses extend backwards on the inferior surface of the velum interpositum, one on either side of the median line. They form the choroid plexuses of the third ventricle, and lie superficial to the ependymal roof of that cavity.

The principal veins in connection with the velum interpositum are the two choroid veins and the two veins of Galen. The choroid vein of each side is situated in the choroid plexus of the lateral ventricle. It passes forwards and inwards to a point behind the corresponding foramen of Monro, where it joins the vein of the corpus striatum, which lies between the optic thalamus and the corpus striatum. In this manner the vein of Galen of one side is formed.

The veins of Galen are two in number, right and left. Each vein is formed, as just stated, by the union between the choroid vein and the vein of the corpus striatum behind the corresponding foramen of Monro. The two veins of Galen pass backwards within the velum interpositum, one on either side of the median line. At first they are near each other; then they diverge; but subsequently they again come together and unite to form one vessel, called the vena magna Galeni, which opens into the anterior extremity of the straight sinus. Each vein of Galen receives numerous tributaries from the corresponding choroid plexus of the third ventricle, optic thalamus, corpus callosum, corpora quadrigemina, and pineal body. Near its termination it is joined by the large basilar vein, which is formed at the locus perforatus anticus by the union of the anterior cerebral vein with the deep Sylvian vein. The vena magna Galeni receives tributaries from the upper surface of the cerebellum and from the occipital lobes of the cerebral hemispheres.

\section{Lateral Ventricles.}

The lateral ventricles are the ventricles of the cerebral hemispheres, and are two in number, right and left. They are of irregular shape, and each occupies about two-thirds of the length of the corresponding hemisphere. They are lined with ependyma (epithelium), and contain a certain amount of cerebro-spinal fluid. Each ventricle communicates with the third ventricle by the foramen of Monro of its own side, which is situated between the 
anterior pillar of the fornix and the front part of the optic thalamus. The lateral ventricle of either side consists of a body and three cornua-anterior, middle or descending, and posterior.

The body extends from the foramen of Monro to the level of the splenium of the corpus callosum. The anterior cornu is situated in front of the foramen of Monro, and curves forwards and outwards into the frontal lobe. The middle or descending cornu

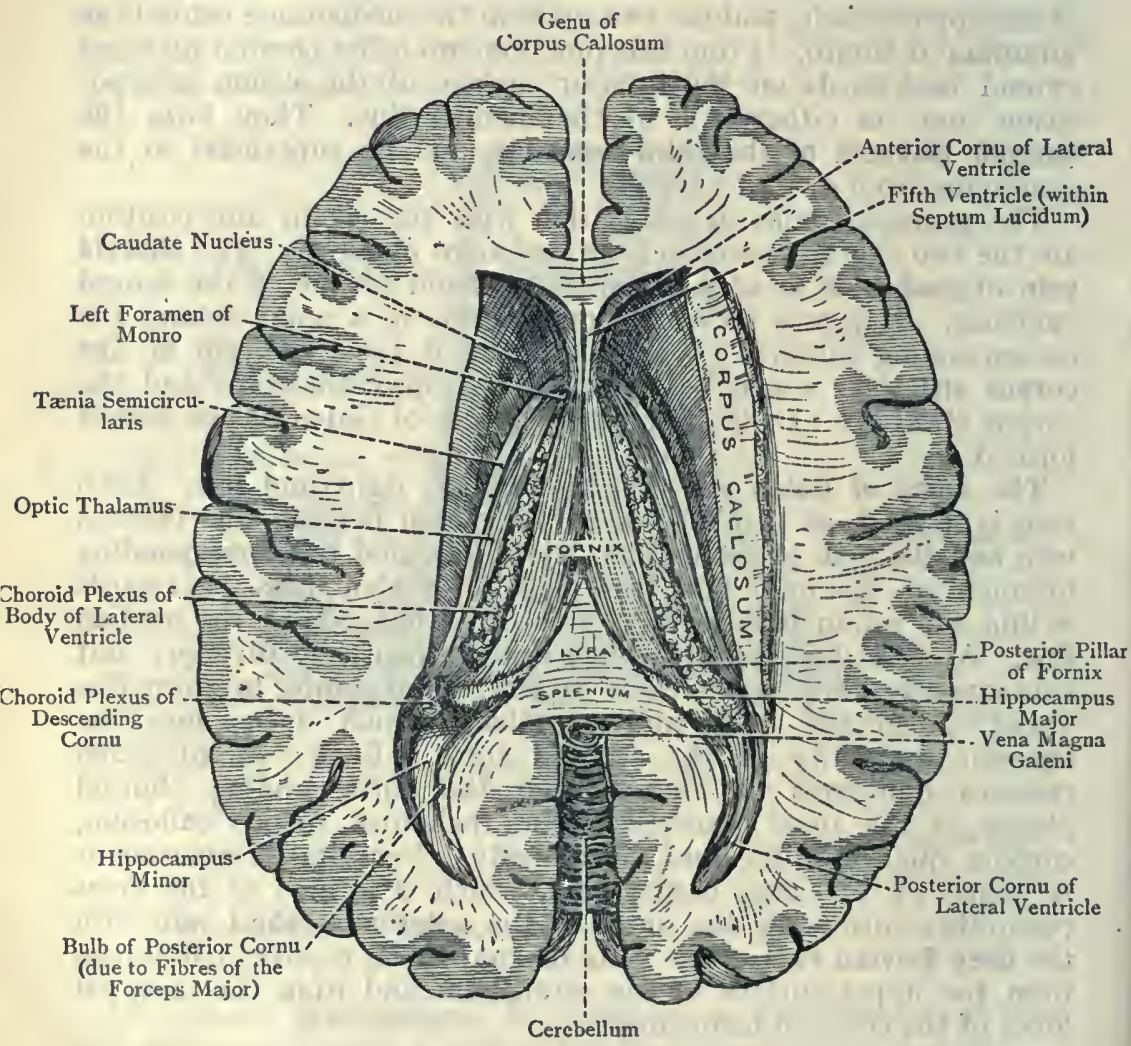

Fig. 591.-The Lateral Ventricles of the Cerebrum (after HiRSCHFELd AND LeVEILlé).

enters the temporal lobe, and describes a remarkable curve as it sweeps round the posterior extremity of the optic thalamus. Its direction is backwards, outwards, downwards, forwards, and finally inwards to a point about I inch from the temporal pole. The posterior cornu curves backwards and inwards into the occipital lobe.

The body of the lateral ventricle presents a roof, an inner wall, 
and a floor. The roof is formed by the corpus callosum (tapetum). The inner wall is formed by the posterior part of the septum lucidum, and, behind this, by the attachment of the body of the fornix to the under surface of the corpus callosum. Externally the body is limited by the meeting of the roof and floor. The floor presents the following structures, in order from within outwards: (I) the sharp lateral border of the fornix; (2) the choroid plexus of the lateral ventricle; (3) a portion of the upper surface of the optic thalamus; (4) an oblique groove, extending forwards and inwards

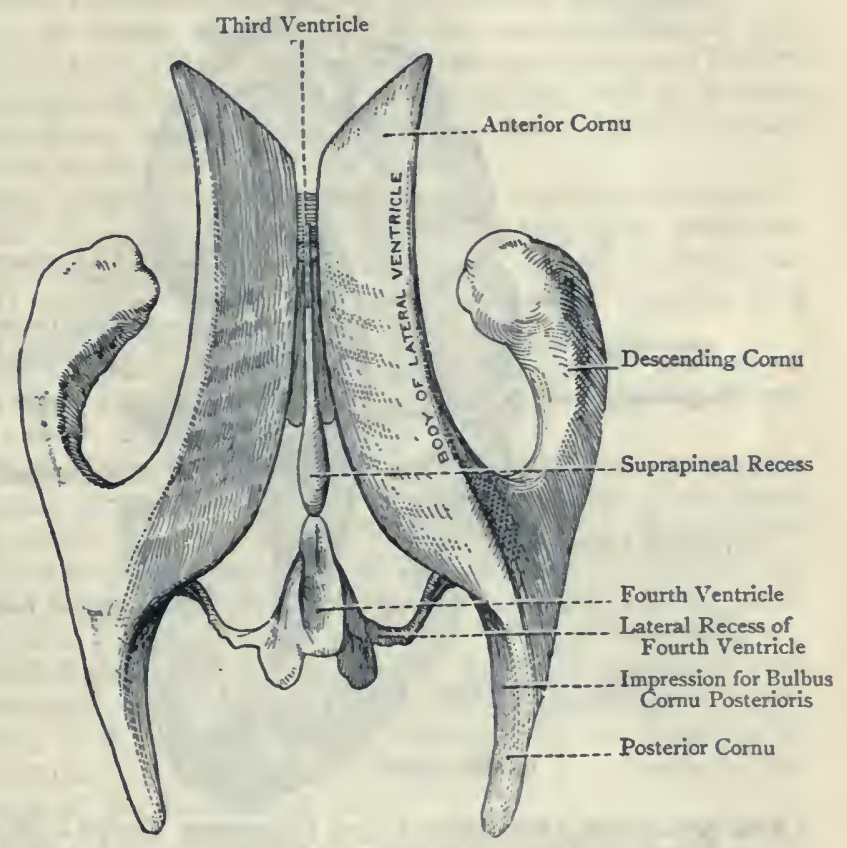

Fig. 592.-Drawing of a Metal Cast of the Ventricles of the Brain of an Adult (SUPERIOR View) (RetziUs).

between the optic thalamus and nucleus caudatus, in which there are $(a)$ a white band, called the tænia semicircularis, and (b) the vein of the corpus striatum, the latter being the more superficial of the two; and (5) the narrow part of the nucleus caudatus of the corpus striatum.

The anterior cornu has its roof formed by the corpus callosum (forceps minor). Externally it is limited by the meeting of the roof and floor. Anteriorly where it is pointed, it is bounded by part of the posterior aspect of the genu of the corpus callosum. Its inner wall is formed by the anterior part of the septum lucidum. 
The floor presents a prominent swelling, which is the large round extremity of the nucleus caudatus of the corpus striatum.

The posterior cornu has its roof and outer wall formed by the tapetum of the corpus callosum. The inner wall presents two elongated, curved eminences, upper and lower. The upper eminence is produced by the fibres of the forceps major as they sweep backwards from the lower part of the splenium of the corpus callosum to the occipital lobe. It is called the bulb of the posterior cornu. The lower eminence is called the calcar avis or hippocampus minor,

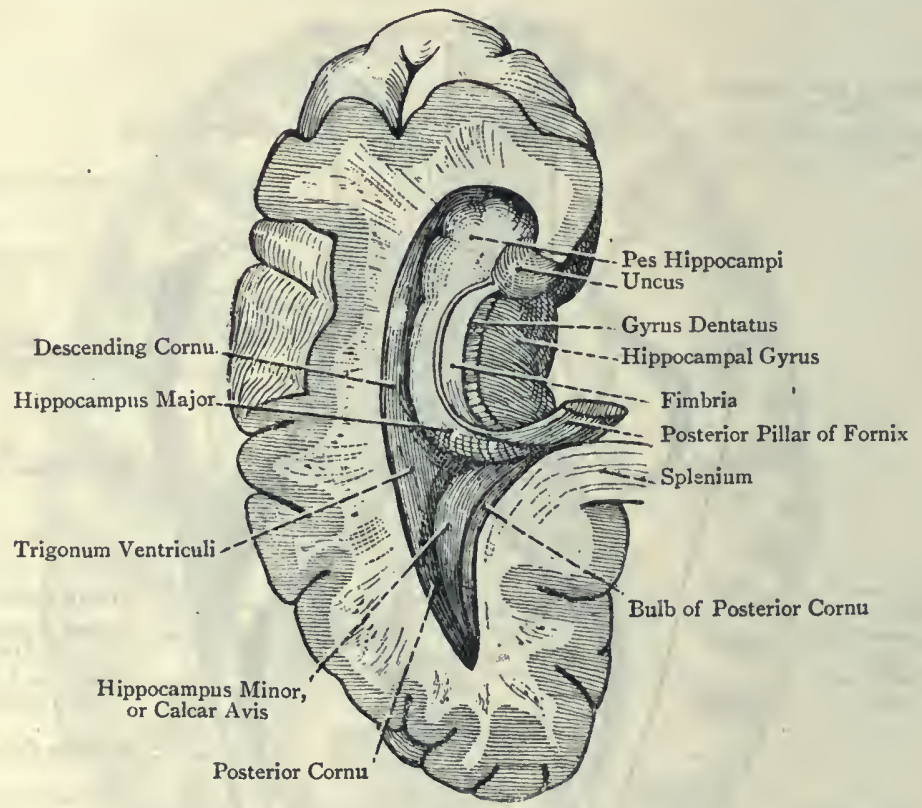

Fig. 593.-The Descending and Posterior Cornua of the Left Lateral Ventricle (After Hirschfeld aNd Leveille).

(The Descending Cornu has been laid open throughout its entire extent).

and is produced by the anterior calcarine fissure on the mesial surface of the cerebral hemisphere.

The middle or descending cornu is situated in the temporal lobe. Its roof is formed chiefly by the tapetum of the corpus callosum, and, at the deep end of the cornu, presents the amygdaloid tubercle, which is produced by a collection of grey matter, called the amygdaloid nucleus. The narrow part or tail of the nucleus caudatus and the tænia semicircularis are prolonged into the roof, and extend in it as far as the amygdaloid nucleus. The floor of the descending cornu presents the following structures: (I) the hippocampus major, or cornu Ammonis; (2) the fimbria or tænia hippo- 
campi; (3) the eminentia collateralis, or pes accessorius; and (4) the choroid plexus of the descending cornu. The hippocampus major, or cornu Ammonis, is a prominent curved elevation which traverses the entire length of the descending cornu, accurately adapting itself to its curves. It enlarges as it descends, and beneath the amygdaloid tubercle it terminates in a swelling, which is notched on the surface. This swelling is called the pes hippocampi.

The hippocampus major is produced by the dentate or hippocampal fissure on the mesial surface of the cerebral hemisphere.

The fimbria, or tænia hippocampi, is the continuation of the posterior pillar of the fornix. It lies along the inner concave border of the hippocampus major, to which it is attached, and it is composed of white fibres, some of which form the layer on the surface of the hippocampus major, called the alveus.

The eminentia collateralis, or pes accessorius, is an elevation which is situated in the angle between the descending and posterior cornua, where there is a small triangular space, called the trigonum ventriculi. It extends backwards into the posterior cornu, and for a variable distance into the descending cornu.

The eminentia collateralis is produced by the central portion of the collateral fissure on the tentorial surface of the cerebral hemisphere.

The choroid plexus of the descending cornu rests upon the surface of the hippocampus major, and is continuous with that of the body of the lateral ventricle. It is covered by the ependyma of the inner wall of the descending cornu, which it invaginates. When the choroid plexus is removed its ependymal covering comes away along with it, and the choroidal fissure then becomes apparent.

The choroidal fissure is situated between the fimbria and the roof of the descending cornu, and, curving round the back part of the optic thalamus, it is traceable as far forwards as the foramen of Monro of the same side. In the other direction it extends to the lower extremity of the descending cornu.

It is produced by an infolding or invagination of the epithelial mesial wall of the cerebral vesicle of one side over the choroid plexus of the descending cornu of the lateral ventricle. On either side it is continuous with the lateral and lower part of the transverse fissure.

When the choroid plexus is withdrawn from the descending cornu of the lateral ventricle, the epithelial or ependymal covering of the plexus comes away with it, or is broken down. Under these circumstances the descending cornu opens freely upon the exterior.

The choroidal fissure is a complete fissure, and the internal prominence corresponding to it is the choroid plexus in the descending cormu of the lateral ventricle.

Development.-The lateral ventricles represent the cavities of the primitive cerebral vesicles. The choroldal fissure is developed as an invagination of the mesial wall of the cerebral vesicle; and the choroid plexus is developed from a growth of mesoblast in to the choroidal fissure. 


\section{Basal Ganglia of the Cerebral Hemispheres.}

The basal ganglia of each cerebral hemisphere are as follows: the nucleus caudatus and nucleus lenticularis of the corpus striatum, the claustrum, and the amygdaloid nucleus.

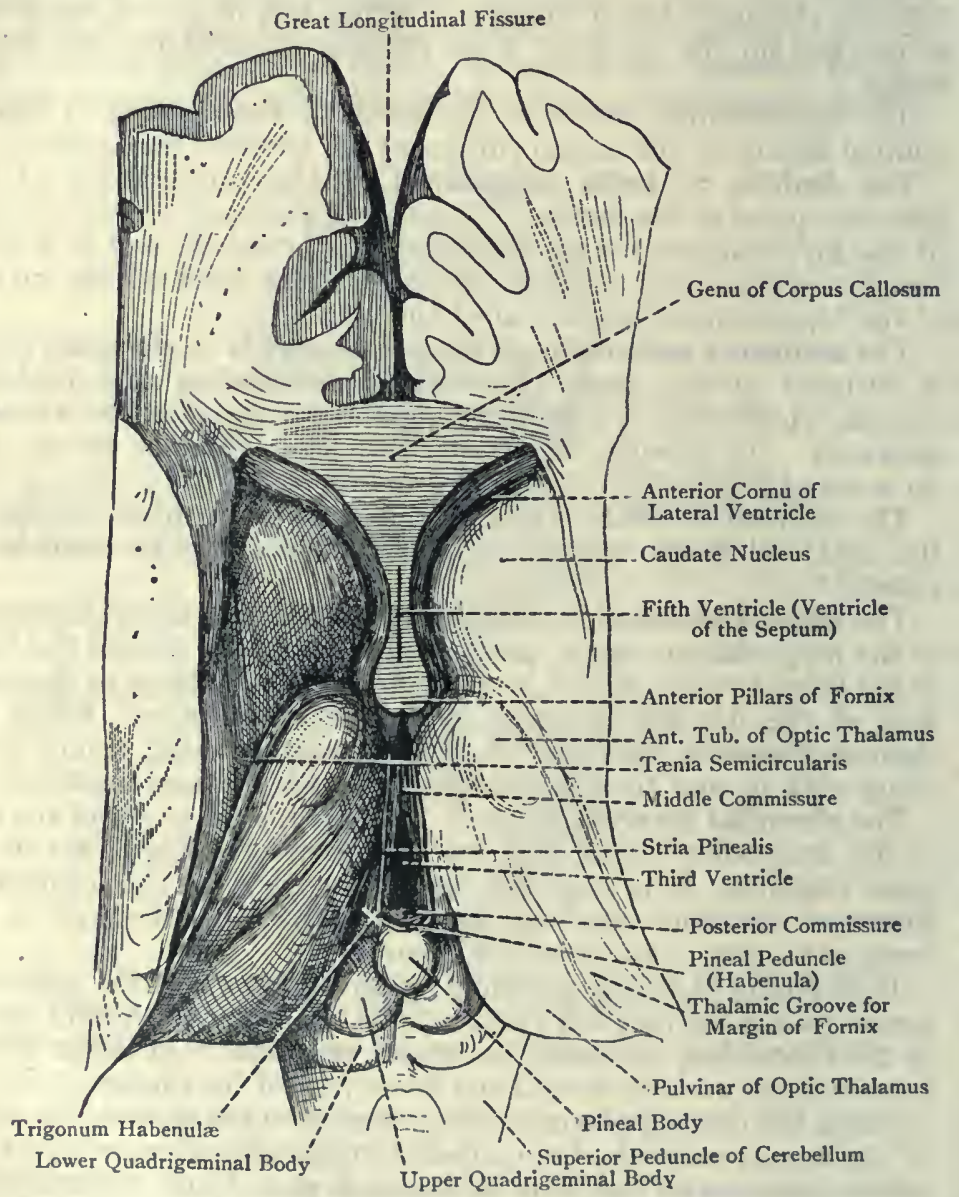

Fig. 594.-The Third Ventricle, Portions of the Lateral Ventricles, Pineal Body, and Corpora Quadrigemina (Superior View) (Henle).

(The Corpus Callosum, Fornix, and Velum Interpositum have been removed).

The corpus striatum is a large ovoid mass, which is situated in front, and on the outer side, of the optic thalamus. It is composed of two collections of grey matter, one of which is intraventricular and the other extraventricular. The intraventricular portion is called 
the nucleus caudatus. The extraventricular portion is embedded in the white matter of the cerebral hemisphere, and is termed the nucleus lenticularis. Between these two nuclei there is a part of the thick tract of white fibres which constitutes the internal capsule; and on the outer side of the nucleus lenticularis there is the thin lamina of white matter, called the external capsule. When a coronal section is made through the corpus striatum on a level with the anterior part of the nucleus lenticularis, the white matter of the front part of the internal capsule is seen to be intersected by striæ of grey matter which pass between the nucleus caudatus and nucleus lenticularis. From the striped appearance thus produced the body has received the name of corpus striatum.

The nucleus caudatus is pyriform. The large round end is directed forwards, and projects into the anterior cornu of the lateral ventricle.

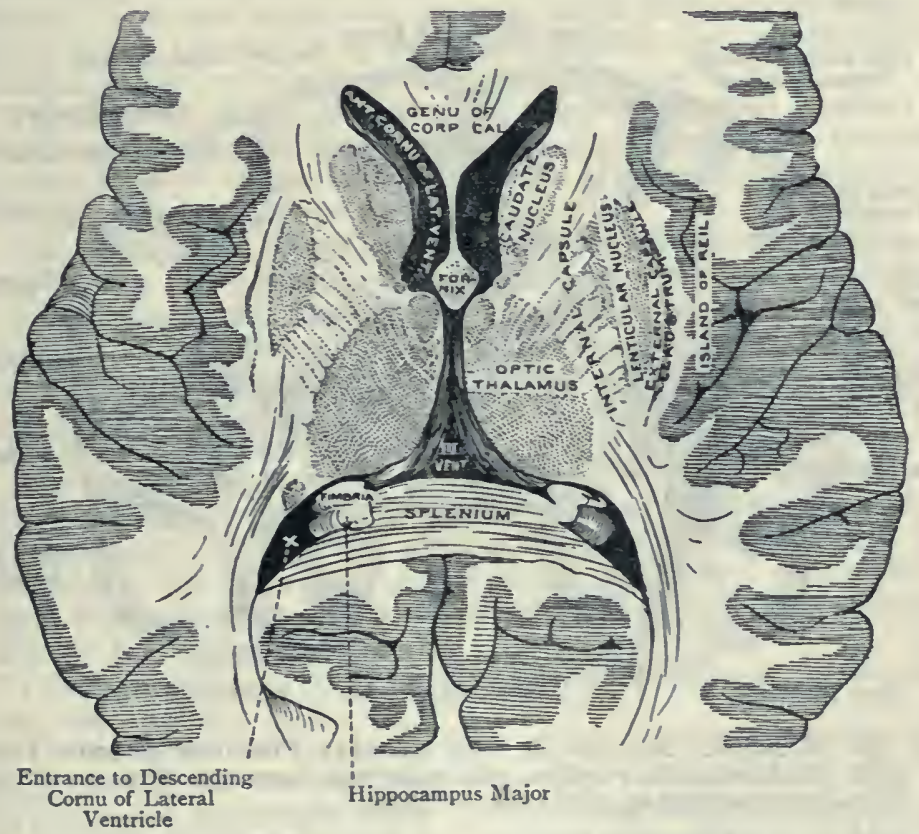

Fig. 595.-Horizontal Section of the Brain through the Genu and Splenium of the Corpus Callosum (Dalton).

The narrow portion is directed outwards and backwards in the floor of the body of the lateral ventricle, where it lies on the outer side of the optic thalamus, from which it is separated by the tænia semicircularis. Its tapering tail is continued into the roof of the descending cornu of the iateral ventricle, and is prolonged in the roof as far as the amygdaloid nucleus, in which it terminates. The 
nucleus caudatus is composed of grey matter, and its cells are of the multipolar variety.

The nucleus lenticularis is embedded in the white matter of the cerebral hemisphere, and lies on the outer side of the nucleus caudatus and optic thalamus, from both of which it is separated by the internal capsule. It is of more limited extent than the nucleus caudatus, and receives its name from the fact that, in horizontal section, it presents the appearance of a biconvex lens, the broadest part being on a level with the front of the optic thalamus. Anteriorly it is closely related to the front part of the nucleus caudatus, being continuous with it inferiorly, and connected with it superiorly by striæ of grey matter which intersect the white matter of the front part of the internal capsule. The anterior part of the nucleus lenticularis, as seen in coronal section,

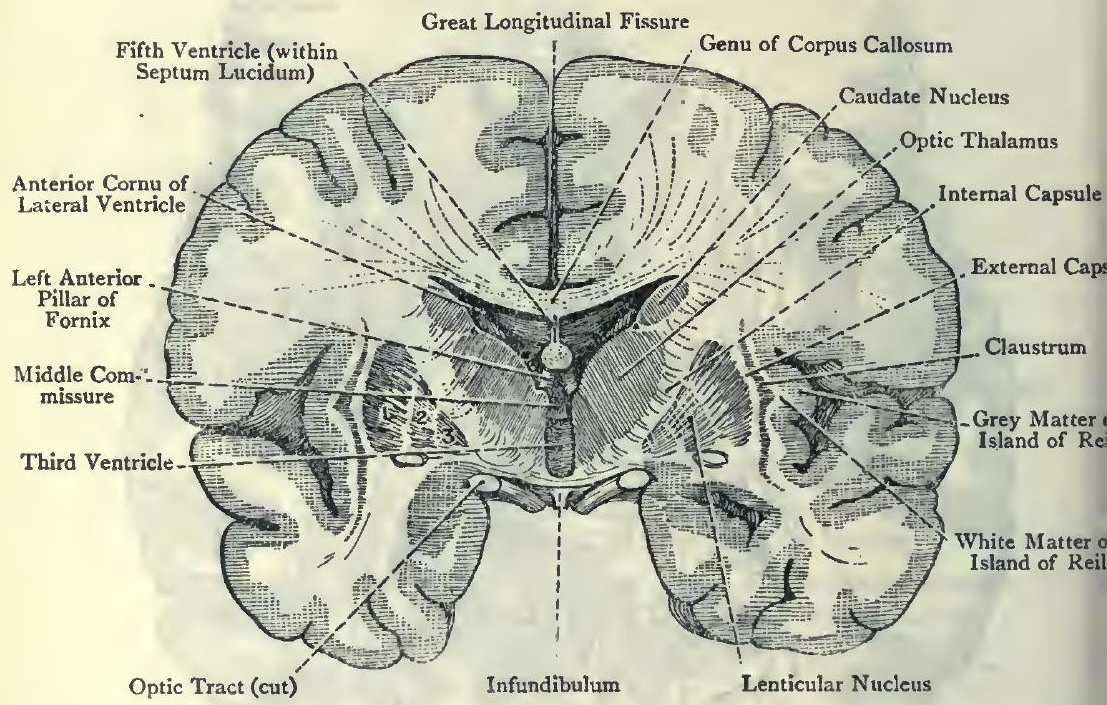

Fig. 596.-Coronal Section of the Frontal Portions of the CerebRAL HEMISPHERES, PASSING tHROUGH THE ANTERIOR CORNUA OF THE Lateral Ventricles (Posterior View).

I, Putamen of Lenticular Nucleus ; 2, 3, Globus Pallidus of Lenticular Nucleus.

is somewhat semilunar. When such a section is made through the centre, the nucleus presents a triangular outline, the base being directed towards the island of Reil, and having the external capsule in loose connection with it, whilst the internal surface is in contact with the internal capsule. In such a section the nucleus is seen to be traversed vertically by two white bands, called the medullary lamina, which divide it into three zones. The outer zone, which has a dark reddish colour, is the largest, and is called the putamen. 
The inner two zones, which are somewhat yellowish, are together known as the globus pallidus. The putamen and globus pallidus, which consist of grey matter, are traversed by white fibres.

The grey matter of the nucleus caudatus and nucleus lenticularis comes to the surface at the base of the brain in the region of the locus perforatus anticus, where it is continuous with the grey matter of the cerebral cortex.

The internal capsule is the thick tract of white matter which lies between the nucleus lenticularis externally, and the nucleus caudatus, tænia semicircularis, and optic thalamus internally. As seen in horizontal section it describes a bend opposite the front part of the optic thalamus. This bend is called the genu, and its convexity is directed inwards. The part of the internal capsule in front of the genu is called the anterior limb. It forms about one-third of the entire capsule, and its direction is forwards and outwards. The part behind the genu is called the posterior limb. It forms about two-thirds of the entire capsule, and its direction is backwards and outwards.

The anterior limb of the internal capsule is situated between the front part of the nucleus lenticularis and the nucleus caudatus. Anteriorly it is intersected by the striæ of grey matter which pass between the two nuclei.

The fibres which compose the anterior limb are partly corticipetal and partly corticifugal. The corticipetal fibres are as follows: (I) thalamo-frontal fibres, which pass from the optic thalamus to the cortex of the frontal lobe; (2) thalamo-striate fibres, which pass from the optic thalamus to the corpus striatum (thalamo-caudate and thalamo-lenticular fibres); and (3) strio-frontal fibres, which pass from the corpus striatum to the cortex of the frontal lobe.

The chief corticifugal fibres constitute the fronto-pontine tract. The fibres of this tract arise in the cortex of the prefrontal region. They traverse the anterior limb of the internal capsule, and then descend in the inner part of the crusta of the crus cerebri to the pons Varolii, within which they terminate in connection with the cells of the nucleus pontis.

Other centrifugal fibres constitute fronto-thalamic, fronto-striate, and strio-thalamic tracts.

The posterior limb of the internal capsule is situated between the back part of the nucleus lenticularis and the optic thalamus, and is prolonged backwards for a little beyond the posterior limit of the nucleus lenticularis. It is therefore conveniently divided into two parts, lenticular, representing the anterior two-thirds, and postlenticular, representing the posterior third.

The lenticular part of the posterior limb, like the anterior limb, is composed of centripetal and centrifugal fibres. The centripetal fibres arise in the optic thalamus, and their destination is the cerebral cortex. The centrifugal fibres represent the pyramidal or motor fibres, and they occupy the anterior portion of the lenticular part of the posterior limb of the internal capsule. These fibres descend from the Rolandic region of the cerebral cortex. Some of them pass to 
the nucleus of the facial nerve; others pass to the nucleus of the hypoglossal nerve; but the majority of them are destined for the motor cells in the anterior grey cornu of the spinal cord. The fibres which pass to the facial nucleus lie close to the genu, and those which pass to the hypoglossal nucleus lie close behind the facial fibres. The fibres of the pyramidal tract occupy the central portion of the crusta of the crus cerebri in their downward course.

The postlenticular part of the posterior limb contains the following sets of fibres: (I) the fibres of the optic radiation, on their way from and to the optic thalamus, external geniculate body, and superior quadrigeminal body; (2) the fibres of the auditory radiation, passing between the auditory region of the temporal lobe and the internal geniculate body; and $(3)^{\circ}$ the fibres of the temporopontine tract (cortico-protuberantial fibres), which pass from the cortex of the temporal lobe through the outer part of the crusta of the crus cerebri to the pons Varolii, where they terminate in the nucleus pontis. The internal capsule is continuous inferiorly with the crusta of the crus cerebri. Superiorly its fibres diverge in a radiating manner on their way to the cerebral cortex, forming the corona radiata, the fibres of which are intersected by those of the radiatio corporis callosi.

The external capsule is a thin lamina of white matter which is situated on the outer side of the nucleus lenticularis, where it lies between that nucleus and the claustrum. In front of and behind the nucleus lenticularis it is continuous with the internal capsule. The external capsule is, as stated, only loosely connected with the putamen of the nucleus lenticularis. The fibres of which it is composed are probably derived from the anterior white commissure and the optic thalamus.

Connections of the Corpus Striatum.-(I) The nucleus caudatus and nucleus lenticularis are partly continuous with each other, and partly connected by striæ of grey matter. (2) The corpus striatum is connected with the optic thalamus by strio-thalamic and thalamo-striate fibres. (3) The nucieus caudatus is said to be connected with the substantia nigra by a tract of fibres known as the stratum intermedium. (4) The nucleus lenticularis is connected with the optic thalamus by the ansa lenticularis. (5) The corpus striatum is connected with the cerebral cortex by cortico-striate fibres.

Development.-The corpus striatum is developed as a thickening of the floor of the cerebral vesicle.

The claustrum is a thin lamina of grey matter which is situated on the outer surface of the external capsule. It lies embedded in the white matter which occupies the region between the lenticular nucleus and the island of Reil. Superiorly it is narrow and tapering, but inferiorly it expands and reaches the surface at the base of the brain, in the region of the locus perforatus anticus. Its outer surface presents elevations and intervening depressions, which correspond to the sulci and gyri of the island of Reil.

The claustrum is regarded as an isolated portion of the grey matter of the island of Reil.

The amygdaloid nucleus is an oval collection of grey matter which is situated in the anterior part of the temporal lobe, where 
it lies in the roof of the deep extremity of the descending cornu of the lateral ventricle. The putamen of the nucleus lenticularis lies above it; anteriorly it is continuous with the cerebral cortex; inferiorly it receives the narrow part or tail of the nucleus caudatus; and the tænia semicircularis arises from it.

The tænia semicircularis is a narrow white band of fibres which, as just stated, arises from the amygdaloid nucleus. The band passes backwards in the roof of the descending cornu of the lateral ventricle, and then it sweeps upwards and forwards into the body of the lateral ventricle, lying between the nucleus caudatus and the optic thalamus. Anteriorly, in the region of the foramen of Monro, it dips downwards towards the anterior pillar of the fornix and the anterior white commissure. In this situation its fibres are variously disposed. Some pass into the anterior pillar of the fornix; others pass in front of the anterior commissure, and enter the grey matter between the head of the nucleus caudatus and the septum lucidum; whilst a few are regarded as entering the nucleus caudatus. The vein of the corpus striatum lies superficial to the tænia semicircularis.

Relation of Structures in the Region of the Corpus Striatum.When a coronal section has been made the relation of structures, from within outwards, is as follows:

I. Nucleus caudatus.

2. Internal capsule.

3. Nucleus lenticularis.

4. External capsule.

5. Claustrum.

6. Island of Reil.

\section{THALAMENCEPHALON OR DIENCEPHALON.}

\section{Third Ventricle.}

The third ventricle is the cleft-like interval which is situated, in the median line, between the two optic thalami. It extends from the pineal body posteriorly to the anterior pillars of the fornix in front, is very narrow from side to side, and is deeper in front than behind. The cavity presents a roof, a floor, two lateral walls, an anterior boundary, and a posterior boundary.

The roof is formed by a delicate layer of epithelium which extends across between the upper margins of the lateral walls, and is continuous with the ependymal lining of the ventricle. Superficial to this epithelial roof, and intimately connected with it, is the velum interpositum, from the under surface of which the two choroid plexuses of the ventricle project downwards, one on either side of the middle line, each invaginating the epithelium of the roof. The epithelium of the roof is so intimately connected with the velum interpositum that, when the latter is removed, the epithelium comes away with it, and the cavity of the ventricle is exposed. Superficial to the velum interpositum is the body of the fornix, and, above this again, is the body of the corpus callosum.

Summary of the Roof.- To expose the ventricle from above the following structures must be removed, in the order named: ( 1 ) the body of the corpus callosum; (2) the body of the fornix; and (3) the velum interpositum, along with the epithelium of the roof. 
The floor, which is sloped downwards and forwards, is formed by the structures which lie within the interpeduncular space at the base of the brain, these being as follows, from behind forwards: the locus perforatus posticus, the corpora mammillaria, and the tuber cinereum, with the upper end of the infundibulum. The tegmenta of the crura cerebri enter, to a certain extent, into the floor posteriorly, and the optic commissure lies across it anteriorly. Above the optic commissure the floor presents a depression, called the optic recess, and behind this there is another depression or diverticulum, called the infundibular recess. The latter forms the upper part of the infundibulum which leads to the posterior lobe of the pituitary body.

The lateral wall is slightly convex, and is formed, for the most part, by the inner surface of the optic thalamus, which has a thick

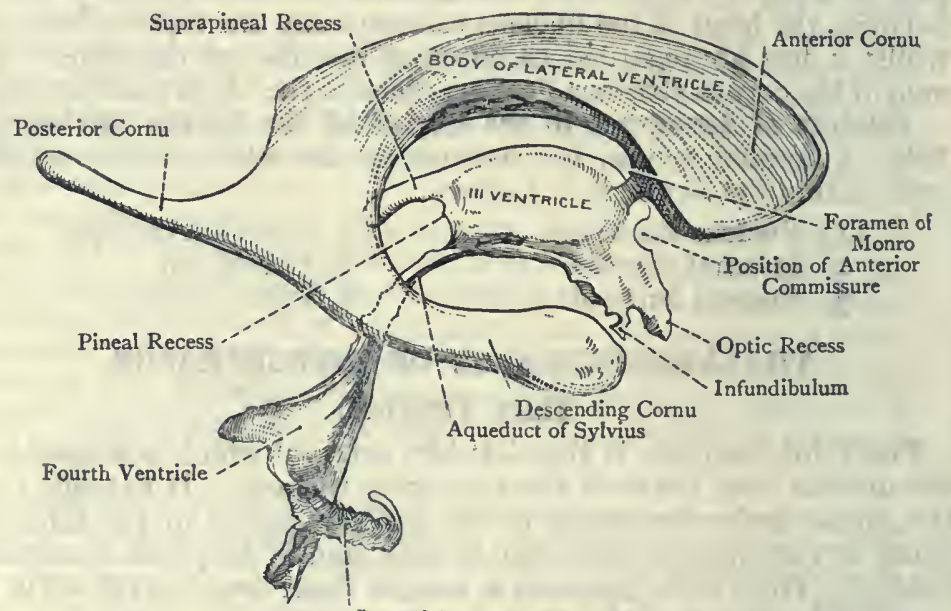

Lateral Recess of Fourth Ventricle

Fig. 597.-Drawing of a Metal Cast of the Ventricles of the Brain of an Adult (Right lateral View) (Retziús).

covering of grey matter. Towards its centre it presents a furrow, which leads from the foramen of Monro in a backward direction towards the upper opening of the aqueduct of Sylvius. This groove is called the sulcus of Monro. At the upper part of the lateral wall there is a delicate band of white fibres, called the stria pinealis or medullaris, which represents the dorsal portion of the stalk of the pineal body, and passes to the anterior pillar of the fornix. Connecting the two lateral walls (optic thalami), in front of the centre of the ventricle, there is a fragile band of grey matter, called the middle or soft commissure. At the anterior part of the lateral wall the corresponding anterior pillar of the fornix passes downwards and backwards.

The anterior boundary is formed inferiorly by the lamina termin- 
abis, which extends upwards from the optic commissure to the rostrum of the corpus callosum, and superiorly by the anterior pillars of the fornix and the central portion of the anterior commissure.

The posterior boundary is formed by the pineal body and the posterior commissure, and under cover of the latter is the upper opening of the aqueduct of Sylvius. The posterior boundary presents two recesses, pineal and suprapineal. The pineal recess passes backwards above the posterior commissure into the stalk of the pineal body, separating the stalk into two portions, dorsal and ventral. The suprapineal recess is connected with the back part of the epithelial roof of the ventricle, and passes backwards over the pineal body. The third ventricle has thus four diverticula, namely, the optic recess, the infundibular recess, (both of which recesses are associated with the floor), the pineal recess, and the suprapineal recess. The cavity communicates with the fourth ventricle by means of the aqueduct of Sylvius, and with the two lateral ventricles by means of the foramina of Monro.

Foramina of Monro.-These are two in number, right and left, and they lead from the lateral ventricles into the third ventricle. Each foramen is situated between the anterior pillar of the fornix in front and the anterior tubercle of the optic thalamus behind. From this point the foramen of each side leads inwards and slightly downwards, and opens into the third ventricle at the anterior and upper part of the corresponding lateral wall. By means of the foramina of Monro the lateral ventricles communicate with the third ventricle, and, through that ventricle, with each other. The choroid plexuses of the lateral ventricles also become continuous with each other and with those of the third ventricle through these foramina. From each foramen a furrow, called the sulcus of Monro, leads, as stated, in a backward direction, over the inner surface of the optic thalamus towards the upper opening of the aqueduct of Sylvius.

Development.-The posterior and greater part of the third ventricle represents the cavity of the thalamencephalon or diencephalon; and the anterior part, in the region of the foramina of Munro, represents the mesial portion of the cavity of the telencephalon.

The foramen of Monro, of each side, represents the original wide communication between the cavity of the cerebral vesicle and the cavity of the mesial portion of the telencephalon.

Optic Thalami. - The optic thalami are two large ovoid masses of grey matter which lie obliquely, with their long axes directed backwards and outwards, for the most part on the sides of the third ventricle. Their anterior extremities are near each other, but their posterior extremities stand apart, the corpora quadrigemina being situated between them. Over their anterior two-thirds they are separated from each other by the third ventricle.

The optic thalamus presents four surfaces-superior, inferior, external, and internal; and two extremities-anterior and posterior.

The superior surface is limited externally by an oblique groove, 
which separates it from the nucleus caudatus, and contains the tænia semicircularis and, anteriorly, the vein of the corpus striatum. Internally it is bounded, from before backwards, by (I) the stria pinealis, (2) the trigonum habenılæ, and (3) the

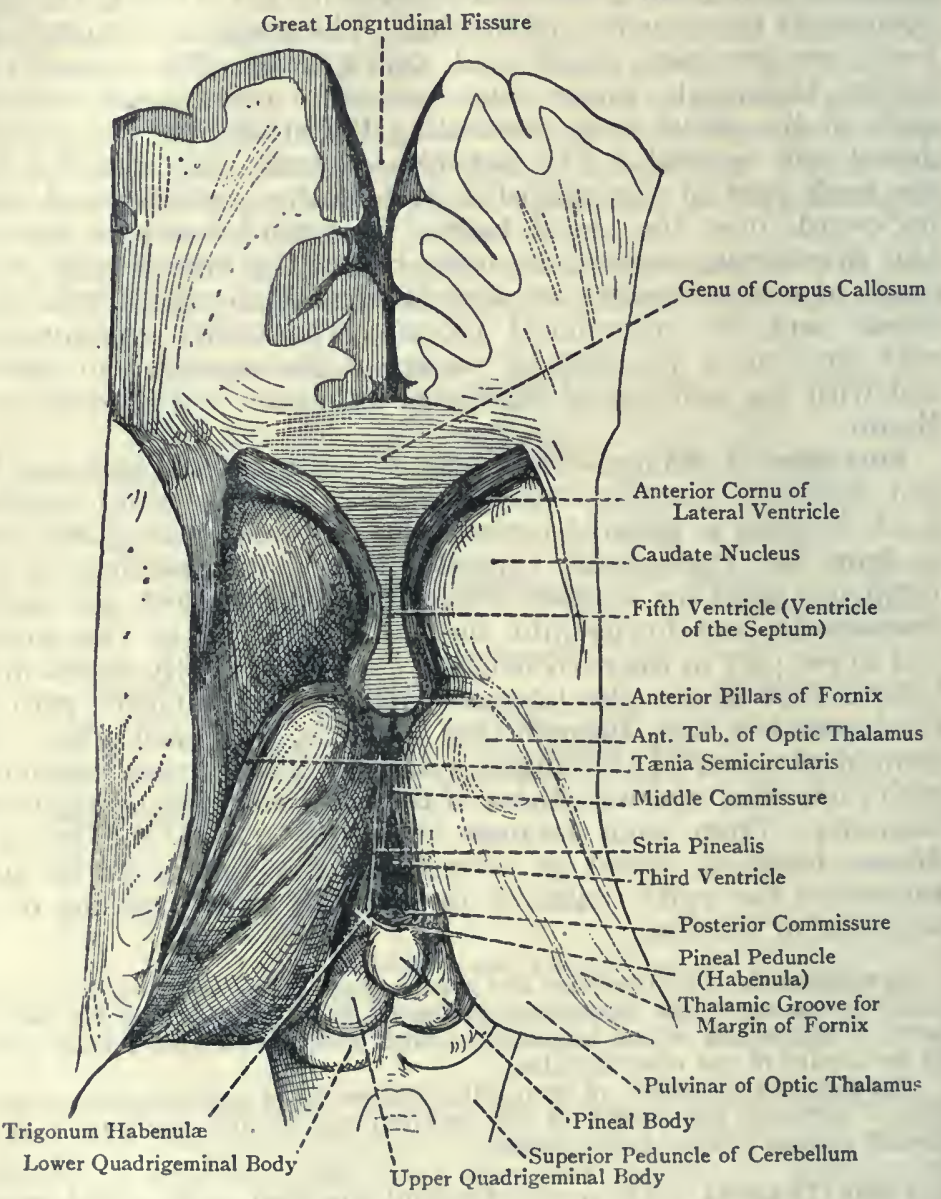

Fig. 598. - The Third Ventricee, Portions of the Lateral Ventricles, Pineal Body, and Corpora Quadrigemina (Superior View) (Henle).

(The Corpus Callosum, Fornix, and Velum Interpositum have been removed).

corpora quadrigemina. It is divided into two areæ, outer and inner, by a groove which is directed backwards and outwards from the inner border, near the anterior extremity, to the outer end of the posterior extremity. This groove corresponds to the 
lateral margin of the body of the fornix. The outer area enters into the body of the lateral ventricle, and is covered by the ependyma of that ventricle. The inner area is excluded from the lateral ventricle, and is covered by portions of the velum interpositum and body of the fornix. The superior surface is covered by a thin layer of white fibres, called the stratum zonale, these fibres being derived from the optic tract and optic radiation.

The inferior suriace lies posteriorly upon the upward prolongation of the tegmental fibres of the crus cerebri, which constitutes the subthalamic tegmental region, but anteriorly it rests upon the corpus mammillare and a portion of the tuber cinereum.

The external surface is directly related to the posterior limb of the internal capsule, which separates it from the nucleus lenticularis. Many fibres emerge from this surface and enter the internal capsule on their way to the cerebral cortex, whilst others from the cerebral cortex enter the optic thalamus through this surface. These fibres constitute the thalamic radiation. On the outer surface the fibres form a well-marked reticular layer of white matter, which is called the external medullary lamina.

The internal surface faces its fellow of the opposite side, with which it is connected by means of the middle or grey commissure. It forms the lateral wall of the third ventricle, and superiorly is limited by the stria pinealis or medullaris. As stated in connection with the third ventricle, it presents the sulcus of Monro. It is covered by a thick layer of grey matter, which is continuous with that around the aqueduct of Sylvius.

The anterior extremity is marked by a prominence, called the anterior tubercle, which enters into the body of the lateral ventricle, and forms the posterior boundary of the corresponding foramen of Monro.

The posterior extremity presents, at its inner end, a well-marked prominence, called the posterior tubercle or pulvinar. It lies over the brachia of the corpora quadrigemina, which it almost conceals. Below and external to the pulvinar there is an oval swelling, called the corpus geniculatum externum. Below this body is the upper brachium of the corpora quadrigemina, and inferior to this is the corpus geniculatum internum.

Metathalamus or Corpora Geniculata. - The corpora geniculata are two in number-external or lateral and internal or mesial. They are associated with the posterior extremity of the optic thalamus, and the internal also with that portion of the mesencephalon which constitutes the corpora quadrigemina.

The corpus geniculatum externum is an oval eminence situated on the posterior extremity of the optic thalamus below, and external to, the pulvinar. Internally it is connected with the upper quadrigeminal body by the superior brachium.

It consists of grey and white curved lamellæ, which alternate with each other. The fibres of the white lamellæ belong to the outer or visual root of the optic tract. The axons of the cells of the grey 
matter enter the optic radiation as corticipetal fibres. The lateral or outer geniculate body is associated with the visual sense.

The corpus geniculatum internum is a small oval eminence, which is situated below the pulvinar and on the lateral aspect of that portion of the mesencephalon which constitutes the corpora quadrigemina. The inferior brachium, which is beneath it, connects it with the lower quadrigeminal body. .

The inner geniculate body contains many nerve-cells, the axons of which become corticipetal fibres, their destination being the cortex of the temporal region of the brain. Through means of the lower quadrigeminal body and the internal brachium the inner geniculate body receives fibres from the lateral or acoustic fillet, which terminate in arborizations around its cells. The axons of these cells become corticipetal fibres, the destination of which is the cortex of the temporal region of the brain. The inner geniculate body is associated with the auditory sense.

Development.-The corpora geniculata appear as elevations on the lateral wall of the thalamencephalon or diencephalon.

Structure of the Optic Thalamus. - The optic thalamus is composed chiefly of grey matter. Its superior surface is covered with a layer of white matter, known as the stratum zonale, and its lateral surface is covered with a reticular layer of white matter, called the external medullary lamina. The mesial surface has a thick coating of grey matter which is continuous with the grey matter around the aqueduct of Sylvius.

The grey matter of the interior of the optic thalamus is traversed by a plate of white matter, called the internal medullary lamina, which divides it into two nuclear areas-lateral and mesial. The lateral nuclear area lies between the internal and external medullary laminx, and extends backwards as far as the pulvinar. The mesial nuclear area lies between the internal medullary lamina and the thick layer of grey matter which coats the mesial surface of the optic thalamus. It extends backwards as far only as the habenular region, and anteriorly it is separated from the anterior tubercle by a lamina of white matter. The region of the anterior tubercle therefore constitutes a third or anterior nuclear area of grey matter. The grey nuclear areas are consequently three in number-lateral, mesial, and anterior.

Lateral Nuclear Area.- This area includes the pulvinar, the geniculate bodies, and the radiate nucleus. The pulvinar and geniculate bodies have just been described. The radiate nucleus is associated with the fibres of the thalamic radiation, to be presently referred to.

Anterlor Nuclear Area.- This area includes the anterior tubercle, and is the chief sensory nucleus. It receives corticifugal fibres, and its cells furnish corticipetal fibres. It also receives many of the fibres of the lateral fillet, as well as those of the superior cerebellar peduncle, and the fibres of the bundle of Vicq d'Azyr.

Mesial Nuclear Area.-This area contains the ganglion habenulæ, to be presently described.

Connections of the Optic Thalamus.-(I) Viewing the optic thalamus as an aggregation of 'cell-stations' in the course of the centripetal fibres of the tegmentum of the crus cerebri, the tegmental fibres probably all terminate in the thalamic cells. (2) Through means of the stratum zonale the optic thalamus is connected with the optic tract and optic radiation. (3) The cells of the anterior nucleus receive the fibres of the bundle of Vicq d'Azyr, which are continuous, through the corpus albicans, with the fibres of the anterior pillar of the fornix. (4) Thalamic Radiation.-This is composed of thalamo-cortical nbres which arise within the optic thalamus as the axons of the thalamic cells. They issue from its external and inferior surfaces, and pass to all parts of the cerebral cortex. They are conveniently arranged 
in four groups or stalks-frontal, parietal, occipital, and inferior or ventral. (a) The fibres of the frontal stalk, having emerged from the front part of the external surface, traverse the anterior limb of the internal capsule, and most of them pass to the cortex of the frontal lobe. Some of these fibres are thalamo-caudate and thalamo-lenticular, as regards their destination. (b) The parietal stalk, having issued from the external surface of the optic thalamus, passes for the most part through the internal capsule, but also, to a certain extent, through the external capsule, to the cortex of the parietal lobe, and the Rolandic region of the frontal lobe. (c) The occipital stalk issues from the outer part of the pulvinar, and, having traversed the postlenticular portion of the posterior limb of the internal capsule, it passes backwards and outwards, external to the posterior cornu of the lateral ventricle, and so reaches the cortex of the occipital lobe. (d) The inferior or ventral stalk emerges from the front part of the inferior surface of the optic thalamus, and its fibres arise as the axons of the cells of the outer and inner nuclei. The most superficial of these fibres constitute a band, called the ansa lent $i$ cularis, which enters the nucleus lenticularis, where it terminates. The remaining fibres pass outwards beneath the nucleus lenticularis to the cortex of the temporal lobe and island of Reil.

Besides the thalamo-cortical fibres there are cortico-thalamic fibres, which pass from the various parts of the cerebral cortex into the optic thalamus, where they terminate in arborizations around the thalamic cells.

Development.-The optic thalamus is developed as a thickening of the lateral wall of the thalamencephalon or diencephalon.

Subthalamic Tegmental Region. - This region represents the upward prolongation of the tegmental fibres of the crus cerebri beneath the posterior portion of the optic thalamus. The parts to be noted are as follows: upward prolongations of the red nucleus and substantia nigra of the tegmentum of the crus cerebri; the mesial fillet; the fibres of the superior peduncle of the cerebellum; and the corpus subthalamicum, or nucleus of Luys. The red nucleus and the substantia nigra gradually disappear, and are no longer visible at the level of the corpus albicans. The mesial fillet lies on the superficial and outer aspects of the red nucleus. The fibres of the superior peduncle of the cerebellum partly terminate in connection with the cells of the red nucleus, but many of them surround it in the form of a capsule. Beyond the red nucleus the mesial fillet, fibres of the superior cerebellar peduncle, and fibres which issue from the red nucleus enter the inferior surface of the optic thalamus, and terminate in connection with the thalamic cells. Some of these fibres may pass through the optic thalamus into the internal capsule, and thence to the cortex of the Rolandic region of the cerebral hemisphere. The corpus subthalamicum or nucleus of Luys is a small lenticular mass of grey matter, surrounded by white fibres, which lies on the outer side of the substantia nigra.

Epithalamus.-The epithalamus includes the following parts:
I. Pineal body.
2. Striæ medullares.
3. Trigonum habenulæ.
4. Posterior commissure.

Pineal Body, or Epiphysis Cerebri.-The pineal body is so named because it resembles a pine-cone. It is situated on the dorsal or superior surface of the mesencephalon, and occupies the depression between the upper quadrigeminal bodies. It is of small size, dark 
red in colour, and somewhat conical in shape. Superiorly it is intimately related to the pia mater, as that membrane passes through the transverse cerebral fissure to form the velum interpositum, and the splenium of the corpus callosum lies above it with the intervention of the pia mater. Inferiorly it is in contact with the depression between the upper quadrigeminal bodies. Its apex, which is directed downwards and backwards, is free. Its base is directed upwards and forwards, and contains the pineal recess, which is continuous anteriorly with the cavity of the third ventricle. The portion of the base which lies below this recess is connected with the posterior commissure, which separates it from the upper opening of the aqueduct of Sylvius. The portion above the recess contains the commissura habenularum and the superior commissure. Connected with either side of the base of the pineal body there is a bundle of. white fibres. These two bundles constitute the peduncles or stalks of the pineal body. Each stalk passes outwards, and soon terminates in the trigonum habenulæ.

Structure of the Pineal Body.-The pineal body is free from nervous constituents. It consists of a number of follicles, lined with epithelial cells, and containing a variable amount of calcareous matter, called acervulus cerebri or brain-sand, which is composed of calcium phosphate, calcium carbonate, magnesium phosphate, and ammonium phosphate.

Development.-The pineal body is developed as a diverticulum of the posterior part of the dorsal aspect of the thalamencephalon or diencephalon. This diverticulum for the most part becomes solid, but a portion of it persists as the pineal recess of the third ventricle.

The pineal body is regarded as representing the pineal eye of lizards.

Striæ Medullares or Pineales.-Each stria medullaris is a narrow strip of white longitudinal fibres, lying along the upper part of the mesial surface of the corresponding optic thalamus. It constitutes the habenula. Anteriorly, most of its fibres are derived from the olfactory lobe, more particularly the olfactory bulb and locus perforatus anticus. Some, however, may be derived from the anterior pillar of the fornix, and through the fornix from the cells of the hippocampus major. Posteriorly, the fibres are disposed in two ways. (I) The lateral fibres enter the ganglion habenulæ, and terminate in connection with its cells. (2) The mesial fibres curve inwards towards the base of the pineal body, in which they cross to the ofposite side, lying above the pineal recess. As they cross the median line they decussate with the mesial fibres of the opposite stria medullaris, and they terminate in the ganglion habenulæ of the side to which they have crossed. Their decussation is known as the commissura habenularum.

Trigonum Habenulæ. - This is a small triangular area which is bounded posteriorly by the upper quadrigeminal body, internally by the posterior part of the stria medullaris, and externally by the adjacent part of the optic thalamus. It contains an important group of multipolar nerve-cells, known as the ganglion habenulæ. This ganglion belongs to the mesial area of the optic thalamus. It receives some of the fibres of the stria medullaris, which come from 
the olfactory lobe, and, it may be, from the anterior pillar of the fornix. The axons of the ganglionic cells issue from the ventral surface of the ganglion and form a bundle, called the fasciculus retroflexus. This bundle passes downwards and forwards in the tegmentum of the crus cerebri, lying on the mesial side of the red nucleus. Its fibres terminate in connection with the cells of the ganglion interpedunculare, which is situated in the lower part of the locus perforatus.posticus, directly above the pons Varolii.

The ganglia habenularum are connected with each other by fibres which constitute the superior commissure or commissure of the habenular ganglia. These fibres cross in the dorsal part of the base of the pineal body, and are on a higher plane than the posterior commissure.

The striæ medullares, or habenulæ, and the ganglia habenularum are associated with the rhinencephalon or olfactory brain.

Posterior Commissure.-This is a band of white fibres which is situated at the back part of the third ventricle. It lies in the posterior wall of the ventricle, directly above the upper opening of the aqueduct of Sylvius, and underneath the base of the pineal body. Its fibres are regarded as arising from a nucleus in the grey matter of the lateral wall of the third ventricle, near the upper opening of the aqueduct of Sylvius. Some of the fibres of either side, after crossing, may descend in the tegmentum of the crus cerebri, as part of the dorsal longitudinal bundle of that side, and so reach the medulla oblongata.

Hypothalamus. - The hypothalamus consists of two partsmammillary and optic. The pars mammillaris hypothalami represents the two corpora mammillaria. The pars optica hypothalami includes the following structures:

I. Tuber cinereum.

2. Infundibulum.

3. Posterior or cerebral lobe of the pituitary body.

4. Optic commissure.

5. Lamina terminalis.

The corpora mammillaria are two small, white, pea-like bodies, which lie side by side directly in front of the locus perforatus posticus. Each is composed of white matter externally, and of a grey nucleus internally. The white matter is derived from the corresponding anterior pillar of the fornix, the fibres of which terminate in connection with the cells of the grey nucleus. This grey nucleus contains many cells, the axons of which give rise to two fasciculi, namely, the bundle of Vicq d'Azyr, and the peduncle of the corpus mammillare. The bundle of Vieq d'Azyr, which is apparently a continuation of the anterior pillar of the fornix, enters the optic thalamus, and its fibres terminate in connection with the cells of the anterior nucleus. The peduncle of the corpus mammillare passes downwards and backwards in the grey matter of the floor of the third ventricle to the tegmental region of the mesencephalon, but the mode of termination of its fibres is not known. 
Development.-The corpora mammillaria are developed from the ventral aspect of the thalamencephalon or diencephalon. Up to the fourth month of intra-uterine life they are represented by a single corpus mammillare, but after that period this divides into two corpora.

The tuber cinereum is an elevated area of grey matter which lies in front of the corpora mammillaria and behind the optic commissure, the anterior portion of each optic tract being on either side. It is continuous anteriorly with the lamina termilialis, and on either side with the grey matter of the locus perforatus anticus.

In the outer part of the tuber cinereum, in the vicinity of the optic tract. there is a collection of nerve-cells, which is variously spoken of as the basal gangllon of Meynert or the supra-optic nucleus of Cajal, and which is connected with the fibres of the commissure of Gudden.

Behind the tuber cinereum, and in front of the corpora mammillaria, there is a small prominence, mesially placed, called the eminentia saccularis of Retzius, who regards it as the homologue of the saccus vasculosus of some lower vertebrates, e.g., fishes.

Along the outer side of the tuber cinereum, close to the innermost fibres of the optic tract, there is a strand of grey matter containing nerve-cells. This collection of cells is known as the basal optic ganglion, or supra-optic nucleus.

The infundibulum is a funnel-shaped stalk which extends downwards from the anterior part of the inferior surface of the tuber cinereum to the posterior lobe of the pituitary body. Its upper part is hollow, and contains the infundibular recess or diverticulum of the cavity of the third ventricle. The infundibulum is the peduncle of the posterior lobe of the pituitary body.

Lamina Terminalis. - This is a thin plate of grey matter which extends between the upper surface of the optic commissure and the rostrum of the corpus callosum near the genu. On either side it is connected with the grey matter of the locus perforatus anticus. It forms the lower part of the anterior wall of the third ventricle.

Development.-The lamina terminalis represents the terminal part of the ventral wall of the embryonic neural tube, and it originally connects the anterior wall of the telencephalon with that of its fellow.

For the pituitary body and infundibulum, see Index.

\section{Optic Nerve, Optic Commissure, Optic Tract, and Optic Radiation.}

The optic nerves, or nerves of sight, in the cranial cavity are connected together at the optic commissure, where some of the fibres decussate. From the back part of the commissure each nerve, under the name of the optic tract, passes backwards round the crus cerebri to its cerebral connections.

The optic commissure or chiasma is somewhat quadrilateral, and rests upon the olivary eminence and optic groove of the sphenoid bone. It lies in front of the tuber cinereum and infundibulum, and its superior surface is connected with the lamina cinerea, and is intimately related to the anterior part of the floor 
of the third ventricle. Each extremity is connected anteriorly with the optic nerve, and posteriorly with the optic tract. On either side of the commissure is the locus perforatus anticus. The most of the fibres of the commissure proceed from each retina in the corresponding optic nerve, being afferent or centripetal; but at the back part of the commissure there are the fibres of the inner roots of the optic tracts, which have no connection with either retina. The decussation of fibres in the commissure is only partial. The fibres which arise in the nasal or inner half of the retina cross and enter the optic tract of the opposite side. The fibres which arise in the temporal or outer half of the retina take no part in the decussation, but pass directly backwards into the optic tract of the same side.

Occupying the back part of the commissure there are, as stated, some fibres which have no connection with either retina. These fibres constitute the commissure of Gudden. They lie behind the decussating fibres, and represent the fibres of the inner root of the optic tract of each side. They constitute the innermost fibres of each optic tract, and they connect the internal geniculate body of one side with its fellow of the opposite side.

Summary. - The fibres which arise in the nasal or inner half of one retina cross in the optic commissure, and enter the optic tract of the opposite side. The fibres which arise in the temporal or outer half of one retina pass directly backwards into the optic tract of the same side. The fibres of the inner root of each optic tract cross in the back part of the commissure, and form the commissure of Gudden, the fibres of which have no connection with the optic nerves, but connect the two internal geniculate bodies, right and left. The optic commissure therefore consists of the following groups of fibres: (I) The crossed fibres, which arise in the nasal or inner portion of each retina; (2) the uncrossed fibres, which arise in the temporal or outer portion of each retina, and occupy the outer part of the commissure; and (3) the fibres of the commissure of Gudden, which occupy the back part of the commissure.

The optic tract of each side is a flattened white band which passes backwards from the optic commissure. It curves round the crus cerebri, and in the region of the posterior extremity of the optic thalamus it divides into two roots, outer and inner. The outer or visual root is the larger of the two. It is chiefly composed of afferent fibres, which pass from the retina to the brain; but it also contains efferent fibres, which pass from the brain to the retina. The afferent fibres are derived from (I) the temporal or outer half of the retina of the same side, and (2) the nasal or inner half of the retina of the opposite side, the latter having crossed in the optic commissure. The fibres of the outer root terminate in the external geniculate body, the pulvinar of the optic thalamus, and the upper quadrigeminal body, reaching the last-named body through the superior brachium. They form arborizations around the cells of these bodies which constitute the terminal nuclei or lower visual centres of the outer or visual root. These lower visual centres are connected with the higher or cortical visual centre by 


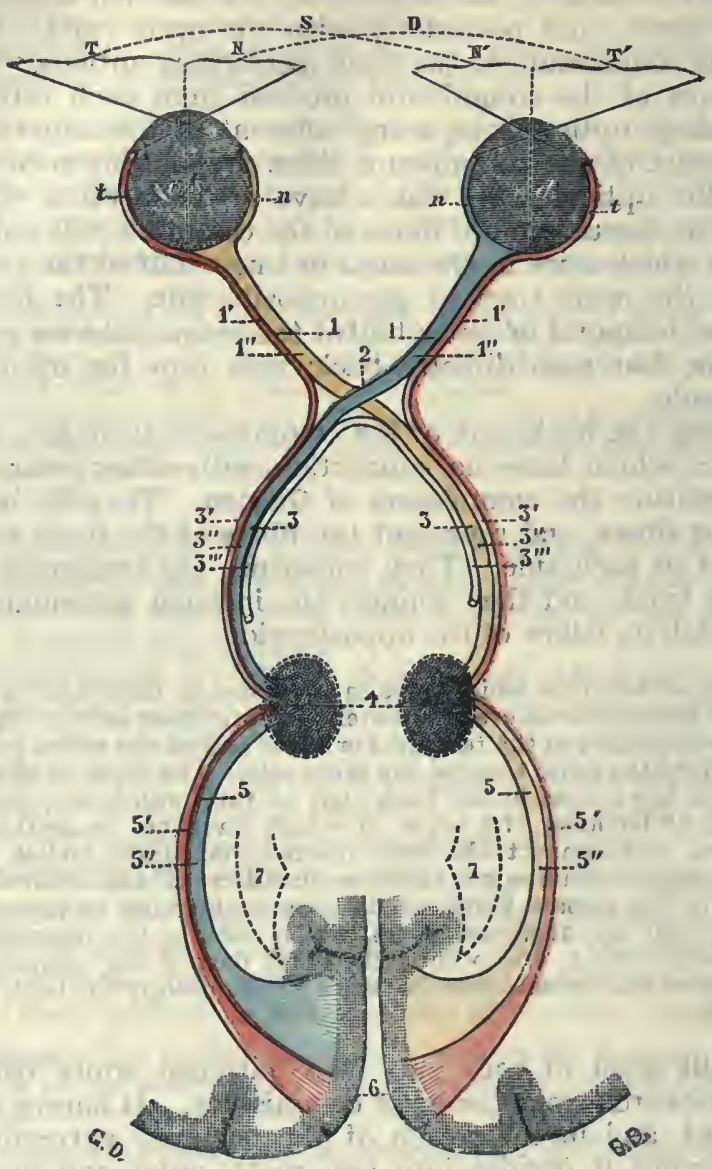

Fig. 599-Scheme indicating the Course of the OPtic Nerve-fibres from the Retina to the Cerebral, Cortex. (L.. Testut's ' Anatomie Humaine ').

\section{o.s. Left Eye}

o.d. Right Eye

$t$. Temporal Zone of Retina

n. Nasal Zone of Retina .

T.N. Temporal and Nasal Portions of the Field of $\mathrm{Vi}$. sion for the, Left Eye

$T N^{\prime}$. The corresponding portions of the Right Eye -

D. The Right Half, and $\mathrm{S}$, the Left Half, of the Field of Vision
I. Optic Nerve

r. Its Direct Fibres (in red) I". Its Crossed Fibres (in yellow on left side, and blue on right side)

2. Optic Commissure

3. Optic "Tract

3. Direct Fibres of Optic Tract.

3". Crossed Fibres of Optic Tract

$3^{\prime \prime \prime}$. Commissure of Gudden
4. Nuclei of Interruption

5. Optic Radiation

5'. Direct Fibres

5 ". Crossed Fibres

6. Cerebral Cortex (Mesial Aspect of Occipital Lobe) ?

7. Posterior Cornu of Lateral Ventricle 
the strand of fibres forming the optic radiation, the higher visual centre being situated in the cortex of the cuneate and lingual gyri of the mesial surface of the occipital lobe. The inner or commissural root of the optic tract passes beneath the internal geniculate body, which represents the nucleus of most of its fibres. As stated, these fibres have no connection with the optic nerve. Having: traversed the inner part of the optic tract, they cross in the back part of the optic commissure, behind the decussating fibres; and are continuous -with the corresponding fibres of the opposite side. These are the fibres which constitute the commissure of Gudden.

Summary of the Optic Tract.-The outermost fibres of each optic tract form the outer portion of the optic nerve of the same side. Most of the other fibres cross in the optic commissure, and form the inner portion of the optic nerve of the opposite side. The innermost fibres cross in the back part of the optic commissure, and form the commissure of Gudden.

Optic Radiation.-The strand of fibres which forms the optic or thalamo-occipital, radiation of either side establishes a connection between the lower visual centres (external geniculate body, pulvinar, and upper quadrigeminal body) and the higher or cortical visual centre, which is situated (I) on the mesial surface of the occipital lobe, close to the calcarine fissure in the region of the cuneus and lingual gyrus, and (2) on the adjacent part of the external surface of the occipital lobe. The strand passes through the post-lenticular part of the internal capsule, and then passes backwards in the medullary substance of the occipital lobe; lying on the outer side of the posterior cornu of the lateral ventricle. Thereafter the fibres pass in a radiating manner to the higher or cortical visual centre.

The optic radiation consists of afferent or corticipetal and efferent or corticifugal fibres. The corticipetal fibres, for the most part, arise as the axons of the nerve-cells within the pulvinar and external geniculate body, which are terminal nuclei of the retinal nervefibres, and they end in the higher or cortical visual centre. Some corticipetal fibres arise in the higher or cortical visual centre of the opposite side and cross in the splenium of the corpus callosum. These fibres are of a commissural character. The corticifugal fibres arise as the axons of the pyramidal cells of the cortex of the visual area of the occipital lobe, and they terminate in the pulvinar and upper quadrigeminal body.

The lower visual centres are regarded as being connected with the nuclei of origin of the nerves which supply the ocular muscles, probably through means of the posterior longitudinal bundle.

\section{Mesencephalon.}

The mesencephalon is composed of the corpora quadrigemina, which form its upper or dorsal portion; the crura cerebri, which form its lower or ventral portion; and the aqueduct of Sylvius, which passes through it from the fourth ventricle below to the third ventricle above. 
Corpora Quadrigemina.-These are four rounded eminences, which, as just stated, form the dorsal portion of the mesencephalon. They are covered by the splenium of the corpus callosum, and are arranged in pairs, upper and lower, the upper pairs (nates) being larger than the lower pair (testes), but not quite so prominent. The four eminences are separated from each other by two grooves, longitudinal and transverse, which are arranged in a crucial manner. The longitudinal groove extends upwards as far as the posterior commissure, and it separates the upper and lower quadrigeminal bodies of one side from those of the other side. Its upper part lodges the pineal body, and from its lower part a band of white

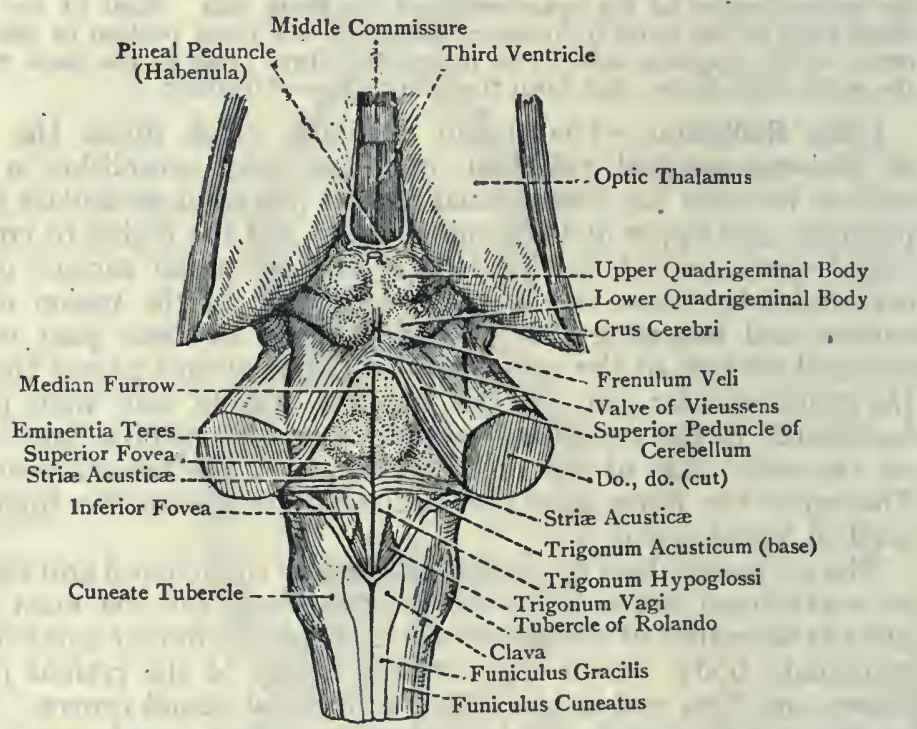

Fig. $600 .-$ The Floor of the Fourth Ventricle and Adjacent Parts.

fibres, called the fremulum veli, passes downwards to the superior medullary velum or valve of Vieussens, which lies below the lower pair of eminences. The transverse groove separates the upper pair of quadrigeminal bodies from the lower pair. Laterally each eminence is connected with a white band, called the brachium, the two brachia being separated by a continuation of the transverse groove.

The superior brachlum extends outwards and forwards from the upper quadrigeminal body to the external geniculate body and the outer root of the optic tract. It passes between the pulvinar of the optic thalamus and the internal geniculate body. Its fibres terminate within the internal geniculate body. These fibres are derived from the lateral or acoustic fillet, partly directly, and partly through the intervention of the lower quadrigeminal body. 
The superior brachium is associated with the visual apparatus. The inferior brachium, though connected with the internal geniculate body, with which body the inner or commissural root of the optic tract (commissure of Gudden) is also connected, is associated with the acoustic apparatus.

The superior brachium contains two sets of fibres, namely, retinal fibres, derived from the outer root of the optic tract, and (2) occipital fibres, from the cortex of the occipital lobe of the cerebrum.

The inferior brachium passes upwards from the lower quadrigeminal body to the under aspect of the internal geniculate body,

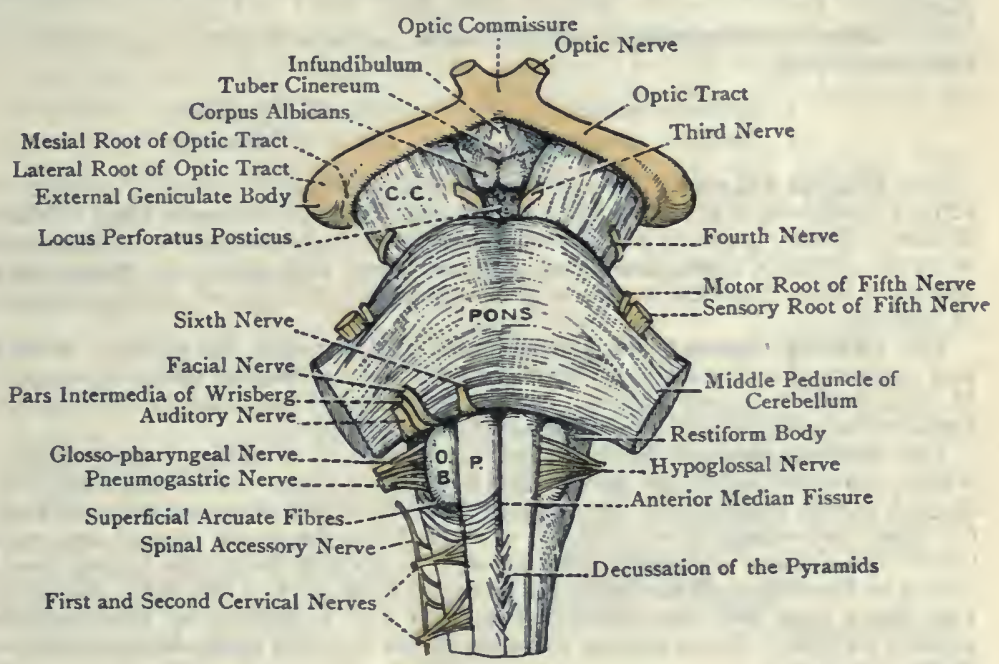

Fig. 6oi.-The Medulla Oblongata, Pons Varolit, and Interpedunctilar REGION.

C.C., Crus Cerebri ; P., Pyramid ; O.B., Olivary Body.

which is a small oval mass on the lateral aspect of the mesencephalon, under cover of the pulvinar of the optic thalamus. Though the inner root of the optic tract is connected with the internal geniculate body, the inferior brachium passes clear of it, and the most of its fibres are traceable to the optic thalamus through the tegmentum.

Structure of Corpora Quadrigemina.-The lower quadrigeminal body (colliculus inferior B.) is composed of the following parts:

I. A central nucleus of grey matter.

2. A dorsal layer of white matter.

3. A ventral layer of white matter.

The central grey nucleus consists of many multipolar cells and nerve-fibres. The axons of the cells pass partly to the dorsal and partly to the ventral layers of white matter. The nerve-fibres are derived from the lateral or 
acoustic fillet, and they terminate in arborizations, around the cells of the central nucleus.

The dorsal white layer derives its fibres from the lateral fillet and from the axons of the cells of the central grey nucleus. The fibres pass into the inferior brachium, by which they are conducted to the internal geniculate body.

The ventral white layer also derives its fibres from the lateral fillet and from the axons of the cells of the central grey nucleus. This layer separates the central nucleus from the subjacent grey matter of the aqueduct of Sylvius. Some of the fibres cross the median plane, and decussate with corresponding fibres of the opposite side, superficial to the roof of the aqueduct of Sylvius. Others enter the tegmentum of the crus cerebri of the same side and also of the opposite side, in which their course is downwards in the lateral fillet.

The lower quadrigeminal body (colliculus inferior B.), which receives its fibres from the lateral or-acoustic fillet, is associated with the acoustic apparatus.

The upper quadrigeminal body (colliculus superior B.) is composed of the following layers:
1. Stratum zonale.
2. Stratum cinereum.
3. Stratum opticum.
4. Stratum lemnisci.

The stratum zonale is the most superficial layer, and probably consists of retinal fibres which are derived from the outer root of the optic tract. Many of these fibres pass into the stratum cinereum and terminate in connection with its cells. Others cross the median plane and decussate with corresponding fibres from the opposite side, superficial to the roof of the aqueduct of Sylvius.

The stratum einereum, or second layer, lies beneath the stratum zonale, and consists of a crescentic layer of grey matter, containing many nerve-cells. It represents the grey nucleus of the upper quadrigeminal body, and the axons of its cells pass to the more deeply seated strata.

The stratum opticum is the third layer, and it consists of grey matter, which contains numerous nerve-cells and nerve-fibres. The fibves are conducted to this stratum by the superior brachium, and they are of two kinds: (1) Many are retinal fibres and are derived from the outer root of the optic tract. (2) Others are corticifugal fibres, which come from the higher visual centre in the cortex of the occipital lobe and form part of the optic radiation. The fibres pass into the stratum cinereum and terminate in arborizations around its cells. The axons of the cells of the stratum opticum pass into the stratum lemnisci.

The stratum lemnisci is the deepest layer. Like the stratum. opticum, it consists of grey matter, which contains numerous nerve-cells and nervefibres. The fibres are derived from the following sources: (I) Many are derived from the mesial or main fillet; and (2) some are the axons of cells belonging to the stratum opticum and stratum lemnisci. The fillet-fibres terminate in the stratum lemnisci. The fibres ${ }^{2}$ formed by the axons of the cells of the stratum opticum and stratum lemnisci cross the median plane and decussate with the corresponding fibres of the opposite.side. This decussation is known as the fountain-docussation of Meynert. The fibres, after crossing, form the ventral longitudinal bundle, or tecto-spinal tract, of that side, and this bundle or tract descends through the pons Varolii and medulla oblongata into the corresponding ventral or anteitor column of the spinal cord.

The upper quadrigeminal body, through means of the superior brachium, is one of the three lower visual centres, the other two being the pulvinar and the external geniculate body, both of which belong to the optic thalamus.

Development of Corpora Quadrigemina.-The corpora quadrigemina are developed from the dorsal wall or roof of the mesencephalon.

Commissure of 'Meynert. - This commissure or decussation consists of fibres which issue from each upper quadrigeminal body and 
cross to the opposite posterior longitudinal bundle, in which they descend towards the pons Varolii.

Crura Cerebri.-The crura or pedunculi cerebri are two large strands which are situated above the pons Varolii. "They lie at first near each other, being separated by the interpeduncular fossa, but afterwards diverge as they pass upwards and outwards to the cerebral hemispheres. The inner surface of each crus looks towards the interpeduncular region, and it presents a furrow, called the oculo-motor sulcus, through which the funiculi of the third cranial, or oculo-motor, nerve emerge, near the pons. The outer surface looks towards the temporal lobe of the brain, which to a large extent overlaps the crus, and this surface also presents a furrow, called the sulcus lateralis. The slender fourth cranial nerve lies upon this surface. Close to the cerebral hemisphere the ventral and outer aspects of the crus are embraced by the optic tract of the corresponding side.

Each crus is composed of two parts-ventral and dorsal. The ventral part is variously known as the crusta, basis, or pes, and the dorsal part is called the tegmentum. The separation between these two parts is indicated superficially by the sulcus lateralis on the outer surface, and the oculomotor sulcus on the inner sur-Aqueduct of face. Within the crus the two parts are separated by a mass of dark grey matter, called the substantia nigra.

The crusta is continuous superiorly with the internal capsule of the corpus striatum, and inferiorly its fibres enter the ventral part of the pons Varolii. The upward prolongation of the tegmentum constitutes the subthalamic tegmental region.

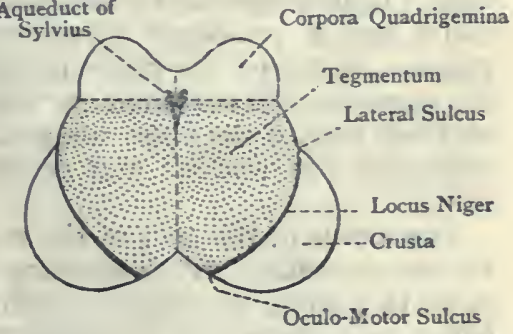

Fig. 602.-TOPOGRAPHY OF THE CRUS CEREBri (AFTER PoIrIER).

Structure of the Crusta, Basis, or Pes.-The crusta, as seen in transverse section, presents a crescentic outline, the concavity of the crescent being occupied by the convexity of the substantia nigra. It consists of longitudinal corticifugal fibres, which arise in the cells of the cerebral cortex. These fibres form two groupspyramidal and cortico-pontine.

The pyramidal fibres, on either side, constitute the motor tract from the pre-Rolandic or motor region of the cortex of the frontal lobe, and they arise, for the most part, from the cells of that region, which control the muscles of the upper and lower limbs, as well as the muscles of the face, though indirectly.

Tegmentum. - The tegmentum is continuous inferiorly with the formatio reticularis of the dorsal portion of the pons Varolii, which in turn, is continuous inferiorly with the formatio reticularis of the medulla oblongata. The two tegmenta, right and left, are separated. 
from each other by a median raphé, which is continuous with that of the pons Varolii. In the lower part of the mesencephalon this raphé is indistinct on account of the decussation which takes place across the median plane between the superior cerebellar peduncles, underneath the lower pair of quadrigeminal bodies.

The dorsal surface of each tegmentum extends on either side of the grey matter of the aqueduct of Sylvius, and becomes continuous with the basal parts of the upper and lower quadrigeminal bodies of the corresponding side. The ventral surface is separated from the crusta by the substantia nigra.

Structure of the Tegmentum.-Each tegmentum, as stated, is continuous inferiorly with the formatio reticularis of the dorsal portion of the pons Varolii. It consists of bundles of longitudinal fibres, separated by bundles of curved fibres which pass transversely. The intervals between these bundles are occupied by grey matter.

Grey Matter.-The grey matter of the tegmentum contains the red nucleus.

The red nucleus (nucleus ruber) is a round reddish mass, which is situated in the centre of the upper. part of the tegmentum, and it lies in the path of the superior cerebellar peduncle of the opposite side. It corresponds to the position of the upper quadrigeminal body, and is prolonged upwards into the subthalamic tegmental region. Some of the fibres of the superior cerebellar peduncle of the opposite side surround the red nucleus in the form of a capsule on their way to the optic thalamus. Other fibres of that peduncle enter the red nucleus and terminate in arborizations around its cells.

The axons of the cells of the red nucleus form two sets of nervefibres-ascending and descending. The ascending fibres pass to the optic thalamus, in company with those fibres of the superior cerebellar peduncle which encapsule the red nucleus. These ascending fibres form relays which carry on those fibres of the superior cerebellar peduncle which terminate within the red nucleus, that nucleus being a cell-station in their path. The descending fibres constitute the rubro-spinal tract, or bundle of Monakow. The fibres of this tract cross the median plane in the raphé, and by their decussation with those of the opposite side they constitute the fountain-decussation of Forel, in contradistinction to the fountain-decussation of Meynert. The latter decussation is on a higher level, and involves the fibres of the ventral longitudinal bundles, or tecto-spinal tracts, which derive their fibres from the cells of the stratum opticum and stratum lemnisci of the upper quadrigeminal bodies. The rubro-spinal tract of either side descends through the pons Varolii and medulla oblongata into the lateral column of the spinal cord, where each constitutes the prepyramidal tract, which lies on the ventro-lateral aspect of the crossed pyramidal tract. (The tectospinal tract, or ventral longitudinal bundle, on either side, descends into the anterior column of the spinal cord.) 
White Matter of the Tegmentum.-The principal tracts of the white matter on either side are as follows:

I. Superior cerebellar peduncle.

2. Dorsal longitudinal bundle.

3. Ventral longitudinal bundle, or tecto-spinal tract.

4. Rubro-spinal tract.

5. Mesial fillet (chief sensory tract).

6. Lateral (acoustic) fillet.

Superior Cerebellar Peduncle.-The fibres of this peduncle emerge, for the most part, through the hilum of the nucleus dentatus in the cerebellar hemisphere. The two peduncles, right and left, having emerged from the hemispheres, pass upwards on the lateral parts of the dorsal surface of the pons Varolii in a converging manner towards the lower pair of the quadrigeminal bodies, being connected by the valve of Vieussens. On entering the mesencephalon, the two peduncles decussate across the raphé beneath the lower quadrigeminal bodies. This decussation extends as high as the upper quadrigeminal bodies, and it involves almost all the fibres of the two peduncles. Each peduncle, having gained the opposite side, ascends in the upper part of the tegmentum as a longitudinal tract, and soon comes into contact with the red nucleus. Many of its fibres enter this nucleus and terminate in aborizations around its cells. Other fibres of the peduncle encapsule the nucleus, and then ascend through the subthalamic tegmental region to the anterior part of the optic thalamus, within which they terminate in arborizations around the cells of the anterior nuclear area (chief sensory nucleus). From the cells of the red nucleus relays of fibres proceed upwards, which carry on those fibres of the peduncle which terminate within the nucleus, and these relays ascend with those fibres of the peduncle which encapsule the red nucleus to the optic thalamus. From the cells of the optic thalamus other relays of thalamo-corticipetal fibres arise, which pass through the internal capsule to the cerebral cortex, more particularly the cortex of the ascending parietal or post-central gyrus, which lies directly behind the fissure of Rolando.

The superior cerebellar peduncle of one side connects the cerebellar hemisphere of that side with the post-Rolandic region of the cerebral cortex of the opposite side, the red nucleus and the optic thalamus being cell-stations in the path of the fibres.

A few of the fibres of each superior cerebellar peduncle do not take part in the decussation beneath the lower pair of quadrigeminal bodies, but ascend to the red nucleus of their own side.

Before the fibres decussate, or after the decussation has taken place, each of them furmishes a descending branch. These descending branches form the descending cerebellar bundle of Cajal, which traverses the dorsal part of the pons Varolii and the medulla oblongata, giving off collaterals to the motor nuclei of these parts. According to Cajal, the fibres of this bundle enter the anterior 
column of the spinal cord, and are connected with the cells of the ventral cornu of grey matter.

The superior cerebellar peduncle is almost entirely an ascending tract, but a few of its fibres are regarded as arising within the optic thalamus and as descending to the cerebellar hemisphere.

Dorsal or Posterior Longitudinal Bundle.-This bundle occupies the dorsal part of the tegmentum, and is intimately related to the grey matter which forms the ventral wall or floor of the aqueduct of Sylvius. It lies close to the median raphé, as does its fellow of the opposite side, and across the raphe an interchange of fibres takes place between the two bundles. In 'the 'spinal cord ' it is represented by the anterior ground-bundle and the anterior marginal bundle of Lowenthal. In the medulla oblongata it traverses the dorsal part of the pyramid, being separated from the,pyramidal (motor) fibres by the mesial fillet or chief sensory 'tract. 'Thereafter it traverses the formatio reticularis of the dorsal part of the pons Varolii, and is continued upwards as one of the tracts of the tegmentum of the crus cerebri.

The fibres of the dorsal longitudinal bundle are regarded as being the axons of cells belonging to ( $r$ ) the nucleus of Deiters, which is one of the terminal nuclei of the vestibular root of the auditory nerve, (2) the formatio reticularis of the medulla oblongata and pons Varolii, (3) the formatio reticularis of the tegmentum, (4) the sensory nucleus of the fifth cranial nerve, and (5) the nucleus of the dorsal longitudinal bundle. 'Inferiorly' the fibres of 'the bundle ramify within the anterior column of the spinal cord in connection with the motor cells of the ventral cornu of grey matter. Superiorly its fibres are intimately related to the following important nucleinamely, (I) the oculo-motor nucleus, or nucleus of the third cranial nerve; (2) the trochlear nucleus, or nucleus of the fourth cranial nerve; and (3) the abducent nucleus, or nucleus of the sixth cranial nerve, these 'being the nuclei which control the muscles of the eyeball and upper eyelid. The bundle furnishes numerous collaterals to each of these nuclei, which terminate in arborizations around their cells. The bundle also establishes connections with the motor nuclei in the pons Varolii and medulla oblongata.

The dorsal longitudinal bundle extends as high as a special nucleus, called the 'nucleus of the dorsal longitudinal bundle, which is situated in the grey matter of the ventro-lateral portion of the third ventricle, near the upper opening of the aqueduct of Sylvius, from the cells of which nucleus some of its' fibres arise.

The dorsal longitudinal bundle consists of ascending and descending association-fibres, which form connections between the important nuclei just referred to. Probably the chief use of the bundle is to maintain a functional association between these nuclei, and insure harmonious action of the muscles which are supplied by the nerves arising from them.

Ventral or Anterior Longitudinal Bundle.-This bundle constitutes the tecto-spinal tract, and it lies on the ventral aspect of the 
dorsal longitudinal bundle. Its fibres are derived from the stratum opticum and stratum lemnisci of the upper quadrigeminal body of the opposite side. These fibres, as stated in connection' with the upper quadrigeminal bodies, cross the median plane and decussate with the corresponding fibres of the opposite side, the decussation being known as the fountain-decussation of Meynert. The fibres descend through the tegmentum, lying close to the red nucleus, to which they furnish collaterals. Thereafter they traverse the formatio reticularis of the pons Varolii and medulla oblongata, still lying on the ventral aspect of the dorsal longitudinal bundle. From the medulla oblongata the fibres pass into the anterior ground-bundle of the ventral column of the spinal cord, in association with the fibres of the dorsal longitudinal bundle, and they form arborizations around the motor cells of the ventral cornu of grey matter.

Rubro-Spinal Tract, or Bundle of Monakow.-The fibres of this tract are derived, as previously stated, from the axons of the cells of the red nucleus. They cross the median plane, decussating with the corresponding fibres of the opposite side, and constituting the forntain-decussation of Forel. The tract then descends through the pons Varolii and medulla oblongata into the lateral column of the spinal cord, in which it constitutes the prepyramidal tract on the ventro-lateral aspect of the crossed pyramidal tract.

Mesial Fillet.-The mesial or main fillet of either side, which represents the chief sensory tract, commences in the lower part of the medulla oblongata. It here constitutes the only fillet on either side, and its fibres are derived from the deep lemniscal arcuate fibres, which arise from the cells of the muclens gracilis and nucleus cuneatus of the opposite side. The main fillet therefore represents the upward continuation of the posterior column of the spinal cord (columns of Goll and of Burdach), and it is spoken of as the chief sensory tract. The deep lemniscal arcuate fibres, as previously stated, cross the median plane directly above the decussation of the pyramids (motor decussation), and they decussate with the corresponding fibres of the opposite side, thus constituting the decussation of the fillets (main fillets, or-chief sensory tracts), or the sensory decussation. The fibres, after crossing the median plane, form the fillet of the side to which they have crossed. 'In the medulla oblongata it lies close to the median raphe, and at first is internal to the dorsal longitudinal bundle. Subsequently, however, the main fillet takes up a position in front of the dorsal longitudinal bundle, and directly behind the pyramid. The main fillet then ascends through the dorsal part of the pons Varolii, its relative position remaining unchanged. In this situation the lateral fillet, to be presently described, takes up its position on the outer or lateral aspect of the main or mesial fillet. The main fillet, on leaving the pons Varolii, enters the ventral part of the tegmentum, still having the lateral fillet on its outer side. As it encounters the red nucleus, it is displaced outwards and back- 
wards, and then occupies the dorso-lateral part of the tegmentum, lying almost beneath the internal geniculate body.

The fibres of the main or mesial fillet (chief sensory tract) terminate in two ways. (I) Some enter the upper quadrigeminal body, and these probably terminate in the stratum lemnisci. (2) Others traverse the subthalamic tegmental region and enter the anterior part of the optic thalamus, within which they terminate in arborisations around the cells of the anterior nuclear area (chief sensory nucleus). From these cells relays of thalamo-corticipetal fibres proceed to the cerebral cortex.

It is convenient to refer to the main or mesial fillet as the sensory fillet.

Lateral Fillet. - The main or mesial fillet being the sensory fillet, it is convenient to refer to the lateral fillet as the acoustic fillet. The fibres of this fillet are derived from the following sources: (I) The corpus trapezoides, the fibres of which come from the ventral cochlear nucleus, the nucleus trapezoides, and the superior olive of the opposite side, as well as from the superior olive of the same side; (2) the striæ acusticæ, which are derived from the lateral cochlear nucleus of the opposite side; and (3) the nucleus of the lateral fillet.

The fibres of the right and left lateral fillets decussate across the median plane. Having crossed to the opposite side, the fibres become longitudinal and form a well-marked ascending tract in the dorsal part of the pons Varolii, which takes up a position on the lateral or outer side of the main or mesial fillet. In this part of its course the lateral fillet encounters a collection of grey matter, called the nucleus of the lateral fillet. Some of its fibres end in this nucleus. Others pursue their upward course, and are reinforced by relays of fibres which arise from the nerve-cells of the nucleus. On leaving the pons Varolii the lateral fillet enters the tegmentum, and its fibres terminate in (I) the nuclei of the lower quadrigeminal body, and (2) the cells of the internal geniculate body. The fibres destined for the lower quadrigeminal body, having curved round the outer aspect of the superior cerebellar peduncle, become superficial on the outer side of the tegmentum. The fibres destined for the internal geniculate body reach it through the inferior brachium, partly directly and partly through the intervention of the lower quadrigeminal body. The axons of the cells of the internal geniculate body form corticipetal fibres which pass to the cortex of the first or superior temporal gyrus of the temporal lobe.

The lateral fillet, as previously stated, is associated with the auditory apparatus. It is chiefly composed of ascending fibres. There are, however, some descending fibres which are probably derived from the lower quadrigeminal bodies.

In addition to the foregoing tracts there other tracts.

Fasciculus Retroflexus. - The fibres of this bundle, already described, arise from the cells of the ganglion habenula. They descend in the upper part of the tegmentum, internal to the red nucleus, and they terminate in arborizations around the cells of the interpeduncular ganglion. 
Central Tract. - The fibres of this tract descend from the optic thalamus, or, it may be, from the nucleus lenticularis of the corpus striatum, through the pons Varolii into the medulla oblongata. Its fibres are regarded as terminating in connection with the inferior olivary nucleus.

Bundle of Munzer. - The fibres of this tract descend from the lower quadrigeminal body to the formatio reticularis of the lateral part of the pons Varolii.

Spino-thalamic Tract. - The fibres of this tract, as stated in connection with the tracts of the spinal cord, arise from the cells of the dorsal grey cornu of the opposite side. Having crossed in the ventral or white commissure, they enter the antero-lateral or indirect cerebellar tract (tract of Gowers) in which they ascend through the medulla oblongata, pons Varolii, and tegmentum of the crus cerebri to the optic thalamus of the side to which they have crossed.

Subthalamic Tegmental Region.-This region represents the upward prolongation of the tegmentum of the crus cerebri beneath the posterior part of the inferior or ventral surface of the optic thalamus. The prolongation contains (I) an upward extension of the red nucleus of the tegmentum, (2) the fibres of the superior peduncle of the cerebellum, and (3) the main or mesial fillet (chief sensory tract).

The upward extension of the red nucleus ceases about the level of the corresponding corpus mammillare. Some of the fibres of the superior peduncle of the cerebellum terminate, as stated, in the red nucleus, and others encapsule it, as they do in the tegmentum. Many fibres issue from the cells of the red nucleus, and these, along with the investing fibres of the superior cerebellar peduncle, enter the inferior or ventral surface of the optic thalamus.

The main fillet (chief sensory tract), which lies on the dorsolateral aspect of the red nucleus, also enters the inferior or ventral surface of the optic thalamus.

Development of the Crura Cerebri.-The crura cerebri are developed from the ventral wall of the mesencephalon.

Crusta or Pes.-The crusta or pes is, as stated, the ventral portion of the crus cerebri, and it is separated from the tegmentum of the crus by a mass of dark grey matter, called the substantia nigra, which is situated in the interior. Externally the separation is indicated on the outer aspect by the lateral sulcus, and on the inner aspect by the oculo-motor sulcus, through which the fasciculi of the oculomotor nerve emerge. The crusta is continuous with the internal capsule of the corpus striatum, and it consists of longitudinal centrifugal fibres, which arise in the cells of the cerebral cortex. These fibres are arranged in two sets, namely, pyramidal and corticopontine. The pyramidal fibres form the motor tract from the Rolandic region of the cerebral cortex; and the cortico-pontine fibres are arranged in two strands, namely, fronto-pontine and temporo-pontine.

The pyramidal fibres form the motor tract from the Rolandic region of the cerebral cortex. They traverse the lenticular portion of the posterior limb of the internal capsule, and then occupy the middle three-fifths of the crusta. Thereafter they descend through the ventral portion of the pons Varolii and the pyramid of the 
medulla oblongata. In the lower part of the pyramid they give rise to the crossed and direct: pyramidal tracts. The crossed pyramidal tract, having taken part in the decussation of the pyramids, descends in the spinal cord, occupying the posterior part of the lateral column of the opposite side. The direct pyramidal tract descends in the spinal cord, occupying the inner portion of the anterior column of the same: side, and forming the column of Türck. Its fibres, however, cross at intervals to the opposite side:

As the pyramidal tract descends through the pons Varolii and medulla oblongata, some of its fibres pass to the motor nuclei of the cranial nerves in these regions.

The cortico-pontine or cortico-protuberantial fibres are arranged in two strands, fronto-pentine: and temporo-pontine. The fibres.

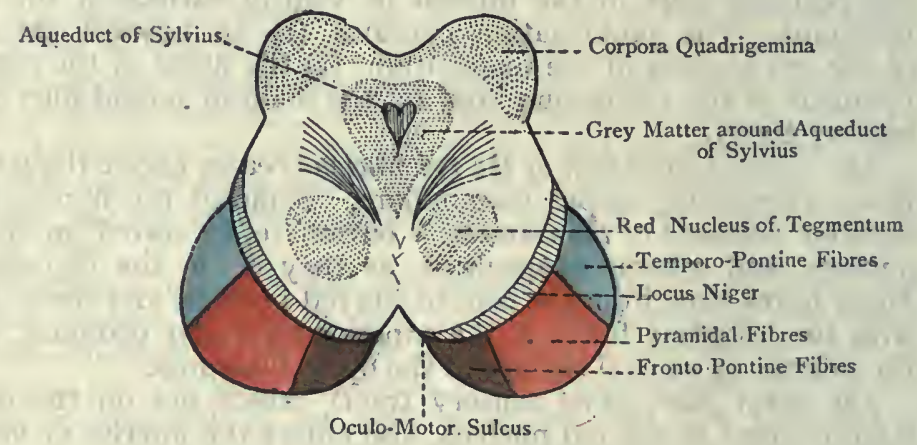

Fig. 603. - Transverse Section of the Crus Cerebri (after Polrier).

of the fronto-pontine' strand arise from the cells of the cortex of the anterior' part of the frontal 'lobe, and, having traversed the anterior limb of the internal capsule, they are regarded as occupying the inner fifth of thie crusta. The fibres' of the temporo-pontine' strand arise from the cell's of the cortex of the temporal lobe; and, having traversed the postlenticular part of the internal capsule, they occupy the outer fifth of the crusta. In the ventral part of the pons both the fronto-pontine and the temporo-pontine fibres terminate in arborizations around the cells of the nucleus pontis; whereas the pyramidal fibres pass uninterruptedly through the ventral part of the pons.

Summary, of the Crusta.-The crusta is divided into three parts. The middle three-fifths contain the fibres of the pyramidal or motor tract, the. inner fifth probably contains the fibres of the fronto-pontine strand, and the outer fifth contains the fibres of the temporo-pontine strand.

Substantia Nigra.-This is a mass of dark grey matter which is situated between the tegmentum and the crusta of the crus cerebri. Like the crusta, it is semilunar or crescentic, as seen in transverse section. It contains many multipolar nerve-cells, which are deeply pigmented, and it extends from the upper border of the pons 
Varolii into the subthalamic tegmental region. Laterally it reaches the lateral sulcus on the outer aspect of the crus, where it is thin, and the oculo-motor sulcus on the inner aspect, where it is thick and is traversed by the fasciculi of the third cranial or oculo-motor nerve. Its tegmental surface is concave, and the surface directed towards the crusta is convex. From the latter surface prolongations extend into the crusta.

Aqueduct of Sylvius.-The aqueduct of Sylvius is the narrow. passage which leads through the mesencephalon from the third to the fourth ventricle (iter a tertio ad quartum ventriculum). It lies nearer the dorsal than the ventral aspect of the mesencephalon; its direction is from above downwards; and its length is rather more than $\frac{1}{2}$ inch. Its upper opening is situated on the posterior boundary of the third ventricle, immediately underneath the posterior commissure, and its lower opening occupies the superior median angle of the floor of the fourth ventricle. In transverse section the aqueduct is T-shaped in its: upper part, near the third ventricle, and triangular in its lower part, near the fourth ventricle. The passage is lined with ciliated columnar epithelium, external to which there is a thick layer of grey, matter, which is spoken of as the Sylvian grey matter. This is continuous superiorly with the grey matter of the floor and lateral walls of the third ventricle, and inferiorly with that which covers the floor of the fourth ventricle. It contains numerous nerve-cells, disposed in a scattered manner, but, in addition to these, there are certain definite cell-groups. These groups constitute the nuclei of origin of the following cranial nerves: the third or oculo-motor, the fourth or trochlear, and the descending or mesencephalic root of the fifth nerve. The oculo-motor nucleus is situated in the ventral portion of the Sylvian grey matter, underneath the upper quadrigeminal body, and it extends upwards into the layer of grey matter on the adjacent portion of the lateral wall of the third ventricle. The trochlear nucleus is also situated in the ventral portion of the Sylvian grey matter, but at a lower level than the oculo-motor nucleus, being placed underneath the upper part of the lower quadrigeminal body. The nucleus of the descending or mesencephalic root of the fifth nerve is extensive, and is situated in the lateral portion of the Sylvian grey matter.

Development.-The aqueduct of Sylvius is the persistent remains of the cavity of the mesencephalon.

Locus Perforatus Posticus.-This space has been previously described, in a general way, in connection with the base of the encephalon. It will here be considered more fully. It lies at the bottom of a deep, depression, called the interpeduncular fossa (fossa Tarini), which forms the back part of the interpeduncular space. The fossa is bounded posteriorly by the median portion of the upper border of the pons Varolii, and laterally by the crura cerebri. Anteriorly it is limited by the corpora mammillaria. 
The locus perforatus posticus is a perforated lamina of grey matter which forms the floor of the interpeduncular fossa, the openings being for the passage of the postero-mesial central or ganglionic branches of the posterior cerebral arteries. This grey lamina extends between the tegmenta of the crura cerebri, and it is hence known as the pons Tarini.

Ganglion Interpedunculare.-This is a collection of nerve-cells, situated mesially in the lower part of the grey lamina which constitutes the locus perforatus posticus. On either side it receives the fibres of the fasciculus retroflexus, which are derived from the ganglion habenulæ.

\section{Structure of the Cerebral Hemispheres.}

The cerebral hemisphere is composed of grey and white matter. The grey matter is disposed externally, and forms the cerebral cortex. The white matter occupies the interior, and constitutes the medullary centre.

Cerebral Cortex.-The grey matter forms a continuous covering to the entire hemisphere, dipping into the sulci, so as to cover the opposed surfaces of the gyri, as well as the bottom of the sulci. It is thicker over the superficial surfaces of the gyri than at the bottom of the sulci, and attains its greatest thickness over the upper portions of the precentral and post-central gyri, whilst it is thinnest over the occipital lobe.

The cerebral cortex is indistinctly divided into strata by means of layers of a whitish substance. When examined on section it therefore presents a stratified appearance, and is seen to consist of successive grey and white layers, alternating with each other. In most parts of the cerebral cortex there are four superimposed strata; but in certain situations, e.g. over the precentral gyrus, there are as many as six. These strata are as follows, from without inwards:

I. Superficial white layer (pale and narrow).

2. Superficial grey layer.

3. Outer white band of Baillarger.

4. Middle grey layer.

5. Inner white band of Baillarger.

6. Inner or deep grey layer, subjacent to which is the white matter of the medullary centre.

The medullated fibres of the medullary centre pass into the stratified grey cortex in a radiating manner, and within the cortex their course, for the most part, is perpendicular to the superficial surface, and between the component cells of the cortex.

Minute Structure of the Cerebral Cortex.-The cortex is composed of nerve-cells and nerve-fibres.

Nerve-cells. - These are arranged in four layers, which are as follows from without inwards; (I) the molecular layer; (2) the 


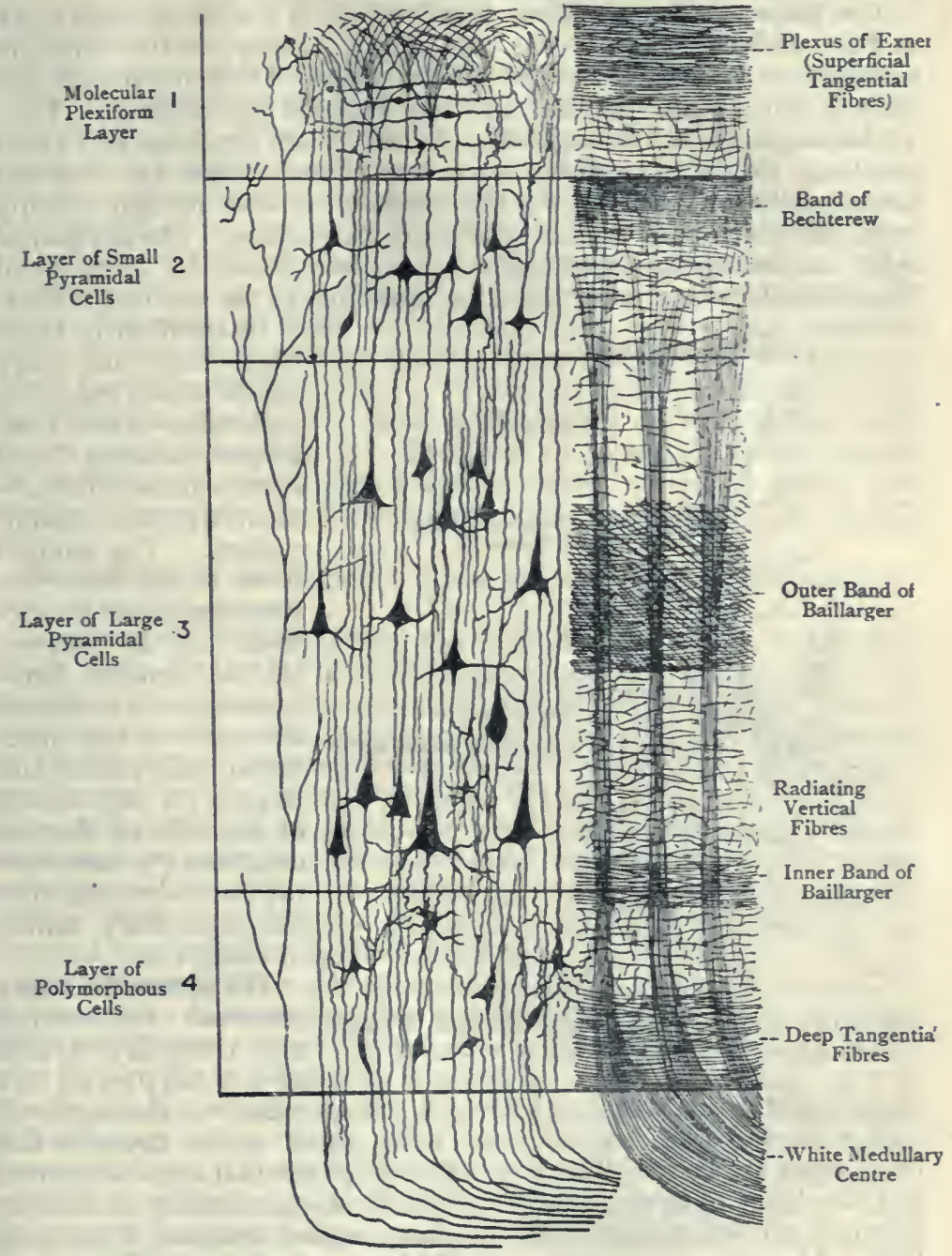

Fig. 604.-Diagram showing the Minute Structure of the Cerebral CORTEX (POIRIER).

The Fibres are shown on the right, and the Cells on the left. 
layer of small pyramidal cells; (3) the layer of large pyramidal cells; and (4) the layer of polymorphous cells.

The molecular or plexiform layer, which is the most superficial, is thin and consists of cells and fibres. Many of the cells are neuroglia-cells, the others being nerve-cells. These nerve-cells are for the most part fusiform, and are disposed horizontally. They are known as the horizontal cells of Cajal. Their dendrons and axons are long, the latter forming medullated fibres which are disposed horizontally or parallel to the surface. These furnish minute branches which pass vertically towards the surface. The horizontal cells, according to Cajal, receive impulses from the corticipetal fibres which extend from the optic thalamus to the cerebral cortex.

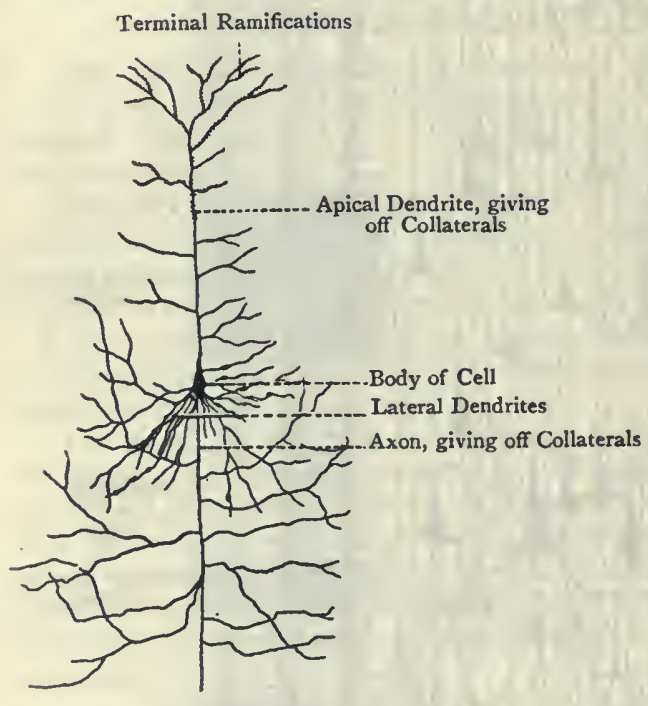

Fig. 605.-A Pyramidal Cell of the Human Cerrebral Cortex (Ramon y Cajal).

In addition to these fibres there are many others which enter the molecular layer from deeper sources, and form a dense interlacement by their ramifications. The sources from which these extraneous fibres are derived are as follows: (I) the terminal ramifications of the apical dendrons of the pyramidal cells (small and large); (2) the axons of the cells of Martinotti; and (3) corticipetal fibres derived from the medullary centre of the gyrus.

The pyramidal layers represent the second and third layers, and are composed of characteristic pyramidal cells which are peculiar to the cerebral cortex, those of the second layer being small, whilst those of the third layer are large. The layer of small pyramidal cells is narrow, but the layer of large pyramidal cells is of considerable thickness. There is no well-marked line of demarcation between these two layers, the one passing imperceptibly into the other. They constitute the chief part of the cerebral cortex.

The apex of each pyramidal cell is directed towards the surface of the gyrus, and is prolonged into a long tapering dendrite, which passes into the molecular layer, giving off delicate collaterals in its course. Near the surface of the molecular layer it divides into terminal filaments, which are disposed horizontally and mingle with 
the tangential fibres. The base of the pyramidal cell is directed towards the medullary centre of the gyrus, and from its centre an axon is given off, which enters the medullary centre, giving off collaterals in its course. From each side of the body of the cell, as well as from each lateral angle of its base, dendrites are given off.

The polymorphous layer is the deepest layer, and is composed of cells which have different shapes. Each cell gives off several dendrites, which pass towards, but do not enter, the molecular layer. The axon of each cell enters the medullary centre as a nerve-fibre.

In addition to the foregoing cells of the cerebral cortex, two other kinds of cells are met with amongst the pyramidal and polymorphous cells, namely, the cells of Golgi and the cells of Martinotti. The cells of Golgi are characterized by the fact that the axon of each almost immediately divides into several branches, which pass towards the surface, but soon terminate without entering the molecular layer. The cells of Martinotti are chiefly met with in the polymorphous layer. The axon of each cell passes towards the surface, and enters the molecular layer, where it divides into terminal branches, which form part of the tangential fibres of this layer.

Nerve-fibres of the Cortex.-These are arranged in two groupsvertical and tangential.

The vertical (radial) fibres are disposed in radiating bundles, which issue from the medullary centre, and traverse the polymorphous and large pyramidal layers, after which they become indistinguishable. The polymorphous and large pyramidal cells lie in the spaces between these bundles, and assume a columnar arrangement. The fibres of the radiating bundles gradually become less numerous, some of them becoming the axons of the polymorphous cells, but most of them becoming the axons of the large pyramidal cells. The radiating bundles contain centripetal cortical fibres, which pass into the molecular layer and end in terminal ramifications, forming part of its tangential fibres.

The tangential fibres are disposed horizontally at different levels, and form the following strata: (I) the superficial tangential fibres (plexus of Exner), which occupy the superficial part of the molecular layer; (2) the band of Bechterew, which is situated in the superficial part of the small pyramidal layer; (3) the outer band of Baillarger (band of Vicq d'Azyr), which intersects the large pyramidal layer; (4) the inner band of Baillarger, which is situated between the large pyramidal and polymorphous layers; and (5) the deep tangential fibres (intra-cortical association fibres), which are situated in the deep part of the polymorphous layer.

The tangential fibres are formed by (I) the collaterals of the polymorphous and pyramidal cells and of the cells of Martinotti; (2) the ramifications of the axons of the cells of Golgi; and (3) association fibres.

Medullary Centre of Cerebral Hemisphere.-The white matter of the medullary centre consists of medullated nerve-fibres, which 
pursue different courses, and are arranged in three groups, namely, projection-fibres, commissural fibres, and association-fibres.

The projection-fibres connect the cerebral cortex with parts at a lower level, and they are of two kinds-corticipetal or afferent, and corticifugal or efferent. The commissural fibres pass from one hemisphere to the other and connect portions of the cerebral cortex of opposite hemispheres. The association-fibres are confined to one side of the median plane, and they bring different parts of the cerebral cortex of the same hemisphere into association with one another.

Projection-Fibres.-These fibres, as stated, are both corticipetal and corticifugal, and the majority of them constitute the internal capsule of the corpus striatum, and the diverging arrangement of its fibres known as the corona radiata, which passes to all parts of the cerebral cortex. Some projection-fibres, however, do not traverse the internal capsule and corona radiata-e.g., the fibres of the ansa peduncularis.

Corticifugal Fibres.- The corticifugal or efferent fibres constitute the following tracts:

I. Pyramidal or motor.

2. Cortico-thaiamic.

5. Optic radiation.

3. Fronto-pontine.

4. Temporo-pontine.

The pyramidal or motor tract derives its fibres from the axons of the pyramidal cells of the cortex of the precentral gyrus, which is situated in front of the fissure of Rolando. Having traversed the corona radiata, these fibres pass, in succession, through (I) the posterior limb of the internal capsule, (2) the middle three-fifths of the crusta of the crus cerebri, (3) the ventral portion of the pons Varolii, and (4) the pyramid of the medulla oblongata. The motor strand enters the spinal cord in three ways - partly as the direct pyramidal tract or column of Turck-partly as the uncrossed lateral pyramidal tract-but chiefly as the crossed lateral pyramidal tract. Ultimately the fibres terminate at different levels in arborizations around the motor-cells of the ventral cornu of grey matter of the opposite side, from which cells the fibres of the motor nerve-roots proceed.

The cortico-thalamic tract extends only between the cerebral cortex and the optic thalamus. Its fibres arise as the axons of the pyramidal cells of various parts of the cerebral cortex, and they terminate in arborizations around the cells of the optic thalamus.

The fronto-pontine tract does not extend lower than the pons Varolii. It consists of fibres which arise as the axons of the pyramidal cells of the cortex of the prefrontal region-that is to say, the region of the frontal lobe in front of the precentral sulcus. These fibres traverse the anterior limb of the internal capsule, and then descend through the inner or mesial fifth of the crusta of the crus cerebri into the pons Varolii. Within the pons they terminate in arborizations around the cells of the nucleus pontis.

The temporo-pontine tract, like the preceding, does not extend 
lower than the pons Varolii. It consists of fibres which arise as the axons of the pyramidal cells of the cortex of the first and second temporal gyri. These fibres traverse the post-lenticular part of the posterior limb of the internal capsule, and then descend through the outer fifth of the crusta of the crus cerebri into the ventral part of the pons Varolii. Within this part of the pons they terminate in arborizations around the cells of the nucleus pontis.

The corticifugal fibres of the optic radiation consist of fibres which arise as the axons of the pyramidal fibres of the cortex of the occipital lobe. They traverse the post-lenticular part of the posterior limb of the internal capsule, and thereafter pass to the lower visual centres, namely, the external geniculate body, the pulvinar of the optic thalamus, and the upper quadrigeminal body. Within these bodies they terminate in arborizations around their component cells.

Corticipetal Fibres.-The corticipetal or afferent fibres belong to the following tracts:

\section{Mesial fillet. \\ 2. Superior cerebellar peduncle. \\ 3. Thalamic radiation. 5. Optic radiation. 4. Auditory radiation.}

The mesial fillet, or principal sensory tract, arises from the nucleus gracilis and nucleus cuneatus of the medulla oblongata, and is the upward prolongation of the posterior column of the spinal cord. Having decussated with its fellow, it ascends through the dorsal part of the pons Varolii, through the tegmentum of the crus cerebri, and through the subthalamic tegmental region to the optic thalamus. Within this body its fibres terminate in arborizations around the thalamic cells. As the mesial fillet ascends towards the optic thalamus some of its fibres enter the upper quadrigeminal body, in which they end. From the optic thalamus the fillet-fibres are continued to the cerebral cortex by relays of thalamo-cortical fibres.

The superior cerebellar peduncle, having decussated with its fellow, soon comes into contact with the red nucleus. Many of the fibres of the peduncle enter this nucleus and terminate in arborizations around its cells. Numerous fibres encapsule the nucleus, and continue their course upwards, traversing the subthalamic tegmental region, and finally entering the ventral aspect of the optic thalamus, within which they terminate in arborizations around the thalamic cells. As in the case of the fillet-fibres, they are continued to the cerebral cortex by relays of thalamo-cortical fibres.

The thalamic radiation is composed of thalamo-cortical fibres which arise as the axons of the cells within the optic thalamus, that body being regarded as an aggregation of cell-stations in the path of such corticipetal fibres as those of the mesial fillet and superior cerebellar peduncle. These thalamo-cortical fibres, as stated in the description of the optic thalamus, issue from that body in four groups or stalks-frontal, parietal, occipital, and inferior or ventral. The fibres of the frontal stalk traverse the 
anterior limb of the internal capsule, and most of them pass to the cortex of the frontal lobe. The fibres of the parietal stalk pass partly through the internal capsule and partly through the external capsule to the cortex of the parietal lobe and of the Rolandic region of the frontal lobe. The fibres of the occipital stalk pertain to the optic radiation, to be presently described. The fibres of the inferior or ventral stalk form the ansa lenticularis and ansa peduncularis. The ansa lenticularis enters the nucleus lenticularis, within which its fibres terminate. The ansa peduncularis passes beneath the nucleus lenticularis and traverses the external capsule, the destination of its fibres being the cortex of the temporal lobe and island of Reil.

The auditory radiation consists of fibres which arise as the axons of the cells of the internal geniculate body. Having issued from that body they traverse the post-lenticular part of the posterior limb of the internal capsule, and pass to the cortex of the middle part of the first temporal gyrus of the temporal lobe.

The corticipetal fibres of the optic radiation are associated with the corticifugal fibres, already described. The corticipetal fibres arise as the axons of the cells of the pulvinar, corpus geniculatum externum, and upper quadrigeminal body. They traverse the post-lenticular part of the posterior limb of the internal capsule, and thereafter pass to the cortex of the occipital lobe.

Commissural Fibres.-These fibres are disposed transversely, and serve to connect the grey cortex of one hemisphere with that of the other. They constitute the following commissures: (I) the corpus callosum; (2) the anterior commissure ; and (3) the lyra or psalterium, which is known as the hippocampal commissure. The fibres of the corpus callosum, as they enter each hemisphere, are disposed so as to form an extensive callosal radiation, and serve to connect the cortex of one hemisphere with that of the other. The individual portions of cortex so connected may be symmetrical, but to a large extent they are asymmetrical. The fibres arise on one side as (I) the axons of pyramidal or of polymorphous cells, or (2) collaterals of projection or of association fibres ; and on the opposite side they terminate in delicate arborizations.

The anterior commissure, which crosses from side to side in front of the anterior pillars of the fornix, divides on either side into two parts, olfactory and temporal. The olfactory portion enters the olfactory tract. Some of its fibres serve to connect the olfactory bulb of one side with that of the other side ; and other fibres connect the olfactory bulb of one side with the temporal lobe of the opposite. The temporal portion enters the white matter of the temporal lobe on either side.

The lyra or psalterium consists of transverse fibres which connect the hippocampus major of one side with its fellow of the opposite side; hence the name hippocampal commissure.

Association Fibres. - These fibres serve to connect different parts 
of the cortex of the same hemisphere, and they are of two kinds, short and long.

The short association fibres pass between contiguous gyri, extending in their course across the bottom of the sulci. Some of them lie beneath the grey cortex, whilst others are contained within its deep part.

The long association fibres pass between portions of the grey cortex, which are at some distance from each other. They are arranged in bundles, the chief of which are as follows: (I) the superior longitudinal fasciculus; (2) the inferior longitudinal fasciculus; (3) the perpendicular fasciculus; (4) the uncinate fasciculus; (5) the cingulum; (6) the occipito-frontal fasciculus; and $(7)$ the fornix.

The superior longitudinal fasciculus consists of fibres which extend from the frontal to the occipital lobe. Posteriorly many of its fibres sweep downwards and forwards into the temporal lobe, and from this circumstance it is sometimes spoken of as the arcuate fasciculus.

The inferior longitudinal fasciculus connects the occipital and temporal lobes, its fibres being disposed upon the outer walls of the posterior and descending cornua of the lateral ventricle.

The perpendicular fasciculus connects the inferior parietal gyrus with the occipito-temporal gyrus.

The uncinate fasciculus crosses the stem of the fissure of Sylvius, and connects the frontal and temporal lobes.

The cingulum is connected with the limbic lobe, and lies upon the under surface of the callosal gyrus and the upper surface of the hippocampal gyrus. Its fibres connect the gyri of the limbic lobe with the cerebral cortex.

The occipito-frontal fasciculus connects the frontal with the occipital and temporal lobes. According to Dejerine the tapetum of the corpus callosum belongs to this fasciculus, and not to the corpus callosum. The fasciculus lies internal to the corona radiata, in intimate relation to the nucleus caudatus, and, as the fibres pass backwards, they lie on the outer walls of the descending and posterior cornua of the lateral ventricle.

The fornix connects the hippocampus major of one side with the corresponding corpus albicans, and, through the latter, with the optic thalamus by means of the bundle of Vicq d'Azyr, the fibres of. which arise in the corpus albicans.

Peculiarities of the Cerebral Cortex-r. Calcarine Area.-This area is situated on the mesial surface of the occipital lobe, in close proximity to the calcarine fissure, and it is known as the visual area. In this region the outer band of Baillarger is very conspicuous, whilst the inner band of Baillarger is absent.

2. Rolandic Area.-In this region, more especially in the cortex of the precentral gyrus, there are groups of very large pyramidal cells, which are known as the giant-cells of Betz, and nerve-fibres are present in large numbers.

3. Hippocampal Area.-The hippocampus major corresponds to the hippocampal or dentate fissure, and is produced by an infolding of the cerebral 
cortex. It is therefore composed chiefly of grey matter, and is covered superficially by a thin layer of white matter, called the alveus, which is continuous with the fimbria. The hippocampus major is composed of the following layers, named in order from the ventricular surface outwards : ( 1 ) the alveus, composed of white matter, and covered by the ventricular ependyma ; (2) neurogliar layer, consisting of neuroglia fibres and cells; (3) pyramidal layer, composed of large pyramidal cells; (4) stratum radiatum, which is the outer part of the pyramidal layer, and is composed of the dendrites of the apical parts of the pyramidal cells, being thereby rendered striated in appearance ; $(5)$ stratum laciniosum, composed of the ramifications of the foregoing apical dendrites, intimately intermixed; (6) stratum granulosum, composed of many small cells; and $(7)$ the involuted medullary lamina, consisting of white fibres.

Olfactory Tract and Olfactory Bulb.-These are developed as a hollow outgrowth from the anterior cerebral vesicle, more particularly from the part of it which ultimately gives rise to the lateral ventricle, and is known as the telencephalon. In many animals the central cavity persists, and maintains its connection with the lateral ventricle; but in man the cavity disappears, though traces of its ependymal lining remain. External to the vestigial ependyma there is a layer of white matter, and, superficial to this, there is a layer of grey matter. In the olfactory tract the layer of grey matter is very thin over the ventral or inferior aspect, but over the dorsal or superior aspect it is fairly thick. In the bulb the reverse is the case, the grey matter being thick over the ventral aspect, where it receives the olfactory filaments, but thin over the dorsal aspect.

Structure of the Ventral Grey Matter of the Olfactory Bulb.The grey matter consists of three layers, namely (I) the nervefibre layer, (2) the glomerular layer, and (3) the granular layer.

The nerve-fibre layer is the most superficial layer, and is composed of olfactory nerve-fibres. These fibres are non-medullated, and arise as the axons of the olfactory cells of the olfactory mucous membrane of the nasal fossa. Having passed through the foramina of one half of the cribriform plate of the ethmoid bone, they enter the grey matter on the ventral aspect of the bulb, where they break up and form arborizations. These intermingle with the arborizations formed by the dendrites of the mitral cells, to be presently described.

The glomerular layer is composed of round bodies or glomeruli, which are formed by the interlacements between the arborizations of the olfactory nerve-fibres and those of the dendrites of the mitral cells.

The granular layer lies next to the layer of white matter, and is chiefly characterized by the presence of large mitral cells. These are pyramidal, and one dendrite from each cell passes into the glomerular layer, where it gives rise to a glomerulus in the manner just described in connection with the glomerular layer. Other dendrites intermingle with those of adjacent mitral cells. The axon of each mitral cell enters the white layer of the bulb, and passes along the olfactory tract to the cerebrum. 
Weight of the Brain.-The average weight of the brain of the adult male is about 48 ounces, and that of the adult female about 44 ounces.

\section{Arteries of the Encephalon.}

Cerebral Part of the Internal Carotid Artery.-The internal carotid artery, having pierced the roof of the cavernous sinus, internal to the anterior clinoid process of the sphenoid bone, ascends between the second and third cranial nerves to the inner end of the stem of the fissure of Sylvius. Here it divides into its terminal branches, namely, anterior cerebral and middle cerebral.

Branches.-These are as follows: posterior communicating anterior choroidal, anterior cerebral, and middle cerebral.

The posterior communicating artery arises from the back part of the internal carotid, and passes backwards to anastomose with the posterior cerebral artery. It is usually of small size, but is occasionally large, and it furnishes twigs to the crus cerebri and inner aspect of the optic thalamus.

The anterior choroidal artery arises from the back part of the internal carotid close to its termination. It passes backwards and outwards between the crus cerebri and the hippocampal gyrus, and it enters the lower and anterior extremity of the descending cornu of the lateral ventricle by passing through the choroidal fissure. In its course it gives twigs to the hippocampal gyrus and crus cerebri, and it terminates in the choroid plexus of the lateral ventricle.

Anterior Cerebral Artery.- This is the smaller of the two terminal branches of the internal carotid artery, and it bas a more limited distribution than the other terminal branch, namely, the middle cerebral artery. It passes forwards and inwards, above the optic nerve, to the commencement of the great longitudinal fissure, where it is connected with its fellow of the opposite side by a short transverse vessel, called the anterior communicating artery. Thereafter it enters the great longitudinal fissure, along with its fellow, turns round the genu of the corpus callosum, and passes backwards over the upper surface of that body to the splenium, where it anastomoses with the posterior cerebral artery.

Branches. - These are arranged in two groups, antero-mesial or ganglionic and cortical.

The antero-mesial or ganglionic branches, small in size, pass through the lamina cinerea, along with twigs from the anterior communicating artery, and supply the front part of the caudate nucleus.

The cortical branches are as follows: ( $($ ) internal orbital, to the internal orbital gyrus, olfactory lobe, and gyrus rectus ; (2) anterior internal frontal, to the superior frontal gyrus, the anterior twothirds of the middle frontal gyrus, and the anterior part of the marginal gyrus; (3) middle internal frontal, to the callosal gyrus, the posterior part of the marginal gyrus, and the upper part of the precentral gyrus ; and (4) posterior internal frontal, to the quadrate 
lobule and the corpus callosum, the branch to the latter being known as the artery of the corpus callosum.

Anterior Communicating Artery.-This vessel connects the two anterior cerebral arteries at the entrance to the great longitudinal fissure, and lies over the lamina terminalis, in front of the optic commissure. It gives off a few twigs, which accompany the anteromesial branches of each anterior cerebral artery.

Middle Cerebral Artery.-This is the larger of the two terminal branches of the internal carotid artery, and it has a wider distribution than the anterior cerebral artery. It enters the stem of the fissure of Sylvius, in which it passes outwards.

Branches.-These are arranged in two groups, antero-lateral or

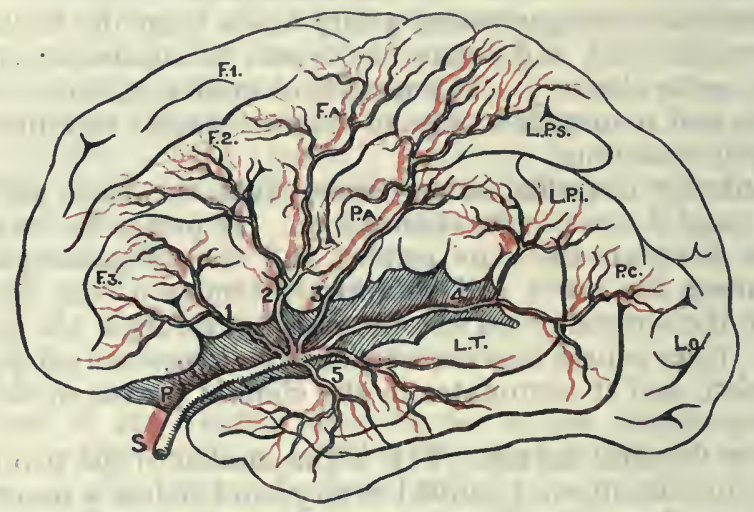

Fig. 606.-Distribution of the Left Middle Cerebral Artery (Charcot).

F.r. Superior Frontal Gyrus

F.2. Middle Frontal Gyrus

F.3. Inferior Frontal Gyrus

F.A. Ascending Frontal Gyrus

P.A. Ascending Parietal Gyrus

I.P.S. Superior Parietal Gyru

L.P.1. Inferior Parietal Gyrus

P.C. Angular Gyrus

L.O. Occipital Lobe

L.T. Temporal Lobe
S. Middle Cerebral Artery entering Fissure of Sylvius

P. Lenticulo-striate Arteries

I. Artery to Broca's Gyrus (Inferior Frontal Gyrus)

2. Ascending Frontal Artery

3. Ascending Parietal Artery

4. Parieto-Temporal Artery

5. Arteries to Temporal Lobe

ganglionic and cortical. The antero-lateral or ganglionic branches pass through the locus perforatus anticus, and form two sets, internal striate and external striate. The internal striate arteries represent the lenticular arteries of Duret, and they supply the globus pallidus (inner part) of the lenticular nucleus, the internal capsule, and the caudate nucleus. The external striate art ries represent the lenticulo-striate and lenticulo-optic arteries of Duret. The lenticulo-striate arteries supply the putamen (outer part) of the lenticular nucleus and the external capsule. One of the lenticulostriate arteries is larger than the others, and is subject to rupture in cases of cerebral hæmorrhage. It is known as the artery of cere- 
bral hemorrhage (Charcot). Its course is outwards and upwards round the outer aspect of the lenticular nucleus, between it and the external capsule, and then through the internal capsule to the caudate nucleus. The lenticulo-optic arteries supply the posterior part of the lenticular nucleus and the anterior part of the optic thalamus on its outer aspect.

The cortical branches arise in the vicinity of the island of Reil, and are as follows : (I) inferior external orbital, to the orbital surface of the frontal lobe external to the internal orbital sulcus, and to the inferior frontal gyrus; (2) ascending frontal, to the posterior

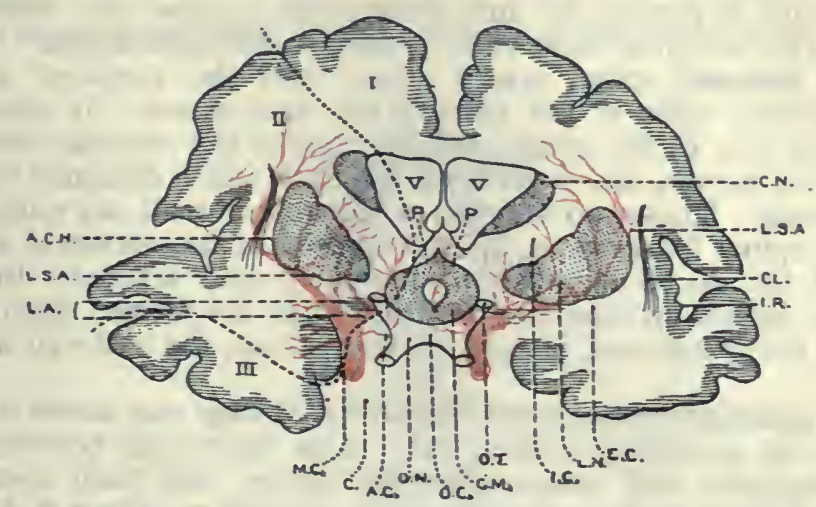

Fig. 607.-Coronal Section of the Cerebral Hemisphęres made One Centimetre behind ihe Optic Commissure (Charcot, From Duret).

(The Arteries of this region are shown).

I. Area of Anterior Cerebral Artery

II. Area of Middle Cerebral Artery

III. Area of Posterior Cerebral Artery

V.V. Sections of Anterior Cornua of Lateral Ventricles

P.P. Anterior Pillars of Fornix

C.N. Candate Nucleus

I. S.A. Lenticulo-Striate Arteries

CL. Claustrum

I.R. Island of Reil

E.C. External Capsule
L.N. Lenticular Nucleus

1.C. Internal Capsule

O.T. Optic Tract (cut)

G.M. Grey Matter of Third Ventricle

O.C. Optic Commissure

O.N. Optic Nerve

A.C. Anterior Cerebral Artery

C. Internal Carotid Artery

M.C. Middle Cerebral Artery

L. A. Lenticular Arteries

A.C.H. Artery of Cerebral Hemorrhage

third of the middle frontal gyrus, and to the lower and greater part of the precentral gyrus; (3) ascending parietal, to the postcentral gyrus and superior parietal gyrus; (4) parieto-temporal, which traverses the posterior horizontal limb of the fissure of Sylvius, and supplies the inferior parietal gyrus, and the posterior parts of the superior and middle temporal gyri; and (5) the temporal branches, which emerge from the posterior horizontal limb of the fissure of Sylvius, and supply the anterior and greater parts of the superior and middle temporal gyri.

Fourth or Intracranial Part of the Vertebral Artery.-The verte. 
bral artery, on leaving the suboccipital triangle, pierces the dura mater and arachnoid, and enters the cranial cavity through the foramen magnum. As it ascends, it lies at first on the side of the medulla oblongata, between the hypoglossal nerve and the anterior root of the suboccipital nerve. It then passes upwards on the ventral surface of the medulla, and, on reaching the lower border of the pons Varolii, it unites with its fellow of the opposite side to form the basilar artery.

Branches.-These are as follows : posterior meningeal, posterior spinal, posterior inferior cerebellar, anterior spinal, and bulbar.

The posterior meningeal artery arises from the vertebral artery just before it pierces the dura mater, and it enters the cerebellar fossa of the occipital bone where it supplies the dura mater.

The posterior spinal artery arises from the vertebral artery immediately after it has pierced the dura mater. It descends upon the side of the spinal cord, in front of the posterior roots of the spinal nerves, and it gives off a branch which descends behind these roots. These two arteries, in themselves small, are reinforced by the spinal branches of the second part of the vertebral artery and of. the dorsal branches of the intercostal arteries. In this manner lateral anastomotic arterial chains are formed upon each side of the spinal cord, in front of, and behind, the posterior nerveroots.

The posterior inferior cerebellar artery, of large size, arises a little above the preceding branch. It passes backwards between the pneumogastric and spinal accessory nerves, and then over the restiform body to the vallecula of the cerebellum, where it divides into branches. Some of these supply the inferior vermis, and others ramify on the inferior surface of the cerebellar hemisphere, at the periphery of which they anastomose with branches of the superior cerebellar artery. The artery furnishes branches to the corresponding choroid plexus of the fourth ventricle and to the medulla oblongata.

The anterior spinal branch of the vertebral artery arises from that vessel near its termination. It passes obliquely downwards and inwards over the ventral aspect of the medulla oblongata, and at the median line it unites with its fellow of the opposite side to form the anterior spinal artery of the spinal cord. It furnishes twigs to the medulla oblongata.

The bulbar branches are distributed to the medulla oblongata.

Basilar Artery. - This vessel is formed by the union of the two vertebral arteries. It extends from the lower to the upper border of the pons Varolii, occupying the median basilar groove on its ventral surface. It lies beneath the arachnoid membrane, within the cisterna pontis, and at the upper border of the pons it divides in to the two posterior cerebral arteries.

Branches.-These are as follows, on either side : transverse, internal auditory, anterior inferior cerebellar, superior cerebellar, and posterior cerebral. 
The transverse or pontine arteries are numerous, and pass outwards on either side to supply the pons, the sensory and motor roots of the fifth cranial nerve, and the middle peduncle of the cerebellum.

The internal auditory artery, long and slender, accompanies the auditory nerve through the internal auditory meatus, and is distributed to the internal ear.

The anterior inferior cerebellar artery arises from the centre of the basilar, and passes backwards to be distributed to the anterior part of the inferior surface of the cerebellar hemisphere. It anastomoses with the posterior inferior cerebellar artery, which is a branch of the vertebral.

The superior cerebellar artery arises from the basilar close to its

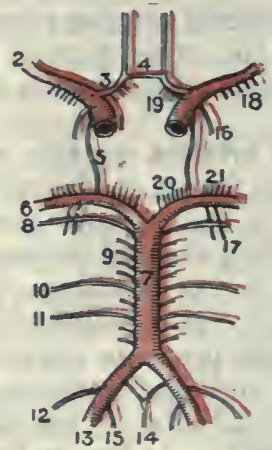

Fig. 608.-The Arteries at the Base of the Brain, and the Circle of Willis.

$\begin{array}{lll}\text { r. Internal Carotid } & \text { 9. Transverse Pontine } & \text { 17. Posterior Choroid } \\ \text { 2. Middle Cerebral } & \text { ra. Internal Auditory } & \text { 18. Central or Ganglionic } \\ \text { 3. Anterior Cerebral } & \text { I1. Anterior Inferior Cerebellar } & \text { - 19. Central or Ganglionic } \\ \text { 4. Anterior Communicating } & \text { 12. Posterior Inferior Cerebellar } & \text { 20. Central or Ganglionic } \\ \text { 5. Posterior Communicating } & \text { 13. Vertebral } & \text { (Postero-mesial) } \\ \text { 6. Posterior Cerebral } & \text { 14. Anterior Spinal } & \text { 21. Central or Ganglionic } \\ \text { 7. Basilar } & \text { 15. Posterior Spinal } & \text { (Postero-lateral) } \\ \text { 8. Superior Cerebellar } & \text { 16. Anterior Choroid } & \end{array}$

termination. It passes outwards, parallel to the posterior cerebral artery, from which it is separated by the third cranial nerve. It then winds round the outer side of the crus cerebri, below the fourth cranial nerve, and so reaches the superior surface of the cerebellar hemisphere, where it divides into branches. These supply the superior vermis, the valve of Vieussens, the velum interpositum, and the superior surface of the cerebellar hemisphere, at the periphery of which they anastomose with branches of the inferior cerebellar arteries.

Posterior Cerebral Artery. - This is one of the terminal branches of the basilar artery, at the upper border of the pons Varolii. It passes at first outwards, beneath the crus cerebri, and parallel to the superior cerebellar artery, from which it is separated by the 
third cranial nerve. It then winds round the outer side of the crus cerebri, lying between it and the hippocampal gyrus, and above the fourth cranial nerve. In this manner it reaches the tentorial or inferior and mesial surfaces of the occipital lobe of the cerebral hemisphere. It receives, not far from its commencement, the posterior communicating artery.

Branches.-These are arranged in two groups-ganglionic and cortical.

The ganglionic group includes three sets of branches, namely, postero-mesial, postero-lateral, and posterior choroidal.

The postero-mesial ganglionic arteries pass internal to the crus cerebri, and pierce the locus perforatus posticus. They supply the inner part of the crus cerebri and the posterior part of the optic thalamus.

The postero-lateral ganglionic arteries pass on the outer side of the crus cerebri, and supply the outer part of the crus, the posterior part of the optic thalamus, the corpora quadrigemina, and the corpora geniculata.

The posterior choroidal arteries are two or three in number, and pass through the choroidal fissure to the velum interpositum, which they supply, together with the choroid plexus of the lateral ventricle, and the corresponding choroid plexus of the third ventricle.

The cortical branches are as follows: (I) anterior temporal, to the anterior parts of the occipito-temporal and hippocampal gyri ; (2) posterior temporal, to the posterior parts of the occipito-temporal and hippocampal gyri, and the inferior temporal gyrus; and (3) occipital, to the occipital lobe. One of the occipital branches is called the calcarine artery. It lies in the calcarine fissure, and supplies the lingual or infracalcarine gyrus and the cuneus.

Circle of Willis. - This circle is formed by (I) the anastomoses which take place between $(a)$ the internal carotid and posterior cerebral arteries, through means of the posterior communicating arteries, and $(b)$ the anterior cerebral arteries, through means of the anterior communicating artery ; and (2) the connection between the two posterior cerebral arteries which is established by the basilar artery. It is situated at the base of the brain, and, though spoken of as a circle, it is really a heptagon-that is to say, it has seven sides and seven angles.

The circle is formed in front by the anterior communicating artery, which connects the two anterior cerebral arteries; behind, by the basilar artery, as it divides into the two posterior cerebral arteries ; and on either side by (I) the anterior cerebral artery, (2) the trunk of the internal carotid artery, (3) the posterior communicating artery, and (4) the posterior cerebral artery, in this order from before backwards. The circle of Willis furnishes twigs to the grey cortex of the interpeduncular region. It serves to equalize the blood-pressure in the cerebral arteries, and it also provides for the regular supply of blood to the brain in cases where one of the main arterial trunks may be obstructed. 
The following parts are contained within the circle of Willis, in order from behind forwards: (I) the locus perforatus posticus; (2) the corpora albicantia or mammillaria; (3) the tuber cinereum and infundibulum; and (4) the optic commissure or chiasma.

\section{Veins of the Encephalon.}

The cerebral veins are arranged in two groups-superficial and deep.

The superficial cerebral veins are divided into two sets-superior and inferior.

The superior cerebral veins return the blood from the upper parts of the outer surfaces of the cerebral hemispheres. They lie in the pia mater, and pierce the arachnoid membrane and inner layer of the dura mater, after which they open into the superior longitudinal sinus, having previously received the veins from the mesial surface of either hemisphere. Their direction, for the most part, is forwards and inwards, whilst the direction of the blood-current in the superior longitudinal sinus is backwards.

The inferior cerebral veins return the blood from the lower parts of the cerebral hemispheres, and they terminate in the cavernous, superior petrosal, and lateral sinuses. One of these veins is known as the middle cerebral, or superficial Sylvian, vein. It passes along the fissure of Sylvius, and opens into the front part of the cavernous sinus. This vein communicates posteriorly with (I) the superior longitudinal sinus, through means of the great or superior anastomotic vein ; and (2) the lateral sinus, through means of the posterior anastomotic vein.

The deep cerebral veins are as follows: (I) the choroidal veins; (2) the veins of the corpora striata ; (3) the veins of Galen; (4) the anterior cerebral veins; (5) the deep Sylvian veins; and (6) the basilar veins.

The choroidal vein of each side commences in the choroid plexus of the descending cornu of the lateral ventricle. It ascends at first, and then passes forwards in the lateral margin of the velum interpositum to the foramen of Monro, where it unites with the vein of the corpus striatum to form the corresponding vein of Galen.

The vein of the corpus striatum is formed by branches which issue from the corpus striatum and optic thalamus. It runs forwards in the groove between these two bodies, lying superficial to the tænia semicircularis, and at the foramen of Monro it joins the choroidal vein to form, as stated, the corresponding vein of Galen.

The vein of Galen, of each side, is formed close to the foramen of Monro by the union of the choroidal vein, and the vein of the corpus striatum. The two veins of Galen, right and left, pass backwards between the two layers of the velum interpositum, and they unite, beneath the splenium of the corpus callosum, to form one vessel, called the vena magna Galeni, which opens into the front part of the straight sinus. Each vein of Galen receives tributaries from 
the optic thalamus, the corresponding choroid plexus of the third ventricle, the corpus callosum, and the corpora quadrigemina ; and, prior to joining its fellow, it takes up the basilar vein of its own side. The vena magna Galeni receives tributaries from the upper surface of the cerebellum.

The anterior cerebral vein of each side is situated in the great longitudinal fissure, along with the corresponding artery. Having curved round the genu of the corpus callosum it passes to the region of the locus perforatus anticus, where it joins the deep Sylvian vein to form the basilar vein.

The deep Sylvian vein returns the blood from the island of Reil, and lies deeply within the stem of the fissure of Sylvius. At the locus perforatus anticus it joins the anterior cerebral vein to form, as stated, the basilar vein.

The basilar vein of each side commences at the locus perforatus anticus, where it is formed by the union of the anterior cerebral and deep Sylvian veins. It passes backwards round the crus cerebri, and opens into the vein of Galen of its own side, just before that vessel joins its fellow to form the vena magna Galeni. The basilar vein receives, close to its commencement, one or more inferior striate veins, which descend from the corpus striatum through the locus perforatus anticus. It also receives tributaries from the parts within the interpeduncular space.

The cerebellar veins are arranged in two groups-superior and inferior.

The superior cerebellar veins terminate in the vena magna Galeni, and in the straight, lateral, and superior petrosal sinuses. The inferior cerebellar veins pass to the lateral, inferior petrosal, and occipital sinuses.

\section{Blood-supply of the Different Parts of the Encephalon.}

The medulla oblongata is supplied by the vertebral, anterior spinal, and posterior inferior cerebellar arteries.

The pons Varolli is supplied by the transverse or pontine branches of the basilar artery.

The cerebellum is supplied inferiorly by the posterior inferior cerebellar branches of the vertebral arteries, and the anterior inferior cerebellar branches of the basilar artery. Superiorly it is supplied by the superior cerebellar branches of the basilar artery, and, to a limited extent, by the posterior inferior cerebellar arteries.

The crus cerebrl is supplied by the postero-mesial and postero-lateral branches of the posterior cerebral artery, and by the posterior communicating artery.

The locus perforatus posticus is pierced by the postero-mesial branches of the posterior cerebral arteries.

The corpora quadrlgemina are supplied by the postero-lateral branches of the posterior cerebral arteries.

The optic thalamus is supplied posteriorly by the postero-mesial and posterolateral branches of the posterior cerebral artery. Anteriorly its outer part is supplied by the lenticulo-optic branches of the middle cerebral artery, and its inner part by the posterior communicating artery.

The locus perforatus anticus is pierced by the antero-lateral ganglionic branches of the middle cerebral artery. 
Frontal Lobe.-The superior frontal gyrus, the anterior two-thirds of the middle frontal gyrus, and the upper portion of the precentral gyrus are supplied by cortical branches of the anterior cerebral artery. The posterior third of the middle frontal gyrus, the inferior frontal gyrus, and the lower and greater part of the precentral gyrus are supplied by cortical branches of the middle cerebral artery. On the orbital surface the internal orbital gyrus, olfactory lobe, and gyrus rectus are supplied by the anterior cerebral artery, whilst the remainder is supplied by the middle cerebral artery.

Parietal Lobe.-The whole of this lobe is supplied by the middle cerebral artery.

Occipital Lobe.-This lobe is supplied by the posterior cerebral artery.

Temporal Lobe.-The superior and middle temporal gyri are supplied by

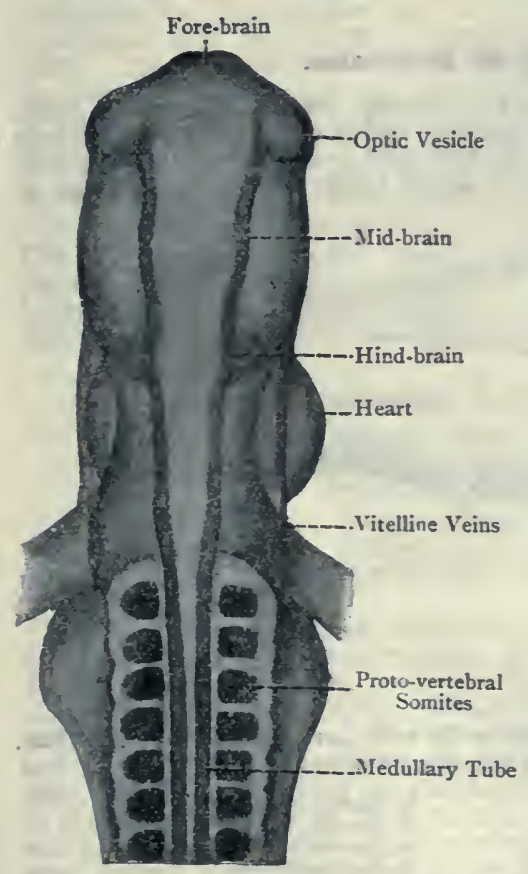

Fig. 6ag.-ANTERIOR PART OF AN EMBRYO CHICK AT THE END OF THE SECONd Day (DORSAL VIEw) (KöLLIKER). round the outer aspect of the lenticular nucleus, between it and the external capule and the external lenticulo-optic lenticulo-optic arteries supply the posterior part of the lenticular nucleus, and the anterior part of the optic thalamus on its outer aspect.

The front part of the caudate nucleus is supplied by the antero-mesial branches of the anterior cerebral and anterior communicating arteries.

The larger arteries occupy the subarachnoid space, where they divide into branches which enter the pia mater. These in turn give off smaller branches, which enter the cerebral substance, some of them being cortical, and others medullary, in their distribution. remainder is supplied by the posor cerebral artery.

Hemisphere.-The anterior cerebral artery has an extensive distribution to this surface, which it supplies as far back as the internal part of the parieto-occipital fissure. The parts behind this fissure, namely, the cuneus and the parts around the calcarine fissure, are supplied by the posterior cerebral artery.

The corpus callosum is supplied by the anterior cerebral arteries.

Corpus Striatum. - The nucleus caudatus and nucleus lenticularis are supplied, for the most part, branches of the middle cerebral artery, which pass through the locus perforatus anticus. According to Duret they form three sets-lenticular, lenticulo-striate, and lenticulo-optic. The lenticular (internal striate) arteries supply the globus pallidus (inner part) of the lenticular nucleus, the internal capsule, and the caudate nucleus. The lenticulostriate (external striate) arteries supply the putamen (outer part) of the lenticular nucleus, and the external capsule. One of the lenticulo-striate arteries is larger than the others, and is subject to rupture in cases of cerebral hæmorrhage. It is known as the artery of cerebral hemorrhage (Charcot). Its course is outwards and upuards

the middle cerebral artery, and the 
Blood-supply of the Choroid Plexuses.-The choroid plexus of the lateral ventricle derives its blood from (I) the anterior choroidal artery, which is a branch of the internal carotid; and (2) the posterior choroidal arteries, which are branches of the posterior cerebral. The choroid plexuses of the third ventricle derive their blood from the posterior choroidal arteries. The chorold plexuses of the lourth ventricle derive their blood from the posterior inferior cerebellar arteries.

The velum interpositum derives its blood from the posterior choroidal arteries and from the superior cerebellar arteries.

Lymphaties of the Brain.- There are no lymphatic vessels in the brain. Their place is taken by spaces in the outer coat of the arteries, called perivascular spaces, which are in communication with the subarachnoid space.

\section{Development of the Encephalon.}

The encephalon is developed from the cephalic part of the neural tube. This part of the tube undergoes enlargement, and is divided by constrictions into three parts, called the anterior, middle, and posterior primary cerebral vesicles. The anterior primary cerebral vesicle forms the prosencephalon or

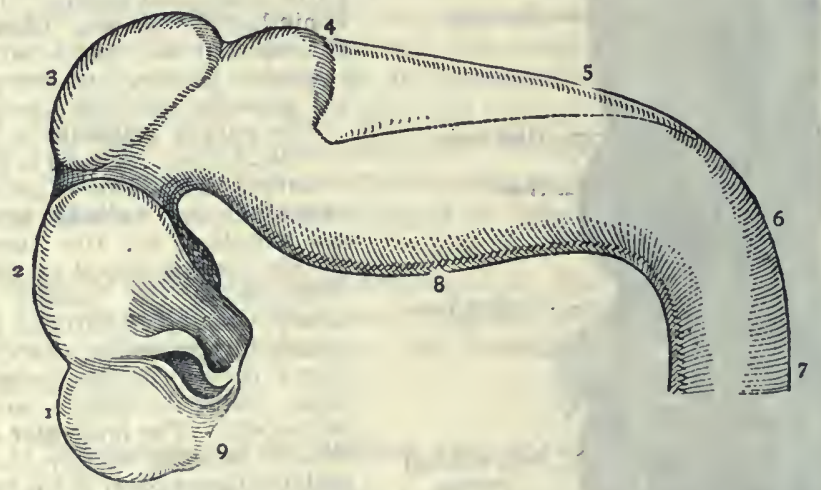

Fig. 6ro.-Brain of an Embryo about Three and a Half Weeks Old. From Quain's 'ANatomy' (His).

3. Telencephalon
2. Thalamencephalon
3. Mesencephalon
4. Metencephalon
5. Myelencephalon

6. Cervical Flexure

7. Spinal Cord

8. Pontine Flexure

9 Olfactory Lobe

s, $2=$ Prosencephalon ; $3=$ Mesencephalon ; 4, $5=$ Rhombencephalon

fore-brain; the middle primary cerebral vesicle constitutes the mesencephalon or mid-brain; and the posterior primary cerebral vesicle gives rise to the hind-brain, or rhombencephalon (rhomb-brain, from the rhomboid character of the floor of the fourth ventricle). The constriction between the mesencephalon and rhombencephalon is called the isthmus. It gives rise to the valve of Vieussens.

From the lower part of each lateral wall of the anterior primary cerebral vesicle (telencephalon) a hollow protrusion is developed. These two protrusions constitute the optic vesicles.

Flexures of the Primitive Encephalon.-Prior to the subdivision of the three primary cerebral vesicles three flexures make their appearance, called cephalic 
pontine, and cervical. The cephalic flexure appears between the prosencephalon and mesencephalon, and by it the prosencephalon is bent ventralward; round the cephalic end of the notochord, on to its ventral aspect. By reason of this flexure the prosencephalon is lowered, and the mesencephalon occupies the summit of the encephalon. The cephalic flexure coincides with the head-fold of the embryo, and the concavity of the flexure is directed ventralwards. The pontine flexure, the concavity of which is directed dorsalwards, appears on the ventral wall of the metencephalon, or cephalic subdivision of the hind-brain, in the region of the future pons Varolii. The cervical flexure appears between the myelencephalon, or caudal subdivision of the hind-brain, and the commencement of the spinal cord. Its concavity is directed ventralwards, like that of the cephalic flexure.

Secondary Cerebral Vesicles.- The middle primary cerebral vesicle remains undivided, but the anterior and posterior primary cerebral vesicles are each subdivided into two parts by constrictions which appear on their lateral walls. The subdivisions of the anterior primary cerebral vesicle are known as the first and second secondary cerebral vesicles, the former being the anterior of the two. The middle primary cerebral vesicle, as stated, remains undivided, and forms now, in series, the third secondary cerebral vesicle. The subdivisions of the posterior primary cerebral vesicle are known as the fourth and fifth secondary cerebral vesicles. There are thus five secondary cerebral vesicles, named first, second, third, fourth, and fitth, of which the third is really not secondary. The first secondary cerebral vesicle represents the telencephalon (end-brain), the second the diencephalon (inter-brain) or thalamencephalon (bedchamberbrain), the third the mesencephalon (mid-brain), the fourth the metencephalon (hind-brain), and the fofth the myelencephalon (marrow-or after-brain).

\section{Secondary Cerebral Vesicles.}

$\begin{array}{lll}\text { First } & \ldots & \begin{array}{l}\text { Telencephalon (end-brain). } \\ \text { Siencephalon (inter-brain); or }\end{array} \\ \text { Second } & \ldots & \begin{array}{l}\text { Thalamencephalon (bedchamber-brain). } \\ \text { Third }\end{array} \\ \text { Fourth } & \ldots & \text { Mesencephalon (mid-brain) } \\ \text { Fifth } & \ldots & \text { Metencephalon (hind-brain). }\end{array}$

The constrictions, which subdivide the anterior primary cerebra vesicle into telencephalon and diencephalon, are anterior to the origins of the optic vesicles, and consequently these vesicles pertain to the diencephalon.

In a general way, the telencephalon gives origin to the cerebral hemispheres and the olfactory lobes. The diencephalon gives origin to the optic stalks and the structures around the third ventricle-e.g., the optic thalami, pineal body, and posterior lobe of the pituitary body. The mesencephalon gives origin to the structures around the aqueduct of Sylvius-namely, the crura cerebri and corpora quadrigemina. The metencephalon gives origin to the cerebellum, pons Varolil, and pontine part of the fourth ventricle. The myelencephalon gives origin to the medulla oblongata and bulbar part of the fourth ventricle.

The following table represents the formations just referred to:

$\left.\begin{array}{c}\text { Anterior primary } \\ \text { cerebral vesicle }\end{array}\right\}=\left\{\begin{array}{c}\text { Prosencephalon, } \\ \text { or fore-brain }\end{array}\right\}=\left\{\begin{array}{c}\text { Telencephalon }=\text { First secon- } \\ \text { and } \\ \text { dary vesicle } \\ \text { Thalamencephalon } \\ \text { or } \\ \text { Diencephalon }\end{array}\right\} \begin{gathered}\text { Second } \\ \text { secondary } \\ \text { vesicle }\end{gathered}$

$\left.\begin{array}{l}\text { Middle primary } \\ \text { cerebral vesicle }\end{array}\right\}=\left\{\begin{array}{c}\text { Mesencephalon, } \\ \text { or mid-brain }\end{array}\right\}=$ Third secondary vesicle

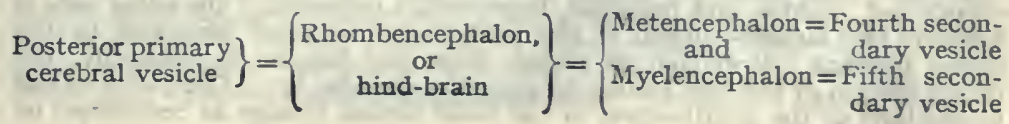


The parts of the encephalon, formed from the foregoing subdivisions, are as follows:

Telencephalon.

Cerebral hemispheres.

Lateral ventricles.

Anterior part of third ventricle.

Foramina of Monro.

Olfactory lobes.
Thalamencephalon, or Diencephalon. Posterior part of third ventricle. Optic thalami. Pineal body. Interpeduncular structures. Pituitary body. Optic nerve and retina.
Mesencephalon.

Corpora quadrigemina. Crura cerebri. Aqueduct of Sylvius.
Metencephalon.

Cerebellum. Pons Varolii. Pontine part of the fourth ventricle.
Myelencephalon.

Medulla oblongata or bulb. Bulbar part of the fourth ventricle.

\section{Metamorphoses of the Secondary Cerebral Vesicles.}

Myelencephalon.-This is the fifth secondary cerebral vesicle, being the caudal subdivision of the rhombencephalon or posterior primary cerebral vesicle. Its walls give rise to the medulla oblongata or bulb, and its cavity forms the bulbar part of the fourth ventricle. The lateral walls of the myelencephalon correspond for the most part with those of the spinal portion of the neural tube, each consisting. of three zones-ependymal, mantle, and marginal, from within outwards: Moreover, the lateral walls are connected by two plates-dorsal or roof-plate, and ventral or floor-plate. Each lateral wall is composed of a ventral or basal lamina and a dorsal or alar lamina, these being separated by a longitudinal groove, called the sulcus limitans, as in the spinal portion of the neural tube.

In the myelencephalon certain changes are effected.

I. The roof-plate becomes much widened.

2. The basal and alar laminæ of each lateral wall, which primarily occupy an almost vertical plane, spread outwards and occupy an almost horizontal plane, the roof plate meanwhile expanding. The basal and alar laminæ now form the floor of the bulbar part of the fourth ventricle, the basal lamina being mesial to the dorsal lamina.

3. The lumen of the neural tube, which is a mere dorso-ventral cleft in the spinal portion of the tube, becomes wide in the region of the fourth ventricle.

Roof-Plate.-This forms the roof-epithelium or ependyma of the bulbar part of the fourth ventricle, and it becomes thickened in certain situations (mesial and lateral), thus giving rise to (I) the obex (mesial), and (2) the ligula, right and left (lateral).

The roof-plate is connected, on either side, with the dorsal margin of the corresponding alar plate, which margin becomes everted and forms the rhombic lip. Subsequently this lip fuses with the contiguous part of the outer surface of the alar lamina.

The widened roof-plate is covered by vascular mesenchyme, representing pia mater. At the level of the pontine flexure the ependymal or epithelial roof is invaginated into the fourth ventricle in the form of a transverse foldplica choroidea, containing pia mater, which extends between the lateral recesses of the ventricle. From this transverse fold two vertical folds, plicas choroidea, likewise containing pia mater, extend vertically downwards into the ventricle, close to the median line. These ependymal folds, containing pia mater, form the two choroid plexuses of the lateral ventricle, thus- $\Pi$.

Lateral Walls.-Each lateral wall, as stated, consists of three zonesependymal, mantle, and marginal, from within outwards. On either side it presents two laminæ-dorsal or alar, and ventral or basal, separated by the sulcus limitans. Moreover, as stated, these two laminx lie vertically, the dorsal margin of the alar lamina forming the rhombic lip. The axons of the neuroblasts of the mantle-layer of the basal lamina emerge as centrifugal or 
efferent nerve-fibres. The alar lamina, ventral to the rhombic lip, receives the centripetal or afferent fibres of the glosso-pharyngeal and vagus nerves, which terminate in a prominent oval area, representing the fasiculus solitarius, at first placed superficially. Subsequently, the two laminæ spread outwards and then occupy a horizontal plane, the roof-plate meanwhile expanding. The basal and alar lamina now form, as stated, the floor of the bulbar part of the fourth ventricle, the basal lamina being mesial to the dorsal lamina. The two basal laminæ, right and left, are connected by the floor-plate. The trigonum hypoglossi in the bulbar part of the floor of the fourth ventricle pertains to the basal lamina, whilst the trigonum vagi and trigonum acusticum belong to the alar lamina.

When the rhombic lip has fused with the contiguous part of the alar lamina, a prolific nest of neuroblasts is formed in this region. Many of these neuroblasts pass ventralwards, embracing the fasciculus solitarius, which now lies deeply. These neuroblasts, on reaching the basal lamina, become differentiated into the olivary body. Other neuroblasts pass into the alar lamina, where they give rise to the nucleus gracilis and nucleus cuneatus.

Each pyramid is a ventral bulging of that part of the basal lamina which is on the mesial side of the olivary body, and it is produced by the motor tracts as they descend from the Rolandic area of the cerebral cortex through the pons Varolii.

The arcuate nucleus is developed from neuroblasts derived from the rhombiclip-area of the alar lamina.

The formatio reticularis, which lies on the dorsal aspects of the pyramid and olivary body, is developed in connection with the mantle-layer of the basal lamina prior to the development of the pyramid.

As the pyramids, right and left, bulge ventralwards, the floor-plate, which connects the basal laminæ, sinks, and the anterior median fissure is formed, as in the development of the spinal cord. The spongioblastic floor-plate is now invaded by nerve-fibres, most of which cross from one side to the other, these fibres being (I) the anterior superficial arcuate fibres, (2) the deep arcuate fibres, and (3) the cerebello-olivary fibres. In this manner the raphe of the bulb is formed, as in the development of the anterior or white commissure of the spinal cord.

The cavity of the medullary or bulbar portion of the neural tube extends in early life as low as the cervical flexure, where it is continuous with the cavity of the spinal portion of the neural tube, and the calamus scriptorius of the fourth ventricle is, at this stage, on a level with the cervical flexure. As development proceeds, the basal and alar lamina, which had spread outwards so as to lie horizontally on the ventral wall, resume their original position in the lateral wall of the lower half of the medullary portion of the neural tube on either side. In this manner the lower half of the bulb becomes closed dorsalwards, and so the lower half contains a prolongation of the central canal of the spinal cord.

Metencephalon. - The metencephalon, which represents the fourth secondary cerebral vesicle, is the cephalic subdivision of the rhombencephalon, hindbrain, or posterior primary cerebral vesicle. From it are developed ( $I$ ) the pons Varolii, (2) the cerebellum, (3) the superior and middle peduncles of the cerebellum, and (4) the superior medullary velum or valve of Vieussens and the inferior medullary velum. The cavity of the metencephalon forms the upper or pontine part of the fourth ventricle.

The pons Varolii is developed from the ventral wall of the metencephalon.

The cerebellum is developed from the dorso-lateral walls of the metencephalon.

The rudiments of the cerebellum appear as thickenings of the right and left dorsal or alar lamina of the lateral walls of the metencephalon. These thickened alar laminæ constitute the two lateral eerebellar plates, the cerebellum being originally composed of two symmetrical halves, separated by a median furrow of the roof-plate of the metencephalon. The two cerebellar plates subsequently fuse across the median dorsal line, and, being now transverse, they form one transverse cerebellar plate. The central part of this plate 
gives rise to the vermis or middle lobe of the cerebellum, and the lateral parts give rise to the cerebellar hemispheres, or lateral lobes.

The surface of the cerebellum is originally smooth and unbroken, but fissures or sulci afterwards appear. The earliest of these is the floccular fissure, of which there are two right and left. At a later period many other fissures make their appearance, which impart a laminated character to the lateral hemispheres and vermis. The floccular fissure of either side cuts off the dorsal part of each lateral cerebellar plate (lateral cerebellar hemisphere), and this part constitutes the flocculus. The paraflocculus on either side is developed from the lateral part of each lateral cerebellar plate, close to the rhombic lip. The two floccular fissures meet across the anterior part of the inferior vermis, giving rise to the post-nodular fissure, which cuts off the nodule from the anterior part of the inferior vermis.

The flocculus and nodule are developed from the rhombic lip-the flocculus from the lateral part and the nodule from the median part.

The cephalic portion of the roof-plate of the metencephalon, close to the isthmus, gives rise to the superior or anterior medullary velum (valve of Vieussens), and lower down it forms the inferior or posterior medullary velum, which is continuous with the ependymal or epithelial roof of the bulbar part of the fourth ventricle.

On either side of the valve of Vieussens the roof-plate is thickened by the superior cerebellar peduncles.

The superior peduncles of the cerebellum, right and left, arise from the cells of the nucleus dentatus of the corresponding lateral cerebellar hemisphere. Emerging from the anterior parts of the dentate nuclei, the peduncles give rise to two thickenings of the roof-plate of the metencephalon, one on either side of the valve of Vieussens. Thereafter they enter the mesencephalon or mid-brain, and after decussating, each passes to the corresponding red nucleus of the tegmentum of either crus cerebri, which constitutes its lower cellstation.

The middle peduncles of the cerebellum (brachia pontis) are developed from the cells of the pontine nuclei of the pons Varolii, right and left. The fibres of each peduncle issue from the lateral portion of the corresponding pontine nucleus and enter the adjacent cerebellar hemisphere.

The inferior peduncles of the cerebellum or restiform bodies are very complex, inasmuch as they derive their fibres from several sources. These sources are as follows:

I. The olivo-cerebellar fibres of the inferior olivary nucleus of the opposite side.

2. The direct cerebellar traet of the lateral column of the spinal cord of the same side.

3. The anterior superficial arcuate fibres from the nucleus gracilis and nucleus cuneatus of the opposite side.

4. The posterior superficial arcuate fibres from the nucleus gracilis and nucleus cuneatus of the same side.

5. Vestibular fibres from the vestibular nuclei of the vestibular division of the auditory nerve.

Mesencephalon. - The mesencephalon represents the third secondary cerebral vesicle, which also constitutes the middle primary cerebral vesicle. Its thickened walls give rise to the parts around the aqueduct of Sylviusnamely, (1) the crura cerebri, (2) the posterior perforated space, and (3) the corpora quadrigemina. Its cavity, very much diminished, persists as the aqueduct of Sylvius, or aqueductus cerebri.

The crura cerebri and posterior perforated space are developed from the ventral wall or floor of the mesencephalon.

The alar plates of the lateral walls encroach so much upon the roof-plate as to reduce it to a mere line. It presents a median longitudinal groove. which separates two rounded eminences, known as the corpora bigemina. At a later period a transverse groove appears, which divides each of the corpora bigemina into two, thus giving rise to the corpora quadrigemina. 
The cavity of the mesencephalon is largely encroached upon by the thickenings undergone by its walls. A minute canal, however, is left, called the aqueduct of Sylvius, or aqueductus cerebri, which constitutes a part of the cephalic prolongation of the central canal of the spinal cord. It is continuous below with the fourth ventricle, and above with the third ventricle.

Dieneephalon or Thalamencephalon.-The diencephalon represents the second secondary cerebral vesicle, which is one of the subdivisions of the prosencephalon, fore-brain, or anterior primary cerebral vesicle. Its walls give rise to the parts around the third ventricle-namely, (I) the greater part of the ependymal roof, with the velum interpositum and pineal body: (2) the optic thalami; and (3) the tuber cinereum, infundibulum, posterior lobe of the pituitary body, and corpora mammillaria.

The diencephalon also gives origin to each optic stalk, from which the optic nerve is developed, the retina being developed from the optic vesicle. The cavity of the diencephalon forms the greater part of the third ventricle. The roof remains very thin, and consists merely of ependymal epithelium. This forms the direct roof of the third ventricle, and it is covered by two layers of pia mater, adherent to each other, and closely connected to the ependymal roof. One of these layers of pia mater belongs to the diencephalon, whilst the other belongs to the under surfaces of the developing cerebral hemispheres, which have expanded backwards so as to overlap the diencephalon. The two layers of pia mater constitute the velum interpositum, or tela choroidea superior, and, with the ependymal lamina, upon which they lie, they form the roof of the third ventricle.

The pineal body, or epiphysis cerebri, is developed from the dorsal part of the ependymal roof of the third ventricle. It appears as a diverticulum of the ependymal roof, close to the mesencephalon, and it is directed backwards, so that it comes to lie over the corpora quadrigemina. The distal end is blind, and, in connection with it, a number of closed follicles are formed which contain calcareous particles, forming the acervulus cerebri, or brainsand. The proximal part of the diverticulum forms the stalk of the pineal body, which contains the pineal recess and opens into the third ventricle.

The lateral walls of the diencephalon become much thickened and give rise to the optic thalami, the cavity of the third ventricle being thereby converted into a narrow cleft.

The floor forms the tuber cinereum, and from the tuber cinereum a diverticulum, called the infundibulum, passes downwards. The lower part of the infundibulum becomes solid and forms the posterior lobe of the pituitary body, the anterior lobe of that body being developed from the pouch of Rathke, which is an upward diverticulum of the stomodcal ectoderm.

Caudal to the infundibulum the floor forms the mammillary recess, from the thickened walls of which the corpora mammillaria are formed.

The optic vesicles are important appendages of the diencephalon, each vesicle growing out from the lateral part of its floor, in front of the level of the infundibulum. For a description of these vesicles see the Development of the Eye.

Telencephalon.-The telencephalon represents the first secondary cerebral vesicle, which is one of the subdivisions of the prosencephalon, fore-brain, or anterior primary cerebral vesicle. It extends forwards and downwards from the diencephalon, and from it are developed the cerebral hemispheres and their associated structures, including the lateral ventricles, as well as the anterior portion of the third ventricle.

The cerebral hemispheres make their appearance as hollow protrusions which grow out from the dorsal zones or alar laminæ of the lateral walls of the telencephalon. These two lateral protrusions constitute the vesicles of the cerebral hemispheres, and their cavities are lateral extensions of the cavity of the telencephalon. This cavity, mesially placed, forms the anterior portion of the third ventricle.

The vesicular cerebral hemispheres grow with great rapidity, and are separated from each other by a cleft called the interhemispherical fissure. which is the rudiment of the great longitudinal fissure. The direction of their 
growth is forwards, upwards, and backwards from the position of the foramen of Monro on either side. They soon cover the diencephalon, and, as their backward growth continues, they cover in succession the mesencepalon and rhombencephalon. The mesial wall of each vesicle is intimately related to the lateral wall of the diencephalon on either side.

As the vesicles of the cerebral hemispheres grow backwards they are still separated by the interhemispherical fissure, and the roof of the diencephalon lies at the bottom of this fissure over a limited area, the corpus callosum and fornix eing as yet undeveloped.

The cavity of each vesicle, which, as stated, is a lateral extension on either side of the cavity of the telencephalon, is the rudiment of the corresponding lateral ventricle; and the cavity of the telencephalon, mesially placed, forms; as stated, the anterior portion of the third ventricle. The communication between the cavity of the vesicle of the cerebral hemisphere and the cavity

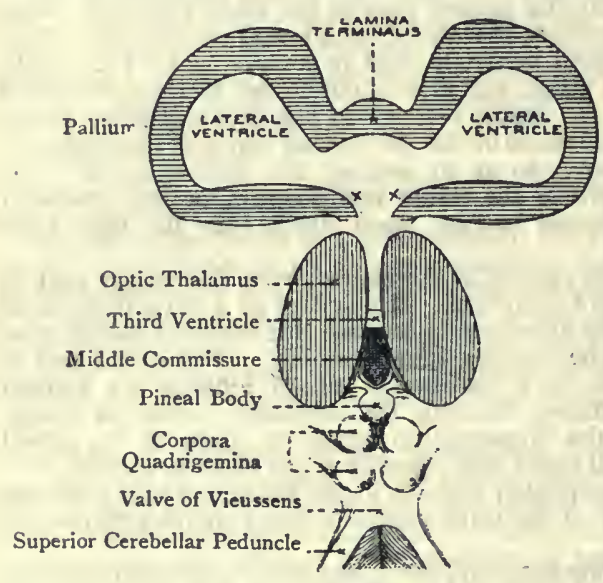

XX Foramina of Monro

Fig. 6it.-Diagram of Primitive ForeBrain and Contiguous Structures. of the telencephalon persists throughout life on either side? It is the rudiment of each foramen of Monro, and is at first of lairge size.

The front part of the telencephalon now presents the anterior portions of the developing cerebral hemispheres right and left, and between them is the thin anterior wall of the telencephalon. This wall represents the terminal cephalic boundary of the embryonic neural canal, and it is known as the lamina terminalis, which connects the antero-mesial parts of the developing cerebral hemispheres. Inasmuch as the cavity of the telencephalon forms the anterior portion of the third ventricle; the lamina terminalis forms the anterior wall of that ventricle, the anterior pillars of the fornix and the anterior white commissure being subsequently developed in connection with it. The roof-plate of the telencephalon forms the anterior part of the roof of the third ventricle.

The walls of the primitive cerebral hemispheres are very thin and consist of neuro-epithelium, the cells of which multiply very freely, the cavity (lateral ventricle) meanwhile diminishing. The innermost cells form the ependyma, which lines the lateral ventricles, but the majority form an extensive superficial mantle-layer, known as the neopallum, or mantle, which constitutes the grey cortex. The neuroblasts of the mantle multiply freely, the ventricular cavity meanwhile becoming diminished. Many of the axons of the neuroblasts pass towards the centre, and they give rise to the white matter which constitutes the medullary centre. The grey matter is not confined to the cortex, but exists also in the basal ganglia of each hemisphere-namely, the corpus striatum, claustrum, and amygdaloid nucleus of each side.

The surface of each hemisphere-vesicle is at first smooth and unbroken. At a very early period, however, a depression makes its appearance at the lower part of each lateral surface of the vesicle. This depression, which is triangular, is called the Sylvian, or lateral cerebral, fossa. The cortical substance over the floor of this fossa becomes much thickened, and its deep part projects into the cavity of the vesicle (lateral ventricle), giving rise to the 
basal ganglia of the cerebral hemisphere-namely, the corpus striatum, claustrum, and amygdaloid nucleus of either side. The superficial part of the cortical substance represents the primitive insula, or island of Rell. The Syl rian fossa gradually extends across the ventral aspect of the hemispherevesicle towards the lower margin of its mesial surface, and the temporal lobe grows forwards and downwards towards it. In this manner the stem of the Sylvian fissure is formed. The fossa also extends in a backward direction upon the lateral surface of the vesicle, and in this manner the rudiment of the posterior horizontal limb of the Sylvian fissure is formed. The anterior ascending and anterior horizontal limbs are formed at a later period. The elevated margins of the Sylvian fossa and of its backward extension, which gradually approach each other, and meet over the insula, represent the opercular region.

The cerebral hemisphere on either side is disposed in the form of an incomplete ring around the Sylvian fossa, the break in the ring being formed by that fossa. The stalk of the hemisphere is located at the fossa, and corresponds to the corpus striatum, with which is associated the optic thalamus. The remaining, and much larger, part of the hemisphere constitutes the neopallium.

The insula, or island of Reil, as stated, is developed from the superficial part of the cortical substance which forms the floor of the Sylvian fossa. The grey matter becomes almost completely surrounded by a limiting fissure, and is then broken up by sulci into the component parts of the adult insula.

The anterior part of the hemisphere-vesicle corresponds to the

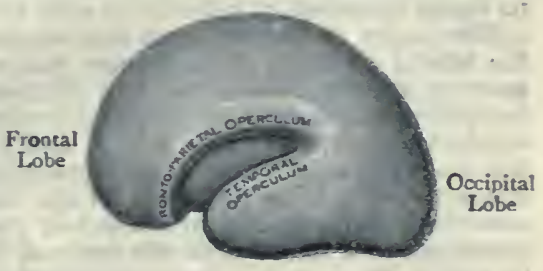

Fig. 612.-The Primitive Fissure of Syzvius. frontal lobe ; the lower part, as far forwards as the stem of the Sylvian fissure, becomes the temporal lobe, and the upper and posterior part represents the parietal lobe. The occipital lobe is formed at a later period as the hemisphere grows backwards. The limbic lobe is developed in connection with the mesial surface of the hemisphere. The olfactory lobe is developed as a hollow protrusion from the ventral aspect of the frontal portion of the hemisphere-vesicle.

The surface of each hemisphere becomes very much broken up into gyri or convolutions, this being effected by means of fissures. The fissures are of two kinds-complete and incomplete. The incomplete fissures are merely sulci produced by the growth of the gyri, and they do not involve the entire thickness of the walls of the cerebral hemispheres. The complete fissures are infoldings of the walls of the cerebral hemispheres, and involve their entire thickness. They. consequently give rise internally to certain prominences upon the wall of each lateral ventricle-namely, the corpus striatum, lateral choroid plexus, hippocampus major, calcar avis, and eminentia collateralis. The complete fissures are as follows:

Sylvian.

Choroidal.

Hippocampal.
Calcarine (anterior part).

Parieto-occipital.

Collateral (central part).

With the exception of the Sylvian fissure, already described in connection with the Sylvian fossa, the other complete fissures appear on the mesial surface of the vesicle of the cerebral hemisphere.

The choroidal fissure is not really a fissure, but merely a groove or sukus produced by an infolding of the vesicular wall, which is here composed entirely of ependyma. It commences above and behind the foramen of Monro of the corresponding side, and it terminates close to the tip of the temporal pole, where the inferior or descending cornu of the lateral ventricle ends. Between these two points it passes backwards, downwards, and then forwards into 
the future temporal lobe, in a crescentic manner, so as to embrace the stalk of the cerebral hemisphere. After the ependymal infolding has taken place, vascular mesenchyme dips in between its two folds, and so a plica choroidea is formed. From this choroidal fold the lateral choroid plexus of the corresponding side is formed. This plexus projects into the lateral ventricle, but is excluded from the ventricular cavity by the ependyma of the wall, previously infolded. When the lateral choroid plexus is withdrawn, the thin ependymal covering of the plexus comes away with it, or is broken down. Under these circumstances the choroidal fissure is really a fissure, inasmuch as the lateral ventricle now opens upon the surface through it. The internal prominence produced by the choroidal fissure is the lateral choroid plexus, covered by ependyma, this prominence being very conspicuous.

The other complete fissures will be found described in connection with the cerebral hemispheres.

The Incomplete fissures are very numerous. The first two to make their appearance are the calloso-marginal fissure, or sulcus cinguli, on the mesial surface of the cerebral hemisphere, and the central sulcus or fissure of Rolando on the external surface. These, along with the other incomplete fissures, will be found described in connection with the cerebral hemispheres.

The cavity of the vesicle of the cerebral hemisphere forms on either side the lateral ventricle, which is very much curtailed by the thickening undergone by the vesicular walls, and the internal prominences produced by the complete fissures. As the frontal lobe undergoes development the body of the ventricle extends forwards into it, and so the anterior cornu of the ventricle is formed. As the temporal lobe grows in a downward and forward direction, it carries with it an extension of the body of the ventricle, this extension constituting the inferior or descending cornu. As the occipital lobe becomes developed at a later period the body of the ventricle extends backwards into it, and so the posterior cornu is formed. Meanwhile the foramen of Monro on either side, originally large, is being gradually much diminished in size.

Basal Ganglia.--The basal ganglia of each cerebral hemisphere are as follows:

Corpus striatum.

Claustrum.

Amygdaloid nucleus.

They are all developed from the deep part of the much thickened cortical substance which forms the floor of the Sylvian fossa. The claustrum and amygdaloid nucleus remain of small size, but the nucleus caudatus of the corpus striatum forms a conspicuous prominence in the lateral ventricle, as it bulges into the anterior cornu of that cavity.

Commissures.- The commissures are as follows:
1. Corpus callosum.
2. Fornix.
3. Hippocampal.

\section{Habenular.}

4. Anterior.

5. Posterior.

6. Middle.

At an early period in the development of the cerebral hemispheres the interhemispherical (great longitudinal) fissure leads directly down to the roof of the diencephalon. At a later period the roof of the diencephalon is separated from the great longitudinal fissure by two commissures, placed one above the other. The upper commissure is the corpus callosum, and the lower one is the fornix.

The corpus callosum and fornlx are formed in connection with the lamina terminalis. This lamina, which is originally thin, becomes thickened, and the mesial walls of the hemispheres come together and fuse directly over and anterior to it. At the same time the united walls of the hemispheres blend with the upper margin of the lamina terminalis. The area of fusion of the walls of the hemispheres now extends both anteriorly and posteriorly, and this area forms a basis or plate for the formation of the corpus callosum and fornix. Transverse fibres are developed over the dorsal aspect of the area, which cross from the neopallium of one hemisphere to that of the other, 
and these transverse fibres constitute the corpus callosum. The fibres of the fornix appear on the ventral aspect of the plate formed by the fused areas of the cerebral hemispheres. On either side they are developed from the hippocampus major, and they form, in succession, the fimbria, or tania hippocampi, the posterior pillar, one half of the body, and the anterior pillar. of the fornix. The anterior pillar passes downwards through the lamina terminalis to the base of the brain, where it becomes twisted in the form of a loop, and forms the white substance of the corresponding corpus mammillare. Thereafter its fibres terminate in the grey nucleus of that body, and from this nucleus the bundle of Vleq d'Azyr passes upwards and backwards into the optic thalamus. Each half of the fornix is therefore a commissure between the hippocampus major and the optic thalamus of the same side.

The hippocampal commissure appears on the back part of the ventral aspect of the plate formed by the fused areas of the cerebral hemispheres. Its fibres pass across from one hippocampus major to the other, and they correspond to the region known as the lyra or psalterium.

The anterior commissure is formed in connection with the dorsal part of the lamina terminalis, over and anterior to which the mesial walls of the cerebral hemispheres fuse.

The posterior commissure is formed in connection with the back part of the roof of the diencephalon, behind the pineal diverticulum.

The so-called middie or grey commissure is not a commissure properly so called, but is formed by the fusion, over a limited area, of the grey matter of the mesial surfaces of the optic thalami.

The habenular commissure is produced by the decussating fibres of the strix pineales, these fibres, as they decussate, forming the dorsal part of the pedicle of the pineal body.

Septum Lueidum and Ventriele of the Septum.-The anterior part of the corpus callosum and its genu and rostrum are separated from the anterior part of the fornix by a cleft-like interval, which is a part of the great longitudinal fissure. This interval is bounded on either side by a limited portion of the thin mesial wall of the cerebral hemisphere. These portions of the mesial walls of the cerebral hemispheres, known as the trapezoid plates, constitute the two laminx of the septum lueldum, which septum forms a partition between the adjacent portions of the lateral ventricles. The interval between the two layers of the septum lucidum is called the ventricle of the septum, or the fifth ventricle. It is destitute of an ependymal lining, and its cleft-like space is entirely disconnected with the cephalic prolongation of the central canal of the spinal cord. It therefore differs from the ependymal ventricles of the brain. As stated, it is an isolated portion of the great longitudinal fissure of the brain.

Lumen of the Neural Tube.-In the development of the spinal cord from the spinal part of the nenral tube it has been stated that the ventral portion of the lumen (neural canal) of that part of the neural tube, having become modified, persists as the central eanal of the spinal cord. The lumen of the cephalic part of the neural tube, from which the various parts of the encephalon are developed, also persists and remains in direct continuity with the central canal of the spinal cord. In the cephalic part of the neural tube, however, the lumen undergoes certain modifications.

At the calamus scriptorius of the fourth ventricle the central canal of the spinal cord opens into the fourth ventricle, thus pertaining to the myelencephalon and metencephalon.

In the mesencephalon it becomes very narrow, and forms the aqueduct of Sylvius, or iter a tertio ad quartum ventriculum, which leads from the fourth to the third ventricle.

In the diencephalon it forms the third ventricle. The third ventricle communicates by the foramina of Monro with the two lateral ventricles, which belong to the telencephalon.

The central canal of the spinal cord is thus in communication with all the cerebral ventricles, properly so called. As previously stated, it is not related to the ventricle of the septum (fifth ventricle), this not being an ependymal 
ventricle, as the others are, but merely an isolated portion of the great longitudinal fissure.

Meninges of the Encephalon.-The walls of all the cerebral vesicles are invested by mesoderm, and this tissue becomes differentiated into the three meninges-namely, the dura mater, arachnoid, and pia mater.

Choroid Plexuses. - The choroid plexuses of the two lateral, third, and fourth ventricles are developed as infoldings of the ependymal walls of the ventricles. Vascular mesenchyme (mesoderm) dips in between the two layers of each infolding, and in this manner plic $x$ choroidece are formed. These choroidal folds give rise to the choroid plexuses which, as they project into the ventricles, carry the ependymal walls, already infolded, before them.

\section{Development of the Peripheral Nervous System.}

The peripheral nerves are arranged in two groups-namely, spinal, which are derived from the spinal cord, and cranial, which arise from the brain.

The spinal nerves are composed of two kinds of fibres-efferent, centrifugal, or motor, and afferent, centripetal, or sensory.

A motor spinal nerve-fibre arises as the axon of a neuroblast or nerve-cell in the mantle-layer of the neural tube. (See Development of the Spinal Cord.)

A sensory spinal nerve-fibre is developed from a cell of a spinal ganglion, and these ganglia are developed from the corresponding neural crest.

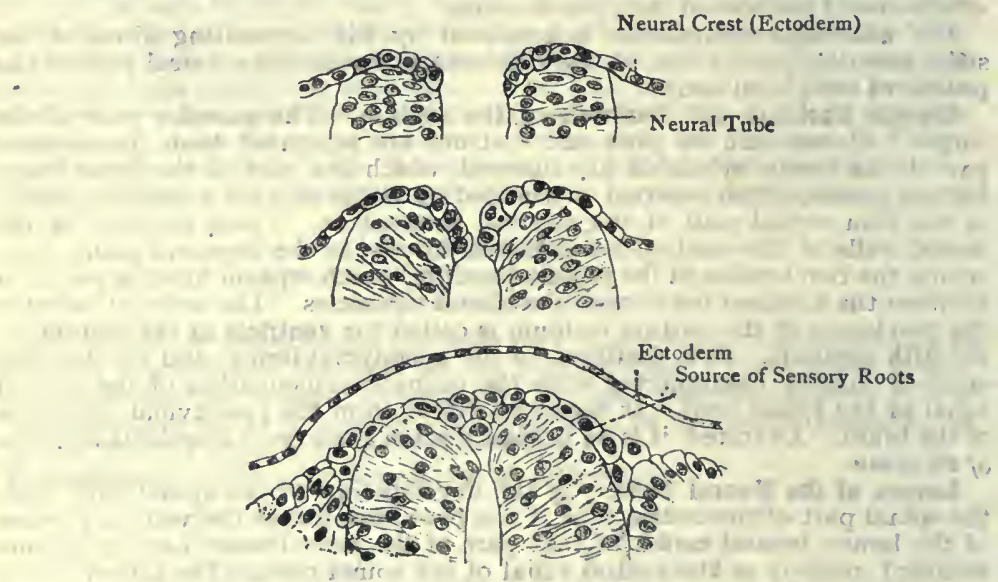

Fig. 613.-Development of the Neural or Ganglion Crest (Keibel and Mall) (AFter Von Lenhossek and Kollmann).

Neural Crests.-The neural or ganglionic crests, right and left, $\mathrm{are}$ ridges of ectodermic cells, which lie on either side of the neural tube. They are derived from a single crest of ectoderm, which is formed by the fusion iof the ectoderm over each neural fold, this single crest being situated mesially on the dorsal aspect of the neural tube, along the line of fusion of the neural folds to close the tube. Subsequently the mesial crest divides into right and left halves, which migrate to the lateral aspects of the neural tube.

Each neural crest becomes broken up into a number of segments, or ganglia, there being four pairs for the head-region, and thirty-one pairs for the region of the trunk.

Spinal Ganglia.-The spinal ganglia are arranged in thirty-one pairs, right and left.

Each cell of a ganglion acquires two poles-afferent or centripetal, and efferent or centrifugal, and, at this stage, it is consequently a bipolar cell. 
The centripetal or proximal pole, which is the axon of a ganglionic cell, grows into the dorsal part of the wall of the neural tube and forms part of the dorsal or posterior root of a spinal nerve. Within the marginal layer of the neural tube the centripetal pole or nerve-fibre divides into two branchesascending and descending, which give off collaterals and terminate in arborizations. The centrifugal or distal pole joins the ventral or anterior nerve-root of the same segment of the spinal cord, on the distal side of the ganglion, to form a spinal nerve.

Though the cells of a spinal ganglion are originally bipolar, they become in the course of growth unipolar, the single pole having a T-shape. This is brought about by an excessive growth of one wall of the bipolar cell, which brings the two original poles into contact, when they fuse, and are now connected with the cell by one stalk or pole, which divides into a centripetal and a centrifugal process.

Whilst the fibres of the dorsal or posterior roots of the spinal nerves grow into the mantle-layer of the neural tube from the cells of the spinal ganglia, the fibres of the ventral or anterior roots arise within the mantle-layer as the axons of its neuroblasts or nerve-cells. The fibres of the anterior roots therefore grow out from the neural tube.

Cranlal Nerves. - The development of the cranial nerves, with the exception of the olfactory and optic nerves, corresponds for the most part with the development of the spinal nerves. The motor cranial nerve-fibres arise as the axons of nerve-cells of the brain and grow into the brain, whereas the sensory cranial nerve-fibres grow into the brain from the cells of the cephalic ganglia.

Cephalic Ganglia.-The cephalic ganglia, like the spinal ganglia, are developed from the neural crests, and they constitute four pairs of ganglionic groups-namely, trigeminal, acoustico-facial, glosso-pharyngeal, and vagal. They are all comparable to the spinal ganglia.

The trigeminal or Gasserian ganglion is connected with the sensory root of the fifth cranial nerve. The centripetal poles of its nerve-cells pass inwards into the brain, forming the large sensory root of the nerve, and the centrifugal poles of its cells pass peripherally, forming the ophthalmic, superior maxillary. and sensory part of the inferior maxillary nerves. The Gasserian ganglion is thus clearly comparable to a spinal ganglion. The small motor root of the fifth nerve is homologous to the motor or anterior root of a spinal nerve, inasmuch as its fibres arise as the axons of nerve cells within the brain.

The acoustico-facial ganglion resolves itself into facial and acoustic parts.

The facial ganglion, known as the geniculate ganglion, is connected with the genu of the facial nerve in the aqueduct of Fallopius. The centripetal poles of the nerve cells of this ganglion form the sensory root of the facial nerve-namely, the pars intermedia of Wrisberg, which passes inwards to the fasciculus solitarius and upper part of the glosso-pharyngeal nucleus. Many of the centrifugal poles of the cells issue from the ganglion as the chorda tympani nerve, which passes to the anterior two-thirds of the tongue as a nerve of special sense.

The acoustic ganglion is represented by the vestibular and cochlear ganglia in connection with the auditory nerve.

The vestibular ganglion is connected with the vestibular division of the auditory nerve in the internal auditory meatus. As in the other ganglia, the centripetal poles of the cells of this ganglion form the centripetal fibres of the vestibular nerve, which pass inwards to the brain. The centrifugal poles of the cells leave the ganglion and form the peripheral part of the nerve as regards its distribution.

The cochlear ganglion, known as the ganglion spirale, is connected with the cochlear division of the auditory nerve, and is situated in the spiral canal of the modiolus. Its nerve-cells are related to nerve-fibres as in the case of the vestibular ganglion.

The glosso-pharyngeal ganglion, which is broken up into a jugular ganglion and a petrous ganglion, is comparable to a spinal ganglion. The centripetal poles of the nerve-cells, which issue from the ganglion, represent the centripetal sensory fibres of the glosso-pharyngeal nerve, passing into the brain. 
The centrifugal poles of the nerve-cells, issuing from the ganglion, represent the peripheral sensory fibres of the nerve.

The vagal ganglion, which is broken up into the ganglion of the root and the

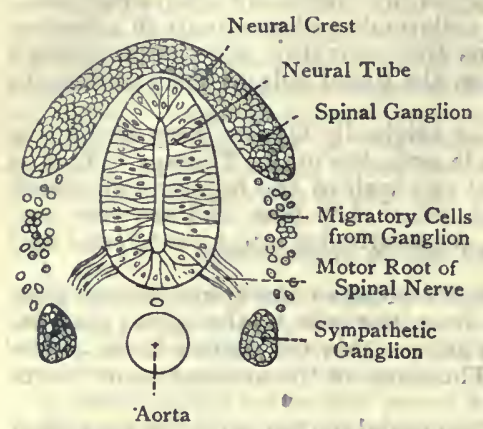

Fig. 614.-DevelopMENT OF SYMPathetic Ganglia. (Schematrc.)

ganglion of the trunk, is disposed towards the sensory fibres of the vagus or pneumogastric nerve as in the case of the other ganglia.

\section{Development of the Sympathetic Nervous System.}

The sympathetic ganglia may be regarded as being developed from the ventral aspects of the neural crests and spinal ganglia, and they are therefore of ectodermic origin, according to this view. Certain cells become detached from the neural crests and spinal ganglia, and migrate ventralwards towards the region of the aorta, where they form the ganglionic sympathetic chain. The ganglionic cells proliferate and are furnished with processes which become fibrillar. These fibrillar processes give rise to the chain which connects the ganglia, and also to the grey rami communicantes and the various visceral branches.

\section{MENINGES OF THE ENCEPHALON.}

\section{Dura Mater.}

The dura mater is a strong fibrous membrane which surrounds the encephalon, and is composed of two layers-outer and inner. The outer or periosteal layer serves as the internal periosteum or endocranium of the cranial bones, and the inner or sustentacular layer supports the encephalon. It is more firmly adherent to the bones forming the base of the skull than over the cranial vault, and it is also firmly attached along the course of the sutures. At the various openings it is prolonged outwards, blending with the sheaths of the transmitted nerves, and also becoming continuous with the external periosteum or pericranium. At the sphenoidal fissure it passes into the orbit to form the orbital periosteum. At the lower margin of the foramen magnum the two layers of which the dura mater is composed separate. The external layer blends with the periosteum of the occipital bone around the margin of the foramen magnum. The internal layer is prolonged into the spinal canal, and forms the theca of the spinal cord. The outer surface of the dura mater is rough and flocculent, owing to fibrous processes which connect it to the inner surfaces of the cranial bones. Its inner surface is smooth and covered by endothelium. Superiorly, on either side of the superior longitudinal sinus, there are several small granular nodules, called Pacchionian bodies, which are best marked in old age. They indent the parietal bone, and protrude into the superior longitudinal sinus, carrying with them prolongations from the endothelial lining of the sinus, which separate them from the blood. 
The Pacchionian bodies are enlargements of the villi of the arachnoid membrane (see p. I48I).

Structure.-The dura mater consists of fibrous and elastic tissues, arranged as parallel bundles.

The intracranial dura mater differs from the dura mater of the spinal cord in the following respects : (I) it consists of two layersouter or periosteal, and inner or sustentacular-whereas the spinal dura mater has only one layer, representing the sustentacular layer; (2) it furnishes certain processes or septa, which project into the cranial cavity, and separate parts of the encephalon from each other, whereas the spinal dura mater sends no septa into the spinal cord; and (3) it contains venous sinuses, which are absent in the spinal dura mater.

Subdural Space. - This is the interval between the dura mater and the arachnoid membrane. There is really no space, but simply sufficient interval to contain a minute quantity of serous fluid for lubricating purposes. The dura mater and the arachnoid are therefore practically in contact with each other.

Blood-supply. - The cranial dura mater is supplied by the meningeal arteries, which are also distributed to the inner table and diploe of the cranial bones. These vessels are very numerous, and the chief are as follows on either side, from before backwards: (I) anterior meningeal, two in number, from the anterior and posterior ethmoidal arteries; (2) meningeal, from the cavernous part of the internal carotid artery; (3) small meningeal, from the middle meningeal, or from the first part of the internal maxillary artery; (4) middle meningeal, from the first part of the internal maxillary ; (5) meningeal branches of the ascending pharyngeal artery; (6) posterior meningeal branch of the occipital artery; and (7) posterior meningeal, from the vertebral artery.

The anterior meningeal branch of the anterior ethmoidal artery arises from that vessel as it accompanies the nasal nerve on the cribriform plate of the ethmoid bone, and it takes part in the supply of the dura mater of the anterior fossa.

The anterior meningeal braneh of the posterior ethmoidal artery arises from that vessel after it has entered the cranial cavity through a minute foramen between the cribriform plate of the ethmoid and the sphenoid. It has a limited distribution to the dura mater in this region.

The meningeal branch of the internal carotid artery arises from the cavernous part of that vessel, and enters the middle fossa to supply the dura mater.

The small meningeal artery is usually a branch of the middle meningeal, but it may arise from the first part of the internal maxillary artery. It enters the cranial cavity through the foramen ovale, and supplies the adjacent dura mater and the Gasserian ganglion.

The middle meningeal artery, as stated, is a branch of the first part of the internal maxillary artery. It is of large size, and enters the cranial cavity through the foramen spinosum in the sphenoid bone, after which it divides into two branches, anterior and posterior. The anterior branch passes forwards, outwards, and upwards in a groove on the upper surface of the great wing of the sphenoid bone to the inner aspect of the antero-inferior angle of the parietal bone, where there is a groove, or sometimes a short canal. The position of the middle meningeal artery at this level is ascertained by taking a point on the exterior of the skull $1 \frac{1}{2}$ inches behind the external angular process of the frontal bone, and $I \frac{1}{2}$ inches above the zygomatic arch. From this point the artery ascends in a branching meningeal groove near the anterior border of the parietal bone, as high as the superior longitudinal sinus. 
In this part of its course it furnishes numerous branches forwards and backwards.

The posterior branch passes outwards and upwards on the squamous part of the temporal bone, and then on to the inner aspect of the inferior border of the parietal bone, where there is a meningeal groove about the centre. From this point it ascends in a branching groove as high as the superior longitudinal sinus, giving off branches forwards and backwards.

The distribution of the middle meningeal artery extends as high as the superior longitudinal sinus, forwards on to the frontal bone, and backwards on to the occipital bone. Besides supplying the dura mater and the inner table and diploe of the bones, the vessel furnishes the following branches: (I) Gasserian to the Gasserian ganglion; (2) a petrosal branch, which passes through the hiatus Fallopii to supply the facial nerve in the aqueduct of Fallopius, and anastomose with the stylo-mastoid branch of the posterior

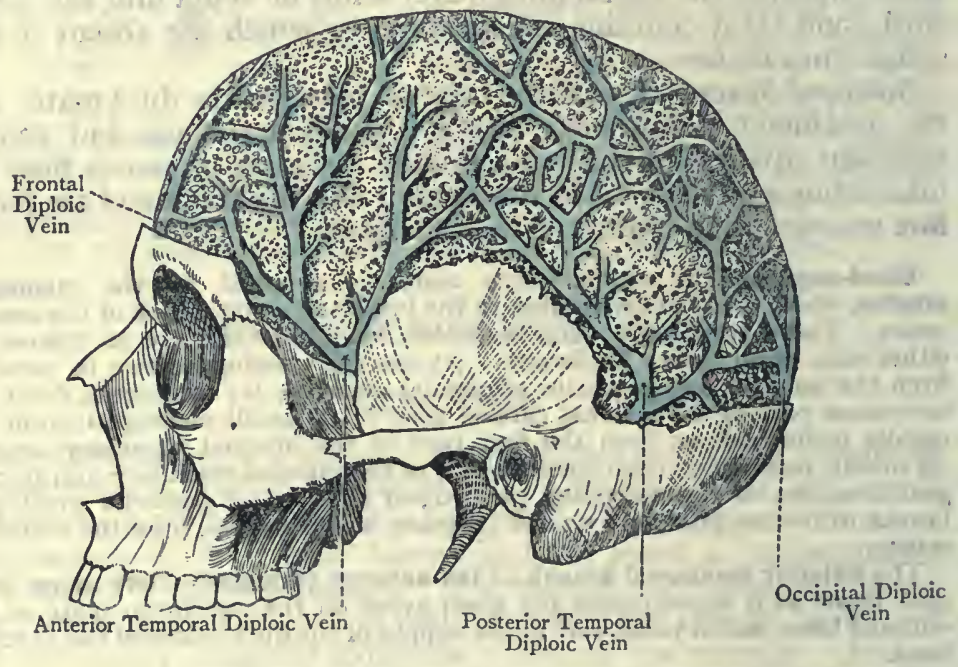

Fig. 6I5.-The VerNs of THE Diplö̈.

(The outer tables of the Cranial Bones have been removed).

auricular artery; and (3) an orbital branch, which enters the orbit through the sphenoidal fissure, and anastomoses with the ophthalmic artery.

The meningeal branches of the ascending pharyngeal artery, which is a branch of the external carotid, are three in number. One passes through the foramen lacerum medium into the middle fossa; another passes through the jugular foramen into the posterior fossa; and the third passes through the anterior condylar foramen, likewise into the posterior fossa:

The posterior meningeal branch of the occipital artery passes through the jugular foramen into the posterior fossa.

The posterior meningeal branch of the vertebral artery passes through the foramen magnum into the cerebellar fossa of the occipital bone.

Meningeal Veins. - The veins accompanying the middle meningeal artery are two in number. They pass through the foramen spinosum, and terminate in the pterygoid plexus of veins. The other meningeal veins are disposed in one of two ways. Some of them accompany the corresponding arteries and terminate in extracranial veins; whilst others terminate in the various intracranial venous sinuses, in part directly and in part through means of venous lacunæ. 
Veins of the Diploë.--These vessels are situated in the cancellated tissue between the outer and inner plates of the cranial bones, and are exposed after removal of the outer plate. They are destitute of valves, and are arranged in the form of a network, from which the blood is returned by four diploic veins on either side, namely, frontal, anterior temporal, posterior temporal, and occipital. These terminate partly in extracranial veins, and partly in the intracranial venous sinuses and meningeal veins.

The frontal diploic vein passes downwards, and escapes through an opening in the outer plate of the frontal bone at the supra-orbital notch, where it joins the communicating vein which passes between the supra-orbital and ophthalmic veins. It receives radicles from the frontal air-sinus of the same side.

The anterior temporal diploic vein is confined to the back part of the frontal and anterior part of the parietal regions, and descends to terminate in two ways. It partly joins one of the extracranial deep temporal veins, by passing through an opening in the outer plate of the great wing of the sphenoid, and in part it ends in a meningeal vein, or in the spheno-parietal, or, it may be, the cavernous, sinus.

The posterior temporal diploic vein is confined to the parietal region, and descends to the postero-inferior angle of the parietal bone, where it pierces the inner plate of that bone, and terminates in the lateral sinus.

The occipital diploic vein occupies the occipital region, and pierces the inner table of the occipital bone, to terminate in the lateral sinus.

Nerves of the Dura Mater.-The dura mater receives nerves from (I) the sympathetic plexuses which accompany the arteries; (2) the three divisions of the fifth cranial nerve; (3) the ganglion of the root of the pneumogastric nerve; and (4) the hypoglossal nerve.

Processes of the Dura Mater.-The dura mater, as stated, is composed of two layers-outer or periosteal, and inner or sustentacular. The processes are formed by the inner or sustentacular layer, and are four in number, namely, the falx cerebri, tentorium cerebelli, falx cerebelli, and diaphragma sellæ.

The falx cerebri is an extensive falciform process, which occupies the great longitudinal fissure of the cerebrum, where it lies between the two cerebral hemispheres. Anteriorly it is almost pointed, and is attached to the crista galli of the ethmoid bone. Posteriorly it is broad, and is attached to the upper surface of the tentorium cerebelli along the median line, the straight sinus being situated at the place of junction. The superior border is convex, and is attached in the median line to the frontal, parietal, and occipital bones, extending upon the latter bone only as low as the internal occipital protuberance. The superior longitudinal sinus is situated along this border. The inferior border is concave and free. It overhangs the upper surface of the corpus callosum, from which it is separated by a slight interval. The inferior longitudinal sinus is situated within this border. The lateral surfaces face the mesial surfaces of the cerebral hemispheres.

The tentorium cerebelli is an extensive crescentic sheet which covers the cerebellum. Superiorly it supports the posterior parts of the cerebral hemispheres, and is elevated along the median line, whence it slopes towards the attached borders. Anteriorly it presents a free, sharp, concave border, which forms, with the dorsum sellæ of the sphenoid bone anteriorly, an oval opening, called the incisura tentorii, within which the mesencephalon is 
placed. Posteriorly and laterally the tentorium cerebelli is convex, and is attached as follows, from behind forwards: (I) to the horizontal ridge on the inner surface of the tabular part of the occipital bone, where the process contains the lateral sinus; $(2)$ to the inner aspect of the postero-inferior angle of the parietal bone, where the process also contains the lateral sinus; and (3) to the superior border of the petrous part of the temporal bone, where the process contains the superior petrosal sinus. Close to the apex of the pars petrosa the outer and anterior borders of the tentorium cerebelli cross, the outer border passing inwards to be attached to the posterior clinoid process of the sphenoid bone, and the anterior

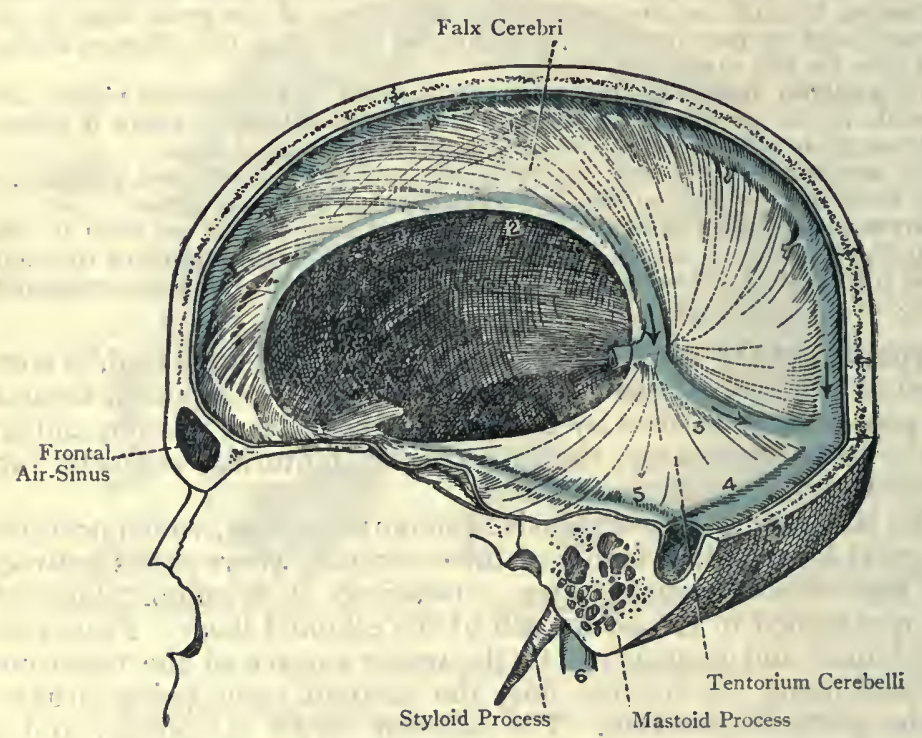

Fig, 6i6.-The Falx Cerebri, Tentorium Cerebelli, and Venous Sinuses of the Dura Mater (Left View).

r. Superior Longitudinal Sinus

2. Inferior Longitudinal Sinus

3. Straight Sinus
4. Lateral Sinus

5. Superior Petrosal Sinus

6. Internal Jugular Vein

border passing forwards to be attached to the anterior clinoid process of that bone.

The falx cerebelli extends forwards into the posterior cerebellar notch, where it lies between the two cerebellar hemispheres. It is falciform in outline. Superiorly it.is attached to the posterior part of the inferior surface of the tentorium cerebelli in the median line. Posteriorly it is attached to the internal occipital crest, and this portion bifurcates inferiorly, the two divisions being connected to the lateral margins of the vermiform fossa. Along this posterior 
attachment it contains the occipital sinus and its two divisions. Anteriorly it ends in a free border.

The diaphragma sellæ is a small circular fold, horizontally placed, which forms a roof for the sella turcica or pituitary fossa of the sphenoid tone, and almost entirely covers the pituitary body. At its centre there is a small opening for the passage of the infundibulum.

Venous Sinuses of the Dura Mater.-These are blood-channels or spaces situated between the two layers of the dura mater, and lined with endothelium. They are as follows :

Snperior longitudinal sinus.

Inferior longitudinal sinus.

Straight sinus.

Lateral sinuses (two).

Occipital sinus.

Cavernous sinuses (two).
Spheno-parietal sinuses (two).

Circular sinus.

Superior petrosal sinuses (two).

Inferior petrosal sinuses (two).

Transverse or basilar sinus.

Petro-squamous sinuses (two).

The superior longitudinal sinus is situated in the median line within the superior convex border of the falx cerebri. It extends from the crista galli of the ethmoid bone to the internal occipital protuberance, where, as a rule, it turns sharply to the right, and opens into the right lateral sinus. It sometimes, however, turns to the left, and opens into the left lateral sinus. Its posterior extremity is dilated, and forms the torcular Herophili, which usually occupies a depression on the right side of the internal occipital protuberance, and is connected with the dilatation at the posterior extremity of the straight sinus by a transverse vessel. It increases in size as it passes backwards, and its shape is triangular, the base being directed towards the cranial vault. The apex is directed downwards, and in this region the sinus is crossed by a number of delicate fibrous bands, called the chordæ Willisii. Opening into the sinus on either side there are venous spaces, called lacunæ laterales, which are situated within the dura mater, and projecting into these lacunæ from below, or into the sinus itself, there are several Pacchionian bodies, covered by the endothelial lining. The sinus receives the superior cerebral veins and some of the meningeal veins of the falx cerebri. The former open into it from behind forwards, so that the blood-flow in these veins is.opposed to the current of blood in the sinus, which is from before backwards.

The superior longitudinal sinus frequently communicates anteriorly with the veins of the roof of the nose through the foramen cacum, and it communicates with the veins of the scalp by means of an emissary vein, which passes through the parjetal foramen of each side, when that is present.

The inferior longitudinal sinus (vein) is of small size, ard is situated in the lower free border of the falx cerebri over its posterior two-thirds. Its direction is backwards, and it opens into the front part of the straight sinus at the anterior margin of the tentorium cerebelli. It is circular, and increases in size as it passes 
backwards. Its tributaries are derived from the lower part of the falx cerebri, and from the adjacent parts of the mesial surfaces of the cerebral hemispheres.

The straight sinus is situated at the junction of the falx cerebri with the tentorium cerebelli, and is formed by the union between the inferior longitudinal sinus and the vena magna Galeni at the anterior margin of the tentorium. It is triangular, and increases in size from before backwards. Its course is downwards and backwards in the median line to the left side, as a rule, of the internal occipital protuberance, where it becomes slightly dilated, and is continued into the left lateral sinus. Its terminal dilatation is connected with the torcular Herophili by a transverse vessel. In those cases where the superior longitudinal sinus passes into the left lateral sinus, the straight sinus passes into the right lateral sinus. In addition to the inferior longitudinal sinus and the vena magna Galeni, the straight sinus receives some of the superior cerebellar veins and tributaries from the falx cerebri and tentorium cerebelli.

The lateral sinuses are two in number, right and left. Each sinus extends from the internal occipital protuberance to the postero-external compartment of the jugular foramen, through which it passes, to terminate in the bulb of the internal jugular vein. The sinus pursues a curved course. It passes outwards in the transverse groove on the inner surface of the tabular part of the occipital bone as far as the inner surface of the posteroinferior angle of the parietal bone. Here it ascends for a little in a groove, and, having described a sharp curve, it passes downwards and inwards in the sigmoid groove on the inner surface of the mastoid portion of the temporal bone. Finally, it turns forwards in the groove on the upper surface of the jugular process of the occipital bone to the jugular foramen. As far as the posteroinferior angle of the parietal bone the lateral sinus is contained within the attached border of the tentorium cerebelli, and, just. before leaving this, it receives the superior petrosal sinus. The right lateral sinus is, as a rule, formed by the superior longitudinal sinus, and, in these circumstances, is larger than the left, which is usually formed by the straight sinus. The reverse, however, may be the case. As stated, the torcular Herophili and the terminal dilatation of the straight sinus are connected by a transverse branch.

The lateral sinus of each side, in addition to the superior petrosal sinus, receives tributaries from the posterior part of the cerebrum and the superior and inferior surfaces of the cerebellum. It also receives the posterior temporal and occipital diploic veins, and the petro-squamous sinus, when that sinus is present. Near its termination it communicates with the marginal sinus of the same side. The sinus communicates with extracranial veins by means of two large emissary veins, posterior condylar and mastoid, which pass through the corresponding foramina. These foramina, 
or inferior occipital sinuses remain separate, and then each opens into the corresponding lateral sinus. The occipital sinus receives tributaries from the falx cerebelli and the inferior surface of the cerebellum, and it establishes a communication between the commencement and termination of the lateral sinuses.

The cavernous sinuses are two in number-right and left-and are so named because the interior of each is broken up by fibrous filaments, which impart to it a reticular appearance. Each sinus is situated on the side of the body of the sphenoid bone, and extends from the inner extremity of the sphenoidal fissure to the apex of the petrous part of the temporal bone. Anteriorly it receives the ophthalmic vein, or veins, from the orbit, and posteriorly it terminates by dividing into the superior and inferior petrosal sinuses. In addition to the ophthalmic vein, or veins, the cavernous sinus receives the spheno-parietal sinus and some of the inferior cerebral veins. It communicates with the angular vein of the face through the superior ophthalmic vein; with its fellow of the opposite side through the circular sinus; with the lateral sinus by the superior petrosal sinus ; with the internal jugular vein by the inferior petrosal sinus; with the pterygoid plexus through the inferior ophthalmic vein, and by an emissary vein which passes through the foramen ovale, or through the foramen Vesalii; and with the pharyngeal p'exus by an emissary vein which passes through the foramen lacerum medium. The internal carotid artery, with the cavernous sympathetic plexus, lies in contact with the inner wall of the sinus, and the sixth cranial nerve is close to the outer side of the artery. In connection with the outer wall of the sinus there are the third, fourth, and ophthalmic and superior maxillary divisions of the fifth, cranial nerves.

The spheno-parietal sinuses are two in number-right and left. Each is of small size, and is situated on the inferior surface of the small wing of the sphenoid bone. It generally commences in connection with the anterior temporal diploic vein, and terminates in the anterior part of the cavernous sinus.

The circular sinus surrounds the pituitary body in the pituitary fossa. It is composed of two transverse sinuses-anterior intercavernous and posterior intercavernous-which lie in front of and behind the pituitary body, and establish communications between the cavernous sinuses.

The superior petrosal sinus is situated along the superior border of the petrous part of the temporal bone, and lies within the attached margin of the tentorium cerebelli. It commences at the back part of the cavernous sinus, and, having passed outwards and backwards, it opens in to the lateral sinus as that is about to enter the sigmoid groove of the pars mastoidea. It receives tributaries from the cerebellum and tympanum.

The inferior petrosal sinus occupies the groove at the junction of the basilar process of the occipital bone with the petrous part of the temporal bone. It commences at the back part of the cavernous sinus, and, passing backwards and slightly outwards, it leaves the 
cranial cavity through the antero-internal compartment of the jugular foramen, to terminate in the bulb of the internal jugular vein. It receives tributaries from the inferior surface of the cerebellum, and from the internal ear.

The transverse or basilar sinus is really a plexus of veins, situated within the dura mater over the basilar process of the occipital bone, and extending as low as the anterior margin of the foramen magnum, where it communicates with the anterior intraspinal veins. It connects the anterior ends of the inferior petrosal sinuses with each other.

The petro-squamous sinuses are two in number-right and leftand are situated, on either side, along the junction of the petrous and squamous parts of the temporal tone. The direction of each is backwards, and it opens into the lateral sinus as that is entering the sigmoid fossa of the pars mastoidea.

Each petro-squamous sinus represents the continuation of the lateral sinus, in early life, to terminate in the primitive jugular vein. Sometimes there are no traces of these petro-squamous sinuses.

Emissary Veins. -These are vessels which pass through foramina in the cranial wall, and serve to establish communications between the intracranial venous sinuses and the extracranial veins. The principal emissary veins are as follows: mastoid, nasal, posterior condylar, parietal, and occipital. In addition to these there are emissary veins, which pass through (I) the foramen ovale, (2) the foramen Vesalii (when present), and (3) the foramen lacerum medium.

The lateral sinus, in many cases, has two emissary veins, mastoid and posterior condylar.

The mastoid emissary vein is of large size. It passes through the mastoid foramen, and usually opens into the posterior jugular vein, but it may open into the posterior auricular vein.

The posterior condylar emissary vein passes through the posterior condylar foramen, and opens into the suboccipital plexus, from which the blood is carried away by the vertebral and deep cervical veins.

The mastoid and posterior condylar veins are not constant.

The superior longitudinal sinus has three emissary veins-nasal, parietal, and occipital.

The nasal emissary vein passes through the foramen cæcum, in front of the crista galli of the ethmoid bone, and it establishes a communication between the superior longitudinal sinus and the veins of the roof of the nose.

The parietal emissary vein passes through the parietal foramen, and opens into the occipital plexus, or into radicles of the superficial temporal vein.

The occipital emissary vein passes from the torcular Herophili to the occipital plexus, being transmitted through a minute foramen which pierces the external occipital protuberance.

The nasal, parietal, and occipital emissary veins are inconstant.

As previously stated, the cavernous sinus communicates with (I) the angular vein of the face, (2) the pterygoid plexus, and (3) the pharyngeal plexus. The marginal or inferior occipital sinus communicates with the posterior intraspinal veins, and the transverse or basilar sinus communicates with the anterior intraspinal veins.

\section{Arachnoid Membrane.}

This is a very delicate membrane which loosely surrounds the encephalon, and is situated between the dura mater and pia mater. 
It does not dip in to the fissures, except in the case of the great longitudinal fissure, its general course being over the gyri and other eminences and depressions of the encephalon. It is conspicuous at the base of the encephalon in the region of the inter-peduncular space, pons Varolii, and medulla oblongata. Its outer surface is practically in close contact with the inner surface of the dura mater, the extremely slight interval containing a very little lubricating serous fluid, and being known as the subdural space. Between the arachnoid and the pia mater there is the interval known as the subarachnoid space. The membrane furnishes sheaths to the various cranial nerves.

Subarachnoid Space.-This space, as stated, is situated between the arachnoid and the pia mater. It is crossed by delicate trabeculæ of connective tissue, which pass between the two membranes in a reticular manner. The meshes of this reticulum contain the cerebrospinal fluid.

The subarachnoid space communicates with the ventricles of the brain by means of five openings. Three of these communicate with the fourth ventricle, namely, the foramen of Magendie, and the foramina of Luschka, the former being situated in the median line of the roof of the ventricle a little above the calamus scriptorius, and the latter being situated each in the roof of the lateral recess of the ventricle. The other two openings communicate with the descending cornua of the lateral ventricles, each being situated at the extremity of the corresponding cornu. In some situations-as, for example, over the gyri-the arachnoid and pia mater are in close contact; but in other localities the two membranes are more or less widely separated by intervals, called cisternæ. These are principally three in number, namely, the cisterna magna, cisterna pontis, and cisterna basalis.

The cisterna magna lies between the posterior part of the inferior surface of the cerebellum and the medulla oblongata. It is of large size, the arachnoid, which here passes from cerebellum to medulla, being widely separated from the pia mater. It is continuous through the foramen magnum with the posterior part of the subarachnoid space of the spinal cord.

The cisterna pontis is situated on the ventral aspect of the pons Varolii. Inferiorly it is continuous with the anterior part of the subarachnoid space of the spinal cord, and in the region of the medulla oblongata it is continuous posteriorly with the cisterna magna. It contains the basilar artery.

The cisterna basalis is situated in front of the pons Varolii, in which situation the arachnoid extends over the interpeduncular space from one temporal lobe to the other. It contains the arteries which form the circle of Willis. This cisterna is prolonged outwards on each side into the stem of the fissure of Sylvius, each of these prolongations containing the middle cerebral artery. Anteriorly it extends in front of the optic commissure into the great longitudinal fissure over the upper surface of the corpus callosum, this prolongation containing the anterior cercbral arteries.

The subarachnoid fluid can be drained away in two directions. 
It can enter the lymph-spaces of the cranial nerves upon which the arachnoid is prolonged outwards in the form of sheaths; and it can enter the lacunæ laterales through the intervention of the Pacchionian bodies, and from these lacunæ it can make its way into the superior longitudinal sinus.

Structure of the Arachnoid Membrane.-The arachnoid consists of fine fibrous tissue, arranged in interlacing bundles, the intervals between these bundles being occupied by delicate cellular membranes. Several such layers, intimately blended together, form the membrane.

Beneath the arachnoid, and constituting a part of it, there is a reticulum of subarachnoid trabecula. These trabeculx consist, as in the case of the arachnoid proper, of fine fibrous tissue, but the intertrabecular spaces, instead of being occupied by cellular membranes, contain cerebro-spinal (subarachnoid) fluid. The trabecular reticulum connects the arachnoid with the subjacent pia mater. The superficial surface of the arachnoid is covered with a delicate layer of endothelium.

The nerves of the arachnoid are derived from the sympathetic system.

Pacchionian Bodies.-These are small fleshy-looking bodies which are situated along the course of the superior longitudinal sinus, into which some of them project. They are also met with in connection with other sinuses, e.g., the lateral and straight sinuses. Each Pacchionian body is a localized projection of the arachnoid membrane, with which it is connected by a narrow pedicle. As stated, some Pacchionian bodies project into the superior longitudinal sinus; others project from below into the lacunæ laterales. In all cases the bodies carry before them the lining of the sinus. Superficially the bodies give rise to the Pacchionian depressions on the internal surface of the parietal bone near the superior border. Each Pacchionian body, as stated, is a localized projection of the arachnoid membrane (enlarged villus--Luschka). It contains a prolongation of the subarachnoid space and reticulum. This is surrounded by a prolongation of the arachnoid membrane, and external to this is the endothelial lining of the sinus or of the lacuna. The Pacchionian bodies constitute channels through which the subarachnoid fluid can be drained away from the subarachnoid space into the lacunæ laterales, and thence into the superior longitudinal sinus, as well as into the other sinuses, e.g., the lateral and straight sinuses. They are quite rudimentary in early life, and are most conspicuous in advanced life.

\section{Pia Mater.}

The pia mater is the most internal covering of the encephalon. It is a very vascular membrane, which invests, and is closely adherent to, the entire surface. From its internal surface delicate processes pass into the cerebral substance, which represent the minute bloodvessels, surrounded by pia-matral sheaths. The pia mater not only invests the external surface, but also dips into the sulci, and covers the opposed surfaces of the gyri. It also furnishes 
sheaths to the various cranial nerves, which blend with their perineurium. It gives rise to two web-like expansions, namely, the tela choroidea inferior and tela choroidea superior.

The tela choroidea inferior is situated in the lower part of the roof of the fourth ventricle, and from it are derived the choroid plexuses of that ventricle. The tela choroidea superior, or velum interpositum, is an invagination of the pia mater through the transverse fissurc beneath the splenium of the corpus callosum. It lies underneath the body of the fornix, and its deep surface is covered by the ependymal lining of the third ventricle, the latter forming the roof of that cavity. The tela choroidea superior furnishes the choroid plexuses of the two lateral and third ventricles.

The pia mater of the encephalon differs from the pia mater of the spinal cord in being thinner and less adherent to the nervous substance: The greater thinness is due to the fact that it is destitute of the outer layer which characterizes the spinal pia mater.

Structure. - The pia mater of the encephalon consists of a single layer of areolar tissue, which contains a great many small bloodvessels, these being derived from the larger vessels, lying in the subarachnoid space.

\section{The Cranial Nerves.}

The cranial nerves are arranged in twelve pairs. They have received numerical names according to the order in which they leave the cranial cavity from before backwards, and they are also known by functional names. "The different pairs of nerves are as follows, in order from before backwards :

First, or olfactory.

Second, or optic.

Third, or oculo-motor.

Fourth, or trochlear (pathetic).

Flfth, or trigeminal (trifacial).

Sixth, or abducent.

\author{
Seventh, or facial. \\ Eighth, or auditory. \\ Ninth, or glosso-pharyngeal. \\ Tenth, or pneumogastric (vagus). \\ Eleventh, or spinal accessory. \\ Twelfth, or hypoglossal.
}

The cranial nerves are connected to certain parts of the encephalon. and these connections constitute their superficial or apparent origins. The fibres, however, can be traced to certain collections of grey matter, which are called nuclei. From the deep positions occupied by these nuclei they constitute the deep origins of the nerves.

First or Olfactory Nerve.-This nerve is a complex structure, and consists of $(x)$ the olfactory lobe, and (2) the olfactory filaments or nerves, which are about twenty in number.

The olfactory lobe has been already described (see p. I397).

The olfactory nerves are non-medullated. They arise as the axons of the olfactory cells of the olfactory mucous membrane of the nasal fossa, and enter the cranial cavity through the foramina of one half of the cribriform plate of the ethmoid bone. Thereafter they enter the grey matter on the ventral or inferior aspect of the olfactory bulb, and terminate in arborizations which intermingle 
with the arborizations formed by the dendrites of the mitral cells, situated in the granular layer of the bulb (see p. I448).

The olfactory nerve is the nerve of smell.

Second or Optic Nerve. - This nerve arises from the brain by means of the optic tract, the deep connections of which have been already described (p. I424). Each optic tract passes forwards and inwards to the optic commissure or chiasma, which is situated in

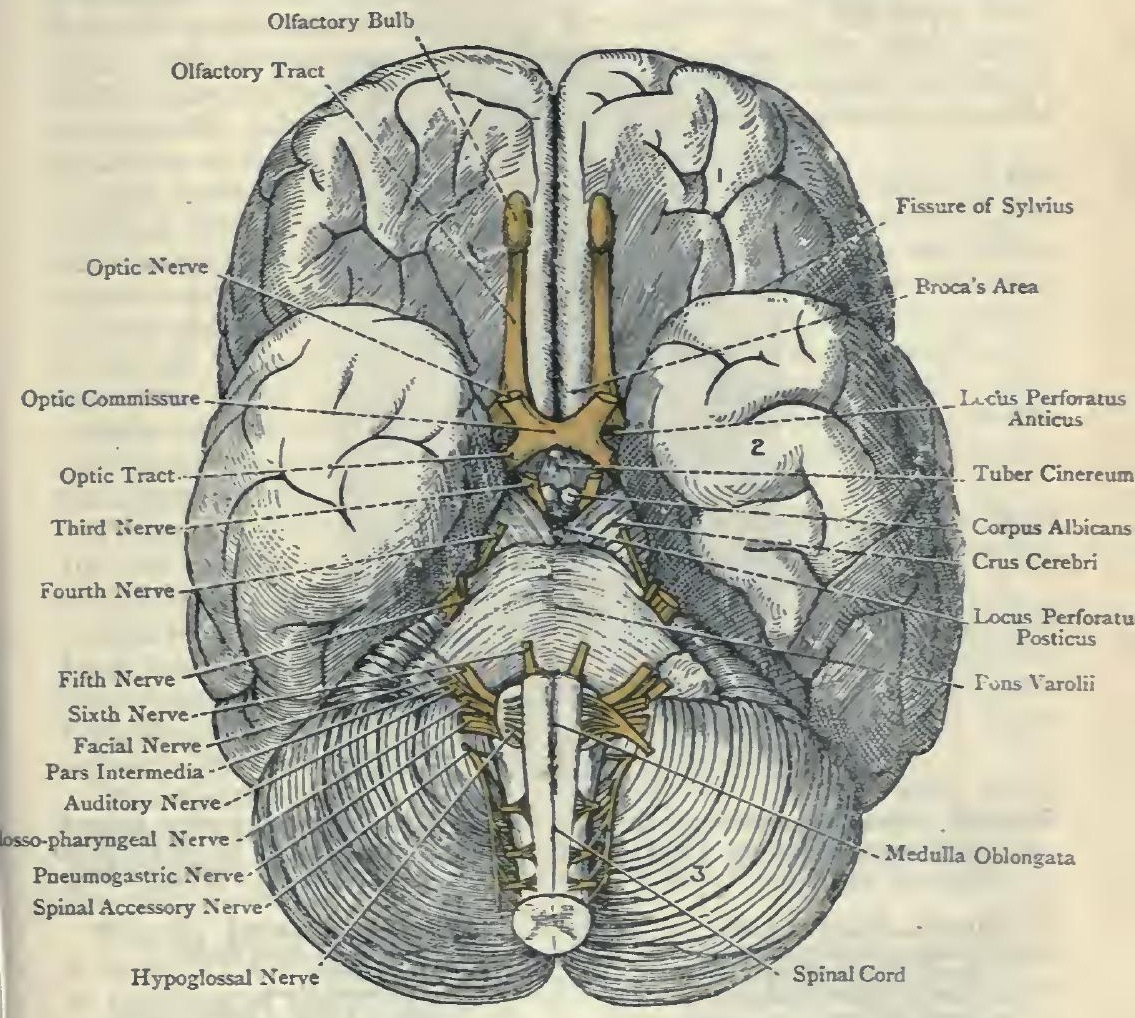

Fig. 6is.-The Base of the Excephalox, and the Cranial Nerves.

I, Frontal Lobe (Orbital Surface) ; 2, Temporal Lobe : 3, Cerebellum.

front of the interpeduncular space. The optic nerve of each side arises from the anterior part of the optic commissure. It courses forwards and outwards to the optic foramen, through which it passes into the orbit, piercing the dura mater, and receiving a sheath from it, as well as from the arachnoid membrane. Having reached the back part of the eyeball, it pierces the sclerotic and choroid coats $\frac{1}{8}$ inch to the nasal or inner side of the axis 
of the eyeball, and terminates in an expansion which forms the most internal layer of the retina, called the nerve-fibre or optic layer.

The optic nerve is the nerve of sight.

Third or Oculo-motor Nerve.-The fibres of this nerve arise from the oculo-motor mucleus, which is situated in the grey matter of the ventral aspect (floor) of the aqueduct of Sylvius, on a levei with

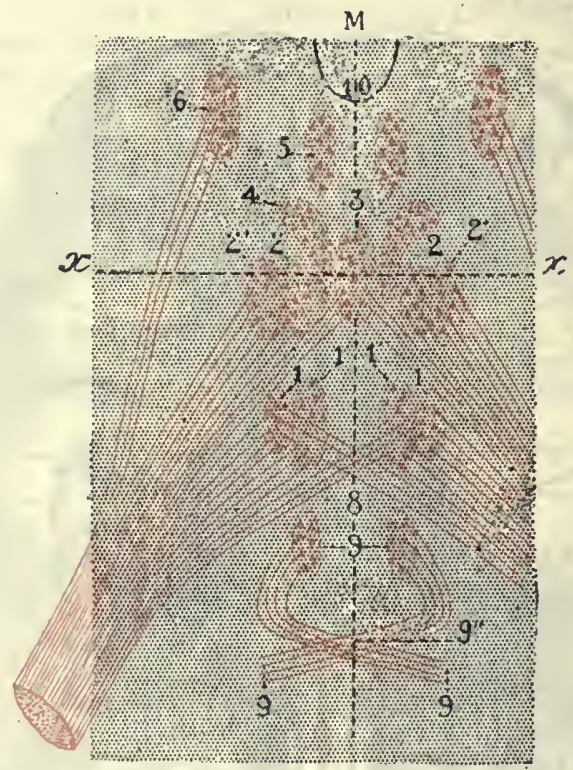

Fig 6r9.-Scheme showing the Different Cell-groups Which constitute, according to Perlia, the Nucleus of Origin of the THird Cranial or Oculo-motor Nerve (L. Testut's 'Anatomie Humaine').

1. Posterior Dorsal Nucleus

$\mathbf{I}^{\prime}$. Posterior Ventral Nucleus

2. Anterior Dorsal Nucleus

$2^{\prime}$. Anterior Ventral Nucleus

3. Central Nucleus

4. Nucleus of Edinger and Westphal

5. Antero-internal Nucleus
6. Antero-external Nucleus

7. Trunk of Oculo-Motor Nerve

8. Crossed Fibres

9. Fourth Cranial Nerve

9ucleus of Origin of Fourth Nerve

$9^{\prime \prime}$. Intercrossing of Fourth Cranial Nerves ro. Third Ventricle

M. Middle Line

the upper quadrigeminal body, and extends superiorly, for a short distance, on to the lateral wall of the third ventricle. The nucleus is intimately related to the posterior longitudinal bundle, through means of which it is connected with the trochlear and abducent nuclei. All three nuclei receive collaterals from the posterior longitudinal bundle; and in this manner a functional association between these nuclei is maintained, and harmonious action is insured on the part of the muscles which are supplied by the nerves 
arising from them. It consists of several groups of cells. As many as seven groups are ascribed to each oculo-motor nucleus by Perlia, which correspond to the seven muscles supplied by the oculo-motor nerve, and are disposed symmetrically. In addition to these, there is a mesially-placed group, the cells of which furnish fibres to both oculo-motor nerves. The fibres which arise from the individual groups of each nucleus are regarded as supplying particular orbital muscles. Certain of the oculo-motor fibres of one side arise from the nucleus of the opposite side, the fibres from either side decussating at the median line. Moreover, each oculomotor nerve is said to receive fibres from the abducent nucleu; of the opposite side, which ascend in the posterior longitudinal bundle and cross to the other side.

Two views are entertained in regard to the nerve supply of the internal rectus muscle. According to one view, the muscle of one side is supplied by those fibres which have crossed from the oculomotor nucleus of the opposite side. The other view is that the muscle of one side is supplied by those fibres which have crossed from the abducent nucleus of the opposite side. According to this latter view the nerve-fibres which supply the external rectus muscle of one side and those which supply the internal rectus muscle of the opposite side arise from the same nucleus, namely, the abducent nucleus, and vice versâ.

Fibres are conveyed by the posterior longitudinal bundle from the oculo-motor nucleus to the facial nerve, and thence to the orbicularis palpebrarum and corrugator supercilii muscles.

Course of the Fibres of the Third Nerve. - The fibres pass forwards from their origin through the tegmentum, the red nucleus, and the inner portion of the substantia nigra, and thereafter make their superficial appearance at the oculo-motor sulcus on the inner aspect of the crus cerebri.

The third or oculo-motor nerve supplies the following seven muscles : the levator palpebræ superioris; the superior, inferior and internal recti ; the inferior oblique ; the sphincter pupillæ ; and the ciliary muscle.

Fourth, Trochlear, or Pathetic Nerve.-The fibres of this nerve arise from the trochlear nucleus, which is situated in the grey matter of the ventral aspect (floor) of the aqueduct of Sylvius, on a level with the upper part of the lower quadrigeminal body. The nucleus is intimately related to the posterior longitudinal bundle, through means of which it is connected with the oculo-motor nucleus.

The fibres are at first directed outwards and backwards, and then downwards and inwards to the upper part of the superior medullary velu $m$, or valve of Vieussens, which they enter. Here the nerve crosses to the opposite side, decussating with its fellow, after which it emerges from the upper end of the superior medullary velum, close below the lower quadrigeminal body, and by the side of the frenulum veli. Thereafter the nerve turns outwards over the superior peduncle of the cerebellum, and is then directed forwards 
round the outer aspect of the crus cerebri, between which and the temporal lobe it makes its superficial appearance.

The fourth nerve supplies the superior oblique muscle of the eyeball.

Fifth Cranial, Trigeminal, or Trifacial Nerve.-The fifth cranial nerve resembles a spinal nerve in having two roots-sensory and motor-the former being large, and having a ganglion, called the Gasserian ganglion.

Sensory Root.-The fibres of this root are derived from the central poles of the bipolar cells of the Gasserian ganglion. After entering the pons Varolii each fibre divides into two branchesascending and descending, as in the case of the fibres of the dorsal or sensory root of a spinal nerve. The terminal nuclei of these ascending and descending sensory fibres are two in number-upper and lower.

The upper sensory nucleus is situated in the outer portion of the dorsal part of the pons Varolii, where it lies close to the outer side of the pontine or principal motor nucleus of the nerve. The ascending sensory fibres, after a short course, enter this nucleus and terminate in arborizations around its cells.

The lower sensory nucleus, which is a continuation of the upper sensory nucleus, is an upward prolongation of the substantia gelatinosa of Rolando from the tubercle and funiculus of Rolando in the medulla oblongata. The nucleus is traceable as low as the dorsal grey cornu of the spinal cord on a level with the second cervical spinal nerve, where it is close to the substantia gelatinosa of Rolando. The descending sersory fibres, which are numerous and constitute the spinal or descending sensory root of the fifth nerve, pass downwards through the pons and medulla oblongata into the spinal cord, as low as the level of the second cervical spinal nerve. They are accompanied by the lower sensory nucleus, and at different levels they enter this nucleus and terminate in arborizations around its cells.

The most of the axons of the cells of the terminal sensory nuclei pass inwards to the raphé and cross to the opposite side. They then become longitudinal and ascend in company with the mesial fillet or chief sensory tract, their destination being the optic thalamus of the side to which they have crossed. They thus constitute a trigemino-thalamic ascending tract. From these fibres collaterals are furnished to (I) the facial nucleus, and (2) the ventral vago-glossopharyngeal nucleus, or nucleus ambiguus, from the cells of which latter the efferent or motor fibres of the pneumogastric or vagus nerve arise.

A few of the axons, however, enter the pontine or chief motor nucleus, and also the mesencephalic or accessory motor nucleus, of the nerve, and terminate in arborizations around its cells.

Motor Root.-The fibres of this small root are derived from two nuclei-pontine and mesencephalic.

The pontine or chief motor nucleus is situated in the outer portion 
of the dorsal part of the pons Varolii, close to the inner side of the upper sensory nucleus, and beneath the floor of the upper or pontine part of the fourth ventricle. The axons of the cells of this nucleus form the larger part of the small motor root. The mesencephalic or accessory nucleus is situated in the grey matter on the lateral wall of the aqueduct of Sylvius. The fibres which arise from the axons of the cells of this nucleus constitute the mesencephalic, Sylvian, or descending motor fibres of the fifth nerve. They enter the pons Varolii, and join the more numerous fibres from the pontine or chief motor nucleus to form the motor root of the fifth nerve. As the mesencephalic fibres descend they furnish many collaterals to the pontine nucleus.

The large sensory and small motor roots appear, close together, on the lateral aspect of the ventral surface of the pons Varolii, the motor root lying above, and slightly internal to the sensory root. The sensory root enters, and the motor root emerges from, the pons Varolii.

Distribution.-The fifth cranial nerve has an extensive distribution through means of its three divisions-ophthalmic, superior maxillary, and inferior maxillary.

Ophthalmic Nerve (Sensory).--(I) The front part of the cranium; (2) the integument of $(a)$ the upper eyelid and $(b)$ the root and tip of the nose; (3) the anterior part of the nasal mucous membrane, and the conjunctiva; (4) the eyeball; and (5) the lachrymal gland.

Maxillaiy or Superior Maxillary Nerve (Sensory).-(I) The integument of the malar and anterior part of the temporal regions; (2) the integument of $(a)$ the lower eyelid, (b) the side of the nose, (c) the upper lip, and (d) the part of the face between the lower eyelid and the upper lip; (3) the pulps of the upper teeth, and the mucous membrane of the upper gum; (4) a large part of the nasal mucous membrane; (5) the mucous membrane of the maxillary airsinus or antrum of Highmore; (6) the mucous membrane of $(a)$ the naso-pharynx, and $(b)$ the soft and hard palate and the tonsil.

Mandibular or Inferior Maxillary Nerve (Sensory and Motor).The sensory distribution of this nerve is as follows: (I) The integument of $(a)$ the temporal region, $(b)$ the outer surface of the pinna, and $(c)$ the external auditory meatus; (2) the integument of the lower lip, and that which covers the mandible; (3) the temporomandibular joint; (4) the parotid salivary gland; (5) the mucous membrane lining the buccinator muscle, and the integument covering that muscle (through means of the long or sensory buccal nerve); (6) the mucous membrane (fungiform and conical papillæ) of the anterior two-thirds of the tongue (common sensation); $(7)$ the submaxillary and sublingual salivary glands; and (8) the pulps of the lower teeth, and the mucous membrane of the lower gum.

The motor distribution of the inferior maxillary nerve is as follows: (I) The muscles of mastication-namely, (a) the masseter, (b) the temporal, and $(c)$ the external and internal pterygoid muscles; (2) the mylo-hyoid muscle and anterior belly of the digastric; (3) the 
tensor tympani muscle by means of a branch from the otic ganglion; and (4) the tensor palati muscle, through the otic ganglion.

Sixth or Abducent Nerve.- The fibres of this nerve arise from the abducent nucleus, which is situated in the dorsal part of the pons Varolii, close to the median line. It lies above the striæ acusticæ on the floor of the fourth ventricle, subjacent to the eminentia teres. The fibres emerge from the inner part of the nucleus, and pass through the lower part of the pons in a forward and slightly downward and outward direction to the point where the nerve makes its superficial appearance, namely, the lower border of the pons, just external to the pyramid of the medulla oblongata.

The abducent nucleus receives collaterals from the dorsal or posterior longitudinal bundle, and a functional connection is thereby established between that nucleus and the oculo-motor nucleus. The internal rectus muscle of one side and the external rectus of the other side are thus associated muscles.

The sixth nerve supplies the external rectus muscle of the eyeball.

Seventh or Facial Nerve.-The facial nerve is composed of two parts. One of these consists of efferent or motor fibres, and is known as the facial nerve proper. The other part, of small size, consists of afferent or sensory fibres, and is called the pars intermedia of Wrisberg. The facial nerve proper arises from the facial mucleus, which is situated deeply in the dorsal part of the lower portion of the pons Varolii. The fibres of the nerve pursue an intricate course before appearing superficially. They at first pass backwards and inwards to the floor of the fourth ventricle. Here they turn upwards, lying close to the median line in the form of a single bundle. The nerve then makes a sharp bend outwards, and passes forwards through the pons in a downward and outward direction to its place of emergence. In its course within the pons the nerve is intimately related to the dorsal aspect of the abducent nucleus.

The intrapontine part of the facial nerve proper is intimately related to the following structures:

I. The abducent nucleus.

2. The superior olive.

3. The corpus trapezoides.
4. The spinal root of the fifth nerve.

5. The dorsal or posterior longitudinal bundle.

The motor facial nucleus receives fibres from the following sources: (I) The corpus trapezoides, being thereby brought into connection with the cochlear division of the auditory nerve; $(2)$ the spinal root of the fifth cranial or trigeminal nerve, which is the sensory nerve of the face; and (3) the pyramidal tract of the opposite side, being thereby brought into connection with the pre-Rolandic motor area of the cerebral cortex.

All the foregoing fibres terminate within the nucleus in arborizations around its component cells. 
The pars intermedia of Wrisberg or sensory portion of the facial nerve arises from the central poles of the bipolar cells of the geniculate ganglion on the facial nerve in the aqueduct of Fallopius. This ganglion resembles the Gasserian ganglion of the fifth rierve and the spinal ganglia, and most of the peripheral poles of its bipolar cells give rise to the chorda tympani nerve. The pars intermedia emerges from the aqueduct of Fallopius into the internal auditory meatus. After issuing from that meatus it passes to the lower border of the pons Varolii, where it lies between the facial nerve proper and the auditory nerve. The nerve then enters the medulla oblongata, and passes downwards to the upper part of the nucleus of the fasciculus solitarius (see Glosso-pharyngeal Nerve), and its fibres terminate in arborizations around the cells of the upper part of that nucleus. In this situation it is closely associated with the terminal afferent or sensory fibres of the glosso-pharyngeal nerve.

The facial nerve proper emerges from the brain at the lower border of the pons Varolii in front of, and internal to, the auditory nerve; and the pars intermedia of Wrisberg enters between the facial nerve proper and the auditory nerve.

Distribution, Motor Part (Facial Nerve Proper).--(I) The muscles of the face, including the buccinator; (2) the occipito-frontalis; (3) the muscles of the auricle; (4) the posterior belly of the digastric and the stylo-hyoid; (5) the platysma myoides; and (6) the stapedius muscle, within the tympanic cavity.

Sensory Part (Pars Intermedia of Wrisberg and Chorda Tympani). -The anterior two-thirds of the tongue (sense of taste).

The chorda tympani nerve conveys secretory and vaso-dilator fibres from the facial nerve proper to the submaxillary and sublingual salivary glands.

"The large superficial petrosal nerve, from the geniculate ganglion of the facial nerve, is concerned in the supply of the mucous membrane of the soft palate, the path being as follows: (I) Large superficial petrosal nerve (facial fibres); (2) the Vidian nerve; (3) Meckel's ganglion; and (4) the descending palatine nerves.

Eighth, Auditory, or Acoustic, Nerve.-The auditory nerve is the nerve of hearing and of equilibrium. It is an afferent or centripetal nerve which conducts impressions from the membranous labyrinth (cochlea and vestibule) to the medulla oblongata and pons Varolii, and thence to the cerebrum and cerebellum. It consists of two divisions-namely, the cochlear nerve or root, and the vestibular nerve or root.

The fibres of the cochlear nerve arise from the bipolar cells of the spiral ganglion in the spiral canal of the modiolus, the modiolus being the central pillar of the osseous cochlea. The fibres of the vestibular nerve arise from the bipolar cells of the ganglion of Scarpa at the deep end of the internal auditory meatus.

The two nerves or roots reach the brain at the lower border of the pons Varolii, external to the facial nerve and ventral to the restiform body. They have different central connections, and 
consequently take different courses. The cochlear nerve passes round the outer side of the restiform body, whilst the vestibular nerve passes backwards on the inner side of that body, and each root has special terminal nuclei.

Cochlear Nerve.-The terminal nuclei of the cochlear nerve, which is the nerve of hearing, are two in number-ventral and lateral.

The ventral or accessory nucleus lies on the ventral aspect of the restiform body, between the cochlear and vestibular nerves. The

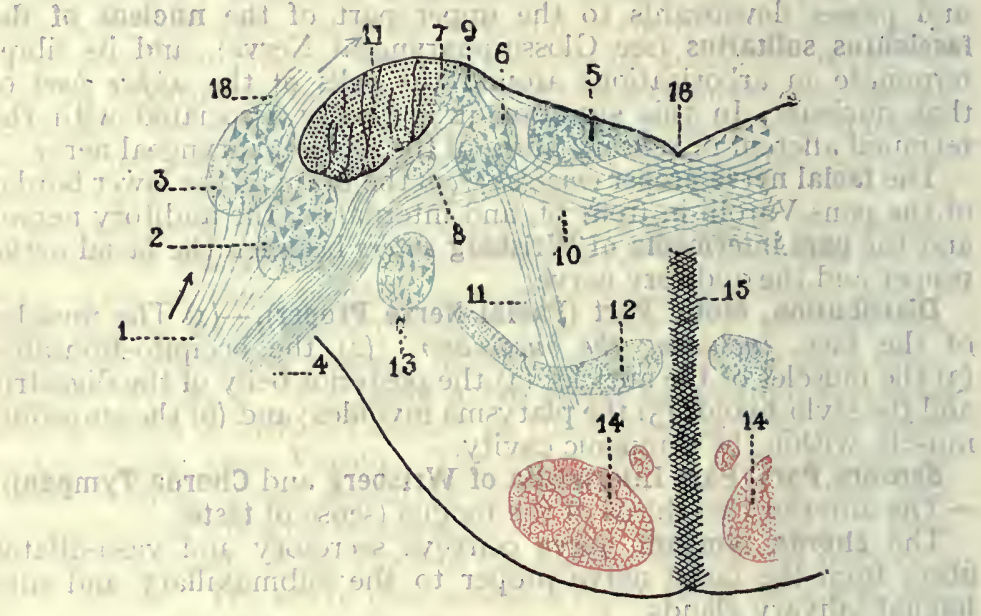

Fig. 620.-Terminal NUClei OF the, Vestibular Nerve, with their Superior Connections (Schematic) (L. Testur's 'Anatomie Humaine ').

r. Cochlear Root, with its Two Nuclei

2 Accessory Nucleus

3. Lateral Nucleus, or Tuberculum Acusticum

4. Vestibular Root

5. Internal Dorsal Nucleus

6. External Dorsal Nucleus, or Nucleus of Deiters

7. Nucleus of Bechterew

8. Inferior or Descending Root of Auditory Nerve

9. Ascending Cerebellar Fibres ro. Fibres passing to Rapbe

Ir. Oblique Fibres

I2. Fillet

13. Inferior Sensory Root of Fifth Cranial Nerve

14. Pyramidal Fibres

I5. Raphé.

I6. Fourth Ventricle

17. Inferior Peduncle of Cerebellum (Restiform Body)

s8. Origin of Striæ Actistica

lateral or dorsal nucleus, or tuberculum acusticum, is situated on the lateral and dorsal aspects of the restiform body. The fibres of the cochlear nerve or root enter these two nuclei, and terminate in arborizations around their component cells.

Central Connections of the Ventral and Lateral Cochlear Nuclei.The ventral and lateral nuclei constitute cell-stations in the path of the fibres of the cochlear nerve, and from these cell-stations two fresh nerve-tracts arise, one being ventral, which constitutes the corpus trapezoides; and the other dorsal, which forms the strix acusticæ. 
Ventral ochlear Tract.-The relays, or fresh supplies, of nervefibres for this tract are furnished by the cells of the ventral mucleus. The axons of these cells give rise to the trapezium, or corpus trapezoides, which is reinforced by the axons of the cells of the nucleus trapezoides, and fibres from the superior olive of the same side. The trapezoidal fibres cross the median plane, and thereafter constitute the lateral or acoustic fillet, being further reinforced by fibres from the superior olive of the side to which they have crossed, and from the nucleus of the lateral fillet. The fibres of this fillet terminate in the lower quadrigeminal body and internal geniculate body, both of the same side.

Dorsal Cochlear Tract.-The relays of fibres for this tract are furnished by the cells of the lateral mucleus. The axons of its cells form the striæ acusticæ, which cross the dorsal aspect of the restiform body and the floor of the fourth ventricle. At the median line they pass forwards, and then cross to the opposite side. Thereafter they join the lateral or acoustic fillet of the side to which they have crossed.

The lateral or acoustic fillet, as previously stated, derives its fibres from the following sources: (I) The corpus trapezoides; (2) the striæ acusticæ; (3) the superior olive of both sides; and (4) the nucleus of the lateral fillet. It serves as a path of connection between the ventral and lateral cochlear nuclei of one side, and the lower quadrigeminal body and internal geniculate body of the opposite side.

It is to be noted that the ventral and lateral cochlear nuclei are slightly connected with the lower quadrigeminal body of the same side, but not with the corresponding internal geniculate body.

The axons of the cells of the internal geniculate body form a corticipetal tract which passes to the cortex of the first or superior temporal gyrus of the temporal lobe of the brain.

The complex nervous chain associated with the cochlear nerve may be tabulated as follows:
I. The bipolar cells of the spiral ganglion.
2. The fibres of the cochlear nerve.
3. The ventral and lateral cochlear nuclei.
4. The fibres of the corpus trapezoides, reinforced as stated.
5. The striæ acusticæ.
6. The internal geniculate body.
7. The corticipetal tract from the internal geniculate body to the superior temporal gyrus.

= The cell-stations connected with this nervous chain are as follows:

I. The ventral cochlear nucleus.

2. The lateral cochlear nucleus.

3. The nucleas trapezoides.

4. The superior olive of each side.
5. The nucleus of the lateral fillet.

6. The lower quadrigeminal body.

7. The internal geniculate body.

Some of the fibres of the chain terminate in these cell-stations, and others are derived from the axons of the cells which compose the stations. 
Vestibular Nerve.-The terminal nuclei of the vestibular nerve, which is the nerve of equilibrium, are three in number-namely, (I) the dorsal or principal nucleus, (2) the descending nucleus, and (3) the nucleus of Deiters, associated with which there is the nucleus of Bechterew.

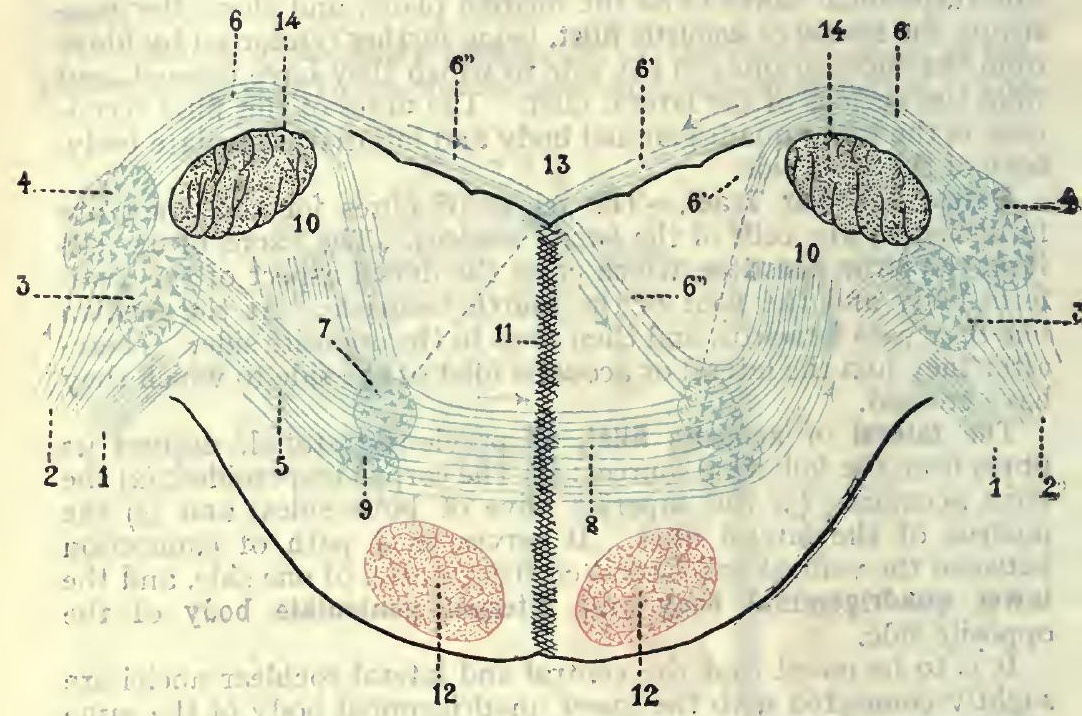

Fig. 621.-Terminal Nuclei of the Cochlear Nerve, with their Supe. Rior Connections (Schematic) (L. Testút's 'Anatomie Humaine ').

(The vestibular root and its terminal nuclei, with the efferent fibres of these latter, have been suppressed. In order not to obscure the trapezoid body. the efferent fibres of the terminal nuclei of the right side have been in a great part of their extent resected. The trapezoid body is consequently composed of only half of its fibres-namely, those which come from the left).

I. Vestibular Root of Auditory Nerve

2. Cochlear Root

3. Accessory Nucleus of Auditory Nerve

4. Lateral Nucleus, or Tuberculum Acusticum

5. Efferent Fibres of Accessory Nucleus

6. Effercnt Fibres of Lateral Nucleus, forming the Strix Acustica

6:. Direct Fibres of the Striæ Acusticæ going to the Superior Olivary Body of the same side
6". Crossed Fipres of the Stria Acustica going to the Superior Olivary Body of the opposite side

7. Superior Olivary Body

8. Corpus Trapezoides

9. Trapezoid Nucleus

Io. Lateral Fillet

II. Raphé

12. Pyramidal Tract

13. Fourth Ventricle

14. Inferior Peduncle of Cerebellum (Restiform Body)

The dorsal or principal nucleus is situated in the floor of the fourth ventricle underneath the area acustica and strice acustica. The descending nucleus is continuous with the lower end of the dorsal nucleus, and it accompanies the descending fibres of the vestibular nerve into the medulla oblongata. The nucleus of Deiters is situated external to the dorsal and descending nuclei, and the 
nucleus of Bechterew represents the upper and outer part of the nucleus of Deiters.

As the vestibular nerve passes backwards on the inner side of the restiform body, some of its fibres, to be presently described, turn downwards. The majority, however, pass to the dorsal or principal nucleus, the nucleus of Deiters, and the nucleus of Bechterew, and terminate in arborizations around the cells of these nuclei. A few of the vestibular fibres are regarded as passing directly to the cerebellum (superior vermis).

Central Connections of the Nuclei of the Vestibular Nerve.-The fibres of the vestibular nerve terminate in the nuclei just stated.

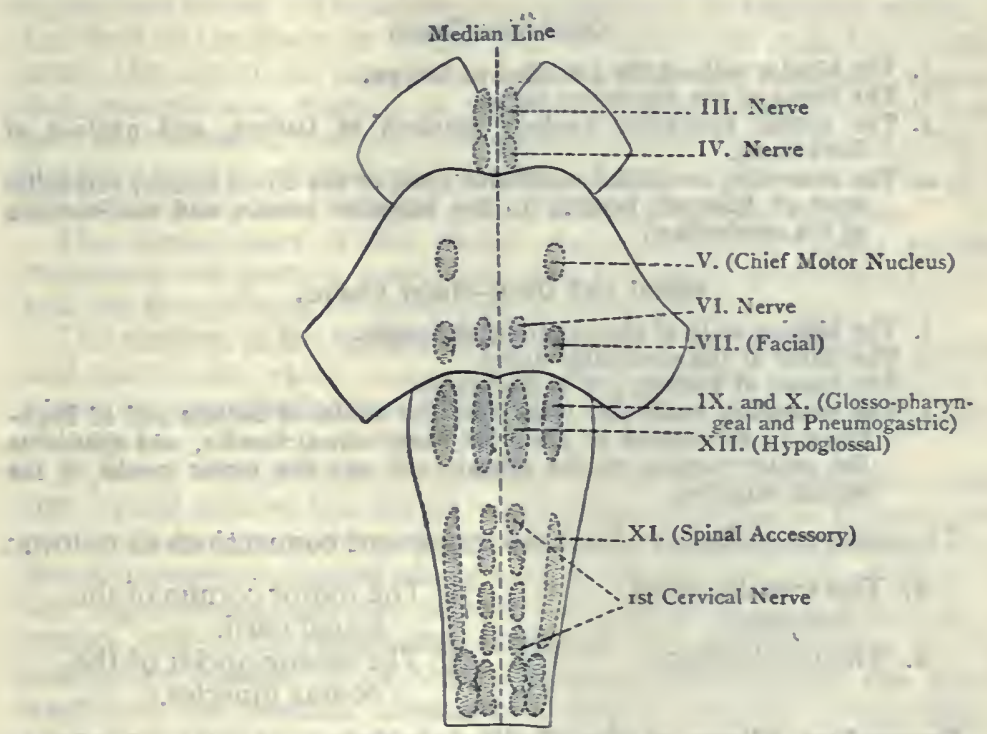

Fig. 622.-Scheme showing the Continuation of the Grey Matter of the Spinal Cord into the Medulla Oblongata, Pons Varolit, and Crura Cerebri (Potrier).

(The separation into two columns on either side and the breaking up of these into Cranial Motor Nuclei are indicated).

The dorsal or principal nucleus and the nucleus of Deiters (including the nucleus of Bechterew), are intimately related to the superior vermis of the cerebellum, and especially to the roof-nucleus, by means of cerebellar fibres. This communication represents the direct sensory cerebellar tract of Edinger, and it is contained within the inferior peduncle of the cerebellum. The axons of many of the cells of the nucleus of Deiters and nucleus of Bechterew pass into the dorsal or posterior longitudinal bundle, within which they divide into ascending and descending branches. In this manner the dorsal 
longitudinal bundle is brought into communication with the vestibular nerve. Through means of the dorsal longitudinal bundle, which represents the ventral ground-bundle and anterior marginal bundle of Lowenthal in the spinal cord, the nucleus of Deiters is brought into communication with the anterior or motor cornua of the spinal cord. Through means of this bundle the nucleus of Deiters is also brought into communication with the nuclei which control the ocular muscles-namely, the oculo-motor, trochlear, and abducent nuclei.

The complex nervous chains associated with the vestibular nerve may be tabulated as follows:

\section{Cerebellar Chain.}

I. The bipolar cells of the ganglion of Scarpa.

2. The fibres of the vestibular nerve.

3. The dorsal vestibular nucleus, nucleus of Deiters, and nucleus of Bechterew.

4. The secondary cerebellar vestibular tract, or the direct sensory cerebellar tract of Edinger, Ieading to the superior vermis and roof-nucleus of the cerebellum.

\section{Spinal and Oculo-Motor Chains.}

I. The bipolar cells of the ganglion of Scarpa.

2. The fibres of the vestibular nerve.

3. The nuclei of Deiters and of Bechterew.

4. The secondary vestibular tract from the nuclei of Delters and of Bechterew to the dorsal or posterior longitudinal bundle, and thence to the motor cornua of the spinal cord and the motor nuclei of the ocular muscles.

The nucleus of Deiters thus has important connections as follows:

I. The membranous vestibule.

2. The cerebellum.
3. The motor cornua of the spinal cord.

4. The motor nuclei of the ocular muscles.

Descending Fibres of the Vestibular Nerve.-As the vestibular. nerve passes backwards on the inner side of the restiform body, some of its fibres, as stated, take a downward course. These descending fibres constitute the so-called descending root of the vestibular nerve. They descend through the lower part of the pons Varolii into the medulla oblongata as low as the level of the cuneate tubercle. They are accompanied throughout by the descending nucleus, and they are usually regarded as terminating in aborizations around the cells of that nucleus. Superiorly they are related to the nucleus of Deiters, and some authorities have regarded them as fibres passing between the nucleus of Deiters and the cuneate nucleus.

The cochlear and vestibular nuclei originally form one acoustic or auditory nucleus, which is developed from the rhombie lip.

Ninth or Glosso-pharyngeal Nerve. -This nerve consists chiefly of afferent or sensory fibres, which grow into the medulla oblongata, 
but it also contains a few efferent or motor fibres, which arise within the medulla oblongata.

Afferent or Sensory Fibres.-These fibres arise from the central poles of the bipolar cells of the jugular and petrous ganglia, which are situated on the glosso-pharyngeal nerve as it passes through the jugular foramen. These ganglia resemble the Gasserian ganglion of the fifth nerve and the spinal ganglia. Having entered the medulla oblongata, the afferent fibres end in two terminal sensory nuclei-namely, the dorsal vago-glosso-pharyngeal nucleus, and the nucleus of the fasciculus solitarius.

The dorsal vago-glosso-pharyngeal nucleus consists of two partsupper and lower. The upper part is situated in the grey matter of the floor of the lower or bulbar part of the fourth ventricle, underneath the superficial area known as the trigonum ragi (ala cinerea), and immediately external to the hypoglossal nucleus. The lower part is situated in the lower or closed part of the bulb, and lies in the grey matter which forms the lateral wall of the central canal of the bulb, being here situated behind the hypoglossal nucleus.

The highest part of the dorsal vago-glosso-pharyngeal nucleus represents the portion associated with the glosso-pharyngeal nerve, and the remaining and greater part belongs to the vagus nerve.

The nucleus of the fasciculus solitarius is a column of grey matter and nerve-cells which accompanies the fasciculus solitarius, to be presently described, throughout the whole length of the medulla oblongata.

$A$ few of the afferent fibres of the glosso-pharyngeal nerve enter the highest part of the dorsal vago-glosso-pharyngeal nucleus, and terminate in arborizations around its cells. The most of the afferent fibres, however, descend along with a few of the afferent fibres of the vagus nerve, the two sets of descending fibres constituting a strand, called the fasciculus solitarius (tractus solitarius B.) or respiratory bundle. This strand descends throughout the whole length of the medulla oblongata. It lies external to the dorsal vago-glosso-pharyngeal nucleus, inclining towards the ventral aspect of the upper part of that nucleus, and towards the dorsal aspect of its lower part. It is accompanied throughout by the nucleus of the fasciculus solitarius, and the glosso-pharyngeal afferent fibres of the fasciculus solitarius terminate, at different levels, in arborizations around its cells. The fasciculus solitarius is formed chiefly, if not entirely, by glosso-pharyngeal fibres. The fasciculus solitarius and its nucleus are comparable to the spinal or descending fibres (root) and lower sensory nucleus of the fifth nerve.

Efferent or Motor Fibres.-These fibres arise within the medulla oblongata as the axons of some of the cells of the ventral vagoglosso-pharyngeal nucleus or nucleus ambiguus. This nucleus is situated in the formatio reticularis grisea of the medulla oblongata, and is in line with the facial motor nucleus which is placed in the dorsal part of the lower portion of the pons Varolii. Both of these nuclei correspond to the motor cells of the ventral grey cornu of 
the spinal cord. The nucleus ambiguus is ventral in position to the dorsal vago-glosso-pharyngeal nucleus, and the axons of its cells, some of which form the glosso-pharyngeal efferent or motor fibres, pass dorsalwards towards the more superficially placed dorsal vago-glosso-pharyngeal nucleus. They then alter their course and pass ventralwards and outwards, and associate themselves with the afferent or sensory glosso-pharyngeal fibres.

The tuniculi of the glosso-pharyngeal nerve appear in the dorsolateral sulcus of the medulla oblongata, between the olivary and restiform bodies, and immediately below the facial nerve.

Distribution.-The glosso-pharyngeal nerve is distributed to (I) the mucous membrane of the posterior third of the tongue, of which part it is the nerve of taste, as well as of common sensation; (2) the mucous membrane of the pharynx, tonsil, and fauces; (3) the mucous membrane of the tympanum; and (4) the stylo-pharyngeus muscle. It also furnishes secretory and vaso-dilator fibres to the parotid gland through means of (I) its tympanic branch (Jacobson's nerve), (2) the tympanic plexus, (3) the small superficial petrosal nerve, (4) the otic ganglion, and (5) the auriculo-temporal nerve.

Tenth, Pneumogastric, or Vagus Nerve.-This nerve consists of afferent or sensory fibres, which grow into the medulla oblongata; and efferent or motor fibres, which arise within the medulla oblongata.

Afferent or Sensory Fibres.-These fibres arise from the central poles of the bipolar cells of the ganglion of the root and the ganglion of the trunk of the nerve, which resemble the glosso-pharyngeal ganglia (jugular and petrous), the Gasserian ganglion of the fifth nerve, and the spinal ganglia. Having entered the medulla oblongata, the afferent fibres pass to the same two terminal sensory nuclei as do the afferent fibres of the glosso-pharyngeal nervenamely, the dorsal vago-glosso-pharyngeal nucleus and the nucleus of the fasciculus solitarius - which have just been described in ccnnection with the glosso-pharyngeal nerve. The most of the afferent fibres of the vagus nerve pass to the vagal portion of the dorsal vagoglosso-pharyngeal nucleus, which represents its greater and lower part, the highest part of the nucleus receiving, as stated, a few of the afferent fibres of the glosso-pharyngeal nerve. Within the vagal part of the nucleus the afferent fibres of the vagus terminate in arborizations around its : cells. A few of the afferent fibres, however, descend along with most of the afferent fibres of the glossopharyngeal nerve, the two sets of descending fibres constituting the strand called the fasciculus solitarius, already described in connection with the glosso-pharyngeal nerve. These descending afferent vagal fibres terminate, like the corresponding glosso-pharyngeal fibres, in the nucleus of the fasciculus solitarius, which has been described in connection with the glosso-pharyngeal nerve.

The dorsal vago-glosso-pharyngeal sensory nucleus, and the nucleus of the fasciculus solitarius, also sensory, are therefore shared in common by the afferent or sensory fibres of the glosso- 
pharyngeal and vagus nerves, but in unequal proportions. Only a few glosso-pharyngeal afferent fibres go to the dorsal vago-glossopharyngeal nucleus, whereas most of the vagal afferent fibres pass to that nucleus. In the case of the nucleus of the fasciculus solitarius it is the reverse.

Efferent or Motor Fibres.-These fibres arise within the medulla oblongata as the axons of most of the cells of the ventral vagoglosso-pharyngeal nucleus or nucleus ambiguus, which has been described in connection with the glosso-pharyngeal nerve. The fibres pass dorsalwards to the more superficially placed dorsal vagoglosso-pharyngeal nucleus. They then alter their course and pass ventralwards and outwards, and associate themselves with the afferent or sensory vagal fibres.

The ventral vago-glosso-pharyngeal nucleus or nucleus ambiguus, which is a motor nucleus, is shared in common by the efferent or motor fibres of the glosso-pharyngeal and vagus nerves, especially the latter.

The funiculi of the pneumogastric or vagus nerve appear in the dorso-lateral sulcus of the medulla oblongata, between the olivary and restiform bodies, and immediately below the funiculi of the glosso-pharyngeal nerve.

Distribution.-The vagus nerve has a very extensive distribution on either side, of which the following is a summary:

Motor Distribution.-(I) The muscles of the soft palate (except the tensor palati); (2) the constrictor muscles of the pharynx; (3) the intrinsic muscles of the larynx; (4) the muscular tissue of the osophagus and stomach; and (5) the muscular tissue of .(a) the trachea, $(b)$ the bronchi, and $(c)$ the bronchial tubes.

Sensory Distribution.-(I) The pharynx, œsophagus, and stomach; (2) the larynx, trachea, and bronchial tubes to their terminal ramifications; and (3) the skin on the inner surface of the pinna, as well as of the lower and back part of the external auditory meatus.

Cardiac Fibres.-The cardiac fibres of the reve are inhibitory (efferent) and depressor (afferent).

The most important connection of the vagus nerve is that which is established with the bulbar or accessory portion of the spinal

\section{accessory nerve.}

Glosso-pharyngeal and Vagal Nuclei.-These two nerves, as stated, consist of afferent or sensory and efferent or motor fibres. The afferent fibres of both nerves share in common two terminal muclei-namely, the dorsal vago-glosso-pharyngeal nucleus and the nucleus of the fasciculus solitarius. Most of the glosso-pharyngeal afferent fibres terminate in the nucleus of the fasciculus solitarius, and most of the vagal afferent fibres terminate in the dorsal vagoglosso-pharyngeal nucleus. According to the description which has been given of the dorsal vago-glosso-pharyngeal nucleus, it is a nucleus of termination, or sensory nucleus. According to certain authorities, however, it is a mixed nucleus - that is to say, it is both a nucleus of termination, or sensory nucleus, and a nucleus of origin, 
or motor nucleus. In accordance with this view, the nucleus contains two sets of cells-sensory and motor-some of the afferent glosso-pharyngeal and most of the afferent vagal fibres terminating in arborizations around the sensory cells, and some of the efferent fibres of each nerve arising as the axons of the motor cells.

The nucleus of the fasciculus solitarius is a nucleus of termination, or sensory nucleus.

The fibres of the pars intermedia of Wrisberg or sensory portion. of the facial nerve terminate in the upper part of the nucleus of the fasciculus solitarius.

The efferent or motor fibres of the glosso-pharyngeal and vagus nerves arise as the axons of the motor cells of the ventral vagoglosso-pharyngeal nucleus, or nucleus ambiguus, which is a nucleus of origin, or motor nucleus. According to the description which has been given of this nucleus, it gives origin to all the motor fibres of the two nerves. If, however, the dorsal vago-glosso-pharyngeal nucleus is a mixed nucleus, then some of the efferent or motor fibres of the two nerves arise as the axons of its motor cells.

The axons of the cells of the terminal sensory nuclei are disposed like those of the cells of the terminal sensory nuclei of the fifth nerve. They cross to the opposite side, become longitudinal, and ascend in company with the mesial fillet or chief sensory tract to the optic thalamus of the side to which they have crossed. They constitute the vago-glosso-pharyngeal ascending thalamic tract.

Eleventh or Accessory (Spinal-Accessory) Nerve.-This is a motor nerve, which is really a continuation of the pneumogastric or vagus: Its fibres arise from the accessory nucleus (dorsal vago-accessory nucleus), which is situated partly within the medulla oblongata or bulb, and partly within the cervical part of the spinal cord. This nucleus consists of a column of large cells which is continuous with the dorsal vago-glosso-pharyngeal nucleus. The column extends from the level of the lower part of the olivary body to the level of the sixth cervical nerve. The bulbar portion of the nucleus is situated on the dorso-lateral aspect of the hypoglossal nucleus. The spinal portion is situated in the lateral part of the anterior grey cornu of the cervical spinal cord, and its cells lie directly behind the motor cells which give origin to the anterior roots of the upper five cervical nerves.

The fibres which arise from the bulbar portion of the accessory nucleus constitute the bulbar part of the accessory nerve, and are accessory to the vagus nerve. The fibres which arise from the spinal portion of the accessory nucleus constitute the spinal part of the accessory nerve.

Bulbar Part.-The fibres of this part arise as the axons of the cells of the bulbar portion of the accessory nucleus. They are directed at first dorsalwards, and then outwards through the lateral part of the medulla oblongata or bulb, from which they emerge in the form of about five funiculi, placed below, and in line with, the funiculi of the pneumogastric nerve. They then pass outwards, lying within 
the cranial cavity, and they join the spinal part of the accessory nerve (which has entered the cranial cavity through the foramen magnum), the accessory nerve subsequently emerging from the cranial cavity through the jugular foramen.

Spinal Part. - The fibres of this part arise as the axons of the cells of the spinal portion of the accessory nucleus. They are directed at first dorsalwards, and then outwards through the lateral column of the spinal cord, from which they emerge as a series of funiculi which succeed to the funiculi of the bulbar part, the lowest spinal funiculus being on level with the fifth cervical nerve. The funiculi of the spinal part ascend, lying in the subdural space between the ligamentum denticulatum and the posterior roots of the upper five cervical nerves. They enter the cranial cavity through the foramen magnum, and join the funiculi of the bulbar part, to form the accessory nerve, which, as stated, emerges from the cranial cavity through the jugular foramen.

Distribution of Accessory Nerve.-After emerging from the jugular foramen, the accessory nerve divides into two branches-internal and external - the internal branch containing the fibres of the bulbar part, whilst the external branch contains the fibres of the spinal part.

Spinal Distribution.-The external or spinal branch supplies the sterno-cleido-mastoid and trapezius muscles.

Bulbar Distribution. - The internal or bulbar branch passes over, and in close contact with, the ganglion of the trunk of the pneumogastric nerve. Its fibres are continued into (I) the pharyngeal and superior laryngeal branches of the ganglion of the trunk of the vagus, and (2) the trunk of the vagus beyond the ganglion.

The bulbar fibres, through their connection with the vagus nerve, are probably distributed to $(\mathrm{I})$ the muscles of the soft palate, excluding the tensor palati; (2) the constrictor muscles of the pharynx; and (3) the intrinsic muscles of the larynx. The bulbar fibres may also furnish $(a)$ the inhibitory fibres to the heart, $(b)$ the motor fibres to the oesophagus, and $(c)$ the motor fibres to the stomach.

The bulbar fibres of the accessory nerve are regarded by some authorities as arising from the column of cells which constitutes the ventral vago-glosso-pharyngeal nucleus, or nucleus ambiguus.

Twelfth or Hypoglossal Nerve.-The fibres of the hypoglossal nerve arise from the axons of the cells of the hypoglossal nucleus, which is situated within the medulla oblongata. This nucleus represents a column of large multipolar motor-cells, which extends .from the level of the striæ acusticæ superiorly to the level of the upper part of the decussation of the pyramids inferiorly. The lower part of the nucleus is situated within the lower or closed part of the medulla oblongata, and its upper part lies within the upper, open, or ventricular part. The lower part lies in the grey matter which forms the ventro-lateral aspect of the central canal of the medulla oblongata. The upper part lies in the grey matter which covers the bulbar part of the floor of the fourth ventricle, and is underneath the area known as the trigonum hypoglossi. 
The nerve-fibres issue from the ventral aspect of the nucleus, and the nerve-funiculi pass through the medulla oblongata in a dorsoventral direction, lying between its anterior and lateral areas, and between the formatio reticularis alba and formatio reticularis grisea. Having reached the bottom of the ventro-lateral sulcus between the pyramid and the olive, they emerge from the medulla oblongata in line with the sixth cranial nerve superiorly.

The two hypoglossal nuclei, right and left, are connected with each other by commissural dendrons; and each nucleus receives collaterals from the pyramidal tract of the opposite side, being thereby brought into connection with the pre-Rolandic motor area of the opposite cerebral hemisphere.

Distribution.-The hypoglossal nerve is the motor nerve of the tongue, and supplies (I) the stylo-glossus, (2) the hyo-glossus, (3) the genio-hyo-glossus, and (4) the intrinsic muscles of the tongue.

\section{Cranio-cerebral Topography.}

The auricular point is the centre of the orifice of the meatus auditorius externus.

The pre-auricular point is situated in the depression between the tragus of the auricle and the condyle of the mandible.

The bregma, or point of junction of the sagittal and coronal sutures, corresponds to the centre of a line connecting the two auricular points (the centre of the orifice of the meatus auditorius externus).

The lambda, or meeting of the sagittal and lambdoidal sutures, is situated about $2 \frac{3}{4}$ inches above the inion, or external occipital protuberance.

The pterion, or region of the spheno-parietal suture, is situated about $I_{2} \frac{1}{2}$ inches behind the external angular process of the frontal bone, and about $I \frac{3}{4}$ inches above the zygomatic arch.

The asterion, or point where the parieto-mastoid, occipitomastoid, and lambdoid sutures meet, is situated about 2 inches behind the auricular point, very nearly in line with the upper border of the zygomatic arch.

The sagittal line represents the line which connects the nasion, or meeting of the two fronto-nasal sutures, with the inion.

The sagittal suture corresponds to that part of the sagittal line which extends from the lambda to the bregma.

The coronal suture is indicated on either side by a line extending from the bregma to the pterion.

The lambdoid suture (occipito-parietal) corresponds to a line extending from the lambda to the asterion.

The squamo-parietal suture, and its continuation backwards as the parieto-mastoid suture, are indicated by a curved line, with the convexity upwards, extending from the pterion to the asterion, the highest part of the curve being about 2 inches above the zygomatic arch. 
The superior longitudinal sinus corresponds to a line drawn from the glabella to the inion, or external occipital protuberance. For the most part it occupies the median line, but, as it grooves the upper portion of the tabular part of the occipital bone, it deviates to one side, most commonly the right side.

The torcular Herophili is usually situated on the right side of the inion.

The occipital sinus corresponds to a line drawn downwards from the inion.

The transverse sinus on either side is indicated by a line drawn outwards from a point immediately above, and external to, the inion to a point immediately above the asterion. This line is slightly curved, the convexity being upwards. The sinus grooves the inner surface of one-half of the tabular portion of the occipital bone, along the line of attachment of the tentorium cerebelli; and in the region of the asterion, where the sinus, in altering its course, describes a curve, it grooves the inner aspect of the parietal bone, close to the postero-inferior angle, for a very short distance.

Inasmuch as the superior longitudinal sinus usually opens into the right transverse sinus, the right sinus is usually larger than that of the left side.

The sigmoid part of the transverse sinus grooves the inner surface of the mastoid portion of the temporal bone, and the superior surface of the jugular process of the occipital bone. It consists of two limbs and a genu. The superior limb passes horizontally forwards, and the inferior limb passes downwards and inwards. The genu intervenes between the two limbs, and its convexity is directed forwards. The following line indicates approximately the course of the sigmoid part of the transverse sinus: (I) Draw a line horizontally forwards from the asterion for fully $\frac{3}{4}$ inch to a point on the root of the mastoid process in line with the upper part of the meatus auditorius externus; (2) the line now curves, and passes downwards and inwards on the front part of the mastoid process towards its tip for $\frac{3}{4}$ inch, lying close to the groove between the back of the pinna and the mastoid process (the level to which this line descends is $\frac{1}{4}$ inch below the lower margin of the orifice of the meatus auditorius externus); and (3) the line finally passes forwards for $\frac{1}{2}$ inch to meet the jugular foramen, through which the sigmoid part of the transverse sinus leaves the cranial cavity to become the internal jugular vein.

Part (I) of the preceding line indicates the superior horizontal limb of the sigmoid sinus; part (2) the inferior descending limb; and part (3) the terminal portion of the sinus passing forwards to the jugular foramen.

The genu and descending limb of the sigmoid sinus lie directly behind the mastoid or tympanic antrum, being separated from it only by a very thin plate of bone. In pyogenic affections of the tympanum and antrum the genu and descending limb of the sigmoid sinus are consequently liable to become affected with thrombosis. 
The convexity of the genu of the sigmoid sinus is on a level with the temporo-mandibular joint, and lies from $\frac{1}{8}$ to $\frac{1}{4}$ inch behind the base of the suprameatal triangle, or $\frac{3}{4}$ inch behind the pre-auricular point.

The right sigmoid sinus is usually larger than the left. In many cases the genu is scarcely perceptible, and the horizontal and vertical limbs under these circumstances are practically almost in direct continuity with each other. $\$$ The genu lies at a depth from the surface varying from $\frac{1}{4}$ to $\frac{1}{2}$ inch.

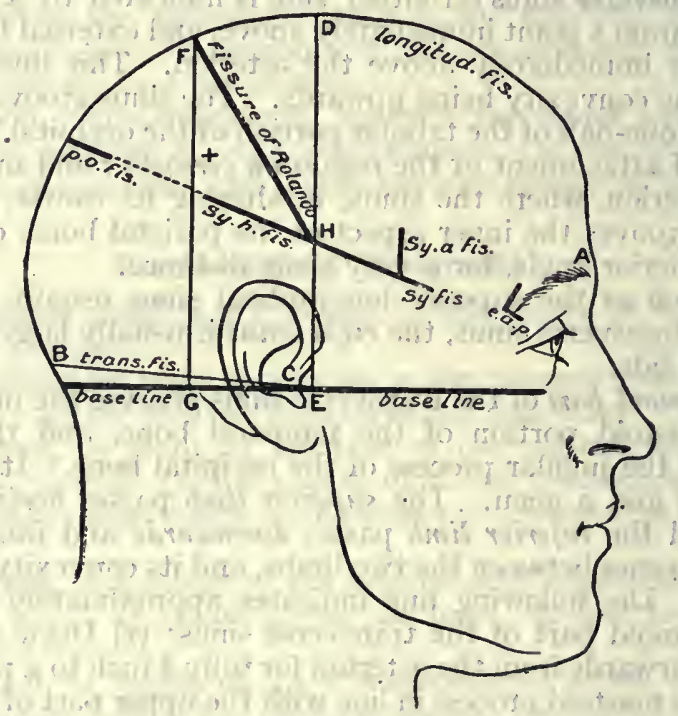

Fig. 623.-Diagram showing the Relations of the Chief Cerebral FISSURES TO THE EXTERIOR OF THE HEAD (REID).
A. Glabella
B. Inion
C. Auricular Point
E. Pre-auricular Point
F. Superior Rolandic Point G. Posterior Border of Root of Mastoid
Process
H. Inferior Rolandic Point

B.C. Transverse Fissure

D.E., F.G. Reid's Perpendicular Lines Sy. Fis. Sylvian Fissure

Sy.a.Fis. Anterior Limb of Sylvian Fissure

Sy.h.Fis. Posterior Horizontal Limb of Sylvian Fissure

p.o. Fis. External Parieto-occipital Fissure + Parietal Eminence

The middle meningeal artery corresponds to the centre of the zygomatic arch. At a point from $\frac{1}{2}$, to $\frac{3}{4}$ inch above the centre of the arch it divides into its two terminal branches -anterior and posterior.

The large anterior division of the middle meningeal artery, as it lies in the groove, or, it may be, short canal, on the internal aspect of the antero-inferior angle of the parietal bone, is indicated by taking a point $I_{2}^{\frac{1}{2}}$ inches behind the external angular process of the frontal bone, and $I \frac{1}{2}$ inches above the zygomatic arch. From this 
point it ascends almost vertically towards the sagittal suture, lying about $\frac{1}{2}$ inch behind the coronal suture.

The small posterior division of the middle meningeal artery lies about 2 inches behind the anterior division.

The Sylvian point coincides with the pterion, and is situated about $I_{2}^{1}$ inches behind the external angular process of the frontal bone, and about 2 inches above the zygomatic arch. It indicates the division of the stem of the fissure of Sylvius into its three limbsanterior, ascending, and posterior. The anterior limb of the fissure

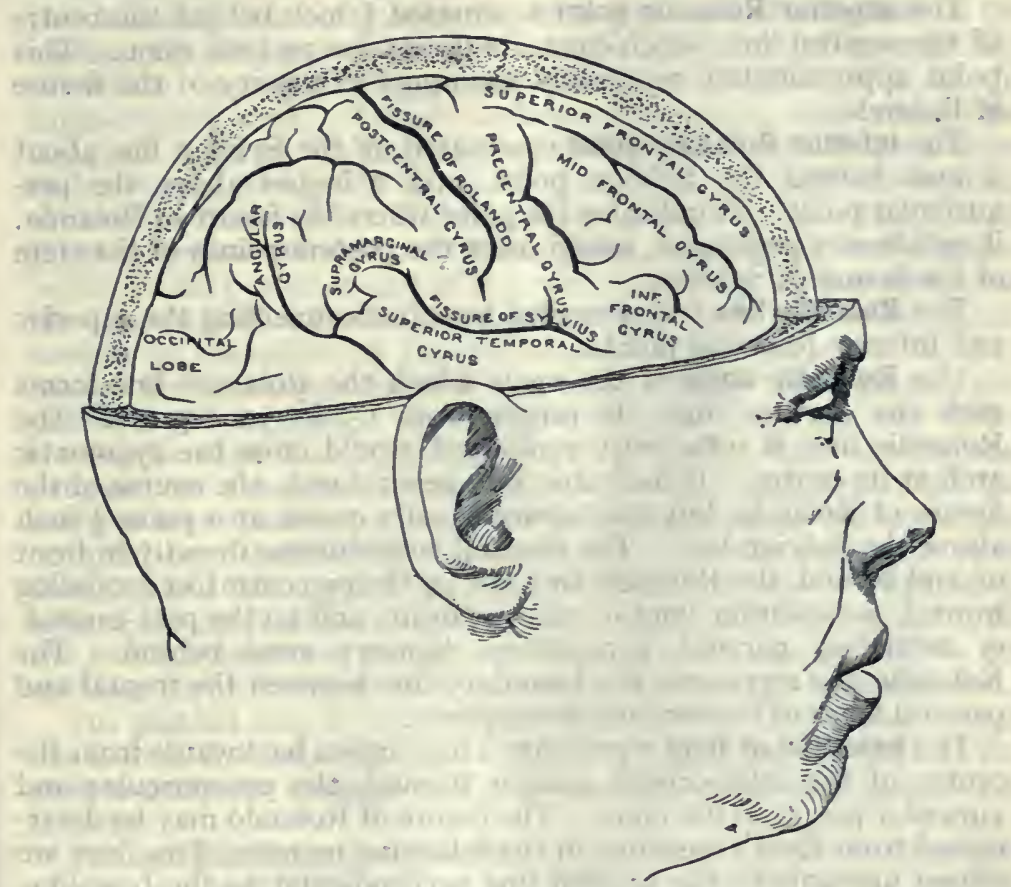

Fig. 624.-The OUter Surface of the Right Cerebral Hemisphere, SHOWING THE RELATIONS OF ITS GYRI AND FISSURES TO THE EXTERIOR of the Cranium (From a PReparat'on made by the AUthor).

passes horizontally forwards for about I inch, and the ascending limb upwards and slightly forwards for a variable distance, from the Sylvian point.

The pars orbitalis of the frontal lobe of the cerebrum lies below the anterior limb; the pars triangularis of the frontal lobe is situated between the anterior and ascending limbs; and the pars basilaris of the frontal lobe is situated directly behind the ascending limb.

The pars triangularis of the left side (le cap of Broca) is the seat of the speech-centre. 
The posterior limb of the stem of the fissure of Sylvius is long, and is directed, for the most part, horizontally backwards for fully 2 inches, after which it turns upwards into the parietal lobe for a short distance. The line which indicates the course of the posterior limb is called the Sylvian line. It extends from the Sylvian point backwards for fully 2 inches, and then vertically upwards for about $\frac{1}{2}$ inch towards the region of the parietal eminence. The parietal lobe and a small portion of the frontal lobe lie above the Sylvian line, and the temporal lobe lies below it.

The superior Rolandic point is situated $\frac{1}{2}$ inch behind the centre of the sagittal line, which connects the nasion and the inion. This point approximately represents the upper extremity of the fissure of Rolando.

The inferior Rolandic point is situated on the Sylvian line about $I$ inch behind the Sylvian point, and 2 inches above the preauricular point. It indicates the point where the fissure of Rolando, if sufficiently prolonged, would meet the posterior limb of the stem of the fissure of Sylvius.

The Rolandic line is represented by a line connecting the superior and inferior Rolandic points.

The Rolandic angle is the angle which the Rolandic line forms with the sagittal line. It ranges from 65 to 70 degrees. The Rolandic line, if sufficiently prolonged, would cross the zygomatic arch at its centre. It indicates, in a general way, the course of the fissure of Rolando, but this fissure usually ceases at a point $\frac{1}{2}$ inch above the Sylvian line. The cerebral convolutions directly in front of, and behind, the Rolandic line are ( $I$ ) the precentral, or ascending frontal, convolution (motor area) in front, and (2) the post-central, or ascending parietal, convolution (sensory area) behind. The Rolandic line represents the boundary-line between the frontal and parietal lobes of the cerebral hemisphere.

The base-line of Reid represents a line drawn backwards from the centre of the infra-orbital margin through the pre-auricular and auricular points to the inion. The fissure of Rolando may be determined from Reid's base-line in the following manner: Two lines are drawn upwards to the sagittal line perpendicular to the base-line, one from the pre-auricular point and the other from the posterior border of the mastoid process close to its root. These two lines, together with the sagittal and Sylvian lines, enclose a quadrilateral area, and the diagonal connecting the postero-superior and anteroinferior angles represents the fissure of Rolando except at its superior and inferior limits.

The parletal eminence, which is usually indefinite, corresponds to the region situated $\frac{1}{2}$ inch behind the centre of a line connecting the pre-Rolandic and pre-auricular points. It indicates the position of the supramarginal gyrus of the parietal lobe of the cerebral hemisphere.

The frontal eminence corresponds to the middle frontal convolution. 
The foramen magnum, through which the medulla oblongata is continuous with the spinal cord, lies midway between the mastoid processes. Its posterior margin is 2 inches from the inion in a downward and forward direction.

The tentorium cerebelli and superior surface of the cerebellum practically coincide with the level of the transverse sinus, as indicated by a line, slightly curved upwards, connecting the inion and asterion.

The lower level of the cerebral hemisphere may be indicated by the following line: Commencing at a point $\frac{1}{2}$ inch external to the nasion, the line passes outwards in an arched manner, with the convexity upwards, lying about $\frac{1}{3}$ inch above the centre of the supra-orbital arch. It then inclines downwards and crosses the temporal ridge of the frontal bone about $\frac{1}{2}$ inch above the frontomalar suture, the guide to which is a point just above the outer canthus of the eyelids. Thereafter the line passes backwards and slightly downwards to the Sylvian point, and thence to the upper border of the posterior part of the zygomatic arch. From this point the line passes backwards, lying about 1 inch above the upper margin of the orifice of the meatus auditorius externus. It then crosses the supramastoid crest (posterior root of the zygoma), and passes to the asterion. Thereafter it nearly follows the line of the transverse sinus from the asterion to a point a little above, and external, to the inion. In other words, speaking generally, the cerebral hemisphere extends as low as the superior curved line of the occipital bone posteriorly, the upper border of the zygomatic arch laterally, and the upper part of the eyebrow anteriorly. The frontal lobe of the cerebral hemisphere is to a large extent in contact with the frontal portion of the frontal bone, but it is also related to the anterior part of the parietal bone as far back as the Rolandic line.

The parietal lobe is related to the part of the parietal bone which lies behind the Rolandic line. The lobe extends as far back as the parieto-occipital fissure, which, is usually situated opposite the lambda.

The occipital lobe occupies the cerebral fossa of the tabular part of the occipital bone, its limits being the level of the lambda superiorly and the level of the inion inferiorly.

The temporal lobe is under cover of the squamous portion of the temporal bone and the postero-inferior part of the parietal bone. The parallel sulcus, which separates the first and second temporal convolutions, is indicated by a line drawn from the lambda to the marginal tubercle on the temporal or posterior border of the malar bone, about $\frac{1}{2}$ inch below the fronto-malar suture. 


\section{THE EYE}

THE eyeball is almost spherical. It consists of the segments of two spheres, namely, a large posterior or sclerotic segment, which is opaque, and a small anterior or corneal segment, which is transparent. The sclerotic segment forms five-sixths of the eyeball, and the corneal segment forms one-sixth. The centre of the

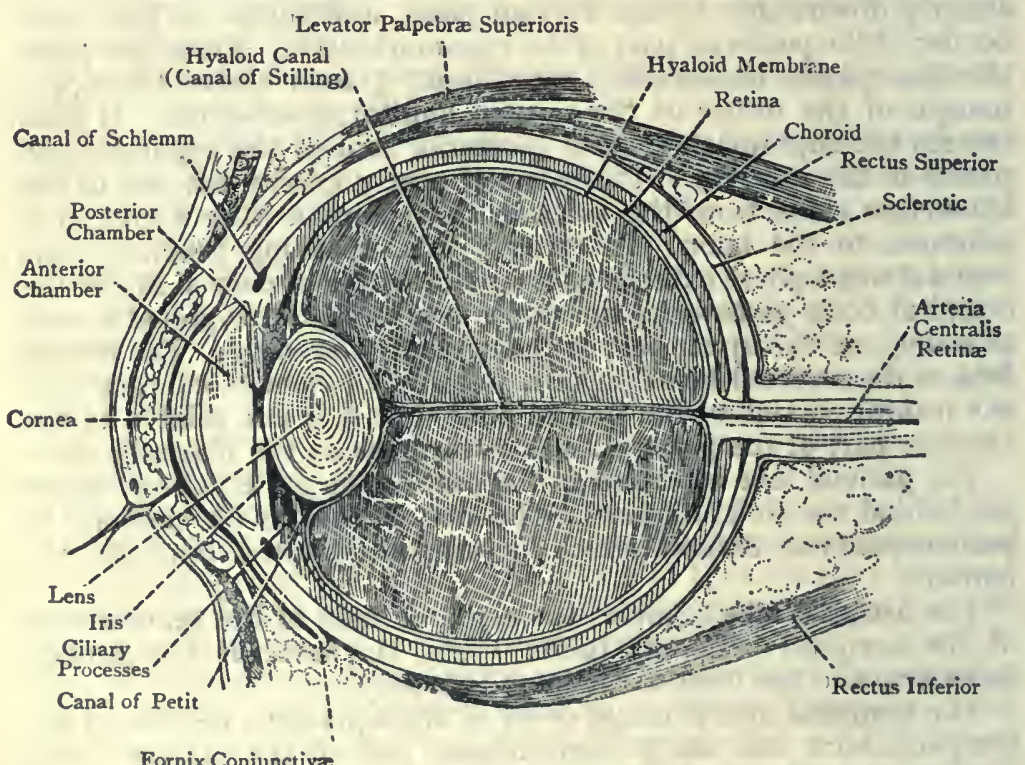

Fornix Conjunctive

Fig. 625.-Vertical Sagittal Section of the Eye and its APPENDAGES (HIRSCHFELD AND LEVEILLE).

corneal segment is called the anterior pole, and the centre of the sclerotic segment is known as the posterior pole. The sagittal (antero-posterior) axis, or axis of vision, of the eyeball is represented by a line connecting the anterior and posterior poles. The equator is represented by a line encircling the eyeball opposite the centre of the axis of vision, the diameter of the circle being I 506 
about I inch. The plane of this circle would therefore divide the eyeball into two halves-an anterior half, consisting of the corneal segment and the front part of the sclerotic segment, and a posterior half, consisting of the back part of the sclerotic segment. The meridian is represented by a line encircling the eyeball at right angles to the equator, and passing through the anterior and posterior poles.

Posteriorly the eyeball receives the optic nerve, which pierces the sclerotic coat at a point about $\frac{1}{8}$ inch to the inner side of, and about $\frac{1}{24}$ inch below, the posterior pole.

The eyeball is composed of three coats, concentrically arranged, as follows : (I) an external coat, consisting of an opaque part, called the sclerotic, and a transparent part, called the cornea ; (2) a middle coat, which is vascular, pigmented, and muscular, and consists of (a) a posterior part, called the choroid coat, (b) an anterior part, called the iris, and $(c)$ an intermediate part, representing the ciliary body ; and (3) an internal coat, called the retina.

These three coats enclose a cavity which contains the refracting media, these being three in number, as follows: (I) a fluid, called the aqueous humour, which lies between the cornea and the crystalline lens, where it occupies the anterior and posterior chambers, into which this region is divided by the iris ; (2) a solid body, called the erystalline lens, which lies behind the aqueous humour; and (3) a soft gelatinous body, called the vitreous body, which occupies the large space behind the crystalline lens.

\section{Coats of the Eyeball.}

External =sclerotic and cornea.

Middle " = choroid, ciliary body, and iris.

Internal $=$ retina.

\section{Refracting Media.}

Aqueous humour. Crystalline lens.

Vitreous body.

\section{External Coat.}

Sclerotic Coat.-The sclerotic (white of the eye) is a strong white fibrous coat of great density, which surrounds the posterior fivesixths of the eyeball, and maintains the shape of the organ. Anteriorly it unites, and becomes continuous, with the cornea, which it slightly overlaps. The junction of the two is indicated by a slight groove, called the sulcus sclera, and the union is known as the corneo-scleral junction. Posteriorly the sclerotic is pierced by the optic nerve, this part being known as the optic entrance. It is situated, as stated, at a point about $\frac{1}{8}$ inch to the inner side of, and about $\frac{1}{24}$ inch below, the posterior pole of the eyeball. The part of the sclerotic corresponding to the optic entrance is pierced by a number of openings for the passage of the fasciculi of the optic nerve, and is hence called the lamina cribrosa.

Around the optic entrance there are numerous minute openings for the ciliary vessels and nerves, and in this situation the duramatral sheath of the optic nerve blends with the sclerotic. About midway between the optic entrance and the corneo-scleral junction 
the sclerotic is pierced by four openings for the passage of the vene vorticose of the choroid.

The sclerotic is thickest posteriorly around the optic entrance. It is also thick near the corneo-scleral junction, where it gives insertion to the recti muscles, from the tendinous expansions of which it receives accessions of fibres.

The outer surface of the sclerotic is covered by a membranous investment, called the capsule of Tenon, and between the two there is the perisclerotic lymph-space or Tenon's space, which is broken up into a reticulum by processes of connective tissue which pass between the capsule of Tenon and the sclerotic. This space com. municates with the subdural and subarachnoid spaces. The inner surface is of a dark brown colour, and presents grooves for the ciliary vessels and nerves. It is lined with connective tissue containing pigment cells, this layer being called the lamina fusca. Processes from this layer pass to be attached to the choroid coat, and these processes, together with vessels and nerves, traverse an interval, which represents the perichoroidal lymph-space. This space communicates with the perisclerotic lymph-space through the vascular openings in the sclerotic. Anteriorly the sclerotic blends with the cornea at the corneo-scleral junction, the sclerotic slightly overlapping the cornea. Posteriorly, around the optic entrance the sclerotic blends with the dura-matral sheath of the optic nerve.

Structure.-The sclerotic is composed of fibrous tissue, mixed with elastic fibres, and it contains many connective-tissue corpuscles. The fibres are arranged in bundles, which are disposed longitudinally and transversely, and interlace with one another. The connective-tissue corpuscles occupy spaces between the fibres, which may be regarded as lymph-spaces.

Arteries.--These are the short ciliary group of posterior ciliary arteries, and the anterior ciliary arteries, which are branches of the ophthalmic artery. The vessels belonging to the former group are disposed in the form of capillary networks ; whilst the vessels derived from the latter form a ring near the corneo-scleral junction beneath the conjunctiva, to which ring they converge in the substance of the sclerotic.

The sclerotic veins open into the anterior ciliary veins, and into the vena vorticose of the choroid.

Nerve-supply. - The ciliary nerves.

The capsule of Tenon will be found described on p. II5r.

Cornea.-The cornea is the transparent part of the external coat of the eyeball, of which it forms the anterior sixth, and it serves to transmit light. It is almost circular, its transverse measurement being slightly greater than the vertical. At its circumference it is continuous with the sclerotic, by which it is slightly overlapped. The anterior surface is convex. The posterior surface is concave, and forms the anterior boundary of the anterior chamber of the eye. 
Structure.-The cornea consists of five layers, which are as follows, from before backwards :

I. The conjunctival epithelium.

2. The anterior elastic lamina.

3. The substantia propria.

4. The posterior elastic lamina.

5. A layer of endothelium.

The conjunctival epithelium is of the stratified variety, there being not less than five strata of cells, and it is continuous with the epithelium which covers the free surface of the conjunctiva. The

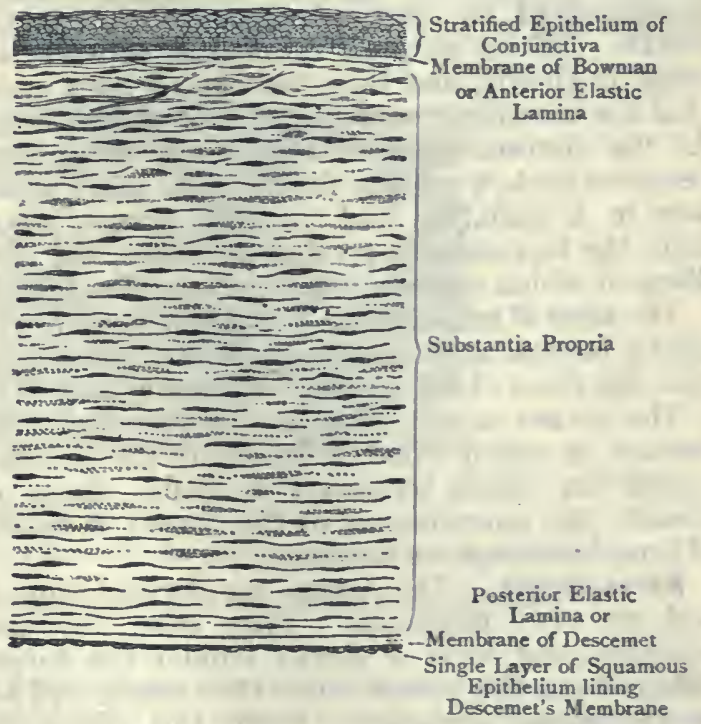

Fig. 626.-Vertical Section of the Cornea (Magnified).

cells of the deepest stratum are columnar; succeeding to these there are layers of polygonal cells; and these in turn are overlaid by layers of squamous cells.

The anterior elastic lamina (Bowman) is probably of the same nature as the fibrous portion of the substantia propria. It is closely connected with the substantia propria, is thin, and contains no corpuscles.

The substantia propria is composed of modified connective tissue, arranged in bundles which form superimposed laminæ. These laminæ amount in number to about sixty: The fibres of alternate laminz cross each other at right angles, and at the circumference of the cornea they are continuous with the fibres of the sclerotic. 
The successive laminæ are connected by cement substance, and within this substance there are branched spaces, called the corneal spaces or lacunæ, which communicate with each other by very delicate canaliculi. Each of these spaces contains a nucleated connective-tissue corpuscle, called the corneal corpuscle. These corpuscles, like the spaces which they occupy, are branched, and the offsets of adjacent corpuscles communicate with one another. As seen in vertical sections of the cornea the corpuscles are spindleshaped, but in horizontal sections they appear flattened out, and give off their branches.

The posterior elastic lamina, or membrane of Descemet (membrane of Demours), covers the posterior surface of the substantia propria. It is thicker than the anterior elastic lamina, and is composed of an elastic homogeneous membrane, which is very brittle. When stripped from the substantia propria it comes away in shreds, and these curl up at their ends in such a manner that the anterior or attached surface of each shred is turned inwards. At the circumference of the cornea the posterior elastic lamina becomes broken up into fibres. The most posterior of these fibres pass in a radiating and reticular manner into the iris, and they form the ligamentum pectinatum iridis, the intervals between the fibres of which represent the spaces of Fontana.

The layer of endothelium lines the posterior surface of the posterior elastic lamina, and consists of one stratum of cells. It is continued over the front of the iris, and into the spaces of Fontana.

The cornea in the adult is non-vascular, except at the circumference, in which situation there are the conjunctival and sclerotic capillaries, which terminate in loops. Being destitute of bloodvessels, the nourishment of the cornea is maintained by the flow of lymph through its substance.

Nerve-supply.- The nerves are derived from the ciliary nerves, and are very numerous. They enter the anterior part of the sclerotic, and form a plexus around the corneo-scleral junction. Offsets from this plexus enter the cornea, and form what is known as the plexus annularis. From this plexus delicate offsets are given off, which traverse the substance of the cornea and pass through the anterior elastic lamina. They then give rise to a fine plexus upon the surface of that lamina, called the subepithelial plexus. From this plexus, in turn, minute fibrils are given off, which pass amongst the cells of the conjunctival epithelium, and almost reach the surface, forming an intra-epithelial plexus.

Ligamentum Pectinatum Iridis.-As stated, the posterior elastic lamina at its circumference breaks up into fibres. The most posterior fibres, which pass in a radiating and recticular manner into the iris, constitute the ligamentum pectinatum iridis, and they are covered by a prolongation of the endothelial layer of the cornea.

Spaces of Fontana.--These spaces represent the irregular intervals which lie between the radiating and reticular fibres of the ligamentum pectinatum iridis. They are lined by a prolongation 
of the endothelial layer of the cornea, and they communicate internally with the anterior chamber and the lymph spaces within the iris, and externally with the canal of Schlemm.

Canal of Schlemm (canal of Fontana, simus circularis iridis, or sinus venosus sclerce). - This canal is situated deeply in the sclerotic, close to the corneo-scleral junction. It communicates internally with the anterior chamber through means of the spaces of Fontana, and with the veins of the iris, and externally with the veins of the sclerotic.

\section{Middle Coat.}

I. Choroid Coat.-This is a very vascular, deeply pigmented tunic of a dark brown colour, which lies between the sclerotic and the retina. It extends over the posterior five-sixths of the eyeball,

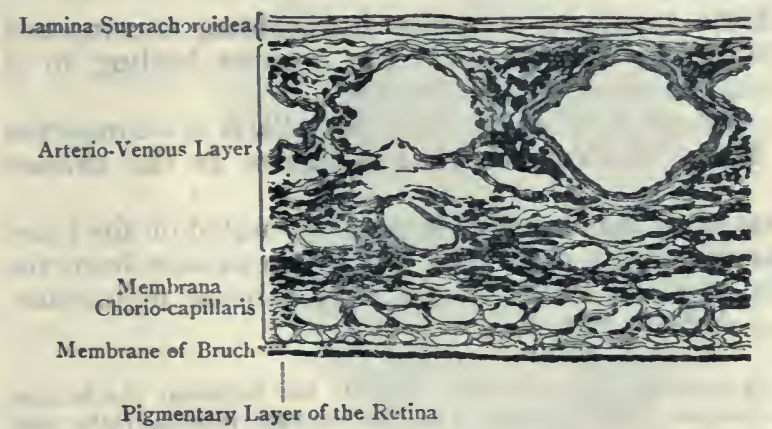

Pigmentary Layer of the Rutina

Fig. 627.-Vertical Section of the Choroid Coat.

(The Pigmentary Layer of the Retina is also shown).

and reaches as far forwards as the ora serrata of the retina. Anteriorly it is connected with the circumference of the iris, and posteriorly it is pierced by the optic nerve. Its outer surface is connected to the inner surface of the sclerotic by means of the lamina fusca and its processes, as well as by vessels and nerves which cross the perichoroidal lymph-space. Its inner surface is in contact with the pigmentary layer of the retina.

Structure.-The choroid coat consists of connective tissue, bloodvessels, and branched pigment cells. It is composed of three layers, which are as follows, from without inwards: $(\mathrm{I})$ the lamina suprachoroidea ; (2) the choroid proper ; and (3) the lamina basalis, or membrane of Bruch.

The lamina suprachoroidea is composed of delicate, non-vascular lamellæ, each of which is made up of elastic fibres, arranged in a reticular manner, and of branched pigment cells.

The choroid proper consists principally of bloodvessels and pig- 
ment-cells, supported by connective tissue. The bloodvessels are arranged partly as arteries and veins, and partly as capillaries. The choroid proper is therefore composed of two layers, namely, external or lamina vasculosa, and internal or lamina choriocapillaris.

The lamina vasculosa (arterio-venous layer) is composed of (I) branches of the short ciliary group of the posterior ciliary arteries, which pass forwards before they turn inwards to terminate in capillaries; and (2) veins, which form the chief part of the lamina vasculosa, and are called the venæ vorticosæ. These veins are very closely set, and are arranged in a whorled manner. They ultimately converge and form about four vessels, which pierce the sclerotic nearly midway between the optic entrance and the corneo-scleral junction, at points equally distant from each other. Scattered throughout the lamina vasculosa there are branched pigment-cells.

The lamina chorio-capillaris, or tunica Ruyschiana, is composed of a plexus of capillary bloodvessels, the arteries leading to it being derived from the short ciliary arteries.

The lamina vasculosa and lamina chorio-capillaris are connected by fine elastic fibres, which form what is known as the stratum intermedium.

The lamina basalis, or membrane of Bruch, is situated on the inner surface of the lamina chorio-capillaris, which it separates from the pigmentary layer of the retina. It is a very delicate membrane, without any very definite structure.

Tapetum.-This is present in certain animals. It lies between the lamina vasculosa and the lamina chorio-capillaris in the stratum intermedium, and it gives rise to a rainbow-like appearance. In some animals it is fibrous in structure, and in others cellular.

2. Ciliary Body.-The ciliary body, as stated, connects the anterior part of the choroid to the circumference of the iris. It is composed of (I) the orbicularis ciliaris, (2) the ciliary processes, and (3) the ciliary muscle.

The orbicularis ciliaris is a narrow zone which lies immediately in front of the anterior part of the choroid, with which it is continuous. It presents plications which are radially disposed, and it separates the ciliary processes from the ora serrata of the retina.

The eiliary processes, which are about seventy in number, are projections or infoldings of the anterior part of the choroid, and consist of the choroid proper and the membrane of Bruch. They constitute a series of rays, arranged in a circular manner, and converge as they pass inwards and forwards to the periphery of the crystalline lens on its anterior aspect. They are somewhat conical in outline. Their free extremities, which are round and prominent, lie bchind the circumference of the iris upon the anterior aspect of the periphery of the crystalline lens. Their attached extremities are connected with the orbicularis ciliaris. 
Anteriorly they are related to the posterior chamber of the eyeball at its circumference. Posteriorly they rest upon the zonule of Zinn, the folds of which fit closely in between them.

Structure.-The ciliary processes are similar in structure to the choroid, but the pigment-cells are not so numerous. On their deep or posterior surfaces the processes are covered by the pars ciliaris retinæ, which is prolonged from the pigmentary layer of the retina, and is continuous with the pars iridica retinæ (uvea) on the posterior surface of the iris.

The arteries of the ciliary processes are derived from those of the anterior part of the choroid, and from the anterior ciliary arteries. The veins pass to those of the choroid.

Ciliary Muscle.-This muscle is composed of unstriped fibres. It forms a greyish-white ring, about $\frac{1}{10}$ inch broad, which is

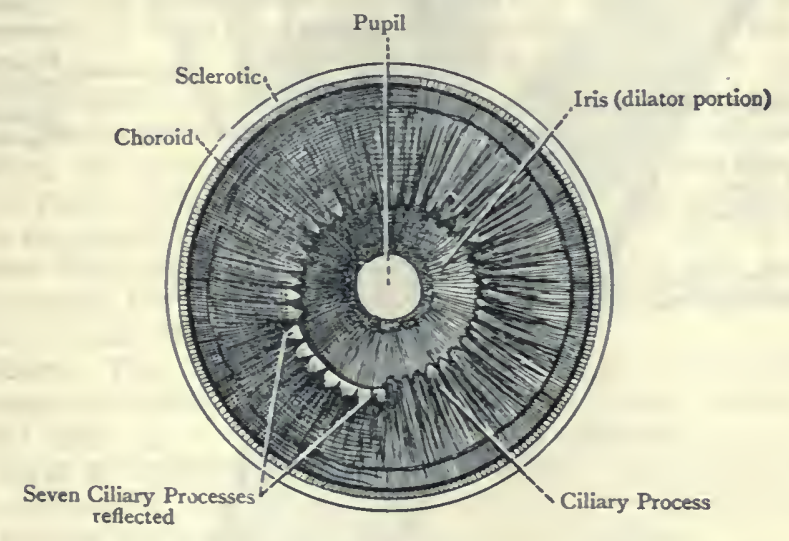

Fig. 628.-The Iris and Cillary Processes (Posterior View) (HiRschFeld AND LeVEILle).

situated at the anterior part of the choroid, opposite the ciliary processes. The fibres are arranged in two sets-radial and circular. The radial fibres arise from the deep surface of the sclerotic, close to the corneo-scleral junction, and behind the canal of Schlemm. From this origin they pass backwards in a radiating manner, and are inserted into the orbicularis ciliaris and the attached ends of the ciliary processes. The circular fibres form a ring around the circumference of the iris, internal to the radial fibres.

The ciliary muscle is supplied by the ciliary nerves, which are branches of the ciliary ganglion.

Action.-The ciliary muscle is the muscle of accommodation, and adjusts the eye to the vision of near objects. When it contracts it draws forwards the choroid and the ciliary processes ; the suspensory ligament of the crystalline lens is thereby relaxed; and, as a consequence, the anterior surface of the lens is rendered convex. 
Anterior Wall of Capsule of Lens

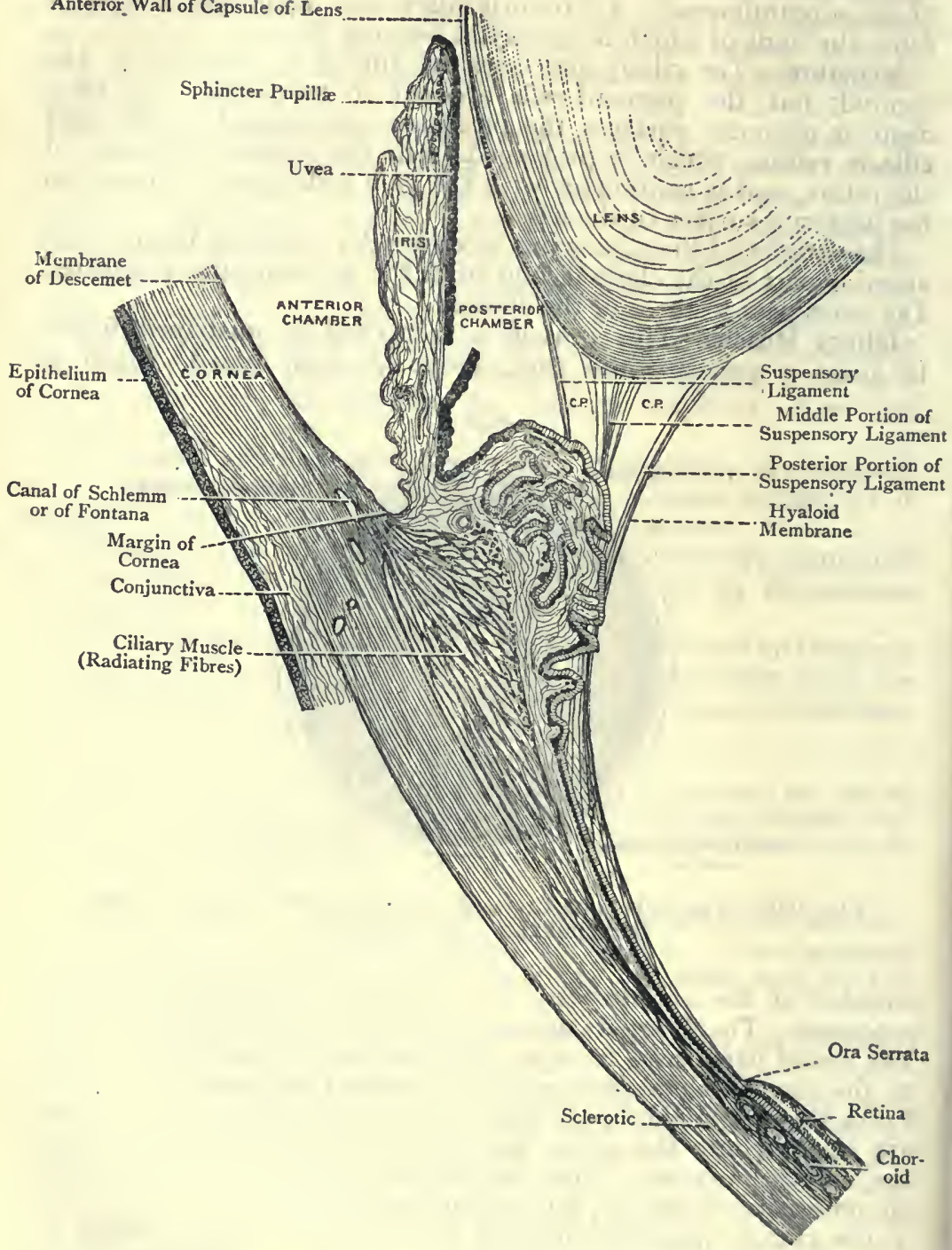

Fig. 629.-Meridional Section through the Anterior Portion of THE EYE (MAGNIFIED $16 \times$ I) (FUCHS). C.P., C.P., Canal of Petit. 
The circular fibres of the ciliary muscle are well developed in cases of hypermetropia, but are deficient in cases of myopia.

3. Iris.- The iris forms the anterior part of the middle coat of the eyeball. It is a coloured contractile curtain, which is suspended in the aqueous humour between the cornea and the crystalline lens. It is perforated by an almost circular aperture, called the pupil, which is situated slightly to the nasal or inner side of its centre, and serves for the transmission of light. The margin which surrounds the pupil is known as the pupillary margin. Its circumference is continuous with the ciliary body, and is connected with the posterior elastic lamina of the cornea by means of the ligamentum pectinatum iridis. The circumference is known as the ciliary margin. The surfaces of the iris are anterior and posterior. The anterior surface is directed towards the cornea. Its colour varies in different individuals, and it presents a striated appearance, the strix converging towards the margin of the pupil, and being produced by the underlying vessels. The posterior surface is directed towards the crystalline lens and ciliary processes. It has a purple colour, and is covered by two layers of columnar epithelium, the cells of which contain dark pigment. These two layers of pigmented cells constitute the pars iridica retinæ (uvea), which is continuous with the pars ciliaris retinæ. The iris divides the space between the cornea and the crystalline lens into two compartments, the anterior chamber and posterior chamber, both of which contain the aqueous humour.

Structure.-The component parts of the iris are as follows: (I) a layer of endothelium; (2) a connective-tissue stroma, with branched cells; (3) muscular tissue ; and (4) pigment.

The layer of endothelium covers the anterior surface of the iris, and is continuous with the endothelium which lines the posterior elastic lamina (membrane of Descemet) of the cornea.

The connective-tissue stroma is composed of fibres which, for the most part, pass in a radiating manner towards the pupillary margin. Some, however, are disposed circularly at the ciliary margin. They support the bloodvessels and nerves, and scattered between their bundles there are branched cells. These cells contain pigment in dark coloured eyes (black, grey, or brown), but in blue eyes they are destitute of pigment.

The muscular tissue is of the unstriped variety, and its fibres are arranged in two sets, circular and radiating. The circular fibres form a ring round the pupil, and are nearer the posterior surface than the anterior. They are known as the sphincter pupillæ. The radialing fibres converge from the ciliary margin of the iris towards the pupillary margin, where they blend with the circular fibres. The radiating fibres constitute the dilator pupilla. Some authorities regard the radiating fibres as being elastic, and not muscular.

The pigment of the iris is variously situated, according to the colour of the eye. In the eyes of albinos there is no pigment. 
In other eyes pigment is contained in the cells of the two layers of columnar epithelium which line the posterior surface of the iris, and form the pars iridica retinæ (uvea). In blue eyes the pigment is confined to this region, but in dark coloured eyes (black, grey, or brown), it is also present in the branched cells of the connective-tissue stroma.

Blood-supply-Arteries.-The arteries of the iris are derived from (I) the long ciliary, and (2) the anterior ciliary, vessels.

The long ciliary arteries are two in number, and belong to the posterior ciliary group of branches from the ophthalmic artery. They pierce the back part of the sclerotic, one on each side of the optic nerve, and pass forwards between the sclerotic and the choroid towards the ciliary margin of the iris. Here each vessel divides into two branches, upper and lower, which anastomose with those of the opposite side to form an arterial ring round the ciliary margin of the iris, called the circulus major. This ring is joined by some

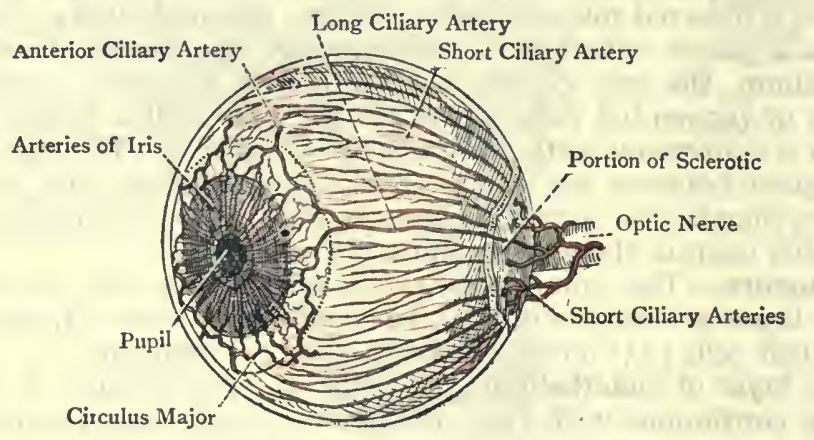

Fig. 630.-The Arteries of the Choroid and Iris (Lateral View).

of the anterior ciliary arteries, and it gives offsets to the ciliary muscle and iris. The branches which enter the iris are supported by the connective-tissue stroma, and converge towards the pupillary margin, near which they form by their anastomoses another arterial ring, called the circulus minor.

The anterior ciliary arteries are about six in number, and are derived from the muscular and lachrymal branches of the ophthalmic artery. They are of small size and pierce the anterior part of the sclerotic, close to the corneo-scleral junction. Some of them supply the ciliary processes, and others join the circulus major.

The veins of the iris accompany the arteries, and are in communication with the canal of Schlemm.

Nerves of the Choroid Coat and Iris.-These are derived from the ciliary nerves, short and long, the former coming from the ciliary ganglion, and the latter from the nasal branch of the 
ophthalmic or first division of the fifth cranial nerve. They are about sixteen in number, and pierce the back part of the sclerotic around the optic nerve. They then pass forwards between the sclerotic and choroid, giving branches to the latter coat, which become disposed in a plexiform manner amongst the bloodvessels. Having reached the corneo-scleral junction, the nerves enter the ciliary muscle, in which they form a plexus. From this plexus branches enter the iris at the ciliary margin. These branches accompany the vessels, and, by their subdivisions and communications, they form a copious plexus of non-medullated fibres in the connective-tissue stroma of the iris. The sphincter pupillæ is supplied by fibres which are derived from the oculo-motor or third

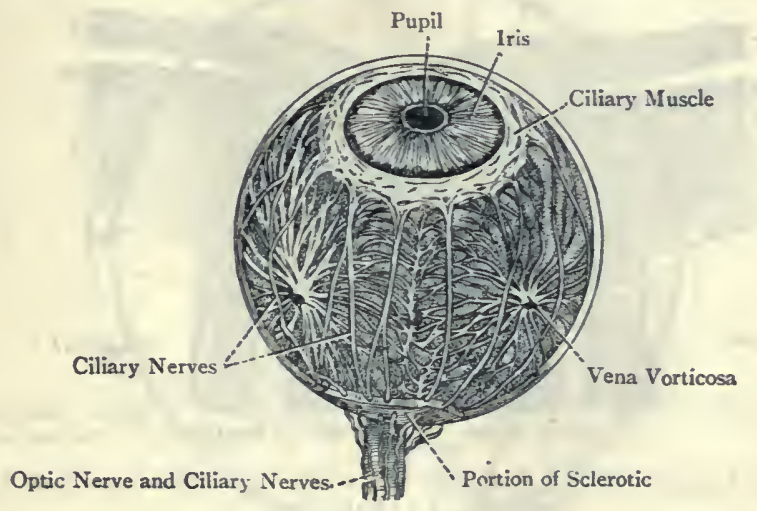

Fig. 63x.-The Choroid ANd IRIS, Showing The Venæ Vorticos.e and Ciliary Nerves (AFter Hirschfeld and Leveillé).

(The Sclerotic and Cornea have been removed).

cranial nerve, through means of the motor root of the ciliary ganglion. The dilator pupillæ is supplied by fibres which are derived from the sympathetic through means of the sympathetic root of the ciliary ganglion.

Membrana Pupillaris.-During intra-uterine life the pupil is closed by a delicate membrane, called the membrana pupillaris. This membrane is vascular, except at its centre, the arteries being derived from the circulus minor, and from those of the front part of the capsule of the crystalline lens. The vessels converge from the circumference of the membrane to near its centre, and terminate by forming loops.

The membrana pupillaris disappears shortly before birth.

\section{Internal Coat.}

Retina. - The retina is the internal or nervous tunic of the eyeball. It is soft in consistence, translucent, and of a pinkish colour. Its internal surface is in contact with the hyaloid membrane, 
which encloses the vitreous body, and its external surface is in contact with the choroid coat. Posteriorly it receives the fibres of the optic nerve. Anteriorly it extends almost to the ciliary body, where it presents a notched border, called the ora serrata. Here its nervous elements terminate, but its pigmentary layer is continued over the deep or posterior surfaces of the ciliary processes on to the posterior surface of the iris, forming, with the addition of a layer of columnar epithelial cells, the pars ciliaris retinæ and pars iridica retinæ (uvea), respectively. The retina diminishes in thickness from behind forwards.

The external surface is formed by a stratum of hexagonal pigmentcells, which send processes into the adjacent layer. When the

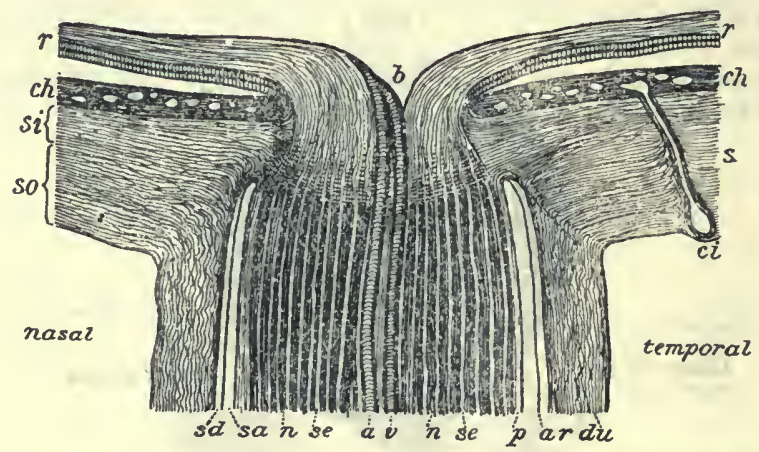

Fig. 632.-Longituninal Section through the Head of the Optic NERVE $(14 \times$ I) (FUCHS).

\author{
r. Retina \\ b. Centre of Porus Opticus \\ cb. Choroid \\ s. Sclerotic \\ so. Outer Part of Sclerotic \\ si. Inner Part of Sclerotic \\ ci. Ciliary Artery (in longi- \\ tudinal section)
}

nasal, Inner Side

\author{
sd. Subdural Space \\ sa. Subarachnoid Space \\ n. Bundles of Nerve-fibres \\ se. Septa between tbe Nerve-bundles \\ a. Arteria Centralis Retinæ \\ v. Vena Centralis Retinæ \\ p. Sheath formed by Pia Mater \\ ar. Sheath formed by Arachnoid \\ du. Sheath formed by Dura Mater \\ temporal, Outer Side
}

choroid is separated from the retina these processes are torn, and the stratum of pigment-cells remains attached to the choroid, being apparently a part of it. The pigmentary layer, however, really belongs to the retina.

The internal surface presents, in the line of the visual axis of the eyeball, the macula lutea or yellow spot, where vision is most distinct. This spot is transversely oval, and measures about $\frac{1}{12}$ inch in the transverse diameter. At its centre there is a slight depression, called the fovea centralis. In this situation the retina is thinnest, and the dark colour of the hexagonal pigmentcells is visible through it, imparting to it the appearance of a foramen. About $\frac{1}{8}$ inch to the inner side of the posterior pole of the 
eyeball, and about $\frac{1}{9+4}$ inch below its level, is the porus opticus, or optic disc. This is circular in outline, and its circumference is slightly elevated. It represents the point of entrance of the fibres of the optic nerve, and the centre of the disc is pierced by the arteria centralis retinæ, which immediately divides into two branches-upper and lower. The optic disc consists entirely of nerve-fibres, and is known as the 'blind spot,' vision being absent in this situation.

Structure of the Retina.-The retina consists of eight superimposed layers, seven of which are nervous and one pigmentary.

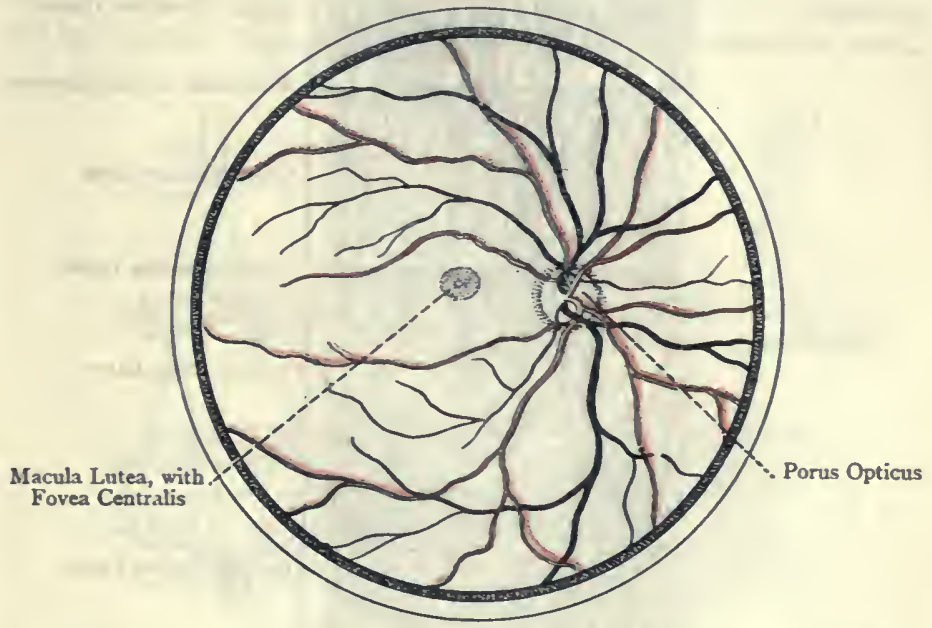

Fig 633.-The Posterior Portion of the Right Retina (ANTERIOR VIEW).

In addition to these, there are sustentacular fibres. The eight layers are as follows, from within outwards :

I. Stratum opticum, or layer of nerve-fibres.

2. Ganglionic layer, or layer of nerve-cells.

3. Inner molecular (inner plexiform) layer.

4. Inner nuclear or granular layer.

5. Outer molecular (outer plexiform) layer.

6. Outer nuclear or granular layer.

7. Layer of rods and cones, or bacillary layer.

8. Pigmentary layer.

In addition to the foregoing layers, there are two very delicate membranes, which really belong to the sustentacular fibres of the retina, but are known as the membrana limitans interna and membrana limitans externa. The membrana limitans interna covers the retina on its internal surface, and the membrana limitans 
externa intervenes between the outer nuclear layer and the bacillary layer. The layers of the retina are supported by fibres called the sustentacular fibres.

I. Stratum Opticum.-This layer consists of the fibres of the optic nerve, and it extends from the optic disc to the ora serrata. The fibres are non-medullated, and are chiefly centripetal, but some are centrifugal. The centripetal fibres arise mainly as the axons of

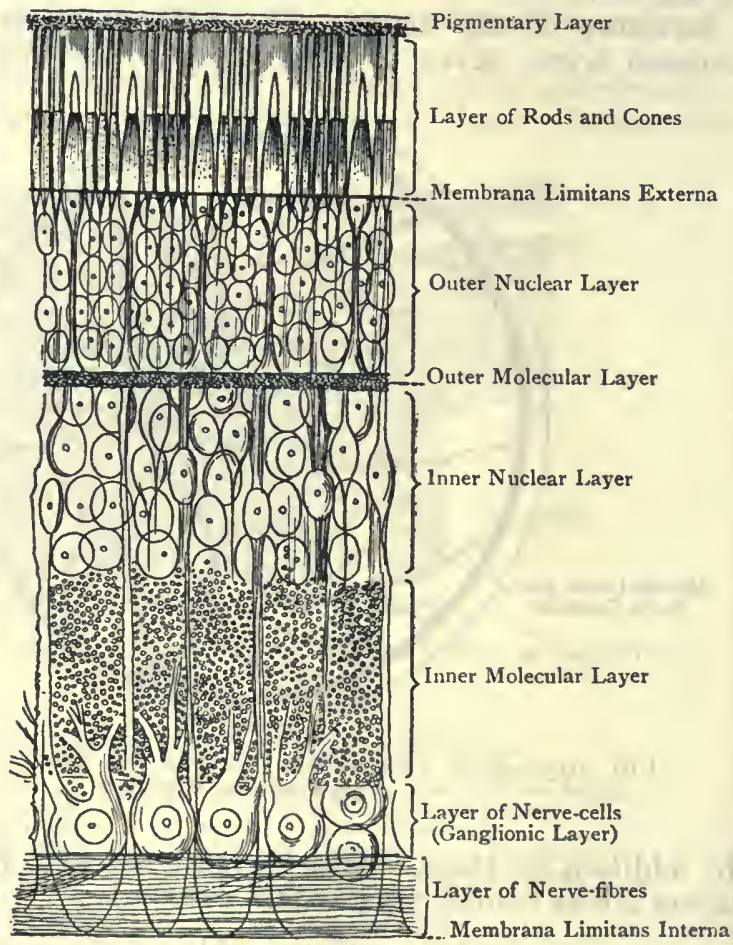

Fig. 634.-Diagrammatic Section of the Human Retina (Schultze).

(Copied from Quain's 'Anatomy').

the cells of the ganglionic layer. The centrifugal fibres pass towards the inner molecular and inner nuclear layers.

2. Ganglionic - Layer.-This layer consists of large, somewhat flask-shaped, multipolar ganglion-cells, which for the most part form a single layer. In the macula lutea, however, they form several layers. The round ends of the cells rest upon the stratum opticum, and from each of these ends an axon is given off, which enters the stratum opticum obliquely, and forms one of its component fibres. The tapering end of each cell sends off several dendrites, which enter 
the inner molecular layer, within which they terminate in arborizations.

3. Inner Molecular (Inner Plexiform) Layer.-This layer consists of the arborizations of the dendrites of $(I)$ the cells of the ganglionic layer, and (2) the bipolar cells of the inner nuclear layer. The intercommunications between these two sets of dendrites give rise to five strata, according to Ramon y Cajal. Besides these, there are the arborizations of the processes of the spongioblasts of the inner nuclear layer, which are likewise arranged in strata.

4. Inner Nuclear or Granular Layer.-This layer consists of cells which are arranged in three groups, namely, (I) bipolar cells, (2) horizontal cells, and (3) spongioblasts, or amacrine cells. The

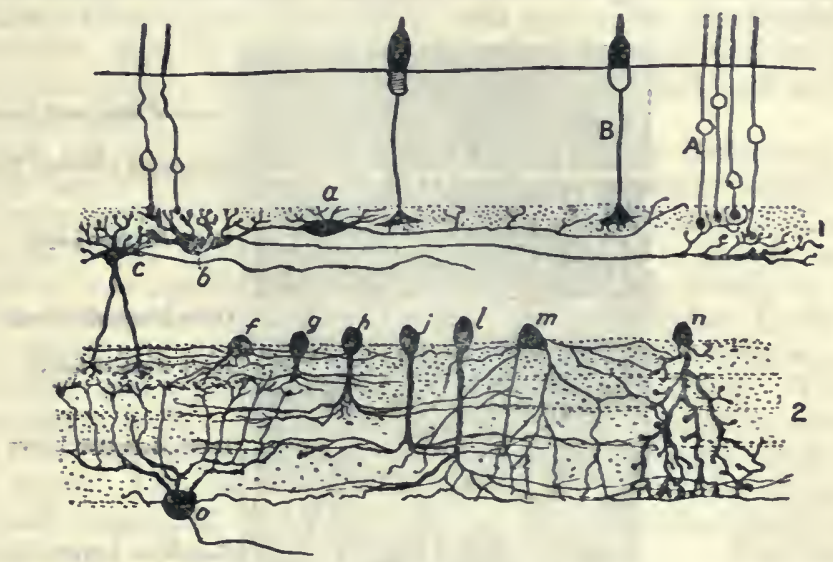

Fig. 635.-Scheme of the Horizontal Cells and Spongloblasts of the RETINa (RAMON Y Cajal).
A. Rod-fibres
B. Cone-fibres
r. Outer Molecular Layer
$a, b$. Horizontal Cells, with arborizations
c. Horizontal Cell, with deep processes
2. Inner Molecular Layer
$f, g, k$,$\} Spongioblasts extending to$
$j, l$.$\} different depths$
$m, n$. Spongioblasts with diffuse processes
o. Ganglionic Nerve-cell

bipolar cells are the most numerous, and are nucleated. Each cell gives off two processes-internal and external. The interial processes of the cells enter the inner molecular layer, and terminate at different levels in arborizations. The external processes pass into the outer molecular layer, and terminate in arborizations in its outermost part, which are closely related to the terminal parts of the rods and cones of the bacillary layer. According to Cajal, the bipolar cells are of two kinds-rod-bipolars and cone-bipolars. The external processes of the rod-bipolars ramify around the terminal parts of the rod-fibres, and the internal processes arborize around the cells of the ganglionic layer. The external processes of the cone-bipolars form horizontal arborizations around the terminal 
parts of the cone-fibres, and the internal processes terminate in arborizations in the inner molecular layer at different levels.

The horizontal cells occupy the outer part of the inner nuclear layer. Their dendrites enter the outer molecular layer, and come into relation with the terminal parts of the cone-fibres, whilst their axons run in a horizontal direction.

The spongioblasts are situated in the innermost part of the inner nuclear layer. They are destitute of axons, and have been called amacrine cells, because each cell is ' without a long fibre or process.' Their dendrites enter the inner molecular layer, and terminate in arborizations, which are arranged in strata.

5. Outer Molecular (Outer Plexiform) Layer.-This layer is composed of the following structures: (I) the external processes of the

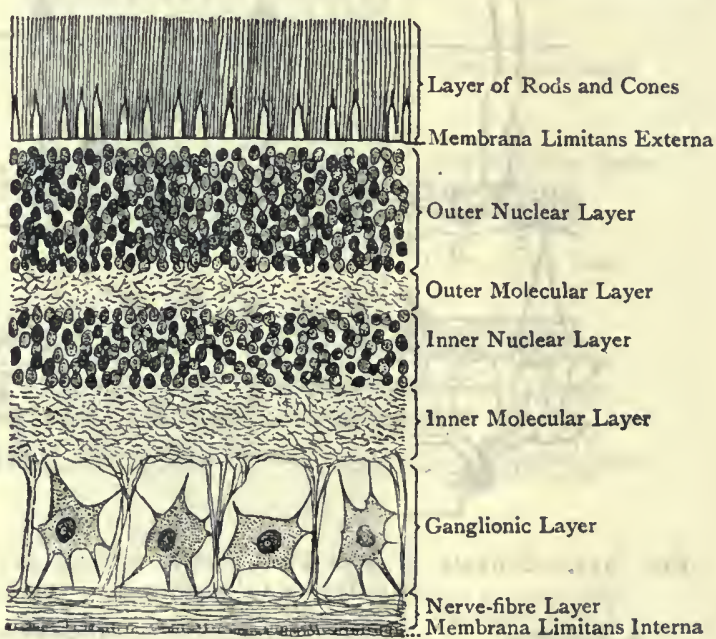

Fig. 636.-Section of the Retina as seen under the Microscope (MAGNIFIED).

rod-bipolars and cone-bipolars of the inner nuclear layer; (2) the dendrites of the horizontal cells of the inner nuclear layer; and (3) the terminal parts of the rod-fibres, and filaments from the footplates of the cone-fibres.

6. Outer Nuclear or Granular Layer.-This consists of granules, which are of two kinds, namely, rod-granules and cone-granules. The rod-granules are the more numerous, and are oval enlargements in the course of the rod-fibres, as these pass to the outer molecular layer. Each rod-fibre has only one rod-granule, and the granules lie at different levels. Each granule has a nucleus, which presents transverse striations, there being at least two clear bands." The external process of each rod-granule is continuous with one of the rods of the bacillary layer, and the internal process passes into the 
outer molecular layer, where it comes into relation with the arborizations of the external process of a rod-bipolar.

The cone-granules are larger than the rod-granules, but not so numerous, and each contains an oval nucleus. They are situated in the outermost part of the outer nuclear layer, close to the membrana limitans externa. The outer end of each granule is continuous with one of the cones of the bacillary layer. The inner end is prolonged into a cone-fibre, which passes into the outermost part of the outer molecular layer, where it expands into a footplate, from which filaments are given off. These filaments come into relation with the arborizations of the external process of a conebipolar cell.

7. Layer of Rods and Cones, or Bacillary Layer.-This layer consists of two elements, namely, rods and cones, the former being cylindrical, and the latter flask-shaped. The rods are much more numerous, longer, and narrower than the cones, and both are placed perpendicularly.

Each rod and cone consists of two segments-outer and inner. In the case of the rods the two segments are of almost equal length, the inner segment being rather larger than the outer. The outer segment is the only seat of the colouring matter known as visual purple or rhodopsin. In the case of the cones, which, as stated, are flask-shaped, the inner segment of each forms two-thirds of the cone, and is of large size ; whilst the outer segment forms one-third, is narrow, and represents the tapering part of the flask. The outer segments of both rods and cones present faint transverse striations. The inner segments of both are subdivided into two parts-outer and inner. The outer part is composed of delicate fibrils, longitudinally arranged, and therefore presents a longitudinally striated appearance. The inner part is faintly granular. The rods and cones are continued at their inner ends through the membrana limitans externa into the rod-fibres and cone-fibres, which belong to the outer nuclear layer. The outer ends of the rods project into the pigmentary layer.

8. Pigmentary Layer.-This is the most external layer of the retina, and it is in close contact with the choroid coat. It consists of a single layer of hexagonal epithelial cells, which contain pigment. The deep surfaces of the cells give off processes which extend into the intervals between the outer ends of the rods and cones.

Sustentacular Fibres, or Fibres of Müller.-These fibres constitute the supporting tissue of the retina, and extend from its internal surface to the boundary-line between the outer nuclear layer and the layer of rods and cones. The inner ends of the fibres are expanded, and cohere by their edges so as to present the appearance of a distinct retinal layer, which is called the membrana limitans interna. Their outer ends, which are very numerous owing to the breaking up of the fibres, also expand, and form what is known as the membrana limitans externa, which lies between the outer nuclear layer and the layer of rods and cones.

(The membrana limitans 
interna and membrana limitans externa are sometimes counted as layers of the retina, under which circumstances the retinal layers would be ten in number, instead of eight, as stated). From the membrana limitans externa delicate offsets enter the layer of rods and cones, in the innermost part of which they form fibre-baskets in connection with the deep ends of the rods and cones. As the sustentacular fibres pass through the inner nuclear layer each presents an oval nucleus, which contains a nucleolus. This nucleus is variously described as being situated on one side of the fibre, or as involving its whole circumference. Throughout their course the sustentacular

B A

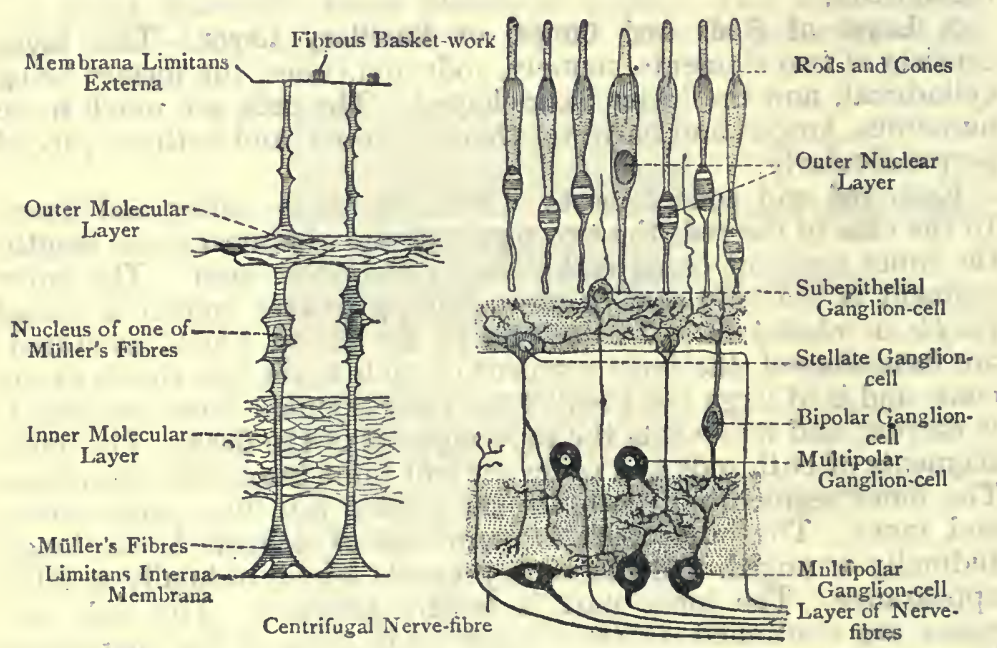

Fig. 637.-Diagram of the Elements of the Retina (Wiedersheim, AFTER PH. STÖHR).

A, Nervous Elements; B, Supporting Elements.

fibres give off lateral offsets, which increase in number from within outwards.

Structure of the Macula Lutea and Fovea Centralis.-The chief structural characters of the macula lutea and fovea centralis may be stated in the following tabular manner :

Macula Lutea.

I. Cones only.

2. Outer nuclear layer has only conefibres, disposed obliquely.

3. Ganglionic ayer very thick, cells being several layers deep.

4. Stratum opticum not continuously disposed.

\section{Fovea Centralis.}

I. Thinnest part of the retina.

2. Pigmentary layer thick.

3. Cones only.

4. Outer nuclear layer has only cone-fibres.

5. Ganglionic layer absent.

6. Stratum opticum absent. 
Structure of the Ora Serrata.-At the ora serrata, as previously stated, the nervous elements of the retina terminate, and its pig: mentary layer is continued over the deep or posterior surfaces of the ciliary processes. In this latter situation there is superadded to its deep or posterior surface a layer of columnar epithelial cells, and the two layers form the pars ciliaris retinæ, which is continued

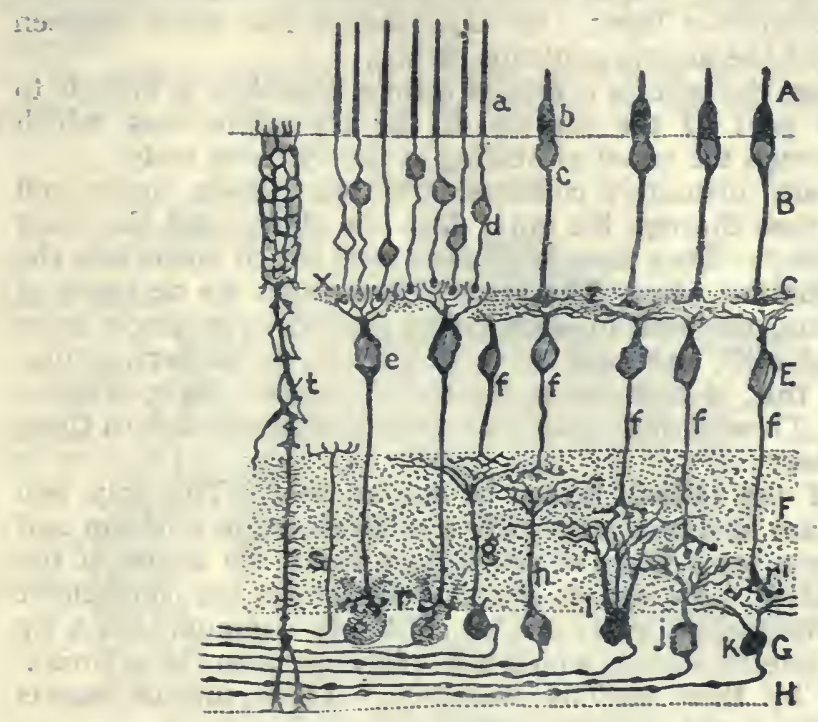

Fig. 638.-Scheme of THE RetiNA, showing the ConNection Between THE LAYER of Rods aNd Cones and the Ganglonic Layer (RAMON Y CAJAL).
A. Layer of Rods and Cones
B. Outer Nuclear Layer
C. Outer Molecular Layer
E. Inner Nuclear Layer
F. Inner Molecular Layer
G. Ganglionic Layer
H. Layer of Nerve-fibres
M. Múller's Fibre
a. Rods
b. Cones
c. Granule of Cones
d. Granule of Rods

into the pars iridica retinæ (uvea). In the latter the cells of both layers are pigmented.

Blood-supply of the Retina.-The retina is supplied with blood by the arteria centralis retinæ, which is a branch of the ophthalmic artery. Within the orbit the artery pierces the under aspect of the optic nerve a little behind the eyeball, and passes forwards in the centre of the nerve. It makes its appearance at the centre of the optic disc, and then divides into two branches, upper and lower. 
Each of these breaks up into two branches, nasal or internal, and temporal or external. The temporal branches keep clear of the macula lutea, but furnish small twigs to it, which, however, do not enter the fovea centralis, this part being non-vascular. As the branches pass inwards and outwards, respectively, towards the periphery of the retina, they ramify freely, and ultimately terminate in capillary networks. The arteries do not extend farther outwards than the inner nuclear layer. No anastomoses take place between the branches of the arteria centralis retinæ.

In the fotus the arteria centralis retinæ furnishes a branch to the posterior part of the capsule of the crystalline lens, which reaches it through the canal of Stilling in the vitreous body.

The veins are ultimately collected into two vessels, upper and lower, which pass through the optic disc, one above, and the other below, the artery. They then form one vessel which opens into the superior ophthalmic vein. The veins of the retina are destitute of muscular tissue, the wall of each being formed by a single layer of endothelial cells, external to which there is a perivascular lymph-space, this, in turn, being limited by another layer of endothelial cells. These lymph-spaces are in communication with those of the optic nerve.

Relation of the retinal layers to one another.-The only two layers which are in direct continuity are the stratum opticum and ganglionic layer, some fibres of the former being the axons of the cells of the latter. As regards most of the strata, the constituent elements of successive layers are brought into communication by means of the interlacements which take place between the arborizations formed by their various processes. These interlacements occur in the inner and outer molecular layers.

In the inner molecular layer there are several strata of interlacements, by means of which the dendrites of the cells of the ganglionic layer are brought into communication with the internal processes of the bipolar cells of the inner nuclear layer. In the outer molecular layer there is a free intermingling between the external processes of the bipolar cells of the inner nuclear layer and the rodfibres and cone-fibres.

Nerve-cells of the Retina.-These are arranged in three strata, and are in communication with one another through interlacing arborizations. The outermost stratum consists of the rods and cones; the middle stratum is formed by the bipolar cells; and the innermost stratum represents the cells of the ganglionic layer. The axons of the ganglionic cells enter the stratum opticum as centripetal fibres, which pass in the optic nerve to the brain. The centrifugal fibres of the stratum opticum ramify in the inner molecular or inner nuclear layer. 


\section{Refracting Media.}

Aqueous Humour and Chambers of the Eye.-The aqueous humour occupies the space between the cornea and the front of the crystalline lens, which is divided by the iris into two chambers, anterior and posterior. It is a clear fluid, having an alkaline reaction, and is composed of $\mathrm{H}_{2} \mathrm{O}$, holding in solution a very small amount of sodium chloride.

The anterior chamber is bounded anteriorly by the cornea, and posteriorly by the iris and the central portion of the crystalline

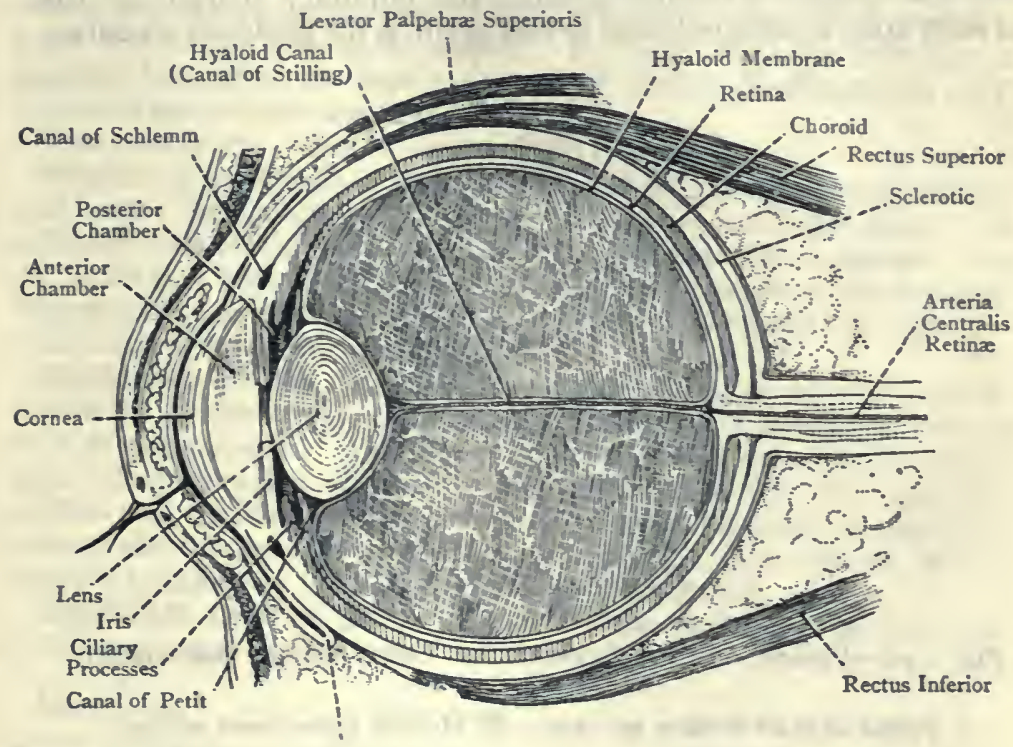

Fornix Conjunctive

Fig. 639.-Vertical Sagittal Section of the Eye and its ApPENDAges (HiRschfeld aNd LeVeillé).

lens, enclosed within its capsule. The anterior chamber communicates with the spaces of Fontana, through them with the canal of Schlemm, and through this canal with the veins of the sclerotic.

The posterior chamber, which is of limited extent, is bounded anteriorly by the iris, and posteriorly by the peripheral part of the crystalline lens and its suspensory ligament, and by the ciliary processes. The anterior and posterior chambers communicate with each other through the pupil ; with lymph-spaces in the iris; and, through the latter spaces, with the perichoroidal lymph-space. 
Crystalline Lens.-The crystalline lens is situated directly behind the pupil and iris, from which latter it is separated by the posterior chamber. It is a solid, transparent, biconvex body, the posterior surface being more convex than the anterior, and it is enclosed within a homogeneous, transparent envelope, called the capsule of the lens. The centre of the anterior surface is called the anterior pole, and that of the posterior surface the posterior pole. The line connecting these two poles constitutes the axis of the lens, and a line surrounding the periphery represents the equator. The transverse measurement of the lens is about $\frac{1}{3}$ inch, and its axis measures about $\frac{1}{6}$ inch. The anterior surface at its central part faces the pupil. External to this, the pupillary margin of the iris rests upon it, and, external to this again, is the posterior chamber,

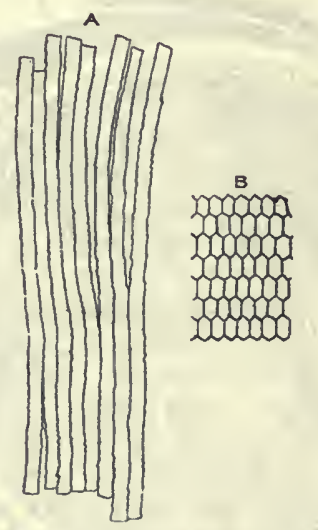

Pig. 640.-Fibres of the Crystalline Lens (highly Magnified) (AfTer Kolliker).

A, Fibres of the Ox (seen on edge); B, Human fibres (seen on end).

with part of the aqueous humour. The posterior surface is received into the patellar fossa on the anterior aspect of the vitreous body. The periphery is related to the suspensory ligament, the canal of Petit, and the ciliary processes. From the anterior and posterior poles delicate lines radiate towards the equator. In early life these are three in number on each surface. Those on the anterior surface are directed as follows : one vertically upwards, and the other two downwards and outwards in a diverging manner, thus $\lambda$. Those on the posterior surface are directed as follows : one vertically downwards; and the other two upwards and outwards in a diverging manner, thus $Y$. These lines represent the free margins of septa within the lens, upon which the ends of the lens-fibres terminate.

Structure.-The lens is laminar in structure. The outer laminæ are soft in consistence, but the succeeding ones gradually become 
firmer, and the central portion, which constitutes the nucleus, is very firm and hard. The laminæ are arranged concentrically, and, after boiling or immersion in alcohol, they may be peeled off, like the coats of an onion. The fibres of which the lamina are composed terminate upon septa within the lens, of which septa the radiating lines on the surfaces, already referred to, are the free margins. The concentric laminæ are therefore not continuous all round, but are split up along these lines. The lens-fibres, which are disposed in a curved manner, are of small size, and have serrated edges, which fit closely to each other. In transverse section the fibres appear as hexagonal prisms. The fibres are the elongated cells which line the posterior part of the epiblastic vesicle (lens-vesicle), from which the lens is developed. In éarly life each fibre has a nucleus, but, after the lens has attained its full development, only the outermost fibres are nucleated.

The lens is non-vascular in the adult.

Capsule of the Lens. - This is a transparent, homogeneous, elastic, and brittle membrane, which surrounds and encloses the lens. Its anterior wall is thicker and more elastic than the posterior. In the adult it is non-vascular, but in the fœtus it receives the hyaloid branch of the arteria centralis retinæ, which reaches it through the canal of Stilling in the vitreous body.

Epithelium of the Lens.-The posterior surface of the lens is devoid of epithelium, and is in direct contact with the posterior wall of the capsule. The anterior surface is covered by a single layer of columnar cells, which intervenes between the anteriorsurface and the anterior wall of the capsule. Towards the equator these cells become elongated, and pass into short fibres, which become continuous with the superficial lens-fibres.

Crystalline Lens at Different Ages.-The characters of the lens at different ages are as follows :

\section{Fœtal Lens.}

Almost spherical.

Pinkish colour.

Semitransparent.

Soft in consistence.

\section{Adult Lens.}

Biconvex.

Colourless.

Transparent.

Firm in consistence.
Lens in old Age.

Flattened.

Amber colour.

Opaque, more or less.

Very firm in consistence.

Vitreous Body. - This body occupies about four-fifths of the space within the eyeball, and is situated between the crystalline lens and the retina. It is transparent, and gelatinous in consistence, and is composed of $\mathrm{H}_{2} \mathrm{O}$, holding in solution a small quantity of sodium chloride and albuminous matter. It is surrounded by a transparent, homogeneous envelope, called the hyaloid membrane. This membrane is in contact with the retina, except anteriorly, in which latter situation it presents an excavation called the fossa patellaris, into which the posterior surface of the crystalline lens is received.

Towards its circumference the vitreous body is laminated, the laminæ being arranged concentrically. Laminæ are also said to 
radiate from its antero-posterior axis towards the circumference: Scattered throughout the vitreous body there are some amoboid corpuscles, and it is traversed from behind forwards by a minute passage called the hyaloid canal, or canal of Stilling (canal of Cloquet). This canal extends from the centre of the optic disc to the posterior wall of the capsule of the lens, and posteriorly it communicates with the lymph-spaces of the optic nerve. In the fotus the canal transmits a branch of the arteria centralis retinæ, called the hyaloid artery, which supplies the capsule of the lens.

No vessels enter the vitreous body, its nutrition being derived from the vessels of the retina and ciliary processes.

Zonula of Zinn.-The zonula of Zinn, or zonula cillaris, is the thickened portion of the hyaloid membrane which is situated in front of the ora serrata of the retina. From this point it extends inwards behind the ciliary processes towards the periphery of the crystalline lens. Behind the ciliary processes it presents radial folds with intervening depressions. The depressions receive the ciliary processes, and the radial folds are separated from the intervals between, the ciliary processes by lymph-spaces, which communicate with the posterior chamber of the eye.

Suspensory Ligament of the Lens, and Canal of Petit.-The zonula of $Z$ inn, as it approaches the periphery of the lens, divides into three layers, posterior, middle, and anterior. The posterior layer lines the fossa patellaris on the anterior aspect of the hyaloid membrane. The middle layer consists of a few scattered fibres which pass to the equator of the lens. The anterior layer is the thickest, and constitutes the suspensory ligament of the lens, which is attached to the anterior wall of the capsule of the lens not far from the equator. When the radiating fibres of the ciliary muscle contract the suspensory ligament is relaxed, and the convexity of the anterior surface of the lens is increased.

Behind the suspensory ligament of the lens there is a sacculated lymph-space, called the canal of Petit, which surrounds the equator of the lens.

\section{Development of the Eye.}

The retina, optic nerve, and crystalline lens are developed from the ectoderm, the retina and optic nerve being derived from the ectoderm of the anterior primary cerebral vesicle, whilst the crystalline lens is developed from the ectoderm of the side of the head. The accessories of the eye-ie.g., the sclerotic, cornea, choroid, ciliary body, and iris, are all developed from the mesoderm. The vitreous body, though developed to a certain extent from the mesoderm, is principally formed from the ectoderm.

The earliest indication of the development of the eye is the formation of a hollow protrusion on either side, from the lower and lateral portion of that part of the anterior primary cerebral vesicle which forms the diencephalon or thalamencephalon, anterior to the level of the infundibulum. This protrusion, therefore, is an adjunct of the cavity of the diencephalon, which gives rise to the third ventricie. On the ventral aspect of the diencephalon, between the openings of the optic vesicles, there is a depression, called the optie recess, in the posterior margin of which the optie chlasma or commissure is developed. The direction of each protrusion is outwards towards the ectoderm of the side of the head. Towards its distal end it becomes enlarged, this enlarge- 
Anterior Wall of Capsule of I ens
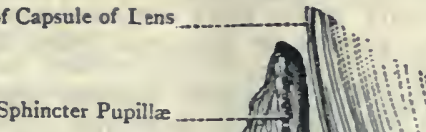

Sphincter Pupillæ

\section{Membrane}

i Descemet

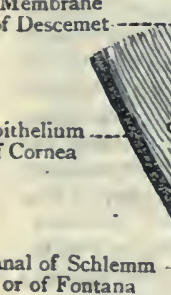

or of Fontana

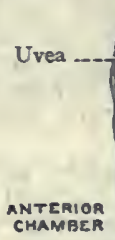

\section{Margin
Cornea}

Conjunctiva

Ciliary Muscle CHAMBER (Radiating Fibres)
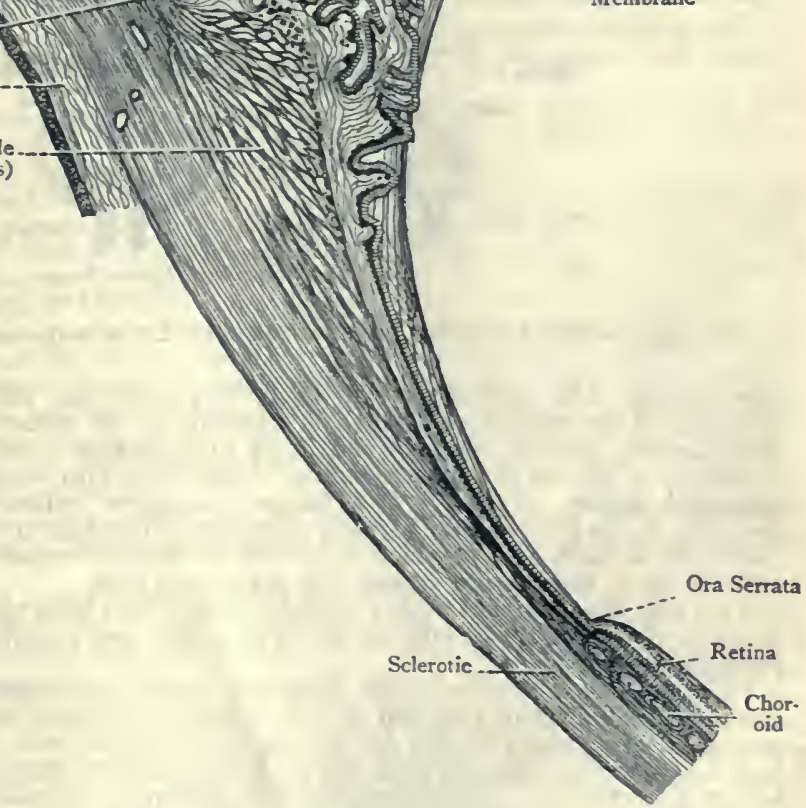

Fig. 64t.-Meridional Section through the ANterior Portion of The Eye (Magnified I6 6 I) (Fuchs).

C.P., C.P., Canal of Petit. 
ment being called the optic vesicle. The remainder of the protrusion constitutes a narrow, hollow pedicle, called the optic stalk, which is attached to the wall of the diencephalon, the cavity of the stalk communicating with the third ventricle. The enlargement formed by the optic vesicle lies beneath the ectoderm of the lateral surface of the head.

The ectoderm corresponding to the optic vesicle becomes thickened and depressed, this depressed portion constituting the lens-area. The depressed ectoderm is deepened and converted into a kind of cup, and, the mouth of the fossa becoming constricted, its lips unite. In this manner the lens-area becomes transformed into a closed ectodermic sac, called the lens-vesicle, from which the crystalline lens is differentiated. The lens-vesicle now becomes completely separated from the surface-ectoderm, with which it was originally continuous.

Coincident with the formation of the lens-vesicle, an important change takes place in the optic vesicle. The distal or outer wall of the optic vesicle is invaginated or pushed inwards towards the inner wall until the two walls come into contact, this being effected by the lens-vesicle. The optic vesicle thereby becomes transformed into the optic cup, the wall of which is composed of two layers-outer and inner. The lens-vesicle lies just within the mouth of the cup. The portion of the cup close to the margin represents the ciliary reglon, and the fundus of the cup forms the retinal reglon. The cup becomes surrounded by mesodermic tissue.

During the formation of the optic cup it is not only the distal or outer wall of the optic vesicle which is invaginated, or pushed inwards, by the

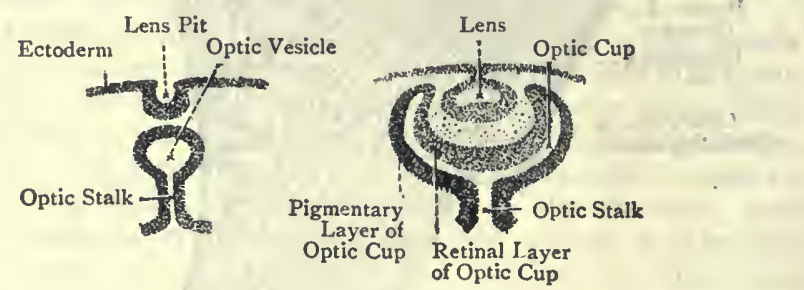

Fig. 642,-Development of Crystalline Lens and Optic Vesicle.

lens-vesicle. The ventral wall of the optic vesicle, and the ventral wall of the part of the optic stalk, which is adjacent to the optic vesicle, are also invaginated. As the result of this extra invagination a cleft, of temporary duration, is left on the under surface of the optic cup, which is called the choroidal fissure. Through this fissure mesoderm enters the optic cup. posterior to the lens-vesicle. In this mesoderm a nutrient artery is developed, called the hyaloid artery, which furnishes the minute vessels conferring vascularity upon the capsule around the developing lens.

As a rule the choroidal fissure becomes closed, and then the hyaloid artery is enclosed within the cavity of the optic stalk, and constitutes the arterla centralis retinæ.

In cases of non-closure of the choroidal fissure, the region of the fissure remains unpigmented, and one of two congenital deficiencies in the eye are met with, each being known by the general term coloboma. If the patent fissure affects the ventral wall of the optic cup, then the deficiency in pigment affects the choroid, and the condition is known as coloboma choroidea. If the patent fissure affects the lower margin of the optic cup, then the deficiency affects the lower part of the iris, and the condition is known as coloboma iridis.

As stated, the wall of the optic cup consists of two layers. The outer iayer, which is comparatively simplc, gives rise to the plgmentary layer of the retina. The inner layer is, on the other hand, very complicated. After much differentiation it gives rise to all the other layers of the retina. The 
mesodermic tissue, which invests the optic cup, gives rise to the sclerotic, cornea, choroid, ciliary body (including the ciliary processes and ciliary muscle), and iris.

Crystalline Lens. - The lens is of ectodermic origin. The surface-ectoderm on the lateral aspect of the head opposite the optic vesicle becomes thickened and depressed to form, as stated, the lens-area. The depressed ectoderm is deepened and converted into a kind of cup. The mouth of the cup becomes constricted, and its lips unite. In this manner the lens-area becomes transformed into a closed ectodermic sac, called the lens-vesicle, from which the crystalline lens is differentiated. The lens-vesicle becomes completely sep. arated from the surface-ectoderm, with which it was originally continuous. It is now received into the optic cup, which has been formed in connection with the optic vesicle, its position being just within the mouth of the cup, the circumference of the margin projecting slightly in advance of the vesicle.

The anterior and posterior walls of the lens-vesicle at this stage consist of several layers of cylindrical cells, and the vesicle contains a small central cavity. The anterior wall becomes gradually thin, and is ultimately formed of one layer of flattened cells, these cells constituting the anterior epithelium of the adult crystalline lens. The cells of the posterior wall become elongated in a forward direction, obliterating the cavity of the vesicle, and coming into contact with the anterior wall. By this process of cell-elongation the lensfibres are formed. At the equator of the lens the cells of the anterior and posterior walls merge gradually into one another through the medium of a transitional zone of columnar cells.

At this stage in its development the crystalline lens consists of ( 1 ) an anterior epithelial wall, and (2) a posterior wall composed of elongated cells forming the lens-fibres.

As development proceeds, additional lens-fibres are formed by the proliferation of cells at the equator of the lens. These fibres are laid down in successive layers, which are arranged concentrically.

Capsule of the Crystalline Lens.-At an early period in its development the lens becomes invested by a mesodermic capsule, freely supplied with bloodvessels derived from the hyaloid artery and anterior ciliary arteries. This capsule is known as the tunica vasculosa. It persists throughout the period of active growth of the lens, and then undergoes retrogression to form the permanent lens-capsule. The portion of the tunica vasculosa which covers the front part of the lens is called the membrana prpillaris, but this usually disappears prior to birth. It may, however, be present at birth, giving rise to the condition known as atresia pupilla. Towards the end of intra-uterine life the tunica vasculosa undergoes retrogression, and becomes transformed, as stated, into the permanent lens-capsule, which is a transparent, homogeneous, elastic membrane.

According to some authorities the lens-capsule is of mesodermic origin, whilst according to other authorities it is derived from the cells of the crystalline lens.

Development of the Optie Cup and Optic Stalk.-The optic cup, as stated. is formed by the invagination by the lens-vesicle of the distal or outer wall of the optic vesicle, the invagination also affecting the ventral wall of the optic vesicle and the ventral wall of the part of the optic stalk which is adjacent to the optic vesicle, thereby giving rise to the choroidal fissure. The mouth of the optic cup is directed towards the lateral aspect of the head, and the lens-vesicle lies just within the mouth. The margin of the cup projects slightly over the lens-vesicle and the circumference of this margin represents the outline of the pupil. The wall of the cup consists of two layersnamely, inner and outer, the inner representing the distal or outer wall of the optic vesicle, which has now become invaginated, or pushed inwards: The cup is divisible into two regions-namely, (I) the ciliary region, adjoining the margin of the cup, and (2) the fundus. The line of separation between these two regions corresponds to the ora serrata of the adult eye,

The ciliary region of the optic cup is associated with the ciliary body. (including the ciliary processes and ciliary muscle) and the iris, which are 
developed from the thickened anterior part of the choroid. The outer layer of the ciliary portion, as elsewhere, forms the pigmentary layer of the retina. The inner layer of the ciliary portion, which is very thin, forms (I) the pars ciliaris retinæ, on the posterior surfaces of the ciliary processes, and (2) the pigmented pars iridica retinn (uvea) on the posterior surface of the iris.

The fundus of the optic cup is the proper retinal region. The outer layer forms, as in the ciliary region, the pigmentary layer of the retina. The inner or retinal, layer becomes differentiated into all the layers of the retina except the pigmentary layer. The changes which it undergoes are very complicated. Its thickness is considerably increased, and it subdivides into two layersouter and inner-from which the various retinal strata (except the outer pigmentary layer) are specialized.

The optic stalk is transformed into the optic nerve. The stalk is at first hollow, its cavity communicating with that of the optic vesicle on the one hand, and with the third ventricle of the brain on the other. As stated, the choroidal fissure involves the under surface of the optic stalk near the optic vesicle, as well as the under surface of the optic vesicle itself. When the choroidal fissure undergoes closure, the hyaloid artery, which passed through that fissure, becomes enclosed within the optic stalk, and forms the arteria centralis retine of adult life. By the closure of the choroidal fissure, and the consequent enclosure of the hyaloid artery, the cavity of the distal portion of the optic stalk becomes obliterated. Inasmuch as the ventral or lower wall of this part of the stalk has been previously invaginated, the wall of the stalk is now composed of two layers-outer and inner-the inner being formed by the invaginated ventral or lower wall. The outer layer of the optic stalk is now continuous with the outer layer of the optic cup, whilst the inner layer of the optic stalk is continuous with the inner layer of the optic cup. As regards the proximal part of the optic stalk, its cavity becomes gradually closed. The wall of the optic stalk becomes thickened, its cells proliferate, and they give rise to the neuroglial or sustentacular tissue of the future nerve. The nerve-fibres, which build up the optic nerve, are regarded as having two sources. The majority of them represent the axons of the ganglion-cells of the retina, which pass in the optic stalk to the diencephalon and mesencephalon. These are therefore centripetal fibres. Other fibres are regarded as being centrifugal, these arising in connection with the diencephalon and mesencepalon.

Vitreous Body.-This body is formed within the optic cup, for the most part posterior to the lens-vesicle. It is principally developed from the ectoderm, but the mesoderm also takes part in its formation. The ectodermic fibres are derived from those cells which pertain to the sustentacular fibres of the retina, or fibres of Muller. These ectodermic fibres form a very delicate reticulum, which constitutes the primary vitreous body. In all probability the vitreous body is reinforced by fibres from the mesodermic tissue which enters the optic cup, along with the hyaloid artery, through the choroidal fissure on the ventral or under surface of the optic cup. The peripheral part of the vitreous body gives rise to the hyaloid membrane.

The vitreous body soon becomes very vascular. The chief artery is the hyaloid artery, which enters the optic cup, along with mesoderm, through the choroidal fissure. The anterior ciliary arteries also contribute to its vascularity, these vessels gaining entrance between the margin of the optic cup and the lens-vesicle. The hyaloid artery occupies the centre of the vitreous body, lying in a passage, called the hyaloid canal, or canal of Stilling. On reaching the posterior part of the lens-capsule it breaks up into a number of branches. The arteries of the vitreous body at this stage confer great vascularity upon the lens-capsule, hence the name tunica vasculosa, and through it they contribute to the nourishment of the lens-vesicle during the period of its active development. The anterior part of this tunica vasculosa, as stated, gives rise to the membrana pupillaris.

Towards the latter part of intra-uterine life the bloodvessels disappear, the hyaloid canal, or canal of Stilling, remaining permanent. The part of the hyaloid artery which becomes enclosed within the optic stalk forms the arteria centralis retine. 
Derivatives of the Mesodermic Envelope of the Optic Cup.-These are as follows: (1) Sclerotic, (2) cornea, (3) choroid, (4) ciliary body (including the ciliary processes and ciliary muscle), and (5) iris.

The mesoderm which invests the outer surface of the optic cup is disposed in two layers-outer and inner. The outer layer has a fibrous character, and gives rise to the sclerotic, of which the cornea is a forward extension. The inner layer is vascular, and gives rise to the choroid, ciliary body, and iris. The outer dense fibrous layer of the mesoderm of the outer surface of the optic cup, as stated, gives rise to the sclerotic. From its anterior margin a thick lamina of mesoderm is prolonged between the lens-vesicle and the surface ectoderm. This lamina divides into two layers-superficial and deep. The superficial layer becomes differentiated into the cornea, which is thus genetically continuous with the sclerotic. The decp layer becomes the anterior $w$ all of the lens-capsule. Between these two layers there is an interval, which represents the aqueous chamber, this chamber being subsequently divided by the iris into the anterior and posterior aqueous chambers.

The inner vascular layer of the mesoderm of the outer surface of the optic cup, as stated, gives rise to the choroid. The anterior margin of the chorold, which adjoins the margin of the optic cnp (ciliary region) becomes thickened, and gives rise to the ciliary body, in connection with which the cillary processes and ciliary muscle are developed. The ciliary processes become covered posteriorly by the pars ciliaris retine, which is a thin retinal expansion from the ciliary region of the optic cup. The iris is also developed at the anterior margin of the choroid in the form of a ring of mesoderm. In this mesoderm the fibres forming the dilator pupille and sphincter pupille muscles are formed, and the back of the iris receives a pigmentary covering (uvea) from the pars iridica retine, which is a thin retinal extension from the ciliary region of the optic cup. 


\section{THE EAR}

THE organ of hearing is divided into three parts, namely, the external ear, the middle ear, and the internal ear.

\section{External Ear.}

The external ear consists of the auricle, or pinna, and the meatus auditorius externus. The auricle, or pinna, has been already described (see Index).

The external auditory meatus extends from the bottom of the concha to the membrana tympani, and is about I inch in length. It consists of two parts-outer, or cartilaginous, and inner, or osseous. The cartilaginous part, which also consists of fibrous tissue, is about $\frac{1}{3}$ inch in length, and the osseous part, which lies within the petrous portion of the temporal bone, is about $\frac{2}{3}$ inch long: The widest part of the meatus is its orifice, which is oval, the long measurement being vertical. The narrowest part is situated in its osseous portion, about $\frac{1}{5}$ inch from the membrana tympani, and it is known as the isthmus. There is another constriction of the canal situated near the deep end of the cartilaginous part, and produced by a projection which is placed antero-inferiorly. The chief direction of the canal is inwards and slightly forwards. At first it is also inclined upwards, then backwards, and finally downwards.

The cartilaginous part is continuous with the cartilage of the auricle, and is attached to the external auditory process of the temporal bone. Its cartilage is folded so as to form a deep groove, which is open at its upper and back part, the cartilaginous deficiency being completed by fibrous tissue. The anterior wall of the cartilaginous part presents two clefts, called the fissures of Santorini, which are occupied by fibrous tissue.

The osseous part has been described in connection with the temporal bone. At its deep end there is a narrow groove, called the sulcus tympanicus, which forms about five-sixths of a circle, the deficiency being placed superiorly, at the notch of Rivinus, where the ring is completed by the squamous part of the temporal bone. The membrana tympani is set obliquely within the sulcus tympanicus, being inclined downwards and towards the median line. The floor and anterior wall of the meatus are consequently longer than the roof and posterior wall.

The meatus is lined with skin, which is continuous with that of the auricle. In the osseous part of the canal the skin is very thin, and is provided with vascular papillæ, but is destitute of glands and hairs. It is reflected over the outer surface of the membrana tympani, of which it forms the outer layer. In the cartilaginous part of the canal the skin is thicker, and is provided with hairs, connected with the follicles of which are sebaceous glands. In 
addition to these sebaceous glands, there are convoluted tubular glands, similar in structure to sweat-glands, and called the ceruminous glands, which secrete the ear-wax.

Blood-supply.-The arteries are derived from the posterior auricular of the external carotid, the deep auricular of the first part of the internal maxillary, and the anterior auricular branches of the superficial temporal. The veins follow the course of the arteries.

Lymphatics. - These pass to the mastoid glands and to the parotid lymphatic glands.

Nerves.-(I) The auriculo-temporal nerve furnishes two branches to the meatus, upper and lower, which enter it by passing between

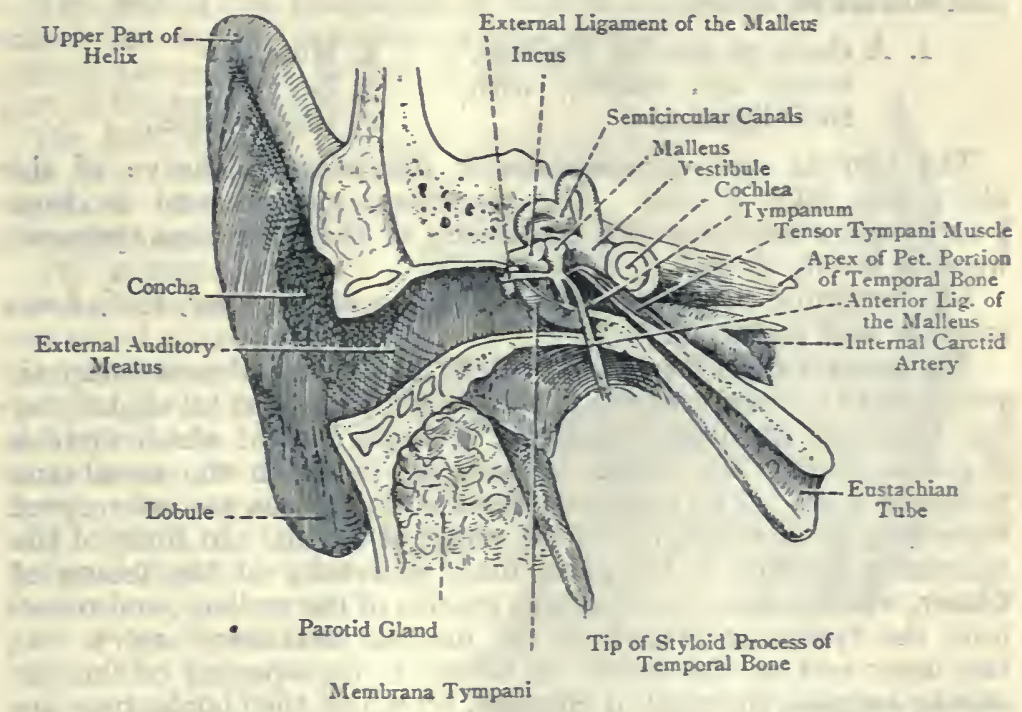

Fig. 643.-General View of the Right Organ of Hearisg (after HiRsChfeld ANd Leveillé).

(The External Ear and Middle Ear are seen in section).

the cartilaginous and osseous walls. The upper branch furnishes twigs to the skin over. the upper part of the membrana tympani. (2) The auricular branch (Arnold's nerve) of the pneumogastric supplies the skin of the osseous part of the canal over its lower and back part, and also the skin over the lower part of the membrana tympani.

Early Condition of the Meatus. - At birth the osseous part of the canal is represented merely by the tympanic annulus and a small portion of the squamous part of the temporal bone. It is connected by fibrous tissue to the cartilaginous framework of the auricle, and within this fibrous tissue the osseous canal becomes formed by two oatgrowths from the tympanic annulus. 


\section{Middle Ear.}

The middle ear, or tympanum, is an irregular space within the petrous part of the temporal bone, which lies between the membrana tympani externally and the outer osseous wall of the internal ear or labyrinth internally. It is lined with mucous membrane, and it communicates with the naso-pharynx by means of the Eustachian tube, through which it receives air. It presents three parts, namely; (I) the tympanum proper, or cavum tympani; (2) the attic, or epitympanic recess; and (3) the tympanic or mastoid antrum.

The tympanum proper, or cavum tympani, is situated between the membrana tympani and the outer wall of the internal ear. Its contents are as follows:

I. A chain of ossicles (malleus, incus, and stapes), with their ligaments.
2. Muscles.

3. Nerves.

4. Bloodvessels.

The vertical and antero-posterior diameters (inclusive of the attic) are fully $\frac{1}{2}$ inch. The transverse measurement is from $\frac{1}{4}$ to $\frac{1}{6}$ inch, except opposite the centre of the membrana tympani, where it is only $\frac{1}{12}$ inch.

The tympanic cavity presents six walls-outer, inner, roof, floor, anterior, and posterior.

The outer wall is formed ( $\mathrm{I}$ ) chiefly by the membrana tympani, which has the handle of the malleus fixed to it, and (2) slightly by the tympanic annulus, within the circumference of which there is a groove, called the sulcus tympanicus, in which the membrana tympani is set. The tympanic annulus and sulcus are interrupted superiorly by a notch, called the notch of Rivini. In front of the tympanic annulus is the open, inner extremity of the fissure of Glaser, which lodges the processus gracilis of the malleus, and transmits the tympanic branch of the internal maxillary artery. At the inner end of the fissure of Glaser is the opening of the iter chordce anterius, or canal of Huguier, by which the chorda tympani nerve leaves the tympanum.

The inner wall separates the tympanum from the internal ear or labyrinth. It is very irregular, and presents the following parts :

I. The fenestra ovalis.

2. Projection formed by part of the aqueduct of Fallopius.
3. The promontory.

4. The fenestra rotunda.

5. The sinus tympani.

The fenestra ovalis is situated in a depression, called the fossula ovalis, at the upper part of the inner wall, and it leads into the cavity of the vestibule. It is irregularly oval, and is elongated from before backwards. It is occupied by the footpiece of the stapes, and the annular ligament which connects the circumference of the footpiece to the margin of the opening, the margin being covered by cartilage.

The projection formed by part of the aqueduct of Fallopius is situated above the fenestra ovalis. The aqueduct, which contains the facial nerve, is here directed backwards. 
The promontory (tuber cochlece) is situated below the fenestra ovalis, between it and the fenestra rotunda, and slightly anterior to both. It is a rounded prominence which is produced by the first turn of the cochlea, and it presents. grooves for the nerves of the tympanic plexus.

The fenestra rotunda is situated in a funnel-shaped depression, called the fossula rotunda, below and behind the promontory: It leads into the scala tympani of the cochlea, and in the recent state is closed by the secondary membrane of the tympanum.

The sinus tympani is a depression situated behind the promontory, and between the fossula ovalis and fossula rotunda.

The roof of the tympanum is formed by a thin plate of bone, called the tegmen tympani, which forms part of the superior surface of the petrous. part of the temporal bone?

The floor is formed by a thin plate of bone which separates the tympanum from the jugular fossa.

The anterior wall is narrow, owing to the descent of the roof, and the inclination towards each other of the outer and inner walls. It presents the orifices of two canals, the upper of which lodges the tensor tympani muscle, whilst the lower is the osseous part of the Eustachian tube. The two orifices are separated by the margin of the processus cochleariformis.

- The posterior wall is formed by the anterior or tympanic surface

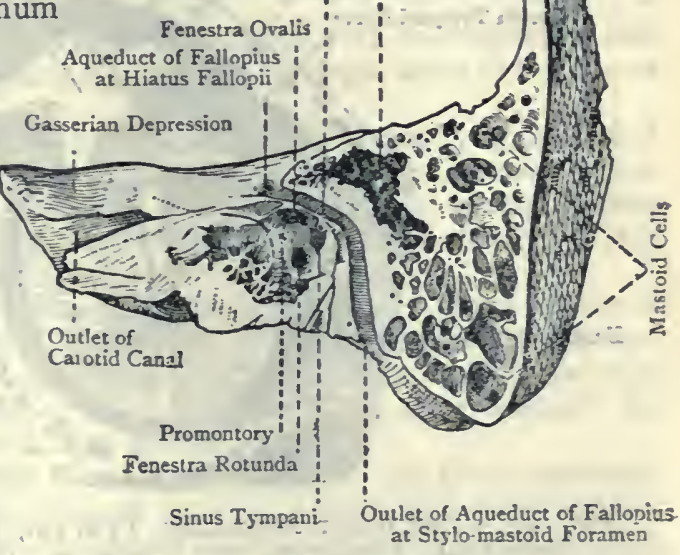

Fig. 644--Section THRóugh THE Petrous and Mastoid Portions of the Temporal BONE, SHOWING THE TYMPANUM AND MAStoid Celis.

of the petrous part of the temporal bone. It presents, from above downwards, the following parts: (I) the opening of the mastoid antrum, which communicates with the attic of the tympanum, or epitympanic recess; (2) a depression called the fossa incudis, which receives the short process of the incus ; (3) a small conical projection, called the pyramid, the summit of which presents an opening for the passage of the tendon of the stapedius muscle; (posteriorly the canal within the pyramid, which contains the stapedius muscle, passes downwards in the posterior wall of the tympanum, and communicates with the descending part of the aqueduct of Fallopius, which contains the facial nerve, thus explaining how the branch of that nerve to the stapedius reaches the 
muscle) ; and (4) the iter chordæ posterius, for the passage of the chorda tympani nerve.

Membrana Tympani. - This is the membrane which closes the inner extremity of the meatus auditorius externus. It is situated on the outer wall of the tympanum, of which it forms the chief part, and it is set, for the most part, in the sulcus tympanicus, which marks the inner surface of the tympanic annulus. Superiorly, however, where the annulus is deficient, the membrane is attached to the notch of Rivini. This part of it is thinner and looser than the rest, and is called the membrana flaccida, or Shrapnell's membrane. The attachment of the membrana tympani to the sulcus tympanicus is effected by means of a thickened band of fibres, disposed in the form of a ring, called the annulus fibrosnis. "This annulus passes

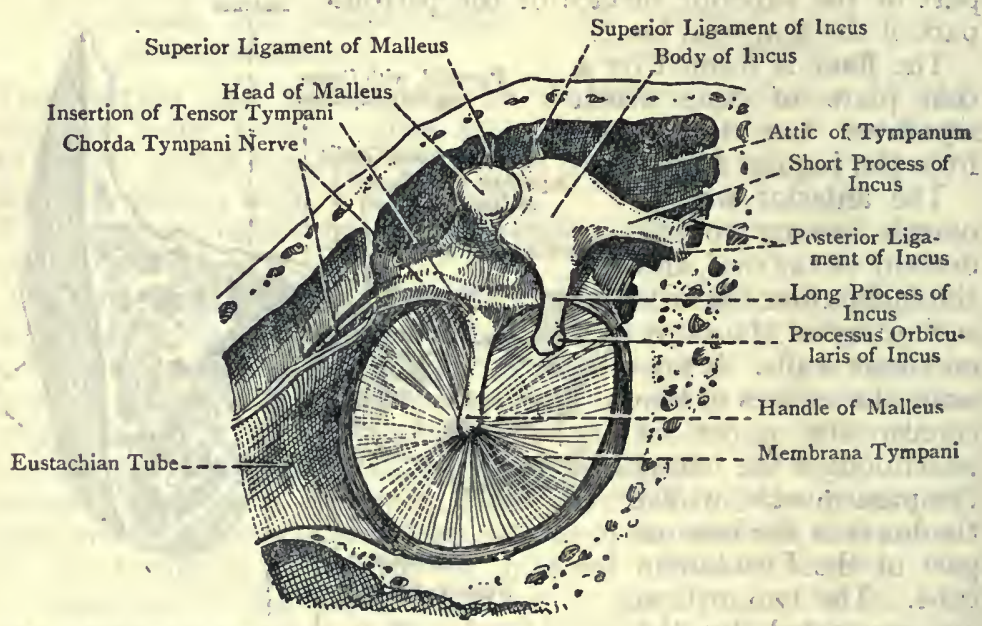

Fig. 645.-The Right Membrana Tympani, Malleus, and Incus (INTERNal, POSTERIOR, aNd SUPERIOR VIew) (SPalteholz).

from the extremities of the notch of Rivini to the short process of the malleus in the form of two bands, which are known as the anterior and external malleolar ligaments, and which form the lower boundary of the membrana flaccida. Opposite the notch of Rivini these annular fibres separate the membrana flaccida from the rest of the membrana tympani. The membrane is set obliquely in the sulcus tympanicus, so that its lower part forms an acute angle with the floor of the meatus auditorius externus, and its upper part an obtuse angle with the roof of the passage.

The membrana tympani is somewhat oval. In the vertical direction it measures about Io $\mathrm{mm}$., and horizontally from 8 to $9 \mathrm{~mm}$. The handle of the malleus lies between the mucous and fibrous layers of the membrane, and descends to a point a little below its centre, where it ends in a small knob, from which the radi- 
ating fibres of the membrane proceed. This knob is firmly attached, and, being directed inwards, the membrane is consequently drawn inwards at that point, and its outer surface presents a slight conical depression, the deepest part of which is called the umbo.

Structure.-The membrana tympani consists of three layersexternal, middle, and internal.

The external or cutaneous layer is very thin, and is derived from the skin of the meatus auditorius externus. It contains no glands, is freely provided with bloodvessels and nerves, and is covered by stratified squamous epithelium.

The middle or fibrous layer forms the proper substance of the membrane, and consists of fibrous tissue. The fibres are arranged in two sets-radial and circular. The radial fibres lie beneath the cutaneous layer, and radiate from the handle of the malleus to the annulus fibrosus. The circular fibres are situated within the radial fibres, and are most numerous towards the circumference of the membrane. Both sets of fibres are absent from the membrana flaccida.

The internal or mucous layer is continuous with the mucous membrane of the tympanum, and is covered by a single layer of squamous epithelium.

The membrana flaccida, or Shrapnell's membrane, is composed of two layers-cutaneous and mucous, there being no fibrous layer. These two layers are united by connective tissue, which is very loosely arranged, and this renders the membrane flaccid. This part is very liable to perforation.

Cone of Light.-Exteriding from the knob, in which the handle of the malleus terminates, downwards and inwards to the anteroinferior margin of the membrana tympani there is seen a specially bright reflection, triangular in outline, with the apex towards the umbo. This is called the cone of light.

Arterial Supply of the Membrana. Tympani.-(I) Deep auricular branch of the internal maxillary artery. This vessel, which passes through the anterior wall of the meatus auditorius externus, supplies the cutaneous layer. It descends from the skin of the roof of the meatus along the course of the handle of the malleus to the umbo, where it divides into branches which radiate towards the circumference of the membrane. (2) The stylo-mastoid branch of the posterior auricular, and (3) the tympanic branch of the internal maxillary. The former artery enters the tympanum from the aqueduct of Fallopius, and the latter through the fissure of Glaser. Branches from them supply the mucous layer, and form an anastomotic ring around the circumference of the membrane. The fibrous layer receives its arterial supply from the vessels of the cutaneous and mucous layers.

The veins pass to the external jugular and internal jugular veins.

Nerve-supply.-The nerves are derived from (I) the auriculotemporal of the inferior maxillary, (2) the auricular branch (Arnold's 
nerve) of the pneumogastric, and (3) branches from the tympanic plexus.

Secondary Membrane of the Tympanum.-This membrane closes the fenestra rotunda on the inner wall of the tympanum, and separätes the tympanic cavity from the scala tympani of the cochlea. It is concave towards the tympanum, and, like the membrana tympani, it consists of three layers. The external layer is formed by the tympanic mucous membrane; the middle layer is fibrous ; and the internal layer is formed by the lining membrane of the cochlea.

The mastoid antrum and mastoid air-cells.will be found described in connection with the temporal bone, and a description of the Eustachian tube will be found on p. I274.

\section{Ossicles of the Tympanum.}

The tympanum contains three small bones, arranged in the form of a chain which extends from the membrana tympani to the fenestra ovalis. The bones are the malleus, the incus, and the stapes.

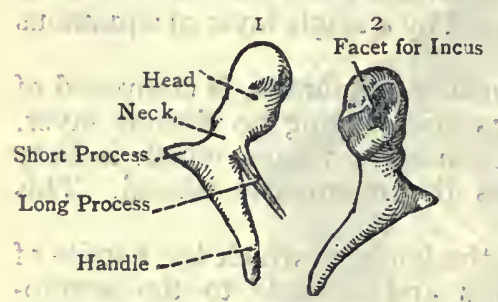

Fig, 646.-The Malleus.

I, Anterior View ; 2, Posterior View. The malleus is related to the membrana tympani, the stapes to the fenestra ovalis, and the incus occupies an intermediate position between these two.

The malleus is so named from its resemblance to a hammer. It is composed of a head, neck, handle, and two processes, namely, long and short. The head is the upper, enlarged, rounded end. Posteriorly it presents a saddle-shaped facet, directed obliquely downwards and inwards, for articulation with the incus in a synovial joint. The neck is the constricted part below the head. The handle (manubrium) is directed downwards, inwards, and backwards from the neck; it is compressed from before backwards; and slightly curved, and it terminates in a slight knob. It lies between the fibrous and mucous layers of the membrana tympani, descending, to a point a little below the centre of the membrane. "It is firmly attached to the fibrous layer by its periosteum. The tensor tympani muscle is inserted into its inner part, close to its root. The long process (processus gracilis), which is slender, springs from the front of the neck, and is directed forwards and downwards to the fissure of Glaser, where it is embedded in fibres which form part of the anterior ligament of the malleus, and connect it to the margins of the fissure. The long process is in the adult for the most part replaced by fibrous tissue, except close to the neck of the malleus. In early life it is continuous with Meckel's cartilage. The short process (processus brevis) is situated immediately below the long process. It is directed outwards to the upper part of the membrana 
tympani, with which it is connected by the annulus fibrosus. It is also connected with the extremities of the notch of Rivini by those bands of the annulus fibrosus which are known as the anterior and posterior malleolar ligaments.

The incus has been so named from its resemblance to an anvil. It consists of a body and two processes-short and long. The body is thick, somewhat four-sided, and laterally compressed. Anteriorly it presents a saddle-shaped articular surface for the head of the malleus, with which it forms a synovial joint. The short process is directed backwards, is tipped with cartilage, and articulates with the fossa incudis on the posterior wall of the tympanum. The long process is directed downwards and inwards, behind and parallel to the handle of the malleus. Its lower extremity is bent inwards, and becomes

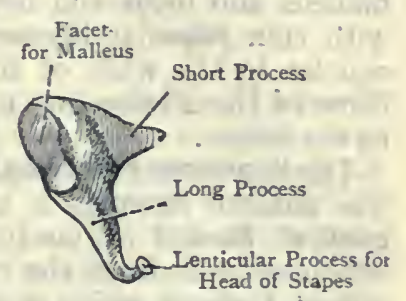

Flg. 647.- The Isces.

(Postero-internal View). narrowed into a neck, upon which is placed a disc-like knob of bone, called the processus lenticularis seu orbicularis, which is covered by cartilage for articulation with the head of the stapes: In early life, and up to the sixth month of intra-uterine life, this process forms a separate ossicle, called the os orbiculare.

The stapes is so named from its resemblance to a stirrup. It consists of a head, neck, two crura, and a foot-piece or base. The head is directed outwards, is concave and covered by cartilage, and articulates with the processus lenticularis of the incus. The neck is the constricted part which lies immediately internal to the head. Posteriorly it gives insertion to the stapedius muscle. The crura are anterior and posterior respectively, and spring from the neck. They diverge as they pass inwards, and are attached to the footpiece near its extremities. The anterior crus is straighter and shorter than the posterior. The foot-piece or base is somewhat oval, is directed inwards, and occupies the fenestra ovalis, which it almost completely fills; its circumference

Fig. 648.-(I) The Stapes, A.ND (2) TYMPANic OssiCLES IN POSITION.

is covered by cartilage, being attached to the margins of the fenestra by annular ligamentous fibres. The arch formed by the crura and foot-piece is occupied by a delicate membrane, which is attached to a slight groove on the inner aspect of the arch.

Development of the Tympanic Ossicles.-The malleus and incus are usually regarded as being developed from the proximal end of Meckel's cartilage, 
which forms the cartilaginous bar of the first or mandibular arch. According. to some authorities, however, the incus is developed from the hyoid bar. The stapes is developed from the dorsal part of the hyoid bar of the second visceral arch, its foot-piece being derived from the labyrinth-cartilage within the fenestra ovalis.

Ligaments of the Ossicles. - The synovial joints between the malleus and incus and between the incus and stapes are provided with thin capsular ligaments. The ligaments which connect the ossicles to the walls of the tympanic cavity are five in number, three of them belonging to the malleus, one to the incus, and one. to the stapes.

The ligaments of the malleus are anterior, posterior, and superior. The anterior ligament is arranged in two parts, as follows: (I) the band of Meckel (formerly known as the laxator tympani muscle), which extends from the root of the long process to the fissure of Glaser, through which it passes to be attached to the spine of the sphenoid bone; and (2) the so-called anterior malleolar fold, which extends from the short process to the anterior extremity of the notch of Rivini. The posterior ligament extends from the short process to the posterior extremity of the notch of Rivini. The superior ligament extends from the head of the malleus to the roof of the attic or epitympanic recess.

The ligament of the incus connects the short process, near its posterior extremity, to the fossa incudis.

The annular ligament of the stapes connects the circumference of the foot-piece, which is covered by cartilage, to the margin of the fenestra ovalis, which is also covered by cartilage.

Muscles of the Tympanum.-These are two in number, namely, the tensor tympani and the stapedius.

Tensor Tympani-Origin.-(I) The cartilaginous part of the Eustachian tube, superiorly; (2) the adjacent part of the great wing of the sphenoid; and (3) the wall of the osseous canal through which the muscle passes.

Insertion. - The inner aspect of the handle of the malleus, close to its root.

Nerve-supply. - A branch from the otic ganglion, and, through it, from the internal pterygoid branch of the inferior maxillary division of the fifth cranial nerve.

The muscle consists of a fleshy belly, about $\frac{1}{2}$ inch long, and a delicate tendon. In passing backwards to the tympanum it lies. in a canal, the entrance to which is situated within the petrosquamous angle of the temporal bone. The canal is placed above the osseous part of the Eustachian tube, from which it is separated by the processus cochleariformis. On entering the tympanum the tendon of the muscle bends sharply over the edge of the processus cochleariformis, and then passes outwards to reach its insertion. The tendon forms very nearly a right angle with the fleshy belly. Action.-To render tense the membrana tympani by drawing. 
inwards the handle of the malleus, and, along with it, the membrane.

Stapedius-Origin.-The wall of the canal within the pyramid, and of the continuation of this canal in front of the descending part of the aqueduct of Fallopius.

The tendon emerges from the canal within the pyramid through a small orifice on the apex.

Insertion.-The posterior aspect of the neck of the stapes.

Nerve-supply. - The facial nerve.

Action.-To draw the head of the stapes backwards. The result is that the front part of the foot-piece of the stapes is tilted away from the vestibule, and its back part is pressed inwards towards the restibule.

Movements of the Ossicles. - The malleus and incus move simultaneously, the movement being one of rotation round an axis extending from the tip of the long process of the malleus to the tip of the short process of the incus. During the action of the tensor tympani muscle, when the handle of the malleus and the membrana tympani are drawn inwards, the long process of the incus is pushed inwards, and the stapes is thereby pressed inwards. The result is that the foot-piece of the stapes presses upon the perilymph of the labyrinth, and the secondary membrane of the tympanum is pressed outwards. Probably the stapedius tends to prevent undue pressure upon the perilymph of the labyrinth during strong contraction of the tensor tympani by the tilting action already ascribed to it.

Mucous Membrane of the Tympanum.-The tympanic mucous membrane is continuous anteriorly with that of the naso-pharynx through the Eustachian tube. Posteriorly it is prolonged into the mastoid antrum, and thence into the mastoid cells. It forms the internal layer of the membrana tympani, and the external layer of the secondary membrane of the tympanum. It also furnishes sheaths for the tendons of the tensor tympani and stapedius muscles, and for the chorda tympani nerve. Two folds extend downwards from the roof of the attic or epitympanic recess, one in front of, and the other behind, the superior ligament of the malleus. The former is connected with the head of the malleus, and the latter (sometimes described as the superior ligament of the incus) with the incus.

Attic or Epitympanic Recess, and its Pouches.-The part of the tympanic cavity which lies above the level of the upper margin of the membrana tympani is called the attic or epitympanic recess, as distinguished from the atrium or tympanum proper. It contains the head and neck of the malleus, and the body and short process of the incus. These divide it incompletely into two compartments-outer and inner. The outer attic is subdivided into two pouches-superior and inferior. The superior pouch is partially separated from the inner attic by the two mucous folds which have been already referred to as descending from the roof 
of the attic in front of and behind the superior ligament of the malleus. The inferior pouch of the outer attic is known as the pouch of Prussak. It is bounded externally by the membrana flaccida, superiorly by the external ligament of the malleus, which partially separates it from the superior pouch, and inferiorly by the short process of the malleus. The pouch communicates posteriorly with the tympanic cavity by an opening which is situated a little above the level of the bottom of the pouch. If fluid therefore should accumulate in Prussak's pouch, it may readily lead to perforation of the membrana fiaccida or Shrapnell's membrane.

Two other pouches are present, namely, the anterior and posterior pouches of Troltsch. These lie one in front of, and the other behind; the handle of the malleus, and are produced by the fold of mucous membrane which invests the chorda tympani nerve.

The tympanic mucous membrane is covered, for the most part, by columnar ciliated epithelium, except over the ossicles and membrana tympani, where the epithelium consists of a single layer of squamous, non-ciliated cells.

The tympanic or mastoid antrum is supplementary to the tympanum proper, or cavum tympani, behind which it is situated. It communicates by a large irregular opening with the attic, and is lined with mucous membrane, which is continuous with that of the attic and cavum tympani. Opening from the antrum there are the mastoid cells, which are lined with mucous membrane, continuous with that of the antrum.

The average measurements of the antrum are as follows: vertical, about 9 millimetres; antero-posterior, about II millimetres; and transverse, about 8 millimetres. The roof is formed by the thin tegmen tympani, which enters into the formation of the middle fossa of the base of the skull, and is consequently related to the temporal lobe of the cerebrum and its meninges. The genu and descending limb of the sigmoid sinus lie behind the antrum, and a little farther back is the cerebellum. The facial nerve, as it traverses the descending part of the aqueduct of Fallopius, lies in the posterior wall of the cavum tympani, close to the inner wall and in front of the mastoid antrum. This part of the nerve is on a plane anterior to the mastoid process, and is nearly flush with the opening of the antrum. The ampulla of the external semicircular canal of the internal ear gives rise to a slight eminence on the inner wall at its anterior part.

The outer wall corresponds with the area of the suprameatal triangle of Macewen, and is formed by the postmeatal plate of the squamous portion of the temporal bone. For a description of this triangle, which is the region selected for mastoidectomy, and also for the mastoid cells, see the description of the Temporal Bone. 


\section{Summary of Important Structures closely related to the Mastoid Antrum.}

I. Temporal lobe of cerebrum and its meninges (roof).

2. Genu and descending limb of sigmold sinus, and farther back the cerebellum (posterior wall).

3. Facial nerve (posterior wall of cavum tympani, close to inner wall, and nearly flush with antral opening).

4. External semicircular canal (anterior part of inner wall).

The upper part of the antrum communicates, as stated, with the attic of the cavum tympani, but the lower part is shut off by bone from the cavity of the cavum tympani.

The mucous membrane of the mastoid antrum is continuous with that which lines the mastoid cells. Anteriorly it is also continuous, as stated, with the mucous membrane of the attic and cavum tympani or tympanum proper. The mucous membrane of the cavum tympani is continuous anteriorly with that of the Eustachian tube, and the mucous membrane of the Eustachian tube is continuous with that of the naso-pharynx. This extensive and continuous tract of mucous membrane is covered by columnar ciliated epithelium, except in the following regions: (I) the promontory; (2) the tympanic ossicles; (3) the membrani tympani; (4) the mastoid antrum; and (5) the mastoid cells. In these regions the epithelium consists of a single layer of squamous non-ciliated cells.

It is of considerable importance to note that micro-organisms may pass from the naso-pharynx through the Eustachian tube into the cavum tympani and attic, and thence into the mastoid antrum and mastoid cells. Purulent affections of these regions may therefore readily be caused in this manner. Such affections may subsequently involve (I) the temporal lobe of the cerebrum and its meninges, (2) the genu and descending limb of the sigmoid sinus, and $(3)$ the internal ear or labyrinth.

Arteries of the Tympanum.-The principal arteries are two in number, namely, ( $\mathrm{I}$ ) the tympanic branch of the internal maxillary, and (2) the stylomastoid branch of the posterior auricular. The tympanic artery enters through the fissure of Glaser, and supplies the membrana tympani and front part of the tympanum. The stylo-mastoid artery enters the aqueduct of Fallopius through the stylo-mastoid foramen, and passes from the descending part of the aqueduct into the tympanum. It supplies the back part of the cavity and the mastoid cells, and it forms, with the tympanic artery, a ring round the circumference of the membrana tympani.

In addition to the foregoing two arteries, the following three arteries enter the tympanic cavity: (1) the petrosal branch of the middle meningeal, which -enters from the aqueduct of Fallopius, into which it'passes through the hiatus Fallopii; (2) the tympanic branch of the ascending pharyngeal, which accompanies Jacobson's nerve through the tympanic canaliculus; and (3) the tympanic branch of the internal carotid, which enters by a minute foramen on the posterior wall of the ascending part of the carotid canal, in company with a sympathetic twig from the carotid plexus.

The veins of the tympanum pass to the pterygoid plexus, the superior petrosal sinus, the internal jugular vein, and the pharyngeal plexus. 
Nerves of the Tympanum- $\mathrm{r}$. Tympanic Plexus.-This plexus is situated on the inner wall of the tympanic cavity, more particularly over the promontory. It is formed by (a) the tympanic branch (Jacobson's nerve) of the petrous ganglion of the glosso-pharyngeal, and $(b)$ the carotico-tympanic branch of the carotid plexus of the sympathetic.

Branches.-These are distributed to the mucous membrane of the tympanum, Eustachian tube, and mastoid cells. Besides these, there are two named branches, called the small superficial petrosal and the small deep petrosal. The small superficial petrosal nerve is to be regarded as the continuation of Jacobson's nerve. After issuing from the tympanic plexus it leaves the tympanum at the upper part of the anterior wall, and traverses a small canal in the petrous part of the temporal bone; passing beneath the upper portion of the canal which lodges the tensor tympani muscle. Whilst in this canal it is joined by a small branch from the geniculate ganglion of the facial nerve, and it emerges from the canal through the accessory hiatus on the superior surface of the pars petrosa. It then passes forwards and inwards beneath the dura mater, and leaves the cranial cavity through the canaliculus innorinatus, or through the fissure between the pars petrosa and the great wing of the sphenoid, or through the foramen ovale. Having entered the zygomatic fossa, it joins the otic ganglion, to which it conducts glossopharyngeal fibres.

The small deep petrosal nerve passes forwards through a minute canal within the processus cochleariformis to the upper aspect of the foramen lacerum medium, where it joins the carotid plexus of the sympathetic and the great deep petrosal, or, it may be, the great superficial petrosal, nerve. In this manner some of its fibres pass in the Vidian nerve to Meckel's ganglion.

2. Chorda Tympani Nerve.-This nerve arises from the facial nerve in the lower portion of the descending part of the aqueduct of Fallopius. Having ascended for a little in the aqueduct, it leaves it by passing forwards through a minute canal, called the iter chordae posterius, which opens on the posterior wall of the tympanum, below the level of the pyramid. The nerve, invested by a fold of mucous membrane, now passes forwards internal to the membrana tympani, and over the upper part of the handle of the malleus. It then leaves the tympanic cavity through a passage situated at the inner end of the fissure of Glaser; called the iter chorde anterius, or canal of Huguier, after which it joins the lingual nerve.

\section{Internal Ear.}

The internal ear is the essential part of the organ of hearing, and is known as the labyrinth from its remarkable complexity. It consists of two parts, namely, the osseous labyrinth and the membranous labyrinth.

\section{Osseous Labyrinth.}

The osseous labyrinth is a cavity situated within the petrous part of the temporal bone, and it is divided into three parts, namely, the vestibule, the semicircular canals, and the cochlea. These divisions are lined with a delicate periosteum, between which and the contained membranous labyrinth there is a clear fluid, called the perilymph.

Vestibule.-The vestibule is the central division of the osseous labyrinth. The semicircular canals lie behind it, and the cochlea is situated in front of it. The outer wall presents the fenestra ovalis, which is occupied by the foot-piece of the stapes and its annular 
of the superior and external semicircular canals. Below the fovea elliptica is the opening of the aqueductus vestibuli, which leads to the posterior surface of the petrous part of the temporal bone, about $\frac{1}{4}$ inch external to the orifice of the meatus auditorius internus. It transmits the ductus endolymphaticus and a minute vein.

Anteriorly the vestibule communicates with the scala vestibuli of the cochlea, and posteriorly it presents the five openings of the semicircular canals.

Semicircular Canals. - The osseous semicircular canals are situated behind the vestibule. They are three in number-superior, posterior, and external-and they open into the vestibule by five circular apertures, the contiguous ends of the superior and posterior canals having a common orifice. Each canal forms about twothirds of a circle, and each presents at one end an enlargement, called the ampulla. The superior semicircular canal occupies a vertical position, and lies transversely as regards the long axis of the petrous part of the temporal bone, giving rise to the eminentia arcuata on its superior surface. Its ampullary end (antero-external) opens independently into the upper part of the vestibule, above the ampullary orifice of the external canal. Its non-ampullary end unites with the non-ampullary end of the posterior canal, and the two open by a common orifice into the vestibule. The posterior semicircular canal arches backwards towards the posterior surface of the pars petrosa, with which it is almost parallel, and, like the superior, it occupies a vertical position. Its ampullary end (inferior) opens independently into the lower and back part of the vestibule, and its non-ampullary end, as just stated, joins that of the superior canal. The external semicircular canal arches outwards, and occupies a horizontal position. Its extremities are independent of those of the other two canals, and they open by separate apertures in to the upper and back part of the vestibule. Its ampullary end is external.

Cochlea.-The osseous cochlea is situated in front of the vestibule. It consists of a tube coiled spirally upon itself, like a snail's shell, and is of conical outline. Its base is opposite the lamina cribrosa at the deep end of the meatus auditorius internus; and its apex, known as the cupola, is directed outwards and slightly forwards towards the canal which contains the tensor tympani muscle. Its length from base to apex is about $\frac{t}{5}$ inch. It consists of $(I)$ a winding tube, called the spiral canal of the cochlea; (2) a central pillar, called the modiolus, round which the spiral canal turns; and (3) a thin plate of bone, called the lamina spiralis, which winds spirally round the modiolus and projects into the spiral canal of the cochlea.

The spiral canal of the cochlea (cochlear canal or tube) winds round the modiolus, which forms its inner wall. It describes two and three-quarter turns, and its basal turn or coil gives rise to the' promontory on the inner wall of the tympanum proper. At the. 
cupola it ends in a blind extremity. It gradually diminishes in size from base to cupola; its length is about 32 millimetres; and its diameter is about 2 millimetres at the base, where it is greatest.

The modiolus is the central pillar round which the spiral canal of the cochlea turns, and it forms the inner wall of that canal. It commences at the cochlear area of the lamina cribrosa at the deep end of the internal auditory meatus, and extends almost to the cupola, gradually tapering. It is traversed by minute canals for branches of the cochlear division of the auditory nerve. One of these canals occupies the centre of the modiolus, and is called the central canal of the modiolus. This canal commences at the foramen centrale of the cochlear area of the lamina cribrosa, and it transmits the nerve-filaments for the apical coil. The other canals, which have no special name, commence at the tractus spiralis

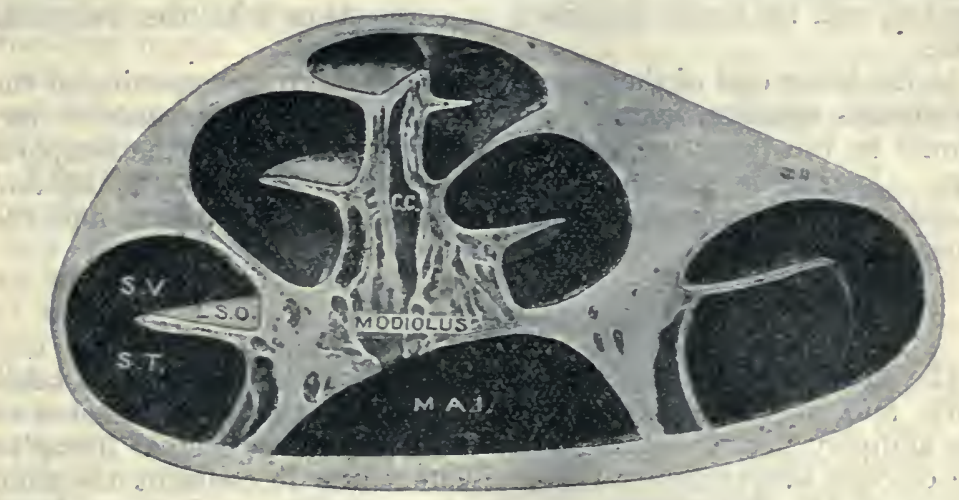

Fig. 650.-Miedian Section of The Left Osseous Cochlea of MaN FROM APEX TO BASE (ARNOLD).

S.V. Scala Vestibuli

S.T. Scala Tympani II.A.1. Meatus Auditorius Internus
L.S.O. Lamina Spiralis Ossea C.C. Central Canal of Modiolus

foraminosus of the cochlear area of the lamina cribros 3 , and they transmit the nerve-filaments for the other coils-middle and basal. At successive levels these canals change their direction, and pass outwards to the attached margin of the lamina spiralis, to be presently described. . Here they coalesce and form a winding canal, called the spiral canal of the modiolus, which lodges the spiral ganglion or ganglion of Corti. From this canal secondary canals for nerve-filaments pass into the lamina spiralis.

The lamina spiralis ossea is a thin plate of bone, which winds spirally round the modiolus, to which it is attached. It projects from the modiolus into the spiral canal of the cochlea throughout the windings of the latter, and it extends for about half-way towards the outer wall of the cochlear canal. It divides that canal incompletely into two passages or scale-an upper, or scala vestibuli, 
and a lower, or scala tympani, the commencement of which is at the fenestra rotunda. Close to the cupola the lamina spiralis terminates in a hook-like process, called the hamulus. The spiral lamina consists of two plates of bone, between which there are canals for nerve-filaments, these canals being offsets of the spiral canal of the modiolus, which, as stated, contains the spiral ganglion or ganglion of Corti. They extend to the free margin of the spiral lamina. The free margin of the lamina spiralis is, in the recent state, attached to the outer wall of the spiral canal of the cochlea by means of the basilar membrane, and the scala vestibuli and scala tympani are now completely separated, except in the region of the hamulus, where they communicate through an opening, called the helicotrema.

The lamina cribrosa, at the deep end of the internal auditory meatus, will be found described in connection with the Temporal Bone.

At the lower end of the scala tympani is the upper opening of the aqueductus cochleæ, which passes downwards and inwards to terminate on the posterior border of the petrous part of the temporal bone. It transmits a small vein to the inferior petrosal sinus, and establishes a communication between the scala tympani and the subarachnoid space.

\section{Membranous Labyrinth.}

The membranous labyrinth is situated within the osseous labyrinth, and its constituent parts receive the terminal branches of the auditory nerve. It is separated from the periosteal lining of the osseous labyrinth by the perilymph, and it contains the fluid known as the endolymph. In the case of the vestibule and the osseous semicircular canals the membranous labyrinth corresponds, more or less, with them; but in the case of the osseous cochlea it forms part of the septum between the scala tympani and scala vestibuli, and contains a passage called the membranous canal of the cochlea.

Vestibular Part of the Membranous Labyrinth.-The vestibule contains two membranous sacs, namely, the utricle and the saccule, which are in close contact; but do not communicate with each other directly. These sacs contain endolymph.

The utricle is the larger of the two sacs, and into it the membranous semicircular canals open. It occupies the upper and back part of the vestibule, a portion of it, known as the recessus utriculi, lying in the fovea elliptica. 'In the vicinity of the crista vestibuli the wall of this recess receives fibres of the auditory nerve and is thickened, this portion of it being called the macula acustica utriculi. From the anterior and inner part of the utricle a minute canal, called the ductus utriculi (ductus utriculo-saccularis), passes to join the ductus sacculi, and so form the ductus endolymphaticus. 
The saccule, which is somewhat oval, lies in front of the utricle, and occupies the fovea spherica, where it is contiguous to the opening leading into the scala vestibuli of the cochlea. Through the openings of the fovea spherica it receives filaments of the auditory nerve, and this portion of the saccule being thickened is known as the macula acustica sacculi. Inferiorly the saccule is connected with a small canal, called the canalis or ductus reuniens of Hensen, which opens into the canal of the cochlea, or ductus cochlearis, not far from its closed vestibular end. From the posterior part of the saccule a minute canal, called the ductus sacculi, passes off, which is soon joined by the ductus utriculi, and so the ductus endolymphaticus is formed. This latter duct traverses the aqueductus vestibuli, and, having reached the posterior surface of the petrous part of the temporal bone, it ends in a small blind dilatation, called the sacculus endolymphaticus, which lies beneath the dura mater. The saccule and utricle are thus indirectly connected by means of the ductus sacculus and ductus utriculus; and the saccule communicates with the ductus cochlearis by means of the canalis or ductus reuniens of Hensen.

Semicircular Canals. - The membranous semicircular canals correspond in outline to the osseous semicircular canals, within which they lie; they form about two-thirds of a circle; and each presents an ampulla at one extremity, which is situated within the ampulla of the osseous canal. They are elliptical in transverse section, and open into the utricle by five orifices, the non-ampullary ends of the superior and posterior canals being united, so that these two ends open by a common orifice. The convex wall of each canal is attached to the periosteal lining of the osseous canal, whilst the concave wall is practically free from the osseous wall, and is bathed by the perilymph. These canals contain endolymph.

Structure.-The walls of the utricle, saccule, and membranous semicircular canals consist of three layers, namely, (I) an outer or fibrous layer, which is vascular ; (2) a middle layer, or membrana propria, which is translucent ; and (3) an inner or epithelial layer. In each ampulla the middle layer, or membrana propria, projects in to the cavity of the canal from the peripheral wall, this projecting part being known as the septum transversum. It partially divides the interior of the ampulla into two compartments, and its free margin, which is covered by the auditory epithelium, is called the crista acustica. The epithelial layer consists of a single stratum of squamous cells, except in those regions to which the filaments of the auditory nerve are distributed. These regions are as follow:s : (I) the macula acustica utriculi ; $(2)$ the macula acustica sacculi ; and (3) the crista acustica of each ampulla.

The macula acustica utriculi is the thickened part of the anteroinferior wall of the recessus utriculi, and is lined with auditory epithelium. The macula is covered by calcareous particles, called otoliths or otoconia, which consist of crystals of calcium carbonate. The macula acustica sacculi is the thickened part of the anterior 
wall of the saccule, and is also lined with auditory epithelium, covered by otoliths. The crista acustica, as stated, is the free margin of the septum transversum in each ampulla, and is covered by auditory epithelium.

The auditory epithelium is of the columnar variety, and consists of two kinds of cells, auditory and sustentacular. The auditory cells are nucleated, and each is provided at its free extremity with a slender, tapering, hair-like filament, which projects into the cavity. These filaments are sometimes spoken of collectively as auditory hairs, and the cells are hence called hair-cells. : Their deep extremities fall short of the membrana propria. The sustentacular cells lie between the hair-cells, and are elongated and nucleated. Their deep extremities are attached to the membrana propria, and their free extremities give rise to a kind of limiting membrane. The auditory nerve-fibres pierce the membrana propria, and, having

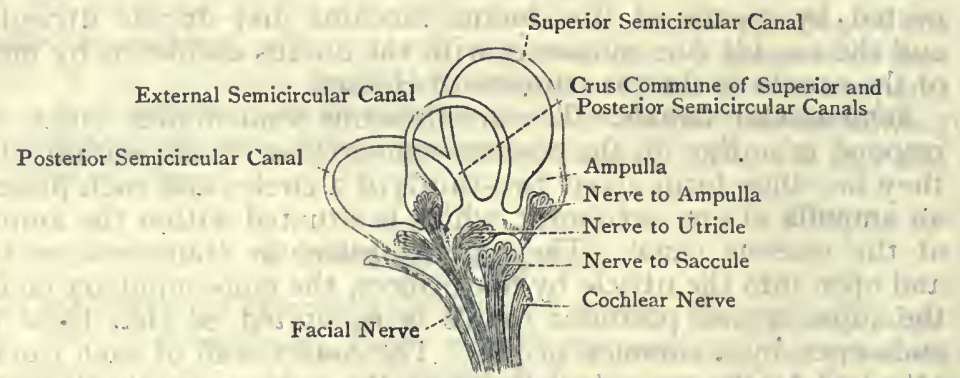

Fig. 651.-The Membranous Semicircular Canals, showing the Distribution OF THE BRANCHES OF THE AUditory NERVE TO THEIR Ampulla: (BREschet).

lost their medullary sheaths, the axons terminate in arborizations around the deep ends of the auditory or hair cells.

Membranous Cochlea. - The membranous cochlea is situated within the osseous cochlea, and completes the deficiency which is left by the lamina spiralis. It consists of two membranes, namely, the basilar membrane and the membrane of Reissner, which enclose between them the ductus cochlearis, or scala media. The osseous cochlea in the recent state is therefore divided into three spiral passages, namely, (I) the scala tympani, (2) the scala vestibuli, and (3) the scala media. The scala vestibuli is continuous with the scala tympani at the cupola through means of an aperture, called the helicotrema; and at the base of the cochlea it opens upon the anterior wall of the vestibule. The scala tympani commences at the fenestra rotunda, and in the recent state is separated from the tympanic cavity by the secondary membrane of the tympanum. The scala media, or ductus cochlearis, communicates, near its lower end, with the saccule by means of the canalis or ductus reuniens of Hensen. The scala vestibuli and scala tympani contain peri- 
lymph, which is continuous with the perilymph of the restibule and osseous semicircular canals. The scala media contains endolymph, which is continuous with that of the saccule.

Basilar Membrane.-The basilar membrane extends from the free margin of the lamina spiralis to the crista basilaris, or lower

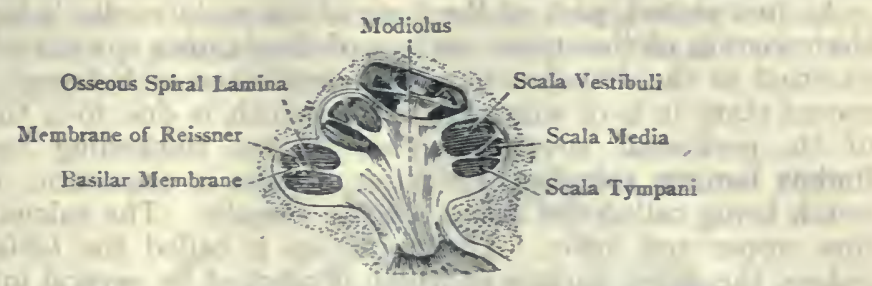

Fig. 652.-Vertical Section of the Cochlea of a Fotal Calf, showing the Scale and Modiolus (Kolliker).

part of the spiral ligament, which ligament is a thickening of the periosteum of that part of the outer wall of the cochlea which forms the outer wall of the scala media, or ductus cochlearis. It separates the scala media from the scala tympani, and is divisible into two zones, inner and outer. The inner zone is called the zona arcuata, and it supports the organ of Corti. The outer zone is called the zona pectinata, and it extends from the foot-plates of the outer rods of Corti to the crista basilaris. The basilar membrane consists of a homogeneous membrana propria, with fibres embedded in it, the fibres being most numerous in the zona pectinata.

Membrane of Reissner.--This is a delicate membrane which extends from the upper surface of the lamina spiralis, a short distance from its free margin, to the outer wall of the cochlea, where it is attached to the periosteum at a point a short distance above the outer attachment of the basilar membrane. It separates the scala media from the scala vestibuli, and consists of a very delicate connective-tissue membrane, lined on each side with a single layer of squamous epithelium.

The scala media, or ductus cochlearis, is situated between the basilar membrane and the membrane of Reissner. It is triangular in transverse section, and presents a roof, an outer wall, and a floor. The roof is formed by the membrane of Reissner, wbich, as just stated, separates the scala media from the scala vestibuli. The outer wall is formed by the outer wall of the cochlea and its periosteum, between the external attachments of the basilar membrane and the membrane of Reissner. The periosteum in this region is much thickened, and forms the spiral ligament of the cochlea, the lower part of which gives rise to the crista basilaris. The floor is formed by (I) the basilar membrane, and (2) the portion of the upper surface of the lamina spiralis which lies external to the attachment of the membrane of Reissner. As stated, the basilar membrane separates the scala media from the scala 
tympani. The scala media terminates above in a closed extremity at the cupola, and it has a similar mode of termination at the base of the cochlea. Near its lower blind extremity it receives the canalis or ductus reuniens of Hensen, by which it communicates. with the saccule.

As just stated, part of the floor of the scala media is formed by that portion of the upper surface of the lamina spiralis which lies. external to the attachment of the membrane of Reissner. In the recent state it is of some thickness, which is due to a thickening. of its periosteal covering. This fibrous thickening forms the limbus laminæ spiralis. Its outer margin is crescentic, the deep notch being called the sulcus lamina spiralis. The sulcus has two. lips, upper and lower. The upper lip is called the labium vestibulare, the upper surface of which is marked by several interlacing. prominences and grooves. At the free margin of this labium the prominences assume the form of tooth-like projections, which are known as the auditory teeth. The lower lip of the sulcus is called the labium tympanicum. It is continued into the basilar membrane, and is perforated by a great number of apertures for the branches of the cochlear division of the auditory nerve.

Organ of Corti.-Over the upper surface of the inner part (zona. arcuata) of the basilar membrane the epithelium undergoes remarkable modification, and gives rise to the organ of Corti. The constituent parts of this very complicated organ are as follows :

I. The rods of Corti.

2. The auditory or hair-cells.

3. The cells of Deiters.
4. The cells of Hensen and of Claudius.

5. The lamina reticularis.

6. The membrana tectoria.

The rods of Corti are arranged in two rows, inner and outer. The rods of the inner row are spoken of as the inner rods, and those of the outer row as the outer rods. Each rod consists of a foot-plateor base, an intermediate portion, and a head, which latter represents the upper extremity. The foot-plate, which is expanded, rests upon the zona arcuata of the basilar membrane, and the foot-plates of the inner rods are separated from those of the outer rods by a slight interval. As the rods rise the intermediate portions of the inner and outer rods incline towards each other, and the heads of the two sets of rods come into contact. In this manner a triangular tunnel is enclosed between the two sets of rods and the basilar membrane, which is called the tunnel of Corti. This tunnel extends along the entire length of the scala media, or ductus cochlearis.

The inner and outer rods present certain differences.

The inner rods are more numerous than the outer, there being from 5,000 to 6,000 of the former, and about 4,000 of the latter. They incline upwards and outwards. The head of each is directed outwards and presents a concavity, above and below which there is a projecting portion, so that it resembles, somewhat, the upper extremity of the ulna, with its great sigmoid cavity and olecranon and coronoid processes. The concavity on the head of 
the inner rod receives the round portion of the head of the outer rod. Finally, the inner rods are shorter than the outer rods. The outer rods are less numerous and longer than the inner, and they incline upwards and inwards. The bead of each is divisible into two parts -inner and outer. The inner part is round, and is received into the concavity on the outer aspect of the head of the inner rod. The outer part is prolonged into a beak-like projection, called the phalangeal process, which forms part of the lamina reticularis, to be presently described.

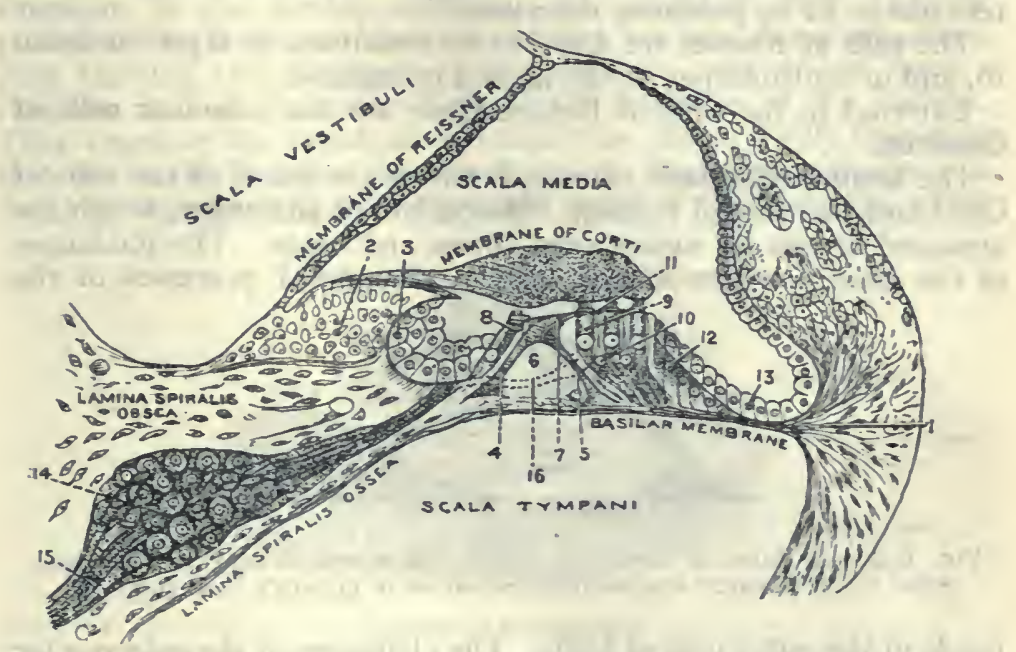

Fig. 653.-The Organ of Corti (Wiedersheim, after Lavdowsky).
I. Spiral Ligament
2. Limbus
3. Sulcus Spiralis
4. Inner Rod of Corti
5. Outer Rod of Corti
6. Tunnel of Corti
7. Phalangeal Process of Outer Rod
8. Inner Hair-cells

\author{
9. Outer Hair-cells \\ I0. Cells of Deiters \\ II. Lamina Reticularis \\ 12. Cells of Hensen \\ 33. Cells of Claudius \\ I4. Spiral Ganglion \\ 15. Cochlear Nerve \\ 16. Nerve-fibres to Hair-cells
}

The auditory or hair-cells are arranged in two sets-inner and outer. The inner hair-cells are situated internal to the row of inner rods, and are disposed in a single row. They are from 3,000 to 4,000 in number. Their free extremities, which lie close to the heads of the inner rods, are each provided with a tuft of short, hair-like filaments. The deep, nucleated ends of the cells are related to the terminal arborizations of nerve-fibres. Internal to the row of inner hair-cells there are two or more rows of columnar cells, which are continuous with the columnar epithelium of the sulcus spiralis laminæ. The outer hair-cells are disposed in three or four rows external to the outer row of rods. They are much more numerous than the inner hair-cells. Their 
free extremities, like those of the inner cells, are each provided with a tuft of short, hair-like filaments, and their deep, nucleated ends are related to the terminal arborizations of nerve-fibres.

The cells of Deiters, which are sustentacular, are situated between the rows of outer hair-cells. Each cell is nucleated and contains a slender filament, known as the sustentacular flament or phalangeal process. This filament is attached by its base to the basilar membrane, and it is prolonged into the tapering upper end of the cell. It terminates in an expansion, which forms a phalanx of the lamina reticularis, to be presently described.

The cells of Hensen are disposed as a continuous layer, external. to, and in continuation of, the lamina reticularis.

External to the cells of Hensen there are the columnar cells of Claudius.

The lamina reticularis extends between the heads of the rods of Corti and the cells of Hensen. It consists of phalanges, which are arranged in two (or more) rows-inner and outer. The phalangesof the inner row are formed by the phalangeal processes of the

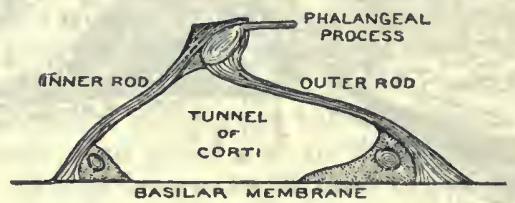

Fig. 654.-A Pair of Rods of Corti, from the Rabbit's Cochlea,

Side View (HIGHLy MAgNified) (Schäfer in Quain's 'ANATOMY.').

heads of the outer rods of Corti. The phalanges of the outer row (or rows) are formed by the phalangeal processes of the cells of Deiters. Between the phalanges there are openings through which the outer ends of the outer hair-cells, with their crescentic tufts of hair-like filaments, project.

The membrana tectoria, or membrane of Corti, which is elastic, is the most superficial structure in connection with the organ of Corti. It extends from the limbus laminæ spiralis near, and external to, the attachment of the membrane of Reissner, to the region of the outer hair-cells. It covers (I) the limbus laminæ spiralis; (2) the labium vestibulare, and the auditory teeth; (3) the sulcus laminæ spiralis; (4) the inner hair-cells; (5) the inner and outer rods of Corti; (6) the cells of Deiters; and (7) the lamina reticularis.

Auditory Nerve.- The auditory nerve, within the meatus auditorius internus, breaks up into two divisions - vestibular and cochlear.

The vestibular nerve, as it traverses the meatus auditorius internus, presents a gangliform enlargement, called the gangiion of Scarpa, and it divides into three branches. These enter the vestibule through the foramina in the superior vestibular area of the 
lamina cribrosa at the deep end of the internal meatus. They are. distributed to the macula acustica utriculi and to the cristæ acusticæ of the ampullæ of the superior and external semicircular canals.

The cochlear nerve, in the meatus auditorius internus, furnishes two branches-one to the macula acustica sacculi, and the other to the crista acustica of the ampulla of the posterior semicircular canal. The filaments of the former, which has a gangliform enlargement, pass through the foramina in the inferior vestibular area of the lamina cribrosa, and the latter passes through the foramen singulare in the lamina cribrosa. The cochlear nerve, having parted with these two branches, breaks up into filaments which pass through the foramina of the cochlear area of the lamina cribrosa, and so reach the base of the modiolus of the cochlea. They traverse the canals of the modiolus, from which they pass into the canals between the two layers of the lamina spiralis. In doing so they have to cross the spiral canal of the modiolus, which

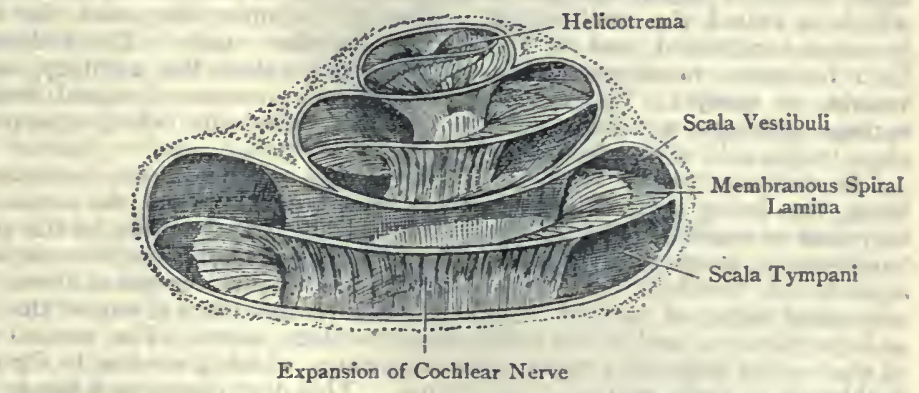

Fig. 655.-Section of the Cochlea, showing the Distribution of the Cochlear Branch of the Auditory Nerve (Magnified) (Hirschfeld AND LEVEILIÉ).

is situated close to the attached margin of the lamina spiralis. This canal contains a ganglion, called the spiral ganglion, which pursues the windings of the canal, and consists of bipolar nerve-cells. As the auditory fibres pass from the canals of the modiolus into the canals of the lamina spiralis, the course of each fibre is probably interrupted by a bipolar cell of the spiral ganglion in the spiral canal. The nerve-fibres, having emerged from these bipolar cells, traverse the canals between the two layers of the lamina spiralis. Having lost their medullary sheaths, they emerge through the foramina of the labium tympanicum on the outer margin of the limbus laminæ spiralis, and enter the basilary membrane, where they terminate in arborizations which are connected with the inner and outer hair-cells.

Blood-supply of the Labyrinth.-The labyrinth derives its blood from the internal auditory artery, which is a branch of the basilar artery. The vessel traverses the meatus auditorius internus, and 
divides at its deep end into two branches-vestibular and cochlear. The vestibular artery supplies the utricle, saccule, and semicircular canals, and the cochlear artery supplies the cochlea.

The veins of the labyrinth ultimately join, for the most part, to form one vessel, called the internal auditory vein, which opens into the inferior petrosal sinus. The aqueductus cochleæ and the aqueductus vestibuli each transmit a vein, that which passes through the former terminating in the inferior petrosal sinus or in the bulb of the internal jugular vein, and that which passes through the latter terminating in the superior petrosal sinus.

\section{Development of the Ear.}

Internal Ear-Membranous Labyrinth.-The membranous labyrinth is developed from the surface-ectoderm in a manner similar to the development of the crystalline lens. Over a circumscribed area, corresponding to the upper. end of the first visceral cleft, and upon the side of the hind-brain, the ectoderm becomes thickened and invaginated. A depression is thus formed. which is called the auditory pit. This pit becomes deepened, its mouth becomes constricted, and its lips, coming together, unite. The auditory pit then becomes transformed into a closed sac, called the auditory, or otic, vesicle, or otocyst. The auditory vesicle now becomes isolated from the surface-ectoderm, and sinks into the adjacent mesoderm, taking up a position close to the side of the hind-brain.

The auditory vesicle, which is at first almost spherical, soon becomes pyriform, this being due to the formation of a process, called the recess of the labyvinth or vestibule, which is prolonged from its dorsal wall. As this process lengthens it gives rise to the ductus endolymphaticus, which occupies the aqueductus vestibuli of the petrous portion of the temporal bone. The expanded terminal part of the ductus endolymphatious is called the saccus endolymphaticus, and it lies underneath the dura mater. The ventral aspect of the vesicle gives off a tapering diverticulum, which gives rise to the ductus cochlearis, or scala media, of the cochlea. This duct describes a bend, within which lies the cochlear ganglion of the cochlear division of the auditory nerve. As the duct elongates, it continues to bend in a spiral manner, and so the ductus cochlearis is formed. The cochlear ganglion elongates, and follows the spiral turns of the duct, from which circumstance the ganglion is known as the gangllon spirale.

The three semicircular canals are developed from tise upper or cephalic part of the auditory vesicle, this part representing the future utricle. This portion of the vesicle presents two folds-vertical and horizontal. From the vertical fold the superior and posterior semicircular canals are formed, whilst the horizontal fold gives rise to the external semicircular canal.

The auditory vesicle now becomes transversely constricted, and is divided into two sacs-large and small. The large sac is called the utricle, from which the semicircular canals have just been developed. The sinall sac is called the saccule, from which the ductus cochlearis has been developed. The portion of this duct which communicates with the saccule becomes constricted, and forms the canalis reuniens of Hensen.

The constriction which completely divides the auditory vesicle into utricle and saccule also involves the vesicular end of the ductus endolymphaticus, and divides it into two ducts, called the ductus utriculi and ductus sacculi. This arrangement constitutes the only communication which now exists between the utricle and the saccule.

As stated, the cochlear ganglion, or ganglion spirale, of the cochlear division of the auditory nerve lies within the spiral turns of the ductus cochlearis. As the osseous cochlea becomes formed, the ganglion spirale comes to occupy the spiral canal of the modiolus. The vestibular ganglion of the vestibular 
division of the auditory nerve lies in the internal auditory meatus after the completion of ossification.

The membranous labyrinth, hitherto considered, is entirely epithelial Certain of its epithelial cells undergo important specializations to fit them for sensorial purposes. These cells form six groups in definite regions. These groups are as follows: (1) The crista acustica ampullaris, of which there are three, one in the ampulla of each of the three epithelial semicircular canals; (4) the macula acustica utriculi; (5) the macula acustica sacculi; and (6) the organ of Corti, specialized from the epithelial ductus cochlearis. The groups connected with the ampullae of the semicircular canals, the utricle, and the saccule, receive their nerve-fibres from the vestibular ganglion of the vestibular division of the auditory nerve. The organ of Corti receives its nerve-fibres from. the cochlear ganglion, or ganglion spirale, of the cochlear division of the auditory nerve.

Osseous Labyrinth. - The membranous labyrinth is surrounded by mesodermic tissue, which becomes disposed in four layers. "These layers, from within outwards, may be-spoken of as (I) the connective-tissue layer, (2) the gelatinous layer, (3) the perichondrial layer, and (4) the cartilaginous layer. The connective-tissue layer forms the connective tissue of the varions parts of the epithelial labyrinth. The fibrous tissue of the gelatinous layer acquires vacuoles, and gives place to the various perilymphatic spaces, which contain a fluid called the perilymph. In the case of the cochlea the scala vestibuli and scala tympani alone contain perilymph, the scala media containing endolymph. The perichondrial layer forms the lining perichondrium of the periotic cartilaginous capsule, and subsequently becomes the lining periosteum of the osseous labyrinth. The cartilaginous layer forms the periotic cartilaginous capsule, which undergoes ossification, and gives rise, amongst other parts, to the osseous labyrinth, which is of larger size than the membranons labyrinth. The osseous semicircular canals conform in shape to the membranous semicircular canals, but are of larger size. The osseous vestibule differs from the membranous vestibule in being a single osseous case, whereas its contents are the utricle and the saccule.

Whilst the perilymph lies external to the membranous labyrinth, the interior of the semicircular canals, utricle, saccule, and scala media contain endolymph, the latter being in communication with the saccule by means of the canalis reuniens.

Middle Ear and Eustachian Tube.-The middle ear, or tympanum, and the Eustachian tube are developed from the entoderm of the upper end of the first visceral cleft, which is an evagination or diverticulum of the entoderm - of the pharyngeal portion of the alimentary tube. The entoderm of the upper end of the cleft is elongated dorsalwards and outwards, and its dorsal extremity, becoming expanded, forms the cavity of the middle ear, or tympanum, whilst the ventral portion of the entodermic diverticulum, remaining tubular, gives rise to the Eustachian tube, which necessarily opens from the pharynx. The entire entodermic diverticulum is invested by mesodermic tissue. The tissue surrounding the enlargement which forms the tympanım, as well as that surrounding the tympanic portion of the Eustachian tube, forms part of the periotic cartilaginous capsule, and when ossified, constitutes the osseous wall of the tympanum, and the osseous upper part of the Eustachian tube. The mesodermic tissue surrounding the lower part of the Eustachian tube gives rise to the eartilage which characterizes that portion of the tube.

Inasmuch as the tympanum is developed from the upper end of the first visceral cleft, it is intimately related to the upper ends of the first and second visceral arches. From the upper end of the skeletal bar of cartilage (Meckel's cartilage) of the first visceral, or mandibular, arch the malleus and the incus are developed; and from the upper end of the skeletal bar of cartilage (hyoid bar, or Reichert's cartilage) of the second visceral, or hyoid, arch the greater part of the incus is developed. These ossicles-namely, the malleus, incus, and stapes, constitute the tympanic ossicles of adult life.

These three ossicles, together with the chorda tympani nerve, all lie external 
to the epithelial wall of the primitive tympanic cavity. After the periotic cartilaginous capsule has undergone ossification and formed the osseous wall of the tympanum, the malleus, incus, stapes, and chorda tympani nerve lieembedded in the mesodermic tissue which intervenes between the epithelial or mucous roof of the membranous tympanum and its osseous roof. This. mesodermic tissue disappears, and the mucous (orginally epithelial) lining of the tympanum now comes into direct contact with the malleus, incus, stapes, and chorda tympani nerve, all of which it encloses within folds. Though these structures apparently lie within the tympanic cavity, this is not their actual position. They are really outside the cavity, inasmuch as they lie external to the mucous lining of the cavity. This may be illustrated by stating that the handle or manubrium of the malleus and the chorda tympani nerve do not lie in the tympanic cavity, but are placed between the middle, or fibrous layer and the internal, or mucous, layer of the membrana tympani.

External Ear.-The external ear consists of (I) the external auditory meatus, including the membrana tympani, and (2) the pinna.

The exiernal auditory meatus is developed from the upper part of the first external or ectodermic furrow, which corresponds, in position, to the first. internal visceral cleft.

The membrana tympani is developed from the closing membrane which separates the first internal visceral cleft from the first external ectodermic. furrow. This closing membrane is a trilaminar structure. Its inner layer consists of entoderm; its middle layer of mesoderm; and its outer layer of ectoderm. The membrana tympani, which is developed from it, is therefore trilaminar. The outer layer is ectodermic, and is continuous with the cuticular lining of the external auditory meatus; the middle layer is mesodermic, or fibrous; and the inner layer is entodermic, or mucous, and is continuous with the mucous lining of the tympanic cavity.

The component parts of the pinna are developed from six projections, consisting of mesoderm, covered by ectoderm. These appear on the upperends of the first and second visceral arches, where these bound the upper part of the first external ectodermic furrow, which gives rise to the external auditory meatus. The helix is developed from two of these tubercles, and each of the other four gives rise to the antihelix, tragus, antitragus, and lobule. The mesodermic tissue of the projections becomes differentiated into connective tissue and cartilage, and the ectoderm forms the integument. 


\section{GUIDE 'TO THE HEAD AND NECK.}

Back of the Scalp and Neck.-The landmarks having been studied, the skin is to be removed by making the following incisions : one is to be carried along: the median line from the level of the seventh cervical spinous process to the vertex of the skull, and this incision may be prolonged to the root of the nose ; a second is to be made from the upper part of the anricle, and a third from the acromial region, both of these meeting the first incision. On reflecting the flaps of skin thus marked out great care has to be exercised, as the skin and superficial fascia are very closely adherent. The cutting edge of the knifeshould be directed towards the skin, so as to score it, and in doing so the roots of the hairs will be severed. At the same time the dissector will note the strong fibrous prooesses which bind the superficial fascia to the skin.

The cutaneous nerves are to be displayed at once, and followed to their terminations. The great occipital nerve, with the occipital artery lying external to it, will be met with piercing the complexus, and, it may be, the trapezius, about $\frac{1}{2}$ inch from the median line, and the occipital venous plexus is to be kept in view. The least, or third, occipital nerve, very minute, will be found internal to the great occipital, and the small occipital nerve is to be found along the posterior border of the sterno-cleido-mastoid muscle. The great auricular nerve lies immediately below the small occipital, and turns upwards and forwards over the upper part of the sterno-cleido-mastoid towards the lobule of the auricle, where its mastoid, auricular, and facial branches are to be shown. The superficial or transverse cervical nerve can cnly be shown initially. It appears immediately below the great auricular, and at once passes horizontally forwards over the sterno-cleido-mastoid, where it must be left in the meantime. The spinal accessory nerve is to be caught where it appears from beneath the posterior border of the sternocleido-mastoid, about its centre and below the superficial cervical. It is to be followed downwards and ontwards across the posterior triangle to the anterior border of the trapezius, beneath which it disappears. Accompanying it, and lying on a lower level than it, an effort should be made to show the two branches which the anterior primary divisions of the third and fourth cervical nerves furnish to the trapezius. The descending superficial branches of the cervical plexus will be met with at the posterior border of the sternocleido-mastoid, immediately below the spinal accessory. If they appear as one trunk they will soon divide into suprasternal, supraclavicular, and supraacromial; but these nerves, in this position of the subject, can only be seen at their commencement.

The internal branches of the posterior primary divisions of the third, fourth, and fifth cervical nerves are to be displayed as they pierce the trapezius close to the median line, and they are to be followed outwards.

Behind the auricle, over the mastoid process, the mastoid glands are to be carefally looked for, and the occipital gland, or glands, will be met with over the cranial insertion of the trapezius, or, it may be, the complexus. Along the posterior border of the sterno-cleido-mastoid the glandulæ concatenatæ are to be looked for. 
The cervical part of the trapezius is to be dissected, and its anterior border clearly defined, with the spinal accessory nerve and branches of the third and fourth cervical nerves passing beneath it. The posterior portion of the upper part of the sterno-cleido-mastoid is also to be clearly defined. The interval between the trapezius and the sterno-cleido-mastoid, partially visible in this position of the subject, is the posterior triangle. Within it are to be partially dissected the following structures: a small angle of the complexus muscle pierced by the great occipital nerve, provided the trapezius is not well developed; a portion of the splenius muscle, flat and broad, and directed upwards and outwards ; and a portion of the levator anguli scapulæ.

The occipitalis muscle is next to be dissected, and the epicranial aponeurosis, upon which its fibres end, is to be displayed. The close connection between this aponeurosis and the superficial fascia is to be noted, whereas it is only loosely connected to the subjacent periosteum or pericranium. Behind the auricle the retrahens auriculam muscle is to be shown passing between the mastoid process and the convexity of the concha. Lying deeply in the hollow between the mastoid process and the auricle careful dissection will bring into view the posterior auricular nerve and artery, both of which are to be followed to their distribution. The posterior auricular vein is of importance, because, in conjunction with the posterior division of the temporo-maxillary vein, it forms the external jugular vein. Above the auricle the attollens auriculam muscle is to be shown, lying upon the temporal fascia. In front of the auricle, and continuous with the preceding muscle, will be found the attrahens auriculam. The latter two muscles will be found to receive twigs from the temporo-facial division of the facial nerve.

Frontal Region.-The dissection of the frontal region is next to be overtaken, the head being well raised upon a block. The frontalis muscle and front part of the epicranial aponeurosis are to be dissected. The supraorbital nerve, of large size, is to be carefully displayed. It will be found ascending in line with the junction of the inner third and outer two-thirds of the supra-orbital arch, in company with the supra-orbital artery, and it lies at first beneath the upper part of the orbicularis palpebrarum and the frontalis muscles. Its twigs to the upper eyelid are to be looked for, and its main branches are to be followed towards the vertex of the skull. The supratrochlear nerve, of small size, will be found internal to the supra-orbital, and in line with the inner canthus of the eye. It is accompanied by the frontal artery. The supra-orbital vein is to be shown passing downwards and inwards to join the frontal vein in the region of the inner canthus, the resultant vessel being the angular vein, which is the commencement of the facial vein. A communicating vessel is to be looked for passing from the supra-orbital vein to the supra-orbital notch, after traversing which it communicates with the superior ophthalmic vein within the orbit.

Temporal Region.- The attollens and attrahens auriculam muscles have been already referred to. Directly in front of the tragus of the auricle there will be found the auriculo-temporal nerve and the superficial temporal artery and vein. The nerve lies between the tragus and the artery, and its meatal, anterior auricular, and temporal branches are to be shown, the first two sets of branches requiring considerable care in their display. The temporal branches of the temporo-facial division of the facial nerve are to be kept in view at this stage. The superficial temporal artery, in two divisions, anterior and posterior, is to be dissected, and special attention is to be given to the tortuous anterior division, which is of some surgical importance. The temporal branch of the temporo-malar or orbital nerve (from the superior maxillary division of the fifth cranial nerve) will be met with piercing the temporal fascia about I inch above the anterior part of the zygoma, and close behind the marginal process on the temporal border of the malar bone. Its cutaneous distribution is somewhat limited.

The temporal fascia, or aponeurosis, is next to be dissected. In the region of the zygomatic arch it will be found to consist of two laminæ, between which, in addition to fat, will be found the orbital branch of the superficial temporal artery on its way to the outer part of the orbicularis palpebrarum 
muscle. In removing this fascia the dissector will note that its deep surface gives origin to the superficial fibres of the temporal muscle superiorly. The upper portion of the temporal muscle is then to be studied, but it cannot be followed at the present time beyond the level of the zygomatic arch.

At this stage the dissector, in association with his companion, may take the steps necessary for the removal of the brain, the subject being now assumed to have lain for two days face downwards. It may, however, be preferable to complete the dissection of the back of the neck.

The trapezius is to be divided by a vertical incision about I inch from the spines of the vertebræ, in association with the dissector of the upper limb. The spinal accessory nerve is then to be dissected along the deep surface of the muscle, in company with the superficial cervical artery, and the plexiform communication between the spinal accessory and the branches from the anterior primary divisions of the third and fourth cervical nerves is to be shown. The insertion of the sterno-cleido-mastoid is to be in great part detached and turned forwards towards the auricle. Assuming that the dissector of the upper limb has paved the way by dividing and reflecting the rhomboid muscles and the serratus posticus superior, the splenius muscle is then to be fully dissected in co-operation with the dissector of the upper limb. Its twofold condition at its insertion, namely, the splenius capitis and the splenius colli, is to be clearly brought out. The muscle is thereafter to be divided by a vertical incision about $\mathrm{I}$ inch from the spines of the vertebræ, a procedure which will enable the dissector to display more fully the twofold condition of the muscle at its insertion.

The trachelo-mastoid and complexus muscles fall next to be dissected, the former being very narrow and ribbon-like, whilst the latter is a large and powerful muscle, and is pierced by the great occipital nerve. Tendinous intersections are to be looked for in these muscles, one in each, and in the case of the complexus the condition of its mesial portion is to be carefully looked to. The dissector will note that this portion presents an intervening tendon, with a fleshy belly at either end, thus accounting for the name biventer cervicis, by which the inner portion of the complexus is known.

At this stage the second part of the occipital artery falls to be dissected. Its princeps cervicis branch (arteria princeps cervicis) is to be dissected very carefully, and preserved for immediate further dissection. The trachelomastoid is to be divided, and the relation of structures in the mastoid region from the surface downwards is to be carefully noted. The complexus is to be divided about 2 inches below its insertion, and on reflecting the muscle the dissector should follow downwards the arteria princeps cervicis. About the level of the spine of the axis he will find that it anastomoses with the deep cervical artery, which, he will note, ascends beneath the complexus. The subsequent and final stage of this dissection consists in displaying the structures of the infra-axial and supra-axial regions. Below the level of the spine of the axis the semispinalis colli muscle, as regards its fourfold insertion, is to be made clear. The origin of this muscle must be dissected in concert with the dissector of the upper limb. Ascending upon it there is, as stated, the deep cervical artery. Passing inwards upon it will be found the internal branches of the posterior primary divisions of the third, fourth, and fifth cervical spinal nerves, whilst those of the sixth, seventh, and eighth nerves pass inwards underneath the muscle. When the semispinalis colli is removed the upper part of the multifidus spinæ falls to be dissected. The interspinales muscles, along with the ligamentum nuchæ, are next to receive attention, and the tendinous slips of insertion of the cervicalis ascendens and transversalis cervicis into tubercles (posterior tubercles) of transverse processes are to be made evident. At this stage the dissector should familiarize himself with the relative position of muscles, proceeding from the tubercles (posterior tubercles) of cervical transverse processes, and passing backwards to the cervical spines.

Above the level of the spine of the axis four muscles and the suboccipital triangle, with its contents, require very careful dissection. The obliquus capitis inferior will be found extending obliquely outwards and upwards from 
the spine of the axis to the transverse process of the atlas, and the dissector will note that the great occipital nerve, preparatory to piercing the complexus, winds round the lower border of the inferior oblique in a backward direction. The obliquus capitis superior will be found extending upwards and inwards from the transverse process of the altas to the occipital bone between the outer parts of the superior and inferior curved lines. The rectus capitis posticus major is to be shown extending from the spine of the axis upwards and outwards to the outer part of the inferior curved line of the occipital bone and the subjacent area. The rectus capitis posticus minor is partially visible internal to the preceding muscle, but is also under cover of it. It will be recognised as a fan-shaped muscle, lying very deeply, and extending from the posterior arch of the atlas, close to the posterior tubercle, in a radiating manner upwards to the inner third of the inferior curved line of the occipital bone and the subjacent area. The suboccipital triangle will now be exposed, lying between the inferior oblique, superior oblique, and rectus capitis posticus major. Within this triangle the dissector, by exercising great care, should expose and study the following structures : ( 1 ) the posterior primary division (undivided) of the first cervical or suboccipital nerve, lying in close contact with the vertebrarterial groove of the atlas; (2) the third part of the vertebral artery; (3) the posterior occipito-atlantal ligament (one half), and one half of the posterior arch of the atlas. The suboccipital plexus of veins also occupies this triangle, from which the blood is conveyed away by the vertebral and deep cervical veins.

Up to this stage of the dissection it may be presumed that the period allowed for the subject to lie with its face downwards has expired.

Removal of the Brain.-The brain may be removed whilst the subject lies upon its face, but there is no objection to removing it whilst the subject lies upon its back. The scalp having been previously dissected, together with the temporal fascia and the origin of the temporal muscle, the cranium is to be cleared for the saw by carrying a scalpel around it, passing about I inch above the orbits, and about $\frac{1}{2}$ inch above the external occipital protuberance. The external table of the cranial bones is then to be sawn through, and thereafter the internal table is to be cracked by means of a chisel and mallet. The calvaria is then to be eased all round by inserting the chisel, and forcibly raised from the dura mater, which is adherent to it. The dura mater, being now exposed, is to be carefully sponged and studied in situ. The superior longitudinal sinus, which runs in an antero-posterior direction in the median line, is to be laid freely open, and its interior examined. On either side of this sinus the outlines of the Pacchionian bodies are to be noted, and the ramifications of the middle meningeal artery are to receive attention.

The dura mater is then to be divided on a level with the sawn margin of the skull, its mesial attachments in front and behind being left intact. On being raised towards the median line, the subdural space will be freely exposed, and the arachnoid membrane and pia mater are to be examined. The cerebral veins, as they pass to the superior longitudinal sinus, are to receive attention, and it should be noted that the direction of these veins is from behind forwards, so that the current of blood in thern is opposed to that in the superior longitudinal sinus. The cerebral hemisphere is now to be slightly pulled away from the median line in order to expose the falx cerebri. When this process has been studied, its attachment to the crista galli is to be severed, and the process is to be thrown backwards towards the tentorium cerebelli, after which the brain is to be removed.

Assuming the subject to be lying upon its face, the posterior cerebral lobes are to be raised, which will expose the tentorium cerebelli. This is to be divided near the superior border of the petrous part of the temporal bone, and for a short distance along the course of the lateral sinus on either side, but the sinus is not to be laid open. At the same time the fourth and fifth pairs of nerves are to be divided. Exactly in the median line the vena magna Galeni is to be cut as it is about to enter the straight sinus. The tentorium cerebelli, along with the falx cerebri, is then to be turned backwards, which will expose the cerebellum. The cerebellar hemispheres are next to be care- 
fully raised from the cerebellar fossæ of the occipital bone, and the spinal cord, vertebral arteries, and spinal accessory nerves are to be divided as low down in the spinal canal as possible.

The hypoglossal, pneumogastric, glosso-pharyngeal, auditory, facial, sixth and third nerves are next to be divided, as near as possible to where they leave the cranial cavity. The infundibulnm, in the median line, is to be cut, and anterior to it on either side the internal carotid artery is to be divided, along with the optic nerve, which lies directly in front of it. The brain is now to be entirely removed, care being taken to remove along with it the olfactory lobes.

Assuming the subject to be lying upon its back, the mode of procedure will be the reverse of the foregoing.

Before placing the brain in hardening fluid the base of the encephalon is to be carefully studied. Its component parts are to be observed as follows: the medulla oblongata, the pons Varolii, the cerebellum, and portions of the cerebral hemispheres. The crura cerebri will be seen diverging from each other above the pons; anteriorly the optic tracts will be seen to converge and form the optic commissure; and within the enclosed rhomboidal area. there will be seen the locus perforatus posticus, the corpora albicantia, the tuber cinereum, and a tag of the infundibulum. The stem of the fissure of Sylvius is to be noted, with the middle cerebral artery traversing it, and the locus perforatus anticus at its inner end. In front of the fissure is the orbital area of the frontal lobe of the cerebral hemisphere, and behind it the prominent temporal lobe. The arachnoid and pia mater are to be carefully examined. Over the gyri they will be found to be in close contact, but in certain situations intervals, called cisternæ, exist between them. These are as follows: (1) cisterna magna, between the cerebellam and the medulla oblongata; (2) cisterna pontis, on the ventral aspect of the pons Varolii, in which the basilar artery lies; and (3) cisterna basalis, over the interpeduncular region, in front of the pons, and containing the circle of Wvillis. The superficial origins of the cranial nerves, including the olfactory lobe, are to be accurately observed, as well as the arrangement of the arteries. The arteries should be studied in the following order: the two vertebrals, the basilar, the two posterior cerebrals, the trunk of each internal carotid, the two anterior cerebrals, joined by the anterior communicating, the middle cerebrals, and the two posterior communicating arteries. In connection with the foregoing arteries the circle of Willis is to receive carefnl attention. The anterior and posterior choroidal arteries, as well as the lenticulo-striate arteries, are to be kept carefully in view.

The arachnoid and pia mater are then to be removed as carefully as possible, in order to expose the gyri and sulci to the influence of the hardening fluid. In doing so a gentle stream of water may be advantageous, but the cranial nerves are not to be interfered with, though the arteries may now be taken away. Thereafter the brain, base upwards, is to be carefully packed in a crock containing hardening fluid, and laid aside for future dissection.

Cranial Cavity.- The venous sinuses, cranial nerves, and arteries at the base of the skuil are next to be dissected. The superior longitudinal sinus along the upper border of the falx cerebri has been already studied. Along the lower border of the falx cerebri the inferior longitudinal sinus, of small size, is to be followed backwards into the straight sinus, which latter is situated along the line of junction of the falx cerebri and tentorium cerebelli. The occipital sinns is to be shown within the falx cerebelli along the course of the internal occipital crest, as are also the inferior occipital or marginal sinuses on either side of the vermiform fossa. The torcular Herophili is to be laid open. It will usually be found upon the right side of the internal occipital protuberance. The lateral sinus on either side is to receive very careful attention, and in studying it the dissector should make himself familiar with the line, upon the exterior of the head, which indicates its winding course. This sinus should be laid open quite up to the postero-external compartment of the jugular foramen.

The cavernous sinus is then to be dissected with the greatest care, keeping 
in view the third, fourth, ophthalmic, superior maxillary, and sixth nerves, as well as the cavernous sympathetic plexus. Anteriorly the sinus will be found to receive its blood from the orbit through means of the ophthalmic vein or veins. It is connected with its fellow of the opposite side by two venous channels, namely, the anterior and posterior intercavernous sinuses (so-called circular sinus), lying in front of and behind the pituitary body. The cavernous. sinus is to be shown dividing posteriorly into the superior and inferior petrosal sinuses. The superior petrosal sinus is to be followed along the superior border of the pars petrosa to the point where it opens into the lateral sinus, and the inferior petrosal sinus is to be laid open between the pars petrosa and the basilar process of the occipital bone as far as the antero-internal compartment of the jugular foramen, through which it passes independently. The transverse or basilar sinus is represented by several veins arranged in a plexiform manner, and lying over the basilar process of the occipital bone. It forms a connection between the right and left inferior petrosal sinuses.

The cranial nerves are next to receive attention, as they make their exit. from the cranial cavity. The olfactory lobes have been removed along with. the brain, and the olfactory filaments traverse the foramina in the cribriform plate of the ethmoid bone. Lying upon each half of this plate there is the nasal nerve, accompanied by the anterior ethmoidal artery, both lying beneath the dura mater, and passing forwards and inwards towards the fore part of the side of the crista galli, where they disappear through the nasal slit. The optic nerve will be found at the optic foramen, where it has the internal carotid artery directly behind it. The third, fourth, ophthalmic, superior maxillary, and sixth nerves are to be met with in close relation to the cavernous. sinus. The first four of these nerves will be found in relation to the outer wall of the sinus, and the sixth nerve in close contact with the outer wall of the cavernous part of the internal carotid artery within the sinus. The dissection of the cavernous sympathetic plexus, in close relation to the artery, requires considerable care, and the artery itself, with its branches, is to beshown.

The Gasserian ganglion on the large sensory root of the fifth nerve is to be carefully displayed, the small motor root of the nerve lying underneath the ganglion. The locality of the ganglion is the Gasserian depression on the superior surface of the pars petrosa near the apex. From the ganglion the ophthalmic nerve is to be followed forwards to the outer wall of the cavernous. sinus, the superior maxillary nerve forwards to the foramen rotundum, and the inferior maxillary nerve, along with the motor root of the fifth nerve, downwards to the foramen ovale. At the entrance to the meatus auditorius. internus will be found the seventh and eighth nerves, with the internal auditory artery. At the jugular foramen will be found the ninth, tenth. and eleventh nerves, as well as the lateral and inferior petrosal sinuses, and the relative position of the structures at this foramen should receive careful attention. The twelfth nerve will be met with at the anterior condylar foramen, and it is to be noted that it pierces the dura mater in two bundles.

The various structures passing through the foramen magnum must be mastered.

The different meningeal arteries will be found fully described in the text. The sphenoidal fissure and the transmitted structures should be postponed until the dissection of the orbit is being overtaken.

The pituitary body is now to receive attention. It will be found in the pituitary fossa or sella turcica of the sphenoid bone, where it is largely covered by the fold of dura mater called the diaphragma sellæ. If in favourablecondition, it should be carefully removed, and studied according to the description given of it in the text.

The interior of the skull, having been carefully cleaned, is now to be protected by placing upon the interior of the base pieces of cotton-wool, soaked in preservative fluid. Thereafter the calvaria is to be replaced, or, if it is. not now available for use, the saturated cotton-wool is to be protected fron. evaporation by the usual means familiar to all.

Side of the Neck. - The subject being now placed with its face upwards, 
it is essential that the dissector should, in the first place, make himself thoroughly familiar with the landmarks of the side and front of the neck, particularly the latter in connection with goitre, laryngotomy, and tracheotomy.

The skin is to be removed by means of the following incisions : a mesial incision is to be carried from the chin to the upper border of the manubrium sterni; another from the lower end of the preceding outwards along the clavicle to the acromion process of the scapula; and a third from the chin along one half of the base of the inferior maxilla to the angle of that bone, and thence to the mastoid region. These incisions have to be made with great care, on account of the thinness of the skin and the important subcutaneous structures.

The muscular sheet, called the platysma myoides, requires very careful dissection, inasmuch as its fasciculi are very pale and delicate, but principally because these fasciculi are embedded in the superficial fascia. Inexperienced dissectors may be surprised when informed that they have removed the muscle in their haste to expose what lies nnderneath it. The muscle, after examination, is to be reflected from below upwards, and laid over the side of the face. This must be done with the greatest care. Thereafter the following veins are to be dissected, namely, the anterior jugular, near the median line of the neck; the external jugular, crossing the sternocleido-mastoid; and the posterior jugular, which is the most external occipital vein. The dissector must be prepared for finding these veins not quite normal. Along the course of the external jugular vein the superficial cervical glands are to be displayed. The nerves which fall to be dissected at this stage are as follows: (I) the great auricular, passing upwards and forwards over the sterno-cleido-mastoid towards the lobule of the auricle; (2) the superficial or transverse cervical, passing forwards over the sternocleido-mastoid, and beneath the external jugular vein; and (3) the suprasternal, supraclavicular, and supra-acromial, which three take a downward course. The inframandibular or cervical branch of the facial nerve must also be dissected.

Posterior Triangle.-The dissection of this region should be accomplished in the course of the first four days after the subject has been placed with its face upwards, in order that the dissectors of the neck and upper limb may work in concert. In his mode of procedure the dissector must keep carefully before him the disposition of the deep cervical fascia, which undoubtedly is of a very complicated and, it may be, perplexing nature. But, perhaps, if he masters the description given in the text as a preliminary, it may clear up matters. The boundaries of the posterior triangle are now to be clearly defined, though in the dissection of the back of the neck they would be apparent to a certain extent. The deep cervical fascia, as it extends from the anterior border of the trapezius to the posterior border of the sternocleido-mastoid, is to be studied, and its deep expansion to embrace the posterior belly of the omo-hyoid is to be kept in view. This belly is to be at once laid bare and studied. The dissector will note that it divides the posterior triangle into two, a superior or occipital triangle, and an inferior or subclavian (supraclavicular) triangle. The occipital triangle should receive attention first. The structures within it have been already referred to, whilst the subject lay upon its face, e.g., the superficial group of branches of the cervical plexus of nerves, the spinal accessory nerve, the branches from the third and fourth cervical nerves to the trapezius, the glands known as the glandulæ concatenatæ, and the following muscles in part: a small angle of the complexus, provided the trapezius is not well developed at its occipital origin; the splenius capitis; the levator anguli scapulæ; and the scalenus medius. If the posterior border of the sterno-cleido-mastoid be pulled forwards and maintained in that position by hooks, there is no objection to the dissection of the very complicated cervical plexus being partially made at this stage. The dissector, however, must first master the description which is given of the plexus in the text, from which he may see that the evolution of its branches is a complicated process. No hard-and-fast lines 
can therefore be laid down for the guidance of the dissector. $\mathrm{He}$ will certainly find the phrenic nerve descending upon the scalenus anticus muscle. Piercing the scalenus medius he should find the following nerves, in order from above downwards: (I) the nerve to the rhomboids; and (2) the upper two roots of the posterior thoracic nerve, or external respiratory nerve of Bell.

The subclavian or supraclavicular triangle now falls to be dissected. Its boundaries will by this time be self-evident, as well as the structures forming, and related to, its roof, in connection with which the supraclavicular glands are to be noted. The third part of the subclavian artery must be carefully dissected, as well as the nerve-trunks of the brachial plexus, all emerging from behind the scalenus anticus muscle. Possibly the posterior scapular artery may be found to arise from the third part of the subclavian. The subclavian vein will not be visible, inasmuch as it lies under cover of the clavicle; neither will the suprascapular vessels. The transverse cervical vessels, however, will be found within the triangle, where the posterior belly of the omo-hyoid is about to disappear beneath the posterior border of the sterno-cleido-mastoid. The relative positions of the upper, middle, and lower nerve-trunks of the brachial plexus to the third part of the subclavian artery are to be made perfectly clear. The formation of the entire plexus is to be made quite manifest. Arising from the front of the upper nervetrunk will be found the nerve to the subclavius muscle, which is of small size, and descends over the artery and behind the clavicle. The suprascapular nerve, of large size, is to be found arising from the back of the upper nervetrunk, and taking a course downwards and outwards.

At this stage the dissectors of the head and upper limb should work in concert, and the clavicle and subclavius muscle should be divided about their centre. After a certain amount of dissection an instructive view will be obtained of (I) the continuity between the third part of the subclavian artery and the first part of the axillary; (2) the subclavian vein; (3) the suprascapular vessels; (4) the cords of the brachial plexus; and (5) the infraclavicular branches of the plexus.

Anterior Triangle of the Neck. - The deep cervical fascia is to be dissected as far as possible, without going too deeply at the present stage. The suprasternal space, or space of Burns, is to be examined, and the portion of the deep cervical fascia which extends between the upper part of the anterior border of the sterno-cleido-mastoid and the angle of the inferior maxilla is to be noted. The sterno-cleido-mastoid muscle is to be completely dissected, and its complicated relations studied. The sterno-hyoid, sterno-thyroid, and the anterior belly of the omo-hyoid are also to be dissected, always keeping in view the disposition of the deep cervical fascia. In the dissection of these muscles the descendens cervicis (hypoglossi) nerve is to be exposed. It will be met with either within, or upon, the carotid sheath over the line of the common carotid artery, and in connection with it the loop known as the ansa cervicis (hypoglossi) is to be exposed. It is situated about the centre of the neck, and taking part in it there will be found, coming from behind, a nerve formed by the union of the two rami communicantes cervicis (hypoglossi) from the second and third cervical nerves. These two rami, however, may join the descendens cervicis separately, and before receiving them it parts with its branch to the anterior belly of the omo-hyoid. The loop itself is to be shown furnishing branches to the sterno-hyoid, sterno-thyroid, and posterior belly of the omo-hyoid. The thyro-hyoid muscle is also to be dissected, and the thyro-hyoid branch of the hypoglossal nerve is to be shown. entering the muscle beneath its posterior border. Passing beneath this border there will also be found the internal laryngeal nerve and superior laryngeal artery, which here pierce the thyro-hyoid membrane on their way to the interior of the larynx.

The structures along the median line of the neck are to be carefully disrected at this stage, in connection with the operations of laryngotomy and tracheotomy. In doing so the disposition of the deep cervical fascia must be constantly kept in view, and the following groups of lymphatic glands may as well be disposed of at once, namely, the submaxillary lymphatic 
glands, the suprahyoid or submental glands, and the prelaryngeal glands, the latter being met with in the vicinity of the cricoid cartilage, but being sometimes absent. The structures to be attended to along the median line of the neck are as follows, in order from above downwards: (1) the submental triangle; (2) the body of the hyoid bone, with the great cornu on either side; (3) the thyro-hyoid membrane; (4) the isthmus (pomum Adami) of the thyroid cartilage, above which is the well-marked V-shaped thyroid notch, whilst on either side of it is the expanded ala of the thyroid cartilage : (5) the crico-thyroid membrane, largely concealed on either side by the cricothyroid muscle, and crossed at its centre by the crico-thyroid arterial arch ; (6) the narrow anterior part of the cricoid cartilage; $(7)$ the trachea; $(8)$ the isthmus of the thyroid body, usually lying upon the second and third tracheal rings; and (9) the inferior thyroid plexus of veins, from which there pass downwards the right and left inferior thyroid veins. The superficial muscular relations of the trachea are to be carefully noted. The region concerned in laryngotomy is that occupied by the crico-thyroid membrane. The high operation of tracheotomy affects the trachea above the isthmus of the thyroid body, and the low operation is performed upon the trachea below the isthmus. In connection with the latter part of the trachea the dissector should carefully note the following points: (1) its gradually increasing depth: (2) the presence of the inferior thyroid plexus of veins; (3) the relation to it of the innominate and right common carotid arteries; and (4) the possible presence of an arteria thyroidea ima at or close to the median line.

The carotid sheath is next to be dissected, keeping in view that the descendens cervicis (hypoglossi) nerve may lie upon it, or be within it. The sheath will be found to be derived from the posterior lamina of the sheath of the sterno-cleido-mastoid muscle. It is to be carefully opened, having previously noted the sterno-cleido-mastoid artery, and its contents displayed, namely, the common carotid artery, the internal jugular vein, and the pneumogastric nerve, all in separate compartments. Possibly the descendens cervicis nerve may be within it. The contents of the sheath are to be fully dissected, bearing in mind that the gangliated cord of the sympathetic lies close behind the sheath. In dissecting the internal jugular vein the superior and middle thyroid veins are to be borne in mind as tributaries of it, and the deep cervical glands will be found more or less along its course. An effort should be made to expose the carotid body, which will be found behind the common carotid artery close to its bifurcation. On a level with the thyroid attachment of the thyro-hyoid muscle the external laryngeal nerve will be found, which is to be traced downwards to its distribution, chiefly to the crico-thyroid muscle.

The digastric muscle is next to be dissected, along with the stylo-hyoid muscle, which lies above its posterior belly, and the hypoglossal nerve, which lies along its lower border, and here gives off the descendens cervicis and thyro-hyoid nerves. In connection with the $\mathbf{V}$-shaped arrangement of the tendon of the digastric and the hypoglossal nerve, which has now passed beneath the digastric tendon, the triangle of Lesser is to be carefully studied. It contains a portion of the lingual artery, lying under cover of the hyo-glossus muscle. The three subdivisions of the anterior triangle of the neck are now to be reviewed, namely, the muscular (inferior carotid), carotid (superior carotid), and digastric or submaxillary triangles, and their contents noted. The first parts of the external and internal carotid arteries are to be dissected, the former as high as the point where it enters the parotid gland. The anterior branches of the external carotid are to be displayed, namely, the superior thyroid, lingual, and facial, in this order from below upwards. The superior thyroid artery is to be completely dissected, and the following branches of it are to be specially noted, namely, the crico-thyroid artery, and the artery of the isthmus of the thyroid body. The facial artery is only to be dissected as far as the base of the inferior maxilla. Its relation to the submaxillary gland is to be noted, and its cervical branches shown, namely, inferior palatine, tonsillar, glandular, and submental. The lingual artery is, meanwhile, only to be dissected at its origin, and in the first part of its course. 
During the present stage of the dissection the facial vein is to be kept in view ; its union with the anterior division of the temporo-maxillary vein to form the common facial vein is to be shown; and the ending of the common facial vein and of the lingual veins in the internal jugular are to be displayed.

The posterior branches of the external carotid, namely, the occipital and the posterior auricular, are also to be fully dissected, and as many of their branches as possible shown. The ascending pharyngeal branch of the external carotid artery is placed too deeply for complete dissection at this stage, but the dissector should bear in mind that it arises from the deep surface of the external carotid near its commencement, and he may expose it by separating the external and internal carotid arteries. The terminal branches of the external carotid, namely, the superficial temporal and the internal maxillary, will be dissected at a later stage in connection with the parotid gland.

At this stage the dissector should direct his attention to the thyroid body. This should be dissected with the greatest care, its lateral lobes and isthmus being clearly defined, together with their blood-supply, the inferior thyroid artery reaching the lateral lobe from below. The not infrequent presence of a pyramidal or middle lobe is to be borne in mind, and in connection with it the fibrous band and levator glandulæ thyroideæ muscle, which pass from its apex to the body of the hyoid bone. When present, it usually springs from the upper border of the isthmus close to the left lobe. The disposition of the thyroid veins is to be noted. The cervical parts of the trachea and œsophagus are also to be fully dissected. The manner in, and the extent to, which the œsophagus projects to the left of the trachea at the root of the neck is to be specially noted, and the inferior or recurrent laryngeal nerve and inferior laryngeal artery are to be carefully displayed as they ascend in the groove between the trachea and œsophagus.

Root of the Neck. - The deep dissection of the root of the neck is now to be overtaken. The scalene muscles-anticus, medius, and posticus-are to be dissected, and their relations are to be carefully noted, more especially those of the scalenus anticus, in connection with which the scalene tubercle or spine (Lisfranc's tubercle) on the inner border of the first rib is to be examined. The first part of the subclavian artery is to be dissected, and its relations mastered. The junction of the internal jugular and subclavian veins to form the innominate vein on either side is to be shown. Opening into the angle of junction of these two veins on the right side the short right lymphatic duct is to be looked for, and on the left side the thoracic duct, the latter arching outwards, forwards, and downwards. The left subclavian artery and the left common carotid at the root of the neck are to be contrasted with the corresponding vessels of the right side, and the differences noted, not only as regards their origins, but also as regards their relations. The right pneumogastric nerve is to be shown crossing the first part of the right subclavian artery, and parting with its recurrent laryngeal branch opposite the lower border of the vessel, that branch turning up behind the artery. The left pneumogastric nerve will be found parallel with the first part of the left subclavian artery, its recurrent laryngeal branch having been given off within the thorax, opposite the lower border of the arch of the aorta. The recurrent laryngeal nerve on either side is] then to be followed upwards in the groove between the trachea and cesophagus, in company with the inferior laryngeal artery. The sympathetic loop, known as the ansa Vieussenii, is to be shown in connection with the first part of the subclavian artery, and the sympathetic cord will be found behind it. The branches of this part of the vessel are now to be dissected. The vertebral artery is to be dissected in the first part of its course, and the inferior cervical ganglion of the sympathetic, which lies behind it, is to be kept in view. The mode of termination of the vertebral vein is to be shown. The internal mammary artery is to be followed downwards as far as its entrance upon its thoracic course, and the phrenic nerve is to be shown crossing it superficially from without inwards. The thyroid axis is next to receive attention, and its three branches, namely, inferior thyroid, transverse cervical, and suprascapular, 
are to be dissected. The tortuous course of the inferior thyroid artery inwards behind the lower part of the carotid sheath is to be noted. and its ascending cervical branch is to be shown. The proximity of the middle cervical ganglion of the sympathetic to the artery is to be borne in mind. The suprascapular artery is to be followed outwards, and its medullary branch to the clavicle is to be looked for. The transverse cervical artery, like the suprascapular, above which it lies, is to be followed outwards. The suprascapular and transverse cervical have been in part previously dissected.

The second part of the subclavian artery is to be exposed by dividing and reflecting the scalenus anticus muscle, behind which it lies, whilst the subclavian vein lies in front of that muscle. At this stage the mode of formation of the brachial plexus, and the relations of its nerve-trunks to the subclavian artery are to be revised. The only branch from the second part of the subclavian artery to be dissected is the superior intercostal artery, which, however, usually arises from the first part of the artery on the left side. To see this vessel on the right side it will be necessary to draw the second part of the subclavian artery well forwards and downwards, because the superior intercostal arises from its posterior and upper aspect. Thereafter the vessel descends in front of the neck of the first rib to supply the first and second intercostal spaces, but, before it does so, the deep cervical artery is to be shown arising from it, and taking a backward course between the seventh cervical transverse process and the neck of the first rib. In an earlier stage of the dissection this vessel has been seen ascending upon the semispinalis colli muscle, under cover of the complexus, and anastomosing with the ramus princeps cervicis of the occipital from the external carotid.

The apex of the lung, covered by the dome of the pleura, is to be very carefully noted, and attention is to be given to Sibson's fascia. This fascia will be found to extend from the front of the transverse process of the seventh cervical vertebra to the inner border of the first rib.

Suprahyoid Region.- The dissection of the digastric or submaxilary triangle is now to be overtaken in so far as it can be prior to section of the mandible. The superficial part of the submaxillary gland, of considerable bulk, will first receive attention. Passing downwards over it the dissector will find the facial vein, and more or less embedded in its upper and outer part he will find the facial artery on its way to the face. The superficial part of the gland is to be turned outwards, and kept out of the way by hooks. Thereafter the mylo-hyoid muscle is to be dissected, along with the mylo-hyoid nerve and submental artery resting upon it. The distribution of the mylohyoid nerve to the mylo-hyoid muscle and anterior belly of the digastric is to be clearly shown, and the median raphé between the two mylo-hyoid muscles is to be made evident. The posterior border of the mylo-hyoid muscle is next to receive attention. Passing beneath this border will be found, in order from below apwards, the hypoglossal nerve, the deep part of the submaxillary gland, and the lingual (gustatory) nerve. In the back part of the digastric triangle a portion of the parotid gland will be met with, which is separated from the submaxilary gland by the process of the deep cervical fascia known as the stylo-maxillary ligament.

Structures beneath the Mylo-hyoid Muscle. - To expose these structures requires very careful dissection. The mylo-hyoid muscle is to be separated from its fellow along the median raphe, care being taken not to injure the genio-hyoid muscle. It is also to be detached from the body of the hyoid bone, and then the entire muscle is to be reflected apwards towards the mandible. The subsequent steps of this dissection will no doubt be simplified at a later stage when it is in the power of the dissector to divide the mandible at certain points, but no definite hard-and-fast lines can be laid down at this stage. After reflection of the mylo-hyoid muscle the hyo-glossus will be exposed, as well as the stylo-glossus and genio-hyoid. The hyo-glossus is the dissector's landmark. Resting upon its surface he will find the following structures: (1) the hypoglossal nerve, along with the ranine vein, lying close to the hyoid bone : (2) the deep part of the submaxillary gland and Wharton's duct, lying above the hypoglossal nerve; and (3) the lingual nerve, highest 
of all. Between the lingual nerve and the deep part of the submaxillary gland he will, without much difficulty, be able to display the submaxillary ganglion, and show its connections and the branches proceeding from it.

In front of the hyo-glossus" muscle the genio-hyo-glossus will be seen, and resting upon it the dissector will find the sublingual gland, to which he should give his attention. The genio-hyo-glossus cannot be dissected in full until the mandible has been divided, and the tongue put upon the stretch by tying its tip to the point of the nose. The following structures passing beneath the posterior border of the hyo-glossus muscle are to be shown, in the following order from above downwards: (1) the glosso-pharyngeal nerve; (2) the stylohyoid ligament; and (3) the lingual artery.

The Face.-In the dissection of the face the dissectors of the head should, if possible, dissect in concert. The muscles of expression should be shown upon one side, together with the chief nerves and vessels. On the other side a detailed dissection of all the nerves and vessels is to be made, which will involve the division of certain of the muscles. The frontal and temporal regions having been previously dissected, the skin is to be removed from the face in an outward direction, for which purpose a median incision is to be made from the root of the nose downwards to the symphysis of the inferior maxilla. This incision is to embrace the nostril and one half of the oral aperture. The reflection of the skin will be facilitated by making one or more transverse incisions at the option of the dissector. An incision should also be made around the orbit. The structures already dissected in thr. frontal and temporal regions are to be revised. The orbicularis palpebrarum is to be dissected by removing the skin very carefully from it in a direction from the circumference to the ciliary margin of each eyelid, along which the skin is to be cut. At the inner canthus the tendo palpebrarum or internal tarsal ligament is to be displayed, and its relation to the lachrymal sac noted. The corrugator supercilii is to be looked for arising from the inner end of the superciliary ridge, and passing outwards and upwards under cover of the frontalis.

The pyramidalis nasi admits of easy dissection as it ascends from the aponeurosis of the compressor naris over the nasal bone to the skin over the glabella. The muscles between the eye, side of the nose, and upper lip are next to be dissected, such as the compressor naris, levator labii superioris alæque nasi, levator labii superioris, depressor alæ nasi, dilator naris anterior, dilator naris posterior, levator anguli oris, and the two zygomatic muscles, major and minor. The risorius of Santorini and the buccinator next fall to be dissected, and in connection with the latter muscle the buccal pad of fat is to receive attention, as well as the buccal lymphatic glands. The muscles between the lower lip and the inferior maxilla come next in order, these being the depressor anguli oris, depressor labii inferioris, and levator menti. The last muscle is most easily shown by (I) everting the lower lip and dividing the mucous membrane in the region of the incisor fossa of the inferior maxilla : and (2) dividing the skin at the median line close down to the bone, but leaving the lower lip uncut. The dissection of the orbicularis oris requires great care. The lips should be well everted, and maintained in this position. The dissector is then to remove the mucous membrane, when the labial mucnus glands will be exposed. The deepest stratum of the orbicularis oris is then to be dissected, and an effort made to expose the special muscles which compose it, namely, (I) the musculus incisivus superior, coming from the incisor fossa of the superior maxilla; (2) the musculus naso-labialis, coming from the back part of the nasal septum ; and (3) the musculus incisivus inferior, coming from the incisor fossa of the inferior maxilla. The lips being replaced in position, the superficial stratum of the orbicularis oris is to be examined. It will be found to be composed of fibres derived from the elevators and depressors of the angles of the mouth and lips, these fibres stopping at the median line of each lip. The intermediate stratum of the muscle will be seen to consist of buccinator fibres, those of opposite sides being directly continuous at the median line.

The nerves, of the face are now to be fully dissected. The supra-orbital. 
the supratrochlear, the temporal branch of the orbital or temporo-malar, and the auriculo-temporal have been already dissected. The numerous branches of the temporo-facial and cervico-facial divisions of the facial nerve are to be followed from the parotid gland to their distribution, care being taken to note that the facial is the motor nerve of the buccinator muscle. The infratrochlear branch of the nasal nerve is to be met with in the region of the inner canthus, where it emerges from the orbit below the pulley of the superior oblique muscle. The external terminal branch of the nasal nerve will be found emerging between the lower border of the nasal bone and the upper lateral nasal cartilage, after which it descends beneath the compressor naris muscle to the tip of the nose. The infra-orbital nerve will be found emerging through the infra-orbital foramen, but to display this nerve it is necessary to divide the levator labii superioris muscle. Under cover of this muscle the nerve communicates with the infra-orbital branches of the facial to form the infra-orbital plexus. The inferior palpebral, nasal, and superior labial branches of the infra-orbital nerve are to be followed out. The malar branch of the orbital or temporo-malar nerve will be found emerging through the malar foramen in the malar bone. The long buccal nerve emerges from beneath the anterior border of the masseter on to the buccinator, where its branches communicate with the buccal branches of the facial nerve to form the buccal plexus. The facial branches of the great auricular nerve ramify in the integument over the parotid gland, and send offsets into the gland. The mental nerve will be found emerging through the mental foramen in the inferior maxilla, where it is under cover of the depressor anguli oris, and in line with the interval between the two lower bicnspid alveoli.

The chief arteries to be dissected are the facial and transverse facial. The facial artery is easily found, and is to be followed from the base of the inferior maxilla to the region of the inner canthus. As many of its branches as possible are to be shown, more particularly the two coronary arteries. The position of the facial vein on the outer side of the artery upon the body of the inferior maxilla, in front of the masseter, is to be noted, and a lymphatic gland is to be looked for in this situation. The facial vein will be found to receive a fairly large tributary, the deep facial vein, which comes from the pterygoid plexus, and emerges from behind the anterior border of the ramus of the inferior maxilla and of the masseter muscle. The transverse facial artery will be met with emerging from the anterior border of the parotid gland, after which it crosses the masseter immediately above Stensen's duct. The infra-orbital and mental arteries admit of easy dissection, The other arteries, though numerous, are for the most part of small size, and for them the dissector is referred to the description given of them in the text.

The parotid gland is to be carefully dissected, and all its relations displayed. The strong capsule which invests it, and which is derived from the deep cervical fascia, is to be noted. The lower part of the gland is to be shown occupying the back part of the digastric triangle, where it is separated from the submaxillary gland by the stylo-maxillary ligament. Upon the gland, but beneath the parotid fascia (anterior wall of the capsule), and also within the gland, will be found the parotid lymphatic glands. Emerging from the anterior border of the gland, and passing forwards over the masseter muscle on to the buccinator, the parotid or Stensen's duct is to be shown, with the transverse facial artery above it, and the infra-orbital branches of the facial nerve below it. The pars or glandula socia parotidis is to be shown accompanying Stensen's duct for a variable distance, and lying above it. The branches of the facial nerve are now to be followed into the parotid gland, cutting away the glandular substance in doing so. This will enable the dissector to trace the trunk of the facial nerve to the stylo-mastoid foramen, and to show its posterior auricular, digastric, and stylo-hyoid branches. The auriculo-temporal nerve is also to be shown within the upper part of the gland, and communications between this nerve and the temporo-facial division of the facial, as well as between the great auricular nerve and the cervico-facial division of the facial, are to be kept in view. The external 
carotid artery is to be shown within the gland, dividing into its superficial temporal and internal maxillary branches. The superficial temporal vein is to be followed downwards within the gland until it is joined by the internal maxillary vein, and the resultant vessel, namely, the temporo-maxillary vein, is thereafter to be followed downwards within the gland in front of the external carotid artery. The mode of termination of the temporo-maxillary vein is also to be shown, its anterior division joining the facial vein to form the common facial vein, and its posterior division joining the posterior auricular vein to form the external jugular vein. The foregoing dissections will have led to the removal of a large part of the parotid gland. The dissector will therefore be able to see that it extends inwards behind the ramus of the inferior maxilla, and deeply into the posterior part of the glenoid fossa of the temporal bone.

Ocular Appendages. - The eyelids or palpebræ, with their tarsal plates, are to receive attention, as well as the eyelashes or cilia, and the canthi, outer and inner. At the inner canthus the lacus lacrimalis, caruncula lacrimalis, and plica semilunaris are to be studied, as are also the papillæ lacrimales and puncta lacrimalia. The lachrymal canaliculi are to be opened up, and the lachrymal sac is to be shown lying in the lachrymal groove of the lachrymal bone and nasal process of the superior maxilla. The sac should be laid open, and a probe passed down the nasal duct. The superior and inferior palpebral ligaments, as well as the external tarsal ligament, are to be dissected. The internal tarsal ligament or tendo palpebrarum has been already seen. The conjunctiva, palpebral and ocular, is to be carefully studied, and the superior and inferior fornix noted. On the internal surface of each tarsal plate, between it and the conjunctiva, the outlines of the Meibomian glands will be seen, and their orifices may be visible, arranged in a row a little behind each ciliary margin. In this situation the glands of Moll are to be borne in mind.

Auricle (Pinna). - The auricle is to be carefully studied and dissected. Its extrinsic muscles, namely, attrahens, attollens, and retrahens, auriculam have been already attended to. The component parts of the auricle are to be mastered, namely, the concha, helix, crus helicis, antihelix, tragus, antitragus, incisura intertragica, lobule, tubercle of Darwin, fossa of the helix (scaphoid fossa), and fossa of the antihelix (triangular fossa). The ligaments and intrinsic muscles are to be dissected, according to the description of them which is given in the text; the blood-supply and nerve-supply of the auricle are to be reviewed; and thereafter an effort is to be made to dissect the plate of yellow elastic fibro-cartilage which forms its framework. The meatus auditorius externus is to receive very careful study.

Nose.-The cartilaginous framework of the nose is now to be dissected, and this should be overtaken by the two dissectors working in concert. The upper lateral nasal cartilages will be found immediately below the nasal bones. The lower lateral nasal cartilages, or cartilages of the apertures, are situated below the preceding, and each is bent so as to lie in front, and on each side, of the nostril. The crus laterale and crus mediale of each cartilage of the aperture are to be noted. Two or more sesamoid, or minor, cartilages are to be looked for in the fibrous tissue which connects the crus laterale to the superior maxilla. In dissecting the foregoing cartilages the dissectors should have before them the description given of them in the text. The cartilage of the septum is mesially placed, and can only be fully studied in connection with the interior of the nasal fossæ. It is sufficient meanwhile to note that its anterior border is visible between the anterior borders of the upper lateral nasal cartilages inferiorly.

Masseter Muscle.-Before leaving the dissection of the face the masseter is to be dissected. Its superficial and deep parts are to be clearly shown, and its relations thoroughly mastered.

Orblt. - To prepare the cavity of the orbit for dissection the osseous roof must be removed. For this purpose a vertical cut is to be made with the saw through the frontal bone as low as a point just external to the fovea trochlearis. A second cut is to be made with the saw through the lateral wall of the skull from a point on a level with the auricle downwards and 
forwards to the sphenoidal fissure. A large portion of the malar bone is to be removed, for which purpose the bone-pliers may suffice. In his further mode of procedure the dissector should be careful to leave intact the portion of bone immediately surrounding the optic foramen. The inner and outer saw-cuts are to be connected by using the chisel and mallet, one or two smart knocks being sufficient for this purpose. Thereafter the osseous roof of the orbit is to be tilted forwards by a smart knock with the mallet. The small wing of the sphenoid bone is next to be removed with the bone-pliers. In the foregoing manner the cavity of the orbit will be exposed, and the sphenoidal fissure will be opened from above.

The periosteum of the orbit is to be examined, and then laid open. Exactly in the median line, and embedded in the soft fat of the orbit, the dissector will find the frontal nerve, which anteriorly is accompanied by the supraorbital artery, and divides into supra-orbital and supra-trochlear. The supratrochlear nerve, before leaving the orbit, will be found to give off a small twig, which descends to form a loop with the infratrochlear branch of the nasal nerve. More deeply placed than the frontal nerve, but also in the median line, the levator palpebræ superioris muscle is to be dissected, and immediately beneath it the superior rectus. The branches of the upper division of the third or oculo-motor nerve to these two muscles are to be found entering the deep or ocular surface of each muscle.

The superior oblique muscle is to be dissected along the inner wall of the orbit, where it lies superficially, and the dissector is to show the fourth nerve entering its superficial or orbital surface far back. The muscle is to be followed forwards, and its tendon traced through the trochlea or pulley, and thereafter to its insertion. Underneath the superior oblique muscle will be found the internal rectus, in dissecting which the branch from the lower division of the third nerve is to be shown entering its deep or ocular surface. Between the superior oblique and internal rectus muscles will be found the nasal nerve and the anterior and posterior ethmoidal vessels. The lachrymal gland will be found at the anterior and outer part of the orbit. The lachrymal nerve, accompanied by the lachrymal artery, is to be found along the outer wall of the orbit, and is to be followed forwards to its distribution. A branch is to be looked for passing downwards from the lachrymal nerve to join the temporal branch of the orbital or temporo-malar nerve. Beneath this nerve the dissector will find the external rectus muscle.

The frontal nerve and levator palpebræ superioris are to be divided, and to facilitate the dissection of the ocular muscles the eyeball may be infiated. This must be done with very great care, in order to leave intact important structures not yet dissected. The optic nerve is to be exposed by carefully pushing aside the yielding structures which cover it. Having slipped a ligature round the nerve not far from the eyeball, a puncture is to be made in the nerve behind the ligature, and a blow-pipe is to be pushed into the nerve as far as its centre. The blow-pipe is then to be pushed forwards into the eyeball, and when the eyeball has been inflated through it the pipe is to be withdrawn, and the ligature made fast. It is possible to destroy the ciliary ganglion in inflating the eyeball, but that ganglion could easily be dissected on the outer side of the optic nerve before taking the steps necessary for inflation.

In the dissection of the ocular muscles the capsule of Tenon is to be kept in view. It forms a connective-tissue covering for the sclerotic coat of the eyeball, and extends from the point of entrance of the optic nerve to near the margin of the cornea, where it joins the ocular conjunctiva. Between the capsule of Tenon and the sclerotic a space can easily be demonstrated by puncturing the capsule, and introducing the handle of a scalpel. This is the perisclerotic space, or Tenon's space. The suspensory ligament of Lockwood requires great care in dissection. It is a thickening of the lower part of Tenon's capsule, and extends between the malar and lachrymal bones. The capsule of Tenon is pierced by the ocular muscles, to which it furnishes sheaths.

In connection with the sheaths of the recti muscles important forward 
expansions are to be noted. Those which are connected with the external and internal recti are strong, and are attached, respectively, to the orbital process of the malar bone and to the lachrymal crest of the lachrymal bone. The expansion connected with the superior rectus blends with the deep layer of the tendon of the levator palpebræ superioris, and that which is connected with the inferior rectus passes to the inferior tarsal plate.

The external rectus muscle is to be fully exposed, with the sixth nerve entering its deep or ocular surface. The two heads of the muscle are to be made quite evident, with the following structures passing between them, in order from above downwards: (1) the upper division of the third nerve, (2) the nasal nerve, (3) the lower division of the third nerve, (4) the sixth nerve, and (5) the ophthalmic vein or veins.

The superior rectus is to be divided and thrown forwards and backwards. On pushing aside and removing a quantity of fat the optic nerve will be exposed, and crossing it from without inwards is the ophthalmic artery. An effort should be made to show the numerous branches of this artery as detailed in the text. The nasal nerve is to be shown crossing the optic nerve from without inwards to the anterior ethmoidal canal, through which it leaves the orbit. The branches of the nerve to be looked for are as follows: (1) the long or sensory root of the ciliary ganglion, which arises from the nasal as it passes between the two heads of the external rectus ; (2) two long ciliary nerves, which communicate with the short ciliary nerves, and accompany them ; and (3) the infratrochlear nerve. The latter nerve is given off by the nasal at the anterior ethmoidal foramen, and it is to be followed forwards to near the pulley of the superior oblique, where it receives a twig from the supratrochlear nerve. Thereafter it passes beneath the pulley, and emerges from the orbit at the inner canthus. The ciliary or lenticular ganglion will be found on the outer side of the optic nerve. Its long or sensory root will be found coming from the nasal nerve, and its sympathetic root, from the cavernous plexus, usually reaches it through the sensory root. Its short or motor root comes from that branch of the lower division of the third nerve which supplies the inferior oblique muscle. Passing forwards from the ganglion are the short ciliary nerves, which will be seen to range themselves above and below the optic nerve, by which they are conducted to the back part of the sclerotic. The third cranial nerve is to be fully dissected. Its upper and lower divisions are to be shown, and the distribution of their branches made evident, those of the upper division supplying the levator palpebræ superioris and the superior rectus, and those of the lower division supplying the internal rectus, inferior rectus, and inferior oblique. The motor root of the ciliary ganglion comes from the branch to the last-named muscle. The dissector is to note that the branches of the third nerve enter the muscles which they supply on their deep or ocular surface, except in the case of the branch to the inferior oblique, which enters the posterior border of that muscle.

In connection with the origins of the recti muscles the fibrous ring in the region of the optic foramen is to be examined ; and its upper part, or superior tendon of Lockwood, and lower part, or ligament of Zinn, are to be noted.

The inferior oblique muscle is situated below the eyeball and also on its outer aspect. In order to expose this muscle the lower eyelid is to be everted, and the conjunctiva divided along the inferior fornix or line of reflection from the lower eyelid on to the eyeball. The origin of the muscle will be found at the anterior and inner part of the floor of the orbit, immediately external to the opening of the lachrymal canal.

The orbital or temporo-malar nerve, which enters the orbit through the spheno-maxillary fissure, is to be looked for in two divisions-temporal and malar. The temporal branch receives a communicating twig from the lachrymal nerve, and ascends upon the outer wall of the orbit to the temporal canal. The malar branch passes forwards in the angle between the outer wall and floor to the malar canal.

During the dissection of the orbit the ophthalmic artery and its branches. as well as the ophthalmic vein or veins, are to be kept in view. 
The structures which pass through the sphenoidal fissure are next to be carefully dissected, and their relative positions noted.

Pterygo-maxillary Reglon.-The temporal fascia and the part of the temporal muscle above the zygomatic arch have been already dissected. The masseter muscle has also been dissected. In order to expose the pterygomaxillary region the dissector must proceed in a definite order. The zygomatic arch is to be divided in front of, and behind, the origin of the masseter. This is to be done at first with the saw and then with the bone-pliers. The zygomatic arch, bearing the origin of the masseter, is to be turned-downwards until the sigmoid notch of the inferior maxilla is exposed. At this stage the dissector is to show the masseteric nerve and artery emerging from the pterygo-maxillary region over the sigmoid notch and entering the deep surface of the masseter. The nerve and artery having been divided, the masseter is to be stripped from the outer surface of the ramus of the inferior maxilla as low as the angle of the bone. The extensive fleshy origin of the deep part of the muscle from the inner surface of the zygomatic arch is to be carefully noted. The dissector is next to turn his attention to the insertion of the temporal muscle, noting how it extends downwards within the anterior border of the mandibular ramus to the region of the third molar alveolus. The long buccal nerve and the buccal artery are to be caught just within the anterior border of the mandibular ramus as they pass forwards and downwards, or, at all events, they are to be separated from the deep aspect of the insertion of the temporal muscle by pressure with the handle of the scalpel. The coronoid process of the mandible is then to be got rid of by means of the saw and bone-pliers in the following manner : (I) make a vertical cut from the centre of the sigmoid notch downwards for fully $\frac{1}{2}$ inch; and (2) make a horizontal cut from the anterior border of the mandibular ramus backwards to meet the vertical cut at a right angle. In addition to these cuts through the bone, the strongly tendinous fibres of the temporal muscle must also be divided. The coronoid process, bearing part of the insertion of the temporal muscle, is then to be turned upwards, and the muscle is to be stripped gradually from the lower part of the floor of the temporal fossa. The deep temporal nerves, three in number, are to be looked for, as well as the anterior and posterior deep temporal arteries. An effort should also be made to expose the middle temporal artery, which, being a branch of the superficial temporal, has had to pierce the temporal fascia and temporal muscle to reach the temporal fossa. This artery ascends in the groove familiar to the dissector as marking the outer surface of the squamous part of the temporal bone.

Two other horizontal cuts with the saw and bone-pliers still require to be made through the mandibular ramus. One is to be made immediately below the neck of the inferior maxilla, thus leaving intact the insertion of the external pterygoid muscle. The other cut is to be made across the ramus from its anterior to its posterior border on a level with the summit of the crown of the third or last molar tooth, or, if this tooth be absent, as near that level as possible. This will keep the dissector free of the inferior dental foramen on the inner surface of the ramus, and will consequently leave intact the inferior dental nerve and artery.

The structures to be dissected in connection with the pterygo-maxillary region are as follows: (I) the external and internal pterygoid muscles, the former passing horizontally to the front of the neck of the inferior maxilla, and the latter extending downwards and outwards to the inner surface of the mandibular ramus between the inferior dental foramen and the angle of the bone; (2) the first and second parts of the internal maxillary artery, the second part of the vessel usually lying upon the external pterygoid muscle, but sometimes passing beneath it ; (3) the pterygoid plexus of veins around the external pterygoid muscle ; (4) the spheno-mandibular ligament (internal lateral ligament of the temporo-mandibular joint), emerging from beneath the external pterygoid muscle and resting upon the internal pterygoid; (5) the inferior dental and lingual nerves, emerging from beneath the lower border of the external pterygoid and passing downwards, the former, which is posterior in position, parting with it: mylo-hyoid branch close to 
the inferior dental foramen; (6) the long buccal nerve emerging between the two heads of the external pterygoid, and giving upwards the anterior deep temporal nerve ; and (7) the middle deep temporal nerve and the masseteric nerve, emerging from beneath the upper border of the external pterygoid, the latter parting with the posterior deep temporal nerve.

The first and second parts of the internal maxillary artery are to be dissected, as are also the pterygoid muscles. In dissecting the artery the internal maxillary lymphatic glands are to be borne in mind. The first part of the artery will be found to give off (I) the deep auricular, (2) the tympanic, (3) the middle meningeal, furnishing the small meningeal, and (4) the inferior dental, giving off its mylo-hyoid branch. The second part gives off masseteric, deep temporal (anterior and posterior), pterygoid, and buccal branches. The posterior dental artery will be found coming off from the vessel as it is about to enter the spheno-maxillary fossa. The third part of the artery must be deferred to the time when the spheno-maxillary fossa is being dissected.

The inferior dental (with its mylo-hyoid branch) and lingual nerves having been dissected in situ, the inferior dental canal is to be laid open, and the inferior dental nerve fully dissected to its distribution. In opening the canal the saw, chisel and mallet, and bone-pliers are to be used. This dissection will show the dissector how the pulps of the lower teeth are supplied with nerves, and it will also make evident the origin of the mental nerve at the level of the mental foramen.

Before reflecting the external pterygoid muscle certain details may be looked to in connection with the temporo-mandibular joint. A complete dissection, however, of this articulation can only be made at the sacrifice of other structures. The dissector is therefore recommended to perform this dissection upon a specially reserved part. He should have little difficulty in studying the external lateral ligament and the capsule. The capsule is to be opened and the interarticular fibro-cartilage noted. This will at once make evident the existence of two synovial membranes, upper and lower. Beyond this the dissector can hardly go, but he should read the description which is given of this joint in the text.

The mandibular condyle is now to be disarticulated and turned forwards, along with the interarticular fibro-cartilage. In this manner the external pterygoid muscle will be reflected; but, in disarticulating the condyle, the dissector should keep a sharp look-out for the auriculo-temporal nerve, which is in intimate contact with the back of the temporo-mandibular joint. The reflection of the external pterygoid muscle will enable the dissector to expose the following structures: ( $I$ ) the inferior maxillary division of the fifth cranial nerve and its branches; (2) the otic (Arnolu's) ganglion; (3) the chorda tympani nerve, passing forwards and downwards, and applying itself to the lingual nerve at an acute angle ; (4) the middle meningeal artery, parting, as a rule, with the small meningeal; and (5) the upper part of the sphenomandibular ligament.

The middle meningeal artery will be found to pass, as a rule, between the two roots of origin of the auriculo-temporal nerve. The foregoing structures are to be dissected, as nearly as possible, in agreement with the description which has been given of them in the text. Admittedly the dissector vill find the otic ganglion extremely difficult of dissection, but he should not refrain from attempting it. The ganglion lies behind the undivided trunk of the inferior maxillary nerve close to the foramen ovale, and the best plan is to trace upwards the internal pterygoid nerve, with which the ganglion is closely connected.

The pterygo-mandibular ligament is to be examined, which separates the buccinator and superior constrictor muscles, giving origin to fibres of both muscles.

Sublingual Region.-The structures in the submaxillary region have been previously dissected, e.g., the mylo-hyoid muscle, the structures covered by it, the genio-hyoid, and the hyo-glossus. This being so, the dissector is advised to saw through the inferior maxilla, say between the lateral incisor and canine alveoli. This should also be done on the other side by his partner. 
because at this stage it is most desirable that the two dissectors should work in concert. The foregoing cuts will isolate the symphysial portion of the inferior maxilla, and so preserve the attachments of the anterior belly of the digastric, genio-hyoid, and genio-hyo-glossus muscles. By a few touches of the knife the mucous membrane on the inner surface of the mandible can be cut. Thereafter a needle, threaded with a ligature, is to be pushed through the tip of the tongue and through the tip (or columella) of the nose. The needle having now been dispensed with, the ends of the ligature are to be tightly drawn and fixed. The object in this procedure will be at once obvious to the dissector, namely, to put the tongue as much upon the stretch as possible. In the further stages of this dissection the ligature can easily be undone so as to allow the tongue to retire to its normal position. The dissector will now recognise structures which he has been advised to dissect partially at a previous stage. The structures covered by the mylo-hyoid muscle are now to be completely dissected and mastered, namely, (1) the lingual nerve, with the submaxillary ganglion; (2) the deep part of the submaxillary gland and Wharton's duct; (3) the hypoglossal nerve; and (4) the sublingual gland, for the ducts of which the dissector is referred to the text. The genio-hyoglossus muscle now admits of complete dissection, and it will well repay the dissector to study it fully. Immediately external to its extensive insertion into the side of the tongue he should expose the tortuous ranine artery. The genio-hyoid and hyo-glossus muscles have been already referred to. This region is admittedly one of extreme complication and perplexity, so that the dissector should not be surprised that no hard-and fast instructions can be submitted for his guidance. He may, however, show the stylo-hyoid ligament, the stylo-glossus muscle (already referred to at an earlier stage), the stylo-pharyngeus muscle, and, in connection with that muscle, the glossopharyngeal nerve.

The hyo-glossus muscle is to be divided near its origin and reflected towards the tongue. This will expose a portion of the lingual artery, which is now to receive the attention of the dissector, and may be fully studied with advantage. The branches of the vessel are to be dissected in accordance with the text, and special attention is to be directed to the ranine artery.

Superior Maxillary Nerve, Meckel's Ganglion, and Thlrd Part of Internal Maxillary Artery. - This dissection is one of considerable difficulty, and the dissector is advised to have before him for his guidance a section of the skull bearing upon this region. The contents of the orbit having been entirely removed, the skull is to be sawn through in a direction downwards and forwards from a point above the meatus auditorius externus to the region of the inner end of the sphenoidal fissure. This cut will pass through the squamous part of the temporal bone and the great wing of the sphenoid. A second cut with the saw is to be made in advance of the preceding, on a level with the anterior border of the great wing of the sphenoid, and this is to meet the first cut. The portion of bone included between these two cuts is to be removed. The foramen rotundum is to be carefully laid open with the bonepliers, and the infra-orbital canal is to be opened with equal care. To open the posterior part of this canal the bone-pliers will be sufficient, but the anterior part of the canal, being somewhat deeply placed, will require the saw, or the chisel and mallet. The superior maxillary nerve is now to be studied, its straight course forwards through the foramen rotundum, upper part of the spheno-maxillary fossa, infra-orbital canal and infra-orbital foramen being conspicuous. Its orbital or temporo-malar branch is to be shown, as are also the two short spheno-palatine branches which descend to Meckel's ganglion. A little farther forwards the posterior superior dental nerve is to be shown. This at once divides into two branches which descend upon the zygomatic surface of the superior maxilla. Sometimes these two branches arise separately from the parent trunk. Within the infra-orbital canal, in order from behind forwards, the middle and anterior superior dental branches are to be shown by carefully raising the infra-orbital nerve. The dissector should exercise his own discretion as to the removal of bone to expose the course of these two nerves. 
Meckel's ganglion is now to be dissected. It is suspended from the trunk of the superior maxillary nerve in the spheno-maxillary fossa by means of the two short spheno-palatine nerves, and lies close to the spheno-palatine foramen on the inner wall of the fossa. To understand the course of the branches of this ganglion it is absolutely necessary that the dissector should have an accurate knowledge of the foramina and canals which communicate with the spheno-maxillary fossa. With this knowledge his path will be comparatively smooth, but without it he is certain to become befogged. The spheno-maxillary fossa is so much hemmed in by bone that the dissection of Meckel's ganglion and its branches is one of the nicest dissections in the body. The dissector must act with discretion and osteological knowledge, and he will no doubt clear paths with the bone-pliers which will show the course of the nerves now to be mentioned.

The spheno-palatine nerves have been already shown descending from the superior maxillary nerve to the ganglion, to which they convey sensory fibres. To understand the courses taken by the branches of the ganglion, it may be said that four paths lead from the spheno-maxillary fossa, namely (I) internal, representing the spheno-palatine foramen; (2) inferior, representing the posterior palatine canal and its two byways - the posterior and external accessory palatine canals ; (3) posterior, representing $(a)$ the Vidian or pterygoid canal and $(b)$ the pterygo-palatine canal; and (4) antero-superior, representing the spheno-maxillary fissure. The dissector will now, it is to be hoped, be able to follow the branches of Meckel's ganglion. Taking the internal path through the spheno-palatine foramen, he will find (I) the superior nasal nerves, and (2).the naso-palatine nerve, or nerve of Cotunnius, all of which enter the nasal fossa. Traversing the inferior path and its two byways, he will find the three descending palatine nerves-great or anterior, small or posterior, and external. In following out the great or anterior descending palatine nerve through the posterior palatine canal the dissector is to show the two inferior nasal nerves which it gives off, and these can be traced without much difficulty to the nasal fossa by removing with the bone-pliers whatever of the vertical plate of the palate bone may be necessary. Pursuing the posterior paths, the dissector should look for (I) the Vidian nerve, which takes the Vidian or pterygoid route, and (2) the pharyngeal nerve, which passes through the pterygo-palatine canal. The orbital branch (or branches) of the ganglion takes the antero-superior path, and, passing through the spheno-maxillary fissure, enters the orbit.

The branches of the third part of the internal maxillary artery fall in the way of being dissected in the course of the foregoing very complicated dissection. The dissector cannot possibly hope to show everything, so that, to a certain extent, he should rest satisfied with the description given in the text. The posterior dental artery has been already dissected. The infraorbital artery may be available for dissection. If so, it will have become evident in the course of dissecting the infra-orbital nerve, of which it is the companion in all respects. The spheno-palatine, descending palatine, Vidian, and pterygo-palatine branches may be left with this enumeration of them.

The course of the Vidian nerve, just referred to, may be exposed by removing the portion of bone which lies external to the foramen ovale, and laying open the Vidian canal. Like the preceding dissection, this is one of much nicety. The Vidian nerve, when followed backwards to the region of the foramen lacerum medium, will be found to divide into the great superficial petrosal and great deep petrosal nerves. The great superficial petrosal nerve may be followed beneath the Gasserian ganglion to the hiatus Fallopii, through which it enters the aqueduct of Fallopius to reach the geniculate ganglion of the facial nerve. The great deep petrosal nerve passes to the carotid plexus of the sympathetic.

The foramen ovale may now be laid open and the inferior maxillary nerve, with the motor root of the fifth cranial nerve, exposed. The internal pterygoid nerve is to be shown arising from the back part of the inferior maxillary immediately after the latter has received the motor root, and, in intimate 
relation with that branch of the parent trunk, the otic (Arnold's) ganglion falls to receive consideration. The relation of the ganglion to the small superficial petrosal and auriculo-temporal nerves is to be borne carefully in mind. The ganglion lies immediately below the foramen ovale, between the inferior maxillary nerve and the cartilaginous part of the Eustachian tube, and the middle meningeal artery is situated behind it. The source of the motor root of the ganglion from the nerve to the internal pterygoid muscle will be readily understood, but the dissector must not overlook the small superficial petrosal nerve, which conducts to the ganglion fibres of the glossopharyngeal and facial nerves. The cartilaginous part of the Eustachian tube in this vicinity may be made apparent by removing, at the discretion of the dissector, the portion of the great wing of the sphenoid bone which lies internal to the foramen ovale. This dissection will allow of the branches of the otic ganglion being displayed. One branch will be found to pass upwards and backwards to the tensor tympani muscle; a second, downwards and forwards to the tensor palati muscle; branches to the auriculo-temporal nerve ; and a branch to the chorda tympani nerve. These, however, are extremely difficult of dissection; nevertheless, an effort made will not be without its reward.

Deep Dissection of the Neck. - A very complicated dissection has now to be overtaken. It is impossible to lay down hard-and-fast instructions for the guidance of the dissector. He is advised to make himself master of what has been carefully stated in the text. The structures referred to are (I) the glosso-pharyngeal, (2) the pneumogastric, (3) the spinal accessory, (4) the hypoglossal, (5) the superior cervical ganglion of the sympathetic, nerves, and (6) the cervical part of the internal carotid artery, along with the upper part of the internal jugular vein. After he has done his best to study these very important structures the dissector is to revise the styloid muscles and ligaments, and thereafter the styloid process of the temporal bone is to be nipped through, and turned downwards and forwards. This procedure may be advisable in the course of the immediately preceding dissection. Without doubt the dissector will find himself in very deep-water here. He should, however, try his best. When he gets clear of the difficulties in the upper part of the neck near the base of the skull, his course is fairly clear.

The spinal accessory nerve is to be completely mastered, and its bulbar part, of considerable importance, is to be shown applying itself to the ganglion of the trunk of the pneumogastric. The distribution of the spinal part of the nerve must by this time be familiar to the dissector.

The glosso-pharyngeal nerve is to be followed in accordance with the description which has been given of it in the text.

The ganglion of the trunk of the pneumogastric nerve admits of easy dissection, but must receive careful study. Its pharyngeal and superior laryngeal branches demand most careful attention, and the relation to it of the bulbar part of the spinal accessory nerve is to be specially noted. The pharyngeal branch, conducting fibres of the bulbar part of the spinal accessory, will be found to contribute largely to the pharyngeal plexus, the other contributory nerves being derived from the glosso-pharyngeal and the superior cervical ganglion of the sympathetic. The superior laryngeal nerve has already been followed out in its internal and external laryngeal branches, but the dissector should note that it must contain some of the fibres of the bulbar part of the spinal accessory nerve. The parent trunk of the pneumogastric is now to be carefully studied in its course down the neck. The dissector will be already quite familiar with it. A superior and an inferior cervical cardiac branch are to be kept in view.

As regards (I) the auricular branch of the pneumogastric, colloquially referred to as Arnold's nerve, and (2) the tympanic branch of the glossopharyngeal nerve (Jacobson's nerve), the dissector is recommended to accept these as described in the text, unless he desires to pursue a dissection of the most intricate nature possible.

The sympathetic cord is now to be dissected. The superior cervical ganglion. of great length and elliptical shape, can easily be shown, and an effort 
should be made to show all its connections, especially its upward continuation into the carotid canal of the pars petrosa. The middle and inferior cervical ganglia will probably have been met with in a previous dissection, the former being associated with the inferior thyroid artery, and the latter with the vertebral artery. From each ganglion, respectively, in order from above dormwards, there come the superior, middle, and inferior cervical cardiac branches; but, though these are actually present, the dissector should not feel disheartened if he cannot verify their existence.

Carotid Canal and its Contents. - The dissector is now getting into as deep water as it would be safe for him to enter. His knowledge of osteology should come to his aid. His object is to display the intrapetrous part of the internal carotid artery, and for this purpose he will remove whatever bone he deems desirable. In intimate association with this part of the internal carotid he will find the upward continuation of the cervical sympathetic cord. He may be able to show how this upward prolongation divides into two branches, external and internal, the former giving rise to the carotid plexus, and the latter going on to form the cavernous plexus. The dissector should carefully read the description given of these plexuses in the text.

The foramen lacerum posterius, or jugular foramen, is next to be dissected. Its three compartments, already familiar to the dissector, namely (I) anterointernal, transmitting the inferior petrosal sinus; (2) middle, transmitting the glosso-pharyngeal, pneumogastric, and spinal accessory nerves; and (3) postero-external, transmitting the lateral sinus, may now be completely verified by opening up the foramen referred to.

Pharynx, Solt Palate, and Mouth.-To prepare these important regions for dissection, the skull must be divided into two parts. The leading bloodvessels and nerves, as well as the trachea and œsophagus, are to be divided close to the root of the neck. The pharynx is then to be carefully separated from the prevertebral muscles quite up to the base of the skull, and the basilar region of the skull is to be divided with the chisel and mallet, aided by the saw. The front part of the skull) bearing the pharynx, along with the great bloodvessels and nerves, is to be separated from the back part, which bears the cervical portion of the vertebral column, with the prevertebral muscles. The pharynx having been distended with tow, the superior, middle, and inferior constrictor muscles are to be dissected, and whilst doing so the pharyngeal plexus of veins is to be noted. The manner in which the constrictor muscles overlap one another from below upwards is to be noted. The stylo-pharyngeus muscle and glosso-pharyngeal nerve are to be shown in the interval between the lower border of the superior constrictor and the upper border of the middle constrictor. Upon the surface of the middle constrictor muscle the dissector should make an effort to show the pharyngeal plexus of nerves, formed by the pharyngeal branches of the glosso-pharyngeal and pneumogastric, and branches from the superior cervical ganglion of the sympathetic. Between the lower part of the middle constrictor and upper part of the inferior constrictor there will be found the internal laryngeal nerve and the superior laryngeal artery. The relation of the lower border of the inferior constrictor to the oesophagus is to be noted, and passing beneath this border of the muscle there are the inferior or recurrent laryngeal nerve and artery. The pharyngeal branch of the external carotid artery and the inferior palatine and tonsillar branches of the cervical part of the facial artery may admit of being dissected at this stage, but the injection often fails to enter these arteries. Above the upper border of the superior constrictor muscle the dissector is to note the sinus of Morgagni and the pterygo-mandibular ligament, the latter separating the superior constrictor from the buccinator muscle. The wall of the pharynx, atove the superior constrictor muscle, is then to be carefully opened, when the levator palati and tensor palati muscles will admit of dissection.

The dissection of the exterior of the pharynx having been completed, the interior is to be shown by opening the pharynx by means of (I) a median 
vertical incision along its posterior wall, and (2) a transverse incision close to the base of the skull. The soft palate is to be studied, and the divisions of the pharynx noted, namely, the naso-pharynx, above the soft palate, and the buccal and laryngeal divisions below it. In connection with the nasopharynx the posterior nares and the orifices of the two Eustachian tubes are to receive careful attention. The lateral pharyngeal recess, or fossa of Rosenmuller, on the lateral wall of the naso-pharynx, is to be noted, and the pharyngeal tonsil at the upper and back part, unless the median vertical incision has interfered with it. The pharyngeal bursa, below the pharyngeal tonsil, is also to be examined. The isthmus faucium, superior aperture of the larynx, and œsophagus are next to be examined.

The soft palate is now to receive careful attention. The anterior and posterior pillars of the fauces are to be carefully examined, together with the uvula. The tonsil, lying between the anterior and posterior pillars, is to receive attention, and the supratonsillar fossa may be noted. The actual dissection of the tonsil, however, is to be postponed till the muscles of the soft palate have been dissected. The levator palati and tensor palati muscles are now to be fully dissected. The relation of the levator palati to the membranous floor of the lower part of the Eustachian tube is to be carefully studied. The relation of the tensor palati to the hamular process of the internal pterygoid plate of the sphenoid bone is to be specially noted. The palato-glossus and palato-pharyngeus muscles are to be dissected, and in connection with the latter muscle the salpingo-pharyngeus is to be looked for, descending from the lower part of the cartilage of the Eustachian tube. The dissection of the muscles of the soft palate is to be completed by showing the azygos uvula, and the tonsil is then to be dissected.

The mouth is to be carefully examined in detail. Its two divisions, namely, the vestibule and mouth proper, will have been under observation at a previous stage of the dissection. The tongue is to receive very careful attention. At its root will be seen the epiglottis and the folds of mucous membrane in this region, namely, median and two lateral, with the glosso-epiglottidean pouch, or vallecula, on either side of the median fold, are to be noted. The fold, known as the frenum linguæ, should be carefully examined. It will be found on the under surface of the tongue a little distance from the tip. Close to each side of the frenum will be seen a small papilla, bearing the orifice of Wharton's duct, and near to this the orifice of the duct of Rivini or of Bartholin. A little posterior to this is the fold, known as the plica sublingualis, produced by the sublingual gland, and bearing the orifices of the majority of the ducts of Walther (sublingual ducts). In connection with the tongue the three sets of papillæ, namely, conical (and filiform), fungiform, and circum, vallate are to be noted. The $V$-shaped arrangement of the latter will attract notice, and attention is to be given to the foramen cæcum. The - limitation of the papilla to the anterior two-thirds of the tongue will be evident. The extrinsic muscles of the tongue are now to be carefully revised, and their dissection completed if necessary. The intrinsic muscles of the organ fall now to be dissected, namely, the lingualis superficialis, lingualis inferior, lingualis transversus, and lingualis verticalis. The ranine artery having been already dissected, the dissector should revise with the utmost care the nerves which are distributed to the tongue.

Nasal Cavities. - To expose these cavities the skull is to be sawn through vertically on one side of the median line. The septum nasi now admits of full study. Descending upon it, in a forward direction, will be found the nasopalatine nerve, or nerve of Cotunnius, from Meckel's ganglion, and accompanying this nerve may be seen the naso-palatine branch of the spheno-palatine artery. An effort should be made to follow the palatine branch of the nasopalatine nerve through the foramen of Scarpa to the anterior part of the hard palate.

The outer wall of the nasal fossa should be studied with the closest attention. The three meatûs-superior, middle, and inferior-and the three conchæ (turbinate bones) admit of easy examination. The openings into the various meatûs are to be inspected and compared with the descriptions given 
of them in the text. To expose the middle meatus completely the middle concha is to be removed with the scissors. The dissector will then see the deep curved groove, called the hiatus semilunaris, at the upper and anterior part of which will be found the infundibulum. The bulla ethmoidalis will be evident, and the atrium and vestibule are to receive attention. By clipping away the anterior part of the inferior concha a view will be obtained of the lower orifice of the nasal duct, with its imperfect mucous fold, known as the valve of Hasner. The distance of the orifice of the nasal duct from the anterior nasal aperture should be carefully inspected. A probe should be passed through the nasal duct, and the direction taken by the probe noted. A Eustachian catheter should also be passed straight backwards through the inferior meatus, and the mode of introducing it into the lower orifice of the Eustachian tube should be inspected and practised.

The dissection of the otic ganglion and of Meckel's ganglion may now be completely overtaken, it being left to the discretion of the dissector to remove whatever portions of bone may be necessary, after a perusal of the descriptions which are given of these ganglia and their branches in the text.

Larynx. - The larynx, with the tongue, is to be separated from the superior maxilla and the lateral parts of the inferior maxilla. The superior aperture of the larynx and the epiglottis are to be carefully studied, and, on looking down through the superior aperture, the rima glottidis is to be inspected, with a true vocal cord on either side of it. A little above each true vocal cord the less distinct false vocal cord may be seen. In connection with the superior aperture, the cornicula laryngis, or cartilages of Santorini, are to be noted, as well as the cuneiform cartilages, or cartilages of Wrisberg. Between the arytenoid cartilage and the back part of the ala of the thyroid cartilage the recess known as the sinus pyriformis is to be looked for, and its downward direction is to receive attention, which accounts for a foreign body being liable to become lodged within it. The position of the great cornu of the hyoid bone on either side should also receive attention, inasmuch as from an external examination of the upper part of the neck it might possibly be taken for, say, a fishbone. In the dissection of the larynx, the dissectors, who must act in concert, should keep in view (I) the superior laryngeal nerve, its external, as well as its internal, branch; (2) the inferior or recurrent laryngeal nerve; and (3) the superior and inferior laryngeal arteries; but the latter may not be sufficiently injected for dissection. The internal laryngeal nerve will be found piercing the fthyro-hyoid membrane, and the external laryngeal nerve is traceable to the crico-thyroid muscle and the lower part of the inferior constrictor muscle of the pharynx. The recurrent laryngeal nerve will be found close behind the crico-thyroid joint.

The larynx being placed with its posterior surface upwards, the two posterior crico-arytenoid muscles will admit of easy dissection by removal of the mucous membrane. They will be found upon the extensive posterior surface of the cricoid cartilage. The median vertical ridge on the posterior surface of the cricoid cartilage will be found to give origin superiorly to the longitudinal muscular fibres of the asophagus. In dissecting the arytenoid muscle, which extends between the posterior surfaces of the arytenoid cartilages, the dissector should first show the two decussating arytæno-epiglottidei muscles. To follow out each of these muscles, the outer layer of the aryteno-epiglottidean fold of mucous membrane requires to be carefully removed. The cornicula laryngis may now be shown. They will be found to cap the arytenoid cartilages, and not far from them the cuneiform cartilages may be exposed in the back part of the aryteno-epiglottidean folds of mucous membrane, already familiar to the dissector in connection with the superior aperture of the larynx.

At this stage it may be said that the dissectors cannot possibly expect to obtain anything like a satisfactory knowledge of such an important structure as the larynx from the dissection of one specimen. The larynx should be dissected several times. However, assuming that the time and opportunities of the dissectors are limited, the cricoid and thyroid cartilages may now be studied, followed by the crico-thyroid and crico-arytenoid joints. The thyro-hyoid membrane is to be dissected, and the epiglottis is to be 
examined. The crico-thyroid muscle is to be studied in so far as it can be seen without interfering with the thyroid cartilage, and its nervesupply, namely, the external laryngeal nerve, is to be carefully noted. The part of the crico-thyroid membrane between the two crico-thyroid muscles has been already studied in connection with the operation of laryngotomy. The laryax being now placed upon its side, the greater part of the ala of the thyroid cartilage is to be removed by making (I) a vertical cut through it not far from the median line, and stopping short of the lower border, and (2) a horizontal cut extending backwards from the lower end of the vertical cut. A complete view will now be obtained of the crico-thyroid muscle, which should be removed. Thereafter the following muscles fall to be dissected, namely, the lateral crico-arytenoid, the thyro-arytenoid, and the thyro-epiglottideus. An effort should be made to show the two portions of the thyro-arytenoid muscle, namely, internal and external; and the close relation of the internal portion to the true vocal cord is to be noted. The lateral crico-arytenoid, and the whole of the thyro-arytenoid, muscles are to be very carefully removed. This will expose the lateral portion of the crico-thyrold membrane, the inferior thyro-arytenoid ligament or true vocal cord, and the wall of the ventricle of the larynx. Above the wall of the ventricle the fibres of the superior thyro-arytenoid ligament, which enter into the false vocal cord, will be found. The mucous membrane above the true vocal cord may now be removed on the side which is being dissected, when the true and false cords of the opposite side may be examined, with the ventricle of the larynx lying between them. The saccule, which is connected with the anterior part of the ventricle, is to be explored with a probe. Dnring the course of the foregoing dissections it is presumed that the dissectors, as recommended, have been keeping in view the nerves and arteries of the larynx.

The ligaments of the larynx will have been displayed in the course of the dissection, these ligaments being the thyro-hyoid membrane, the cricothyroid membrane, the superior thyro-arytenoid ligament, and the inferior thyro-arytenoid ligament. Two joints require close attention, namely, the crico-thyroid and the crico-arytenoid joints, and the movements allowed at these articulations are to be carefully studied. The varying conditions assumed by the rima glottidis will be found described in the text. The actions of the laryngeal muscles are to be studied with the closest attention. and the function of the epiglottis is to be considered.

Prevertebral Muscles. - The scalene muscles-anticus, medins, and posticus, if not previously dissected, should now be examined, followed by the rectus capitis anticus major, rectus capitis anticus minor, rectus capitis lateralis, and longus colli.

The vertebral artery, as it traverses the costo-transverse foramina of the upper six cervical vertebræ, is to be dissected, and the plexiform arrangement of veins around it is to be noted. The vertebral plexus of the sympathetic is also to be borne in mind.

The Ear. - The meatus auditorius externus is to be laid open with the chisel and bone-pliers, and the membrana tympani is to be carefully examined from the outside. The upper part of the membrane, called the membrana flaccida, or Shrapnell's membrane, which is attached to the notch of Rivini, is to receive special attention. The handle of the malleus will be observed extending between the mucous and fibrous layers of the membrane to a point a little below its centre, where the membrane is drawn inwards so as to give rise ex. ternally to a slight depression, known as the umbo. To expose the cavity of the tympanum, an opening is to be made through the thin lamina of bone, called the tegmen tympani, which is situated on the superior surface of the petrous portion of the temporal bone. If this opening is made external to the eminentia arcuata, it will expose the mastoid antrum. The opening is then to be enlarged with the bone-pliers, and the canal containing the tensor tympani muscle is to be laid open. The meatus auditorius internus may also be laid open. The tympanic cavity, with its intricate contents, the tensor tympani muscle, and the osseous part of the Eustachian tube, are now available for examination. The contents of the meatus auditorius internus are to be held over in the meantime. The ossicles of the tympanum, namely, 
the malleus, incus, and stapes, are to be studied in situ. The insertions of the tensor tympani and stapedius muscles are to be made evident, and their actions studied. The stapedius muscle will be found to enter the tympanum through an opening on the summit of the pyramid on the posterior wall. The dissector need not expect to be able to show the ligaments of the tympanic ossicles, but he may make an effort to show the chorda tympani nerve. This nerve enters the tympanum through the iter chordx posterius on the posterior wall. It then passes forwards upon the membrana tympani, lying between its mucous and fibrous layers, and it leaves the tympanic cavity by passing through the iter chordæ anterius, or canal of Huguier, at the inner end of the fissure of Glaser. The inner wall of the tympanum, with the fenestra ovalis, occupied by the foot-piece of the stapes and annular ligament, the promontory, and the fenestra rotunda, closed by the secondary membrane of the tympanum, is to receive careful attention. The dissector may note the position of the attic or epitympanic recess, which lies above the level of the upper margin of the membrana tympani, and which is divided incompletely into two compartments, outer and inner, by the head and neck of the malleus, and the body and short process of the incus. In connection with the outer compartment, he may try and familiarize himself with the pouch of Prussak, which is bounded externally by the membrana flaccida, or Shrapnell's membrane. For the pouches of Troltsch, see the description of the tympanum in the text. The osseous part of the Eustachian tube is to be carefully studied, and the mastoid cells are to be examined. For the latter purpose the mastoid process of the temporal bone is to be laid open.

The internal ear or labyrinth can only be effectually studied from specimens which have been specially prepared. By means of the chisel and bone-pliers, however, the dissector will obtain a fair amount of information regarding this exceedingly complicated region. The meatus auditorius internus having been already laid open, the roof of the labyrinth is to be removed in a fragmentary fashion. The coiled cochlea, with its two and a half turns, is to be noted, its base being directed towards the deep end of the meatus anditorius internus. Behind the cochlea will be found the vestibule, and at the back part of the vestibule the three semicircular canals are to be noted.

Contents of the Meatus Auditorius Internus.-This meatus having been already laid open, the auditory nerve, the pars intermedia of Wrisberg, the meatal portion of the facial nerve, and the internal auditory artery are to be studied. The auditory nerve will be found to break up into two divisions - vestibular and cochlear. The meatal portion of the facial nerve will be found on the upper and anterior aspect of the auditory nerve; and the pars intermedia of Wrisberg lies between the two. The auditory and facial nerves are connected by two branches. The internal auditory artery is seldom injected.

Facial Nerve in the Aqueduct of Fallopius. - To make a complete dissection of this very intricate aqueduct, the temporal bone requires to be decalcified. An effort should, however, be made to display the aqueduct in the natural bone. The tympanic cavity having been already exposed, the position of the second portion of the aqueduct will be recognised by a ridge on the upper part of the inner wall of the tympanum above the fenestra ovalis. It is here to be laid open with the chisel and mallet. The first portion of the aqueduct, which leads from the deep end of the meatus auditorius internus, may also be laid open with the chisel and mallet in such a manner as to follow the course of the facial nerve, which is horizontally outwards between the cochlea and vestibule to the inner wall of the tympanum. The third or vertical part of the aqueduct, which descends behind the tympanum, to the stylo-mastoid foramen, can best be laid open by removing the mastoid process of the temporal bone. This process is to be sawn across in the vertical-transverse direction, posterior to the level of the stylo-mastoid foramen, into which a probe may be passed for guidance. Another cut is to be made from behind forwards, also in the vertical direction, to meet the preceding cut, close to the stylo-mastoid foramen. The included bone having been removed, the third part of the aqueduct may be reached and laid open with the chisel. In dissecting the facial nerve in the aqueduct, the following structures are to be 
shown : (1) the geniculate ganglion, giving off $(a)$ the great superficial petrosal nerve, $(b)$ a communicating branch to the small superficial petrosal nerve, and (c) the external superficial petrosal nerve (inconstant), all these being situated in the first portion of the aqueduct; and (2) the following branches, arising in the third part of the aqueduct, namely, $(a)$ the nerve to the stapedius muscle, (b) the chorda tympani nerve, and (c) a communicating branch to the auricular branch (Arnold's nerve) of the pneumogastric.

Articulations of the Atlas, Axis, and Occipital Bone.-The ligaments of these joints are to be dissected according to the descriptions given of them in the text, and the movements allowed between the occipital bone and the atlas, and between the atlas and axis, are to be carefully studied. In particular, the following ligaments require special attention : (I) the transverse ligament of the atlas, with the extensive synovial membrane between it and the odontc id process of the axis; (another synovial membrane is to be noted between the odontoid process and the anterior arch of the atlas) : (2) the cruciform ligament, of which the preceding forms a part; (3) the lateral odontoid or alar ligaments; and (4) the posterior occipito-axial ligament, or membrana tectoria, which covers the other ligaments just enumerated.

Certain of the ligaments under consideration admit of being dissected from the exterior. These are as follows: (1) the atlanto-axial-anterior, postei ior, and capsular; and (2) the atlanto-occipital-anterior, posterior, and capsular. The others, however, are situated within the spinal canal, in relation to its anterior wall. These are as follows: (1) the posterior occipito-axial, or membrana tectoria; (2) the accessory atlanto-axial ligaments; (3) the cruciform ligament, of which the transverse ligament of the atlas constitutes a part ; (4) the lateral odontoid or alar ligaments; and (5) the middle odontoid, or suspensory, ligament. In order to display the ligaments which are situated within the spinal canal, the posterior arch of the atlas and the neural arch of the axis should be cut through with the saw. The tabular part of the occipital bone is to be sawn across a little behind the occipital condyles, and the posterior arch of the atlas and the neural arch of the axis are also to be sawn through. After removal of the dura mater, the first ligament to be dissected is the posterior occipito-axial, or membrana tectoria, which, as stated, covers the others, and should be detached from the axis and turned upwards after it has been examined. The cruciform ligament having been studied, the final stage in this intricate dissection consists in detaching the superior crus of the vertical part of the cruciform ligament from the basilar groove of the occipital bone, and turning it carefully downwards.

The Eyeball. - The dissection of the human eyeball can only be accomplished on perfectly fresh specimens. In order to prepare himself for this the dissector is recommended to obtain several eyeballs of the ox, say five or six. The fat, ocular muscles, conjunctiva, and capsule of Tenon are to be removed by dividing, with the scissors, the capsule of Tenon and the conjunctiva in a circular manner at the corneo-scleral junction. The adjuncts of the sclerotic can then be stripped off in a backward direction towards the optic nerve. In detaching the capsule of Tenon from the sclerotic the perisclerotic lymphspace, or Tenon's space, is to be noted. The venæ vorticosæ will probably also be seen. These are usually four in number, and they pierce the sclerotic about midway between the optic entrance and the corneo-scleral junction, at points equally distant from each other. Near the corneo-scleral junction the anterior ciliary arteries may be seen piercing the sclerotic, and around the entrance of the optic nerve the ciliary nerves and posterior ciliary arteries may be seen piercing the sclerotic.

It is well that the eyeballs should be prepared for dissection by keeping them in a ro per cent. solution of formalin, which may sufficiently harden them. There being several ox's eyeballs at the disposal of the dissector, it will be to his advantage to make use of two of them in the following manner: (I) divide one eyeball into two halves, inner or nasal, and outer or temporal, by making an antero-posterior cut along the sagittal axis, or axis of vision-that is to say, a line connecting the anterior and posterior poles of the eyeball; and (2) divide another eyeball into two halves, anterior and posterior, by making a cut along the course of the equator. These two sections 
will afford the dissector a general knowledge of the component parts of the eyeball.

Upon another ox's eyeball the sclerotic and cornea are to be examined. The sclerotic is to be carefully opened at a point in the course of the equator down to the level of the chocolate-coloured choroid. Thereafter the sclerotic is to be clipped completely round the eyeball in the course of the equator, which will divide it into two parts-anterior and posterior. These two parts are then to be carefully reflected, one forwards and the other backwards, which may be facilitated by clipping each into flaps. The dark brown tint of the inner surface of the sclerotic is to be noted, this being due to the connective-tissue layer, called the lamina fusca. Processes passing from the lamina fusca to the subjacent choroid coat will have been observed, these processes traversing the perichoroidal lymph-space.

The sclerotic having been removed in the foregoing manner, the dissector will now obtain a view of the choroid or vascular coat, easily recognised by its chocolate colour. If the dissector can succeed in washing out the pigment from the choroid, he may obtain a view of the closely-set, whorled venæ vorticosæ, which converge to four points, and form four vessels. These vessels will then present a white appearance.

Ciliary Process. - Upon another ox's eyeball a vertical-transverse section is to be made, anterior to the line of the equator. The posterior segment is to be laid aside in the meantime, for the examination of the posterior half of the retina. The vitreous body having been removed from the anterior segment, a good view will be obtained, from behind, of the ciliary processes, which radiate from the periphery of the crystalline lens.

Iris.-To obtain a view of the iris, and also of the ciliary processes, from before, the cornea is to be clipped in another eyeball around the corneo-scleral junction, and removed. This opens up the anterior part of the aqueous chamber, and the iris can now be examined. The shape of the pupil in the ox's eye is to be noted, it being much elongated in the transverse direction.

Flaps of the anterior part of the sclerotic are then to be made with the scissors, by clipping from before backwards, and thereafter they are to be turned backwards. This may enable the dissector to obtain some knowledge of the ciliary muscle, which may be recognised as a white ring in the region of the corneo-scleral junction. In front of the ciliary muscle is the canal of Schlemm. To demonstrate the canal of Petit a blow-pipe may be introduced, close to the periphery of the crystalline lens, through the posterior layer of the zonula of Zinn, behind the ciliary processes (see the text). By blowing air in, the canal of Petit may become evident, and it may then present a sacculated appearance. The suspensory ligament of the lens will be recognised as lying in front of the canal of Petit.

Crystalline Lens.-The iris having been removed, the anterior wall of the capsule of the crystalline lens is to be laid open, when the lens will escape. The outer portion of the lens will be felt to be soft, but the central portion, which constitutes the nucleus, is of firm consistence.

The posterior segment of the eyeball, recently laid aside, is now to be examined by carefully removing the vitreous body. This will enable the dissector to examine the posterior part of the retina from the front.

The foregoing dissections having been made upon the ox's eyeballs, the dissector should avail himself of the first opportunity of repeating them upon the human eyeball. For this purpose the eye of a child will suffice, or a human eye may possibly be obtained from the post-mortem room of a hospital.

The Spinal Oord.-To expose the spinal cord within the spinal canal, the vertebral and sacral grooves of the vertebral column are to be cleaned as well as possible by scraping away the muscles which occupy them. The laminze of the vertebra, having been thereby exposed, are to be sawn through on either side in an inwardly slanting direction, and the ligamenta subflava, which connect the laminæ, are to be divided. The chisel and mallet should also be used in this dissection. This will enable the dissector to remove the posterior wall of the spinal canal, which will be available for the dissection 
of the ligamenta subflava, and the interspinous and supraspinous ligaments. The spinal canal having been laid open, the dissector will expose the epidural space, occupied by loose areolar tissue, embedded in which there is a copious plexus of veins and spinal arteries. The arteries of the spinal canal and spinal cord, though usually uninjected, are to be studied from the description given of them in the text, and, in association with the arteries, the veins are to receive attention.

Meninges of the Cord.-The dura mater, forming the theca, the arachnoid, and the pia mater, are to receive the most careful attention. The outer surface of the dura mater will be readily exposed by removing the areolar tissue and plexus of veins already referred to. The dissector will easily be able to show the tubular sheaths which the dura mater furnishes on either side to the spinal nerves as these pass throngh it, these sheaths being prolonged into the intervertebral foramina.

The dura matral theca is now to be carefully laid open along the middle line with a pair of scissors. This will expose the narrow interspace known as the subdural space. This space having been studied, attention is to be directed to the arachnoid membrane, beneath which is a wide interspace, called the subarachnoid space. In studying this space the dissector is to make himself familiar with its division into three compartments by means of the septum posticum, posteriorly, and the ligamenta denticulata, one on either side. The arachnoid membrane having been carefully removed, the pia mater is to be studied. Its very intimate relation to the spinal cord will be at once evident, and in connection with it the ligamenta denticulata, right and left, should receive careful attention. The manner in which the ligamenta denticulata act as suspensory ligaments to the spinal cord will be evident.

The spinal cord, along with the dura mater, is now to be removed from the spinal canal. In doing so the dissector should be careful to remove, at the same time, the lateral prolongations of the dura mater into the intervertebral foramina, containing the spinal nerves. For this purpose the intervertebral foramina may be laid open with the bone-pliers, and the actual dissection of a spinal nerve may be accomplished at this stage, if the dissector prefers it.

The external characters of the cord are then to receive the most careful attention. The glistening band, called the linea splendens, is to be noted on the anterior surface of the cord, over the course of the anterior median fissure. The cervical and Iumbar enlargements are to be noted. The mode of origin of a spinal nerve by two roots is to be made evident, and the ganglion on the posterior root is to be shown. The union of the two roots immediately outside the ganglion, to form a spinal nerve, shonld be shown, as well as the division of the spinal nerve into anterior and posterior primary divisions. The anterior median and posterior median fissures, as well as the postero-lateral sulcus, are to receive attention. The antero-lateral and posterior columns are to be studied, as well as the columns of Goll and of Burdach. At the lower end of the cord the conus medullaris will be seen, and the arrangement of nerves, known as the cauda equina, will attract attention, in the centre of which leash the filum terminale can be shown without difficulty.

When the dissector has studied the external characters of the cord, he should endeavour to harden it by immersion in the ordinary fluid used for the preservation and hardening of the brain. This process will occupy three or four weeks, after which time transverse sections should be made through the cord at various levels. This will gire the dissector some knowledge of the internal structure of the cord.

The Brain.-The brain, it is assumed, has been sufficiently hardened by immersion in the fluid used for this purpose. In their operations upon this organ it is absolutely necessary that the dissectors should treat it with the most scrupulous gentleness, supporting it on all sides where necessary, and always keeping those parts which are not under immediate dissection or observation covered with cloth saturated with the hardening fluid. With these general precantions the brain should be placed on a wooden platter, 
the base being at first downwards, and a kind of nest having'been made for it on the platter with pieces of cotton wadding, saturated with the hardening fluid. Two small wedge-shaped blocks should also be in constant use, each being insinuated gently underneath the lateral aspect of the corresponding cerebral hemisphere. These blocks the dissectors will find to be of great utility in keeping the parts of the brain together.

The superior surface of the brain is to receive the most careful attention, and the dissectors are recommended to study the description given of it in the text.

The brain is now to be reversed, and placed in its nest with the base upwards. In doing so it must be handled with the greatest gentleness. The dissectors have been already advised to study the base immediately after the removal of the brain from the cranial cavity. It will, however, well repay them to renew this examination in view of the importance of the parts here exposed for their careful study, e.g.; (I) the component parts of the encephalon; (2) the superficial origins of the cranial nerves, including the olfactory lobes ; (3) the arteries, including the circle of Willis ; (4) the cisternæ beneath the arachnoid, and so on (see the description given of all these in the text).

A careful effort may be made to follow ont the leading arteries, but this is on no account to be pushed, as, to do so, might endanger structures of considerable importance. The branches of each vertebral artery may admit of reasonable dissection; so also may those of the internal carotid. The dissectors will find a description of the arteries in the text; and their special attention is drawn to the lenticulo-striate arteries. If the fissure of Sylvius has not been already opened up, that should now be carefully done, in order to see the gyri operti of the insula or island of Reil, lying deeply at the bifurcation of the fissure.

The bloodvessels and membranes are now to be carefully removed from the base of the brain, except in the interval between the cerebellum and medulla oblongata. For this purpose the forceps will be sufficient, aided occasionally by a pair of scissors. In doing so there is considerable risk of taking away the cranial nerves, but this must not be done. The inte:peduncular region may now be studied, and the superficial origins of the cranial nerves revised, the olfactory lobe also receiving attention.

The dissectors are now advised to reverse the position of the brain, and to proceed with a systematic dissection of all its parts; but they must be prepared to encounter considerable difficulties, as it is not an easy matter to lay down hard-and-fast directions. It may be further stated that the brain should be dissected several times, and in different ways.

The brain being now placed with its base downwards, the fissures; lobes, and gyri of the cerebral hemispheres, in so far as they are visible, are to be thoroughly mastered, special attention being directed to (I) Broca's lobe; (2) the fissure of Rolando, and the gyri which bound it in front and behind; (3) the parieto-occipital fissure; and (4) the three limbs of the fissure of Sylvius. The opercula insulæ are also to be studied.

The cerebral hemispheres are then to be sliced away with a long knife until the level of the corpus callosum is reached. In doing so the dissector is to note the white matter in the centre, surrounded by the grey cortex. The corpus callosum is to be carefully inspected from above, the raphe, striæ longitudinales mesiales, and strix longitudinales laterales being noted. The anterior cerebral arteries will also be seen sweeping backwards upon the corpus callosum. The forward and backward extent of the corpus callosum is also to be attended to.

Lateral Ventricles.-These ventricles are to be opened by means of two longitudinal cuts through the corpus callosum, each cut being made about $\frac{1}{2}$ inch from the median line. Each ventricle is then to be laid completely open, so as to show the anterior and posterior cornua, by removing as much of the hemisphere as may be necessary. The various structures exposed in the floor of the lateral ventricle are to be carefully studied, namely, (I) the nucleus caudatus of the corpus striatum in the anterior cornu ; (2) the trinia semicircularis, and the vein of the corpus striatum: (3) a portion of the 
upper surface of the optic thalamus, resting upon which there are $(a)$ the sharp lateral border of the fornix, and $(b)$ the choroid plexus of the lateral ventricle; and (4) the bulb of the posterior cornu, produced by the fibres of the forceps major, and beneath this the calcar avis, or hippocampus minor, produced by the anterior calcarine fissure on the mesial surface of the cerebral hemisphere.

The middle or descending cornu of the lateral ventricle, situated within the temporal lobe, is to be laid freely open by removing a large portion of that lobe. The remarkable course of this cornu is to be noted, and the following structures, which occupy its floor, are to be studied: (I) the hippocampus major, or cornu Ammonis; (2) the fimbria, or tænia hippocampi; (3) the eminentia collateralis, or pes accessorius : and (4) the choroid plexus of the descending cornu. The swelling in which the hippocampus major terminates, called the pes hippocampi, is to be noted ; and the choroidal fissure which extends from the foramen of Monro to the lower extremity of the descending cornu is to receive attention.

The corpus callosum is now to be divided transversely about its centre. On raising the anterior part the septum lucidum will be exposed, as it extends vertically between the under surface of the corpus callosum and the upper surface of the body of the fornix. The relation of the septum lucidum to the lateral ventricles having been studied, the septum is to be carefully divided with a pair of scissors, and the interval between its two layers, called the fifth ventricle, or ventricle of the septum, is to be examined. It will be evident to the dissectors that this ventricle is a closed space.

The posterior part of the corpus callosum may now be turned backwards.

The fornix falls to be studied at this stage. Thereafter it is to be divided transversely about its centre, and the two parts are to be carefully raiser from the subjacent velum interpositum, and turned forwards and backwards. The two posterior pillars of the fornix will be seen to be at first connected with the under surface of the corpus callosum, but subsequently each enters the descending cornu of the lateral ventricle under the name of the fimbria, or tænia hippocampi. On the under surface of the corpus callosum, between the diverging posterior pillars of the fornix, the dissector is to note the lyra, or psalterium. On turning forwards the anterior part of the fornix, its two anterior pillars will be seen descending towards the base of the brain, and in the interval between them a partial view will be obtained of the anterior commissure. After reflection of the fornix the velum interpositum is exposed, in connection with which the two veins of Galen are to be studied, these subsequently joining to form the vena magna Galeni. The transverse fissure of the brain, by which the pia mater enters, to form the velum interpositum, may be studied at this stage, its lateral portions being the choroidal fissures.

Third Ventricle. - To expose this ventricle at the present stage the velum interpositum is to be removed with the greatest care. It is to be laid hold of with the forceps anteriorly, and turned backwards. In doing so the two choroid plexuses of the third ventricle on the under surface of the velum interpositum, as well as the ependymal roof of the third ventricle, are necessarily removed. In reflecting the back part of the velum interpositum the dissector must be careful not to disturb the pineal body, which rests upon the mesencephalon at the back part of the ventricle. This dissection will expose fully (I) the upper surface of each optic thalamus; (2) the chink-like cavity of the third ventricle, lying in the mesial line between the two optic thalami ; and (3) the middle, or grey, commissure, which extends between the inner surfaces of the optic thalami. This commissure, however, being very soft, often gives way. At this period the dissectors should make themselves familiar (by revision) with the various structures which form the roof of the third ventricle. This ventricle itself is to be fully studied, and the relation of its sloping floor to the interpeduncular region at the base of the brain is to be carefully noted. The optic thalamus is to be studied, and in connection with it the following parts are to be noted: (I) the anterior tubercle: (2) the posterior tubercle, or pulvinar; (3) the corpus geniculatum externum; and (4) the stria pinealis, or medullaris, as well as the sulcus of 
Monro, on its internal surface. The anterior pillars of the fornix and the anterior commissure are to be again looked to. The two foramina of Monro are to receive careful consideration, each being situated between the anterior pillar of the fornix in front and the anterior tubercle of the optic thalamus behind. It will be evident to the dissectors that the third ventricle communicates freely with the two lateral ventricles by means of the foramina of Monro. If attention is now directed to the posterior part of the third ventricle, the dissectors will see the upper opening of the aqueduct of Sylvius, by which aqueduct the third ventricle communicates with the fourth ventricle. Above the upper opening of this aqueduct, and beneath the stalk of the pineal body, the dissectors will have no difficulty in seeing the posterior commissure.

Pineal Body.-This body is situated upon the mesencephalon at the posterior part of the third ventricle, and should receive careful attention. In connection with it the following parts are to be noted : (I) the stalk, peduncle, or habenula, the dorsal portion of which forms the stria pinealis, or medullaris, already referred to in connection with the internal surface of the optic thalamus; (2) the pineal recess, which passes backwards above the posterior commissure into the stalk, or habenula, of the pineal body (but this recess, like the optic, infundibular, and suprapineal recesses, can best be studied from plaster, or metal, casts of a mesial section through the brain); and (3) the trigonum habenulæ, which will come more fully into view presently.

Mesencephalon, or Mid-brain. - The mid-brain may be exposed from above by displacing backwards the anterior part of the cerebellum until the corpora quadrigemina are sufficiently exposed. This should be done with the greatest care, in order not to injure (I) the valve of Vieussens, immediately below the lower pair of quadrigeminal bodies, with the superior peduncle of the cerebellum on either side; and (2) the slender fourth cranial nerves, which issue from the valve of Vieussens, and thereafter turn round the crura cerebri, one on either side.

The corpora quadrigemina now fall to be studied. They will easily be recognised as four rounded eminences, arranged in pairs, upper and lower, and the crucial groove which separates them from one another will attract attention. The internal geniculate body will be recognised as a small oval mass on the lateral aspect of the mesencephalon, under cover of the pulvinar of the optic thalamus. The superior and inferior brachia are to be examined, also upon the lateral aspect of the mesencephalon. A. reference to the text may guide the dissectors in their examination of these brachia.

At this stage the dissectors are advised to read the description of the optic tract and its connections as given in the text. They are to make themselves familiar with the mesial and lateral roots of the optic tract, and are to note very carefully that (1) the pulvinar of the optic thalamus, (2) the external geniculate body, and (3) the upper quadrigeminal body constitute the lower visual centres, as distinguished from the higher, or cortical, visual centre in the cortex of the cuneate and lingual gyri close to the calcarine fissure on the mesial surface of the occipital lobe. The trigonum habenulæ, previously referred to, will now be fully exposed.

To complete the dissection of the mesencephalon the crura cerebri are to receive the most scrupulous attention at this stage, and it will be necessary to sarefully reverse the preparation for this purpose-at all events, for revision.

The mesencephalon is now to be divided transversely between the upper and lower pairs of quadrigeminal bodies. This procedure will enable the dissectors to remove the following parts, namely, the pons Varolii, cerebellum, and medulla oblongata. On examining the cut surface the aqueduct of Sylvius will be seen in section. The division of each crus cerebri into two by the crescentic mass of grey matter, called the substantia nigra, may be evident, the upper or dorsal part being the tegmentum, and the lower or ventral part the crusta or pes. The oculo-motor sulcus on the inner surface of the crus for the third or oculo-motor nerve is to be noted, and on the outer surface will be seen the lateral sulcus. The description given of the crus cerebri in the text is to be carefully read. 
Basal Ganglia of the Cerebral Hemispheres.-The pons Varolii, cerebellum, and medulla oblongata are to be laid aside in the meantime, and the dissection of what is left of the cerebral hemispheres is to be overtaken. This should be divided into two parts by a mesial, antero-posterior incision, and one of these parts is to be used for the examination of the inferior surface of the cerebral hemisphere. (The mesial surface of the cerebral hemisphere should be studied on another brain.) In this connection the orbital surface of the frontal lobe will fall to be examined, with its olfactory sulcus, triradiate sulcus, and gyri. The inferior surfaces of the temporal and occipital lobes will also admit of being examined.

The basal ganglia are then to be attended to, these being the nucleus caudatus and nucleus lenticularis of the corpus striatum, the claustrum, and the amygdaloid nucleus. Several sections through the cerebral hemispheres require to be made to obtain a knowledge of these important ganglia. On one side coronal, that is to say, vertical-transverse sections are to be made through the cerebral hemisphere from the level of the front part of the nucleus caudatus backwards to the posterior part of the optic thalamus. On the other side several horizontal sections are to be made through the structures which lie upon the floor of the lateral ventricle. In connection with these sections the dissectors are advised to read the description given of the basal ganglia, and also of the optic thalami, in the text.

Medulla Oblongata. - The dissectors are to note carefully the superficial origins of the seventh, eighth, ninth, tenth, eleventh, and twelfth cranial nerves. The anterior median fissure, with the pyramid on either side, is to be examined, and an effort is to be made to show the decussation of the pyramids. The olivary body and anterior superficial arcnate fibres are to receive attention. In connection with the posterior area of the medulla oblongata it is to be noted that it is divided into two portions, lower or closel, and upper or open. The lower portion presents in the median line the posterior median fissure, on either side of which are the following structures, in order from within outwards: (I) the funiculus gracilis, which is the continuation of Goll's column of the spinal cord, and which terminates superiorly in the clava ; $(2)$ the funiculus cuneatus, or continuation of Burdach's column of the spinal cord, terminating superiorly in the cuneate tubercle; and (3) the funiculus of Rolando, terminating superiorly in the tubercle of Rolando. The calamus scriptorius, between the two clavæ, is to be noted; bnt the upper or open part of the medulla had better be postponed until the floor of the fourth ventricle is under consideration. Suffice it to say that it presents on either side the restiform body. The dissectors should at this stage make themselves familiar with the cisterna magna, in connection with the subarachnoid space, this cisterna lying between the cerebellum and the medulla oblongata. Further dissection of the medulla oblongata should only be overtaken witn proper assistance.

Pons Varolii.-This will admit of easy examination, except as regards its dorsal surface and internal structure. The points to be noted are as follows: (1) the basilar groove; (2) the crura cerebri, emerging from its upper part; (3) the middle peduncles, emerging from it laterally; (4) the stout sensory, and slender motor, roots of the fifth cranial nerve, also emerging from it laterally; and (5) the sixth, seventh, and eighth cranial nerves at its lower border. The dorsal surface of the pons is to be postponed for a short time, until the floor of the fourth ventricle is nnder examination.

Cerebellum.-Like other parts of the encephalon, the cerebellum requires to be examined from several points of view. Assuming, however, that the dissectors desire to get as much as possible out of one brain (a desire to be strongly deprecated), the component parts of the cerebellum are to be studied. On the superior surface the superior vermis and the upper surface of each hemisphere are to be examined. On the under surface the vallecula is to be noted, with the inferior vermis lying deeply in it. In connection with the inferior vermis the following parts are to be studied: ( 1 ) the tuber valvulæ; (2) the pyramid; (3) the uvula, with the furrowed band on either side of it, covered by the amygdala; and (4) the nodule, with the lateral portion of the inferior medullary velum on either side of it. On the under surface of each 
cerebellar hemisphere the following parts are to be noted : (I) the posteroinferior lobule, composed of $(a)$ the lobulus gracilis, and $(b)$ the inferior semilunar lobule; (2) the biventral lobule; (3) the amygdala; and (4) the flocculus.

The dissectors should also make themselves familiar with the peduncles of the cerebellum, namely, the superior, middle, and inferior peduncles, right and left. The superior peduncles will be seen to converge towards the lower pair of quadrigeminal bodies, after they emerge from the cerebellar hemispheres, the valve of Vieussens having been already observed extending between them. The middle peduncles, formed by the transverse fibres of the pons Varolii, sink into each cerebellar hemisphere. The inferior peduncles, which constitute the restiform bodies, connect the cerebellar hemispheres with the upper or open part of the medulla oblongata posteriorly.

Fourth Ventricle. - This ventricle is to be laid open by making a median incision through the vermis of the cerebellum from above. This will bring into view the central collection of white matter called the corpus trapezoides. The two parts of the cerebellum are then to be gently separated, so as to expose the superior medullary velum, or valve of Vieussens, the inferior medullary velum, and the tela choroidea inferior, which lies lower down than the inferior medullary velum, and presents in the median line the foramen of Magendie. The obex is to be looked for in the lower part of the roof, where it overhangs from behind the upper opening of the central canal of the spinal cord, and on either side the ligula may be seen projecting over the epithelium of the roof for a short distance. When these structures have been divided, the floor of the fourth ventricle will be exposed. Its rhomboidal shape is to be noted. At the lower end the dissectors will observe the opening of the central canal of the spinal cord and the calamus scriptorius, and at the upper end the lower opening of the aqueduct of Sylvius. The longitudinal groove, traversing the floor between these two openings, is to be noted, and the striæ acusticæ crossing the floor towards its centre, on either side of this groove, are to receive attention. The floor is thus divided into two portions, lower and upper. The lower or medullary portion will be seen to be bounded on either side, from below upwards, by the clava, the cuneate tubercle, and the restiform body, which latter should now receive full consideration. The parts to be noted in connection with each half of the lower portion of the floor are as follows: (I) the fovea inferior, (2) the trigonum vagi, (3) the trigonum hypoglossi, and (4) the trigonum acusticum. The trigonum acusticum may be seen to be continued superiorly into a prominence called the tuberculum acusticum, over which the striæ acusticæ pass.

The upper or pontine portion of the floor will be seen to be bounded on either side by the superior peduncle of the cerebellum, and the parts to be noted in connection with each half are as follows: (I) the fovea-superior; (2) the eminentia teres, produced by the subjacent fasciculus teres; (3) the locus cæruleus ; and (4) an upward prolongation of the tuberculum acusticum.

The lateral recess of the fourth ventricle is to be examined. It will be found as a lateral prolongation of the ventricle, on either side, round the upper part of the restiform body. In the roof of each lateral recess is the foramen of Kay and Retzius.

The great horizontal fissure of one cerebellar hemisphere is now to be opened up, and the upper half of the hemisphere is to be removed. This will give the dissectors a view of the three cerebellar peduncles as they enter the white medullary substance in the centre of the hemisphere. This substance contains the corpus dentatum, which is a wavy lamina of grey matter enclosing white matter, and open at the upper and inner part. Through this opening many of the fibres of the superior cerebellar peduncle emerge. To expose the corpus dentatum a cerebellar hemisphere has to be cut through horizontally a little above the level of the centre. Sagittal sections are also to be made across the direction of the cerebellar laminæ, when the appearance known as the arbor vitx cerebelli will become evident.

Lastly, with proper assistance, sections should be made through the pons Varolii, with the view of obtaining some knowledge of its internal structure. 


\section{GENERAL EMBRYOLOGY.}

EMBRYOLOGY is a treatise on the embryo and the development of its tissues and organs from the stage of the fertilized ovum to their mature condition.

Two factcrs are concerned in the formation of the embryonamely, (I) the male pronucleus, formed by the head and a portion of the middle piece of a spermatozoon or male germ-cell, and (2) the female pronucleus, which represents the mature ovum or female germ-cell.

\section{The Animal Cell.}

The animal cell is a mass of a living substance called protoplasm. The essential component parts of the cell are (I) a cell-body, and (2) a nucleus. The nucleus may contain one or more nucleoli, but these are not essential elements. The protoplasm of the cell-body is called the cytoplasm, or cell-protoplasm, and it may be enclosed (as in the ovum) within an envelope, called the cell-membrane, which is simply a condensation of the peripheral cytoplasm. The protoplasm of the nucleus is called the karyoplasm, or nucleoplasm, and it is enclosed within an envelope, called the nuclear membrane. The animal cell is therefore "a mass of protoplasm, containing a nucleus.'

Cytoplasm. - The cytoplasm is the protoplasm of the cellbody, as distinguished from the karyoplasm, which is the protoplasm of the nucleus. It is viscid, translucent, and more or less granular. At the periphery it may be condensed to form a

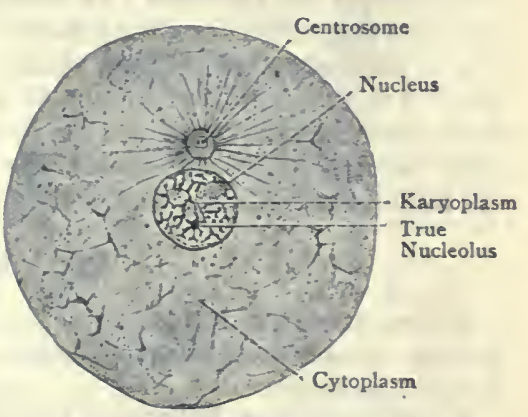

Fig. 656.-The Animal Cell. cell-membrane. The basis of the cytoplasm consists of a network of slender filaments, which is known as the spongioplasm. The meshes of this reticulum are occupied by a semifluid substance called the hyaloplasm.

The cytoplasm contains granules, which are called cyto-microsomes. The hyaloplasm, in addition to the cyto-microsomes, contains several non-protoplasmic bodies-e.g., food-particles and pigment-granules-which are known as the dentoplasm. 
In most cells, usually close to the nuclear membrane, but external to it, there is a small spherical area of cytoplasm, from which lines radiate outwards into the cell-protoplasm. This area is called the centrosome or attraction-sphere, and the protoplasm around the area is known as the archoplasm. At the centre of the centrosome there are usually two small nodules of protein matter, called the central or attraction-particles, from which lines radiate outwards into the archoplasm and cytoplasm. The centrosome thus constitutes the aster, and it plays an important part in nuclear division by mitosis.

The cell-nembrane, when present, is a condensation of the peripheral cytoplasm. In many cells, however, it is absent.

The Nucleus.-The nucleus is usually situated eccentrically in the cytoplasm. Its protoplasm is called karyoplasm, and the nuclear elements are as follows:

\section{x. Nuclear membrane.}

2. Nuclear reticulum.

\section{Karyoplasm.}

4. Nucleoli.

The nuclear membrane is a well-defined envelope which surrounds the nuclear contents and separates them from the cytoplasm. It consists of the elements of the nuclear reticulum-namely, nuclein containing chromatin, and linin.

The nuclear reticulum, which corresponds to the spongioplasm of the cell-protoplasm, consists of nuclein, containing a stainable material called chromatin, arranged in granules. These granules are connected by threads of linin.

The karyoplasm, which corresponds to the hyaloplasm of the cell-protoplasm, occupies the meshes of the nuclear reticulum, and contains granules, known as karyosomes.

The nucleolus (sometimes absent) may be one or more in number. There are two kinds of nucleoli-true and false. The true nucleoli lie in the nuclear reticulum, or, it may be, in the karyoplasm. The false nucleoli are nodes which are connected with the filaments of the nuclear reticulum, where they intersect.

\section{Cell-Divislon,}

There are two kinds of cell-division-namely, karyokinetic or mitotic, which is indirect division, and akinetic or amitotic, which is direct division.

Karyokinesis or Mitosis. - This kind of cell-division is of a very complicated nature, and the changes involved affect both the nucleus and the centrosome. It is convenient to consider it under four phases-namely, (I) the anaphase, (2) the metaphase, (3) the kataphase, and (4) the telophase.

Anaphase.-The anaphase constitutes the preparatory stage, and it includes three phenomena, all of which lead ' up ' to the metaphase, as follows:

I. Formation of spireme.

2. Formation of chromosomes.

3. Formation of spindle. 
Spirome.-The chromatin and linin of the nuclear reticulum and nuclear membrane become transformed into a coiled thread, called the spireme or skein.

Chromosomes. - The spireme is broken up transversely into an even number of segments, called chromosomes, the number of these being constant and characteristic of the species of animal. These chromosomes usually assume the form of short rods, which resemble a V.

Spindle.-Whilst the chromosome-stage is in progress, important changes take place in the stellate centrosome or aster. It divides into two segments, each division taking up a central or attractionparticle, and being furnished with radiating fibres. In this manner two centrosomes or asters are formed. Certain of the radiating fibres extend from one centrosome to the other in a fusiform manner, and these connecting fibres, called the spindle-fibres, constitute the spindle, which has a centrosome or aster at either pole. As the nucleus becomes elongated transversely, the two centrosomes take up positions one at either pole of the somewhat elliptical nucleus, the spindle-fibres becoming gradually elongated. Up to this point the spindle, with an astral centrosome at either pole, is external to the nuclear membrane, but when this membrane disappears the spindle becomes intranuclear, and the spindle-fibres extend from one pole of the nucleus to the other, where they are connected with the two astral centrosomes respectively.

The foregoing phenomena conclude the anaphase or preparatory stage.

Metaphase.-After the disappearance of the nuclear membrane, the chromosomes are brought into direct contact with the spindle, and lie at first scattered between the spindle-fibres. Very soon, however, they congregate at the equatorial plane of the spindle, which corresponds to its widest part. Here they are arranged in a stellate manner, which constitutes the aster according to some authorities. Each chromosome now splits longitudinally into two equal parts, called daughter-chromosomes, the original number of parent-chromosomes being thereby doubled. The formation of daughter-chromosomes constitutes the metaphase or chief stage.

Kataphase. - The daughter-chromosomes at first form two rows at the equatorial plane of the spindle, lying close to each other. They soon, however, separate, those of each row travelling along (metakinesis) the corresponding spindle-fibres to either pole of the spindle, where they enter the centrosome or aster. These phenomena conclude the kataphase, or leading ' down ' stage.

Telophase. - The daughter-chromosomes within each aster now unite end to end, and form a spireme, round which a new nuclear membrane is formed. The spireme gradually assumes the form of a chromatic reticulum, characteristic of a normal nucleus, and karyoplasm is formed within the meshes of the reticulum. Two daughter-nuclei are thus constructed, one in either centrosome, each of which contains one-half of the parent-chromosomes belong- 
ing to the original cell. The cytoplasm of the parent-cell now becomes constricted at the equatorial plane, and by the deepening
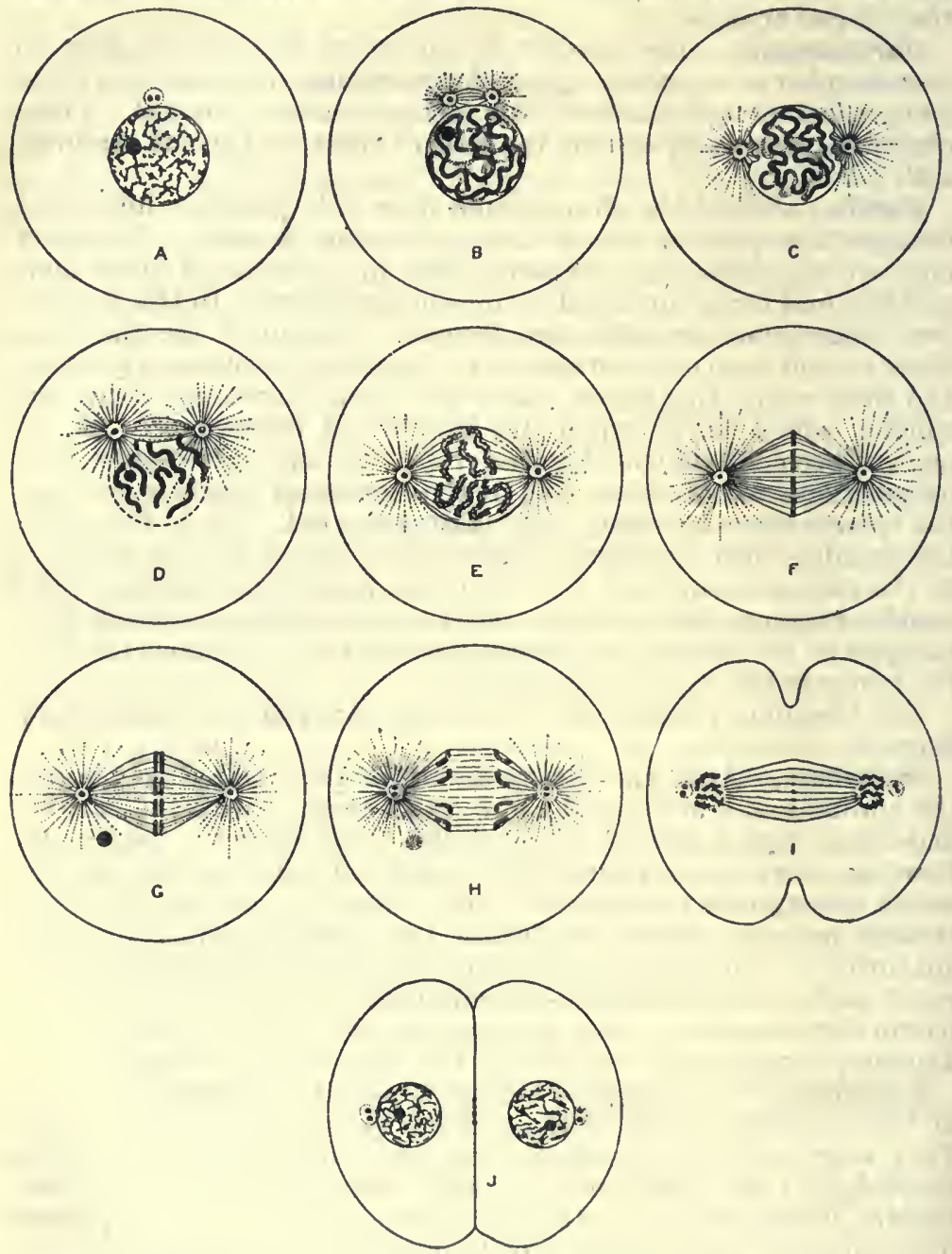

Fig. 657--Stages of Karyokinesis (From E. B. Wilson's "Cell," By Permission of the Macmillan Company, New York).
A, Resting-Cell
B, Early Anaphase
C, Later Anaphase
D, Later Anaphase
E, Latest Anaphase

$\mathrm{H}$, Kataphase

F, Cell ready for

J, Division complete

of this constriction the cytoplasm is divided into two halves, which separate frem each other, each lialf surrounding the nuclear mem- 
brane of the corresponding daughter-nucleus. Two complete daughter-cells are thus formed, and the telophase or concluding stage is finished.

The complex changes concerned in the mitotic division of the parent-cell are concluded with the formation of two complete daughter-cells.

Summary of Karyokinesis, or Mitosis.-There are four phases - namely, anaphase, metaphase, kataphase, and telophase.

The anaphase consists in (I) the conversion of the linin-and chromatin-reticulum of the nucleus into a spireme, or skein; (2) the breaking up of this spireme into chromosomes; and (3) the formation of a spindle from the spindle-fibres which connect the two centrosomes, these centrosomes gradually separating from each other, and the nuclear membrane disappearing.

The metaphase consists in the congregation of the chromosomes. at the equatorial plane of the spindle.

The kataphase consists in (I) the splitting of each chromosome into two daughter-chromosomes, and (2) the migration of these daughter-chromosomes from either side of the equatorial plane of the spindle along the corresponding spindle-fibres to either pole of the spindle where they enter the aster.

The telophase consists in (I) the formation of a daughter-nucleus within each aster, and (2) the cleavage of the cytoplasm of the parent-cell into two halves, each of which surrounds the corresponding daughter-nucleus, two daughter-cells being thereby formed.

Amitosis. - This is direct cell-division. The nucleus is simply cleft into two daughter-nuclei, and this is accompanied by cleavage of the cytoplasm.

\section{The Spermatozoon.}

The spermatozoon is the germ-cell of the male, distinguished from the ovum, which is the germcell of the female.

A spermatozoon is essentially a cell, though it has undergone considerable modifications from the usual cell-type. It is an elongated body, which is endowed with remarkable power of movement, the movement being of a lashing or vibratory nature. It consists of the following parts:

\section{A head. 2. A middle piece. 3. A flagellum or tail:}

The head is short, and, as seen on the flat, is oval. When, however, it is viewed in profile it is pyriform, the narrow. end being directed forwards. It is provided with a head-cap, which covers its anterior part. Over the apical, somewhat pointed, anterior part of the head this cap, is 
arranged in a lanceolate manner, and forms the perforaculum, which is well adapted for perforating. The head represents the nucleus of the parent-spermatid, the nucleus being elongated and oval. It consists of a dense collection of chromatin, and external to it there is a delicate layer of cytoplasm. The archoplasm of the parent-spermatid gives rise, at the anterior end of the nucleus, to the head-cap.

The middle piece lies behind the head, and from its posterior end the flagellum, or tail, is prolonged. The middle piece is developed from the cytoplasm of the parent-spermatid. It contains a centrosome, which is situated near the head.

The flagellum or tail, is the slender filament of lashing movement, and is much elongated. It consists of a central fibril, or axial filament, which is prolonged from the centrosome in the middle piece. The filament is surrounded by a delicate sheath, which disappears towards the end of the tail. The relation of a spermatozoon to its parent-spermatid may be stated as follows:

Spermatozoon.

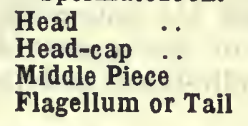

Parent-Spermatid.

Nucleus.

.. Archoplasm.

.. Cytoplasm.

.. Centrosome and Cytoplasm.

\section{Spermatogenesis.}

Spermatogenesis is the begetting of spermatozoa, which are formed in immense, numbers within the tubuli seminiferi of the

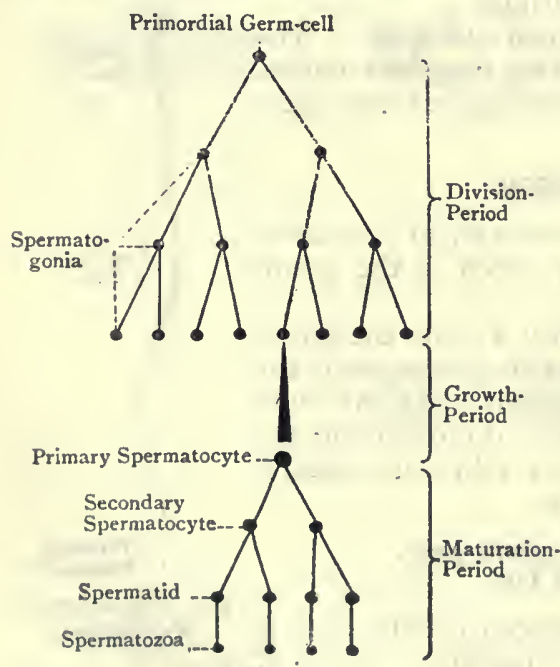

Fig, 659.-Diagram showing SpermatoGENESIS (AFTER BOVERI). testes. Each. spermatozoon is developed from the germinal epithelium, its original source being known as the primordial germ- or spermeell, which is of large size. These cells undergo several mitotic divisions, and from thelast generation spermatogonia are developed, which correspond to the oogonia of the female. These spermatogonia divide, by mitosis, and give rise to primary spermatocytes, two for each spermatogonium, and these correspond to the female primary oöcytes. Each primary spermatocyte divides, by mitosis, into two cells, which are known as the secondary spermatocytes, and correspond to the female secondary oöcytes.

Each secondary spermatocyte, in turn, divides, by mitosis, into two cells, which are called spermatids, and each of these corresponds 
to the mature ovum of the female. Each spermatid now undergoes transformation into a spermatozoon, the change taking place within a cell or column of Sertoli (sustentacular cell). Prior to its full development a spermatozoon has passed through four stagesnamely, (I) a spermatogonium; (2) a primary spermatocyte; (3) a secondary spermatocyte; and (4) a spermatid. In all cases the celldivisions are of the nature of a somewhat modified mitosis or karyokinesis, consisting in a reduction-division-that is to say, a reduction in the number of chromoses at each cell-division.

From one primary spermatocyte (mother-cell) there thus result four grand-daughter cells of equal size, each of which is a spermatid. These spermatids subsequently undergo transformation, each into an active spermatozoon, capable of fertilizing a mature ovum.

\section{The Ovum.}

The ovum or oöcyte, which is the female germ-cell, has all the characters of a typical cell, being specially remarkable for the large

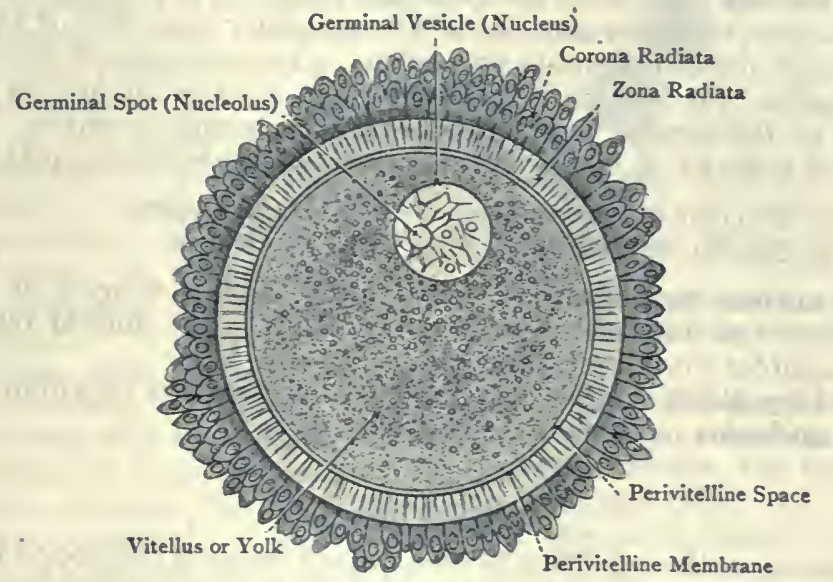

Fig. 660.-The OVUM (MODIFIEd AFTER STRICKER).

size of its nucleus and nucleolus. It is formed within a Graafian follicle of the ovary, and it has a diameter of $\frac{1}{125}$ inch. Its component parts are as follows:
I. Cell-wall.
2. Cell-body.
3. Nucleus.
4. One or more nucleoli.

The cell-wall is known as the vitelline membrane.

The vitelline membrane surrounds the vitellus, of which it is a peripheral condensation. External to the vitelline membrane is the zona pellucida, which is separated from the vitelline membrane by a narrow interval, called the perivitelline space. When examined 
under a high power of the microscope, it presents very delicate striæ, which radiate across its breadth, and from this circumstance it is known as the zona radiata. These strix are regarded as minute pores or passages.

External to the zona radiata there are several layers of cells, which are disposed in a radiating manner and constitute the corona radiata. These cells, like the zona radiata, are derived from the discus proligerus within the Graafian follicle, and the innermost cells send processes through the pores of the zona radiata to the cytoplasm of the ovim.

The cell-body, as in an ordinary cell, consists of cytoplasm (oöplasm), and this presents the usual reticulum or spongioplasm, the meshes of which are occupied by hyaloplasm. The oöplasm constitutes the vitellus or yolk. Embedded in it there are several fat-globules and albuminoid granules. These granules constitute the dentoplasm or nutritive yolk. According to some authorities, the vitellus contains, in the earlier stages, an attraction-sphere and centrosome, situated close to the nuclear membrane.

The nucleus represents the germinal vesicle, and constitutes the essential part of the ovum. As will be presently described, it forms. the mature ovum or female pronucleus, after extrusion of the two polar bodies. It is a large spherical body, situated at first at the centre of the ovum, but subsequently becoming eccentric. Its. diameter is about $\frac{1}{300}$ inch, and it consists of the following parts:

I. Nuclear membrane.

2. Nuclear reticulum.

3. Karyoplasm.

4. Nucleolus.

The nuclear membrane is well marked, and is formed by the chromatin and linin of the nuclear reticulum. The nuclear reticulum resembles that of a typical cell.

The karyoplasm occupies the meshes of the nuclear reticulum.

The nucleolus represents the germinal spot.

\section{Oögenesis.}

Oögenesis is the begetting of ova. Each ovum is developed from the germinal epithelium, the remnant of: which epithelium covers the adult ovary. The original source of the ovum is known as the primordial germ-cell. These cells undergo many mitotic divisions, and from the last generation oögonia are developed, which correspond to the spermatogonia of the male. These ooggonia divide by mitosis, and give rise to primary oöcytes. Each primary oöcyte represents the ovum as it leaves the Graafian follicle, and it corresponds to a male primary spermatocyte. In the process of development each primary oöcyte undergoes two mitotic divisions, one after the other, both of which are unequal. In the first division the primary oöcyte (mother-cell) extrudes the, first polar body, and then it becomes a secondary oöcyte, which corresponds to a secondary, spermatocyte of the male. In other words, the primary oocyte 
divides by mitosis into two cells-namely, ( $\mathrm{I}$ ) the first polar body, of small size; and (2) the secondary oöcyte. In the second division the secondary oöcyte (daughter-cell) extrudes the second polar body, and then it becomes a mature ovum (female pronucleus). In other words, the secondary oöcyte divides, by mitosis, into two cellsnamely, ( $\mathrm{r}$ ) the second polar body, of small size; and (2) the mature ovum (female pronucleus), which latter only undergoes further division if fertilized. Prior to its maturátion, the ovum has passed through three stages-namely, (I) oögonium, oöcyte, and

(2) primary oöcyte. The mature ovum corresponds to a male spermatid, the difference, in the case of the latter, being that the spermatid undergoes further transformation into a spermatozoon.

In all cases the cell-divisions are of the nature of a somewhat modified mitosis or karyokinesis, consisting in a reduction-division-that is to say, a reduction in the number of chromosomes at each celldivision.

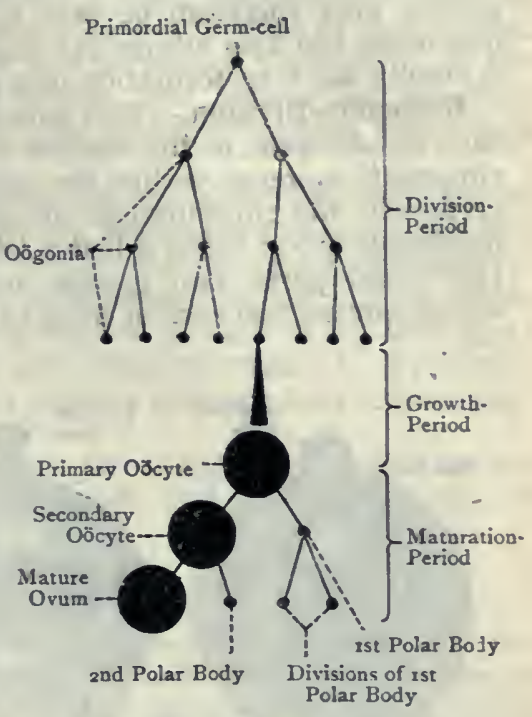

Fig. 66r.-Diagram showing OöGENESIS (AFTER BOVERI).

From one primary oöcyte (mother-cell) there thus finally result four grand-daughter cells, one large and three small-namely, the mature ovum (female pronucleus) of large size, and three small polar bodies, the first polar body, as a rule, having divided into two small cells. The mature ovum is capable of fertilization, but the polar bodies (abortive ova) are inactive and disappear.

\section{Table of Comparison between the Male and Female Germ-cells.}

\section{Male.}

Spermatogonium $\quad \ldots=$ Oögonium.

Primary spermatocyte.. = Primary oöcyte.

Secondary spermatocyte = Secondary oöcyte.

Spermatid .. .. = Mature ovum.

Though there is a great resemblance between spermatogenesis and oögenesis, two differences are to be noted: (I) The final result in oögenesis is the formation of four cells-namely, $(a)$ the mature ovum, of large size, and capable of fertilization; and $(b)$ three, as a rule, polar bodies, all small, quite inactive, and subsequentry 
disappearing. In spermatogenesis, on the other hand, though four spermatids are formed at the same stage of cell-division as in oögenesis, they are all equal in size. (2) The mature ovum undergoes no further change, unless it becomes fertilized. Each spermatid, on the other hand, becomes transformed into an active spermatozoon, capable of fertilizing a mature ovum. Spermatogenesis may therefore be said to comprise one stage more than oögenesis, but this additional stage is not one of cell-division, but is simply the transformation of a spermatid into a spermatozoon.

Reduction-Division.-This process consists in the reduction, at each cell-division, of the number of chromosomes or segments of the chromatic spireme within the nucleus. In the male and female germ-cells the chromosomes are arranged in groups of four, each quadruple group being called a tetrad. When a tetrad is split into two equal parts, two groups are formed, each containing two chromosomes, and each of these pairs is called a dyad. The number

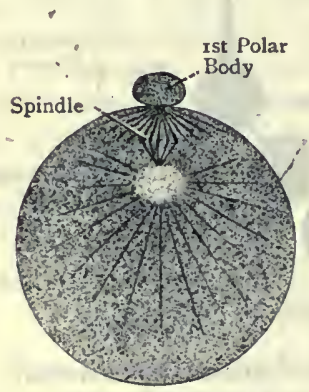

First Stage
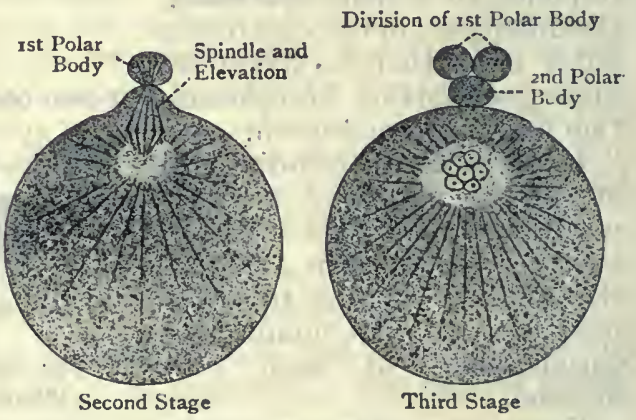

Fig. 662.-Extrusion of Polar Bodies (Modified after Hertwig).

of individual chromosomes, which is always an even number, is constant and characteristic of each species of animal.

Primary Oöcyte (Ovum prior to Maturation).- The number of single chromosomes in the germinal vesicle, for the purpose of illustrating reduction-division, may be assumed to be eight, these being disposed as two tetrads. At the first unequal cell-division, or extrusion of the first polar body, each of these two tetrads is split into a dyad, four dyads being thus formed. Two dyads go with the first polar body, and two remain within the germinal vesicle: Though, therefore, the cell-division is unequal, the reduction and distribution' of the chromosomes is equal. When the first polar body subdivides (during the extrusion of the second polar body) one dyad goes with each subdivision.

At the second unequal cell-division, or extrusion of the second polar body, each of the two dyads within the germinal vesicle splits into two chromosomes, and one chromosome of each dyad goes with. 
the second polar body, and one of each remains within the nucleus or germinal vesicle of the ovum, which latter is nowmature.

Each of the four cells which finally result from the primary oöcyte -namely, the mature ovum and the three polar bodies-contains two single chromosomes.

The preceding remarks, as illustrating reduction-division in chromosomes in the primary oöcyte, or ovum prior to maturation, may be taken as applicable, for the purpose of illustration, to the primary spermatocyte. Each of the four spermatids, resulting from a primary spermatocyte, will therefore contain two single chromosomes.

Each of the two single chromosomes of the mature ovum and of the spermatid respectively represents one-quarter of one of the two tetrads present in the primary oöcyte and primary spermatocyte. Moreover, the number of single chromosomes in the mature ovum and in the three polar bodies, as well as in each of the four spermatids-namely, two-represents the number of tetrads of chromosomes in the primary oöcyte and primary spermatocyte respectively -namely, two.

Reduction-division, therefore, in the sexual germ-cells consists in (I) the division of tetrads into dyads, and (2) the allocation of dyads to cell-divisions.

\section{Ovulation.}

The ovum lies for some time within a Graafian follicle. At this period it is embedded within a heap of cells, known as the discus proligerus. The innermost cells of this discus, which are in direct contact with the ovum, form the zona pellucida or zona radiata, and two or three layers of the succeeding cells give rise to the corona radiata. Within the Graafian follicle, besides the discus proligerus and ovum, there is some fluid, called the liquor folliculi.

Ovulation is the extrusion of the ovum from the Graafian follicle. As a follicle becomes mature, it approaches the surface of the ovary, and, when quite mature, it lies close beneath the surface. This part of the follicle presents a slight projection, on which there is a pale spot, called the stigma. The stigma, becoming very much attenuated, ruptures. The liquor folliculi then escapes, carrying with it the ovum, surrounded by the corona radiata and the zona pellucida or radiata, these, as stated, being derived from the discus proligerus. The expelled ovum is, as a rule, conducted by the grooved ovarian fimbria of the corpus fimbriatum to the ostium abdominale of the Fallopian tube. Here it enters that tube, and is gradually conveyed into the cavity of the body of the uterus, where, if previously fertilized, it undergoes development into the embryo, and then into the fotus.

Abnormal Conditions.-(I) The ovum may never leave the Fallopian tube. and, if fertilized, it would give rise to hubal pregnancy. (2) When expelled from the Graafian follicle and ovary, the ovum may drop into the abdominal cavity, and, if fertilized under these conditions, it would give rise to abdominal 
pregnancy. (3) In extremely rare cases the ovum may not leave the Graafian follicle, even though that follicle and the ovary should rupture in the usual way. If fertilized under these conditions, it would give rise to ovarian pregnancy. These three abnormal conditions are spoken of as cases of extrauterine pregnancy.

The periods of ovulation, or extrusion of the ovum from the Graafian follicle and ovary, which occur at more or less regular successive intervals, are attended by certain changes in the mucous membrane of the cavity of the body of the uterus. These changes constitute menstruation.

\section{Maturation of the Ovum.}

The ovum, as it leaves a Graafian follicle, represents a primary oöcyte. In order to become mature and capable of being fertilized it has to undergo certain changes which affect primarily the germinal vesicle or nucleus, these changes being of the nature of karyokinetic or mitotic cell-division. Briefly stated, the changes consist in the ovum undergoing two unequal cell-divisions. In other words, the ovum extrudes two polar bodies, one after the other, these being minute spherical globules, which are abortive ova.

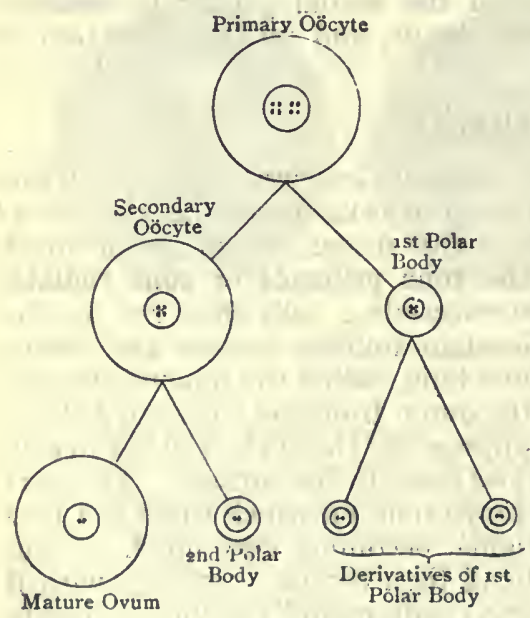

Fig. 663.-Maturation OF THE Ovum.

When the first cell-division takes place the primary oocyte (ovum) divides into two cells of unequal size. The large cell is the secondary oöcyte, and the small one is the first polar body. At the second cell-division the secondary oöcyte likewise divides into two cells, of unequal size. The large cell is the mature ovum, or female pronucleus, and the small one is the second polar body. The first division may take place whilst the ovum is in a Graafian follicle, or, like the second, in the Fallopian tube.

The polar bodies are extruded from the ovum, and, prior to the extrusion of the second, the first polar body usually undergoes an equal division, so that u timately there are three polar bodies. These, being abortive ova, so mn disappear.

The final result of the foregoing two divisions is the formation of four cells. One of these, of large size, is the mature ovum, or female pronucleus, which is capable of activity, and the other three, of small size, are inactive polar bodies, or abortive ova, the first polar body having, as a rule, divided into two. Prior to the first celldivision, or extrusion of the first polar body, the germinal vesicle or nucleus moves towards the periphery of the primary oöcyte. 
After the second polar body has been extruded from the secondary oöcyte the mature ovum, or female pronucleus, passes from the periphery to the centre, and there awaits the male pronucleus, provided fertilization is to take place.

Nature of the Changes. - The germinal vesicle, or nucleus, having moved to the upper surface (animal pole) of the primary oöcyte, undergoes division, of the nature of karyokinesis, or mitosis, slightly modified. Four phases have to be considered-namely, the anaphase, metaphase, kataphase, and telophase.

Anaphase.--The anaphase comprises four stages. (I) A spireme, or skein, is formed from the chromatic reticulum of the germinal vesicle. (2) This spireme breaks up into chromoses, which are dis-
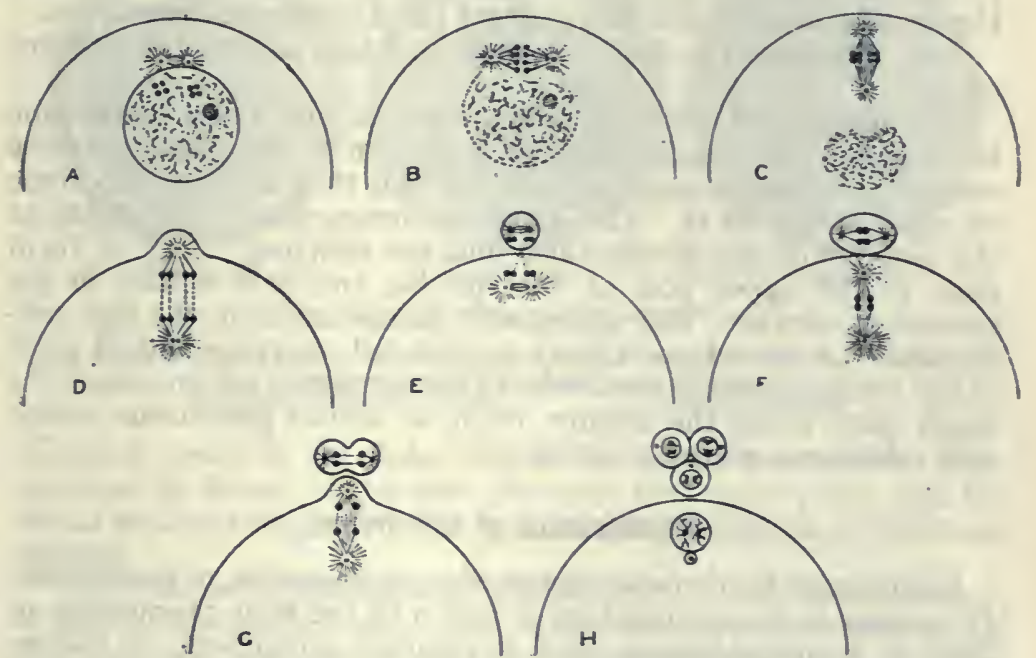

Fig. 664.-Maturation of Ovum (Wilson, By Permission of the Macmillan Company, New York).
A, Initial Phase
B, Equatorial Plate
C, Rotation of Spindle
D, Formation of First Polar Body E, Dyads G, Second Polar Body
F, Division of First Polar Body H, Three Polar Bodies

posed as short rods. (Meanwhile the nuclear membrane of the vesicle begins to disappear; the germinal spot or nucleolus becomes disintegrated; and the karyolymph flows into the vitellus, or cytoplasm.) (3) A spindle is formed, with a centrosome at each pole, and the rod-like chromosomes lie parallel to the spindle-fibres. (4) The chromosomes congregate at the equator of the spindle.

At this stage the spindle lies parallel to the upper surface (animal pole) of the ovum. It now, however, undergoes rotation, and takes up a position at right angles to the animal pole of the ovum. The superficial or peripheral pole of the spindle produces a slight 
projection of the vitellus, or cytoplasm, which is known as the polar bud.

Metaphase.-The chromoses, being now close to the equator of the spindle, are broken up into daughter-chromoses.

Kataphase.-The daughter-chromosomes now separate, half of them travelling along (metakinesis) the spindle-fibres to the upper pole of the spindle, where a centrosome awaits them, and half remain in the primary oöcyte.

Telophase.-Cell-division now takes place in the primary oöcyte. The upper pole of the spindle, with its contained daughter-chromosomes, is cut off and extruded as the first polar body, after the entrance of a spermatozoon. This takes with it the vitellus, or cytoplasm, forming the polar bud, as well as some filaments from the spindle, and those which connect the daughter-chromosomes.

This concludes the first cell-division, which affects the primary oöcyte.

The interrupted spindle is now completed, and a new centrosome is formed. The spindle takes up a position at first parallel to the animal pole of the secondary oöcyte, and then rotates to become at right angles to it. The daughter-chromosomes congregate at the equator of the spindle, and undergo splitting. Half of them pass to the upper pole of the spindle, and half remain in the secondary oöcyte. The subsequent phases are as in the first celldivision. A second polar body is extruded, carrying with it some of the vitellus, and the remainder of the secondary oöcyte, much the larger part, forms the mature ovum, or female pron'ucleus, which now takes up a central position.

\section{Fertilization of the Ovum.}

Fertilization is otherwise spoken of as impregnation, or fecundation. It zonsists in the conjugation or fusion of the male pronucleus, or head of a spermatozoon, with the female pronucleus, or mature ovum, and it constitutes the commencement of the development of a new individual to propagate the species. As a general rule, conjugation takes place in the outer part of the Fallopian tube, or oviduct, into which spermatozoa have made their way through the vagina and uterus by the lashing movement of their tails.

When the spermatozoa come into contact with the mature ovum one of them as a rule passes through the zona pellucida, or radiata, into the yolk. At the point of entrance the yolk forms a conical protuberance, called the receptive, or entrance cone. As the spermatozoon passes through this cone it parts with its tail, the surrounding vitellus becoming disposed in a radiating manner. Meanwhile a delicate membrane is formed round the yolk, called the vitelline membrane, which prevents the entrance into the yolk of other spermatozoa as a rule.

The head, or nucleus, of the spermatozoon now constitutes the male pronucleus, or sperm-nucleus, and the middle piece contair.s 
a centrosome, called the spermo-centre. The male pronucleus advances towards the centre of the ovum, near which, up till now, the mature ovum, or female pronucleus, is lying quiescent, being destitute of its original centrosome, which has disappeared. As the male pronucleus, along with its centrosome, advances, the centrosome leading the way, the female pronucleus shows receptive signs, and moves slightly to meet the approaching visitor. The two pronuclei then come into very near contact, not far from the centre of the ovum, but they do not as yet fuse. The male centrosome, or spermo-centre, now divides, and two centrosomes are formed, one

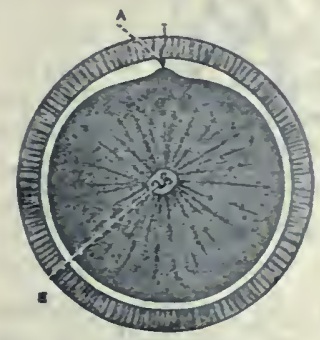

First Stage

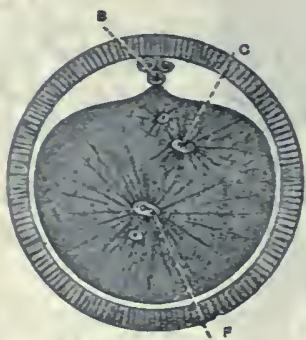

Second Stage

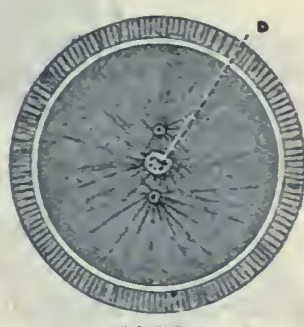

Third Stage

Fig. 665.-Fertilization of Ovus.

A, Entrance of Spermatozoon B, Extrusion of Polar Bodies C, Male Pronucleus D, Componnd Nucleus (Male and Female Pronucleus) E, Female Pronucleus (Ovum)

F, Female Pronucleus

of which passes to the distal side of the female pronucleus. Conjugation or fusion of the two pronuclei now takes place, and the mixed nucleus thus produced is called the segmentation, or cleavagenucleus.

This completes the stage of fertilization. A chromatic spireme, or skein, chromosome, and a spindle are subsequently formed within the segmentation-nucleus, and the segmentation-stage is entered upon-that is to say, cell-division of the nature of mitosis, or karyokinesis, takes place.

Certain authorities maintain that the ovum, after parting with the second polar body, retains a centrosome, known as the ovo-centre. The male pronucleus, as stated, brings with it a centrosome or spermo-centre. Each ovocentre and spermo-centre divides into two, and each division of the ovocentre joins a division of the spermo-centre. When, therefore, fusion of the two pronuclei has been effected, the resultant segmentation-nucleus has two mixed centrosomes (male and female), one on either side, or at each pole.

\section{Segmentation of the Ovum.}

Segmentation consists in the division of the fertilized ovum into a mass of cells.

After the mature ovum, or female pronucleus, has been fertilized by fusion with the male pronucleus, mitotic or karyokinetic celldivision commences, and the ovum is ultimately transformed into 
a great number of cells, which are called blastomeres, or segmentation-cells. At the first division, which affects primarily the segmentation nucleus, the ovum is cleft into two cells, which lie close together, the opposed surfaces being flattened. At the second division each of these cells is cleft into two, so that four blastomeres now occupy the interior of the ovum. Each of these, in turn, divides into two, thus giving rise to eight blastomeres. This process

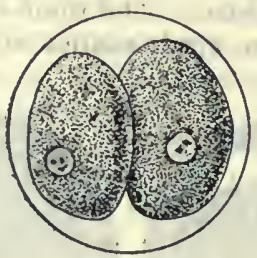

First Division

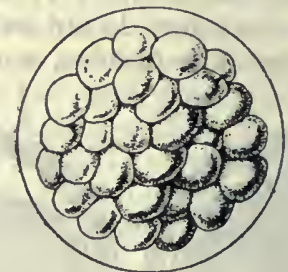

Final Division

Fig. 666.-Segmentation of Ovum.

of cell-division goes on, sixteen blastomeres being formed, succeeded by thirty-two, and so on. Finally, the ovum, originally simple, becomes transformed into a heap of nucleated blastomeres, or segmentation-cells, the superficial cells being clear, whilst the more deeply placed cells are granular. These constitute a solid, spherical, mulberry-like mass, called the morula, and this stage is hence known as the morula-stage.

\section{Formation of the Blastodermic Vesicle and Blastoderm.}

After the morula-stage has been completed, fluid accumulates within the morula and a cavity is formed, called the segmentationcavity or blastocele. The ovum is now a vesicle, and is known variously as the blastula, or blastodermic vesicle. During the formation of the segmentation-cavity the blastomeres or cells of the morula become arranged in two groups-outer and inner. The cells of the outer group, which are clear, spread out and form a membrane, which is known as the enveloping layer, or trophoblast. This layer represents the primitive ectoderm, and it encloses (I) the inner group of cells, and (2) the segmentation-cavity. That portion of the enveloping layer which covers the inner group of cells at the upper pole of the blastodermic vesicle is known as Rauber's covering layer. The primitive ectoderm (enveloping layer), being extraembryonic, gives rise to the fœtal part of the placenta and the fœtal membranes.

The cells of the inner group, which are granular and spherical, and which originally filled the morula, become gradually collected into a round heap, known as the inner cell-mass. This mass is situated at the upper, embryonic, or germinal pole of the blasto- 
dermic vesicle, where they are in intimate contact with the enveloping layer (this part of the enveloping layer being, as stated, Rauber's covering layer). Elsewhere, the inner cell-mass is separated from the enveloping layer by the segmentation-cavity and its fluid.

The enveloping layer constitutes the blastoderm, which, at this stage, is unilaminar.

The cells of the inner cell-mass, which lie next the segmentationcavity, now begin to spread out, and they ultimately form a layer which lines the enveloping layer. This lamina represents the primitive entoderm. The blastoderm is now bilaminar, its outer, or enveloping, layer representing the primitive ectoderm, whilst its inner layer corresponds to the primitive entoderm.

Prior to the completion of the entodermic layer, certain changes take place within the remainder of the inner cell-mass. The lower cells form a thickened group, known as the embryonic bud, or disc, from which the embryo is developed. Within the upper cells vacuoles appear, which rapidly increase and coalesce so as to form a cavity within the upper part of the inner cell-mass, which is called the amniotic cavity. The roof of this cavity is formed directly by the superficial cells of the inner cell-mass. Superficial to this lamina, at first, there is that part of the enveloping layer, or extraembryonic ectoderm, which is known as Rauber's layer. The floor of the amniotic cavity is formed by a layer of cells, derived from those which form the amniotic surface of the embryonic bud, the

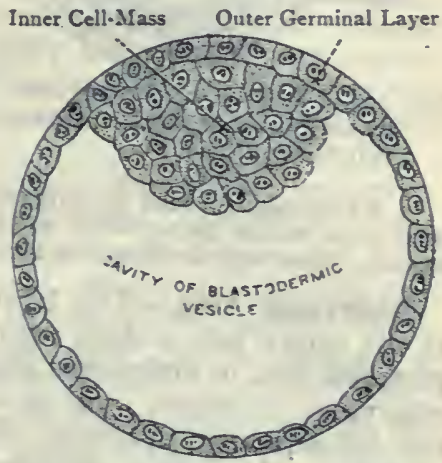

Fig. 667.-ThB SegMentation CAvity OF BLASTOderMic VESICLE. cells of that bud being derived from the inner cell-mass. This floorlayer of cells and the direct roof-layer of cells, both of which are derived from the inner cell-mass, constitute the embryonic ectoderm, as distinguished from the extra-embryonic ectoderm, which is represented by the enveloping layer. The embryonic ectoderm, which surrounds the amniotic cavity, becomes continuous with the extraembryonic ectoderm, or enveloping layer, at the periphery of the roof of the amniotic cavity; and that part of the enveloping layer which enters into the roof of the cavity (Rauber's layer) disappears. The upper pole of the blastodermic vesicle is now covered by embryonic ectoderm.

Gastrulation.-In Amphioxus the blastomeres or segmentation-cells of the fertilized ovum are disposed at an early period in one layer, and there is no morula-stage. This single layer of cells constitutes the unilaminar blastoderm, which encloses a cavity, and the ovum is now a blastula or blastodermic vesicle. 
The unilaminar blastoderm becomes invaginated at one pole of the blastula, and the original cavity, being thereby gradually encroached upon, ultimately disappears. Another cavity, however, is formed within the invaginated portion of the unilaminar blastoderm, and this cavity is the archenteron or primitive intestinal cavity. The opening by which the archenteron com-

Blastopore or Primitive Mouth

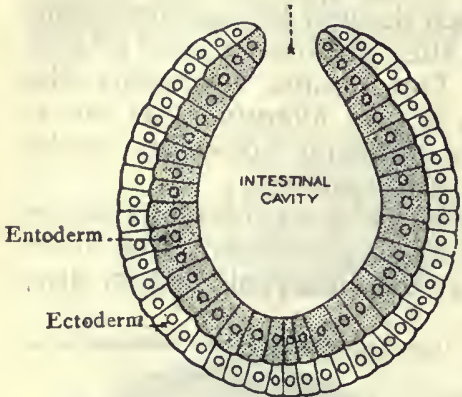

Fig, 668.-The Gastrula of AMPHIOXUS (MODIFIED AFTER WIEDERSHEIM). municates with the exterior, and which is situated at the place where invagination occurs, is called the blastopore. The result is that a double-walled sacciform body is formed, called the gastrula. The inner wall of the gastrula is formed by the invaginated portion of the unilaminar blastoderm, and this constitutes the entoderm. The outer wall of the gastrula consists of ectoderm. At the margins of the blastopore these two germinal layers are continuous with each other. This constitutes the gastrula-stage or gastrulation.

In such animals the entoderm is entirely the result of a process of invagination. Moreover, the mesoderm is subsequently developed at the margins of the blastopore, and gradually insinuates itself between the ectoderm and entoderm.

In Mammalia no such invagination takes place. . The entoderm is formed from cells which are entirely within the blastula or blastodermic vesiclenamely, those which constitute the inner cell-mass.

Embryonic Area.-The bilaminar blastodermic vesicle presents at the upper pole a dark region, which is called the embryonic or germinal disc, or embryonic shield. It is due to a thickening of the embryonic ectoderm, and its shape is at first circular, then oval, and subsequently pyriform, the narrow end being directed caudalwards. Over its caudal or posterior half a longitudinal streak appears, called the primitive streak, which extends forwards in the median line to the centre of the embryonic area. The streak is due to a proliferation of the deep cells of the ectoderm. Along the primitive streak a furrow is formed, known as the primitive groove, and immediately beneath the primitive streak there is entoderm.

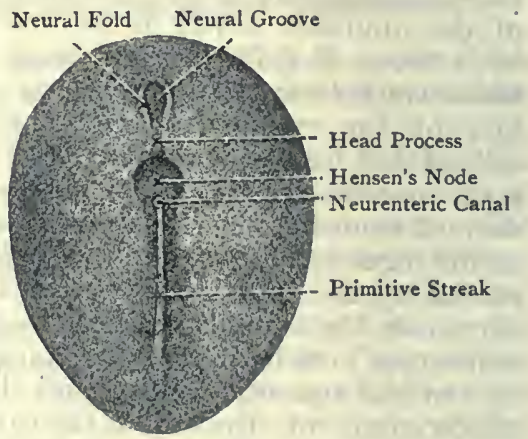

Fig. 669.-The Embryonic Area.

As stated, the primitive streak extends only to the centre of the embryonic area. At the anterior end of the streak there is a definite dark spot, which is due to an accumulation of cells on the under aspect of the ectoderm, and is known as Hensen's node. In front of this node there is another dark linear marking, mesially placed 
and in line with the primitive streak, which constitutes the headprocess of the streak.

Signiffeance of the Head-Process and Hensen's Node.-The ectodermic cells, which lie over the head-process, give rise to the medullary plate, neural or medullary groove, medullary folds, and neural or medullary canal, the last named being the rudiment of the entire cerebro-spinal nervous system. The posterior end of the neural groove embraces the anterior end of the primitive streak and primitive groove. In this situation, where Hensen's node is located, a somewhat wide communication exists at an early period between the posterior end of the neural canal and the primitive intestinal cavity. This communication, which is temporary, is known as the neurenteric canal. It passes through Hensen's node, and it corresponds to the blastopore of Amphioxus.

Along the under aspect of the ectodermic cells, which give rise to the neural canal, the cells of the subjacent entoderm become thickened and differentiated to form the notochordal plate. This plate gives rise to the cylinder of cells, called the notochord, which is the axis round which the vertebral column and its ligaments are developed.

The posterior part of the primitive streak gives rise to the cloacal membrane, which is bilaminar, one layer consisting of ectoderm, and the other of entoderm. It forms the posterior boundary of the primitive alimentary tube, and is subsequently concerned in the formation of the anus.

Formation of the Mesoderm.-On either side of the primitive groove the cells of the primitive streak become proliferous. Cellproliferation also occurs on either side of the head-process. The new cells, thus formed, gradually extend, on either side, into the blastoderm, and insinuate themselves between its two layers, which consist of ectoderm and entoderm respectively. In this manner a third or middle layer is formed for the blastoderm, and this layer, which is of secondary formation, represents the primitive mesoderm. It is chiefly derived from the primitive streak and its head-process, which consist of embryonic ectoderm; but it is reinforced by cells from the subjacent entoderm. The mesoderm is thus a secondary formation, whilst the ectoderm and entoderm are of primary origin.

Inasmuch as the embryonic ectoderm and entoderm are derived from the inner cell-mass, it follows that the inner cell-mass is concerned in the formation of all three germinal layers of the embryo.

The blastodermic vesicle has now a trilaminar blastoderm, the layers, from without inwards, being the ectoderm, the mesoderm, and the entoderm.

\section{Summary of the Formation of the Germinal Layers.}

The germinal layers are three in number-namely, ectoderm, entoderm, and mesoderm. The ectoderm and entoderm are of primary origin, whilst the mesoderm is of secondary origin. The ectoderm is developed from the outer layer of blastomeres or segmentation-cells, which result from the segmentation of the fertilized ovum, and the entoderm is developed from the inner cell-mass. The mesoderm is developed chiefly from the primitive streak on the embryonic area of the blastodermic vesicle, the cells of which streak consist of ectoderm, but it also derives cells from the entoderm which lies close beneath the primitive streak. The mesoderm is also, in part, derived from the head-process of the primitive streak. 
The blastoderm is the wall of the blastodermic vesicle, this vesicle representing the fertilized ovum after segmentation has been completed, and after a cavity, called the segmentation-cavity or blastocœle, has been formed within the morula or mulberry-like mass of cells which results from the segmentation of the fertilized ovum. The blastoderm is at first unilaminar, and is formed by the outer cells of the morula, which spread out and form the enveloping layer. This layer represents the primitive ectoderm, and, being extra-embryonic, it gives rise to the fotal part of the placenta and the fœtal membranes. Meanwhile, during the formation of the cavity of the blastodermic vesicle, the remaining cells of the morula become collected into a round heap, called the inner cell-mass, which is situated at the upper embryonic, or germinal pole of the blastoderm. The cells of the inner cell-mass which lie next the segmentation-cavity, spread out and ultimately form a layer, which lines the primitive ectoderm or enveloping layer. This lamina. represents the primitive entoderm, and the blastoderm is now bilaminar. Prior to the completion of this entodermic lamina, certain changes take place within the remainder of the inner cell-mass. Some of its cells give

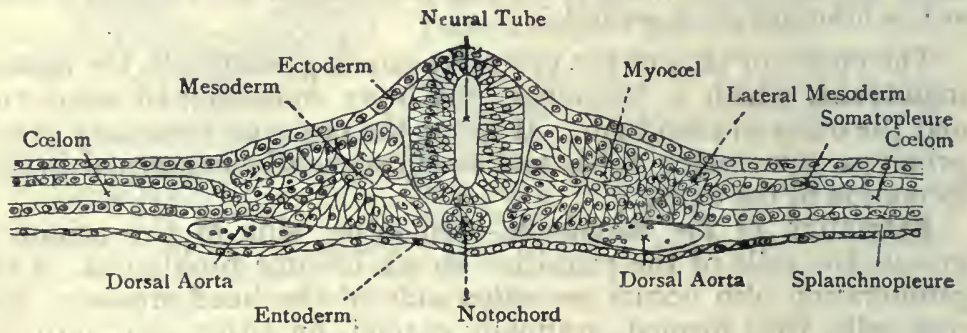

Fig. 670.-The Germinat Layers.

rise to the embryonic bud or disc, which is the rudiment of the embryonic body. In others vacuoles appear, which coalesce and form a cavity within the upper. part of the inner cell-mass, beneath Rauber's layer, called the amniatic cavity. The floor of this cavity is formed by cells derived from the embryonic bud. This layer of cells represents the embryonic ectoderm, which becomes continuous with the extra-embryonic ectoderm forming the enveloping layer. Up to this stage the blastoderm has remained bilaminar.

Formation of the Mesoderm. - On either side of the primitive streak and its head process, cell-proliferation takes place, and the new cells thus formed gradually extend, on either side, into the blastoderm, insinuating themselves between its two layers-ectodermic and entodermic. In this manner a third or intermediate layer is formed, which represents the primitive mesoderm, this being secondary as regards development. It receives its cells chiefly from the ectoderm of the primitive streak and its head-process, but it is reinforced by cells derived from the subjacent entoderm.

The blastoderm is now trilaminar, being composed, from without inwards, of ectoderm, mesoderm, and.entoderm.

Characters of the Germinal Layers.-The cells of the ectoderm are of an epithelial nature. In the region of the embryonic or germinal pole of the blastoderm they are somewhat columnar, but elsewhere they are flattened. The cells: of the entoderm are also of an epithelial nature, and are all flattened in the first instance; but subsequently they become columnar. The cells of the mesoderm are at first separated by intercellular vacuoles, containing fluid, and they are branched. 


\section{Structures derived from the Germinal Layers.}

\section{Ectoderm.}

I. The nervous system-that is to say, the spinal cord and encephalon, the peripheral nerves, and the sympathetic system.

2. The epithelial elements of the organs of sense, except the tongue-e.g., the epithelial elements of the olfactory region, internal ear, optic nerve, and retina.

3. The epithelial elements of the posterior lobe of the pituitary body, and those of the pineal body.

4. The crystalline lens.

5. The epidermis and its appendages - e.g., the hairs and nails.

6. The epithelial elements of the sebaceous glands, sweat-glands, and mammary glands.

7. The plain muscular tissue connected with the hair-follicles, and arranged as the musculi arrectores pilorum, as well as the plain muscular tissue of the sweat-glands.

8. The epithelium of the roof and sides of the mouth, but not that which covers the tongue and back part of the floor of the mouth; the epithelium of the salivary glands; the enamel of the teeth; and the anterior lobe of the pituitary body.

9. The epithelium of the nasal fossæ and of the air-sinuses which communicate with them, as well as of the naso-pharynx.

Io. The epithelium of the external auditory meatus and outer layer of the membrana tympani.

II. The epithelium of the conjunctiva and front part of the cornea.

I2. The epithelium (modified epidermis) of the anal canal below the anal valves.

I3. The epithelium of the spongy part of the male urethra.

\section{Entoderm.}

I. The epithelium of the alimentary canal, except the following parts: (a) The rcoi and sides of the mouth; $(b)$ the naso-pharynx; and (c) the anal canal below the anal valves.

2. The epithelium of the tongue (including that of the taste-buds) and of the back part of the floor of the mouth.

3. The epithelium of the glands which open into the alimentary canal (except the salivary glands)-e.g., the liver and pancreas. The epithelium of the gall-bladder is included.

4. The epithelium of the Eustachian tube and tympanum.

5. The epithelium of the thyroid and thymus bodies.

6. The epithelium of the respiratory tract-e.g., the larynx, trachea, bronchial tubes, and air-cells of the lungs.

7. The epithelium of the urinary bladder, of the prostatic and membranous parts of the male urethra, and of the whole of the female urethra. 


\section{Mesoderm.}

I. The various connective tissues-e.g., bone, cartilage, dentine, cement, areolar tissue, fibrous tissue, and blood.

2. Muscular tissue, striated and plain, except the muscular tissue of the sweat-glands, that which constitutes the musculi arrectores pilorum in connection with the hair-follicles, and that of the iris.

3. The vascular and lymphatic systems, together with their endothelial linings.

4. The serous and synovial membranes, together with their endothelial linings, including all bursal sacs.

5. The kidneys and ureters.

6. The testes, and their complicated excretory equipments.

7. The ovaries, Fallopian tubes, uterus, and vagina.

8. The spleen.

The mesoderm exists under two forms, called mesothelium and mesenchyme.

Mesothelium is that form of mesoderm in which the cells are flattened and form a definite epithelial membrane or layer, known as endothelium, there being only a very small amount of intercellular substance. It lines serous membranes, as well as the chambers of the heart, the bloodvessels, and the lymphatic vessels.

Mesenchyme is that form of mesoderm in which the cells are more or less scattered in a homogeneous ground-substance or matrix, as occurs, say, in the various connective tissues. The cells are stellate and non-epithelial.

\section{Formation of the Embryo.}

\section{Early Stages.}

The early stages in the formation of the embryo take place in that part of the embryonic area which lies in front of the primitive streak. These stages are three in number-namely, (I) the formation of the neural canal; (2) the formation of the notochord; and (3) the formation of the mesodermic somites.

Neural Canal. - The ectodermic cells of the head-process, in front of the primitive streak, give rise to the neural plate, which very soon presents a median longitudinal furrow, called the neural groove. This groove is bounded on either side by a longitudinal ridge of ectoderm, these two ridges being known as the neural or medullary folds. Anteriorly, these folds are continuous with each other in front of the neural groove; but, posteriorly, they are separate, and embrace between them the anterior end of the primitive streak and Hensen's node, through which node the neurenteric canal passes. 'That canal is therefore originally in direct communication with the neural groove, subsequently the neural canal, its other communication being with the archenteron or primitive intestinal cavity.

The neural folds, becoming elevated, incline towards each other. and eventually they unite over the neural groove. In this manner 
the groove is converted into a tube, which constitutes the neural canal. This canal forms the rudiment of the whole cerebro-spinal nervous system, and it consists entirely of ectoderm. The cephalic end of the canal becomes dilated, and gives rise to the three primary cerebral vesicles, which are the rudiments of the fore-brain, mid-brain, and hind-brain, including the cavities of the cerebral ventricles. The remainder of the canal, from the wall of which the spinal cord is developed, persists as the central canal of the -spinal cord. As previously stated, the neural canal originally communicates with the primitive intestinal cavity by means of the neurenteric canal. At this point the ectoderm of the neural canal becomes continuous with the entoderm of the primitive intestinal cavity. The neurenteric canal, however, is only of temporary duration.

Notochord or Chorda Dorsalis. Along the ventral aspect of the neural groove the cells of the entoderm become thickened and differentiated to form the notochordal plate, which constitutes the first stage in the formation of the notochord from the entoderm. For a description of the notochord, which forms the axis round which the vertebral column and its ligaments are developed, see Index.

Mesodernic Somites. - After the formation of the mesoderm it becomes disposed in four parts. Two of these form longitudinal columns, which lie not far from the median or axial line, one being placed on either side of the neural canal and notochord. These two columns constitute the paraxial mesoderm. The other two parts are disposed in the form of sheets, which are known as the lateral plates, each plate lying external to the corresponding paraxial column, with which it is at first in continuity.

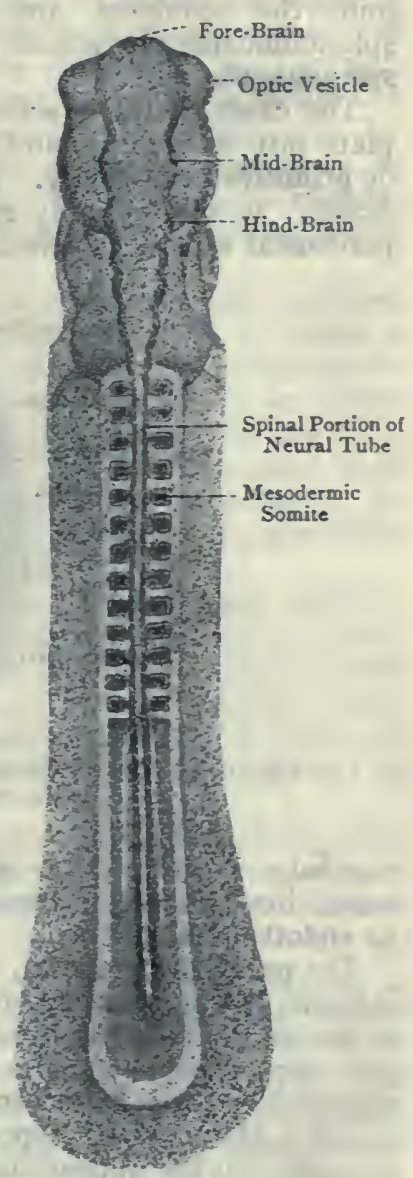

FIG. 671. - EMrRYo ChICK, SHOWING CRANIAL aND SPINAL Portions of NeURal TUbe AND Mesodermic SOMITES (KOLLIKER).

The lateral plates of mesoderm are each composed of several strata of cells, which form, on either side, a single compact sheet. Within each plate vacuoles form, which coalesce and so give rise to a cleftlike space. In this space fluid soon accumulates, and a cavity is 
formed. The mesoderm of each lateral plate becomes thereby disposed in two lamellæ-outer or parietal, and inner or visceral. The parietal layer is called the somatic mesoderm, and the visceral layer is known as the splanchnic mesoderm. The somatic mesoderm joins the ectoderm, and so the somatopleure is formed. The splanchnic mesoderm joins the entoderm, and the two constitute the splanchnopleure.

The cavity formed within each lateral plate, and dividing that plate into its somatic and splanchnic lamellæ, is called the cœlom or primitive body-cavity. It is also known as the pleuro-peritoneal cavity. It subsequently gives rise to the pleural, pericardial, and peritoneal serous cavities, and also to the cavity of each tunica

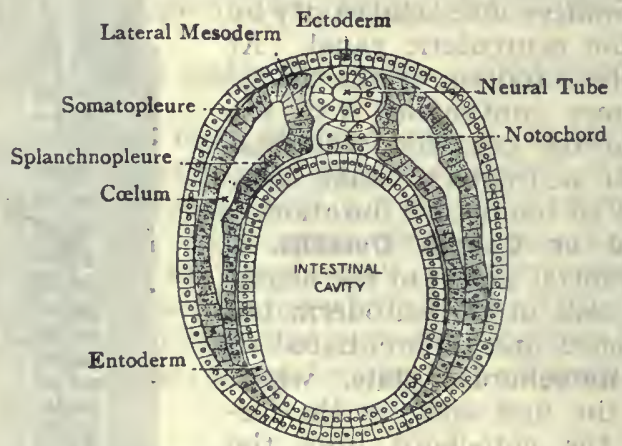

Fig. 672.-Transverse Section of Body of an Amphioxus (MODIFIED AFTER HATSCHEK).

vaginalis in connection with the testes. The mesodermic cells which line the cœlom represent mesothelium, subsequently known as endothelium.

The paraxial mesoderm, as stated, is disposed as two longitudinal columns of thickened mesoderm, one being situated on either side of the neural canal and notochord. Each of these columns undergoes segmentation, and is broken up into a number of primitive segments, called mesodermic somites, cervical, thoracic, lumbar, sacral, and coccygeal respectively. The process of segmentation is preceded by the appearance of a series of transverse furrows along each column. In this manner there is formed, in each longitudinal column of paraxial mesoderm, a series of cubical somites. There are thus two parallel rows of mesodermic somites, one right and one left, situated one on either side of the neural canal and notochord.

The somites on either side become gradually separated from the contiguous lateral plate of mesoderm by a longitudinal groove. Along this groove a row of cells appears, known as the intermediate cell-mass by which the somites are for some time connected with 
the contiguous lateral plate. Eventually, however, the lateral plate becomes detached. The intermediate cell-mass on either side gives rise to the internal genital and the renal organs.

Each mesodermic somite consists of cells, which are arranged in a laminar manner around a central cavity or myoccele, which is of temporary duration. These cells become disposed in three groups, two being internal, and one external. Of the two inner groups the mesial one, or that nearest to the neural canal and notochord, by its mesenchyme, constitutes a sclerotome, and the lateral group, or that nearest to the temporary cavity of the somite, forms a muscleplate. The external group of cells is generally regarded as giving rise to the cutis-plate.

The successive sclerotomes on either side proliferate and surround the notochord and superjacent neural canal, forming for them a sclerotogenous or skeletogenous sheath of mesenchyme. This investment, as regards the notochord, represents the membranous vertebral column, and from it are developed (I) the vertebræ and their ligaments, and (2) the intervertebral discs, except the central pulp of each, this pulp being a persistent part of the notochord. As regards the neural canal, the sclerotogenous investment gives rise to the meninges of the brain and spinal cord.

The successive muscle-plates give rise to myotomes (musclesegments), and from these the striated muscles of the trunk, at least, are developed. According to some authorities, they also give rise to the muscles of the limbs; but another view is that the limbmuscles are developed from the core of mesenchyme in the centre of each limb-bud, which core gives rise to the skeletal framework of the limb.

The cutis-plate, formed by the external group of somitic cells, gives rise to the cutis vera or corium, the epidermis being of ectodermic origin. According to some authorities, however, this group of cells is concerned in the formation of muscular tissue.

Mesodermic Somites of the Head.-The first or most anterior primitive or trunk mesodermic somite, derived as stated from the division of the column of paraxial mesoderm, occupies the future occipital region. In front of this region several head-somites or segments appear in lower vertebrates. They are regarded as being nine in number, but they are less numerous in the human embryo, there being probably three. They are formed by a segmentation of the mesoderm of the head, as distinguished from the paraxial mesoderm.

\section{Later Stages in the Formation of the Embryo.}

The early stages in the formation of the embryo, just described, are threefold, as follows: (I) The formation of the neural canal from ectoderm, this canal being the rudiment of the entire cerebro-spina! nervous system; (2) the formation of the notochord or chorda dorsalis from entoderm, this chord constituting the axis round which the vertebral column and its ligaments are developed from the sclerotogenous or skeletogenous sheath of the notochord, the chord 
itself only persisting as the central pulp of each intervertebral disc; and (3) the formation of the mesodermic somites from the paraxial mesoderm, these somites being arranged in two longitudinal rows, right and left, one on either side of the neural canal and notochord. The lateral plate of mesoderm on either side has been divided into two lamellæ-namely, somatic and splanchnic, this being effected by the formation, within each lateral plate, of vacuoles, which coalesce and so give rise to a cleft-like space. Within this space fluid accumulates and a cavity is formed, called the colom, primitive body-cavity, or pleuro-peritoneal cavity. The somatic mesoderm joins the ectoderm, and forms the somatopleure, whilst the splanchnic mesoderm joins the entoderm, and forms the splanchnopleure. The cavity of the cœlom is therefore bounded on the one hand by somatopleure, and on the other by splanchnopleure.

The cavity of the blastodermic vesicle, which is situated on the ventral aspect of the notochord, is single at this stage, and is bounded by "splanchnopleure. This cavity represents the primitive intestinal cavity.

Separation of the Embryo. - The primitive embryo, developed. from the embryonic area of the blastodermic vesicle, becomes. separated from the extra-embryonic part of that vesicle by a process of infolding of somatopleure ventralwards around the embryonic area. This infolding gives rise to certain grooves and folds. The grooves are cephalic, lateral, and caudal. In the chick. (to which the following description applies) the cephalic or anterior groove lies, in a crescentic manner, in front of the head-end of the embryo, the concavity of the crescent being directed towards the head. The lateral grooves are disposed one on either side of the embryonic area. The caudal or posterior groove is situated, in a crescentic manner, behind the tail-end of the embryonic area, the concavity of the crescent being directed towards the tail. By the coalescence of these four grooves one continuous groove is formed, which surrounds the entire embryonic area, and is known as the marginal or circular groove.

The folds lie external to the grooves, of which they are the elevated. outer lips, and they are spoken of as head-fold, lateral (right and left), and tail-fold. They consist of two layers of somatopleure, inner and outer, and constitute the amniotic folds.

As the circular groove deepens, it encroaches, from all sides, upon. the ventral aspect of the embryonic area as far as the central part, which coincides with the site of the future umbilicus. The head-. tail-, and lateral amniotic folds become more elevated, the embryo sinks slightly, and the amniotic folds approach one another over the embryo. Subsequently these folds unite over the dorsal aspect of the embryo, and give rise to a sac, called the amnion, which surrounds the embryo. (In man the amnion is formed in a different manner, as will be presently described.)

After fusion, of the amniotic folds has taken place to form the amnion, the inner layer of somatopleure separates from the outer 
layer, except at the caudal or posterior extremity of the embryo. The inner layer constitutes the amnion, whilst the outer layer, which is part of the general or extra-embryonic somatopleure, forms the chorion.

As the circular groove encroaches from all sides upon the ventral aspect of the embryonic area, the somatopleure of the inner wall of the groove forms the lateral and ventral body-walls of the embryo, and, at the site of the future umbilicus, this somatopleure becomes continuous with that of the amnion.

The subjacent splanchnopleure meanwhile encroaches upon the blastodermic cavity, and, as a result of this constriction, from all sides, the archenteron is incompletely divided into, two unequal parts. The small dorsal compartment gives rise to the mid-gut (primitive gut), and the large ventral compartment constitutes the yolk-sac or umbilical vesicle. These two compartments communicate with each other at the site of the constriction by a narrow canal, called the vitelline duct or yolk-stalk. The walls of the mid-gut and yolk-sac consist of splanchnopleure, and, like their cavities, they are continuous with one another.

The mid-gut, which lies along the ventral aspect of the notochord, is originally a straight groove called the intestinal groove, which extends towards the cephalic and caudal extremities of the embryo. It is, however, subsequently converted into a tube. At first it has only one opening-namely that of the vitelline duct or.

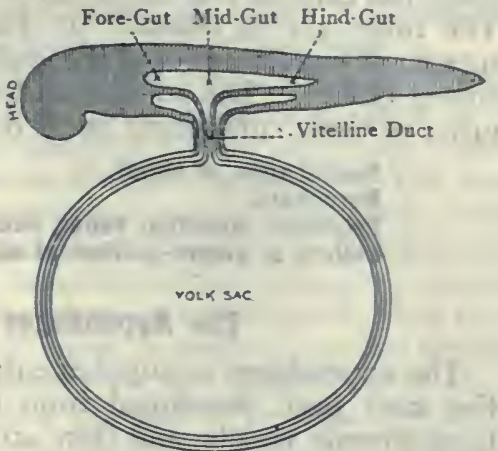

Fig. 673.-Yolk-SAc, Vitelline Duct, AND EMBRYo (ScheMaTIC). yolk-stalk-through which it communicates with the yolk-sac or umbilical vesicle. Its cephalic and caudal extremities are closed.

The fore-gut is formed as a tubular recess or diverticulum from the anterior part of the blastodermic vesicle, and it lies within the downward bend formed by the head of the embryo. Posteriorly it opens into the mid-gut compartment of the blastodermic vesicle at the intestinal orifice of the vitelline duct, the opening being known as the anterior intestinal portal. Anteriorly it is, at this stage, closed by the bucco-pharyngeal membrane, which consists of two layersan outer or ectodermic, and an inner or entodermic.

The hind-gut is formed, similarly to the fore-gut, as a tubular recess or diverticulum from the posterior part of the blastodermic vesicle; and it lies within the tail-fold. Anteriorly it opens into the mid-gut compartment of the blastodermic vesicle at the intestinal orifice of the vitelline duct, the opening being known as the posterior intestinal portal. The cephalic and caudal ends of the 
embryo become bent ventralwards, and these bent ends form the cephalic or head-fold and the caudal or tail-fold. Posteriorly the hind-gut is, at this stage, closed by the cloacal membrane, which, like the bucco-pharyngeal membrane at the cephalic end of the alimentary tract, consists of two layers-an outer or ectodermic, and an inner or entodermic. The entire primitive intestinal canal is therefore closed at either end, cephalic and caudal, by bilaminar membranes-bucco-pharyngeal and cloacal - the internal lamina of each of which consists of entoderm.

The wall of the primitive intestinal canal is formed by splanchnopleure-that is to say, entoderm and the splanchnic lamella of the lateral plate of mesoderm-the tube being lined with entodermic cells.

The unfolding processes, just described, accomplish the separation of the embryonic area on the dorsal aspect of the blastodermic vesicle, and that area now represents the primitive embryo. At this stage the embryo consists of three tubes and a solid cylinder of cells. The tubes are as follows:- (I) The neural tube; (2) the primitive intestinal canal; and (3) the cœlom, body-cavity, or pleuro-peritoneal cavity. The solid cylinder of cells is the notochord. The order of these component parts dorso-ventralwards is as follows:

\section{Neural tube or eanal. \\ Notochord. \\ Primitive intestinal canal, enclosed by splanchnopleure. \\ Cœlom or pleuro-peritoneal cavity, enclosed by somatopleure.}

\section{The Appendages of the Embryo.}

The appendages or supplementary organs of the embryo are, for the most part, developed from the extra-embryonic area of the blastodermic vesicle, and they are as follows:

1. The amnion.

2. The yolk-sac and vitelline duct.

3. The chorion (representing a large part of the placenta).

4. The allantois (diverticulum and stalk).

5. The umbilleal cord.

Functionally they are (I) protective, (2) nutritive, (3) respiratory, and (4) excretory. All these appendages are dispensed with at birth, the umbilical cord being then divided.

\section{The Amnion.}

The amnion is one of the two fretal deciduous membranes, the other being the chorion. It is a closed membranous sac, the cavity of which, called the amniotic cavity, contains a clear albuminous fluid, known as the liquor amnii. The amnion surrounds the embryo, which floats in the liquor amnii. When this fluid is present in excessive quantity, the condition is known as hydramnios, or dropsy of the amnion. Rupture of the amnion and escape of the liquor amnii usually precede the birth of the child. 
The following account of the mode of formation of the amnion in the chick, although referred to already, may here be re-stated. The amnion is developed from the somatopleure which forms the outer elevated lip of the marginal or circular groove round the embryonic area of the blastodermic vesicle, the appearance of which groove constitutes the first stage in the separation of the germinal disc. This somatopleure, which is disposed in two layers, inner and outer, gives rise to the amniotic folds. The circular groove deepens and encroaches, from all sides, upon the ventral aspect of the embryonic area as far as the site of the future umbilicus; and the somatopleure of the inner wall of the groove forms the lateral and ventral body-walls of the embryo. Meanwhile the amniotic folds gradually encroach, from all sides, upon the dorsal aspect of the embryonic area in the form of cephalic, lateral, and caudal hoods. Subsequently these hoods meet and fuse. When this fusion has taken place, the inner layer of somatopleure separates from the outer layer (except at the caudal or posterior extremity of the embryo), and it constitutes a closed sac, which is the amnion, the outer layer of somatopleure giving rise to the chorion.

The wall of the amnion is formed by somatopleure-that is to say, ectoderm and somatic mesoderm. The ectodermic layer is the inner of the two, and forms the lining of the amniotic cavity, whilst the layer of somatic mesoderm is situated externally. The bodywall of the embryo, as stated, also consists of somatopleure; but in this case the ectoderm is external, and the somatic mesoderm lies on its inner aspect. The ectodermic layer of the wall of tise amnion, which is the inner layer, therefore faces the ectodermic layer of the body-wall of the embryo, which layer is situated on the exterior. At the site of the future umbilicus, on the ventral aspect of the embryo, the somatopleure forming the wall of the amnion becomes continuous with the somatopleure which constitutes the body-wall of the embryo.

In the human embryo the amnion is formed in a manner different from that which has just been described as applicable to the chick. In the chick the amnion, briefly stated, is formed from the amniotic folds, representing the outer elevated lips of the circular groove around the embryonic area. The four amniotic folds-namely, cephalic, two lateral, and caudal - come together over the embryonic area, and by their fusion give rise to the amnion, which is therefore not a closed sac at first. In the human embryo it is different. From the very commencement the amnion is a closed sac, and is formed within the inner cell-mass of the blastodermic vesicle. Vacuoles form within the uppermost of these cells, the remainder of the cells forming the embryonic bud or disc. By the coalescence of these vacuoles the amniotic cavity is formed, which is thus a closed cavity from the first. The cells surrounding this cavity, and therefore forming its lining, are derived from the embryonic ectoderm, which forms the inner layer of the wall of the amnion. The outer layer of the wall, formed by somatic mesoderm, is derived 
from the somatic mesoderm of the embryo, as well as from the mesoderm of the allantoic or body-stalk. The embryonic bud liesoriginally below the amniotic cavity. As the periphery of the bud curves downwards or ventralwards, it carries with it the wall of the amnion where that is connected with the periphery of the bud. The roof of the amniotic cavity is formed by the superficial cells of the inner cell-mass, covered by that portion of the enveloping layer which is known as Rauber's layer.' The floor is formed by the embryonic bud or disc. The cells on the amniotic surface of this bud spread out over the wall of the cavity and become continuous with the roof-cells derived from the inner cell-mass, forming the embryonic ectoderm, as distinguished from the extra-embryonic éctoderm, which is represented by the enveloping layer. The: embryonic ectoderm thus lines the amniotic cavity, and it becomes continuous with the extra-embryonic ectoderm or enveloping layer at the periphery of the roof of the amniotic cavity, that part of the enveloping layer which forms Rauber's layer having disappeared. External to the lining layer of embryonic ectoderm there is a layer of somatic mesoderm, which is derived from the somatic mesoderm of the embryo, as well as from the mesoderm of the allantoic or belly-stalk. The wall of the amniotic cavity therefore consists of two layers - an inner, formed by embryonic ectoderm; and an outer, formed by somatic mesoderm-both connected together.

The embryonic bud or disc, as stated, lies originally in the floor of the amniotic cavity. As the periphery of the bud curves ventralwards, it carries with it the wall of the amnion, where that is connected with the periphery of the bud.

At the caudal or posterior extremity of the embryo the inner and outer layers of the amnion still remain connected. In, this region; the outer layer of the amnion, formed by somatic mesoderm, is: continuous with the inner layer of the chorion, which also consists of somatic mesoderm. This connection gives rise to the allantoic or belly-stalk, and, as the amnion is continuous with the body-wall of the embryo, the allantoic stalk connects the embryo with that part of the chorion which subsequently forms, along with the mesoderm of the allantoic stalk, the fotal part of the placenta.

The amnion is peculiar to Mammals, Birds, and Reptiles, these three classes of Vertebrata forming the group of Amniota. In Amphibia and Fishes the amnion is absent, and these two classes are consequently grouped as

\section{Anamnia.}

Pro-Amnion.-In front of the embryonic area of the blastodermic vesicle is the pro-amniotic area, the blastoderm of which is bilaminar, the two layers consisting of ectoderm and entoderm. As the head of the embryo grows forwards, it comes to lie over the pro-amniotic region, and the embryo is meanwhile sinking slightly. The bilaminar blastoderm of the pro-amniotic region thus forms a hood for the head of the embryo, this hood being known as the pro-amnion. At a later period the pro-amnion is invaded by somatic and splanchnic lamellx of mesoderm, and then it consists of somatopleure and splanchnopleure. Its somatopleuric constituent fuses with that division. of the amniotic folds which is called the head-fold, and therefore forms part of the amnion. The pro-amnion is well marked in the chick, but in the human embryo, if present at all, it is only for a very brief period. 


\section{The Yolk-Sac and Vitelline Duct.}

The yolk-sac is the ventral compartment of the cavity of the original blastodermic vesicle. As the formation of the amnion is being completed, an infolding of splanchnopleure takes place. The blastodermic vesicle is thereby gradually constricted, and its cavity is divided, at first incompletely, into two compartments-namely, dorsal and ventral. The dorsal compartment, which is the smaller of the two, gives rise to the mid-gut (primitive gut), and is situated within the body of the embryo. It is at first a groove, known as the intestinal groove, but it is subsequently converted into a tube. The ventral compartment which is large and capacious, forms the yolk-sac or umbilical vesicle, and is extra-embryonic.

The yolk-sac is at first in wide communication with the centre of the primitive mid-gut by a passage called the vitelline or vitellointestinal duct (yolk-stalk) which passes through the umbilical opening, and is connected with the ventral aspect of the mid-gut. The wall of the yolk-sac and vitelline duct is continuous with that of the mid-gut, both consisting entirely of splanchnopleure-that is to say, entoderm and splanchnic mesoderm, the entoderm forming the lining membrane.

In the human embryo the yolk-sac contains fluid. Its wall presents a well-marked vascular area, with which the primitive bloodvessels are associated. These vessels are called the vitelline or omphalo-mesenteric vessels and are four in number, two arteries and two veins, which will be again referred to.

The yolk-sac-undergoes atrophy, but a remnant of it persists in the umbilical cord. The vitelline duct also undergoes atrophy, and its lumen disappears. The part of the duct, however, which is connected with the mid-gut sometimes remains persistent, and so gives rise to an intestinal protrusion, known as Meckel's diverticulum.

\section{The Chorion.}

The chorion is the outer of the two fœtal deciduous membranes, the one internal to it being the amnion. It is developed from the outer layers of the amniotic folds, and, like the amnion, is composed of somatopleure. Its external surface consists of extra-embryonic ectoderm, and the internal surface is formed by somatic mesoderm.

The chorion is situated external to the amnion, and the somatic mesoderm of its inner surface faces the somatic mesoderm which forms the outer surface of the amnion. The interval between the chorion and the amnion is known as the chorionic cavity, and the embryo is now surrounded by two membranes, the inner of which is the amnion.

The chorionic cavity represents the extra-embryonic portion of the ccelom or body-cavity, and it contains (I) the embryo, enclosed within the amnion; and (2) the yolk-sac or umbilical vesicle. It is 
further traversed by the allantoic stalk or belly-stalk, as that structure extends from the umbilical orifice of the embryo to the placental area of the chorion, with which area the mesoderm of the allantoic stalk blends, and to which area that mesoderm conducts bloodvessels.

The chorionic cavity becomes gradually obliterated as the amniotic sac expands, the chorion and amnion then being intimately related to each other.

From the outer or ectodermic surface of the chorion small budlike processes grow into the contiguous maternal blood-lacunæ. These processes represent the rudimentary chorionic villi, and are at first entirely composed of ectoderm. Subsequently the mesoderm, which forms the inner layer of the chorion, grows outwards into these rudimentary villi, and forms for each of them a core. In this mesodermic core capillary bloodvessels are developed, which are continuous with the allantoic vessels through the mesoderm of the allantoic stalk, which fuses with the placental area of the chorion. The villi are now complete, and are somewhat clubshaped. A complete villus consists of the following parts: (I) An external envelope of ectoderm, the superficial layer of which consists of syncitium; (2) a vascular core of mesoderm; and (3) an arteriole, capillaries, and a venous radicle.

The villi at first beset the entire outer surface of the chorion, and are of equal size. Subsequently, however, the villi of one particular area undergo enlargement and ramifications, whilst the others undergo retrogression, and become very small and scattered, ultimately becoming atrophied. The area over which retrogression of villi takes place becomes comparatively smooth, and this part of the chorion is consequently called the chorion leve. The limited area over which the villi become enlarged is rough and shaggy, and is known as the chorion frondosum or leafy chorion. This area of the chorion becomes intimately connected with the uterine mucosa, usually at the upper part of the posterior wall of the uterine cavity, close to the fundus. Along with the mesoderm of the adjacent part of the allantoic stalk, it forms the fœtal part of the placenta.

The villi of the chorion frondosum are numerous and large. Moreover, they ramify and so become arborescent. Some of them penetrate by filamentous processes into the uterine mucosa (decidua basalis or serotina) in the placental region, and these processes, or calkers, not only grip but become continuous with it. Such villi are known as fixation- or root-villi. Others project into, and lie free within, the maternal blood-lacunæ, which are formed in connection with the decidua basalis or serotina of the uterine mucosa, where they are freely bathed by the maternal blood.

The uterine surface of the chorion frondosum has an epithelial covering consisting of ectoderm. This ectoderm is disposed at an early period in two layers - superficial and deep. The deep layer is distinctly cellular, and is known as the layer of Langhans. The 
villi pass through it, but have no connection with it. Subsequently its cells become grouped into patches, and finally disappear.

The superficial layer becomes converted into a tissue, composed of nucleated protoplasm, but without any recognisable cells. This layer is known as the syncitial layer. It is also spoken of as the syncitium, being so named because it has no recognisable cells.- In the case of the fixation-or root-villi, this syncitium accompanies them in the form of filiform processes, as they penetrate into the uterine mucosa.

The villi of the chorion frondosum, as they project from the surface, are separated by intervals, which constitute the maternal blood-spaces, -sinuses, or -lacunæ. These intervals are also known as the intervillous spaces, and those villi which are free project into them. These spaces are lined with syncitium, which tissue consequently covers the surface of the chorion frondosum between the villi, as well as the villi themselves.

\section{The Allantois.}

The allantois extends as a hollow tubular diverticulum from the dorsal wall of the yolk-sac to the inner surface of the chorion frondosum. The hind-gut has not been differentiated at this stage, but when this takes place the allantois becomes connected with the ventral wall of the hind-gut. At this point it is placed on the cephalic side of the cloacal membrane. It soon acquires an important relation to the cloaca, this latter being a blind chamber into which the hind-gut and urogenital ducts open. The allantois becomes connected with the ventral wall of the ventral or urogenital compartment of the cloaca, the dorsal compartment pertaining to the rectum.

The allantois consists of splanchnopleure (entoderm and splanchnic, or visceral mesoderm), which is continuous with the splanchnopleure of the wall of the gut. It soon elongates towards the umbilical orifice, through which it leaves the body of the embryo, lying on the caudal side of the vitelline duct. At this opening the allantoic diverticulum enters the allantoic, or body-stalk. This stalk traverses the chorionic cavity, and ends by blending with the inner surface of the chorion frondosum, which gives rise to the fœtal part of the placenta. The allantois consequently establishes an important connection between the body of the embryo and the placenta.

In order to understand the functions of the allantois it may be divided into two parts-intra-embryonic and extra-embryonic. The intra-embryonic part is distinguished as the allantoic diverticulum, and the extra-embryonic part is known as the allantoic, or body-stalk. The allantoic diverticulum has just been described.

The allantoic stalk extends from the umbilical orifice to the placental area of the chorion. As has been incidentally stated, the amnion and chorion do not become completely separated at the 
caudal or posterior end of the embryo, their opposed surfaces, which consist of mesoderm, being here connected. From this mesoderm a peduncle is developed, and this mesodermic peduncle constitutes the allantoic stalk, in the centre of which is the remnant of the allantoic diverticulum, now without a lumen. After having traversed the chorionic cavity it blends, as stated, with the chorion frondosum, which gives rise to the fotal part of the placenta. The mesodermic tissue of the allantoic stalk contains the allantoic or umbilical bloodvessels (two arteries and two veins). This vascular stalk therefore establishes an important communication between the caudal or posterior extremity of the embryo and the fotal (chorionic) part of the placenta. Its vessels render the placental area of the chorion, and also the chorionic villi, vascular.

Whilst the amnion and chorion are extra-embryonic, the allantois is both intra-embryonic and extra-embryonic.

Functions of the Allantois. - The extra-embryonic part or allantoic stalk is concerned with respiration and nutrition, as regards the human embryo, through means of the allantoic arteries and veins. In the embryos of oviparous animals it also serves the purpose of excretion (urinary).

Subsequent History of the Allantois.-The extra-embryonic part contributes to the formation of the chorionic or fœtal part of the placenta. The intra-embryonic part, or allantoic diverticulum, which is originally tubular, is connected, as stated, with the ventral or urogenital compartment of the cloaca. The urinary bladder is to be regarded as developed from the anterior or cephalic portion of this compartment, and perhaps from the root or cloacal end of the allantoic diverticulum. The portion of the allantoic diverticulum between the apex of the urinary bladder and the umbilicus loses its tubular character, and persists in the form of a fibrous cord, called the urachus.

\section{The Umbilicus and Umbilical Cord.}

The umbilicus is the opening in the ventral body-wall, through which originally the vitelline duct or yolk-stalk enters the colom or body-cavity, of the embryo. The allantois also leaves the fotal body through it. As the somatopleure (somatic mesoderm and ectoderm) closes the ventral body-wall it divides the embryonic portion of the body-cavity from the extra-embryonic portion. When the somatopleure reaches the situation of the vitelline duct an aperture is left in it for the duct, this aperture being the primitive umbilicus. After entering the embryonic body-cavity, the vitelline duct is attached to the ventral aspect of the mid-gut. At the margins of the umbilicus the somatopleure becomes continuous dorsalwards with that of the amnion, and ventralwards it ensheathes the vitelline duct for a short distance towards the yolk-sac, which is extra-embryonic.

The umbilical cord connects the fotus with the placenta, and, 
arnongst other structures, it contains the bloodvessels which convey (I) the impure blocd from the body of the fotus to the placenta for purification, and (2) the blood, when purified in the placenta, from that organ back into the fœtal body. Functionally it replaces the allantoic stalk. It extends, as a ' cord,' between the umbilical orifice and the fœtal part of the placenta. In the later stages of pregnancy its average length is about 22 inches. This length is only attained after expansion of the amniotic sac, and it permits of ample freedom of movement on the part of the foetus in the amniotic fluid. Under these circumstances the placenta is not subjected to any influence of a dragging nature. The average length, however, may be exceeded, or it may be very much curtailed. The cord presents a spiral appearance, principally due to the winding of the two umbilical arteries around the umbilical vein.

The constituents of the umbilical cord are as follows:
I. Two umbilical arteries.
2. One umbilical vein.
3. A remnant of the allantoic stalk.
4. A remnant of the yolk-sac.
5. A remnant of the vitelline duct.
6. Mesoderm and Wharton's jelly.
7. An ectodermic envelope.

As stated in connection with the allantois, the allantoic stalk consists of mesoderm, and contains two allantoic arteries and two allantoic veins, which bloodvessels it serves to conduct tc and from the placental area of the cherion (chorion frondosum). The two umbilical arteries replace the allantoic arteries; and the single umbilical vein of the umbilical cord represents the two allantoic veins.

The umbilical arteries, within the abdomen of the fœtus, are

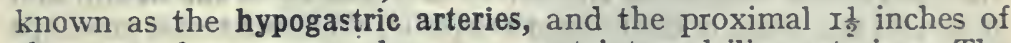
these vessels represent the permanent internal iliac arteries. The remainder of each vessel, for the most part, constitutes the permanent obliterated hypogastric artery. The hypogastric and umbilical arteries carry impure blood from the foetus to the placenta. The umbilical vein undergoes no change in name on entering the abdomen of the fœtus. It conveys pure blood from the placenta to the fœtus, and, after entering the abdomen, it passes to the umbilical fissure of the liver. As regards its subsequent history, it is represented by (I) the round ligament of the liver, and (2) the ligamentum ductus venosi.

The remnant of the allantoic stalk blends with the chorion frondosum. In rare cases the lumen of the allantoic diverticulum may persist for some time, thus giving rise to the condition which is known as an umbilical urinary fistula.

The yolk-sac and vitelline duct are present as mere remnants.

Mesoderm forms a large part of the umbilical cord. It supports and connects the various constituent elements, and consists of stellate cells, so arranged as to form a reticulum. The meshes of 
this reticulum are occupied by Wharton's jelly, which is composed of connective tissue, pervaded by a gelatinous fluid.

The external epithelial covering of the cord consists of ectoderm, and at the placental end of the cord this ectoderm is continuous with that of the amnion.

In early fœtal life one or more loops of intestine protrude into the umbilical cord for a short distance, but this condition is of temporary duration.

\section{The Uterine Mucosa.}

The uterine mucosa, or mucous membrane of the cavity of the body of the gravid uterus, having undergone hypertrophy, is known as the membrana decidua. It consists of three parts-namely, decidua vera, decidua capsularis, or reflexa, and decidua basalis, or serotina.

The ovum, after entering the cavity of the body of the uterus, becomes embedded in the uterine mucosa, the lining ciliated columnar epithelium of this part having disappeared, as well as the basement membrane and some of the superficial cells of the corium. The mucosa thus becomes excavated at the place where the ovum sinks into it, and the connection between the two is established through means of the fixation or root villi of the chorion frondosum. The usual site of attachment of the ovum is the upper part of the posterior uterine wall, close to the fundus uteri.

The decidua vera represents the greater part of the uterine mucosa or membrana decidua. It lines the cavity of the body of the uterus, except where the ovum is attached, and it has no direct connection with the ovum.

The decidua capsularis, or reflexa, is derived from the margins of the excavation formed at the site of attachment of the ovum, and it invests the ovum in the form of a capsule. This maternal envelope lies outside the chorion leve, but does not encroach upon the chorion frondosum. It is at first separated from the decidua vera by a space. As, however, the embryo increases in size the decidua capsularis, or reflexa, comes into contact with, and joins, the decidua vera, one deciduous membrane being thereby formed, and the cavity of the body of the uterus being obliterated.

The decidua basalis, or serotina, is that portion of the uterine mucosa which forms the floor of the excavation formed at the site of attachment of the ovum. It corresponds to the region of the chorion frondosum, with which it becomes incorporated, and it gives rise to the maternal part of the placenta, as distinguished from the fretal part.

Structure of the Decidua Vera.-The mucosa which forms the decidua vera has undergone certain modifications in structure. (I) It has become very much thickened, and the covering layer of ciliated columnar epithelium has disappeared. (2) The uterine glands have undergone enormous development, and are now much elongated, dilated, and tortuous. (3) In the interglandular spaces 
there are many large spherical cells, called the decidual cells. These cells are present throughout the whole thickness of the decidua vera, but they are most numerous in its superficial stratum.

The decidua vera is composed of three layers-namely, stratum compactum, stratum spongiosum, and stratum profundum.

The stratum compactum is the superficial layer, and lines the uterine cavity. The uterine glands, as they traverse it, are elongated, somewhat dilated, slightly tortuous, and parallel with each other. The epithelium lining them consists of cubical or flat cells.

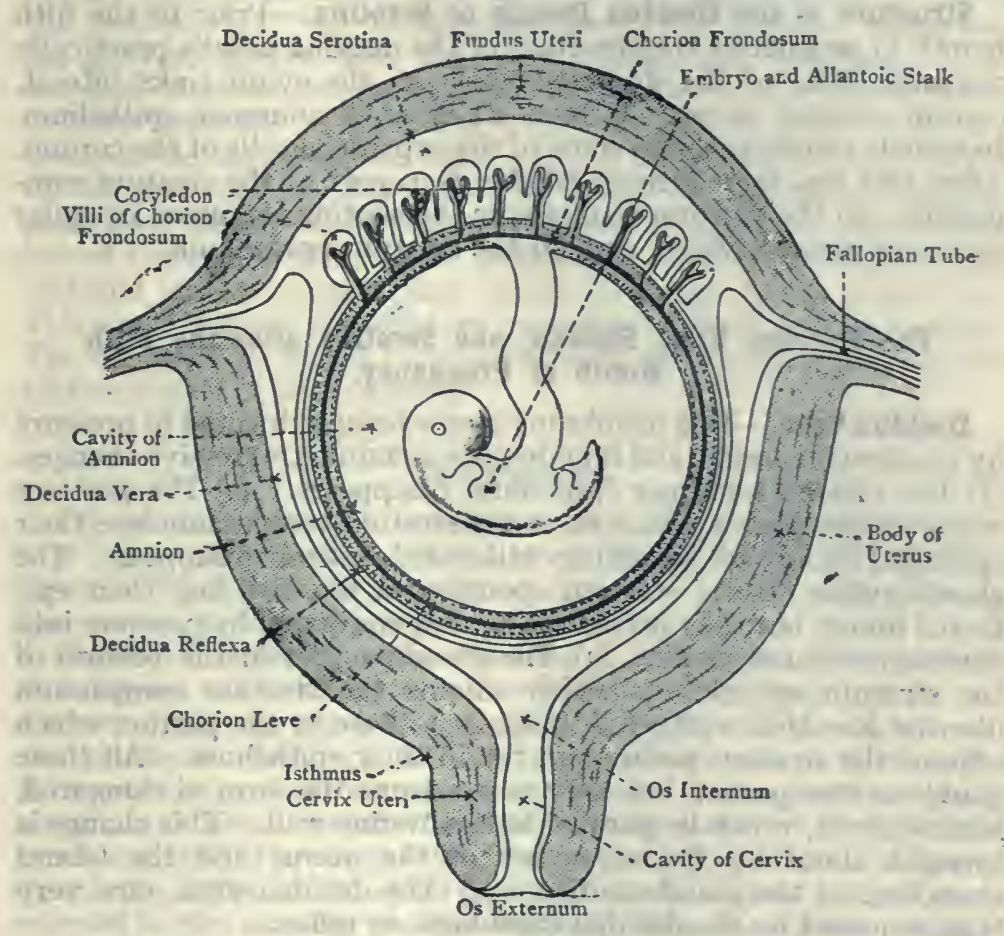

Fig. 674.-The Gravid Uteros.

This stratum contains many decidual cells, and has a compact structure.

The stratum spongiosum, or cavernosum, is more deeply placed than the stratum compactum. The uterine glands, as they traverse it, are much dilated, and very tortuous. It contains few decidual cells, and has a spongy or cavernous structure. As seen in section it presents a number of irregular spaces, or areolæ, which represent the gland-cavities. The epithelium lining these cavities consists of cubical or flat cells. 
The stratum profundum lies next the muscular coat, there bcing no submucosa in the uterine wall. It contains the deep parts of the uterine glands, the blind ends of which project into the inner muscular layer, after having undergone in many cases dichotomous division.

Structure of the Decidua Capsularis or Reflexa.-This contains no uterine glands, except where it is in continuity with the decidua vera. Essentially it consists of a matrix of connective tissue, permeated by decidual cells, and it is devoid of epithelium.

Structure of the Decidua Basalis or Serotina.-Prior to the fifth month of pregnancy the structure of the decidua basalis practically resembles that of the decidua vera. As the ovum sinks into it, certain changes occur. It loses its ciliated columnar epithelium, basement membrane, and some of the superficial cells of the corium. After this loss there remain (I) the deep part of the stratum compactum, (2) the stratum spongiosum, presenting the usual irregular spaces or gland-cavities, and (3) the stratum profundum.

\section{The Decidua Vera, Reflexa, and Serotina after the Fifth Month of Pregnancy.}

Decidua Vera.-This membrane is now being subjected to pressure by the growing foetus, and it undergoes certain retrogressive changes. (I) The ciliated columnar epithelium disappears. (2) The portions of the uterine glands which lie in the stratum compactum lose their epithelial lining and, becoming obliterated, entirely disappear. The gland-cavities in the stratum spongiosum likewise lose their epithelial lining, but they persist, being now converted by pressure into narrow elongated clefts. (3) The gland-cavities in the portion of the stratum spongiosum which adjoins the stratum compactum likewise lose their epithelial lining, but those in the portion which adjoins the stratum profundum retain their epithelium. All these gland-cavities persist, but they now assume the form of elongated, narrow clefts, which lie parallel to the uterine wall. This change is brought about by the expansion of the uterus and the lateral stretching of the gland-cavities. (4) The decidua vera, now very thin, is joined by the decidua capsularis, or reflexa.

Decidua Capsularis or Reflexa.-This membrane gradually expands after the fifth month of pregnancy in adaptation to the growing fotus, and it comes to press closely upon the decidua vera. The uterine cavity thereby becomes gradually occupied by (I) the foetus and its membranes, and (2) the placenta. Whatever glandular elements the decidua capsularis contains disappear, the membrane becomes extremely thin, and it fuses with the decidua vera. According to some authorities, the membrane entirely disappears, in which case the chorion leve would come into contact with the decidua vera.

Decidua Basalis, or Serotina.-The changes which this membrane has undergone prior to the fifth month of pregnancy have just been 
stated. As it is the source from which the maternal part of the placenta is formed, its future intra-uterine history differs materially from that of the decidua vera and decidua capsularis. The changes, therefore, which this membrane undergoes after the fifth month are associated with the full development of the placenta, which see. They may, however, be stated here in continuity with the changes undergone by the decidua vera and decidua capsularis.

(I) The gland-cavities of the stratum spongiosum undergo changes similar to those which affect the corresponding stratum of the decidua vera. (2) In the remainder of the stratum compactum the uterine glands lose their epithelium and disappear. This vestigial stratum acquires great vascularity, and becomes consequently increased in thickness. (3) Superficially-that is to say. towards the region of the fœtal part of the placenta-it gives rise to the maternal blood-lacunæ, into which the maternal arterioles pour their blood. (4) Underneath - that is to say, on the uterine side of this region of maternal blood-lacunæ-there is a layer of the vestigial stratum compactum, which is known as the basal layer. This layer adjoins the stratum spongiosum, and is very vascular. The decidua basalis will be again referred to in connection with the maternal part of the placenta.

\section{The Placenta.}

The placenta is a very vascular organ, which establishes an important connection between the body of the fœtus and the maternal uterus. Its function is respiratory, nutritive, and excretory, during the last six months of intra-uterine life.

Physical Characters of the Placenta at Full Term.-The placenta at full term has the form of a discoid cake, which is spongy in consistence. Its average width is about 7 inches, and its thickness at the centre is about $I_{\frac{1}{2}}$ inches, the circumference, which is the thinnest part, measuring about 23 inches. Its average weight is about 20 ounces. In all these respects, however, it is subject to much variety. It presents two surfaces - namely, fotal and maternal or uterine. The foetal surface is smooth and concave, and is loosely covered by the amnion, through which the branches of the umbilical arteries can be seen as they divide and subdivide before entering the organ. It is connected with the abdominal wall of the fœetus at the umbilical orifice by means of the umbilical cord, which is attached to the placenta at, or near, the centre, as a rule. Sometimes, however, the umbilical cord is attached to some part of the margin of the placenta, which then bears a resemblance to a battledore. Under these circumstances it is known as a battledoreplacenta.

The maternal or uterine surface is convex, and constitutes the vault of the placenta. It is very irregular, being broken up into a number of polygonal masses or lobes, called cotyledons, which are arborescent tufts of chorionic villi. 
At its circumference the placenta is connected with the chorion leve, decidua capsularis or reflexa, and decidua vera.

The usual site of the placenta is the posterior wall of the cavity of the body of the uterus near the fundus. It may, however, be attached to any part of the internal surface of the body of the uterus. When it is implanted over the os uteri internum, it forms the lowest of the contents of the gravid uterus, and under these circumstances it gives rise to the condition called placenta pravia, or placental presentation. In some of these cases the placenta is attached round the entire circumference of the os uteri internum, this condition being known as complete placenta pravia, or placenta centralis. In other cases the attachment of the placenta in this region is incomplete, and such a condition is spoken of as partial placenta pravia or placentà lateralis.

The placenta consists of two parts-namely, fœetal and maternal or uterine, which cannot be separated from each other.
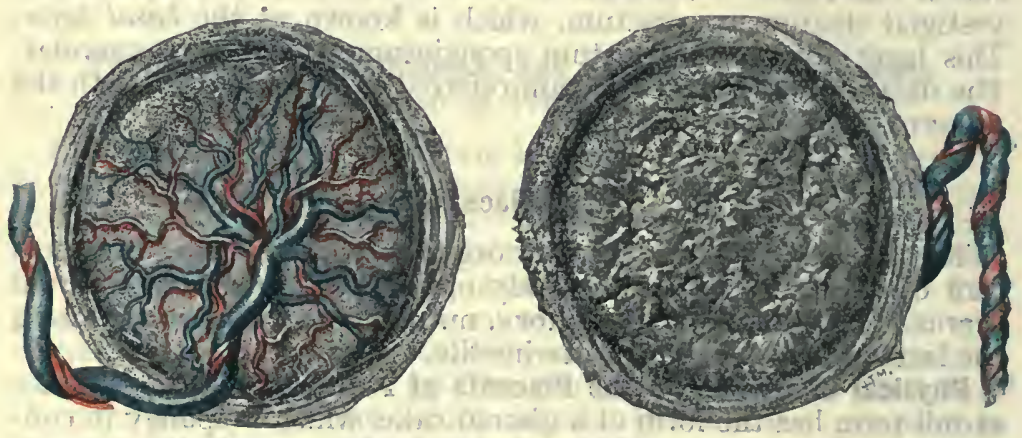

Fig. 675--Placenta: Fctal and Maternal Súraces.

Fœtal Part of the Placenta. - The fotal or chorionic part constitutes the largest portion of the placenta. It is formed by the chorion frondosum, which is joined by the chorionic end of the allantoic stalk. The chorion leve takes no part in its formation, the villi of the chorion leve, at first few and scattered, becoming atrophied. The villi of the chorion frondosum are of large size, and they undergo ramifications, forming, as stated, arborescent tufts or cotyledons. Some of these villi are attached to the maternal part of the placenta, with which they become continuous. Hence the fœtal and maternal parts cannot be separated from each other. These attached villi are, as previously stated, called fixation-or rootvilli. Other villi project into, and lie free within, the maternal blood-lacunæ, where they are bathed by the maternal blood.

Each villus, as stated in connection with the chorion, consists of the following elements: (I) An external covering of ectoderm, the superficial lamina of which is formed by syncitium, and (2) a core of 
somatic mesoderm. This core contains an arteriole, capillaries, and a venous radicle. As the arteriole traverses the mesodermic core of the villus it gives off minute twigs, which form a rich capillary network, lying close beneath the surface, from which the blood passes into the venous radicle. At the free extremity of the villus the arteriole is connected with the commencement of the venous radicle by a capillary loop.

Maternal Part of the Placenta. - The maternal or uterine part forms a small portion of the entire placenta, corresponding to the convex uterine surface or vault, and it is developed from the decidua basalis or serotina of the uterine mucosa. The decidua basalis or serotina consists, at an early period, of the three strata which characterize the decidua vera-namely, the stratum compactum, stratum spongiosum, and stratum profundum. As the ovim sinks into the decidua basalis, in order to grip the uterine mucosa, the decidua basalis undergoes certain important changes in giving rise to the maternal part of the placenta. Its ciliated columnar epithelium disappears, as well as the superficial part of the stratum compactum and the portions of the uterine glands contained therein. There are this left ( $I$ ) the deep part of the stratum compactum, (2) the stratum spongiosum, and (3) the stratum profundum.

The deep pari of the stratum compactum gives rise to the maternal part of the placenta. The portions of the uterine glands within it disappear, and the bloodvessels become distended, a condition of hypertrcphy being the result. This deep part of the stratum compactum (the only part of it now left) consists of two constituents. The deepest portion of it - that is to say, the portion contiguous to the stratum spongiosum - is called the basal layer, and it is very vascular. The superficial portion of the deep part of the stratum compactum is now transformed into blood-spaces, known as the maternal blood-lacunæ or intervillous spaces, which receive their blood from the maternal arterioles. The structure, therefore, of the decidua basalis or serotina becomes modified in the latter half of "pregnancy, and it consists of the following parts, in order from without inwards: (I) The stratum profundum, unaltered; (2) the stratum spongiosum, the gland-cavities of which have lost their epithelial lining, and have become flattened out; (3) the basal layer of the stratum compactum; and (4) the maternal blood-lacunæ, or intervillous spaces.

The basal layer of the stratum compactum is, according to some authorities, composed of syncitium-that is to say, nucleated protoplasm without any recognisable cells. Vertical septa, composed of syncitium, extend between it and the surface of the chorion between the villi. These septa separate the placental cotyledons from one another, and they traverse the maternal blood-sinuses or intervillous spaces, which are thus broken up into blood-lacunæ. These septa contain maternal arterioles and venous radicles. The blood-lacunæ, now separated by these septa, are limited, on the maternal side, by the basal layer, and, on the foetal side, by the 
surface of the chorion covered by syncitium beneath which is the celliular layer of Langhans.

The blood-lacunæ are situated entirely within the maternal placenta, and are filled with maternal blood, by which the cotyledons are freely bathed. They are lined with syncitium, and are by some regarded as dilated capillaries, their syncitial lining being continuous with the endothelium of the maternal arterioles in the basal layer of the decidua basalis. More probably, however, the syncitial lining is derived from the syncitium which forms the superficial layer of epithelium of the chorion frondosum.

The arteries of the maternal part of the placenta are derived from the uterine and ovarian arteries.

Placental Circulation.-Both fœtal and maternal blcod circulate through the placenta. The impure blood of the fotus is conveyed to the placenta by the two hypogastric and umbilical arteries, which are represented, at an earlier period, by the two allantoic arteries. After being purified in the placenta, the blood is conveyed from that organ into the body of the fœtus by the umbilical vein, which is represented, at an earlier period, by the two allantoic veins. The arteries of the maternal part of the placenta are derived, as just stated, from the uterine and ovarian arteries. The maternal arterial blood eventually leaves the capillaries and enters the maternal blood-lacunæ, which are formed in connection with the stratum compactum of the decidua basalis or serotina, and into which the capillaries open. The blood is conveyed away from the intervillous spaces by minute venous radicles which open into veins in the deep part of the decidua basalis. It thus freely bathes the chorionic villi, each of which contains, as stated, an arteriole, a venous radicle, and a rich network of capillary bloodvessels. There is no direct intermingling of the fœtal and the maternal blood. The two are separated by (I) the ectodermic (syncitial) envelope of each villus, and (2) the core of mesoderm within. This mesoderm, however, really represents the endothelial walls of the capillary ramifications of the arteriole within the villus. These tissues, however, are so thin that interchanges readily take place through them, by osmosis, between the fotal and the maternal blood. These interchanges are of a threefold nature-namely, respiratory, nutritive, and excretory. (I) The fotal blood is here supplied with oxygen and deprived of carbon dioxide; (2) it is furnished with nutritive materials; and (3) excretory products of the fotus are removed from it. Being now purified, it is conveyed into the body of the fœetus by the umbilical vein.

It is to be noted, in connection with the placental circulation, that the fœtal blood never leaves closed vessels, whilst the maternal blood does do so, and enters blood-lacunæ. It is right, however, to state that these blood-sinuses are regarded by some authorities as greatly enlarged capillaries.

The veins of the maternal part of the placenta commence as venous radicles which lie partly in the basal laycr, and partly in the syncitial. 
septa between the blood-lacunæ; and they receive their blood from these sinuses through minute pores, by which the basal layer and septa are perforated. Some of these veins pass to the uterine surface or vault of the placenta, and thence to the veins of the uterine wall. Most of them, however, pass to the periphery of the placenta, where they open into a channel, called the circular or marginal venous sinus, from which it passes into the veins of the decidua vera.

The Placenta and Deciduous Membranes at Full Term.-At the period of birth (full term) rupture of the amnion takes place as a rule, and this is accompanied by the discharge of the liquor amnii. Expulsion of the child then takes place, but the placenta and the deciduous membranes usually remain within the uterus for a certain period.

The umbilical cord having been divided, the next stage consists in the expulsion of the placenta and membranes. These membranes are as follows: (I) The amnion; (2) the chorion leve; and (3) the united decidua capsularis or reflexa, and stratum compactum of the decidua vera. The parts of the uterine mucosa which remain in situ or in utero are (I) the stratum spongiosum, in whole or in part, and (2) the stratum profundum of the decidua vera and of the decidua basalis or serotina, with the parts of the glands contained within these two strata.

The uterine mucosa therefore undergoes a splitting, usually at the line of junction of the stratum compactum and stratum spongiosum, the former being expelled, whilst the latter remains in silu. The splitting, however, may take place through the stratum spongiosum.

The impoverished uterine mucosa now undergoes regeneration, the cast-off stratum compactum being formed anew by the epithelial cells which line the gland-cavities within the stratum spongiosum and the deep ends of the uterine glands within the stratum profundum.

The body of a child at birth is usually smeared with a white cheesy coating, which is called the vernix caseosa (smegma embryonum). This is formed by the sebaceous glands of the skin, and it contains epidermic scales, which have become detached from the superficial layer of the epidermis, known as the epitrichium. It acts as a kind of ointment, which protects the epidermis from the softening action of the liquor amnii, and it occurs in greatest abundance in the following regions: the head and face, the flexures of the joints, the palms of the hands, the soles of the feet, and the natal cleft.

The fine soft down or hair of the child at birth, some of which is shed prior to birth, imparts a somewhat woolly appearance to the regions provided with it. This fœtal down or hair is called the lanugo.

Relative Position of Structures within the Gravid Uterus.-When the wall of the body of the gravid uterus is divided in the operation 
known as hysterotomy, the following structures are met with, in order from without inwards:

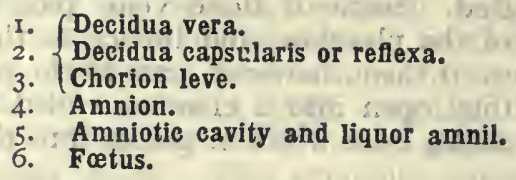

\section{The Vascular System.}

The vascular system consists of (I) the heart, (2) the bloodvessels, and (3) the blood, all of which are developed from the mesoderm. The heart is developed from the embryonic mesoderm, whilst the primitive bloodvessels and the blood are derived from the extraembryonic mesoderm. The earliest form assumed by the vascular system is associated with the vitelline circulation. This gives place to the allantoic circulation, and this, in turn, is supplanted by the placental circulation.

Bloodvessels. - The earliest bloodvessels, called vitelline or omphalo-mesenteric, are four in number - two arteries and two veins. They are developed in connection with the wall of the yolk-sac, which consists of splanchnopleure-that is to say, splanchnic mesoderm and entoderm. The region of the yolk-sac in which they appear is called the vascular area, which is disposed in the form of a band or strip surrounding the embryo.

The first stage consists in the formation of cells, called angioblasts or vessel-formers, which are arranged as cords or cylinders, known as cell-cords. These cell-cords are disposed in a reticular manner, the reticulum being called the angioblastic reticulum, and within its meshes there are groups of cells.

The second stage consists in the flattening and extension of thr superficial cells of the cell-cords to form continuous endothelia] walls, which represent the lining walls of the future bloodvessels, and which enclose the remainder of the cell-cords.

The primitive bloodvessels, therefore, are at first solid cords or cylinders, each representing a cell-cord, surrounded by an endothelial wall.

The third stage consists in the hollowing out of these solid cellcords, so that each now acquires a lumen. Each contained cellcord becomes permeated by a clear fluid, which has entered from the surrounding mesoderm, and the cells are pressed towards the walls of the vesscls. In these situations they form groups, called the blood-islands of Pander, which are nests or 'cradles ' of primitive red corpuscles. These groups encroach more or less upon the lumen of the vessel, and may even temporarily occlude it. The individual cells of each group increase in number by mitotic or karyokinetic division, and, entering the blood-stream, they represent the first corpuscles of the embryonic blood, which are called erythroblasts. The blood-islands, being thus gradually deprived 
of their cells, eventually disappear. The erythroblasts are at first colourless, but soon assume a straw-colour (pale yellow), and they constitute the primitive red corpuscles, being the only globules which the blood contains at this stage. Each has a large nucleus at this period. Being nucleated cells, they undergo mitotic division for some time, and so multiply.

In the second month of intra-uterine life, however, non-nucleated red corpuscles appear, known as erythrocytes, which rapidly increase in number. Many of the erythroblasts lose their nuclei, probably by a process of extrusion, and then become non-nucleated red corpuscles. It is to be noted that, though in early life the red corpuscles are nucleated, in adult life they are non-nucleated in Mammalia.

The white corpuscles or leucocytes are formed outside the bloodvessels, and therefore enter the blood-stream by immigration. The sources from which they are derived are as follows: (I) The spleen; (2) the marrow of bone; (3) the lymphatic glands, lymphoid follicles, tonsils, and thymus; and (4) the embryonic liver.

The blood-platelets may represent portions of disintegrated leucocytes, or they may be fragments of the multi-nucleated giant-cells of bone-marrow.

The organs concerned in the formation of blood-corpuscles are spoken of as hamatopoietic (blood-forming) organs.

The process of vascularization invades the entire wall of the yolksac, and is brought about in a manner similar to that which affects the vascular area. The splanchnopleure is invaded by cell-cords; the superficial cells of these cords form the endothelial linings of the vessels; and the contained cell-cord, in each case, becomes followed out to form the lumen of the vessel.

This process of vascularization extends along the vitelline duct, and in this manner the vessels are brought within the body of the embryo. Thereafter they become connected with the primitive heart, which at this stage is undergoing development. When this connection has been effected-direct as regards the vitelline veins, but indirect as regards the vitelline arteries - the vitelline circulation is established, by means of which nutritive materials are conveyed from the yolk-sac to the embryo (chick or rabbit).

\section{The Vitelline Circulation.}

The bloodvessels concerned in the vitelline circulation are four in number - namely, (I) the two vitelline or omphalo-mesenteric veins, and (2) the two vitelline or omphalo-mesenteric arteries-there being an intervening system of vessels, of the nature of capillaries, and a blood-channel, called the sinus terminalis.

The sinus terminalis surrounds the vascular area of the yolk-sac, and the numerous capillary vessels proceeding from it give rise by their confluence to the two vitelline veins. These veins, having entered the body of the embryo, pass along the primitive intestinal 
tube to the sinus venosus, which is situated close to the caudal or posterior end of the developing heart.

The two vitelline arteries are branches of the two primitive dorsal aorta, and are therefore connected with the developing heart only indirectly.

The vitelline circulation is of little importance in Man, but it is a considerable factor in the case of oviparous animals-e.g., Birdsthe yolk in such animals being very abundant and specially nutritive.

\section{The Allantoic Circulation.}

As retrogression of the yolk-sac and vitelline circulation takes place, the nutritive and respiratory functions of the fotus are assumed by the allantois and allantoic circulation. The left vitelline artery becomes entirely obliterated, but the proximal or intra-embryonic portion of the right vitelline artery persists, and gives rise to the superior mesenteric artery.

As previously stated, the allantois is a blind diverticulum from the ventral wall of the hind-gut. This diverticulum consists of splanchnopleure - that is to say, entoderm and splanchnic mesoderm-which is continuous with the splanchnopleure of the wall of the gut. This part of the allantois is known as the allantoic diverliculum. At the umbilical orifice it enters the allantoic stalk, which extends from the umbilicus to the placental area of the chorion, or chorion frondosum. The mesodermic tissue of the allantoic stalk contains the two allantoic arteries and the two allantoic veins.

The allantoic arteries grow from the caudal ends of the two primitive dorsal aorta. "In the region of the chorion frondosum they divide into branches which enter the cotyledons or tufts of arborescent chorionic villi, and render these important structures vascular. In this manner the peripheral part of the allantoic stalk and the chorion frondosum are brought into continuity. The blood is conveyed from the primitive dorsal aortæ to the chorion frondosum by the allantoic arteries, which leave the body of the fotus through the umbilical orifice; and, after having undergone the necessary changes, it is conveyed back into the body of the fœtus by the allantoic veins. These veins enter the foetal body through the umbilical orifice, and, after passing along the intestinal tube, they open into the sinus venosus at the caudal or posterior end of the developing heart.

The foregoing constitutes the allantoic circulation, which is of special importance in Birds, Reptiles, and Fishes. In Man, however; this circulation is of a transitory nature, and soon gives place to the placental circulation.

\section{The Placental Circulation.}

The nature of the placental circulation has been described in connection with the placenta. It is sufficient to state in this place 
that the bloodvessels concerned in it are the two umbilical arteries and the two umbilical veins (there being two such veins at an early period).

The umbilical arteries replace the allantoic arteries, and they originally grow from tile caudal ends of the two primitive dorsal aorta. They carry impure blood from the fœetus to the placenta, where it is purified and furnished with nutritive materials from the maternal blood by a process of osmosis.

The umbilical veins replace the allantoic veins, and they convey pure blood from the placenta to the body of the fœtus. At an early stage, to which this description only applies, they terminate in the sinus venosus. These umbilical veins have been already referred to in connection with the development of the veins.

\section{Development of the Pericardial, Pleural, and Peritoneal Cavities, Septum Transversum, and Diaphragm.}

The cœlom, body-cavity, or pleuro-peritoneal cavity is the space which lies between the somatopleure of the body-wall and the splanchnopleure of the primitive alimentary tube. It is originally a single cavity, but is subsequently divided into (I) the pericardial cavity; (2) the pleural cavities, right and left; and (3) the peritoneal cavity. The pericardial and pleural cavities are situated in the thorax, and the peritoneal cavity is situated in the abdomen, the septum between the thorax and the abdomen being formed by the diaphragm.

Pericardial Cavity. - In connection with the development of the heart, it has been stated that the rudiments of that organ take the form of two tubes, which are hollowed out in the splanchnic mesoderm. These two tubes, approaching each other, fuse and form a single longitudinal tube, or primitive heart, which lies mesially along the ventral aspect of the pharyngeal part of the fore-gut. It has been further stated that the primitive heart is connected with the ventral body-wall by a bilaminar fold of splanchnic mesoderm, called the ventral meoscardium, and with the pharyngeal part of the foregut by another similar fold, called the dorsal mesocardium. These folds soon disappear-the ventral mesocardium entirely, and the dorsal mesocardium to a large extent. There are also two lateral mesocardia-right and left-which are continuous with the ventral margin of the septum transversum, each of these containing a duct of Cuvier on its way to the sinus venosus of the heart.

The heart is developed in the splanchnic mesoderm of the pericardial region (cardiac portion of the ventral mesentery), the portion of that mesoderm which is ventral to the primitive heart being the ventral mesocardium, whilst the portion dorsal to the primitive heart forms the dorsal mesocardium. The part of the cœlom which corresponds to the pericardial region is known as the pericardiothoracic cavity, and it gives rise to the pericardial eavity. This latter cavity is subsequently shut of from the peritoneal cavity by the 
septum transversum, which gives rise to the ventral portion of the diaphragm. The pericardial cavity is also shut off from the two pleural cavities by the formation of the pleuro-pericardial meimbrane. Finally, the pleural cavities are shut off from the peritoneal cavity by the pleuro-peritoneal membranes, which give rise to the dorsal part of the diaphragm.

Demarcation of the Pericardial Cavity. - The pericardial cavity is the first of the divisions of the coelom to be differentiated. As stated, it is cut off from the peritoneal cavity by the septum transversum, which gives rise the ventral portion of the diaphragm.

Septum Transversum.-This is a thick, incomplete lamina of mesoderm, which is situated at the caudal end of the pericardial region on a level with the third and fourth cervical somites. It separates the sinus venosus of the primitive heart within the pericardial portion of the colom or body-cavity from the primitive stomach and intestinal tube; within the peritoneal portion of the colom. At first it occupies an almost horizontal plane, but, as the heart extends dorsalwards, it gradually assumes an oblique plane, the ventral attachment of the septum descending, so that the direction of the plane is dorso-ventralwards. As just stated, the septum is incomplete, the deficient part being situated dorsally, and its attachments are as follows: Ventrally (anteriorly) it is attached to the ventral wall of the cœlom, in which situation it receives the vitelline and umbilical veins on their way to the sinus venosus of the primitive heart. Laterally it is attached to the lateral wall of the cœlom on either side (lateral mesocardia), where it receives the corresponding duct of Cuvier, these two ducts being likewise on their way to the sinus venosus. Dorsally the margin of the septum may be divided into three parts - mesial and two lateral, right and left. The mesial part faces, and is soon closely connected with, the ventral aspect of the primitive duodenum, at the place where the hepatic diverticulum makes its appearance. The lateral parts of the dorsal margin are free and form the ventral boundarics of the peritoneal openings of the two thoracic (pleural) recesses of the peritoneum.

As development proceeds, the septum transversum becomes separated into three layers - cephalic, mesial, and caudal. The cephalic or pericardial layer becomes the diaphragmatic portion of the pericardial wall. The middle or diaphragmatic layer gives rise to the ventral portion of the diaphragm. The caudal or hiepatic layer becomes separated from the middle or diaphragmatic layer, as the development of the liver from the hepatic diverticulum proceeds within it, and this layer gives rise to the connecting tissue of the liver.

The deficiency left by the septum transversum dorsally is filled up by. the two pleuro-peritoneal membranes, right and left, to be presently described, and in this manner the dorsal part of the diaphragm is formed.

As stated, the septum transversum is incomplete dorsally. On 
either side of the fore-gut and its dorsal mesentery there is a prolongation of the peritoneal cavity into the thorax. These thoracic prolongations are narrow cleft-like passages, known as the thoracic or pleural recesses, and they represent the primitive pleural cavities. They communicate ventralweards with the dorsal aspect of the pericardial cavity by the pleuro-pericardial openings, and caudalwards with the peritoneal cavity by the pleuro-peritoneal openings.

The lung-buds of the pulmonary diverticula abut against these dorsally placed thoracic or pleural recesses in a ventro-lateral direction. As the lung-buds grow, the primitive pleural cavities (thoracic recesses) extend ventro-laterally, gradually displacing the parietal pericardium from the body-wall in a mesial direction. Subsequently they surround the pericardial cavity, except on its ventral and cauda! aspects.

The coelom, or body-cavity, originally single, is now divided into four compartments - pericardial, two pleural, and peritoneal-all of which communicate with one another.

The pericardial cavity is shut off from the pleural cavities by the formation of the pleuro-pericardial membrane. This membrane is derived from (I) the right and left lateral mesocardia, and (2) a portion of the dorsal mesocardium. The lateral mesocardia (pleuropericardial folds) grow in the form of two curtains towards the mesial line, and unite with a portion of the dorsal mesentery to form the pleuro-pericardial membrane. The pericardial cavity is now completely isolated from the pleural cavities. At an earlier date it is shut off from the peritoneal cavity by the septum transversum.

The parietal pericardium is derived from (I) the somatic mesoderm of the somatopleure of the body-wall, which has been displaced mesially by the growth of the lungs; (2) the cephalic or pericardial layer of the septum transversum; and (3) the pleuro-pericardial membrane. The viseeral pericardium is derived from the splanchnic mesoderm of the primitive cardiac tube.

Pleural Cavities. - These cavities are formed by the thoracic or pleural recesses of the peritoneal cavity, on either side of the foregut and its dorsal mesentery. They originally communicate ventrally with the dorsal aspect of the pericardial cavity by the pleuropericardial openings, and with the peritoneal cavity by the pleuroperitoneal openings. As the lung-buds of the pulmonary diverticula grow, each carries along with it splanchnic mesoderm continucus with that of its diverticulum, and the superficial part of this splanchnic mesoderm ultimately forms the visceral pleura. This explains why the lungs lie behind the pleural sac. The parietal pleura is derived from the somatic mesoderm of the somatopleure of the body-wall.

Closure of Pleuro-Peritoneal Openings.-The closure of these openings is effected by the pleuro-peritoneal membranes, each of which is formed by the growth ventralwards of the mesoderm of the dorsal body-wall. When this mesoderm reaches the ventral boundary of the pleuro-peritoneal opening on either side, it joins 
the free lateral portion of the dorsal margin of the septum transversum. In this manner the pleural compartments of the cœlom are shut off from the peritoneal compartment, and the diaphragm is completed dorsally. At an earlier date the pleural cavities are shut off from the pericardial cavity by the pleuro-pericardial membrane. The four divisions of the originally single cœlom or bodycavity now constitute four serous sacs, quite independent of each other-namely, pericardial, pleural, right and left, and peritoneal.

From what has been stated it will be evident that the pleural sacs or cavities are originally thoracic prolongations of the peritoneal cavity, from which they are shut off by the pleuro-peritoneal membranes. At the caudal end of the peritoneal cavity there are two somewhat similar scrotal prolongations, called the processus vaginales, one on either side. These prolongations are, as a general rule, shut off from the peritoneal cavity by the closure of their upper ends. The lower part of each process forms the tunica vaginalis.

The corresponding prolongations in the female are known as the eanals of Nuck.

The diaphragm, as stated, is developed in two parts, ventral and dorsal. The ventral portion is formed from the middle layer of the septum transversum, whilst the dorsal portion is developed from the two pleuro-peritoneal membranes. In very rare cases the dorsal portion of the diaphragm may fail to unite with the ventral portion on one or both sides, due to an arrest in the development of one or both pleuro-peritoneal membranes, and this condition is spoken of as an arrest in the development of the diaphragm. Under such circumstances a communication exists between the peritoneal and thoracic cavities. If a part of an abdominal viscus should protrude into the thoracic cavity through such an aperture, the condition is known as a congenital diaphragmatic hernia.

Musculature of the Diaphragm.-As stated, the septum transversum is, at first, on a level with the third and fourth cervical somites. The muscular tissue of the diaphragm is, in part, derived from the myotomes of these segments, especially the fourth segment, and, it may be, the fifth also, which explains why the muscle is supplied by the phrenic nerve, the two chief roots of that nerve being derived from the anterior primary divisions of the third and fourth cervical spinal nerves. 


\section{A P P E N D IX.}

\section{ANATOMICAL NOMENCLATURE.}

\section{OSTEOLOGY.}

(Skeletal System).

\section{Vertebral Column.}

ENGLISH.

Odontoid facet of Atlas. Axis.

Lateral mass of Sacrum. Auricular surface of ",
LATIN (BASLE).

Fovea dentis.

Epistropheus.

Pars lateralis.

Facies auricularis.

\section{Thorax.}

False, or asternal, Ribs. Ensiform process of Sternum. Interclavicular notch of ,
Costæ spuriæ.

Processus xiphoideus. Incisura jugularis.

\section{Bones of the Head.}

Occipital Bone.

Anterior condylar foramen. Jugular process. Superior curved line. Inferior Groove for läteral sinus.

\section{Parietal Bone.}

Internal surface.

Posterior border.

Anterior

Superior

Inferior

Parietal eminence.
Os Occipitale.

Canalis condyloideus. Tuberculum jugulare. Linea nuchæ superior. ," inferior. Sulcus transversus.

\section{Os Parietale.}

Facies cerebralis. Margo occipitalis. $"$ frontalis. " sagittalis. , squamosus. Tuber parietāle. 
ENGLISH.

\section{Frontal Bone.}

Frontal eminence. Superciliary ridge. Supra-orbital arch. External angular process.

\section{Temporal Bone.}

Fissure of Glaser. Glenoid fossa.

Articular eminence.

Mastoid Portion.

Digastric groove.

Petrous Portion.

Aqueduct of Fallopius.

Gasserian depression.

Vaginal process.

Aqueduct of the cochlea:

Eustachian canal.

\section{Sphenoid Bone.}

Pituitary fossa.

Olivary eminence.

Carotid groove.

Sphenoidal turbinate bones.

Optic groove.

External pterygoid plate. Internal

Eustachian groove.

\section{Ethmoid Bone.}

Lateral mass.

Os planum, or orbital platc.

\section{Superior Maxillary Bone.}

Orifice of the antrum.

Lachrymal groove.

Nasal process.

Malar process.

Incisor canal.

Alveolar border.

Malar Bone.

Malar canal.

\section{Lachrymal Bone.}

Hamular process.
LATIN (BASLE).

Os Frontale.

Tuber frontale. Arcus superciliaris. Margo supra-orbitalis. Processus zygomaticus.

Os Temporale (Temporis).

Fissura petrotympanica.

Fossa mandibularis.

Tuberculum articulare.

Pars Mastoidea.

Sulcus mastoideus.

\section{Pars Petrosa.}

Canalis facialis.

Impressio trigemina.

Vagina processûs styloidei.

Canaliculus cochleæ.

Semicanalis tubæ auditoriæ.

\section{Os Sphenoidale.}

Fossa hypophysea.

Tuberculum sellæ.

Sulcus caròticus.

Conchæ sphenoidales.

Sulcus chiasmătis.

Lamina lateralis.

$$
.2 \text { medialis. }
$$

Sulcus tubæ auditoriæ.

Os Ethmoidale.

Labyrinthus ethmoidalis. Lamina papyracea.

\section{Maxilla.}

Hiatus maxillaris.

Sulcus naso-lacrimalis.

Processus frontalis.

Canalis incisivus. zygomạticus.

Limbus alveolaris.

\section{Os Zygomaticum.}

Canalis zygomatico-orbitalis.

Os Lacrimale.

Hamulus lacrimalis. 
ENGLISH.

Inferior Turbinate Bone.

Palate Bone.

Inferior turbinate crest.

Superior ,

Inferior Maxillary Bone. Condyle.

Sigmoid notch.

Inferior dental foramen.

Hyoid Bone.

Ivory.

Enamel.

Nasmyth's membrane.

Dental arch.

Wisdom tooth.
LATIN (BASLE).

Concha Inferior.

Os Palāti.

Crista conchalis.

" ethmoidalis.

\section{Mandibula.}

Processus condylaris.

Incisura mandibularis.

Foramen mandibulare.
The Teeth.

Os Hyoideum.

\section{Teeth.}

\section{Dentes.}

Substantia eburnea.

Cuticula dentis. adamantina.

Arcus dentalis.

Dens serōtínus.

\section{Bones of the Upper Limb.}

\section{Humerus.}

Bicipital groove.

\section{Radius.}

Bicipital tuberosity. Sigmoid cavity.

\section{Ulna.}

Great sigmoid cavity.

\section{Hand.}

\section{Carpus.}

Scaphoid bone:

Semilunar bone.

Cuneiform or pyramidal bone.

Pisiform bone.

Trapezium.

Trapezoid.

Os magnum.

Unciform bone.

Thumb.

Index finger.

Middle ,

Ring ",

Little ,

\section{Humerus.}

Sulcus intertubercularis.

\section{Radius.}

Tuber radii.

Incisura ulnaris.

\section{Ulna.}

Incisura semilunaris.

\section{Manus.}

\section{Carpus.}

Os naviculare manûs.

Os lunatum.

Os triquetrum.

Os pisiforme.

Os multangulum majus.

Os multangulum minus.

Os capitatum.

Os hamatum.

Pollex.

Index.

Digitus medius. annularis.

Digitus minimus. 


\section{Bones of the Lower Limb.}

ENGLISH.

Hip-bone.

Articular surface of acetabulum. Cotyloid notch.

Ilium.

Ilio-pectineal line.

\section{Ischium. \\ Os Pubis.}

Pubic spine.

Pelvis.

Brim.

Outlet.

\section{Femur.}

Depression for ligamentum teres. Outer lip of linea aspera.

Inner

Popliteal' surface.

Foot.

\section{Tarsus.}

Scaphoid bone.

Great toe.

Sinus pedis.
IATIN (BASLE).

Os CoXæ.

Facies lunāta.

Incisura acetabuli.

Os Ilii.

Linea arcuata.

Os Ischii.

Os Pubis.

Tuberculum pubǐcum.

Pelvis.

Apertũra superior. " inferior.

\section{Os Femoris.}

Fovea capitis.

Labium laterale. mediale. Trigōnum femoris.

\section{Pes.}

Tarsus.

Os naviculare pedis.

Hallux.

Sinus tarsi.

\section{ARTHROLOGY.}

(Articulations or Joints).

$\left(L_{0}=\right.$ Ligamentum).

\section{Vertebral Column.}

Intervertebral discs.

Circumferential fibro-laminar part of disc.

Central pulp of disc.

Anterior common ligament.

Pasterior

Occipito-atlantal ligaments.
Fibro-cartilagines interverte. brales.

Annulus fibrosus.

Nucleus pulposus.

L. longitudinale anterius.

L. posterius.

Membrānæ atlanto-occipitales. 
EXG_ISH.

LATIN (BASLE).

Lateral odontoid, or alar, liga- Ligamenta alaria. ments.

Middle odontoid ligament.

Cruciform ligament.

L. apicis dentis.

L. cruciatum.

\section{Thorax.}

Anterior costo-central, or stel- L. capitis costæ radiatum. late, ligament.

Interarticular ligament.

Middle costo-transverse ligament. Anterior and posterior costosternal ligaments.

L. capitis costæ interarticulare.

L. cervicis costæ.

Ligamenta sterno-costalia radiata. .

\section{Upper Limb.}

Interarticular meniscus.

Suprascapular ligament.

Spino-glenoid ligament.

Glenoid ligament of shoulderjoint.

Internal lateral ligament of elbow-joint.

External lateral ligament of elbow-joint.

Pisi-uncinate ligament.

Pisi-metacarpal ligament.
Discus articularis.

L. transversum scapulæ superius.

L. transversum scapulæ inferius.

Labrum glenoidale.

L. collaterale ulnare.

L. collaterale radiale.

L. piso-hamatum.

L. piso-metacarpāle.

\section{Pelvis.}

Great sacro-sciatic ligament.

Small

Inferior or subpubic ligament.

Interpubic disc.
L. sacro-ischio-tuberans.

L. sacro-ischio-spinosum.

L. arcuatum.

Lamina fibro-cartilaginea interpubǐca.

\section{Lower Limb.}

Cotyloid ligament of hip-joint.

Pubo-femoral ligament.

External lateral ligament of knee-joint.

Internal lateral ligament of kneejoint.

Mucous ligament (ligamentum mucosum).

Alar ligaments.
Labrum glenoidale.

L. pubo-capsulare.

L. collaterale fibulare.

L. collaterale tibiale.

Plica synovialis patellaris.

Plicæ alares. 


\section{MYOLOGY.}

(Muscular System).

(M.= Musculus).

\section{Muscles of the Back.}

ENGLISH.

Complexus.
LATIN (BASLE).

Semispinalis capitis.

\section{Muscles of the Upper Limb.}

Serratus magnus.

Biceps flexor cubiti.

Brachialis anticus.

Triceps extènsor cubiti.

Long head of ," ,

External ", ,

Internal ,", ,

Pronator radii teres.

Supinator radii longus.

Supinator radii brevis.
M. serratus antīcus.

M. biceps brachii.

M. brachialis.

M. triceps brachii.

Caput.longum. , - laterale.

", "mediale.

M. pronator teres.

M. brachio-radialis.

M. supinator.

\section{Muscles of the Lower Limb.}

Quadriceps extensor cruris.

Vastus externus.

Crureus.

Vastus internus.

Subcrureus.

Biceps flexor cruris.

Flexor, or musculus, accessorius:
M. quadriceps femoris.

M. vastus lateralis.

M. " intermedius.

M. ", medialis.

M. articularis genūs.

Biceps femoris.

M. quadratus plantre.

\section{Muscles of the Abdomen.}

Poupart's ligament.

Gimbernat's ligament.

Triangle of Petit.
Ligamentum inguinale.

Trigōnum lumbale.

\section{Muscles of the Thorax.}

Triangularis sterni.

Foramen quadratum of dia- Foramen venæ cavæ inferioris. phragm.

\section{Muscles of the Head.}

Epicranial aponeurosis.

Compressor naris.

Dilator ale nasi.
Galéa aponeurotica.

M. nasalis transversus.

M. " alaris. 
ENGLISH.

Depressor alæ nasi.

Orbicularis palpebrarum.

-Tensor tarsi.

Depressor labii inferioris. Palato-glossus.

" pharyngeus.

Azygos uvulæ.

Lingualis superficialis.

" inferior.
LATIN (BASLE).

M. depressor septi.

M. orbicularis oculi.

Pars lacrimalis musculi orbicu. laris oculi.

M. quadratus labii inferioris.

M. glosso-palatinus.

M. pharyngo-palatinus.

M. uvulæ.

M. longitudinalis superior.

M.

inferior.

\section{ANGEIOLOGY.}

(Vascular and Lymphatic Systems).

\section{Heart.}

Auriculo-ventricular groove. Antero-superior surface.

Inferior surface.

Vestigial fold of Marshall.

\section{Auricle.}

Interauricular septum.

Columnæ carneæ.

Annulus ovalis.

Foramina Thebesii.

Tubercle of Lower.

Eustachian valve.

Thebesian valve.

Interventricular Septum.

Pars membranacea.

Tricuspid Valve.

Right cusp.

Left or infundibular cusp.

Posterior or septal cusp.

Mitral Valve.

Semilunar or Sigmoid Valves. Corpus Arantii.

\section{Cor.}

Sulcus coronarius.

Facies sterno-costalis. diaphragmatica.

Ligamentum venæ cavæ sinistræ.

\section{Atrium.}

Septum atriorum.

Trabeculæ carneæ.

- Limbus fossæ ovalis.

Foramina venarum minimarum. Tuberculum intervenosum.

Valvula venæ cavæ inferioris. , sinus coronarii.

\section{Septum Musculare Ventricu-} lorum.

Septum membranaceum ventriculorum.

Valvula Tricuspidalis. Cuspis medialis.

, anterior.

" posterior.

Valvula Mitralis vel Bicuspidalis.

Valvula Semilunares.

Nodulus valvulæ semilunaris. 
ENGLISH.

Arteries.
IATIN (BASLE).

Arteriæ.

(A. = Arteria vel Arteriæ).

Pulmonary artery.

Arch of the Aorta.

Right coronary artery.

Left

Innominate artery.

Common carotid artery.

\section{External Carotid Artery.}

Ranine artery.

Facial

Inferior coronary artery.

Superior . "

\section{Internal Maxillary Artery.}

Vidian artery.

Circle of Willis.

\section{Subclavian Artery.}

Internal mammary artery.

Superior phrenic artery.

Thyroid axis.

Suprascapular artery.

\section{Axillary Artery.}

Superior thoracic.

Acromio-thoracic artery.

Long thoracic artery.

Dorsalis scapulæ artery.

Anterior circumflex artery.

Posterior

$$
\text { " }
$$

,"

\section{Brachial Artery.}

Superior profunda artery.

Inferior Anastomotic" artery.

\section{Radial Artery.}

Superficial volar artery.

Anterior radial carpal artery.

Radialis indicis artery.

\section{Ulnar Artery.}

Common interosseous artery.

Anterior

Postcrior
A. pulmonalis.

Arcus Aortæ.

A. coronaria dextra.

A. sinistra.

A. anonyma.

A. carōtis communis.

\section{A. Carōtis Externa.}

A. profunda linguæ.

A. maxillaris externa.

A. labialis inferior.

A. labialis superior.

\section{A. Maxillaris Interna.}

A. canalis pterygoidei. Circulus arteriosus.

\section{A. Subclavia.}

A. mammaria in terna.

A. pericardiăco-phrenica.

Truncus thyreo-cervicalis.

A. transversa scapulæ.

\section{A. Axillaris.}

A. thoracalis suprēma.

A. thoraco-acromialis.

A. thoracalis lateralis.

A. circumflexa scapulæ.

A. circumflexa humeri anterior.

A.

" posterior.

\section{A. Brachialis.}

A. collateralis superior.

A. media.

A., inferior.

\section{A. Radialis.}

A. volaris superficialis.

A. radialis carpea volaris.

A. radialis volaris indicis.

\section{A. Ulnaris.}

A. interossea communis.

A. volaris.

A., dorsalis. 
EXGLISH.

Anterior ulnar carpal artery. Posterior.

Profunda artery.

Superficial palmar arch.

Deep palmar arch.

\section{Abdominal Aorta.}

Capsular arteries.

Spermatic "

Coliac axis.

Gastric (coronary) artery.

\section{Splenic Artery.}

Vasa brevia.

\section{Hepatic Artery.}

Pyloric artery.

\section{Superior Mesenteric Artery.}

Rami intestini tenuis.

Right colic artery.

Middle ," ,

Inferior Mesenteric Artery. Left colic artery.

Sigmoid arteries.

Internal Iliac Artery. Gluteal artery.

Sciatic Artery of the vas deferens.

Internal Pudic Artery.

Superficial perineal artery. Artery of the bulb.

" " corpus caver-

nosum.

\section{External Iliac Artery.}

Deep epigastric artery.

Cremasteric artery.

\section{Femoral Artery.}

Anastomotic artery.
LATIN (BASLE).

A. ulnaris carpea volaris.

A. dorsalis.

A. volaris profunda. Arcus volaris superficialis. Arcus " profundus.

\section{Aorta Abdominalis.}

A. suprarenales.

A. spermaticæ internæ.

A. cœliaca.

A. gastrica sinistra.

\section{A. Lienalis.}

A. gastricæ breves.

\section{A. Hepatica.}

A. gastrica dextra.

A. Mesenterica Superior.

A. intestinales.

A. colica dextra.

A. ", media.

\section{A. Mesenterica Inferior.}

A. colica sinistra.

A. sigmoideæ.

\section{A. Hypogastrica.}

A. glutæa superior.

A. " inferior.

A. deferentialis.

\section{A. Pudenda Interna.}

A. perinæi.

A. bulbi urethræ.

A. profunda penis vel clitoridis.

A. Iliaca Externa.

A. epigastrica inferior.

A. spermatica externa.

A. Femoralis.

Arteria suprema genūs. 
ENGLISH.

Popliteal Artery.

Superior external articular artery. Superior internal articular artery. Middle or azygos articular artery. Inferior external articular artery. Inferior internal articular artery. Lower muscular or sural arteries.

\section{Plantar Arteries.}

Internal plantar artery. External

Dorsal Artery of Foot.

Tarsal artery.

Metatarsal artery.

Veins.
LATIN (BAsLe).

\section{A. Poplitea.}

A. superior lateralis genüs.

A.

A. media genüs. medialis ",

A. inferior lateralis genūs.

A. " medialis "

A. surales.

\section{A. Plantares.}

A. plantaris medialis.

A. " lateralis.

\section{A. Dorsalis Pedis.}

A. tarsěa lateralis.

A. arcuata.

$$
\text { (V. = Vena vel Venæ). }
$$

Venæ.

Great cardiac or coronary vein. Small cardiac, or right conorary, vein.

Middle cardiac vein.

Smallest cardiac veins.

Innominate veins.

Vena azygos major, or Right azygos vein.

Vena azygos minor inferior, or Lower left azygos vein.

Vena azygos minor superior, or Upper left azygos vein.

Facial vein.

Temporo-maxillary vein.

Common facial vein.

Portal vein.

Splenic vein.

Internal iliac vein.

Internal or long saphenous vein.

External or short ", ",

Sinuses of the Dura Mater.

V. magna cordis.

V. parva

V. media

V. minimæ ,

V. anonymæ, dextra et sinistra.

V. azygos.

V. hemi-azygos.

V. , accessoria.

V. facialis anterior.

V. " posterior.

V. " communis.

V. portæ.

V. lienalis.

V. hypogastrica.

$V$. saphēna magna.

V. " parva.

\section{Sinûs Duræ Matris.}

Superior longitudinal sinus.

Inferior

Straight sinus.

Circular "

Basilar ",

Lateral ",

Cavernous sinus.
( $\mathrm{S}_{0}=$ Sinus).

S. sagittalis superior.

S. " inferior.

S. rectus.

Sinûs intercavernosi.

Plexus basilaris.

S. transversus.

S. cavernosus. 
ENGLISH.

Lymphatic System.

Thoracic duct.

Receptaculum chyli.

Right lymphatic duct.
LATIN (BASLE).

\section{Systēma Lymphaticum.}

Ductus thoracicus.

Cisterna chyli.

Ductus lymphaticus dexter.

\section{NEUROLOGY.}

(The Nervous System).

Spinal Cord.

Cervical enlargement.

Lumbar

Anterior column.

Lateral

Posterior

Column of Goll.

Burdach.

Anterior cornu.

Posterior ,

Lateral

Central canal.

Clarke's column.

\section{Medulla Spinalis.}

Intumescentia cervicalis. lumbalis.

Funiculus anterior.

" lateralis.

, posterior.

,, gracilis.

, cuneatus.

Columna grisea anterior.

" " posterior.

, , lateralis.

Canalis centralis.

Nucleus dorsalis.

\section{Tracts in the Spinal Cord.}

Tract of Goll.

Burdach.

Direct cerebellar tract.

Tract of Gowers.

Pyramidal tract, lateral or crossed part.

Pyramidal tract, anterior or direct part (column of Türck.) Antero-lateral descending cerebellar tract.

Anterior ground-bundle. Lateral

3)

\section{Encephalon.}

Bulb.

Pyramid.

Olivary body or eminence. Restiform Body. Fillet.
Funiculus gracilis. cuneatus.

Fasciculus dorso-lateralis cerebello-spinalis.

Fasciculus antero-lateralis cerebello-spinalis.

Fasciculus cerebro - spinalis lateralis.

Fasciculus cerebro-spinalis anterior.

Fasciculus cerebello-vestibulospinalis.

Fasciculus anterior proprius. , lateralis proprius.

Medulla Oblongata.

Pyramis.

Corpus olivare vel Oliva.

Corpus Restiforme.

Lemniscus. 
ENGLISTH.

Decussation of the Fillets.

Superior medullary velum

(Valve of Vieussens).

Inferior medullary velum.

Red nucleus.

Superior quadrigeminal bodies.

Inferior

Stratum cinereum.

Aqueduct of Sylvius.

Crusta or Pes of the Crus Cerebri.

Pineal body.

Ganglion habenulæ.

Middle or grey commissure.

Corpora albicantia.

Bundle of Vicq d'Azyr.

Optic tract.

Optic commissure.

Nucleus of Luys.

Great longitudinal fissure.

Fissure of Sylvius.

Fissure of Rolando.

Quadrate lobule of Parietal Lobe.

Island of Reil.

Threshold of the Island of Reil.

Convolution of the Corpus Callosum.

Limbic Lobe.

Uncus.

Hippocampus major.

Dentate Gyrus.

Lyra or Psalterium.

Cuneate Lobule.

Radiation of the Corpus Callosum.

Forceps minor.

Forceps major.

Anterior pillars of the Fornix.

Posterior

Septum lucidü.

\section{Lateral Ventricles.}

Anterior horn.

Middle or Lateral horn.

Posterior horn.
LATIN (BASLE).

Decussatio lemniscorum.

Velum medullāre superius vel anterius.

Velum medullāre inferius vel posterius.

Nucleus ruber.

Colliculi superiores. inferiores.

Stratum griseum.

Aquæductus cerebri.

Basis pedunculi.

Corpus pineāle.

Nucleus habenulæ.

Commissüra mollis.

Corpora mammillaria.

Fasciculus thalamo-mammillaris.

Tractus opticus.

Chiasma opticum.

Corpus subthalamicum vel $\mathrm{Nu}$ cleus hypothalamicus.

Fissura interhemispherica.

Fissura lateralis.

Sulcus centralis.

Præcuneus.

Insula.

Limen insulæ.

Gyrus fornicatus.

Gyrus fornicatus et gyrus hippocampi.

Uncus gyri hippocampi.

Hippocampus.

Fascia dentata hippocampi.

Commissura hippocampalis.

Cuneus.

Radiatio corporis callosi.

Pars frontalis.

, occipitalis.

Columnæ fornicis.

Crura fornicis.

Septum pellucidum.

\section{Ventriculi Laterales.}

Cornu anterius.

" inferius.

" posterius. 
EXGIISH.

Third Ventricle.

Fourth Ventricle.

Fifth Ventricle.

\section{Meninges.}

Epidural space.

Subdural

Subarachnoid space.

Pacchionian Bodies.
LATIN (BASLE).

Ventriculus tertius.

Ventriculus quartus.

Ventriculus quintus vel Cavum septi pellucidi.

\section{Meninges.}

Cavum epidurale. " subdurale. ", subarachnoidale.

Granulationes arachnoidales.

\section{Cerebro-spinal Nerves.}

(N. $=$ Nervus vel Nervi).

Cranial Nerves.

First or Olfactory nerve.

Second or Optic nerve.

Third or Oculo-motor nerve.

Fourth or Trochlear nerve.

Fifth or Trigeminal nerve.

Superior maxillary nerve.

\section{Inferior}

Sixth or Abducent nerve.

Seventh or Facial nerve.

Eighth or Auditory nerve.

Ninth or Glosso-pharyngeal nerve.

Jugular ganglion of

Petrous ganglion of ",

Jacobson's nerve.

Tenth or Pneumogastric nerve.

Ganglion of Root of

Ganglion of Trunk of

Arnold's nerve.

Eleventh or Spinal Accessory nerve.

Twelfth or Hypoglossal nerve.

Descendens cervicis (hypoglossi).

Cervical Plexus.

Small occipital nerve.

Great auricular nerve.

Superficial or Transverse cervical nerve.

First cervical nerve.

Great occipital nerve.

Third or Least occipital nerve.

Phrenic nerve.
N. Cerebrales.

N. Olfactorius.

N. Opticus.

N. Oculo-motorius.

N. Trochlearis vel Patheticus.

N. Trigeminus.

N. Maxillaris.

N. Mandibularis.

N. Abducens.

N. Facialis.

N. Acusticus.

N. Glosso-pharyngeus.

Ganglion superius.

N. T'ympanicus.

$\mathrm{N}$. Vagus.

Ganglion jugulare.

Ganglion nodosum.

N. Auricularis.

$\mathrm{N}$. Accessorius.

N. Hypoglossus.

N. cervicalis descendens.

Plexus Cervicalis.

$\mathrm{N}$. occipitalis minor.

$\mathrm{N}$. auricularis magnus.

$\mathrm{N}$. cutaneus colli.

N. suboccipitalis.

$\mathrm{N}$. occipitalis major.

$\mathrm{N}$. , minimus.

N. phrenicus. 
ENGLISH.

\section{Brachial Plexus.}

Nerve to the Rhomboid Muscles. Long thoracic nerve, or External respiratory nerve of Bell.

Anterior thoracic nerves.

Median nerve.

Ulnar

Circumflex nerve.

Long subscapular nerve.

Twelfth thoracic ,

\section{Lumbar Plexus.}

Genito-crural nerve.

Crural branch of

Genital branch of ",

External cutaneous nerve.

Obturator nerve.

Anterior crural nerve.

Internal or long saphenous nerve.

\section{Sacral Plexus.}

Small sciatic nerve.

Great

External "popliteal, or Peroneal, nerve.

Lateral cutaneous branch of peroneal nerve.

Fibular communicating nerve.

Tibiai

Musculo-cutaneous nerve.

Anterior tibial nerve.

Internal popliteal, or Tibial, nerve.,

External or short saphenous nerve.

Internal plantar nerve.

External

Deep division of plantar nerve.

Pudic nerve.
LATIN (BASLE).

\section{Plexus Brachialis.}

N. dorsalis scapulæ.

$\mathrm{N}$. thoracalis longus.

N. thoracales anteriores.

N. mediānus.

N. ulnaris.

$\mathrm{N}$. axillaris.

N. thoraco-dorsalis.

N. subcostalis.

Plexus Lumbalis.

N. genito-femoralis.

$\mathrm{N}$. lumbo-inguinalis.

N. spermaticus externus.

N. cutaneus femoris lateralis.

N. obturatorius.

$\mathrm{N}$. femoralis.

N. saphēnus.

\section{Plexus Sacralis.}

N. cutaneus femoris posterior.

$\mathrm{N}$. ischiadicus.

N. peronæus communis.

N. cutaneus suræ lateralis.

$\mathrm{N}$. anastomoticus peronæus.

$\mathrm{N}$. communicans tibialis.

N. peronæus superficialis.

N. , profundus.

N. tibialis.

$\mathrm{N}$. cutaneus suræ medialis vel N. suralis.

N. plantaris medialis.

N. plantaris lateralis.

Ramus profundus.

N. pudendus.

\section{SPLANCHNOLOGY.}

(The Visceral System).

\section{Digestive System.}

Mouth.

Lips.

Cheeks.
Cavum oris. Labia oris. Buccæ. 
ENGLISH.

Gums.

Hard palate.

Soft

Anterior pillar of the fauces.

Posterior

Tongue.

Dorsum of the tongue.

Inferior surface of the tongue.

Parotid gland.

Parotid, or Stensen's, duct. Glandula socia parotidis.

Wharton's duct.

Pharynx.

Tonsils.

Crypts of the tonsil.

Pharyngeal tonsil.

Fossa of Rosenmüller.

Orifice of the Eustachian tube.

Eustachian cushion.

Gullet (Esophagus).

\section{Stomach.}

Fundus (Cardiac end).

Esophageal orifice.

Pyloric orifice.

Plicæ villosæ.

\section{Small Intestine.}

Valvulæ conniventes.

Glands of Brunner.

Crypts of Lieberkühn.

Solitary glands.

Agminated glands, or Peyer's patches.

\section{Large Intestine.}

Ileo-colic, or ileo-cæcal, valve.

Upper segment of ", ,

Lower segment of ,",

Retinacula of

Vermiform appendix.

Ascending colon.

Hepatic flexure.

Transverse colon.
LATIN (BASLE).

Gingivæ.

Palātum durum. dulum palāti.

Arcus glosso-palatinus.

Lingua.

pharyngo-palatinus.

Dorsum linguæ.

Facies inferior.

Glandula parōtica.

Ductus parōticus.

Glanđula parōtica accessoria.

Ductus submaxillaris.

Pharynx.

Amygdalæ, vel Tonsillæ palatinæ.

Fossulæ tonsillares.

Tonsilla pharyngea.

Recessus pharyngeus.

Ostium pharyngeum tubæ auditorix.

Torus tubæ auditoriæ.

Gula.

\section{Ventriculus.}

Fundus ventriculi.

Cardia.

Pylorus.

Plicæ gastricæ.

Intestinum Tenue.

Plicæ circulares.

Glandulæ duodenales.

Glandulæ intestinales.

Noduli lymphatici solitarii.

s)

aggregati.

\section{Intestinum Crassum.}

Valvula coli.

Labium superius. inferius.

Frenula valvulæ coli.

Processus vermiformis.

Colon vel Colum dextrum.

Flexūra dextra coli.

Colon transversum vel medium 
ENGLISH.

Splenic flexure.

Descending colon.

Iliac colon.

Pelvic colon.

Sacculi of colon.

Crescentic folds between the sacculi.

Rectum.

Rectal valves, or Valves of Houston.

Anal canal.

Columns of Morgagni.

\section{Liver.}

Suprarenal impression.

Gastric

Colic

Renal

Duodenal

Omental tuberosity.

Quadrate lobe.

Caudate lobe.

Fossa of the inferior vena cava. , (fissure) of the ductus venosus.

Umbilical fissure.

Portal, or Transverse, fissure.

Fossa of the gall-bladder.

Round ligament.

Hepatic duct.

Capsule of Glisson.

\section{Gall-bladder.}

Cystic duct:

Common bile-duct.

\section{Spleen.}

Diaphragmatic surface.

Gastric surface.

Renal

Basal

$$
\text { ", }
$$

\section{Kidneys.}

Muscular surface.

Visceral

Pyramids of "Malpighi.

Columnæ Bertini.
LATIN (BASLE).

Flexūra sinistra coli.

Colon sinistrum.

Colon iliăcum.

Colon pelvĭcum.

Haustra coli.

Plicæ semilunares coli.

Intestinum rectum.

Plicæ transversales recti.

Pars analis intestini recti.

Columnæ canalis analis.

\section{Hepar.}

Impressio suprarenalis.

" gastrica.

" colica.

"renalis.

" duodenalis.

Tuber omentale:

Lobus quadratus.

Lobus vel Processus caudatus.

Fossa venæ cavæ inferioris.

, ductūs venosi.

" venæ umbilicalis.

Porta hepătis.

Fossa vesıcæ fellěæ.

Ligamentum teres hepătis vel Ligamentum venosum.

Ductus hepaticus.

Capsula fibrosa.

\section{Vesica Fellĕa.}

Ductus cysticus.

, communis cholèdochus.

\section{Lien.}

Facies diaphragmatica.

$$
\begin{array}{ll} 
& \text { gastrica. } \\
" & \text { renalis. } \\
\text { basalis. }
\end{array}
$$

Rēnes (singular, Rēn).

Facies muscularis. visceralis.

Pyramides renales.

Columnæ renales. 
ENGLISH.

Medullary rays.

Labyrinth.

Malpighian corpuscles.

Uriniferous tubules.

Renal arteries.

Renal veins.

\section{Urinary Bladder.}

Base of bladder.

Summit, or Apex, of bladder.

Body of bladder.

Urethral orifice of bladder.

Fibrous cord of the urachus.

Peritoneal coat.

External muscular coat.

Middle

Internal "

"

Sphincter muscle.

Submucous coat.

Mucous coat.

Male Reproductive Organs. Testicles.

Tunica vaginalis testis.

Tunica , scroti.

Globus major of Epididymis.

Globus minor ,"

Organ of Giraldès.

Digital fossa.

Coni vasculosi.

Vas deferens.

Spermatic cord.

\section{Penis.}

Upper surface of penis.

Under

Body of penis.

Prepuce.

Glands of Tyson.

Corpora cavernosa.

Corpus spongiosum.

\section{Prostate Gland.}

Base of prostate gland. Apex of ", ,
LATIX (BASLE).

Pars radiata.

, convolūta.

Corpuscula renis.

Tubuli renales.

Arteriæ renis.

Venæ renis.

\section{Vesica Urinaria.}

Fundus vesicæ.

Vertex vesicæ.

Corpus vesicæ.

Orificium internum urethræ.

Ligamentum umbilicale medium.

Tunica serosa.

Stratum musculare externum. medium.

$" \quad " \quad$ internum.

Annulus urethralis. .

Tela submucosa.

Tunica mucosa.

\section{Orgăna Genitalia Virilia. Testes.}

Lamina visceralis. parietalis.

Caput epididymis.

Cauda

Paradidymis.

Sinus epididymis.

Lobuli epididymis.

Ductus testis.

Funiculus spermaticus.

Penis.

Dorsum penis.

Facies urethralis.

Corpus penis.

Præputium.

Glandulæ præputiales.

Corpora cavernosa penis.

Corpus cavernosum urethræ.

Glandula Prostatica vel

Prostadica.

Basis prostadis. Apex prostadis. 
ENGLISH.

Male Urethra.

Prostatic part.

Membranous part.

Spongy part.

Meatus urinarius.

Glands of Cowper.

Glands of Littré.

\section{Female Reproductive Organs.}

Ovary.

Upper, or Tubal, pole of ovary.

Lower, or Uterine, pole of ovary.

Anterior border of ovary.

Peritoneal fold of ovary.

Ligament of ovary.

Peritoneal fossa of ovary.

Graafian follicles.

Discus proligerus.

\section{Fallopian Tube.}

Duct of Gartner.

Hydatids of Morgagni.

\section{Uterus.}

Anterior surface.

Posterior ,

Cavity of the cervix.

Plicæ palmatæ.

Broad ligament.

Round

Peritoneal "coat.

\section{- Vagina.}

Lower part of anterior column.

\section{Vulva, or Pudendum.}

Fourchette.

Female urethra.

Meatus urinarius.

Mammary Gland.

Glands of Montgomery. Galactophorous ducts.

Ampulla of galactophorous duct.
LATIN. (BASLE).

\section{Urethra Virilis.}

Pars prostatíca vel prostadica. " membranacea.

", cavernosa.

Orificium externum urethræ.

Glandulæ bulbo-urethrales.

$$
\text { " urethrales. }
$$

Orgăna Genitalia Muliebria vel Feminěa.

Ovarium.

Extremitas tubalis.

$$
\text { ", uterina. }
$$

Margo mesovarii.

Mesovarium.

Ligamentum proprium ovarii.

Fossa ovarii.

Folliculi vesiculosi oöpherii.

Cumŭlus oöpherii.

\section{Tuba Uterina.}

Ductus epoöpherii longitudi nalis.

Appendices vesiculosi.

\section{Uterus.}

Facies vesicalis. intestinalis.

Canalis cervicis uteri.

Arbor vitæ uterina.

Ligamentum latum uteri.

Tunica serosa. teres ",

\section{Vagina.}

Carina urethralis.

Pudendum muliebre vel feminĕum.

Frenulum labiorum pudendi.

Urethra muliebris vel feminěa. Orificium externum urethræ.

\section{Mamma.}

Glandulæ areolāres. Ductûs lactiferi. Sinus lactiferus. 
ENGLISH.

Larynx.

Thyroid cartilage.

Oblique line.

Cricoid cartilage.

Arytenoid cartilages.

Cartilages of Santorini.

Cartilages of Wrisberg.

Epiglottis.

Tubercle, or Cushion, of epiglottis. Superior aperture of the larynx.

Cavity of the larynx.

Superior thyro-arytenoidligament.

Inferior thyro-arytenoid ligament.

False vocal cords.

True

False glottis.

True

Vocal glottis.

Respiratory glottis.

Ventricle of the larynx.

Saccule

,, 9

\section{Organs of Sense.}

\section{Eye.}

Eyeball.

Capsule of Tenon.

Sclerotic coat.

Canal of Schlemm.

Membrane of Bowman.

Membrane of Descemet, or of Demours.

Choroid coat.

Membrane of Bruch.

Ciliary body.

Zonula of Zinn.

Valve of Hasner.

Ear.

\section{External Ear (Auricle).}

Upper portion of concha, above crus helicis.

Lower portion of concha, below crus helicis.

Tubercle of Darwin.
IATIN (BASLE).

\section{Larynx.}

Cartilago thyroidea.

Linea obliqua.

Cartilago cricoidea.

Cartilagines arytænoideæ. corniculatæ.

Epiglottis. cuneatæ.

Pulvinar epiglottideum.

Aditus laryngis.

Cavum laryngis.

Ligamentum ventriculosum. vocale.

Plicæ ventriculosæ.

Glottis spuria.

Glottis vera, vel Rima glottidis.

Pars intermembranacea. intercartilaginea.

Ventriculus laryngis.

Appendix ventriculi.
Oculus vel Orgănum visûs. Globus oculi. Fascia bulbi. Sclera.

Sinus venosus scleræ.

Lamina elastica anterior. $, \quad, \quad$ posterior.

Tunica chorioidea. Lamina basalis. Corpus ciliare. Zonula ciliaris. Plica lacrimalis.

Auris Orgănum Auditûs. Auris Externa (Auricula).

Cymba conchæ.

Cavum conchæ.

Tuberculum auriculæ. 
ENGLISH.

Fossa of the helix.

Fossa of the antihelix.

Eminence on cranial aspect of concha.

Hairs of tragus and antitragus.

External auditory meatus.

Sweat-glands of auditory meatus.

\section{Middle Ear.}

Tympanic cavity.

Outer wall of ,

Inner ", ",

Anterior wall ",

Posterior ", ,"

Roof ", ,

Floor ", ,"

Tympanic Membrane.

Shrapnell's membrane.

External layer of tympanic membrane.

Middle layer of tympanic membrane.

Internal layer of tympanic mem- Stratum mucosum. brane.

Fenestra ovalis.

Fenestra rotunda.

Secondary membrane of the Membrāna tympani secundaria. tympanum.

Mastoid antrum.

Eustachian tube.

Tympanic orifice of tube.

Pharyngeal ,

,

Tympanic Ossicles.

Malleus.

Handle.

Long process.

Short ",

Incus.

Long process.

Short ",
Scapha.

Fossa triangularis.

Eminentia conchæ.

Barba hircina.

Meatus acusticus externus.

Glandulæ ceruminosæ.

\section{Auris Media.}

Cavum tympani.

Paries membranacea. labyrinthica. carotica. mastoidea. tegmentalis. jugularis.

\section{Membrāna Tympani.}

Membrāna vel Pars flaccida. Stratum cutaneum.

Membrāna propria (Stratum radiatum et stratum circulare).

Fenestra vestibuli. cochleæ.

Antrum mastoideum vel tympanicum.

Tuba auditoria.

Ostium tympanicum.

„ pharyngeum.

\section{Ossicula Auditûs.}

\section{Malleus.}

Manubrium.

Processus longus.

" brevis.

\section{Incus.}

Crus longum.

"breve. 
ENGLISH.

Stapes.

Anterior crus.

Posterior,

Foot-plate.

Internal Ear.

Osseous Labyrinth.

\section{Vestibule.}

Fovea spherica.

" elliptica.

\section{Cochlea.}

Base of cochlea.

Apex

Modiolus.

Spiral canal of the modiolus.

Internal auditory meatus.

Membranous Labyrinth.

Canal of Hensen.

Membranous cochlea.

Membrane of Reissner.

Basilar membrane.

\section{Nose.}

Cartilage of the septum.

Vomerine cartilage.

Upper lateral nasal cartilage.

Lower lateral nasal cartilage.

Outer plate of nasal cartilage. Inner plate of , ,

Superior meatus.

Middle

Inferior "

Inferior ,

Glands of Bowman.
LATIN (BASLE).

Stapes.

Crus anterius.

Basis. posterius.

Auris Interna.

Labyrinthus Osseus.

Vestibulum.

Recessus sphericus.

ellipticus.

Cochlea.

Basis.

Cupola.

Columella.

Canalis spiralis modioli.

Meatus acusticus internus.

Labyrinthus Membranaceus.

Ductus reuniens. media.

Membrana vestibularis.

, basilaris.

\section{Nasus.}

Cartilago septi nasi.

Cartilago vomero-nasalis Jacobsoni.

Cartilago nasi lateralis superior. " , ", inferior vel Cartilago apertūræ nasi.

Crus laterale.

Crus mediale.

Meatus nasi superior.

" , medius.

") " inferior.

Glandulæ olfactoriæ. 


\section{GLOS S A R Y}

Abdōmen, probably from abdo, I hide or conceal; perhaps a corruption of adipōmen, from adeps, the soft fat of animals.

Aberrant, wandering from the normal source.

Acervǔlus, a little heap.

Acetabulum, a vessel for holding vinegar ; a juggler's cup.

Acinus, any juicy berry with stones, e.g., the grape ; the kernel in the berry.

Acrocephalus, pointed or conical head.

Acromion, the point or summit of the shoulder.

Acusticus, $-\mathrm{a},-\mathrm{um}$, pertaining to sound, or to the sense of hearing.

Adamantoblast, enamel-germ.

Adenoid, glandular.

Aditus, an approach or access.

Adrenal, near to, or pertaining to, the kidney.

Advehens, carrying to.

Afferent, carrying to.

Agger, a mound or rampart.

Agminated, in groups.

Ala, a wing.

Albicans, white.

Albuginea, whitish.

Allantois, sausage-like.

Alveolus, a little trough.

Alveus, a trough.

Amacrine, without a long fibre.

Ambiguus, dark, obscure.

Ameloblast, enamel-germ.
Ammōnis, cornu, horn of Ammon, who was represented as having the head of a ram.

Amphiarthrosis, literally, articulation on both sides.

Ampulla, a flask.

Amygdala, an almond.

Anastomosis, literally, an outlet; the communication of branches of vessels with one another.

Anconeus, pertaining to the elbow.

Ankylosis, bony union between two bones which are normally separate.

Annulus, a little ring.

Ansa, a handle, haft, or brace.

Anserinus, pertaining to a goose. Antecubital (Anticubital), before the elbow.

Anticus, in front, anterior.

Antinion, facing the inion.

Antrum, a cave or cavity.

Anus, a ring.

Aorta, literally, the lower end of the trachea; a carrier.

Aponeurosis, an expansion from a tendon.

Apophysis ('grow from '), a process or swelling on a bone.

Arachnoid, like a spider's web.

Archenteron, primitive intestine.

Arcuatus, curved.

Areola, a small open place.

Artery, literally, the trachea; a bloodvessel which carries the blood from the heart. 
Arthrodia, from a Greek word meaning 'a joint'; gliding is implied.

Arytenoid, pitcher-like.

Aspera, rough.

Asterion, a star.

Astragalus, the ankle-bone; a die (pl. dice).

Atlas, a support.

Atresia, imperforation.

Atrium, the hall in a Roman house.

Attollens, raising up, elevating. Attrahens, drawing to or towards.

Auditory, pertaining to the organ, or sense, of hearing.

Auricle, the external ear.

Azygos, without a yoke, single.

Bacillary, pertaining to a small staff or rod.

Balănus, an acorn.

Barba, a beard.

Basilar, belonging to the base.

Basilie, royal, important.

Basion, base.

Biceps, having two heads.

Bicornis, two-horned.

Biventer, having two bellies.

Brachium, the arm.

Brachycephalic, short-headed.

Bregma, from a Greek verb meaning 'to moisten.'

Bronchial, pertaining to the windpipe.

Bronchiole, a little bronchus.

Bronchus, literally, a draught; the windpipe.

Bubonocele, a tumour in the groin.

Buccinator, a trumpeter.

Bulla, a knob.

Bursa, a sac containing fluid.

Cacūmen, tip, peak, or end.

Cæcum, blind.

Cæruleus, dark blue.

Calamus, a reed-pen.
Calcaneum, the heel.

Calcar, a spur.

Calcination, reduction to a powder (or lime) by heat.

Calcis, of the heel.

Calix, a cup or goblet.

Callosum, hard, thick.

Calvaria (calva, the bald scalp), the upper part of the skull.

Cancellated, lattice-formed, re ticulated.

Caninus, canine.

Conthus, the angle of the eye.

Capillary, pertaining to the hair : a vessel of hair-like minuteness.

Capitellum, a small head.

Cardia, the opening of the stomach; the heart.

Cardiac, pertaining to the heart.

Cardinal, principal or chief.

Carina, a keel.

Carneæ, pertaining to flesh.

Carōtid, stupefying ; or perhaps from two Greek words meaning ' head' and 'ear.

Carpus, the wrist.

Caruncula, a little piece of flesh. Cauda, a tail.

Caudal, pertaining to a tail.

Caudate, pertaining to a tail.

Cavernous, full of hollows or cavities.

Centimetre (cm.), $\frac{2}{5}$ of an English inch.

Cephalic, pertaining to the head. Cerăto, horny.

Ceruminous, pertaining to wax.

Chiasma, two lines placed like an $\mathbf{X}$.

Choāna, a funnel.

Choledŏchus, bile-receiving.

Chondral, pertaining to carti. lage.

Choroid (Chorioid), like skin.

Cinereus, ash-coloured.

Cingulum, a small girdle.

Circumflexus, bent around.

Cisterna, a cistern or reservoir. 
Claustrum, a bolt, barrier, or inclosure.

Clava, a club.

Clavicle, from clavis, a key.

Cleido-, pertaining to the clavicle.

Clinoid, like the knob of a bedpost.

Clitoris, from a Greek verb meaning 'I shut up' or 'enclose.'

Clivus, a slope.

Cloāca, a sewer or drain.

Coccyx, a cuckoo.

Cochlea, a snail.

Cochleariformis, spoon-like.

Coliac, pertaining to the belly.

Collicŭlus, a little hill.

Colon, the great gut.

Comes, a companion.

Complexus, literally, folded together; encompassing.

Concatenatæ, chained together.

Concha, a shell.

Condyle ('knuckle '), a small round prominence covered by cartilage.

Conjunetiva, connecting.

Conniventes, winking or blinking.

Conoid, cone-like.

Convolūta, rolled together.

Coracoid, like a crow or raven.

Cordiform, heart-shaped.

Cornea, horny.

Corniculum, a little horn.

Corōnal, literally, pertaining to a crown ; transverse.

Coronary, encircling.

Coronoid, like a crooked beak.

Corrugator, a wrinkler.

Cortex, the bark or outer covering.

Costal, pertaining to a rib.

Cotyloid, cup-like.

Coxa, the hip.

Cranium, the skull.

Crassum, thick, dense, or bulky.

Cremaster, a suspender.

Cretin, a simple-minded person.

Cribriform, sieve-like.
Cribrosa, perforated with sievelike pores.

Cricoid, like a ring.

Crucial, pertaining to, or shaped like, a cross.

Crural, pertaining to the leg.

Cryptorchismus, concealment of the testis.

Cryptozygous, hidden arches.

Cubitum, the elbow.

Cucullaris, pertaining to a cowl or hood.

Culmen, the top or summit.

Cuneate, wedge-shaped.

Cuneus, a wedge.

Cupola, a dome.

Cymba, a boat or skiff.

Cystic, pertaining to the gallbladder.

Cytoplasm, formative yolk.

Dacryon, a tear.

Dartos, skinned or flayed.

Deciduous, falling away.

Deferens, carrying away.

Detrüsor, from detrūido, I drive away.

Deutoplasm, nutritive yolk.

Dia-, through or between.

Diaphragm, a partition.

Diaphysis ("grow between '), the shaft of a bone, or the part which grows between the epiphyses.

Diarthrosis, an 'apart' joint (without intervening substance).

Diencephalon, the 'tween-brain or inter-brain.

Digastric, having two bellies.

Diploë, a doubling.

Diverticulum, from diverto, 'I separate,' or 'part,' or 'go a different way.'

Dolichocephalic, long-headed.

Dorsal, pertaining to the back aspect.

Dorsum, the back.

Duodēnum, twelve. 
Ebur, -ŏris, ivory.

Eburnea, pertaining to ivory.

Ectopia, a displacement.

Efferent, carrying out.

Emboliformis, beak-shaped or wedge-shaped.

Emissary, sent out.

Emulgent, milking, straining out.

Encephalon, the contents of the head or skull.

Endocardium, 'within the heart'; the lining membrane of the cardiac chambers.

Endognathion, literally, inner jaw.

Endosteum, 'within a bone'; the medullary membrane.

Ensiform, sword-like.

Entomion, a notch.

Ependyma, from a Greek word meaning ' clothing.'

Ephippium, a saddle.

Epi-, upon or over.

Epicardium, upon the heart.

Epididymis, upon the testicle.

Epiotic, upon or over the ear.

Epiphysis ("grow upon '); a process of a bone which has a secondary centre of ossification.

Epiploön, from a Greek verb meaning 'I sail upon.'

Epipteric, upon a wing.

Enoöphoron, upon the egg-bearing organ.

Erythröblast, red germ.

Ethmoid, sieve-like.

Exognathion, literally, outer jaw.

Exomphalos, out of the navel.

Facet (French, facette, a little face), a small plane articular surface.

Falciform, sickle-like.

Falx, a sickle.

Fascia, a band.

Fasciculus, a small bundle.

Fastigium, a roof.

Fauces, the throat.

Fel, fellis, the gall-bladder.
Femur, the thigh.

Fenestra, an opening, a window.

Ferruginea, pertaining to ironrust.

Fibula, a buckle, clasp, or brace. Filum, a thread.

Fimbria, a fringe.

Fimbriatum, fringed.

Fissure, a cleft or slit.

Fistula, a pipe or tube.

Flocculus, a little lock of wool

Foilicle, a small bag or sac.

Fontanelle, a small spring.

Foramen, an aperture or a hole.

Forceps, a claw of a beetle.

Fornicatus, pertaining to an arch.

Fornix, an arch or a vault.

Fossa, a ditch or trench.

Fourchette, a fork.

Fovea, a small pit.

Foveola, a small pit.

Frenum, a bridle.

Frenulum, a small bridle.

Frontal, pertaining to the forehead.

Fundiform, sling-like.

Funicular, pertaining to a cord.

Funiculus, a slender rope, a cord.

Furcalis, pertaining to a twopronged fork.

Furcula, a small two-pronged fork.

Fusca, dark or dusky.

Galactophorous, milk-carrying. Galěa, a helmet.

Gallinaginis, of a woodcock.

Gallus, a cock.

Ganglion, a swelling or excres cence.

Gastric,pertaining to thestomach. Gastrocnemius, the belly of theleg. Gemellus, paired or double. Geminus, double or twofold Geniculate, knee-like.

Genio-, pertaining to the chin.

Genu. the knee. 
Ginglymus, a hinge.

Glabella, without hair ; smooth.

Gladiolus, a small sword.

Grenoid, like a shallow socket.

Globosus, round or spherical.

Globus, a globe or sphere.

Glomerulus, a small ball of thread.

Gloss0-, pertaining to the tongue.

Glottis, the mouthpiece of a flute.

Gluteal, pertaining to the buttock.

Gnathic, pertaining to the jaw.

Gnathion, the jaw.

Gomphosis, a bolting together.

Gonion, an engle.

Gracilis, slender.

Grisea, grey.

Gubernaculum, a rudder.

Gula, the gullet.

Gustatory, pertaining to taste.

Guttural, pertaining to the throat.

Gyrus, a circle ; a crook.

Habenula, a small rein.

Hallux, the great toe.

Ham, see p. 440 (footnote).

Hamular, hook-shaped.

Harmonia, a fitting together.

Haustrum, a machine for drawing water.

Helicine, spiral.

Helicotrēma, hole of a spiral.

Helix, a coil or spiral.

Hemorrhoidal, pertaining to hemorrhoids or piles.

Hepar, the liver.

Hepatic, pertaining to the liver.

Hernia, a sprout ; a rupture.

Hiatus, a gap.

Hilum, a little thing, a trifle.

Hippocampus, a seahorse.

Hircina, pertaining to a goat.

Homodynamie, having the same force or value.

Homogenesis, one and the same generation.

Homologous, agreeing in position or structure.
Humerus, the upper part of the arm ; the shoulder.

Hyaline, glassy.

Hyaloid, like glass.

Hydatid, a watery vesicle.

Hydrocele, a watery tumour.

Hymen, a thin skin or membrant. Hyoid, like the Greek letter upsilon.

Hypo-, beneath or under.

Hypophysis, ' grow beneath.'

Hypothenar, beneath the palm of the hand.

Ileum, implying twists or coils. Ilium, the flank.

Ima, lowest.

Impar, dissimilar (in number), unequal.

Incisivus, cutting into.

Infundibulum, a funnel.

Infundibuliform, funnel-shaped.

Inguinal, pertaining to the groin.

Inion, literally, the occiput.

Innominatum, unnamed.

Insula, an island.

Intercalary, inserted.

Internodium, between two joints. Interpositum, placed between.

Interstitial, belonging to interstices or small intervals separating the minute parts of bodies.

Iris, the rainbow.

Ischiatic, pertaining to the hip.

Ischium, the hip.

Iter, a passage or road.

Jejunum, empty.

Jugal, yolking.

Jugular, pertaining to the throat. Jugum, a yolk.

Labrum, a basin.

Lachrymal, pertaining to tears.

Laciniosum, full of folds, in. dented, jagged.

Lacteal, pertaining to milk.

Lactiferous, milk-carrying. 
Lacuna, a hollow or cavity.

Lacunar, pertaining to a hollow or gap.

Lamella, a small plate.

Lamina, a platè.

Lateral, external.

Latissimus, broadest.

Latum, broad.

Lemniscus, a ribbon.

Leptorhine, having small narrow nostrils.

Levator, a lifter or raiser.

Lien, the spleen.

Lienno-, pertaining to the spleen.

Ligament, a band.

Ligula, a little tongue.

Limbous, pertaining to a border.

Limbus, a border.

Limen, a threshold.

Linea, a line.

Lingual, pertaining to the tongue.

Lingula, a little tongue.

Longissimus, longest.

Lumbar, pertaining to the loin.

Lumbricalis, pertaining to an earth-worm.

Lunar, pertaining to the moon.

Lunula, a little moon.

Luteum, muddy, like clay.

Lymphatic, from lympha, pure or spring water; lymph.

Lyra, a lyre.

Macula, a spot.

Malar, pertaining to the cheek.

Malleolus, a small hammer or mallet.

Malleus, a hammer or mallet.

Mamma, a breast or pap.

Mammilla, a little breast or pap.

Mandible, the chewing bone.

Manubrium, a handle or hilt.

Massēter, the chewing muscle.

Mastoid, breast- or pap-like (nipple-like).

Maxilla, jaw.

Meatus (pl. meatûs), a passage or canal.
Mediastinum, standing in ths middle.

Medulla, marrow.

Megacephalic, having a large head.

Megaseme, great index.

Meninges, membranes.

Meniscus, a crescent.

Mental, pertaining to the chin.

Mesaticephalic, pertaining to a:I intermediate head.

Mesencephalon, the mid-brain.

Mesentery, middle intestine.

Mesial, internal.

Meso-, middle.

Mesocephalic, pertaining to an intermediate head.

Mesogastrium, middle belly.

Mesognathion, middle jaw.

Mesometrium, middle of womb.

Meso-nephros, mid-kidney.

Mesorhine, pertaining to an intermediate nose.

Mesosalpinx, middle of trumpet or tube.

Mesoseme, intermediate index.

Meta-, after or beyond.

Meta-nephros, hind-kidney.

Metencephalon, the after-brain.

Metopic, pertaining to the forehead.

Metopism, persistence of the metopic or frontal suture.

Microcephalic, pertaining to a small head.

Microseme, small index.

Millimetre $(\mathrm{mm}$.$) , slightly less$ than $\frac{1}{25}$ of an English inch.

Minimæ, least, smallest.

Mitral, resembling an Asiatic head-dress.

Modiolus, the nave of a wheel.

Molar, pertaining to a mill, or to grinding.

Montānum, pertaining to a mountain.

Monticulus, a small mountain.

Morbus, a disease.

Muliebris, pertaining to a woman, feminine. 
Multifidus, many cleft; divided into many parts.

Myelencephalon, marrow-brain.

Myeloplaxes, marrow-plates.

Myentericus, pertaining to the muscular tissue of the bowel.

Mylc-, pertaining to a mill.

Myccardium, the muscular tissue of the heart.

Mystiform, like a myrtle-bcrry.

Naris (pl. nares), a nostril.

Nasal, pertaining to the nose.

Natal, pertaining to the buttock.

Natis (pl. nates), the buttock.

Navicular, pertaining to a boat.

Nephros, a kidney.

Neural, pertaining to a nerve.

Neuroglia, ' nerve and glue.'

Nictitans, winking.

Norma, a rule or measure, (aspect).

Natochord, string or cord of the back.

Nucha, the nape of the neck.

Nucleus, a kernel.

Nymphæ, demi-goddesses, or jnferior deities, of the fountains.

Obelion, a horizontal line (perhaps a little spit).

Obex, a bolt.

Obturator, a stopper-up.

Occipital, against the head.

Odontoblast, a tooth-germ.

Odontoid, tooth-like.

Odoriferæ, carrying odours.

Esophagus, food-carrier.

Olecranon, head of the elbow.

- Olfactory, pertaining to smell.

Olivary, pertaining to an olive.

Omentum, a caul or net; the fat-skin.

Omo-, pertaining to the shoulder. Omphalo-, pertaining to the navel.

Operculum, a covering.

ophryon, the eyebrow.

Ophthalmic, pertaining to the eye. Opisthion, hinder or rear.

Opisthotic, behind the ear.

Optic, pertaining to sight.

Ora, a border or margin.

Orthognathous, pertaining to a straight or upright jaw.

Os, oris, a mouth.

Os, ossis, a bone.

Osteoblast, bone-germ.

Osteoclast, bone-destroyer.

Osteogenetic, bone-begetting.

Ostium, a door, entrance, or exit.

Otic, pertaining to the ear.

Otoconia, ear-dust.

Otoliths, ear-stones.

Ovary, egg-former.

Oxyntic, producing acid.

Palātum, the palate.

Pallium, a covering.

Palmar, pertaining to the palm.

Palpebra, an eyelid.

Pampiniform, tendril-like.

Pancreas, literally, all flesh.

Para-, near, by the side of.

Paracidymis, near the testis.

Parametrium, near the womb.

Parietal, pertaining to a wall.

Paroophoron, near the egg-bearing organ.

Parōtid, near the ear.

Patella, a small dish; a plate.

Patheticus, affecting.

Pecten, the os pubis.

Pectinatus, pertaining to a comb.

Pectiniform, comb-like.

Pectinēal or Pectinēus, pubic.

Pectoralis, pertaining to the breast.

Pelvis, a basin.

Penicillus, a painter's brush.

Penis, a tail.

Peri-, around, about, or near.

Pericardium, around the lieart.

Perineum, from a Greek verb meaning 'I dwell, or' am situated, around.'

Đeriosteum, around a bone. 
Periotic, around the ear.

Peritoneum, from a Greek verb meaning 'I stretch around.'

Peroneal or Peroneus, pertaining to a buckle, clasp, or brace.

Petrous, rocky.

Phalans, a rank of soldiers.

Pharynx, the throat.

Phenozygous, having visibls arches.

Philtrum, a love potion.

Phrenic, pertaining to the diaphragm.

Pinēal, belonging to, or like, a pine-nut or pine-cone.

Pinna, a kind of shell-fish; a feather or wing.

Pisiform, like a pea.

Pituitary, pertaining to phlegm or mucus.

Placenta, a flat cake.

Plagiocephalous, pertaining to an oblique or twisted head.

Planta, the sole of the foot.

Plantar, pertaining to the sole of the foot.

Platyenèmism, broadness of leg.

Platyrhine, pertaining to a broad nose.

Platysma, a broad sheet.

Pleura, a side, or a rib.

Plexus, a twining or network.

Plica, a fold.

Pneumogastric, pertaining to the breathing organs and stomach.

Pocularis, pertaining to a cup.

Pollex, the thumb.

Pons, a bridge.

Poplitēal or Poplitēus, pertaining to the ham.

Porta, a gate.

Portal, pertaining to a gate.

Postaxial, distal or caudal.

Posticus, posterior.

Præputium, the prepuce.

Preaxial, proximal or cephalic.

Proctodæum, the threshold of the anus.
Prognathous, pertaining to a projecting lower jaw.

Proligerus, bearing offspring ; germinating.

Pro-nephros, fore-kidney.

Pro-otic, before the ear.

Prosencephalon, the fore-brain.

Prostate, standing before; or, more probably, pertaining tc a porch or vestibule.

Psalterium, a psaltery or instrument of the lute kind.

Psoas, the loin.

Pterion, a wing.

Pterotic, pertaining to a wing.

Pterygoid, wing-like.

Pubes, the hair which appears on the external genital organs at the age of puberty.

Pubic, pertaining to the os pubis.

Pudendal, pertaining to the pudendum.

Pudendum, "of which one ought to be ashamed.'

Pudic, modest or chaste.

Pulmo, a lung.

Pulmonary, belonging to the lungs.

Pulvinar, a couch or cushion.

Putamen, that which is pruned off ; chippings.

Pylōrus, literally, a gate-keeper.

Pyriformis, pear-shaped.

Quadrätus, square.

Quadriceps, having four heads.

Quadrigeminus, fourfold, four.

Racemose, pertaining to a cluster of grapes; full of clusters ; clustering.

Radius, a staff or rod; the spoke of a wheel.

Ramus, a branch.

Ranine, pertaining to a frog.

Raphé, a seam. 
Receptaculum, a receptacle.

Rectus, straight.

Recurrent, running back.

Refractory, breaking up.

Ren, a kidney.

Restiform, like a rope or cord.

Rete, a net.

Retina, from rete, a net.

Retrahens, drawing back.

Revehens, carrying back.

Rhinencephalon, the 'nose' or olfactory brain.

Rhinion, a nose.

Rhombencephalon, the rhombbrain (hind-brain).

Rima, a cleft or chink.

Risorius, laughing.

Rostrum, a beak.

Rotula, a little wheel.

Rugæ, wrinkles.

Sacrum, sacred.

Sagittal, pertaining to an arrow ; antero-posterior.

Salpinx, a trumpet or tube.

Salvatella, saving, or making well.

Saphēnous, manifest.

Sartorius, pertaining to a tailor.

Scala, a ladder, flight of steps, or staircase.

Scalēnus, of unequal sides.

Scansorius, of, or for, climbing.

Scaphocephalous, pertaining to a head like a boat.

Scaphoid, like a boat.

Scapula, a spade ; probably from a Greek verb meaning 'I dig.'

Schindylesis, a splitting or cleavage.

Sciatic (identical with Ischiatic), pertaining to the hip.

Sclera, hard.

Sclerotic, hard.

Scrobiculus, a little ditch or trench.

Scrotum, a skin-bag or -pouch ; a hide.

Sebaceous, pertaining to grease.
Sella, a seat ; a saddle.

Seminalis, pertaining to semen.

Septum, a fence or barrier.

Serotinus, that comes or happens late.

Serrātus, saw-shaped.

Sesamoid, like sesamé (a kind of grain).

Sigmoid, like the Greek letter $\Sigma$ (sigma).

Sinus, a cavity or hollow.

Smegma, a cleanser.

Solar, relating to the sun.

Soleus, a sole or sandal ; a soleSperma, seed or semen. [fish. Spermatic, pertaining to semen. Spermatoblast, a seminal bud.

Spermatozoa (plural), semina] animals.

Sphenoid, wedge-like.

Sphenotic, pertaining to the sphenoid bone and ear-capsule.

Sphincter, binding or closing tight.

Splanchnic, pertaining to viscera. Splenium, a bandage or compress.

Splenius,pertaining to a bandage. Squamous, scaly.

Stapes, a stirrup.

Stellātum, starry.

Stephanion, a crown or wreath.

Sternebra, a segment of the sternum.

Sternum, the breast or chest.

Stomata, mouths or pores.

Stomatodæum or Stomodæum, the threshold of the mouth.

Styloid, pen-like.

Subflava, somewliat yellow.

Subiculum, an underlayer or support.

Sudoriferous, sweat-carrying.

Sulcus, a furrow.

Supercilium, an eyebrow.

Sural, pertaining to the calf of the leg.

Sustentaculum, a prop or support. Suture, a sewing together, a seam. 
Symphysis, growth together.

Syn-, with; together with, (union or harmony may be implied).

Synarthrosis, literally, a 'together with ' (direct) joint.

Synchondrosis, ' with cartilage.'

Syndesmosis, "with a band " or ' ligament.'

Synovia, resemblance to the white of an egg.

Tænia, a band or ribbon.

Talus, a die (pl. dice) ; the anklebone.

Tapētum, a carpet or coverlet.

Tarsus, a broad flat surface; the instep.

Tectorius, pertaining to a cover.

Tegmen, a covering.

Tegmentum, a covering.

Tela, a web.

Telencephaion, the end-brain.

Temporal, pertaining to time.

Tendon, from tendo, 'I stretch.'

Tentorium, a tent.

Tenuis, slender, small.

Teres, rounded.

Testis, a witness.

Thalamencephalon, the bedchamber-brain, or inter-brain.

Thalamus, a bed-chamber, situated between two other rooms ; a marriage-bed.

Theca, a cover, case, or sheath.

Thenar, pertaining to the palm.

Thorax, the breast or chest; a breast-plate.

Thymus, thyme.

Thyroid, like a shield.

Tibia, a pipe or flute; the shinbone.

Tinca, a small fish, perhaps the tench.

Torcular, a wine-press (twisting is implied).

Torus, a protuberance.

Trabecula, a little beam.
Trachea ('rough'), the wind-pipe.

Trachēlo-, belonging to the neck.

Tragus, a goat.

Trapezium, a table; a four-sided figure, no two sides of which are parallel to one another.

Trefoil, having three leaves.

Triceps, having three heads.

Trigeminus, threefold, triple.

Trigonocephalus, a triangular head.

Trigōnum, a triangle ; triangular.

Triquetrum, three-cornered ; triangular.

Triticěa, wheaten.

Trochanter, from a Greek verb meaning 'I roll, turn, or revolve.'

Trochlea, the wheel of a pulley.

Trochlear, pulley-shaped.

Trochlearthrosis, a pulley-joint.

Trochoides, wheel-like.

Tuba, a trumpet.

Tubarius, pertaining to a trumpet. Tubercle, a small swelling.

Tuberosity, an exaggerated tubercle.

Turbinate, whirled or coiled; like a top.

Turbo, a whirl or coil ; a top.

Turcica, Turkish.

Tympanum, a dru:ı.

Ulna, the elbow.

Umbilicus, the navel.

Umbo, a boss or knob.

Unciform, hook-like.

Uncinate, furnished with a hook.

Ungual, relating to a nail.

Unguis, a nail.

Unicornis, one-horned.

Urăchus, urine-holder.

Urĕter, from a Greek verb mean. ing 'I pass urine.'

Urethra, the canal by which urine is passed.

Uriniferous, urine-carrying.

Uterus, the womb or matrix. 
Utricle, a little womb or matrix. Uvæa, from $u v a$, a bunch of grapes ; a cluster.

Uvula, a small bunch of grapes.

Vagina, a scabbard or sheath.

Vagus, strolling about, wandering, vagrant.

Valgus, bent or turried outwards.

Vallecula, a little valley.

Vallum, a rampart.

Varus, bent or turned inwards.

Vas (pl. vasa), a vessel.

Velum, a curtain or veil.

Veneris, 'of Venus.'

Ventral, pertaining to the belly.

Vermiform, like a worm.

Vertebra, from verto, 'I turn.'

Vertex, the top or crown of the head.

Veru, a dart, javelin, or spear.

Vesica, the urinary bladder.

Vescial, pertaining to the urinary bladder.
Vespertilio, a bat.

Vestigial, pertaining to a trace.

Vestigium, a trace or vestige.

Vibrissa, a stiff hair of the nostril.

Villus, shaggy hair; a tuft of hair.

Vinculum, a band or bond.

Vitelline, pertaining to the yolk of an egg.

Vitellus, the yolk of an egg.

Vitreous, like glass, glassy.

Vola, the palm of the hand.

Volar, pertaining to the palm; palmar.

Vomer, a ploughshare.

Vorticosæ, full of whirlpools, eddying, coiled.

Vulva, a wrapper or covering.

Xiphoid, like a sword.

Zygōma, a yoke. 


\section{INDEX}

AbDomex, 6II

division into regions, 689

superficial view of contents, 690

Abernethy, fascia of, 777

Aberrans, vas, of Haller, 684

Accessory lachrymal glands, i i 89

$$
\text { thyroids, I } 122
$$

Acervulus cerebri, or brain sand, I.422 Acetabulum, 226

Achillis, tendo, 520

Acromio-clavicular joint, 326

Acustica, area, 1378

Acusticæ, striæ, 1377

Acusticum, trigonum, 1378 tuberculum, ${ }_{37} \mathrm{~S}$

Adamantoblasts, 173

Adductor tubercle, 236

Air sinuses, I 39

Ala cinerea, 1378

Alæ vespertilionis, 880

Alar thoracic artery, 3 II

Alcock, canal of, 623

Allantoic stalk, I629 veins, $163 \mathrm{I}$

Allantois, 870,1629

Alveus, 1409

Ambiguus, nucleus, 1495

Amitosis, r6or

Ammonis, cornu, 1409

Amnion, 1624

Amphiarthrosis, 280

Ampulla of Vater, 710

Amygdala of cerebellum, I37 I

Amygdalæ, or tonsils, 1253

Amygdaloid nucleus, $\mathrm{I}_{4} \mathrm{I}_{4}$

tubercle, 1408

Anal canal, of male, 86 I development of, 877 of female, 890 lymphatics of, 877

fascia, 840 structure of, 874

Anaphase, 1598

valves of Morgagni, 875

Anastomosis, digital (trochanteric), $4^{8} 3$

femoral, 483

round elbow-joint, 362

knee-joint, 508

scapular, 323
Anastomotica magna of brachial, 339

Anconeus muscle, 356 of femoral, 484

Angle, sacro-vertebral, 38

sternal, 48

Angular movement $28 \mathrm{r}$

Animal cell, I597

matter of bone, 3

Ankle-joint, 569

Annular ligament, anterior, of ankie, 500

external, of ankle, 498 internal, of ankle, 499 anterior, of hand, 376 posterior, of hand, 390

Annular (orbicular) ligament, 404

Annulus fibrosus, I540

ovalis, 977

tympanicus, 80

Ansa cervicis, $\mathrm{IIO}_{7}$

hypoglossi, I IoS

lenticularis, $I_{+} 2 \mathrm{I}$

subclavia, $1 \geq 35$

Vieussenii, I 235

Anterior triangle of neck, logs

Antibrachium, 175 subdivisions of, 1099

Anticubital space, 340

Antinion, 133

Antrum of Highmore, 102 mastoid, 73

pylori, 693

Anus, 6 I I

imperforate, 878

Aorta, abdominal, 750

arch of, 958

development of, 963

primitive, dorsal, rooo

ventral, 1002

thoracic, descending, 1023

Aortic arches, I000

isthmus, 960

orifice, 983

plexus, 738

position of, $9 S_{5}$

spindle, 960

valves, $98_{3}$

vestibule, 981

Aortico-renal ganglion, 736

Aponeurcsis, epicranial, 1069 intercostal, anterior, 927 
Aponeurosis intercostal, posterior, 927

lumbar, 289, 765 posterior lamina of, 289

pharyngeal, I266

of soft palate, I250

vertebral, 289

Apparatus, lachrymal, I I90

Appendices epiploicæ, 699, 794

Appendicular mesentery, 697

skeleton, I

Appendix, vermiform, 696

Aqueduct of Sylvius, I 439 development of, 1439 grey matter of, $\mathrm{I}_{439}$

Aqueductus cochleæ, 78, I $55^{2}$

Fallopii, 75

vestibuli, 76

Arachnoid membrane, cranial, 1479

nerves of, $\mathrm{I}_{4} 8 \mathrm{I}$

spinal, 1309

structure of, $148 \mathrm{I}$

Arantii, corpus, 984

duct of, roog

Arbor vitæ cerebelli, I 373

uterina, 888

Arc, longitudinal, of skull, $15^{8}$

Arch, dental, I68

dorsal venous, of foot, $5 \mathrm{I} 4$

femoral, deep, 460,652 superficial, $460,65 \mathrm{I}$

hyoid, I27 I

mandibular, I27 I

palmar, deep, 380

plantar, 563 superficial, 370

thyro-hyoid, I27I

Arches, arterial, Iooo

dental, r68, r 238

of foot, 577

longitudinal, 577

transverse, $57^{8}$

visceral, I27I

first or mandibular, $16_{3}$

second or hyoid, 165

third or thyro-hyoid, I 65

development of, I 27 I

Neckel's cartilage, 1272

metamorphoses of, 1272

first or mandibular, 1272

second or hyoid, 1272

third or thyro-hyoid, 1272

fourth or fifth, 1273

sixth, 1273

morphology of, 1273

Arcuate fasciculus, I 447

fibres of medulla, deep, I 359 superficial, I359
Arcuatum, ligamentum, 903

Arcus parieto-occipitalis, 1389

Area, cochlear, 75

embryonic, I6I4

inferior vestibular, 75

superior vestibular, 75

Areas, of Flechsig, I 360 dorsal, 1361

lateral, 1361

medulla, I 360

ventral, I 36 I

Areola of nipple, 300

Arm, 329

Arnold, nerve of, 1073,1226

Arteria centralis retinæ, II59, I525

princeps cervicis, 1062 deep branch of, I062. superficial branch of, I062 thyroidea ima, I 123

Arteriæ rectæ, 824

Artery or Arteries-

acromial rete, 324

acromio-thoracic, 310

alar thoracic, 3 II

allantoic, I63I

anastomosis, crucial, 483

digital (trochanteric), 483

round elbow-joint, 362

knee-joint, 508

scapular, 323

anastomotica magna of brachial, 339 of femoral, 484

angular, II 79

aorta, abdominal, $75^{\circ}$ branches of, $75 \mathrm{I}$ guides to bifurcation of, 642

arch of, $95^{8}$ development of, 963

ascending, 957 branches of, 958 development of, 958 great sinus of, 957

descending, ro23 development of, 1027

thoracic, 957, I023

varieties of, $96 \mathrm{I}$

appendicular, 727

arch, palmar, deep, 380 superficial, 370

plantar, 563

articular, of popliteal, $44^{2}$

auditory, internal, $\mathbf{1 4 5 3}$, I 559

of auricle, 1 I95

auricular, deep, 1203

posterior, I073, II 20

branches of, 1073, I120

axillary, 308

first part, 308

branches of, 3 ro

nucleus, I 355 
Artery or Arteries (continued) axillary, second part, 309 branches of, 3 II third part, 3 Io branches of, 3 II varieties of, 312

axis, thoracic, 3 Io

azygos, 442

basilar, I 342,1452 branches of, 1342,1452 brachial, 338

branches of, 338

collateral circulation after occlusion of, $34^{\circ}$

varieties of, 339

bronchial, I025

buccal, I I 82,1205

of bulb, female, $63 \mathrm{~s}$ male, 624

cacal, 727

calcarine, 1454

capsular, middle, 752

of liver, 804

carotid, common, I I Io

collateral circulation, after ligation of, I I 12 left, in thorax, 96I surgery of, III2

external, I I I 4

branches of, I I 6 development of, II 66 relations of, III 5

internal, at base of brain, I342, I449

cavernous part of, 1078 cervical part of, 1222 development of, 1223 petrous part of, 1296

carpal, anterior radial, 357

posterior radial, 392

anterior ulnar, 362

posterior ulnar, 362

cerebellar, anterior inferior, $\mathbf{1 4 5 3}$ posterior inferior, $\mathbf{1 4 5 2}$ superior, 1453

cerebral, anterior, I 343, I449 branches of, r449

middle, I 343, I $45^{\circ}$

branches of, I $45^{\circ}$

posterior, 1453

branches of, 1454

of cerebral hemorrhage, I $45^{\circ}$

cervical, ascending, II 42

deep, ro63, II44

branches of, 1063

superficial, I I 43

transverse, 285, 1096, I I 43

choroidal, anterior, I449

posterior, I 454

ciliary, anterior, I 5 I 6

long, I I 59,1516
Artery or Arteries (continued) -

ciliary, posterior, I I59

short, II 59

circle of Willis, I 343 , I 454

parts contained within, $\mathbf{I} 455$

circulus major, I5I 6

minor, 1516

circumflex, anterior, of axillary, 3 II

posterior, of axillary, 312 varieties of, 312

external, of profunda femoris, $48 \mathrm{I}$

internal, of profunda femoris, $4^{8} 3$

iliac, deep, $66_{3}$ superficial, of femoral, 479,646

clitoris, dorsal artery of, 638

cochlear, I 560

coliac axis, $739^{\circ}$

colic, left, 729

middle, 727

right, 727

comes nervi ischiadici, 433.

mediani, 36r

phrenici, 93 I

communicating, anterior, 1450

posterior, I 449

coronary, of face, I26I

of heart, $95^{8}$

of corpus callosum, I450 .

cavernosum clitoridis, 638 penis, 624

spongiosum penis, 624

crico-thyroid, I I 16

cutaneous, anterior, of abdominal wall, 646

lateral, of abdominal wall, 646

plantar, $54^{8}$

cystic, 742

deferential, 669, 84 I

dental, anterior, 1205

inferior, 1204 branches of, $\mathrm{I}_{2} \mathrm{O}_{4}$

middle, 1205

posterior, I 205

digital, of foot, 564 superficial, $56 \mathrm{I}$

of hand, 370

dorsal, of penis, 624

dorsalis linguæ, I I I 8 scapulæ, 3 I I

of dura mater, cranial, I 47 I spinal, I308

of encephalon, I 449

at base of, 1342

epigastric, deep, 662 branches of, 662

guide to, 642 
Artery or arteries (continued) epigastric, lateral or intermuscular, 664

superficial, 479,646

superior, 663,932 branches of, 663

ethmoidal, anterior, I I60, 1260 posterior, I 160, 1 261

of Eustachian tube, 1275

of face, $\operatorname{Ix}_{7} 8$

facial, in neck, I I 9

branches of, $\mathrm{x} x \mathrm{I} 9$

on face, $x \geq 78$

branches of, Ix 79

transverse, 1 I 8 I

femoral, 477

first part, $47^{8}$

second part, 478

branches of, 479

collateral circulation after occlusion of, 485

deep, $48 \mathrm{r}$

varieties of, 485

fibular superior, 507

of frenum linguæ, 4 I8

frontal, ro68, I r6I

ganglionic, I449, 1454

gastric, 739

gastro-duodenal, 74I

gastro-epiploic, left, $74^{\circ}$ right, $74^{\mathrm{I}}$

gluteal, 430,844

hallucis, arteria dorsalis, $5 \times 2$

helicine, 869

magna or princeps, 565

hemorrhoidal, inferior, 623,876

middle, 842,876

superior, 875

hepatic, $74 \mathrm{r}$

branches of, 74I, 804

humeri, transversalis, 320

hypogastric, 840

iliac, circumflex, deep, $66_{3}$

branches of, 663

superficial, 479,646

common, 774

branches of, 776

collateral circulation,

after ligation of, 776

guide to, 642,775

varieties of, 776

external, 777

branches of, 778 collateral circulation after ligation of, 778 development of, 778

guide to, 642,777

varieties of branches, $77^{8}$

internal, 840

branches of, $84 \mathrm{I}$
Artery or Arteries (continued) -

iliac, internal, fœtal condition of, $84^{\circ}$ varieties of, 840

ileo-cæcal, 727

ileo-colic, 727

ilio-lumbar, 843

incisor, 1205

indicis, dorsalis, 393

radialis, $38 \mathrm{I}$

infra-hyoid, $x \geq 16$

infra-orbital, I I 82, I 205

branches of, 1205

innominate, 960 varieties of, $96 \mathrm{I}$

intercostal, 929, rO25 anterior, 930

aortic, 929

collateral, ro26

lower two, 664

superior, I027, I $x_{43}$

interlobar of kidney, 822

interlobular of kidney, 823 of liver, 804

interosseous, common, 360 anterior, $36 \mathrm{r}$

posterior, $36 x$

first dorsal, of hand, 392

of iris, $15 \times 6$

of kidney, 822

afferent, 823

efferent, 823

labial, inferior, I I 79

of labyrinth, $\mathbf{5 5 9}$

lachrymal, II 59

laryngeal, inferior, I I 42

superior, II 16

of larynx, 1293

lenticular, of Duret, $145^{\circ}$

lenticulo-optic, of Duret, $145 \mathrm{r}$

lent:culo-striate, of Duret, $\mathrm{r}_{45}$

lingual, $x \times x 7$

branches of, $\operatorname{Ir} 8$

lumbar, 772

abdominal branches of, 664 branches of, 772

malar, I 182

malleolar, external, 508

internal, of anterior tibial, 507

of posterior tibial, 498

mammary, extern?1, 3 II

internal, cervical part of, I 42

thoracic part of, 93I

branches of, $93 \mathrm{I}$

masseteric, 1205

mastoid, 1062

maxillary, internal, 1202

branches of, 1203

first part, 1203 
Artery or Arteries (continued) maxillary, internal, second part, I 205

median, $36 \mathrm{r}$

$$
\text { third part, } 120_{5}
$$

medullary, of clavicle, I I43

$$
\text { of femur, } 484
$$

of fibula, 526

of humerus, 339

of radius, $36 \mathrm{I}$

of tibia, 525

of ulna, 36r

of membrana tympani, ${ }_{54}$ I

meningeal, anterior, of ethmoidal, I 47 I

of ascending pharyngeal, I I 2 I, I 472

of internal carotid, I 47 I

middle, I 203 , I $47 \mathrm{I}$ guide to, $\mathrm{I}_{502}$

posterior, of occipital, 1062, 1472

Small, I $204, I_{47}$ I

of vertebral, I $452, I_{472}$

mental, I I82

mesenteric, inferior, 729

branches of, 729

superior, 725

branches of, 726

metatarsal, 512

of mouth, I 239

musculo-phrenic, 93 I

mylo-hyoid, 1204

of nasal fossæ, 1260

nasal, I I8I

lateral, II 79

naso-palatine, 1260

of nose, facial aspect of, I 196

obturator, 488,843 aberrant or abnormal, 462

occipital, 1062, I 120

branches of, 1062, I I 20

olecranon rete, 363

ophthalmic, I I 59

branches of, I I 59

ovarian, 755,890

branches of, 755, S9o

of palate, soft, 1252

palatine, descending, 1205 , I260 inferior, III9

of ascending pharyngeal, I I 2 I

palmar arch, deep, 380

branches of, 381

interosseous of, $38 \mathrm{I}$

summary of, 382

superficial, 370

branches of, palpebral, external, $\begin{aligned} & 370 \\ & \text { I } 82\end{aligned}$

internal, I I6I, I I8I
Artery or Arteries (continued) -

pancreatic magna, 740

pancreatico-duodenal, inferior, 727

superior, $74 \mathrm{I}$

pedis, dorsalis, 5 Io

varieties of, 512

perforating of profunda femoris, 484

perineal, superficial, of female,

$$
638
$$

of male, 623

transverse, 623

peroneal, 526

anterior, 509, 526

posterior, 527

varieties of, 527

petrosal, 1297

pharyngeal, ascending, I I 2 I

of pharynx, 1269

branches of, II 2 I

phrenic, inferior, 752

superior, 93 I

of pia mater, I 3 II, I 482

plantar or perforating, 565

external, 562 varieties of, 565

internal, 56r varieties of, 565

pollicis, dorsales, 393

princeps, 380

pontine, 1453

popliteal, $44^{\circ}$

branches of, 442

varieties of, 443

profunda, inferior, of brachial, 339

profunda, superior, of brachial, $33^{8}$

femoris, $48 \mathrm{I}$

of ulnar, 370

pterygo-palatine, 1206

pudic, accessory, 624

pudic, external, inferior, of femoral, $48 \mathrm{I}$

superior, of femoral, 479,645

internal, $433,622,843$

branches of, 623

varieties of, 624

pulmonary, 963

development of, 964

pyloric, $74 \mathrm{I}$

radial, 356

first part, 356

branches of, 356

second part, 39I

branches of, 392

third part, 280

branches of, 380

varieties of, 382 
Artery or Arteries (continued)rami intestini tenuis, 726 ranine, $\operatorname{Ir} 8$

recurrent, of forearm, posterior interosseous, $36 \mathrm{r}$ radial, 356 anterior ulnar, 360 posterior ulnar, 360 anterior, of tibial, 507 posterior, of tibial, 507

renal, 752

aberrant, 753

accessory, 753

branches of, 753,822

rete, acromial, 324 olecranon, 363

sacral, lateral, 844 middle, $86 x$

saphenous, external, 442 internal, 484

of scalp, 1067

scapulæ, dorsalis, 3 I r

scapular, posterior, 287

sciatic, 432,843

extrapelvic branches of, 432

of sclerotic, 1508

sigmoid, 730

spermatic, 754 terminal párt of, 670 varieties of, 754

spheno-palatine, 1206,1260

branches of, 1206

spinal, anterior, 1332, I452

lateral, 1332

poster or, I332, I 452

of spinal canal, r 33 I

of spinal cord, $\mathrm{r} 332$

anterior, 1332

posterior, I 332

splenic, 739

sterno-cleido-mastoid, $x \times 16$

striate, external, $\mathbf{r}_{450}$

internal, 1450

stylo-mastoid, I I 2 I, 1547

subclavian, first and second parts of, II 38 . I I 40

branches of, $\mathrm{I}_{4}{ }^{\circ}$

collateral circulation after ligation of first part of, I 45

third part of, I095'

collateral circulation after

ligation of third part of, I 45

left, in thorax, 961

subcostal, 773,1027

sublingual, II 8

submental, I I 19

subscapular, 3 I I

suprahyoid, $\operatorname{x} 1 \mathbf{1} 8$

supra-nrbital, 1067, I 160
Artery or Arteries (continued)-

suprascapular, 320, rog6

branches of, $32 \mathrm{r}$

temporal, anterior, deep, 1205

of lachrymal, i i 82

posterior, deep, I 205

superficial, ro 70

branches of, 107I

thoracic, alar, 3 II

axis, 3 ro

inferior or long, $3 \mathrm{xI}$

superior or short, 3 IO

thyroidea ima, 960

thyroid, inferior, II 42 - branches of, II 42

superior, I 16

branches of, I I I 6

tibial, anterior, 505

branches of, 507

recurrent, 507

varieties of, 508

posterior, 523

branches of, 525 recurrent, 507

varieties of, 527

of tongue, 1246

of tonsils, I253

tonsillar, of facial, IIIO

transversalis humeri, 320

tympanic, $\mathrm{r} 547$

of ascending pharyngeal,

\section{121}

of internal carotid, r 296

of internal maxillary, 1203

ulnar, $35^{8}$

first part, $35^{8}$

branches of, 360

second part, 364

third part, $37^{\circ}$ branches of, 370

varieties of, 363 in hand, 382

umbilical, 840

uterine, $89 \mathrm{I}$

branches of, 891

vaginal, 892

azygos, 892

of liver, 804

vasa brevia, of splenic, $74^{\circ}$ recta, false, of kidney, 823 true, of kidney, 824

vertebral, at base of brain, I 342

first part of, $\mathrm{II}_{4} \mathrm{I}$ second part of, II 4 I branches of, II $4 \mathrm{I}$ third part of, 1065 branches of, 1066

fourth part of, r45I branches of, 1452

vesical, inferior, $84^{2}$ 
Artery or Arteries (continued)vesical, superior, $84 \mathrm{I}$ vestibular, 1560

Vidian, I 206, I260 branches of, I 206

volar, superficial, 357

Arthrodia, 280

Arthrology, 279

Articulations. See Joints

Arytæno-epiglottidei muscles, I 290

Arytenoid cartilages, 1279

muscle, 1290

Ary-vocalis muscle, 1289

Aspera, linea, 236

Association fibres of cerebrum, I 446

Asterion, I 42

Astragalo-calcaneal joint, anterior, 579 posterior, 578 .

Astragalo-navicular joint, $5^{80}$

Astragalus, 254

Atlanto-axial joints, I3or

ligaments of, 1302 accessory; $\mathrm{I}_{3} \mathrm{O} 3$

-occipital joints, 1303

ligaments of, $\mathrm{I}_{3} \mathrm{O}_{3}$ accessory, 1303

Atlas, I4

ossification of, 29

transverse ligament of, I 302

Atresia ani, 878

Atria of lung, 951

Atrium of heart, 973

of middle meatus, 1255

Attic of tympanum, 1545

Attollens auriculam muscle, Io 72

Attrahens auriculam muscle, I072

Auditory artery, internal, I 453, I 559

cells, I 554

epithelium, I554

hairs, I 554

meatus, external, $78,1_{536}$ internal, 75

nerve. See Cranial Nerves

process, external, 79

radiation, 1446

teeth, 1556

vesicle, ${ }_{5} 60$

Auerbach, plexus myentericus of, 793

Auricle, II92

blood-supply of, II95

ligaments of, I I93

lymphatics of, 1195

muscles of, extrinsic, 1072 intrinsic, I I 93

nerve-supply of, I 195

veins of, II 95

Auricular appendix, 973

artery, deep, 1203

posterior, I073, II 20

branches of, II 20
Auricular canaliculus, 76

fissure, 77

glands, posterior, 1073

nerve, great, ro59 branches of, I059, I 178

posterior, I073, II 73 branches of, I I 73

point, $\mathbf{r}_{40}$

vein, posterior, ro73, I I 2 I

Auriculo-temporal nerve, I070, I209

branches of, 1070, 1209

Anriculo-ventricular bundle, 990

Axial skeleton, I

Axillary artery, 308

varieties of, $3 \mathrm{I} 2$

first part, 308

branches of, 3 Io

second part, 309

branches of, 3 II .

third part, 310

branches of, 3 II

Axillary lymphatic glands, 317 afferent vessels, 317

efferent vessels. $3 \mathrm{I} 7$

sheath, 312

trunk, 3 I 7

space, 306

vein, $3 \mathrm{I} 2$

contents of, 307

Axis, 17

ossification of, 29

basi-bregmatic, 149

basi-cranial, I 49

basi-facial, I 49

cerebro-spinal, I 306

coliac, 739

thoracic, or acromio - thoracic artery, 310

Axon, 1320

cylinder process, I335

Azygos artery, $44^{2}$

uvulæ muscle, $125^{\circ}$

veins, 762

Bacillary layer of retina, $\mathbf{I}_{523}$

Back, the, 283

Back of scalp and neck, Io $_{5} 8$

landmarks of, 1058

Baillarger, inner band of, I440, I 443

outer band of, I $440, I_{443}$

Ball-and-socket joint, 280

Bar, hyoid, 1272

Bartholin, glands of, 633 development of, 633

Basi-bregmatic axis, 149

Basi-cranial axis, I 49

Basi-facial axis, 149

Basi-hyal, I6 5

Basi-occipital, 59

Basilar artery, I.342, I452 
Basilar artery, branches of, I 342,1452 border, striated, 739

groove, 59

membrane, I 555

process, 59

sinus, 1479

vein, 1456

Basilic vein, 334

Basion, I 48

median, 332

Basket-cells of cerebellum, I 375

Bechiterew, band of, $\mathrm{x} 443$

nucleus of, 1493

Bell, external respiratory nerve of, 3 I 3

muscles of, 867

nerve of, 1098

Bellini, duct of, 820

Bergmann, fibres of, 1376

Bertin, bones of, 90

Bertini, columnæ, 818

Betz, giant cells of, 1447

Biceps femoris (flexor cruris), 437 flexor cubiti, 336

Bicipital (semilunar) fascia, 336 groove, I 86

hollow of ulna, 199

tuberosity of radius, I9I

Bicuspid teeth, 167

valve, 982

Bigelow, $Y$ ligament of, 489

Bile-canaliculi, 806

capillaries, 806

duct, common, 7 IO

ducts, 806

interlobular, 806

Biventer cervicis muscle, 293

Biventral lobule, 137I

Bladder, urinary, male, $85^{\circ}$ development of, 870

female, 889

infantile, 867

ligaments of, false, 836,867 true, 867

lymphatics of, 868

orifices of, 866

peritoneal relations of, $85 \mathrm{I}$

structure of, 865

trigone, external, of, $85 \mathrm{I}$ internal, of, 866

Blandin, glands of, I 243

Blastoderm, I6 13,1616

Blastodermic vesicle, I 6 I 2

Bochdalek, ganglion of, 12 I 8

Body cavity, primitive, I620

Bone-cell, 7

Bones, classification of, 7

astragalus, 254

atlas, 3 ?

axis, I-

of Bertin, 90
Bones, calcaneum or os caícis, $25^{6}$

carpal, $20 \mathrm{r}$

clavicle, 175

$\operatorname{coccyx}, 36^{\circ}$

cuboid, 263

cuneiform of carpus, 202 of tarsus, 260

endochondral, I 62

epipteric, $\mathbf{1}_{42}, \mathrm{I}_{55}$

ethmoid, 92

femur, 232

fibula, $25^{\circ}$

frontal, 65

of head, 56

humerus, I 84

hyoid, I 2 I

ilium, 220

innominate, 220

interparietal, 57, 6I

ischium, 223

lachrymal, 108

of limbs, homologies of, 277

of lower limb, 220

lunar, 202

magnum, 205

malar, 104

maxillary inferior, II 5 superior, 97

membranous or tegmental, 162

metacarpal, 208

metatarsal, 265

nasal, ro7

navicular, 258

occipital, 57

palate, I IO

parietal, 62

patella, 24I

phalangeal of foot, $27 \mathrm{I}$ of hand, $2 I_{3}$

pisiform, 203

pubic, 225

pyramidal, 202

radius, I9I

ribs, $4 \mathrm{I}$

sacrum, 30

scaphoid of carpus, 201 of tarsus, 258

scapula, I 78

semilunar, 202

sesamoid, of foot, 272 of hand, $2 \pi 6$

sphenoid, 82

sternum, 47

supra-occipital, 57

tarsal, 253

temporal, $\epsilon_{9}$

tibia, 242

trapezium, 203

trapezoid, 204

triquetral, I55

of trunk, II 
Bones, turbinate, inferior, Ios sphenoidal, 90

ulna, 197

unciform, 206

of upper limb, I 75

vomer, $\mathrm{II}_{4}$

Wormian, 155

Bowman, capsule of, 819,824 glands of, 1257

Brachia of corpora quadrigemina, I 428, I 429

Brachial artery, 338 branches of, 338 varieties of, 339

Brachial plexus, 312 , $10 y 6$ infraclavicular branches of, 3 I 4 supraclavicular branches of, 313, 1098 branches of inner cord, 315 of outer cord, $3 \mathrm{I}_{4}$ of posterior cord, 315

region, back of, $34^{2}$

front of, 334

Brachialis anticus, 337

Brachio-radialis, $3^{8} 3$

Brachium, I75

Brachycephalic skulls, 132,158

Brain, I 337 development of, 1458

lymphatics of, I 458 weight of, I 449

Eregma, I24

Bregmatic bone, 69, ז 55

Broca, area of, I 397

le cap of, 1387

Bronchial tubes, 952, 1020

Bronchioles, 952

Bronchus, eparterial, 1020

hyparterial, 1020

left, I020

right, 1020

Bruch, membrane of, I 5 I 2

Brunner, glands of, 790

Bryant's test-line, 447

Bubonocele, 678

Buccal artery, I 82 , 1205 lymphatic glands, i 8 ;

nerve, long, II 77,1208 short, I I69

Buccal fad of fat, I I 70

Buccinator muscle, I 169

Bucco-pharyngeal fascia, 1087

Budin, hinge-joint of, 62

Bulb, I345 olfactory, 1397

Bulbi vestibuli, 632

Bulla ethmoidalis, I 255

Bundle, anterior longitudinal, I 359 posterior longitudinal, 1358

Burdach, column of, 1315,1326
Burns, falciform process of, 460 space of, 1086

Bursa, great palmar, 374

olecranon, $33 \mathrm{I}$

pharyngeal, 1269

popliteal, 542

prepatellar, $464,54 \mathrm{I}$

subacromial, 320

subscapular, 346,347

suprapatellar, $54 \mathrm{I}$

Bursæ, at ankle-joint, 573

at elbow-joint, $40 \mathrm{r}$

at hip-joint, 495

at knee-joint, 54 I

at shoulder-joint, 347

plantar, 544

Cacuminis, folium, 1368

Cxcum, 695 peritoneal relations of, 696 varieties of, 696

Cæruleus, locus, 1378

Calamus scriptorius, I 377

Calcaneo-cuboid joint, 58 ?

Calcaneo-navicular ligaments, 580

Calcaneo-plantar nerve, 548

Calcaneum, 256 ossification of, $25^{8}$

Calcar avis, 1408 femorale, 240

Calcarine fissure, I391

Calcination, 4

Calcis, os, 256 tuber, 256

Calices of kidney, 826

Callosal gyrus, I 394 radiation, I 401,1446

Calloso-marginal fissure, $I_{3} 8_{3}$

Calvaria, 149

Camper, fascia of, 643

Canal or canals-

of Alcock, 623

carotid, 74

central, of medulla, 1360 of spinal cord, I3 19

cranio-pharyngeal, 82,92

dental, anterior and middle, 98 inferior, 118 posterior, 97

ethmoidal anterior, 68, 95 posterior, 68, 95

femoral, 46r

of Fontana, 15 I I

Haversian, 5

hepatic, 805

of Huguier, 72, 1538

Hunter's, 475 .

hyaloid, of Stilling, 1530

incisor, IOI

infra-orbital, $98,{ }_{3} 6$

inguinal, 666 
Canal or Canals (continued) intestinal, 694

malar, ro5 naso-palatine, Ior

of Nuck, $88 \mathrm{I}$

palatine, posterior, or palatomaxillary, I 12

accessory, external, II 2

of Petit, 1530 posterior, II 2

portal, 804

pterygoid or Vidian, 89

pterygo-palatine, 84 , I I 3

spinal, 40

of Stensen, ror

temporal, I05

Volkmann's, 6

Canaliculi of bone, 6

Canaliculus, auricular, 76,148

innominatus, 87

lacrimalis, I 190

tympanic, 77

Canalis reuniens of Hensen, I 553

Cancellated bone, 7

Canine fossa, 97

ridge, 97

teeth, 167

Canthus, inner, II 87

outer, I 87

Capitellum of humerus, I 89

Capsule, external, of brain, I4 I4

internal, of brain, 1413

centrifugal fibres of, $\mathrm{I}_{4} \mathrm{I} 3$

centripetal fibres of, I 4 I 3

genu of, I4I 3

limb, anterior of, I 4 I 3

Caput gallinaginis, 855 posterior, of, I $4 \mathrm{I} 3$

Cardia, 693

position of, 693

Cardiac nerves, of pneumogastric, cervical, 1229

thoracic, 967,968

of sympathetic, cervical, I 234,1235

plexus, 967

deep, 968

superficial, 968

Carotico-clinoid foramen, 86

Carotid artery, common, I I ro collateral circulation after ligation of, I I 12 development of, III 3 left, in thorax, $96 I$ surgery of, III 2

external, I I I 4

branches of, III 6 development of, I I 16 relations of, III 5

internal, at base of brain,

I 342, 1449
Carotid artery, internal, branches of, I 449

cavernous part of, 1078 branches of, 1079

cervical part of, 1222

development of, 1223

petrous part of, 1296

Carotid body, II 3 branches of, 1296

Carotid triangle, II 00

groove of sphenoid, 84

notch of sphenoid, 85

tubercle, 20

Carpal artery, anterior radial, 357 posterior radial, 392 anterior ulnar, 362

Carpal joints, 406 posterior ulnar, 362

Carpi, extensor, radialis brevior, 383

extensor, ulnaris, 386 lnngior, 383

flexor, radialis, 352 ulnaris, 353

Carpus, 201

as a whole, 207

ossification of, 208

varieties of, 208

Cartilage, basal, I6 I basi-occipital, I6I basi-sphenoid, $16 \mathrm{r}$ ethmoidal, I6I Reichert's, 165

Cartilages of larynx, I275 of Santorini, I280 of Wrisberg, I280

Cartilago triticea, 1280

Caruncula lacrimalis, II 89

Carunculæ myrtiformes, 632

Casserius, perforating nerve of, 3 I 5

Cauda equina, 1314

Caudatus, nucleus, I 4 II

blood-supply of, 1457

Cavernous groove, 84

plexus, 1234

$$
\begin{aligned}
& \text { sinus, I } 478 \\
& \text { communications of, } \\
& \text { I } 479 \\
& \text { interior of, } 1078
\end{aligned}
$$

Cell division, 1598

Cells, ethmoidal, 96

Cement, I 72

Centrale, os, 208,278

Centro-acinar cells of Langerhans, 8 ro

Cephalic index, $15^{8}$

vein, 334 median, 332

Cerato-hyal, I 65 
Cerebellar tract, antero-lateral ascending, or indirect, of Gowers, 1330

antero-lateral descending, 1329

dorso-lateral ascending, or direct, 1328

Cerebellum, $\mathrm{r}_{367}$

association and commissural

fibres of, 1373

blood-supply of, r 456

development of, 1376

fissures of, $137 \mathrm{I}$

great horizontal, 1367

hemispheres of, 1338,1367

lobes and lobules of under surface of, $137^{\circ}$

lobes and lobules of upper surface of, 1368,1369

monticulus, 1367

peduncles of, 1372

inferior, 1372

middle, 1372

superior, 1372,1433

structure of, I 375

vallecula of, I 369

veins of, 1456

vermis, inferior, 1370

superior, 1367

Cerebral hemispheres, $I_{3} 8 \mathrm{I}$

arteries of, $145^{\circ}$

artery of cerebral hemorrhage, $145^{\circ}$

commissure, anterior, $\mathrm{I}_{403}$

hippocampal, I 403

middle or grey, 1416

posterior, I 423

convolutions or gyri of, I 382

cortex, 1440

cells of, r.440

Golgi, I443

of Martinotti, I 443

corticefugal fibres, I 444 molecular layer, I 442 pyramidal layer, I 442

development of, 1396

fibres of, I 443

association, 1446

commissural, I 446

projection, I 444

fissures (interlobar) of, 1382

calloso-marginal, $\mathrm{I}_{3} \mathrm{8}_{3}$

circular or limiting, I 384

collateral, 1384

parieto-occipital, 1383

of Rolando, 1383

of Sylvius, 1382

(For other fissures see special names)
Cerebral ganglia, basal, of, r410

lobes of -

frontal, 1385

island of Reil, I393

limbic, 1394

occipital, I 390

parietal, 1388

temporal, I 39 I

lobules of-

cuneus, I39I

paracentral, I 388

postcentral, I 393

præcuneus, I 390

precentral, I 393

quadrate, 1390

blood-supply of, 1457

pole, frontal, of, I $3 \&$ I occipital, of, $138 r$ temporal, of, I 392

veins, I 455

vesicles, primary, I $45^{8}$ secondary, I 459

Cerebri, crura, I338, I 431

Cerebro-spinal axis, 1306

Ceruminous glands, 1537

Cervical fascia, deep, 1085 compartments of, 1087

muscular, ro88 space of Burns, ro87 suprasternal space, ro86, ro87

vertebral, I088

visceral, Io8 8

laminæ of, 1086

carotid, 1086

pretracheal, Io87

prevertebral, 1087

- visceral, ro87

parotid process of, 1088

Cervical glands, deep, 1102 superficial, 1085

nerve, superficial, 106o, I093

plexus, rog I

branches of, deep, 1093 superficial, 1092 ascending, 1059 descending, ro6I

spinal nerves, origins of, I 3 I 5

posterior primary divisions of, 1066

Cervical vertebræ, 12

Cervicalis ascendens muscle, 290

Cervicis, transversalis, muscle, 292

Charcot, artery of cerebral hemorrhage of, I450

Chassaignac, carotid tubercle of, 20, 108 I

Check ligaments of eyeball, I 152

Cheeks, 1238

Chemical composition of bone, 3

Choanæ, $4_{4}^{\circ}$ 
Chondrocranium, 160

Chondro-glossis muscle, II 33

Chorda tympani nerve, 1 246, 1298

Chordæ tendineæ, 979

Willisii, I 475

Chorion, I627

Choroid coat of eyeball, I 5I I

structure of, I5I I

choroid proper, I5I I

lamina siprachoroidea, I 5 I I chorio - capillaris or tunica Ruyschiana, I 5 I 2

lamina vasculosa, I5I2 membrane of Bruch, or

tapetum, 1512

lamina basalis, 1512

Choroid plexus or plexuses -

of lateral ventricle, $\mathrm{I}_{405}$

of descending cornu of, I 409

blood-supply of, I 458

of fourth ventricle, I 380

blood-supply of, I 458

of third ventricle, I 405 , I 4 I 5

Choroidal fissure, 1409 blood-supply of, $145^{8}$

vein, 1455

Chromosomes, 1599

Chyli, receptaculum, 762

Cilia or eyelashes, I I 87

Ciliaris, orbicularis. I 512

pars, retinæ, I 5 I 3

Ciliary body of eye, 1512

ganglion, I 156

branches of, II 57

summary of, II $58^{\circ}$

muscle, 1513

nerves, long, I I 56

short, 1157

processes, I 512

Cinerea, ala, 1378

Cinercum, tuber, 1424

Cingulum, 1395

Circle of Willis, I 343, I 454

Circular fissure of brain, 1384 sinus, 1478

Circulation, allantoic, 1642 course of, $97 \mathrm{I}$

placental, 1642

vitelline, I64I

Circulus major, 1516

minor, 1516

Circumduction, 282

Circumferential lamellæ, 6

Circumflex artery, anterior, 311 posterior, $3 I_{2}$ external, 481 iliac artery, deep, 663
Circumflex iliac artery, superficial, of femoral, 479,646

nerve, 316

Circumflexus palati muscle, 1252

Cisterna basalis, 1480

chyli, 762

magna, I 480

pontis, 1480

Clarke, Lockhart, intermedio-lateral, tract of, 1322

posterior vesicular column of, 1322

Classification of bones, 7

of joints, 279

of movements, $28 \mathrm{I}$

Claudius, cells of, $155^{8}$

Claustrum, I4I 4

Clava of medulla oblongata, $1350^{\circ}$

Clavicle, I 75

ossification of, ro7

Clavi-pectoral fascia, 305

Cleft palate, varieties of, I04

Clefts, viszeral, $127^{\circ}$

Clinoid process, anterior, 85

middle, 86

Clitoris, 630 posterior, 83

frenulum, 630

glans, 630

præputium, 630

septum pectiniformc, 630

Clivus monticuli, 1368

of sphenoid, 83

Cloaca, 870

Cloacal fossa, 870 membrane, 870

Cloquet, fascia of, $46 \mathrm{I}$

Club-foot, tendons involved in, 565

Coaptation, 282

Coats of eyeball, 1507

Coccygeal body of Luschka, 862

Coccygeus muscle, 863

Coccyx, 36 ossification of, 37

Cochlea, I $55^{\circ}$

canal of, I 550

lamina spiralis of, 1550

modiolus of, I 55 I

Cochlex, aqueductus, 1552

tuber, or promontory, 1539

Cochlear area, 75

artery, 1560

nerve, I 301,1559

nucleus, lateral, I 491

ventral, r 491

Cochleariformis, processus, 78,1539

Coliac axis, 739

plexus, 736

Coelom, I622

Collagen, ?

Collateral fissure, $I_{3} 84$ 
Colles, fascia of, 614

ligament of, 653

Colliculus seminalis, 855

Colon, ascending or right, 697 peritoneal relations of, 697

descending or left, $69 \mathrm{~s}$ peritoneal relations of, 699

flexure of, hepatic, 697

iliac, 699 splenic, 698

pelvic, 859 peritoneal relations of, 699 peritoneal relations of, 859

transverse or middle, 698 peritoneal relations of, 698 tæniæ of, 699

Columella nasi, i 196

Column, vertebral, 38

Columnæ carneæ, 977

Bertini, 8r 8

rugarum, 898

Columns of Morgagni, 875

Comma tract of spinal cord, 1327

Comes nervi ischiadici, 433 mediani, $36 \mathrm{I}$

Commissures of brainanterior, $\mathrm{I}_{4} \mathrm{O} 3$ corpus callosum, I400

Gudden, of, I 425

habenular, 1422

hippocampal, I403

Meynert, of, I430

middle or grey, 1416

posterior, I 423

Commissures of spinal cord-

grey, I31 7,1322

white, 1317, 1322

Communicating artery, antericr, I 450

Compact bone, 5 posterior, I 449

Complexus muscle, 292

Compressor sacculus laryngis muscle, 1291

Conarium, I42I

Concatenatæ, glandulæ, 1058

Concentric lamellæ, 6

Concha, inferior, 109 middle, 95 superior, 95

Conchæ, 1254

Condylar foramen, anterior, 60 joint, 280

Condylarthrosis, 280

Condyles, occipital, 59 third, 61

Cone-bipolar cells of retina, $\mathbf{I}_{52} \mathbf{I}$

Cone of light, I 54 I

Cones of retina, 1523

Coni vasculosi of testis, $68_{4}$

Conical cells of olfactory region, 1258

Conjoined tendon, $65^{\circ}$
Conjunctiva, I I 89

epithelium of, I I 89

ocular, I I 89

palpebral, I I 89

plica semilunaris of, I I90

Conniventes, valvula, 788

Conoid ligament, 327

tubercle, $1 ; 6$

Constrictor isthmi faucium muscle, I 250

Constrictor muscle, inferior, 1264

middle, 1264

superior, 1265

nerve-supply of, 1265

Conus arteriosus of heart, 975, 977

Conus medullaris of spinal cord, I 312

Convoluted tubule, distal, 8 I9

proximal, 8 I 9

Cooper, fascia propria of, 464,649 . 671

ligamenta suspensoria of, 300

pubic ligament of, $46 \mathrm{I}, 780$

reflected tendon of, 658

tunica vasculosa of, 683

Copula, 165

Coraco-acromial ligament, 328

brachialis muscle, 335

clavicular ligament, 327

Coraco-humeral ligament, 344

Coracoid process, I 82

Cordiform tendon of diaphragm, 759

Cords, vocal, false and true, 1284 .

Cornea of eyeball, I 508

nerve-supply of, 15 Io

structure of, I 509

Corneal corpuscles, I 5 Io

Corneo-scleral junction, I 507

Cornicula laryngis, I 280

Cornu Ammonis, 1409

anterior, of spinal cord, I3 I 7

lateral, I3 8

posterior, $\mathrm{I}_{3} \mathrm{I}_{7}$

Cornua of lateral ventricles, 1406

anterior, 1407

middle or descending, I foS

amygdaloid tubercle of, I 408

nucleus of, I 408

posterior, I 408

bulb of, 1408

calcar avis, 1408

hippocampus minor, 1408

Corona glandis, $64 \mathrm{~S}$

radiata, $\mathrm{I}_{4}$ O I, I $4 \mathrm{I} 4$

Coronal section of skull, I 49

Coronary arteries, of face, I 26 I

of heart, 958

ligaments of knee, 535

plexuses, 970

sinus, 986 
Coronary valve, 976

Coronoid fossa of humerus, I 89 process of ulna, 197

Corpora albicantia, $I_{42} 3$

geniculata, 1420

mammillaria, 1423

peduncles of, $I_{42} 3$

quadrigemina, 1428

blood-supply of, 1456

brachium, inferior, $I_{429}$

superior, 1428 occipital fibres of, I 429

retinal fibres of, 1429

development of, 1430 .

structure of, 1429

Corpus albicans of ovary, 893

Corpus Arantii, 984

Corpus callosum, I400

blood-supply of, I 457

corona radiata, I 4 OI

destination of fibres of, I 401

forceps major, $\mathrm{I}_{4} \mathrm{O} 2$

forceps minor, I 402

geniculate gyrus, I 4 OI

genu, I 401

peduncles of, I 4 or

radiatio corporis callosi, I 401

rostrum, I 401

splenium, I 4 or

stria longitudinalis lateralis, I 4 Or

mesialis, I 400

subcallosal gyrus, I 401

tapetum, I 401

Corpus dentatum of cerebellum, I373

fimbriatum, $88_{5}$

geniculatum externum, I 4 I 9

internum, 1420 development of, I 42 I

Highmorianum, 683

luteum, 893

striatum, I4Io

blood-supply of, 1457

connections of, I $4 \mathrm{I}_{4}$

development of, I 4 I 4

external capule of, I 4 I 4

internal capsule of, I 4 I 3

extraventricular portion of, I 4 II

intraventricular portion of, 1410

relation of structures in region of, I 415

vein of, I 455

subthalamicum, I 421 .

trapezoides, of cerebellum, 1372 of pons Varolii, $1_{3} 63$
Corrugator supercilii muscle, I 166 Corti, organ of, I 556 .

auditory of hair cells of, I 557

cells of Claudius, 1558

of Deiters, $155^{8}$

of Hensen, $155^{8}$

lamina reticularis, 1558

membrana tectoria, I $55^{8}$

membrane of Corti, I $55^{8}$

rods of, 1556

phalangeal processes of, 1557

tunnel of, 1556

Cortico-medullary arterial arches of kidney, 822

venous arches of kidney, 826

Costal cartilages, 46

Costo-clavicular ligament, 326

Costo-coracoid ligament, 305

membrane, 305

Cotunnius, nerve of, 1220,1260

Cotyloid cavity, 226

ligament, 492

notch, 226

Cowper, glands of, 621

Coxæ, os, 220

Cranial cavity, dissection of, 1074

Cranial nerves- .

first, or olfactory. See Olfactory Bulb

second, or optic. See Optic Nerve

third, or oculo-motor, at base of skull, I074

in outer wall of cavernous sinus, 1078

in orbit, II 53

at sphenoidal fissure, I I 62

fibres of, course of, ${ }_{4} 85$

nucleus of, $\mathrm{I}_{4} 84$

origin of, deep, $\mathrm{I}_{4} 84$ superficial, I 34 I

summary of, $\mathrm{I}_{4} 8_{5}$

fourth, trochlear, or pathetic, at base of skull, I075

in outer wall of cavernous sinus, 1078

in orbit, $\mathbf{I} 53$

at sphenoidal fissure, 1162 fibres of, course of, $\mathrm{I}_{4} 85$ nucleus of, $\mathrm{I}_{4} 85$ origin of, deep, 1485

superficial, I 34 I

summary of, I 486

fifth, trigeminal, or trifacial, at base of skull, I075

distribution of, 1487

Gasserian ganglion, 1076 branches of, 1077

origin of, deep, 1486 motor root, $I_{4} 86$ 
Cranial nerves (continued)fifth, origin of motor root, nuclei of $1486, \mathrm{I}_{4} 87$ sensory root, $I_{4} 86$ nuclei of, $1_{4} 86$ superficial, I34I summary of, $\mathrm{r}_{4} 87$

sixth, or abducent, at base of skull, 1075

in cavernous sinus, 1078

in orbit, II53

at sphenoidal fissure, I 162

fibres of, course of, $\mathrm{I}_{4} 88$

nucleus of, $\mathrm{I}_{4} 88$

origin of, deep, 1488

superficial, I34 I

summary of, 1488

seventh, or facial, at base of skuIl, 1076

distribution of, $\mathbf{I}_{4} 89$

in Fallopian aqueduct, 1297

after emerging from Fal. lopian aqueduct, 1172

meatal portion of, 1297

cervico-facial division of, II 74

fibres of, course of, 1488

geniculate ganglion, 1299

nucleus of, $\mathrm{I}_{4} 88$

origin of, deep, 1489 superficial, I $34 \mathrm{I}$

pars intermedia of Wrisberg, I 299, I 4 89

sensory root of, 1299

summary of extracranial part of, II 75

temporo-facial division of, II 73

eighth, or auditory, at base of skull, I076

cochlear division of, 1559 nervous chains of, I49I

fibres of, course of, 1490 meatal portion of, 1300

origin of, deep, ${ }^{4} 49$ cochlear root, 1490 nuclei of, 1490 vestibular root, 1492 nuclei of, 1492

superficial, I 34 I

vestibular division, nervous chains of, I 494

ninth, or glosso-pharyngeal, at base of skull, 1076

distribution of, 1496

extracranial portion of, 1223

branches of trunk, 1224
Cranial nerves (continued)-

- ninth, jugular ganglion of, 1223

petrous ganglion of, 1223

branches of, 1223

origin of, deep, I 495

superficial, $\mathrm{r} 34 \mathrm{I}$

summary of, 1226

tenth, pneumogastric, or vagus, in abdomen, 786

at base of skull, 10 76

cervical portion of, 1226

cardiac branches of, I 229

ganglion of root, 1226

branches of, 1226

of trunk, 1227

branches of, 1227

distribution of, 1497

inferior laryngeal branch of, 966, 1228 , I 292

lingual branch of, 1227

origin of, deep, I496

superficial, I34I

summary of, in neck, 1229

thoracic portion of left, 965 of right, 964

cardiac branches, 967,968 pulmonary plexuses of, 967

eleventh, or spinal accessory, at base of skull, 1076

bulbar or accessory part of, 1229

distribution of, I 499

extracranial portion of, I 229

spinal part of, 1060, 1230

origin of, deep, 1498

superficial, $134 \mathrm{I}$

summary of, I 23 I

twelfth, or hypoglossal, at base of skull, I076

distribution of, I 500

extracranial portion of, 1231

branches of, I23I

fibres of, course of, I 500

- origin of, deep, 1500

superficial, I 34 I

summary of, 1232

Cranial nerves at base of brain, $134^{\circ}$ at base of skull, 1074

Cranio-cerebral topography, I 500

Cranio-iacial angle, I 49

Craniometrical terms, I57

Cranium, 56

development of, 159

Cremaster muscle, external, $656,67 \mathbf{r}$

internal, $67 \mathrm{I}$

middle, 671 
Cremasteric fascia, 656

Crest, falciform, 75 reflex, 656

incisor, ror

of ilium, 220

infratemporal of sphenoid, 87

malar of sphenoid, 87

pubic, relations of structures at, 660

sphenoidal, 84

supramastoid, 70

of urethra, female, 899

male, 855

Cretinism, 159

Cretin skull, I 59

Cribriform plate of ethmoid, 92

Cribrosa, lamina, 75

Crico-arytenoid joint, I 282 - muscle, lateral, I288 posterior, I 288

Crico-thyroid joint, 1282 membrane, 1280 muscle, 1287

Cricoid cartilage, 1278 development of, 1279

Crista acustica, 1554

basilaris, I 555

galli, 92

terminalis, 975

vestibuli, 1549

Crucial ligaments of knee, 532

Cruciform ligament, I 305 transverse portion of, I 306

Crura cerebri, I 338

Crural (femoral) arch, deep, 460 superficial, 460

canal, 46r

nerve, anterior, 470

ring, $46 \mathrm{I}$

sheath, 462

Crurale, septum, 46I

Crureus muscle, 467

Crus cerebri, blood-supply of, $x_{45} 6$ crusta or pes, I 43 I, I 437 cortico - pontine fibres of, $\mathrm{r} 43^{8}$

fronto-pontine strand of, 1438 temporo - pontine strand of, 1438 pyramidal fibres of, 1437

summary of, 1437

development of, 1437

lateral sulcus of, $\mathrm{r} 43 \mathrm{r}$

nucleus of, $x_{432}$

oculo-motor sulcus of, $\mathbf{1 4 . 3 \mathrm { I }}$

posterior longitudinal bundle

in, $\mathbf{r} 434$

red nucleus of, I 432

structure \& ternal, of, I43I
Crus cerebri, substantia nigra of, I43I superior cerebellar peduncle in 1433

summary of, I 435

tegmentum, I 43I

Crus cerebri, in ternal structure of, 143 I superior cerebellar peduncle in, 1433

nucleus of tegmentum, 1432 posterior longitudinal bundle in, 1434 red nucleus of, 1432

Crusta of crus cerebri, I 43 I, I437 petrosa, 172

Cryptorchismus, 676

Cryptozygous skull, $\mathbf{I}_{32}$

Crypts of Lieberkühn, 79r

Cubo-cuneiform joint, 585

Cuboid bone, 263

Cubo-metatarsal joint, 587

Cucullaris muscle, 285

Culmen monticuli, ${ }_{3} 68$

Cuneate nucleus, accessory or ex. ternal, I 354

tubercle, 1350

Cuneatus, nucleus, I 354

Cuneiform bone of carpus, 202

of tarsus, 260 external, 262

internal, 260

middle, $26 \mathrm{r}$

Cuneiform cartilages, I 280

Cuneus of brain, I 391

Cup, palmar, 408

Cupola of cochlea, I $55^{\circ}$

Cutaneous nerves of arm, internal, $315,331,340$

lesser internal, 3 I 5, 33 I of musculo-cutaneous, 332

of musculo-spiral, external, 332

internal, 331, 343

of back, 283

of foot, 547

of forearm and hand, $34^{8}$

of gluteal region, 424

of pectoral region, 298

of sacral plexus, perforating, 425

of scapular region, 319

of thigh, external, 449

internal, 449

middle, 449

of thorax, lateral, 298

of ulnar nerve, 332,349

Cuvier, ducts of, Ior I

Cystic duct, 7 ro

notch, 708

structure of, 809

Cytoplasm, 894, I 597 
Dacryon, 133

Dartos, tunica, of scrotum, 650

Darwin, tubercle of, 1193

Deferens, vas, 668 development of, 686 structure of, 669

Decidua basilis, 1632,1634 capsularis, I 632,1634 reflexa, 1632 scrotina, 1632 vera, I632, 1634

Deciduous membrane, 1632

Deiters, cells of, 1558 process of, 1320 nucleus of, 1492

Deltoid, 319 spine, 175

Demours, membrane of, I 5 Io

Dendrites, 1320

Dental arches, 168, 1238 arteryanterior, 1205 inferior, 1204 middle, 1205 posterior, I 205

canals, anterior and middle, 98 inferior, I I 8 posterior, 97

foramen, inferior, II 8

nerves, inferior, 1209 superior, anterior, 1218 middle, I2I 8 posteror, 1218

papilla, 172

sac, 172

Dentate fissure, I 396

Dentated suture, 279

Dentatum, corpus, of cerebellum, I 373

Dentinal tubules, 172

Dentine, I 72

Depressor alæ nasi muscle, I 166 anguli oris muscle, II 70 labii inferioris muscle, I I 70

Descemet, membrane of, ${ }_{5}$ Io

Descendens cervicis nerve, 1107 hypoglossi nerve, 1107

Descent of testis, 671,673 abnormal conditions of, 675 positions, 676

gubernaculum testis, 672 mesotestis, 67I metamorphoses of folds, 674 of processus vaginalis, 675

plica gubernatrix, 672

urogenital fold, 67 I

Descriptive terms, I mesentery, 672

Deutoplasm, 894

Development of arteries of lower $\operatorname{limb}, 568$
Development of arteries of upper limb, 397

of cranium, I59

of diaphragm, $76 \mathrm{I}$

of external genital organs, 633 female, 634

of eye, 1530 male, 635

optic cup, 1532

stalk, I 534

of face, 162

vesicle, 1532

of heart, 991

mesocardial folds, 992

transformation of aortic bulb, 994 of auricular canal, 993 of single heart, 093 ventricle, 994

of hyoid bone, $16_{5}$

of internal sexual organs, $6 \delta_{\bar{j}}$ genital ridge of, 685 indifferent organ of, 685 sex-cords of, 685

of limb bones, 591

of limb-buds, 59 I

of lymphatic system, Ior6

jugular sacs, Ior6

lymphatic vessels, roI7 structure of, Ior 8

lymph glands, IoI 7 Sacs, Ior6

retroperitoneal sacs, IoI6 sciatica sacs, IoI 7

metamorphosis of structures concerned in development of external genital organs, 636

of ovary, 687

egg-tubes of Pflüger, 688

mesovarium, 688

rete-cords of, 687

summary of, 688

of principal arteries, 999

cervical arte-ies, 1002

primitive dorsal aortæ, 1000 ventral aortæe, 999

segmental arteries, I000

subclavian arteries, 1002 summary of, 1003

vertebral arteries, 1003

visceral arch arteries, I000

of principal veins, 1005

anterior cardinal veins, 10 ro metamorphoses of, Io ro azygos veins, 1030

inferior vena cava, Ior 5

lumbar veins, ror 5

posterior cardinal veins, IOI I

metamorphoses of, IOI 4 
Development of priscipal renal veins, Ior 5

subcardinal veins, ror 2 metamorphoses of, I I I4

suprarenal veins, Ior 5 '

umbilical or allantoic veins, 1008

summary of, I009

vena portæ, roo5

vitelline or omphalo-mes-

of skull, I59

enteric veins, 1005

of teeth, I 72

of testis; 686

rete cords of, 686

Wolffian duct of, 686

of valves of heart, 995

of veins, I005

of lower limb, 569

of upper limb, 397

vitreous body, 1534

Diaphragm, $75^{8}$

openings of, 760

ligaments of, $76 \mathrm{I}$

pelvic, 864

Diaphragma, oris, II 3 I

sellæ, I 475

Diaphragmatic plexus of nerves, 736

Diarthrosis, 280

Diencephalon, I337, I4I 5

Digastric muscle, II 29

nerve, II 73

triangle, I ror

contents of, $\mathrm{IIO}_{2}$

Digital arteries, palmar, 370 plantar, 564

fossa of peritoneum, 676 of tunica vaginalis, $68 \mathrm{I}$

Digiti, abductor minimi, of foot, 551

extensor minimi, 384 of hand, 379

flexor brevis minimi, of foot, 556 of hand, 380

opponens minimi, of foot, 556 of hand, 380

Digitorum, extensor brevis, 509 communis, 384 longus, 504

flexor brevis, 549

longus, $52 \mathrm{I}$ tendon of, $55 \mathrm{I}$

profund $\mathbf{u} ; 365$

sublimis, 354

Dilator naris anterior, muscle, 1167

pupillæ muscle, I 5 I 5 posterior, muscle, 1167

Diploê, 7

Diploic veins, 1473

Disc, germinal, 1614

Discs, intervertebral, 1038

Discus proligerus, 894
Dolichocephalic skull, I 32, I 58

Dorsalis linguæ artery, I I 8 pedis artery, 510 nerve, $5 \mathrm{I} 3$

scapulæ artery, 3 I I

Dorsum ephippii of sphenoid, 83 sellæ of sphenoid, 83

Dorsum ilii, 222

Douglas, pouch of, 879 recto-uterine folds of, 879 semilunar fold of, 660

Duct or ducts-

Bartholin, of (sublingual), II 36

bile, 806 common, 710

cystic, 7 10

ejaculatory, common, 852

galactophorous, 3 or

hepatic, 708

lymphatic, right, I I 46

mesonephric, 830

Müllerian, 898

nasal, II9I

naso-lachrymal, I I9I

parotid, 1185

pronephric, 829

prostatic, 855

Rivini, of, great, I I 36

segmental, 829

Steno or Stensen, of, I I 85

sublingual, I 136

submaxillary, I I 34

thoracic, in abdomen, 762

in neck, II 45

in thorax, I03I

umbilical, 798

vitelline, 798

vitello-intestinal, 798

Walther, of, I 136

Wharton, of, I I 34

Wolffian, 8II

Ductus arteriosi, ligamentum; 964

Ductus arteriosus, 997

cochlearis, 1555

Ductus communis choledochus, 7 10

endolymphaticus, 76,1553

perilymphaticus, 78

reuniens of Hensen, I 553

sacculi, 1553

thyro-glossus, 1124

utriculi, 1552

utriculo-saccularis, 1552

venosus, 997

Duodeno-jejunal flexure, 695

Duodenum, 694, 73I

first part of, $73 \mathrm{I}$

second part of, $73 \mathrm{I}$

third part of, 732

fourth part of, 732

Dura mater, of brain, 1470

blood-supply of, $147 \mathrm{r}$ 
Dura mater, nerves of, 1473 processes of, 1473 sinuses of, 1475 structure of, $\mathrm{I}_{47} \mathrm{I}$ of spinal cord, 1306 blood-supply of, r 308 nerves of, I 308

Duret, arteries of, $\mathbf{I} 45^{\circ}$

Duverney, glands of, 633 Dyad, I606 development of, 633

Ear, development of, 1560 external, 1536 internal, $154^{8}$ middle, 1538

Earthy matter of bone, 4

Ebner, glands of, $12+3$

Eboris, membrana, I 7 I

Ectopia cordis, 50

Efferentia, vasa, 684

Ejaculatory ducts, common, 852 development of, 872 structure of, 872

Elbow-joint, 398

Emboliformis, nucleus, 1373

Embryo, appendages of, 1624 formation of, 1618

Embryology, general, I 597

Eminentia arcuata, 75,1550 articularis, 70

collateralis, 1409 teres, $137 \mathrm{~S}$

Emissary veins, 1479

Enamel, I72

cells, I 73

cuticle, I $72_{2}$

germ, common, $\mathbf{I}_{72}$ special, 172

organ, $\mathrm{I}_{72}$

prism, 17.3

Enarthrosis, 280

Encephalon, 1337

base of, 1337 arteries at base of, 1342

development of, $\mathbf{r}_{45} 8$

flexures of primitive, $145^{8}$ meninges of, I $47^{\circ}$

metamorphoses of secondary vesicles, 1460

- rhombic lip of, 1460

of basal ganglia, I 466

of mesencephalon, 1462

of metencephalon, $146 \mathrm{r}$

of telencephalon, $\mathrm{I}_{4} 63$

of thalamencephalon, 1463

secondary cerebral vesicles, I459 three primary cerebral vesicles, $145^{8}$

veins of, I 455

Endocardium, 988
Endognathion, 104

Endolymph of internal ear, 1552

Endolymphaticus, ductus, 76

Endoskeleton, I

Endosteum, II

Ensiform process, 47,49

Entomion, I 42

Ependyma, 1464

Ephippii, dorsum, of sphenoid, $\delta_{3}$

Epicardium, 944, 988

Epicondyle, external, of humerus, I 89 internal, of humerus, 189

Epicranial aponeurosis, 1069

Epididymis, 682 development of, 686 structure of, $68_{3}, 68_{4}$

Epidural space, spinal, I 308

Epigastric artery, deep, 662

Epiglottis, I275

superficial, 479,646

frenum, 1276

functions of, 1292

Epi-hyal, I 65

Epiotic centre of temporal bone, $\delta$ r

Epiphysis cerebri, $\mathbf{r}_{42} \mathbf{I}$

Epiploicæ, appendices, 699, 794

Epiploōn, 7 I 6

Epipteric bone, I42, I 55

Episternal bones, 5 I

Epithalamus, I 42I

Epitympanic recess and its pouches, I545

Epoöphoron, 884

development of, $88_{3}, 898$

Equina, cauda, $\mathrm{r}_{3} \mathrm{I} 4$

Erector spinæ muscle, 290 inner column, 292 middle column, 291 outer column, 290

Eruption of teeth, I 70

Erythroblasts, Io

Ethmoid bone, 92 ossification of, 96

Ethmoidal arteries, 1260

canal, anterior, 68,95 posterior, 68, 95

cells, 96

spine, 82

Ethmo-turbinals, 94

Eustachian groove, 147

orifice, 78

spine of sphenoid, 89

tube, 1274

development of, 1275

muscles connected with, I 275

valve, 976,997

Exner, plexus of, I 443

Exoccipitals, 59

Exognathion, 104

Exomphalos, 680 
Exoskeleton, I

Eye, $x$ 506

chambers of, 1527

coats of, 1507

development of, I 530

equator of, 1506

meridian of, $\mathrm{I}_{507}$

movements of, II5I

muscles of, II 47

Eyelashes, I I 87

Eyelids, I 187

development of, II90

structure of, 1 I 87

Face, 56

arteries of, $\operatorname{I} 178$

development of, I62

landmarks of, I 163

muscles of, I I65

sensory nerves of, 1 I 76

Facial artery, cervical part of, I I I 9

branches of, III9

facial part of, $I I 78$

branches of, II 79

transverse, I I 8I

nerve. See Cranial Nerves

vein, $\operatorname{I} 79$

deep, 1206

transverse, I I 8 I

Falciform crest, $75^{\circ}$

process of Burns, 460

Fallopian tubes, $88_{4}$

development of, 898

lymphatics of, 896

structure of, 895

Fallopius, aqueduct of, 75

Fallopius hiatus, 74

Fạlx cerebelli, I 474

cerebri, I 473

Fascia of Abernethy, 777

anal, 840

of anterior abdominal wall, deep, 643
superficial, 643

axillary, 300

of back, deep, 283 superficial, 283

bicipital or semilunar, 336

of brachial region, deep, 334

bucco-pharyngeal, 1087

of Camper, 643

cervical, deep, 1085

clavi-pectoral, 305

of Cloquet, $46 \mathrm{I}$

of Colles, 6 I 4

of Cooper, propria, 649, 671

dentata of Tarinus, I 296

of dorsum of foot, deep, 509

of forearm, deep, $35 \mathrm{I}$

gluteal, 425

deep, 426
Fascia of hand, back of, 390 interosseous, 394

iliaca, 764

infundibuliform, 665, 671

intercolumnar, 651

lata, 456

iliac lamina, 457

processes of, 458

pubic lamina, 457

of leg, deep, 497

lumbar, 765

masseteric, 1238

obturator, 837

of palm, superficial, 368

palmar, 369

parotid, II 84

of pectoral region, 299

pelvic, parietal, $8_{36}$ visceral, 838

perineal, deep, 6 I9

pharyngeal, 1263

plantar, 545

propria of Cooper, 464

of pyriformis, 837

of scapular region, deep, 319

of Scarpa, 643

of Sibson, 937 , I 146

spermatic, external, $65 \mathrm{I}$ internal, 649 middle, 656

subpubic, 6 r9

superficial, of front of thigh, 452

temporal, 1074

transversalis, 665

triangular, 653

Fasciculus, arcuate, 1447

longitudinal, inferior, I 447 superior, 1447

occipito-frontal, 1447

perpendicular, I 447

retroflexus, 1447

solitarius, 1495

uncinate, 1447

Fastigii, nucleus, 1373

Fauces, I 249

pillars of, anterior, 1249 posterior, 1249

Femoral artery 477 first part, 478 second part, 478

branches of, 479 varieties of, 485 deep, $48 I$

canal, $46 \mathrm{I}$

hernia, parts concerned in, 460 course of, 462 coverings of, 462

ligament of Hey, 460

opening, $47^{2}$

ring, 461

sheath, 460 
Femoral vein, 486

tributaries of, 486

varieties of, 488

external cutaneous, 455

internal cutaneous, 455

Femur, 232

female, 240

ossification of, 240

trigone of, 236

Fenestra ovalis, 1538 rotunda, I 539

Ferrein, pyramids of, 8r 9

Ferruginea, substantia, 1378

Fibres, perforating, of Sharpey, 7 pyramidal or motor, $\mathrm{I}_{4} \mathrm{I} 3$

thalamo-frontal, I 413

thalamo-striate, I 4 I 3 of Tomes, 172

Fibro-cartilages, in terarticular, 281 acromio-clavicular, 327

of knee-joint, 534

in lower limb, 591

of radio-ulnar, inferior, 405

sterno-clavicular, 325

Fibula, 250 in upper $\operatorname{limb}, 4$ II

$$
\text { ossification of, } 253
$$

Fibular artery, superior, 507 nerve, 445

Fillet, $r_{360}$ decussation of, 1359

lateral, 1367,1436 mesial, r $367,1_{435}$

Filum terminale of spinal cord, I 3 I 3

Fimbria, of brain, I 396, I 409 ovarian, 885

Fimbriæ, 884

Fimbriata, plica, of tongue, 124 I

Fimbriatum, corpus, 885

Fimbrio-dentate sulcus, I 396

Fissura palpebrarum, II 87

Fissure, auricular, 77

of Glaser, 72

petro-tympanic, 72

pterygo-maxillary, I 44

sphenoidal, 88,136

spheno-maxillary, I 36, I 43

sternal, 50

tympano-mastoid, 77

Fistula in ano, 6I 4

umbilical urinary, 870

Flaccida, membrana, I 540

Flat bones, 7

Floccular fossa, 76,82

Flocculus, I 37 I

Flood's ligament, 345

Fœtal circulation, 997 changes in, at birth, 999 peculiarities of, 996

Folds, neural, 16 I 8

Folinm cacuminis, I 368
Follicles of Lieberkühn, 791

Folliculi, liquor, 894 theca, 894

Fontana, canal of, 15I: spaces of, 1510

Fontanelles, I 55 sagittal, 65

Foot, arches of, 577 dorsum of, 509 as a whole, 274

Foramen or foraminacxcum of frontal bone, 67 carotico-clinoid, 86 carotid, 76,148 condylar, anterior, $60, I_{4} S$ dental, inferior, I I of Huschke, 79,82 infra-orbital, 97 jugular, 148,1076 lacerum anterius, 8S, I 36 medium, $\mathbf{I}_{4} \mathrm{~S}, \mathbf{I}_{52}$ orbitale, 88 posterius, $\mathbf{I}_{4} 8, \mathbf{I}_{54}$

of Luschka, I 380

of Magendie, I 380 magnum, 6I, I48 structures passing through, 1076

malar, 104

mastoid, 73

mental, II 6

of Monro, $\mathrm{I}_{4} \mathrm{I} 7$

obturator, 227

optic, 86

ovale, 86,996

of capsule of shoulder-joint, 344

valve of, 996

palatine, accessory, external, I 47 posterior, 147

parietal, 63

pterygo-spinous, 89

quadratum of diaphragm, 760

rotundum, 86

sacro-sciatic, great, 904 compartments of, 429

small, 904 structures passing through, 430

of Scarpa, 102, I 45

singulare, 75

spheno-palatine, I 40,144

sphenotic, ${ }_{4} 8$

spinosum, 87

of Stensen, 102, 145

sternal, 50

stylo-mastoid, 77

supra-orbital, I34

supratrochlear, I 89

thyroid, 227 
Foramen or foramina (continued) Vesalii, 86

of Winslow, $72 x, 802$

Forearm, front of, 351

and hand, $34^{8}$

back of, 382

Fore-gut, 798, 1623

Fore-kidney, 829

Formatio reticularis of medulla, $\times 35^{2}$ alba, $x 353$

grisea, 1352

Fornicatus, gyrus, I 394

Fornix, anterior, of vagina, 889

posterior, of vagina, 889

of brain, I 402

development of, $\mathbf{1} 403$

conjunctivæ, Ix 89

Fossa or fossæ-

of antihelix, $x x 93$

of base of skull, anterior, 150

middle, + I 5 I

canine, 97

posterior, I 54

coronoid, $\mathrm{x} 89$

floccular, 76,82

glenoid, $7 x$

guttural, I 47

of helix, II93

iliac, 223

incisor, 97

incudis, I 539

infraclavicular, 297

infraspinous, $\times 78$

intercondylar, 239

jugular, 76

lachrymal, 67

nasal, I 37, I 254

navicularis, of male urethra, 857 of pudendum, 632

olecranon, 189

ovalis, 977

palatine, anterior, Ior, 145

patellaris, $\times 529$

peritoneal-

digital, 676

duodenal, $72 \mathrm{x}$

duodeno-jejunal, 722

ileo-cæcal, 722

ileo-colic, 722

inguinal, 676

intersigmoid, 722

para-duodenal, 722

peri-cæcal, 722

retro-cæcal, 722

retro-duodenal, 722

pituitary, 82

pterygoid, 88,147

scaphoid, of auricle, Ir93

of sphenoid, 89

spheno-maxillary, 144

supraspinous, 178
Fossa or fossæ (continued) -

supratonsillar, 1253

of Tarinus, I 339

temporal, 142

triangular, of auricle, I 193

trochlear, 67

vermiform, 58

zygomatic, $\mathrm{I}_{\mathbf{4}} 2$

Fossula ovalis, 1538

rotunda, I 539

Fourchette, 632

Fovea centralis retinæ, 1518

elliptica, I 549 structure of, $\mathrm{r}_{524}$

inferior, of fourth ventricle, 1377

spherica, $x 549$

superior, of fourth ventricle, I 378

Foveola of kidney, 821

Frenulum clitoridis, 630

Giacomini, 1396

labii, 1236

lingulæ of cerebellum, 1368

veli of superior medullary velum,

I $379, \quad 1427$

Frenum epiglottidis, 1276

linguæ, $1237,124 \mathrm{x}$

præputii, 647

Frontal artery, I068, I I6I bone, 65

air sinuses of, 68 ala of, 67

nasal notch of, 67

orbital plates, 67

ossification of, 69

lobe of brain, 1385

nerve, I 154

vein, 1068

diploic, $x_{473}$

Fronto-pontine tract, $x_{4} \times 3, x_{444}$

Fronto-nasal process, $x 63$

Fundiform ligament of Retzius, 50I

Funiculus cuneatus, I 354

gracilis, 1353

Furcula, I270

Furrow, nuchal, 1058

Furrows, palmar, 367

Fusca, lamina, 1508

Galactophorous ducts, 30I

Galen, veins of, 1455

Gall-bladder, 709

development of, 809

structure of, 808

Gallinaginis, caput, 855

Ganglia, basal, $x_{4} 66$

Ganglion, aortico-renal, 736

Arnold, of, I2I I

basal, of Meynert, I 424

Bochdalek, of, $x 218$

cervical, inferior, 1235 middle, 1235 
Ganglion, cervical, superior, 1232 ciliary, I 56 branches of, $I 157$ summary of, $115^{8}$

coccygeal, 862

diaphragmaticum, 758,942

Gasserian, 1076 branches of, 1077

geniculate, of facial, I 299

habennlæ, I 422

branches of, 1299

impar, 862

interpedunculare, $\mathbf{1} 44^{\circ}$

jugular, 1223

Meckel, of, I 2 I9

mesenteric, inferior, $73^{8}$ superior, 738

nodosum, 1226

otic, I2II

petrous, 1223

phrenic, 736

Scarpa, of, I 300, I 558

semilunar, 736

spheno-palatine, I 219

spinal, 1325

spiral, I 559

splanchnic, ro35

stellatum, 1034

submaxillary, I 212

Valentin, of, 1218

Wrisberg, of, 968

Ganglionic layer of retina, $\mathbf{I}_{520}$

Gärtner, duct or canal of, 883

Gasserian depression, 74

- ganglion, 1076 branches of, 1077

Gastric sympathetic plexus, 736

Gastrocnemius, 518

Gastro-phrenic ligament, 720

Gastrula, I6I 4

Gemellus, inferior, 430

superior, 429

Genial spines, II 7

Geniculate bodies, 1420

ganglion, $\mathrm{x} 299$

gyrus of corpus callosum, I 4 OI

Genio-hyo-glossus muscle, I I 3 I

Genio-hyoid muscle, II 3 I

Genital cord, 685

eminence, $6_{34}, 635,87 x$

fold, 634,87 I

ridge, 685

Genito-femoral nerve, 424, 770

Gerlach, valve of, 796

Germinal epithelium, 685

layers-

structures derived from, I $6 \mathrm{I} 7$

summary of formation of, spot, 894
Germinal vesicle, 894

Giacomini, frenulum, I 396

Gimbernat's ligament, $+34,653$

Ginglymus or hinge-joint, 280

Giraldès, organ of, 682

Girdle, pectoral, morphology of, 277

Glabella, 65, 133

Gladiolus, 47

Gland, Haversian, of hip-joint, 492

tarsal, I I 89

thymus, 945

thyroid, II 22

Glandis, corona, 648

Gland or glands -

agminated, 79 I

axillary, $3^{I} 7$

Bartholin, of, 633

Blandin, of, 1243

Bowman, of, 1257

bronchial, ro 34

Brunner, of, 790

buccal, lymphatic, I 183

mucous, I I 7 I

cardiac, of stomach, $78_{3}$

carotid, II I 3

caval, I034

ceruminous, $\mathbf{I}_{537}$

cervical, deep, I I02

superficial, 1085

coccygeal, 862

coeliac, 742

concatenatx, I062

Cowper, of, 621

Duverney, of, 633

Ebner, of, 1243

femoral, deep, 455

gastric, 783

superficial, 453

lymphatic, 742

hepatic, 742

iliac, common, 777 external, 778

internal, 845

infraclavicular, $3 I_{7}$

inguinal, 453

deep, 455

superficial, 453,455

intercostal, anterior, 933

labial, II 7 I posterior, I032

Littré, of, $8_{5} 8$

of lower limb, summary of, 568

lumbar, 773

mastoid, 1073

mediastinal, anterior, ro32 posterior, I034 superior, I032

Meibomian, I 88

mesenteric, 728

molar, I I 7 I

Moll, of, I I 87 
Gland or glands (continued) of Montgomery, 300

Nuhn, of, 1243

occipital, I063

odoriferæ, 647

palatal, 1248

pancreatic, $74^{2}$

parotid, lymphatic, II 83 salivary, 1183

popliteal, 445

prelaryngeal, 1 IO2

pretracheal, I I02

prostate, 853

pyloric, 784

sacral, 862

saphenous, 453

solitary, $79 \mathrm{I}$

splenic, 742

sternal, 933

submaxillary, lymphatic, I Ior salivary, I 34

submental, I IOI

suprahyoid, I IOI

suprascapular, 1094

tibial, anterior, 509

tongue, of, I 243

Tyson, of, 647

of upper limb, 396

uterine, or utricular, 897

Weber, of, $\tau 243$

Glandula, of pars, socia parotidis, I I 84

Glandulæ odoriferæ, 647

Glans clitoridis, 630

penis, 648

Glaser, fissure of, 72,1538

Gleno-humeral bands, 345

ligament, 345

Glenoid cavity, I80

fossa, $7 \dot{r}$

ligament, 345

Gliding joint, 280

movement, 282

Glisson, capsule of, 803

Globosus, nucleus, I 373

Globular processes, I62, I 240

Globus pallidus, I 4 I 3

Glomerulus of kidney, 824

Glossary, I 668

Glosso-epiglottidean fold, 1276

Glosso-pharyngeal nerve. See Cranial Nerves

Glottidis, rima, 1285

Glottis, false, 1284

respiratoria, 1285

vera, or true, 1285

vocalis, 1285

Gluteal artery, 430

lines, 222

nerve, inferior, 434

superior, 434
Gluteal region, 424

landmarks of, 424 vein, 432

Gluteus maximus, 426

medius, 428

minimus, 428

quartus, 429

Gnathic index of skull, $I_{5} 8$

Gnathion, I33

Golgi, cells of, $\mathbf{r} 443$

Goll, column of, I $315, x_{326}$

Gomphosis, I 72

Gonion, $\mathrm{I}_{4} 2$

Gowers, intermediate process of, 1322 tract of, r 330

Graafian follicles, 893 structure of, 893

Gracilis, adductor, muscle, $47^{\circ}$ nucleus, 1353

Granular layer of Purkinje, 172

Granulosa, membrana, 894

Grey matter of spinal cord, varieties of, 1317

Groove, basilar, 59

bicipital, 186

carotid, 84

cavernous, 84

digastric, 72

infra-orbital, 98

nasal, of ethmoid, 93

neural, I6r 8

nuchal, 283

olfactory, 82

optic, of sphenoid, 82

primitive, I $6 \mathbf{I}_{4}$

pulmonary, of thorax, 53

radial, I95

sigmoid of temporal. 73

spinal, 283

spiral, I 88

subcostal, 43

ulnar, of humerus, I 89

Grooved suture, 280

Ground-bundle, anterior, 1329

lateral, I 331

Gubernaculum testis, $6_{72}$

Gudden, commissure of, $1_{42} 2$

Guérin, sinus of, 858 valvule of, 858

Guide to digital arteries of hand, 367 to dissection of abdomen, 905 of brain, I $59 \mathrm{I}$ of ear, 1587 of eye, 1589 of head and neck, ${ }_{5} 6_{3}$. of lower limb, 592 of nasal cavities, $\mathrm{I}_{5} 85$ of spinal cord, 1590 of thorax, 1046 of upper limb, 4I I

to gluteal artery, 432 
Guide to palmar arch, deep, 367 superficial, 367 to removal of brain, 1566

Gulæ, plexus, 965

Gustatory cells, 1247 pore, 1247

Guthrie, muscle of, 6 i 7

Guttural fossa, I 47

Gyrus or gyriangular, 1390 breves, 1393 callosal, I 394 cinguli, 1394 cuneus, r 391 dentatus, I 396 fornicatus, 1394 frontal ascending, 1385 inferior, 1386 pars basilaris, $\times 387$ orbitalis, 1387 triangularis, 1387 middle, 1386 superior, 1386 geniculate, I 401 hippocampal, I 395 infracalcarine, I 391 lingualis, I 391 longus, $x 393$ marginal, i 388 occipito-temporal, r393

orbital, anterior, I 388 external, I 388 internal, 1387 posterior, I 388

parietal, ascending, 1389 inferior, 1389 superior, 1389

precentral, 1385 postcentral, I 389 postparietal, 1390 subcallosal, r.40I supracallosal, I 4 or supramarginal, I 390 temporal, first, $x 392$ second, I 392 third, I 392

Habenula of pineal body, I 422

Habenulæ, ganglion, 1422 trigonum, 1422

Hair-cells, 1554

Haller, vas aberrans of, 684

Hallucis, abductor, 549

adductor obliquus, 555 transversus, 555

arteria dorsalis, 5 I 2 magna or princeps, ${ }_{5} \boldsymbol{6}_{\mathbf{j}}$

extensor hrevis, 510 proprius or longus, 504

flexor brevis, 555
Hallucis, flexor longus, 523

tendon of, $.55 \mathrm{I}$

Ham, $44^{\circ}$

Hamstring muscles, 437

Hamular process of lachrymal, xo8 of sphenoid, 89

Hamulus of lamina spiralis, $155^{2}$

Hand, I 75

as a whole, 216

Harmonic suture, 279

Hasner, valve of, I I 92 , I 255

Hassall, concentric corpuscles of, 946

Haversian canals, 5 gland of hip-joint, 492 of knee-joint, 537

system, 7

Head and neck, 1058

bones of, 56

of tibia, structures attached to 245

process, I6 5

Heart, 970

apex of, 974

auricular portion of, 973

auricle, left, 980

$$
\text { right, } 975
$$

base of, 974

development of, 991

exterior of, 972

interior of, 975

structure of, 988

topography of, 970 of orifices of, 985

ventricular portion of, 973

ventricle, left, 98 I right, 977

Heister, valves of, Sog

Helicis major muscle, I 193

minor muscle, I 193

Helicotrema, I554

Helix, I 192

Henle, band of, 660

cremaster muscle, internal, of. 671

ligament of, 658

tubule of, 8rg

Hensen, canalis or ductus reuniens of, I 553

cells of, 1558

Hensen's node, I6I $4, I_{6} I_{5}$

Hepatic artery, 74I

canals, 805

cells, 805

duct, 708

flexure of colon, 697

lobule, $\mathrm{So}_{4}$

plexus, 736

veins, 805

Hepatis, pons, 706

sustentaculum, 697

Hernia, appendicular, 7 r 8 
Hernia, congenital, 679

diaphragmatic, 759,761

femoral, 462 parts concerned in, 460

infantile, 680

inguinal -

direct, internal, 678

oblique, external, 677 internal, 679

mesenteric, $7 \mathrm{I} 7$

meso-colic, 718

retro-peritnneal, 721

umbilical, 680 congenital, 680

Herophili, torcular, 59, I475

Hesselbach, ligament of, 658

triangle of, 668 guide to, 642

Hey, femoral ligament of, 460

Hiatus, accessory, 75

Fallopii, 74

semilunaris, I 255

subarcuatus, 76

Highmore, antrum of, IO2 corpus of, 683

Hilton, muscle of, 1291 white line of, 875

Hilton's law, 316

Hilum pulmonis, 949

Hind-gut, 798, I623

Hind-kidney, 830

Hinge-joint, 280

Hip-joint, 488 movements of, 493

Hippocampal commissure, I 403 fissure, I 396

gyrus, 1399

Hippocampi, pes, 1409 tænia, I405, I409

Hippocampus major, I 409 minor, $\mathrm{I}_{4} \mathrm{O} 8$

Holden's guide to great trochanter, 447

Homodynamic, 3

Homogenetic, 3

Homologies, approximate, of bones of limbs, 277

Homology, 3

Horizontal section of skull, I49

Horner, muscles of, I 165

Houston, muscle of, $6 \mathbf{I}_{7}$ valves of, 874

Huguier, canal of, 72,1538

Humeri, transversalis, artery, 320

Humerus, 184 anatomical neck of, 184 ossification of, 190 surgical neck of, 186

Hunter's canal, 475

Huschke, foramen of, 79,82 vomerine cartilages of, II99
Hyaloid artery, 1530

canal of eye, I530

membrane, I 529

Hyaloplasm, I 597

Hydatids of Morgagni, in male, 682

Hydrocele, encysted, of spermatic cord, 676

Hymen, 631

development of, 632

Hyo-epiglottidean ligament, 1276

Hyo-glossus muscle, I I 33

Hyoid arch, 165,1272

bar, 163,1272

bone, I $2 \mathrm{I}$

development of, I 65 ossification of, 123

Hypochordal bar, 55

Hypogastric sympathetic plexus, 738 zone, 690

Subdivisions of, 690
Hypoglossal nerve. See Cranial Nerves

Hypoglossi, trigonum, 1378

Hypophysis cerebri, 1079

Hypothalamus, I 423

Hypothalmi, pars mammillaris, 1423 pars optica, $\mathrm{I}_{423}$

Hypothenar muscles, 379

Ileo-cæcal fold, 722

valve, 797

Ileum, 695

peritoneal relations of, 695

Iliac arteries. See Arteries

fossa, 223

left, contents of, 642 right, contents of, 642

veins. See Veins

Ilii, dorsum, 222

Ilio-costalis muscle, 290

Ilio-hypogastric nerve, 425, 661, 769

Ilio-inguinal nerve, $449,66 \mathrm{I}, 77^{\circ}$

Ilio-pectineal line, relation of structures at pectineal portion of, 660

septum, 779

Ilio-psoas muscle, 465

Ilio-tibial band of fascia lata, 457

Ilium, 220

anterior superior spine of, 641

Impar, tuberculum, I 270

Incisor canal, IoI

crest, IOI

fossa, 97

teeth, I 66

Incudis, fossa, 1539

Incus, I 543

Index, alveolar, 158

cephalic, 158

gnathic, $15^{8}$

megaseme, 1.58 
Index, mesoseme, 158 microseme, $15 \mathrm{~S}$ nasal, I 58 orbital, ${ }_{5} 8$

Indicis, abductor, muscle, 394 dorsalis, artery, 393 extensor, muscle, 388 radialis, artery, $38 \mathrm{I}$

Infracalcarine gyrus, I 391

Infraglenoid ridge, I 80

Infrahyoid muscles, I I02 region, I 106

Infra-orbital artery, I I82, I205 branches of, 1205

canal, $98,1_{3} 6$

foramen, 97

groove, 98

nerve, I I 77, I 2 I 7

branches of, II 17, I 217 plexus, I I 74

vein, II 82

Infrapatellar pad of fat, 537 tendon, 470

Infraspinatus muscle, 320

Infraspinous fossa, $x 78$

Infratemporal crest of sphenoid, 87

Infratrochlear nerve, II 56

Infundibuliform sheath, 460

Infundibulum, 69, 94, I 39

of Fallopian tube, $88+$

of heart, 975,977

of kidney, 827

of lung, $95^{2}$

of nasal fossa, 1254

of pituitary body, 1080

Inguinal canal, 666

fossæ, 676

$$
\text { position of, } 642
$$

nerve, $449,66 \mathrm{I}, 770$

Inion, 132

Innominatum, os, 220

Inscriptions or intersections of rectus abdominis muscle, 659

Insula of cerebral hemisphere. See Island of Reil

Interarticular fibro-cartilages, 28I menisci, 28 I

Intercellular passages of liver, 806

Intercolumnar fibres, 651

Intercostal nerves, 927, I03 I spaces, 55

Intercosto-humeral nerve, 31 7, 33 I

Intercuneiform joints, 584

Interior of cranium, I 49

Interlobar fissures of brain, I 382 calloso-marginal, $\mathrm{I}_{3} \delta_{3}$ circular or limiting, $\mathrm{I}_{3} 84$ collateral, $\mathrm{I}_{3} 84$ parieto-occipital, $I_{3} 83$ Rolandic, I 383 Sylvian, I 382
Interlobar notch of liver, 708

Interlobular plexuses of liver, $\mathrm{SO}_{4}$

Intermaxillary bone, $\mathrm{IO}_{4}$

Intermediate septum, posterior, of spinal cord, I3I 5

Intermetatarsal joints, 588

Interosseous artery, anterior, of forearm, 36r

common, of forearm, 360

posterior, of forearm, 36I

membrane of forearm, 405

of leg, 577

muscles of foot, 556

dorsal, 557

plantar, $55^{6}$

of hand, 393

dorsal. 394

palmar, 394

nerve, posterior, of forearm, $35^{8}$

Interparietal bone, 57, 6I

Interpeduncular space, I339

structures within, $\mathrm{I}_{42} 23$

corpora albicantia vel mammillaria, 1423

peduncles of, 1423

development of, $\mathrm{r}_{424}$

infundibulum, IOSO, I 424

locus perforatus posticus, I 439

pituitary body, I079

tuber cinereum, I.424

Interpositum, velum, $\mathrm{I}_{4} \mathrm{O} 4$ blood-supply of, I 458

Interspinales muscles, 294

Interstitial lamellæ of bone, 6

Intertransversales muscles, 295

Intertubercular line, 689 plane, 689

Interventricular septum, $9 \delta_{5}$

Intestinal canal, 694 blood-supply of, 725

Intestine, large, 695 development of, 724

small, 694 structure of, 794

structure of, 787

Intrajugular process, $6 \mathrm{I}$

Intralobular plexuses of liver, 804

Iris, I 515

arteries of, $I_{516}$

ciliary margin of, 1515

muscles of, I5I 5

nerves of, 1516

pars iridica retinæ, I 5 I 5

pupil, I5I5

pupillary margin of, 1515

structure of, I 5 I 5

uvea, I 5 I 5

veins of, 1516

Irregular bones, 7

Ischio-rectal fossa, $6 \mathrm{I} 2$ 
Ischio-rectal fossa, cortents of, $6 I_{3}$

Ischium, 223 diverticula of, $6 \mathrm{I}_{3}$

spine of, structures on back of, 434

tuberosity of, 224

Island of Reil, or insula, r 393

development of, I 396

gyri breves, I 393

gyrus longus, I 393

limen of, I 393

lobule precentral, I 393

postcentral, I 393

sulcus centralis, I 393

Isthmus faucium, 1249

pharyngeal, 1268

Iter a tertio ad quartum ventriculum, I 439

Iter chordæ anterius, 72, I 538

Ivory, I $7 \mathrm{I}$ posterius, 75 , I $54^{\circ}$

Jacobson, cartilages of, Ir99

organ of, 1262

development of, $\mathrm{I}_{2} 6_{3}$

nerve of, 1224

Jejunum, 695

Joints, acromio-clavicular, 326

amphiarthrosis, 280

ankle, 569

arthrodia, 280

astragalo-calcaneal, 578

anterior, 579

posterior, 578

astragalo-navicular, 580

guide to, 543

atlanto-axial, I 30I

atlanto-occipital, I 303

calcaneo-cuboid, 582 guide to, 543

carpal, 406

first row, 406

second row, 407

carpo-metacarpal, 408 of thumb, 408

chondro-sternal, ro.43

classification of, 279

condylarthrosis, 280

costo-central, IO4 I

costo-chondral, ro 43

costo-transverse, $\mathrm{IO}_{4} 2$

costo-vertebral, 10.41

crico-arytenoid, 1282

crico-thyroid, 1282

cubo-cuneiform, 585

cubo-metatarsal, $5^{87}$

diarthrosis, 280

elbow, 398

enarthrosis, 280

femoro-patellar, 540

of foot, 578
Joints, ginglymus, 280

gliding, 280

of hand, 406

hinge, 280

hip, 488

nerve-supply of, 493

interchondral, I044

intercoccygeal, 900

intercuneiform, 584

intermetacarpal, 409

intermetatarsal, 588

interphalangeal (of foot $59 I$

(of hand), 4 ro

knee, 528 nerve-supply of, 538

lumbo-sacral, 899

metacarpo-phalangeal, 410

metatarso-phalangeal, 590

naviculo-cuboid, 583

naviculo-cuneiform, 584

of occipital bone, I30I

of pelvis, 899

pisiform, 406

pivot, 280

radio-carpal or wrist, 402

radio-ulnar, inferior, 404 intermediate, 405 superior, 404

of ribs, IO4I

sacro-coccygeal, 900

sacro-iliac, 900

shoulder, 344

sternal, 1045

sterno-clavicular, 324

suture, 279

dentated, 279

false, 279

harmonic, 279

limbous, 279

serrated, 279

squamous, 279

true, 279

symphysis, 280 pubis, 903

synarthrosis, 279

synchondrosis, 279

syndesmosis, 280

tarsal, 578

tarso-metatarsal, 586

external, $5^{87} 7$

internal, 580

middle, 586

temporo-mandibular, $\mathrm{I}_{2} \mathrm{I}_{4}$

tibio-fibular, inferior, 576

intermediate, 576

superior, 575

transverse carpal, 407

trochleartlirosis, $2 \mathrm{SO}$

trochoides, 280

varieties of, 279 
Joints of vertebral column, 1036 wrist, 402

Jugal point, I 42 process of temporal, 70

Jugular foramen, $\mathrm{I}_{4} 8$ fossa of temporal, 76 notch of occipital, 6I process of occipital, 60

vein, anterior, 1083 external, 1084 development of, 1084 internal, II 13 development of, I I I 3 posterior, 1063

Jugum sphenoidale, 82

Karyokinesis, 1598

Karyoplasm, I 597

Kataphase, 1599

Kerkring, valves of, 788

Kidneys, 744

arteries of, 822

cortical part of, 8 I 9

development of, 829

early condition of, 829

hilum of, $74^{8}$

medullary part of, 8 r 8

sinus of, 748,818

structure of, $8 \mathrm{I}_{7}$

varieties of, 748

veins of, 826

Knee-joint, 528

arterial supply, 538

movements, 539

nerve-supply, 538

Kobelt, pars intermedia of, 633 parovarium of, 884

Krause, ulnar collateral nerve of, 316 , 364

ventriculus terminalis of, I3 I9

Labia majora, 628

development of, 630

minora, 629

development of, 630

Labial mucous glands, r237

Labium tympanicum, 1556

vestibulare, 1556

Labyrinth of ear, membranous, 1552 blood-supply of, 1559 osseous, I 548

of kidney, 8I 9

Lachrymal apparatus, I I90 development of, 1 r9I artery, I I 59

bones, ros

ossification of, 109

canaliculi, II90

gland, 1146

nerve, II 55

punctum, II87
Lachrymal papilla, $118_{7}$

sac, I I 90

structure of, II9I

Lacrimalis, caruncula, I I 89

lacus, II 87

Lacteals of small intestine, 728,790

Lactiferous ducts, $30 \mathrm{O}$

Lacuna or lacunx-

laterales, 1475

magna of Morgagni, 858

of Morgagni, 858

muscular, 779

vascular, 780

Lacunx of bone, 6

Lacunar region, 779

Lacus lacrimalis, 1187

Lambda, 124

Lambdoid ligament, 501

Lamellæ, circumferential or peripheral, 6

concentric, of bone, 6

interstitial, of bone, 6

pressure, 7 of femur, 240

tension, 7 of femur, 240

Lamina basalis, 1512

chorio-capillaris, $\mathrm{I}_{5} \mathrm{I} 2$

cribrosa of sclerotic, $\mathrm{I}_{507}$

of internal auditory meatus, I55I of temporal bone, 75

dental, true, I 73

elastic, anterior, of Bowman, I 509

posterior, of Descemet, I 5 ro

fusca, 1508

labio-dental, ${ }_{72}$

reticularis, $155^{8}$ phalangeal process of, $155^{8}$

spiralis of cochlea, I $55 \mathrm{I}$

suprachoroidea, I $_{5}$ II

terminalis of brain, $\mathbf{1 4 2 4}$

vasculosa, I 5 I 2

Lancisi, nerves of, $140 \mathrm{r}$

Landmarks of abdominal wall, 639

of arm, 329

of back, 283

of scalp and neck, 1058

of thigh and popliteal space,

$$
435
$$

of face, $\mathrm{II}_{3}$

of gluteal region, 424

of knee, 447

of leg, 496

of male perineum, $61 \mathrm{I}$

of side of neck, Ioso

of sole of foot, 543

of front and inner side of thigh, $44^{6}$

of front of wrist and palm, 367 
Langerhans, centro-acinar cells of, 8 ro islets of, $8 \mathrm{ro}$

layer of, 1628

Laryngeal artery, inferior, II 42 superior, I I 6

nerve, inferior or recurrent, 966 , I 228 , I 292

superior, 1227,1292 external branch of, I 228,1292 internal branch of, I 228, I 292

summary of, I 293

Larynx, aperture, superior, of, 1283

arteries of, I 293

cartilages of, 1275

arytenoid, 1279

cornicula, I 280

cricoid, 1278

cuneiform, I 280

epiglottis, 1275

development of, 1294

glottidis, rima, 1285

glottis, 1285

interior of, 1283

ligaments of, I 280

lymphatics of, I293

mucous membrane of, 1286

muscles of, 1287

actions of, summary of, I 29 I

nerves of, 1292

pouch of, 1283

saccule of, $\mathrm{I}_{28}$

sinus of, I 283

sinus pyriformis, $\mathrm{I}_{28} 8_{3}$

structure of, I 275

veins of, I 293

ventricle of, 1283

vestibule of, 1283

vocal cords, false and true, 1284

Lateral sinus, $\mathrm{I}_{47} 6$ course of, 1477

emissary veins of, 1479

guide to, I 501

Latham, circle of, 97 I

Latissimus dorsi muscle, 286

Law, Hilton's, 3 I 6

of ossification, 178,190

Leg, the, 496

anterior aspect of, 503

external aspect of, 5 I 5

internal aspect of, 502

posterior aspect of, 517

Lemniscus, 1360

Lens, crystalline, 1528

capsule of, I 529

structure of, I 528

vesicle, I 532

Lenticular ganglion, I I 56

branches of, II 57

summary of, II 58
Lenticularis, ansa, of optic thalamus, I42I

nucleus, I 4 I 2

blood-supply of, I 457

processus, of incus, I 543

Leptorhine skulls, I 58

Lesser, triangle of, I 30

Levator anguli scapulæ muscle, 287 ani muscle, 862 palati muscle, I25I

Levatores costarum muscles, 296 longiores, 296

Lieberkühn, crypts or follicles of, $79 x$ Lienis, sustentaculum, 698, 720

Ligament or ligaments-

accessory, of atlas and axis, $\mathbf{I} 302$ acromio-clavicular, 327

alar, of axis, 1305

of ankle-joint, 569

annular, 404

of ankle, anterior, 500 external, 498 internal, 499

of wrist, anterior, 376 posterior, 390

arcuate, of diaphragm, $76 \mathrm{I}$

of astragalo-calcaneal joint, anterior, 579

posterior, 578

of astragalo-navicular joint, 580 atlanto-axial, $\mathrm{I}_{3} \mathrm{O} 2$

atlanto-occipital, I3O3

of auricle, II 93

of bladder, false, 867 true, 867

of Bigelow, 489

of calcaneo-cuboid joint, 582

calcaneo-navicular, inferior, 580

central, of spinal cord, I 313

check, of axis, 1305

chondro-sternal, I044

Colles, of, 653

common, anterior, of vertebræ, I036

posterior, of vertebræ, 1037

conoid, 327

coraco-acromial, 328

coraco-clavicular, 327

coraco-humeral, 344

coronary, of knee, 535

costo-central, anterior, IO4I interarticular, $\mathrm{IO}_{42}$

costo-clavicular, 326

costo-colic, 698, 720

costo-transverse, middle, I043 posterior, IO 43 superior, 1043

cotyloid, 492

crico-thyroid membrane, I 280

crucial, 532 
Ligament or ligaments (continued) cruciform, I 305

of cubo-cuneiform joint, 585

of cubo-metatarsal joint, $5^{87}$

discs, intervertebral, 1038

of elbow, 398

femoral, of Hey, 460

Flood's, 345

gastro-phrenic, 720

Gimbernat, of, 653

glenoid, 345

gleno-humeral, 345

Henle, of, 658

Hesselbach, of, $65^{8}$

of hip-joint, 488

hyo-epiglottidean, 1276

ilio-femoral, 489

ilio-lumbar, 900

ilio-trochanteric, 490

incus, of, I 544

interclavicular, 326

of intercuneiform joints, $5^{84}$

of intermetatarsal joints, $5^{88}$

internal brachial of Struthers, 335

interosseous, of forearm, 405 inferior, of leg, 576

membrane of leg, 577

of interphalangeal joints (of hand), 4 IO

(of foot), 59I

in terspinous, of vertebræ, $\mathrm{IO}_{4} \mathrm{O}$ in tertransverse, of vertebræ, 1040 ischio-capsular, 490

of knee-joint, 528

accessory, 535

of laminæ of vertebræ, I039

larynx, of, 1280

lieno-phrenic, 720

lieno-renal, 720

liver, of, 718

coronary, 7 r9

falciform, 718

lateral, 7 I9

posterior, 7 I 9

round, 719

suspensory, 7 I 8

triangular, 7 I9

lumbo-sacral, lateral, 900

malleus, of, $154^{\circ}$

metacarpo-phalangeal, 4 ro

metatarso-phalangeal, 590

of naviculo-cuboid joint, 583

of naviculo-cuneiform joint, $5^{84}$

oblique, of forearm, 405

occipito-axial, I304

odontoid, of axis, lateral, I 305

orbicular, 404

middle, 1305

palpebral, i i 88

patellar, lateral, 531
Ligament or ligaments (continued) pelvic, transverse, 619

perineal, transverse, 6 I9

phreno-colic, 698, 720

phreno-splenic, 720

plantar, long, 582

short, 582

Poupart, of, 651

pterygo-mandibular, I088

pterygo-spinous, ro 88

pubic, 903 of Cooper, $46 \mathrm{I}, 780$

pubo-femoral, 490

pubo-prostatic, 839

radiate, IO4I

radio-ulnar, superior and inferior, 404

rhomboid, 326

sacro-coccygeal, 900

sacro-iliac, 901

sacro-sciatic, great, $90 \mathrm{I}$

Schlemm's, 345 small, 902

of shoulder-joint, $3+4$

spheno-mandibular, I2I 4

spino-glenoid, $32 S$

spiral, of cochlea, I 555

spleen, of, 720

spring, $5^{80}$

stapes, annular of, 1544

stellate, I04I

sterno-clavicular, 324

sterno-pericardial, 944

stylo-hyoid, I I 34

stylo-mandibular, I 2 I 5

subpubic, 903

suprascapular (transverse), 328

supraspinous, of vertebræ, 1040

suspensory, of axis, 1305

of eye (Lockwood's), I I 5 I

of lens, I 530

of penis, 647

tarsal, I 165 , I I 88

of tarso-metatarsal joints, external, $5^{87}$

internal. 586

middle, $5^{86}$

temporo-mandibular, I 2 I 4

teres, 49I

thyro-arytenoid, I28 I

thyro-epiglottidean, 1275

thyro-hyoid membrane, $1280^{-}$

of tibio-fibular joint, inferior, 576 superior, 575

transverse, of atlas, I 302

transverse, deep (of hand), 409

superficial (of hand), 368

transverse, of hip-joint, 492

transverse, humeral, 345

of knee-joint, 535

of leg, 576 
Ligament or ligaments (continued) -

transverse, metacarpal, 409 metatarsal, 589

trapezoid, 327

triangular, of urethra, 619

of uterus, broad, 880 round, $88 \mathrm{r}$

vaginal, of hand, 375

of vertebræ, 1036

of wrist-joint, 402

$Y$-shaped, of Bigelow, 489

Zinn. of, I I 50

Ligamentum or ligamenta-

alaria, 537

arcuatum, 903

externum, $76 \mathrm{I}$

internum, 76r

brevia (of hand), 376

ductûs arteriosi, 964

venosi, 999

latum pulmonis, 937

longa (of hand), 376

mucosum, 537

nuchæ, 286, 106I

patellæ, 470,528

pectinatum iridis, 15 Io

posticum Winslowii, 529

subflava, 1039

suspensoria of Cooper, 300

teres, 491

Ligula of fourth ventricle, 1378

Limb, lower, 424

bones of, 220

divisions of, 220

guide to dissection of, 592 summary of veins of, 566

upper, 283

bones of, $\mathrm{r} 75$

divisions of, I 75

guide to dissection of, 4 I I

Limbic lobe of brain, 1394

Limbous suture, 279

Limbus laminæ spiralis, 1556

sphenoidalis, 82

Vieussenii, 993

Linea alba, 639

aspera of femur, 236

splendens, I3 I I

Linex semilunares, 640

transversæ, $64^{\circ}$

Lingual artery, I I I 7

branches of, $\operatorname{III} 8$

nerve, 12 Io

branches of, I 2 II

veins, I 118

Lingula of cerebellum, 1368

of mandible, 118

sphenoidalis, 84

Lips, 1237

Liquor folliculi, 894

Lisfranc's ligament. .587
Lisfranc's operation, 587

tubercle, 44

Lissauer, tract of, 1327

Lithotomy, lateral, structures avoided in, 628

Littré, glands of, 858 divided in, 627

Liver, 702

borders of, 708

cells of, $80_{5}$

component parts of, 702

Liver, connections of, 702

development of, 807

duct of, 708

early life, in, 710

excretory apparatus of, 708

fissures or fossæ of-

caval, 708

cystic, 705

portal, 706

transverse, 706

umbilical, 706

venosal, 707

impressio cardica, 704

colica, 706

duodenalis, 706

gastrica, 705

renalis, 706

suprarenalis, 708

licaments of, 718

loives of -

caudate, 706

left, 705

quadrate, 705

right, 705

Spigel's, 707 tuber papillare of, 706

lobules of, 804

notches of-

cystic, 708

interlobar, 708

umbilical, 708

œsophageal groove of, 707

outline of, summary of, 702

peritoneal relations of, 708

position of, 702

surfaces of, 703

structure of, 803

topography of, 702

tuber omentale, 705

uncovered area of rightlobeof, 708

Lobes and lobules of cerebral hemi-

spheres. See Cerebral Hemispheres

of cerebellum. See Cerebellum

Lobuli testis, $68_{3}$

Lockwood, superior tendon of, I I 49

suspensory ligament of, I I 5 I

Locus cæruleus, 1378

perforatus anticus, $134^{\circ}$

blood-supply of, 1.456 
Locus perforatus posticus, I 439 blood-supply of, 1456

Longbones, 7

Longissimus dorsi muscle, 29 I

Longitudinal fissure, great, 1344 sinus, inferior, -1475 superior, 1475 emissary veins of, I 479

Lowenthal, anterior marginal bundle of, 1329

Lower, tubercle of, 977

Lucidum, septum, 1403

Lumbar aponeurosis, 765 plexus, 768 posterior lamina of, 289 vertebræ, 25

Lumbo-sacral cord, 77 I

Lumbrical muscles (of hand), (of foot), 553

Luschka, cartilage of, i 282 coccygeal gland of, 862 sterno-pericardial ligaments of, 944

Lung-buds, 954

Lungs, 946 cervical part of, $\operatorname{II}_{4} 6$

development of, 954

in fœetus, 955

root of, $95 \mathrm{I}$

structure of, $95 \mathrm{I}$

Lunulæ of aortic valves, $9 S_{4}$

Lutea, macula, 1518

Luteum, corpus, 893

Luys, nucleus of, I 42 I

Lymphatic glands-

aortic, 757

anticubital, 335

axillary, 317

bronchial, 1034

buccal, I $8_{3}$

caval, I034

cervical, deep, 1102

colic, $73 \mathrm{I}$ superficial, $108_{5}$

femoral, deep, 455 superficial, 453

ileo-colic, 729

iliac, common, 777 external, 778 internal, $8+5$

infraclavicular, 317

inguinal, 452

deep, 455

superficial, $45^{2}$

intercostal, anterior, 933 posterior, $\mathrm{IO}_{2} 2$

intestine, of large, 73 I of small, 728

jugular, right and left trunk, I 102
Lymphatic glands (continued) -

of lower limb, summary of, 568

lateral aortic, 757

lingual, I I I 9

lumbar, lateral, 774 median, 773

maxillary, internal, 1206

mediastinal, anterior, 1032 posterior, 1034 superior, 1032

mesenteric, 728

meso-colic, 731

occipital, 1063

parotid, I I 83

popliteal, 445

pre-aortic, 757

pretracheal, I I 27

pubic, 453

rectal, 877

retro-aortic, 758

retro-pharyngeal, I 270

saphenous, 453

sternal, 933

stomach, of, 785

submental, I IO5

submaxillary, r ror

thoracic, 957

tibial, anterior, 509

thyroid, inferior, I 29

of upper limb, 396

system, development of, ror 6

trunk, axillary, 317

vessels of-

abdominal wall, deep, $66_{4}$ superficial, 646

anal canal, 877

auricle, II 95

bladder, 868

colon, ascending, 729 descending, $73 \mathrm{I}$

iliac, $73 \mathrm{I}$

pelvic, 731

transverse, 729

face, deep, 1183 superficial, I 182

Fallopian tube, 896

gluteal region, 435

gall-bladder, 809

heart, 988

intercostal spaces, 930

intestine, large, 731 small, 728

kidney, 826

larynx, 1293

liver; 806

lower $\operatorname{limb}, 567$

lungs, 954

nasal fossa, 126 I

nose, I I 96

œsophagus, 1022 
Lymphatic glands (contimued) ovary, 894 penis, deep, 649 superficial, 648 pericardium, 945 perineum, female, $63^{8}$ male, deep, 625 superficial, 625

pharynx, I 269

pia mater, I3I I

pleura, 939

rectum, 877

scrotum, 650

spinal cord, I334

stomach, 785

testis, 683

tongue, 1246

tonsils, 1254

upper limb, deep, 396

ureter, 827 superfícial, 395

urethra, female, 899 male, 858

uterus, 897

vagina, 898

vesiculæ seminales, 872

Lymphatics, axillary, 30I

epicondylar, internal, 335

infraclavicular, 302

internal mammary, 302

of mamma, zor

of scalp, frontal region, 1074

subareolar, 30I

Lyra, I403, I 446

McBurney, point of, 642

Macewen's triangle, 72

Macula acustica sacculi, I 553

lutea, 15 I 8 utriculi, I 552 2. I 553

$$
\text { structure of, } 1524
$$

Magendie, foramen of, 1380

Malar artery, II 82

bone, 104

ossification of, 107 marginal process of, I06

Malleolar folds of malleus, I 540

Malleolus, external, 250 internal, 247

Malleus, 1542

Malpighian corpuscles of kidney, 824 of spleen, $8 \mathrm{I}_{4}$

pyramids of kidney, 818

Mamma, 300

development of, 303

lymphatics of, 302

nerves of, 302

structure of, 302

vessels of, 301

Mammæ, accessory, 302

Mainmary artery, external,' 3 II
Mammary artery, internal, cervical part, II 42

Mammillary process, 25 thoracic part, 931

Mandible, II 5

Mandibular arch, I63, I272

Mantle of brain, I 393

Manubrium sterni, 47

Marginal bundle, anterior, of Lowenthal, I329

Marrow, ro

posterior, of Lissauer, 1327

Marshall, oblique vein of, 987 vestigial fold of, 944

Martinotti, cells of, I 443

Masseter muscle, I 199

Masseteric fascia, 1238

nerve, 1207

Mastication, muscles of, I 199

Mastoid antrum, 73, I 546, I 547 cells, 73

foramen, 73

portion of temporal, 72

Mater, dura, cranial, I470

spinal, 1306

pia, cranial, I48I

spinal, I3Io

Maturation of ovum, 1608

Maxillary air-sinus, I02

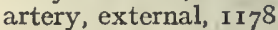

internal, 1202

first part of, $\mathrm{I}_{2} \mathrm{O} 3$ branches of, $\mathrm{I}_{203}$ second part of, 1205 branches of, I205

third part of, 1205 branches of, 1207

bone, inferior, II 5 ossification of, I I9

superior, 97 ossification of, 103

nerve, inferior, 1077,1207

anterior division of, 1207

posterior division of, 1209

summary of, $\mathrm{I}_{2} \mathrm{I}_{4}$

superior, 1077, I 217

branches of, I I 58,1217

summary of, I22I

process of mandibular arch, 1272

processes, 163

vein, internal, 1206

Maxillo-turbinal, 109

Meatus auditorius externus, I536

bloodvessels of, I 537

lymphatics of, 1537 nerves of, 1537

auditory, external, 78 internal, 75

inferior; 139 
Meatûs, middle, 95,139

superior, 95, I 39

urinarius, female, 631,890 male, 609, 854

Meatûs of nasal fossæ, 1254 inferior, 1255 middle, 1254 atrium of, 1255 bulla ethmoidalis of, 1255

hiatus semilunaris of, 1255

vestibule of, I255 superior, 1254

Meckel, cartilage of, 163,1272

cave of, 1076

diverticulum of, 695,799

ganglion of, 1219

branches of, 1220

summary of, 1221

Median artery, 361

nerve, 340,365

inner root of, 315

outer root of, 3 I 5

branches of, 365

in hand, 372

digital brạnches of, 372 summary of, 373

vein, 332

deep, 332

basilic vein, 332

cephalic vein, 332

Mediastinal space, 939

Mediastinum, anterior, 940

middle, $94 \mathrm{I}$

posterior, $94 \mathrm{I}$

superior, 940

testis, 683

thoracis, 939

Medulla, Io

Medulla oblongata, I 345

oblongata, anterior area of, $x 347$ lateral area of, $134^{8}$ posterior area of, 1350

arcuate fibres of, superficial,

$$
\text { I } 359
$$

deep, 1359

nucleus of, I 355

blood-supply of, $1+56$

central canal of, 1360

clava of, $135^{\circ}$

closed or lower part of, 1346 cuneate nucleus of, external, I 354 accessory, 1354

tubercle, $135^{\circ}$

decussation, motor, 1348

of pyramids, 1348

sensory, 1359

development of, 136r

fillets, 1360
Medulla oblongata (continued) -

formatio reticularis, 1352

alba, 1353

grisea, 1352

funiculus cuneatus, $135^{\circ}$

gracilis, 1350

Rolando, of, I $35^{\circ}$

longitudinal bundle, posterior, $135^{8}$

nucleus cuneatus, I $35^{\circ}$

gracilis, $135^{\circ}$

olivary body of, I 348

nucleus, I 354

dorsal accessory, I355

inferior, 1354

mesial, I 355

open or upper part of, 1346

pyramid of, 1347

decussation of, $\mathrm{r}_{34} 8$

pyramidal tract, crossed, I 348

raphé of, 1360

direct, 1348

relation of chief nerve-

fasciculi of spinal cord to, ${ }_{3} 6 \mathrm{r}$

restiform body of, 1357

structure, internal, of, I 35 I

substantia gelatinosa of Ro-

lando of, 1353

tubercle of Rolando of, $135^{\circ}$

Medullaris, conns, 1312

stria, I 4 I 6

Medullary artery of femur, 484

of fibula, 526

of humerus, 339

of radius and ulna, 361

of tibia, 525

laminæ of lenticular nucleus, I4I 2

membrane, II

nerve of tibia, 443

rays of kidney, 822

summary of, 822

spaces, 7

Medulla spinalis, 1312

Megacephalic skulls, 157

Megaseme, ${ }_{5} 8$

Meibomian glands, I I 88

Meissner, plexus of, 787,793

Membrana eboris, 171

flaccida, $154^{\circ}$

granulosa, 894

nictitans, 1 rgo

pupillaris, ${ }^{5}{ }^{7} 7$

sacciformis, 405

tectoria, 1558

tympani, I $54^{\circ}$

secondary, 1542 
Membrane of Reissner, 1555

Meningeal artery, middle, I203, I47I guides to divisions of, $\mathrm{I}_{5} \mathrm{O} 2$

small, I 204, I 47 I

Meninges of encephalon, I 470 of spinal cord, I 306

Menisci, interarticular, and marginal, 281

Mental artery, II 82

foramen, I 16

point, 133

protuberance, II 6

tubercle, I 6

Mercier, bar of, 866

Meridian of eyeball, $x_{507}$

Mesaticephalic skulls, $132, I_{5} 8$

Mesencephalon, 1337,1427 .

Mesenteric glands, 728

hernia, 717

holes, 717

plexus, inferior, 738

- superior, 738

Mesentery, definition of, 7 I I

primitive ventral, 724 . dorsal, 724

proper, $7 \mathbf{I} 7$

ventral gastro-duodenal, 724

Mesethmoid, 93

Meso-appendix, 697, 7I 7

Mesocardium, 992

Mesocephalic skulls, I 57

Meso-colon, pelvic, 7 I8, 859 transverse, 718

Mesoderm, formation of, I6I5 somatic, 1620

splanchnic, 1620

Mesogastrium, 724, 802 .

Mesognathion, IO 4

Mesognathous skulls, I 34, I58

Mesometrium, 887

Meso-nephron, 748

Meso-nephros, 829

Mesorchium, 671, 688

Mesorhine skulls, $x_{58}$

Mesosalpinx, 881

Mesoseme, 158

Mesosternum, 49

Mesovarium, 688, 88I

Metacarpal bone, first; 209 . second, 209 third, 2 Io fourth, 2II

Metacarpus, 208 fifth, 2 II

ossification of, 214

as a whole, 213

Metanephric blastema, 830

Meta-nephros, $8_{30}$

Metaphase, I 599

Metasternum, 49 .
Metatarsal artery, 512

bone, first, 266 second, 266

third, 267

fourth, 268 fifth, 269

Metatarsus, 265 ossification of, 272

as a whole; $27 \mathrm{I}$

Metathalamus, I 419

Metencephalon, I 337

Metopic suture, 125

Metopism, 69

Meynert, commissure of, 1430

Microcephalic skulls, I57

Microseme, 158

Mid-gut, 798, I 623

Mid-kidney, 829

Milk teeth, I69

Mitosis, I 598

Mitral cells, 1448

orifice, 980 position of, 985

valve, 982

Moderator band of heart, 978

Modiolus, I $55 \mathrm{I}$ spiral canal of, I 55 I, I 559

Molar teeth, I 68

Molecular layer, of cerebellum, 1375

inner, of retina, $152 \mathrm{I}$

outer, of retina, 1522

Moll, glands of, I 187

Monro, foramina of, $14 \mathrm{I} 7$ point of, 642 sulcus of, I 416

Mons Veneris, 628

Montgomery, glands of, 300

Morbus cæruleus, 999

Morgagni, anal valves of, 876 columns of, 875

hydatids of, 682

lacunæ of, 858

sinus of, 1265

Morphology, 3

of pectoral girdle, 277

Morula, I6I 2

Moss-fibres of Cajal, 1376

Mouth, I 236

development of, 1239

stomodæum, I 239

bucco - pharyngeal membrane, 1239

globular process, 1240

labial ridge, $124^{\circ}$

maxillary process of first visceral arch, 1240

primitive oral cavity, I $24^{\circ}$

vestibule of, 1236

Movements, angular, 282

circumduction, 282 
Movements, coaptation, 282

gliding, 282

pronation, 406

of ribs, 1045

rotation, 282

supination, 406

of temporo-mandibular joint, I 216

of vertebral column, I04\%

Muliebres, testes, 894

Müller, ducts of, 830,898

fibres of, in retina, 1523

Multifidus spinæ muscle, 294

Muscles-

abductor hallucis, 549

indicis, 39

minimi digiti (of foot), $55 \mathrm{I}$

(of hand), 379

ossismetatarsiquinti, $55 \mathrm{I}$

pollicis, 377

accelerator urinæ, 6 I6

accessorius ad ilio-costalem, 290

adductor brevis, $47 \mathrm{I}$

gracilis, $47^{\circ}$

longus, $47 \mathrm{I}$

magnus, $47 \mathrm{I}$

minimus, $47^{2}$

obliquus hallucis, 555 pollicis, $37^{8}$

transversus hallucis, $\mathbf{5 5 5}$ pollicis, $37^{8}$

anconeus, 356

arytæno-epiglottideus, I $290^{\circ}$

arytenoid, 1290

attollens auriculam, 1072

attrahens auriculam, 1072

auricle of, extrinsic, 1072

intrinsic, II 93

biceps femoris (flexor cruris), 437

flexor cubiti, 336

biventer cervicis, 293

brachialis anticus, 337

brachio-radialis, 383

bulbo-cavernosus, of female, 637 of male, 616

buccinator, $1 \times 69$

cervicalis ascendens, 290

ciliary, I 513

circumflexus palati, 1252

coccygeus, $86_{3}$

complexus, 292

compressor sacculus laryngis, I 291

naris, I 166

urethræ, of female, 637 of male, $6 I_{7}$

venæ dorsalis penis, $6 \mathrm{I} 6$

constrictor, inferior, of pharynx, 1264
MIuscles (continued)-

constrictor, isthmi faucium, I 250

middle, of pharynx, 1264

superior, of pharynx, 1265

urethræ, of female, 637 of male, $6 \mathrm{I}_{7}$

coraco-brachialis, 335

corrugator cutis ani, 6I 2

cremaster, 656 supercilii, I I66

crico-arytenoid, lateral, I 288

crico-thyroid, 1287

$$
\text { posterior, I2 } S 8
$$

crureus, 467

cucullaris, 285

deltoid, 319

depressor alæ nasi, I 166

anguli oris, II 70

labii inferioris, I 70

detrusor urinæ, 865

diaphragm, $75^{8}$

central tendon of, 759

development of, 761

foramina of, 760

digastric, II 29

dilator naris anterior, 1167 posterior, I 167

ejaculator urinæe, $6 \mathrm{I} 6$

erector clitoridis, 637

penis, 6I 5

spina, 290

inner column, 292

middle column, 29 I

outer column, 290

extensor brevis digitorum, 509

hallucis, 5 Io pollicis, 388

carpi radialis brevior, $3^{S_{3}}$

longior, $3 \delta_{3}$

ulnaris, 386

communis digitorum, 384

indicis, 358

longus digitorum, 504

hallucis, $50_{4}$

pollicis, 388

minimi digiti, $38_{4}$

ossis metacarpi pollicis, 388

primi internodii - pollicis, 388

proprius hallucis, " $5 \mathrm{O}_{4}$

secundi internodii pollicis, ${ }_{3} 88$

flexor accessorius (of foot), 553

brevis digitorum, 549

hallucis, 555

minimi digiti (of.foot),

pollicis, 378

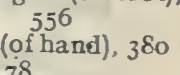

carpi radialis, 352

ulnaris, 353 
Muscles (continued) -

flexor longus digitorum, 521 tendon of, $55 \mathrm{I}$

hallucis, 523 tendon of, $55 \mathrm{I}$

pollicis, 366

profundus digitorum, 365

sublimis digitorum, 354

frontalis, 1068

gastrocnemius, 518

gemellus, inferior, 430

superior, 429

genio-hyo-glossus, I I 3 I

genio-hyoid, I I 3 I

gluteus maximus, 426

medius, 428

minimus, 428

quartus, 429

gracilis, $47^{\circ}$

Guthrie, of, 617

hamstring, 437

Horner, of, I $16_{5}$

Houston, of, 617

hyo-glossus, i 33

hypothenar, 379

iliacus, 766

minor, 7 ( 7

ilio-capsularis, 767

ilio-costalis, 290

ilio-psoas, 465,766

infrahyoid, I IO2

infraspinatus, 320

intercostal, external, 926 internal, 927

interosseous (of foot), 556 (of hand), 393

primus volaris, 378

interspinales, 294

intertransversales, 295

ischio-cavernosus, of male, $6 \mathrm{I}_{5}$

larynx, of, 1287 of female, 637

summary of actions of, I 291

latissimus dorsi, 286

levator anguli oris, II68

ani, 862 scapulæ, 287 .

glandulæ thyroidex, 1122

labii inferioris, $I \geq 7^{\circ}$ superioris, I I68 alæque nasi, I I66

menti, I I 70

palati, I 25 I

palpebræ superioris, I I47 prostatx, 863

levatores costarum, 296

urethræ, 617

longissimus dorsi, 291

longus colli, 1294

lumbricales (of foot), 553
Muscles (continued) -

lumbricales (of hand), 376

masseter, I I99

mastication, of, I I 99

multifidus spinæ, 294

mylo-hyoid, I I 30

obliqui, of eye, II 50

obliquus externus abdominis, $65^{\circ}$ internus abdominis, 654

inferior, of eye, II 50

superior, of eye, I I 50

obturator externus, 473 internus, 429

occipitalis, 1068

occipito-frontalis, 1068

omo-hyoid, $\mathrm{IIO}_{2}$

posterior belly of, 320

opponens minimi digiti (of foot),

$$
\begin{aligned}
& 556 \\
& \text { (of hand), } 380
\end{aligned}
$$

pollicis, 377

orbicularis oris, I I 70 palpebrarum, I I 65

palato-glossus, 1250 pharyngeus, 1250

palmaris brevis, 368 longus, 352

pectineus, 465

pectoralis major, 303

minor, 306

perinzi transversus, profundus, 617 superficialis, 6 I 5

peroneus brevis, $5 \mathrm{I} 7$ tongus, 5 I 5 tertius, 505

plantaris, 520

platysma, 1082

popliteus, $52 \mathrm{I}$

prevertebral, 1294

pronator quadratus, 366 radii teres, 35 I

psoas magnus, 766 parvus, 766

pterygoid, external, I 200 internal, $\mathbf{1} 202$

pubo-vesical, 865

pyramidalis abdominis, 659 nasi, I 166

pyriformis, 429,864

quadratus femoris, 430

lumborum, 767

menti, I I 70

quadriceps extensor femoris, 466 recto-coccygei, 875

rectus abdominis, $65^{8}$ sheath of, 659

capitis lateralis, 1294 anticus major, 1294 minor, 1294

femoris, 466 
Muscles (continued) -

rectus, external, of eye, I I 49 inferior, of eye, I $\mathbf{I}_{49}$

rectus, internal, of eye, $\mathrm{I}_{4} 8$ superior, of eye, $\mathrm{II}_{+7}$

respiration, of, 1045

rhomboideus major, 287 minor, 287

Retrahens auricalam, 1072

rider's muscle, 473

risorius of Santorini, I 68

rotatores spinæ, 294

salpingo-pharyngeus, 1275 ]

sartorius, 464

scalenus anticus, $\operatorname{II}_{36}$ medius, I I 36 posticus, I $_{3} 8$

scansorius, 429

semimembranosus, 438

semispinalis colli, 294 dorsi, 294

semitendinosus, 437

serratus magnus, 318

posticus, inferior, 258 superior, 288

soft palate, of, $125^{\circ}$

soleus, 519

sphincter ani externus, 612 internus, 875

pupillæ, I 5 I 5

vaginæ, 637

vesicæ, 865

spinalis dorsi, 292

splenius, 289

capitis, 290

colli, 290

stapedius, 1545

sternalis, 304

sterno-cleido-mastoid, 1088

posterior border of, 1061

sterno-hyoid, $\mathrm{IrO}_{3}$

sterno-thyroid, $\mathrm{I} \mathrm{IO} 3_{3}$

stylo-glossus, I 133

stylo-hyoid, I I 30

stylo-pharyngeus, I 221

subanconeus, $3+2$

șubclavius, 306

subcostal, 1035

subcrureus, 469

subscapularis, 323

supinator radii brevis, 386

supraspinatus, 320 longus, $3^{8} 3$

temporal, 1074, 1200

tensor fasciæ femoris, 464

palati, 1252

tarsi, I I 65

tympani, I544

teres major, $32 \mathrm{I}$

minor, 321

thenar, 377
Muscles (continued) -

thyro-arytenoid, 1289

thyro-hyoid, I IO3

tibialis anticus, 504

posticus, 522

tongue, of, intrinsic, I 244

lingualis inferior, 1245 superficialis, 1244

transversus, 1245

verticalis, 1245

trachelo-mastoid, 292

transversalis abdominis, 656 cervicis, 292 pedis, 555

transversus nuchæ, I06I

trapezius, $28_{5}$ in neck, ro6r

triangularis menti, I I 70 sterni, 933

triceps extensor cubiti, 342

trochlearis, of eye, I 150

tympanum, of, 1544

vastus externus, 467 internus, 468

Wilson, of, $6 \mathbf{I} 7$.

Wood's muscle, 551

zygomaticus major, I 168 minor, I $6 \mathrm{~S}$

Musculi papillares, 977

pectinati, 975

Musculus accessorius ad ilio-costalem, 290

antitragicus, I 194 of foot, 553

helicis major, I 194 minor, I 194

incisivus, inferior, II $7 \mathrm{I}$ superior, I I $7 \mathbf{I}$

naso-labialis, $\mathbf{1} \mathbf{7} \mathbf{r}$

obliquus of auricle, 1194

suspensorius duodeni, 732

tragicus, II9f

transversus of auricle, I I 94

Musculo-cutaneous nerve of arm,

$$
315,34^{\circ}
$$

of leg, 503 summary of, 504

Mnsculo-spiral nerve, $316,3 f^{2}$

branches of, $3+3$

Myelencephalon, I337, I 345

Myeloplaxes of Robin, 10

Myentericus, plexus, 787,793

Mylo-hyoid muscle, I 130

Myocardium, 988

structure of, 991

Myrtiformes, caruncula, 632

Myrtiform fossa, 97

Nabothi, ovula, 897

Nares, anterior, $4_{4} \mathrm{O}$

posterior, $1_{40}$ 
Nasal artery, II $8 \mathbf{I}$

bones, 107

- ossification of, 108

cartilages, I 197

duct, I I9I development of, I I9I

Nasal fossæ, I 37

arteries of, 1260

development of, I26I

floor of, 1256

lymphatics of, I $26 \mathrm{I}$

meatûs of, 1254

mesial nasal process, 1262

mucous membrane of, 1256

nasal capsule, 1262

nerves of, 1259

regions of, 1256

roof of, 1256

wall of, inner, 1256

index, $x_{5} 8$ outer, 1254

point, I 33

process, lateral, I62 mesial, 162

spine, anterior, 97, ror

posterior, III I

vein, transverse, II8I

Nasion, I 33

Nasmyth's membrane, I 72

Naso-palatine artery, 1260 canal, ror

Naso-pharynx, I 268

Natal cleft, 424

Nates of corpora quadrigemina, $\mathbf{I}_{42} 28$

Natis, fold of, 424

Navicular bone (of foot), $25^{8}$

Navicularis, fossa, 857

Neck of humerus. anatomical, $\mathrm{I}_{4}$ surgical, I 86

landmarks of, I080

side of, 1080

Nélaton's line, 447

Neopallium, I 464

Nerves-

abdominal wall, of, $645,66 \mathrm{I}$

abducent. See Cranial Nerves

anterior femoral, $47^{\circ}$ interosseous, 365

Arnold, of, 1073, I226

auditory. See Cranial Nerves

a uricle, of, I I 95

auricular, great, I059 branches of, $\operatorname{IO}_{59}, \operatorname{II}_{7} 8$

posterior, I073, II 73 branches of, IO73, II 73

auriculo-temporal, I070, I 209

branches of, 1070, I209

13ell, ex ternal respiratory, of, 1098

brachial plexus, 312, 1096 infraclavicular branches of, 3 I 4
Nerves (continued) -

brachial supraclavicular branches of, 313 , I098 plexus, branches of inner cord, 3I 5 outer cord, $3 \mathbf{I} 4$ posterior cord, 315

buccal, long, I I 77, I 208

plexuș, I I 75

short, I 169

calcaneo-plantar nerve, 528,548 Casserius, perforating nerve of, $3 \mathbf{I} 5$ cervical plexus, I09I

branches of, deep, 1093 superficial, ascending, 1092

descending, I06 I, 1093

cervical spinal, posterior primary divisions of, 1066

superficial, I060, 1093

transverse, 1060

chorda tympani, I 246,1298

circumflex, 316

coccygeal, 845

Cotunnius, of, 1260

crural, anterior, $77 \mathrm{I}$

cutaneous, of back, 283

of foot, 547

of forearm and hand, $34^{8}$

of gluteal region, 424

internal, of arm, 315, 331, $34^{\circ}$

lesser, of arm, 3I 5, 33I

of pectoral region, anterior, 298

lateral, 298

perforating, of sacral plexus, 425

of scapular region, 319

of thigh, external, 449 internal, 449 middle, 449

dental, inferior, I 209 branches of, I 2 Io

superior, anterior, I 218 middle, 1218 posterior, 1218

descendens cervicis, I IO7

hypoglossi, 1107

digastric, 1173

dorsal, last, $66 \mathrm{I}$

of penis, 627

dorsalis pedis, 5 I 3

dorso-lumbar, 768

eighth cranial. See Cranial Nerves

eleventh cranial. See Cranial

Nerves

external respiratory of Bell, 3 I 3 
Nerves (continuced) -

face, of, motor, I 72

sensory, I I 76

facial. See Cranial Nerves

fibular, 445

first cranial, 1258,1482

fourth cranial. See Cranial Nerves

fifth cranial. See Cranial Nerves

genito-femoral, $77^{\circ}$

crural (femoral) branch of, $45^{\circ}$

glosso-pharyngeal. See Cranial Nerves

gluteal, inferior, 434,847

gluteal, superior, 434,847

hemorrhoidal or perineal branch of fourth sacral, 6II, 849 inferior, 612, 626

hypogastric, of ilio-hypogastric, 645

hypoglossal. See Cranial Nerves

ilio-hypogastric, 66r, 769

iliac branch of, 425

ilio-inguinal, $449,66 \mathrm{r}, 770$

infra-orbital, I I 77

branches of, 1177,1217

inguinal, $449,661,77^{\circ}$

intercostal, 927, 103I

branches of, 929

lower five, 66I

in tercosto-humeral, $317,33 \mathrm{I}$

Krause, ulnar collateral nerve of, 316,364

laryngeal, inferior or recurrent, $966, \mathbf{1} 228, \mathbf{1} 292$

left in thorax, 966 summary of, 1293

superior, 1227,1292 external branch of, I228, 1292

internal branch of, 1228,1292

summary of, 1293

lingual, of fifth, I 2 10, I 246

of glosso-pharyngeal, 1246

of pneumogastric, 1227

lumbo-sacral cord, $77 \mathbf{I}$

masseteric, 1207

maxillary, inferior, 1077,1207

anterior division of, 1207 branches of, 1207

posterior division of, I 209 branches of, 1209

recurrent meningeal branch of, 1077

motor root of, 1207

sensory root.of, 1207

summary of, 1214

maxillary, superior, 1077, 1217

branches of, II 58, I2I 7
Nerves (continued) -

maxillary, in infra-orbital canal, I2I 7

in spheno-maxillary fossa, $\mathrm{I}_{217}$

recurrent meningeal, branch of, 1077

summary of, I 221

median, 340,365

inner root of, 315

outer root of, 3 I 5

in hand, 372

digital branches of. 372

palmar cutaneous of, 349

median, summary of, 373

medullary, of tibia, 443

mental, I 177

musculo-cutaneous, of arm, 3 I5. $34^{\circ}$

cutaneous branch of, 332

musculo-cutaneous, of leg, 503

summary of, 504

musculo-spiral, 316,342

branches of, 343

cutaneous, external, 332 internal, 332, 344

mylo-hyoid, 12 10

nasal, in cranial cavity, 1074

in orbit, I 155

in nasal fossa, I 156

infratrochlear branch of, I 776

nasal branches of, 1259

terminal branch of, I 777

nasal inferior, 1221, 1259

superior, I220, 1259

naso-palatine, 1220, 1260

ninth cranial. See Cranial Nerves

obturator, $450,474,771$

accessory, $475,77 \mathrm{I}$

geniculate branch of, 474

internus muscle, to, 434,847

occipital, great, I059

least, 1059

small, ro59

oculo-motor. See-Cranial

Nerves

olfactory, I $258, I_{4} 82$

ophthalmic, 1077

optic. See Optic Nerve

orbital, of superior maxillary.

I 58

pars intermedia of Wrisberg,

1076, 1299

patellar plexus, 450

perineal, 626

superficial, 626

deep, 627

of fourth sacral, 6I I, 849

peroneal, 445 
Nerves (continued) -

peroneảl, deep, 509

petrosal, deep, great, I 234 small, 1548

superficial, external, I 078 , 1298

great, 1078,1297

small, 1078, I 224

I $54^{8}$

summary of, I 300

pharyngeal, I227

plexus, 1227

branches of, 1227

phrenic, in neck, 1093

in thorax, 942

plantar, $55^{8}$

external, 560

sum*nary of, $56 \mathrm{I}$

internal, $55^{8}$

summary of, 559

pneumogastric. See Cranial

Nerves

popliteal, external, 445,848

branches of, 445

internal, 443,848

branches of, 443

to popliteus, 445

posterior interosseous, $35^{8}$

pudendal, long, of Soemmering, 436,627

pudic, 625,848

to quadratus femoris, 435

radial, 350,357

palmar cutaneous of, 349

rami communicantes, abdominal, . $76 \mathrm{I}$

cervical, $x 107$

thoracic, I03 I

ramus communicans fibularis, 445

tibialis, 443

rhomboids, to, 313, 1098

sacral, 845

saphenous, external or short, $5 \times 8$

internal or long, $45^{\circ}$

! sciatic; great, 439,848 .

branches of, 439

guide to, 439

small, 436,848

branches of, 436

recurrent branches of, 425

scrotal, long, 627,650

second cranial. See Optic

\section{Nerve}

seventh cranial. See Cranial

Nerves

sixth cranial. See Cranial Nerves Soemmeing, long pudendal of, 436,627
Nerves (continued) -

spinal accessory. See Cranial Nerves

posterior primary divisions of, 296

splanchnic, 1035

pelvic, 847

stylo-hyoid, I I 73

subclavius muscle, to, 314,1098

subcostal, 66r, 773

iliac branch of, 425 .

suboccipital, posterior primary division of, 1066

anterior primary division of, IO9I

subsartorial plexus, 452

subscapular, 3 I 5

supra-acromial, 298, 3 I9

supraclavicular, 298

supra-orbital, 1067

suprascapular, 3I4, 32 I, Iog8

suprasternal, 298.

supratrochlear, 1067

sural, 445

sympathetic, gangliated cord of in abdomen, 761

in neck, 1232

constitution of, 1235

cardiac accelerator fibres, 1236

dilator pupillæ fibres, I 235

ganglia of -

cervical, inferior, 1235

branches of, 1235

middle, 1235

branches of, I 235

superior, 1232

branches of, 1232

in pelvis, 862

in thorax, 1034

ganglion impar of, 862

plexuses-

cardiac, 968

carotid, 1234

cavernous, I079, I 234

epigastric, 734

hypogastric, 738

pelvic, 840

solar, 7.34

temporal, deep, 1207

temporo-malar, I070, I I 58

tenth cranial. See Cranial Nerves

third cranial. See Cranial Nerves

thoracic, anterior, external, 3 I 4 internal, 3 I 5

posterior, I 3 I 3

thyro-hyoid, I Io8

tibial, 443

anterior, 509

posterior, 527 
Nerves (conitinued) -

tibial, posterior, fibular branch of, 527

twelfth cranial. See Cranial Nerves

tongue of, 1246

trifacial or trigeminal. See

Cranial Nerves

trochlear. See Cranial Nerves

tympanic, 1224

thyro-hyoid, I 108

trigeminal, 1075

ulnar, 315,364

branches of, 364

collateral of Krause, 316 , 364

cutaneous of, 332, 349

ulnar, dorsal branch of, 350

in hand, 374

deep division, 374

superficial division, 374

palmar cutaneous, 349

summary of, 374

vagus. See Pneumogastric, under

Nerves

Vidian, I 219

formation of, I 219

of Wrisberg, 315, 33I

Nerve-plexuses, annular, of eye, 1510 aortic, 738

brachial, I096

buccal, II 75

cardiac, deep, 968 superficial, 968

carotid, I 234

cavernous, 1234

cervical, I09I

coliac, 736

coronary, 970

diaphragmatic, 736

epigastric, 7.34

gastric, 736

gulæ, 965

hepatic, 736

hypogastric, 738

intra-epithelial, of eye, I5 5 Io

lumbar, 768

Meissner, of, 787,793

mesenteric, inferior, 738 superior, 738

myentericus, of Auerbach, 787 , 793

ovarian, 738

pelvic, $84^{\circ}$

pharyngeal, I 227

phrenic, 736

pudic, 847

renal, 738

sacral, 846

sacro-coccygeal; 849

sciatic, 847
Nerve-plexuses, solar, 734

spermatic, $73^{8}$

splenic, 736

subepithelial, of eye, I5 Io

suprarenal, 736

tympanic, 1548

vertebral, I 235

Nerve-roots of spinal nerves, anterior, I 322

Nervous system, I 306 posterior, I 322

cephalic ganglia, 1469

cerebro-spinal, I306

development of, ${ }_{4} 68$ peripheral, $I_{4} 68$

neural crests, I468

spinal ganglia, 1468

sympathetic, 1306 ganglia, I 470

Neural folds, I6I 8

tube, 16 I 8

Neurenteric canal, I6r 8

Neuroglia of spinal cord, I 320, I 335 glia-cells of, 1320 neuroglial fibres of, I 322

Neuron, 1320

Nictitans, membrana, I 190

Nidus avis of cerebellum, I37 I

Nipple avis of cerebellum, I37I

Nipple, 300

Nomenclature, anatomical, I647

Norma basilaris, I 45

frontalis et facialis, I 32

lateralis, I 40

occipitalis, I3 I

verticalis, 132

Nose, I I 96

apertures, of, $\mathrm{I} 198$

arteries of, I 196

cartilages of, II 97

crus laterale, I 98 mediale, II 98

Huschke, of, II 99

Jacobson, of, I I 99

lateral, lower, 1 I 98 upper, I 197

minor, 1198

septum, of, II99

sesamoid, I I98

vomerine, I I 99

development of, Iren

lymphatics of, I 1, 6

nerves of, I I 96

Notch, great scapular, 79

jugal, $6 \mathrm{I}$

lachrymal, of superior maxilla, 99

popliteal, 243

pterygoid, 88

spheno-palatine, I I4

supra-orbital, 66 
Notch, suprascapular, I79

Notochord, 53, 1519

Nuchæ, ligamentum, 286, I06I

Nuchal furrow, Io5$_{5}$ groove, 283

Nuck, canal of, $88 \mathrm{I}$

Nucleus or nucleiambiguus, $\mathrm{r} 495$ amygdaloid, I 4 I 4 arcuate, I 355 auditory, cochlear, lateral, I490 ventral, I 49 I dorsal or principal, $\mathrm{I}_{492}$ external, 1492 internal, or of descending root, 1492

Bechterew, of, I 493

caudatus, I4II

cuneate, accessory or external, I 354

cuneatus, 1354

Deiters, of, I 492

emboliformis, 1373

fastigii, 1373

globosus, I 373

gracilis, I 353

late:alis, I 356

lenticularis, I4II, I4I2 globus pallidus of, I4I 3 medullary laminæ of, $\mathrm{r}_{4} \mathrm{I} 2$ putamen of, I4 12

oculo-motor, I 439

olivary, accessory, dorsal, I354 inferior, $\mathrm{r} 355$ mesial, I354

pontis, 1362

red, of tegmentum, 1432

of trigeminal nerve, 1365 of mesencephalic root of, I 439

motor of, 1365 sensory of, 1365

trochlear, I 439

Nuhn, glands of, I 243

Nymphæ, 629

Obelion, I 24

Obex, I 378

Obliquus capitis inferior muscle, I064 superior muscle, ${ }^{106} 4$

externus abdominis muscle, $65^{\circ}$ internus abdominis muscle, 654 inferior muscle of eyeball, $\mathrm{II}_{5} \mathrm{O}$ superior muscle of eyeball, I I5O musculus of auricle, II 94

Oblongata, medulla, 1345

Obturator artery, 488

abnormal or aberrant, 462 canal, 904 externus muscle, 473
Obturator fascia, 837

foramen, 227

internus muscle, 429,864 nerve to, 434

membrane, 904

nerve, 450,474

vein, 488 accessory, 475

Occipital artery, first part of, I I 20 second part of, 1062 third part of, 1062

bone, 57 ossification of, 6 I

glands, 1063

lobe of brain, $\mathrm{r} 390$

nerve, great, 1059 least, 1059 small, 1059

point, 132

sinus, 1477 guide to, 1501

sulcus, lateral, I 39 r transverse, I 39 I

triangle, rogr

veins, ro63

Occipito-axial ligaments, 1304

Occipito-temporal gyrus, I 393

Ocular appendages, II 87

Oculi, tendo, II 88

Oculo-motor nerve. See Cranial

Nerves

Odontoblasts, I 7 I

Odontoid process of axis, I7

Odoriferæ, glandulæ, 647

Esophagus, cervical part of, I I 29

development of, ro23

lymphatics of, 1022

structure of, I022

thoracic part of, IO2I

Olecranon rete, 363

Olfactorium, trigonum, 1397

Olfactory areas, development of, I26r bulb, 1397 structure of, 1448

hairs, 1258

lobe, I 397 development of, $\mathrm{x} 397$

lobules of, 1397

mucous membrane, I 257

nerves, course and distribution of, $125^{8}$ origin of, 1482

organ, development of, 1398

primitive nasal fossæ, $126 \mathrm{I}$

region of nasal fossa, 1256

sulcus of frontal lobe, ${ }_{3} S_{7}$ tract, 1397

Olivary body, $134^{8}$

eminence, 82

nuclei, 1354

peduncle, 1354 
Omentale, tuber, of liver, 705

Omental tuberosity of pancreas, 734

Omentum, gastro-colic, 690,716

gastro-hepatic, 7 I 6

gastro-splenic, 7 or, 7 I 7

great, 690, 7 r6

small, 716

Omo-hyoid muscle, I 102

posterior belly of, 320

Oöcyte, primary, I606

Oögenesis, 1604

Opening, femoral, 472

saphenous, $45^{8}$

Openings into orbit, I 36

Opercula insulæ, 1394

Ophryon, I33

Ophthalmic artery, I 159

ganglion, 1 I 56

branches of, I 157

summary of, II 58

nerve, 1077 branches of, I I 54

veins, 1 I $6 \mathrm{r}$ summary of, I I 58

Opisthion, 148

Opisthotic centre, $8 \mathrm{I}$

Optic chiasma or commissure, 1424

cup, 1532

disc of retina, 1519

foramen, 86

nerve in cranial cavity, 1424

course of, $\mathrm{I}_{4} 83$

development of, 1530

in orbit, $1 \mathrm{I} 52$

origin of, deep, 1425 superficial, $\mathrm{I}_{4} 8_{3}$

radiation, $\mathrm{I}_{42} 2, \mathrm{I} 446$

stalk, I532, I 534

thalami, $\mathrm{r}_{4} \mathrm{r} 7$

blood-supply of, I 456

connections of, $1_{420}$

development of, $\mathrm{I}_{42} \mathrm{~T}$

nuclei of, 1420

structure of, I 420

tract, I 425

roots of, 1425

inner or commissural, 1427

outer or visual, 1425

summary of fibres of, I 427

vesicle, prii.ary, I 458, I 532

secondary, or optic cup,

I 532

Opticus, porus, of retina, r 519

Ora serrata of retina, 1518

structure of, I 525

Orbicularis ciliaris muscle, I 5 I 2

oris muscle, II 70

palpebrarum muscle, I 65

processus of incus, 1543
Orbit, II 46

contents of, I I 46

fascia of, II 5 I

muscles of, I I 47

nerves in, II 52 ciliary ganglion, I $_{56} 6$ nerves, long, I 156

short, I 157

fourth cranial, II 53

frontal, II 54

lachrymal, I I 55

nasal, I I 55

optic, II 52

orbital, II 58

sixth cranial. II 53

supra-orbital, II 54

supratrochlear, II 54

temporo-malar, I 158

third cranial, I I 53

periosteum of, $\mathrm{II}_{4} 6$

Orbital index, 158

nerve, II 58,1217

malar branch of, II 77

temporal branch of, ro7o, I 77

sulcus, 1387

Orbito-sphenoids, 84

Orbits, I 34

openings into, 136

Orthognathous skulls, $134,{ }_{5} S$

Os calcis, 256

centrale, 208

ossification of, $25^{8}$

cox 2,220 ossification of, 227

innominatum, 220 ossification of, 227

japonicum, ro7

magnum, 205

planum of ethmoid, 94

pubis, 225 spine of, 226

tinca, 886

trigonum, 256, 264

unguis, 108

uteri externum, 886 internum, 888

Ossification, 8

centres of, 8

in cartilage, 9

in membrane, 8

law of, 178

Osteoblasts, ro

Osteoclasts, 9

Osteogenetic fibres, 8

layer, 9

Otic ganglion, I 2 I I

branches of, 1212

Otoconia, r 553

Otoliths, I 553

Ovarian plexus, 738 
Ovary, 88r

abnormal positions of, 884

descent of, 883

development of, 687

ligament of, 883

structure and development of, 892

suspensory, of, 883

poles of, 882

structure of, 892

Oviduct, 884

Ovulation, 1607

Ovum, 894, r603

fertilization of, I610

maturation of, 1608

Oxyntic cells, 783

Pacchionian bodies, $1_{4} 8 \mathrm{I}$

depressions, 64

Pacinian bodies of foot, 559

Palate bones, I Io of hand, 373

soft, 1248 ossification of, I I 4

glands of, $125^{\circ}$

muscles of, I250

nerves of, 1252

relation of component parts of, $125^{2}$

Palatine artery, descending, I205, I 260

branch of ascending pharyngeal, I I2I

canal, posterior, II 2

accessory, external, II2 posterior, II 2

fossa, anterior, Ior, I 45

foramen, accessory, external, I47 posterior, 147 posterior, 147

Palatine nerves, 1221

Palato-glossus muscle, r 250

Palato-maxillary canal, i 12

Palato-pharyngeus muscle, $1250^{\circ}$

Pallidus, globus, I4I 3

Pallium of brain, I393

Palmar arch, deep, 380 superficial, 370

branches of, $38 \mathrm{I}$

summary of, 382

bursa, great, 374

cup, 408

fascia, 369

furrows, 367

interosscous arteries, $38 \mathrm{r}$

veins, deep, 382

Palmaris brevis muscle, 368

longus muscle, 352

Palmatæ, plicæ, 888

Palpebræ, I 187

Palpebral arteries, external, I 82
Palpebral arteries, internal, I I 8 I ligaments, II 88 veins, 1182

Palpebrarum, fissura, I I 87 tendo, I 188

Pampiniform plexus, 670

Pancreas, 732 connections, position, and relations of, 732

development of, $8 \mathrm{I} 2$

lymphatics of, $8 \mathrm{I} 2$

structure of 809

Pancreatic duct, 8I I

Papilla, bile, 789 accessory, 8I I

lacrimalis, I 87

Papillæ of tongue, 1242

Paracentral lobule, I 388

Paradidymis, 682

Paraflocculus, 1462

Parametrium, 887

Pararectal fossa, 836

Parathyroids, I I 25

Paravesical fossa, 836

Paravesicular fossa, 836

Parietal bones, 62 ossification of, 65

foramen, 63

lobe of brain, 1388

Parieto-occipital fissure of brain, 1383

Paroöphoron, 884 development of, 687,883

Parotid gland, i 83 development of, I I 86

duct, I I 85

lymphatic glands, 1 I 83

Parotidis, glandula socia, I I 84

Parovarium, 884

Pars basilaris of frontal lobe, $\mathrm{I}_{3} 87$

ciliaris retinæ, $\mathrm{x}_{5} \mathrm{I} 3$

intermedia of Kobelt, 633 of Wrisberg, I 299, I 489

iridica retin $x$, I 515

membranacea septi of heart, 985

orbitalis of frontal lobe, I 387

triangularis of frontal lobe, 1387

Patella, 24I

ossification of, 242

Patellaris, fossa, I 529

Patellar plexus of nerves, $45^{\circ}$

Pathetic (trochlear) norve. See Cranial Nerves

Pavilion of Fallopian tube, 885

Pecquet, cistern of. See Receptaculum Chyli

Pectineus muscle, 405

Pectiniforme, septum, of clitoris, 630 of penis, 868

Pectoral girdle, morphology of, 277 region, 297

Pectoralis major muscle, 303 
Pectoralis minor muscle, 306

Pelvic fascia, in female, visceral, 879 in male, parietal, 836 girdle, 220 visceral, 838

Pelvis, 228, 833

axes of, $23 \mathrm{I}$

of child, 232

contents of, 834

diameter, conjugate, 230

false, 228

transverse, 230

fermale, 8,8 peritoneum of, 879 viscera, position of, 878

inclination of, 230

of kidney, 826

male, 834 peritoneum of, 834 viscera, position of, 834

sexual differences of, $23 \mathrm{I}$

true, 228

Penis, 647

angle of, 858

composition of, 648

corpora cavernosa, 868

corpus spongiosum, 869 bulb of, 869

coverings of, 647

crus, 6 I 8

fascial sheath of, 647

glans, 648

præputium, 647

structure of, 868

Perforaculum, 1602

Perforating arteries of profunda femoris, 484

fibres of Sharpey, 7

Pericardium, 942

development of, 945

sinus, transverse, of, 944

structure of, 945

Perichoroidal lymph-space, I508

Peridental membrane, 172

Periglottis, 1276

Perilymph of internal ear, I $55^{2}$

Perilymphaticus, ductus, 78

Perineal body, 636

fascia, deep, 619

fold, $87 \mathrm{I}$

pouch, $6 I_{4}$

triangle, deep, 6 I 8

Perineum, female, 628

male, 6ri central tendinous point of, 618

Periosteum, 9

Periotic cartilaginous capsules, $\mathrm{I} 60$

Perisclerotic lymph-space, I15I, I508

Peritontum, 7 ro
Peritoneum, course of, transverse, $7 \times 3,715$

vertical, 7 II

development of, 725. Sor

folds of, 7 I $\mathrm{r}$

ligaments, 7 I 8

mesenteries, 717

omenta, 716

fossa, digital, of, 676

fossa, pelvic, of, 836

in fœetus, $164+1645$

pelvic, in female, 879 in male, 834

plicæ of, on anterior abdominal wall, 676

pouches of, on anterior ab-

dominal wall, $72 \mathrm{I}$

primitive, of testis, 674

sac, great, of, 720 small, of, 720

structure of, 725

Permanent teeth, I 70

Peroneal artery, 526 anterior, 509, 526 posterior, 527

bone, 250 varieties of, 527

groove, $251 ; 264$

nerve, 445 deep, 509

spine, 258

Peroneus brevis muscle, $5^{1} 7$

longus muscle, 5 I 5

tertius muscle, 505

Pes accessorius, 1409

anserinus, II 73

crus cerebri, of, 1431,1437

hippocampi, I 409

Petit, canal of, I 530

triangle of, 286,654

Petrosal nervesguide to, 642

deep, great, 1234

small, $154^{8}$

superficial, external, ro 78 , I 298

great, 1078, 1297

small, $1078,1224, x_{548}$

summary of, 1299

sinus, inferior, $x_{47} \mathrm{~S}$

superior, $I_{478}$

Petro-squamous sinus, 1479

Petro-tympanic fissure, 72

Petrous ganglion of glosso-pharyngeal, 1223

Peyer, patches of, 79 I

Pflüger, egg-tubes of, 687,688

Phalanges of foot, $27 \mathrm{I}$

muscular attachments of, 27 I

ossification of, 272 
Phalanges of hand, 213

muscularattachments of, 2 I 5 ossification of, 216

Pharyngeal aponeurosis, I 266 artery, asćending II 2 I

bursa, I 269 branches of, II 2 I

isthmus, I 268

nerve, I220

pouches, 1270

development of, i2 270

metamorphoses of, 1270 of first, 1270 second pouch, 1270 third pouch, 1270 fourth pouch, I270 tuberculum impar, 1270 furcula, 1270 sinus arcuatus, I270

recess, lateral, 1269

tonsil, I 269

tubercle, 59

Pharyngo-epiglottidean folds, 1276

Pharynx, I 263

blood-supply of, 1269

development of, I270

lymphatics of, 1269

mucous membrane of, 1267.

muscles, constrictor, of, 1264

relations of, 1263

Phenozygous skulls, $\mathbf{I}_{\mathbf{3}} \mathbf{2}$

Philtrum, I 164,1237

Pia mater, cranial, I48I structure of, $I 482$

spinal, I3ro blood-supply of, r 311 lymphatics of, I3II nerves of, I 3 I I structure of, r $_{3}$ I I

Pigmentary layer of retina, ${ }_{523}$

Pineal body, I 42 I development of, I 422

habenula, peduncle, or stalk of, I 422

stria medullaris vel pinealis, I 422

structure of, 1422

Pisiform bone, 203

Pituitary body, I079

development of, ro8o

structure of, Ioso'

fossa, 82

membrane, 1256

Pivot joint, 280

Placenta, 1635

circulation of, 1642

Plagiocephalus, I59

Plantar artery, external, 562. varieties of, 565 internal, $56 \mathrm{I}$ varieties of, 565
Plantar branch of dorsalis pedis, 565 fascia, 545

muscles, 549

nerve, external, 560 summary of, 561 internal, $55^{8}$

triangle, 556

summary of, 559

Plantaris muscle, 520

Platycnemism, 249

Platyrhine skulls, ${ }_{5} 8$

Platysma muscle, 1082

Pleura, 935

Pleuræ, blood-supply of, 939

development of, 939

lymphatics of, 939

reflections of, 936

lines of, 937

Plexus, subsartorial, 452

Plica epigastrica, 676

hypogastrica, 676

semilunaris of conjunctiva, I 190

sublingualis, I I 35,1237

urachi, 676

ureterica, 866

Plicæ palmatæ, 888.

villosæ, 783

Pneumogastric nerve. See Cranial Nerves

Pocularis, sinus, 855

Point, auricular, I 40 central tendinous, of perineum, 6 I 8 jugal, 142

Polar bodies, 1608 extrusion of, 1608

Pollicis, abductor, 377

adductor obliquus, 378

transversus, 378

dorsales, arteriæ, 393

extensor brevis (extensor primi internodii), 388

longus (extensor secundi internodii), 388

ossis metacarpi, 388

flexor brevis, 378

longus, 366

opponens, 377

princeps, arteria, 380

Pomum Adami, 1277

Pons hepatis, 706

Tarini, I $44^{\circ}$

Varolii, I 338, I 362

blood-supply of, 1456

corpus trapezoides of, I 263

nuclei of, 1365

ascending root of fifth nerve, I 365

facial nerve, 1365

sixth nerve, I 365 
Pons Varolii, nuclei of, olivary, superior, $I_{3} 65$

posterior longitudinal bundle in, 1365

structure of, 1362

Pontis, cisterna, I 480

nucleus; 1362

Popliteal artery, $44^{\circ}$ branches of, 442

nerve, external, 445 varieties of, 443

internal, 443 branches of, 445

notch, 243 branches of, 443

space, $435,44^{\circ}$

boundaries of, $44^{\circ}$

vein, 443

contents of, $44^{\circ}$

Popliteus muscle, $52 \mathrm{I}$

nerve to, $4+5$

Portæ, vena, 742

branches of, in liver, $80_{4}$

sinus, of, 743

Portal canals, 804

fissure, 706

structures at, 706

sinus, 743

vein, 742

Porus opticus, I 519

Postcentral sulcus of brain, ${ }_{389}$

Posticum, tuber, 1370

Post-sphenoid, 91, 160

Poupart, ligament of, $460,65 \mathrm{I}$

Præcuneus of brain, 1390

Præputium clitoridis, 630 penis, 647

Precentral sulcus of brain, 1385

Prechordal cartilages and plate, I60

Preglenoid tubercle, 7 !

Pre-interparietal bone, I 55

Prelaryngeal lymphatic glands, 1 I 27

Premaxilla, 103

Presphenoid, 91, r6o

Presternum, 47

Pretracheal lymphatic glands, II 27

Prevertebral muscles, 1294

Process, accessory, of lumbar vertebra, 26

acromion, I 82

basilar, 59

clinoid, anterior, 85

middle, 86

coracoid, 182 posterior, $8_{3}$

coronoid, of mandible, II 8 of ulna, 197

ensiform, 47,49

external auditory, 79

fronto-nasal, 162
Process, globular, $16_{3}$

hamular, of sphenoid, 89

intrajugular, 6r

jugal, of temporal bone, 70

jugular, 60

lateral nasal, 162

marginal, of malar, 106

maxillary, 163

of first or mandibular arch, $127^{2}$

mesial nasal, I 62

muscular, of arytenoid cartilage, 1279

nasal, of frontal, 67

orbital, of malar, 104

paramastoid, $6 \mathbf{r}$

postglenoid, 70

styloid, of temporal bone, 79

supracondylar, $\mathrm{I} S \delta$

uncinate, of ethmoid, 96

vaginal, of sphenoid, 84

of temporal bone, 79

vocal, of arytenoid cartilage, I279

xiphoid, 47,49

Processus cochleariformis, $78,1539$.

dentatus, 17

reticularis of spinal cord, 13 I 8

tubarius, 89

vaginalis, 675

abnormal conditions of, 676

Proctodæum, $\delta_{7} \mathbf{r}, \delta_{7} \delta$

Profunda artery of brachial, superior,

$$
\begin{aligned}
& 338 \\
& \text { inferior, } 339 \\
& \text { of ulnar, } 370
\end{aligned}
$$

femoris artery, $4 \mathrm{~S} \mathrm{I}$

$$
\text { vein, } 487
$$

Prognathous skulls, I $34, I_{58}$

Proligerus, discus, 894

Pronation, 406

Pronator quadratus muscle, 366

radii teres muscle, 351

Pronephros, 829

Pronucleus, female, r 605

Pro-otic centre, or

$$
\text { male, 16ro }
$$

Prosencephalon, I 337

Prostate gland, 853

blood-supply of, $\$ 73$

development of, 873

lymphatics of, $S_{73}$

structure of, 872

Prostatic fissure, 853

sinus, 855

Prussak, pouch of, ${ }_{546}$

Psalterium, I 403,1446

Psoas magnus muscle, 766

parvus muscle, 766

sheath, 764

Pterion, I26 
Pterotic centre, 8r

Pterygoid bones, 92

$$
\text { canal, } 89
$$

fossa, $88, x 47$

muscle, external, I 200 in ternal, 1202

notch, 88

plate of sphenoid, external, 89 internal, 89

plexus of veins, 1206

processes, 88

tubercle of sphenoid, 89

Pterygo-mandibular ligament, ro88

Pterygo-maxillary fissure, 144 region, I I 99

Pterygo-palatine artery, I 206 bar, 164 canal, 84 , II 3

Pterygo-spinous foramen, 89

Pubic angle, 226 ligament, ro88

crest, 226 relation of structures at, 660 spine, 226

Pubis, os, 225

Pudendal nerve, long, of Soemmering, 436,627

Pudendum, 628

frenulum, 632

rima, 629

vestibule of, 632

Pudic artery, inferior external, $48 \mathrm{I}$ internal, 433 guide to second part, 434

superior external, 479

Pulmonary alveoli, 952

groove of thorax, 53

orifice of heart, 978

position of, 985

valve of heart, 980

Pulp cavity, 17 I dental, I $7 \mathrm{I}$

Pulvinar of epiglottis, 1276 of optic thalamus, 1419

Punctum lacrimale; 1587

Pupil, $\times 5 \times 5$

Pupillaris, membrana, I 517

Purkinje, cells, of, 1375

fibres of, 990

granular layer of, 172

Putamen of lenticular nucleus, $\mathbf{4}_{4} 12$

Pylori, antrum, 693 sphincter, 782,786

Pyloric valve, 786

Pylorus, 693, 786 position of, 694

Pyramidal tract, direct, I $328, \times 348$ crossed, 1327

Pyramids, Ferrein, of, 822 Malpighi, of, 8 r 8
-Pyramids, medulla oblongata, of, I 347 decussation of, 1348

Pyriformis muscle, 429,864

sinus of larynx, 1283

Quadrangular muscular space of arm, 323

Quadrate lobule of brain, 1390

lobe of liver, 705

Quadratus femoris muscle, 430

menti muscle, I I 70

nerve to, 435

Quadriceps extensor femoris, 466

Quadrigemina, corpora, 1428

lower pair of, 1428

structure of, 1429

upper pair of, I 428

structure of, I 430

Radial artery, 356

first part of, $35^{6}$

branches of, 356

second part of, 39 I

branches of, 392

third part of, 380

branches of, 380

varieties in hand, 382

nerve, $350 ; 357$

vein, 334

Radiata, corona, I4I4

Radiatio corporis callosi, I 40 r

Radiation, optic, 1427,1446

Radius, I9 I

grooves of, 195

ossification of, 196

Ramus communicans fibularis nerve, 445

Ranine artery, I I I 8, I 246

tibialis nerve, 443 .

Rathke, pouch of, 92

Rauber's layer, I6 22

Rays, medullary, of kidney', 822

Receptaculum chyli, 762

Recess, infundibular, of third ven-

- tricle, r4 16, I4I 7

lateral, of fourth ventricle, 1380

optic, of third ventricle, 1416

pineal, of third ventricle, $\mathrm{r}_{4} \mathrm{I} 7$

spheno-ethmoidal, 84

Recessus cochlex, I 549

utriculi, I 549, I552

Rectal pits, 874

Recti muscles of eyeball, I I 47

Recto-coccygei muscles, 875

Recto-uterine folds, 879 pouch, 879

Recto-vesical pouch, $8{ }_{3} 6$.

Rectum, female, 800

male, 860

blood-supply of, 875

development of, 877 
Rectum, lymphatics of, 877 peritoneal relations of, 835 structure of, 873 valves of, 874

Rectus abdominis muscle, $65^{8}$ capitis anticus major muscle, I 294 minor muscle, I 294 posticus major muscle, 1064 minor muscle, 1064 lateralis muscle, I 294

femoris muscle, 466

Recurrent laryngeal nerve, 966, I 228, I 292

Red nucleus of the tegmentum, 1432 Reduction-division, 1606

Refracting media of eyeball, 1527

Reid, base line of, $\mathrm{I}_{504}$

Reil, island of, I 393

development of, 1396

Reissner, membrane of, I 555

Relation of muscles at back of upper end of femur, 494

along linea aspera, 495

on front of body of os pubis, 494

Renal sympathetic plexus, 738

Respiratory apparatus, development of, 954

Restiform body, I35I, r 357 arcuate fibres, $135^{8}$

cerebello-olivary fibres, 1358 cerebellar tract, direct, I 358

Rete, acromial, 324

olecranon, 363

testis, $68_{4}$

Rete-cords, 686

Reticularis, processus, of spinal cord, I31 8

Retina, 1517

arteria centralis retinæ, $\mathbf{I} 525$

cone-granules, 1523

fibre-baskets of, 1524

fovea centralis, 1518

macula lutea, 1518

nerve-cells of, I 526

ora serrata, I 5 I8

porus opticus, 1519

rod-granules, 1522

structure of, 1519

bacillary layer, $15 \circ 3$

ganglionic layer, I 520

granular layer, inner, 1521

outer, 1522

molecular layer, inner, $\mathbf{I}_{52} \mathrm{I}$ outer, I 522

nuclear layer, inner, $1_{52} \mathrm{I}$ outer, 1522

optic layer, I 520

pigmentary layer, 1523
Retina, structure of, plexiform layer, inner, $152 \mathrm{x}$ outer, 1522

rods and cones layer. I 523

sustentacular fibres of, I 523

veins of, 1526

Retrahens auriculam muscle, ro 2

Retro-pharyngeal space, 1087

Retro-pubic pad of fat, 836

Retzius, fundiform ligament of, $50 \mathrm{r}$

Rhinencephalon, 1400

Rhinion, I 33

Rhombencephalon, I337, I 345

Rhombic lip, 1460

Rhomboideus major muscle, 287

minor muscle, 287

Rhomboids, nerve to, 313

Rib, first, 43

second, 45

tenth, 45

eleventh, 45

twelfth, 45

Ribs, 4 I

development of, 56

ossification of, 46

Rider's bone, 473

muscles, 473

Rima glottidis, $\mathrm{I}_{2} \mathrm{~S}_{5}$

oris, 1236

pudendi, 629

Ring, abdominal, external, 653 guide to, $64 \mathrm{x}$

internal, -665

crural, $46 \mathrm{I}$ guide to, 642

crural or femoral, $46 \mathrm{I}$ guide to, $64 \mathrm{I}$

Risorius mnscle, 1168

Rivinus, great duct of, I I 36 notch of, 1538

Robin, myeloplaxes of, ro

Rod-bipolar cells of retina, I52I

Rods of retina, 1523

Rolandic angle, 1504

line, 1504

Rolando, fissure of, 1383

funiculus of, 1350 guide to, $\mathrm{I}_{5} \mathrm{O}_{4}$

substantia gelatinas of, 1353

tubercle of, I35I

Rosenmüller, fossa of, 1269 organ of, $88_{4}$

Rostrum, 84

Rotation, 282

Rotatores spinæ muscles, 294

Rotula, 24 I

Rotunda, fenestra, $\mathbf{1} \mathbf{5 3 9}$

fossula, 1539

Rugarum, columnæ, of vagina, 898

Ruyschiana, tunica, ${ }_{5} 12$ 
Sac, lachrymal, I 190 development of, IIgI

Sacciformis, membrana, 405

Saccule of internal ear, 1553 laryngeal, 1283

Sacculus endolymphaticus, 1553

Saccus reuniens, 973, 995

Sacral canal, 35 plexus, 846

Sacro-uterine folds, 879

Sacro-vertebral angle, 38

Sacrum, 30 ossification of, 36

Sagittal fontanelle ${ }_{i} 6$ line, 1378

Salpingo-pharyngeus muscle, I250, I 275

Santorini, cartilages of, I 280 duct of, $8 \mathrm{II}$

fissures of, 1536

Saphenous artery, external, 442 internal, 484

nerve, external or short, 518 internal or long, $45^{\circ}$

opening, $45^{8}$ guide to, 641

vein, anterior, 455 external or short, $5 \mathrm{I} 4$ internal or long, 455 guide to, 456

posterior, 455

Sartorius muscle, 464

Scala media, 1554

tympani, 1554

vestibuli, I 554

Scalene tubercle or spine, 44

Scalenus anticus muscle, II 36 medius muscle, I 136 posticus muscle, II 38

Scansorius muscle, 429

Scaphocephalus, 158

Scaphoid bone of carpus, 201 of tarsus, 258

fossa of sphenoid, 89

Scapula, I $^{8} 8$

ossification of, 184

Scapular artery, posterior, 287, II 43 notch, great, I79 region, 319

Scarpa, fascia of, 643 foramina of, 102,145 ganglion of, 1300 triangle of, 475

Schindylesis, $\mathbf{I} 3 \mathbf{I}, 280$

Schlemm, canal of, 15 I I

ligament of, 345

Schneiderian membrane, 1256

Sciatic artery, 432 extra-pelvic branchos of, 432

nerve, great, 439
Sciatic nerve, great, branches of, 439

small, 436 branches of, 436

recurrent branches of, 425

notch, great, 227

small, 224, 227

vein, 433

Sclerotic coat of eyeball, I507

a. blood-supply of, 1508

\&, nerve-supply of, 1508

structure of, 1508

Scrobiculus cordis, 639

Scrotum, 649

arteries of, 650

development of, 635

lymphatics of, 650

nerves of, 650

septum, $65^{\circ}$

-structures forming wall, 649

Segmental tubes of Wolffian body, 829

Segmental type, 3

Segmentation vavity, $16 \mathbf{I}_{3}$

of ovum, I6rI

Sella turcica of sphenoid, 82 dorsum of, 83

Sellæ, diaphragma, I 475

Semicircular canals, membranous, I 553 structure of, 1553 osseous, I 550

Semicircularis, tænia, I 4 I 5

Semilunar bone, 202

fascia of biceps, 336

ganglion, 736

Semilunaris, hiatus, 1255

Semimembranosus muscle, 438

Seminales, vesiculæ, 852 blood-supply of, 872 development of, 872 structure of, $87 \mathrm{I}$

Seminiferous tubules, 683

Semispinalis colli muscle, 294 dorsi muscle, 294

Semitendinosus muscle, 437

Sensory nerves of face, I 176

Septa, intermuscular, of brachial region, 334

of foot, 547

of leg, 498

of thigh, 458

Septula renum, 8 i 8

Septum, femorale, $46 \mathrm{I}$

lucidum, $\mathrm{I}_{4} \mathrm{O} 3$

orbitale, I 88

pectiniforme of clitoris, 630 of penis, 868

posticum of spinal cord, I3IO

primum, 993 
èptum, secundum, 993

transversum, 1644 ierrata, ora, of retina, I $5 \mathbf{1} 8$ jerratus magnus muscle, 318 posticus inferior muscle, 288 superior muscle, 288

Sertoli, cells of, 684,686

Sesamoid bones of foot, $27^{2}$

$$
\text { of hand, } 216
$$

Sexual eminence, 634,872

Sharpey, perforating fibres of, 7

Sheath, axillary, 3 I 2

crural, 460

fe noral, 460

infundibuliform, 460

of flexor tendons of hand, 375

Short bones, 7

Shoulder-girdle, I75

Shoulder-joint, 344

Shrapnell, membrane of, I $54^{\circ}$

Sibson, fascia of, II 46

Sigmoid cavity of radius, 195 great, of ulna, I98 small, of ulna, rgs

groove of temporal, 73

Sinus arcuatus, 1270

basilar, I479

cavernous, ${ }_{4} 78$

circular, $147^{8}$

circularis iridis, I 5 I I

coronary, 986

Guérin, of, 858

laryngeal, 1283

lateral, 1476 guide to, 1501

longitudinal, inferior, 1475 superior, 1475 guide to, I50

Morgagni, of, 1265

occipital, I477

petrosal, inferior, 1478 superior, $147^{8}$

petro-squamous, 1479

pocularis, 855

portal, 743

prostatic, 855

pyriformis, $12 \mathrm{~S}_{3}$

spheno-parietal, $\mathrm{I}_{47} 8$

straight, 1476

transverse, 1479

tympani, I 539

venosus, 992 scleræ, I 5 II

Sinusoids, 1005

Sinus, annularis, 1005

tarsi, 277

uro-genital, 635

Skeleton, I visceral arch, I59, I6I

Skull, anterior region of, 132 at birth, 155
Skull, brachycephalic, I 32, I 58

of cretin, I 59

deformities of, I $5 \mathrm{~S}$

development of, 159

dolichocephalic, 132 , I 58

of female, 156

horizontal section of, I 49

inferior region of, I 45

internal base of, $15^{\circ}$

lateral region of, $1_{4} \mathrm{O}$

leptorhine, 158

longitudinal arc of, ${ }_{5} 8$

megacephalic, I 57

mesaticephalic, 132, $15^{8}$

mesocephalic, I 57

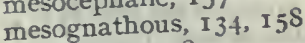

mesorhine, 158

microcephalic, 157

orthognathous, ${ }_{5} 8$

platyrhine, $15^{8}$

posterior region of, I 3 I

prognathous, 134,158

racial peculiarities of, I 56

sagittal section of, 149

superior region of, 132

as a whole, 123

Smegma præputii, 647

Snuff-box, anatomical, 368

Soemmering, long pudendal nerve of, 436,627

Solar plexus, 734

Sole of foot, $5+3$ landmarks of, 543

Soleus muscle, 519

Solitarius, fasciculus, I 495

Somites, mesodermic, I6I9

Speech-centre, $\mathrm{I}_{3} \mathrm{~S}_{7}$

Spermatic artery, 754

terminal part of, 6 ;0

cord, 668 varieties of, 754 coverings of, $67 \mathrm{I}$

fascia, external, 651,671 internal, 649, 67 r middle, 656,67 I

plexus of nerves, $73^{8}$ of veins, 754

Spermatoblasts, 1602

Spermatogenic cells, $160 \%$

Spermatogenesis, I 602

Spermatozoa, 1603

Spheno-ethmoidal recess, 90, I 254

Spheno-mandibular ligament, I II4

Spheno-maxillary fissure, I36, I43

fossa, 144

Spheno-palatine artery, I 206, 1260

foramen, I40, I 44

ganglion, 1219

branches of, 1220

roots of, I 219

nerves, 1217 
Spheno-palatine notch, II 4

Spheno-parietal sinus, 1478

Sphenoidal air sinuses, 90

crest, 84

fissure, 88 , I 36

structures passing through, I 62

process of palate bone, II 3

turbinate or spongy bone, 90

Sphenoid bone, 82 ossification of, 9 I

Sphenotic foramen, $1_{4} 8,152$

Sphenotics, 9 I

Sphincter ani externus muscle, 6 r2 internus muscle, 875

pupillæ muscle, 1515

pylori muscle, 782,786

vaginæ muscle, 637

vesicæ muscle, 865

Spigel, lobe of, 707 tuber papillare of, 706

Spinal accessory nerve. See Cranial

Nerves

canal, 40 arteries of, I 33 I

cord, I 306

arteries of, I 332

central canal of, I 319

characters, external, of, I 312

distinguishing, in different regions, I 320

columns of, I 3 I 4 commissures of, $\mathrm{I}_{3} \mathrm{I} 7, \mathrm{I}_{322}$ conus medullaris of, I 312 development of, I 334 ependymal zone, I 335 marginal zone, I 335 metamorphoses of primitive spinal cord, I 336

lumen of neural tube, 1336

myelospongium, I334

neural fold, I 334

groove, I 334

plate, I 334

tube, I334

neuroglia, I 335

glia or spider cells, I 335

neuroblasts, 1335

axons of cells, I 335

dorsal or posterior roots, 1323

enlargements of, I 3 i 2

filum terminale of, 1313

fissures of, I 314

furrow, posterior intermediate of, I 315

grey matter of, $\mathbf{r}_{3} \mathbf{r}_{7}$
Spinal cord, grey matter of, cells of, I 320,1322

substantia gelatinosa of

Rolando, I 3 I 7, I 322

Gowers, intermediate process of, 1318

ground-bundle, anterior, of, I 329

lateral, of, I 33 I

ligament, central, of, I 3 I 3

ligamenta denticulata of, I 3 I I

lymphatics of, I 334

marginal bundle, anterior, of, 1329

marginal bundle, posterior, of, 1327

meninges of, $\mathbf{x} 306$ arachnoid, I 309 dura mater, I 306 pia mater, I3 Io

nerve-cells of, I 320 of anterior cornu, I $32 \mathrm{I}$ Clarke's column, I 322 intermedio-lateral cellcolumn, I32I

posterior vesicular cellcolumn, I 322

Stilling, dorsal nucleus of, I 322

nerve-fibres of, course of,

1323

processus reticularis of, 1318 structure of, internal, ${ }_{3}{ }_{1} 7$ minute, 1320

tracts of. See Tracts

veins of, I 333

ventral or white commissure, 1323

white matter of, I319, I 323

ganglia, I 3 I 5, I 325

groove, 283

nerves, I 315

divisions, primary, of, I 316 groups of, I 3 I 5

origin of, $\mathrm{I}_{3} \mathrm{I} 5$

relation of vertebræ to, I 3 I 6

posterior primary divisions of, 1066

roots of, I 315

Spinalis dorsi muscle, 292

Spindle, 1599

Spine, genial, I I 7

iliac, anterior and posterior, 220,222

nasal, anterior, 97, Ior of frontal, 67 posterior, I I I

palatal, I I

peroneal, $25^{8}$ 
Spine, of tibia, 244

Spino-thalamic fibres, $i 330$

Spino-umbilical lines, 6.40

Spiral ganglion, I 559 groove of humerus, 188

line of femur, 236

Spireme, 1599

Splanchnic ganglion, I0 35 nerves, thoracic, ro35 pelvic, 862

Spleen, component parts, connections, and position of, 699 accessory, 70I

development of, 8i 5

ligaments of, 720

lymphatics of, 8 I $_{5}$

nerves of, $8 \mathrm{I}_{5}$

peritoneal relations of, 701 structure of, $8 \mathrm{I}_{3}$

Splendens, linea, I 3 I I

Splenic dulness, area of, 702 flexure of colon, 698 sympathetic plexus, 736

Splenium of corpus callosum, I4OI

Splenius muscle, 289

capitis, 290

colli, 290

Spongy bone, inferior, I09

middle, 95

superior, 95

Spring ligament, 580

tubercle of navicular, 259

Stage of irruption in ossification, 9

Stapedius muscle, 1545 nerve to, 1298

Stapes, 1543

Stellatum, ganglion, 1034

Steno or Stensen, duct of, 1 I 85

canal of, Ior

foramina of, I02, 145

Stephanion, I 42

Sternal angle, $4^{8}$

fissure, $5^{\circ}$

foramen, 50

Sternalis muscle, 304

Sternebræ, 47

Sterno-cleido-mastoid muscle, I088 posterior border of, I06I

Sterno-hyoid muscle, I I03

Sterno-pericardial ligaments of

Luschka, 944

Sterno-thyroid muscle, I 103

Sternum, 47

development of, 56

ossification of, 50

Stilling, canal of, $x 530$ dorsal nucleus of, 1322

Stomach, component parts, connections, and position of, $69 \mathrm{I}$

curvatures of, 692

blood-supply of, $7^{85}$
Stomach, development of, 798

divisions of, 693

gastro-phrenic ligament of, 720

lymphatics of, $78_{5}$

Meckel's diverticulum of, 799

nerves of, 786

orifices of, 692

peritoneal relations of, 694

structure of, $78 \mathrm{I}$

topography of, 693

trigone or uncovered area of, 694

U-loop, 800

Stomatodæum, 798

Stomodæum, I239

Straight sinus, 1476

Stratum intermedium, I 5 I2

Striæ acusticæ, I 377

Stria longitudinalis lateralis, I 401 mesialis, 1400

medullaris vel pinealis, $I_{422}$

Striatum, corpus, I 410

Structure of bone, 5

of a vertebra, $\mathrm{I}_{2}$

Structures derived from germinal layers, 16 i 7

in middle line of neck, $108 \mathrm{I}$. IIO4

Stylo-glossus muscle, I I 33

Stylo-hyal, 82

Stylo-hyoid muscle, I I 30

ligament, II 34

nerve, I I 73

Styloid process of fibula, 250

of radius, 193

of third metacarpal bone, 210

of temporal bone, 79

of ulna, 200

Stylo-mandibular ligament, I088, I 215

Stylo-mastoid foramen, 77

Stylo-pharyngeus muscle, I 221

Subanconeus muscle, 342

Subarachnoid space of brain, $\mathbf{r}_{4} 80$

Subarcuatus, hiatus, 76 of spinal cord, I 309

Subcallosal gyrus, r4or

Subclavian artery, $\mathrm{I}_{3} \mathrm{~S}$

left, first part of, in neck, I I 40

in thorax, $96 \mathrm{r}$

right, first part of, I I 38 second part of, I I 40

groove, I76 third part of, 1095

triangle, 1094

vein, 1096, I 144

Subclavius muscle, 306

Subcostal groove, 4.3

nerve to, 31 \&, 1098

line, 689 
Subcostal nerve, iliac branch of, 425 plane, 689

zone, 690 subdivisions of, 690

Subcrureus muscle, 469

Subdural space of brain, I $47 \mathrm{I}$ of spinal cord, I 309

Subflava, ligamenta, I039

Subiculum, I 395

Sublingual artery, I Ir 8

ducts, I I 36

gland, I I 35

development of, I I 36

ducts of, $\operatorname{Ir} 36$ structure of, I I 36

Submaxillary duct, I I 34

gland, I 34

development of, I I 35

duct of, $\mathbf{I}_{34}$

structure of, II 35

ganglion, I 212

branches of, 1213

roots of, I $2 \mathrm{I} 3$

lymphatic glands, I ror

triangle, I Ioo contents of, 'I I IOI:

Submental glands, Iror

triangle, I 105

Subnasal point, r 33

Suboccipital nerve, anterior primary division of, I OgI

posterior primary division of, 1066

region, 1064

muscles of, 1064

triangle, 1065

venous plexus, 1066

Subperitoneal areolar tissue or fat, 676

Subsartorial plexus, 452

Subscapular artery, 3 I I nerves, 3I5

Subscapularis muscle, 323

Substantia ferruginea, I 378 gelatinosa of Rolando, 1317 , I 353 nigra, 1438 propria of cornea, 1509

Subthalamic tegmental region, 142 I

Subthalamicum, corpus, I 42 I

Sulcus-

callosal, I 395

centralis insulæ, 1393

fimbrio-dentate, 1396

frontal, inferior, I 385

superior, I 385

intraparietal, 1389

laminæe spiralis, 1556

lateral, of crus cercbri, I 43 I

occipital, lateral, I 39 I

transverse, I39I

occipito-temporal, I 392
Sulcus (continued) -

oculo-motor, of crus cerebri, I43r olfactorius, roo

olfactory, 1387

orbital, I 387

postcentral, I 389

postcero-lateral, of spinal cord. I3 I 5

precentral, I 385

scleræ, 1507

temporal, 1392

terminalis of right auricle, 973 of tongue, I24I

triradiate, 1387

tympanicus, 79,1538

valleculæ, 1338

Supination, 406

Supinator radii brevis muscle, 386

radii longus muscle, 383

ridge of ulna, 200

Supra-acromial nerve, 298, 319

Supracallosal gyrus, I 4 or

Supraclavicular nerve, 298

triangle, 1094

Supracondylar process of humerus, I 88

Suprahyoid glands, I ror

region, IIO4, II29

Supramastoid crest of temporal, 70

Suprameatal triangle, 72

Supra-occipital bone, 57

Supra-orbital artery, I067, I 160

foramen, I 34

nerve, 1067, I 154

notch, 66

vein, 1068

Suprapatellar pad of fat, $54^{2}$

tendon, 469

Suprarenal capsules, 749

development of, $8 \mathrm{I} 7$

lymphatics of, 8I 7

structure of, 816

plexus, 736

Suprascapular artery, 320, rog6, I I 43

branches of, 321

nerve, 3I4, 32I, I098

notch, 179

vein, I096, I 145

Supraspinatus muscle, 320

Supraspinous fossa of scapula, 178

Suprasternal nerve, 298

space, 1086

Supratrochlear foramen of humerus, $\mathrm{r} 89$.

nerve, I067, II 54

Sural nerves, 445

Suspensoria, ligamenta, of Cooper, 300

Suspensory ligament of axis, 1305

of lens, 1530

of penis, 647 
Sustentacular fibres of retina, 1523

Sustentaculum hepatis, 720 lienis, 720

tali, $25^{8}$

Sutures, 279

coronal, I 24

dentated, 279

false, 279

fronto-ethmoidal, 130

fronto-lachrymal, I 30

fronto-malar, 127, 129

fronto-maxillary, 127

fronto-parietal, 124

fronto-squamosal, 126

groovel, 280

harmonic, 279

intermaxillary, 127

interparietal or sagittal, $\mathrm{I}_{2} 4$

lachrymo-ethmoidal, I30

lachrymo-maxillary, I 30

limbous, 279

malo-maxillary, 127

metopic, or frontal, 69,125

occipito-mastoid, 124

occipito-parietal, 123

orbital, 129

orbital-palato-ethmoid, I 29

orbito-malo-maxillary, 129

orbito-palato-maxillary, 129

orbito-spheno-frontal, 129

palato-maxillary, 128

parieto-mastoid, 125

parieto-squamosal, 125

petro-squamosal, 128

premaxillary, 128

pterygo-maxillary, 128

schindylesis, 131,280

serrated, 279

special, I 21

spheno-frontal, 126

spheno-malar, 129

spheno-parietal, 126

spheno-squamosal, I27

squamo-mastoid, 128

squamous, ' 279

Sylvian border of sphenoid, 85

fossa, 1464

line, 1503

point, 1382

guide to, $\mathrm{r}_{5} \mathrm{O}_{3}$

vein, deep, I 456 superficial, I 455

Sylvius, aqueduct of, 1439 development of, 1439 grey matter of, 1439 nerve-nuclei related to, 1439

fissure of, 1382 development of, 1464 limbs of, 1383

Sympathetic gangliated cord and plexuses. See Nerves
Symphysis, 280

Synarthrosis, 279

Synchondrosis, 279

Syncitium, 1629

Syndesmosis, 280

Synovia, 281

Synovial membrane, $28 \mathrm{I}$

of tarsal and tarso-metatarsal joints, summary of, 590

Tabular bones, 7

Tæniæ coli, 794

Tænia hippocampi, I 409

semicircularis, I415

teeta, 1401

Tali, sustentaculum, 258

Talipes, calcaneo-valgus, 566

calcaneus, 566

equino-varus, 566

equinus, 565

valgus, 566

varus, 565

Talus, 254

Tapetum of corpus callosum, I 4 or of choroid, I 512

Tarinus, fascia dentata of, 1396 fossa of, 1339

pons of, 1440

Tarsal glands, posterior, I 189

ligament, external, of eyelids, I 65, I 88

internal, of eyelids, 1165 , I 188

plates, of eyelids, II 88

Tarso-metatarsal joints, surgical anatomy of, 588

Tarsus, 253

ossification of, 265

varieties of, 264

as a whole, 264

Taste-buds, 1247

development of, 1247

structure of, 1247

Tectoria, membrana, of organ of Corti, $155^{8}$

(posterior occipito-axial

Teeth, 165 ligament), I 304

alveoli of, ror

bicuspid, 167

canine, 167

development of, 172

eruption of, I 70

incisor, 166

of temporary, 174

molar, 168

present at birth, 174

permanent, 166

structure of, I7 I

temporary or milk, 169 
Tegmen antri, 73 tympani, 75

Tegmentum of crus cerebri, r $_{43}$ I red nucleus of, $\mathbf{I}_{432}$. structure of, $\mathrm{I}_{432}$ white matter of, $\mathrm{r}_{433}$

Telachoroidea inferior, I $379, I_{4} 82$

Telence superior, $\mathrm{I}_{482}$ superior, I 482
halon, $\mathrm{r} 337, \mathrm{I} 38 \mathrm{I}$

Telophase, I 599

Temporal artery, superficial, Io70 arteries, deep, 1205 branches of ro7r

bones, 69 fascia, ro74 ossification of, 80

fossa, $\mathrm{r}_{42}$

gyri of cerebrum, 1392

lobe of cerebrum, r 39 r

muscle; 1074, 1200

nerves, deep, 1207

pole of cerebrum, I 392

region, 1070

vein, superficial, ro72

veins, deep, 1206 diploic, 1473

Temporo-facial division of facial nerve, II73

Temporo-malar nerve, I $_{5} 58$, I 217 malar branch of, II 58, I I 77 temporal branch of, 1070 I I 58, II 77

Temporo-mandibular joint, $\mathrm{r} 2 \mathrm{I} / 4$

Temporo-pontine tract, $\mathrm{r}_{43} 8, \mathrm{I}_{444}$

Tendo Achillis, 520

Tendon, conjoined, 658

infrapatellar, 470

reflected, of Cooper, 658

suprapatellar, 469,058

Tendo oculi, 1 165, I 188 palpebrarum, I 165, I 188

Tenon, capsule of, I I $5 \mathrm{I}$ space of, II 5 I

Tensor fasciæ femoris muscle, 464 palati muscle, 1252,1275 tarsi muscle, I 165

Tentorium cerebelli, I 473

Teres, eminentia, $x 378$ major muscle, 321 minor muscle, 321

Terminale, filum, of spinal cord, $x_{3} x_{3}$

Terms, craniometrical, $x_{57}$ descriptive, I

Testes of corpora quadrigemina, $\mathrm{I}_{428}$ abnormal positions of, 676 . descent of, $67 \mathrm{I}$

development of, 686

lymphatics of, 683

structure of, 683,684

Thalamencephalon, $x_{337}$, I4r5
Thalamic radiation, $\mathrm{I}_{420}$

Thalami optic, I 4 I 7

blood-supply of, I 456

Thalamo-cortical fibres, 1420

Thalamo-olivary tract, I 355

Thebesii, foramina, 976 venæ, 987

Thebesius, valve of, 976

Theca folliculi, 894 of spinal cord, 1308

Thenar muscles, 377

Thigh, 435

back of, 435

front and inner side of, 446

Thoracic artery, alar, 3 II inferior or long, 3 II

axis, 310 superior or short, 310

cavity, 934 contents of, 934 diameters of, ro46

duct, abdominal part of, 762 cervical part of, I I 45 thoracic part of, 103I

vertebræ, 20 receptaculum chyli, 762

wall, 926

Thorax, diameters of, 53

as a whole, 5 I

Thymus body, 945 development of, 946 in adult, 946 structure of, 945

Thyro-arytenoid ligaments, I28 I muscles, r289

Thyro-epiglottidean ligament, I275

Thyro-hyals, 165 muscle, I 289

Thyro-hyoid arch, 1273

bar, 1273

membrane, I280

muscle, $\mathrm{IIO}_{3}$

Thyroid artery, inferior, I I 42

axis, II42 superior, I I 16

body, II 22 development of, $\mathrm{Ir} 24$ structure of, $1 \times 24$

cartilage, 1277 development of, 1278

foramen, 227

nerve, I 108

veins, 1123

Tibia, 242

ossification of, 249

tuberosities of, 243

Tibial artery, anterior, 505

branches of, 507

varieties of, 508

posterior, 523

branches of, 525 
Tibialartery, posterior, varieties of, 527 nerve, 443

anterior, 509

posterior, 527

fibular branch of, 527

Tibialis anticus muscle, 504

posticus muscle, 522

Tincæ, os, 886

Tomes, fibres of, 172

Tongue, I24I

arteries of, 1246

development of, 1247

frenum of, I24I

glands of, 1243

lymphatics of, 1246

mucous membrane of, 1241

muscles of, extrinsic, II 3 I

nerves of, 1246

intrinsic, I244

plica fi mbriata of, I 24 I

veins of, 1246

Tonsil, pharyngeal, 1269

Tonsils, 1253

structure of, 1254

Tooth, structure of, I $7 \mathrm{I}$

Torcular Herophili, 59, I475

guide to, $I_{5}$ OI

Torus occipitalis transversus, 61 nterinus, 879

Trabeculæ cranii, 160

Trachelo-mastoid muscle, 292

Trachea, cervical part of, I I25

development of, II29

lymphatics of, I 127

structure of, 1128

thoracic part of, IoI9

Tract, olfactory, I 397

roots of, 1397

structure of, 1448

Tracts of spinal cord-

of antero-lateral column, I327

ascending tracts-

dorsal spino-cerebellar, I $329,135^{6}$

spino-tectal; I330

spino-thalamic, $\mathbf{I} 330$

ventral spino - cerebellar, or tract of Gowers, I 330, I 357

association fibres-

antero-lateral marginal or ground-bundle, I 33 I

lateral marginal, I33I

descending tracts-

anterior marginal, or ground - bundle of Lowenthal, I 329

bulbo-spinal, 1329

crossed or lateral pyramidal, 1327
Tracts of spinal cord (continued) -

descending tracts -

direct pyramidal, $\mathbf{x} 328$

prepyramidal or rubrospinal, I 329

uncrossed lateral pyramidal, I 329

arcuate, $\mathbf{I} 359$

deep fibres of, I 359

superficial fibres of, I 359

olivo-cerebellar, I359, I 360

of posterior column, I325

Burdach, column of, I 326

comma tract, 1327

Goll, column of, 1326

Lissauer, tract of, 1327

posterior marginal

bundle, I 327

ventral tract, 1327

Tragicus, musculus, I 194

Transversalis cervicis muscle, 292

fascia, 665

humeri artery, 320

pedis muscle, 555

Transverse, or basilar, sinus, 1479

Transversus, musculus, 1194

nuchæ, I06I

Trapezium, 203

Trapezius muscle, 285 cervical portion of, I06I

Trapezoid bone, 204

tuberosity, 204

Trapezoides, corpus, of pons Varolii, I 363

of cerebellum, 1372

Trefoil tendon of diaphragm, 759

Treitz, musculus suspensorius duodeni of, 695

Triangle or triangles-

anterior, of neck, rog8

carotid (superior carotid), of neck, I I Oo

digastric, of neck, r IoI

Hesselbach, of, 668

Lesser, of, I I 30

Macewen's, 72

muscular (inferior carotid), of neck, I099, I I00

occipital, of neck, rogI

perineal, deep, 6I 8

Petit, of, 286, 654

plantar, 556

posterior, of neck, I090

Scarpa's, 475

subclavian, of neck, 1094

submaxillary, I 100

submental, I ro5

suboccipital, ro6 5

supraclavicular, 1094

suprameatal, 72 
Triangular fascia, 653

ligament, of female, 636 of male, 6I9

muscular space of arm, 323

Triangularis menti muscle, I 70

Triceps extensor cubiti muscle, $34^{2}$

Tricuspid orifice, 976,979 valve, 979 position of, 985

Trifacial or trigeminal nerve. See Cranial Nerves

Trigone, external, of bladder, $85 \mathrm{I}$ internal, of bladder, 866

Trigonocephalus, $\mathbf{5} 59$

Trigonum acusticum, 1378

femoris, 236 .

-. habenulæ, I422

hypoglossi, 1378

olfactorium, 1397

os, 256,264

vagi, 1378

ventriculi of lateral ventricle, I 409

Triradiate sulcus, $\mathrm{I}_{387}$

Trochanter, great, 234

small, 234

Trochlea of humerus, 189

Trochlear fossa, 67 nerve. See Cranial Nerves .

Trochlearthrosis, 280

Trochoides, 280

Troltsch, pouches of, $\mathbf{I}_{546}$

True vertebræ, II

Tubarius, processus, 89

Tube, Eustachian, 1274 development of, 1275 muscles connected with I 275

Tuber calcis, 256

cinereum, I 424

cochleæ, I539

ischii, 224

omentale, 705

posticum, $\mathrm{r} 37^{\circ}$

valvulæ, I 370

Tubercle, adductor, of femur, 236

amygdaloid, 1408

carotid, 20

Chassaignac's, 20

Darwin's, II93

lachrymal, roo

Lisfranc's, 44

malar, I05

mental, I16

pharyngeal, 59

preglenoid, $7 \mathbf{I}$

pterygoid of sphenoid, S9

radial, 195

scalene, 44

Tuberculum impar, 1270

Tuberosity, great, of humerus, $\mathbf{r} 86$
Tuberosity, small, of humerus, $\mathbf{8} 86$

of malar, $\mathrm{rO}_{4}$

omental, of pancreas, 734

of palate, $\mathrm{II}_{2}$

of superior maxilla, 97

Turbinate bones, inferior, rog

ossification of, r ro

sphenoidal, 90

process, inferior, of ethmoid, 95

superior,' of ethmoid, 95

Tubules, seminiferous, 683

uriniferous, 819

Tunica albuginea, 683

dartos, 650

vaginalis, $68 \mathrm{I}$

vasculosa of Cooper, 683

Türck, column of, 1328

Tympani, membrana, I540

sinus, 1539

secondary, I542

tegmen, I539

Tympanic annulus, 80

artery, 1203

attic, 73, I 545

canaliculus, 77

nerve, 1224

plate of temporal, 79

plexus of nerves, 1548

sulcus, 79

Tympanicus, sulcus, 1538

Tympano-hyal, 82

Tympano-mastoid fissure, 77

Tympanum, $\mathrm{I}_{53} 8$

arteries of, 1547

mucous membrane of, 1545

muscles of, I 544

nerves of, 1548

ossicles of, $\mathrm{I}_{542}$

development of, $\mathrm{I}_{543}$

ligaments of, I 544

movements of, 1545

Type, segmental, 3

Tyson, glands of, 647

Ulna, 197

coronoid process of, 197

olecranon process of, 197

ossification of, 200

Ulnar artery, $35^{8}$

first part of, 358

branches of, 360

second part of, 364

third part of, 370

branches of, 370

varieties of, 363

in hand, 382

collateral nerve of Krause, 316 ,

364

nerve, 315,364

branches of, 364 
Ulnar nerve, dorsal branch of, $35^{\circ}$ in hand, 374 nerve, summary of, 374 vein, anterior, 334

U-loop, 800 posterior, 334

Umbilical cord, I630 duct, 798 notch of liver, 708 urinary fistula, I631 zone of abdomen, 690

Umbilicus, 640 , 1630 subdivisions of, 690

Umbo, I 54 I

Unciform bone, 205

Uncinate process of pancreas, 733

Uncus of brain, $x 395$

Unguis, os, 108

Upper limb, bones of, I 75 divisions of, $\mathrm{I} 75$ guide to dissection of, 4 I I

Urachus, 870

Ureter, in abdomen, 826

-7) in pelvis, 849

development of, 829

structure of, 826

Urethra, female, 890

male, 854 development of, 899 lymphatics of, 899

bulb of, 618,869

development of, 870

ligament, triangular, of, 6 I9 lymphatics of, 858 membranous part of, 856 prostatic part of, 854 spongy part of, $8_{5} 6$

Urinarius, meatus, female, 631, 890 male, 647,854

Uriniferous tubules, 819 structure of, $82 \mathrm{I}$ summary of, $82 \mathrm{I}$

Uro-genital fold, 8,30

Uro-genital sinus, 830,870

Utero-vesical folds, 879

Uterus, 885 pouch, 879

anteflexion of 887

anteversion of, 887

birth, at, 889

body of, 886 cavity of, 888

broad ligament of, 880

cervix of, 886 cavity of, 888

development of, 898

fundius of, 885

gravid, I633

isthmus of, 886

lymphatics of, 897

position of, 887
Uterus, relations, general, of, 887 peritoneal, of, 887

structure of, 896 varieties of, 889,899

Uterus masculinus, 855

Utricle of internal ear, $155^{2}$ of male urethra, 855

Uvea of iris, I 515

Uvula of cerebellum, $137^{\circ}$ of soft palate, 1248 vesicæ, 866

Vagi, trigonum, 1378

Vagina, 889 arteries of, 898 development of, 898 fornix of, 889 lymphatics of, 898 orifice, external, of, $63 \mathrm{I}$ structure of, 897

Vaginal ligaments, 375

Vaginale, ligamentum, 675

Vaginalis, processus, 675

Vagus (pneumogastric) nerve. See Cranial Nerves

Valentin, ganglion of, 1218

Vallecula of cerebellum, 1369

Sylvii, 1382

of tongue, I24I

Valsalva, sinuses of, 984

Valve or valves-

anal, 875

aortic, 983

bicuspid, 982

Gerlach, of, 796

Guérin, $8_{5} 8$

Hasner, of, I 192

Heister, 809

Houston, of, 874

ileo-cæcal or ileo-colic, 797

mitral, 982

pulmonary, 980

pyloric, 786

rectal, 874

tricuspid, 979

Tulpius, of, 797

Vieussens, of, 1379

Valvulæ conniventes, $7^{58}$ tuber, $137^{\circ}$

Valvule, Guérin, of, 858

Varieties of joints, 279

Varolii, pons, 1362

blood-supply of, $\mathrm{r}_{456}$

Vas aberrans, of Haller, 684 deferens, 668

ampulla of, 852

development of, 686

pelvic portion of, 852

structure of, 669

Vasa efferentia of testis, $68_{4}$ recta, false, of kidney, 823 
Vasa recta, true, of kidney, 824

Vasculosi, coni, 684

Vastus externus muscle, 467 internus muscle, 468

Vater, ampulla of, 7IO

Veins-

anal, 876

angular, II79

dorsal arch of foot, 5 I 4

auditory, internal, $\mathbf{1 5 6 0}$

auricle, of, II 95

auricular, posterior, 1073, II2I

axillary, 3 I2

azygos, left, lower, in abdomen,

$$
762
$$

in thorax, 1029

upper, ro3o

right, in abdomen, 762

in thorax, 1028

transverse, 1030

basilar, 1456

basilic, 334

median, 332

bronchial, ro25

capsular, of liver, 804

cardiac or coronary, 985,987

cardinal, roro

central, of liver, 805

cephalic, 334 median, 332

cerebellar, 1456

cerebral, 1455

cervical, deep, I 45 transverse, 1096, II 45

choroidal, I 455

clitoris, dorsal, of, 638

coronary sinus, 986

corpus striatum, of, 1405,1455

cystic, 742

deferential, 670

development of, 1005, 1010

diploic, 1473

emissary, I479

epigastric, deep, 663

superficial, 646

superior, $66_{3}$

extraspinal, anterior, 1333

facial, common, II 20

deep, 1206

superficial, on face, II79

in neck, II 20

transverse, I I 8 I

femoral, $4^{86}$

tributaries of, 486

varieties of, 488

cutaneous, external, 455

internal, 455

frontal, 1068

diploic, 1473

Galen, of, I405, I 455

gastric, 739
Veins (continued)-

gastro-epiploic, left, 74 I

gluteal, 432

right, 742

hemorrhoidal, inferior, 625,876 middle, 876

superior, 876

plexus, external, 876 internal, 876

hepatic, 805

iliac, circumflex, deep, 664 common, 776 development of, 776 external, 778 development of, 779 internal, 844 development of, 845

infra-orbital, I 182

innominate, 955

intercostal, 930, 1027 collateral, 930, 1027

superior, 1028

intralobular, of liver, 805

intraspinal, anterior longitudinal, 1333

posterior longitudinal, I 333

iris, of, $15 \mathrm{I} 6$

jugular, anterior, 1083

external, ro8.

internal, I I I 3

posterior, 1084 mastoid emissary tributary of, I 479

primitive, roro

labyrinth, of, I 560

larynx, of, I 293

lingual, I I 8

dorsal, I I 9

of lower limb, summary of, 566

lumbar, 773 ascending, 772

mammary, internal, 933

Marshall, oblique, of, 987

maxillary, internal, I 206

median, 332

deep, 332

meningeal, 1472

mental, I 82

mesenteric, inferior, 730

superior, 728

nasal fossa, of, r 26 r

transverse, I I 8 I

obturator, 488

occipital plexus, 1063 occipital emissary tributary of, 1479

parietal emissary tributary of, 1479

occipital diploic, I 473

omphalo-mesenteric, 1005

ophthalmic, II6I 
Veins (continued) -

ovarian, 890 plexus, 890

of palm, 372 deep, 382

palpebral, II 82

pampiniform plexus, 670

pancreatico-duodenal, 742

parumbilical, 719

penis, dorsal, of, 647

pharynx, of, I 269

phrenic, inferior, 752

plexus, dorsal of hand, 350 palmar, $35 \mathrm{r}$

popliteal, 443

portal, 742,804

profunda femoris, 487

pterygoid plexus, 1206

pudic, internal, 625

pulmonary, 953, 964

pyloric, 742

radial, 334

ranine, II 8

renal, 753

retina, of, 1526

saphenous, anterior, 455 external or short, 5 I 4 internal or long, 455 guide to, 456 posterior, 455

sciatic, 433

sclerotic, of, 1508

spermatic, 754

spinal column, of, 1332 dorsal plexus, 1332 cord, of, 1333

splenic, $74 \mathrm{I}$

subcardinal, 101 2

subclavian, Io96, I I 44

subcostal, 773

sublobular of liver, 805

suboccipital plexus, 1066

posterior condylar emissary, tributary of, I 479

superficial, of sole of foot, 548

supra-orbital, 1068

suprarenal, 752

suprascapular, I096, I I 45

Sylvian, deep, I 456

superficial, 1455

temporal, superficial, ro72 deep, I 206

temporo-maxillary vein, II 85

thyroid, II 23

tonsils, of, I 254

tympani, membrana, of, I54I

tympanum, of, 1547

ulnar, anterior, 334

umbilical, roo 8
Veins (continued) -

uterine, $89 \mathrm{I}$

vaginal, 892 of liver, $\mathrm{SO}_{4}$

vertebral, I I 44 anterior, I I 45

vitelline, 1005

Veli, frenulum, $1_{42} 7$

Velum interpositum, $\mathbf{I}_{4} \mathrm{O}_{4}$ arteries of, $145^{8}$ veins of, 1405

medullary, inferior, 1379 superior, 1379

pendulum palati, 1248

Vena cava, inferior, 755 development of, 757 , IOI 5 tributaries of, 756

superior, 956 development of, 957 IOI I

magna Galeni, I 405, I 455

portæ, 742

development of, 744, 1005

sinus of, 743

tributaries of, sources of, 743.744 summary of, 744

within the liver, $\mathrm{SO}_{4}$

salvatella, 351

Venæ advehentes, 1008

basis vertebra, $\mathrm{I} 333$

comites, brachial, 340

palmar, 372

radial, 357

tibial, anterior, 506 posterior, 525

ulnar, 364

minimæ cordis, 976,987

rectæ of kidney, 825

revehentes, 1008

stellatæe of kidney, 825

Thebesii, 987

vorticosæ, I 5 I 2

Ventricle or ventricles-

fifth, $\mathrm{I}_{4} \mathrm{O} 4$

development of, $\mathrm{r}_{4} \mathrm{O}_{4}$

fourth, 1376

choroid plexuses of, $\mathrm{I}_{3} 80$

development of, 1380

floor of, 1377

summary of, 1377

roof of, 1378

summary of, 1379

lateral, I 405

choroid plexuses of, I405, I 409

cornua of, 1406,1408

development of, 1409

septum lucidum, $\mathrm{I}_{403}$

third, 1415 
Ventricle or ventricles (continued) third, choroia plexuses of, I 405 . I 4 I 5 development of, $\mathrm{r}_{4} \mathrm{I} 7$ floor of, $\mathrm{I}_{4} 16$ recesses of, $I_{4} 16, I_{4} I 7$ roof of, I4I5

larynx, of, 1283 summary of, 1415

Ventriculus terminalis of Krause, I 319

Vermiform appendix, 696 mesentery of, 697

positions of, 697

fossa, 58 structure of, 796

process of cerebellum, ${ }^{3}{ }_{37}$

Vermis of cerebellum-

inferior; 1370

nodule of, $137 \mathrm{r}$

pyramid of, I 370

tuber posticum vel valvulæ of, $137^{\circ}$

uvula of, I 370

superior, I 368

central lobule of, 1368

clivus monticuli, I 368

culmen, 1368

folium cacuminis, 1368

lingula, $x_{3} 68$

Vertebra, atlas, I4

axis, 17 ossification of, 29

cervical, I2 ossification of, 29

sixth, 20

classification of, I I

component parts of, i I

false, I I, 30

lumbar, 25

fifth, 27

ossification of true, 27 .

prominens, I9

thoracic, 20 peculiar, 23

Vertebral aponeurosis, 289

arteries, at base of brain, 1342

artery, development of, $\mathbf{I r}_{42} 2$

first part of, $\operatorname{Ir}_{4} \mathrm{I}$

second part of, I $1_{4}$ I

third part of, 1065

fourth part of, $145 \mathrm{I}$

bow, 55

column, I r, 38

development of, 54

sympathetic plexus, 1235

vein, I I 44 anterior, II 45

Vertex of skull, I 32

Verumontanum, 855

Vesalii, foramen, 86
Vesicæ, uvula, 866

Vesicles, cerebral, I458, I 459

Vesicula prostatica, 855

Vesicula seminales, 852

blood-supply of, 872 development of, 872 lymphatics of, 872 structure of, 87 I

Vespertilionis, alæ, 880

Vestibular area, inferior, 75 superior, 75

artery, 1560

nerve, I 300, I $55^{8}$

Vestibule of internal ear, 1548

of middle meatus, of nasal fossa 1255

of mouth, 1236

of pudendum, $63 \mathrm{I}$

Vestigial fold of Marshall, 944

Vibrissæ of nostrils, I 196

Vicq d'Azyr, bundle of, I402, I423

Vidian artery, I206, I260 canal, 89

nerve, I2I 9

Vieussenii, ansa, 1235

limbus, 993

Vieussens, valve of, $\mathbf{1} 379$

Villi of small intestine, 789

Villosæ, plicæ, of stomach, $7^{8} 3$

Villous coat of small intestine, 788

Vincula accessoria tendinum, 376

Visceral arches, I 27 I clefts, 1270

Visual centre, higher, $\mathbf{1}_{427}$ centres, lower, $\mathrm{I}_{427}$

Visual purple or rhodopsin, $\mathbf{I}_{52}$

Vitelline duct, 695 membrane, 894

Vitello-intestinal duct, 695

Vitellus, 894

Vitreous body, I529

Vocal cords, false and true, 1284

i. process of arytenoid cartilage, I 279

Volar artery, superficial, 357

Volkmann's canals, 6

Vomer, I I 4 ossification of, $\mathrm{I} 15$

Vorticosæ, venæ, I 512

Vulva, 633

Waldeyer, paroöphoron of, 884

Weber, glands of, 1243

Wharton, duct of, I I 34

Wharton's jelly, 16 6 I

White line of Hilton, 875 of pelvic fascia, 838

matter of spinal cord, I3r9

Willis, circle of, I 343,1454 parts within, I 455 
Willisii, chordæ, 1475

Wilson, muscles of, $6 \mathrm{I}_{7}$

Winslow, foramen of, 721,802 posterior ligament of, 529

Wirsung, duct of, 8 I I

Wolffian body, 684, 829 duct, 686,829 tubules, 829

Wood's muscle, $55 \mathrm{I}$

Wormian bones, 155

Wrisberg, cartilages of, 1280 ganglion of, 968 nerve of, 315.331 pars intermedia of, I299, I489

Xiphisternum, 47, 49

Xiphoid process, 47,49
Yolk, 894

Yolk-sac, I 627

Yolk-stalk, I627

Zinn, ligament of, II 50 zonula of, 1530

Zona arcuata, 1555 orbicularis, 489 pectinata, I 555 pellucida, 1603 radiata, 894

Zonula ciliaris, 1530 Zinn, of, $153^{\circ}$

Zygomatic fossa, $\mathbf{x}_{42}$

Zygomaticus major muscle, II 68 minor muscle, I 168

Zygoma, 70 


\section{. $+4=$}

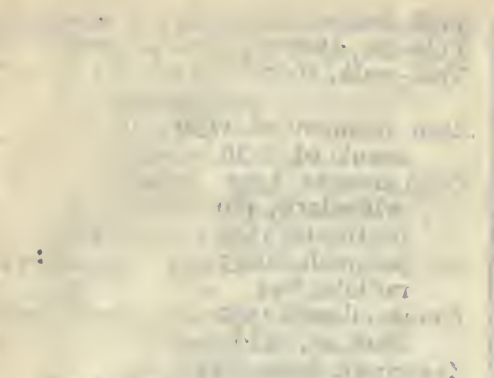

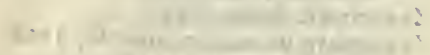

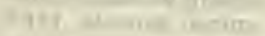
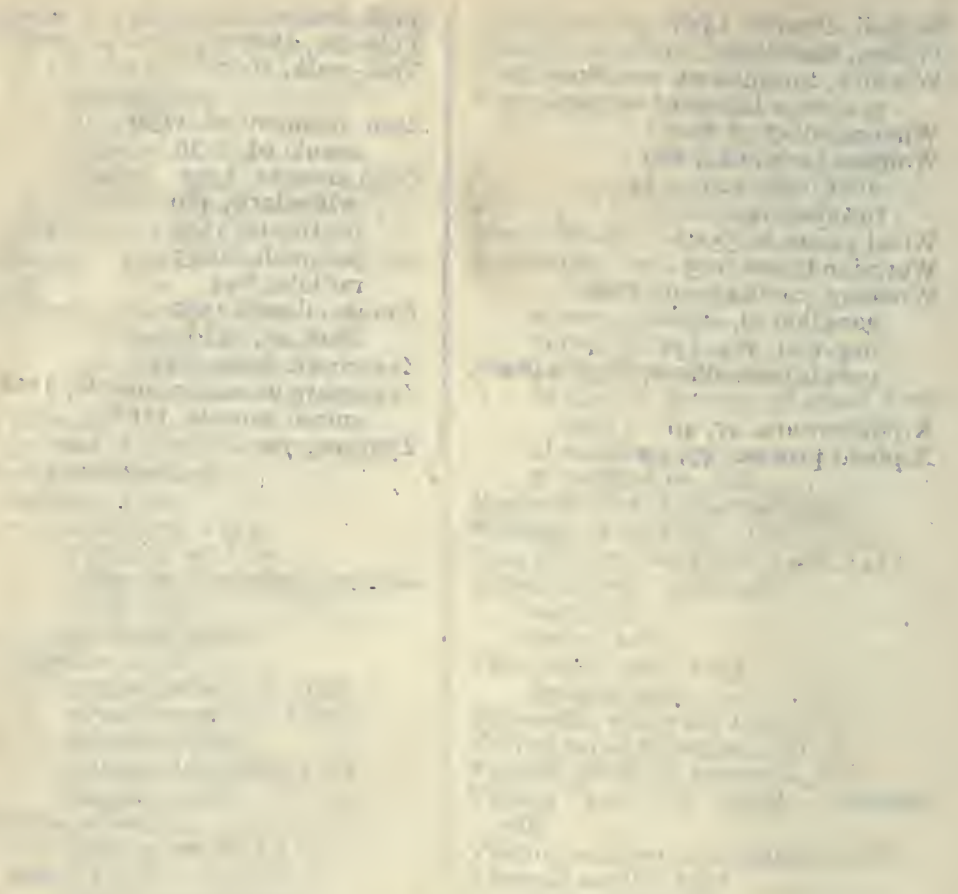

Q.? 12.

$100\}^{n+1}$ 



BioMed

\section{PLEASE DO NOT REMOVE CARDS OR SLIPS FROM THIS POCKET}

\section{UNIVERSITY OF TORONTO LIBRARY}


